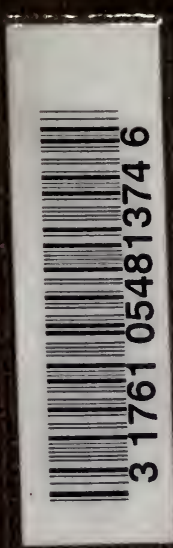


Digitized by the Internet Archive in 2007 with funding from Microsoft Corporation 


\section{PRINCIPLES OF}

\section{HUMAN PHYSIOLOGY \\ 166}

\section{BY}

ERNEST H

M.D. (Lond.), F.R.C.P., F.R.S., Hon. M.D. (Breslau)

Hon. Sc.D. (Cambridge and Dublin)

Jodrell Professor of Physiology in University

College, London

SECOND EDITION

With 566 Illustrations, 10 in Colour

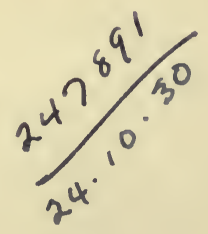

PHILADELPHIA

LE A \& F E B I G E R

706 SANSOM STREET

I9I5 
$30.4018=030$

sulitionse

(स)

$1 y=11$

7)

40111
7.11 .11

Printed in Great Britain

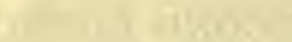




\section{PREFACE 'TO THE SECONI) EDITION}

DURING the short time that has elapsed since the publication of the first edition of this work physiology has made some considerable advances, and I have found it necessary to re-write much of the sections dealing with voluntary muscle and with the circulation, in addition to making many modifications in other parts of the work. New sections have also been introduced dealing with the nutrition of the brain and with the innervation of the bronchi.

I am much indebted to many friends, known and unknown, who have pointed out mistakes and omissions in the first edition. I shall be glad to receive any suggestions as to points in which this text-book may be made more useful to students.

Universin $\mathbf{x}$ College, Loxdon 



\section{PREFACE TO THE FIRS'T EDITION}

Physiology, though dealing with the phenomena of living organisms, has to use the same tools, whether material or intellectual, as the sciences of physics and chemistry. Any advances which are made in these sciences not only increase our powers of attack upon physiological problems but at the same time alter the intellectual standpoint from which we view them. On the other hand, the investigation of the phenomena of living beings is continually attracting our attention and that of workers in the other branches of science to unexplored regions in physics and chemistry. This mutual stimulation and co-operation among the different sciences have as their result a continual modification of our attitude with regard to the fundamental problems of physiology. The present time has seemed to me, therefore, fitting for the production of a textbook which, while not neglecting the data of physiology, should lay special stress on the significance of these data, and attempt to weave them into a fabric representing the principles which are guiding physiologists and physicians of the present day in their endeavours to extend the bounds of the known and to increase their powers of control over the functions of living organisms.

In a science such as physiology, based on so wide a discipline and with so diverse a technique, it is almost impossible for any one man to attain to a personal acquaintance with all its branches. In the present book I have therefore not hesitated to avail myself of the work of masters of the science in fields which I had not myself explored. Thus, in the physiology of the nervous system, which has been transformed and built up on a new basis by the researches of Sherrington, I have endeavoured to follow this author as closely as possible. I am also deeply sensible of my obligations to the writings of Tigerstedt, Leathes, and Lusk on general metabolism, of Abderhalden and Plimmer on physiological chemistry, of Bayliss on general physiology, as well as to various authors of articles in the "Ergebnisse der Physiologie," in Nagel's " Handbuch der Physiologie," and in Dr. L. E. Hill's "Recent and Further Advances in Physiology."

Although I have endeavoured to confine my demands on the previous knowledge of the student within the narrowest possible limits, I should recommend him in every case to read some primer on physiology in order to obtain a bird's eye view of the subject before beginning the study of this work. He will then be able to vary the order of chapters in this book according to the part of physiology which he is hearing about in his lectures or working at in his practical classes. Those of my readers who are entirely unacquainted with physiology might do well on a first perusal to omit Book I., dealing with the general concepts of the science. 
I have deemed it a hopeless and indeed a useless task to give any full account of the multifarious methods employed in the experimental investigation of the different organs of the body. In most cases I have consigned to small type a description of one or two typical methods, which would suffice to show how the questions may be approached from the experimental side.

Throughout the work I have sought to show that the only foundation for rational therapeutics is the proper understanding of the working of the healthy body. Until we know more about the physiology of nutrition, quacks will thrive and food faddists abound. Ignorance of physiology tends to make a medical man as credulous as his patients and almost as easily beguiled by the specious puffings of the advertising druggist. I trust, therefore, that the following pages will be found of value not only to the candidate for a university degree but also to the practitioner of medicine in equipping him for his struggle against the factors of disease.

In the selection of diagrams for the illustration of this book I am especially indebted to Professor Schäfer and to his publishers, Messrs. Longmans, for the permission to make use of a large number from Quain's "Anatomy" and from Schäfer's "Essentials of Histology." I must also express my obligation to Professor Wilson for the use of certain figures from his admirable work on the cell, to the publishers of Cunningham's "Anatomy," and to many physiological friends, especially to Dr. Mott and Dr. Gordon Holmes, for the use of original diagrams. The index was kindly made for me by Mr. Lovatt Evans.

\section{University College, London}

ERNEST H. STARLING May 1912 


\title{
CONTENTS
}

\author{
CHAPTER I \\ INTRODUCTION
}

BOOK I

\section{GENERAL PHYSIOLOGY}

\author{
CHAPTER II \\ THE STRUCTURAL BASIS OF THE BODY
}

CHAPTER III

SECTION

I. The Elementary Constituents of Living Cells

II. The Proximate Constituents of the Animal Body

THE MATERIAL BASIS OF THE BODY

III. THe Fats

IV. The Carbohydrates

V. The Proteins

VI. The Mechanism of Organic Synthésis

CHAPTER IV

THE ENERGETIC BASIS OF THE BODY

I. The Energy of Molecules in Solution

II. The Passage of Water and Dissolved Substances across Membranes

IV. The Mechanism of Chemical Changes in Living Matter. Ferments 153

V. Electrical Changes in Living Tissues

\section{BOOK II}

\section{THE MECHANISMS OF MOVEMENT AND SENSATION}

\section{CHAPTER V}

THE CONTRACTILE TISSUES

I. The Structure of Voluntary Muscle

II. The Excitation of Muscle

III. The Mechanical Changes that a Muscle undergoes whex it Cox- 


\section{CHAPTER V (continued)}

rION PAGE

IV. The Conditions affecting the Mechanical Response of a Muscle 205

V. The Chemical Changes in Muscle 212

VI. The Production of Heat in Muscle 219

ViI. Electrical Changes in Muscle 224

VIII. The Intmate Nature of Muscular Contraction 234

IX. Voluntary Contraction $\quad 239$

X. Other Forms of Contractile Tissue 243

\section{CHAPTER VI}

NERVE FIBRES (CONDUCTING TISSUES)

I. The Structure of Nerve Fibres 250

II. Propagation along Nerve Fibres 253

III. Events accompanying the Passage of a Nervous Impulse 256

IV. Conditions affecting the Passage of a Nervous Impulse 258

V. The Excitation of Nerve Fibres $\quad 262$

VI. The Conditions which Determine Electrical Stimulation 270

VII. The Neuro-Muscular Junction $\quad 275$

VIII. Polarisation Phenomena in Nerve 280

IX. The Nature of the Excitatory Process 284

\section{CHAPTER VII}

\section{THE CENTRAL NERVOUS SYSTEM}

I. The Evolution and Significance of the Nervous System 288

II. The Nervous System of Vertebrates 297

III. General Characteristics of Reflex Actions 303

IV. Nature of the Connection between Neurons 307

V. Functions of the Nerve-Cell 312

\section{THE SPINAL CORD}

VI. Structure of the Spinal Cord

VII. The Spinal Cord as a Reflex Centre 322

VIII. The Mechanism of Co-ordinated Movements 338

IX. Trophic Functions of the Cord 359

X. The Spinal Cord as a Conductor 351

\section{THE BRAIN}

XI. The Structure of the Bratn Stem

XII. The Functions of the Brain Stem 192

XIII. The Functions of the Cerebellum 397

XIV. Visual Reflexes $\quad 406$

XV. Summary of the Connections and Functions of the Cranial Nerves 410 
XVI. General Structural Arrangements of the Cerebrum

XVII. The Functions of the Cerebral Hemispheres

XVIII. The Nutritive and Vascular Arrangements of the Central Nervous System

THE AUTONOMIC SYSTEM

XIX. The Visceral or Autonomic Nervous System

CHAPTER VIII

THE PHYSIOLOGY OF SENSATION

I. On the Relation of Sensation to Stimulus

II. Cutaneous Sensations

III. Sensations of Smell and Taste

IV. Auditory Sensations

V. VoICE ANd SPEECH

\section{VISION}

VI. Dioptric Mechanisms of the Eyebali

VII. The Retinal Chavges involved in Vision 558

VIII. Visual Sensations $\quad 568$

IX. Movements of the Eyeballs _ . 585

X. Visual Judgments 559

XI. The Nutrition of the Eyeball $\quad \cdot 594$

\section{THE ORGANIC SENSATIONS}

XII. Sensations of Movement and Posttion 598

XIII. The Labyrinthine Sensations

BOOK III

\section{THE MECHANISMS OF NUTRITION}

CHAPTER IX

THE EXCHANGES OF MATTER AND ENERGY IN THE BODY (GENERAL METABOLISM)

I. Methods employed in Determinting the Total Exchanges of the BODY

II. The Metabolism durina Starvation

III. The Effect of Food on the Metabolism of the Body

IV. The Effect of Muscular Work on Metabolism

V. The Significance of the Food-stuffes 


\section{CHAPTER $\mathrm{X}$}

\section{THE PHYSIOLOGY OF DIGESTION}

SECTION

Changes undergone by the Food-stuffs in the Alimentary Canal

PAGE

I. Digestion in the Mouth

661

II. The Passage of Food from the Mouth to the Stomach - 676

III. Digestion in the Stomach

IV. The Movements of the Stomach

V. The Pancreatic Juice

VI. The Liver and BiLe

VII. The Integstinal Juice

VIII. Functions of the Large Intestine

IX. Movements of the Intestines

X. The Absorption of the Food-stuffs

XI. The F ÆCES

\section{CHAPTER XI}

THE HISTORY OF THE FOOD-STUFFS

I. Protein Metabolism

II. Nuclein or Purine Metabolism

III. The History of Fat in the Body

IV. The Metabolism of Carbohydrates

\section{CHAPTER XII}

THE BLOOD

General Characters of the Blood

I. The White Blood-Corpuscles $\quad 813$

II. The Red Blood-Corpuscles $\quad 818$

III. The Blood-Platelets $\quad 836$

IV. The Coagulation of the Blood 839

V. The Quantity and Composition of the Blood in Man

CHAPTER XIII

\section{THE PHYSIOLOGY OF THE CIRCULATION}

I. General Features of the Circulation

II. The Blood-Pressure at Different Parts of the Vascular Circuit

III. The Velocity of the Blood at Different Parts of the Vascular SYSTEM

IV. The Mechanism of the Heart Pump 
V. The Flow of Blood throdah the Arteries

VI. The Flow of Blood in the Veins

VII. The Pulmonary Circulation

viII. The Causation of the Heart-Beat

IX. The Nervous Regulation of the Heart

X. The Nervous Control of the Blood-Vessels

XI. The Effect of Muscular Exercise on the Circulation

XiI. The Influence on the Circulation of Variations in the Total QUANTITy OF BLOOD

CHAPTER XIV

LYMPH AND TISSUE FLUIDS

CHAPTER XV

THE DEFENCE OF THE ORGANISM AGAINST INFECTION

I. The Cellular Mechanisms of Defence

II. The Chemical Mechanisms of Defence

\section{CHAPTER XVI}

\section{RESPIRATION}

I. The Mechanics of the Respiratory Movements

1039

II. The Chemistry of Respiration

III. The Regulation of the Respiratory Movements

The Chemical Regulation of the Respiratory Movements

The Reflex Nervous Regulation of Respiration

IV. The Effects on Respiration of Changes in the Air Breathed

V. The Mechanisms of Oxidation in the Tissues

\section{CHAPTER XVII}

\section{RENAL EXCRETION}

I. The Composition and Characters of the Urine

II. The Secretion of Urine

III. The Physiology of Micturition 
CHAPTER XIX

SECTION

CHAPTER XX

THE DUCTLESS GLANDS

BOOK IV

\section{REPRODUCTION}

\section{CHAPTER XXI}

\section{THE PHYSIOLOGY OF REPRODUCTION}

I. The Essential Features of the Sexual Process 1199

II. Development and Heredity 1212

$\begin{array}{ll}\text { III. RePROduCtion IN MAN } & 1217\end{array}$

IV. Pregnancy and Parturition 1230

$\begin{array}{ll}\text { V. The Secretion and Properties of Milk } & 1137\end{array}$

$\begin{array}{ll}\text { INDEX } & 1247\end{array}$ 


\section{CHAP'TER I}

\section{N T ROD UCT ION}

Physiology in its widest sense signifies the study of the phenomena presented by living organisms, the classification of these phenomena, and the recognition of their sequence and relative significance. Such a range would include many studies which are not generally grouped under the term physiology, and would in fact correspond to the comprehensive science of biology. Thus the study of the relations of living beings to one another and to their surroundings is the special object of the science of œcology. The aims of physiology in its restricted sense are the description, analysis, and classification of the phenomena presented by the isolated organism, the allocation of every function to its appropriate organ, and the study of the conditions and mechanisms which determine each function.

The fundamental phenomena of life are essentially identical throughout the whole series of living organisms. This continuity of function is the necessary correlation of the continuity of descent, which brings into relation all members of the animal and vegetable kingdoms. No living organism can therefore be regarded as outside the sphere of our investigations. The interest of mankind in this subject was, however, naturally awakened in connection with his own body, and the science, growing up as ancillary and preliminary to medical studies, has always taken man as its chief type of study. In the present work the elucidation of the functions of man will also be our first concern, and this for two reasons. In the first place, in physiology, as in all other sciences, the motive of man's activity is his social instinct to increase the power of his race in the struggle for existence, by the acquisition of control, either over the external forces of nature, which may be turned to his own benefit, or over the factors, intrinsic and extrinsic, which tend to his enfeeblement or extirpation by disease and death. Consciously or unconsciously, all our researches on physiology, whether on the higher animals or on the lowest protozoa, have the welfare of man as their ultimate object. In the second place, the choice of the higher animals as our chief objects of study receives justification from the fact that whereas morphology, or the science of structure, must proceed from the lowest to the highest organisation, the science of function presents its problems in their simplest form in the most highly differentiated organisms. In the unicellular animal all the essential functions which we associate with living beings are carried out, often simultaneously, in one little speck of protoplasm. An analysis of these functions, the determination of their conditions 
and mechanism, is obviously impossible under such circumstances. It is only when, as in the higher animals, one part of the living body is differentiated into an organ which has one function and one function only as the outlet for its activities, that it becomes possible to peer into the details of the function with some chance of discovering its ultimate mechanism.

Our especial preoccupation with the physiology of man will not prevent our employment of examples from any part of the animal or vegetable kingdom, when light can be thrown by their study on fundamental physiological phenomena common to the whole of the living world. In many cases such a study will enable us to separate the essential features in a process from those which have been added as auxiliary, with increasing complexity of the structures concerned.

What are the fundamental phenomena which are wrapt up in our conception of living beings? When dealing with the higher animals, we are inclined to lay greater stress on the phenomena involving a discharge of energy. Thus we should say that a man was alive if his body were warm and if he were presenting spontaneous movements, such as those of respiration or of the heart. The life of a man in the ordinary sense of the term is made up of those movements which place him in relationship with his environment. For the production of these movements, as for the maintenance of a constant body-temperature, a continual expenditure of energy is necessary. Experience teaches us that these movements come to an end in the absence of food or of oxygen, and that an increased call on the energies of the body must always be met by a corresponding increase in the air and in the food supplied. Two further processes must therefore be included among those making up our conception of life, viz. the function of assimilation (the taking in and digestion of food), and the function of respiration, in which oxygen is absorbed and carbon dioxide is excreted into the surrounding atmosphere.

The substances which make up our food-stuffs are all capable of oxidation. Composed chiefly of carbon and hydrogen, with some oxygen, nitrogen, and sulphur, they yield on complete combustion carbon dioxide, water and small amounts of ammonia or allied bodies, and sulphates. In this process of oxidation there is liberation of heat. In the body a similar oxidation occurs, the products of oxidation being discharged into the surrounding medium. An amount of energy is thus set free which is available for the activities of the living organism. Before we can make any accurate investigations of the conditions which determine these activities, we must know whether the two great laws of chemistry and physics, viz. the conservation of mass and the conservation of energy, hold good for the processes within the living body. The many experiments which have been made on this point have decided the question in the affirmative. Thousands of experiments have been made, both on man and on animals, in which the total income of the body, viz. food and oxygen, has been weighed, and compared with the total output, viz. carbon dioxide, water, and bodies allied to ammonia (urea, \&c.). In every case complete equality has been 
obtained, and we can be certain that any matter found in the body must have been derived from without. There is no creation or destruction of matter in the body.

The determination of the equation in the case of the total energy of the body is rather more difficult. We have, in the first place, to measure the total income and output of the body, and to determine the total heat which would be evolved by the oxidation of the food-stuffs taken into the carbon dioxide, water, \&c., that are given out. We must then compare the figure so obtained with the actual output of energy by the body. The latter can be measured in terms of heat by placing the animal inside a calorimeter. Many practical difficulties arise in the performance of the experiment, in consequence of the necessity of providing the animal with a constant supply of air to breathe, and of allowing for the continual loss of water by evaporation which is going on at the surface of the animal. The first accurate experiments of this nature were made by Rubner. This observer determined by means of the calorimeter the total heat loss of dogs. In the same animals the material income and output of the body were measured, and a calculation was made as to the amount of energy which would be set free in the body by the processes of oxidation involved in the change of material observed. The following Table represents a summary of Rubner's results :

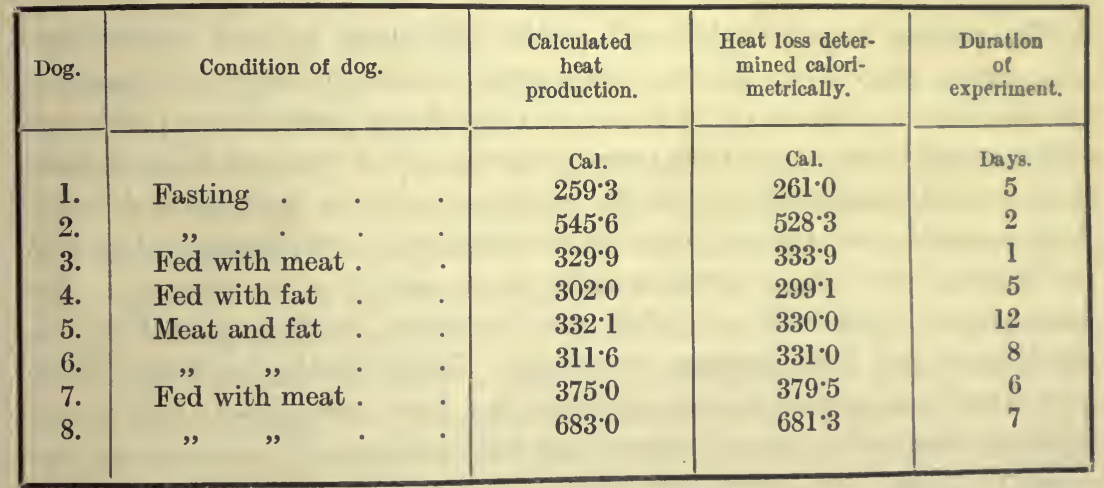

It will be seen that the average difference between the calculated and observed results amounts only to 1.01 per cent.-an amazing agreement considering the extreme difficulties of the experimental methods involved. The important deduction to be drawn from these observations is that the food-stuffs which are oxidised in the body develop in this process exactly the same amount of energy as when they are burnt up outside the body.

From one aspect, therefore, the animal body may be looked upon as a machine for the transformation of the potential energy of the food-stuffs into kinetic energy, represented by the warmth and movements of the body as well as by other physical changes involved in vital processes. In the living organism we cannot, however, distinguish between the source of energy and the machinery, as we can in the case of our engines. When we endeavour to trace the food-stuffs after their entry into the body, we lose sight of 
them at the point where they are built up to form apparently an integral part of the living framework. During activity there is a discharge of the products of oxidation of the food-stuffs from this living matter, which therefore becomes reduced in mass. This reduction, or disintegration of the living matter, associated with activity, is always followed by a period of increased integration, during which the organism grows by the assimilation of more food. Our conception of life must therefore involve the idea of a constantly recurring cycle of processes, one of building up, repair, or integration, and the other, associated with activity, of destruction or disintegration. If the former process predominates, we obtain a steady increase in the mass of the organism, an increase which we speak of as growth, and in many cases, as in that of plants, it is this power of growth which we take as our criterion of the existence of life. In fact, the possession by the green parts of plants of the power of utilising the energies of the sun's rays for the integration of food-stuffs, such as starch, with a high potential energy, is the necessary condition for the existence of all higher forms of life on this earth.

Closely associated with the property of growth is the power possessed by all living organisms of repair, i.e. the replacement by newly formed healthy living material of parts which have been damaged by external events.

The process of growth does not, in the individual, proceed indefinitely. At a certain stage in its life every organism divides, and a part or parts of its substance are thrown off to form new individuals, each of them endowed with the same properties as the parent organism, and destined to grow until they are indistinguishable from the organism whence they were derived. In the lowest forms of life, the unicellular organisms, these processes of growth and division may go on until brought to an end by some change in the environment which will not allow the necessary conditions of life, viz. assimilation and disintegration, to proceed. In all the higher forms, however, after the process of reproduction has been completed, the parent organism begins to undergo decay, and the processes of assimilation and repair no longer keep pace with those of destruction, however favourable the environment, until finally death of the organism takes place.

All these phenomena, viz. assimilation, respiration, activity associated with the discharge of energy, growth, reproduction, and death itself, are bound up in our conception of life. All have one feature in common, viz. they are subject to the statement that every living organism is endowed with the power of adaptation. Adaptation may indeed receive the definition which Herbert Spencer has applied to life-" the continuous adjustment of internal relations to external relations." A living organism may be regarded as a highly unstable chemical system which tends to increase itself continuously under the average of the conditions to which it is subject, but undergoes disintegration as a result of any variation from this average. It is evident that the sole condition for the survival of the organism is that any such act of disintegration shall result in so modifying the relation of the 
system to the environment that it is once more restored to the average in which assimilation can be resumed. Every phase of activity in a living being must be not only a necessary sequence of some antecedent change in its environment, but must be so adapted to this change as to tend to its neutralisation, and so to the survival of the organism. This is what is meant by 'adaptation.' Not only does it involve the teleological conception that every normal activity must be for the good of the organism, but it must also apply to all the relations of living beings. It must therefore be the guiding principle, not only in physiology with its special preoccupation with the internal relations of the parts of the organism, but also in the other branches of biology, which treat of the relations of the living animal to its environment, and of the factors which determine its survival in the struggle for existence. The origin of new species and the succession of the different forms of life upon this earth depend on the varying perfection of the mechanisms of adaptation.

We may imagine that the first step in the evolution of life was taken during the chaotic chemical interchanges which accompanied the cooling down of the molten mass forming the earth, when some compound was formed, probably with absorption of heat, endowed with the property of continuous polymerisation and growth at the expense of surrounding material. Such a substance could continue to exist only at the expense of the energy derived from the surrounding medium, and would undergo destruction with any stormy change in its environment. Out of the many such compounds which might have come into being, only such would survive in which the process of exothermic disintegration tended towards a condition of greater stability, so that the process.would come to an end spontaneously, and the organism or compound be enabled to await the more favourable conditions necessary for the continuance of its growth. With the continued cooling of the earth, the new production of endothermic compounds would become rarer and rarer; and in all probability the beginning of life, as we know it, was the formation of some complex substance, analogous to the present chlorophyll corpuscles, with the power of absorbing the newly penetrating sun's rays and utilising them for the endothermic formation of further unstable compounds. Once given an unstable system, such as we have imagined, the great principle laid down by Darwin, viz. survival of the fittest, will suffice to account for the production from it by evolution of the ever-increasing variety of living beings which have appeared in the later history of this globe. The 'adaptation,' i.e. the reactions of the primitive living material to changes in its environment, must become ever more and more complex, since only by means of increasing variety of reaction is it possible to provide for the stability of the system within greater and greater range of external conditions. The difference between higher and lower forms is therefore one of complexity of reaction, or of range of adaptation.

In all the physiological processes which we shall study in the course of this work, adaptation will be found the constant and guiding quality. The 
naked protoplasm of the plasmodium of Myxomycetes, if placed on a piece of wet blotting-paper, will crawl towards an infusion of dead leaves, or away from a solution of quinine. It is the same property of adaptation, the deciding factor in the struggle for existence, which impels the greatest thinkers of our time to spend long years of toil in the invention of the rneans for the offence and defence of their community, or for the protection of mankind against disease and death. The same law which determines the downward growth of the root in plants is responsible for the existence to-day of all the sciences of which mankind is proud.

This " adjustment of internal to external relations" is possible, however, only within strictly defined limits, limits which increase in extent with rise in the type of organism, and in the complexity of its powers of reaction. Some of these limiting conditions we shall have to study in the next chapter. Among the chief of them are temperature, and the presence of food material and of oxygen. At the present time the limits of temperature may be placed between $0^{\circ}$ and $50^{\circ} \mathrm{C}$. Many organisms, however, are killed by the alteration of only a few degrees in the temperature of their environment. Every shifting of a cold or warm current in the Atlantic, in consequence of storms on the surface, leads to the destruction of myriads of fish and other denizens of the sea. In the higher animals a greater stability in face of such changes has been accomplished by the development of a heat-regulating mechanism, so that, provided sufficient food is available, the temperature of the body is maintained at a constant level, which represents the optimum for the discharge of the normal functions of the constituent parts of the body. The presence of food material in the environment of the living organism is a necessary condition for its continued existence. In some cases, and this we must assume to be the primitive condition, the food material must be of a given character and form a constant constituent of the surrounding medium. In the higher forms, the development of a complex digestive system has enabled the organism to utilise many different kinds of food, while the storage of any excess of food as reserve material, either in the form of fats or carbohydrates, provides for a constant supply of food to the constituent cells of the body, even when it is quite wanting in the environment. Since plants depend for their food in the first place on the carbohydrates produced within the chlorophyll corpuscles out of the atmospheric carbon dioxide by the energy of the sun's rays, necessary conditions for their existence will be sunlight and the presence of this gas in the surrounding atmosphere.

One other necessary condition for the existence of life is the presence of water. Although this substance cannot furnish any energy to the complex molecules of which the living matter is composed, it is an essential constituent of all living matter, and takes part in all the changes which determine the transformations of matter and energy in the organism.

This short summary of the chief characteristics of living beings would be incomplete without the mention of what is perhaps their distinctive feature, namely, organisation. Although little marked in the lowest members 
of the living kingdom, where we can detect only a speck of structureless material containing a few granules, of which one or more, in consequence of it 3 reaction to stains, is distinguished by the name of a nucleus, in the higher members this organisation becomes more and more marked. This increased complexity of organisation, which we often speak of as histological differentiation, runs parallel with increasing range of power of adaptation, and with increasing efficiency of adaptive reactions attained by the setting apart of special structures (organs) for the performance of definite functions. This parallelism between the development of function and structure justifies us in the assumption generally, though often only tacitly, made by physiologists, that the structure is the determining factor for the function. We might regard the histological differentiation as representing merely a continuation of the increasing molecular complexity, which we assumed must accompany and determine every widening in the range of the adaptive power of the organism.

To sum up :- our objects in the study of physiology include the description of the chief reactions of the body to changes in its environment, the analysis of these reactions into the simpler reactions of which they are made up, and the assignment to each differentiated structure of the organism its part in every reaction. We must determine the conditions under which each reaction takes place, so that we may learn to evoke any part of it at will by application of the appropriate stimulus, i.e. by effective change of environment.

A reaction involves expenditure of energy, and this can be derived only from chemical change in the reacting organ, and ultimately from the disintegration or oxidation of the food-stuffs. Our next task must be, therefore, the analysis of the energetic and material changes, with a view to determining the whole sequence of events, from the occurrence of the external exciting change to the finished reaction, which will alter in the direction of protection the relation of the organism to its environment. In short, it is the office of physiology to discover the routine sequence of events in the living organism under all manner of conditions. In attacking this problem our methods cannot differ fundamentally from those of the physicist and chemist. In every case our experiments will consist in the observation and measurement of movements of one kind or another which we shall interpret in terms of mass or energy. Physiology, if it could be completed, would therefore describe the how of every process in the body. It would state the sequence of events and would summarise these as so-called 'laws.' These laws would, however, no more explain the phenomena of life than does the 'law of gravitation' explain the fact that two masses tend to move towards one another with uniform acceleration. Nor can we hope to explain physiological phenomena by reference to the laws of physics and chemistry, since these themselves are only expressions of sequences, and not explanations. With every growth in science, however, its generalisations become wider and its laws summarise ever more extensive groups of phenomena. We have no reason for asserting that, in the course of research, 
we may not finally succeed in describing vital phenomena in the " conceptual shorthand "* used by the physicist, involving his ideal world of ether, atom, and molecule. At present we are far from such a consummation. The principle of adaptation is the only formula which will include all the phenomena of living beings, and it is difficult to see how this principle can be expressed by means of the concepts of the physicist.

This difficulty, which must be felt with greater force the more deeply the physiologist endeavours to peer into the processes within the living cells, has led some, even at the present day, to the assumption of some special quality in living organisms which is designated as 'vital force' or 'vital activity.' Such views are classified together under the term vitalism. From his beginning man has been accustomed to draw a sharp line of distinction between those phenomena which by their constant occurrence seemed to him natural, and therefore explicable, and those phenomena of which he could not see the determining antecedent, and which were to him, therefore, anomic and capricious. To the latter he set up graven images, and not perceiving his own springs of action, endowed them with a self-determining personality such as he imagined himself to possess. This procedure, though possessing certain advantages in allowing him to perform his common duties free from the ever-lurking fear of supernatural interference, suffered from the great drawback that it fenced off unknown phenomena as unknowable and not to be known. It has therefore acted as a continual check on the growth of man's knowledge and control of his environment. Such a graven image is vitalism. As a working hypothesis it must be sterile. Just as the hypothesis of special creation would impede all research into the relationships of animals and plants, so vitalism would stay the hand of the physiologist in his endeavours to determine the changes which occur within the living organism. In many cases, however, the terms 'vitalism' and its antithesis 'mechanism' are used unjustifiably. The production of energy within the body is due to the oxidation of the food-stuffs. In certain functions it is not yet fully established whether the changes involved take place at the expense of the energy, hydrostatic pressure or otherwise, of the fluids outside the cells, or whether energy is supplied to the process by the cells themselves at the expense of oxidative or other changes occurring in their living substance. Both views are possible, but the adoption of either by a physiologist does not justify the statement that he is a 'vitalist,' 'neo-vitalist,' or ' mechanist.' The office of the physiologist is the determination of the changes which occur in the living body and the establishment of the causal nexus (i.e. the routine of sequences) between them. For such a man to describe himself as a vitalist or mechanist is as germane to the subject as if he were to call himself a Trinitarian or a Plymouth Brother.

Throughout this chapter we have assumed no necessary dividing line between the different classes of phenomena in the conceptual universe, although in the present state of our knowledge we are far from being able to include the whole of them under the same general laws. It might be

* Karl Pearson, "Grammar of Science," p. 328 et seq. (2nd ed.) 


\section{INTRODUCTION}

objected that in taking up this attitude we had left out of account one supreme fact, viz. the existence of consciousness in ourselves. As a comparative and objective study, however, physiology is concerned, not with the study of consciousness, but with the conceptions in consciousness of the phenomena presented by living beings. Consciousness, in fact, we know only in ourselves. From the actions of other living beings similarly organised, we infer in them the existence of a similar consciousness. Again, from the fact that the reactions of the higher mammals are evidently determined, not by immediate impressions, but largely by stored-up impressions of past stimuli we credit them also with a certain but lower degree of consciousness. As we descend the scale of animal life, evidence of the existence of consciousness, as we know it, rapidly diminishes and finally disappears, though it is impossible to draw a sharp line between those animals which possess consciousness and those in which it is absent. That it is a necessary accompaniment of life is certainly not the case. A man is living though he is asleep, anæs. thetised, or stunned, and it would be absurd to speak of the consciousness of a cabbage. Consciousness is, in fact, connected with the possession of a highly developed central nervous system, and its activity is in proportion to the complexity of this system. Since the brain with all the other organs of the body is derived from a simple cell, the fertilised ovum, similar in its absence of differentiation to the lowest organisms, it might be argued that all types of life are endowed with something which is not consciousness, but which has the potentiality of developing into consciousness. To such a hypothetical property Lloyd Morgan has given the name ' metakinesis.' We have, however, no means of judging of the presence or absence of this hypothetical quality and still less of determining whether it is a property only of living substance, or is shared also by the atoms of so-called dead material. 



\section{BOOK I}

\section{GENERAL PHYSIOLOGY}





\section{CHAPTER II}

\section{THE STRUCTURAL BASIS OF THE BODY}

\section{THE CELL}

ALL the higher animals and plants, when submitted to microscopic examination, are seen to consist of structural units which are spoken of as cells. In each organ we find a mass of these cells closely resembling one another in all respects, and we may therefore regard the function of any organ as the sum of the functions of its constituent cells. Indeed, any given reaction of the whole body is the resultant of the reactions of the unlike cells of which the body is composed. The cell is therefore the physiological as well as the structural unit, and it is necessary to commence our study of the functions of the animal body with some consideration of the functions and reactions which are common to all the structural units.

This composite structure is peculiar to the higher forms of life. Amongst the lower forms, both animal and vegetable, an immense number of organisms consist only of a single cell. In this cell are represented all the phenomena of life, all the adapted reactions which we associate with the life of the higher organisms. That the unicellular condition represents the more primitive stage from which the higher organisms have been evolved in the course of ages is indicated by the fact that every one of these higher organisms in the course of its development passes through a unicellular stage, namely, the fertilised ovum. We may assume that the series of changes attending the development of the higher organism from the egg is a repetition in summary of the changes which have determined the evolution of the species from the primitive unicellular type.*

The general characteristics of the cell present important similarities, whether we are considering a cell which forms the whole of an organism or a cell which is but an infinitesimal part of a highly developed animal.

The name 'cell' was first applied by botanists to the structural units found by them in plant tissues, and involved therefore the idea of certain qualities which do not enter into our present conception of the term. A section through the stem of a growing plant shows it to be made up of an aggregation of cells in the etymological sense of the word, i.e. small sacs bounded by a wall of cellulose and containing cell sap. Immediately inside the cellulose wall is a thin layer, the primordial utricle, which encloses at one point a spherical or oval structure known as the nucleus. If the section be 
taken from the growing tip of a plant (Fig. 1), the cell sap is found to be wanting and the cells consist only of the substance known as protoplasm, which later on will form the primordial utricle. This with a nucleus is enclosed in a delicate cellulose wall. The wall is not an essential constituent, since it is absent from many vegetable cells at some period of their life and from animal cells generally.

A better conception of the essentials of a cell can be obtained by the study

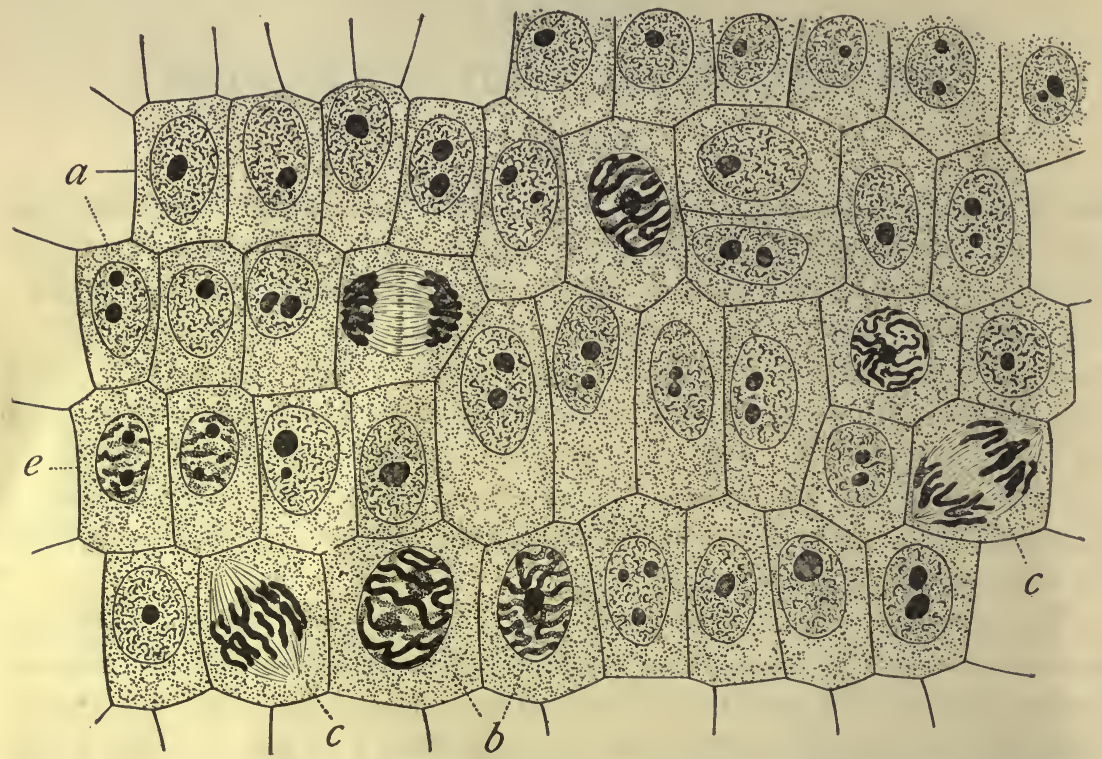

FIG. 1. General view of cells in the growing root-tip of the onion, from a longitudinal section, enlarged 800 diameters. (WILSON.)

$a$, non-dividing cells, with chromatin-network and deeply stained nucleoli; $b$, nuclei preparing for division (spireme-stage); $c$, dividing cells showing mitotic figures; $e$, pair of daughter-cells shortly after division.

of a unicellular animal such as an amœba (Fig. 2). This is an organism frequenting stagnant pools, of varying size (from $0 \cdot 1$ to $0.3 \mathrm{~mm}$. in diameter), apparently of a semi-fluid consistence. When first examined it is generally spherical, but in a short time begins to change its form, putting out processes known as pseudopodia. By shifting the distribution of its material among these processes, it is able to move about and also to ingest particles of food or pigment with which it may come in contact. Near its centre a differentiated portion can be distinguished which is known as the nucleus. The rest of the amœba, the protoplasm or cytoplasm, often presents further differentiation into an outer clear layer and an inner finely granular substance. The latter may contain coarser granules, some of food material, others apparently formed in situ by the surrounding protoplasm, and often small vacuoles ('contractile vacuoles') which are continually altering their size and serve to keep up a circulation of fluid in the interstices of the cytoplasm. In all cells, whether animal or vegetable, with which we are acquainted, this 

twofold structure is also found. So we may define a cell as a small mass of
protoplasm containing a nucleus.

Doubt has often been expressed whether a nucleus is to be regarded as essential latter, no distinct nucleus can be demor the Cyanophycers and Bacteria among the sions of the whole organism are too minuted. In many of these forms the dimen.

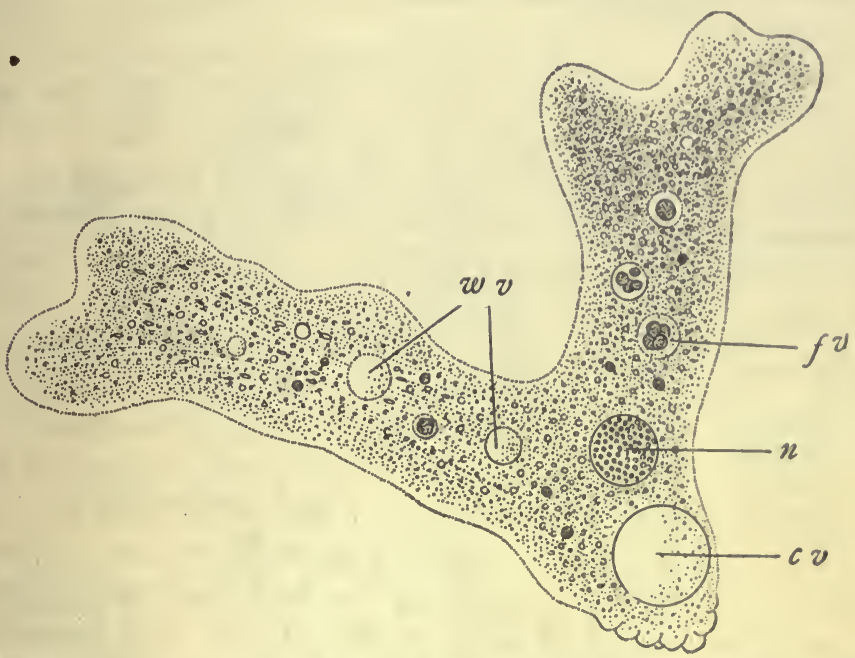

FiG. 2. Amoeba proteus, an animal consisting of a single naked cell, $\times 280$ (From SELGWICK and WILSON's Biology.)

$n$, the nucleus ; $w v$, water-vacuoles ; $c v$, contractile vacuole ; $f v$, food-vacuole.

made as to the presence or absence of nuclear material. In the larger of them, how. ever, the cytoplasm of the cell contains numerous scattered granules which stain with dyes in the same way as do the nuclei of the cells of higher animals, and these granules possess the resistance to the action of certain digestive fluids which is typical of nuclei. They may therefore be taken as representing the nucleus in the higher forms. Even in the latter, at certain stages, namely, during the division of the oell, the nucleus breaks up into discrete parts, and there is no reason for believing that sueh a scattered condition of the nuclear material may not last throughout the whole life of the cell.

We have defined a cell as a snall mass of protoplasm containing a nucleus. Since we shall have to use the term 'protoplasm' on many occasions in the course of this work, we must have a definite conception of what we mean by it. The term is often used by histologists as implying a substance of certain definite chemical and staining characters. When employed by physiologists it generally implies any material which we can, on a study of its behaviour to changes in its environment, regard as endowed with life. Huxley has defined it aș " the physical basis of life." Though it may be convenient to have a word such as protoplasm signifying simply 'living material,' it is important to remember that there is no such thing as a single substance - protoplasm. The reactions of every cell as well as its organisation are the resultant of the molecular structure of the matter of which it is built up. The gross methods of the chemist show him that the composition of the 
'protoplasm' of the muscle cell is entirely different from that of a leucocyte or white blood corpuscle. The finer methods of the physiologist show him that every sort of cell in the body has its own manner of life, its own peculiarities of reaction to uniform changes in its surroundings. No individual will react in exactly the same manner as another individual, even of the same species, and the reactions of the whole organism are but the sum of the

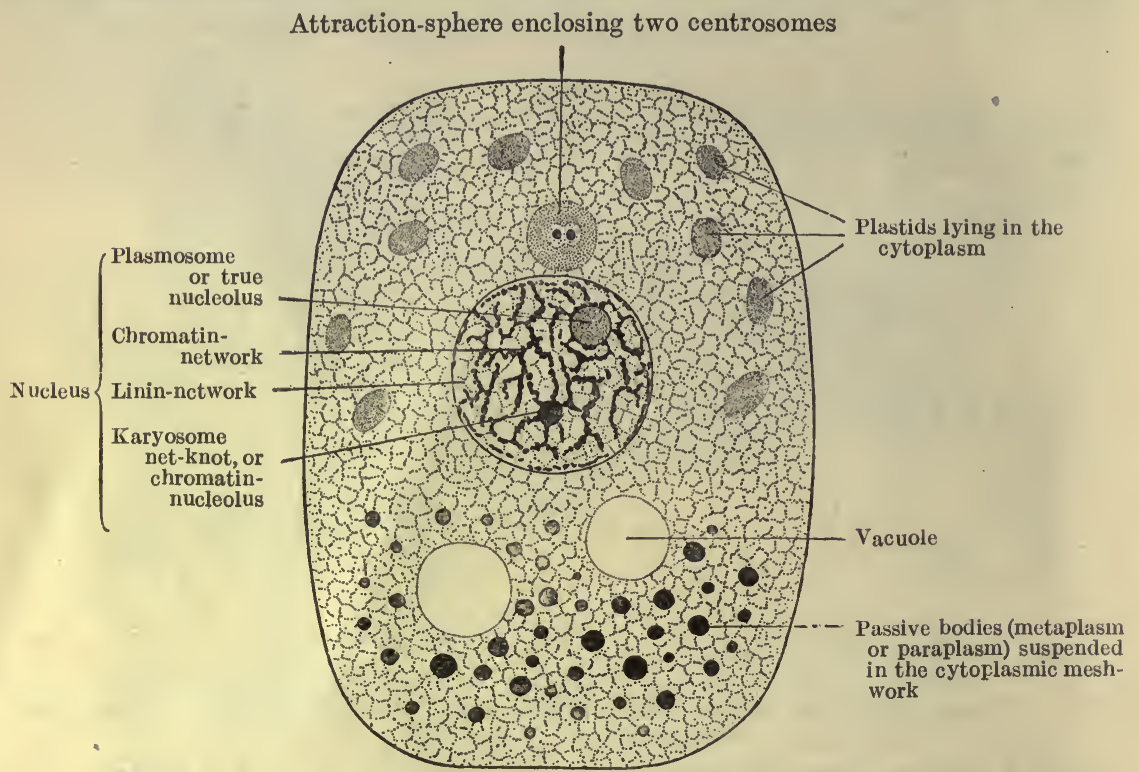

FIG. 3. Diagram of a cell. Its basis consists of a meshwork containing numerous minute granules (microsomes) and traversing a transparent ground-substance. (WILson.)

reactions of its constituent cells. There is not one protoplasm, therefore, but an infinity of protoplasms, and the use of the term can be justified only if we keep this fact in mind and use the word merely as a convenient abbreviation for any material endowed with life. Even in a single cell there is more than one kind of protoplasm. In its chemical characters, in its mode of life, and in its reactions, the nucleus differs widely from the cytoplasm. Both are necessary for the life of the cell and both must be considered, according to our present ideas, as ' living.' In the cytoplasm itself we find structures or substances which we must regard as on their way to protoplasm or as products of the breakdown of protoplasm; but in many cases it is impossible to say whether a given material is to be regarded as lifeless or as reactive living matter. Even in a single cell we may have differentiation among its different parts, one part serving for the process of digestion while others are employed for the purpose of locomotion. Here again there must be chemical differences, and therefore different protoplasms. In this work the term protoplasm will be used in its broadest sense, namely, as the physical basis of living organisms.

STRUCTURE OF THE CELL. In every cell can be distinguished the two parts-nucleus and cytoplasm. The nucleus is generally an oval or 
spherical body lying near the centre of the cell and bounded by a definite contour or nuclear membrane. In its interior it contains masses or filaments of a material known as chromatin, which are strung, so to speak, on a fine network of material known as linin. Besides the granules of chromatin, other masses are sometimes found which stain in a different manner and are called nucleoli. The material filling up the meshes of the network is the nuclear sap or nucleoplasm. The cytoplasm, which varies greatly in extent in different cells, varies also in its appearance, being sometimes homogeneous, sometimes alveolar, sometimes granular in structure. In it can be often distinguished differentiated parts which may be regarded as organs of the cell. Thus in the amœba we have the contractile vacuoles already mentioned. In the green parts of plants the cytoplasm contains green granules, the cbloroplasts, whose special function it is to assimilate carbon dioxide from the atmosphere, and by means of the energy of the sun's rays to convert this into starch with the evolution of oxygen. Other parts of the plant have similar granules, the leucoplasts, whose office it is to build up sugar into starch, and it is possible that other kinds of these 'plastids' with special chemical functions are present in the cytoplasm of many cells. In addition to these cell organs, the cytoplasm may contain granules which represent stages in the metabolism of the cell and are either food material which is being assimilated or products of the disintegration of the protoplasm, formed either for the service of the cell itself or, in the case of the multicellular animals, for the service of other cells of the organism. Others of these granules may represent reserve material, i.e. excess of nourishment which has been put aside by the cell in an insoluble form, to serve for its subsequent needs in times of scarcity.

THE PHYSICAL STRUCTURE OF PROTOPLASM. Owing to the close similarities which exist between the fundamental properties of all living organisms, histologists have sought to discover some corresponding uniform morphological organisation of the physical basis of these phenomena, namely, protoplasm.

It is often impossible, even under the highest powers of the microscope, to make out any structure whatsoever in the cytoplasm, which is spoken of then as hyaline. In most cases examination of a cell, even unstained, shows some differentiation between a more or less regular framework or meshwork and a more fluid portion filling up its interstices, and these appearances are still more manifest when the cells have been fixed by various hardening fluids. All the results obtained in this manner must be regarded with some suspicion, since, as has been shown by Fischer and by Hardy, it is possible to imitate artificially the various structures, which have been assigned as characteristic of protoplasm, by hardening a homogeneous colloidal solution such as egg-white by different methods and with different agents. The theories of protoplasmic structure can be classified under three heads :

1. The Granular Theory of Altmann. By the use of certain hardening reagents, a dense mass of spherical or rod-shaped granules may be demonstrated in almost all cells of the body (Fig. 4). These granules have been 
regarded by Altmann as the elementary particles of life, and he locates in them the various vital functions, the sum of which make up the life of the cell. According to Altmann these granules can only arise from the division of pre-existing granules, and he has formulated the phrase omne granulum $e$ granulo, which is a further extension of Virchow's sentence omnis cellula $e$ cellula. It is probable that a number of different kinds of structures of varying importance are included among Altmann's granules. In some cases they

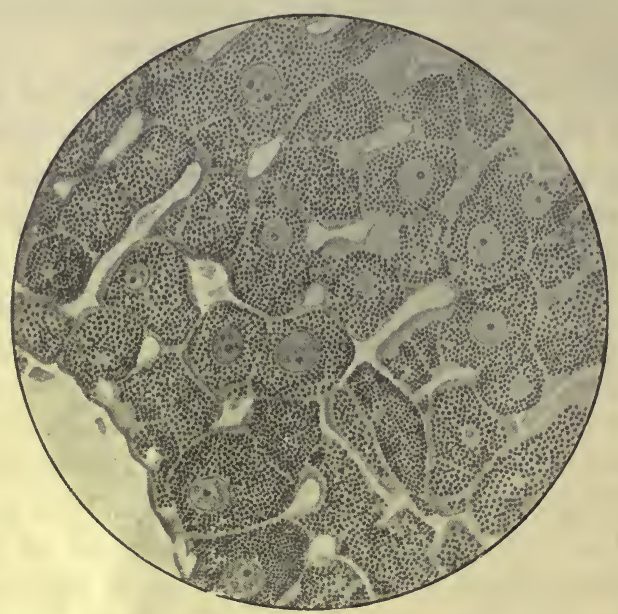

Fig. 4. Section of liver stained to show granules. (Altmann.)

are the products of the activity of the cytoplasm and, as in secreting cells, will be later on cast out with water and salts as the specific secretion. In other cases they may be cell organs or plastids with the special metabolic functions assigned to all granules by Altmann. In some cases no treatment whatever will display the existence of granules.

2. The Fibrillar Theory. By the employment of appropriate methods of hardening, it is easy in most cells to demonstrate a network or clusters of fibrils which form, so to speak, a denser part of the cell. This fibrillar network has been named the 'spongioplasm' in contra-distinction to the structureless material filling its meshes known as ' hyaloplasm.' A network is, however, one of the commonest pseudostructures produced in the coagulation of an albuminous fluid by any means whatever, and it is probable that in most cases the network which is seen in hardened cells is simply an artefact. Sometimes a large portion of the protoplasm may take a fibrillar form which can be detected even in the unstained and unfixed cell, and there is no doubt that, in certain phases at any rate, the fibrillar structure of the protoplasm is really present.

3. The Alveolar Theory of Bütschli. This theory may be looked upon as corresponding morphologically to the granular theory of Altmann. If we imagine a hyaline protoplasm which is continually manufacturing metaplasmic products and storing them up in its protoplasm, these products will be deposited as spherules gradually increasing in size, so that the proto- 
plasm between them will be converted into alveolar partitions between the droplets. In many an egg cell, where there is a growth of protoplasm from this building up of food into reserve materials, the development of such

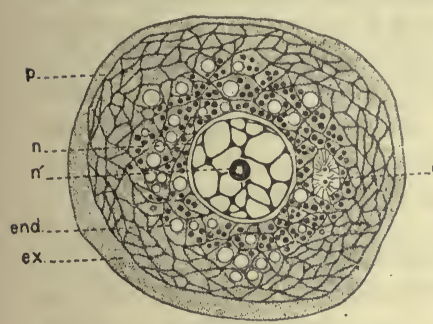

FiG. 5. Diagram of a cell, highly magnified. (SсHÄFER.)

$p$, protoplasm, consisting of hyaloplasm and a network of spongioplasm ; ex, exoplasm ; end, endoplasm, with distinct. granules and vacuoles; $c$, double centrosome; $n$, nucleus ; $n^{\prime}$, nucleolus.

an alveolar structure can be followed in the living protoplasm, and such cells when mature show a marked alveolar structure whether examined fresh or in the hardened and stained condition. Such a protoplasm would be practically an emulsion of one fluid in another and according to Bütschli artificial emulsions, made by mixing rancid oil with sodium carbonate solutions, may show under the microscope a very close resemblance to cell protoplasm (Fig. 6), and may even exhibit amoboid changes of form in consequence of the diffusion currents set up at the surface of the drop between its contents and the surrounding water. Most histologists are in accord that none of the above theories can be regarded as applicable to all forms of protoplasm, but that during the life of a cell its protoplasm, as observed under the microscope, may be either hyaline and structureless or may present any of the structural modifications described above, according to its state of nutrition and the form in which its metabolic products are laid down in the cell. Of course it is possible that, even in the apparently hyaline protoplasm, a structural differentiation is still present, but is invisible owing to the minute size of its constituent parts or an identity of refractive index between the alveolar walls and their contents. The fact that every chemical differentiation occurring within the colloidal mass will tend to cause differences of surface tension, and therefore formation of droplets, shows that an alveolar structure, i.e. one in which there is a large number of surfaces separating heterogeneous mixtures inside the cells, must be of very common
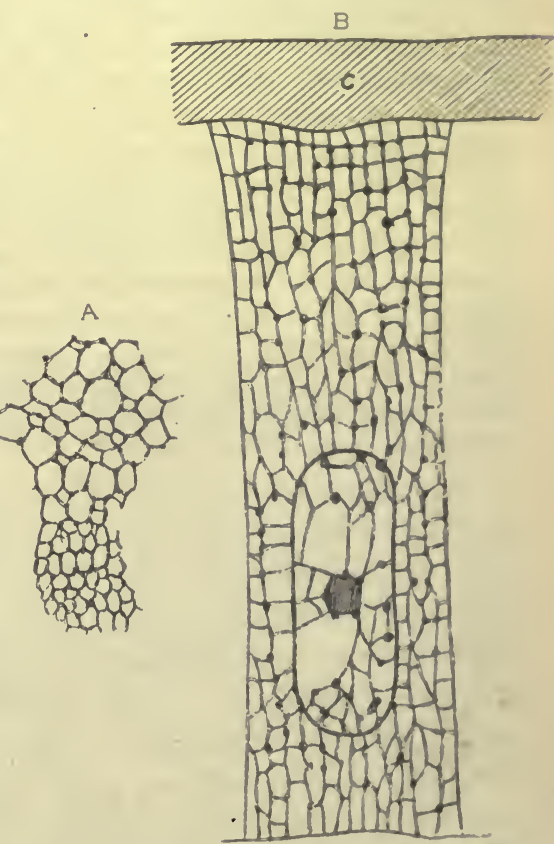

Fig. 6. A, protoplasm of an epidermal cell of the crayfish; B, foam-like appearance of an emulsion of olive oil. (BÜTSCHLI.) occurrence, even in cases where it is not detectable under the microscope. Such a structure must be present, at any rate, in those cases where, apart 
from the existence of a solid cell wall, the cell presents a certain degree of rigidity and resistance to deforming stress.

ULTRAMICROSCOPIC STRUCTURE OF PROTOPLASM. Since the study of the behaviour of the cell shows that it must possess a much more complex structure or organisation than that which is revealed by the microscope, one, that is to say, which permits of the spatial differentiation of the different chemical processes that may occur at one and the same time in the protoplasm, many theories have been put forward of an ultramicroscopic cell structure. Though Spencer in 1864 spoke of physiological units out of which protoplasm could be regarded as made up, and Darwin (1868) conceived ultramicroscopic particles-gemmules-which might be discharged from every cell in the body and, passing into the reproductive organs, serve as the material basis of heredity, the first elaborate conception of such a structure was worked out by Nägeli (1884). According to Nägeli all organised structures are made up of micellæ, minute particles arranged in definite order and surrounded with water. For growth to take place it was necessary that the system should be in a condition of 'turgor,' which was determined by the amount of water between the micellæ. These micellæ arose in every case from the division of pre-existing micellæ, and the vital properties of the protoplasm were to be regarded as the sum of the changes taking place in the individual micellæ. Similar conceptions have been put forward by numerous other observers, each of whom has applied a different name to the elementary living particle, such as 'pangene,' ' plasome,' 'biophor,' ' biogen-molecule,' and many others. The resemblance of these theories to that of Altmann is obvious, though the latter regarded the elementary particle as in many cases of microscopic size and capable of demonstration by appropriate methods of staining. That the cell possesses organs of smaller dimensions than itself, which may give rise to like organs by division, is shown by Schimper's observations on the plastids of plant cells. These apparently are not formed by a process of differentiation of the protoplasm, but are continuous from one generation to another and are reproduced by division. There is no doubt, however, that most of the granules to be observed in the cytoplasm are not of this character, but are elaborated by the general cytoplasm out of the foodstuffs which are supplied to it; and though conceptions such as those of De Vries and Verworn are often of value as a means of describing certain phenomena in the life of the cell and have played a great part in the description of the phenomena of heredity, they cannot be regarded as having any'serious justification in fact. At the present time our knowledge of the properties of the colloidal and capillary systems, which must play so great a part in the organisation and reactions of living protoplasm, is much too meagre to justify weight being laid on any theory of the ultramicroscopic structure of protoplasm that can at present be put forward.

One question which has been much discussed relates to the physical condition of protoplasm. Is it to be regarded as a viscous fluid or as a soft solid? The perfect potential mobility of the protoplasm of many cells, as instanced by the flow of a substance of an amœba into its pseudopodia, or the occurrence of rapid streaming movements in the threads of protoplasm found in many plants, e.g. the root hairs of tradescantia, indicates a fluid character for the protoplasm. Against such a character has been urged the fact that in protoplasm we may have shape, organisation, and power of resistance to deformation-qualities which are generally associated with the possession of solidity. It must be remembered, however, that the absence of resistance to deformation, which is characteristic of a liquid, applies only to the internal molecules, and that the surface of any liquid is in a condition of tension which not only limits deformation, but presents considerable resistance to any enlargement of the surface. Small water animals take 
advantage of this resistance to run freely over the surface of water, although their specific gravity may be greater than that of water. The continued existence of protoplasm in a watery environment shows that not only must its composition be different from that of its environment, but that there must be a distinct surface separating the two. The superficial layers of the protoplasm must therefore be in a condition of tension, and exercise pressure on the internal portions of the cell, which will tend to diminish the surface of the cell to the smallest possible extent, i.e. to bring it into the spherical form.

This form is characteristic of free cells in their conditions of inactivity, and the smaller the mass of protoplasm, supposing it to be homogeneous, the greater will be the pressure exerted by its surface layer on its contents and the greater resistance will it present to deformation of the spherical form. A fluid drop, if suspended in a fluid with which it is immiscible, will present greater rigidity the smaller its dimensions. Almost any degree of rigidity can also be imparted to larger masses of fluid protoplasm if their interior has undergone chemical differentiation so as to be made up of two or more im. miscible fluids arranged as droplets within alveoli, as in Bütschli's theory. In such a case every droplet will present resistance to deformation and every surface will resist penetration or extension. The resistance of the surface in colloidal fluids is still further increased by a property common to all these fluids, namely, the aggregation in the surface of a greater concentration of the dissolved substance than is present in the underlying fluid. If, for instance, we take a beaker containing egg-white diluted 100 times, and drop a steel magnetised needle on to the surface, it will float although it is much heavier than the fluid, in consequence of the resistance of the surface. If the needle be greasy the same thing will occur on a glass of water. In this case the needle will lie N. and S. On the albumen solution, however, the needle will lie in the position in which it has been dropped. The aggregation of the albumen molecules on the surface of the fluid is such that it is practically solid and resists any turning of the needle. In consequence of the surface aggregation and solidification of the colloidal molecules, it is possible to throw out the greater part of the albumen in a solid form from a solution of this substance, if it be shaken up in a bottle with a little air so as to make a surface. As the fluid is shaken fresh surfaces are always being formed, and the albumen aggregating in each of these surfaces has not time to redissolve before a fresh aggregation occurs on a new surface, and the films thus produced gradually collect to form a solid mass of insoluble protein. Protoplasm may be regarded as essentially fluid in character, the form and rigidity which are acquired by most cells being due to chemical and physical differentiation occurring in the fluid.

THE SURFACE LAYER OF CELLS. Since it is by means of its surface layer that the organism enters into relation with its environment, this layer acquires a prime importance for the life of the cell, and we may therefore consider here at greater length some of the properties of this layer, the Plasmahaut, as it has been called.

The superficial layer of the protoplasm is not to be confounded with the 
cell wall. The latter, which plays a great part in the building up of vegetable tissues, is formed by a process of secretion from the living protoplasm and is situated altogether outside the superficial Plasmahaut. The cell wall differs considerably in its chemical composition from the protoplasm out of which it has been formed. In most plants it consists of cellulose, a substance belonging to the carbohydrate group, and with a composition represented by some multiple of the formula $\mathrm{C}_{6} \mathrm{H}_{10} \mathrm{O}_{5}$. In other cells the wall may be built up from calcium carbonate or other lime salts, from silica, from chitin. In many cases it is perforated to allow the passage of communicating strands of protoplasm between adjacent cells. It is generally freely permeable to all kinds of solutions, and in this case plays no part in regulating the interchanges of the cell with the environment.

The superficial layer of protoplasm represents that part of the living substance which stands in immediate relationship to the environment. Every change in the latter can only influence the living cell through this layer, and it is through this layer that substances must pass on their way into the cell for assimilation, or out of the cell for excretion. The retention of an individuality by the cell must be determined by chemical and physical differences between this layer and the surrounding fluid. Since it differs from the rest of the protoplasm in the changes to which it is subject, it must also differ in its chemical composition, apart altogether from the factors which, as we saw above, determine molecular differences between the surface and the interior of any colloidal solution. On this account one must assume the existence of a definite boundary layer of the protoplasm, even where it is impossible to see any differentiation between this layer and the deeper parts under the highest powers of the microscope.

A (living) cell, which leads its life in a liquid environment, must take up the greater part of its food material in the form of solution, and it is the permeability of the superficial protoplasm which will determine the passage of food substances from the surrounding medium into the body of the cell. The immiscibility of the protoplasm with the surrounding fluid shows that the permeability of the membrane must be a limited one. The qualitative permeability can be easily studied in vegetable cells. These present within a cellulose wall a thin layer of protoplasm (the primordial utricle), enclosing a cell sap. If the root hairs of tradescantia be immersed in a 10 per cent. solution of glucose or in a 2 to 3 per cent. solution of salt, a process of plasmolysis takes place. The cell sap diminishes in amount by the diffusion of water outwards so that the primordial utricle shrinks (Fig. 7). On immersing the cells in distilled water, water passes into the cell sap until the further expansion of the protoplasmic layer is prevented by the tension of the surrounding cell walls. This behaviour can be explained only on the assumption that the protoplasm is impermeable both to sugar and to salt, but is freely permeable to molecules of water, i.e. it behaves as a semipermeable membrane. Similar experiments can be made on animal cells. The most convenient for this purpose are the red blood corpuscles. These also shrink when immersed in salt solutions with a greater molecular 
concentration than would correspond to the plasma of the blood from which the corpuscles were derived, whereas if placed in weak salt solutions or distilled water they swell up and burst, discharging their hæmoglobin in solution into the surrounding fluid. By comparison of various salts it is found that the strength of each salt solution which is just necessary to cause plasmolysis or hæmolysis, as the case may be, is determined entirely by its molecular concentration, i.e. a decinormal solution of sodium chloride will be equivalent

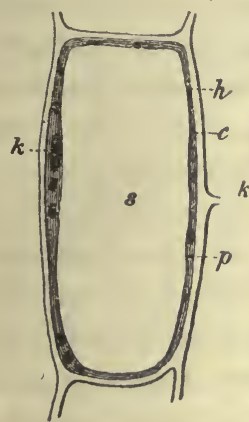

1

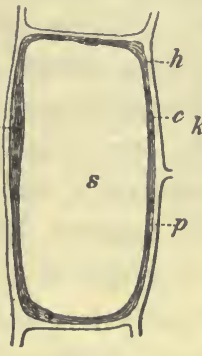

2

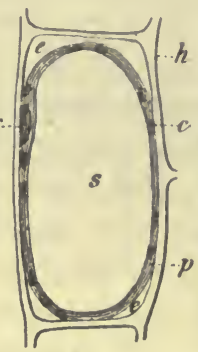

3

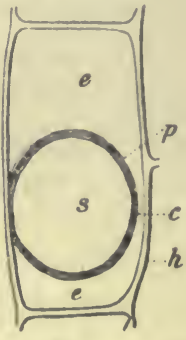

4

FiG. 7. Vegetable cells, showing varying degrees of plasmolysis. (DE VrIEs.)

in its effect on the cells to a decinormal solution of potassium nitrate or of potassium chloride. The impermeability of the plasma skin does not apply to all dissolved substances. Overton has found that, whereas this layer is practically impermeable to salts, sugars, and amino-acids, it, permits the easy passage of monatomic alcohols, aldehydes, alkaloids, \&c. All these substances are more soluble in ether, oil, and similar media than they are in water. The passage of dissolved substances through a membrane wetted by the solvent depends on the solubility of these substances in the membrane, and Overton therefore concludes that the superficial layer of protoplasmic cells must itself partake of a 'lipoid' character, and that cholesterin and lecithin probably enter largely into its composition. Thus only those aniline dyes which are soluble in a mixture of melted lecithin and cholesterin have the property of penetrating the living cell, and only these dyes, such as methylene blue, neutral red, can be used for intra vitam staining. For the same reason substances which have the power of dissolving lecithin and cholesterin, such as ether or bile salts, also act as hæmolytic agents, i.e. they cause a destruction of the red blood cells by dissolving the superficial layer which is necessary for their preservation from the solvent effects of the surrounding fluid.

The semi-permeability of the plasma skin can be altered by changes in the saline concentration or other factors of the surrounding medium. Overton has shown that, whereas a 7 per cent. solution of saccharose produces plasmolysis in living cells, no plasmolysis is observed if they are treated with a solution containing 3 per cent. methyl alcohol plus 7 per cent. cane sugar. The superficial layer, therefore, is able to dissolve a mixture of methyl alcohol and cane sugar, although it has no solvent power on cane sugar in 
pure watery solutions. It is possible that, in order to serve the nutrient needs of the cells, more extensive changes may take place in the permeability of the surface layer under limited conditions of time and space. There is no doubt, for instance, that dextrose, to which the surface layer is apparently impermeable, can yet serve as a very efficient food for the cell, and one might ascribe the fact that the cell assimilates only the food which it requires and no more, to such limited changes in permeability. An important factor in the process of assimilation, at any rate by lowly organised cells, must be the relative solubility of the absorbed substances in the cell and its surrounding medium respectively. When a watery solution of iodine is shaken up with chloroform, the latter sinks to the bottom, carrying with it the greater part of the iodine. If a watery solution of organic acid be shaken with ether, the latter fluid will extract the greater quantity of the acid. In no case will the extraction be complete, but there will be a definite ration between the amount dissolved by the ether and the amount dissolved by the water, the so-called 'coefficient of partage,' depending on the different solubilities of the dissolved substance in the two menstrua. In the same way a mass of protoplasm will tend to absorb from the surrounding medium and to concentrate in itself all those substances which are more soluble in the colloidal system of the protoplasm than in the surrounding fluid, and this process of absorption may be carried to a very large extent, if the dissolved substances meet in the cell with any products of protoplasmic activity with which they form insoluble compounds so that they are removed from the sphere of action. It is probably by such a process as this that we may account for the accumulation of calcium or silicon in such large quantities in connection with the bodies of various minute organisms.

Whereas assimilation by a living cell is ultimately conditioned by the permeability of the surface protoplasm, its form is determined by the tension of this layer. If the tension is uniform at all parts of the surface the form of the cell will be spherical. Any diminution of the surface tension at one point must tend to cause a bulging of the fluid contents at this point, just as on distending a rubber tube with one weak spot in its wall this suddenly gives way with the production of a large balloon, which rapidly extends in size and ruptures unless the pressure be diminished. Diminution of the surface tension at one point of the cell will be attended by a contraction of all the rest of the surface and a driving out of the contents through the weak part. This process will not as a rule result in destruction of the cell ; the resulting protrusion will be limited by the distortion of the internal alveolar structure of the protoplasm caused by any alteration of the spherical form of the cell. Change of form in living structures thus depends ultimately on alterations in surface tension, return to normal being affected by the elastic reaction of the structural arrangement of the protoplasm. This point we shall have to consider more fully when dealing with muscular contraction. At present it is sufficient to see how any slight alteration in the chemical environment, such as might be due to the presence of a particle of food-stuff, may cause local variations in the surface tension of the plasma skin and 
thus result in the protrusion of pseudopodia and the ingestion of the food

VITAL PHENOMENA OF CELls. A. Assimilation. The activity of every living being, whether uni- or multicellular, can be regarded as compounded of two phases, assimilation and dissimilation. By assimilation we mean the building up of the living substance at the expense of material obtained from the external world. In this process substances are formed of high potential energy, and this energy can be obtained only at the expense either of energy imparted to the system at the moment of assimilation, as, e.g. in the assimilation of carbon from carbon dioxide under the influence of the sun's rays, or of energy contained in the food-stuffs themselves. In all living organisms, except those provided with chlorophyll corpuscles, it is the latter method which is adopted, and a food-stuff therefore connotes some substance which can be taken in by the cell and can serve to it as a source of chemical energy. The evolution of energy, which is required for the movements and other vital activities of the cell, is derived from a disintegration or dissimilation of the protoplasm and is generally associated with the process of oxidation. In assimilation, besides the building up of living protoplasm, there may also be a synthesis of more complex from less complex compounds, without their necessary entry into the structure of the living molecule. In the absence of any definite criteria by which we may judge as to the living or non-living condition of parts of the cell, it is a little dangerous to draw any hard-and-fast distinction between these two sets of processes. Assimilation requires the ingestion of food into the organism, and in the second place its digestion, i.e. its solution in the juices of the cells. These two processes are succeeded, through stages which we cannot trace, by an actual growth in the living material. In naked cells ingestion may occur either at any part of the surface, as in the amœba, or at a specialised portion, so-called 'mouth,' as in many of the infusoria. Digestion is apparently effected in most cases by the production and secretion around the ingested food particle of solutions containing ferments, i.e. agents which have the power of hydrolysing the different food-stuffs and rendering them soluble.

In the vast majority of living organisms the energy for their activities is derived from the oxidation, ultimately of the food-stuffs, but immediately of molecules attached to the living protoplasm. A necessary condition, therefore, for the life of these cells is the presence of oxygen in the surrounding medium, from which it is taken up in the molecular form. We may therefore speak of an assimilation of oxygen; but it is still a matter of dispute whether the oxygen is built up as such in the living molecule (so-called intramolecular oxygen) to be utilised for the formation of carbon dioxide when a discharge of energy is necessary, or whether it is only taken in at the moment when the combustion of the carbon and hydrogen constituents of the food or protoplasm is necessary for the supply of energy. However this may be, products are formed as a result of this oxidation which are of no further value to the cell and are therefore excreted, i.e. turned out of the cell. The chief of these are the products of oxidation of carbon and hydrogen, namely, 
carbon dioxide and water. There are also many substances resulting from the oxidation of the nitrogenous portions of the protoplasm, which have to be excreted in the solid or dissolved form.

Although the assimilation of oxygen is so general a quality of living protoplasm, the presence of this gas, at any rate in the free form, does not seem to be necessary for all kinds of life. Thus a number of the bacteria are known which are anaerobic, i.e. exist only in the absence of oxygen. Examples of such are bacillus tetanus, and the bacillus of malignant œdema. In order to cultivate them it is necessary to displace all the air in the cultivating vessels by means of a current of hydrogen. It has been supposed that the ultimate source of the energy of these organisms is also derived from a process of oxidation, and that they differ from other organisms in being able to utilise for this purpose oxygen which is built up into the structure of their food substances. It is possible, however, that these organisms derive the energy for the building up of their protoplasm, for their movements, \&c., not from a process of oxidation at all, but from processes of disintegration of the substances which they utilise as food. It is by such means that in all probability the intestinal worms. fairly highly organised animals, are able to exist in the intestine in a medium containing no oxygen, but rich in carbon dioxide. Here they are plentifully supplied with food-stuffs and can afford to adopt a wasteful method of nutrition, in which only a sinall fraction of the energy is obtained which would be produced by a total oxidation of the food.

B. The Phenomena of Dissimilation. The activities of a living cell or organism can be regarded in every case as dependent originally on environmental change, and are adapted to this change, i.e. are of such a nature that they tend to preserve the organism intact, to favour its growth, or prevent its destruction. The property of reacting in such a manner to changes in the environment is fundamental to all protoplasm and is spoken of as excitability, and the change which will influence an organism and cause a corresponding adaptive change in it is known as a stimulus. Stimuli may be of various kinds. Thus mechanical, thermal, chemical, electrical changes, light, and so on, may act as stimuli. The reactions which they evoke involve in every case chemical changes in the protoplasm, i.e. changes in the metabolism of the cell. Sometimes this change may be assimilatory in character, leading to an increased growth of the protoplasm, or at any rate to a cessation of dissimilation. In such a case the stimulus is spoken of as inhibitory, because it diminishes or prevents the output of energy by the organism. The frequent result of a stimulus is an increased output of energy, which may appear in the form of movement, in the form of heat, or as chemical change.

A common feature of all dissimilatory changes evoked by the application of a stimulus is that the energy of the reaction is always many times greater than the energy represented by the stimulus, the excess, of course, being supplied at the expense of the potential energy of the food material which has been stored up in or built up into the living protoplasm. This disproportion between stimulus and reaction can be well illustrated on an excitatory tissue such as muscle. Thus in one experiment the gastrocnemius muscle of a frog was loaded with a weight of $48 \mathrm{gms}$. The nerve running to the muscle was placed on a hard surface and a weight of half a gramme was allowed to fall upon it from a height of $10 \mathrm{~mm}$. The muscle contracted in response to this mechanical stimulus applied to the nerve and raised the weight $3.8 \mathrm{~mm}$. 
In this case the work performed by the muscle was $48 \times 3.8=182.4$ grm. mm., while the potential energy of the stimulus represented only $0.5 \times 10 \cdot 0=5 \cdot 0 \mathrm{grm} . \mathrm{mm}$. Thus the work performed by the muscle was thirty-six times larger than the energy of the stimulus applied to the nerve.

In the ease of unicellular organisms, definite elasses of motor reaction to stimulus have been deseribed. The ordinary retraetion of a unicellular organism, sueh as the vortieella, in response to a toueh is ealled thigmotaxis. Certain eells are influenced by gravity, tending to rise or fall in the surrounding medium aeeording to the conditions which favour their existenee. A similar sensitiveness to gravity is obscrved in the growing parts of plants, where the root always grows downwards and the stem upwards. This reaction to gravity is known as geotaxis, which is distinguished as 'negative ' or 'positive' respeetively, aecording as the plant grows in opposition or in obedienee to the gravitational attraetion. If growing plants be placed on the rim of a wheel and rotated so that the centrifugal foree is greater than that of gravity, the stems all grow towards the centre of the wheel while the rootlets grow outwards. In the same way the reaction of miero-organisms to light is known as phototaxis, some organisms seeking the light while others shun it. Among the primitive reactions of eells perhaps the most important in the life of higher animals are those grouped under the term chemiotaxis. The fertilisation of the ovum in the prothallus of ferns is effected by the penetration of the antherozoids produced in the male organs at some little distanee from the female organs. It was shown by Pfeffer that the movement of the antherozoids towards the ova is effeeted in response to a chemieal stimulus, probably malic aeid, sinee he found that antherozoids suspended in a fluid will always swim towards any locality where there is a greater eoneentration of this aeid. In the same way aerobie baeteria are attracted by the presenee of oxygen. If such bacteria are present in a solution with an alga, on exposure of the fluid to light there is an evolution of oxygen by the green alga, and a consequent eongregation of the baeteria round the seat of production of the oxygen. The movements of the white corpuseles of the blood of the higher animals are also largely determined by their chemieal sensibility, and various substances ean be divided into $(a)$ those which exereise positive and $(b)$ those which exereise negative ehemiotactie influenee on the leueoeytes. Thus the introduetion under the skin of an animal of a eapillary tube eontaining a solution of substanees of the first elass, sueh as peptone, tissue extraets, or the ehemical products of eertain bacteria, leads to an aceumulation within the tube of leueoeytes which pars to it from all the surrounding tissues. Other substanees; sueh as quinine, exert a negative ehemiotaxis. Tubes filled with these, after introduetion into the subcutaneous tissue of a mammal, will be found many hours later to eontain no leueoeytes at all.

THE RELATIONS OF THE NUCLEUS TO THE CYTOPLASM. The universal existence in living cells of a differentiated nucleus indicates that the life cycle of assimilation and dissimilation must depend on an interaction between the nucleus and cytoplasm, and that each plays a distinct part in the sum of the changes which make up the life of the cell. The different staining reactions of nucleus and cytoplasm suggest a corresponding difference in their chemical composition, a suggestion which is confirmed by analysis. In the building up of protoplasm proteins play an important part. They are not present, however, as simple proteins, but built up with other complex bodies to form conjugated proteins. Whereas in the cytoplasm these conjugated proteins consist chiefly of compounds of protein and lecithin, in the nucleus the chief constituents belong to the class of nucleo-proteins. The nucleoproteins are of varying composition, and are distinguished chiefly by the 
large amount of phosphorus in their molecule. A nucleo-protein can be broken down into nuclein and protein. Nuclein can be broken down into nucleic acid and a protein-like substance, protamine. Nuclei differ among each other and at different periods of their existence or in different conditions of activity according to the greater or less amount of protein which is com-
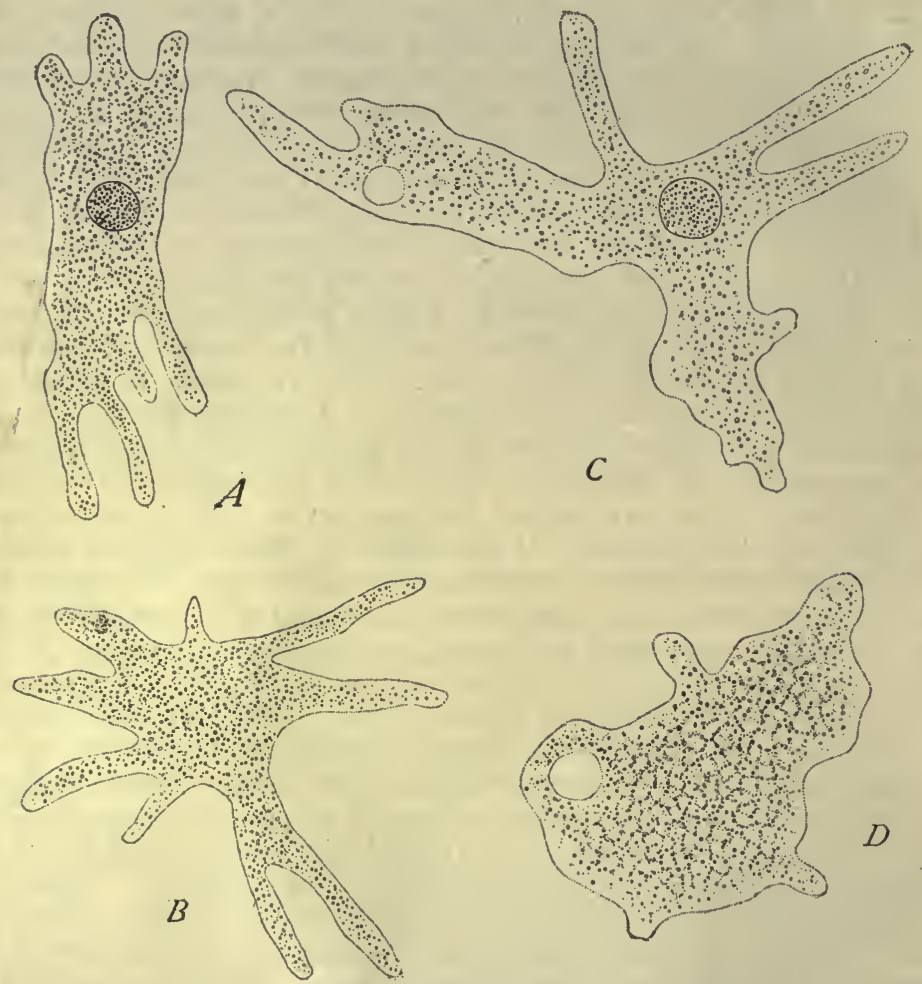

Fir. 8. Nucleated and non-nucleated fragments of Amoba. (Wirson after Hofer.)

$A, B$. An Amaba divided into nucleated and non-nucleated halves, five minutes after the operation. $C, D$. The two halves after eight days, each containing a contractile vacuole.

bined with the nuclein. The latter seems to be the essential constituent of cell nuclei and to be present in only small quantities in the cytoplasm. The properties and reactions of these bodies will be dealt with at greater length in the next chapter.

In order to appreciate the part played by the nucleus in the ordinary cell processes, we must study the behaviour of cells or parts of cells deprived of a nucleus and compare it with that of similar cells or parts of cells still containing a nucleus. By means of a fine needle it is possible to divide the larger protozoa into two pieces, one with and one without a nucleus. Hofer, experimenting on the amœba, found that the fragment containing the nucleus quickly regenerated the missing part and pursued a normal existence. On the other hand, the non-nucleated fragments showed no signs of regeneration. They might, indeed, live as long as fourteen days after the operation (Fig. 8). 
Their movements continued for a short time and then ceased, though the pulsations of the contractile vesicle were but little affected. The power of digestion of food was completely lost. Other observers have shown that Stentor, an infusorium which possesses a fragmented nucleus, may be broken up into fragments of all sizes. Nucleated fragments as small as one-twenty-seventh the volume of the entire animal are still capable of
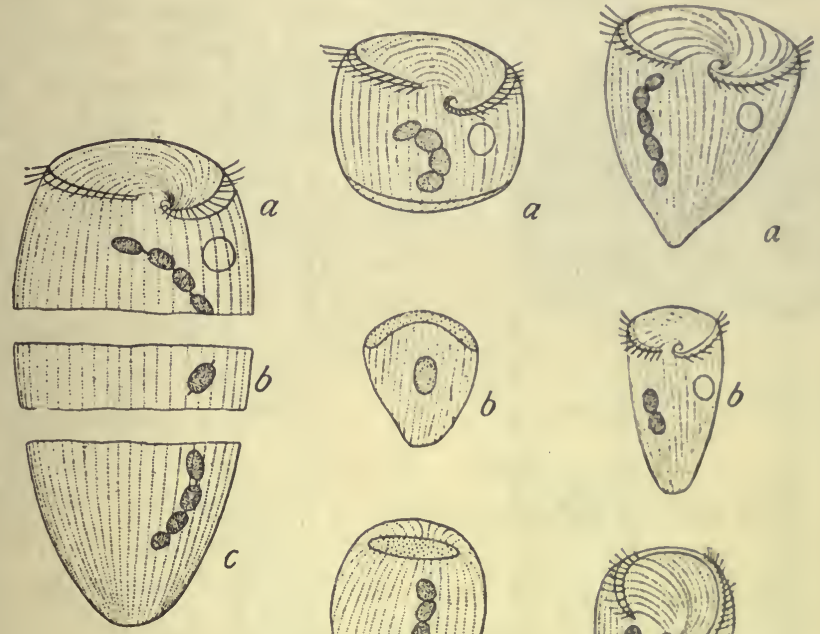

$A$
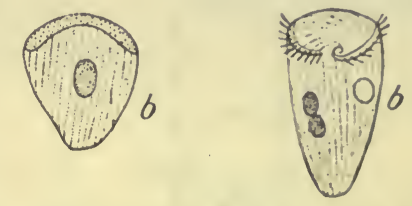

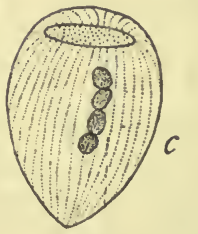

B

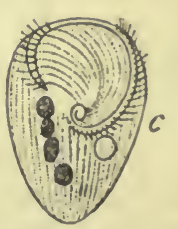

C

F1G. 9. Regeneration in the unicellular animal stentor. (From Grober after Balbusi.)

$A$. Animal divided into three pieces, each containing a fragment of the nucleus, $B$. The three fragments shortly afterwards. $C$. The three fragments after twenty-four hours, each regenerated to a perfect animal.

regeneration. The wound quickly heals and the special organs-the mouth, with its surrounding cilia, and the contractile vacuole - are regenerated, but all non-nucleated fragments quickly perish (Fig. 9).

Many similar observations have shown that the non-nucleated cytoplasm, though it may survive for some time and perform normal movements in response to stimuli, such as those of ingestion of food particles, loses entirely the power of digestion, secretion, and growth. In animals possessing a shell, a small secretion of the lime salts may occur on the surface, but this process rapidly comes to an end as the store of material in the cytoplasm is exhausted. In vegetable cells it is possible to break up the protoplasm by means of plasmolysis into nucleated and non-nucleated parts. The nucleated part quickly forms a new cell wall. The non-nucleated part is unable to effect this formation, and soon dies unless it is in connection with an adjacent cell containing a nucleus by means of fine threads of protoplasm which pass through pores in the intercellular septa (Fig. 10). In the higher animals 
we have, in the case of the nerve-cell, an example of the necessity of the nucleus for growth. Here division of the nerve fibre causes degeneration of the whole fibre separated from the cell containing the nucleus, and regeneration of the fibre, when it occurs, is effected by a down-growth of that part of the fibre which is still in connection with the nucleus. All these facts show
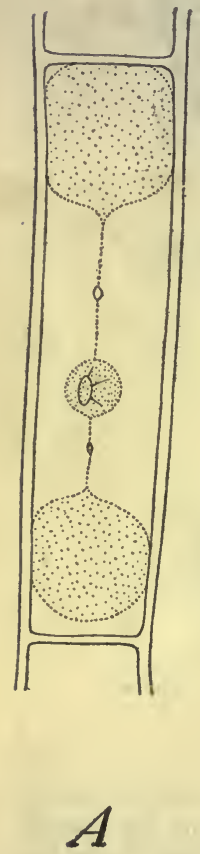
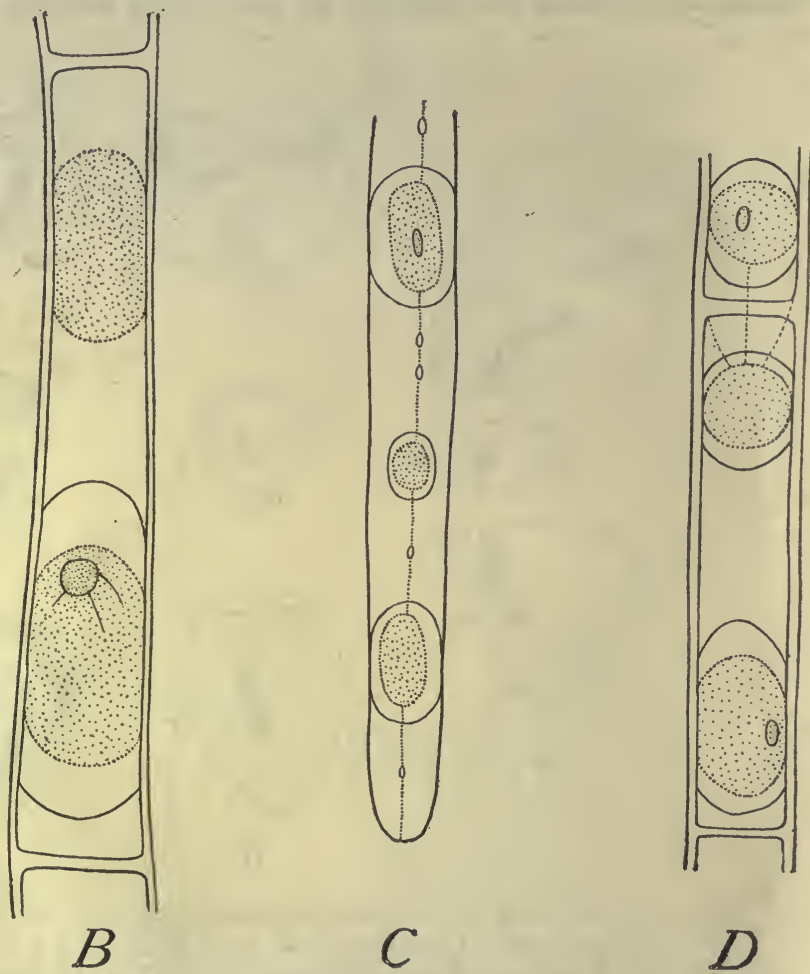

FiG. 10. Formation of membranes by protoplasmic fragments of plasmolysed cells.

(WILSON after TOWNSEND.)

A. Plasmolysed ccll, leaf-hair of Cucurbita, showing protoplasmic balls connected by strands. B, Calyx-hair of Gaillardia; nucleated fragment with membrane, nonnucleated one naked. C. Root-hair of Marchantia; all the fragments, connected by protoplasmic strands, have formed membrancs. D. Leaf-hair of Cucurbita; nonnucleated fragment, with membrane, connected with nucleated fragment of adjoining cell.

that the power of morphological as well as of chemical synthesis depends on the presence of a nucleus. On this account the nucleus, as we shall learn later on, must be regarded as the especial organ of inheritance. The transmission of the paternal qualities from one generation to the next is effected by the entrance simply of the nuclear material of the male cell, the spermatozoon, into the ovum. In the words of Claude Bernard, " the functional phenomena in which there is expenditure of energy have their seat in the protoplasm of the cell (i.e. the cytoplasm). The nucleus is an apparatus for organic synthesis, an instrument of production, the germ of the cell."

Similar conclusions may be drawn from a study of the changes in the nucleus which accompany different phases in the activity of the whole cell. 
Thus in growing plant cells the nucleus is always situated at the point of most rapid growth. In the formation of epidermal cells the nucleus moves towards the outer wall and remains closely applied to it so long as it is growing in thickness. When this growth is finished the nucleus moves to another part of the cell. In the formation of root hairs the outgrowth always takes place in the immediate neighbourhood of the nucleus, which is carried forward and remains near the tip of the growing hair. The active growth of cytoplasm, which accompanies the activity of secreting cells, is always associated with changes in the position and in the size of the nucleus. Where the nutritive activity of the cell is very intense, as in the silk glands of various lepidopterous larvæ, the nucleus is found to be very large and much branched (Fig. 11) so as to present the

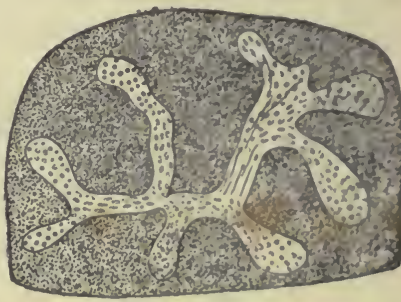
greatest possible extent of surface through Fir. 11. Branched nucleus from which interchanges can go on between nucleus and cytoplasm. the spinning gland of butterfly larva (Pieris). (Konscher.t.)

The important changes which the nucleus undergoes in the process of cell division we shall have to consider more fully in the later chapters of this work. In the function of assimilation it is natural to assume that it is those constituents of the nucleus which are peculiar to it both morphologically and chemically, namely, the chromatin filaments, which are most directly concerned. This assumption receives support from the changes which have been observed to occur in these filaments during various phases of nutritive activity of the cell. The staining powers of chromatin are in direct proportion to the amount of nuclein it contains. In the eggs of the shark it has been shown that the chromosomes undergo characteristic changes during the entire growing period of the egg. At first they are small and stain deeply with ordinary nuclear dyes, but during the period of growth they undergo a great increase in size and at the same time lose their staining capacity, ${ }^{*}$ their surface being increased by the development of long threads which grow out in every direction from the central axis. As the egg approaches its full size, the chromosomes diminish in size and are finally reduced to minute intensely staining bodies which take part in the first division of the egg preparatory to its fertilisation (Fig. 12). We must conclude that whereas the processes of destructive metabolism or dissimilation, which determine the activity of the cell, have their immediate seat in the cytoplasm, the processes of constructive metabolism which lead to the formation of new material, to the chemical and morphological building up of the cell, are carried out in or by the intermediation of the-nucleus.

HISTOLOGICAL DIFFERENTIATION OF CELLS. Even within the limits of a single cell, differentiation of structure can take place by the setting apart of distinct portions of the cell for isolated functions. Thus in an organism such as vorticella the cell is shaped somewhat like a wine-glass,

* Rückert, cited by Wilson. 
the stem being composed of a spiral contractile fibre which has the function of withdrawing the rest of the organism when necessary towards its point of attachment. The main portion of the cell presents at its free extremity a part which is the seat of ingestion of food, and is therefore spoken of as the 'mouth.' This is surrounded by a circle of cilia whose function it is to set up currents in the surrounding fluid and so favour the passage of food particles towards the mouth. Food when ingested at this end passes only a short

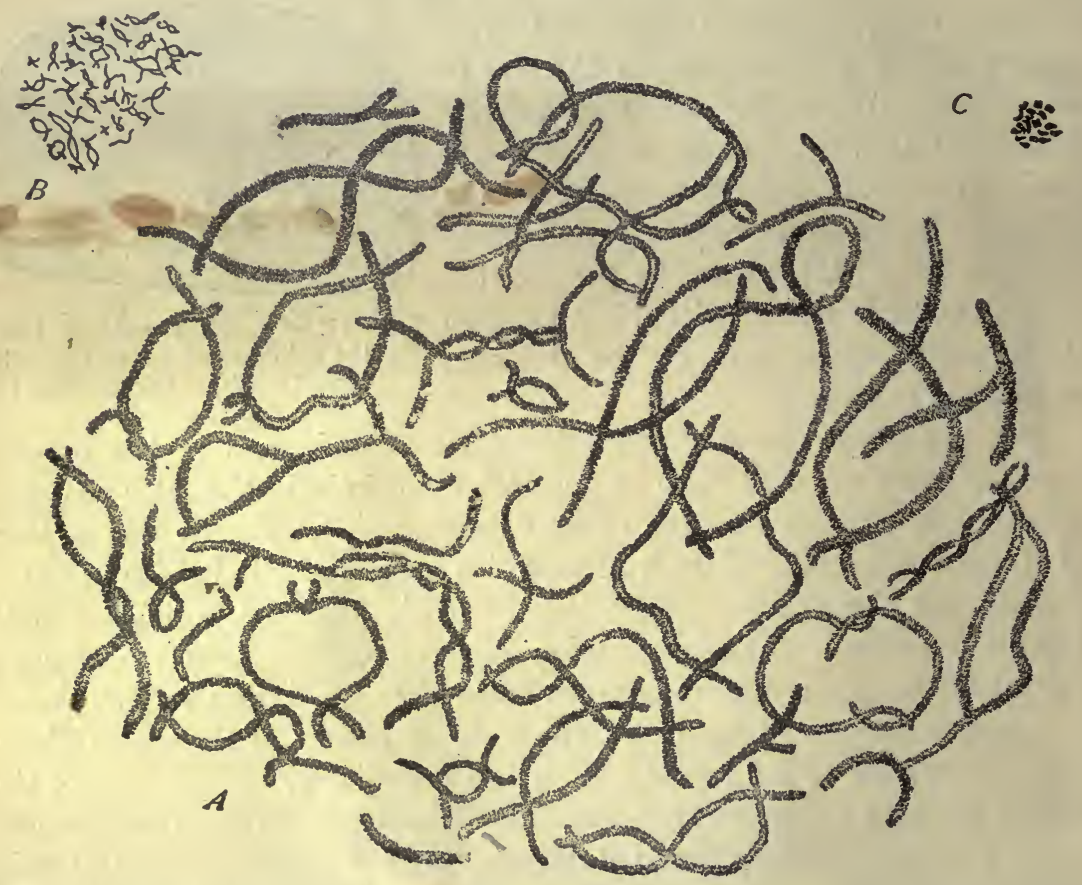

FiG. 12. Chromosomes of the germinal vesicle in the shark Pristiurus, at different periods, drawn to the same scale. (RückERT.)

$A$. At the period of maximal size and minimal staining-capacity $(\mathrm{egg} 3 \mathrm{~mm}$. in diameter). $B$. Later period (egg $13 \mathrm{~mm}$. in diameter). $C$. At the close of ovarian life, of minimal size and maximal staining-power.

distance into the body of the vorticella. Here fluid is secreted around it which serves for its digestion. This portion of the cell may therefore be regarded as the alimentary canal or stomach. The indigestible residue of the food is excreted in close proximity to the mouth. In addition to these organs we have the usual differentiation of the protoplasm into an external and internal layer, and the development within the protoplasm of contractile vacuoles which serve to keep up a circulation of fluid and therefore to pass the products of digestion through all parts of the cell body. Within the limits of the single cell which forms the vorticella we may therefore speak of organs for contraction, for digestion, for circulation, and so on.

The organs which are thus formed in unicellular animals or plants can be divided into two classes, namely (1) temporary organs, which are formed out 
of a common structural basis and can therefore be replaced at any time by the cytoplasm if destroyed. Examples of such organs are the cilia, the commonest motor apparatus of unicellular organisms; the pseudopodia, which, as we have seen, can be made and destroyed at will; the mouth of animals such as Volvox or Vorticella; and the stinging cells or nectocysts, which surround the mouth of many of these animals and serve to paralyse or kill the smaller living organisms which are brought by the cilia within reach in order that they may serve as food. In contradistinction to these organs are (2) a number of others which must be regarded as permanent. These cannot be formed by differentiation from the cytoplasm of the cell, but are derived by the division of pre-existing organs of the same character, and are therefore transmitted from one generation to another. As examples of such cell organs may perhaps be mentioned the nucleus, with its chromosomes, and the plastids, of which the chloroplasts of vegetable cells are the most conspicuous. Certain cell organs may fall into either class. Thus, the contractile vacuoles are sometimes derived by the division of the preexisting vacuoles in a previous generation, at other times are certainly formed out of the common cytoplasm. The centrosome, a small particle generally situated in the cytoplasm, which plays an important part in cell division, is generally derived by the division of a pre-existing centrosome, but under certain conditions and in some organisms can be developed in situ in the cytoplasm itself.

The possibility of histological differentiation and of the adaptation of structure to definite functions becomes much more pronounced as we pass from the unicellular to the multicellular organisms or metazoa. The lowest of the metazoa, such as the sponges, consist of little more than an aggregation or colony of cells. All the cells are still bathed with the outer fluid, and any differentiation of structure or function seems to be entirely conditioned by the position of the cell. In the cœlenterata the differentiation is already much more marked. The hydra, one of the simplest of the group, consists of a sac formed of two layers of cells and attached by a stalk to some firm basis. Round the mouth of the sac is a circle of tentacles. The inner layer, or hypoblast, represents the digestive and assimilatory layer, while the epiblast, or outer layer, is modified for the purposes of protection, of reception of stimuli, and of motor reaction. In the jelly-fish the differentiation of the outer layers leads to the formation of the first trace of a nervous system, i.e. a system fitted especially for the reception of stimuli and for their transmission to the reactive tissues, namely, the muscles.

In all these classes of animals the external medium of every cell forming the organism is the sea-water or other medium in which they live. This can penetrate through the interstices between the cells, and every cell is therefore exposed to all the possible variations which may occur in the composition of the surrounding medium. A great step in evolution was accomplished with the formation of the cœlomata, the class to which all the higher animals belong. In these, by the formation of a body cavity containing fluid, an internal medium is provided for all the working cells of the body. The com 
position of this internal medium is maintained constant by the activity of the cells in contact with it, and the stress of sudden changes in the chemical composition of the surrounding medium is borne entirely by the outer protective layer of epiblast cells. These are rendered more or less impermeable by the secretion on their surfaces of a cuticular layer, and only such of the constituents of the surrounding medium are allowed to enter the organism as can be utilised by it for building up its living protoplasm. Out of the cœlom is later on formed a circulatory system which, by the circulation of the cœlomic fluid or of blood throught the whole body, can procure a still more perfect uniformity in the chemical conditions to which every cell is exposed. It is not till much later that the organism achieves an independence of external conditions of temperature.' In the mammalia, by means of the reactive nervous system, the heat produced in every vital activity by the chemical changes of combustion and disintegration is so balanced against the heat lost through the external surface to the environment that the temperature of the internal fluid is maintained practically constant. One of the main results of the differentiation of function and structure is therefore a gradual setting free of the majority of the cells of the body from the influence of variations in the environment ; and in the highest type of all animals, in man, this independence of external conditions is carried to a much further extent by conscious adaptations, such as the use of clothes, dwellings, artificial heating, and so on.

The differentiation of the cells which compose the organs of the body is determined in the first place by the different conditions to which they are exposed in virtue of their positions in the course of development. All the higher animals may be considered as built in the form of a tube, the external surface of which is modified for the purpose of defence and for adaptation to changes in the environment. From this layer there are developed not only the protective cuticle, but also the organs of motor reaction, namely, the special senses and the nervous system. The internal surface of the tube is modified for purposes of alimentation. From it are developed all those structures which serve for the digestion of the food-stuffs, for their absorption into the common circulating fluid, for their elaboration after absorption, and their preparation for utilisation by other cells of the body. Between these two surfaces are situated the supporting tissues of the body as well as the organs for the conversion of the potential energy of the body into motion and work, namely, the muscles. Here also is the cœlom or body cavity, represented in the higher animals by the pleural and peritoneal cavities. The alimentary canal projects for a considerable part of its course into this cœlom, being attached to the body wall only by one side. From the cœlom is also developed the blood vascular system, surrounded by contractile and connective cells which maintain a constant circulation of the blood throughout the body. By this differentiation the body becomes divided into a number of organs, each of which is composed of like cells, modified for a common function and bound together by connective tissue, the latter serving also to carry the blood-vessels which convey the common medium for the working 


\section{THE STRUCTURAL BASIS OF THE BOJY}

cells. In the study of physiology our task consists, firstly, in the description of the special part taken by each organ in the general functions of the body, and, secondly, in the determination of the limiting conditions of such func. tions and of the physical and chemical factors which determine them. Finally, we have to endeavour to form a complete conception of the chain of events concerned in the discharge of each function and of their causal nexus.

We have compared the higher animal in the foregoing lines to a colony of cells, and we often speak of an isolated cell of the body as if it were an independent elementary organism. A better term for such an aggregation of cells as presented by the higher animals is not however 'cell colony,' but ' cell state,' since, just as in the state politic, no cell is independent of the activities of the others, but the autonomy of each is merged into the life of the whole. With increasing differentiation there is increasing division of function among the various members of the state, and each therefore becomes less and less fitted for an independent existence or for the discharge of all its vital functions. The more highly civilised a man becomes and the greater his specialisation in the work of the community, the smaller chance would he have of existing on a desert island. Thus the life of the organism is essentially composed of and determined by the reciprocal actions of the single elementary parts. It is evident that, if the process of specialisation has gone far enough, a discussion whether each unit has or has not an independent life is beside the mark, since it cannot possibly exist apart from the activities of the other cells. Of late years histologists have brought forward evidence which seems to imply that an actual structural interaction exists, in addition to the functional dependence which is a necessary resultant of specialisation. Even in the case of plant cells with their thick cellulose walls, fine bridges of protoplasm can be made out passing from one cell to another through pores in the cellulose wall. In animals protoplasmic bridges are known to exist joining up adjacent cells in unstriated muscle, epithelium and cartilage cells, and in some nerve-cells. The conclusion has therefore been drawn that the morphological unit is not the cell, but the whole organism, and that the division of the common cytoplasm into cells is merely a question of size and convenience. There can be no doubt that the determining factor in the division of cells is their growth; the cell divides because it grows. With increased mass of living substance it is necessary to provide for increase of surface both of cytoplasm and of nucleus. Whether all the tissues of the higher animals remain in structural continuity by protoplasmic bridges, \&c., must be to us a matter of indifference, since all that is necessary for the interdependent working of the different cells of the body is a functional continuity, and this in the higher animals is effected by the presence of a common circulating fluid and a reactive nervous system connected by conducting strands with all the cells of the body. 


\section{THE MATERIAL BASIS OF THE BODY}

\section{SECTION I \\ THE ELEMENTARY CONSTITUENTS OF PROTOPLASM}

THE material basis of which living organisms are built up is derived from the surrounding medium, and the elements which compose the framework of the body must therefore be identical with those found in the earth's crust. Not all the elements are so utilised in the formation of living matter. Every living organism without exception contains the following elements : carbon, hydrogen, oxygen, nitrogen, sulphur, phosphorus, chlorine, potassium, sodium, calcium, magnesium, and iron. In addition to these twelve elements others are found in certain organisms, sometimes to a large extent, but it is not known how far they are necessary to the proper development of these organisms, and it is certain that they do not form an integral constituent of all organisms. Of these elements we may mention especially silicon, iodine, fluorine, bromine, aluminium, manganese, and copper. Dealing with the first class, i.e. those which are essential to all forms of life, we find that their relative proportions in living organisms have little or no relation to their proportions in the environment of the organisms. Their presence, however, in the latter is a necessary condition of life. In the case of plants which have a fixed habitat and cannot move in search of food, the growth of the plant is limited by the amount of the necessary element which is present in smallest quantities in the surrounding medium. This is what is meant by the agriculturist's ' Law of the Minimum.' Of the elements derived from the earth's crust, those present in the smallest amounts in most soils are potassium, nitrogen, and phosphorus. The growth of a crop in any given soil is determined by the amount of that one of these three substances which is present in smallest quantities, and the aim of agriculture is to supply to every soil the ingredient which is present in minimal amount.

Carbon forms the greater part by weight of the solid constituents of living protoplasm. The proximate constituents of living organisms are practically all carbon compounds, so that organic chemistry, which was originally the chemistry of substances produced by the agency of living organisms, has come to be synonymous with the chemistry of carbon com- 
pounds: The carbon compounds which make up the living cell are com. bustible, i.e. they can unite with oxygen to form carbon dioxide with the evolution of heat. In the inorganic world practically all the carbon occurs in a completely oxidised form, namely, carbon dioxide. A small amount, 4 parts in 10,000 , is present in the atmosphere, while vast quantities are buried in the crust of the earth as carbonates of the alkaline earths, \&c., in the form of chalk and limestone. In this condition the carbon dioxide is practically removed from the life cycle, the whole of the carbon contained in the tissue of living beings, whether plant or animal, being derived from the minute proportion of carbon dioxide present in the atmosphere. The energy for the conversion of carbon dioxide into the oxidisable forms with high potential energy, which make up the tissues of plants and animals, is furnished by the sun's rays. The machine for the conversion of the radiant energy into the potential chemical energy of the carbon compounds is represented by the chlorophyll corpuscles in the green parts of plants. In these corpuscles, under the influence of the sun's rays, the carbon dioxide of the atmosphere, together with water, is converted into carbohydrates, viz. starch $\left(\mathrm{C}_{6} \mathrm{H}_{10} \mathrm{O}_{5}\right)$, and the oxygen liberated in the process is set free into the surrounding atmosphere.

$$
6 \mathrm{CO}_{2}+5 \mathrm{H}_{2} \mathrm{O}=\mathrm{C}_{6} \mathrm{H}_{10} \mathrm{O}_{5}+6 \mathrm{O}_{2} \text {. }
$$

In this process a large amount of energy is absorbed, an energy which can be set free later by the oxidation of the starch to carbon dioxide. In the oxidation of one gramme of starch about 4500 calories are evolved, and this represents also the measure of the solar energy which must be absorbed by the chlorophyll corpuscle in the process of formation of starch from the carbon dioxide of the atmosphere. By this means the world of life is provided with a source of energy. At the expense of the energy of the starch further synthetic processes are carried out. By the oxidation of a part of the carbohydrates; sufficient energy may be supplied to deoxidise other portions of the carbohydrates with the production of fats. Thus

$$
\underset{\text { (Glucose) }}{3 \mathrm{C}_{6} \mathrm{H}_{12} \mathrm{O}_{6}}-8 \mathrm{O}_{2}=\underset{\text { (Stearic acid) }}{\mathrm{C}_{18} \mathrm{H}_{36} \mathrm{O}_{2}}
$$

The potential energy of a fat is still greater than that of a carbohydrate, one gramme of fat giving on complete combustion to carbonic acid and water as much as 9000 calories. By the introduction of ammonia groups $\left(\mathrm{NH}_{2}\right)$ into the molecules of fatty acids, amino-acids may be formed, from which the complex proteins are built up to form the chief constituents of the living protoplasm.

The synthesis of carbon compounds from the inert carbon dioxide of the atmosphere can be effected only by chlorophyll corpuseles. All animals take in carbon, hydrogen, nitrogen, oxygen, and sulphur in the form of the carbohydrates, fats, and proteins which have been built up in the living plants. In the anima lorganism these food-stuffs serve as sources of energy. They undergo a gradual oxidation, and finally leave the body in the form of 
carbon dioxide, water, ammonia or some related compound, and sulphates. A sharp distinction has therefore often been drawn between the metabolism of plants and animals, plants being regarded as essentially assimilatory in character while animals are dissimilatory, utilising the stores of energy which have been accumulated by the plant. There is, however, no definite line of demarcation. Although, generally speaking, the green plant breaks up carbon dioxide, giving off oxygen and storing up carbon compounds, and the animal taking in carbon compounds oxidises them with the help of the oxygen of the atmosphere to carbon dioxide, which is redischarged into the surrounding medium and is available for further assimilation by plants, yet this process of respiration is common to all living organisms, whether plants or animals. In the green plant it may be masked by the assimilatory process occurring under the influence of the sun's rays, but in the dark all parts of the plant, and in the light all parts which are free from chlorophyll, display a process of respiration, i.e. they are constantly taking up oxygen from the atmosphere and using it for the oxidation of carbon compounds in their tissues, with the production of carbon dioxide.

The sum total of the processes of life tend, therefore, to maintain a constant proportion of carbon dioxide and oxygen in the atmosphere, the decomposition of carbon dioxide by the green plants being balanced by the oxidation of the carbon compounds and the continual discharge of carbon dioxide by animals. It is not certain, however, that this balance will be maintained throughout all time. As Bunge has pointed out, there are cosmic factors at work which are apparently tending to cause a constant diminution in the quantity of carbon dioxide in the atmosphere, which alone is of value to the plant. One of these factors is the variable affinity of the silica and carbon dioxide respectively for the chief bases of the earth's crust. At a high temperature silica can displace carbon dioxide from its compounds. Thus chalk heated with silica will give rise to calcium silicate with the evolution of carbon dioxide. At an early geological epoch, therefore, it is probable that the greater part of the silica was present in combination with bases and that the proportion of carbon dioxide in the atmosphere was very much higher than it is now. At temperatures at present ruling on the earth's surface carbon dioxide is a stronger acid than silica. The action of water charged with carbon dioxide on a silicate is to cause its gradual decomposition with the formation of carbonate and silica. Both these products, being insoluble, are deposited as part of the earth's crust, the silica in the form of sandstone, the carbonate as chalk or limestone. The carbon dioxide is being constantly removed by water from the atmosphere and being locked up in this way in the earth's crust, the process of separation of calcium carbonate being aided to a marked extent by the agency of living organisms themselves. The whole of the extensive deposits of limestone and chalk have been separated from the sea-water by the action of living organisms. With the cooling of the earth's crust which is supposed to be going on, the discharge of carbon dioxide by volcanoes must get less and less, so that one can conceive a time when the whole of the carbon dioxide will be bound up 
with bases in the earth's crust, and life, without any source of carbon, must become extinct.

Hydrogen exists almost exclusively in the form of water. In this form it is taken up by plants and animals, with the exception of a small proportion absorbed in the form of ammonia. In this form too it is discharged by living organisms. Oxygen is the only element which, in all the higher organisms at any rate, is taken up in the free state. It forms one-fifth of the atmosphere and, as the oxides of the various metals, a considerable fraction of the earth's crust. It takes a position apart from the other food-stuffs in that its presence is the essential condition for the utilisation of their potential energy. In the living cells it combines with the oxidisable compounds formed by the agency of the living protoplasm, with the production of carbon dioxide and water, and the evolution of energy. This process is spoken of as respiration.

Like the three elements we have already considered, nitrogen is also derived directly or indirectly from the surrounding atmosphere. In consequence of its feeble combining power for other elements and the instability of its compounds, very little nitrogen is to be found in the combined state in the earth's crust, whereas it constitutes four-fifths of the atmospheric gases. It can be taken up by most plants only in the form of ammonia, nitrites, or nitrates. To animals these compounds are useless, and the only source of nitrogen to this class is the protein which has been built up by the agency of the plant cell. Since nitrogen in the free state is useless to nearly all living organisms, the existence of life must depend on the amount of combined nitrogen which is available. In view of the small tendency presented by this element to enter into combination, it becomes interesting to inquire into the source of the combined nitrogen which is the common capital of the living kingdom. There are certain cosmic factors which result in the production of combined nitrogen. The passage of electric sparks or of the silent discharge through moist air leads to the production of ammonium nitrite.

$$
\mathrm{N}_{2}+2 \mathrm{H}_{2} \mathrm{O}=\mathrm{NH}_{4} \mathrm{NO}_{2} \text {. }
$$

Every thunderstorm, therefore, will result in the production of small quantities of ammonium nitrite, which will be washed down with the rain and serve as a source of combined nitrogen to the soil. Every decaying vegetable or animal tissue serves as a source of ammonia, so that from various causes the soil may contain nitrogen in the form of ammonia or of ammonium nitrite. These forms of combined nitrogen are not, however, suitable for all classes of plants. Most moulds can assimilate ammonia as ammonium carbonate or as amino-acids or amines, provided that they are supplied at the same time with sugar, the oxidation of which will serve them as a source of energy. Some moulds, many of the higher plants, and especially the Gramineæ, which include the food-producing cereals, require their nitrogen in the condition of nitrates. It is necessary, therefore, that the ammonia or nitrites in the soil shall be converted into this highly oxidised form. This 
conversion is effected by a group of micro-organisms. There are a number of bacteria (bacterium nitrosomonas) which have the power of converting ammonia into nitrites. Others (bacterium nitro-

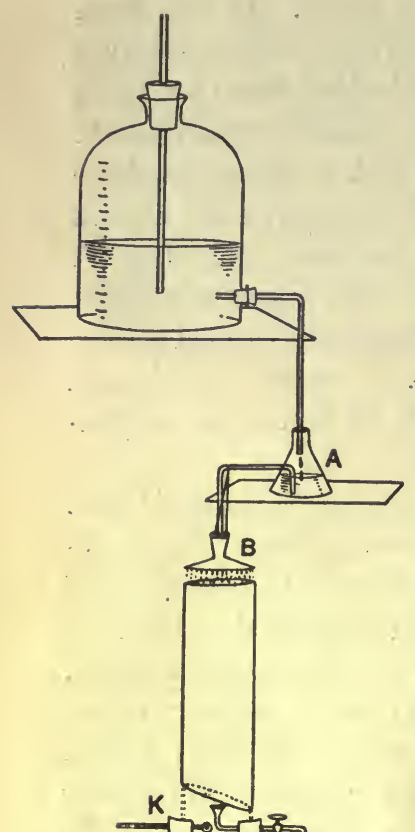
monas) convert nitrites into nitrates. If sewage matter rich in ammonia is allowed to percolate through a cylinder packed with coke and the process be continued for several weeks, it is found after a time that in its passage through the filter the fluid has lost its ammonia and contains the whole of its nitrogen in the form of nitrate. If the cylinder be tapped (Fig. 13) half-way down, say at K, the fluid will be found to contain, not nitrates, but nitrites. In this conversion the two kinds of microbes mentioned above are concerned. At the top of the cylinder the nitrous bacterium is present, in the bottom of the cylinder the nitrate bacterium is present. The conversion of ammonia into nitrates by the agency of bacteria has been made the basis of a method of treatment of sewage which is now very largely employed. These different bacteria play an important part in all soils in preparing them for the cultivation of crops.

Is the total capital of combined nitrogen which is worked over by these bacteria and utilised by the whole living world confined to the small quantities produced by atmospheric discharges? Of late years definite evidence has been brought forward that such is not the case and that organisms exist which can utilise and bring into combination the free atmospheric nitrogen itself. Thus certain soils have been found to undergo a gradual enriching in nitrogen although no nitrogenous manure has been applied to them. Winogradsky has shown that this fixation of nitrogen by soils is effected by a distinct micro-organism, which may be isolated by growing it on gelatinous silica free from any trace of combined nitrogen, so that the organism has to procure its entire nitrogen from the atmosphere. Under such conditions the numerous other micro-organisms of the soil die of nitrogen starva-

Fig. 13. Arrangement for studying the nitrification of sewage. (Mrss Н. Сніск.) 
the surrounding oxygen. The mechanism by which this organism is able to fix free nitrogen, and the nature of the first product of the assimilation are not yet ascertained. Such an assimilation will serve to the organisin as a source of energy, since the application of heat is necessary for the dissociation either of ammonium nitrite or of nitrous acid into nitrogen and water, as is seen from the following equation:

$$
\begin{gathered}
\mathrm{HNO}_{2} \text { Aq. }+308 \mathrm{Cal} .=\mathrm{H}+\mathrm{N}+\mathrm{O}_{2}+\mathrm{Aq} . \\
\mathrm{NH}_{4} \mathrm{NO}_{2} \text { Aq. }+602 \text { Cal. }=2 \mathrm{~N}+4 \mathrm{H}+2 \mathrm{O}+\mathrm{Aq} .
\end{gathered}
$$

In addition to this spontaneous fixation of nitrogen by humus, a method has long been known to farmers by which the fertility of a soil can be increased without the application of nitrogenous manures. If a plot of land is to be left fallow it is a very usual custom to sow it with some leguminous crop such as sainfoin. Careful experiments by Boussingault, Lawes and Gilbert, and others have shown that the growth of almost any leguminous crop in a soil poor in nitrogen may result not only in the production of a crop containing much combined nitrogen, but also in an actual increase of nitrogen in the soil from which the crop is taken. It was then shown by the last two observers, as well as by Schloesing and Laurent, that the power of a leguminous crop to enrich the soil with nitrogen was dependent on the presence on the roots of certain small nodules which had been described long before by Malpighi (Fig. 14). They showed also that the production of these nodules took place only as a result of infection. Beans grown in sterilised sand produced a plant free from nodules, which, however, grew very scantily unless nitrogenous manure were added to the sand. Such a crop derived the nitrogen for its growth from the added nitrogen, the total amount of which in the soil was therefore diminished by the crop. If, however, the sterilised sand were treated with an infusion of root nodules from another plant without the addition of any combined nitrogen at all, the beans developed nodules on their roots and grew luxuriantly, and at the termination of their

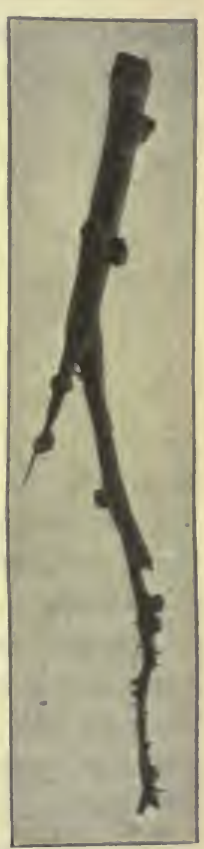

Fia. 14. Root of vetch with nod. ules. growth the soil was richer in nitrogen than at the commencement. On microscopic examination the protoplasm which makes up these nodules is found to be swarming with small rods (Fig. 15), and it was shown by Beyerinck that these rods are bacteria and can be cultivated in media apart altogether from the plant. We have thus an example of a class of bacteria which, like those of humus, are able to assimilate the free nitrogen of the atmosphere, but, unlike them, can only effect this assimilation in a condition of symbiosis, i.e. living in the growing tissues of a leguminous plant. Similar nodules have been described on the roots of other plants which can grow in a 
soil free from combined nitrogen, e.g. conifers, but it is in the leguminosæ that their presence is most widespread.

The source of the combined nitrogen, which can be built up by plants into proteins and utilised in this form by animals, is thus not only the ammonium nitrite produced by the agency of electric discharges in the atmo-

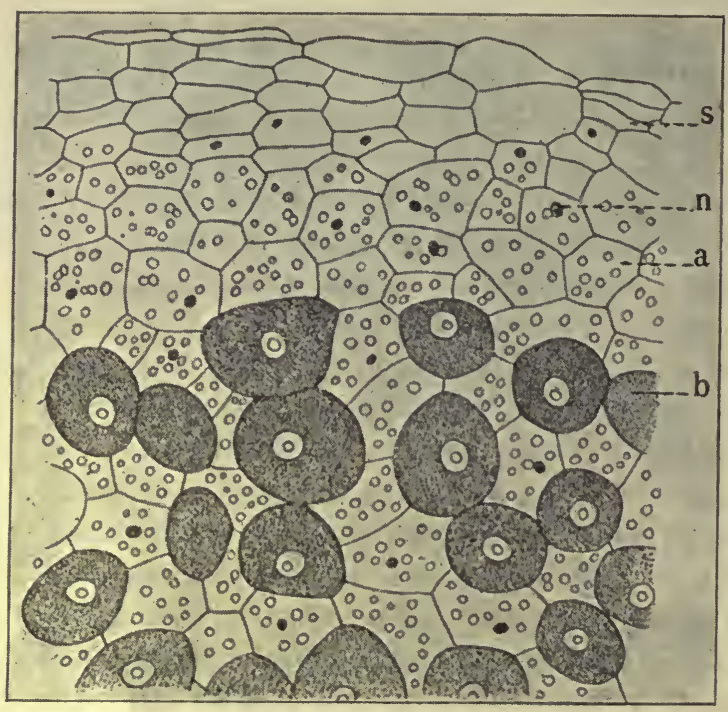

Fig. 15. Section of a root nodule of Dorychnium. (VuILLemin.) $a$, cortical tissue; $b$, cells containing bacteria.

sphere, but also the free nitrogen of the atmosphere assimilated by various types of bacteria.

Sulphur is found in all soils in the form of sulphates, generally of lime. As sulphates it is taken up by plants. In the plant cell a process of deoxidation takes place at the expense of the energy derived either from the starch or, in the case of bacteria, from other ingredients of their food-supply. It is built up, together with nitrogen, carbon, and hydrogen, to form sulphur derivatives and amino-acids such as cystine, and these, together with other amino-acids, are synthetised to form proteins. Practically the whole of the sulphur taken in by animals is in the form of proteins. It shares the oxidation of the protein molecule in the animal body which it leaves in the form of sulphates. The output of sulphates by an animal can therefore be regarded, like the nitrogen output, as an index of the protein metabolism. It is returned to the soil in the form in which it was taken by the plant, and the cycle can be continuously repeated.

Iron, although forming but a minute proportion of the material basis of living organisms (the whole body of man contains only six grammes), is nevertheless indispensable for the maintenance of life. It is necessary, for instance, in two important functions, viz. the formation of chlorophyll in the green plant and the respiratory process in the higher animals. Although iron forms no part of the chlorophyll molecule, plants grown in the absence of this 
substance remain etiolated, but form chlorophyll if the smallest trace of iron is added to the soil in which they are growing or even if the leaves are washed with a very dilute solution of an iron salt. In animals iron forms an essential constituent of hæmoglobin, the red colouring-matter of the blood, whose office it is to carry oxygen from the lungs to the tissues. It is probable too that the minute traces of iron in protoplasm exercise an important function in the processes of oxidation which are continually going on. Even in the inorganic world iron plays the part of an oxygen carrier. In the earth's crust it occurs as ferrous salts and as ferric oxide. The ferrous silicate, for instance, may be decomposed by water containing carbon dioxide into silica and ferrous carbonate; the latter then absorbs oxygen from the atmosphere, liberating carbon dioxide and forming ferric oxide. In the presence of decomposing organic matter, the ferric oxide parts with its oxygen to oxidise the organic substances and is converted once more. into ferrous carbonate, and this may be decomposed by the oxygen of the air as before. In the presence of sulphates and decomposing organic matter ferrous sulphate, which is first formed, undergoes deoxidation to ferrous sulphide, and this may again be oxidised to sulphates and ferric salts on exposure to the atmosphere, so that both the sulphur and the iron act as oxygen carriers between the atmosphere and the organic matter. Iron is obtained by plants from the soil as ferrous or ferric salts. In the protoplasm it is built up into highly complex organic compounds, and in this form is taken up by animals. It is probable that the main requirements of the animal for iron, which are very small, may be satisfied entirely at the expense of these organic compounds, but there can be little doubt that the animal can, if need be, also utilise the iron salts present in its food. The animal proceeds extremely economically with its supply of iron. Any excess of iron above that needed to supply the iron lost to the body, as well as the latter, is excreted almost entirely with the fæces in the form of sulphide. In the soil this undergoes oxidation and returns once more to the form in which it was originally taken up by the plant.

Phosphorus is absorbed by the plant as phosphates. In the cell protoplasm it is built up with fatty acids and other organic radicals to form complex compounds such as lecithin, a phosphorised fat, and nuclein, a combination of phosphorus with nitrogenous bases of great variety. Both lecithin and nuclein are essential constituents of living protoplasm. Practically the whole of the phosphorus income of animals is represented by these lecithin and nuclein compounds. After absorption into the animal body they are broken down by processes of dissociation and oxidation, with the pro. duction, as a final result, of phosphates, which are excreted with the urine or fæces and return to the soil.

Chlorine, potassium, sodium, calcium, and magnesium are taken up by the plants in the form of salts. Although playing an essential part in all vital processes, they do not seem to be built up into organic combination with the protein and other constituents of the cell protoplasm. They are therefore taken up also by animals in the form of salts, and as such are again excreted with the urine. 
Little is known about the significance, if any, of the other elements which I have mentioned as occasional constituents of living beings. Silicon, which is of universal distribution, is assimilated as silica, probably in colloidal solution, and is distributed in minute quantities through all plant and animal tissues. It forms a very large percentage of the mineral basis of grasses, but even here it does not seem to be indispensable, since these will grow in a medium devoid of silica as luxuriantly as under normal conditions.

Fluorine is found in the enamel of the teeth and in minute traces in other tissues of the body.

Bromine, though present in quantity in some seaweeds, appears to play no part in the economy of higher animals.

Iodine is found in large quantities in many seaweeds and is present as an organic iodine compound in the skeleton of certain horny sponges. An organic iodine compound is also found in the thyroid gland of the higher animals, and may possibly be the active principle by means of which these glands are able to affect the nutrition of the whole body. Iodine, therefore, would seem to be an essential constituent of the higher animals.

Aluminium is found in large quantities in certain lycopods. Whether it is essential to their growth is not known.

Copper is certainly not a necessary constituent of a large number of plants and animals. In one class, the cephalopods, it appears to take the part of iron in the formation of a blood pigment. The hæmocyanine, which was described by Frédéricq, plays the same part in the blood of cephalopods that is played by hæmoglobin in the blood of vertebrates. When oxidised it is of a blue colour, but gives off its oxygen and is reduced to a colourless compound on exposure to a vacuum.

Among these elementary constituents of the body, a definite line of demarcation can be drawn between the carbon and hydrogen on the one hand and all the other constituents on the other. The first two elements are built up in a deoxidised form into the living structure of the protoplasmic molecule. The products of their complete oxidation are volatile, namely, carbon dioxide and water, and leave the body in these forms. The nitrogen set free by the breaking down of the proteins will pass off as free nitrogen or as ammonia. The sulphuric acid formed by the oxidation of the sulphur combines with the bases to form non-volatile salts. We may therefore divide the ultimate constituents of the body into those which are combustible and are driven off on heating, and those which are left behind as the ash. 


\section{SECTION II \\ THE PROXIMATE CONSTITUENTS OF THE ANIMAL BODY}

IN spite of the enormous variety of the proximate constituents of living organisms, they are all members or derivatives of three classes of compounds. Since living organisms form the entire food of the animal kingdom, a study of these proximate constituents includes the study of all the food-stuffs. These classes are :

(a) Proteins, containing the elements carbon, hydrogen, nitrogen, oxygen, and sulphur; in some cases also phosphorus.

(b) Fats, containing carbon, hydrogen, and oxygen.

(c) Carbohydrates, containing carbon, hydrogen, and oxygen, the two latter elements being present in the proportions in which they form water.

\section{THE CHIEF TYPES OF ORGANIC COMPOUNDS OCCURRING IN THE ANIMAL BODY}

The full consideration of the various modifications undergone by these three classes of food-stuffs in the body, especially if we include the by-products occurring both in plants and in animal metabolism, involves a wide knowledge of organic chemistry which indeed at its origin was simply the chemistry of the products of living (i.e. organised) beings. The most important substances with which we shall have to deal belong to a comparatively restricted number of groups. For the convenience of the reader a short summary of the relationships of these groups to one another and to the hydrocarbons is given here.

THE HYDROCARBONS (FATTY SERIES). These form a continuous homologous series, and may be saturated or unsaturated. Examples of the saturated series are :

$$
\begin{array}{ll}
\mathrm{CH}_{4} & \text { methane } \\
\mathrm{C}_{2} \mathrm{H}_{6} & \text { ethane } \\
\mathrm{C}_{3} \mathrm{H}_{8} & \text { propane } \\
\mathrm{C}_{4} \mathrm{H}_{10} & \text { butane, and so on, }
\end{array}
$$

the general formula for the group being

$$
\mathrm{C}_{\mathrm{n}} \mathrm{H}_{2 \mathrm{n}+2} \text {. }
$$

These paraffins, the lower members of which are gaseous, while the higher members form the petroleum ether, the heavy petroleums, vaseline, and the paraffin wax with which we are all familiar, are entirely inert in the animal body. If taken with the food they pass through the alimentary canal unchanged. In order to render them accessible to the action of the living cell they must first undergo oxidation.

The unsaturated hydrocarbons have the general formuli $\mathrm{C}_{n} \mathrm{H}_{2 n}, \mathrm{C}_{n} \mathrm{H}_{2 n-2}$, $\mathrm{C}_{\mathrm{n}} \mathrm{H}_{2 \mathrm{n}-4}$, \&c. 
Examples of the first two groups are ethylene $\mathrm{CH}_{2}$

\section{$\mathrm{CH}_{2}$}

and acetylene

Derivatives of all these groups occur in the body.

THE ALCOHOLS. The first product of the oxidation of hydrocarbons is the series of bodies known as the alcohols. Examples of these are :

$\mathrm{CH}_{3} \mathrm{OH}$ methyl alcohol
$\mathrm{C}_{2} \mathrm{H}_{5} \mathrm{OH}$ ethyl ,
$\mathrm{C}_{3} \mathrm{H}_{7} \mathrm{OH}$ propyl,
$\mathrm{C}_{4} \mathrm{H}_{9} \mathrm{OH}$ butyl ",
$\mathrm{C}_{5} \mathrm{H}_{11} \mathrm{OH}$ amyl ",
$\mathrm{C}_{6} \mathrm{H}_{13} \mathrm{OH}$ capryl ", and so on,

the general formula for the group being

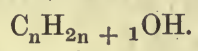

In all these alcohols the $\mathrm{OH}$ group is, so to speak, more mobile than the other atoms connected with the carbons, and can therefore be replaced by other substances or groups with comparative ease. In this respect therefore an alcohol can be compared to water $\mathrm{HOH}$ or to an alkaline hydroxide $\mathrm{NaOH}$ or $\mathrm{KOH}$. The best-known example of the group is ethyl alcohol, the ordinary product of fermentation of sugar. In these alcohols the $\mathrm{H}$ of the $\mathrm{OH}$ group can be replaced by Na. Thus, water with metallic sudium gives sodium hydroxide and hydrogen as follows :

$$
2 \mathrm{HOH}+2 \mathrm{Na}=2 \mathrm{NaOH}+\mathrm{H}_{2} \text {. }
$$

In the same way alcohol treated with metallic sodium gives off hydrogen, and the remaining fluid contains sodium ethylate, thus :

$$
2 \mathrm{C}_{2} \mathrm{H}_{5} \mathrm{OH}+2 \mathrm{Na}=\underset{\text { (sodium ethylate) }}{2 \mathrm{C}_{2} \mathrm{H}_{5} \mathrm{ONa}+\mathrm{H}_{2}}
$$

On the other hand, the $\mathrm{OH}$ group may be replaced by acid radicals. Thus, if ethyl alcohol be treated with phosphorus pentachloride, ethyl chloride is formed together with phosphorus oxychloride and hydrochloric acid. Thus :

$$
\begin{aligned}
& \mathrm{Et} . \mathrm{OH}+\mathrm{PCl}_{5}=\mathrm{POCl}_{3}+\mathrm{HCl}+\text { (ethyl chloride) } \\
& \text { Et. }
\end{aligned}
$$

With concentrated sulphuric acid the reaction is similar to that which obtains between sodium hydrate and this acid, and we have formed ethyl hydrogen sulphate and water. Thus :

$$
\text { Et. } \mathrm{OH}+\mathrm{H}_{2} \mathrm{SO}_{4}=\mathrm{Et} . \mathrm{HSO}_{4}+\mathrm{HOH}
$$

If alcohol be warmed with acetic acid and strong sulphuric acid, among the products of the reaction is ethyl acetate, which is volatile, and therefore passes off. Thus :

$$
\text { Et. } \mathrm{OH}+\mathrm{HC}_{2} \mathrm{H}_{3} \mathrm{O}_{2}=\text { Et. } \mathrm{C}_{2} \mathrm{H}_{3} \mathrm{O}_{2}+\mathrm{HOH} \text {. }
$$

These compounds of the hydrocarbon group of the alcohol, such as methyl, ethyl, propyl, \&c., with an acid, in which the ethyl takes the part of a base, are known as esters.

An ester treated with an alkali is decomposed with the formation of an alkaline salt of the acid, and the corresponding alcohol which, being volatile, is given off on warming the mixture. Thus :

$$
\underset{\text { (ethyl acetate) }}{\mathrm{Et} . \mathrm{C}_{2} \mathrm{H}_{3} \mathrm{O}_{2}}+\underset{\text { (potasium acetate) }}{\mathrm{NaHO}}=\mathrm{NaC}_{2} \mathrm{H}_{3} \mathrm{O}_{2}+\mathrm{Etcohol} \text { ) }
$$

This process of decomposition of an ester withe formation of the alkaline salt of an acid is often spoken of as saponification, i.e. soap formation, though the term 'soap' 
is applied only to the compounds of alkalies with the higher fatty acids. The series of alcohols we have just dealt with containing one $\mathrm{OH}$ group replaceable by metals or acid radicals are known as monatomic alcohols. If in the molecule of the paraffin two or more atoms of hydrogen have been replaced by the group $\mathrm{OH}$, we speak of diatomic or polyatomic alcohols. Thus, derived from the paraffin propane $\mathrm{C}_{3} \mathrm{H}_{8}$ we may have the monatomic alcohol $\mathrm{C}_{3} \mathrm{H}_{7} \mathrm{OH}$, propyl alcohol, or the triatomic alcohol $\mathrm{C}_{3} \mathrm{H}_{5}(\mathrm{OH})_{3}$. which is known as glycerin, or glycerol.

Other alcohols of physiological importance are cholesterol and cetyl alcohol. Cholesterol is a monatomic alcohol with the formula $\mathrm{C}_{27} \mathrm{H}_{45} \mathrm{OH}$. It is very complex in structure, and belongs to the aromatic series. Recent work points to an affinity of cholesterol with the terpenes, which have hitherto been found only as the product of the metabolism of plant cells. Cholesterol is a constant constituent of protoplasm. It occurs in large quantities in the medullary sheath of nerves; it is a normal constituent of bile and may form concretions (biliary calculi) in the gall bladder. In combina. tion with fatty acids it is an important constituent of sebum and of wool fat.

Another alcohol-cetyl alcohol $-\mathrm{C}_{16} \mathrm{H}_{34} \mathrm{O}=\left(\mathrm{CH}_{\mathrm{CH}_{2}} \mathrm{OH}\right.$

the duck and forms an important constituent of the wax, spermaceti, obtained from a cavity in the skull of the sperm whale.

ALDEHYDES. By oxidation of any of the alcohols we obtain another group of compounds-the aldehydes. From ethyl alcohol, for instance, by warming with potas. sium bichromate and dilute sulphuric acid, ethyl aldehyde is produced and given off. In

these aldehydes the group $\mathrm{C}=\mathrm{H}$ is converted into the group $\mathrm{C}=0$, and it is the possession of this group which determines the aldehyde character of any compound, as well as the reactions which are typical of this class of compounds.

Some of the typical reactions of aldehydes may be here shortly summarised:

(1) They act as reducing agents, the CHO group being converted into the group $\mathrm{COOH}$, which is distinctive of an acid. We may therefore say that on oxidation aldehydes are converted into the corresponding fatty acids as follows:

$$
\left.\right|_{\text {(ethyl aldehyde) }} ^{\mathrm{CH}_{3}}+\mathrm{O}={\underset{\text { (acetic acid) }}{\mathrm{COOH}}}_{\mathrm{CH}_{3}}^{\mathrm{CH}_{3}}
$$

On account of the ease with which this oxidation takes place, aldehydes act as strong reducing agents. Warmed with an alkaline solution of cupric hydrate, they take up oxygen, reducing the cupric to a red precipitate of cuprous hydrate. If warmed with an ammoniacal solution of silver (i.e. silver nitrate solution to which ammonia has been added until the precipitate first formed is just redissolved), they reduce the silver nitrate with the formation of a mirror of metallic silver on the surface of the glass vessel in which they are heated.

(2) On warming with phenyl hydrazine, they give the typical compounds, hydrazones and osazones, which are also given by the sugars and will be mentioned in con. nection with these bodies.

(3) They also form addition products. With ammonia, they yield the group of compounds known as aldehyde ammonia. Thus :

$$
\left.\right|_{\mathrm{CHO}} ^{\mathrm{CH}_{3}}+\mathrm{NH}_{3}=\left.\right|_{\mathrm{C}} ^{\mathrm{CH}_{3}} \frac{\mathrm{OH}}{\mathrm{H}}_{\mathrm{NH}}^{\mathrm{NH}_{2}}
$$


With sodium hydrogen sulphite the following reaction takes place:<smiles>CC[18O]S(=O)(=O)c1ccccc1S(=O)(=O)[O-]</smiles>

These compounds of aldehydes with sodium sulphite can be readily obtained in a crystalline form and furnish a convenient means of separating the aldehydes from their solutions.

(4) All the aldehydes possess a strong tendency towards polymerisation. Ethyl or acetic aldehyde treated with strong sulphuric acid gives the compound paraldehyde. Thus :

$$
\underset{\text { (acetic aldehyde) }}{3 \mathrm{C}_{2} \mathrm{H}_{4} \mathrm{O}}=\underset{\text { (paraldehyde) }}{\mathrm{C}_{6} \mathrm{H}_{12} \mathrm{O}_{3} .}
$$

If warmed with strong potash the polymerisation occurs to a still further extent with the formation of resinous substances of unknown composition, but at any rate of a very high molecular weight, the so-called 'aldehyde resin.' Formic or methyl aldehyde, $\mathrm{CH}_{2} \mathrm{O}$, may in the same way undergo polymerisation with the formation of a mixture of substances belonging to the group of sugars, namely, the hexoses, as follows :

$$
6 \mathrm{CH}_{2} \mathrm{O}=\mathrm{C}_{6} \mathrm{H}_{1.2} \mathrm{O}_{6} \text {. }
$$

This formation of sugar from formic aldehyde probably plays an important part in the assimilation of the carbon from the carbonic acid of the atmosphere by the green parts of plants.

ACIDS. By the oxidation of the group $\mathrm{CHO}$ of the aldehydes we obtain the group $\mathrm{COOH}$, which is characteristic of an organic acid. Thus, formic aldehyde on oxidation gives the compound $\mathrm{HCOOH}$, formic acid. Ethyl or acetic aldehyde, $\mathrm{CH}_{3} \mathrm{CHO}$, with an atom of oxygen, gives the compound $\mathrm{CH}_{3} \mathrm{COOH}$, acetic acid.

$$
\left.\right|_{\mathrm{CHO}} ^{\mathrm{CH}_{3}}+\mathrm{O}=\left.\right|_{\mathrm{COOH}} ^{\mathrm{CH}_{3}}
$$

Since these acids are derived from the paraffins a whole series of them exists corresponding to the series of paraffins, and known as the fatty acids. Examples of this group are :

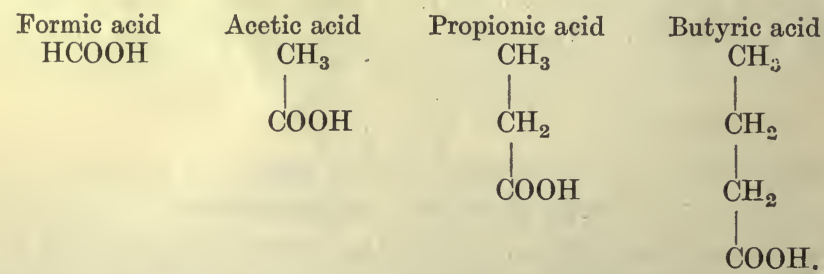

In addition to these fatty acids, there are also unsaturated acids, derived from the unsaturated hydrocarbons.

\section{DERIVATIVES OF THE FATTY ACIDS}

AMINO-ACIDS are derived from the fatty acids by the replacement of one atom of hydrogen by the group $\mathrm{NH}_{2}$.

Thus from propionic acid we may have :

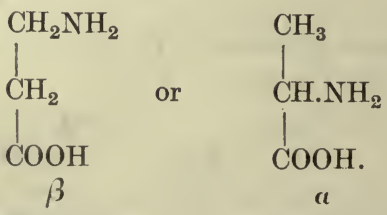

The second form, the a-amino acid, is the only one which occurs in the body. 

Thus :

OXYACIDS are formed by the replacement of one $\mathrm{H}$ atom by the group $O H$.

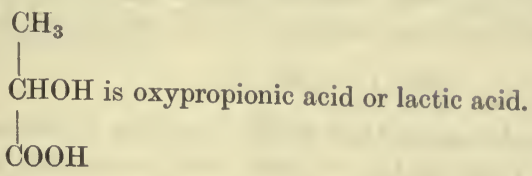

KETO-ACIDS. Oxyacids are formed by the oxidation of the group $\mathrm{CH}_{2}$ or $\mathrm{CH}_{3}$. If at the same time the $\mathrm{H}_{2}$ group be removed by oxidation a keto-acid may be formed. This is probably the manner in which such acids arise in the body, though it is more usual to regard a keto-acid as the result of oxidation of a ketone. Thus :<smiles>CC(=O)C(C)(C)C</smiles><smiles>CC(=O)CO</smiles><smiles>CC(=O)OC1(C(=O)O)CC1</smiles>

ACID AMIDES are formed from a fatty acid by replacing the - $\mathrm{OH}$ of the - $\mathrm{COOH}$ group by $-\mathrm{NH}_{2}$, e.g. :

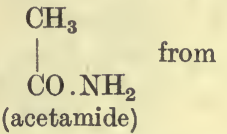<smiles>CC(=O)O</smiles>

AMINES. These may be regarded as formed from ammionia $\mathrm{NH}_{3}$ by replacing one or more of the $\mathrm{H}$ atoms by an organic radical. Thus we may have :

$$
\begin{aligned}
& \mathrm{N}-{ }_{\mathrm{H}}^{\mathrm{CH}_{3}} \\
& \text { (methylamine) } \\
& \mathrm{N}-{ }_{\mathrm{H}}^{\mathrm{CH}_{3}} \\
& \mathrm{~N} \div \frac{\mathrm{CH}_{3}}{\mathrm{CH}_{3}}
\end{aligned}
$$

Under the action of living organisms primary amines may be formed from a-amino acids by a process of decarboxylation. Thus :<smiles>CC(N)C(=O)OC(C)C(C)N</smiles>

\section{AROMATIC COMPOUNDS}

These all contain a nucleus, made up of six carbon atoms, which is extremely stable, so that processes of oxidation, reduction, \&c., can be carried out in the compound without destruction of the nucleus. The simplest aromatic compound is benzene $\mathrm{C}_{6} \mathrm{H}_{6}$. It behaves as a saturated compound. It is represented as a hexagon with a hydrogen atom at each angle.<smiles>CCCC</smiles> 
All the hydrogen atoms are of equal value. They may be replaced by other groups, such as $\mathrm{OH}, \mathrm{Cl}, \mathrm{NH}_{2}$, or by more complex groups belonging to the fatty series, e.g. $\mathrm{CH}_{3}, \mathrm{C}_{2} \mathrm{H}_{5}$, \&c. Monosubstitution derivatives exist only in one form :

$$
\mathrm{C}_{6} \mathrm{H}_{5} \cdot \mathrm{X}
$$

Disubstitution compounds exist in three forms, according to the relative position of the substituted $\mathrm{H}$ atoms. These are known as the ortho, meta, and para compounds, and have the formulæ:<smiles>[X]C1CCCCC1</smiles>

ortho-<smiles>[X]c1ccccc1</smiles>

meta-

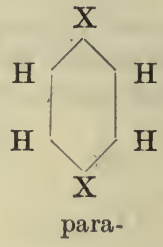

The following are some of the most important monosubstitution derivatives of benzene :

$\begin{array}{ll}\text { Nitrobenzene } & \mathrm{C}_{6} \mathrm{H}_{5} \cdot \mathrm{NO}_{2} \cdot \\ \text { Aniline } & \mathrm{C}_{6} \mathrm{H}_{5} \cdot \mathrm{NH}_{2} \cdot \\ \text { Benzene sulphonic acid } & \mathrm{C}_{6} \mathrm{H}_{5} \cdot \mathrm{SO}_{3} \mathrm{H} . \\ \text { Phenol } & \mathrm{C}_{6} \mathrm{H}_{5} \cdot \mathrm{OH} \text {. } \\ \text { Toluene } & \mathrm{C}_{6} \mathrm{H}_{5} \cdot \mathrm{CH}_{3} . \\ \text { Benzyl alcohol } & \mathrm{C}_{6} \mathrm{H}_{5} \cdot \mathrm{CH}_{2} \mathrm{OH} \text {. } \\ \text { Benzylaldehyde } & \mathrm{C}_{6} \mathrm{H}_{5} \cdot \mathrm{CHO} \text {. } \\ \text { Benzoic acid } & \mathrm{C}_{6} \mathrm{H}_{5} \cdot \mathrm{COOH} .\end{array}$

Of the disubstitution compounds, we need only mention the following:

The dihydroxybenzenes:

Pyrocatechin or catechol<smiles>OC1CCCCC1</smiles>

ortho-

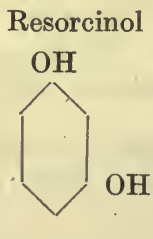

meta-
Hydroquinone

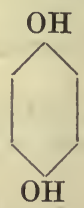

para-

Salicylic acid (o-hydroxybenzoic acid)<smiles>O=C(O)c1ccccc1</smiles>

Tyrosin (parahydroxyphenyl alanine):

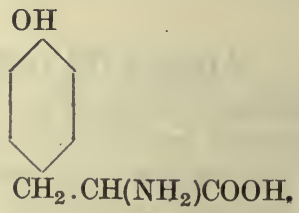

Examples of trisubstitution derivatives of benzene are :

Pyrogallol

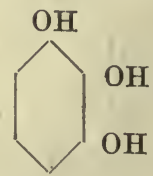




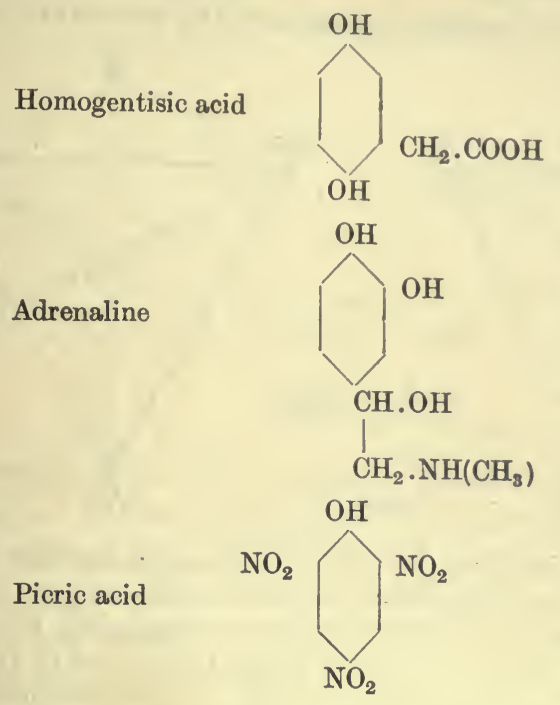

\section{OPTICAL ACTIVITY}

Most of the compounds produced by the agency of living organisms exhibit optica activity, i.e. have the property of rotating the planc of polarised light either to the right or to the left.

In an ordinary wave of light the vibrations of the waves takc place in all planes perpendicular to the direction of its propagation. When such a ray is passed through a Nicol's prism (made of Iceland spar) it emerges as a plane polarised beam, i.e. waves in one plane only are transmitted. Another Nicol's prism will allow such a ray to pass only if it is parallel to the first prism. If it is rotated through a right angle, no light will pass. A Nicol's prism may thus be used to determine the plane of polarisation of any beam of light.

In the polarimeter two Nicol's prisms"mounted parallel to one another are employed. One of them (the polariser) is fixed; the other (the analyser) can be rotated round

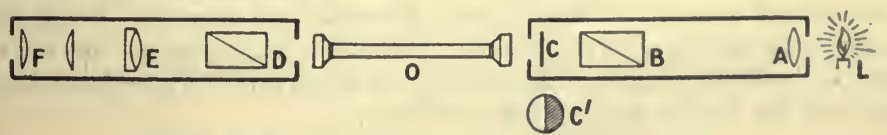

Fic. 16. Diagram of polarimeter.

B, polariser ; $\mathrm{D}$, analyser ; $\mathrm{O}$, tube containing solution under examination.

the axis of the beam of light passing through the first. When both prisms are parallel light passes through the analyser. On interposing a solution of an optically active substance between the two prisms, the plane of polarisation of the beam is rotated, so that the light passing through the analyser is diminished. The light may be brought to its original intensity by rotating the analyser either to the right (clockwise) or to the left. In this way the direction and degree of the optical activity may be determined. Optical activity is connected with the molecular arrangement of the substance exhibiting this property, and depends on the presence of one or more 'asymmetric carbon atoms ' in the molecule.

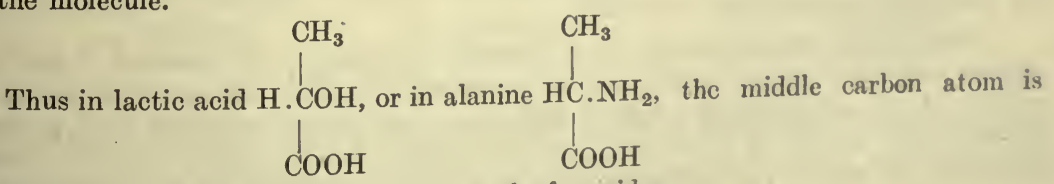

asymmetric, i.e. it is unequally loaded on the four sides. 
We can imagine such a carbon atom as occupying the interior of a tetrahedron.

A

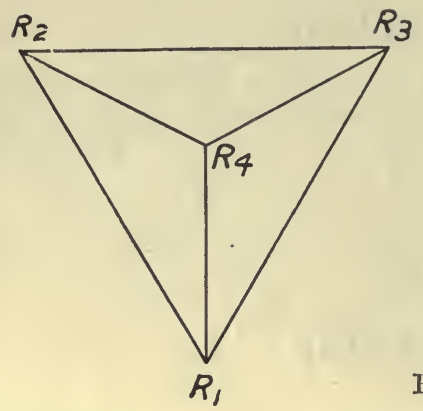

B

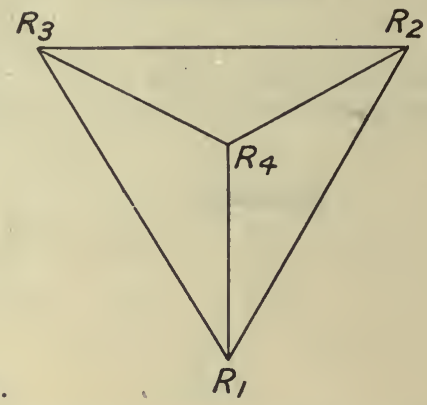

In this tetrahedron, if we represent the four groups combining with the carbon by $R_{1}, R_{2}, R_{3}, R_{4}$, they can be arranged either as in $A$ or $B$. It is evident that no amount of turning about will convert the tetrahedron $\mathrm{A}$ into tetrahedron $\mathrm{B}$, but that, if we hold A before a mirror, its image in the mirror will be represented by B. One arrangement is therefore the mirror image of the other, and a compound containing one such carbon atom will be capable of existing in two forms, namely, one form corresponding to $\mathrm{A}$, the other form corresponding to $\mathrm{B}$. It is found that the unequal loading of the carbon atom, which is present in such an asymmetric arrangement, causes the compound containing the asymmetric carbon to have an action on polarised light. One of the varieties will rotate polarised light to the right, while its mirror image will rotate polarised light to the left. A mixture of equal parts of the two compounds will rotate equally to left and right, i.e. will have no action on polarised light.

The variety rotating to the right is dextrorotatory, and the other lævorotatory,* while the mixture of the two is known as the racemic or inactive variety. The three forms are said to be stereoisomeric, and are distinguished as the $d, l$, and $i$ forms respectively. If two asymmetric carbon atoms are present in a compound, we may have four stereoisomers; and generally if there are $n$ asymmetric atoms in a molecule, there will be $2^{n}$ possible stereoisomers. These will not all be necessarily optically active, since the dextrorotation due to one asymmetric carbon atom may be exactly neutralised by the lævorotation due to another, so that 'internal compensation' takes place and the substance is optically inactive. Thus in tartaric acid four forms are known, namely, $d, l$, racemic or $i$, and mesotartaric, also inactive, in which internal compensation occurs. These four varieties may be represented as follows :

\section{$\mathrm{COOH}$ $\mathrm{HCOH}$ $\mathrm{HOCH}$} $\mathrm{COOH}$

$d$-tartaric acid

\section{$\mathrm{COOH}$ \\ $\mathrm{HOCH}$ \\ $\mathrm{HCOH}$}

$\mathrm{COOH}$

$l$-tartaric acid

\section{$\mathrm{COOH}$ \\ $\mathrm{HCOH}$ \\ $\mathrm{HCOH}$ \\ $\mathrm{COOH}$}

mesotartaric acid

inactive tartaric acid

Several methods may be employed to separate the racemic form into its two optically active components. One of these methods, first employed by Pasteur, is to grow moulds in the solution. One of the optical isomers is destroyed, leaving the other unchanged. Another method is the fractional crystallisation of the salts with alkaloids, e.g. strychnine in the case of lactic acid.

* The specific rotatory power of a substance is equal to the number of degrees through which the plane of polarisation is rotated when it passes through a 100 per cent. solution of the substance in a tube 1 decimetre long. Thus polarised light passing through such a tube of 10 per cent. glucose solution would show a rotation of $5 \cdot 25$ degrees, i.e. its specific rotatory power is $+52 \cdot 5$. 


\section{SECTION III}

\section{THE FATS}

THESE substances are widely distributed throughout the animal and vegetable kingdoms. In the higher animals they form the main constituents of the fatty or adipose tissue lying under the skin and between the muscles and often forming large accumulations around the viscera. In the marrow of bones they may amount to 96 per cent. They also occur in fine particles distributed through the protoplasm of cells and probably also in combination with the other constituents which make up protoplasm. Large amounts are also found in certain members of the vegetable kingdom, as, for instance, in the fatty seeds and nuts, e.g. linseed, olives, Brazil nuts.

\section{CHEMISTRY OF THE FATS}

The fats are esters of glycerol and the fatty acids. Glycerol is a trihydric or triatomic alcohol and can therefore form esters with one, two, or three of its hydroxyl groups; thus with acetic acid the following compounds are known :

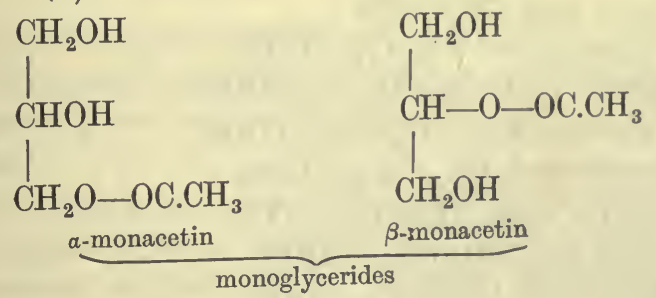

(3)

(4)

(5)
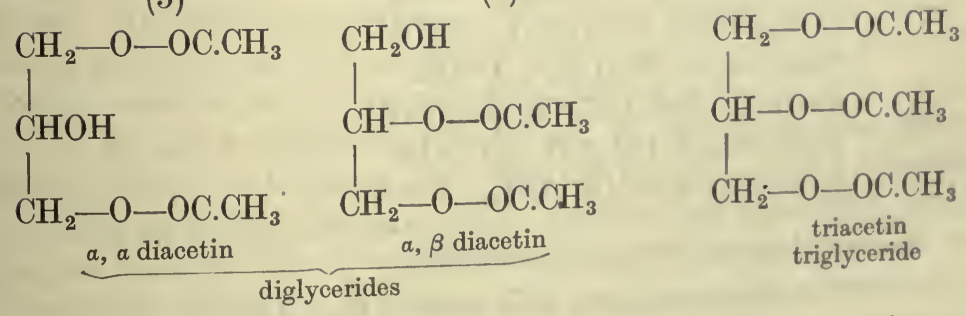

In these compounds the phenomenon of isomerism occurs owing to the presence of primary and secondary alcohol groups in glycerol. In the case 
of the diglycerides and the triglycerides mixed esters, in which the fatty acid radical varies, are possible :

(6)

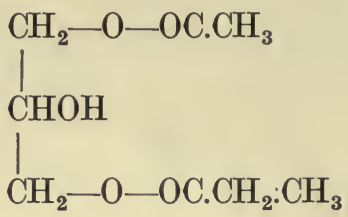

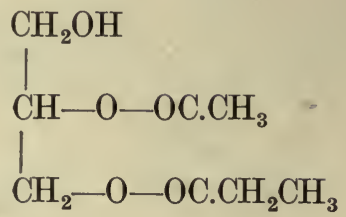

(8)<smiles></smiles>

The glyceryl esters which compose the fatty material of living matterwhether animal or plant - are mainly triglycerides, the monoglycerides and diglycerides being seldom found in nature. The natural fat is usually found to consist of a mixture of triglycerides; these triglycerides, instead of being mixed esters as in formula (8), are generally simple esters as in formula (5). The differences in the composition of the natural fats depend therefore on the variety of the fatty acid radical combined with the glycerol.

The fatty acids which enter into the composition of the triglycerides belong to two main homologous series :

A. The saturated fatty acids, namely :

Formic acid, H.COOH

Acetic acid, $\mathrm{CH}_{3} \cdot \mathrm{COOH}$

Propionic acid, $\mathrm{CH}_{3} \cdot \mathrm{CH}_{2} \cdot \mathrm{COOH}$

Butyric acid, $\mathrm{CH}_{3} \cdot \mathrm{CH}_{2} \cdot \mathrm{CH}_{2} \cdot \mathrm{COOH}$

Valerianic acid, $\mathrm{CH}_{3} \cdot\left(\mathrm{CH}_{2}\right)_{3} \cdot \mathrm{COOH}$

Caproic acid, $\mathrm{CH}_{3} \cdot\left(\mathrm{CH}_{2}\right)_{4} \cdot \mathrm{COOH}$

Caprylic acid, $\mathrm{CH}_{3} \cdot\left(\mathrm{CH}_{2}\right)_{6} \cdot \mathrm{COOH}$

Capric acid, $\mathrm{CH}_{3}\left(\mathrm{CH}_{2}\right)_{8} \cdot \mathrm{COOH}$

Lauric acid, $\mathrm{CH}_{3}\left(\mathrm{CH}_{2}\right)_{10}$. $\mathrm{COOH}$

Myristic acid, $\mathrm{CH}_{3}\left(\mathrm{CH}_{2}\right)_{12} \cdot \mathrm{COOH}$

Palmitic acid, $\mathrm{CH}_{3}\left(\mathrm{CH}_{2}\right)_{14} \cdot \mathrm{COOH}$

Stearic acid, $\mathrm{CH}_{3}\left(\mathrm{CH}_{2}\right)_{16}$. $\mathrm{COOH}$

Arachidic acid, $\mathrm{CH}_{3}\left(\mathrm{CH}_{2}\right) 18 . \mathrm{COOH}$

Behenic acid, $\mathrm{CH}_{3}\left(\mathrm{CH}_{2}\right)_{20} \cdot \mathrm{COOH}$

Lignoceric acid, $\mathrm{CH}_{3}\left(\mathrm{CH}_{2}\right)_{22} \cdot \mathrm{COOH}$

B. The unsaturated fatty acids, namely :

(1) Acrylic series, e.g. oleic acid $\left(\mathrm{C}_{n} \mathrm{H}_{2 n-2} \mathrm{O}_{2}\right)$

(2) Linoleic series, e.g. linoleic acid $\left(\mathrm{C}_{n} \mathrm{H}_{2 n-4} \mathrm{O}_{2}\right)$

(3) Linolenic series, e.g. linolenic acid $\left(\mathrm{C}_{n} \mathrm{H}_{2 n-6} \mathrm{O}_{2}\right)$ 
Of the long list of fatty acids given above only a few occur to any extent in the animal body. In milk, although the greater part of the fat consists of the triglycerides of oleic, palmitic, and stearic acids, other members of the series given above are present in small amounts. On the other hand, the adipose tissue, strictly so called, consists almost exclusively of the fats derived from the fatty acids palmitic, stearic, and oleic, i.e. tripalmitin, tristearin, and triolein. The great differences in the appearance of the fat of different animals are due to the varying amounts in the relative quantities of these three fats which may be present. While triolein is liquid at $0^{\circ} \mathrm{C}$, tristearin and tripalmitin are solid at the temperature of the body. According to the relative amounts of these three substances, therefore, we may have a fat which, like mutton suet, is solid at the body temperature, or a fat con. taining much olein which is still fluid and runs away when the body is opened after death, even when it has already cooled.

PROPERTIES OF THE FATS. The fats are colourless substances devoid of smell. They are insoluble in water, in which they float. They are soluble in warm absolute alcohol, but separate out into crystalline form on cooling. They are easily soluble in ether. If they are strongly heated with potassium bisulphate they give off pungent vapours of acrolein derived from the decomposition of the glycerin of their molecule.

$$
\mathrm{C}_{3} \mathrm{H}_{5}(\mathrm{OH})_{3}-2 \mathrm{H}_{2} \mathrm{O}=\mathrm{C}_{3} \mathrm{H}_{4} \mathrm{O}
$$

If they are heated with water or steam or submitted to the action of certain ferments, they undergo hydrolysis, taking up three molecules of water, and are split into three molecules of fatty acid and one molecule of glycerin, e.g.,

$$
\underset{\text { (neutral fat-tripalmitin) }}{\mathrm{C}_{3} \mathrm{H}_{5}\left(\mathrm{C}_{16} \mathrm{H}_{31} \mathrm{O}_{2}\right)_{3}}+\underset{\text { (palmitio acid) }}{3 \mathrm{H}_{2} \mathrm{O}}=\underset{\text { (glycerin) }}{3 \mathrm{HC}_{16} \mathrm{H}_{31} \mathrm{O}_{2}}+\underset{\mathrm{C}_{3} \mathrm{H}_{5}(\mathrm{OH})_{3}}{\text { (n) }}
$$

This process may occur spontaneously when fat is left exposed to the air. Fat which has been artificially split in this way is said to be rancid. Most natural fats generally contain a small amount of fatty acid which gives them an acid reaction.

On boiling a neutral fat for a long time with an aqueous solution of potassium or sodium hydrate, or better still with an alcoholic solution of potassium or sodium ethylate, the fat undergoes saponification, giving the alkaline salt of a fatty acid and glycerin. The former compound is spoken of as a soap. In water the soaps form a sort of pseudo-solution on heating which sets to a solid jelly on cooling. From a dilute watery solution the soap can be thrown down in the solid form by the addition of neutral salts. Fats are insoluble in and non-miscible with water. If shaken up with water the droplets rapidly run together and rise to the surface, forming a continuous layer of the oil or fat. The same thing happens if an absolutely neutral fat be shaken up with a dilute solution of sodium carbonate. If, however, the fat be slightly rancid, i.e. if fatty acid be present, the latter combines with the alkali with the expulsion of $\mathrm{CO}_{2}$ to form a soap. The presence of soap in colloidal solution in the water at once diminishes or abolishes the surface 
tension between the neutral fat and the water. Like many other colloidal solutions, a soap solution presents the phenomenon of surface aggregation, i.e. the concentration of the soap at the surface is increased to such an extent as to form practically a solid pellicle of molecular dimensions on the surface of the fluid. The same pellicle formation occurs at the surface of every oil globule, so that on shaking up rancid oil with dilute sodium carbonate, the whole of the oil is broken up into fine droplets which show no tendency to run together again, and remain in suspension in the water. The suspension of fine oil droplets, which has the appearance of milk, is spoken of as an emulsion. It can be at once destroyed by adding acid. This decomposes the soap, setting free the fatty acids, which are insoluble in the water. The pellicle around each globule is destroyed, and the globules run together as neutral oil would in pure water.

In order to characterise any given animal fat or mixture of fats the following reactions are made use of :

(1) The 'acid number' of the fat, i.e. its content in free fatty acids, is determined by titrating it in ethyl alcohol solution with $\frac{\mathrm{N}}{10}$ alcoholic solution of potash, using phenolphthalein as an indicator.

(2) The 'saponification number.' This represents the number of milligrammes of potassium hydrate necessary to saponify completely one gramme of fat.

(3) The percentage of volatile fatty acids is determined by saponifying the fat, then treating it with a mineral acid to set free the fatty acids and distilling over the volatile acids in a current of steam.

(4) The iodine number is the amount of iodine which is taken up by a given weight of fat. It is a measure of the amount of unsaturated fatty acid present, i.e. in ordinary fat, oleic acid.

Besides the glycerides, a certain number of substances occur in the body derived, not from a combination of fatty acids with glycerol, but from a formation of esters of the fatty acids and other alcohols, e.g. cholesterol or cetyl alcohol. Thus, spermaceti is a mixture of cetyl palmitate with small quantities of other fats. The fatty secretion of the sebaceous glands in man and the higher animals, which furnishes the natural oil of hair, wool, and feathers, consists, with small traces of glycerides of cholesterol esters. Lanoline, which is purified wool fat, consists almost entirely of cholesteryl stearate and palmitate. These cholesterol fats are attacked with extreme difficulty by ferments or micro-organisms. It is probably on this account that they are manufactured in the body for protective purposes. So far as we know, when once formed, they are incapable of further transformation in the body. They are not appreciably altered by the digestive ferments of the alimentary canal, and the cholesterol is said to pass through the latter unaltered.* Cholesterol is also found in combination with fatty acids in every living cell. Whenever protoplasmic structures are extracted with boiling ether, a certain amount of cholesterol is present with the fats which are so extracted. In view of the great stability of this substance when exposed to the ordinary mechanisms of chemical change in the body, it seems

* According to Gardner, cholesterol may be absorbed from the intestine. 
probable that the part played by cholesterol is that of a framework or skeleton, in the interstices of which the more labile constituents of the proto. plasm can undergo the constant cycle of changes which make up the phenomena of life.

\section{PHOSPHOLIPINES OR PHOSPHATIDES}

The fats form the chief constituent of the deposited and reserve fat throughout the animal kingdom and are also contained in the protoplasm of the living cell. The chief fatty constituents of protoplasm differ from the above fats in the following particulars : they contain phosphoric acid and an amine. On this account they have been called phosphorised fat's. Thudichum, who isolated various compounds of this nature from brain, suggested the term phosphatides as a general name for them. The term lipoid has also been used, but it includes all the substances composing a cell which are soluble in ether, e.g. cholesterol, cetyl alcohol, and the fats. Leathes has suggested the term phospholipine for those compounds, for it denotes that the compound is partly fat (lip), that it contains phosphorus, as well as a nitrogenous basic radical (ine). The phospholipines comprise the substances lecithin, cephalin, cuorine, sphingomyeline. In brain and other tissues similar compounds, which contain no phosphorus, occur, and in the place of glycerol we may find galactose. Leathes has proposed calling these compounds lipines and galactolipines.

Lecithin, the chief phospholipine, is an ester compounded of two fatty acid radicals, phosphoric acid, glycerol, and the amine, choline. The various lecithins may be distinguished, according as they contain different fatty acid radicals, as oleyl-lecithin, stearyl-lecithin. The following formula represents distearyl-lecithin :

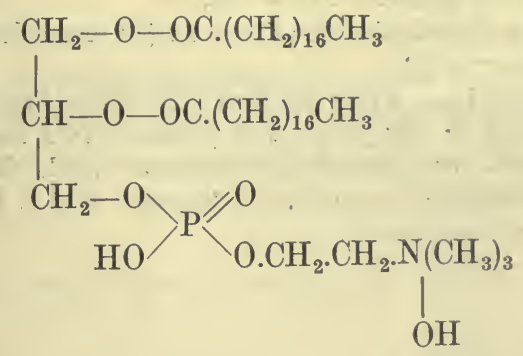

On warming with baryta water lecithin is broken down into fatty acid, glycerophosphoric acid, and choline. The latter base, which is trimethyloxethyl-ammonium hydrate, $N\left\{\begin{array}{l}\mathrm{C}_{2} \mathrm{H}_{4} \mathrm{OH} \\ \left(\mathrm{CH}_{3}\right)_{3} \\ \mathrm{OH}\end{array}\right.$ must be distinguished from neurine, $\mathrm{N}\left\{\begin{array}{l}\mathrm{C}_{2} \mathrm{H}_{3} \\ \left(\mathrm{CH}_{3}\right)_{3} \\ \mathrm{OH}\end{array}\right.$ which is trimethyl-vinyl-ammonium hydrate, and is much more poisonous than choline. Choline forms a salt with hydrochloric acid, which, with platinum chloride, yields a double salt of characteristic crystalline 
form, insoluble in absolute alcohol. The universal distribution of lecithin seems to indicate that it plays an important part in the metabolic processes of the cell. There is no doubt that it may serve, inter alia, as a source of the phosphorus required for building up the complex nucleo-proteins of cell nuclei. It seems to represent an intermediate stage in the utilisation of neutral fats by protoplasm, and its occurrence in the brain as a constituent of more complex molecules, which contain also a carbohydrate nucleus (galactosides, such as cerebrin), might be interpreted as indicating some share also in the metabolism of carbohydrates.

Lecithin may be extracted from tissues by boiling with absolute alcohol. On cooling the alcoholic extract in a freezing mixture, the lecithin separates out as granules or semi-crystalline masses. When dried in vacuo, it forms a waxy mass, which melts at $40^{\circ}$ to $50^{\circ} \mathrm{C}$. In water it swells up to form a paste, which, under the microscope, is seen to consist of oily drops or threads, the so-called myelin droplets. In a large excess of water it forms an emulsion or a colloidal solution. Its power of taking up water on the one hand, and its solubility in alcohol and similar media on the other, give it an intermediate position between the water-soluble crystalloids and the insoluble fats, and enable it to play an important part both as a vehicle of nutritive substances and as a constituent of the lipoid membrane, which bounds and determines the osmotic relationships of all living cells.

The phospholipines are provisionally classified according to the proportions of $\mathrm{N}$ and $\mathrm{P}$ in their molecule, as follows :

(a) Mono-amino-monophosphatides, $\mathrm{N}: \mathrm{P}=1: 1$ (including lecithin and cephalin).

(b) Diamino-mono-phosphatides, $\mathrm{N}: \mathrm{P}=2: 1$ (e.g. sphingomyelin).

(c) Mono-amino diphosphatides, $\mathrm{N}: \mathrm{P}=1: 2$ (e.g. cuorin, a lipine extracted from heart muscle by Erlandsen).

(d) Diamino-diphosphatides, $\mathrm{N}: \mathrm{P}=2: 2$.

(e) Triamino-monophosphatide, $\mathrm{N}: \mathrm{P}=3: 1$ (an example has been reported as occurring in egg yolk).

All these bodies (except cuorin) are obtained by the extraction of the brain or of nerve fibres. Many also occur in egg yolk. The galacto-lipines include two substances extracted from the brain, viz. phrenosin and kerasin. Both these on decomposition yield galactosc, a nitrogenous base called sphingosine and a fatty acid. We know little or nothing of their significance. 


\section{SECTION IV}

\section{THE CARBOHYDRATES}

THE carbohydrates are a group of bodies or wide distribution and great importance in both the vegetable and animal kingdoms. In plants the first product of assimilation of carbon is a carbohydrate, and in animals these substances form one of the most important sources of energy. They consist of the elements carbon, hydrogen, and oxygen, the two last-named being almost invariably in the proportions necessary to form water. It is on this account that the term carbohydrate has been given to the group. Their general formula might be expressed $\mathrm{C}_{n} \mathrm{H}_{2 n} \mathrm{O}_{n}$. Certain derivatives of the group, obtained by the substitution of methyl and other radicals for a hydrogen atom, though necessarily classified with carbohydrates on account of their reactions, do not conform to this general formula, e.g. rhamnose, $\mathrm{C}_{6} \mathrm{H}_{12} \mathrm{O}_{5}$. All the carbohydrates which are of importance in the animal economy contain six carbon atoms or a multiple of this number. Analogous substances, however, can be prepared containing less or more than this number of carbon atoms. A series of compounds exist which contain in their molecule $2,3,4,5,6,7,8,9$ carbon atoms, and are termed dioses, trioses, tetroses, pentoses, hexoses, heptoses, and so on ; the termination 'ose ' with the Greek numeral prefixed, indicating the number of carbon atoms, gives them a distinct designation. These are all oxidation products of polyatomic alcohols, being either ketones or aldehydes of these alcohols. Thus from $\mathrm{COH}$

glycerol we may obtain glyceryl aldehyde $\mathrm{CHOH}$ and dioxyacetone

\section{$\mathrm{CH}_{2} \mathrm{OH}$} $\mathrm{CH}_{2} \mathrm{OH}$

CO. Both these substances behave as sugars and belong to the group of | $\mathrm{CH}_{2} \mathrm{OH}$

trioses. They are generally obtained together and are called glycerose. $\mathrm{CH}_{2} \mathrm{OH}$

From the hexatomic alcohol $(\mathrm{CHOH})_{4}$ we may obtain either the aldehyde 


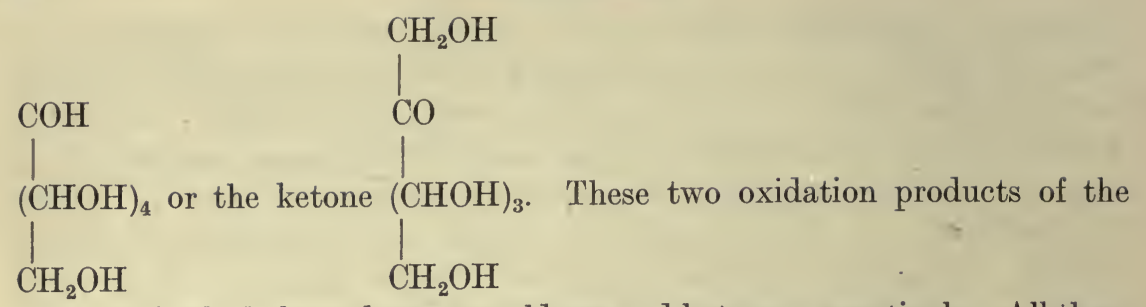
polyatomic alcohols are known as aldoses and ketoses respectively. All these compounds are distinguished by the termination 'ose.' It is convenient to call those compounds containing six carbon atoms the sugars, because it is to this group that the natural sugars belong.

Stereoisomerism in the Sugars. It will be noticed that of the six carbon $\mathrm{CH}_{2} \mathrm{OH}$

atoms contained in the sugar molecule, e.g. the aldose $(\mathrm{CHOH})_{4}$, four

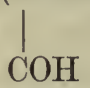

are asymmetric, i.e. their four combining affinities are saturated with groups of different kinds, viz. several carbon atoms, one $\mathrm{H}$ atom, and one $\mathrm{OH}$ group :

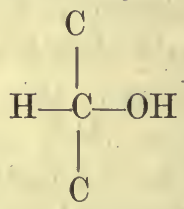

They muist therefore present many stereoisomeric forms. . If $n$ represent the number of asymmetric carbon atoms in a compound, the possible number of stereoisomers is $2^{\mathrm{n}}$. Thus an aldehexose with four asymmetric carbon atoms $(\mathrm{CHOH})_{4}$ must present $2^{4}$ isomers, i.e. sixteen isomeric compounds, so that there must be sixteen sugars all possessing the formula $\mathrm{CH}_{2} \mathrm{OH}(\mathrm{CHOH})_{4} \mathrm{COH}$, in addition to the inactive sugars obtained by a mixture of two oppositely active members of this group. Of the sixteen possible sugars of this formula, as many as fourteen have been found or have been artificially prepared. Only a small number are, however, of any physiological importance. These include the aldoses, glucose, mannose, and galactose, and the ketose, fructose or levulose. All the other sugars are unassimilable by the animal cell and are not manufactured by plants.

Since these sugars can be divided into the optically active and the inactive varieties, an obvious mode of designation would be to represent them as d-, l-, and i- varieties respectively, i.e. dextro-rotatory, lævo-rotatory, and inactive. On Fischer's suggestion, however, this mode of nomenclature has been altered in favour of representing, by the letter prefixed, not the optical qualities of the substance in question, but its relation to other substances, especially glucose. Thus, $d$-fructose means that fructose is the ketose corresponding to the dextro-rotatory glucose, d-fructose itself being lævo-rotatory, though its active asymmetric $\mathrm{C}$ atoms are identically arranged with those in 
glucose. With this limitation one may say that it is only the d-hexoses of a particular form which are assimilable, and therefore of physiological importance. The small differences in the configuration of the four d-sugars can be readily seen if their graphic formulæ be compared :

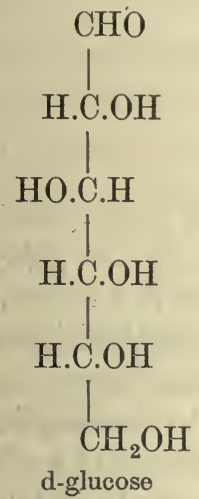

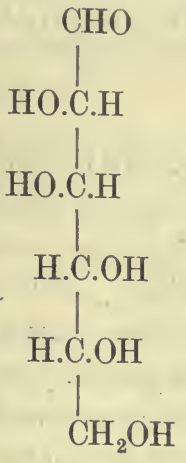

d-mannose
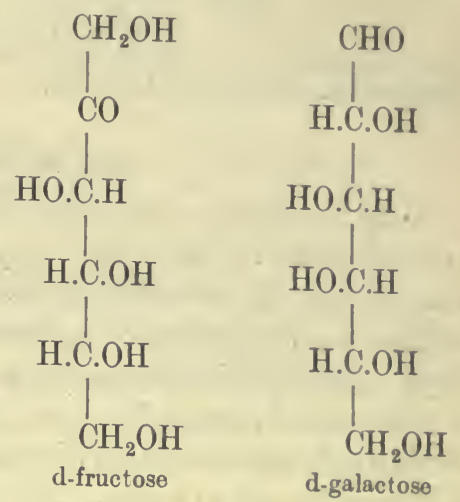

THE PENTOSES. $\mathrm{C}_{5} \mathrm{H}_{10} \mathrm{O}_{5}$

These bodies occur largely in plants in the form of complex polysaccharides, the pentosanes, which give pentoses on hydrolysis with acids. Two forms of pentose have been found in the animal body, namely, $\mathrm{i}$-arabinose, which has been isolated from the urine in cases of pentosuria, and l-xylose (or d-ribose, Levene), which occurs built up into the nucleic acid molecule of the pancreas and perhaps other organs.' The pentoses can apparently be utilised by herbivora as food-stuffs. We know nothing as to the part they play in the animal body or as to the causation of the rare condition of pentosuria. Since, however, they are reducing substances and the presence of pentose in urine might therefore lead to a suspicion of diabetes, it is necessary to mention the tests by which the presence of pentoses may be detected. The two following are the chief tests for pentoses:

(1) The solution supposed to contain a pentose is mixed with an equal volume of concentrated hydrochloric acid. To the mixture is added a small quantity of solid orcin and the whole is heated. If pentose is present the solution becomes at first reddish-blue and later bluish-green. The colour can be extracted on shaking the fluid with amyl alcohol, the solution, on spectroscopic examination, showing in absorption band between $\mathrm{C}$ and $\mathrm{D}$.

(2) Instead of adding orcin, we may add phloroglucin to the mixture of hydrochloric acid and pen'ose. The solution on heating becomes first cherry red and then cloudy. On shaking with amyl alcohol a red solution is obtained which shows a band between $\mathrm{D}$ and $\mathrm{E}$.

\section{THE HEXOSES AND THEIR DERIVATIVES}

The most important of the carbohydrates belong to this class and are either hexoses or formed by a combination of two or more hexose molecules. They are divided into three main groups :

(1) Monosaccharides, with the formula $\mathrm{C}_{6} \mathrm{H}_{12} \mathrm{O}_{6}$, examples of which are glucose, fructose, \&c.

(2) Disaccharides, which are derived from two molecules of a monosaccharide with the elimination of a molecule of water, as follows:

$$
2 \mathrm{C}_{6} \mathrm{H}_{12} \mathrm{O}_{6}-\mathrm{H}_{2} \mathrm{O}=\mathrm{C}_{12} \mathrm{H}_{22} \mathrm{O}_{11} \text {. }
$$

(Examples, maltose and cane sugar.) 
(3) Polysaccharides, composed of three or more molecules of a monosaccharide. The number of molecules which are associated in the compounds of this group may be very large. We can regard their general formation as represented by the following equation :

$$
\mathrm{nC}_{6} \mathrm{H}_{12} \mathrm{O}_{6}-\mathrm{nH}_{2} \mathrm{O}=\left(\mathrm{C}_{6} \mathrm{H}_{10} \mathrm{O}_{5}\right)_{n} \text {. }
$$

(Examples, starch, dextrin, \&c.)

\section{THE MONOSACCHARIDES}

Only four hexoses out of the large number which have been synthetised are assimilable by the animal body. These are mannose, glucose, galactose, and fructose, the three former being aldoses, while the last is a ketose. All of them are derivatives of d-glucose. They may be synthetised in several ways. The most interesting, because it probably represents the mechanism of synthesis of hexoses in plants, is the formation from formaldehyde. In alkaline solutions formaldehyde polymerises with the formation of a mixture of hexoses known as acrose. From this mixture $\alpha$-acrose can be isolated by the formation of its osazone and the reconversion of this osazone into sugar. It is found to be identical with i-fructose. If a solution of this i-fructose be treated with yeast, d-fructose is fermented, leaving l-fructose behind. For the preparation of $d$-fructose it is necessary to convert the inactive sugar into the corresponding acid, mannonic acid. This with strychnine or morphia forms salts which can be separated into the d- and l- groups by fractional crystallisation. From the d- modification d-mannose can be obtained, and this by conversion into the osazone and reconversion into a sugar gives d-fructose.

All the monosaccharides, however many carbon atoms they contain, present certain general reactions determined by their chemical composition.

(a) Like ordinary aldehydes and ketones, the sugars act as strongly reducing substances, and, like aldehydes, reduce ammoniacal solution of silver to metallic silver, and many of the higher oxides of metals to lower oxides. On this behaviour is founded the commonest of all the tests for the presence of reducing sugar-Trommer's test.

(b) On oxidising a monosaccharide the $\mathrm{COH}$ group becomes converted to $\mathrm{COOH}$. Thus, glucose on oxidation gives gluconic acid :

$$
\mathrm{COH}(\mathrm{CHOH})_{4} \mathrm{CH}_{2} \mathrm{OH}+\mathrm{O}=\mathrm{COOH}(\mathrm{CHOH})_{4} \mathrm{CH}_{2} \mathrm{OH} \text {. }
$$

On further oxidation the end group $\mathrm{CH}_{2} \mathrm{OH}$ also is affected, and we obtain a dibasic acid. Thus glucose gives saccharic acid.

(c) By means of nascent hydrogen the monosaccharides can be reduced to the corresponding polyatomic alcohol. Thus the three hexoses, glucose, fructose, and galactose, give the corresponding three alcohols, sorbite, mannite, and dulcite $\mathrm{C}_{6} \mathrm{H}_{14} \mathrm{O}_{6}$.

(d) Another important general reaction of the monosaccharides depending on the $\mathrm{COH}$ or the $\mathrm{CO}$ group is the reaction with phenyl hydrazine. On warming a solution of sugar with a solution of phenyl hydrazine in acetic 
acid, the following reactions take place. The first reaction results in the production of a hydrazone :

$$
\begin{aligned}
& \mathrm{CH}_{2} \mathrm{OH}(\mathrm{CHOH})_{3} \mathrm{CHOHCHO}+\mathrm{H}_{2} \mathrm{~N} . \mathrm{NH} \cdot \mathrm{C}_{6} \mathrm{H}_{5}= \\
& \mathrm{CH}_{2} \mathrm{OH}(\mathrm{CHOH})_{3} \mathrm{CHOH} . \mathrm{CH}: \mathrm{N} . \mathrm{NH} \cdot \mathrm{C}_{6} \mathrm{H}_{5}+\mathrm{H}_{2} \mathrm{O} \text {. }
\end{aligned}
$$

The hydrazone then reacts with another molecule of phenyl hydrazine with the production of an osazone :

$$
\begin{array}{r}
\mathrm{CH}_{2}(\mathrm{OH})(\mathrm{CHOH})_{3} \mathrm{CHOH} . \mathrm{CH}: \text { N.NH.C } \mathrm{H}_{5}+\mathrm{H}_{2} \mathrm{~N}_{2} \mathrm{NHC}_{6} \mathrm{H}_{5}= \\
\mathrm{CH}_{2} \mathrm{OH}(\mathrm{CHOH})_{3} \mathrm{C} . \mathrm{CHN} . \mathrm{NH} \cdot \mathrm{C}_{6} \mathrm{H}_{5} \\
\| \\
\text { N.NH.C. } \mathrm{C}_{6} \mathrm{H}_{5}+\mathrm{H}_{2} \mathrm{O}+\mathrm{H}_{2} \text {. }
\end{array}
$$

The hydrogen formed in this reaction acts upon a second molecule of phenyl hydrazine, splitting it into aniline and ammonia. On this account it is always necessary to have an excess of phenyl hydrazine in the operation.

The osazones form well-defined crystalline products which are generally yellowish in colour and differ in their melting-point and in their crystalline form. They are therefore of extreme value in the separation and identification of different carbohydrates. They can be also used for the artificial preparation of certain sugars. Under the influence of acetic acid and zinc dust they form osamines, which on treatment with nitrous acid are reconverted into the corresponding sugar, generally a ketose.

GLUCOSE, DEXTROSE or GRAPE SUGAR, is the chief constituent of the sugar of fruits, especially of grapes. It occurs in the body as the endproduct of the digestion of starch. When pure it forms white crystals which melt at $100^{\circ} \mathrm{C}$., and lose the one molecule of water of crystallisation at $110^{\circ}$ C. It is easily soluble in water, and the solution shows bi-rotation. Its final specific rotatory power at $20^{\circ} \mathrm{C}$. is $52 \cdot 74$.

TESTS FOR GLUCOSE. Trommer's test depends on the power possessed in common with the other sugars of reducing cupric hydrate to cuprous oxide. The sugar solution is made alkaline with caustic potash or soda, and a few drops of copper sulphate solution added. On heating the blue solution thus obtained to boiling, it turns yellow, and a yellowish-red precipitate of cuprous hydrate is produced. This test is generally performed with Fehling's solution, which consists of an alkaline solution of cupric hydrate in Rochelle salt. The proportions in making the solutions are so arranged that 10 c.c. of Fehling's solution are completely reduced by .05 gramme glucose. This reaction is made use of for the quantitative determination of glucose in solution. The determination may be carried out either volumetrically, as in Fehling's or Pavy's method, or gravimetrically, as in Allihn's method.

Moore's Test. A solution of glucose treated with a little strong caustic potash or soda and warmed, becomes first yellow and then gradually dark brown, and gives off a smell of caramel.

With ordinary yeast, glucose solutions ferment readily, giving off $\mathrm{CO}_{2}$, and form alcohol with small traces of amyl alcohol, glycerin, and succinic acia.

With phenyl hydrazine glucose gives well-marked needles of glucosazone. These are precipitated when the liquid is still hot, the precipitate being increased as the liquid cools. The crystals form bundles of fine yellow needles which are almost insoluble in water, but are soluble in boiling alcohol. When purified by recrystallisation they melt at $204-205^{\circ} \mathrm{C}$.

On treating a watery solution of glucose with benzoyl chloride and caustic soda 
and shaking till the smell of benzoyl chloride has disappeared, an insoluble precipitate is produced of the benzoic ester of glucose. This method has been often used for isolating glucose from fluids in which it occurs in minute quantities.

Molisch's Test. On treating 0.5 c.c. of dilute glucose solution with one drop of a 10 per cent. alcoholic solution of $\alpha$-naphthol, and then pouring 1 c.c. of concentrated sulphuric acid gradually down the side of the tube, a purple ring is produced at the junction of the two fluids, which on shaking spreads over the whole fluid. This reaction depends on the formation of furfurol from the glucose.

In order to identify glucose in a normal fluid, the following tests may be applied, after removing any protein which may be present :

(1) Reduction of cupric hydrate or Fehling's solution.

(2) Estimation of reducing power of solution.

(3) Estimation of rotatory power of solution on polarised light.

(4) Formation of osazone crystals with phenyl hydrazine. These crystals must come down while the fluid is still hot. They must be purified and their melting-point taken. A determination by combustion of their nitrogen content will give direct information whether the sugar is a monosaccharide or disaccharide.

(5) The solution is made acid and boiled for some time. . It is then made up to its former volume and its reducing power and effect on polarised light once more taken. In the case of a disaccharide, which would be converted into monosaccharide by boiling in acid solution, these two readings would be altered, whereas neither the rotatory power nor the reducing power of glucose would undergo any change.

(6) Fermentation with ordinary yeast.

A positive result would exclude glycuronic acid.

D-FRUCTOSE, or LEVULOSE, occurs mixed with dextrose in honey and in fruit sugar. It is also, with glucose, formed by the digestion or inversion of cane sugar. It is crystallisable with difficulty. Its watery solution is lævo-rotatory; and reduces Fehling's solution somewhat less strongly than glucose, its reducing power being 92, if we take that of glucose as 100 . It ferments readily with yeast; with phenyl hydrazine it gives the same osazone as is formed from glucose.

GALACTOSE is formed by the digestion or hydrolysis of milk sugar or lactose. It is also obtained on hydrolysing cerebrin, a constituent of the brain, with dilute mineral acids, and by the hydrolysis of certain vegetable gums. It is much less soluble in water than glucose. It is dextro-rotatory and shows marked bi-rotation. With ordinary yeast it ferments but extremely slowly. One species of yeast is known, namely, saccharomyces apiculatus, which, while fermenting d-fructose and glucose, has no effect on galactose. This yeast can therefore be used to isolate galactose from a mixture of the monosaccharides. It reduces Fehling's solution, its reducing power being somewhat less than that of glucose. Yeasts can be trained to ferment galactose:

MANNOSE. Mannose, though an assimilable sugar, is of such rare occurrence in our food-stuffs that it plays practically no part in animal physiology. It is dextrorotatory, reduces Fehling's solution, ferments easily with ordinary yeast, and gives an osazone which is identical with that derived from glucose.

\section{DERIVATIVES OF THE HEXOSES}

Two derivatives of glucose are of considerable physiological importance; namely; glucosamine and glycuronic acid. 
Glucosamine, $\mathrm{C}_{6} \mathrm{H}_{13} \mathrm{NO}_{5}$, has the structural formula :

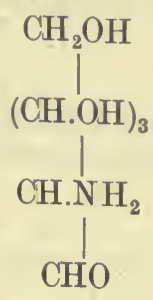

It is obtained from chitin, which forms the exoskeleton of large numbers of the invertebrata; by boiling this with concentrated hydrochloric acid. It is stated to have been obtained as a decomposition product of certain proteins and their derivatives, such as the mucins. It is of special interest as affording an intermediate product between the carbohydrates and the oxy-amino acids which can be obtained by the disintegration of proteins. In solution it is dextro-rotatory, reduces Fehling's solution, and gives an osazone resembling that derived from glucose.

GLYCURONIC ACID, $\mathrm{C}_{6} \mathrm{H}_{10} \mathrm{O}_{7}$, may be regarded as one of the first results of oxidation of the glucose molecule. The group which has undergone oxidation is not the readily oxidisable $\mathrm{CHO}$ group, but the $\mathrm{CH}_{2} \mathrm{OH}$ group at the other end of the molecule. The formula of this acid is therefore:

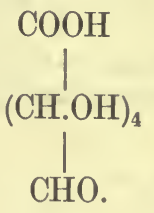

In the free state it does not occur in the animal body. It is constantly found in the urine after administration of certain drugs such as phenol, camphor, or chloral, and then occurs as a conjugated acid with these substances. These conjugated acids are lævo-rotatory, though the free acid is dextro-rotatory. In the free state it reduces Fehling's solution and gives an osazone which is not sufficiently characteristic to distinguish from glucosazone. It does not undergo fermentation with yeast. This test is therefore the best means of distinguishing the acid in urine from glucose.

\section{THE FORMATION OF GLUCOSIDES}

The graphic formulæ given on p. 61 do not explain all the possible modes of arrangement of the groups of the sugar molecules. Many of these sugars, when dissolved in water, present the phenomenon known as multi-rotation. If their rotatory power be taken immediately after solution, it is found to be greater or less than the rotatcry power taken some hours or days later. Glucose, for instance, immediately after solution, has a high specific rotatory power, which dimninishes rapidly if the solution bo boiled, and more slowly if it be allowed to stand. Finally, the specific rotatory power becomes constant at $+52 \cdot 5^{\circ} \mathrm{D}$. This change in rotatory power seems to be associated with a change in the arrangement of the groups, the aldose, for example, assuming, by the shifting of a mobile oxygen atom, what is known as a lactone arrangement. 
Thus glucose $\mathrm{COH}(\mathrm{CHOH})_{2} \mathrm{CHOH}$. $\mathrm{CHOH} . \mathrm{CH}_{2} \mathrm{OH}$ becames $\mathrm{CHOH} .(\mathrm{CHOH})_{2}$. $\mathrm{CH} . \mathrm{CHOH} . \mathrm{CH}_{2} \mathrm{OH}$

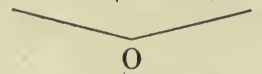

This change in the arrangement of the molecule renders a further stereoisomerism possible, owing to the fact that now the end group which was formerly $\mathrm{COH}$ becomes<smiles>CC(O)CO</smiles>

so that now there are five instead of four asymmetric carbon atoms. The two isomers of glucose, which are thus rendered possible, are represented by the following structural formulæ:
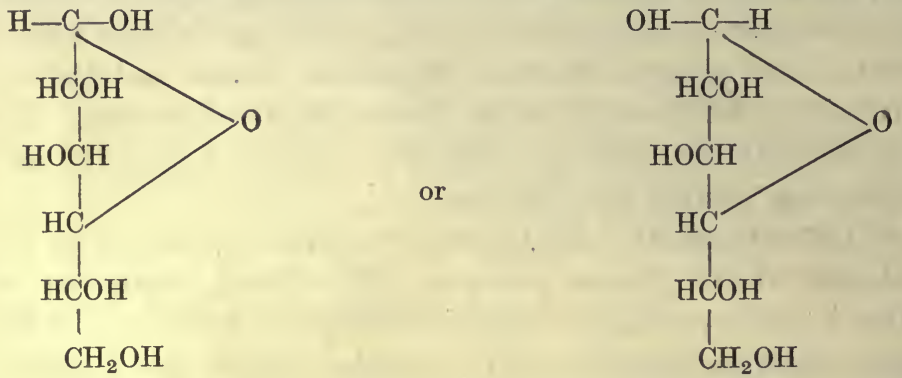

In these molecules the $\mathrm{OH}$ attached to the end group can be replaced by other radicals, including other sugar molecules. In this way we get the formation of glucosides. Thus, if glucose be dissolved in methyl alcohol and be treated with hydrochloric acid, we obtain $\alpha$ and $\beta$ methyl glucosides, the formulæ of which would bə represented as follows :
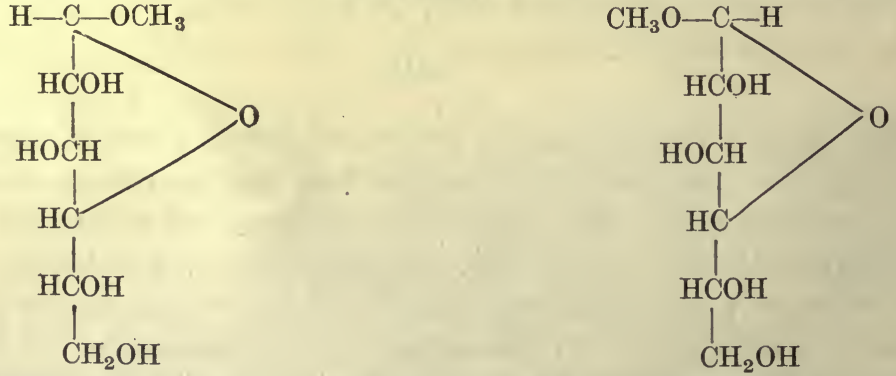

Instead of methyl we might insert other groups, and even other hexose groups, such as glucose or galactose, obtaining the two sugars maltose and lactose, which may thus be regarded as glucosides-maltose as the $\alpha$ glucoside of glucose, lactose as the $\beta$ galactoside of glucose. The mode of combination of the two hexose groups to form these disaccharides may be represented as follows :

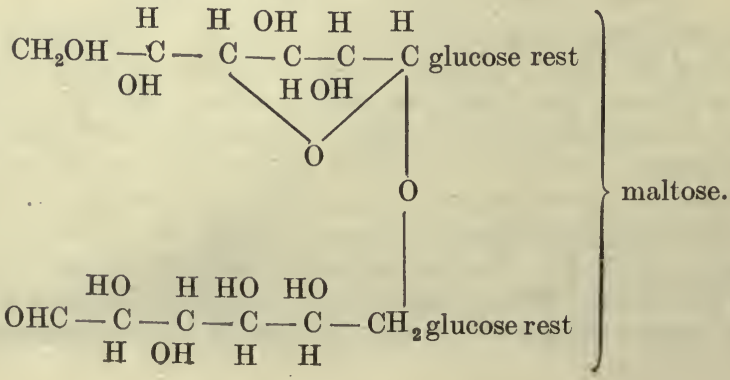




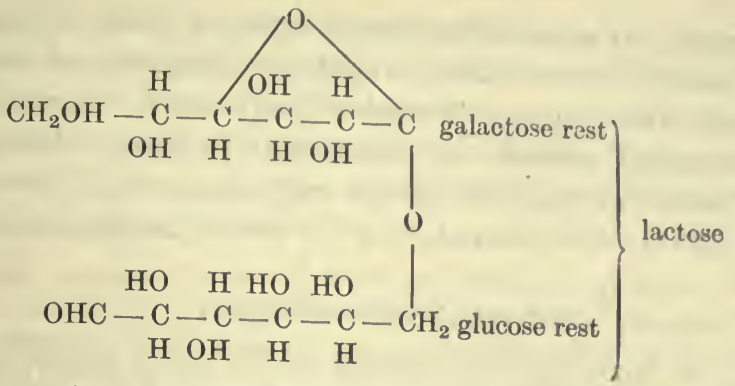

A very large number of glucosides occur as plant products. Among these wo may
mention amygdalin, salicin, phloridzin, indican, \&c.

\section{THE DISACCHARIDES}

The disaccharides are formed by the union of two molecules of mono. saccharides with the elimination of one molecule of water, and can be regarded, according to the manner in which the molecules are combined, as glucosides, galactosides, \&c. On hydrolysis, e.g. on heating with acids, they take up one molecule of water and are split up into the corresponding monosaccharides. Thus, cane sugar gives equal parts of glucose and fructose, maltose gives equal parts of glucose and glucose, while milk sugar or lactose gives equal parts of glucose and galactose.

CANE SUGAR, sometimes known as saccharose, is widely distributed throughout the vegetable kingdom, and forms an important article of diet. It has no reducing power on Fehling's solution. It is strongly dextro-rotatory and has a specific rotatory power of $+66 \cdot 5^{\circ}$. On hydrolysis it is converted into equal molecules of glucose and fructose. Owing to the fact that fructose rotates polarised light more strongly to the left than glucose does to the right, the mixture of the two monosaccharides so obtained is lævo-rotatory. On this account the change from free cane sugar to the mixture of monosaccharides is known as inversion, and the mixture is often spoken of as 'invert sugar.' The term 'inversion ' has since been loosely applied to the process of hydrolysis itself, so that we often speak of the inversion of maltose or of lactose, meaning thereby the hydrolysis of these sugars with the production of their constituent monosaccharides. With yeast, cane sugar first undergoes inversion by a special ferment present in the yeast (invertase), and the mixture of fructose and glucose is then fermented.

MALTOSE is formed during the hydrolysis of starch by acids or by digestive ferments, and is also the chief sugar in germinating barley or malt. It is strongly dextro-rotatory, ferments easily with yeast, and reduces Fehling's solution; its reducing power is about 70 per cent. of that of glucose. With phenyl hydrazine it gives phenyl maltosazone, which forms definite yellow crystals with a melting-point of $206^{\circ} \mathrm{C}$.

MILK SUGAR or LACTOSE is found only as a constituent of milk. It forms colourless rod-like crystals, which are much less soluble in water than are the two other disaccharides. On account of this solubility it is much less sweet than either cane sugar or maltose. It is dextro-rotatory and 
shows bi-rotation. It is not fermented by ordinary yeast. Before fermentation can occur the lactose must be split by the agency of acids or by a ferment, lactase, which occurs in the animal body and in certain moulds, into the monosaccharides glucose and galactose. Lactose reduces Fehling's solution and gives with phenyl hydrazine lactosazone, which is easily soluble in hot water and therefore does not come down until the fluid is cold.

\section{THE POLYSACCHARIDES}

These play an important part throughout the whole vegetable kingdom, where all the supporting tissues of the plants, their protective substances, and many of their reserve materials consist of members of this group. In the animal body, where the supporting tissues are composed chiefly of derivatives of proteins, the sole significance of polysaccharides lies in their value as food-stuffs. In plants, anhydrides both of hexoses and pentoses occur in bewildering variety. Here, however, we may confine our attention to those members of the group of polysaccharides which are important as food-stuffs.

STARCH $\left(\mathrm{C}_{6} \mathrm{H}_{10} \mathrm{O}_{5}\right)$ is present in large quantities in nearly all vegetable foods, and is an important constituent of the cereals, from which flour and bread are derived, as well as of tubers, such as the potato. In the plant cells it occurs as concentrically striated grains within minute protoplasmic structures - the amyloplasts, the office of which it is to manufacture starch from the glucose present in the cell sap. When freed, by breaking up the cells and washing with water, it forms a white powder consisting of microscopic grains, each of which presents the characteristic concentric striation. It is insoluble in cold water. In hot water the grains swell up and burst, forming a thick paste, which sets to a jelly on cooling. This semi-solution, as well as the original starch-grains, gives an intense blue colour on the addition of iodine. On treating starch with cold alkalies or cold dilute acid, it is converted into a soluble modification, the so-called soluble starch or amylodextrin, which also gives a blue colour with iodine. This modification is also produced as the first stage of the action of diastatic ferments upon starch. On boiling with dilute acids, starch is converted first into a mixture of dextrins, then into maltose; and finally into glucose. On acting upon starch with various ferments, such as the diastase which may be extracted from malt or germinating barley, or with the amylase occurring in saliva or pancreatic juice, it undergoes hydrolysis, the final result of the action being a mixture of four parts of maltose to one part of dextrin. As to the intermediate stages in this reaction opinions are still divided. The first product is soluble starch, amylodextrin, giving a blue colour with iodine. This breaks up into a reducing sugar, and another dextrin, erythrodextrin, which gives a red colour with iodine, and this dextrin, on further hydrolysis, yields reducing sugar and achroodextrin, which is not coloured by the addition of iodine. Thus there are a series of successive hydrolytic decompositions of the molecule, each resulting in the splitting off of a molecule of sugar and the production of a lower dextrin.

The DEXTRINS are ill-defined bodies which are difficult to separate. 
They are a morphous white powders, easily soluble in water, forming solutions which, when concentrated, are thick and adhesive. They are insoluble in alcohol and ether. With cupric hydrate and caustic alkali they form blue solutions, which reduce slightly on boiling. They are not precipitated by saturation with ammonium sulphate. On boiling with dilute acids, they are converted entirely into glucose.

The changes undergone by starch during its hydrolysis by means of diastase have been used by Brown and his co-workers as a method of arriving at some idea of the size and structure of the starch molecule. Proceeding from the discovery that the end-products of this reaction consisted of 81 per cent. maltose and 19 per cent. dextrin, they concluded that starch must consist of five dextrin-like groups, four of which are arranged symmetrically round the fifth. At each stage one of these groups is split off and hydrolysed to form malto-dextrin: $\left\{\begin{array}{c}\mathrm{C}_{12} \mathrm{H}_{22} \mathrm{O}_{11} \\ \left(\mathrm{C}_{12} \mathrm{H}_{20} \mathrm{O}_{10}\right)_{2}\end{array}\right\}$ one molecule of water being taken up. The malto-dextrin group is then changed into maltose by the further assimilation of two molecules of water. The central dextrin-like group is attacked with great difficulty by the ferment, and therefore remains at the end of the reaction as achroodextrin. The malto-dextrin, the penultimate stage in the action of diastase, can be regarded as formed by the condensation of three molecules of maltose attached by the oxygen of two $\mathrm{CHO}$ groups, so that one $\mathrm{CHO}$ group remains free and determines the reducing power of the malto-dextrin molecule. Its formula may therefore be represented as follows:<smiles>CCOC(CC)OCCOC</smiles>

the sign

They further found that the stable dextrin remaining at the end of the diastatic hydrolysis of starch probably had the formula of $40 \mathrm{C}_{6} \mathrm{H}_{10} \mathrm{O}_{5} \mathrm{H}_{2} \mathrm{O}$, and might be regarded as a condensation of forty glucose molecules with the elimination of thirty-nine moleculcs of water. The starch molecule cannot be less than five times that of the stable achroodextrin. Since the latter has a molecular weight of 6498 , the molecular weight of starch cannot be less than 32,400 , and its empirical formula can be represented by:

$$
100 \mathrm{C}_{12} \mathrm{H}_{20} \mathrm{O}_{10} \text {, or }\left(80 \mathrm{C}_{12} \mathrm{H}_{20} \mathrm{O}_{10} \cdot 40 \mathrm{C}_{6} \mathrm{H}_{10} \mathrm{O}_{5}\right) \text {. }
$$

INULIN. Another kind of starch, known as inulin, occurs in dahlia tubers. It is easily hydrolysed by weak acids, and is entirely converted into d-fructose, or levulose.

GLYCOGEN, or animal starch, is found in the liver, muscles, and other tissues of the body, and occurs in large quantities in all fœtal tissues. It is a white powder, soluble in water, forming an opalescent solution. It is precipitated from its solution on the addition of alcohol to 60 per cent., or by saturation with solid ammonium sulphate. On boiling with acids, it is entirely converted into glucose. It is affected by the ferments diastase and amylase, in the same way as vegetable starch, giving first dextrins and finally a mixture of maltose and dextrin. With iodine it gives a mahogany-red colour, which, like the blue colour produced in starch, is destroyed by boiling, to return again on cooling. We shall have occasion to consider its properties more fully when we are dealing with the functions of the liver. 
THE CELlulOSES. Cellulose $\left(\mathrm{C}_{6} \mathrm{H}_{10} \mathrm{O}_{5}\right)_{\mathrm{x}}$ is a colourless, insoluble material, or mixture of materials, which compose the cell walls of the younger parts of plants, and therefore forms a constituent of most of our vegetable foods. It is insoluble in water or dilute acids or alkalies, its only solvent being an ammoniacal cupric oxide solution. On boiling with strong acids, it gradually undergoes hydrolysis and yields sugar, the nature of which varies according to the source of the cellulose. In herbivorous animals cellulose undergoes digestive changes and forms an important constituent of their food. The solution of the cellulose in this case is effected by the agency, not of ferments secreted by the wall of the gut, but of micro-organisms which swarm in the paunch of ruminants and in the cæcum of other herbivora. In some cases the effective agent is a cytase present in the vegetable cells themselves. Since this ferment is destroyed by boiling, cooked hay is much less digestible than in the raw condition. In certain invertebrata it seems probable that a true cellulose-digesting ferment, or cytase, is secreted by the walls of the alimentary canal. In man cellulose undergoes practically no change in digestion, and serves merely by its bulk to promote peristalsis and the normal evacuation of the bowels. A further consideration of its chemical properties, as well as of the closely allied vegetable materials, gums, pectins, mucilages, derived for the most part from the condensation of pentose molecules, may be dispensed with here. 


\section{SECTION V}

\section{THE PROTEINS}

As sources of energy to the organism all three classes of food-stuffs are valuable in proportion to their heat equivalents, and it is often a matter of indifference whether the main bulk of the energy required is supplied at the expense of fat or at the expense of carbohydrate. The proteins, however, form the most important constituent of living protoplasm. On this account protein must always be present in the food to supply the material necessary for building up new protoplasm in the growing animal and for replacing the waste of living material which is taking place in the discharge of its normal functions. Regarding the complexity of reaction presented by living protoplasm as determined in the first instance by the chemical and physical complexity of this material itself, we should expect to find that the proteins forming its main constituents, would themselves partake of some of this quality. The carbohydrates and fats, although in many cases made up of huge molecules, are nevertheless built up on a very simple type. Starch, for instance, with a molecular weight of over 30,000 , is formed simply by the polymerisation of glucose molecules. The ordinary fats, stearin and palmitin, consist of fatty acids with long straight chains of $\mathrm{CH}_{2}$ groups combined with the glyceryl radical. Their molecular weight is very large, but their molecules are simple in structure. When, however, we break up a protein molecule we meet with a great number of subsidiary groups, the presence of which is essential to the making of a nutritive protein.

Owing to this complexity of structure it is not easy to give a simple definition in chemical terms of what we mean by the term 'protein.' It is necessary rather to describe certain of the qualities presented by this group, the possession of which we regard as essential to the conception of a protein.

Elementary Composition. All proteins contain oxygen, hydrogen, nitrogen, carbon, and sulphur. The proportion of these elements in the various proteins may be represented as follows :

C $50 \cdot 6-54 \cdot 5$ per cent.

H $6 \cdot 5-7 \cdot 3,,$,

N $15 \cdot 0-17 \cdot 6$, , ,

S $0 \cdot 3-2 \cdot 2, "$,

$021 \cdot 5-23 \cdot 5, "$,

Nearly all the proteins contain a small trace of phosphorus varying from 
0.4 to 0.8 per cent. It is doubtful, however, how far this phosphorus forms an integral part of the protein molecule.

Physical Characters. The proteins are amorphous indiffusible substances belonging to the class of bodies known as colloids. Most of them are soluble either in water, weak salt solutions, or in dilute acids or alkalies. They are inert bodies and tasteless. Although they form compounds with various metallic salts, acids, or alkalies, these compounds are but ill defined, and the relative proportions of the ingredients vary according to the conditions under which the compound was formed. As is the case with most colloids when in solution or pseudo-solution, they can be brought into an insoluble form by various simple agencies, such as shaking, change of temperature, alteration of reaction, or addition of neutral salts. Coagulation by heat forms a distinguishing feature of a number of members of this class, which are therefore spoken of as 'coagulable proteins.' For instance, white of egg is a solution of different proteins. On diluting it with weak salt solution no precipitation takes place. If, however, the solution be heated to about $80^{\circ} \mathrm{C}$ : a precipitate of coagulated protein is formed. If a strong solution be boiled the whole fluid sets to a solid white mass (hydrogel). This change is irreversible, i.e. it is not possible by lowering the temperature to bring the white of egg again into solution, and many. properties of the protein have been changed in the act of coagulation. With certain proteins and their allies the coagulation on change of temperature is a reversible process. Thus an alkaline solution of caseinogen, the chief protein of milk, if treated with a little calcium chloride and heated, undergoes coagulation and sets into a jelly, but on cooling the mixture the coagulum once more enters into solution. Ordinary gelatin, which is closely allied to the proteins, with water forms a solid jelly below $20^{\circ} \mathrm{C}$., and a fluid solution above this temperature.

If a protein be heated in a current of air or oxygen it undergoes combustion. In all cases a certain amount of incombustible material is left, consisting of inorganic salts which were closely attached to the protein molecule. If a solution of protein be subjected to long-continued dialysis, the proportion of ash may be diminished very largely, but in no case has any experimenter succeeded in obtaining a preparation of protein absolutely ash-free. On this account it.has been thought that the salts of the ash must be in chemical combination with the protein; but having regard.to the physical character of colloidal solutions, which we shall study in the next chapter, and the power of adsolption of substances possessed by such solutions, there is no need to regard these salts as essential constituents of the protein.

Crystallisation of Proteins. Although the indiffusibility of protein solutions differentiates them from the crystalloid substances such as sugar or sodium chloride, under certain conditions it is possible to obtain crystals consisting, largely at any rate, of proteins. Thus in the seeds of certain plants, e.g. hemp seeds, Brazil nut, pumpkin and castor-oil seeds, the so-called aleurone crystals may be seen under the microscope enclosed in the protoplasm of the cells. These crystals consist of proteins belonging to the class of 
globulins. By chemical means they can be separated from the surrounding tissues and, after washing, dissolved in a solution of magnesia. Drechsel showed that on dialysing such a solution against alcohol, the fluid undergoes gradual concentration, and crystalline granules of the magnesia compound of the protein separate out. These crystals contain 1.4 p.c. MgO. A better method of obtaining such crystals has been devised by Osborne. The ground seeds are extracted with 10 per cent. sodium chloride solution, and filtered. The filtrate is diluted with water heated to $50^{\circ}$ or $60^{\circ} \mathrm{C}$. until a slight turbidity forms. After warming the diluted solution until this turbidity disappears, and then allowing it to cool slowly, the protein separates in well-developed crystals. It is possible also to obtain crystals of animal proteins. Hæmoglobin, the oxygen-carrying protein of the red blood corpuscles, can be made to crystallise with extreme ease. With some animals, such as the rat, it is only necessary to bring the hæmoglobin into solution, by the addition of a little distilled water and ether to the blood, to cause the crystallisation of the liberated hæmoglobin.

Egg albumin and serum albumin may also be crystallised with ease by a method devised by Hofmeister and improved by Hopkins. If, for instance, we wish to crystallise egg albumin, white of eggs is treated with an equal bulk of saturated solution of ammonium sulphate in order to precipitate the globulin. It is then filtered, and the filtrate is treated with saturated ammonium solution until a slight permanent precipitate is produced. This precipitate is then just redissolved by the cautious addition of water, and dilute acetic acid (10 per cent.) is added drop by drop until a slight precipitate is produced. The flask is now corked and allowed to stand for twenty-four hours, when the precipitate, which will have increased in quantity, will be found to consist entirely of acicular crystals. A similar method may be used for serum albumin. In each case the crystals contain a considerable proportion of ammonium sulphate. This may be replaced by sodium chloride by washing the crystals with a saturated solution of this salt. By absolute alcohol the crystals may be coagulated and may be then washed practically free from salt, but it is not possible to obtain crystals of coagulable protein free from the presence of some salt.

Although by repeated crystallisation of egg albumin a product may be obtained which is absolutely constant in both its physical and chemical characters, we cannot ascribe to crystallisation the same importance in securing purity and homogeneity of the substance that we can when we are dealing with inorganic salts. This is due to the fact that these crystals take up other colloids with great ease. When hæmoglobin, for instance, is crystallised from blood, the first crop of crystals, although thoroughly washed from their mother liquor, always contain a considerable proportion of serum albumin. Indeed, the presence of colloidal material seems to render the production of the so-called mixed crystals much more easy. Thus Schultz has shown that in urine mixed inorganic crystals can be obtained. Human urine is allowed to stand twenty-four to forty-eight hours with dicalcium phosphate and then filtered. On allowing the filtrate to evaporate slowly, 
a crystalline precipitate is produced consisting of whetstone-shaped crystals which are doubly refracting. On treating these crystals with dilute acetic acid this acid extracts calcium phosphate from the crystals. The original shape of the crystals is, however, retained. The only difference under the microscope consists in the fact that they have now lost their doubly refracting power on polarised light. They consisted of a mixture of calcium sulphate and calcium phosphate, from which, on treatment with acid, only the calcium phosphate was dissolved out.

The Molecular Weight of Proteins. We may arrive at an approximate idea of the minimum size of the protein molecule in various ways, though in all cases our calculations are apt to be vitiated by the difficulty of obtaining a preparation which is homogeneous, i.e. chemically pure, and by the ease with which molecules of the size which we must assume for proteins form adsorption combinations in varying proportions with other substances. If we assume that each molecule of the respective protein contains only one atom of sulphur, we can calculate its molecular weight. It is evident that the protein which contains 1 per cent. of sulphur will have a molecular weight of 3200 . In this way the following molecular weights have been arrived at (Abderhalden) :

\begin{tabular}{|c|c|c|c|c|c|}
\hline & & \multicolumn{2}{|c|}{ Sulphur per cent. } & \multicolumn{2}{|c|}{ Molecular weight. } \\
\hline Edestin & . & . & $0 \cdot 87$ & .. & 3680 \\
\hline $\begin{array}{l}\text { Oxyhæmoglobin } \\
\text { (horse) }\end{array}$ & . & • & $0 \cdot 43$ & .. & 7440 \\
\hline $\begin{array}{l}\text { Serum albumin } \\
\text { (horse) }\end{array}$ & - & - & $1 \cdot 89$ & .. & 1700 \\
\hline Egg albumin & $\bullet$ & $\bullet$ & $1 \cdot 30$ & -. & 2460 \\
\hline Globulin & - & - & $1 \cdot 38$ & .. & 2320 \\
\hline
\end{tabular}

The greater part at any rate of the sulphur in the protein molecule occurs as a constituent of a substance, cystine, each molecule of which contains two atoms of sulphur. Each molecule of protein must also contain two atoms of sulphur, and we must regard double the molecular weight given in this Table as the minimum molecular weights of these various proteins. Some idea of the molecular complexity represented by these weights may be gained by writing out the empirical formulæ of the various proteins, e.g.,

\begin{tabular}{|c|c|c|}
\hline $\begin{array}{l}\text { Egg albumin . } \\
\text { Protein in hæmoglobin (from horse) } \\
\text { Protein in hæmoglobin (from dog) } \\
\text { Crystallised globulin (from pumpkin }\end{array}$ & $\begin{array}{ll}\cdot & \cdot \\
\cdot & \cdot \\
\cdot & \cdot \\
\text { seeds) }\end{array}$ & $\begin{array}{ll}\text { - } & \mathrm{C}_{204} \mathrm{H}_{322} \mathrm{~N}_{52} \mathrm{O}_{66} \mathrm{~S}_{2} \\
\cdot & \mathrm{C}_{680} \mathrm{H}_{1098} \mathrm{~N}_{210} \mathrm{O}_{241} \mathrm{~S}_{2} \\
\text {. } & \mathrm{C}_{725} \mathrm{H}_{1171} \mathrm{~N}_{194} \mathrm{O}_{214} \mathrm{~S}_{2} \\
\text {. } & \mathrm{C}_{292} \mathrm{H}_{481} \mathrm{~N}_{20} \mathrm{O}_{83} \mathrm{~S}_{2}\end{array}$ \\
\hline
\end{tabular}

With some proteins we may make use of other elements to arrive at an idea of the approximate molecular weight. Thus, oxyhæmoglobin contains between 0.4 and 0.5 per cent. iron. If we assume that each molecule of oxyhæmoglobin contains one atom of iron, its molecular weight must be from 11,200 to 14,000 .

Attempts have been made to solve the same question by studying the compounds of proteins with inorganic salts or oxides. Thus, the crystals of globulin from pumpkin seeds prepared with magnesia contain $1 \cdot 4$ per cent. 
$\mathrm{MgO}$. Assuming that one molecule of protein has combined with one molecule $\mathrm{MgO}$, the molecular weight of the protein must be about 2800 .

(If $x$ be the molecular weight

$$
\begin{aligned}
\frac{x}{40} & =\frac{100-1 \cdot 4}{1 \cdot 4} \\
\therefore x & =2817)
\end{aligned}
$$

Harnack has shown that many proteins are precipitated from their solutions as copper compounds by the addition of copper sulphate. Harnack found that this precipitate of copper contained either 1.34-1.37 Cu. or $2 \cdot 48-2 \cdot 73$ per cent. $\mathrm{Cu}$. The smaller percentage would correspond to a molecular weight of 4700 , while the second number might be accounted for on the hypothesis that each molecule of protein was combined with two atoms of copper. Similar attempts have been made by determining the amount of acid or alkali necessary to keep certain types of protein in solution. We shall see later on, however, that the amounts vary largely with the physical condition and previous history of the colloidal substance. We are dealing here not with compounds in the strict chemical sense of the term, but with adsorption compounds, where the quantities taken up are determined not only by the chemical nature of the protein itself, but by the state of aggregation of its molecules. It is therefore impossible to lay any great stress on the determinations of the molecular weight which have been effected in this way.

Some clue to the size of the protein molecule is afforded by determinations of the osmotic pressure or molecular concentration of their solutions by physical methods. When we determine the freezing-point or boiling-point of protein solutions, the depression of freezing-point, or elevation of boilingpoint is so small that it falls within the limit of experimental error or is no greater than can be accounted for by the inorganic salts present in the solution. Since, however, colloidal membranes, such as films of gelatin or vegetable parchment, are impervious to proteins, we can directly determine the osmotic pressure of their solutions. In many cases no osmotic pressure whatever is found. In other cases, e.g. egg albumin, or serum, the colloidal constituents of these solutions are found to give an osmotic pressure of such a height that 1 per cent. protein corresponds to about $4 \mathrm{~mm}$. $\mathrm{Hg}$. pressure. Such an osmotic pressure would indicate a molecular weight for the serum proteins of about 30,000 . A determination of the osmotic pressure of hæmoglobin by Hüfner gave a molecular weight about 16,000 . These results, however, must be received with caution, since we are not justified in applying to these gigantic molecules data derived from a study of smaller molecules such as salt or sugar. Even if we accept these determinations of osmotic pressure as indicating the molecular weights I have just quoted, it is evident that a very slight degree of aggregation of the molecules into larger complexes will bring the osmotic pressure below the point at which it is measurable, and would transform the solution into a suspension of particles in which one could not expect to find any osmotic pressure whatsoever. 


\section{THE STRUCTURE OF THE PROTEIN MOLECULE}

We can arrive at some idea of the manner in which the protein molecule is built up only by breaking it down bit by bit, employing methods which, while resolving the large molecule into its proximate constituents, will not act too forcibly in changing the whole arrangements of these constituents. The relation of the starches or polysaccharides to the sugars was found by studying the hydrolysis of the former, and it is by the hydrolysis of the proteins that we have arrived at most of our present knowledge of their constitution. Contributory evidence may also be gained by the use of oxidising agents or by employing the refined methods of analysis possessed by certain living organisms-bacteria, by which means we can effect limited oxidations or reductions or can replace an $\mathrm{NH}_{2}$ group by $\mathrm{H}$, or a $\mathrm{COOH}$ group by $\mathrm{H}$.

ACID HYDROLYSIS OF PROTEINS. For this purpose rather stronger acids are used than for the hydrolysis of starch. The protein is heated for twenty-four hours in a flask fitted with a reflux condenser either with concentrated hydrochloric acid or with a 25 per cent. sulphuric acid. Hydrochloric acid was first made use of by Hlasiwetz and Habermann, who added a certain amount of stannous chloride to the mixture in order to prevent any oxidation taking place. We obtain in this way an acid fluid containing an extremely complex mixture of various substances, all of which belong to the class of amino-acids, and must be regarded as the proximate constituents of the protein molecule.

A similar hydrolytic change may be effected by the use of digestive ferments obtained either from the alimentary canal of higher vertebrates or from certain plants. Thus we may use pepsin, the active constituent of the gastric juice, trypsin, the proteolytic ferment secreted by the pancreas, papaine, or other vegetable ferments obtained from papaya, from pineapple juice, and so on. These ferments are all milder in their action than the strong acids. Pepsin, for instance, only effects a partial decomposition of the protein molecule. Its action results in the formation of substances which still present all the protein reactions and are classified as hydrated proteins or as proteoses and peptones. Trypsin carries the protein a stage further and gives a mixture of amino-acids. Certain groups, however, of the protein molecule present a considerable resistance to the action of trypsin, so that when its action is complete these groups are still found not yet broken down into their constituent amino-acids.

The putrefactive processes determined by the process of bacteria in solutions of proteins are somewhat too complicated in their results to throw much illumination on the structure of the protein molecule itself. This method is, however, of extreme value when it is applied to isolated constituents of the proteins. Under the action of these bacteria we may have a process of deamination which may be accompanied by simple hydrolysis or by reduction. In the former case an amino-acid may be converted into an oxyacid, in the latter case into a fatty acid. 
Thus tyrosine under the action of bacteria of putrefaction may split up into ammonia and oxyphenyl propionic acid.

$$
\begin{gathered}
\mathrm{OH} \cdot \mathrm{C}_{6} \mathrm{H}_{4} \cdot \mathrm{CH}_{2} \cdot \mathrm{CHNH}_{2} \cdot \mathrm{COOH}+\mathrm{H}_{2}= \\
\mathrm{HO} \cdot \mathrm{C}_{6} \mathrm{H}_{4} \cdot \mathrm{CH}_{2} \cdot \mathrm{CH}_{2} \cdot \mathrm{COOH}+\mathrm{NH}_{3}
\end{gathered}
$$

Under the action of yeasts an amine may become an alcohol.

$$
\underset{\text { (amylamine) }}{\mathrm{C}_{5} \mathrm{H}_{11} \cdot \mathrm{NH}_{2}}+\mathrm{H}_{2} \mathrm{O}=\underset{\text { (amylalcohol) }}{\mathrm{C}_{5} \mathrm{H}_{11} \cdot \mathrm{OH}}+\mathrm{NH}_{3}
$$

On the other hand, the effect of the bacteria may be to split off carbon dioxide from the amino-acids. Thus, the diamino-acid, lysine,

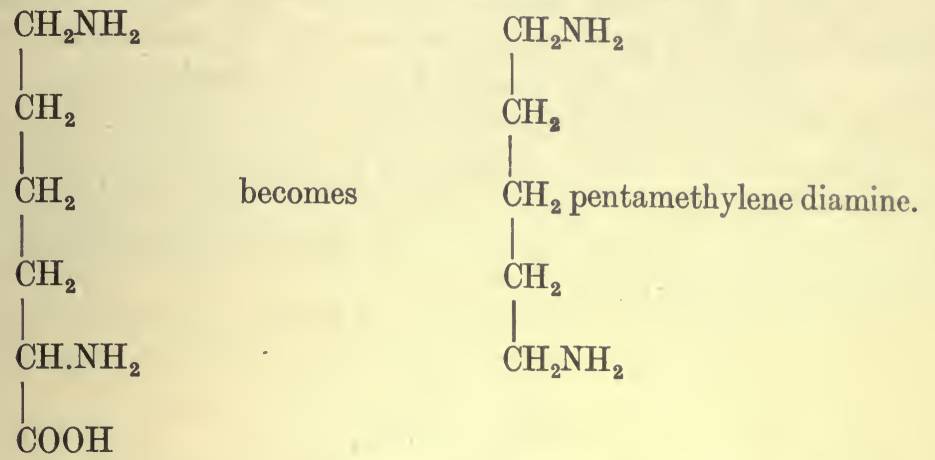

Tyrosine becomes p. oxyphenylethylamine, a substance having marked physiological effects, and an important constituent of ergot. Phenylalanine $\mathrm{C}_{6} \mathrm{H}_{5} \cdot \mathrm{CH}_{2} \cdot \mathrm{CH} \cdot \mathrm{NH}_{2} \cdot \mathrm{COOH}$, becomes phenylethylamine $\mathrm{C}_{6} \mathrm{H}_{5} \cdot \mathrm{CH}_{2} \cdot \mathrm{CH}_{2} \cdot \mathrm{NH}_{2}$. These reactions are therefore of value in determining the exact grouping of the atoms in the more complex of the proximate constituents of the proteins.

Since all the known disintegration products of the proteins belong to the class of amino-acids, it may be of value to point out some of the distinguishing features of this class of bodies.

PROPERTIES OF AMINO-ACIDS. An amino-acid is derived from an organic acid by the replacing of one atom of hydrogen by the amino group $\mathrm{NH}_{2}$. Thus from the acids,

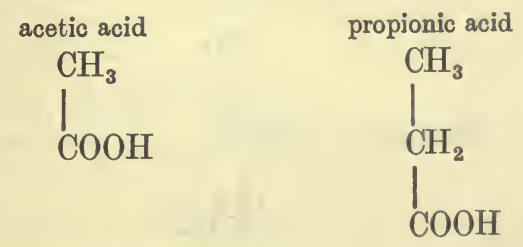

we may obtain the mono-amino-acids,

amino-acetic acid

$\mathrm{CH}_{2} \mathrm{NH}_{2}$

$\mathrm{COOH}$ alanine or a-amino-propionic acid

$\mathrm{CH}_{3}$

$\left.\right|_{\mathrm{CH} . \mathrm{NH}_{2}}$

$\mathrm{COOH}$ 
It will be noticed that in the fatty acids with more than two atoms of carbon the position of the $\mathrm{NH}_{2}$ group may be varied. Thus, instead of alanine we may have another amino-propionic acid, namely:

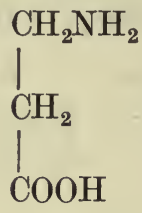

This acid would be spoken of as $\beta$-amino-propionic acid, alanine being $\alpha$-amino-propionic acid. This nomenclature is always used to distinguish the position of the $\mathrm{NH}_{2}$ group, so that we may have mono-amino-acids $\alpha$, $\beta, \gamma, \delta, \epsilon \ldots$ and so on. Practically all the amino-acids which occur as constituents of the protoplasmic molecule belong to the $\alpha$ group.

On inspection of the formula of glycine it is evident that only one isomer of this body is possible. In alanine, however, the carbon atom to which $\mathrm{NH}_{2}$ is attached, is asymmetric, since its four combining affinities are each attached to different groups. Thus :<smiles>CC(C)N</smiles>

In this case, therefore, there is a possibility of stereoisomerism, and alanine must have an influence on polarised light. If the compound

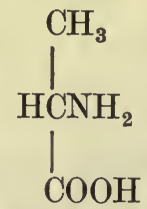

is dextro-rotatory, then its stereoisomer<smiles>C[CH]C(=O)O</smiles>

will be lævo-rotatory, and it will be possible to obtain a racemic modification without any influence on polarised light by mixing equal molecules of these two isomeric forms. All the amino-acids derived from proteins are optically active, whereas those obtained by synthesis are inactive, and special means have to be devised in order to obtain from the artificially formed racemic amino-acid either the $d$ - or $l$-amino-acid.

If more than one hydrogen atom in an organic acid be replaced by $\mathrm{NH}_{2}$ 
we obtain diamino- and triamino-acids. Thus, ornithine, obtained by the splitting up of arginine, one of the commonest disintegration products of protein, is $\alpha$ - $\delta$-diamino-valerianic acid.

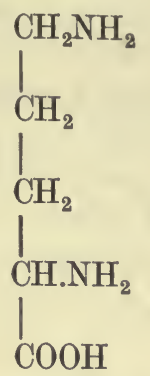

The presence in the amino-acids of the basic radical $\mathrm{NH}_{2}$ and of the acid group $\mathrm{COOH}$ lends to these bodies a double character. In themselves devoid of strong chemical qualities, possessing neither acid nor alkaline reaction, they are able in the presence of strong acids or bases to act either as base or acid. When in solution by themselves it is possible that there is an actual closing of the ring by a soluble union between the $\mathrm{NH}_{2}$ group and the $\mathrm{COOH}$ group, so that, e.g. the formula of glycine may be :<smiles>O=C1CNO1</smiles>

When such a neutral compound is treated with acid this bond is loosed and we have the salt of the amino-acid. Thus, with hydrochloric acid, glycine forms glycine hydrochlorate :

$$
\prod_{\mathrm{COOH}}^{\mathrm{CH}_{2} \mathrm{NH}_{2} \mathrm{HCl}}
$$

a salt which still possesses an acid group and which is therefore capable of combining with ethyl to form the hydrochlorate of the ester of the amino-acid. Thus :

$$
\left.\right|_{\mathrm{COOC}_{2} \mathrm{H}_{5}} ^{\mathrm{CH}_{2} \cdot \mathrm{NH}_{2} \mathrm{HCl}}
$$

With bases the amino-acids form salt-like compounds such as potassium amino-acetate :

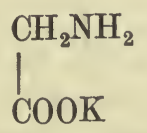

Amino-acids also combine with one another. This power of combination much increases the difficulty of separating the constituents from a mixture of amino-acids. Amino-acids, which singly are extremely insoluble, are readily soluble when in the presence of other amino-acids.

On account of the dual nature of the amino-acid molecule, these sub- 
stances act as feeble conductors of the electric current, i.e. as electrolytes. The charge carried by an amino-acid and its ionisation depends upon the conditions in which it is placed. Since it may act either as the cation or the anion, it is spoken of as an amphoteric electrolyte.

One reaction of the amino-acids is of special interest in connection with the respiratory functions of the body, namely, the formation of carbaminoacids. If a stream of carbon dioxide be passed into a mixture of an aminoacid, e.g. glycine, with lime, the carbon dioxide is taken up. On filtering the mixture a clear liquid passes through which gradually in course of time deposits a precipitate of calcium carbonate. The filtrate first obtained contains a compound of calcium, calcium glycine carbonate. The formula is as follows :

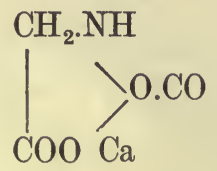

METHODS OF SEPARATING AMINO-ACIDS. By the hydrolysis of protein by means of acid or of trypsin, we obtain a complex mixture of amino-acids. From this mixture certain amino-acids are separated with ease. Thus, tyrosine, which is extremely insoluble, crystallises out on concentrating the fluid, and further concentration leads to the separation of leucine. The other acids, which keep each other mutually in solution, are however very difficult to isolate. We owe to Fischer the first general method for their separation. We may take one experiment as an example.

Five hundred grammes of casein are heated for some hours under a reflux condenser with $1 \frac{1}{2}$ litres of strong hydrochloric acid. The liquid is then saturated with gaseous hydrochloric acid and allowed to stand for three days in the ice-chest. Crystals of hydrochlorate of glutamic acid separate out. The filtrate from these crystals is evaporated at $40^{\circ} \mathrm{C}$. under diminished pressure to a syrupy consistence, and is then dissolved in $1 \frac{1}{2}$ litres of absolute alcohol. Hydrochloric acid is then passed into the solution to complete saturation, the mixture being warmed for a short time on the water bath, and the mixture is once more evaporated to a syrupy consistence. By this treatment all the amino-acids have been converted into the hydrochlorates of their esters, e.g. :
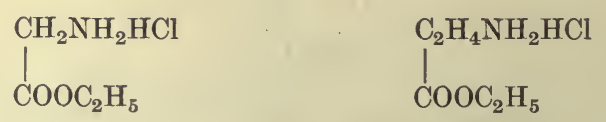

\&c.

From the hydrochlorates the esters are set free by the addition of potassium carbonate, the mixture being cooled in a freezing mixture. By this means the esters of aspartic and glutamic acids are separated and are extracted by shaking with ether. The remaining esters are then liberated by the addition of 33 per cent. caustic soda together with potassium carbonate, and are again extracted by ether. The combined ethereal solutions are dried by standing over fused sulphate of soda and then evaporated, when a residue containing the free esters is obtained. These esters are then separated by fractional distillation under a very low pressure obtained by means of the Fleuss pump, the second receiver of the apparatus baing cooled in liquid air. The various fractions of aminoesters obtained in this way are hydrolysed-the lower fractions by boiling for some hours with water, the higher fractions by boiling with baryta. The 
acids obtained by the hydrolysis can then be further purified by means of fractional crystallisation.

\section{THE DISINTEGRATION PRODUCTS OF THE PROTEINS}

By the methods just described the following substances have been isolated from proteins :

\section{A. FATTY SERIES}

\section{(1) Mono-amino-acids (Monobasic)}

GLYCINE or GLYCOCOLL. This, the simplest member of the group, is amino-acetic acid :

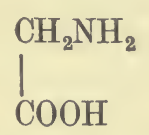

It occurs in considerable quantities among the disintegration products of gelatin and to a slight extent among those derived from certain of the proteins. Like the other $\alpha$-amino-acids, it has a sweetish taste, whence its name was derived ( $\gamma \lambda \nu \kappa o \sigma=$ sweet, $\kappa \circ \lambda \lambda \eta=$ glue).

ALANINE is $\alpha$-amino-propionic acid:

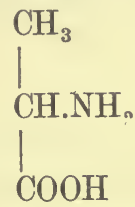

It is optically active, the alanine derived from proteins being dextrorotatory.

Closely allied to alanine is the amino-acid SERINE, which was first obtained by the hydrolysis of silk and has since been found as a constituent of a large number of proteins. Its formula is :

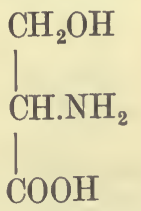

i.e. it is amino-oxypropionic acid. Its special interest lies in the fact that it was one of the first of the amino-oxyacids to be isolated, and it is possible in these acids that we must seek the intermediate stages between carbo. hydrates and proteins.

AMINO-VALERIANIC ACID has the formula

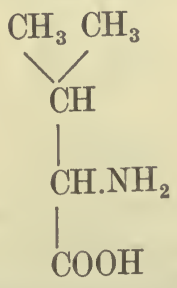

It occurs only in small quantities in the protein molecule. 
LEUCINE, one of the oldest known members of the group of amino-acids, is obtained in large quantities from the disintegration of nearly all the animal proteins, of which in some cases it may form as much as 20 per cent. It has the formula

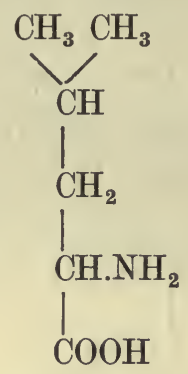

i.e. it is amino-isobutyl acetic acid. On evaporating a tryptic digest of protein, impure leucine crystallises out in the form of imperfect crystals, the so-called 'leucine cones.'

Lately another isomer of leucine has been discovered, namely, $a$-amino-methyl ethyl propionic acid. This is called isoleucine.

\section{(2) Mono-amino Derivatives of Dibasic Acids}

Of these two are known, namely, aspartic and glutamic acids.

ASPARTIC ACID is $\alpha$-amino-succinic acid :

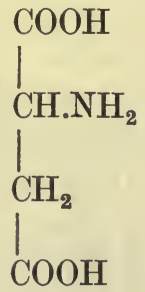

and glutamic acid is the next homologue, namely, $\alpha$-amino-glutaric acid :

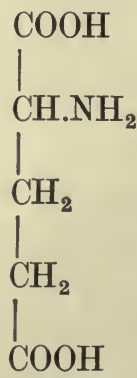

Owing to the possession of two carboxyl groups these amino-acids have a much more pronounced acid character than is the case with the other members of the group which we have been studying. 
Aspartic acid was first found in the shoots of asparagus in the form of the amide, asparagine :

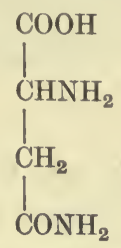

This substance is very widely distributed throughout the vegetable kingdom and is present in seedlings in very large quantities, as much as 25 per cent. of the dried weight. In plants it apparently serves either as a reserve material or as the form in which the greater part of the nitrogen is conveyed from the reserve organs to be built up into the protoplasm of the growing parts of the plant.

\section{(3) Diamino-acids}

Of these two are known, namely, lysine and ornithine. Owing to the presence of two $\mathrm{NH}_{2}$ groups in their molecule, they possess marked basic characters, and are precipitated from the acid solution obtained by the hydrolysis of proteins on adding phosphotungstic acid. Since lysine, arginine, and histidine (another amino-acid which will be described later) all contain six carbon atoms in their molecule, these three bodies were classed together by Kossel as the 'hexone' bases. Apart, however, from their high content in nitrogen, the chemical resemblance between these bodies is no closer than between them and the other members of the amino-acid series.

Another body isolated by Fischer in small quantities is supposed to belong to this class and to have the composition diamino-trioxydodecoic acid.

LYSINE $\mathrm{C}_{6} \mathrm{H}_{14} \mathrm{~N}_{2} \mathrm{O}_{2}$ is $\alpha$ - - -diamino-caproic acid having the formula

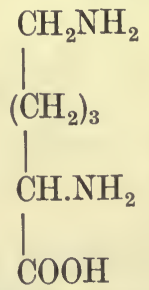

ARGININE, which was first discovered in plants (the cotyledons of lupins), is not a simple amino-acid, but a compound of an amino-acid with guanidin. If boiled with baryta water it splits up into urea and a substance reacting as a base which was called ornithine.*

ORNITHINE, diamino-valerianic acid, has the formula

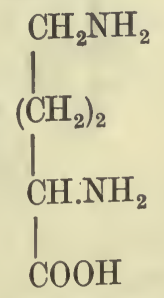

* Ornithine had bzen previously discovered in the urine of fowls, after the administration of benzoic acid, in the form of an acid known as ornithuric acid. 
The constitution of arginine is analogous to that of creatine, one of the most abundant nitrogenous extractives of muscle, which has the formula

$$
\mathrm{HN}=\underset{\mathrm{H}_{2} \mathrm{~N}}{\mathrm{C}}-\mathrm{N}\left(\mathrm{CH}_{3}\right) \mathrm{CH}_{2} \mathrm{COOH}
$$

It is methyl guanidine acetic acid. On boiling creatine with baryta water it takes up a molecule of water and splits in the situation of the dotted line in the formula, giving

$$
{ }_{\mathrm{H}_{2} \mathrm{~N}}^{\mathrm{H}_{2} \mathrm{~N}} \mathrm{CO} \text { (urea) and } \mathrm{NH}\left(\mathrm{CH}_{3}\right) \mathrm{CH}_{2} \mathrm{COOH} \text { (methyl glycine). }
$$

This later substance is known as sarcosine and is derived from glycine by the replacement of one atom of hydrogen by a methyl group $\mathrm{CH}_{3}$.

Arginine has a similar formula. On the left-hand side of the dotted line the formula would be identical with that of creatine. On the right-hand side the sarcosine group is replaced by a diamino-acid of the fatty series, diamino-valerianic acid or ornithine.

DIAMINO-TRIOXYDODECOIC acid is, as its name implies, a derivative of a twelve carbon acid. Its constitutional formula has not yet been made out.

B. AMINO-ACIDS CONTAINING AN AROMATIC NUCLEUS

The best known of these is TYROSINE, which has the formula

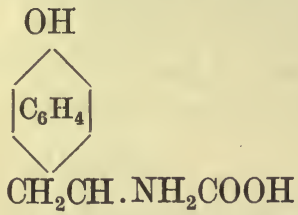

It is paraoxyphenyl $a$-alanine. It is one of the first of the amino-acids to be split off from the protein molecule under the influence of hydrolytic agents. Owing to its insolubility it rapidly separates out as bundles of fine needle-shaped crystals at the sides and bottom of the vessel.

When tyrosine is treated with an acid solution of mercuric nitrate containing a little nitrous acid, a precipitate is produced, and on boiling, the precipitate and the supernatant fluid assume a deep red colour. This reaction is given by all benzene derivatives in which one atom of hydrogen in the ring is replaced by one $\mathrm{OH}$ group. This

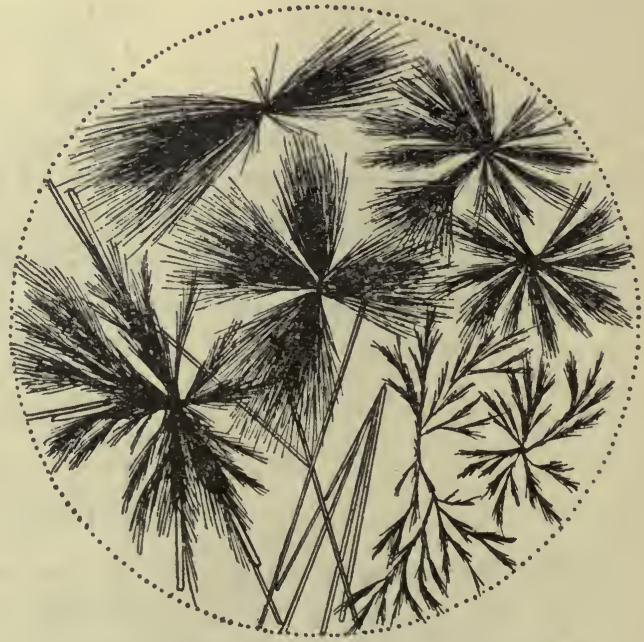

Fra. 18. Tyrosine crystals. (Plimmer.) 
is known as Hoffmann's test, but is identical with Millon's reaction, which is given by all proteins containing tyrosine in their molecules.

Closely allied to the foregoing compound is another aromatic amino-acid, namely, PHENYL $\alpha$-ALANINE :

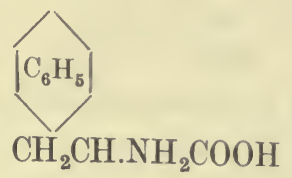

It is an almost constant constituent of proteins.

TRYPTOPHANE was known long before it had been isolated, owing to the fact that with bromine water it gives a rose-red colour. It had long been observed that this substance was to be obtained at a certain stage in the digestion of proteins by pancreatic juice, but nothing was known about its constitution until Hopkins succeeded in isolating it by precipitation with mercuric sulphate dissolved in 5 per cent. sulphuric acid. Cystine is also precipitated by this reagent, but comes down with a less concentration of the salt than trytophane, so that it is possible to separate the two substances by a species of fractional precipitation. Tryptophane can be isolated by decomposing the mercury salt with sulphuretted hydrogen, and is obtained in a crystallised form. On distillation it gives an abundant yield of indol and skatol, bodies also obtained during the putrefaction of proteins. Tryptophane itself is indol amino-propionic acid :

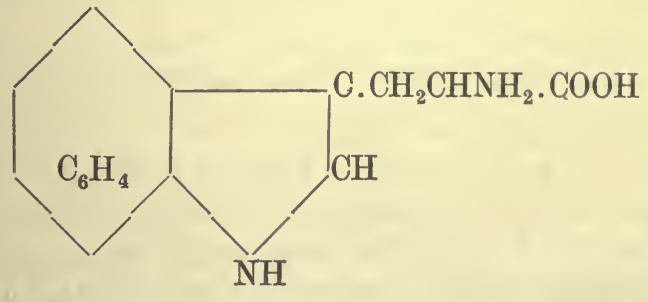

\section{AMINO-ACIDS OF HETEROCYCLIC COMPOUNDS}

Three of the disintegration products of proteins can be grouped in this class. Two of them contain the pyrrol ring, namely, proline and oxyproline.

PROLINE, which was first isolated by Fischer, is $\alpha$-pyrrolidin carboxylic acid and has the formula

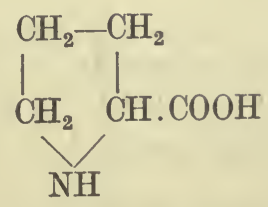

OXYPROLINE is the oxy-derivative of this body and has the formula $\mathrm{C}_{5} \mathrm{H}_{9} \mathrm{NO}_{3}$, the exact position of the oxy-group having not yet been determined. Doubts have been expressed whether the pyrrol group is present as such in the protein molecule, or whether proline, for example, is 
not formed by the closing of an open chain of a compound belonging to the amino-acids in the fatty series. Thus from an oxy-amino-valerianic acid $\mathrm{CH}_{2} \mathrm{OH} \cdot \mathrm{CH}_{2} \cdot \mathrm{CH}_{2} \cdot \mathrm{CH} \cdot \mathrm{NH}_{2} \cdot \mathrm{COOH}$ we can by dehydration make the compound $\mathrm{CH}_{2} \mathrm{CH}_{2} \cdot \mathrm{CH}_{2} \cdot \mathrm{CH} \cdot \mathrm{COOH}$, the formula of which will be seen

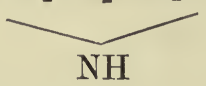

to be identical with that given for proline.

-The third member of this group contains the iminazol ring:<smiles>c1ncncn1</smiles>

and is known as HISTIDINE. Its structural formula is as follows :

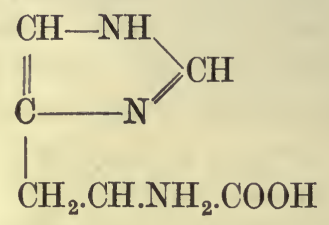

i.e. it is iminazol $\alpha$-amino-propionic acid or iminazol alanine. Since it occurs in the phosphotungstic precipitate from the products of acid disintegration of proteins and contains six carbon atoms, it was formerly classified with lysine and arginine as a hexone base.

\section{SULPHUR-CONTAINING AMINO-ACIDS}

Sulphur forms an integral part of the molecule of all classes of proteins except protamines. In some substances allied to proteins, such as keratin, it may occur to the extent of 3 per cent. On boiling proteins with caustic potash or soda, a portion of the sulphur is split off to form a sulphide, which gives a black precipitate on addition of copper salts. On this account it was formerly thought that the sulphur must be present in two forms, the oxidised and the unoxidised, in the protein molecule. Recent investigation has shown, however, that practically the whole of the sulphur is present in the form of CYSTINE, and that this body on boiling with alkaline solutions gives up only a little more than half its content in sulphur.

This substance, which has been known for many years as the chief constituent of a rare form of urinary calculus and as occurring in the urine in certain cases of disordered metabolism, is again a derivative of the threecarbon propionic acid. On reduction it gives a body known as cysteine, which is $\alpha$-amino-thiopropionic acid.

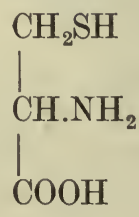


Cystine itself is compounded of two cysteine molecules joined together by their sulphur atoms and has the formula

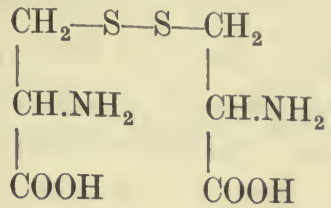

\section{E. OTHER CONSTITUENTS OF THE PROTEIN MOLECULE}

When we add together the total amino-acids obtainable by the acid disintegration of any given protein, a considerable proportion of the original protein remains unaccounted for. This remainder must have a greater content in hydrogen and oxygen than the amino-acids enumerated above, and it has been suggested that among the missing unascertained constituents of proteins may be oxyamino-acids, of which serine would form one of the lowest members. The isolation of such substances would present considerable interest, in that it would supply the intermediate stages between the constituent groups of the protein molecule and the carbohydrates, the first product of assimilation by living organisms. Only one such intermediate body has so far been isolated, namely, glucosamine, an amino-derivative of glucose. It was first shown by Pavy that from the products of disintegration of a protein such as egg-white it was possible to obtain a reducing substance and to isolate an osazone resembling in its characters those derived from the sugars. Since then various observers have shown that this reducing substance is most probably glucosamine :

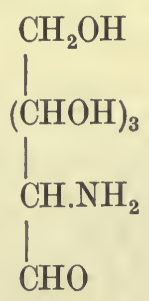

Although this substance may be obtained from crystallised egg albumin or crystallised serum albumin, authorities are not yet convinced that it forms an integral part of these proteins. Both egg-white and serum contain proteins belonging to the class of mucins, ovomucoid and serum mucoid, each of which yields on acid hydrolysis from 16 to 30 per cent. glucosamine. Since various observers have obtained results varying from 1 to 16 per cent. glucosamine for crystallised egg albumin, it seems possible that in every case the crystals carried down with them some of the carbohydrate-rich mucoid, and that the varying results were due to the different amounts of mucoid present in the crystals. By our ordinary methods it is impossible to prepare a specimen of either egg albumin or serum albumin which is entirely free from this amino-derivative of carbohydrate.

Connected with this group of proteins may be reckoned the diamino- 
trioxydodecoic acid already mentioned as occurring among the disintegration products of proteins.

\section{THE BUILDING UP OF THE PROTEIN MOLECULE}

By simple hydrolysis the protein molecule may be broken down into a large number of amino-acids. Analyses of various proteins show that these amino-acids are present in different proportions in the individual proteins, so that in many cases a large number of identical amino-acid groups must be present in the protein molecule with smaller numbers of other groups. In endeavouring to form an idea of the manner in which the amino-acids can be linked together into one gigantic molecule, Hofmeister first put forward the idea that the linkage follows the general formula :

$$
\text { or } \quad-\mathrm{CH}_{2}-\mathrm{NH}-\mathrm{CO}-
$$

This theory of the constitution of proteins was based on the fact that a similar grouping was known to occur in leucinimide, obtained by the condensation of two molecules of leucine,

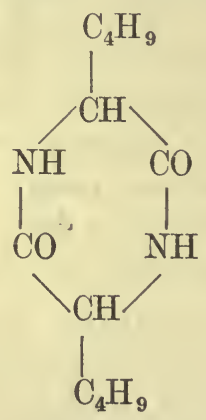

and also by the fact that only a small proportion of the $\mathrm{NH}_{2}$ groups present in the separated amino-acids exist free in the protein molecule. By the action of nitrous acid the terminal $\mathrm{NH}_{2}$ groups are split off and replaced by $\mathrm{OH}$. When proteins are treated with nitrous acid only a small proportion of the total nitrogen is split off in this way. The linking of the amino groups must therefore take place by means of the nitrogen, i.e. by $\mathrm{NH}$ groups. Synthetic experiments have fully confirmed this hypothesis. In 1883 Curtius obtained a substance giving the biuret reaction, the so-called 'biuret base,' by the spontaneous polymerisation of glycocoll ester. This base has been shown by recent researches to consist of four glycine molecules arranged together in an open chain. The clue to the structure of this base was given by Fischer, who has devised a number of ingenious methods for combining together amino-acids of any character and in any number. Thus from two molecules of glycine we may obtain the compound glycyl glycine, as follows :

$$
\begin{gathered}
\mathrm{NH}_{2} \cdot \mathrm{CH}_{2} \cdot \mathrm{COOH}+\mathrm{HNH} \cdot \mathrm{CH}_{2} \cdot \mathrm{COOH}-\mathrm{H}_{2} \mathrm{O}= \\
\mathrm{NH}_{2} \cdot \mathrm{CH}_{2} \cdot \mathrm{CO} \cdot \mathrm{NH} \cdot \mathrm{CH}_{2} \cdot \mathrm{COOH}
\end{gathered}
$$


This may be prepared in various ways. In one method glycine is converted into its ester $\mathrm{CH}_{2} \cdot \mathrm{NH}_{2} \cdot \mathrm{CO} \cdot \mathrm{OCH}_{3}$. In a watery solution this undergoes spontancous conversion into glycine anhydride which belongs to the class of bodies known as diketo.
piperazins, as follows:

$$
2 \mathrm{NH}_{2} \cdot \mathrm{CH}_{2} \mathrm{CO} \cdot 0 \mathrm{CH}_{3}=2 \mathrm{CH}_{3} \mathrm{OH}+\mathrm{NH}\left\langle\stackrel{\mathrm{CO}-\mathrm{CH}_{2}}{\text { methyl alcohol }}\right\rangle_{\mathrm{NH}}
$$

On treating this with dilute alkali it takes up water, splitting in the situation of the dotted line and forming glycyl glycine, $\mathrm{NH}_{2} \mathrm{CH}_{2} \mathrm{CO} \cdot \mathrm{NH}$. $\mathrm{CH}_{2} \mathrm{COOH}$. More general methods have been devised by Fischer for the same purpose, depending
on the use of the halogen acyl chlorides.

Thus chloracetylchloride and alanine yield chloracetalanine:

$$
\begin{aligned}
& \mathrm{Cl} \cdot \mathrm{CH}_{2} \cdot \mathrm{COCl}+\mathrm{NH}_{2} \cdot \mathrm{CH}\left(\mathrm{CH}_{3}\right) \cdot \mathrm{COOH}= \\
& \mathrm{Cl} \cdot \mathrm{CH}_{2} \cdot \mathrm{CO}-\mathrm{NH} \cdot \mathrm{CH}\left(\mathrm{CH}_{3}\right) \mathrm{COOH}+\mathrm{HCl} \text {. }
\end{aligned}
$$

By the subsequent action of ammonia, the halogen group is replaced by the amino group, and a dipeptide results :

$$
\begin{aligned}
& \mathrm{Cl} . \mathrm{CH}_{2} \cdot \mathrm{CO}-\mathrm{NH} \cdot \mathrm{CH}\left(\mathrm{CH}_{3}\right) \mathrm{COOH}+2 \mathrm{NH}_{3}= \\
& \mathrm{NH}_{2} \cdot \mathrm{CH}_{2} \cdot \mathrm{CO}-\mathrm{NH} \cdot \mathrm{CH}\left(\mathrm{CH}_{3}\right) \mathrm{COOH}+\mathrm{NH}_{4} \mathrm{Cl} \text {. }
\end{aligned}
$$

Different halogen acyl chlorides are used for introducing the various amino-acid radicals, e.g. chloracetylchloride for glycyl, $\boldsymbol{a}$-bromopropionylchloride for alanyl, \&c.

By various such methods Fischer has succeded in combining compounds containing as many as eighteen amino-acids, e.g. alanyl leucine, glycyl tyrosine, dialanyl cystine, dileucyl cystine, leucyl pentaglycyl glycine, and so on. The last named would be built up out of one molecule of leucine and six molecules of glycine. These compounds have been designated by Fischer as polypeptides, to signify their close connection with the peptones produced by the agency of digestive ferments on the proteins. He distinguishes di-, tri-, tetra-, \&c., peptides according to the number of individual amino-acids taking part in the formation of the compound. The polypeptides resemble in all respects the peptones. Most of them, even if derived from relatively insoluble amino-acids, are soluble in water, insoluble in absolute alcohol. They dissolve in mineral acids and in alkalies with the formation of salts, thus resembling in their behaviour the amino-acids. They have a bitter taste, although the amino-acids from which they are formed have a slightly sweet taste, in this way again resembling the natural peptones. The higher members of the series give certain reactions, such as the biuret reaction, which are regarded as characteristic of peptones, and like the latter are precipitated by phosphotungstic acid. Their behaviour with trypsin depends on the optical behaviour of the amino-acids from which they are formed. If synthetised from the amino-acids identical with those occurring in the disintegration of natural proteins, they resemble the peptones in undergoing hydrolysis and disintegration into their constituent amino-acids. Trypsin, however, has no influence on polypeptides compounded of the inactive amino-acids, or of the amino-acids which are the optical opposites of those which form the constituents of normal proteins. Though most of the amino-acids which occur naturally are lævo-rotatory, the polypeptides formed from them are generally strongly dextro-rotatory. 
Thus in the building up of the protein molecule there is an almost indefinite coupling up of numerous amino-acid groups, the connecting element in each case being the nitrogen. Of the two or more optical isomers possible of each amino-acid containing more than two carbon atoms, only one is made use of for this purpose. A still further flexibility in its reactions to its environment is conferred on the protein molecule by changes occurring with great readiness in the intra-molecular grouping of its constituent atoms. Thus, if we take the simplest member of the class of polypeptides, glycyl glycine, four structural formulæ are possible, namely :

$$
\begin{aligned}
& \mathrm{NH}_{2} \mathrm{CH}_{2} \mathrm{CO}-\mathrm{NH} \cdot \mathrm{CH}_{2} \cdot \mathrm{COOH} \\
& \mathrm{NH} \cdot \mathrm{CH}_{2} \cdot \mathrm{CO} \\
& \mathrm{CO} \cdot \mathrm{CH}_{2} \cdot \mathrm{NH}_{3} \\
& \mathrm{NH}_{2} \cdot \mathrm{CH}_{2} \cdot \mathrm{C}(\mathrm{OH})=\mathrm{N} \cdot \mathrm{CH}_{2} \cdot \mathrm{COOH} \\
& \mathrm{N} \cdot \mathrm{CH}_{2} \cdot \mathrm{CO} \\
& \| \\
& \mathrm{C}(\mathrm{OH}) \mathrm{CH}_{2} \cdot \mathrm{NH}_{3}
\end{aligned}
$$

(2) and (4) being the intramolecular form of the formulæ (1) and (3). (3) and (4) are sometimes spoken of as the enolic form. If we consider that perhaps some hundred of the amino-acid groups may go to making up a single protein molecule, it is possible to form some conception of the enormous variability in reaction possible to such a compound.

\section{THE CONSTITUTION OF DIFFERENT PROTEINS}

All the proximate constituents of proteins, so far as we know, are aminoacids. Of these the following have been isolated, namely, glycine, alanine, amino-valerianic acid, leucine, isoleucine, proline, oxyproline, serine, phenyl alanine, glutamic acid, aspartic acid, tyrosine, tryptophane, cystine, lysine, histidine, arginine, and 'di-amino-trioxydodecoic' acid.

The question now arises whether all the different varieties of protein owe their peculiarities to the presence of different amino-acids or whether the greater number of the amino-acids above mentioned are present in all proteins, the differences between the latter being determined by differences in the arrangement and relative amounts of their proximate constituents. A large number of analyses of different proteins have been made by Abderhalden, Osborne, and others, utilising the methods for the isolation of amino-acids devised by Fischer. The constitution of some representative proteins as determined in this way are given in the Table opposite.

These results show that all the proteins contain a very considerable proportion of the total number of amino-acids which have as yet been isolated from acid digests of proteins. The differences in various proteins cannot therefore be determined by qualitative differences in their constituent molecules, but must depend on the relative amounts of the amino-acids which are present and on their arrangement in the whole molecule. As regards relative amounts of amino-acids we find very striking differences. Thus, 


\begin{tabular}{|c|c|c|c|c|c|c|c|c|c|c|c|}
\hline & & 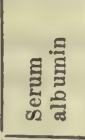 & 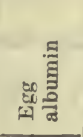 & 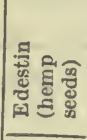 & : & 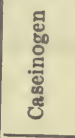 & 흉 & हू हूँ & & $\begin{array}{l}\text { 몰 } \\
\text { 递 }\end{array}$ & 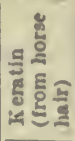 \\
\hline Glycine & & 0 & 0 & $3 \cdot 8$ & 0.9 & 0 & 0 & 0 & - & 16.5 & $4 \cdot 7$ \\
\hline Alanine . & & $2 \cdot 7$ & $8 \cdot 1$ & $3 \cdot 6$ & $2 \cdot 7$ & $1 \cdot 5$ & $4 \cdot 2$ & - & - & $\begin{array}{r}0.8 \\
0.8\end{array}$ & 1.5 \\
\hline $\begin{array}{l}\text { Serine } \\
\text { Amino - valeri }\end{array}$ & & 0.6 & - & 0.33 & 0.12 & 0.5 & $0 \cdot 6$ & $7 \cdot 8$ & - & 0.4 & $0 \cdot 6$ \\
\hline $\begin{array}{l}\text { Amino - valeri } \\
\text { anic acid }\end{array}$ & & 一 & - & present & 0.3 & $7 \cdot 2$ & - & $4 \cdot 3$ & - & 1.0 & 0.9 \\
\hline Leucine . & & $20 \cdot 0$ & $7 \cdot 1$ & 20.9 & $6 \cdot 0$ & $9 \cdot 35$ & $29 \cdot 0$ & 0 & - & $2 \cdot 1$ & $7 \cdot 1$ \\
\hline Proline . & & $1 \cdot 0$ & $2 \cdot 25$ & $1 \cdot 7$ & $2 \cdot 4$ & $6 \cdot 70$ & $2 \cdot 3$ & $11 \cdot 0$ & - & $5 \cdot 2$ & $3 \cdot 4$ \\
\hline Oxyproline & & - & 一 & $2 \cdot 0$ & - & $0 \cdot 23$ & $1 \cdot 0$ & 一 & - & $3 \cdot 0$ & - \\
\hline Glutamic acid & & $7 \cdot 7$ & $8 \cdot 0$ & $6 \cdot 3$ & 36.5 & $15 \cdot 55$ & $1 \cdot 7$ & - & - & 0.88 & $3 \cdot 7$ \\
\hline Aspartic acid & & $3 \cdot 1$ & $1 \cdot 5$ & $4 \cdot 5$ & $1 \cdot 3$ & $1 \cdot 39$ & $4 \cdot 4$ & - & - & 0.56 & $0 \cdot 3$ \\
\hline Phenylalanine & • & $3 \cdot 1$ & $4 \cdot 4$ & $2 \cdot 4$ & $2 \cdot 6$ & $3 \cdot 2$ & $4 \cdot 2$ & - & - & $0 \cdot 4$ & 0 \\
\hline Tyrosine & & $2 \cdot 1$ & $1 \cdot 1$ & $2 \cdot 1$ & $2 \cdot 4$ & $4: 5$ & $1 \cdot 5$ & - & - & 0 & $3 \cdot 2$ \\
\hline Tryptophane & & present & present & present & 1.0 & $1 \cdot 50$ & present & - & - & 0 & - \\
\hline Cystine & & $2 \cdot 3$ & 0.2 & $0 \cdot 25$ & $0 \cdot 45$ & $?$ & 0.3 & - & - & 一 & ov. $10^{3}$ \\
\hline Lysine & • & - & $2 \cdot 15$ & $1 \cdot 0$ & 0 & $5 \cdot 95$ & $4 \cdot 3$ & 0 & $12 \cdot 0$ & $2 \cdot 75$ & $1 \cdot 1$ \\
\hline Arginine . & & - & $2 \cdot 14$ & $11 \cdot 7$ & $3 \cdot 4$ & $3 \cdot 81$ & $5 \cdot 4$ & $87 \cdot 4$ & $58 \cdot 2$ & $7 \cdot 62$ & $4 \cdot 5$ \\
\hline Histidine . & . & - & 一 & $1 \cdot 1$ & $1 \cdot 7$ & $2 \cdot 5$ & $11 \cdot 0$ & 0 & 12.9 & $0 \cdot 4$ & $0 \cdot B$ \\
\hline
\end{tabular}

glutamic acid, which forms 8 per cent of egg albumin and only 1.7 per cent. of globin (derived from hæmoglobin), amounts to 36.5 per cent. in gliadin, the protein extracted from wheat flour. Striking differences are also noticeable in the relative proportions of the diamino-acids and bases, the so-called hexone bases. Whereas in casein they form about 12 per cent. of the total molecule, in globin they form about 20 per cent., and in the protamines, salmine and sturine, about 85 per cent. of the total molecule consists of these bodies. On this account the two last-named bodies have a strongly basic character. From these figures it is evident also that certain of the aminoacids must occur many times over in the protein molecule. Thus in globin, if we assume the presence of one tyrosine molecule, there must be at least thirty-two leucine and ten histidine molecules. On these data the molecular weight of hæmoglobin would come out at about 14,000, a figure which agrees with that derived from a study of the amounts of sulphur and iron respectively in its molecule.

\section{THE DISTRIBUTION OF NITROGEN IN THE PROTEIN MOLECULE}

Attempts have been made to differentiate among the proteins by a method which, while less laborious than the isolation and recognition of the individual amino-acids, may yet throw some light on the manner in which the nitrogen is combined within the molecule, and on the relative amounts of the different classes of nitrogen groups which may be present. One method, which was devised by Hausmann, is carried out as follows. One gramme of the protein is dissociated by boiling with strong hydrochloric acid. The nitrogen, which has been split off as ammonia and is present in the solution 
Table I.

\begin{tabular}{|c|c|c|c|c|c|c|c|}
\hline Group & Protein & Source & $\begin{array}{c}N \text { per } \\
\text { cent. }\end{array}$ & $\underset{\mathbf{N}}{\text { Amide }}$ & $\underset{N}{\operatorname{Amino}}$ & $\underset{N}{\text { Basic }}$ & $\underset{N}{\mathrm{Humin} *}$ \\
\hline Protamines & $\left\{\begin{array}{l}\text { Salmine } \\
\text { Sturine }\end{array}\right.$ & $\begin{array}{l}\text { Salmon-roe } \\
\text { Sturgeon-roe }\end{array}$ & - & $\begin{array}{l}0 \\
0\end{array}$ & & $\begin{array}{l}87 \cdot 8 \\
83 \cdot 7\end{array}$ & \\
\hline Histones & Histone & Thymus & - & $3 \cdot 3$ & & $38 \cdot 7$ & \\
\hline $\begin{array}{l}\text { Albumins } \\
\text { and }\end{array}$ & Ovalbumin & Egg-white & $15 \cdot 51$ & $8 \cdot 64$ & $68 \cdot 13$ & $21 \cdot 27$ & $1 \cdot 87$ \\
\hline $\begin{array}{l}\text { phospho- } \\
\text { proteins }\end{array}$ & Caseinogen & Milk & $15 \cdot 62$ & $10 \cdot 36$ & $66 \cdot 00$ & $22 \cdot 34$ & $1 \cdot 34$ \\
\hline Globulins & $\left\{\begin{array}{l}\text { Globulin } \\
\text { Edestin }\end{array}\right.$ & $\begin{array}{l}\text { Wheat } \\
\text { Hemp seed }\end{array}$ & $\begin{array}{l}18 \cdot 39 \\
18 \cdot 64\end{array}$ & $\begin{array}{r}7 \cdot 72 \\
10 \cdot 08\end{array}$ & $\begin{array}{l}53 \cdot 40 \\
57 \cdot 83\end{array}$ & $\begin{array}{l}37 \cdot 10 \\
31 \cdot 70\end{array}$ & $\begin{array}{l}1 \cdot 52 \\
0 \cdot 64\end{array}$ \\
\hline $\begin{array}{l}\text { Alcohol- } \\
\text { soluble } \\
\text { proteins }\end{array}$ & $\begin{array}{l}\text { Zein } \\
\text { Gliadin }\end{array}$ & $\begin{array}{l}\text { Maize } \\
\text { Wheat and rye }\end{array}$ & $\begin{array}{l}16 \cdot 13 \\
17 \cdot 66\end{array}$ & $\begin{array}{l}18 \cdot 40 \\
23 \cdot 78\end{array}$ & $\begin{array}{l}77 \cdot 56 \\
70 \cdot 27\end{array}$ & $\begin{array}{l}3 \cdot 03 \\
5 \cdot 54\end{array}$ & $\begin{array}{l}0.99 \\
0.79\end{array}$ \\
\hline Albumoses & $\left\{\begin{array}{l}\text { Prot- } \\
\text { albumose } \\
\text { Hetero- } \\
\text { albumose }\end{array}\right.$ & $\begin{array}{l}\text { Witte's } \\
\text { peptone } \\
\text { Witte's } \\
\text { peptone }\end{array}$ & - & $\begin{array}{l}7 \cdot 14 \\
6 \cdot 45\end{array}$ & $\begin{array}{l}68 \cdot 17 \\
\text { ธั7 } 7\end{array}$ & $\begin{array}{l}25 \cdot 42 \\
38 \cdot 93\end{array}$ & - \\
\hline
\end{tabular}

Table II.-Distribution of the Nitrogen in "Various Proteins (VAN SLYKE)

\begin{tabular}{|c|c|c|c|c|c|c|c|}
\hline 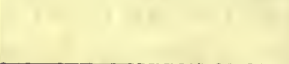 & Gliadin & Edestin & $\begin{array}{l}\text { Hair } \\
(\text { dog) }\end{array}$ & Gelatin & Fibrin & $\begin{array}{l}\text { Hæmo- } \\
\text { cyanin }\end{array}$ & $\begin{array}{l}\text { Ox hæmo- } \\
\text { globin }\end{array}$ \\
\hline Ammonia $\mathrm{N}$. & $25 \cdot 52$ & $9 \cdot 99$ & $10 \cdot 05$ & $2 \cdot 25$ & $8 \cdot 32$ & $5 \cdot 95$ & $5 \cdot 24$ \\
\hline Melanine N . & 0.86 & 1.98 & $7 \cdot 42$ & 0.07 & $3 \cdot 17$ & $1 \cdot 65$ & $3 \cdot 60$ \\
\hline Cystine N . & $1 \cdot 25$ & $1 \cdot 49$ & $6 \cdot 60$ & 0 & 0.99 & 0.80 & 0 ? \\
\hline Arginine $\mathrm{N}$. & $5 \cdot 71$ & $27 \cdot 05$ & $15 \cdot 33$ & $14 \cdot 70$ & $13 \cdot 86$ & $15 \cdot 73$ & $7 \cdot 70$ \\
\hline Histidine $\mathrm{N}$. & $5 \cdot 20$ & $5 \cdot 75$ & $3 \cdot 48$ & $4 \cdot 48$ & $4 \cdot 83$ & $13 \cdot 23$ & $12 \cdot 70$ \\
\hline $\begin{array}{l}\text { Lysine } \mathrm{N} \\
\text { Amino } \mathrm{N} \text { of the }\end{array}$ & 0.75 & $3 \cdot 86$ & $5 \cdot 37$ & $6 \cdot 32$ & $11 \cdot 51$ & $8 \cdot 49$ & $10 \cdot 90$ \\
\hline $\begin{array}{c}\text { filtrate } \\
\text { Non-amino } \mathrm{N} \text { of the }\end{array}$ & $51 \cdot 98$ & $47 \cdot 55$ & $47 \cdot 50$ & $56 \cdot 30$ & $54 \cdot 30$ & $51 \cdot 30$ & $57 \cdot 00$ \\
\hline $\begin{array}{l}\text { filtrate (proline, } \\
\text { oxyproline, } \frac{1}{2}\end{array}$ & & & & & & & \\
\hline tryptophane) & $8 \cdot 50$ & $1 \cdot 70$ & $3 \cdot 10$ & $14 \cdot 90$ & $2 \cdot 70$ & $3 \cdot 80$ & $2 \cdot 90$ \\
\hline Sum & $99 \cdot 77$ & $99 \cdot 37$ & $99 \cdot 85$ & $99 \cdot 02$ & $99 \cdot 58$ & $100 \cdot 95$ & $100 \cdot 00$ \\
\hline
\end{tabular}

as ammonium chloride, is then distilled off with magnesia and received into decinormal acid, where its amount can be determined by titration. This nitrogen is variously designated as amide nitrogen, ammonia nitrogen, or

* When a protein is boiled for a long time with strong acid, a black precipitate may occur which contains Nitrogen. This is known as humin nitrogen. 
easily displaceable nitrogen. The remaining fluid, freed from ammonia, is precipitated with phosphotungstic acid. By this means all the diamino-acids and bases are thrown down. The nitrogen in the preciptate is determined by Kjeldahl's method and is called diamino- or basic nitrogen. In the remaining fluid, which contains mono-amino-acids, the total nitrogen, the mono-amino-nitrogen, is determined by Kjeldahl's method. Table I., p. 92, gives some of the results obtained in this manner, and shows that there are considerable differences in the distribution of the different kinds of nitrogen among the various classes of proteins. The method is, however, only a rough one as compared with the separation of the individual amino-acids.

An improved means of determining the distribution of nitrogen in the protein molecule has been devised by Van Slyke. Some of his results are given in Table II., p. 92.

\section{TESTS FOR PROTEIN}

\section{A. COLOUR REACTIONS OF THE PROTEINS}

These are of importance since in many cases they are an.indication of the nature of the groups present in the protein molecule.

(1) THE BIURET REACTION. When a solution of a protein is made strongly alkaline. with caustic potash or soda, and dilute copper sulphate added drop by drop, a colour varying from pink to violet is produced. In the case of the proteoses and peptones (the hydrated proteins) the colour is pink ; in the case of the coagulable proteins, violet. According to Schiff this colour is given by all compounds containing the following groups :

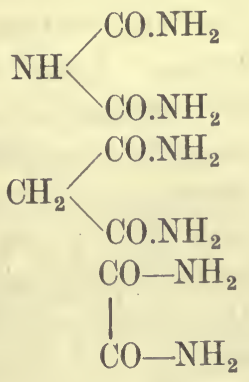

and the group

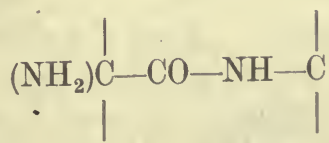

We have already seen that this grouping is typical of the protein molecule.

(2) THE XANTHO-PROTEIC REACTION. On adding strong nitric acid to a solution of protein and boiling, a yellow colour is produced which turns to a deep orange when excess of caustic alkali or ammonia is added. The production of this reaction points to the existence of benzene derivatives in the protein molecule, and it is therefore a general test for the presence of aromatic groups. 
(3) MILLON'S REACTION. Millon's reagent is a solution of mercuric nitrate in water containing free nitrous açid. On adding a few drops of this to a protein solution a white precipitate is produced which turns a brick-red colour on boiling. It depends on the presence in the protein of a hydroxyderivative of benzene, and is determined in the protein by the tyrosine, which is oxyphenylalanine.

(4) SULPHUR REACTION. On warming a solution of protein with caustic soda in the presence of lead acetate a black colour is produced owing to the precipitation of lead sulphide. The depth of coloration gives a rough indication of the amount of sulphur in the protein under investigation.

(5) THE HOPKINS-ADAMKIEWICZ REACTION. It was stated by Adamkiewicz that on the addition of acetic acid and concentrated sulphuric acid to protein, a violet colour was produced. Hopkins and Cole showed that the success of this reaction depended on the presence of glyoxylic acid $\mathrm{CHO} . \mathrm{COOH}$ as an impurity in the acetic acid used. The test is therefore performed now as follows :

Glyoxylic acid is prepared by the action of sodium amalgam on a solution of oxalic acid. A few drops of this solution are added to the solution of protein, and strong sulphuric acid poured down the side of the tube. A bluish violet colour is produced at the junction of the two fluids. This reaction is due to the presence in the protein of tryptophane.

The so-called Liebermann's reaction has been shown by Cole to be essentially a modification of the above, and is due also to the presence of tryptophane. In this test the protein is precipitated by alcohol, washed with ether, and heated with concentrated hydrochloric acid, when a blue colour is produced, glyoxylic acid being derived from the alcohol and ether.

(6) REACTIONS INDICATING THE PRESENCE OF CARBOHYDRATES. Molisch's test is applied as follows. A few drops of alcoholic solution of $\alpha$-naphthol and then strong sulphuric acid are added to a protein solution. A violet colour is produced, which on addition of alcohol, ether, or potash turns yellow. The reaction is determined by the presence, either as an impurity or a constituent part of the molecule, of a carbohydrate radical which, under the influence of strong sulphuric acid, is converted into furfurol. The furfurol gives the colour reaction with the $\alpha$-naphthol.

Another test for the carbohydrate radical is the orcin reaction. A small quantity of the dried albumin is added to 5 c.c. of fuming hydrochloric acid, and the mixture is then warmed. When the albumin is nearly all dissolved a little solid orcin is added on the point of a knife, and then a drop of ferric chloride solution. After warming this mixture for some minutes a green colour is produced which is soluble in amyl alcohol and gives a definite absorption spectrum.

\section{B. MET ALLIC SALTS}

The following metallic salts form double insoluble compounds with proteins, and therefore cause a double precipitation when added to solutions of these bodies : ferric chloride, copper sulphate, mercuric chloride, lead acetate, zinc acetate. 


\section{ALKALOIDAL REACTIONS}

Proteins, like the polypeptides and the amino-acids of which they are composed, may function either as weak acids or as weak bases, according as they are treated with bases or acid radicals respectively. In the presence of strong acids, therefore, proteins act like organic bases, and are thrown down in an insoluble form by the various alkaloidal precipitants. With certain proteins, such as the protamines, where there is a preponderance of basic groups, it is not necessary to add mineral acid in order to ensure the precipitation. The following are the principal alkaloidal precipitants which may be employed :

(a) Phosphotungstic acid.

(b) Phosphomolybdic acid.

(c) Tannic acid.

(d) Potassium mercuric iodide.

(e) Acetic acid and potassium ferrocyanide.

(f) Trichloracetic acid. (In order to precipitate all the coagulable proteins from a solution it is treated with an equal volume of 10 per cent. trichloracetic acid, well shaken and filtered.)

(g) Metaphosphoric acid.

(h) Salicyl-sulphonic acid.

These two latter are generally employed in a 5 per cent. solution.

(i) Picric acid.

A mixture of picric and citric acids is largely employed, under the name of Esbach's reagent, as a precipitant for coagulable proteins in the urine.

\section{TESTS DEPENDING ON THE COLLOIDAL CHARACTER OF THE PROTEIN}

(1) HEAT COAGULATION. On boiling proteins in a very slightly acid solution some are coagulated and form an insoluble white precipitate. This test is applicable to albumins, globulins, and under certain conditions to the derived albumins. In order that the separation of protein in this way may be complete it is necessary to provide for the presence of neutral salts and also for the maintenance of a slight acidity. The best method of carrying out this test, therefore, is to boil the protein in slightly alkaline or neutral solution after the addition of $2-5$ per cent. of sodium chloride or sodium sulphate. While the solution is in active ebullition 1 per cent. acetic acid is added drop by drop until the reaction is just acid to litmus. By this means a nearly perfect separation of all the coagulable proteins may be effected.

(2) HELLER'S TEST. On pouring a solution of protein carefully down the side of a test-tube containing strong nitric acid so as to form a layer on the top, a white layer of coagulated protein is produced at the junction of the two fluids. A similar coagulative effect is produced by other strong mineral acids. 
(3) PRECIPITATION BY NEUTRAL SALTS. On addition of a neutral salt in excess to a colloidal solution the relation between the solvent and the particles which are in suspension or pseudo-solution are altered. It is therefore possible in many cases by the addition of neutral salts to separate out the dissolved colloid without otherwise altering its characters in any way, so that, on collecting the precipitate and separating the salt carried down with it, it can be dissolved again by adding water. Some classes of proteins can be salted out very readily, while others require a much higher concentration of salt before they are precipitated.

The salts which are generally employed for salting out proteins have been divided by Schryver into three classes :

Class $I$.

Sodium chloride.

Sodium sulphate.

Sodium acetate.

Sodium nitrate.

Magnesium sulphate.
Class II.

Potassium acetate.

Calcium chloride.

Calcium nitrate.
Class III.

Ammonium sulphate.

Zinc sulphate.

The two calcium salts are, however, rarely employed, as they tend to render the precipitated protein insoluble.

The salts of the first class require much higher concentration for the precipitation of the proteins than those of the second, and these than those of the third. Since the degree of concentration of any salt necessary for the precipitation of any particular protein is characteristic for this body, it is possible to employ a fractional process of salt precipitation in order to separate mixtures of proteins into their components. Owing, however, to the tenacity with which different colloids adhere to one another it is difficult, even after many repetitions of the process of fractional salting out, to obtain products which can be regarded as free from admixture. For the purpose of fractional precipitation the salts most frequently employed are those of the third class, namely, ammonium sulphate and zinc sulphate. We shall have to deal with results obtained by this method when treating of the separation of albumoses and peptones. The precipitability of different proteins with neutral salts serves also as the basis of the ordinary classification of these bodies.

\section{THE CLASSIFICATION OF PROTEINS}

It is possible that in the future, when we know all the disintegration products of the various proteins and the manner in which they are arranged in the molecule, the classification of these bodies will be based on their constitution. At the present time it is obviously impossible to make any classification on such a basis, since the necessary knowledge is wanting, and we have therefore to use a purely artificial classification, such as that adopted by the Chemical and Physiological Societies in 1907, based chiefly on the solubilities of the various proteins in water and salt solutions. We shall here only indicate the characters of the main groups into which proteins 
are conventionally divided, and leave the closer study of the individual proteins to be dealt with in connection with the organs or tissues in which they occur.

(1) THE PROTAMINES. These occur in the body only in combination with other groups. They are obtained from the ripe spermatozoa of certain fishes, where they occur in combination with nucleic acid. They are characterised by the very large amount of bases contained in their molecule, amounting to 85 per cent. of the total substance. It was formerly thought by Kossel that the protamines contained only diamino-acids and bases, but it has been shown later that a small proportion of mono-amino-acids may also be obtained from their disintegration ( $v$. Table, p. 91). On account of their constitution they possess strongly basic characters and form wellmarked salts, e.g. sulphates and chlorides, as well as double salts with platinum chloride. They contain no sulphur and do not coagulate on heating.

(2) HISTONES. This class of proteins, like the protamines, only occurs in combination with other groups, such, for instance, as nuclein and hæmatin. They may be obtained from red blood-corpuscles, where they form the globin part of the hæmoglobin molecule, or from the leucocytes of the thymus gland, or from the spermatozoa of fishes. The histones are precipitated from their watery solutions by addition of ammonia, but are soluble in excess of this reagent. In the presence of salts they are coagulated on boiling. With cold nitric acid they give a precipitate which dissolves on warming, but is thrown down again on cooling: The most characteristic feature of this class of bodies is, however, the high proportion of diamino-acids and bases contained in their molecule.

(3) ALBUMINS. These are soluble in pure water and are precipitated by complete saturation with ammonium sulphate, zine sulphate, or sodiomagnesium sulphate.

EGG Albumen forms the greater part of the white of egg. It gives the ordinary protein tests, coagulates on heating at about $75^{\circ} \mathrm{C}$., and is precipitated from its solutions if shaken with a drop of dilute acetic acid in excess of ether. It is lævo-rotatory, its specific rotatory power being $-35 \cdot 5^{\circ}$.

Serum Albumen occurs in large quantities in the blood plasma, serum, lymph, and tissue fluids of the body. It coagulates at $75^{\circ} \mathrm{C}$., and is distinguished from egg albumen by its greater specific rotatory power, $-56^{\circ}$, and by the fact that it is not precipitated by ether and sulphuric acid. Some vegetable proteins belong to this class, e.g. the leucosin of wheat.

(4) GLOBULINS. These bodies are insoluble in pure water and require the presence of a certain amount of neutral salt to dissolve them. They are precipitated from their solutions by complete saturation with magnesium sulphate or by half-saturation with ammonium sulphate. The chief members of this class are :

CRYstallin, obtained from the crystalline lens by passing a stream of carbon dioxide through an aqueous extract of this body. 
Serum Globulin or Paraglobulin, a constituent of blood plasma and blood serum.

Fibrinogen, which occurs in blood plasma and is converted into fibrin when the blood clots.

Paramyosinogen, a normal constituent of muscle.

Midway between these two groups may be placed the muscle protein, myosin (or myosinogen), which, though soluble in pure water, resembles the class of globulins in the ease with which it is precipitated by the addition of neutral salts.

In addition to the members of the globulins named above and derived from the animal body, proteins allied to this class form an important constituent of plants, and are found in large quantities in many seeds used as articles of food. These are vegetable globulins. Prominent members of the group are the edestins, which may be obtained from hemp seeds, cotton seeds, and sunflower seeds, zein from maize, legumin from beans.

(5) GLIADINS, contained in cereals, and soluble in alcohol.

(6) GLUTELINS, proteins also obtained from cereals and soluble in weak alkalies.

(7) DERIVATIVES OF PROTEINS. A. METAPROTEINS. These may be regarded as compounds of the protein molecule or of part of the molecule with acid or basic radicals.

Acid Albumin, or acid metaprotein, is formed by the action of warm dilute acids or of strong acids in the cold on any of the preceding bodies. If a weak alkali be added so as to nearly neutralise the solution of acid metaprotein, this latter is precipitated. If the precipitate be suspended in water and heated, it is coagulated and becomes insoluble in dilute acids or alkalies.

Alkali Albumin, or alkaline metaprotein, is formed by the action of strong caustic potash on white of egg or on any other protein, or by adding alkali in excess to a soluion of acid metaprotein. It is precipitated on neutralisation of its solution.

In close association with this group may be included the proteins as they occur in combination with the metallic salts, such as copper sulphate. On splitting off the copper moiety from these compounds, the protein left is practically free from ash, and behaves in many respects like an albuminate, being insoluble in absolutely pure water, but easily dissolved by the addition of. a trace of free acid or alkali.

A group of protein derivatives described by Hopkins is produced by the action of the free halogens on protein solutions. We get in this way two definite classes of compounds. One class, which contains the largest percentage of halogen, is obtained by treating a protein solution with chlorine, bromine, or iodine, dissolving up the resultant precipitate in alcohol and pouring the alcoholic solution into ether, when the halogen compound is thrown down as a fine white precipitate. By dissolving this precipitate in weak soda and precipitating with acid, we obtain a series of compounds containing only about one-third as much of the halogen as is contained in the first precipitate, suggesting that the halogen forms both substitution and additive compounds with the protein molecule.

Albumins, globulins, and metaproteins are often associated together as the coagulable proteins, since they may be thrown down entirely from their solution on boiling in slightly acid medium in the presence of neutral salts. 
B. HYDRATED PROTEINS. When proteins are subjecterl to the

action of superheated water or steam, or heated with acids, or acted on at the body temperature by certain ferments, e.g. pepsin, trypsin, or papain, they undergo a change which is attended by the addition of a number of molecules of water to the protein molecule (hydrolysis). This action, when carried to its end, results in the production of the amino-acids which we have already dealt with.

These hydrolytic changes proceed by a series of stages, so that the intermediate products still present many of the protein reactions. The hydrated proteins are divided into two groups, proteoses and peptones. The formation of these intermediate products is especially marked with the proteolytic ferments. Pepsin with hydrochloric acid, the ferment of the gastric juice, for example, only breaks down the protein molecule as far as the proteoses and peptones. Trypsin also gives rise to both proteoses and peptones as intermediate products. The action of these ferments on proteins is in fact closely analogous to the action of diastase on the great polysaccharide molecule of starch. In this case, as intermediate products we have first dextrins of various complexity, secondly maltose, and finally, if the ferment maltase be also present, dextrose. The monotony of the starch molecule determines a great similarity of composition between its various disintegration products. It may be regarded as an anhydride of many (100 or more) molecules of a hexose, and the intermediate stages in this hydrolysis are also hexoses and their anhydrides. The protein molecule is distinguished by the variety of the groups which enter into its formation, and this heterogeneous character of the molecule renders possible a much greater variety of intermediate products than we find in the starches. Thus a protein molecule may consist of the groups A, B, C, D, E, F, G, H, \&c. When hydrolysis occurs it may result in the immediate splitting off, say, of part of group A, while the residue breaks up into a series of proteoses whose composition may be represented as $\mathrm{ABF}, \mathrm{ABC}, \mathrm{DFG}, \mathrm{BDEF}$, \&c. With further hydrolysis these groups are broken into still smaller ones, and the penultimate stages of the hydrolysis will be polypeptides similar to those which have been synthetised by Fischer from the ultimate products of protein hydrolysis. No sharp dividing line can be drawn between the proteoses, peptones, and polypeptides. Of the last group we have already seen that the higher members give the biuret reaction as well as the other protein reactions, if the necessary groups, e.g. tyrosine, tryptophane, are present in the molecule. The proteoses and peptones are, however, ill-defined bodies. We have at present no satisfactory means of isolating the different members of these groups and obtaining them in a state of chemical purity. Their classification is therefore, like that of the proteins generally, a conventional one, depending on their solubilities and their precipitability by neutral salts, especially ammonium sulphate. Both proteoses and peptones give the xanthoproteic and Millon's reactions common to all proteins, and, like these, are precipitated by such reagents as mercuric chloride, potassio-mercuric iodide, or phosphotungstic acid. On adding excess of caustic potash and a drop of dilute 
copper sulphate to solutions of either of these classes of bodies, a pink colour is produced which deepens to a violet on addition of more copper (the biuret reaction). Their solutions can be boiled without undergoing coagulation. Many of them may be thrown down from their solutions by absolute alcohol, but are not rendered insoluble even by prolonged standing under the alcohol. The characters of the different members of these groups will be considered at greater length when dealing with the changes undergone by the proteins during the process of digestion. At present we may merely summarise the distinguishing features of these two classes.

(a) Proteoses, e.g. albumose from albumin, caseose from casein, elastose from elastin. All of these are precipitated from their solutions on saturation with ammonium sulphate. In the presence of a neutral salt they give a precipitate on the addition of nitric acid. This precipitate is dissolved on heating the solution, but reappears on cooling. All, with the exception of heteroalbumose, are soluble in pure water, and all are soluble in weak salt solutions or dilute acids or alkalies. They are slightly diffusible through animal membranes.

(b) Peptones, e.g. fibrin peptone, gluten peptone. These are all soluble in pure water, diffuse fairly readily through animal membranes, but otherwise give the same reactions as albumoses. From the latter class peptones are distinguished by the fact that they are not precipitated on saturation of their solutions either in acid or alkaline reaction with ammonium sulphate or any other neutral salt. Many of them are soluble in alcohol.

(8) THE PHOSPHOPROTEINS. In this class may be grouped a number of substances of very diverse properties which, however, resemble one another in containing phosphorus as an integral part of their molecule. When subjected to digestion with pepsin and hydrochloric acid they are dissolved, but a small quantity of a phosphorus-containing complex may remain behind undissolved. This residue has been called paranuclein or pseudonuclein. It is in reality derived from nucleoprotein, which is present in the phosphoprotein as impurity and should be called simply nuclein. The phosphoproteins have markedly acid characters. They are insoluble in pure water, easily soluble in alkalies and ammonia from which the original body is thrown down again on addition of acid. Their solutions in alkali are not coagulated by heating. To this class belong caseinogen, the chief protein of milk, vitellin, the main protein in the yolk of egg, and the vitellins in the eggs of fishes and frogs. The vitellins are generally associated with a large amount of lecithin. The phosphoproteins differ from the nucleoproteins, which also contain phosphorus, in the facts that they are readily decomposed by caustic alkali with the liberation of phosphoric acid, and do not contain purine bases. The phosphorus of the nucleoproteins is not split off by alkali ( 1 per cent.), and on hydrolysis the nucleic acid constituent gives rise to purine bases.

(9) CONJUGATED PROTEINS. Various complex bodies which play an important part in building up cells and in the various processes of the body make up this group of compounds. They resemble one another only in the 
fact that in each of them a protein radical is combined with some other body, often spoken of as the prosthetic group.*

(a) Chromoproteins. Of this class, consisting of a colouring-matter combined with a protein, the most important is homoglobin. This substance, which is the red colouring-matter of the red corpuscles of the blood and plays an important part in the processes of respiration, acting as an oxygen carrier from the lungs to the tissues, is composed of the protein, globin, united with an iron-containing body, hæmatin. Oxyhæmoglobin contains from 4-5 per cent. hæmatin $\left(\mathrm{C}_{32} \mathrm{H}_{32} \mathrm{~N}_{4} \mathrm{O}_{4} \mathrm{Fe}\right)$. It is easily crystallisable, and its physical and chemical characters have therefore been more precisely determined than is the case with most other members of the group of conjugated proteins. We shall have to deal more fully with its properties in the chapters on Blood and Respiration.

(b) The Nucleoproteins. These are formed by the combination of a phosphorised organic acid, nucleic acid, with a protein which may belong to any of the classes we have enumerated above. Some of the best-marked members of this group consist of compounds of nucleic acid with basic histones or protamines. The combination between protein and the prosthetic group seems to take place in two stages. If a nucleoprotein be subjected to gastric digestion a large amount of the protein goes into solution as proteose or peptone, leaving an insoluble remainder. This precipitate is not, however, nucleic acid, but still contains a protein group, the compound being spoken of as nuclein. From the latter nucleic acid can be split off by heating with strong acids or other means. The nucleoproteins are soluble in water and salt solutions, and are easily soluble in dilute alkalies. They have acid characters and are precipitated by the addition of acids. The nucleins, on the other hand, are insoluble in water and salt solutions, but are easily dissolved by dilute alkalies. The nucleins and nucleoproteins form the chief and invariable constituent of cell nuclei. They may be therefore prepared from the most diverse organs. The heads of the spermatozoa of the salmon consist entirely of nuclein. Miescher and Schmiedeberg found that the nuclein obtained from this source contained 60.5 per cent. nucleic acid and $35 \cdot 56$ protamine, and was in fact a nucleate of protamine. The nuclein derived from the spermatozoa of echinoderms has been found to be a compound of nucleic acid and histone. From organs rich in cells, such as the thymus and the pancreas, and from nucleated red blood-corpuscles, nucleoproteins may be obtained which can be broken down into nuclein and protein, the nuclein again being composed of a protein residue with nucleic acid.

As first extracted from the animal cell the nucleoproteins are associated with a considerable proportion of lecithin, and in this labile compound form the 'tissue fibrinogen ' of Wooldridge. To prepare this substance an organ rich in cells, such as the thymus, is minced and extracted with water or normal salt solution. After

* By the Germans the term 'proteid' is often applied to this group. In English, however, the term 'proteid' has been generally used for the simple protein known to the Germans as 'Eiweisskörper.' On account of the confusion which has risen from this double use of the term 'proteid,' I have attempted to avoid it altogether in this volume. 
separating the cells by means of the centrifuge, the clear fluid is decanted off and acidified with acetic acid. A precipitate is produced consisting of 'tissue fibrinogen.' This substance is soluble in excess of acid and is easily soluble in alkalies. All the tissue fibrinogens are highly unstable bodies and undergo changes in the mere act of precipitation and re-solution. When injected into the blood they cause intravascular clotting. On digestion with gastric juice they yield a precipitate of nuclein, and this precipitate contains a large proportion of the lecithin present in the original substance. In the nucleoproteins nucleic acid is combined with proteins in two degrees, a large portion of the protein being separable by gastric digestion, while the remainder needs stronger reagents for its dissociation. The relation of the two portions of the nucleoprotein may be represented therefore by the following schema:

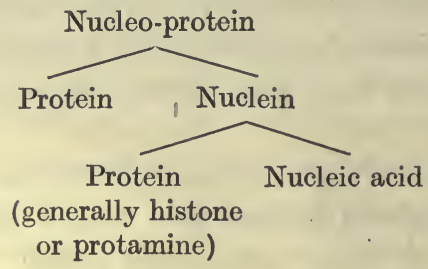

By various means, all of which involve hydrolysis, the nucleic acid may be broken up into its proximate constituents. These differ according to the source of the nucleic acid. Whatever the source, the disintegration products belong to closely allied groups of substances. These may be grouped as follows :

(1) Phosphoric Acid. The proportion of phosphorus varies within but narrow limits in the different nucleic acids, the average being about 10 per cent. It is probable that the phosphoric acid represents, so to speak, the combining medium for the groups contained in the nucleic acid molecule, as is the case with the various groups which make up the lecithin molecule.

(2) The Purine Bases. Among the products of disintegration of nucleic ard we find constantly one of the bases adenine, $\mathrm{C}_{5} \mathrm{H}_{5} \mathrm{~N}_{5}$, and guanine. $\left(\mathrm{C}_{5} \mathrm{H}_{5} \mathrm{~N}_{5} \mathrm{O}\right)$. These substances, with the products of their oxidation, xanthine, $\mathrm{C}_{5} \mathrm{H}_{4} \mathrm{~N}_{4} \mathrm{O}_{2}$, hypoxanthine, $\mathrm{C}_{5} \mathrm{H}_{4} \mathrm{~N}_{4} \mathrm{O}$, have long been known to be clusely allied to uric acid, $\mathrm{C}_{5} \mathrm{H}_{4} \mathrm{~N}_{4} \mathrm{O}_{3}$, but their true relationships have only been thoroughly known since the researches of Fischer on this group. According to Fischer they can be all regarded as derivatives of the body purine,

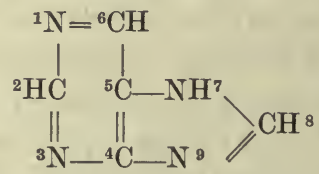

Each group in this purine ring is generally designated with a number indicated in the structural formula, in order that it may be possible to represent the position of any substituted groups in its derivatives. Uric acid itself is 2-6-8-trioxypurine with the following formula :

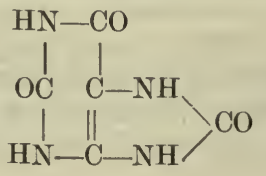


It can be synthetised by fusing together in a sealed tube trichlorolactamide and urea. Thus:

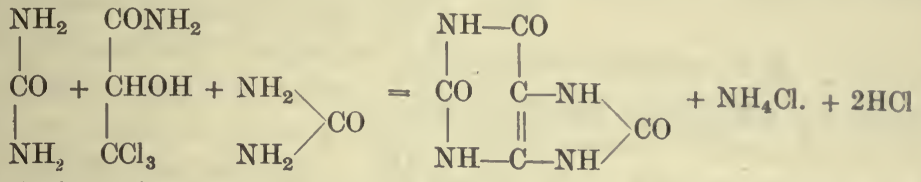

The relation of xanthine, hypoxanthine, guanine, and adenine to uric acid is shown by the following formulæ :<smiles>O=c1[nH]c(=O)[nH]c2c(=O)[nH]c=2[nH]1</smiles>

Uric acid 2-6-8-trioxypurine<smiles>O=C1NC2CNC1N2</smiles>

Xanthine 2-6-dioxy purine<smiles></smiles>

Hypoxanthine 6-oxypurine<smiles>Nc1cncnc1N</smiles>

Closely allied to this group of bodies are the chief constituents of tea, coffee, and cocoa, namely caffeine, which is trimethyl dioxypurine, and theobromine, which is dimethyl dioxypurine. From the structural formulæ given it will be seen that the purine radical contains two nuclei. The nucleus<smiles>C1CNCCN1</smiles>

is spoken of as the pyrimidine nucleus, pyrimidine having the formula<smiles></smiles>

The other is the radical which we have met with already in histidine, a disintegration product of proteins, namely iminazol :<smiles>c1c[nH]cn1</smiles>

Besides the purine bases proper, we find among the disintegration products of nucleic acid a series of bases derived from the pyrimidine ring. These are uracil, thymine, and cytosine.

URACIL is 2-6-dioxypyrimidine, $\mathrm{NH}-\mathrm{CO}$<smiles>CC1CCCC1=O</smiles> 
Thy mine is 5-methyl uracil,<smiles>CNC(=O)NC=CC(C)=O</smiles>

while CYTosine is 6-amino-2-oxypyrimidine,<smiles></smiles>

Besides these two groups of nitrogenous compounds derived from the purine and pyrimidine rings, many nucleic acids yield on hydrolysis a carbohydrate. Thus, Hammarsten has isolated a pentose, from the nucleoproteins of the pancreas. It is supposed that the nucleic acid of the thymus gland contains a hexose, since it is possible to split off from it lævulinic acid, which is one of the first products of the decomposition of a hexose. The complex constitution of the nucleic acids and nucleoproteins may be rendered clearer from the following schema :

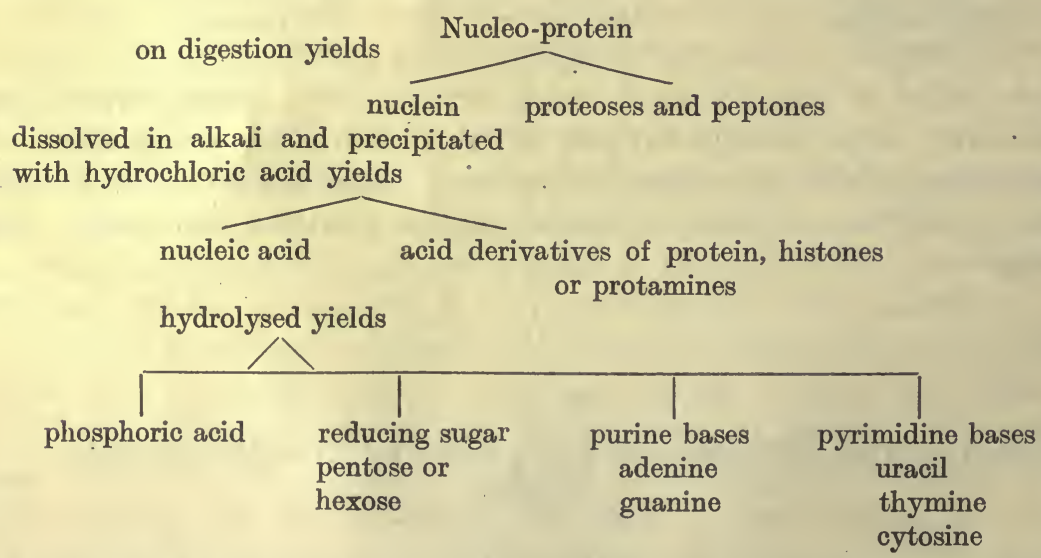

It must not be imagined, however, that all these disintegration products are present in all nucleic acids. Thus the nucleic acid derived from the pancreas, the so-called guanylic acid, yields of the purine bases only guanine, and of the pyrimidine bases only thymine and uracil, and every variety is met with as we analyse the nucleic acids of different origin. The fact that nucleic acid is a characteristic and necessary constituent of all nuclei adds interest to the divergence of its constituent radicals from those which distinguish the proteins of the cell protoplasm. Further importance is lent to this section of the chemistry of the body by the close relationship which we shall have to study later between the nuclein metabolism of the body and the production and excretion of uric acid.

(c) The Glycoproteins. In the glycoproteins the prosthetic group is represented by a carbohydrate radical, generally containing nitrogen, such as glucosamine or galactosamine. They are split into their two constituents, 
protein and carbohydrate radical, on prolonged boiling with dilute mineral acids or by the action of alkalies. They may be divided into the two main groups of mucins and mucoids.

The mucins play a large part in the animal kingdom as protective agents. They form the slimy secretion which covers the inner surface of the mucous membranes and the outer surface of many marine animals, and is secreted either by the goblet cells of the epithelium or by special groups of cells collected together to form a mucous gland. They may be precipitated from their solutions or semi-solutions by the addition of acids, and after precipitation need the addition of alkalies for their re-solution. They are not coagulable by heat. The presence of their protein moiety causes them to give the various typical protein tests, such as the xanthoproteic, Millon's, the biuret reaction, and so on. Prolonged boiling with acids splits the molecule, with the production of acid metaprotein and albumoses and glucosamine. From the mucin of frogs' eggs a similar treatment results in the production of galactosamine.

With the mucins may be classified certain bodies which have been derived from ovarian cysts, namely, pseudomucin and paramucin. Pseudomucinoccurs as a constituent of the colloid material from ovarian tumours. It forms slimy solutions which do not coagulate by heat and are not precipitated by acetic acid. It is precipitated by alcohol, the precipitate being soluble in water even after standing a long time under the alcohol. On boiling with acid it gives a reducing substance. Paramucin differs from the above in reducing Fehling's solution before boiling with acids. Otherwise it resembles pseudomucin. Leathes, in investigating this body, isolated from it a reducing substance which apparently was an amino-derivative of a disaccharide, perhaps in combination with glycuronic acid.

The mucoids include a number of substances which may be extracted from various tissues by the action of weak alkalies, e.g. from tendons, bone, and cartilage. The best studied example of this group is the chondromucoid which, with collagen, forms the ground substance of cartilage. Chondromucoid is especially rich in sulphur and gives protein by long treatment with weak alkali. On boiling for a short time with acid it is decomposed into sulphuric acid and chondroitin, and this latter, on further action of the acid, is converted into a substance chondrosin, which is certainly an aminoderivative of a polysaccharide containing the elements of glycuronic acid and an amino-disaccharide. Chondroitin-sulphuric acid occurs not only in cartilage but also in bone, yellow elastic tissue, white fibrous tissue, and as a constant constituent of the lardacein or amyloid substance which occurs as a deposit in the middle coat of the blood-vessels as the result of syphilis or long-continued suppuration, and gives rise to the condition known as 'lardaceous disease.' Another example of this class of mucoids is ovomucoid, which is a constituent of egg-white. In order to prepare ovomucoid the globulin and albumin are precipitated by boiling diluted egg-white. From the filtrate ovomucoid can then be thrown down by alcohol. A similar body has been prepared from blood serum. Both these mucoids yield a large amount of reducing substance on hydrolysis. Thus from $100 \mathrm{grm}$. of ovomucoid it is possible to prepare $30 \mathrm{grm}$. of glucosamine. 
(10) THE ALBUMINOIDS OR SCLERO-PROTEINS. Under this heading are grouped a number of diverse substances which play an important part in building up the framework of the body. Their value as skeletal tissue seems to be determined by their insoluble character. On this account it is practically impossible to speak of purifying them. In every case we can simply take the residue of a skeletal tissue which is left after extraction of the soluble constituents. When broken down by the action of strong acids they yield a series of disintegration products which are included among those we have already studied as the disintegration products of proteins. Their difference from the proteins which are employed in metabolism for their nutritive value is caused either by the absence of certain groups common to all the nutritive proteins, by the presence of an excess of one or two groups, or by the presence of certain polypeptides which present considerable resistance to the action of digestive ferments. This class plays the part in the animal economy which in the vegetable kingdom is filled by the anhydrides of the hexoses and pentoses, e.g. the celluloses, lignin, the pentosanes, \&c. Collagen forms the main constituent of white fibrous tissue and the ground substance of bone and cartilage. It is insoluble in water, hot or cold, and in trypsin. Under the action of acids or when subjected to prolonged boiling with water, especially under pressure, it is converted into gelatin, which is soluble in hot water, forming a colloidal solution liquid at high temperatures, but setting to a jelly when cold. When subjected to acid hydrolysis it gives a series of amino-acids from which tyrosine and tryptophane are wanting. On this account gelatin does not give any reaction either with Millon's reagent or with glyoxylic acid. On the other hand, there is a preponderance of such groups as glycine and phenylalanine, and it is probable that glycine, phenylalanine, and leucine are joined together, perhaps with other aminoacids, to form a polypeptide which is not attacked by digestive ferments, and therefore determines the resistance of the original collagen molecule to solution. Gelatin is precipitated by tannic acid, but not by acetic acid. It is dissolved with hydrolysis by gastric juice or by pancreatic juice, whereas collagen, its anhydride, is unaffected by the latter. On prolonged boiling in water it is converted into a modification which does not form a jelly on cooling. Under the action of formaldehyde it is converted into an insoluble modification which does not melt on warming.

Reticulin. This name has been applied to the tissue which forms the supporting network of adenoid tissue, and has also been described in the spleen, the mucous membrane of the intestine, liver, and kidneys. It differs from collagen in resisting digestion by gastric juice, and also in containing phosphorus in organic combination. According to Halliburton there is no essential difference between reticulin and collagen.

The keratins are produced by the modification of epithelial cells and form the horny layer of the skin as well as the main substance of hairs, wool, nails, hoofs, horns, and feathers. They are distinguished by their insolubility in water, dilute acids or alkalies, and in the higher animals pass through the alimentary canal unchanged. Although differing in their elementary composition, according to the tissue from which they are pre- 
pared, they are all distinguished by the very large amount of sulphur present in their molecule. The greater part of this sulphur is in the form of cystine, of which as much as 10 per cent. can be extracted from keratin. They also yield, on acid hydrolysis, tyrosine in larger quantities than is the case with the ordinary proteins.

Neurokeratin, which forms the basis of the neuroglial framework of the central nervous system, must be grouped by its general behaviour as well as by its origin with the keratins. It resembles the other members of this class in its insolubility and in its high content in sulphur. It is extracted from nervous tissues by boiling these with alcohol and ether and then submitting the tissue to prolonged tryptic digestion, which leaves the neurokeratin unaffected.

Elastin is a constant constituent of the connective tissues, where it forms the elastic fibres. In some localities, as in the ligamentum nuchæ, practically

\begin{tabular}{|c|c|c|c|c|c|c|c|c|c|}
\hline & & & & $\begin{array}{l}\text { Fibroin } \\
\text { of } \\
\text { silk }\end{array}$ & Elastin & $\begin{array}{c}\text { Keratin } \\
\text { from } \\
\text { horn }\end{array}$ & $\begin{array}{c}\text { Keratin } \\
\text { from } \\
\text { horsehair }\end{array}$ & $\begin{array}{c}\text { Keratin } \\
\text { from } \\
\text { feathers }\end{array}$ & Gelatin \\
\hline Glycine. & . & . & - & $36 \cdot 0$ & $25 \cdot 75$ & 0.45 & $4 \cdot 7$ & $2 \cdot 6$ & 16.5 \\
\hline Alanine & - & . & - & $21 \cdot 0$ & $6 \cdot 6$ & $1 \cdot 6$ & $1 \cdot 5$ & $1 \cdot 8$ & 0.8 \\
\hline Amino-valeris & anic & cid & . & 0.0 & $1 \cdot 0$ & 4.5 & 0.9 & 0.5 & $1 \cdot 0$ \\
\hline Proline . & . & . & . & present & $1 \cdot 7$ & & & & $5 \cdot 2$ \\
\hline Leucine. & . & . & . & 1.5 & $21 \cdot 4$ & $15 \cdot 3$ & $7 \cdot 1$ & $8 \cdot 0$ & $2 \cdot 1$ \\
\hline Phenylalanine & & . & 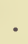 & 1.5 & $3 \cdot 9$ & 1.9 & 0.0 & 0.0 & 0.4 \\
\hline Glutamic acid & & . & & 0.0 & $0 \cdot 8$ & $17 \cdot 2$ & $3 \cdot 7$ & $2 \cdot 3$ & 0.88 \\
\hline Aspartic acid & & . & . & present & present & $2 \cdot 5$ & 0.3 & $1 \cdot 1$ & 0.56 \\
\hline Cystine & - & . & ${ }^{\circ}$ & - & - & $7 \cdot 5$ & & - & - \\
\hline Serine . & . & . & . & $1 \cdot 6$ & - & $1 \cdot 1$ & 0.6 & $0 \cdot 4$ & 0.4 \\
\hline Tyrosine & . & . & & 10.5 & $0 \cdot 34$ & $3 \cdot 6$ & $3 \cdot 2$ & $3 \cdot 6$ & 0.0 \\
\hline Tryptophane & . & . & & - & - & - & - & - & 0.0 \\
\hline Lysine. & . & . & & traces & 一 & 0.2 & $1 \cdot 1$ & - & $2 \cdot 75$ \\
\hline Arginine & . & . & $\bullet$ & $1 \cdot 0$ & 0.3 & $2 \cdot 7$ & $4 \cdot 5$ & - & $7 \cdot 62$ \\
\hline Histidine & . & . & & $\begin{array}{l}\text { small } \\
\text { amount }\end{array}$ & - & - & 0.6 & - & $0 \cdot 4$ \\
\hline Oxyproline & • & . & . & - & - & - & - & - & $3 \cdot 0$ \\
\hline
\end{tabular}

the whole tissue is made up of these fibres. Elastin is insoluble in water, alcohol, or ether, or in dilute acids and alkalies. It is slowly dissolved on prolonged treatment with gastric juice, but is practically unaffected in the alimentary canal. It gives the xanthoproteic and Millon's tests.

Other members of this group are fibroin, which forms the main substance of silk, spongin, the horny framework of sponges, conchiolin, the ground substance of shells, and perhaps the amyloid substance or lardacein which we have already mentioned in connection with the mucoids." All these scleroproteins present considerable differences in their qualitative and quantitative composition in amino-acids. Their proximate composition is shown in the Table given above (Abderhalden).

We have finally to mention a miscellaneous collection of bodies which are allied to the proteins and are distinguished by their extreme insolubility. 
They are often designated as albumoids. Of their composition we know practically nothing. Under this name are grouped such substances as those forming the membrana propria of glands, the sarcolemma of striated muscle, the albumoid of the crystalline lens, the ground substance of the chorda dorsalis, the organic basis of fish scales, and many similar substances. In every case the substance is characterised necessarily according to its place of origin, little or nothing being known as to its chemical composition. 


\section{SECTION VI \\ THE MECHANISM OF ORGANIC SYNTHESIS}

\section{THE ASSIMILATION OF CARBON}

THE building up of protoplasm from the material which is available at the earth's surface must be an endothermic process. The food presented to the plant contains the necessary elements, but as a rule in a state of complete oxidation. The energy of the living plant, as of animals, is derived almost entirely from the oxidation of its constituents. The building up of unorganised into organised material must therefore be effected at the expense of energy supplied from without. The source of this energy is the sun's rays. The machine for the conversion of solar radiant energy into the chemical potential energy of protoplasm is the green leaf. Here a deoxidation of the carbon dioxide of the atmosphere takes place, with the production of carbohydrates, generally in the form of starch. The formation of starch must be regarded as the first act in the life-cycle, since this substance serves as a source of energy to the already formed protoplasm in its work of building up all the other constituents of the living cell. It is the solar energy captured by the green leaf which is utilised by all plants devoid of chlorophyll, as well as by the whole animal kingdom.

There are one or two exceptions to this statement. Thus the bacterium nitrcsomonas, described by Winogradsky, grows on a medium devoid of all organic constituents, and derives the energy for its constructional activity from that set frce in the conversion of ammonia into nitrites. The sulphur bacteria apparently derive their energy from the decomposition of hydrogen sulphide and the liberation of sulphur.

The fundamental importance of this process of assimilation for the whole of physiology justifies some account of the researches which have been directed to the elucidation of its mechanism. The production of oxygen by the green plant was disovered by Priestley in 1772, and a few years later Ingenhaus showed that this production occurred only in the light and was effected only by green plants. De Saussure (1804) pointed out that the essential process concerned was a setting free of the oxygen from the carbon dioxide of the atmosphere, and recognised that the co-operation of water was also necessary. Mohl in 1851 observed the formation of starch grains in the chlorophyll corpuscles, and regarded these as the first products of 
assimilation. The organs of carbon dioxide assimilation are the chloroplasts. These, which are responsible for the green colour of plants, are generally small oval bodies embedded in the cytoplasm, but sometimes, as in spirogyra, may have the form of spiral bands. In a plant which has been kept for some time in the dark, or in an atmosphere free from carbon dioxide, they present no enclosed granules. Within three to five minutes after exposure to light in the presence of carbon dioxide, starch granules make their appearance within them, and grow rapidly, assuming the typical laminated structure. Engelmann has pointed out a means by which it can be proved that the chloroplasts carry out this process without the co-operation of the rest of the cytoplasm. Certain bacteria have a great avidity for oxygen and present movements only in the presence of this gas. If a filament of spirogyra be placed in a suspension of these bacteria and be examined under a microscope, the bacteria will be seen to congregate in the immediate neighbourhood of the chlorophyll bands. The same phenomenon is observed in the case of chlorophyll corpuscles isolated by breaking up the cells in which they were contained. These corpuscles therefore take up carbon dioxide and water, and form carbohydrate and oxygen, as follows :

$$
\mathrm{n}\left(6 \mathrm{CO}_{2}+5 \mathrm{H}_{2} \mathrm{O}\right)=\left(\mathrm{C}_{6} \mathrm{H}_{10} \mathrm{O}_{5}\right)_{\mathrm{n}}+\mathrm{n}\left(6 \mathrm{O}_{2}\right)
$$

The whole structure of the green leaf is directed to the furthering of this process. Its cells contain chlorophyll corpuscles, which change their position according to the intensity of the illumination. A free supply of air to all the cells is provided by means of the stomata on the under surface of the leaf. Horace Brown has shown that the rate at which carbon dioxide diffuses through such fine openings is as great as if the whole leaf were an absorbing surface. We get, therefore, optimum absorption of carbon dioxide by the leaf, with the maximum protection of the absorbing tissue and the necessary limitation of loss of water by transpiration.

In view of the very small amount of carbon dioxide in the atmosphere, the extent of the assimilatory process is remarkable. One square metre of leaf of the catalpa can lay on 1 grm. of solid per hour, using up for this purpose $784 \mathrm{ccm}$. carbon dioxide. The rapidity of assimilation is increased within limits by increasing the intensity of the light falling on the plant, though an over-stimulation of the process is prevented by the movements of the chloroplasts just mentioned. It is also increased by raising the percentage of carbon dioxide in the atmosphere supplied to the leaf. The optimum percentage of carbon dioxide will of course vary with the other conditions of the leaf. In certain experiments Kreusler found the optimum to be about 1 per cent. Taking the amount of assimilation in normal air with .03 per cent. carbon dioxide at 100 , the assimilation in an atmosphere containing 1 per cent. was 237 , and was not increased by raising the percentage of carbon dioxide to 7 per cent. Owing to the decomposition of the organic matter of the soil, the percentage of carbon dioxide near the ground is always greater than in the higher strata of the atmosphere-a fact which is taken 
advantage of by the low-growing plants and herbage. Other necessary conditions of assimilation are the presence of water and the maintenance of a certain external temperature. The absorption of the sun's rays by the leaf raises the temperature of the later above that of the surrounding medium, and so quickens the process of assimilation.

The assimilation of carbon dioxide, the formation of starch, and the evolution of oxygen will go on in the isolated chloroplast. In the absence of chlorophyll, as in an etiolated leaf, the formation of starch will take place if the plant be supplied with a sugar such as glucose, and this conversion represents the main function of the leucoplasts present in all the cells of the reserve organs of plants. In the absence of chlorophyll no decomposition of carbon dioxide takes place, so that this pigment is evidently essential for the utilisation of the sun's energy. Chlorophyll may be extracted from leaves by means of absolute alcohol. A solution is thus obtained which is green by transmitted and red by reflected light, i.e. chlorophyll is a fluorescent substance. It presents four absorption bands, the chief being an intense black band between Fraunhofer's lines B and C. If the chlorophyll is the means of conversion of the solar into chemical energy, the conversion must take place at the expense of the light which is absorbed by the pigment. One would expect, therefore, the process of assimilation to be most pronounced in those parts of the spectrum corresponding to the absorption bands - an expectation which has been realised by experiment.

As to the exact chemical changes effected by these absorbed rays physiologists are still undecided. There can be no doubt that an early product of the process is a hexose, which is rapidly converted into cane sugar or into starch. It was suggested by Baeyer in 1870 that carbon dioxide was reduced to formaldehyde, which later by condensation yielded sugar. We know that formaldehyde easily polymerises to form a mixture of hexoses, but until recently no evidence had been brought forward of its presence as an intermediate product in the assimilatory process. For most plants, indeed, formaldehyde is extremely poisonous, though certain algæ, as well as the water-plant, Elodea, can stand a solution containing 001 per cent. formaldehyde. Bokorny stated that spirogyra could form starch out of such derivatives of formaldehyde as sodium oxymethyl-sulphonate, or from methylal. The difficulty in these cases is that possibly a spontaneous formation of sugar from the formaldehyde had taken place in the solution and that the plants were using up the sugar rather than the formaldehyde as the source of their starch.

One must assume, with Timiriazeff, that the function of chlorophyll in the process of assimilation is that of a sensitiser. Just as the addition of eosin to the emulsion used for coating photographic plates will render these sensitive to the red and green parts of the spectrum, i.e. will excite change in the silver salt when light from these parts of the spectrum falls upon it, so the chlorophyll serves as a means by which the absorbed solar energy can be utilised for the production of chemical change in the chloroplast. Attempts 
have been made to imitate this process outside the plant. Thus Bach passed a stream of carbon dioxide through a 1.5 per cent. solution of a fluorescent substance, uranium acetate, in sunlight. As a result there was a precipitate of uranium oxide and peroxide, with the formation of traces of formaldehyde. Usher and Priestley, on treating a solution of carbon dioxide with 1.5 per cent. uranium acetate or sulphate in bright sunlight, obtained uranium peroxide and formic acid, but no formaldehyde. The formation of peroxides in these conditions suggests that the first change in the chloroplast may be as follows :

$$
\mathrm{CO}_{2}+3 \mathrm{H}_{2} \mathrm{O}=2 \mathrm{H}_{2} \mathrm{O}_{2}+\mathrm{CH}_{2} \mathrm{O}
$$

Such a reaction must be regarded as reversible since the hydrogen peroxide first formed would tend to oxidise the formaldehyde again. Moreover it would have a destructive influence on the chlorophyll itself, which is easily oxidised. In order, therefore, that the reaction should go on in one direction only, i.e. that of assimilation, means must be present in the chlorophyll corpuscles for the removal of both hydrogen peroxide and formaldehyde as soon as they are formed. The removal of the hydrogen peroxide can be effected by a catalase, which is fairly widely distributed in plants and has been shown by the last-named authors to be present in the chloroplasts. In order to demonstrate the production of the first result of assimilation, i.e. formaldehyde, the further stages in its conversion must be stopped by killing the plant and the catalase it contains. They therefore placed leaves, which had been boiled, in water saturated with carbon dioxide and exposed them to bright sunlight. The leaves were bleached by the oxidation of the chlorophyll, and some substance of an aldehydic nature was produced, as shown by the red colour obtained on placing them in rosaniline, previously decolorised with sulphurous acid.

Two proofs were brought forward that this substance was formaldehyde :

(a) Some of the bleached leaves were soaked for twelve hours in aniline water. The chloroplasts under the microscope were seen to contain crystals resembling methylene aniline.

(b) The leaves were distilled in a current of steam. The distillate was shown to contain formaldehyde by the formation of methylene aniline crystals on treatment with aniline, and by the preparation from it of the characteristic tetrabrome derivative of hexamethylenetetramine.

Usher and Priestley conclude that the first products of the photolysis of carbonic acid are hydrogen peroxide and formaldehyde. Both these substances are rapidly removed from the reaction. The hydrogen peroxide is broken up by the catalase into water and oxygen which is turned out by the plant. The formaldehyde is at once polymerised in the protoplasm of the chloroplast with the formation first of a hexose and then of starch. The formaldehyde, if not removed in this way, destroys the catalase. The hydrogen peroxide, if not broken up by the catalase, destroys the chlorophyll. 
The relations between the various factors in this process may be diagrammatically expressed thus :

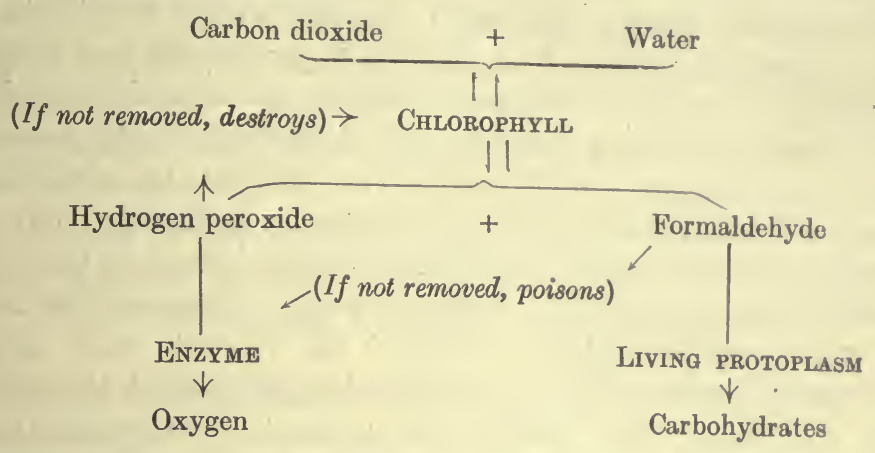

In thus reducing certain of the stages in the assimilation of carbon to phenomena which can be imitated outside the living organism we have made considerable strides in the ' understanding' of the process. The stage for which the vitality of the chloroplast is absolutely essential is the formation of starch from formaldehyde. Outside the body, our polymerisation of formaldehyde results in the formation of a mixture of sugars which are optically inactive. The same process, in the living cell, leads to the production of optically active sugars which are connected stereochemically and mutually convertible one into the other, e.g. fructose and glucose. The derivatives of protoplasm, containing asymmetric carbon atoms, are in the same way optically active, and it seems that the asymmetry of the protoplasmic molecule conditions a corresponding asymmetry in the substance which it builds on to itself. The protoplasm furnishes, so to speak, a mould in which polymerisation of formaldehyde can result only in the production of sugars of certain definite stereochemical configurations.

Few, if any, chemical reactions are pure. Nearly all are attended with by-reactions, so that the yield of end product never attains 100 per cent. of the theoretical yield. Even if the above mechanism be regarded as the chief one, it is probable that side reactions take place at the same time, so that we may have the formation of substances such as glyoxylic acid and other derivatives of the fatty acid series. Such by-products might play an important part in the other synthetic activities of the cell, and especially in the formation of fats and proteins.

\section{THE FORMATION OF PROTEINS}

Our knowledge of the mechanism by which proteins are synthetised in plants is still more incomplete than that of the synthesis of carbohydrates, and we are reduced in most cases to a discussion of the possible ways in which, from our knowledge of the chemical behaviour of the constituents of the protein molecule, we might conceive of its formation. We can at any rate state the problems which have to be solved and study the conditions under which the synthesis of protein is possible in plants and in animals. 
We know that plants are independent of any organic food for building up their various constituents, whether carbohydrate, protein, or fat, provided only that they possess chlorophyll corpuscles and so are able to utilise the energy of the sun's rays. Most plants will grow in the dark if supplied with sugar and with combined nitrogen either in the form of ammonia or of nitrates. The higher plants are especially dependent on the presence of nitrogen in the latter form, and it is on this account that the nitrifying bacteria of the soil acquire so great an importance for agriculture. From the carbon dioxide of the atmosphere or from the hexose formed by the assimilation of carbon, and from nitrogen, in the form either of ammonia or nitrates, together with inorganic sulphates, the plant cell is able to build up all the various types of protein which are distributed throughout the vegetable kingdom. Our study of the disintegration products of proteins has shown that this class of bodies contains a large number of the most diverse groups, having as a common character the possession of nitrogen in their molecule, generally as an $\mathrm{NH}_{2}$ or $\mathrm{NH}$ group. These disintegration products can be classified as follows :

(a) Open chain amino-acids.

(b) Heterocyclic compounds, including :

(1) Pyrrol derivatives.

(2) Pyrimidine derivatives.

(3) Iminazol derivatives.

These two last groups co-exist in all the purine compounds.

(c) Benzene derivatives.

(d) Indol derivatives.

The first step in the synthesis of proteins is probably the formation of these constituent groups. Just as in digestion the protein molecule is taken to pieces with the formation of the different amino-acids, so in the synthetic action of protoplasm the reverse process of dehydration occurs, resulting in a coupling up of the different groups, as has been effected by Fischer in the case of the polypeptides. Wherever transport of protein from one part of the organism to another is necessary the protein is carried, not in its original form, but in the hydrolysed condition of amino-acids. Thus the germination of seeds which contain rich stores of protein is accompanied by a liberation of proteolytic ferments within the cells of the seeds, and the breakdown of the reserve protein into its constituent amino-acids. As amino-acids it is transported into the growing tip and leaves of the seedling, analysis of the latter showing a very large percentage of nitrogen in the form of amino-acids. This is especially the case if the synthetic functions of the growing tip are hindered by interference with assimilation, as, e.g. by keeping the plant in the dark. Under these circumstances, asparagine may form as much as 25 per cent. of the total dried weight of the seedling. In animals the greater part of the protein of the food is broken down into its constituent aminoacids in the intestine. These are absorbed and probably carried to the 
different organs of the body, where they are resynthetised, generally in different proportions from those of the original protein, into the protein specific for the organ or tissue. The same process of hydrolysis and subsequent synthesis occurs whenever the transport of protein is neces. sary from one organ to another. We shall later on have to discuss the possibility of synthesis of the different amino-acids in animals. We need, therefore, at present only deal with the possible methods by which, from the glucose or substances produced in the assimilation of carbon and from the ammonia or nitrates derived from the soil, the plant is able to make the different groups which go to the building up of the protein molecule.

All the amino-acids contain the $\mathrm{NH}_{2}$ group in the $u$ position. We can therefore consider them as formed by the interaction of an $u$-oxyacid and ammonia. Thus :

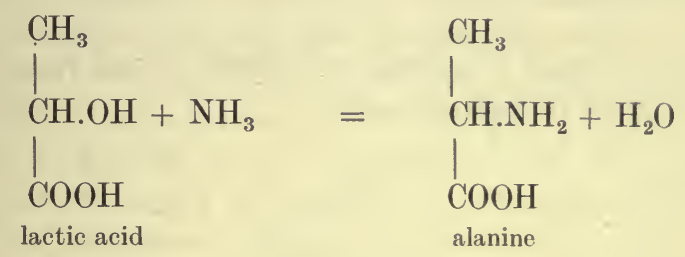

This particular example, namely, the formation of alanine, may occur at the expense of the glucose produced as the first product of assimilation of carbon dioxide. If a solution of glucose together with lime be exposed to sunlight for a considerable time it undergoes decomposition with the formation of lactic acid. Thus :

$$
\underset{\text { glucose }}{\mathrm{C}_{6} \mathrm{H}_{12} \mathrm{O}_{6}}=\underset{\text { lactic acid }}{2 \mathrm{C}_{3} \mathrm{H}_{6} \mathrm{O}_{3}}
$$

This change of glucose to lactic acid under the catalytic influence of the alkaline calcium hydrate probably occurs by means of a shifting of the elements of the water, a process which in many long chains seems to occur with considerable facility, and is dependent on the spatial configuration of the molecule involved. Thus the change of sugar to lactic acid is readily effected by means of many micro-organisms in the case of glucose, fructose, and mannose, but with considerable difficulty in the case of galactose. In the three former sugars the atoms round the two middle carbon atoms of the chain are disposed thus :

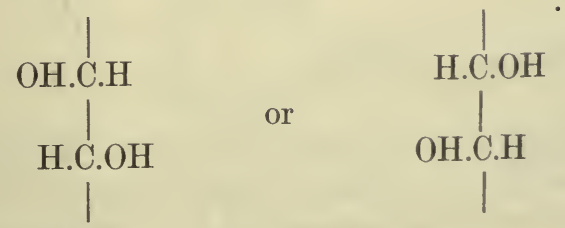


When either of these arrangements reacts with water, thus :
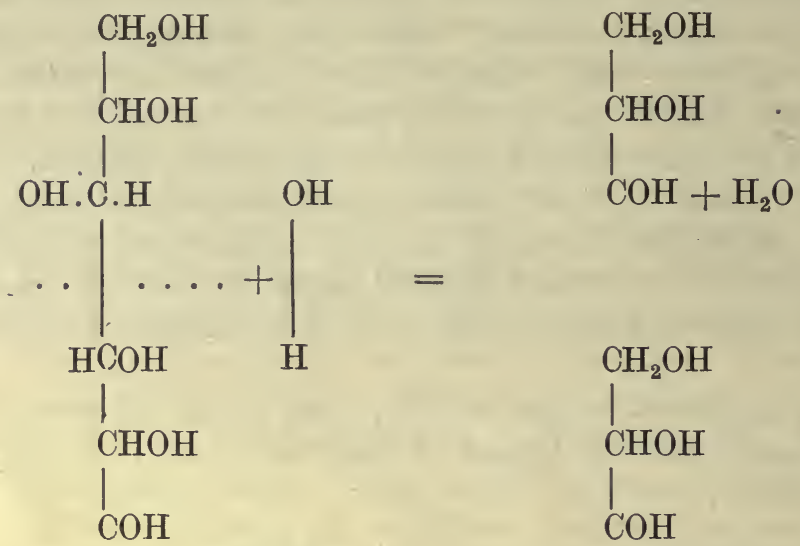

we obtain two molecules of glyceric aldehyde, which then by a further shifting of the $\mathrm{OH}$ and $\mathrm{H}$ groups becomes

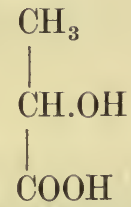

lactic acid

Lactic acid with ammonia and some dehydrating agent will give aninopropionic acid or alanine. The formation of the higher amino-acids involves a process of reduction of the sugar first formed in the chlorophyll granules. It is possible, however, that the starting-point for the amino-acid synthesis may be, not a hexose itself, but some other substances, formed, so to speak, as by-products in the assimilation of sugar from carbon dioxide. We have seen reason to believe that the first result of the action of the sun's rays within the chlorophyll corpuscle is formaldehyde. This substance in the presence of calcium carbonate when exposed to the light gives a mixture of glyceryl aldehyde and dihydroxyacetone. If we can assume that acetone is formed from the latter by a process of reduction, we might possibly derive leucine from an interaction of this substance with lactic acid and ammonia. Thus :

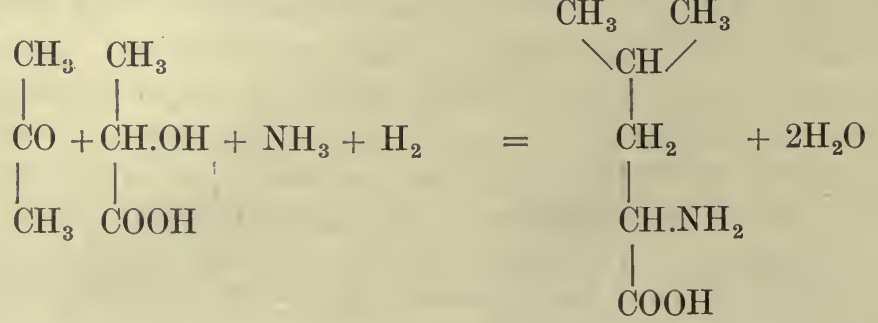


As an intermediate product in the synthesis of starch, glyoxylic acid CHO $\mathrm{COOH}$

has been described as occurring in the green parts of plants. This

substance with ammonia gives formyl glycine, and by the splitting off of formic acid, glycine or amino-acetic acid. Why nitrates are necessary for certain forms of plants is not at present understood. In the proteins nitrogen always occurs in an unoxidised form as $\mathrm{NH}$ or $\mathrm{NH}_{2}$, and the nitrates taken up from the soil must therefore undergo reduction before they can be built into the protein molecule. It is supposed that they may pass through a series of reductions, namely:

$\begin{array}{cccl}\mathrm{HNO}_{3} & \mathrm{HNO}_{2} & \mathrm{HNO} & \mathrm{H}_{2} \mathrm{~N}-\mathrm{OH} \\ \text { nitric acid } & \text { nitrous acid } & \text { hyponitrous acid } & \text { hydroxylamine }\end{array}$

and that the latter substance then reacts with formaldehyde or other substance derived from the carbon dioxide assimilation to form amino-compounds. In general we may say that the probable mechanism of formation of amino-acids is the production of $\alpha$-oxyacids, which then react with ammonia to form the amino-acids of the protein molecule; but of the exact steps in this process we are at present ignorant. Knoop's work would point to the ketonic acids as forming one step, and as interacting with ammonia, with simultaneous reduction, to form amino-acids.

The pyrrol ring which occurs in proline and in oxyproline may possibly be derived from an open chain amino-acid, and it has, in fact, been suggested that the proline found in the products of the acid digestion of proteins is derived from ornithine by a process of condensation with the loss of ammonia. Thus :

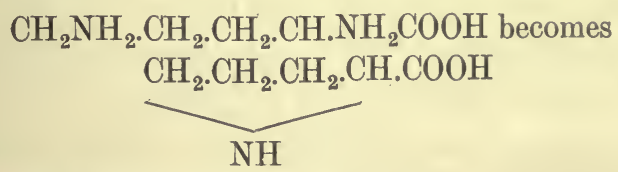

or, as it is generally written :

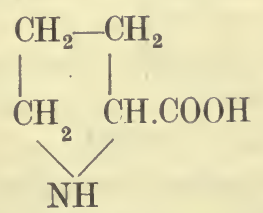

Its pre-existence in the protein molecule is, however, practically assured, and it plays an important part in the building up both of chlorophyll and of hæmatin, the prosthetic group of hæmoglobin.

Iminazol

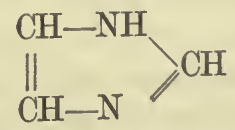

occurs in histidine (which is iminazol alanine), and can be formed fairly readily by the action of certain catalytic agents on a mixture of glucose and ammonia. Thus, if a solution of glucose with ammonia and zine oxide be 
exposed to light, methyl iminazol is formed in large quantities. Windaus and Knoop imagined that in this process glyceric aldehyde and formaldehyde are first formed, and that these then interact with ammonia to form methyl iminazol.

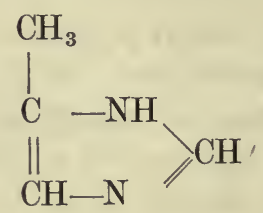

It is interesting to note that if we attach to this compound carbamide or urea we obtain a body belonging to the class of purines. Xanthine, for instance, would have a formula

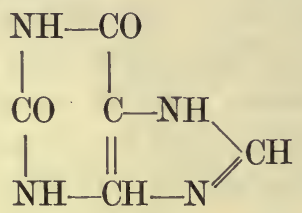

Thus by the action of simple catalytic agencies on sugar and ammonia we can obtain the iminazol nucleus, and by easy transitions pass through this to the purine nucleus with its contained ring, the pyrimidine nucleus, found in the bases cytosine, uracil, \&c., which occur in the nucleins.

With regard to the formation of the aromatic constituents of the protein molecule, i.e. those containing the benzene and indol rings, we have at present very little indication even of the lines along which it might be possible to prosecute our researches. It has been suggested that inosite may represent some stage in the formation of the benzene ring from the open chain found in the carbohydrates. Inosite has the same formula as glucose, namely, $\mathrm{C}_{6} \mathrm{H}_{12} \mathrm{O}_{6}$, but is a saturated ring compound :

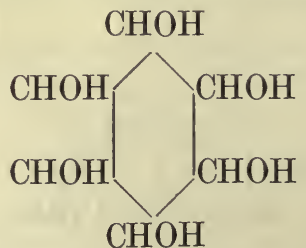

and may be expected to be formed as a result of polymerisation of formaldehyde. We have no evidence, however, of the possibility of such a formation, and the relations of this substance with the benzene compounds are by no means intimate. It is of such universal occurrence, both in plants and animals, that it is difficult to refrain from the suspicion that it may play some part as an intermediate stage between the fatty and the aromatic series.

Since plants are able to manufacture all these varied substances out of the products of assimilation of carbon and ammonia or nitrates, they must also find no difficulty in transforming one amino-acid into another, and we know that most plants can procure their nitrogen from a solution of a single amino-acid as well as from a nutrient fluid containing the nitrogen in the form 
of ammonia. In animals the power of transforming one amino-acid into another, of one group into another, is probably strictly limited. So far as we know, nearly all the amino-acids utilised in the building up of the animal proteins are derived directly from those contained in the food. On the other hand, we have evidence in the animal body of synthesis of the purine bodies, and therefore of the pyrimidine and iminazol rings. The hen's egg at the beginning of incubation contains very little nuclein, nearly the whole of its phosphorus being present in the form of phosphoproteins and lecithin. As incubation proceeds these substances disappear, their place being taken by the nucleins which form the chief constituent of the nuclei of the developing chick. In the same way the ovaries and testes of the salmon are formed during their sojourn in fresh water at the expense of the skeletal muscles, especially those of the back. Here again there is a transformation of a tissue poor in purine bases into a tissue which consists almost exclusively of nucleins and protamines. Whether in this case there is a direct conversion of the mono-amino-acids of the muscle proteins into the diamino-acids and bases typical of protamines, we do not know. It is more probable that only diamino-acids and bases previously existing in the muscle are utilised for the formation of the generative glands, the other amino-acids being oxidised and utilised for the ordinary energy requirements of the animal.

\section{THE SYNTHESIS OF FATS}

In some plants fat globules have been stated to appear as the first products of the assimilation of carbon dioxide under the influence of sunlight, but there is no doubt that as a rule the formation of fats as reserve material in seeds or fruits occurs at the expense of carbohydrates. In the higher animals, too, although a certain amount of the fat of the body is derived from the fat taken up with the food, the organism can also manufacture neutral fat out of the carbohydrates presented to it in its food. The problem, therefore, of the synthesis of the fats is the problem of the conversion of a sugar such as glucose into glycerin and the fatty acids. Although this conversion is apparently so easily effected by the living organism, it is one which from the chemical standpoint involves considerable difficulties. On account of the fact that the higher fatty acids consist largely of oleic and stearic acids, i.e. acids containing eighteen carbon atoms in their chain, it has been thought that the synthesis might be brought about by the linking together of three molecules of a hexose. Such a change would involve a series of difficult chemical transformations. For instance, no less than sixteen out of the eighteen oxygen atoms present in the three glucose molecules would have to be dislodged in order to convert the chain into stearic acid. Moreover, although these two acids contain a multiple of six carbon atoms, a whole array of fats are found both in plants and animals which could not be derived by a simple aggregation of glucose molecules, and it is worthy of note that, of all the fatty acids which occur in nature, all those with more than five carbon atoms contain an even number of carbon atoms. Thus in milk, in addition to the three common fats, tristearin, tripalmitin, and triolein, we find the 
glycerides of caproic, caprylic, capric, lauric, and myristic acids, i.e. acids with $6,8,10,12$, and 14 carbon atoms. In all cases these acids are the normal acids with straight unbranched chains. It seems probable that in the transformation of carbohydrate into fatty acid the latter is built up, not by six carbon atoms, but by two carbon atoms at a time. It has been suggested by Magnus Lévy and by Leathes that the transformation may occur by way of lactic acid. We have seen already that glucose and the sugars of analogous composition may be converted under the influence either of sunlight or of micro-organisms into lactic acid. Lactic acid breaks down with readiness into aldehyde and formic acid.

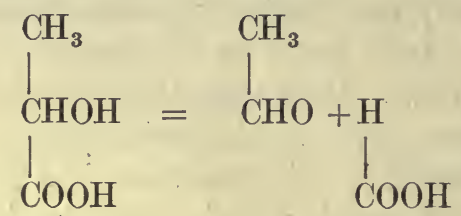

Aldehyde undergoes condensation to form aldol.

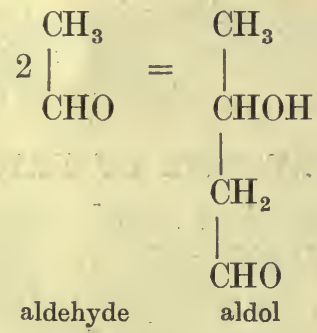

Aldol reacts with water and undergoes a shifting of its $\mathrm{OH}$ and $\mathrm{H}$ groups, in a manner with which we are already familiar as occurring in the conversion of glucose into lactic acid, forming butyric acid. We may represent the reaction in the following way, placing the water molecules opposite those groups of the aldol molecule with which they react :

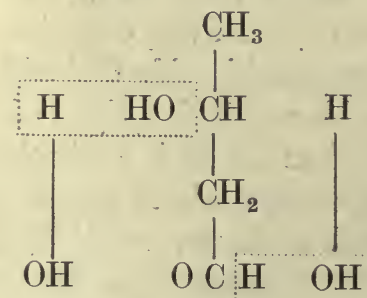

gives

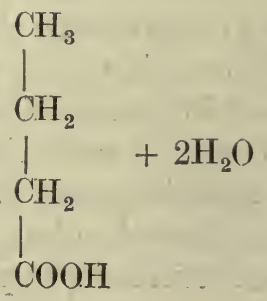


It will be seen that although water must enter into reaction there is no addition of water to the aldol in order to form the butyric acid.

It has been suggested that similar reactions might account for the formation of the higher fatty acids, in which case one molecule of acetic aldehyde would be added to the fatty acid in order to build up the acid which is next highest in the series. Although certain of the higher acids have been prepared in this way, proof is still wanting that a continuous series of syntheses may be effected by the continuous addition of aldehyde. Such a hypothesis is, however, more probable than the direct conversion of three molecules of sugar into one molecule of stearic acid. The latter change would be associated with a very great absorption of energy, whereas a continuous building up of fatty acids by the addition of aldehyde obtained through lactic acid from the disintegration of hexose molecules only requires a small expenditure of energy, which could be obtained by the combustion of the formic acid formed as a by-product in the process. If we suppose that the synthesis of the higher fatty acids from sugar is carried out in this way, the energy equations would be as follows (Leathes) :

1 g. mol. glucose $\left.\begin{array}{c}677 \cdot 2 \text { cals. }\end{array}\right\} \rightarrow\left\{\begin{array}{c}2 \text { g. mols. aldehyde }+2 \text { g. mols. formic acid. } \\ 2 \times 275.5+2 \times 61 \cdot 7\end{array}\right.$ $=674.4$ cals.

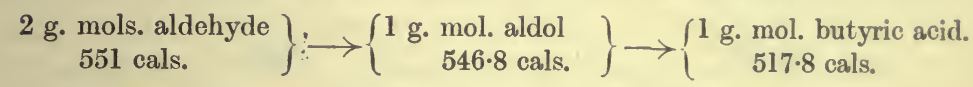

Or, tracing the same change on as far as palmitic acid:
4 g. mols. glucose $\} \rightarrow\{1$ g. mol. palmitic acid +8 g. mols. formic acid. 2708 cals. 2362 cals. +494 cals. $=2856$ cals.

In the first stage of the synthesis, the reaction leading to butyric acid, the net result would be, supposing the formic acid to be oxidised, that some 160 calories, or nearly 25 per cent. of the whole energy, would be rendered available for other purposes. In the latter stages leading to palmitic acid some of the energy derived from the oxidation of the formic acid would be required for effecting the synthesis, and only about 12.5 per cent. of the original amount contained in the sugar would be set free. It is worth noting that in the butyric fermentation of sugar by micro-organisms there is a production first of lactic acid, and this substance then disappears to give place to butyric acid. At the same time carbonic acid and hydrogen are evolved, both gases being derived from the decomposition of the formic acid. In the process a certain amount of caproic acid is always produced, and the crude butyric acid of fermentation is used as the source from which commercial caproic acid is derived.

Attempts to produce the higher fatty acids by the condensation of successive molecules of aldehyde have so far only resulted in the production of branched chains Raper has shown that the normal caproic acid may be formed by the condensation of aldol with itself. Miss Smedley has suggested that a more probable line of synthesis lies through pyruvic acid. Pyruvic acid, which may be produced in the body from 
lactic acid, and so from carbohydrate, is fermented by yeast with the prcducticn of acetaldehyde and carbon dioxide, by means of a ferment carboxylase. If we assume the existence of a similar ferment in the cells of the body, it would split this acid into aldehyde and $\mathrm{CO}_{2}$. Aldehyde, however, combines with a molecule of pyruvic acid to form a higher keto=acid, which might either be oxidised to the fatty acid containing one carbon atom less, or might be again transformed by enzymes into an aldehyde capable of reacting with another molecule of pyruvic acid. These changes are represented in the following equations :

$$
\begin{aligned}
\mathrm{CH}_{3} \mathrm{CO} \cdot \mathrm{COOH} & =\mathrm{CH}_{3} \mathrm{CHO}+\mathrm{CO}_{2} \\
\mathrm{CH}_{3} \mathrm{CHO}+\mathrm{CH}_{3} \mathrm{CO} \cdot \mathrm{COOH} & =\mathrm{CH}_{3} \mathrm{CHOH} \cdot \mathrm{CH}_{2} \cdot \mathrm{CO} \cdot \mathrm{COOH} \\
\mathrm{CH}_{3} \mathrm{CHOH} . \mathrm{CH}_{2} \cdot \mathrm{CO} \cdot \mathrm{COOH}+\mathrm{O} & =\mathrm{CH}_{3} \mathrm{CHOH} \cdot \mathrm{CH}_{2} \mathrm{COOH}+\mathrm{CO}_{2}
\end{aligned}
$$

$\beta$-oxyacids would thus be a normal stage in the building up as well as in the breaking down of fatty acids.

The glycerin which enters into the formation of the ordinary neutral fats can be synthetised by both plants and animals, and there is every ground for believing that it, like the fatty acids, may be derived from carbohydrates. We have already seen that in the conversion of glucose into lactic acid the first step is the formation of glyceric aldehyde,
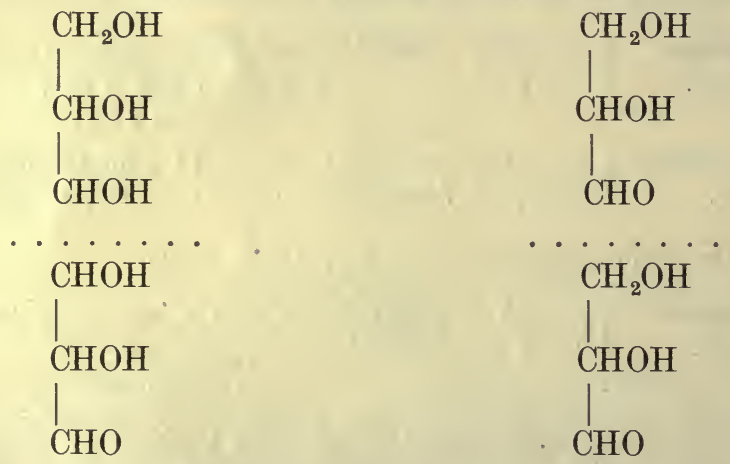

and it is easy to understand how by a process of reduction the aldehyde is converted into the corresponding alcohol, namely, glycerin. The synthesis of the neutral fat from glycerin and fatty acid is a change which can be accomplished by many ferments. It is one involving practically no absorption or expenditure of energy. The change is a reversible one, and we find both in plants and animals that a hydrolysis of neutral fat into fatty acid and glycerin always occurs when a transport of the fat is required, while the laying down of fat as a store of energy is always preceded by a resynthesis of the neutral fat. We shall have occasion to deal in greater detail with these questions when we have to discuss the formation and fate of the fat in the animal body. 


\section{CHAPTER IV}

\section{THE ENERGETIC BASIS OF THE BODY}

\section{SECTION I}

\section{THE ENERGY OF MOLECULES IN SOLUTION}

EVERY vital act involves at the same time a transformation of the material basis of the living cell and a transformation of energy. The ultimate source of the energies displayed by the animal organism is the chemical energy of the substances taken in as food. In all the changes undergone by either matter or energy in the body there is neither destruction nor creation. The living organism may therefore be regarded in one sense as a machine, that is to say, a system for the conversion of one form of energy into another. Thus the steam-engine converts the potential energy of overheated steam into mechanical work; a gas-engine the chemical energy of an explosive mixture of gases into heat and mechanical energy ; in a battery there is a transformation of chemical into electrical energy ; in a dynamo, of mechanical into electrical energy, and so on. In the living cell the chemical energy of the food may undergo conversion into any of the other forms mentioned above, i.e. heat, work, electrical difference of potential, or it may be used for the production of other chemical substances possessing perhaps as much potential energy as or more than the food-stuffs themselves.

Protoplasm, which is the seat of all these changes in both plants and animals, is active only within fairly narrow limits of temperature, approximately between $5^{\circ}$ and $40^{\circ} \mathrm{C}$. In consistence it is slimy and wet, water forming from 70 to 95 per cent. of its bulk. No substance introduced into the protoplasm has any influence on it, unless it be soluble, and the first stage in the preparation of food-stuffs for assimilation always consists in a process of solution. The sole source of energy to the body being that conveyed with the food, it follows that all the energy with which we have to deal is the energy of molecules in watery solution, the playground of whose activities is a jelly-like mass of colloidal material, heterogeneous yet structurally continuous. It is important, therefore, at the outset to inquire into the nature of this energy and the methods by which it may be measured.

OSMOTIC PRESSURE. If we place two gas jars together, mouth to mouth, as in Fig. 19, the upper jar containing hydrogen and the lower jar some heavier gas, such as oxygen or carbon dioxide, within a very short time 
the gases will have become intimately mixed, and each jar will contain an equal amount of both gases. We say that each gas has diffused into the other, and ascribe the diffusion to the movement of the gaseous molecules. In closed vessels the rapidly moving molecules are continually impinging on the walls of the vessels and rebounding, and it is this bombardment by the gaseous molecules which is responsible for the pressure

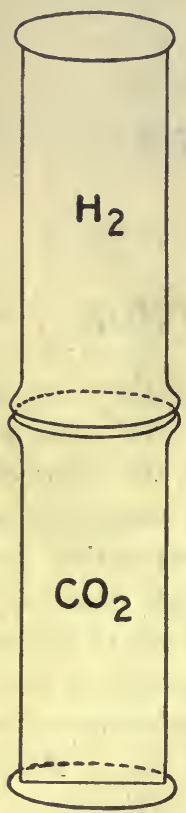

FIG. 19. exerted by a gas on its containing walls. If we double the amount of gas in a given space, we double the amount of molecules which strike a unit area of the wall in unit time, and therefore double the pressure exerted by the gas on the vessel wall. In this way we may explain the law of Boyle that the pressure of a gas is inversely proportional to its volume, or the product of pressure and volume at a given temperature is a constant, $\mathrm{PV}=\mathrm{C}$, or since the energy of the molecules is proportionate to the absolute temperature, $\mathrm{PV}=\mathrm{RT}$, the familiar gas equation.

The molecules of substances in solution behave, within the limits of the solution, in a manner precisely similar to the free molecules of a gas. Thus, if a vessel be half filled with a 10 per cent. solution of sugar and be then filled up by carefully pouring distilled water, so as to form a distinct layer on the heavier sugar solution, the sugar at once begins to move upwards into the distilled water. In consequence of the resistance offered to the movement of the sugar molecules through the water, this process of diffusion is slow, but if the vessel be left undisturbed and free from any agitation for two or three months the sugar will be found to spread gradually throughout the liquid, so that at the end of this time all parts of the fluid contain a uniform amount of sugar.

This process of diffusion, like that of gases, must be ascribed to a continuous translatory movement of the dissolved molecules. Since the molecules possess mass and are endowed with a velocity, it is evident that they can exercise a pressure on any membrane or dividing surface which tends to hinder their free passage within the limits of the solvent. Thus if we take a pig's bladder containing a 20 per cent. solution of dextrose and immerse it in distilled water, water will pass in and distend the bladder to such an extent that it may burst from the rise of pressure in its interior. This swelling of the bladder is due to the fact that the molecules of sugar pass through it only with difficulty, and therefore in their passage outwards towards the confines of the water exert a pressure on the walls, driving them apart and so causing a distension of the bladder. It is impossible, however, by this means to obtain the full osmotic pressure due to the pressure exerted by the sugar molecules, since the bladder wall itself is not absolutely impermeable to sugar. If we imagine the sugar solution confined in a cylinder and covered with a layer of distilled water, the movement of the sugar 
molecules will cause them to wander from the lower to the upper part, and this process of diffusion will cease only when the concentration has become the same in all parts of the solution. Supposing, however, the two fluids are separated by a piston, P (Fig. 20), which is 'semi-permeable,' i.e. allows free passage to water, but not to the dissolved sugar, the molecules of sugar will now exert a pressure on the piston similar to that exerted on the walls of the containing vessel, and will tend to drive it upwards. The force which it is necessary to apply to the piston to prevent its upward movement will be the measure of the osmotic pressure of the sugar in the solution. If the piston be pressed down with a greater force, the sugar molecules alone are pressed together, since water can pass freely through the surface of the piston, and the sugar solution is therefore rendered more concentrated. Since force must be applied to the piston in order to press it down, work is done in the process, so that the concentration of any solution involves the performance of an amount of work determined by the initial and final osmotic pressures of the solution. If, on the other hand, a weight be applied to the piston which is less than the osmotic pressure exerted by the sugar solution, the piston with its weight will be moved upwards, and the solution will undergo dilution until its osmotic pressure exactly balances the weight on the piston. We see that the osmotic pressure of a solution represents a certain amount of

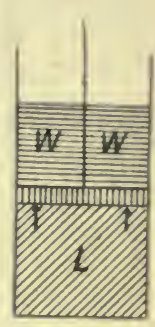

Fig. 20 potential energy, which can be utilised in an osmotic machine, such as that represented in the diagram, for the performance of work.

THE MEASUREMENT OF THE OSMOTIC PRESSURE. By a method differing but little from the one just sketched out, Pfeffer succeeded directly in measuring the osmotic pressure of certain solutions. For this purpose Pfeffer took advantage of the fact, discovered by Traube, that various precipitates, if deposited in the form of membranes, were impermeable to the substances producing them as well as to some other dissolved substances, though allowing a free passage of water. Thus, if a drop of a concentrated solution of potassium ferrocyanide suspended to a glass rod be introduced carefully into a more dilute solution of copper sulphate, it will be observed that at the junction of the drop and the surrounding fluid there is a brown membranous precipitate of copper ferrocyanide. In consequence of the greater concentration of the fluid in the drop, a constant passage of water takes place from without inwards through the membrane, and the drop therefore grows continually in size, sometimes sending out branches as a result of slight currents in the fluid set up by accidental vibrations. Sugar introduced into such a drop, although quickening its rate of growth, does not pass out into the surrounding copper sulphate solution, nor is there any passage of copper sulphate inwards or potassium ferrocyanide outwards. Pfeffer conceived the idea of depositing such a semi-permeable membrane within the interstices of a clay cell. Strengthened in this way, it is able to afford a resistance to pressure, and therefore to permit of the contained fluid reaching 
its full osmotic pressure. For this purpose a porous jar carefully cleansed and containing a solution of sugar mixed with a little copper sulphate is dipped into a weak solution of potassium ferrocyanide. A semi-permeable membrane of copper ferrocyanide is thus produced in the pores of the filter, and this, while allowing the passing of water, is impermeable to the sugar. The tube is then fitted with a cork provided with a closed mercurial manometer and is immersed in distilled water, when it is found that water passes into the cell until the pressure within the latter is equal to the osmotic pressure of the dissolved substances. By this means Pfeffer obtained the following results with a 1 per cent. solution of cane sugar at different temperatures :

\begin{tabular}{|c|c|c|}
\hline \multirow{2}{*}{ Temp. ${ }^{\circ} \mathrm{C}}$. & \multicolumn{2}{|c|}{ Pressure in atmospheres } \\
\cline { 2 - 3 } & & Calculated \\
\hline & & Atm. \\
$6 \cdot 8$ & $0 \cdot 664$ & $0 \cdot 665$ \\
$13 \cdot 7$ & $0 \cdot 691$ & $0 \cdot 681$ \\
$22 \cdot 0$ & $0 \cdot 721$ & $0 \cdot 701$ \\
$32 \cdot 0$ & $0 \cdot 716$ & $0 \cdot 725$ \\
$36 \cdot 0$ & $0 \cdot 746$ & $0 \cdot 735$ \\
\hline
\end{tabular}

It is always possible to calculate the pressure of a gas when its nature, its mass, and its volume are known. By Avogadro's hypothesis, equal volumes of gases at the same pressure contain equal numbers of molecules. On this account the molecular weight of any gas can be reckoned directly from its density. The figures obtained by Pfeffer show that the same laws apply to the osmotic pressure of substances in solution as to the pressure of gases in their free state. It is therefore possible to reckon the osmotic pressure which would be exerted by 1 per cent. sugar in solution at a given temperature.

This calculation is carried out as follows: A gramme molecule of any gas at $0^{\circ} \mathrm{C}$. and $760 \mathrm{~mm} . \mathrm{Hg}$ has a volume of 22.4 litres, therefore 342 grammes of cane sugar (the molecular weight of $\mathrm{C}_{12} \mathrm{H}_{22} \mathrm{O}_{11}=342$ ), if it could be converted into a gas at $0^{\circ} \mathrm{C}$. and $760 \mathrm{~mm} . \mathrm{Hg}$, would have a volume of $22 \cdot 4$ litres. One gramme of sugar therefore at the same temperature and pressure would have a volume of $\frac{22 \cdot 4}{342}$ litres $=65 \cdot 5$ c.c. $\quad$ In Pfeffer's experiment the gramme of sugar was dissolved in 100 grammes of water, making a total volume at $0^{\circ} \mathrm{C}$. of $100 \cdot 6$ c.c. The gaseous pressure of the sugar molecules in this solution will therefore amount to $\frac{65 \cdot 5}{100 \cdot 6}=0.651$ atmosphere. At a temperature of 6.8 the pressure would be $0 \cdot 667$ atmosphere, as against the observed 0.664 atmosphere.

Pfeffer's method is difficult to carry out and is not applicable to all dissolved substances, since the cupric ferrocyanide membrane is permeable for many substances, such as potassium nitrate or hydrochloric acid. Other 
indirect methods have therefore been applied to the comparison of the osmotic pressures of different solutions.

DETERMINATION OF THE OSMOTIC PRESSURE BY PLASMOLYSIS. Solle tions which have the same osmotic pressure are spoken of as isosmotic or isotonic, The method of plasmolysis, which we owe to the botanist De Vries, consists essentially in the comparison of the osmotic pressure of solutions with that of the cell sap of certain plant cells, and depends on the fact that the ' primordial utricle,' the layer of protoplasm enclosing the cell sap, while freely permeable to water, is impermeable to a large number of salts and other crystalloids, such as sugar. It is therefore, so far as concerns these substances, 'semi-permeable.' The cells which have been most used for this purpose are the cuticular cells on the mid-rib of the lower surface of the leaves of tradescantia discolor. If some of these cells are brought into a concentrated salt solution, which is 'hypertonic' as compared with the cell sap, water passes out of the cell into the salt solution, until the contents of the cell attain a molecular concentration equal to that of the surrounding medium. The protoplasmic layer therefore shrinks, leaving a space between it and the cell wall (Fig. 7, p. 23). If the outer solution has a smaller molecular concentration than the cell sap, water passes into the cell and causes here a rise of pressure. which simply presses the protoplasm still more closely against the cell wall. If we determine the concentration of the salt solution at which the shrinkage of the protoplasm, the plasmolysis, just occurs, and another smaller concentration at which plasmolysis is absent, we know that the concentration of the cell sap lies between those of the two salt solutions. Thus, if plasmolysis occurs in a solution containing 0.6 per cent. sodium chloride and is absent in a solution containing 0.59 per cent. of the same salt, the concentration of the cell sap must be about equivalent to a 0.595 per cent. $\mathrm{NaCl}$ solution. Solutions of different salts, in which plasmolysis just occurs, must also be isotonic with one another. Thus a 1.01 per cent. solution of $\mathrm{KNO}_{3}$ is found to be isotonic with a 0.58 per cent. $\mathrm{NaCl}$ solution.

DETERMINATION BY HAMBURGER'S BLOOD-CORPUSCLE METHOD. The limiting external layer of red blood-corpuscles resembles the primordial utricle of plant cells in being impermeable to a number of dissolved substances. If, therefore, it be placed in a solution of smaller concentration than the corpuscle contents, it will swell up and, since it has no supporting cell wall, the increase in size will go on until the corpuscle bursts, and its contained red colouring-matter, hæmoglobin, passes into solution in the surrounding fluid. If the corpuscles be then allowed to settle or be centrifuged, the fact that hæmolysis has occurred is shown by the red colour of the clear supernatant fluid. With a given sample of blood, the concentration of a potassium nitrate solution is found at which the first traces of hæmolysis occur. In order to determine the osmotic pressure of a solution, say, of sugar or of sodium chloride, these are also added in various dilutions to blood corpuscles until we get solutions in which hæmolysis just occurs. These solutions will then be isotonic with the first determined potassium nitrate solutions. As an example of this method may be adduced the following results :

\begin{tabular}{|c|c|c|c|c|}
\hline 1 & 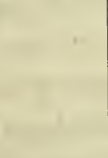 & $\begin{array}{l}\text { Concentration } \\
\text { of the solution } \\
\text { in which the } \\
\text { blood corpuseles } \\
\text { do not lose } \\
\text { hæmoglobin }\end{array}$ & $\begin{array}{l}\text { Concentration } \\
\text { of the solution } \\
\text { in which the } \\
\text { blood corpuseles } \\
\text { begin to lose } \\
\text { hæmoglobin }\end{array}$ & $\begin{array}{c}\text { Mean } \\
\text { concentration }\end{array}$ \\
\hline $\begin{array}{l}\text { Potassium nitrate } \\
\text { Sodium chloride } \\
\text { Cane sugar } \\
\text { Potassium iodide } \\
\text { Sodium iodide. } \\
\text { Potassium bromide }\end{array}$ & $\begin{array}{ll}\cdot & \cdot \\
. & \cdot \\
. & . \\
\cdot & . \\
. & .\end{array}$ & $\begin{array}{c}\text { Per cent. } \\
1 \cdot 04 \\
0 \cdot 60 \\
6 \cdot 29 \\
1 \cdot 71 \\
1 \cdot 54 \\
1 \cdot 22\end{array}$ & $\begin{array}{c}\text { Per cent. } \\
0.96 \\
0 \cdot 56 \\
5 \cdot 63 \\
1 \cdot 57 \\
1 \cdot 47 \\
1 \cdot 13\end{array}$ & $\begin{array}{l}\text { Per cent. } \\
1 \cdot 00 \\
0 \cdot 585 \\
5 \cdot 96 \\
1 \cdot 64 \\
1 \cdot 505 \\
1 \cdot 17\end{array}$ \\
\hline
\end{tabular}


OSMOTIC PRESSURE OF ELECTROLYTES. It will be noticed in the last Table that the isotonic solutions of different salts contain these salts in the proportion of their molecular weights, i.e. each solution contains the same number of molecules of dissolved salt. For the term isotonic we might therefore employ equimolecular. When, however, these salts are compared with solutions of sugar, it is found that the osmotic pressures of the salt solutions are double or nearly double those of equimolecular solutions of sugar. The osmotic pressure of a sugar solution is equal to the pressure which its molecules would exert if they occupied the same space in a gaseous form. A dilute salt solution therefore acts as if every one of its molecules were doubled. This deviation of salt solutions from solutions of sugar is bound up with the power of the former to conduct an electric current. A sugar solution conducts electricity little better than pure water. On the other
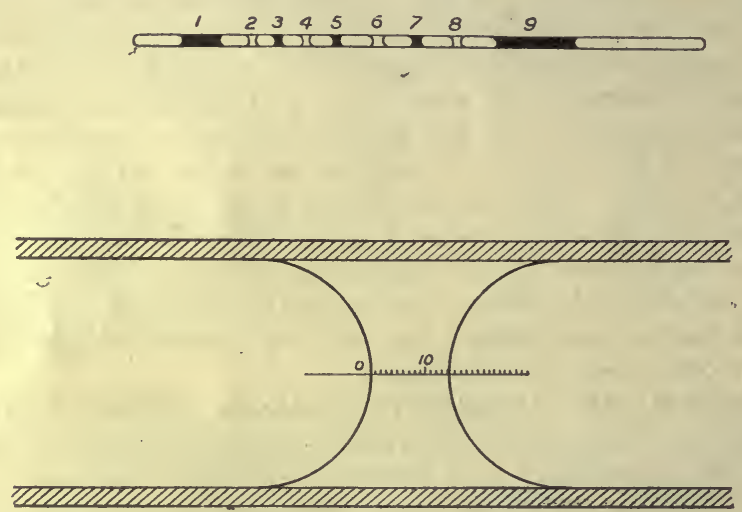

FIG. 21. Diagram to illustrate Barger's method of determining osmotic pressure. The upper figure shows the capillary tube with nine alternate drops of cane sugar and the substance under investigation.

hand, the smallest trace of salt added to distilled water enormously increases its conducting power. As Arrhenius has shown, this increase of the osmotic pressure of a salt solution is determined by the dissociation which all these salts undergo in watery solution. A dilute solution of sodium chloride contains, not the molecule $\mathrm{NaCl}$, but an equal number of the ions $\mathrm{Na}$ and $\mathrm{Cl}, \mathrm{Na}$ carrying a positive charge while the $\mathrm{Cl}$ ions carry a negative charge. As regards osmotic pressure and various other properties, each of these charged ions acts as a whole molecule. It is the existence of these ions which confers on the salt solution the power of conducting electricity - a power the exercise of which is attended with a dissociation (an electrolysis) of the salt into its constituent ions, the electro-positive ion being deposited at the negative pole while the electro-negative ion is deposited at the positive pole. The molecular weight of $\mathrm{NaCl}$ is 58.5. The molecular weight of glucose is 180 . If there were no dissociation, a 0.58 per cent. solution of $\mathrm{NaCl}$ would be isotonic or isosmotic with a 1.8 per cent. solution of glucose. On account of the ionic dissociation or ionisation, it is actually isosmotic with a glucose solution of about $3 \cdot 5$ per cent. 
INDIRECT METHODS OF MEASURING OSMOTIC PRESSURE. Equimolecular solutions have the same osmotic pressures. Since the osmotic pressure of a solution is therefore directly dependent on the number of molecules it contains in unit space, any method which will give us informa. tion as to the number of molecules present will also enable us to determine the osmotic pressure. Other properties of solutions which, like the osmotic pressure, are functions of the number of molecules present, are vapour-tension, boiling-point, freezingpoint. The presence of a substance in solution in water diminishes its vapour-tension at any given temperature, raises its boiling-point, and depresses its freezing-point, and the extent of the deviation from distilled water is proportional to the number of dissolved molecules present. The determination of the rise of boiling-point, though much employed by chemists, is of very little value in physiology, owing to the fact that nearly all the fluids of the body are seriously modified in character by a rise of tem. perature to $100^{\circ} \mathrm{C}$. On the other hand, Barger has suggested an ingenious method in which the alteration of vapour-tension is made the basis of a method for determining the osmotic pressure of small quantities of fluids at ordinary temperatures. And this method may find important applications in physiology.

BARGER'S METHOD. Drops of the fluid, the vapourtension of which it is desired to ascertain, are drawn up into a tube $(1.5 \mathrm{~mm}$. in diameter), so as to alternate with small drops of cane sugar solution of known content (Fig. 21). Water in a state of vapour will pass from the solution of which the vapour-tension is the higher. By observing the edge of a drop under a magnification of 65 diams., it can be casily seen whether it has grown or diminished in size. If the edge of the drop remains stationary, it shows that the vapour-tension and the osmotic pressure of the two fluids are equal. A series of trials is made with different strengths of salt solution until this equality is established. In this method only minimal quantities of material are required, and the determination of the aqueous tension is made at ordinary temperatures.

The method, however, which is of greatest value

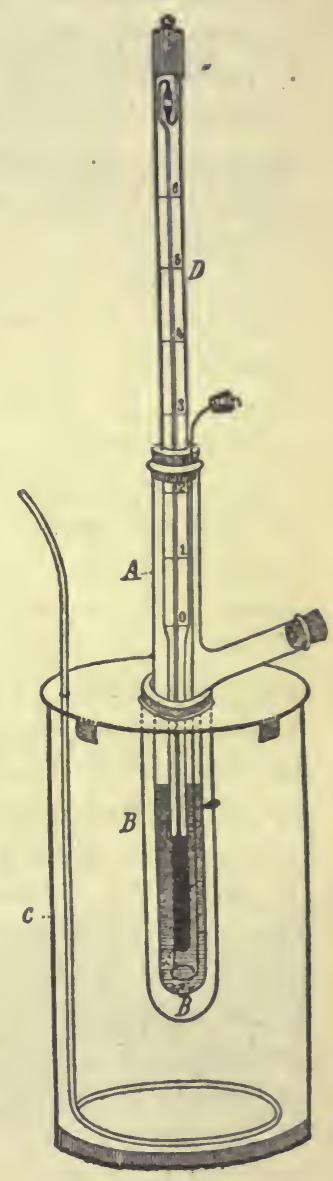

FIG. 22. Beckmann's a pparatus for determination of freezing-point. in physiology is the measurement of the depression of freezing-point. The depression of freezing-point can be converted directly into osmotic pressure by multiplying the depression of freezing-point observed by the factor $122 \cdot 7$. Thus a 1 per cent. solution of sodium chloride with $\Delta=0.61$ will have an osmotic pressure of $0.61 \times 122.7=74.847$ metres of water.

The determination is carried out in a Beckmann's apparatus with a thermometer reading to $\frac{1}{100}^{\circ}$ C. (Fig. 22). A solution freezes at a lower temperature than pure 
water, and the depression of freezing-point is proportional to the number of molecules present. Thus the freezing-point of a 1 per cent. solution of $\mathrm{NaCl}$ is $-0 \cdot 61^{\circ} \mathrm{C}$. The depression of freezing-point is generally represented by the Greek letter $\Delta$. This method has the advantage that the fluids are in most cases in no wise altered by the process of freezing, and it can be applied to solutions containing coagulable proteins which would be irretricvably altered by any considerable rise of temperature.

Every substance in solution possesses, therefore, a certain amount of potential energy in the form of osmotic pressure. This pressure is independent of the nature of the substance dissolved and is determined merely by its molecular concentration. It can be used as a driving force for the movement by diffusion of the molecules themselves, or, by the use of appropriate mechanisms or 'machines,' for the performance of mechanical work, or, as will be seen later, for the production of electrical differences of potential.

In addition to this osmotic or volume energy every molecule in solution can be regarded as endowed with a chemical energy, which is dependent not only on the number of molecules present, but also on the nature of the molecules. In the case of electrolytes and of substances which are susceptible of ionisation, the potential or intensity of the chemical energy of each molecule is capable of measurement. On the other hand, the chemical energy of a substance such as glucose cannot be definitely expressed apart from consideration of the conditions under which it is present. If we take the whole course of transformations undergone by glucose in the body, we may speak of it as having a potential energy, which is measured by the total heat energy given out by this substance on its complete combustion with oxygen to carbon dioxide and water. In the intermediate changes which it undergoes during its metabolism in the cells of the body, this energy is probably set free by degrees, but its chemical energy in any given phase cannot be measured unless the conditions and the end results of the chemical changes which it is undergoing are known. This chemical energy may be utilised for the production of heat, for the performance of chemical work in the building up of other substances, or by the multiplication of the number of molecules in a solution, for the production of increased osmotic pressure, which in its turn may be converted into the energy of movement either of masses or of molecules. 


\section{SECTION II \\ THE PASSAGE OF WATER AND DISSOLVED SUBSTANCES ACROSS MEMBRANES}

WE have already seen that if, in a solution, the concentration of the dissolved substance or solute is not uniform, there is a movement of the substance from the place of higher to the place of lower concentration, and this movement proceeds until the concentration is equal throughout the fluid. This movement of dissolved substances through a fluid is spoken of as diffusion, and is analogous in all respects with the process by which the intermixture of gases is attained. The movement in the case of dissolved substances, as of gases, takes place from the region of higher to the region of lower (osmotic) pressure. It can therefore be ascribed to differences of pressure, or rather to the factor which we regarded as responsible for the production of the pressure, viz. the movement of the molecules themselves. The rate of diffusion is not the same for all substances. In gases the rate varies inversely as the square root of the density of the gas. Thus hydrogen (density =1) diffuses four times as rapidly as oxygen (density $=16)$. We find similar differences between the rates of diffusion of dissolved substances-differences which also are determined in all probability by the weight and size of the individual molecules, although the relation between molecular weight and rate of diffusion is not so simple as the ratio between these two quantities in gases. The diffusibility of a substance is given by its diffusion coefficient. The amount of dissolved substance, which diffuses in a unit of time across a given area of fluid, is proportional to the difference between the osmotic pressures at two cross-sections of the column of fluid at an infinitesimally small distance apart. If we take a cylindrical mass of solution which is one centimetre long and has a sectional area of one square centimetre (Fig. 23), and maintain a constant difference of concentration between $\mathrm{A}$ and $\mathrm{B}=1$, the diffusion coefficient is the amount of substance which diffuses in a unit of time from A to B. Thus the statement that the diffusion co-efficient of urea is 0.810 at $7 \cdot 5^{\circ} \mathrm{C}$. denotes that if $\mathrm{A}$ be con-

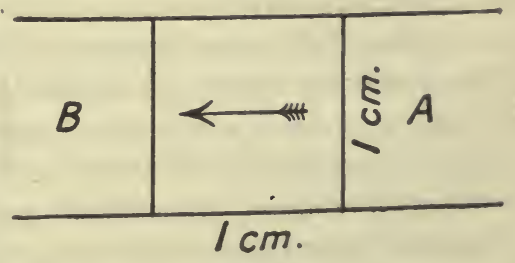

FIG. 23. tinually filled with a 1 per cent. solution of urea, while in B a constant current of distilled water is kept up so as to maintain the concentration at zero, in the course of a 
day 0.810 gramme of urea will pass from A to B through the cylinder of one centimetre in length and one square centimetre in cross-section. The determination of these diffusion coefficients presents many difficulties. The task is, however, rendered easier by the fact, first ascertained by Graham, that diffusion of salts occurs as rapidly through a solid jelly of gelatin or agar-agar as through water. It is therefore possible to make the plug in the diagram solid by the admixture of one of these two substances, and to maintain a constant concentration on the two sides of it by the circulation of fluid without affecting the rate of diffusion through the cylinder by setting up accidental currents.

More important from the physiological point of view than diffusion through fluids is the exchange of fluids (water and dissolved substances), which may take place across membranes. Such processes are of constant occurrence in all parts of the body and are concerned in such functions as the formation and absorption of lymph, the absorption from and secretion into the intestines, absorption from serous cavities, and so on. In many of these functions we shall have to consider later whether the transference across the membrane is determined solely by the nature and concentration of the fluids on the two sides of it or is effected by the active intervention, involving the expenditure of energy, on the part of living cells forming constituent elements of the membrane itself. It is worth our while, therefore, to consider at some greater length the purely physical factors which may be concerned in the passage of water and dissolved substances across membranes.

In the case of fluids containing only one substance in solution, the exchange across the membrane will be determined entirely by the osmotic pressures. Thus, if two watery solutions, with the same osmotic pressure, are separated by a membrane through which diffusion can take place, no change in volume occurs on either side of the membrane. If the solutions on either side of the membrane are of unequal osmotic pressure, water passes from the side where the pressure is lower to the side where it is higher, and there is a simultaneous passage of the solute from the side of greater to the side of less concentration.

If, however, the solutions on the two sides contain dissimilar substances, with different diffusion coefficients, the conditions are more complicated, and may tend even to produce a movement of fluid in apparent opposition to the difference of osmotic pressure. Under these circumstances the nature of the membrane itself is all-important. We may therefore shortly consider the various modes in which interchanges may take place across membranes of varying permeability. We shall see that the close analogy which exists between substances in solution and gases, when dealing with 'semipermeable ' membranes, isalso borne out by experiment when used to predict the behaviour of solutions separated by such permeable membranes as occur in the body.

The simplest case is that in which two fluids are separated by a perfect semi-permeable membrane that permits the passage of water but is absolutely 
impermeable to dissolved substances. In this case the transference of water from one side to the other depends entirely on the difference of osmotic pressure between the two sides.

If we suppose two vessels, A and B (Fig. 24), separated by such a mem. brane, A containing a solution of $\alpha$ and $B$ a solution of $\beta$, water will pass from $A$ to $B$ so long as the osmotic pressure of $\beta$ is greater than the osmotic pressure of the solution of $\alpha$. If $B$ be subjected to a hydrostatic pressure greater than the osmotic difference between the two fluids, water will pass from $\mathrm{B}$ to $\mathrm{A}$ until the force causing filtration or transudation (the hydrostatic pressure) is equal to the force causing absorption into $\mathrm{B}$ (the difference of osmotic pressures). Under no circumstance will there be any transference of salt or dissolved substance between the two sides. Such semi-permeable membranes as this, however, rarely occur in the body over any extent of

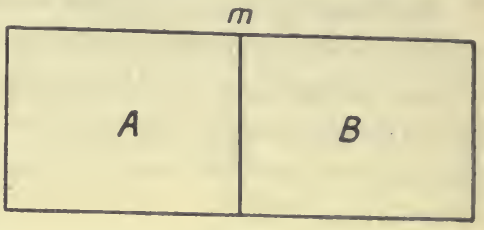

FIG. 24. surface. The external layer of the cell protoplasm may resemble the protoplasmic pellicle of plant cells in possessing this 'semi-permeability'; but in nearly all cases where we have a membrane made up of a number of cells, it can be shown to permit the free passage of at any rate a large number of dissolved substances.

Let us now consider what will occur when the two solutions A and B are separated by a membrane which permits the free passage of salts and water. If the osmotic pressure of $\mathrm{B}$ be higher than $\mathrm{A}$ at the commencement of the experiment, the force tending to move water from $A$ to $B$ will be equal to this osmotic difference. But there is at the same time set up a diffusion of the dissolved substances from $\mathrm{B}$ to $\mathrm{A}$ and from $\mathrm{A}$ to $\mathrm{B}$. The result of this diffusion must be that there is no longer a sudden drop of osmotic pressure from $\mathrm{B}$ to $\mathrm{A}$, and the result of the primary osmotic difference on the movement of water will be minimised in proportion to the freedom of diffusion which takes place through the membrane. Now let us take a case in which A and $B$ represent equimolecular and isotonic solutions of $\alpha$ and $\beta$. It is evident that the movement of water into $\mathrm{A}$ will vary as $\mathrm{A} p-\mathrm{B} p^{*}=0$. But diffusion also occurs of $\alpha$ into $B$ and of $\beta$ into $A$. Now the amount of substance diffusing from a solution is proportional to the concentration, and therefore to its osmotic pressure, as well as to its diffusion coefficient.

Hence the amount of $\alpha$ diffusing into B will vary as $\mathrm{A} p . \alpha k$ (when $k$ is the diffusion coefficient).

In the same way the amount of $\beta$ diffusing into $A$ will vary as $B p . \beta k^{\prime}$.

Hence, if $\alpha k$ is greater than $\beta k^{\prime}$, i.e. if $\alpha$ is more diffusible than $\beta$, the initial result must be that a greater number of molecules of $\alpha$ will pass into $B$ than of $\beta$ into $A$. The solutions on the two sides of the membrane will thus be no longer equimolecular, but the total number of molecules of $\alpha+\beta$ in $B$ will be greater than the number of molecules of $\alpha+\beta$ in $A$, and this differ- 
ence will be most marked in the layers of fluid nearest the membrane. The result, therefore, of the unequal diffusion of the two substances is to upset the previous equality of osmotic pressures. The layer of fluid on the B side of the membrane will have an osmotic pressure greater than the layer of fluid in immediate contact with the A side of the membrane, and there will thus be a movement of water from A to B. Hence if we have two equimolecular and isotonic solutions of different substances separated by a membrane permeable to the solutes, there will be an initial movement of fluid towards the side of the less diffusible substance.

We have an exact parallel to this in Graham's familiar experiment, in which a porous pot filled with hydrogen is connected by a vertical tube with a vessel of mercury. In consequence of the more rapid diffusion outwards of the hydrogen than of atmospheric air inwards, the pressure within the pot sinks below that of the surrounding atmosphere and the mercury rises several inches in the tube.

We must therefore conclude that, even when the two solutions on either side of the membrane are isotonic, there may be a movement of fluid from one side to the other with a performance of work in the process. In fact, osmosis may occur from a fluid having a higher towards a fluid having a lower osmotic pressure. If, for example, equimolecular solutions of sodium chloride and glucose be separated by a peritoneal membrane, the osmotic flow will take place from the fluid having the higher osmotic pressuresodium chloride.* We might compare with this experiment the results of separating hydrogen at one atmosphere's pressure from oxygen at two atmospheres' pressure by means of a plate of graphite. In this case the initial result will be a still further increase of pressure on the oxygen side of the diaphragm - a movement of gas against pressure taking place in consequence of the greater diffusion velocity of hydrogen.

So far we have considered only the behaviour of solutions when separated by a membrane, the permeability of which to salts is comparable to that of water; so that the passage of salts through the membrane depends merely on the diffusion rates of the salts. There can be no doubt, however, that we might get analogous movements of fluid against total osmotic pressure determined, not by the diffusibility of the salts, but by the permeability of the membrane for the salts - a permeability which may depend on a state of solution or attraction existing between membrane and salts. We have an analogue to such a condition of things in the passage of gases through an india-rubber sheet. If two bottles, one containing carbonic acid, the other hydrogen, be separated by a sheet of india-rubber, carbon dioxide passes into the hydrogen bottle more quickly than hydrogen can pass out into the carbon dioxide bottle, so that a difference of pressure is created, and the rubber bulges into the carbon dioxide bottle. We might, in the same way, conceive of a membrane which permitted the passage of dextrose more

* In consequence of ionic dissociation of the sodium chloride, a decinormal solution of this salt will have an osmotic pressure nearly twice as great as that of a similar solution of the non-ionised glucose. 
easily than that of urea. The importance of the membrane in determining the direction of the osmotic passage of fluid is well illustrated by Raoult's experiments. When alcohol and ether were separated by an animal membrane, alcohol passed into the ether, whereas if vulcanite were employed for the diaphragm, the osmotic flow was in the reverse direction, and an enormous pressure was set up on the alcohol side of the diaphragm. *

The next point to be considered is the passage of a dissolved substance across membranes, in consequence of differences in the partial pressure of the substance in question on the two sides of the membrane. Stress has been laid by Heidenhain and others on the fact that in the peritoneal cavity, as well as from the intestine, salt may be taken up from fluids containing a smaller percentage of this substance than does the blood plasma, and they regard this absorption as pointing indubitably to an active intervention of living cells in the process. This argument requires examination. Let us suppose the two vessels A and B (Fig. 25) to be separated by a membrane which offers free passage

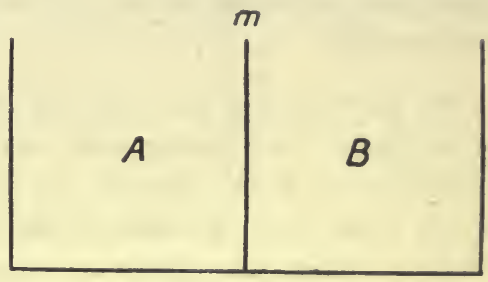

FII. 25.

to water and a difficult passage to salts. Let A contain 0.5 per cent. salt solution and $\mathrm{B}$ a solution isotonic with a 1 per cent. $\mathrm{NaCl}$, but containing only 0.65 per cent. of this salt, the rest of its osmotic tension being due to other dissolved substances. If the membrane were absolutely 'semi-permeable,' water would pass from A to B until the two fluids were isotonic, i.e. until A contained 1 per cent. $\mathrm{NaCl}$ (we may regard volume $\mathrm{B}$ as infinitely great to simplify the argument). If, however, the membrane permitted passage of the dissolved substances, the course of events might be as follows : At first water would pass out of $\mathrm{A}$, and salt would diffuse in until the percentage of $\mathrm{NaCl}$ in $\mathrm{A}$ was equal to that in $\mathrm{B}$. There would now be an equal partial pressure of $\mathrm{NaCl}$ on the two sides of the membrane, but the total osmotic pressure of $B$ would still be higher than $A$. Water would therefore still continue to pass from A to B more rapidly than the other ingredients of B could pass into A. As soon, however, as more water passed out from $\mathrm{A}$, the percentage of $\mathrm{NaCl}$ in $\mathrm{A}$ would be raised above that in B. The extent to which this occurs will depend on the impermeability of the membrane. As the $\mathrm{NaCl}$ in $\mathrm{A}$ reaches a certain concentration it will pass over into B, and this will go on until equilibrium is established between $\mathrm{A}$ and B. Extending this argument to the conditions obtaining in the living body, we may conclude that neither the raising of the percentage of a

* Here we have a possible clue to the explanation of some phenomena of cell activity, to which the term 'vital' is often assigned. In the swimming-bladder of fishes, for instance, we find a gas which is extremely rich in oxygen, and the oxygen is said to have been secreted by the cells lining the bladder. It is, however, possible that the processes here may be analogous to Graham's atmolysis, and that the bladder may represent a perfected form of Graham's india-rubber bag. 
salt in any fluid above that of the same salt in the plasma, nor the passage of a salt from a hypotonic fluid into the blood plasma, can afford in itself any proof of an active intervention of cells in the process.

In the case of the pleura, for example, we seem to have a membrane which is very imperfectly semi-permeable. It is permeable to salts, but presents rather more resistance to their passage than to the passage of water. Hence on injecting 0.5 per cent. $\mathrm{NaCl}$ solution into the pleural cavity, water passes from the pleural fluid into the blood, until the percentage of sodium chloride in the fluid is raised perceptibly above that in the blood plasma. The limit of the resistance of the pleural membrane to the passage of salt is, however, soon reached, and then salt passes from pleural fluid into blood; but in every case this passage is from a region of higher to a region of lower partial pressure. Hence at a certain stage of the experiment we find a higher percentage of salt in the pleura than in the blood-vessels, although the total amount of salt in the pleural fluid is less than that originally put in, or, in other words, salt has been absorbed.

We have already seen that the effective osmotic pressure of a substance, i.e. its power of attracting water across a membrane, varies inversely as its diffusibility, or as the permeability of the membrane to it. What, then, will be the effect if on one side of the membrane we place some substance in solution to which the membrane is impermeable?

We will suppose that $\mathrm{A}$ and $\mathrm{B}$ both contain 1 per cent. $\mathrm{NaCl}$, but that $\mathrm{B}$ contains in addition some substance $x$ to which the membrane is impermeable. Since the osmotic pressure of $B$ is higher, by the partial pressure of $x$, than that of $\mathrm{A}$, fluid will pass from $\mathrm{A}$ to $\mathrm{B}$ by osmosis. But the consequence of this passage of water will be to concentrate the $\mathrm{NaCl}$ in $\mathrm{A}$, so that the partial pressure of this salt in $\mathrm{A}$ is greater than in $\mathrm{B}$. $\mathrm{NaCl}$ will therefore diffuse from $\mathrm{A}$ to $\mathrm{B}$, with the result that the former difference of total osmotic pressure will be re-established. Hence there will be a continual passage of both water and salt from $A$ to $B$, until $B$ has absorbed the whole of $\mathrm{A}$. This result will be only delayed if the osmotic pressure of $\mathrm{A}$ is at first higher than $\mathrm{B}$, in consequence of a greater concentration of $\mathrm{NaCl}$ in $\mathrm{A}$. There may be at first a flow of fluid from $\mathrm{B}$ to $\mathrm{A}$, but as soon as the $\mathrm{NaCl}$ concentration on the two sides has become the same by diffusion, the power of $x$ to attract water from the other side will make itself felt, and this attraction will be proportional to the osmotic pressure of $x$. We shall have occasion to discuss a specific instance of this case when dealing with the mechanism of absorption of fluid by the blood-vessels from the connective tissue spaces.

A more familiar example is afforded by the process known as dialysis. Many animal membranes, all of which are colloidal in character, and others such as vegetable parchment, while freely permeable to salts, are impermeable to dissolved colloids. If, therefore, a fluid containing both colloids and crystalloids in solution, e.g. blood-serum, be enclosed in a tube of vegetable parchment, which is hung up in a large bulk of distilled water (Fig. 26), all the salts diffuse out, and if this be frequently changed, we obtain finally a fluid within the dialyser free from salts and other crystalloid substances, but containing the whole of the colloidal proteins originally present. 
Thus the transference of fluids and dissolved substances across membranes is determined not only by the osmotic pressure of the solutions, but also by the diffusion coefficient of the solutes and the permeability of the membrane. This permeability may be of the same character as the permeability of water, in which case the rates of passage of the dissolved substances across the

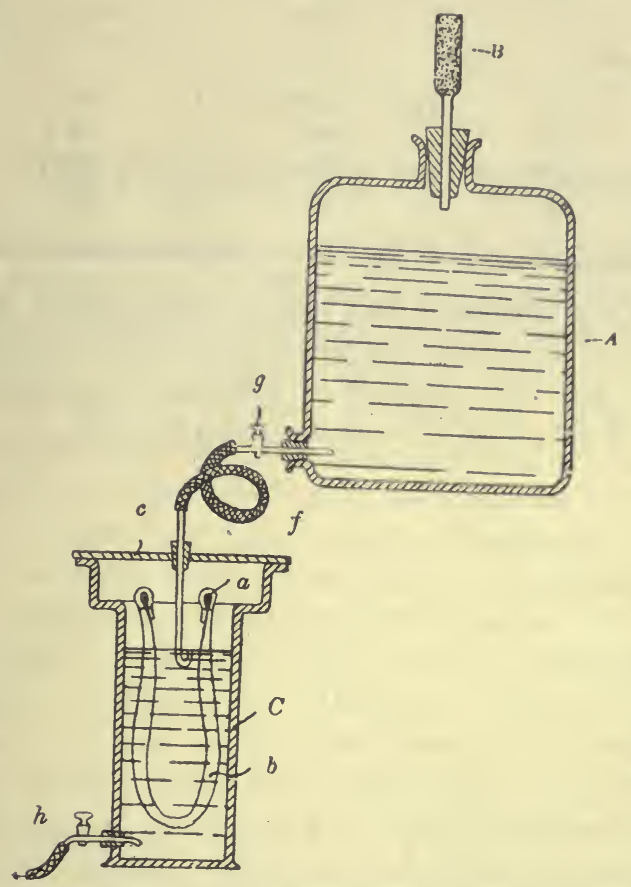

FIG. 26. Dialyser, consisting of a tube of parchment paper immersed in a vessel through which a constant stream of sterile distilled water can bo passed. (WROBLESKI.)

membrane vary as their diffusibilities, and are therefore probably some function of their molecular weights. On the other hand, the membrane may exhibit a certain attraction for, or power of dissolving, some of the solutes to the exclusion of others, in which case there will be no relation between the diffusibilities and the rates of passage of the dissolved substances.

In a recent paper Bayliss has drawn attention to certain other factors which may determine permanent inequality of distribution of a salt on the two sides of a membrane permeable to the salt. If Congo red, which is a compound of an indiffusible colloid acid with sodium, be placed in an osmometer which is immersed in water, a certain osmotic pressure is developed. On adding sodium chloride either to the inner or outer fluid, there is a fall in the osmotic pressure if time be allowed for equilibrium to be established. At this point it is found that the outer fluid, which is free from dye, contains a larger percentage of sodium chloride than the inner solution of dye. This difference is permanent and is more marked the greater the concentration of the dye salt. In the following Table is given the concentrations of the two 
fluids with different percentages of salt. The numbers indicate the litres to which each gramme molecule of the salt is diluted. Apparently the difference depends on the fact that the non-dissociated salt must be equal on

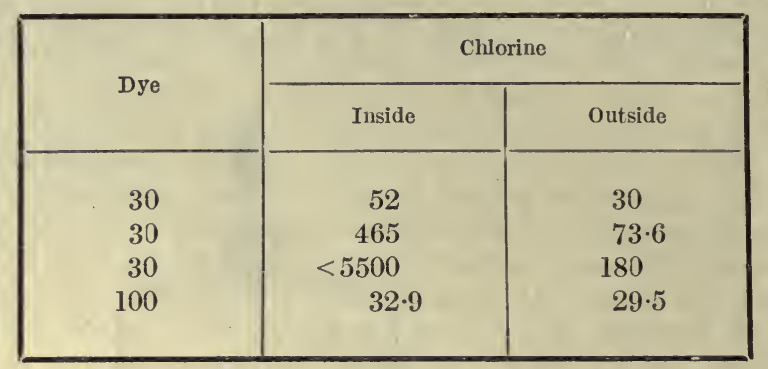

the two sides of the membrane and that the dissociation is much impeded on the inner side on account of the presence there of another salt of sodium. A sodium salt of any other indiffusible substance, e.g. of a protein such as caseinogen, would behave in a precisely similar fashion. 


\section{SECTION III \\ THE PROPERTIES OF COLLOIDS}

Although the chemical changes involved in the various vital phenomena occur between substances in watery solution, the solution in every case is bound up within the meshes or adsorbed by the surfaces of a heterogeneous mass of colloids. The complex chemical molecules which make up protoplasm itself are all colloidal in character. The participation of colloids in chemical reactions introduces conditions and modes of reaction differing widely from those which have been studied in watery solutions. Our knowledge of these conditions is still very imperfect, but the important part played by colloids in the processes of life renders it necessary to discuss in some detail their properties and modes of interaction.

The term colloid, from $\kappa \sigma \lambda \lambda \eta$, glue, was first introduced by Thomas Graham, Professor of Chemistry at University College from 1836 to 1855. Graham divided all substances into two classes, viz. crystalloids, including such substances as salt, sugar, urea, which could be crystallised with ease, diffused rapidly through water, and were capable of diffusing through animal membranes; and colloids, which included substances such as gelatin or glue, gum, egg-albumin, starch and dextrin, were non-crystallisable, formed gummy masses when their solutions were evaporated to dryness, diffused with extreme slowness through water, and would not pass through animal membranes. The process of dialysis was therefore introduced by Graham for the separation of crystalloids from colloids. Although the broad distinction drawn by Graham between colloids and crystalloids still holds good, some of the criteria by which he distinguished the two classes are no longer strictly applicable. For instance, it has been shown that many typical colloidal substances, such as hæmoglobin, can be obtained in a crystalline form. On the other hand, all gradations exist between substances, such as egg-albumin, which are practically indiffusible, and those, such as common salt, which are very diffusible. Graham pointed out that colloids exist under two conditions :

(1) In a state of solution or pseudo-solution, in which'they form sols, and are distinguished as hydrosols, when the solvent is water; and

(2) In a solid state, in which a relatively small amount of the colloid sets with a large amount of a fluid, such as water, to form a jelly. This solid form is known as a gel. The most familiar instance is the jelly which is obtained on dissolving a little gelatin in hot water and allowing the mixture 
to cool. Such a jelly is known as a hydrogel. In many of these gels the water can be replaced by other fluids, such as alcohol, without any alteration in the appearance of the solid, which is then known as an alcogel. An example of an alcogel is the jelly which can be made by dissolving soap in warm alcohol and allowing the mixture to cool.

A number of these colloidal substances can be shown on purely chemical grounds to consist of monstrous molecules. Thus the molecular weight of hæmoglobin is at least 16,000 , and one must ascribe similar high molecular weights to such substances as egg-albumin and globulin. Still greater must be the molecular size of such substances as the cell proteins, which may be made up of more than one type of protein built up with various nucleins, with lecithin and cholesterin, to form a gigantic complex, to which it would probably not be an exaggeration to ascribe a molecular weight of over 100,000 . This chemical complexity is not, however, a necessary condition of the colloidal state, as is shown by the existence of colloidal silica, of colloidal ferric hydrate and alumina, and even of colloidal metals.

On neutralising a weak solution of sodium silicate or water-glass by means of $\mathrm{HCl}$, we obtain a solution which contains sodium chloride and silicic acid. On dialysing this mixture for some days against distilled water, the whole of the $\mathrm{NaCl}$ passes out, and a solution of silicic acid or colloidal silica is left in the dialyser. This solution can be concentrated over sulphuric acid. When concentrated to a syrupy consistence it becomes extremely unstable. The addition of a minute trace of sodium chloride or other electrolyte to the solution causes it to set at once to a solid jelly (gelatinous silica), the change being accompanied by an appreciable rise of temperature. The change is irreversible, in that it is not possible to bring the silicic acid into solution again by removal of the electrolyte by means of dialysis. If, however, it be allowed to stand with weak alkali for some time, it gradually passes into solution. Analogous methods are employed for the preparation of colloidal $\mathrm{Fe}_{2} \mathrm{O}_{3}$ and $\mathrm{Al}_{2} \mathrm{O}_{3}$.

Of special interest are the colloidal solutions of the metals. Faraday long ago pointed out that, on treating a weak solution of gold chloride with phosphorus, it underwent reduction with the formation of metallic gold. The gold was not precipitated, but remained in suspension or pseudosolution, giving a deep red ${ }^{*}$ or a blue liquid, according to the conditions under which the reaction was effected. This solution was homogeneous in that it could be filtered without change, and could be kept for months without deposition of the gold. The latter was, however, thrown down on addition of mere traces of impurity, though greater stability could be conferred on the solution by adding to it a little ' jelly,' i.e. a weak solution of gelatin. In 1899 Bredig showed how similar hydrosols might be prepared from a number of different metals, viz. by the passage of a small arc or electric sparks between metallic terminals submerged in distilled water. If, for example, the terminals be of platinum, the passage of the current is seen to be accompanied by the giving off of brown clouds, which spread into the surrounding fluid. These clouds consist of particles of platinum of all sizes. The larger settle at the bottom of the vessel, the smaller-

* Ruby glass is a colloidal 'solid ' solution of gold in a mixture of silicates. 
which are ultra-microscopic in size, i.e. from $5 \mu \mu$ to $40 \mu \mu^{*}$-remain in suspension, and we obtain a brown fluid which can be filtered through paper or even through a Berkefeld filter without losing its colour. It may be kept for months without any deposit taking place. The addition of minute traces of electrolytes precipitates the platinum particles, leaving a colourless fluid. We shall have to return later on to the consideration of the behaviour of these metallic sols.

PROPERTIES OF GELS. A typical hydrogel is the firm mass in which a solution of gelatin sets on cooling. It is clear, hyaline, apparently structureless, and possesses considerable elasticity, i.e. resistance to deforming force. It may be regarded as formed by the separation of the warm pseudo-solution of gelatin into two phases : first a solid phase, rich in gelatin and forming a tissue or meshwork, in the interstices of which is embedded the second phase, consisting of a very weak solution of gelatin.

If the process be observed under the microscope, according to Hardy, minute drops first appear, which, as they enlarge, touch one another and form networks. In stronger solutions the first structures to make their appearance consist, not of the more concentrated phase, but of droplets of the dilute solution of gelatin; the stronger solution collects round these drops and solidifies to a honeycomb structure.

In many cases the more fluid part of the gel is practically pure water. In such a case immersion in alcohol causes a diffusion outwards of the water, which is replaced by alcohol with the formation of an alco-gel. In a dry atmosphere the gel loses water and becomes shrivelled and dry, but in some cases, e.g. gelatin, it can resume its former size and characters on immersion in water. Other gels, such as silicic acid or ferric hydrate, lose the power of swelling up after drying. The change in them is therefore irreversible. A gel adheres to the last traces of water with extreme tenacity. In consequence of its structure, it presents an enormous extent of surface on which adsorption can take place. At this surface the vapour-tension of fluids is diminished, as well as the osmotic pressure of dissolved substances. On this account gelatin must be heated for many hours at a temperature of $120^{\circ} \mathrm{C}$. in order to be thoroughly dried. When dry, it, as well as other solid colloids, can exert a considerable amount of energy when caused to swell up by moistening. This energy was made use of by the ancient Egyptians in the quarrying of their stone blocks by the insertion of wedges of wood; water was poured on the wood, and the swelling of the wedges split the rock in the desired direction. $\uparrow$

On account of the extent of surface it is practically impossible to wash out the inorganic constituents from a gel. The diminution of the osmotic pressure of many dissolved substances at surfaces causes the concentration at the surface of the solid phase to be greater than that in the surrounding medium. Thus, if dry gelatin be immersed in a salt solution it will swell

* One $\mu$ is one-thousandth of a millimetre ; one $\mu \mu$ is one-thousandth $\mu$, i.e. oncmillionth of a millimetre.

$\dagger$ According to Rodewald, the maximal pressure with which dry starch attracts water amounts to 2073 kilo. per sq. $\mathrm{cm}$. 
up, but the solution which it absorbs will be more concentrated than the solution in which it is immersed, so that the proportion of salt in the latter will be diminished. When, however, equilibrium is established between a gel and the surrounding fluid, it is found to présent no appreciable resistance to the passage of dissolved crystalloids. Thus salt or sugar diffuses through a column of solid gelatin as if the latter were pure water. On the other hand, gels are practically impermeable to other colloids in solution. This impermeability is made use of in the separation of crystalloids from colloids by dialysis, membranes used in this process being generally irreversible and heterogeneous gels (i.e. vegetable parchment, animal membranes). Other. gels, such as tannate of gelatin or copper ferrocyanide, are not only impermeable to colloids, but also to many crystalloid substances. These membranes, therefore, were used by Pfeffer for the determination of the osmotic pressure of such crystalloids as cane sugar.

PROPERTIES OF HYDROSOLS. Substances such as dextrin or eggalbumin may be dissolved in water in almost any concentration. If a solution of egg-albumin be concentrated at a low temperature, it becomes more and more viscous and finally solid. But there is no distinct point at which the fluid passes into the solid condition. Such solutions are known as hydrosols. Much discussion has arisen whether they are to be regarded as true solutions or as pseudo-solutions or suspensions. The chief criterion of a true solution is its homogeneity. In a solution the molecules of the solute are equally diffused throughout the molecules of the solvent, and it is impossible, without the application of energy, to separate one from the other. Thus filtration, gravitation leave the composition of the solution unchanged. It is true that, by the employment of certain kinds of membrane, e.g. the semi-permeable copper ferrocyanide membrane, it is possible to separate solute from solvent, but in this case the force required to effect the filtration is enormous and grows with every increase in the strength of the solution. The measure of the force required is the osmotic pressure of the solution, and it becomes natural therefore to regard the possession of an osmotic pressure as a distinguishing criterion of a true solution. Is there any evidence that colloid solutions also display an osmotic pressure?

I have shown that it is possible to determine the osmotic pressure of colloidal solutions directly, taking advantage of the fact that colloidal membranes, while permitting the passage of water and salts, are impermeable to colloids in solution.

The method originally adopted was as follows: In order to determine the osmotic pressure of the colloidal constituents of blood-serum, 150 c.c. of clear filtered serum are filtered under a pressure of 30-40 atmospheres through a porous cell which has been previously soaked with gelatin. The first 10-20 c.c. of filtrate, which contain the water squeezed out of the meshes of the gelatin and have also lost salt in consequence of absorption by the gelatin, are rejected. The filtration is allowed to go on for another twenty-four hours, when about 75 c.c. of a clear colourless filtrate is obtained, perfectly free from all traces of protein, but possessing practically the same freezing-point as the original serum. (Although the colloids, if they possess an osmotic pressure, must 
also cause a depression of the freezing-point, any such depression would be within the errors of observation, since a pressure of $45 \mathrm{~mm}$. $\mathrm{Hg}$. would correspond only to $0.005^{\circ}$ C.) The concentrated serum left behind in the filter is then put into the os. mometer, the filtrate being used as the imner fluid. The construction of the osmometer
is shown in the diagram (Fig. 27).

The tube BB is made of silver gauze, connected at each end to a tube of solid silver. Round the gauze is wrapped a piece of peritoneal membrane, as in making a cigarette. This is painted all over with a solution of gelatin (10 per cent.) and then a second layer of membrane applied. Fine thread is now twisted many times round the tube to

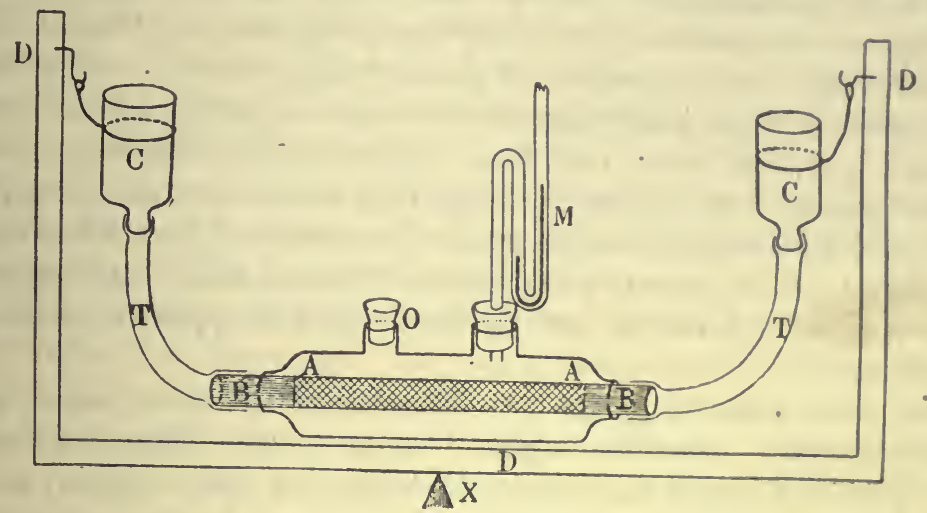

Fig. 27.

prevent any disturbance of the membranes, and the whole tube is soaked for half an hour in a warm solution of gelatin. In this way one obtains an even layer of gelatin between two layers of peritoneal membrane and supported by the wire gauze. The tube so prepared is placed within a wide tube, $\mathrm{AA}$, which is provided with two tubules at the top. One of these, $\mathrm{O}$, is for filling the outer tube; the other is fitted with a mercurial manometer, M. Two small reservoirs, CC, are connected with the outer ends of BB, by means of rubber tubes. The whole apparatus is placed in a wooden cradle, DD, pivoted at $\mathrm{X}$, and provided with a cover so that it may be filled with fluids at different temperatures if necessary. The colloid solution is placed in AA, and the reservoirs, $\mathrm{CC}$, and inner tube, $\mathrm{BB}$, are filled with the filtrate, i.e. with a salt solution approximately or absolutely isotonic with the colloid solution. The apparatus is then made to rock continuously for days or weeks by means of a motor. In this way the fluid on the two sides of the membrane is continually renewed, and the attainment of an osmotic equilibrium facilitated. With this apparatus I found that the colloids in blood-serum, containing from 7 to 8 per cent. proteins, had an osmotic pressure of 25 to $30 \mathrm{~mm}$. $\mathrm{Hg}$., which would correspond to a molecular weight of about 30,000 .

A more convenient form of osmometer has been devised by B. Moore, using parchment paper as the membrane. With this osmometer, the existence of an osmotic, pressure in colloidal solutions has been definitely established both by Moore in the case of hæmoglobin, proteins, and soaps, and by Bayliss in the case of colloidal dyes, such as Congo red. The osmotic pressure of hæmoglobin was found by Hüfner to correspond to a molecular weight of about 16,000 , i.e. a molecular weight already deduced from its composition and its combining powers with oxygen. Often, however, the osmotic pressure is very much smaller than would be expected from the molecular weight of the substance, owing to the fact that colloids in solution 
may be in many different conditions of aggregation. Thus the molecule of colloidal silica must be many, probably thousands of times larger than the molecule as represented by $\mathrm{H}_{2} \mathrm{SiO}_{3}$. The osmotic pressure being proportional to the number of molecules in a given volume of solution, the larger the aggregate the smaller would be the total number of molecules, and the smaller therefore the osmotic pressure of the solution.

It is in consequence of the huge size of the molecular aggregates that colloidal solutions, such as starch or glycogen, and probably globulin, display no appreciable osmotic pressure. We cannot divide colloidal solutions into two classes, viz. those which form true solutions and present a feeble osmotic pressure, and those which only form suspensions and therefore exert no osmotic pressure. In inorganic colloids, such as arsenious sulphide, Picton and Linder have shown that all grades exist between true solutions and suspensions. With increasing aggregation of the molecules, the suspension becomes coarser and coarser until finally the sulphide separates in the form of a precipitate.

The measurement of the osmotic pressure of the colloids of serum points to their having a molecular weight of about 30,000. Chemical evidence shows that hæmoglobin has a molecular weight of about 16,000 , and we have every reason to believe that the much more complex molecules forming the cell proteins may have molecular weights of many times this amount. When, however, we arrive at molecular weights of these dimensions, the disproportion between the size of the molecules and those of the solvent, water, becomes so great that a homogeneous distribution of the two substances, solute and solvent, is no longer possible. The size of a molecule of water has been reckoned to be $.7 \times 10^{-8} \mathrm{~mm}$. A molecule 10,000 times as large would have a diameter of $\cdot 7 \times 10^{-4} \mathrm{~mm} .=\cdot 07 \mu$, a size just within the limits of microscopic vision. Long before molecules attained such a size they would no longer react according to the laws which have been derived from the study of the behaviour of the almost perfect gases, but would possess the properties of matter in mass. They have a surface of measurable extent, and their relations to the molecules of water or solvent will be determined by the laws of adsorption at surfaces rather than by the laws of interaction of molecules. As a matter of fact we find that such solutions present an amazing mixture of properties, some of which betray them as mechanical suspensions, while others partake of the nature of the chemical reactions such as those studied in the simpler compounds usually dealt with by the chemist.

OPTICAL BEHAVIOUR OF HYDROSOLS. Nearly all colloidal solutions present what is known as the Faraday-Tyndall phenomenon. When a beam of light is passed through an optically homogeneous fluid, the course of the beam is invisible. A beam of sunlight falling into a dark room is rendered visible by impinging on and illuminating the dust particles in its course. Each of these particles, being illuminated, acts as a centre of dispersion of the light, so that the course of the beam is apparent to a person 
standing on one side of it. Tyndall showed that, if the particles were sufficiently minute, the light dispersed by them at right angles to the beam was polarised. This can be easily tested by looking at the beam through a Nicol's prism. If the prism be slowly rotated, it will be found that, while at one position the light is bright, in the position at right angles to this it becomes dim or is extinguished. The production of the Tyndall phenomenon may therefore be regarded as a test for the presence of ultra-microscopic particles, varying in size from 5 to $50 \mu \mu$. The phenomenon is perhaps too sensitive to be taken as a proof that a fluid presenting it is a suspension rather than a solution. It is shown, for instance, by solutions of many bodies of high molecular weight, such as raffinose (a tri-saccharide) or the alkaloid brucine (Bayliss).

A particle having a diameter less than half the wave-length of light, i.e. about $300 \lambda$ or $\cdot 3 \mu$, cannot be clearly distinguished under any power of the microscope. The fact that an ultra-microscopic particle may serve as a centre for dispersal of light may be used for rendering such particles visible under the microscope. For this purpose a strong beam of light is passed in the plane of the stage of the microscope through a cell containing the hydrosol, which is then examined under a high power. The arrangement for this purpose was first devised by Zsigmondy and Siedentopf. On examining with this apparatus a dilute gold sol, we see a swarm of dancing points of light, 'like gnats in the sunlight,' which move rapidly in all directions, rendering it almost impossible to count their number in the field. The coarser particles present slight oscillations similar to those long known as the Brownian movements. The smallest particles which can be seen show a combined movement, consisting of a translatory movement, in which the particle passes rapidly across the field in one-sixth to one-eighth of a second, and a movement of oscillation of much shorter period. The representation of the course of such a particle is given in Fig. 28.

The size of the smallest particles seen in this way may amount to $\cdot 005 \mu$. Not all colloidal solutions show these particles in the ultra-microscope. In some cases this is due simply to the small size of the particles, and the addition of any substance, which causes aggregation and therefore increase in the size of the particles, will bring them into view. In others the absence of optical inhomogeneity may be due to the coincidence of the refractive indices of the two phases of the hydrosol, or to the absence of any surface tension and therefore dividing surfaces between the two phases.

\section{ELECTRICAL PROPERTIES OF COLLOIDS}

In the case of many hydrosols the ultra-microscopic particles of which they are composed carry an electric charge which, according to the nature of the solution, may be either positive or negative. On this account, the particles move if placed in an electric field, and the direction of their movement reveals the nature of their change. Thus colloidal ferric hydrate is 
electro-positive and travels from anode to cathode. Silicic acid, in the presence of a trace of alkali, is electro-negative, and the same is true of a hydrosol of gold. When a current is passed through these hydrosols, the colloidal particles travel to the anode, where they are precipitated. In certain colloids the charge varies according to the conditions under which they are brought into solution. If, for instance, egg-white be diluted ten times with distilled water, filtered and boiled, no precipitate occurs, but

A.
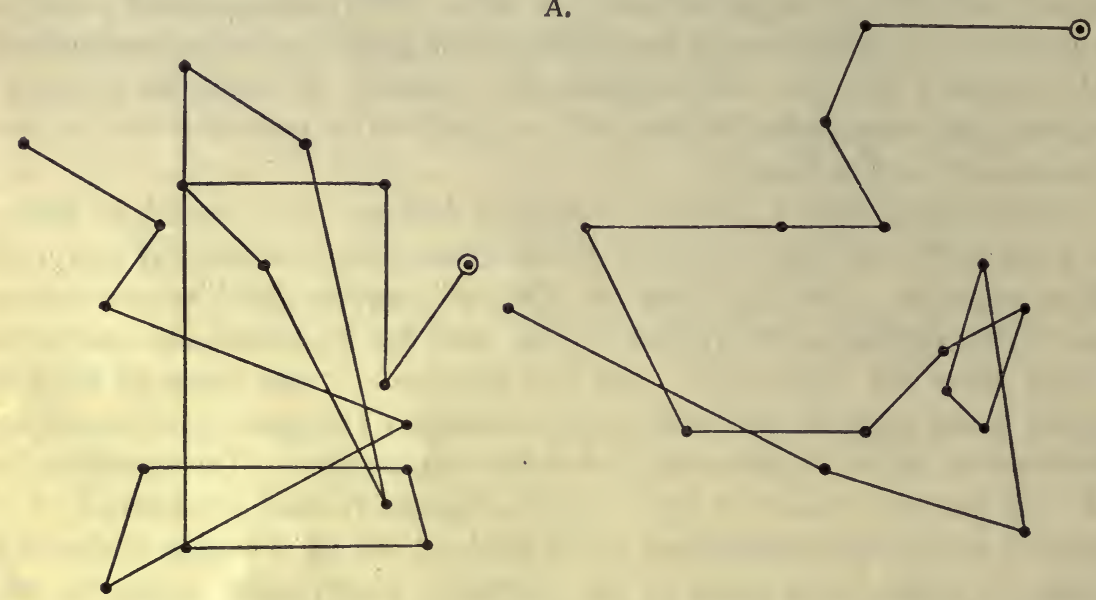

FIG. 28. Movements of two particles of india-rubber latex in colloidal solution, recorded by cinematograph and ultra-microscope. (HENRI.)

we obtain a colloidal suspension of albumin. When thoroughly dialysed, this protein is insoluble in pure water, but is soluble in traces of either acid or alkali. In acid solution the protein particles carry a positive charge, whereas in alkaline solution their charge is negative. The charged condition of these particles must play a considerable part in keeping them asunder and therefore in preventing their aggregation and precipitation. This is shown by the fact that any agency which will tend to discharge them will cause precipitation and coagulation. Among such agencies is the passage of a constant current, just mentioned. To the same action is due the coagulative or precipitating effects of neutral salts. Thus any of the colloids we have mentioned, ferric hydrate, silica, gold, or boiled albumen, are thrown down by the addition of traces of neutral salts, and it is found that in this process they carry with them some of the ion with the opposite charge to that of the colloidal particle. Thus, in the precipitation of the electro-positive ferric hydrate the acid ion of the salt is the determining factor, the coagulative power increasing rapidly with the valency of the acid. On the other hand, in the precipitation of a gold sol the electro-positive ion is the effective agent, and here again the coagulative effect is enormously increased by a rise in valency. This is shown in the following Tables, where it will be seen that, in the coagulation of gold, barium chloride, with the divalent $\mathrm{Ba}^{\prime \prime}$, is seven times as powerful as $\mathrm{K}_{2} \mathrm{SO}_{4}$, containing the univalent $\mathrm{K}^{\prime}$. On the other hand, in the precipitation of the electro-positive 
ferric hydrate, $\mathrm{K}_{2} \mathrm{SO}_{4}$, with a divalent $\mathrm{SO}_{4}{ }^{\prime \prime}$, is 400 times as effective as $\mathrm{BaCl}_{2}$.

Amount of Salt necessary to precipitate Colloidat Solutions

To coagulate $\mathrm{Fe}_{2} \mathrm{O}_{3}$

$\mathrm{K}_{2} \mathrm{SO}_{4} 1 \mathrm{~g}$ mol. in $4,000,000$ c.c

$\mathrm{MgSO}_{4}$," , , 4,000,000 ,

$\mathrm{BaCl}_{2} \quad$, , , 10,000 ,

$\mathrm{NaCl}, \quad, \quad, \quad 30,000$,
To coagulate Gold

$\mathrm{BaCl}_{2} 1$ g. mol. in 500,000 c.c.

$\mathrm{NaCl},, \quad, \quad 72,000$,

$\mathrm{K}_{2} \mathrm{SO}_{4}, \quad, \quad, \quad, 75,000$,

The presence of a charge is not, however, a necessary condition for the stability of a colloidal solution. Thus the proteins of serum, globulin in a weak saline solution, or gelatin, present no drift when exposed to a strong electric field. In such cases one must assume the stability of the solution to be determined by the absence of any surface tension between the two phases in the solution, or between the particles of solute and the solvent. Thus no force is present tending to cause aggregation of the particles.

The charged condition of a colloidal particle makes it behave in an electric field in much the same way as a charged ion of an electrolyte, and this similarity extends also to its chemical behaviour, so that we have a class of compounds formed resembling in many respects chemical combinations, but differing from these in the absence of definite quantitative relations between the reacting substances. This class of continuously varying chemical compounds has been designated by Van Bemmelen absorption compounds. Since, however, the interaction must take place at the surface layer bounding the charged particles, it will be perhaps better, as Bayliss has done, to use the term adsorption. The huge molecules or aggregates of molecules which distinguish the colloidal state form a system with a considerable inertia, so that we have a tendency to the establishment of conditions of false equilibrium. Once a configuration is established, it is necessary, in consequence of the inertia, to overstep widely the conditions of its formation in order to destroy it. Thus a 10 per cent. gelatin solution sets at $21^{\circ} \mathrm{C}$., but does not melt until warmed to $29 \cdot 6^{\circ} \mathrm{C}$. Solutions of agar in water set at about $35^{\circ} \mathrm{C}$, but do not melt under $90^{\circ} \mathrm{C}$. A gel of gelatin takes twenty-four hours after setting to attain a constant meltingpoint.

The factors involved in the formation of adsorption or absorption combinations are therefore :

(1) Extent of surface. In a colloidal solution this must be enormous in proportion to the mass of substance in solution. Thus a 10 c.c. sphere with a surface of $22 \mathrm{sq}$. $\mathrm{cm}$., if reduced to a fine powder consisting of spherules of $\cdot 00000025 \mathrm{~cm}$. in diameter, will have a surface of $20,000,000 \mathrm{sq}$. cm., i.e. nearly half an acre. At the whole of this surface adsorption may take place, involving the concentration of dissolved electrolytes, ions, or gases.

(2) Chemical nature of particle.

(3) Electric charge on the surface. The sign of this may be determined 
by the chemical nature of the colloid and its relation to the electrolytes in the surrounding medium.

Another factor which may determine the character of the charge on the particles has been pointed out by Coehn. This observer finds that, when various non-conducting bodies are immersed in fluids of different dielectric constants, they assume a positive or negative charge according as their own dielectric constants are higher or lower than the fluid with which they are in contact. For instance, glass (5 to 6) is negative in water (80) or alcohol (26), whereas in turpentine $(2 \cdot 2)$ it is positive. In water, as Quincke has found, nearly all non-conducting bodies take on a negative charge. Among these are cotton-wool and silk. Particles of these in water, exposed to an electric field, move towards the anode. The same is true, as Bayliss has shown, of paper.

The conditions which determine the formation of these adsorption compounds can be studied in their simplest form on the adsorption of dyestuffs by substances such as paper. If we take a series of solutions of a dye, such as Congo-red, in progressively diminishing concentration, and place in each solution the same amount of filter-paper, we find that a part of the dye is taken up by the paper, and the proportion taken up is larger the more dilute the solution. This relation has been spoken of by Bayliss as the law of adsorption. This is illustrated by the following Table of results of such an experiment :

\begin{tabular}{|cc|c|c|}
\hline \multicolumn{2}{|c|}{$\begin{array}{c}\text { Concentration of } \\
\text { solution }\end{array}$} & $\begin{array}{c}\text { Proportion of dye } \\
\text { in solution }\end{array}$ & $\begin{array}{c}\text { Proportion of dye } \\
\text { in paper }\end{array}$ \\
\cline { 1 - 2 } Initial & Final & Per cent. & Yer cent. \\
0.014 & 0.0056 & 40 & 60 \\
0.012 & 0.0024 & 20 & 80 \\
0.010 & 0.0009 & $9 \cdot 3$ & $90 \cdot 7$ \\
0.008 & 0.0003 & 4 & 96 \\
0.006 & 0.00008 & $1 \cdot 3$ & $98 \cdot 7$ \\
0.004 & - & trace & practically all \\
0.002 & - & trace & practically all \\
\hline
\end{tabular}

If put into the form of a curve, where the ordinates represent the percentage of dye left in solution, and the abscissæ the original concentration of the solution, the curve only approaches the axis (i.e. zero concentration) asymptotically. In other words, however dilute the original solution may be, there will always be a certain amount of the dye left unabsorbed by the paper. Similar relations are found to exist between proteins and electrolytes. By continuously washing a protein or gelatin with distilled water, the removal of electrolytes becomes slower and slower, but it is practically impossible within finite time to get rid in this way of the last traces of ash.

Although the chemical behaviour of colloids is largely determined by surface phenomena, it presents at the same time analogies with more strictly chemical reactions, since it is conditioned by the chemical structure of the colloid molecule as well as by the charge carried by the latter. A good example of these adsorption combinations is presented by globulin, the behaviour of which has been studied by Hardy. This may be obtained from diluted blood-serum by precipitation with acetic acid. Four states 
can be recognised in both the solid condition and in solution, viz.globulin itself, compounds with acid or with alkali, and compounds with neutral salt. The amount of acid and alkali combining with the globulin is indeterminate, the effect of adding either acid or alkali to the neutral globulin being to cause a gradual conversion of an oqaque, milky suspension into a limpid, transparent solution. On drying $\mathrm{HCl}$ globulin, the dried solid is found to contain all the chlorine used to dissolve it. The acid may therefore be regarded as being in true combination. Both acid and alkali globulins act as electrolytes, the globulin being electrically charged and taking part in the transport of electricity. In order to produce the same extent of solution, the concentration of the alkali added must be double that of the acid. The relation of globulin to acids and alkalies is similar to that of the so-called amphoteric substances, such as the amino-acids. An amino-acid, such as glycine, can react as a basic anhydride with other acids, thus :

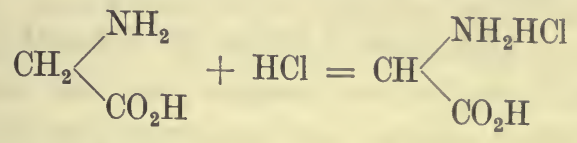

or as an acid anhydride with bases :

$$
\left.\right|_{\mathrm{COOH}} ^{\mathrm{CH}_{2} \cdot \mathrm{NH}_{2}}+\mathrm{NaHO}=\left.\right|_{\mathrm{COONa}} ^{\mathrm{CH}_{2} \cdot \mathrm{NH}_{2}}+\mathrm{H}_{2} \mathrm{O}
$$

Like these too, globulin forms soluble compounds with neutral salts. An amphoteric electrolyte thus acts as a base in the presence of a strong acid, and as an acid in the presence of a strong base.

From true electrolytes, colloidal solutions differ in the fact that their particles are of varying size according to the conditions in which they exist and carry varying charges of electricity, whereas an ion such as $\mathrm{Na}$ or $\mathrm{Cl}$ has a mass which is constant for the ion in question, and always carries the same electric charge. The charged particles of an acid- or alkali-globulin may be distinguished therefore as pseudo-ions.

In these adsorption combinations, although the chemical nature of the colloidal molecules is concerned, there is an absence of definite equilibrium points, such as we are accustomed to in most chemical reactions. The inertia of the system and the large size of the molecules determine the occurrence of false equilibria and of delayed reaction, so that the condition and behaviour of a colloidal system at any moment are determined, not entirely by the quantitative relations of its components, but also by the past history of the system.

\section{COMBINATIONS BETWEEN COLLOIDS•}

Besides the compounds between colloids and electrolytes, combination, or at least interaction, takes place between different colloids. Many colloids are precipitated by other colloidal solutions. This effect is always found to occur when the colloidal solutions carry different charges. Thus ferric hydrate in colloidal solution is precipitated by colloidal silica or colloidal 
gold, both colloids being thrown out of solution. On the other hand, certain colloids may exercise a protective influence on other colloidal solutions. Thus, as Faraday first showed, colloidal gold is much more stable in the presence of a little gelatin. The colloids of serum can dissolve a considerable amount of purified globulin. Although the latter in solution shows a drift in the electric field, the resulting solution is quite unaffected by the passage of a current through it. In these cases the protective colloids carry no charge, but the same protective effect may be observed if a large excess of, e.g. an electro-positive colloid be added to an electro-negative colloid. This interaction between different colloids probably plays an important part in many physiological phenomena. We have reason to believe that the reactions between toxin and antitoxin, and between ferment and substrate, which we shall study later, are of this character, and that the compounds formed belong to the class of adsorption combinations.

\section{THE COAGULATION OF COLLOIDS}

Most colloidal solutions are unstable, and the relations between the suspended particle or molecule and the surrounding fluid may be upset by slight changes of reaction or the presence of minute traces of salts. As a result the hydrosol is destroyed, the suspended particles aggregating to form larger complexes. These aggregations may settle to the bottom of the fluid as a precipitate, or may form a species of network, the result varying according to the nature of the colloid and its concentration. Thus gelatin changes from the condition of hydrosol to hydrogel with fall of temperature. The same is true of agar. On the other hand, by adding calcium chloride to an alkaline solution of casein, we obtain a mixture which sets to a jelly on warming, but becomes fluid again on cooling. Other agencies may lead to the production of changes which are irreversible. Thus a strong solution of colloidal silica sets to a solid jelly on the addition of a trace of neutral salt, and it is not possible to reform the hydrosol, however long the jelly is submitted to dialysis.

Two methods of bringing about coagulation of hydrosols deserve special mention. The first of these is heat-coagulation. If a solution of eggalbumin or globulin be heated in neutral or slightly acid medium and in the presence of neutral salt, the whole of it is thrown down in an insoluble form. This coagulated protein is insoluble in dilute acids or alkalies. The same coagulative effect of heating is observed in the metallic sols. With concentrated solutions of protein, heat coagulation results in the formation of a gel, i.e. a network of insoluble protein, containing water or a very dilute solution of protein in its meshes. In dilute solutions the result is the production of a flocculent precipitate.

Another method is the so-called mechanical coagulation. If a solution of globulin or albumin be introduced into a bottle, which is then violently shaken, a shreddy precipitate makes its appearance in the solution, and this precipitate increases, so that by prolonged shaking it is possible to throw down 80 or 90 per cent. of the dissolved protein in the coagulated form. 
Ramsden has shown that this mechanical coagulation is a surface phenomenon. It depends on the fact that a large number of substances in solution (viz. any which lower the surface tension of their solutions) undergo concentration at the free surface of the fluid. Such substances are proteins, bilesalts, quinine, saponin, \&c. In the case of proteins the concentration reaches such an extent, and the molecules at the surface are so closely packed together, that they form an actual solid pellicle, which hinders the movement of any object, such as a compass needle, suspended in the surface. When the solution is violently shaken, new surfaces are constantly being formed, and as the older surfaces are withdrawn into the fluid, the solid pellicle on them is rolled up into a fine shred of coagulated protein, and this process will continue until there is no protein left to form a pellicle.

We must conclude that colloidal solutions, although differing so widely from true solutions in many of their properties, are connected with these by all possible grades. In a solution of an ordinary crystalloid or electrolyte the molecules of the dissolved substance are distributed equally and homo. geneously among the molecules of the solvent. In the various grades of solution a colloid solution or hydrosol may be assumed to begin when the size of the molecule is increased out of all proportion to that of the molecules of the solvent. The ' dissolved ' molecules now begin to have the properties of matter in mass and to present surfaces with all their attendant attributes. The same sort of solution may be formed with smaller molecules, such as $\mathrm{SiO}_{2}$, when these are aggregated together with adsorbed water into huge molecular complexes, or, as in metallic sols, by the division of the solid metal into ultra-microscopic particles. The distinguishing features of a colloidal solution are due to this lack of homogeneity, and to the fact that in every solution there are two phases - a fluid phase, and a second phase, which is either solid or a concentrated or supersaturated solution of the colloid. The huge size of the molecules and the development of surface not only determine the formation of adsorption combinations, but, on account of the inertia of the system, cause a delay in changes of state, and tend to the formation of false equilibria dependent on the past history of the system.

\section{IMBIBITION}

All colloids, even those such as starch or gelatin, which are insoluble in cold water, exhibit a phenomenon, viz. 'Quellung' or imbibition, which in many cases it is impossible to distinguish from the process of solution. This phenomenon, which was long ago studied by Chevreul and has lately been the subject of a series of careful experiments by Overton, is exhibited by all animal tissues and all colloids. Thus elastic tissue dried in vacuo absorbs from a saturated solution of common salt 36.8 per cent. of water and salt. If dried colloids be suspended in a closed vessel over various solutions, they will take up water in the form of vapour from the solution, and the osmotic pressure of the solution in question will inform us as to the amount of work which would be necessary in order to separate the water again from the colloids. 
Thus it has been reckoned that to press out water from gelatin containing 28.4 parts of water to 100 parts of dried gelatin would require a pressure of over two hundred atmospheres. The imbibition pressure of colloids increases rapidly with the concentration of the colloid and at a greater rate than the latter. In this respect, however, imbibition pressure resembles osmotic or indeed gaseous, pressure. At extreme pressures the pressure of hydrogen rises more rapidly than its volume diminishes. In solutions this effect is more marked the larger the size of the molecule. Thus a 6.7 per cent. solution of cane sugar has the same vapour-tension, and therefore the same osmotic pressure, as a $\cdot 67$ per cent. $\mathrm{NaCl}$ solution. A 67 per cent. cane-sugar solution has, however, the same osmotic pressure as an $18 \frac{1}{2}$ per cent. solution of common salt. It is impossible to draw any hard line of distinction between imbibition pressure and osmotic pressure, or to say where a fluid ceases to be a solution and becomes a suspension. All grades are to be found between a solution such as that of common salt with a high osmotic pressure and optical homogeneity, and a solution such as that of starch, which scatters incident light and is therefore opalescent, and has no measurable osmotic pressure.

The close connection between the processes of imbibition and of solution is shown also by the fact that the latter occurs only in certain media, the nature of the media being dependent on the chemical character of the substances in question. Thus all the crystalline carbohydrates-such as grape sugar and cane sugar-are easily soluble in water, only slightly soluble in alcohol, and practically insoluble in ether and benzol. The amorphous carbohydrates, which must be regarded as derived by a process of condensation from the crystalline carbohydrates-e.g. starch, cellulose, gum arabic, \&c.- -have a strong power of imbibition for water. This power may be limited, as in the case of cellulose, or may be unlimited, as in the case of gum arabic, so that a so-called solution results. On the other hand, they swell up but slightly in alcohol, and are unaffected by ether and benzol. In the same way proteins all take up water, and in many cases form a so-called solution, but are unaffected by ether and benzol-a behaviour which is repeated in the case of the amino-acids, out of which the proteins are built up, and which are easily soluble in water, but are practically insoluble in ether and benzol. On the other hand, india-rubber and the various resins take up ether, benzol, and turpentine often to an indefinite extent, while they are untouched by water. With this behaviour we may compare the easy solubility of the hydrocarbons, the aromatic acids, and esters in ether and benzol, and their insolubility in water. As Overton has pointed out, the power of amorphous carbohydrates to take up fluids is modified by alteration of their chemical structure in the same direction as the solubility of the corresponding crystalline carbohydrates. Thus, if the hydroxyl groups in the sugars be replaced by nitro, acetyl, or benzoyl groups, they become less soluble in water, while their solubility in alcohol, acetone, \&c., is increased. In the same way the replacement of the hydroxyl groups in cellulose by $\mathrm{NO}_{2}$ groups diminishes the power possessed by this substance of taking up water, but renders it capable of swelling up or dissolving in alcohol and acetone. 


\section{SECTION IV}

\section{THE MECHANISM OF CHEMICAL CHANGES IN LIVING MATTER. FERMENTS}

ALL the events which make up the life of plants and animals are accompanied and conditioned by chemical changes of the most varied character. In a previous chapter we have endeavoured to form an idea of the ways in which some of the synthetic processes that occur in the living body may be effected. We saw that, although it was possible to imitate in many respects the vital syntheses by ordinary laboratory methods, the imitation fell far short of the process as it actually occurs in the living cell, both in completeness of the reaction and in the ease with which it could be effected. We can, for instance, by passing carbon dioxide over red-hot charcoal, convert it into carbon monoxide, and this gas, acting on dry potassium hydrate, forms potassium formate. Formate of lime, on dry distillation, gives a small proportion of formaldehyde which, under the influence of dilute alkalies, will condense to the mixture of sugars known as acrose. The green leaf in sunlight absorbs the minimal quantities of carbon dioxide present in the atmosphere and converts it almost quantitatively into starch within a few minutes, and this change is effected in the absence of any concentrated reagents and at the ordinary temperature of the atmosphere. Many of the chemical transformations effected by living cells we have so far been quite unable to imitate. The problem of the synthesis of camphor, of the terpenes, of starch, of cellulose, is still unsolved, and even in the case of those substances which we can manufacture outside the living cell our methods involve the use of powerful reagents and of high temperatures, and result in most cases in the production of many side reactions, besides that which it is our special object to imitate. The distinguishing characteristics of the chemical changes wrought by the living cell are :

(1) The rapidity with which they are effected at ordinary temperatures.

(2) The specific direction of the process, which is therefore almost complete, with a surprising absence of the side reactions which interfere to such an extent with the yield of the methods employed in a chemical laboratory.

This second characteristic may, however, be regarded as a consequence of the first, since an increase in the velocity of any given reaction will determine a preponderance of this reaction over all other possible ones. A funda- 
mental question, therefore, in physiology must relate to the manner in which the cell is able to influence the velocity of some specific reaction.

In spite of the enormous diversity of chemical reactions occurring in the body, they may be divided into a relatively small number of types. Nearly all the reactions are reversible. The chief types of chemical change are as follows :

(1) HYDROLYSIS. In most cases this involves the taking up of water and a decomposition into smaller molecules. Thus the proteins are broken down in the intestine into their constituent amino-acids. The disaccharides, such as maltose or lactose, take up one molecule of water and give rise to two molecules of monosaccharide. The fats take up three molecules of water with the formation of fatty acid and glycerin. Hippuric acid is broken down into benzoic acid and glycine." The reverse change, that of dehydration, is also effected, apparently with equal facility, by the living cell, the hexoses losing water and being converted into a complex starch or glycogen molecule, while the amino-acids are built up first into polypeptides, and these again into the complex proteins of the cell. Besides the reactions in which there is a difference in the amount of free water on the two sides of the equation, it seems probable that hydrolysis and simultaneous dehydrolysis at different parts of the molecule determine a number of chemical transformations, which at first sight seem to involve a simple splitting of the molecule. An example of such a process is afforded by the conversion of glucose into lactic acid described on p. 116.

(2) DEAMINATION. This process involves the splitting off of an $\mathrm{NH}_{2}$ group from an amino-acid as ammonia, and its replacement by $\mathrm{H}$ or $\mathrm{OH}$. Many tissues of the body appear to have this power. In most cases the nature of the change in the remaining fatty moiety of the molecule has not yet been ascertained. If, for instance, to a mass of liver cells some aminoacid, such as glycine, alanine, or leucine, be added, ammonia is set free in proportion to the amount of amino-acid which was added. This ammonia is therefore assumed to be derived from the amino-acid, and it has been suggested that here also the process of splitting off ammonia is a hydrolytic one and that the $\mathrm{NH}_{2}$ group is replaced by $\mathrm{OH}$. Thus-

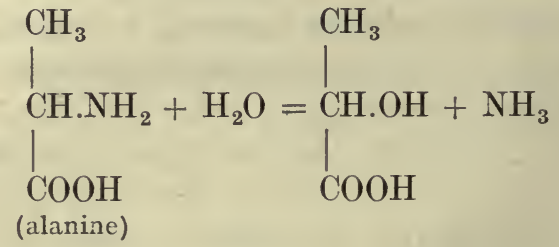

Recent work by Neubauer tends to show that deamination is accompanied in the first place by oxidation, so that the first intermediate product formed is not an $\alpha$ oxy-acid, but an $\alpha$ ketonic acid. A second atom of oxygen is then taken up, and carbon dioxide is split off, with the production of the next lower acid of the series. 
We might represent these changes as follows :

(1)<smiles>CC(CC(=O)O)C(C)([NH3+])OC(=O)O</smiles>

(2)

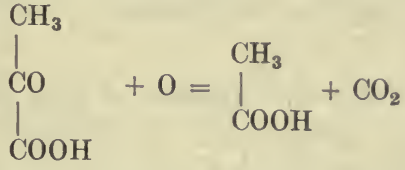

Is the reverse change ever effected in the animal body? If it were possible to replace the $\mathrm{OH}$ group in an oxy-fatty acid by $\mathrm{NH}_{2}$ or the $\mathrm{O}$ in an $\alpha$ ketonic acid by $\mathrm{HNH}_{2}$, it ought also to be possible to nourish an animal from a mixture of carbohydrates and ammonia, or at any rate by supplying him with a mixture of the appropriate oxy-acids or ketonic acids and ammonia. Until recently there was no evidence that the animal body is able to utilise nitrogen, except in organic combination as amino-acids or the complex aggregate of amino-acids known as proteins. In the plant the process of synthesis of protein from ammonia and a carbohydrate such as hexose is continuously going on, and it is probable that the formation of amino-acid occurs by a process the reverse of that which we have just been studying. Knoop has shown that the same reversed change may occur even in a mammal, and that here again the intermediate substance is an $\alpha$ ketonic acid. On administering benzylpyruvic acid $\left(\mathrm{C}_{6} \mathrm{H}_{5} \cdot \mathrm{CH}_{2} \cdot \mathrm{CH}_{2} \cdot \mathrm{CO} \cdot \mathrm{COOH}\right)$ to a dog, a certain amount of benzylalanine $\left(\mathrm{C}_{6} \mathrm{H}_{5} \cdot \mathrm{CH}_{2} \cdot \mathrm{CH}_{2} \cdot \mathrm{CHNH}_{2} \cdot \mathrm{COOH}\right)$ appeared in the urine. The first phase of the oxidative deamination of amino-acids is thus a reversible one and may be represented :

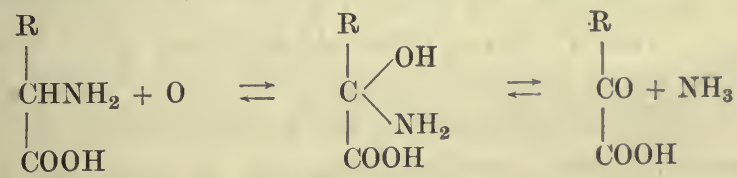

(3) DECARBoxylation. Many amino-acids when subjected to the agency of bacteria lose a molecule of carbon dioxide and are converted into a corresponding amine.

For instance, lysine, which is diamino-caproic acid, is converted into pentamethylene diamine or cadaverine. Thus :

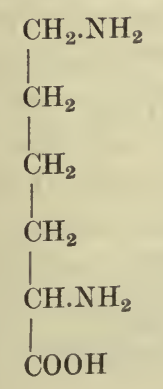

becomes<smiles>NCCCCN</smiles> 
In the same way ornithine derived from the breakdown of arginine is converted by putrefactive bacteria into tetra-methylene diamine or putrescine. Other examples of this process of decarboxylation are :

Isoamylamine from leucine, $\left(\mathrm{CH}_{3}\right)_{2} \cdot \mathrm{CH} \cdot \mathrm{CH}_{2} \cdot \mathrm{CH}_{2} \cdot \mathrm{NH}_{2}$.

$\beta$ phenylethylamine from phenylalanine, $\mathrm{C}_{6} \mathrm{H}_{5} \cdot \mathrm{CH}_{2} \cdot \mathrm{CH}_{2} \cdot \mathrm{NH}_{2}$.

Para-oxyphenylethylamine from tyrosine, $\mathrm{OH} \cdot \mathrm{C}_{6} \mathrm{H}_{4} \cdot \mathrm{CH}_{2} \cdot \mathrm{CH}_{2} \cdot \mathrm{NH}_{2}$.

A similar process has been supposed to take place as a step in the successive oxidation of the carbon atoms in the long chain fatty acids-or carbohydrates, but a thorough study of this process as it occurs in the higher animals is still wanting, and its very existence is indeed still hypothetical. In the case of the fats the oxidation takes place chiefly or entirely in the $\beta$ position. On the other hand, decarboxylation certainly takes place in substances such as the $\alpha$ amino-acids, where the first oxidation change occurs in the $\alpha$ group, and probably closely follows this oxidation change. The reverse reaction, namely, the insertion of the group CO.O at the end of the long carbon chain, is not known to take place, but would furnish a means by which the organism with apparent simplicity could build up long carbon chains and so imitate the process which in the laboratory is generally effected by attaching a $\mathrm{CN}$ group to the end of the molecule. In the case of the fats the building up, like the oxidative breakdown, appears to occur by two carbon atoms at a time; hence all the fatty acids met with in the body have an even number of carbon atoms in their chain.

It is worthy of note that all the changes which we have been considering - changes which not only account for the greater part of the chemical reactions of the living body, but may lead to the production of the most complex substances known-are performed with little expenditure or evolution of energy. This is evident if we examine the heat evolved by the total combustion of one gramme molecule of the initial and final substances in a number of typical reactions. In the following Table these are given for the substances involved in typical instances of the three classes of chemical change that we have just been considering :

(1) Hydrolysis

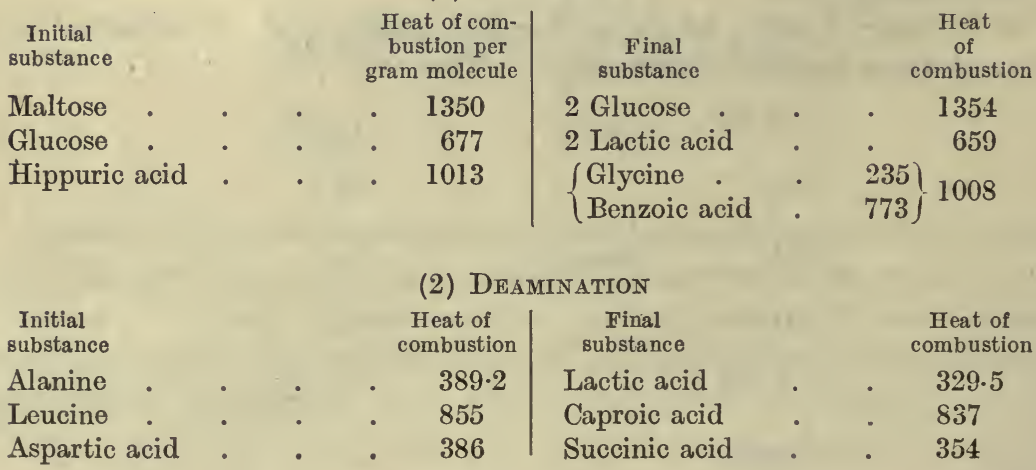


Initial

substance

Alanine

Leucine
(3) Decarboxylation

\begin{tabular}{c|c}
$\begin{array}{c}\text { Heat of } \\
\text { combustion }\end{array}$ & $\begin{array}{c}\text { Final } \\
\text { substance }\end{array}$ \\
389 & Ethylamine. \\
855 & Isoamylamine.
\end{tabular}

Ileat of

combustion

409

867

(4) OXIDATION AND REDUCTION. The fourth class of chemical reactions differs from those just described in being attended with a very considerable energy change. This class involves the processes of oxidation and reduction. In almost every living cell by far the largest amiount of the energy available for the discharge of the functions of the cell is derived from the oxidation of the food-stuffs, and even in the plant the energy is obtained from the oxidation of the food-stuffs, built up in the first instance at the cost of the energy of the sun's rays. If we take the final changes in the food-stuffs, the very large evolution of energy attending their oxidation is at once apparent. Thus in the conversion of glucose into $\mathrm{CO}_{2}$ and water there is an evolution for each gramme molecule of 677 calories. In the combustion of glycerin 397 calories are evolved. In the oxidation of a fat such as trimyristin there are 6650 calories evolved. The change does not, in the living cell, occur all at once, but the molecule is oxidised step by step. In each step the heat change will, however, be probably greater than the heat changes in the other types of chemical change which we have been considering.

Since the mechanism of oxidation in the animal body will have to be discussed at length in a subsequent part of this work, we may at present confine our attention to the other types of chemical change. Of these, all which involve a splitting of a large molecule into smaller ones with the taking up of one or more molecules of water, as well as, in all probability, those in which the reverse change of dehydration and synthesis occur, are effected in the body by means of ferments. To the same agency are also ascribed the process of deamination, which takes place in many organs of the body, and, though with less certainty, the processes which involve decarboxylation.

\section{FERMENTS}

Under the name ferments we include a number of substances of indefinite composition whose existence is chiefly known to us by their action on other substances. A ferment has been defined as a body which on addition to a chemical system is able to effect changes in this system without supplying any energy to the reaction, without being used up, and without taking any part in the formation of the end products. It differs therefore from the reacting substances in the absence of any strict quantitative relationships between it and the substances included in the system in which its effects are produced. Minimal quantities of ferment can induce chemical changes involving almost indefinite quantities of other substances, the only result of increasing the quantity of ferment being to quicken the rate of the change. Since they are effective in minimal doses they occur in living tissues in minute quantities, and it is partly due to this fact that it has 
hitherto proved impossible to obtain any preparation of a ferment which could be regarded as a pure substance. The difficulty in their isolation is increased by the fact that all of them are colloidal or semi-colloidal in character, and present, therefore, the tendency common to all colloids of adhering to other colloidal matter as well as to surfaces such as those presented by a precipitate. A common method of isolating, or rather obtaining a concentrated preparation of a ferment is to produce in its solution an inert precipitate such as cholesterin or calcium phosphate. The ferment is carried down on the precipitate and may be obtained in solution on washing the precipitate with water. A further difficulty in their preparation lies in the unstable character of many members of the group. Although they are not coagulated by alcohol, they are nevertheless gradually changed, so that every act of precipitation of a ferment tends to rob it of some of its powers, i.e. of the only characteristic by which we can establish its identity.

Of these ferments a large number have already been described as taking part in the ordinary chemical processes of life. So wide is their dominion in cell chemistry that many physiologists have thought that the whole of life is really a continual series of ferment actions. The following list represents some of the ferments whose existence has been definitely established in the animal body. The greater part of them are involved in the processes of digestion in the alimentary canal. The preponderance, however, of digestive ferments in the list is due to the fact that we know more about digestion than about the other chemical processes taking place within the cells of the body.

List of Ferments

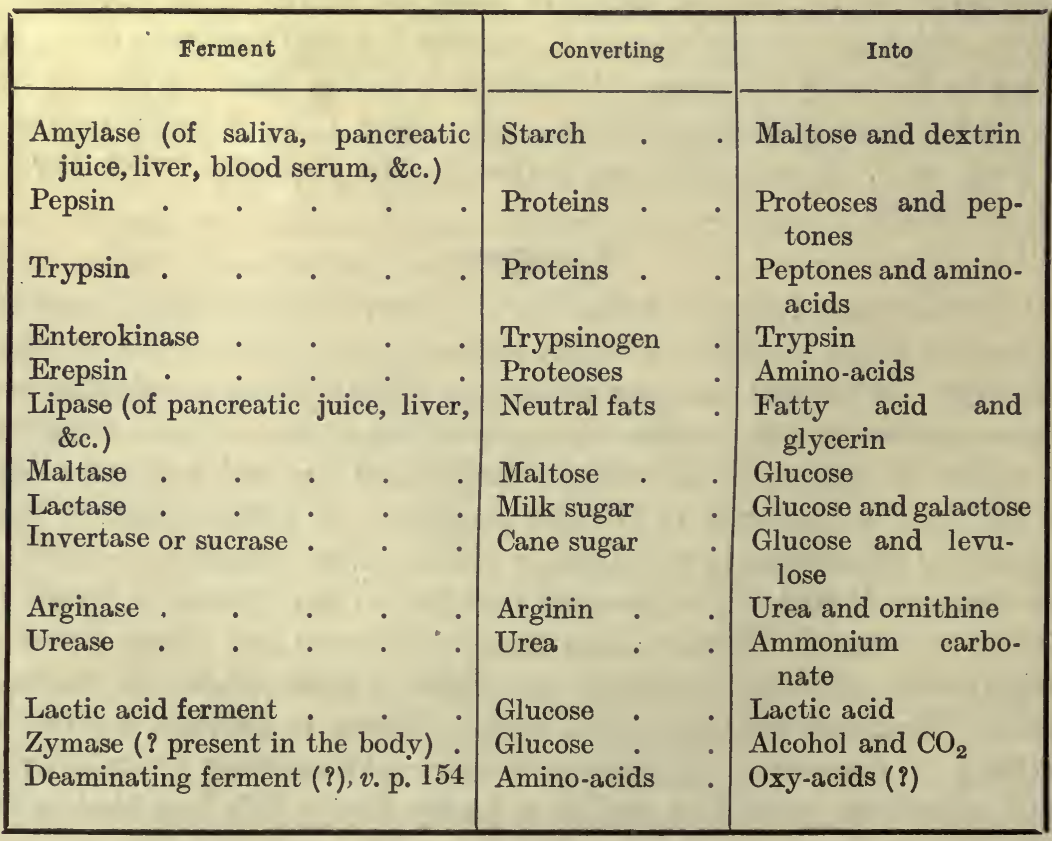


Many other ferments will probably be distinguished with increase in our knowledge of cellular metabolism. The long list which is here given suffices to show how great a part these bodies must play in the normal processes of life. A study of the conditions of ferment actions is therefore essential if we would form a conception of the chemical mechanisms of the living cell.

It is important to note that all the changes wrought by ferments can be effected by ordinary chemical means. Thus the disaccharides can be made to take up a molecule of water and undergo conversion into monosaccharides. If a solution of maltose be taken and bacteria be excluded from the solution, it undergoes at ordinary temperatures practically no change. If the solution be warmed, a slow process of hydration takes place which is quickened by rise of temperature, so that if the solution be heated under pressure to, say, $110^{\circ} \mathrm{C}$., hydrolysis occurs with considerable rapidity. If, however, a little maltase be added to the solution, the change of maltose into glucose takes place rapidly at a temperature of $30^{\circ} \mathrm{C}$. In the same way a solution of protein may be kept almost indefinitely without undergoing hydrolysis, which, however, can be induced by heating the solution under pressure. The action of the ferments in these two cases is to quicken a process of hydrolysis which without their presence would take an infinity of time for its accomplishment.

In this respect their action is similar to that of acids, and indeed of a whole class of bodies which are spoken of as catalysers or catalysts. A catalyser is a substance which will increase (or diminish) the velocity of a reaction without adding in any way to the energy changes involved in the reaction, or taking any part in the formation of the end-products. Since the catalyser is unchanged in the process, a very small quantity is able to influence reactions involving large quantities of other substances. By adding acids to a watery solution of the food-stuffs, the process of hydrolysis is quickened in proportion to the strength and concentration of the acid. The effective catalytic agents in this process appear to be the hydrogen ions of the free acid. There are many other bodies, besides the free acids, which may act as catalysers, and a study of the conditions under which catalysis takes place may throw some light on the essential nature of the action of ferments.

The velocity of almost any reaction in chemistry can be altered by the addition of some catalytic agent, and there are few of the ordinary reactions in which catalysis does not play some part. Among such processes we may instance the action of spongy platinum on hydrogen peroxide. Hydrogen peroxide undergoes slow spontaneous decomposition into water and oxygen. If a little spongy platinum be added to it, it is at once seen to decompose rapidly with the evolution of bubbles of oxygen, and the action does not cease until the whole of the hydrogen peroxide has been destroyed. Spongy platinum is able in the same way to quicken a very large number of chemical reactions. Thus sulphur dioxide and oxygen when heated together will combine very slowly; the combination becomes rapid if a mixture of the 
two gases be passed over heated platinum. The same reaction, namely, the combination of sulphur dioxide with oxygen, may be quickened by the addition of a small trace of nitric oxide, and this fact is made use of in the manufacture of sulphuric acid on a commercial scale by the ordinary leadchamber process. Hydrogen peroxide and hydriodic acid slowly interact with the formation of water and iodine. This reaction may be quickened by the addition of many substances, among which we may mention molybdic acid.

There is moreover a specificity in the action of catalysers, though not so well marked as with ferments. Whereas all the disaccharides are converted by acids into the corresponding monosaccharides, a ferment such as invertase acts only on cane sugar, and has no action on maltose or lactose, each of which requires a specific ferment (maltase, lactase) to effect their ' inversion.' But we find many examples of a restricted action even among inorganic catalysers. Thus potassium bichromate will act as the catalyser for the oxidation of hydriodic acid by bromic acid, but not for the oxidation of the same substance by iodic acid. Iron and copper salts in minute traces will quicken the oxidation of potassium iodide by potassium persulphate, but have no influence on the course of the oxidation of sulphur dioxide by potassium persulphate. Tungstic acid increases the velocity of oxidation of hydriodic acid by hydrogen peroxide, but has no effect on the velocity of oxidation of hydriodic acid by bromic acid, and these examples may be multiplied to any extent. One cannot therefore regard the limitation of action of the ferments as justifying any fundamental distinction being drawn between the action of this class of substances and catalysts.

Whereas the influence of most catalysers on the velocity of a reaction increases rapidly with rise of temperature, in the case of ferments this increase occurs only up to a certain point. This point is spoken of as the optimum temperature of the ferment action. If the mixture be heated above this point the action of the ferment rapidly slows off and then ceases. This contrast, again, is only apparent. The ferments are unstable bodies easily altered by change in their physical conditions, and destroyed in all cases at a temperature considerably below that of boiling water. Thus ferment actions, like catalytic actions, are quickened by rise of temperature, but the effect of temperature is finally put a stop to by the destruction of the ferment. The same applies to those inorganic catalysers whose physical state is susceptible, like that of the ferments, to the action of heat. Thus the colloidal platinum 'sol' exerts marked catalytic effects on various reactions, e.g. on the decomposition of hydrogen peroxide and on the combination of hydrogen and oxygen. The reaction presents an optimum temperature, owing to the fact that the colloidal platinum is altered, coagulated, and thrown out of solution when this is heated to near boiling-point: We may therefore employ either class of reactions in trying to form some conception of the processes which are actually involved.

Very many theories have been put forward to account for this action of catalysers or of ferments. Many of them are merely transcriptions in 
words of the processes which actually occur, and fail to throw any light on their real nature. The essential phenomena involved fall directly into two classes. In the first class we must place those which are determined by the influence of surface. In many cases the combination of gases can be hastened by increasing the surface to which they are exposed, as by passing them over broken porcelain or over powdered charcoal. This catalytic effect is certainly connected with the power of a solid to condense gases at its surface, and is therefore proportional to the extent of surface exposed. Thus the efficacy of platinum in hastening the combination of hydrogen and oxygen is in direct proportion to its fineness of sub-division, and is best marked when the metal is reduced to ultra-microscopic dimensions, as in the colloidal solution of platinum. Every colloidal solution must be regarded as presenting an enormous surface in proportion to the mass of substance in solution. There is therefore a direct proportionality between the power of a substance to condense a gas on its surface and its power to quicken the velocity of chemical changes in which the gas is involved. The same process of condensation may occur with dissolved substances. In all cases where the presence of a substance in solution diminishes the surface tension of the solvent, there is a diffusion of dissolved substances into the surface, i.e. a concentration of dissolved substances at the surface of contact. It was suggested by Faraday that the catalytic property of surfaces was due to this condensation of molecules, and the consequent bringing of the two sets of molecules within each other's sphere of influence. Whether this is the sole factor involved is doubtful, since mere compression of gases or increased concentration of solutions does not in the majority of cases result in such a quickening of the velocity of reaction as is brought about by the effect of the surface.

It is possible that this condensation effect or adsorption may be in every case combined with the second factor which we must now consider, namely, the formation of intermediate products. If we boil an alkaline solution of indigo with some glucose, the indigo is reduced with oxidation of the glucose. The mixture therefore becomes colourless. On shaking up with air the colourless reduction product of the indigo absorbs oxygen from the atmosphere, and is re-transformed into indigo. These two processes can be repeated until the whole of the glucose is oxidized, and the process can be made continuous if air or oxygen be bubbled through a heated solution of glucose containing a small trace of indigo. In this case the indigo does not add to the energy of the reaction. It appears unchanged among the final products and a small amount may be used to effect the change of an infinite quantity of glucose. It therefore may be said to act as a ferment or catalytic agent. Instead of an alkaline solution of indigo, we may use an ammoniacal solution of cupric oxide for the purpose of carrying oxygen from the atmosphere to the glucose. This is reduced to cuprous hydrate on heating with the sugar, but cupric hydrate can be at once re-formed by shaking up the cuprous solution with air. It has been thought that many or all of the catalytic reactions occur in the same way by two stages, i.e. 
by the formation of an intermediate product. Thus, in the old lead chamber process for the manufacture of sulphuric acid, the nitric oxide may be supposed to combine with the oxygen of the air to form nitrogen peroxide. This interacts with sulphur dioxide, giving sulphur trioxide and nitric oxide once more. The nitric oxide, which we alluded to before as the catalyser, may in this way be regarded as the carrier of oxygen from air to sulphur dioxide. It has been suggested that the action of spongy platinum or colloidal platinum rests on the same process, and that in the oxidation of hydrogen, for instance, $\mathrm{PtO}$ or $\mathrm{PtO}_{2}$ is formed and at once reduced by the hydrogen with the formation of water.

There is a certain amount of experimental evidence in favour of this hypothesis. According to Engler and Wöhler,* platinum black, which has been exposed to oxygen, in virtue of the gas which it has occluded, has the power of turning potassium iodide, and starch blue. This power is not destroyed by heating to $260^{\circ}$ in an atmosphere of $\mathrm{CO}_{2}$, or by washing with hot water. On exposure of the platinum black to hydrochloric acid, a certain amount is dissolved, and the substance loses its effect on potassium iodide. The amount dissolved corresponds with the amount of iodine liberated from potassium iodide, and also with the amount of oxygen occluded, the (soluble) platinum and oxygen being in the proportions necessary to form the compound PtO.

But why should a reaction take place more quickly if it occurs in two stages instead of one? As Ostwald has pointed out, the formation of an intermediate compound can be regarded as a sufficient explanation of a catalytic process only when it can be demonstrated by actual experiment that the rapidity of formation of the intermediate compound and the rapidity of its decomposition into the end-products of the reaction are in sum greater than the velocity of the reaction without the formation of the intermediate body. In the case of one reaction this requirement has been fulfilled. The catalytic effect of molybdic acid on the interaction of hydriodic acid and hydrogen peroxide has been explained by assuming that the first action which takes place is the formation of permolybdic acid, and that this then interacts with the hydrogen iodide to form water and iodine. Now it has been actually shown-(1) that permolybdic acid is formed by the action of hydrogen peroxide on molybdic acid;(2) that permolybdic acid with hydriodic acid produces water and iodine; (3) that the velocity with which these two reactions occur is much greater than the velocity of the interaction of hydrogen peroxide and hydriodic acid by themselves.

Although we may find it difficult to explain why a reaction should occur more quickly in the presence of a catalyser by the formation of these intermediate bodies, certain simple analogies may help us to comprehend how a factor which introduces no energy can yet assist the process. Thus a man might stand to all eternity before a perpendicular wall twenty feet high. Since he cannot reach its top at one jump, he is unable to get there at all. The introduction of a ladder will not in any way alter the total energy he must expend on raising his body for twenty feet, but will enable him to attain the top. Or we might imagine a stone perched at the top of a high hill. The passive resistance of the system, the friction of the stone, and its inertia will tend to keep it at rest, even though it be on a sloping surface and therefore tending to slide or roll to the bottom. If, however, it be rolled to a point where there is a sudden increase in the rapidity of slope, it may roll over, and having once started * Quoted by Mellor, "Chemical Statics." 
its downward course, its momentum will carry it to the bottom. The amount of energy set free by the stone in its fall will not vary whether the course be a uniform one, or whether it falls over a precipice at one time and rolls down a gentle slope at another. It is evident that by a mere alteration of the slope, or, in the case of a chemical reaction, of the velocity of part of its course, a change in the system may be initiated and brought to a conclusion which without this alteration would never take place.

Since the action of ferments, like that of catalysts, consists essentially in the quickening up of processes which would otherwise occur at an infinitely slow velocity, it is possible that in their case also the formation of an intermediate compound may be involved in the reaction. Light may be thrown upon this question by a study of the velocity of the reaction induced by the action of a ferment.

It is well known that the velocity of a reaction depends on the number of molecules involved. As an illustration, we may take first the case of a reaction involving a change in one substance. If arseniuretted hydrogen be heated, it undergoes decomposition into hydrogen and arsenic. This decomposition is not immediate, but takes a certain time, and the velocity with which the change occurs depends on the tempera. ture. At any given temperature the amount of substance changed in the unit of time varies with the concentration of the substance. If, for instance, one-tenth of the gas be dissociated in the first minute, in the second minute a further tenth of the gas will also be dissociated. Thus, if we start with 1000 grammes of substance, at the end of the first minute 100 grammes will have been dissociated, and 900 of the original substance will be left. In the second minute one-tenth again of the remaining substance will be dissociated, i.e. 90 grammes, leaving 810 grammes. In the third minute 81 grammes will be dissociated, leaving 729 grammes. The amount changed in the unit of time will always bear the same ratio to the whole substance which is to be changed, and will therefore be a function of the concentration of this substance. Put in the form of an equation, we may say that $\phi$, the amount changed in the unit of time, will be equal to $\mathrm{KC}$, where $\mathrm{K}$ is a constant varying with the substance in question and with the temperature, and $\mathrm{C}$ represents the concentration of the substance. The equation $\phi=\mathrm{KC}$ applies to a monomolecular reaction.

If two substances are involved, the equation will be rather different. In this case the amount of change in a unit of time will be a function of the concentration of each of the substances, and the form of the equation will be $\phi=\mathrm{K}\left(\mathrm{C}_{\mathrm{x}} \times \mathrm{C}_{\mathrm{y}}\right)$. In the case of the unimolecular reaction, halving the concentration of the substance will halve the a mount of substance changed in the unit of time. In the case of a bimolecular reaction, halving each of the substances will cause the amount of change in the unit of time to be reduced to one-quarter of its previous amount. If now either a unimolecular or a bimolecular reaction be quickened by the addition of a catalyser or ferment, and the ferment enter into combination with one of the substances at some stage of the reactioll, it is evident that our equation must take account also of the concentration of the ferment or catalyser. In the case of the catalytic effect of molybdic acid on the interaction between hydrogen peroxide and HI, there was definite evidence of a reaction taking place between the molybdic acid and the peroxide, resulting in the formation of an intermediate compound, namely, permolybdic acid. Brode has shown that the interaction of the molybdic acid is revealed in the equation representing the velocity of the reaction. Without the addition of molybdic acid the equation would be :

$$
\phi=\mathrm{K}\left(\mathrm{C}_{\mathrm{H}_{2} \mathrm{O}_{2}} \times \mathrm{C}_{\mathrm{HI}}\right) \text {. }
$$

After the addition of molybdic acid, the equation becomes :

$$
\phi=\mathrm{K}\left(\mathrm{C}_{\mathrm{H}_{2} \mathrm{O}_{2}}+\gamma \mathrm{C} \text { molybdic acid }\right) \mathrm{C}_{\mathrm{H} 1},
$$

when $\gamma$ is another constant depending on the molybdic acid. If ferments act in a similar way by the formation of intermediate compounds, this fact should be revealed by a study of the velocity at which the ferment action takes place. 
Various methods may be adopted for the study of the velocity of ferment action. If, for instance, we are investigating the action of diastase upon starch, we should take solutions of starch and of diastase of known concentrations, keep them in a water bath at $38^{\circ} \mathrm{C}$, and at a certain point add, say, 20 c.c. of ferment solution to every 100 c.c. of the starch solution. At periods of five or ten minutes after the addition had been made, 5 c.c. of the mixture might be withdrawn by a pipette and at once run into boiling Fehling's solution. The precipitated cuprous oxide would be dried and weighed, and would give directly the amount of sugar formed by the action of the ferment. After obtaining a series of data in this way, a curve could be drawn, showing the amount of change of starch which had occurred in each unit of time. In the case of the action of invertase on cane sugar the investigation is still easier. Since the change from cane sugar to invert sugar is accompanied by a change in the rotatory power of the solution on polarized light, it is only necessary to put the mixture of ferment and cane sugar into a polarimeter tube, which is kept at a constant temperature by means of a water jacket, and read off at intervals of a few minutes the change in the rotatory power of the solution. From this change can be easily calculated the percentage of cane sugar still present, and therefore the total amount which has been converted into fructose and glucose.

In investigating the action of proteolytic ferments, as, e.g. that of trypsin on caseinogen, samples are taken at five-minute intervals and run into some substance such as trichloracetic acid, which will precipitate all the unchanged protein, but will leave in solution the products of hydration of the protein. From the amount of nitrogen in the filtrate from the precipitate can be determined the total amount of protein which has undergone hydration in the sample under observation. Or the amount of albumoses and peptones present in each sample may be estimated by the intensity of the biuret reaction which can be obtained. This method, however, suffers from the drawback that the albumoses and peptones, at any rate in the action of trypsin, are formed merely as a stage in the process, and the intensity of the reaction will first rise to a maximum and then gradually disappear. A very convenient method is that employed by Henri and by Bayliss in the investigation of the kinetics of tryptic action, namely, the determination of the conductivity of the solution. In the disintegration of the molecule caused by the action of the ferment, there is a continuous increase in the conductivity of the solution, and this increase can be regarded as an index to the rate of change in the substances undergoing disintegration.

By such methods it has been found that, when the quantity of ferment employed is very small in comparison with the substrate (the substance acted upon), the amount of change in a given time is proportional to the amount of ferment present, and is (within limits) independent of the concentration of the substrate. This is well shown by the two following Tables representing the action of lactase upon lactose (E. F. Armstrong) : 
Proportions Hydrolysed in 100 c.C. OF a 5 PER Cent.

Soldtion of LACTOSE

\begin{tabular}{|c|c|c|c|}
\hline Solutions containing-- & 1.5 hours & 20 hours & 45 hours \\
\hline 1 c.c. lactase & $0 \cdot 15$ & $2 \cdot 2$ & $3 \cdot 9$ \\
\hline 10 c.c. , & $1 \cdot 6$ & $23 \cdot 3$ & $38 \cdot 6$ \\
\hline 20 c.c. " & $3 \cdot 2$ & $45 \cdot 8$ & - \\
\hline
\end{tabular}

Amount of Sugar (Lactose) Hydrolysed

\begin{tabular}{|c|c|c|c|c|}
\hline \multirow{2}{*}{ Solutions containing - } & \multicolumn{2}{|c|}{24 hours } & \multicolumn{2}{|c|}{46 hours } \\
\hline & Proportion & Weight & Proportion & Weight \\
\hline 10 per cent. lactose & $14 \cdot 2$ & $1 \cdot 42$ & $22 \cdot 2$ & $2 \cdot 22$ \\
\hline $20 \quad$ & $7 \cdot 0$ & $1 \cdot 40$ & $10 \cdot 9$ & $2 \cdot 18$ \\
\hline $30 \quad, \quad$, & 4.8 & $1 \cdot 44$ & $7 \cdot 7$ & $2 \cdot 21$ \\
\hline
\end{tabular}

Moreover, if we take only the earlier stages of the ferment action, it is found that, with small proportions of ferment, equal amounts of substrate are changed in successive intervals of time until about 10 per cent. has been hydrolysed. This is shown in the following Table:

\section{PER CENT. LACTOSE WITH LACTASE}

Time
$\frac{1}{3}$ hour
$\frac{2}{3}$,
1 "
2 hours
3 ",

$$
\begin{aligned}
& \text { Amount hydrolysed } \\
& 3 \cdot 2 \\
& \text { 6. } 4 \\
& 9 \cdot 6 \\
& 16 \cdot 4 \\
& 20 \cdot 8
\end{aligned}
$$

These results can be interpreted only by assuming that the first stage in the reaction is a combination of ferment with substrate. It is only this compound which represents the active mass of the molecules, i.e. the molecules of substrate which are undergoing change. This compound, as soon as it is formed, takes up water and breaks down, setting free the hydrolyzed substrate and the ferment, which is at once ready to combine with a further portion of the substrate. In such a case the velocity of reaction must be directly proportional to the amount of ferment, and the same absolute quantity of substance will continue to be changed in succeeding units of time. Supposing, for instance, we had a load of bricks at the bottom of a hill which had to be transferred to the top, and five men to effect the transference. The rate of transference would be directly proportional to the number of men employed; we could double the rate by doubling the men. 
Moreover, the number of bricks carried in each unit of time would be the same. Five men would carry as many bricks in the second ten minutes as they would in the first, and so on. On the other hand, the velocity with which the transference was effected would be independent of the numberthat is, the concentration - of the bricks at the bottom of the hill. The active mass of bricks could be regarded as that number carried at any moment by the transferring factor, namely, the men. The equation of change would be $\phi=\mathrm{KC}$, where $\mathrm{C}$ is the concentration of the ferment. This concentration is always being renewed, and kept constant by the breaking down of the intermediate product, so that the rate of change would be continuous, throughout the experiment.

On the other hand, when the amount of ferment is relatively large, the rate of change, though at first very rapid, tends continuously to diminish. This is shown by the following Table representing the rates of change, during succeeding intervals of ten minutes, in a caseinogen solution to which a strong solution of trypsin had been added (Bayliss) :

\section{Velocity of Trypsin Reaction}

6 c.c. 8 per cent. caseinogen +2 c.c. $\frac{N}{10} A m H O+2$ c.c. 2 per cent.

trypsin at $39^{\circ} \mathrm{C}$.

1st 10 minutes
2nd ,"
3rd
4th ,
5th ,"
7th _-",

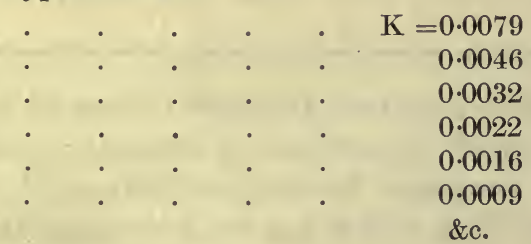

The cause of this rapid diminution in the velocity of change is probably complex. One factor may be an auto-destruction of the ferment, which is known to occur in watery solution. That this is not the only, or even the chief, factor involved is shown by the fact that, when the action of trypsin on caseinogen has apparently come to an end, it may be renewed by further dilution of the mixture or by removal of the end-products of the action by dialysis. It is evident that, in this retardation of the later stages of ferment action, the end-products are concerned in some way or other, and the retardation can be augmented by adding to the digesting mixture the boiled endproducts of a previous digestion. The retarding effect of the end-products resembles in many ways that observed in a whole series of reactions which are known as reversible.

As an example of such a reaction we may take the case of methyl acetate and water. When methyl acetate is mixed with water, it undergoes decomposition with the formation of methyl alcohol and acetic acid. On the other hand, if acetic acid be mixed with alcohol, an interaction takes place with the formation of methyl acetate and water. These changes are represented by the equation :

$$
\underset{\text { methylacetate }}{\mathrm{MeC}_{2} \mathrm{H}_{3} \mathrm{O}_{2}}+\underset{\text { water }}{\mathrm{HOH}} \underset{\text { methylalcohol acetic acid }}{\rightleftharpoons} \mathrm{MeOH}+\mathrm{HC}_{2} \mathrm{H}_{3} \mathrm{O}_{2} .
$$

Each of these changes has a certain velocity constant, and, since they are in opposite directions, there must be some equilibrium point where no change will occur, and 
there will be a definite amount of all four substances present in the mixture, namely, water, alcohol, ester, and acid. This equilibrium point can be shifted by altering the amount of any of the four substances. Thus the interaction of methyl acetate and water can be diminished to any desired extent by adding to the mixture the products of the interaction, namely, methyl alcohol and acetic acid.

There is evidence that some of the ferment actions are reversible. Thus maltase acts on maltose with the formation of two molecules of glucose. If the maltase be added to a concentrated solution of glucose, we get a reverse effect, with the production of a disaccharide which has been designated as isomaltose or revertose. To this reverse action may be due a certain amount of the retardation observed in the action of trypsin on coagulable protein. A more important factor is probably the combination of the ferment itself with the end-products and the consequent removal of the ferment from the sphere of action. Several facts speak for such a mode of explanation. Thus the action of lactase on milk sugar is not retarded by both its end-products, namely, glucose and galactose, but only by galactose. In the same way the action of invertase on cane sugar is retarded by the end-product fructose, but not at all by the other end-product, glucose.

So far, therefore, a study of the velocity of ferment actions would lead us to suspect that the ferment combines in the first place with the substrate, and that this combination is a necessary step in the alteration of the substrate. In the second place, the ferment is taken up to a certain extent by some or all of the end-products, and this combination acts in opposition to the first combination, tending to remove the ferment from the sphere of action, and therefore to retard the whole reaction. Other facts can be adduced in favour of these conclusions. Thus it has been shown that invertase ferment, which is destroyed when heated in watery solution at a temperature of $60^{\circ} \mathrm{C}$., can, if a large excess of its substrate, cane sugar, be present, be heated $25^{\circ}$ higher without undergoing destruction. The same protective effect is observed in the case of trypsin. Trypsin in watery or weakly alkaline solutions undergoes rapid decomposition. At $37^{\circ} \mathrm{C}$. it may lose 50 per cent. of its proteolytic power within half an hour. If, on the other hand, trypsin be mixed with a protein such as egg albumin or caseinogen, or with the products of its own action, namely, albumoses and peptones, it can be kept many hours without undergoing any considerable loss of power.

It has been found that, whereas maltase splits up all the $a$-glucosides, it has no power on the $\beta$-glucosides; that is to say, maltase will fit into a molecule of a certain configuration, but is powerless to affect a molecule which differs from the first only in its stereochemical structure. On the other hand, emulsin, which breaks up $\beta$-glucosides, has no influence on $a$-glucosides. This specific affinity of the ferments for optically active groups of bodies suggests that the ferment itself may be optically active. We cannot of course isolate the ferment and determine its optical behaviour; but that it is optically active is rendered probable both by these results and certain results obtained by Dakin on lipase, the fat-splitting ferment. Dakin carried out his experiments on the esters of mandelic acid. Mandelic acid is optically inactive, but this optically inactive modification consists of a mixture of equal parts of dextro-rotatory 
and lævo-rotatory mandelic acid. The esters prepared from the optically inactive acids are themselves optically inactive. Dakin found that, when an optically inactive mandelic ester was acted upon by a lipase prepared from the liver, the final results of the action were also inactive ; but if the reaction were interrupted at the half-way point, the mandelic acid which had been liberated was dextro-rotatory, while the remainder of the ester was lævo-rotatory. Thus the rate of hydrolysis of the dextrocomponent of the ester is greater than that of the lævo-component, a result which can be best explained by the assumptions $(a)$ that the enzyme or a substance closely associated with it is a powerfully optically active substance; $(b)$ that actual combination takes place between the enzyme and the ester undergoing hydrolysis. Since the additive compounds thus formed in the case of the dextro- and lævo-components of the ester would not be optical opposites, they would be decomposed with unequal velocity, and thus account for the liberation of the optically active mandelic acid.

We may conclude that in the action of ferments on the food substances, whether carbohydrate or protein, an essential factor is the combination of the ferment with the substrate. Only the part of the substrate, which is thus combined with the ferment, can be regarded as the active mass and as undergoing the hydrolytic change. What is the nature of this combination? Ferments, which are all of a colloid or semi-colloid character, cannot be dealt with in the same way as the catalysts of definite chemical composition, such as molybdic acid or nitric oxide. In many cases the substrate, e.g. starch or protein, is also colloidal, and the combination therefore falls into the class of combinations between colloids. In this we have an interaction between two substances in which the adsorption by the surfaces of the molecules of one or both substances plays an important part, though this adsorption is itself determined or modified by the chemical configuration of the molecules. The combination of ferments with their substrates belongs, therefore, to that special class of interactions, not entirely chemical and not entirely physical, but depending for their existence on a co-operation of both chemical and physical factors, which we have discussed earlier under the name of adsorption compounds.

\section{FERMENTS AS SYNTHETIC AGENTS}

If maltase, obtained from yeast, or from the so-called takadiastase (prepared from Aspergillus oryzoc), be added to a solution of maltose, the latter is hydrolysed to glucose. The process of hydrolysis stops short of complete inversion at a point varying with the concentration of the sugar solution. Thus in a 10 per cent. solution of maltose, inversion proceeds until 98 per cent. of the maltose is converted into dextrose, whereas in a 40 per cent. solution the change stops short when 85 per cent. sugar has undergone inversion. Croft Hill showed that if the maltase were added to a 40 per cent. solution of dextrose, a change took place in the reverse direction, which proceeded until 85 per cent. of the glucose was left. The sugar formed, which is a disaccharide, was regarded by Croft Hill as maltose. According to Emmerling, however, it is the stereoisomeric sugar, iso-maltose, which is formed; and Croft Hill in his later papers spoke of the sugar as revertose.

In،the same way it has been shown by Castle and Loewenhart that the 
hydrolysis of esters by lipase is a reversible reaction, the action of lipase being simply to hasten the attainment of the equilibrium point between the four substances - ester (or neutral fat), water, fatty acid, and alcohol. Similar reversible effects have been described for other ferments. Thus the addition of pepsin to a strong solution of albumoses causes the appearance of an insoluble precipitate, which is called plastein, and has been regarded as produced by the resynthesis of the original protein molecule.

If all ferment actions are in this way reversible, a possibility is opened of regarding the synthetic processes occurring in the living cell, as well as the processes of disintegration, as determined by the action of enzymes. It must be noted that these effects are only obtained with distinctness when dealing with concentrated solutions. The degree of synthesis which would be produced in the very dilute solutions of glucose, \&c., occurring in the animal cell would therefore be infinitesimal. But if a mechanism were provided for the immediate separation of the synthetical product from the sphere of reaction, either by removing it to a different part of the cell or by building it up into some more complex body which was not acted on by the ferment, the process of synthesis might go on indefinitely, and the infinitesimal quantities be summated to an appreciable amount.

Some experiments by Bertrand on fat synthesis have been interpreted as showing that the process of synthesis by ferments is not the mere attainment of an equilibrium point in a reversible reaction. It has long been known that watery extracts of the fresh pancreas split neutral fats into the higher fatty acids and glycerine. This observer has shown that if the pancreas be dried with alcohol and ether and powdered, addition of the dry powder to a mixture of the higher fatty acids and glycerine brings about a rapid synthesis of neutral fat. The process of synthesis is at once stopped by the addition of water. In this case either there are two ferments present, one a synthetising, the other a hydrolysing, ferment, differing in their conditions of activity, or there is one ferment which may act either as a fatsplitting or fat-forming agent according to the conditions under which it is placed. In the latter case the effect of the addition of water would be simply to alter the equilibrium point of the mixture. It has been shown that in all reversible reactions the equilibrium position is the same from whichever side it be approached. The action of the ferment is to hasten the attainment of equilibrium, the position of the latter being determined by the relative concentration of the reacting molecules. 


\section{SECTION V}

\section{ELECTRICAL CHANGES IN LIVING TISSUES}

THE material composing living cells and tissues is permeated throughout with water containing electrolytes in solution. All salts, as we have seen, undergo ionic dissociation in watery solution-a dissociation which, in the concentrations occurring in the animal body, must be nearly complete. When an electric current passes through the living tissues it is carried by the charged ions formed by the dissociation of the salts. Thus, $\mathrm{n} / 10$ solution of sodium chloride contains almost entirely $\stackrel{+}{\mathrm{Na}}$ and $\overline{\mathrm{Cl}}$ ions. In addition to these charged inorganic ions, the cell protoplasm contains in solution or suspension various colloidal particles which in many cases are themselves charged. By the presence of these colloidal particles marked differences may be caused in the distribution of the inorganic ions owing to the power of adsorption possessed by the colloids for many inorganic salts. It is evident that any unequal distribution of the charged ions or colloidal particles in a tissue or on the two sides of a membrane may give rise to corresponding unequal distribution of electric charges, and therefore differences of potential between different parts of the tissue, which under suitable conditions may find their expression in an electric current. It is therefore not surprising that practically every functional change in a tissue has been shown to be associated with the production of differences of electrical potential. Thus all parts of an uninjured muscle are isopotential, and any two points may be led off to a galvanometer without any current being observed. If, however, one part of the muscle be strongly excited, as, for instance, by injury, so that it is brought into a state of lasting excitation, it will be found that, on leading off from this point and a point on the uninjured surface to a galvanometer, a current flows through the latter from the uninjured to the injured surface. Every beat of the heart, every twitch of a muscle, every state of secretion of a gland, is associated in the same way with electrical changes. In most cases the electrical changes associated with activity have the same general character, the excited part being found to be negative in reference to any other part of the tissue which is at rest. The uniform character of the electric response in different kinds of tissues suggests that an accurate knowledge of the changes in the distribution of charged ions responsible for the response ought to throw important light on the intimate nature of excitation generally. It may be therefore advisable to consider more closely the 


\section{ELECTRICAL CHANGES IN LIVING TISSUES}

conditions which determine differences of potential in a complex system of
electrolytes.

As a simple case we may take an ordinary concentration cell. Two vessels (Fig. 29), A and B, are united by a glass tube C. A contains a 10 per cent, solution of zinc sulphate and B a 1 per cent. solution of the same salt. A rod of pure zinc is immersed in each limb. On connecting the zinc by a zinc wire to a galvanometer a current is observed to flow from $\mathrm{A}$ to $\mathrm{B}$ through the galvanometer, and therefore from B to A through the cell. A

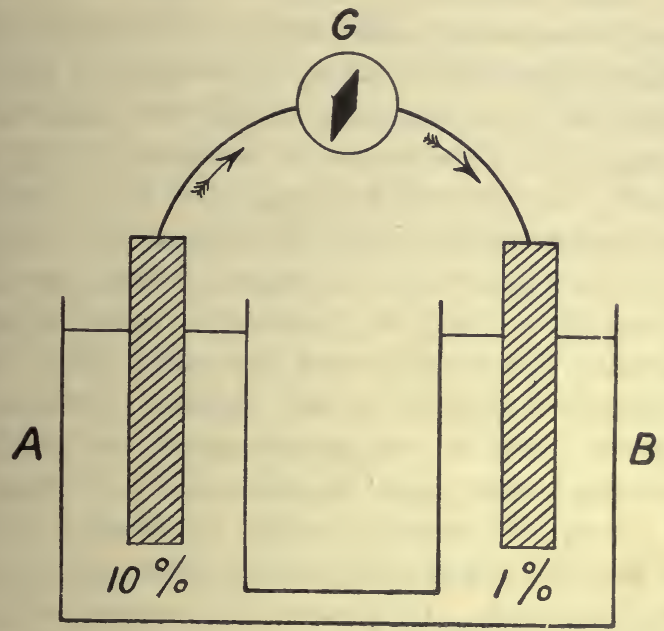

FIG. 29.

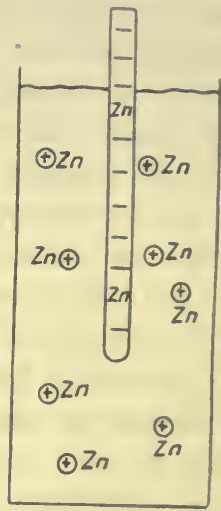

Fig. 30.

solution of zinc sulphate contains partly undissociated $\mathrm{ZnSO}_{4}$ and partly dissociated $\stackrel{+}{\mathrm{Z}} \mathrm{n}$ and $\overline{\mathrm{SO}}_{4}$ ions. If a rod of zine be immersed in a watery fluid the zine tends to dissolve. The $\mathrm{Zn}$ passing into the fluid is, however, directly ionised, and therefore carries a positive charge into the fluid, leaving the zinc negatively charged (Fig. 30 ). This process of solution will rapidly come to an end, since the positively charged ions in the fluid will repel back into the zinc any ions which may be escaping from the zinc. The amount of zine actually dissolved in the fluid is infinitesimal, the process of solution ceasing when the pressure (osmotic pressure) of the $\mathrm{Zn}$ ions in the fluid equals what may be called the 'electrolytic solution pressure' of the zinc. The continued solution of the zine is therefore only possible when means are supplied for the $\mathrm{Zn}$ ions in the fluid to get rid of their positive charges.

In an ordinary Daniell cell the $\mathrm{Zn}$ ions which leave the zinc are discharged by combining with the $\mathrm{SO}_{4}$ ions passing to the zinc from the copper sulphate in the outer cell. It is a well-known fact that pure zinc does not dissolve in acid until some other metal, such as copper, is brought into contact with it, so as to set up an electric couple, i.e. to provide means for the discharge of the $\mathrm{Zn}$ ions passing into the solution. When the zinc is immersed in the two solutions of zinc sulphate in the concentration battery, the same change will occur. The $\mathrm{ZnSO}_{4}$ solution in the two limbs of the 
concentration cell already contains $\mathrm{Zn}$ ions. Since their pressure in the 10 per cent. solution is greater than in the 1 per cent. solution, fewer $\mathrm{Zn}$ ions will leave the zinc in A than in B. The negative charge on the $\mathrm{Zn}$ in A will therefore be less than that on the rod in B, and positive electricity will therefore flow from $A$ to $B$. This will disturb the equilibrium at the surface both of $B$ and $A$, so that $\mathrm{Zn}$ ions will be deposited from the fluid on the surface of the zinc in $\mathbf{A}$ and will continue to pass from zinc into solution in $\mathrm{B}$. At the same time there is a movement of $\mathrm{SO}_{4}$ ions, set free at the surface of $\mathrm{A}$ towards B. The ultimate result, therefore, is that the zinc in B dissolves and the same amount of zinc is deposited on A. The solution of zinc sulphate on A becomes progressively weaker, while that in B becomes stronger, until finally the concentrations in the two limbs are identical and the current ceases. In this process no chemical energy is involved, the energy set free by the conversion of zinc into zinc sulphate in $B$ being exactly balanced by the energy lost by the deposition of zinc from zinc sulphate in A. Yet the current which is produced has a certain amount of energy which can be utilised for heating a wire through which it is made to pass. Since this energy must be taken from the cell, the cell is cooled during the passage of the current. We have here a close analogy with the case of compressed gases. If the 10 per cent. and 1 per cent. solutions were mixed together in a calorimeter, no change of temperature would be produced, since no work is done in the process. In the same way no cooling effect is observed if compressed gas be allowed to expand into a vacuum. If, however, the compressed gas be allowed to expand from a narrow orifice against the pressure of the external air, so that it does work in the process, it is cooled, and this cooling effect is made use of in the working of refrigerating machines or for the liquefaction of gases. We may therefore regard the concentration battery as a machine for making the substances in solution do work as they expand from a strong into a dilute solution.

The differences of potential obtained from an ordinary concentration

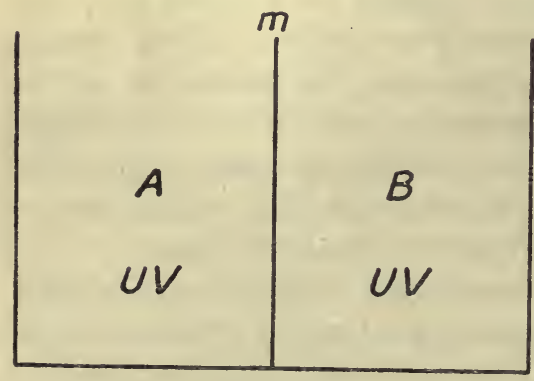

FIG. 31. cell are very small and would not suffice to account for such a high electromotive force as is set up, e.g. in the contraction of a muscle. We have seen earlier, however, that even in isosmotic solutions differences of pressure may be brought about by differences in diffusibility of the substances in solution, especially if the two solutions be separated by a membrane. Very large differences may be produced if this membrane be practically impermeable to one or other of the dissolved substances. In the same way a semipermeable membrane, i.e. a membrane with different permeabilities for the different ions of the two solutions, may suffice to bring the 
differences of potential of a concentration cell up to and beyond the extent which is observed in living tissues. Supposing we have (Fig. 31) two solutions, $\mathrm{A}$ and $\mathrm{B}$, each containing an electrolyte, UV, in different concentrations separated by a membrane $m$. If $u$ represents the velocity of transmission of $U$ through $m$, and $v$ the velocity of $V$, then the electromotive force of the cell is given by the formula

$$
\frac{u-v}{u+v} 0 \cdot 0577 \cdot \log \cdot 10 \frac{c_{2}}{c_{1}} \text { Volt. }
$$

If $v$ is taken as very small, the membrane maybe regarded as semipermeable for the corresponding ion V. Supposing we take potassium chloride as the solution, we should have to make the concentration in B eight times that in $A$, in order to get a current of a strength equal to that obtained from the olfactory nerve of the pike, for example. Macdonald has made such an assumption in order to explain the normal nerve current. He suggests that the axis cylinder contains an electrolyte which is equivalent to a $2 \cdot 6$ per cent. solution of potassium chloride. It is unnecessary, however, to assume such great differences of concentration if we regard the membrane as itself a solution of electrolytes, as has been suggested by Cremer, or if we take different substances on the two sides of the membrane. In the case of two electrolytes, $\mathrm{U}_{1} \mathrm{~V}_{1}, \mathrm{U}_{2} \mathrm{~V}_{2}$ ( $\mathrm{U}$ being the cation in each case), separated by a membrane with varying permeability for the different ions, the electromotive force of the cell is given by the following formula :

$$
0 \cdot 0577 \cdot \log \cdot 10 \frac{u_{1}+v_{2}}{u_{2}+v_{1}}
$$

where $u_{1}, v_{1}, u_{2}, v_{2}$, are the velocities of the corresponding ions. We assume that the concentrations of the two solutions are identical. Now it is evident that by making $u_{2}$ and $v_{1}$ very small, the expression $\log _{10} \frac{u_{1}+v_{2}}{u_{2}+v_{1}}$ may be made to attain any quantity, and in the same way by making $u_{1}+v_{2}$ infinitesimally small the electromotive force of the combination will also become correspondingly small. The thickness of the membrane does not come into the formula, so that membranes of microscopic or even ultramicroscopic thickness, which we have seen reason to assume as present in and around cells and their parts, could perform all the functions required of the hypothetical membrane in the above example. This is also the case when $\mathrm{V}_{1}$ is the same as $\mathrm{V}_{2}$ - that is to say, there is a common anion or a common cation on the two sides of the membrane.

It must be remembered that the passage of a current through a membrane impermeable to one or other ion in the surrounding fluid will cause an accumulation of the ion at the surface of the membrane, so that this will become polarised. Such an accumulation at any surface will naturally alter the properties of the surface, including its surface tension. The construction of the capillary electrometer depends on this fact. When mercury is in contact with dilute acid or mercuric sulphate solution it takes a positive charge from 
the fluid, and the state of stress at the surface of contact between the mercury and the negatively charged fluid diminishes the surface tension of the mercury. If the mercury be in the form of a drop in a tube drawn out to a capillary, the mercury will run down the capillary and the drop will be deformed until the surface tension tending to pull the mercury into a spherical globule is just equal to the force of gravity tending

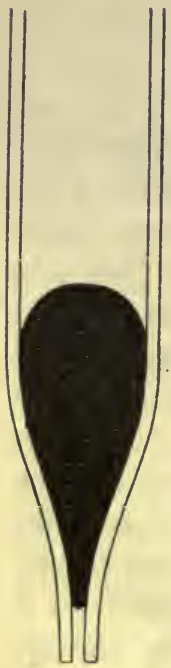

FIg. 32 . to make the mercury run out through the end of the capillary (Fig. 32). If the mercury be immersed in sulphuric acid it will descend to a lower level in the capillary owing to the diminution of its surface tension. If now the acid and the mercury be connected with a source of current so as to charge the mercury negatively, the effect will be to diminish the charge previously taken up by the mercury. The state of tension at the contact with the acid is therefore diminished, the surface tension is increased, and the mercury withdraws itself from the point of the capillary. If, however, the mercury be connected with the positive pole, its charge will be increased and its surface tension correspondingly diminished, so that the meniscus will move towards the point of the capillary. The movement of the meniscus to or away from the point may thus be used, as in the capillary electrometer, to show the direction and amount of any moderate electric change occurring in a tissue, two points of which are connected with the mercury and the acid respectively. It is possible that this electrical alteration of surface tension may be a determining factor in many of the phenomena of movement observed in the animal body. We shall have occasion to discuss this question more fully when endeavouring to account for the ultimate nature of muscular contraction. 


\section{BOOK II}

\section{'THE MECHANISMS OF MOVEMEN'T AND SENSA'TION}





\section{CHAPTER V. \\ THE CONTRACTILE TISSUES}

\section{SECTION I \\ THE STRUCTURE OF VOLUNTARY MUSCLE}

THE most striking features in the continual series of adaptations to the environment, which make up the life of an individual, are the movements carried out by contractions of the skeletal muscles. In fact, all the mechanisms of nutrition can be regarded as directed to the maintenance of the neuro-muscular apparatus, i.e. of the mechanism for adapted movement. With the growth of the cerebral hemispheres, which determines the rise in the scale of animal life, the skeletal muscles become more and more the machinery of conscious reaction. Even the highest of the adaptations possessed by man, those involving the use of speech, are impossible without some kind of movement. A man's relation to his fellows, and his value in the community, are determined by these higher muscular adaptations. It is not, therefore, surprising that the organs of the body which present in the highest degree the reactivity characteristic of all living things should have early attracted the attention of physiologists and have been the object of numberless researches directed to determining the ultimate nature of the processes generally described as vital.

The movements of the muscles are carried out in response to changes aroused in the central nervous system by events occurring in the environment and acting on the surface of the body. Every movement of an animal is thus in its most primitive form a reflex action, and involves changes in a peripheral sense organ, in an afferent nerve fibre, in the central nervous system, and in an efferent nerve fibre, before the actual process of contraction occurs in the muscle itself and gives rise to the resultant movement (Fig. 33). If we are to determine the nature of the changes involved in this reflex action, we must be able to study them as they progress along the different elements which make up the reflex arc. This analysis is facilitated by the fact that we are able to arouse a condition of activity in the different parts of the arc, even when isolated from one another. Thus we can excite any given reflex movement by stimulation of the periphery of the body, or of the afferent nerve passing from the surface to the central nervous 
system. We can proceed further and cut the efferent nerve away from the central nervous system and still succeed in exciting a condition of activity in the efferent nerve or in its attached muscle. All parts of the reflex arc possess the property of excitability, and we are thus able to arouse the activity of each part in turn, to study its conditions, its time relations, and the physical and chemical changes concomitant with the state of activity.

It will be convenient for our analysis to begin with the tissue whose

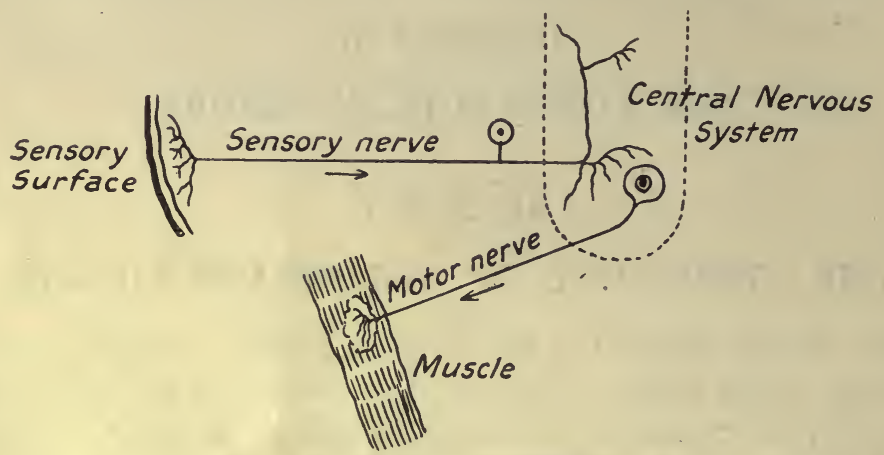

Fig. 33. Diagram of a reflex arc.

reaction forms an end link in the reflex chain, namely, the muscle, and to proceed from that to the consideration of the processes occurring in the conducting strand between central nervous system and muscle, namely, the nerve fibre, postponing to a future chapter the treatment of the more complex processes associated with the central nervous system.

In the higher animals we may distinguish several varieties of muscle. All movements that require to be sharply and forcibly carried out are effected by means of striated muscular tissue, and as these movements are in nearly all cases under the control of the will the muscles are generally spoken of as voluntary. Unstriated or involuntary muscles from sheets or closed tubes surrounding the hollow viscera. By their slow, prolonged contractions they serve to maintain and regulate the flow of the contents of these organs. Such fibres are found surrounding the blood-vessels, the alimentary canal, the bladder, \&c. Intermediate in properties as well as structure between these two classes is the heart muscle. This, like voluntary muscle, is striated, but presents considerable variations both in structure and function from ordinary skeletal muscle. Many of its properties will be considered in treating of the physiology of the heart. The properties of contractile tissues have been most fully investigated in the voluntary muscles, almost exclusively on the muscles of cold-blooded animals, such as the frog. The choice of skeletal muscles for this purpose is justified by the fact that a function is most easily investigated in the organs in which it is most highly developed. The choice of cold-blooded animals is guided by the fact that it is possible to isolate the muscle from the rest of the body and to study its reactions during a considerable time 
without the research being interfered with by the death of the tissue. We may therefore deal at length with the properties of the skeletal muscles, pointing out incidentally in what respects the heart muscle and involuntary muscle differ from the skeletal muscle.

The voluntary or striated muscles form a large part of the body, and are known as the flesh or meat. Each muscle is embedded in a layer of connective tissue, and is made up of an aggregation of muscular fibres, which are united into bundles by means of areolar connective tissue. The individual fibres vary much in length, and may be as long as 4 or $5 \mathrm{~cm}$. At each end of the muscle the fibres are firmly united to tough bundles of white fibres, which form the tendon of the muscle, and are attached as

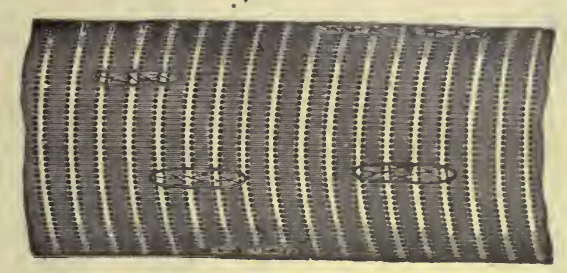

FIG. 34. Muscular fibre of a mammal, examined fresh in serum, highly magnified. (SCHÄFER.)

a rule to bones. Running in the connective tissue framework of the muscle we find a number of blood-vessels, capillaries and nerves.

On examination of a living muscle, each fibre is seen to consist of a series of alternate light and dark striæ, arranged at right angles to its long axis, and enclosed in a structureless sheath-the sarcolemma. Lying under the sarcolemma are a number of oval nuclei embedded in a small amount of granular protoplasm. In some animals these nuclei occupy a central position in the fibre. Each band may be considered to be made up of a number of prisms (sarcomeres) side by side, with interstitial substance (sarcoplasm) between them. The muscle prisms of adjacent discs are connected to form long columns (primitive fibrillæ, or sarcostyles). Each muscle prism is more transparent at the two ends than in the middle, thus giving rise to the appeareance of light and dark striæ. In the middle of the light band is a line or row of dots (often appearing double), called Krause's membrane.

The development of this regular cross and longitudinal striation is closely connected with the evolution and specialisation of the muscular function, i.e. contraction. Contractility is among others a function of all undifferentiated protoplasm. Undifferentiated cells, such as the amœba, can effect only slow and weak contractions. Directly a. specialisation of function is necessary and some cell or part of a cell has to contract rapidly in response to some stimulus from within or without, we find a differentiation both of form and of internal structure. In many cases, as in the developing muscle of the embryo or the adult muscles of many invertebrates, this differentiation affects only part of the cell, so that while one part presents the ordinary granular appearance, the other half is finely and longitudinally 
striated, the striation being apparently due to the development of special contractile fibrillæ. In the slowly contracting unstriated muscle of the vertebrate intestine, the longitudinal striation is with difficulty made out, but as the muscle rises in the scale of efficiency, the longitudinal striation becomes more apparent, and in the striated muscle of vertebrates, and still more in the wonderful wing-muscles of insects, which can perform three hundred complete contractions in a second, the longitudinal is associatert

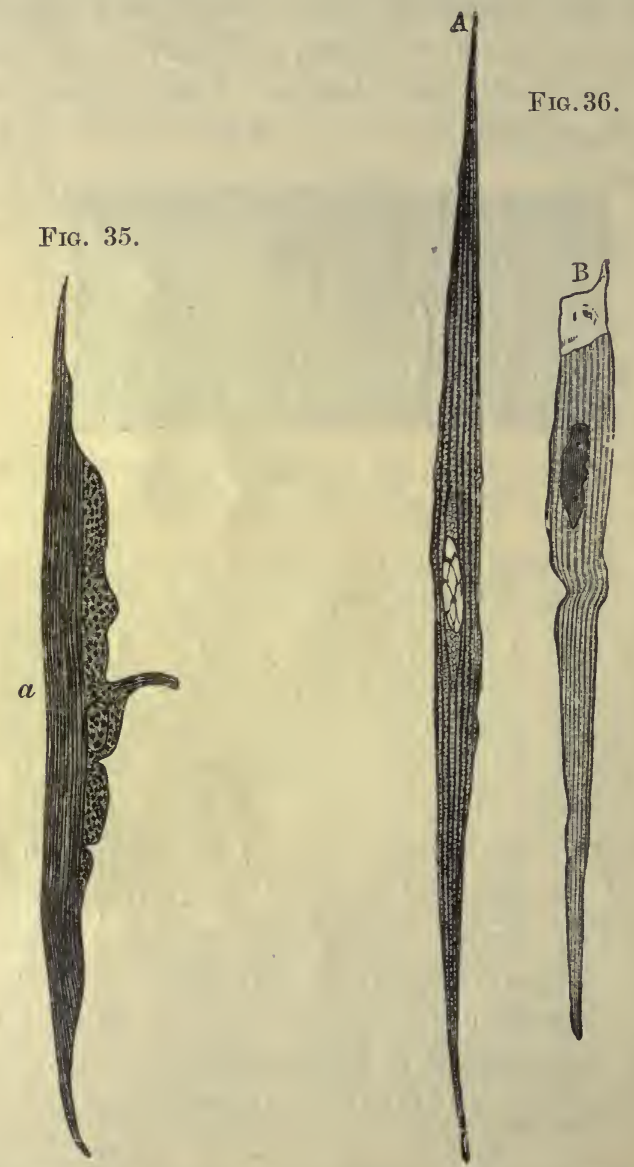

FIG. 35. Muscle fibre of an ascaris. $a$, the differentiated contractile portion of the cell. (After HeRTwi(..)

FIG. 36. Muscle fibres from the small intestine, showing the fine longitudinal striation. (SCH ÄFER.)

with and often apparently subordinated to a transverse striation, due to the regular segmentation of the contractile fibrillæ or sarcostyles. Every muscular fibre, which presents any trace of histological differentiation, may be said to consist of contractile fibrillæ (sarcostyles), each composed of a series of contractile elements (sarcous elements or sarcomeres), and embedded in a granular material known as sarcoplasm. The great divergence in the aspect of muscular fibres from different parts of the animal kingdom is 
largely conditioned by the varying relations, spatial and quantitative, of the sarcoplasm to the sarcostyles. Thus in the higher vertebrates, two types of voluntary muscular fibref are distinguished, according to the
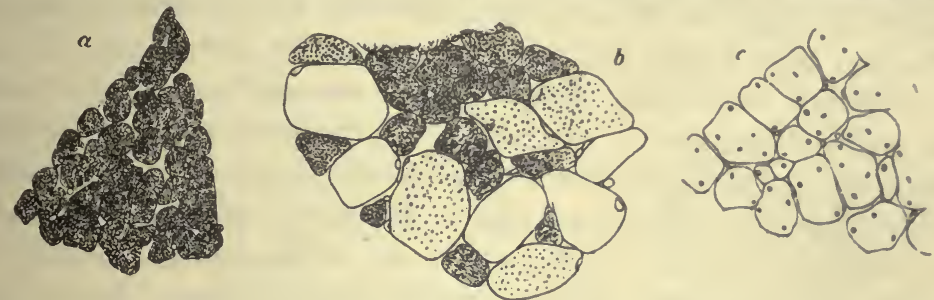

Fig. 37. Transverse sections of the pectoral muscles of $a$, the falcon, $b$, the goose, and $c$, the domestic fowl. It will be noticed that the relative amount of granular or red fibres present varies directly as the bird's power of sustained flight. (After KNoll.)

amount of sarcoplasm they contain : one rich in sarcoplasm, more granular in cross-section, and generally containing hæmoglobin ; and the other poor in sarcoplasm, clear in cross-section, and containing no hæmoglobin. From the fact that the granular fibres are found chiefly in those muscles which have to carry out long-continued and powerful contractions, it seems reasonable to regard the interstitial sarcoplasm as the local food-supply of the active sarcostyles, although some authors have endowed the sarcoplasm with a contractile power of its own, differing only by its extremely prolonged character from the quick twitch of the sarcostyles. The connection between structure and activity of the muscle-fibres is well shown by Fig. 37.

In some animals, such as the rabbit, we find muscles consisting almost entirely of one or other of these varieties; but in most animals (amongst which we may reckon frog and man) the two varieties occur together in one muscle, so that what we have to say about the properties of voluntary muscle, which rests nearly entirely on experiments with frog's muscle, really has reference to a mixed inuscle, i.e. muscle containing both red and white fibres.

Since the sarcous element represents the contractile unit of the muscle, a knowledge of its intimate structure should be of great importance for the theory of muscular contraction. Unfortunately, howprepared by Rollet's method. Highly magnified. (E. A. SCHÄFER.)

$\mathbf{A}$, a contracted fibril. B, a stretched fibril, with its sarcous elements separated at the line of Hensen. C, an uncontractcd fibril, showing the porous structure of the sarcous elements.

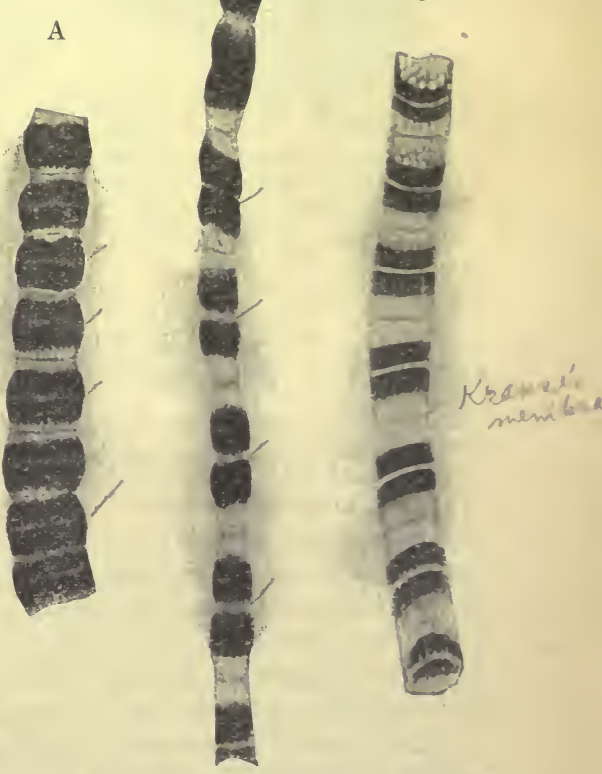


ever, we are here at the limits of the demonstrably visible. It becomes difficult to determine how far the appearances observed under the microscope are due to actual structural differences or are produced by the unequal diffraction of light by the various elements of the muscle fibre. All observers are agreed that the essential contractile element is the row of sarcous elements forming the muscle fibril or sarcostyle. Schäfér, working on the highly differentiated wing-muscle of the wasp, concludes that each sarcostyle is divided by Krause's membranes (the lines in the middle of each light stripe) into sarcomeres. Each sarcomere contains a darker substance near the centre divided into two parts by Hensen's disc. At each end of the sarcomere the contents are clear and hyaline. In the act of contraction, the clear material flows, according to Schäfer, into tubular pores in the central dark material.

Most histologists agree in assigning to the middle part of the sarcous element (the sarcomere) a denser structure than to the two ends. According

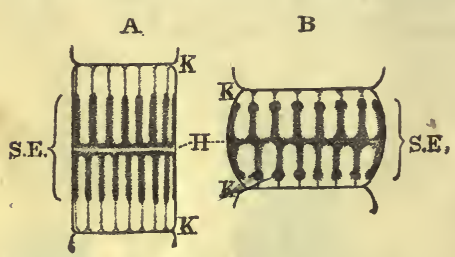

Fra. 39. Diagram of a sarcomere in a moderately extended condition, $A$, and in a contracted condition, $\mathrm{B}$; $\mathrm{K}, \mathrm{K}$, membranes of Krause; $\mathrm{H}$, line or plane of Hensen; SE, poriferous sarcous element. (SCHÄFER.) to Macdougall, however, the lighter appearance at each end of the sarcomere is an optical illusion. He regards the sarcous element as a cylindrical bag with homogeneous contents, crossed only by one or three delicate transverse membranes. Krause's membrane would be rigid, while the lateral wall of the sarcous element is extensible, and is folded longitudinally, so that it can bulge out and produce a shortening and thickening of the whole sarcous element if by any means the pressure be raised in its interior. In favour of a differentiation within the sarcomere itself is the fact that under certain conditions it is possible to produce a precipitate, limited only to central part, i.e. to the sarcous element to which Schäfer assigns a tubular structure.

When a muscle fibre, killed by osmic acid or alcohol, is examined under the microscope by polarised light, it is seen to be made up of alternate bands of singly and doubly refracting material. The doubly refracting (anisotropous) substance corresponds to the dark band, and the singly refracting (isotropous) to the light band. If the living fibre be examined in the same way, it is found that nearly the whole of it is doubly refracting, the singly refracting substance appearing only as a meshwork with long parallel meshes corresponding to the muscle prisms. In short, in a living fibre the muscle prisms are anisotropous, the sarcoplasm isotropous.

When a muscle fibre contracts, there is an apparent reversal of the situations of the light and dark stripes, owing to the fact that the interstitial sarcoplasm is squeczed out from between the bulging sarcomeres, and accumulates on each side of the membranes of Krause. The accumulation of sarcoplasm in this situation makes the previously light striæ appear dark, and the dark striæ by contrast lighter than they were before. That there is no true reversal of the strix is shown by examining the muscle by polarised light, the two substances, isotropous and anisotropous, retaining their relative positions.

Every skeletal muscle is connected with the central nervous system by nerve fibres, some conveying impressions from the muscle to the centre, 
the others acting as the path of the motor impulses from the centre to the muscle. These latter-the motor nerves - end in the muscular fibre itself, by means of a special end-organ - the motor end-plate. The neurilemma of the nerve fibre becomes continuous with the sarcolemma, the medullary sheath ends suddenly, while the axis cylinder ramifies in a mass of undifferentiated protoplasm, containing nuclei, and lying in contact with the contractile substance of the muscle immediately under the sarcolemma (Fig. 40). This mass of protoplasm is known as the 'sole plate.' It is not marked in all animals. Thus in the frog the axis cylinder ends in a series of branches at right angles to one another, distributed over a considerable length of the muscle fibre. The sole plate in this case seems to be limited to scattered nuclei lying in close contact with the terminal branches of the nerve fibre. So far as we can tell at present, the ultimate ramifications of the axis-cylinder end freely and do not enter into organic connection with the contractile substance itself.

Most of our knowledge on the subject of muscle has been derived from the study of the gastrocnemius and sartorius muscles of the frog. The position of these muscles is shown in the accompanying diagram (Fig. 41). The gastrocnemius, which, with the attached sciatic nerve, is most frequently employed as a nerve-muscle preparation, forms a thick belly immediately under the skin at the back of the leg, and arises by two tendons from the lower end of the femur and the outer side of the kneejoint. The two tendons converge towards the

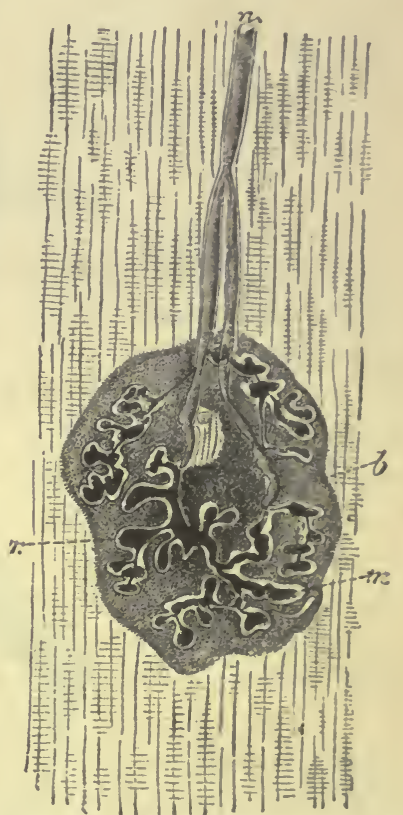

Fia. 40. Moter end-ørgan of a lizard, gold preparation. (KüHNE.) $n$, nerve fibre dividing as it ap. proaches the end-organ; $r$, ramification of axis cylinder upon $b$, granular bed or sole of the end-organ; $m$, clear substance surrounding the ramifications of the axis cylinder. centre of the muscle, uniting about its middle, and from them a number of short muscular fibres arise, passing backwards and dorsally to be inserted into a flat aponeurosis covering the lower half of the muscle, which ends in the tendo Achillis. On account of this irregular arrangement of the muscular fibres, the gastrocnemius can only be employed when the contraction of the muscle as a whole is the object of investigation. The effective cross-area of the fibres is much greater than the actual cross-section of the muscle, so that, while the actual shortening of the gastrocnemius is but small, its strength of contraction is considerable.

The sartorius muscle consists of a thin band of muscle fibres running parallel from one end of the muscle to the other. It lies on the ventral surface of the thigh, arising from the symphysis pubis by a thin flat tendon, and is inserted by a narrow tendon into the inner side of the head of the tibia. On account of the regularity with which its fibres are disposed, this muscle is of especial value in experiments on the local conditions of a muscle fibre accompanying its activity. When a greater mass of approximately parallel fibres is necessary, recourse may be had to a preparation consisting of the gracilis and semi-membranosus muscles together. This latter muscle lies dorsally to the gracilis muscle which is shown in the illustration. 
Other muscles in the frog used for particular purposes are the mylohyoid and the dorsocutaneous muscles. The mylohyoid muscle of the frog, which lies on the ventral surface of the tongue, has the advantage that its fibres lie in close contact with a

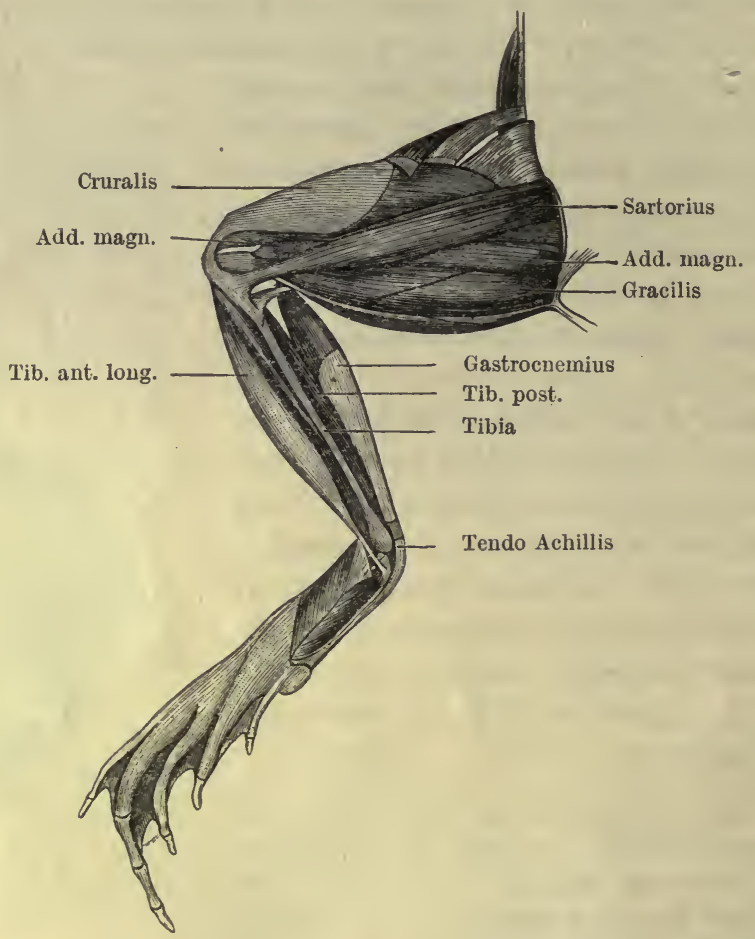

Fig. 41. Muscles of hinder extremity of frog. (After EckER.)

lymph-space occupying the centre of the tongue. If any drug be injected into this lymph-space it acts with extreme rapidity on the muscle fibres, so that the tonguepreparation of the frog is a useful one for the study of the action of different substances on muscle fibres. 


\section{SECTION II}

\section{EXCITATION OF MUSCLE}

A MUSCLE may be caused to contract in various ways. Normally it contracts only in response to impulses starting in the central nervous system and transmitted down the nerves. But contraction may be artificially excited in various ways in a muscle removed from the body. If we make a muscle-nerve preparation (i.e. a muscle with as long a piece of its nerve as possible attached to it), such as the gastrocnemius of the frog with the sciatic nerve, we find we can cause contraction by various forms of stimulimechanical, thermal, or electrical-applied to the muscle or the nerve (direct and indirect stimulation). Thus the muscle responds with a twitch if we pass an induction shock through it or its nerve, or pinch either with a pair of forceps. Or we may use chemical stimuli, and cause contraction by the application of strong glycerin or salt solution to the nerve.

These experiments do not prove conclusively that muscle itself is irritable. It might be urged that, when we pinched or burnt the muscle we stimulated, not the muscle substance itself, but the terminal ramifications of the nerve in the muscle, and that these in their turn incited the muscle to contract. But the independent excitability of muscle is shown clearly by the following experiment by Claude Bernard.

A frog, whose brain has been previously destroyed, is pinned on a board, and the sciatic nerves on each side exposed. A ligature is then passed round the right thigh underneath the nerve, and tied tightly so as effectually to close all the blood-vessels supplying the limbs, without interfering with the blood-supply to the nerve. Two drops of a 1 per. cent. solution of curare are then injected into the dorsal lymph-sac. After the lapse of a quarter of an hour it is found that the strongest stimuli may be applied to the left sciatic nerve without causing any contraction of the muscles it supplies. On the right side, stimulation of the nerve is as efficacious as before. Both gastrocnemii respond readily to direct stimulation, showing that the muscles are not affected by the drug. Since both sciatic nerves have been exposed to the influence of the curare, it is evident that the difference on the two sides cannot be due to any deleterious effect on them by the curare. We have also excluded the muscles themselves; so we must conclude that the curare paralyzes the muscles by affecting the terminations of the nerve within the muscle, and probably the end-plates themselves. 
This experiment teaches us that muscle can be excited to contract by direct stimulation, even when the terminal ramifications of the nerve within it are paralyzed, so that stimulation of them would be without effect.

The same fact may be demonstrated in a different way by means of chemical stimuli. It is found that whereas strong glycerin excites nerve fibres, it is without effect on muscle fibres, while on the other hand weak ammonia is a strong excitant for muscle, but is without effect on nerve. If the frog's sartorius be dissected out and the lower end dipped in glycerin, no twitch is produced. On snipping off the lower third of the muscle and then immersing the cut end in glycerin, a twitch at once occurs. The lower

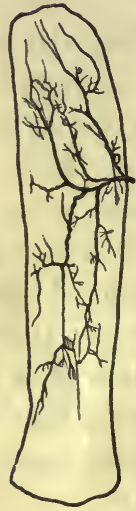

Fig. 42. The ramification of the nerve fibres within the sartorius muscle of the frog, showing the freedom of the lower portion of the muscle from nerve fibres. (KÜHNE.) end contains no nerve fibres (Fig. 42), and it is only when a section containing nerve-fibres is exposed to the action of glycerin that contraction takes place. On the other hand, mere exposure of muscle to the vapour of dilute ammonia causes contraction (and subsequent death), although the nerve to the muscle can be immersed in the solution without any excitation being produced.

Of all the different stimuli capable of exciting muscular contraction, the electrical is that most frequently employed. It is easy, using this form, to graduate accurately the intensity and duration of the stimulus. At the same time the stimulus may be applied many times to any point on the muscle or nerve without killing the part stimulated, whereas with other forms of stimulus it is difficult to obtain excitatory effects without injuring to a greater or less extent the part stimulated.

\section{METHODS EMPLOYED FOR THE STIMULATION OF MUSCLE AND NERVE.}

The two commonest forms of electrical stimuli employed are (1) the make and break of a constant current, (2) the induction currents of high intensity and short duration obtained from an induction coil.

(1) Constant Current. As a source of constant current a Daniell's cell is generally employed. This consists of an outer pot containing a saturated solution of copper sulphate, in which is immersed a copper cylinder. To the cylinder at the top a binding screw is attached, by which the connection of the copper with a wire terminal is effected. Within the copper cylinder is a second pot of porous clay, filled with dilute sulphuric acid, in which is immersed a rod of amalgamated zinc. In this cell the zinc is the positive and the copper the negative element. Hence the current flows (in the cell) from zinc to copper, and if the binding screws of the two elements are connected by a wire, the current flows in the wire (outer circuit) from copper to zinc, thus completing the circuit. Since in the outer circuit the current flows from copper to zinc, the terminal attached to the copper is called the positive pole, and that to the zinc the negative pole. When the current is required to be very constant, the zine may be immersed in a saturated solution of zinc sulphate instead of dilute sulphuric acid. A Daniell's cell, though very constant, gives only a small current, owing to its small electromotive force and high internal resistance.

When a stronger current is required it is best to use a storage battery. In this, 
when charged, the two elements are lead and lead oxide, $\mathrm{PbO}_{2}$. It has the advantage that it may be used over and over again, being recharged through a resistance from the electrical mains when it has run down.

Another useful type of cell is the Leclanché cell. This consists of a glass jar containing a solution of sal-ammoniac. Into this dips an amalgamated rod of zinc, which is the positive plate. A piece of gas carbon forms the negative plate. This is surrounded by peroxide of manganese $\left(\mathrm{MnO}_{2}\right)$ which is kept in contact with the surface of the carbon by being placed in a porous pot. In some forms of Leclanché the manganese and carbon are ground up together and pressed into a cylinder which surrounds the zine rod. When the cell is on open circuit-that is, when the terminals are not connected and no current is passing-very little action takes place ; but when the circuit is closed and the current passes, the zinc dissolves in the sal-ammoniac, forming a double chloride of zine and ammonia, while ammonia gas and hydrogen are liberated at the carbon pole. The nascent hydrogen reduces the peroxide of manganese and so polarisation is prevented. On account of its great solubility in water the ammonia has no polarising action. The Leclanché is a convenient form of cell, as when once set up it requires a minimum of attention. If it is worked through a considerable resistance, it will keep in order for some time, particularly if the work is intermittent ; but if it is used with a small resistance in circuit it polarises very rapidly. The E.M.F. of one Leclanché cell is 1.4 volt in the external circuit. The positive current is conventionally said to run from the zine to the carbon in the cell, and from the carbon to the zinc in the circuit outside. The wire attached to the carbon is the positive pole, that to the zinc the negative pole. Dry cells are usually Leclanché cells, in which the solution of sal-ammoniac is prevented from spilling by absorption with sawdust or plaster of Paris. The E.M.F. is the same as the Leclanché, but they polarise much more readily.

If the poles of a Daniell's cell be connected by wires with a nerve or muscle of a nerve-muscle preparation (as in Fig. 43), the current will flow from copper to the nerve at $\mathrm{A}$, and along the nerve from $\mathrm{A}$ to $\mathrm{K}$. At $\mathrm{K}$ the current will leave the nerve to flow to the zinc of the battery, so completing the circuit. The point at which the current enters the nerve (i.e. the point of the nerve connected with the positive pole of the battery) is called the anode, and the point at which the current leaves the nerve is called the cathode. The wires by which the current is conducted

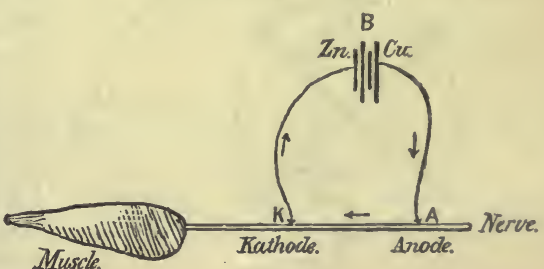

Fic. 43. to and from the nerve are called the electrodes. As electrodes we generally employ two platinum wires mounted together on a piece of vulcanite.

For the purpose of making or breaking the current at will, various forms of keys are employed. The ordinary make and break key consists of a hinged wire dipping into a mercury cup. When the wire is depressed so that it dips into the mercury, the circuit is complete. On raising the wire by means of the handle, the circuit is broken.

Du Bois Reymond's key consists of two pieces of brass, each of which has two binding screws for the attachment of wires. These are connected by a third piece, or bridge, which is jointed to one of the two side bits, so that it may be raised or lowered at pleasure ( $v$. Fig. 44). It may be used either as a simple make-and-break key, or, as is more usual, as a short-circuiting key. In the first case one brass bank is attached to one terminal, the other to the other terminal. If the bridge be now lowered, the connection is made and the current passes. If the bridge be raised, the current is broken. Fig. $44 \mathrm{~A}$ and B show the way in which the key is arranged for short-circuiting. It will be seen that four wires are attached to the key; two going to the battery, and two we may suppose going to a nerve. When the bridge is down, as in Fig $44 \mathrm{~A}$, the current from the cell on coming to the key has a choice of two routes. It may either go through the brass bridge, or through the other wires and nerve. The resistance of 
the nerve however is about $100,000 \mathrm{ohms}$, whereas that of the bridge is not the thousandth part of an ohm. When a current divides, the amount of current that goes along any branch is inversely proportional to the resistance. Here the resistance in the nervecircuit is practically infinite compared with that in the brass bridge, and so all the

A

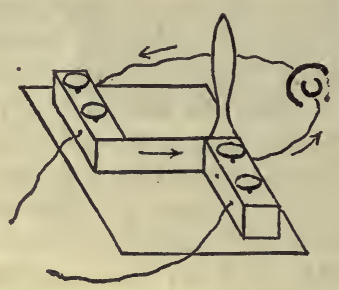

B

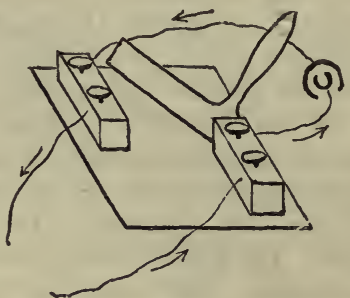

Fia. 44. Du Bois key, closed. Du Bois key, open.

current goes through the bridge and none through the nerve. We say then that the current is short-circuited.

It is often necessary to reverse the direction of a current through a nerve-muscle preparation or a galvanometer in the course of an experiment. For this purpose Pohl's reverser may be used. It consists of a slab of ebonite or paraffin or other insulating material, in which are six small holes filled with mercury. A binding screw is in connection with the mercury in each of these holes. Two cross-wires (not in contact with one another) join two sets of pools together, as shown in Fig. 45. A cradle consisting of two wires joined by an insulating handle carries two arcs of wire by which the pools at $a$ and $b$ may ba put into connection with either $x$ and $y$, or the corresponding
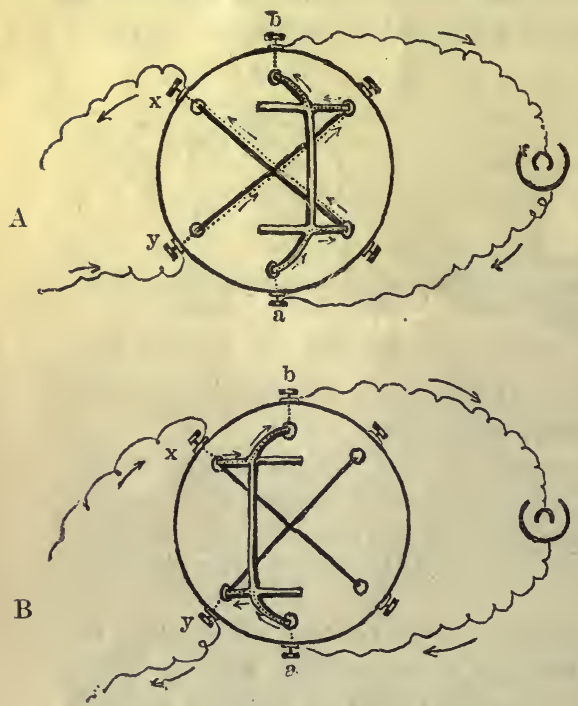

Fig. 45. Diagram of Pohl's reverser. pools on the opposite side. It will be seen that with the cradle tipped to one side, as in Fig. $45 \mathrm{~A}$, the current from the battery enters the reverser at $a$; this proceeds up the wire of the cradle, down towards the right, then along the cross-wire to the pool at $x . \quad x$ is therefore the anode, and $y$ the cathode. In Fig. $45 \mathrm{~B}$ the cradle has been swung over to the other side. Here the cross-wires are not used at all by the current, which passes from $a$ up the sides and down the curved wire to $y$. In this case $y$ is now the anode and $x$ the cathode, and the direction of the current through the circuit connected with $x$ and $y$ is reversed. By taking out the cross-wires, Pohl's reverser may be used as a simple switch, by which the current may be led into two different circuits in turn.

With this form of reverser difficulty is often experienced owing to dirt accumulating on the mercury and forming an insulating layer between it and the binding screw or copper wire. Several improved forms of reverser are now made where the mercury poles are replaced by brass banks, and these are generally to be preferred in practice.

(2) InduCEd CuRREnts. In using these the muscle or nerve is stimulated by the current of momentary duration produced in the secondary circuit of an induction-coil by the make or break of a constant current in the primary.

The construction of the induction-coil or inductorium is founded on the fact that if a coil of wire in connection with a galvanometer be placed close to (but insulated from) 
another coil through which a current may be led from a battery, it is found that on make and break of the current of the second coil a momentary current is induced in the first. The induced current on make is in the reverse direction, that on break in the same direction as the primary current. The electromotive force of the induced current is proportional to the number of turns of wire in the coils. The induction-coil consists of two coils, each containing many turns of wire. The smaller coil $\left(\mathrm{R}_{1}\right.$, Fig. 46), consisting of a few turns of comparatively thick wire, is the primary coil, and is put into connection with a battery. It has within it a core of soft iron wires, which has the effect of attracting the lines of force, concentrating them, and so increasing its power of inducing secondary currents. The secondary coil, $R_{2}$, of a large number of turns of very thin wire, is arranged so as to slide orer the primary coil. It is provided with two terminals, which may be connected with the nerve or other tissue that we wish to stimulate. Since the electromotive force of

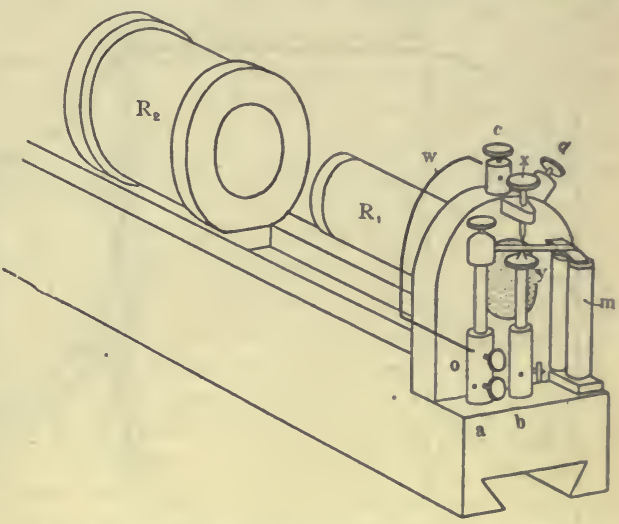

Fig. 46. Diagram of inductorium. $\mathrm{R}_{1}$, primary ; $\mathrm{R}_{2}$, secondary coil. $m$, electro-magnet of Wagner's hammer. w, Helmholtz's side wire. the induced current is proportional to the number of turns of wire, it is evident that the electromotive force of the current delivered by the induction coil may be many thousand times that of the battery current flowing through the primary coil. The induced currents increase rapidly in strength as the coils are approached to one another; the strength of these therefore may be regulated by shoving the secondary up to or away from the primary coil.

A short-circuiting key is always placed between the secondary coil and the nerve to be stimulated. If only single induction shocks are to be used, a make-and-break key is put in the primary battery circuit, and the two wires from the battery and key are attached to the two top screws of the primary coil ( $c$ and $d$, Fig. 46). It is then found that the shock given by the induced current on break of the primary current is much stronger than that on make.

In endeavouring to explain this difference in the intensity of the make-and-break induction shocks, it must be remembered that the intensity of the momentary current induced in the secondary coil at make or break of the primary current is proportional (1) to the number of turns of wire in each coil ; (2) inversely to the mean distance between the coils (i.e. the nearer the coils, the stronger the induced current); $(3)$ to the rate of change in strength of the primary current. I Now, when a current is made through the primary coil, induction takes place, not only between primary and secondary coils. but also between the individual turns of the primary coil itself. This current of selfinduction, being opposed in direction to the battery current, hinders and delays the attainment by the latter of its full strength, and so slows the rate of change of current in the primary coil. Hence the intensity of the momentary current induced in the secondary coil is less than it would have been without the retarding effect of self-induction. At break of the current, an extra current is also produced in the primary coil in the same direction as the battery current, and therefore tending to reduce the rate of change of the current from full strength to nothing. In this case, however, the primary circuit being broken, the current of self-induction cannot pass without jumping the great resistance offered by the air, so that its retarding effect on the rate of disappearance of the primary current may be practically disregarded. In Fig 47 the line, $a, b, c, d$, will represent the changes occurring in the primary current at make and break, $a b$ corresponding to the make and $c d$ to the break. The lower line represents 
the momentary currents induced in the secondary circuit, $m$ being the current of low intensity and long duration produced by the make, and $\mathrm{B}$ the shock of high intensity and short duration caused by the sharp break of the primary current.

When we desire to use faradic stimulation-that is, secondary induced shocks rapidly repeated 50 to 100 times a second-we make use of the apparatus attached

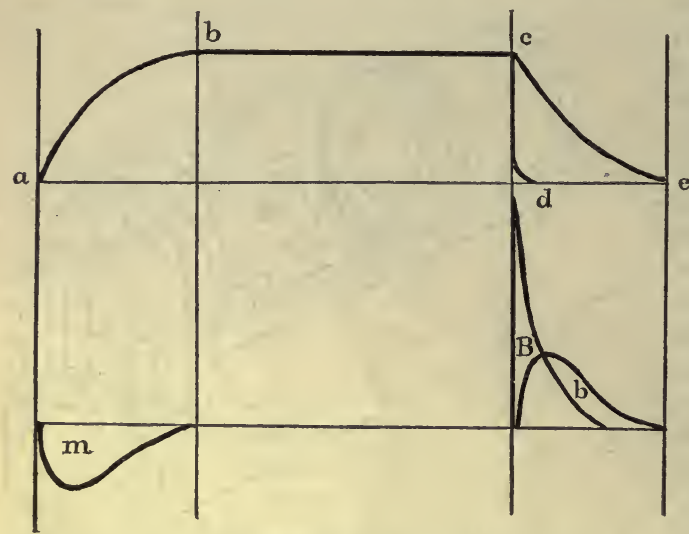

FIG. 47. to the coil, known as Wagner's hammer (Figs. 48A and 48B). In this case the wires from the battery are connected to the two lower screws ( $a$ and $b$, Fig. 46). Fig. 48A shows the direction of the current when Wagner's hammer is used. The current enters at $a$, runs up the pillar and along the spring to the screw $x$. Here it passes up through the screw, and through the primary coil $\mathrm{R}_{1}$. From the primary coil it passes up the small coil $m$, and from this to the terminal $b$ and back to the battery. But in this course the coil $m$ is converted into an electro-magnet. The hammer $h$ attached to the spring is attracted down, and so the spring is drawn away from the screw $x$, and the current is therefore broken. The break of the current destroys the magnetic power of the coil, the spring jumps up again and once more makes circuit with the screw $x$, only to be drawn down again directly this occurs. In this way the spring is kept vibrating, and the primary circuit is continually made and broken, with the production at each make-and-break of an induced current in the secondary coil.

It is evident that, when the primary current is made and broken fifty times in the second, there will be a hundred momentary currents produced during the same period in the secondary coil. Every alternate one of these produced by the break of current

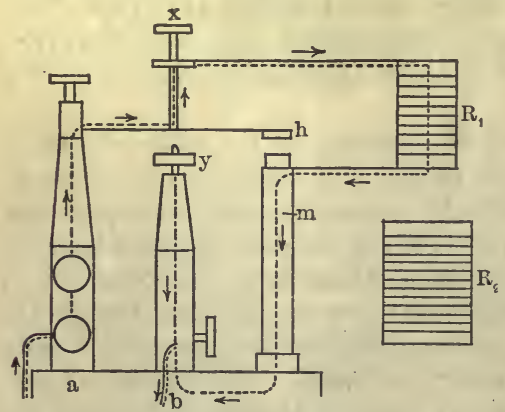

FIG. 48A. Diagram showing course of current in inductorium when Wagner's hammer is used.

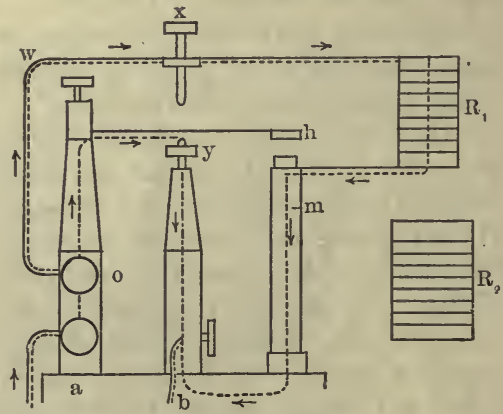

FIG. 48B. Diagram showing course of current when the Helmholtz side wire is used.

in the primary will be much stronger than the intervening currents produced by the make. In order to equalise make and break induction-shocks, so that a regular series of momentary currents of nearly equal intensity may be produced, the arrangement known as Helmholtz's is used. In this arrangement the side wire $w$, shown in Fig. 46, and diagrammatically in Fig. 48B, is used to connect the binding screw 0 with the binding screw $c$ at the top of the coil. The screw $x$ is raised, so as not to touch the spring, and the lower screw $y$ is moved up till it comes nearly in contact with the under 
surface of the spring. If we consider the direction of the current now, we see that it enters as before at the terminal, travels up the Helmholtz wire $w$ to the serew $c$, thence through the primary coil $\mathrm{R}_{1}$, then through the coil $m$ of the Wagner's hammer, and so back to the battery. The coil $m$, thus becoming an electro-magnet, draws down the hammer $h$. In this act the under surface of the spring comes in contact with the screw $y$. The current then has a choice of two ways. It may either go through the coil as before, or take a short cut from the terminal $a$, up the pillar, along the spring, through the screw $y$, and down to the terminal $b$ back to the battery. As the resistance of this. latter route is very small compared with the resistance of the primary coil, \&c., the greater part of the current takes this way. The infinitesimal current which now passes through the coil of Wagner's arrangement is insufficient to magnetise this, and the hammer springs up again; thus the process is restarted, and the spring vibrates rhythmically. With this arrangement the primary current is never broken, but only short-circuited, and so diminished very largely. Hence the retarding influence of self-induction is as potent with break as with make of the current, and the effects on the secondary coil in the two cases are approximately equal. In Fig. 47 ce represents the change in the primary current when the current is short-circuited instead of being broken, and $b$ represents the effect produced in the secondary coil. It will be seen that the currents $m$ and $b$ are practically identical in intensity and duration.

When the induction-coil is used for stimulating, it is usual to graduate the strength of the shock administered to the excitable tissue by moving the secondary coil nearer to or further away from the primary coil. It must be remembered that the strength of the induced current does not vary in numerical proportion with the distance of the two coils from one another. If one coil is some distance, say, $20 \mathrm{cms}$. from the primary coil, the induced current produced by make or break of the primary current is very small, and on moving the secondary from $20 \mathrm{up}$ to $10 \mathrm{cms}$. the increase in strength of the current will not be very rapid. The increase will however become more and more rapid as the two coils are brought closer together. Using the same strength of eurrent in the primary coil and the same resistance in the secondary coil, we can say that the make or break current will be uniform so long as the distance of the coils remains constant. We are not able, however, to say by how much the current will increase as the secondary coil is moved, say, from 11 to $10 \mathrm{cms}$. distant from the primary coil. If it is required to know the exact increment in the exciting current which is uscd, it is necessary to graduate the induction-coil by sending the induction shocks, obtained at different distances of secondary from primary coil, through a ballistic galvanometer.

Another method which may be adopted for the excitation of muscle or nerve is the discharge of a condenser. The advantage of this method is that we can determine not only the amount of electricity discharged through the preparation, but the actual energy employed. If two plates of metal separated from one another by a thin insulating layer of dielectric such as air, glass, mica, or paraffined paper, be connected with the two poles of a battery, each plate acquires the potential of the pole of the battery with which it is connected, and receives thcrefrom a charge of electricity (positive or negative). If the connections be broken the two plates retain their charge. If now they be connected by a wire they discharge through the wire, and if a nerve be inserted in the course of the wire, it may be excited by the discharge.

The amount of electricity, that may be stored up in this way, will depend on the extent of the plates and their proximity to one another, as well as on the E.M.F. of the charging battery. In order to get great extent of surface, a condenser is built up, as in the diagram

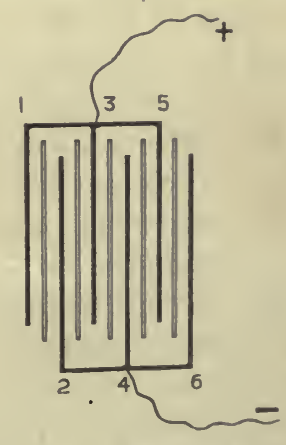

Fig. 49. Diagram to show the mode of construction of a condenser.

(Fig. 49), of a very large number of plates of tinfoil, separated by dises of mica or paraffined paper. Alternate dises are connected together: thus, $1,3,5$ are connected to one pole, while $2,4,6$ are connected to the other. 
The rheocord is used to modify the amount or strength of current flowing through a preparation. One form of it is represented in Fig. 50. A constant source of current at $\mathbf{B}$ causes a flow of electricity from $a$ to $b$ through a straight wire. As the resistance of this wire is the same throughout its length, the fall of potential from $a$ to $b$ must be constant. The nerve, or whatever preparation is used, is connected with the straight wire at two points, at $a$ and at $c$, by means of a sliding contact or rider. Supposing that there is an electromotive difference of one volt between $a$ and $b$, it is evident that if $c$ is pushed close to $b$, the E.M.F. acting on the nerve will be also one volt. The E.M.F., however, may be made as small as we like by sliding $c$ nearer to $a$. Thus if

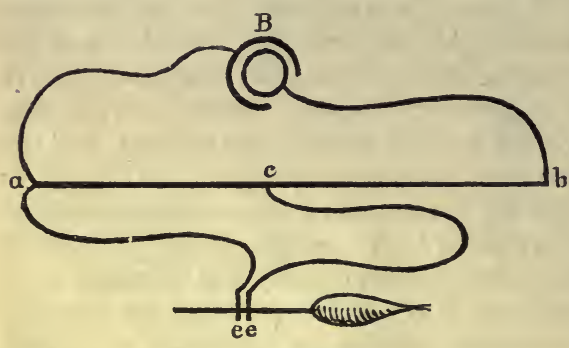

FIG. 50. $a b$ is one metre, and there is a difference of one volt between the two ends, then if $c$ be one centimetre from $a$, the E.M.F. acting on the nerve will be $\frac{1}{100}$ volt. Thus we alter the current passing through the nerve by altering the E.M.F. which drives the current.

If a weak current from a Daniell's cell (or any other form of battery) be passed through a muscle or any part of its nerve, at the make of the current the muscle gives a single sharp contraction-a muscle-twitch. In this contraction the whole of the muscle fibres may be involved. During the passage of the current no effect is apparently produced and the muscle seems to be quiescent, though on careful observation we may see that there is a state of continued contraction limited to the immediate neighbourhood of the cathode, which lasts as long as the current is passing through the muscle, and is not propagated to the rest of the muscle. If the current be now broken, the muscle may remain quiescent. If however the current is above a certain strength, the muscle responds to the break of the current with another single rapid contraction. With a current of moderate strength we may get a contraction both at make and break of the current, but the makecontraction may be stronger than the break-contraction. Thus stimulation is caused by the make and break of a constant current, the make-stimulus being more effective than the break-stimulus. If the duration of the passage of the current is sufficiently short, no contraction is produced at the break of the current, however strong this may be. The same phenomenon of a single twitch may be evoked by the passage of an induction shock. This is the current of momentary duration produced in the second circuit of an induction-coil by the make or break of a constant current in the primary. Using this mode of stimulus, it is found that the contraction on break of the primary current is much stronger than that on make. It must not be imagined, however, that there is any contradiction between this and the fact that the make of a constant current is a stronger stimulus than the break.

When we put a muscle in the secondary circuit and make a current in the primary, there is a current of momentary duration induced in the secondary, so that there is a current made and broken through the muscle; and the same thing takes place again when the primary circuit is broken. It has been shown that, when we use currents of such short duration, the break 
stimulus is ineffective; so in both cases, whether we make or break the current in the primary circuit, we are dealing with a make stimulus in the muscle. The difference in the efficacy of make and break induction shocks is purely physical, and depends on the fact that the current induced in the secondary coil on make is of slower rise and smaller potential than that produced at break.

In using either of these modes of stimulation we find that there is a certain intensity which the stimulating current must possess in order that any effect shall be produced. Any strength of stimulus below this is known as a subminimal stimulus. A minimal stimulus (sometimes known as liminal or threshold stimulus) is the weakest stimulus that will produce any result, i.e. in muscle, a contraction. A maximal stimulus is one that produces the strongest contraction a muscle is capable of under the effects of a single stimulus. A submaximal stimulus is any strength of stimulus between these two extremes. 


\section{SECTION III}

\section{THE MECHANICAL CHANGES THAT A MUSCLE UNDERGOES WHEN IT CONTRACTS}

IF a skeletal muscle, such as the gastrocnemius, be stimulated either directly or by the intermediation of its nerve by any of the means mentioned in the foregoing chapter, it responds by a single short sharp contraction, followed immediately by a relaxation. The volume of the muscle does not alter in the slightest degree, but each muscle-fibre and the whole muscle become shorter and thicker. At the same time, if a weight be tied on to the tendon of the muscle, the muscle during contraction may raise the weight and thus perform mechanical work. In order to determine the time relations of the simple muscle contraction or the muscle-twitch, and to study its conditions, it is necessary to employ the graphic method, so as to obtain a record of the changes in shape of the muscle during contraction. We may use the graphic method either for recording the changes in shape or for registering changes in tension of a muscle which is prevented from contracting.

In order to record the contraction of the frog's gastrocnemius, the muscle is excised together with a portion of the femur to which it is attached, and the whole length of the sciatic nerve from its origin in the spinal canal to its insertion into the muscle. The femur, to which the gastrocnemius is attached, is clamped firmly, and the tendo Achillis attached by a thread to a light lever, free to move round an axis at one end. The point of this lever is armed with a bristle (anything that is stiff and pointed will do), which just touches the blackened surface of a piece of glazed paper. This paper is stretched round a cylinder (drum) which can be made to rotate at any constant speed required. If the drum is moving, the point of the bristle draws a horizontal white line on the smoked paper.

If a single induction shock be sent through the nerve of the preparation the lever is jerked up, falling again almost directly, and a curve is drawn like that shown in Fig. 52. A similar curve is obtained if the muscle be stimulated directly.

In all such graphic records we should have also-

(1) A time record. This is furnished by means of a small electro-magnet, armed with a pointed lever writing on the smoked surface. This electro-magnet (time marker or signal) is made to vibrate 100 times a second (more or less as may be required) by putting it in a circuit which is made and broken 100 times a second by means of a tuning-fork vibrating at that rate. The tuning-fork is maintained in vibration in the same way as the Wagner's hammer of an induction-coil.

(2) $A$ record of the exact point at which the nerve or muscle is stimulated. This may be obtained in two ways :

(a) When using the pendulum or trigger myograph, in both of which the recording surface is a smoked flat surface on a glass plate, this latter is so arranged that it knocks 
over a key as it shoots across, and so breaks the primary circuit and excites the nerve when it knocks over the key, we can mark on the contraction curve the exact moment

(b) If we wish to make and break the primary circuit at will by means of a key, a small electro-magnetic signal, interposed in the circuit, is arranged to write on the revolving drum, and so mark the point of stimulation.

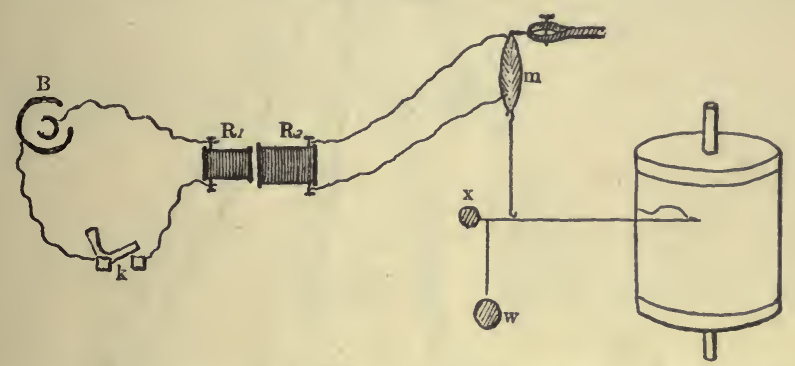

FIG. 51. Arrangement of apparatus for recording simple muscle-twitch.

In the figure (Fig. 52) the upper line is the curve drawn by the lever of the muscle as it contracts ; the small upright line shows the point at which the muscle was stimulated ; and the second line is the tracing of the chronograph, every vibration representing $\frac{1}{444}$ of a second.

In the pendulum myograph (Fig. 53) a smoked glass plate is carried on a heavy iron pendulum. At each side the pendulum is armed with a catch, which fits on to other catches at the side of the triangular box, from the apex of which the pendulum

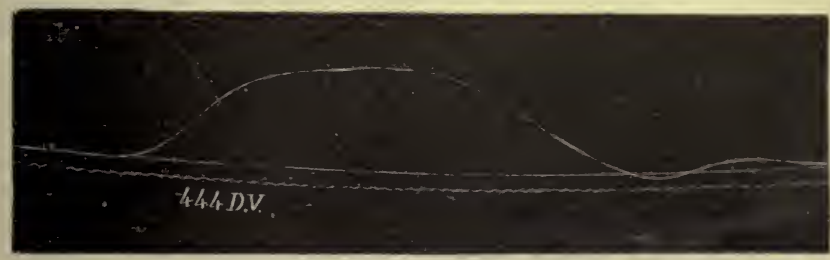

FiG. 52. Curve of single muscle-twitch taken on a rapidly moving surface . (pendulum myograph). (Y Eо.)

is suspended. At its lower part the pendulum carries a projecting piece which can knock over the 'kick-over' key $\mathbf{K}$, thus breaking a circuit in which is included the primary coil of an induction-coil. The lever attached to the muscle is arranged so as to write lightly on the glass plate. Everything being ready, and the key $\mathrm{K}$ closed, the pendulum is raised to $A$, the catch $A$ is then released, and the pendulum falls at an ever-accelerating rate and then rises again, gradually slowing off·until it is caught again at $\mathbf{B}$. As it passes by the key it breaks the circuit. A break induction shock is sent into the muscle or nerve, which contracts, and a curve is obtained similar to that shown in Fig. 52. Since the rate of the pendulum is constantly varying throughout its course, it is necessary to have a tuning-fork, or time-marker actuated electrically by a tuning-fork, writing just below the muscle-lever.

In the spring myograph, otherwise known as the trigger or shooter myograph (Fig. 54), a smoked glass plate is also used. "The frame supporting the glass plate slides on two horizontal steel wires. To make the instrument ready for use, the frame is moved to one side, which compresses a short spring. When the catch holding it in this position is released by the trigger, the spring, which only acts for a short space, 
gives the frame and the glass plate a rapid horizontal motion; and the momentum carries the glass plate through the rest of the distance, till stopped by the buffers. The

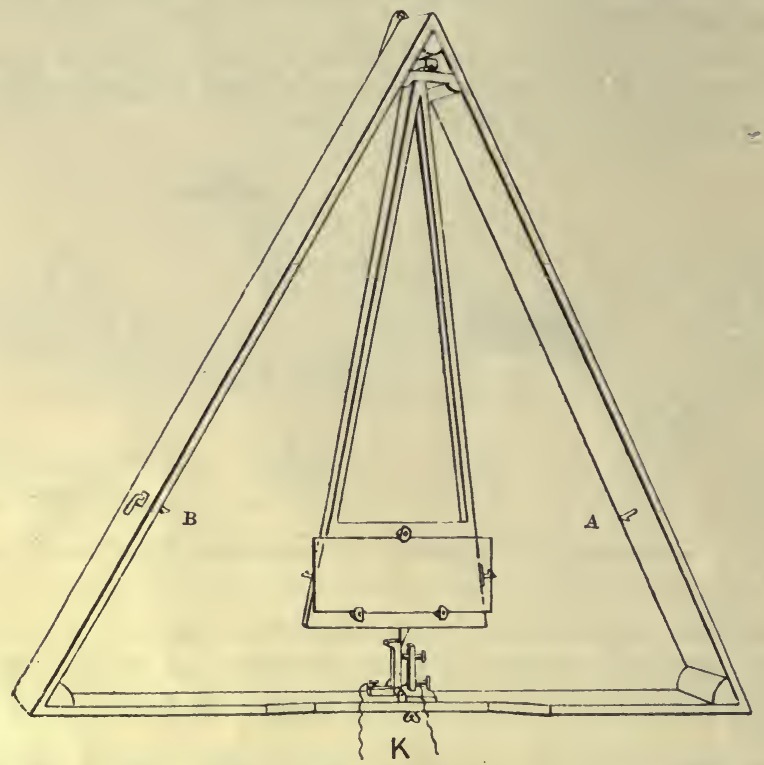

Fig. 53. Simple form of pendulum myograph.

velocity during this time is nearly constant, as the friction of the guides is small. Two keys are knocked over by pins on the frame and break electric circuits. The relative positions at which the circuits are broken can be altered by a convenient adjustment.

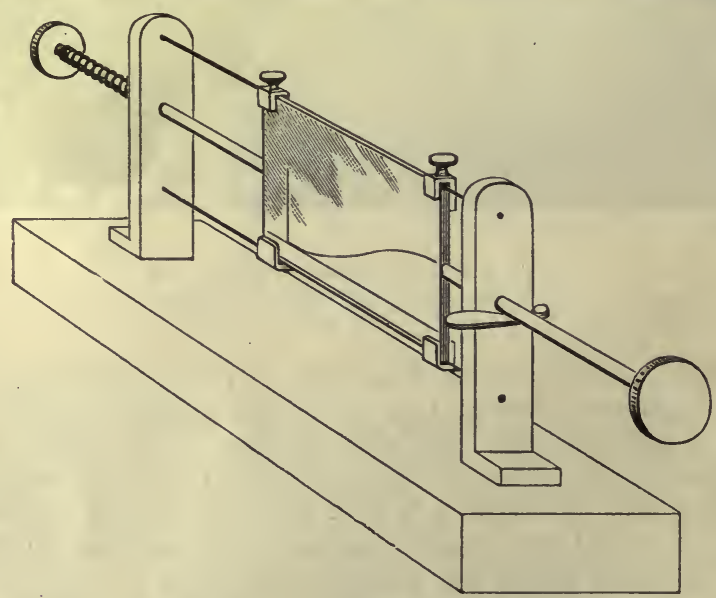

FIG. 54. Diagram of spring myograph, or ' shooter.'

A tuning-fork vibrating about 100 per second fixed to the base of the instrument marks the time; its prongs are sprung apart by a block between their ends, and the same action which releases the glass plate also frees the fork by removing the block and allows it to vibrate; a writing style then draws a sinuous line on the smoked surface of the moving glass plate. A muscle lever with a scale-pan attached also forms part of the instrument."

The record obtained in either of these ways may, in consequence of instrumental 
inertia, be a very inaccurate reproduction of the true events occurring in the muscle itself. When the muscle begins to contract it imparts a very rapid movement to the lever, which therefore tends to overshoot the mark and deform the curve. This sourco of error may be almost avoided by making the lever as light as possible, and hanging the extending weight in close proximity to the axle of the lever, as shown in Fig. 55. Since the energy of a moving mass is proportional to the square of the velocity $\left(=\frac{1}{2} m r^{2}\right.$, and the tension due to the weight as well as the velocity on contraction is directly proportional to the distance of the weight from the axis, it follows that it is better to

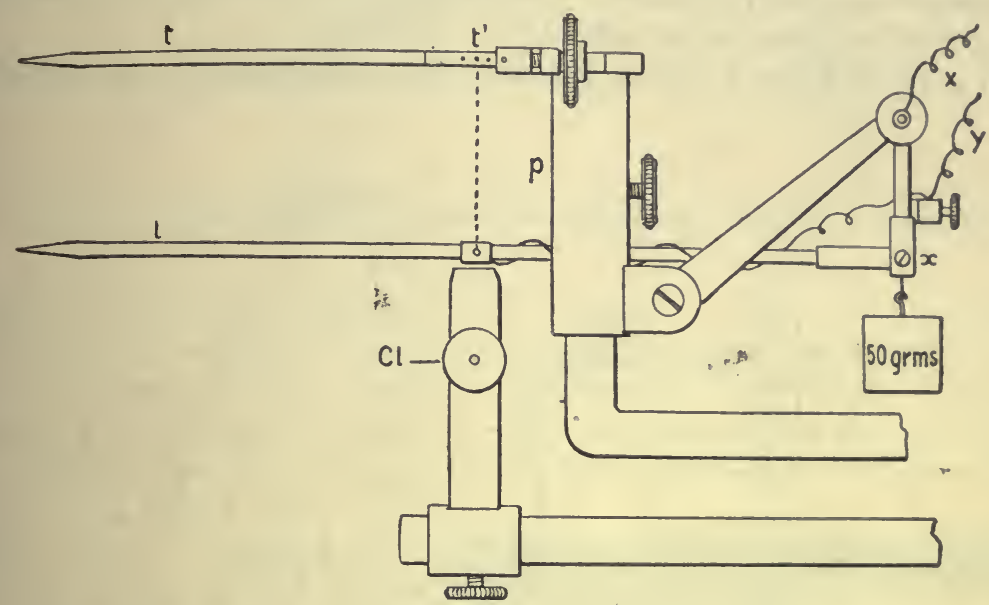

FIG. 55. Blix apparatus for recording isometric and isotonic curves synchronically. (M ISS Buchanan.) $p$, the steel cylindrical support with jointed steel arm to bear the isotonic lever $l$, which consists of a strip of bamboo with an aluminium tip. $t$, the isometric lever, also of bamboo, except for a short metal part $t$ ', in which are holes for fixing the muscle. The two wires from an induction coil are brought, one to $x$, which is in connection with the support and hence with the metal bar $t^{\prime}$, the other to $y$, which is insulated from the support but connected by a copper wire with a thin piece of copper surrounding the isotonic lever at the point where the muscle is attached to it. $C l$, clamp for fixing the lower end of the muscle when an isometric curve is to be taken. The axis of the isotonic lever is at $x$, close to. which is hung the weight of $50 \mathrm{grm}$.

load the muscle with 40 grams 1 millimetre from the axis than with 1 gram 40 millimetres from the axis, though the tension put on the muscle will be the same in both cases.

In the first case the energy of the moving mass will be proportional to $\frac{40 \times(1)^{2}}{2}=20$, and in the second to $\frac{1 \times(40)^{2}}{2}=800$, and it is this energy which determines the overshooting of the lever and the deformation of the curve. Since throughout the contraction in the latter arrangement the lever follows the muscle in its movement, the tension on the muscle remains the same throughout, and the method is therefore known as the isotonic method.

It is of importance to be able to record the development of the energy (i.e. the tension) of the active muscle apart from any changes in its length. / For this purpose the muscle is allowed to contract against a strong spring, the movements of which are magnified by means of a very long lever. Thus the shortening of the muscle is almost entirely prevented, but the increase in its tension causes a minute but proportionate movement of the spring, which is recorded by means of the lever. Since in this case the length or measurement of the muscle remains approximately constant, while the tension is continually varying throughout the contraction, it is known as the isometric method. The great magnification necessary in this method introduces serious sources of error ; but it seems that if all due precautions be taken to avoid these errors, the isometric 
curve differs very little in form from the isotonic, displaying only a somewhat quicker development of energy at the beginning of contraction. / It is better to eliminate the lever altogether and magnify the minute movements of the spring by attaching to it a small hinged mirror by which a ray of light is reflected through a slit on to a travelling photographic plate. Since the ray of light has no inertia, magnification of the movements may be carried to any extent without increasing the instrumental deformation of the curve (Fig. 56).

A simple muscular contraction or twitch, such as that in Fig. 52, produced by a momentary stimulus, consists of three main phases :

(1) A phase during which no apparent change takes place in the muscle,

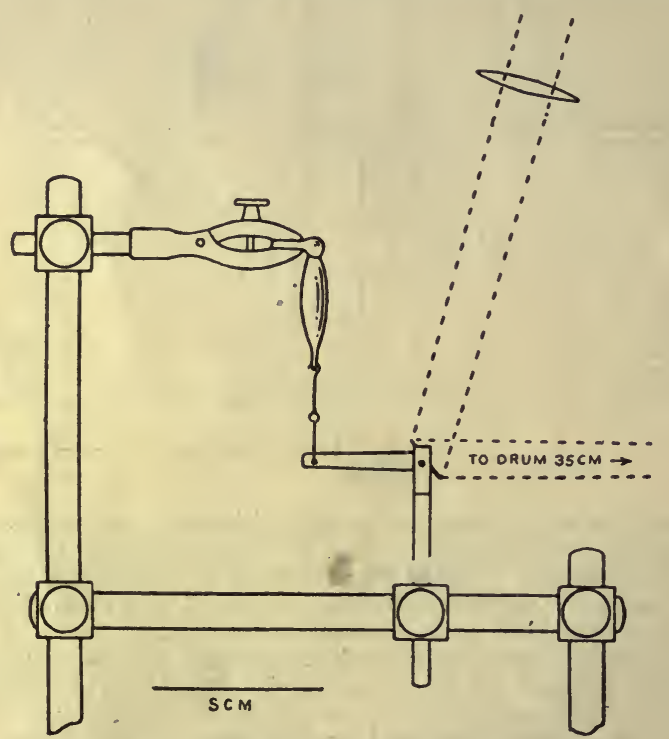

Fig. 56. Myograph for optical registration of muscular contraction. (K. LUCAS.)

or at any rate none which gives rise to any movement of the lever. This is called the latent period.

(2) A phase of shortening, or contraction.

(3) A phase of relaxation, or return to the original length.

The small curves seen after the main curve are due to elastic vibrations of the lever, and do not indicate any changes occurring in the muscle itself. From the time-marking below the tracing we see that the latent period occupies about $\frac{1}{100}$ second, the phase of shortening $\frac{4}{100}$, and the relaxation $\frac{5}{100}$ second.

Thus a single muscle-twitch is completed in about $\frac{1}{10}$ second. It must be remembered, however, that this number is only approximate, and varies with the temperature of the muscle and its condition, being much longer in a fatigued muscle. Moreover, it is almost impossible to avoid some deformation of the curve due to defects of the recording instruments used. Thus the relative period during which no mechanical changes are taking place in the muscle must always be shorter than is apparent from a curve obtained 
by the foregoing method. The elasticity and extensibility of the muscle must prolong the apparent latent period, since the first effect of contraction of any part of the muscle will be to stretch the adjacent part, and only later to

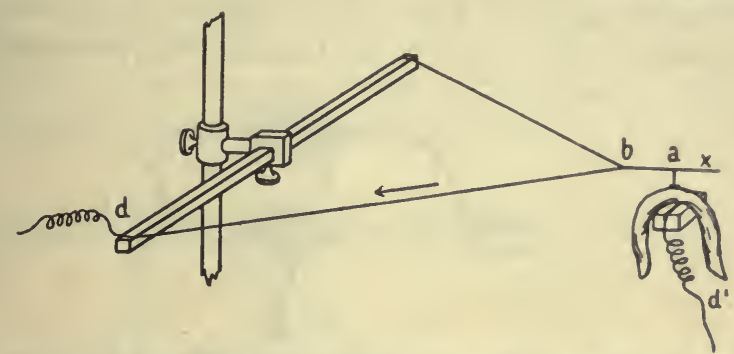

FiG. 57. Burdon Sanderson's method for photographic record of muscle-twitch. The exciting shock is sent into the muscle by the wires $d$ and $d^{\prime}$.

move the tendon to which the lever is attached. Thus if we have a weight supported by a rigid wire, and suddenly pull the upper end of the wire so as to raise the weight, the latter will rise instantaneously. If, however, the weight be suspended by a piece of elastic, it will not follow the pull exactly, but will lag behind, the first part of the pull being occupied with stretching the india-rubber, and only when this is stretched to a certain degree will the weight begin to rise. The same retardation of the pull would be observed if, instead of indiarubber, we used a piece of living muscle.

It is possible to obviate this instrumental inertia by employing solely photographic methods for the record and magnification of the muscletwitch. Thus in the experiments of Sanderson and Burch the thickening of the muscle at the point stimulated was recorded graphically by photographing the movement on a slit (Fig. 57), behind which was a moving sensitive plate. Thus avoiding all instrumental

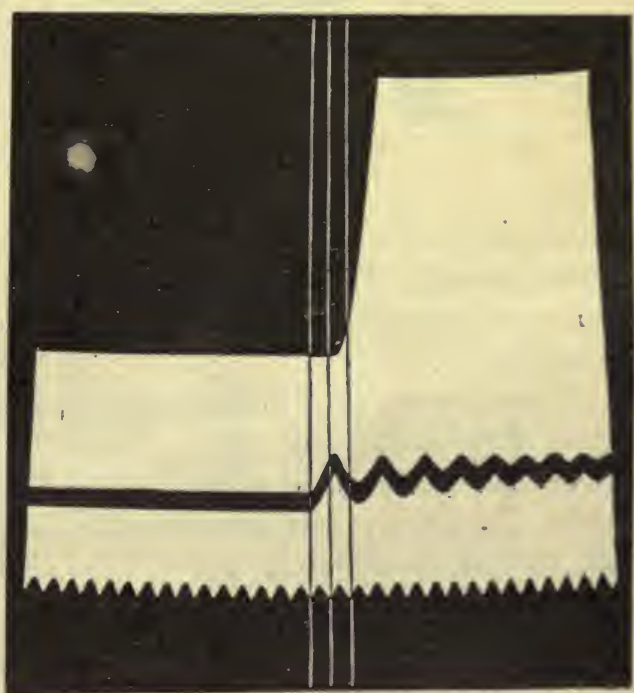

FIG. 58. Photographic record of muscle-twitch. (B. SANDERSON.) The upper curve is the movement of the muscle, the middle curve the signal showing the moment of excitation, and the lower curve is that of a tuning-fork vibrating 500 times a second. inertia, and diminishing the inertia of the muscle to a minimum, the mechanical latent period was found to be only 0.0025 second (Fig. 58). This figure we can take as the average latent period for the skeletal muscle of the frog at the ordinary temperature of the laboratory (about $16^{\circ} \mathrm{C}$.). We shall have occasion later on 
to consider the changes which occur in the muscle between the application of the stimulus and the moment at which the first mechanical change makes its appearance.

The relaxation of muscle is helped by a moderate load, and in a normal condition is complete. It is not active-that is to say, is not due to a con-

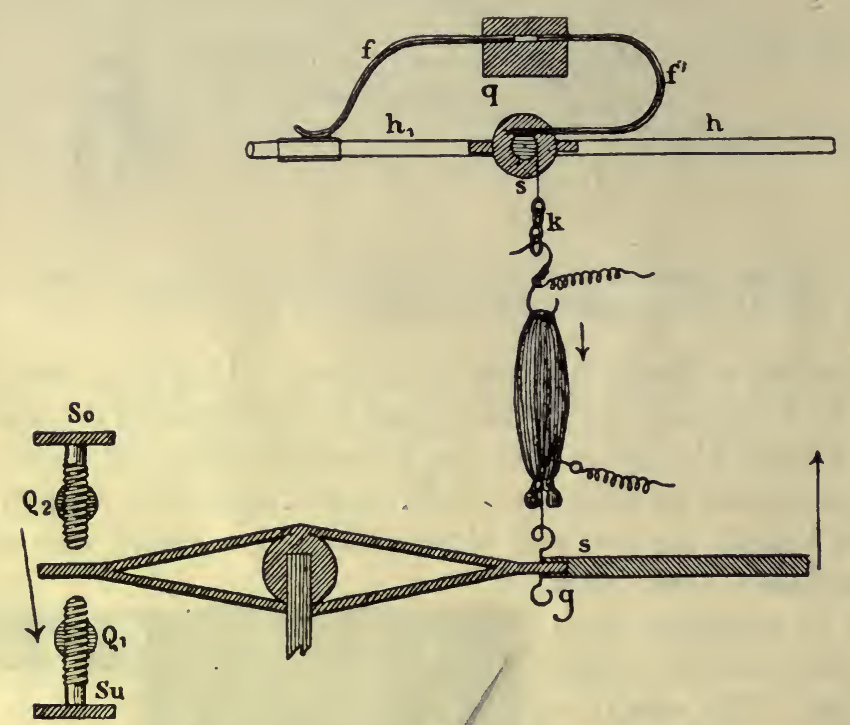

FIG. 59. V. Kries' apparatus for taking ' after-loading' and 'arrested contraction curves.'

traction in the transverse direction-but is a passive effect of extension and elastic rebound. This may be shown by allowing a muscle to contract while floating on mercury. The subsequent lengthening on relaxation is very incomplete.

Even with the most careful arrangements for securing isotonicity in the record of the contraction there is probably a certain amount of over-shoot of the lever whenever, as at high temperatures, the contraction is sufficiently rapid. The effect of this is that one cannot assume the existence of an actual pull on the lever during the whole time of the ascent of the latter. We can therefore speak of a period during which there is contractile stress-that is to say, when the muscle is actually pulling on the lever, which will occupy only a part of the ascent of the curve. The duration of this period of contractile

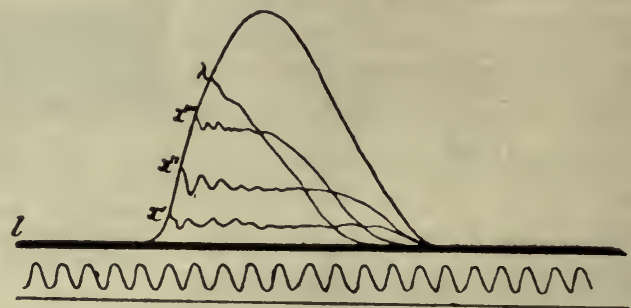
stress may be shown by recording what is known as 'arrested' contractions. One mechanism for this purpose is shown in the figure (Fig. 59). The stop Su is used 
simply for after-loading the muscle so that the weight shall not act upon the muscle until it begins to contract. The stop So may be regulated so that it suddenly checks the movement of the lever at any desired height above the base line. We may thus get a series of contractions such as those shown in Fig. 60. It will be seen that at the points $x^{\prime}$, $x^{\prime \prime}$, and $x^{\prime \prime \prime}$ the muscle was still pulling on the lever, and therefore held it up against the stop. At the point $\lambda$ the arrested twitch returns rapidly to

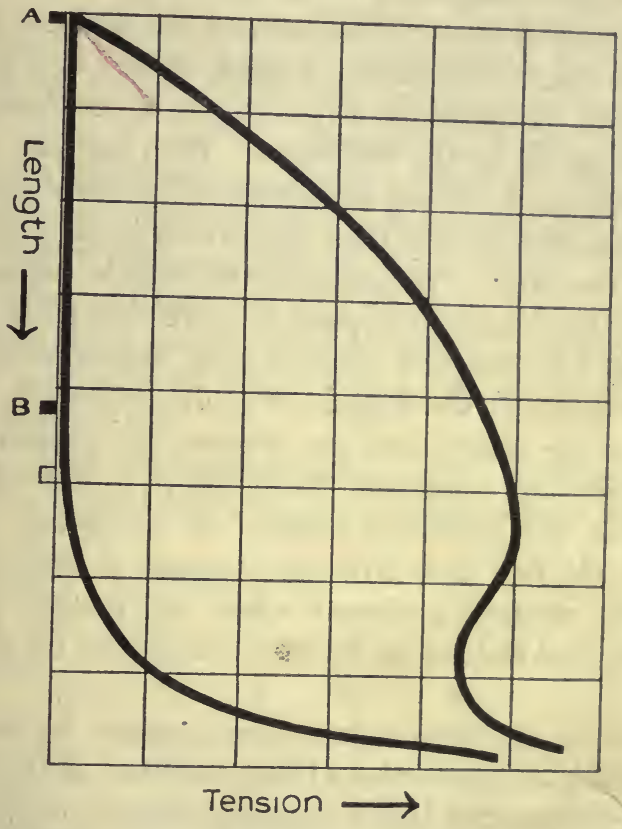

FIG. 61. Diagram to show the relation between the initial length of a muscle and the tension developed in it during excitation (as measured by the isometric method). The tension developed at each initial length is measured by the horizontal distance between the two thick lines, the left line representing the resting muscle, and the curved thick line on the right the contracted muscle. (From BuIx.)

the base line, showing that the movement of the lever in the unarrested curve above this point was due to the inertia of the moving parts and not to the actual pull of the muscle.

THE ENERGY OF CONTRACTION. When a muscle contracts we may conceive of it as converted into a body with elastic properties other than those which it possesses during rest. Directly after it has been excited it possesses potential energy which can be measured as tension by the isometric method and which will degenerate in a.few hundredths of a second into heat, or can be turned into work by allowing the muscle to shorten and to raise a weight, as in the isotonic method of recording muscular contractions. Under the conditions of an ordinary physiological experiment, a contracted muscle loaded only by a light lever is shorter than the non-contracted, but can be stretched to the 
length of the latter by a certain weight, when it will be in a condition of tension. In their natural position in the body muscles may possess any length between extreme shortening and extreme elongation whether they are in a resting or in an excited condition. Since the relaxed muscle requires only a minimal force to extend it to the maximal length possible in its natural relationships in the body, it is usual to speak of the different lengths of an excited and unexcited muscle, the lengths being in this case those which are impressed on the muscle by a minimal load. When we measure by means of the isometric method the maximum energy set free in a muscle as the result of excitation, we find, as Blix first pointed out, that this energy depends on the length of the muscle fibres during the period of contractile stress set up by the excitation. With increase in the length of the muscle the tension developed on excitation increases until the length of the muscle is somewhat greater than that which it possesses in its normal relationships in the body. To lengthen the muscle beyond this point a certain stretching force must be applied to it which rapidly increases. The tension developed on excitation however soon begins to diminish. These relationships are shown by the diagram (Fig. 61 ), where the ordinates represent the length of the muscle and the abscissæ the tension on the muscle. The left-hand thick line represents the muscle in a state of rest, the righthand curved line the muscle in a state of excitation. The horizontal distance between the two lines gives the increase of tension (as measured by the isometric method) produced when the muscle passes from the resting into the excited state as the result of stimulation by a single induction shock.

Since the tension set free on excitation deppends on the length of the muscle fibres during the production of the condition of tension, the tension developed will be diminished if the muscle be allowed to shorten before its maximum tension has been reached./ This is the case with all isotonic records of muscular contraction, so that it becomes difficult to give any exact expression for the total energy changes in a muscle which is allowed to shorten. As A. V. Hill has pointed out, a muscle is a machine primarily for developing tension, and the potential energy thus set up may be used for the production of work to any degree the conditions of loading allow.

The work done by a muscle when it contracts is measured by multiplying the weight lifted by the height through which it is lifted, $w \times h$. Since however, the result will vary according to the conditions of loading of the muscle, a much more useful quantity is obtained by measuring the tension produced in a muscle which is stimulated but not allowed to shorten. The potential energy available due to the new elastic conditions of the fibres is found to be approximately $\frac{1}{6} \mathrm{~T} l$, where $\mathrm{T}$ is the maximum tension developed in the twitch and $l$ is the length of the muscle (A. V. Hill). 


\section{THE EXTENSIBILITY OF MUSCLE}

Living muscle in a perfectly normal condition is distinguished by its
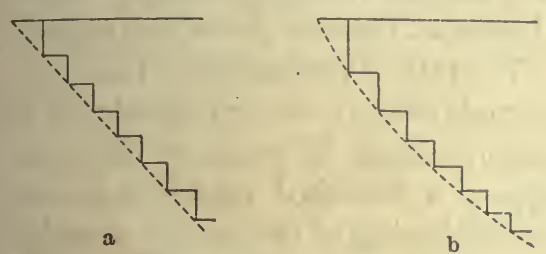

slight but perfect elasticity; that is to say, it is considerably stretched by a slight force (in the longitudinal direction), but returns to its original length when the extending weight is removed. The length to which muscle

Fic. 62. Extensibility of india-rubber $(a)$ is stretched is not proportional to compared with that of a frog's gastroc- the weight used, but any given in-
nemius muscle $(b)$.

crement of weight gives rise to less elongation the more the muscle is already stretched. The accompanying curves show diagrammatically the elongation of muscle as compared with a piece of india-rubber when the weight on it is uniformly increased.

Dead muscle is less extensible and its elasticity is less perfect. A given weight àpplied to a dead muscle will not stretch it so much as when the muscle was alive, but the dead muscle does not return to its original length when the weight is removed.

A contracted muscle, on the other hand, is more extensible than a muscle at rest. A gramme applied to a contracted gastrocnemius will cause greater lengthening than if it were applied to the same muscle at rest. The relation between the excitability of a muscle under the two conditions of contraction and rest are shown in the diagram in Fig. 63.

At the Point $y$ the muscle is unable to shorten at all against a weight. It is evi-

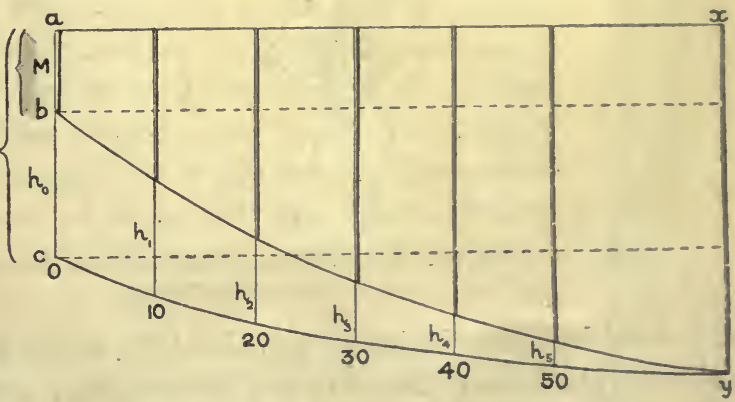

FIa. 63. Curve showing the length of a muscle under various loads in the contracted condition by, and uncontracted condition $c y$. The double lines $a b$, \&c., represent the contracted muscle, while the long single lines $a c$, \&c., show the length of the inactive muscle. dent from this diagram that the height of contraction of a muscle diminishes as the load is increased, very rapidly if the muscle is after-loaded, less rapidly if the weight applied to the muscle be allowed to extend it at rest. It is evident however that in either case the diminution in height is not in proportion to the load and that the work done by the muscle, $w \times h$, as the weight is.incréased, rises at first quickly, then more slowly to a maximum to sink finally to zero. By inspection of diagram (Fig. 63) it will be seen that

$$
0 . h_{0}<10 . h_{1}<20 . h_{2}<30 . h_{3}>40 . h_{4}>50 . h_{5},
$$

so that in this case the maximal mechanical work is obtained when the muscle is loaded with about $30 \mathrm{gms}$. 


\section{PROPAGATION OF CONTRACTION. THE CONTRACTION WAVE}

The whole muscle does not as a rule contract simultaneously. When excited from its nerve the contraction begins at the end-plates and spreads in both directions through the muscle. The rate of propagation of the contraction wave can only be measured by employing a curarised muscle, so as to avoid the wide spreading of the excitatory change by means of the intramuscular nerve-endings. For this purpose a curarised sartorius muscle is taken, stimulated at one end, and the thickening of the muscle recorded by means of two levers placed, one near the exciting electrodes and the second at the other end of the muscle, as shown in the diagram (Fig.64). The

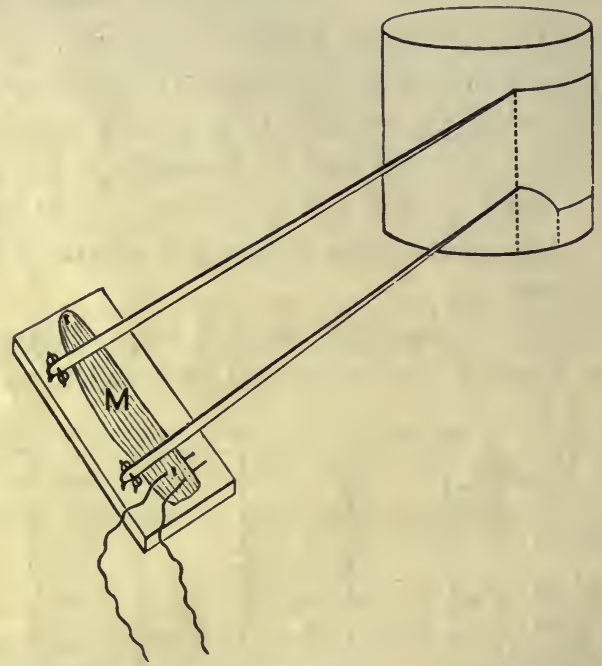

FIG. 64. Diagram of arrangement for recording the contraction wave in a curarised sartorius.

difference between the latent periods of the two curves represents the time taken by the contraction wave in travelling from $a$ to $b$. By measurements carried out in this way it is found that the rate of propagation of the contraction in frog's muscle is 3 to 4 metres per second; in the muscle of warm-blooded animals it may amount to 6 metres.

The actual duration of the shortening at any given point is necessarily smaller than that of the whole muscle, and amounts in frog's muscle to only $0.05-0.09 \mathrm{sec}$., about half the duration of the contraction of a whole muscle of moderate length. The length of the wave is obtained by multiplying the rate of transmission by the duration of the wave at any one point. It varies therefore in frog's muscle between $3000 \times \cdot 05(=150)$ and $4000 \times \cdot 09$ $(=360)$ millimetres. Thus the muscle fibres in the frog are much too short to accommodate the whole length of the wave, and the contraction of the whole muscle must be made up of the summated effects of the contraction wave as it passes from point to point. Hence the longer the muscle, the more must the contraction be lengthened by the time taken up in propagation from one end to another. 


\section{SECTION IV}

\section{THE CONDITIONS AFFECTING THE MECHANICAL RESPONSE OF A MUSCLE}

STRENGTH OF STIMULUS. If a series of single break-shocks be applied to a muscle or nerve at intervals of not less than five seconds, it will be found that beyond a certain distance of the secondary from the primary coil no effect at all is produced. The shocks are said to be subminimal. On pushing the secondary coil nearer the primary a point will be reached at which a small contraction will be observed. On then pushing in the coil a millimetre at a time the contraction will become greater for the next couple of centimetres (e.g. as the coil is moved from 12 to $10 \mathrm{~cm}$. distance). Further increase of current by approximation of the coils is without effect, although the current actually used may be increased a hundred times in moving the coil from 10 to 0 . It was formerly thought that this limited gradation of the muscular response according to strength of stimulus was due to a similar gradation in the response of each individual muscle fibre of which the muscleis composed. It seems more probable, however, that, when a minimal or subminimal response is obtained, not all the fibres making up the muscle are contracting. A minimal contraction is in fact a contraction in which some fibres of the whole muscle are stimulated. A maximal contraction is one in which all the fibres are stimulated. So far as concerns each individual muscle fibre every contraction is a maximal contraction. The fibre either contracts to its utmost or it does not contract at all. The rule of 'all or none' which was first enunciated for heart-muscle is probably true for every contractile element. The difference between skeletal and heart

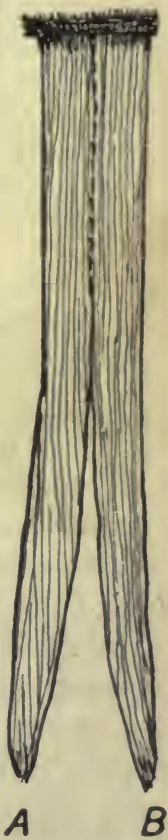

FIG. 65 . muscle lies in the fact that in the former the excitatory process does not spread from one fibre to its neighbours. If, for instance, we take a curarised sartorius and split its lower end, as in Fig. 65, the stimulus.applied to A causes a contraction only of the left-hand side of the muscle, while a stimulus applied to $\mathrm{B}$ is in the same way limited to the right-hand side. If a piece of ventricular or auricular muscle of the frog or tortoise were treated in the same way, a stimulus applied at $\mathrm{A}$ would cause a contraction which would travel across the bridge at the upper end and extend to $\mathrm{B}$. 
It was shown by Gotch that, if each of the three roots which make up the sciatic nerve and send fibres to the gastrocnemius be stimulated in turn, it is often impossible to evoke a maximal contraction of the gastrocnemius, however strongly each root be stimulated. Keith Lucas has shown that if stimuli in gradually increasing strength be applied to the motor nerve (containing only seven to nine fibres,) which supplies the dorso-cutaneus muscle of the frog, the contraction of the muscle increases, not gradually, but by a series of steps. This can be explained only by assuming that the smallest effective stimulus excites perhaps four out of the seven nerve fibres, those immediately in contact with the electrodes. With increasing strength of current the stimulus becomes effective for the three fibres lying next to these, and finally still further increase of current may excite all the fibres making up the nerve (Fig. 66).

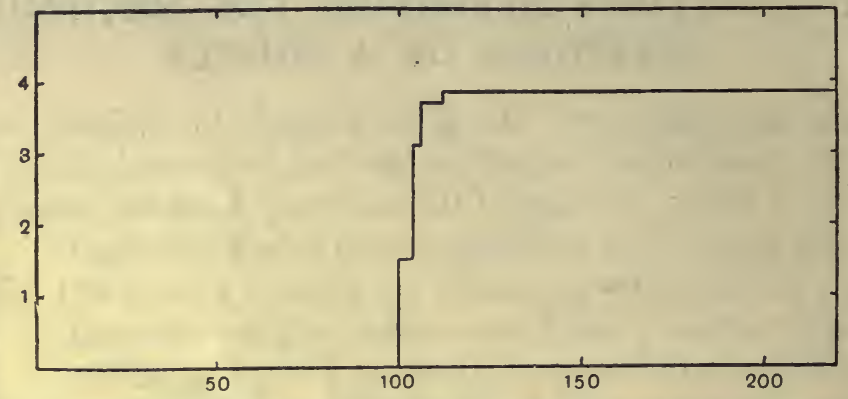

Fig. 66. Curve showing relation of height of contraction of dorso-cutaneus muscle to strength of stimulus. Ordinates = height of contraction; abscissa $=$ strength of stimulus. (K. LUCAS.)

\section{THE REPETITION OF STIMULUS}

SUMMATION. The response of a muscle fibre to a single shock, whether measured by the isotonic or the isometric method, i.e. as shortening or as tension, is independent of the strength of stimulus and varies only with the length of the fibre during the rise of the excitatory condition. If, however, a second shock is sent in during this period a further evolution of energy is possible, and the effect is still further increased by putting a series of stimuli into the muscle or its attached nerve before the development of the contractile stress due to the first stimulus has reached its maximum. If two shocks at intervals of one hundredth of a second be sent into a muscle, the response, whether shortening or rise of tension, will be greater than that produced by one shock. If a series of shocks be sent in, the excitatory condition is maintained, so that instead of a simple muscle twitch rising to a maximum and then falling, the muscle lever rises to a given point, which in the muscle contracting isometrically may be double that due to a single stimulus, and then remains at this height during the continuation of the repeated excitations. If the muscle be allowed to contract isotonically, the continued contraction produced by a series of stimuli may with a heavy load be three or four times as considerable as that produced by a single stimulus. This condition of apparently continued stimulation brought about by continued application of stimuli is said to be summated.

REFRACTORY PERIOD. If the interval between two stimuli sent into a muscle be successively shortened in a series of observations we finally arrive at a point at which summation is no longer apparent, i.e. the effect of 
the two stimuli is no greater than the effect of a single stimulus. This means that the second stimulus has become ineffective, and this ineffective. ness we must ascribe to the condition set up in the muscle as the result of the first stimulus. / For a very short period of time after stimulation a muscle is inexcitable to a second stimulus. The period during which it is inexcitable is known as the refractory period and amounts in skeletal muscle to about $\cdot 0015$ second. ( The same phenomenon is better marked in certain other excitable tissues, such as the heart muscle, but it seems to be a common property of excitable tissues generally.

When a loaded muscle is made to record its contractions isotonically we may get summation of effects, though the interval between the stimuli is greater than that which corresponds to the duration of the rise of contractile stress. Thus if the interval is just so long that the second becomes effective just as the contraction due to the first has commenced to die away, the second contraction seems to start from the point to which the muscle has been raised by the first (Fig. 67). By repeating these stimuli in a heavily loaded muscle, the contraction may be made three or four times as extensive as a single twitch. With slow stimuli the

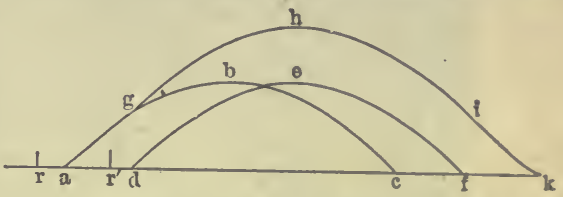

Fig. 67. Muscle curves showing summation of stimuli. $r$ and $r^{\prime}$, the points at which the stimuli were sent into the nerve. From the first stimulus alone the curve $a b c$ would bo obtained. From $r^{\prime}$ the curve def is obtained. These two curves are summated to form the curve aghik when both stimuli are sent in at the interval $r r^{\prime}$. summation is, however, rather mechanical than physiological. The period of contractile stress, which lasts only about .03 second, is so short that it has no time to raise the weight to the maximum height before it has passed away. This is shown by the fact that if the muscle be afterloaded, so that the lever is raised to the top of the curve of a single twitch, application of the stimulus will make it shorten still more, and by repeated after-loading in this fashion, it is possible to make the muscle raise a weight in response to a single stimulus

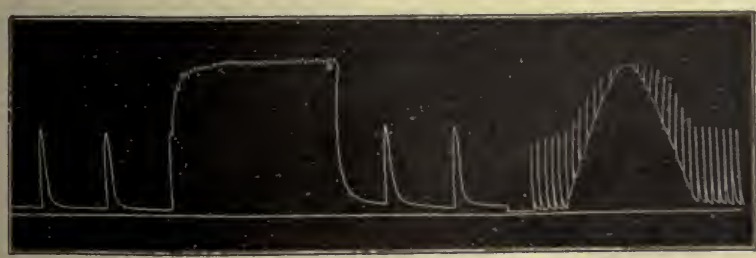

Fig. 68. Contractions of a frog's muscle. Two single twitches are followed by a tetanus, which is almost twice as high as a single contraction. After two more single twitches, the drum was made to rotate more slowly, and single shocks employed, at the same time as the 'after-loading, was continually increased. It can be seen that the curve obtained in this way is as high as the original tetanus. (V. FREY.)

to the same height that it would if excited by a series of stimuli. This mechanical factor in sum. mation is shown in Fig. 68. It will be noted, however, that the tetanus is not a steady one and is probably due to stimuli repeated at intervals of about $\frac{1}{10}$ of a second. If the rate of stimulation were increased to 50 or 100 per second, a tetanus would be produced and the curve would be probably twice as high as that represented in the figure. We thus see that for the orercoming of a resistance a single twitch is not economical. It is doubtful whether any contractions of muscles which occur in the body are other than tetani of varying duration.

TEMPERATURE. Speaking generally, the effect of warming a muscle is to quicken all its processes. The latent period becomes shorter and the muscle curve steeper and shorter. 
It is very often observed that the height of contraction of the warmed muscle is greater than that obtained at ordinary temperatures. It seems that this apparent increase in height is really instrumental in origin, the quicker-moving muscle jerking the lever beyond the real extent of the contraction. If proper means are taken to eliminate this overshooting of the lever, it is found that the height of contraction is unaltered between $5^{\circ}$ and $20^{\circ} \mathrm{C}$., the only change being in the timerrelations of the curves. This is especially well shown in the so-cal'ed 'arrest' curves (Fig. 69).

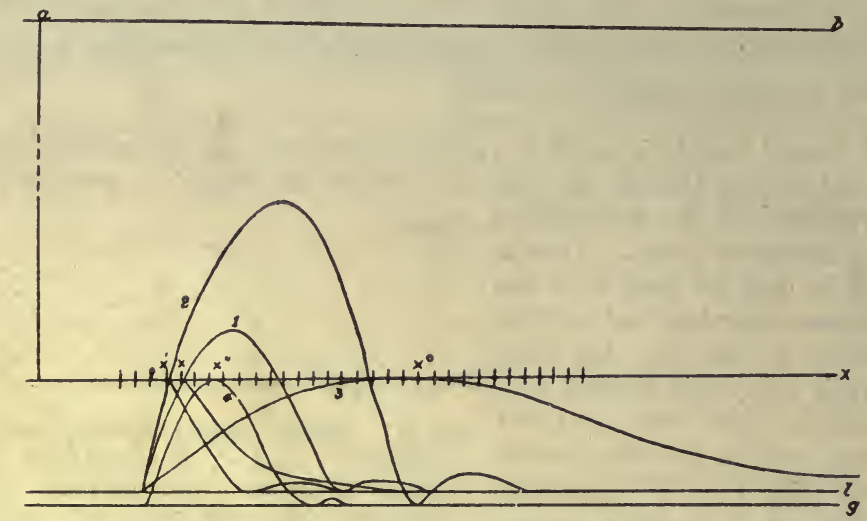

Fig. 69. Isotonic and 'arrest' curves of muscle-twitch : (1) unloaded at $14^{\circ} \mathrm{C}$. ; (2) at $25^{\circ} \mathrm{C}$. ; (3) at $0^{\circ} \mathrm{C}$. ; (4) loaded at $14^{\circ} \mathrm{C}$. Note that the arrest curves attain the same height throughout. (KAISER.)

If a muscle be heated gradually (without stimulation) up to about $45^{\circ} \mathrm{C}$., it begins to contract slowly at about $34^{\circ} \mathrm{C}$, and this contraction reaches its maximum at $45^{\circ} \mathrm{C}$., at which point the muscle has entered into pronounced rigor mortis.

Cold has the reverse effect. The intra-molecular processes which lie at the root of the muscular activity are slowed, so that the latent period and the contraction period are prolonged. The action of cold on the excitability of muscle is to increase it, so that any form of stimulus is more effective at $5^{\circ} \mathrm{C}$. than at $25^{\circ} \mathrm{C}$. Moreover, when maximal stimuli are being used, and the muscle is heavily loaded, the first effect of the application of cold may be to increase the height as well as the duration of contraction, for the same reason that a gentle push is more efficacious in closing a door than would be a heavy blow with a hammer. If, however, a muscle be cooled for a short time to zero or a little below, it loses its irritability, which returns if the muscle be gradually warmed again. Prolonged exposure to severe cold irrevocably destroys its irritability. Warming the muscle will now simply bring about rigor mortis.

FATIGUE. A muscle will not go on contracting indefinitely. If it be repeatedly stimulated, changes soon become apparent in the curve of contraction. The latent period is prolonged, as well as the length of the contractions; the absolute height and work done are diminished. At the same time the muscle does not return to its original length ; the shortening which remains is spoken of as 'contraction remainder.' After an initial rise during the first few contractions, these diminish uniformly in height 
till they are no longer apparent, so that the muscle is now said to have lost its irritability. At the same time there is a great prolongation of the curve, occasioned almost entirely by a retardation of the relaxation, so that after forty or fifty contractions several seconds may elapse before the lever returns to the base line (Fig. 70).

The fact that the relaxation part of the muscle curve is affected by various conditions, especially fatigue, apparently independently of the contraction part, led Fick to put forward a theory that two distinct processes were concerned in the response of a

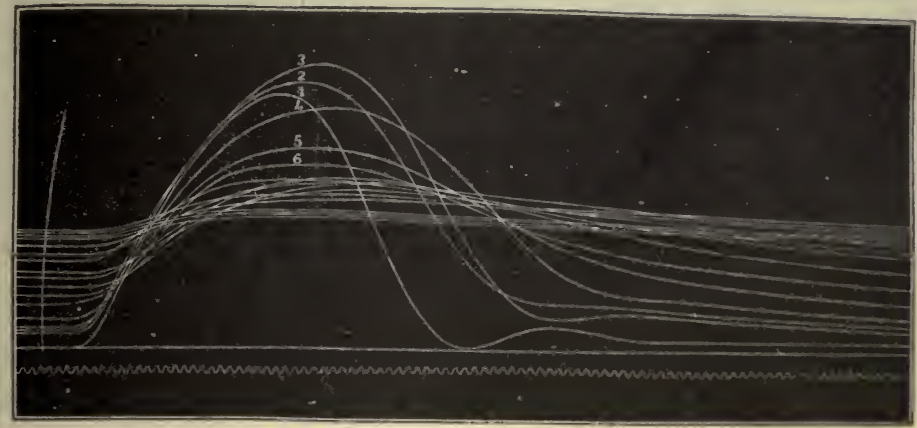

FIG. 70. Muscle curves showing fatigue in consequence of repeated stimulation. The first six contractions are numbered, and show the initial increase of the first three contractions. (BRoDIE.)

muscle to excitation, one process causing the active shortening and the other the relaxation. (It must be noted that this is not the same as saying that the lengthening is an active process, a statement negatived by the behaviour of a muscle when caused to contract on mercury.) He suggested that the disintegration associated with activity might be conceived as occurring in two stages: the first resulting in the production of sarcolactic acid and the active shortening of the muscle; the second in the further conversion of the acid into $\mathrm{CO}_{2}$, with a consequent relaxation. A retardation of this second phase would cause the prolonged curve with ' contraction remainder ' observed in a fatigued muscle. We shall return to this point when discussing the chemical and heat changes which accompany contraction.

If left to itself, the muscle which has been exhausted by repeated stimulation will recover. The recovery is hastened by passing a streum of blood, or even of salt solution, through the blood-vessels of the muscle. Recovery in a muscle outside the body is never complete.

The phenomena of fatigue probably depend on two factors :

(1) The consumption of the contractile material or the substances available for the supply of potential energy to this material.

(2) The accumulation of waste products of contraction. Among these waste products the lactic acid is probably of great importance. Fatigue I may be artificially induced in a muscle by 'feeding' it with a dilute solution of lactic acid, and again removed by washing out the muscle with normal saline solution containing a small percentage of alkali. 


\section{THE ACTION OF SALTS}

The action of sodium salts on muscle is of considerable interest. We are accustomed to use a 0.6 per cent. solution of $\mathrm{NaCl}$ as a 'normal fluid' to keep muscle preparations moist. If, however, the solution be made with distilled water, it has a distinctly excitatory effect upon the muscle,

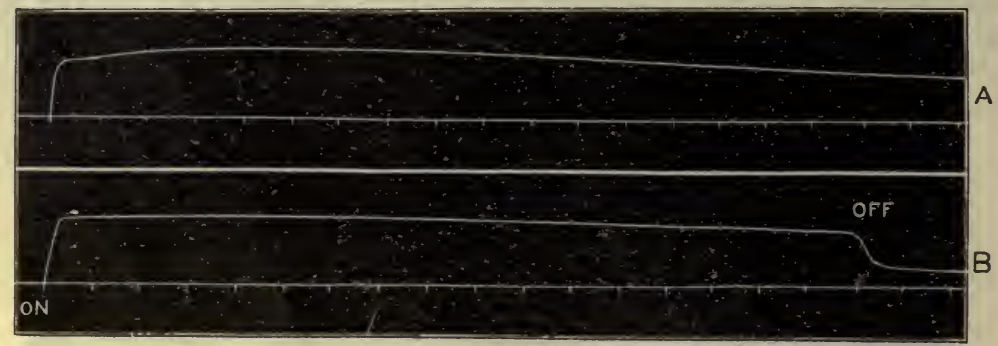

FIG. 71. A: Tracing of the contraction of a frog's sartorius, poisoned with veratrin, in response to a momentary stimulus. The time-marking indicates seconds.

B. Tetanic contraction of normal sartorius in response to rapidly interrupted stimuli. (The duration of the stimulus is indicated by the words ' on ' and 'off.') It will be noticed that the two curves are practically identical. (Miss Buchanan.)

so that single induction shocks may cause tetaniform contractions. The same excitatory effect is still better marked with solutions of $\mathrm{Na}_{2} \mathrm{CO}_{3}$. If a thin muscle, such as a frog's sartorius, be immersed in a solution containing 0.5 per cent. $\mathrm{NaCl}, 0.2$ per cent. $\mathrm{Na}_{2} \mathrm{HPO}_{4}$, and 0.04 per cent. $\mathrm{Na}_{2} \mathrm{CO}_{3}$ (Biedermann's fluid), the muscle enters into a series of frequent contractions,

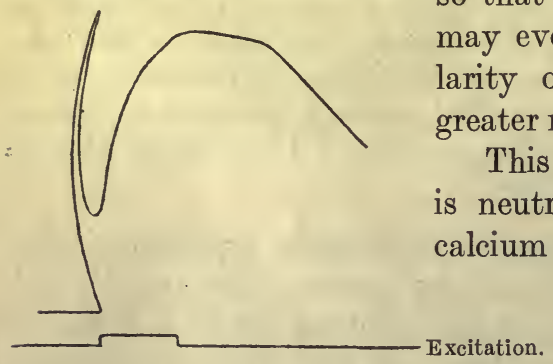

uruhurharaharahurar Seconds.

FIG. 72. Tracing of the contraction of a muscle poisoned by the injection of a strong solution of veratrin, showing the double contraction due to unequal poisoning of different fibres. (BIEDERMANN.)

so that it may wriggle from side to side, or may even 'beat' for a time with the regularity of heart-muscle, though at a much

This excitatory action of sodium salts is neutralised by the addition of traces of calcium salts. Hence the normal saline used in the laboratory should always be made with tap water, containing calcium salts.

Potassium salts, although forming so important a constituent of the ash of muscle, act as muscle poisons, quickly and permanently destroying its irritability. If a muscle be transfused with normal fluids containing minute traces of potassium salts, it.at once shows all the signs of fatigue, signs which may be removed by washing out the potassium salts by means of 0.6 per cent. $\mathrm{NaCl}$ solution. It is possible that the setting free of potassium salts may be one of the factors involved in the development of the normal fatigue of muscle. 


\section{THE ACTION OF DRUGS}

Of the drugs that have a direct action on muscle, the most remarkable is veratrin, which causes an excessive prolongation of a muscular contraction (produced by a single stimulus). Thus the 'twitch' of a muscle poisoned with veratrin may last fifty or sixty seconds, instead of the normal one-tenth of a second (Fig. 71).

Barium salts have a similar, though less marked effect.

In order to carry out the poisoning with veratrin, very weak solutions ( 1 in 100,000 or 1 in 1,000,000 of normal saline) should be used and the muscle exposed to its action for some time. We get then on a single stimulus a response lasting many seconds and exactly similar in height and form to a tetanus obtained by discontinuous stimulation. If stronger solutions be used, the action of the drug is apt to affect the fibres unequally, so that we may have a sharp normal twitch preceding the prolonged contraction (Fig. 72). If the muscle be excited several times immediately after the prolonged contraction has passed away, it responds with twitches like those of a normal muscle, but if allowed to rest a few minutes, stimulation is again followed by the peculiar long-drawn-out contraction. 


\section{SECTIUN V}

\section{CHEMICAL CHANGES IN MUSCLE}

\section{CHEMICAL COMPOSITION OF VOLUNTARY MUSCLE}

Voluntary muscle consists of elongated cells, the muscle fibres being embedded in a connective tissue framework, and, as in all cellular tissues, proteins form its chief chemical constituents. The contents of the fibres are semi-fluid and can be expressed from the finely divided muscle as a viscous fluid known as muscle plasma.

Muscle plasma is obtained in the following way. The living muscle of frogs is frozen, minced with ice-cold knives and pounded in a mortar with four times its weight of sand containing $\cdot 6$ of common salt. The mixture is then thrown on to a filter kept at $0^{\circ} \mathrm{C}$. when an opalescent fluid filters through. The filters soon become clogged and therefore must be frequently changed, and their temperature must not be allowed to rise above $2^{\circ}$ to $3^{\circ} \mathrm{C}$.

If the temperature of the muscle plasma be allowed to rise, clotting takes place, the clot later on contracting and squeezing out a serum, as is the case with blood plasma.

The muscle-plasma is neutral or slightly alkaline. When coagulation takes place, however, it becomes distinctly acid, and this acidity is due to the formation of sarcolactic acid in the process. Arguing chiefly from analogy with the blood-plasma, the muscle-plasma has been said to contain a body, myosinogen, which is converted when clotting takes place into myosin.

The exact nature of the proteins in muscle-plasma, as well as of the protein constituent of the clot, which we have called myosin, is still a subject of debate. Kühne, to whom we owe our first acquaintance with muscle-plasma, described the clot as consisting of myosin, a globulin, soluble in 5 per cent. solutions of neutral salts, such as $\mathrm{NaCl}$ or $\mathrm{MgSO}_{4}$, precipitated by complete saturation with $\mathrm{MgSO}_{4}$, and coagulated on heating to $56^{\circ} \mathrm{C}$. In the muscle-serum, obtained after separation of the clot, he found three proteins, one coagulating at $45^{\circ} \mathrm{C}$., one he called an albumate (i.e. a derived albumen or metaprotein), and the third coagulating about $75^{\circ} \mathrm{C}$., and apparently identical with serum albumen. Halliburton extended these researches to the muscles of warm-blooded animals. He described four proteins as existing in muscle-plasma, of which two, paramyosinogen and myosinogen, gave rise to the clot of myosin.

In no case, however, is it possible entirely to dissolve up the clot when once formed, and it seems that the so-called solution in dilute salt solutions was merely an extraction of still soluble protein in the meshes of the clot. Von Fürth has shown that if the muscles of a mammal are washed free of adherent lymph and blood, the plasma obtained by extraction with normal salt solution contains only two proteins. These proteins are extremely unstable, and are gradually transformed on standing into insoluble 
protein, giving rise to a precipitate in dilute solutions, or forming a jelly-like clot in strong solutions. The properties of these proteins may be summarised as follows: $50^{\circ} \mathrm{C}$., precipitated by half saturation Halliburton). A globulin, coagulating at about $47^{\circ}-$ slowly in solution, rapidly on preipith ammoniumsulphate or on dialysis. Transformed

(2) Myogen (myosinogen of Halliburton) an insoluble protein. myosin fibrin.

that it is not precipitated by dialysis. Coagul. A protein allicd to the albumens in slowly into an insoluble protein, myogen soluble stage called soluble myogen fogen fibrin, but passes through an intermediate $40^{\circ} \mathrm{C}$, , being instantly converted at hedy coagulates on heating to It does not seem that any ferm at this temperature into insoluble myogen fibrin. may represent by the following schema:

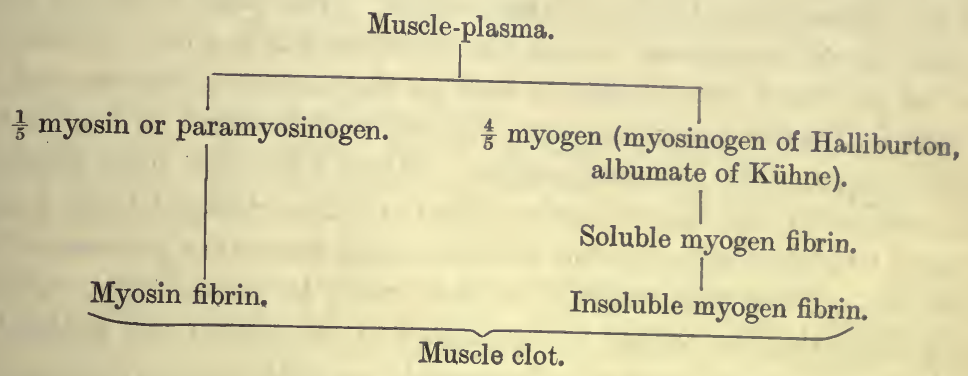

Soluble myogen fibrin, which in mammalian muscle-plasma forms only on standing, exists apparently preformed in frog's muscle. Hence the instantaneous clotting of frog's muscle-plasma on warming to $40^{\circ} \mathrm{C}$.

The residue left after the expression of the muscle-plasma consists chiefly of connective tissue, sarcolemma, and nuclei, and as such contains gelatin (or rather collagen), mucin, nuclein, and adherent traces of the proteins of the muscle-plasma itself.

The muscle-selum contains the greater part of the soluble constituents of muscle.

OTHER CONSTITUENTS OF MUSCLE. A number of other substances are found in muscle in small quantities. those which are soluble being contained to a great part in the muscle serum. It will suffice here to enumerate the chief of these.

(a) Colouring-matter. All red muscles contain a considerable anount of hæmoglobin. A special muscle pigment allied to hæmoglobin has been described by MacMunn as myohæmatin. The only evidence for its existence is spectroscopic.

(b) Nitrogenous extractives. Of these, the most important is creatine $\left(\mathrm{C}_{4} \mathrm{H}_{9} \mathrm{~N}_{3} \mathrm{O}_{2}\right)$ of which 0.2 to 0.3 per cent. may be found in muscle. Its significance will be the subject of consideration later. Other nitrogenous bodies occurring in smaller quantities are hypoxanthine, xanthine, and traces of urea and amino-acids.

(c) Non-nitrogenous constituents.

Fats, in variable amount.

Glycogen. This substance is invariably found in healthy muscle. Fresh skeletal muscle contains about 1 per cent. In the embryo the muscles may contain many times this quantity of glycogen.

Glucose is present in fresh muscle in minimal quantities, about 01 per cent.

When muscle is allowed to stand, especially in a warm place, the glycogen undergoes partial conversion into glucose, so that the latter increases at the expense of the former. 
Inosit $\left(\mathrm{C}_{6} \mathrm{H}_{12} \mathrm{O}_{6} 2 \mathrm{H}_{2} \mathrm{O}\right)$ or 'muscle sugar' occurs in minute traces in muscle. It does not belong to the group of carbohydrates at all, being a hexahydrobenzene. It is nonfermentable and does not rotate polarised light nor does it reduce Fehling's solution. Its significance is quite unknown.

(d) Inorganic constituents. Muscle contains about 75 per cent. of water. Ash forms 1 to 1.5 per cent. and consists chiefly of potassium and phosphoric acid, with traces of calcium, magnesium, chlorine and iron.

\section{RIGOR MORTIS}

All muscles after removal from the body, or if left in the body after general death, lose after a time their irritability, and this loss is succeeded by the phenomenon known as rigor mortis. The muscle, which was pre: viously flaccid, contracts, though the shortening is not very powerful and can be prevented by a moderate load on the muscle. Whereas the living muscle is translucent, supple, and extensible, it becomes in the process of rigor opaque, rigid and inextensible. When rigor has been established, the reaction of the muscle is also found to have changed from a slightly alkaline to a distinctly acid one, the acid being due to the presence of sarcolactic acid. From this condition of rigor there is no recovery. There can be no doubt that the change in consistence of the muscle and probably also its shortening in rigor are due to the coagulation of the muscle proteins. Both changes can be imitated by heating the muscle, as is indicated by Brodie's experiments. This observer found that, if a living muscle be lightly loaded and then warmed very gradually, a series of stages in the heat-contraction could be distinguished corresponding to the coagulation temperatures of the different proteins described by von Fürth in muscle-plasma. It seems likely, however, that the main contraction at all events, that which comes on spontaneously after death or immediately on warming the muscle to $45^{\circ} \mathrm{C}$., has another component. In the coagulation of the separated muscleproteins there is no evidence of any appreciable formation of sarcolactic acid, whereas the formation of this substance seems to bear an important relation to the occurrence of rigor. Thus after severe muscular fatigue, as in hunted animals, where there has already been a considerable formation of the waste products of muscular contraction, rigidity may come on almost immediately after death. If a thin living muscle be plunged into boiling water, it undergoes instant coagulation, but no chemical change. The reaction of the scalded muscle, like that of fresh muscle, is slightly alkaline to litmus. No sarcolactic acid or carbonic acid is produced. On the other hand, in surviving muscle, after the cessation of the circulation, there is a steady formation of lactic acid which accumulates in the muscle. The actual coagulation of the muscle-proteins occurring in rigor is largely, if not entirely, determined by the increasing acidity of the muscle thereby produced. In fact, it is the production of the acid which causes the onset of rigor, and not the rigor which causes a sudden formation of acid. Hence if the accumulation of lactic acid be prevented by perfusing the muscle with salt solutions, the onset of rigor may be postponed indefinitely, and the muscle may begin to putrefy without having undergone rigor. 
THE PRODUCTION OF LACTIC ACID IN SURVIVING MUSCLE

The lactic acid formed in muscle (sarcolactic acid) is a physical isomer of the lactic acid formed in the fermentation or souring of milk. They both have the formula $\mathrm{CH}_{3} \cdot \mathrm{CH}(\mathrm{OH}) \cdot \mathrm{COOH}$, i.e. they are ethylidene lactic acids. The lactic acid of fermenta. tion is optically inactive ; sarcolactic acid rotates polarised light to the right; while a third isomer which is lævo-rotatory is produced by the action of various bacilli and vibriones on cane sugar. The sarcolactic acid can be extracted from the muscle by means of alcohol.

It was pointed out by Hopkins and Fletcher that most of the methods previously used for the extraction of lactic acid from muscle caused the formation of lactic acid in this tissue. To obviate this difficulty, they adopted the precaution of cooling the muscles before cutting them out of the body and then dropping them into alcohol cooled to $0^{\circ} \mathrm{C}$. While in this ice-cold alcohol they were finely divided with scissors and then pounded up in a cooled mortar. In this way the tissue was destroyed at a temperature which did not allow of the changes responsible in surviving muscle for the production of lactic acid. It is generally separated in the form of the zinc sarcolactate, by boiling its partially purified solution with zinc carbonate. Its presence may be tested for by means of Uffelmann's reagent, which is made by the addition of ferric chloride to dilute carbolic acid. The purple solution thus produced is at once changed to yellow by the addition of even traces of lactic acid.

A much more definite colour reaction for lactic acid has been introduced by Hopkins. The test is carried out in the following way. About 5 c.c. of strong sulphuric acid are placed in a test-tube together with one drop of saturated solution of copper sulphate, which serves to catalyse the oxidation that follows. To this mixture a few drops of the solution to be tested are added, and the whole well shaken. The test-tube is now placed in a beaker of boiling water for one or two minutes. The tube is then cooled under a water-tap, and two or three drops of a very dilute alcoholic solution of thiophene (ten to twenty drops in 100 c.c.) are added from a pipette. The tube is replaced in the boiling water and the contents immediately observed. If lactic acid is present the fluid rapidly assumes a bright cherry red colour, which is only permanent if the tube be cooled the moment after its appearance.

A study of the lactic acid content of muscle by Fletcher and Hopkins, using the precautions described above, has shown that fresh muscle contains only minimal amounts of lactic acid, the quantity being smaller, the greater the care that is taken to avoid injury to the muscle and to keep its temperature low until sufficient time has elapsed for its vital chemical processes to be destroyed by the action of the cold alcohol. If the muscle be left in the body after the death of the animal or be excised, a steady formation of lactic acid takes place, which is more rapid in the first few hours after death, but continues until the muscle passes into rigor. With the complete onset of rigor, frog's muscles are found to contain about 44 per cent. lactic acid. After this time the amount does not increase. The onset of rigor and the rate of production of lactic acid are quickened if the muscle be kept warm. It is interesting to note that the amount of lactic acid found in rigid muscle is almost invariable whatever the previous history of the muscle. Thus, if the muscle be finely minced and then extracted with cold alcohol, it is found to contain about $\cdot 2$ per cent. lactic acid. If, however, it be allowed to stand after mincing, there is a slow production of lactic acid up to the maximum 4 per cent. Again, a muscle which has been tetanised to exhaustion contains about $\cdot 2$ per cent. lactic acid. When allowed to undergo rigor, the amount rises to about 44 per cent. 
It has long been known that the onset of rigor is associated with an evolution of carbonic acid by the muscle. Fletcher has shown that this increased output of carbonic acid by a surviving muscle is due simply to the driving off of carbonic acid from the carbonates in the muscle as a result of the production of lactic acid. There is no evidence of a new formation of carbonic acid in the dying muscle as a result, for instance, of oxidative changes.

\section{THE CHEMICAL CHANGES WHICH ACCOMPANY ACTIVITY}

The principle of the conservation of energy teaches us that the energy of the contraction of muscle must be derived from chemical changes, probably processes of decomposition and oxidation, occurring in the muscle itself. In seeking out the nature of these changes three methods are open to us :

(1) We can examine the changes in the muscle itself, avoiding so far as possible reintegrative changes by working on excised muscles.

(2) We can investigate the changes in the medium surrounding the muscle. Muscle may be exposed in a vacuum or in a confined space of air, and its gaseous interchanges during rest and activity compared. Or we may lead a current of defibrinated blood through excised muscles, and determine the change in the composition of the blood in passing through the muscle under various conditions.

(3) A method which, although apparently complex, has rendered the utmost service to the physiology of muscle is to use the changes in the total metabolism of the animal during rest and muscular work as a clue to the muscular metabolism itself. In such a case the respiratory exchanges of the animal are determined (viz. its oxygen intake and its $\mathrm{CO}_{2}$ output), and the urine and fæces are carefully analysed, in order to judge of the action of muscular work on the carbon and nitrogen metabolism of the body.

By the third of these methods we may show that muscular exercise increases largely the intake of oxygen and the output of carbon dioxide by the body. No corresponding changes are found in the nitrogenous metabolism, so that ultimately we may regard the energy of the muscular contraction as derived from the oxidation of the food-stuffs and especially the carbohydrates. That it is this class of bodies which is the immediate, or at any rate the most accessible, source of muscular energy, is shown by the rise in the respiratory quotient which occurs during muscular exercise. When the exercise is moderate there is no evidence of the production of any other substance than carbon dioxide as a result of the muscular metabolism, but with violent exercise it can be shown that lactic acid is not only produced in the muscle, but appears in the blood and is excreted in the urine.

It has been shown by Ryffel that no:mal urine contains $3-4 \mathrm{mg}$. of lactic acid per hour. In one experiment the urine passed after running one third of a mile with the production of severe breathlessness contained $454 \mathrm{mg}$. of lactic acid. In another experiment blood obtained before running contained $12.5 \mathrm{mg}$. per 100 c.c., and that obtained immediately after running one third of a mile contained $70 \mathrm{mg}$. lactic acid per 100 c.c. On the other hand, the examination of the urines of competitors in a twenty-four hours track walking race showed no increase in the output of lactic acid above the normal $4 \mathrm{mg}$. per hour. 
The appearance of lactic acid thus seems to be attendant on a relative deficiency in the oxygen supply to the contracting muscle. The same conclusion may be drawn from experiments made many years ago by Araki, in which lactic acid was observed in quantities in the urine in cases where the oxidative processes of the body were interfered with by $\mathrm{CO}$ poisoning.

Similar results are obtained when we investigate the chemical changes accompanying the contraction of excised muscles of the frog. If frogs' muscle be hung up in an atmosphere of nitrogen and stimulated repeatedly with single shocks, it will give a series of contractions gradually diminishing in size (v. p. 209). After a time the muscle is completely fatigued, and no further response can be elicited on stimulation. On now examining it, it is found to be acid in reaction and to contain about $\cdot 2$ per cent. lactic acid. There is no evidence that under these conditions any carbonic acid is produced, though a certain amount may be liberated in consequence of the acidification of the muscle. Almost the same results are obtained when the muscle is stimulated in ordinary atmospheric air. The penetration of oxygen from the air through the body of the muscle is so slow that all the muscle except the thin layer on the surface may be regarded as cut off from the action of oxygen. By hanging the muscle, especially a thin muscle such as the sartorius, in an atmosphere of pure oxygen, the results are quite different. In the first place the muscle does not fatigue so soon. Moreover, a muscle which has been stimulated to exhaustion in an atmosphere of nitrogen, if restored to one of pure oxygen, will rapidly recover its power of contraction. In pure oxygen no lactic acid is produced, and a muscle stimulated to exhaustion contains very little more lactic acid than does resting muscle. On the other hand, the intake of oxygen and the output of carbonic acid by the muscle is increased at each contraction. We thus find that a muscle during contraction may produce lactic acid or carbonic acid according as oxygen is absent or present. In both cases contraction takes place apparently normally, but fatigue supervenes much more rapidly in the absence of oxygen. The question arises whether we should regard the formation of lactic acid and carbonic acid as alternative processes, or whether lactic acid is first formed and is then removed under the action of oxygen, undergoing partial or complete oxidation to carbonic acid in the process. The evidence is distinctly in favour of the second hypothesis. Thus Hopkins and Fletcher have found that muscle possesses in itself a chemical mechanism for the removal of lactic acid. If a fatigued muscle be exposed to pure oxygen, 30 per cent. of the lactic acid present in the muscle may disappear within two hours and 50 per cent. within six to ten hours. Thus, even apart from the circulation which of course would remove large quantities of any lactic acid which might be produced in the muscles, these can deal with this metabolite locally. It has been found that a muscle may be fatigued several times and then placed in oxygen to recover, so that lactic acid is produced and removed also several times. If at the end the muscle be allowed to undergo rigor, it is found to contain $\cdot 4$ per cent. lactic acid, i.e. exactly the same amount as if it had 
given no contractions at all. Fletcher and Hopkins interpreted this result as showing that under the influence of oxygen, lactic acid is put back into the precursor from which it arose, and would assume that part of the lactic acid is completely oxidised to carbonic acid and water, the energy so evolved being employed in the building up of the precursor from the rest of the lactic acid. On the other hand it is possible that the lactic acid produced in the initial stage of contraction may be under normal circumstances completely removed by oxidation, and that the energy or part of the energy so made available is used to build up some precursor substance, not out of the lactic acid, but out of the glycogen already present in the muscle (Parnas). It is certain that prolonged activity of muscle, especially in the presence of oxygen, may be associated with a diminution in the glycogen store of the muscle. We cannot, however, discuss this question further without reference to the total energy changes in muscle contracting with or without oxygen, and the clue to these changes is given by a study of the heat production in muscle. 


\section{SECTION · VI}

\section{THE PRODUCTION OF HEAT IN MUSCLE}

THE experience of everyday life teaches us that muscular exercise is associated with increased production of heat. Thus a man walks fast on a frosty day to keep himself warm. In large animals the production of heat in muscular contraction can be easily shown by inserting the bulb of a thermometer between the thigh muscles, and stimulating the spinal cord. The rise of temperature produced in this way may amount to several degrees. This observation is confirmed when we investigate the contraction of an isolated muscle outside the body. If a frog's muscle is tetanised, its temperature rises from $0 \cdot 14^{\circ}$ to $0 \cdot 18^{\circ} \mathrm{C}$, , and for each single twitch from $0.001^{\circ}$ to $0.005^{\circ} \mathrm{C}$.

It is evident that such small changes in temperature as $0.001^{\circ}$ cannot be estimated by ordinary thermometric methods. By converting a heat change into an electrical change, however, we can estimate differences of temperature with much greater accuracy and fineness than by the use of a thermometer. Two main principles are employed in measuring temperature by electrical methods. The thermoelectrical method depends on the fact that, when the junctions of a circuit made of two metals are at different temperatures, a current of electricity generally flows through the circuit. This current can be measured by means of a galvanometer, and is proportional to the difference of temperature between the two junctions. Thus in the circuit (Fig. 73)

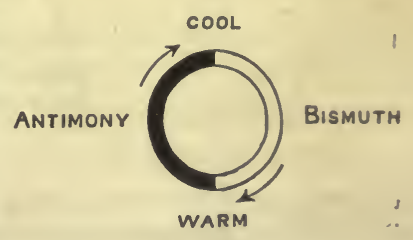

Fig 73. composed of two metals, antimony and bismuth, if the upper junction be cooled, there will be a current flowing from antimony to bismuth in the direction of the arrow, and this current will within limits be proportional to the difference of temperature.

To measure the production of heat during muscular contraction, a small flat thermopile (containing four or six elements composed of iron and German silver, or copper and 'constantan') is fixed with one of its ends between two frog's gastrocnemii. Another exactly similar pile, but reversed, is placed between two other gastrocnemii, which are kept resting and at a perfectly constant temperature. So long as the two piles are at the same temperature no current flows; but, with a sensitive galvanometer, the slightest difference of temperature, such as that caused by the contraction of one pair of muscles, at once causes a deflection of the galvanometer, the extent and direction of which enable us to estimate exactly the seat and amount of heat produced.

When we are using such delicate detectors of temperature difference, we are met by the difficulty that every junction in the circuit tends to become the seat of an electromotive force in consequence of slight changes of temperature due to currents of air, \&c. It is therefore advisable to use a plan adopted by Blix, of placing all the apparatus, the muscle included, within the galvanometer case. The arrangements of such an experiment as employed by A. V. Hill are shown in the diagram (Fig. 74). 
In this instrument the junction of copper with the alloy constantan constitutes a thermo-electric couple. The magnet and mirror chamber is entirely separated off from the rest of the instrument by the walls of the tube containing the magnet. The grooves are usually filled with plasticine, and into them fit the edges of an outer case of brass constituting the walls of the muscle chamber. The inside of this case is lined with wet blotting-paper. The copper coil consists of many more turns than are shown in the figure : its ends, $\mathbf{A A}$ and $\mathbf{B B}$, are separated by the celluloid plate, and are connected by the constantan plug; the points where the copper meets the constantan constitute the thermo-electric junctions. The tube containing the magnet hangs

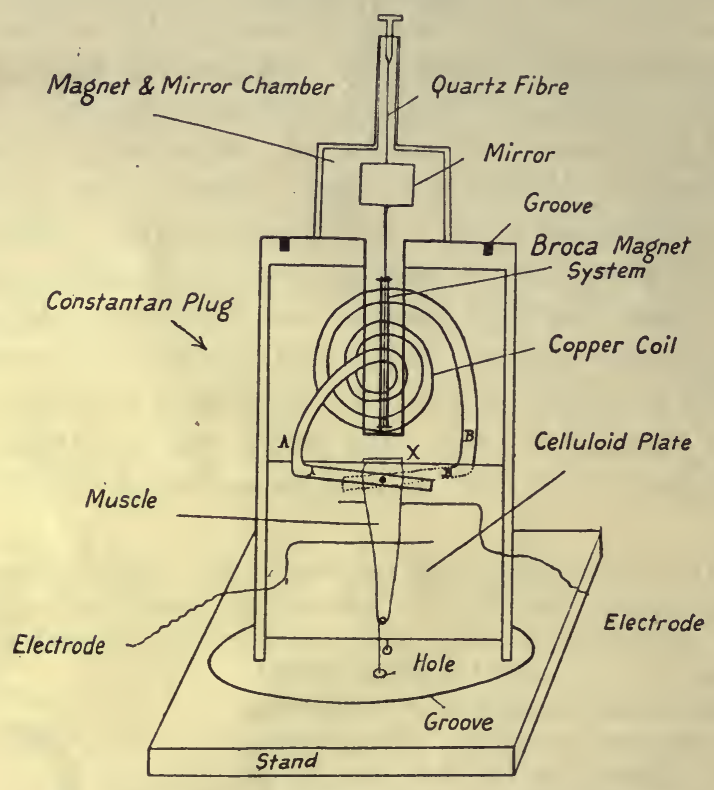

FIG. 74.

down through holes bored in the broad copper coil. The two semimembranosus muscles ride astride of the celluloid plate, one in contact with each end of the constantan plug. The small pieceof bone at their upper ends which has been left connecting thetwo muscles is placed exactly on the top of the celluloid plate at $\mathrm{x}$ and held in position by a clamp (not shown in the figure). The copper terminals of the coil are coated with celluloid varnish to prevent short circuiting of the thermo-electric currents, and to prevent poisoning of the muscles. Each muscle is in contact with a pair of electrodes, made of fine platinum wire; the muscle lies over the upper and beneath the lower of these electrodes, as shown in the figure. The tendons at the lower ends of the muscles are tied to silk threads which pass through holes in the base of the instrument. These are then attached to recording levers which write on a drum beneath the table. When all is ready three heavy soft-iron cylinders are placed over the instrument : the latter is screwed to a wooden block which is fixed to a thick iron plate attached to the table. In the cylinders holes are bored to admit and let out after reflexion the light from a Nernst lamp. The lamp, which is about three metres away, shines upon the mirror, and a line in it, after reflexion, is focussed on to the screen, which is also three metres away. The line is brought on to the scale by the small field exerted by a control magnet placed outside the cylinders at a suitable position on the table : its position may be read easily to half a millimetre, and the movement due to a twitch is usually of the order of $80 \mathrm{~mm}$. These soft-iron cylinders cut off entirely all external magnetism sufficient to cause harmful disturbance during an experiment. They lower very largely the strength of the constant external field in which the magnet lies, and leave it chiefly 
supported in any position by the quartz fibre. Thus all the movements set up in the magnet by the thermo-electric currents are working against little more than the torsion of a quartz fibre only $6 \mu$ thick. This explains the great sensitivity of the
instrument.

A second method depends on the fact that rise of temperature increases the resistance of a wire to the passage of an electric current. A current detector consists of a Emall grid of fine platinum wire which is placed against the muscle between two muscles.
This grid is then made one limb of a Wheatstone's bridge (Fig. 74A). A small current is passed through the circuit, and the resistances are so adjusted that no current flows through the galvanometer. Any alteration in temperature of the grid will alter the balance of the resistance and will cause a current to flow through the galvanometer in a direction which will vary according as the resistance in the grid is increased or diminished. It

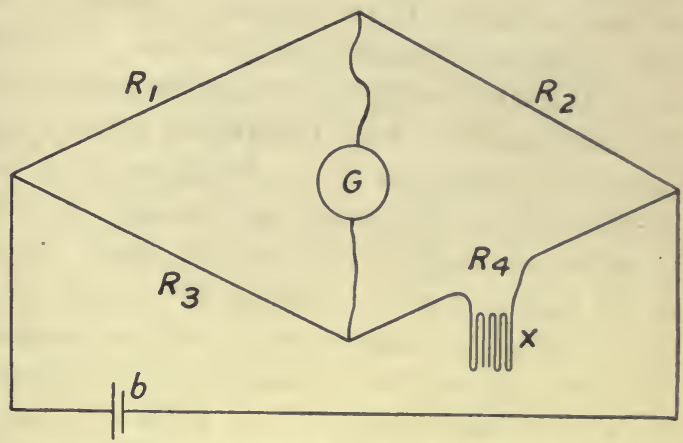

Fig. 74A. Arrangement of apparatus for measuring small differences of temperature. is possible to calibrate the arrangement so that a deflection of the galvanometer over one degree will correspond to a certain fraction of a degree of difference in temperature of the grid. This method is employed in Callender's recording thermometers, and has been made by Gamgee the basis of an arrangement for the continuous record of the temperature of the human body.

Most of the earlier work on the development of heat in muscle had as its leading motive the discovery of the relation between the heat produced and the work performed by a muscle under varying conditions of load. When a loaded muscle contracts, however, it is not easy to analyse its mechanical conditions, since part of the shortening of the muscle during contraction can be regarded merely as a recovery from the condition of extension induced by the weight, and the amplitude of the excursion may be largely conditioned by the inertia of the weight moved. Working on these lines, Heidenhain discovered that the heat production in muscle during contraction is not an invariable quantity, but varies according to the condition of the muscle and especially according to the tension developed in it during contraction. It was therefore at its maximum under isometric conditions when it was not allowed to shorten at all during contraction. As we have seen, the muscle changes, as the result of excitation, from a body having certain elastic properties to one having other elastic properties. The whole energy of the contraction is converted for a short period into a state of tension which can be used to do work by raising a weight. If it be not allowed to shorten, the state of tension passes off and the whole energy which has been set free must appear as heat. The potential energy developed in a muscle twitch is approximately equal to $\frac{1}{6} \mathrm{Tl}$, where $\mathrm{T}$ is the tension developed and $l$ the length of the muscle, and it is this amount which must be compared with the heat production measured in the muscle 
by one of the methods described. A. V. Hill has shown that the heat production in a contracting skeletal muscle occurs in two phases, a rapid production of heat which apparently is synchronous with the contraction itself, and a slow production of heat which continues for some time after the muscle has relaxed. The second phase of heat production depends on the presence of oxygen, and is observed at its best when the muscle is kept in pure oxygen. If the muscle be allowed to contract in nitrogen only the initial heat production is observed. The heat production of the second phase is stated by Hill to be approximately equal to that in the first phase. These results have been interpreted as showing that the initial change in muscular contraction is the development of lactic acid. The appearance of this lactic acid in some way changes the muscle and sets up potential energy at the surface of its ultimate fibrils, which will result in a shortening of the muscle if any movement of its ends be allowed. A comparison of the energy of the tension set up with the actual heat evolved in the initial stage when a muscle is not allowed to contract shows that the two quantities are approximately equal. In a series of experiments Hill found that the ratio $\frac{1}{6} \mathrm{Tl}: \mathrm{H}$ in the sartorius muscle under low initial tensions and in comparatively weak contractions approximated to the value 1 , the mean value being 91 . Under high initial tensions and in strong contractions of the sartorius muscle, it is lower, being roughly from 0.4 to $0 \cdot 6$. $\mathrm{He}$ concludes from this that under certain conditions the initial process of contraction consists largely, if not entirely, of the liberation of free potential energy manifested as tension in the muscle. This potential energy may be used for the accomplishment of work or for the production of heat. The efficiency of the initial stage of contraction is therefore almost 100 per cent.

If, however, a muscle is to go on contracting without rapidly showing signs of fatigue, it must be kept in oxygen, so that the processes of replacement or of removal of the lactic acid may take place. Under these circumstances there is a further evolution of heat after the contraction, equal to that set free during the initial stage. So that the total efficiency of a muscle kept in oxygen would not be more than 50 per cent.

This is assuming that the process of oxidation of the lactic acid and its replacement in whole or in part in the muscle molecule is completely carried out during the time of the observation. It is improbable that such is the case, and it seems possible that the evolution of heat during the so-called recovery stage of the muscle has been under-estimated.

If a series of observations of the heat production and tension developed during isometric contractions be made with varying initial tension on the muscle, it is found that while the ratio of tension developed to heat produced is approximately constant, both these quantities first increase and then finally diminish. The optimum of the heat production in some experiments seems to fall later than the optimum of the tension developed. It seems probable that in this case the essential factor is not so much the tension exerted on the muscle previous to its excitation, but the length of the muscle fibre during the time that it is excited. The longer the muscle fibre, within 


\section{PRODUCTION OF HEAT IN MUSCLE}

limits, when it is excited, the greater the tension and the greater the heat production developed, i.e. we may assume that increased length of muscle fibre increases the chemical changes, ensuing on excitation, which are responsible both for the development of mechanical energy and the production of heat. The significance of these results for the essential nature of muscular contraction we shall discuss in a later chapter, 


\section{SECTION VII}

\section{ELECTRICAL CHANGES IN MUSCLE}

IF a current from a battery be passed between two plates of platinum immersed in acidulated water or salt solution, electrolysis of the water takes place, bubbles of oxygen appearing on the positive plate (anode), and bubbles of hydrogen on the negative plate (cathode). If now we remove the battery, and connect the two plates (electrodes) by wires with a galvanometer, a current passes through the galvanometer and water

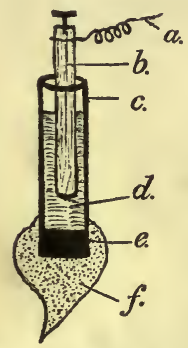

Fig. 75. Diagram of nonpolarisable electrode.

$a$,covered wire; $b$, amalgamated zinc rod; $c$, glass tube; $d$, saturated $\mathrm{ZnSO}_{4}$ solution; $e$, plug of zinc sulphate clay; $f$, plug of normal saline clay. in the reverse direction to the previous battery current. This current is called the polarisation current, and is due to the electrolysis of the water that has taken place. The vessel in which the electrodes are immersed has in fact become a galvanic cell, the platinum covered with oxygen bubbles being the positive element, and that covered with hydrogen bubbles the negative element. Exactly the same process of electrolysis or polarisation takes place when we pass currents through the tissues of the body by means of metallic electrodes.

Hence before we can study accurately the delicate electrical changes that may occur normally in living tissues, it is necessary to have some form of electrodes in which this polarisation will not occur. The 'non-polarisable' electrodes which are most generally used for this purpose are made in the following way. A glass tube (Fig. 75) is closed at one end with a plug of kaolin made into a paste with a saturated solution of zinc sulphate. The rest of the tube is filled with a similar solution. Dipping into the zinc sulphate solution is a rod of pure zinc, amalgamated. Just before use, a plug of china clay made with normal saline solution is put on the end of the tube, so as to effect a connection between the zinc sulphate clay and the nerve or muscle which it is desired to stimulate or lead off. In these electrodes there is no contact of metals with fluids that can produce dissimilar ions (e.g. hydrogen or oxygen) at the surface of contact, and hence they may be regarded as practically non-polarisable. A more convenient form is that employed by Burdon Sanderson, in which the glass tube is bent into a $\mathrm{U}$ (Fig. 76). The mouth of the tube is closed by a smaller glass tube plugged with clay, and bearing a plug of normal saline clay.

In such electrodes the conduction of the current through the nerve or muscle to the metallic

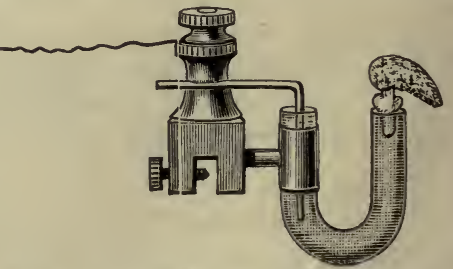

FIG. 76. U-shaped non-polarisable electrodes. part of the circuit may be represented as shown on the opposite page (see Fig. 77).

If a muscle such as the sartorius be removed from the body, and two nonpolarisable electrodes connected with a delicate galvanometer be applied to two points of its surface, there will be a deflection of the mirror attached to the galvanometer, showing the presence of a current in the muscle from 
the ends to the middle, and in the external circuit from the middle (or equator) to the ends. It was formerly thought that this current was always present in all normal muscles, and it was spoken of as the 'natural muscle current' ; the muscle was said to be made up of a series of electromotive molecules, the equator of each molecule being positive to the two poles (du Bois Raymond). It has been conclusively shown, however (by Hermann and others), that this

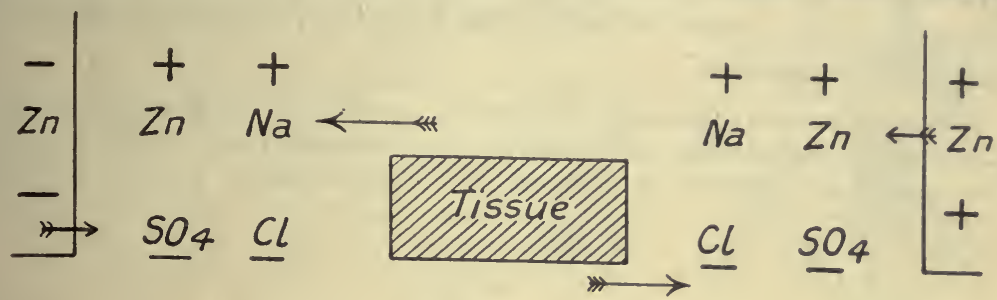

FIG. 77.

current of resting muscle is not a natural current at all, but is due to the effects of injury in making the preparation. The less the preparation is injured, the smaller is the current to be obtained from it, and in some contractile tissues, such as the heart, there may be absolutely no current during quiescence.

Hermann describes the fact of the existence of currents of rest thus : "In partially injured muscles every point of the injured part is negative towards the points of the uninjured surface." Fig. 78 shows the direction of the current in a muscle with two cut ends When the whole muscle is quite dead, this current of rest, or 'demarcation current' (Hermann), disappears. The current is due to the electrical differences at the junction of living and dying (not dead) tissue. If the sartorius of the frog be cut out and immersed for twenty-

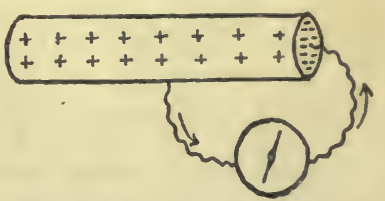

FiG. 7z. Current of rest. four hours in 0.6 per cent. $\mathrm{NaCl}$ solution made with tap water (i.e. containing lime), all the injured fibres die, and the uninjured fibres are then found to be iso-electric and therefore currentless.

The existence of this current may be demonstrated without using a galvanometer. If the nerve of a sensitive muscle-nerve preparation ( $a$, Fig. 80$)$ be

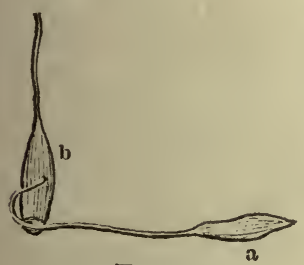

Fig. 79.

Rheoscopic frog. allowed to fall on an excised muscle $b$, so that two points of the nerve are in contact with the cut end and with the surface of the second muscle $b$, the muscle $a$ will contract each time the nerve touches $b$ so as to complete the circuit.

Whatever be the explanation of this current of resting muscle, there is no doubt that a very definite electrical change occurs in a muscle when it contracts. To show this change, we may lead off two points, one on the cut end and one on the surface of the muscle of a muscle-nerve preparation, to a galvanometer. We shall then obtain a deflection of the mirror 
of the magnet, due to the current of rest or demarcation current. If now the nerve be stimulated with an interrupted current so as to throw the muscle into a tetanus, the ray of light from the galvanometer mirror swings back towards the zero of the scale, showing that the current which was present before is diminished. When the excitation of the nerve is discontinued, the galvanometer indicates once more the original current of rest. This diminution of the current of rest during activity of a muscle is spoken of as the 'negative variation.'

In carrying out this experiment it is usual to compensate the demarcation current by sending in a small fraction of the current from a constant cell. The arrangement of the apparatus is represented in the accompanying diagram. Two non-polarisable

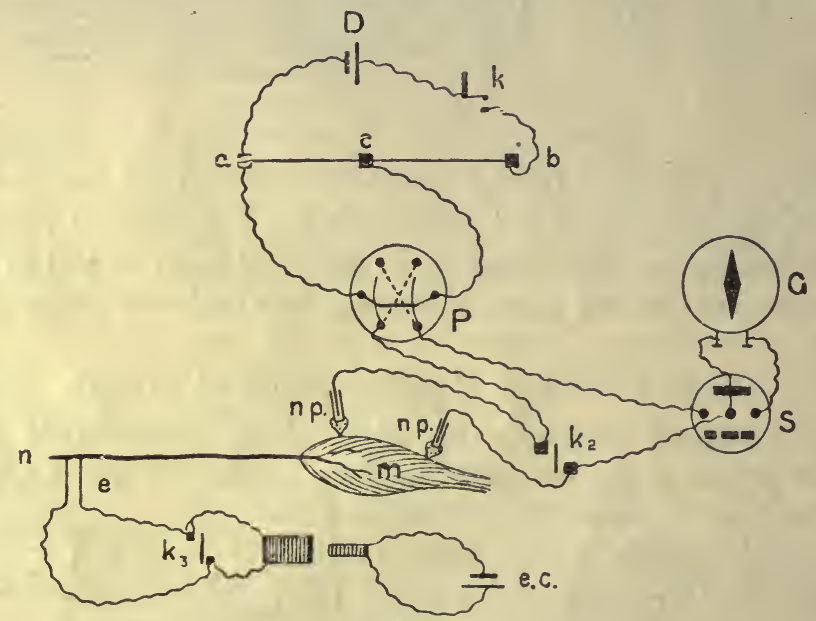

FIG. 80.

electrodes $n p$ are applied to the surface and cross-section of a muscle $m$. These are connected with the shunt of the galvanometer, one of the wires, however, being connected with a Pohl's reverser $P$, and this in its turn with the shunts. The two endterminals of the reverser are connected with a rheochord, through the wire of which $a b$ a constant current is passing from the Daniell cell D. By means of the rider $c$ the fraction of current passing through the reverser can be modified to any extent. The key $k$ being open, the muscle is connected with the shunt and galvanometer, and the direction and extent of the swing noticed. The key $k$ is then closed, and by means of the reverser the current is sent through the galvanometer in the opposite direction to the demarcation current, and the rider $c$ shifted until the two currents exactly balance one another, and the needle of the galvanometer returns to zero of the scale. This adjustment is first made, using only $\frac{1}{1000}$ of the total current, and then by means of the shunt, $\frac{1}{100}, \frac{1}{10}$, and finally the whole current is thrown into the galvanometer. If this precaution be not taken, much too large a current may in the first case be sent through the galvanometer, to the detriment of the instrument. If we know the difference of potential between the two ends of the wire, the proportion $\frac{a c}{a b}$ will give us the E.M.F. of the demarcation current. The galvanometer needle having by compensation been brought to zero, stimulation of the nerve at $e$ by interrupted currents causes the needle to swing at once in the opposite direction to the first variation. This swing is the measure of the negative variation or current of action.

In order to study the electrical changes accompanying a single muscle twitch, it is necessary to employ some instrument which can react much more rapidly than the 
ordinary galvanometer. For this purpose we may employ either the capillary clectro. meter or the string galvanometer of Einthoven.

The capillary electrometer is an instrument for recording and measuring difference of potential. That is to say, if connected with two points, it measures the force which would make a current flow between these two points if they were connected by a wire. Its structure is very simple. It consists of a glass tube drawn out to a fine capillary point. This tube with the capillary is filled with mercury. The point dips into a wide tube containing dilute sulphuric acid, at the bottom of which is a little mercury. Two platinum wires fused into the glass and dipping into the mercury serve as terminals. When the instrument is used, the meniscus of the mercury in the capillary at its junction with the acid is observed under the microscope, or a magnified image of it is thrown on a screen with the aid of the electric light. If now the capillary and acid be connected with two points, it will be observed that any difference in the potential of these two points causes a movement of the meniscus. If the point connected to acid be negative as compared with the point connected to mercury in capillary, the meniscus moves towards the point of the capillary. If the acid be positive as compared with the capillary, the meniscus moves away from the point. The extent of the excursion is proportional to the difference of potential. Since the capillary electrometer appears to have no latent period, and is free from instrumental vibrations, it is extremely useful in recording the quick changes in potential occurring in the diphasic electrical changes that accompany every contraction-wave in the body. The excursions lend

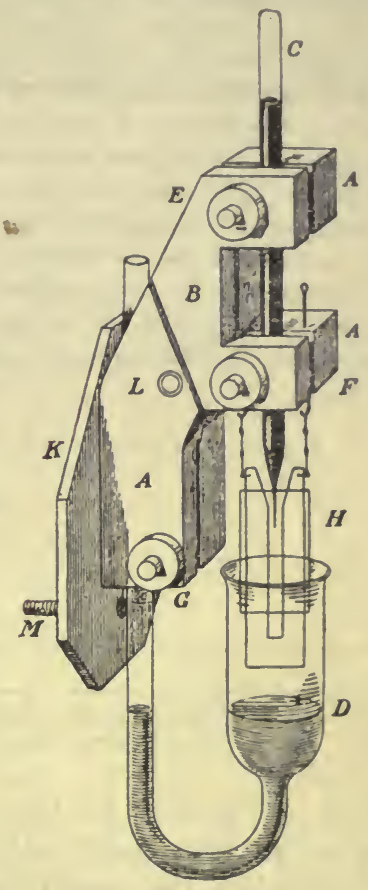

F1G. 81.

Capillary electrometer. (Burcr.) themselves well to photography, so that we may obtain a graphic record of every electrical variation, and thus determine its extent and its time-relations.

It must be remembered that this instrument is an electrometer (measurer of difference of potential), and not a galvanometer (current measurer). When the electrometer

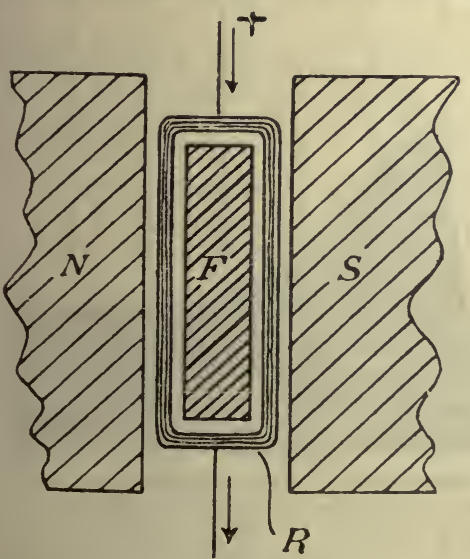

FIG. 82 .

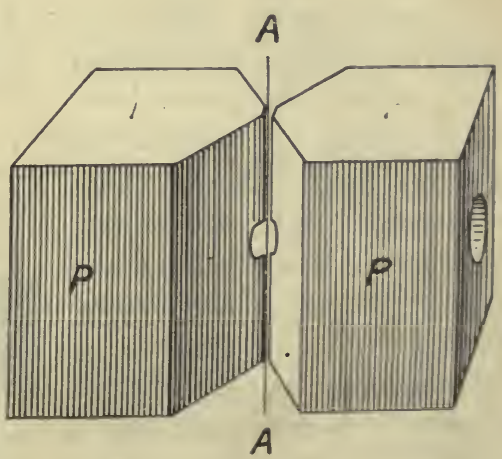

Fig. 83.

is connected with two points at different potential, current passes into it for a fraction of a second, and polarises the surface of the mercury, so that it takes up a new position 
in the capillary. This polarisation causes an electromotive force which exactly balances the E.M.F., setting up the polarisation so no current passes the surface. Hence the use of non-polarisable electrodes is not so essential in experiments with this instrument as when we make use of the galvanometer.

In the $D^{6}$ Arsonval galvanometer (Fig. 82) the current is sent through a coil of fine wire hung between the poles of a permanent magnet. The same principle is made use of in the string galvanometer of Einthoven (Fig. 83). In this a very delicate thread of silvered quartz or of platinum is stretched between the poles of a strong magnet. The poles of the magnet are pierced by holes so that the thread may be illumined by an electric light from one side, and from the other may be observed by means of a microcope ; or a magnified image of the thread may be thrown upon a screen. Whenever a current passes through the thread it moves laterally, and the lateral movement may be photographed on a moving photographic screen. Owing to the minute dimensions of the thread the instrument is one of extreme delicacy. It will detect very minute currents and will respond accurately to very rapid changes in potential.

If a perfectly uninjured regular muscle (Fig. 84), such as the sartorius,

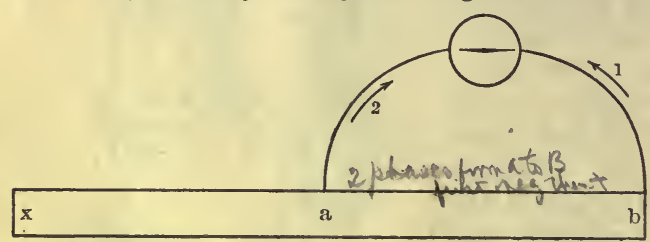

FIG. 84. Diagram showing diphasic variation of uninjured muscle. be stimulated with a single induction shock at one end, $x$, and two points, $a$ and $b$, be led off to a capillary electrometer, each stimulus applied at $x$ gives rise to an excursion of the meniscus of the electrometer, known as a 'spike,' and shown in Fig. 85. Knowing the constants of the instrument used, we can analyse this spike, and we find that it represents a diphasic change. Our study of the mechanical changes in muscle has shown that, when the muscle is stimulated at $x$, a contraction wave commences which travels down the muscle through $a$ and $b$. The electrical investigation of the muscle shows that excitation of $x$ arouses an electrical change which also passes down the muscle at the same rate as the mechanical change which it precedes. If we are leading off from $x$ and $a$, the electrical change ensues immediately upon stimulus, i.e. there is no latent perind to the electrical change. On leading off from $a$ and $b$ there is a latent period between the stimulus and the

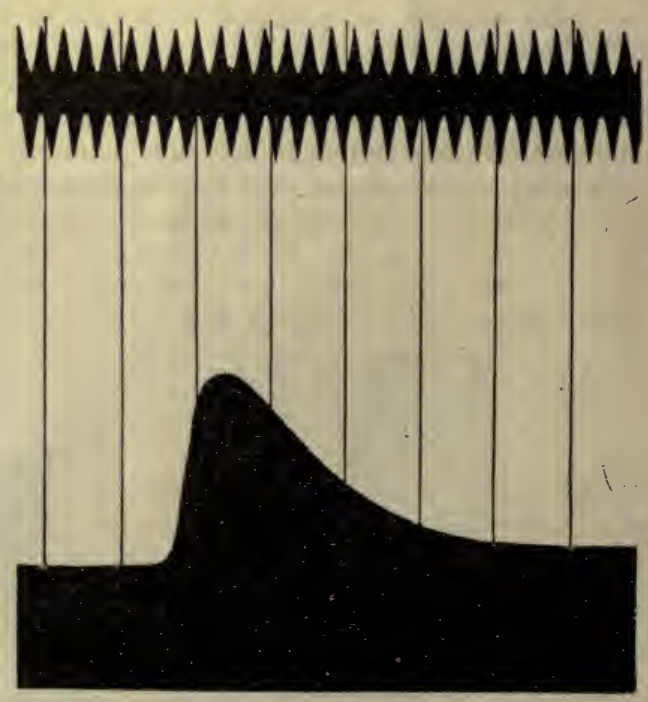

FiG. 85. A typical electrometer record from a sartorius muscle excited by a single induction shock. Time-marking $=200 \mathrm{D} . \mathrm{V}$. $\quad$ (KEITH LUCAS.) first change, representing the time taken from the change to travel from $x$ to $a$. When the change reaches $a$ this becomes the seat of an electro- 


\section{ELECTRICAL CHANGES IN MUSCLE}

motive force of such a direction that the current would pass in the outer circuit from $b$ to $a$. We may say, therefore, that $a$ is negative to $b$. A fraction of a second later the excitatory change has passed on to $b$ and has died away at $a$. Now $b$ is negative to $a,{ }^{*}$ and the current therefore passes in the opposite direction. Between $a$ and $b$, therefore, there is a diphasic current, the first phase representing negativity of $a$ to $b$, and the second phase A

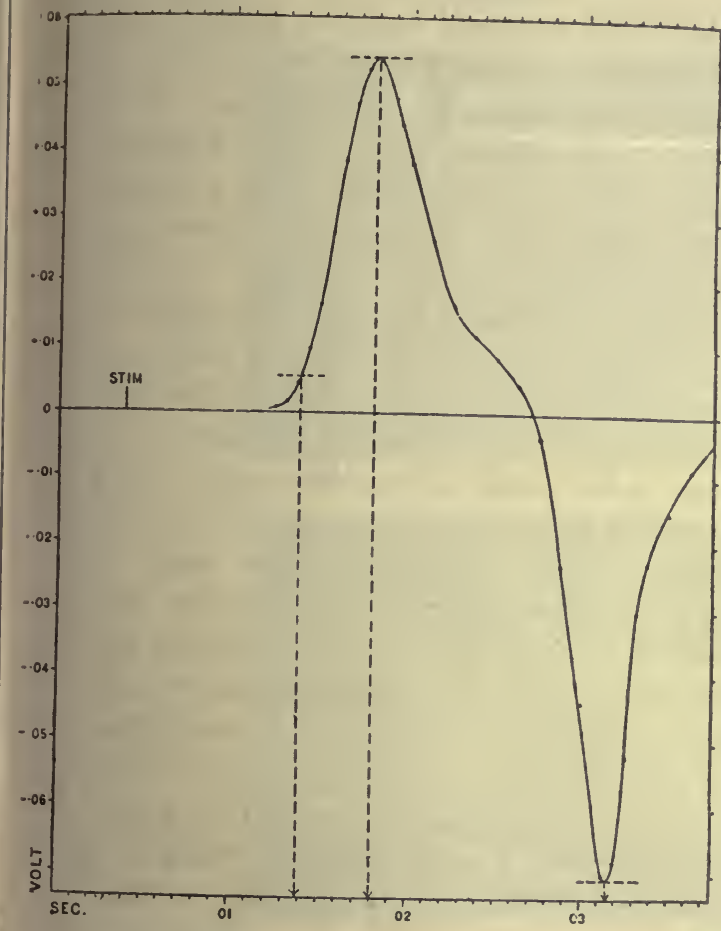

B

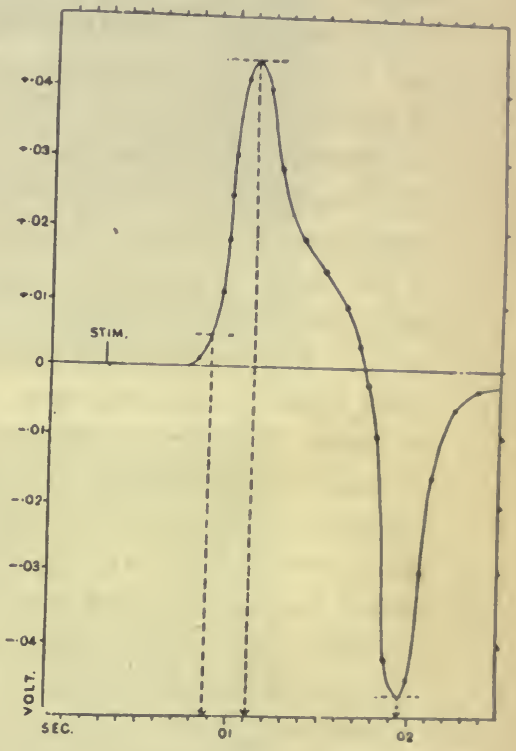

Fra. 86. Diphasic response of uninjured sartorius (obtained by analysis of curves such as Fig. 85). A, at $8^{\circ} \mathrm{C}$; B, at $18^{\circ} \mathrm{C}$. (KeITH LuCAS.)

representing negativity of $b$ to $a$. A diphasic change is thus also a sign of a propagated change. Every excitation of a normal muscle gives rise to a diphasic variation of such a direction that the point stimulated first becomes

* The statement that the excited portion of the muscle becomes 'negative,' though sanctioned by long usage, is not very exact and may give rise to misconception. When we lead off the terminals of a copper-zinc couple or cell to a galvanometer, a current flows outside the cell from copper to zinc and inside the cell from zinc to copper. In this case the zinc is said to be electropositive to the copper, and in the same way we must assume that the excited portion of a muscle is electropositive to the unexcited portions. When, therefore, we speak of any part of a tissue being negative, we are using a conventional expression to indicate the direction of the current in the outer circuit, and not the electrical condition of the tissue itself. In order to avoid the confusion which might result from an attempt to replace the loose expression 'negative' by the more correct expression 'electropositive,' Waller has suggested the employment of the term 'zincative' to indicate the electrical condition accompanying excitation. This term would also serve to emphasise the fact that the excited portion, like the zinc in a zinc-copper cell, is the chief seat of chemical change. 
negative to all other points of the muscle, and this 'negativity', to use a loose but convenient expression, passes as a wave down the muscle, preceding the wave of contraction and travelling at the same rate.

If one leading-off point be injured, e.g. at $b$, the change accompanying excitation is absent at that point. A single stimulus applied at $x$ will in this case give only a monophasic variation in which $a$ is relatively negative to $b$.

When we study the time relation of the electrical variation ensuing on a single stimulus, we find that the electrical change under the electrodes begins at the moment that the stimulus is applied. It takes about $\cdot 0025$ sec. to attain its culminating-point. At this point the mechanical change or contraction of the muscle begins. These time-relations vary with the temperature of the muscle. We have already seen that the effect of lowering the temperature is to increase the latent period of the contraction. In the same way it slows the rise of the electrical change and the rate of propagation of the wave of electrical change. This is shown in Fig. 86, in which are given the diphasic response of the sartorius first at $8^{\circ} \mathrm{C}$. and secondly at $18^{\circ} \mathrm{C}$. We are therefore justified in regarding the electrical change as an index to the chemical changes evoked in the muscle as the direct result of the stimulus. The flow of material, which is responsible for the change in form of each contracting unit, is secondary to these changes. As the result of stimulation, a chemical change is aroused at the point of excitation and travels thence along the muscle fibres at a rate of about three metres per second, i.e. the same rate as that of the following wave of mechanical change and, like this, varying with the temperature. Under certain conditions an excitatory condition may be propagated without the presence of a visible contraction. Thus, if the middle third of the sartorius be soaked for a time in water, it passes into a condition known as 'water rigor,' in which it is incapable of contracting, although capable of transmitting an excitation from one end of the muscle to the other.

The connection of a diphasic current of action with an excited condition of the tissues passing as a wave from one end to the other is shown still more clearly on a slowly contracting tissue, such as the ventricle of the frog or tortoise. Fig. 87, A, is a photographic record of the variation obtained from the tortoise ventricle, which is led off to a capillary electrometer, one (acid) terminal being connected with the base of the ventricle, the other (mercury) with the apex. Each part of the ventricle remains contracted for a period of $1 \frac{1}{2}$ to 2 seconds, and then the contraction passes off, first at the base and later at the apex. The electrical events are an exact replica of the mechanical. Directly after the stimulus has been applied, the base becomes negative and the column of mercury moves up. A moment later the excitatory condition extends to the apex. There is thus a sudden equalisation of potential between the two terminals, and the mercury comes back quickly to the base line. Here it stays for $1 \frac{1}{2}$ to 2 seconds. During this time the whole heart is in an excited condition. Both base and apex are equally excited, and there can be no difference of potential between them. The excitatory condition 
then passes off, first at the base and then at the apex. There is thus a small period of time in which the apex is still contracted or excited while the base is relaxed, and the apex is therefore negative to the base. This terminal negativity of the apex is shown on the photograph by the excursion of the column of mercury away from the point of the capillary. If one terminal, e.g. the apex, be injured, we obtain quite a different variation, which is shown in Fig. 87, B. It is evident from this figure that the electrical sign lasts practi-

A
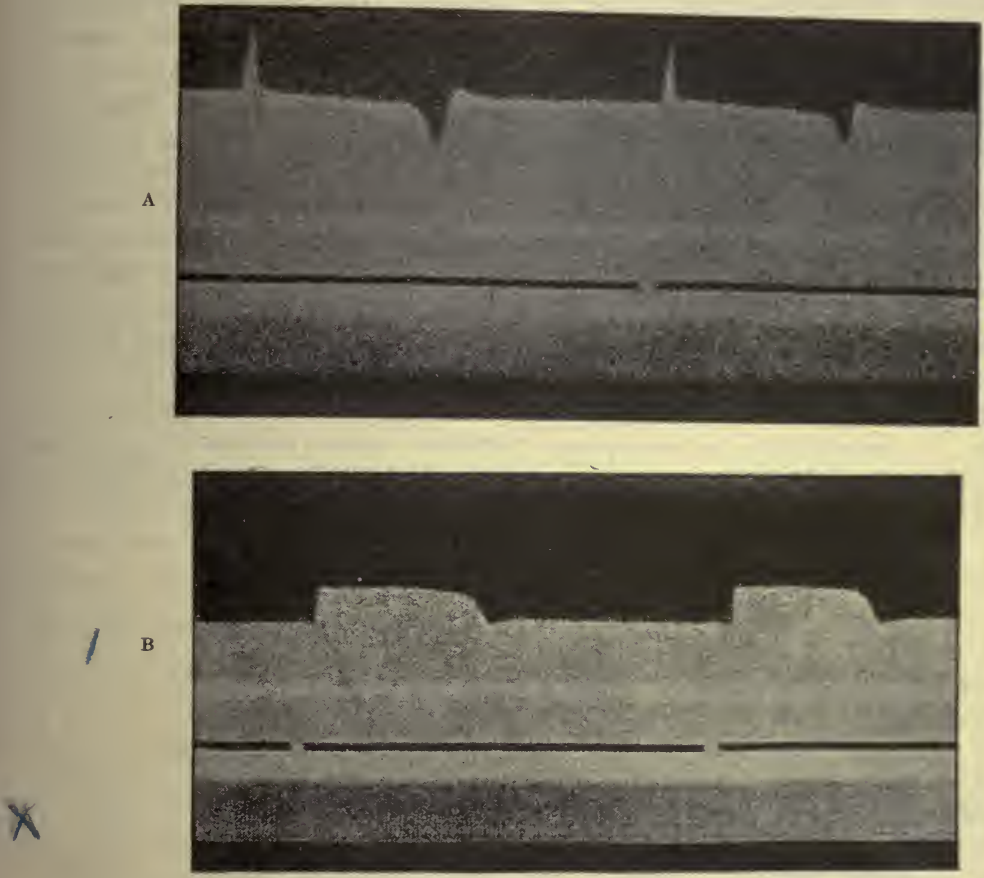

FIG. 87. Electrometer records of the electrical variations in a tortoise ventricle, excited to beat rhythmically by single shocks.

A, Ventricle uninjured. B. One leading off spot injured. (B. SANDERSON.)

cally as long as the mechanical sign of the excited state, and that we are not justified in regarding the first spike of the diphasic variation as indicative of an excitatory wave attended by an electrical change which is independent of the succeeding mechanical change.

The only difference between the electrical changes in this case and in that of voluntary muscle is that in the latter all processes are very much quicker, so that as a rule the point $a$ (Fig. 84) has ceased to be negative before the negativity of $b$ has attained its full height, and there is thus no prolonged equipotential stage.

Although in the case of the slowly contracting ventricle of the tortoise, the record obtained of the electrical changes accompanying its contraction by means of the capillary electrometer shows with great clearness the diphasic nature of the variation, and therefore the wave character of the electrical change, considerable difficulty is experienced sometimes in recognising that the 'spike' record of the electrical change in roluntary muscle or in nerve is also due to a diphasic variation. In this case the electrical change 
at any spot lasts only about $\frac{1}{200}$ second, and there is not a prolonged equipotential period, as in the case of the heart. The nature of the variation is, however, obvious, if we compare the electrometer record of an intact and therefore currentless muscle with that of a muscle in which one of the leading-off points has been injured, so as to give rise to a demarcation current. The two curves are given in Fig. 88, the upper shadowy tracing being that obtained from the injured muscle. It will be seen that the dis-

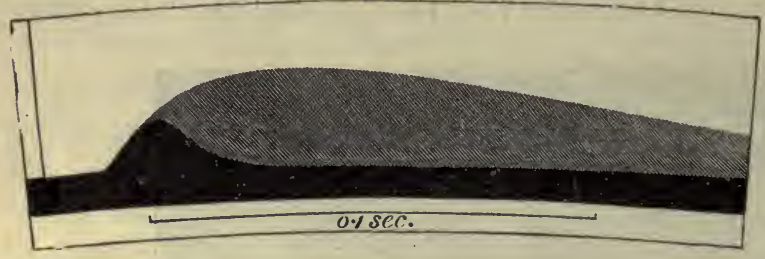

F1G. 88. Superimposed photographs of the electrical variation of the sartorius in response to a single stimulus. (BuRdoN SANDERSON.)

duced by the gradual leakage of the charge imparted to the instrument back through theelectrodes and muscle. When such a record is analysed, we obtain a curve similar to those in Fig. 89, which represent monophasic variations of a sartorius injured at one end, under different conditions of temperature. A similar curve to the diphasic variation can be obtained by putting in a current of similar E.M.F. from a battery, first in one direction for $\frac{1}{2} \frac{1}{00}$ second, and then in a reverse direction for another $\frac{1}{200}$ second. It must be remembered that a diphasic variation does not mean that one part of a muscle changes from normal in one direction, and then swings back past the normal in another direction, but that a change in one direction at one electrode dies away and is succeeded by a similar change in the same direction, which also dies away, at the second electrode: that is to say, a diphasic variation implies the progression of a wave of electrical change between the leadingoff points. Using a string galvanometer, which reacts much more rapidly, the diphasic nature of the variation is immediately apparent from the photographic record, even with voluntary muscle, or nerve.
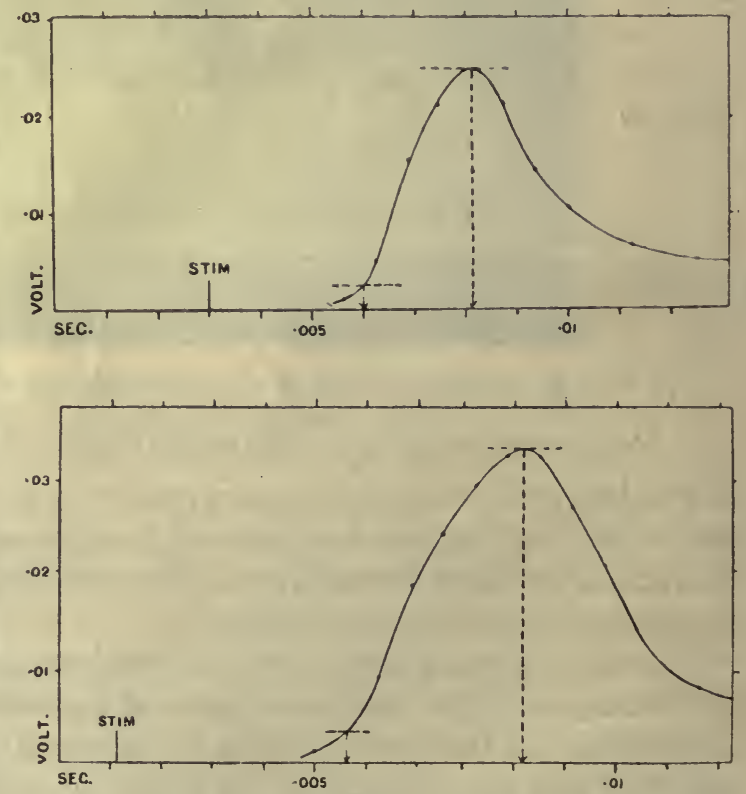

FIG. 89. Monophasic variations of an injured sartorius. $\mathrm{A}$, at $18^{\circ} \mathrm{C}$. ; B, at $8^{\circ} \mathrm{C}$. (KE1TH LUCAS.)

The electrical variation obtained by leading off a heart beating normally is a much more complex affair, and even now physiologists are not agreed as to its interpretation. Gotch has suggested that the complex character of the variations obtained both from the spontaneously beating frog's heart as 
well as from the mammalian heart is due to the twisting and alteration in direction of the fibres and of the course of the contraction wave which have occurred in the evolution of the heart from a simple tube. The question wil. be discussed more fully in chapter xiii.

\section{THE DEMARCATION CURRENT OR CURRENT OF INJURY}

According to Hermann, muscle or nerve may become negative under two conditions : (1) During activity; (2) when dying as the result of injury. It is doubtful, however, whether these two conditions are really distinct. Section or injury of a muscle causes a prolonged stimulation of the adjacent parts of the muscle fibres. These parts, therefore, being excited, must be negative to the unexcited parts which are further away from the seat of injury so that a demarcation current is really an excitatory current. We thus come to the conclusion, only paradoxical in terms, that the so-called currents of rest are really currents of action and are due to excitation around the injured spot.*

\section{SECONDARY CONTRACTION. RHEOSCOPIC FROG}

The negative variation of one muscle may be used to make another contract.

If the nerve of the preparation $a$ (in Fig. 90) be laid so as to touch at two points the cut end and surface of the muscle $b$, and the nerve of $b$ then stimulated with single induction shocks, every contraction of $b$ will be attended by a contraction of $a$, excited by the negative variation of the current passing through its nerve from the point touching the cut end to that in contact with the equator of $b$.

If the nerve of $b$ is tetanised, $a$ as well as $b$

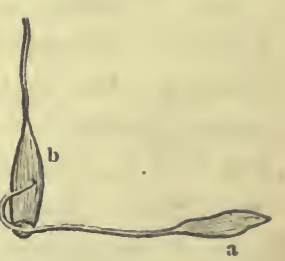

FIc. 90.

Rheoscopic frog. enters into a continued contraction. This 'secondary tetanus' is of interest as showing that, although the contractions of $b$ are fused, the excitatory process and negative variations are still quite distinct.

* If the demarcation current is really only due to excitation, we should expect to find it weaker than the action current obtained by exciting the whole muscle to contract. And this is the case. The E.M.F. of the demarcation current of a sartorius equals about 0.05 of a Daniell cell. The action current of the same muscle may attain to an E.M.F. $=0.08$ of a Daniell cell (Gotch). 


\section{SECTION VIII}

\section{THE INTIMATE NATURE OF MUSCULAR CONTRACTION}

Experiments on the metabolism of the body as a whole show that the energy of muscular work is derived from the oxidation of the food-stuffs. In man the performance of work involves an increase of the oxidative processes of the body with a corresponding evolution of energy, of which four-fifths will appear as heat while one-fifth may be transformed into mechanical work. In this respect the physiological mechanisms for the production of mechanical energy resemble the greater number of the machines employed by man for the same purpose. In nearly all these the prime source of energy is the oxidation of carbon and hydrogen in the form of coal or oil. In the steam-engine and internal-combustion engine the whole energy set free by the process of oxidation appears first as heat, and then a certain portion of the heat is converted into mechanical work. There is a limit to the efficiency of such heat engines, depending on the maximum differences of temperature available between the two sides of the working part of the machine. The efficiency of any heat engine is expressed by the formula $\mathrm{E}=\frac{\mathrm{T}-\mathrm{T}^{1}}{\mathrm{~T}}$, where $\mathrm{T}$ is the highest temperature (in absolute measurement) obtained by the working substance and $\mathrm{T}^{1}$ is the lowest temperature of the same substance. Ordinary engines rarely attain more than half this ideal efficiency, but it is evident that the greater the difference of temperature available the greater will be the efficiency of the machine. Internal-combustion engines, such as the gas engine or the oil-engine, therefore give a greater percentage of the total energy of the fuel out as mechanical energy than is the case with the steam-engine.

Engelmann has maintained that in muscle there is a similar transformation of heat into mechanical energy. He has found that non-living substances, which contain doubly refractive particles and possess the property of imbibition (e.g. catgut) when soaked with water, will contract on heating and relax again on cooling. He has constructed a model in which a thread of catgut in water, surrounded by a platinum coil, can be made to simulate muscular contractions and relaxations by passing a heating current through the platinum coil. He imagined that the chemical changes in the muscle liberate heat and that the effect of this heat upon the doubly refractive particles is to make them imbibe the surrounding water so that they change 
from an oval to a spherical shape. It would be impossible, however, for any large changes of temperature to take place in the muscle without entirely destroying its chemical character, and with small differences of temperature it would be impossible to attain the efficiency of 12 to 20 per cent. which characterises muscle.

Under certain conditions we may obtain by a machine almost the entire energy of a chemical change. The condition is that the chemical change shall be susceptible of taking place in a galvanic battery. We may use, for instance, a series of Daniell cells to drive an electric motor and allow the motor to perform mechanical work. Under these circumstances we could theoretically obtain 100 per cent. of the total chemical energy available, and in conditions of practice the efficiency of the machine may attain to 70 or 80 per cent. A similar arrangement might be present in the ultimate contracting elements of the muscle fibre. The mechanism in the fibre must be one which will provide for a more or less direct transformation of chemical energy into mechanical energy without a previous conversion of the chemical into heat energy. In the living body, where everything is in solution, all the energies may be reduced to one of two kinds, osmotic energy and surface energe contractile machine must therefore be one which employs one o-ther, or both, of these forms of energy. We might with Macdougall, regard the contractile element as a cylindrical structure differing in its contents from the surrounding sarcoplasm. When the muscle is at rest the contents of the muscle prism will be in equilibrium with the surrounding sarcoplasm. We might imagine the excitatory process to consist in a sudden chemical change occurring in the contents of the muscle prism. The production of a number of new molecules within the muscle prism (e.g. of lactic acid) would raise the osmotic pressure within the prism and occasion a rapid flow of water from the sarcoplasm. As a result the pressure in the muscle prism would rise and cause a bulging of its lateral wall and a shortening of the whole element. The subsequent phase of relaxation may be due either to a secondary change, e.g. oxidation, leading to the formation of a substance to which the walls of the prism are freely permeable, or to the gradual leak of the primary products of oxidation or disintegration into the sarcoplasm. The substance or substances giving rise to the osmotic differences which determine contraction may be either products such as lactic acid and carbon dioxide, which are formed during contraction, or may possibly be of the nature of neutral salts set free from some condition of combination with the proteins of the sarcous element. Macdonald has brought forward micro-chemical evidence of the appearance of potassium salts in the sarcous element during the state of activity of the muscle.

On the other hand, Bernstein has suggested that the changes during muscular contraction are determined by alterations in surface tension. If a little mercury be spilt on a plate the particles form globules. They are kept from spreading themselves out in a thin film under the influence of gravity in consequence of the surface tension of the mercury. Any modification of the surface will alter the tension, and therefore state of expansion, of the 
globule. Thus, if the globule be in sulphuric acid it undergoes a certain amount of polarisation, and becomes positively charged. By altering the charge of such a globule we can change its shape, as is shown diagrammatically in Fig. 91. If в represents the shape of the globule lying on the plate in some weak sulphuric acid, A will represent the shape of the globule when it is connected with the negative pole of a battery, while $\mathrm{c}$ will repre-

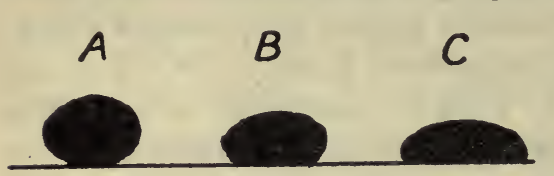

FIG. 91. sent its shape when it is connected with the positive pole of a battery, the other pole in each case being connected with the acid. If we consider muscle as made up of a series of chains of oval particles, a chemical change in the surface of these particles, causing an increase of surface tension, will tend to make them assume the globular shape, and will therefore cause a shortening and thickening of the whole fibre.

According to Schäfer, contraction is associated with a flow of the outer hyaline contents of the sarcous element into the tubular structure forming the middle portion. Such a flow may be determined either by osmotic differences between the centre and periphery of the sarcous element, or by a change in the surface tension obtaining between the isotropic fluid at the ends and the anisotropic structures in the centre of the huscle prism.

The tendency of recent investigation is all in favour of the second hypothesis, namely, that the essential factor in the processes of excitation and contraction is an alteration of surface. In the first place the electrical changes accompanying the excitatory process denote a polarisation or accumulation of ions on the surfaces situated in the excited area. The chemical change which is responsible for the current of action, or the negative charge at the exeited spot, takes place almost instantaneously and disappears somewhat more slowly. It would seem that the excitatory process consists essentially in the setting free of certain ions on the surface or surfaces in the contractile tissue, and that the passing away of the excitatory state is due to the disappearance of these ions, either by diffusion away into the surrounding fluid or by further chemical changes, such as oxidation. A study of the development of tension and of heat production in a muscle on excitation has shown that in both cases the yield of energy on excitation is increased by lengthening and diminished by shortening the muscle. Now alteration in length of the muscle will not alter its volume, but will alter the extent of its longitudinal surfaces, and it appears therefore that the production of heat as well as of mechanical energy is not a volume, but a surface effect. Finally the work of A. V. Hill on the heat production in muscle seems to show that the rise of tension in a muscle on excitation is due to the liberation of chemical bodies, of which lactic acid is certainly one, in the neighbourhood of certain longitudinal surfaces or membranes, and that the presence of these bodies changes the tension at such surfaces and thereby the longitudinal tension of the fibre. The extent and intensity of the production of these bodies must depend on the area of the chemically 
active surfaces. The muscle reacts at the end of the excitatory stage, not by any active process of lengthening, but by neutralisation, or simply physical diffusion of the active chemical bodies away from the interfaces or mem. branes. Later on, lactic acid is removed or replaced by its previously unstable precursor under the influence of oxygen with the production of some carbon dioxide and a certain amount of heat. We have seen already that the efficiency of the initial chemical change in which lactic acid is set free may approximate 100 per cent.

It must be noted that although the oxidative processes are responsible ultimately for all the energies of the higher animal, no oxidative change is involved in the production of lactic acid from e.g. glucose, nor is the presence of oxygen necessary for the contraction of muscle to take place. On the other hand, if we wish to obtain the maximum amount of work from a muscle, we must supply it richly with oxygen, the presence of which seems essential not to the contractile process but to the stage of recovery. In this stage a certain amount of heat is evolved, set free by the oxidation of the lactic acid, and we must assume that part of the energy so available is utilised for building up the precursor from which the lactic acid is derived. It is as if the process of oxidation furnished the energy for winding up a spring, whereas excitation removed a catch and allowed the spring to run down, setting free this energy for the performance of work or for conversion into heat.*

For many years it was imagined, as a result of experiments by Hermann, Pflüger, and others, that the oxygen supplied to a muscle was built up with its other constituents, especially carbohydrates, into a complex 'inogen' molecule. On stimulation this molecule underwent an explosive rearrangement, the carbohydrate and oxygen parts of the molecule combining to form carbonic acid, another product of the decomposition being lactic acid. The careful experiments of Fletcher have shown, however, that in the absence of oxygen there is no evidence of the formation of carbonic acid during contraction, and therefore no reason to assume the presence of oxygen in the muscle in an intramolecular form. Everything points to oxygen being taken in and applied forthwith to the purposes of oxidation, so that the output of carbon dioxide and water keeps pace with the intake of oxygen.

It is at present quite impossible to come to any conclusion as to the nature of the precursor from which the lactic acid is derived. The immediate precursor cannot be glucose or glycogen since the heat evolved in the initial stage of contraction is two or

* Peters has shown that if a muscle be stimulated to exhaustion under anaerobic conditions, about $0 \cdot 2$ per cent. lactic acid is formed with the evolution of 9 calories per gramme of muscle substance. The production of $1 \mathrm{gm}$. of lactic acid is therefore accompanied by the evolution of 450 calories. According to A. V. Hill the 'recovery heat production' in oxygen is of about the same order as the initial heat production, so that in the oxidative removal of $1 \mathrm{gm}$. of lactic acid there would also be an evolution of about 450 calories. The oxidation of $1 \mathrm{gm}$. of lactic acid produces 3700 calories, about eight times as much as the quantity observed. Hill considers this amount far too large to have escaped detection in his experiments, and therefore concludes that the lactic acid is not oxidised but replaced in its previous position under the influence and with the energy of the oxidation either; $(a)$ of a small part of the lactic acid itself, or $(b)$ some other body. He regards the latter alternative as the more probable, and concludes therefore, that the lactic acid is part of the machine and not part of the fuel of the muscle. 
three times as great as could be derived from the mere conversion of either of these substances into lactic acid. We must, therefore, conclude that the oxidation of lactic acid which goes on during the process of recovery is used to yield the energy necessary for building up the active molecules, which are the precursors of lactic acid and which have a higher potential energy than glucose itself, so that when it rapidly decomposes sufficient energy is set free to account for the observed heat production. Some such utilisation of the energy of oxidation of the lactic acid is indicated by the results of Parnas, who found that the heat evolved during this recovery process corresponded to only about one half the heat which would be evolved by the formation of the carbon dioxide output of the muscle during the same time as a result of the oxidation of lactic acid. 


\section{SECTION IX}

\section{VOLUNTARY CONTRACTION}

THE whole of our analysis of the processes accompanying the contraction of a skeletal muscle has so far had reference merely to the contractions evoked by artificial stimuli, mainly electric. These contractions have either been the simple twitch, with a duration of about one-tenth of a second, evoked by a momentary stimulus, or the tetanus, a continued contraction composed of a number of single twitches, summated and fused together. Under normal circumstances the contraction of skeletal muscles is brought about either reflexly, or in response to some stimulus descending from the cerebral cortex, the so-called 'voluntary contraction.' These contractions may have a duration of almost any extent. The quickest contractions carried out by man have a duration of about $0.1 \mathrm{sec}$. Considerable effort and training are required to reduce a muscular movement to this degree, and nearly all contractions, even the rapid ones, last considerably over $0 \cdot 1$ sec. Since we have no certain means of producing contractions of any given length, except by means of repeated stimuli, it is natural that physiologists have regarded voluntary contractions as similar to the artificial tetanus, and as, like this, composed of fused single contractions, and have endeavoured to determine the number of contractions per second, i.e. the natural rhythm of the tetanus. If, however, every muscular contraction in the body is to be regarded as of the nature of a tetanus, effected by rapidly repeating stimuli sent down the motor nerve from the central nervous system, we must assume a similar discontinuity for the process underlying the normal tone of muscles, and for the continued contraction of unstriated muscles, e.g. of the arteries. Is this discontinuity of muscles really essential for the production of a prolonged contraction? So far as our present knowledge of the intimate nature of muscular contraction goes, it would seem quite possible that the continuous state of contraction is dependent on a continuous evolution of energy in the muscle. We have seen reason to regard the chemical processes in a contracting muscle as presenting two phases, namely, (1) the production of a substance which increases the osmotic pressure within the sarcous elements, or raises the surface tension of the ultimate contractile elements of the muscle, thus causing a shortening and thickening of those elements; and (2) the further change of this substance into one which can escape by diffusion, or into a substance with a low surface tension, so that now the muscle relaxes and can be stretched by any extending 
force. If these two phases went on continuously, but the first phase kept ahead of the second one, a continuous state of contraction would be produced in the muscle. Since the contraction of the muscle only occurs in response to impulses from the central nervous system, we should have to imagine also a continuous stream, e.g. of negatively charged ions, descending the nerve and evoking an excitatory change in the muscle fibres as they impinge on the neuro-muscular junction. We have evidence that a state of excitation of a nerve, which is apparently continuous, may excite a correspondingly continuous state of excitation in the muscle attached. During the passage of a constant current through muscle there is a continuous contraction in

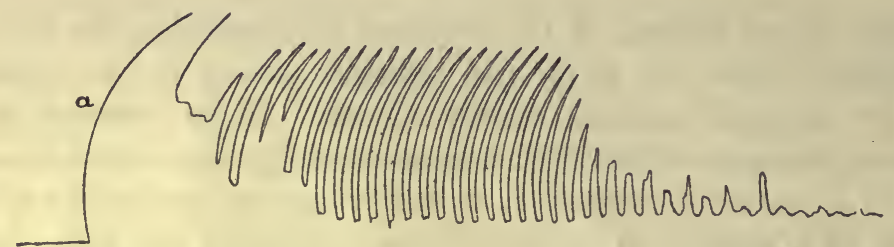

Fig. 92. Continued contraction followed by rhythmic contractions of a muscle in response to a constant stimulas. (BIEDERMENN.)

The muscle was excited by the passage of a constant current, the cathoda end having been moistened with a weak solution of $\mathrm{NaCO}$.

the neighbourhood of the cathode. If the irritability of the muscle at this point be increased by the application of a solution of sodium carbonate, Biedermann has shown that this excitation is propagated to the rest of the muscle, and on closure of the current we obtain a prolonged contraction followed by rhythmic contractions (Fig. 92). Moreover in frogs, the excitability of which has been heightened by keeping them at $2^{\circ}$ to $3^{\circ} \mathrm{C}$. for some days, the closure of a descending current through the sciatic nerve causes a prolonged contraction of the gastrocnemius; and in the same way there may be a prolonged contraction produced by the opening of an ascending current through the nerve.

The question, however, can only be decided by experiment. If a voluntary or reflex contraction is of the nature of a tetanus, we should be able, by a study of the mechanical and electrical phenomena combining the contraction, to obtain distinct evidence of this causation. It was shown by Wollaston that, on listening to a contracting muscle, a low sound was heard which, according to him, corresponded to a vibration frequency of 36 to 40 per second. The same observation was made by Helmholtz, and can be repeated by any one who will place the end of a stethoscope on a muscle, e.g. the biceps, and listen to the sound produced when it contracts. Helmholtz pointed out, however, that the tone heard corresponded to the resonance tone of the external ear, and was the same as that noted when listening to any irregular sound of low intensity. Thus the roar of London that we hear in the middle of Hyde Park has the same pitch as the muscle sound of the contracting biceps. The muscle sound, therefore, teaches us nothing as to the pitch or number of contractions per second making up the 
voluntary tetanus. It merely points to an irregularity or discontinuity in this contraction. By bringing vibrating reeds of different frequency in contact with the contracting muscles of the frog, Helmholtz came to the conclusion that the chief element in the muscle sound was the first over-tone of a sound with a vibration frequency of 18 to 20 per second, which, according to him, was to be taken as representing the number of single contractions in every voluntary muscular contraction.

Nearly all voluntary contractions present a certain degree of irregularity, and the same irregularities are observed when a tetanic spasm in the muscle of the body is caused by strong excitation of the cerebral cortex, as in epilepsy. On taking a record of such contractions, Schaefer and Horsley showed that in nearly all cases the tracing presents superposed undulations repeated at the rate of eight to twelve per second. These observers concluded that this was the normal rate at which the impulses descend the nerve to arouse a voluntary contraction. One difficulty in this conclusion is that when human muscle is excited by eight to twelve stimuli per second, we obtain, not a tetanic contraction with a few irregularities superposed on it, but a series of single contractions, the so-called clonus. In order to produce a nearly continuous contraction we must employ a vibration frequency of about 30 per second. It has been suggested to get over this difficulty that under normal circumstances the discharge does not travel along all the nerve fibres at the same time, so that the different muscle fibres composing the muscle will be in different phases of contraction, and there will be never any large degree of relaxation between the individual contractions of the whole muscle. Von Kries has found that the duration of a muscle twitch may be lengthened by lengthening the duration of the electrical change used to excite the nerve, and has suggested that the normal excitatory process may resemble the prolonged electrical change which can be produced electro-magnetically, rather than the short sudden shock represented by the induced current of an induction-coil. Attempts have been made to decide the question by recording the electrical changes accompanying the natural contractions of a muscle, i.e. those excited reflexly from the central nervous system. It was long ago shown by Lovèn that a certain discontinuity could be seen in records of the electrical changes obtained from a frog's muscle in the tetanic spasms produced by an injection of strychnine, but according to Burdon Sanderson this discontinuity represents a series of spasms discharged from the central nervous system. Each discharge produces, not a twitch, but a continued contraction of short duration. On photographing the electrical changes of strychnine spasm as obtained by a capillary electrometer, he found that each individual spasm could only be compared to a short tetanus. The most recent investigations of the question we owe to Piper, who made use of the string galvanometer, an instrument much more delicate in the reproduction of rapid changes than is the capillary electrometer. Piper led off two points in the fore-arm, one electrode being placed about two inches below the bend of the elbow, and the other about four inches above the wrist. A single stimulus of the median nerve was found 
by him to give a typical diphasic variation in the muscles. When the muscles were contracted voluntarily, well-marked oscillations of the galvanometer wire were obtained, indicating the existence in the muscle of fortyeight to fifty complete diphasic variations in the second (Fig. 93). Piper obtained similar records on leading off other muscles of the body when these were placed voluntarily in a state of contraction, and he concludes therefore that each voluntary contraction, short or long, is a tetanus composed of about fifty fused twitches per second. These results would indicate that

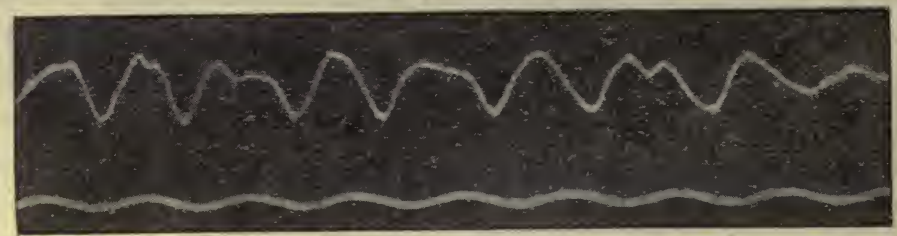

FIG. 93. Electrical variations produced by voluntary contractions of human muscle. (PIPER.)

the impulse, which normally travels down the motor nerve from the anterior cornual cell to the muscle, is discontinuous, and therefore that on leading off a motor nerve to a galvanometer we ought to obtain electrical oscillations of fifty distinct stimuli per second. Dittler has investigated by means of the string galvanometer the electrical changes accompanying the ordinary contractions of the diaphragm, and also those occurring in the phrenic nerve. He finds that both in the muscle. and in the nerve there is evidence that each contraction is a fused series of single contractions, evoked by the discharge along the nerve of between fifty and seventy excitations per second. So far therefore the evidence is in favour of the view that voluntary contraction, and, one must add, the tonic contractions of all skeletal muscles, are discontinuous in nature and analogous to the tetanus which we may evoke artificially by rapid stimulation either of muscle or of its motor nerve. 


\section{SECTION $\mathrm{X}$ \\ OTHER FORMS OF CONTRACTILE TISSUE SMOOTH OR UNSTRIATED MUSCLE}

THE little we know about the physiology of unstriated muscle is derived chiefly from experiments on the intestine, ureter, bladder, and retractor penis.* This tissue differs from voluntary muscle in containing numérous plexuses of nerve fibres (non-medullated) and ganglion-cells, so that in all our researches it is difficult to be certain whether the results are due to the muscle fibres-themselves, or to the nerves and nerve-cells which are so intimately connected with them; especially as we have as yet no convenient drug like curare, by aid of which we might discriminate between action on muscle and action on nerve.

The differences between unstriated and voluntary muscle, although at first sight very pronounced, on further investigation prove to be in most cases differences of degree only, qualities and reactions which are marked in involuntary muscle being also present in a minor degree in the more highly differentiated tissue.

The contraction of smooth muscle is so sluggish that the various stages of latent period, shortening, and relaxation can be easily followed with the eye. The latent period may be from 0.2 to 0.8 second, and the contraction may last from three seconds to three minutes.

Smooth muscle preserves many of the properties of undifferentiated protoplasm, especially an automatic power of contraction, which is regulated by the condition of the muscle. Thus whereas the voluntary muscle is intimately dependent on its connection with the central nervous system, and in the absence of this is reduced to a flabby inert tissue, the smooth muscle, isolated from all its nervous connections, presents in many cases rhythmic contrastions, and can carry out a peripheral adaptation to its environment. These rhythmic contractions are almost invariably observed

* The retractor penis, which is found in the dog, cat, horse, hedgehog (but not in rabbit or man), is a thin band of longitudinally arranged unstriated muscle, which is inserted at the attachment of the prepuce, and is continued backwards in a sheath of connective tissue to the bulb, where it divides into two slips, which pass on either side of the anus. It is innervated from two sources, the motor fibres being derived from the lumbar sympathetic and running to the muscle in the internal pudic nerve, while the inhibitory fibres run in the pelvic visceral nerves (nervi erigentes) and are derived from the second and third sacral nerve-roots. 
if the muscular tissue be subjected to a certain amount of tension, after separation from the central nervous system. The rhythm of the contractions may vary from one (spleen) to twelve (small intestine) contractions in the minute.

The stimuli for smooth muscle are essentially the same as for striated. As we should expect, however, from the sluggish response of this kind of contractile tissue, the optimum rate of change of current which excites is very much slower than in the case of striated muscle. Thus in many instances a single induction shock, even if very strong, is powerless to excite contraction, and the make-induction shock of long duration and low intensity is always more efficacious than the short sharp break-induction current. A still better stimulus is the make or break of a constant current. When the latter form of stimulation is used, response occurs at the make sooner than at the break, and, just as in the voluntary muscle, the make excitation starts from the cathode and the break excitation from the anode.

An apparent exception to this statement is afforded by the behaviour of certain forms of involuntary muscle. In the intestine, in the skin of worms, and in many

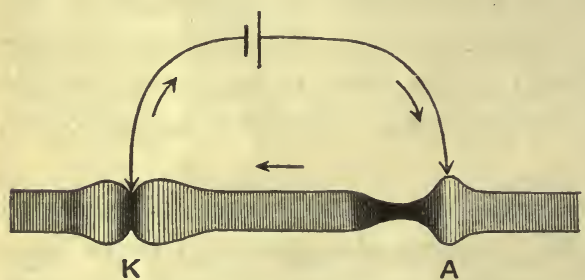

FIG. 94. At the cathode $\mathrm{K}$ there is a small line of constriction, surrounded by an area of relaxation. At the anode itself the muscle is relaxed, but is strongly contracted on each side of the anode, so that on rough observation it would be thought that contraction occurred at the anode itself. other muscular tubes the smooth muscle-fibres are arranged in two different sheets, one consisting of longitudinal, the other of circular fibres. If non-polarisable electrodes, connected with a constant source of current, be applied to the surface of the small intestine, when the current is made there will be apparently a strong contraction of the circular coat at the anode, which spreads up and down the intestine, and a linear contraction of the longitudinal coat at the cathode. The same result is observed in the earthworm and leech. But careful observation shows in each case that the irregularity is really only apparent, and that in the immediate neighbourhood of the anode there is relaxation of both coats, with a contraction of the circular coat on each side, and that at the cathode there is a contraction of both coats. The accompanying diagram (Fig. 94) will serve to show the condition of the circular coat at each electrode.

As a matter of fact, in consequence of the arrangement of the fibres, we have in the neighbourhood of the anode a number of places (virtual cathodes)where the current is leaving the musclecells to enter inert conducting tissues, and in the same way there will be in the neighbourhood of the cathode a number of virtual anodes (Fig. 95). Thus if we take the ureter and lead a current through it while it is slung up in thread loops serving

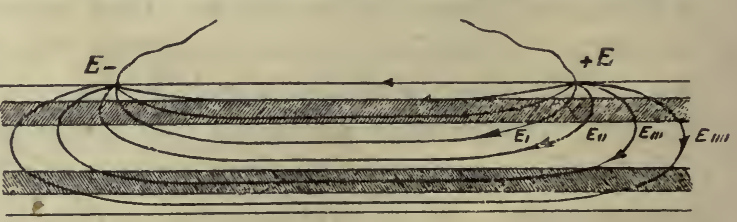

FIG. 95. Diagram to show the spread of current which occurs. when a current is led through a tube such as the ureter by means of two electrodes applied to its surface. It will be noticed that while $+\mathrm{E}$ is the anode, there are immediately below and around it a number of cathodes, $\mathrm{E}, \mathrm{E}_{1,}, \mathrm{E}_{\prime \prime \prime}, \mathrm{E}_{\prime \prime \prime \prime}$ due to the current leaving the muscle to flow through indifferent tissues. (BIEDERMANN.)

as electrodes, there is contraction of both coats at the cathode and relaxation of both at the anode. If, however, the ureter be packed in a pulp of blotting-paper 
moistened with normal saline, thus allowing the current to leave the contractile tissues anywhere along the ureter, we get the same aberrant results of stimulation as are obtained with the intestine.

In voluntary muscle, if one stimulus follows another at an interval which is not too large, a summated contraction is produced which is greater in amplitude than that due to a single stimulus. This summation may be mechanical or physiological, the former being observed when the stimulus is repeated during the decline of the excitatory process and being due simply to the after-loading of a muscle by the first contraction. It is best marked when the muscle is heavily loaded. If, however, the stimuli be sent in at sufficiently short intervals so that two stimuli fall within the period of rise of contractile stress, an increased height of contraction is obtained under all conditions, and under isometric conditions the tension developed is greater than that with a single stimulus. If the interval between two stimuli be so short that the second falls within what we have called the refractory period due to the first stimulus, no summation is obtained, the second stimulus being ineffective.

In the slow contraction of involuntary muscle we could hardly expect mechanical summation to come into play. Most types of this tissue show, however, the true summation, i.e. the increased liberation of energy due to repetition of the stimulus during the rise of the excitatory condition. As might be expected the refractory period is also longer in involuntary muscle, since all the processes of this muscle are slowed in comparison with those of voluntary muscle. In certain types of tissue, and especially in heart muscle, the refractory period lasts during the whole of the period of contraction. During this time therefore a second shock will be ineffective. As the contraction dies away the muscle fibre gradually recovers its susceptibility to stimulation, but it does not recover its full irritability until it has entirely relaxed. On this account it is impossible to obtain summation in or to tetanise heart muscle, the application of interrupted currents to this tissue producing only a series of rhythmic contractions.

In all involuntary muscle we may observe summation of the effects of stimuli even when the individual stimuli are insufficient to produce any excitation. Thus in a muscle such as the retractor penis, we may find a strength of induction shock which, applied singly, is just insufficient to evoke any response. If, however, the shocks are repeated at intervals of a second, it will be found that the first three or four stimuli are ineffective and then the muscle enters into a contraction which increases with each succeeding stimulus until it has attained its maximum. There is thus summation before any contraction has occurred, a summation of stimuli. Each stimulus, in fact, alters the state of the contractile tissus and makes it more ready to respond to the next stimulus, so that the stimuli become more and more effective. If time is allowed for the muscle to relax between successive stimuli, this summation is evidenced by a continually increasing height of contraction, the so-called 'staircase.' The same initial increase of 
effect is observed when voluntary muscle is excited by continually recurring stimuli (v. Fig. 70, p. 209).

We shall meet with other examples of this summation of stimuli when dealing with the physiology of the central nervous system. It is indeed a fundamental phenomenon in the physiology of excitation.

CHEMICAL STIMULATION. Strong salt solution excites contractions just as in the case of skeletal muscle. Many drugs, such as physostigmine, ergot, salts of lead and barium, digitalis, may act directly on smooth muscle and cause contraction. As one would expect, however, from the greater independence of the smooth muscle, the action of these drugs varies from organ to organ, muscle-fibres, which apparently are histologically identical, reacting diversely according to their origin.

MECHANICAL STIMULATION. Smooth muscle may react to a local pinch or blow with a local or a general (propagated) contraction. The most important form of mechanical stimulation is that produced by tension. The effect of increasing the tension on smooth muscle may be twofold: causing in the first place relaxation and in the second excitation with increased contraction. These two effects may be illustrated by taking the case of the bladder. If this viscus (which is surrounded by a complete coat of smooth muscle) has all its connections with the central nervous system severed, it is when empty in a state of tonic contraction. If fluid be injected into it rapidly there is a great rise of pressure in its cavity, due to the forcible distension. If, however, the fluid be injected slowly the bladder muscle relaxes to make room for it, so that a considerable amount of fluid may be accommodated in the bladder without any great rise of pressure. This process of relaxation has its limit. If the injection of fluid be continued the walls begin to be stretched passively, and this increased tension acts as a stimulus causing marked rhythmic contractions of the whole bladder.

In the same way the response of a smooth muscle to an electrical stimulus is much increased by previous increase of the tension on the muscle fibres.

PROPAGATION OF THE EXCITATORY STATE, OR WAVE OF CONTRACTION. On stimulating any part of a voluntary muscle fibre, a wave of contraction is started which travels to each end of the fibre, but no further. There ị no propagation from muscle fibre to muscle fibre, the synchronous contraction of the whole muscle being brought about by simultaneous excitation of all its fibres. It is doubtful whether this isolation of the excitatory state is found in smooth muscle. As a rule a stimulus applied to any part of a sheet of smooth fibres may travel all over the sheet just as if it were a single fibre. It seems probable indeed that there is protoplasmic continuity by means of fine bridge-like processes between adjacent muscle cells. And even in the absence of such bridges the propagation of the contraction could be easily accounted for. Although in the case of voluntary muscle the rule is isolated contraction, yet a very small change in the muscle, such as that produced by partial drying or by pressure, is sufficient to cause the contraction to spread from one fibre to another. 
Indeed by clamping two curarised sartorius muscles together, as in the diagram (Fig. 96), it is found that stimulation of the muscle A causes contraction of the muscle $\mathrm{B}$. The current of action of $\mathrm{A}$ in this case has served to excite a contraction in $\mathrm{B}$.

It must be remembered that in all unstriated muscle the fibres are surrounded by a network of non medullated nerve fibres. Some physiologists are inclined to ascribe to these fibres an important part in the propagation of the contraction wave. In the case of the heart muscle, however, it can be shown almost conclusively that the propagation take splace independently of nerve fibres, and probably the same is true for many kinds of involuntary muscle.

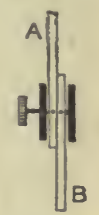

Fra. 96.

INFLUENCE OF TEMPERATURE. Smooth muscle is extremely susceptible to changes of temperature; as a rule warming causes relaxation, while application of cold causes a tonic contraction. The condition of the muscle at any given time depends not only on its actual temperature, but also on the rapidity with which this temperature has been reached. Thus a rapid cooling of the retractor penis muscle of a dog from $35^{\circ}$ to $25^{\circ}$ may cause a contraction as extensive as would be produced by a slow cooling to $5^{\circ} \mathrm{C}$. On warming a muscle from $30^{\circ}$ to $50^{\circ} \mathrm{C}$. it lengthens gradually up to about $40^{\circ}$, and it_may then undergo a marked heat contraction (varying in degree in different muscles) at about $50^{\circ} \mathrm{C}$., which may pass off at a somewhat higher temperature. It is killed somewhere between $40^{\circ}$ and $50^{\circ} \mathrm{C}$. It seems very doubtful whether any true rigor mortis occurs in smooth muscle. The hard contracted appearance of the smooth muscle in a recently dead animal is chiefly conditioned by the fall of temperature. On excising the muscle and warming it up to body temperature it may again relax and show signs of irritability two or three days after the death of the animal. Different smooth

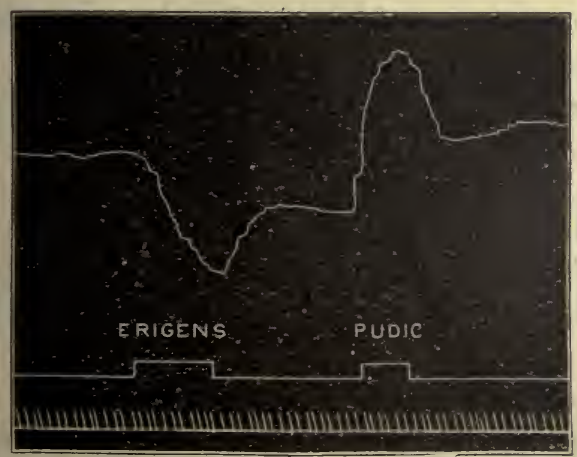

Fig.'97. Tracing from the retractor penis muscle of the dog, showing lengthening (inhibition) on stimulation of the nervus erigens, and a smart contraction on stimulating the pudic (motor) nerve. (Movements of muscle reduced $\frac{1}{2}$.) fibre terminating in an end-plate. muscles, however, vary very much in their tenacity of life.

DOUBLE INNERVATION. Voluntary muscle is absolutely dependent for its activity on the central nervous system. Cut off from this it is flabby and motionless. Its sole function is to contract efficiently and smartly on receipt of impulses arriving along its nerve. It is only necessary therefore that these impulses should be of one character-motor, and we know that each fibre of a muscle, such as the sartorius, receives one efferent nerve In the case of smooth muscle we have a tissue which has an activity and reactive power of its own, and apart from its innervation may be at one time in a state of relaxation, at another in a state of tonic contraction. 
In order that the central nervous system should have efficient control over such a tissue, it must be able to influence it in two directions : it must be able to induce a contraction or increase a contraction already present, and it must also be able to put an end to a spontaneous contraction, i.e. to induce relaxation. In order to carry out these two effects, smooth muscle receives nerve fibres of two kinds from the central nervous system, one-kind motor, analogous to the motor nerves of skeletal muscle, the other kind inhibitory, causing relaxation or cessation of a previous contraction. All these fibres belong to the visceral or 'autonomic' system. They are connected with ganglion-cells in their course outside the central nervous system, and their ultimate ramifications in the muscle are always non-medullated. A typical tracing of the opposite effects of these two sets of nerves is given in Fig. 97.

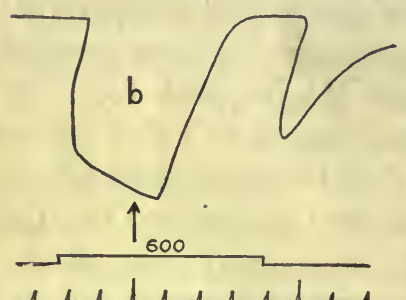

FIG. 98. Tracing of contraction of adductor muscle of claw of crayfish, showing inhibition resulting from stimulation of its nerve $(a t b)$ by means of a constant current. The break of the current causes a second smaller inhibition. (BIEDERMANN.)

In the invertebrata many 'voluntary' striated muscles probably possess a double innervation. Thus in the crayfish the adductor muscle of the claw consists of striated muscular fibres, every fibre of which is supplied with two kinds of nerve fibres. By exciting these fibręs one may get, according to the conditions of the experiment, either contraction of a relaxed muscle or relaxation of a tonically contracted muscle (Fig. 98).

\section{AMCEBOID MOVEMENT}

Amœboid movement is seen in the unicellular organisms such as the amœba and in the white blood corpuscles. It can occur only within certain limits of temperature (about $0^{\circ} \mathrm{C}$. to $40^{\circ}$ ); within these limits it is the more active the higher the temperature. At about $45^{\circ}$ the cell goes into a condition resembling heat rigor.

The fluid in which the corpuscles are suspended is of great importance. Distilled water, almost all salts, acids and alkalies, if strong enough, stop the action and kill the cell.

The movements are also stopped by $\mathrm{CO}_{2}$ or by absence of oxygen.

Artificial excitation, whether electrical, chemical, or thermal, causes universal contraction of the corpuscle, which therefore assumes the spherical form.

\section{CILIARY MOVEMENT}

Cilia are met with in man in nearly the whole of the respiratory passages and the cavities opening into them, in the generative organs, in the uterus and Fallopian tubes of the female, and the epididymis of the male, and on the ependyma of the central canal of the spinal cord and its continuation into the cerebral ventricles.

The cilia (Fig. 99) are delicate tapering filaments which project from the hyaline border of the epithelial cells. There are about twenty or thirty to each cell. The hyaline border is really made up of the enlarged basal portions of the cilia. 
In action the cilia bend suddenly down into a hook or sickle form, and then return slowly to the erect position. This movement is repeated many (twelve to twenty) times a second, and thus serves to move forward mucus, dust, or an ovum, as the case may be. The movement seems to be entirely automatic, and it is quite unaffected by nerves, at any rate in all the higher animals.

There is, however, a functional connection between all the cells of a ciliated epithelial surface, so that movement of the cilia, started in one cell, spreads forward as a wave, just as, when the wind blows, waves of bending pass over a field of corn.

The conditions of ciliary action are the same as those for amœboid movement of naked cells.

The minuteness of the object has up to now prevented us from deciding whether the

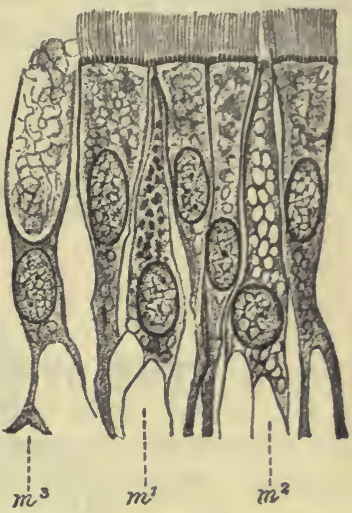

FIG. 99. Ciliated columnar epithelium from the trachea of a rabbit; $m^{1}, m^{2}, m^{3}$, mucus-secreting cells. (SCHAFER.)

cilium is itself actively contractile, or whether it is simply passively moved by the action of the basal part situated in the hyaline border of the cell. 


\section{CHAPTER VI}

\section{NERVE FIBRES (CONDUCTING TISSUES)}

\section{SECTION I}

\section{THE STRUCTURE OF NERVE FIBRES}

ON stimulating the nerve of a nerve-muscle preparation at any part by electrical, thermal, or mechanical means, the stimulus is followed, after

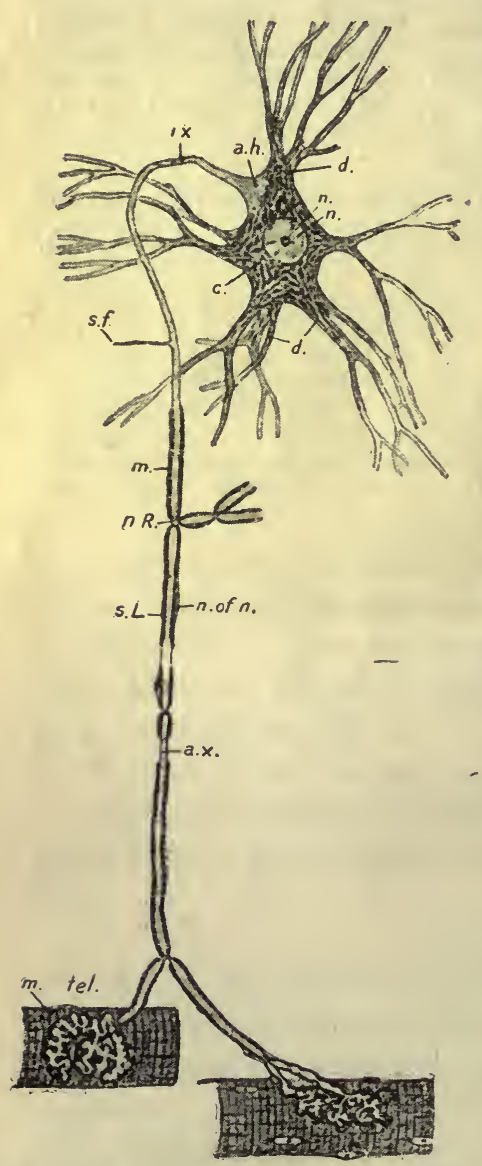

FIG. 100. Diagram of a motor nervecell with its nerve fibre. (After BARKER.)

$a . h$, axon hillock; $d$, dendrites ; $a . x$, axis cylinder; $m$, medullary sheath; n.R , node of Ranvier. a very short interval, by a contraction of the muscle. This observation illustrates the two functions of nerve fibres, irritability and conductivity - that is to say, a suitable stimulus can set up changes in any part of the nerve, which are transmitted down the nerve without any visible effects occurring in it, and it is not until this nervous change has reached the muscle that a visible effect takes place in the shape of a contraction. In the animal body a direct excitation of the nerve fibre in its course never takes place under normal circumstances. The only function the nerve fibre has to perform is that of conducting impulses from the sense organs at the periphery to the central nervous fsystem, and efferent impulses - from this to the muscles and other of its servants. Hence it is absolutely essential that there should be vital continuity along the whole length of the fibre. Damage to any part, such as by crushing, heat, or any other injurious condition, infallibly causes a block to the passage of an impulse.

A nerve fibre is essentially a long process or arm of a nerve-cell (Fig. 100). The cell may either be situated on the surface of the body or, as in most cases in the higher animals, may be withdrawn from the surface into a special collection of cells such 
as the posterior root ganglion, or may be one of the mass of cells and interlacing processes making up a central nervous system. All nerves are alike in possessing as their conducting part the continuous strand of protoplasm produced from the nerve-cell and known as the axon or axis cylinder. By special methods the axon may be shown to be made up of fibrillæ or neuro-fibrils, embedded in a more fluid material (Fig. 101). These neuro-

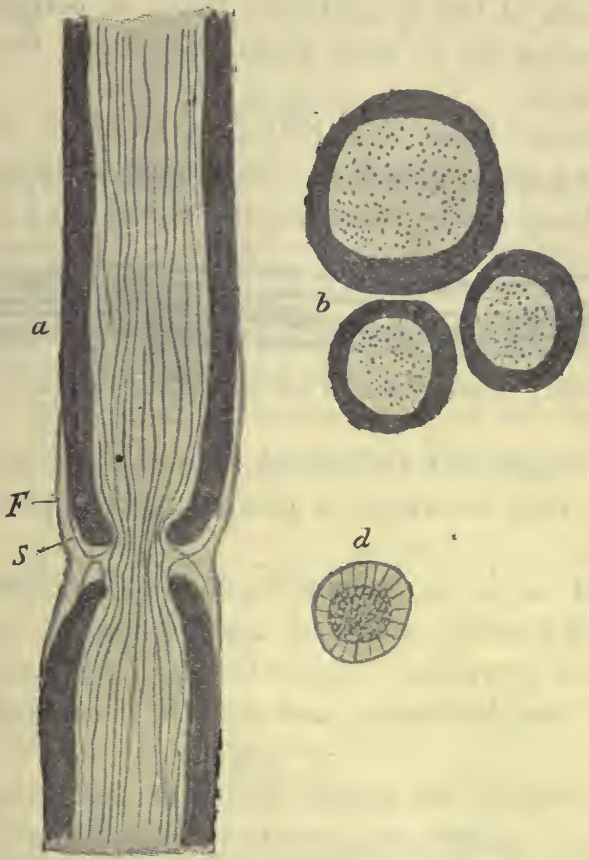

Fig. 101. Medullated nerve fibres, showing continuity of the neuro-fibrils across the node of Ranvier. (BETHE.)

$a$, longitudinal ; $b$, transverse section.

fibrils are supposed to be continuous throughout the cell and the axis cylinder and to represent the essential conducting constituents of the nerve. In the course of growth the nerves develop certain histological differences, which appear to bear some relation to the nature of the processes they conduct or to the character of their parent cell. Thus all the fibres which are given off from and which enter the central nervoussystem, i.e. the brain and spinal cord, belong to the class known as medullated. In this type the conducting core or axis cylinder is surrounded with a layer of apparently insulating material known as myelin, forming the medullary sheath, or the sheath of Schwann. This sheath consists of a fatty material composed largely of lecithin, and staining black with osmic acid, supported in the interstices of a network formed of a horny substance known as neurokeratin. The medullary sheath is surrounded by a structureless membrane, the primitive sheath or neurilemma. At regular intervals a break occurs in the medullary sheath, the neurilemma coming in close contact with the axis cylinder. This 
break is the node of Ranvier, the intervening portions of medullated nerve being the internodes. In each internode, lying closely under the neurilemma, is an oval nucleus embedded in a little granular protoplasm. The medullated nerve fibres vary considerably in diameter, the largest fibres being distributed to the muscles and skin, the smallest carrying impulses from the central nervous system to the viscera. The latter all come to an end in some collection of ganglion-cells of the sympathetic chain or peripheral ganglia, the impulses being carried on to their destination by a fresh relay of nonmedullated nerve fibres.

The non-medullated fibres (Fig. 102) differ from the medullated simply in the absence of a medullary sheath. They possess, in many cases at any rate, a primitive sheath, under which we find nuclei lying closely on the side

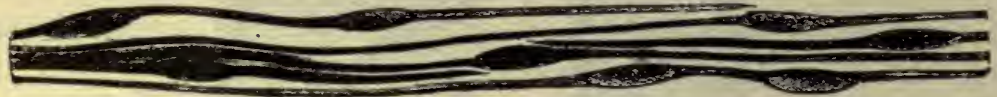

Frg. 102. Non-medullated nerve fibres. (ScHAFER.)

of the fibre and bulging out the sheath. In their ultimate ramifications they tend to form close networks or plexuses and appear to lose the last traces of a sheath.

The medullated nerves are bound together by connective tissue (endoneurium) into small bundles, which are again united by tougher connective tissue into larger nerve-trunks. These fibres as a rule branch only when in close proximity to their destination, and then the branching always occurs at a node of Ranvier.

As to the functions of the myelin sheath in the medullated nerve fibre very little is known. It does not make its appearance until the axis cylinder is formed, and is apparently derived from a series of cells which grow out from the spongioblasts of the central nervous system and form a chain surrounding the out-growing axons. In the regeneration of a nerve fibre after section the myelin sheath appears later than the axon in the peripheral part of the nerve. It has been supposed by some to act as a sort of insulator ensuring isolated conduction within any given nerve fibre. We have, however, no proof that equally isolated conduction is not possible in the non-medullated fibres of the visceral system, although it is certainly true that a finer ordering of movements is required in the skeletal muscles than in the visceral unstriated muscles. Moreover in the central nervous system the main tracts cannot be shown to be functional before the date at which they acquire their medullary sheaths, suggesting that previously any impulse making its way along the tract underwent dissipation before arriving at its destination. It is possible too that the myelin sheath may serve as a source of nutrition to the enclosed axis cylinder, which, in the greater part of its course, is far removed from its trophic centre, namely, the cell of which it is an outgrowth. This trophic function of the myelin sheath has a certain basis of fact in that the myelin sheath is as a rule larger in those fibres which take the longer course. 


\section{SECTION II \\ PROPAGATION ALONG NERVE FIBRES}

THE velocity of propagation along a nerve fibre may be measured, although in early times it was thought to be as instantaneous as the lightning flash. To measure the velocity of propagation in a motor nerve, a frog's gastrocnemius is prepared, with a long piece of sciatic nerve attached. The muscle is arranged (Fig. 103) so that its contraction may be recorded on a rapidly moving surface, on which are also recorded, by means of electro-magnetic

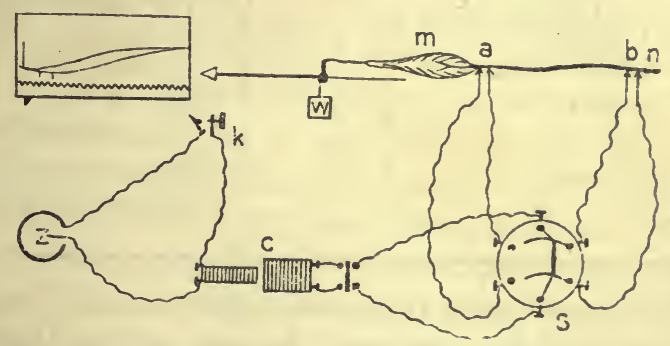

FIG. 103. Diagram of arrangement of experiment for the determination of the velocity of transmission of a motor impulse down a nerve.

The battery current passes through the primary coil of the inductorium $c$, and $\mathrm{a}$ 'kick over' key $k$. By means of the switch $\mathrm{s}$, the break shock in the secondary circuit can $b$ s sent through the nerve $n$, either at $b$ or at $a$. The muscle $m$ is arranged to write on the blackened surface of a trigger or pendulum myograph, and is excited during the passage of the recording surface by the automatic opening of the key $k$. (The time-marker is not shown.)

signals, the moment at which the stimulus is sent into the nerve, and also a time-marking showing $\frac{1}{250}$ sec. Tracings are now taken of the contraction of the muscle : first, when the nerve is stimulated at its extreme upper end ; secondly, as close as possible to the muscle. It will be found that the latent period, which elapses between the point at which the stimulus is sent into the nerve and the point at which the lever begins to rise, is rather longer in the first case than in the second. The difference in the two latent periods gives the time that the nervous impulse has taken to travel down the length of nerve between the two stimulated points. Calculated in this way, the velocity of propagation in frog's nerve is about 28 metres per second.

In man and in warm-blooded animals the velocity has been variously estimated at from 60 to 120 metres per second. The higher of these figures is probably nearer the truth. 
On the other hand, in invertebrata the velocity of propagation along nerve fibres may be quite slow. The following Table represents the velocity of transmission along a number of different fibres, as determined by Carlson, compared with the duration of a single muscle-twitch in the same animal.

\begin{tabular}{|c|c|c|c|c|c|c|c|}
\hline \multirow{2}{*}{\multicolumn{2}{|c|}{ Species }} & & \multicolumn{3}{|c|}{ Muscle } & \multicolumn{2}{|l|}{ Nerve } \\
\hline & & & \multicolumn{2}{|c|}{ Muscle } & $\begin{array}{l}\text { Contrac- } \\
\text { tion } \\
\text { time in } \\
\text { seconds }\end{array}$ & Nerve & $\begin{array}{c}\text { Rate of } \\
\text { the impulse } \\
\text { in metres } \\
\text { per seconó }\end{array}$ \\
\hline Frog & . & & \multicolumn{2}{|c|}{ Gastrocnemius } & $0 \cdot 10$ & $\begin{array}{l}\text { Sciatic } \\
\text { (medullated) }\end{array}$ & $27 \cdot 00$ \\
\hline Snake & & & \multicolumn{2}{|c|}{ Hyoglossus } & $0 \cdot 15$ & $\begin{array}{l}\text { Hyoglossal } \\
\text { (medullated) }\end{array}$ & $14 \cdot 004$ \\
\hline $\begin{array}{l}\text { Lobster } \\
\text { (Homar }\end{array}$ & rus) & . & \multicolumn{2}{|c|}{$\begin{array}{l}\text { Adductor of } \\
\text { forceps }\end{array}$} & 0.25 & $\begin{array}{l}\text { Ambulacral } \\
\text { (non-medullated) }\end{array}$ & $12 \cdot 00$ \\
\hline Hagfish & . & . & \multicolumn{2}{|c|}{ Retractor of jaw } & $0 \cdot 18$ & $\begin{array}{l}\text { Mandibular } \\
\text { (non-medullated) }\end{array}$ & $4 \cdot 50$ \\
\hline Limulus & - & . & \multicolumn{2}{|c|}{$\begin{array}{l}\text { Adductor of } \\
\text { forceps }\end{array}$} & $1 \cdot 00$ & $\begin{array}{l}\text { Ambulacral } \\
\text { (non-medullated) }\end{array}$ & $3 \cdot 25$ \\
\hline Octopus & . & . & Mantle & . & 0.50 & $\begin{array}{l}\text { Pallial } \\
\text { (non-medullated) }\end{array}$ & $2 \cdot 00$ \\
\hline Slug (Lim & $\operatorname{nax})$ & . & Foot & . & $4 \cdot 00$ & $\begin{array}{l}\text { Pedal } \\
\text { (non-medullated) }\end{array}$ & $1 \cdot 25$ \\
\hline Limulus & . & . & Heart & . & $2 \cdot 25$ & $\begin{array}{l}\text { Nerve plexus in heart } \\
\text { (non-medullated) }\end{array}$ & $0 \cdot 40$ \\
\hline
\end{tabular}

The velocity of propagation in sensory nerves is more difficult to determine owing to the fact that a sensory impulse, on arrival at the receiving organ-i.e. some part of the central nervous system-does not at once give rise to some definite recordable mechanical change, such as a muscular contraction. There is another method of determining the velocity of conduction, which may be used also with sensory fibres. The passage of a nerveimpulse down a nerve, just as the passage of a wave of contraction along a muscle fibre, is immediately preceded or accompanied by an electrical change, which also travels along the nerve as a wave of 'negativity.' The velocity of propagation of this wave may be measured, and is found to give the same numbers as the velocity determined by the preceding method.

The existence of this electrical change enables us to show that a nerveimpulse, excited at any point in the course of a nerve fibre, travels in both directions along the fibre. The power of nerves to transmit impulses in either direction is shown further by the experiment known as Kühne's gracilis experiment. The gracilis muscle of the frog is separated into two portions by a tendinous intersection, so that there is no muscular continuity between the two halves. The nerve to the muscle divides into two branches, one to each half, and at the point of junction there is division of the axis cylinders themselves. If the section $a$ in the diagram (Fig. 104), which is quite isolated from the rest of the muscle, be stimulated, as by snipping it with scissors, 
the whole muscle contracts. If the portion of the muscle which is free from nerve fibres be stimulated in the same way, the contraction is limited to the fibres directly stimulated, showing that in the first case the stimulus excited nerve fibres which transmitted the impulse up the nerve to the point of division and then down again to the other half of the muscle.

Since nerves have this power of conduction in both directions, it might be thought that a single set of nerve fibres might very well subserve both afferent and efferent functions, at one time conducting sensory impulses from periphery to cord, at another time motor impulses from cord to muscles. But this is not the case. As a matter of fact we find in the body a marked differentiation of function between various nerve fibres. Thus Bell and Majendie showed that the spinal roots might be divided into afferent and efferent, the anterior roots carrying only impulses from spinal cord to periphery, while the posterior roots carried impulses from periphery to central nervous system. The law known by the name of these observers states indeed that a nerve fibre cannot be both motor and sensory. We may find both kinds of fibres joined together into a single nerve-trunk, but the fibres in each case are isolated and conduct impulses only

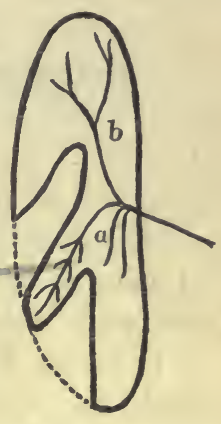

Fig. 104. Kühne's gracilis experiment. in one or other direction. Under normal conditions the afferent fibres are excited only at their endings on the surface of the body, while the efferent fibres are excited only at their origin from the spinal cord. The difference in the function of different nerve fibres depends therefore not so much on the structure of the nerve fibre itself as on the connections of the fibre. We can show this experimentally by grafting one set of nerve fibres on to another. If the cervical sympathetic be united to the lingual nerve, stimulation of the sympathetic, instead of causing, as usual, constriction of the vessels of the head and neck, will cause dilatation of the vessels of the tongue and secretion of watery saliva. In the same way the finer functional differences between the various forms of sensory nerves seem to be determined by their connections within the central nervous system. Stimulation of the optic nerve by any means whatsoever evokes a sensation of light. One and the same stimulus applied to different nerves will evoke different sensations, e.g. a tuning-fork applied to therskin will give a sensation of vibration, to the ear a sensation of sound. We shall have occasion to return to this question of the restricted function of nerve fibres when we deal with Müller's ' law of specific irritability' in the chapter on Sensations. 


\section{SECTION III}

\section{EVENTS ACCOMPANYING THE PASSAGE OF A NERVOUS IMPULSE}

In muscle we saw that the passage of an excitatory wave was accompanied or followed by electrical changes, production of heat, and mechanical change, all pointing to an evolution of energy from the explosive breaking-down of contractile material.

In nerve, however, which serves merely as a conducting medium, we should not expect so much expenditure of energy, or in fact any expenditure at all. All that is necessary is that each section of the nerve should transmit to the next section just so much kinetic energy as it has received from the section above it. And experiment bears out this conclusion. The most refined methods have failed to detect the slightest development of heat in a nerve during the passage of an excitatory process, and we know already that there is no mechanical change in the nerve. The only physical change in a nerve under these circumstances is the development of a current of action. A nerve becomes, when excited at any point, negative at this point to all other parts of the nerve, and, just as in muscle, this ' negativity' is propagated in the form of a wave in both directions along the nerve.

That the excitatory process in nerves is probably accompanied by certain small chemical changes is indicated by the facts that, in the complete absence of oxygen, the nerve fibres lose their irritability, and that this loss of irritability is hastened by repeated stimulation of the nerve. When the irritability has been abolished by stimulation in the absence of oxygen, it may be restored within a few minutes by readmission of oxygen to the nerve.

If we connect a galvanometer to two points of an uninjured nerve, no current is observed, all points of a living nerve at rest being isoelectric. On making a cross-section of the nerve at one leading-off point, a current is at once set up, which passes from the surface through the galvanometer to the cross-section. This is a demarcation current, set up at the junction between living and dying nerve. This current rapidly diminishes in strength and finally disappears, owing partly to the fact that the dying process started in the nerve by the section extends only as far as the next node of Ranvier and there ceases, so that after a short time the electrode applied to the crosssection is simply leading off an intact living axis cylinder through the dead portion of the nerve, which acts as an ordinary moist conductor. On making a fresh section just above the previous one, the process of dying is again set 


\section{EVENTS ACCOMPANYING A NERVOUS IMPULLSE}

up, and the demarcation current is restored to its original strength. If, while the demarcation current is at its height, we stimulate the other end of the nerve with an interrupted current, the needle of the galvanometer swings back towards zero, i.e. there is a negative variation of the resting current.

In order to demonstrate the wave-like progression of the electrical change from the excited spot along the nerve, it is necessary, as in the case of muscle, to make use of a very sensitive capillary electrometer or a string galvanometer. It is then found that the change progresses along the nerve at the same rate as the nervous impulse, i.e. 28 to 33 metres per second in the frog. But it lasts only an extremely short interval of time at each spot, viz. six to eight ten-thousandths of a second. Thus the length of the excitatory wave in nerve is about $18 \mathrm{~mm}$. 


\section{SECTION IV}

\section{CONDITIONS AFFECTING THE PASSAGE OF A NERVOUS IMPULSE}

TEMPERATURE. Below a certain temperature the propagation of the excitatory process in the nerve is absolutely abolished. The exact temperature at which this occurs varies according as we use a warm- or a cold-

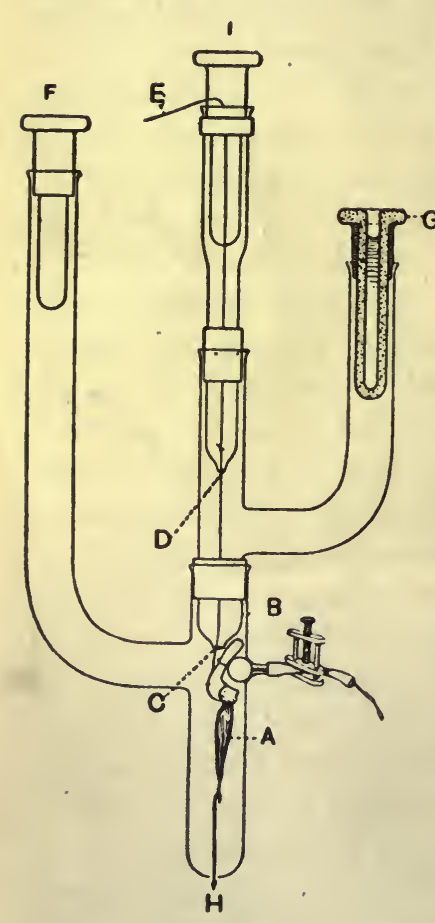

Fig. 105. blooded animal. In the frog it is necessary to cool the nerve below $0^{\circ} \mathrm{C}$. before conduction is abolished, whereas in the mammal it is sufficient to cool the nerve to somewhere between $0^{\circ}$ and $5^{\circ}$ C. Since cooling the nerve does not excite it, this procedure forms a convenient method for blocking the passage of impulses along a nerve without using the irritating procedure of section. On warming the nerve again the conductivity returns. The rapidity with which the excitatory process is propagated along either a nerve or a muscle fibre depends on the temperature. Thus the mean rate of conduction in the frog's nerve at $8^{\circ}$ to $9^{\circ} \mathrm{C}$. is about 16 metres per second. The temperature coefficient of the velocity of nerve propagation, i.e. $\frac{\text { velocity at } \mathrm{Tn}+10}{\text { velocity at } \mathrm{Tn}}$ has been found by Lucas to be about $1 \cdot 79$. The same value was found by Maxwell for conduction in molluscan nerve, and in frog's striated muscle Woolley found the temperature coefficient for conduction of the excitatory process to vary between $1 \cdot 8$ and 2 .

An ingenious method (Fig. 105) has been used by Keith Lucas for the determination of the conduction rates in nerve at different temperatures. The glass vessel represented in the figure is filled with Ringer's solution, in which the whole nerve-muscle preparation is immersed. The muscle used was the flexor longus digitorum, so that the whole length of the sciatic, tibial, and sural nerves could be used. The nerve is passed up through the constrictions in the inner glass vessels at $\mathbf{C}$ and $\mathbf{D}$, and is attached to the thread E, F, I, and $\mathrm{G}$ are three non-polarisable electrodes composed of porous 
clay, containing saturated zinc sulphate, in which a zinc rod is immersed. If the current is passed in at $G$ and out at $F$ the effective cathode is at the lower end of the constriction C, and similarly if the current is passed in at I and out at $G$, the effective cathode is at D. The tendon of the muscle $A$ is attached by a thin glass rod $\mathrm{H}$ to a very light recording lever, the movement of which is magnified by placing it in the focal plane of a projecting eye-piece and recording its image on a moving sensitive plate. The whole apparatus, with the exception of the glass rod at $\mathrm{H}$, can be immersed in a water bath at any given temperature. Two records are taken with the whole apparatus, first stimulating at $\mathrm{c}$, and sccondly stimulating at $\mathrm{D}$. The difference between the latent periods in these two cases is the time taken for the excitatory wave to travel from $\mathrm{D}$ to $\mathrm{C}$. The rate of propagation is similarly recorded when the water bath is raised to $18^{\circ} \mathrm{C}$. or to any desired temperature. Since we are only dealing with differences in latent periods the effect of the rise of temperature on the latent period of the muscle itself does not affect the determinations.

THE INFLUENCE OF FATIGUE.- In the description of the phenomena of muscular fatigue given in the last chapter it was assumed that the muscle was being ex-

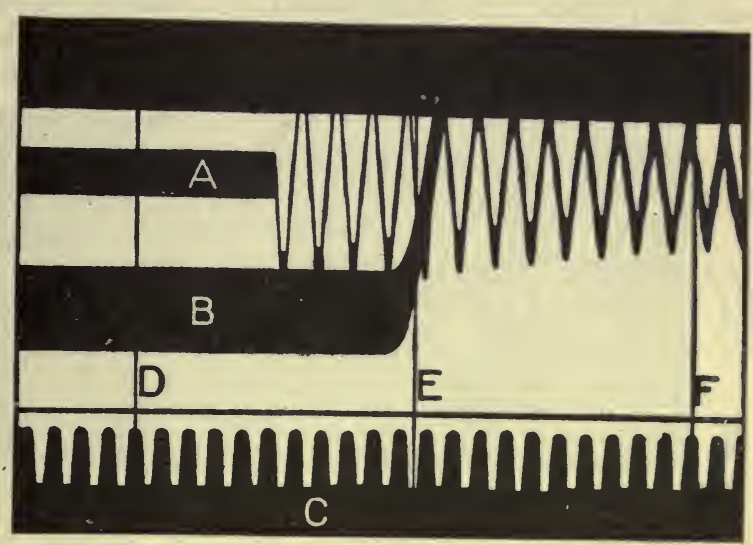

FIG. 106. Curve of muscle-twitch obtained by foregoing method. (KerTH LuCAS.) $\mathbf{A}=$ moment of excitation. $\mathbf{B}=$ movement of muscle. $\mathrm{C}=$ time-marker. cited directly. The same phenomena are observed when the muscle is excited through its nerve, though in this case fatigue comes on much more quickly. If, after the muscle has been excited in this way until exhausted, it be excited directly, it will respond with a contraction nearly as high as at the beginning of the experiment. We see therefore that the nervous structures are more susceptible to the influences causing fatigue than the muscle itself, and it can be shown that the weak point in the nervemuscle preparation is not the nerve, but the end-plates. In fact it is not possible to demonstrate any phenomena of fatigue in the nerve-trunk.* This fact can be shown in mammals by poisoning the animal with curare, and then stimulating a motor-nerve continuously while the animal is kept alive by means of artificial respiration. As the effect of the curare on the endplates begins to wear off in consequence of its excretion, the muscles supplied by the stimulated nerve enter into tetanus. The action of the curare may be cut short at any time by the injection of salicylate of physostigmine, when the muscles will at once begin to react to the excitation.

The same fact may be shown on the excised nerve-muscle preparation of the frog. The gastrocnemii of the two sides with the sciatic nerves are dissected out, and an exciting circuit is so arranged that the interrupted

* Unless it be asphyxiated by total deprivation of oxygen. 
secondary currents pass through the upper ends of both nerves in series (Fig. 107). At the same time a constant cell is connected with two non-polarisable electrodes $(n p, n p)$ placed on the nerve of $\mathrm{B}$, so that a current runs in the nerve in an ascending direction. The effect of passing a constant current through a nerve is to block the passage of impulses through the part traversed

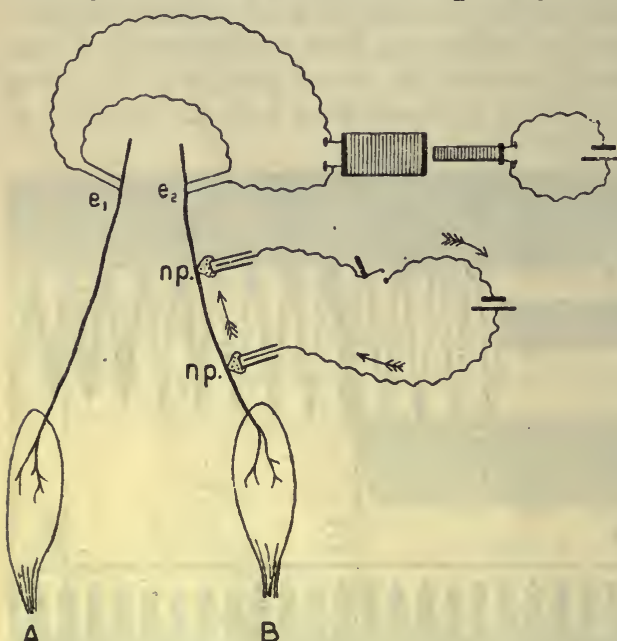

FIG.107. Arrang ement of experiment for demonstrating the absence of fatigue in medullated nerve fibres.

EC, exciting circuit ; $\mathrm{CP}$, polarising circuit.

by the current. When the constant polarising current is made, the muscle may give a single twitch, and then remains quiescent. The exciting current is then sent through both nerves by the electrodes $e_{1}$ and $e_{2}$. The muscle A enters into tetanus, which gradually subsides owing to "fatigue." When a no longer responds to the stimulation, the constant current through the nerve of $\mathrm{B}$ is broken. B at once enters into tetanus, which lasts as long as the contraction did in the case of $\mathbf{A}$, and gradually subsides as fatigue comes on. Since both nerves have been excited throughout, it is evident that the fatigue

does not affect the nerve-trunk. We have already seen that a muscle will respond to direct stimulation when stimulation of its nerve is without effect: and must therefore conclude that the first seat of fatigue is the junction of nerve and muscle, i.e. the end-plates.

In the normal intact animal the break in the neuro-muscular chain which is the expression of fatigue occurs still higher up, i.e. in the central nervous system, and is probably due to some reflex inhibition of the central motor apparatus from the muscle itself. Thus after complete fatigue has been produced in a muscle so far as regards voluntary efforts, direct stimulation of the muscle itself or its nerve will produce a contraction as great as would have been the case at the beginning of the experiment.

THE INFLUENCE OF DRUGS, The most important drugs with an influence on nerve fibres are those belonging to the

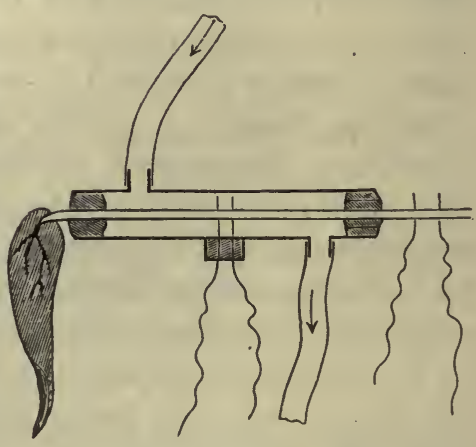

Fig. 108. class of anæsthetics. Of these we may mention carbon dioxide, ether, chloroform, and alcohol.

The action of any of these substances on the excitability and conductivity of a nerve may be studied by means of the simple apparatus represented in Fig. 108. The nerve 
of a nerve-muscle preparation is passed through a glass tube which is made air-tight by plugs of normal saline clay surrounding the nerve at the two ends of the tube. By means of two lateral tubulures a current of $\mathrm{CO}_{2}$, or air charged with vapour of ether or other narcotic, can be passed through the tube. The nerve is armed with two pairs of electrodes which are stimulated alternately, the pair within the tube serving to test the action of the drug on the excitability, while the pair outside the tube show the presence or absence of any effect on the conducting power of the nerve below it.

Of the gases and vapours mentioned above, $\mathrm{CO}_{2}$ and ether both diminish and finally abolish the excitability and conductivity of the nerve fibres. The conductivity, however, persists after all trace of excitability has disappeared, before in its turn being also abolished. On removing the gas

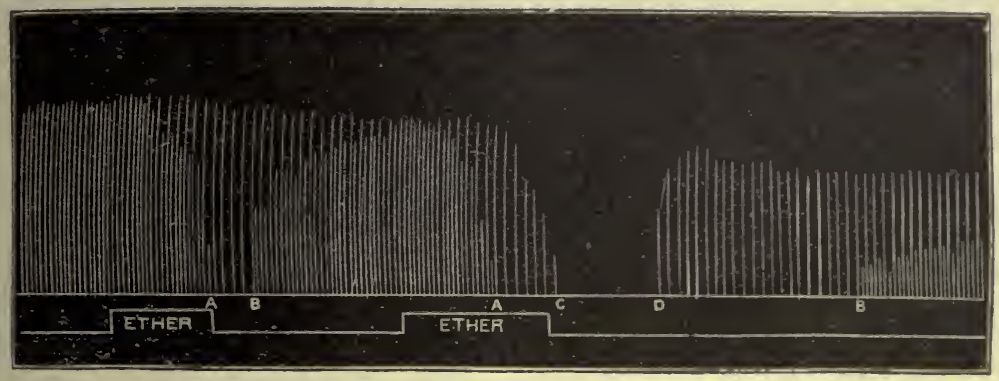

FIG. 109. Tracing to show the effect of ether on excitability and conductivity of nerve. Nerve excited by single induction shocks alternately within and above ether, chamber. The vertical lines indicate contractions of the muscle (gastrocnemius.) The lower line indicates the periods during which the nerve was exposed to the action of ether.

A, disappearance of excitability; $\mathbf{B}$, reappearance of excitability; $\mathbf{c}$, disappearance of conductivity; D, reappearance of conductivity. (From a tracing kindly lent by Prof. Gotch.)

or vapour by blowing air over the nerve, the conductivity and excitability gradually return in the reverse order to their disappearance (Fig. 109).

Alcohol is said to increase the excitability or leave it unaffected, while diminishing the conductivity of the nerve.

Chloroform rapidly abolishes both excitability and conductivity. It is a much more severe poison than the drugs just mentioned, so that in many cases its effects are permanent, and no, or only a very partial, recovery of the nerve is obtained on removal of the chloroform vapour from the apparatus. 


\section{SECTION V}

\section{THE EXCITATION OF NERVE FIBRES}

MANY different forms of stimuli may be used to arouse the activity of an excitable tissue such as muscle or nerve. Thus we may use thermal, mechanical, or chemical stimuli. If the temperature of a motor nerve be gradually raised, no effect is noticed till about $40^{\circ} \mathrm{C}$. is reached, when the muscle may enter into weak quivering contractions. Sudden warming of the nerve always gives rise to excitation. At about $45^{\circ} \mathrm{C}$. the nerve loses its irritability and dies. On the other hand, a nerve may be rapidly cooled without any excitation taking place.

A nerve may be excited mechanically by crushing or cutting. These methods destroy the nerve. It is possible to excite a nerve mechanically, without any serious injury to it, by carefully graduated taps, and this method has been used in investigating the phenomena of electronus.

All chemical stimuli applied to the nerve have a speedy effect in destroying its irritability. The chemical stimuli most used are strong salt solutions, glycerin, or weak acids. If any one of these be applied to a motor nerve, the muscle enters into an irregular tetanus, which lasts till the irritability of the nerve is destroyed at the part stimulated.

None of these forms of stimuli can be adequately controlled either as to strength or duration. Moreover, owing to their destructive effects, any repetition of the stimulus will fall on a nerve or muscle more or less altered by the first stimulus. We are therefore justified in the use of electrical stimuli not only for arousing the activity of excitable tissues, but also for determining the conditions of excitation of muscle and nerve. For this purpose we may use either the make and break of a constant current, the induced current of short duration produced in a secondary coil of an inductorium by the make or break of the primary circuit, or the discharge of a condenser.

The last-named method of stimulation is especially useful when we desire to determine the total amount of energy involved in the electrical stimulation of a nerve or muscle. The arrangement of such an experiment is shown in Fig. 110. By means of the switch $\mathrm{S}$ the condenser can be put into connection either with the battery from which it receives its charge or with the nerve through which it can discharge. By knowing the capacity of the condenser and the electromotive force by which it is charged, we can estimate the energy of the charge sent through the nerve.

$\mathrm{E}$ (energy in ergs) ${ }^{*}=5 \mathrm{FV}^{2}$

( $\mathrm{F}=$ capacity in microfarads ; $\mathrm{V}=$ electromotive force in volts).

* An erg is the amount of work produced or energy expended by the action of one dyne through one centimetre. A dyne is the force which will give to a mass of one gram an acceleration of one centimetre per second per second. 

In this way it has been found that the energy of a minimal effective stimulus for frog's
nerve is about $\frac{1}{1000}$ of an erg.

The amount of energy necessary to excite the nerve will vary with the rate at which the condenser is allowed to discharge through the nerve. Its rate can be modified by altering the resistance in the discharging circuit or by altering the electromotive force of the charge. This method has been adopted by Waller in determining the rate of change at which excitation is obtained with a minimal expenditure of energy, which he calls the "characteristic " of the tissue in question. To this point we shall have occasion to refer later.

When using the make and break of a constant current as a stimulus, the first fact of importance is the relation of the seat of excitation to the poles by which the current is led into or out of the excitable tissue. We have already seen that when a current is passed through a muscle or nerve the muscle contracts only at make or at break of the current, no propagated excitatory effect being produced during the passage of the current. The excitation at make is obtained with a smaller current than the excitation at break.

Besides this difference in intensity, there is a difference in the point from which excitation starts. A make contraction starts from the cathode, a break contraction from

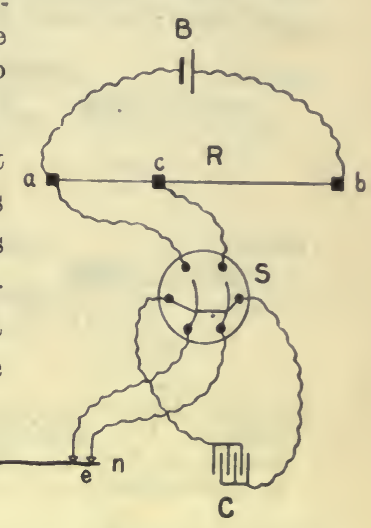

Fig. 110. Arrangement of apparatus for the excitation of a nerve by means of condenser discharges.

$\mathrm{B}$, battery; $\mathrm{R}$, rheochord; $c$, rider of rheochord; s, switch (Pohl's reverser without cross wires); c, condenser; $n$, nerve ; $m$, muscle; $e$, nonpolarisable electrodes. the anode. This is well shown by the two following experiments:

(a) A curarised sartorius muscle of the frog (Fig. 111), with its bony insertions still attached, is fastened at the two ends to two electrodes, which are able to swing when the muscle contracts, and are attached by threads to levers which serve to record the contraction. The middle of the muscle is

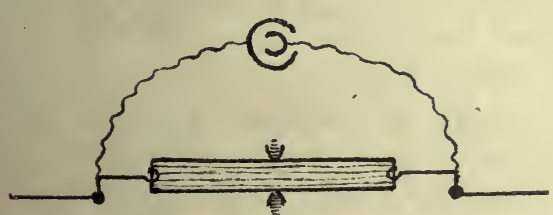

Fic. 111. Sartorius clamped in middle and attached to levers at either end. muscle-rises before the other lever. On the other hand, on breaking the current the lever at the anode rises first, showing that the anodic half of the muscle contracts before the cathodic half.

(b) The irritability of a muscle, i.e. its power of responding to a stimulus by contracting, is intimately dependent on the life of the muscle. If the muscle be injured or killed at any spot, its irritability at this spot will be therefore diminished or destroyed. Hence, if we stimulate a muscle at the injured spot, no contraction will ensue. This fact may be used to demon- 
strate the production of excitation at cathode on make, and at anode on break of a constant current.

A muscle with parallel fibres, such as the sartorius, is injured at one end, and a constant current passed, first from the injured to the uninjured end, and then in the reverse direction (Fig. 112). It is found in the former case, when the anode is on the injured part (which is therefore less excitable),

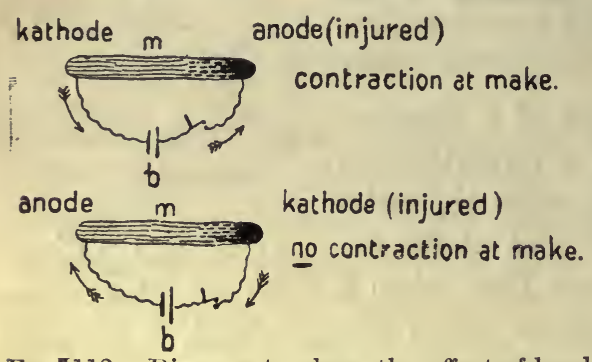

Fig. 1112 . Diagram to show the effect of local injury on the excitability of a muscle. $b$, at break. Every induction shock battery; $m$, muscle. The arrows indicate can be therefore regarded as a
the direction of the current. the direction of the current. that break of the current is ineffective, and in the latter, when the cathode is on the injured surface, that the make stimulus is ineffective, showing that the part excited corresponds to the cathode at make and to the anode at break.

With a current of very short duration no excitation is produced make stimulus, and when such a shock is led through a muscle the contraction in each case will start at the cathode, i.e. the point at which the induction shock leaves the muscle. The results of stimulating nerve fibres are similar to those obtained by stimulating muscle fibres directly.

Under normal circumstances, if a constant current be passed through the nerve of a nerve-muscle preparation for a short time, the muscle responds only at the make and the break of the current, remaining perfectly quiescent all the time the current is passing. If the nerve be in a very excitable condition, it is possible that the muscle may be thrown into a tetanus or continued contraction during the whole time that the current is passing ('closing tetanus'). On the other hand, if a strong ascending current be passed through the nerve for a considerable time, the muscle when the current is broken may go into continued contraction, which may last some time. Normally, however, the muscle simply responds with a single twitch at the make and break of the current, although, on investigating the condition of the nerve during the passage of the current, we find that it is considerably modified. This modification in the condition of the nerve is spoken of as electrotonus, and includes changes in its irritability and its electrical condition.

To investigate these changes the following apparatus is necessary: two constant batteries, induction-coil, a reverser and keys, a pair of non-polarisable electrcdes, and a pair of ordinary platinum electrodes. Fig. 113 represents roughly the arrangement of the experiment. A constant current from the battery is led through a part of the nerve by means of non-polarisable electrodes, which are about one inch apart. In this circuit we put a reverser, by means of which the direction of the current of the nerve may be changed at will, and a key to make or break the current. This is the polarising circuit. The other battery is arranged in the primary circuit of the coil, a key being interposed, so that we may use make or break induction shocks, which are applied to the nerve by means of the small platinum electrodes. The tendon of the muscle is connected by a thread with a lever, which is arranged to write on a smoked surface, so that the height of the contraction can be recorded. 


\section{'THE EXCITATION OF NERVE FIBRES}

We first find the position of the secondary coil, at which the broak induction shock is a submaximal stimulus, and we employ this strength of stimulus throughout the experiment. The make induction shock is prevented from acting on the nerve by closing a shortcircuiting key in the circuit of the secondary coil. The nerve is now stimulated at various points with a single break induction shock, and the contractions recorded. The heights of these contractions serve to indicate the irritability of the nerve at the point stimulated. We now throw the polarising current into the nerve. At the make of this current the muscle will probably respond with a twitch which is not recorded. We then test once more the irritability of different points of the nerve, and we find that, when the stimulus is applied near $a$, the point where the current enters the nerve (anode), the stimulus, which before gave a moderately large contraction of the muscle, now has either no effect or else produces a very weak contraction. On the other hand, in the region of the cathode the stimulus, which before was submaximal, has now become maximal, as is shown by the increase in the height of the contraction evoked by the induction shock.

We now reverse the direction of

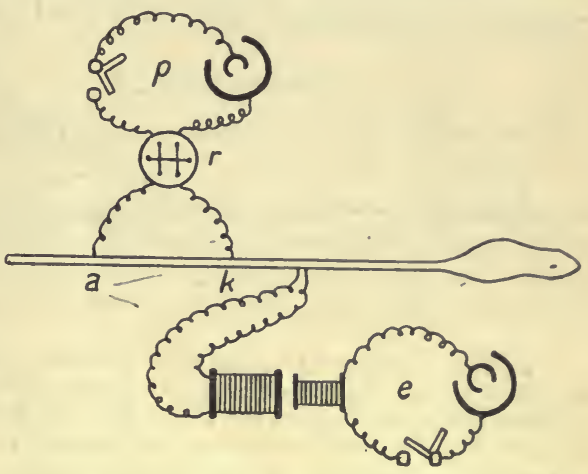

FIG. 113. Arrangement of apparatus for showing electrotonic changes in irritability. $e$, exciting current; $p$, polarising current ; $r$, Pohl's reverser. the polarising current, so that the current of the nerve runs from $k$ to $a$. With this reversal of current there is also a reversal of the changes in the nerve; that is to say, the normally submaximal stimulus is maximal when applied near $a$, and minimal when applied near $k$. On break of the polarising current the condition of the nerve returns to normal, and the submaximal stimulus is once more submaximal throughout.

This return to normal conditions, however, is not immediate, since the first effect of breaking the current is a swing-back, so to speak, past the normal, the diminished irritability at the anode giving place to an increased irritability, which only gradually subsides. In the same way, immediately after the polarising current has ceased to flow, the neighbourhood of the cathode acquires a condition of diminished irritability, and this only gradually gives place to a normal condition.

This experiment teaches us that, when a constant current is passed through a nerve, there is increase in the irritability in the nerve near the cathode, and a diminution in irritability near the anode. These conditions of increased and diminished irritability are spoken of as catelectrotonus and anelectronus respectively. In muscle we have seen that a make contraction always starts from the cathode, and a break contraction from the anode. Now the event that takes place at the cathode on make and at the anode on break of a constant current is, as the last experiment shows us, a rise in irritability, in the former case from normal to above normal, in the latter from subnormal to normal. Hence we may say that the excitation is caused by a sudden rise of irritability, which may be due either to a sudden appearance of catelectrotonus, or a sudden disappearance of anelectrotonus. I have said sudden because the steepness of the rise of irritability is a necessary factor in causing excitation. If the polarising current passing through a nerve be slowly and gradually increased to considerable strength, it will 


\section{PHYSIOLOGY}

give rise to no contraction. The degree of suddenness of the rise, which is most beneficial in causing contraction, varies with the nature of the tissue stimulated. Thus it is more rapid in nerve than in muscle, and in pale muscle than in red muscle, and in voluntary muscle than in unstriated muscle.

It is evident that there must be, somewhere between the anode and cathode, an indifferent point-that is to say, a region where the irritability

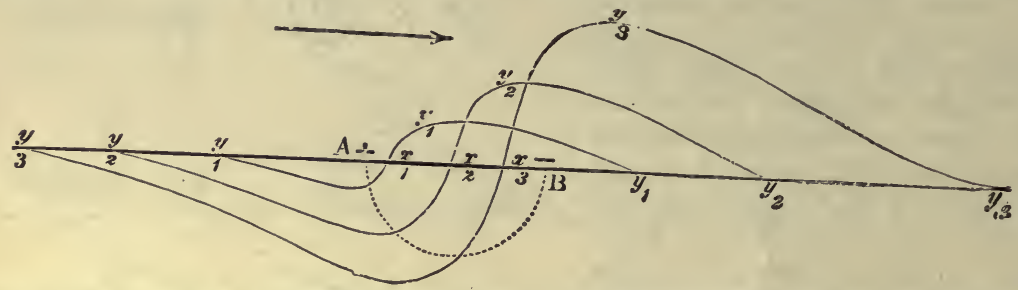

Fig. 114. Diagram to show the variations of irritability in a nerve during the passage of polarising currents of different strengths. The degree of change is represented by the distance of the curves from the base line; the part of the curve below the line signifying decrease, that above the line increase of irritability.

A, anode ; B, cathode ; $y_{1}$, effect of weak current ; $y_{2}$, medium current ; $y_{3}$, strong current. It will be noticed that the indifferent point, $x$, where the curve crosses the horizontal line, approaches nearer and nearer the cathode as the currcnt is increascd in strength. (From FOSTER, after PFLÜGER.)

is neither increased nor diminished. We find experimentally that this indifferent point is nearer the anode when the polarising current is weak, and approaches the cathode as the current is strengthened, so that with very strong currents nearly the whole intrapolar length is in a condition of anelectrotonus (Fig. 114). When a strong polarising current is used, the depression of irritability at the anode is so marked that no impulse can pass
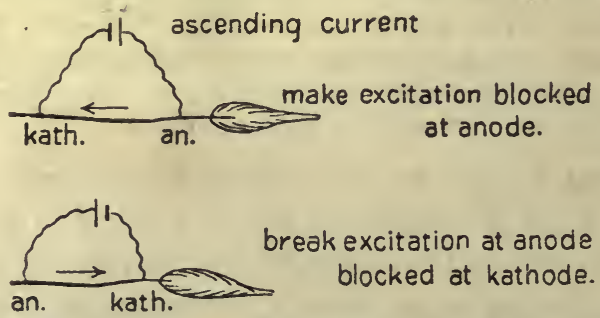

FIG. 115. Diagram to show the blocking effect of a strong constant current passed through the nerve of a nerve-muscle preparation.

this region. Thus if we send a very strong ascending current through the nerve, there is no contraction at make. This is owing to the fact that the impulse started at the cathode on make of the current cannot reach the muscle, its passage down the nerve being blocked in the region of the anode (Fig. 115, A).

The results of stimulating motor nerves by means of constant currents were studied by Pflüger and, embodied in a Table, make up what is known as Pflüger's law. The result of stimulating varies with the strength of a current. 
LAW of Contraction

\begin{tabular}{|c|c|c|c|c|c|c|c|}
\hline \multicolumn{4}{|c|}{ Strength of current } & \multicolumn{2}{|c|}{ Ascending } & \multicolumn{2}{|c|}{ Descending } \\
\hline Weak & $\cdot$ & • & - & $\begin{array}{c}\text { Make } \\
\text { c }\end{array}$ & $\begin{array}{c}\text { Break } \\
\text { O }\end{array}$ & $\begin{array}{l}\text { Make } \\
\text { c }\end{array}$ & $\begin{array}{c}\text { Break } \\
\text { O }\end{array}$ \\
\hline Medium & • & · & - & c & c & C & c \\
\hline Strong & & . & . & $\mathrm{O}$ & $\mathrm{C}$ or $\mathrm{T}$ & $\mathrm{C}$ or $\mathrm{T}$ & 0 \\
\hline
\end{tabular}

$\mathrm{c}=$ contraction. $\quad \mathrm{C}=$ strong contraction. $\mathrm{T}=$ tetanus. $\mathrm{O}=$ no effect.

With the weakest currents excitation occurs only at make, since a make-stimulus, i.e. the rise of catelectrotonus, is always more effectual than a break-stimulus, i.e. the disappearance of anelectrotonus. With currents of moderate strength excitation occurs both at make and break, being better marked at make, especially in the case of

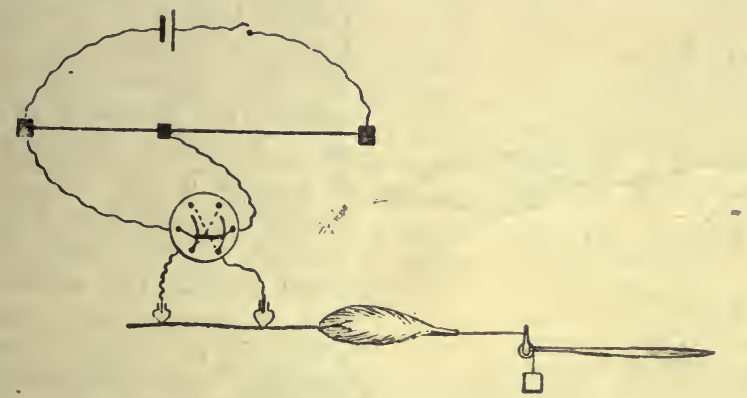

FIG. 116. Arrangement of experiment to demonstrate Pflüger's law of contraction.

descending currents. With very strong currents we get a contraction at make only when the current is descending, since, when the current is ascending, the excitation started at the cathode cannot pass the block at the anode. For the same reason a break contraction is obtained only with an ascending current, since at the break of a descending current there is a swingback of the nerve at the cathode to a condition of diminished irritability, which effectually blocks the excitation started higher up the nerve at the anode.

The arrangement of the experiment for demonstrating Pflüger's law is shown in Fig. 116. The strength of the current is graduated by means of the rheochord, the current being led into the nerve by means of nonpolarisable electrodes. It is extremely important in these experiments to avoid any injury or drying of the nerves at either of the two electrodes, since the excitatory effect either at make or break would be abolished by local injury.

These results, worked out chiefly on motor nerves, have been confirmed as far as possible experimentally on sensory nerve, and on muscle and contractile tissues generally, and probably hold good for all irritable living tissues.

It is said that an anelectrotonus takes some time to attain its full height, and a catelectrotonus reaches its maximum almost directly after the current is made, and that it is on this account that a current of very short duration excites only at the make, the break occurring before the anelectrotonus is developed enough for its disappearance to cause a stimulus.

Other things being equal, a current of given strength causes a stronger 
excitation the greater the length of nerve that it flows through. It must be remembered, however, that the nerve offers considerable resistance to the passage of the current, and so, to keep the current constant while increasing the length of intrapolar nerve, we must largely increase the electromotive force employed.

A very convenient method of showing the effect of the length of intrapolar nerve on excitation has been suggested by Gotch. The two sciatic nerves of a frog are dissected

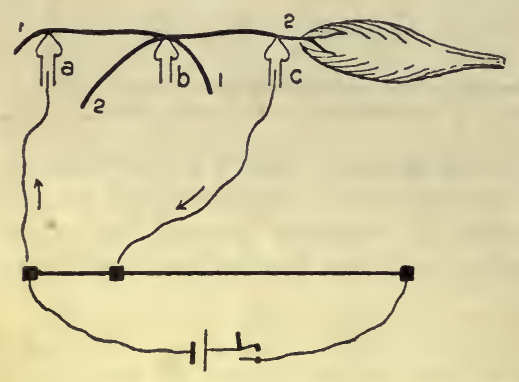
out, one of them being in connection with the gastrocnemius. These are first arranged as in Fig. 117. $a . b$, and $c$ are three non-polarisable electrodes, the terminals of a constant battery being connected to $a$ and c. The position of the rider on the rheochord is then ascertained at which make of the current just excites contraction in the muscle of nerve 2, the current in this case passing from $a$ to $b$ along nerve 1 , and from $b$ to $c$ along a small piece of nerve 2 . We will suppose that eleven units of current are necessary to produce excita. tion. $b$ is then withdrawn and the nerve 2 laid on a (Fig. 117, B), so that the current can now pass from $a$ to $c$ entirely through a long stretch of nerve 2. On again seeking the minimal stimulus, it will be found that a smaller current is sufficient to excite, contraction being obtained with seven units. Since the FIg. 117.

length of nerve traversed, and therefore the resistance to the ourrent, are the same in both cases, it is evident that a current is more effective the greater the length of excited nerve that it traverses.

A nerve cannot be excited by currents passed transversely across it, since in such cases the anode and cathode lie so close to one another in a nervefibril, as it is traversed by a current, that their effects counteract one another.

\section{ELECTRICAL STIMULI AS APPLIED TO HUMAN NERVES}

When we attempt to apply the results gained on frog's nerves to man, we are met at once by the difficulty that we cannot dissect out the nerves and apply stimuli to them directly. So usually unipolar excitation is used, one electrode, either anode or cathode, being applied to the skin over the nerve to be stimulated, and the other to some indifferent point, such as the back. It is evident under these circumstances that the current is concentrated as it leaves the anode and reaches the cathode, and diffuses widely in the body, seeking the lines of least resistance. Thus it is impossible to get pure anodic or cathodic effects. If the anode be applied over the nerve, the current enters by a series of points (the polar zone), and leaves by a second series (the peripolar zone). The polar zone will thus be in the condition of anelectrotonus, and the peripolar zone in that of catelectrotonus. The current, however, will be more concentrated at the polar than at the peripolar zone, and so the former effect will predominate. These restrictions in the applica- 
tion of the current cause slight apparent irregularities in the law of contrac. tion as tested on man.

In stimulating the nerves of man for the purpose of determining the conditions of the different muscles, we may use either induced currents (generally called faradic stimulation) or the make and break of a battery current (galvanic stimulation). The electrodes are covered with chamois leather moistened with salt solution in order to diminish the resistance of the skin. When it is desired to stimulate any given muscle the stimulating electrode is brought as nearly as possible over the spot where the muscle receives its motor nerve. These 'motor points' have been mapped out, and reference is generally made to a diagram in determining the point for any given muscle. By reversing the current the stimulating electrode may be made either anode or cathode. It is found that stimulation occurs most easily on closure of the current when the stimulating electrode is the cathode; with the greatest difficulty when the current is broken and the stimulating electrode is the cathode. These different contractions are. generally represented by capital letters, and the usual relationship is expressed by the statement that $\mathrm{CCC}$ is obtained most easily, then ACC and AOC, and finally COC.

$\mathrm{CCC}=$ cathodal closing contraction

$\mathrm{ACC}=$ anodal closing contraction.

$\mathrm{AOC}=$ anodal opening contraction.

$\mathrm{COC}=$ cathodal opening contraction.

When the motor nerve to a muscle has undergone degeneration the muscle also begins to
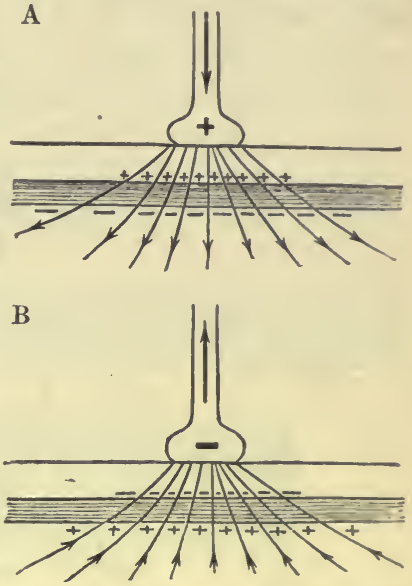

FIG. 118. Electrodes applied to the skin over a nerve-trunk. In A the polar area is anelectrotonic and the. peripolar catelectrotonic. The former condition therefore preponderates, since the current here is more concentrated. In $\mathrm{B}$ the conditions are reversed, the polar zone corresponding in this case to the cathode. (WALLER.) degenerate, and we find certain alterations in its response to artificial stimulation. In the first place, the muscle may fail to respond to induction shocks, while it may show an increased irritability for galvanic shocks. In the second place, qualitative alterations in irritability may be present, so that ACC may be obtained with a smaller current than CCC. These alterations are spoken of as the ' reaction of degeneration.' 


\section{SECTION VI}

\section{THE CONDITIONS WHICH DETERMINE ELECTRICAL STIMULATION}

For every tissue traversed by a current there is a minimum rate of change at which the current through the tissue must be increased or diminished in

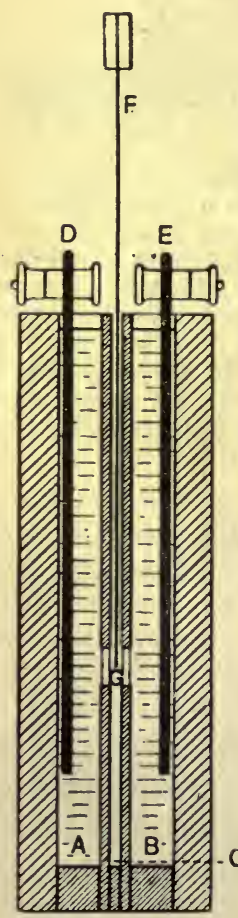

FIG. 119.

Rheonome of Keith Lucas. order to cause excitation. If instead of suddenly making and breaking the current passing through an irritable structure we carry out the change gradually, no excitatory effect is produced, even although the current may finally attain a considerable strength. This fact may be demonstrated by the use of an apparatus known as the rheonome.

A useful form of rheonome is that devised by Lucas (Fig. 119). Two zinc plates $\mathbf{D}$ and $\mathbf{E}$, immersed in a saturated solution of zinc sulphate contained in a rectangular cell, are separated from one another by a vulcanite diaphragm. In the diaphragm is a hole $\mathrm{a}$ by which the two sides of the vessels are connected. This hole can be closed at any desired rate by a shutter F. When the hole is closed no current can pass between the plates, and the amount which can pass will depend on the extent to which the shutter has been raised. By giving the hole the right shape it is possible to diminish the resistance of the apparatus regularly. If this rheonome be placed in circuit with a battery and an excitable tissue, such as the nerve of a nerve-muscle preparation, we can make a current or break a current through the tissue at any desired rate. Thus the course of the current through the tissue will be represented, not by a vertical line, but by a sloping line which may be given any desired degree of steepness (Fig. 120).

If the current be slowly increased through the nerve or be slowly cut off from the nerve, no excitatory effect takes place, while quickly opening or closing the shutter will cause excitation. It might be concluded that the excitatory effect of a current increases with

1. The intensity of the current.

2. The rate of change of the current.

The second of these conditions needs, however, some correction. As we increase the rate of change of current, by employing in the case of induced currents more and more rapid alternations, we find that the excitatory effect, 
instead of increasing, begins to diminish and finally disappears, so that highfrequency currents of enormous tension can, as in Tesla's experiments, be led through the body without any apparent physiological effect. On the other hand, by using more sluggish forms of irritable tissue, we may find that even induction shocks are too rapid for effective excitation. Thus the red muscles of the slow-moving tortoise react better to the slow make than to the sudden break induction shock, and many forms of unstriated muscle are unaffected by either make or break shock. There is in fact for each tissue an optimum rate of change varying with the character of the tissue, at which the current necessary to produce a response is at a minimum. This optimum rate of change is spoken of by Waller as the 'characteristic' of an irritable tissue.

A further investigation of the time relations of electrical stimuli by Keith Lucas has thrown important light on the character of the excitatory

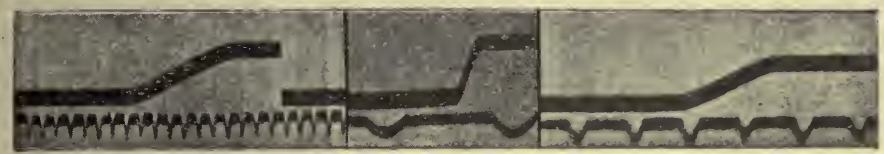

FIG. 120. String galvanometer records of the change of current obtained by opening the diaphragm in the rheonome (Fig. 119) at different rates. (K. LUCAS.)

response itself. The difference between various excitable tissues is perhaps best brought out by finding the minimum strength of current which will excite at make and then determining how much this current must be increased when it is broken at a very short interval of time after it has been made. The following Table represents the relation between duration and strength of current necessary to stimulate in the case of the sciatic nerve of the toad :
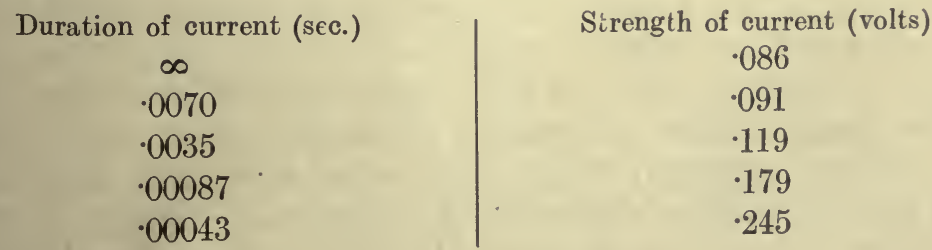

If we slightly alter the use by Waller of the word ' characteristic' we may take as the characteristic of the tissue the duration of the stimulus at which the current necessary to stimulate was just double the minimum. In the above case the minimal stimulating current was approximately doubled when the duration of the current was limited to about $001 \mathrm{sec}$. From a number of experiments of this description, Lucas gives the following as the characteristic, or what he terms the "excitation times," of muscle and of nerve : 


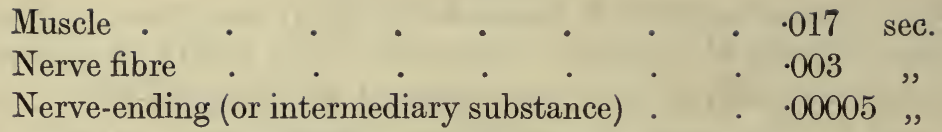

Similar differences are obtained when we attempt to determine by means of the rheonome the minimal gradient of current necessary to excite a nerve. In the case of the toad's nerve the minimal gradient must be ten times as steep as in the case of the toad's muscle, and is such that in one second the current must reach a strength forty-five times the minimal strength which is necessary to excite when the current is made instantaneously. In the frog's nerve the minimal gradient is still steeper, so that in one second the current must reach sixty times the strength of the minimal exciting current. We may interpret these results as signifying that the excitatory state produced in an irritable tissue involves the production of some change which passes away spontaneously. The rate of production of the change, and still more the rate of its spontaneous disappearance, differ from tissue to tissue. If the gradient of a current which is made through the tissue is too slight, the spontaneous disappearance of the excitatory change goes on as rapidly as the production in consequence of the rise of current. No excitation therefore takes place. The "excitation time" of the tissue will thus be proportional to the duration of the excitatory change produced in the tissue as a result of the stimulus. We may compare the excitation time of three tissues with the duration of the electrical change produced in the same tissues by a single stimulus.

The excitation times were:

Frog's nerve fibre

Muscle fibre of sartorius

Ventricular muscle of frog
.003 sec.

.017,

$2 \cdot 000$,

In the case of muscle, according to Burdon Sanderson, the electrical change reaches its culminating-point in .0025 sec., and may take perhaps eight times this interval before it dies away. In the cardiac muscle of the tortoise Sanderson found the electrical change to last between two and three seconds.

SUMMATION OF STIMULI. Closely associated with the excitation time of the tissues are the phenomena of 'summation of stimuli' and ' refractory period.' If two subminimal stimuli are sent in within a sufficiently short interval of time, their effect is summated, so that two stimuli, each of which would be ineffective, may together produce an excitation. In the case of striated muscles, in order that mechanical summation of contraction may take place, the second stimulus must become effective before the muscle has completely relaxed; the second contraction, that is to say, starts from the height to which the first contraction has brought the muscle. A similar condition of things appears to hold for summation of stimuli, if we substitute for mechanical change in muscle the molecular change which accompanies the excitatory state. For summation of two stimuli to take place, the second stimulus must occur at a time before the condition excited by the first stimulus has passed away. The maximum time at which summation of two stimuli can take place will therefore vary from tissue to tissue and will bear a relation to what we have designated the 'excitation time" of the tissue and also to the rapidity of current gradient necessary to excite the tissue. This will be evident if we compare the maximum summation intervals for different tissues with the excitation time of the same tissues. 


$$
\begin{aligned}
& \text { Frog's nerve } \\
& , \quad \text { sartorius } \\
& ,, \quad \text { heart }
\end{aligned}
$$

Stimulating current $5 \%$ above minimal stimulus

$$
\text { Summation interval "Excitation time" }
$$

sec.

.0005

.0015

.0080 sec.

.003

.017

$2 \cdot 000$

REFRACTORY PERIOD. The phenomenon of a refractory period has long been known in connection with the heart muscle and has often been regarded as characteristic of this muscle. If, in the isolated ventricle, a beat be evoked by a single minimal stimulus, subsequent repetition of the stimulus during the course of the contraction is ineffective, and becomes effective only when the contraction has passed away. The heart is said to be refractory to stimuli during this period. The duration of the refractory period is a question of the strength of the stimulus used. With strong stimuli the heart may be made to contract when the relaxation has only progressed half way, and with very strong stimuli one contraction may be made to follow the last at such a short interval that hardly any trace of relaxation is observable between the beats. The phenomenon seems to be common to all excitable tissues. Thus if two stimuli are applied to a nerve within a sufficiently brief interval, the second stimulus is ineffective, so far as can be determined by the response of an attached muscle or by means of a capillary electrometer. The period is longer the lower the temperature and varies from $.0006 \mathrm{sec}$. at $40^{\circ} \mathrm{C}$. to $.002 \mathrm{sec}$. at $12^{\circ} \mathrm{C}$. This critical interval is lengthened if the irritability of the nerve is depressed by narcotics. We may ascribe it to the second stimulus being applied before the excitatory change due to the first stimulus has reached its culminating-point.

THE EFFECT OF TEMPERATURE ON EXCITABILITY. It was found by Gotch that the excitability of a nerve within certain limits was increased by cooling the nerve and diminished by raising its temperature (Fig. 121). Thus, if a frog be cooled to $2^{\circ}$ C. or $3^{\circ} \mathrm{C}$. for a day, it will be found that simple section of the sciatic nerve may'suffice to send the gastrocnemius into continued contraction, and under these circumstances 'closing tetanus ' may be obtained with the greatest ease. This increase of excitability does not apply to all kinds of stimuli. In the case of nerve its irritability was found to be increased by warming, and diminished by cooling for induction shocks and for all galvanic currents of less duration than .005 sec. In skeletal muscle Gotch found the excitability for all forms of stimuli increased by cooling. Lucas has shown that these paradoxical effects in nerve, namely, increase of excitability towards currents of long duration and the simultaneous decrease towards currents of short duration, are conditioned by two opposed changes in the tissue. The fall of temperature delays the subsidence of the excitatory process, but at the same time renders more difficult the initiation of a propagated disturbance. The first of these effects reduces the current required for excitation in a ratio which is greater the greater the duration of the current. The latter increases the 
current required in the same ratio for all durations. If then the change of temperature is such that the two opposite effects are exactly balanced at a certain medium duration of current; it follows that for currents of longer duration the net result will be to reduce the current required for excitation, while for currents of shorter duration the net result will be to increase the current required. The effect of temperature therefore on the minimum exciting current will vary from tissue to tissue according as the two factors, rate of subsidence of excitatory change and the initiation of a propagated

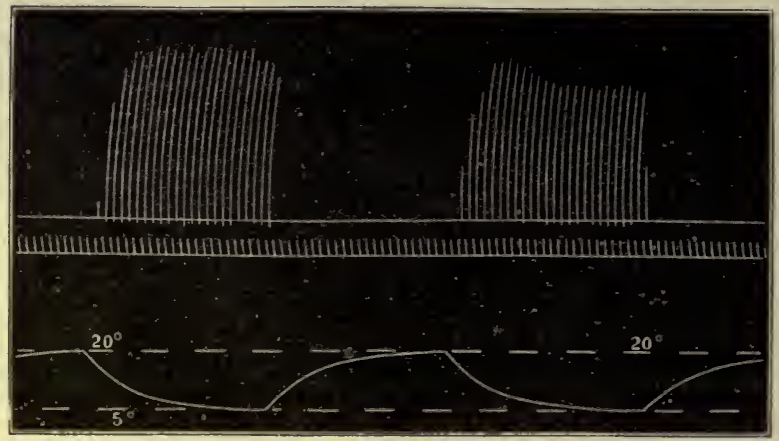

FiG. 121. Tracing of muscle contractions to show effect of cooling a nerve on its excitability. The lower line indicates the changes in temperature of the excited part of the nerve. The muscle responded only when the nerve was cooled, the stimulus becoming ineffectual when the nerve was warmed. (Gотсн.)

disturbance as a result of the excitatory change, are relatively affected by change of temperature.

THE EFFECT OF INJURY. The irritability of the nerve of a musclenerve preparation is not equal in all parts of its course, but is greater at the upper end, probably in consequence of the proximity of the cross-section.

Some time after a motor nerve is divided the increased irritability at the upper end gives way to a decreased irritability, and this decrease goes on till the nerve is no longer excitable. The diminution in excitability gradually extends down the nerve fibre, so that the part of the nerve nearest the muscle remains excitable the longest. This progressive change in the irritability of a nerve after section is spoken of as the Ritter-Valli law. It is soon followed by definite histological changes in the nerve, which we shall describe later. 


\section{SECTION VII}

\section{THE NEURO-MUSCULAR JUNCTION}

THE excitatory process travelling down a motor nerve has to be transmitted to the muscle by the intermediation of the nerve-ending or end-plate. We have learnt to regard the axis cylinder as the seat of the propagated excitatory process. In the end-plate, however, the axis cylinder comes to an end. When stained by methylene blue or by impregnation with chromate of silver or mercury, the axis cylinder, after passing through the sarcolemma of the muscle fibre, is seen to break up into a number of branches (in some cases forming a typical endarborisation), which lie on or are embedded in a small amount of undifferentiated protoplasm containing nuclei (the 'sole plate'). A similar break in structural continuity seems to occur in the central nervous system wherever an impulse is propagated from the axon process of one nerve-cell to the body or dendrites of another nerve-cell. The end-processes of the axon come in contact with the next member in the chain of neurons, but no anatomical continuity is to be made out, at any rate in the higher animals. In the central nervous system the area of contiguity, where an impulse passes from one neuron to another, is spoken of as a synapse. The presence of the synapse, or end-plate, between muscle and nerve imposes certain new conditions on the conduction of the excitatory impulse. One

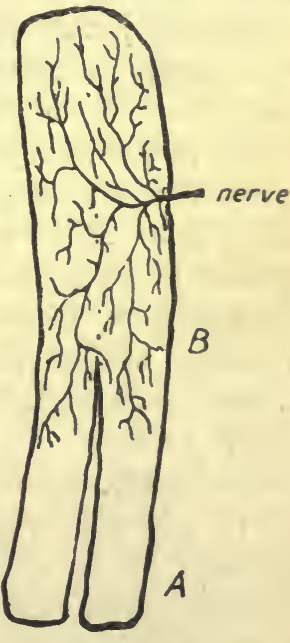

HIG. 122. of the most important of these lies in the fact that the conduction across the end-plate, and probably across the synapse of the central nervous system, is irreciprocal. An excitatory process started in the nerve travels easily across the end-plate to the muscle. On the other hand, an excitatory process started in the muscle does not extend through the end-plate to the nerve fibre. This fact may be shown on the frog's sartorius. If the lower tibial end of the muscle be split, as in Fig. 122, a mechanical stimulus, such as a snip with the scissors, applied to the lower nerve-free end of one of the limbs, e.g. at A, causes a contraction of the corresponding half of the muscle, which does not extend to the other half. On snipping the muscle a little higher up at $\mathrm{B}$, where nerve-endings are present, the resulting 
contraction involves the whole of the muscle, owing to the fact that the excitation started in the nerve-endings spreads in both directions through the branching nerve fibres. It is possible that this irreciprocity of conduction may be of comparatively late appearance in evolution. So far as we know, an excitatory process in a sheet of muscle and nerve fibres, such as we find in lower invertebrata, e.g. in medusa, may travel with equal facility in all directions. We are probably not warranted from our experiments on skeletal muscle in concluding that the contraction of a cardiac musclecell may not set up an excitatory process in the surrounding network of nerve fibres. It is impossible, however, to put such a suggestion to experimental test, since in the heart there is no portion of muscle fibre sufficiently removed from nerves to allow of an excitation being applied which might not at the same time affect the nerve fibres.

There is evidence that the transmission of the excitatory condition across the end-plate, from nerve to muscle, involves a special excitatory process and the expenditure of energy. Thus there is a period of delay between the arrival of an excitatory impulse at the terminations of the motor nerve and the beginning of the electrical change which marks the moment of stimulation of the muscle fibre. If we compare the latent period of a muscle stimulated directly with its latent period when excited through the nerve, we find that there is an increased period of delay in the latter which is not wholly accounted for by the time taken for the impulse to travel from the stimulated spot down the nerve fibres to the muscle. The extra delay is due to the processes occurring in the end-plate. This endplate delay amounts to $0013 \mathrm{sec}$. We may take it roughly at a thousandth of a second. The end-plate seems to be the weakest point in the neuromuscular chain. We have already seen that, when a nerve of a nervemuscle preparation is stimulated repeatedly, the muscle very soon shows signs of fatigue, and that the seat of this fatigue is not in the nerve, nor in the muscle, but in the end-plate.

It has been suggested that the excitation of muscle through nerve depends on an electrical change or discharge at the nerve-ending. This discharge must originate in the terminations of the axon and must influence, in the first instance, the substance which forms the intermediary between the axon and the contractile material of the muscle. We have indeed direct evidence of the existence of a third substance, neither nerve nor muscle, at the point of junction of these two tissues. Thus, curare is generally said to paralyse the end-plates. Evidence for this statement has been given in the previous chapter. Kühne has shown that when the irritability of the frog's sartorius is tested at different points it is greater in the situation of the end-plates. This might be ascribed to the presence of the more irritable nerve-fibres passing into the muscle-fibres at these points. The unequal distribution of irritability is not, however, changed when the muscle is fully poisoned with curare, so as to block entirely the passage of any impulse from the nerve to the muscle. We must therefore regard curare as acting, not on the axon terminations, but on the substance 
intervening between these terminations and the contractile substance of the muscle. Additional evidence of the existence of such a "receptor" substance, as he calls it, has been furnished by Langley. Nicotine resembles curare in blocking the passage of impulses from the motor nerve to skeletal muscle, though inferior to curare in this respect. If $4 \mathrm{mg}$. of nicotine be injected into the vein of an anæsthetised fowl, the hind limbs become gradually stiff and extended in consequence of a tonic contraction of all their muscles. The effect slowly passes off, but can be reinduced by a second dose of nicotine. It is worthy of note that the stimulating effect of nicotine occurs even when sufficient is given entirely to paralyse the motor nerves. It might be thought that the stimulating effect of nicotine was a direct one upon the muscle fibre, but experiment shows that curare has a marked antagonising action on the contraction produced by nicotine. A sufficient dose of curare annuls the contraction produced by a small amount of nicotine and diminishes that caused by a large amount. The point of action of the nicotine must therefore be the same as that of the curare. After a muscle has been relaxed by curare it can be still made to contract by direct stimula. tion. On the other hand, nicotine will produce its stimulating effect when injected into a bird in which degeneration of all the nerve fibres of the muscle has been produced by previous section of the nerve-trunks. It is evident therefore that nicotine, like curare, acts, not on the axon terminations, but on a receptor substance, an intermediary substance intervening between the axon terminations and the contractile substance of the muscle.

Evidence in favour of such an intermediary substance has been brought by Keith Lucas from an entirely different standpoint. In determining the optimal electrical stimuli or the 'characteristic' of muscle and nerve by the condenser method (v. p. 263), Lucas finds that, even after moderate doses of curare sufficient to abolish the possibility of excitation through the nerve-trunk, the muscles show two optimal stimuli, pointing to the existence in them of two excitatory substances, one of which is not paralysed by moderate doses of curare. This result was confirmed when the tissue was investigated by determining the relation of current duration to the liminal current strength necessary to excite. In a normal sartorius he finds three substances, each distinguished by its own 'excitation time.' In the pelvic nerve-free end of the sartorius there is only one substance with an excitation time of $\cdot 017 \mathrm{sec}$. This may be regarded as the muscle substance proper. In the sciatic nerve-trunk there is a second substance with a much steeper characteristic and with an excitation time of $.003 \mathrm{sec}$. On experimenting on the middle region of the sartorius we find not only these two substances but a third substance, which Lucas calls the substance $\beta$, with an extremely rapid excitatory process. Its excitation time is 00005 sec. The presence of these three substances in the middle part of the toad's sartorius is shown in the diagrams (Fig. 123), which represent the relation of strength to duration of the currents necessary to evoke a contraction. In this curve $\alpha$ represents the muscle material, $\gamma$ the nerve material, and $\beta$ the curve of the intermediary substance. 
Similar conditions are found in the visceral neuro-muscular system. Here the nerve fibres leaving the central nervous system do not pass direct to the muscle fibres, but end in arborisations round ganglion-cells, which are collected to form the ganglia of the sympathetic chain or ganglia situated more peripherally and nearer the reacting tissue. Relays of fibres, for the most part non-medullated, arise from these ganglion-cells and pass to the unstriated muscles of the blood-vessels and viscera, where they end in plexuses or networks among the muscle fibres, possibly connected by short branches with the fusiform muscle fibres themselves. No structure is

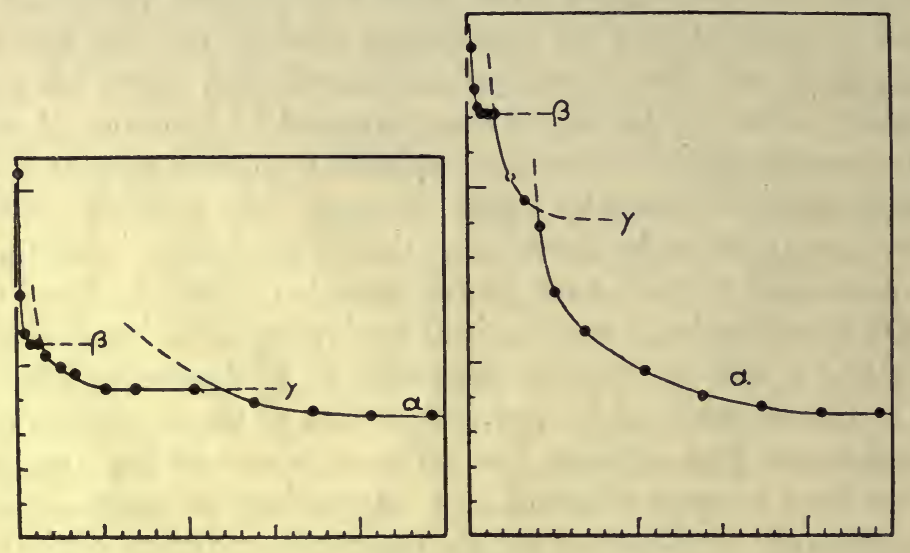

Fig. 123.

present at the periphery exactly analogous to the end-plate, and it is possible that as Elliott suggests, the end-plate is really homologous with the whole of the sympathetic ganglion with its post-ganglionic fibres passing to the visceral muscles. At any rate, the action of curare and of nicotine on these peripheral ganglia is very similar to their action on the skeletal end-plates, nicotine, however, having a relatively stronger action than curare. Injection of nicotine stimulates and then paralyses the peripheral nerve-cells of the visceral system; curare in sufficiently large doses paralyses them. More instructive in relation to the presence of receptor substances is the action of adrenalin. This substance, which is produced by the medulla of the suprarenal glands, has a specific action on all tissues innervated by the sympathetic system. It causes almost universal constriction of the blood-vessels, dilatation of the pupil, acceleration of the heart, and inhibition of the intestinal muscles, with the exception of the ileo-colic sphincter, which causes it to contract, all of which effects can also be produced by stimulation of branches of the sympathetic nerve. On the other hand tissues which are not innervated from the sympathetic, such as the bloodvessels of the brain, are unaffected by the drug. This fact, together with the opposite effects of adrenalin on different unstriated muscles, shows that its action cannot be a direct one on muscle-fibre. It presents a marked contrast, for instance, to barium salts, which produce a contraction of every unstriated muscle-fibre in the body. On the other hand, we cannot 
ascribe this action to a stimulation of the sympathetic nerve-endings, since adrenalin is equally effective if applied after the whole of these nerve-endings have been made to degenerate by section of the post-ganglionic sympathetic nerve-trunks. Its action therefore must lie at the junction between nerve and muscle, and must be on some intermediate or receptor substance developed at the myoneural junction, and having for its function the transference of the excitatory process from the nerve fibre to the contractile substance of the muscle fibre. Similar receptor substances may act as intermediaries in every case of propagation of an impulse across a synapse of whatever description, and may by their properties determine the peculiar qualities of the synapse. We may compare them to the fulminating cap which in a shell is used to transfer the process of combustion from the slowmatch to the bursting charge. Their existence is of especial importance when we endeavour to investigate the mode of action of drugs. It is probable that they will be found to play a great part in determining the differential action of drugs on various tissues in the body. 


\section{SECTION VIII}

\section{POLARISATION PHENOMENA IN NERVE}

ELECTROTONIC CURRENT. If a constant current be passed through a nerve fibre through the electrodes $x$ and $y,-x$ being the anode and $y$ the cathode - and the extrapolar portions of the nerve $a b, c d$ be connected with galvanometers, the needles of both are deflected, and the dirertion of the deflection shows the existence of a current in the extrapolar portions of the nerve $a$ to $b$, and from $c$ to $d$.

We thus see that a passage of a current through a part of a nerve gives

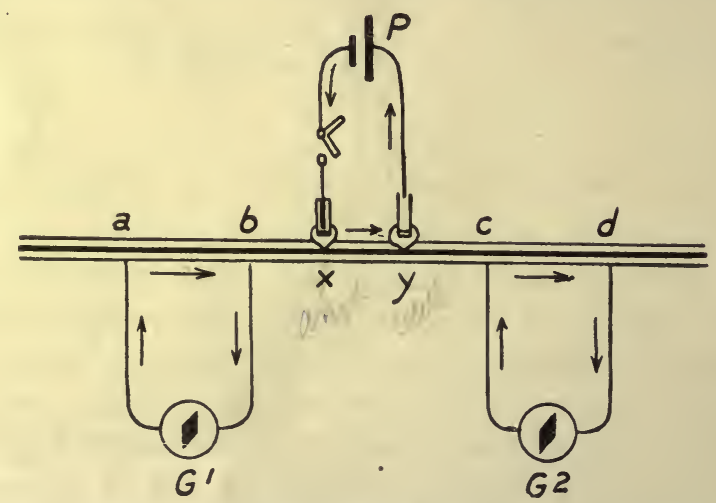

FIG. 124. Diagram showing electrotonic currents. P, polarising circuit ; $G^{1}, G^{2}$, galvanometers.

The galvanometers will indicate, before the passage of the polarising current, the ordinary demarcation current of the nerve resulting from the cross-section at the upper end. This current flows, in the outer circuit, from equator to cut end, and therefore in the nerve fibre from $a$ to $b$, and from $d$ to $c$. The effect of closing the polarising current will be to increase the current of rest between $a$ and $b$, and to diminish that between $c$ and $d$.

rise to a current flowing through a considerable portion of the nerve fibre on each side of the polarising current and in the same direction. This current is called the electrotonic current. It must not be confounded with the current of action, which originates at one of the poles, only at make or break of the current, and is transmitted thence in the form of a wave with a measurable velocity (in the frog) of about 30 metres per second The electrotonic current is developed instantaneously, and lasts the whole time that the current is flowing through the nerve. Its production is dependent 
on the occurrence of polarisation between the sheath and the conducting part of the nerve fibre and may be exactly reproduced on a model consisting of a core of zinc or platinum wire in a casing of cotton soaked with ordinary salt solution. Although thus physical in origin, its production is dependent on the vitality of the nerve, and so is not to be confounded with the simple spread of current.

The polarisation phenomena resulting from the passage of a constant current through a medullated nerve can be studied on a model made up of

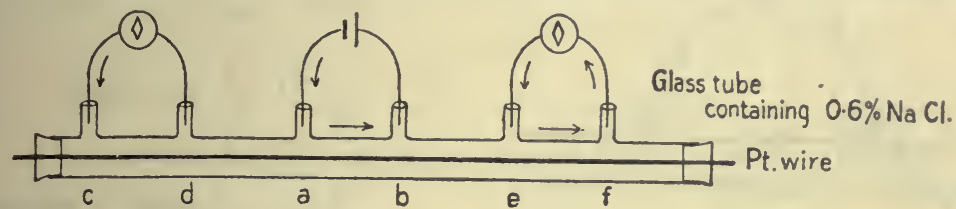

FIG. 125. Apparatus for imitating the polarisation phenomena in medullated nerve ('Kernleiter' model).

a glass tube filled with normal salt solution, containing a platinum or zine wire stretched through it (Fig. 125). On leading a current through $a$ and $b$, and connecting $c$ and $d$ with a galvanometer, a current will be observed in the extrapolar portion of the model in the same direction as in the intrapolar. That this spread of current is due to polarisation is shown by the fact that, if the model be made of zinc wire immersed in saturated zinc sulphate solution, so that no polarisation can occur, the spread of current to the extrapolar area is also wanting. If we examine the phenomena taking place at the anode, we see that a current passes here through an electrolyte to the conducting core. Every passage of a current through an electrolyte must be accompanied by dissociation, the current being carried by the ions. We get therefore a movement of negative ions up into the electrode, and a deposition of electropositive ions on the core (Fig. 127,a). In the same way at the cathode there will be a deposit of electro negative ions on the core (Fig. 126, d), so we may say that the core is positively polarised lat the anode and negatively polarised at the cathode. This polarisation while opposing

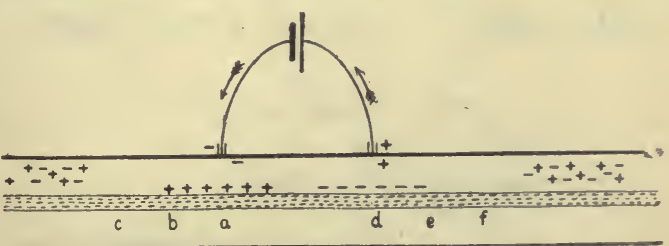

FIG. 126. Diagram to show polarisation at the surface between conducting core and electrolyte sheath in a 'Kernleiter.' the primary current, will set up currents in the surrounding electrolytic sheath, as shown by the arrows in Fig. 127, the current passing from $a$ to $b$ and from $b$ to $c$ in the electrolyte, returning towards $a$ in the core. Hence if we lead off from the sheath in the neighbourhood of the anode from $a$ and $c$, a current will pass in the galvanometer from $a$ to $c$, that is along the core in the same direction as the intrapolar current. The same factors will cause an extrapolar current in the cathodic area, the catelectrotonic current. 
This polarisation will not disappear at once on breaking the polarising current. The nerve or nerve-model will still be positively polarised at the anode and negatively polarised at the cathode. On connecting therefore these two points with the galvanometer, we shall get a current in the opposite direction to the previous polarising current, viz. from anode to cathode (Fig. 128). This is the so-called negative polarisation of nerve. Similarly in the extrapolar regions of the nerve we shall have currents in the same

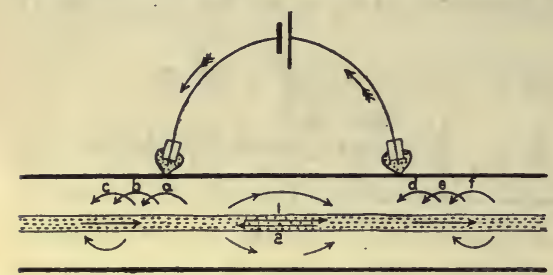

Fig. 127. Diagram to show polarisation currents in a 'Kernleiter,' or in a medullated nerve.

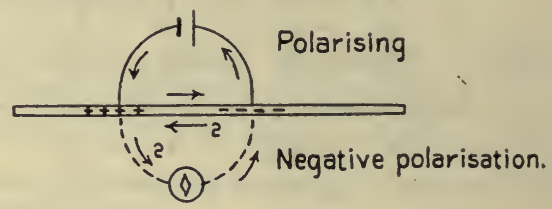

Fig. 128. Diagram to show direction of the negative polarisation current.

direction as the previous polarising current, as shown by the arrows. So far then the nerve behaves exactly like the mechanical model. If, however, we pass a very strong current through a nerve, and then quickly switch the nerve on to a galvanometer, we find a momentary current through the galvanometer in the same direction as the previous polarising current. This is known as positive polarisation of nerve. It is absolutely dependent on the living condition of the nerve, and is in fact an excitatory phenomenon due to the strong excitation which occurs at break of the current at the anode. Thus in the diagram (Fig. 129) a strong current is passing through through the nerve from $a$ to $k$. When this current is broken, excitation occurs, as we have already learnt, at the anode, and this excitatory state may, if the previous currents were strong, last two or three seconds. An excited tissue is, however, always negative towards adjacent unexcited

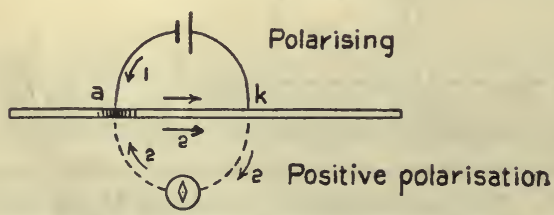

FIG. 129. Diagram to show direction of the positive polarisation current, due to a break excitation at the anode.

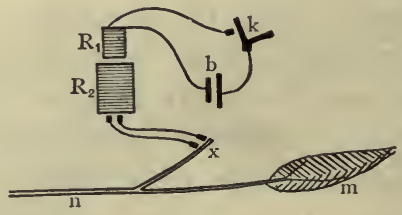

Fic. 130. Diagram of arrangement for showing paradoxical contraction.

tissue, and therefore if we connect $a$ to $k$, there must be a current outside the nerve from $k$ to $a$, and in the nerve from $a$ to $k$, viz. in the same direction as the polarising current. We see therefore that negative polarisation is due to polarisation occurring between an electrolytic sheath and a conducting core, whereas positive polarisation is hardly a polarisation effect at all, but is a current of action. 
PARADOXICAL CONTRACTION. If the sciatic nerve of a frog be dissected out, and one of the two branches into which it divides be cut, and the central end of this branch stimulated, the muscles supplied by the other half of the nerve contract to each stimulus. Ligature or crushing of the nerve $x$ (Fig. 130) between the points stimulated and the point which joins the main trunk puts a stop to this effect, showing that it is not due to a mere spread of current. The fibres passing down $n$ are in fact stimulated by the electrotonic current developed in $x$ during the passage of the exciting current. 


\section{SECTION IX}

\section{THE NATURE OF THE EXCITATORY PROCESS}

UNDER this heading we have really two questions to discuss, namely, $(a)$ the nature of the change excited at the stimulated spot in an excitable tissue, and $(b)$ the propagation of the excitatory change away from the excited spot, e.g. down a nerve fibre. That these two phenomena are more or less independent and may be dealt with separately is shown by the result of passing a constant current through a parallel-fibred muscle, such as the sartorius. In this case, as we have seen (p. 214), at make of the current an excitatory change occurs at the cathode and is transmitted throughout the whole length of the muscle, giving rise to a twitch of the muscle. During the passage of the current there is still an excitatory change at the cathode, but limited to a region within one or two millimetres of the cathode.

An attempt has been made by Boruttau and other physiologists to explain the nerve process, not as a wave of electrical change affecting the substance of the axis cylinder itself, but as a propagated catelectrotonic current. This observer found that, by working with a 'platinum core model' ('Kernleiter') (Fig. 125) of considerable length, the catelectrotonic current was developed at one end of the model some appreciable time after a current had been sent in at the other end, thus resembling a current of action. It is, however, impossible to explain all the electrical phenomena of nerve as due simply to polarisation. We might go so far as to assume that the excitatory effect at the cathode is due to negative polarisation, and that excitation at break, i.e. at the anode, is caused by the sudden coming into existence of a negative polarisation current; but then it would be difficult to understand how the excitation, so produced at the anode, should give rise to a current so much exceeding the current which produced it that it would appear in our external circuit as a current of positive polarisation.

The same objection would hold to the comparison of a nerve-fibre with a submarine cable. An electric disturbance produced at any part of a cable (i.e. a conducting wire in an insulating sheath) is propagated along the cable at a certain finite velocity which can be calculated when we know the conductivity of the core, the capacity of the cable, and the di-electric constant of the sheath. In all these cases there must be a decrement of the change as it is transmitted away from its seat of origin, a decrement for the existence of which there is no evidence in a nerve fibre or other excitable tissue.* Moreover the phenomenon of propagation of an excitatory process

* It might be urgcd, on the other hand, that one would not expect to find any appreciable decrement in a cable only 1 to 3 inches long. 
is equally well marked in tissues, such as muscle and non-medullated nerve fibres, which show very little of the electrotonic effects described in the last section. The absence of decrement in the excitatory process has been taken as an indication that the axis cylinder of the nerve is the seat of energy changes which may be let loose under the influence of chemical or electrical changes, just as the energy of a contracting muscle is set free by the exertion of an infinitesimal force applied as a stimulus. The nerve on this view does not simply transmit the energy which is imparted to it, like a telegraph wire, but itself furnishes the energy of the descending nerve-process.

Against this view might be urged the absence of phenomena of fatigue in nerve, as showing that nervous activity is not accompanied by any expenditure of energy or using up of material. But it must be remembered that this absence of fatigue holds good only for medullated nerve fibres and is not found in non-medullated nerves, ${ }^{*}$ and even in medullated nerves the persistenee of irritability is dependent on the continual supply of a certain small amount of oxygen. It may therefore possibly be explained by a continual process of restitution taking place at the expense of the sheath. Fatigue is absent, not because nothing is used up, but because the assimilative changes exactly balance and make good the dissimilation involved in the propagation of a nervous impulse.

There is thus a certain amount of justification in the comparison of a nerve fibre to a chain of gunpowder, though in the nerve fibre the impetus to disintegration, imparted from each particle to the next in order, consists, not in a rise of temperature at the point of ignition, but in all probability in an electrical change; and the total evolution of energy is so small that it cannot be measured as heat by the most sensitive methods at our disposal. The excited condition at any segment of a nerve is associated with a development of electromotive forces at the junction of the segment with the adjacent resting segments. The current of action thereby produced can pass by the sheath of the nerve, so that it must enter the axon at the excited spot, and leave it at the adjacent unexcited segment. Hermann has suggested that in this way the current of action at any excited spot may excite the adjacent segments or molecules, causing them to become negative and thus setting up a current of action which in its turn excites the succeeding segments. In this way the excitatory process may travel the whole length of the nerve. Propagation would thus involve the successive setting up of an excitatory process all along the nerve or excitable tissue, though it is difficult to see why on this theory every excitatory state should not give rise to a propagated change.

We are as yet a long way from a comprehension of the changes involved in the process of excitation, though we are able to form some idea of many of the factors which must be involved. Any theory of the excitatory process must take into account the following phenomena:

(1) The excitatory state is attended with an electrical change of such

* This statement is based chiefly on experiments on the olfactory nerve of the pike. Halliburton and Brodie found no signs of fatigue in the non-medullated fibres of the sympathetic supply to the spleen, even after several hours stimulation. 
a nature that the excited spot is negative to adjacent unexcited spots. This electrical change rises rapidly to a maximum and dies away more slowly, the rate of its rise, and still more of its subsidence, varying largely according to the nature of the tissue under investigation.

(2) The excitatory change is aroused only at the poles of a current passing through the tissue, i.e. at those places where polarisation can occur in consequence of the electrical movement of ions.

(3) Excitation only occurs at the cathode at make of the current, and only occurs if the current attains a sufficient strength within a certain period of time, the relation of strength of current to rate of change varying in different tissues.

(4) All living tissues are made up of colloids, divided into compartments by membranes of various permeabilities and permeated with salts and other electrolytes in solution.

Disregarding for the moment all considerations of structure, it is possible to form a hypothesis of the nature of electrical excitation which takes into account the facts just mentioned and enables us to give a quantitative or mathematical expression to the factors involved. An electrical current passing through a tissue containing membranes, impermeable to the dissolved ions, will set up differences of concentrations at and near the membranes. Nernst, on the supposition that these differences of concentrations, when sufficiently large, would cause an excitation, arrived at a formula connecting the lowest current required to excite with its duration, and another formula connecting the lowest amplitude of an electrical current with its frequency. The mathematical investigation of the question has been continued by A. V. Hill in conjunction with Keith Lucas. For this purpose we may suppose that the excitable unit is represented by a cylindrical space closed at its two ends by the mem-

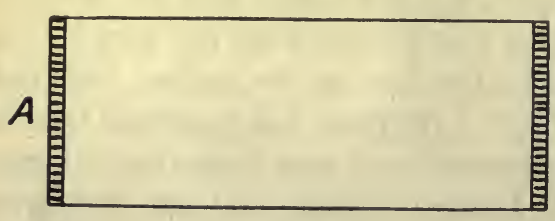

FIa. 131. branes A and B (Fig. 131) and filled with a solution of electrolytes. If a current be passed from $\mathrm{B}$ to $\mathrm{A}$ the positively charged ions will move towards $\mathbf{A}$ and tend to accumulate there. The accumulation of the ions near the membranes will be limited by the tendency of the ions to equalise their concentration in all parts of the cell by diffusion. If we suppose that a necessary condition for excitation is that the concentration of the ions in the neighbourhood of one of the membranes shall reach a certain definite value, it becomes possible to calculate under what conditions of strength, duration, \&c., an electrical current will just produce excitation. The rise of the excitatory state would here be determined by the rate at which the ions accumulate, the subsidence of the excitatory state by the rate of dispersal of the ions by diffusion. The formula arrived at by these observers has this form :

$$
i=\frac{\lambda}{i-\mu \theta^{t}}
$$


where $\quad i$ is the smallest current which will excite, $t$ is duration of the current.

while $\lambda, \mu, \theta$ are constants which depend on :

(1) The distance between the membranes.

(2) The distance from the membrane at which the concentration changes are being considered.

(3) The diffusion constant of the ion.

(4) The number of ions by which a given quantity of electricity is carried.

(5) A constant expressing in general terms the ease with which a propagated disturbance is set up.

Investigation on these lines may give us in future sufficient information to form a material conception of the factors involved in excitation, factors which in the above formula have only a symbolic existence. Thus a determination of the distance between the membranes would give us some clue to the size of the ultimate excitatory units in the tissue involved.* The constant $\mu$ has reference only to the position relative to the membranes at which the changes of concentration are effective. From Lucas's experiments it would seem that the changes of concentration occur in the immediate neighbourhood of one of the membranes. Macdonald has brought forward evidence that the passage of a current through a nerve involves the setting free of certain inorganic ions. The subsidence of the excitatory state depends on the rate of diffusion of ions. If, however, we compare the rates of subsidence of the excitatory state in different tissues we find much greater divergence than would be possible on the assumption that the diffusion is one affecting inorganic ions. Thus between the substance $\beta$ (the intermediate substance) of the frog's sartorius and the ventricular muscle fibre of the same animal, the rate of subsidence of the excitatory state changes in the ratio $4000: 1$. If the ions concerned were simple ions, such as $\mathrm{H} \cdot \mathrm{Ca} \cdot \cdot \mathrm{Na}$, $\mathrm{Cl}^{\prime}$, \&c., it would be impossible to account for this wide variation, since their velocities differ in the ratio of $10: 1$ at most. Moreover the effect of rise of temperature on the rate of subsidence is greater than the effect of a similar rise on ionic velocities. It is evident therefore that the theory is one for use as a working hypothesis only. That excitation is associated with accumulation of ions in the region of the exciting electrode, that the subsidence of of the excitatory state is due to disappearance by diffusion or otherwise of these ions, there can be little doubt. But the questions as to the nature of these ions, and their relation to the colloidal constituents of the excitatory tissue, or to other possible substances, changes in which may form an integral part in the excitatory state, must be left for future investigation.

* It would be premature at present to give any histological significance to Hill and Lucas's diagrammatic cylinder. As Hardy has pointed out, the nerve cannot consist of a row of such cylinders, otherwise excitation would occur throughout the whole intrapolar region, and not be confined to the cathode at make and the anode at break. It may be that we are dealing here again with the polarisable sheath of the 'Kernleiter.' and that the membrane A corresponds to the surface of the axis cylinder or of its neuro-fibrils. 


\section{CHAPTER VII}

\section{THE CENTRAL NERVOUS SYSTEM}

\section{SECTION I \\ THE EVOLUTION AND SIGNIFICANCE OF THE NERVOUS SYSTEM}

EVERY vital phenomenon may be regarded as a reaction conditioned by some change in the environment of the animal and adapted to its preservation. In the community of cells forming the whole organism, the defence of any one part must involve the co-operation of the whole community; no change in a cell of the body can be regarded as a matter of indifference to any of the other cells. For this subordination of the activities of each part to the welfare of the whole, as for the co-operation of all parts in maintaining the welfare of each, a means of communication is necessary between the various cells. For some of the lower functions the channel of communication is the blood, which serves as a medium for carrying food material from one part of the body to another, or for the transmission of chemical messengers, which, elaborated by one set of cells, may affect the mètabolism of cells in distant parts of the body. This method of correlating different activities would, however, be too slow and clumsy for the quick adaptation of the organism to sudden changes of environment. Such a rapid correlation can be effected only by a propagation of some molecular change from the seat of incidence of the stimulus either to all parts of the body, or to some mechanism controlling all parts of the body. The medium for the propagation of a state of excitation is furnished by the nervous system. We have seen that stimuli of various kinds, involving such various forces as thermal, chemical, and electrical energy, are transformed by a muscle or nerve fibre into what we call a state of excitation, which is propagated along the fibres, whether nerve or muscle, at a certain definite rate, its passage in the case of the muscle being followed by a wave of contraction.

In unicellular animals, such as the amoba and vorticella, there is no differentiation of any structure which can be regarded as peculiarly nervous. A stimulus applied to any part of the amœba may evoke responsive activity in all other parts. A slight touch applied to any point on a vorticella will cause an excitation which is rapidly propagated to the stalk, causing this to 
contract and so withdraw the organism from any possible injury. In the lowest metazoa, such as the sponges; we find no special nervous structures. The cells forming the sponge may react to changes in their environment by contraction or by alteration of their relative positions. Many of the cells can move from one part of the sponge to the other in response to chemical changes occurring in the body of the sponge. So far, however, no cells have been distinguished as endowed above their fellows with the property of irritability or the power of reaction to stimulus. It is in the next class, that of the Cœlenterata, where we first find a definite nervous system. The object
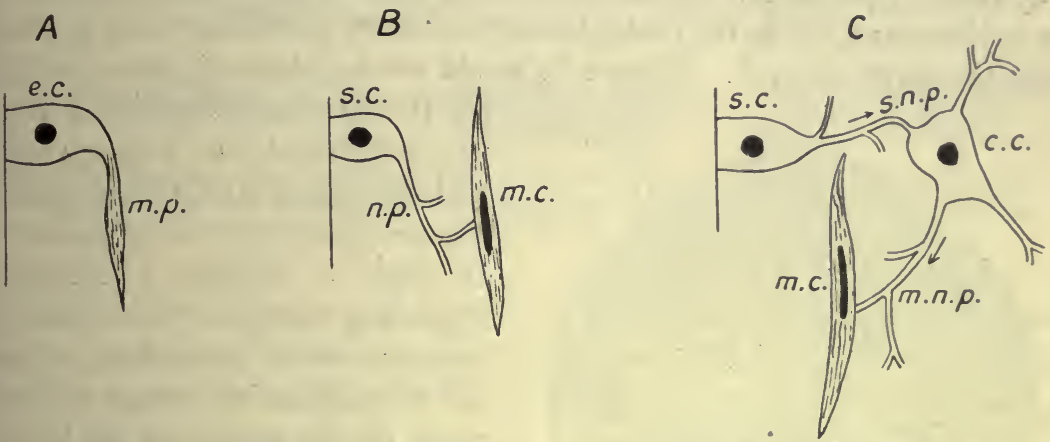

FIG. 132. Diagrammatic representation of evolution of a nervous system.

(Modified from Foster.)

$e c$, epithelial cell; $m p$, muscular process ; $s c$, sensory cell; $n p$, nerve process or fibre; $m c$, muscle-cell ; snp, sensory nerve process ; $m n p$, motor nerve process ; cc, central cell.

of a nervous system is to ensure the co-operation of the whole organism in any reaction to changes in its surroundings. At its first appearance therefore we should expect a nervous system to be developed in connection with that layer of the animal which is in immediate relation to the environment, namely, the epiblast or external layer. In some species of hydra, though no typical nervous tissues have been detected, many of the epithelial cells lying on the surface are prolonged at their inner ends into a long contractile process (Fig. $132, \mathrm{~A})$, so that stimuli applied to the surface and acting on the epithelial cells can cause, as an immediate response, a contraction of the underlying muscular processes. We may easily conceive that in such an animal, among the cells forming the epiblast, certain cells might become endowed with a special sensitiveness to external changes, other cells being developed, like those of the hydra just mentioned, into special contractile structures. If in the course of development the protoplasmic continuity between these two sets of cells had not become interrupted (and we have no ground for assuming that such an interruption occurs under normal circumstances), it is evident that we should have so produced the simplest form of a reflex arc (Fig. 132, B), namely, a sensory cell, which is stimulated by slight physical changes in its surroundings and is thereby thrown into a state of activity similar to that which we have already studied in muscle and nerve. This state of activity. would be propagated by the protoplasmic channels to the muscular cell and 
arouse there the specific function of the muscle, namely, contraction. In such a simple reactive tissue, lines of less resistance would be rapidly laid down through the protoplasmic continuum, and these lines, acquiring a specific structure or composition, would form a network uniting sensory and muscular cells. Thus a stimulus applied to any sensory cell would spread to the adjacent sensory and muscular cells, and the response of the muscle-cells would be greatest near the stimulated spot, gradually dying away as the area of the excitation widened. A further step in the development of such a hypothetical elementary nervous system would occur when certain of the sensory cells (Fig. 132, c) developed a special sensitiveness, not to mechanical changes in the environment, but to the protoplasmic excitatory process arriving at them along the nerve network. These cells would act as relays of force, picking

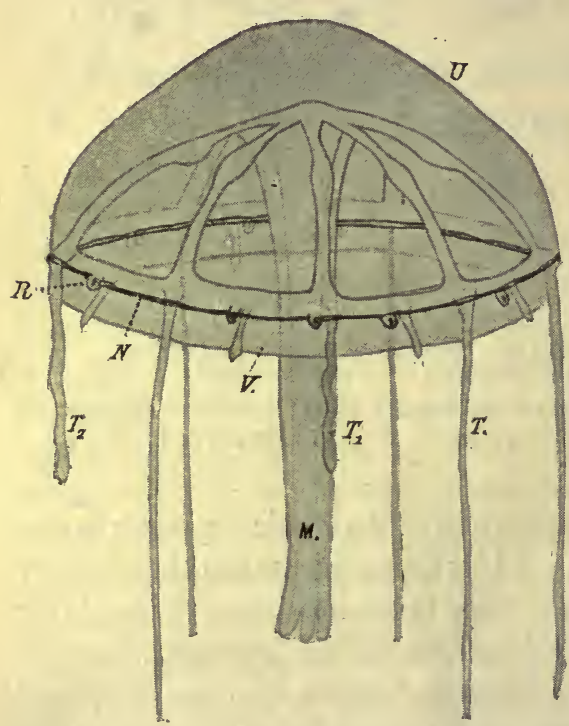

Fig. 133. Diagrammatic view of a jelly-fish. (HeRTwiG.)

U, umbrella ; M, manubrium; $T_{1}, T_{2}$, tentacles ; $\mathbf{v}$, velum ; $\mathrm{N}$, nerve ring ; $\mathbf{R}$, ' marginal body.'

up the excitations arriving from the undifferentiated sensory cells, and sending them on with increased vigour along the nerve network. In such a manner a stimulus applied at one point could be sent on in successive relays from cell to cell throughout the whole reactive tissue on the surface of the body.

We cannot point to any particular animal as presenting instances of either of the two types of elementary nervous system just described. If such exist, they have not yet been investigated, or the undifferentiated character of their nervous tissues has thwarted the efforts of zoologists to display their specific charàcters by staining reagents. In the lowest definite nervous system with which we are acquainted, namely, that of the jelly-fish, all three เtypes of cell, the sensory cell, the reactive or central cell, and the motor cell, are already developed and have undergone among themselves a considerable degree of differentiation. In a jelly-fish or medusa, such as aurelia or sarsia (Fig. 133), the reactive tissue of the body is confined to the under-surface of the so-called umbrella with the tentacles and manubrium. A section through the umbrella shows a layer of epithelium containing differentiated sense-cells, below which is a plexus or rather network of fine nerve fibres with a certain number of nerve-cells at the nodes of the network. From this network fibres pass more deeply to end in a finer network situated among a layer of muscle fibres formed, like the sensory cells, by a differentiation of the primitive epithelium or epiblast (Fig. 134). Besides this diffuse 
nervous system, there is a continuous ring of nerve fibres round the margin of the umbrella, thickened at intervals by the accumulation of nerve-cells, which are in close relation to special collections of sensory cells in the ' marginal bodies.' These sensory cells present a differentiation among themselves, some being apparently determined for the reception of mechanical

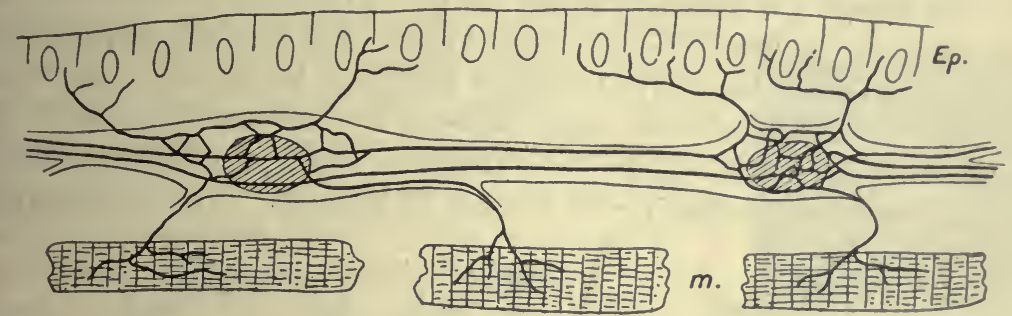

FıG. 134. Diagram of subepithelial plexus of nerve fibres and nerve-cells, communicat. ing on the one side with the sensory epithelium, and on the other side with the subumbrellar sheet of muscle fibres. (After BETHE.)

stimuli, others for the reception of light stimuli, while others again are found in close relation with little masses of calcium carbonate crystals, by the direction of the weight of which the cells are able to react to changes in the position of the animal in space. In the jelly-fish therefore the nervous or reactive system has already acquired a considerable degree of differentiation.

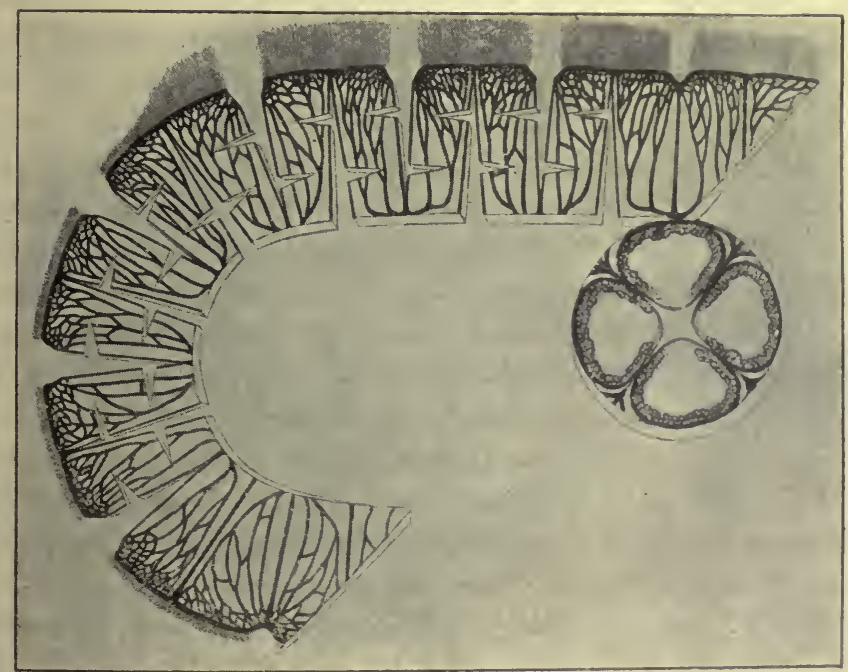

FIG. 135. Figure of a jelly-fish in which all the marginal bodies except one have been removed, and which has been incised in various directions so as to divide the nerve ring and all the 'long paths,' so that only the diffuse nerve network remains functional. (Romanes.)

We may study the behaviour of a more primitive system if we remove the special sense-organs of the medusa by cutting off the whole of the marginal ring with its contained marginal bodies (Fig. 135). We have then a layer 
of contractile tissue, innervated by a nerve network, and covered by a layer of epithelium containing sense-cells. To this network is attached the manubrium, which represents the mouth and stomach of the animal. In such a mutilated jelly-fish it is easy to show that a stimulus applied to one spot on the surface travels outwards from the excited spot to all parts of the bell. The stimulus is propagated also to the manubrium, which in some species bends in the direction of the excited spot-that is to say, in the direction which represents the shortest possible path from the excited spot to the manubrium. This preparation rarely presents any automatic activity. It may react to a constant stimulus by a rhythmic series of contractions, but remains perfectly motionless in the absence of stimulus. The unmutilated

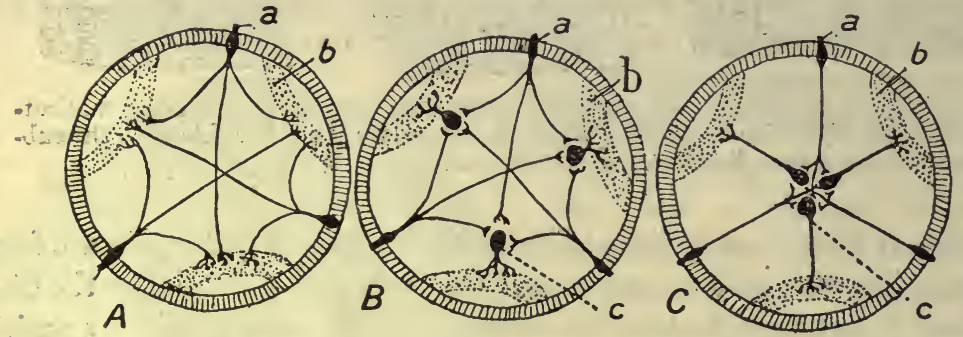

FIG. 136. Schema showing the utility of the multiplication of neurons and their grouping in central ganglia. (CAJAL.)

A, an ideal invertebrate with only cutaneous 'sensory' neurons.

B, invertebrate, such as a medusa, with sensory and motor neurons, but no central nervous system.

c, invertebrate (e.g. Annelid) in which the motor neurons are concentrated in central ganglia.

$a$, sensory neuron; $b$, muscle ; $c$, motor neuron.

jelly-fish presents rhythmic contractions of its sub-umbrella tissue which are inaugurated in any or all of the marginal bodies and serve to drive the animal onwards through the water in which it is immersed. The rhythmic contractions may be initiated, augmented, or diminished, in response to stimuli of light, mechanical irritation, or changes in the position of the whole animal acting on the marginal bodies. In the reaction of an animal to external stimuli it must be an advantage if the energies of the whole can be concentrated in defence of any one part and be evoked by a stimulus applied at one point. Such a co-operation of the whole for the benefit of the part involves the existence of direct paths from the stimulated point to all parts of the animal if the reaction is to take place with any promptitude. In the medusa we find a beginning of such ' long paths.' The general direction of the fibres of the network is radial, and there is a concentration of such fibres in the neighbourhood of the marginal bodies, so that an excitation can pass more readily from a sense-organ to the menubrium than it can laterally along the circumference of the animal. Moreover, a stimulus which is too slight to excite a reflex contraction of the muscular tissue may travel along the nerve tissue to each of the marginal ganglia and arouse these to a discharge of motor impulses. We have therefore in the medusa sensory cells of different sensibilities ; central cells specially adapted to reacting to and reinforcing a 
nerve stimulus started by a small change in the environment; a general nerve network propagating the excitatory changes in all directions, but with special ease in certain directions; and a reactive muscular tissue which carries out movements at the end of the chain of excitation, all the elements forming the chain being derived from epiblastic cells.

A further differentiation of a nervous system, such as that just described, must in the first place involve the laying down of more 'long paths' and the collection of the special 'central' cells into closely connected masses (ganglia), so as to concentrate the control of the reactions of the body, and to permit of the ready subordination of every part to the needs of the whole. A special direction is given to this development by the evolution of animals, such as worms and crustaceans, which are segmented and capable of locomotion. The fact that these animals. are segmented determines the collection of the central cells into a chain of ganglia, one ganglion or pair of ganglia being provided for each segment. In the act of locomotion it is of advantage to the animal that those sense-organs or sensory cells which are projicient, i.e. which are stimulated by changes in the environment originating at a distance from the animal, should be collected together near that part which goes first, namely, the head end. Thus the projected sensations of sight, those which are excited by chemical changes in the surrounding medium and represent the sense of smell, and those which are specially aroused by vibrations in the surrounding medium and correspond to those which we call the sense of sound, are in the majority of these animals subserved by organs situated near the head end.

The wisdom of a man is measured by his foresight. The chances of an animal in the struggle for existence are determined by the degree to which the reactions of the animal to its immediate environment are held in check in response to stimuli arising from approaching events. An animal without power to see, smell, or hear its enemy will receive no impulse to fly until it is already within its

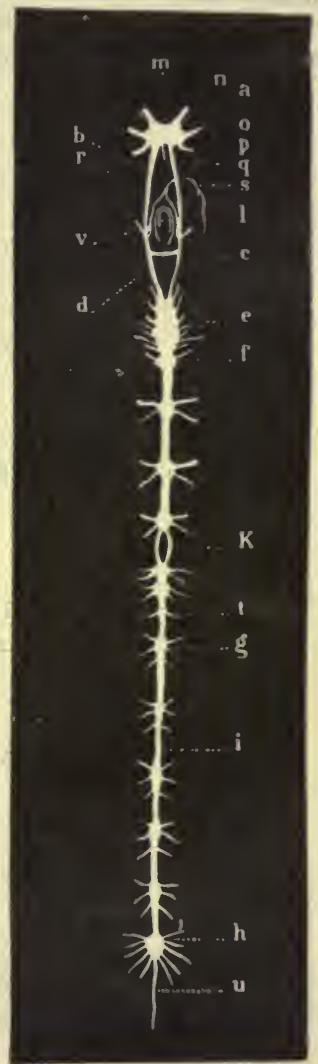

FIG. 137. View of central nervous system of crayfish. (After Y UNG and VoGT.)

$a$, cerebral ganglion.

$b$, commissure.

$e$, subœsophageal ganglion. $g$, first abdominal ganglion. $l$, œsophagus.

$m$, optic nerve.

$p$, antennary nerve.

8 , stomato-gastric nerve. enemy's jaws. It must therefore be of advantage to segmented animal that the activities of the whole chain of segmented ganglia should be subservient to those central nerve-cells which are in direct connection with the projicient sense-organs at the head. The influence exerted by the head ganglia will be in the first place inhibitory of the direct reaction 
excited in each segment by stimulation of its surface, and, for this influence to be propagated, long tracks must be laid down, joining up ganglion to ganglion and propagating impulses from the head ganglia to the most distant part of the chain. As a type of such a system we may refer to the crayfish.

In this animal the central nervous system (Fig. 137) consists of a chain of thirteen ganglia, namely, six abdominal ganglia, six thoracic ganglia, and one supraœsophageal or cerebral ganglion. In the abdomen and thorax the ganglia form a longitudinal series situated in the middle line of the ventral aspect of the body close to the integu-

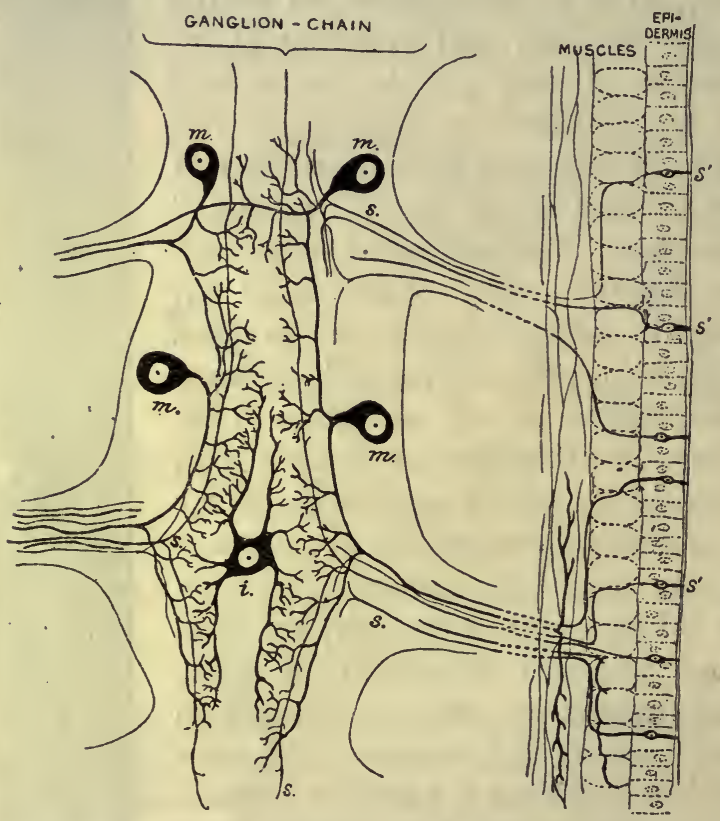

FIG. 138. Diagram of nervous system of a segmented invertebrate (earthworm or crayfish). (From SchäfER, after RETZIUs.)

$s^{\prime}$, sensory cells; $s$, afferent nerve fibres ; $m$, motor neuron ; $i$, central or intermediate cell.

ment. All give origin to a variable number of nerves, which are distributed partly to the muscles, partly to the skin and sense-organs. They are connected by longitudinal bands of nerve fibres or commissures, which are double, each ganglion being bilobed. The most anterior of the thoracic ganglia, which is the largest, is marked at the side by notches, as if it were made up of several pairs of ganglia fused together. From this ganglion two commissures pass forward round the gullet to unite in front of this tube, behind the eyes, with the transversely elongated mass of ganglion cells and fibres called the supraœsophageal ganglion. This ganglion consists of three fused pairs of ganglia, which have been termed the protocerebron, the deuterocerebron, and the tritocerebron. The most anterior gives origin to the optic nerves, which run by the optic stalks to the eyes. From the middle ganglion on each side a tegumentary nerve passes to ramify in the integument and from the inferior surface the antennulary nerves pass to the internal antennæ. From these small branches are distributed to the organ of hearing. The posterior protuberance of the brain gives origin to the antennary nerves which pass to the large external antennæ of the animal. The first thoracic, 
or subœsophageal, ganglion gives origin to ten pairs of nerves which are distributed to the mandibles, to the jaws or maxillæ, to the maxillipedes, and to the branchial appendages of the latter.

When we investigate the structural basis of such a nervous system we find that, as in medusa, the starting-point of the reflex arc is in certain neuro-epithelial cells (Fig. 138) lying on the surface of the body. These cells are spindle-shaped, and have one short process passing to the surface, and a long process which runs in a nerve fibre or collection of fibres towards the ganglion of the segment. Arrived at the ganglion it divides into two branches, which pass towards the two ends of the body and become lost in

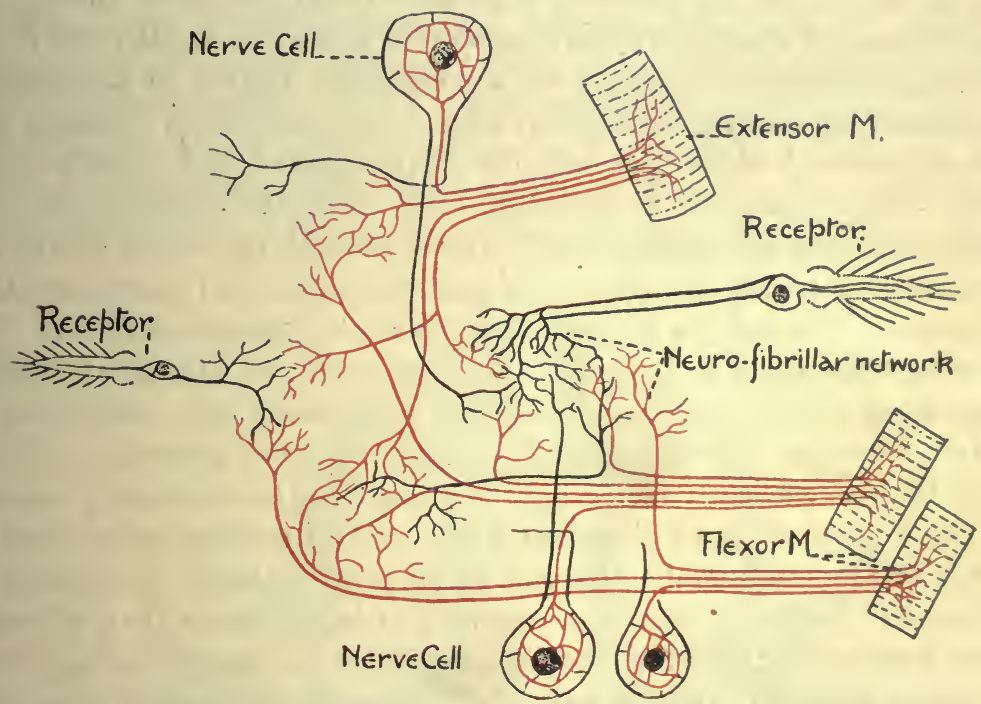

Fig. 139. Diagram of a reflex arc in a (neuro-fibrillar) invartebrate nervous system.

(BETHE.) The efferent paths are coloured red, the afferent black.

the granular material forming the inner part of exch ganglion. The ganglia themselves consist internally of this punctated substance or granular material, and externally of a capsule of ganglion-cells. Each of the ganglioncells sends one thick process towards the centre, which rapidly divides, some of the branches passing into the granular material, while one branch passes outwards in a nerve to end in a network of fine fibrils within the muscles on the surface of the body. The nervous impulse excited in the sensory cell on the periphery travels therefore up a nerve fibre into the granular substance of the ganglion. From this granular substance it is collected by the fine branches of the ganglion-cells and is transmitted by them along the motor nerve fibre to the muscles. The central granular material consists entirely of a close felt-work of fibres, which may be regarded as processes either of the sensory nerve fibres or of the nerve-cells. The typical reflex arc in this case therefore is formed by two nerve-cells with their processes. Such a nerve-cell with its processes is spoken of as a neuron. The first neuron the recipient neuron, or receptor, is represented by the sensory cell with its 
two processes in the granular material. The second neuron is formed by the ganglion-cell with its finely branched dendritic processes in the granular matter and its motor axon, which passes into the muscle fibres. As to the manner in which the impulse passes from the branches of one cell into those of the other, opinions are still divided. The question will have to be more fully considered when we come to deal with the vertebrate nervous system. Many believe that there is no anatomical continuity between the two neurons, and that the excitatory change is transmitted by a mere contiguity, a change in one set of nerve-endings exciting a corresponding change in another set of nerve-endings in immediate contact with them. By certain methods, however, it is possible to show the existence of an anatomical continuum throughout the whole nervous system in these invertebrate animals. Apathy and Bethe have demonstrated the presence of a continuous system of neurofibrils, mucch smaller than an individual nerve fibre, which, starting in a sensory cell, pass into a network of fibrils forming the greater part of the central granular matter. From this network neurofibrils run along the dendrites into the ganglion-cells, forming there a small network through the centre of which a neurofibril is continued down the nerve processes again, and passes out along the motor nerve to end in a network of fibrils among the muscle fibres. In a system so constituted it is evident that, although an excitatory process passing along a given fibril may find certain paths easier than others, and so maintain a constant prescribed path through the nerve system, yet it will be possible, by sufficiently increasing the strength of the excitatory process, to cause it to travel in all directions in the central nervous system and to evoke in this way a general activity of all parts of the body, a condition in fact found to obtain in the normal animal. It is significant that, although a great number of fibrils pass into the bodies of the ganglion-cells, yet in many cases, especially in crustaceans, fibrils are to be found sweeping from the neuropilem or nerve network of the granular substance into a nerve process, and thence into its motor axon without at any time entering the body of the cell (Fig. 139). 


\section{SECTION II \\ THE NERVOUS SYSTEM OF VERTEBRATES}

IN these, as in the invertebrata, the central nervous system is developed by an involution of the epiblast, revealing thereby its primitive relations to the surface of the body. At an early period in fœtal life, shortly after the formation of the two layers of epiblast and hypoblast, a thickening is observed in the epiblast. This thickening soon gives place to a groove, the neural groove (Fig. 140), and the walls of the groove folding over form a

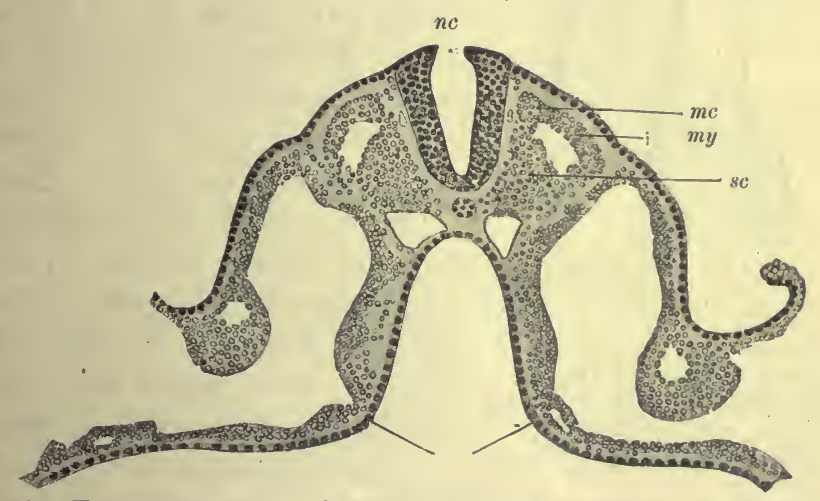

Fig. 140. Transverse scction of human embryo of $2.4 \mathrm{~mm}$. to show developing neural canal. (T. H. BRYCE.) $n c$, neural canal ; $m c$, muscleplate ; $m y$, outer wall of somite ; $s c$, sclerotome.

canal, the neural canal, which is dilated at the head end of the embryo to form three enlargements known as the cerebral vesicles.

When first formed the canal is oval in cross-section, its wall being made up of a layer of columnar cells between the outer extremities of which are seen smaller rounded cells. The internal layer of columnar cells send a process peripherally which branches at the end so as to form a close meshwork of fibres. These fibres branch more and more as development progresses, and eventually form the supporting tissue of the adult central nervous tissue, known as the neuroglia. As the wall of the canal grows in thickness, some of the cells may wander outwards and form neuroglia-cells with numerous radiating branches. In the adult nervous system little is left of these cells except their nuclei, so that the neuroglia appears as a close felt-work of fibres, to which here and there nuclei are attached. These cells 
are formed from the most superficial layer of the invaginated epiblast, and are spoken of as spongioblasts. The deeper layer of cells, which are to give rise to the permanent nerve-cells, and are therefore known as neuroblasts, rapidly divide and form a thick layer surrounding the internal layer of spongioblasts, through which pass the peripheral processes of the latter. When first formed these cells have no processes. Later on each neuroblast acquires a pear shape, the stalk of the pear having a somewhat bulbous extremity (Fig. 142). The stalk continually elongates, and the elongated process may leave the spinal cord altogether and grow outwards to any part

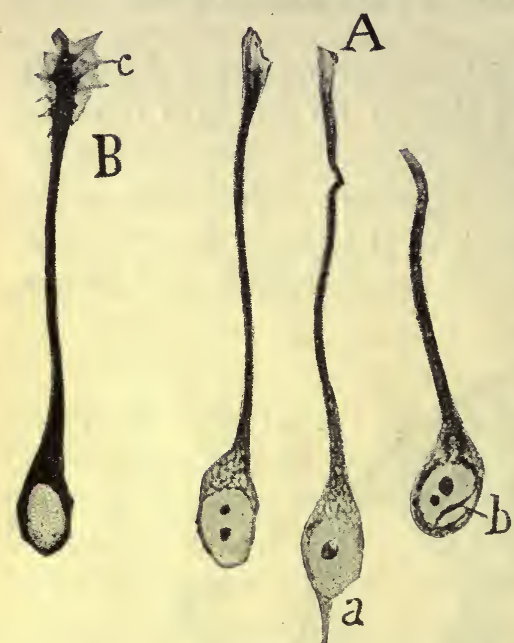

Fig. 141. Neuroblasts from the spinal cord of a chick embryo. (CAJAL.)

A, three neuroblasts stained to show neuro-fibrils ; $a$, a bi-polar cell.

B, a neuroblast showing the 'incremental cone ' $c$. of the body of the embryo, or may pass to other parts of the central nervous system. This long process of the developing nerve-cell is known as the axon. Some time after its formation other processes grow out from the cell, which soon branch and end in the immediate neighbourhood of the cell. The axons of the cells near the ventral part of the neural tube grow out to the different muscles of the body, where they end in close connection with the muscular fibres by an arborisation which forms the end-plate. They provide an efferent path for impulses running from the central nervous system to the musculature of the body. The afferent channel is formed in a somewhat different manner. Even before the neural groove has closed in, a thickening of the epiblast is seen immediately external to the groove on each side. This thickening becomes divided into a series of collections of cells lying immediately under the epiblast on the lateral and dorsal surface of the neural canal. The cells, which are at first round or oval, send off two processes in opposite directions so that they become bi-polar (Fig. 142). One process passes into the central nervous system, where it divides, some of its branches being distributed in the nervous system at the same level, while others run a considerable distance towards the head immediately outside the tube of nervecells. The other process grows downwards, along with the processes from the ventral cells of the tube, towards the periphery of the body, where it ends in close connection with the surface in the various sense-organs of the skin and muscles. These collections of bi-polar cells form the posterior root ganglia. In fishes they retain their primitive character throughout life, but in mammals the bi-polar cell is to be found only in the spiral and vestibular ganglia which give origin to the fibres of the eighth nerve. In all the other ganglia the shape of the cell becomes modified by an approximation of the points of attachment of the two processes until finally the cell 
becomes uni-polar, giving off one process which divides by a T-shaped junction into two, one of which runs towards the spinal cord, while the other takes a peripheral course as the afferent nerve fibre. The central nervous system thus becomes provided with a ' way in ' and a ' way out ' for the chain of impulses concerned in a nervous reaction or reflex action. The further development of the spinal cord is mainly determined by the extension of the

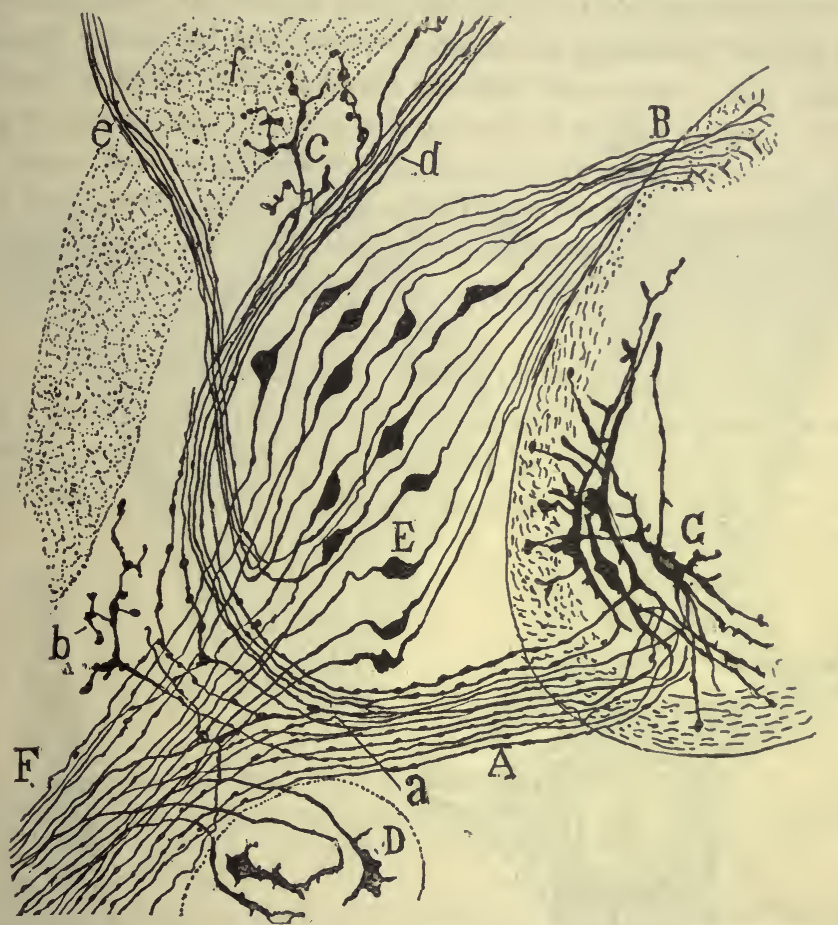

Fig. 142. Section through developing spinal cord and nerve-roots from chick embryo of fifth day. (CAJAL.)

$A$, ventral root ; $\mathrm{B}$, dorsal root ; $\mathrm{C}$, motor nerve-cells ; $\mathrm{D}$, sympathetic ganglioncells; $\mathrm{E}$, spinal ganglion cells still bi-polar; $\mathrm{F}$, mixed nerve; $b, c, d$, motor nerve fibres to $f$, developing spinal muscles; $e$, a sensory nerve-trunk.

axons of the cells outside the tube of cells themselves, and by the provision of the ' long paths' which are a necessary condition of increased efficiency of the reacting organ. Some time after the outgrowth of the axon a medullary sheath is formed, apparently by the agency of the axon itself, so that each group of axons leaving or entering the cord forms a bundle of medullated nerve fibres. The long branches of the posterior or dorsal roots running up towards the head form a mass of fibres behind the tube of cells known as the posterior columns. Fibres starting in the spinal cord itself run upwards and downwards to end in other parts of the cord, or in the more anterior divisions of the central nervous system forming the brain, and surround the neural tube on its ventral and lateral aspects with a sheath of white matter. To these white fibres are added others, which take origin in the brain and pass all the way down the cord. Meanwhile the cells themselves become separated 
by the ramifications between them of the branches of axons entering the cord, as well as of the dendrites of the cells themselves. Thus, in its adult form, the spinal cord consists of a central mass of nerve-cells and fibres, known as the grey matter, which is encased in a sheath of white matter formed of medullated nerve fibres. The cord itself is cylindrical in shape, and is divided into two symmetrical halves by the anterior and posterior fissures. In each half of the cord the grey matter on cross-section is crescentic in shape, presenting an anterior or ventral horn and a posterior or dorsal horn, and is connected with the corresponding mass in the other half of the cord by grey matter known as the anterior and posterior grey commissures. Between the two grey commissures is the central canal, relatively very minute when compared with the condition in the fœtus and lined by a single layer of columnar ciliated epithelium, the cells of which are directly descended from the neural epithelium lining the medullary canal.

\section{THE STRUCTURE OF NERVE-CELLS}

In the adult animal a typical nerve-cell, such as those forming a prominent feature in the anterior horn of the spinal cord, is a large cell with many branches. It has a large vesicular nucleus with very little chromatin,

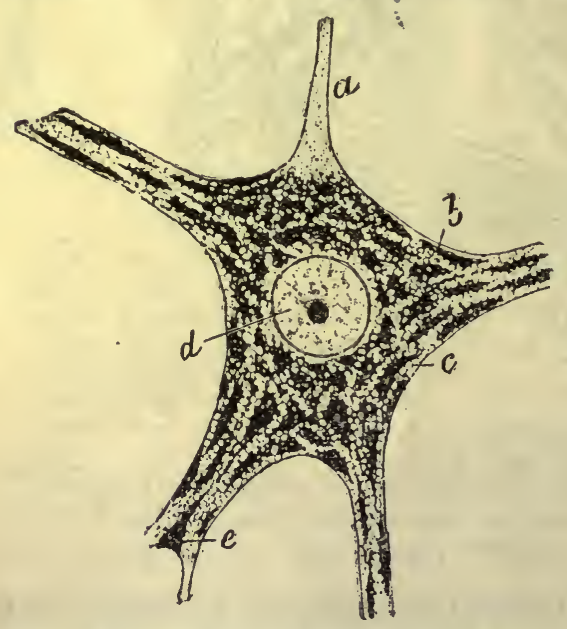

FIG. 143. Nerve-cell from the spinal cord, stained by Nissl's method.

$a$, axis-cylinder process or axon; $b$, protoplasm of cell, consisting of $c$, fibrillated ground substance, and $e$, the granules of Nissl ; $d$, nucleus. (LenHossek.)

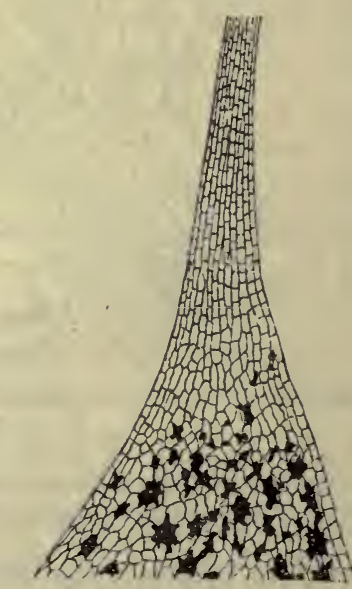

FIG. 144. The point of origin of the axon, the "nervehillock,' highly magnified, to show absence of Nissl's granules from the origin of the process. (HELD.)

which may be collected into one or two nucleoli. The body of the cell presents different appearances according to the manner in which it has been treated for histological examination. When separated from the surrounding tissues by means of dissociating fluids it may present traces of striation, the individual striæ running from one process to another of the cell. When treated fresh "with methylene blue, or hardened by alcohol or corresive sublimate and stained with methylene blue or toluidine blue, 
the protoplasm is seen to contain angular masses which are deeply coloured with the dye (Fig. 143). These masses are known as the Nissl granules or bodies. By other methods it is possible to demonstrate that the whole protoplasm of the cell between the Nissl bodies is pervaded by fine fibrils, which enter the cell from the processes and may run out of the cell by the axon or may run into some of the other shorter processes (Fig. 146). The processes of the cell, as is evident from their development, are of two kinds. The axon which becomes continuous with the axis cylinder of the medullated

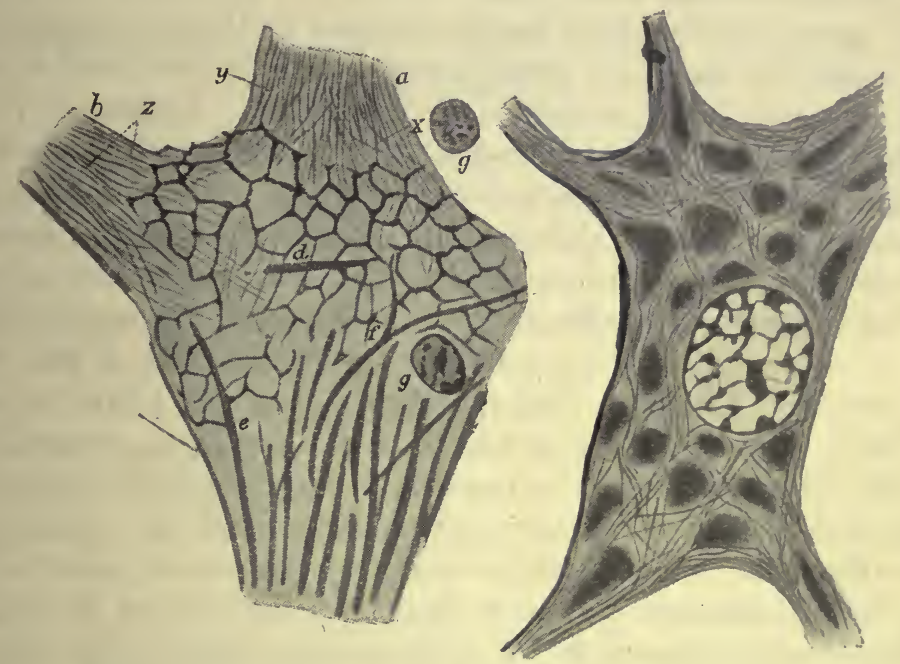

Fias. 145 and 146. Nerve-cells from spinal cord. (BETHE.)

Fig. 145, showing Golgi network, and neurofibrils : $d, e, f$, junctions of axons with Golgi network. Fig. 146, showing neurofibrils and Nissl bodies.

nerve fibre arises from a part of the cell body known as the axon hillock, which is the only part of the cell free from Nissl bodies (Fig. 144). The other processes, which may be very numerous, are known as the dendrites. They are generally thicker than the axon at their origin from the cell, but rapidly diminish in size as they give off branches, the branches apparently terminating freely in the grey matter in the immediate neighbourhood of the cell. In specimens stained by the Golgi method the dendrites may sometimes present a somewhat serrated outline. The Nissl bodies of the cell extend some way into the dendrites.

A nerve cell with all its processes, axon, and dendrites is spoken of as a neuron. From the development of the central nervous system in vertebrates, it is evident that the nervous path of every reaction must be made up of two or more neurons. If we take, for example, the simplest possible reaction which might be effected through a single segment of the spinal cord, we see that the afferent impulse might be started by some stimulus applied to the ramifications in the skin of the distal processes of the posterior root ganglioncell (cf. Fig. 132). The nerve impulse so started is carried by the nerve fibre past the T-shaped junction in the posterior root ganglion into the cord, 
and along a branch of the entering nerve fibre which runs right across the cord to terminate in the neighbourhood of the anterior horn-cells. Here the impulse must be transmitted in some way to the dendrites or body of one of the large motor nerve-cells in the anterior horn, whence it is carried along the axon of the cell, leaving the cord by the anterior root and passing down a peripheral nerve to the end-plate on a muscle fibre. = Here again by some means the arrival of the impulse excites the muscle to contract. This reaction never takes place in the contrary sense, i.e. no impulse started in the motor nerve can travel back through the spinal cord and along the sensory nerve. Although an impulse excited in the nerve passes easily to the muscle, an excitatory process started in the muscle itself is confined to this tissue and never extends to the nerve fibre. Apparently the same rule holds good within the grey matter of the central nervous system, where two neurons come into relation with one another. An impulse passes easily from the axon of one into the dendrites and cell of the other neuron, but, so far as we are aware, it is impossible by exciting an axon to cause a retrograde.wave of excitation to pass through its corresponding cell and into the terminations of the axons in immediate contact with the cell. This statement has been called by Sherrington the 'Law of Forward Direction.' It might be also spoken of as the irreciprocal conduction of the nerve arc. The character of a reaction to any stimulus, applied to the surface of the body, is determined by the course which the impulse, excited in the afferent nerves, takes on entrance into the central nervous system. This course is laid down by the connections of the neurons through which the nerve impulse passes. In the central nervous system therefore, more than in any other part of the body, function is directly dependent on structure. Theoretically if we had a perfect knowledge of the connections of the neurons in the central nervous system and knew the nerve fibres affected by any given stimulus, we should be able to prophesy exactly the result of such stimulus. In the case of the simpler reactions this is already possible, but in the higher parts of the nervous system the enormous complexity of the systems of neurons excludes any possibility of our forming more than a general idea as to the nerve paths traversed in any given reaction; and the variations which exist from individual to individual must always prevent in the intact animal an absolute prediction of the results of any stimulus. 


\section{SECTION III}

\section{GENERAL CHARACTERISTICS OF REFLEX ACTIONS}

THERE are certain features common to alluctions, carried out through the intervention of the central nervous system, which must be regarded as determined by the properties of the neurons, i.e. the conducting links in the chain of excitatory tissues intervening between the stimulated spot on the exterior and the reacting tissue, muscle or gland. These characteristics may be roughly classified as follows:

(1) LOCALISATION. In a simple system of neurons a given stimulus will nearly always produce the same reaction. In a frog possessing-only a spinal cord, the upper parts of the central nervous system having been destroyed, any harmful stimulus applied to a toe will cause a lifting of the leg. If the motor nerve to the gastrocnemius be excited, the whole muscle contracts. If one of the nerve-roots entering into the formation of the sciatic nerve be excited, only certain fibres of the gastrocnemius contract, the locality of the reacting fibres being determined by their connection with the excitednerve fibres. In the same way the contraction of certain muscles of the leg, in response to a stimulus applied to the skin of the foot, is determined by the fact that the nerve fibres, which carry the impulses from the toe into the spinal cord, divide there and make connections with the motor neurons, whose axons are distributed to the several muscles involved in the reaction. The connection of the sensory with the motor neuron may be direct, but in most cases the impulse has to pass through intermediate neurons before arriving at the motor neurons. The path of the impulse, however, in spite of its enormous extension, is as definite as is the path from an excited motor nerve-root to a muscle fibre.

(2) DELAY. Instead of one nerve-ending intervening between the stimulated nerve and the reacting tissue, there will be, in the case of the reflex action, two, three, or more nerve-endings interpolated in the path of the impulse. These nerve-endings are the fields of conjunction, the synapses, between the axon of one neuron and the dendrites and cell-body of the neuron next in the chain.

We have seen that there is a distinct difference between the latent period of a muscle excited through its nerve as compared with the latent period when excited directly, and we ascribed this latent period to a delay in the motor end-plate. We should expect therefore to find that the delay or 
latent period in the case of a reflex action, i.e. the lost time in the conversion of an afferent into an efferent impulse in the central nervous system, would be appreciable and would increase with the complexity of the response-that is, with the number of neurons involved in the reaction. Such is indeed the case.

, In determining the actual 'lost time' in the central nervous system for any given reflex, it is necessary to subtract from the total delay, interposed between the application of the stimulus and the resultant movement, the time taken by the impulse in travelling to and from the central nervous system, as well as the latent period of the muscles themselves. The remainder is known as the 'reduced reflex time.' Wundt found in the frog, when a reflex contraction of the gastrocnemius was excited by a stimulation of a posterior root of the same side, that the reduced reflex time was $.008 \mathrm{sec}$. For a crossed reflex the delay was increased by $\cdot 004 \mathrm{sec}$. If we assume that one additional neuron is involved in the crossed reflex, the lost time at a synapse would be $.004 \mathrm{sec}$; if two cells are intercalated, the synapse delay would be only $\cdot 002 \mathrm{sec}$. Since the uncrossed reflex has a delay of $008 \mathrm{sec}$., at least two, and possibly four, synapses are involved in the path of this simple reflex.

The blinking excited by stimulation of the eyelid has a reduced reflex time of $\cdot 047 \mathrm{sec}$.

(3) SUMMATION. When contractile tissues, such as striated or unstriated muscle, are excited by single shocks, a certain minimal strength of stimulus is necessary in order to produce a contraction. Weaker stimuli are spoken of as sub-minimal, and when applied singly have apparently no effect on the muscle. In dealing with the properties of involuntary muscle we saw that a sub-minimal stimulus is not necessarily devoid of effect because it fails to evoke a contraction, since, if repeated at sufficiently frequent intervals, a summation of stimulus occurs, so that at the fifth or sixth application a stimulus, which was previously ineffective, becomes effective and a contraction results. The muscle will now continue to respond to each stimulus, but, if the excitations be discontinued for a time, reapplication of a stimulus of the same strength becomes once more ineffective. This summation of stimulus is a prominent feature in all reflex actions, so much so that it may be often impossible to evoke a reaction to a very strong single induction shock, whereas the application of a tetanising current too weak to be felt on the tongue may produce a marked reaction. We shall have occasion later on to deal with special examples of this summation of stimulus.

(4) FATIGUE. In the muscle-nerve preparation the weakest point and that which soonest suffers from fatigue is the end-plate, or rather the field of conjunction of nerve fibre and muscle fibre. In the central nervous system the synapses of the different neurons are equally susceptible, and since several of such synapses are involved in every reflex action, we should expect to find that the central.nervous system would show signs of fatigue before the peripheral structures. If a given reaction be repeatedly elicited by applying a stimulus to a certain area of the surface, the reaction becomes 
feebler and finally disappears altogether long before any signs of fatigue in the motor apparatus can be detected by stimulation of the motor nerve itself. This fatigue is produced equally, well if the reaction be excited by stimulating a sensory nerve directly, and since we know that it is practically impossible to fatigue nerve fibres, we must conclude that the seat of fatigue is in the grey matter of the spinal cord itself.

(5) 'BLOCK' OR RESISTANCE. In the central nervous system there is an absolute block to the passage of an impulse backwards through a synapse, i.e. from a nerve-cell or its dendrites into the end ramifications of an axon. The phenomena of fatigue show that there is a certain degree of resistance at the synapse to the passage of an impulse in the normal direction, and that this resistance is rapidly increased under the conditions which produce fatigue. When we study the structure of the central nervous system more fully, we find that although there are certain shortest possible paths, i.e. ones involving few neurons, for every impulse arriving at the central nervous system, yet so extensive is the branching of the entering nerve fibres and so complex are the neuron systems with which they come in connection that an impulse entering along one given fibre could spread to practically every neuron in the spinal cord and brain. Such a result is indeed observed in animals poisoned by strychnine. In such animals the slightest stimulus applied to any part of the skin excites strong tonic spasms in the whole musculature of the body. Every single nerve fibre, that is to say, can discharge into every motor neuron of the cord. That this result does not ensue on localised stimulation in a normal animal is dependent on the varying resistance to the passage of an impulse into the several neurons with which the entrant fibre comes in relation. A small stimulus will discharge therefore only along the few neurons where the resistance is lowest. Increase of the stimulus, either by increase of its strength or by summation of weak stimuli, will enable the impulse to spread along more neurons and therefore will elicit a more widespread response. Only when the blocks are entirely removed by the administration of strychnine, or when the stimuli are abnormally powerful and long continued, will the impulse spread to all regions of the central nervous system, so that response becomes general and inco-ordinate instead of local and adapted to the stimulus.

(6) FACILITATION OR 'BAHNUNG.' The passage of a nervous impulse across a synapse or series of synapses in the central nervous system has a twofold effect. If the passage be too often repeated phenomena of fatigue are produced, and there is an increase of the block at each synapse. If, however, the stimulus be not excessive and the reaction not too frequently evoked, the effect of passage of an impulse once is to diminish the resistance, so that a second application of the stimulus evokes the reaction more easily. The process of summation in fact is chiefly in the direction of removal of block. We have a close analogy to this process of facilitation in the "staircase phenomenon observed in cardiac and unstriated muscle. In these tissues the repetition of a sub-minimal stimulus renders it in time effective, and then repetition of the now effective stimulus causes a gradually 
increasing height of contraction, which depends on the state of the contracting tissue itself and cannot be evoked by changes in the strength of the stimulus. This process of facilitation or 'Bahnung' is of great interest in connection with the development of 'long paths' in the central nervous system, and more especially with the acquirement of new reactions by the higher animals. The Law of Facilitation is really the Law of Habit. When an impulse has passed once through a certain set of neurons to the exclusion of others it will tend, other things being equal, to take the same course on a future occasion, and each time that it traverses this path the resistances in the path will be smaller. Education is the-laying down of nerve-channels in the central nervous system, while still plastic, by this process of 'Bahnung' along fit paths, combined with inhibition (by pain) in the other unfit paths. Memory itself has the process of 'facilitation' as its neural basis.

(7) INHIBITION. The constant occurrence of a reaction in response to a given stimulus is obtained only if care be taken to isolate the segment of the central nervous system involved from the entry of other afferent stimuli. As a rule, if two stimuli be applied simultaneously at different points, the reaction which ensues will not be a combined one, the resultant of the reactions which would be normally conditioned by each single stimulus, but will be a response to one of the stimuli, which we must therefore regard as the more effective. The reaction to the other stimulus is either abolished altogether or comes on after a considerable period of delay. The central nervous system can apparently attend to only one thing at a time. In physiological terms we should say that every effective reaction inhibits every other reaction. In the spinal cord of the frog the normal withdrawal of the foot in response to stimulation of the toe of the same side can be inhibited by strong stimulation of the other sciatic nerve, by stimulation of the spinal cord at a higher level, or by stimulation of the optic lobes. Immediately after pithing the brain of the frog, the whole animal becomes flaccid and motionless, and for the next few minutes it is impossible to elicit any reaction by stimulation, however strong, applied to the skin of the body. In the production of this condition of 'shock' the inhibition of all the spinal centres, produced by the strong stimulation of the injury to the brain and medulla, plays at any rate an important part. (We may say that the passage of an impulse through a chain of neurons diminishes the block for subsequent impulses at each synapse that it traverses, but increases during its passage the block in all the adjacent synapses.)

In dealing with the special reactions of the spinal cord we shall have occasion to refer more fully and in greater detail to many of these properties which are characteristic of all reflexes. Before, however, treating of the functions of the separate parts of the central nervous system in the higher mammals, it may be of interest to consider the exact nature of the structure intervening between neuron and neuron at each field of conjunction or synapse, as well as the significance of the two chief elements of the central nervous system, nerve-cell and nerve fibre, in the production of co-ordinated purposive reactions. 


\section{SECTION IV \\ NATURE OF THE CONNECTION BETWEEN NEURONS}

THE study of the development of the central nervous system in higher animals has shown that this system is made up of neurons, the connections of which determine the possible paths of impulses in the adult cord. The first stage in the development of the neuron is a single cell without processes, and it is only by the growth of these processes out from the cell that the spinal cord becomes capable of serving as an aggregate of conducting paths. Moreover the deferred acquisition of an influence of one neuron on the next neuron in the line of impulse, or at any rate on the peripheral tissue which receives the end arborisation of its axon, is shown by the fact that entire destruction of the spinal cord in the embryo at an early stage in its development does not prevent in any way the development of the voluntary muscles (Harrison); although, after birth, a severance of the connection between spinal cord and skeletal muscle leads to a rapid degeneration and atrophy of the latter. In the muscle-nerve preparation there is an apparent break of structure at the termination of the nerve in the muscle fibre, any continuity between nerve-ending and contractile substance being subserved by undifferentiated protoplasm. There is therefore no difficulty in conceiving a propagation across a similar nerve-ending or synapse, between the axon of one neuron and the cell body or dendrites of another neuron. If, however, the conception we have formed above of the evolution of a nervous system from a continuous conducting protoplasmic network, by a process of facilitation or 'Bahnung' attended by histological differentiation, be correct, we should expect to find in the fully developed brain and spinal cord some traces at any rate of continuity throughout the whole system of neurons. The question as to the existence of anatomical continuity from neuron to neuron has been hotly discussed both for vertebrates and invertebrates. In the case of the latter, evidence in favour of the continuity of neuro-fibrillæ from sensory surface to reacting tissue is very strong. Many observers, especially Apathy, Bethe, and Held, have described a similar continuity in the nervous system of mammals. The last-named observer regards this continuity as a product of later development and as due to a process of concrescence occurring between the axon terminations and the bodies of the nerve-cells with which they come in contact. It is easy to show the existence of a fibrillar structure both in the nerve-cell and in the 
nerve fibre (Fig. 147). The axis cylinder of the nerve fibre can be regarded as made up of fine fibrillæ embedded in an interfibrillar substance. At the nodes of Ranvier the interfibrillar substance is interrupted, the fibrillæ alone extending into the next internode and representing the continuous structure which determines the conducting power of the nerve fibre. In the nervecell the fibrillæ occupy all the space between the Nissl bodies, passing from dendrite to dendrite, and many of them from all the dendrites and all parts of the cell sweeping through the axon hillock to form the fibrillæ of the nerve fibre. The existence of these fibrillar structures in nerve-cell and nerve

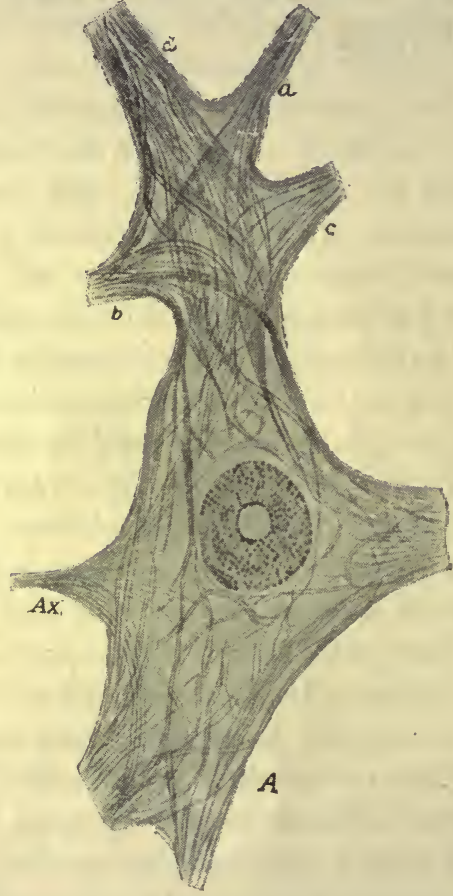

Fig. 147. Part of an anterior cornual cell from the calf's spinal cord, stained to show neuro fibrils. (BETHE.)

$A x$, axon; $a, b, c$, dendrites.

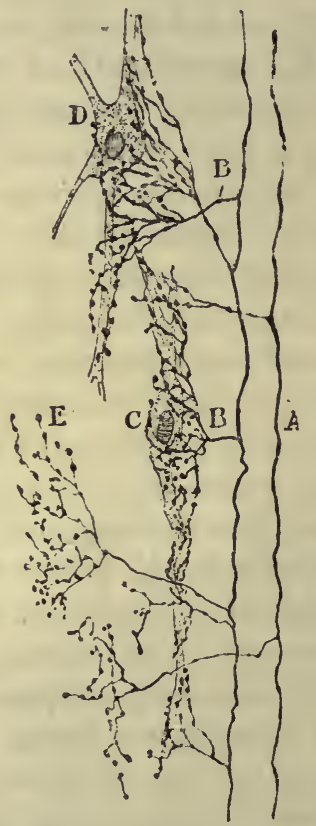

FIG. 148. Arborisation of collaterals from the posterior root-fibres round the cells of the posterior horn. (RAMÓN Y CAJAL.)

fibre is accepted by most histologists. The question, however, of the connection between the fibrillæ of one axon and those of the next neuron, i.e. the histology of the synapse, presents much greater difficulties and has excited much difference of opinion. If we examine a nerve-cell such as a cell of Purkinje of the cerebellum, or a cell of Clarke's column in the cord, we find that it is surrounded by a thick basket-work of fibres which are the arborisations or end terminations of the axons which pass to the cell to enter into functional relationships with it (Figs. 148 and 149). This pericellular network is of great extent and may equal in total diameter the diameter of the cell itself. Whether the basket-work is really a network, 


\section{NATURE OF CONNECTION BETWEEN NEURONS}

or merely a felt-work in which the fine fibres intertwine among each other without becoming actually continuous at any points, is difficult to make out. On the periphery of the cell itself another network has been described and is known as the Golgi network (Fig. 150). This has been displayed both by the process of impregnation with silver chromate (Golgi method), as well as by staining with methylene blue. Some authors have regarded this network as an artefact due to precipitation of albuminous fluids on the surface of the cell. According to Bethe, however, the Golgi network on the one hand receives fibres from the encircling pericellular basket-work of axons,

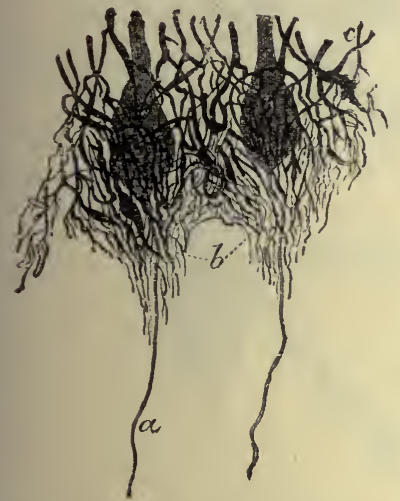

FIG. 149. Basket-work of fibres around two cells of Purkinje. (CAJAL.)

$a$, axis-cylinder or nerve-fibre process of one of the corpuscles of Purkinje; $b$, fibres prolonged over the beginning of the axiscylinder process ; $c$, branches of the nerve-fibre processes of cells of the molecular layer, felted together around the bodies of the corpuscles of Purkinje.
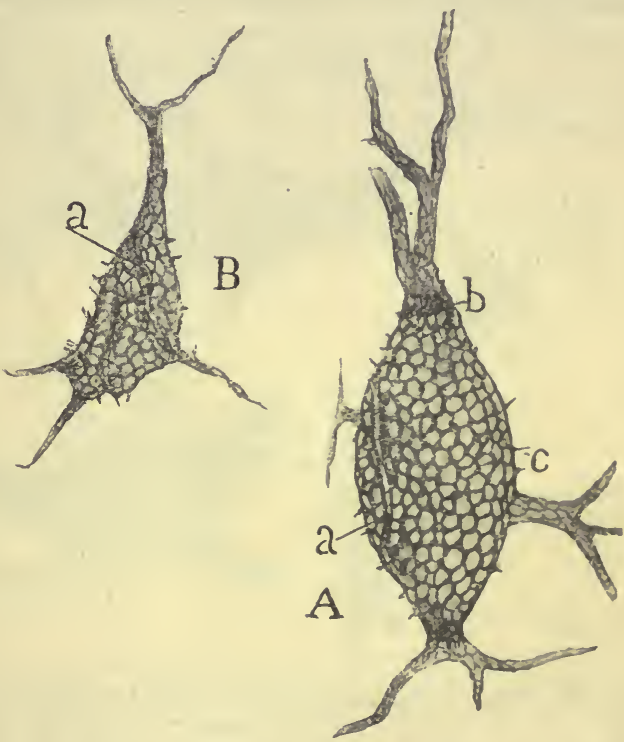

FIG. 150. Superficial network of Golgi surrounding two cells from the cerebral cortex of the cat; Erlich's method. (CAJAL.)

$\mathbf{A}$, large cell ; $\mathrm{B}$, small cell ; $a, a$, folds in the network; $b$, a ring-like condensation of the network at the poles of the larger cell; $c$, spinous projections from the surface.

and on the other hand gives off towards the interior of the cell fine fibrils, which are continuous with the neurofibrillæ of the cell and pass out in its axon. The diagrammatic course of a nerve impulse according to Bethe is represented in the accompanying diagram (Fig. 151). An impulse starting from the periphery of the body travels up the distal process of the posterior root ganglion-cell, passes either through the cell or directly to the central process, and travels along this to the terminations of the posterior root fibres round a posterior horn-cell. Here it passes into the peri-cellular basket-work or axon network, thence into the Golgi network and along the fibrillæ of the cell out by the fibrillæ of the axon and so to a fresh synapse with a cell of the anterior horn.

There are certain physiological difficulties in the acceptance of this 
doctrine of continuity through the central nervous system. Even if it be true, it would not in any way upset the importance of the neuron theory. Every plant or animal individual must be regarded as a protoplasmic continuum. With growth of the living matter, its metabolic functions demand the dispersion of nuclear material through the protoplasm, and this is effected by division of the nucleus. Considerations of strength and rigidity demand the division of the protoplasm into compartments or cells, which, at first at any rate, remain in protoplasmic continuity. This division has probably a further advantage in that lesions of parts of the individual entail merely the death of the cells immediately affected and do not necessarily

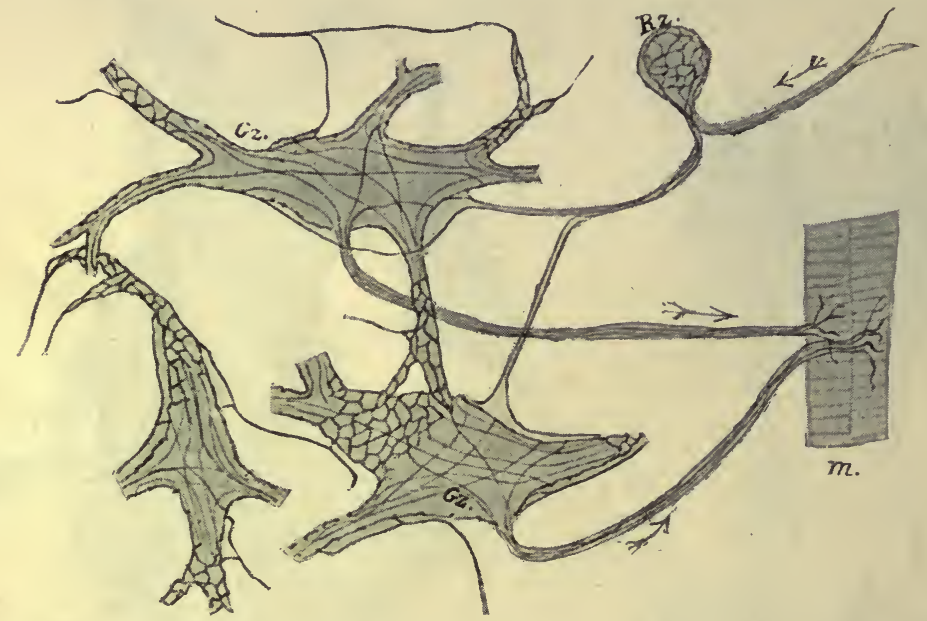

FIG. 151. Schema of the neurofibrillar continuum, involved in an ordinary reflex act, in a vertebrate nervous system. (Ветне.)

spread to the whole organism. Thus in the central nervous system injury to one axon causes degeneration of the axon below the point of section, but the degeneration stops short at the end arborisation and does not spread into the next neuron. If we assume that, in consequence of the straitness of the path, the propagation through the fibrillæ is especially difficult in the synapse, most of the phenomena described above as characteristic of the reactions which take place in the central nervous system can be easily explained on the theory of continuity of the fibrillæ. The serious difficulty in the acceptance of this theory is, however, the 'Law of Forward Direction,' i.e. the fact that an impulse will pass from an axon to the next neuron, but will not pass backwards across the synapse from the cell body to the contiguous axon. Bethe suggests that this rule of Forward Direction, which is possibly present only in the more highly developed nervous systems, may be due to a species of "polarity" of the nerve-fibril, of such a nature that an impulse is strengthened and so assisted on its passage in the normal direction, but is diminished and finally abolished when it passes in the opposite direction. Such an explanation is unsatisfactory, since there is absolutely no experimental evidence of the existence of such polarity in a 
nerve fibre; all the evidence that we have at present points to a nerve fibre having the power of propagating equally well in either direction. It is certainly more useful to regard a synapse as of the nature of a motor nerveending, in which an impulse arriving along the branches of an axon excites a fresh impulse in the excitable tissue, i.e. the nerve-cell, with which the branches of the axon come in contact. Moreover the neurons are formed without any structural connection with the future destination of their axons. These grow out as processes with thickened amœboid extremities. Harrison has shown that the growth of the axon from the cell may be observed under the microscope in a neuroblast separated altogether from the body, and kept on a warm stage in a thin layer of coagulated lymph. It is possible that we may have to distinguish two types of nervous system, viz. :

(a) A neurofibrillar type, peculiar to invertebrata, with conduction in all directions.

(b) A synaptic type, in which the Law of Forward Direction holds, of later evolution, and forming the greater part of the nervous system of vertebrata. 


\section{SECTION V \\ FUNCTIONS OF THE NERVE-CELL}

WHEN a unicellular organism, containing a single nucleus, is cut into two parts, both continue to live for some time, each performing active movements and evincing all the phenomena which we associate with activity and therefore with destructive katabolism. For the continued existence of a cell the processes of constructive metabolism, or anabolism, must take place pari passu with those of disintegration, and for this the presence of the nucleus is necessary. Hence, in a few days, the half cell with the nucleus has repaired its loss and become once again a normal individual, whereas the half without a nucleus undergoes degeneration and death. The axon of a nerve-cell can be regarded as analogous to a long pseudopodium of an amœba. Like this, if cut away from that part of the cell containing the nucleus, though capable for a time of discharging its active function of propagation of excitatory impulses, yet it finally dies, death of the nerve fibre occurring in the mammal within three to five days after separation of the axon from the cell. Every nerve-cell therefore may be looked upon as a trophic centre of the nerve fibre proceeding from it as well as of the medullary sheath, which is practically a product or secretion of the axis cylinder. But has the nerve-cell any more important functions to discharge? It has long been customary to endow the nerve-cell with all the properties which are distinctive of a nervous system, and to ascribe to it the active part in the origination of automatic actions, in the reflection of afferent impulses, and in the supply of energy to all nervous processes. That the passage of impulses through the nerve-centres requires the expenditure of energy by these centres can be proved in various ways. In the first place, we have the fact that in all nervous systems, at any rate of the higher animals, arrangements are made for their free supply with oxygen Very short deprivation of oxygen causes a complete block throughout the system, in many cases preceded by a short period of increased excitability or ease of transmission. If, in the rabbit, the thoracic aorta be clamped for a few minutes, the hind limbs become paralysed, and if the obstruction be continued for half an hour, there is widespread degeneration and death of the cells with their fibres in the grey matter of the lumbar and sacral cord. In the second place, the ready production of fatigue of the nervous system points to a considerable using up of material as a condition of the passage of nerve impulses. In many instances, moreover, an infinitesimal stimulus travelling up a few nerve fibres may excite widespread activity of the whole central nervous system with the discharge of impulses along 
practically every nerve of the body. Thus the presence of a crumb on the larynx will excite impulses travelling up the superior laryngeal nerve, which in themselves can involve but little expenditure of energy. The result, however, of their arrival at the central nervous system is the discharge of impulses along the motor nerves causing spasmodic contractions of almost every muscle in the body. It seems beyond doubt then that energy is evolved in the central nervous system as a result of metabolic changes, and that energy may be added to impulses passing through the central nervous

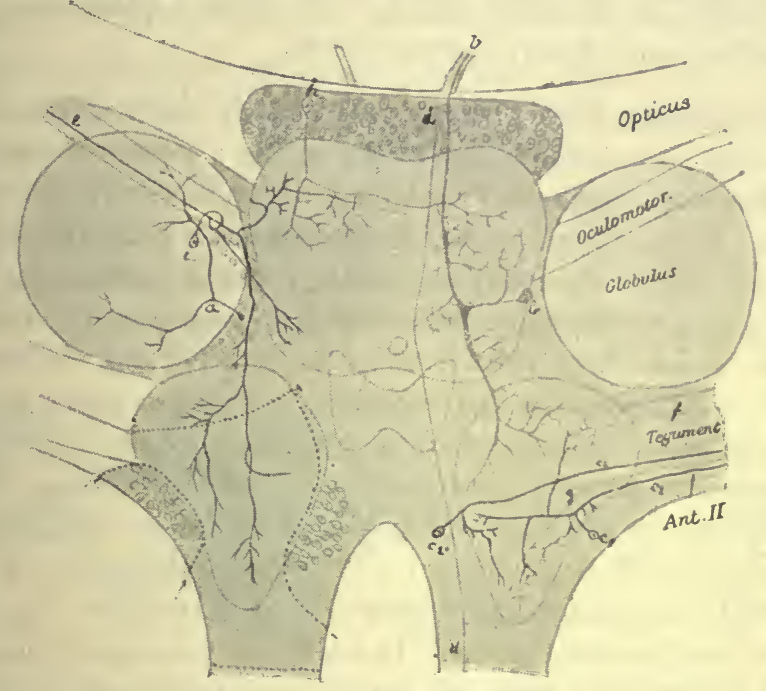

FIG. 152. Diagrammatic representaticn of the brain of Cacrinus to show the parts involved in Bethe's experiment. The dotted line $x$ shows the incision employed to isolate the neuropilem of the ganglion of the second tentacle.

system, which therefore acts as a relay of force. But this activity does not necessarily require the presence or co-operation of nucleated cells. In dealing with the nature of a nerve impulse we had reason to conclude that there may be an actual, though minimal, liberation of energy in the axis cylinder with the passage of each nerve impulse. The non-nucleated parts of a cell, whether the axon or the cell body, are equally capable of this evolution of energy, and we might conceive therefore of a nervous system which, existing for a few days, might act as a normal reflex centre in the entire absence of the nucleated cell bodies.

This conception has been realised by Bethe in an experiment on the crab (Carcinus menas). In this animal the reflex movements of the tentacle are carried out by a ganglion situated at its base. As in the other crustacea, the cell bodies in this ganglion lie outside the mass of neuro-fibrils in the centre, forming a sort of capsule (Fig. 152). Bethe was able, under the dissecting microscope, to remove the cell bodies without interfering with the nerves entering or leaving the central mass of fibrils. All the nerve processes with their connections were therefore left intact. In animals, operated in this way, Bethe found that for two or three days the tentacle reacted normally to stimuli applied to its surface. The reflex functions of the ganglion were not in any way affected by the removal of the nucleated bodies of the cells. A similar 
experiment would be impossible in the central nervous system of vertebrates, since impulses must of necessity pass through the cell body on their way from the termination of one axon to the beginning of the next. In the spinal root ganglion, however, most of the cells lie on the surface. In the rabbit Steinach exposed a posterior root ganglion, separating it from all its vascular supply, but leaving its nervous attachments intact. The wound was opened every day for the next few days and an instrument passed under the ganglion so as to divide any newly forming vessels. As a result of the deprivation of blood-supply the ganglion-cells died. But Steinach found that nerve impulses were still conducted perfectly well through the ganglion at a time when microscopic examination showed a complete atrophy of all cells. It is therefore only in virtue of the fact that the nerve-cell is the seat of the nucleus, and therefore of the assimilative functions of the neuron, that any pre-eminent importance can be ascribed to it in the building up of a reactive nervous system.

Prominent among the functions with which the nerve-cell has been endowed is that of automaticity of action in the absence of stimulus other than that supplied by its own metabolism or by the fluids which bathe it. A priori there is no reason to deny to the neuron a property which is possessed by other cells of the body, such as the muscular cells of the heart, and is a fundamental quality of undifferentiated protoplasm. The purpose, however, for which these cells have been evolved and differentiated is that of reaction, of adapting the organism to changes in its environment, and it is doubtful whether, in this differentiation, it has retained any automatic properties whatsoever. In the absence of any afferent impulse the whole central nervous system would probably be inert. In a frog retaining only the spinal cord Hering divided all the posterior roots. The frog remained flaccid and motionless. Injection of strychnine was powerless to evoke the usual tetanic spasms. In such a strychninised frog, however, it was only necessary to open the wound and touch one of the divided posterior roots to throw the whole body into convulsions. As shown by Sherrington and Mott, division of all the afferent nerves coming from the upper limb in monkey or man entirely abolishes all such contractions of the limb, as are usually affected through the intermediation of the cerebral cortex. Cutting off the major portion of the afferent impulses to the respiratory centre does not, it is true, abolish all respiratory discharges, but converts the rhythmic respirations into a series of inspiratory spasms which are repeated at long intervals and are entirely inadequate for the proper aeration of the blood. According to Sherrington a repetition on the mammal of Hering's experiment does not lead to the same results, since a spasmodic discharge is produced from the isolated spinal cord as a result of asphyxia. But it is doubtful whether in this case there was not some continuous excitation of the cord going on, as a result of the closure of the demarcation current in the cut ends of the posterior roots by the body fluids. It is possible that the neurons possess some automatic power, i.e. some power of initiating nervous processes, as a result of changes in the fluids surrounding them. This automaticity, however, is not a prominent feature of the nervous system, which has been evolved as a purely reactive mechanism to the afferent impulses resulting from the material changes continually taking place in the environment of the animal. 


\section{SECTION V1 \\ STRUCTURE OF THE SPINAL CORD}

In the higher representatives of the invertebrate class the central nervous system consists, as we have seen, of a chain of ganglia, each ganglion or pair of ganglia presiding over the reactions of its own segment, but connected by long paths with the other ganglia and with the head ganglia. The latter, being especially developed in connection with the organs of special sense which are projicient in function, acquire a control over the rest of the ganglia (Fig. 153). The vertebrate spinal cord may be looked upon as a chain of ganglia which
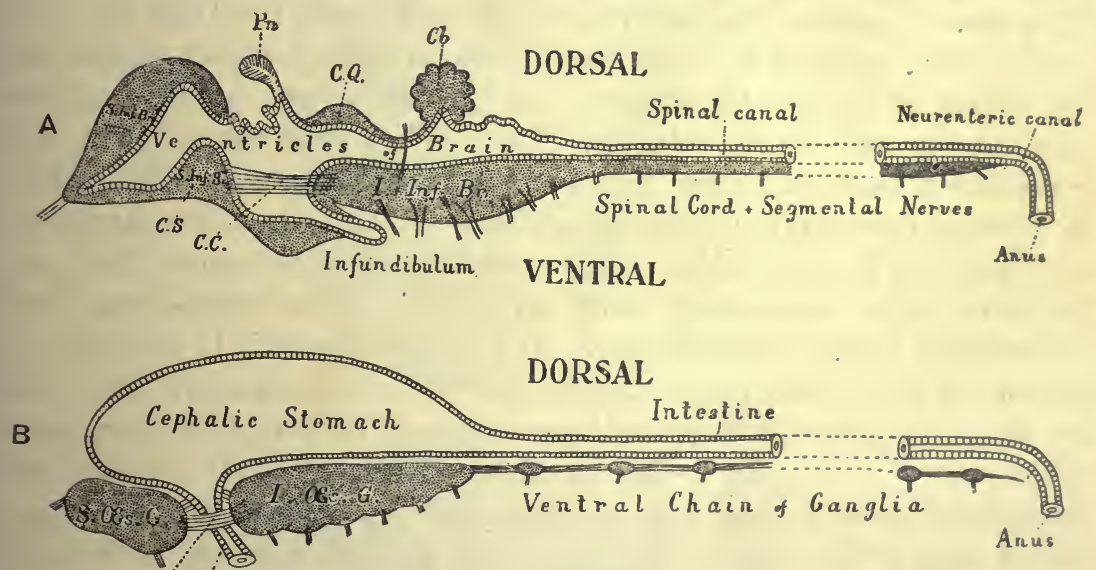

$$
n_{s} \text { Com. Oisophagus }
$$

FiG. 153. Vertebrate central nervous system compared with that of the arthropod. (GASKELL.) (Note that according to Gaskell the ventricles of the brain and the primitive neural canal correspond to the invertebrate stomach and intestine.)

have become fused concurrently with a diminution in the importance of the local segmental reactions and with a growth in the solidarity of the whole system; so that in the higher vertebrates, at any rate, little trace of the primitive segmental arrangement is evident in the internal structure of the cord. Some remains of this arrangement still persist, however, in the origin from the cord of nerve roots, which are distributed roughly within the area of the corresponding segment of the body.

In man the spinal cord is an elongated cylindrical structure slightly flattened from before backwards and about eighteen inches long. It gives off a series of nerve-roots, which are arranged in thirty-one pairs and are distributed symmetrically to the two sides of the body. Each 
nerve arises by two roots, an anterior and a posterior, the anterior being composed of a series of rootlets spread over a considerable area of the cord, while the posterior roots arise as a compact bundle from a groove on the postero-lateral aspect of the cord. The posterior nerve-roots pass through a ganglion and join the anterior roots in the intervertebral foramina to form the mixed nerve. On section the cord is seen to consist of a core of grey matter surrounded on all sides by white matter. The white matter is made up of medullated nerve fibres which are devoid of a neurilemma, and run within tunnels or tubes in the supporting neuroglia. The grey matter has roughly the form of a letter $\mathrm{H}$, and consists, in cross-section, of a comma-shaped mass on each side of the cord, joined across the middle line by a band of grey matter. On the anterior aspect of the cord is a furrow, the anterior fissure, which contains a process of the enveloping membrane of the cord, the pia mater, and is limited at its bottom by a band of white matter, the anterior white commissure, which unites the anterior columns of white matter.

On the hinder aspect of the cord is another fissure, the posterior fissure, which is very narrow and is built up chiefly by neuroglia. A third fissure at the point of origin of the posterior nerve-roots serves to divide the white matter of the cord into an antero-lateral column and a posterior column, and the former is imperfectly separated by the spread-out anterior rootlets into anterior and lateral columns. The cord in cross-section (Fig. 154) is circular in the dorsal region and oval in the cervical and lumbar regions. It presents two marked enlargements, namely, the cervical enlargement, corresponding to the outflow of the nerves going to the upper limb, and the lumbo-sacral enlargement, which gives off the nerves to the lower limb. In the sacral region it rapidly tapers off to a blunt point. In the centre of the band of grey matter, connecting the two masses on each side of the middle line, is the central canal of the cord, the remains of the primitive neural canal of the embryo. The grey matter.in front of it is called the anterior grey commissure, that behind the posterior grey commissure. The commashaped mass of grey matter on each side of the cord presents in front the broad anterior cornu, and behind the narrower posterior cornu, which extends up to the postero-lateral groove in the line of emergence of the posterior roots. In the dorsal region of the cord the grey matter projects into the lateral column of white matter to form the lateral horn. The grey matter consists of a supporting tissue of neuroglia in which are embedded nerve-cells and their processes and the endings of nerve fibres. The neuroglia is formed of a thick felt-work of fibres with here and there nuclei applied to the fibres. Occasionally we may meet cells provided with a very large number of branches and representing the cells from which all the fibres of the neuroglia have been derived. The neuroglia is present in specially large amount in two situations, namely, immediately around the central canal and as a capsule to the enlargement of the posterior cornu, known as the head or caput cornu posterioris. In this latter situation the neuroglia contains a large number of small richly branched nerve-cells and is spoken of as the substantia gelatinosa of Rolando. The nerve-cells are arranged in 


\section{STRUCTURE OF THE SPINAL CORD}

distinct groups. In the anterior horn we may distinguish three groups, a median group of cells near the middle line, many of which send their processes across to the other side in the anterior white commissure, and an external
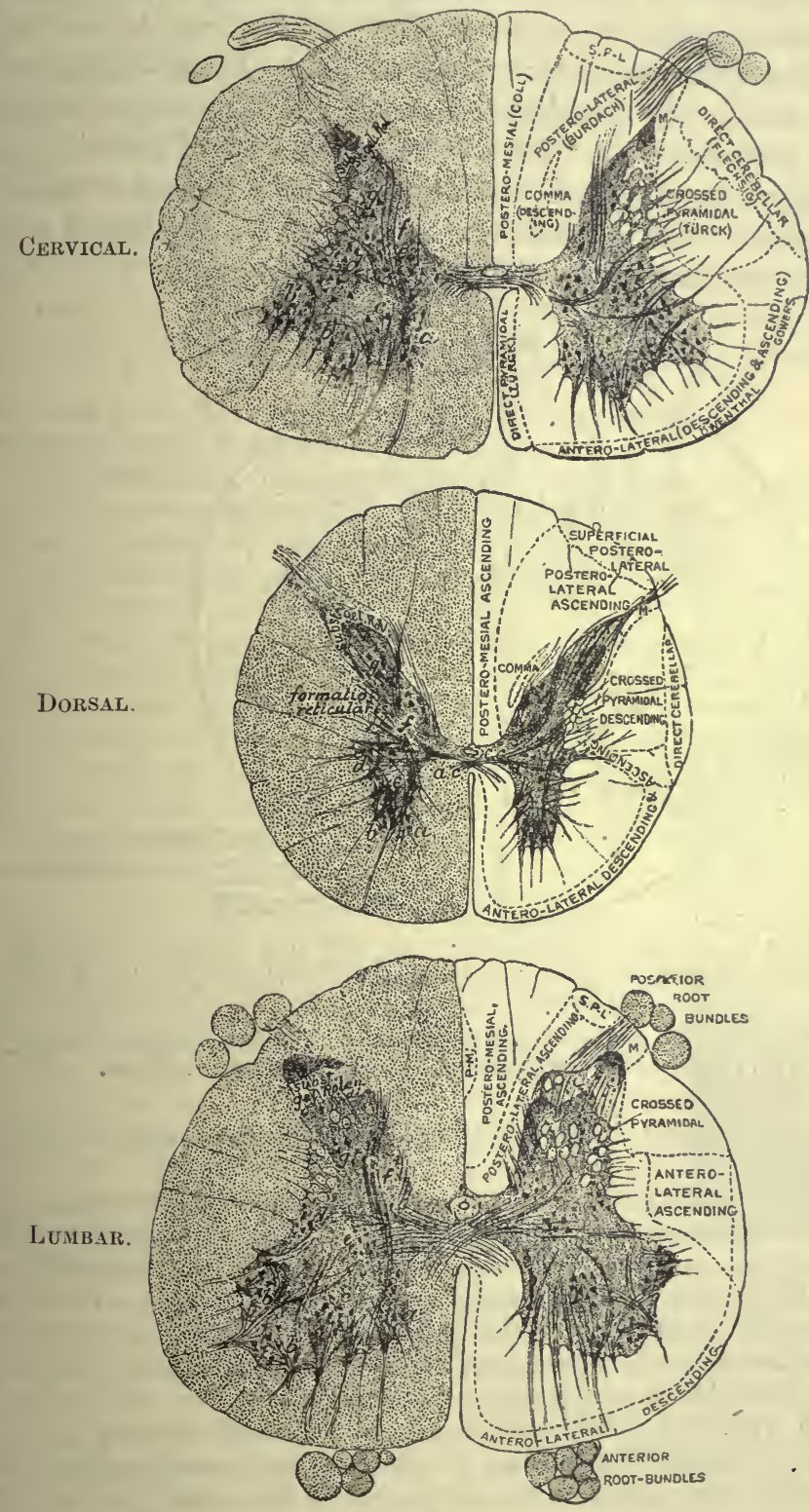

Fig. 154. Sections of human spinal cord from the lower cervical, mid-dorsal, and mid-lumbar regions, showing the principal groups of nerve-cells, and on the right side of each section the conducting tracts as they oscur in the several regions (magnified about 7 diameters). (E. A. SCHÄFER.)

$a, b, c$, groups of cells of the anterior horn ; $d$, cells of the lateral horn; $e$, middle group of cells ; $f$, cells of Clarke's column; $g$, cells of posterior horn ; $c c$, central canal ; ac, anterior commissure. 
group often subdivided into a postero-external and an antero-external. The latter group is especially developed in the regions of the cervical and lumbar enlargements and consists of very large multipolar cells with many dendrites which send their axons into the anterior roots and by these to the muscles of the limbs. Another group of rather smaller cells is found in the lateral horn, in that region of the cord where this is marked. A very definite group of cells may be seen in the dorsal region of the cord in the inner aspect of the root of the posterior horn. This, which is known as Clarke's column, is formed by large cells elongated in the longitudinal direction of the cord. Besides these definite columns a number of nerve-cells are distributed irregularly through the grey matter, especially of the posterior.horn.

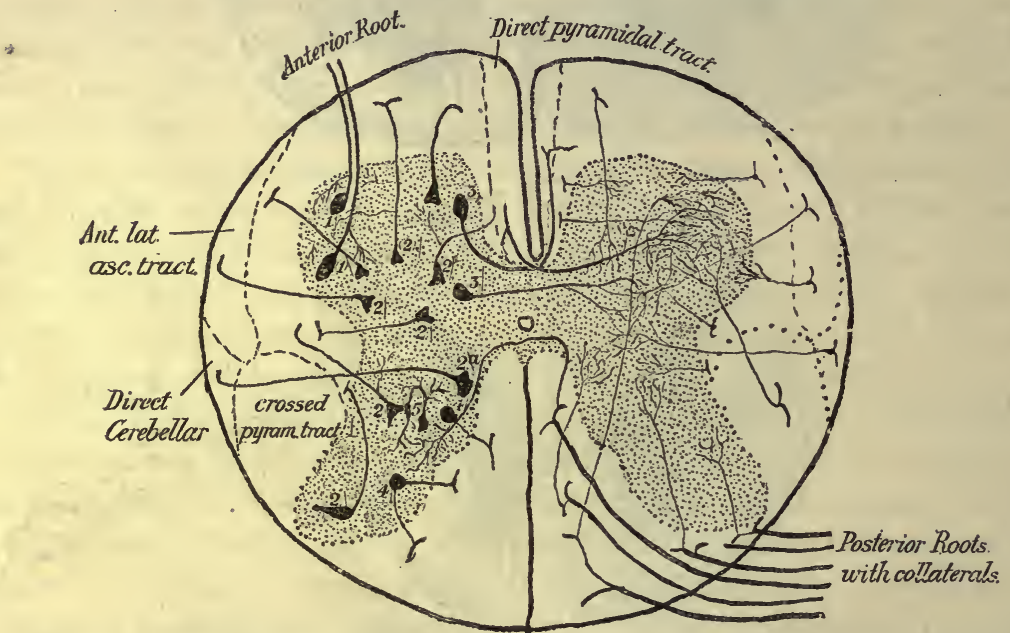

Fig. 155. Spinal cord. (After Lenhossex.) On left side of figure are shown the nerve-cells with their axis-cylinder processes. On the right side the distribution of the chief collaterals.

1 , motor cells ; 2 , cells of the columns ; $2 a$, cells of Clarke's column, sending processes across into direct cerebellar tract; 3,4 , and 5 , commissural cells.

According to the destiny of their axons these nerve-cells may be divided into four groups (Fig. 155).

(1) THE MOTOR CELLS, the largest of all, which send their axons into the anterior roots, where they run to supply skeletal muscle fibres. A s a sub-group of these cells we may class the somewhat smaller cells of the lateral horn, which in all probability send their axons by the anterior roots to supply visceral muscles. Their axons can be distinguished from the motor axons by the smaller diameter of the nerve fibres they form. They pass later from the mixed nerve along a white ramus communicans into the sympathetic system, in the ganglia of which they end.

(2) CELLS OF THE COLUMNS. As typical of these cells we may take those which form Clarke's column. Their axons do not leave the central nervous system, but pass out into the white matter to some other part of the central nervous system, contributing thus to form the white columns of the cord.

(3) COMMISSURAL CELLS. These cells send their axon across the middle line to the opposite side of the cord, making up a great part of the anterior white commissure.

(4) CELLS OF GOLGI. These cells are found chiefly in the posterior horn. They are multi-polar and are distinguished from all the other cells by the fact that their 
axon does not pass far from the cell, but rapidly breaks up into a number of branches which terminate in the near neighbourhood of the cell giving off the axon. They may be regarded as association cells, i.e. as serving to establish a functional connection between many different cells at any given level of the grey matter.

The white matter of the cord is divided by the fissures already described into anterior, lateral and posterior columns. The nerve fibres of which it is composed are all of them axons of nerve-cells situafed at different levels of the central nervous system or outside the cord. Since the whole object of the study of the anatomy of the cord is the tracing out of the systems of neurons of which it is made up, and therefore of the possible paths of any reflexes or nerve impulses through the cord, a mere anatomical differentiation of different columns is quite useless unless we can determine in each column the origin and destination of the fibres of which it is composed.

For tracing out the course of the different axon systems in the central nervous system several methods are available.

(a) HISTOLOGICAL. Two methods may be employed for staining a nerve-cell with all its processes, namely, the intravitam staining with methylene blue and the impregnation method invented by Golgi. In the latter method, of which there are many modifications, the nervous tissue is hardened in some chromate or bichromate, and is then soaked in a solution of silver nitrate or mercuric chloride. In this way a precipitate of silver or mercuric chromate is formed within the nerve-cells and their processes ; but for some unexplained reason the impregnation is not general, and is confined to a small percentage of the neurons. If the precipitate were diffuse, even a thin section would be absolutely opaque; since it is partial, thick sections may be cut and, after clearing, allow the tracing of the processes of the few impregnated nerve-cells through the whole thickness of the section. We may in this way get sections $0.1 \mathrm{~mm}$. thick at the point of entrance of a posterior nerve-root and trace out the course and ending of a large number of the fibres composing the nerve-root, or we may in a section involving the anterior nerve-root trace the course of an axon of an anterior cornual cell out of the cord into the root. This method is of no use in tracing any given nerve fibre through the whole length of the cord. For this purpose, however, several methods are available.

(b) MYELINATION METHOD OF FLECHSIG. Nerve fibres at their first formation as axons of a nerve-cell are non-medullated, the medullary sheath being formed later with the beginning of function of the nerve. It has been shown by Flechsig that the myelination does not occur simultaneously through all parts of the central nervous system, but that it is later in proportion as the nerve fibre is more recent in the phylogenetic history of the animal. The cord in its most primitive form can be regarded

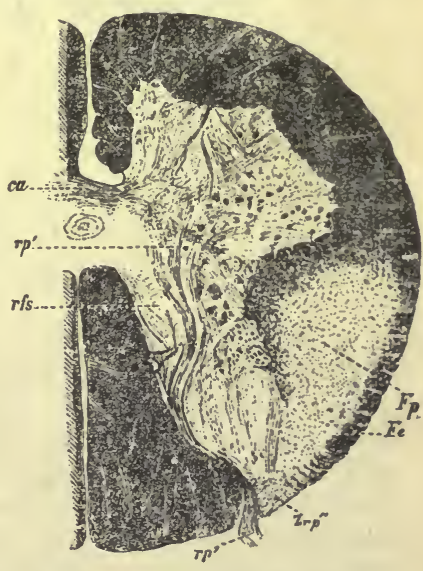

Fig. 156. Section through the cervical spinal cord of a new-born child, stained by Weigert's method, to show absence of medullation in pyramidal tract.

$c a$, anterior commissure; $F p$, crossed pyramidal tract; $F e$, direct cerebellar tract; Z $\mathrm{Zrp}$, posterior root zone; $r p^{\prime}$, posterior rootfibres. (BECHTEREW.) as a series of ganglia presiding over the different segments of the body. The most primitive fibres therefore would be those which run from the periphery of the body to each segment and from each segment out to the muscles, and so a medullary sheath is first formed in a number of the fibres entering and leaving the cord in the nerve-roots. Next in order of myelination are those 
fibres which connect different segments of the cord, the internuncial or intra-spinal fibres. Next come those fibres which connect the spinal cord with the cerebellum. Last of all to receive a medullary sheath are the fibres which take a direct course from the cerebral cortex to the spinal cord. These are called the pyramidal tracts, and in man are not medullated until the first month after birth (Fig. 156).

(c) THE WALLERIAN METHOD. A nerve fibre, when cut off from the nervecell of which it is a process, degenerates. This degeneration is marked by a breaking up of the medullary sheath and a conversion of the phosphorised fat, myelin, of which it is composed, into ordinary fat. Later on the fat is absorbed and the nerve becomes replaced by a strand of fibrous tissue in the case of peripheral nerves, of neuroglia in the central nervous system. If the white matter of one half of the spinal cord be divided in the dorsal region, and the animal be killed about three weeks after the operation, sections of the cord both above and below the lesion will show the presence of degenerated fibres. In order to display these fibres pieces of the cord are hardened in a solution containing bichromates and are then immersed in a mixture of osmic

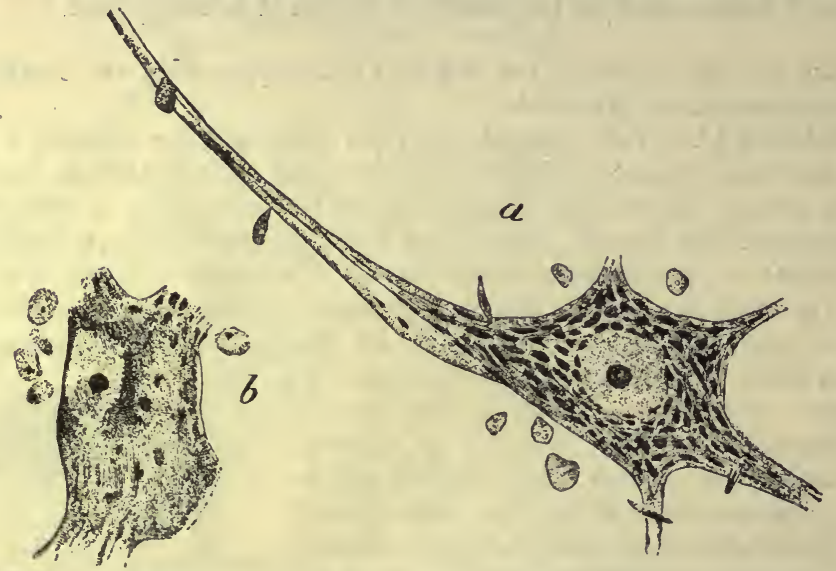

Fig. 157. Cells from the oculo-motor nuclei thirteen days after section of the nerve on one side.

$a$, cell from healthy side; $b$, cell from side on which nerve was divided. (FLATAU.)

acid and bichromate. By this method ordinary fat is stained, but myelin is left unstained (Marchi's method). Degenerated fibres are therefore stained black in virtue of their content in fat. The black staining has different distribution according as we take a section of the cord above or below the lesion. The existence of the degeneration shows that those fibres which are degenerated in the cervical region are axons of nerve cells situated below the lesion, while the fibres in the lumbar cord which are degenerated must have their nerve-cells in some part of the nervous system which is above the lesion. If the animal be kept alive for a considerable time, six months or more, before being killcd, the occurrence of degeneration in any given area of the cord will be shown by the absence of normal nerve fibres in this area. In such a case some method of staining the medullary sheath, such as that of Weigert or Heller, is employed, when the degenerated area will be evident owing to its inability to take the stain. This method, however, is not so satisfactory as the Marchi method, since it is impossible in this way to detect in a section the presence of one or two degenerated nerve fibres, whereas by the use of the Marchi method they would appear as black dots in the unstained section (cp. Fig. 164).

(d) METHOD OF RETROGRADE DEGENERATION. When a nerve fibre is divided there is no degeneration as a rule in the part of the nerve fibre central to the lesion. The nerve-cell is, however, affec'ed, and the extent to which this occurs is more pronounced according as the lesion is nearer to the cell (Fig. 157). If, for instance. 
an anterior root be divided and three weeks later the animal be killed and sections made of the corresponding segment of the cord and stained with toluidine blue or methylene blue, a striking difference will be observed between the cells of the anterior horn of the two sides of the cord. On the side of the lesion the nucleus of the cells will be somewhat swollen, and may be displaced towards the periphery of the cell. The Nissl granules are no longer distinct, but the whole cell is diffusely stained blue. In some cases this change may go on to complete atrophy of the cell and consequent degenera. tion of the whole of its axon. Generally, however, the cell gradually recovers, so that six months after the lesion no difference will be observable between the cells on the two sides of the cord. This method must be used with some caution as a means of tracing out the connections of any given neurons in the central nervous system, since it has been shown by Warrington that somewhat similar changes may be produced in the anterior horn-cells by division of the posterior roots, thus cutting off those impulses by which their activity is normally excited. Here we have a lesion applied to one neuron exciting a histological change in the cell body of another neuron which is next in the chain of the nervous arc.

The structure of the cord is closely connected with and determines its twofold function, namely, as a series of reflex centres for the different segments of the body, and as a means of communication between the trunk and limbs and the higher parts of the central nervous system. An examination of the relative area of the white matter at different levels of the cord shows a steady increase from the lower to the upper end. The increase is not, however, proportional to the number of fibres which enter or leave the cord in the various spinal nerve-roots. Of these fibres therefore a certain proportion are destined to serve merely the local segmental reflexes, while others are continued directly upwards to the brain or are connected with cells which themselves send their axons up to the brain (cells of the columns). All the motor fibres in the nerve roots arise from cells in the spinal cord near the point of origin of the root. Any direct influence of the brain on the motor mechanisms of the body is therefore effected through the intermediation of the segmental neural mechanisms of the grey matter of the cord. We will consider the function and related structure of the cord in these two aspects : first, as a reflex centre, and, secondly, as a conductor of impulses to the higher parts of the central nervous system. 


\section{SECTION VII}

\section{THE SPINAL CORD AS A REFLEX CENTRE}

IN the evolution of the cord the primitive segmental arrangement has been especially interfered with by the development of the four limbs. Since the reactions of the limbs transcend in importance and complexity those of the rest of the body, a great enlargement of the cord has occurred in the region of the nerve-roots which supply the limbs. Each limb must be considered as produced by the fusion of a number of body segments, in which the morphological segmental arrangement has entirely given place to a physiological one. Thus no single muscle of the limbs is innervated from one nerve-root, every muscle being formed from elements belonging to several segments and innervated from several nerve-roots. The segmental arrangement of the cord is hidden moreover by the increasing complexity of the spinal reflexes and the consequent involvement of many segments in even the simplest reactions. As we shall see later, practically no reflex can be evoked, even by stimulation of one nerve fibre or nerve-root in any of the vertebrata, which does not involve in its response elements belonging to many segments.

Since the reactions, which can be carried out by any part of the nervous system, depend on the neurons of which the part is composed, it is necessary, before treating of the reactions of the spinal animal, to consider the " way in " to and the "way out" of the centre, as well as the connections between the entering and issuing paths. Each segment of the cord gives off a pair of nerve-roots, subdivided into an anterior and a posterior root (Fig. 158). In mammals it is easy to show that the posterior root is exclusively afferent in function. Section of the root, either distal or proximal to the ganglion, produces no paralysis of any description. It may cause diminished sensation in the area supplied by it, and if two or three adjacent posterior roots be divided, complete anæsthesia results in the central part of the skin area supplied from these roots. Stimulation of the central end of a divided posterior root evokes in a conscious animal signs of pain. In an animal possessing only spinal cord and bulb, reflex effects are produced, i.e. movements of skeletal muscles as well as effects on visceral muscles, such as constriction of bloodvessels, relaxation of intestinal muscle, and so on. On the other hand, section of an anterior root causes paralysis of muscles or parts of muscles. Section of all the anterior roots going to a limb will produce complete motor paralysis of the limb. Stimulation of the central end of a divided 
anterior root has no effect. Stimulation of the peripheral end evokes conEraction of muscles, and if the root experimented on be in the upper dorsal region of the cord, certain visceral effects, e.g. dilatation of the pupil or augmentation of the heart beat, may result.

To this general law, the law of Bell and Magendie, which affirms the purely afferent function of the posterior roots and the purely efferent function of the anterior roots, certain exceptions must be noted. In the first place, in the lower vertebrata the separation of afferent from efferent fibres seems to be not so complete as in the higher vertebrates. Thus in the chick Cajal and others have described fibres given off as axons from the cells of the grey matter and leaving the cord by the posterior root. The function of these fibres is unknown. In the frog Steinach has stated that visceral

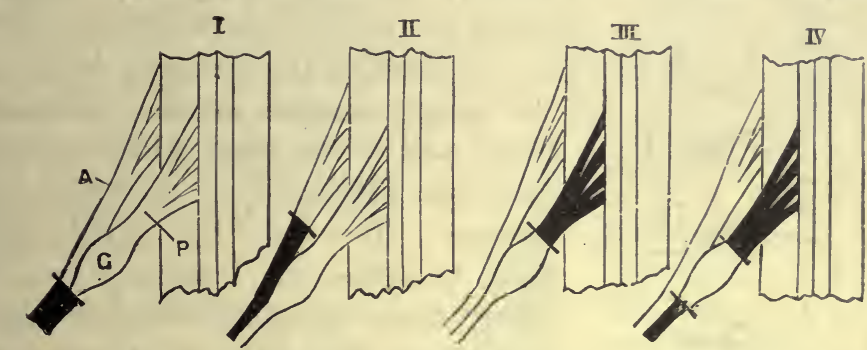

FIG. 158. Figures (from YEo) to illustrate the degree and direction of degeneration as a result of section of the spinal roots.

I, division of whole nerve below ganglion. II, division of anterior root. III, division of posterior root above ganglion. IV, division of posterior root above and below ganglion.

effects may ensue on stimulation of the lower posterior roots. This statement is controverted by Horton-Smith, who, however, has noticed contractions of fibres of voluntary muscles as the result of stimulating these roots.

In a class by themselves we must place the vasodilator effects observed by Stricker, Dastre and Morat, and Bayliss to follow excitation of the peripheral ends of the posterior roots. Bayliss has shown that the fibres, through which the vasodilatation is produced, must have their cell-station in the posterior root ganglia. It seems therefore that the same fibres provide for carrying both afferent impulses from skin to cord, and vasodilator impulses from the cord to the vessels of the skin. Bayliss has designated the impulses which effect the vasodilatation as antidromic, since they are opposed in direction to the normal impulses of the nerve fibre. Of the same nature are the curious trophic impulses which extend along the posterior roots and which must come into play when eruptions of erythema or herpes occur as the result of inflammation or hæmorrhages in the substance of the posterior loot ganglia. Both these phenomena are at present but imperfectly understood; and their anomalous character is only intensified by the further fact elicited by Bayliss, viz. that it is possible, by stimulation of afferent nerves, to excite reflexly vasodilatation through the intermediation of the posterior roots. Unless this reflex dilatation is simply an example of an 'axon reflex' (v. p. 275) it would furnish an exception to the otherwise universal law of forward direction in the mammalian nervous system.

A third exception to the law of Bell and Magendie is only apparent. It is sometimes found that excitation of the peripheral end of a divided anterior root gives rise to manifestations of pain or to reflex movement. This has been shown by Schiff to be due to the presence, in the sheaths of the anterior roots, of fine fibres derived from the posterior roots and taking a recurrent course to end probably in the membranes of the cord. This recurrent sensibility is at once abolished by section of two or three adjacent posterior roots. 


\section{THE WAY IN}

We may now consider the possible ways open to a nerve impulse entering the cord. Each posterior root on entering the cord divides into two bundles. The smaller bundle passes to the outer side of the tip of the posterior horn, where its fibres bifurcate (Fig. 159), giving

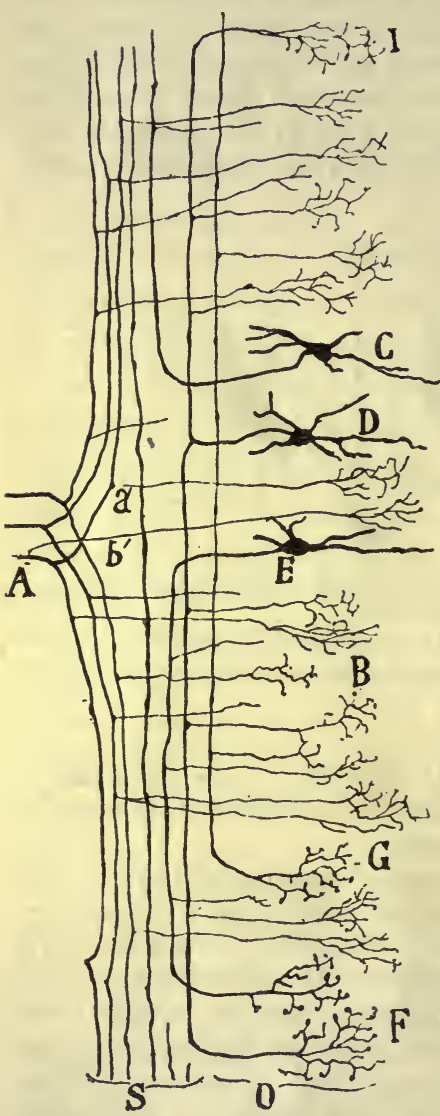

FIG. 159. Longitudinal section of spinal cord of chick, showing bifurcation of dorsal rootfibres, and the passage of their collaterals into the grey matter. Three cells of the dorsal horn are also seen sending their axons into the dorsal columns. (CAJAL.) rise to fibres which pass up and down the cord in a small longitudinal band of fibres known as Lissauer's tract. The fibres run only a short distance before turning into the grey matter, and terminate in arborisations round the cells of the substantia gelatinosa in the head of the posterior horn. By far the greater number of the posterior rootfibres pass to the inner side of the posterior horn into Burdach's or the postero-external column. Here they also divide into two main branches, one running up and the other down in the white matter. The descending branch passes through two or three segments before turning into the grey matter of the posterior horn of a lower segment. Of the ascending branches, some end at different levels of the cord, but a certain proportion of the fibres from every root traverse the whole length of the cord in the posterior columns to terminate in the posterior column nuclei (nuclei gracilis and cuneatus) in the medulla oblongata. As we proceed up the cord the entering posterior root fibres displace the long fibres of those below towards the middle line, so that in a section through the cord in the upper cervical region the posterior median column, or column of Goll, is made up almost exclusively of fibres from the hind limb, while the postero-external column consists of fibres from the fore limb.

Besides these distant connections, every entering nerve fibre makes connection with

all parts of the grey matter in and about its level of entrance by means of collaterals (Fig. 160). Five groups of these collateral branches can be distinguished, i.e.,

(1) Fibres which arborise round cells in the posterior horn of the same side.

(2) Fibres which pass through the dorsal grey commissure to the grey matter of the opposite side of the cord. 
(3) Fibres terminating round the median group of cells of the anterior horn.

(4) Fibres which end in a rich basket-work round the cells of Clarke's column.

(5) The sensori-motor bundle, which passes forwards through the grey matter to end round the cells in the anterior horn of the same side. of the cord.

Each entering posterior root fibre, besides these collaterals in the neighbourhood of its entrance, gives but few to higher segments of the cord before it terminates in the posterior column nuclei. Sherrington suggests that the cells of Clarke's column receive fibres mainly from the ascending branches of the nerve roots from the posterior limb, a corresponding station for the nerve fibres of the anterior limb being represented by the cells of the nucleus cuneatus.

That several different systems of fibres are included in these roots is shown by the different periods at which they acquire their myelin sheath. Among the earliest to acquire a sheath are the fibres which end in the posterior horn and those which pass to the anterior horn, while the long fibres in the dorsal columns do not become medullated until much later in fœtal life. Since the nerve fibres of the central nervous system do not become functional until they have acquired a medullary sheath,

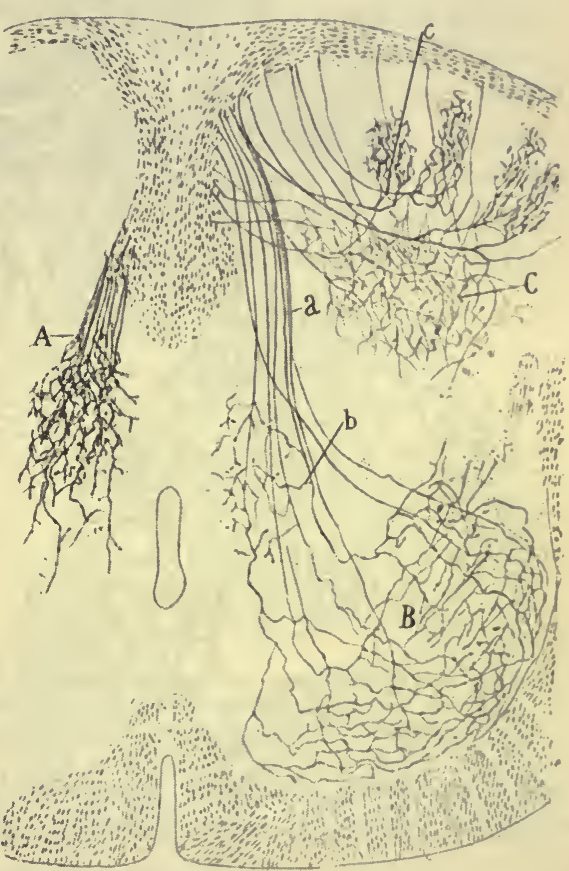

Fra. 160. Chief collaterals of dorsal column fibres from new-born mouse. (CAJAL.) $\mathbf{A}$, intermediate nucleus; $\mathbf{B}$, anterior (ventral) cornu ; c, dorsal or posterior cornu ; $c$, substance of Rolando. we must conclude that the reflex responses affecting the segment in which the fibres enter are developed earlier than those which involve also the activity of the cerebellum and medulla.

The primitive segmental character of the central nervous system is retained in its pure form only in the segmentation of the dorsal spinal root ganglia. Each of these ganglia or afferent roots consists of the fibres from the sense-organs in a segmental area of the body surface as well as from the muscular and visceral apparatus in the same segment. Section of one dorsal posterior nerve-root will cause a diminution of sensibility over a band-like area corresponding to the distribution of the fibres of the root, though to produce complete insensibility the two adjacent nerve-roots must 
be divided, in consequence of the overlap of fibres at the periphery. In the limbs the segmental distribution of the sensory fibres is made out with more

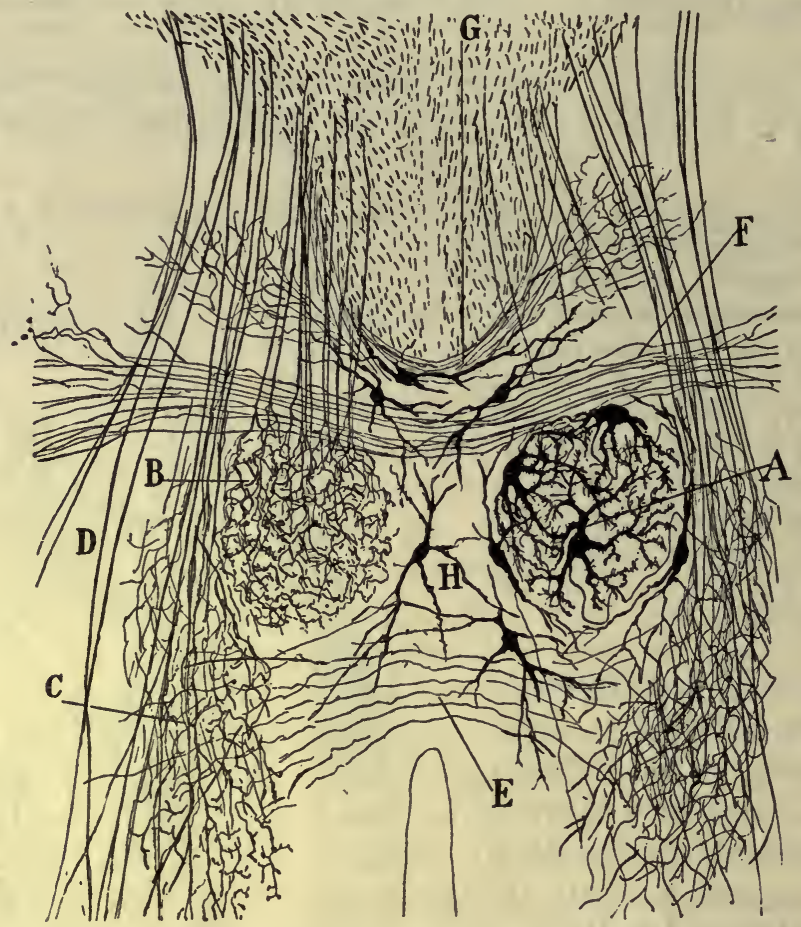

FIG. 161. Transverse section of spinal cord, showing collaterals terminating in a rich arborisation round the cells of Clarke's column $(\mathbf{A}, \mathbf{B})$, as well as others passing to the anterior cornua, and through the commissures. (CAJAL.)

difficulty. Each limb must be regarded as made up from a series of fused segments, from five to seven in number. The accompanying diagram (Fig. 162)

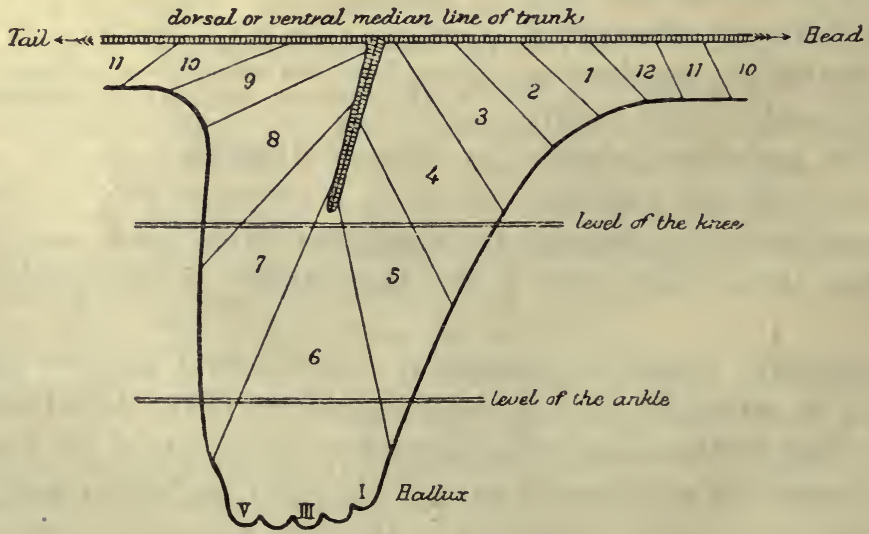

FIG. 162.

from Sherrington shows the manner in which the skin fields of these segments are combined to make up the total skin area in the hind limb of the monkey. 


\section{THE WAY OUT}

Primitively the motor nerves also represent fibres passing from a collection of ganglion-cells to the muscles of the corresponding body segment. In the dorsal region this segmental arrangement of motor nerve fibres is still traceable in the adult animal. In all other parts the morphological has become subservient to a physiological arrangement. Every muscle of the limbs contains elements from several segments, and is innervated therefore from several anterior spinal roots. Hence it follows that stimulation of one anterior root produces no definite movement of a group of muscles, but partial contraction of a number of muscles which do not normally contract simultaneously. Thus stimulation of a sensory nerve may evoke either flexion or extension of a limb, but not both simultaneously. Stimulation of the motor roots will cause simultaneous contraction of both flexor and extensor muscles. It is this subordination of morphological to physiological arrangement in the limbs which has necessitated the formation of limb plexuses. The nerve-root is a morphological collection of fibres; the nerve issuing from a limb plexus and passing to a group of muscles is a physiological collection. When it is stimulated it evokes a contraction of a group of muscles which are normally synergic, i.e. cooperate in various movements.

The fibres passing to the skeletal muscles are large, about $14 \mu$ to $19 \mu$ in diameter, and their axis cylinders represent the axons of large nervecells in the anterior horn. In the dorsal region of the cord in man, from the second dorsal to the second lumbar nerve-roots, the anterior roots contain, besides these coarse

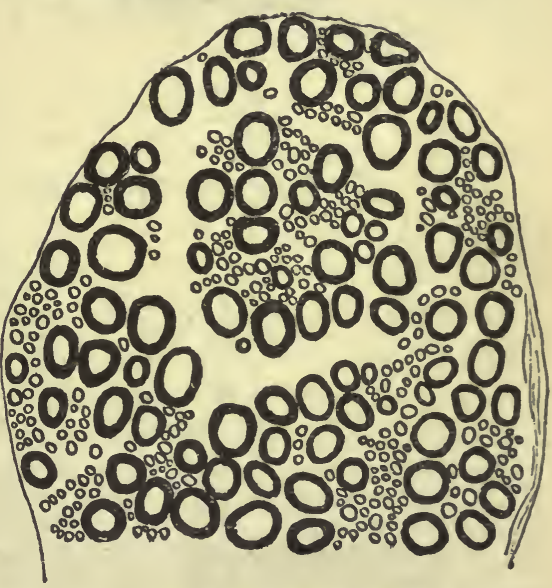

FIG. 163. Section across the second thoracic ventral nerve-root of the dog (stained with osmic acid) to show varying sizes of the constituent fibres. (GASKELL.) fibres, a number of fine fibres about $1.8 \mu$ to $3 \cdot 6 \mu$ in diameter (Fig. 163). These fine fibres were shown by Gaskell to leave the nerve shortly after the junction of the two roots, to pass as a white ramus communicans to the sympathetic. Excitation of the white rami evokes various visceral effects, such as dilatation of the pupils, augmentation of the heart, contraction of bloodvessels, inhibition of the gut, erection of hairs, \&c. Gaskell pointed out that the outflow of these fine fibres coincided with the existence of a prominent lateral horn in the grey matter, and suggested that cells of the lateral horn might be regarded as the origin of the visceral nerve fibres. This suggestion has been confirmed by Anderson, who has shown that section of the white rami communicantes.brings about an alteration in the cells of the lateral horn as a result of retrograde degeneration. 


\section{CENTRAL PATHS OF SPINAL REFLEXES}

The impulse entering the cord is thus able to affect immediately a number of systems of neurons, namely, cells in the anterior horn, in the posterior horn, in Clarke's column, in the substantia gelatinosa, in the lateral column of the same side of the cord, and the corresponding groups of cells on the opposite side of the cord either directly by crossing collaterals or indirectly through
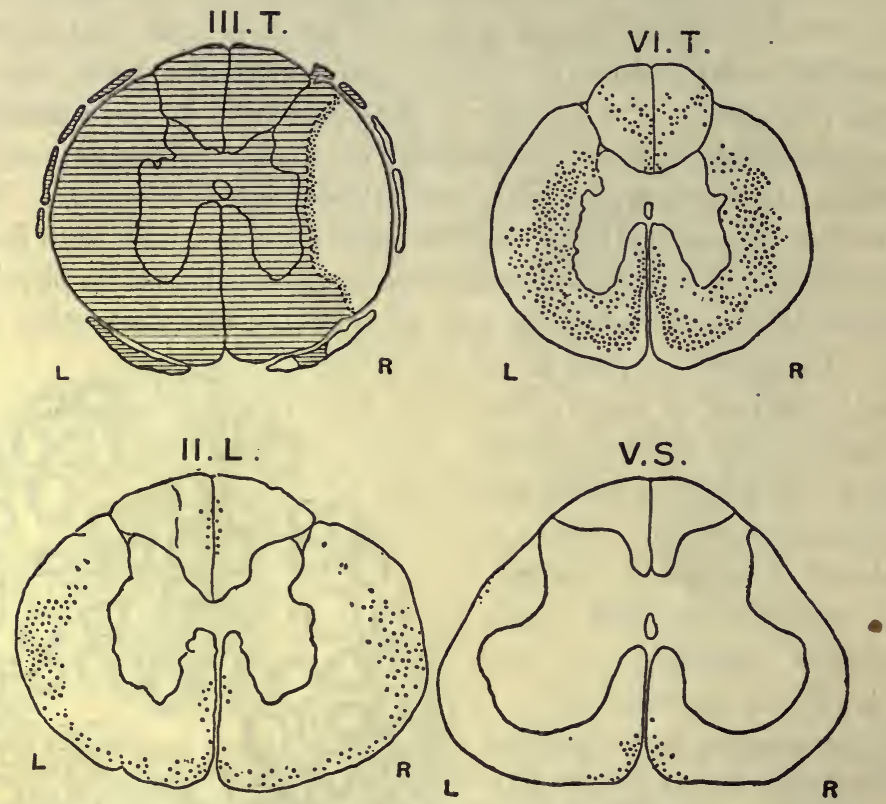

FIG. 164. Cross-sections of spinal cord of a dog, showing the descending nervetracts originating in the first three thoracic segments (method of 'successive degeneration'). The eighth cervical segment had been excised and 568 days later a cross-cut was made at level of the third thoracic nerve. The extent of the lesion is shown in the first figure (III. T). The other sections show the degenerations as revealed three weeks later by Marchi's method. (ShERrington.)

cells which send their axons across the middle line. Through the ascending and descending fibres of the posterior columns it can also set into action the reflex mechanisms of adjacent segments of the cord. In addition to this direct spread of afferent impulses up and down the cord there is an anatomical basis for a co-ordination between the grey matter of different levels. This co-ordination is effected through the intermediation of the internuncial or intra-spinal fibres which pass up and down the cord from segment to segment. The course of the descending fibres may be studied by carrying out a total transection of the spinal cord at the sixth cervical vertebra, and six months later, when all the fibres degenerating as a result of the section have disappeared, carrying out a further transection or hemisection a few segments below the first transection. If the animal be killed two or three weeks after the second operation it will be found that a number of fibres in the white matter are degenerated below the second section (Fig. 164). These fibres 
therefore must be derived from cells of the grey matter situated between the levels of the first and second sections, and they can be traced down the cord through a large number of segments. Analogous methods may be used for tracing the course of the ascending intra-spinal fibres. These intra-spinal fibres occur in the following situations :

(1) In the lateral columns immediately outside the grey matter, in the bay between the anterior and posterior horns.

(2) Close to the grey matter in the anterior basis bundle.

(3) In the posterior columns, united with the descending branches of the entering posterior roots in the comma tract, and also in the immediate periphery of the cord and abutting on the posterior fissure in the septomarginal tract.

(4) Mingled with the fibres of the pyramidal tract.

All these tracts are mixed, i.e. contain both ascending and descending fibres. As a rule, the longer the course of a fibre the more peripherally does it lie in the cord. The shortest of the fibres may only unite segment to segment, while the longest fibres may run through the greater part of the cord.

\section{THE SPINAL ANIMAL}

An animal possessing only a spinal cord contains a reflex neural apparatus which can be excited to activity by impulses of various qualities and from any part of the skin. Thus the afferent impulse may correspond to what in ourselves we call tactile and be provoked by mechanical stimulation, or may result from changes of temperature and correspond to those producing sensations of heat and cold. Strong stimuli of any kind may give rise also to afferent impulses which in the intact animal would have the quality of pain. Since these stimuli are such as to produce injury if continued, they may be named, when applied to the spinal animal, pathic or nocuous. The spatial distribution of the stimulus will determine the situation and number of nerve fibres set into action, so that there will be a great variation in the distribution of the excited neurons of the central grey matter according to the quality, distribution, and intensity of the stimulus. The efferent part of the reflex is provided for by the connection of the anterior cornual cells to the whole skeletal musculature of the body, as well as by the distribution of the axons of the lateral horn-cells to the sympathetic system and through this to the viscera. On the other hand, if the spinal cord be separated from the medulla oblongata and higher parts of the brain, it is deprived of all connection with the most highly elaborated sense-organs of smell, sight, hearing, and equilibration, and also of the important afferent and efferent impulses which pass between brain and viscera through the vagus nerves. In studying the reaction of the isolated spinal cord we are studying a nervóus system cut off from its most complex components, but at the same time deprived of the initiation and guidance which it must normally be continually receiving from the higher sense-organs through the brain. A study of the spinal animal will therefore be instructive as a study of the mammalian nervous system in its simplest possible aspect. It will, however, in all cases be the study of 
an incomplete and maimed system, the incompleteness increasing as we ascend the scale of animals in our experimentation, owing to the increasing subordination of the lower to the higher centres, and of the immediate reflexes to the educated reactions of the anterior part of the brain.

\section{SPINAL SHOCK}

If the spinal cord of the frog be divided just below the medulla, for some minutes after the section all four limbs are perfectly flaccid, and it is impossible to evoke any reaction by the application of the strongest stimuli. If the animal be left to itself for half an hour there is a gradual return of reflex tone; the animal draws up its legs and assumes a position not far removed from that of the normal frog, the head being lower than under normal conditions. We may say that the phenomena of shock in the frog last only a short time. With increasing complexity of the nervous system the phenomena of shock become more lasting, so that among laboratory animals it is in the monkey that spinal shock is most apparent. It is interesting to note, as pointed out by Sherrington, that shock appears to take effect only in the aboral direction. Thus, even in the monkey, section through the lower cervical region, though causing profound paralysis of the lower limbs and part of the trunk, apparently has no influence at all on the reactions of the nervous system above the section. "The animal immediately after the section will contentedly direct its gaze to sights seen through the window, or, if the section have been below the brachial region, may amuse itself by catching flies on the pane. This is the more remarkable since the profound depression of the nerve-centres below the point of section extends also to the blood-vessels and viscera, so that there is a great fall of blood pressure and diminished production with increased loss of heat. The sphincters are flaccid or patulous, the skeletal muscles are toneless, and no reaction is evoked by the strongest stimulus to the skin or to a sensory nerve."

Much discussion has arisen as to the duration of shock. Goltz and others imagined that the phenomena of shock may persist for months or even years. According to Sherrington, in the higher animals the phenomena of shock are complicated by the onset of an "isolation dystrophy" which may occur before the condition of shock has entirely disappeared. In order therefore to examine the capabilities of the isolated spinal cord at their best, a time must be chosen when the sum of shock and isolation dystrophy together is at its minimum.

The occurrence of shock after complete transection of the cord in the cervical region cannot be ascribed to the fall of blood pressure which ensues as a result of the severance of the efferent vaso-motor tracts from the vaso-motor centre in the medulla. The centres above as well as those below the transection are equally exposed to the effects of the lowered blood pressure, but it is only those below the section which show signs of shock. Nor can it be regarded as operative shock due to the severity of the lesion; such an operative shock would be effective in either direction, and we do not 
find that the method of transection, whether by tearing across the cord or cutting it with a minimum disturbance, alters appreciably the amount of shock displayed by the segment of the cord situated below the lesion. On the other hand, if in a dog, which has undergone transection of the cord in the lower cervical region and has been allowed sufficient time to recover from the shock, a second transection be carried out two or three segments below the site of the first operation, the influence of the second section is hardly noticeable on the lower segment of the cord. Apparently then the chief factor in determining shock in all those centres situated aborally of the lesion is the cutting off of the impulses which are continually streaming down from the higher centres and from the great sense-organs connected with the anterior portions of the nervous system. With every rise in the animal scale the impressions received by the special senses take an increasing part in the determining of all the reactions of the body, so that we might expect the effect of cutting off the impulses from the higher centres to be greater, the higher in the scale of animal life is the animal on which the experiment is carried out.

The state of profound shock produced in the spinal cord by the operation passes off gradually. The blood pressure, which may have fallen to 40 or $50 \mathrm{~mm}$. Hg., rises within two or three days to its normal height, i.e. 90 to $110 \mathrm{~mm}$. Hg. The sphincter muscles of the anus gradually recover their tone, and within a short time the reflex evacuation of the bladder and rectum may occur as in a normal animal. The skeletal muscles recover their tone within a few days, and after a short time co-ordinated movements can be brought about in the trunk and limbs by appropriate stimulation of sensory surfaces. At first the reactions thus produced are feeble and the reflex is rapidly fatigued. Of these reflexes those excited by nocuous or painful stimuli are the first to make their appearance; a little later are seen those due to stimuli affecting the tactile organs in the skin, or the sense-organs of deep sensibility situated round the bones and joints and excited by deep pressure or changes in posture of the limbs.

In a dog which has undergone complete cervical transection two or three months previously, the tone of the muscles is somewhat increased. Although the dog is unable to walk, if it be raised and given a little push forward, so as to stretch the extensor muscles of its hind limbs, it may take two or three steps forward before its legs collapse. Although the locomotor apparatus is present, the nexus is lacking which determines the regulation of these movements through the organs of static sense, so that the spinal movements are insufficient to maintain the animal in such a position that a line drawn vertically from its centre of gravity shall fall between its points of support. On the other hand, swimming movements may be carried out regularly. The frog deprived of its brain can swim like a normal animal, but in consequence of the depression of its head tends to swim ever deeper in the water. If a ' spinal' dog be held up by the fore limbs, the hind limbs nearly always enter into alternating movements of flexion and extension (' mark time' movements), the two limbs acting alternately as in normal progression. The stimuli in this case seem to be started by the stretching of the skin and other 
structures at the front of the thighs. In such animals three reflexes, amongst others, can be excited almost invariably, viz. :

(1) Scratch reflex. Gentle stimulation, mechanical or electrical, of any point over a saddle-shaped area on the dorsum behind the shoulders (Fig. 165) causes rhythmic movements of flexion and extension of the hind limb of

THE SIMPLE REFLEX
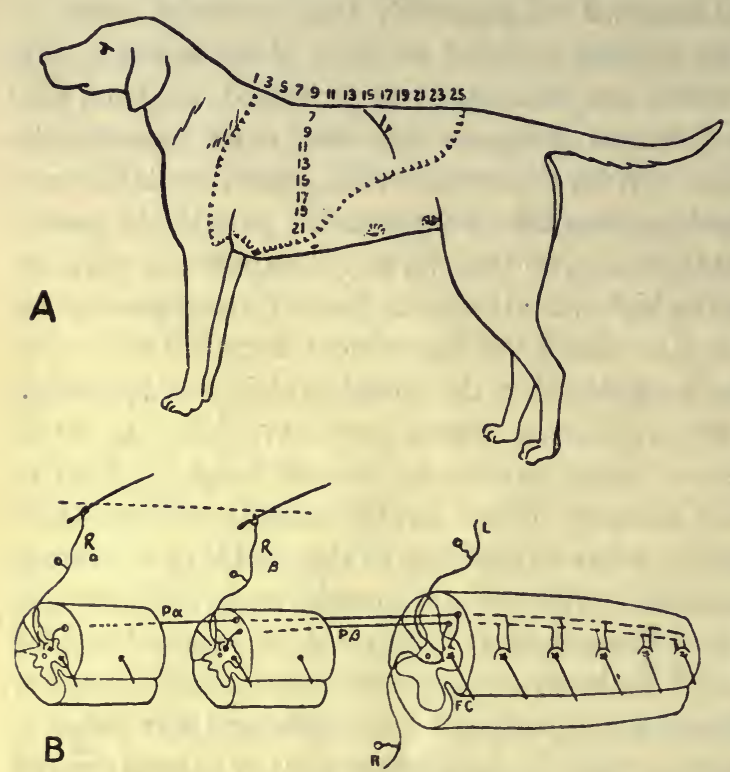

Fic. 165. A. The receptive field, whence the scratch reflex of the left hind limb can be evoked.

B. Diagram of spinal ares involved. L, afferent path from left foot; $B$, afferent path from right foot; $\mathrm{R} a, \mathrm{R} \beta$, receptive paths from hairs on 'scratch area'; FC, final common path (motor neuron); $\mathrm{Pa}, \mathrm{P} \beta$, proprio-spinal neurons. (Sherrington.) the same side, the effect of which would be to scratch away the irritant object. These movements are repeated at the rate of about four per second.

(2) Flexor reflex. Nocuous stimuli, such as the prick of a needle applied to any part of the foot, causes flexion of the leg and thigh, often accompanied by extension of the opposite hind limb.

(3) Extensor or 'step. ping' reflex. Gentle pressure applied to the plantar surface of the hind foot, especially if the limb is somewhat flexed, causes a movement of extension of the limb accompanied sometimes by a flexion of the opposite hind limb.

In such an animal the carrying out of the vis-

ceral reflexes may be very efficient. The blood pressure has attained its normal height and may be altered reflexly in very much the same way as in a normal animal, although the medullary vaso-motor centre can no longer be concerned. Thus in the diagram (Fig. 166) is represented the effect on the blood pressure of exciting the central end of the digital nerve in a spinal dog. The pressure rises from 90 to $208 \mathrm{~mm}$. $\mathrm{Hg}$. - a pressor effect as great as any which can be obtained in an animal still possessing all the connections of the vascular system with the vaso-motor centre. The height of the rise shows that as regards the influence on the blood pressure the spinal cord must be acting as a whole. No effect on the blood-vessels confined to the segment, or segments, adjacent to that of the nerve stimulated would suffice to cause a rise of more than a few $\mathrm{mm}$. $\mathrm{Hg}$.

The reflex apparatus for other visceral functions seems to be equally perfect. The urinary bladder, when sufficient urine is accumulated, con- 
tracts forcibly, the contraction being accompanied by relaxation of the sphincter and followed by rhythmic contractions of the urethral muscles; accumulation of fæces in the rectum leads to their normal evacuation. With a little assistance impregnation may be effected in or by such a maimed

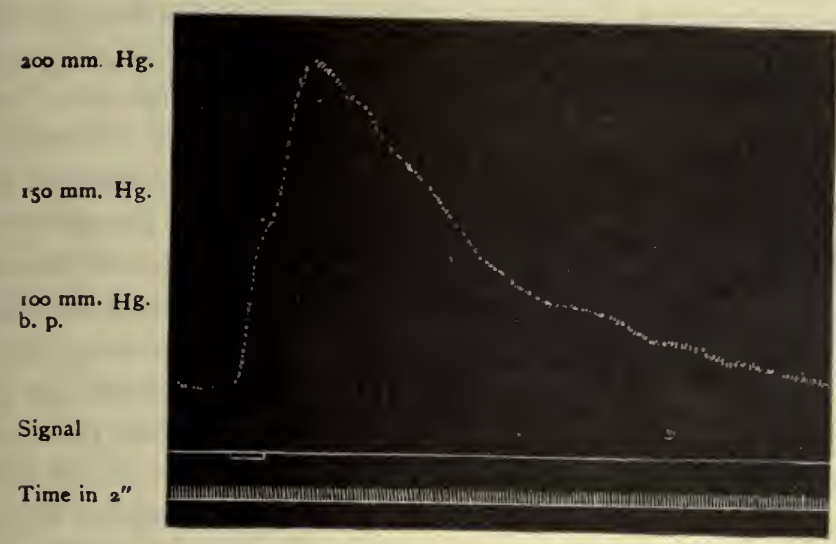

Fig. 166. Blood pressure tracing from a spinal dog. The signal indicates the time during which the afferent nerve was stimulated. (SHERrivg Ton.)

animal, and in the female may terminate at full term in normal parturition. Pregnancy is accompanied by hypertrophy of the mammary glands and is followed by secretion of milk, so that the young may be suckled as in a normal animal. Similar phenomena have been observed in the human subject.

Such an animal furnishes us with an opportunity of analysing the factors which are involved in the maintenance of muscle tone, as well as in the carrying out of the simplest reflexes involving contractions of the skeletal muscles.

\section{MUSCULAR TONE}

Every muscle in the body is in a condition of slightly continued contraction which keeps it tense, so that when it contracts in response to a stimulus there is, so to speak, no 'slack' to be taken up before the muscle begins to pull on its attachments. This tone is seen in the retraction undergone by muscles or tendons when they are divided in the living animal.

If a frog possessing only spinal cord be hung up by its jaw, the limbs will be observed to occupy a position which is short of complete extension. The tone of the muscles which is concerned in the maintenance of this attitude is at once abolished by the destruction of the spinal cord. It may be abolished on one side by section either of the anterior roots going to the muscles, or of the posterior roots coming from the muscles (Fig. 167). In the intact animal muscle tone is diminished by disease and may be abolished during profound anæsthesia, as it is indeed in the condition of shock.

Much light has been thrown on the factors which determine muscular tone by a study of the 'tendon phenomena' of which the knee-jerk is the 
most familiar example. If the leg is allowed to hang loosely in a position of slight flexion at hip and knee and the patellar tendon be struck, the extensor muscles of the thigh contract and raise the leg. This phenomenon is known as the knee-jerk. Similar 'tendon reflexes' can be obtained in other muscles, such as the tendo Achillis, the triceps, and the extensor muscles of the wrist, but with not so great ease as is the case with the knee. The knee-jerk is not altered by rendering the tendon anæsthetic by section

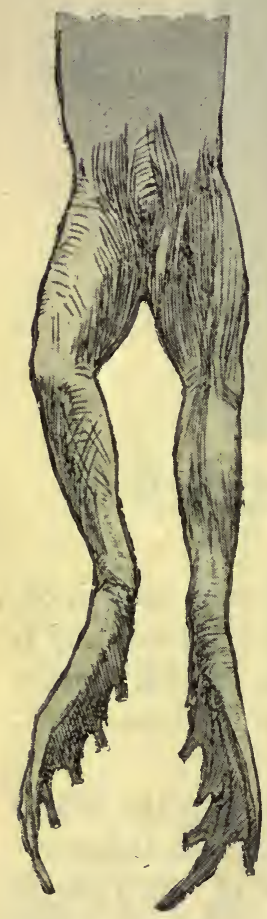

Fig. 167. Hind part of a spinal frog, hung up by the jaw. The posterior roots of the nerves to the left hind limb have been divided.

(BECHTEREW.) of all its nerves. The essential feature is a slight passive increase of the tension to which the muscle is already subjected. Since the jerk is abolished by severance at any point of the reflex are, viz. muscle spindle, cord, muscle, it was thought at first to be of the nature of a reflex action. The interval, however, which elapses between the moment at which the tendon is struck and the response of the muscle is generally considered to be too short to allow of an impulse to travel from the tendon, or muscle, up to the cord and back again to the muscle. The interval was found by Gotch to be about $005 \mathrm{sec}$., whereas the latent period of contraction which ensues upon direct stimulation of the vastus internus is also .005 sec. On the other hand, the latent period when the nerve to the muscle was stimulated was 01 sec. The lost time of the knee-jerk is less than one-quarter of that of the briefest reflex time. The contraction has therefore been thought to be due to the direct stimulation of the muscle by the sudden stretching produced on striking its tendon. Mere tension of the muscle is not, however, the only factor. The tone which is reflexly maintained in the muscle is necessary for this response to direct stimulation to take place, and it seems to keep the muscles in a state of wakefulness ready to respond to the slightest local stimulation. The knee-jerk is therefore of special importance as an index to the tonic condition of the muscles concerned, being brisk and easily elicited when the tonus is pronounced, and slight or absent when the tone of the muscle is depressed.

Especially interesting is the relation shown by Sherrington to exist between the tonic condition of antagonistic muscles, e.g. between the hamstrings and the vastus internus of the quadriceps extensor muscle. Section of the hamstring muscles (so as to relax them) or even section of their nerve causes at once great increase in the jerk elicited by tapping the patellar tendon. On the other hand, the knee-jerk is abolished by stretching the hamstring muscles, or by weak stimulation of the central end of the cut nerve to the hamstrings (Fig. 168).

In this way a voluntary flexion of the knee by contraction of the hamstrings automatically abolishes the resistance which would be offered by 
the tonic contraction of the extensor muscles. In the absence of such an arrangement every movement of a joint, by stretching the antagonistic muscles, would automatically increase their tone, and thus set up a resistance to itself. The subject would thus be muscle-bound.

Very great exaggeration of the tendon phenomenon is observed in cases where the pyramidal tracts are degenerated, and indicates a heightened reflex excitability of the lower spinal centres, perhaps reinforced by impulses from the cerebellum. The importance of the latter impulses in determining the myotatic irritability of the muscles is especially marked in man, where total transverse lesion of the upper part of the spinal cord often abolishes permanently the tone of the muscles innervated from the lower portion of the cord, and especially the kneejerk. How far this absence of tone is due to collateral changes in the cord has not yet been determined. In animals complete transverse section of the cord of the cervical region is followed by increase of the kneejerk, which in the rabbit may be elicited within a quarter of an hour after the section has been carried out. In the increased myotatic irritability observed after removal of the cerebral cortex, or after degeneration of

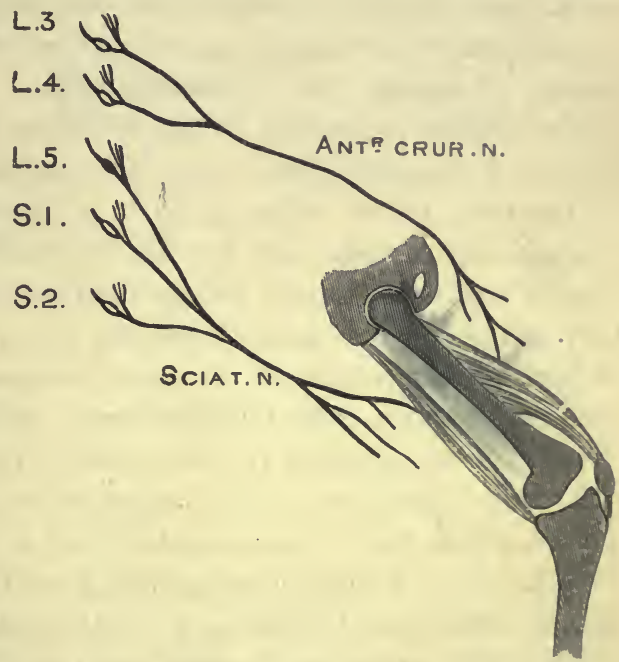

FIG. 168. Diagram to show muscles and nerves concerned in Sherrington's experiment on the reciprocal innervation of antagonistic muscles.

L3, L4, L5, third, fourth, and fifth lumbar roots; s1, s2, first and second sacral roots. the pyramidal tracts coming from the motor cortex, a single tap on the patellar tendon may evoke a series of contractions of the extensor muscles of the thigh, giving rise to what is known as knee clonus. In the same way forcible flexion of the ankle causes a series of rhythmic contractions of the calf muscles (ankle clonus), varying in rhythm from six to ten per second. The heightened tone of the muscles under these conditions, and the ease with which any slight increase in their tension gives rise to clonic contractions, cause such patients to have a peculiar dancing gait, characteristic of pyramidal degeneration, and known as the 'spastic' gait; it is generally associated with a certain loss of voluntary control of the movements of the limbs, so that the whole complex of symptoms is called 'spastic paraplegia.'

The value of the tendon phenomena as a means of diagnosis has tended to obscure their great importance in the normal individual. Every joint is protected by inextensible ligaments and by muscles. A sudden strain on a ligament either will have no effect, or will rupture some of its fibres and perhaps injure the adjacent joint surfaces. An ordinary reflex contraction 
would be powerless to prevent this, since the mischief would be done before the reaction could take place. But the central nervous system confines itself to keeping the muscles awake, so that they themselves may react to any sudden increase in their tension by an equally sudden contraction, which saves the joint before the central nervous system has even become aware of the strain.

The tone of the muscles, as well as the consequent tendon phenomena, is dependent on the integrity of the reflex arc governing the muscles in question. It has been shown by Sherrington that the afferent part of the arc is represented by the afferent nerves from the muscle itself, and that these nerves receive their sense impressions from the special nerve-endings characteristic of muscle-the 'muscle-spindles.' Even in the purely muscular nerves a large proportion of the fibres are afferent in function, and, after section of the appropriate posterior roots distal to the ganglia, as many as 40 per cent. of the fibres going to a muscle may be found degenerated. Though most of these have the muscle-spindles as their destination, a certain number pass to the tendon and aponeuroses connected with the muscle, where they. end in the end-organs known as the organs of Golgi and the organs of Ruffini. After section of the motor nerves the muscle fibres degenerate, with the exception of the modified fibres, which, enclosed in a connected tissue sheath, are concerned in the formation of the muscle-spindles. Muscle tone and tendon phenomena may therefore be abolished by lesions of afferent nerves, which leave a considerable part of the cutaneous sensibility of the limb intact. In man the spinal reflex mechanism connected with the kneejerk is situated in the third and fourth lumbar segments. The jerk may be abolished by section of the third and fourth posterior nerve-roots, although to render the whole hind limb anæsthetic it would be necessary to divide all the roots from the second lumbar to the fourth sacral inclusive.

Recent researches by Snyder and by Jolly indicate that the reflex nature of the knee-jerk cannot be entirely excluded. Jolly, using the string galvanometer, has taken the current of action in the vastus internus muscle as an index of the commencing contraction of this muscle in the knee-jerk. He has also by the same method, by leading off the afferent and efferent nerves respectively, measured the lost time in the sense-organs and in the motor end-plates of the muscle. In the spinal cord he obtained the following electrical latencies in one case :

$$
\begin{aligned}
& \text { Latency of knee-jerk _ . . . . . . } 5 \cdot 3 \sigma^{*} \\
& \text { Afferent endings _. . . . . . } 0.4 \sigma \\
& \text { Nerve conduction . } \quad . \quad \text {. } \quad . \quad \text {. } 1.4 \\
& \text { Motor endings and action current } \quad \text {. } \quad ~ \quad 1 \cdot 3 \\
& \text { Synapse time } \quad . \quad \text {. . . } \frac{3 \cdot 1}{2 \cdot 2 \sigma}
\end{aligned}
$$

In this case the shortest latency determined for nerve-endings has been deducted from the shortest latent period obtained from the knee-jerk in the spinal cat. On the other hand, some decapitated preparations have been found to present considerably longer latent periods, e.g. 11 and $12 \sigma$. This variation in the latent period supports the view that the knee-jerk is a reflex of which the synapse time is very short, about $2 \sigma$, 
and that in certain cases there may be increased delay in the spinal cord. When the latencies of the knee-jerk and the homonymous flexor reflex are compared by the electrical method, it is found that the latter is roughly double the former, the average latency of the knce-jerk in the spinal cat being $6 \cdot 6 \sigma$, and of the homonymous flexor reflex $13 \cdot 2 \sigma$. Jolly suggests that this difference may be duc to the fact that the kneejerk mechanism involves only one spinal synapse or set of synapses, while the flexor reflex may involve two. In these estimates the rate of conduction in mammalian nerve has been taken at 120 metres per second. 


\section{SECTION VIII}

\section{THE MECHANISM OF CO-ORDINATED MOVEMENTS}

Tre detailed study of the chief reflexes obtainable from a spinal animal has, in Sherrington's hands, yielded much information as to the linking of the various events which are concerned in the carrying out of every co-ordinated movement, and as to the conditions which determine the sequence and extent of the activities involved.

We may take, as the type of such a reflex, the flexion of the leg and thigh which ensues on the application of a painful stimulus to the ball of the foot, such as pricking with a needle, or the application of the faradic current. Of course, in the spinal animal no pain can result from stimulation of any part below the level of section of the cord, and it is better therefore under such circumstances to speak of nocuous or pathic stimuli, since all stimuli which cause pain are such that, if their operation continued, they would result in damage to the material structure of the animal. This flexor reflex is also easily obtainable in the frog as a result of stimulating one of its toes by mechanical or chemical stimuli, but it is easier to analyse the different events involved in the reaction in the case of the larger animal.

The effect varies with the strength of the stimulus. The minimal effective stimulus causes simply movement of the foot. As its strength is increased this movement is attended by flexion of the leg on the thigh, and finally by flexion of the thigh on the body. With still further increase there is a spread to the opposite hind limb, which, however, performs the opposite movement of extension. Increase in the strength of stimulus causes not only an increase in the strength of contraction of the reacting muscles, but also an extension of the reaction to more and more muscles, or groups of muscles. The spread occurs always in definite order. The stimulus when represented by the prick of a needle can affect only one or two nerve fibres. The impulse carried along these fibres through a posterior root to the cord spreads in the cord, affects the motor neurons of the anterior horns, and causes these to discharge. The first discharge is as a rule limited to those in the immediate proximity of the entering impulses, but even when minimal, involves the simultaneous action of more than one anterior root. We may say that the motor response is determined to a certain extent by the spatial proximity of the afferent to the efferent tracts, but that it is always pluri- 
segmental, the most important determining factor being the adaptation of the movement to the stimulus which is applied.

The gradual spread of the response with-increasing strength of stimulus is spoken of as 'irradiation.' The nature of the response is determined by the locus or place of application of the stimulus and by the quality of the latter. While a painful stimulus causes flexion of the leg, deep pressure on the plantar surface of the paw causes extension-the 'stepping' reflex. However extensive the irradiation, the muscles which are set into action are always such that their actions co-operate towards a given end. Thus, when the impulse spreads to the opposite limb and produces an extension, the reaction is such as would ensue when the dog steps on a sharp point and immediately retracts the irritated limb away from the injurious agent while it extends the other limb in the first act of progression or movement away from the dangerous spot.

A superficial study of this reflex would therefore lead us to the conclusion that, by the varying resistance in the different synapses on the course of the connections of the stimulated afferent nerves, the impulses are directed so as to affect solely and exclusively the muscles whose activity will cooperate and aid the primary reflex. Such a description would, however, only represent one half of the process. Every muscle in the body is in a state of tone varying with its extension. If this tone is not to interfere with the carrying out of a reflex movement, there must be some means by which it can be inhibited. Such an inhibition we have seen occur as the result of contraction of antagonistic muscles; but the remarkable fact has been brought out by Sherrington that the impulses, which start on the surface of the body and set loose a chain of motor impulses resulting in the co-ordinated contraction of certain muscles, spread at the same time to the motor mechanisms governing the muscles antagonistic to the movement, and exercise on these an inhibitory effect.

This inhibition can be easily shown in a spinal animal in the following way. The anterior thigh muscles are cut away from their attachments to the tibia and the patellar tendon is connected by a thread with a recording lever. On then exciting the flexor reflex by nocuous stimulation of the foot, the lever attached to the patellar tendon falls (Fig. 169в), showing that the extensor muscles have undergone actual elongation. The same effect is observed even when the hamstrings, the flexors of the knee, have been divided. The inhibition of the extensor tone is thus not only determined by the increased tension of the flexors, but is a direct result of the primary cutaneous stimulus.

From a broad standpoint the function of the nervous system is the co-ordination of all the activities of the body so that these may be combined to one common end, viz. the preservation of the organism. For this purpose there must be no clashing between opposing activities of different parts. If one part is engaged in any action this action must be the policy of the body as a whole. Yet the surface of the body is
being continually played upon by ever-changing stimuli, tending to excite 
first one reflex and then another, and the activities so excited would produce confusion in the conduct of the animal, if there were not some means by which at any one time only one reaction should be in the act of being carried out. The imperative stimulus should dominate the actions of the body as a

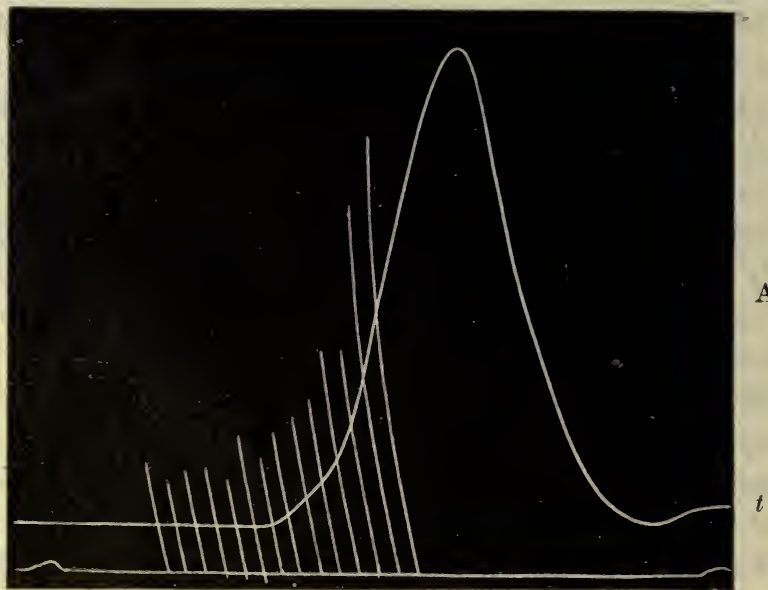

A

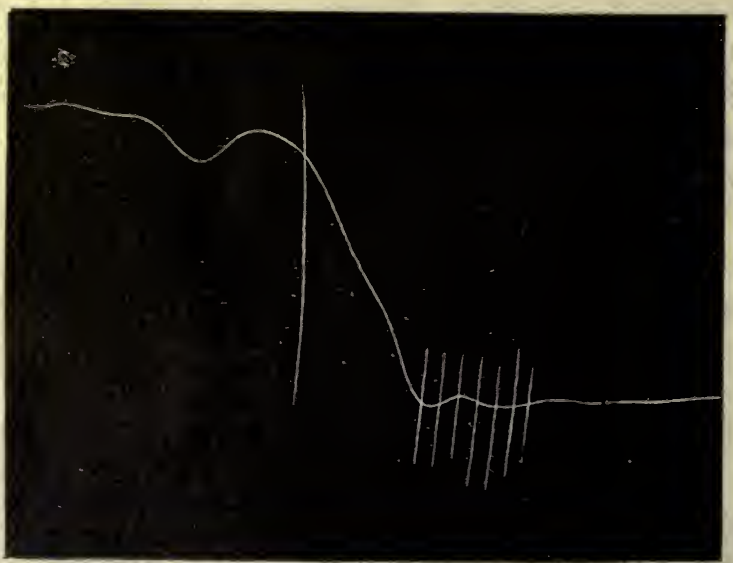

B

Fig. 169, A and B. (Sherrington.)

The flexion reflex observed as reflex contraction (excitation) of the flexor muscles of the knee (A), and as reflex relaxation (inhibition) of the extensor muscle (B). The stimulus was a series of weak break induction shocks applied to a twig of the internal saphenous nerve below the knee. Observation B was made four minutes after $A_{\text {. N N }}$ Note the summation of stimuli, in each case six stimuli being required before the reaction was evoked.

whole. Just as, in the mental world, attention must be undivided if we are to avoid confusion of judgment, so in the lower nervous activities there must always be concentration on one act or another. There may be a struggle of different stimuli, but one must finally be prepotent and annul altogether the influence of the others. The study of the spinal animal shows that this concentration of energy is obtained by the process of inhibi- 
tion. Every successful reflex, i.e. one which actually occurs, inhibits all other reflexes which are not co-operative with the one which is taking place. We may, for instance, stimulate the area of skin which gives rise to the scratch reflex, and at the same time apply a painful stimulus to the foot. The result is not a movement compounded of the two reflexes, but, as a rule, the flexor reflex preponderates. If, for instance, the scratch reflex be proceeding and then the foot be pricked, the scratch reflex immediately comes to an end, and the flexor reflex occurs. When this in its turn has come to an end, the scratch reflex may be once more resumed (Fig. 170).

One stimulus may reinforce another if the reactions ensuing on the two stimuli are allied-i.e. tend to co-operate one with another. In every other case, however, an afferent impulse entering the cord and spreading to a motor mechanism, so as to produce a co-ordinate contraction of various muscles, causes at the same time inhibition of the muscles antagonistic to the movement, and a block, or inhibition, in all other reflex arcs of the cord.

The anatomical basis of the various events involved in the carrying out of such a reflex as that just studied is shown in the diagram (Fig. 171). In this diagram the nerve-fibre $\alpha$ represents the pain-receiving or nociceptive nerve from the skin of the foot. This passes by a posterior root into the spinal cord, where it divides and gives off a number of collaterals. These collaterals, as we have already seen, pass in various directions; some to the neighbouring grey matter, some to the centres in the higher parts of the nervous system. Neglecting the latter and any intermediate

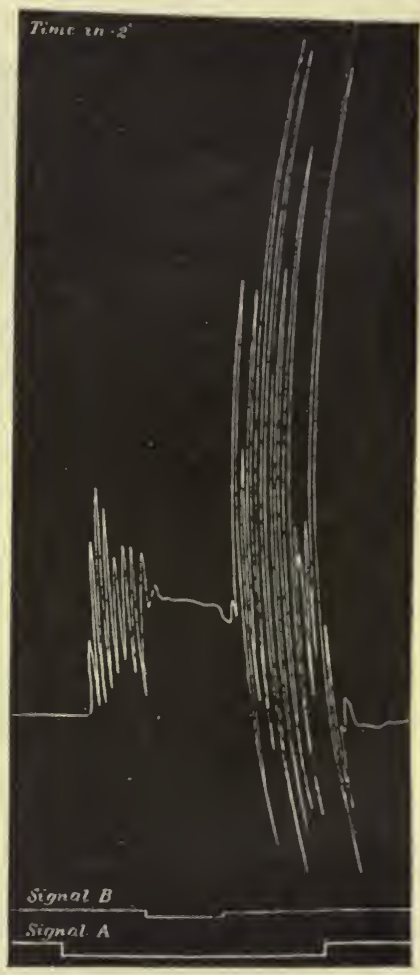

Fig. 170. Scratch reflex temporarily inhibited by application of a pathic stimulus to foot.

Signal A, stimulation of scratch area. Signal B, stimulation of paw by strong induction shock. neuron which may be intercalated between the afferent fibre and the motor cell, we see that those collaterals which affect the motor cells of the muscles of the two hind limbs can be divided into two sets, one of which always produces during activity excitation in certain efferent neurons, whilst the other produces inhibition of the efferent neurons of the antagonistic muscles. The single afferent nerve fibre is therefore, with regard to one set of its central terminal branches, specifically excitor, and, in regard to another set of its central endings, specifically inhibitor. In the case in point the central terminal branches of the nerve $\alpha$ are excitor for the flexor muscles of the same side and inhibtor 
for the extensor muscles of the same side and for the flexor muscles of the opposite side.

The ascending branches of the nerve fibre in the same way will have endings which, while inhibitor for the greater number of other possible reflex changes, will be excitor in a slight degree for certain efferent neurons whose action is allied to that of the primary reflex. The diagram shows also that the contraction of the flexor muscle, set up as the result

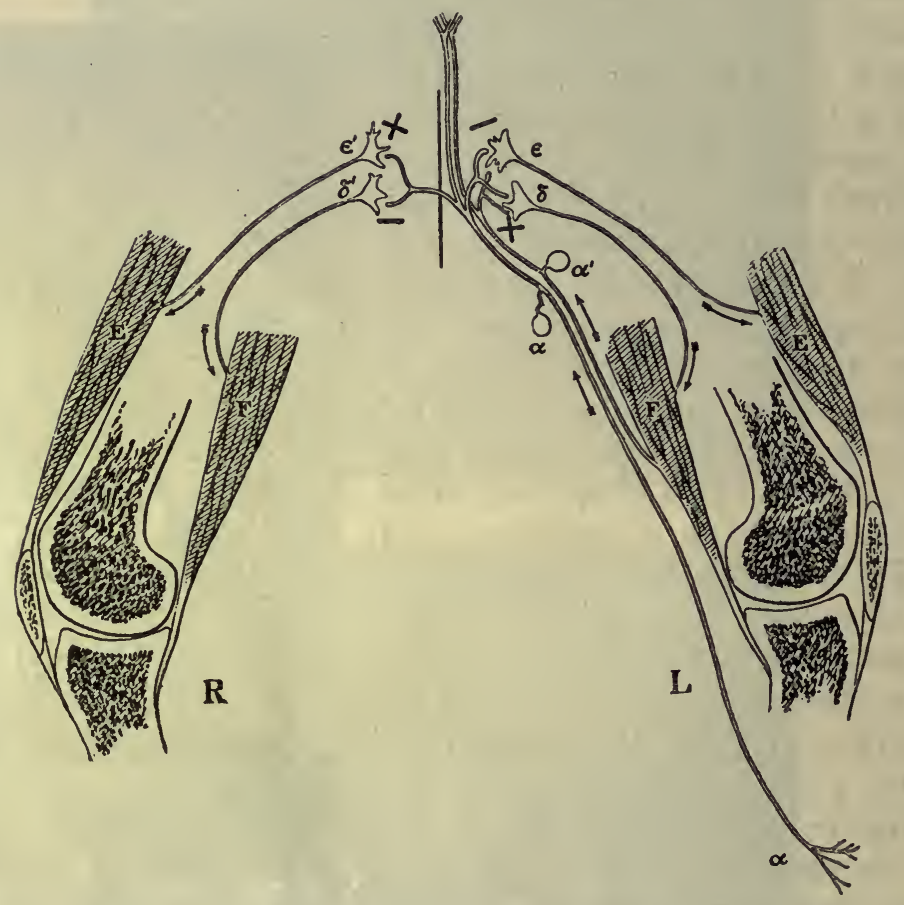

FIG 171. Diagram indicating connections and actions of two afferent spinal root-cells $a$ and $a^{\prime}$, in regard to their reflex influence on the extensor and flexor muscles of the two knees. The sign + indicates an excitatory effect, the sign - an inhibitory effect. (SHERRINGTON.)

of stimulating $\alpha$, itself initiates a secondary reflex process from muscle up the nerve fibre $\alpha^{\prime}$ and back again to the muscle by the efferent neuron. This muscular afferent nerve also has central terminations of two signs-excitor to itself and inhibitor to the antagonistic muscles. For the sake of clearness the diagram omits a number of other channels coming from other regions of the cord, or from other efferent nerves, the sign of which would be negative, i.e. which would tend to inhibit the activity of the whole reflex arc.

We see therefore that from every sensitive point on the surface of the body impulses can be initiated which will set into action whole chains of neurons, and will have a widespread influence throughout the central nervous system. It is important to note that the efferent path innervating, say, the flexor muscles of one side is common to many reflexes. It is used, for instance, by mutually antagonistic reflexes such as the scratch reflex and the flexor or 


\section{THE MECHANISM OF CO-ORDINATED MOVEMENTS}

pain reflex. We must assume therefore that the mutual inhibition of different reflexes occurs, not in the 'final common path' -i.e. in the motor neurons, which must always remain open-but further back in the arc, probably near its afferent side.

We have reason to believe that the propagation of impulses through the central nervous system involves expenditure of energy, and that the seat of this expenditure may be located in all probability at the synapses. It follows that the result of any particular sensory stimulation will not be absolutely invariable, but that the spread of the nerve process in the nervous system,

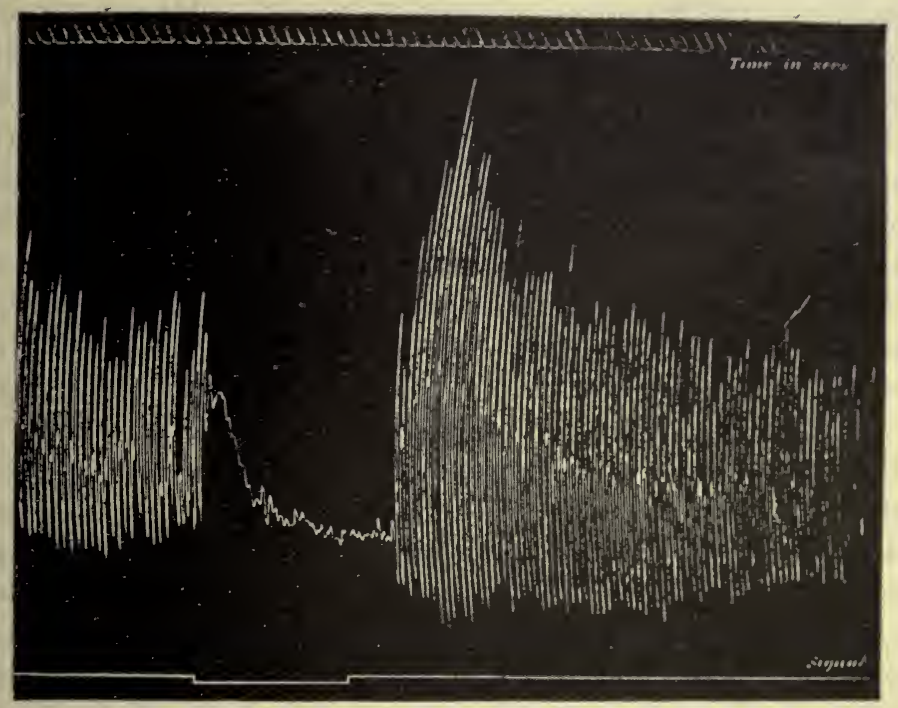

Fig. 172. 'Mark-time' reflex in spinal dog, inhibited by slight stimulation of the tail (duration of stimulation shown by signal). Note the augmentation of the mark-time reflex following the inhibition (successive spinal induction). (ShERringtoN.)

and the degree of block presented by the various synapses and determining the potency of any given reaction, will depend on the condition of the various synapses at the time of the stimulation.

This condition may be altered in various ways. Repeated excitation causes in the synapses, just as in the nerve-endings of the skeletal muscle, a condition of fatigue. Stimulation confined to a single point in the "scratch area ' of the spinal dog excites a scratch reflex which rapidly dies away. On shifting the exciting electrodes a little to one side the reflex act begins again, often with greater force than at first, and a very prolonged reaction can be induced by gradually moving the electrodes along the surface of the skin. A reflex arc therefore rapidly shows signs of fatigue, and the minute change in locus of stimulus which is required to reinduce a practically identical action shows that the seat of fatigue must lie chiefly on the afferent side of the arc; perhaps in the first synapses through which the impulse has to pass. This easy incidence of fatigue tends to cut short any given reaction and to render it easier for other reactions to take its place. 
Just as excitation causes fatigue and therefore furnishes a hindrance to repetition of the same act, so the reverse process of inhibition, which is a large component of every reaction, is followed by a condition of increased excitability, or diminished resistance to the passage of impulses. In each case there is a tendency for a 'swing-back' to take place from inexcitability, to over-excitability, from excitation to inexcitability. This 'successive spinal induction,' as it has been termed, may be seen on inhibiting some movement by the excitation of an independent reflex. Thus the scratch reflex is excited, and then while the excitation is still continued the reaction is inhibited by excitation of the extensor or stepping reflex. As soon as the 'stepping ' reflex has passed off, the scratch reflex returns with an intensity greater than before. This successive spinal induction explains the tendency which exists in the spinal cord to an alternation of response ; every act tending to come to an end by fatigue and, as a result of negative spinal induction, inducing the opposed or antagonistic act. Thus if a spinal dog be held up in the vertical position, so that the hind limbs hang freely, these latter execute a series of alternate movements of flexion and extension. The starting-point of these is the stretching of the anterior thigh muscles. Once started they continue of themselves, each act exciting the alternating antagonistic act.

A reflex act has often been distinguished from other reactions, described as conscious or purposive, by its fatality-i.e. by the invariability with which it results on a given stimulus, whether the reaction be for the good of the animal as a whole or not. Thus a decapitated eel will wind itself with equal readiness around a stick or a hot poker. All reactions are, however, purposive. The machinery for them has been evolved and the paths laid down in the spinal cord under the action of natural selection, so that they must act, at any rate in the average of cases, towards the well-being of the animal as a whole. Since the nerve path involved in any reaction includes a number of synapses, each of which may be influenced from other parts of the body in a positive or negative direction, an absolute uniformity of response cannot be predicated for any one reaction. There will be changes in the facility with which it is evoked and changes in its extent, and these will become the more operative the greater the complexity of the arc, and the larger the number of other impulses to which it may be subject. The fatality of response is therefore only shown at its best in the very simplest of reflexes, or the most lowly organised nervous systems.

The purposive character of the reflexes obtained from the spinal frog has sometimes led writers, especially in pre-Darwinian days, to endow the spinal cord with a guiding intelligence. At the present time we recognise that every reaction of a living being must be purposive, in the sense of being adapted to the preservation of the species, if the latter is to survive in the struggle for existence. The question as to whether we are justified in predicating the existence of even a germ of consciousness or volition in the spinal animal must be decided in the negative. "Associative memory would seem to be a postulate for the very existence of perception. Where even simplest ideas are not, there cannot be consciousness. Animal movements that are appropriate not only for an immediate but also for a remote end indicate associative memory. 
The approach of a dog in answer to the calling of its name, the return of an animal when hungry to the place where it has been wont to receive food, such movements may be taken as indicative of consciousness since they indicate the working of associative memory. Examined by this criterion all purely spinal reactions fail to evince features
of consciousness" (Sherrington).

THE PART PLAYED BY AFFERENT IMPRESSIONS IN THE COORDINATION OF MUSCULAR MOVEMENTS. Every reflex act is initiated in the first place by some form of sensory stimulus. In the carrying out of the muscular contractions and the resultant movements of the limbs, other impulses are set up in the structures which subserve deep sensibility, including those of muscles, which in their turn affect the excitability and the activity of the motor neurons. These secondary afferent impulses are important whether the movements be aroused by immediate sensory stimulation of the surface of the body, or through the higher parts of the brain, as in volitional movements.

Their significance is shown by the marked disorders of movement produced in a limb by section of some or all of its afferent nerves. Thus if all the posterior roots supplying one hind limb of the frog be divided the posture of the-desensitised limb is abnormal, whether the frog be suspended or be in a sitting posture. Such a frog generally swims with the desensitised limb in permanent extension. The complete absence of muscular tone under these circumstances has already been mentioned. When a contraction of the quadriceps extensor is induced by a single shock applied to the intact motor nerve, the curve obtained shows a relaxation line much slower and more prolonged than when the cut nerve is similarly excited. In the latter case, or when the posterior roots alone are divided, the lever at the end of relaxation dips below the base line with an inertia fling, which is never present while the nerve is intact. The contraction of the muscle, when its afferent path is intact, seems to develop reflexly in the muscle itself a condition of tone which damps the inertia swing of the contraction. In the dog, after section of the afferent nerves of one hind limb, this limb is not at first used for walking; it is kept more or less flexed at hip and knee, and later, when it is employed in walking, it is lifted too high with each step. After division of the afferent fibres of both limbs these appear as if they were affected with motor paralysis. At first, during walking, the fore limbs simply drag the hind limbs after them, though later, as the hind limbs are drawn along, they make alternate movements and may ultimately afford a certain amount of support to the body.

Still more striking effects are observed in complete apæsthesia of the fore limb in monkey or man. The limb is permanently paralysed; it is never used in climbing, or in the taking of food. That the peripheral motor mechanism is intact is shown by the fact that stimulation of the appropriate area of the cerebral cortex in such animals elicits at once a perfectly normal movement of the hand or limb. It seems, however, impossible for the cortex to initiate such movements in the absence of all afferent impulses arriving from the limb. Similar paralysis was observed by Chas. Bell in the upper lip of the ass after 
section of the corresponding branches of both fifth nerves, and was interpreted by him as indicating a possible motor function for these nerves.

In these phenomena of sensory paralysis we are dealing with the effects produced by the deprivation of two distinct classes of afferent impressions, viz. those from the skin, and those from the deep structures and muscles. The phenomena due to these two factors may be studied separately If in the monkey all the afferent brachial roots except the last cervical, which supplies cutaneous sensations to the whole hand, be divided, the monkey uses the arm and hand both in climbing and in taking food. A marked ataxy of the movement is, however, observed. Whereas the normal monkey, in taking grains of rice out of the observer's hand, exhibits perfect precision of movement so that he rarely touches the hand on which the grains are lying, the monkey with only cutaneous sensibility remaining grasps clumsily with the whole hand, and the arm sways as it is put out, often missing the object aimed at altogether. Cutaneous insensibility of the hind limb causes very little disturbance of locomotion, the alternate movements of which seem to be started by the stretching of the structures at the front of the thigh. On the other hand, a patient affected with such a loss may be the subject of ' static ataxy;' i.e. he is unable to stand with his feet together and his eyes shut. The afferent impressions from the skin of the feet appear therefore to be necessary for the maintenance of static equilibrium.

In the carrying out of co-ordinated movements, such as those of locomotion, the impressions from the muscles play a more important part. Division of the afferent nerves from the muscles gives rise to a condition of tonelessness, and the passive mobility of the joints is greater than usual, so that the hip with the limb extended at the knee may be flexed to an abnormal extent. The effect of this loss of tone is more apparent in the case of certain muscles. The disturbance of co-ordination resulting from the cutting off of afferent muscular impressions is well seen in cases of tabes dorsalis, or locomotor ataxy, in man, and to a slighter extent in cases of peripheral neuritis affecting chiefly the sensory nerves of muscles. The ataxic gait of such patient is characteristic. There is no loss of power in the muscles, but there is loss of control. The patient is unaware of the position of his limbs and has to guide his walk by visual impressions ; even then the movements are inco-ordinated. The contraction of every muscle is exaggerated, so that in walking the leg is first raised too high and then is brought down on to the ground with a stamp. As the disease progresses the loss of control becomes more and more pronounced, so that attempts to walk simply give rise to a profusion of disordered movements, the legs being thrown in all directions with the patient's efforts, but with no effective result. The centres are no longer informed of the degree to which each muscle is contracted, and the impressions are wanting which should cut short the contraction of a muscle when it has attained its optimum, and which should inhibit the antagonists during the contraction and induce activity of the antagonists in successive alternation to those of the other muscles. In such a patient therefore walking finally becomes impossible, and, with well- 

the rest of his days in bed.

\section{THE EFFECT OF POISONS ON THE SPINAL CORD}

The reflex functions of the spinal cord may be abolished by the same drugs, such as ether, chloral, \&c., which abolish conductivity in a nerve fibre. The central effect of these drugs is obtained with much smaller con-
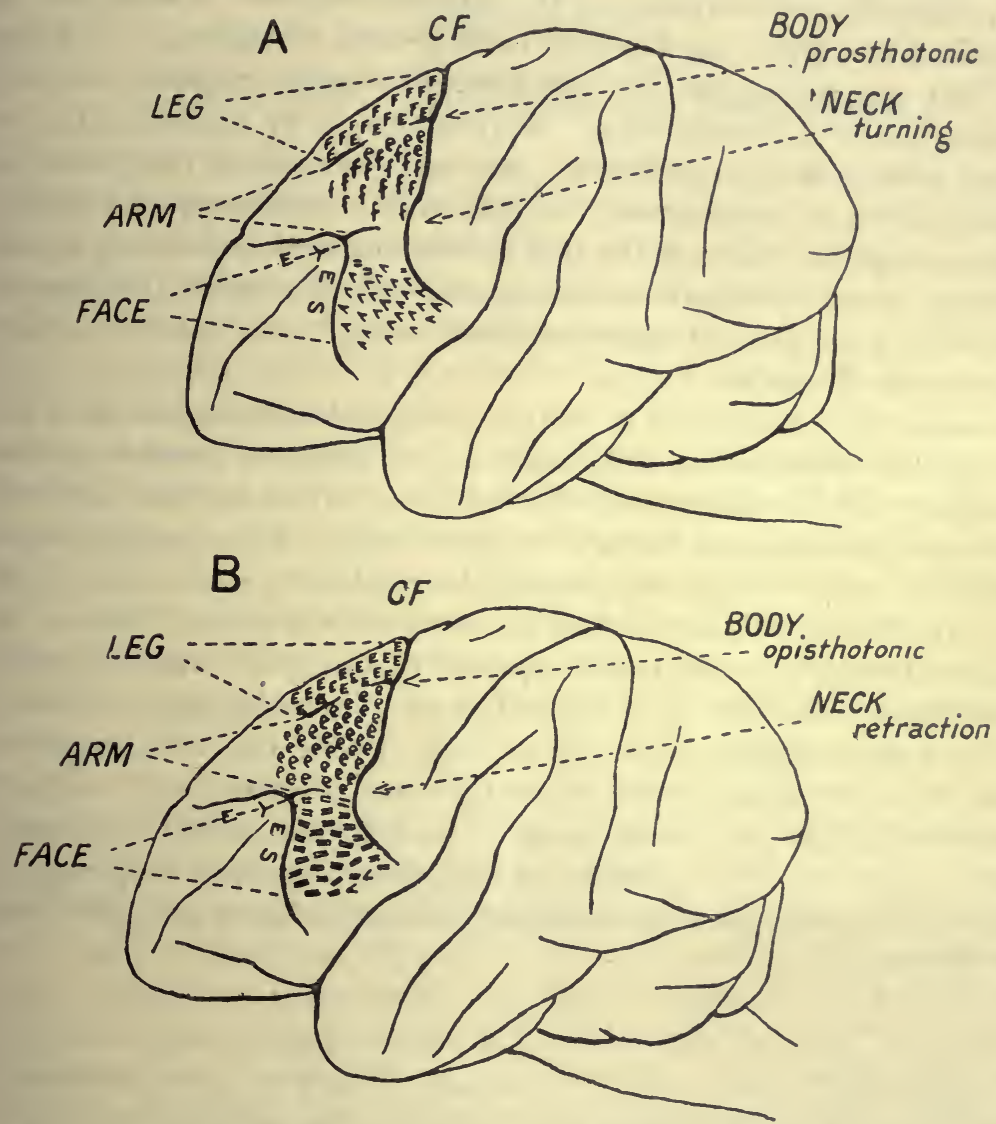

Fig. 173. Diagram by Sherrington to show influence of tetanus toxin on the response to excitation of the motor area of the cortex in the monkey.

A, normal animal. B, after poisoning with tetanus, $\quad F$ and $f=$ flexion of leg and arm respectively. $\mathrm{E}$ and $e$ signify extension. < signifies opening of mouth; = signifies closing of mouth.

centrations than is the case with the peripheral nerves and is the cause of their anæsthetic effect.

More interesting from the point of view of the physiologist is the action of such a drug as strychnine, or the somewhat similar action of the toxin formed by the tetanus bacillus. If a small dose of strychnine be injected into a spinal frog, after a short period of heightened irritability the slightest stimulus applied to the surface will cause spasms, which may affect every 
muscle in the body. Pinching the foot, instead of causing it to be drawn up now causes the legs, arms and back to be rigidly extended. The extension is not a co-ordinated act, but is associated with strong contraction of the flexors, the final position of the limbs being determined by the preponderating strength of the extensor muscles. The real meaning of this condition is seen if, in a spinal mammal, the extensor muscles be connected with a lever and the flexor muscles cut. On exciting the flexor reflex by pricking the foot, there is instantaneous relaxation of the extensor muscles. A small dose of strychnine is now given, insufficient to cause general convulsions. It is now found that on pricking the foot the extensor muscles respond, not with inhibition, but with a contraction. Strychnine acts by abolishing the inhibitory side of every co-ordinated act and converting the process of inhibition into one of excitation. Co-ordination therefore becomes an impossibility, and stimulation of any spot excites contractions not only of the appropriate muscles but also of the antagonists of these muscles, the direction of the resulting movement being determined simply by the relative strength of the two sets of muscles.

The same effect is produced by tetanus toxin, and, since the action of this toxin may be confined in its early stages to one limb, it is possible to show the abolition of the inhibitor side of the reflexes in this one limb while the limb of the other side reacts normally to the stimulus. The same abolition of inhibition is found whether the response be excited by stimulation of the skin or by voluntary excitation from the cortex of the brain. Thus in the monkey, on stimulating the cortex, opening of the mouth may be excited from all the spots marked " $<$ " in the diagram, closure being only obtained from those spots marked "=" (Fig. 173). Under the influence of the tetanus toxin excitation of every one of the spots, whether " $<$ " or " $=$," causes closure of the jaw. It is impossible for a patient under these circumstances to open his mouth, because every willed impulse for opening innervates at the same time the stronger masseter muscles and effectively closes the mouth. 


\section{SECTION IX}

\section{TROPHIC FUNCTIONS OF THE CORD}

THE reflexes which are excited by painful or nocuous stimuli must be regarded as prepotent in that their inhibitory effect on other reflexes is more marked than that produced by any other quality of stimulus. In the struggle for existence the reaction to nocuous stimuli must predominate over those due to any other kind, since it is essential for the survival of the animal that the stimulus should be removed or avoided, so that the animal should escape from its injurious effects.

It is natural therefore that after complete section of the afferent nerves from any part of the surface of the body there should be a tendency to trophic disturbances, such as the formation of ulcers, \&c. Such ulceration is frequently observed in patients suffering from spinal disease. After section of the first division of the fifth nerve ulceration of the cornea is often produced. These effects are, however, merely due to the absence of the normal protective reactions of the part, and can be prevented by scrupulous cleanliness and protection of the apæsthetic part from all possible injuries. There are other trophic effects caused by nerve lesions which cannot be ascribed to the mere absence of protective reflexes. Thus inflammation of the posterior root ganglia often sets up herpes zoster, or 'shingles,' in the region of cutaneous distribution of the corresponding sensory nerve. Changes in the skin (' glossy skin ') nails and hair are often seen after irritative injuries of nerves to the part. Section of a motor nerve causes rapid changes in the skeletal muscles supplied, which become smaller and after months or years may disappear altogether, being replaced by connective tissue. The changes in the excitability of the muscles produced under these circumstances have already been described.

It seems that the nutrition of a tissue is determined by its activity, and this in turn is under the control of some nerve path. Section of the nerve path, by cutting away the impulses which normally maintain the activity of the part, must at the same time seriously affect its nutrition. Thus the muscles which, though striated, are not so immediately under the control of the central nervous system, such as the sphincter ani, do not undergo degeneration after section of their nerves, or after extirpation of the lower part of the spinal cord.

On the other hand, it is only during post-fœtal life that the activity of the skeletal muscles is determined by the motor nerves of the cord. Thus 
they may be developed normally even in the complete absence of a central nervous system. Whether we are justified in assuming the existence of trophic nerves exercising an influence on the nutrition of the part they supply, apart from any influence on its other functions, the experimental evidence before us is not sufficient to decide; nor can we as yet give a physiological analysis of the changes in nutrition which may be brought about in hysterical patients under the influence of emotion. 


\section{SECTION X}

\section{THE SPINAL CORD AS A CONDUCTOR}

THE nervous system is built up of chains of neurons which subserve reactions of varying complexity. The complexity increases with the interference of the higher parts of the brain in the reactions and becomes, therefore, more and more marked as we ascend the animal scale. Whatever the course taken by the impulses in the central nervous system they must all finally make use of the motor common path, represented by the anterior spinal roots and by the motor roots of the cranial nerves.

The co-operation in any co-ordinated movement of widely separated portions of the central nervous system necessitates the existence of long paths, i.e. the axons of certain nerve-cells must extend through a considerable distance in the central nervous system before they arrive at the next relay in the chain of which they form part. During this course the axons run in the white matter of the central nervous system, and are surrounded by medullary sheaths. The white matter of the cord consists almost exclusively of medullated nerve fibres running for the most part longitudinally. These are of various sizes, some of the smaller fibres being collaterals, which have been given off from the larger ones and which will shortly turn into the grey matter. In section they resemble closely the fibres of an ordinary peripheral nerve, but differ from these in that they have no primitive sheath or neurilemma. Each consists of an axis cylinder surrounded by a thick sheath of myelin, the whole embedded in a tube formed by the neuroglia.

Of these fibres part belong to the spinal cord, the proprio-spinal or internuncial fibres, which we have studied previously. The greater number serve to establish connection between the grey matter of the cord or the afferent roots entering the cord, and the different levels of the brain, and these fibres may carry impulses either up towards the brain or down towards the spinal cord; they may be ascending or afferent, so far as the brain is concerned, or descending and efferent. No fibre takes an isolated course on its way through the cord; practically every one sends off fine branches or collaterals, which run into the grey matter at various levels, there making connection or having synapses with the local reflex mechanisms contained in each segment.

On inspection the white matter is seen to be divided by the anterior and posterior fissures of the cord into two symmetrical halves, and the nerve-roots divide each half into anterior or ventral, lateral, and posterior 
or dorsal columns. On account of the scattered distribution of the anterior root fibres over a considerable area of the surface of the cord, the division between the anterior and the lateral columns is ill defined, and the whole region is often defined as the antero-lateral column. In the cervical and upper dorsal region of the cord slight grooves on the surface of the cord indicate a division of the anterior column into the antero-median and anterolateral columns, and of the posterior column into the postero-median and postero-lateral columns. These two posterior columns are often designated as the columns of Goll and Burdach. In order to determine the origin, course, and destination of the fibres which make up these white columns we must have recourse to the indirect methods of development and of degeneration which were described on p. 319. By these means we may divide the white matter into ascending and descending tracts. An 'ascending' tract means, not that the direction of conduction of the impulse is necessarily in the upward direction, i.e. from spinal cord to brain, but that the nerve-cell which gives off the fibres sends its axons towards the brain, while a descending fibre in the cord is the axon of a nerve-cell situated in the upper part of the cord, or in some part of the brain. If the assumption which we have made as to the normal direction of conduction in axons and dendrites be correct, an ascending fibre will also conduct impulses in an ascending direction. After section of the cord, say in the mid-dorsal region, transverse sections of the cervical and lumbar regions of the cord, taken at the appropriate period after the lesion has been inflicted, show patches of degenerated fibres in the white matter. The fibres which are degenerated above the section represent the ascending tracts, whereas those which degenerate below the section, i.e. in the lumbar region, are the descending tracts of the cord ( $c p$. Figs. 174 and 175). In this way the following tracts have been distinguished :

\section{A. DESCENDING TRACTS}

(1) PYRAMIDAL TRACTS. If the spinal cord be divided in the upper cervical region, degeneration of two distinct tracts on each side, in the anterior and postero-lateral columns, is produced. These are the anterior or direct and the crossed pyramidal tracts. The fibres composing these tracts are derived from large nerve-cells in the motor area of the cerebral cortex, and therefore degenerate if the motor area of the cortex is destroyed. The pyramidal tracts are derived from the cerebral cortex of the opposite side, having crossed the middle line at the lower level of the medulla oblongata in the pyramidal decussation. The anterior pyramids represent a certain number of fibres which have not crossed with the others, but continue the course of medullary pyramids for a time, crossing gradually by the anterior commissure on their way down the cord, so that, as a rule, they come to an end in the mid-dorsal region, all the fibres having passed into the lateral columns of the opposite side. A few fibres of the pyramids on their way from the cerebral cortex pass into the lateral columns of the same side; these are the uncrossed pyramidal fibres. The greater number of the fibres, however, finally reach the crossed pyramidal tracts, in which they can be traced as far 
as the lower end of the cord. They end in the spinal cord by turning into the grey matter where they break up into a fine bunch of fibrils in close connection with the motor cells of the anterior horn, or, according to Schäfer, with the cells of the posterior horn.

On their way down the cord they give off fine side branches or collaterals, which run into the grey matter, thus establishing connections between one cortical cell and the anterior cornual cells of several different segments of the spinal cord. These fibres carry voluntary motor impulses from the cerebral cortex to the reflex motor mechanisms of the cord. Their destıuction by disease, or otherwise, causes the abolition of voluntary control over the muscles, without, however, interfering with the reflex motor functions of the cord, which, as a matter of fact, are increased in cases where these tracts have undergone degeneration.

(2) R U B R O-S PINAL O R PREPYRAMIDAL TRACT (also called Monakow's Bundle). This is a fairly compact group of fibres which degenerate downwards after section of the cord. It is situated, in cross-section, ventral to the pyramidal tracts. Its fibres can be traced up to the cells in the red nucleus, a mass of grey matter in the midbrain lying ventrally to the nucleus of the third nerve.

(3) VE S T I B U LO-S P I N A L TRACT. This consists of scattered fibres in the antero-lateral column, which degenerate in the

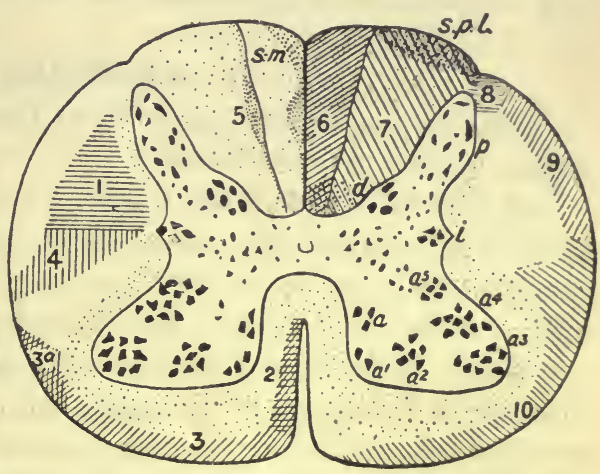

FIG. 174. Diagram (from ScHÄFER) showing the ascending (right side) and the descending (left side) tracts in the spinal cord.

1 , crossed pyramidal; 2, direct pyramidal; 3 , antero-lateral descending: $3 a$, spino-olivary descending (bundle of Helweg); 4, pre-pyramidal (rubro-spinal); 5, comma; 6, postero-mesial ; 7 , postero-lateral; 8 , Lissauer's tract ; 9 , dorsal (ascending) cerebellar; 10, antero-lateral ascending; $s m$, septo-marginal; $s p l$, dorsal root zone; $a$, anterior horn-cells; $i$, intermedio-lateral horn; $p$, cells of posterior horn; $d$, Clarke's column. The fine dots represent the situation of the 'internuncial' or 'endogenous' fibres of the spinal cord.

downward direction. They were formerly supposed to be derived from the cerebellum of the same side, but it has been shown that they are in all probability derived from Deiters' nucleus in the medulla-an important transmitting station between the cerebellum and cord.

(4) OLIVO-SPINAL AND THALAMICO-SPINAL TRACTS (Bundle of Helweg). This tract is also situated in the antero-lateral column, opposite the head of the anterior horn. It consists mainly of fibres which pass from the thalamus (the fore brain) through the inferior olive of the medulla downwards in the cord as far as the lower cervical region.

(5) COMMA TRACT. This tract lies in the posterior columns at the junction of the postero-median and postero-lateral portions. It consists for the most part of the descending branches of the afferent dorsal nerveroots which enter the cord. These divide as they enter the cord, and their 
descending branches pass down for two or three segments in the comma tract before turning into the grey matter. The tract, however, contains fibres of other origin, some of which begin and end in the spinal cord itself.

(6) TRACT OF MARIE. This, also in the anterior column, contains both descending and ascending fibres and is largely a continuation of the posterior longitudinal bundle, the connections of which we shall have to study later on. A small tract of fibres, which degenerate in the descending direction, is also found in the posterior part of the cord adjoining the posterior longitudinal fissure.

(7) SEPTO-MARGINAL BUNDLE. This is largely proprio-spinal, but may contain fibres coming from the mid-brain.

\section{B. ASCENDING TRACTS}

These may be divided according as they are situated in the posterior, the lateral, or the anterior columns.

(a) THE POSTERIOR COLUMNS. Almost the whole of the fibres making up these columns are exogenous, being axons of cells in the posterior root ganglia. They can be divided into long, medium, and short fibres, all of which, on their way up, give off collaterals, which pass into the grey matter and ramify round nerve-cells, especially in the posterior horns (cp. Fig. 160). The longest fibres pass to the upper end of the cord, where they end in the posterior column nuclei, the nucleus gracilis and the nucleus cuneatus of the medulla. These fibres remain entirely on the side of the cord on which they have entered. As they pass up they are displaced towards the middle line by each incoming and higher placed root. Thus in the cervical region, and indeed from the fifth dorsal segment upwards, two columns can be distinguished in the posterior part of the cord, viz. the postero-median and postero-lateral columns, the division between which is indicated by a small groove on the surface. The postero-median column contains from within outwards the fibres from the sacral region, those from the lumbar region, and those from the inferior dorsal region. The postero-lateral column, or column of Burdach, contains mesially the four upper dorsal root fibres and more laterally the fibres from the cervical nerves.

(b) THE LATERAL COLUMNS. In these columns are found the two cerebellar tracts, as well as scattered fibres passing to the fore- and midbrain.

(1) The Direct or Dorsal Cerebellar Tract arises from the cells of Clarke's column on its own side. It consists of large fibres, which pass through the grey matter to the lateral columns of the same side, and ascend in the cord immediately ventral to the incoming posterior root fibres, and external to the crossed pyramidal tract. In the medulla they are joined by a bundle of fibres from the opposite inferior olive and pass with the restiform body into the cerebellum, where they terminate in the superior vermis of this organ.

(2) The Ventral or Anterior Cerebellar Tract, often called the tract of Gowers, arises in cells scattered through the grey matter, chiefly of 

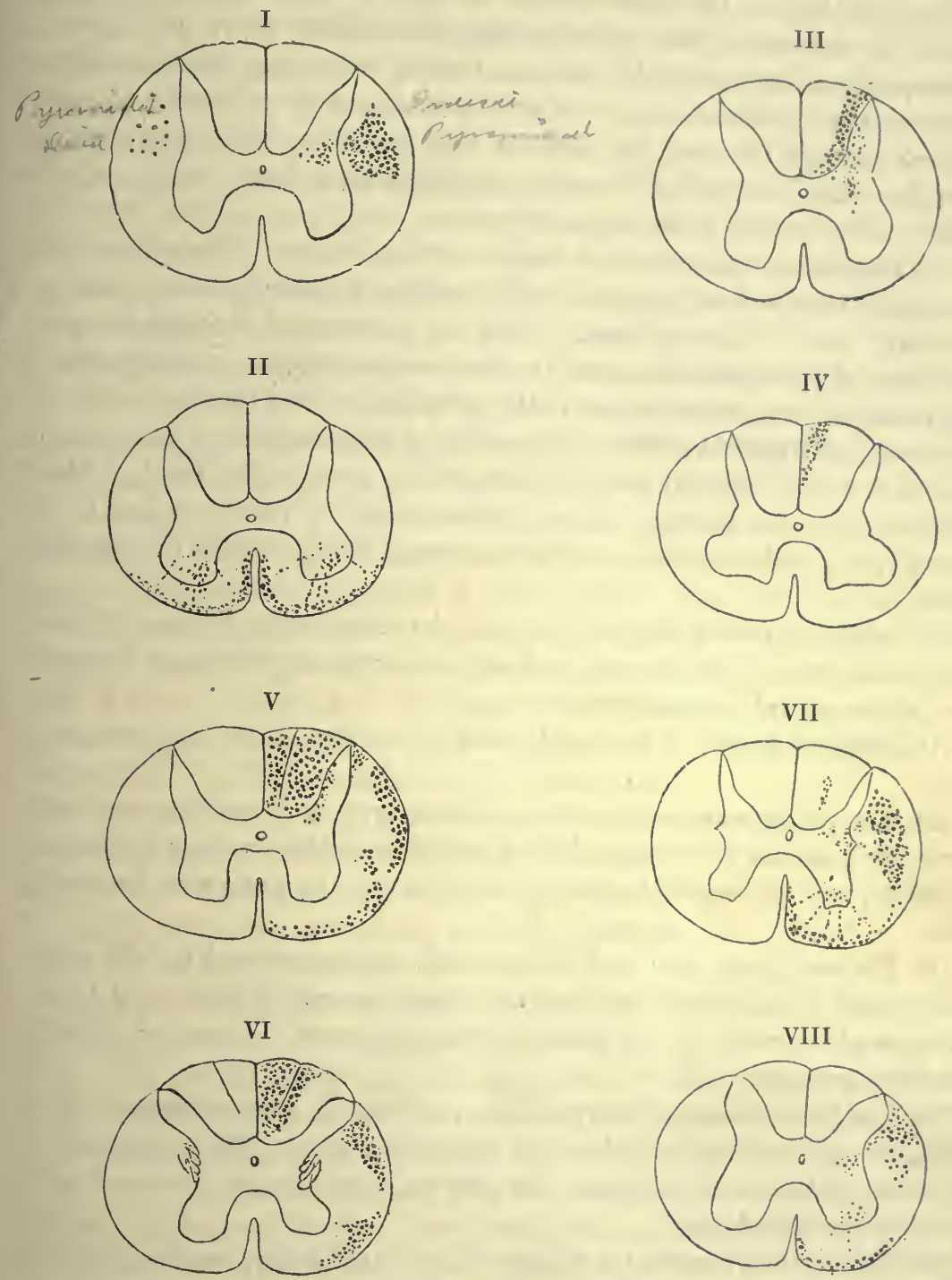

FIG. 175. Diagram of sections of the spinal cord of the monkey showing the position of degenerated tracts of nerve fibres after specific lesions of the cord itself, the afferent nerve-roots, and of the motor region of the cerebral cortex. (SснӓғER.) (The degenerations are shown by the method of Marchi.) The left side of the cord is at the reader's left hand.

I. Degenerations resulting from extirpation of the motor area of the cortex of the left cerebral hemisphere.

II. Degenerations produced by section of the posterior longitudinal bundles in the upper part of the medulla oblongata.

III and IV. Result of section of posterior roots of the first, second, and third lumbar nerves on the right side. Section III is from the segment of cord between the last thoracic and first lumbar roots; section IV from the same cord in the cervical region.

$V$ to VIII. Degenerations resulting from (right) semi-section of the cord in the upper region thoracic. $V$ is taken a short distance above the level of section; VI higher up the cord (cervical region); VII a little below the level of section; VIII lumbar region. 
the posterior horn of the opposite side, though a few fibres are derived from cells of the same side. The tract consists of fine fibres which pass upwards in the peripheral margin of the lateral column, extending from the direct cerebellar tract behind to the level of the anterior roots in front; it passes upwards through the cord, the medulla, and the pons, then turns round to enter the cerebellum through the superior cerebellar peduncle, ending chiefly in the ventral portion of the superior vermis.

(3) The Spino-thalamic and Spino-tectal Tracts. These fibres form a scattered bundle lying internally to the anterior cerebellar tract, and are practically part of Gowers' tract. They may be traced through the cord, medulla, and pons and end, partly in the anterior corpora quadrigemina of both sides, but to a greater extent in the optic thalamus of the same side.

(c) ANTERIOR COLUMNS. A number of scattered fibres pass up the anterior columns, mingled with the descending fibres of the tract of Marie in the angle of the anterior fissure. Others pass up partly to end in the olivary body, partly to run on with the mesial fillet towards the thalamic region.

The white matter of the cord can thus be regarded as made up of short and of long tracts, which maintain direct connection between the following parts of the central nervous system :

(1) Different levels of the cord itself by means of the proprio-spinal fibres.

(2) Hind-brain and spinal cord, by the anterior and posterior cerebellar tracts, the posterior columns, and the spino-olivary fibres among the ascending tracts, and the vestibulo-spinal and olivo-spinal among the descending tracts.

(3) The mid-brain and cord connections are represented by the spinotectal tracts in the lateral columns as a direct ascending path, and by the rubro-spinal tract which furnishes a direct efferent connection between mid-brain and cord.

(4) The fore-brain, viz. the thalamus, receives the spino-thalamic fibres, which, though scattered, are of considerable importance. They run chiefly in the lateral and anterior columns. Its efferent fibres cannot be traced below the lower cervical region.

(5) The cerebral cortex, the master tissue of the body, receives no fibres directly from the cord or periphery of the body, but by the pyramidal tracts is able to influence directly the activities of the motor mechanisms at every level of the cord. These fibres, so far as is known, exist only in mammals, and show a great increase in relative extent when traced from lower to higher types. While in the rabbit the pyramidal tract is hardly perceptible, in the monkey it is the best marked of all the tracts, and in man is still more highly developed. This relative increase, which is probably associated with the shunting of more and more of the reactions of the body from the region of the unconditioned reflex to that of the educatable reaction, is shown not merely by the tract occupying a larger proportion of the transverse area of the cord, but by its fibres being more densely set within that area. 


\section{THE PATHS OF IMPULSES IN THE CORD}

The greater part of the white matter is thus concerned in transmitting impulses to nerve-cells in the brain, and from the brain towards the cord. The complex reactions determined by these impulses are in many cases as unconscious and automatic as those we have studied in the spinal cord, even though they may involve the activity of the cerebral cortex itself. Others, however, influence consciousness, so that their afferent side appears in consciousness as sensations of various qualities, and their efferent side as the result of volition, i.e. as willed or emotional movements.

The posterior spinal (sensory) roots at their entrance into the cord divide into two bundles. The smaller of the two, situated more laterally and consisting of fine fibres, enters opposite the tip of the posterior horn and turns up at once in Lissauer's tract, a bundle of fine longitudinal fibres close to the periphery of the cord. The fibres seem to pass into and end in the substance of Rolando. The larger median bundle of coarse fibres passes into the postero-external column. Here each fibre divides into a descending and an ascending branch, the former running in the comma tract, the latter in the posterior columns up as far as the gracile and cuneate nuclei of the medulla. Both of these branches give off collaterals in the whole of their course, most numerous near the point of entry of the nerve. These collaterals may be divided into four sets according to their destination :

(1) Fibres ending round cells of anterior horn on same side or crossing by posterior commissure to grey matter on other side.

(2) Fibres ending in grey matter of posterior horns.

(3) Fibres ending round cells of Clarke's column.

(4) Fibres to lateral horn.

Since the motor nerves arise from the anterior horn-cells, the first set, the 'sensori-motor' collaterals, represent the shortest possible spinal reflex path. The second group may also represent a spinal reflex path with two relays of cells, and therefore greater choice of response and longer reaction time. The third set puts into action the cerebellar tracts which arise from the cells of Clarke's column, and therefore call into play a much more complicated mechanism, the limits of whose action it would be difficult to define. The collaterals to the lateral horn probably represent the afferent tracts of the various visceral and vaso-motor reflexes which we shall study later.

We find no special tracts devoted to those impulses which affect consciousness as sensations. All tracts going towards the cerebral hemispheres are interrupted by cell relays, in the medulla or cerebellum, and must serve as afferent channels for unconscious as well as for conscious reactions. The quality of an afferent impulse can only be defined by its origin, or by its effect on consciousness, and much discussion has arisen as to the exact path of the various cutaneous and muscular sensations in the cord.

It is evident that an impulse may travel to the cortex by way of the two cerebellar tracts through the cerebellum, or by way of the posterior columns through the intermediation of the bulbar nuclei, or by a series of relays from 


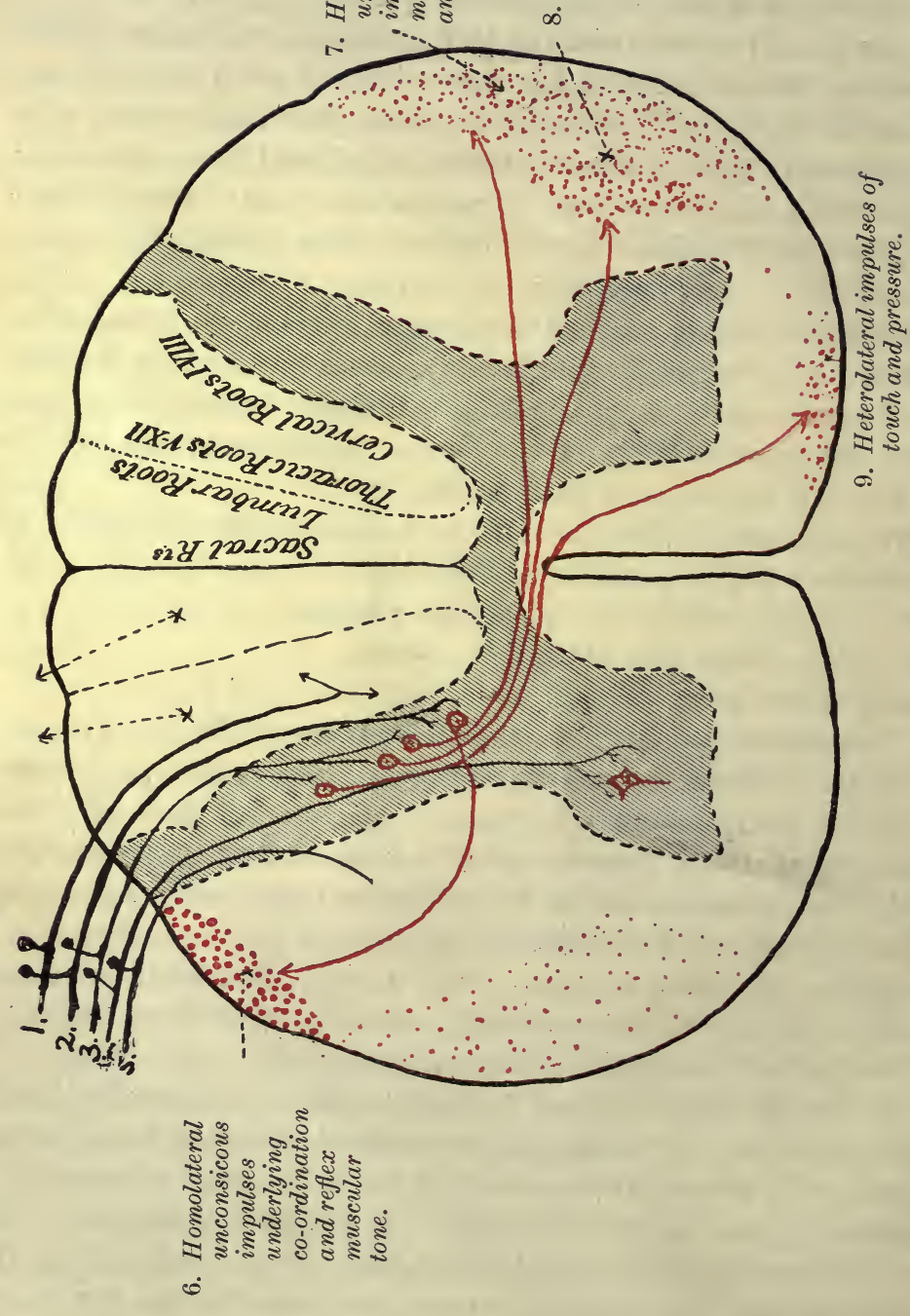

速过

ต ริ

.. 娄 $\infty$

苑

จิ ปี

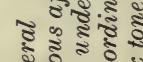

离范

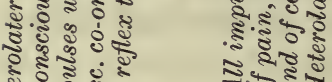

స.. है

证

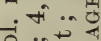

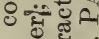

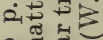

$\circ$ ह

.

-

‡ 8.

है

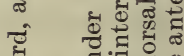

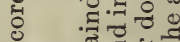

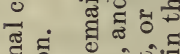

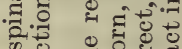

๖

मै

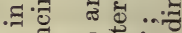

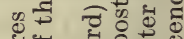

हैं

त्ठ है क्ष

है है

क

డ

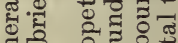

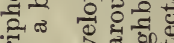

영

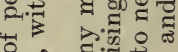

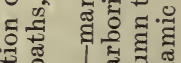

. ำ

욤

象융용

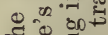

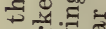

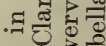

क्षेत कू

- 00 운

. 듀

क

$0 \quad$ काष

$\therefore \quad$ o

๒ 
one segment of the cord to another through grey and white matter alternately. It is supposed that all of the ascending tracts may convey afferent impulses from the posterior spinal roots to the brain, although evidence as to the part taken by each tract is very conflicting. The following account represents the views which may be regarded as the most probable (Page May) (Fig. 176) : Pain impulses, on entering the cord by the posterior roots, cross to the other side at once, and then pass up, chiefly in the antero-lateral column, by the spino-thalamic fibres, as far as the optic thalamus. Sensations of heat and cold take a very similar course. Hence they are generally affected by lesions of the cord in the same way as pain sensations. Impulses of touch and pressure, after entering the cord, pass up in the posterior column of the same side for four or five segments, then cross gradually and pass up in the opposite anterior column. Impulses serving muscular sensibility, including the impulses from joints and tendons, take two courses. Those which do not reach consciousness, and are involved in the involuntary guidance of muscular movements, run up chiefly in the anterior and posterior cerebellar tracts of the same side. Those which furnish the material for conscious sensations and give information as to the position of the limbs, \&c., are entirely homolateral, and travel up in the posterior columns of the same side of the cord. All impulses which reach the brain cross finally to the optic thalamus and thence to the cerebral cortex of the opposite side.

Hemisection of the cord on one side, as was first pointed out by Brown Sequard, causes the following symptoms :

(1) Paralysis of the voluntary motor conductors on the same side.

(2) A paralysis also of the vaso-motor conductors on the same side, and, as a consequence, a greater afflux of blood, and a higher temperature. There may be some degree of hyperæsthesia on this side.

(3) There is anæsthesia affecting all kinds of sensibility, excepting the muscular sense, in the opposite side to that of the lesion, owing to the fact that the conductors of sensitive impressions from the trunk and limbs decussate in the spinal cord; so that an injury in the cervical region of that organ in the right side, for instance, alters or destroys the conductors from the left side of the body.

(4) There is some degree of anæsthesia also on the side of the lesion, in a very limited zone, above the hyperæsthetic parts, and indicating the level of the lesion in the cord. This anæsthesia is due to the fact that the conductors of sensory impressions, reaching the cord through the posterior roots, at the level or a little below the seat of the alteration, have to pass through the altered part to reach the other side of the cord.

The only direct unbroken cortico-spinal fibres are those contained in the pyramidal tracts. Motor impulses, which start from the cerebral cortex on one side, pass down that side till they reach the lower part of the medulla. Here the greater number of the fibres cross over in the pyramidal decussation to run down in the crossed pyramidal tract on the other side of the cord. The few fibres which do not cross over in the pyramidal decussation are continued as the direct or anterior pyramidal tract. These, however, also 
cross to the other side in their passage down the cord before becoming connected with the anterior cornual cells. Hemisection therefore of the spinal cord in the dorsal region will produce paralysis of motion and loss of or impaired muscular sensation in the parts supplied by the nerves on the same side below the lesion.

A great part of the white matter of the cord is concerned then in maintaining connection between the brain and higher parts of the nervous system and the periphery, through the intermediation of the cells of the grey matter of the cord. Corresponding to this function we find a gradual increase in the number of fibres in the white matter as we ascend from the sacral part of the cord to the medulla, the white matter being continually reinforced as it ascends the cord by fibres establishing connection with the ganglion-cells forming the nuclei of the nerve-roots.

Vaso-motor impulses to the limbs travel down the lateral columns of the cord on the same side. 


\title{
THE BRAIN
}

\author{
SECTION XI
}

\section{THE STRUCTURE OF THE BRAIN STEM}

THE physiology of the brain falls naturally into two main divisions, namely, that of the brain stem, including the medulla, the pons, Sylvian iter, corpora quadrigemina and third ventricle, and that of the cerebral hemispheres. - It is usual, in treating of the structure of the brain stem, to consider it as a prolongation forwards of the spinal cord and as consisting, like this, of a central tube of grey matter surrounded by a tube of white matter. Like the spinal cord, the brain stem may be regarded as originating primitively by the fusion of a series of ganglia presiding over the local reactions of their respective somites. The modifications in this segmental arrangement, which have occurred in the course of evolution, have been so profound that little trace of the primitive segmental arrangement is to be observed. At the fore end of the body have been developed the organs of special sense, which are the most important in determining the reactions of the animal in response to present or approaching changes in its environment. Indeed the whole course of evolution is conditioned by the evolution of the brain stem in the first place, and of its outgrowth, the cerebral hemispheres, in the second. Hence we cannot expect to find in the brain stem the regularity of arrangement of grey and white matter that we have studied in the cord. The typical division of the grey matter into cornua becomes altogether lost. While some nerves take their origin from or terminate in the central tube of grey matter, in other cases the collections of nerve cells and fibres forming the nuclei of the cranial nerves have become more or less separated from the central axis. Moreover the central grey matter is by itself quite inadequate to deal with the flood of afferent impressions entering the central nervous system through the organs of special sense, or to co-ordinate these with one another or with those arriving from the skin and lower part of the body. Masses of grey matter, which have no representative in the cord, make their appearance, and may be regarded as additional sorting stations or fields of conjunction for the afferent efferent impulses which determine the nervous activities of the animal.

The general features of the structure of the brain will be best understood by reference to the mode of development of this part of the central nervous 
system. At the front end of the body, the primitive neural tube, formed by the invagination and growing over of the epiblast, is somewhat enlarged and is marked off by two constrictions into the three primitive cerebral vesicles, which are named respectively the fore-, the mid-, and the hind-brain, or the prosencephalon, the mesencephalon, and the rhombencephalon (Fig.

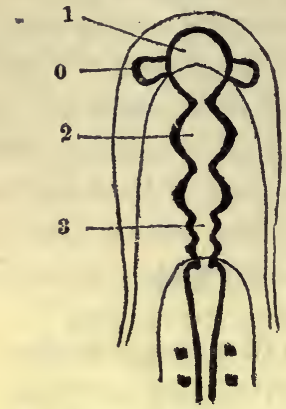

Fra. 177. Diagram of the cerebral vesicles of the brain of a chick at the second day. (СADIAT.)

$1,2,3$, cerebral vesicles; 0 optic vesicles.

177). At their first formation the walls of these vesicles are composed of simple epithelial cells, and show no trace of nervous structure. A little later the cells forming the walls present a differentiation into neuroblasts and spongioblasts, the former developing into nerve-cells, while the latter form the neuroglial supporting tissues of the brain and probably also furnish the cells of the sheath of Schwann to the outgrowing cranial nerves. In some places the wall of the vesicles remains undifferentiated; no nervous tissues develop in it, and it forms a layer of epithelium known as ependyma. By the varying growth of nervous tissue in different parts of the wall, the typical structure of the adult brain is brought about (Fig. 178). Thus in the hindbrain, or rhombencephalon, the roof of the neural canal posteriorly fails to develop, so that in the adult brain there is merely a layer of epithelium covering the expanded central canal, here known as the fourth ventricle. This back part of the hind-brain is often called the myelencephalon, the anterior portion being the metencephalon. The floor of the myelencephalon undergoes considerable thickening and forms the future medulla oblongata. In the metencephalon, nervous tissue is developed all round the canal, the floor of the canal forming the pons Varolii, while the cerebellum is developed by an outgrowth of the dorsal wall. In the region of the constriction between the hind- and mid-brain known as the isthmus, the roof or dorsal wall forms the superior cerebellar peduncles at

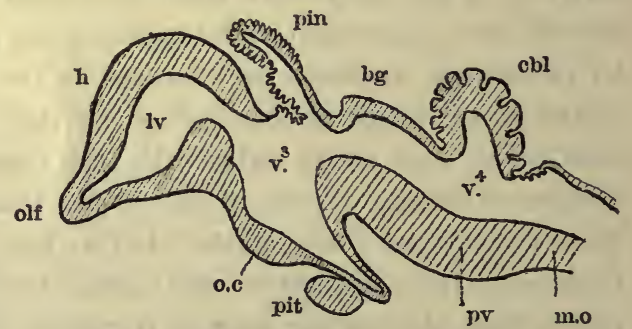

FIG. 178. Longitudinal section through brain of chick of ten days. (After MrHalkovicz.)

olf olfactory lobes; $h$, cerebral hemisphere; $l v$, lateral ventricle ; $p i n$, pineal gland; $b g$, corpora bigemina; $c b l$, cerebellum; oc, optic commissure; pit, pituitary body; $p v$, pons Varolii; mo, medulla oblongata; $v^{3}, v^{4}$, third and fourth ventricles. the side, and between them a thin layer of nervous matter known as the valve of Vieussens, or superior medullary velum. The cavity of the third vesicle corresponds in the adult brain to what is known as the fourth ventricle.

The mesencephalon, or second cerebral vesicle, takes a relatively small part in the formation of the adult human brain, though very conspicuous in many of the lower types of brain. The whole of its wall is transformed 
into nervous tissue, the roof or dorsal wall forming the corpora quadrigemina, while the two crura cerebri are developed in its ventral wall. The cavity of the second cerebral vesicle is retained as a narrow canal known as the aqueduct of Sylvius, and connects the fourth ventricle with the third ventricle.

Very soon after its first appearance the first cerebral vesicle is modified by the formation of lateral expansions, known as the optic vesicles, which later on are constricted off from the central part of the cavity so as to be connected with this by two short tubular passages, the optic stalks. From the optic vessels are ultimately developed the retinæ of the eyes. By the development of nerve-cells in the optic cup the ganglion-cell layer of the retinæ is produced, and from these cells fibres grow back along the optic stalk and make connection with the grey matter developed in the lateral wall of the fore-brain and with the adjacent parts of the mid-brain, viz. the superior corpora quadrigemina. The large masses of nervous tissue developed in the lateral walls of the fore-brain are the optic thalami, which represent the head ganglia of the brain stem. The front portion of the first cerebral vesicle expands in a forward and downward direction, and from the upper and lateral aspects of the outgrowth thus formed the cerebral hemispheres are produced as two hollow pouches. The original back part of the fore-brain is sometimes spoken of as the diencephalon, while the anterior part of the cerebral hemisphere growing from it is the telencephalon. The floor or ventral wall of the fore-brain undergoes moderate thickening to form the nervous structures which occupy the 'interpeduncular space' at the base of the brain, viz. the posterior perforated spot, the corpora mammillaria and the tuber cinereum. The roof of the first cerebral vesicle remains thin and in its primitive epithelial condition, like the roof of the back part of the fourth ventricle.

In the course of development the cerebral hemispheres become larger than the whole of the rest of the brain put together, growing backwards over the latter as far as the middle of the cerebellum (Fig. 179). Their dorsal and lateral walls become much thickened and consist of white matter internally and grey matter externally. The part of the hemisphere which lies over the first cerebral vesicle is undifferentiated and remains as a simple epithelial layer. This becomes closely applied to the similar layer forming the roof of the third ventricle, from which it is separated only by a process of the pia mater carrying numerous blood-vessels (the velum interpositum). In the adult brain the cavities of the cerebral hemispheres are known as the lateral ventricles, the remains of the first cerebral vesicle receiving the name of the third ventricle. The lower and outer part of the hemispheres, i.e. the part which is first formed, becomes much thickened and forms the corpus striatum, which is closely applied to the front and outer part of the optic thalamus. In the corpus striatum two masses of grey matter are developed, namely, the nucleus caudatus and the nucleus lenticularis. A layer of nerve fibres ascends from the brain stem to be distributed throughout the whole of the cerebral hemispheres. This forms a sort of capsule to the optic thalamus, lying between this body and the corpus striatum behind, but in 
front piercing the corpus striatum between its two nuclei. It is called the internal capsule.

The development of the different parts of the brain stem from the three cerebral vesicles and their gradual subordination and overshadowing in the course of development by the cerebral hemispheres is well shown if we

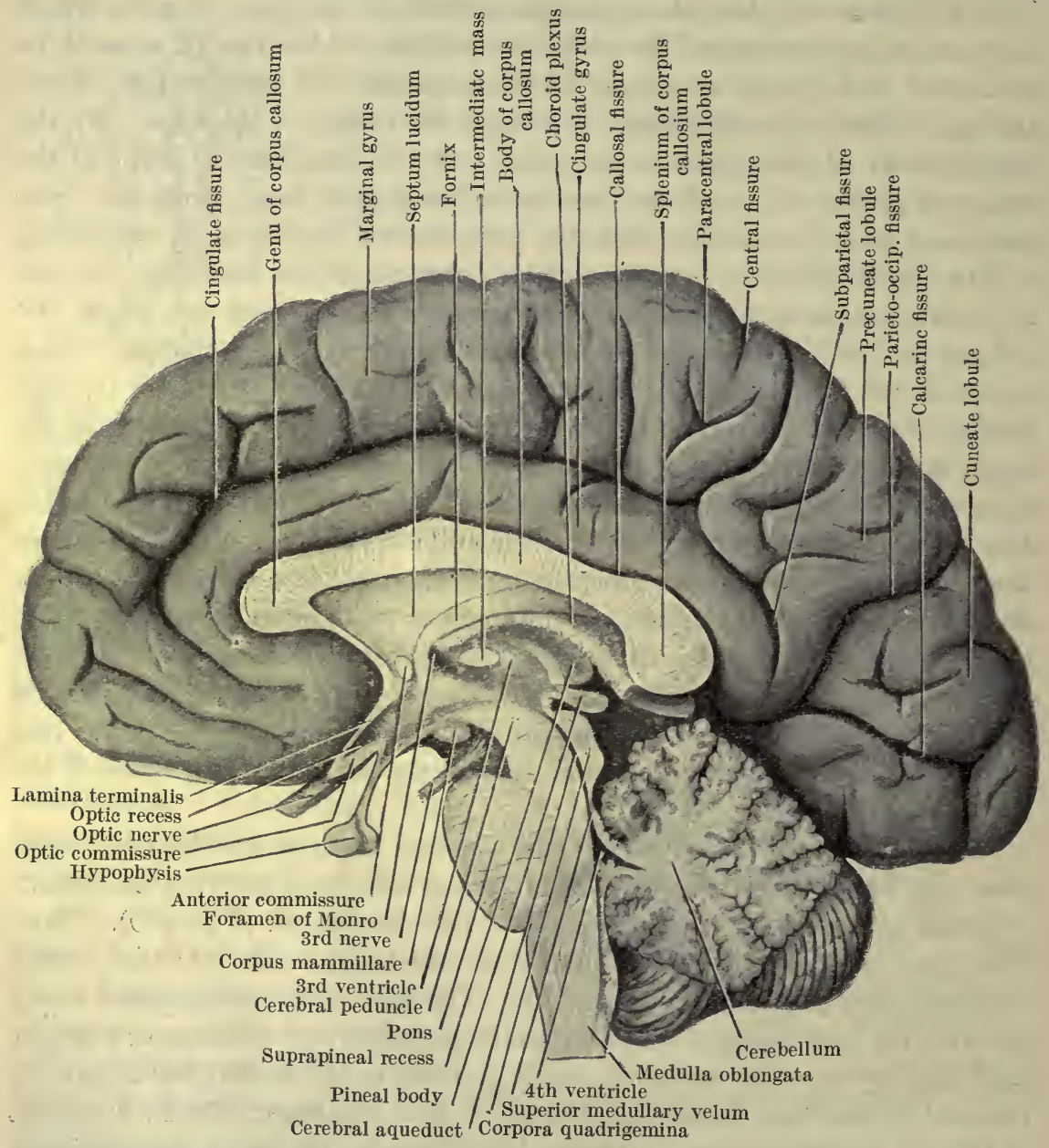

Fia. 179. Median section of an adult human brain. (J. Symington.)

compare the brain of a fish with that of a reptile and again with that of a mammal (F'ig. 180). Man's position in the scale of animal life is determined not by increasing complexity of the structures forming his brain stem, but by the gradual subordination of these to the latest formed cerebral hemispheres, and the enormous growth of his capacity to adapt himself to a varying environment consequent on the increase in size of his cerebral hemispheres. 

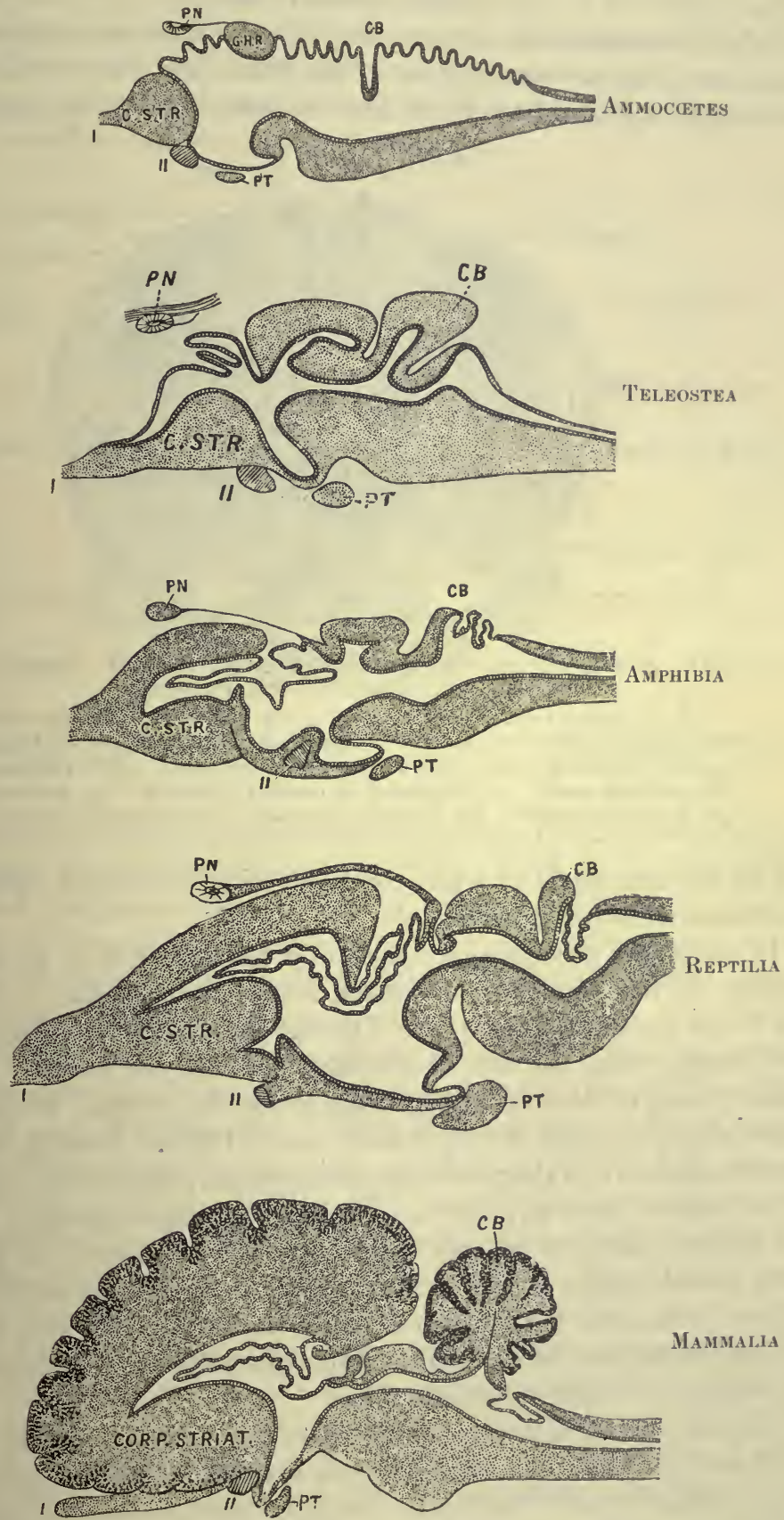

Mammalia

FIG. 180. Diagrammatic view of the brain in different classes of vertebrates.

(GASKELL.)

CB, cerebellum ; PT, pituitary body ; PN, pinea body ; C.STR, corpus striatum ; GHR, right ganglion habenulæ; I, olfactory; II, optic nerves. 


\section{THE HIND-BRAIN}

It will be convenient to trace first the modifications undergone by the axial part of the nervous system in the brain, and then to deal with the new masses of grey matter which have no homologies in the spinal cord, as

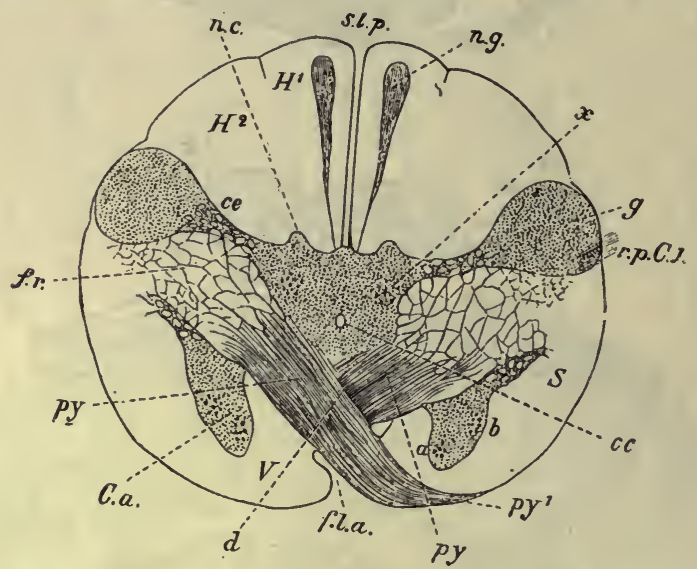

Fig. 181. Section through the lower border of the medulla oblongata, at the pyramidal decussation. (BECHTEREW.)

$f l a$, anterior fissure ; $d$, decussation of the pyramids ; $V$, anterior columns ; $C a$, anterior cornu ; $c c$, central canal ; $S$, lateral columns ; $f r$, formatio reticularis ; ce, neck, and $g$, head of the posterior cornu ; $r p C 1$, posterior root of first cervical nerve ; $n c$, beginning of nucleus cuneatus ; $n g$, nucleus gracilis ; $H^{1}$, funiculus gracilis ; $H^{2}$, funiculus cuneatus ; $s l p$, posterior fissure.

well as the long tracts of white matter serving to connect different levels or different sides of the brain.

In examining successive sections from the spinal cord up through the medulla, the first change which makes its appearance is due to the decussation of the pyramids (Fig. 181). Throughout the spinal cord, fibres have been crossing from one side to the other through the anterior white commissure, many of them belonging to the pyramidal system. But at the lower border of the medulla we see a large mass of fibres crossing between the anterior columns and the postero-lateral columns, at first cutting off the head of the anterior horn and later on breaking this up altogether, so that the only definite collection of grey matter left in this situation is a small part of the lateral column of grey matter known as the lateral nucleus. In this way are formed the big anterior columns of the medulla, which are known as the pyramids, and contain all the fibres that in the cord are represented by the direct and crossed pyramidal tracts.

The next change is due to the ending of the posterior columns (Fig. 182). These are the central ascending branches of dorsal nerve roots, having therefore an origin outside the cord. On their way up the cord they send in collaterals to end in the grey matter of the posterior horn. The main mass terminates in the medulla, just above the pyramidal decussation, in two collections of grey matter-the nucleus gracilis and the nucleus cuneatus- 
which are formed by a great hypertrophy of the grey matter at the root of the posterior horn. The effect of this development in the dorsal region of the medulla is to push the head of the posterior horn outwards. At the same time this mass of gelatinous substance becomes enlarged, so that in section we have three grey masses from within outwards, the nucleus gracilis, the nucleus cuneatus, and the nucleus of Rolando.

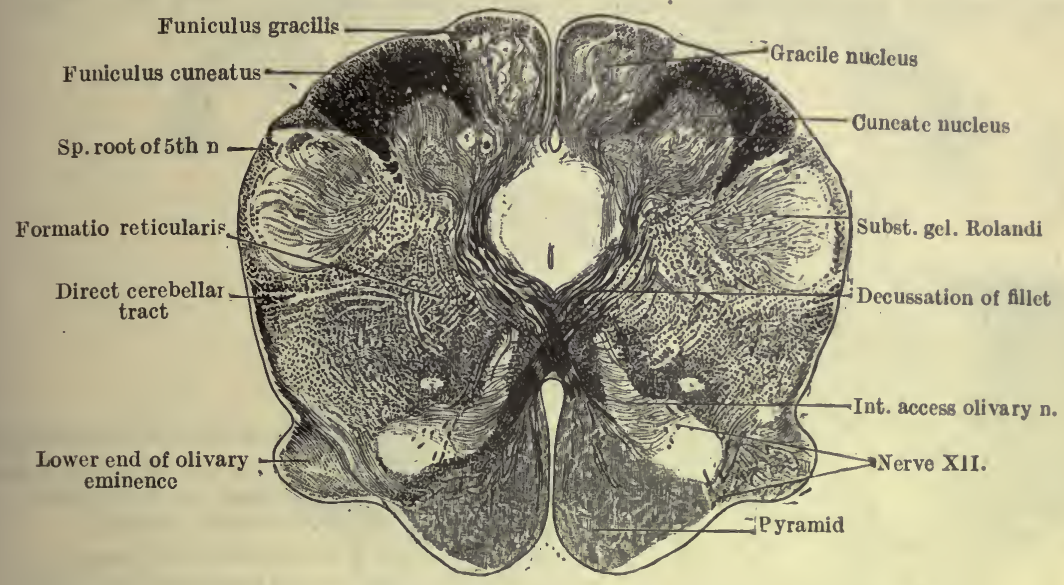

FiG. 182. Transverse section through medulla of fœtus, immediately above pyramidal decussation. (Cunningham.) Stained by Pal-Weigert method.

The fibres of the postero-median column, which are derived chiefly from the lower limb, end in arborisations round the cells of the nucleus gracilis, while those of the postero-external column, or column of Burdach, of which the majority is formed by fibres from the upper limbs, terminate in the grey matter of the nucleus cuneatus. The cells of these two masses of grey matter of course give off axons, which can carry on the impulses brought to them by the fibres of the posterior columns. These axons speedily leave the dorsal aspect of the medulla, bending round, as the arcuate fibres, to the deeper parts of its structure. Thus nothing is left to take the place of the posterior columns on the posterior aspect of the cord. With the disappearance of these columns and the development of the pyramids we get a practical obliteration of the anterior fissure and a displacement of the central canal towards the dorsal surface. A little higher up (Fig. 183) the canal opens out altogether, forming the fourth ventricle, covered on its dorsal surface only by a thin layer of ependyma, a simple epithelium representing all that is left of the dorsal wall of the primitive cerebral vesicle. The appearance of the section is now modified by two structures. In the first place, a new mass of grey matter, consisting of a thin layer shaped like a flask with its orifice directed inwards, is developed in the lateral part of the medulla, between the pyramids in front and the tubercle of Rolando behind. This is the olivary body, and has on its inner and dorsal sides two little grey masses which are the accessory olivary bodies. The other feature is the new 
relay of sensory fibres which start from the dorsal nuclei, the nuclei gracilis and cuneatus. These fibres run outwards and forwards from the nuclei right round the medulla. Some fibres pass into the restiform body of the same side. A larger number, forming the superficial arcuate fibres, pass superficially to the olive to join the restiform body of the opposite side, while others, the deep arcuate fibres, pass deeply to the olives, and crossing in the median raphe turn upwards in the broken mass of grey and white

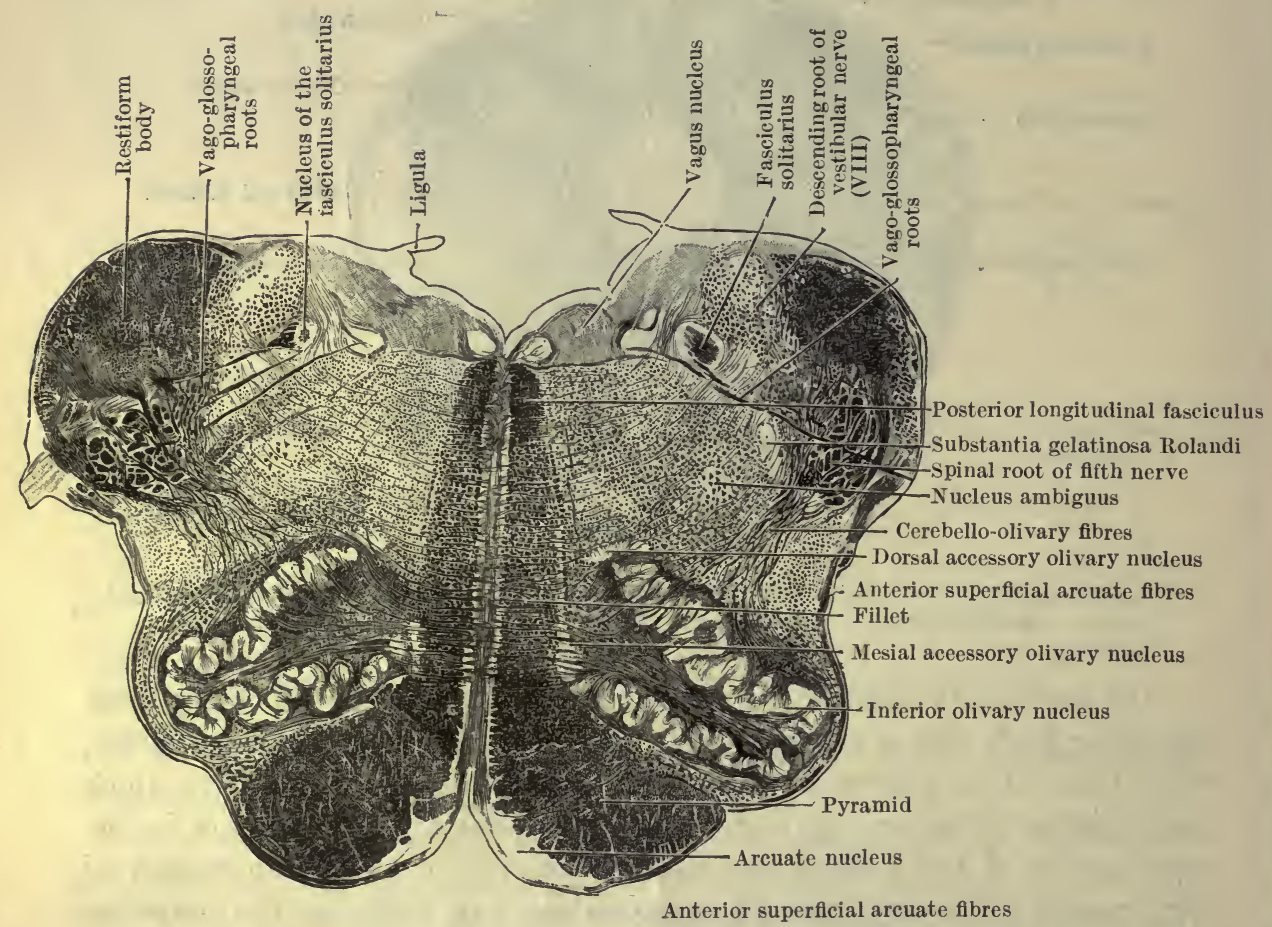

Fia. 183. Transverse section through the middle of the olivary region of the human medulla. (CUNNINGHAM.)

matter which lies between the olives and the superficial grey matter of the fourth ventricle. This decussation, which is known as the 'decussation of the fillet' or the sensory decussation, takes place immediately above the level of the decussation of the pyramids. In its upward course it forms a conspicuous strand of fibres, lying close to the mesial plane and separated from its fellow of the opposite side simply by the median raphe. To this collection of fibres is given the name of the fillet or lemniscus. It is perhaps the most important of the afferent tracts of the brain stem, receiving as it does continuations of the posterior columns of the cord as well as contributions from the various sensory cranial nerves. It may be traced forwards as far as the thalamus and subthalamic region, where its fibres terminate. The region corresponding to the anterior column of the spinal cord is thus invaded in the medulla by two great longitudinal tracts of fibres namely, the pyramids and the tracts of the fillet. The region corre- 
sponding to the anterior basis bundle, i.e. that part of the anterior columns occupied chiefly by intra-spinal fibres, is thus pushed further backwards and finally comes to lie immediately beneath the grey matter of the floor of the fourth ventricle. Immediately dorsally to the fillet is to be seen another

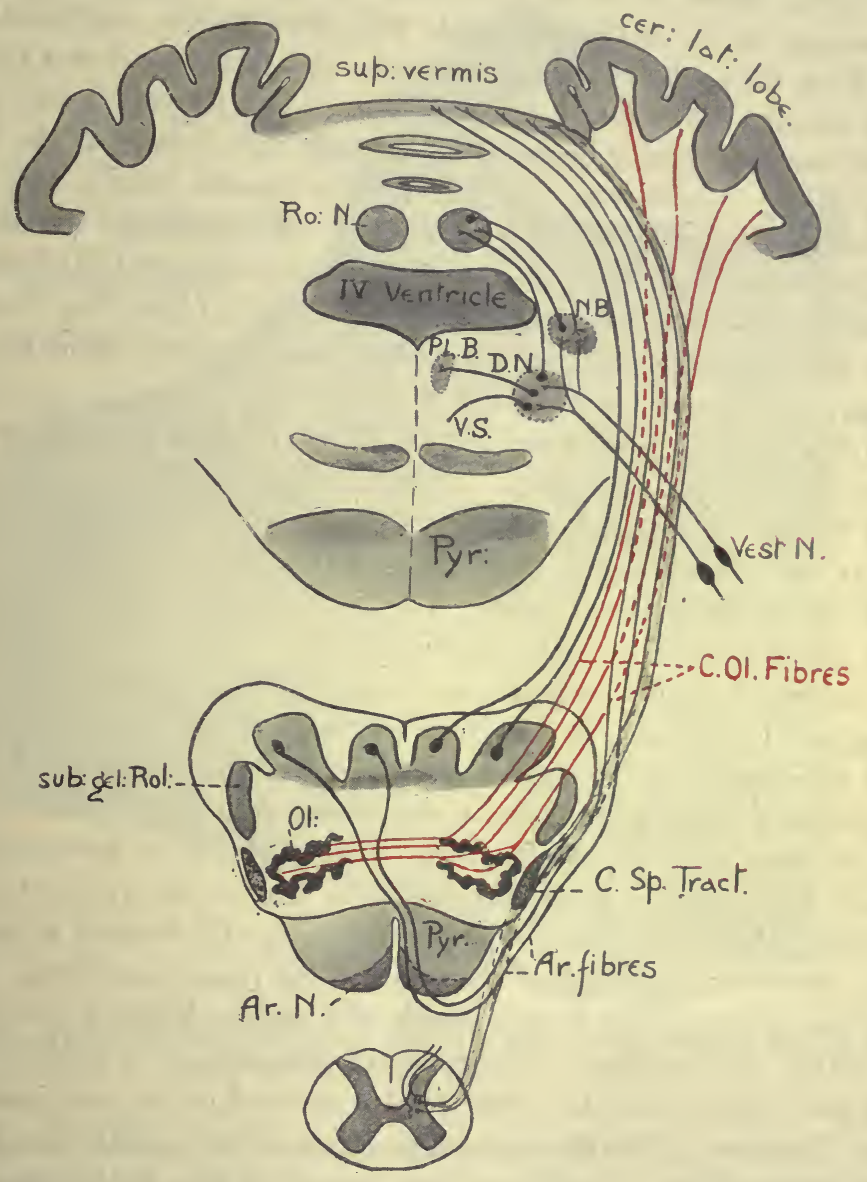

FIG. 181. Diagram to show the sources of the fibres making up the restiform body. Ar.N, arcuate nucleus; Ar fibres, arcuate fibres; Pyr, pyramid; C.Sp. Tract, direct cerebellar tract; C.Ol fibres, cerebello-olivary fibres; PI. B, posterior longitudinal bundle ; DN, nucleus of Deiters ; NB, nucleus of Bechterew ; Ro.N, roof nuclei ; Vest. N, vestibular nerve.

well-marked bundle of longitudinal fibres, known as the posterior longitudinal bundle. These fibres, which serve to connect the nuclei of many of the cranial nerves, can be regarded as analogous to the constituent fibres of the anterior basis bundle in the cord, and can in fact be traced into this part of the anterior columns in the first and second cervical segments of the cord.

The fourth ventricle is covered in by the cerebellum, which is attached to the axial part of the brain by three peduncles, the inferior peduncles or restiform bodies, the lateral peduncles, which form the great mass of transverse fibres known as the pons Varolii, and the superior 
peduncles, which run forward to the posterior corpora quadrigemina. The restiform bodies can be regarded as the direct continuation forwards of the lateral columns of the cord, minus the pyramidal tracts, the chief remaining tract therefore being the posterior or direct cerebellar tract. In the region of the dorsal nuclei, however, it receives accession of fibres from the gracile and cuneate nuclei of the same side and, through the superficial arcuate fibres, from the nuclei of the opposite side, and thus passes as a thick white bundle into the cerebellum. Among these arcuate fibres are also a number derived from the olivary body of the opposite side, known as the cerebelloolivary fibres. On its way it is joined by a smaller bundle, the 'internal restiform body,' which conveys fibres from the vestibular division of the eighth nerve and also serves to connect Deiters' nucleus with the cerebellum. The restiform body is thus made up of the following fibres (Fig. 184) :

(1) The direct or posterior cerebellar tract, derived from the cells of Clarke's column on the same side of the cord.

(2) The posterior superficial arcuate fibres, derived from the gracile and cuneate nuclei of the same side.

(3) The anterior superficial arcuate fibres, from the gracile and cuneate nuclei of the opposite side.

(4) The cerebello-olivary fibres.

(5) The vestibulo-cerebellar fibres.

A section through the pons shows the fourth ventricle widely dilated, with a floor formed of grey matter as in the medulla. The chief difference in the appearance of the section is due to the great masses of transverse fibres which pass into the pons by the lateral peduncles of the cerebellum, cross by the median raphe, and turn either upwards or downwards on the opposite side or end in connection with the nerve-cells which are scattered throughout the white fibres. The pyramids can still be seen as thick longitudinal bundles on each side in the midst of the transverse fibres. They are considerably larger than in the medulla and become larger as we trace them up towards the mid-brain, owing to the presence of a number of fibres which are derived from the cortex cerebri and end in the grey matter of the pons. The tract of the fillet lies on each side of the middle line dorsally to the transverse fibres. A little to the outside of the fillet is seen a special mass of grey matter, known as the superior olive. The nervous mass lying behind the transverse fibres of the pons, between them and the grey matter of the floor of the fourth ventricle, is known as the formatio reticularis. It is divided into a lateral and mesial part by the fibres of the hypoglossal nerve. In the lateral portions there is a considerable quantity of grey matter, which can be regarded as continuous with the grey matter of the lateral horns of the cord. The 'lateral nucleus' is simply a condensed part of this grey matter, lying between the olive and the gelatinous substance of Rolando. The mesial part of the formatio reticularis is almost free of nervecells. The reticular appearance of this part of the pons is due to the intersection of fibres which run longitudinally and transversely. The transverse fibres are a continuation of the deep arcuate fibres. The longitudinal fibres 
in the outer part of the formatio reticularis are the representative of the lateral columns of the cord after the removal of the direct cerebellar and the crossed pyramidal tracts. They include therefore the antero-lateral ascending tract (tract of Gowers) and a number of other fibres corresponding to the

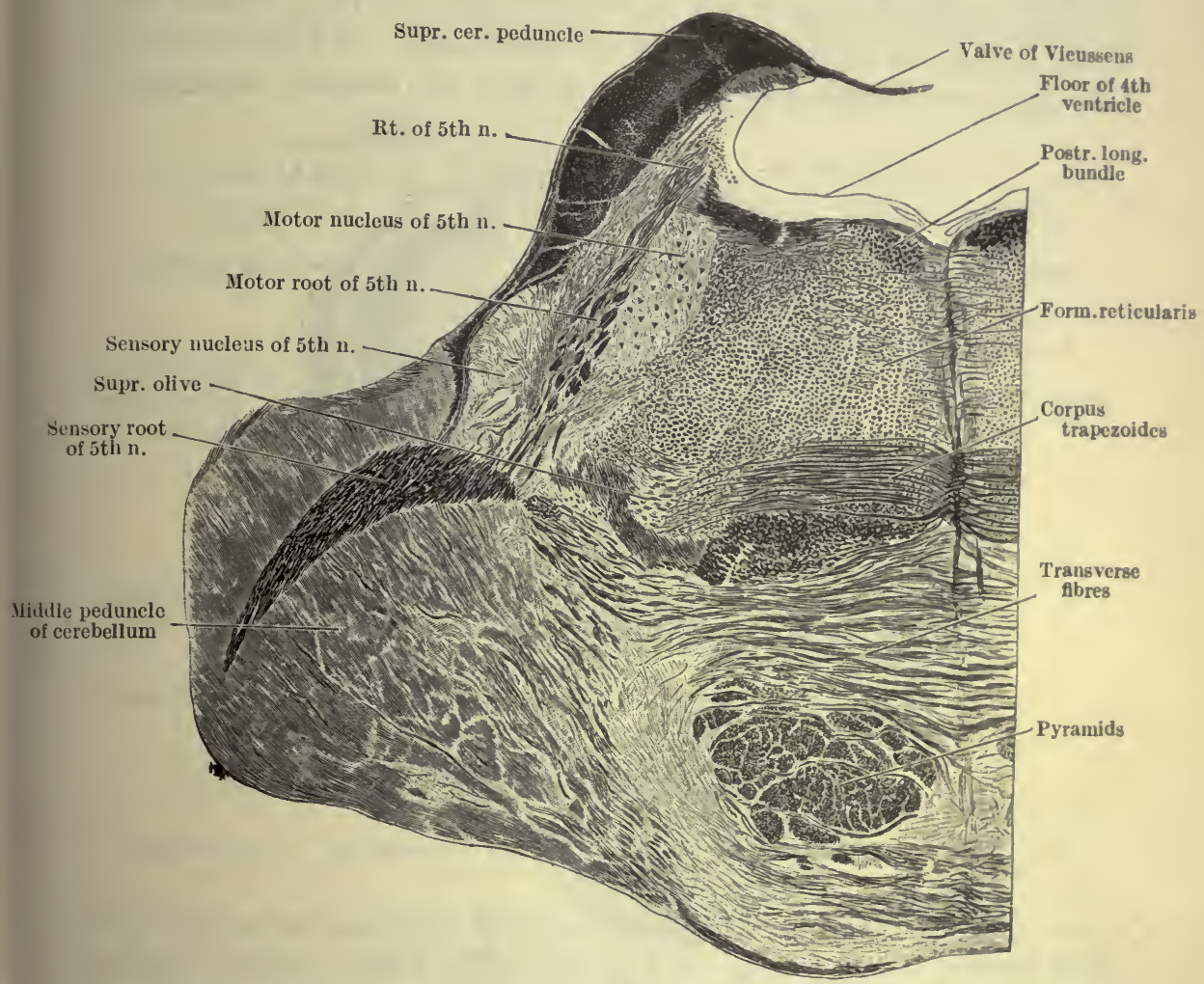

FIG. 185. Transverse section through middle of pons Varolii of orang on level of nuclei of fifth nerve. (CunNingham.)

lateral basis bundle in the cord. In the mesial part of the formatio reticularis the longitudinal tracts are the tract of the fillet and the posterior longitudinal bundle on each side of the middle line. In the upper part of the pons Varolii a well-marked collection of transverse fibres are to be seen lying dorsally to the tracts of the fillet. This collection is called the corpus trapezoides and is made up of ascending fibres derived from the nuclei of the cochlear nerve, the auditory part of the eighth nerve.

A little further forward a section will escape the cerebellum altogether, being bounded ventrally by the upper or anterior part.of the pons and dorsally by a thin mass of grey matter, the valve of Vieussens (Fig. 186). On each side of the valve of Vieussens may be seen the superior peduncles of the cerebellum. As these peduncles are traced upwards they sink gradually deeper into the pons until they lie on the outer side of the tegmental region or formatio reticularis. They are made up of fibres which run from the 
dentate nucleus of grey matter in the cerebellum to the mid-brain, where they decussate below the Sylvian iter and end in the red nucleus and in the thalamus of the opposite side. They also contain the continuation upwards of the antero-lateral ascending tract, which, passing up in the superior peduncles, bends dorsally round the fourth nerve and then, turning backwards, ends in the superior vermis of the cerebellum. In a section through the upper part of the pons the division into the formatio reticularis or

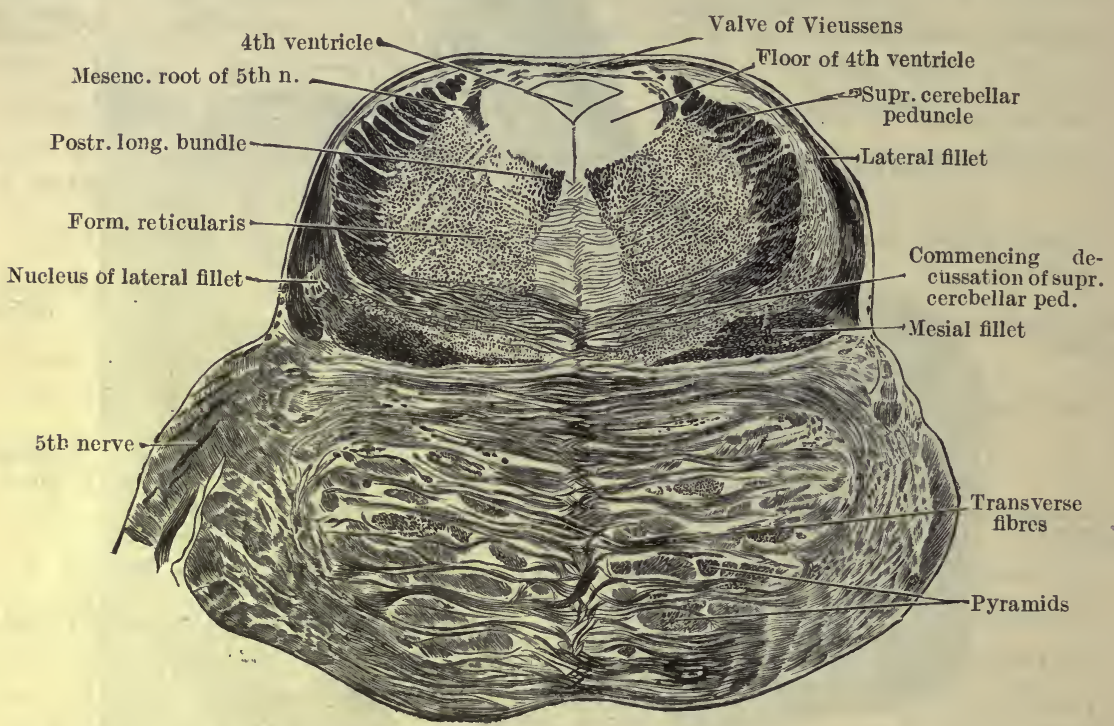

Fig. 186. Section across upper part of pons Varolii of the orang. (Cunningham.)

tegmentum and the part made up of transverse and longitudinal fibres, the pedal portion, is well marked ( $v$. Fig. 186). The fourth ventricle has now become constricted to a narrow canal triangular in section and closed above by the valve of Vieussens. It is surrounded, especially on its ventral side, by grey matter containing the cells of origin of the fourth nerve. In the tegmental portion we may distinguish on each side the superior cerebellar peduncle. Outside the longitudinal fibres of this peduncle are a number of transverse fibres derived from the corpus trapezoides seen in the previous section. To these fibres is given the name of the 'lateral fillet.' 'They are on their way to end in the roof of the mid-brain in the posterior corpora quadrigemina. The posterior longitudinal bundle lies near the middle line, immediately under the grey matter of the floor of the fourth ventricle, while the longitudinal fibres of the fillet, now called the mesial fillet, form a distinct mass in the ventral portion of the formatio reticularis. The pedal portion contains the longitudinal fibres of the pyramids, now much increased in amount, cut up into bundles by transverse fibres derived from the middle peduncles of the cerébellum.

The cerebellum, which covers in the fore part of the fourth ventricle, will have to be described in greater detail later on. At present it will suffice 
to say that it consists of a middle and two lateral lobes. The surface of the middle lobe turned towards the fourth ventricle is known as the inferior vermis, the dorsal surface forming the superior vermis. Each vermis and each lateral lobe is subdivided into a number of smaller lobes. The intimate structure of all parts of the cerebellum is, however, very uniform. It consists of a mass of white matter internally, covered by a layer of grey matter, the

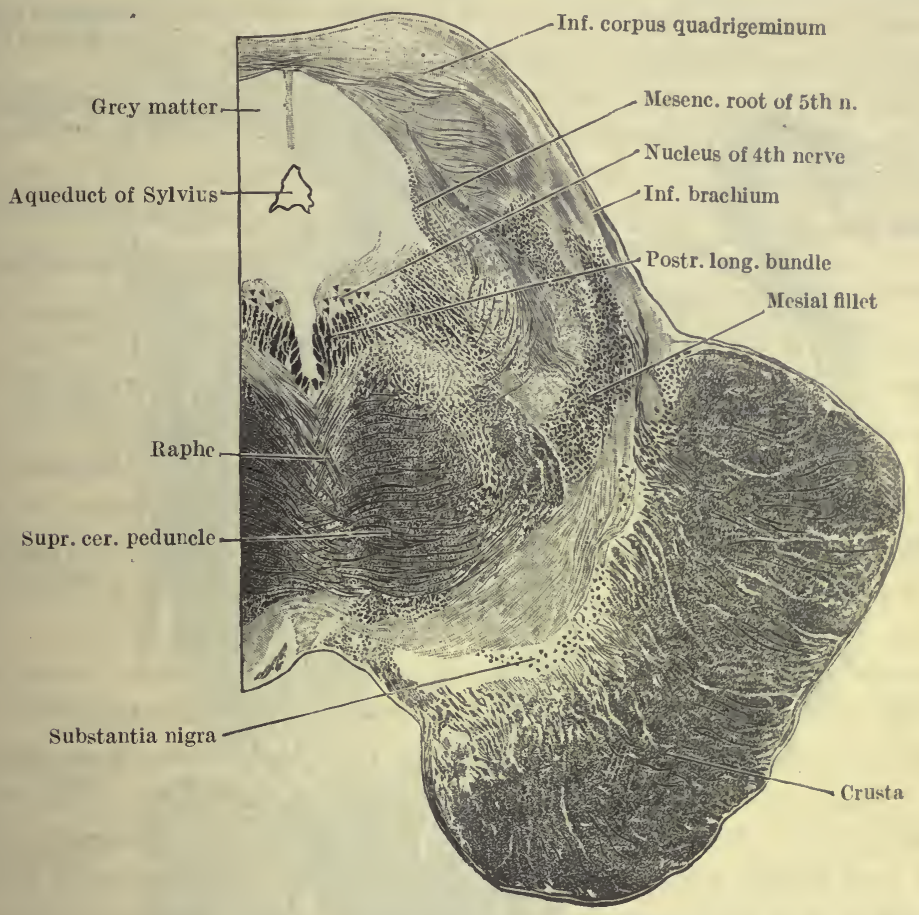

FIa. 187. Transverse section through human mid-brain, on level of the inferior corpora quadrigemina. (CUNNINGHAM.)

extent of grey matter being largely increased by the formation of numerous parallel and more or less curved grooves or sulci which give the whole organ a laminate appearance. In the mass of white matter, which forms the core of each lateral hemisphere, is an isolated nucleus of grey matter known as the corpus dentatum. In the white matter of the middle lobe is another mass of grey matter known as the roof nucleus or nucleus fastigii. Between the nucleus fastigii and the nucleus dentatum are two other nuclei, the nucleus globosus and the nucleus cmboliformis.

\section{THE MID-BRAIN}

A little further forward the fourth ventricle comes to an end, and the section passes through the mid-brain (Fig. 187), the cavity of the second cerebral vesicle being represented by the narrow Sylvian aqueduct, bounded dorsally by the corpora quadrigemina and ventrally by the crura, the stalks 
of the brain. The crura are divided by an irregular mass of grey matter, the substantia nigra, into two parts. The ventral portion is known as the pes or crusta. It is composed almost entirely of longitudinal white fibres, among which is the continuation forwards of the pyramids of the medulla. The pyramids, however, form only about two-fifths of the total mass of white fibres, the rest consisting of fibres which run from the different parts of the

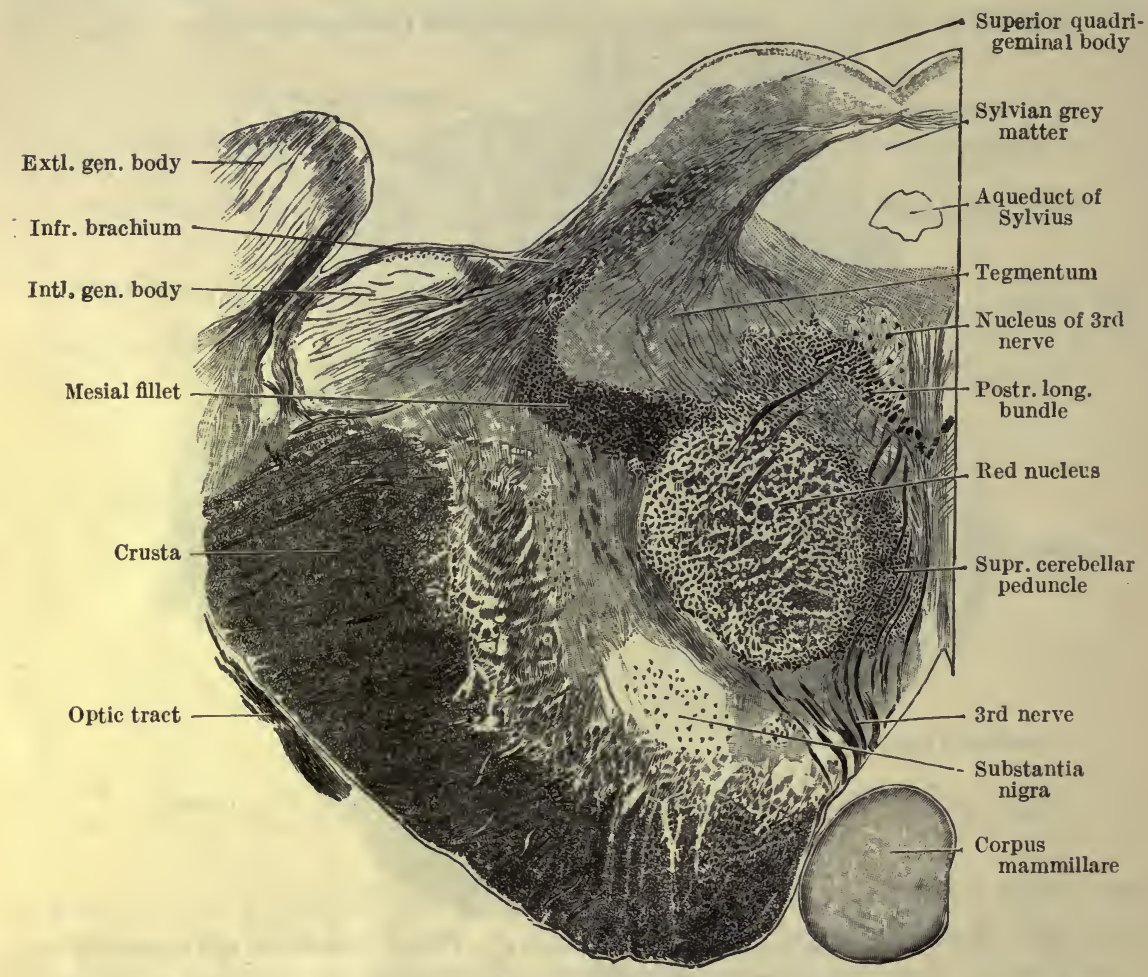

Fia. 188. Transverse section through human mid-brain at the level of the superior corpus quadrigeminum. (CUNNINGHAM.).

cerebral cortex, especially from the frontal and temporal lobes, to end in the formatio reticularis of the pons, probably in relation with the grey matter in this situation and with the endings of the transverse fibres derived from the cerebellum and forming the middle peduncles of the cerebellum. The dorsal part, the tegmentum, is a direct prolongation forwards of the formatio reticularis of the medulla and pons, and like this contains much scattered grey matter. On a level with the inferior corpora quadrigemina a number of decussating fibres are to be seen in the tegmentum, which are derived from the superior cerebellar peduncles. Their decussation is complete at the level of the upper border of the inferior corpora quadrigemina. Here each peduncle turns upwards, and a large proportion of its fibres end in the red nucleus (Fig. 188), a mass of grey matter forming a conspicuous feature of sections through the anterior part of the mid-brain. Many of the fibres pass round the red nucleus, forming a sort of capsule over it, to the ventral 
part of the optic thalamus, in which they probably end. It is possible that a certain proportion pass through the optic thalamus and run straight to the cerebral cortex of the Rolandic area. The lateral fillet has disappeared from the region of the tegmentum and passed into the inferior corpora quadrigemina. The mesial fillet forms a flat band lying to the outer side of the red nucleus and comes into close relation with a ganglion of the fore-brain, known as the internal geniculate body. The roof of the mid-brain is formed by the corpora quadrigemina. The inferior corpora quadrigemina are composed of central grey matter encapsulated by white matter, derived chiefly from the lateral fillet. The superior corpora quadrigemina are composed of several layers of grey matter traversed by nerve fibres, derived partly from the fillet, partly from the optic tract, and partly from the occipital lobe of the cerebral hemisphere.

\section{THE FORE-BRAIN}

In the fore-brain the most important feature is the optic thalami, the two head ganglionic masses of the brain stem (Fig. 189). In this region the central neural canal, which in the mid-brain forms the Sylvian iter, widens out to the third ventricle, in the lateral walls of which are developed the two optic thalami. It is a narrow cleft, rapidly deepening in depth from behind forwards. As we trace sections forwards we see that the two crura cerebri diverge from one another. The floor of the third ventricle is thus left thin. It is formed from behind forwards by a thin layer of grey matter with numerous vessels, the locus perforatus posticus, two small eminences, the corpora mammillaria, and in front of these another lamina of grey matter known as the tuber cinereum. In front of the tuber cinereum is the infundibulum, which leads to the posterior lobe of the pituitary body. In front of the infundibulum the optic chiasma is closely attached to the lowest part of the anterior wall of the ventricle. The front wall is formed by a thin layer of nervous matter, the lamina cinerea, at the upper border of which, projecting slightly into the ventricle, is a strand of white fibres connecting the anterior parts of the two optic thalami and known as the anterior commissure. The roof of the third ventricle is formed entirely of epithelium, the ependyma, along the upper surface of which is the layer of pia mater, the velum interpositum. The roof is invaginated into the cavity by two delicate vascular fringes, the choroid plexuses. At the back part of the roof is attached the stalk of the pineal body, and behind this stalk, between the anterior parts of the anterior corpora quadrigemina, is a small space known as the trigonum habenula, which contains a well-marked collection of nerve-cells known as the ganglion habenulce. The lateral walls are formed entirely by the optic thalami. The upper surface of the optic thalamus looks into the lateral ventricle of the cerebral hemispheres, from which it is 'separated by the velum interpositum and by the ependyma, the epithelium completing the inferior wall of the lateral ventricle in this region. It consists of three masses of grey matter-the anterior nucleus, the lateral nucleus (the largest of the three), and the mesial nucleus. Its outer surface is in contact with the layer of nerve fibres formed by the crusta of each crus cerebri as it diverges 
from its fellow to pass up into the cerebral hemispheres. Into this layer, 'the internal capsule,' fibres proceed from all parts of the thalamus to pass to the cerebral cortex. The anterior extremity of the thalamus, known as the anterior tubercle, forms a marked projection into the lateral ventricle. In

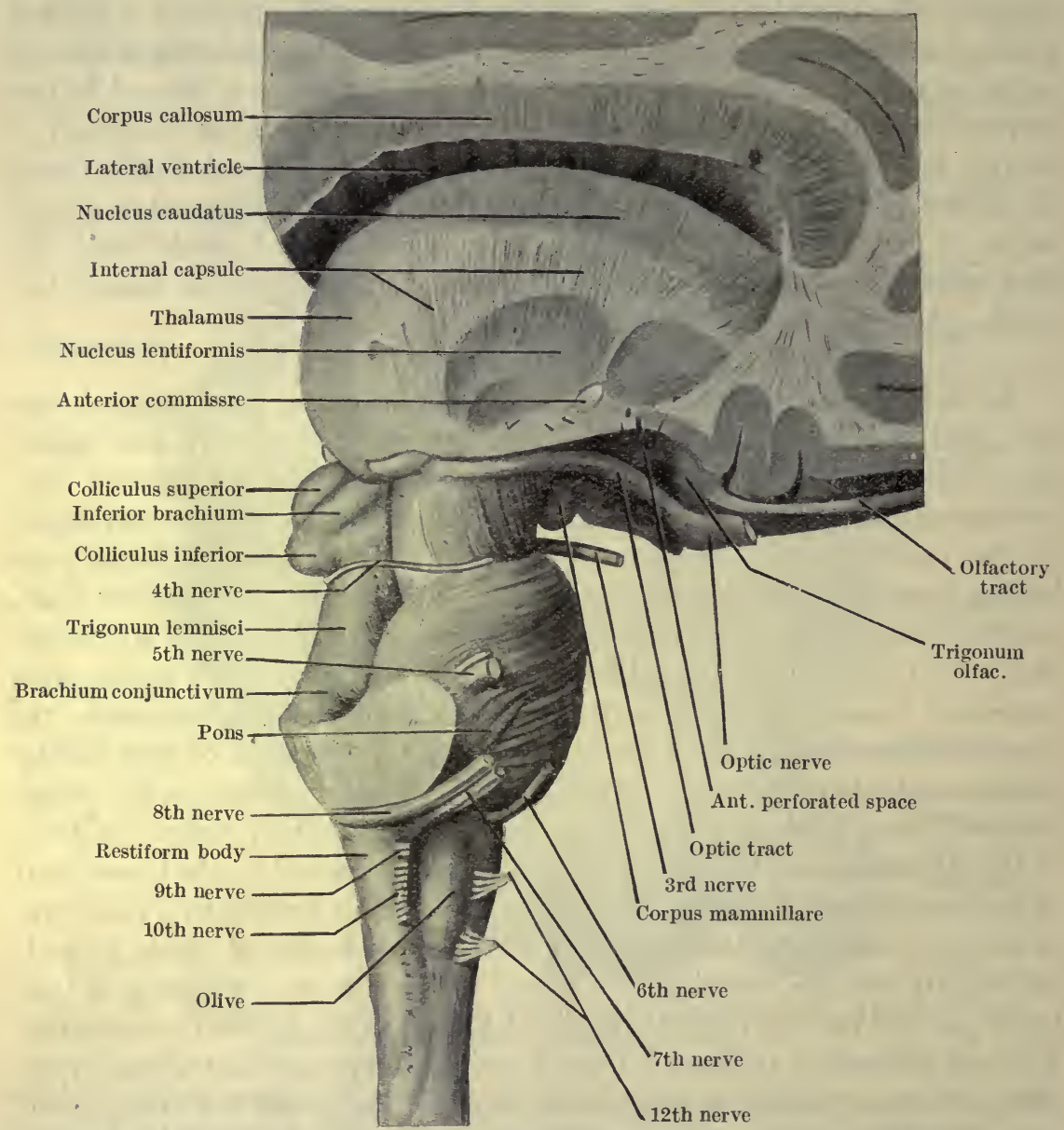

Fig. 189. Right lateral aspect of brain stem, with a part of the cerebrum. (J. Symingtox.)

front of this, the foramen of Monro leads from the third ventricle into the lateral ventricle. This foramen is bounded anteriorly by a strand of fibres, known as the 'anterior pillar of the fornix,' which lies just behind the anterior commissure and forms a conspicuous feature in the anterior part of the lateral wall of the third ventricle. It passes in the wall down to the corpus mammillare. From the corpus mammillare a well-marked bundle of fibres passes up into the optic thalamus to end round the large cells in the anterior nucleus of the thalamus. The posterior extremity of the thalamus forms a definite prominence, the pulvinar. To the outer and back part of the pulvinar two bodies are developed, known as the geniculate 
bodies. These may be regarded as special outgrowths of the grey matter of the optic thalamus, one of which, the external geniculate body, is in close connection with the fibres from the optic tracts, while the other, the internal geniculate body, receives fibres from the lateral fillet ultimately derived from the organ of hearing. In a section through the fore part of the mid-brain (Fig. 190) these two bodies may be seen lying to the outer side of the anterior corpora quadrigemina, so that the fore-brain, to a certain extent, enfolds the anterior part of the mid-brain. Below the thalamus at its back part is the prolongation forwards of the tegmentum of the crus. This is often spoken of as the subthalamic region. The red nucleus is a conspicuous object in

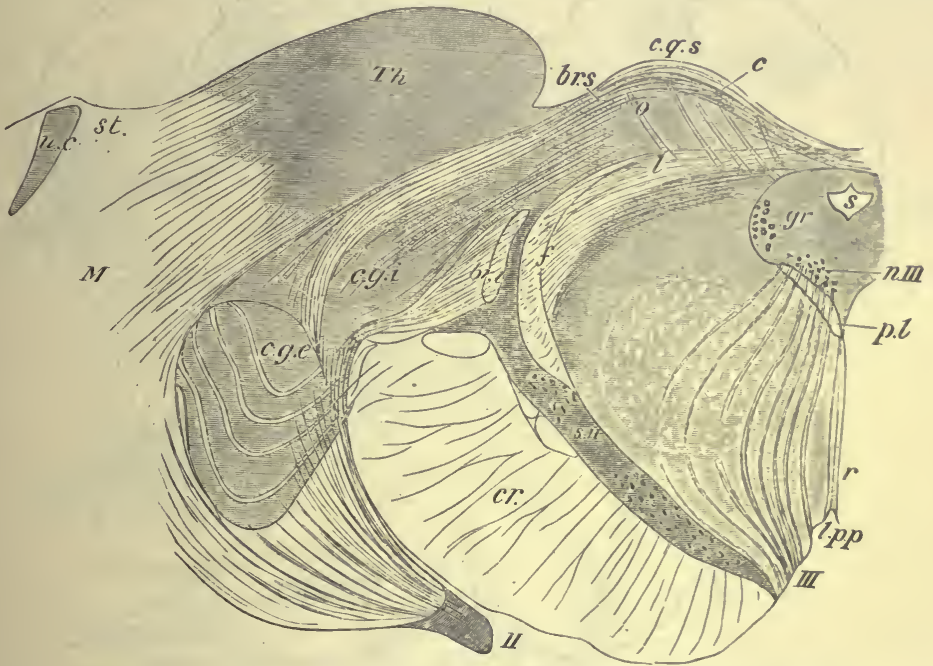

FIG. 190. Transverse section through upper part of mid-brain.

$T h$, thalamus; brs, brachium superior; cqs, anterior (or superior) corpus quadrigeminum ; cgi, cge, internal and external geniculate bodies; $f$, fillet ; $s$, aqueduct; $p l$, posterior longitudinal bundle; $r$, raphe; $I I I$, third nerve; $n I I I$, its nucleus ; lpp, posterior perforated space; $s n$, substantia nigra ; $c r$, crusta; $I I$, optic tract; $M$, medullary centre of the hemisphere; $n c$, nucleus caudatus; $s t$, stria terminalis.

sections through the back part of this region, but gradually diminishes as we proceed forwards, and disappears before the level of the corpora mammillaria is reached. The mesial fillet, which in the mid-brain lies on the lateral and dorsal aspect of the red nucleus, is prolonged upwards together with fibres from the superior cerebellar peduncle into the ventral part of the thalamus, where probably all of the fibres end in connection with the thalamic cells. The substantia nigra gradually disappears. Before it has disappeared we may see on its outer side a special collection of grey matter called the nucleus of Luys or the corpus subthalamicum. In addition to the anterior and posterior commissures already described as connecting the two optic thalami at the front and back of the third ventricle, the two sides are connected about the middle of the cavity by the middle or soft commissure. The optic thalamus is often described together with the corpus striatum as forming the basal ganglia. The corpus striatum is, however, genetically, and 
probably functionally, part of the cerebral hemispheres, and its connections will therefore be best dealt with when describing the latter bodies.

\section{THE AXIAL GREY MATTER}

In the spinal cord we could distinguish between the anterior grey matter giving origin to the motor nerves, the posterior grey matter serving as an end station for a number of the sensory posterior root fibres, and a lateral horn,

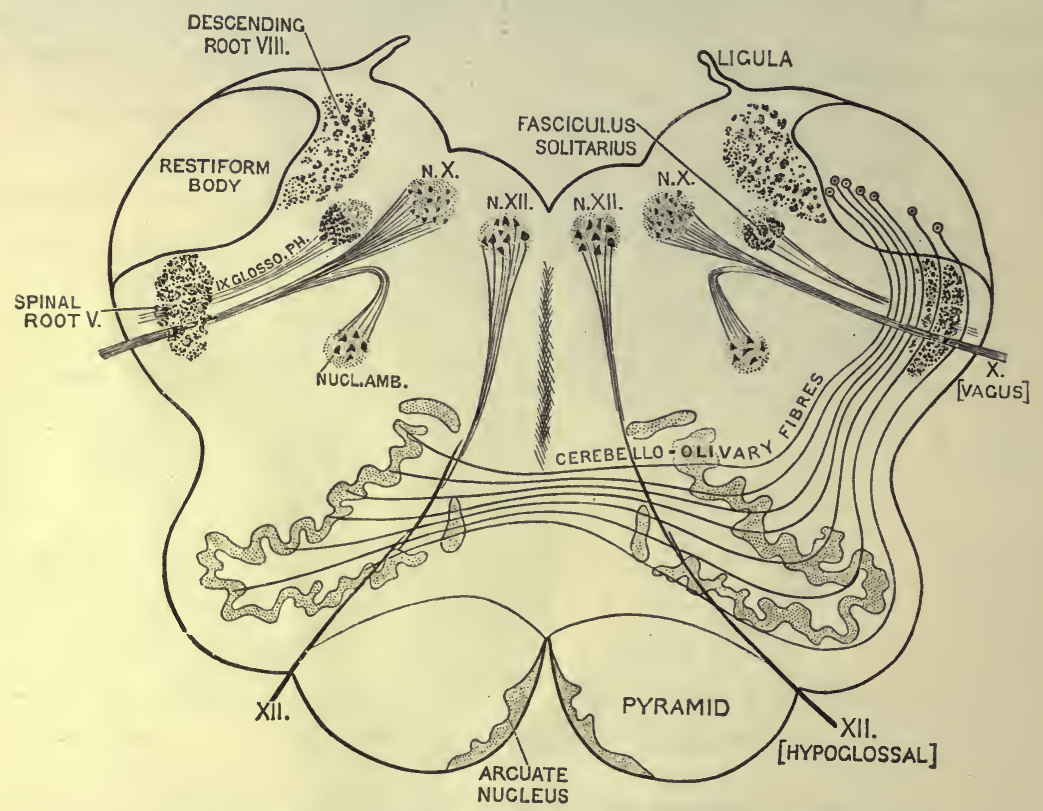

FiG. 191. Cross-section of medulla showing nuclei of nerves $\mathrm{x}$ and $\mathrm{xII}$.

(CUNNINGHAM.)

less well marked, probably giving origin to the visceral system of nerves. As the central canal widens out to form the fourth ventricle, the relative position of these various parts becomes altered, the anterior grey matter being now nearest the median line, while the posterior grey matter lies more laterally. Part of the lateral grey matter seems to lie deeper than the rest, from which it is separated by the tangle of fibres and cells known as the formatio reticularis. All the cranial nerves from the third to the twelfth arise or end in the axial grey matter, or in close proximity to it. So great, however, is the complexity of this part of the nervous system, and so involved are the genetic relations of the various nerves, that it is difficult or impossible in many cases to state definitely the spinal analogies of these nerves.

The cranial nuclei (of origin or termination) may be roughly classed as follows :

(1) Motor Somatic Nuclei. These consist of an almost continuous column of multipolar cells, lying close to the middle line on each side in the floor of 
the fourth ventricle, the Sylvian iter, and the back part of the third ventricle. From below upwards these groups of cells give origin to the fibres of :

(a) The hypoglossal nerve.

(b) The sixth nerve.

(c) The fourth nerve.

(d) The third or oculo-motor nerve.

(2) Splanchnic Sensory Nuclei. Immediately outside the column of motor cells is a column of grey matter which receives the terminations of

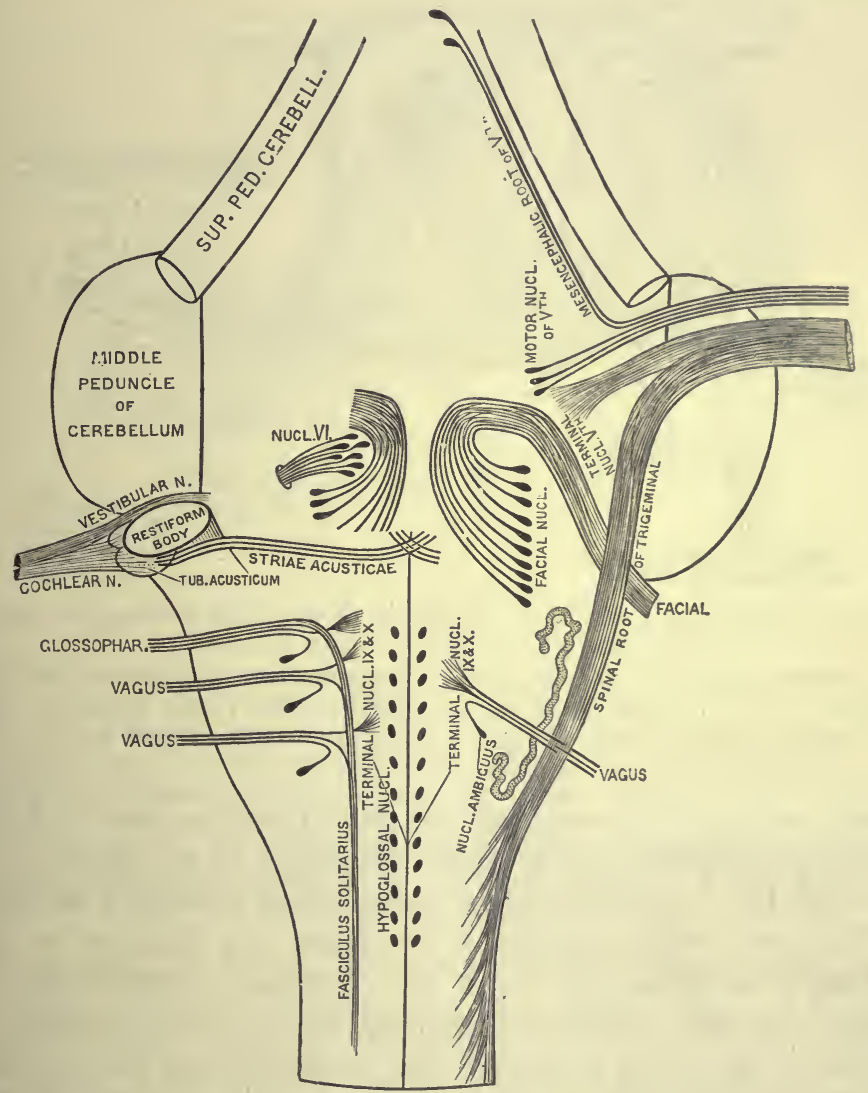

FIG. 192. Diagram showing the brain connections of the vagus, glosso-pharyngeal auditory, facial, abducent, and trigeminal nerves. (CUNNINGHAM after OBERSTEINER.)

the afferent fibres belonging to the ninth, tenth, and eleventh nerves, and is sometimes called the vago-glossopharyngeal-accessory nucleus. This grey matter of course does not give rise to the fibres of these nerves, which, like other sensory nerves, are axons of ganglion-cells lying outside the central nervous system.

(3) Splanchnic Motor Nuclei. These lie more deeply at some distance from the middle line, and include the nucleus ambiguus for the efferent fibres of the vago-glossopharyngeal, the nucleus of the seventh or facial nerve 
(originally splanchnic or branchial, now typically somatic), and the motor nucleus of the fifth nerve with its prolongation into the mid-brain.

(4) Sensory Somatic Nuclei. The chief representative of this group is the great sensory root of the fifth nerve. The fibres of this nerve arise from the Gasserian ganglion, pierce the fibres of the pons Varolii, and run to the dorso-lateral part of the pons, where they divide into ascending and descending fibres. These fibres form a cap to the substantia gelatinosa, the descending branches, which are longer, being conspicuous in sections of

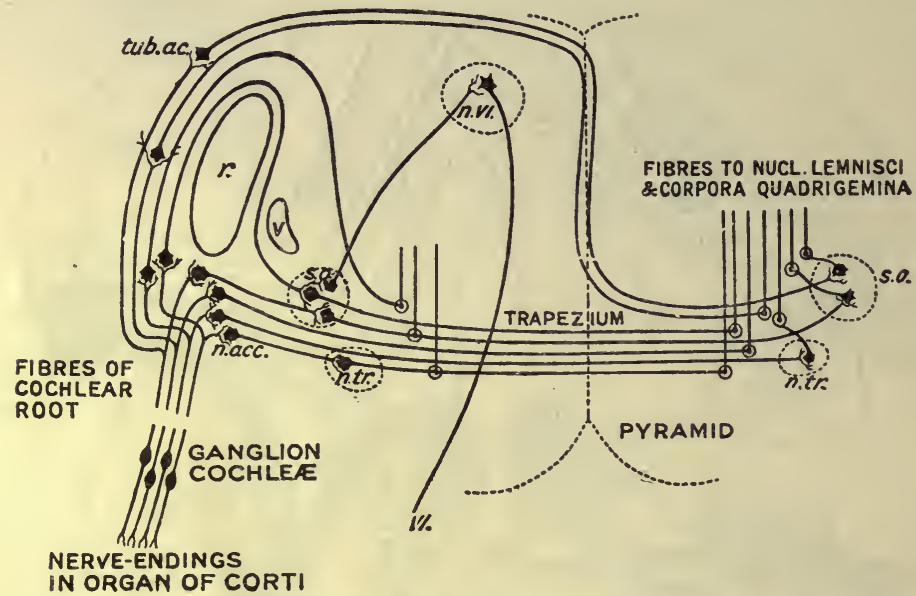

Fia. 193. Plan of the course and connections of the fibres forming the cochlear root of the auditory nerve. (SCḦ̈ FER.)

$r$ restiform body; $V$, descending root of the fifth nerve; tub.ac, tuberculum acusticum; n.acc, accessory nucleus; s.o, superior olive; n.tr, nucleus of trapezium ; $n . V I$, nucleus of sixth nerve; $V I$, issuing root-fibre of sixth nerve.

the medulla as low down as the first or second cervical nerve. This nerve gives common sensation to practically the whole of the head.

It is doubtful in what group we should place the fibres of the eighth nerve. This nerve really consists of two parts very different in function, the cochlear or auditory nerve, and the vestibular or labyrinthine nerve. The fibres of each are derived from ganglion-cells in the internal ear, pass to the medulla at its widest part, and then, dividing into two, terminate in masses of grey matter situated at the extreme lateral part of the floor of the fourth ventricle.

The branches of the cochlear nerve (Fig. 193) make connection with two collections of cells, the dorsal nucleus, apparently embedded in the fibres of the root itself, and the accessory nucleus, a little triangular mass of grey matter situated in the angle between the cochlear and vestibular nerves. From these nuclei fibres are given off which take two courses. Some, following the previous course of the cochlear nerve, pass across the surface of the fourth ventricle as the strice medullares or strice acousticoe, and then bending inwards pass into the tegmentum of the opposite side. Others pass deeply and form a mass of transverse fibres in the ventral part of the tegmentum, the 
corpus trapezoides or trapezium. After making connections with the superior olivary body and a special nucleus, they join the superficial set of fibres, and run up in the tegmentum to the inferior corpora quadrigemina, forming the lateral fillet.

The vestibular nerve (Fig. 194) also has two nuclei of termination, the median nucleus with small cells, and the lateral or Deiters' nucleus with large cells. Some fibres pass also to the nucleus of Bechterew, which is in close relation with the roof nuclei of the cerebellum. The descending fibres end

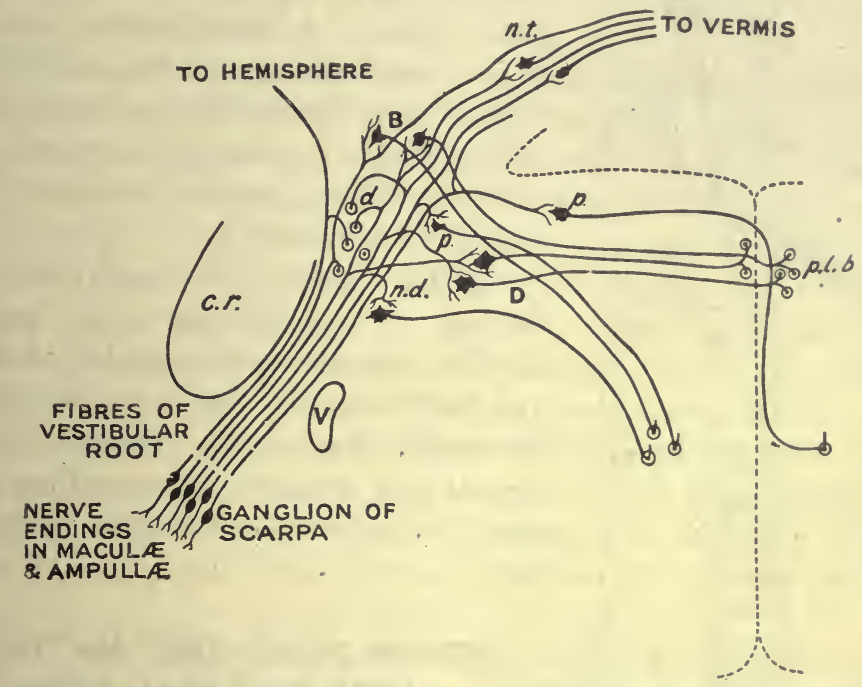

FIG. 194. Plan of the course and connections of the fibres forming the vestibular root ${ }^{-1}$ of the auditory nerve. ( $\mathrm{SCH} \ddot{\mathrm{A} F E R}$.)

$r$, restiform body ; $\mathrm{v}$, descending root of fifth nerve ; $p$, cells of principal nucleus of vestibular root; $d$, fibres of descending vestibular root ; $n d$, a cell of the descending vestibular nucleus; D, cells of nucleus of Deiters; B, cells of nucleus of Bechterew; $n t$, cells of nucleus tecti (fastigii) of the cerebellum; plb, fibres of posterior longitudinal bundle. No attempt has been made in this diagram to represent the actual positions of the several nuclei. Thus a large part of Deiters' nucleus lies dorsal to and in the immediate vicinity of the restiform body.

chiefly in the median nucleus, while the ascending fibres end in Deiters' nucleus. From the latter a distinct band of fibres passes up to the cerebellum, forming the median division of the restiform body, while other fibres run across to the tegmentum of the opposite side, where they take part in the formation of the posterior longitudinal bundle.

In a section through the fourth ventricle through the middle of the pons, a group of large cells is seen in the position occupied by the nucleus of the hypoglossal below. These cells give rise to the fibres of the sixth nerve. Another group is seen lying laterally and more deeply, evidently belonging to the lateral horn system. This is the nucleus of the seventh or facial nerve, the fibres of which pass dorsally and anteriorly, looping round the sixth nerve-nucleus, before issuing as the root of the seventh nerve.

In the upper part of the pons we find the fifth nerve (Fig. 195) with its two roots. The fibres of the senisory root derived from the cells of the 
Gasserian ganglion bifurcate. The upper divisions, which are short, end in a mass of grey matter at the lateral part of the formatio reticularis, the so-called sensory root, while the descending divisions form a long strand of white fibres passing down as far as the second cervical nerve and lying over the substantia gelatinosa of Rolando, around the small cells of which the

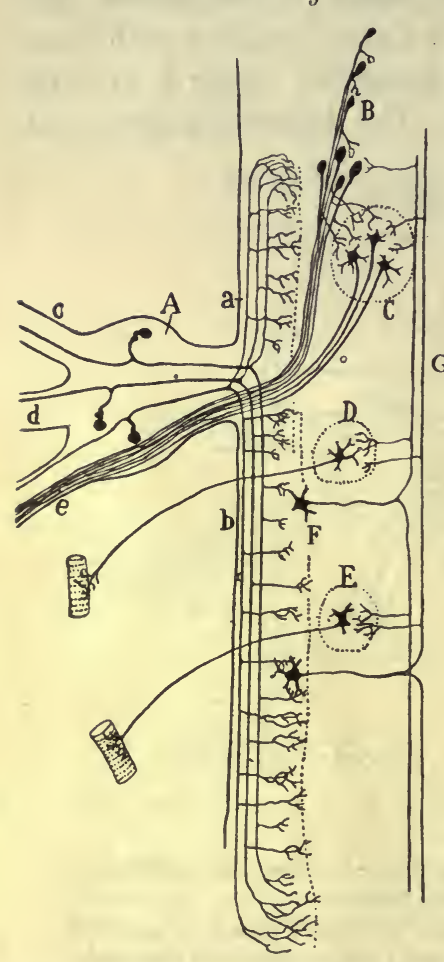

FIG. 195. Diagram showing central connections of fifth nerve. (CAJAL.)

A, Gasserian ganglion; B, accessory motor nucleus; c, main motor nucleus; D, facial nucleus; $\mathrm{E}$, nucleus of hypoglossal; F, sensory nucleus of fifth nerve; $G$, cerebral tract (fillet) of fifth nerve. fibres finally terminate. The motor fibres arise partly from the motor nucleus, a mass of cells lying internally to the sensory nucleus, and belonging probably to the lateral horn system. A large number are derived from a long column of cells, which stretches forward from the nucleus as far as the level of the anterior corpora quadrigemina. These fibres are known as the descending motor root of the fifth nerve.

In the region of the mid-brain, besides the root of the fifth nerve just mentioned, we find only the motor nuclei of the third and fourth nerves, which are situated near the median line in the ventral part of the central grey matter, corresponding in situation to the sixth and twelfth nerves lower down.

\section{INTERMEDIATE GREY MATTER OF THE CEREBRAL AXIS}

The masses of grey matter which are found throughout this region may be regarded as extra shunting stations (or association centres for various systems of nuclei and conducting paths), which have arisen in consequence of the great complexity of reaction required of the nerve mechanisms in connection with the organs of special sense. We must confine ourselves here to little more than the enumeration of the chief masses, though we shall have occasion to refer to some in more detail when dealing with the co-ordinating mechanisms of the cerebral axis. From below upwards we may enumerate the following grey masses :

In the medulla is the large olivary body, with the accessory olive lying on its inner side. Each olive sends fibres across the middle line to the opposite cerebellar hemisphere, and must be regarded as connected with this body in its functions, since atrophy or removal of one side of the cerebellum is followed by atrophy of the opposite olive.

In the pons we find a similar but smaller body, the superior olive, in the neighbourhood of the nucleus of the seventh nerve. The superior olive is 
closely connected with the co-ordination of visual and auditory impressions with the eye movements.

Deiters' nucleus, which occurs in the same region, although described as one of the nuclei of the eighth nerve, might equally well be included in this class owing to its manifold connections with both afferent and efferent mechanisms.

In close connection with Deiters' nucleus are a number of grey masses in the cerebellum, the roof nucle $i$ in the roof of the fourth ventricle.

In the mid-brain we must mention the superficial grey matter covering the corpora quadrigemina.

On the ventral side of the Sylvian iter are the various masses of grey matter in the crura, the red nucleus, a large mass in the tegmentum just below the oculo-motor nucleus, and the substantia nigra, which divides each crus into two parts, the dorsal tegmentum and the ventral pes or crusta.

Finally at the fore part of the cerebral axis we come to the great ganglionic mass already described, the optic thalamus and the geniculate bodies. The geniculate bodies may be regarded as outgrowths of the optic thalamus which have developed in connection with the terminations of the auditory and the optic nerve fibres. The optic thalamus is connected by fibres with all parts of the cortex and represents the termination of the whole tegmental system, so that in many ways it may be regarded as a sort of foreman of the central nervous system, controlling the activities of the lower level centres and bringing all parts of this system in relation with the supreme cerebral cortex.

\section{THE CHIEF LONG PATHS IN THE BRAIN STEM}

In dealing with the spinal cord we were able to treat it as one organ, very largely on account of the uniformity of the afferent and efferent mechanisms connected with its various segments. Every afferent impulse arriving at the cord has many possible paths open to it, on account of the branching of the nerve fibres as they enter the cord and the connection of these branches with different neurons of varying destination. The exact path taken by any given impulse under any given set of circumstances is determined by the varying resistance at the synapses which intervene between the terminations of the afferent fibres conveying the impulse and the next relay of neurons. These resistances in their turn are altered by the processes of facilitation and inhibition, which may be due to contemporaneous or previous events. A conspicuous example of these conditions is afforded by the phenomena of simultaneous and successive spinal induction.

The uniformity of afferent and efferent mechanisms disappears when we include the brain stem with the spinal cord. The main efferent channel of impulses is still through the spinal cord, since here are found the efferent mechanisms for all the skeletal muscles of the trunk and limbs, the chief servants of the central nervous system in the daily events of life. Other efferent channels are added, which acquire special importance with the growth of the upper brain or cerebral hemispheres. These mechanisins 
include those for the movements of the eye muscles, those concerned in facial expression, and those responsible for the movements of the mouth in mastication and deglutition, and in man, in speech. Important visceral efferent fibres are also contained in the vago-glossopharyngeal nerves, which leave the brain stem at its hindmost part in the region of the medulla oblongata, and influence the condition of the heart and the alimentary canal with its accessory organs. On the other hand, the afferent mechanisms of the brain stem far transcend in importance, i.e. in their influence on the reactions of the animal, those of the spinal cord. Among these afferent mechanisms are those which we have spoken of as 'projicient' sense organs or organs of foresight, the impulses from which must predominate over all reactions determined by the immediate environment of the animal. Into the medulla oblongata are poured the impulses from the greater part of the alimentary canal and from the heart (the chief factor in the circulation) and the lungs. At the junction of the medulla and pons is the great eighth nerve, really consisting of two, one of which, the cochlear nerve, carries impulses from the projicient sense-organ of hearing, while the other, the vestibular nerve, has its terminations in the labyrinth, the sense-organ of equilibration. To the impressions received from this organ all the complex co-ordinating motor mechanisms of the spinal cord have to be subordinated, in order that they may co-operate in the maintenance of the equilibrium of the body as a whole. Into the pons enters the fifth nerve, carrying sensory impressions from the whole of the head, while in the mid- and fore-brain we find the endings of the optic tracts derived from the eyes and carrying visual impressions. From the front of the fore-brain are produced the olfactory lobes.

At each segment or level in the brain stem the afferent fibres from these various sense-organs enter and join afferent tracts, carrying impulses on from the spinal cord-impulses originally derived from the muscles and skin of the trunk and limbs. At each level there may be an immediate 'reflection' back to the cord, so that the spinal afferent impressions may co-operate with the cranial afferent impressions in the production, through the spinal cord, of reactions affecting the viscera or the skeletal muscles. On the other hand, both kinds of afferent impressions may pass on up the brain stem to involve higher centres, and, mingling with impulses from other afferent nerves or from the projicient sense-organs, may result at some higher level in an efferent discharge, which may include reactions not represented in the cord, or reactions of far greater complexity than are possible in the purely spinal animal.

In consequence of the endless complex intermingling of afferent impulses, any diagrammatic representation of tracts is apt to be misleading unless it be remembered that at each break or synapse in the chain of neurons there are numerous possibilities of branching discharge, and that in our diagrams we can only give the course of such impulses as, by the frequency of repetition in the average life of the animal, have involved the grouping of a large number of nerve paths of similar function into tracts. The constituent elements of these tracts will present similar destinations and possi- 
bilities of interruption, i.e. of reactions involving the motor mechanisms at the different levels in the brain stem. It is thus much more difficult in the brain stem than in the spinal cord to describe a 'way in ' and a 'way out.' In a chain consisting, say, of six neurons, $a, b, c, d, e, f$ (Fig. 196), though $a$ is certainly afferent and $f$ efferent, it must always be more or less a question of words whether we regard neurons $c$ and $d$ as afferent or efferent in character. It is usual in our classifications to be guided chiefly by the direction of such impulses in relation to the cerebral hemispheres. All tracts going up to the cerebral hemispheres may be involved more or less in the production of such changes in the nervous matter of these hemispheres as are associated with conscious sensation. In the same way there is a possibility that the chains of neurons which carry impulses in a descending direction may be involved in the production of voluntary movement. It is therefore usual to classify these two sets of tracts as ascending and descending, or as afferent and efferent. If we adopt such a classification it must be with a distinct reservation that tracts which apparently are going downwards may play a greater part in the determination of sensation than in the determination of movement, and that there may, and indeed must, be a reverberation of impulses through these ascending and descending tracts,

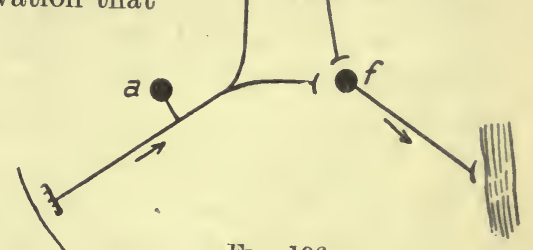

Fig. 196. so that it must be difficult to dissociate the various elements in the extremely complex neural events which are involved, say, in the simplest kind of conscious sensation.

As we trace out the evolution of the brain we find an ever-increasing subordination of the lower to the higher centres, so that in man himself many reactions which in the lower animals are carried out by the spinal cord alone, involve the educated co-operation of the cerebral hemispheres. With this increased control there is a corresponding increase in the development of long paths. In the brain of a fish, for instance, the cerebral hemispheres are connected only with the fore-brain; a little higher up there are connections between the hemispheres and the mid-brain as well. The chief long tracts are those which run between the thalamus, the mid-brain or the hind-brain, and the spinal cord. With the huge development of the cerebral hemispheres in man there is also development of long paths, the pyramidal tracts, from the hemispheres down to all the motor mechanisms of the cord, and of tracts which connect all parts of the cortex with the grey matter of the pons and indirectly with the cerebellum. The tracts which in the lower animals were of supreme importance in determining subordination of lower to higher centres, of immediate reactions to those determined by the organs of foresight, dwindle therefore in importance. Those tracts, such as the thalamo-spinal, tecto-spinal, vestibulo-spinal, which form the main mass 
of the white matter of the brain stem in lower types of vertebrates, become reduced to a few scattered fibres in the brain of man and are insignificant as compared with the great cerebro-bulbar and cerebro-spinal tracts.

\section{ASCENDING TRACTS}

The Tracts of the Fillet. The fibres which enter the spinal cord by the posterior roots pass into the posterior columns and along these to the dorsal column nuclei, the nucleus gracilis and the nucleus cuneatus, where they end by arborisations among the cells composing these nuclei. From these nuclei the axons of the cells pass in various directions, the chief mass of them forming the deep arcuate fibres. These emerge from the inner side of the nuclei and pass through the raphe to the other side of the medulla where they join the spino-thalamic fibres and form the definite collection of longitudinal fibres, lying dorsally to the pyramids, which is known as the main tract of the fillet, or, often, the mesial fillet. As these inbres traverse the pons they are joined at the outer side by a number of bundles which are derived from the central continuation of fibres connected with those derived from the cochlear nerve. This part is known as the lateral fillet. The cells of the accessory and lateral nuclei of the cochlear nerve send their axons by the trapezium to the superior olivary nucleus and other small masses of grey matter on the other side. In these nuclei the fibres for the most part terminate, but a fresh relay of neurons carries on the impulses and forms the main part of the lateral fillet. These pass up, getting more dorsal as they ascend, and finally terminate in the inferior corpora quadrigemina. The mesial fillet, which we can regard as a continuation of certain spinal tracts upwards, is reinforced throughout the whole extent of the medulla and pons by fibres originating from the masses of grey matter in which the sensory cranial nerves terminate. Certain of these fibres may form a distinct tract in the formatio reticularis, known as the central or thalamic tract of the cranial nerves. Another similar tract in the formatio reticularis is derived from the central terminations of the fifth nerve, and is known as the trigemino-thalamic tract. All these fibres pass up in the tegmentum of the mid-brain and finally end, partly in the grey matter of the subthalamic region and partly in the grey matter of the thalamus itself. To the thalamus are also continued a few fibres from the lateral fillet. By this means the head ganglion of the fore-brain is in a position to receive, so to speak, samples of the afferent impressions derived from every sense-organ of the body.

The Visual Paths. Two classes of afferent impressions which arrive at the optic thalamus are probably of equal importance to all the other afferent impressions taken together. These are impulses derived from the organs of vision and of smell. The greater part of the fibres composing the optic nerves arise as axons of the ganglion-cells of the retinæ. Passing backwards, the nerves of the two sides join in the optic chiasma, which is closely attached to the floor of the third ventricle. After joining in the chiasma the optic nerves are apparently continued round the crura cerebri as the optic tracts. 


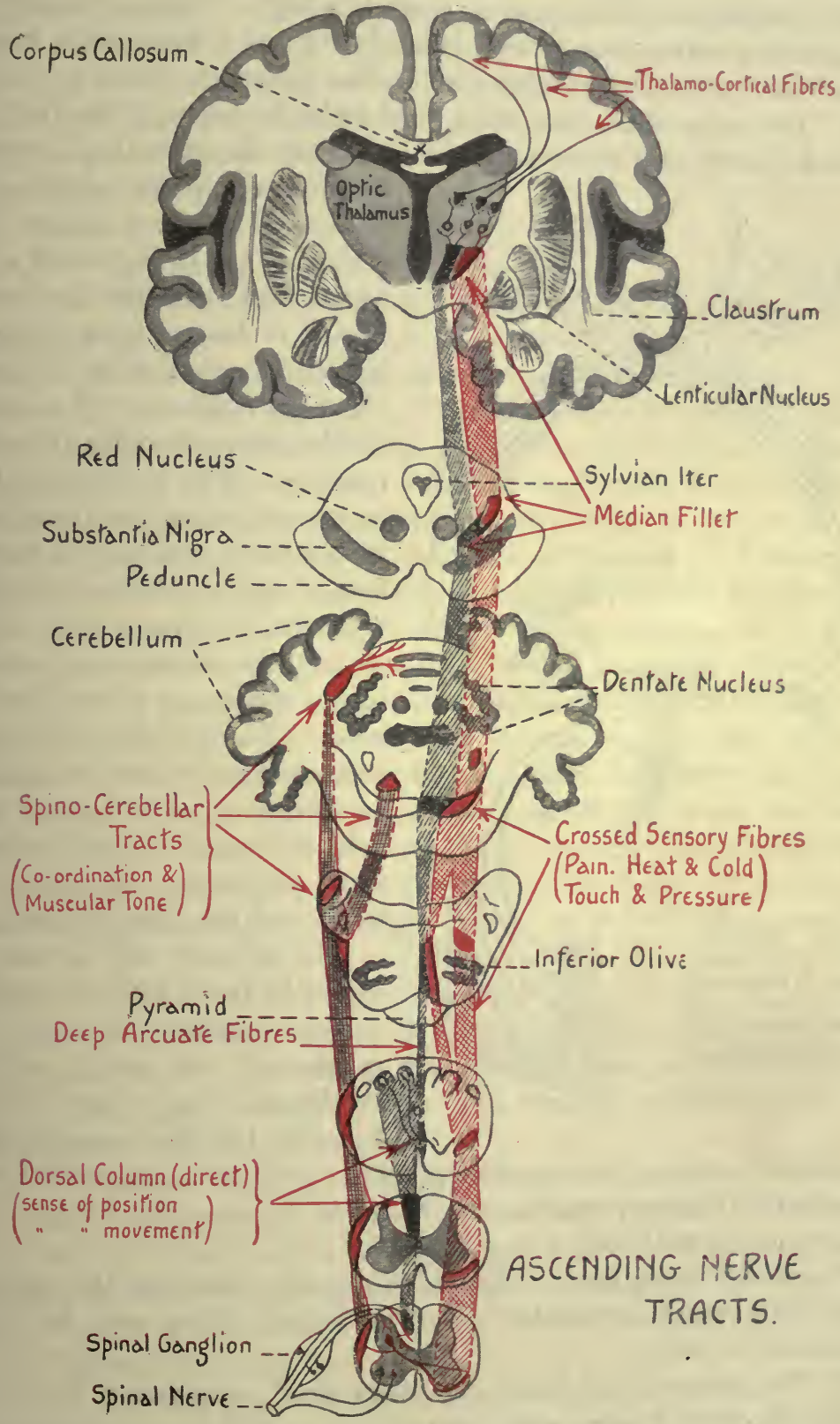

FIG. 197. Diagram of ascending tracts between the spina cord and brain (GoRDos HoLMES), with the probable path of sensory impulses. 
These pass round on each side and can be seen to make connection with the back part of the thalamus, the external geniculate body, and the superior corpus quadrigeminum. Part of the tract, which is sometimes called the mesial root, passes into the internal geniculate body. This part of the tract has probably nothing to do with vision and forms a commissure running in the optic chiasma connecting the internal geniculate bodies of the two sides. The course of the optic fibres is shown in the diagram (Fig. 198). In man and in 'some other mammals, e.g. dog, monkey, the nerve fibres decussate

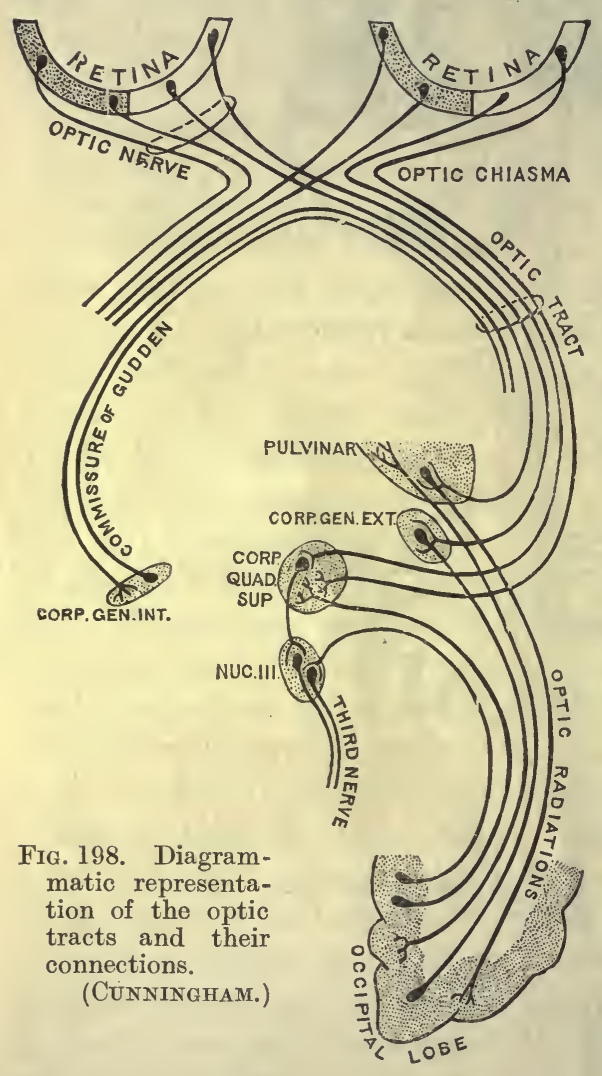
incompletely in the chiasma. The uncrossed bundle is derived from the outer half of the retina of the same side, whereas the crossed bundle is derived from the mesial half of the retina on the other side. The right optic nerve thus carries all the impulses originating in the right eye. The right optic tract carries all the impulses originating from stimuli occurring in the left field of vision. It must be remembered that vision in man is binocular, both retinæ being concerned in the perception of each field of vision. The external and internal geniculate bodies may be regarded as extensions of the optic thalamus, the former in special relation with the organ of vision, the latter with the organ of hearing.

The olfactory bulb is also connected by tracts with the thalamic region, probably through the column of the fornix and the bundle of Vicq d'Azyr. Since, however, the chief connections of the olfactory lobe are with the more primitive portions of the cerebral hemispheres, the olfactory tracts will be more conveniently treated of in connection with the latter.

The Cerebellar Paths. We have already traced out the course of spinal fibres which terminate in the cerebellum. They may be shortly summarised as follows :

(1) The posterior or direct cerebellar tract, originating in Clarke's column of cells of same side, passing up in the lateral columns and by the restiform body into the superior vermis of the middle lobe of the cerebellum.

(2) The anterior cerebellar tract or tract of Gowers, originating in the grey 
matter of both sides of the cord and passing in the lateral columns through the lateral part of the medulla and pons, and finally attaining the superior vermis through the superior cerebellar peduncles.

(3) The posterior columns, ending chiefly in the homolateral posterior column nuclei. From these nuclei, though the great mass of fibres passes into the fillet, a certain number from the nuclei of both sides join the restiform body to pass into the middle lobe of the cerebellum.

In the medulla these afferent tracts of the cerebellum are joined by the following sets of fibres:

1. The olivo-cerebellar.

2. The vestibulo-cerebellar.

3. A few fibres from the chief sensory nuclei, including those of the vago glossopharyngeal nerves.

All these fibres terminate in the cortex, chiefly of the middle lobe. From the cortex of this lobe fibres pass to the central and roof nuclei of the cerebellum, namely, the nucleus dentatus, the nucleus emboliformis, the nucleus fastigii, and the nucleus globosus. The efferent tracts of the cerebellum start from these central nuclei, no fibres which originate in the cortex of the cerebellum apparently leaving the precincts of this organ. Some of these efferent fibres of the cerebellum will be better described with the descending tracts of the brain stem. Of those which take an ascending direction, the great bulk are contained in the superior cerebellar peduncles. These originate for the most part in the dentate nucleus and the nuclei emboliformis and globosus. As the superior peduncles run forwards they sink below the posterior corpora quadrigemina, and in the tegmentum, below the Sylvian iter, decussate with the tract of the opposite side to pass to the red nucleus. In the red nucleus many of the fibres end, some, however, passing through the nucleus together with fibres derived from the cells of the red nucleus itself to end in the thalamus and in the grey matter of the subthalamic region.

\section{DESCENDING TRACTS}

The chief descending tracts having their origin in the brain stem are the rubro-spinal bundle or bundle of Monakow, the complex system of fibres known as the posterior longitudinal bundle, and the vestibulo-spinal fibres from the upper part of the medulla.

(1) The RUBRo-SPINAL FIBRES originate in the red nucleus. They cross the median line and run down, at first in the tegmentum and later in the lateral column of the medulla oblongata and cord. In their passage they communicate with the various motor nuclei of the cranial nerves. They can be traced to all segments of the cord, where they terminate in connection with the anterior horn-cells.

(2) The posterior longitudinal Bundle. This bundle is to be seen in all sections through the brain stem below the level of the oculo-motor nucleus. It consists of fibres, some of which pass upwards, while others pass downwards. Most of the fibres take origin in the cells of Deiters' nucleus and of the reticular formation of the pons, medulla, and mid-brain, as well as from 


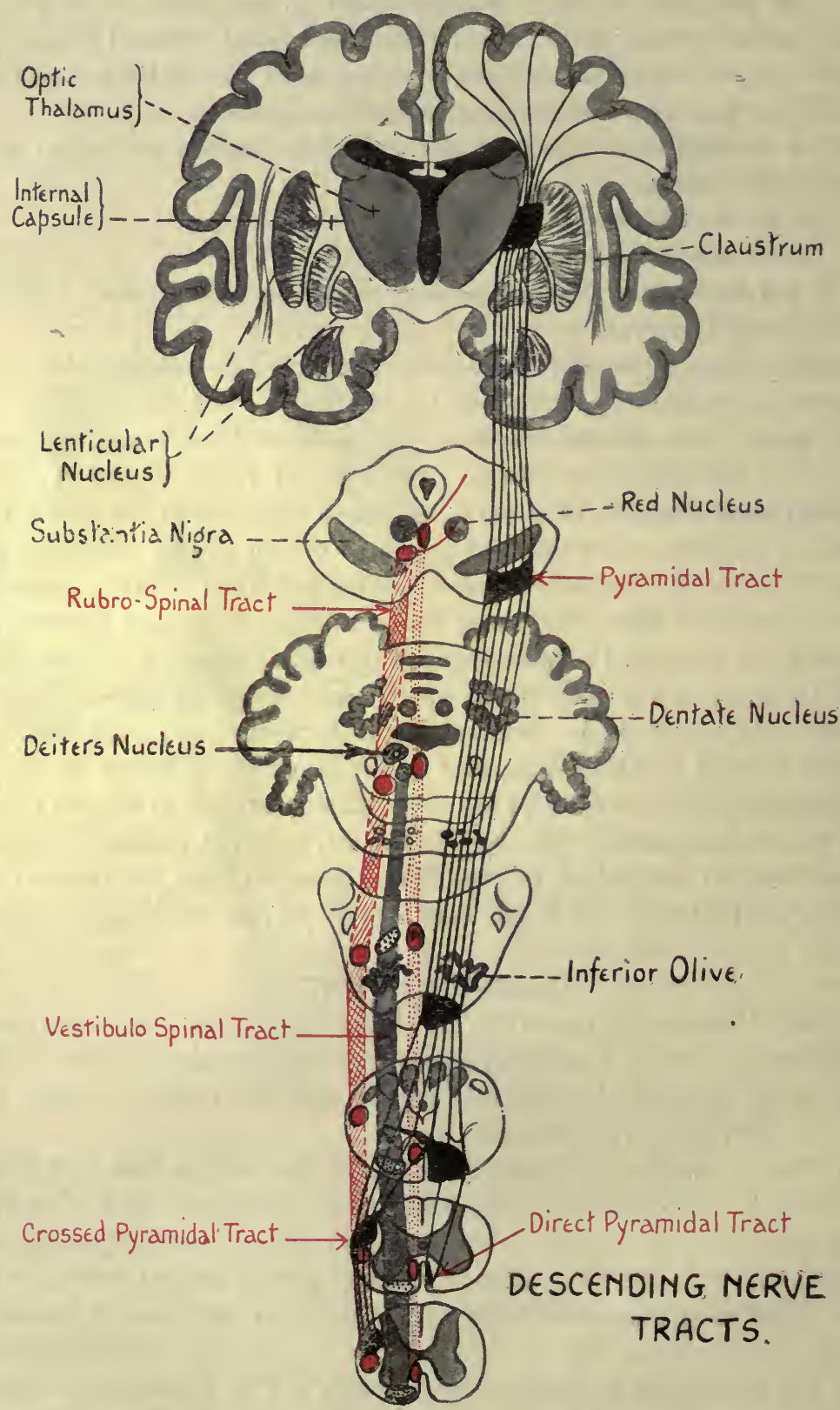

FIG. 199. Schema of course taken by chief descending tracts of brain stem. (Gordon Holmes). The tract in red, to the right of the rubro-spinal tract, includes the posterior longitudinal bundle, together with the fibres of the thalamo-spinal and tecto-spinal tracts. 
certain cells in the sensory nucleus of the fifth nerve. The fibres traced upwards can be seen to send collaterals to end in the various parts of the nuclei of the third, fourth, and sixth nerves. Lower down it becomes continuous with the anterior basis bundle of the spinal cord and merges in the internuncial fibres which serve to connect the various levels of the cord. Some of the fibres, which are descending, are derived from a small nucleus, the so-called nucleus of the posterior longitudinal bundle, which is found in the grey matter at the side of the posterior part of the third ventricle. This bundle also receives fibres from the superior olivary body. It is one of the earliest to undergo myelination in the fœtus ( $c p$. also Fig. 205, p. 408).

(3) The vestibulo-SPINAL TRACT takes origin for the most part in the cells of Deiters' nucleus. The fibres pass down in the anterior part of the spinal cord and terminate in the anterior horns. They are sometimes known as the antero-lateral descending tract. It is probably through this tract that the cerebellum is able to affect indirectly the activity of the motor mechanisms of the cord.

Two other descending tracts which are important in the lower vertebrates are insignificant in man. These are the thalamo-spinal tract, consisting of descending fibres derived from the optic thalamus, and the tecto-spinal tract, containing fibres derived from the roof of the mid-brain. In the mid-and hind-brain these fibres run in the tegmentum. In the cord they are found in the anterior columns. The olivo-spinal tract, which is supposed to originate in the olivary body, forms a small tract in the cervical region near the surface, opposite the lateral angle of the anterior horn. 


\section{SECTION XII}

\section{THE FUNCTIONS OF THE BRAIN STEM}

THE brain stem may be taken to include all those parts lying between the cerebral hemispheres and the spinal cord, from the optic thalamus in front to the medulla oblongata behind. The brain may be divided into the following parts from before back :

(1) Thalamencephalon, including the corpus striatum, the cerebral hemispheres and rhinencephalon, or olfactory lobes.

(2) Diencephalon, i.e. the fore-brain, especially the optic thalamus.

(3) Mesencephalon, or mid-brain, including the quadrigemina, the iter of Sylvius, and the crura cerebri.

(4) Metencephalon, composed of the pons Varolii, the upper part of the fourth ventricle, and cerebellum.

(5) Myelencephalon, or bulb, consisting of the medulla oblongata.

We may get some idea of the part played by these different regions of the brain in determining the reactions of the individual as a whole by examining the behaviour of the animals in whom all the rest of the brain in front of the part in question has been removed. If, however, we take into account the numberless connections existing between the different levels in the central nervous system, the interdependence between the different portions, and the subordination, especially in the higher animals, of the functions of the lower to those of the higher levels, we must acknowledge that such experiments can only give us an imperfect idea of the possibilities of each level when in connection with all other portions of the nervous system.

\section{THE FUNCTIONS OF THE MEDULLA OBLONGATA OR MYELENCEPHALON}

The possibilities of any given nervous centre are determined by the afferent impressions which enter it, and by the connections made by the nerves carrying these impulses with the motor tracts within the centre. The bulb receives afferent impressions of 'taste' from the tongue through the nervus intermedius, from the alimentary canal as low as the ileocolic sphincter: from the lungs, the heart, and the larger blood-vessels, i.e. from the most important of the viscera of the body, by the fibres of the vago-glossopharyngeal nerves. Its only skeleto-motor centre is that for the muscles of the tongue (the hypoglossal). It sends also efferent fibres to the viscera, which arise from cells in the nucleus ambiguus. These fibres carry motor impulses 
to the muscles of the larynx and bronchi, and to the osophagus, stomach, and intestines, secretory fibres to the stomach and inhibitory fibres to the heart.

At the upper border of the bulb enter also the fibres of the eighth nerve, carrying important impressions from the organ of hearing and the organ of static sense. These will be in all probability divided or injured in isolating the bulb from the higher portions of the brain. While in connection with the upper portions of the brain, the bulb receives also afferent impressions from the skin of the face, and the mucous membrane of the nose and mouth through the descending branches of the root of the fifth nerve, which pass down superficially to the tubercle of Rolando. When in connection with the cord the medulla receives afferent impressions from the whole surface of the body and from all the muscles and joints through the posterior column nuclei.

The bulbo-spinal animal, i.e. one in whom a section has been carried out at the upper boundary of the medulla, differs from the spinal animal chiefly in the maintenance of the nexus between the visceral functions and the skeleto-motor functions of the body. After removal of all the brain in front of the bulb, the animal still continues to breathe regularly and automatically. The blood pressure and the pulse rate remain normal, and all three mechanisms, respiration, pulse rate, klood pressure, may be affected reflexly by appropriate stimuli, or may be altered in consequence of central stimulation of the medulla.

In addition to the reflex mechanisms of locomotion, which are evident in the spinal animal, the bulbo-spinal animal shows a greater degree of solidarity in its responses. It is easier to evoke movement of all four limbs. In the frog, if the eighth nerve has been left intact, there is a certain power of equilibration left, and the animal when laid on its back tries to right itself and usually succeeds.

It is in this portion of the central nervous system that have been located the great majority of the so-called centres. By a statement, that the centre of such-and-such movement or function is situated in the medulla, we mean merely that the integrity of the medulla, or certain parts of it, is essential for the carrying out of the function. Every function, for instance, in which impulses passing up the vagus nerves are involved, is necessarily dependent on the integrity of these nerves and their central connections, and, since these are situated in the medulla, the centres for these functions are also located in this region. From a broad standpoint the medulla or bulb may be looked upon as a ganglion, or a collection of ganglia, whose main office is to guard and preside over the working of the mechanisms at the anterior opening of the body; by means of which food is seized, tasted, taken into the alimentary canal, and finally digested. The respiratory apparatus belongs to the same system and is innervated through the same nerve channels. Hence the various events in alimentation, such as deglutition, vomiting, mastication, or in the allied respiratory functions, such as phonation, coughing, and respiration itself, are endowed with centres in this part of the brain. In 
connection with the termination of the vagus nerves of this part of the brain is the location here of the chief vaso-motor centre, i.e. in a region which is in close proximity to the endings of the chief afferent nerves from the heart and larger blood-vessels and to the nucleus of the efferent controlling nerve to the heart.

\section{THE METENCEPHALON (PONS VAROLII AND CEREBELLUM)}

Destruction of the brain at the front of the fourth ventricle and just behind the posterior quadrigemina will leave the animal with a central nervous system, which is in connection by efferent nerves with the whole musculature of the body (with the exception of certain eye muscles) and which receives impressions through the spinal cord from the whole surface of the trunk and limbs, and through the fifth nerve from the face and head, and also the higher specialised impressions from the organ of hearing and the organ of static sense. The impressions from the two great projicient senses of smell and sight would be wanting.

Such an animal presents considerable advance in the complexity of its reactions above one possessing only spinal cord and bulb. The frog, for instance, after such an operation, can still walk, spring, and swim; when placed on a turntable it reacts to passive rotation by turning its head in the opposite direction. On stroking its back it croaks. If the cerebellum be also removed the animal becomes spontaneously active and crawls about until it is blocked by some obstacle. In this condition there is great activity of the swallowing reflex. Anything which touches the mouth is snapped at. If placed on its back the frog at once rights itself.

In the mammal a similar increase of reflex activity is observed though the power of progression is not retained.

\section{THE MESENCEPHALON OR MID-BRAIN}

A section in front of the anterior corpora quadrigemina would leave the animal with the nervous system receiving all normal sensory impressions, with the exception of the olfactory, and with efferent paths to all the muscles of the body, including those of the eye. In the mammal such an operation brings about a condition known as 'decerebrate rigidity.' Though respiratory movements continue normally, the whole musculature is in a cataleptic condition, the elbows and knees being extended and resisting passive flexion ; the tail is stiff and straight, the neck and head retracted. This condition seems to depend on an over-activity of the reflex tonic functions of the lower centres. That it is reflex is shown by the fact that the rigidity is at once abolished in a limb on dividing the appropriate posterior roots. The position of the limbs may be also modified by sensory stimuli. A similar condition of increased tonus is observed in the frog.

The apparatus for emotional expression is still intact though somewhat modified, and an impression which would give rise to pain in the intact animal may cause vocalisation in an animal in whom the brain above the mesencephalon has been destroyed. 


\section{THE BRAIN STEM AS A WHOLE (INCLUDING THE THALAM- ENCEPHALON, OR OPTIC THALAMI)}

The introduction of the head ganglia of the brain stem, viz. the optic thalami, completes in the lower animals at all events the apparatus for immediate response to stimulus. The powers of such an apparatus may be studied by examining the behaviour of an animal in whom the cerebral hemispheres have been destroyed. The result of this operation varies according to the type of animal chosen, though all types present certain common features. When a frog's cerebral hemispheres have been excised, a casual observer would not at first notice anything abnormal about the animal. It sits up in its usual position, and on stimulation may be made to jump away, guiding itself by sight, so that it avoids any obstacles in its path. Movements of swallowing and breathing are normally carried out. The animal thrown on to its back, immediately turns over again. If put into water, it swims about until it comes to a floating piece of wood or any support when it crawls out of the water and sits still. If it be placed on a board and the board be inclined, it begins to crawl slowly up it, and by gradually increasing the inclination may be made to crawl up one side and down the other. But a striking difference between it and a normal frog is the almost entire absence of spontaneous motion-that is to say, motion not reflexly provoked by changes immediately taking place in its environment. All psychical phenomena seem to be absent. It feels no hunger and shows no fear, and will suffer a fly to crawl over its nose without snapping at it. "In a word, it is an extremely complex machine, whose actions, so far as they go, tend to self-preservation; but still a machine in this sense, that it seems to contain no incalculable element. By applying the right sensory stimulus to it we are almost as certain of getting a fixed response as an organist is when he pulls out a certain stop."

According to Schrader and Steiner, if care be taken not to injure the optic thalami, spontaneous movements may be occasionally observed after removal of the cerebral hemispheres. On the approach of winter such a frog has been observed to bury itself in order to hibernate, and with spring to resume activity and to feed itself by catching insects. The behaviour of such decerebrate animals depends on the part taken in the initiation of movement and adapted reactions by stimuli entering through the higher sense-organs. Thus an ordinary bony fish after ablation of the cerebral hemispheres maintains its normal equilibrium in water. It is continually swimming about, stopping only when it reaches the side of the vessel or when worn out by fatigue. Here, again, if the thalami and optic lobes be intact the fish has been observed to show very little difference from a normal animal and to possess the power of distinguishing edible from non-edible material. On the other hand, in the elasmobranch fishes, which depend mainly upon their olfactory apparatus as a guide to movement, the removal of the cerebral hemispheres with the olfactory lobes, or of the latter alone, produces complete immobility and absence of 
spontaneous movement, even though the optic thalami and optic lobes may be intact.

In the bird the cerebral hemispheres may be removed with ease. A decerebrate pigeon, if its optic lobes be intact, walks about avoiding all obstacles, and may even fly a short distance. In the dark, i.e. in the absence of visual impressions, it remains perfectly still. The bird, however, is unable to recognise food, or enemies, or individuals of the opposite sex; it shows no fear and responds to stimuli like the brainless frog described above.

Goltz has succeeded in the dog in removing the whole of the cerebral hemispheres in three operations. The dog was kept alive for eighteen months after the final operation. It was able to walk in normal fashion and spent the greater part of the day in walking up and down its cage. At night it would sleep and then required a loud sound to awaken it. It reacted to stimuli in a normal fashion, shutting its eyes when exposed to a strong light, shaking its ears in response to a loud sound. On pinching its skin it attempted to get away, snarling or turning round and biting clumsily at the experimenter's hand. It had no power to recognise food and had to be fed by placing food in its mouth, though, if this food were mixed with a bitter substance, such as quinine, it was at once rejected. The dog never showed any signs of pleasure, or recognition of the persons that fed it, or of fear. Removal of the hemispheres had thus produced loss of all understanding and memory. There was no sign of conscious intelligence, and all the actions of the animal must be regarded as reflex responses to immediate excitation.

With the development of the cerebral hemispheres in the higher mammals there is a considerable shifting of motor reactions from those which are immediate and 'fatal' or inevitable to those which are educatable. The cerebral hemispheres in man take a large part in the determining of even the common reactions of everyday life. Ablation of the hemispheres therefore, or even part of the hemispheres, in the ape and man gives rise to much more lasting symptoms than is the case in the animals we have just studied. These defects we shall have to consider more fully later. The results, however, obtained on the lower animals, from the dog downwards, show that the brain stem, from the head ganglion of the optic thalamus back to the medulla, with the spinal cord, represents a complex mechanism which can be played upon by impulses received through all the sensory apparatus of the body, and is able to adjust the motor and visceral reactions to the immediate environment of the animal.

Certain of these immediate reactions are susceptible of further physiological analysis. We have seen that the spinal cord contains the co-ordinated mechanism for the movement of the limbs. We may now discuss how the movements of the limbs are co-ordinated with those of the trunk and head in the maintenance of the unstable position of the animal in standing and in locomotion. For this purpose there has been developed the great mass of nerve matter in the roof of the metencephalon, viz. the cerebellum. 


\section{SECTION XIII}

\section{THE FUNCTIONS OF THE CEREBELLUM}

THE carrying out of co-ordinated movements is associated with and regulated by afferent impressions which can be divided into two main groups.

In the first group may be placed those due to the changes in the environment of the animal, working on sensory structures or 'receptors,' of varying qualitative sensibility, in the surface of the body. These receptors may be excited by the mechanical stimuli of pressure, by changes of temperature, or by nocuous or harmful impressions, such as would, in the presence of consciousness, give rise to pain. At the fore end of the body we have in addition the special receptor organs excited by waves of light or of sound. The action of any of these impressions, if of sufficient intensity, is to evoke an appropriate reflex movement, such as the flexor reflex in response to nocuous stimulus applied to the foot, or the stepping, or extensor, reflex excited by steady pressure on the sole of the foot.

The integrity of the nerve paths carrying these afferent impressions and of the motor paths to the muscles is not, however, sufficient. A secondary set of afferent impulses is essential in order to guide and regulate the extent of the resultant discharge. These secondary afferent impulses start in the deep tissues, viz. the muscles, joints, and ligaments, which are provided with special sense-organs capable of being stimulated by the mechanical changes of tension or pressure set up by the movements themselves. The importance of these impressions for the carrying out of muscular movements is shown by the ataxia which is the result of injury to the corresponding afferent nerves. Degeneration of the nerves to muscles, or section of the afferent roots, causes marked ataxia of the movements of the limb, whereas no such result follows section of all the cutaneous nerves supplying the surface of the limb with sensibility. To this system of afferent nerves Sherrington has given the name of the ' proprioceptive ' system, since it is excited, not directly by changes in the environment, but by alteration in the animal itself. It is responsible for reactions differing in many respects from those which are the immediate result of stimulation of the other system, the 'exteroceptive,' which is distributed over the surface of the body. Since it is excited by the movement of the muscles themselves, i.e. by the first result of the reaction to external stimulus, it serves as a governing mechanism to regulate the extent of each motor discharge. Its excitation not only prevents over-action of the muscles, but may evoke a compensatory reflex in an opposite direction to the reflex immediately excited from the skin. A marked feature of this 
system is its tendency to continued or tonic activity. The steady slight contraction, or 'tone,' which is observable in most skeletal muscles, is independent of the surface sensibility and depends entirely on the proprioceptive system of the muscles and their accessory structures.

In the decerebrate animal the rigidity of a limb disappears at once after section of its afferent roots, though it is unaltered by division of the main skin nerves. This tonus does not affect all muscles to an equal degree. In every limb there is a predominance of tonus in certain muscles, so that the result on the whole limb is an attitude or posture which is typical of the limb or the animal. Thus the spinal frog takes up an attitude which is very different from that which would be impressed on it by gravity in the absence of muscular activity. If one of its hind limbs be extended gently, it soon draws it up to reproduce the same crouching position. The posture of the limb is therefore a result of afferent impressions continually ascending its proprioceptive nerves and exciting a tonic activity which predominates in certain definite muscles. This posture, as carried out by the spinal cord, is a segmental response. It determines the relation of the limb to the trunk, and to a less extent of the four limbs to one another. It is not concerned with the relation of the animal as a whole to its environment, and only to a slight extent with the maintenance of equilibrium in the presence of the continually acting force of gravity.

In the evolution of the nervous system there has been a continual subordination of the hinder parts to the head end, in consequence of the development at this end of the all-important distance receptors, the impulses from which take a predominating part in determining the reactions of the body as a whole. In fact the subordination of one part of the central nervous system to another is in direct relation to the importance of the afferent impulses arriving at each portion of the system. Thus the vaso-motor centres segmentally distributed throughout the spinal cord are subject to the vaso-motor centre in the medulla, which is developed at the point of entry of the vagus nerves, i.e. the chief afferent nerves from the heart and large blood-vessels. The collections of grey matter presiding over the segmental reactions of the intercostal muscles are entirely subordinated to the grey matter in the medulla around the entry of the vagus fibres from the lungs.

This subordination of the hinder to the anterior sense-organs is paralleled in the case of the proprioceptive system. Entering the hind-brain at the upper border of the medulla is the eighth nerve, composed of two parts which differ widely in functions, viz. the cochlear division and the vestibular division. The former is entirely concerned with the reception of sound waves, and is therefore the auditory nerve. The vestibular nerve, which is distributed to the rest of the membranous labyrinth, must be assigned to the proprioceptive system. The labyrinth is practically a double organ. The primitive auditory sac arises as a simple involution of the surface. In the course of development the front part is modified to form the canal of the cochlea, which is set apart entirely for the reception of sound. From the 
back part there are formed two sacs - the saccule and utricle- and the three semicircular canals. The saccule and the utricle, which receive each a larg: branch of the vestibular nerve, represent the otolith organ, which is found in almost all classes of animals. The crayfish, for instance, at the base of its antennæ presents a small sac lined with hairs and richly supplied with nerves. In this sac a small calcareous particle rests on the hairs. It is evident that the incidence of the pressure of the small stone or otolith on the hairs will vary according to the position of the animal (Fig. 200), so that any change in the position of the head will be attended by alteration in the nerve fibres which have been stimulated by the
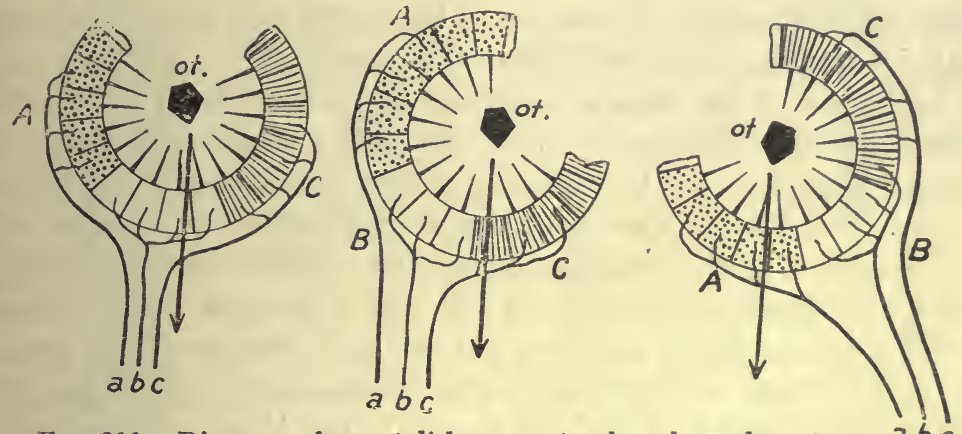

FiG. 200. Diagram of an otolith organ, to show how alterations in its position will cause the weight of the otolith (ot.) to press on different sense-cells, and therefore to affect different nerve fibres.

pressure of the otolith, and therefore in the nature of the impulses flowing to the central nervous system. The importance of these impulses in regülating the locomotion and the maintenance of the equilibrium of the animal is well shown if the otolith be replaced by a small fragment of iron. Under normal circumstances the iron particle will act quite as well as an otolith. If, however, a powerful magnet be brought in the neighbourhood of the animal, the pressure of the particle will not be determined simply by gravity and therefore by the position of the animal, so that there will be a dissonance between the impulses arriving from the otolith organ and those arising from the sense-organs of the body, and marked disorders of equilibrium are the result.

In the saccule and utricle the vestibular nerve ends in similar otolith organs known as the maculæ acousticæ. These are small elevations covered with long hairs and supplied with nerves. One or two calcareous secretions or otoliths are embedded in the hairs, so that any change in position will cause a corresponding change in the nerve fibres which are being excited by the weight of the otoliths. The semicircular canals, which lie in the three planes of space, are also provided with end-organs, somewhat similar in structure to the maculæ acousticæ, but devoid of otoliths. They are excited by mass movements of the fluid endolymph, filling the canals, which are set up by rotation of the head.

Since the nervous apparatus of the labyrinth is excited not by changes in the environment, from which it is carefully shielded, but by changes in the 
animal itself, we are justified in assigning it to the proprioceptive system, of which indeed it represents the most important receptor. Just as the proprioceptive nerves of a limb are responsible for the tonus of the limb muscles, so, as Ewald has shown, each labyrinth is responsible to a considerable degree for the tonus of the corresponding side of the body. Extirpation of one labyrinth causes a lasting loss of tone in the muscles of the same side. A further functional resemblance lies in the part played by the labyrinth in the determination of posture. The resultant effect of the impulses arising in it is to maintain a reflex posture of the head and eyes, so that the optic axes in a position of rest are directed towards the horizon. Stimulation of the labyrinth causes therefore movements of the eyes which may or may not be associated with correlated movements of the head.

As in the case of the other sense-organs of the anterior end of the body, the reflexes excited from the labyrinth dominate over those evoked by proprioceptive impulses from the hinder portions of the body. At the entry of its nerve into the brain stem a mass of grey matter is developed, which must be regarded as the head ganglion of the proprioceptive system, and the chief co-ordinating organ of all the reflex systems which determine posture of the limbs and of the whole animal, and therefore the maintenance of equilibrium both at rest and during locomotion. This organ is the cerebellum, associated with the grey matter in the upper part of the fourth ventricle at the point of entry of the vestibular nerves. The cerebellum commences in early fœtal life as a small elevation in the dorsal wall of the neural tube, where the eighth nerve enters. Simple in structure and small in extent in most of the fishes and amphibia, it grows in extent with increasing complexity of the animal's motor reactions, and attains its greatest development in the mammalia. In this class the cerebellum, like the cerebrum, is most highly developed in man and the higher apes. It is generally described in man as consisting of a middle lobe, composed of the superior and inferior vermis, with two lateral hemispheres, and these are subdivided by anatomists according to the situation of the chief sulci. From the physiological point of view the structure of the organ is relatively simple, as is shown by the uniformity of its structure throughout all parts. It may be considered as formed of two main structures, viz. the cortex and the central or roof ganglia.

The surface of the cerebellum is increased by being thrown into folds or laminæ, so that a section of this organ has a tree-like appearance. A section through a lamina shows three distinct zones : an outer molecular layer presenting a granular appearance with a few nuclei ; internal to this a granule layer composed of many nuclei of nervecells; and most deeply a central core of white matter. Between the molecular and granular layers are situated the cells of Purkinje, large flask-shaped cells each with one apical dendrite, distinguished above all other dendrites of the central nervous system by the richness of its branching, and with one axon, which leaves the base of the cell and passes down into the central white matter, giving off collaterals in its course. In preparations made by Golgi's method we are able to distinguish the various elements composing these layers and their relations. The molccular layer, besides neurogliacells and the branching dendrites of the cells of Purkinje, contains certain star-shaped cells ( $a$ Fig. 201), which give off an axon running parallel with the surface in the molecular layer. From this axon branches dip down towards the cells of Purkinje, 
where they end in a rich basket-work of fibres around the body and beginning of the axon of these cells. The nuclear or granular layer presents two kinds of cells. The most numerous is a small cell with a few short dendrites, each of which terminates in a claw-shaped arborisation, and a single long axon, which passes straight up into the molecular layer, where it bifurcates. The two branches run parallel with the surface in a direction at right angles to the plane of expansion of the dendrites of Purkinje's cells, apparently resting against the serrations on the $\epsilon$ dges of these processes. The second kind of cell in the granular layer is the so-called Golgi's cell-a large cell

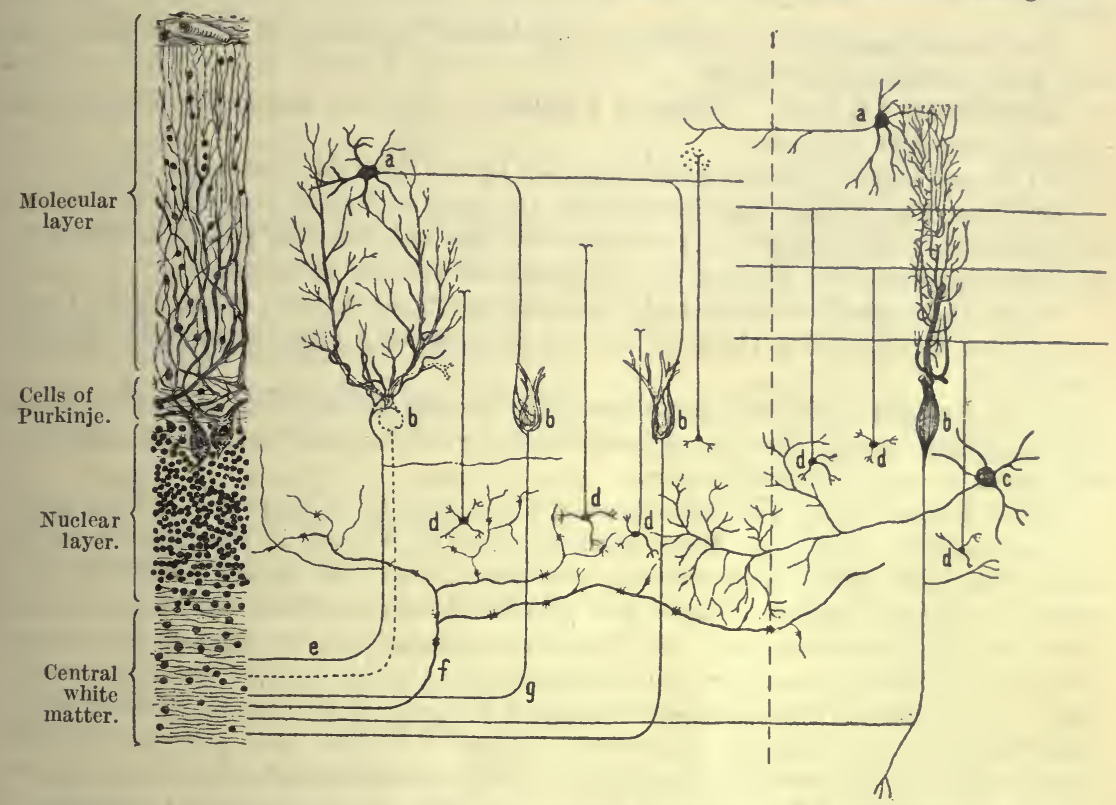

Fig. 201. Schema of constituent elements of cerebellum. (Modified from Вöчм and DAvidofF.) On the left is a section of the cortex as it appears when stained by ordinary methods. The middle portion represents diagrammatically a section at right angles to the laminæ, while to the right of the dotted line the section is taken in the same plane as the laminæ.

$a$, star-shaped cells of molecular layer ; $b, b$, cells of Purkinje; $c$, 'Golgi cell' ;

$d$, small cells of nuclear layer; $e$, 'tendril fibre'; $f$, 'moss fibre'; $g$, axon of cell of Purkinje.

with many dendrites and an axon which terminates by frequent branches in the neighbouring grey matter.

The fibres making up the white matter are of three kinds-two afferent and one efferent. The moss fibres, so called from the curious thickenings they present in the nuclear layer, pass up into the grey matter and terminate by frequent branches in this layer. The tendril fibres, also afferent, end in a rich arborisation which surrounds the distal part of the bodies and the bases of the dendrites of the cells of Purkinje. The efferent fibres are represented by the axons of the cells of Purkinje, which acquire a medullary sheath and, run down into the white matter.

This slight sketch of the anatomy gives us a conception of the extreme complexity of choice presented to nervous impulses traversing the cerebellar cortex. Thus a discharge along an axon of the cell of Purkinje may be excited (1) by an impulse ascending the tendril fibres; or (2) by one ascending the moss fibres through the granule cells, and then passing by their bifurcating axon to the dendrites of the cells of Purkinje; or (3) by the star-shaped cells of the molecular layer and their basket-work round the body of Purkinje's cells.

The roof ganglia consist of the nuclei fastigii near the middle line, the nuclei em- 
boliformes situated just dorsal to these, and the nuclei dentati, large crenated capsules of grey matter lying in the middle of each lateral lobe. The cells composing the grey matter of the central nuclei are large and multipolar, resembling those found in the nuclei of motor nerves.

The cerebellum receives fibres from all the receptor apparatus of the body which can be classed in the proprioceptive system. The greater number of these fibres run directly to the cortex, especially of the vermis, and there is no evidence of the passage of any efferent fibres from the cortex directly to the motor apparatus of the cord.

The connections of the cerebellum are established by means of its three peduncles, and may be classified as follows :

AFFERENT TRACTS. INFERIOR PEDUNCLE. By this peduncle afferent fibres pass to the superior vermis :

(1) From Clarke's column of the same side by the posterior cerebellar tract.

(2) From the dorsal column nuclei, viz. the nucleus gracilis and nucleus cuneatus of each side, so that connection is estabished in this way with the prolongations of the posterior sensory roots which run into the posterior columns of the cord.

(3) By the internal restiform body from the vestibular division of the eighth nerve, part of the fibres passing through, and perhaps making connections with, Deiters' nucleus.

(4) A strong band of fibres passes from the inferior olivary body into the opposite cerebellar hemisphere. Atrophy of one side of the cerebellum induces a corresponding atrophy in the opposite olivary body.

Middle Peduncle. The broad mass of fibres making up these peduncles is partly afferent and partly efferent. Many fibres originate in the cells in the formatio reticularis of the pons, cross the middle line, and pass up into the lateral cerebellar hemisphere of the opposite side. Fibres also pass from the cerebellum to the pons to end round cells in the same region. By this means connection is established between the cerebellar hemispheres and the corticopontine fibres which pass by the crura cerebri between the pons and the frontal and temporal portions of the cerebral cortex of the opposite side. On account of this connection there is a close association between the development of each cerebellar hemisphere and the contralateral cerebral hemisphere. Atrophy of one half of the cerebrum brings about atrophy of the opposite hemisphere of the cerebellum.

The Superior Peduncle. By this path fibres from the superior corpora quadrigemina, i.e. from the terminations of the optic nerve, pass into the cortical grey matter of the cerebellum (Fig. 202).

EFFERENT TRACTS. The cerebellar cortex must be regarded as a receiving rather than as a discharging station. Stimulation of it has little effect unless strong currents are employed, and a motor response is obtained more easily the deeper the electrodes are sunk below the grey matter. The fibres which form the axons of the cells of Purkinje pass partly towards the pons by the middle peduncle, largely, however, towards the roof nuclei, where they terminate. These nuclei form the efferent stations of the cerebellum. From them fibres pass in various directions. A large bundle leaves the dentate nucleus, runs into the superior peduncle, or brachium, and passing deeply across to the tegmentum of the opposite side, traverses the red nucleus to end in the subthalamic region of the opposite side of the brain. A certain number of fibres, chiefly derived from the central nuclei, such as the nucleus fastigii, pass forward to the corpora quadrigemina chiefly on the same side. From the cerebellum itself no direct tract runs into the spinal cord. The nuclei of Deiters and of Bechterew (the paracerebellar nuclei), which are connected with the endings of the vestibular nerve, are, however, closely associated with the roof nuclei, and give rise to descending fibres which pass into the antero-lateral region of the cord as the vestibulo-spinal tract.

The cerebellum is a receiving station not only for impulses which arise in the skin and eyes, i.e. on the surface of the body, but especially for those 
which have been defined as proprioceptive, and originate either in the muscles and tendons or in the labyrinth. Activity of this apparatus is roused as a rule by the movement of the organism itself, and is only a secondary result of the environmental stimulation which provoked the original movement. By its efferent tracts starting in the roof- and paracerebellar nuclei, the cerebellum is able to affect the musculature of the same side of the body by a direct influence on the anterior horns. It also enters to a much greater extent into relation with the opposite cerebral hemispheres, so that it is in a position to control or modify the activity of these, whether excited on their sensory or on their motor sides.

STIMULATION OF THE CEREBELLUM. It was first shown by Ferrier that movements of the same side of the body can be excited by stimulation either of the cerebellar hemispheres or of the superior vermis. These results have been confirmed by subsequent observers, and point to each half of the cerebellum being connected functionally with the skeletal muscular apparatus of the corresponding side of the body. The cortex cerebelli is not excited with ease. To evoke movements much stronger stimuli are necessary than, e.g. for the excitation of the motor area of the cerebral cortex. This again is in accordance with what we should expect from the anatomy of the organ, knowing as we do that the cortex is an end-station for a number of afferent paths, but has no direct efferent paths from it to the lower motor mechanisms

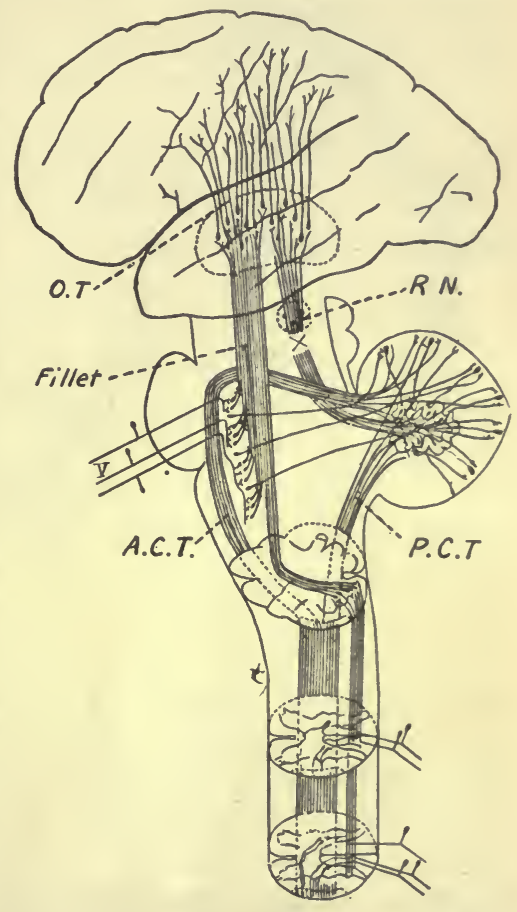

Fig. 202. Diagram of afferent and efferent tracts of cerebellum.

(After v. GeHUCHTEN.)

от, optic thalamus; RN, red nucleus; PĆT, posterior cerebellar tract; $\mathrm{ACT}$, anterior cerebellar tract; $v$, fifth nerve. of the cord. On the other hand, movements are excited by minimal stimuli from the intrinsic nuclei of the cerebellum.

As a result of his experiments Horsley has concluded that the cortex cerebelli must be regarded as an afferent receptive centre from which axons pass to the ventrally placed efferent nuclei, viz. the nuclei dentati, fastigii, emboliformes, as well as Deiters' nuclei. Whereas excitation of the roof nuclei produces more especially movements of the eyes and head, the paracerebellar (e.g. Deiters' nucleus) are responsible more especially for the movements of the trunk and limbs. The movements of the body which are thus evoled are those concerned in maintaining equilibrium and are involved in every alteration in the position of the body. 
EFFECTS OF ABLATION OF THE CEREBELLUM. Complete unilateral extirpation of the cerebellum, after the irritative effects of the lesion itself have passed away, brings about a condition of the animal characterised by :

(1) Slight loss of power on the same side of the body.

(2) Considerable loss of tone on the same side.

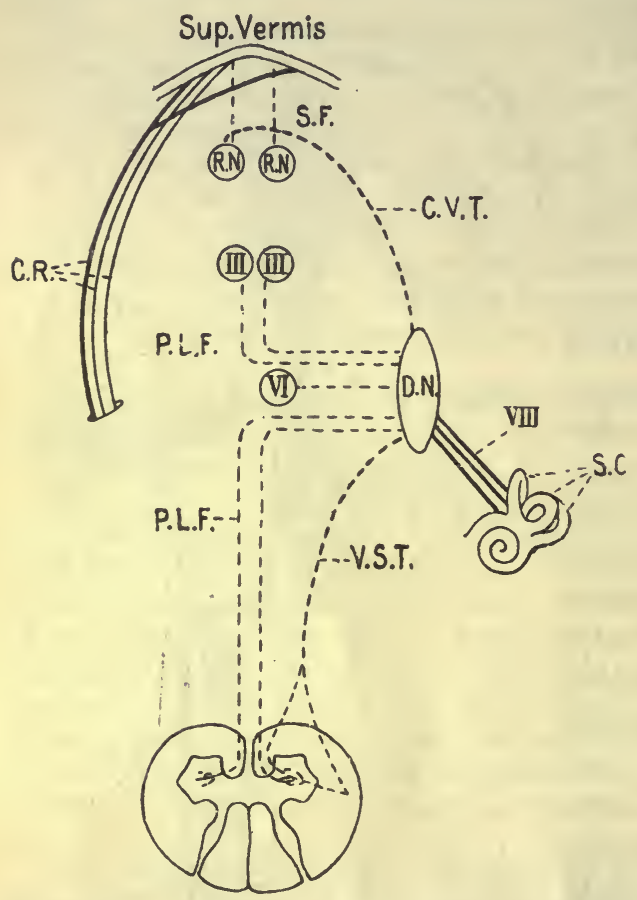

FIG. 202A. Schema of connections of Deiters' nucleus. (BRUCE.)

CR, restiform body; RN, roof nuclei; SF, sagittal fibres from cortex to roof nuclei; CVT, cerebellovestibular tract; DN, Deiters' nucleus; III, VI, nuclei of third and sixth nerves; PLF, posterior longitudinal bundle; vIII, vestibular division of eighth nerve; sc, scmicircular canals; vst, vestibulo spinal fibres.

(3) Tremors or rhythmical movements of the muscles on the same side accompanying any willed movements.

These three symptoms are denoted by Luciani as asthenia, atonia, and astasia. At first the animal is quite unable to stand, and lies on the side of the lesion with neck and trunk curved in the same direction; when it attempts to stand it always falls to the same side. After two or three weeks the power to stand is regained, though when it attempts to walk the hindquarters drag and tremors accompany every effort. The animal attempts to correct the tendency to fall towards the side of the lesion by an exaggerated abduction of the limbs to that side, and is always ready to take advantage of the support of a wall to enable it to maintain its equilibrium. Swimming is much better carried out than walking, the contact of the water with the skin furnishing guidance to the spinal mechanism which is lacking when the animal attempts to walk.

When the whole cerebellum is removed the animal is unable to walk, sometimes for months. After a time it gradually learns to walk, but this is carried out by an alteration of the method of progression. The disorders of locomotion are quite distinct from the spinal ataxia observed after interference with the afferent tracts from the muscles. The difficulty now is that each diagonal movement of the limbs in progression tends to throw the centre of gravity to one side or other of the basis of support, and it is the mechanism for maintaining the right position of the centre of gravity, i.e. the posture of the body as a whole in relation to its environment, which is at 
fault. The animal, in the case of the dog, therefore attempts to correct the tendency to fall to one side or other at each step by making its basis of support as wide as possible, and gradually acquires a peculiar gait, consisting of a series of springs, in which the two fore limbs and two hind limbs act together, the diagonal movements of the fore limbs being practically abandoned.

In lesions of the cerebellum in man the most marked symptoms are the cerebellar ataxy and the occurrence of tremors, ' astasia,' on the performance of willed movements. The ataxy has the same origin as that in the dog ; each spinal act of locomotion tends to throw the centre of gravity outside the line of support, and the tendency to fall thus brought about is voluntarily compensated by abduction of the corresponding limb. A staggering gait is thus produced, which is practically identical with that of a drunken man, and presents no trace of the over-action of muscles so characteristic of spinal ataxy. That the compensation, which is slowly acquired after extirpation of the cerebellum, is of cerebral origin is shown by the fact that extirpation of the cerebral hemispheres, or even of the motor areas of the hemispheres, after extirpation of the cerebellum, at once abolishes the power of movement which has been reacquired, and after the motor areas are destroyed on both sides the loss of power of progression is permanent.

These experiments show that the cerebellum, in Sherrington's words, must be regarded as the head ganglion of the proprioceptive system, acting as a centre where arrive the afferent impulses from the cord, the fifth nerve and especially from the labyrinth. It influences, through the superior peduncle, the cerebral cortex and furnishes the subconscious basis for the guidance of the motor functions of the latter organ. Through its connections with the nuclei of the bulb and the efferent tracts arising therefrom, it augments the tonic activity of all the muscles of the body, an effect which is especially marked in the absence of the cerebral hemispheres and is responsible for the condition known as decerebrate rigidity. As a centre of conjunction for the afferent impressions from the muscles and those from the labyrinth it co-ordinates the segmental reflexes, which determine the relative posture of each limb, with those originating in the labyrinth and determining the position of the head. Thus the whole mechanism provides for a maintenance of equilibrium of the body as a whole, and for the proper balancing of the reflex movements of the different limbs with those of the trunk during all the changes in the position of the centre of gravity attending locomotion.

The view here put forward really includes the various descriptions of the functions of the cerebellum which have been given by different authorities. Thus Luciani describes the cerebellum as an organ which by unconscious processes exerts a continual reinforcing action on the activity of all the spinal centres. Munk ascribes to the cerebsllum the function of maintaining bodily equilibrium. Lewandowsky regards the cerebellum as the central organ of the muscular senses. Hughlings Jackson expressed many years ago an important characteristic of the cerebellum when he wrote that the cerebellum is the centre for continuous movements, and the cerebrum for changing movements. All these descriptions come under Sherrington's conception of the cerebellum as head ganglion of the proprioceptive system. 


\section{SECTION XIV}

\section{VISUAL REFLEXES}

Foremost among the afferent impulses determining the reactions of higher animals are those arising in the eyes. Each retina, or rather the two retinæ acting together as a single organ, can be regarded as a sensory surface, every point of which corresponds to a point, or series of points, lying in a given direction outside the body. Each optic nerve contains about half a million nerve fibres, i.e. as many as enter the cord by the posterior roots from the whole of the body. The two optic nerves coming from the retinæ meet together in the floor of the fore-brain and form the chiasma. At the chiasma a decussation of fibres takes place, which, in animals such as the rabbit, with no fusion of the fields of the two eyes, is practically complete. In man only those fibres which arise in the mesial half of each retina cross the mesial plane; these, together with the uncrossed fibres from the temporal half of the other retina, form the optic tract of the opposite side (Fig. 203). The optic tract passes backwards across the crus cerebri and finally divides into three branches, in the roof of the mid- and fore-brain, which end in the grey matter of the anterior corpora quadrigemina and in the external geniculate body and the pulvinar of the optic thalamus. Running in the optic tract are also fibres which are simply commissural; these form the mesial root of the optic tract. They cross in the optic chiasma and serve to connect the

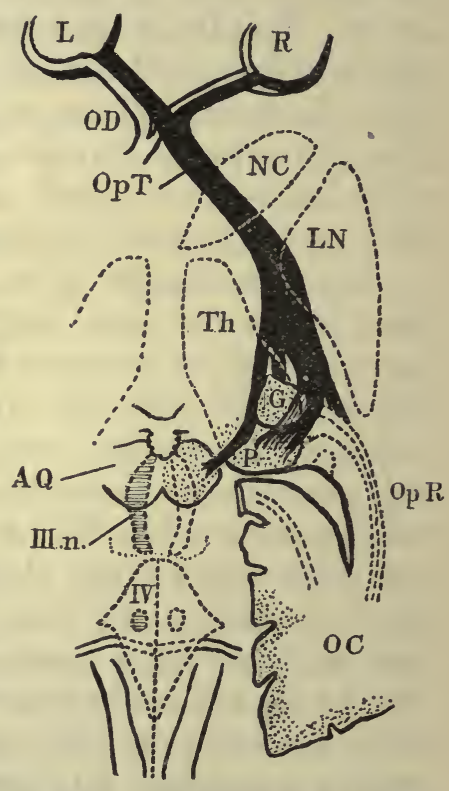

Fig. 203. Diagram to show connections of optic tracts. (After Sherrington.)

$\mathrm{L}$, left, and $\mathrm{R}$, right retina; OD, optic decussation (chiasma); OpT, optic tract; NC, nucleus caudatus; LN, lenticular nucleus; Th, optic thalamus; $G$, external geniculate body; $\mathbf{A Q}$, anterior corpus quadrigeminum; $\mathrm{P}$, pulvinar; $\mathrm{OpR}$, optic radiations running to $O C$, the occipital cortex ; IIIn, nucleus of third nerve in floor of Sylvian aqueduct; IV, fourth ventricle. two internal geniculate bodies. In addition to the afferent fibres from the retina to the brain the optic tract contains a certain number of efferent fibres which pass out and end in the retinæ.

It is evident from these connections that whereas section of one optic 
nerve, say the right, will only cause loss of vision in the right eye, section of the right optic tract will divide the fibres coming from the right halves of both retinæ. This portion of the retina in each eye is stimulated in the normal position of the eyes by rays of light coming from the objects lying to the left of the field of vision. Section of the right optic tract therefore causes blindness to all objects to the left of the median line, left hemianspia. Section of both optic tracts of course causes complete blindness.

Every movement of the head involves compensatory movements of the eyes, and conversely, in any change in the environment of the animal which demands its attention, there is a movement of the eyes so as to turn the gaze on to the origin of the disturbance as an antecedent to any body movement. In the absence of normal regulative impulses from the skin, or from the semicircular canals, the afferent impressions from the eyes may serve for the maintenance of fairly well co-ordinated movements - a compensation which is rendered possible by the power of the cerebral cortex to learn new reactions by experience.

The centres for the eye movements are contained in the grey matter in the floor of the back part of the third ventricle and of the iter of Sylvius. Here we find the nucleus of the third or oculo-motor nerve. The oculo-

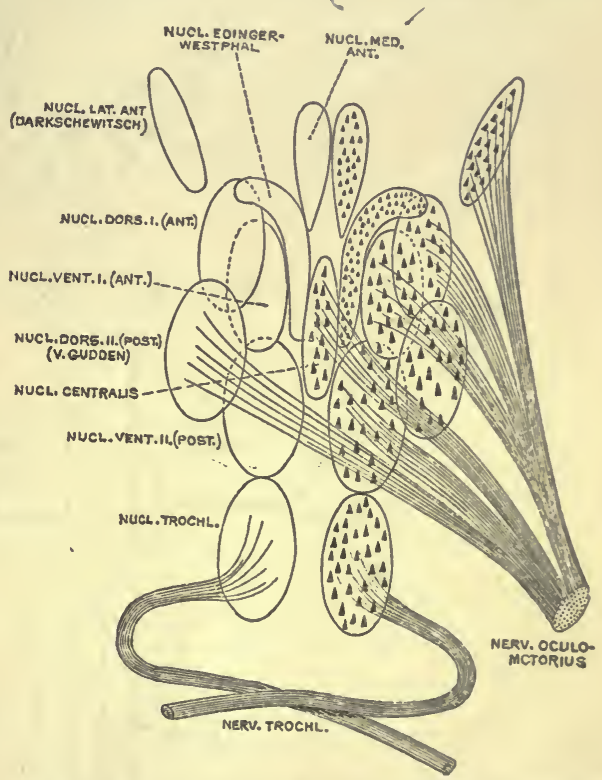

FIG. 204. Diagram to show origin of the different fibres of the third and fourth nerves from the oculo-motor nuclei.

motor nucleus consists of several divisions, viz. a lateral part containing large motor cells, a superficial median nucleus with small cells, and a deeper median nucleus with large cells. By localised stimulation it has been found possible to differentiate the functions of the different parts of the nucleus (Fig. 204). Stimulation of the back part of the third ventricle causes contraction of the ciliary muscles, and a little behind this contraction of the pupil. On stimulating the floor of the iter, from before backwards we obtain contractions in order of the rectus internus, the rectus superior, the levator palpebræ superioris, the rectus inferior, and the inferior oblique muscle. On stimulating more laterally, or exciting the corpora quadrigemina, dilatation of the pupil was obtained.

It seems probable that the optic thalamus and the closely related external geniculate body are mainly concerned with the reception of visual impulses and their forwarding to the cerebral cortex. On the other hand, the anterior or superior corpora quadrigemina are mainly concerned with the co-ordination 
of visual impressions and visual movements with the movements of every part of the body, and especially with the complex mechanism we have already studied in connection with the labyrinth and cerebellum. Stimulation of the corpora quadrigemina therefore evokes movements of the eyes and of the head; extirpation of this part, though it may interfere with co-ordination, does not necessarily involve loss of sight, even when the extirpation is bilateral.

The multifarious intercourse which is continually taking place between

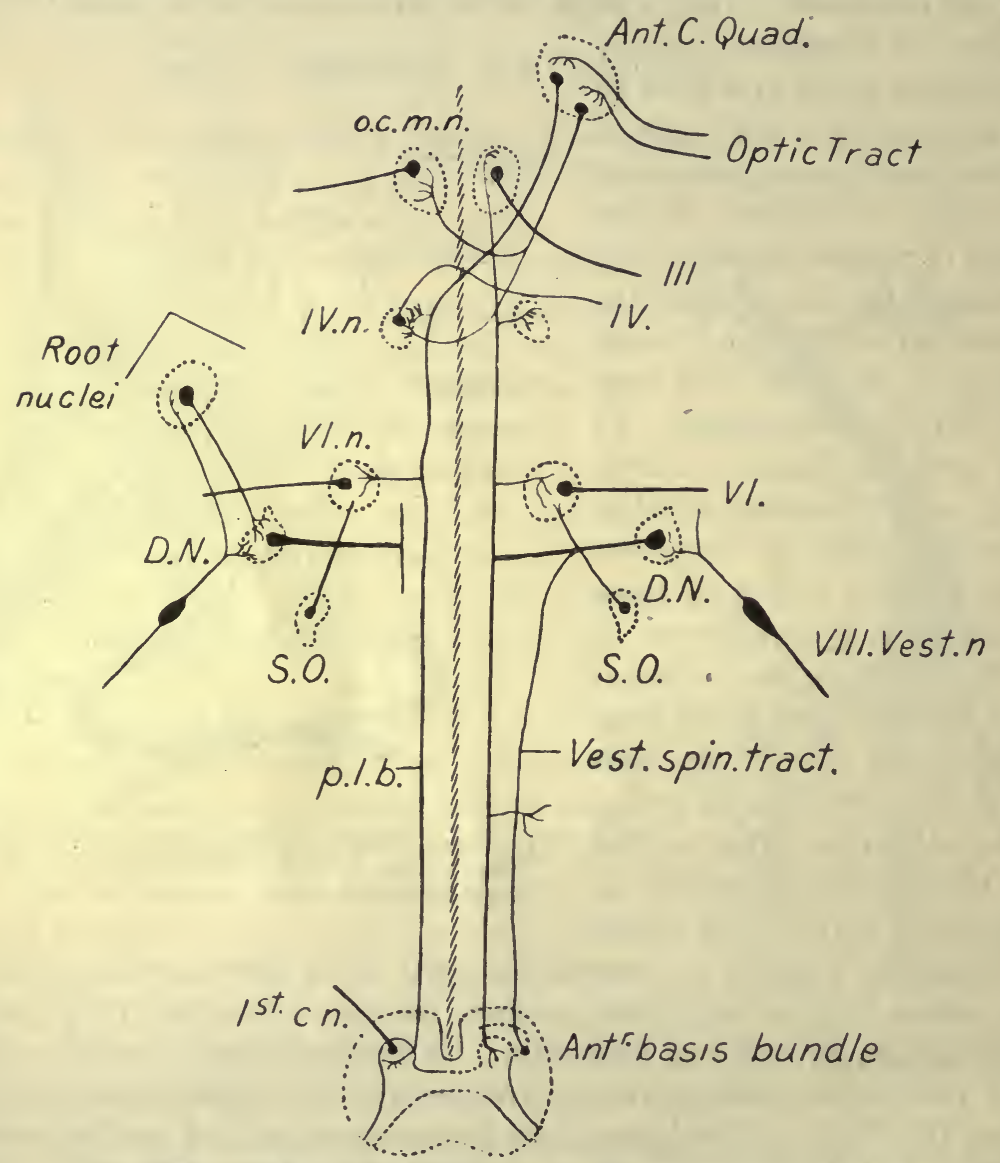

FIG. 205. Diagram of connections of posterior longitudinal bundle.

Ant.C.Quad, anterior corpus quadrigeminum; oc.m.n, oculo-motor nucleus; IV.n, nuclcus of fourth nerve ; VI.n, nucleus of sixth nerve; D.N, Deiters' nucleus ; S.O, superior olive; VIII. Vest.n, vestibular nerve; p.l.b, posterior longitudinal bundle; 1st c.n, first cervical nerve.

the eye centres and those for the movements of the body, and between afferent impressions from the eyes and those from the semicircular canals and the proprioceptive system generally, is effected to a large extent through the intermediation of the posterior longitudinal bundle, which extends throughout the whole length of the mid-brain and the hind-brain, and in the spinal cord becomes continuous with the anterior basis bundle of the anterior 
columns. Receiving fibres above through the anterior commissure from the optic thalamus, and from the superior corpora quadrigemina, it is associated in its course with the three motor nuclei that give origin to the nerves supplying the muscles of the eyeball, viz. the third, fourth, and sixth nerves. Fibres enter the posterior longitudinal bundle from the auditory system, and from the superior olive, and connections are also established between this bundle and the facial nucleus; and the nucleus of Deiters, representing the central station of impulses from the labyrinth. The general connections of the bundle are shown in Fig. 205. 


\section{SECTION XV}

\section{SUMMARY OF THE CONNECTIONS AND FUNCTIONS OF THE CRANIAL NERVES}

Cranial nerves. The cranial nerves are generally reckoned as twelve in number : 1st, olfactory ; 2nd, optic ; 3rd, oculo-motor ; 4th, or trochlear ; 5th, or trigeminus ; 6th ; 7th, or facial ; 8th, auditory ; 9th, glossopharyngeal ; 10th, vagus or pneumogastric ; 11th, spinal accessory ; 12th, hypoglossal.

Of these the first two stand on a different footing from the rest, which, like the spinal nerves, are outgrowths of nerve fibres from the central tube of grey matter surrounding the neural canal or from ganglia corresponding to the spinal posterior root ganglion.

The olfactory bulb and the retinæ, from which the majority of the fibres forming the olfactory tract and the optic nerve respectively take their origin, are analogous rather to lobes of the brain than to peripheral senseorgans. Thus in the retina there are three relays of neurons through which the visual impulse must pass before it arrives at the optic nerve. The olfactory tract and optic nerve are thus comparable with the association or commissural fibres connecting different parts of the central nervous system. The connections of these sensory fibres have already been fully dealt with, and the structure of the peripheral sense-organ will be treated of under the physiology of the special senses. Among the cranial nerves proper we may therefore reckon the third to the twelfth.

The third or oculo-motor arises from an extensive nucleus which extends on either side along almost the whole length of the ventral part of the aqueduct of Sylvius close to the middle line, the most anterior part lying in the back part of the third ventricle (Fig. 204). The anterior part is composed of small cells which give origin to the fibres innervating the intrinsic muscles of the eye, namely, the ciliary muscle and the sphincter pupillæ. The rest of the nucleus is made up of large multipolar cells, arranged in - groups, and gives origin to the fibres passing to most of the extrinsic muscles of the eye. The fibres of the third nerve pass through the tegmentum to emerge at the inner margin of the crusta of the same side. The fibres from the posterior large-celled nucleus supply the following muscles: levator palpebrarum, superior rectus, inferior rectus, internal rectus, and inferior oblique.

Stimulation of the trunk of the third nerve causes the eyeball to look 
upwards and inwards, with contraction of the pupil and spasm of accommodation.

The nucleus of the fourth nerve is situated, just behind that for the third, in the floor of the Sylvian aqueduct, on a level with the inferior corpora quadrigemina. The fibres run from here down towards the pons, then turn sharply backwards to pass into the valve of Vieussens, which they cross horizontally, decussating with the nerve of the opposite side. The superficial origin is therefore from the valve of Vieussens. This nerve supplies the superior oblique muscle of the eyeball. Its stimulation causes the eyeball to look downwards and outwards.

The sixth nerve, the motor nerve for the external rectus muscle of the eyeball, arises from a group of large multipolar cells lying on each side of the middle line in the floor of the fourth ventricle. The fibres of the nerve pass directly outwards to emerge from the anterior ventral surface of the medulla between the pyramids and the olivary eminence, at the lower border of the pons. Stimulation of this nerve causes the eyeball to look directly outwards. All these three oculo-motor nuclei receive collaterals from the fibres forming the posterior longitudinal bundle, many of which are axons of cells in Deiters' nucleus. It is by this means that the contractions of the muscles moving the eyeball are co-ordinated. Sherrington has shown that although the third, fourth, and sixth nerves arise directly from the brain stem and have no ganglion on their course, they are really mixed afferent-efferent nerves. Their afferent fibres, which must arise from the cells in the central nervous system itself, run to the receptor nerve endings with which all the extrinsic eye muscles are richly provided. They are exclusively proprioceptive, and supply no organs outside the muscles innervated by the motor fibres. The occurrence of afferent fibres in these nerves explains the fact previously observed by Sherrington, that after total desensitisation of the eyeball by means of cocaine, or by section of the first division of the fifth nerve, the ocular movements are carried out with as much precision as in the normal animal. As we have seen, such precision of movement requires the co-operation of afferent impressions from the muscle, and the only possible channels for these impressions are the proprioceptive sense-organs and the afferents of the third, fourth, and sixth nerve pairs themselves.

The fifth nerve, or trigeminus, resembles a spinal nerve in that it has a motor as well as a sensory root. The motor root is much the smaller of the two. The fibres of the sensory root take their origin in the cells of the Gasserian ganglion, which is in all respects similar to the ganglion of a posterior spinal nerve-root. The sensory root represents the somatic afferent part of all the motor cranial nerves from the third to the hypoglossal and has a correspondingly wide field of ending in the brain stem. The afferent fibres of the fifth nerve, as they enter the pons, bifurcate, like a spinal afferent nerve, into ascending and descending branches. The ascending branches are short and pass to an upper sensory nucleus, situated below the lateral part of the fourth ventricle in the upper part of the pons. The 
descending branches, which are much longer, are collected into one or more bundles which pass downwards in the lateral part of the reticular formation, accompanied by the downward extension of the sensory nucleus known as the substantia gelatinosa. The descending root can be traced down in the upper part of the cervical cord, its fibres in this region forming a cap to the gelatinous substance of Rolando. From the cells of the sensory nucleus fibres pass towards the median raphe, crossing to the other side to take part in the formation of the tract of the fillet (the trigemino-thalamic tract). The efferent fibres forming the motor root arise from two nuclei. The chief motor nucleus consists of large pigmented multipolar cells situated just below the surface of the lateral margin of the fourth ventricle at the upper part of the pons. The accessory or mesencephalic nucleus is composed of large unipolar cells, situated in the central grey matter along the lateral aspect of the anterior end of the fourth ventricle, and in a corresponding position in mid-brain as far as the upper border of the inferior corpora quadrigemina.

The fifth nerve is the motor nerve for the muscles of mastication, and for the tensor tympani and tensor palati muscles. It is the sensory nerve for the whole of the face (including eyeball, mouth, and nose). It also contains dilator fibres to blood-vessels derived from the chorda tympani, and is said to have trophic functions. The latter conclusion is from the fact that section of the fifth nerve in the skull is followed by ulceration and sloughing of the cornea, and finally by destructive changes involving the whole eyeball. Since, however, these results may be prevented by carefully shielding the eye from all dust and deleterious influences, it is probable that the ulceration is merely a secondary consequence of the anæsthesia. The cornea being anæsthetic, foreign objects that fall on its surface are allowed to remain there, and so give rise to injurious changes and ulceration.

The fifth is also said to be the nerve of taste for the anterior third of the tongue, but it is probable that the taste fibres which run in the fifth are derived from the glossopharyngeal or from the nervus intermedius.

The eighth nerve and its connections have been discussed already on several occasions. We may here briefly summarise what has already been stated. In describing the eighth nerve it is necessary to consider separately its two divisions, the dorsal or cochlear division and the ventral or vestibular nerve. The fibres of the cochlear nerve originate in the bipolar cells of the spiral ganglion of the cochlea. They carry impulses from the auditory endorgan. On entering the medulla they bifurcate into ascending and descending branches which terminate in two nuclei, the ascending branches in the ventral nucleus, the descending branches in the dorsal nucleus. The ventral or accessory nucleus lies between the cochlear and vestibular divisions ventrally to the restiform body. The dorsal nucleus, often called the acoustic tubercle, forms a rounded projection on the lateral and dorsal aspects of the restiform body. From these two nuclei new relays of fibres start, pass to the other side, by crossing the median raphe (where they form the trapezium) to run up in the lateral fillet of the opposite side. From the ventral nucleus the fibres pass directly to the opposite side, forming the greater part of the 
trapezium, making connection on their way with the nucleus of the trapezium and with the superior olive. From the dorsal nucleus most of the axons pass dorsally, forming the striæ acousticæ at the middle of the floor of the fourth ventricle. On arriving at the middle line they dip down and join the fibres of the trapezium of the opposite side. 'The further course of these fibres up to the internal geniculate body, the posterior corpora quadrigemina, and the auditory radiations of the cerebral cortex, have been described on p. 380 .

The ventral division of the eighth nerve, or vestibular nerve, originates in the bipolar cells of the vestibular ganglion or ganglion of Scarpa. These cells, like those of the spiral ganglion, retain the primitive bipolar character. The fibres divide into ascending and descending branches which become connected with two nuclei. The dorsal or vestibular nucleus, or principal nucleus, which receives the ascending fibres, is a mass of grey matter lying laterally of the vago-glosso-pharyngeal nucleus and corresponding to the lateral triangular area, the trigonum acoustici, which is seen on the surface of the fourth ventricle outside the ala cinerea. The descending vestibular nucleus, receiving the descending branches of the vestibular nerve, lies below but continuous with the principal nucleus. The fibres of the vestibular nucleus send also collaterals to the nucleus of Deiters and the nucleus of Bechterew, two accumulations of large multipolar cells lying ventrally and internally to the vestibular nucleus, both nuclei being in close relation to the roof nuclei of the cerebellum. Many fibres of the vestibular nerve pass apparently through these various nuclei on the inner side of the restiform body into the cerebellum, where they make connection with the roof nucleus or nucleus fastigii. By the nuclei of Deiters and Bechterew the vestibular nerve is connected through the dorsal longitudinal bundle and the descending vestibulo-spinal tract with the motor nuclei of the cranial and spinal nerves.

The use of the vestibular nerve is entirely connected with the function of equilibrium. It is probably not concerned in conveying auditory impressions, all its nerve fibres being derived ultimately from the nerve-endings in the saccule and utricle and semicircular canals.

The seventh cranial nerve or facial nerve emerges from the brain at the inferior margin of the pons, lateral to the point of exit of the sixth nerve. It is almost entirely a motor nerve, but carries also some sensory fibres for taste and general sensibility which it receives from the nervus intermedius of Wrisberg. The motor nucleus of the seventh nerve lies in the reticular formation, dorsally to the superior olive, at some depth below the floor of the fourth ventricle. From this nucleus the fibres first pass inwards and dorsally towards the floor of the ventricle, where they collect to form a bundle which runs upwards in the grey matter for a short distance and then turns sharply in a ventro-lateral direction to emerge on the lateral aspect of the pons. The fibres from the motor nucleus supply the muscles of the face, the scalp, and the ear. Secretory fibres also run in the chorda tympani, which is a branch of the facial. These, however, are probably derived, like the sensory fibres, from the nerve of Wrisberg. The sensory fibres of the nerve of Wrisberg originate in the nerve-cells of the geniculate ganglion, and 
passing inwards with the main root of the facial, divide into ascending and descending branches and end in the upper part of the column of grey matter which receives also the sensory fibres of the ninth and tenth cranial nerves.

The ninth and tenth cranial nerves arise by a series of bundles of nerve fibres from the side of the medulla. Both the ninth and tenth are mixed visceral sensory and motor nerves. The sensory nucleus is a column of grey matter lying laterally to the hypoglossal nucleus just below the promin-

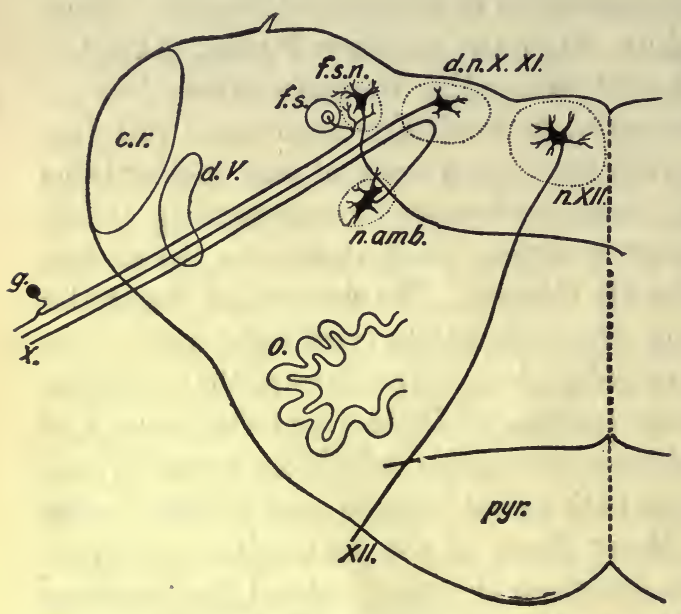

FIG. 206. Plan of the origin of the tenth and twelfth nerves.

pyr, pyramid; $n X I I$, nucleus of hypoglossal ; $X I I$, hypoglossal nerve; $d n X, X I$, dorsal nucleus of vagus and accessory; n.amb, nucleus ambiguus; $f s$, fasciculus solitarius (descending root of vagus and glossopharyngeal); $f s n$, its nucleus ; $X$, crossing motor fibre of vagus; $g$, cell in ganglion of vagus giving origin to a sensory fibre; $d V$, descending root of fifth; $c r$, corpus restiforme. grey"matter lying deeper in the medulla (Fig. 206). ence on the floor of the fourth ventricle known as the ala cinerea. The descending fibres of these nerves form a wellmarked bundle of white fibres known as the fasciculus solitarius, or sometimes, from its supposed connection with the regulation of respiration, the ' respiratory bundle of Gierke.' It may be traced down as far as the uppermost part of the cervical cord, its fibres losing themselves on their way down among the cells of the enclosing grey matter. The efferent fibres of the ninth and tenth nerves are derived partly from the dorsal nucleus of the vagus and accessory nerves lying externally to the nucleus of the twelfth nerve, and partly from the nucleus ambiguus, a mass of

* The ninth or glossopharyngeal nerve supplies motor fibres to the muscles of the pharynx and the base of the tongue, and secretory fibres to the parotid gland. The sensory fibres convey impulses from the tongue, the mouth, and pharynx, the fibres originating outside the central nervous system in the ganglion-cells of the ganglion petrosum and the ganglion superius. It also contains inhibitory fibres to the respiratory centre.

The tenth nerve, vagus or pneumogastric, is joined by the accessory part of the spinal accessory, so that the two nerves may be considered together. It has both afferent and efferent functions :

\section{Efferent functions :}

Motor to levator palati and three constrictors of pharynx.

Motor to muscles of larynx.

Inhibitory to heart.

Motor to muscular walls of œesophagus, stomach, and small intestine. 
Motor to unstriated muscle in walls of bronchi and bronchioles.

Secretory to glands of stomach and to pancreas. Afferent functions :

Regulate respiration. Stimulation of central end may quicken respiration and promote inspiration, or may inhibit inspiration. Stimulation of central end of superior laryngeal branch causes stoppage of inspiration, expiration, cough.

Depressor and pressor (from heart to vaso-motor centre).

Reflex inhibition of heart.

Its afferent fibres arise from cells in the ganglia on the trunk of the vagus, namely, the jugular ganglion and the ganglion trunci vagi. The spinal accessory nerve arises partly in connection with the vagus, partly by a series of roots from the lateral region of the spinal cord as low as the sixth cervical segment. The spinal portion of the nerve is purely motor and supplies fibres to the sterno-mastoid and trapezius muscles.

The twelfth or hypoglossal nerve arises from a collection of large multipolar cells in the floor of the fourth ventricle at its lower end close to the middle line. The nerve-trunk issues from the ventral part of the medulla in the groove between the anterior pyramid and the olivary body. The hypoglossal is purely motor in function, supplying the muscles of the tongue, the extrinsic muscles of the larynx, as well as those moving the hyoid bone.

Since the integrity of the nuclei of the cranial nerves is a necessary condition for the carrying out of various reflex acts in which these nerves are involved, the grey matter of the fourth ventricle and aqueduct is often spoken of as if it were cut up into a series of centres distinct for every act. The chief of these are the respiratory and the vaso-motor centres. Other centres that may be enumerated are :

Centres for movements of intrinsic and extrinsic ocular muscles.

Cardiac inhibition.

Mastication, deglutition.

Sucking.

Convulsive (connected with respiratory).

Vomiting.

Diabetic (connected with vaso-motor).

Salivary.

Centres of phonation and articulation.

We shall have to consider the action of these centres more fully in treating of the several functions of the body. It must be remembered, however, that when a dozen or more centres are enumerated as being situated in the fourth ventricle, it is not meant that we can anatomically distinguish a group of cells for each act or group of actions named. When we say that a part of the nervous system is a centre for any action, we merely mean that this part forms a necessary link, or meeting of the ways, in the complicated directing of nerve impulses that takes place in every co-ordinated act. 


\section{THE CEREBRAL HEMISPHERES}

\section{SECTION XVI \\ GENERAL STRUCTURAL ARRANGEMENTS OF THE CEREBRUM}

THE cerebral hemispheres form the most important part of the brain. It is to the development of this part that is due the rise in type in vertebrates. In development they are formed as two diverticula from the front part of an outgrowth of the first cerebral vesicle. In the lowest vertebrates these outgrowths are connected entirely with the olfactory sense-organs, and we

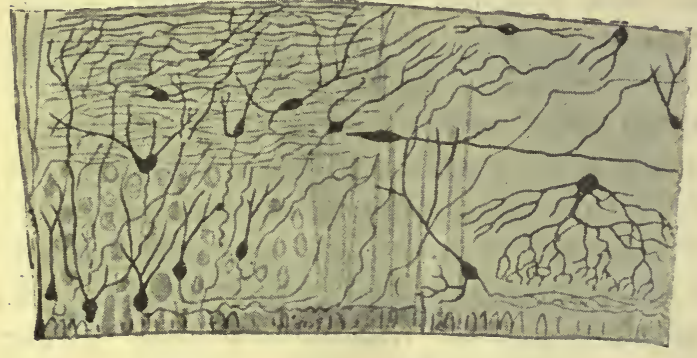

FIG. 207. Section through cerebral cortex of the frog. (After EDINGER.) may regard the olfactory part of the brain as a fundamental part on which has been built up all the rest of the cerebral hemispheres. In a cartilaginous fish the whole of the upper brain is connected with the organ of smell, and consists of a thickening in the floor of the outgrowth from the fore-brain. The roof of the outgrowth is formed of simple epithelium. With the development of the visual sensations in the bony fishes there is still very little corresponding growth of the fore-brain, most of the fibres from the optic nerves going to the roof of the mid-brain (the optic lobes). The beginning of the cerebral hemispheres is associated with the development of nervous tissue in the roof of the prosencephalon. At its first appearance this higher brain material still receives chiefly olfactory impressions. But the structure of the cerebral cortex thus laid down differs from that of the centres forming the brain stem or the olfactory lobe itself in that it provides for a very rich association of impulses between all its parts. The fibres entering the cortex break up into a fine meshwork of fibres which run tangentially to the surface and come in contact with innumerable dendrites of nerve-cells situated at some little distance below the surface (Fig. 207). We have here the first germ of an apparatus in which the nerve-paths can be determined by education, i.e. in consequence of inhibitions by pain, rather than by the limits set by heredity. In the amphibian brain, and still more in the brain of the reptile, the cerebral 
cortex extends over the whole of the roof of the cerebral hemispheres, though even here a very large proportion of it is devoted to the association of olfactory impulses. The importance of these olfactory association fibres is well shown in the figure (Fig. 208) of a diagrammatic section through a lizard's brain. Above the reptiles there is a divergence in the course of development. The wider reactive powers of birds are based chiefly on an enormous development of the corpus striatum, whereas in mammals the corpus striatum remains relatively small and the chief development occurs in the roof of the cerebral hemispheres, the so-called pallium or mantle. With the increased entry of fibres from the optic thalamus into the cerebral hemispheres, carrying impulses from the eyes, ears, and all the other senseorgans of the body, the olfactory part of the brain diminishes in importance,

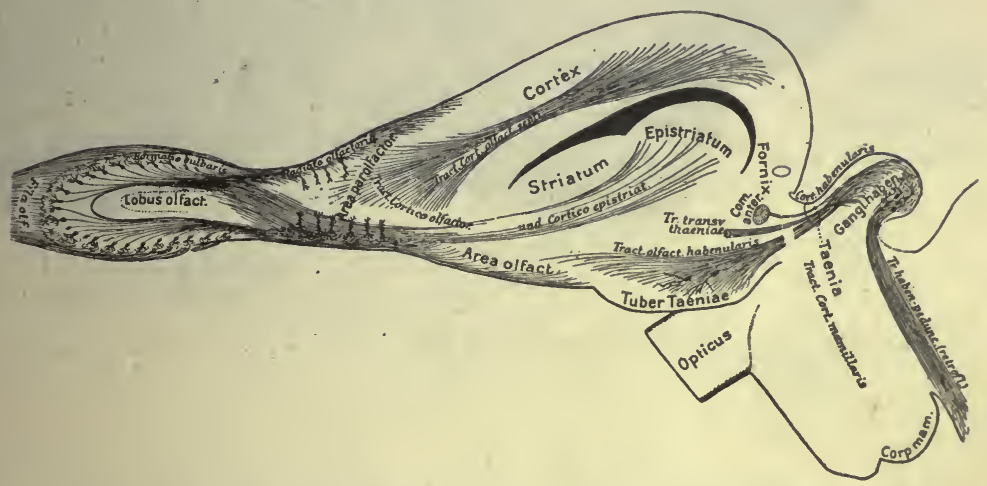

FIG. 208. Schematic section through brain of lizard showing the chief nerve-tracts. (After EDINGER.)

and in the higher mammals and man is altogether overshadowed by the newly formed part of the pallium. On this account those parts of the cerebral hemispheres in special connection with the olfactory sense-organs are often spoken of as the archipallium, in distinction to all the rest of the more newly formed brain substance, known as the neopallium.

In man the cerebral hemispheres form a great ovoid mass exceeding in size all the rest of the brain put together. The two hemispheres are separated by a deep fissure, the great longitudinal fissure. Before and behind, this fissure extends to the base of the cerebrum, but in the middle the two hemispheres are connected by a mass of transverse fibres known as the corpus callosum. On the outer side each cerebral hemisphere presents a deep cleft, the Sylvian fissure. The whole surface of the brain is thrown by fissures (or sulci) into convolutions, by which means a very large increase of the surface grey matter is obtained. By these fissures the brain surface is divided into lobes. The general arrangement is shown in Figs. 209 and 210. The chief lobes are the frontal, the parietal, the occipital, the temporal, the insular, the limbic, and the olfactory. On the inner side, from before backwards, we have the marginal, the paracentral, the pre-cuneus, the cuneus; and in close proximity to the corpus callosum, the cingulum or 


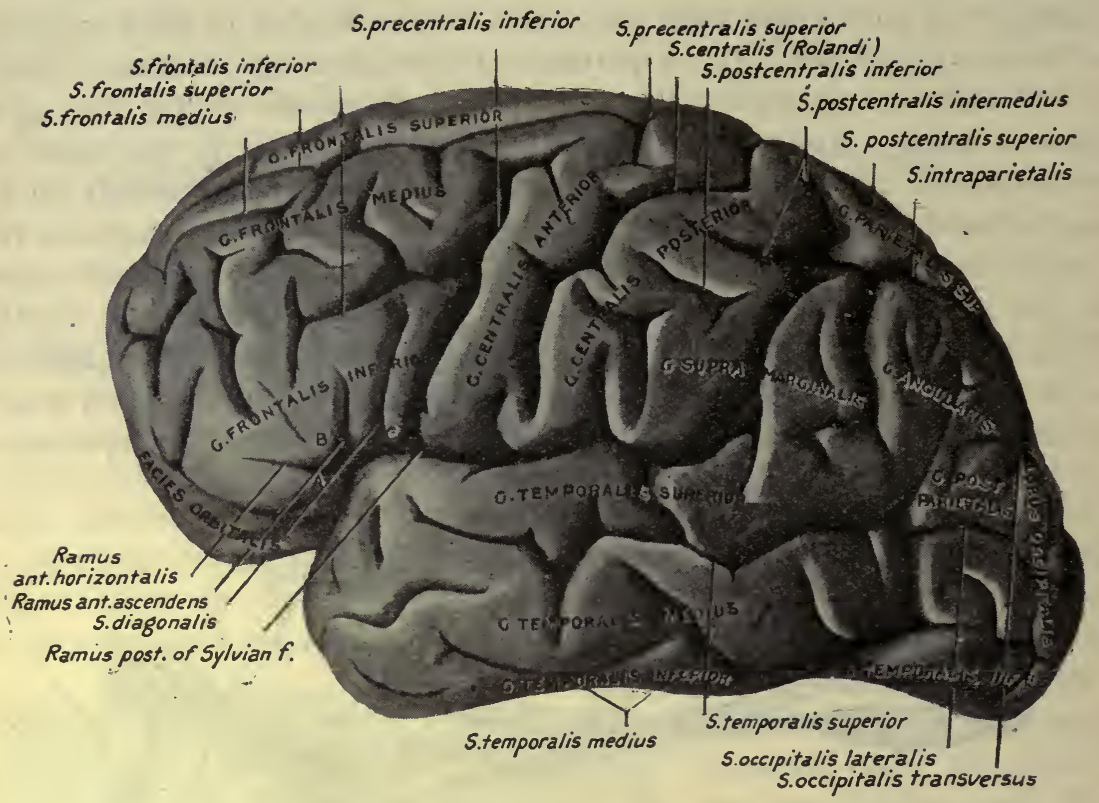

Fig. 209. Left cerebral hemisphere of man, lateral aspect. (Symington.)

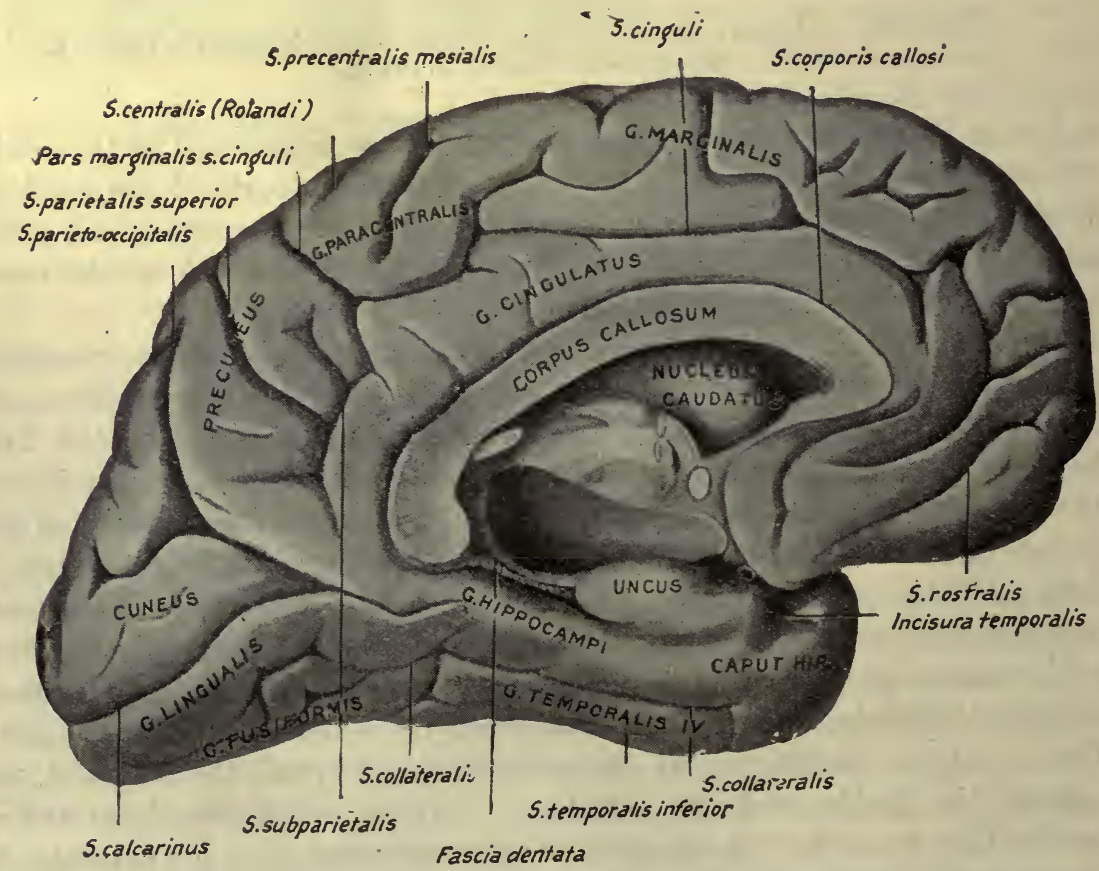

Fig. 210. Left cerebral hemisphere of man, from the mesial aspect. (Srmington.) 


\section{STRUCTURAL ARRANGEMENTS OF CEREBRUM}

supra-callosal convolution above, and the hippocampal convolution and the uncus below. The chief fissures separating these are the Sylvian fissure, the

A

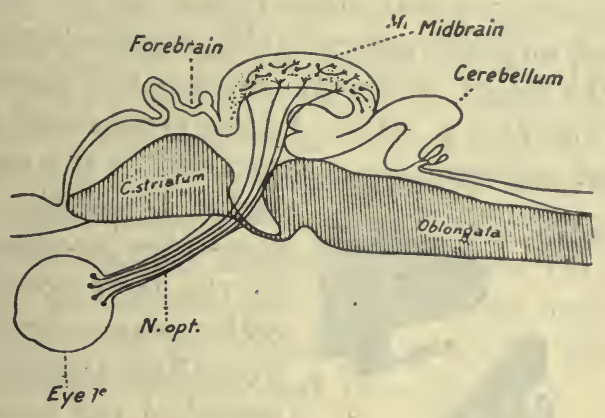

B

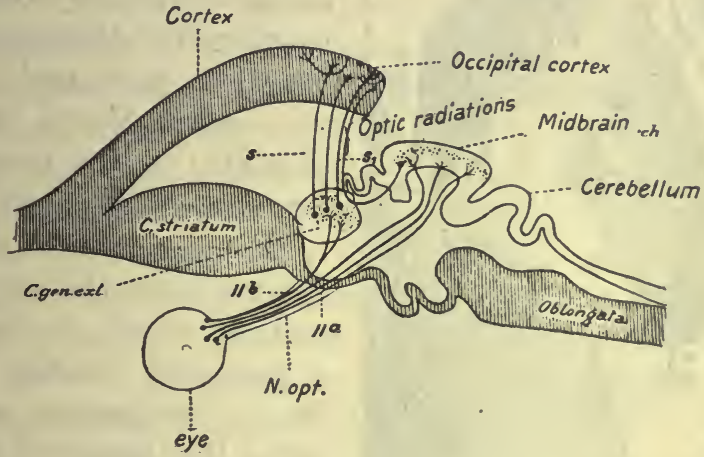

C

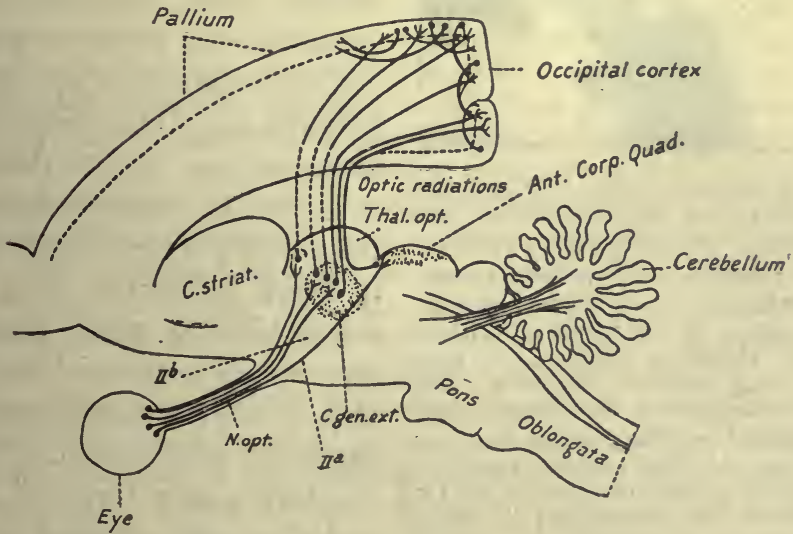

Fig. 211. Diagrams from Monakow, showing the evolution of the neopallium, and the gradual shifting of the visual sensory tracts from the mid-brain to the forebrain, and thence to the occipital cortex.

A, a bony fish. B, brain of a lizard. C, brain of a mammal (cat).

central sulcus or fissure of Rolando, the parieto-occipital fissure, the calcarine fissure, the collateral fissure, and the calloso-marginal fissure. Each of the main lobes (or gyri) mentioned above is further subdivided 
by smaller fissures. The extent of these secondary fissures varies from brain to brain, the higher types of brain being richer in convolutions than those of the more primitive races.

The gradual evolution of the cerebral cortex, and the concomitant shifting of the chief afferent impulses, arising in the projicient sense-organs, from the lower ganglia to the higher educatable cortex, is well shown in the diagrams from Monakow (Fig. 211, p. 419). In the lower fishes practically all the reactions to visual impressions are carried out by the optic lobes. In the

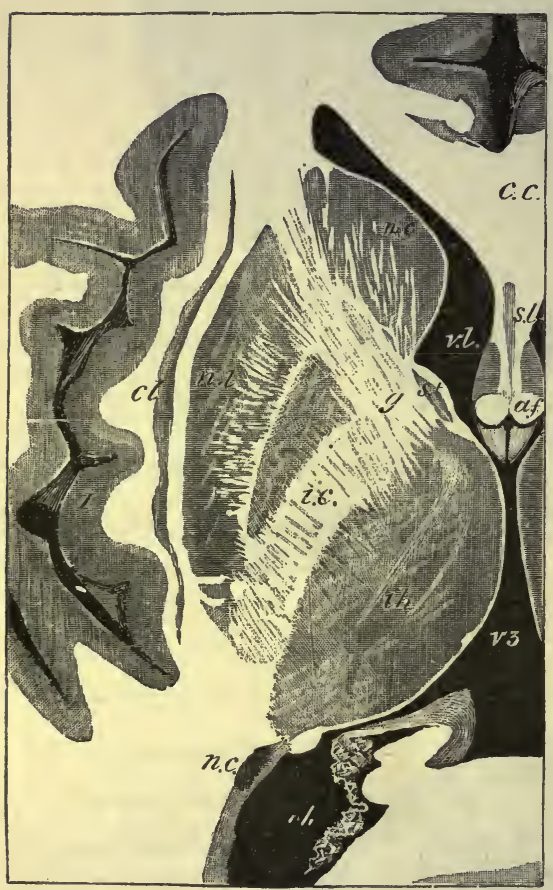

Fig. 212. Horizontal section through the optic thalamus and corpus striatum, the ' basal ganglia.' (Natural size.) (QuAIN.) $v l$, lateral ventricle, its anterior cornu; $c c$, corpus callosum; $s l$, septum lucidum; $a f$, anterior pillars of the fornix ; $v 3$, third ventricle; $t h$, thalamus opticus; st, stria medullaris; $n c$, nucleus caudatus, and $n l$, nucleus lenticularis of the corpus striatum ; ic, internal capsule; $g$, its angle or genu; $n c$, tail of the nucleus caudatus appearing in the descending cornu of the lateral ventricle; $c l$, claustrum ; $I$, island of Reil.

is opened, so that in this region the wall of the cerebral hemispheres, like the roof of the third ventricle, is limited to a simple layer of ependyma. At the margin of the choroid plexus can be seen a part of the superior surface of the optic thalamus, separated, however, from the cavity of the ventricle by a layer of ependyma. Outside and in front of the optic thalamus are the these lobes become subordinated, first to the more complex organ of the optic thalamus (where representatives from all the afferent tracts of the body assemble), and later to the still more complex occipital cortex, when the reactions are determined not only by inherited nerve-paths but also by the various blocks and facilitations imprinted on the nerve-paths by the experience of the individual himself.

The original cavities of the hemispheres form the lateral ventricles, each of which, in the adult brain, is prolonged into the main divisions of the hemispheres as the anterior horn, the posterior horn, and the inferior horn. Each lateral ventricle is roofed over by the corpus callosum and the adjoining white matter of the hemispheres. On opening the ventricle we see on its floor the body of the fornix, a flattened tract of white matter with longitudinal fibres, which in front bifurcates into two cylindrical bundles which pass vertically downwards in front of the foramen of Monro into the mesial part of the sub. thalamic tegmentum. Internal to the fornix is a layer of pia mater, including the choroid plexus. On removing this the third ventricle

higher types the reflexes through 
masses of nervous material constituting the corpus striatum. These present two nuclei of grey matter, known as the nucleus caudatus and the nucleus lenticularis (Fig. 212). The crusta of the crura cerebri as it ascends to the cerebral hemispheres passes behind between the optic thalamus and the corpus striatum, and in front between the nucleus lenticularis and nucleus caudatus of the corpus striatum. Outside the corpus striatum we find another mass of white fibres, known as the external capsule, and this is separated from the white matter of the cortex cerebri by a thin layer of grey matter known as the claustrum. In a horizontal section through the brain, the part of the internal capsule which pierces the corpus striatum forms an angle with the posterior part separating the optic thalamus from the lenticular nucleus. The part where the two limbs come in contact is known as the genu of the internal capsule (Fig. 212).

\section{THE OLFACTORY APPARATUS OF THE BRAIN}

In man the olfactory sense is but feebly developed, and the parts of the brain connected therewith are inconspicuous in comparison with those engaged in the reception of impressions from the other two main projicient sense-organs, namely, sight and hearing. On this account it is not easy to make out the connections of the olfactory lobe proper, the rhinencephalon, with the primitive part of the cortex, the archipallium, subserving the olfactory sense and probably the allied sensations derived from the mouth cavity. The wide connections of the olfactory. sense-organs with the different parts of the brain in the lower vertebrate are shown in the diagrammatic figure of the brain of a reptile (Fig. 208, p 417).

It is interesting to note that the olfactory nerve fibres are derived from cells situated actually on the surface of the body. These are bilateral, spindle-shaped cells, lying in the olfactory mucous membrane at the upper part of the nasal cavity. The peripheral process is short and passes towards the surface, while the deep process passes as a non-medullated nerve-fibre through the cribriform plate of the ethmoid to sink into the olfactory bulb. The bulb, in man, is a greyish enlargement at the anterior end of the olfactory tract. In sections stained by Golgi's method of impregnation it may be seen that the olfactory fibres terminate in an arborisation in close connection with a thick end arborisation derived from a dendrite of a large nerve-cell, known as a mitral cell. The synapses between these two sets of fibres are prominent objects in a section through the olfactory bulb and form the 'olfactory glomeruli' (Fig. 213). The axons of the mitral cells pass back in the olfactory tracts. Each olfactory tract divides posteriorly into two roots, the mesial root which curves inwards behind Broca's area and passes into the end of the callosal gyrus, and the lateral root which runs backwards and over the outer part of the anterior perforated spot. Its filores pass into the uncinate extremity of the hippocampal gyrus. The small triangular field of grey matter between the diverging roots of the olfactory tract is known as the olfactory tubercle. The primitive rhinencephalon includes in the adult human brain the olfactory bulb and tract, together with the anterior perforated space, the anterior part of the uncinate gyrus, the subcallosal gyrus, the septum lucidum, and the hippocampal convolution. The two sides of the rhinencephalon are united by fibres passing through the anterior commissure. Other tracts subserving this apparatus include the habenula passing from the fornix to the ganglion of the habenula, the fasciculus retroflexus passing from this to the interpeduncular ganglion, and the corpus mammillare which is connected with the column of the fornix on the one hand and through the bundle of Vicq d'Azyr with the thalamus on the other. 


\section{THE CHIEF TRACTS OF THE CEREBRAL HEMISPHERES}

We may divide the tracts of the upper brain or cerebral hemispheres into three classes :

I. Tracts connecting the brain with lower levels of the central nervous system.

II. Tracts connecting different parts of the cortex of one hemisphere and serving as a means of association between these different parts.

III. Tracts (commissural) connecting the two cerebral hemispheres together.

\section{THE PROJECTION FIBRES}

These are the fibres which connect the cerebral cortex with the different lower levels of the central nervous system. They form a great part of the

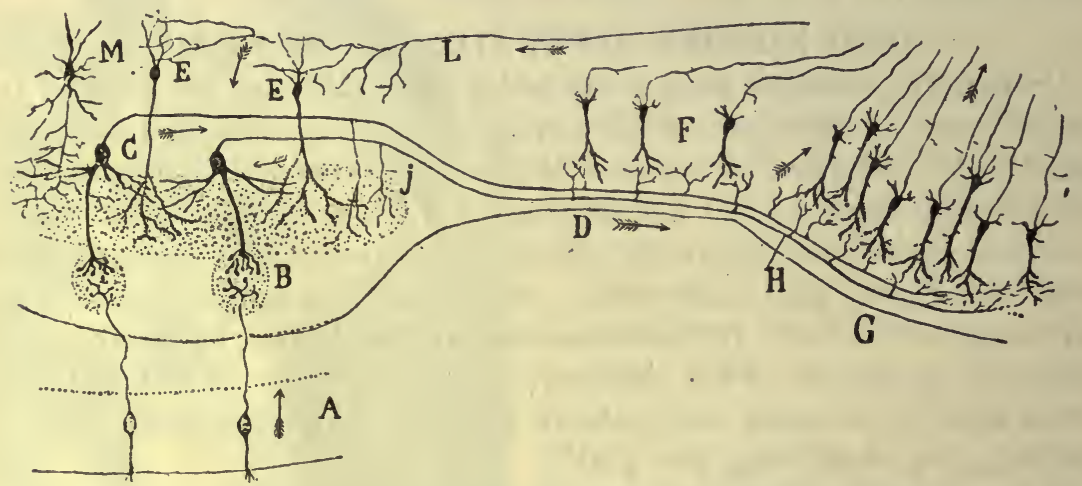

Fig. 213. Schema of course of olfactory impulses. (RAmón y CAJAL.)

A, olfactory mucous membrane; B, olfactory glomeruli; C, mitral cells; $\mathrm{E}$, granule cells ; $\mathrm{D}$, olfactory tract ; L, centrifugal fibres.

fibres of the corona radiata and are condensed at the base of the brain into the broad band of fibres known as the internal capsule. A few of the fibres of the projection system may gain the cortex through the lenticular nucleus and by the external capsule. The projection fibres may be divided into two groups according as they conduct impulses to or away from the cerebral cortex : the afferent or corticipetal, and the efferent or corticifugal.

\section{A. AFFERENT TRACTS OF THE CEREBRUM.}

(1) The thalamo-cortical. From all parts of the optic thalamus fibres arise as axons of the cells of its grey matter and streaming out from its outer and under surfaces pass to every part of the cortex. Although there is no division of them into distinct groups as they leave the thalamus, they are often described as constituting a frontal, a parietal, an occipital, and a ventral stalk. The front fibres pass through the anterior limb of the internal capsule to reach the cortex of the frontal lobe, many of the fibres, however, terminating in the caudate and lenticular nuclei. The parietal fibres issuing from the lateral surface of the thalamus pass through the internal capsule to be distributed chiefly to the parietal lobe. The occipital fibres issue from 
the outer part of the pulvinar and the external geniculate body and constitute the so-called 'optic radiation,' passing outwards and backwards to be distributed to the cortex of the occipital lobe. The ventral fibres pass downwards and outwards below the lenticular nucleus and end partly in the latter nucleus and partly in the cortex of the temporal lobe and of the insula or island of Reil.

(2) The fillet system of Fibres. This great mass of ascending fibres has been already described (cp. Fig. 197) as gathering up the impulses from the different sensory nerves of the cerebro-spinal system and terminating in the thalamus and subthalamic region.

(3) The SUPERIOR CEREBELLAR PEdUNCle. These fibres, from the central ganglia of the cerebellum, terminate for the most part in the thalamus and subthalamic region. It is possible that some of them may pass through the hinder end of the internal capsule, without interruption in the thalamus, to end in the Rolandic area.

(4) The optic Radiation. These diverging fibres in the back part of the coronaradiata are mixed up with fibres which are partly corticifugal. The corticipetal fibres arise in the pulvinar and the external geniculate body and end in the occipital cortex.

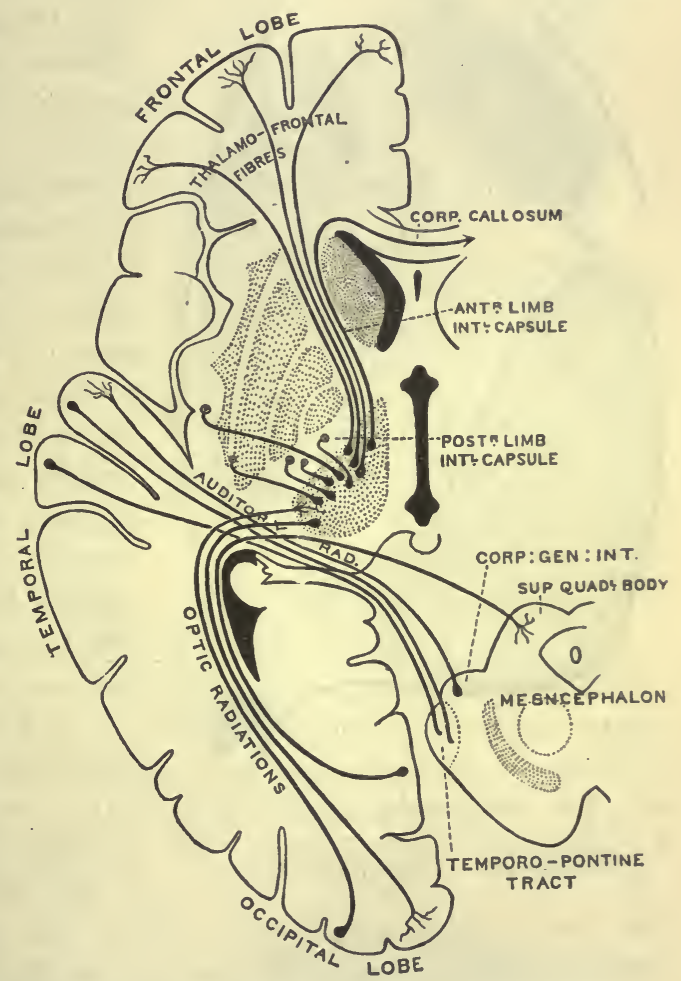

FIG. 214. Schema of projection fibres of cortex. (CUNNINGHAM.)

(5) The auditory radiation. These fibres consist of the axons of cells situated in the internal geniculate body. They pass through the posterior limb of the internal capsule under the lenticular nucleus to end in the temporal lobe.

\section{B. THE EFFERENT PROJECTION FIBRES.}

(1) The pyramidal tract. This is composed of fibres which arise from the large Betz cells in the ascending frontal convolution, the 'motor area.' They pass through the corona radiata into the internal capsule, where they occupy the genu and the anterior two-thirds of the posterior limb. Hence they pass into the crusta, where they occupy the middle two-fifths of this 
structure, and are continued as the pyramids of the pons and medulla to she upper part of the spinal cord, where most of them decussate to the other tide to form the crossed pyramidal tracts. Some of the fibres do not cross at the pyramidal decussation, but are continued down in the same position in the anterior columns of the spinal cord of the same side, forming the direct or anterior pyramidal tracts. These fibres cross for the most part lower down in the cord, so that the direct pyramidal tract is not seen below the cervical

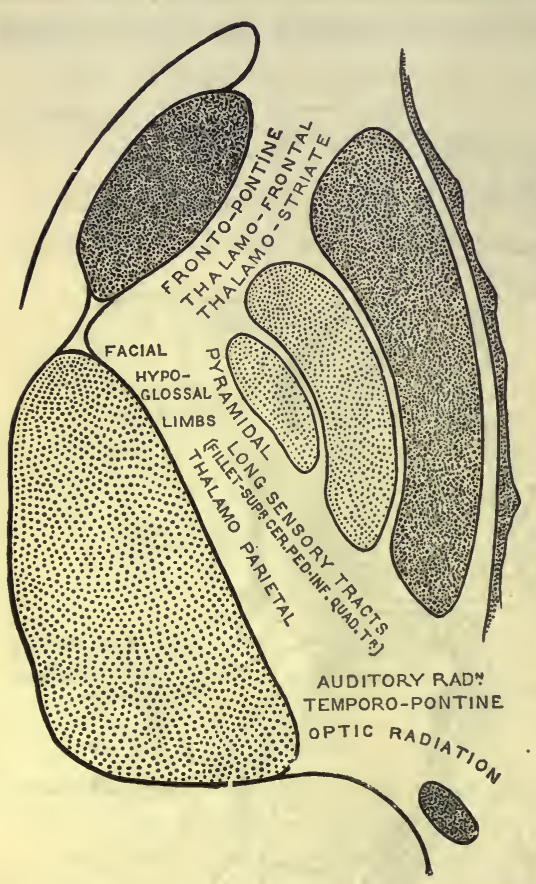

Fig. 215. Diagrammatic representation of the internal capsule, as seeen in horizontal section. (CUNNINGHAM.) region. The pyramidal tracts are not found in lower vertebrates, and make their first appearance in the mammalia. Their development corresponds with the gradual increase in the direct interference of the cerebral cortex in the reactions of the organism as a whole and are an index to the gradual shifting of these reactions from the inevitable to the educated reflex. The fibres of the pyramidal tract end at various levels of the spinal cord and can be traced to the lower end of the sacral region. According to Schäfer they end in the posterior cornua, so that their action is to set going a reaction which could otherwise be elicited by stimulation of the afferent fibres entering by the posterior root at the level of the cord where they end.

(2) The Fronto-Pontine Fibres. These arise from cells in the cortex of the frontal lobe, and pass down in the anterior limb of the internal capsule to gain the mesial part of the crusta of the crus cerebri. The fibres end in the grey matter of the formatio reticularis of the pons, the nucleus pontis.

(3) The temporo-pontine fibres. These arise from the two upper temporal convolutions, especially from that area which is associated with hearing. They pass inwards under the lenticular nucleus through the hinder limb of the internal capsule to gain the outer part of the crusta. In this situation this tract passes down into the pons, where it ends in the nucleus pontis.

As part of these projection fibres we ought probably to reckon the fibres which take origin or end in the corpus striatum. The afferent fibres of this body are derived chiefly from the thalamus, forming the thalamo-striate fibres. Other fibres arise in the nuclei of the corpus striatum and pass down in the dorsal portion of the crusta to end for the most part in the pons, the strio-pontine fibres.

The relative position of these various fibres in the internal capsule 
and in the crusta is shown in the accompanying diagrams (Figs. 215 and 216).

The fronto-pontine and temporo-pontine fibres, which end in the nucleus pontis, come there in relationship with the fibres forming the middle peduncles of the cerebellum and derived chiefly from the lateral lobes of the cerebellum. These fibres may therefore be regarded as the efferent side of the great cerebro-cerebellar connections of which the afferent side is represented by the fibres - efferent so far as concerns the cerebellum-which pass from the cerebellar cortex to the dentate nucleus and thence by a fresh relay in the superior cerebellar peduncles to the red nucleus, optic thalamus, and cortex of the opposite side. The development of these fibres, as of the lateral lobes of the cerebellum, is largely proportional to the growth of the cerebral hemispheres. In cases where there has been congenital atrophy of one cerebral hemisphere the crusta of the same side and the lateral lobe of the cerebellum of the opposite side also fail to develop.

\section{ASSOCIATION FIBRES}

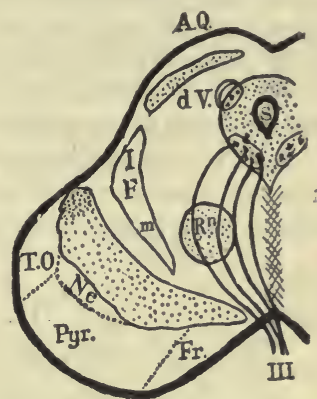

Fig. 216. Transverse section through mid-brain to show position of fillet and pyramid.

$A Q$, anterior corpus quadrigeminum ; $d V$, descending root of fifth nerve; $\mathrm{F}$, fillet (I, lateral, and $\mathrm{m}$, mesial fillet); Pyr, pyramid; Fr. fibres from frontal lobe to pons; TO, fibres from tem. poral and occipital lobes to pons; Ne, fibres from nucleus caudatus to pons; III, root of third nerve; S, Sylvian iter; Rn, red nucleus.

These fibres serve to unite different portions of the cortex of the same hemisphere and may be classified into short and long association fibres. The short association fibres pass round the bottom of the sulci in $\mathrm{U}$-shaped loops connecting adjacent convolutions.
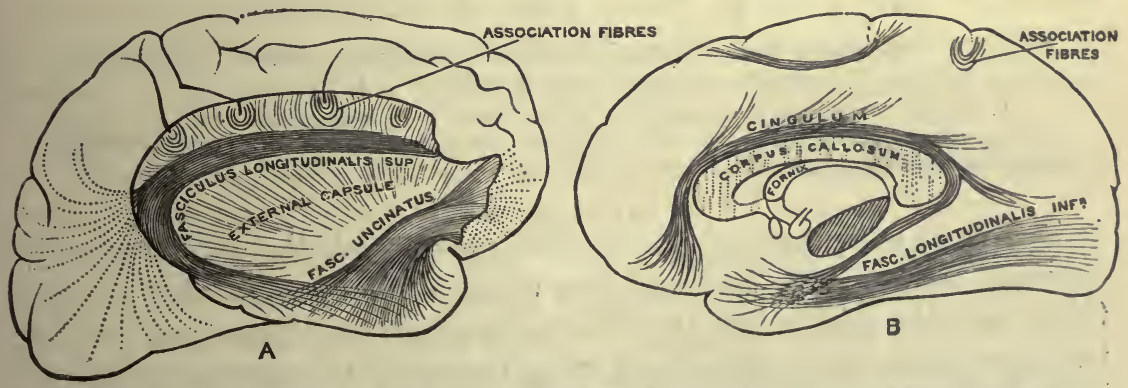

Fig. 217. Chief association bundles of the cerebral hemispheres. (CUnNingham.)
A. Outer aspect of hemisphere.
B. Inner aspect of hemisphere.

These fibres are some of the latest to acquire a medullary sheath and probably first become functional as associated activity between the various portions of the cortex is gradually acquired by education.

The long association fibres may be divided into five groups as follows :

(a) The uncinate fasciculus passes from the orbital convolutions of the frontal lobe to the front part of the temporal lobe round the stem of the Sylvian fissure (Fig. 217). 
(b) The cingulum is closely associated with those parts of the cerebral cortex known together as the limbic lobe. In front it originates in the neighbourhood of the anterior perforated space, passes round the genu of the corpus callosum, and then is carried backwards over the upper surface of this body to its hinder end, where it turns round and is distributed to the hippocampal gyrus and to the temporal lobe.

(c) The longitudinal superior fasciculus lies in the base of the frontal and parietal lobes, and passing from before backwards connects the frontal occipital, and temporal parts of the cerebral cortex.

(d) The longitudinal inferior fasciculus runs along the whole length of the occipital and temporal lobes, being situated behind on the outer aspect of the optic radiation.

(e) The occipito-frontal fasciculus lies on the inner aspect of the corona radiata in intimate relation to the caudate nucleus, and projects out over the upper and outer aspect of the lateral ventricle immediately outside the ependyma.

\section{THE COMMISSURAL FIBRES}

These are arranged in three groups :

(a) The corpus callosum forms a great mass of white fibres passing transversely in both directions between the two hemispheres. Its fibres are

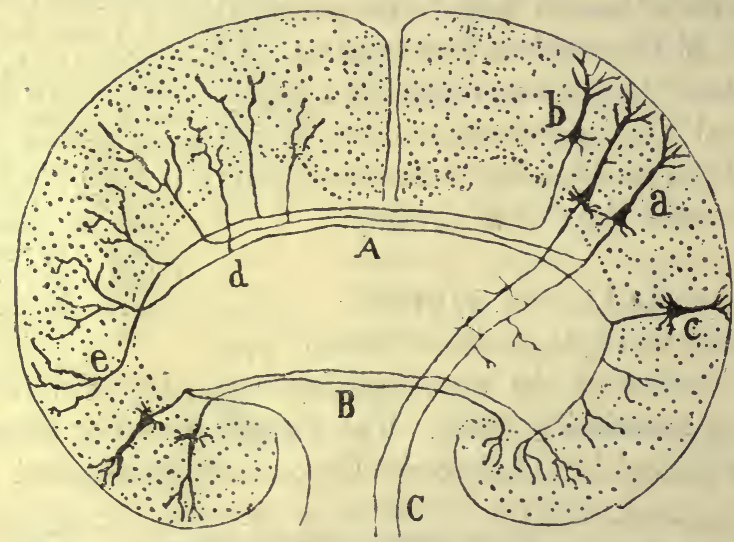

Fig. 218. Schematic section through cerebral hemispheres, to show chief classes of nerve tracts: (After RAMón Y CAJAL.)

A, corpus callosum; $\mathbf{B}$, anterior commissure; $\mathbf{C}$, pyramidal tract; $a$, cell giving off projection fibre; $b$, cell giving off commissural fibre ; $c$, cell with axon forming association fibres.

derived from every part of the cerebral cortex with the exception of the olfactory bulb and the hind and fore parts of the temporal lobe. As the fibres cross the middle line they become gradually scattered, so that they tend to connect wholly dissimilar parts of the cortex of opposite hemispherés. Each callosal fibre arises in one hemisphere and ends by fine arborisations in the opposite hemisphere. It may represent either the axon of one of the cortical cells or a collateral from a fibre of association or a collateral from a projection fibre (Fig. 218).

(b) The anterior commissure is situated in the anterior wall of the third ventricle in front of the two pillars of the fornix. It connects together the two olfactory lobes and portions of the opposite temporal lobes. In lower vertebrates it is almost entirely olfactory in function, but in man the olfactory 
fibres only form a small proportion of the total number making up the bundle.

(c) The psalterium or hippocampal commissure is a thin lamina formed of transverse fibres filling up the small triangular space on the under surface of the hinder part of the corpus callosum formed by the divergence of the posterior pillars of the fornix. Like the anterior commissure, the hippo: campal commissure is closely associated with the sense of smell. Its fibres arise from the pyramidal cells in the cornu ammonis or hippocampus and pass for the greater part to the cornu ammonis of the opposite side.

\section{MINUTE STRUCTURE OF THE CEREBRAL CORTEX}

The cortex of the cerebral hemispheres consists of a layer of grey matter covering a central mass of white fibres. With the growth in size of the brain, which accompanies the development of increased intelligence and powers of adaptation the necessary increase in cortex is rendered possible by the folding of the surface into convolutions and fissures. The chief of these convolutions have already been indicated in the sketch of the anatomy of the brain (Fig. 209).

On section the grey matter is seen to consist of many layers of nerve-cells embedded in neuroglia and nerve fibres, both medullated and non-medullated. The nerve-cells vary in size and shape ; one kind of cell is, however, typical of this part of the central nervous system. This is the pyramidal cell (Fig. 219), a cone-shaped or pear-shaped cell with one large apical dendrite which runs towards the surface and breaks up in the most superficial layer into a number of branches. Dendrites are also given off from the sides and lower angles of the cell. The axon, which arises from the axon hillock in the middle of the base of the cell, passes downwards into the white matter, giving off collaterals in its course. Some of these axons pass by the corona radiata into the internal capsule and into the crura cerebri, including those which form the pyramidal tracts ; others, or their collaterals, may pass into the adjacent regions of the cortex, or across by the corpus callosum into the opposite hemisphere.

Although varying in structure at different parts, it is generally possible to distinguish four or five layers in the cortex.

(1) The most superficial layer, known as the outer fibre lamina, or molecular layer, contains very few cells. It is composed generally of the dendrites of cells from the deeper layers. It contains a few cells which are spindle-shaped and are provided with several processes running parallel to the surface. These are sometimes called association cells. It is probable that afferent fibres, entering the cortex, pass up towards the surface and end for a large part in this molecular layer.

(2) Below this is a layer of pyramidal cells, the outer cell lamina, which is divided by some observers, e.g. Campbell, into three, viz. :

(a) The small pyramidal cells.

(b) Medium-sized pyramidal cells.

(c) Internal layer of large pyramidal cells. 
(3) Below the pyramidal layer we find a stratum of small cells, most of which are stellate.in form. This is known as the stellate or granule layer, or middle cell lamina.

(4) Internal to the granule layer is the inner fibre lamina. In the motor cortex and in certain other parts of the brain this contains large solitary cells, which in the motor area receive the name of the cells of Betz.

(5) Most internal of all, lying next to the white matter, is the poly-

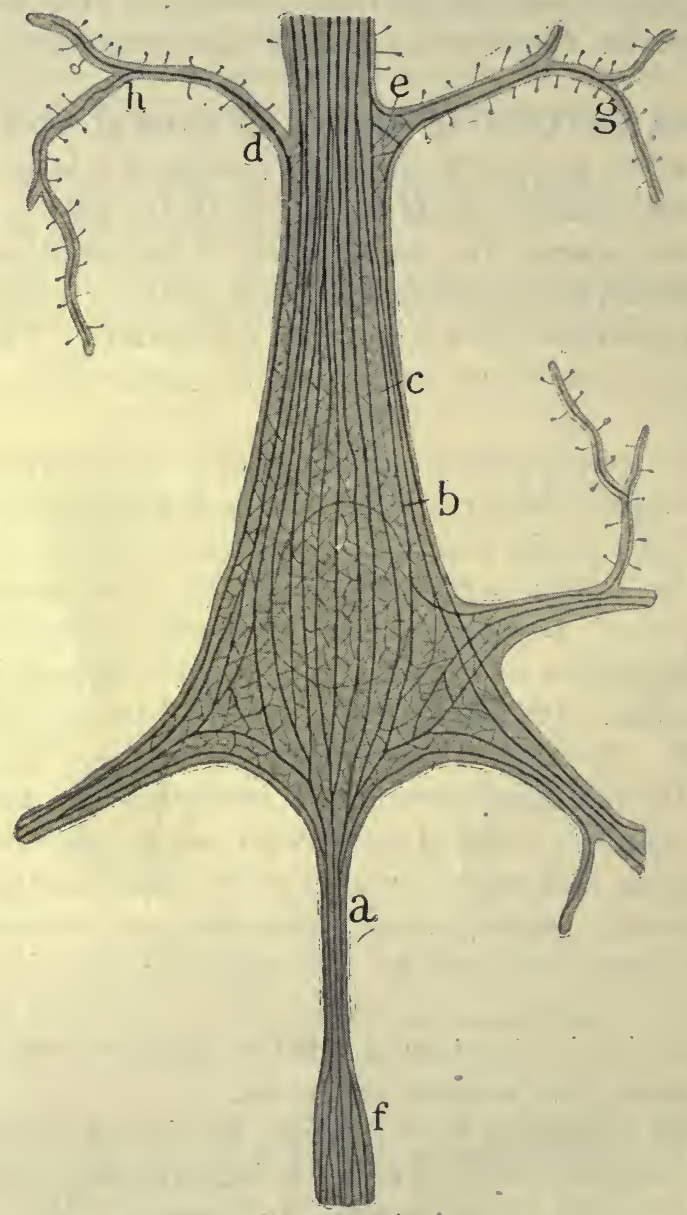

Fic. 219. Schematic representation of the neuro-fibrillar apparatus of a cortical pyramidal cell. (After CAJAL.)

$a$, axon; dh, dendrites.

morphous layer or inner cell lamina, composed of many types of cells, among which spindle-shaped cells predominate. Other cells are also found resembling pyramidal cells of the more superficial layer, but directed in the reverse direction, so that their axons take a course towards the surface. These are the cells of Martinotti. We also find Golgi cells with a freely branching axon, which terminates in the adjacent grey matter. 
If sections of the cortex be stained by some method such as Weigert's, which displays medullated nerve fibres, sheaves of radial fibres may be seen running from the white centre towards the surface and giving off a rich

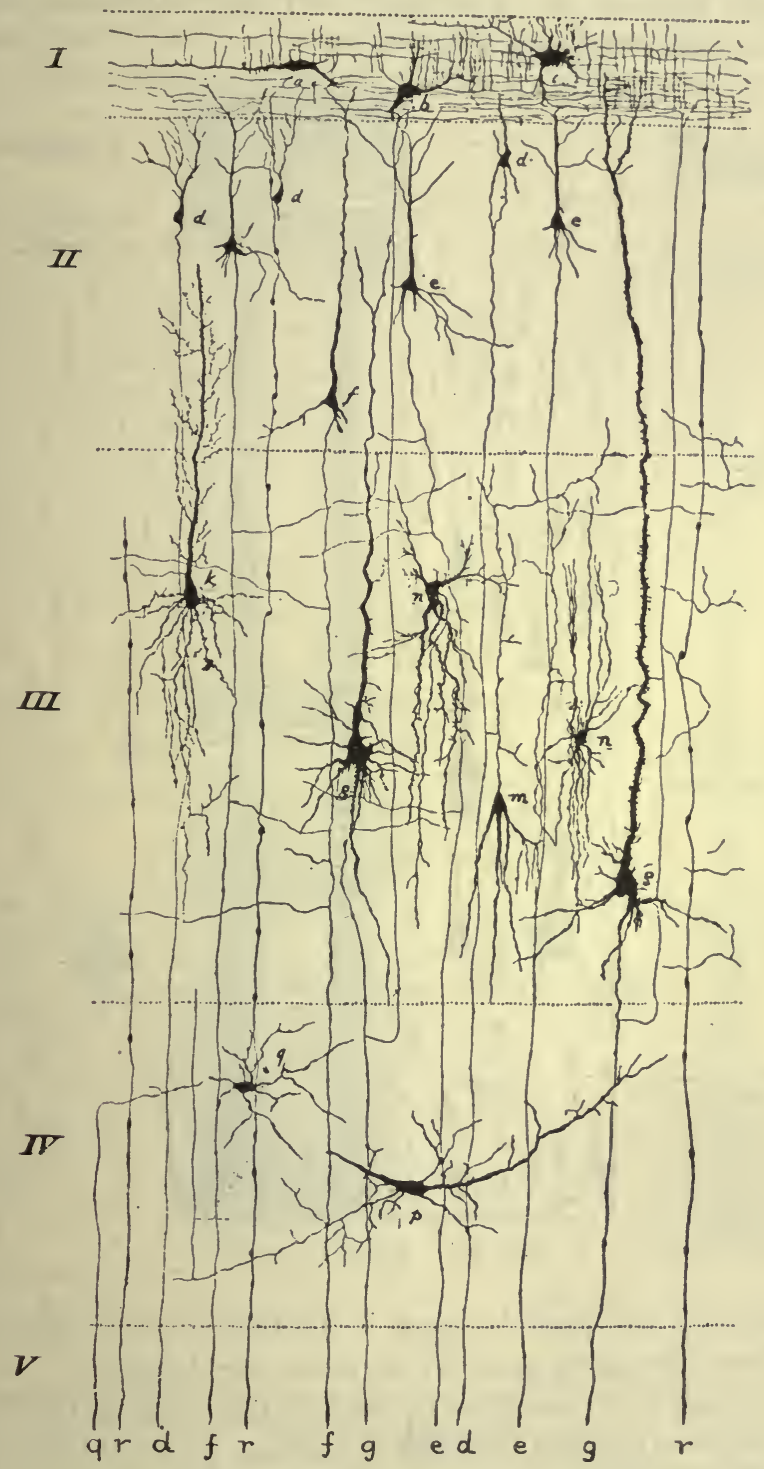

Fig. 220. Diagrammatic section of cerebral eortex. (From BARKER after STARR, Strong, and Leaming.)

I, molecular layer with $a$, bi-polar cell; II, layer of small pyramidal eells; III, layer of large pyramidal cells; IV, polymorphous layer; $V$, white matter.

meshwork of fibres to the intervening portions of the grey matter. In addition bands of tangential fibres are seen running parallel to the surface in certain situations, viz. : 
(a) A layer of very fine fibres just under the surface of the cortex. This layer is especially marked in the hippocampal convolution and is but slightly developed in other regions of the cortex.

(b) A layer between the molecular layer and the layer of pyramidal cells, known as the outer line of Baillarger.

(1) Molecular or outer flbre lamina. $0.34 \mathrm{~mm}$.

(2) Pyramidal or outer cell lamina. $0.90 \mathrm{~mm}$.

(3) Granular layer or middle cell lamina. $0.22 \mathrm{~mm}$.

(4) Inner fibre lamina. $0.22 \mathrm{~mm}$.

(5) Polymorphic orinner cell lamina. $0.31 \mathrm{~mm}$.

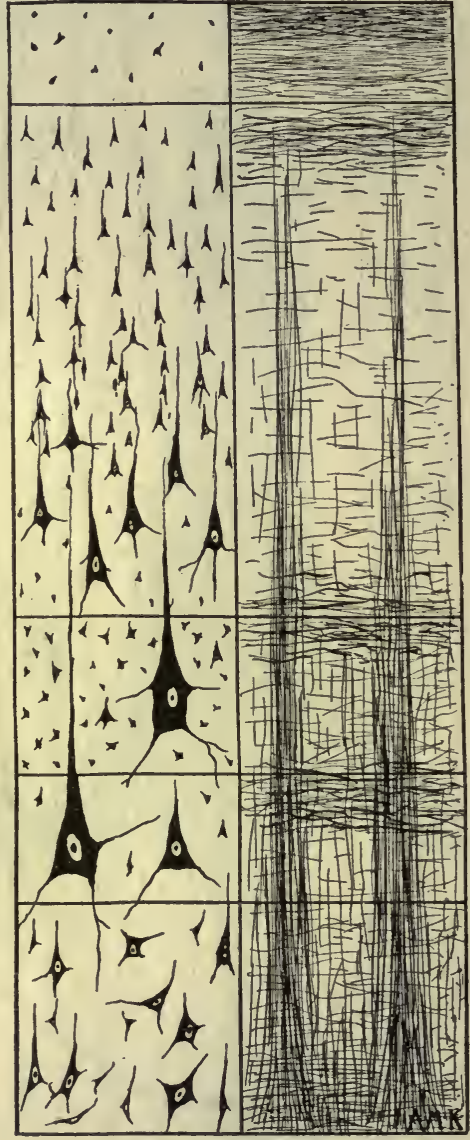

Fig. 221. Motor leg area. a. Tangential layer.

b. Outer line of Baillarger. c. Inner line of Baillarger.

(c) Internal to the granule layer is another zone of fibres, the inner line of Baillarger, giving its name to the inner fibre lamina.

(d) In the part of the occipital cortex, distinguished as the visuo-sensory area, which receives fibres of the optic radiations, a special layer of tangential fibres is observed running through the middle of the granular layer and dividing it into two parts. This is known as the line of Gennari (Fig. 222).

A careful study of the distology of the different parts of the cortex in man enables us to distinguish certain types of structure characteristic of various regions of the grey matter. In attempting by such means an histological localisation of functions we have to take into account:

(a) The thickness of the cortex. 
(b) The relative thickness of the various layers.

(c) The character of the cells found in the various layers.

(d) The arrangement and degree of development of the systems of medullated fibres, both radial and transverse.

The possibilities in such a method are at once apparent if, as in Figs. 221, 222 and 223 , we compare the structure of the cortex from, e.g. the pre-central

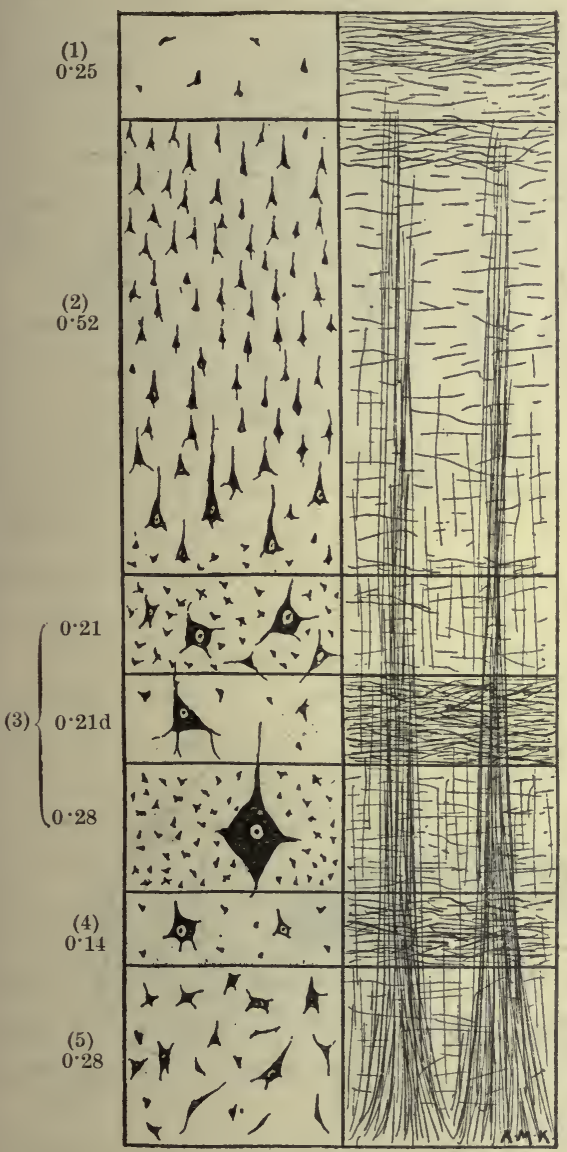

Fig. 222. Visuo-sensory.

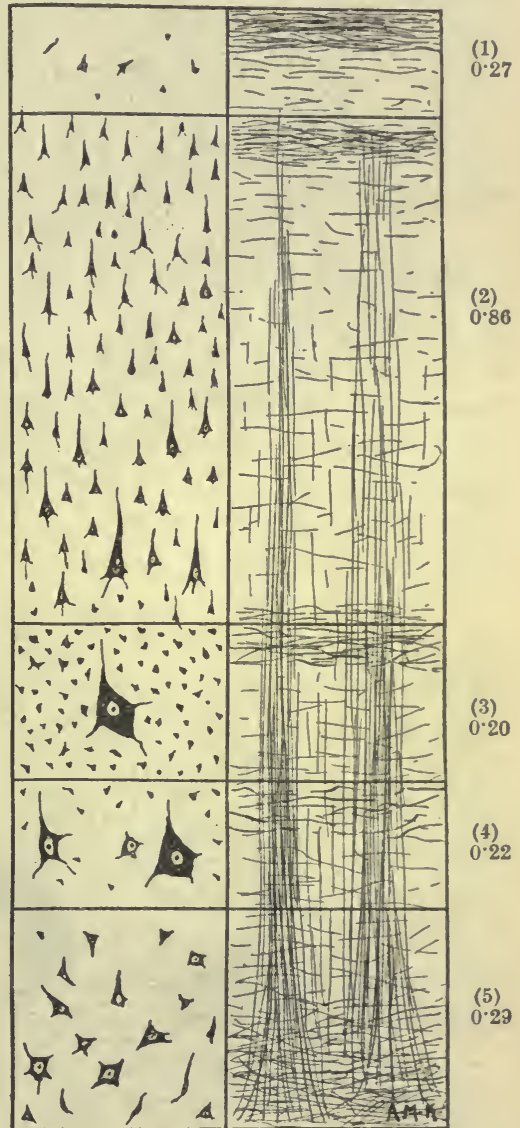

FIg. 223. Visuo-psychic.

motor convolution, the visuo-sensory area of the occipital convolution, and the 'visuo-psychic' area. The finer differences are not so readily perceptible without careful study and measurement of the various layers and the elements constituting them. By this method we can mark out the cortex into areas, which agree closely with the localisation of functions as arrived at by experimental methods or by a study of the systems of fibres in the brain or of the functional defects observed under pathological conditions.

The localisation arrived at in this way is represented in the diagrams taken from Campbell (Fig. 224). Thus in the motor area, the precentrul 
or ascending frontal convolution, we find below the granular layer the wellmarked pyramidal cells or Betz cells, which are larger than any other element in the cerebrum. The layer of pyramidal cells is also very thick, while

A
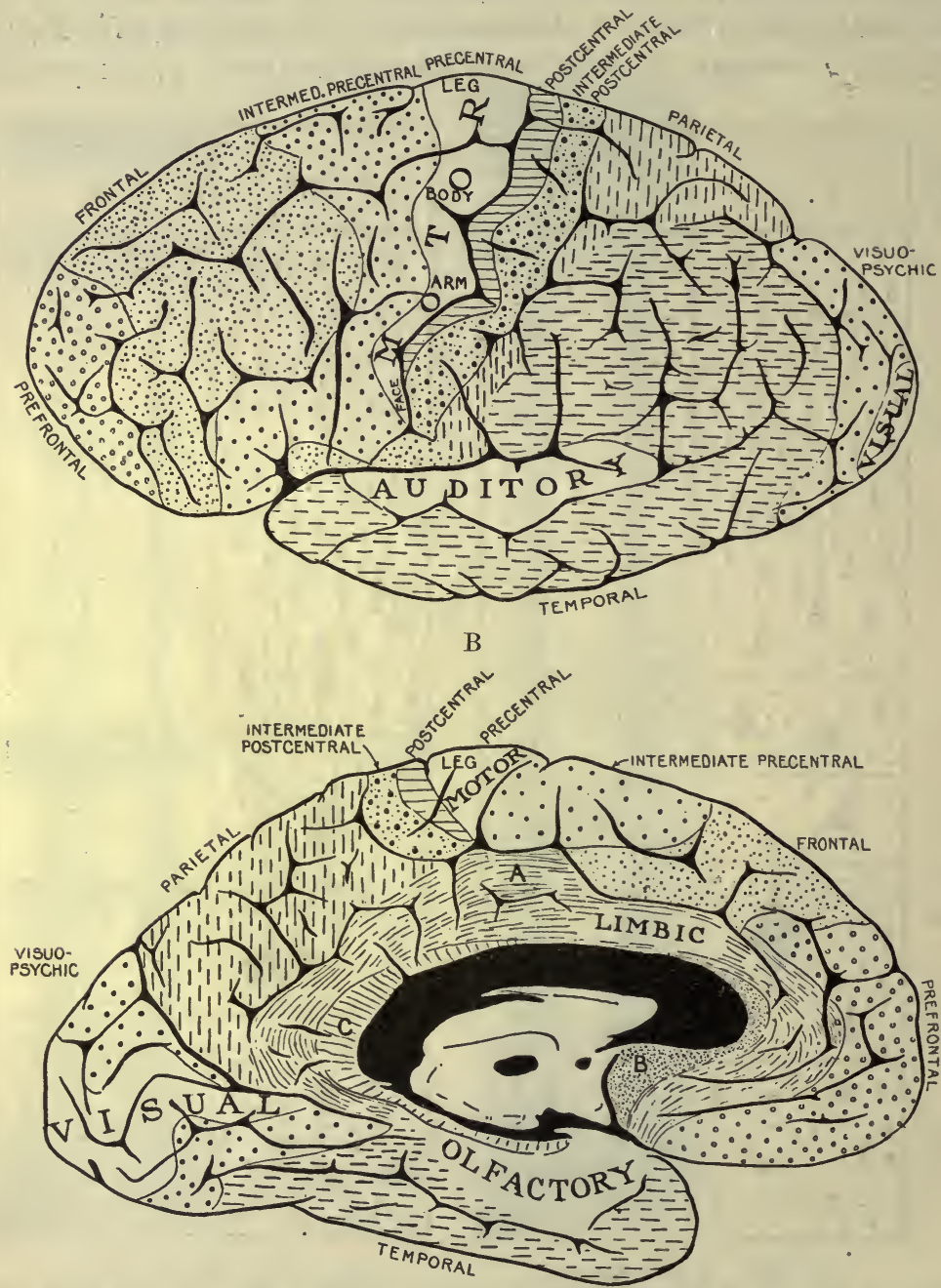

FIG. 224. Human brain showing outer (A) and mesial (B) surfaces, and the situation of the chief motor and sensory areas. The different shading represents the extent of each of these areas as determined by a study of the histological structure of the cortex. (CAMPBel.L.)

the granular layer is but slightly developed. In this area the actual average thickness of the different layers is as follows :

Molecular or outer fibre lamina .

- $0.34 \mathrm{~mm}$.

Pyramidal or outer cell lamina .

- $0.90 \mathrm{~mm}$.

Granular or middle cell lamina .

. $0.22 \mathrm{~mm}$.

Betz or inner fibre lamina

Polymorp̧hous layer or inner cell lamina

. $0.22 \mathrm{~mm}$.

. $0.31 \mathrm{~mm}$. 
In the visuo-sensory area the granular layer is the thickest, and is divided into two layers by the band of tangential fibres forming the line of Gennari. In the association areas, both those, such as the intermediate precentral and visuo-psychic, which are normally associated with motor or sensory processes, as well as the higher association centres of Flechsig, i.e. the frontal, parietal and temporal lobes, the most marked feature in the section is the great development of and the large number of cells observed in the outer cell lamina or pyramidal cell layer. It will be noticed, too, that the audito-sensory area is but small in extent and lies almost entirely within the lips of the fissure of Sylvius, while the greater part of the superior temporal convolution, which on the left side is associated with the auditory images and associations necessary for the comprehension of speech, partakes of the character of an intermediate or psychic sensory area.

The same method may be applied to a comparison of the relative development of the cerebral functions in different types of animals. A comparison of the brain of the dog, ape, and man shows that while the absolute amount of brain substance devoted to the elementary functions of movement and sensation remains practically the same throughout, in man these areas are, relaticely to the whole brain, very much diminished in size, the greater part of the brain surface being taken up with the nervous material of the type which is connected with the functions of association involved in the higher processes of reflection, intelligence, and volition.

If we draw still lower animals into the sphere of our observations we are enabled to form some idea as to the relative significance of the various elements of the cortex. Thus in an animal, such as the rabbit, the polymorphous layer is three times the thickness of the pyramidal layer; whereas in man, with an infinitely greater range of reaction, it is only one-third of the thickness of this layer. If we may roughly assign a function to each of the types of cells found in the cortex, we may say that the pyramidal cell layer is generally associative in functions. The large pyramidal cells of Betz are motor ; the granular layer is sensory, while the polymorphous layer presides over the lowest cortical functions, such as those concerned in the getting of food, the sexual instincts, and so on.

The description given above applies to the whole of the neopallium. In the more primitive part of the brain, the archipallium, represented by the hippocampus, we find only two cell laminæ which are homologous with the middle (granule) and the inner polymorphous cell layers of the neopallium. 


\section{SECTION XVII \\ THE FUNCTIONS OF THE CEREBRAL HEMISPHERES}

IN an animal possessing cerebral hemispheres it is impossible to foretell with certainty what particular reaction may be evoked by any stimulus. The animal which has been deprived of its hemispheres can, as we have seen, be played on at will, whereas the intact animal is an individual whose actions - to judge by our own experience-are guided by intelligence, and influenced by motives or by feelings of fear, hunger, pain, and the like. In short, its behaviour is analogous to that which in man we associate with conscious feeling and volition. This association of the volitional manifestations with the cerebral hemispheres has long been assumed, and is borne out by the exact parallelism existing between the degree of intelligence with which an animal is endowed and the extent of development of its cerebral hemispheres. Moreover in man himself there is a proportionality between the average size of the brain, i.e. of the cerebral hemispheres, and the average intelligence of the race.

Earlier attempts to analyse the factors entering into the sphere of consciousness and to associate with these factors localised parts of the brain failed, largely on account of a faulty psychological analysis and the absence of any proper experimental groundwork for the conclusions put forward. Gall, the founder of phrenology, recognised more clearly than previous authors that the cerebral hemispheres must be regarded as the material basis of consciousness. Impressed, however, by the fact that there was no proportionality between the acuteness of the senses and the degree of development of the cerebral hemispheres, he considered that any division of functions among different parts of the hemispheres must relate to highly complex psychical conditions, and therefore on very slender grounds allotted to parts of the brain functions such as those of intelligence, memory, judgment, amativeness, and so on. These conclusions of Gall were overthrown by Flourens on both theoretical and experimental grounds. In the first place, Flourens pointed out that the mental faculty of man cannot be divided up into a number of different independent qualities or faculties, such as those proposed by Gall. In the second place, he showed that in the pigeon although loss of the whole cerebral hemispheres destroyed intelligence and, associative memory with the actions founded on such endowments, removal of portions of the brain caused simply a lowering of these functions, and it 
was a matter of indifference whether the brain substance was taken from the anterior or from the posterior portions of the hemispheres. Flourens therefore concluded that the cerebral hemispheres acted as a whole as the seat of the will and intelligence. There is no doubt that Flourens was so far perfectly correct, since all parts of the brain must co-operate in determining the psychical condition of any individual in any given moment. He was, however, as later researches showed, in error in thinking that no difference could be distinguished between the parts contributed by the various convolutions of the brain to the organic whole which is called consciousness.

As we have seen, histological evidence, which in the case of the cerebellum displays a marked uniformity throughout the whole cortex, in the case of the cerebrum reveals striking differences between its various areas. The demarcation of the cerebral cortex into areas according to the histological structure of their grey matter agrees with, and in many cases supplements, the results procured by an experimental inquiry into the functions of the different parts.

That there is a localisation of function in the cortex so far as concerns the movements. of the two sides of the body was known to Galen, who mentions the occurrence of paralysis on one side of the body as a re-

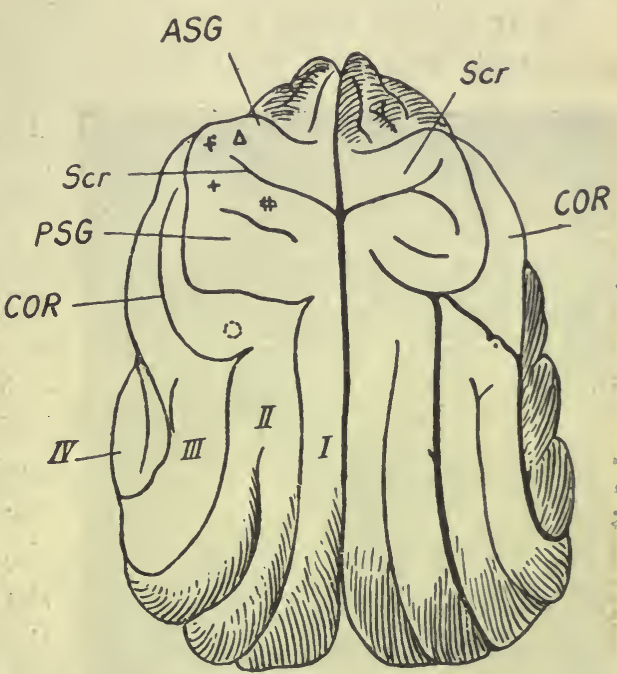

FIa. 225. Upper surface of dog's brain, showing results of excitation. (Fritsch and Hitzig.)

$\Delta$, neck muscles; + , movements of fore limb; $\rightarrow$; movements of hind limb; 0 , movements of face; $A S G$, anterior sigmoid gyrus; $P S G$, postcrior sigmoid gyrus; $C O R$, coronary fissures; $S c r$, crucial sulcus.

sult of lesions in the brain of the opposite side. In 1861 a French physician, Broca, confirming older statements by Dax and Bouillaud, maintained that aphasia, i.e. loss of power of speech, when it occurred in right-handed people was always associated with a lesion of the third frontal convolution of the left hemisphere, which has ever since that time been known as Broca's convolution. Hughlings Jackson in 1864 drew attention to the connection of localised spasms (Jacksonian epilepsy) with lesions of certain parts of the central convolutions. On anatomical grounds Meynert considered that the posterior portions of the hemispheres were probably mare nearly connected with sensation, and the anterior with the power of movement; but direct evidence of motor localisation was first brought by Fritsch and Hitzig in 1870. These observers pointed out, in contradiction of the then received idea, that the grey matter of the cortex was excitable, and that it was possible to evoke co-ordinated movements of the limbs on stimulating the 
front part of the hemispheres in dogs with weak currents. The results of their experiments are shown in Fig. 225. These experiments were soon after repeated and confirmed by Ferrier, who extended his observations to the monkey, and more lately by Horsley, Schäfer, Beevor, Sherrington, and others in the higher apes and man. It was formerly a subject of dispute whether the movements resulting from stimulation of the cortex were due to the excitation of the grey matter or of the underlying white matter. The following facts show that the seat of the excitation is in the grey matter :

(1) A smaller strength of current is required to excite the grey matter than the underlying white matter, after removal of the grey matter.

(2) In animals poisoned by chloral the grey matter is inexcitable, though movements can still be aroused on stimulating the white matter. A

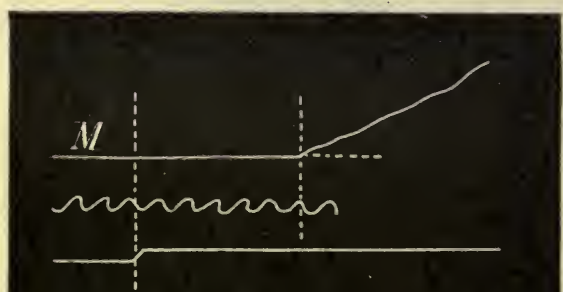
similar inexcitability of the grey matter can be produced by painting it with cocaine.

(3) The latent period elapsing between the beginning of the stimulation and the occurrence of the movement in the corresponding limb is longer when the grey matter is excited than when the stimulus is applied to the white matter. The results obtained by François Franck give a latent period of 065 sec. for the grey matter and $045 \mathrm{sec}$. for the white matter (Fig. 226).

Whether the stimulus acts directly on the pyramidal cells of the cortex, or whether, as seems more likely, it is the endings of the afferent nerves to the cortex which are really excited by the stimulus, we cannot at present determine.

Fig. 226. Tracings to show latent periods of movements obtained by stimulating:

$A$, grey matter; $B$, underlying white matter of cortex. Time-marking $=\frac{1}{100}$ sec.

(F. Franck.)

When we compare different animals, such as the dog, monkey, and man, we find there is a much finer differentiation of movements evoked by stimulation of the cortex in the higher than in the lower type. Whereas in the dog the excitable areas shade into one another, in the higher ape and man the areas are much more circumscribed and are often separated from adjoining areas by an inexcitable zone. The localisation of motor functions in the cortex of the chimpanzee is indicated in the accompanying diagrams by Sherrington (Figs. 227, 228). It will be seen that the motor cortex is limited, on the convex side of the brain, to the precentral convolution, or ascending frontal convolution, situated immediately in front of the fissure of Rolando. On the inner aspect of the hemispheres only the corresponding part of this convolution gives motor responses on excitation. We may say broadly that, from above downwards, by stimulation of the precentral convolution we get movements of the leg, arm, and face ; though, as is shown in the diagram, 
within these larger areas smaller areas can be distinguished for definite co-ordinated movements of the different parts of the body.

NATURE OF MOVEMENTS EXCITED. The movements obtained by excitation of these areas resemble in every respect the co-ordinated move-

FIa. 227.

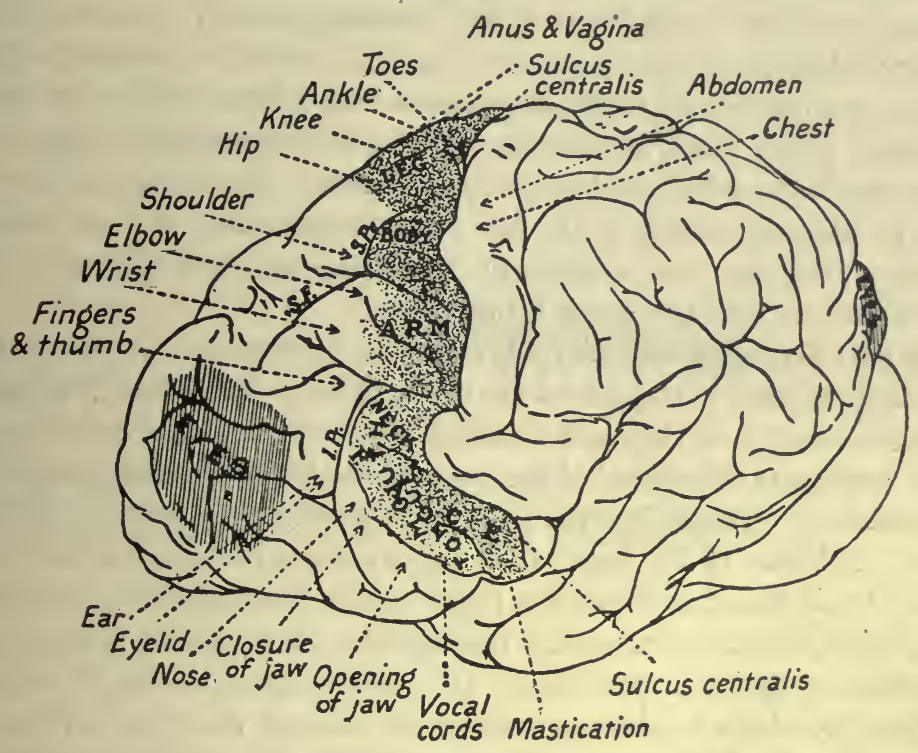

FIG. 228.

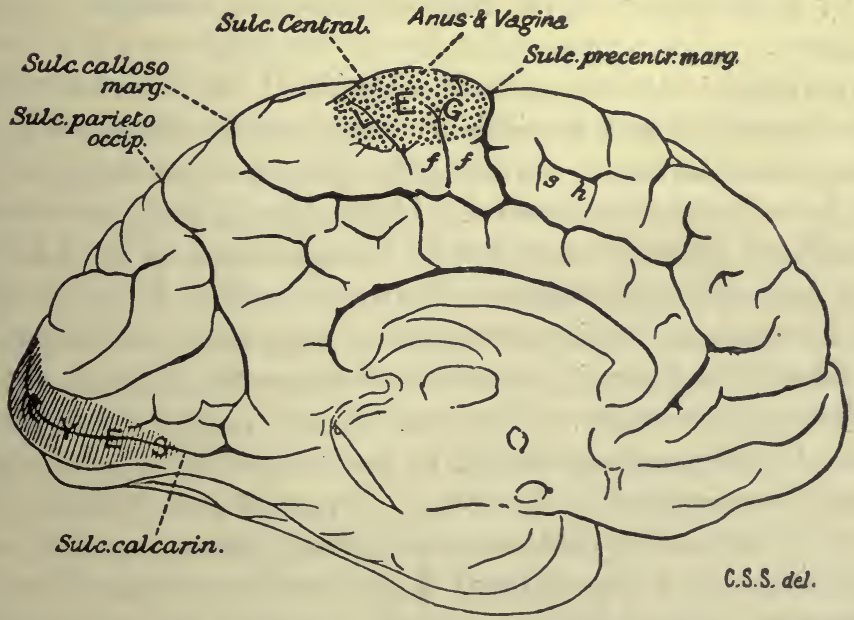

FIG. 227, outer surface; FIG. 228, inner surface of brain of chimpanzee, showing movements obtained by excitation of the motor areas. (SHERRINGTON.)

ments observed during the normal willed or spontaneous activity of the animal. Like the movements evoked by stimulation of a sensory surface they involve therefore the reciprocal innervation of antagonistic muscles. Never do we find simultaneous contractions of antagonists, even where two 
opposing centres are excited simultaneously; one reaction is prepotent, as is the case with cutaneous excitation, and this reaction is attended and brought about by ordered contraction of certain muscles accompanied by an ordered relaxation of their antagonists. Thus the movement of opening the jaw, which can be excited from a fairly large area of the cortex, involves a relaxation of the normal tone of the masseter muscle. Flexion of the leg demands relaxation of the extensor muscles. As in the case of the spinal reflexes, this relaxation, or inhibition, can be abolished under the action of strychnine, or the toxin of tetanus. After administration of either of these it is impossible to evoke inhibition of any muscle. Excitation of the cortical centre for the movements of the jaw causes contraction of both closers and openers of the jaw, i.e. a strife in which the stronger masseter muscles predominate, so that the jaw is firmly closed.

The part played by muscular relaxation in the response to cortical stimulation is also well seen in the case of the eye muscles. Stimulation of the centre for eye movements on the convex surface of the frontal lobes on the right side causes ' conjugate deviation' of both eyes to the left. This movement involves contraction of the right internal rectus and left external rectus, and a simultaneous inhibition of the tone of the right external rectus and left internal rectus. If all the muscles of the right eye be divided except the external rectus, this eye looks permanently towards the right side, i.e. a right external strabismus or squint is produced. On now exciting the right cortex both eyes move to the left, although the right internal rectus is divided. The movement of the right eye stops at the middle line, and is brought about simply by a relaxation of the tone of the right external rectus muscle (Sherrington).

This movement of both eyes on stimulation of one side of the brain shows that the function of each hemisphere is not entirely unilateral with regard to the muscles of the body. As a rule the response to excitation of the motor area for limbs is strictly unilateral. In the case of those movements, however, which are normally carried out by co-operation of the muscles of the two sides, such as the movements of the trunk, neck, and eyes, stimulation of the motor area in one hemisphere evokes a movement involving the muscles of both sides of the body, i.e. the cortical representation is one of movement rather than one of muscles. Where an action is carried out by similar contractions of corresponding muscles on the two sides, the movement itself is bilaterally represented in the cortex. Types of such reactions are found in closure of the mouth, contraction of the abdominal muscles, erection or flexion of the trunk. It seems that under such circumstances there is a free communication between the lower motor centres of the two sides, since the bilaterality of the response is not altered by extirpation of the cortex of the opposite hemispheres to that which is being stimulated.

CORTICAL EPILEPSY. When electrical excitation, of any strength over the minimum effective stimulation, is applied to the motor area of the cortex, the movements evoked tend to persist for a short time beyond the duration of the stimulus. On still further increasing the strength of the 
current, the contraction spreads to adjoining muscles, and finally may affect all parts of the body, giving rise to the phenomenon known as an epileptic convulsion. The same effect may often be caused by weak stimuli, if the irritability of the cortex be raised in consequence of repeated previous stimulation. A typical fit consists of two parts. The first effect of the stimulation is a strong tonic contraction; this outlasts the stimulus for some time, and then gives way to a series of clonic contractions, repeated at first at intervals of from six to ten per second, but gradually getting slower as the fit dies away. The tracing of such a contraction is given in Fig. 229.

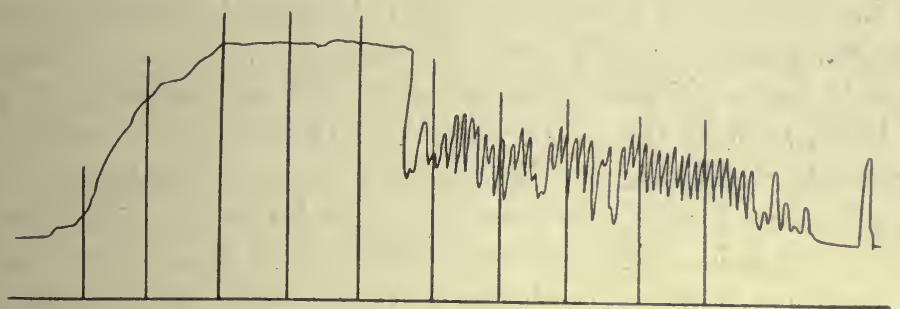

Fia. 229. Tracing of muscular contractions during an epileptic convulsion aroused by strong stimulation of the motor area. (HORSLEY and SoHÄFER.)

The main phenomena of a fit, due to irritation of any portion of the motor area, were described by Hughlings Jackson in 1864, even before the experimental proof of cortical localisation had been brought forward by Fritsch and Hitzig. A similar condition may occur in the human subject as a result of irritative lesions of this part of the cortex, such as that due to the presence of a tumour or a spicule of bone pressing on the brain. Jackson showed that in this condition the convulsive movements follow a certain order or 'march.' Thus if the thumb area be the seat of stimulation, the fit begins by a contraction of the thumb muscles, then spreads to the miscles of the hand, fore-arm and shoulder muscles of the same side, and then to the face, trunk, and leg. If it begins in the toes the order would be up the leg and down the arm. The same ' march' is observed in artificial stimulation of the motor area. If the convulsions are very strong they spread to the leg of the opposite side and then to the whole body. The spread to the other side of the body is not prevented by division of the corpus callosum, nor by isolating the centres from one another, so that the sequence seems to be maintained through the mediation of the sub-cortical centres. Complete excision of the cortical centre for any given movement excludes this movement from participation in the fit. In man this type of epilepsy is, in the milder cases at any rate, generally unattended with loss of consciousness. In animals epileptic convulsions can be excited by stimulation of any portion of the cortex, though it is obtained by a weaker stimulus applied to the motor cortex than to any other part. Jacksonian epilepsy is often preceded by a sensation of numbness or tingling, the 'aura,' in the part in which such convulsions begin. In ordinary idiopathic epilepsy tactile or visual sensory auræ may precede the attack; but in this case loss of consciousness is always a prominent symptom, even in the milder form of the disease. Universal 
epileptic convulsions can be excited in animals by the injection of absinthe into a vein. During the convulsion there is a rise of blood pressure and a quickening of the pulse; the respiration is very often stopped during the tonic part of the spasm, so that the patient becomes livid. The universal condition of excitation affects also the centres from which the secretory nerves originate, so that there is an excessive flow of saliva, which, in the idiopathic case, is responsible for the characteristic frothing at the mouth.

\section{EFFECTS OF ABLATION OF THE MOTOR CENTRES}

We have seen that a dog may preserve complete power of movement after a total ablation of both cerebral hemispheres. We should not expect therefore to find any lasting paralysis as a result of extirpation of portions of the brain, such as the motor centres. Ablation of the motor areas in these animals, during the first few weeks after the operation, gives rise to considerable disorders of movement, the muscles on the side of the body opposite to the lesion being markedly weaker than those on the same side. These symptoms, however, gradually pass off, so that after a time not only are both limbs employed in the ordinary automatic movements of progression, but the animal can be taught new movements in the limb, the cortical centre for which has been excised. We must conclude therefore that in the dog all the movements; including those which are voluntary and conscious, can be carried out in the absence of the motor centres, although destruction of these centres may impair the accuracy with which some of the finer movements are regulated.

In the monkey (Macacus) the effect of ablation is more marked, corresponding to the greater degree of localisation in these animals. If the whole of the motor area on the external surface of the brain be excised, e.g. on the right side, there will be almost complete paralysis of the left arm and the left side of the face, and weakness of the muscles of the left leg. The animal will continue to use the leg in walking and in climbing. If the lesion extends to the medial side of the hemisphere paralysis of the leg is more marked, and the muscles of the left side of the trunk are also affected. Many of these symptoms disappear in the course of time. In a monkey in which Goltz had destroyed the greater part of the left side of the cerebral hemispheres it was found that the right arm and hand could be still employed alone for such purposes as taking food, although the movements were much more awkward than those of the left hand.

Still less complete is the recovery from lesions of the motor area in man. We possess now a considerable number of typical histories of cases in which part of the motor cortex has been destroyed by disease or by operation, and the seat of the lesion verified by post-mortem examination. In all these cases there has been a loss of voluntary movement corresponding in distribution to the seat of the lesion and proportionate in its severity to the extent of the lesion. On the other hand, equally extensive lesions outside the ascending frontal convolution have been shown to have no effect on voluntary movements. The loss of movement is chiefly confined to those which we 
regard as volitional. Although, for instance, the arm may be paralysed, it can be still raised in association with a movement involving the other arm. A certain degree of recovery from the immediate effects of the lesion may be observed, but the recovery is never complete.

The difference in the reaction of various animals to lesions of the motor cortex is connected with the gradual shifting of functions from the sphere of fatal necessary reactions to the sphere of educatable adaptations (i.e. from the lower centres to the cerebral cortex), which is a characteristic of the evolution of the higher type of nervous system, and is a concomitant of the increased adaptability which distinguishes man from all the lower animals. In the animal without hemispheres the motor mechanisms for all the movements of the body are present and can be set into action from any point on the sensory surface of the body. The first effect of adding the cerebral hemispheres to this mechanism is to increase the range of reactions, to modify them or to inhibit them, by diverting the stream of nervous impulses into channels which have to a large extent been laid down in the cortex by the past experience of the individual. In the frog and bird we notice an automaticity and a 'conscious' adaptation of movements to purpose, although the hemispheres have no direct connection with the motor centres of the cord, and present no areas which we can designate as motor. In the dog, although a portion of the brain is in direct connection with the spinal motor centres, and can therefore initiate movements without making use of the mid-brain motor machinery, these movements play only a small part in the motor life of the animal, and the removal of the corresponding centres takes away but little of the conscious functions of the animal. In man the enormous power of acquisition of new movements is rendered possible by the shifting of one motor function after another to the sphere of influence of the cerebral hemispheres. Almost every act of life in man has become one involving co-operation of the cerebral cortex. For many years after birth man is helpless and far inferior, as a reactive organism, to animals much lower in the scale. Even the lower motor functions, such as those of locomotion or defence, have to be painfully learnt, and this learning implies the laying down of paths (Bahnung) in the cortex. On this account the subcortical centres in man are no longer complete. Acting in every instance of life as a subordinate or adjunct to the cerebral hemispheres, they are unable to carry out even the simpler motor reactions of the body after removal of those portions of the hemispheres especially engaged in the control of voluntary movement. The motor defect therefore which is brought about in man, as a result of destruction of one or more of the motor centres, is to a large extent permanent.

If the lesion in man be strictly limited to the motor areas in the ascending frontal convolution, it is impossible to detect any loss of sensation in the affected parts of the body. On the other hand, some loss of sensation is often found where the paralysis is widespread and occasioned by extensive lesion in the neighbourhood of the Rolandic area. Moreover, even in localised lesions in man, an epileptic fit may be preceded by a sensory aura in the part 
which is the starting-point of the convulsive movements. Much discussion has taken place as to the exact significance to be assigned to these slight sensory phenomena. By some observers, e.g. Munk, it has been thought that the motor centres were the end-stations of the fibres subserving muscular sensations, and that the movements resulting from their stimulation were due to the revival of such sensations. Bastian insisted on the important part played in voluntary actions by afferent impressions, and these centres have sometimes been spoken of as 'kinæsthetic' or sensori-motor. The discussion has, however, now resolved itself practically into one of terms. There is no doubt that, when the lesion is strictly localised in the motor area, paralysis may be present without any loss of sensation whatsoever. The paralysis therefore cannot be classed with the sensori-motor paralysis distinguished earlier as the result of division of sensory roots. On the other hand, when we say that this part of the brain represents a 'centre for voluntary movements,' we do not mean that the volitional motor impulses arise de novo from the pyramidal cells in its grey matter. Every neuron in the nervous system is part of an are, and it is generally difficult to label any given neuron as definitely sensory or motor. In a reaction involving a chain of neurons we can assign the name of motor to that neuron which sends its axon to the muscle, and of sensory or afferent to that neuron which receives the impulses at the periphery of the body. Where in the chain we are to draw the dividing line and to say these neurons are sensory and those motor, it is difficult to decide. The motor areas in the cortex give origin to the long fibres of the pyramidal tract, which passes right through the central nervous system to the segmental centres of the cord. We know that the integrity of these tracts is essential for the carrying out of voluntary movement. It is therefore convenient to speak of them as motor or efferent tracts, and their origin as motor centres; although these tracts have the same relation to the motor-cells of the spinal segment as have the afferent fibres from the posterior roots by which similar movements may be evoked.

On the other hand, the activity of the pyramidal cells of the cortex, like those of the motor-cells of the spinal cord, is determined by the arrival at them of afferent impressions. In the absence of these afferent impressions no spontaneous discharge of motor impulses takes place. Thus in the spinal frog we have seen that complete inactivity is brought about by section of all the posterior roots. In the same way paralysis of the arm is induced by section of all its posterior roots, although it can be shown that the motor cortex is still excitable, and that the application of an induced current to the motor centres of the arm evokes a movement as easily as in the normal animal. The motor-cells in the cortical motor centres are normally played upon and aroused by impressions arriving at them from all other parts of the brain and nervous system, and determined originally by impressions falling on the surface of the body. 


\section{THE FUNCTIONS OF THE CORPUS STRIATUM}

The mass of grey matter known as the corpus striatum, which consists of the nucleus lenticularis and the nucleus cordatus, is the basal part of the outgrowth from which each cerebral hemisphere is formed and in the lowest vertebrata represents almost the whole of the telencephalon. For many years the corpus striatum was classed with the optic thalamus as the 'basal ganglia,' and these two ganglia were regarded as relay stations between the cerebral cortex and the lower parts of the csntral nervous system. This view was correct so far as concerns the optic thalamus, in which end all the afferent tracts and from which afferent impressions are carried on by fresh relays of fibres to the cortex. In the higher mammals the motor cortex has a direct connection with the motor nuclei of the bulb and spinal cord through the pyramidal tracts, which are not interrupted anywhere on their course. On destroying the corpora striata degenerated fibres are found running to the optic thalamus, to the red nucleus, and from the latter to the posterior longitudinal bundle. On the other hand the corpus striatum receives fibres from the olfactory tracts and from the optic thalamus. These connections would tend to show that the corpus striatum serves in no way as an intermediary between the cortex and the lower parts of the central nervous system, but is an independent mass of grey matter, receiving impulses from the same source as the cortex and sending impulses which may join in the stream of impressions which play upon the lower motor mechanisms of the bulb and cord.

Isolated excitation of the caudate and lenticular nuclei has no visible effect, provided the current is not so strong as to spread to the adjoining pyramidal fibres in the internal capsule. A study of the evolution of the central nervous system in different classes of animals points to a diminishing importance of these bodies in the normal life of the animal. In the cartilaginous fishes it probably serves to a greater or less degree the same functions in the determination of educated reflexes as the cerebral hemispheres in mammals. In birds the corpus striatum attains its greatest relative development, the increased powers of adaptation in these animals being apparently procured by development of the corpus striatum instead of as in mammals by increased development of the pallium or cerebral hemispheres. In the monkey Kinnear Wilson found no definite results to follow destruction of the grey matter in these bodies. The animals were, however, only allowed to survive the operation of destruction three weeks, and the same observer has pointed out that destruction of the corpora striata in man may give rise to a morbid condition, characterised by tremor in the execution of willed movements and increased tonicity of the muscles. He therefore ascribes to these bodies, or rather to the sensori-motor mechanism which has its chief meeting-place in their nuclei of grey matter, a steadying effect on the motor system, and places this system by the side of the other systems which we have already studied, namely, the vestibular, the cerebellar, and the pyramidal system. 
According to Meyer and Barbour, the anterior part of the corpus striatum plays an important part in the regulation of body temperature. In the experiments a metal tube, closed at one end, was introduced through the brain so as to lie in or on the corpus striatum. Through this tube water at any temperature could be passed. It was found that cooling the water gave rise to shivering and increased heat production in the body with a rise of body temperature, while warming the water had the reverse effect. $\mathrm{He}$ is therefore inclined to regard this part of the nervous system as representing the chief thermo-taxic mechanism of the body.

\section{THE LOCALISATION OF SENSORY FUNCTIONS IN THE CORTEX}

It was pointed out by Ferrier that movements might be obtained on electrical excitation of regions of the cortex cerebri other than those we have described as motor. Thus excitation of the superior temporal convolution on the right side causes the animal to turn its head and eyes to the left and to prick up its ears. In the same way stimulation of the right occipital lobe causes movement of both eyes and head to the left side. These portions of the brain cannot be regarded as having a direct relationship to the motor mechanisms involved in the above movements, since their ablation leads to no defect of movement, but does, in many cases, lead to defect of sensation. Thus excision of the right occipital lobe in the monkey, though leaving the eye movements intact, causes a loss of power to discern objects lying to the left of the middle line. The obvious explanation therefore of the movements, obtained on excitation of this portion of the cortex, is that they are due to the revival or arousing of sensory impressions, that these portions of the cortex represent the cortical receiving-stations for the impulses from definite sense-organs, and that the movements obtained are simply those which are normally associated with a corresponding sensory excitation.

This conclusion is borne out by the fact that it requires a greater strength of stimulus to excite movement on stimulation of the sensory areas than is necessary if the stimulus be applied to the Rolandic area. Moreover Schäfer has shown that the latent period which intervenes between the stimulus and the resulting movement is considerably longer when the stimulus is applied to the sensory centre than when it is applied to the motor centre, suggesting that more neurons are interpolated between the point of stimulus and the discharging motor neuron in the first case than in the latter. Thus in one experiment the latent period between the stimulus and the resulting movement of the eyes amounted to $0 \cdot 2 \mathrm{sec}$. when the frontal lobes were stimulated and $0.4 \mathrm{sec}$. when the occipital lobes were stimulated. Finally the anatomical investigation of the course of the fibres in the white matter of the cerebral hemispheres points to a concentration of sensory fibres from different senseorgans towards certain regions of the cortex. The diagrams (Figs. 230 and 231) show those portions of the brain to which the endings of the sensory tracts of the central nervous system are directed.

From the purely anatomical standpoint we may designate as 'sensory areas' of the cortex : 
(1) An area including both central convolutions, i.e. the ascending frontal and the ascending parietal, and spreading forward into the frontal lobes.

(2) An area occupying the hinder portion of the occipital lobe and the greater part of its inner surface.

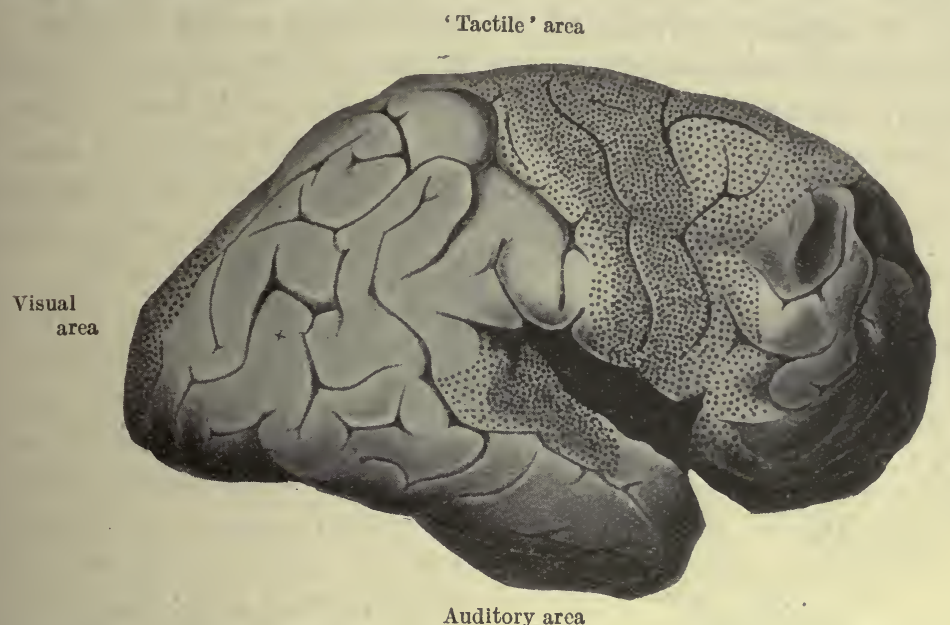

Fia. 230. Outer side of right cerebral hemisphere, according to Flechsig. The dotted surface indicates the regions where the majority of the afferent (sensory) fibres end.

(3) An area occupying the superior temporal convolution and extending well into the fissure of Sylvius.

(4) An area on the inner side of the hemisphere, occupying the hippo-

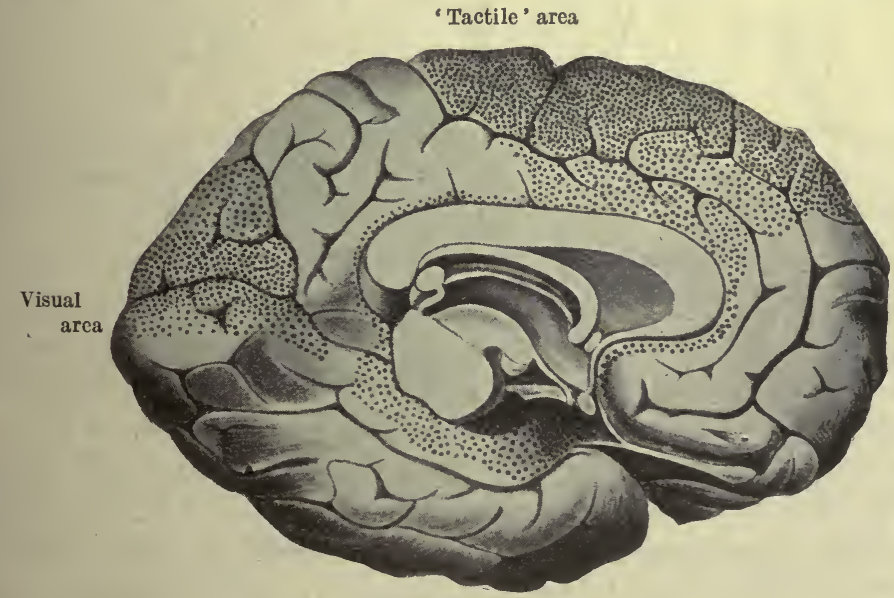

Olfactory area

Fig. 231. Inner surface of the same hemisphere. (FLECHSIG.)

campal gyrus and the margin of the gyrus fornicatus close to the corpus callosum.

Let us see how far experimental evidence bears out this localisation. 
(a) TACTILE AND MOTOR SENSIBILITY. A lesion limited to the ascending frontal convolution may produce paralysis of definite movements or groups of muscles without any detectable interference with sensation. When, however, in man a widespread injury, involving both the Rolandic area and the adjacent portions of the brain, occurs as the result of some morbid condition, such as blockage of the middle cerebral artery, the resulting hemiplegia is almost always associated with a greater or lesser degree of hemiancesthesia. We are therefore justified in locating tactile and muscular

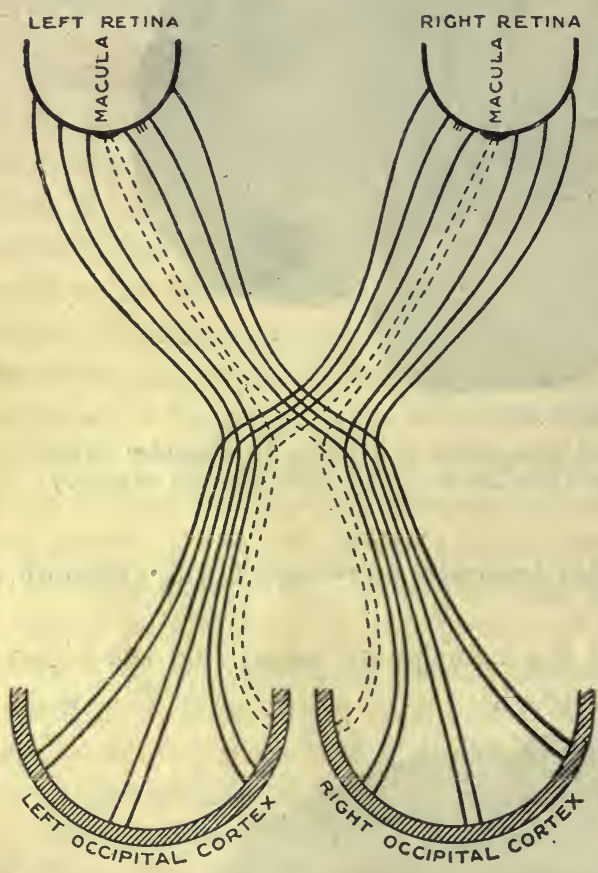

Fig. 232. Diagram showing the probable relations -between the parts of the retinæ and the visualarea of the cortex. (SCHÄFER.) sensibility somewhere in the region of the central convolutions, and it is probable that while it may include the motor area its chief representation is to be found in the post-central gyrus, i.e. the ascending parietal convolution.

The sensory aura which precedes an attack of Jacksonian epilepsy points to the motor area itseif having some degree of sensory functions, and it has been observed that faradisation of the central convolution in man may produce tingling sensations in the part of the body which is the seat of the muscular contractions induced by stimulation. No pain is, however, felt as a result of the stimulation. The impulses which subserve cutaneous and muscular sensibility travel up to the brain in the mesial fillet. This tract comes to an end in the ventro-lateral portion of the thalamus and the subthalamic region. The new relays of fibres, which carry on impulses to the cortex, arise in the thalamus and pass through the hinder limb of the internal capsule to be distributed to the central convolutions. Their area of distribution is, however, much wider than the area of origin of the pyramidal fibres. We may therefore conclude that tactile and muscular sensibility are chiefly subserved by the central convolutions, including the motor area, but are especially dependent on the integrity of the post-central gyrus. Flechsig has shown that fibres from the thalamus, which may.probably be regarded as continuations of the fillet system, are also distributed to other portions of the cortex, i.e. the temporal, the frontal, and the occipital lobes. It is therefore not surprising that the hemianæsthesia produced by lesions in the cetral convolutions is rarely or never complete. 


\section{VISUAL IMPRESSIONS}

Each optic tract, carrying impulses arising as a result of events occurring in the opposite field of vision, ends in the pulvinar of the optic thalamus, the external geniculate body, and the superior corpora quadrigemina. The last named is apparently not concerned in vision, but represents a centre for the co-ordination of visual impressions with those from other regions of the body in influencing bodily movements. From the pulvinar and external geniculate body arises a sheaf of fibres, which pass through the extreme hinder end of the posterior limb of the internal capsule and diverge in the centrum ovale to be distributed to the occipital lobes, being here known as the optic radiations. The anatomical connection of the occipital lobes with vision is confirmed by evidence derived from experiment. Movements of the eyes result from stimulation of almost any part of this lobe. If the upper surface of the right occipital lobe be stimulated, both eyes move downwards and towards the left. Excitation of the posterior part causes movement of the eyes up and to the left; while between these two parts there is an intermediate zone, most marked on the mesial surface, stimulation of which evokes a purely lateral deviation of the eyes to the left. It is therefore concluded not only that there is representation of visual impressions in the occipital lobes, but that there is a certain amount of localisation within the visual area itself, as is represented in the diagram (Fig. 232).

These conclusions are fully borne out by the results of ablation. While extirpation of the whole occipital lobe on one side in animals causes crossed hemianopia, i.e. has the same effect as division of the corresponding optic tract, extirpation of these lobes on both sides causes complete blindness. It seems that the fovea centralis-the region of distinct vision-is bilaterally represented, so that central vision is usually retained in both eyes after destruction of one occipital lobe (Fig. 233).

The area connected with vision seems to be smaller in man than in the ape, and in the ape than in the dog. Thus in man complete blindness has been observed as the result of localised bilateral lesions of the internal surfaces of the occipital lobes, and we find the same relative limitation of area as we proceed from lower to higher forms in the case of the other sensory areas of the cortex.

\section{THE AUDITORY AREA}

Anatomical study indicates a connection of auditory sensations with the superior temporal lobe. The impulses, started by the arrival of sound waves at the ear, travel by the cochlear nerve to the medulla. From the two auditory nuclei a well-marked set of fibres passes across to the opposite side in the corpus trapezoides, then turns up into the tegmentum of the oppsite side to form the tract known as the lateral fillet. The fibres of this tract end partly in the inferior corpora quadrigemina, partly in the internal geniculate body. From the latter, fibres pass into the internal capsule, and thence as 'auditory radiations' directly to the superior temporal convolution.

In the monkey stimulation of the upper two-thirds of this lobe of the 
brain causes pricking of the opposite ear, dilatation of the pupils, and rotation of the head and eyes to the opposite side. It was stated by Ferrier that ablation of the superior temporal convolution causes deafness, but Schäfer found that, even after extirpation of the superior temporal convolutions of both sides, monkeys showed signs of hearing quite distinctly, and of understanding the nature of the sounds heard. One must conclude therefore that the function of auditory perception is not entirely confined to the temporal lobe, though its focal point may be located in the superior temporal convolution, especially in that part which is seated within the fissure of Sylvius.
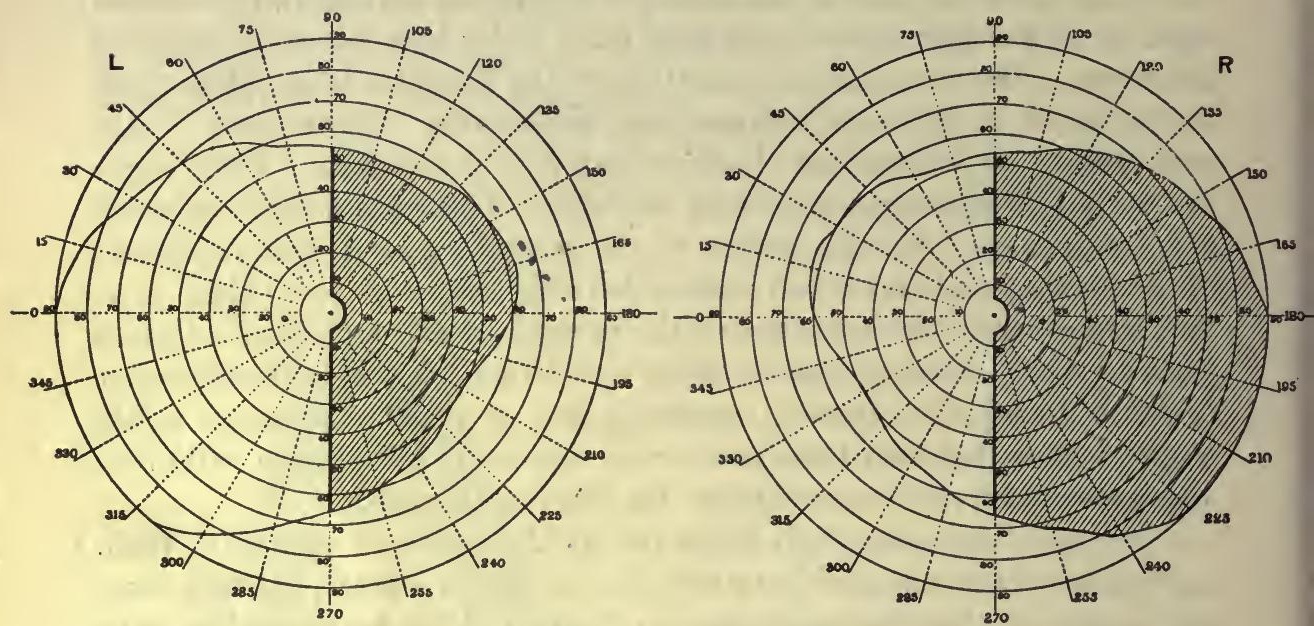

Fig. 233. Perimeter charts from right and left eye, showing the limitation of the field of vision (right hemianopia) produced by a lesion of the left occipital cortex. (BECHTEREW.)

This conclusion is strengthened by the results of clinical evidence in man, in whom cerebral lesions, which have produced disturbances of auditory perception, are found almost invariably to be closely associated with the superior temporal convolution.

\section{SMELL AND TASTE}

The course of the fibres from the olfactory lobe may be used to throw light upon the localisation of olfactory sensation in the cerebral cortex. There is a great divergence between different animals in the degree to which the olfactory sense is developed, and with this divergence we find corresponding variations in the development of certain portions of the brain. In those species with highly developed olfactory sense the following parts of the brain show special growth :

(1) The olfactory lobe, including the olfactory bulb, and the olfactory tract.

(2) The posterior part and the inferior surface of the frontal lobe.

(3) The hippocampal gyrus and the dentate convolution.

(4) A convolution termed the gyrus supracallosus and forming that part of the gyrus fornicatus closely encircling the corpus callosum.

(5) The anterior commissure. 
The olfactory lobe is connected almost exclusively with the cerebral hemispheres of the same side. Ferrier found that electrical excitation of the hippocampal region causes contortion of the lip and nostril on the same side, i.e. a reaction such as that actually induced in these animals by application of an irritative or pungent odour direct to the nostril. Ablation experiments have not yielded very definite evidence on the question of localisation of the olfactory sense. So widespread are the connections of the olfactory tract throughout the brain that it would be extremely difficult, if not impossible, to extirpate all those paris which receive fibres from this tract. It is usual to regard the sense of taste as associated with that of smell, but here again experiment and clinical evidence have yielded very little that is definite.

\section{GENERAL CHARACTERISTICS OF CORTICAL MOTOR FUNCTIONS}

The motor phenomena, which may be observed as the result of artificial excitation of the motor and sensory areas in the cortex, constitute a very small fraction of the activities which must be associated with the cerebral hemispheres. An animal with its cerebral hemispheres intact differs markedly from a decerebrate animal in the variety of combined movements which it may exhibit, either spontaneously or in response to external stimuli. When, however, we excite the motor areas directly, we obtain movements which are practically identical with those which we may elicit from a bulbospinal animal by appropriate peripheral stimulation. The movements thus excited from the skin may be looked upon as variations from the tonic postural activity of the musculature of the body. We have seen that from the endorgans subserving deep and muscular sensibility (the proprioceptive system), as well as from the labyrinth, impulses are continually arising which travel up to the spinal cord, bulb, cerebellum, and mid-brain, and excite a tonic activity of these centres. The normal attitude of the animal depends on the tonus thereby produced in certain muscles. Muscular tone is indeed a quality specially found in certain groups of muscles. If the cerebral hemispheres be removed, as by a section through the crura cerebri or in front of the mid-brain, this postural tonus is increased and the animal enters into the condition of ' decerebrate rigidity.' Destruction of one labyrinth diminishes the tone on the same side of the body; section of all the afferent nerves from a limb abolishes the tone in that limb, so that its posture thereafter depends entirely on gravity.

The movements which are excited in such animals by cutaneous stimulation involve as a necessary factor inhibition of the postural tone as well as co-operative inhibition of the antagonistic muscles. In the same way excitation of the motor area of the cortex has as its most essential feature an inhibitory action on the postural tonus in addition to its excitatory action on the muscles concerned in the movement. A certain antagonism is evident between the total action of the cerebral hemispheres and that of the proprioceptive part of the central nervous system. Whereas in the decerebrate animal there is increased tonus in the masseters, in the neck muscles, 
the muscles of the trunk, and the extensor muscles of the limbs, stimulation of the cortex produces opening of the mouth, flexion of the fore limb or of the hind limb, more easily than any other movements. That an essential part of this action is inhibitory is shown by the effects of exciting the motor area of the cortex after exhibition of strychnine or during the local action of

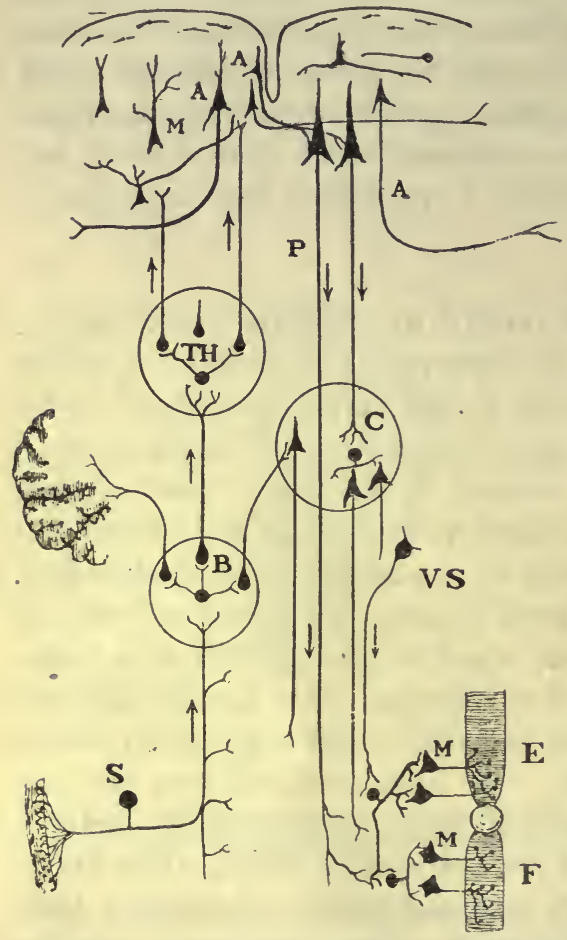

Fic. 234. Diagram (from Мотт after MoNAKOw) to show the interaction of the different levels in the central nervous system in the production of co-ordinated 'volitional' movements.

$\mathrm{S}$, sensory neuron; $\mathrm{B}$, bulb; $\mathrm{TH}$, thalamus; MA, motor area; P, pyramidal fibre; c, cerebello-pontine nuclei ; vs, vestibular neuron (Deiters' nucleus)

tetanus toxin. Whereas in the normal animal closure of the jaw and extension of the fore limb are only obtainable from one or two points on the surface of the brain, after the injection has taken place, every part of the jaw area gives closing of the jaw, every part of the arm area gives extension of the limb (cp. Fig. 173).

Since the predominant influence of the motor cortex is therefore inhibitory of the stronger muscles of the body, as well as of the tonus, which is continually and reflexly maintained, it is not surprising that excision of both hemispheres should give rise to decerebrate rigidity, or that destruction or division of the chief direct tracts from the cortex to the motor spinal mechanisms, viz. the pyramidal tracts, should determine increased tonus and rigidity of the limbs-the so-called 'spastic' condition observed in cerebral paralyses.

Two separable systems of motor innervation appear thus to control two sets of musculature. One system exhibits the transient phases of heightened reaction which constitute reflex movements; the other maintains that steady tonic response which supplies the muscular tension necessary to attitude. Hughlings Jackson long ago called attention to this contrast between the two systems. He pointed out that while the cerebrum innervates the muscles in the order of their action from the most voluntary movements (the limbs) to the most automatic (trunk), the cerebellum, or, as we should say now, the whole proprioceptive system, innervates them in the opposite order. The cerebellum therefore he regarded as the centre for continuous movements and the cerebrum for changing movements. The increased tone of the paralysed muscles, observable after hemiplegia, he ascribed to unbalanced cerebellar influence. While there is no doubt that the cerebellum must play, and does play, a considerable part in the production of decerebrate rigidity 
and of the spastic condition of hemiplegia, it is not the only element involved ; nor is it essential, since decerebrate rigidity may continue after extirpation of the cerebellum and an exaggerated knee-jerk may result from section of the spinal cord in the lower cervical region.

\section{HIGHER ASSOCIATIVE FUNCTIONS OF THE CORTEX}

The simple and uncomplicated nature of the movements elicited on cortical stimulation shows that we cannot regard these motor centres as responsible for the whole, or even the greater part, of the motor functions of the cortex. They are in fact simply the starting-point for the motor impulses which run down the long pyramidal tracts, but which result from the activities of the cerebral hemispheres as a whole. In the lower mammals they do not even represent the only starting-point, as is shown by the almost perfect recovery of volitional motor power in a dog deprived of its motor cortex. The distinguishing feature of the response of an animal possessing cerebral hemispheres is that it is not determined solely and exclusively by the nature and position of the peripheral stimulation, but involves elements connected with the past experiences of the animal, and including therefore the results of previous stimulation of many of the sense-organs, either directly, or indirectly as a result of reflex movements. The animal's reactivity is determined by the past history of the animal, and this modifying influence on the brain must involve parts connected with all its sense-organs. In any conscious motor act we may say therefore that the brain acts as a whole, or nearly as a whole.

In endeavouring to arrive at some idea of the neural processes concerned in volitional movements, i.e. movements of the intact animal, we are dealing with events which in ourselves come within the sphere of consciousness, so that some assistance is derived by appealing to our own mental experiences. Especially is this necessary in the case of the sensations. It might be imagined that a simple sensation would ensue as the result of local stimulation, say of the visual centre on one side. Our knowledge of the properties of the systems of neurons composing the central nervous system would teach us that no excitatory process could remain confined to one portion of the brain, but must diverge in many directions. It is true that excision of the occipital lobes on one side causes blindness to objects in the opposite half of the field of vision. This is, however, merely a result of localisation of the end of visual fibres, and the same effect can be brought about by division of the right optic tract, or damage to the right half of both retinæ.

On the other hand, an appeal to our own experience shows that no sensation can be regarded as simple, i.e. as following merely stimulation of visual fibres or visual centres. Thus the sensation of a luminous point has connected with it not only luminosity but also colour and intensity. Moreover the apparent position of the luminous point comes into consciousness at the same time as the consciousness of the luminosity itself, and this location of the stimulation involves muscular impressions from the eyeballs and an association between certain points on the retina and certain corre- 
sponding muscular movements of the eye muscles, of the head and neck, and even of the body and arm-movements which would be necessary to bring the image of the spot on to the fovea centralis and to approach the whole body to the site of the stimulating object.

As the visual sensation becomes more complex the associated sensations and experiences which it evokes become more numerous. Thus the image of a chair falling on the retina excites a long train of nervous processes. At once we become aware not only of a visual impulse but of an object which possesses colour, extension, or size in three dimensions, solidity, hardness, distance or position in space, \&c. These qualities are founded on past experiences-visual, muscular, and tactile. Moreover we are at once aware of the uses of the chair, and of its name both spoken and written, a mental activity connoting revival of higher visual and auditory sensations. The higher in the scale of intelligence, the greater is the development of the cerebral hemispheres and the more extensive are the associations arising in connection with any single sense impression.

Besides the portions of the brain which send out the motor paths and which receive the endings of the sensory paths, there may be whole regions taken up by the interconnecting neurons which subserve the association of the activities of all parts of the cerebral hemispheres, and the higher the animal is in the scale of intelligence the larger must be the relative amount of brain substance set apart for these functions of association. This is very evident if we compare the brain of three animals, such as the dog, the ape, and man. Although as we ascend to man there is an absolute increase in the amount of brain substance involved-say in the motor areas or in the sensory areas - the increase is very small as compared with that in those portions of the brain which give no response on stimulation, and in man these 'silent' parts of the brain form the greater part of the cerebral cortex. Although every phase of cerebral activity, every conscious event, involves co-operation of a large number of distant portions of the brain substance, in most of them there will be some seat of sense impressions which will be predominant, and a train of ideas may be specially visual, or auditory, or tactile. It is therefore not surprising that in the immediate neighbourhood of the cortical areas which receive the endings of the sensory tracts association areas are developed which may be labelled according to the sense-organ with which they are most nearly in relation. Thus we may speak of the visual-sensory and the visual association, or psychical area, the auditorysensory and the auditory-psychical, and so on. The limits of these areas are indicated in Fig. 224, p. 432.

Conditioned reflexes. Until recently, our study of the processes of association and therewith all the higher functions of the cerebral homispheres was chiefly carried out in man, and in most cases by the introspective method. Even when carried out on other men, it was chiefly by using speech as an index to the introspective experiences of those who were being investigated. During the last few years a method has been introduced by Pawlow, for investigating the higher cerebral functions by an objective method which is capable of very wide application. When an animal which is hungry is shown food, we say that 'its mouth waters,' i.e. there is a secretion of 
saliva; and if the animal be provided with a salivary fistula the extent of the emotion of appetite may be gauged in cc. of saliva flowing from the fistula. It is found in such an animal that a flow of saliva may be excited, not only by the sight or administration of food, but also by any other event which has become associated as the result of experience with the taking of food. Thus we may use this method in order to determine the sensitiveness of the animal's perception of pure tones. Thus if we wish to know whether the animal can recognise the difference between middle $\mathrm{C}$ and middle $\mathrm{C}$, as produced by tuning-forks, we can for some days or weeks allow him to hear both these sounds frequently but always follow up one of them, say C, by giving him a piece of meat. After a time it is found that not only can he distinguish between the two sounds, but that he has a memory of the absolute pitch, so that whenever the note middle $\mathrm{C}$ is sounded or any note differing from it by not more than $8 \mathrm{~d}$.v. per second, there is a flow of saliva from the fistula, whereas the note $\mathrm{C}-$ is heard without producing any response. Such an acquired reaction is designated by Pawlow, a "conditioned reflex ' and the method has been applied by him to study the association between the most widely different impressions and the condition which we can regard as appetite and which is associated psychically with the idea of food.

\section{THE FUNCTION OF SPEECH}

The acts of a conscious individual, i.e. one possessing cerebral hemispheres, are determined by his experience. The wider the range of past sense impressions which can be called up and taken into the chain of processes involved in any reaction - the more, that is to say, the individual weighs his acts in the light of past experience - the more fitted will these acts be to his maintenance amid the ever-changing stresses of the environment. In this guiding of behaviour by experience man, as well as the higher mammals, may profit also from accumulated racial experience. The increased complexity of the neural processes concerned in every reaction of the body, and the excessive lost time brought about by the intercalation of one neuron after another in the chain of the excitatory process, would finally counteract the advantages derived from the growth in complexity of the brain, were it not that, as a result of education or training, short cuts are laid down, by means of which reactions adapted to the maintenance of the individual can be carried out immediately, without thought and without correlated calling up of numberless sense impressions. Education in fact consists in laying down these 'short cuts' which, as habits, are the basis of the behaviour of the animal. The more complex the central mechanism and the wider the range of environmental change to which adaptation is necessary, the longer must be the time involved in this process of road-making within the cerebral hemispheres. The behaviour of man is therefore a product of many years' training, during which time he is in a state of subjection and unfit, from the absence of habit, to maintain himself as a unit in the human community. The neural short cuts of habit are, however, only of advantage to the individual in dealing with those events which are of everyday occurrence. Every novel circumstance must involve a revival of past sense impressions and a calling up of antivities of the most diverse portions of the brain in order to arrive at the safest or most advantageous mode of action adapted to the circumstances. Here again the complexity of the process would, by the very delay involved, put a stop to a further 
rise in intellectual, i.e. associative, capacities, were it not for the invention of SPEECH.

In speech we have a symbolism which acts as an economy of thought or of cerebral activities. An object, such as a table, with its associated properties of colour, consistence, spatial extension, and resistance, with the connoted acts associated with its use, can now be evoked as a word, involving comparatively simple auditory and motor processes, which itself may be employed as a unit of thought and brought into connection with other words, each of which in the same way is the symbol for a whole series of sensory and motor processes. The training of the cultivated man consists in a constant extension of the range of this symbolism, and the acquisition of words including wider and wider groups of neural processes, so that finally we arrive at those short verbal collections which, as the so-called natural laws, summarise the experience not only of the individual but such as is common to the whole race of mankind. All science may in fact be regarded as an extension of the process of representation of neural experience in symbolic shorthand, which in the child begins with the utterance of such a simple word as 'mamma,' and from which speech has arisen. A study of the nervous mechanisms involved in speech is therefore of interest in its relations to the development of the intelligence, and helps us to realise more completely the conditions which determine the activity and functioning of the cerebral hemispheres. Much light is thrown upon this mechanism by the study of disorders in man grouped together under the name Aphasia.

It has been usual to divide the disorders of speech known as aphasia into various groups, as follows :

(1) Motor aphasia, or aphasia of Broca. In this condition, which was described fully by Broca and referred by him to a lesion of the third left frontal convolution, the patient is unable to speak, although he understands what is said to him and has been stated to suffer from no impairment of his intelligence.

(2) Sensory aphasia, or aphasia of Wernicke. This condition was connected by Wernicke with the existence of lesions in a fairly wide area, known as the area of Wernicke, which involves the supramarginal and angular gyri and the hinder portions of the first and second temporo-sphenoidal convolutions. In these cases there may be limited power of speech, but there is serious impairment of the intelligence and especially of the power of appreciation of spoken words, so that the patient does not understand what is said to him. This condition may or may not be attended with alexia, loss of power to read. Any impairment of the motor processes of speech which is present is due rather to the inability of the patient to appreciate what he himself is saying, so that there is here a species of sensory paralysis in the higher sphere of neural processes.

(3) Anarthria. This is a condition in which there is marked impairment of the motor powers of expression, although intelligence and appreciation of speech, both spoken and written, may be unaltered. This condition is generally associated with lesion of the white matter of the external capsule as it passes round the lenticular nucleus. 
There are, however, considerable difficulties in the acceptation of this traditional classification. Microscopic examination of Broca's convolution shows a type of cortex entirely different from that part, viz. the psychomotor area of the ascending frontal convolution, which is concerned with the higher cerebral processes resulting in movement. Its structure is in fact identical with that described by Campbell as the 'intermediate precentral area ' and regarded as characteristic of the association areas. Moreover it is difficult to comprehend how a function such as speech, with its enormously complex mechanism, could be limited to so small a portion of the brain as Broca's convolution. The neural basis of language must in fact be coextensive with the sensory centres (the projection spheres) and with the whole region of lower association. We might indeed speak of auditory and visual word-centres as located in the visuo-psychical and auditory psychical centres. There is probably, however, no word, still less a collection of words, expressing an idea, which does not involve the activity of practically all parts of the cerebral cortex. As Bolton * points out, " a word, such as ' mouse,' at once sets in effect processes of association which pass to every projection sphere with the solitary exception of the gustatory, and even this may be aroused in a person who has eaten a fried mouse in the hope of thereby recovering from an attack of whooping-cough."

A careful examination of an extensive series of cases by Marie has shown, in fact, that Broca's aphasia does not exist as a result of lesions of Broca's convolution. This part of the brain may be destroyed without any disorder of speech. The cases described by Broca of motor aphasia are really cases of sensory aphasia from lesion of Wernicke's area, combined with anarthria due to subcortical injury of the fibres of the internal capsule. The statement that there is no loss of intelligence in these cases of so-called motor aphasia does not bear investigation. Although as patients they may comport themselves reasonably, as soon as they have to perform any duties which have been learnt by them in connection with their ordinary avocations they show their deficiency. They are incapable of transacting ordinary business, at any rate to the extent to which they were before the lesion. The amount of impairment of intelligence will vary in different cases according to the extent of the lesion. Thus softening generally affecting the occipital lobe may, with hemianopia, cause 'word-blindness,' or alexia, a loss of power of appreciating the meaning of pronounced or written words. In most individuals, and certainly in the uneducated, this power may be cut out altogether without interfering considerably with the mental powers. On the other hand, from babyhood upwards we have learnt the meaning of words and their grouping by auditory impressions. If the whole of the auditory associations be destroyed by an extensive lesion in the first and second temporal convolutions the resulting loss of word appreciation, sensory aphasia, will be attended with great diminution of mental powers. It must be remembered that the area of Wernicke is not a sensory centre, but a centre of association between the various sense-impressions, especially those of

* In his admirable article in Hill's "Further Advances in Physiology." 
hearing and sight. It may therefore be spoken of as an intellectual centre. Pure motor aphasia of course exists, but is always anarthria and is due to a lesion in the lenticular zone, i.e. in the lenticular nucleus and its neighbourhood, in the anterior part and the genu of the internal capsule, and possibly in the external capsule.

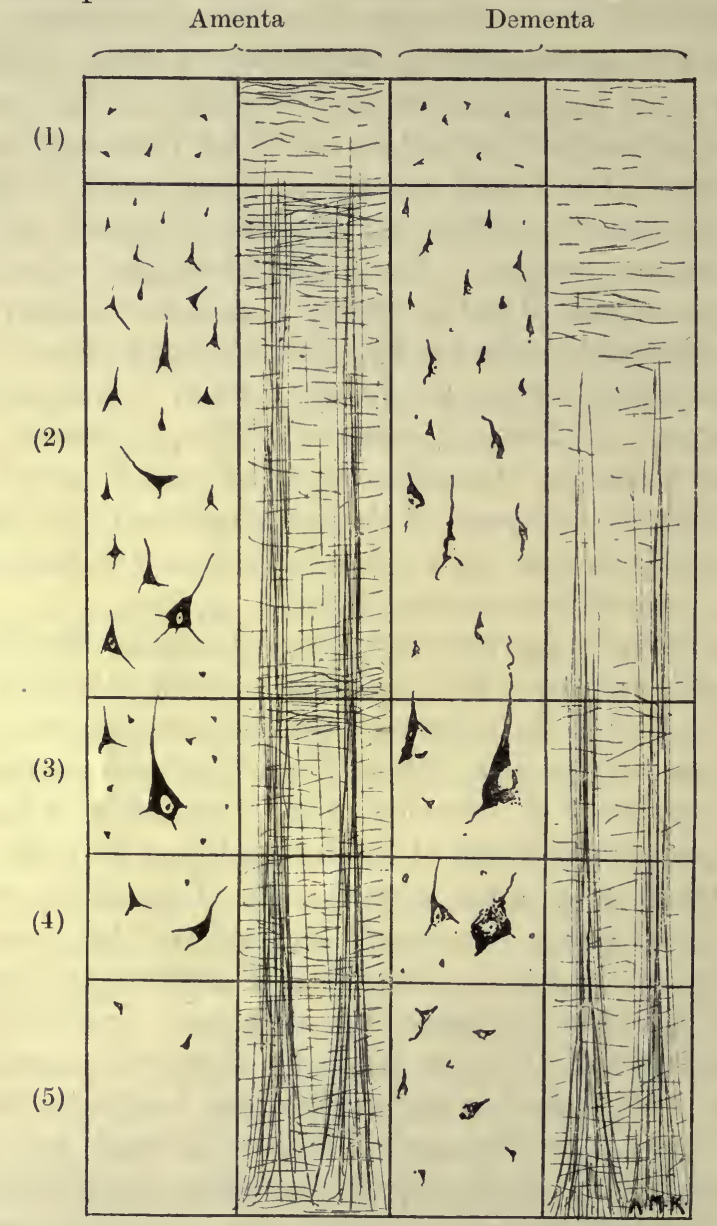

Fig. 235. Types of lesions giving rise to deficient intellectual power. In amentia, the deficiency is due to failure of development ; in dementia, to atrophy of the cells (especially small pyramidal) previously present in the cortex. (Мотт.)

It is important to make a distinction between loss of sanity and loss of intellectual powers. The essential factor of sensory aphasia is the existence of intellectual impairment, though in his behaviour the patient may appear perfectly normal. On the other hand, in insanity there may be perfect retention of the intellectual processes, which depend on the proper working of the lower association centres. The personality of the individual, and therefore finally his behaviour, involves a further association on a higher plane of these intellectual processes and therefore control in accordance with the relation, past, present, or future, of the individual to his environment. 
The prefrontal region is in all probability the seat of this highest plane of association. Insanity always involves alteration of personality and depends on failure of development or on disintegration processes (subevolution or dissolution of this region) (Fig. 235). In monkeys and cats Franz has found that destruction of the frontal lobes causes a loss of recently formed habits. He concludes from his experiments that the frontal lobes are the means by which we are able to learn and to form habits, $i e$. to regulate our behaviour in accordance with the needs of our position in society.

\section{THE TIME RELATIONS OF CENTRAL NEURAL REACTIONS}

In the spinal animal a stimulus of any particular quality and localisation always evokes an appropriate reaction. A certain period of time necessarily elapses between the moment at which the stimulus is applied and the moment at which the resulting reaction takes place. This interval is spoken of as the simple reaction time, and in the spinal animal is entirely independent of consciousness. Many reactions, even in the intact animal, are also, as we may say, involuntary and are not modified perceptibly by our consciousness of their occurrence: such reflexes as the withdrawal of the hand when it comes in contact with a hot surface, the shutting of the eyelid when the conjunctiva is touched, the drawing up of the leg when the sole of the foot is tickled. Not only are these carried out in the absence of voluntary impulses, but in many cases it is almost, if not quite, impossible to check the reaction by any effort of the will.

When the leg is drawn up in response to a painful or nocuous stimulus applied to the foot, a certain amount of time is involved in each of the following links in the chain of processes which determine the reaction :

(1) The conversion in the peripheral sense-organ of the mechanical stimulus into a nerve process.

(2) The passage of a nerve impulse up the nerve from the end organ to the spinal cord.

(3) The passage of the impulse across two or more synapses in the grey matter of the cord.

(4) The passage of the impulse down the motor nerve fibres from the spinal cord to the muscles.

(5) The processes occurring in the end-organs of the muscle.

(6) The latent period in the muscle fibre itself.

With a weak stimulus No. 1 is impossible to measure. With a strong stimulus it may be so short as to be practically negligible. (2), (4), (5), and (6) represent quantities for the measurement of which we have all the necessary data.

In any given reflex therefore we may add these periods together and subtract them from the total reaction time; we thus get a 'reduced reaction time,' which represents the time involved in the passage of the impulse through the central nervous system, and in the conversion of an afferent impulse into an aggregate of co-ordinated motor impulses. It is found that the reduced reaction time accounts for the greater part of the total 
reaction time. Since we have no reason to assume that the rate of passage of an impulse through the intra-spinal course of a nerve fibre differs appreciably from the rate at which it is conducted by the same nerve fibre outside the cord, the extra delay which occurs in the passage of the impulse through the cord must take place either in the nerve-cells themselves, or in the synapses, through which the impulse passes from one neuron to the next in the chain of reflex elements.

The rate of passage of an impulse through the nerve-cell can only be determined in one part of the body, viz. in the posterior spinal root ganglia,

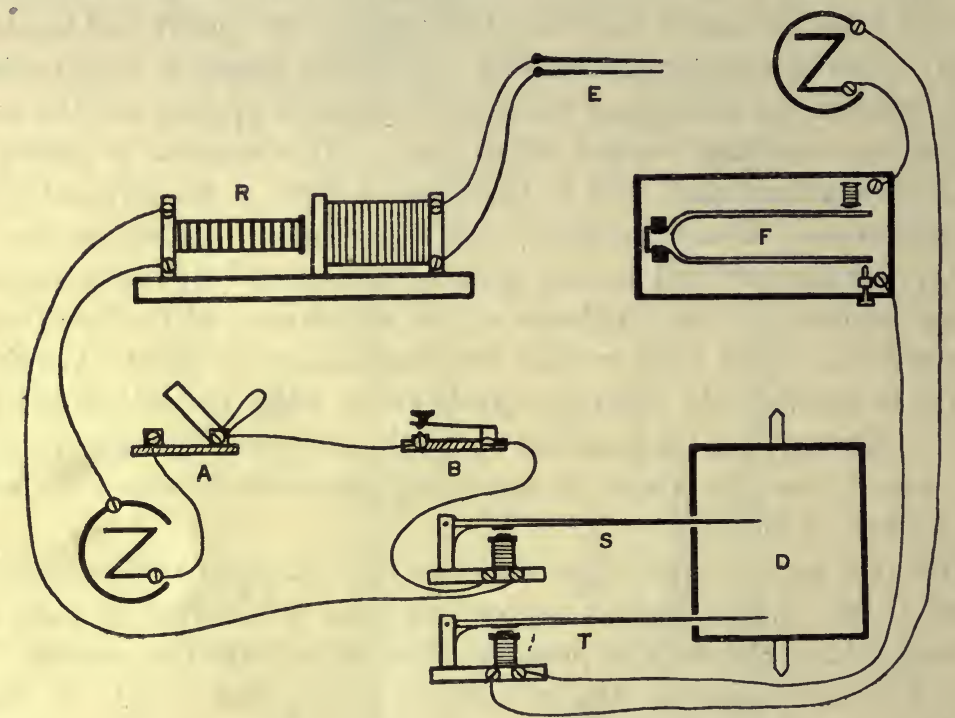

FIG. 236. Arrangement of apparatus for determination of reaction time. (Alcock and Ellison.)

R, coil ; E, exciting electrodes ; F, tuning-fork ; A, B, keys ; S, T, electromagnetic signals; $\mathbf{D}$, drum.

since only in these is it possible to detect the moment of passage of an impulse across a given section of a nerve fibre on both sides of the ganglion-cell in which the nerve fibres arise. Experiments on this point have been made by Steinach and by Moore. In each case the time occupied in the passage of the impulse through the ganglion was not appreciably longer than if the impulse had passed through a corresponding stretch of uninterrupted nerve fibre. We are therefore justified in concluding that the relatively great delay in the passage of an impulse through the central nervous system has its seat in the synapses across which the impulse has to pass. This conclusion is in accordance with our experience on the latent period of muscle, the greater part of which is due to changes occurring in the nerve-endings, i.e. in the synapses between motor nerve and muscle. The greater the number of synapses involved in any given reaction, i.e. the greater the complexity of the reaction, the longer will be the period which elapses between the moment of application of the stimulus and the moment at which the 
response takes place. Especially is this the case when the complex meshwork of neurons of the cerebral hemispheres is involved, or when the occurrence of the reaction is associated with the conscious processes of sensation and volition. In the latter case the determination of the reaction time has the added interest that it gives information as to the time-relations of the psychical processes which are the representation in consciousness of the physiological changes occurring in the neurons of the central nervous system.

Many methods are employed for the measuring of the reaction time of conscious processes. In most methods the application of the stimulus is arranged so as to close the circuit of a current which flows through an electro-magnet activating a lever which writes on a rapidly moving blackened surface. The reaction of the individual who is the subject of experiment is arranged so that the resulting movement activates a key by which the same current is opened. We thus obtain a tracing on the blackened surface showing the moment of application of the stimulus and the moment at which the reaction takes place. Thus, if the reaction time for an auditory stimulus is to be determined, the electric current is arranged so as to pass through :

(1) A spring contact key which can be pressed so as to make a noise.

(2) An electric signal writing on a rap:dly moving surface.

(3) A second key, which the subject will release as soon as he hears the noise of the first key and so break the current.

The recording surface may be a drum, a pendulum myograph, or a spring myograph, such as the 'shooter' of du Bois-Reymond. If the sensory impression is to be from the skin the current may be made to pass through the primary coil of an inductorium and wires be taken from the second coil to some part of the surface of the skin. In this case the signal may be started by opening the circuit, and the subject of the experiment will respond by closing the circuit by means of a spring key directly he feels the shock caused by the break of the primary circuit. If the reaction period is to be determined for sight a white piece of paper may be placed on an electro-magnet in the primary circuit and the person will respond directly he sees this move. Many other instruments have been devised for the same purpose.

The average reaction times obtained with the different senses are as follows :

Sight

$0 \cdot 186$ to $0 \cdot 222 \mathrm{sec}$.
Hearing

$0 \cdot 115$ to $0 \cdot 182 \mathrm{sec}$.
Electrical stimulation of skin

The two figures given for each case are the extremes obtained in different series of observations.

The times vary according to the condition of the person that is the subject of the experiment. They are lengthened by fatigue; they are shortened up to a certain point by continued practice. Within limits also they are shortened by increase of the strength of the stimulus.

DILEMMA. When the subject has to make a deliberate choice between the parts of the body stimulated the reaction time is considerably longer. To show this, the wires from the secondary coil are connected by a switch to two pairs of electrodes which are applied, one to the right and one to the left half of the body. It is agreed beforehand that the subject shall react only to stimulation, say, of the right side. The switch is removed from the observation of the subject and the stimulus is applied irregularly to one side or to the other. It is found that the additional neural processes 
involved in determining whether the stimulus is on the right side, and therefore should be followed up as agreed, adds considerably to the length of the reaction time (on an average $\cdot 066$ sec.). It is possible to complicate the dilemma to almost any extent. Thus the experiment may be so arranged that either a red or a white disc appears and the subject has to react with the right hand to the red disc and with the left hand to the white disc. In such an experiment the reaction time was found to be 0.154 sec. longer than the simple reaction time. A still more complex process would be involved in the experiment in which a word was spoken, and the subject had to speak some other word which had some association with the word which formed the stimulus, e.g. horse-mammal ; paper-pen, \&c. In such an experiment the reaction time was found to be as long as 0.7 to $0.8 \mathrm{sec}$.

We see that the recording of the time of occurrence of any physical event can only occur after a certain lost time, which represents the observer's reaction time for the stimulus in question. This only applies, however, to movements carried out in response to single stimuli, or to stimuli repeated at irregular intervals. When the stimuli are rhythmic the lost time only applies to the first one or two of the stimuli. The observer or subject is conscious of the interval elapsing between the physical event and his reaction, and anticipates the later stimuli so that his reaction becomes synchronous with the stimulus. This synchronism of stimulus and reaction characterises all ryhthmic movements, such as dancing or the playing of an orchestra in time with the beat of the conductor's bâton. 


\section{SECTION XVIII}

\section{THE NUTRITIVE AND VASCULAR ARRANGEMENT OF THE CENTRAL NERVOUS SYSTEM}

THE brain and spinal cord are enclosed within three membranes or meninges, named from without inwards, the dura mater, the arachnoid membrane, and the pia mater. The dura mater consists of a strong fibrous membrane, smooth and lined with endothelial cells on its inner surface. In the head its outer surface is closely attached to the bones forming the cranium, of which it represents the periosteum. Strong fibrous partitions are sent from the dura mater into the cavity of the cranium to support the chief parts of the brain. One of these, the falx cerebri, supports the two cerebral hemispheres, a second, the tentorium cerebelli, forms a horizontal division between the cerebral hemispheres and the cerebrum, and a smaller one, the falx cerebelli, passes a short distance inwards between the cerebellar hemispheres. In the spinal canal the bones have their own periosteum and the dura mater, which is closely attached round the margins of the magnus, forms a loose sheath round the spinal cord, being slung up in the vertebral canal by the tubular prolongations which it sends along each nerve root to form the outer sheath of the nerve. The dura mater in the cranium may be separated with greater or less difficulty into two layers, and between these two layers are found the venous sinuses, which receive the whole of the blood returned from the brain. These venous sinuses are angular clefts, the chief of which lie along the attached margin of the falx cerebri and the tentorium cerebelli. Most of the blood leaves the skull by the internal jugular veins. In the spinal cord the place of these venous sinuses is taken by a plexus of thinwalled veins, imbedded in fat, lying on the outside of the dura. Under the dura mater we find the subdural space, which is rather potential than actual. It can be regarded as a large lymph space and any contents are drained off into the lymph spaces of the nerve roots and adjoining tissues.

The arachnoid is a delicate transparent membrane which covers the whole of the surface of the brain and spinal cord. Superficially it is covered with endothelial cells which bound the subdural space. On its deep surface it is connected with the pia mater by fine fibres. It bridges over the inequalities in the surface of the brain so that in various localities a space is left which is filled with cerebro-spinal fluid and is known as the subarachnoid space. In certain situations it sends prolongations into the fissures of the brain. Thus a marked expansion passes by the transverse fissure between the 
cerebral hemispheres and the third ventricle, sending prolongations into the lateral ventricles. This layer of connective tissue is covered on one surface by the ependyma of the ventricles, on the other surface by the ependyma forming the roof of the third ventricle. It carries a rich plexus of blood vessels known as the choroid plexus, and the ependyma covering the vascular fringes which dip into the cerebral ventricles consist of clear columnar or cubical cells, often spoken of as the epithelium of the choroid plexus. Similar vascular fringes are found in the roof of the fourth ventricle.

The pia mater is a layer of connective tissue which serves to carry the blood-supply to the whole surface of the brain. It is closely applied to the surface and follows all the irregularities of the latter, dipping down into the various fissures and crevices on the brain. In the spinal canal the pia mater sends out a series of processes on each side of the spinal cord, the ligamentum denticulatum, the outer extremities of which are attached to the dura mater and serve to sling the spinal cord in its dural sheath.

The brain is richly supplied with blood. Its chief supply is derived from the two carotids and the two vertebral arteries. The vertebrals unite on the lower surface of the bulb to form the basilar artery, which divides again at the anterior extremity of the pons varolii into two branches which unite with the two carotid arteries to form the circle of Willis, so that the pressure. in this arterial circle can be maintained indifferently by any three out of the four arteries by which it is supplied. From these vessels three main arteries, the anterior, middle and posterior cerebral, pass up to supply the corresponding regions of the outer surface of the brain, while the inner parts of the brain, e.g. the corpus striatum, optic thalamus, \&c., are supplied from arteries arising from the circle of Willis and passing straight into the substance of the brain. The connection between the vascular supply of the different parts of the brain is slight and effected only by the capillaries; hence obstruction of any one vessel, such as the middle cerebral, permanently cuts off the blood-supply to the greater part of the area supplied by it and the result is death and softening of the brain substance. The arteries supplying the surface of the brain divide up into arterioles and capillaries within the pia mater, and the capillaries run into the brain substance surrounded by a so-called lymphatic sheath, which apparently communicates with the subarachnoid space.

In certain cases of disease these perivascular sheaths may be found to contain leucocytes often filled with products of disintegration of the nervous tissues.

\section{THE CEREBRO-SPINAL FLUID}

The subarachnoid space contains a thin transparent colourless fluid, known as the cerebro-spinal fluid. In composition this fluid resembles blood plasma minus its protein constituents. It contains a mere trace of coagulable proteins but it has the same molecular concentration as the blood plasma and its salts are identical with those of the blood plasma. It also contains other diffusible constituents of blood plasma, e.g. small traces of sugar and of urea. It may be collected by introducing a cannula through the 
atlanto-occipital membrane into the ample subarachnoid space lying over the fourth ventricle. Another method is to introduce a glass cannula through a slit in the sheath of a nerve root up into the subarachnoid cavity of the spinal canal. In man it may be obtained in small quantities for examination by introducing a hollow needle directly into the spinal canal in the lumbar region between the laminæ of the vertebræ. On introducing a cannula into the subarachnoid space, the fluid may spurt out, showing that it is under a certain pressure (about $100 \mathrm{~mm} . \mathrm{H}_{2} \mathrm{O}$ ). After the first rush the fluid begins to drop away, at first rapidly, but becoming slower with lapse of time. If the fluid be allowed to drain off for some hours, signs of interference with the functions of the central nervous system are evinced. The cerebro-spinal fluid appears to be formed chiefly in the neighbourhood of the choroid plexus. Although its composition would suggest that it was merely a transudation from the blood, the amount formed does not seem to run parallel with the pressure in the capillaries of the brain. Moreover, it has been shown by Dixon and Halliburton that a considerable increase in the flow of cerebrospinal fluid may be brought about by injecting an extract of the choroid plexus itself. It has therefore been concluded that this fluid is really a secretion by the modified ependyma cells covering the fringes of the choroid plexus.

Although the method of formation of the cerebro-spinal fluid is still not clear, there is no question that its removal from the subarachnoid space is brought about by simple physical factors. The subarachnoid space communicates with the ventricles by means of openings in the roof of the fourth ventricle. The pressure of the fluid is ordinarily about equal to the pressure in the venous sinuses of the cranium. If salt solution be injected into the subarachnoid space, it escapes with extreme ease and it is found that its channel of escape is into the veins and especially into the venous sinuses of the dura mater. Its removal by these sinuses is facilitated by the existence of peculiar structures, known as the Pacchionian bodies. Each of these bodies is a bulbous protrusion of the arachnoid membrane into a blood sinus. It remains "connected with the arachnoid by a narrow pedicle, through which a continuation of the sub-arachnoid space is prolonged into the interior of the sinus. It is therefore a little sac of arachnoid membrane separated from the blood stream only by an invagination of the endothelium lining the sinus. Filtration of the cerebro-spinal fluid will occur into the venous sinuses whenever the pressure of the fluid rises above that of the blood in the sinus. The fluid can also escape, but with greater difficulty, along the sheaths of the spinal nerve roots, by which it will pass into the lymphatic space outside the spinal canal.

THE NUTRITION OF THE BRAIN. The grey matter of the brain is very richly supplied with blood vessels. Any interference with the blood flow through the brain rapidly checks the functions of the central nervous system in consequence of deprivation of oxygen. Although so susceptible to slight deprivation of oxygen it does not seem that the brain tissues have a very rapid gaseous metabolism; that is, they need oxygen supply at high tension but do 
not deprive the blood of any very large amount of the oxygen which it contains. Nor does it seem probable that the brain requires a large supply of food material. It must be remembered that in all parts of the brain a peri-vascular lymphatic intervenes between the capillary and the brain tissue. Since these 'lymphatics' communicate with the subarachnoid space they must contain a fluid which differs little if at all from the composition of the cerebro-spinal fluid obtained from the subarachnoid space. The nutrient fluid of the brain is therefore practically salt solution with a trace of sugar and possible minute traces of amino acids.

Our study of the events which accompany the propagation of a nervous impulse down a nerve fibre has prepared us for the conclusion that very little energy is involved in ordinary nerve activity. It is true that extreme fatigue causes changes in the initial granules of the nerve cells and is therefore associated with the using up of some material constituent. But even though material changes in the nerve-cells and in the synapses may be larger in amount than those in nerve fibres, they are probably not to be compared in extent with those taking place in a contracting muscle or in an active liver cell.

\section{THE CEREBRAL CIRCULATION}

In all higher animals the brain is enclosed in a rigid case formed by the bony cranium. In the child, before the cranial vault is fully ossified, part of this vault consists of membrane, known as the anterior fontanelle. It is easy to see that the fontanelle pulsates with each heart-beat as well as with rise of venous pressure, such as that produced during strong expiratory efforts. When ossification is complete, such alterations in the volume of the cranial contents are impossible. And yet the pressure in the arteries within the cranium must be still pulsatile, the rise of pressure at each heart-beat must make the arteries expand, but room for this expansion has to be found by contraction of some other part of the cranial contents. We find that each arterial beat is associated with a corresponding expulsion of some of the contents of the veins and a contraction of these vessels. If, for instance, a cannula be introduced through the occipital bone into the torcular Herophili, the venous blood is seen to pulsate and to be pressed out with each beat of the heart. If there is a rise of arterial pressure, although the arteries may expand somewhat at the expense of the veins, there can be no dilatation of the whole organ. The only effect of the rise of pressure will be to cause an increased pressure fall in the cranial vascular system, and therefore augmented velocity of flow through the system. A prolonged rise of pressure may cause a certain amount of dilatation of the vessels, but only at the expense of the cerebro-spinal fluid. Since this is only small in amount, any expansion of the brain due to vascular causes must be very limited.

BRAIN PRESSURE. If by means of a trephine an opening be made into the cranial vault, the brain bulges into the opening. By screwing a tube covered with a membrane into the trephine opening, we can find the pressure necessary to force the brain back to its previous position. This is known as the brain pressure and is approximately equal, as might be expected, to the 
cerebro-spinal pressure and to the pressure in the venous sinuses. It is closely dependent on the latter. Forced expiratory efforts, such as may occur in the convulsions of strychnine poisoning, may raise the pressure from 30 to $50 \mathrm{~mm}$. $\mathrm{Hg}$. In the vertical position in man the pressure may be slightly negative in consequence of the tendency of the venous blood to run downwards towards the heart.

\section{REGULATION OF THE BLOOD-SUPPLY TO THE BRAIN}

No satisfactory evidence has been brought forward of the existence of vaso-motor nerves controlling the calibre of the cerebral blood vessels. Nor indeed are such nerves necessary. The brain, as the master tissue of the body, controls through the medullary centres the circulation through all other parts of the body. It is therefore able to regulate the blood-supply through its arteries by allowing less or more blood to pass through other parts of the body. For the exercise of its normal functions it requires a certain blood-supply, which again will depend simply on the pressure in the carotid arteries and circle of Willis. If this pressure fails, the functions of the brain are affected and loss of consciousness rapidly ensues. This is what occurs when a person who is weak from long illness faints on suddenly getting up from bed. In the normal individual the change in the circulation with alteration of bodily position, which would be produced by the action of gravity, is at once counteracted through the vaso-motor system. The splanchnic area is contracted or dilated according to the necessities of the case, but the pressure in the carotid and the circulation of the brain remains unaltered. Even when the heart in consequence of disease is scarcely able to carry on the circulation, the arterial pressure undergoes little or no alteration. Any other tissue of the body, even the heart itself, may suffer, but the brain at all costs must receive its proper supply of blood. 


\section{SECTION XIX}

\section{THE VISCERAL OR AUTONOMIC NERVOUS SYSTEM}

IN the medulla oblongata it is easy to differentiate the central grey matter connected with the peripheral nerves into two categories, viz. splanchnic and somatic. Each of these two sets of nerves possesses both afferent and efferent fibres. Gaskell has suggested that the same arrangement would hold for any typical segmental nerve, which would therefore have four roots, viz. two somatic-the motor and sensory roots distributed to the skin and skeletal muscles - and two splanchnic roots, also motor and sensory, and composed of small fibres distributed to the viscera or structures which are visceral in origin (e.g. developed from the branchial arches). In the medulla the somatic efferent fibres, such as the sixth and twelfth nerves, arise from the column of large cells lying in the floor of the fourth ventricle close to the middle line. The splanchnic fibres, e.g. those of the facial and vago-glossopharyngeal nerves, arise from a column of cells-the nucleus ambiguos and facial nucleus, lying more laterally and deeper, below the surface of the ventricle. The motor root of the fifth would also belong to the same system. In the spinal cord the visceral fibres arise in the cells of the lateral horn, i.e. from a situation corresponding to the splanchnic motor nuclei of the pons and medulla. Whereas, however, the splanchnic afferent nerves, such as the glossopharyngeal, and perhaps the sensory nucleus of the fifth, form a wellmarked splanchnic system of nuclei in the medulla, in the cord the afferent fibres from the viscera pass in with the other afferent somatic fibres, and their immediate connections in the cord are as yet unknown.

The autonomic system of nerves includes the sympathetic system (properly so called) and some of the cranial and sacral nerves. The sympathetic system (Fig. 237) is composed of a chain of ganglia lying each side of the vertebral column, there being as a rule one ganglion to each spinal nerve-root. In the cervical region these ganglia are condensed into two, the superior and inferior cervical ganglia, united by the cervical sympathetic trunk; and the upper three or four thoracic ganglia on each side are condensed to form the 'stellate' ganglion. At the bottom of the chain there is only one coccygeal ganglion for the coccygeal vertebræ.

In the abdomen is a second system of ganglia, in special connection with the abdominal viscera, lying in front of the aorta and surrounding the origins of the large arteries to the alimentary canal. These are the semilunar or solar ganglia, the superior mesenteric and the inferior mesenteric ganglia. 


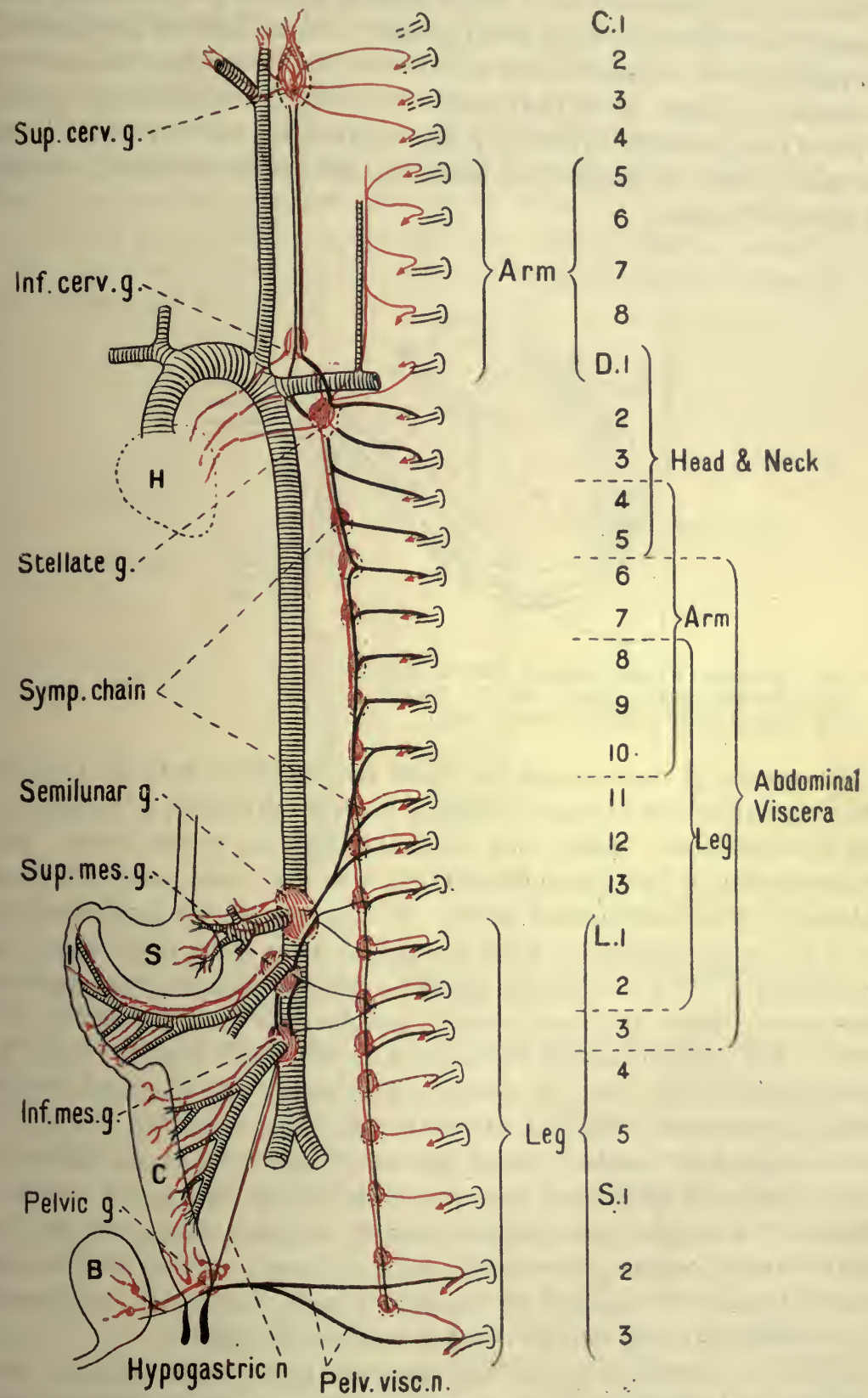

FIG. 237. Diagrammatic representation of the distribution of the sympathetic system.

The black lines represent the medullated pre-ganglionic fibres, such as those making up the white rami communicantes, while the post-ganglionic fibres are printed in red. On the extreme right of the figure is indicated the general distribution of the white rami arising from the several nerve-roots, while the double brackets point to the nerveroots making up the limb plexuses. H, heart ; s, stomach; I, small intestine ; $\mathrm{C}$, colon ; B, bladder. 
In the organs themselves we find a third system of ganglion-cells, either scattered or collected to form small ganglia. These isolated ganglion cells as a rule have no connection with the fibres of the sympathetic system, but, as we shall see later, lie on the course of the impulses descending by other nerves of the autonomic system, e.g. the vagus or the pelvic visceral nerves. The three systems of ganglia have been distinguished as the lateral, collateral, and terminal ganglia.

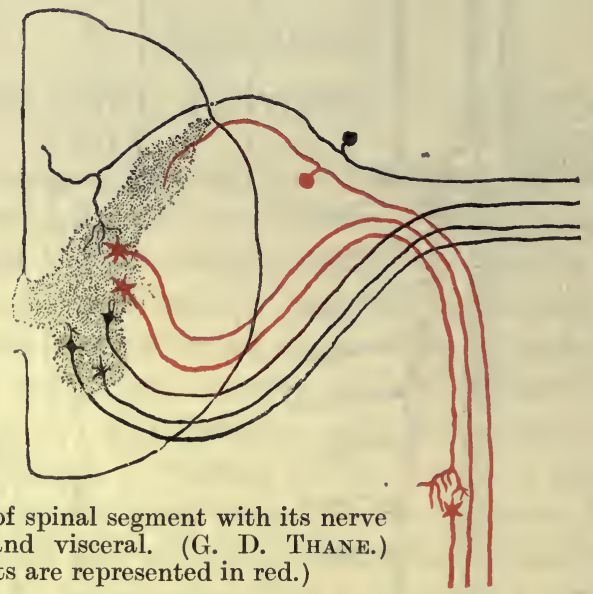

FIG. 238. Diagram of spinal segment with its nerve roots, somatic and visceral. (G. D. Thane.)

(The visceral roots are represented in red.)

The ganglia of the sympathetic chain are connected with all the spinal nerves, just after they have given off their posterior divisions, by means of the rami communicantes. These rami communicantes are of two kinds: white rami consisting of small medullated fibres, and grey rami composed almost exclusively of non-medullated nerves. It has been shown by Gaskell that the white rami are formed by fibres which have their origin in the spinal cord and perhaps in the posterior root ganglia; whereas the grey rami represent fibres which, arising in the sympathetic ganglia, run back to join the spinal nerves. The visceral outflow represented by the white rami is limited to a distinct region of the cord, viz. from the first thoracic to the third or fourth lumbar nerve-roots; whereas the grey rami pass from the sympathetic to all the spinal nerve-roots. It is found by experiment that stimulation of a limited number of white rami produces all the effects that can be evoked by stimulation of the grey rami, showing that the impulses leaving the cord pass upwards and downwards in the sympathetic system and are broken somewhere in their course, being transferred to a fresh relay which, by means of non-medullated nerves, carries them on to their destination.

Finally, in certain organs of the body are to be found sheets of nerve structures, including both ganglion cells and fibres, which must be regarded as local nerve-centres, capable of carrying out co-ordinated acts in response to stimuli, independently of the central nervous system. It seems probable that these systems are to be regarded as analogous rather to the diffuse neurofibrillar system of an animal, such as the medusa, than to the synaptic nervous structures characteristic of the central nervous system of vertebrates. 
In the latter the direction and effect of any impulses are determined by the synapses intervening between various systems of neurons and allowing the passage of the impulse only in one direction. This law of forward direction has not been proved to hold good for the primitive nerve systems; an impulse apparently spreads equally well in either direction. As a type of this peripheral diffuse nerve system may be cited the Auerbach's and Meissner's plexuses in the wall of alimentary canal. How far we are to regard the nerve-nets in other viscera, such as the heart and the bladder, as conforming to this type is still a moot point, and will be discussed in dealing with the origin of the heart-beat.

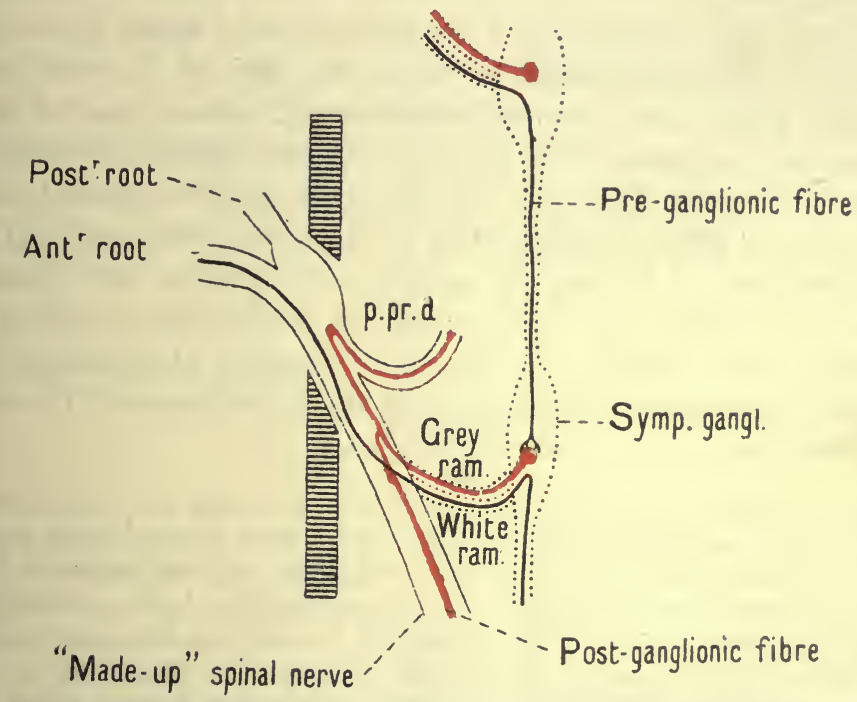

Fig. 239. Diagram (after LANGLEY) to show the manner in which a spinal nerve is completed by the entry of a grey ramus, containing fibres derived from cells in the sympathetic chain.

p.pr.d, posterior primary division. ('The post-ganglionic fibres are represented red.)

The relationships of the white and grey rami are strikingly illustrated in the case of the pilomotor systems of nerves. These in the cat arise from the cord by the anterior roots from the fourth thoracic to the third lumbar inclusive. Passing by the white rami to the sympathetic system, they travel upwards and downwards and end by arborisations in the various ganglia of the main chain. From the cells of each ganglion a fresh relay of fibres starts, which runs as a bundle of non-medullated nerves (the grey ramus) to the corresponding spinal nerve, with which it is distributed to its peripheral destination. Each grey ramus causes erection of the hairs above one vertebra, whereas stimulation of one white ramus causes erection over three or four vertebræ, showing a distribution of the fibres of the white ramus to the cells in several successive ganglia.

These pilomotor fibres in the cat have the following distribution :

(1) For the head and upper part of the neck the fibres arise by the fourth to the seventh thoracic anterior roots, and have their cell stations in the 
superior cervical ganglion. They travel as small medullated nerve fibres from the white rami up the sympathetic chain, through the stellate ganglion and ansa Vieussenii and up the cervical sympathetic.

(2) The next set of nerve fibres have their cell station in the stellate ganglion. The white rami arise from the fifth to the eighth thoracic nerves, while the grey rami pass to the nerve-roots from the third cervical nerve to the fourth thoracic nerve.

(3) The remaining nerves, supplying all the rest of the body and tail, arise by the white rami from the seventh thoracic to the third or fourth lumbar nerve, and are distributed as grey rami to all the spinal nerves below the fourth thoracic.

We thus see that, in speaking of the functions of a spinal nerve-root, we must clearly distinguish whether we mean the root as it arises from the spinal cord, in which case its visceral functions will include those of its white ramus, or whether we mean the made-up or complete spinal nerve after it has received its grey ramus (Fig. 239). In the latter case the visceral functions of the root will be more restricted than in the former case, and will have a different distribution. In stimulating the nerve-roots in the spinal canal it is sometimes possible, by weak stimuli, to display the functions of the corresponding white ramus, and then by increasing the stimulus to get superadded the effects due to the excitation of the grey ramus in the made-up nerve, in consequence of the spread of current.

"When, for example, the eleventh thoracic anterior roots are stimulated in the spinal canal with weak shocks, a fairly long strip of hairs in the lumbar region will be erected, the maximum movement of the hairs being near the middle of the strip. This marks the area of distribution of the pilomotor nerves given by the eleventh thoracic nerve to the sympathetic. If then the strength of the shock be increased to a certain point, the hairs in the long strip will of course be erected as before, but in addition there will be energetic erection of hairs in a short strip a little distance above the long strip, and separated from it by a quiescent region. This short strip is the same as that affected by stimulating the grey ramus or the dorsal cutaneous branch of the eleventh thoracic nerve. It marks the area of distribution of the pilomotor fibres received by the spinal nerve from the sympathetic." (LANGLEY.)

We may now indicate briefly the main course and functions of the fibres of the sympathetic system.

(1) The head and neck are supplied by fibres leaving the spinal cord by the first five dorsal nerves (chiefly by the second and third). They all have their cell station in the superior cervical ganglion. They convey:

Vaso-constrictor impulses to the blood-vessels.

Dilator fibres to the pupil.

Secretory fibres to the salivary glands and sweat glands.

Vaso-dilator fibres to the lower lip and pharynx (?).

(2) The thoracic viscera (heart and lungs) are supplied by the same five nerve-roots. The cell station of these fibres is, however, situated in the stellate ganglion: They convey:

Accelerator or augmentor impulses to the heart.

(3) The abdominal viscera receive fibres from the lower six dorsal nerves 
and the upper three or four lumbar. Most of these fibres run through the sympathetic chain without making any connection with the ganglia, and have their cell stations in the collateral ganglia of the solar plexus, the semilunar and superior mesenteric ganglia. On their way to these ganglia they form the greater and lesser splanchnic nerves. Their functions are :

Vaso-constrictor for stomach and small intestine, kidney, and spleen.

Probably vaso-dilator for the same viscera.

Inhibitory for both muscular coats of stomach and small intestine.

Motor for ileocolic sphincter.

(4) The pelvic viscera are supplied by the lower dorsal and upper three or four lumbar nerve-roots. These fibres also pass by the main chain to

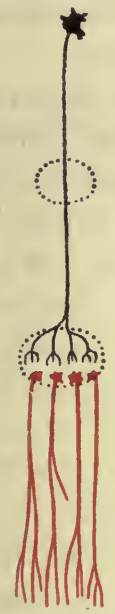

A.

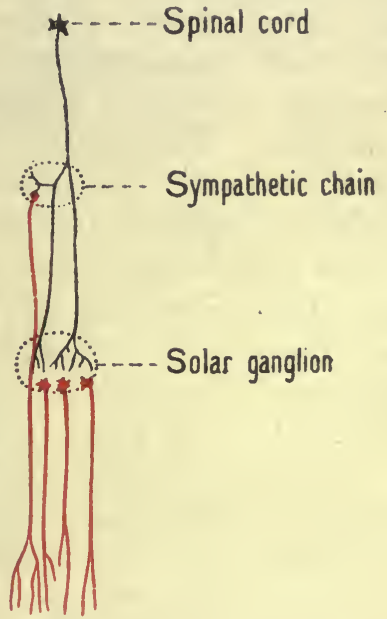

B.

Fig. 240. Figure (after LANGLEY) to show the probable mode of connection of the fibres of the splanchnic nerve with nerve-cells.

A, usual type, all the fibres passing through the lateral chain to end in the collateral ganglia of the solar plexus; $\mathrm{B}$, alternative condition, in which a small minority of the fibres have their cell-stations in the sympathetic chain. The pre-ganglionic fibres are black, the post ganglionic red.

make connections with the cells chiefly in the inferior mesenteric ganglia. They convey :

Vaso-constrictor impulses to pelvic viscera.

Inhibitory fibres to colon (both coats).

Motor and also inhibitory fibres to bladder.

Motor fibres to retractor penis.

Motor and inhibitory fibres to uterus and vagina.

(5) The fore limb receives nerves from the white rami of the fourth to the tenth thoracic nerves. All these fibres are connected with cells in the stellate ganglion. They convey :

Vaso-constrictor impulses to the blood-vessels.

Secretory nerves to the sweat glands.

(6) The hind limb is supplied by the nerve-roots from the eleventh 
thoracic to the third lumbar inclusive. The cell stations of these fibres are situated in the sixth and seventh lumbar and first sacral ganglia. They convey :

Vaso-constrictor impulses.

Secretory nerves to the sweat glands.

Every fibre of the sympathetic system is thus in some point of its course interrupted by a nerve-cell, and Langley has shown that this is the only cellbreak in the fibre, i.e. every fibre is connected with one cell and one cell only. This law applies not only to the sympathetic fibres but also to the fibres of the other visceral nerves. Each fibre therefore can be regarded as made up of two sections - a pre-ganglionic fibre arising in the central nervous system and passing down to a ganglion as a fine medullated nerve fibre, and a post-ganglionic fibre arising in this ganglion and continued generally as a non-medullated fibre to its peripheral distribution.

This transference from one system to another involves the passage across a synapse and a nerve-cell. The situation of this nerve-cell may be easily revealed by utilising the action of nicotine, first studied by Langley. If nicotine be applied to a sympathetic ganglion, it first stimulates and then paralyses any junction between axon termination and nerve-cell which may lie in the ganglion. Intravenous injection of nicotine therefore causes a primary general excitation of all visceral ganglion-cells. There is an enormous rise of blood pressure, which may be accompanied by other sympathetic effects, such as dilatation of the pupil, secretion of saliva, erection of the hairs, and so on. This rise rapidly passes off, and it is then found impossible to evoke any reflex visceral effects or any contraction, e.g. of the blood-vessels, by stimulation of the spinal cord ; the passage of the impulses is blocked in every one of the visceral ganglia. By observing the effects of stimulation of a nerve before it enters a ganglion and then painting the ganglion with nicotine and again trying the effects of excitation, it is easy to determine whether the nerve fibres which were excited in the first case form any connections with the nerve-cells of the ganglion. In the strength in which it is usually applied nicotine is without effect on nerve fibres.

\section{THE CRANIAL AUTONOMIC FIBRES}

In the course of development the greater part of the fibres of the facial nerve have lost their special visceral functions and have the aspect and functions of ordinary somatic fibres. Visceral fibres are contained in the following cranial nerves - the third, seventh, ninth, tenth, and eleventh.

Third nerve. The visceral fibres of this nerve pass to the ciliary ganglion in the orbit where they end; the post-ganglionic fibres from this ganglion form the short ciliary nerves which innervate the sphincter pupillæ and the ciliary muscles.

Seventh nerve. The autonomic fibres of the facial arise from the medulla in the intermediate nerve of Wrisberg, which is practically the anterior continuation of the ninth, tenth, and eleventh nerves. From the seventh nerve is derived the chorda tympani nerve, which supplies vaso-dilator nerves 
to the tongue, the submaxillary and sublingual glands, and secretory fibres to these glands.

The cell stations of these nerves lie peripherally, those of the sublingual gland in the so-called submaxillary ganglion; those of the submaxillary gland in the hilus of this organ. It is probable that the seventh sends also pre-ganglionic visceral fibres to the spheno-palatine ganglion, whence a fresh relay of fibres-post-ganglionic-run with the branches of the fifth nerve, to supply secretory fibres and possibly vaso-dilator fibres to the mucous membrane of the nose, soft palate, and upper part of the pharynx. The glossopharyngeal or ninth nerve sends fibres, which evoke secretion as well as vaso-dilatation in the parotid gland, viâ the otic ganglion. Probably also dilator fibres leave this nerve to supply vessels at the back of the tongue.

\section{THE VAGUS}

The efferent visceral fibres of the tenth and eleventh nerves arise in the same column of cells as the two nerves just considered. Most of the fibres run in the vagus. They include motor fibres to the œsophagus, stomach, and small intestines as far as the ileocolic sphincter; inhibitory fibres to the heart, motor fibres to the unstriated muscles of the bronchi, and secretory fibres to the gastric glands. The cell stations of these fibres are apparently situated peripherally, the jugular ganglion, and the ganglion of the trunk of the vagus being in all likelihood responsible only for the afferent fibres in this nerve. Nicotine therefore abolishes any effect of stimulating the vagus in the neck, though inhibition of the heart can still be produced on excitation of the post-ganglionic fibres arising from the cells in the sinus venosus.

\section{SACRAL AUTONOMIC FIBRES}

These all run in the pelvic visceral nerve; also called nervus erigens. This nerve is connected with a collection of ganglia lying in the hypogastric plexus at the base of the bladder. It has the following functions :

Dilator to vessels of the penis (hence its name of nervus erigens).

Motor to bladder, colon, and rectum.

Inhibitory to sphincter muscle of bladder.

Inhibitory to retractor penis.

It will be observed that in many cases the viscera get their nerve-supply from both sets of visceral nerves, and that in such cases the two sets of nerves are antagonistic in function. It is impossible, however, to draw a sharp line between the functions of the two sets, since the same nerve may be motor for one set of muscular fibres and inhibitory for another set in the same viscus. Thus the colonic branches of the inferior mesenteric ganglion are motor (constrictor) for the blood-vessels and inhibitory for the muscular walls of the colon. While the sympathetic nerve-supply is inhibitory for nearly the whole intestinal muscles, it produces strong contraction of the band of muscle forming the ileocolic sphincter. In the bladder there is no doubt that the sympathetic supply includes both inhibitory and 
motor fibres, the predominating effect on excitation of the nerve varying from one species of animal to another.

\section{FUNCTIONS OF THE SYMPATHETIC AND PERIPHERAL GANGLIA}

These ganglia consist of a mass of nerve-cells embedded in connective tissue, each cell being surrounded by a special capsule of endothelial cells. The nerve-cells; though in section resembling those in a posterior root ganglion, differ from these in being multipolar, each cell probably possessing one axon and several dendrites. The dendrites end in little arborisations round adjacent cells.

Since the main nervous system is characterised by the possession of nerve-cells, it was formerly thought that any collection of nerve-cells must partake of the co-ordinating and reflex functions of the central nervous system, i.e. must act as local nervous centres. All efforts have failed, however, to prove the existence of such a function, and we must conclude that the sole use of these ganglia is to serve as distributing-centres. We may assume that one pre-ganglionic fibre divides, and the branches arborise round several cells (Fig. 240), whence new fibres arise to carry the impulse to the periphery - an impulse in the case of which there is no need for any minute localisation. Indeed the essential part of a nerve-centre is not the nerve-cells at all, but the presence of a complex tangle of fibres, rendering possible the passage of impulses in all directions, the passage of an individual impulse being limited by reason of the varying strength of the impulse and the varying resistance of the many possible tracts. In many invertebrata the nervous system consists of a punctated material composed of a dense interlacement of fibrils, while the cells lie outside the centres and have one thick process dipping into the nervous mass, from which process both axon and dendrites arise. In this case, as we have seen, extirpation of the cell bodies does not destroy the capacity of the remaining fibrillar substance to act as a reflex centre. Such a complex of fibres is found in mammals in the plexuses of Auerbach and Meissner, which act as local nerve-centres for the intestine. But all such mechanism is wanting in the sympathetic ganglia, which contain neither association fibres between different cells of a ganglion nor commissural fibres between the cells of adjacent ganglia. All the fibres in a sympathetic ganglion have either entered it from the white rami or are destined to leave it as fibres of grey rami.

Several reflexes formerly described in peripheral ganglia, as, e.g. the 'submaxillary' ganglion, have been proved to be fallacious. There is, however, a certain group of phenomena which can be elicited in sympathetic ganglia, and which have been termed by Langley and Anderson pseudoreflexes, or, better, axon refflexes. If, for instance, we divide all the nerves going to the inferior mesenteric ganglion, leaving the bladder connected with the inferior mesenteric ganglion only by the hypogastric nerves, and then after dividing the left nerve stimulate its central end, we obtain a contraction of the right half of the bladder. This effect is abolished by 
painting the inferior mesenteric ganglion with nicotine, showing that the activity of the cells of this ganglion is involved in the process. It has been shown, however, by Langley and Anderson that this is not a true reflex, but is rather analogous to Kühne's gracilis experiment (cf. p. 255). A preganglionic fibre arriving at the inferior mesenteric ganglion branches, one branch ending round the cells of the ganglion, while the other branch passes down in the left hypogastric nerve to a cell situated near the base of the bladder (Fig. 241). When therefore we stimulate this nerve we are stimula-

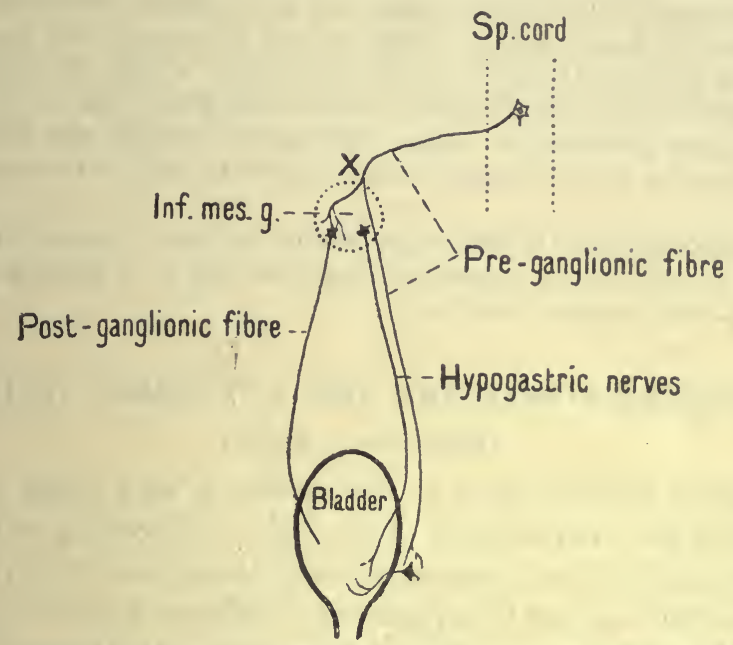

FiG. 241. Diagram to illustrate Langley and Anderson's explanation of the hypogastric reflex as an axon reflex.

The division of the axon where the propagation or ' reflexion' takes place is at $\mathbf{X}$.

ting a pre-ganglionic fibre, and the excitation spreads up to the point of junction of the two branches and then down the other branch to excite the cell in the inferior mesenteric ganglion. We thus obtain an apparent motor reflex by stimulation of a nerve which is itself motor. Similar pseudoreflexes can be obtained along the abdominal chain on the pilomotor nerves but furnish no grounds for ascribing the property of reflex centres to peripheral ganglia. On the other hand, these axon reflexes are very similar to the spread of the excitatory process which occurs in the diffuse nerve network of an animal such as the medusa. Irreciprocity of conduction would seem therefore to be the most useful criterion of a true reflex.

\section{INHIBITION IN PERIPHERAL GANGLIA}

The existence of ganglion-cells in the course of the nerves to visceral muscles has often been supposed to account for certain peculiarities in the innervation of visceral, as compared with skeletal, muscle. Chief among the differences between these two kinds of muscle is the frequency with which inhibition may be brought about in visceral muscle by stimulation of peripheral efferent nerves. In skeletal muscle inhibition is only known as the result of alteration of the activity of the motor centres from which it is supplied. It has therefore been throught that the peripheral ganglia of visceral muscle play the part of the motor spinal centres of skeletal muscle, and that when we excite an inhibitory nerve, say to the intestine, we are interfering with and diminishing 
a tonic state of activity which has its seat in a peripheral ganglion-cell connected with the visceral muscle.

This view, which was first put forward by Claude Bernard, has been specially defended by Dastre and Morat. These observers point out that vaso-dilator action diminishes or disappears as nerve-strands are stimulated more and more peripherally, and conclude that the vaso-dilator fibres run to the sympathetic ganglion and inhibit their tonic action. The untenability of this view has been demonstrated by Langley. Thus the chorda tympani fibres run to a local nerve-' centre 'in the hilum of the submaxillary gland. On Bernard's theory stimulation of the fibres peripherally to the centre should cause contraction of the arteries; but it is found that, after paralysis of the ganglion by nicotine, stimulation of the post-ganglionic fibres causes dilatation, so that the nerve fibres given off from the local centre are not vaso-constrictor but vaso-dilator. Moreover it can be shown that the sympathetic fibres which do cause constriction make no connection with the cells in the hilum of the gland, but run on the walls of the arteries to their distribution. These fibres are connected, not with cells of the submaxillary ganglion (the local nerve-centre), but with cells in the superior cervical ganglion.

We must conclude that the inhibitory nerves in these visceral structures exert their influence directly on the peripheral tissue, and not by a diminution of activity of a tonically acting peripheral centre.

\section{AFFERENT FIBRES AND THE AUTONOMIC SYSTEM}

\section{(Referred Pain)}

The supply of afferent fibres to the viscera is very small in proportion to the supply to the outer surfaces of the body. According to Langley, in a visceral nerve, such as the hypogastric, only about one-tenth of the medullated fibres are afferent, and the proportion of afferent fibres in the splanchnic nerves is probably not very different from this. At the two ends of the alimentary canal, i.e. at the mouth and anus, the afferent visceral fibres become of more importance, since through their means co-operative somatic reflexes have to be excited as well as the simple visceral reflexes. Thus in the pelvic visceral nerve about one-third of the fibres are afferent, and the vagi contain a large number of afferent fibres from the lungs as well as from the other viscera innervated by it.

According to Dogiel, afferent visceral fibres arise from sensory cells of the sympathetic ganglia and, passing in to the posterior spinal ganglia, divide and form pericellular endings round a special type of posterior root-cells, the axon from which divides again into a number of branches which end in connection with typical unipolar cells. Thus, according to this observer, a few afferent sympathetic fibres can stimulate a considerable number of posterior root-cells. Langley, however, has not been able to obtain any experimental confirmation of the origin of branching afferent fibres from cells of the sympathetic system.

It is probable that the afferent fibres of the visceral nerves arise, like those of the somatic system, from ganglion-cells of the posterior spinal ganglia. Every white ramus contains afferent fibres, stimulation of which may evoke a rise of blood pressure as well as movements of the skeletal muscles. In spite of the supply of afferent fibres to the viscera, most of these organs are very insensitive to ordinary stimulation such as handling or cutting. In operations on man for resection of the gut, if the abdominal cavity be opened under local or general anæsthesia, cutting and suturing may be 
conducted without any anæsthetic and without causing any pain to the patient. On the other hand, impulses may arise in the afferent nerveendings of the viscera, as a result of disease or certain forms of stimulation, e.g. stretching or compression, which may reach consciousness and give rise to the sensation of pain. This pain is not localised in the viscera, but is referred to certain parts of the surface of the body. When the afferent autonomic fibres of a nerve are the seat of pain, the primary referred pain is in the area of the cutaneous somatic fibres of the nerve. It has been shown by Mackenzie and by Head that visceral disease may cause hyper-sensitivity of the corresponding areas of the skin, and a method has been elaborated by these observers for utilising this referred pain or skin tenderness as a means of localising the site of the disease. 


\section{CHAPTER VIII}

\section{THE PHYSIOLOGY OF SENSATION}

\section{SECTION I}

\section{ON THE RELATION OF SENSATION TO STIMULUS}

Up to the present we have dealt with the central nervous system as a machine for the conversion of afferent into appropriate efferent impulses, and have regarded the sense-organs simply as mechanisms by means of which stimuli of various quality, arising from events in the environment of the animal, could give rise to nerve impulses. We have seen reason to assign these afferent impressions to various classes, according to the physical character of the stimulus involved and according to the quality of the response. The nature of the physiological processes evoked in the organism depends on the physical nature of the stimulus and the locus of its incidence on the surface of the body. Thus a nocuous stimulus applied to the foot causes flexion of the leg, whereas steady pressure on the sole evokes a 'stepping' reflex with extension of the leg. A beam of light falling on the eye calls forth movements involving contractions of the intrinsic and extrinsic ocular muscles. The same beam of light falling on the skin is devoid of effect unless it is so strong as to burn the skin, when a reflex is excited similar to that evoked by a nocuous stimulus. As our study of the adaptive nerve mechanisms becomes more detailed, and especially when we take into account the activities of the association centres in the cortex, it becomes more and more difficult to follow the chain of processes which lead to any given reaction; in order to advance further in our knowledge of the activity of the receptor organs, we have frankly to abandon the objective method which has served us in the study of all the other functions of the body, and appeal to our own consciousness for information as to the effects of their excitation. Each one of us is aware that stimulation of an afferent nerve may cause a change in consciousness which we denote as a sensation, and we attribute to other living organisms, presenting similar reactions to ourselves, similar changes in consciousness in consequence of like stimuli.

Certain sensations differ one from the other to such an extent that comparison among them becomes impossible. Thus in the skin and underlying parts we have, as a result of stimulation, sensations of touch and pressure, sensations of heat and of cold, and sensations of pain. The contact of 
certain dissolved substances with the end-organs of the gustatory nerves excites in us a sensation of taste. Other substances diffused in the air and carried by it to the olfactory terminations give us sensations of smell. Vibrations of a certain frequency, transmitted by the air and by the auditory ossicles to the endings of the auditory nerve, produce sensations of sound, while light falling on the retina evokes visual sensations.

Besides these sensations resulting from stimulation of the exteroceptive system of nerves, we are aware of the existence of a number of organic sensations-some derived from the viscera (enteroeeptive), others caused by stimulation of the proprioceptive system. As examples of the latter we may mention the muscular sense, by which we judge of the amount of tension exerted by a contracting muscle ; the sense of position of the limbs; and the sense of position of the head, resulting from stimulation of the labyrinthine organ.

How the physiological excitatory process in nerve fibres, with its concomitant chemical and electrical phenomena, is able on arrival at the brain to excite a conscious sensation we are unable to decide, or even to discuss, since we are dealing here with processes of two different orders. We should not arrive any nearer to the solution of this riddle if we were able to follow out the whole of the events occurring in the body as the result of the application of any given stimulus to its surface. We might under these circumstances be able to predict with certainty the behaviour of any animal, if we knew its past history and the comparative resistance of every path in its central nervous system which might possibly be traversed by any given nerve impulse. Such knowledge would be purely objective and could not be used to explain the 'epi-phenomenon' of consciousness. One might in fact imagine a machine which would react like a living animal, but would be perfectly devoid of self-consciousness, and we should be unable in such a case to decide whether consciousness were or were not present. Each one of us only knows consciousness as it exists in himself.

Before therefore we can employ our conscious sensations as a means of throwing light on the conditions of action of the receptor organs of the body, we must have some idea as to how far our sensations correspond to the stimuli, i.e. the physical events by which they have been evoked. Appeal to our own experience shows the existence of two kinds of difference between various sensations. The greatest difference is found between those sensations which are normally evoked by different sense-organs. Thus we are all aware of the meaning attached to such qualities or such sensations as sweet, red, hard, high-pitched (of sound), \&c. It would be absolutely impossible to compare these sensations among themselves. We cannot say, for instance, that this sound is louder than that colour is red. Such fundamental differing qualities of sense are spoken of as the modality of the sensation.

On the other hand, within the sensation evoked by any one sense-organ we find differences of quality which are more comparable among themselves. Thus we can compare the pitch of various sounds, or the colour of various objects seen with the eye. We can even say that the bitter taste of any 
substance is more marked than the sweet taste of another. The question arises how far these differences in sensation correspond to and are a measure of differences in the physical events by which they have been evoked. A very little consideration suffices to show that there is no resemblance between a sensation and the stimulus, and that one and the same physical event applied to different sense-organs will evoke absolutely distinct sensations; while different modes of stimuli applied to one sense-organ will always evoke the same sensation. Thus if light falls on the retina it causes a sensation of light. If the same radiant energy, consisting of transverse vibrations in the ether, be allowed to fall on the skin, it either produces no sensation at all, or, if concentrated by means of a burning-glass, may give rise to a sensation of warmth, heat, or pain. If we take a tuning-fork which is vibrating 100 times per second and apply it to the surface of the skin, we get simply a sensation of vibration, i.e. a series of tactile impressions repeated at rapid intervals. If the same tuning-fork be applied to the head, its vibrations are imparted to the bones of the skull and thence to the auditory nerve-endings and arouse in our consciousness a tone-sensation of a certain note. The same thing happens if the vibrations of the tuning-fork are conducted by the ear to the auditory nerve-endings in the ordinary way through the external and middle ear. On the other hand, a sensation of light may be aroused not only by the incidence of radiant energy of a certain wave length on the retina, but also by electrical or mechanical stimulation of the retina. If the eye be turned inwards and the finger be pressed on the eye through the outer canthus of the lids, a sensation of light is aroused and we see a coloured circle which we refer to some spot lying to the nasal side of the eye stimulated. The character of the sensation bears therefore no resemblance to the physical events by which the sensation is evoked, but depends entirely on the nature of the sense-organ which is stimulated. A sensation of light may be produced by any stimulation of the retina, or of the optic nerve, or of the terminations of the optic nerve in the brain. In the same way stimulation of an auditory nerve or its intracranial endings gives rise to sensations of sound.

Where the question has been investigated it has not been found possible to evoke different qualities of sensation by different modes of stimulation of nerve fibres, and it has therefore been concluded that the quality of any sensation depends simply and solely on the termination of these nerves in the central nervous system, and that where sensations of different quality are produced there must be also difference of nerve fibres. This idea was formulated by Müller, and is often alluded to as Müller's 'law of specific irritability.' The law states that every sensory nerve reacts to one form of stimulus and gives rise to one form of sensation only, though if under abnormal conditions it be excited by other forms of stimuli, the sensation evoked will be the same.

Although the different forms of sensation must be regarded as dependent on the integrity of the brain, and of its connections with the peripheral senseorgans, sensations are not referred to the brain, but are localised as proceed- 
ing from some part of the body or from some region outside of the body. Thus the sensation of taste is always localised in the mouth; sensation of touch at the skin or surface of the body; while the sensations of hearing and of sight are 'projected,' i.e. are interpreted as coming from the environment outside ourselves. Even the organic sensations of posture or fatigue are referred to the peripheral reacting parts of the body and not to the central nervous system. A sensation therefore cannot be interpreted as a reproduction of external events, but as a symbol of these events evoked by stimulation of the sense-organs of the body.

It is indeed impossible by a purely intuitive study of sensations to arrive at any correct idea of their origin or of the factors concerned in their production. No sensation is the immediate and sole product of a stimulus applied to the peripheral end of a nerve fibre, but the simplest sensation involves a judgment, i.e. complex neural activities which are the resultant of innumerable past and present streams of nervous impulses aroused by peripheral events and poured into the central nervous system. It is important therefore not to regard a sensation as in any way constituting an elementary unit, by the aggregation of a number of which a conscious state is produced. As we have seen, the primitive function of the whole nervous system is reaction. The neural life of an animal is composed of a series of reactions, some simple, some complex, and becoming ever more complicated as we ascend the animal scale. The first reactions of a baby, for instance, will be those by which it procures nourishment and satisfies a need. The earliest event in its dawning consciousness will be, not a sensation of sweetness or of colour, but that of a thing which can satisfy its needs. It will have had to try many gustatory experiments before, out of the sum of its material experiences, it will be able to choose a number of like factors which can be grouped together as 'sweet.' Judgment of quality of sensation involves a power of abstraction and of classifying similar elements in different neural events or reactions and the referring of these elements to the external world. It is very difficult, however, to divest ourselves of the mental standpoint reached as the result of many years' continual trials, successes and failures, and constant care has to be exercised if we are not to fall into the common conception of the ego, the personality, or soul, as a sort of sentient god sitting somewhere in the brain, or, as Descartes suggested, in the pineal gland, and receiving by means of one part or other of his servile material brain a blue sensation from the eyes, or an auditory inipression, or a tactile impression, and then, if he feels so inclined, pressing the stop in a pyramida cell to let out a voluntary motor response. An elementary unit in psychical life, as in neural life, must be a complete reaction. It is from the reaction and not from the sensation that a constructıve psychology will have to be built up.

Although any given sensation may be produced by many forms of stimulation of the sense-organ, under normal circumstances each sense-organ is so arranged and protected that it is only stimulated by one kind of physical process, i.e. by the one for which its liminal or threshold excitability is at a minimum. Thus the retina, though it may be stimulated mechanically 
or electrically, is in the normal individual very thoroughly protected from the possibility of such excitation, so that all impulses arising in the retina may be almost certainly referred to changes in the light-waves which fall on the eye, and by means of the dioptric mechanism of this organ are thrown in a distinct pattern upon the retina. Although the sensation is not a reproduction of the stimulus, it is a symbol of the stimulus, and can be used to inform us of events occurring in the world around. Like stimuli, falling on the same end-organ, always evoke like sensations, other conditions being equal. An orderly sequence of sensations may therefore be interpreted as indicating a corresponding orderly sequence of physical occurrences in the world around us.

\section{THE QUANTITATIVE RELATIONSHIPS BETWEEN STIMULUS AND SENSATION}

Since our sensations are merely symbols of the physical conditions which give rise to them, it is important to inquire how far they correspond quantitatively to differences in the energy of the afferent stimuli, i.e. how alterations in the strength of stimulus will affect the intensity of the resulting sensation. Whatever form of stimulus be applied and whatever sense-organs be affected, a certain minimum intensity of stimulus is necessary for it to be effective, i.e. to produce a minimum sensation. This strength, which varies with different sense-organs, is spoken of as the 'liminal intensity,' or 'threshold value ' of stimulus or sensation respectively. As the strength of the stimulus is increased above this minimal amount the resulting sensation also increases. The change in intensity of sensation is not, however, indefinite. When the stimulus is increased to a certain amount the resultant sensation becomes maximal, and a further increase in the stimulus evokes no further increase in sensation. In fact fatigue of the sense-organs or recipient centres of the brain rapidly sets in, so that the sensation diminishes even with increasing strength of stimulus. In each sense-organ we can measure the amount of energy which must be applied to it in order to evoke a minimum sensation. This figure varies considerably with the physiological condition of the animal. In dealing with reflexes we have seen that the motor result of stimulation of a receptor organ varies in the same manner. Thus a minimal stimulus is more effective if repeated a few times at definite intervals (summation of stimulus) : the stimulus which is subminimal may become minimal and effective as a result of repetition.

Another factor which intervenes is that known as 'adaptation'-a process associated to a certain extent with the phenomenon of fatigue. Adaptation is best studied in the case of the eye. Here the dark-adapted eye, i.e. one that has been kept from light for half an hour, will react, and give a visual sensation, to a strength of stimulus which is only one-fiftieth of the minimal stimulus required to evoke sensation in the eye that has been lately exposed to light.

Another phenomenon which may alter the strength of the liminal intensity 
of stimulus is that known as 'contrast.' A finger plunged into mercury feels a ring of constriction at the level of the surface of the mercury, i.e. where there is a contrast between the pressure of the mercury and the absence of pressure as the finger emerges into the air.

The strength of the effective stimulus depends also on the number of nerve-endings simultaneously excited. Thus when dealing with tactile sensations, or sensations of pressure, in determining the minimal stimulus we must take into account the area stimulated, and we express the stimulus, just sufficient to produce a threshold sensation, as ' weight per square millimetre of surface.' Moreover the rapid 'fatigability' or adaptation of all sense-organs makes the rate at which the stimulus is applied of considerable importance. Thus when weight was applied to the skin of the ball of the thumb at the rate of 0.75 gramme per second over a surface of $21.2 \mathrm{sq} . \mathrm{mm}$., the minimum load necessary to evoke a distinct sensation was 2.5 grammes. When, however, the rate of application of the weight was increased to 5 grammes per second, distinct sensation was produced with a load of 0.25 gramme. It is evident that the larger the area of the skin stimulated the greater will be the minimal weight required, since this has to be distributed over all the nerve-endings contained in the area of skin which is subjected to pressure, and the larger the area the smaller will be the stimulus applied to each nerve-ending. The following figures were obtained by von Frey on different regions of the skin :

Stimulated surface $21.2 \mathrm{~mm} .2$

Volar side of wrist (Subject K) . Ball of thumb (Subject K) Volar side of wrist (Subject F) .

Stimulated surface $35 \mathrm{~mm} .2$

Ball of thumb (Subject F)

Tip of finger (Subject F) .

Volar side of wrist (Subject F) .
Rate of loading $1.7 \mathrm{grm}$. per second. Threshold value of stimulus per mm.2

$0.024-0.038$ grm.

$>0.189-0.039$,

$>0.236-0.055$

Rate of loading $3 \mathrm{grm}$. per second Threshold value of stimulus. $0.200-0.045 \mathrm{grm}$. $0.170-0.028$, $0.640-0.028$,

It will be noticed that when the excited surface is small much greater variations are found from spot to spot in the size of the minimum stimulus. This is probably connected with the fact that the sense-organs for pressure are distributed in points or spots over the surface of the skin, so that when the stimulated surface is small the threshold value of the stimulus will be determined by the number of the tactile spots which are included in the stimulated area. The minimal effective stimuli in the case of the other senses have been determined as follows :

(a) HEARING. A musical tone can be heard when the variations of pressure in the air ammount to $00000059 \mathrm{~mm}$. Hg. with an amplitude of vibrations of $.000000066 \mathrm{~mm}$. It has been calculated that the intensity of the work performed on the drum of the ear by such a minimal tone represents an average of $5 \cdot 1 \times 10^{-15} \mathrm{ergs}$. In the case of noise the amount of energy required to produce a minimum sensation is still smaller. A distinct 
sound was heard when a weight 6.7 milligrammes was allowed to fall a distance of $1.2 \mathrm{~mm}$. on to an iron plate at a distance of $500 \mathrm{~mm}$.

(b) VISION. The minimum intensity of light necessary to arouse sensation in a dark-adapted eye is, according to Aubert, equal to about one three-hundredth of the intensity of the light reflected from a piece of white paper which is being lit by the light of the full moon. The amount of energy involved in such a stimulus is much smaller even than that determining a sensation of touch or hearing.

\section{WEBER'S LAW}

It is an interesting question how far the strength of sensation may be regarded as an index to the strength of stimulus. Although it is easy to measure in absolute terms the intensity of a stimulus, i.e. of a purely physical process, there is no means by which we can express in absolute measure the strength of a sensation. We cannot even compare the strengths of two sensations differing in quality or modality; and although we can say that such and such a light is stronger than another light, it is impossible to say that the sensation resulting from the stronger is two, three, or more times that of the weaker. In measuring the effect on sensation of increasing the stimulus we are therefore reduced to using the smallest appreciable increase of sensation as our unit of sensation. The question as to the relation between the intensity of stimulus and the intensity of sensation resolves itself into an inquiry as to what increase in a given stimulus is necessary in order that it may evoke an appreciable increase in sensation. Weber's law states that the increase of stimulus which is necessary to produce an appreciable increase in sensation must always bear the same ratio to the whole stimulus. Thus if we found that we could just distinguish the difference between a weight of $10 \mathrm{oz}$. and a weight of $9 \mathrm{oz}$., it would not be sufficient to add one ounce to a weight of $10 \mathrm{lb}$. in order to produce a distinct difference in sensation. In the latter case we should not be able to appreciate any difference until we had added a pound, i.e. one-tenth of the whole stimulus to the weight. We can distinguish between $10 \mathrm{oz}$. and $11 \mathrm{oz}$., or between $10 \mathrm{lb}$. and $11 \mathrm{lb}$., but not between $10 \mathrm{lb}$. and $10 \mathrm{lb} 1 \mathrm{oz}$.

Several methods have been proposed for testing the limits of the applicability of this law. Of these the most important are :

(1) The method of minimal difference.

(2) The method of average error.

In the first method we find by trial how much a given stimulus must be increased in order to evoke an appreciable increase of sensation, and this determination is made for a number of stimuli of different intensity. In the second method it is sought to find a strength of stimulus which is just equal to another stimulus of given intensity. It will be found that errors will be made on both sides, and the average error is taken as representing the minimum difference, which is just sufficient to cause a distinct difference of sensation.

In all sense-organs Weber's law is only applicable between limits, which 
vary with each sense-organ, and it does not hold either for very weak or for very strong stimuli. Within these limits the ratio which an increase of stimulus must bear to the whole stimulus to produce an increase of sensation may be given approximately as follows for the different sense-organs:

When weights are placed on corresponding points of two sides of the body, e.g. on the two hands, we can appreciate differences of about one-third; if the contrast be successive, i.e. if the weights be placed on the same spot in succession, we can appreciate differences between one-fourteenth and one-thirtieth. The range over which this amount of accuracy is attained extends from 50 to 1000 grammes. In judging of weights with the help of movement (the method one ordinarily adopts) the limit of accuracy is about one-twentieth ; for sounds the appreciation of difference amounts to about one-ninth. The organ which is most susceptible to slight changes of intensity is the eye ; by this organ we can appreciate differences of one one-hundredth to one one-hundred-and-twentieth in the total illumination.

FECHNER'S LAW. Fechner has endeavoured to give a mathematical expression to the facts described under Weber's law. According to Weber the proportion between the increase of stimulus necessary to cause increase of sensation and the whole stimulus is a constant for all intensities of excitation. Thus if $\mathrm{C}$ is a constant

$$
k=C \frac{\Delta}{R}
$$

where $\mathrm{k}$ represents the smallest appreciable increase of sensation evoked by the minimal increase of stimulus, $\mathrm{R}$ is the stimulus, and $\Delta \mathrm{R}$ is the minimal increase of stimulus.

If the same relation may be allowed to hold for infinitesimally small differences of sensation and infinitely small differences of stimulus, this formula may be expressed by the equation:

$$
\mathrm{dE}=\mathrm{C} \frac{\mathrm{dR}}{\mathrm{R}}
$$

By integration we obtain the expression :

$$
\mathrm{E}=\mathrm{C} \text { log. nat. R. }
$$

i.e. the sensation is proportional to the natural logarithm of the stimulus, which is Fechner's psycho-physical law.

In view of the fact, however, that Weber's law only holds good between certain limits, not much practical value can be attached to such a mathematical expression. Moreover Fechner's calculation is based on the unprovable and unjustifiable asst mption that, within the limits of applicability of Weber's law, the smallest appreciable increase in sensation is always the same, i.e. that the increased sensation which is evoked by the addition of 6 grammes to a weight of 100 grammes is identical with the increased sensation called forth by adding 60 grammes to an initial weight of 1000 grammes. Such an assumption does not, as a matter of fact, agree with our own experience ; and it is probably premature here, as in many other departments of biology, to attempt to include the complex of variable phenomena presented by animal functions within the Procrustean bed of a mathematical formula. 


\section{SECTION II}

\section{CUTANEOUS SENSATIONS}

THE skin, being the outermost layer of the body, represents the tissue or organ by which the organism is brought into relationship with its environment. In the widest sense of the term the skin is protective. This function it discharges by virtue not only of its physical properties but also of its rich endowment with sense-organs, by means of which the intracorporeal events can be correlated with those occurring outside and immediately affecting the organism.

We are accustomed to distinguish several qualities of sensation among those having their origin in the skin, the chief of which are the sense of touch, including that of discrimination, the sense of pain and the sense of temperature. The very different qualities of sensation included under these three classes suggest that there may be a special mechanism, or class of mechanism, for each sense, and a careful investigation of the sensory qualities of the skin surface bears out this idea. Isolated stimulation of minute areas on the skin does not excite all the sensations together, but only a sense of touch or of pain, or a sense of cold or warmth. We are therefore justified in dealing with each of these sensations separately.

THE TEMPERATURE SENSE. By means of the skin we can appreciate that a body coming in contact with the skin is either cold or warm. If the body is at the same temperature as the skin, as a rule no sensation of temperature is excited. It was formerly thought that the sensations both of heat and cold were determined by the excitation of one and the same end-organ. Warming of this end-organ would produce a sensation of warmth, while a diminution of its temperature would produce the sensation of cold. Careful investigations by Blix and Donaldson of the distribution of the temperature sense has shown that this opinion cannot be maintained. If a small surface warmed to a few degrees above the temperature of the skin be moved over. any part of the surface of the body, e.g. the back of the hand, it is found that the warmth of the instrument is not appreciable equally at all parts of the surface of the skin. At some points the sensation of warmth will be very pronounced, but between these points the sensation of warmth may be entirely wanting and the instrument may be judged to be of the same temperature as the hand itself. In this way a series of 'warm points' may be mapped out. On now cooling the instrument a few degrees below the temperature of the surface of the body and then moving it over the surface in 
the same way, it will be found again that the coolness of the instrument is only appreciated at certain points which can be regarded as ' cold points ' and as containing the nerve-endings by the excitation of which the sensation of cold is produced. If the warm points be pricked out in red ink and the cold points in blue ink it will be seen that they do not in any way correspond.

A convenicnt instrument for this purpose is the one invented by Miescher, consisting of two tubes cemented together and communicating at a small flattened extremity, which is applied to the surface of the skin; through the tubes water can be led at any desired temperature, which is read off by a thermometer placed within the tube. Having mapped out the warm spots it may be shown that they are excitable by means of mechanical or electrical stimuli and that the sensation produced is the same as if they had been excited by their adequate stimulus, viz. rise or fall of temperature.

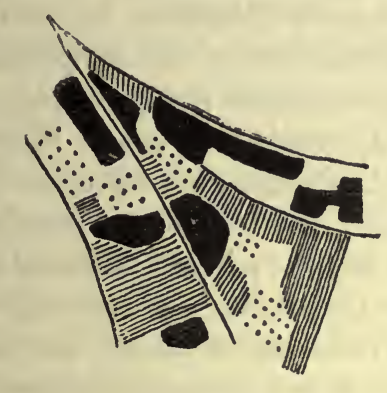

Cold spots.

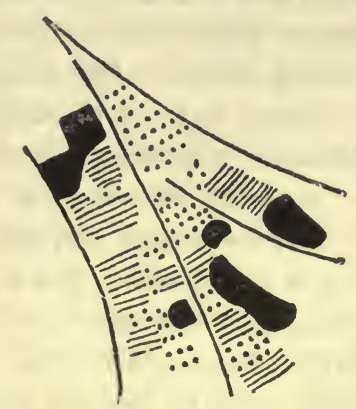

Heat spots.

FIG. 242. Heat and cold spots on part of palm of right hand.

The sensitive points are shaded, the black being more sensitive than the lined, and these than the dotted parts. The unshaded areas correspond to those parts where no special sensation was evoked. (GoLDSCHEIDER.)

The mapping out of the spots is rendered difficult by the irradiation of the sensation produced so that it is difficult to refer the sensation of warmth or cold definitely to the point stimulated. An investigation of the topography of these warm and cold spots shows that the apparatus for the appreciation of cold is much more extensively distributed over the body than that for the appreciation of warmth, as is evidenced from the diagram (Fig. 242) giving the topographic distribution of the cold and warm senseorgans on the palm of the hand. The temperature sense is best marked in the following regions of the body: the nipples, chest, nose, the anterior surface of the upper arm and the anterior surface of the fore-arm, and the surface of the abdomen. It is much less marked on the exposed parts of the body, such as the face and hands, and is but slight in the mucous membranes. Thus it is possible to drink hot fluid, such as tea, at a temperature which would be painful to the hand, and still more to any other part of the body. The scalp is also very insensitive to changes of temperature. The acuteness of the temperature sense varies considerably with the condition of the skin and with the previous stimulation of the sense-organs. The sense is most acute at about ordinary skin temperature, i.e. between $27^{\circ}$ and $32^{\circ} \mathrm{C}$. At this temperature the skin can appreciate a difference of $\frac{1}{5}^{\circ} \mathrm{C}$. When the skin is very cold or very hot the temperature sense is not nearly so delicate. 
This sense presents the phenomenon of adaptation in a marked degree. It is a familiar experience that on coming from the external air on a cold day into a warm room a sensation of warmth is experienced all over the body. In a few minutes this sensation wears off. On now leaving the room to go outside again, the sensation of cold is at once appreciated, to disappear in its turn after a few minutes. The effect of adaptation is still better shown by the experiment of taking three basins of water $a, b$, and $c: a$ contains cold water, $b$ tepid water, $c$ hot water. The left hand is immersed in the cold water and the right hand in the hot water for a few minutes. On now placing both hands into the basin of tepid water it feels hot to the left hand and cold to the right hand. Such experiences as this led Weber to the conclusion that the essential stimulus for the temperature sense was not the actual temperature to which the sense-organs were subjected, but the fact of a change of temperature. Hé imagined that while the temperature senseorgans were being warmed a sensation of warmth was produced, and when their temperature was being lowered, a sensation of cold. Such a theory would not, however, account for the fact that, above a certain temperature, water may feel warm and the feeling may continue so long as the skin continues to be stimulated. On a cold day the air may feel cold to the face and the feeling may last the whole time that the face is exposed. Moreover we have in the temperature sense conditions which remind one of the after images which occur in the eye and which will form the subject of a later section. If a penny be pressed on the forehead and then removed the sensation of cold lasts some little time after the penny has been removed. In this case a sensation of cold is produced although the end-organs are being gradually warmed up after the removal of the penny. In order to account for these facts Hering, at a time when the differentiation of hot and cold spots had not yet been effected, suggested that the temperature sense-organs could be regarded as having a zero-point at which no sensation was produced. If their temperature was raised above this point a sensation of warmth was produced and vice versa. The zero-point, however, was not a fixed one, but could move upwards to a certain extent on prolonged exposure to high temperature, or downwards on prolonged exposure to a low temperature. In the light of the researches of Blix and Goldscheider we should have to apply Hering's theory of a zero-point to each of the temperature end-organs separately.

A cold pencil passed over a warm spot evokes no sensation whatsoever. If, however, a pencil considerably warmer than the skin be passed over a cold spot this may be excited, so that the paradoxical result is produced of a sensation of cold as the result of stimulation with a warm body. It is a familiar fact that the immediate effect of entering a hot bath is very much the same as that of entering a cold bath, viz. a rise of blood pressure and contraction of the unstriated muscles of the skin and hair follicles with the production of 'goose skin.' It has been suggested that the distinctive quality of a sensation of hot as compared with that of warm is due to the simultaneous stimulation of warm spots and cold spots. When testing the 
distribution of the temperature sense it is found that the sense of cold is evoked more promptly than that of warmth. This is interpreted as showing that the end-organs for the warm sense are situated more deeply than those for cold. We have no evidence as to the histological identity of these organs.

\section{THE SENSE OF TOUCH}

By means of the sense of touch we arrive at a conclusion as to the qualities, such as shape, texture, hardness, \&c., of the bodies with which the skin is in contact. In this judgment, however, very many other sensations are involved besides those which can be regarded as strictly tactile. Thus the hardness of an object signifies its resistance to deformation, besides its power of deforming the skin surface with which it is in contact; the former quality, i.e. of resistance, is one which involves the muscular sense, since we judge of it by the extent to which we can move our muscles without causing any alteration of the surface of the body.

The tactile sensibility of the skin as a whole, like its temperature sensibility, is due to the presence in it of a number of touch spots, i.e. small areas which are extremely sensitive, separated by areas almost or entirely insensitive to pressure. The tactile sensibility of any part is proportional to the number of such touch spots present. If the calf of the leg be shaved and then tested by pressing on it with a fine bristle or hair it will be found that the minimal stimulation used evokes sensation only at certain definite points, the ' touch spots.' In a square centimetre of such skin there may be about fifteen touch spots. On thrusting a fine needle into one of these spots a sharply localised sensation of pressure is produced unaccompanied by any painful quality and often describedas having a "shotty' character, as of a little hard object embedded in the skin and there pressed upon. These touch spots are arranged chiefly around the hairs, lying usually on the side from which the hair slopes. They vary in number according to the part of the body which is the subject of investigation. Thus the dorsal surface of the finger contains about seven times as many touch spots as an equal area between the shoulders. In some regions, such as the skin over subcutaneous surfaces of bone, as much as one centimetre may intervene between two neighbouring touch spots. They have no relation to the warm and cold spots; they are entirely absent from the cornea, the glans penis, and the conjunctiva of the upper lid.

The adequate stimulus for these tactile nerve-endings is not so much pressure as deformation of surface. It appears to matter little whether the surface be deformed by pulling it or by pushing an instrument into it. The ineffectiveness of mere pressure is shown by dipping the finger into a vessel of mercury. The sensation of pressure is only noted at the point where the finger passes through the surface of the mercury, and this is the only part where there is an actual deformation of the skin, due to the sudden passage from the pressure of the mercury to the negligible pressure of the outside air. The tactile apparatus is smarter in its response than any other of the sense-organs. On this account stimuli are still perceived as discrete, 
when they are repeated at a rhythm which would result in complete fusion in the case of any of the other sense-organs. Thus if a bristle be attached to a tuning-fork and allowed to press on the skin, the vibrations of the fork are perceived by the ear as a continuous sound and by the skin as a series of discontinuous taps. Faradic currents when applied to the skin can be perceived as separate when repeated at the rate of 130 per second. The sensations evoked by placing the finger against the edge of a cog-wheel do not become continuous until the wheel is revolving at such a rate that the stimulation on the skin by the serrations occurs at a greater rate than 500 or 600 per second. The tactile apparatus resembles all the other skin sense-organs in showing adaptation. A stimulus after continuing for some time may become ineffective. We are usually entirely unaware of the stimulation of our skin by the pressure of the clothes, and even an unwonted stimulation, such as that of the mucous membrane of the mouth by a plate carrying artificial teeth, though almost unbearable

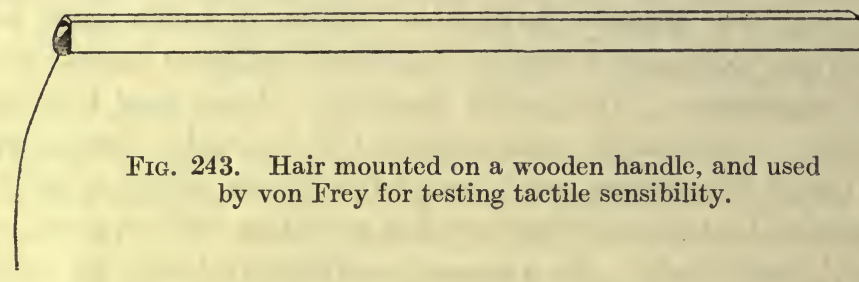

during the first day, rapidly becomes less, and in a few days it is not perceived at all.

In order to test the sensitiveness of touch we may use the method introduced by Hensen, viz. the bending of a glass-wool fibre. We could determine the pressure at which any given fibre will bend, and if we find by trial the fibre which just evokes sensation when pressed on the skin, we know exactly the force which we are applying to the skin. Von Frey employed hairs of different thickness for the same purpose (Fig. 243). The following represents the minimal excitability of the surface of different parts of the body when tested in this way.

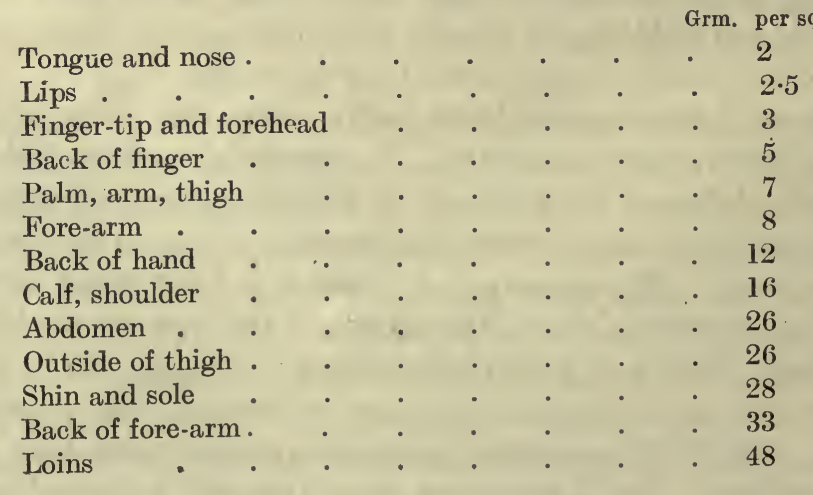


The sensitiveness of the sense-organs in the skin is probably much greater than that of the nerve-trunks themselves. Thus Tigerstedt found that the minimal mechanical stimulus necessary to excite the exposed nerve amounted to $0.2 \mathrm{grm}$. moving at $140 \mathrm{~mm}$. per second. For the touch spots von Frey found that $0 \cdot 2 \mathrm{grm}$. moving at $0 \cdot 17 \mathrm{~mm}$. a second is an adequate stimulus.

In testing the sensibility of any surface it is important to remember that the hairs themselves form very effective tactile organs. The touch spots are distributed in greatest profusion around hair follicles, and there is a rich plexus of nerve fibres round the root of each hair. A slight touch applied to the hair acts on these as on the long end of a lever, the hair being pivoted at the surface of the skin, so that pressure on the hair is transmitted, increased five or more times in force, to the hair follicle and the surrounding nerve-endings. The actual sensibility of any part is therefore much diminished by removal of the hairs. On 9 sq. $\mathrm{mm}$. of the skin, from which the hairs had been shaved, the minimal stimulus necessary to evoke a tactile sensation was found to be $36 \mathrm{mg}$., whereas on the same surface before it was shaved $2 \mathrm{mg}$. was effective.

WEBER'S LAW. The smallest increment or decrement of stimulus which determines a perceptible difference of sensation must, according to Weber's law, always bear the same ratio to the whole stimulus. In measuring such differences it is best to apply the stimulus successively to the same surface of the skin rather than simultaneously to adjoining areas. The time interval between two successive stimuli should not be more than five seconds and the duration of the stimuli should be equal. Weber found that in the terminal phalanx of the finger the minimal perceptible difference was about one-thirtieth, but the ratio was not the same for all regions of the skin nor for all individuals. The following represents the liminal difference in various skin regions :

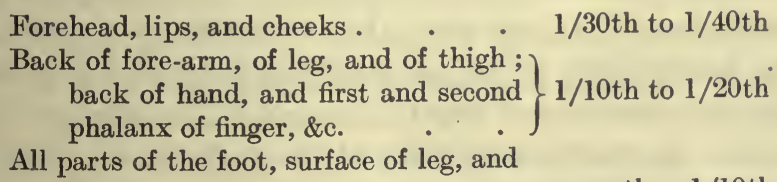
thigh

more than $1 / 10$ th

THE SPATIAL QUALITY OF TOUCH. DISCRIMINATION. If any part of the skin be stimulated the subject of the experiment can tell at once the exact situation of the excited spot. If two points be stimulated simultaneously excitation is perceived as double, i.e. as proceeding from two points, provided the distance between the points exceeds a certain amount, varying in different parts of the body. The power of discrimination, i.e. of judging whether a stimulus is single or double, can be tested by arming the points of a pair of compasses with small pieces of cork and then seeing how far apart the points must be when pressed on the skin in order that the 
stimulus may be perceived as double. The following Table represents this distance for various regions of the body :

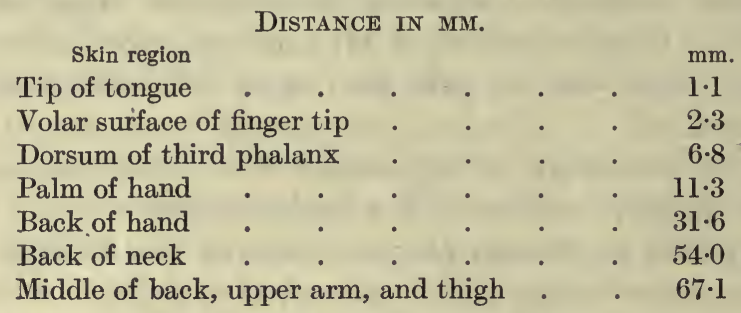

When touch spots are sought out for stimulation with the points of a compass, the distance at which the excitation is perceived as double is much diminished, as is shown by the following Table of distances for the touch spots in millimetres:

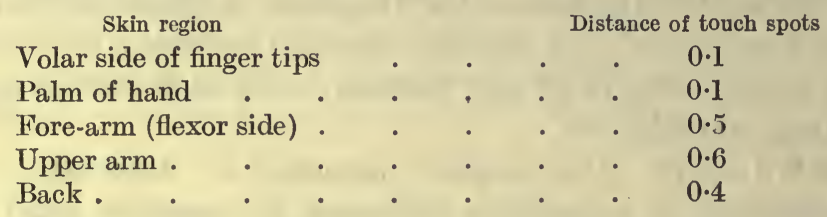

The compass points are perceived to lie apart with a special distinctness when they are applied to touch spots lying on different lines which radiate from the hair follicles. The figures given in the first Table have no relation to touch spots, but show the average distance over which an excitation can be perceived as double.

The delicacy of discrimination of any part is largely associated with its mobility. Thus in the arm the delicacy increases continuously from the shoulder to the finger-tip. If the localising power for touch on the shoulder be taken as 100, that of the finger-tips will be represented by 2582 . In the same way there is a continuous decrease of the distances of discrimination as we pass along the cheek from the ear to the lip, i.e. from the nonmobile to the mobile part. The power of discrimination is increased to a certain extent by practice and largely diminished by fatigue. Any factor which diminishes the tactile sensibility of the part, such as cold, will also diminish the power of discrimination.

The fact that we can localise the point of stimulation shows that every factile sensation derived from the surface of the body, besides the qualities of intensity and extensity, has also associated with it a characteristic quality dependent on its position. This localised quality of a tactile sensation was called by Lotze 'local sign.' Among psychologists there has been much discussion as to how far this 'local sign' is an inborn attribute of the sensation of every point on the body surface, or how far it is acquired by experience and based on memory of movements and muscular impressions. In the retina we have a sense-organ which, like the skin, possesses local sign, but in far higher degree, the power of discrimination of the retina being three thousand times as great as that of the most sensitive part of 
the skin. Cases of congenital cataract occur in which the subjects have been blind from birth. By extraction of the cataract we can give such persons the power of sight. It is found that at first there is no power of localising visual impressions. The 'local sign' is only developed in response to experience, by comparing simultaneous visual, tactile, and motor sensations. By analogy we might ascribe the local sign of cutaneous sensations to a similar causation. Our study of the spinal animal has indeed given us a physical or histological conception of local sign. We know that stimulation of any part of the body evokes an appropriate reaction, the nature of which is determined by the central connections of the entering nerve fibres. A fibre entering at one segment must therefore come into relation with a different set of motor cells from those which are set into action by a fibre entering one segment lower down. Every nerve fibre from the skin will therefore have an appropriate complex of motor paths in functional connection with its central endings, and when the activity of these reflex paths comes to be represented in consciousness it is evident that the sensation derived from each point must differ from that derived from any other point of the skin by virtue of the differing motor events actually or potentially excited from the two points. In ascribing therefore 'local sign' to coincident muscular sensations, and to the memory and experience of past movements, we are giving but an imperfect explanation ; since the difference between the sensations from different parts, which are at the bottom of our powers of localisation, has its origin in the structure of the central nervous system itself and is present from the very beginning of the evolution of a reactive nervous system.

PROJECTION OF TOUCH. Since the alterations in the surface of the skin which give rise to tactile sensations are habitually caused by contact with external objects, we come to regard the sensations themselves, not as changes in the skin, but as qualities of the object which touch the skin, i.e. we project the sensation. The projection is, however, not so great as in the case of visual sensations. Cutaneous sensations we always consider as qualities of an object immediately affecting and altering the condition of ourselves, whereas the visual sensations are referred at once to objects lying right away from ourselves, so that we are not aware that any change has taken place in our bodies as a result of the entering of rays of light into the eye.

It is remarkable to what extent projection of touch sensation may occur. Thus a surgeon actually lengthens his fingers by using a probe. When he is probing for dead bone he feels the grating of the bone, not at his finger-tips, but he projects the sensation to the end of the probe. In the same way tactile sensations evoked by the contact of bodies with the insentient endings of hair are referred to the ends of the hairs rather than to the hair follicles where the nerve impulses actually come into being.

The dependence of local sign on habitual experience is well shown. by the various tactile illusions, such as the well-known experiment of Aristotle. If we cross the first and middle fingers and bring them in this 
position in contact with a pea, if the eyes are shut, we should at once say that two peas lay under the fingers. This is especially marked if the pea be rolled between the fingers. The two sides of the fingers which come in contact with the pea usually touch two different objects, and these parts of the skin would have to be re-educated, i.e. their local sign would have to be changed in accordance with the changed conditions, before the pea would be perceived in its true state as single.

\section{THE PAIN SENSE}

When the pressure of a hard object on the skin is increased beyond that necessary to evoke a tactile sensation, at a certain pressure the quality of sensation changes and it becomes painful. For the evolution of the race as well as. for the preservation of the individual this pain sense is allimportant; it is the expression in consciousness of the reflexes of selfpreservation which can be evoked in the spinal animal by stimuli which are nocuous, i.e. calculated to do actual damage to the tissues of the body. Thus when a sharp point is pressed on the skin the sensation becomes painful just before the pressure is sufficient to cause penetration. The so-called trophic lesions which occur in parts devoid of sensation are determined for the most part by the lack of the pain sense and the consequent failure of the preservative reflexes of the part. It is remarkable that pain may result from changes in organs which are devoid of ordinary sensibility. Thus the intestine may be cut, sewn, or handled without arousing any sensation whatsoever. A strong contraction of the muscular wall or increased distension of the gut will, however, evoke a griping pain. In the same way the ureters, which are devoid of sensation, can give rise to excruciating agony when they are contracted firmly on a retained calculus.

We are accustomed to distinguish many different qualities of pain, but on analysis it will be found that these qualities depend on the nature of the sense-organ which is simultaneously stimulated. Thus a burning pain denotes simultaneous stimulation of the pain sense and of the nerve-endings to the warm spots. A throbbing pain results when the vessels of the part are dilated and the part is tense with effused lymph, so that each pulse of the vessels causes an exacerbation of the painful stimulation and perhaps also stimulation of the tactile end-organs.

The sense of pain has often been ascribed to over-maximal stimulation of any form of sensory nerve. Although it is true that over-stimulation of the auditory or optic nerve by a loud sound or a bright light may be extremely unpleasant, the sensations evoked do not partake of the characters of painful sensations such as would be produced by pricking or burning the skin. Moreover a careful investigation of the sensory points on the skin brings out the fact that there are besides the tactile and temperature spots, other spots from which only painful sensations can be evoked. We have seen already that over-stimulation of a touch spot does not, as a matter of fact, cause pain. The pain spots which are distributed among the touch and temperature spots are insensitive to a low grade of stimulus. As the 
strength of the stimulus is increased a point is suddenly reached at which the sensation evoked is painful. Moreover in parts of the body tactile and temperature sense are entirely wanting, though painful impressions can be easily evoked. The best example of this is seen in the cornea, minimal stimulation of which evokes pain, but nothing which can be regarded as a tactile sensation. The specific quality of pain sensation is shown moreover by the fact that in many cases of disease the sense of pain may be abolished without the sense of touch. Such a patient is said to suffer from analgesia, but not anæsthesia. When pricked on an analgesic part the patient can say that he is pricked, but has no objection to any amount of repetition of the stimulus, since the sensation is entirely devoid of painful character.

\section{THE WORK OF HEAD ON CUTANEOUS SENSIBILITY}

In a long series of researches on man Head has shown that three different classes of sensations may be evoked by stimuli applied to the surface of the body. In order to study the functions of the afferent nerves Head has investigated not only the condition of patients, the subjects of accidental division of cutaneous or other nerves, but also (in conjunction with Rivers) the effects of nerve section on himself. In the first place, it is necessary to differentiate deep sensibility from cutaneous sensibility proper. After desensitisation of any given area of the skin it is still possible in this area to appreciate deep pressure and pain, and the localisation of the situation of the pressure is fairly accurately carried out. On the other hand, the sensations of light touch, as well as of temperature and the pain evoked. by a light pin prick, are absent. The sensations of pressure, as well as of deep pain or -pressure pain, are therefore carried by the nerves of deep sensibility. These nerves are not the cutaneous nerves, but are derived from the sensory elements in the muscular nerves. To the fingers, for instance, they run in the tendons of the muscles. Simultaneous division, as by a circular-saw cut, of the cutaneous nerves and tendons to the fingers will abolish deep as well as superficial sensibility. Deep sensibility must therefore be classified, anatomically at any rate, with the 'organic sensations' of muscular effort and of position, which will be dealt with in a subsequent section.

Cutaneous sensibility proper Head divides into two categories, namely, protopathic and epicritic sensibility. These two forms of sensibility may be studied separately on an area of skin, which has been desensitised by section of its cutaneous nerves, during the process of regeneration of these nerves.

PROTOPATHIC SENSIBILITY returns to the skin at an interval of seven to twenty-six weeks after the nerve section. At this time it is possible to appreciate in the area under investigation the sensation of pain, and to recognise roughness of an object rubbed on the skin. Localisation is still somewhat diffuse and inaccurate, so that the sensation evoked by stimulation of the protopathic area may be referred to some adjoining normal 
part of the skin. The temperature sense is also present, but of a low grade. Thus heat over $38^{\circ} \mathrm{C}$. and cold under $24^{\circ} \mathrm{C}$. can be appreciated as such, but the intervening temperatures produce no sensation. Sensations evoked in the protopathic area are strongly endowed with what may be termed 'affective' character. Thus painful stimulation is much more unpleasant when applied to this area than would a similar stimulation be when applied to a normal area of skin.

In contradistinction to the deep sensibility which is diffuse, protopathic sensibility is distributed in spots, so that heat and cold spots, for instance, may be distinguished as on the normal skin. It is interesting that the glans penis is normally provided only with protopathic sensibility.

EPICRITIC SENSIBILITY does not return to the desensitised area until one to two years have elapsed since the division of the nerves. With its return the affective character of the protopathic sensations at once disappears and is replaced by an accurate discrimination of the nature and extent of the stimulus; the tactile sense proper, i.e. the appreciation of the lightest touch applied to the skin and its accurate localisation, belonging entirely to the epicritic sensations. The power of discriminating the distance between two points applied to the skin simultaneously is also a function of the epicritic sensibility.

With the discriminating tactile sense returns also the power of appreciating fine differences of temperature, i.e. differences between $26^{\circ}$ and $37^{\circ} \mathrm{C}$.

This classification may be summed as follows :

\begin{tabular}{|c|c|c|}
\hline Deep sensibility & . including & $\left\{\begin{array}{l}\text { Pressure sense } \\
\text { Pressure pain }\end{array}\right.$ \\
\hline $\begin{array}{l}\text { Protopathic sensibility } \\
\text { (strongly affective) }\end{array}$ & . $\quad$ & $\left\{\begin{array}{l}\text { Skin pain } \\
\text { Heat over } 38^{\circ} \mathrm{C} \text {. } \\
\text { Cold under } 24^{\circ} \mathrm{C} \text {. }\end{array}\right.$ \\
\hline $\begin{array}{l}\text { Epicritic sensibility } \\
\text { (accurately localised) }\end{array}$ & . $\quad$ & $\left\{\begin{array}{l}\text { Tactile sense proper } \\
\text { Pain localisation } \\
\text { Discrimination } \\
\text { Heat and cold between } \\
26^{\circ} \text { and } 37^{\circ} \mathrm{C} \text {. }\end{array}\right.$ \\
\hline
\end{tabular}

Head and Thompson have shown that on entering the cord these various sensations undergo a new grouping. Thus the pain impulses, which arise in and are carried by the muscular nerves, the nerves of deep sensibility, unite with those which run in the protopathic system, so that a lesion of the cord which abolishes the sense of pain will abolish all forms of pain, whether arising from the skin or from the underlying tissues. In the same way all temperature sensations, whether the fine ones of the epicritic system or the coarser ones of the protopathic system, run together in the cord. If the heat sense is affected by a lesion of the cord all forms and all degrees of the sensation are affected in like measure, and the same applies to the sensations of cold. 


\section{THE HISTOLOGICAL CHARACTER OF THE ELEMENTS INVOLVED IN CUTANEOUS SENSATIONS}

A very large number of different forms of sensory nerve-endings have been described in relation to the skin. Their exact allocation among the different cutaneous senses presents considerable difficulties.

As regards touch, two kinds of elements are probably involved. In the first place, the most sensitive tactile apparatus are the follicles of the short hairs. Around these follicles we find a sheaf of nerve fibres, some of which end in the hair papilla and others form a ring near the level of the openings of the sebaceous glands. The other tactile end-organ is Meissner's corpuscle. The distribution of these in the skin is not, however, dissimilar to that of the power of discrimination, with which they may be specially connected. Other end-organs which are supposed to be stimulated by changes of pressure, and therefore to be tactile, are the organs of Ruffini which occur in the papillæ of the palm and fingers, and, lying more deeply, the elastic tissue spindles as well as the Golgi corpuscles and the Pacinian corpuscles in the subcutaneous tissue.

As regards pain, we known that in the cornea, which possesses only the pain sense, the sensory nerve-endings are in the form of branches of axis cylinders among the epithelial cells. Similar free nerve-endings occur in the epidermis all over the body, and it is therefore imagined that these have the special function of subserving the pain sense. We have at present no evidence as to the histological character of the organs by which the sensations of heat and cold are aroused. 


\section{SECTION III}

\section{SENSATIONS OF SMELL AND TASTE}

Every living organism shows a susceptibility, i.e. a power of reaction, to chemical stimuli. Thus the plasmodium of myxomycetes, placed on a strip of filter-paper of which one end is immersed in an infusion of dead leaves and the other in distilled water, will crawl along the paper towards the infusion of leaves. If the infusion of dead leaves be replaced by a weak solution of quinine, the plasmodium will be repelled and will travel along towards the vessel of water. These movements of attraction and repulsion are spoken of as positive and negative chemiotaxis respectively. A similar chemical sensibility accounts for the clustering of aerobic bacteria towards the surface of a fluid, i.e. where the density of oxygen is greater, or around chlorophyll-containing algæ which are giving off oxygen in the sunlight. The aggregation of leucocytes round microbes or other foreign particles in the tissues is also determined by their chemiotactic sensibility. Chemiotaxis then represents the faculty by means of which these minute organisms are able to adapt themselves to chemical changes in their environment and to react to chemical substances at a considerable distance from themselves. If we could endow these elementary organisms with conseiousness and with a sense of their surroundings, we should have to say that they became aware of the presence of some harmful or attractive material at some distance from themselves. The sensation they received from these distant objects would be therefore a projected sensation.

On the other hand, a chemical sensibility of the body surface or part of it furnishes the criterion by which particles are accepted and ingested as food or rejected as useless or harmful. Consciousness in this case would be of something affecting and in contact with some part of the organism itself. The sensation would not be projected further than the periphery of the body.

These two kinds of chemical sense-the projected and the surface sense-are found throughout almost all classes of the animal kingdom, and in the higher animals at least are known as the senses of smell and taste. The former sense in many animals attains a high degree of complexity and is prepotent in determining the behaviour of an animal in response to the changes in its surroundings. In the elasmobranch fishes the olfactory lobes form the greater part of the higher brain, and extirpation of them produces a loss of spontaneity and of delayed reactions similar to 
that which can be brought about in higher types by extirpation of the whole of the cerebral hemispheres.

The sense of taste, on the other hand, is only used for sampling the nature of substances taken into the mouth and determining their ingestion or rejection. It is therefore much simpler in its extent and more susceptible of analysis.

\section{THE SENSE OF TASTE}

The end-organs which subserve the function of taste are represented by the taste-buds. These are oval bodies (Fig. 244) embedded in the stratified epithelium, which occur scattered over the tongue, a few being also found on the hard palate, the anterior pillars of the fauces, the tonsils, the back of the pharynx, the larynx, and the inner surface of the cheek. On the tongue they are found chiefly in the grooves around the circumvallate papillæ of man, and in the grooves of the papillæ foliatæ of rabbits. A few are also present on many of the fungiform papillæ. They consist of medullary and cortical parts, the former being composed of columnar or sustentacular cells, the latter of thin fusiform cells, the tastecells proper. The nerve fibres concerned with taste end in arborisations among these tastecells. The peripheral end of the fusiform cell projects as a delicate process through the orifice of the taste-bud, so that it can come in contact with the fluids contained in the cavity of the mouth. A sapid substance, to stimulate these organs, must be in solution; hence quinine in powder is almost tasteless, owing to its slight solubility in neutral or alkaline fluids.

The number of different tastes is very limited.

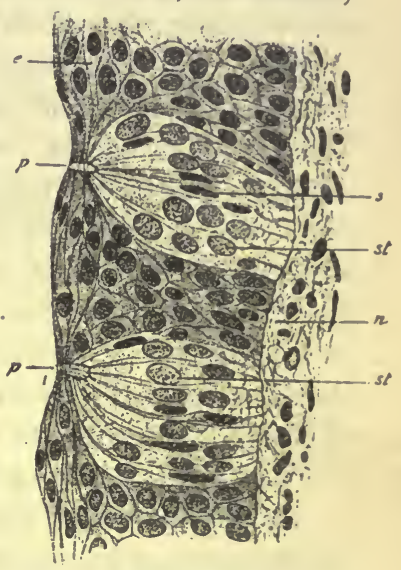

FIG. 244. Two taste-buds from the tongue.

$e$, Stratified epithelium; $p$, opening or pore of tastebud ; $s$, gustatory cells; st, sustentacular cells.

(KöLLIKER.)

We distinguish four primitive taste sensations, viz. sweet, sour, bitter, and salt, some authors adding to this an alkaline taste and a metallic taste. Many substances owe their distinctive character when taken into the mouth to the fact that they stimulate not only the taste-nerves but also the nerveendings of common sensation. Thus acids, when in weak solution, have an astringent character besides their sour taste, and if strong produce a burning sensation. The primitive taste sensations can affect one another if excited simultaneously. With weak stimulation one taste may practically annul another. Thus a dilute solution of sugar is rendered almost tasteless by the addition to it of a few grains of common salt. If the primitive taste sensations are more strongly excited we get a mixed sensation, in which the components can still be distingushed. Thus, adding sugar to lemon juice not only diminishes its acidity but produces a mixed sensation, the quality of which is pleasant and in which the components, sour and sweet, can be easily distinguished. We get no such fusing of sensations 
as in the eye, where a sensation of white light may result from stimulation of the retina by two complementary colours. Stimulation of one kind of tasteorgan heightens the sensibility of the other taste-organs. Thus after the application of salt, distilled water may taste sweet.

That these primitive taste sensations are served by different nerveendings is shown by the following facts :

(a) The tongue is not equally sensitive at all points to all four tastes. Thus the back of the tongue is more sensitive to bitter, while the tip and sides of the tongue react more easily to sweet and sour substances. A difference may be detected between even the circumvallate papillæ themselves; a mixture of quinine and sugar applied to one papilla may excite chiefly a bitter taste, while with an adjacent papilla'a sweet taste may predominate.

(b) By certain drugs we can depress the sensibility of the taste-organs, and we then find that the various tastes are affected to different degrees. Thus on painting the tongue with cocaine the first effect is a diminution of tactile and pain sensibility, so that the application of acid evokes a very sour taste without any of the astringent or stinging sensations normally aroused by the contact with the acid. After this point the taste sensations are also abolished. The bitter sensation disappears first, then the sweet, and then the sour, while the taste of salt appears to remain unaffected. On the other hand, if the leaves of Gymnema sylvestre be chewed, the sensations of bitter and sweet are abolished, leaving intact the acid and salt tastes, and also the general sensibility of the mucous membrane.

There is no doubt that the stimulating effect of any chemical substance on the taste-nerves has relation to its chemical constitution. Thus a sour taste is determined by the presence of $\mathrm{H}$ ions; the alkaline taste by that of $\mathrm{OH}$ ions. The fact that certain acids, e.g. acetic, have a stronger sour taste than would correspond to their dissociation, i.e. to the number of $\mathrm{H}$ ions present, is due to the fact that these acids penetrate more easily into the gustatory cells than the mineral acids with a larger dissociation coefficient. All the $\alpha$-amino-acids have a sweet taste. On the other hand, the polypeptides produced by the combination of these amino-acids, as well as the peptones derived from the hydrolysis of proteins, have a bitter taste. Most of the alcohols and sugars have a sweet taste, while the metallic derivatives of these substances are bitter. We do not yet understand the law which determines whether any given substance shall have a taste at all, and what its taste should be.

The nerves of taste are the glossopharyngeal, which supplies the back part of the tongue, and the lingual branch of the fifth nerve and the chorda tympani, which supply the front part. All these fibres are probably connected with a continuous column of grey matter in the brain stem, which represents the splanchnic afferent nucleus of the fifth nerve, the nervus intermedius, and the glossopharyngeal. Some authors have stated that all the taste fibres of the fifth nerve are derived from the glossopharyngeal by the communication through the tympanic plexus and the chorda tympani nerve, while Gowers has recorded a case of complete unilateral loss of taste 
in which there was a lesion destroying the fifth nerve, the glossopharyngeal being intact. It seems possible that the actual region of the taste-nerve may vary, the fibres running to the splanchnic column of grey matter being contained sometimes in the fifth, sometimes in the glossopharyngeal, and sometimes in both.

Most of our so-called tastes should rather be designated flavours, and are dependent, not on the gustatory nerves, but on the sense of smell.

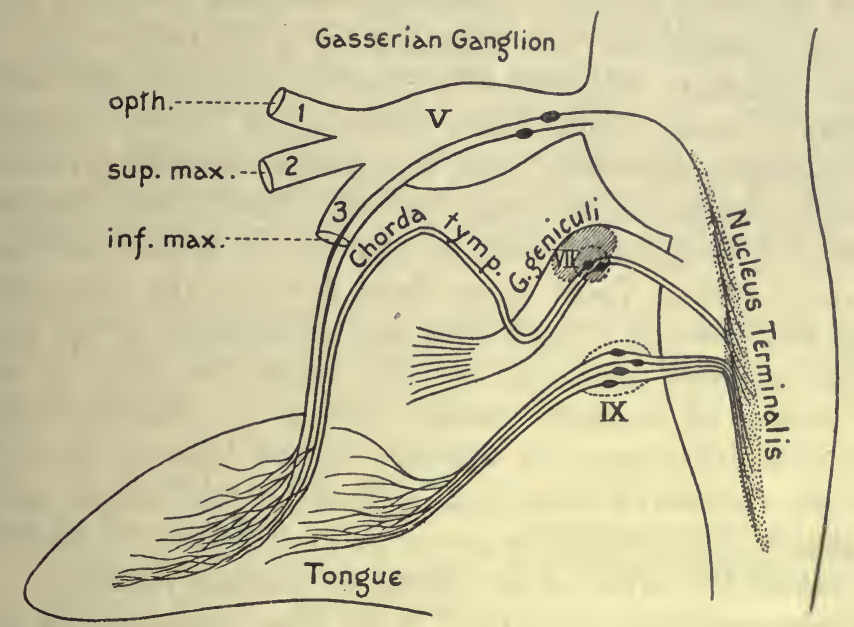

FIG. 245. Diagram showing origin and course of the nerve fibres of taste.

When the olfactory sense is destroyed very little difference is to be perceived between an onion and an apple. The epicure with a fine palate has really educated his sense of smell and would be but little satisfied with the simple sensations derived from his four sets of gustatory end-organs.

\section{THE SENSE OF SMELL}

The psychical analysis of olfactory sensations is rendered difficult by the fact that this sense in man plays but a small part in his usual adaptations. We have thus to deal with a sense which is in many respects vestigial. We see traces of great complexity in its possibilities of performance, but are baffled in our endeavours to reduce the whole of the phenomena to the simpler factors of which they are composed. Moreover, like all vestigial functions, the extent to which the sense is developed varies from one individual to another. Many, for instance, are unable to appreciate the smell of vanilla, of hydrocyanic acid, or of violets. On the other hand, in animals, such as the dog, the olfactory sense seems to play a great part in determining behaviour, and the nervous associations, which are the physiological basis of ideas, must in these animals be largely connected with olfactory impressions. Another factor which diminishes the importance of olfactory sensations in man is the ease with which the sense-organ becomes fatigued. It often happens that the inmates of a room are perfectly comfortable 
and may perceive no fault in the ventilation, although a new-comer from the outside at once remarks that the air is foul.

The organ of smell is situated at the upper part of the nasal cavities. Here the mucous membrane covering the superior and middle turbinate bones and the corresponding part of the septum is different from that covering the rest of the nasal passages. Over the lower parts of the nasal cavities the mucous membrane is of the ordinary respiratory type, and is composed of ciliated columnar epithelium containing a number of gobletcells. In the olfactory part the epithelium is much thicker, of a yellow colour, and apparently composed of a layer of columnar cells resting on several layers of nuclei. These nuclei belong to the olfactory cells proper, true spindle-shaped nerve-cells with one process extending towards the mucus covering the free surface, while the other is continued along channels in the bone, and through the cribriform plate as one of the non-medullated olfactory nerve fibres. These nerve fibres dip into the olfactory lobes, where they terminate by a much-branched arborisation or end basket in the so-called olfactory glomeruli, in close connection with a similarly branched dendrite of the large ' mitral' cells of the olfactory lobe. The axons from these latter carry the olfactory impulse towards the rest of the brain. In the connective tissue basis (dermis) of the mucous membrane are a number of small mucous or serous glands (Bowman's glands) whose office it is to keep the surface of the membrane constantly moist.

In ordinary respiration the stream of air never passes higher than the anterior inferior border of the superior turbinate bone, so that it does not come in contact with the olfactory mucous membrane. The sensations of smell which are aroused during ordinary respiration depend on diffusion from the respiratory air into the still air of the upper olfactory portion of the nasal cavity. The direction of olfactory attention is achieved by sniffing; in this act the nostrils are dilated and the direction of the anterior part of the nasal respiratory chamber altered, so that the stream of entering air is directed towards the upper olfactory portion of the cavity.

The fact that we are able to perceive smells when breathing normally shows that the odorous substance must be diffusible, i.e. gaseous in form. The amount of substance necessary to excite sensation is extremely minute. 'Thus $01 \mathrm{mg}$. of mercaptan diffused in 230 cubic metres of air is still distinctly perceptible. In this case a litre of air would contain only $00000004 \mathrm{mg}$. of the substance, and the amount actually in contact with the olfactory epithelium would be still smaller. It is possible, however, to show the presence of these odorous substances in air by physical means. Tyndall pointed out that air containing a small proportion of odorous substances absorbed radiant heat to a much greater degree than did pure air. Thus in one experiment air containing patchouli absorbed radiant heat thirtytwo times as strongly as the pure air. Most odorous substances possess large molecules and have therefore high vapour densities. On this account the smell tends to hang about objects, the rate of diffusion of the vapour being only small. 
Since the endings of the olfactory cells are bathed in fluid, it is evident that the odorous substances must be dissolved by this fluid before they can excite the olfactory nerve fibres, and in the case of aquatic animals we know that the projected chemical sense, which we call smell, can only be aroused by substances in solution. It is difficult to show in man that the nerveendings can be excited by solutions. Most of the experiments have been made with solutions which had an injurious effect upon the olfactory epithelium. According to Aronsohn it is possible to excite sensations of smell if the nasal cavity be filled with normal saline fluid, containing a very small proportion of the odorous substance. To this experiment it has been objected that it is almost impossible to fill the nasal cavities without leaving some air spaces so that the olfactory sensation obtained might have been due to stimulation of the olfactory cells in such a space. There is, however, no a priori reason to deny the probability of Aronsohn's conclusions.

Many olfactory stimuli owe their peculiar character to the simultaneous stimulation of other kinds of nerve-endings. Thus a pungent smell, as that of ammonia, chlorine, \&c., involves stimulation of the nerves of common sensibility, i.e. the fifth nerve, besides stimulation of the olfactory nerve.

No satisfactory classification of smells has yet been made. The following facts tend to show that there are a number of primitive sensations of smell, as of other sensations :

(a) Certain individuals, whose olfactory sense is in other respects normal, have no power of distinguishing some odours.

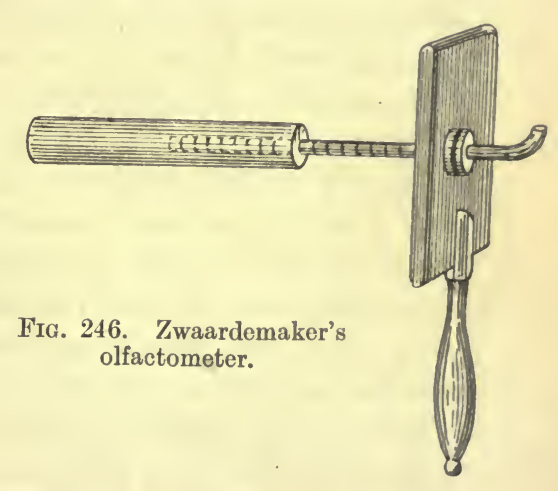

(b) The olfactory sense is easily fatigued. If it be fatigued so as to be absolutely insensitive for one kind of smell, it is still normally excitable for other smells.

(c) It is possible by mixing odoriferous substances in certain proportions to annul their effect on the olfactory organ. Thus $4 \mathrm{grm}$. of iodoform in $200 \mathrm{grm}$. of Peruvian balsam is almost odourless, and the same neutralisation of odours is obtained if the odour of each substance be allowed to act separately on each side by tubes inserted into each nostril.

For this purpose we may use the instrument invented by Zwaardemaker, called the olfactometer. This consists of a porous cylinder into which is inserted a tube. The porous cylinder is first immersed in the fluid whose porous qualities are to be tested, and when it is thoroughly soaked it is taken out, dried outside by a cloth, and inside by drawing air through it for a short time. One end of the bent tube is then inserted into the cylinder, which it must accurately fit, while the other end is placed in one nostril. The small wooden screen shown in Fig. 246 serves to shut off the smell of the fluid from the other nostril. When the observer breathes through the bent tube the amount of vapour taken up from the cylinder will depend on the amount 
of surface exposed, and therefore can be diminished or increased by pushing the bent tube further in, or by drawing it out. If the tube is pushed in so far that the smell is only just perceptible the length of the tube may be measured and taken as the liminal intensity of stimulus for the given substances, in its action on the olfactory nerveendings. This unit was called by the inventor of the instrument an 'olfactie.' By this means it is possible to make quantitative estimations of the olfactory sense on one individual and to compare them with observations made on other individuals. By using two such instruments it is possible to present different smells to the two nostrils. One obtains in this way combination effects which can be compared to the phenomenon which we shall study later in dealing with binocular contrast. 


\section{SECTION IV}

\section{AUDITORY SENSATIONS}

By means of our auditory sensations we are made aware of such changes in our environments as are capable of giving rise to a disturbance which can be propagated through the surrounding elastic medium, the air, to our ears. Any sudden jar given to a solid body sets up vibrations which are propagated to the surrounding air as sound waves, i.e. a series of acts of condensation and rarefaction spreading out from the centre of disturbance, like the waves which are caused on the surface of a pond by throwing a stone into its middle. With a delicate tambour we can record these changes of pressure and convert them, by means of a lever writing on a blackened surface, into movements at right angles to the direction of movement of the surface. The amplitude of vibration of the membrane will be proportional to the amount of compression and expansion occurring at each wave. These waves travel through the air at the rate of 1100 feet per second, their wave length varying with the number of vibrations per second. It is of course possible to get vibrations of almost any number per second. Only when the number of vibrations fall within distinct limits are they effective in producing a sensation of sound. Before we can discuss the physiological mechanism of hearing we must have a clear idea of the character of the physical change - the stimulus - which is effective in evoking an auditory sensation and determining its quality.

Sounds may be divided into noises and musical tones. If the vibrations or series of vibrations arriving at the ear are irregular in character, such as those produced by striking the table or the floor with a stick, we speak of the resultant sensation as a noise. If, on the other hand, the vibrations follow one another at a regular sequence and possess a rhythm-if, for instance, a series of vibrations be imparted to the air by a tuning-fork vibrating at 100 times per second - the effect on consciousness is that of a musical tone. Of course there is no hard-and-fast line between the two kinds of sound; even when the prevalent impression is that of a noise it is often possible to pick out some series of vibrations which predominate among the irregular ones with which they are accompanied. When we strike a single stick with a hammer the effect is that of a noise. If, however, we take a series of sticks of different lengths and strike them in succession, it will be noticed that the sound produced by each stick corresponds to a distinct note, and tunes may be played on such a collection of sticks. On the other hand, 
the tone of a musical instrument may be so harsh that there is very little difference between it and a noise.

In a musical tone we can distinguish various characters or qualities :

I (1) The loudness of a tone is determined by the amplitude of the vibrations of which it is composed. If a violin string be bowed forcibly the excursion of its string at each vibration is greater than when it is bowed gently, and the amplitude of the corresponding alternating waves of sound varies in proportion to that of the vibrating body by which they are started. By attaching a pointed slip of paper to the end of a tuning-fork and so recording its vibrations on a blackened surface, it is easy to see the connection which exists between the amplitude of vibrations and the loudness of the sound produced by the vibrating. fork.

(2) The pitch of a musical tone depends on the frequency of the vibrations of which it is composed. By means of a siren we can determine the number of vibrations corresponding to each note. As the speed of revolution of the siren is increased, and therefore the number of interruptions per second of the stream of air passing through the holes in its disc, the note appears to us to rise continuously. If we take two tuning-forks, one vibrating at 100 times per second and the other vibrating 200 times per second, the pitch of the latter is observed to be considerably higher than that of the former. In fact it forms the octave. If the number of vibrations is less than about thirty per second no musical tone is produced, the individual vibrations being perceived as a series of pulses in the surrounding air, and it is only when we increase the number to about forty per second that we are able to appreciate the pitch of the note produced. As the number of vibrations per second is increased the note rises steadily without break till we arrive at 40,000 to 50,000 vibrations per second. Above this number of vibrations the human ear is incapable of perceiving any note at all, though it is probable that small animals can perceive notes still higher in the scale. In music neither the lowest nor the highest tones are used. The lowest tones of the large organs, that of the sixty-four foot pipe, is 16 vibrations per second, and one can hardly speak of its effect as that of a musical tone. The highest notes employed in music are $a 4$ and $c 5$ with 3520 and 4224 vibrations per second on the piano, and $d 5$ with 4752 vibrations on the piccolo flute. In music therefore we only employ between 40 and 4700 vibrations per second, i.e. about seven octaves. The manner of construction of the musical scale will be dealt with later.

(3) Timbre or quality of musical sounds.

When the same note is sounded on different instruments, i.e. tuning-fork, violin, piano, trumpet, human voice, every person, whether he has an educated musical ear or not, can say at once what kind of instrument is being used. This fact shows that the sound wave produced by these instruments must differ, altogether apart from any differences in amplitude or in number of vibrations per second, and if the sound waves produced by these instruments be recorded an actual difference is found in the shape of the curve. 


\section{AUDITORY SENSATIONS}

If a stretched wire be plucked so as to set it into transverse vibrations it will give out a certain note, dependent on its length, its thickness, and the tension to which it is subjected. If its length be halved it will give out a note which is of double the number of vibrations per second. If only onethird of the wire be set into vibrations the sound wave produced will have three times the number of vibrations of that of the whole string. When the string is free to vibrate as a whole the segments of it tend to vibrate even while the whole string is vibrating. If therefore we take the note given out by the whole string, the 'fundamental tone,' as corresponding to 132 vibrations per second, there will also be a series of notes superadded to the fundamental tone with vibrations per second in the ratio of $1,2,3,4,5$, and 6 , \&c. Thus if the fundamental tone be $c$, the overtones, or harmonics, will be produced as is shown below :

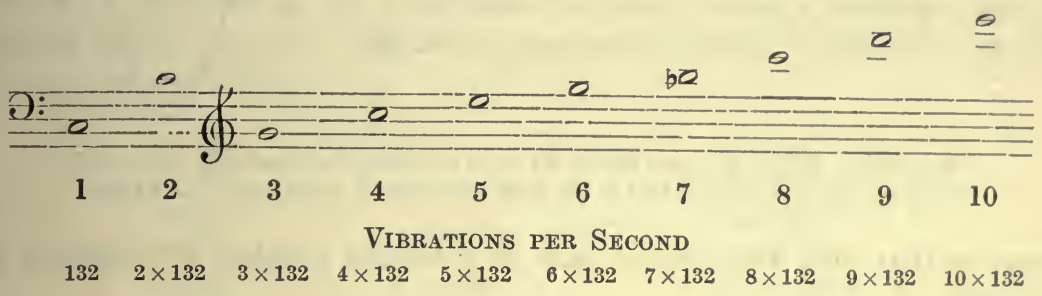

Nearly all musical instruments, as well as the apparatus for producing the human voice, resemble a stretched wire in giving out overtones in addition to the fundamental tones, and the difference in the quality of various instruments is chiefly determined by the varying predominance of the different overtones. In some the higher overtones may be most marked, in others only the lower overtones. The tuning-fork is practically the only instrument the note of which is pure, i.e. free from harmonics or overtones. It must be remembered that these different tones arrive at the external ear simultaneously. We do not have some particles of air vibrating at one rate and other particles at another rate, but all the simple vibrations of which the component tone is composed are combined together to form a compound wave, the shape of which differs according to the constituent vibrations of which it is made up.

Thus in the diagram (Fig. 247) the wave shown by the continuous line is compounded of the series of simple vibrations represented by the different dotted lines. The components of such a compound curve can be detected if the sound be analysed by allowing it to act on resonators, i.e. on instruments which can be set into vibration by certain simple tones of which the component tone is made up. The stretched strings of a piano may be used as a battery of such resonators. If the dampers of the strings be raised, by depressing the loud pedal, and a note be then sounded into the piano, it may be noticed that the piano gives back the sound, and on attaching pieces of straw to the various strings it will be seen that only certain straws vibrate, i.e. those on the strings which are vibrating to the fundamental tone or overtones contained in the sound received by the piano. For the analysis 
of sounds the resonators devised by Helmholtz are generally employed. These consist of hollow vessels, with an opening at one end, made of different

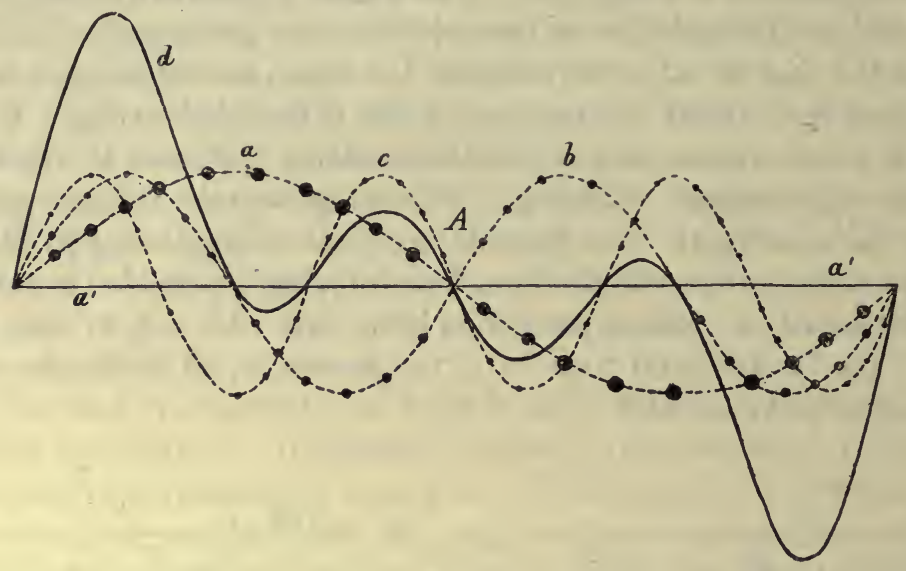

Fig. 247. $d$, a compound sound wave, which may be analysed into $a$, the fundamental tone, and $b$ and $\mathrm{t}$, the first and second overtones. (HENSEN.)

sizes, so that each will resound only to a definite number of vibrations per second.

BEATS AND DISSONANCE. The overtones of any sound, at any rate the lower ones, are at considerable distance from one another on the musical scale, and therefore differ considerably in the number of vibrations of which they are composed. If two tuning-forks be sounded, the vibrations of which differ only by one or two per second, the phenomenon known as 'beats' is produced. This is due to the summation or interference of the waves from the two tuning-forks. Supposing we have tuning-forks vibrating one at 100 and the other at 101 times a second, and they start vibrating together. At first the waves of compression started by each fork will coincide, so that the total compression of the air at each beat will be the compound effect of the compression produced by the two forks. The two forks therefore will reinforce one another. After the lapse of half a second the tuning-forks will be at different phases of their excursion. The 101 fork will be moving in one direction while the 100 fork is moving in the other, so that the compression produced by one fork coincides with the expansion of the air produced by the moving backwards of the other fork. The sound produced by one fork is therefore diminished by the sound produced by the other fork, and the total sound is less than either of the two forks. At the end of one second, the phases of the two forks once more corresponding, we shall get the sound increased in loudness; thus there is an alternate waxing and waning of the sound which recurs once a second and is spoken of as a 'beat.'

The number of beats per second may be used to determine the differences in the vibration frequencies of two forks. Thus two forks vibrating one at 100 and the other at 110 will give ten beats per second. As the number of beats increases the effect produced on the ear becomes more and more dis- 
agreeable, just as the rapid alternation of illumination produced by a flickering light is disagreeable to the eye. This objectionable character of the sound is most marked when the beats recur at about thirty-three times per second; the individual beats are not then distinguished, but we speak of the sound as discordant or dissonant.

CONSONANCE. The opposite condition of consonance or harmony involves therefore, in the first place, an absence of beats, i.e. of rhythmic oscillations of amplitude of sound waves which reach the ear. The constituent tones and overtones must be capable of being combined into a compound wave of regular amplitude and rhythm. In the most complete consonance the component notes are identical as concerns at any rate the greater number of their overtones. The most complete consonance is attained when the two notes which are sounded together are identical. Almost as complete is the consonance obtained when a note is sounded together with its octave. The other consonant intervals which are employed in music are as follows :

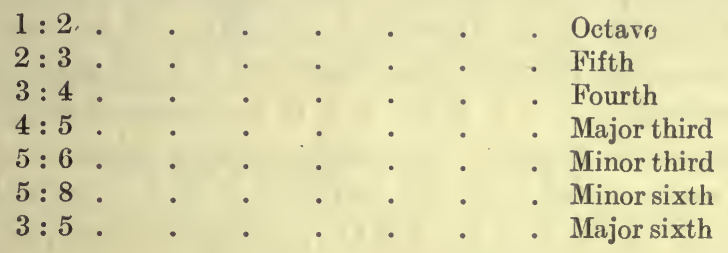

It will be noticed that in all these consonant combinations the vibration frequencies of the notes are in proportion to small whole numbers. If we put down not only the fundamental tones of these notes but also their overtones, we shall see that there is considerable identity as regards the latter. In the case of the octave the two are almost identical, the only difference being the ground tone of the lower note, and the identity diminishes as we pass from the octave through the thirds to the sixths. The overtones which are identical are shown by black type :

\begin{tabular}{|c|c|c|c|c|c|c|c|c|c|}
\hline Fundamental tone & & & & Over & rtone & & & & \\
\hline Octave $1: 2$ & $\left\{\begin{array}{ll}1: & 2 \\
& 2\end{array}\right.$. & 3 & $\begin{array}{l}4 \\
4\end{array}$ & - 5 & - 6 & 7 & $\begin{array}{l}8 \\
8\end{array}$ & & . \\
\hline Fifth $2: 3$ & $\begin{cases}2 & 4 \\
3 & \end{cases}$ & $\begin{array}{l}6 \\
6\end{array}$ & 8 & 10 & $\begin{array}{l}12 \\
12\end{array}$ & 14 & 16 & $\begin{array}{l}18 \\
18\end{array}$ & \\
\hline Fourth $3: 4$. & $\left\{\begin{array}{rrrr}3 & & 6 & \\
& 4 & & 8\end{array}\right.$ & 9 . & $\begin{array}{l}12 \\
12\end{array}$ & .15 & 16 18 20 & $0^{21} \cdot$ & $\begin{array}{l}24 \\
24\end{array}$ & . 27 & $7.8^{3}$ \\
\hline Major third 4:5 & 4.8 & $12 \dot{15}$ & 16 . & $\begin{array}{l}20 \\
20\end{array}$ & . 24 . & $5^{28} 30$ & & & \\
\hline Minor third $5: 6$ & $\left\{\begin{array}{rrr}5 & . & 10 \\
& 6 & 12\end{array}\right.$ & 15 is & 20 & $24^{25}$ & $\begin{array}{c}30 \\
30\end{array}$ & - 35 & $6^{40}$ & $4^{45}$ & 58 \\
\hline Major sixth 3 & $\left\{\begin{array}{lll}3 & & 6 \\
& & \\
& 5 & \end{array}\right.$ & 9 & 12 & $\begin{array}{l}15 \\
15\end{array}$ & . 18 . & $0^{21}$ & 24 & $\dot{25}^{27}$ & \\
\hline
\end{tabular}


Fundamental tone

Overtone

Second $8: 9$

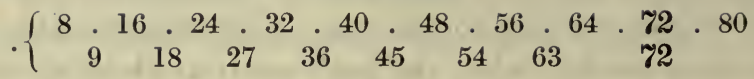

Seventh $8: 15$

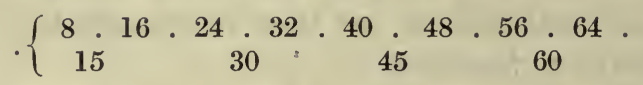

In the second and seventh, which are discordant, it is only the eighth overtones which are identical, while the fundamental tones will, as a rule, be so close together that beats will be produced of a number calculated to give dissonance. Since the phenomenon of beats depends on the absolute number of vibrations per second they are more easily produced by two notes near together at the lower end of the scale than at the upper end. Thus the dissonance is quite perceptible in a major third at the lower end of the piano, but disappears at the upper part, since here the beats produced are so rapid that they become imperceptible.

The various notes used in music are obtained by employing the consonant intervals which we have given above. The major chord is composed of the fundamental tone, the major third and the fifth. If we take ' $c$ ' as the fundamental tone, the notes of the chord are $c, e, g$, with vibration frequencies corresponding to $1, \frac{5}{4}, \frac{3}{2}$, i.e. $4,5,6$, the major chord from $g$ is $g, b$, $d$, i.e. three notes with vibration frequencies corresponding to $\frac{3}{2}, \frac{15}{8}, \frac{9}{4}$, i.e. $4,5,6$. The major chord from the fourth, $f$, is $f, a, c$, with the vibration frequencies $\frac{4}{3}, \frac{20}{12}, \frac{12}{6}$, i.e. $4,5,6$. The $\mathrm{C}$ major scale is therefore as follows :

$$
\begin{array}{cccccccc}
\text { C } & \text { D } & \text { E } & \text { F } & \text { G } & \text { A } & \text { B } & \text { C } \\
\underline{1} & \frac{9}{8} & \frac{5}{4} & \frac{4}{3} & \frac{3}{2} & \frac{5}{3} & \frac{15}{8} & 2
\end{array}
$$

Different instruments are tuned to one normal note, i.e. to A with 440 vibrations per second (this note varies somewhat in different countries).

\begin{tabular}{|c|c|c|c|c|c|c|c|c|c|}
\hline \multicolumn{3}{|c|}{ Notes } & \multicolumn{7}{|c|}{ Vibrations per second } \\
\hline C & . & . & 33 & 66 & 132 & 264 & 528 & 1056 & 2112 \\
\hline D & . & & $37 \cdot 125$ & $74 \cdot 25$ & 148.5 & 297 & 594 & 1188 & 2376 \\
\hline $\mathrm{E}$ & . & & $41 \cdot 25$ & $82 \cdot 5$ & 165 & 330 & 660 & 1320 & 2640 \\
\hline $\mathbf{F}$ & . & . & 44 & 88 & 176 & 352 & 704 & 1408 & 2816 \\
\hline G & . & . & $49 \cdot 5$ & 99 & 198 & 396 & 792 & 1584 & 3168 \\
\hline A & . & . & 55 & 110 & 220 & 440 & 880 & 1760 & 3520 \\
\hline B & . & • & $61 \cdot 875$ & $123 \cdot 75$ & $247 \cdot 5$ & 495 & 990 & 1980 & 3960 \\
\hline
\end{tabular}
Taking this as the normal, the vibration frequencies of the various notes used in music are given in the following Table :

COMBINATION TONES. If two tuning-forks, with an interval of one-fifth between them, are sounded together, we may hear a weak lower tone, the pitch of which is an octave below that of the lower fork. This is known as a 'combination tone.' The combination tones are divided into two classes : 
(1) 'difference tones,' in which the frequency is the difference of the frequencies of the generating tones; (2) 'summation tones,' which have a pitch corresponding to the sum of the vibrations of the tone of which they are composed. By means of appropriate resonators these tones can be reinforced, showing that they have an objective existence and are not produced in the ear itself.

Not only can the ear appreciate differences between different musical instruments, dependent on the varying overtones present in the sound produced by each instrument, but, when a number of these instruments are sounded simultaneously, the ear can pick out from the compound sound the notes due to the individual instrument, and a person with a trained ear can with ease name notes composing any chord struck on an instrument such as the piano. This power of analysis, which is possessed by the ear, or at any rate by the auditory apparatus, may be stated in the form of the law, known as Ohm's law, which is as follows:

"Every motion of the air which corresponds to a composite mass of musical tones is capable of being analysed into a sum of simple pendular vibrations, and to each single vibration corresponds a simple tone, sensible to the ear and having a pitch determined by the periodic time of the corresponding motion of the air."

\section{THE PHYSIOLOGY OF HEARING}

The organ of the ear may be considered as consisting of an accessory part and an essential part. The latter is formed by the terminal expansion of the auditory nerve. The accessory part is constructed so as to bring the waves of sound to act on the end organs. The ear is divided anatomically into three parts - the external ear, consisting of the pinna with the external auditory meatus ; the middle ear, consisting of the tympanic cavity ; and the internal ear, consisting of the osseous and membranous labyrinths with the terminal branches of the auditory nerve.

The external ear in the lower animals is fashioned so as to collect sound waves from different directions. To this end it is provided with muscles and in many cases is very movable. In such animals the immediate response to a slight sound is a pricking up of the ears and a direction of their orifices towards the source of sound, a reflex direction of attention which in man is replaced by a conjugate deviation of the two eyes towards the side from which the sound comes. The collecting function of the pinna in man is rudimentary; in fact a man can hear almost as well with his ear cut off as normally.

The form of the pinna in man may have a slight influence in the judgment of the direction from which sounds proceed. It has been noticed that a compound tone changes slightly in quality as its position in relation to the ear is altered. This is partly due to the fact that the auricle may reflect a fundamental tone more strongly than the partial or the converse. According to Rayleigh this difference in quality is determined chiefly by the fact that diffraction of the sound waves occurs as they pass round the head to the ear remote from the source of the sound, so that the partial tones reach the two ears in different degrees of intensity and determine a difference in quality of the sound as heard by the two ears. 
The external auditory meatus in man is about one inch long and directed forwards, inwards, and slightly upwards. Its general function, other than as a mere conductor of the sound vaves, is to protect the delicate vibrating membrana tympani which closes its inner end. Opening on the skin of the meatus are special sebaceous glands which secrete a yellow wax (cerumen) with bitter taste and peculiar odour. The wax not only protects the cuticle of the ear and the membrana tympani from drying, but, together with the hairs at the orifice of the meatus, serves to repel insects and prevent their entering. By the length of the meatus moreover the drum is protected from draughts and its temperature is maintained constant.

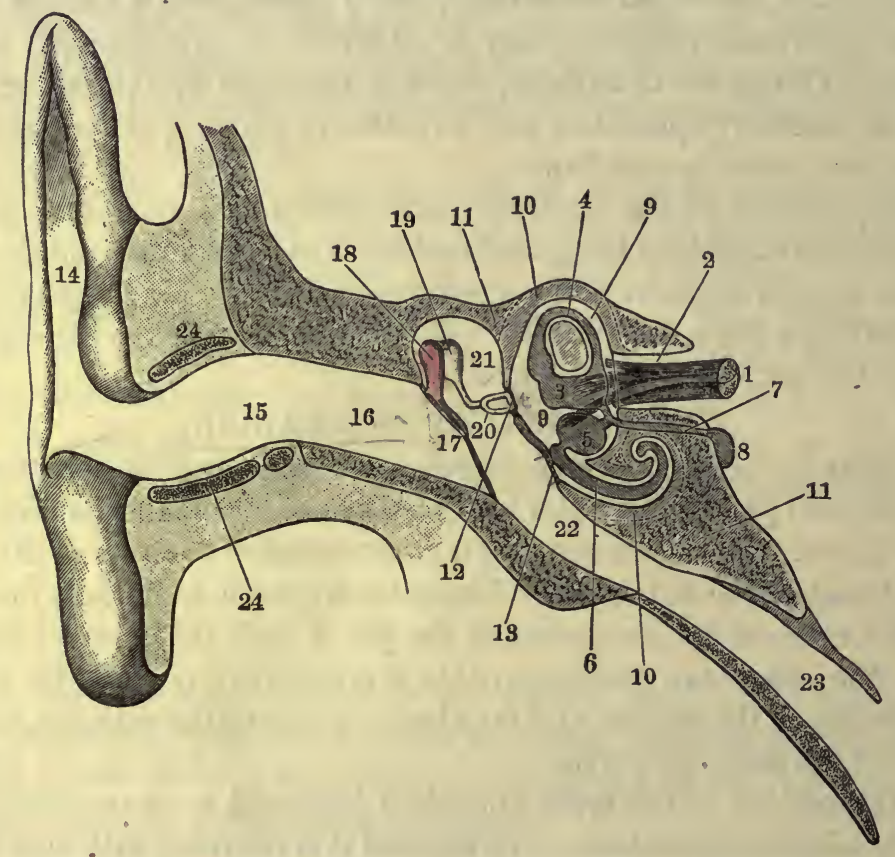

FIG. 248. Diagrammatic view of auditory organ. (After ScHäFER.)

1 , auditory nerve $; 2$, internal auditory meatus $; 3$, utricle $; 5$, saccule $; 6$, canalis media of cochlea; 9 , vestibule containing perilymph; 12 , stapes; 13 , fenestra rotunda; 19 , incus ; 18 , malleus ; 17 , membrana tympani ; 16 , external auditory meatus; 14, pinna of external ear ; 23, Eustachian tube.

The sound waves which pass down the external meatus impinge on the drum of the ear and set this into vibration. The vibrations are thence transmitted by a chain of three small bones, the auditory ossicles, across the cavity of the tympanum to the fluid which bathes the terminations of the auditory nerve in the internal ear. Since the drum of the ear has to pick up and transmit vibrations of every frequency, and to reproduce accurately in its movement the finest variations of pressure in the course of the wave, it is essential that it should be devoid of any periodicity, i.e. a tendency to vibrate at a certain frequency. If such periodicity were present the ear would pick out and magnify some particular overtone present in the compound tones reaching the ear and magnify it to the exclusion of the other 
overtones. The perfect aperiodicity of the tympanic membrane is secured by its structure and attachments. The membrane is composed of a thin layer of fibrous tissue covered externally with skin and internally by the mucous membrane of the tympanum. To its inner surface is attached the handle of the malleus, the first of the auditory ossicles, along its whole length. This attachment of an elastic membrane to a mass of bone would itself tend to damp any vibrations of the membrane. By the attachment of the tendon of the tensor tympani muscle to the inner surface of the handle of the malleus the middle of the membrane is drawn inwards, so that it forms a cone whose walls are convex outwardly. The membrane is built up of circular and radial fibres, the circular being best marked towards the periphery. By the dragging inwards of its central part it follows that the tension of its constituent fibres varies from point to point so that each bit of the membrane would have a different periodicity, and the membrane as a whole is aperiodic.

By exposing the tympanum from above it is possible with a microscope to observe the actual movements of the handle of the malleus when sound waves fall on the tympanic membrane. The maximum movements at the apex $\cap$ the cone may be taken as about $.04 \mathrm{~mm}$., but sounds are easily audible which would produce movements of the tympanic membrane quite imperceptible under this method of examination. On the inner side of the tympanic membrane is the tympanic cavity, which is connected in front with the pharynx by means of the Eustachian tube. This is opened by each movement of swallowing, so that the pressure in the tympanum is kept equal to that of the outside air. When the Eustachian tube is blocked or diseased the air in the tympanum is gradually absorbed and the patient becomes deaf on that side. Stretching across the tympanum, from the membrana tympani to the outer wall of the internal ear, is a chain of ossicles, which are named respectively the malleus, the incus, and the stapes. These ossicles are articulated together, so that a movement inwards of the malleus causes a movement inwards of the base of the stapes. The malleus, or hammer bone, consists of a thickened head, from which two processes run, viz. the manubrium, which is attached to the tympanic membrane, and the processus gracilis, by which it is anchored to the walls of the tympanic cavity. By means of three ligaments it is so fixed that it is capable only of rotating around a horizontal axis, which passes through the anterior ligament, the head of the malleus, the body of the incus, and the short process of the incus When the manubrium is pushed inwards, a part of the malleus above this axis must move outwards. The incus, sometimes known as the anvil bone, is articulated with both the stapes and the malleus, and a ligament passes from its short process to the posterior wall of the tympanic cavity. The posterior surface of the rounded head of the malleus fits into the saddleshaped cavity on the anterior surface of the incus, while the tip of the long process of the incus is articulated with the stapes. Movement inwards or outwards of the head of the malleus causes rotation of the incus round an axis which passes from the tip of the short process through its body. Thus when the handle of the malleus moves inwards the greater part of the body of 
the incus and of the head of the malleus move outwards together, while the long process of the incus moves inwards. The stapes, or stirrup bone, is fixed in the fenestra ovalis of the internal ear, in the inner surface of the tympanum, by the annular ligament. It is placed almost at right angles to the long process of the incus, and therefore is pressed into the foramen ovale when this process moves inwards. The breaking up of the connection between the tympanic membrane and the foramen ovale into three bones, connected by joints, must tend to prevent any propagation of sound by direct continuity of substance. The vibrations of the membrane result in actual movements of the whole bones, which represent a chain of levers reproducing exactly the movements of the membrane. In the transmission of a sound wave from the membrana tympani to the labyrinth there is a change in the amount of force as well as in the amplitude of the movement. The three bones can be regarded as forming a lever with two arms, one of which is the manubrium of the malleus, and the other the long process of the incus. The length of the former is to that of the latter as 3 to 2 , so that the movements transmitted from the tympanic membrane to the base of the stapes are diminished in the proportion of 3 to 2 and have their force increased in the proportion of 2 to 3 . Moreover, as the drum of the ear has an area which is about twenty times that of the foramen ovale, the energy of its movements is concentrated on an area twenty times smaller. Hence the pressure of a sound wave acting on the tympanic membrane is increased thirty-fold $\left(\frac{3}{2} \times 20\right)$ when it acts on the base of the stapes.

The computation of the actual energy, involved in the movement of these structures by sound waves, which are just perceptible to the ear, yield striking results as to the extreme sensitiveness and efficiency of this apparatus. Lord Rayleigh has estimated that the amplitude of the movement of an aerial particle involved in the propagation of sound at the limits of audibility is less than one ten-millionth of a centimetre. By other methods it has been calculated that the ear is affected by vibrations of molecules of the air not greater than $\cdot 0004 \mathrm{~mm}$., which is equal to $0 \cdot 1$ of the wave length of green light. These results show that the amounts of energy, required to influence the eye and the ear respectively, are of the same order of magnitude.

Two muscles are found in the tympanum, viz. the tensor tympani and the stapedius muscles. When the tensor tympani contracts it draws the handle of the malleus inwards and so increases the tension of the tympanic membrane. Direct observation has shown that a contraction of this muscle occurs whenever sounds fall on the membrane, and that this reflex contraction is bilateral even when the stimulation of the ear is unilateral. The stapedius muscle tilts the base of the stapes and at the same time draws it slightly outwards, so relaxing the tympanic membrane. It acts therefore as an antagonist to the tensor tympani. It is not yet known what exact part this muscle plays in audition.

\section{THE END-ORGANS OF HEARING}

The movements of the stapes are communicated to a fluid, the perilymph, and by this to the endolymph, which immediately bathes the end-organ of 
hearing. The internal ear consists essentially of a membranous sac, formed originally by an involution of the epithelium covering the surface of the embryo. In the course of development the sac,. which is filled with the endolymph, becomes much modified in shape, forming from before backwards the scala media of the cochlea, the saccule, the utricle, and the three semicircular canals. At certain parts of its inner surface thickenings of the epithelium occur, which become connected with the terminations of the eighth nerve. The membranous labyrinth lies inside a bony case, the osseous

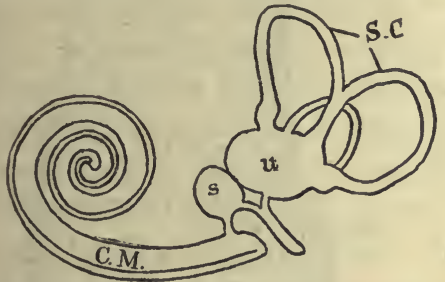

Fig. 249. The membranous labyrinth. CM, canalis or scala media of the cochlea; $s$, saccule; $u$, utricle; so, semicircular canals.

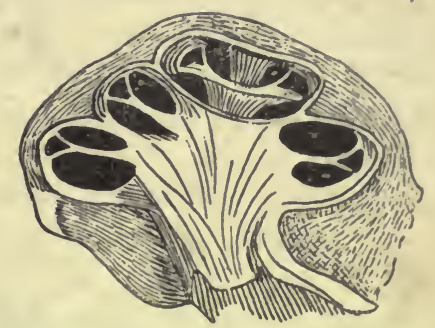

FIG. 250. Vertical section through the cochlea.

labyrinth, from which it is separated by the perilymph. The osseous labyrinth is formed from before backwards by the cochlea, the vestibule, and the semicircular canals.

The utricle, the saccule, and the semicircular canals are concerned with the functions of equilibration. At present we have only to deal with the structure of the cochlea and the end-organs contained in the scala media. The cochlea is a spiral tube of bone 20 to $30 \mathrm{~mm}$. long, divided by the scala media into two parts, viz. scala vestibuli and scala tympani, which are continuous at the apex of the spiral (helicotrema). The essential part of the organ of hearing is contained in the scala media. The sound waves falling on the ear and striking the membrana tympani are transmitted with diminished amplitude but increased force by the chain of ossicles to the fenestra ovalis; through these they are communicated to the perilymph which fills the vestibule. The vibrations travel from the vestibule to the scala vestibuli. Every rise of pressure in this canal will cause an actual movement of fluid and will push the scala media towards the scala tympani. The increased pressure thus communicated to the scala tympani causes a bulging of the membrane closing the foramen rotundum. Movement inwards of the stapes causes therefore a movement outwards of this membrane and vice versa, and the wave of pressure in passing from one aperture to the other must be communicated to the scala media with all the sensitive structures which it contains.

The scala media is triangular in cross-section,-having its apex at the spiral lamina and its base at the outer wall of the cochlea. It is separated by the membrane of Reissner from the scala vestibuli and by the basilar membrane from the scala tympani. The basilar membrane is composed of a number of elastic fibres, which pass in a radial direction from the spiral 
lamina to the spiral crest on the outer wall of the cochlea. The length of these fibres increases from $0.041 \mathrm{~mm}$. at the base of the cochlea to $0.495 \mathrm{~mm}$. at the helicotrema.

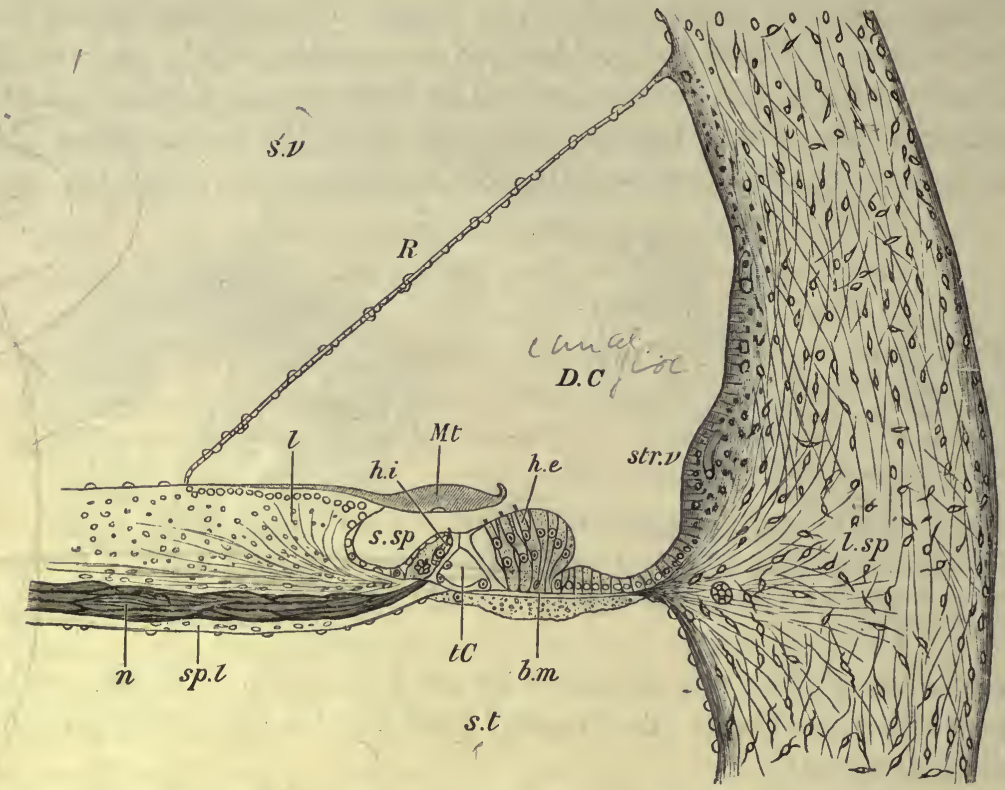

Fig. 251. Vertical section of the first turn of the human cochlea. (G. Retzius.) $s . v$, scala vestibuli ; s.t, scala tympani ; d.c, canal or duct of the cochlea; sp.l, spiral lamina ; $n$, nerve fibres; $l . s p$, spiral ligament ; str. $v$, stria vascularis; $s . s p$, spiral sulcus ; $R$, section of Reissner's membrane ; $l$, limbus laminæ spiralis ; $m . t$, membrana tectoria ; $t C$, tunnel of Corti ; b.m, basilar membrane; h.i, h.e, internal and external hair-cells.

The end-organ of the auditory nerve is represented by the organ of Corti, which rests on the basilar membrane (Fig. 252). It consists of a double

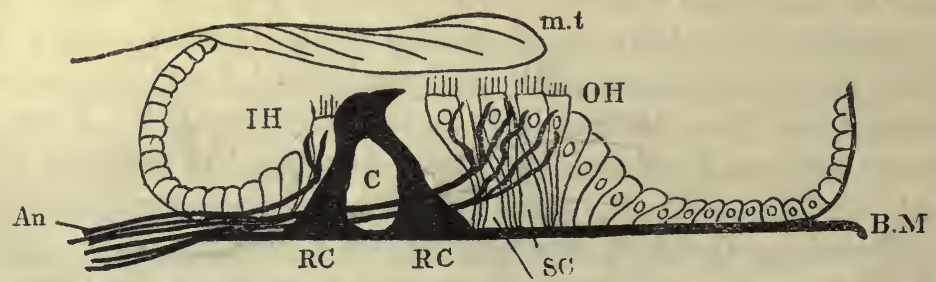

FIG. 252. Section through the end-organ of the auditory nerve in the cochlea (organ of Corti).

BM, basilar membrane ; $\mathrm{c}$, canal of Corti ; RC, rods of Corti ; IH and $\mathrm{OH}$, inner and outer hair-cells; sc, sustentacular cells ; An, auditory nerve; mt, membrana tectoria.

row of stiff cells, the inner and outer rods of Corti, which run throughout the whole length of the scala media and are surrounded by sense epithelium, the hair-cells. On the inner side of the rods of Corti there is a single row, on the outer side three rows of hair-cells. Between the hair-cells are the sustentacular cells, or cells of Deiters, the peripheral processes from which join 
together so as to form a reticulate membrane over the hair-cells, the hairs themselves projecting through orifices in the membrane. Resting on the upper surface of the membrana reticularis is the membrana tectoria. To this membrane is often ascribed a damping effect on the vibrations of the structures below. Any movement of the basilar membrane would be transmitted to the rods of Corti, and by these to the overlying hair-cells. With every vibration these would move in the line of their.long axis so that their hairs would move up and down in the membrana reticularis and possibly strike against the under surface of the membrana tectoria. The fibres of the auditory nerve pass up through the column of the cochlea, through the bipolar ganglion-cells which form the spiral ganglion, and then out along grooves in the spiral lamina to end in arborisations, partly in the inner haircells and partly among the outer hair-cells.

The complexity of the structure above described suggests that a large amount of discriminating and analysing power possessed by the ear for sounds of different qualities is determined by the differentiation of the endorgan itself. Not only are we able to appreciate differences in amplitude and pitch of the sound waves which arrive at the ear, but we are also capable of analysing the compound sounds and determining the simple tones out of which they have been composed. This power of analysis must be due either to the presence of some battery of resonators in the end-organs of the auditory nerve, or to the existence of a large number of different nerve fibres, each of which is excited only by a distinct number of vibrations per second, or, finally, we must assume that the end-organ of hearing is affected as a whole and that the nerve fibres transmit to the brain the different forms of wave caused by various complex sounds, the analysis being carried out in the cerebral cortex itself. This last hypothesis, the relegation of the powers of analysis to the cerebral cortex, is, at the present time at any rate, equivalent to giving up any attempt to explain the power of analysis possessed by the organ of hearing. On the other hand, the complex structure of the organ of Corti suggests that here we have an actual battery of resonators, by means of which sense waves are analysed into their components. This is Helmholtz's theory of the function of the cochlea. It assumes that in the organ of Corti there are vibrating structures tuned to frequencies within the limits of hearing, viz. from 30 vibrations to about 4000 vibrations per second. We can distinguish notes in the middle of the musical scale which differ from one another only by 0.3 to 0.5 vibration per second. Within the limits of this scale we should therefore require about 4200 resonators in the ear. In order to account for the sensitiveness of the ear to sounds below 40 vibrations and above 4000 per second, we might allow another 300 vibrators, so that 4500 different resonators would be necessary altogether. Helmholtz at first thought that these resonators were represented by the arches of Corti, but on Hensen pointing out that the basilar membrane was composed of fibres varying from 0.041 to $0.495 \mathrm{~mm}$. in length, he concluded that it was probably the breadth of the basilar membrane which determined the tuning to any particular note. This membrane from its structure behaves like 
a system of stretched strings bound together by a semi-fluid substance. The parts of the membrane near the fenestra rotunda would be adapted for the higher notes, while those near the helicotrema would vibrate to deep tones. Each fibre would, by its viloration, set the overlying part of the organ of Corti into vibration, so that each nerve fibre composing the auditory nerve would be stimulated by a different note. Müller's law of specific irritability is thus observed, each nerve fibre transmitting an impulse which excites one quality, and only one quality, of sensation. When a compound wave falls on the organ of Corti, it is actually resolved into its simple component waves by the fibres of the basilar membrane, and we therefore get stimulation of a number of nerve fibres and a sensation produced which is a true mixed sensation compounded of a number of simple tone sensations. By an effort of attention therefore it is possible to pick out from a mixed sensation its different components, and in this way we may explain the analytic powers of the ear.

Certain observations on fatigue of the auditory fibres support the notion that each fibre reacts to notes of one pitch, and only to these notes. If the vibrations of a tuning-fork be conducted by two telephones to both ears the sound appears to come from somewhere in front of the middle line of the body. If the sound be transmitted to one ear for some time so as to produce a condition of slight fatigue, and then the other telephone be held up to the other unfatigued ear, the sound is at once referred to the unfatigued side. In this localised projection of the sound waves we have a simple means of judging of the presence or absence of the apparatus in one ear or the other. It was pointed out by Donders that, when one ear was fatigued by a note of 360 vibrations per second, and immediately afterwards a note of 365 vibrations per second was conducted equally to both ears, no trace was perceptible of fatigue, the sound being located exactly in the median line. We are therefore justified in concluding that the end-organ of the nerve fibre which carries the nerve impulses corresponding to a vibration frequency of 360 per second is not the same as the nerve fibre or end apparatus which evokes the sensation corresponding to a note of 365 vibrations per second. If this theory is correct, destruction of the lower part of the cochlea should abolish the power of appreciation of high notes, while damage to the region of the helicotrema should impair sensibility to low notes. Certain results of experiments on dogs afford confirmation of this view, although all such results involving judgment of the powers of appreciation of high or low sounds respectively possessed by animals must be received with caution. A few isolated cases have been recorded in man in which atrophy of the nerve fibres supplying the lower whorl of the cochlea was attended by total want of perception of high tones.

According to Rutherford, the whole of the basilar membrane, and therefore of the auditory hairs, vibrates equally to every note, just as the plate of a telephone receiver reproduces faithfully the shape of the vibrations impinging on the transmitter of the telephone. This 'telephone theory,' as it has been called, relegates the whole work of analysis to the central nervous system, and gives us no clue as to how such an act of analysis may take place. One would imagine that a much simpler apparatus than 


\section{AUDITORY SENSATIONS}

that represented by the organ of Corti would be sufficient, if no analysis of the stimulus took place in the peripheral organ.

When a bow is drawn against the edge of a plate the vibrations affect different parts of the plate unequally, so that lycopodium powder sprinkled on such a plate assumes a complicated pattern. Waller suggests that the basilar membrane vibrates as a whole to every tone, but that it presents nodal and internodal points, like the vibrating plate. Since the hair-cells move with the basilar membrane they produce what may be called ' pressure patterns ' against the membrana tectoria, so that different combinations of nerve fibres are stimulated according to the pattern, i.e. according to the shape of the compound wave. A somewhat similar hypothesis has been put forward by Ewald, but neither of these theories presents any advantages over the resonator theory of Helmholtz, nor does it account satisfactorily, as the Helmholtz theory does, for the remarkable powers we possess of analysing all kinds of complex sounds. The cochlea becomes more elaborate in structure as we ascend the animal scale, and there is no doubt that this elaboration attains its greatest height in man, who possesses greater powers of analysing sounds than are possessed by any other animal. 


\section{SECTION V \\ VOICE AND SPEECH}

THE development of the analytical powers of the auditory apparatus is so closely connected with that of the faculty of speech that we may conveniently deal with the latter at this point rather than relegate it to a chapter on special muscular mechanisms. We may first consider the mechanism of production of voice, which man shares with many other animals, before discussing the mechanism of the wholly human faculty of speech.

Voice is produced in the larynx, a modified portion of the wind-pipe, by the vibrations of two elastic bands, the vocal cords, which are set into action by an expiratory current of air from the lungs. In many respects the larynx resembles a reed instrument, in which a current of air is caused to vibrate by the vibrations of an elastic tongue. Whereas, however, the period of the vibrations in such an instrument, and therefore the note, is determined by the length of the tube which is attached to the reed, in the larynx the note produced by the blast of air is modified partly by alterations in the tension of the vocal cord, and partly by varying the strength of the blast of air.

ANATOMICAL MECHANISM OF THE LARYNX. The essential framework of the larynx is formed by four cartilages, viz. the cricoid, the thyroid, and the two arytenoid cartilages. The cricoid cartilage, which lies immediately over the uppermost ring of the trachea, is shaped like a signet ring, the small narrow part being directed forwards and the broad plate backwards. The thyroid cartilage consists of two parts or alæ, joined together in front and forming the prominence known as Adam's apple ; behind, it presents four processes or cornua, the superior of which are attached by ligaments to the hyoid bone, while the inferior cornua articulate with the posterolateral portion of the cricoid cartilage. By means of this articulation very free movement is permitted between the two cartilages, the general direction of movement being one of rotation of the cricoid cartilage on the thyroid, round a horizontal axis directly through the two articular surfaces between the two cartilages, while movements of the thyroid upon the cricoid are also possible in the upward, downward, forward, and backward directions. The two arytenoid cartilages are pyramidal in shape. By their bases they articulate at some distance from the middle line with convex articular surfaces situated in the upper margin of the plate of the cricoid cartilage. The anterior angle of the base is the vocal process, while the external angle is the muscular process of the arytenoid. The crico-arytenoid joints permit of two kinds of movements of the arytenoid cartilages, viz. :

(1) Rotation on their base around their vertical long axis, so that the anterior vocal process is rotated outwards and the muscular process backwards and inwards or conversely. 
(2) Sliding movements of the whole arytenoid cartilagc either outwards or inwards, so that their inner margins may be drawn apart or approximated.

The larynx is covered internally by a mucous membrane continuous with that of the trachea. It is lined with ciliated epithelium, except over the vocal cords, where the epithelium is stratified. The two vocal cords, or thyro-arytenoid ligaments, consist of elastic fibres which run from the middle of the inner angle of the thyroid cartilage to be inserted into the anterior angle of the arytenoid cartilages. Their length in man is about $15 \mathrm{~mm}$., in woman about $11 \mathrm{~mm}$. The cleft between them is known as the glottis, or rima glottidis.

Two ridges of mucous membrane above and parallel to the vocal cords are the false vocal cords (Fig. 253). Between the true and the false vocal cords on each side is a recess known as the ventricle of Morgagni. This ventricle permits the free vibration of the vocal cords. The false cords take no part in phonation, but help to keep the true cords moistened by the secretion of the numerous mucous glands with which they are provided. The position and tension of the vocal cords are determined by the action of the various intrinsic muscles of the larynx. The part taken by the various muscles in each movement cannot be directly ascertained. We can in most cases only study the direction of the fibres, and judge, from this direction and consequent isolated action of the muscles, the part taken by any given muscle in the production of voice. The chief muscles (Fig. 254) are as follows :

(1) The crico-thyroid muscle is a short triangular muscle attached below to the cricoid cartilage and above to the inferior border of the thyroid cartilage; the fibres pass from below upwards and backwards. When this muscle contracts, the cricoid cartilage is drawn up under the anterior part of the thyroid cartilage so that its broad expansion behind with the arytenoid cartilages, is drawn downwards and backwards, thus putting the vocal cords on the stretch. This muscle is probably the most important in determining the tension of the vocal cord.

(2) The posterior crico-arytenoid muscle arises

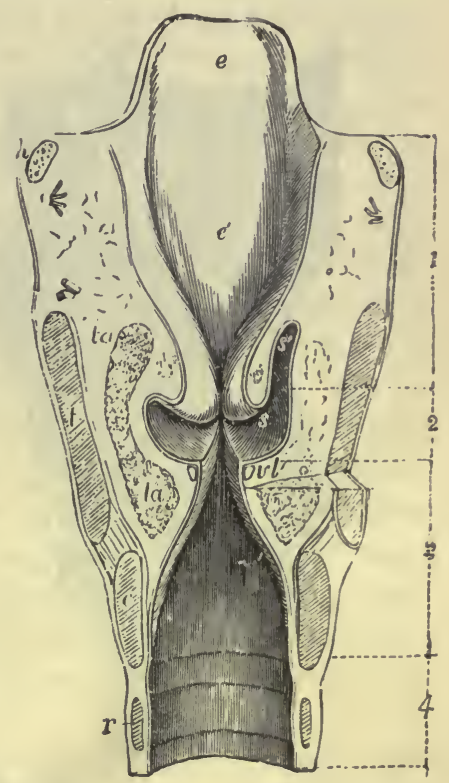

Fig. 253. Anterior half of the larynx, seen from behind. The section on the right side is somewhat in front of the left side.

$e$, epiglottis; $e^{\prime}$, cushion of epiglottis ; $t$, thyroid cartilage ; $s, s^{\prime}$, ventricle of larynx; $h$, great cornu of hyoid bone; $t a$, thyro-arytenoid muscle; $v l$, vocal cords. Above the ventricles are the false vocal cords. $r$, first ring of trachea.

(A. Thomson.) from a broad depression on the corresponding half of the posterior surface of the cricoid cartilage. It passes upwards and outwards, its fibres converging, to be inserted into the outer angle of the arytenoid cartilage. These muscles rotate the outer angle of the arytenoid cartilages backwards and inwards. They thus cause a movement outwards of the anterior angles, so that the glottis is widened. During every act of inspiration there is a widening of the glottis, which is probably effected by contraction of these muscles. If they are paralysed the vocal cords are approximated and tend to come together during inspiration, so that dyspnœea may be produced.

(3) The lateral crico-arytenoid muscle arises from the upper border of the cricoid cartilage and passes backwards to be inserted into the muscular process of the arytenoid cartilage. These muscles when they contract pull the muscular process of the arytenoid cartilage forwards and downwards, thus approximating the vocal cords at their posterior ends and antagonising the action of the posterior crico-arytenoid muscles.

(4) The arytenoid muscles consist of transverse fibres, some of which decussate, 
uniting the posterior surface of the two arytenoid cartilages. When they contract they draw the arytenoid cartilages together.

(5) The thyro-arytenoid muscles consist of two portions. The outer fibres rise in front from the thyroid cartilage and pass backwards to be inserted into the lateral border and the muscular process of the arytenoid cartilage. Some of the fibres pass obliquely upwards towards the aryteno-epiglottidean folds. These are often spoken of as a separate muscle, the thyro-epiglottidean. By their action they tend to draw
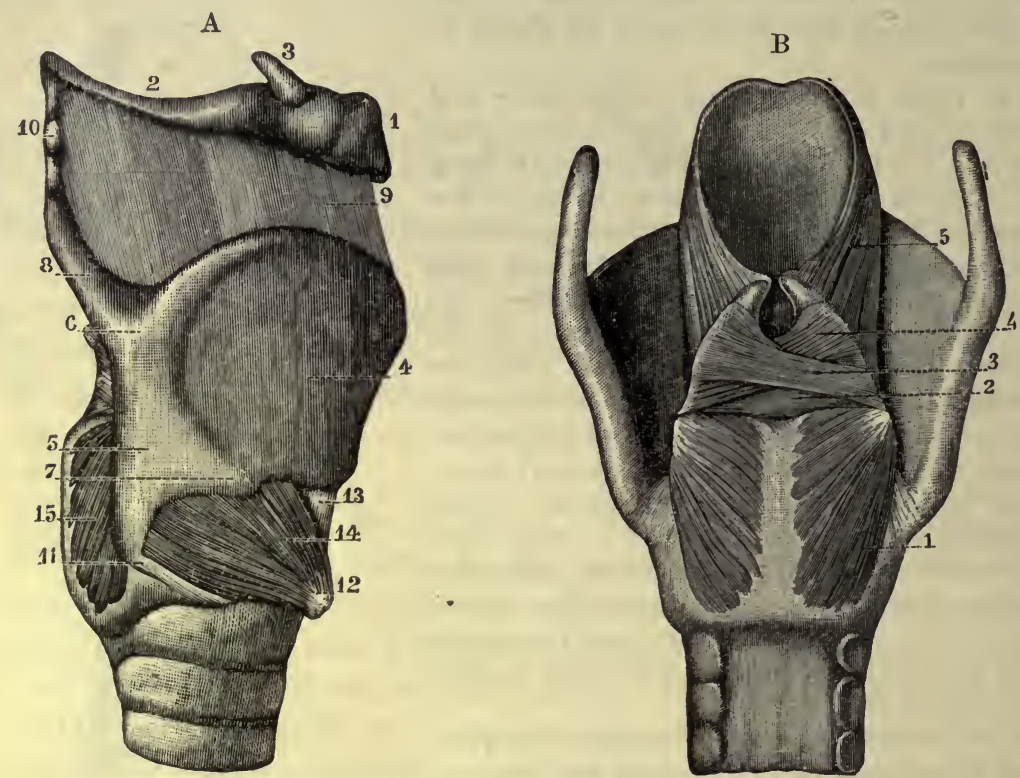

FIG. 254. Muscles of the larynx. (SAPPEX.)

$A$, as shown in a view of the larynx from the right side.

1 , hyoid bone; 2,3 , its cornua ; 4, right ala of thyroid cartilage ; 5 , posterior part of the same separated by oblique line from anterior part; 6,7 , superior and inferior tubercles at ends of oblique line ; 8 , upper cornu of thyroid; 9 , thyro-hyoid ligament; 10 , cartilago triticea; 11, lower cornu of thyroid, articulating with the cricoid; 12, anterior part of cricoid; 13, crico-thyroid membrane; 14, crico-thyroid muscle; 15, posterior cricoarytenoid muscle, partly hidden by thyroid cartilage.

$B$, as seen in a view of the larynx from behind.

1, posterior crico-arytenoid; 2, arytenoid muscle; 3,4 , oblique fibres passing around the edge of the arytenoid cartilage to join the thyro-arytenoid, and to form the arytenoepiglottic, 5.

the arytenoid cartilages forwards and to relax the vocal cords. The upper fibres may also assist in depressing the epiglottis. The inner fibres are called the musculus vocalis. They arise from the lower half of the angle of the thyroid cartilage, and passing backwards in the vocal cords are attached to the vocal processes and to the adjacent parts of the outer surfaces of the arytenoid cartilages. Many fibres do not run the whole distance, but end in an attachment to some part of the vocal cord. Although their action must be to draw the arytenoid cartilages forwards, yet, since they are contained in the vibrating portion of the vocal cords, they cannot by their contraction relax these cords. It is probable that they play a great part in determining the tension of the vocal cords after these have been put on the stretch by the action of the cricothyroid muscles. They may possibly act as a sort of fine adjustment of the tension, the coarse adjustment being represented by the crico-thyroids. 


\section{THE PRODUCTION OF VOICE}

In order to study the changes in the larynx which are associated with voice production we must make use of the laryngoscope. The principle of this instrument is very simple. A large concave mirror with a central aperture is fixed before one eye of the observer, sitting in front of the patient or person to be observed. The latter is directed to throw his head slightly backwards and to open his mouth. In order to keep the tongue out of the way the patient is made to hold the end of it by means of a towel. The mirror is then so arranged as to reflect light from a lamp into the cavity of the mouth. A small mirrer fixed in a handle is then warmed, so as to prevent the condensation of the patient's breath, and passed to the back of the mouth until it rests upon and slightly raises the base of the uvula. By this mirror the light reflected into the mouth from the large mirror is again reflected down on to the larynx, and a reflection of the larynx and trachea is seen in the mirror. By laryngoscopic examination we can see the base of the tongue, behind which is the outline of the epiglottis. Behind this again in the middle line are seen the two vocal cords, white and shining (Fig. 255). The cords appear to approximate posteriorly; between them is a narrow chink, the diameter of which varies with each respiration, being wider during inspiration. On each side of the true vocal cords are seen the pink false vocal cords. In some cases the rings of the trachea, and even the bifurcation of the trachea itself (Fig. 255, c), may be seen in the interval between the vocal cords.

In order that the vocal cords may be set into vibration, they must be put into a state of tension and the aperture of the glottis narrowed, so as to afford resistance to the current of air. In the dead larynx it is possible to produce sounds by forcing air from bellows through the trachea, after the vocal cords have been put on the stretch by pulling the arytenoid cartilages backwards. By experimenting on patients on whom tracheotomy has been performed, it has been found that the pressure of air in the trachea, necessary to cause production of voice, is, for a tone of ordinary loudness and pitch, between 140 and $240 \mathrm{~mm}$. of water, and with loud shouting the pressure rises to as much as $945 \mathrm{~mm}$. of water. This pressure is furnished by the contraction of the expiratory muscles, i.e. of the abdomen and of the thorax. Since the pitch of the note produced rises with increasing force of the blast, while the tension of the cords remains constant, it is evident that, in the act of 'swelling' on a note, the increased pressure necessary for the crescendo must be associated with diminishing tension of the cords. It is the failure to secure this muscular relaxation that so often causes a singer to sing sharp when swelling on any given note.

The voice, like the sound produced on any musical instrument, may vary either in pitch, loudness, or in quality or timbre. The range of any individual voice is generally about two octaves. The pitch of the voice usually employed is determined chiefly by the length of the vocal cords. Thus in children the voice is high-pitched. Before and at puberty there 
is a considerable development in the size of the larynx in both sexes. This is especially marked in the male, and accounts for the sudden drop in pitch ('breaking') of the voice. In the female the increased size of the larynx is chiefly perceptible in the increase in fulness and richness of the voice which occurs at this age. Even when we take all the voices together,
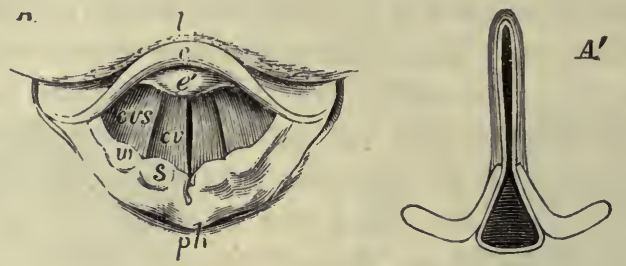

B
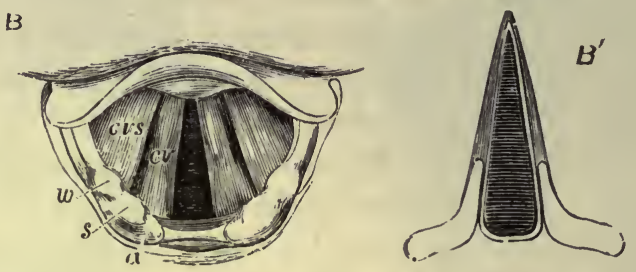

$C$

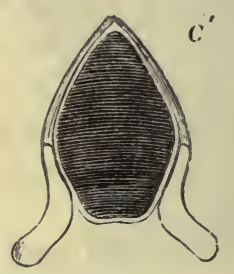

Fig. 255. Three laryngoscopic views of the superior aperture of the larynx and surrounding parts in different states of the glottis during life. (From Czermak.)

A, the glottis during the emission of a high note in singing. B, in easy or quiet inhalation of air. $\mathrm{C}$, in the state of widest possible dilatation, as in inhaling a very deep breath. The diagrams A', B', $\mathrm{C}^{\prime}$ have been added to Czermak's figures to show in horizontal sections of the glottis the position of the vocal ligaments and arytenoid cartilages in the three several states represented in the other figures. In all the figures so far as marked, the letters indicate the parts as follows, viz. : $l$, the base of the tongue; $e$, the upper free part of the epiglottis ; $e^{\prime}$, the tubercle or cushion of the epiglottis ; $p h$, part of the anterior wall of the pharynx behind the larynx ; in the margin of the aryteno-epiglottidean fold $w$, the swelling of the membrane caused by the cuneiform cartilage ; $s$, that of the corniculum; $a$, the tip of the arytenoid cartilages; $c v$, the true vocal cords or lips of the rima glottidis ; $c v s$, the superior or false vocal cords; between them the ventricle of the larynx ; in C, $t r$ is placed on the anterior wall of the receding trachea, and $b$ indicates the commencement of the two bronchi beyond the bifurcation, which may be brought into view in this state of extreme dilatation.

bass, tenor, alto, and soprano, the total range for ordinary individuals does not exceed three octaves. In singing the voice may be produced in various ways, i.e. in different registers. Thus we distinguish the chest register, the middle register, and the head register. The deeper notes of any individual voice are always produced in the chest register. Observation of the vocal cords shows that when producing such notes the glottis forms an elongated slit, all the muscles which close the glottis and increase the tension of the cords being in action. The vocal cords are relatively thick and broad and can be seen to vibrate over their whole extent. When singing with the head voice 
the vibrations of the cord are apparently confined to their inner margins ; the aperture of the glottis is wider in front than behind, so that more air escapes during phonation by this method than in the production of the chest voice.

In order to change the pitch of the note the following means are probably employed in the larynx:

(1) Alteration in the tension of the vocal cords.

(2) Alteration in the length of the part of the vocal cords which is free to vibrate, which can be accomplished by the approximation of the arytenoid cartilages to one another, or by their approximation to the thyroid cartilage.

(3) The alteration in the shape of the vocal cords, which is determined by the activity of the different portions of the internal thyro-arytenoid muscles.

(4) The varying pressure of the blast of air passing through the glottis.

The loudness of the tone produced is practically proportional to the force of the blast of air employed. The quality or timbre of the voice depends not so much on the vocal cords as on the accessory resonating apparatus, represented by the trachea and chest and by the cavity of the mouth. The greater part of the education involved in voice training is directed to the modification of the shape of the mouth cavity, so as to secure the greatest possible fulness, i.e. richness in overtones, of the tone produced in the larynx.

\section{THE MECHANISM OF SPEECH}

The sounds employed in speech, viz. vowels and consonants, are produced by modifying the laryngeal tones by changes in the shape of the mouth and nasal cavities. In whispering speech there is no phonation at all, but the sound is produced by the issue of a blast of air through a narrow opening between the lips, between the tongue and soft palate, or between the tongue and the teeth.

The vowel sounds are continuous, whereas the consonants are produced by interruptions, more or less complete, of the outflowing air in different situations. The vowel sounds, $\mathrm{u}, \mathrm{o}, \mathrm{a}, \mathrm{e}, \mathrm{i}$ (pronounced oo, oh, ah, eh, ee), are tones, i.e. are produced by a regular series of vibrations. These tones take their origin in the mouth cavity, as can be shown easily by the fact that we can whisper these sounds distinctly without any phonation whatever. To each of them corresponds one or two distinct notes, the pitch, i.e. the resonance, of which is regulated by the shape of the cavity in which they are produced. It is possible to determine these notes by means of resonators. The pronunciation even of the simplest vowel sounds differs in different individuals. For instance, those pronounced by a Londoner differ from those pronounced by a man from Manchester or from Yorkshire, and the French vowels differ somewhat in pitch from those employed by the German, and these again from those employed by the average Englishman. 
The characteristic notes were given by Helmholtz as follows :

$$
\begin{aligned}
& \mathrm{U}=\mathrm{f} \\
& \mathrm{O}=\mathrm{b}^{\mathrm{I}} \\
& \mathrm{A}=\mathrm{b}^{\mathrm{II}} \\
& \mathrm{E}=\mathrm{f}^{\mathrm{I}}, \mathrm{b}^{\mathrm{II}} \\
& \mathrm{I}=\mathrm{f}, \mathrm{d}^{\mathrm{IV}}
\end{aligned}
$$

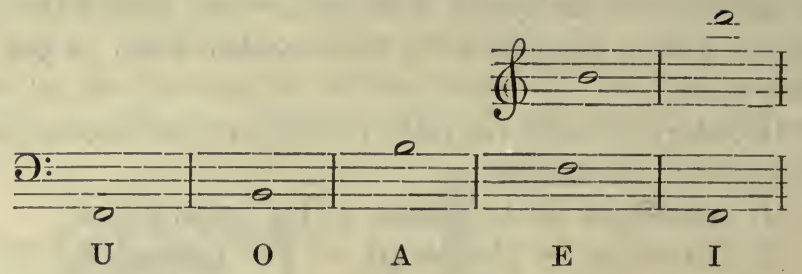

If the five vowels are whispered loudly, the gradual rise in pitch of the tone is easily perceptible. We do not in this way, however, note the lower component of the sound in the $\mathrm{E}$ and $\mathrm{I}$; this can be brought out by a simple device (Fig. 256). If we place the mouth in the position necessary to produce these different vowels, and then percuss over the cheek, we obtain the typical note for each vowel, the air in the mouth cavity being set into vibration by the percussion. Now shift the finger, which is to be percussed, so that it lies over the pharynx, just behind the angle of the jaw, and percuss again. The note will be observed to rise with $\mathrm{U}, \mathrm{O}, \mathrm{A}$,

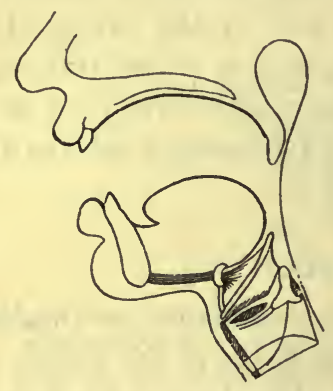

$A(a h)$

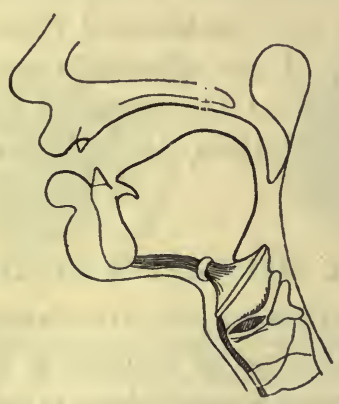

$U(\overline{o o})$

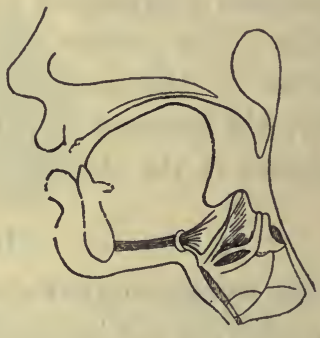

$I(\overline{e e})$

Fra. 256. Shape of the oral cavity in the production of the vowel sounds, $A, U, I$. (GR ÜTZNER.)

and then fall with $\mathrm{E}$. I. With the three vowels $\mathrm{U}, \mathrm{O}, \mathrm{A}$, we have a single cavity formed by the lips, the palate, and the tongue; this cavity is longest and narrowest with $U$ and shortest and most open with $\mathrm{A}$. With $\mathrm{E}$ and I the dorsum of the tongue comes up against the front part of the soft palate, so that the mouth cavity is divided into two, the anterior short narrow cavity, and the posterior broader cavity between the soft palate and the base of the tongue. We therefore have two notes produced, one in each cavity. The change in shape of the mouth cavity is shown in the figures. With $U$ and $A$ the cavity seems to be single; with I the development of a pharyngeal resonating cavity is well shown. Diphthongs are produced by changing the form of the mouth cavity from that of one vowel sound to another, thus AI (the English i) = ah-ee run together and abbreviated.

Consonants are sounds produced by a sudden check being placed in the course of the expiratory blast of air by closure of some part of the pharynx 
or mouth. They are classified into labials, dentals, or gutturals, according as the check takes place at the lips, between teeth and tongue, or between back of tongue and soft palate. Each of these again can be divided into soft and hard consonants as they are accompanied or not with phonation. Thus when we pronounce $\mathbf{D}$ the production of the laryngeal sounds goes on during the check of the sound produced at the teeth, whereas with $\mathrm{T}$ there is an absolute interruption of phonation during the pronunciation of the consonant. It is thus practically impossible to make any marked difference between hard and soft consonants when whispering.

In the production of nasal sounds such as NG the mechanism is the same as for the production of B, D, G, except that the posterior opening of the nares is not kept shut by the soft palate, so that part of the sound comes continually through the nasal passages, when it acquires a peculiar resonance. These sounds are on this account often spoken of as ' resonants.' The aspirates are produced by the passage of a simple blast of air through a narrow opening which may be at the throat as in $\mathrm{H}$, between tongue and teeth as in $\mathrm{TH}$, or between lips and teeth as in $\mathrm{PH}$ or $\mathrm{F}$.

The vibratives, such as $\mathrm{R}$, are formed by placing the tip of the tongue or the uvula, or the lips, in the path of the blast of air so that they are set into vibration by the blast. In English the vibrative $\mathrm{R}$ employed is entirely due to the tongue.

The sibilants, which may be voiceless as in ' $\mathrm{S}$ ' or accompanied with phonation as in ' $\mathrm{Z}$,' consist of continuous noises produced by a narrowing of the path of the air between the tongue and the hard palate. They are therefore similar in production to the aspirates. In the production of the sound ' $\mathrm{L}$ ' the tongue is applied by its edge to the alveolar process of the upper jaw, so that the air or voice escapes by two small apertures in the region of the first molar and between the inner side of the cheek and the teeth. The acoustic characters of these various consonants are still but imperfectly studied. 


\section{VISION}

\section{SECTION VI}

\section{DIOPTRIC MECHANISMS OF THE EYEBALL}

WHEN light falls on any object a certain proportion of it is reflected and scattered, and will affect any organism in the neighbourhood possessing sensibility to light. Mere sensibility of the surface to light would not, however, suffice to arouse projected sensations, since the rays of light from a number of different objects would interfere with one another. An animal with such sensibility would be aware of or be able to react to differences of light and darkness, but could not direct its movements in accordance with the nature of the objects from which the light proceeded. For this purpose there must be not only a surface sensitive to light impressions but also dioptric mechanisms, by means of which a real image of external objects, in their proper spatial relationships, is thrown on to an extended sensory surface. Each point in this surface will correspond to a point lying outside the body and serving as a source of light, and the sensations evoked, since they correspond to the rays of light coming from external objects, can be projected, and referred to the objects themselves lying outside and at some distance from the body.

The organ of vision, the eye, consists of two parts, viz. :

(a) The sensory surface or retina, composed of a number of areas, each of which can be separately stimulated by light.

(b) A dioptric mechanism for projecting a real image of external objects on this sensory surface.

We have in fact an arrangement very analogous to a photographic camera, where a real image is thrown by a lens on to a sensitive plate, each point of which undergoes chemical change in proportion to the amount of incident light, so that a photographic record is the result.

\section{THE FORMATION OF AN IMAGE BY A LENS}

We may confine our attention to the case of a bi-convex lens, and we may assume in the first place that the thickness of the lens is negligible. In Fig. $257 \mathrm{C}$ and $\mathrm{C}^{\prime}$ are the centres of the sperical surfaces bounding the lens ; the line joining them is the optical axis of the lens. The surface at $Q$ is parallel to the surface at $\mathrm{R}$, so that a ray of light, such as $\mathrm{PQ}$ falling on the 
lens at $\mathrm{Q}$, will leave the lens at $\mathrm{R}$ in direction $\mathrm{RS}$ parallel to $\mathrm{PQ}$. The point $o$ where the ray cuts the optical axis is known as the optical centre of the lens. This optical centre in a bi-convex lens lies within the lens, its distance from the two surfaces being practically as their radii.

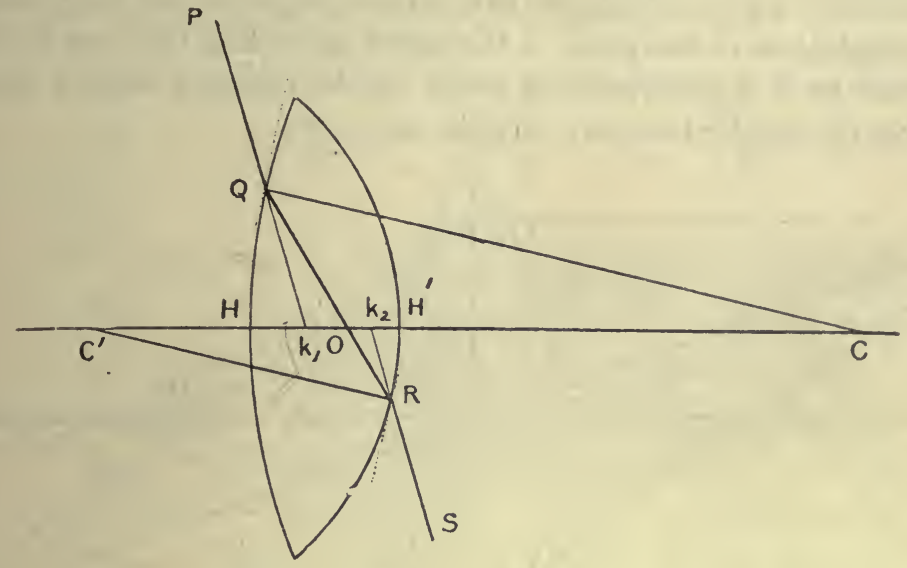

Fig. 257.

Since we are neglecting the thickness of the lens the line PQRS may be regarded as straight, so that we may say that the rays which pass through the centre of a lens do not deviate. If a pencil of parallel rays falls upon the lens, while those rays which pass through the optic centre undergo no deviation, all the others on leaving the lens will be convergent towards a point which is known as the principal focus of the lens (Fig. 258). Con-

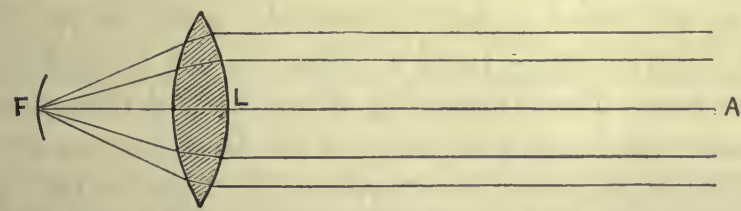

Fic. 258. Diagram of the course of parallel rays through a bi-convex lens by which they are converged to the principal focus, $\mathrm{F}$.

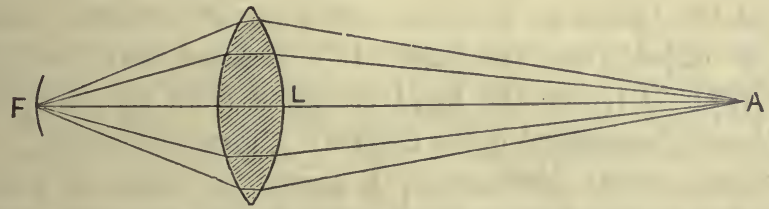

FIG. 259. The rays of light from A converge on passing through the lens to the secondary focus, $\mathrm{F}, \quad \mathrm{F}$ and $\mathrm{A}$ are conjugate foci.

versely, if a point of light be placed at the principal focus the rays of light passing through it to the lens will take the reverse course and leave the lens as a bundle of parallel rays. Any point of light situated between infinite distance and the principal focus will have a corresponding point on the other side of the lens to which its rays will converge. Such corresponding points are known as a conjugate foci (Fig. 259). In a thin lens, 
with the same media on each side, the anterior and posterior focal distances are the same, so that from which ever direction a parallel beam falls on the lens the point to which its rays are converged on the other side of the lens is constant.

If instead of a point of light we have a series of points such as that coming from a bright line in the plane of the paper (as in Fig. 260), each of these points will have a corresponding point on the opposite side of the lens. Thus from the point $P$ three rays may be taken, viz. :

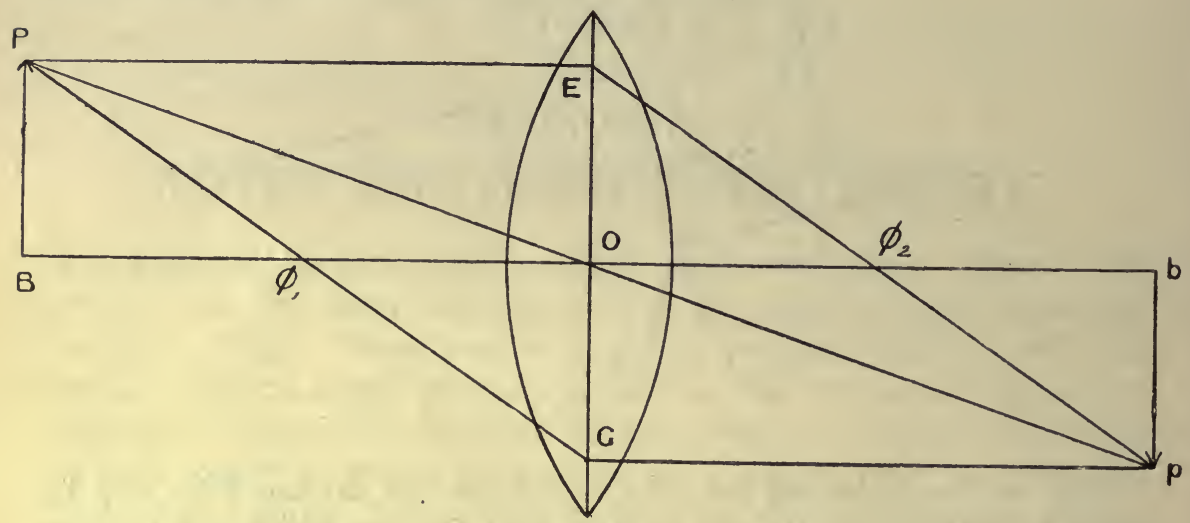

FIG. 260 .

(1) The ray Po, which passes through the centre of the lens and does not deviate.

(2) The ray $\mathbf{P E}$, which is parallel to the axis and therefore is converged to the principal focus $\phi_{2}$.

(3) The ray $\mathbf{P G}$, which passes through the focus on the front surface of the lens $\phi_{1}$ and therefore takes a course on the other side of the lens parallel to the axis. The intersection of any two of these three lines will be the situation of the image $p$ to the point $P$. In the same way all the other points in the object will have a corresponding image on the opposite side, so that an inverted real image of the luminous object is formed on this side.

The size of the image as compared with the object will depend on the distance of the object from the lens. It is greater than the object if the latter is less than $2 f$ (twice the focal distance) from the lens, equal if the distance is $2 f$, and diminished if the distance is greater than $2 f$.

A lens with a focal distance of 1 metre is taken as unit strength. Such a lens is said to have a strength of one dioptre; a lens of two dioptres would have a focal distance of $\frac{1}{2}$ metre; of four dioptres one of $\frac{1}{4}$ metre, and so on.

\section{DIOPTRIC MECHANISMS OF THE EYEBALL}

The eye is not directly comparable to a camera in its optical arrangements, since the image is formed, not in air, but in the fluids of the eye itself. Moreover the conditions are complicated by the facts that it is 
impossible to neglect the thickness of the refractive media, and that these are many in number.

If we take the simplest case, where there are only two media separated from one another by a spherical surface of contact, we can easily determine the course takcn by any ray in passing from the first to the second medium.

In Fig. 261 (from Landois) let $\mathrm{L}$ be the first (e.g. air) and $\mathrm{a}$ the second (e.g. glass). These are separated by the spherical surface $a b$, with its centre at $m$. Since all the

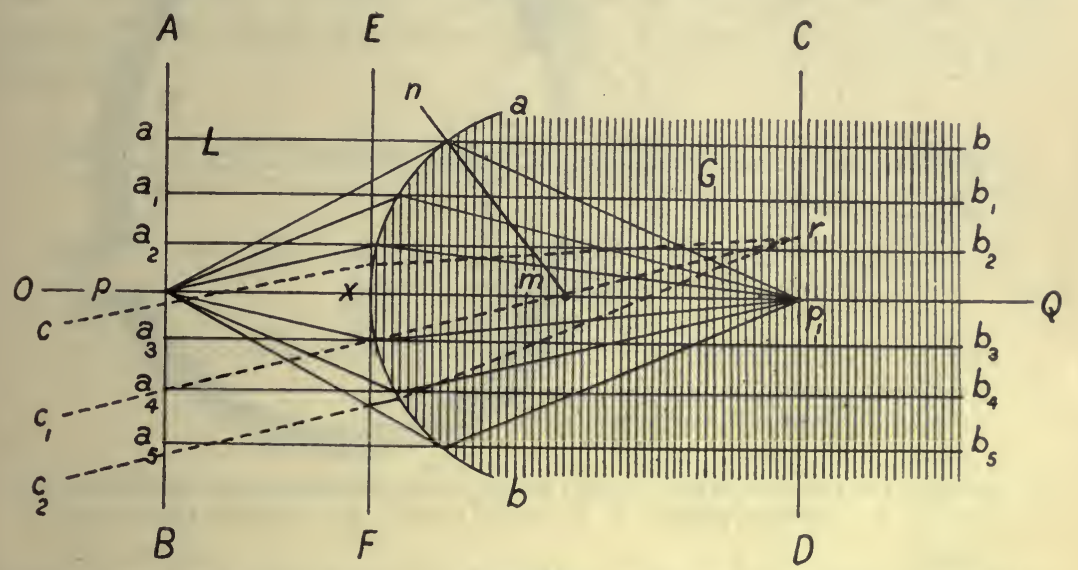

FrG. 261.

radii drawn from $m$ to $a b$ are perpendicular to the surface all rays falling in the direction of the radii must pass unrefracted through $m$. All rays of this sort are called rays or lines of direction; $m$, as the point of intersection of all these, is called the nodal point. The line which connects $m$ with the vertex of the spherical surface, $x$, and which is prolonged in both directions, is the optic axis, $\mathrm{OQ}$. A plane (EF) in $x$, perpendicular to $\mathrm{OQ}$, is called the principal plane, and in it $x$ is the principal point. The following facts have been ascertained: (1) All rays ( $a$ to $\left.a_{5}\right)$, which in the first medium are parallel with each other and with the optic axis, and fall upon $a b$, are so refracted in the second medium that they are all again united in one point $\left(p_{1}\right)$ of the second medium. This is called the second principal focus. A plane in this point, perpendicular to $\mathrm{OQ}$, is called the second focal plane (CD). (2) All rays $\left(c\right.$ to $\left.c_{2}\right)$, which in the first medium are parallel to each other, but not parallel to $\mathrm{OQ}$, reunite in a point of the second focal plane $(r)$, where the non-refracted dircetive ray $\left(c_{1} m r\right)$ meets this. (In this case the angle formed by the rays $c$ to $c_{2}$ with $\mathrm{OQ}$ must be very small.) The propositions 1 and 2 , of course, may be reversed ; the divergent rays proceeding from $p k$ towards $a b$ pass into the first medium parallel to each other, and also with the axis oQ $\left(a\right.$ to $\left.a_{5}\right)$; and the rays proceeding from $r$ pass into the first medium parallel to each other, but not parallel to the axis oQ (as $c$ to $c_{2}$ ). (3) All rays, which in the second medium are parallel to each other $\left(b\right.$ to $\left.b_{5}\right)$ and with the axis $O Q$, reunite in a point in the first medium $(p)$ called the first focal point; of course, the converse of this is true. A plane in this point perpendicular to $\mathrm{OQ}$ is called the first focal plane (AB). The radius of the refractive surface $(m x)$ is equal to the difference of the distance of both focal points $\left(p\right.$ and $\left.p_{1}\right)$ from the principal focus $(x)$; thus $m x=p_{1} x-p x$.

In compound systems composed of several refractive media with spherical surfaces of contact, such as the eye, we may proceed from medium to medium with the same methods as those just described. Since, however, such a procedure would be very tedious, the method first proposed by Gauss is usually adopted. Gauss showed that if the several media are 'centred '-i.e. if all have the same optic axis-then the refractive indices of such a centred system may be represented by two equally strong refractive 
surfaces at a certain distance apart. The rays falling upon the first surface are not refracted from it, but are regarded as projected forwards parallel with themselves to the second surface. Refraction is then considered to take place at the second surface just as if that were the only surface present (represented by the dotted line $l l$ in Fig. 262).

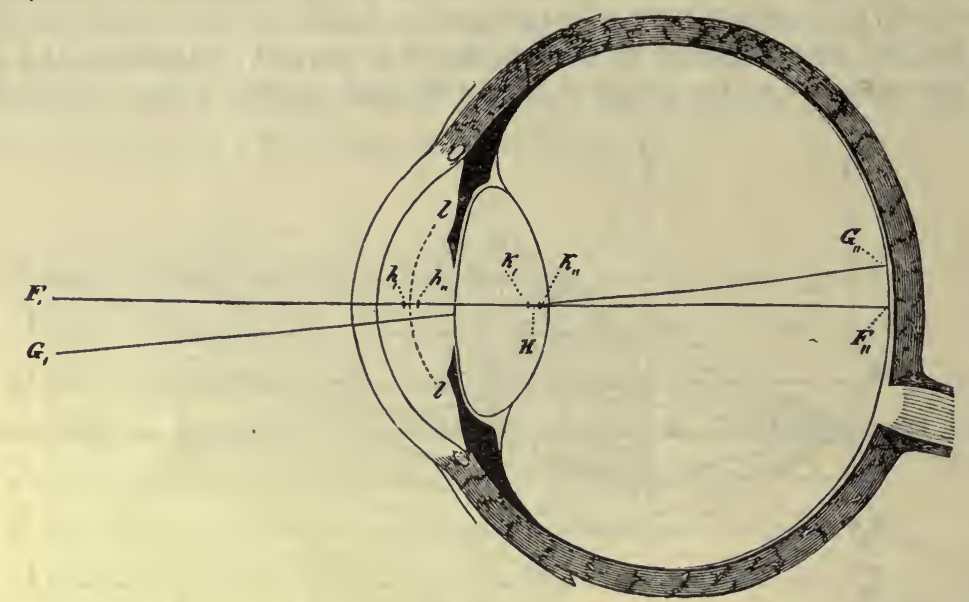

Fig. 262. The position of the cardinal points in the schematic eye. (HeLMHoLtZ.) $h_{\ell}, h_{\iota,}$, principal points ; $k_{\ell}, k_{\iota,}$, nodal points ; $\mathbf{F}_{/,}$, posterior focus.

All such systems possess three pairs of cardinal points, viz. two principal foci, two principal points, and two nodal points. These points may be defined by the following principles :

(a) Rays which pass through the principal focus are, after refraction, parallel to the optic axis.

(b) Rays which pass through the first principal point, after refraction, pass through the second.

(c) Rays which pass through the first nodal point, after refraction pass through the second, and the direction of the refracted ray is parallel to the direction of the incident ray.

In Fig. 262 the situation of these cardinal points is shown in the human eye. The rays of light pass through the following media, cornea, aqueous humour, lens, and vitreous humour, and may be refracted at the following surfaces ; anterior and posterior surfaces of the cornea, anterior and posterior surfaces of the lens. Since, however, the refractive indices of cornea, aqueous humour, and vitreous humour are practically identical, we can reduce the refractive media to two, viz. cornea, aqueous, and vitreous in the one case, and lens in the other; and the refractive surfaces to three, viz. anterior surface of cornea, anterior surface of lens, and posterior surface of lens.

In order to determine the path of the rays in the eye we have to determine the following data, viz. :

(1) The radius of curvature of the anterior surface of the cornea.

(2) The distance of the anterior surface of the cornea from the anterior surface of the lens. 
(3) The radius of curvature of the anterior surface of the lens.

(4) The thickness of the lens.

(5) The radius of curvature of the posterior surface of the lens.

In addition we must know the refractive index of the cornea and aqueous or vitreous humour, and also the average refractive index of the lens. There is a considerable difference between the refractive indices of the outer and the inner portions of the lens, the inner part being much denser and having a higher refractive index than the periphery. The following Table represents the constants of a human eye as determined by Helmholtz :

Refractive index of aqueous and vitreous humours

Total refractive index of lens

$1 \cdot 3365$

$1 \cdot 4371$

$\mathrm{mm}$.

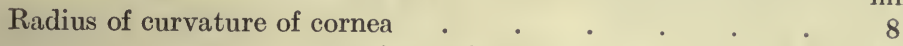

, $\quad$, anterior surface of lens . . . 10

" , posterior surface of lens . . . 6

Distance from anterior surface of cornea to anterior surface of lens

, posterior surfacc
of lens

Anterior focus of cornea

Posterior focus of cornea

Focus of lens

Posterior focus of eye

Anterior focus of eye .

posterior
of lens

$23 \cdot 692$

$31 \cdot 692$

$43 \cdot 707$

$19 \cdot 875$

$14 \cdot 858$

Distance from anterior surface of cornea to :

First principal point

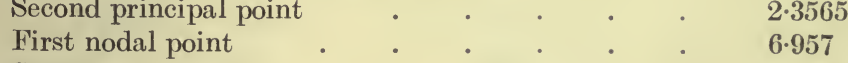

Second nodal point . . . . . . . $7 \cdot 373$

Anterior focus of eye . . . . . . . 12.918

Posterior focus of eye . . . . . . . $22 \cdot 231$

It will be seen that both the principal points lie in the anterior chamber, while the nodal points fall in the back part of the lens. The posterior focus of the eye falls upon the retina. For many purposes we may simplify our calculations by running the two principal points and the two nodal points into one. In such a reduced eye the single principal point is situated $2.3 \mathrm{~mm}$. behind the anterior surface of the cornea, and the single nodal point $0.47 \mathrm{~mm}$. in front of the hinder surface of the lens. If a circle be drawn from the single nodal point as a centre through the single principal point as a circumference we get a surface $l l$ in the figure, which represents the anterior refracting surface of such a reduced eye.

A reference to the Table of Constants of the human eye shows that, whereas the anterior focal distance of the cornea is $23 \mathrm{~mm}$., that of the whole eye is $15 \mathrm{~mm}$. and that of the lens $44 \mathrm{~mm}$. It is evident from this that the anterior surface of the cornea is the most important refractive surface of the eye, and that, in the convergence of rays necessary for the formation of an image on the retina, it is the refraction at this surface which plays the 
greatest part. Under water this refraction is of course abolished, since the refractive indices of the aqueous humour and cornea are practically identical with that of water. The eye therefore becomes long-sighted; it is impossible to get any clear image of near objects, and distant objects can only be seen with a strong effort of accommodation. A smaller effect is produced by removal of the lens, an operation often undertaken when this body has become opaque as a result of cataract. Such an operation not only abolishes the power of accommodation of the eye, but diminishes the refractive power of the eye at rest by ten dioptres, so that a lens of this power has to be placed at the front of the eye in order to render possible clear vision of distant objects.

\section{PART OF THE RAYS IN THE FORMATION OF THE RETINAL IMAGE}

In the reduced eye the construction of the path of the rays is very simple. When the eye is focused for the object which is being looked at, the rays from any point of the object come to a point on the retina. All that is necessary therefore is to draw lines from points of the object through the single nodal point to the retina, as is shown in Fig. 263. The image thus produced, like

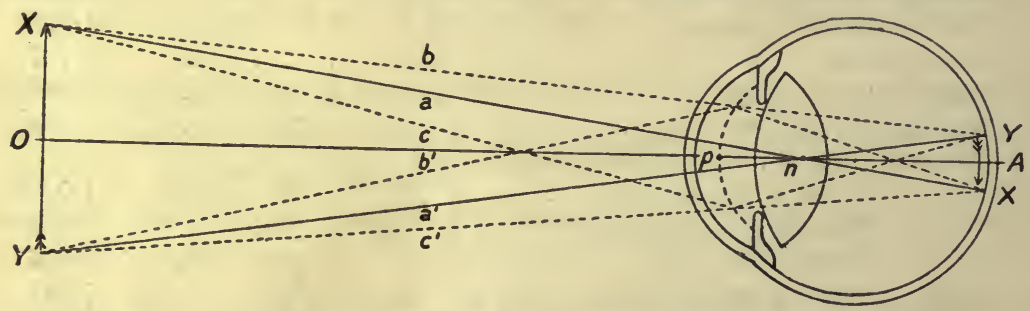

Fig. 263. Path of the rays in the formation of an image on the retina.

that produced by a bi-convex lens, is real, inverted, and diminished in size. The further the distance of the object from the retina the smaller will be its image on the retina. The angle formed at the junction of two lines drawn from the extreme points of an external object to the nodal point is the 'visual angle.'

In the schematic eye a visual angle of sixty seconds corresponds to a distance on the retina between the two ends of the image of $00438 \mathrm{~mm}$. Few people can distinguish two points of light the line joining which subtends a smaller visual angle than sixty seconds. If we measure the histological elements in the centre of the retina which are responsible for distinct vision, viz. the cones, we find that in the yellow spot each cone is about $\cdot 002$ to $.005 \mathrm{~mm}$. thick. The limits of our power of distinguishing two luminous points is approximately in agreement with the diameter of each end-organ of vision. In order that the images of the two points may give rise to distinct sensations their images must fall upon different cones. This fineness of vision exists only at the yellow spot, the accuracy of vision in the peripheral parts of the retina being very much lower. Not only is the image less 
perfectly focused, but the number of sensory elements in a given area of the retina diminishes steadily as we pass from centre to periphery.

\section{OPTICAL DEFECTS OF THE EYE}

The normal eye is so constructed that parallel rays come to a point on the retina. Rays which are divergent when they fall on the anterior surface of the cornea are brought therefore to a focus behind the retina.

In such an eye any object lying at a distance nearer than five metres would give rise to an image behind the retina, were it not for the fact that the eye possesses means of altering its focus and so of bringing divergent rays to a focus on the retina; this means is known as accommodation. In a photographic camera the process of focusing is carried out by altering the distance between the lens and the sensitive plate. The same method is adopted in the eyes of certain animals. In the mammalian eye, however, accommodation for near objects, i.e. the focusing of divergent rays on to the retina, is accomplished by a change in the curvature of the lens. The lens becomes more convex on its anterior surface, so that its refractive power is increased and the hinder focus of the dioptric mechanism of the eye is therefore diminished. Every eye possesses a certain definite range of vision, which, in a normal person, has its far point at infinite distance and its near point

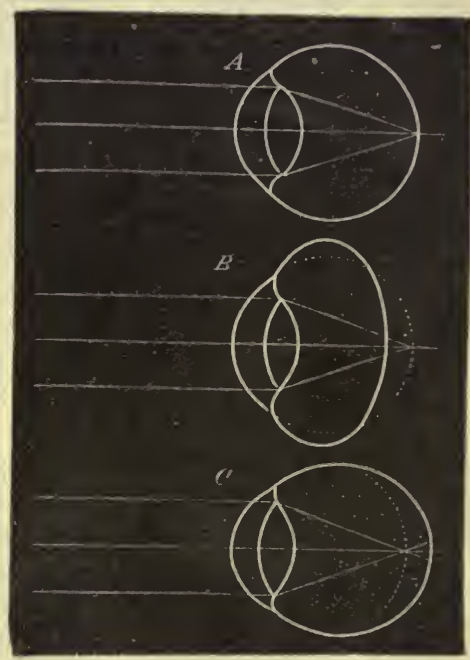

Fig. 264. Diagrams of course taken by parallel rays in entering normal (emmetropic) eye (A), hyper-metropic eye (B), and myopic eye (c).

at a distance from the eye which depends on the elasticity of the lens and the range through which this can alter its curvature.

The normality of an eye is determined by the fact that parallel rays come to a focus on the retina when the apparatus of accommodation is at rest. Two classes of deviation from this normal, or emmetropic, eye are common. In the first class parallel rays come to a focus in front of the retina. In such eyes, which are designated myopic, it is impossible to get a clear image on the retina of distant objects. In order that the rays may be focused on the retina they must be divergent, so that even when accommodation is paralysed the far point of distant vision lies at some finite distance from the eye, varying with the extent of the disorder. If in such eyes the range of accommodation is normal, the near point will be much nearer to the eye than in the emmetropic eye (Fig. 264).

The second class of abnormal eyes are known as hypermetropic. These eyes can be regarded as too short for their refractive media. Parallel rays 
are brought to a focus at a point behind the retina. Persons so affected can see objects at a distance, but always with some effort of accommodation. If accommodation be paralysed by means of atropine everything will appear blurred. Since accommodation is required even for infinite distance, the greatest possible effort will be insufficient to bring near objects into accurate focus, and the near point of such eyes will be greater than normal. Persons with hypermetropia, or long-sightedness, can therefore see objects at a distance perfectly well, but are unable to read small print, since the point of near vision is too far from the eye to allow the small letters to subtend a sufficiently large angle.

Both these disorders can be corrected by suitable spectacles. In the case of the myopic eye we need lenses which will convert the parallel rays into divergent rays; such cases are treated therefore with concave lenses. Conversely, hypermetropia is treated with convex lenses, which will aid the too feeble refractive power of the eye, and so bring parallel rays to a focus on the retina without any effort of accommodation. The degree of myopia or hypermetropia is denoted by the refractive power of the lens which is necessary to make the eye emmetropic.

In order to determine the refractive power of any eye it is usual to employ Snellen's test typs. This consists of a series of letters which are placed at a distance of five metres from the eye. At this distance the visual angle subtended by each of these letters is so small that a clear retinal image is necessary for their recognition. This is easy in the case of a normal eye. After allowing the patient to attempt the recognition of the type without spectacles he is then made to regard it through a weak convex lens. If the patient can now read as well as or better than before he is hypermetropic, since it is only the hypermetropic eye which is able to unite convergent rays of light on to the retina. If, on the other hand, the reading of the type is made more difficult the patient is either normal (emmetropic) or myopic. In the latter case a concave lens is tried. If the reading is rendered more easy by this means the patient is myopic.

In prescribing the lenses for hypermetropia, the strongest lens with which the patient is able to see represents the degree of hypermetropia. Since now the mechanism for accommodation must be relaxed as far as is possible, the strength of such a lens serves as a measure of the degree of hypermetropia. On the other hand, in myopia the degree of the disorder is determined by the weakest lens, by means of which the patient is able to see distant objects.

In a perfect dioptric mechanism the media through which the light passes must be perfectly transparent, and the centres of curvature of the various refracting surfaces must lie in one straight line, i.e. the system must be properly centred. In neither of these respects can the eye be regarded as perfect. If a strong beam of light be thrown into the eye, the refraction of the beam caused by the slight difference in structure between adjacent portions of the cornea and lens makes these objects immediately visible, and the field of vision is filled with diffused light arising from the illuminated points in these structures. Under normal circumstances, however, these slight differences in the regularity of the refracting media do not make any appreciable difference to our vision. More easily detected are the opacities due to structures in the vitreous humour. These opacities can be seen, when the eyes are turned towards a uniformly illuminated surface, as small dark 
points or strings of beads, which, since they alter their position with changes in the direction of the eyes, are often spoken of as musca volitantes. The centring of the eye is also never perfect. In the horizontal meridian the optic axes of the cornea only diverge about $0.3^{\circ}$ from the axis of the lens, but in the vertical meridian there is as much as $1.3^{\circ}$ difference between the two axes. Moreover the visual axis does not correspond exactly with the optic axis of the eye. The fovea centralis, the point of distinct vision on which the image of any object must be brought in order to see it as distinctly as possible, always lies outside and somewhat below the point at which the optic axis strikes the retina. The angle between the two axes is often spoken of as the angle $a$. This angle in the horizontal meridian varies between $3.5^{\circ}$ and $7^{\circ}$, and in the vertical meridian is about $3.5^{\circ}$.

Although this divergence of the axes causes a certain amount of astigmatism (v. p. 538), it is too small to interfere appreciably with the sharpness of vision.

SPHERICAL ABERRATION. When a beam of parallel rays falls on to the surface of a spherical lens those rays which pass through the circumference are converged to a focus which lies nearer to the lens than the focus of those rays which pass through its centre. In an optical instrument the blurring of the image thus produced is counteracted in two ways :

(1) By making the curvature in the middle of the lens greater than at its periphery.

(2) By stopping out the peripheral rays by means of a diaphragm, or by using only a cylinder cut from the centre of the lens. It is familiar to every photographer that where sharpness of definition is required it is necessary to use a small stop, giving a corresponding increased exposure.

In the eye spherical aberration is diminished by both these means. The curvature of the lens is greater towards its centre than at its circumference, and the peripheral rays of light are shut out by a circular diaphragm, the iris, the diameter of the aperture in which varies according to the amount of light falling into the eye, and according to the nearness of the object which is the point of regard.

CHROMATIC ABERRATION. The refraction of light in passing from a lighter to a denser medium is due to the fact that in the latter the velocity of propagation of the light is less than in the former. This diminution of the velocity of the propagation affects rays of various wave-lengths differently, so that of the various rays which make up white light those at the red end with a long wave-length are refracted least, and those at the violet end with a short wave-length are refracted the most. On this account, when light passes into a prism it is split up into its component rays with the production of a spectrum. The same splitting up of rays occurs when light passes through a simple lens. As is shown in Fig. 265, the violet rays come to a focus at a point nearer the lens than the red rays. A screen held at $v$ will therefore show a bluish-violet centre with a red margin ; at $\mathrm{R}$ the centre will be reddish and the margin violet.

In optical instruments this chromatic aberration is corrected by 
combining glasses of different powers of dispersion, with the production of socalled achromatic lenses. In the eye achromatism is practically uncorrected. The difference in the focus of red and violet rays in the eye amounts to about $0.5 \mathrm{~mm}$. ; hence, if we are looking at a red and a violet spot situated close together, it requires a greater act of accommodation to bring the image of the red spot on the retina than is the case with the violet spot. The red spot therefore looks nearer, i.e. more prominent. It may be this special

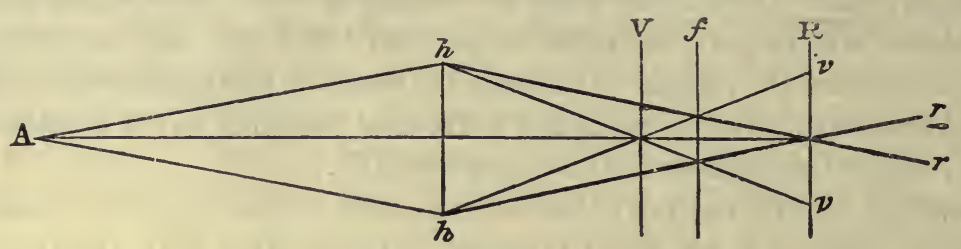

Fig. 265.

effort of accommodation necessary for the appreciation of red that makes this colour stand out, so to speak, and be conspicuous as compared with the other colours of the spectrum.

The fact that as a rule we do not see coloured fringes around every object that we look at is due, not to the optical, but to the physiological qualities of the eye. When white light falls on the eye and is focused by the latter on to the retina, it will be the rays of medium refrangibility which come to a point at the retina; these optically will be surrounded with a halo composed of the red and violet rays, as is shown in the figure at $f$. The retina is, however, comparatively insensitive for rays at the two extreme ends of the spectrum. Moreover the strong illumination produced by the middle rays of the spectrum, i.e. about the yellow, at the centre of the illuminated spot, by contrast depresses the excitability of the surrounding parts of the retina, so that the halo due to the red and violet rays is neglected and does not come into consciousness.

ASTIGMATISM. We have assumed so far that the refracting surfaces of the eye are practically spherical; this does not apply strictly either to the cornea or the lens. The small differences between the curvatures of the cornea in the horizontal and vertical meridian towards its centre do not as a rule give rise to appreciable disorders of vision. In many cases, however, the asymmetry in the anterior surface of the cornea is sufficient to cause a considerable difference in the refraction of rays in the different meridians, and this disturbance is known as astigmatism.

The curvature of the vertical meridian of the cornea is nearly always somewhat greater than that of the horizontal meridian. When this difference is sufficiently pronounced it becomes impossible for a definite image of a point of light to be formed on the retina, since the rays which diverge from the luminous point in the vertical plane are brought to a focus sooner than those in a horizontal plane. Such an eye will therefore possess two posterior foci, one for the vertical meridian, the other for the horizontal meridian. The manner in which such rays diverge is shown in Fig. 266. 
The effects of astigmatism are especially noticeable when the patient is trying to see clearly objects composed of horizontal and vertical lines, such as print. A vertical line can be conceived as made up of a series of points each of which sends out a horizontal sheaf of rays. In the same way a horizontal line is distinguished as a series of points sending out flat sheafs of vertical rays. If the curvature of the cornea in the vertical meridian is greater than in the horizontal it will need a greater effort of accommodation to bring the vertical lines to a focus on the retina than it does to bring the horizontal lines. In reading therefore there is a constant shifting of the focus of the eye, and the mechanism of accommodation becomes rapidly tired and

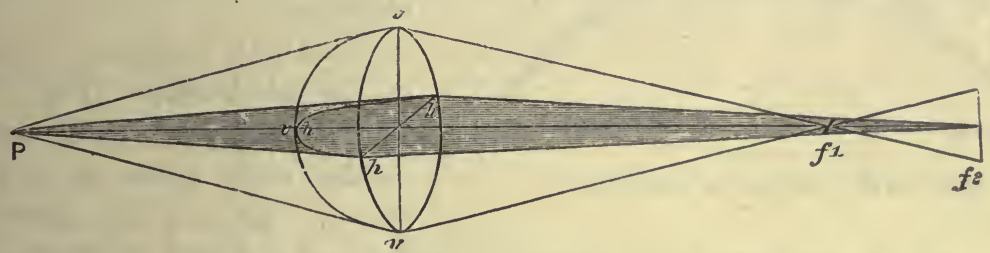

FrG. 266. Diagram showing course of rays in an astigmatic eye. (WALLER.)

The curvature of the cornea is greater in the vertical meridian $v v v$ than in the horizontal meridian $h h h$. Hence the rays of light coming from the point $P$ and passing through the vertical meridian come to a focus at $f^{1}$, while those through the horizontal meridian come to a focus at $f^{2}$. There is thus no point behind the cornea at which all the rays from $\mathbf{P}$ will come to a focus, and the image of the point must be blurred, being elongated in a horizontal direction at $f^{1}$, and in a vertical direction at $f^{2}$.

strained, with the production of pain in the eyes or of headache. In order to correct astigmatism it is necessary to find out first the curvature of the cornea in the different meridians, and then to reinforce the curvature of the weaker meridian by means of a cylindrical lens. If the eye is myopic the cylindrical lens may be concave and placed so that its curvature counteracts that of the cornea in the meridian in which the curvature is greatest.

\section{ACCOMMODATION}

The rays falling on the eye from a point of light at a distance greater than five metres from the eye may be regarded as practically parallel, and are converged in the normal eye to a focus on the retina. As the point of light is moved nearer to the eye the latter is still able to focus the rays on the retina through a considerable range. On approximating the points of light to a distance which is less than the near point of distinct vision, the rays are no longer converged to a point on the retina, and a blurred image is the result. This near point of vision may be determined in any eye by finding out the smallest distance from the eye at which small print can be easily distinguished. The distance is measured by means of a graduated rod between the eye and the printed object. This 'accommodation,' by which the eye is able to focus divergent rays on to the retina, implies either a change in the distance of the refracting surfaces from the retina, or an increase in the total refractive powers of the eye.

In man and the higher animals it is by the latter means alone that 
accommodation is effected. The fact that accommodation, as was shown by Young, may be carried out under water, i.e. under conditions in which the curvature of the cornea does not cause any appreciable deviation of the rays passing through it, shows that the change in the combination cannot be located in the cornea. It was shown by Helmholtz that the essential process

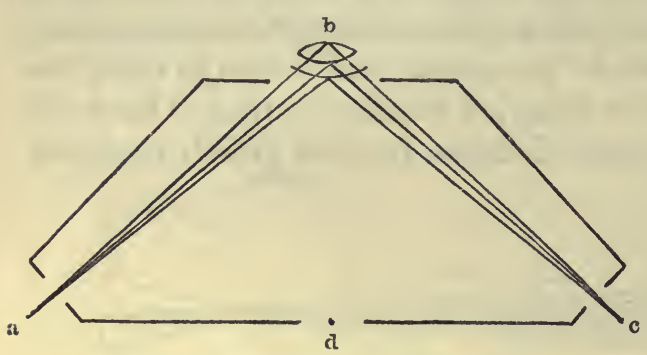

Fig. 267. Diagram of phakoscope.

in accommodation is an alteration in the curvature of the lens, the anterior surface becoming more convex when the eye is accommodated for near objects.

This may be shown by means of the phakoscope (Fig. 267). This is simply a box, blackened inside, with holes at $a, b, c$, and $d$. At $a$ is the observer's eye; at $b$ the observed eye. Across the middle of $d$ a wire is stretched.

A candle is placed at $c$. The observer at $a$ then sees three reflections of the candle from the eye at $b$ : a bright erect image from the anterior surface of the cornea; a larger but dimmer erect image from the anterior surface of the lens; and a small very dim inverted image from the posterior surface of the lens. These images must be observed first when the eye at $b$ is accommodated for a distant object, and then when it is accommodated for the wire stretched across the opening $d$. It will be noticed that the change of accommodation from far to near objects is accompanied by a change in the second image (that from the anterior surface of the lens), which becomes smaller. The change in this image is more easily seen if the candle be made to throw two images on the eye by interposing a double prism at $c$. Then, as the lens becomes more

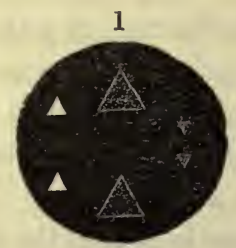

a b 3

FIG. 268. Diagram of reflected images from cornea and lens surfaces seen in phakoscope.

$a$, from anterior surface of cornea; $b$, from anterior surface of lens ; $c$, from posterior surface of lens. 1, during accommodation for distance; 2, during accommodation for near objects.

convex to accommodate for near objects, the two images of the candle reflected from its anterior surface approach one another (Fig. 268).

By measuring the size of the image of the candle-flame produced by the anterior surface of the lens, and knowing the size of the flame itself and the distance from the observed eye, it is possible to calculate the curvature of the lens in the living body.

The radius of curvature of a reflecting surface is given approximately by the following formula:

$$
\mathrm{R}=2 \frac{a \cdot b}{\mathrm{C}}
$$




\section{DIOPTRIC MECHANISMS OF THE EYEBALL}

where $\mathrm{R}$ is the radius of curvature, $a$ the distance of the object, $b$ the size of the image, $\mathrm{C}$ the size of the object. The object generally used is the distance between two lights or two white objects called mires ; the 'image' being the distance between their images. Owing to the movements of the eye the latter cannot be accurately measured by the usual method, employed by physicists, of looking at the images through a telescope which has a micrometer at the focus of the object. This difficulty is overcome by doubling the image. For this purpose Helmholtz devised the ophthalmometer, in which the doubling is brought about by two plane glass plates set at a variable angle to one another. The principle of the instrument can be gathered from the diagram (Fig. 269). We may suppose it is necessary to measure the line $a b$, which may be taken to represent an image reflected from the anterior surface of the cornea or lens. If we look at this line through a plate of glass the plane of which is at right angles to our line of sight, no distortion of the line $a b$ takes place. If, however, the plate be placed

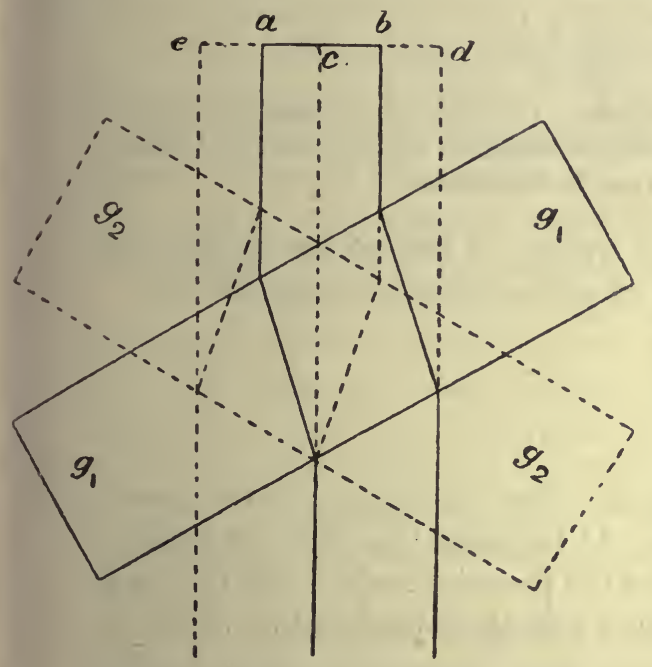

Fic. 269. Diagram to illustrate principle of ophthalmometer. (After ScHENCK.)

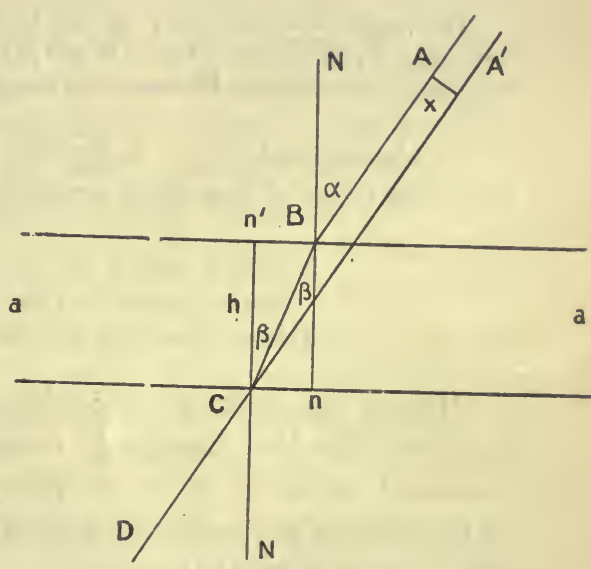

FIg. 270.

obliquely, as at $g_{1} g_{1}$, there will be an apparent shifting of the line sideway to $c d$. In . the ophthalmometer there are two glass discs, $g_{1} g_{1}$, and $g_{2} g_{2}$, one immediately over the other, so placed that the image $a b$ is looked at through the junction between the two plates. The plates are then turned, as in the diagram, until $a b$ appears as two distinct lines $e c$ and $c d$ just touching one another at $c$. At this point each image of the line $a b$ has been shifted through one half the length of $a b$. Knowing the thickness of the plates and their refractive index, it is easy to calculate, from the angle through which the plates have been turned, the apparent shifting of the line $a b$. This lateral movement amounts to $a c$, i.e. to $\frac{a b}{2}$, and we have merely to double this result in order to obtain the actual size of the image on the cornea or lens.

A table is generally supplied with the instrument giving the actual size of theimage corresponding to the angle through which the plates have been turned; the eye always being placed at a constant distance from the instrument and the luminous object always being the same size.

The size of the image is calculated in the following way * :

"Let $a a$ (Fig. 270) be one of the plates, $A B$ the incident, $C D$ the refracted ray. Then, since the refracted ray is parallel to the incident ray, the angle $A B N$ is equal 
to the angle $D C N^{\prime}(=a)$. Similarly the angle of refraction $C B n$ is equal to the angle $B C n^{\prime}(=\beta)$. Let $h$ be the thickness of the glass plate. Produce $D C$ backwards to $A^{\prime}$. It is required to find the perpendicular distance between $A$ and $A^{\prime}(=x)$.

Now

$$
\begin{aligned}
\frac{x}{B C}= & \sin B C A^{\prime} \\
= & \sin \left(n^{\prime} C A^{\prime}-n^{\prime} C B\right) \\
= & \sin (a-\beta) . \\
& \frac{h}{B C^{\prime}}=\cos \beta . \\
& x=h \cdot \frac{\sin (a-\beta)}{\cos \beta} .
\end{aligned}
$$

And

Therefore

If there are two such plates, arranged as in Fig. 269, then

$$
\begin{aligned}
a b & =2 x \\
& =2 h \cdot \frac{\sin (\alpha-\beta)}{\cos \beta}
\end{aligned}
$$

\begin{tabular}{|c|c|c|c|}
\hline Anterior surface of cornea & & & \\
\hline Anterior surface of lens & & & \\
\hline Posterior surface of lens & & & \\
\hline
\end{tabular}

The angle $a$ is measured by the instrument; the angle $\beta$ is calculated from the formula for refraction, $\sin a=n$. $\sin \beta$; and the thickness of the plates, $h$, is known. Therefore the distance between the images can be calculated."

In the normal eye in a position of repose, i.e. focused for parallel rays, the curvatures of the three principal refracting surfaces are as follows :

During maximum accommodation the radius of curvature of the anterior surface of the lens changes to $6 \mathrm{~mm}$. At the same time there is a slightly increased curvature at the periphery of the posterior surface, but the effects of this change are negligible as compared with those produced by the alteration of the anterior surface.

In order to determine the method in which this change in curvature of the anterior surface of the lens is brought about we must refer in some detail to the structure of the anterior half of the eye and the manner in which the lens is hung up between the aqueous and vitreous humours. The outermost layer of the eye is formed by the sclerotic coat, a strong tough membrane of white fibrous tissue. In front this is continuous with the cornea, which, having a smaller radius of curvature than the globe of the eye, protrudes like a watch-glass from its anterior surface. The main substance of the cornea is formed, like the sclerotic, by white fibrous tissue, modified however in its consistence so as to be perfectly transparent instead of white and opaque like the rest of the sclerotic. Internal to the sclerotic is the choroid coat, a membrane with a double pigmented internal layer, and supplied with bloodvessels which furnish the vascular supply to the whole eye. In front the choroid coat presents a series of folds, arranged in a circle around the anterior part of the cavity of the vitreous and known as the ciliary processes. In front of the ciliary processes the choroid leaves the sclerotic and hangs as a curtain into the cavity of the aqueous humour, between the cornea and the lens. The curtain, which is known as the iris, presents a circular orifice at 
its centre, the pupil, and is provided with muscular fibres by means of which the pupil may be constricted or dilated. The posterior surface of the iris, like the inner surface of the choroid generally, is lined by a pigmented epithelium. The posterior layer of the cornea is formed by a tough elastic membrane (Descemet's membrane) which is covered posteriorly by a layer of cubical epithelial cells. At the circumference of the cornea Descemet's membrane, or the posterior elastic lamina, breaks up into a number of fibres, which pass outwards and backwards to be inserted into the anterior part of the choroid and root of the iris. These fibres are the ligamentum pectinatum iridis. Between the fibres of the ligamentum pectinatum are a number of spaces lined with endothelium continuous with the anterior chamber and known as the spaces of Fontana, and outside these spaces, in the corneo-sclerotic junction, is a circular sinus continuous with the venous system, the canal of Schlemm or sinus venosus. The iris rests at its inner margin on the lens, so dividing the cavity of the aqueous humour into two parts. In front is the anterior chamber and behind the posterior chamber. The latter is a small annular space, triangular in cross-section, and bounded by the iris in front, the lens behind, and the ciliary processes externally.

The crystalline lens, made up of radiating lens fibres, each of which is produced by the modification of an epithelial cell, is biconvex, the posterior surface being more convex than the anterior. It is surrounded by a tough structureless membrane, the capsule of the lens, and rests in a cavity hollowed out of the anterior surface of the vitreous humour. At its circumference it is hung up and fastened to the ciliary processes by the suspensory ligament, or zonule of Zinn (zonula ciliaris). (Fig. 271.) This ligament is formed in the following way:

The vitreous humour is bounded externally by the hyaloid membrane, which separates it from the retina. In front the hyaloid membrane passes behind the lens, but as it lies on the ciliary processes is closely adherent to these structures and sends off fibres which pass radially from the ciliary processes to the capsule of the lens and form the zonule, or suspensory ligament. The greater part of the suspensory ligament, i.e. from the ora serrata of the retina to the edge of the ciliary processes, is closely attached to these processes. From their edge a number of fibres pass and fuse at their inner extremities with the lens capsule. These fibres are arranged in three groups:

(1) The anterior group passing to the anterior capsule of the lens.

(2) A middle group passing to the equator of the lens.

(3) A posterior group lying close to the hyoid membrane and passing to the posterior lens capsule.

The suspensory ligament is always in a condition of tension. If the finger be pressed on the outside of the eyeball, it will be felt that this organ presents a resistance to deformation which cannot be ascribed simply to the firmness of the sclerotic coat, but must be determined by the existence of a positive pressure in the fluid filling the eyeball. This pressure, which is known as the intra-ocular pressure, may be measured by means which we shall have to discuss later, and amounts to about $25 \mathrm{~mm}$. Hg. As a result of 
this pressure the membranes which confine the fluids of the eyeball are distended, i.e. pressed outwards, and this pressure keeps the bases of the ciliary processes pressed against the choroid coat and thus enables them to withstand the pull exerted by the tense suspensory ligament.

The pull exerted by the suspensory ligament affects mainly the tough anterior surface of the lens capsule and so has a constant flattening effect on the anterior surface of the lens. This may be proved by measuring the curvature of the lens in a recently excised eye, and then removing the lens altogether from the eyeball and measuring the curvatures of its surfaces again. It

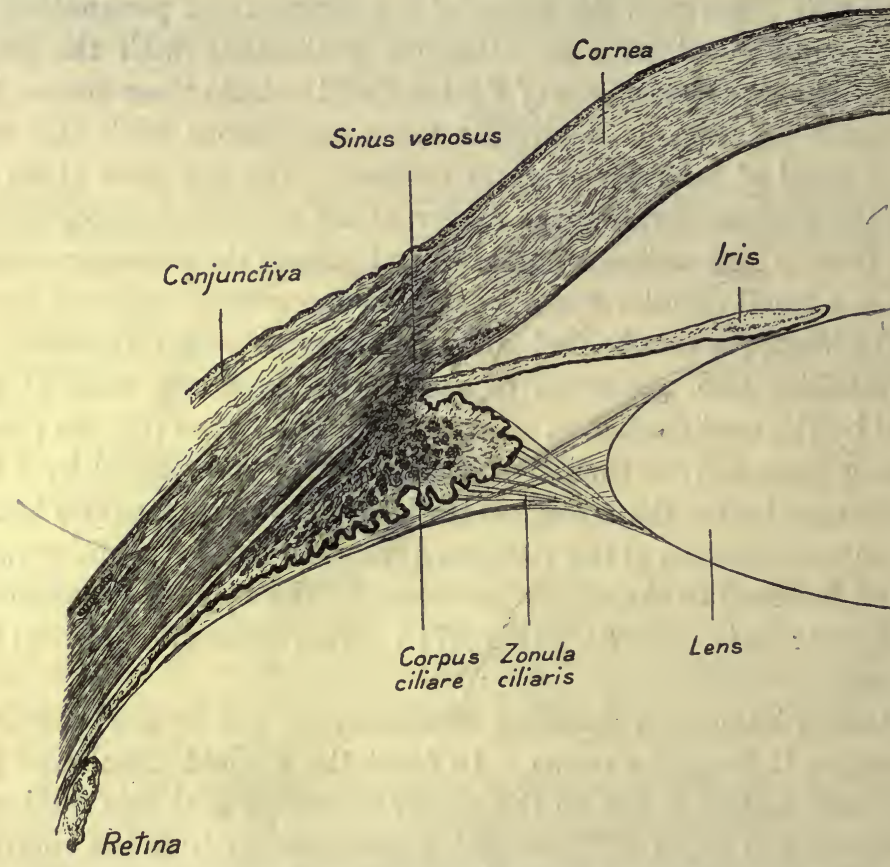

FIG. 271. Section through anterior part of eyeball to show mode of suspension of lens. (After Merkel and Kallius.)

will be found that, as soon as the lens is free from its attachments in the eyeball, the curvature of its anterior surface is increased. The change therefore in the lens, which is responsible for the alterations in its refractive power determining accommodation, may be effected by any means wi.ich will relax the suspensory ligament, such, for instance, as approximation of the ciliary processes to the margin of the lens. This movement of the ciliary processes is effected by the ciliary muscle. The attachments of this muscle are shown in Fig. 271. It forms a circle of unstriated muscle fibres, triangular in cross-section, and extending round the whole circumference of the eyeball. The fibres of the muscle are divided into three groups :

(a) The meridional fibres, which run from the corneo-sclerotic junction backwards and outwards to be attached to the anterior part of the choroid coat behind the ciliary processes.

(b) The radial fibres, which pass from the margins of the canal of Schlemm, 
and from the fibres of the ligamentum pectinatum to be attached behind to the whole extent of the ciliary processes.

(c) The circular bundle, which forms a ring-muscle, composed of fibres running around the circumference of the eye in the inner part of the ciliary processes. This bundle is best marked in hypermetropic eyes and is almost absent in myopic eyes.

When this muscle contracts it draws the anterior part of the choroid and the ciliary processes forwards and inwards, while the ring-fibres approximate the ciliary processes to the margin of the lens. By this approximation of the ciliary processes to the lens the suspensory ligament is relaxed, and the anterior surface of the lens bulges, i.e. becomes more convex as a result of its inherent elasticity (Fig. 272).

This explanation of accommodation, which was first put forward by Helmholtz, is almost universally accepted. According to some the change of shape of the lens during accommodation is brought about by the actual pressure of the ciliary processes on its margin by which the middle of its anterior surface

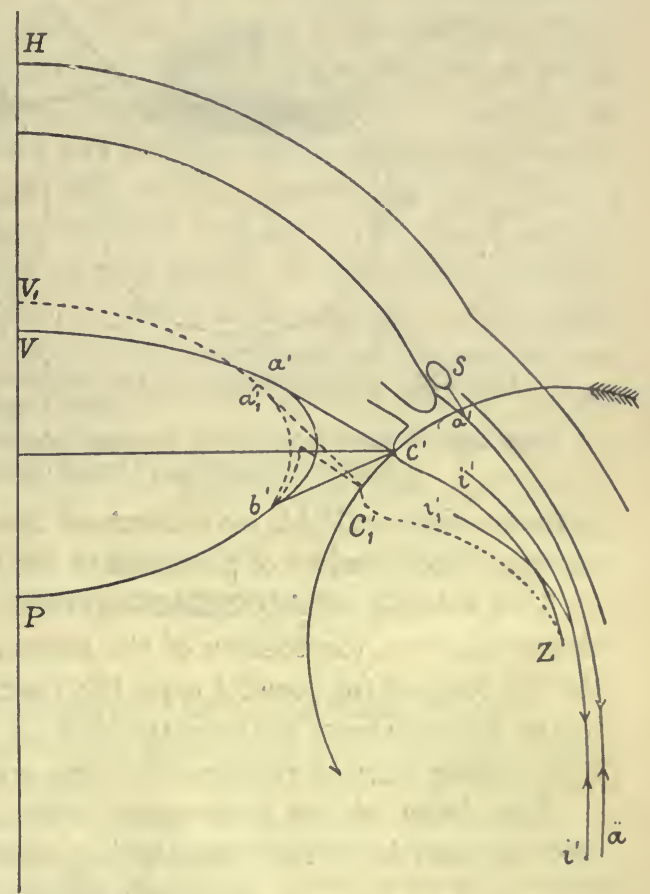

FIG. 272. Diagram of mechanism of accommodation. (TIGERSTEDT after SchöN.)

The dotted line shows the form of the lens during accommodation for near objects. is pressed forwards. According to Tscherning this effect is produced, not by relaxation, but by a tightening of the suspensory ligament through the contraction of the ciliary muscle. There is no doubt, however, that during forced accommodation, such as can be brought about by instillation of eserine into the eye, which produces spasm of the ciliary muscle, the suspensory ligament is so relaxed that the lens lies loosely in the eyeball. Bending the head downwards causes therefore an actual change in the position of the lens, which may drop as much as $1 \mathrm{~mm}$. forwards towards the cornea. Under the same conditions a quick movement of the head causes the lens to shake, and the quiver of the lens can be seen by an external observer and proved by the subjective oscillation of external objects which is noticed after such a movement. Moreover, if a needle be passed through the sclerotic so that its point lies in the ciliary processes, stimulation of the ciliary muscle causes a movement of the outer part of the needle backwards, showing that the point of the needle which is in the ciliary processes has been moved forwards (Fig. 273). The loosening of the lens during spasm of accommodation is well shown in rare cases where there is congenital absence of the whole iris. In such cases the shaking of the patient's head is seen to cause an oscillation of the lens within the eyeball.

During accommodation the increased curvature of the anterior surface of the lens causes an approximation of this surface to the cornea, which may 
be directly observed (cp. Fig. 273), especially in people with somewhat prominent eyes. No change takes place in the intraocular pressure, in either aqueous or vitreous cavities, as the result of accommodation. The passage of fluid takes place with such ease between the fibres of the suspensory

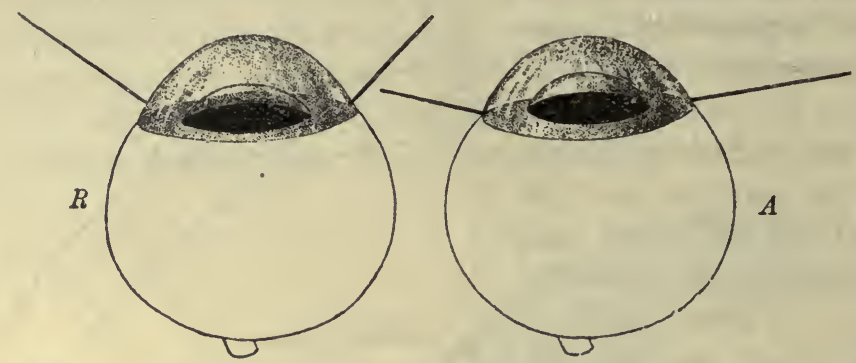

FIG. 273. Accommodation in the cat's eye. R, distance; $A$, for near vision.

(After BeEr.)

Two needles have been passed through edge of cornea into ciliary bodies, to show forward movement of latter during accommodation.

ligament that a slight movement of the lens forwards or backwards does not upset the equality of pressures in the two chambers.

THE RANGE OF ACCOMMODATION. Assuming that, as is the case with the normal eye, the tension of the suspensory ligament is sufficient to keep the eye focused for parallel rays, the near point of vision will be determined by the unconstrained shape of the lens, i.e. the amount by which its curvature can increase when the suspensory ligament is completely relaxed.

The shape of the lens varies with age, its convexity, being greatest directly after birth and diminishing steadily from that time to the age of

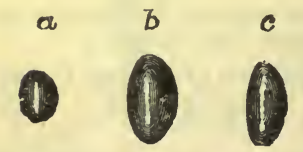

Fic. 274. Lens from human eye at different periods of life. (Allen Thomson.) $a$, at birth; $b$, adult; c, old age. sixty or seventy (Fig. 274). In consequence of the small size of the eyeball, the eye at birth is generally somewhat hypermetropic, but from the age of ten onwards we find that the point of near vision recedes continuously with advancing age. The range of accommodation is measured by the strength of the lens which will give to rays coming from the near point of distinct vision the same direction as if they came from the far point. In a normal eye the far point is at infinite distance and the rays are parallel. The change in the range of accommodation with advancing years is shown in the following Table :

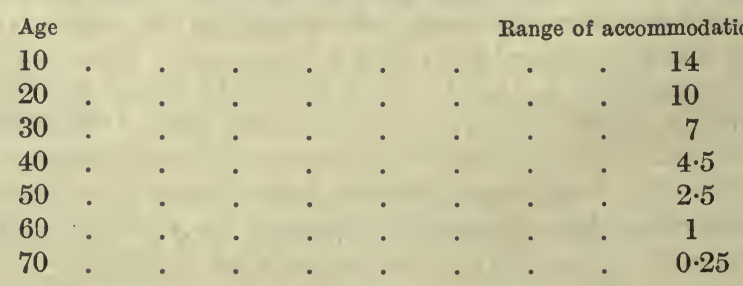

This gradual diminution in the range of accommodation gives rise finally to disturbances of vision which are known as presbyopia. The ordinary reading 
distance is about ten inches. A smaller distance than this is rarely made use of even in youth, when the point of near vision is only three or four inches from the eye, on account of the effort required to converge the axes of the two eyes to this extent. The effect of the gradual diminution in the elasticity of the lens is noticed as soon as the point of near vision recedes beyond ten or twelve inches, i.e. 25 to $30 \mathrm{~cm}$. This occurs as a rule between fortyfive and fifty, when we begin to experience difficulty in reading small print, since the visual angle subtended by such print at a distance over ten or twelve inches is too small to allow of distinct vision. The condition of presbyopia, is remedied by employing reading glasses, i.e. by wearing spectacles which converge the rays of light and so enable small objects to be brought nearer to the eye than its near point. It is evident that these glasses must be strengthened continually with advancing age. No trouble is experienced in seeing distant objects, the refraction of the eye at rest remains as before. The dimness of vision in extreme old age for distant as well as near objects is due to changes, not in the refractive power of the eye, but in the transparency of the refractive media, such as cataract, i.e. opacity of the lens, opacity of the cornea, and so on. The former condition can often be remedied by extracting the lens. Strong convex glasses (ten dioptres) must then be used to take the place of the lens.

\section{THE COMPARATIVE PHYSIOLOGY OF ACCOMMODATION}

The mechan:sm of accommodation which we have studied in man is found with very little modification throughout the whole group of mammalia, though, in the domestic animals at any rate, the range of accommodation is very much less than in man. On examining other types of animals we meet, as was shown by Beer, an amazing variety of methods by which the range of the eye may be altered. In order to bring distinct images of objects at various distances on to the retina, practically every possible focusing method is made use of in one type or another of the animal kingdom. The following details are taken from Beer's papers.

In birds the eye, like that of man, is normally focused for distant objects, and accommodation for near objects is accomplished by a change in curvature of the anterior surface of the lens. Whereas, however, in man the suspensory ligament is relaxed by a drawing forwards of the choroid membrane, in the bird's eye this relaxation is effected by a drawing backwards of the posterior lamina of the cornea, where it breaks up into the ligamentum pectinatum iridis. In these eyes the main attachment of the suspensory ligament is to the ligamentum pectinatum; the retraction of this ligament is effected by a special muscle known as Crampton's muscle, which corresponds to the ciliary muscle in man, but unlike this consists of striated voluntary muscle. This movement of the posterior elastic lamina of the cornea can be easily shown by passing two needles through the corneo-sclerotic junction until their points lie in the anterior chamber. On exciting Crampton's muscle electrically, the outer end of the needle moves forwards, showing that the deeper part of the corneo-sclerotic junction is being pulled backwards towards the ciliary portion of the eye (Fig. 275). The histological character of the muscle of accommodation in birds seems to be connected with the rapid accommodation that is necessary when a bird swoops down towards the ground to pick up some food insect. Moreover, since binocular vision is not present in many birds, and convergence of the optic axes must be minimal, it is probable that the contractions of Crampton's muscle play a great part in guiding the movements of the bird, and especially in aiding it to judge distances. In our selves such judgment is very faulty without the co-operation of the two eyes. 
In amphibia and snakes, which at rest are also focused for distance, active accommodation for near objects is effected, not by change in curvature of the lens, but by an increase in the distance between the lens and the retina. In amphibia the ciliary muscle, which lies between the root of the iris, the sclerotic and choroid, causes a rise of pressure in the vitreous cavity, and the lens, being the most movable part of the boundary wall of this cavity, is pushed forwards towards the cornea. The aqueous humour, which is displaced by this forward movement of the lens, finds a place in the lateral angle of the eye, which is increased in depth by the pull of the muscle fibres.

In snakes the same action is effected by a muscle, often cross-striated, which is situated in the root of the iris. In both these cases the movement of accommodation is unaffected by opening the aqueous cavity, whereas in mammals it is at once rendered impossible if the aqueous cavity be laid open.
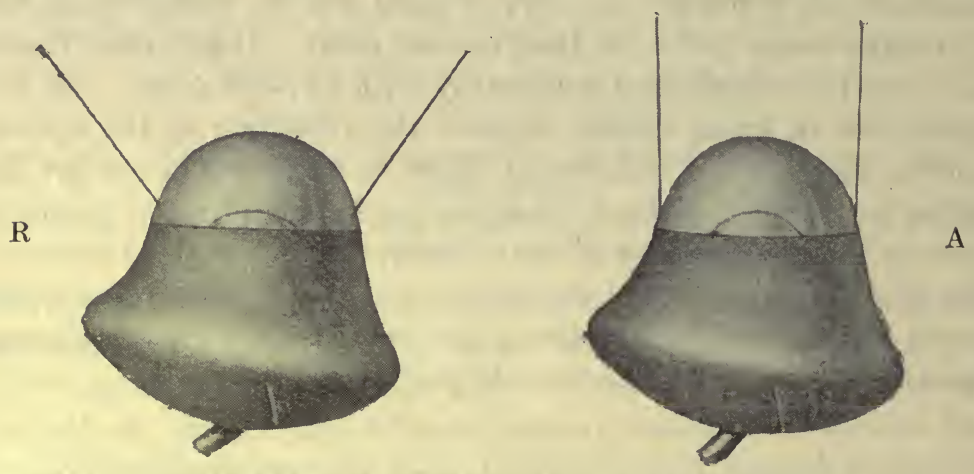

Fia. 275. Accommodation in a bird's eye. (BEER.)

$\mathrm{R}$, rest ; A, accommodation for near objects.

Most of the teleostean fishes are short-sighted, i.e. at rest they are focused for near objects. Active accommodation in these animals diminishes the refractive power of the eye, so that accommodation occurs for distant objects. In the fish's eye there are no ciliary processes, ciliary muscle, zonule of Zinn, or spaces of Fontana, such as are found in the higher vertebrata. The iris only approaches the margin of the lens, and does not shut out its peripheral rays. The lens, which is spherical (Fig. 276), is hung up by means of a flat band attached to its upper pole. This is known as the 'suspensory ligament,' but is quite different in structure and mechanism from the suspensory ligament or zonule of Zinn of the vertebrate eye. From the lower and inner pole of the lens a dark pigmented structure passes backwards; this was described by its first discoverer as the campanula, but since it is muscular in character is better named the 'retractor lentis.' On stimulation this muscle pulls the lens backwards, and so lessens the distance between it and the retina, in this way accommodating the eye for distance.

The eye of the cephalopod mollusc, such as sepia, is also short-sightcd, and actire accommodation, as in the fish's eye, is accommodation for distance. The mechanism is, however, quite different. The globe of the cephalopod's eye has the shape shown in the diagram (Fig. 277). The most resistant part of the globe is formed by a strong ring of cartilage which passes round the equator of the eye. The rest of the sclerotic is formed of delicate membrane, which is thinnest in the ring just behind the cartilaginous ring. In the anterior wall of the eye is a strong muscular ring, composed of meridional fibres, which run from the cartilaginous ring to be inserted into the ciliary processes or corpus ciliare, which is closely attached to the equator of the lens.

When this muscle contracts it pulls back the whole anterior wall of the eye together with the lens, approximating it to the retina. This movement is of necessity accompanied by a rise of ocular pressure, but room for the displaced fluid is found by a bulging of the walls of the eyeball at their thinnest part, i.e. just behind the cartilaginous 
ring, so that there is an actual diminution of the distance between the lens and the retina.

In every class of animals, except in the cephalopod and in birds, species are found which possess no power of accommodation at all, or only to a very slight extent. This
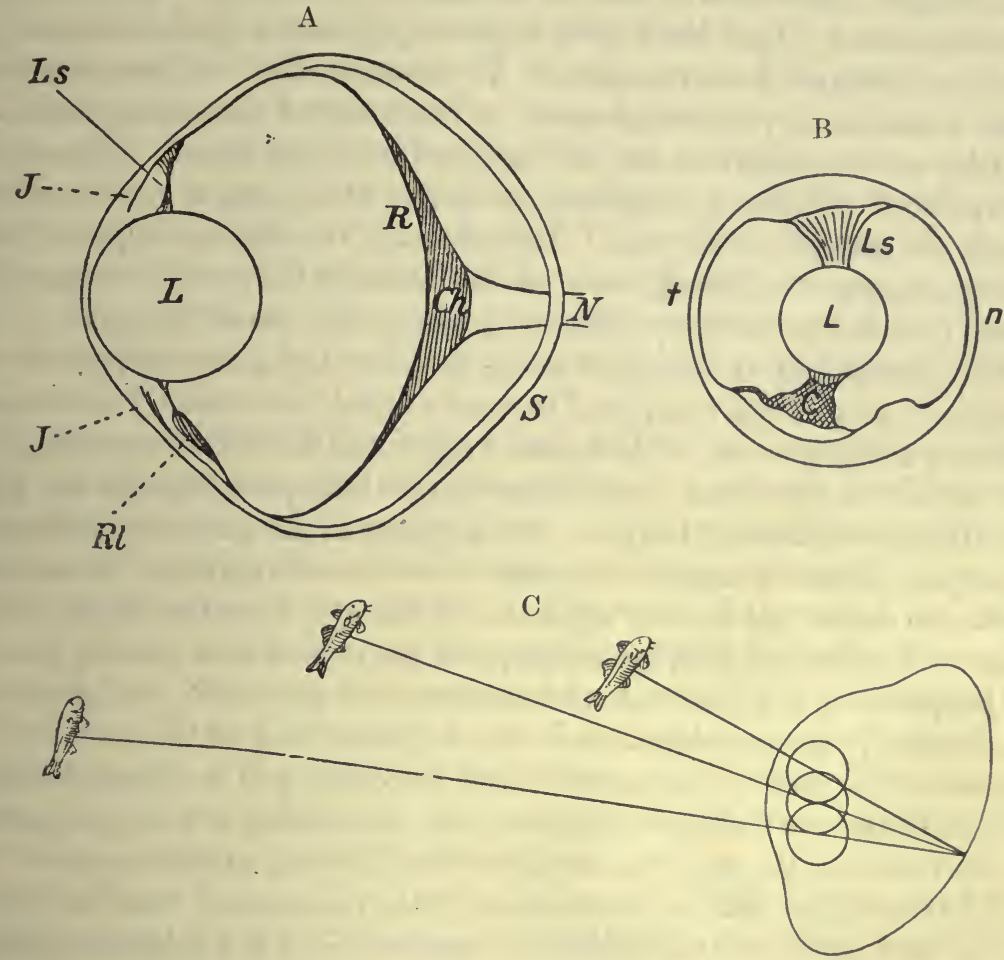

FiG. 2i6. Diagrams from BEER to show mode of accommodation (for distance) in a fish.

A, vertieal section of the eyeball ; B, view of eye from front; $L$, lens ; $L s$, suspensory ligament; $J$, iris ; $R l$, retractor lentis or 'campanula'; C, changes in position of lens when eye is accommodated for an object at varying distances.

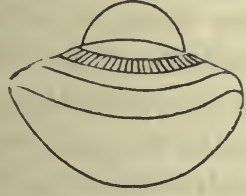

$R$

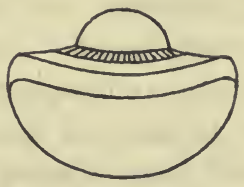

A.

FrG. 277. Accommodation in eye of sepia. (BEER.) $\mathrm{R}$, at rest ; A, during accommodation (for distance).

is the ease in frogs, alligators, vipers, and in many rodents. Many of these animals are distinguished by nocturnal habits, and in daylight their pupils may be constricted to such an extent as to render accommodation unnecessary. In many of them, too, the exact form of an object is not so important as the power to follow its movements. In such cases the movement of the extrinsic ocular muscles or of the head are more important than the exact focusing of the object on the retina. 


\section{THE FUNCTIONS OF THE IRIS}

The iris is the forward prolongation of the pigmented choroid coat. It is covered anteriorly by a layer of epithelium continuous with Descemet's epithelium, and behind by a thick layer of pigmented epithelium which is prolonged forwards from the retina. It is composed of delicate connective tissue, attached at its circumference to the fibres of the ligamentum pectinatum, and contains two sets of unstriated muscular fibres. The one set, the sphincter pupilla, is composed of fibres which run a circular course around the margin of the pupil. The other set, the dilatator pupillac, forms a flattened layer of radiating fibres, which lie close to the posterior surface and extend from the attachment of the iris nearly to the rim of the pupil.

The pigment in the iris and choroid serves the same purpose as the blackened lining, which is supplied to every optical instrument, in preventing reflection and dispersion of the incident light, and therefore preventing any light falling on the retina except those rays which pass through the pupil and refractive surfaces of the eye. The pigment in the iris has an additional importance in that it enables this organ to act as a diaphragm. It not only shields the retina, the sensory apparatus of the eye, from the effects of any excess of illumination, but, by stopping out the rays of light passing through the periphery of the lens, it diminishes spherical aberration and enables a clear image of external objects to be formed on the back of the eyeball. The diameter of the pupil is continually varying, according to the amount of light falling into the eye and the condition of the mechanism of accommodation.

Contraction of the pupil occurs under the following circumstances :

(1) When light falls on the retina. This movement, which is known as ' the light reflex,' is determined by a contraction of the sphincter pupillæ, together with a relaxation of the dilatator muscle. The contraction ensues within a period of 0.04 to $0.05 \mathrm{sec}$. after the moment at which the light has access to the retina, and attains its maximum within $0 \cdot 1$ sec. In man as well as in other animals which have binocular vision, and in which there is a partial decussation of the fibres of the optic nerves in the optic chiasma, the reflex is bilateral, i.e. light falling into one eye causes simultaneous contraction of both pupils. In the higher animals this reaction of the pupil to light demands the integrity of the nervous paths between the eye and the brain ; but in many of the lower animals, e.g. in the frog and eel, the reflex nervous mechanism is aided by a local sensibility of the iris to light. In these animals the contraction of the pupil in response to illumination takes place even in the excised eye, and seems to be determined by a direct stimulation of the pigmented contractile fibres of the sphincter pupillæ by means of the light.

The effect of light on the pupil varies considerably according to the condition of adaptation of the eye. The dilatation of the pupil is maximal when the eye has been in the dark for some time and may amount then to $7 \cdot 3$ to $8 \mathrm{~mm}$. In one experiment, on exposing the eye to a feeble light, e.g. 1.6 candles at a moderate distance, the pupil diminished in size to $6.3 \mathrm{~mm}$. ; 
with an illumination of 50 to 100 candles the size of the pupil was $3.7 \mathrm{~mm}$., and with 500 to 1000 candles, $3.3 \mathrm{~mm}$. This effect was obtained by a rapid change of the illumination of the eye. When the change in illumination is sufficiently slow no alteration of the pupil takes place, and when the illumination, which has at first caused a maximal constriction of the pupil, is continued the pupil gradually relaxes with the adaptation of the retina to light. This relaxation occurs within three or four minutes after exposure to light has taken place. The same influence of adaptation will be observed if two individuals are brought into a moderately lighted room, one from bright daylight and the other from a dark room. The pupils of the first will dilate widely, while those of the second will constrict to their maximum extent. In each case the change will pass off gradually, so that at the end of five or ten minutes there will be no difference observable between the eyes of the two persons.

(2) When vision is directed to a near object the contraction of the ciliary muscle which results in accommodation is accompanied by convergence of the visual axes, brought about by contraction of the two internal rectus muscles. With these two movements is always associated a third, viz. contraction of the sphincter pupillæ, the increased sharpness of the image obtained by this means being an indispensable condition for the fineness of vision which we desire when we examine any object closely. The fact that the amount of light which will fall into the eye from any given object increases inversely as the square of the distance of the object from the eye ensures that sufficient light will pass through the constricted pupil for the appreciation of the finer details of the object.

(3) In sleep the pupils are always contracted. This fact seems at first in opposition to the other conditions regulating the size of the pupil, since during sleep no light is falling into the eye. If the eyelid of a sleeping person be raised the pupil will be found to be constricted; as the person wakes up, in consequence of the interference, the pupil dilates and may then constrict again if the light is held so as to fall into the eye. This behaviour of the pupil may enable us to distinguish feigned from real sleep. The constriction of the pupil is really, like that which accompanies accommodation, an associated condition, and depends on the fact that during sleep the axes of the eyeballs are directed upwards and inwards.

(4) Contraction of the pupil is a marked effect of the action of certain drugs, especially opium and its alkaloid, morphia, as well as of the alkaloids eserine, or physostigmine, and pilocarpine. Contraction of the pupil also occurs in general excitatory conditions of the central nervous system and is therefore found during the stage of induction of chloroform and ether anæsthesia.

Dilatation of the pupil occurs :

(1) On the removal of light stimulus from the eye. If the removal is complete the pupil remains dilated, but if there is any light at all the pupil gradually constricts again as the eye becomes dark-adapted.

(2) Dilatation of the pupil can be reflexly excited by the stimulation of 
many sensory nerves, and is constantly observed as a result of severe pain. The presence or absence of dilated pupils may serve therefore as a means of testing how far an emotional expression of pain is to be credited to a physical cause.

(3) The pupils are often dilated in emotional conditions such as fear.

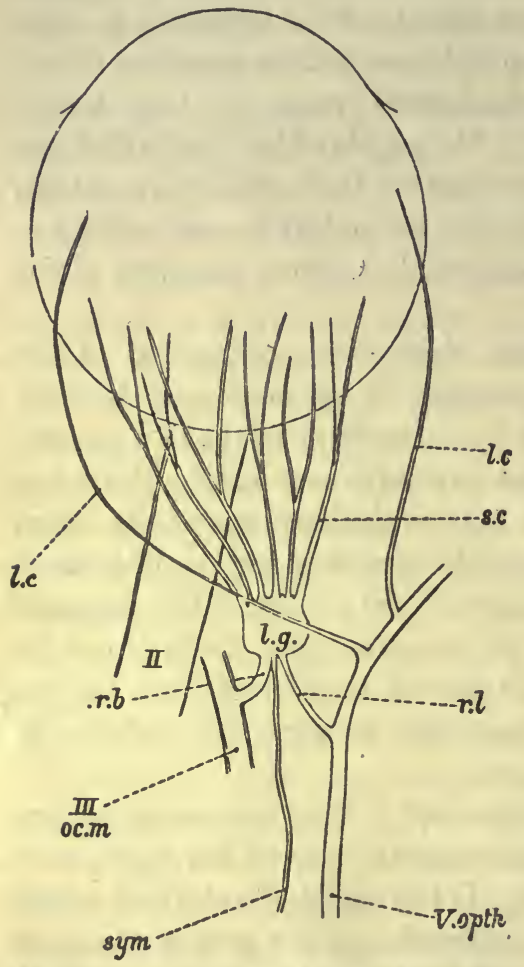

FIG. 278. Nerve-supply to the eyeball. (After Foster.)

l.g, lenticular ganglion with its three roots, viz. : r.b, radix brevis or short root; r.l, radix longus or long root; sym, sympathetic root; $V$. opth. ophthalmic division of $V$ nerve; $I I I$ oc.m, oculo-motor nerve ; II, optic nerve ; l.c, long ciliary nerves; s.c, short ciliary nerves.

through the fibres lying on the internal carotid artery, with the cervical sympathetic nerve.

(3) With the nasal branch of the ophthalmic division of the fifth nerve by means of the 'long root.'

The eyeball is also supplied by the two long ciliary nerves (Fig. 278) which come direct from a branch of the ophthalmic division of the fifth nerve and pass forwards on to the eyeball, piercing the sclerotic coat in front of the point at which this coat is penetrated by the short ciliary nerves. There are thus three nerves by means of which the activity of the 
muscular fibres forming the ciliary muscle, the sphincter, and the dilatator iridis can be influenced, viz. the third nerve, the fifth nerve, and the sympathetic nerve. On exciting the root of the third nerve we obtain :

(a) Constriction of the pupil.

(b) Contraction of the ciliary muscle, i.e. spasm of accommodation.

The same effects are produced by stimulating the lenticular ganglion or the short ciliary nerves.

Excitation of the long ciliary nerves of the ophthalmic division of the fifth nerve, or of the Gasserian ganglion, causes dilatation of the pupil, but is without influence on the ciliary muscle.

Stimulation of the sympathetic in the neck causes maximal dilatation of the pupil accompanied by constriction of the vessels of the iris and the eyeball generally. If the superior cervical ganglion be extirpated so as to cause degeneration of all the sympathetic fibres passing up to the eye, it will be found a fortnight later that stimulation of the Gasserian ganglion has no longer any influence on the size of the pupil. We may therefore come to the following conclusions as to the functions of the nerves supplying the interior of the eyeball :

The third nerve supplies fibres which run through the lenticular ganglion and the short ciliary nerves and cause constriction of the pupil and contraction of the ciliary muscle. These fibres arise in the oculo-motor nucleus, which is situated at the back part of the floor of the third ventricle, immediately below the anterior corpora quadrigemina.

The sympathetic nerve sends fibres which pass to the eye along two routes. A certain number which run on the external carotid artery in the cavernous sinus pass by the sympathetic root to the ganglion and by the short ciliary nerves to the eyeball and cause contraction of the blood-vessels. Other fibres pass from the superior cervical ganglion to the Gasserian ganglion of the fifth nerve, along the nasal branch of its first division and then along the long ciliary nerves to the eyeball. These fibres carry impulses which dilate the pupil. The sympathetic fibres to the eyeball arise in the cord, probably from cells of the lateral column in the lower cervical or uppermost dorsal region. They leave the cord by the first two dorsal anterior roots, pass through the stellate ganglion, the ansa.Vieussenii, and up the cervical sympathetic to the superior cervical ganglion where they terminate. New relays of fibres start in this ganglion and travel direct to their destination in the eyeball. Excitation of the cervical spinal cord easily evokes dilatation of the pupil, and it was on this account that Budge located in this part of the cord a cilio-spinal centre.

The fibres derived from the fifth nerve itself must be looked upon as chiefly afferent or sensory in function. Some observers have ascribed to them a dilatator effect on the blood-vessels of the eye, but confirmation for this view is wanting. The ciliary muscle is normally at rest and is only set into activity as a result of volitional or reflex efforts to direct the gaze to near objects. The iris is under the influence of tonic impulses which arrive at it along both sets of nerve fibres, oculo-motor and sympathetic. Section 
therefore of the sympathetic nerve causes constriction, and section of the third nerve dilatation of the pupil. These tonic influences are probably reflex in origin, since it is found that, after cutting off afferent impressions from the retina by division of the optic nerve, section of the third nerve produces no further dilatation of the pupil.

Since the dilatator muscle is often difficult to demonstrate under the microscope, the view has been put forward that dilatation of the pupil on stimulation of the sympathetic nerve is due merely to the relaxation of the tonic contraction of the sphincter

A

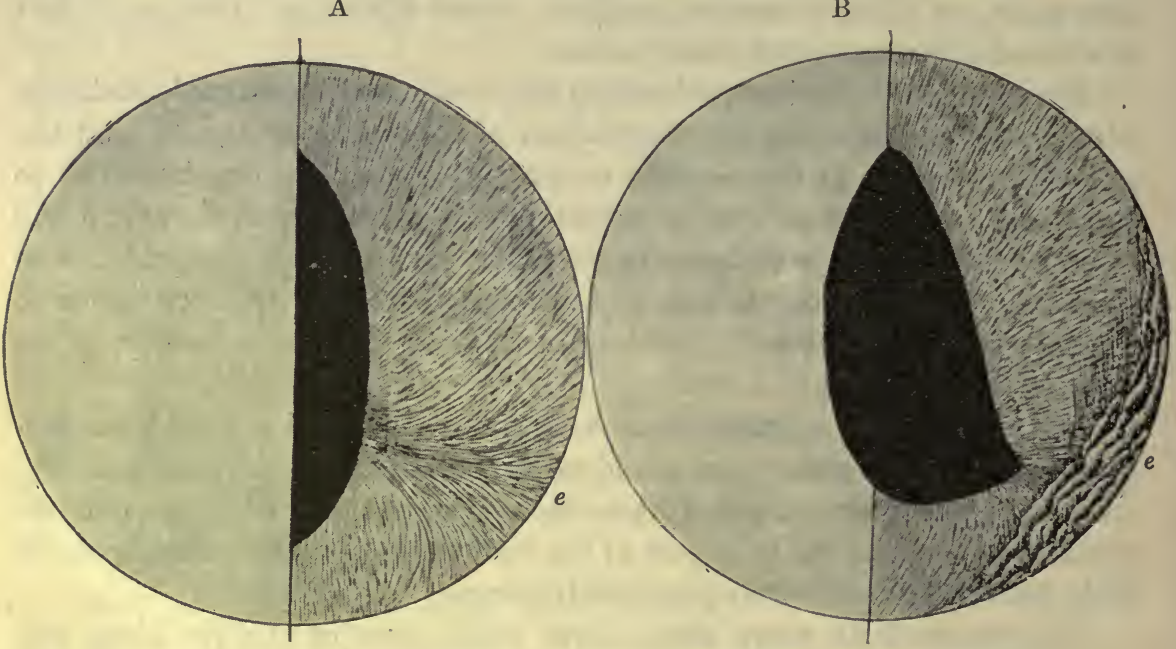

FIG. 279. Effect on iris of cat of local stimulation.

The first effect, as in A, is to cause contraction of the constrictor pupillæ below the electrodes, and this is succeeded in B by a strong localised contraction of the radiating fibres. (LANGLey and ANDERson.)

pupillæ. The following experiments by Langley and Anderson showed definitely the erroneousness of this view :

On stimulating the corneo-sclerotic junction so as to excite a limited portion of the iris, a well-defined local dilatation of the pupil is produced. If the dilatation were due to the relaxation of the sphincter, the dilatation could not be local, but would have extended to the whole circumference of the pupil (Fig. 279). In another experiment they isolated a sector of the iris by two radial cuts ; on exciting this sector it shortened, and the same effect was produced by excitation of the sympathetic in the neck, although any action of the sphincter must have been abolished by the mode of preparation. Section of the sympathetic in the neck causes lasting constriction of the pupil, and the same effect is produced by extirpation of the superior cervical ganglion. After the lapse of some time, however, the muscles, freed from their nervous connections with the ganglion, enter easily into a condition of hypertonus, so that the pupil on the side of the lesion may be more dilated than on the normal side. This hypertonus is especially marked when a slight amount of asphyxia or rise of blood pressure is present.*

\section{THE OPHTHALMOSCOPE}

By means of this instrument we are enabled to obtain a magnified picture of the back of the eyeball, as well as to judge of the presence and degree of abnormalities in the refracting apparatus of the observed eye.

* Probably on account of the escafe of adrenaline into the circulation, and its sympatho-mimetric action on the iris. 
When light falls on the eye through the pupil the greater part of it is absorbed by the pigment of the retina and the choroid coat. A small amount, however, is diffusely reflected, and is sent out by the way it came, viz. through the pupil. If the vision is directed on the luminous point at the source of the rays, the reflected rays leaving the eye will be converged to a point and form an image which will coincide with the source of illumination. It is on this account that the pupil always appears black. When we look at a person's eye, we necessarily interpose our head and eye between the observed eye and the source of light, so that no reflected light can come back to our eyes. Only in albinos, where the pigment of the choroid coat and retina
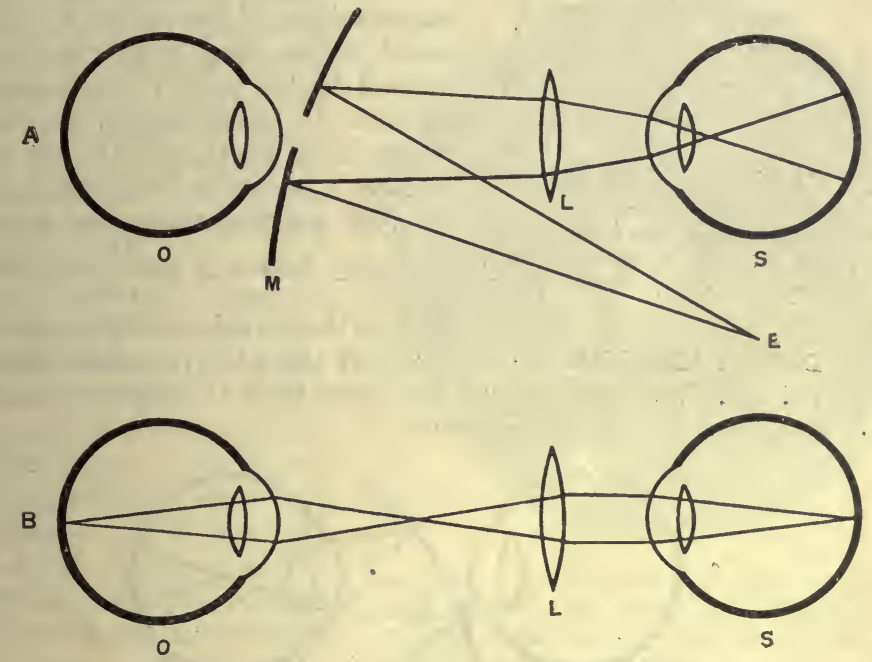

Fig. 280. Indirect ophthalmoscopy.

A, course of rays from source of light $\mathrm{E}$ to observed eye; o, observer's eye; M, mirror; L, lens.

$B$, course of rays from an illuminated spot on the retina of the observed eye to the observer's eye.

is lacking, do we get a red appearance, due to the reflected light passing through the vascular tissues of the choroid and iris.

In a hypermetropic eye at rest only those rays are brought to a focus on the retina which are convergent as they enter the pupil. Light reflected from the retina of such an eye will therefore be divergent as it leaves the pupil, and we may obtain a 'red reflex' by direct observation of the eye.

In order that we may obtain an image of the interior of a normal eye we must arrange that our eye coincides with the source of illumination. For this purpose we use the device invented by Helmholtz, viz. a slightly concave mirror with a hole in the centre. By means of this mirror light is converged on to the pupil, and the light reflected by the retina is brought to a focus at the centre of the mirror, where is placed the observer's eye. This ophthalmoscope may be used in one of two ways :

(a) INDIRECT OPHTHALMOSCOPY. In carrying out ophthalmic observations the examination is much facilitated by instilling atropine into the observed eye, so as to dilate the pupil to the widest extent and paralyse the mechanism of accommodation. If a beam of light be thrown into the pupil, the emergent rays from the eye will be parallel and will give rise to a red reflection seen by the observer's eye at the centre of the ophthalmoscopic mirror. If the eye be myopic, the issuing rays will be convergent and will therefore be brought to a focus at some point in front of the eye, giving rise to a real image of the retina. If the eye be hypermetropic, the issuing rays will be divergent, and the observer will see the red reflection of light from the back of the retina.

If now a lens of low power, say about ten dioptres ( 4 in. focus), be held a few centi- 
metres in front of the observed eye (Fig. 28CB), the reflected rays issuing from the pupil will be brought to a focus at a point between the observer and the lens, so that at this point will be formed a real inverted image of the back of the eyeball. This image, in the case of the normal eye, will lie at the focus of the lens. If the eye be myopic, the convergent rays will be brought to a focus nearer to the lens than its principal focus, while the divergent rays from the hypermetropic eye will give rise to an image in a plane between the principal

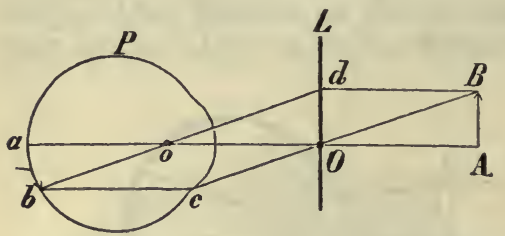

Fig. 281 . To illustrate how the rays from an illuminated point of the retina form a parallel beam on leaving the eye, and are brought to a focus at $B$ by interposing the lens $L$. focus and the observer. From the figure (Fig. 281) it is evident that $\frac{a b}{\mathrm{AB}}=\frac{a o}{\mathrm{AO}}$, i.e. the magnification of the image will be proportional to the focal length of the lens used divided by the posterior focal length of the eyeball. If we are using a bi-convex lens of $10 \mathrm{~cm}$. focal length and the eye be assumed to have a posterior focal length of $1.5 \mathrm{~cm}$., the real inverted image that we see in the bi-convex lens will be $\frac{10}{1 \cdot 5}$, i.e. $6 \cdot 7$ times as large as the retinal structures represented.

(b) THE DIRECT METHOD. In this method the observer places himself close to the observed eye, throwing light into the latter from the mirror, and relaxes by an effort of will his accommodation absolutely.*
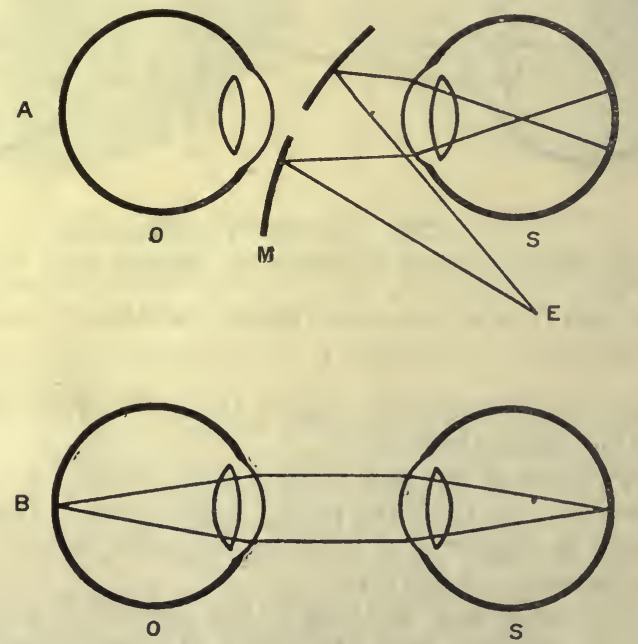

FIG. 282. Path of rays in examination by the direct method.

$\Delta$, path of illuminating rays; $B$, path of rays from illuminated retina to observer's eye.

If both the observer's eye and the observed eye are normal and unaccommodated, i.e. focused for distance, the rays of light, iscuing from any point on the retina of the observed eye, will leave the corneal surface as a beam of parallel rays, which on entering the observing eye will in turn be focused to a point on its retina. The observer therefore sees an erect magnified image of the retina of the observed eye. If we take the

* In the use of the opththalmoscope it is very difficult to relax accommodation when trying to see something which is quite close. The student will find it an advantage to try to imagine that he is looking through a telescope at an object at a considerable distance off. He will then find the picture at the back of the eyeball suddenly come into view. 
focus of the eye as $1.5 \mathrm{~cm}$. the magnification of the image is equivalent to that which would be produced by a lens of the same focus and is equal to $\frac{20}{\mathbf{1 . 5}}$, i.e. about thirteen times.

Since this method gives us a highly magnified image of the back of the eyeball, it is of extreme value in judging of the existence of pathological conditions of the retina or choroid. It is also of value in enabling the oculist to determine by objective methods the existence of any errors of refraction in a patient's eye. On examining the eye by the direct method, if the eye be myopic and the rays leaving it convergent, it will be impossible for the observing eye to bring them to a focus, and it will be necessary to place a concave lens in front of the hole in the ophthalmoscopic mirror in order to bring the back of the observed eyeball into view. The weakest divergentlens through which an image of the observed eye can be obtained will give the degree of myopia of the eya. On the other hand, the rays from a hypermetropic eye, being divergent, will need a certain effort of accommodation to bring them to a focus in the observer's eye, and here the degree of hypermetropia will be given by the focus of the strongest convex lens through which it is just possible to obtain a clear image of the retina and retinal vessels. By the same

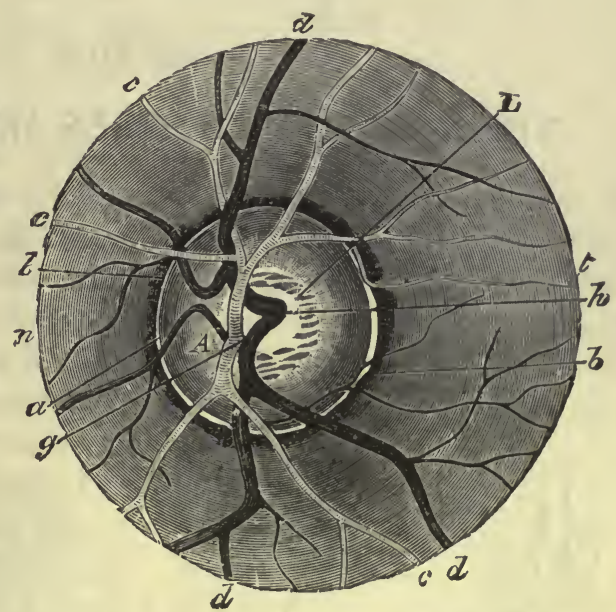

Fig. 283. Ophthalmoscopic view of fundus of eye, showing the optic disc, or point of entry of the optic nerve, with the retinal vessels branching from its centre. means we may judge of the existence of astigmatism and form an idea of the meridians in which the refractive power of the eye is faulty. For this purpose observations are taken of the focus of the eye-firstly, for horizontal retinal vessels; secondly, for vessels which are running vertically.

On examining the back of the eyeball by either of these methods, the most prominent object is the optic disc or optic nerve-papilla, which marks the point of entrance of the optic nerve. It is seen as a pale oval dise surrounded by a deep red background (Fig. 283). From the middle of the papilla the retinal vessels pass into the eyeball, and they are seen diverging from the papilla to ramify over the rest of the retina. The arteries can be distinguished from the veins by their brighter red colour as well as by the stronger reflection of light from their surfaces. The yellow spot is very difficult to see, except in atropinised eyes, since it only comes into view when the observed eye is looking straight into the ophthalmoscope. Under these conditions there is a strong 'light reflex,' and the pupil contracts up to a pin-point, unless paralysed by means of atropine. In order to see the blind-spot, or optic disc, the observed eye must be directed inwards ; thus if $\mathrm{A}$ is looking at the right eye of $\mathrm{B}, \mathrm{B}$ must be told to look over $\mathrm{A}$ 's right s ioulder. 


\section{SECTION VII}

\section{THE RETINAL CHANGES INVOLVED IN VISION}

IN nearly all sense-organs the essential constituent is a bipolar nerve-cell having one process extending towards the surface and ending between epithelium-cells covering that surface, and a central process, which runs towards the central nervous system, where it forms synapses with the processes of other nerve-cells (Fig. 284). In some cases, such as the olfactory cells and the sense-cells embedded in the epidermis of worms and other

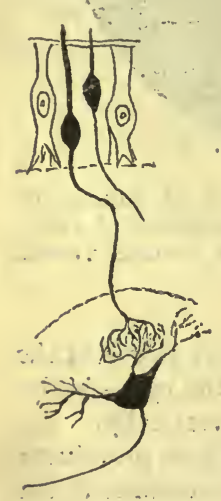

A

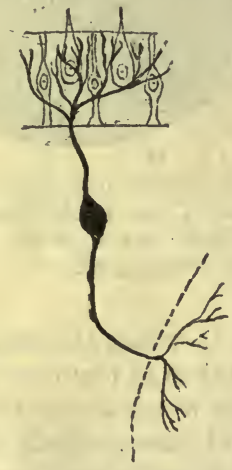

B

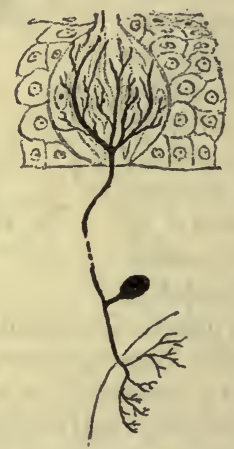

C

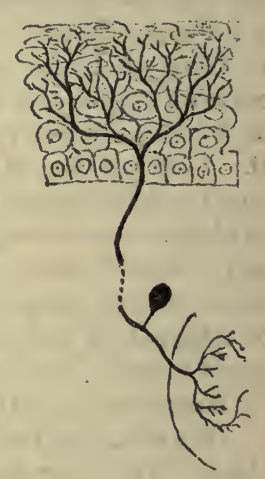

D

FIG. 284.

A, olfactory sense-cell; $\mathbf{B}$, auditory sense-cell; $\mathbf{C}$, connections of gustatory fibres (taste-bud); D, nerve-ending in skin or corneal epithelium (probably pain fibres).

invertebrata, the peripheral process is quite short. In other cases, as in the ordinary posterior root ganglion-cell, the peripheral process may be several feet in length. The retina, however, cannot be regarded as a simple senseorgan, but is homologous with a complete lobe of the brain. It is formed, like the cerebral hemispheres, as a hollowed outgrowth from the fore-brain or anterior cerebral vesicle. The stalk of this outgrowth narrows so as to produce an optic vesicle connected with the rest of the fore-brain by the optic stalk. As the vesicle grows towards the surface its anterior wall is invaginated so that an 'optic cup' is formed, at the mouth of which the lens and other parts of the eye are developed at the expense of the over-lying epiblast and the surrounding mesoblast. The posterior wall of the cup develops into the retinal pigment, while the nervous elements which make up the retina are formed by division and differentiation from the anterior 
wall. That part of the cup originally derived from the external surface of the body is turned towards the posterior layer or retinal pigment. From it is developed the special end-organ of vision, viz. the rod and cone layer of the retina. Besides this special sensory epithelium, the retina presents two other sets of neurons through which impulses generated in the sensory epithelium must pass before they arrive at. the optic nerve. The three relays of nervous elements in the retina have the following arrangement :

(1) The first relay - the sense epithelium-consists of rods and cones with their nuclei (Fig. 285), the latter being situated in the outer nuclear layer. Each rod presents an external $(a)$. and an internal limb (b). The former, in the eye which has been kept in the dark, has a purplish colour from the presence of rhodopsin or visual purple. From the inner end of the inner limb a fine fibre $c$ passes to its nucleus in the outer nuclear layer, and from the nucleus a central process $g$ passes into the outer molecular layer where it ends freely in a little knob $e$. The cones, which are thicker than the rods, also possess outer and inner limbs. From the inner limb a thick process containing a nucleus, passes through the external nuclear layer and ends with a broad base $e$ in the outer molecular layer, from which short fibres are given off to come in contact with the bipolar cells of the inner nuclear layer.

(2) The second relay is formed by the bipolar cells of the inner nuclear layer. Each of these sends off one fibre peripherally to make contact with endings of the rod and cone fibres in

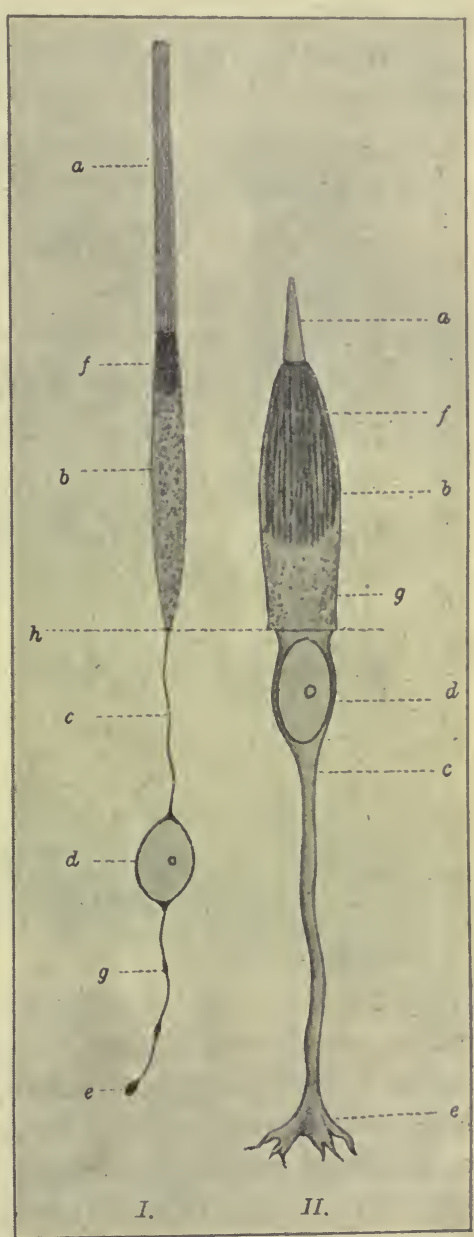

FIG. $2 \& 5$.

I, a rod; II, a cone of mammalian retina; $h$, external limiting membrane. (R. GreefF.) the outer molecular layer, and another process which passes centrally into the inner molecular layer. Here the process of the rod bipolar forms an arborisation around the body of a cell in the ganglion-cell layer, while the processes of the cone bipolars end at various levels in the inner molecular layer, forming synapses with the dendrites of the ganglion-cells.

(3) The ganglion-cells, which represent the third relay, receive the impulses from the more peripheral parts of the retina and send them towards 
the brain along the fibres of the optic nerve, each of which is the axon of one of the ganglion-cells. These axons, which form the inner layer of the retina, the so-called ' nerve-fibre layer,' are non-medullated as they pass over the surface of the retina, but acquire a medullary sheath as they pass out of the eyeball through the cribriform plate of the sclerotic and join to form the optic nerve.

Most of the bipolar cells are connected with several rods or cones; only in the fovea centralis do we find a special bipolar cell provided for every cone.

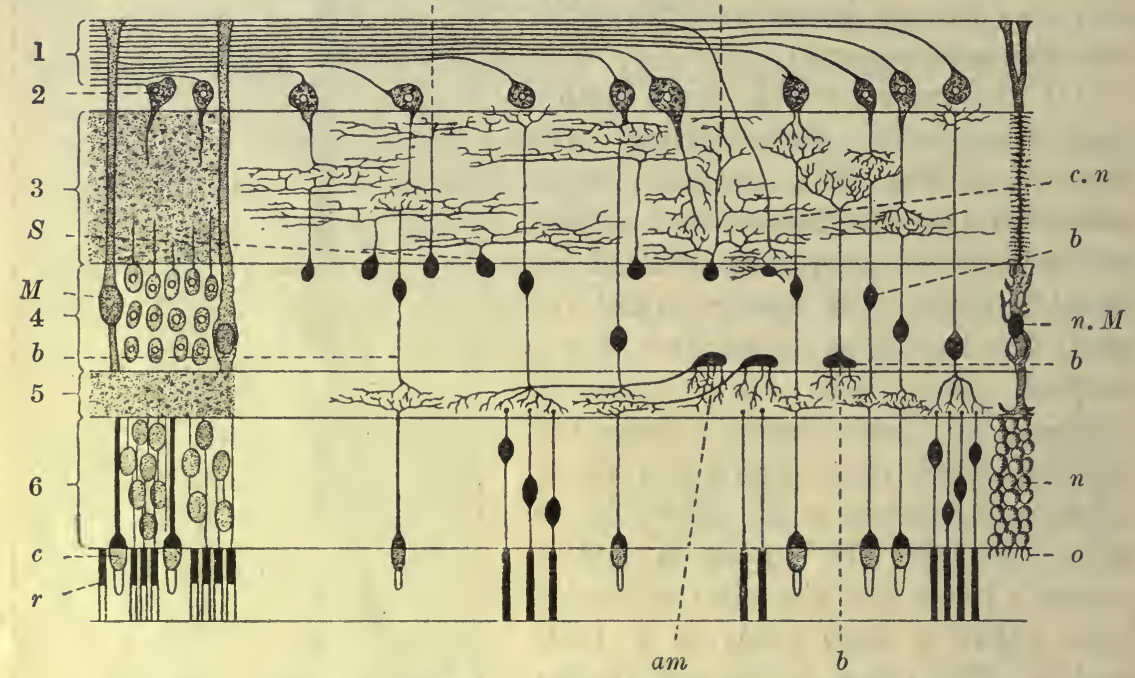

Fı. 286. Schema of retina. (From Bö нт and DAvidoff after $\mathrm{C}_{A} \mathrm{~J} \Lambda \mathrm{L}$. .)

1 , nerve-fibre layer ; 2 , ganglion-cell layer ; 3 , inner molecular layer ; 4 , inner nuclear layer ; 5 , outer molecular layer ; 6 , outer nuclear layer ; $c$, cone ; $r$, rod ; $b$, bipolar cells ; $S$, spongioblast ; am, amacrine cell ; $c . n$, centrifugal nerve fibre ; $M$, fibre of Müller; $n . M$, nucleus of fibre of Müller ; $n$, neuroglia ; $o$, outer limiting membrane.

Every ganglion-cell comes into connection with and receives the impulses from a considerable number of bipolar cells, so that the number of fibres in the optic nerve running centrally is not so great as the number of sense elements in the rod and cone layer of the retina. Besides these cells situated on the direct path of the visual impulse, other cells of a nervous nature are found in the inner nuclear layer (the outer and inner horizontal cells), and also in the inner molecular layer, the so-called 'amacrine' cells. These cells have been imagined to serve as a means of connection or association between different parts of the retina, and may be taken as analogous to the associationcells found in the cerebral cortex. The analogy of the retina with a lobe of the brain is illustrated by the fact that, in addition to the fibres originating in the retina and passing towards the brain, a considerable number of fibres pass from the central nervous system into the retina, where they end chiefly in the two molecular layers. These may have as one of their functions the corre'ation of processes occurring in the retinæ of the two eyes and may be associated with phenomena such as those of binocular contrast, which we shall have to study later on. 
Important differences are found in the structure of the retina in its different parts. At the point of entrance of the optic nerve-the optic disc the only elements present are the nerve fibres, which diverge from this point over the whole inner surface of the retina. A short distance externally to the optic disc is found the macula lutea with a small depression in the middle, the central spot or fovea centralis (Fig. 287). When we fix our gaze on any object the visual axes are so directed that the image of the object falls on the fovea centralis. At this spot the retina is thinned by the gradual disappearance of all its layers except the outermost. The outermost layer is moreover distinguished by the fact that the rods have disappeared and that

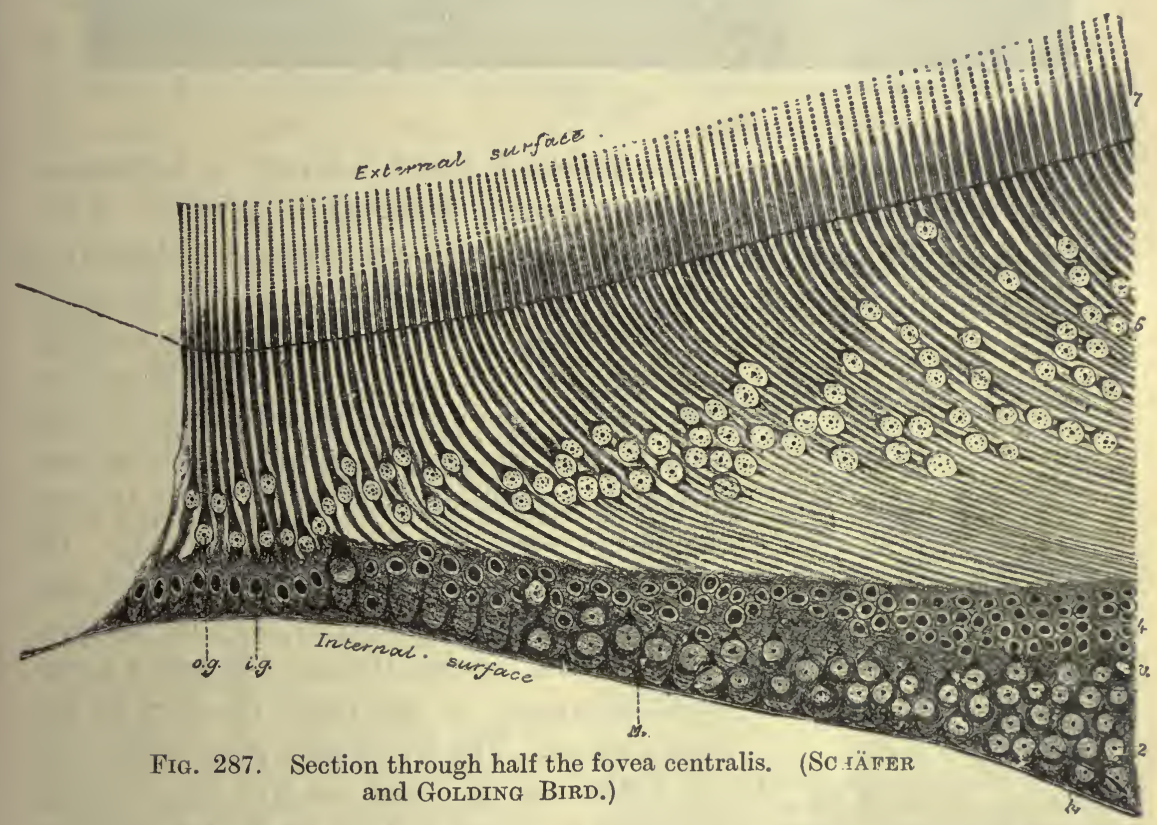

only the cones are present, and are very much larger than the cones in any other part of the retina. The fibres from those cones, passing to the inner nuclear layer, diverge as they leave the fovea centralis, all the layers of the retina being displaced towards the circumference in order to allow the light to fall on the cones without having to pass through any of the other layers of the retina. As we pass from the centre to the periphery of the retina the cones become fewer and the rods more numerous. At the extreme margin the rods also are scattered more diffusely, and at the ora serrata, which lies a short distance behind the ciliary processes, the special nervous elements come to an end, and the retina is continued forwards over the ciliary processes and the posterior surface of the iris as a layer, two cells thick, closely packed with pigment granules (the uvea).

The following facts show that the layer of the rods and cones represents the end-organ of vision, and that for distinct vision to take place the image of an external object must be formed in this layer : 
( $x$ ) The point of entry of the optic nerve, where the whole thickness of the retina is composed of rerve fibres, is absolutely insensitive to light and constitutes the blind-spot (Fig. 288). If the light of a small flame be

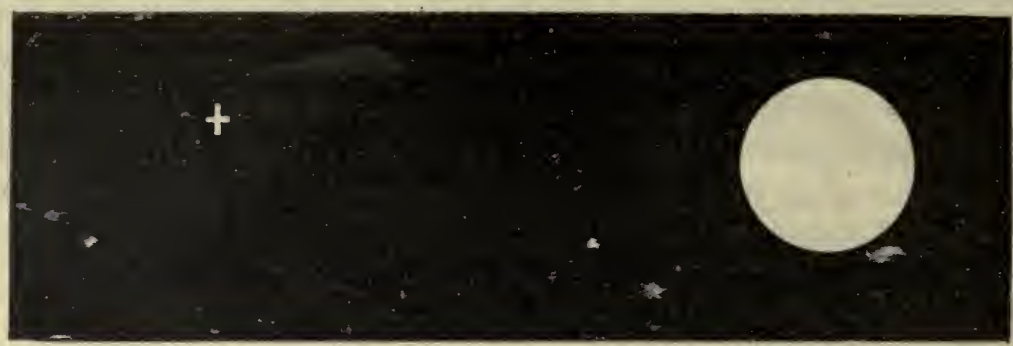

FIG. 288.

directed, by means of a mirror, into the eye so that it falls only on to the optic disc, the individual receives no sensation of light. The existence of the blind-spot is more easily shown by the following experiment: On closing the

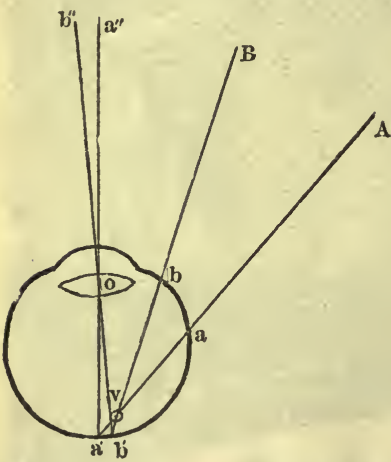

Fic. 2£9. Diagram of the path of the rays of light in the formation of Purkinje's figures.

$v$ represents a retinal vessel. When this is lluminated from $A$, a shadow is formed on the hinder layers of the retina at $a^{\prime}$. This is projected along a line passing through the optic axis, and appears to come from a point $\left(a^{\prime \prime}\right)$ on the wall. On moving the light from $\mathrm{A}$ to $\mathrm{B}$, the image of the vessel appears to move from $a^{\prime \prime}$ to $b^{\prime \prime}$. stare fixedly at a dull background, an arborescent image of the retinal vessels will appear on the background. On moving the illumination the image of the vessels will move in the same direction. Knowing the dimensions of the eyeball and the distance of the background from the eye as well as the angle through which the light is moved and the apparent displacement of the image of the vessels, the distance of the sensory part of the retina behind the vessels may be calculated (Fig. 289). Direct measurements in this way have shown 
that the distance between the vessels and the sensitive elements of the ratina must amount to between 0.17 and $0.36 \mathrm{~mm}$ Anatomical measurements of the thickness of the retina show also that the average distance between the vessels and the layer of rods and cones varies between 0.2 and $0.3 \mathrm{~mm}$., showing that it is in this layer that the actual transformation of a light stimulus into a nerve impulse must take place.

On spreading out the retina under the microscope and looking at its external surface we see that the rods and cones form a sort of mosaic, the thicker cones being surrounded by the smaller circles representing the cross-sections of the rods. Singe each of these is a terminal sense-element the image thrown by the dioptric mechanism of the eye on to the retina must be converted into a mosaic-like expanse of small isolated pictures, and our impression of external objects must be formed by a synthesis of the elementary sensations produced by the stimulation of every single rod or cone cell.

\section{DIRECT AND INDIRECT VISION}

7. If we fix our attention on to an object, we direct our eyes so that the image of the centre of the object falls exactly on the fovea centralis of each ietina. The diameter of the central spot is about 1 to $1.5 \mathrm{~mm}$., which corresponds to a visual angle of $4^{\circ}$ to $6^{\circ}$. This angle therefore represents the extent of the visual field in which we have distinct vision. The light which falls into the eye forms an image of external objects which extends over the whole of the retina. The sensations excited by the stimulation of the periphery of the retina are much more indistinct than those excited by the image on the central spot. The appreciation of external objects, by means of the image they throw on the external parts of the retina, is spoken of as indirect vision in contradistinction to direct vision, which implies fixation of the object and the formation of an image of

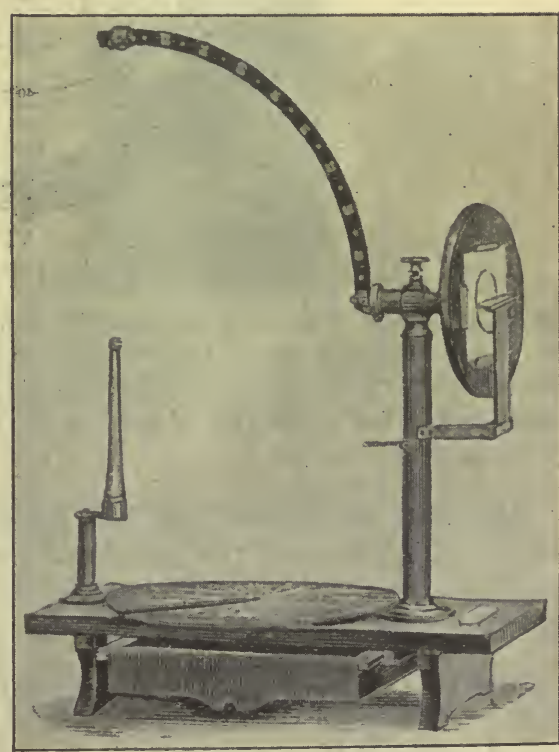

Fig. 29J. Priestley Smith's perimeter. it on the fovea centralis. The whole extent of the objects which we can see by direct and indirect vision is spoken of as the visual field. In order to determine the visual field we make use of a perimeter (Fig. 290).

This instrument consists of a band of metal forming the are of a circle of about $35 \mathrm{~cm}$. radius. At one end this arc is fastened to a pillar, and can be turned through the axis passing through the pillar so as to lie in various meridians. At the centre of the circle is another pillar, which provides a chin-rest, so arranged that the eye 
of the observed person lies exactly at the centre of the circle at the top of the pillar. At the point round which the arc rotates is a small white disc. In using the instrument the person, whose field of vision is to be determined, places his eye at the top of the pillar and gazes fixedly at the white disc; another small white disc is then moved along the curved arc and the point noted at which it is no longer visible, while theobserved person is gazing fixedly at the white disc on the axis of rotation. The are is then moved $20^{\circ}$ and the same experiment carried out, and this is continued until the limit has been determined in every meridian of the visual field. The rotating arc is graduated, the graduations showing the visual angles subtended by any portion of the arc. As

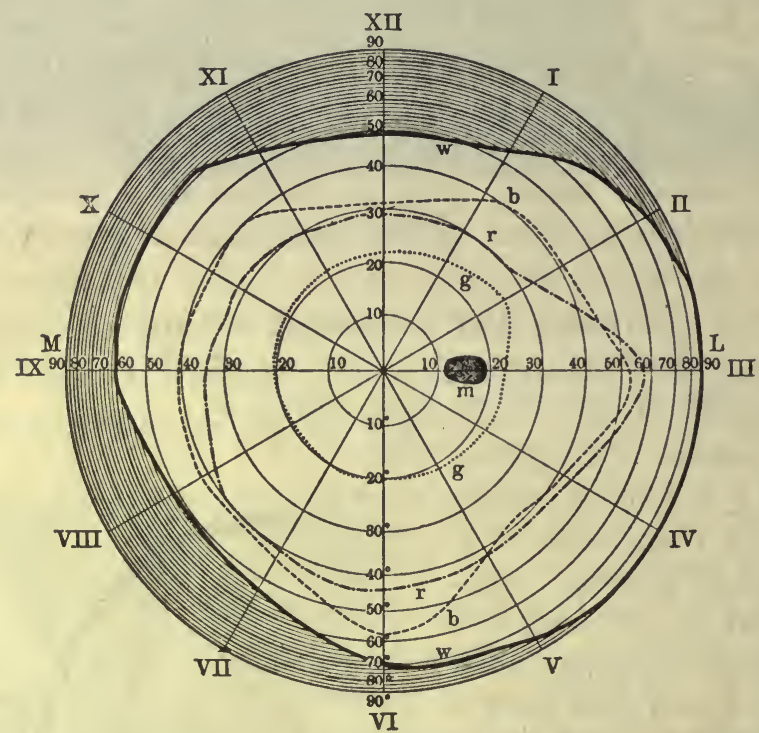

FIG. 291. Perimeter chart showing the field of vision in a normal (right) eye.

the readings are made they are marked down on a chart, such as that shown in Fig. 291 , so that finally a graphic representation of the visual field is obtained. The visual field is more extensive on the outer than on the nasal side of the eye, the latter being contracted by the cutting off of some of the rays falling on the outer side of the retina by means of the nose.

Although stimulation of the peripheral parts of the retina does not give us much idea of the nature of the things we are looking at, yet it is of great importance in informing us of the relation of the object, which is the immediate point of attention, to its surroundings. It therefore plays a great part in regulating the movements of the body. Its importance will be at once appreciated if one eye be closed and the stimulation of the peripheral parts of the retina in the other eye be excluded by allowing this eye only to look through a tube. Although we can then see the objects to which we direct our gaze perfectly distinctly, we find that on trying to move towards any given object our movements are uncertain and misdirected.

\section{CHEMICAL AND PHYSICAL CHANGES IN THE RETINA}

When light falls on the retina chemical and physical changes take place; these either originate or accompany the transmutation of the ether vibrations into the nerve impulses, which ascend the optic nerve. If a frog that has been 
in the dark for some time be killed, an eye taken out, bisected, and the retina removed and examined by a weak light, it will be found to have a purplish-red colour. On microscopical examination this colour is seen to be confined to the outer limbs of the rods. After a very short exposure to diffuse daylight the colour disappears. The colouring-matter (rhodopsin) may be dissolved out by means of a solution of bile salts. The purple-red solution thus formed also bleaches rapidly on exposure to light. By means of this rhodopsin photographs or 'optograms' of external objects may be taken on the retina. The rabbit's eye is cut out and placed in front of a window. After some time the eye is bisected and plunged into a 4 per cent. solution of alum, which partially fixes the optogram, and an inverted picture of the window with its cross-bars is obtained on the retina.

If a retina, which has been bleached by exposure to light, be replaced on the pigment layer lining the choroid, in a short time the colour will be

A

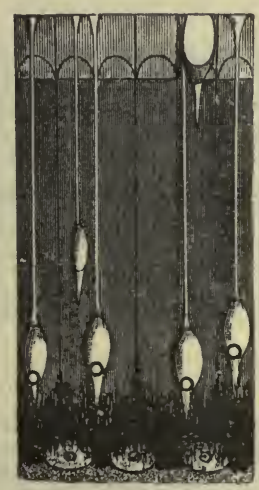

B

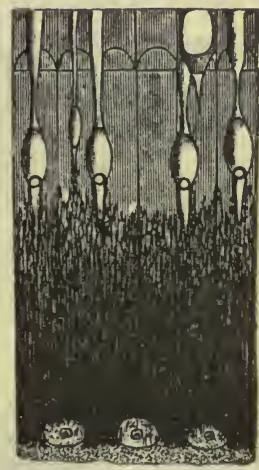

FIG. 292. Sections of the frog's retina.

A, kept in the dark ; B, after exposure to the light, showing retraction of the cones, and protrusion of the pigmented epithelium between the outer limbs of the rods. (Engelmann.)

restored. On examining sections through the retina it is found that, in one which has been exposed to light, the cells of the layer of pigmented epithelium send up fine processes full of pigmented granules between the outer limbs of the rods. In an eye which has been kept in the dark, on the other hand, the cells of the pigment layer are quite flat, so that the front part of the retina, including the rods and cones, can be removed without any difficulty (Fig. 292). Thus the function of the pigmented epithelium is to supply visual purple to the outer limbs of the rods as fast as the pigment already there is bleached by light. It might be thought that this chemical change was the active agent in producing excitation of the optic nerve fibres; but the fact that in the fovea centralis, * the region of most distinct vision, we find only cones which contain no visual purple indicates that this chemical process is not essential for the conversion of light-waves into a nervous impulse.

* According to Edridge Green visual purple diffuses into the fovea centralis, and p'sys an essential part in vision as a sensitiser of the cones. 
When light falls upon the retina the cones are retracted, and lie close upon the external limiting membrane; whereas in an eye that has been kept in the dark they extend down between the rods as far as the pigmented layer.

I.

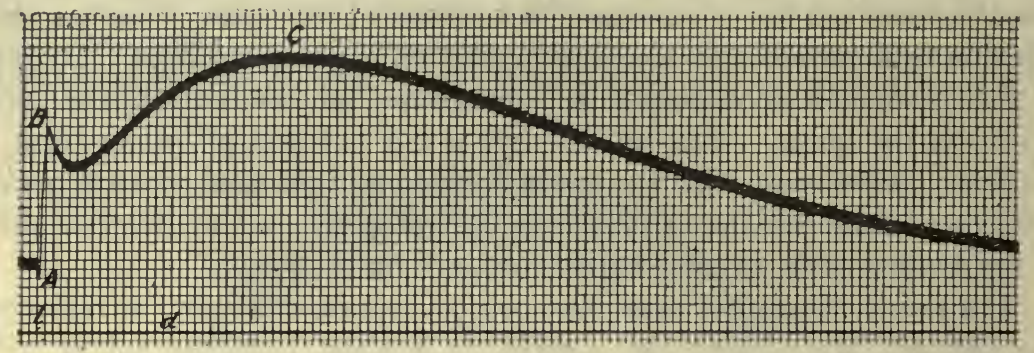

II.

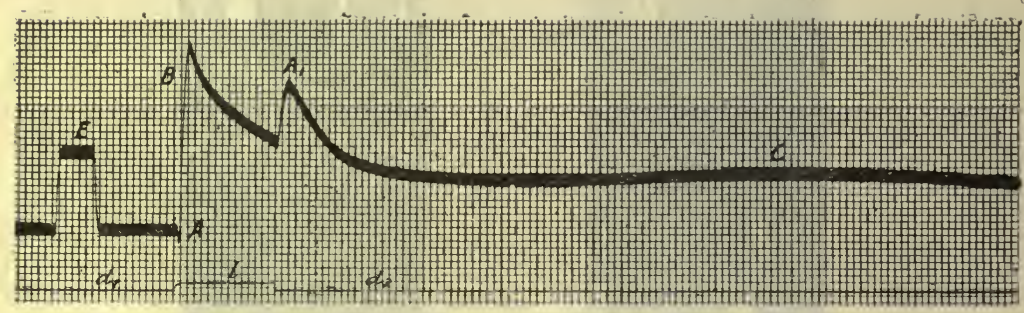

III.

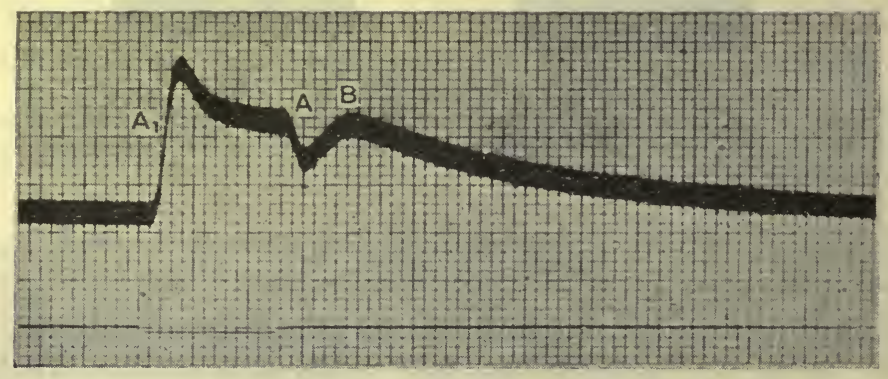

FIG. 293. Electrical variation in frog's eye as recorded by the string galvarometer. (EINTHOVEN and Jolly.)

I, on exposure to a single flash; II, on exposure to light of moderate duration; III, effect on a light eye of momentary darkening.

The falling of light on the retina is also accompanied by an electrical change, which may be regarded as analogous to the current of action in nerve. It is, however, much more complicated than the latter. The eyeball of the frog led off from its anterior and posterior surfaces shows a current directed in the eyeball from behind forwards (the resting or demarcation current). It was first shown by Holmgren that this resting current undergoes modification when light is allowed to fall on the eyeball. Of late years the nature of this modification has been studied especially by Waller with the galvanometer, by Gotch with the capillary electrometer, and by Einthoven and Jolly with the string galvanometer. The nature of the response varies according to the 
strength and duration of the stimulus, and to the conditicn of the eye, whether fatigued or fresh, light- or dark-adapted. A typical response to a momentary flash is shown in Fig. 293, I. Within a very short latent period after the incidence of the flash, i.e. after a latent period of not more than .01 sec., there is a small short negative variation of the resting current, which is immediately followed by a large positive variation, i.e. the resting current is increased. This is followed immediately by a diminution and

I.

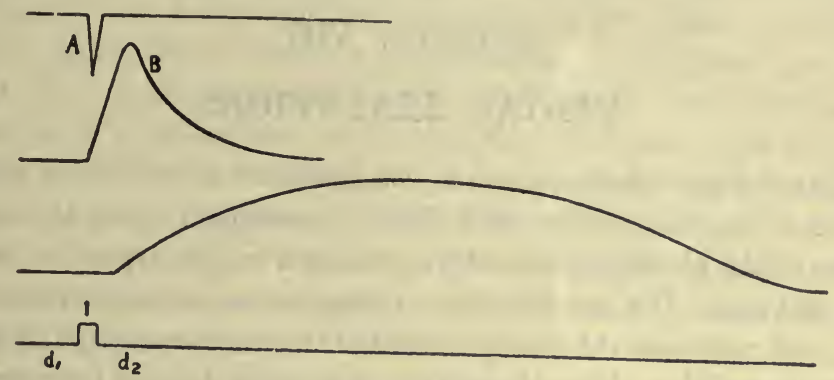

II.

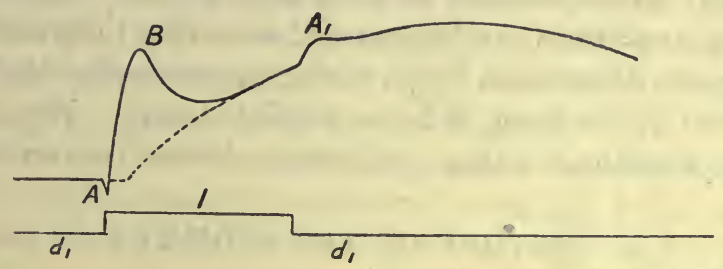

Fig. 294. Diagrammatic representation of the reactions to light of the three hypothetical substances $\mathbf{A}, \mathbf{B}$, and $\mathbf{C}$. (EINTHoven and Jolly.)

I, the three effects shown separately; II, the three effects combined to form a single curve. $A$ is the lighting and $\mathbf{A}^{\prime}$ the darkening effect of the first substance. $l$, light ; $d$, darkness.

then, after a considerable latent period, by a second slow prolonged increase of the current. When the duration of the stimulus is longer the moment of shutting off the light is seen to be followed immediately by a second positive variation. This is shown in Fig. 293, II. It is possible to obtain this response to darkness by shutting off for a short period of time the light falling into the eye. The result of such an experiment is shown in Fig. 293, III. Einthoven and Jolly explain these results by the assumption that three separate processes are concerned when the retina is stimulated. For convenience they speak of the changes in three distinct substances, $\mathbf{A}, \mathbf{B}$, and $\mathbf{c}$. Of these A reacts more rapidly than the other two, and its action is specially marked in a 'light' eye, appearing almost isolated on sudden darkening of short duration (a flash of darkness). On lighting, it develops a negative, on darkening a positive potential difference. The substance $\mathrm{B}$ reacts less rapidly than A. On lighting, it develops a positive, on darkening a negative potential difference. Its action is especially marked in a dark eye which is illuminated for a short time with a weak light. Substance $\mathbf{C}$ reacts in the same sense as $\mathbf{B}$, but much more slowly. Its action is wanting in a completely light eye. The actions of these three substances are represented diagrammatically in Fig. 294, I. and $I$.

It is interesting to note that the latent period of the photo-electric reaction agrees with the latent period of the light perception of the human eye, and may vary from $.01 \mathrm{sec}$. with strong stimuli to as much as 2 sec. with very weak stimuli. 


\section{SECTION VIII}

\section{VISUAL SENSATIONS}

THE retinal changes which we have just described as occurring in the retina on exposure to light give us very little information as to the nature and conditions of the physiological activity excited in this organ by the physical stimulus of light. We are therefore driven to use as our criterion of these physiological processes the changes excited in consciousness, and the greater part of our knowledge of the physiology of vision is derived from examination of such of our own sensations as have their primary origin in the retina. How far these sensations can be regarded as having their seat in the retina, how far they are determined by physiological changes in the visual and adjacent portions of the brain, it is not possible to say. We are only able to deal with the sensations as they spring ready formed into our consciousness.

\section{NATURE OF THE STIMULUS}

The word ' light,' as employed by physicists, implies a particular kind of energy, which, arriving at the retina in a certain way, excites in us a sensation of light. The conception is therefore primarily physiological. Every material substance is endowed with a certain amount of internal energy, the index to which is its temperature. In virtue of this energy it is constantly radiating energy at a greater or less rate through the surrounding ether, its internal energy at any given moment being determined by the balance between the amount of energy it gives off and the amount of energy which it receives from surrounding bodies. This radiant energy is transmitted through space as transverse oscillations of the ether at the rate of about two hundred thousand miles per second and with very variable wave-length and rate of oscillation. The whole energy available to us on the surface of the earth is derived from that portion of the radiant energy of the sun which is intercepted by the earth.

Since the velocities of transmission of rays of various wave-lengths differ as these rays pass through a dense medium, such as glass, it is possible to break up the compound waves of radiant energy arriving at us from the sun, or emitted by any hot body, by allowing them to pass through a prism. When the luminous solar rays are passed in this way through a prism we get, as is known, a spectrum, the rays which are refracted the least being red, while those which are most refracted are violet. Between these two extremes we have rays of the following colours-orange, yellow, green, blue, indigo, which merge one into the other without any perceptible break. The 
different parts of the visible spectrum, when obtained from the sun, show vertical dark lines, which are known as Fraunhofer's lines. These are due to the fact that certain rays emitted by the glowing centre of the sun are absorbed in passing through the gaseous envelope which surrounds the sun. The lines are distinguished by certain letters and have all been assigned to the existence of known elements in a gaseous form in the solar envelope. Any part of the spectrum is distinguished according to its relation to these lines, since each of them has a constant wave-length. The visible spectrum extends from the line $\mathrm{A}$ at the limit of the red, which has a wave-length of 760 millionths of a millimetre, to the line $\mathrm{H}$ at the end of the violet with a wave-length of 397. The visible part of the spectrum does not, however, include even the majority of the rays which arrive at the earth from the sun. Beyond the red we get the ultra-red rays, which have a large amount of energy, so that their presence can be easily detected by their warming effect on blackened bodies, such as the blackened bulb of a thermometer or a thermo-junction, held in this part of the spectrum. In the same way, beyond the violet end there is a long extent of rays with high refrangibility and small wave-length. Though not perceptible to the eye, they reveal their existence by the marked influence they exert on salts of silver; they are therefore often spoken of as the actinic or photographic rays.

If the investigation of the constituent rays of the solar spectrum were carried out at a considerable altitude above the sea and by means of quartz prisms and lenses, the extent of the invisible spectrum would be found to be largely increased, since the ultra-red and ultra-violet rays are absorbed by the constituents, especially the aqueous vapour, of the atmosphere. Why can we not see these rays as well as those in the middle of the spectrum? Is it that they are absorbed by the media of the eye, through which the ray of light has to travel before it reaches the retina, or is their invisibility due to an actual insensibility of the retina to rays of high and low wave-lengths ? On testing the absorption of these rays by the transparent media of the eye, we find that the absorption of the ultra-red rays is but slight and that a considerable proportion of these rays must be always arriving at the retina, so that their invisibility must be determined by the fact that they are unable to excite the special sensory elements of the retina. On the other hand, the absorption of the ultra-violet rays by the eye media is practically complete, although these rays on arriving at the retina have the power of evoking sensation. Thus it has been observed that after extraction of the lens for cataract the visibility of the spectrum, which in the normal eye only extends to the line $\mathrm{H}$ with a wave length of 397 , is increased on the violet side so that the spectrum may be seen as far as a point corresponding to a wave length of 313 . It is evident from this that in the absorption of the ultra-violet rays by the eye the lens takes a preponderating part.

\section{LUMINOSITY OF DIFFERENT PARTS OF THE SPECTRUM}

The physiological nature of our conception of light is shown by the fact that the spectrum differs in its luminosity, i.e. in its total stimulating effect 
on the retina in its different parts, and that the relative luminosities of different parts of the spectrum bear no relation to the amount of radiant energy represented by each kind of wave length. The greatest energy is attained by the ultra-red rays and there is a gradual diminution from here to the violet end of the spectrum. The yellow part of the spectrum, however, appears much brighter than any other part. The relative luminosity of different parts is shown in the following Table by Vierordt:

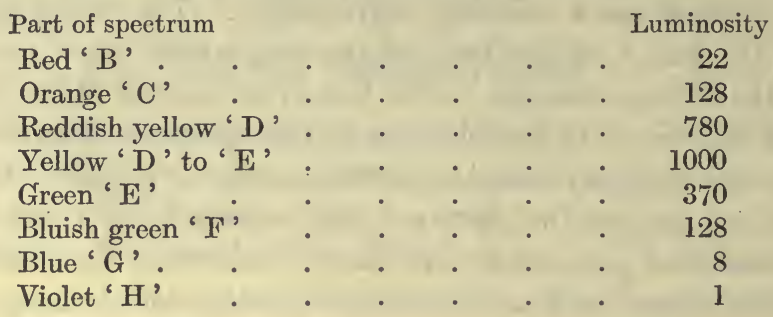

The limited excitability of the retina and its special sensitivity to rays in the middle of the spectrum present considerable advantages for the normal functioning of the optical apparatus. No provision is made for securing achromatism. The dispersion of the ultra-red and ultra-violet rays is so great in passing through the refracting surfaces of the eyeball that, if they all arrived at the retina, and this organ were sensitive to both kinds of rays, it would be impossible to obtain any clear image of external objects. The image formed by the ultra-red rays would be far behind the retina when the ultra-violet rays were focused on the retina and vice versa. As it is, the retina is unstimulated by the two ends of the spectrum, and its stimulation by the red as well as by the blue rays is only minimal, so that, for the excitation of a mosaic of spots on the retina in spatial extension and arrangement corresponding to that of the objects from which the light reaches the retina, practically only the middle part of the spectrum is of importance, and the distance of the image formed by the reddish-yellow rays from that formed by the green rays will not be great enough to cause any appreciable distortion of the exciting image.

\section{THE RELATION OF THE INTENSITY OF SENSATION TO THE STRENGTH OF STIMULUS}

Weber's law, viz. that the increase of stimulus necessary to give an increase of sensation always bears the same ratio to the whole stimulus, holds good for visual sensations. This ratio in the case of white light is about $\frac{1}{100}$. We can thus distinguish between two lights of 20 and $20 \frac{1}{5}$ candle-power, both of them at the same distance from the eye, or between two of 99 and 100 candle-power. If the illumination be excessive the law no longer holds good, and we should be unable to tell the difference between two lights of the latter power if held close to the eye, or between two arc lamps at a considerable distance, even though one might be much stronger than the other. According to some authors our power of distinguishing differences in luminosity varies with different colours. 


\section{TIME RELATIONS OF THE EXCITATORY PROCESS}

When a stimulus of short duration, such as an induction shock, is applied to a muscle, the response of the latter bears no likeness in its intensity and its time-relations to the exciting stimulus. The muscle after a short latent period begins to contract when the stimulus has already ceased to act. It contracts rapidly at first, then more slowly, and then relaxes. In the same way the sensation evoked by a momentary light stimulus takes a certain time to attain its maximum and then dies away slowly, persisting, that is to say, during a certain interval after the stimulus has been entirely withdrawn. This persistence of the visual sensation is experienced whenever we look at a brilliant source of light, such as a candle or lamp, and then either shut the eyes or direct the gaze on to a blackened surface. We then see on the dark background a bright image of the candle or lamp, which gradually fades This phenomenon is often spoken of as a 'positive after-image.' If the object has been very bright the image in fading becomes coloured. It first appears greenish blue, which changes later to violet, rose colour, and finally orange or green.

The slow rise and fall of the sensation evoked by a momentary stimulus, or by a change in the intensity of light falling on the retina, are responsible for the blurred outline of any object that is regarded while in rapid motion. If a disc with alternate sectors of black and white, such as is shown in Fig. 295, be caused to rotate slowly, it is easy to distinguish both black and white sectors. As the speed of rotation increases the margins of the sectors become blurred, and the fact that both margins of each white sector are blurred shows that the sensations produced both by the application and by the shutting off of the stimulus, and due to the white light reflected from the surface, are gradual and not instantaneous. With further

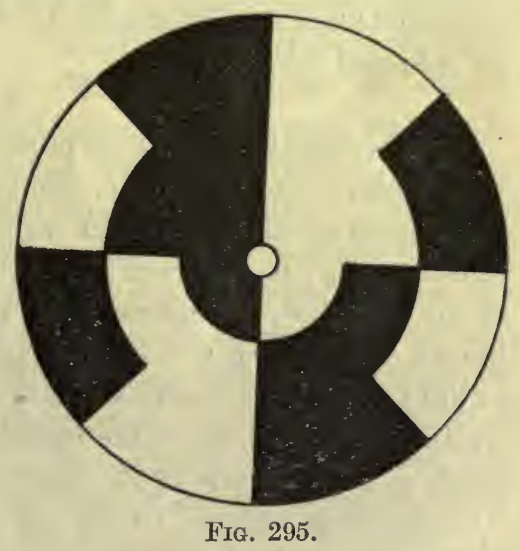
increase in rate the whole disc takes a grey colour, which, however, oscillates or 'flickers,' and with a still further increase the 'flicker' disappears and the disc has a uniform grey appearance. The phenomenon is analogous to the phenomenon observed in muscle as the result of intermittent excitation. Each single shock gives rise to a contraction which is more prolonged than the shock itself. When the shocks are repeated we obtain, according to their frequency, a series of single contractions, a partial fusion of contractions, so that an imperfect tetanus is produced ("flicker '), or finally-with a certain rate of interruption of the stimulus-complete fusion of contraction, i.e. complete tetanus.

We see therefore that when a portion of the retina is excited for a certain 
period by rays of a given intensity during a period $\mathrm{A}$, and is then unilluminated during a period $\mathrm{B}$, if $\mathrm{A}+\mathrm{B}$ is sufficiently small, e.g. in the case of the disc if the rotation is sufficiently rapid, the sensation evoked is a continuous one and is equal to that which would be produced by a continuous stimulation which is equal to $\frac{A}{A+B}$. This fact is spoken of as Talbot's law. It enables us to produce a grey of any desired intensity by varying the relative sizes of black and white sectors on a rotating disc. For complete fusion to take place the period $\mathrm{A}+\mathrm{B}$ need not be less than 04 sec. if we are using light of moderate strength. With low intensity of illumination this value rises, i.e. complete fusion is obtained with a lower rate of rotation of disc than when we are using bright illumination. The value also varies according to the colour of the light. Different colours require different times for their action on the retina in order to produce the maximum sensation. If a spectrum be exposed to the eye for a very short period of time it appears colourless and shortened at the red end. If the period of exposure be increased the red and blue ends are seen, but no other colour is perceptible. The sensations due to the incidence of red rays attain their maximum in the shortest time, then come blue rays, while the green take the longest time to attain their maximum. This difference between the time-relations and the sensations evoked by the various rays accounts for the fact that on rotating a disc of alternate white and black sectors at a certain rate the sectors appear blurred and bounded by coloured fringes.

\section{FATIGUE}

If a constant stimulus be prolonged, the intensity of the resulting sensation rapidly diminishes, i.e. the apparatus concerned in the production of the sensation shows signs of fatigue. This diminution in the intensity of sensation may be observed so early as one-fifth of a second after the beginning of the stimulus. Connected with this fatigue of the retina is the phenomena known as the 'negative after-image.' If we look at a bright spot or source of light for some seconds and then turn our gaze to a uniformly illuminated white surface, we see in the middle of the white surface, i.e. at the point of fixation, a dark image of the bright object which we had previously looked at. The stimulus applied to all parts of the retina in this case is uniform. Certain elements, however, i.e. those which had been previously stimulated by the bright object, are fatigued. Their response is therefore less than that of the surrounding untired retinal elements, and the resulting sensation is a dark image, which is referred to that part of the white surface from which proceeds the light falling on the fatigued elements of the retina.

\section{AD APT ATION}

It is common experience that our eyes have the power of adapting their sensitiveness according to the degree of illumination. When we pass from daylight into a dark room, such as the developing chamber of the photographer, it is at first impossible to distinguish any objects even by the dim red light coming from the photographer's lamp or the window. After a short 
time, however, we begin to distinguish objects more clearly. Any slight defects in the dark chamber begin to make themselves apparent, such as entry of light under the door and through cracks or nail-holes in the ceiling or walls. By direct measurement it can be shown that within ten minutes after passing from daylight into complete darkness the sensitiveness of the retina increases twenty-five-fold, and after two hours' exposure to complete darkness thirty-five-fold. On the other hand, on coming out of the dark room we are at first dazzled by the flood of light with which we appear to be surrounded; the pupils constrict to their utmost; and accurate vision is impossible on account of the excess of light which seems to pour into our eyes. Very shortly this condition passes off, and within five minutes vision is once more normal, and the ordinary size of the pupils reestablished.

The process of adaptation affects not only the quantitative relation between the intensity of the stimulus and the resulting sensation, but determines also a qualitative alteration in the reaction of the retina to light. This is especially marked in the case of colours. On going into a flower-garden on a summer morning, when dawn is just beginning, although all objects in the garden can be clearly distinguished, there is a striking difference in its colour-tone as compared with that which it presents in daylight. The scarlet geraniums have disappeared. On close examination this disappearance is found to be due to the fact that the flowers are dark, i.e. the light from them does not stimulate the retina at all. The other coloured flowers can be distinguished, but only in shades of grey. With a very little increase in illumination the blue flowers come into evidence and the prevailing tone of the garden is cold, made up as it is of greens, blues, and greys. With increasing illumination the reds finally make their appearance. After long exposure to the darkness of night the eyes have become dark-adapted. The same behaviour of the dark-adapted eye may be demonstrated in the laboratory. If a person who has been in a dark room for half an hour observes a spectrum of low intensity the whole spectrum appears colourless, its red end being cut off. The distribution of luminosity over the spectrum is also altered. Whereas the spectrum to the normal light-adapted eye appears brightest in the yellow between the lines $\mathrm{D}$ and $\mathrm{E}$, the spectrum of low intensity to the dark-adapted eye has its point of greatest luminosity in the green.

This striking difference between the light-adapted and the dark-adapted eye does not apply to small objects the image of which, when the vision is directed towards them, will fall entirely on the fovea centralis of the retina. In the dark-adapted eye, the sensitiveness of the central spot of the retina is not nearly so great as that of the more peripheral portion. On a dark night we are often able to distinguish a star which, however, disappears as soon as we turn our eyes so as to bring its image on the central spot of the two retinæ. Moreover the qualitative change in relation to the colours observed in the dark-adapted eye does not apply to the fovea centralis. In a dark room a small spot of light, whatever its colour, when the visual 
axes are directed on it, is seen in its true colour as soon as its intensity is sufficient for it to be seen at all. Before this takes place it may be seen by the peripheral parts of the retina, but as a colourless spot of light which disappears as soon as the gaze is directed on it. This marked difference between the behaviour of the fovea centralis and the more peripheral parts of the retina in the dark-adapted eye has been attributed to the difference we have already studied in the anatomical structure of these parts. The only percipient elements found in the fovea centralis are the cones. In the adjacent portion of the retina we find also the rods which increase in relative number as we pass from the central spot towards the periphery. Schultz long ago pointed out that in the retinæ of many night animals, such as the owl, the mouse, the cat, the rods are the predominating element, the cones being absent or very few in number. Von Kries has suggested that in all probability the retina is endowed with two kinds of vision.

(a) Vision by means of rods, which are colour-blind, so that on stimulation by any rays of the retina a sensation of white or grey is produced. The rods are chiefly excited by the more refrangible rays of the spectrum, being totally unaffected by the red rays. They show great power of adaptation. This form of rod vision may be connected with the visual purple. In the dark-adapted eye this pigment is found pervading the whole of the outer limbs of the rods ; it rapidly fades on exposure of the eye to light, so that it must be absent in the light-adapted eye. On examining its absorption spectrum we find that its absorptive power is greatest for the rays in the green part of the spectrum, and that it allows the red rays to pass almost without absorption, i.e. it absorbs just those rays which experiment shows us have the greatest effect in producing a sensation of light in the dark-adapted eye.

(b) The cones, on this view, would represent a more highly differentiated apparatus of vision. They alone are present in that part of the retina which we use exclusively for distinguishing the finer details of surrounding objects; they are sensitive to all colours, and when stimulated by all the rays of the spectrum simultaneously give rise to a sensation of white light. Their sensitiveness to illumination is, however, inferior to that of the rod apparatus. According to this theory, therefore, whereas in a dim light we determine the position of surrounding objects and differences in their luminosity by means of the rods, the greater part of our visual impressions, including all that we obtain by daylight and our knowledge of the finer visual qualities of things, are brought to us by the intermediation of the cones.

\section{COLOUR VISION}

If a ray of white light be passed through a prism it is widened out into a bright coloured band or spectrum, the red rays, which are least refrangible, being at one end, and the blue rays at the other. It is usual to divide the colours of the spectrum into seven-red, orange, yellow, green, blue, indigo, violet; but the division is an arbitrary one, and the colours shade into one another so gradually that no two observers would agree exactly on the limits between them. The difference of wave-length necessary to give a dis- 
tinct difference in colour varies according to the part of the spectrum which is under observation. In the middle of the spectrum it is between $0.7 \mu \mu$ and $2.0 \mu \mu$. At the red end a difference of $4.7 \mu \mu$ is required to evoke a new quality of sensation, and at the extreme end, both red and violet, there is a section of the spectrum over which no variation in colour can be perceived. By passing the rays forming a spectrum through a similar prism placed in the reverse direction, all these rays can be recomposed to form white light. The sensation of white light is therefore due to the simultaneous incidence on the retina of all the rays of the spectrum. That we have no suspicion of the existence of these rays when we experience a sensation of white shows that our eye does not possess any resolving or analysing apparatus such as exists in the internal ear for the compound wave of sound.

Any part of the spectrum, or any coloured object, may be characterised in three different ways :

(1) LUMINOSITY. The luminosity of different parts of the spectrum varies, being greatest in the yellow for the light-adapted eye. We could, however, match the luminosity of the red of one spectrum with that of the yellow of a second spectrum by increasing the intensity of the beam of light used to produce the first spectrum.

(2) SHADE OR COLOUR. It has been reckoned by König that in the spectrum we can distinguish 165 different shades of colour. Edridge-Green has shown that when a normal observer screens off the rest of the spectrum until the part left appears monochromatic, and repeats this operation through the whole length of the spectrum, he will mark off not more than eighteen to twenty-seven 'monochromatic patches.' There are certain colours which can be appreciated by the eye which are not present in the spectrum, such as the varying shades of purple.

(3) SATURATION. When we look at a coloured surface, e.g. red, our eye is stimulated partly by the white light which is reflected in toto from the surface, partly by the red rays which are specifically reflected and give the colour of the object. According as these red rays are free from mixture with white rays, so their saturation is said to increase. The degree of saturation of any colour can be determined by regarding it through a spectroscope. A completely saturated red would give only rays at the red end of the spectrum. We can, however, speak not only of a physical but of a physiological saturation. According to the condition of the retina and nature of the stimuli to which it has been previously exposed, so does the saturation of any given colour vary.

It might at first be thought that the retina could respond with a simple sensation to a stimulus by any part of the spectrum, a low number of ether vibrations per second producing a sensation of red, a number rather higher a sensation of orange ; so that there might be as many simple colour-sensations as we can appreciate different shades in the spectrum. But a simple analysis of our own sensations seems to show that some of the spectral colours, although simple in so far as the stimuli are concerned, are compound so far as the sensation is concerned. Thus most people would say at once that orange 
is a mixture of red and yellow, and, as a matter of fact, we find that on mixing rays from the red with others from the yellow part of the spectrum we do obtain a sensation of orange. The stimulus obtained by mixing the red and yellow rays is not the same as a stimulus caused by rays from the orange part of the spectrum. In the former case compound waves made up of the two wave-lengths, $656 \mu \mu$ and $564 \mu \mu$, are falling on the retina, in the latter case a simple wave with a length of $608 \mu \mu$, and yet the sensations produced are identical. Experience shows that there are relations between the physiological effects produced by different parts of the spectrum which have no physical analogue in the stimuli themselves. Such, for instance, are the phenomena known as the coloured after-image. We have seen that, as the result of fatigue, stimulation of any part of the retina by a bright object produces, when the stimulation is removed, a dark after-image, which has its seat in the previously stimulated portion of the retina. If we look steadfastly for a minute in a bright light at a red disc on a white ground and then look away at a uniform white surface, we see an after-image of the disc on the surface. This after-image is, however, green, and the white background takes on a reddish tinge. If the disc in the first instance has been a greenish blue the after-image is red, and to every colour in the spectrum we find there corresponds another which represents the after-image evoked by stimulation with the first colour. If the first disc has been green the after-image will be purple, and vice versa. We can therefore arrange the spectrum into a series of pairs of colours which are known as complementary. The following is a list of such pairs, with wave-lengths of the rays involved, as determined by Helmholtz :

\section{Colours}

Red-greenish blue

Orange-blue

Bright yellow-blue

Yellow-indigo

Greenish yellow-violet .

Wave-lengths
$656-492$
$608-490$
$574-482$
$567-465$
$564-433$

If the retina be stimulated by any of these pairs of colours simultaneously the effect is not that of colour, but of white. White light, or the sensation of white, can thus be due either to simultaneous stimulation of the retina by all the rays of the spectrum, or to stimulation of the retina by pairs of colours which are known as complementary. If these pairs be taken rather nearer in the spectrum a colour is obtained representing a part of the spectrum situated between the two constituents of the pair. If the rays are further away than would correspond to the complementary colours we obtain again a coloured sensation, which, however, is unsaturated, being mixed with a certain amount of white light. By taking three colours, such as red, green, and violet, or four colours, such as red, yellow, green, and violet, it is possible by mixing them in various proportions to form either white light or any colour of the spectrum, besides the various purples which do not occur in the spectrum.

These experiments of mixing colours can be carried out in various ways :

In every case we aim at stimulating the retina simultaneously with rays of different 
wave-lengths, or successively at such short intervals of time that there is complete fusion of the sensations resulting from the individual excitations. In order to determine fine differences in shade a coloured surface is always provided with which a colour produced by the fusion of the different rays under experiment may be compared :

(1) The most exact method of mixing colours is to employ a couple of spectra and by means of prisms to bring different parts of the spectra on one and the same white surface on which the result of the mixture can be compared with sample colours.

(2) In Maxwell's ' colour top' discs with a radial slit are placed one over the other on a disc which can be made to revolve with considerable rapidity. By means of the slit two or three dises of different colours can be slid one into the other, so that the disc is composed of sectors of variable extent of the two or three colours which we wish to mix. These discs are generally mounted on a background of a larger disc, which can be used as a sample colour to compare with the result of the mixture of the colours in the centre. If we are determining the relative amount of different colours necessary to produce white, the outside disc would be partly white and partly black, so that on rotation the effect of grey is produced, i.e. a weak white. Thus in one experiment the small dises in the centre were red, green, and blue. It was found that when the sectors were chosen in the following proportion : $165 \mathrm{red}, 122$ green, and 73 blue, a grey colour was produced equal to the grey obtained in the outer disc by mixing 100 white with 260 black.

These methods and all others in which coloured surfaces are used suffer from the defect that no pigments give perfectly pure colour-sensations. On looking at a red painted surface, for instance, in a bright light with a spectroscope, it will be found that the spectrum contains yellow and green rays as well as red. On this account no information as to the effect of mixing colour-sensations can be obtained by mixing the pigments themselves. Thus a familiar way of producing a green pigment is to mix a blue and a yellow pigment. A mixture of blue and yellow rays will give a sensation of white. The fact that blue and yellow pigment mixed together give a green.pigment is due to the fact that the blue pigment cuts off the red and yellow rays, and reflects, or allows to pass, the blue and green rays, while the yellow pigment retains the blue and violet rays, but reflects the red, yellow, and green rays. When we mix the two we get, not an addition, but a subtraction; the blue pigment absorbs the red rays which the yellow pigment allows to pass, and the yellow pigment absorbs the blue rays which the blue allows to pass. The only rays left over are the green, and the mixture of pigment has a green colour.

\section{THEORIES OF COLOUR VISION}

These facts show that in all probability the primitive colour-sensations are few in number, and that the various sensations excited by the different parts of the spectrum are not simple, but are compounded of mixtures of these primary sensations. The two theories which have obtained most vogue, and which enable us to account for a certain number of the phenomena associated with colour-vision, are those known as the Young-Helmholtz theory and the Hering theory.

(a) THE YOUNG-HELMHOLTZ THEORY. This theory, which was first put forward by Young and elaborated by Helmholtz, assumes that there are three primary colour-sensations-red, green, and violet-which are represented by three separate sets of elements in the retina, or in each 
cone, or by separate substances, each of which is affected by one of these colours.

Thus the rays with a longer wave-length excite chiefly the red fibres or elements ; those of medium wave-length the green percipient element; those of short wave-length the violet percipient element of the retina. The excitability of these three elements by the rays of different parts of the spectrum are represented in the figure (Fig. 296). At the extreme red and violet end

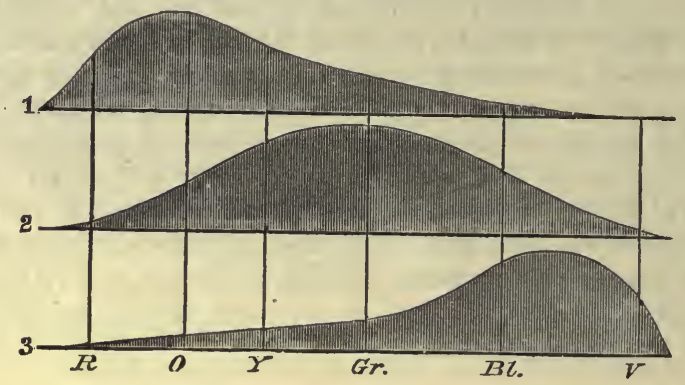

Fig. 296. Curves, showing sensitiveness of the three varieties of nerve fibres to different parts of the spectrum.

1 , red fibres; 2, green fibres; 3 , violet fibres.

of the spectrum alterations of the wave-length cause no alteration in colour, showing that these rays only excite either the red or the violet fibres. All other parts of the spectrum excite the three sets of fibres simultaneously, but to varying degrees. Thus the greater part of the red, e.g. about 'C,' excites the red percipient element strongly, the other two only weakly; the result is a sensation of red. The yellow rays at ' $\mathrm{D}$ ' excite almost equally the red and the green percipient elements; but the violet only slightly. Yellow is therefore a mixed sensation. The green rays excite the green percipient element strongly, the other two slightly, with green sensation as a result. Blue rays excite green and violet percipient elements to a moderate extent, and the red rays to a somewhat less extent; blue is therefore a mixed sensation composed chiefly of green and violet. Simultaneous excitation of all three elements evokes a sensation of white or grey, according to the intensity.

The cases of defective colour-vision which occur-the so-called colourblindness - are regarded under this. theory as due to the absence of one or other of the elements which determine the primary colour-sensations. The normal eye is spoken of as trichromatic since it has three primary coloursensations. Two kinds of dichromatic eyes are described-those in which the red sensation is lacking, and those in which the green sensation is lacking. In either of these cases the subject confuses red and green. The ripe cherry on a tree they may distinguish from the leaves, not by their colour, but by form or difference in their luminosity. It is only when they are tested by means of the spectrum that we find that whereas in the first case (red blindness) there is insensibility to the red end of the spectrum, in the second case the red end of the spectrum is seen as well as by the normal person, so that 
the defect must be located towards the middle of the spectrum. Theoret:cally, of course, violet blindness ought also to exist, but cases of this nature are so rare that their very existence is doubted. Cases have also been recorded with total colour-blindness (so-called monochromatic vision). Such cases have been supposed to be endowed only with vision similar to that found in the dark-adapted eye, i.e. with sensations only of white and black rather than vision determined only by the existence of the violet-perceiving constituent of the cones. Indeed the phenomena we have studied under the heading of dark adaptation would tend to show that, if we accept the Young-Helmholtz theory, we should add to the primary visual sensations those of light and dark, which have their seat in the-rods.

(b) THE HERING THEORY. According to Hering there are four, and not three, primary colour-sensations, viz. red, yellow, green, and blue. In this theory the sensations of white and black are also regarded as primary visual sensations. These sensations are placed in three groups-red and green, yellow and blue, white and black. For each pair of sensations he considers that there is a special substance in the retina, dissimilation or catabolism of which gives rise to one colour-sensation ; anabolism or assimilation to the other. Thus if white light falls on the retina, it causes a breaking down or catabolism of the white-black substance. This breaking down excites certain fibres of the optic nerve, and produces in consciousness a sensation of white. If the light be now removed, this breaking down gives place to anabolism or building up of the white-black substance, which excites the same nerve fibrils in a different way, giving rise to a sensation of black. The white-black substance is affected not only by white light but also by the colours red, green, yellow, blue, and their mixtures. The other two visual substances are affected only by red and green or by yellow and blue respectively. Hence even the spectral colours do not give rise to pure sensations, there being always some mixture of a sensation of white with the proper colour-sensation.

Most of the phenomena of colour-vision that we have mentioned above can be equally well explained on either theory. Thus the fact that blue and yellow together give rise to a sensation of white may be explained on the Young-Helmholtz theory by saying that the stimulation of all three sets of fibrils is equal, as will be seen by adding together the ordinates of each curve in Fig. 296 at yellow and at blue.

Adopting Hering's hypothesis, we may say that, anabolism and catabolism being equally excited in the yellow-blue substance, no change in it takes place, and the sole sensation is that produced by the stimulation of the whiteblack substance. The pairs of colours that we have distinguished are therefore, according to this theory, not in the strict sense of the word complementary, but antagonistic. The fact that white light appears to us as a simple sensation and gives us no suspicion of the coloured rays of which it may be composed is in favour of Hering's theory. Cases of colour-blindness would be reduced by Hering to two classes, viz. those in which the red-green substance is lacking and those in which the yellow-blue substance is lacking. 
Most of the data with regard to colour-blindness have been worked out with reference to the Young-Helmholtz theory, and have therefore been interpreted in accordance with this hypothesis.

It is very difficult, however, to harmonise the facts of colour-blindness either with this or with Hering's hypothesis. It is better therefore to abandon hypotheses altogether and to adopt a purely empirical classification of colour-vision, as has been done by Edridge-Green. This observer points out truly that very marked colour-blindness may be present without any interference with the appreciation of the luminosity of any part of the spectrum. A person may be able to see the spectrum up to its extreme red end, and yet distinguish in the spectrum only two colours, which we may call red and violet. We may, in fact, regard discrimination of colour difference as superadded to and evolved later than the appreciation of light. Discrimination may show various degrees of deficiency without any interference with the appreciation of luminosity. According to Edridge-Green a normal individual will name six distinct colours in the spectrum - red, orange, yellow, green, blue, violet. Such an individual, when made to map out the spectrum in the manner indicated on p. 575, will distinguish about eighteen monochromatic patches. A few individuals will place another colour, which has been called indigo, between the blue and the violet, and will mark out from twenty-two to twenty-nine monochromatic patches.

'Colour-blindness' may be brought about by one of two conditions : (a) a shortening of the red or violet end of the spectrum; $(b)$ absence of power to discriminate between the colours in the spectrum. The former condition may be present with complete power of discrimination between the different parts of the spectrum which are visible. Thus in normal individuals the limit of the visible red spectrum is between $\lambda 760$ and $\lambda 780$. In a certain number of cases it is found that the spectrum is not visible beyond $\lambda 700$ with bright light, or beyond $\lambda 620$ with dim light. Such cases may be said with truth to suffer from red blindness, and they will be unable to see a red lantern or appreciate its colour unless the red light is mixed with a considerable amount of orange. They may be detected by testing their power of mixing colours. A rose colour consists of a mixture of a violet and red light. In an individual with a shortened red end of the spectrum only the violet element of the rose would be visible, so that he would be inclined to class it with the blues rather than with the reds. Cases also occur in which there is a shortening of the violet end of the spectrum, but they have little or no practical importance.

Of the second class of cases, distinguished by deficiency of power of discriminating colours, all grades are known. A very large proportion of individuals, as much as 20 per cent., present a power of distinguishing colours which is below normal, and Edridge-Green distinguishes these various classes (calling the normal person hexachromic) as pentachromic, tetrachromic, trichromic, and dichromic. If we regard a spectrum in very dim. light it appears grey. With a slight increase in luminosity we can make out two colours, red at one end and violet at the other. On further increasing 
the luminosity the spectrum appears trichromic, being composed of the colours red, green, and violet. In colour-blind individuals this limitation of the colours distinguished applies to all strengths of luminosity. Thus the dichromic sees only red and violet, the trichromic sees red, green, and violet. It is the dichromic cases to which the name of 'colour-blind' has been chiefly applied, the trichromic cases being often missed, or classified simply as ' colour weak.'

The name of 'anomalous trichomats' has been applied to people who, while able to discriminate most of the spectral colours, use abnormal proportions of the red and green, when they mix these colours to form yellow.

The ordinary ' red-blind ' person is generally a dichromic with shortening of the red end of the spectrum. The 'green-blind' person is a dichromic without shortening of the red end. It is an instructive experience to make either a dichromic or a trichromic mark out on a spectrum a monochromatic patch. In a dichromic, such a patch at the red end will include red, orange, yellow, and green. In a trichromic, red, orange, and yellow will probably be included in the patch. This method is the most accurate way of determining diminution of the power of colour discrimination and shows with ease even the minor degrees of colour blindness.

\section{CONTRAST PHENOMENA}

Simultaneous Contrast. If a grey disc be placed on a piece of red paper, and the whole covered with tissue paper, the disc will take on a greenish tinge. If the ground colour be green, the disc will appear red; if blue, the disc will appear yellow; in fine, whatever be the ground colour, the colour of the disc will be complementary to it. These effects are spoken of as simultaneous contrast.

Successive Contrast. If, after gazing steadily for some time at a red disc on, a white surface, the eyes be turned towards a plain white surface, a negative after-image of the disc is seen on the paper coloured green, i.e. the complementary colour of the red disc. Surrounding this the paper appears red. If we look at the sun for some time, and then turn our eyes away, there is at first a positive after-image, and we see a bright sun wherever we look. In a short time this disappears and gives way to a black sun (a negative after-image). Thus we may say that stimulation of any part of the retina with any colour is followed by a colour sensation referred to the same part of the visual field and complementary to the first.

It has been much discussed whether these phenomena are simply effects of judgment, or whether they are produced by definite changes taking place in the retina. Helmholtz explains them by the first hypothesis, and looks upon them as cerebral processes. Hering, on the other hand, has extended his theory so as to embrace these phenomena, and ascribes them to definite changes in the retina, or at any rate in the peripheral part of the visual mechanism. A corollary to his theory that we mentioned above is that if dissimilation of a visual substance be excited at any point of the retina, assimilation of the same substance is set up in the parts of the retina 
immediately adjoining that point. To this process the name of ' retinal induction' has been given. In this way the phenomena of simultaneous contrast may be explained. Thus if a ray of red light falls on any spot, it may be supposed to excite dissimilation of the red-green substance at this spot. This sets up assimilation of the same substance in the adjoining parts of the retina, and the red object is therefore surrounded with a green halo, which at once becomes evident if we increase our appreciation for slight colourtones by diminishing the total amount of light by means of tissue-paper.

It has been much debated whether the contrast phenomena depend upon psychical or retinal events. There is no doubt that the question must be answered in the latter sense, and that these phenomena are quite independent of the judgment of the individual. This is shown clearly by two experiments. A box (Fig. 297) is divided into
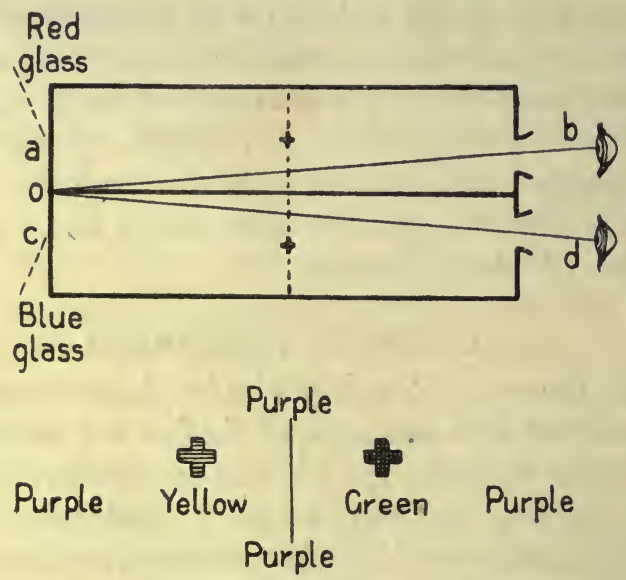

Fig. 297.

two long compartments, $a b$ and $c d$. At $a$ the compartment is closed by a red glassplate and at $c$ by a blue glass-plate. Apertures are proviced at $b$ and $d$ for the observer's eyes. At + and + two small grey crosses are fixed about the middle of the compartment on sheets of transparent glass. On looking through the openings $b$ and $d$ and converging the eyeballs, so as to fix the line $o$, we get a fusion more or less complete of the two colours red and blue, so that the background appears purple; or there may be a struggle between the colours, at one time blue, at another red predominating. To the judgment, however, there is one background and not two, and therefore, according to the theory of Helmholtz, the grey crosses should by contrast both acquire the same induced colour, which would be complementary for purple. But it is found that the two crosses are perfectly distinct in colour, that which is seen by the eye against the blue ground being yellow while that on the red ground is green, showing that the phenomena of simultaneous contrast are peripheral and not central in their causation. The same fact is very definitely established by the following experiment devised by Sherrington. The disc (Fig. 298) presents two rings, each half-blue and half-black. The outer ring is intensified when at rest by simultaneous contrast, the black half being seen against the surrounding yellow, while the luminosity of the blue half is increased by the effect of the surrounding black. In the inner ring the blue half is darkened by contrast with the surrounding yellow, while the black half is not evident at all. If the disc be rotated, we get two concentric rings on an apparently homogeneous field. It is found, however, that the outer ring flickers long after complete fusion has taken place in the inner ring, showing that the stimulation of the retina by the outer ring is increased under the influence of contrast. 
On this theory successive contrast phenomena are analogous to certain phenomena we have already studied in other tissues. If extensive breaking down of the visual stuff has been occurring, when the stimulus is removed there will be a swing-back of the condition of the protoplasm of the nerveendings in the opposite direction, and the catabolic will be replaced by anabolic changes; just as, on breaking a constant current that has been flowing through a nerve, the condition of raised irritability at the cathode gives place to a condition in which the irritability is depressed below the normal. The improving effect on the heart of stimulation of the vagus is also analogous to a successive contrast effect. During stimulation of the vagus the breaking down of the contractile substance is stopped or checked, so that building up or anabolism can go on without interruption. When

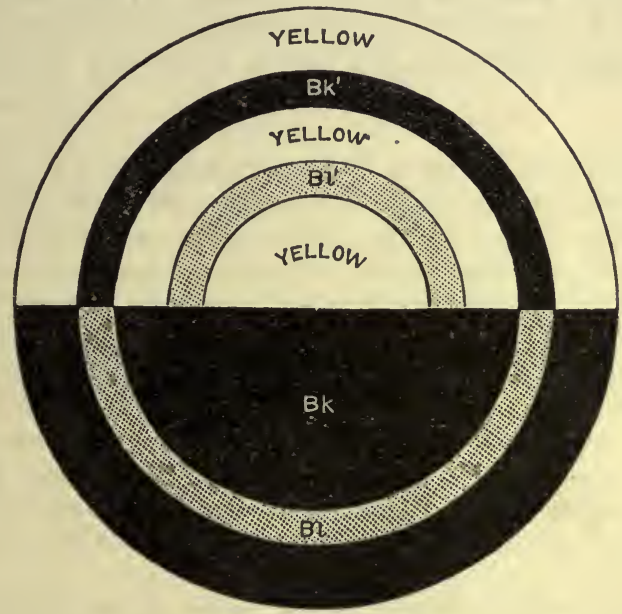

Fig. 298.

the excitation of the vagus ceases there is an extra store of contractile material in the muscle-cells. This causes the beat to be more vigorous, and we may say that the increased anabolism has been followed by a period of increased catabolism, just as strong stimulation of a part of the retina with green (anabolism) gives rise to a red after-image (catabolism).

It seems probable that, as McDougall has pointed out, the examples of simultaneous contrast depend on inhibitory processes analogous to those which we have studied in the spinal cord in dealing with reciprocal innervation and the conditions for the isolation of any effective reflex. Just as the flexor reflex, due to nocuous stimulation of the paw, inhibits the extensor or stepping reflex, by blocking the synapses of those elements on the sensory path which would pour their impulses into the final common path of the extensors, so stimulation of any portion of the retina will tend to inhibit processes of a similar kind occurring in adjacent parts of the retina or their central connections.

Stimulation of one part of the intestine causes inhibition of the activity of the intestine below the point of stimulation. If this inhibition occurred 
on both sides of the stimulated spot we should have a phenomenon exactly analogous to the process of simultaneous contrast. In the same way the increased briskness of the antagonistic reflex which follows the temporary inhibition accompanying the primary reflex can be compared to the negative after-image resulting on prolonged stimulation of any point in the retina, while alternating after-images, positive and negative, which may succeed a single positive stimulation, have their analogue in the alternating rhythmic movements of flexion and extension of the two legs which may follow single stimulation of one of them. The phenomena of binocular contrast show that the retinæ are not alone concerned in the production of these phenomena, but that we are dealing also with the central connections of the optic nerves, the activities of which must be regulated by the same laws as those determined from our study of the more lowly activities of the spinal cord. 


\section{SECTION IX}

\section{MOVEMENTS OF THE EYEBALLS}

IN order to obtain stinct vision of any object, an image of it must be formed on the fovea centralis. Visual attention, i.e. the fixing of the gaze on any object, involves the adjustment of the visual axes by movements of the eyeball, in order that the image of the object of attention shall fall on the central spot in each eye.

The eyeball rests on a pad of fat surrounded by a denser capsule of connective tissue, the capsule of Tenon, from which it is separated by a lymph space. Within this space it is able to rotate around axes which pass through a point almost in the middle of the eyeball. These movements of rotation are carried out by the six ocular muscles, the four recti and the two oblique. The four recti muscles-the superior, inferior, external, and internal-arise from a continuous tendinous oval rin 3 which is attached at the back of the orbit to the margin of the optic foramen and sphenoidal fissure. From this common origin the muscles pass forwards as flat bands close to the walls of the orbit ; they come in contast with the eyeball at its equator, passing through the surrounding lymph space, and are attached to the eyeball about $7 \mathrm{~mm}$. behind the margin of the cornea, their positions being indicated by their names. Of these muscles the internal rectus is the thickest and strongest, the superior rectus is the thinnest and weakest.

The superior oblique muscle arises in a short tendon attached to the back part of the orbit in front of and internal to the optic foramen. The muscle runs forward in the upper and inner angle of the orbit and ends in a round tendon, which passes through a fibrous pulley at the upper and inner angle behind the anterior margin of the orbit. The tendon then makes a sharp bend and passes outwards, backwards, and downwards between the superior rectus muscle and the eyeball, and is attached to the latter just below the outer edge of the superior rectus, behind the attachments of the rectus muscles and about half-way between the anterior and posterior poles of the eyeball.

The inferior oblique muscle rises from the orbital plate of the superior maxilla, just within the anterior margin of the orbit. The muscle forms a flat band and passes upwards, backwards, and outwards between the inferior rectus and the wall of the orbit, and ends in a tendinous expansion, which is inserted under the external rectus muscle into the posterior and outer part of the eyeball, somewhat behind the line of attachment of the superior oblique muscle.

In discussing the actions of these muscles we may assume the eyes to be in what is known as their primary position, i.e. with the visual axes parallel and directed to a point on the horizon. It is evident that if the muscles rotate the eyeball about an axis in a plane at right angles to the visual axes, the pupils will move inwards, outwards, or in any direction, but will not rotate. On the other hand, if the axis of rotation of a movement produced by any muscle lies in a plane which is not vertical to the visual axes, then 
a movement of the pupil will be associated with rotation of the eyeball. The first of these conditions is practically fulfilled by the external and internal rectus muscles, as is shown in the diagram (Fig. 299). These muscles by their contraction will move the pupil directly outwards or directly inwards. The axis of rotation in the diagram would run vertically through the eyeball. The other four muscles produce movements with axes of rotation which are not at right angles to the visual axis. The rectus superior, for instance, produces rotation round the axis joining r.sup. and r.inf., and will therefore produce movement of the eyeball upwards and inwards with a certain amount of rotation of the eyeball on its antero-posterior diameter. The rectus inferior in the same way moves the eyeball downwards and inwards with

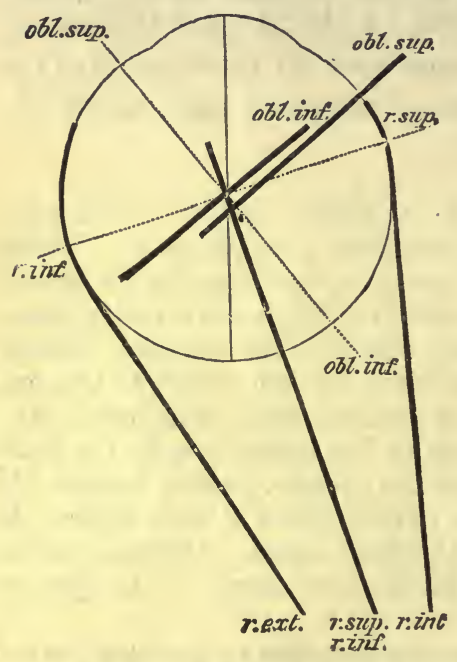

Fig. 299. Diagram to show points of attachment and liens of action of extrinsic ocular muscles.

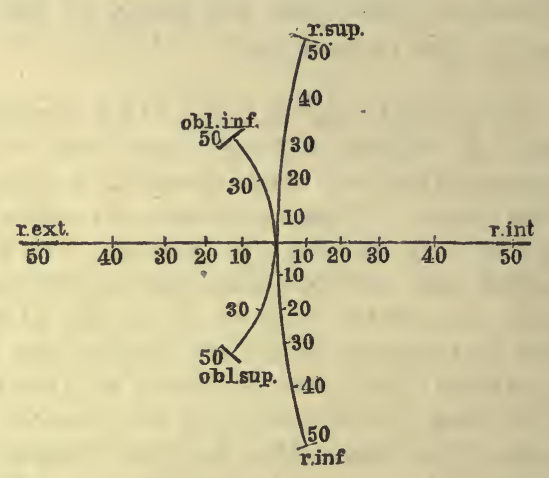

Fig. 300. Diagram to show direction in which pupil will move under the action of the various ocular muscles.

rotation. The obliquus superior moves the eyeball downwards and outwards, and the obliquus inferior upwards and outwards, in both cases with some rotation on its antero-posterior axis (Fig. 300). The only movements of the eyeball therefore which can be carried out by the action of one muscle, or by the reciprocal action of a pair of muscles, are the movements outwards and inwards, which are involved in the common actions of convergence of the visual axes and conjugate deviation of both eyeballs. Movements upwards or downwards will require the co-operation of at least two muscles. In order to direct the gaze upwards a contraction of the superior rectus which moves the eyeball upwards and inwards must be associated with a contraction of the inferior oblique muscle which rotates the eyeball upwards and outwards. In the same way the inferior rectus muscle will be associated with the superior oblique.

As we have seen, four out of six muscles when acting singly cause rotation of the eyeball on its antero-posterior axis. The question arises whether 
these movements of rotation ever occur under normal circumstances. This may be tested in the following way: The gaze is first directed at a brilliant line of light, e.g. a straight electric incandescent filament. The gaze is then directed to a uniform white surface. On this white surface a negative afterimage of the vertical line is seen. It is found that in whatever direction the eyes be turned, upwards, downwards, or obliquely to right or left, the afterimage always retains its vertical direction. If there were rotation of the eyeballs on their antero-posterior diameters, the stimulated portions of the retina, i.e. those which are the seat of the after-image, would lie obliquely, and the apparent direction of the negative after-image would be also oblique. We see therefore that, under normal circumstances, no rotation of the eyeball on its antero-posterior diameter takes place. The actions of the different muscles are always so co-ordinated that all movements of the eyeballs take place round axes, which lie in a plane passing through the centre of rotation of the eyeballs and at right angles to the visual axes.

In man movements of the eyes are always bilateral and take place in such a way that an image of one and the same object will fall on the central spot of each retina. These movements are simple in character and are of only two kinds, viz. :

(1) Movements of both eyes with maintenance of parallelism of the visual axes ; to this class belong the movements of conjugate deviation employed in following the passage of an object across the field of vision from right to left, or vice versa, as well as less extensive upward and downward movements of both eyeballs.

(2) The movement of convergence of the axes of both eyes, which is always associated with accommodation for near objects, and therefore with contraction of the ciliary muscles and of the constrictors of the pupils.

Other movements can be effected, but only with effort. Thus we can converge the axes of the eyes so as to look at a near object lying to one side of us. Such an action is, however, associated with considerable effort, and in nearly all cases is replaced by movements of the head. Whenever we wish to examine an object closely we turn the head so that the object lies between the two eyes, and a simple movement of convergence serves to bring the image of the object on to the two foveæ centrales.

\section{BINOCULAR VISION - CORRESPONDING POINTS}

When we fix our gaze on any object, although an image of the object is formed on each retina, the object appears as single and not as double. On the other hand, gentle pressure on one eyeball, so as to shift its gaze slightly from that imposed on it by the co-ordinated action of the ocular muscles. at once causes the object to appear double. This shows that the single appearance of an object seen with the two eyes is due to the fact that the image from this object must fall upon points in the retina, simultaneous stimulation of which produces only a single sensation. These points are known as 'corresponding points.' It is evident that to each point in one retina only one point can exist in the other retina which corresponds to it, since for every 
position of the eyes a luminous point can only throw an image on a definite point in each retina.

Such corresponding points are, in the first place, the central spots in each retina. When we look at any spot the axes of the above eyes are so directed that the image of the spot falls upon the fovea centralis of the two retinæ. The image of all points to the right of this spot will fall on the nasal side of the right retina and on the temporal side of the left retina, and vice versa. If the right retina were cut out and placed on the left, the corresponding points of the two retinæ would be nearly over one another.

This statement is not absolutely accurate, as is shown by a careful investigation of the corresponding points by means of the haploscope. In this instrument a white screen is placed vertically at the far point of vision of the eyes, which are made somewhat myopic by means of a convex lens. Each eye looks through a cylindrical tube, the axis of which coincides with the visual axes. On each half of the white field is a mark which, however, appears single, since its image falls on the fovea centralis of the two retinæ. If in the left field of vision a line be drawn vertically upwards from the mark, and in the right field of vision another line vertically downwards, the two linss appear as a single line passing through the mark. It will be seen that the upper half of the line is not absolutely continuous with the lower half, but appears to form a slight angle with it, showing that the general statement made above is not absolutely correct. By means of this instrument we can determine the extent and situation of all the points in the two retinæ which correspond. The totality of all the points in space, the lines from which to the eyes will fall on corresponding points, is known as the horopter. Its determination is, however, only of theoretical interest.

It might be thought that single vision with the two eyes was due to the fact that the nerve fibres from corresponding points in the two retinæ pass to and terminate in identical nervous structures in the brain. This is not the case. Single vision is largely the result of experience, and we can show by experiment that both elements are present in the fused sensation obtained from the two eyes. Thus if the left eye is directed on to a red surface and the right eye on to a blue surface, we may obtain either a fusion of the fields with the production of a purple colour, or a struggle of the two fields so that the whole field appears sometimes red and sometimes blue. In the same way if two figures, lying side by side, one ruled with vertical lines and the other with horizontal lines, be looked at so that the image of the right figure falls on the right retina and of the left figure on the left retina, we do not see a single figure with the lines crossing one another so as to form a network, but we again obtain a struggle so that first one figure is seen with the vertical lines prominent, and then this wanes and is succeeded by the other figure in which the horizontal lines are prominent. Which figure we see most easily seems to be dependent on the direction of the eye movement. If the eyes are moved vertically upwards and downwards the figure with the vertical lines comes more into prominence, while the figure with the horizontal lines is seen when the eyes are moved from side to side. 


\section{SECTION $\mathrm{X}$}

\section{VISUAL JUDGMENTS}

LOCALISATION AND PROJECTION. Much discussion has been wasted on the question why we see things upright while the images on our retina are inverted. The answer is a simple one. We do not look at nor are we conscious of the image on our retina. When we say that we see anything we are not expressing merely a sensation, but we are giving an interpretation of certain sensations in the light of long experience which has involved a large number of sensations besides that of vision. Thus a new-born child sees, i.e. receives images on its retina which excite impulses in the brain, but it is unable to interpret anything that it sees. In the first few months there is indeed no connection between the visual sensations and eye movements; it is only about the third month after birth that the child will follow a lighted candle or bright object with its eyes, and this association of ocular movements with retinal impressions gradually extends also to many other movements. The continual and at first apparently aimless movements of the infant bring in a flood of muscular and tactile impressions which only after many trials are recognised as corresponding with sensations arriving from the eyes. It at first finds that with the right hand it can touch objects lying on the right side of the field of vision. It becomes conscious therefore, not of the left side of its retina, but of a series of objects which have distinct relations to its right hand, and of a certain thing seen outside itself. The projection and localisation of visual impressions are therefore not intuitive or innate qualities attached to stimulation of each point of the retina, but are the result of experience, the testing and comparing of visual sensations with tactile and muscular sensations from all parts of the body. From these experiences we learn to associate stimulation, say, of the right side of the retina with the presence of objects lying in front of and to the left of the body, and to project our visual sensations in this direction. If, for instance, we press the finger, with closed eyelids, on the outer side of the right eyeball, a luminous ring, or phosphene, will be seen apparently towards the left, i.e. the region whence the pressed-upon part of the retina will be normally stimulated by rays of light.

The projection of visual impressions is well shown in Scheiner's experiment. Two needles are placed one behind the other on a wooden rod, one at $18 \mathrm{~cm}$. and the other at a distance of $60 \mathrm{~cm}$. from the eye. One eye is closed, and then a card is held before the other. In the card two small holes are pierced by means of a needle at a distance 
from one another less than the diameter of the pupil. On accommodating now for one needle, the other needle appears double. Thus if the eyes are accommodated for the distant needle $\mathrm{F}$ (Fig. 301, II), the image of $\mathrm{N}$ is formed behind the retina, and since only a very narrow bundle of rays can pass through the holes in the card two images
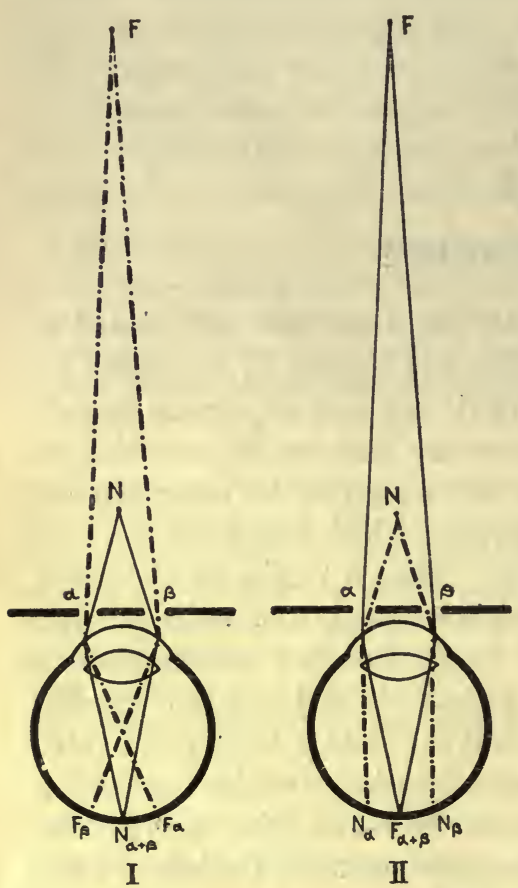

FIG. 301. Diagram to illustrate Scheiner's experiment.

$F$, the far needle ; $\mathrm{N}$, the near needle ; $\alpha$ and $\beta$, two pin-holes in a piece of card. The continuous lines indicate the path of the rays for which the eye is accommodated. of the needle are formed on the retina. In the same way, if the eye be accommodated for the near needle the image of $\mathrm{F}$ falls in front of the retina, and therefore there will be again two images of it on the retina. In the former case, if the hole $\beta$ be covered with a card, the left-hand image disappears. In the latter case, on covering $\beta$ the right-hand image disappears, showing that the apparent position of the object depends on the relation of its image in the retina to the point of fixation, i.e. to the fovea centralis.

JUDGMENT OF SIZE. The apparent size of an object is determined in the first place by the magnitude of its image formed on the retina, and therefore by the visual angle which the object subtends at the optical centre of the eye, as will be evident from the diagram (Fig. 302). The apparent size of any given object varies inversely in proportion to the distance. Thus the size of an image on the retina of an object two inches long at a distance of one foot is equal to the image of an object four inches long at a distance of two feet. An object can be seen if the visual angle subtended by it (the angle $\mathrm{AcB}$ in Fig. 302) is not less than sixty seconds. This is equivalent to an image on the fovea centralis about $4 \mu$ across, which is about the diameter of a cone.

The visual angle is, however, by no means the most important factor in our judgment of size. Thus as a man walks away from us his size does not appear to vary, although the visual angle subtended by him on our retina is continually diminishing. Where the size of an object is known to us, as in the instance just mentioned, it is used as a means of judging the size of surrounding objects. Where the size of the object is unknown our judgment of its size is determined by a comparison of its apparent size, as judged from the size of the retinal image, with the muscular effort of the convergence and accommodation which are present at the same time. Thus if we gaze at the sun for a minute so as to gain a negative after-image the size of this image will be constant. Its apparent size, however, will vary according to the distance of the surface on which we direct our gaze : on looking at a piece of paper held near, it may be about one inch across ; on looking at a distant wall, it may be 
several feet across. If we look through a piece of coarse wire gauze at a distant window, the meshes of the gauze appear to be in the neighbourhood of the window and extremely large ; on directing the gaze then to an object held just in front of the wire gauze, the mesh will look extremely small. On the other hand, if we cut out the movements of accommodation and convergence by looking at a piece of wire gauze through a minute pinhole in a card, the size of the meshes will increase as the gauze is brought near to the eye. In this case we judge of their size entirely by the visual angle they subtend.

In the muscular elements which

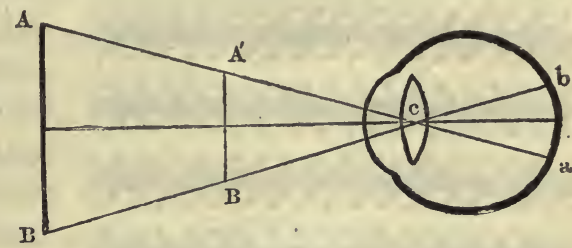

Fig. 302. contribute to this judgment of size, the convergence of the axes is more important than the accommodation of each eye, so that judgment is but little affected if we paralyse accommodation altogether by dropping atropine into the eye. The visual axes may be regarded as practically parallel for any object at a greater distance than five metres ; for such objects no act of accommodation is necessary. In judging of the size of any object beyond this distance we have only the visual angle to go by, which of course gives by itself no information unless we know the distance of the object. Here the obscuration of the outlines of the object in consequence of the deficient transparency of the atmosphere plays a large part in our judgments and may be upset in either direction by changes in transparency of the atmosphere. Thus when walking on the Downs in foggy weather a gigantic object may be seen looming through the mist, which, on advancing a couple of paces, is seen to be a sheep. On the other hand, in the clear air of the Alps the traveller continually under-estimates the size of distant objects, and takes a mass of rocks of the size of St. Paul's for a traveller wending his way up the snow arête.

\section{ILLUSIONS OF SIZE}

The distance between two points appears longer if a number of points be interposed between the two. Thus if two equal quadrilateral figures be

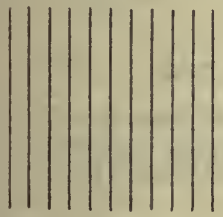

FIg. 303. divided - one by horizontal and the other by vertical lines - the one divided by horizontal lines will appear elongated vertically, and that divided by vertical lines elongated horizontally (Fig. 303). Apparently it requires a somewhat less effort to pass directly from one point to the other than when the gaze has to follow an interrupted line. The eye muscles probably make a separate effort of movement at each interruption of the line. To these 
movements is due the illusion in Fig. 304, where parallel lines seem to diverge or converge. Of two equal lines, one of which is vertical and one horizontal, the vertical line seems the longer. When the eye is

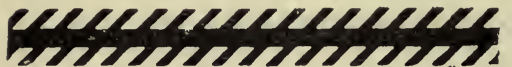
DNNDNONONONIS

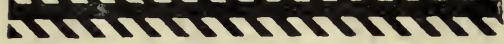

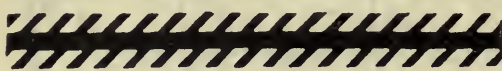
DNONDNDNDND

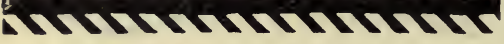

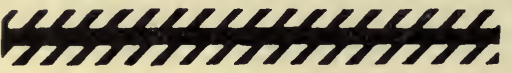
DNONDNMNDNDS ThNTNThNon

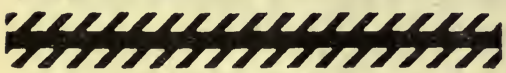

Fig. 304. moved upwards, the tendency of the superior rectus to move the eye inwards has to be counteracted by a pull of the inferior oblique muscle turning the eye outwards. A greater effort is therefore required than when the eye is moved either inwards or outwards, and it has been suggested that it is this greater muscular effort which is responsible for our over-valuation of the length of vertical as compared with that of horizontal lines.

It must not, however, be imagined that an actual movement of the eyes is necessary in order to judge of the size and distance of any object. If a white thread be hung up in a dark room and be illuminated for an instant of time by an electric spark it is impossible for an observer in the dark room to move his eyes so that the

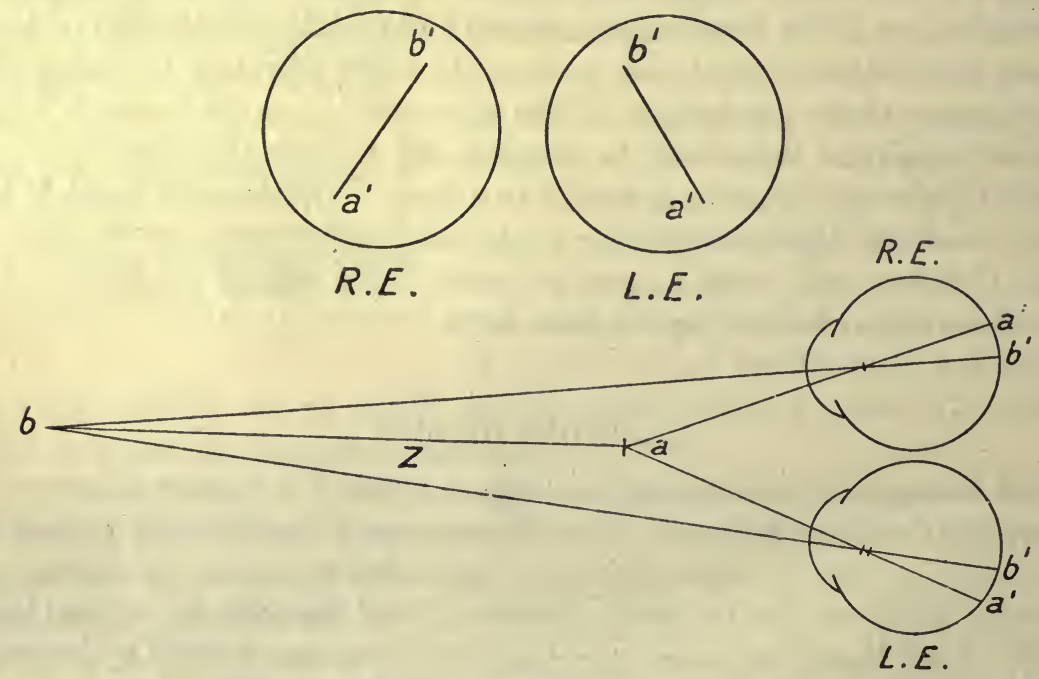

Fig. 305. The eyes are directed to the point $b$. A thread hung obliquely at $a$ under these circumstances gives rise to the images shown in the upper figures -i.e. two images which do not lie on corresponding points. Nevertheless the thread is seen as single.

image of the thread shall fall on the corresponding points of the two retinæ before the illumination has disappeared. In spite of the fact, however, that the image of the thread falls on non-corresponding points, as will be seen from the diagram (Fig. 305), the thread is seen, not as double, 
but as single, and a very correct impression may be obtained of its size and position. In this judgment or interpretation a number of separate processes must be involved. The fact that the eyes are not accommodated for the thread evokes at once the associated movements which would be necessary, if time allowed, to bring the non-corresponding images on to corresponding points of the retina. We do not therefore assume a doubleness of an object, even when its images fall on non-corresponding points: unless our gaze is voluntarily directed on the object.

THE J UDGMENT OF SOLIDITY. The fusion of non-corresponding images to a single visual conception is responsible for our appreciation of solidity. If we look at any object which is not too far from the eyes, first with the right and then with the left eye, we shall see that the images in the two eyes are not identical. If we look, for instance, at a truncated pyramid, we shall see with the right eye rather more of the right side of the pyramid and with the left eye rather more

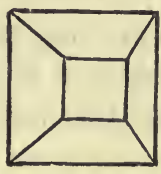

$a$

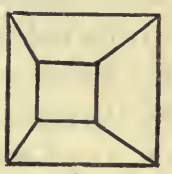

b

Fig. 306.

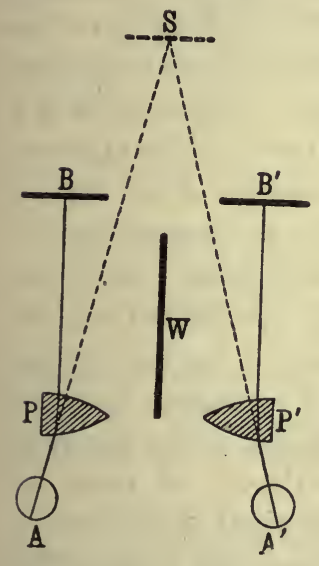

FiG. 307. Brewster's stereoscope. of the left side (Fig. 306). If these two images $a$ and $b$ be so arranged that they fall on corresponding points of the two retinæ, there is no confusion of sensation, but the resulting impression is that of a solid object. This is the principle involved in the stereoscope, which allows us to combine two images of this character so that they fall on corresponding points of the two retinæ.

In Brewster's stereoscope (Fig. 307), which is almost invariably used at the present time, the combination of the two pictures is effected by means of two half-lenses with convex surfaces, and their thinner margins directed inwards so that they act as prisms. A vertical black screen is placed between the two lenses. On looking through the lenses towards the point $\mathrm{s}$, the direction of the rays is changed by the prisms so that the image of the pictures $\mathbf{B}$ and $\mathbf{B}^{\prime}$ fall on corresponding points of the retin $x-B$ into the left eye and $\mathrm{B}^{\prime}$ into the right eye. The ordinary photographs for these stereoscopes are taken by means of a double camera with lenses at a distance apart considerably greater than that between the two eyes. On this account there is actually an exaggeration of the solidity of the combined images.

When only one eye is used the external world has a much flatter appearance. Some idea of solidity is still gained from the fact that the accommodation has to be altered in order to bring different parts of the solid objects into focus. The judgment is also aided by the effects of light and shade. The inadequacy of such means in default of the stereoscopic images in binocular vision is at once appreciated when we try to dissect or to carry out any fine manipulation using only one eye. 


\section{SECTION XI \\ THE NUTRITION OF THE EYEBALL}

THE eyeball is protected in front by the eyelids. These are lined internally with a delicate mucous membrane continuous with the conjunctiva covering the anterior surface of the eyeball. This membrane is kept constantly moist by the secretion of the lacrymal gland, a small acino-tubular gland built up on the type of a serous gland, situated at the upper and outer angle of the orbit. The secretion, 'the tears,' has a slightly alkaline reaction and contains about 98.2 per cent. water and 1.8 per cent. total solids, viz. 0.5 per cent. coagulable protein and 1.3 per cent. inorganic salts, of which sodium chloride is the most important constituent. Since the tears are constantly flowing over the eyes they serve not only to moisten the surface but to wash away any irritant material or bacteria which may be deposited from the air. The tears have a bactericidal power which is lost if the fluid be boiled for two or three minutes. Our knowledge as to the nervous mechanism of the secretion of the tears is still incomplete. Stimulation of the conjunctiva evokes an increased secretion of lacrymal fluid which can also be induced by emotional conditions. It is stated that lacrymal secretion can be produced by the stimulation of the cervical sympathetic as well as by stimulation of certain cranial nerves, e.g. facial and fifth nerve. Structural changes analogous to those to be described in the salivary glands have also been found in the lacrymal gland as a result of secretion. The excess of fluid is drawn off from the conjunctival sac by the nasal duct, which leads to the nasal cavity on the same side. If the eyes be kept open for some minutes, the conjunctiva covering the eyeball becomes dry and irritation is set up. Normally the membrane, and especially that over the cornea, is kept moist and transparent by involuntary movements of the eyelids, which close or blink about twice a minute, so distributing the lacrymal secretion over the whole conjunctival surface. This blinking is a reflex act, the afferent channels being fibres of the fifth nerve, and the efferent the fibres of the facial nerve supplying the orbicularis palpebrarum. It is spoken of as the 'conjunctival reflex,' and is one of the last reflexes to disappear in chloroform or ether narcosis.

Just below the mucous membrane of the lids we find a series of specialised sebaceous glands, the 'Meibomian glands.' The fatty secretion of these glands is poured out at the edge of the lids, keeping these and the eyelashes greasy, and so preventing their being wetted by the tears. Any overflow of tears from the conjunctival sac is thereby prevented, unless the secretion 
becomes excessive; so that the whole of the fluid under normal circumstances is kept within the sac and flows away only through the nasal ducts.

INTRAOCULAR PRESSURE. The eyeball is formed of a tough inextensible capsule, the sclerotic, filled with fluid or semi-fluid contents. In order that the eyeball may be sufficiently rigid to maintain the normal relations of the various refractive media, and to afford a fixed point for the action of the ciliary muscle, this fluid must be under pressure. On connecting

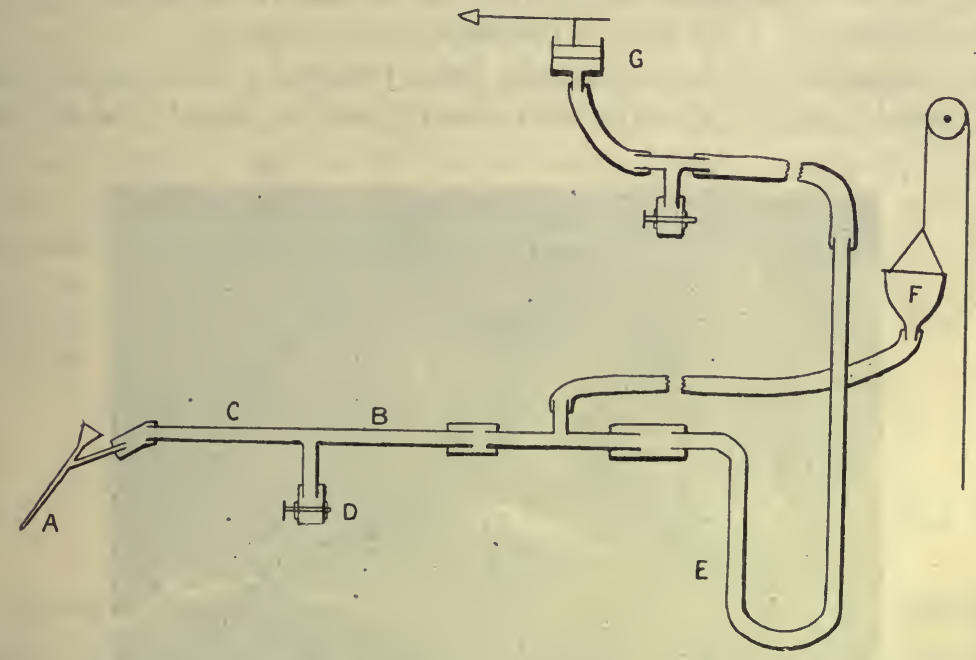

Fig. 308. Arrangement of apparatus for measurement of intraocular pressure.

(Henderson and Starling.)

$G$ is a piston-recorder for recording graphically the changes in pressure.

a small manometer with the anterior chamber, care being taken to prevent any escape of the intraocular fluid, it is found in the normal eye that this pressure is about $25 \mathrm{~mm}$. $\mathrm{Hg}$.

The problem of measuring intraocular pressure is analogous to that of measuring the intracranial pressure. The eyeball represents a cavity into which fluid is continually being poured and from which it is being absorbed, the intraocular tension determining the exact balance between the processes of secretion and absorption. It is therefore necessary in determining the amount of this pressure to take care that no fluid either enters or leaves the eyeball. For this purpose we can make use of the arrangement represented in the accompanying diagram (Fig. 308). The steel needle $\mathbf{A}$ is connected to a capillary glass tube, $\mathbf{C B}$. This has a lateral opening, through which a bubble of air can be introduced into the tube. By means of a $\mathbf{T}$-piece the capillary is connected with a water manometer $\mathrm{E}$, and with a reservoir $\mathrm{F}$, containing 0.9 per cent. salt solution. The pressure in the apparatus is now raised to about $25 \mathrm{~cm}$. of water. While the fluid is dropping from the end of the needle, it is thrust through the lateral part of the cornea, so as to lie in the middle of the anterior chamber. A bubble is then introduced by the side tube, $\mathrm{D}$, into the capillary tube, and the reservoir adjusted to such a height that the bubble remains stationary. We know then that the pressure inside the eye exactly balances the pressure of the fluid in the reservoir, and we have also provided that there shall be no appreciable escape of fluid from the eye or entry of fluid into the eye. If the bubble remains stationary for three or four minutes we know that equilibrium is attained, and we can read off the height of the intraocular pressure on the manometer $\mathrm{E}$ connected with the reservoir. 
On making an opening into the cornea the fluid drains away and the eyeball becomes soft and collapsed, the cornea being folded, and the eye being naturally useless as an optical instrument. The fluid which flows away, and which forms the aqueous humour and also fills the interstices of the gelatinous tissue of the vitreous, contains only a minute trace of protein, consisting, in every 100 parts, of 98.7 parts water and 1.2 to 1.3 total solids, of which only 0.08 to 0.12 part consists of protein. If a cannula be kept in the anterior chamber this fluid rapidly alters its character, becoming coagulable, and containing 3 to 4 per cent. of proteins.

The intraocular fluid is continually being renewed. The eyeball receives a rich vascular supply, which forms a close network of vessels and capillaries

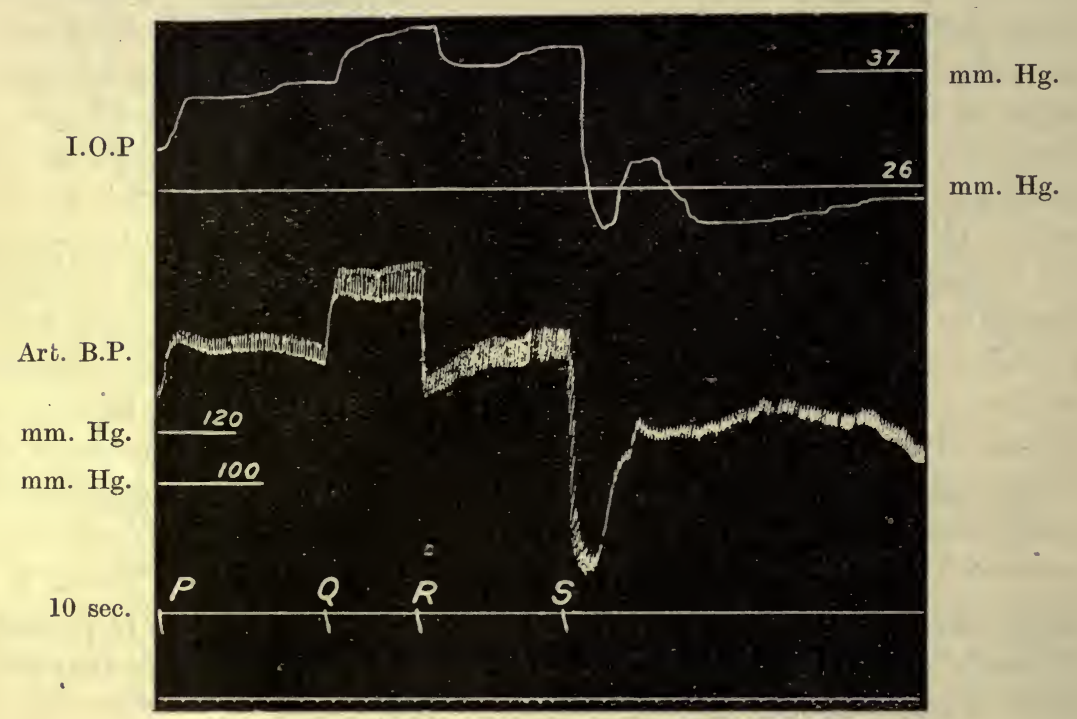

FIG. 309. Curve showing effects on the intraocular pressure (in the dog) of mechani cal interference with the circulation. Blood-pressure measured in left carotid, intraocular pressure in right eye. From $\mathrm{P}$ to $\mathrm{s}$ the descending thoracic aorta was occluded. From $\mathbf{Q}$ to $\mathrm{R}$ the right vertebral and subclavian arteries were also occluded. (Henderson and Starling.)

in the choroid coat, with its prolongations the ciliary processes and iris. The chief seat of formation of the intraocular fluid is the ciliary processes. Here there is a constant transudation of fluid from the blood-vessels into the anterior part of the vitreous cavity, the amount of the transudation varying with the pressure in the blood capillaries (Fig. 309), being increased by any rise in the capillary blood pressure or by any fall in the intraocular pressure. Of the fluid poured out by the ciliary processes a very small proportion (perhaps one-fiftieth) passes backwards into the vitreous humour and gradually drains out of the eyeball by the lymphatic spaces of the optic nerve. By far the larger amount passes forward through the fibres of the suspensory ligament into the posterior chamber (the annular cavity between the iris in front and the lens and ciliary processes behind), and thence round 
the margin of the iris into the anterior chamber. From the anterior chamber it passes into the spaces of Fontana at the outer angle of the chamber, whence, under pressure, it can filter slowly between the endothelial cells lining the canal of Schlemm into this vessel and so drain away into the venous system. A considerable resistance is offered to the passage of fluid into the canal of Schlemm. Hence the constant transudation of fluid from the ciliary processes raises the intraocular pressure to $25 . \mathrm{mm} . \mathrm{Hg}$, and a continuous production of about 6 cubic millimetres of fluid per minute suffices to maintain the pressure at this height.

If the formation of intraocular fluid be increased by rise of blood pressure, the intraocular pressure must also rise until a point is reached at which the increased filtration through the anterior angle is just equal to the increased production resulting from the rise of blood pressure. Within wide limits therefore the intraocular pressure varies with the blood pressure. This is shown by the following records of both pressures in different animals (Henderson and Starling):

$\begin{array}{ccc}\text { Arterial pressure } & \text { Dog } & \\ 128 \mathrm{~mm} . \mathrm{Hg} . & \ldots & \text { Intraocular pressure. } \\ 158 \mathrm{~mm} . \mathrm{Hg} . & \ldots & 34 \mathrm{~mm} . \mathrm{Hg} . \\ 180 \mathrm{~mm} . \mathrm{Hg} . & \ldots & 40 \mathrm{~mm} . \mathrm{Hg} . \\ 70 \mathrm{~mm} . \mathrm{Hg} . & \ldots & 23 \mathrm{~mm} . \mathrm{Hg} .\end{array}$

Conversely the intraocular pressure may be altered by increasing or diminishing the ease with which the fluid escapes through the filtration angle. If the anterior angle becomes blocked as the result of inflammatory changes, or other causes, the intraocular pressure rises gradually until it attains a height far above normal. The eyeball to the finger feels stony hard, and the increased pressure affects seriously the circulation of blood through the retinal vessels, so that atrophy of the retina is produced together with disturbance of the nutrition of the whole eyeball. This condition of raised intraocular tension occurs in the disease known as glaucoma.

The constant renewal of the intraocular fluid is important not only for the maintenance of the intraocular pressure but also for the nutrition of the structures, such as the lens, suspensory ligament, and vitreous humour, which do not receive any vascular supply. 


\section{THE ORGANIC SENSA'TIONS}

\section{SECTION XII}

\section{SENSATIONS OF MOVEMENT AND POSITION}

IN studying the phenomena of reflex movements, as presented by the spinal animal, our attention was drawn to the importance of the afferent impulses transmitted to the central organ by means of a special system of senseorgans, called by Sherrington the proprioceptive system. These afferent impressions intervene at a later period in every reflex action than do the initiating sensory (exteroceptive) impulses. They arise as a result of the reflex movement itself, and serve to regulate the extent of this movement as well as the co-ordinated changes in the other muscles of the body. Whether they be synergic or antagonistic, the abolition of the impulses arising in this system has an effect similar to that of the destruction of the governor of an engine. The movements excited by peripheral stimulation become excessive and conflicting; there is no longer the give-and-take of the antagonistic muscles surrounding the joint, and the result is a state of disorder and inco-ordination, termed ataxy.

Of the proprioceptive impulses a certain proportion reach the cerebral cortex and arouse states of consciousness which we speak of as sensations of position, movement, or resistance, and which form the basis of judgments as to these conditions. In consciousness they are contrasted with the sensations arising from the other sense-organs in the same way as they are in the subconscious regulation of the motor adaptations of the body. All the senses which we have so far considered give us information of things, i.e. of a material world which can affect ourselves, but which we conceive of as existing altogether apart from our sensations of it. Indeed the visual and auditory sensations we project to distances remote from the body. The sensations, on the other hand, which are aroused through the intermediation of the proprioceptive system we refer entirely to ourselves. By them we receive information of the condition of the material 'me,' i.e. of ourselves as things apart from the objects which surround us and the changes in which ordinarily excite our activity.

Consciousness we have seen to be developed in proportion to the differentiation of the educatable association centres, which are responsible for our powers of ideation, and by means of which the different reflex movements 
which we call volitional are carried out, guided, augmented, or inhibited, according to the past experience of the individual. Volitional movement is therefore a movement determined by previous neural events, of which a part at any rate is represented in consciousness as feeling, emotion, or desire. Where an act is involuntary, i.e. does not need the guidance of experience, individual or racial, for its performance, the afferent impulses which arouse it are also, as a rule, devoid of representation in consciousness. Thus we have no sensation of the passage of a bolus along the œsophagus. The proprioceptive impulses also only affect consciousness where they are necessary for the guidance of volitional movement. The tactile and gustatory impressions from the tongue have a very full representation in consciousness. Volition, however, only interferes for the rejection or acceptance of the food taken into the mouth, and is not required for the minute direction of the movements of mastication and deglutition. The muscular sensibility of the tongue, and therefore our voluntary control of its movement, is extremely slight, although there must be a continual flow of afferent impressions from the tongue to the lingual motor centres to guide the complex movements both of mastication and deglutition. In the case of the palate muscles, as of the œsophagus, muscular sensibility is entirely wanting.

It has been suggested that afferent impressions from the muscles can play only a subordinate part in our sensations of movement, since we are not aware of the part taken by each individual muscle in any given movement. Such a statement is absurd. We have no objective phenomenal experience of our muscles. All that we are aware of and can judge of by our other senses is the movement as a whole, and our sensation of movement is therefore referred to the whole movement and not to the individual muscles.

The sensations arising in the proprioceptive system can be divided into two main classes :

(1) The sensation of the relative positions of parts of the body.

(2) The sensations which inform us of the position of the head, with regard to its surroundings, i.e. with regard to the direction of the pull of gravity. (It must be remembered that 'downwards' always means towards the centre of the earth, ' upwards' away from the centre of the earth, i.e. against the gravitational forces.) This orientation sense depends on the integrity of a special sense-organ contained in the labyrinth of the internal ear. It is therefore sometimes spoken of as the labyrinthine sense.

\section{THE SENSE OF RELATIVE POSITION, INCLUDING THE MUSCULAR SENSE}

Without using our eyes we are able at any moment to tell the position of our limbs. If one arm be moved passively into any position we can without difficulty move the other arm into an exactly similar position. We thus know the extent to which we move the limb and the static position attained as the result of the movement. If the movement is resisted, we are able to 
adjust the force of the muscular contraction to the resistance, and to form therefore a fair idea as to the strength of the resistance.

(a) PASSIVE MOVEMENTS. A large number of different sense-organs contribute to the formation of these judgments. In the appreciation of passive movement the chief end-organs involved are those in connection with the joints and their ligaments, though it is probable that the deeper senseorgans in the soft parts around the joints also contribute to the total sensation. Cutaneous sensations apparently play but little part in the judgments of passive movement. It is true that the alternating movements of the hind limbs, which occur in a spinal animal when it is held up by the hands under the fore limbs, are started, partly at any rate, by the stretching of the skin of the thighs ; but this effect is one rather of initiation of movement, and can hardly be regarded as proprioceptive in character.

The strength of the sensation of passive movement depends on the extent of the movement as well as on the rate with which it is carried out. The delicacy of perception varies in different joints. Thus in some joints a movement of $0 \cdot 25^{\circ}$ per second is appreciated as a movement, while in other joints the movement must be as extensive as $1 \cdot 4^{\circ}$ per second. It is more easily appreciated when the joint surfaces are pressed together than when they are pulled apart, showing that the nerve-endings in the joint surfaces play a part in the origination of the sensations.

(b) THE SENSE OF MOVEMENT (MUSCULAR SENSATION). This term is applied to those sensations by which we judge of the extent and force of any active movement which we may have carried out. Many authors have ascribed an important part in this act of judgment to the so-called 'sense of innervation,' i.e. a sense of the actual energy which is being discharged from the motor cells of the central nervous system to the muscles, and have thought that when we raise a weight we judge of its amount, not by the degree of stretching of the muscle or pressure on sensory nerves in the muscle, but by the amount of force we voluntarily put out to raise the weight. The fact, however, that we can judge of weights, when the muscles are made to contract by electrical stimuli and not by voluntary impulses, shows that this sense is in large part, if not entirely, peripheral. It is, however, very complex in nature, and is served by a whole array of different end-organs in the skin, joints, tendons, and muscles. The muscles themselves are known to be well supplied with afferent nerves. Stimulation of the central end of a muscular nerve may reflexly excite or inhibit movements of other muscles. Sherrington has shown that, after section of the motor roots, over one-third of the fibres in a muscular nerve remain undegenerated, proving their connection with the posterior root ganglia. The sensory nerveendings in the muscle are represented partly by the tendon nerve-endings and partly by the muscle-spindles. The former are richly branched endarborisations of nerve fibres on the surface of the tendon bundles. The muscle-spindles consist of one or more muscle fibres, often continuous with normal fibres, enclosed in a sheath composed of several layers of fibrous tissue with intervening lymph-spaces. One or more nerve fibres pierce this 
sheath and, after making many spiral turns round the muscle fibres, branch freely and terminate in little knobs on the surface of the fibres (Figs. 310,311). The cross-striation of the muscle fibres within the spindle is but faintly marked. It is evident that the continuity of these sense-organs with the contracting muscle ensures in the best possible way that the organs should

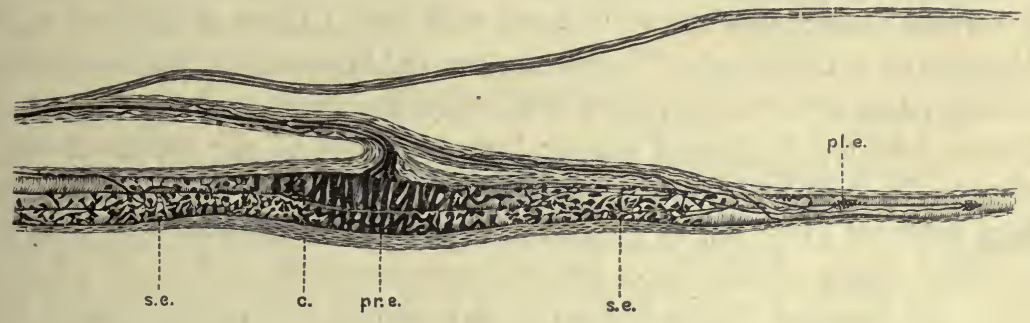

Fig. 310. A neuro-muscular spindle of the cat. (RuFrivi.)

$c$, capsule; pr.e, primary ending; s.e, secondary ending; pl.e, plate ending (all these are probably sensory in function).

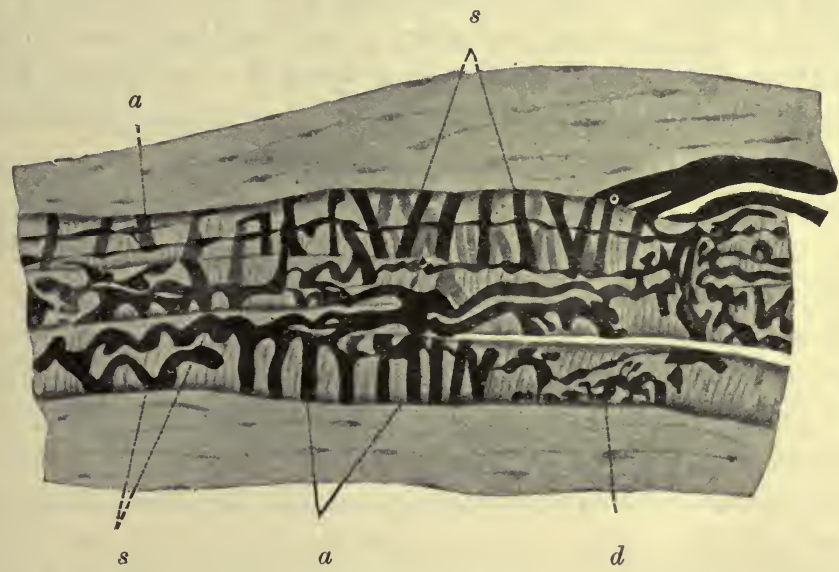

Fig. 311. Part of a muscle-spindle more highly magnified.

$n$, nerve fibres passing to spindle ; $a$, annular endings of axis cylinders ; $s$. spiral endings ; $d$, dendritic endings; $s h$, connective-tissue sheath of spindle. (RurrinI.)

be affected by the slightest change of tension of the muscle, and should transmit information of the state of tension to the central nervous system:

THE PSYCHOLOGICAL SIGNIFICANCE OF SENSATIONS OF MOVEMENT. Not only are these organic sensations of importance as affording us information of the condition of our own bodies as distinct from the objects in the world around, but they enter into and qualify our judgments derived from all the sensations which arise in the special sense-organs.

When we regard the continuous aimless activity of a healthy baby, we see that all ideas of space, of extension, of relative position are wanting, or at any rate are not present to guide the movements. Bit by bit muscular experience is acquired. The child learns that a given movement of the right arm will bring the hand in contact with something which is exciting the left 
side of his retina. The surface of the thing, if of sufficient extension, can excite tactile sensations in all the fingers of the right hand. By moving one finger over the object the tactile sensations are found to be continuous ; by moving the whole hand forwards the thing is found to possess extension in a direction away from the body, and therefore in the third plane of space. Thus gradually are acquired not only ideas of extension, distance, and space, but certain movements are correlated with stimulation of definite regions of the skin or of the retina. Tactile and retinal impressions therefore acquire local sign, and power is acquired of moving the limbs to a degree and in a direction adapted to stimuli arising from any part of the tactile or retinal surfaces. The child gradually acquires the power of following a bright object with its eyes, i.e. of contracting the ocular muscles so as to keep the retinal image of the object on the fovea centralis, and up to adult age we are still engaged in this balancing of muscular movement against sense impressions - a balancing in which the muscular sensations are the constant guide and criterion of success. Only by the muscular sensations are we informed whether our willed movement has been carried out or not. It is in virtue of the muscular and allied sensations that we are able to clothe our visual and tactile sensations with properties of extension, solidity, and resistance, which create them in consciousness as parts of a material world. 


\section{SECTION XIII}

\section{THE LABYRINTHINE SENSATIONS}

Throvghout almost the whole of the animal kindgom, and in practically all freely moving metazoa, we find a sense-organ which has often been designated as an auditory organ. This organ, which is situated in the integument, is in the form of a small sac generally open to the exterior, and lined by cells provided with hairs and richly supplied with nerves. Resting among the hairs is a small concretion, generally of carbonate of lime, which is known as an otolith. These sacs have generally been regarded as auditory in function, hence the term otolith applied to the concretion. The evidence for audition, i.e. the power of appreciating vibrations in the elastic medium surrounding them, is scanty. Thus in fishes this power has been stated to be absent unless the vibrations are of sufficient amplitude to affect the senseorgans of the skin.* On the other hand, there is evidence that these otolith organs are connected with equilibration. Section of the nerves going to them in the crayfish causes disturbance of locomotion. Steinach has succeeded in the crayfish in replacing the concretion by a small particle of iron. The animal's behaviour and movements were perfectly normal until it was brought within a powerful magnetic field. Under the influence of this field the effect of gravity on the iron particle was annulled and replaced by a force of attraction in another direction, and the effect was at once seen as pronounced disorders of locomotion, the animal swimming in an abnormal position.

From a sac, such as that present throughout the lower animals, the organ of hearing in the higher vertebrata is developed. Arising as a pit in the epiblast in the neighbourhood of the hind-brain, the auditory sac becomes shut off from the exterior, and then, by an outgrowth in various directions, forms the complex membranous labyrinth of the internal ear. This membranous labyrinth, as we have seen, can be divided into two parts, viz. the canalis media of the cochlea in front, and the saccule, utricle, and semicircular canals behind. The canalis media of the cochlea is concerned with the reception and analysis of sound waves. In the lower vertebrates in which auditory sensations are wanting the cochlea is absent, and in fishes is represented merely by a small diverticulum known as the lagena. With the development of air-breathing vertebrates we see the first signs of a special

* On the other hand, Piper has succeeded in detecting an electrical variation in the eighth nerve of fishes in response to a sound stimulus. 
organ of hearing. Thus a primitive cochlea is present in the amphibia, and especially in the anura, and in some of the reptiles as well as in birds it acquires a bend and shows the beginning of a spiral arrangement. Only in the mammals does it attain a degree of development at all comparable with that found in man, and characterised by the formation of one and a half to four spiral turns in the cochlea as well as in the canalis media.

This development of auditory functions cannot involve any abrogation of the important part played by the otolith organ throughout all the lower classes of the animal kingdom. In man, as in the crayfish, it is the otolith

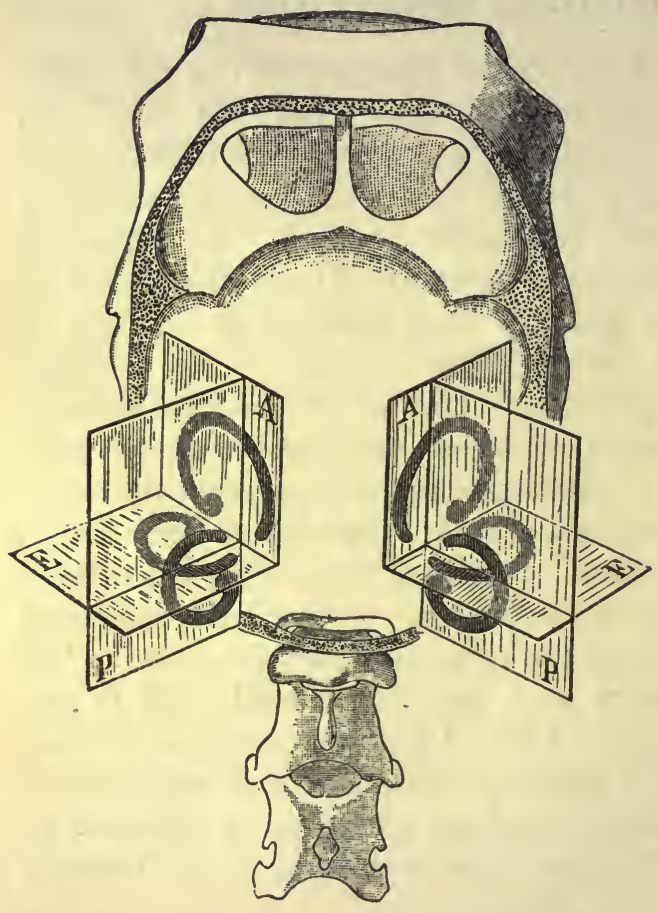

Fig. 312. Figure from Ewald showing the situation of the three semicircular canals in the skull of the pigeon. organ which determines his behaviour in relation to the force of gravity, and is therefore responsible not only for the maintenance of equilibrium but also for the sensations which enable him consciously to orientate himself and to know the position in which he happens to be at any given moment. With the increasing importance of visual sensations in determining the behaviour of the animal, close connections are established between the central connections of the nerves running from the otolith organ and the parts of the brain concerned with the innervation of the eye muscles. By this means the position of the eyes is constantly adapted to the position of the head.

The auditory part of the internal ear has already been described. That part of the labyrinth which represents the primitive otolith organ consists of a bony framework containing perilymph, in which is contained the membranous labyrinth with the endings of the vestibular division of the eighth nerve. The osseous labyrinth consists of a cavity, thes vestibule, into which open behind the three bony semicircular canals. In the vestibule are contained two little membranous sacs, the utricle and saccule, the cavities of which are connected by means of the saccus endolymphaticus. Into the utricle open the three semicircular canals, the three canals having five openings. These semicircular canals are arranged in three planes, each of which is at right angles to the other two, so that in the organ are represented the three planes of space. We may distinguish on each side an external or horizontal canal, an anterior or superior vertical canal, and a posterior vertical canal. The two outer canals lie always exactly in the same plane, which is practically horizontal in the normal position of the head. Each posterior vertical canal lies in a plane which is parallel to that of the superior vertical canal of the opposite side. We thus see that these semicircular canals form 
together three planes-one horizontal and two vertical, the two latter being at righ. angles to one another (Fig. 312). The membranous canal lies within the osseous canal, a considerable space intervening between the two canals. At one end the osseous canal is dilated and the membranous canal undergoes a corresponding dilatation so as to fill up the whole bony canal. In this dilatation, which is known as the ampulla, we find the ending of a branch of the vestibular nerve in a special sense epithelium forming the crista acustica (Fig. 313). The crista is composed of hair-cells with sustentacular cells between them. The fibres of the vestibular nerve end in arborisations

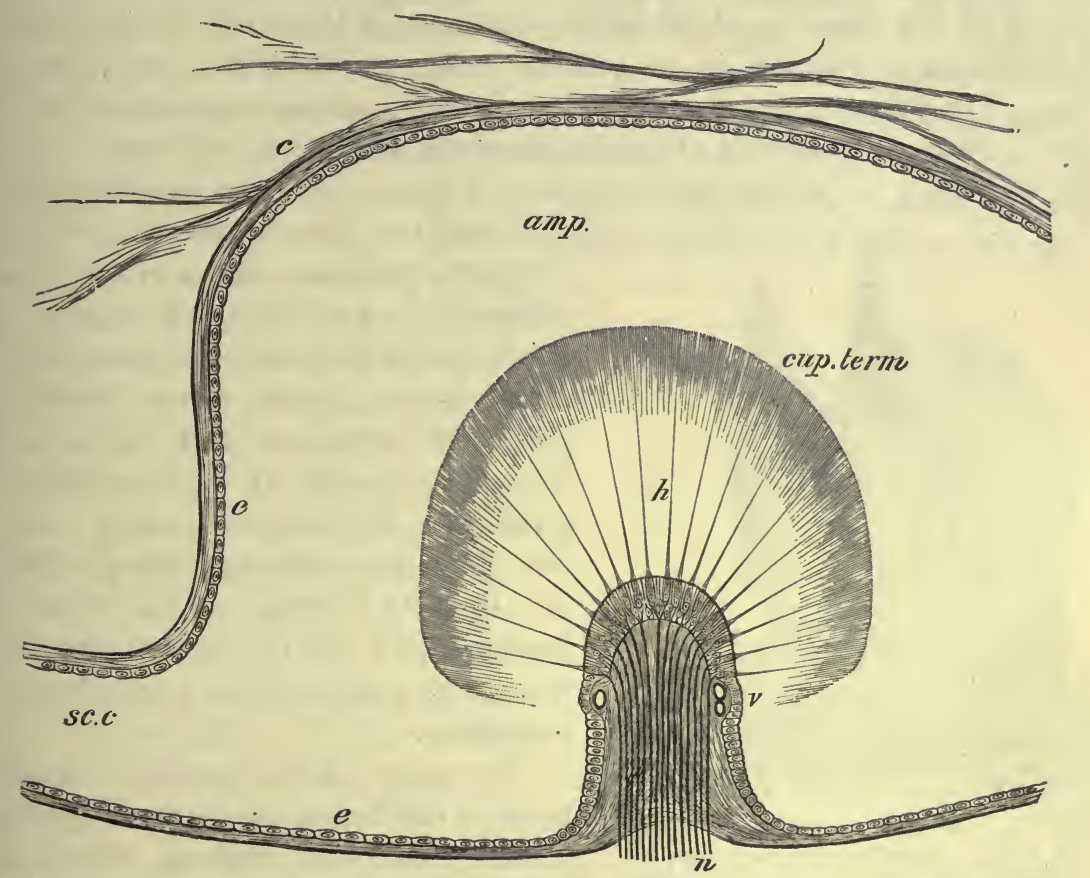

FIG. 313. End-organ of vestibular nerve in ampulla of semicircular canal (' crista acustica ').

among the hair-cells, the hairs of which project into the endolymph filling the ampulla. In the utricle and saccule we also find special sense-organs, known as the macula acustica, the structure of which is very similar to that of the crista in the ampullæ. Among the hairs, however, of the macula is found a small concretion of carbonate of lime, the otolith.

The first accurate experimental investigation of the functions of these different parts we owe to Flourens. This observer showed that, whereas extirpation of the cochlea caused deafness, extirpation of the vestibule and semicircular canals left the auditory sense intact, but caused marked disorders of equilibration. That the peculiar arrangement of the semicircular canals in the three planes of space was connected in some way with the functions of these structures was also indicated by Flourens' observation that destruction of the horizontal canals on each side gave rise to continual nodding movements of the head in the plane of the injured canals. By many physiologists the results obtained by Flourens were ascribed to continued irritation of the peripheral sense-organs or of the central parts of the brain in consequence of the lesion. The accurate experiments of Goltz, and especially 
those of his pupil Ewald, showed that these effects might last twelve to eighteen months, or be permanent, and must therefore be regarded as an Ausfallserscheinung, i.e. as due to abolition of a function and not to the arousing of a function by abnormal stimulation.

Most of the experiments on this subject have been carried out on pigeons on account of the easy accessibility of their semicircular canals. Confirmatory observations have, however, been made on mammals. After destruction of all the canals or of the whole membranous labyrinth on both sides, disturbances of equilibrium are aroused which may last for a considerable time. The animal can neither stand, nor fly, nor maintain any fixed attitude, but is constantly moving about incoherently and often so violently that it is necessary to pad its cage in order to prevent it from injuring itself.

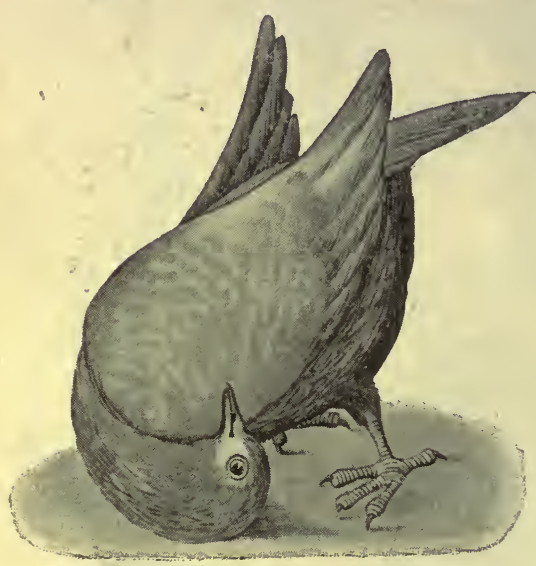

Fig. 314. Abnormal posture of pigeon, in which the labyrinth had been extirpated on one side) five days previously. (EWALD.) Although the movements are so violent, very little guidance suffices to stop them altogether. Any support given by the hand enables the animal to rest quietly. After some months these disorders gradually disappear, and the animal learns to guide its movements by sensations of touch and sight alone; but they are instantly. brought back in all their severity if the eyes be bandaged, so as to deprive the coordinating centres of the guiding visual sensations.

The same effect is produced if that part of the brain which alone is educatable, viz. the cerebral cortex, be excised. Extirpation of the cerebral hemispheres in pigeons causes no disorders of equilibrium, but extirpation, after destruction of the labyrinth, brings back the disorders which were noted during the first days after the operation, and these disorders are now permanent. Recovery even in the presence of the cerebral hemispheres is, however, never really complete. Although the animal may be able to walk and fly very fairly, it suffers from a loss of power and loss of tone which affect all its muscles, but especially those moving the trunk and neck. If the labyrinth has been extirpated only on one side, then this loss of tone is noticed chiefly on the opposite or contralateral side of the body (Fig. 314). Loss of tone after complete destruction is well shown in the following experiment devised by Ewald :

A small lead bullet.is hung by a thread to the beak of the pigeon. As the bird moves about the bullet swings, the head following its movements ; finally the bullet happens to fall over the beak of the animal - the head is now found to be fixed in the position shown in the figure (Fig. 315). The anterior muscles of the neck are too weak and toneless to restore the head to its 
normal position against the weight of the bullet. No such phenomena are presented by a normal bird.

The same absence of tone is seen in mammals. A dog with both labyrinths destroyed may jump down from a table once, but will not repeat the experiment, since the muscles of the fore limbs are too toneless to support the head against the shock of the jump, and he knocks his head against the ground as his legs collapse under him. If only one canal be put out of action, as, for instance, by stopping it with dentist's amalgam, the head is thrown into oscillations in a corresponding plane, or perhaps rather we should say that when the head oscillates in this plane there are no corresponding sensations set up which tend to inhibit the movements. The same effect may be produced temporarily by painting any one of the canals with cocaine so as to paralyse its nerve-endings. The converse experiment of isolated stimulation of one canal has also been effected by Ewald. For this purpose Ewald, by means of a dentist's burr, opened one bony canal at two spots. By the hole furthest away from the ampulla he introduced an amalgam stopping, so as to prevent any current of fluid backwards through the canal. Over the second hole he fixed, by means of plaster of Paris, a tube which was connected by a flexible rubber tube with a rubber ball. By this means, while the bird was sitting quietly on its perch, he could suddenly blow upon the exposed membranous canal without disturbing the bird in any way.

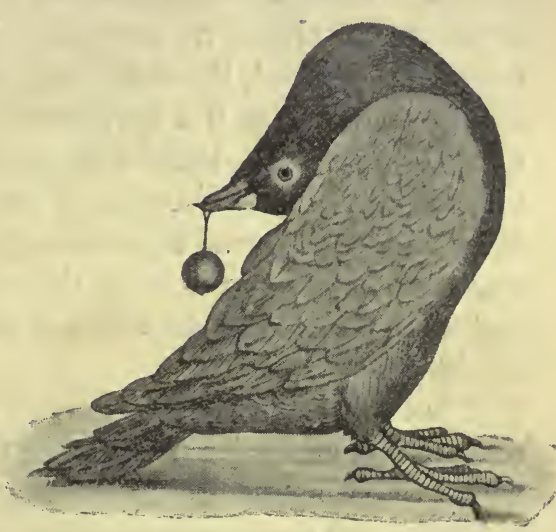

FIG. 315. By the air pressure thus produced on the canal a stream of endolymph was caused in the direction of the ampulla. Every time this was done he found that the animal moved its head and eyes in the direction of the current and always exactly in the plane of the canal which was being stimulated. By this means proof was brought of the correctness of the theory put forward by Breuer and Mach, viz. that the specific stimulus of the nerve-endings in the ampulla is afforded by the current of the endolymph in the semicircular canals.

Since the endolymph is a fluid with inertia it will not immediately follow a rotational movement of the bony walls of the semicircular canals. Thus a sudden turning of the head from left to right will cause movement of endolymph towards, and therefore increased pressure on, the ampullary nerveendings of the right horizontal canal, and movement of endolymph away from, and therefore diminished pressure on, the corresponding ampulla of the left side. In this way, for movement in any given plane, the two corresponding semicircular canals of the two sides are synergic, and unite in sending impulses which guide the equilibrating centres, and inform us of 
the position of our head in space. "One canal can be affected by and transmit the sensation of rotation about one axis in one direction only : and for complete perception of rotation in any direction about any axis six semicircular canals are required in three pairs, each pair having its two canals parallel (in the same plane), and with their ampullæ turned opposite ways. Each pair would thus be sensitive to any rotation about a line at right angles to its plane or planes, the one canal being influenced by rotation in the one direction, the other by rotation in the opposite direction "(Crum Brown). These reflex movements of head and eyes are the invariable result of movements set up in the endolymph, and occur equally well in the absence of the cerebral hemispheres. If an animal or man be placed on a turntable and rotated, his first tendency will be to turn his head and eyes in the opposite direction to that of rotation. If the rotation be continued, the endolymph gradually takes up the movement of the surrounding parts of the head, and if the eyes be closed, no movement of head or eyes is observed. If now the rotation is stopped, the endolymph will tend to go on moving, and the effect will be the same as if a movement of rotation were suddenly begun in the opposite direction. Head and eyes will now be turned, without any voluntary impulse, in the direction of the previous rotation, and in consciousness there will be an actual sensation of rotation in the opposite direction. This sensation is in opposition to the sensations derived from other parts, and hence the feeling of giddiness and the actual disorders of equilibrium which are its concomitants.

That this feeling of giddiness on rotation is due to impulses started in the semicircular canals is shown by the fact that, in a large number of deaf-mutes where these organs are imperfectly developed, it is impossible to produce giddiness and the associated eye movements by passive rotation.

\section{THE FUNCTION OF THE OTOLITHS}

The semicircular canals are, as we have seen, a higher development of the otolith organ. The primitive part of this organ is represented by the maculæ in the utricle and saccule. It is to these organs that we must ascribe our powers of appreciating the static position of the head, as well as, to a slight degree, movements, not of rotation, but in one plane forwards or backwards.

A consideration of the structure of the otolith organ shows at once that the incidence of the weight of the otoliths on the hairs of the macula will vary according to the position of the head. Thus in the diagram (Fig. 200, p. 399) in $\mathrm{A}$ (normal position) the chief weight of the otolith falls on the hairs from $b$ to $c$, whereas, when the head has been rotated round a right angle so that the man, for instance, is lying on his right side, the chief weight of the otoliths will fall on the hairs at $\mathrm{c}$. The nerve-endings stimulated by the weight of the otoliths will therefore vary according to the position of the head. The cerebellum and its associated structures represent a mechanism for the regulation of the movements of the trunk as a whole and the position of its centre of gravity in relation to the position of the head. 
The beginning and ending of all movements of the head, or any change in the rate at which it is moving, must cause a momentary alteration of the incidence of pressure of the otoliths on the sensory hairs. Any translatory movements of the head must therefore excite a set of nerve fibres which will be constant for each direction. We are therefore justified in ascribing to these organs the functions possessed by the otolith organ throughout the animal kingdom, viz. the transmission of impulses to the central nervous system which are aroused by the position or movements of the head, and, like the sensations from the muscles, regulate and govern any motor reaction to a sensory timulus. Of these afferent impulses a certain proportion will arrive at the cerebral cortex, and in consciousness will inform us of our position in space and of the direction and extent of any movement, active or passive, of the head. 



\section{BOOK III}

\section{THE MECHANISMS OF NU'TRITION}




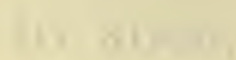

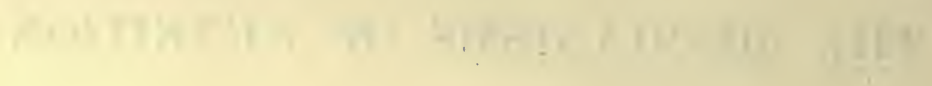




\section{CHAPTER IX}

\section{THE EXCHANGES OF MATTER AND ENERGY IN THE BODY}

\section{GENERAL METABOLISM}

ALL the energy which leaves the body as heat or work is derived from processes of oxidation, the carbon, hydrogen, nitrogen, and sulphur of the foodstuffs uniting with oxygen in the body and being eliminated in the form of carbon dioxide, water, urea and other substances, and sulphates. In a starving animal this discharge of energy must be associated with a loss of body substance. The necessity for taking food is determined by the need of replacing this loss. The food-stuffs cannot, like the coal or fuel of a steamengine, be utilised directly as a source of energy, but must be built up to a greater or less degree into the structure of the living protoplasm. The total amount of living material in the body, though maintained fairly constant in the adult animal, may yet undergo alterations under varying conditions, and these alterations are naturally more marked in the growing animal. We have in this chapter to inquire into:

(1) The nature and amount of the substances which may serve as foodstuffs and are necessary for maintaining the weight of the body constant or providing for its growth ;

(2) The relation between the total amount of material taken up by the body and the total amount given out;

(3) The variations in the total chemical exchanges determined by variations in the output of energy by the body; and

(4) The significance of the various classes of food-stuffs as sources of energy and in the replacing of tissue waste.

We have therefore to make balance-sheets of two kinds, namely: (1) an accurate comparison of the ingesta (food and oxygen) and the egesta (carbon dioxide, water, urea, \&c.) ; and (2) one showing the amount of potential energy introduced into the body $c$ smpared with the amount of energy set free in the body. 


\section{SECTION I}

\section{METHODS EMPLOYED IN DETERMINING THE TOTAL EXCHANGES OF THE BODY}

THE determination of the material exchanges of the body involves an accurate comparison of its income and output. The income consists of the food-stuffs and oxygen. The food-stuffs may be divided into two classes, namely, (1) the organic food-stuffs, which on oxidation may serve as sources of energy, and (2) the inorganic food-stuffs, such as salts and water.

The latter class neither add to nor subtract from the total energy of the organism, but their presence is a necessary condition of all vital processes, and as they are contained in the various excreta a corresponding amount must be present in the food in order to make good this loss.

In spite of the bewildering complexity of the nature of the foods taken by man, their essential constituents can always be confined to the three classes, proteins, fats, and carbohydrates, and any analysis of the food must give the relative amounts present of these three classes of substances. The approximate analysis of the food-stuffs presents little difficulty. The nitrogen is determined by Kjeldahl's method. The figure thus obtained is multiplied by the factor $6 \cdot 25$, and the resulting figure is taken to represent the total protein in the food. Of course such a valuation may give too high a value when the food-stuff is one that is rich in nitrogenous extractives. The total fat is determined by extracting the food in a Soxhlet apparatus with ether. It is advisable to precede this extraction by an extraction with boiling alcohol. The total ethereal and alcoholic extract obtained is reckoned as fat. The amount of water is determined by drying the food-stuff at $110^{\circ} \mathrm{C}$, and the amount of inorganic constituents by ashing the dried remainder. Carbohydrates may be determined directly by boiling the food with dilute acids in order to convert all its disaccharides and polysaccharides into hexoses, which are then reckoned as glucose, and estimated by their copper-reducing power. In most cases, however, the total protein, fat, and ash are subtracted from the dried weight of the food and the remainder is taken as carbohydrate.

Although the methods for the analysis of food-stuffs are by no means diffcult, the total analysis of the food during a metabolism experiment may become extremely tedious on account of the very large number of analyses which have to be performed. The labour is lightened by the fact that nearly all the ordinary food-stuffs have been subjected to analysis and their average 
composition published by the Agricultural Board of the United States. Since, however, the foods vary in composition, especially in water content, from time to time, a calculation of the total income of proteins, fats, and carbohydrates from data given by workers in other lands must present a considerable margin of error. In order to attain greater accuracy some observers have made a complete food in the form of biscuits or of preserve which is prepared in large quantities at the beginning of the experiment and used as the sole food throughout the experiment. Pflüger, for instance, converted the horse-flesh, with which he desired to feed his dogs in a metabolism experiment, into sausage meat which was sealed up in cases and sterilised. The sausage meat having been analysed at the beginning of the experiment, it was only necessary thereafter to weigh the amount eaten by the dog in order to know accurately the total amount of protein, fat, and carbohydrate ingested by the animal. In experiments on man it has been endeavoured to obtain the same result by limiting the food to a few articles of diet which could be accurately analysed in each case. The monotony of such a diet tends to interfere with the success of the experiment, since the subject of the experiment loses his appetite and his processes of nutrition are not normally carried out. It is usually possible to steer a middle course between the two extremes of too much and too little variation of diet, and so to obtain values for the composition of the ingesta which cannot differ very largely from their true composition.

The material output of the body consists of the products of combustion of the food-stuffs, which are turned out by the various channels of excretion, namely, the kidneys, the alimentary canal, the lungs, and the skin. These excreta must therefore be collected and analysed. In addition to the main sources of excretion, small quantities of material are lost by the shedding of the cuticle, by the growth and cutting of the hair and nails, and so on. In most cases the losses in this way are so small that they may be disregarded. The nitrogen of the food-stuffs and that derived from the disintegration of the tissues of the body is excreted almost exclusively in the urine, a small amount being thrown out by the alimentary canal. The total nitrogen must be therefore determined both in the fæces and in the urine. The nitrogen in the fæces is derived from two sources. Part represents those nitrogenous constituents of the tissues which have resisted the digestive processes of the alimentary canal. There is in addition a certain amount derived from the intestine itself. During complete starvation fæcal masses are formed in the intestine, and it has been calculated that in a normal individual about one gramme of nitrogen a day is excreted by the mucous membrane of the gut and contributes to the formation of the fæces. It is usual therefore to regard one gramme of the nitrogen of the fæces as belonging to the output of the body and representing the result of nitrogenous metabolism, while the balance is taken as belonging to undigested food-stuffs, and is subtracted from the total nitrogen of the latter in reckoning the real income of the body. A small amount of nitrogen is also lost by sweat, but this can be disregarded unless the sweating is profuse, when the loss of nitrogen by this channel may 
rise to as much as 4 per cent. of the total nitrogenous output of the body. Although a trace of ammonia has been described as occurring in the expired air, the amount is so minute that any loss of nitrogen by the lungs can be neglected. That the loss both by lungs and skin under ordinary circumstances can be disregarded is shown by the fact that it is possible to account directly for the whole nitrogen of the body by a comparison of the composition of food with that in the urine and fæces. If, for instance, an animal is kept on a sufficient diet which contains a perfectly regular amount of nitrogen, after a few days a condition known as nitrogenous equilibrium is set up, i.e. the total nitrogen of fæces and urine is exactly equal to the total nitrogen of the food. The same thing applies to the sulphur, as is shown in the following Table (quoted by Tigerstedt) :

\begin{tabular}{|cc|c|c|c|c|c|}
\hline \multicolumn{2}{|c|}{$\begin{array}{c}\text { Days of } \\
\text { experiment }\end{array}$} & $\begin{array}{c}\text { Nitrogen } \\
\text { of food }\end{array}$ & $\begin{array}{c}\text { Nitrogen } \\
\text { excretcd }\end{array}$ & $\begin{array}{c}\text { Per cent. } \\
\text { difference }\end{array}$ & $\begin{array}{c}\text { Sulphur } \\
\text { ingested }\end{array}$ & $\begin{array}{c}\text { Sulphur } \\
\text { excreted }\end{array}$ \\
\hline $1-7$ & $\cdot$ & $154 \cdot 81$ & 153.02 & -0.51 & - & - \\
\hline $8-17$ & $\cdot$ & 213.72 & $213 \cdot 26$ & -0.21 & 12.77 & 12.79 \\
\hline
\end{tabular}

In order to express the nitrogenous metabolism in terms of protein, we use the factor employed in estimating the amount of protein in the food, i.e. we multiply the total nitrogen of the excreta by $6 \cdot 25$. This will give the total protein which has been broken down during the period of the experiment. Much more important from the energy standpoint is the determination of the total processes of oxidation of the body, information on which is given by a comparison of the oxygen intake with the output of carbon dioxide and water. The estimation of these substances presents much greater difficulties than the investigation of the nitrogenous exchange and involves the use of some form of respiration apparatus.

The following are the chief methods which have been employed for this purpose :

I. THE METHOD OF HALDANE. This method is extremely convenient when dealing with the gaseous exchanges of small animals, such as mice, rats, guinea-pigs

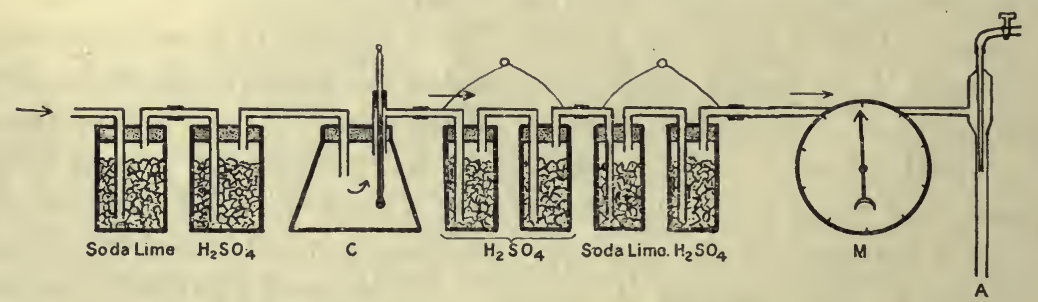

Fic. 316. Haldane-Pembrey respiration apparatus. c, chamber for animal ; $\mathrm{M}$, gas meter.

or rabbits. The animal is placed in the chamber c, which may be simply a widemouthed bottle (Fig. 316). This chamber is supplied with a thermometer, and can be kept at any desired temperature by immersion either in warm or cold water. On the inlet side of the bottle is a series of tubes or bottles, some of which contain sulphuric acid and pumice-stone, while the others contain soda lime. On the outlet 
side of the vessel is a corresponding series of vessels for the absorption of water and of carbon dioxide. On the further side of these vessels is a gas meter. During an cx. periment air is sucked through the whole apparatus by means of an aspirator or a water pump, the amount of air passing through the apparatus being measurcd by the meter. The animal is thus supplied with pure air freed from water vapour and from carbon dioxide. Any water or carbon dioxide produced by the animal is absorbed by the vessels interposed in the course of the outgoing air. These vessels are weighed at the beginning of the experiment and at the end, and the difference in weights will thercfore give the amounts of carbon dioxide and water which have bcen discharged by the animal.

The intake of oxygen by the animal is determincd indirectly. Since it gives off only carbon dioxide and water, and absorbs only oxygen during its stay in the chamber, the loss of weight of the animal during its stay in the chamber, subtracted from the total amount of carbon dioxide plus water it gives off, will represent the amount of oxygen absorbed.

The advantage of this apparatus is that it can be fitted up in any laboratory, and is accurate for the purposes to which it is applied. It is not, however, appropriate for long-continued experiments or for experiments on larger animals or on man himself. Most of the data with regard to the respiratory exchange under various circumstances have therefore been obtained by one of the three following methods :

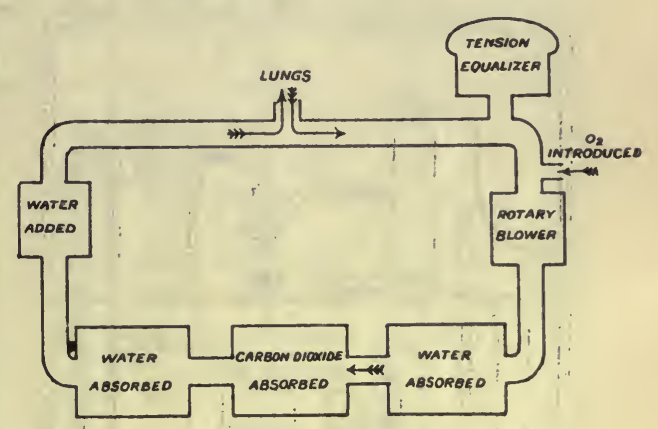

FIG. 317. Air circuit in Benedict's respiration apparatus.

II. THE METHOD OF REGNAULT AND REISET. The principle of this method consists in placing the animal that is to be the subject of investigation in a closed chamber containing a given volume of air. The carbon dioxide produced by the animal is absorbed by means of caustic alkali, and the oxygen consumed by the animal is macie good by allowing oxygen to flow into the chamber from a gasometer. The inflow of oxygen is regulated so as to keep the pressure of air in the chamber constant. At the end of the experiment the alkali is titrated and the amount of carbon dioxide absorbed thus determined. The air in the chamber is also analysed so as to be ccrtain that it contains an excess neither of carbon dioxide nor of oxygen. The amount of oxygen absorbed by the animal is known already, the oxygen which has been allowed to flow in having been measured.

A modification of this method has been deviscd by Benedict and is especially applicable to clinical purposes. In this method the individual who is the subject of the experiment breathes through a nose-piece into a wide metal tube, the mouth being kept closed. The metal tube forms part of a closed system through which a current of air is maintained by means of a pump. In the course of the current of air are interposed vesscls for the absorption of carbon dioxide and of water, and the volume of gas in the system is maintained constant by admitting oxygen to it in proportion as the oxygen of the system is used up in respiration. In Fig. 317 is given a diagrammatic scheme of the air circuit, and in Fig. 318 a diagram of the arrangement of the whole respiration apparatus, showing the nose-piece for breathing, the tension equaliser, the air-purifying apparatus, and the oxygen cylinder. The tension equaliser, $\mathbf{A}$, is attached to the ventilating pipe near the point of entrance of the air into the lungs. It consists of a pan with a rubber diaphragm (which may be conveniently made from a lady's bathing-cap). As the air is drawn into the lungs the rubber diaphragm sinks, to rise again with expiration. The respiratory movements can thus proceed without altering appreciably the pressure within the clcsed system of tubes. By the admission of oxygen the supply of oxygen is adjusted so as to keep the bag from becoming cither too much distended or too much flattened. As the air lcaves the lungs and passes 
into the constantly moving current of air, it is carricd along by the pump and flows through two Wolff's bottles containing strong sulphuric acid and pumice for the removal of watcr vapour. It then passes through a brass cylinder, c, filled with soda lime for the absorption of carbon dioxide. From here it passes again through sulphuric acid in a Kipp generator for the absorption of water given off by the soda lime. Since the air so deprived of moisture would be uncomfortable to breathe, it is then carried through another Kipp generator containing water with a trace of sodium carbonate for the neutralisation of any acid fumes which may be given off by the sulphuric acid. It then passes back to the tube from which the subject is breathing. In this way it is possible to determine very accurately the amount of oxygen used up and the amount

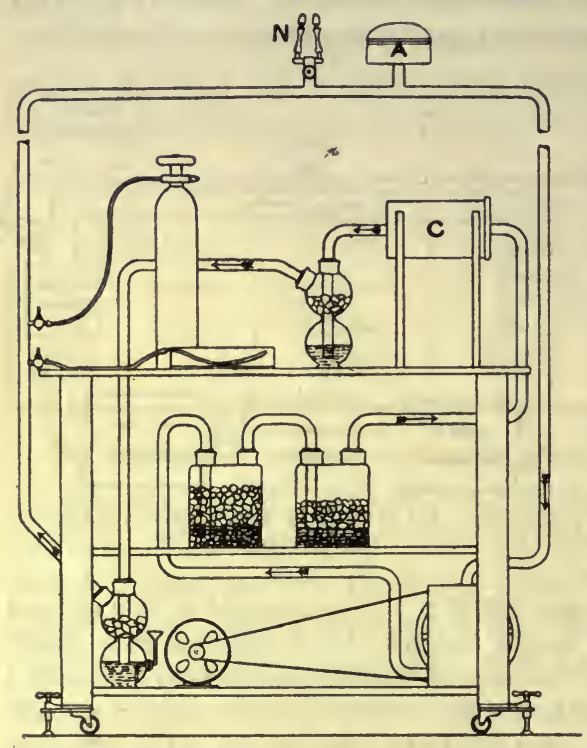

FiG. 318. Arrangement of apparatus in Benedict's method for determination of respiratory exchange.

$\mathrm{N}$, tubes inserted into nostrils of patient; A, tension equaliser; c, cylinder contain. ing soda lime for absorbing $\mathrm{CO}_{2}$. of carbon dioxide given off in the course of an experiment lasting one to three hours or longer. The oxygen consumption is measured by weighing the cylinder of this gas, chosen small for this purpose, bcfore and after the experiment.

III. PETTENKOFER'S METHOD. In the apparatus designed by Pettcnkofer the animal or man was placed in a chamber through which a constant current of fresh air was passed. The amount of air passing through the chamber was measured by means of a meter. Throughout the experiment continuous samples both of the air entering the chamber and of the air leaving the chamber were taken. The analyses of these samples served to show the composition of the whole air entering and leaving the chamber, and therefore the changes in the air caused by the presence of the animal. The advantage of this apparatus is that an adequate ventilation can be kept up, and the apparatus can be built of any size. In the apparatus of Tigerstedt built on this plan the chamber had a capacity of $100 \cdot 6$ cubic metres, and was, in fact, a small room. A small respiratory apparatus has been built by Atwater.

IV. ZUNTZ AND GEPPERT'S METHODS. For many purposes the methods devised by Zuntz and Geppert present many advantages, especially when it is desired to take the respiratory exchanges in man or any animal during a limited period of time. The subject of the experiment has his nostrils clamped and breathes into and out of a face-piece. This face-piece is provided with valves either of aluminium or of animal membrane, which serve to separate the in-going from the out-going current of air, In the course of the out-going current is placed a very delicate gas meter which presents practically no resistance to the air current. A branch from the efflux tube passes to a gas analysis apparatus. By an ingenious method it is arranged that an aliquot part of the whole of the out-going air is drawn off into this apparatus, so that the experiment can be interrupted at any time, and the analysis of this sample will give the average composition of the expired air, and therefore, on multiplication by the total gas passing through the gas meter, the total output of carbon dioxide during the course of the observation. One advantage of this method is that the apparatus is portable, and can be applied to the investigation of the respiratory exchanges of patients in hospitals or of man or animals while they are walking about. It has been used, for instance, by Zuntz and his pupils in an interesting series of researches on the gaseous metabolism of men at high altitudes. 
By means of one or more of these methods we may arrive at a correct idea of the total income and output of an individual for periods of many days. The following details by Tigerstedt may serve as an example of the results obtained in such an experiment. The experiment lasted two days. The subject was a man of twenty-six years of age, weighing about 65 kilos, who had previously taken no food for five days. The following Tables represent his material income and output.

Total Income

\begin{tabular}{|c|c|c|c|c|c|c|c|c|c|c|}
\hline Food & 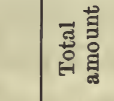 & N. & c. & 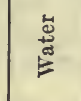 & 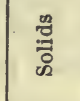 & $\begin{array}{l}\text { a } \\
\text { 莒 } \\
\text { a }\end{array}$ & Fat & 客蒡 & Ash & 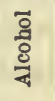 \\
\hline Bread & 373 & $7 \cdot 3$ & & 36 & 337 & 46 & 4 & 278 & 9 & - \\
\hline Butter & 388 & $0 \cdot 4$ & & 37 & 351 & 3 & 337 & 4 & 7 & - \\
\hline Cheese & 116 & $4 \cdot 3$ & & 56 & 60 & 27 & 35 & - & 5 & - \\
\hline Salt meat & 26 & $1 \cdot 1$ & & 16 & 10 & 9 & - & - & 2 & - \\
\hline Milk . & 2313 & $11 \cdot 3$ & & 2047 & 266 & 71 & 85 & 95 & 16 & - \\
\hline Broth & 658 & $11 \cdot 8$ & & 580 & 78 & 74 & - & - & 9 & - \\
\hline Beer & 1413 & $1 \cdot 2$ & & 1273 & 77. & 8 & - & 67 & 3 & 59 \\
\hline Beef steak & 700 & $20 \cdot 6$ & & 533 & 167 & 129 & 33 & - & 7 & - \\
\hline Potatoes & 452 & 0.9 & & 359 & 93 & 6 & 1 & 82 & 5 & - \\
\hline Water & 2335 & - & & 2335 & - & - & - & - & - & - \\
\hline Totals & 8773 & $59 \cdot 3$ & $831 \cdot 6$ & 7275 & 1439 & 371 & 497 & 525 & 61 & 59 \\
\hline
\end{tabular}

Total OUtput

\begin{tabular}{|c|c|c|c|c|c|c|c|c|c|}
\hline & \begin{tabular}{|c|} 
Total \\
amount
\end{tabular} & N. & c. & Water & Solids & Protein & Fat & \begin{tabular}{|} 
Carbo- \\
hydrate
\end{tabular} & Ash \\
\hline Respiration & \begin{tabular}{|l|}
2701 \\
\end{tabular} & - & $45 \cdot 0$ & 2248 & - & - & - & - & - \\
\hline Urine & 2564 & $41 \cdot 5$ & 32.5 & 2490 & - & - & - & - & 21 \\
\hline Fæces & 455 & $4 \cdot 8$ & $43 \cdot 8$ & 363 & $91 \cdot 6$ & 30 & 20 & 27 & 15 \\
\hline Totals & 5720 & $46 \cdot 3$ & $529 \cdot 3$ & 5101 & $91 \cdot 6$ & 30 & 20 & 27 & 36 \\
\hline
\end{tabular}

As we should expect in a man who had fasted five days, this balance-sheet shows a marked retention of the food taken in, i.e. a marked excess of income over output. Thus of the nitrogen ingested, 13 grm., which is equivalent to $81.3 \mathrm{grm}$. of protein, was retained ; of the carbon, $302 \mathrm{grm}$. was retained. Of this $302 \mathrm{grm} ., 42 \cdot 7 \mathrm{grm}$. would be contained in the $81 \cdot 3 \mathrm{grm}$. of protein, so that the rest of the carbon, namely, $259 \cdot 6 \mathrm{grm}$., was probably laid down in the form of fat. This would correspond to $339 \mathrm{grm}$. of fat. Of the salts contained in the ash of the food, $25 \mathrm{grm}$. were retained in the body. The carbon and nitrogen reappearing in the excreta serve as an index of the amount of metabolism of the food-stuffs which had occurred during the two days. In order to supply the energy requirements of the body during the time of the experiment, $498 \mathrm{grm}$. of carbohydrate, $59 \mathrm{grm}$. of alcohol, 
and $138 \mathrm{grm}$. of fat had been completely oxidised. The amount of protein used up during this time can be obtained by multiplying the nitrogen of the urine plus $1 \mathrm{grm}$. of nitrogen of the fæces by the factor $6 \cdot 25$, and is found to amount to $271.9 \mathrm{grm}$.

\section{THE ENERGY BALANCE-SHEET OF THE BODY}

The energy income of the body is measured by the potential energy of the food-stuffs, i.e. the amount of energy which can be evolved, either as heat, work, or in any other form, by the oxidation of the food-stuffs to the endproducts which occur in the body. Since it is convenient to have a uniform method of expressing the total potential energy of a food-stuff, we generally express it in calories, and speak of the heat-value of a food-stuff. The heat-value of any given food is the amount of large calories * which it evolves on complete combustion with oxygen, and is determined by burning a weighed quantity of the dried food-stuff in oxygen in the bomb calorimeter. The following heat values have been obtained for different food-stuffs :

\begin{tabular}{|c|c|c|}
\hline Substance & 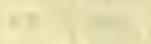 & Heat value \\
\hline Lean meat & . & $5 \cdot 656$ (or $5 \cdot 345$ Rubner) \\
\hline Lard . & . $\cdots$ & . $9 \cdot 423$ \\
\hline Butter & . & . $9 \cdot 231$ \\
\hline Grape sugar & • & . $3 \cdot 692$ \\
\hline Cane sugar & . & . $4 \cdot 116$ \\
\hline Starch & . & . $4 \cdot 191$ \\
\hline
\end{tabular}

In the case of some food-stuffs it is necessary to draw a distinction between the absolute heat-value and the physiological heat-value. Since carbohydrates and fats undergo complete oxidation in the body to carbonic acid and water, their physiological heat-values, i.e. the values of these foodstuffs to the organism, are identical with their absolute heat-values. Proteins, however, do not undergo complete oxidation. When they are oxidised in the bomb calorimeter the nitrogen is set free in a gaseous form. 'In the animal body no nitrogen is eliminated in the gaseous form, the whole of it being excreted as urea and allied substances still endowed with a considerable store of potential energy, which can be set free when their oxidation is completed in a calorimeter. In order to determine the physiological heat-value of protein we must subtract from its absolute heat-value the heat-value of the excretory products in the form of which it leaves the body. The physiological heat value of proteins has been determined by Rubner in the following way: A dog was fed with the same protein which had served for the determination of the absolute heat-value. While the dog was receiving this food its urine was collected, dried, and its heat-value determined by combustion in the calorimeter. It was found that for each gramme of protein which had undergone disintegration in the body an amount of urine was passed corresponding to a heat-value of 1.0945 calories. The heat-value

* A calorie is the amount of heat necessary to raise a gramme of water from $0^{\circ} \mathrm{C}$. to $1^{\circ} \mathrm{C}$. A large calorie is the heat required to raise a kilogramme of water from $0^{\circ} \mathrm{C}$. to $1^{\circ} \mathrm{C}$, and is therefore equal to 1000 small calories. 
of the fæces formed under the same diet was $0 \cdot 1854$ calorie for each gramme of protein. Rubner further reckoned that a certain amount of heat would be required for the solution of the proteins and of the urea, and reckoned this at 0.05 calorie. The reduced or physiological heat-value of protein is therefore equal to $5 \cdot 345-(1 \cdot 0945+0 \cdot 1854+0 \cdot 05)=4 \cdot 015$ calories.

A determination of the heat-values of the various food-stuffs shows minute differences between individual members of the same class. Since it is impossible to reckon out accurately the relative amounts of the different kinds of protein, carbohydrate, \&c., contained in each diet, Rubner has calculated the average physiological heat-values of the three classes of foodstuffs. These figures have been universally adopted, and are as follows :

$\begin{array}{ll}1 \text { grm. protein } & =4 \cdot 1 \text { calories } \\ 1 \text { grm. fat } & =9 \cdot 3, \\ 1 \text { grm. carbohydrate } & =4 \cdot 1,\end{array}$

Careful experiments have shown that just as there is no loss of matter in the body, so also the sum of the energies put out by the body is equal to the sum of the energy obtained by the oxidation of the tissues and of the food-stuffs in the body during the same time. In an earlier chapter I have quoted the results of an experiment by Rubner on a dog, which demonstrated this equivalence, as proving the important fact that the fundamental doctrine of the Conservation of Energy applies to the organised as to the inanimate world. Similar results have been arrived at by Atwater in a series of experiments with a special calorimeter on man. It may be sufficient here to give the figures from one such experiment:

\begin{tabular}{|c|c|c|c|c|c|c|c|c|c|}
\hline & a & b & c & $d$ & e & f & g & $\mathrm{h}$ & i \\
\hline $\begin{array}{r}\text { Date } \\
\text { Dec. } 9-10 . \\
10-11 . \\
11-12 . \\
12-13 .\end{array}$ & $\begin{array}{l}\text { Cals. } \\
2519 \\
2519 \\
2519 \\
2519\end{array}$ & $\begin{array}{r}\text { Cals. } \\
110 \\
110 \\
110 \\
110\end{array}$ & $\begin{array}{r}\text { Cals. } \\
142 \\
133 \\
132 \\
133\end{array}$ & $\begin{array}{l}\text { Cals. } \\
-85 \\
-25 \\
-21 \\
-14\end{array}$ & $\begin{array}{l}\text { Cals. } \\
+3 \\
-44 \\
-93 \\
-55\end{array}$ & $\begin{array}{l}\text { Cals, } \\
2349 \\
2345 \\
2391 \\
2345\end{array}$ & $\begin{array}{l}\text { Cals. } \\
2414 \\
2386 \\
2413 \\
2375\end{array}$ & $\begin{array}{l}\text { Cals. } \\
+65 \\
+41 \\
+22 \\
+30\end{array}$ & $\begin{array}{r}\% \\
+2 \cdot 8 \\
+1 \cdot 7 \\
+0 \cdot 9 \\
+1 \cdot 3\end{array}$ \\
\hline Total 4 days & 10076 & 440 & 540 & -145 & -189 & 9430 & 9588 & +158 & \\
\hline $\left.\begin{array}{l}\text { Average } \\
\text { one day }\end{array}\right\}$ & 2519 & 110 . & 135 & -36 & -47 & 2357 & 2397 & +40 & $+1 \cdot 7$ \\
\hline - & 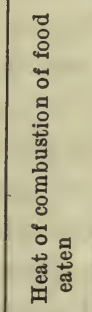 & 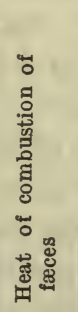 & 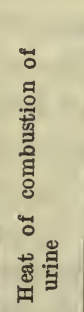 & 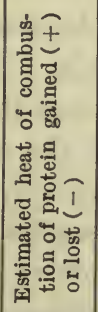 & 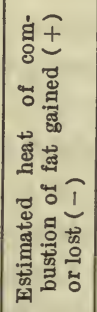 & 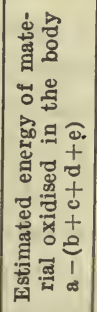 & 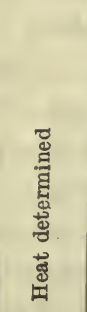 & 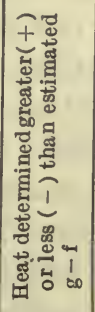 & 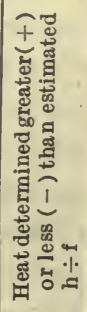 \\
\hline
\end{tabular}


It we take into account the great difficulties of such an experiment, we cannot but be impressed with the closeness of agreement between the total output of energy reckoned as heat and measured by the warming of a given volume of water and the total income of energy as estimated from the chemical reactions involved in the metabolic changes which had taken place during four days of the experiment. The important result which comes out in such experiments is that the food-stuffs produce the same amount of energy

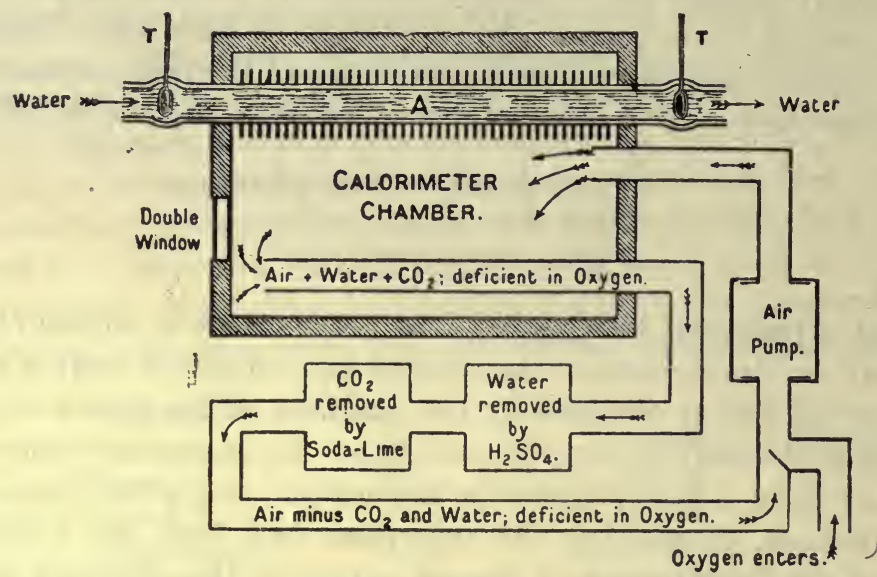

Fig. 319. Diagram to show the principle of the Atwater -Benedict calorimeter.

(After Halliburton.)

- when oxidised in the body as when burnt to the same end-products outside the body, so that it becomes easy in any given research to sum accurately the energy income of the body.

The Atwater calorimeter has been improved to such an extent by Benedict and his fellow workers that it has practically replaced all other forms for physiological purposes. It consists of a room or chamber with double non-conducting walls. All round the inner wall of the room are fitted coils of pipes through which a stream of water flows. The pipes are fitted with discs so as to take up rapidly heat produced in the room. The current of water is accurately adjusted so as to maintain the temperature of the inner wall constant. As the inner wall and outer wall are kept at the same temperature, no heat is lost to the exterior, the whole of the heat produced by the animal or individual in the chamber being communicated to the water passing through the chamber. The temperatures of the entering and leaving water are taken by accurate thermometers reading to a hundredth or a thousandth of a degree Centigrade. Knowing the amount of water that has passed through in a given time and the difference in temperature during the same time, it is easy to calculate the amount of heat given off by the animal under investigation. It is generally convenient to maintain a constant difference of temperature between the entering and leaving water by appropriate adjustment of the amount of water passing through the apparatus. The equality of temperature between the inner and outer casing is recorded 
by electric thermo couples, any difference of temperature being at once compensated by electrically warming the cooler part. The chamber contains a bicycle or other arrangement for the performance of mechanical work. It is adequately ventilated by a current of air passing through an apparatus similar to that of Benedict, described on p. 617. It is thus possible to estimate simultaneously the total heat production of an individual as well as the respiratory exchanges, including both carbon dioxide output and oxygen intake. The general principle of the calorimeter is shown in the diagram (Fig. 319). The calorimeter is also supplied with bed, table, chair, \&c., and food can be introduced through a double window so that an experiment may be continued over two or three days on one and the same individual. 


\section{SECTION II}

\section{THE METABOLISM DURING STARVATION}

IT will tend to simplify our task if we deal first with the results of the experiments which have been made on the metabolic exchanges of animals during starvation, i.e. during a period when the whole energy involved in the maintenance of the movements of respiration and circulation, and in the maintenance of the body temperature, \&c., is derived from the animal's own tissues. It must be remembered that the tissues of an animal comprise two distinct classes. In the first class must be placed the living machinery of the body, generally composed of proteins or their near allies. In the second class are the fatty tissues of the body, which form no part of the ordinary machinery, but function simply as a storehouse of material which can be utilised for the production of energy. In addition to the store of fat there is, in a well-fed animal, a certain reserve of carbohydrate in the form of glycogen, deposited in the liver and muscles of the body. This store of glycogen is drawn upon to a large extent at the beginning of a period of starvation. The total amount of glycogen present at any time is so small in comparison with the possible amount of fat that it cannot provide the energy necessary for the prolonged period during which the maintenance of life is possible in a complete state of inanition, although it plays an important part during the first one or two days of a period of starvation.

Contrary to general belief, the condition of an animal which is completely deprived of food is not a painful one. For this statement we have not only such evidence as can be derived from inspection of animals placed in this condition, but also evidence derived from men who have voluntarily or involuntarily been deprived of food for considerable periods. Especially instructive in this connection are the cases of the so-called professional ' fasting men,' two of whom, Succi and Cetti, have been subjected to complete metabolic investigation during the period of their starvation. During the first day or two there is a craving for food at meal-times. This, however, passes off, and during the later portions of the experiment even the desire for food may be entirely absent. As might be expected, the restriction of food is followed by a diminution in the amount of water required by the animal. The essential characteristic of the state of inanition is an ever-increasing weakness, accompanied by a strong disinclination to undertake any mental or physical exertion whatsoever. The animal passes its time in a state of 
sleep or semi-stupor. In the case of Succi, who fasted for thirty days, considerable muscular exertion was undertaken on the twelfth and on the twenty-third day of starvation without any appreciable ill-effects. A strong effort of the will must have been necessary in his case to overcome the automatic instinct to preservation of life by the utmost economy in the expenditure of energy. The pulse-rate and the body temperature remain nearly normal until a few days before death, which is ushered in by an increase in the somnolent condition of the animal and by a gradual slowing of respiration and fall of temperature. The urine is naturally diminished with diminution in the output of urea and in the amount of water consumed. Some fæces are formed, and may be voided during or at the close of the starvation period. In Succi their amount varied from 9.5 to $22 \mathrm{grm}$. a day and contained from 0.3 to $1.0 \mathrm{grm}$. nitrogen. On microscopic examination they consisted of an amorphous material enclosing a number of crystals of fatty acids.

During the whole of the starvation period energy is being used up in the body for the maintenance of its temperature and the vital movements of respiration and circulation. Since this energy is derived from the destruction and oxidation of the tissues of the body, it is evident that starvation must be associated with a constant and steady loss of body weight. In experiments on man the daily loss of weight during the first ten days amounts to between 1 and 1.5 per cent. of the original total weight. This loss of weight does not affect all parts of the body alike. It might be imagined that, since the loss of weight is determined by the using up of the tissues of the body for the production of energy, those organs which are most active should show also the greatest loss of weight. The very reverse of this is the case, as will be seen from the following Table :

Percen fage Ijoss of Weight of Different Organs and Tissues during StaRvation. (Vort.)

\begin{tabular}{|c|c|c|c|c|}
\hline & Tissue & & $\begin{array}{c}\text { Percentage loss } \\
\text { of weight of fresh } \\
\text { tissue }\end{array}$ & $\begin{array}{l}\text { Percentage loss } \\
\text { of dry tissue }\end{array}$ \\
\hline Fat & & & 97 & - \\
\hline Spleen & & $\cdot$ & 67 & 63 \\
\hline Liver. & . & . $\quad$. & 54 & 57 \\
\hline Testes & . & ..$\quad$. & 40 & - \\
\hline Muscles & & . & 31 & 30 \\
\hline Blood & & . & 27 & 18 \\
\hline Kidneys & . & . & 26 & 21 \\
\hline Skin and hairs & & . . . & 21. & - \\
\hline Intestines & . &.$\quad$. & $18^{\circ}$ & - \\
\hline Lungs & . & . & 18 & 19 \\
\hline Pancreas . & . & . & 17 & - \\
\hline Heart & . & . & 3 & - \\
\hline Brain and spinz & ord . & 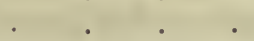 & 3 & 0 \\
\hline
\end{tabular}


Those organs of the body which are most necessary for the maintenance of life, the brain, the heart, the respiratory muscles, such as the diaphragm, undergo very little loss of weight. Of the other tissues the fat, which is a mere reserve to provide for such contingencies, is drawn upon first, and during starvation 97 per cent. of the total fat of the body may be consumed. The nitrogen needs of the body during starvation seem to be supplied chiefly at the expense of the muscles and glands, which waste to a very marked degree. The muscles being used simply as reserve material, it is easy to understand the condition of lethargy and muscular inactivity which characterises the state of inanition. During starvation all tissues of the body undergo a process of autolysis or disintegration, giving up the products of this process to the common circulating fluid. The nutritional demands of a tissue are determined.by its activity. Hence the active tissues of the body take up the material set free from all the other cells of the body and so maintain their weight at the expense of all other parts. A similar predominance of the nutrition of active over inactive tissues is to be observed in cases of partial starvation, i.e. where the deprivation of food only applies to a single food constituent. Thus Voit, in a series of experiments, fed pigeons on a food which, while normal in all other respects, contained a deficiency of calcium salts. On killing the birds after a certain length of time, it was found that while the bones used in the necessary movements of the animals presented a normal appearance, the others, such as the sternum and skull, showed a marked deficiency of lime salts and had undergone a process of rarefaction giving rise to the condition known by pathologists as osteoporosis. Many other instances of the sacrifice of a temporarily useless tissue on behalf of tissue of high physiological value are known. Thus the salmon and its congeners, which live part of the year in the sea, lay their eggs and undergo their early development in the fresh water of the upper reaches of rapid streams. An adult salmon leaves the sea in the early summer months in a magnificent state of muscular development, fit to perform the prodigious feats of swimming which are required in order to get it over the rapids of the river which it has to ascend. It takes no food. In the upper reaches of the stream or river there is a growth of the genital glands, ovaries, or testes. The whole material for the growth of these large organs is derived from the atrophy of the skeletal muscles. In this case we have the growth of an active tissue at the cost of an inactive one, the activity, however, being determined, not by the direct call upon it from the environment, but by what we may speak of as the 'physiological habit' of the animal.

The animal organism, in the complete absence of food, deals with the resources of its bodily tissues with the utmost possible economy. The total metabolism therefore sinks rapidly during the first two days of starvation, and then remains practically constant. There is indeed a slight continuous diminution with the fall in body weight, but if we reckon out the total metabolism per kilo body weight, we find that till within a day or two before death it is a constant quantity. This fact is shown in the following Table of 
THE METABOLISM DURING STARVATION

the output of energy in man during a five days' period of starvation (Tigerstedt) :

Metabolism during Starvation (Man)

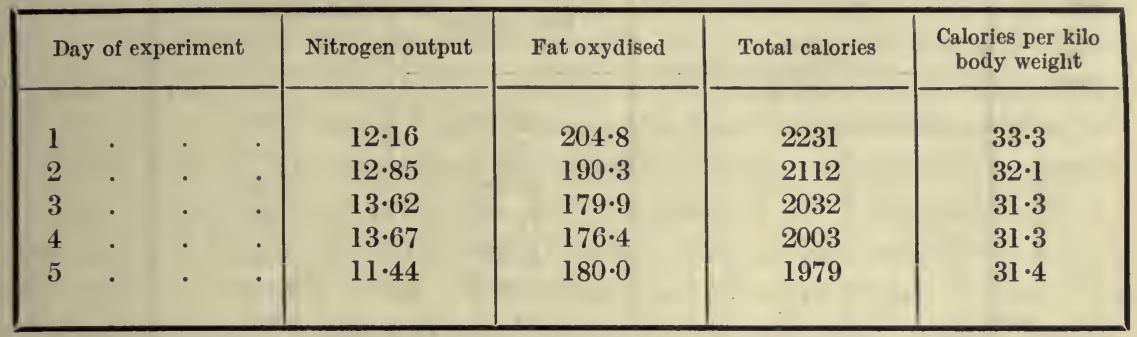

Although in one and the same individual the total metabolism during hunger varies directly with the body weight, this rule does not apply when we compare the metabolism of different animals or different examples of the same species. We find, in fact, that in larger animals the metabolism is relatively less than in smaller animals, so that if we take the evolution of calories per kilo body weight the result is inversely proportional to the body weight. This is shown in the following Table, which represents the total metabolism of a number of animals of different sizes (Rubner):

\begin{tabular}{|c|c|c|c|}
\hline H & & $\begin{array}{l}\text { Body weight, } \\
\text { k:los }\end{array}$ & $\begin{array}{l}\text { Calories per kilo } \\
\text { body weight }\end{array}$ \\
\hline $\begin{array}{l}\text { Man } \\
\text { Dog } 1 \\
\text { Dog } 2 \\
\text { Dog } 3 \\
\text { Rabbit 1 } \\
\text { Rabbit } 2 \\
\text { Guinea pig }\end{array}$ & 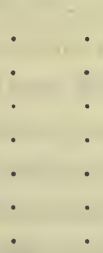 & $\begin{array}{l}70 \cdot 6 \\
30 \cdot 4 \\
17 \cdot 7 \\
3 \cdot 1 \\
2 \cdot 9 \\
2 \cdot 05 \\
0 \cdot 672\end{array}$ & $\begin{array}{r}32 \cdot 9 \\
35 \cdot 3 \\
45 \cdot 0 \\
85 \cdot 3 \\
50 \cdot 2 \\
58 \cdot 5 \\
223 \cdot 1\end{array}$ \\
\hline
\end{tabular}

On account of the greater relative metabolism of smaller animals, their resistance to starvation is less than that of larger animals. A rat or a mouse will only stand total abstinence from food for two or three days. The difference is determined by the fact that a smaller animal has a relatively larger surface per unit body weight than is the case with a larger animal. The greater part of the energy set free during starvation is required for the maintenance of the body temperature. A larger amount of energy per kilo is required in those animals with a relatively larger body surface through which heat loss may occur. That the difference in relative surface is the determining factor for the differences in total metabolism per kilo body weight is shown by the fact that, if we reckon out the amount of surface presented by each of the animals in the above list, we find that the output of energy per square metre of body surface is approximately identical in all cases. This is shown in the following Table, in which the calorie 
output per square metre of surface has been reckoned for a number of animals of different weight:

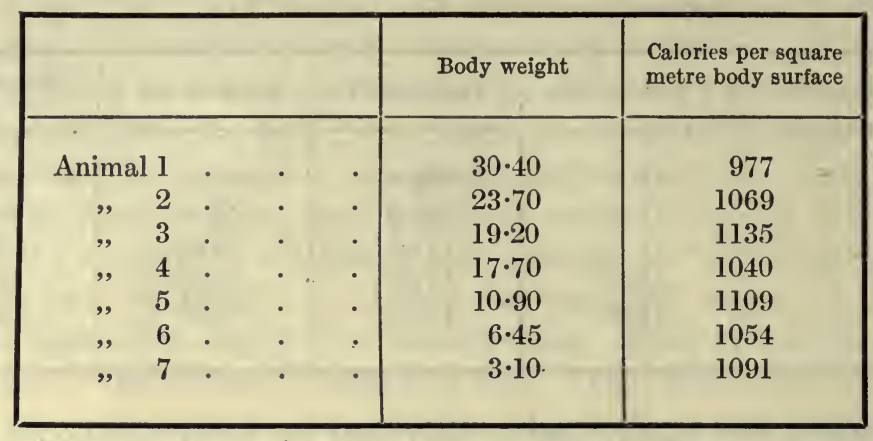

Speaking roughly, we may say that a warm-blooded animal during starvation requires the daily expenditure of 1000 calories per square metre body surface in order to maintain its temperature and carry out such motor processes as are essential to life.

\section{THE METABOLISM OF CARBOHYDRATE, FAT, AND PROTEIN DURING STARVATION}

Since during starvation no energy is supplied to the body from without in the shape of food-stuffs, we can regard the whole expenditure of the animal during its period of starvation as occurring at the expense of its capital. The amount of carbohydrate which can be stored up as glycogen or other forms is strictly limited. In many experiments the glycogen metabolism has therefore been entirely disregarded, and it has been estimated that the chemical capital of the body consisted entirely of proteins and fats. The glycogen metabolism, however, during the first day of a period of starvation may form a considerable fraction of the total metabolism of the body, and can hardly be excluded without introducing serious errors. The relative parts played by protein, carbohydrate, and fat respectively in the chemical exchanges of a starving animal may be determined in the following way: The amount of protein consumed is given by estimating the total nitrogen of the excreta by Kjeldahl's method and multiplying the result by the factor $6 \cdot 25$. The loss of weight of the body minus the protein consumed may be roughly taken as equivalent to the fat plus carbohydrate consumption. But this is only a rough method, since the quantity of water in the tissues may undergo considerable variations, and so affect the total weight of the body. For any accurate results the respiratory exchanges must be measured including both oxygen intake and carbon dioxide output. After deducting the carbon dioxide due to the combustion of the carbon of the proteins which does not appear in the urine in combination with nitrogen, the remainder of the carbon dioxide is derived entirely from carbohydrate and fat metabolism. Knowing the respiratory quotient and the total amount of carbohydrate and fat metabolism, it is possible to come to a conclusion as to how much of the 
carbon dioxide is derived from oxidation of glycogen and how much from the oxidation of fat. A very efficient check on this calculation is furnished if the individual or animal can be placed at the same time in an accurate calorimeter, as in Benedict's experiments, owing to the fact that a gramme of fat when converted into carbon dioxide and water produces more than double the amount of heat which would be evolved by the complete oxidation of glycogen. In Benedict's experiments the heat-value of the metabolism calculated by the above method agreed with the heat as actually measured by the calorimeter within 0.5 per cent., whereas if the total carbon of the first day had been reckoned as fat, the discrepancy would have been as high as 5 per cent. in many cases. The influence of glycogen metabolism on that of protein during the first and second days of fasting is shown in the following experiments (Benedict) :

\begin{tabular}{|c|c|c|c|c|c|c|}
\hline & \multicolumn{3}{|c|}{ First day } & \multicolumn{3}{|c|}{ Second day } \\
\hline & \multicolumn{2}{|c|}{ Glycogen metabolised } & \multirow{2}{*}{$\begin{array}{c}\text { N. } \\
\text { eliminated }\end{array}$} & \multicolumn{2}{|c|}{ Glycogen metabolised } & \multirow{2}{*}{$\begin{array}{l}\text { N. } \\
\text { eliminated }\end{array}$} \\
\hline & Total & Per kilo & & Total & Per kilo & \\
\hline S.A.B. & $181 \cdot 6$ & $3 \cdot 15$ & $5 \cdot 84$ & $29 \cdot 7$ & 0.52 & $11 \cdot 04$ \\
\hline S.A.B. & $135 \cdot 3$ & $2 \cdot 31$ & $10 \cdot 29$ & $18 \cdot 1$ & $0 \cdot 31$ & $11 \cdot 97$ \\
\hline S.A.B. & $64 \cdot 9$ & $1 \cdot 09$ & $12 \cdot 24$ & $23 \cdot 1$ & $0 \cdot 39$ & $12 \cdot 45$ \\
\hline H.C.K. & $165 \cdot 6$ & $2 \cdot 33$ & $9 \cdot 39$ & $44 \cdot 7$ & $0 \cdot 64$ & $14 \cdot 36$ \\
\hline H.R.D. & $32 \cdot 8$ & 0.59 & $13 \cdot 25$ & $41 \cdot 6$ & $0 \cdot 76$ & $13 \cdot 53$ \\
\hline
\end{tabular}

The total metabolism per kilo body weight very soon attains a constant level. The relative part taken in the production of the total energy by fats and proteins respectively may vary from animal to animal according to the amount of fat available in the body. If the animal has been receiving previous to the experiment a diet rich in protein the excretion of nitrogen and urea in the urine diminishes rapidly during the first days of starvation. During the first two days therefore a considerable proportion of the necessary energy is obtained at the expense of protein. Between the third and fifth day, however, the nitrogenous excretion reaches a minimum, at which point it remains approximately constant to within a day or two before death. If the animal has been receiving a diet very poor in protein the excretion of nitrogen may be low throughout the whole course of the experiment. These facts are illustrated by the curves in Fig. 320, which show the output of urea in three experiments on a dog under different conditions of nutrition. In the first experiment the dog, before the experimental period, had.been receiving $2500 \mathrm{grm}$. of meat daily; in the second it had been receiving 1500 grm. of meat, and in the third only a small quantity, which was not accurately measured. Although there is a great difference between the urea output during the first day of the experiments, the urea output during the sixth to eighth days is identical. In many cases for a few days before 
death there is a rise of protein metabolism. This rise is synchronous with a practically complete disappearance of fat from the body. The animal now has to supply all its requirements at the expense of the protein tissues, which

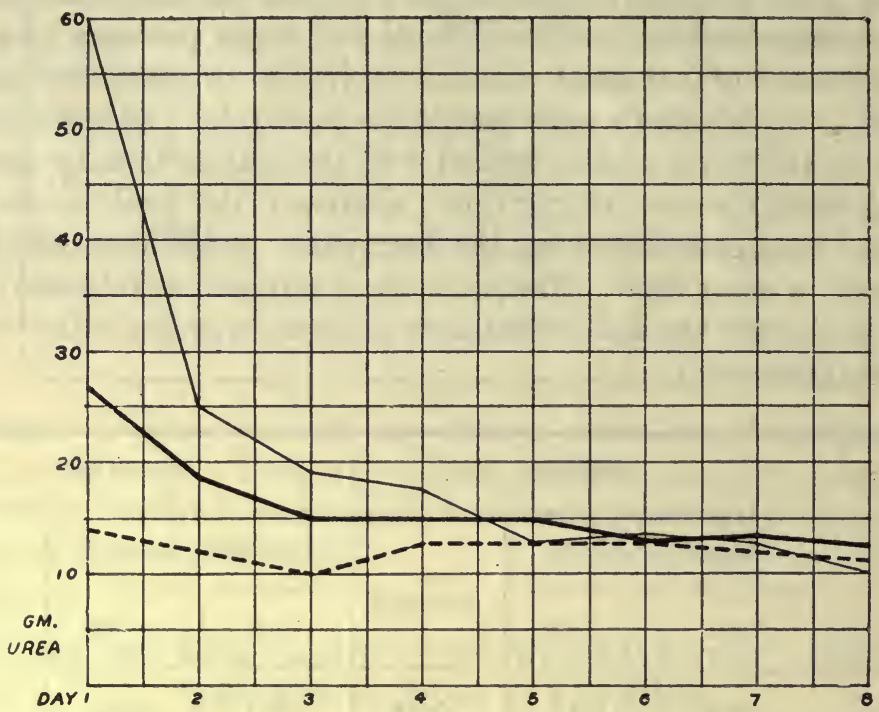

Fig. 320. Three experiments on the output of urea during starvation (dog). (TIGERSTEDT after VoIT.)

In (1) (thin line), the dog received 2500 grm. meat per day before the experiment; in (2) (thick line), the diet was 1500 grm. meat; and in the third experiment the meat was reduced to a minimum.

therefore waste rapidly and account for the increased excretion of nitrogen. This is shown in the following experiment of Rubner on a rabbit :

\begin{tabular}{|c|c|c|c|}
\hline Days & & $\begin{array}{l}\text { Average daily out- } \\
\text { put of nitrogen }\end{array}$ & $\begin{array}{l}\text { Average amount of } \\
\text { fat oxidised daily }\end{array}$ \\
\hline $1-3$ & & $1.67 \mathrm{grm}$ & $1 C \cdot 3 \mathrm{grm}$ \\
\hline $4-5$ & $\cdot$ & $1 \cdot 46$ & $10 \cdot 3$, \\
\hline $6-8$ & . & $3 \cdot 21$ & $2 \cdot 4$ \\
\hline
\end{tabular}

We see therefore that during starvation, apart from the first day or two, the animal derives the main portion of its necessary energy from the combustion of fats, provided that there is a sufficient store of these substances in the body. A certain consumption of protein is unavoidable. Since protein comes from the working tissues of the body they are spared so far as possible, and it is only when the stored fat is used up that any large call is made on the tissue-protein. 


\section{SECTION III}

\section{THE EFFECT OF FOOD ON THE METABOLISM OF THE BODY}

A MARKED contrast exists between protein and the other two classes of food-stuffs in relation to nutrition. Whereas it is possible in the case of many animals to maintain life with a diet consisting of proteins, salts, and water, such as is contained in the leanest possible meat, a diet of pure fat or carbohydrate, or a mixture of the two, is almost equivalent to an absolute abstinence from food, the animal on such a diet surviving only a few days longer than during complete starvation. The primary importance of proteins in nutrition therefore indicates that it is advisable to deal first with the effects on metabolism of a diet consisting of this food-stuff alone. In an animal which has been starved for five or six days the nitrogenous output of the body has attained a practically constant level, varying with the size of the animal and with the relative content of its body in fat. Let us assume that such an animal is excreting $5 \mathrm{grm}$. of nitrogen daily, corresponding to a protein metabolism of $31.25 \mathrm{grm}$. of protein. It might be thought that this loss of protein to the body would be met if we administered to the animal as food a similar amount, i.e. $31.25 \mathrm{grm}$. of protein. On trying the experiment, however, we find the effect of giving protein food is to increase largely the nitrogenous output of the body, so that after receiving this amount of protein the animal's nitrogenous excretion will amount to nearly $10 \mathrm{grm}$. The waste of tissue-protein in the body therefore proceeds. In order to stop this waste, i.e. to ensure that the animal does not lose more nitrogen than it receives in its food, we must give an amount of protein corresponding to between two and a half and five times the amount of protein which undergoes disintegration during starvation. The reason for this is obvious on reference to p. 627. There we see that a man on the fifth day of starvation excreted $11.44 \mathrm{grm}$. of nitrogen, corresponding to $71.5 \mathrm{grm}$. of protein. This protein metabolism did not, however, represent the sole source of the energy output of the body. The total energy output was 1979 calories. Of this amount only 293 calories could be obtained from the combustion of the $71 \mathrm{grm}$. of protein, the balance being due to the oxidation of fat stored up in the body. This signifies that only one-fifth of the total energy requirements of the body were supplied at the expense of protein. We cannot therefore expect to stop loss of kody substance by giving an amount of protein food which would correspond only to one-fifth of the energy requirements. In most cases, 
if we are dealing with an animal with a considerable store of fat in its body, nitrogenous equilibrium, i.e. an equivalence between income and output of nitrogen, is attained with a quantity of protein in the food which is less than five times the amount lost during starvation. In such a case the total energy requirements of the body are met not only at the expense of the protein food but also at the expense of the fat of the tissues. The animal will continue to lose weight and to become thin, although he is in a state of nitrogenous equilibrium.

The protein taken in with the food on a pure protein diet has a twofold function to perform. In the first place, every functional activity of the living tissues is probably associated with a certain amount of wear and tear, and results in the production of disintegration products which are not in a condition to be resynthetised into living working protoplasm. We know, for instance, that from every mucous surface dead cells are being continually cast off and that a constant disintegration of red blood corpuscles goes on, resulting in the production of the bile pigments ; and we are warranted in extending the operation of these changes of which we have ocular evidence to the case of other cells, such as those of the liver and of the muscles, where direct proof of destruction of tissue during normal metabolism is more difficult to obtain. It is certain that some portion of the nitrogen excreted during complete starvation must come from this source, and that one of the functions of protein food is the replacement of tissue which has been lost in this way. When, however, we are feeding an animal on a pure protein diet, by far the larger portion of the food is utilised for meeting the energy requirements of the body. In this function protein food, apart from accidents of digestibility and structural adaptation of the animal's digestive arrangements to its habits of life, presents no apparent advantages over the other two classes of food-stuffs. Its value to the animal is represented by its physiological heat-value. It may be represented therefore numerically as $4 \cdot 1$, and is equivalent to the value of carbohydrate $*$ and is far inferior to the value of fats with a heat equivalent of 9*3. If, instead of giving to the starving animal a pure protein diet, we administer a mixed diet containing a sufficient quantity of fat or carbohydrate, or of both substances, to meet the normal energy requirements of the body, we can restrict the utilisation of protein more nearly to the replacement of tissue waste in the body, and are therefore able to attain nitrogenous equilibrium with a much smaller proportion of protein than is possible when this substance furnishes the whole diet. In carnivora, which have the habit of supplying a large proportion of their energy needs at the expense of protein, the amount of carbohydrate and fat which must be added to protein in order to attain nitrogenous equilibrium with a nitrogenous output equal to that in starvation is very large and corresponds to an energy-value in excess of the total energy expenditure during starvation. In omnivora, such as man, it is easy to attain nitrogenous equilibrium on a mixed diet with a smaller nitrogen turnover

* This may be expressed by saying that protein is isodynamic with an equal weight of carbohydrate. 
than is found during starvation. In the experiment given on p. 627 the average nitrogen output during starvation was about $12 \mathrm{grm}$. of nitrogen. In Succi, the fasting man, the nitrogen output varied from $11.19 \mathrm{grm}$. on the fifth day to $2 \cdot 82 \mathrm{grm}$. on the twenty-first day.

\section{Daily Nitrogen Excretion of Succi in Starvation}

\begin{tabular}{|c|c|c|c|c|c|c|c|c|c|c|}
\hline Day & & N. & Day & & & N. & Day & & & N. \\
\hline 1 & • & . $17 \cdot 0$ & 8 & • & . & $9 \cdot 74$ & 15 & • & • & $5 \cdot 05$ \\
\hline 2 & - & . $11 \cdot 2$ & 9 & . & . & . 10.05 & 16 & & 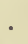 & $4 \cdot 32$ \\
\hline 3 & . & . 10.55 & 10 & - & . & $7 \cdot 12$ & 17 & & 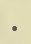 & $5 \cdot 4$ \\
\hline 4 & . & . $10 \cdot 8$ & 11 & . & . & $6 \cdot 23$ & 18 & & $\cdot$ & $3 \cdot 6$ \\
\hline 5 & . & . $11 \cdot 19$ & 12 & . & . & $6 \cdot 84$ & 19 & $\theta^{\circ}$ & . & $5 \cdot 7$ \\
\hline & . & . $11 \cdot 01$ & 13 & . & . & $5 \cdot 14$ & 20 & . & . & $3 \cdot 3$ \\
\hline & . & . 8.79 & 14 & . & . & $4 \cdot 66$ & 21 & & . & $2 \cdot 8$ \\
\hline
\end{tabular}

Chittenden has shown that in man a perfectly normal nutrition may be maintained on a mixed diet containing about $7 \mathrm{grm}$. of nitrogen daily. In the cases investigated by Chittenden the energy output of the men could be regarded as normal, corresponding to 32-35 calories per kilo body weight. If the amount of fat and carbohydrate be very largely increased it is possible to maintain nitrogenous equilibrium on even smaller quantities of protein. Thus nitrogenous equilibrium was attained by Siven on a diet containing $33 \mathrm{grm}$. of protein daily (=5 grm. of nitrogen), but in this case the carbohydrates and fats were increased to such an extent that the man was taking in 78.5 calories per kilo per day. These results suggest that the qualitative metabolism of the body is determined by the relative amount of food-stuff supplied to the body and circulating in its juices at any given time, and that preponderance of one food-stuff will tend to excite the cells of the body to the utilisation of this food-stuff at the expense of the other two. That such is the case is shown by a study of the effect of increasing each class of food on the metabolism of the body as a whole.

\section{EFFECTS OF VARIATIONS IN PROTEIN}

Most of the experiments on the influence of variations in the quantity of protein food have been made on carnivora, such as the dog and cat. Within very wide limits the output of nitrogen is proportional to the intake. This is shown in the Tables (p. 634) by Voit, representing two experiments on dogs.

In Experiment I the animal had been fed for some days with $500 \mathrm{grm}$. of meat per diem. The fact that he was excreting nitrogen corresponding to $547 \mathrm{grm}$. shows that this amount was insufficient and that he was not yet in a condition of nitrogenous equilibrium. Each day he was using up $47 \mathrm{grm}$. of the protein of his body in addition to the $500 \mathrm{grm}$. supplied in the food. On increasing his food three-fold to $1500 \mathrm{grm}$. the nitrogenous output was also increased, but a state of nitrogenous equilibrium was not reached until the eighth day of the experiment. During the six days intervening $778 \mathrm{grm}$. of meat had been retained in the body, i.e. there had been a retention of 
protein, probably in the form of increased muscular substance. The amount is too great to be accounted for by retention of the disintegration products of the protein in the body. It must have been stored up in the form of protein and probably, to a large extent at any rate, as actual living tissue.

\begin{tabular}{|c|c|c|c|c|c|c|}
\hline \multicolumn{4}{|c|}{ EXPERIMENT I } & \multicolumn{3}{|c|}{ EXPERIMENT II } \\
\hline & Day & $\begin{array}{c}\text { Daily meat } \\
\text { ration }\end{array}$ & $\begin{array}{c}\text { Flesh loss } \\
\text { per day }\end{array}$ & Day & $\begin{array}{c}\text { Daily meat } \\
\text { ration }\end{array}$ & $\begin{array}{l}\text { Flesh loss } \\
\text { per day }\end{array}$ \\
\hline 1 & . & 500 & 547 & & 1500 & 1500 \\
\hline 2 & . & 1500 & 1222 & 2 . & 1000 & 1153 \\
\hline 3 & . & 1500 & 1310 & 3. & 1000 & 1086 \\
\hline 4 & . & 1500 & 1390 & 4. & 1000 & 1088 \\
\hline 5 & . & 1500 & 1410 & 5 . & 1000 & 1080 \\
\hline 6 & & 1500 & 1440 & 6. & 1000 & 1027 \\
\hline 7 & 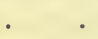 & 1500 & 1450 & & & \\
\hline & . & 1500 & 1500 & & & \\
\hline
\end{tabular}

In the second experiment the diminution of the protein of the food was followed by a loss of protein from the body, the output being greater than the income. The excess, however, was rapidly diminishing and equilibrium had been practically attained on the last day of the experiment. During this time the animal had excreted $14 \cdot 8 \mathrm{grm}$. of nitrogen more than it had received in its food, which would correspond to a diminution of the protein store of its body, reckoned as muscular substance, by $434 \mathrm{grm}$. Many such experiments have been performed, and they all agree in showing that in carnivora a very appreciable storage of nitrogen can take place in the body. In cats it is sometimes possible to double the body weight by administration of a large protein diet. Since no fat is laid on at the same time and the animals are in a fine healthy condition, one must conclude that the greater portion of the storage takes place by a growth of muscle substance. The degree to which the storage can take place is, however, variable and is generally smaller in dogs than in cats. However much protein is given, the limit is finally arrived at where no further laying on of protein tissues of the body is possible, and the animal then enters into a state of nitrogenous equilibrium, when he excretes a quantity of nitrogen exactly equal to that taken in. This equivalence of income and output signifies that the extent of the total metabolism of the body is affected by the amount of protein supplied in the food, and, as a matter of fact, the total energy output of the body rises and falls with the quantity of protein in the food. This is shown in the Table (p. 635) by Pettenkofer and Voit, in which the figures have been recalculated by Pflüger.

We see therefore that carnivorous animals can satisfy their total energy requirements at the expense of protein. When the protein income is in excesss of their requirements a small amount is laid on, probably in the shape of increased muscular tissue. The most marked effect is, however, an 
increased metabolism which rises in proportion to the nitrogenous income. The limit to this increase is set by the powers of the alimentary canal to digest the protein. The rise in metabolism consequent on protein food is very rapid and affects the gaseous exchanges as well as the output of nitrogen. Magnus Levy and Falk found that a large protein meal might increase the respiratory exchanges 40 per cent., an increase which lasted seven hours.

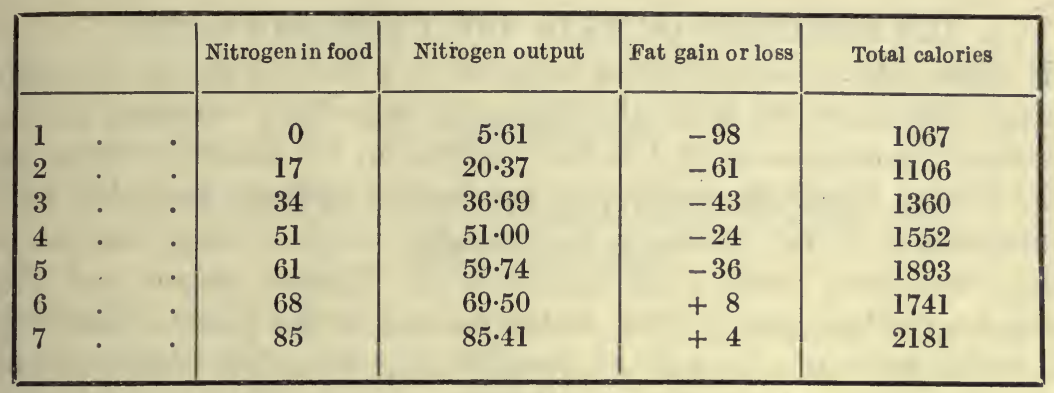

The nitrogenous output also rises immediately after a protein meal, so that 50 per cent. of the nitrogen of the ingesta may appear in the urine within seven hours after the meal.

The whole of these results cannot be strictly applied to omnivorous animals, such as man. In these it is impossible to supply all the energy requirements of the body on a pure protein diet. Even if a man eats as much meat as he can, he will be unable to obtain sufficient energy for his daily requirements. Whereas the average daily requirements of a man amount to about 3000 calories, $1 \mathrm{lb}$. of meat would yield only about 400 calories, and even if he took $4 \mathrm{lb}$. of meat daily, an amount which is impossible for most individuals, he would only be obtaining about 1600 calories. The cures for obesity, in which a large protein diet plays an important part, owe their efficiency to this fact. They are in all cases practically equivalent to a state of semi-starvation. Many experiments have been made on the influence of variations in the quantity of protein in a mixed diet. Within wide limits the output of nitrogen is strictly proportional to the intake. A normal adult man seems to be unable to store up protein in any form, and differs in this respect from carnivora, such as the dog or cat. The only way in which protein can be laid on in the body is by furnishing a physiological stimulus to the growth of muscle, i.e. by constant exercise. Without this it is not possible to produce growth of the muscles of the body, however much protein we may give in the diet. The conditions are, however, different when dealing with an individual in whom from some cause or other the muscular tissues have not attained their full development. Thus in growing individuals a certain amount of the protein of the food is always retained in the body and laid on as tissue-protein. In convalescence after severe fever, during which a great wasting of the muscles has taken place, forced feeding with large amounts of protein has been found to give rise to a considerable retention of protein in the body. This process only goes on until the muscles have attained their normal condition of development. When the tissues 
have, so to speak, reached 'par,' the possibility of laying on protein tissues ceases. On the other hand, protein food has in man, as in animals, a specific stimulating effect on metabolism, so that the respiratory exchanges are largely increased as a result of a heavy protein meal. This effect has been named by Rubner the 'specific dynamic effect' of protein. We shall have occasion later to discuss its significance.

\section{THE INFLUENCE OF FATS AND CARBOHYDRATES}

If either fats or carbohydrates be given to a starving animal a certain sparing of the fat of the body takes place, but this effect, according to some observers, is accompanied by a distinct increase in the metabolic exchanges of the body. As regards the protein metabolism, Cathcart finds that while administration of fat increases the nitrogen output during starvation, carbohydrate food causes a diminution in the nitrogen output, and thus exercises a marked sparing effect on the proteins of the body. Voit found that during starvation or with an insufficient protein diet addition of fat to the food increased the total metabolism. When sufficient protein was being supplied, the addition of fat caused no increase in the total metabolism, the whole of the fat in the food being laid on as fat in the body. The stimulating effect of fat on metabolism is but slight. Magnus Levy found that the increase in the metabolism on the administration of fat to a starving animal was minimal and never exceeded 10 per cent.

Carbohydrates have a somewhat greater influence on metabolism. The administration of a large meal of carbohydrates to a starving animal may raise the respiratory exchanges from 20 to 30 per cent., and the increase may last four hours after the meal. This stimulating influence on metabolism is, however, much less than that observed on the administration of large doses of protein. If carbohydrate be given in excess of the daily energy requirements the greater proportion of it remains in the body, being stored up to a small extent as glycogen, but mainly in the form of fat. 


\section{SECTION IV \\ THE EFFECT OF MUSCULAR WORK ON METABOLISM}

WHEN an animal performs muscular work its total output of energy must be increased, and this must take place at the expense either of an increased intake of food or an increased destruction of the tissues of the body. For many years a theory put forward by Liebig received universal acceptance among physiologists. According to this view the food-stuffs could be divided into two classes, namely, the proteins, which were the plastic food substances and were concerned in the building up of tissues, and, secondly, the fats and carbohydrates, which took no part in the building of the tissues, but were oxidised for supplying heat to the body. Muscular work was supposed to be attended with a breakdown of the muscular tissue, and therefore to be performed at the expense of an increased protein metabolism, which had to be made good by adding to the protein intake in the food. If this view were correct one would expect to find a great increase in the nitrogenous metabolism of the body with every increase in muscular work. Such an increase has, in fact, been found by Pflüger in dogs which were nourished on a purely protein diet. In these animals the sole source of energy to the organism was protein, and therefore any additional call on the energies of the body must be associated with an increased intake of food or an increased loss of material from the body in the shape of protein. In an animal which is enjoying a normal mixed diet, or has a store of carbonaceous material in its tissues in the shape of fat, there is no increase of nitrogenous metabolism during muscular work which would correspond in any way to the amount of work done. This was shown long ago by Voit in experiments on the dog. The following Table represents the nitrogenous metabolism, i.e. the amount of muscular material in the shape of protein metabolised during the day, under varying conditions of rest and activity, during starvation and after food :

\section{Dog I}

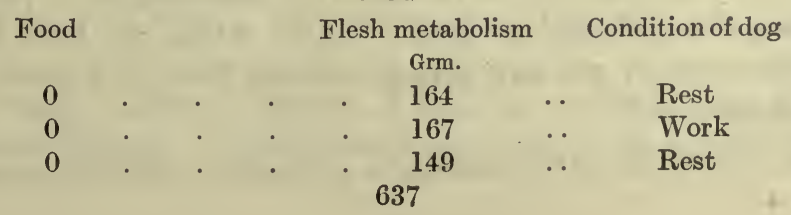




\section{Doa II}

Food

Flesh metabolism

Condition of dog

1500 grm. lean meat
$1500, ", \quad, \quad$,
$1500, ", \quad, \quad$,
$1500 \quad, \quad, \quad$,
$1500 \quad, \quad, \quad$,

Grm.

1522

1625

1526

1583

1535

Rest

Work

Rest

Work

Rest

During the work days the animal performed about 1500 kilogramme metres in the day. The differences in the protein metabolism between the rest and the work days are thus practically insignificant, the nitrogenous output being determined, not by the work done, but by the amount of protein administered in the food. In the second experiment there is an average increase of protein metabolism during the work days amounting to $86 \mathrm{grm}$. of flesh, a quaritity which is insufficient to furnish the energy of the work done. The same results were arrived at in a classical experiment performed by Fick and Wislicenus on themselves, in which they measured their total nitrogenous metabolism during an ascent of the Faulhorn from the Lake of Brienz. The vertical distance traversed was 1956 metres. During the seventeen hours before the experiment, the six hours of the ascent, and the seven subsequent hours they ate food practically free from nitrogen. The urine passed during the ascent and during the next seven hours was collected in each case and its nitrogen determined. Fick passed $5 \cdot 74 \mathrm{grm}$. of nitrogen, which, if the energy of the protein were totally converted into work, would correspond to 63,378 kilogramme metres. In Wislicenus the amount was $5 \cdot 55 \mathrm{grm}$. of nitrogen, equivalent to 61,280 kilogramme metres. Fick, who weighed 66 kilos, in raising himself to a height of 1956 metres, had performed 129,096 kilogramme metres, and Wislicenus, with a weight of 76 kilos, had performed 148,656 kilogramme metres. Even if we assume the possibility of a conversion of the total energy of the protein metabolised during the experiment into mechanical energy, we cannot account for more than half of the total work done. All subsequent experimenters have confirmed the deductions which were drawn from these two researches, namely, that muscular work, while practically without influence on nitrogenous metabolism, increases enormously the carbonaceous metabolism of the body, so that, except in the rare cases where the diet consists of pure protein and the body is practically free from fat, the additional energy output during work is derived from the oxidation of carbon and hydrogen to carbon dioxide and water.

The general nature of the changes in the metabolism of the body during work is well illustrated by the results obtained by Atwater on man. The total energy output of a man was reckoned as heat by means of the calorimeter. The heat equivalent of the external muscular work performed by the man was also reckoned as heat. In the Table (p. 639) we give the total output of energy per day during rest and work, the latter being also expressed in calories.*

* One large calorie, or 'kilo-calorie,' is equivalent to 425 kilogrammetres. 
ENERGY PER DAY

\begin{tabular}{|c|c|c|c|c|c|}
\hline \multirow[b]{2}{*}{ Nature of experiment } & \multicolumn{3}{|c|}{ Heat eliminated } & \multirow{2}{*}{$\begin{array}{l}\text { External } \\
\text { work in } \\
\text { calories }\end{array}$} & \multirow{2}{*}{$\begin{array}{l}\text { Total } \\
\text { in } \\
\text { calories }\end{array}$} \\
\hline & $\begin{array}{l}\text { By radiation } \\
\text { and } \\
\text { conduction }\end{array}$ & $\begin{array}{l}\text { In urine } \\
\text { and } \\
\text { fæces }\end{array}$ & $\begin{array}{l}\text { In water } \\
\text { vaporised } \\
\text { from lungs } \\
\text { and skin }\end{array}$ & & \\
\hline $\begin{array}{l}\text { Rest with food-(average } \\
\text { of four days) }\end{array}$ & 1850 & 26 & 521 & 一 & 2397 \\
\hline $\begin{array}{l}\text { Rest fasting (four experi- } \\
\text { ments) (average of } \\
\text { five days) }\end{array}$ & 1605 & 21 & 561 & 一 & 2187 \\
\hline $\begin{array}{l}\text { Work (fourteen experi- } \\
\text { ments) (average of } \\
\text { forty-six days) }\end{array}$ & 3802 & 29 & 743 & 546 & 5120 \\
\hline
\end{tabular}

If we compare the energy-value of the work done with the excess of the total expenditure of the body over that found during the rest experiments, we find that the performance of muscular work involves an increase in the total energy-expenditure of the body by an amount equal to about five times that of the work done. Of course a certain proportion of this excess of energy over work done is accounted for by the increase in the work which must be performed by the respiratory muscles and heart in the state of greater activity which is imposed upon them by the external work, and is necessary for the proper provision of the active muscles with increased foodsupply and oxygen. Even if we neglect these factors altogether, we see that the efficiency of the body as a machine corresponds to between 16 and 20 per cent., an efficiency which exceeds that of the best of our steam-engines and is only equalled by certain internal-combustion engines.

A comparison of the excreta of the same individual whose energy exchanges are given in the above Table during rest and activity will give us information as to the source of the increased energy put out during the performance of muscular work. Thus during a period of rest and starvation the average output of carbon dioxide during six hours amounted to $189 \cdot 6$ grm. ; during a rest experiment with food the average output for a period of six hours was 230.4 grm. of carbon dioxide. During work the average output in the same individual during six hours rose to $705 \mathrm{grm}$. of carbon dioxide on a carbohydrate diet, and to $634.8 \mathrm{grm}$. on a diet containing a large amount of fat. The oxidation of carbon was therefore increased more than threefold as a result of muscular work. If we compare in the same way the protein metabolism of the same individul during these experiments no such alteration is observed. Thus the average output per day during rest. and starvation corresponded to $82 \mathrm{grm}$. of protein. During rest and with an approximately sufficient amount of food the average amount of protein consumed was $98.8 \mathrm{grm}$. During a work day in which he received practically 
the same amount of protein in the food and a somewhat insufficient amount of carbohydrates and fats, his consumption of protein amounted to $109.4 \mathrm{grm}$. Here, as against a three- to fourfold increase of the gaseous metabolism of the body, we have only a 10 per cent. increase of the protein metabolism.

A still larger difference between the respiratory changes in the resting or working condition is found when the exchanges are taken over shorter intervals. The following Table represents the respiratory exchanges in a trained muscular subject during complete rest and when doing moderate or severe work, namely, riding a bicycle with a brake. Each observation lasted from 10 to 15 minutes.

\begin{tabular}{|c|c|c|c|c|c|c|}
\hline Condition & & $\begin{array}{c}\mathrm{CO}_{2} \text { eliminated } \\
\text { per minute }\end{array}$ & $\begin{array}{c}\text { Oxygen absorbed } \\
\text { per minute }\end{array}$ & $\begin{array}{c}\text { Respiratory } \\
\text { quotient }\end{array}$ & Pulse rate & Rate \\
\hline $\begin{array}{l}\text { Lying } \\
\text { Moderate work } \\
\text { Severe work }\end{array}$ & $\dot{.}$ & $\begin{array}{r}200 \\
1720 \\
2227\end{array}$ & $\begin{array}{r}243 \\
1834 \\
3265\end{array}$ & $\begin{array}{l}.83 \\
.94 \\
.98\end{array}$ & $\begin{array}{r}56 \\
150 \\
166\end{array}$ & $\begin{array}{l}20 \\
32 \\
38\end{array}$ \\
\hline
\end{tabular}

These last figures represent a maximum since the subject worked with great difficulty and was exhausted when he came off the machine.

There is another method by which we can arrive at some idea of the nature of the material which is furnishing by its oxidation the necessary energy for the performance of muscular work. It is evident, if we compare the formulæ of a carbohydrate and a fat respectively, that it will require a relatively larger amount of oxygen to oxidise the fat than is necessary in the case of the carbohydrate. In the latter there is sufficient oxygen to combine with all the hydrogen present and form water. The whole of the oxygen therefore which is caken in is employed in the oxidation of the carbon, and one volume of oxygen will produce one volume of carbon dioxide. Thus : $\mathrm{C}_{6} \mathrm{H}_{12} \mathrm{O}_{6}+6 \mathrm{O}_{2}=6 \mathrm{H}_{2} \mathrm{O}+6 \mathrm{CO}_{2}$. If the whole of the animal's energy requirements were furnished by the oxidation of carbohydrates, the output of carbon dioxide expired would be exactly equal in volume to the oxygen inspired, and the respiratory quotient of the animal, namely, $\frac{\mathrm{CO}_{2} \text { expired }}{\mathrm{O}_{2} \text { inspired }}$, would be equal to unity. In fats the amount of oxygen is only sufficient to combine with four atoms of the hydrogen of the molecule. When fats undergo oxidation, of the oxygen used only a portion is devoted to the formation of carbon dioxide, the rest being employed in the oxidation of hydrogen to water. In an animal using only fats the carbon dioxide output of the body would be considerably less than the oxygen intake and its respiratory quotient would be less than unity. The respiratory quotients for pro- tein, fats, and carbohydrates are given in the following Table (Atwater): 


\section{Material}

Starch

Cane sugar

Glucose

Animal fat

Protein
Respiratory quotient $\frac{\mathrm{CO}_{2}}{\mathrm{O}_{2}}$

$1 \cdot 0$

$1 \cdot 0$

$1 \cdot 0$

$0 \cdot 711$

$0 \cdot 809$

The respiratory quotient in an animal at any given time is therefore determined by the nature of the substances which are undergoing oxidation in its body. If the performance of muscular work involved special chemical processes, a metabolism of one of the main constituents of the body in preference to either of the others, this sudden change in the quality of the metabolism should show itself in the respiratory quotient.

According to Speck and Löwy, moderate muscular work which is not associated with dyspnœa, although attended by a large increase in the carbon dioxide output and the oxygen intake of the body, does not alter the respiratory quotient. This is probably correct if the respiratory changes are taken over a sufficient length of time, when the respiratory quotient must depend on the food which is furnished to the body however the energy of this body is expended. When however, as in Benedict's and Cathcart's experiments, the observations are of short duration, muscular exercise is almost always found to be associated with a rise in the respiratory quotient, which lasts too long to be accounted for by a mere washing out of the carbon dioxide from the blood and the body tissues. After the cessation of the exercise the respiratory quotient sinks below normal. The respiratory quotient however rarely rises even during exercise to 1 as it would if the muscular work were performed solely at the expense of carbohydrates.

These results suggest that while muscular work can be performed at the expense of any of the food-stuffs or of the three classes of constituents of the body, carbohydrate is the immediate or the most readily available source of muscular energy. When the body passes suddenly from a resting to an active condition the first call is therefore on the carbohydrates of the blood and those stored up in the muscles and liver, whereas after exercise these carbohydrate stores are slowly replenished probably at the expense of the proteins. The fact that the body can draw on its fat stores for the performance of muscular work suggests that this substance may also serve as a source of muscular energy, but there is no evidence that the body is able to convert fats under any circumstances into carbohydrates, so that we must assume that under certain conditions the fats or their decomposition products may be directly utilised by the muscles. 


\section{SECTION V}

\section{THE SIGNIFICANCE OF THE FOOD-STUFFS}

WHEN the proteins are taken as food they are rapidly and almost completely metabolised, so that the energy output of the body increases pari passu with the increased protein food. With a large excess of protein, a certain limited storage of this material is possible, but the stored-up protein rapidly disappears on deprivation of food. On this account the course of the metabolism during starvation is the same after the first two or three days, whether the animal has previously received large or small amounts of protein in its diet. In man, even this limited power of storing nitrogen is apparently absent. Under no circumstances can we produce a laying on of fat in the body, even in carnivora, however much the protein income is increased.

The metabolism of fats and carbohydrates, on the other hand, is determined, not by the amount of these substances in the food, but by the energy requirements, i.e. the functional activity of the living tissues. Any excess * of either of these foods simply gives rise to their storage in the body almost entirely in the form of fat. How are we to regard the particular position taken up by the proteins in metabolism? Voit, whose laborious observations form the foundation of all our present knowledge of metabolism, drew a sharp contrast between the proteins which were built up to form parts of the living cells, the tissue or morphotic protein, and those which underwent rapid oxidation in the tissue juices without ever forming an integral constituent of the living protoplasm. The latter he designated circulating protein. The rapid fall in the nitrogenous excretion during the first two days of starvation he ascribed to the using up of the circulating protein. As soon as this was exhausted the animal was reduced to living on its own tissues, so bringing into the metabolic cycle the tissue-proteins themselves. This theory has been energetically attacked of late years by Pflüger, according to whom the whole of the protein, which is broken down and oxidised to urea, must at one time have formed an integral part of a living cell, so that tissueprotein would be the sole source of the urea. He explains the rapid excretion of nitrogen which follows the ingestion of a protein meal by the special avidity of the animal cell for protein. When enough of this is presented to it it feeds upon nothing else, and only when there is a comparative lack of protein will it make use of carbohydrate or fat for its needs. Thus while a dog

* That is, assuming that the animal is able to digest and absorb the excess. On this factor probably depends the possibility of fattening an animal. 
is fed on a rich mixed diet it lives practically on protein alone, storing up the fats and carbohydrates of the food as fat. If food be now withdrawn the animal must live either at the expense of its own living tissue (proteins) or must attack the stored-up fats in its body. The latter alternative, as a matter of fact, takes place. The animal spares the precious protein and lives on the fat of its own body. Hence comes the great fall in the excretion of urea that is observed in starvation, the consumption of proteins sinking to the indispensable minimum. If now a protein meal be given, the cells of the body return to their former way of living, and satisfy as much of their needs as possible at the expense of protein, so that the urea excretion rises almost in proportion to the food given. In order to attain nitrogenous equilibrium on a purely protein diet, it is necessary to give the cells enough protein for their total requirements, $i . e$. three to five times as much as would correspond to the nitrogenous excretion during hunger. If a larger amount of protein be given than is necessary for the maintenance of nitrogenous equilibrium, a certain amount of nitrogen is retained in the body, probably as protein, giving rise to an increase in the total living material of the body, and the animal increases in weight. The amount of urea excreted by an animal is proportional not only to the quantity of protein taken in with the food but also to the weight of the animal; so the animal which has grown heavier in consequence of increased supply of nitrogenous food will need a larger amount of protein to maintain its nitrogenous equilibrium, which will be produced with the same amount of protein as soon as the animal has increased in weight to a certain extent. In order therefore to maintain the increase in weight, it is necessary to give ever-increasing quantities of protein, and the stuffing process is finally put an end to by the refusal of the digestive organs to digest any more.

There can be little doubt, however, that Voit was substantially correct in assigning a twofold fate to the ingested protein, and that, as Speck has pointed out, we can consider protein metabolism under two headings, viz. tissue or nutritional metabolism and energy metabolism. Since the proteins form the main constituent of living protoplasm, the death and destruction of cells, which are constantly going on in the body, must result occasionally in the production of substances which are not available for resynthesis, and are therefore turned out of the body with the urine.* A certain proportion of the proteins of the food must therefore be applied to replacing this waste of tissue. The proportion will be larger in cases where a growth of the nitrogenous tissues is occurring, as in the young animal or during convalescence from a wasting disorder. On the other hand, a certain proportion of the nitrogen in the urine will be derived from the breakdown of tissues, and this in its turn

* Tigerstedt suggests that the irreducible minimal protein consumption may be due, not to a special metabolic cycle of the protein on its way into and out of the living cell, but to the fact that the circulating fluids of the body contain large amounts of protein as essential and constant ingredients, and the living cells therefore, which are bathed by these fluids, cannot refrain, even in times of protein scarcity, from feeding to a certain extent on these the predominant constituents of their nutrient medium. 
will be increased under any conditions which bring about an augmented tissue disintegration, such as the toxæmia of fevers, poisoning by arsenic or phosphorus, or partial asphyxia by deprivation of oxygen, as after inhalation of carbon monoxide gas. In this function protein cannot be replaced by either of the other food-stuffs. With regard to its second fate, viz. the furnishing of energy to the body, protein stands on the same level as carbohydrates or fats, its relative value as compared with these two classes of substances being represented by its physiological heat-value. Owing to the inability of the body to store protein to any marked extent, any protein which is absorbed in the alimentary canal in excess of the nutritional requirements of the body is at once broken down and oxidised to satisfy the energy requirements of the cells. The $\mathrm{NH}$ and $\mathrm{NH}_{2}$ groups in the protein molecule add little or nothing to its chemical or potential value. The energy value of the protein depends on its carbon and hydrogen content, and it seems probable that the greater part of the nitrogen is split off as ammonia from the protein molecule during or shortly after its absorption from the alimentary canal. It is on this account that an increased excretion of urea is the almost immediate consequence of the ingestion of protein. The point made by Pflüger against Voit, viz. that no oxidation occurs in the lymph or blood, is really beside the mark. The living cell is a complex system which may include food in all degrees of oxidation or chemical change, without this food material necessarily forming part of the living framework. Every histologist distinguishes the paraplasma from the more active and essential protoplasm, and we must assume that it is in the ultra-microscopic interstices of the foam-like protoplasm that the chief processes of chemical change and oxidation occur. A proof therefore that oxidation depends on the condition of the cells and not on that of the blood does not justify the conclusion that the whole nitrogenous metabolism of the body is confined to the living protoplasm itself.

Folin has lately brought forward a number of facts which point not only to a twofold origin of the nitrogen of the urine but also to a qualitative difference in the two orders of protein metabolism. Whereas in the urine of man on a normal diet the urea nitrogen forms 85 per cent. or more of the total nitrogen, a reduction of the protein ration to the minimum necessary to meet the nutritional requirements of the body causes not only an absolute diminution of the urea but a large relative diminution when compared with the other constituents of the urine, such as creatinin. He concludes therefore that the nitrogenous end-products of nutritional metabolism are different from those of the energy metabolism. As Speck has pointed out, there is also a difference in the time-relations of the two orders of metabolism. Whereas the nitrogen, which furnishes no energy to the body, is rapidly eliminated when protein is being utilised for the supply of energy to the body, the occurrence of increased tissue waste causes a rise of nitrogenous excretion, which comes on slowly, often after the lapse of a day, and may last two or three days. The process of protoplasmic disintegration appears therefore to occur in a series of stages, which occupy a considerable time and end in the production of substances qualitatively distinct from that 
substance, urea, which is the almost exclusive nitrogenous end-product of the energy metabolism of protein.

\section{THE FOOD-VALUE OF CERTAIN SUBSTANCES ALLIED TO PROTEINS}

PROTEOSES AND PEPTONES. In the digestion of the naturally occurring proteins the first products of hydration consist of a mixture of substances known as proteoses and peptones. In the further processes of digestion, under the influence of the ferments of the pancreas and small intestine, these substances are converted into the amino-acids which we have learnt to regard as the proximate constituents of the protein molecule. Many experiments have been performed in order to determine the nutritive value of these digestive products. In nearly all cases it has been found that the meat in the diet of an animal can be replaced by a corresponding quantity of the products of digestion of the same meat without interfering with the nitrogenous equilibrium of the animal, and Loewi and others have shown that the same result may be attained by feeding an animal on the products of pancreatic digestion of protein, i.e. a mixture consisting almost entirely of amino-acids. Since the proteins of the body differ in their composition from the majority of the proteins of the food, it is evident that each food-protein molecule has to be taken to pieces and reconstructed before it can take its place in the body fabric, and it is therefore only natural that, so far as metabolism is concerned, the results should be identical whether we feed the animal with the ordinary food-protein or with the products of its metabolism. This mode of feeding cannot, however, be regarded as presenting any advantages. Under normal circumstances the food molecules are broken down by degrees. Their products of hydrolysis are set free in small quantities at a time and can be therefore absorbed and disposed of in proportion as they are set free. On the other hand, a sudden flooding of the alimentary canal with a krge quantity of the products of digestion introduces an abnormal factor which must tend to produce wastage of nitrogen in the body and to disturb the normal chain of processes involved in the regular course of digestion.

COLLAGEN AND GELATIN. Among the various sclero-proteins or albuminoids which occur as normal constituents of our foods these two are the only substances which undergo digestion and solution in the alimentary canal to any appreciable extent, other substances, such as elastin and keratin, reappearing for the greater part in the fæces. As we have seen, gelatin, the first product of hydration of collagen, represents, so to speak, an imperfect protein. When it is hydrolysed by acids its disintegration products include the ordinary amino-acids of the fatty series, including a considerable amount of glycine and also a certain amount of phenyl alanine and proline. The oxyphenyl group which occurs in all the food-proteins in the form of tyrosine, as well as the indol-containing group tryptophane, are absent. On this account purified gelatin does not give either Millon's test or the Hopkins-Adamkiewicz test with glyoxylic acid. As might be expected from its composition, 
gelatin cannot entirely replace the proteins of the food, but is able to take the place of part of the proteins. If nitrogenous equilibrium has been attained on a certain amount of protein together with a mixed diet, a considerable proportion of the protein, but not all, can be replaced by gelatin. In an experiment on a dog, in nitrogenous equilibrium on a mixed diet containing $0.6 \mathrm{grm}$. protein per kilo, it was found that fully five-sixths of the protein could be replaced by gelatin without any disturbance of the nitrogenous metabolism. Physiologists have succeeded in maintaining animals for a short time in a state of nitrogenous equilibrium on a diet containing no protein, but in its place a mixture of gelatin with tyrosine and tryptophane. It is doubtful, however, whether such experiments could be continued indefinitely. In most cases the animal after a time refuses to eat the gelatin. A somewhat similar behaviour is found in the case of zein, the crystallised protein from maize, which yields no tryptophane or tyrosine on hydrolysis. Hopkins has shown that animals fed with zein, together with a small proportion of tryptophane, live longer than those fed with zein alone. But in no case could he maintain life on this diet for a period greater than forty-five days. There are evidently other groups in the protein molecule which are essential for the maintenance of life and which were not represented in the mixture of zein and tryptophane.

Experiments have been carried out in order to ascertain whether asparagine, which forms so important a nitrogenous constituent of young plants, can be directly utilised by animals. There is evidence that this substance has a real nutritive value for certain herbivora. The utilisation is not, however, a direct one. The asparagine appears to be taken up by the bacteria which swarm in the paunch or cæcum of these animals. It is built up by these micro-organisms into protein, and it is the protein of the microorganisms and not the asparagine itself which is digested, absorbed, and utilised by the mammal.

\section{OTHER CONSTITUENTS OF THE FOOD}

CELLULOSE. This substance, which forms the cell walls of plants, furnishes an important food-supply to the herbivora. Its digestion is not, however, carried out by the action of juices secreted by the intestinal canal itself. The solution of the cellulose is effected partly under the influence of a cellulose or cytase present in the plant cell themselves, partly under the influence of the micro-organisms living in the paunch or cæcum. Under the influence of these bacteria cellulose is dissolved with the production of carbon dioxide, methane, and butyric and acetic acids. In man the greater part of the cellulose of the food is undigested, and its chief value appears to be that of lending bulk to the indigestible material and so aiding the normal movements of the intestines. In the case of young plant cells, such as those of lettuce or carrots, a certain percentage of the cellulose undergoes solution in the intestine. Here, again, the digestion is probably effected by the agency of putrefactive organisms.

ALCOHOL. When alcohol is taken by man in moderate quantities the greater part of it undergoes oxidation, and leaves the body as carbon dioxide and water. About 10 per cent. which escapes oxidation is excreted unaltered 
by the lungs and urine. This oxidation of alcohol is a result of true utilisation, since the addition of a certain amount of alcohol to the food does not result in an increase of the output of carbon dioxide. In small quantities therefore alcohol can act as a food. This function, however, is quite unimportant, and is overshadowed by the poisonous action of this substance. A man unaccustomed to its action cannot take more than 16 to $25 \mathrm{grm}$. without experiencing its poisonous effects. This amount of alcohol only represents a total heat-value of 112 to 175 calories, i.e. only about 5 per cent. of the total energy requirements of the body. Only very rarely therefore can we be justified in administering alcohol as a food. ' Its value in a diet is entirely that of an accessory or adjuvant in exciting appetite by its taste and smell, an advantage which is largely counterbalanced by the danger of introducing a poison into the body which on long continuance tends to set up various degenerative changes in the tissues, and if taken in any quantity at one time causes a temporary abolition of those processes of inhibition and control which have been the determining factors in the survival of the race throughout the struggle for existence.

\section{THE INORGANIC FOOD-STUFFS}

If an animal be fed with the proper quantities of fats, proteins, and carbohydrates, from which all the salts have been removed as completely as possible, it rapidly shows a distaste for the food, becomes ill, and dies in a shorter time than if it were receiving no food at all. Part of the symptoms which occur in these cases are due to the production of acid substances, e.g. sulphuric acid, in the course of metabolism of the proteins. It is possible to obviate this acid intoxication by administering sodium carbonate with the food. This admixture, however, only suffices to prolong the life of the animal for a short time. It is evident therefore that the inorganic constituents of the food, although yielding no energy to the body, are as essential for the maintenance of life as the energy-yielding food-stuffs, namely, proteins, carbohydrates, and fats. In the course of this work we shall have occasion to study the intimate dependence of the functions of various tissues, such' as skeletal and heart muscle, on the presence of salts in normal proportions in the fluids with which they are bathed. Animals in a state of salt hunger show by the disorders of digestion which occur that the presence of salts is equally requisite for the due performance of the processes of secretion and absorpticn. Towards the end of the experiment the animal vomits its food, which shows no signs of digestion even when it has lain some hours in the stomach. Forster has shown that in salt-hunger the body is continually giving off inorganic constituents in the urine. The amount of these is smallest when it is supplied richly with organic food-stuffs. It seems that the salts of the body exist in a state of unstable combination with the tissue constituents, especially the proteins. If the amount of food supplied is insufficient, the animal lives on its own tissues, thus setting free salts which appear in the urine. The loss of salts to the body will therefore be in direct proportion to the degree in which the animal is living at the expense of its own tissues. 


\section{SECTION VI}

\section{THE NORMAL DIET OF MAN}

THE adult man has to take a certain quantity of food every day in order to furnish an amount of energy equal to that lost from the body as heat and mechanical work, and to replace the waste of tissue. This income is represented, not by the total food taken into the alimentary canal, but by the proportion of the food which is absorbed from the canal. This will vary with the digestibility and nature of the diet, and in any experiments instituted to determine the metabolism of man the first question that must be decided is as to the proportion of food-stuffs actually utilised. Food which is not absorbed will be excreted from the body in the fæces. The degree of utilisation of food-stuffs will therefore be given by an analysis of the fæces passed daily on any given diet. In order to be certain that the fæces passed during a given time correspond to and are derived from the food taken in at the same time, it is usual to give at the beginning and end of the experiment a capsule of lampblack, so as to colour the fæces and delimit those formed during the periad of the experiment. The fæces during starvation contain a certain proportion of nitrogen, carbohydrates, and fats, and in judging of the degree of utilisation of any given food-stuff the amounts of these substances excreted by the alimentary canal during starvation must be deducted from the total amount found in the fæces.

The excretion of nitrogen by the intestines varies between 0.54 and 1.36 grm. per day. If we find an amount of nitrogen in the fæces not exceeding these figures, we are justified in concluding that the utilisation of the nitrogenous constituents of the food is practically complete. This is the case when the man receives as food the animal proteins, such as meat, eggs, or milk. In experiments carried out with these materials the amount of nitrogen of the fæces varied between 0.14 and $1.9 \mathrm{grm}$. Only when excessive amounts of milk are given is the utilisation less complete and the nitrogen in the fæces increased above this amount. If, however, the protein be given in the form of vegetable food, the wastage of nitrogen by the fæces is much greater. It may rise as high as 48 per cent. and amount to 9 grm. per day. Nearly always it exceeds 15 per cent. This greater wastage of the nitrogen of vegetable food depends partly on the fact that certain non-protein and indigestible nitrogenous constituents of seeds and grains are reckoned as protein, partly on the fact that a considerable proportion of the protein may be enclosed in indigestible envelopes, and partly on the greater stimulant. 
action of the vegetable diet on the movements of the alimentary canal, so that the food is hurried through the intestine before the processes of digestion and absorption have had time to attain their limit. This last factor may interfere also with the digestion of animal protein on a mixed diet. The fats and carbohydrates of the ordinary diet of man are also utilised to a very large extent. The fæces passed on a fat-free diet always contain between 3 and $6 \mathrm{grm}$. of ethereal extract which is reckoned as fat. When the fat of the food consists largely of olein and is fluid at the temperature of the body, it is almost totally absorbed, the absorption becoming less as the melting-point of the fat rises. Ordinary carbohydrates are also very well absorbed, but here very large variations may be produced by altering the condition in which they are presented in the food. The following Table shows the relative digestibility of the different food-stuffs in a healthy individual on a normal diet :

\begin{tabular}{|c|c|c|c|c|c|}
\hline \multicolumn{6}{|c|}{ Percentage of Food-stuff Absorbed } \\
\hline 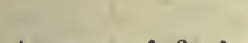 & Protein & Fat & Carbohydrate & Ash & Total energy \\
\hline $\left.\begin{array}{c}\text { Average of five } \\
\text { experiments }\end{array}\right\}$ & $92 \cdot 6$ & 94 & $97 \cdot 7$ & $77 \cdot 4$ & $90 \cdot 5$ \\
\hline
\end{tabular}

In judging therefore of the sufficiency of any given dietary, it is important to remember that on the average only about 90 per cent. of the total energy of the food is available for use by the body. If, for instance, the body requires an amount of energy equivalent to 3000 calories per day, it would be necessary to give food corresponding to 3333 calories per day. If, as is the case with the poorer classes, the food consists mainly of vegetable products it may be necessary to increase still further this allowance, since on a diet such as rye bread the loss of energy in the fæces may amount to as much as 35 per cent. of the total energy of the food.

The quantity of food which is necessary to keep an adult man in a state of health, without loss or gain of weight, is represented by that amount which is sufficient after absorption to supply the total daily output of energy. This output will vary considerably not only from individual to individual but also with the weight and size of the man, and above all with his state of muscular activity. Thus in the case of a woman weighing 49.45 kilos, in a state of hysterical sleep, the total output of energy during twenty-four hours amounted to 1228 calories, i.e. $24 \cdot 8$ calories per kilo body weight. Under more normal conditions, with the increased tone of muscle which is present during waking hours, the evolution of energy by the body is increased above this amount. Pettenkofer and Voit found that a resting individual had an output of 2300 calories during starvation and 2670 calories on a normal diet. A series of experiments by Tigerstedt on individuals kept in a state of rest, but on normal diet, gave results varying between 26 and 36 calories per kilo for the twenty-four hours. We may take therefore 30 cảlories per kilo body weight as the average requirements of a man in a state of rest. This would correspond to 2100 calories for a man weighing 70 kilos. When muscular work is performed the energy output is at once largely increased, and with it the food requirements of the body. The attempts which have been 
made to arrive at some idea of the average amounts of food required by a working man have been based not so much on scientific experiment as on an analysis and comparison of the diets in general use among different classes of men, the amounts of which are determined by the instinct of the man himself, while its quality is limited by his daily earnings. Tigerstedt divided the different diets which have been investigated into six classes, according to the total amount of energy which each of them represents. These are as follows:

\begin{tabular}{|c|c|c|c|c|c|c|}
\hline Group & Protein & Fat & $\begin{array}{c}\text { Carbo- } \\
\text { hydrate }\end{array}$ & $\begin{array}{l}\text { Calories } \\
\text { gross }\end{array}$ & $\begin{array}{c}\text { Calories } \\
\text { net }\end{array}$ & $\begin{array}{c}\text { Calories per } \\
\text { kilo body } \\
\text { weight }\end{array}$ \\
\hline I & 84 & 56 & 599 & 2483 & 2235 & 32 \\
\hline & 88 & 39 & 512 & 2825 & 2538 & 36 \\
\hline III & 130 & 64 & 520 & 3257 & 2932 & 42 \\
\hline IV & 141 & 71 & 677 & 4020 & 3618 & 52 \\
\hline V. & 167 & 89 & 774 & 4685 & 4218 & 60 \\
\hline VI & 152 & 139 & 1062 & 6269 & 5642 & 81 \\
\hline
\end{tabular}

In view of this great variation among the diets of different individuals it becomes difficult to arrive at any conclusion as to the diet which should be regarded as the average and should guide us in drawing up dietary scales for public institutions. Voit, from experiments on ordinary workmen performing eight or nine hours' labour a day, such as a bricklayer or carpenter, has laid down the following as an average diet, namely:

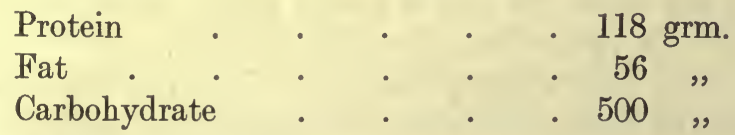

This would correspond to a total calorie value of 3055 , or, subtracting 10 per cent. for the food which is not utilised in the alimentary canal, to 2749 calories. The fat in this diet is rather low, preference being given to the carbohydrate on account of its greater cheapness. It is not advisable to reduce the fat below this limit, since the human body has a need for a certain proportion of fat, in the absence of which the utilisation of the other food-stuffs does not proceed normally. The presence of fat in the diet is especially important in the case of infants and young children, many of the disorders of nutrition prevalent among the children of the lower classes being largely determined by the absence of the proper quantity of fat from the diet. In the case of severe work this diet may not be adequate to supply the necessary quantity of energy. Thus, for soldiers, Voit's ration during manœuvres consists of :

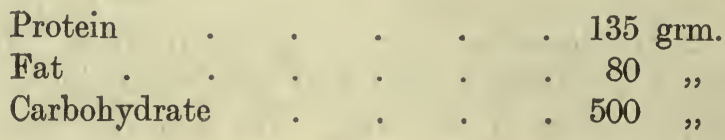


with a total calorie value of 3348 . His ration for war-time consists of :

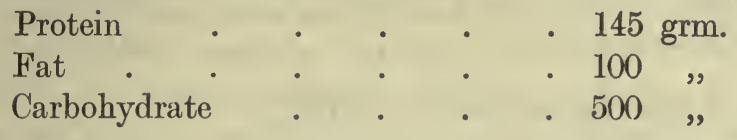

corresponding to 3575 calories. Even this may be insufficient to supply the energy needs during a period of intense muscular activity. In one experiment by Atwater, in which the individual performed muscular work for a period of sixteen hours out of the twenty-four in a calorimeter, the total energy given out amounted to over 9000 calories in the course of the twentyfour hours. It is probable, however, that in all cases where such excessive calls on the energies of an individual are made, one or even two rest days would follow the day of exertion, so that the deficiency of the food on the day of exertion would be made good by an increased intake of food on the following days. Thus three days' intake of 5000 calories would yield sufficient energy for the output of 9000 calories on one day of exertion and of 3000 calories, the normal amount, on each of the two succeeding rest days. The relative part played by the different constituents of a diet in yielding energy to the body has been determined by many observers and especially in a long series of researches by Atwater. In the following Table are given details of the daily food in one such experiment on a man weighing 76 kilos :

\begin{tabular}{|c|c|c|c|c|c|c|c|c|c|}
\hline $\begin{array}{c}\text { Food } \\
\text { material }\end{array}$ & $\begin{array}{c}\text { Weight } \\
\text { per day } \\
\text { grm. }\end{array}$ & $\begin{array}{l}\text { Water } \\
\text { grm. }\end{array}$ & $\begin{array}{c}\text { Protein } \\
\text { grm. }\end{array}$ & $\begin{array}{l}\text { Fat } \\
\text { grm. }\end{array}$ & $\begin{array}{l}\text { Carbo- } \\
\text { hydrate } \\
\text { grm. }\end{array}$ & $\begin{array}{c}\text { N. } \\
\text { grm. }\end{array}$ & $\begin{array}{c}\text { C. } \\
\text { grm. }\end{array}$ & $\begin{array}{c}\text { H. } \\
\text { grm. }\end{array}$ & $\begin{array}{l}\text { Heat } \\
\text { of com- } \\
\text { bustion } \\
\text { calories }\end{array}$ \\
\hline Beef & 35 & $22 \cdot 0$ & $11 \cdot 5$ & $1 \cdot 0$ & - & $1 \cdot 84$ & $6 \cdot 73$ & 0.99 & 76 \\
\hline Butter & 245 & $27 \cdot 2$ & $2 \cdot 9$ & $208 \cdot 3$ & - & $0 \cdot 47$ & $1 \cdot 54$ & $25 \cdot 28$ & 1929 \\
\hline Bread & 350 & $148 \cdot 7$ & $28 \cdot 3$ & $3 \cdot 9$ & $165 \cdot 5$ & $4 \cdot 97$ & $90 \cdot 69$ & 13.55 & 913 \\
\hline Gingersnaps & 60 & $2 \cdot 4$ & $3 \cdot 6$ & $3 \cdot 4$ & $49 \cdot 2$ & $0 \cdot 63$ & $25 \cdot 74$ & $3 \cdot 98$ & 259 \\
\hline Shredded & & & & & & & & & \\
\hline wheat . & 40 & $2 \cdot 5$ & $4 \cdot 1$ & $0 \cdot 6$ & $32 \cdot 2$ & 0.72 & $16 \cdot 70$ & $2 \cdot 54$ & 164 \\
\hline Sugar . & 60 & - & - & - & $60 \cdot 0$ & - & $25 \cdot 26$ & $3 \cdot 89$ & 238 \\
\hline Cereal coffee & 1200 & $1191 \cdot 6$ & 0.7 & 一 & $7 \cdot 2$ & $0 \cdot 12$ & $3 \cdot 96$ & $0 \cdot 60$ & 41 \\
\hline Whole milk & 1355 & $1149 \cdot 0$ & $52 \cdot 9$ & $74 \cdot 6$ & $69 \cdot 2$ & $8 \cdot 40$ & $108 \cdot 00$ & 16.93 & 1247 \\
\hline $\begin{array}{c}\text { Total ration } \\
\text { per day }\end{array}$ & 3345 & $2543 \cdot 4$ & $104 \cdot 0$ & $291 \cdot 8$ & $383 \cdot 3$ & $17 \cdot 15$ & $431 \cdot 08$ & $67 \cdot 76$ & 4867 \\
\hline
\end{tabular}

Very few accurate experiments have been made on the daily requirements of women. Since the average weight of a woman is less than that of a man and the work performed less severe, she will require a smaller amount of food both to meet the energy expenditure of the body and to provide for the repair of her tissues. Voit, under the assumption that the body weight of woman is four-fifths that of man, and that her energy requirements are diminished in the same proportion, has given the following as the daily requirements of a woman engaged in manual labour : 


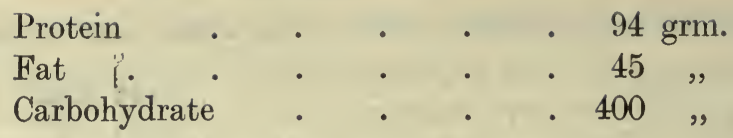

equivalent to a gross calorie value of 2444 .

\section{'VIT AMINES,}

The statement that a diet composed of the five classes of food-stuffs, proteins, fats, carbohydrates, salts and water, is all that is necessary for the maintenance of life is not strictly true. It is found that if rats, for instance, be fed on milk or bread and milk, they can live and grow in a normal manner. If, however, the proteins, fats and carbohydrates be extracted from the milk, purified, and then mixed in the same proportion as they were in the original milk, the animals, if young, cease to grow, and after some days or weeks die. The ordinary nourishing effect may be restored by adding to the artificial mixture an alcoholic extract of milk, or of yeast cells, or of many other living tissues. It was thought by Stepp, who first called attention to this fact, that certain lipoids must be an essential ingredient of the food. Further light has been thrown on the question by the researches of Hopkins and of Funk. The latter has investigated especially the condition known as beri-beri, which is a state of mal-nutrition brought on by an exclusive diet of polished rice, i.e. a rice which has been deprived of its outer coating. This condition does not occur if to the diet be added the dust rubbed off the rice in the process of polishing. A similar condition can be produced in fowls and is rapidly cured by addition to the diet of rice polishings or of an alcoholic extract of these polishings, of yeast cells, or of lime juice. It seems that in addition to the five classes of food-stuffs, minimal quantities of certain other substances are necessary in order that the processes of life may proceed normally. How these substances act we do not know, but we must imagine that they have a druglike effect on some organs of the body and take the place of or give rise to some of the hormones which are essential for the orderly working of the different organs of the body. Funk has given to these substances the name of 'vitamines.'

\section{RELATIVE PROPORTIONS OF THE DIFFERENT FOOD- STUFFS IN THE DIET OF MAN}

Since we have exact determinations at our disposal of the total energy output of a man under various conditions, it is easy to assign a total diet to each class which shall satisfy these energy requirements. In such a diet fat and carbohydrate are mutually replaceable in proportion to their calorie value, though it seems that in most individuals for the conservation of perfect health a certain minimum amount both of fats and carbohydrates is necessary. Some observers, however, have described an increased output of carbon dioxide as the result of the ingestion both of carbohydrates and of fats, pointing to a stimulating effect on metabolism of these food-stuffs themselves. If these results are generally applicable, we cannot regard the total energy output of a man on a given diet as affording a criterion of the amount of food necessary for him per day, since it is possible that with a smaller amount of food the stimulating effect on metabolism might be wanting and that the functions of the body might be normally performed with a greater ecunomy of material. The stimulating effect of fats and carbohydrates on metabolism has not, however, been universally observed, whereas in the case of proteins every worker has noted an increased metabolism in propor- 
tion to the amount of protein in the diet. Especially is this marked in man, where the power of storing protein in the body seems to be minimal or absent in the normal adult. As we have seen, the protein taken in the food has a twofold destiny. Part of it, probably the smaller portion, is needed to be built up into the tissues, and to form living protoplasm in replacement of wear and tear. The other, the larger portion, serves, like the fats and carbohydrates, for meeting the energy requirements of the body. The aminoacids produced by the disintegration of the proteins in the alimentary canal are rapidly absorbed and apparently undergo deamination in the body. The nitrogen so split off is at once excreted in the urine, while the nonnitrogenous moiety is rapidly oxidised to carbon dioxide and water. However much protein is ingested, so long as the digestive powers of the animal are not overtaxed, all that is not required for replacing tissue waste undergoes this fate, and we can therefore attain nitrogenous equilibrium on a diet containing $50 \mathrm{grm}$. or $150 \mathrm{grm}$. of protein daily. The amount of protein taken in the food, and digested and oxidised in the body by any given individual, affords no clue to the amount which is absolutely necessary for the maintenance of life and for the normal discharge of the bodily functions. It is therefore not surprising to find the greatest possible divergences between various classes of men in the quantity of protein taken in their daily food. An average individual in affluent circumstances, eating three meat meals a day, probably takes in from 100 to $160 \mathrm{grm}$. of protein daily, corresponding to a nitrogen content of 16 to $25 \mathrm{grm}$. On the other hand, there is no doubt that an individual can lead a perfectly normal existence with a nitrogen intake as low as 5 or $6 \mathrm{grm}$. a day. It is not possible to explain these differences as determined by individual idiosyncrasies, nor is the appetite of the individual to be taken as a safe guide to the relative composition of the foods. The average diet of any race has been determined up to the present not so much by the physiological requirements of the body as by the nature of the food available. Hence, whereas the races living in tropical climates are mainly herbivorous or frugivorous, the northerners, who have developed their intellectual and bodily superiority in their harder struggle for food amidst more inclement surroundings, have been perforce obliged to satisfy their energy requirements at the expense of animal food. In these days when the products of all climes are at the disposal of civilised man, his food-supply is no longer dependent on the country in which he lives, and it is possible to regulate the composition of his food according to the results of physiological experience. Since the proteins represent the most costly constituents of the food, it becomes important to inquire how much of this class of food-stuffs is essential to the maintenance of health and whether any advantage is given by taking proteins in excess of the physiological minimum. The fact that those nations which hold the highest place in the world are mainly flesheaters cannot be regarded as any proof of the advantage of a flesh diet over a diet poorer in protein. It is the hard struggle for existence which in the northern races has eliminated the weakling and resulted in the production of a superior race." The fact that he is a flesh-eater can also be ascribed to the 
exigencies of climate and does not necessarily prove that a large flesh diet is responsible for his greater efficiency.

Of late years a number of careful experiments have been made to determine the minimum amount of protein which must be present in the daily ration of man. I have mentioned above two experiments in which nitrogenous equilibrium was obtained with much smaller amounts of protein than those given in the normal diets. In these experiments, in one of which the man received $43.5 \mathrm{grm}$. of protein daily, and in the other only $33 \mathrm{grm}$. of protein, it was found necessary to give at the same time amounts of carbohydrates and fats far exceeding those in the normal diet, so that whereas, e.g. the normal individual takes in between 32 and 35 calories per kilo, the man on $43.5 \mathrm{grm}$. of protein needed 47.5 calories per kilo, and the one on $33 \mathrm{grm}$. of protein needed the huge amount of 78.5 calories per kilo.

Other experimenters have, however, succeeded in maintaining perfect health and nitrogenous equilibrium for a considerable time on a diet containing a much smaller amount of protein than has been generally considered necessary without adding to the ration abnormal quantities of fats or carbohydrates. Thus Siven, in an experiment on himself, found that he could maintain nitrogenous equilibrium for thirty-two days on a diet containing only $6.26 \mathrm{grm}$. of nitrogen. The total heat-value of the food per day was only 2444 calories. Folin, in individuals with an insufficient amount of protein containing only $2 \cdot 1$ to $2 \cdot 4 \mathrm{grm}$. of nitrogen, found that the nitrogen output per diem was only 3 to $4 \mathrm{grm}$. It would seem therefore that a healthy adult man, having a sufficient intake of non-nitrogenous food, need not metabolise more protein than suffices to yield 3 to $4 \mathrm{grm}$. of nitrogen per day, i.e. between 25 to $35 \mathrm{grm}$. of protein. Other observations have been made on vegetarians, showing that individuals can maintain perfect health on a diet containing only about $34 \mathrm{grm}$. of protein a day, and with a total calorie value of 1400 to 2000 . A series of experiments were conducted by Chittenden with a view to determining how far such a diet is suitable to the average individual, and especially whether it can be continued for long periods of time without interfering with the well-being of the subject of the experiment. The general results of these experiments show that the physiological needs of the body can be met by greatly reduced protein intake with the establishment of continued nitrogenous equilibrium on a far smaller amount of protein food than is contained in the ordinary dietary tables, and that on this diet the individual in some cases, far from suffering in health, has his physical and mental efficiency increased. The experiments were made on various classes of men: instructors and students in the university, soldiers, and athletes. In the case of Chittenden himself the average daily diet contained about 6 grm. of nitrogen and had a heat-value of about 1600 calories. In the case of another individual the intake of nitrogen per day was $9.5 \mathrm{grm}$. and the heat-value of the food 2500 calories. It is thus possible to reduce the total energy of the food from about 3300 calories to about 2500 calories. 'Of the protein taken in by a normal individual therefore a certain amount which is not needed by the organism is at once broken up and serves 
simply to increase the total metabolism of the body without serving any useful physiological purpose other than heat production.* Many ailments, especially of middle age, have been ascribed to an excess of protein in the food. It has been thought that the kidneys and other organs may suffer from the strain of eliminating excess of nitrogenous waste products. But the energy metabolism of proteins results almost entirely in the formation of urea -an innocuous substance which can have little harmful effect on the kidneys, even if we assume (an assumption hardly justifiable) that these organs (unlike other organs of the body) suffer as a result of their normal functional activity. There is no doubt that many disorders of middle life may be put down to over-feeding and lack of muscular exercise, but there is just as much reason to ascribe these evils to the carbohydrates as to the proteins of the diet. It is indeed possible that an almost exclusive protein diet might be more suitable than carbohydrates for a sedentary life, where the normal stimulus to oxidation of the food-stuffs, viz. muscular exercise, is absent, and that the 'stimulant' effect of proteins on metabolism might have a real value to the organism.

The limitation of protein diet to a minimum is only justifiable in adult healthy men. Where from any cause there has been a loss or destruction of nitrogenous tissue, as after infective diseases, the body possesses the power of storing up nitrogen in the form of flesh or muscular tissue, and it is important under such circumstances to give in the food an excess of protein which can be used for this purpose. Moreover, when a rapid growth of muscle is going on, as during training, an excess of protein in the food is also desirable, though nothing is gained in pushing this administration of protein to an inordinate extent.

The fact that in many cases greater economy and efficiency of nutrition are attained by diminishing the protein of the diet affords no argument for accepting or rejecting any given class of foods. Thus the normal requirements of the individual can be obtained by the administration of a diet containing meat, eggs, vegetables, and cereals, or by a diet derived entirely from the vegetable kingdom. A purely vegetable diet is rendered possible in civilised countries by the ease with which the products from warmer climates can be obtained. A diet composed only of the products of temperate climates would tend to be deficient in fats and oils. In such climates it is therefore necessary to import the oily fruits grown in warmer lands, or to supplement the diet with such animal food as milk or butter or eggs. In drawing up a purely vegetarian diet it is important to remember that its constituents, especially its protein, are digested with greater difficulty than the corresponding ingredients in animal food. A larger quantity therefore has to be given in a vegetable diet in order to allow for the greater loss by the fæces. Any general reform of diet which may be indicated by recent physiological experiments would seem to lie rather in the direction of limitation of the

* But heat production is a very important function of the food, and on the Chittenden diet tends to' be deficient; so that individuals on this régime 'feel the cold' more than they did when on an ordinary diet. 
quantity of different articles of food, perhaps especially of those rich in protein, than in a limitation to foods obtained from the animal or vegetable kingdom. In certain individuals the increased bulk of indigestible residue which is afforded on a vegetarian diet presents a distinct advantage, since it serves to promote peristalsis and the normal evacuation of the large bowel. There is no scientific evidence that for the ordinary person any advantage is to be gained by adherence to a strictly vegetarian diet.

\section{THE DIET OF THE GROWING ANIMAL}

Since the young animal is smaller than the adult, it presents a greater surface in proportion to its bulk, and therefore will need more energy per kilo body weight than is the case with the adult. The processes of growth are attended moreover with an active metabolism, so that the metabolism is more energetic in the young animal than in the adult, even if we take into account the relative surface in the two cases. In the following Table is given a number of observations showing the output of carbon dioxide per square metre body surface at different ages in boys and men :

Effect of Age on Metabolism (Man)

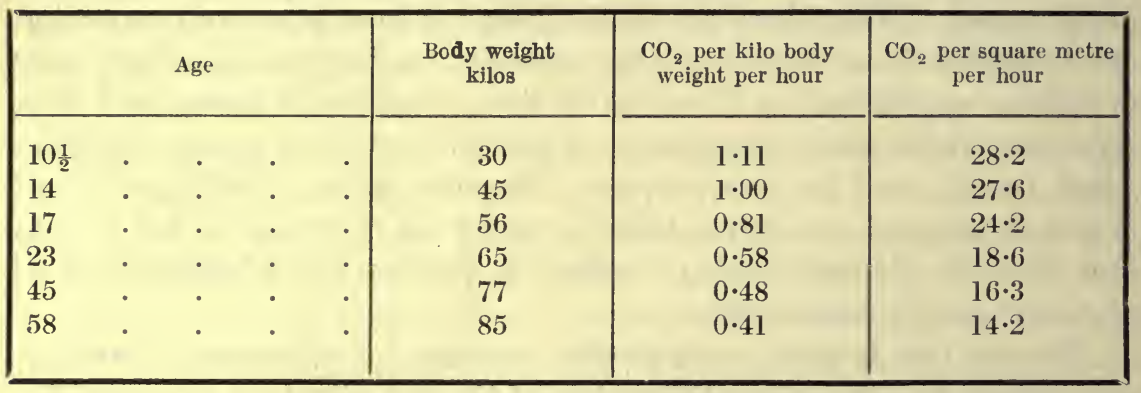

Between the ages of fourteen and nineteen years in boys the carbon dioxide output is absolutely greater than at any other age. It is during these years that the growth of the boy is most marked. A boy between nine and thirteen years old requires just as much food as a full-grown man, and between the ages of fourteen and nineteen he will require more. This great increase between the fourteenth and nineteenth years is not noticed in females. There is a gradual absolute increase in girls up to the eleventh year. At this age and henceforward the total carbon dioxide output and therefore the total food requirements remain constant, being about equal to that of a woman of thirty. The total energy requirements of the growing animal are not, however, the only factor which we have to consider. The protein of the food has not only to replace the wear and tear of tissue, but also to provide material for the growth of new tissues. The protein needs therefore of the growing animal are relatively greater than those of the adult, and absolutely greater during the period of most rapid growth, namely, in boys between the ages of fourteen and nineteen. It is a popular belief that in a working-class family the worker or wage-earner needs a greater protein supply than the rest of the family. This is a mistake. The adult worker can obtain his energy equally well from carbohydrates and fats, whereas 
an excess of protein is absolutely necessary in the case of the children to provide the material for their proper development and growth. The relation between rate of growth and protein content of food is well illustrated by a comparison of the composition of the milk in different animals. In the following Table (Proscher) it will be seen that the more rapidly an animal grows the greater is the protein content of the milk with which it is supplied :

\begin{tabular}{|c|c|c|c|c|c|c|}
\hline & & \multirow{2}{*}{\begin{tabular}{|c|} 
Time in which \\
the body weight \\
of the new-born \\
animal was \\
doubled. \\
Days
\end{tabular}} & \multicolumn{4}{|c|}{100 parts of Milk contain } \\
\hline & & & Protein & Ash & Lime & $\begin{array}{c}\text { Phosphoric } \\
\text { acid }\end{array}$ \\
\hline Man . & . & 180 & $1 \cdot 6$ & $0 \cdot 2$ & $0 \cdot 328$ & 0.473 \\
\hline Horse . & . $\quad$. & 60 & $2 \cdot 0$ & $0 \cdot 4$ & $1 \cdot 240$ & $1 \cdot 310$ \\
\hline Cow . & . & 47 & $3 \cdot 5$ & 0.7 & $1 \cdot 600$ & $1 \cdot 970$ \\
\hline Goat . & . & 19 & $4 \cdot 3$ & $0 \cdot 8$ & $2 \cdot 100$ & $3 \cdot 220$ \\
\hline Pig & . & 18 & $5 \cdot 9$ & - & - & - \\
\hline Sheep . & - & 10 & $6 \cdot 5$ & $0 \cdot 9$ & $2 \cdot 720$ & $4 \cdot 120$ \\
\hline Dog . & . & 8 & $7 \cdot 1$ & $1 \cdot 3$ & $4 \cdot 530$ & $4 \cdot 930$ \\
\hline Cat & . & 7 & $9 \cdot 5$ & - & - & - \\
\hline
\end{tabular}

We should expect then that the milk which is the sole food of the growing infant should contain a relatively greater proportion of protein than is necessary in the case of the adult. In an experiment by E. Feer, quoted by Bunge, a child weighing $8226 \mathrm{grm}$. at the thirtieth week took $951 \mathrm{grm}$. of milk. Human milk contains :

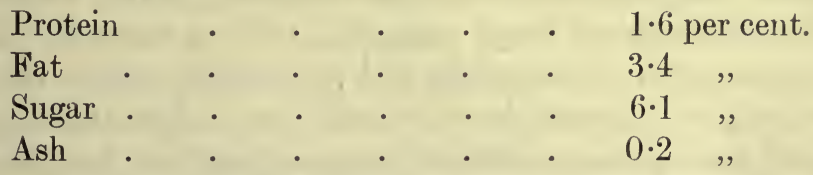

The child was therefore receiving daily :

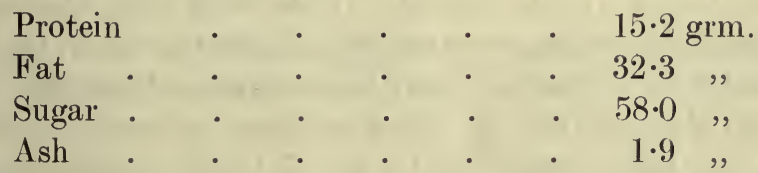

According to the same proportions a man of 70 kilos would take in :

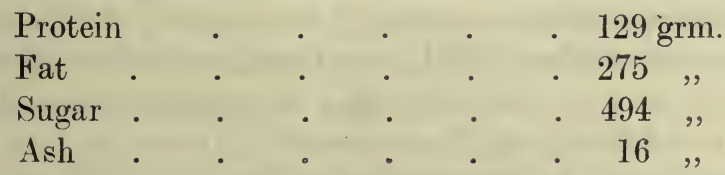

It is interesting to note that the protein of this diet differs but little from that in the diets ordinarily accepted as standard, but there is a large excess in the fat, and in the total calori value, as would be expected from the more rapid metabolism and the relatively larger body surface of the young child. 


\section{CHAPTER X \\ THE PHYSIOLOGY OF DIGESTION}

\section{CHANGES UNDERGONE BY THE FOOD-STUFFS IN THE ALIMENTARY CANAL}

THE use of the process of digestion is to alter the food-stuffs so as to fit them for absorption into the blood, by means of which they may be carried to all parts of the body. In most cases the food-stuffs cannot be utilised in their original form by the living cells. When we nourish ourselves at the expense of an animal or plant, we are taking in not only the current coin of the organism which is being used for the supply of energy to its vital processes, but also, and to a much larger extent, the framework forming the machinery of the organism as well as its stores of carbohydrate or fat. The food-stuffs as we ingest them are in the most inactive form possible. Practically all are colloidal, neutral, and tasteless, and present no tendency to unite with oxygen or, indeed, to undergo any change whatsoever, apart from the interference of living organisms such as bacteria. In a starving animal the stores of carbohydrate and fat and the protein structure of the inactive living cells have to be converted into a soluble form-transformed, so to speak, into currency-before they can be utilised by other living cells, such as those of the heart, for the discharge of their normal functions and the maintenance of the life of the animal. In the same way, when we take these colloidal or insoluble substances into our alimentary canal, they have to be rendered soluble or diffusible, in order to allow of their easy transference across the wall of the gut into the blood and their transport to the tissue-cells. The cells of the body cannot deal with all kinds of carbohydrate. Most animal cells will starve when presented with starch, dextrin, or any of the disaccharides, such as maltose, lactose, or cane sugar. It is necessary therefore that all the carbohydrates shall be reduced in the alimentary canal or in its walls to the form of monosaccharides. As regards proteins, the processes of digestion have a different significance according as we are dealing with their value as givers of energy or their value as builders up of the living protoplasm. If the proteins of the food are to be oxidised and utilised as a source of energy, they must be rendered soluble so as to enable them to be absorbed and carried to those parts of the body where they may undergo deamination and complete oxidation. If they are to be built up as integral parts of living cells, to take the place of molecules which have been destroyed in the 
wear and tear of functional activity, the change must be almost equally profound. The proteins of the cells from different parts of the body have different molecular constitutions. Not only do they differ among themselves, but they differ very largely from many of the proteins which may be taken in with the food. A child is able to obtain material for the growth of his braincells, his muscle-cells, or his liver-cells, from a diet containing protein in the form of caseinogen, or of vegetable gluten, or of meat fibrin. A reference to the Table on p. 91 will show the striking difference in composition between the various proteins of the food and the proteins which have to be formed from them in the living tissues. It is evident that to form serum albumin, for instance, out of wheat gliadin, an entire reconstruction is necessary. This can only be accomplished by taking the protein molecule to bits, and by selecting certain of its constituent parts and building these up in the proper proportions to form a new protein molecule. For the purposes of nutrition therefore the changes in the protein molecule must be greater the more variation there is in the composition of the protein of the food from the composition of the proteins of the tissues.

In primitive alimentary canals every cell lining the canal may be endowed with amœboid properties and capable of devouring the food particles, the subsequent changes in the latter to fit them for their journey through the rest of the body being performed in the body of the cell itself. In all the higher animals, however, including ourselves, the greater part of the preparation of the food is accomplished extracellularly in the lumen of the alimentary canal, and the changes are effected by means of special digestive juices, which are formed by the activity of masses of cells produced as outgrowths from the wall of the canal. The digestive juices attack the food-stuffs by means of ferments, and in every case the action of these ferments is hydrolytic, the food-stuffs taking up one or more molecules of water and undergoing dissociation into simpler molecules. Since each class of food-stuff requires a different ferment, a great variety of ferments is concerned in the processes of digestion.

As the end-result of digestion the many kinds of food taken by man are reduced to a fairly small number of simpler bodies. These end-products are

(1) Carbohydrates.

The monosaccharides : glucose, fructose or levulose, and galactose.

(2) Fats. Fatty acids, or (in alkaline medium) soaps, and glycerin.

(3) Proteins. Here we have a great variety of mono- and diamino-acids, which may be enumerated as follows :

\section{MoNO-AMINO-ACIDS}

Glycine (aminoacetic acid)

Alanine (aminopropionic acid)

Serine or oxyalanine (oxyaminopropionic acid)

Aminovalerianic acid

Monobasic acids

Leucine (aminoisobutylacetic acid)

Isoleucine (aminocaproic acid)

of fatty series 


\section{Mono-AMINo-AcIDS-continued}

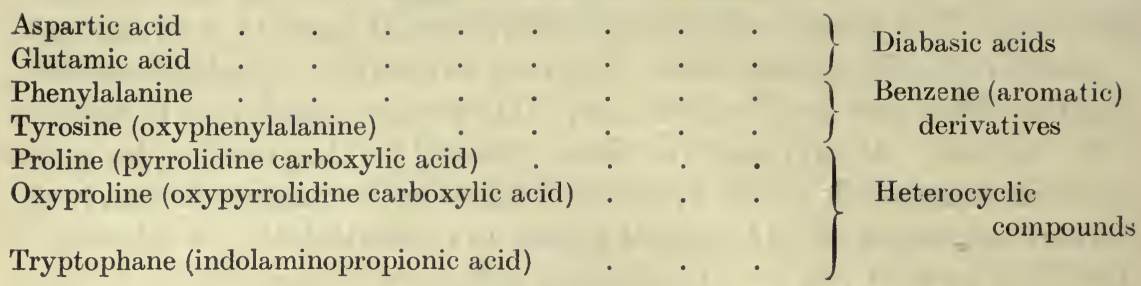

DiAMINO-ACIDS AND THEIR COMPOUNDS

Lysine (diaminocaproic acid)

Arginine (guanidinaminovalerianic acid)

Histidine (iminazolalanine)

'Diaminotrioxydodecoic acid'

Cystine (derived from aminothiopropionic acid)

$$
\begin{gathered}
\cdot \\
\cdot{ }_{\text {derived from a } 12 \text {-carbon acid }}^{\text {The }} \\
\cdot\left\{\begin{array}{c}
\text { Sulphur-containing } \\
\text { body }
\end{array}\right.
\end{gathered}
$$

Those constituents of the food which undergo no oxidation in the body, such as the water and salts, are practically unchanged in the alimentary canal, and are absorbed in their original form into the blood. 


\section{SECTION I \\ DIGESTION IN THE MOUTH}

IT is a common experience that when food is taken into the mouth there is a flow of a liquid, 'saliva,' into this cavity. Saliva is the product of secretion of three pairs of large salivary glands situated in the neighbourhood of the mouth and pouring their secretions into this cavity by means of ducts. It is possible to collect the fluid secreted by each of these glands separately, and it is found that the saliva varies in properties according to the gland from which it is derived. In addition to these large glands the whole mucous membrane of the mouth is beset with small glands.

The saliva is in most cases a mixture of the secretions of all three pairs of salivary glands as well as of the small glands of the mucous membrane. When collected it forms a colourless cloudy liquid, slimy in character. The cloudiness is due to the presence of a number of formed elements consisting of desquamated epithelial cells, disintegrating leucocytes, and gland-cells, as well as coagulated clumps of mucin. Its reaction is in healthy individuals slightly alkaline. Its

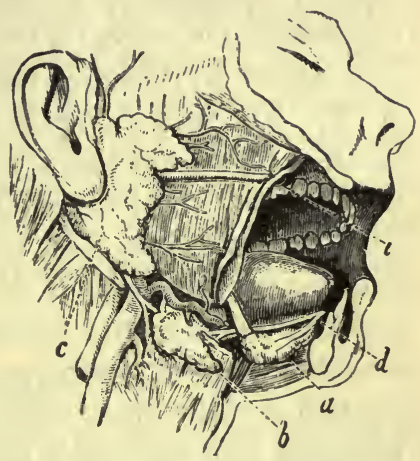

FIG. 321. Dissection to display the salivary glands.

$a$, sub-lingual gland; $b$, submaxillary gland; $c$, parotid gland; $d$, common opening of ducts of sub-maxillary and sublingual glands; $i$, opening of duct of parotid gland. specific gravity varies between 1002 to 1008. Its chief constituents are coagulable proteins, mucin, and in some cases a diastatic ferment, ptyalin, and traces of potassium sulphocyanate. Its average composition is as follows :

100 parts mixed saliva contain :

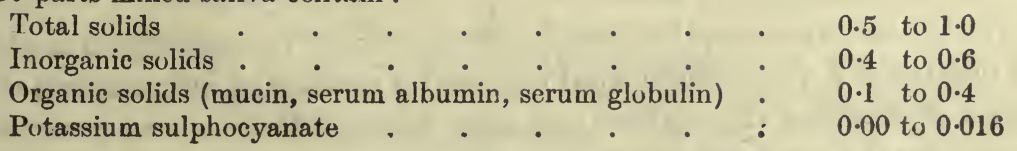

Freezing-point $(\Delta)=-0.07$ to $-0.3 \pm$

Potassium sulphocyanate is an almost constant constituent of human saliva, though it is often absent in that of other animals, such as the dog. It is generally present to the extent of 01 per cent. so that on the addition of a drop of ferric chloride to 
saliva a definite red colour is obtained. So far as we know it is formed in the body whenever cyanides or organic nitriles in small quantities make their appearance in the circulating fluid, either as the result of administration or perhaps as by-products in the normal processes of metabolism. The conversion of the poisonous cyanides into the almost innocuous sulphocyanates seems to be a means by which the organism protects itself against the poisonous effects of the former. The channels of excretion of the sulphocyanate are by the salivary glands, the kidneys, and possibly by the gastric juice.

\section{THE USES OF SALIVA}

The main function of saliva is to moisten the food and so facilitate its mastication and deglutition. The presence of the mucin is of special value for the latter process since it renders the mass of food slippery. In animals, such as dogs, where the saliva is devoid of any digestive ferment, this must represent its sole function. In man and some of the herbivora the saliva exerts a well-marked digestive effect on one of the food-stuffs, namely, starch. If a warm solution of starch be taken into the mouth, kept there for one minute, and then expelled into a test-tube, the starch will be found to have entirely disappeared, its place being taken by a reducing sugar. The stages of the action of saliva on boiled starch can be followed more easily if its action is retarded by keeping the mixture cool, or at a temperature not above $25^{\circ} \mathrm{C}$. The first change is a conversion of the opalescent gelatinising starch solution into a clear solution which no longer sets on cooling, but still gives a blue colour with iodine. The fluid contains what is known as soluble starch. The soluble starch then undergoes hydrolytic dissociation into a dextrin, which gives a red colour with the iodine and is therefore known as erythrodextrin, together with maltose. The erythrodextrin is then hydrolysed into an achroodextrin (giving no colour with iodine) and maltose, and the achroodextrin is still further broken up into dextrin and maltose. The conversion of starch into maltose is never complete, though if the maltose be removed by dialysis as it is formed, it was found by Lee to be possible to convert as much as 95 per cent. of the starch into reducing sugar. The stages in the conversion are represented in the following Table :

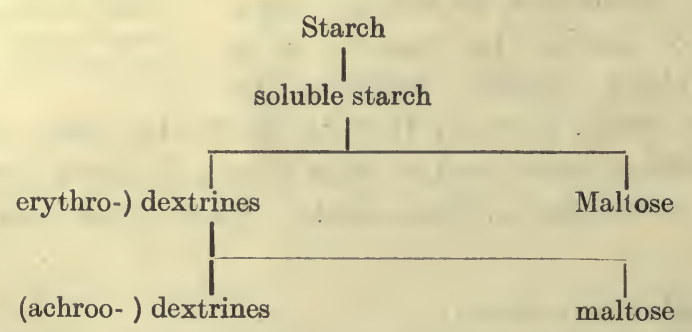

The process by which the huge starch molecule is converted into dextrins and maltose is a very complicated one, and a number of intermediate compounds of dextrins and maltose can exist between those whose presence is revealed by their varying reaction to iodine.

Ptyalin is most active in a neutral medium, so that the addition of minute traces of acid to the saliva increases its diastatic power. In the presence of free mineral acid ptyalin is rapidly destroyed, 003 per cent. 
hydrochloric being sufficient for this purpose. It acts most rapidly at the body temperature. At $0^{\circ} \mathrm{C}$. its action is still just perceptible. If heated to $60^{\circ} \mathrm{C}$. it is destroyed.

We have seen that boiled starch solution is changed by saliva when kept only a few seconds in the cavity of the mouth. When the starch is in the solid condition, as in biscuits and most farinaceous foods, its stay in the mouth during the normal process of mastication is not long enough to allow of any considerable hydrolysis occurring. When a meal is taken the food which is swallowed forms a mass lying in the fundus of the stomach. This mass is penetrated only with difficulty by the acid gastric juice secreted by the mucous membrane of the stomach within five minutes of the taking of food. Even half an hour after a meal the interior of the mass of food in the stomach may be still found to be neutral or slightly alkaline. The food therefore, thoroughly moistened by and mixed with saliva, remains in the stomach for thirty to forty minutes before the salivary ferment is destroyed by the penetration of the acid gastric juice. During this time the ptyalin continues to exert its effect, so that we may say that the chief part of the salivary digestion occurs actually in the stomach, and results in an almost complete alteration of the starch into dextrins and maltose. Unboiled starch is attacked with extreme slowness by the diastatic ferments either of the saliva or the pancreatic juice, so that, if taken by man, large quantities are unutilised and reappear in the fæces. Thirty to forty minutes after a meal the food becomes thoroughly soaked with the acid gastric juice, and salivary digestion gives place to gastric digestion.

\section{THE SECRETION OF SALIVA}

The mucous membrane of the mouth, especially on the under surface of the tongue, presents a number of small glands which contribute by their secretions to the moistening of the mouth. The greater part of the saliva is, however, formed in man by three pairs of glands, viz. the sub-lingual and the sub-maxillary glands, situated in the floor of the mouth below the jaw, and the parotid gland, lying in the cheek over the ramus of the inferior maxilla.

The arrangement of these glands, especially of those in the floor of the mouth, varies somewhat in different animals. In the dog and cat the sub-lingual gland is wanting, its place being taken by a gland situated somewhat further back and known as the retro-lingual gland. In the pig both retro-lingual and sub-lingual glands are present in addition to the sub-maxillary, and one may sometimes find traces of the retrolingual gland in man. Many animals, e.g. the dog, also possess a gland situated in the orbit, which pours its secretion into the mouth - the orbital gland. These glands can be divided, according to their structure and the nature of the secretion which they form, into several classes. Among the salivary glands of the mucous membrane we may distinguish two types, the mucous gland and the serous gland. In specimens hardened and stained by the ordinary methods the mucous gland is distinguished by the fact that its short duct opens into wide alveoli, the lining cells of which are distended with mucin and therefore present a clear unstained space in the section. In the other type, the serous gland, the duct lined with columnar cells branches into a series of acini which present a well-marked lumen and are lined with small granular cells with a very distinct and well-staining nucleus. The same general distinction can be made out in the large salivary glands. The parotid gland in man and in 
all the higher mammalia is a typical serous gland, though here and there a mucous cell may be occasionally seen. The orbital gland of the dog represents practically one of the mucous glands of the general mouth cavity on a large scale. The sub-lingual and sub-maxillary glands in man represent a third type. Most of the alveoli are mucous in character. At the ends of the alveoli are seen crescent-shaped cells between the mucin-distended cells and the basement membrane. These are known as the demilune cells, or the Crescent cells of Gianuzzi. In some cases these mucous alveoli with demilunes may be found alongside of typical serous alveoli.
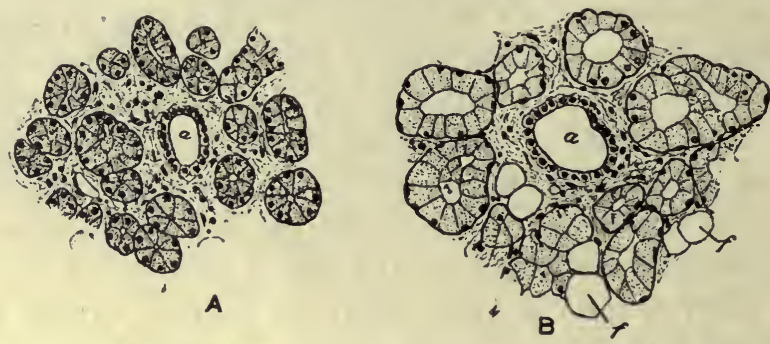

FıG. 322. A, serous gland; B, pure mucous gland from mouth. (KöLLIKER.) $a$, ducts ; $f$, fat-cells.

Thus in man the sub-maxillary gland is usually a mixed gland, the serous alveoli predominating. The sub-lingual gland is also mixed, but with a predominance of the mucous alveoli. In the monkey the sub-maxillary gland is almost entirely serous. In the dog the sub-maxillary gland is a pure mucous gland with demilunes, while the retro-lingual and sub-lingual gland when present are of the mixed type. In the rabbit the sub-maxillary gland is serous, while the sub-lingual gland is mucous. In the cat the sub-maxillary is mucous, the retro-lingual is mixed, and the sub-lingual, when present, is mixed, with predominance of the mucous type.

The normal behaviour of the salivary glands during digestion is best studied by aid of a method used long ago by de Graaf and reintroduced with considerable elaboration of late years by Pawlow. It is possible without any disturbance of the animal's nutrition to transplant the papilla on which the duct opens to the outside so that the saliva from any particular gland shall flow externally instead of into the cavity of the mouth. By attaching a small funnel to the fistulous opening, it is possible to collect the pure saliva unmixed with the secretion of any other of the glands.

By this method it has been found that as soon as food is introduced into the mouth there is a secretion of saliva, the relative extent to which different glands are involved varying according to the nature of the stimulation. Thus with meat there is only a small amount of secretion, which is derived chiefly from the sub-maxillary and sub-lingual glands, and is rich in organic constituents. When dry material, such as dry powdered meat, is introduced the flow of juice is more copious and more watery. The same effect may be produced in the dog by psychic excitation. Thus salivation may be induced by showing food to the dog, or even by the suggestion that dry powder is to be introduced into the mouth. A comparison of the juices obtained from different glands shows that the serous and mucous glands differ, as might be expected, in the nature of their secretion. A serous gland, such as the parotid, gives a thin watery secretion almost free from mucin, but containing small traces of coagulable protein. The mucous gland delivers 
a secretion which is viscid from the presence of mucin, and contains also a small trace of coagulable protein. Both in parotid and mucous saliva the percentage of salts is very low, so that the freezing-point of these fluids is considerably higher than that of the blood-plasma. In the dog the saliva is free from any ferments. In man ptyalin - the starch-splitting fermentis found in the saliva from both kinds of gland, though it predominates in that obtained from the parotid gland. The total amount of saliva which may be obtained varies, of course, in different animals. Each gland may, however, in the course of the day give an amount of juice far exceeding, e.g. ten or twelve times, its own weight. In man it is reckoned that over one litre of

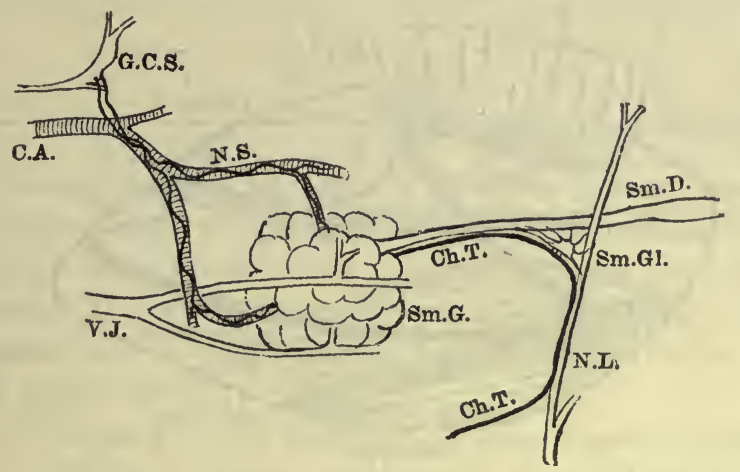

Fig. 323. Diagram of nerve-supply to sub-maxillary gland.

Sm.G, sub-maxillary gland; N.L, lingual nerve; Ch.T, chorda tympani; Sm.Gl, sub-maxillary ganglion; Sm.D, Wharton's duct; V.J, jugular vein; C.A, carotid artery; G.C.S, superior cervical ganglion; N.S, sympathetic fibres ramifying on facial artery. (After Foster.)

saliva may be formed every twenty-four hours, and in the herbivora, such as the horse, the total diurnal production must amount to many litres ; 500 grammes of hay alone may evoke the secretion of a litre of saliva.

The intimate dependence of the secretion of saliva on the events occurring in the mouth shows that the activity of the salivary glands must be excited by reflex means. The afferent nerves in this reflex are those that supply the mucous membrane of the mouth, i.e. the fifth nerve and the glossopharyngeal. The efferent channels of the reflex were discovered by Ludwig. Each one of the large salivary glands receives nerve fibres from two sources, viz. from the cerebro-spinal and from the sympathetic system. It is probable that the cerebrospinal supply is derived always from one part of the cerebral axis, namely, from the filaments which make up the nervus intermedius. From this point they diverge in their course to the glands. The fibres to the sub-maxillary, the sub-lingual, and the retro-lingual glands pass into the facial nerve, and then from this nerve along the chorda tympani to the lingual division of the fifth nerve. The lingual nerve passes below the duct, and just before it crosses the two ducts of the sub-maxillary and retro- or sublingual glands it gives off a small branch backwards, namely, the chorda tympani, which runs along the sub-maxillary duct to be distributed to the glands, and in its course gives off fibres also to the retro-lingual (Fig. 323). 
The fibres are apparently finally distributed to the secreting alveoli, where they end freely on the secreting cells just below the basement membrane. They do not, however, take an uninterrupted course. By means of the nicotine method Langley has shown that all the fibres to the sub-lingual and the sub-maxillary glands end somewhere near the glands in connection with ganglion-cells. From the ganglion-cells fresh relays of fibres are given off which pass to the gland-cells themselves. The fibres going to the sub-maxillary gland are connected with scattered cells lying in the substance of the gland itself; in the cat those passing to the retro-lingual gland are connected for the most part with the ganglion-cells which make up the

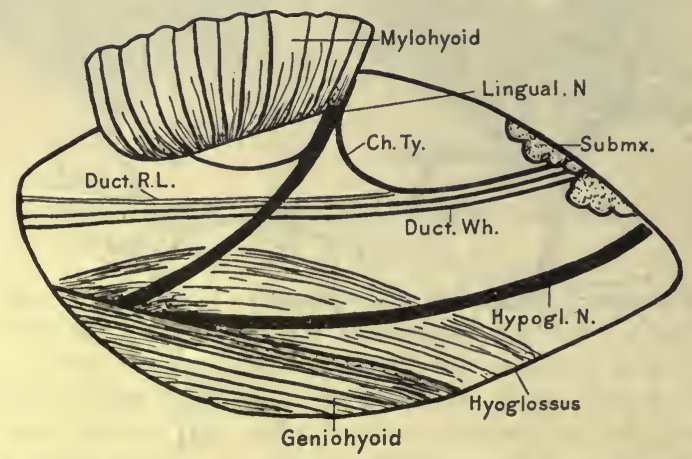

FIG. 324. Diagram of the arrangement of the nerve-supply to the sub-maxillary gland, as exposed in an actual experiment.

Duct,Wh, Wharton's duct (of sub-maxillary); Duct.R.L, retro-lingual duct; Ch.Ty, chorda tympani nerves. (Alcock and Eltison.)

so-called 'sub-maxillary ganglion.' The fibres to the sub-lingual gland in man probably take a similar course.

The fibres to the parotid gland pass along the glossopharyngeal nerve and its tympanic branch (nerve of Jacobson) to the tympanic plexus. Hence the fibres are carried by the small superficial petrosal nerve to the otic ganglion, and thence by the auriculo-temporal, which is a branch of the third division of the fifth nerve.

The sympathetic supply to all these glands is contained in fine filaments on the walls of the arteries with which they are supplied. The fibres are derived ultimately from the spinal cord, whence they issue by the upper three anterior dorsal nerve-roots. They pass into the stellate ganglion, round the ansa Vieussenii to the inferior, and so to the superior cervical ganglion of the sympathetic. Here the fibres end around the cells of the ganglion, and a fresh relay of fibres, chiefly non-medullated, arise from the cells and travel on the walls of the branches of the external carotid artery to their destination.

The effect of stimulating the peripheral ends of the cerebrospinal nerves going to the glands presents a general resemblance, whichever be the gland involved. Within a period of half to two seconds after the stimulation has been applied, a secretion of saliva is produced, presenting similar characters 
to that which would be obtained from the gland under normal conditions if it were provided with a permanent fistula. The concentration of the saliva as well as its rate of secretion depends on the strength of the stimulus. The following Table (Heidenhain) shows the effect on the amount and composition of sub-maxillary saliva obtained by weak and strong stimulation of the chorda tympani nerve :

\begin{tabular}{|c|c|c|c|}
\hline Strength of stimulus & $\begin{array}{l}\text { Quantity in one } \\
\text { minute }\end{array}$ & $\begin{array}{l}\text { Per cent. of } \\
\text { organic solids }\end{array}$ & $\begin{array}{l}\text { Per cent. of } \\
\text { salts }\end{array}$ \\
\hline $\begin{array}{lll}\text { Weak } & & \cdot \\
\text { Strong } & \cdot & \cdot \\
\text { Weak } & \cdot & \cdot\end{array}$ & $\begin{array}{l}0.17 \\
0.72 \\
0.17\end{array}$ & $\begin{array}{l}0 \cdot 84 \\
2 \cdot 06 \\
1 \cdot 67\end{array}$ & $\begin{array}{r}0.20 \\
0.46 \\
0.26\end{array}$ \\
\hline
\end{tabular}

With the strong stimulus the amount of saliva was increased over fourfold, while the percentage of organic substances in the saliva was raised from 0.84 to 2.06 per cent. There was at the same time an increase in the percentage of salts. If the excitation be continued for a considerable time, there is a gradual rise in the percentage of inorganic salts and a fall in the percentage of organic matter.

The cranial nerves going to these glands have another important effect, namely, vaso-dilatation. It was shown by Claude Bernard that if the outflow from the vein of the sub-maxillary gland were measured, on exciting the chorda tympani the flow might increase four to eight times, and indeed to such an extent that the blood passing through the gland did not stay there long enough to lose its oxygen. Moreover the dilatation of the arterioles removes the normal resistance which serves to damp and obliterate the pulse between the arteries and the veins. As the result of exciting the chorda therefore the blood coming from the vein may show distinct pulsation, and may have a brilliant scarlet hue just as if it were derived from an artery. The same dilatation has been observed to attend excitation of the cranial supply to the parotid gland.

The effects of exciting the sympathetic nerve-supply differ according to the gland and the animal which is the subject of experiment. In the dog excitation of the cervical sympathetic causes the secretion of a few drops of thick viscid saliva from the sub-maxillary gland. In this animal no secretion is obtained at all from the parotid gland on exciting the sympathetic, but the influence of the excitation is shown by the occurrence of histological changes in the gland-cells. In the cat the sub-maxillary saliva obtained on sympathetic excitation may be as copious as and even more watery than the saliva obtained from the sub-maxillary on stimulation of the chorda tympani. We shall have later on to discuss how far these results are to be ascribed to a fundamental difference in the point of attack of impulses carried to the secreting cells by the two sets of nerve fibres, and how far to the varying effects of the cranial and sympathetic nerves respectively on the blood-vessels. It must be remembered that the sympathetic nerve 
carries the vaso-constrictor fibres to most or all of the vessels of the head and neck, and therefore in most cases we should expect, and we find, that stimulation of this nerve causes vascular constriction in the gland affected. Before attempting to decide this point we must study in somewhat greater detail the changes which occur in the gland concomitantly with the secretion.

\section{CHANGES IN THE GLAND ACCOMPANYING SECRETION}

The fact that a sub-maxillary gland of the dog under favourable conditions will secrete its own weight of saliva in five minutes, and will continue to secrete for many hours afterwards, shows that there must be a continual

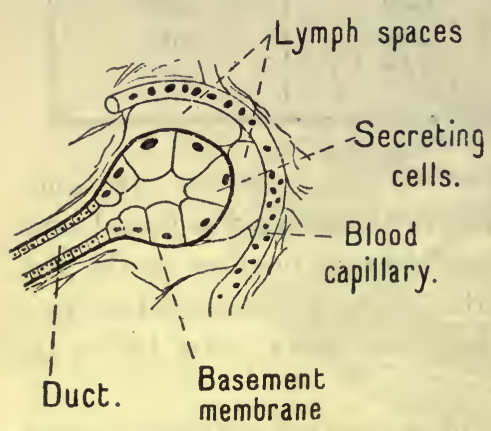

FIG. 325. Diagram to show relation of the secreting cells of a gland to the blood and lymph supply. renewal of the fluid which is turned out in the secretion. The source of this fluid must be the blood which is circulating through the gland. If we refer to the diagrammatic representation of the elements which make up a secreting lobule and which may be involved in the act of secretion (Fig. 325), we see that between the lumen of the duct and the blood, which must be regarded as the source of the fluid, the following layers of cells intervene: (1) the endothelium of the blood capillaries ; (2) the basement membrane ; (3) the epithelial cells of the gland proper. We have, in the first place, to decide to which of these elements can be ascribed the chief part in the act of secretion.

The secretory activity of the sub-maxillary gland, whether evoked reflexly by the introduction of acids into the mouth or directly by the injection of pilocarpine or by stimulation of the peripheral end of the chorda tympani nerve, is always associated with a considerable dilatation of the vessels of the gland, and a consequent large increase in the blood flow through the gland, which may amount to between three and eight times the quantity passing during rest. Such an increase in the supply of blood is necessary in order to afford a source for the large quantity of fluid which is turned out in the saliva. Another effect of the dilatation will be to raise the pressure in the capillaries of the gland. We cannot, however, regard this rise of pressure in the capillaries as an essential factor in the act of secretion. If atropine be administered excitation of the chorda causes the same vasodilatation as before, though no secretion is produced. Moreover Ludwig showed that the force with which the secretion is turned out into the ducts of the gland is greater than that represented by the blood pressure. The blood pressure in the capillaries must be considerably lower even with full vascular dilatation than the pressure in the carotid artery. If two manometers be connected, one with the carotid artery and the other with the duct, it will be found that on stimulating the chorda tympani nerve the secretion will be excited, and the mercury will be driven up by the pressure 
of the secretion in the corresponding manometer until it attains a height which may be double that of the mercury in the manometer connected with the carotid artery, and therefore must be still greater than the pressure in the capillaries of the gland. This experiment, which is easy to repeat, showed the impossibility of the act of secretion being in any way determined by a process of filtration. We have now further evidence that work is done in the production of the salivary secretion, evidence which was not available when Ludwig first carried out the experiment just described. When a fluid containing salts in solution is filtered through a porous membrane the filtrate has the same content in salts as the original fluid. We can effect a separation of dissolved salts from a fluid by filtration under pressure through some membrane which is impermeable to the salts, e.g. a membrane of copper ferrocyanide - a so-called semipermeable membrane. Under these circumstances a very large pressure is necessary in order to cause the filtration of any fluid at all, a pressure which is equal to the osmotic pressure exerted by the substances in solution. Thus if we were filtering a 1 per cent. solution of $\mathrm{NaCl}$ through a semipermeable membrane, we should have to exert a pressure of about seven atmospheres in order to obtain a filtrate free from sodium chloride. To

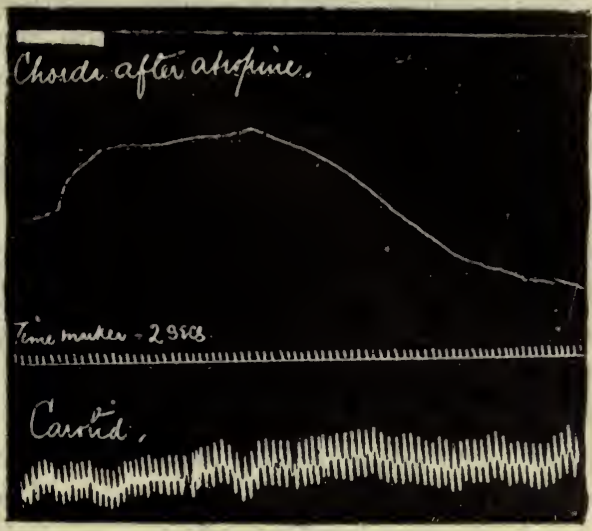

Fig. 326. Tracing of volume of sub-maxillary gland, showing effect of stimulation of the chorda after administration of $10 \mathrm{mg}$. atropine. The blood-pressure (lowest line) was unaltered by the stiumlation. (BUNCH.)

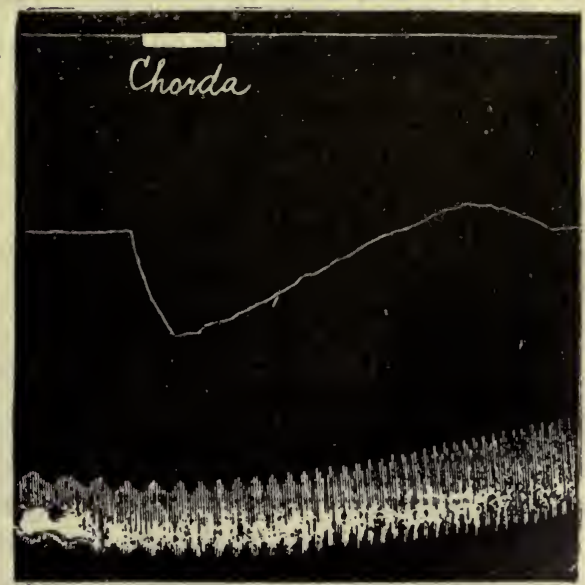

FIg. 327. Tracing of volume of sub-maxillary gland showing decrease on excitation of chorda. (BUNCH.)

obtain a filtrate containing half the amount of sodium chloride, if such were possible, would therefore need a pressure of about three and a half atmospheres. On comparing the osmotic pressures of saliva and blood respectively - and for this purpose we can employ the-depression of freezing-point as our index-we find that the molecular concentration of saliva, and therefore its osmotic pressure, is always very much less than that of the blood plasma, and may vary between half and three-quarters of the latter. Supposing the membrane separating the lumen of the duct from the 
blood-vessels could be regarded as endowed with the properties of a semipermeable membrane, we should need, in order to effect the separation, a pressure ten to twenty times as large as the arterial blood pressure. We must conclude then that work, both osmotic and mechanical, is performed in the separation of the fluid from the blood and its transference in the form of saliva to the duct., A very simple experiment will suffice to show that this work must be effected, not by the endothelial cells of the bloodvessels, but by the gland-cells themselves.- The fluid passes from the blood-vessels first into the lymphatic spaces, whence it is taken up by the secretory cells. If the first act in secretion consisted in an increased exudation of fluid from the blood into the lymph spaces the first effect of exciting the chorda tympani nerve should be to cause an accumulation of fluid in the lymph spaces, some increase in the lymph flow from the gland, and the swelling of the whole gland. By placing the gland in a plethysmograph we can record the actual changes in its volume which ensue on excitation of the chorda tympani. If all secretion be prevented by the previous administration of atropine, stimulation of this nerve produces, as might be anticipated, an increased volume of the gland in consequence of the dilatation of its vessels (Fig. 326). If, however, the gland be allowed to secrete we obtain, in spite of the simultaneous increase in size of the vessels, an actual diminution in the size of the gland itself, showing that the first effect of the stimulation is on the cells of the alveoli (Fig. 327). Under the stimulus these empty themselves of the fluid they contain, replenishing their loss at the expense of the fluid in the lymph spaces. The increased passage of fluid from blood to lymph space is therefore a secondary and not a primary effect of the nerve stimulation, and the first effect on the gland is a diminution of volume and not an increase, as one would expect if the vascular endothelium were primarily responsible for the act of secretion.

\section{HISTOLOGICAL CHANGES DURING SECRETION}

The process of secretion is associated with marked changes in the structure of the cells composing the secretory alveoli. The changes are of the same general character whatever class of glands we investigate, though the ease with which they are to be demonstrated varies with the reactions of the various glands to the hardening fluids usually employed. If a small fragment of a mucous gland be teased in blood serum or in 2 per cent. $\mathrm{NaCl}$ solution, the cells are found to be packed with a mass of coarse highly refractive granules (Fig. 328). If a corresponding specimen be made from a serous gland (Fig. 329) the cells are also packed with granules, which, however, are much finer in structure. On making similar specimens from glands which have been forced to secrete for six or seven hours, the individual "cells are found to be much smaller and the protoplasm of the cell is absolutely or relatively increased in amount, while the granules are much fewer and are now confined almost entirely to the inner margin of the cell. Activity is thus associated certainly with a discharge of granules, and probably with some increased building up of protoplasm. We may regard the act of 
secretion as determined by the alteration of the granules and their discharge, together with water and salts, to form the specific secretion of the gland. During rest the granules are re-formed by precipitation in or modification of the protoplasm surrounding the nucleus. We have evidence that although the granules form the secretion, they represent, not the secretion itself, but a precursor of some at any rate of its constituents. Thus if acetic acid be added to the saliva obtained from the sub-maxillary gland, the mucin is precipitated as threads and films. If the granules in the secreting cells also consist of mucin we should expect acetic acid to have a coagulating effect upon them. We find, on the contrary, that on allowing acetic acid to flow over a section of the fresh gland the granules at once swell up and burst.
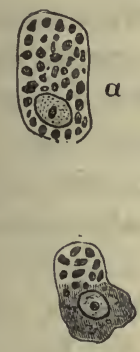

b
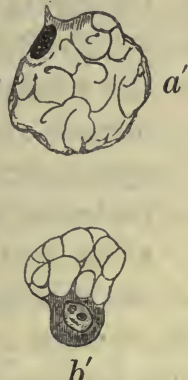

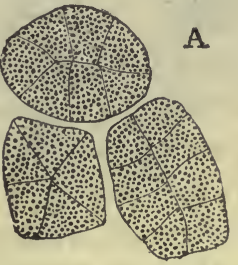

B

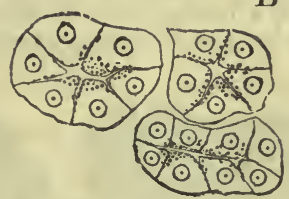

Fic. 329. Acini of a serous salivary gland. (LANGLEY.)

A, resting condition; B, discharged condition.

We must regard these granules therefore, not as mucin, but as a precursor of mucin, mucigen. The effect of ordinary hardening reagents, such as dilute alcohol up to 70 per cent. or Müller's fluid, is to cause these granules to swell up so that the cells become filled with a mass of mucin giving the typical hyaline appearance of ordinary sections of these glands. In the case of the serous gland the granules (Fig. 330) are apparently protein in nature. Where ptyalin is a constituent of the saliva we are probably justified in assuming that it is contained either pre-formed or more probably as a precursor in the granules. In the glands of the stomach we have evidence that the granules are not pepsin - the characteristic ferment of gastric juice-but a precursor of this substance; namely, pepsinogen. It is very customary therefore to speak of the granules in a secreting gland as zymogen granules, i.e. the precursors of zymins or ferments. It is probable that we ought to regard these granules not merely as material precursors of the constituents of the secretion, but as little machines or cell laboratories in which proceed a whole series of chemical and osmotic changes which determine the production of the fully formed secretion directly from the protoplasm and indirectly from the ordinary constituents of the surrounding lymph. 
From an examination of the stained specimens of glands the following stages in the production of secretion have been described :

(1) In the neighbourhood of the nucleus, and probably with its active co-operation, a differentiation of the protoplasm occurs with the production

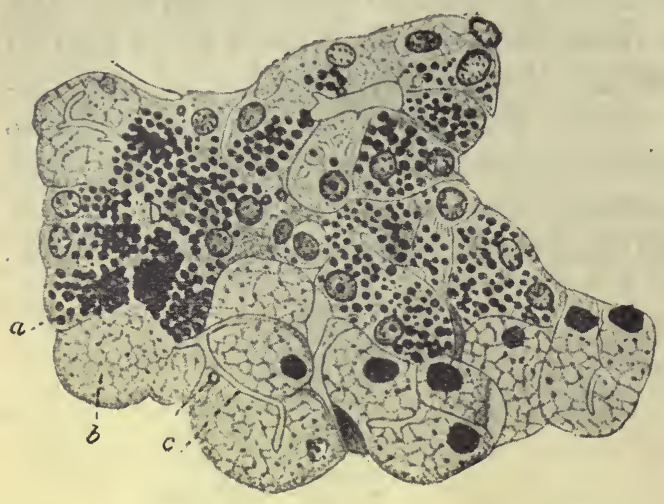

Fig. 330. Sub-maxillary gland of rabbit.

(SCHÄFER after E. MÜLLER.)

The cells, all serous, are in different functional states : $a$, a loaded cell ; $b$, a discharged cell ; $c$, a secretory canaliculus penetrating into a cell.
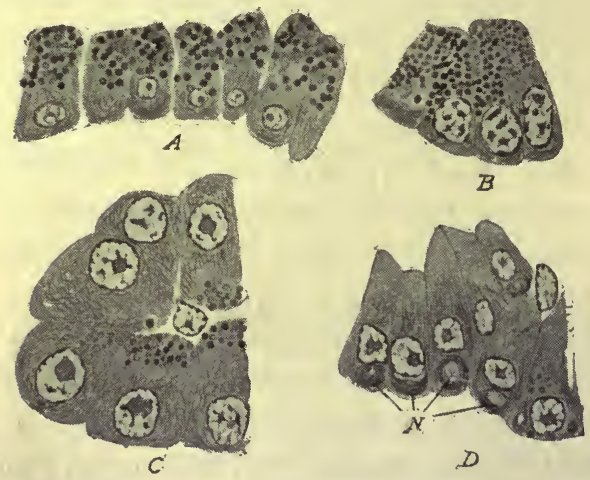

Fig. 331. Cells of pancreas, showing successive stages in activity, A, B,C,D. A, resting; $\mathrm{D}$, discharged gland. (MAтнEws.) in most cases of a basophile substance which in hardened specimens generally takes the appearance of filaments. Since these filaments are sometimes regarded as the working part of the protoplasm, they have been given the name of ergastoplasm (Fig. 331, C and D).

(2) By a modification of the ergastoplasm granules are formed. After their first appearance these granules undergo gradual modification, as is shown by the fact that the staining reactions of the granules near the base of the cell differ from those of the granules at the free margin.

(3) When the secretion is excited, the fully formed granules take up water and salts in varying proportions, swell up, and discharge their contents into the lumen of the alveolus as the secretion proper to the gland.

\section{ELECTRICAL CHANGES}

Every localised chemical change in a system permeated by electrolytes must give rise to electrical differences of potential. It is therefore natural that electrical changes should accompany the intense chemical activity which is associated with secretion. The interpretation of these changes is difficult, owing to the simultaneous operation of another factor which may determine electrical differences of potential, namely, the movement of fluids through porous membranes. If the hilum of the sub-maxillary gland and its outer surface be connected with a galvanometer, a resting difference of potential is nearly always found, generally in such a direction that the outer surface is positive to the hilum. The current through the gland is therefore from within out. On exciting the chorda tympani nerve a diphasic effect is generally obtained, 
the resting difference being first increased and later on diminished. On excitation of the sympathetic nerve we generally obtain a purely negative variation of the resting difference. These results were interpreted by Bayliss and Bradford as due to the co-operation of the two factors, chemical change in the gland-cells and movement of fluid through the cells. The positive variation, i.e. the current from within out, was ascribed to the movement of fluid, whereas the negative variation of the resting difference was thought to be due to the chemical changes in the gland-cells.

\section{THE SIGNIFICANCE OF THE DOUBLE NERVE-SUPPLY TO THE GLANDS}

1ccording to Heidenhain, although the parotid gland gives little or no secretion on stimulation of the sympathetic nerve, prolonged stimulation of this nerve causes histological changes in the gland even more marked than those produced by the cranial nerve. Similar histological changes were found by him in the sub-maxillary gland. He was therefore led to put forward the hypothesis that the salivary glands are supplied by two fundamentally different classes of fibres, namely: (1) trophic fibres, which determine the chemical changes in the gland responsible for the production of the specific constituents of the secretion, and (2) secreto-motor fibres, excitation of which causes the cells to take up water and salts from the lymph and blood, and pass them in large quantities into the duct. According to this view the sympathetic nerve-supply to the gland would consist almost entirely of trophic fibres, whereas secreto-motor fibres would predominate in the cranial nerve-supply. The action of atropine would appear at first sight to favour this hypothesis. In minute doses it entirely annuls the action of the chorda tympani nerve or the corresponding nerve to the parotid, while it is without effect on the sympathetic nerve-supply unless given in huge doses. The preponderating effect of the sympathetic on the histological structure of the gland-cells has not been confirmed by later observers, and the varying effects of atropine on the two sets of nerve fibres may be conditioned by morphological rather than by functional differences between their nerve-endings. According to Langley and Carlson, the difference in the action of the chorda tympani and of the sympathetic on the submaxillary gland is due to the synchronous action of these nerves on the blood-supply to the gland, the sympathetic causing vaso-constriction, while the chorda tympani causes vaso-dilatation. In confirmation of this explanation they have shown that clamping the carotid artery during chorda stimulation diminishes the amount of saliva secreted but increases the percentage of solids in the fluid. This theory is however inadequate to explain the differences observed in the secretion of saliva reflexly aroused by introduction of substances into the mouth. In a dog with a permanent submaxillary fistula a copious flow of saliva may be caused by the introduction of $\cdot 25$ per cent. hydrochloric acid or of meat powder. The amount of saliva secreted under the two circumstances is approximately the same, but that evoked by the introduction of meat powder contains about twice as much solid 
eontents as that which follows the introduction of acid into the mouth. -Babkin has shown that the same differences are found after complete section of the sympathetic and that there is the same acceleration of the circulation through the gland whether the secretion is aroused by introduction of meat powder or of acid. It is impossible therefore to explain the difference in the composition of the saliva obtained under these two circumstances as due to differences in the blood-supply to the gland, and we must conclude either that the chorda tympani contains different kinds of fibres which are excited to varying extent according to the nature of the reflex stimulations, or that one and the same nerve fibre can convey specifically different impulses. The latter explanation would not be in accord with the generally accepted Müller's law of specific irritability but is the explanation to which Babkin himself inclines. At any rate it is certain that according to the nature of the reflex stimulus either the secretion of water and salts or the secretion of organic solids may preponderate, altogether apart from changes in the circulation simultaneously evoked.

\section{THE ENERGY INVOLVED IN THE ACT OF SECRETION}

The source of the energy must be sought in the processes of oxidation occurring in the cells of the gland, and Barcroft has attempted to determine the total amount of energy put out by the gland in the act of secretion by measuring its respiratory exchanges under the conditions of rest and activity. He found that the resting submaxillary gland in a small dog took up 0.25 c.c. of oxygen per minute and put out $0 \cdot 17$ c.c. $\mathrm{CO}_{2}$, while during active secretion it absorbed 0.86 c.c. $\mathrm{O}_{2}$ and gave off 0.39 c.c. $\mathrm{CO}_{2}$. Assuming that the total oxygen taken up is employed in the oxidation of a food substance, such as glucose, and that the whole of the energy of the chemical changes is set free in the form of heat, we find that a resting gland weighing about six grammes produces about $1 \cdot 1$ calories per minute. We know, however, that a certain amount of external work is performed in the secretion of a saliva containing less salts than the original blood, and also, when there is any resistance to the flow of saliva through the duct, in raising the hydrostatic pressure of the saliva in the duct to a height greater than that in the blood capillaries.

Can. we from all these data form a conception of the total changes occurring in the gland and involved in the formation of the secretion? Even during rest, changes are going on in the gland-cells, changes which involve the taking up of food material and its assimilation under the influence of the nucleus, perhaps into the nucleus itself, and certainly into the undifferentiated cytoplasm. In this cytoplasm a further change occurs, leading to its transformatiun into granules. When activity is excited by the stimulation of secretory nerves, rhe primary change appears to involve simply the granules. These structures must absorb water, apparently against osmotic pressure. Those-nearest the lumen swell up, become converted into spheres containing water and salts. in smaller proportion than exists in the lymph bathing the cells (and presumably in the protoplasm surrounding the granules), and in this swollen form are discharged or ruptured on the periphery of the cell 
into the lumen, so giving rise to secretion. This discharge of a fluid with a smaller molecular concentration than the cell or surrounding blood plasma must lead to an increased concentration in the remaining parts of the cell. The increased concentration would naturally induce a flow of water from lymph into cell, and the consequent concentration of the lymph would in the same way cause a flow of water from blood to lymph. This pull of water by the cell from the blood is still further increased in another way. The act of secretion, involving as it does the expenditure of energy, can be carried out only at the expense of chemical changes in the cell. These chemical changes, as in all other metabolic processes of the body, will result in the formation of a number of small molecules from the great colloid molecules of the protoplasm. The products of metabolism, or metabolites, will therefore accumulate in the cell, pass into the lymph, and increase the concentration of the latter. The increased concentration will call forth an increased transudation of fluid, e.g. water, from the blood-vessels, and the transudation thus evoked will be greater than that necessary to provide the water of the saliva, and will therefore produce a distension of the lymphatic spaces of the gland and an increased discharge of lymph along its efferent lymphatics. As a secondary result of the activity, perhaps in consequence of the removal of the products of the resting metabolism of the gland, there is increased growth of protoplasm, increased activity of the nucleus, and therefore a tendency to increased assimilatory changes and a preparation of the cell for further secretory changes either immediately or hereafter.

In the gland, however, as in muscle, when we attempt to form a conception of the mechanism of the chemical machine in the living cell, we are brought up against insuperable difficulties. One might perhaps conceive of the secretory granules being bounded by a membrane impermeable to intermediate metabolites and salts, but permeable to carbon dioxide. If the first effect of stimulation of the secretory nerves were to produce an explosive disintegration of the complex molecules making up the granules, we should have a sudden multiplication of molecules within the granules. This would cause a large rise of the osmotic pressure in these granules and the consequent absorption of water from the surrounding protoplasm. This process, however, could only result in the production of a fluid in the granules having the same osmotic pressure as the surrounding medium, whereas we know that saliva has a molecular concentration which is only one half of that of the blood or lymph. We should therefore have to make a second assumption, namely, that, before the extrusion of the solution from the granules, there is a further breakdown of the metabolites by a process of oxidation, with the production of carbon dioxide which diffuses into the surrounding protoplasm. We have, however, no evidence of either of these processes or for any of these assumptions, and I have only adduced them in order to show how far we are still from the actual comprehension of the events occurring in every living cell, and underlying its conditions of rest and activity. 


\section{SECTION II}

\section{THE PASSAGE OF FOOD FROM THE MOUTH TO THE STOMACH}

THE food after mastication is carried to the stomach by a complex series of co-ordinated movements involving the muscles of the pharynx and

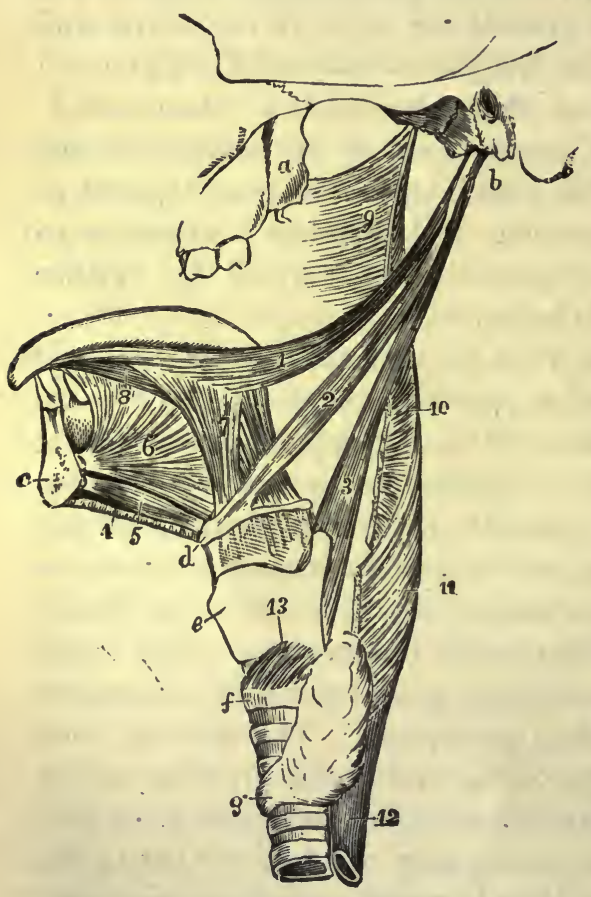

Fig. 332. Dissection to show muscles employed in deglutition.

$b$, styloid process, from which arise 1 , the styloglossus; 2 , the stylohyoid; 3 , the stylopharyngeus muscles; $c$, section of lower jaw ; $d$, hyoid bone; $e$, thyroid cartilage; $g$, isthmus of thyroid gland; 4 , cut edge of mylohyoid muscle ; $5,6,7,8$, muscles of tongue ; $9,10,11$, superior, middle, and inferior constrictors of pharynx; 12, œsophagus. (Allen Thomson.)

obtained by passing sounds provided with rubber balloons to different levels in the tube and connecting these sounds with recording tambours methods have been used to study the process of deglutition in man and the higher animals. Important information can be obtained by allowing a man or animal to swallow either a fluid or solid with which bismuth is mixed and observing the passage of the opaque substance under the Röntgen rays. The subnitrate of bismuth may be mixed with milk for a fluid or with bread and milk for a semi-solid substance, or may be enclosed in a cachet and swallowed as a solid bolus. The time of entry of the food into the stomach may be determined by auscultating with a stethoscope over the region of the cardiac orifice. Since a certain amount of air is always swallowed at the same time as the food, the escape of this air through the small cardiac orifice gives rise to a bubbling noise which can be easily heard. In the horse the movement of a bolus down the œsophagus can be seen or felt from the outside of the neck. The relative timerelations of the events at different parts of the œsophagus may be the œsophagus (Fig. 332). Various 
or piston recorders. This method has been employed both in men and in animals.

When a mouthful of water is taken two sounds may be heard on auscultating over the œsophagus. The first sound immediately follows the beginning of the act of swallowing and is probably due to the impact of the fluid against the posterior pharyngeal wall brought about by the sudden contraction of the mylohyoid and other muscles which throw the fluid from the back of the tongue across the pharynx. The second sound is heard best by listening over the epigastrium. It begins from four to ten seconds after the first sound, and lasts for two or three seconds. The interval between the two sounds is not constant, and may vary in the same individual. If the observation be carried out on a man lying on his back, the trickling second sound is changed into a series of sounds which have been described as squirts, which vary from two to five in number, each lasting about one second. The second sound may be absent when a solid bolus is swallowed. On observing the process by Röntgen rays very much the same time-relations are obtained. If a mouthful of milk mixed with bismuth carbonate be swallowed, it will be seen passing rapidly down the œesophagus to the cardiac orifice of the stomach. Here the passage becomes slow, and the fluid escapes slowly in a narrow stream into the stomach. The average time which elapses between the beginning of deglutition and the disappearance of the last trace of fluid from the cesophagus is about six seconds. The same course of events is induced when the food swallowed is semi-solid. If, however, the bolus be dry, such as a cachet of bismuth carbonate, it may pass down the œsophagus with extreme slowness and may take as much as fifteen minutes to reach the cardiac orifice, although the individual who has swallowed it is quite unaware of its continued presence in the œsophagus. If, as would normally be the case, the cachet be well moistened with saliva or water before swallowing, it passes much more rapidly, the total time taken being between eight and eighteen seconds.

It has been customary since the time of Magendie to divide the act of swallowing into three stages, during the first of which the bolus of food is carried past the anterior pillars of the fauces ; during the second through the pharynx, past the openings of the nasal cavities and of the larynx; and during the third through the œsophagus into the stomach. There is, however, no pause between these various stages. The act of deglutition is one, and the initiation of the first stage inevitably involves the completion of the third. The food is masticated, and is collected as a bolus on the dorsum of the tongue. A pause then takes place in the movements of mastication, a slight movement of the diaphragm usually occurs known as ' respiration of swallowing,' and then a sudden elevation of the tongue throws the bolus back through the anterior pillars of the fauces. In this movement the chief factor is the contraction of the mylohyoid muscle, which presses the tongue against the palate and pushes it backwards. The backward movement of the tongue may also be aided by the contraction of the styloglossus and palatoglossus muscles which pull the base of the tongue suddenly backwards. 
These muscles, especially the palatoglossi, serve to close the isthmus faucium, thus preventing any return of the food towards the mouth.

As the food is passing through the upper part of the pharynx it traverses a region common to the respiratory as well as the digestive passages. Its passage through this region is therefore rapid, and is associated with a closure of the two openings of the air passages into the pharynx. The nasal cavity is shut off by a simultaneous contraction of the levator-palati and palato-pharyngeus muscles and azygos uvulæ, by which means the soft palate is raised (Fig. 333) and the posterior pillars are proximated to the uvula.
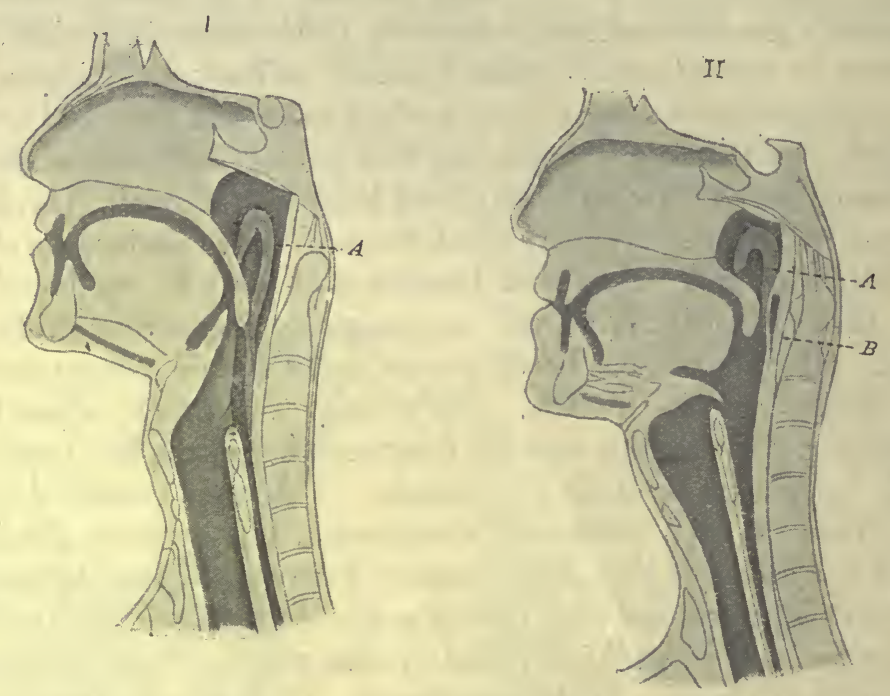

Fig. 333. Diagram (after Tigerstedt) to show the position of the soft palate. I, during rest; II, during the act of swallowing.

The upper and back wall of the palate is thus formed into a tense sloping roof which guides the bolus down the pharynx.

More important is the shutting off of the lower air passages. The contraction of the mylohyoid muscles which initiate deglutition is followed almost immediately (at an interval of $0.47 \mathrm{sec}$.) by an elevation of the larynx, and this elevation is accompanied by closure of the glottis as well as of the superior opening of the larynx. The laryngeal opening is bounded in front by the epiglottis, behind by the tips of the arytenoid cartilages, and at the sides by the aryteno-epiglottidean folds. When deglutition takes place the arytenoid cartilages, which normally lie against the posterior wall of the pharynx, are rotated and move inwards and forwards, so that the laryngeal opening assumes the form of a tri-radiate fissure, the vertical limb being short, while the transverse limb is rounded owing to the pulling inwards of the margins of the epiglottis. At the same time both the true and false vocal cords are approximated, while the movement of the dorsum of the tongue backwards enables the closed laryngeal orifice to lie directly under the back part of the tongue. The muscles which are actively involved in this closure 
of the lower air passages are the external thyro-arytenoid, arytenoid, aryepiglottidean, and the lateral crico-arytenoid muscles. Since the approximation of the posterior to the anterior boundary of the laryngeal opening is only rendered possible by the elevation of the whole larynx under the hyoid bone, the act of deglutition cannot be carried out rnless the larynx is free to move.

The two openings from the back of the pharynx into the air passages being thus closed, the bolus is shot rapidly past them into the region of the middle and inferior constrictors of the pharynx. If the bolus be liquid or semi-fluid, the movement of the back part of the tongue may be sufficient to propel the substance past the constrictors through the lax œsophagus to its lower end. It is on this account that, when corrosive fluids are swallowed by accident, we very often find the damage to the œsophagus limited to the three points where it is narrowed and where, therefore, there is a slight hindrance to the onward flow of fluid. If the bolus be large and solid or semi-solid, it is seized in the grasp of the middle constrictors on passing through the upper part of the pharynx, and is thrust by successive contractions of this muscle and of the inferior constrictor gradually down the œsophagus. The walls of the cervical part of the œsophagus are composed of striated muscle. In the thorax striated and unstriated muscles are associated together, while the lower third, in the neighbourhood of the stomach, consists almost entirely of unstriated muscle. Corresponding to these differences in structure, Kronecker and Meltzer have found differences in the Juration and rapidity of propulsion of the contractional waves in each part. The following Table shows the time-relations of the chief muscles engaged in deglutition as determined by Kronecker and Meltzer and by Marckwald :

\begin{tabular}{|c|c|c|c|}
\hline $\begin{array}{l}\text { Time from } \\
\text { commencement }\end{array}$ & Interval & Muscle movement & $\begin{array}{l}\text { Duration of } \\
\text { contraction }\end{array}$ \\
\hline - & 一 & Mylohyoid & 0.6 sec. \\
\hline 一 & 0.03 sec. & Respiration of swallowing & - \\
\hline 一 & 0.07 sec. & Elevation of larynx & 0.8 sec. \\
\hline $0 \cdot 3$ sec. & $0.2 \mathrm{sec}$. & Constrictors of pharynx & 1.0 to $2.0 \mathrm{sec}$. \\
\hline - & $0.9 \mathrm{sec}$. & First section of œsophagus & $2 \cdot 0$ to $2.5 \mathrm{sec}$. \\
\hline $3 \cdot 0 \mathrm{sec}$. & 1.8 sec. & Second section of œsophagus & 6.0 to $7 \cdot 0 \mathrm{sec}$. \\
\hline 6.0 sec. & $3.0 \mathrm{sec}$. & Third section of œsophagus & about $10 \mathrm{sec}$ \\
\hline
\end{tabular}

The free passage of food down the œsophagus under the influence of the propulsive force exercised by the mylohyoid muscles shows that the walls of this tube must be lax, and in fact one must assume that the first act of deglutition, so far as concerns the osophagus, is an inhibition initiated 
reflexly with the beginning of the act of deglutition. When a second act of deglutition succeeds the first with a sufficiently short interval, the reflex inhibition due to the second act may prevent the development of any wave of contraction in the cesophagus. This tube thus remains in a lax condition
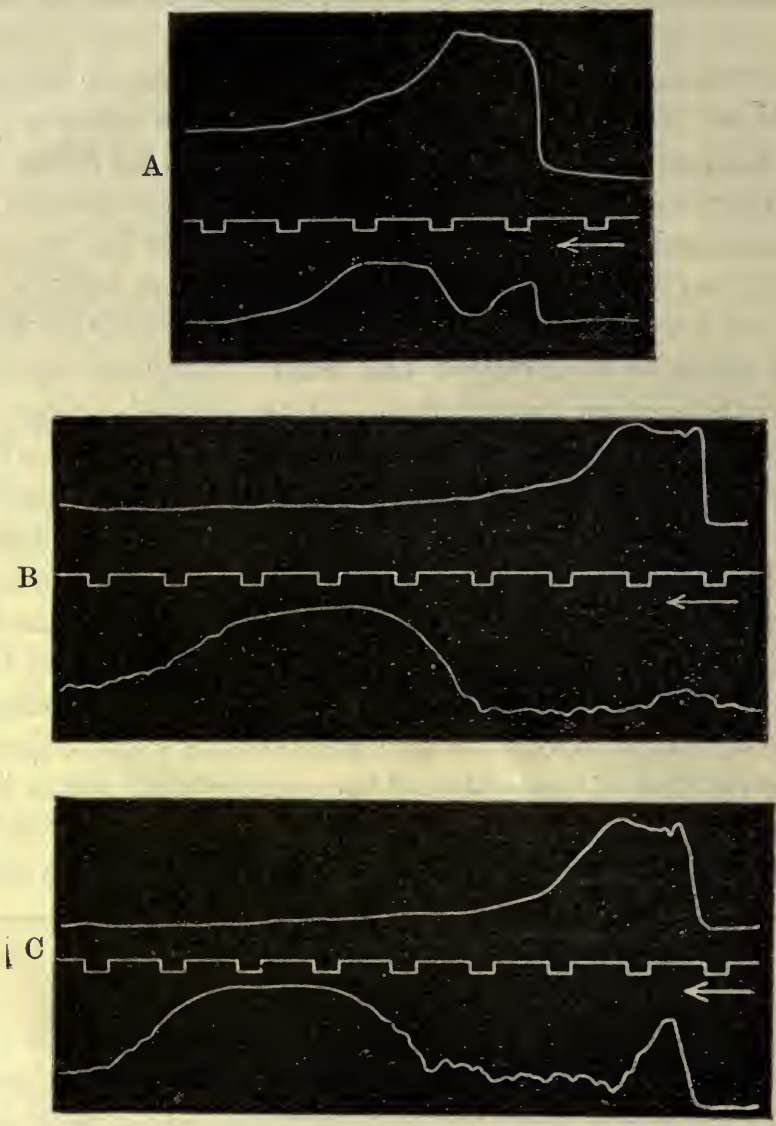

FIG. 334. Curves obtained during swallowing by placing two rubber balloons, one (the upper curve) in the pharynx, the other (lower curve) in the œsophagus. In $\mathrm{A}$ the second balloon was $4 \mathrm{~cm}$.; in $\mathrm{B} 12 \mathrm{~cm}$.; and in $\mathrm{c} 16 \mathrm{~cm}$. from the upper end of the œesophagus. In each curve it will be noticed that the excursion of the upper lever is followed immediately by an excursion of the lower lever (due to passage of the swallowed fluid and transmitted rise of pressure), and then, after an interval of time varying with the distance between the balloons, by another rise due to the peristaltic contraction of the wall of the œesophagus.

and allows the free rapid passage of the food downwards until the movements of deglutition have come to an end, when a peristaltic contraction occurs and sweeps all remaining adherent particles of food into the stomach. The circular fibres of the lower end of the œsophagus which form the cardiac sphincter of the stomach are normally in a state of tonic contraction. When one mouthful of food is swallowed it may be either squirted directly into the stomach, or may remain at the lower end of the œsophagus until the following 
peristaltic wave forces it through the orifice. When several acts of deglutition succeed one another, the cardiac sphincter shares in the inhibition of the osophageal walls, and offers no resistance to the direct propulsion of food from the mouth to the stomach.

Cannon has shown that the relaxation of the cardiac orifice which accompanies swallowing extends also to the cardiac end of the stomach, This relaxation lowers the pressure within the stomach, and makes room for the incoming food.

If the stomach be filled with a fluid such as starch solution, the cardiac sphincter may be seen to relax rhythmically, allowing of the regurgitation of

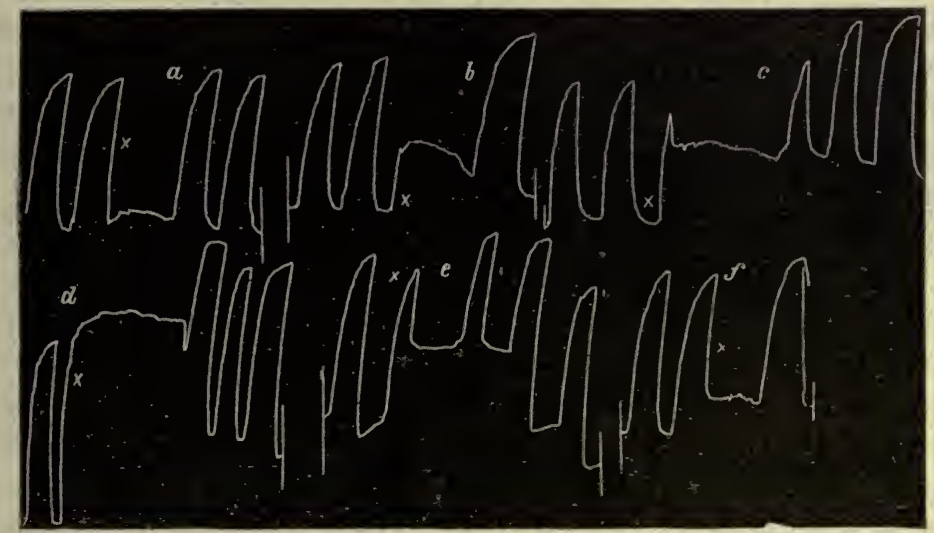

FIG. 335. Tracings of respiratory movements to show the effect of stimulating the central end of the glossopharyngeal nerve. (MarckwaLd.)

The point of stimulation is marked with a cross. Note that the stoppage may occur at any phase of the respiratory movement.

the stomach contents into the lower part of the œsophagus. Their entry into this tube is at once followed by a peristaltic contraction of this part of the œsophagus (apparently entirely unconscious), which drives the fluid back into the stomach. These movements of regurgitation become more and more infrequent as the gastric contents become acid, and are not observed at all if the stomach be filled with a fluid already acid. This phenomenon has been spoken of as the 'acid control of the cardia.'

\section{THE NERVOUS MECHANISM OF DEGLUTITION}

Deglutition is a reflex act. When we swallow voluntarily we supply the necessary initial stimulus either by touching the fauces with the tongue or by forcing a certain amount of saliva into the fauces. The afferent channels of the reflex are contained in the second division of the fifth nerve, the glossopharyngeal nerve, and the pharyngeal branches of the superior laryngeal nerve. We can excite a single act or a whole series of acts of deglutition by electrical stimulation of the central end of the last-named nerve. The efferent fibres which determine the contraction of muscles engaged in the act of deglutition travel by the hypoglossal nerve to the muscles of the 
tongue, by the fifth to the mylohyoid, by the glossopharyngeal, the vagus, and the spinal accessory nerves to the muscles of the fauces and pharynx: The closure of the larynx is effected by impulses which travel through the superior and inferior laryngeal branches of the vagus. The centre for the act is situated in the medulla oblongata, and can be considered as consisting of a chain of centres stimulation of one of which involves the firing off of all the others in orderly sequence. Thus, as Meltzer has shown, the propulsion of the contraction down the œsophagus is determined by the intracentral nervous connections, and does not require the integrity of the muscular tube itself. If the œsophageal nerves be divided, the act of deglutition is abolished, the upper part of the œsophagus becoming permanently relaxed, while the lower part, including the cardiac sphincter, enters into a state of tonic contraction. On the other hand, the œsophagus may be ligatured or cut across without interfering with the propulsion of the wave of contraction. On the other hand, the osophagus may be ligatured or cut across without interfering with the propulsion of the wave of contraction, started in the pharynx, from one end of the tube to the other. Stimulation applied to the mucous surface of the œsophageal tube is without effect.

There is an important interdependence between the functions of respiration and deglutition. If an inspiratory or expiratory movement were going on during the act of deglutition, food might be drawn into the lungs or driven into the nasal cavities. Such an accident is prevented by the fact that every act of swallowing inhibits a respiratory movement. This inhibition is effected reflexly through the glossopharyngeal nerve. Stimulation of the central end of this nerve at once causes cessation of respiration in whatever phase it may happen to be (Fig. 335). This cessation lasts for five or six seconds, i.e. a sufficient length of time for a whole series of acts of deglutition. Respiration then recommences, and the inhibition cannot be prolonged by continuing the stimulation of the glossopharyngeal nerve. This inhibition of the activity of the respiratory centre can be shown on oneself. If the breath be held until the feeling of dyspnœa, i.e. the need to breathe, becomes insistent, relief is at once experienced by swallowing, and the feeling of relief will last for three or four seconds. 


\section{SECTION III \\ DIGESTION IN THE STOMACH}

\section{GASTRIC JUICE}

Within five minutes of the taking of foud into the mouth a secretion of gastric juice begins from the multitude of tubular glands which make up the greater part of the mucous membrane of the stomach. As the food, masticated and thoroughly mixed with saliva, is swallowed in successive portions, it accumulates in a mass in the fundus of the stomach, and the mass thus formed is penetrated with difficulty by the juice which is continually being poured out by the walls of the stomach, so that salivary digestion can be continued for a considerable time.

The gastric juice, which is so poured out, can be obtained in various ways, most of them yielding it mixed more or less with the food-stuffs. In clinical practice it is the custom to give a definite meal, and then at a given interval after the meal to wash out the stomach, so obtaining a mixture of gastric juice and partially digested food.

A method of obtaining the juice in a perfectly pure condition has been devised by Pawlow. A case had been previously described by Richet in which, as the result of the accidental taking of a corrosive alkali, the œsophagus had become occluded by the cicatrisation of the ulcer produced. In order to preserve the individual from starvation, it was necessary to perform gastrostomy, i.e. to make an artificial opening into the stomach through which he could be fed. Although in this patient the passage of the saliva from mouth to stomach was completely prevented, it was observed that merely taking food into the mouth was followed by the secretion of gastric juice. Pawlow produced this condition artificially in dogs. The œsophagus was divided and the two ends brought to the surface of the neck. At the same time an opening was made into the stomach. The animals could be fed either through the opening of the cesophagus in the neck, or with solid food through the gastric fistula. They could eat also and swallow food as usual, but the food thus swallowed simply fell out of the opening in the neck without passing into the stomach. Under these circumstances it is found that the taking of food is quickly followed by a secretion of gastric juice, which can be collected in vessels connected with the fistulous opening. If taken from a fasting animal, such a juice is perfectly free from admixture, and can be regarded as pure gastric juice. It is quite clear, strongly acid, without smell. It contains about 0.3 to 0.6 per 
cent. total solids; it contains no peptone, but traces of protein. The following Table represents its average composition :

Hydrochloric acid
Chlorine .
Total solids
Ash .

0.46 to 0.58 per cent.
0.49 to 0.62 ,
0.43 to 0.60 ",
0.09 to 0.16 ",

If the juice be allowed to stand in the ice chest for a day it becomes cloudy and deposits a fine granular precipitate, which apparently represents the active agent of the juice, and may perhaps be regarded as pepsin in a pure form.

The actions of gastric juice are due partly to the acid, partly to the combined action of the acid and the ferments. The acid of the gastric juice, when obtained free from admixture, is entirely hydrochloric acid. Dog's juice contains on the average about $0 \cdot 6$ per cent. $\mathrm{HCl}$; human gastric juice probably contains less, about $0 \cdot 2$ per cent. When, however, we examine the gastric contents, composed of a mixture of gastric juice and semi-digested food, we always find, besides the hydrochloric acid, other acids present, among which the most prominent is lactic acid. So constantly is this latter acid present that it was formerly thought by some physiologists to be the chief acid of the gastric juice. It is produced by processes of fermentation occurring in the food. Whenever we take carbohydrates we swallow at the same time micro-organisms, and these in the warm moist mass quickly attack the carbohydrates, converting them into sugar and then into lactic acid. As the gastric juice gradually soaks into the food and renders it acid, it stops this lactic acid fermentation, so that whereas in the early stages of gastric digestion both acids are present in considerable quantity, towards the end of gastric digestion lactic acid is almost entirely absent.

In some pathological conditions free hydrochloric acid may be entirely wanting from the gastric juice, and the detection of this acid in gastric juice becomes therefore a matter of considerable clinical importance. For this purpose we can employ various indicators, which change colour in the presence of a free strong acid such as $\mathrm{HCl}$, but are unaffected by weak acids such as lactic acids, or the fatty acids. Chief among these indicators are Congo red, which turns blue with mineral acids, and a slaty colour with lactic acid; and tropaolin 00 , which turns a brilliant red in the presence of a free mineral acid, but is unaltered by lactic acid. The reagent which is most employed is Gunzberg's reagent. This is a mixture of phloroglucin and vanillin dissolved in absolute alcohol. A drop of this is evaporated to dryness in a porcelain capsule. A drop of the fluid suspected to contain free acid is then added, and also evaporated to dryness. If free $\mathrm{HCl}$ be present, the residue on drying becomes a brilliant red colour, an effect which is not produced by the presence of free lactic or free fatty acids.

Great stress has been laid on the determination of the actual amount of free $\mathrm{H}$ ions present, and for this purpose the acidity of gastric juice or of digestive mixtures has been tested by determining its inverting power on cane sugar, or its power to hasten the saponification of ethyl acetate. The acidity estimated in this way is diminished considerably by the presence of albumens, and still more by the presence of albumoses or peptones. But it does not seem that the adjuvant action of the acid on the proteolytic 
powers of the gastric ferment is in any way affected by the diminution of its acidity caused by the presence of peptone. The coloured indicators mentioned above, however, serve as trustworthy indications of the amount of free acid present, considered with regard to its digestive functions.

In order to determine quantitatively the amount of free $\mathrm{HCl}$, the following procedure is employed (Mörner and Sjöqvist): Ten cubic centimetres of the gastric juice are neutralised with barium carbonate (litmus being employed as an indicator). The mixture is dried in a platinum dish, incinerated, and the ash extracted with warm water and filtered. In this process the organic acids are destroyed and converted into barium carbonate. The solution therefore contains merely the barium which was taken up to combine with the free $\mathrm{HCl}$. Estimation of the barium in the filtrate gives the amount of $\mathrm{BaCl}$ present, and therefore the amount of hydrochloric acid in the gastric juice. The barium is determined by titrating in presence of sodium acetate and acetic acid with potassium bichromate solution, 'tetra paper' being used as an indicator. This turns deep blue in the presence of free bichromate in solution.

\section{THE ACTIONS OF GASTRIC JUICE ON FOOD-STUFFS}

By the action of the hydrochloric acid certain changes are induced in the food-stuffs. Cane sugar is inverted to glucose and fructose ; some proteins, such as blood fibrin, are swollen up to form a jelly-like mass. The caseinogen of milk is precipitated, the collagen of the connective tissues is swollen up. It is possible that a certain small amount of hydrolysis also takes place in the dextrins and maltose produced by the action of ptyalin on starch.

The chief digestive function of the gastric juice is dependent on the action of the ferment pepsin. This substance, which is inactive in neutral medium, needs the co-operation of an acid, hydrochloric acid being the most effective, though its place may be taken by phosphoric, sulphuric, or lactic acid. Its main effect is on the proteins of the food. The stages in its action may be best studied by taking as an example its action on blood fibrin. If fibrin be immersed in 0.4 per cent. hydrochloric acid, it swells up to a gelatinous mass. On then stirring in an extract of gastric mucous membrane, or any preparation of pepsin, the gelatinous mass rapidly undergoes solution. If the mixture be boiled and neutralised, immediately after solution has occurred, nearly the whole of the protein is thrown down in a coagulated form. The first effect therefore is the production of coagulable soluble proteins from the insoluble fibrin. If the action be allowed to proceed for some hours, a whole series of products of hydrolysis are found in the mixture. On neutralising the fluid, a precipitate may be thrown down consisting chiefly of acid albumen. The greater proportion of the protein remains in solution. This remainder may be purified from any unaltered coagulable protein by boiling in slightly acid solution and filtering. The filtrate contains a mixture of bodies belonging to the class of hydrated proteins, viz. proteoses and peptones.

By means of fractional precipitation with ammonium sulphate or zinc sulphate, these mixtures can be subdivided into various substances, although in no case can we be certain that we are dealing with chemical individuals. The Table on p. 686 represents the chief bodies obtained by Pick by this 


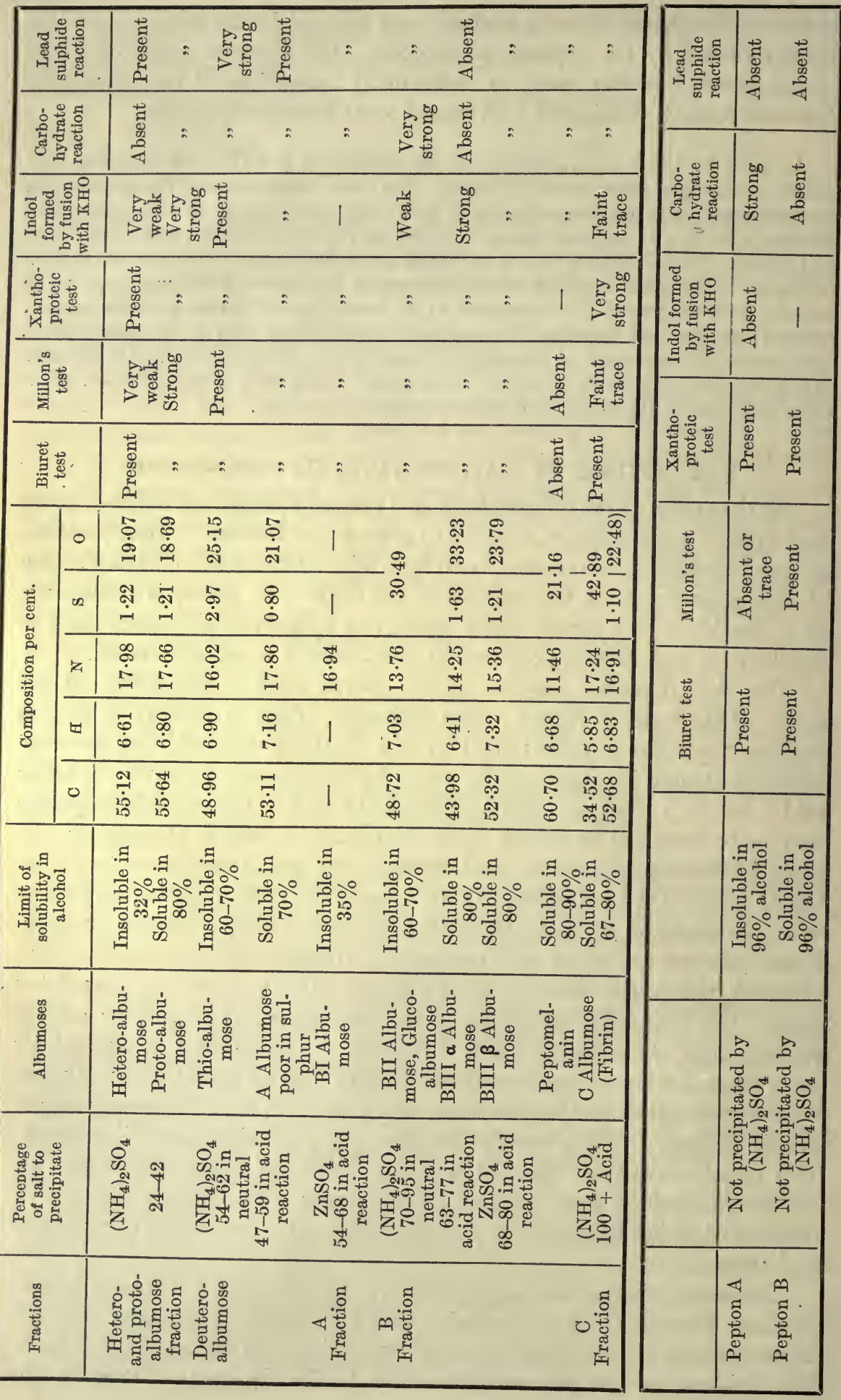


method from 'Witte's peptone,' 'a commercial preparation containing proteoses and peptones.

The following careful analysis of the constituents of protoalbumose and heteroalbumose (or protoproteose and heteroproteose) respectively shows that the different proteoses really correspond to different groupings of the amino-acids making up the original protein molecule :

Results of the Complete Hydrolysis of Hetero- and Protoalbumose

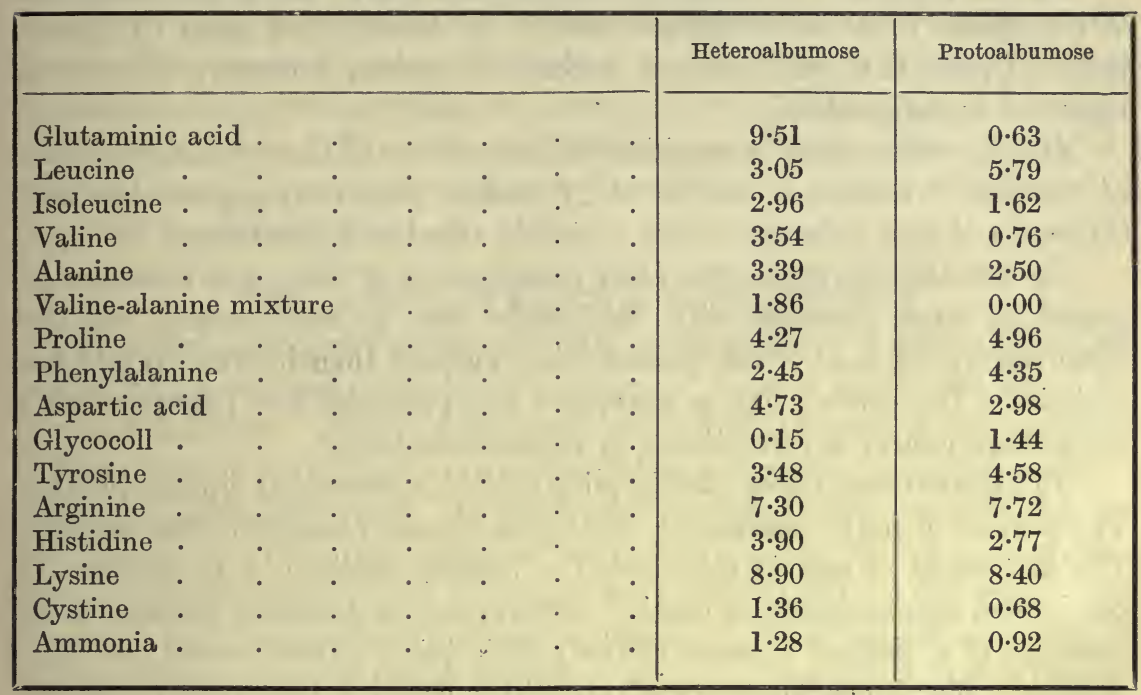

The results obtained by Pick by hydrolysis of these different bodies show that in the breakdown of protein produced by gastric juice there is really a division of the complex molecule into smaller molecules, which are qualitatively different. Thus of the fractions which he obtained, some contain the greater part of the sulphur originally present in the protein molecule, another contains the greater part of the carbohydrate group, while others are free altogether from the tryptophane group which is responsible for Hopkins' reaction obtainable in the original protein.

Proceeding from primary through secondary albumoses to peptones, there is probably a continuous diminution in the size of the molecule. During the time which gastric juice has to exert its influence, a maximum, say, of twelve hours, the breakdown of proteins never passes beyond the albumose and peptone stage, and it is in this form that the proteins of the food pass on through the pyloric orifice into the small intestine.

\section{ACTION ON THE CONNECTIVE TISSUES AND OTHER FOOD-STUFFS ALLIED TO PROTEINS}

Collagen. The connective tissues are made up chiefly of white fibres more or less modified, which consist of collagen. This substance forms the main basis of areolar tissue, of white fibrous tissue, and of bone. On prolonged boiling, it is converted into gelatin. The gastric juice dissolves 
collagen, converting it, probably through the stage of gelatin, into gelatoses and gelatin peptones, bearing the same relation to the original substance as is borne by the proteoses and peptones to the proteins. On account of this action, adipose tissue (which consists of protoplasmic cells distended with fat, and bound together by connective tissue) is broken up into its constituent cells. The protoplasmic pellicle is dissolved, and the fat floats freely in the gastric juice.

ELASTIN, which also occurs in varying amounts as the chief constituent of the elastic fibres of connective tissues, is slowly acted upon by gastric juice. Under the conditions of natural digestion, however, it may be regarded as indigestible.

MucIN, which forms a considerable proportion of the ground substance of connective tissues, is converted by gastric juice into peptone-like substances, and into reducing bodies probably allied to glycosamine.

The NUCLEO-PRoteins, the chief constituents of cells, and therefore ingested in large amounts with food-stuffs such as sweetbreads, are first dissolved by the acid of the gastric juice, and are then broken up into two moieties. The protein half is converted into proteoses and peptones, while the nuclein moiety is precipitated in an insoluble form.

On PHOSPHO-PROTEINS gastric juice acts in a somewhat similar manner. The protein of milk, caseinogen, undergoes special changes in the stomach. The first effect of gastric juice, even in neutral medium, is to convert the caseinogen into an insoluble casein. This action is generally ascribed to the presence of a distinct ferment of the gastric juice, named rennin, or rennet ferment. But, according to some authorities, it is due directly to the pepsin, i.e. rennin and pepsin are identical. For the conversion of caseinogen into the solid clot of casein the presence of lime salts is necessary. The addition of rennet to an oxalated milk apparently produces no effect, but clotting ensues if a soluble lime salt, such as calcium chloride, is then added to the mixture. Under the action of the acid gastric juice the solid clot of casein is dissolved, but a precipitate is left containing a small proportion of the original phosphorus of the caseinogen. This precipitate is sometimes spoken of as para-nuclein, or pseudo-nuclein. It does not yield purine bases on hydrolysis with acids, but contains phosphoric acid in organic combination. By prolonged digestion with strong gastric juice it is possible to dissolve the whole of this precipitate. It is therefore thought that in the clotting of milk the caseinogen under the action of the rennet first undergoes a conversion into a soluble casein, or perhaps a splitting into a soluble casein and some other protein. The soluble casein then, under the influence of the lime salts, forms an insoluble casein which is precipitated, and causes the solidification of the milk. In the absence of lime salts, the conversion or splitting of caseinogen takes place, but the second stage of the process cannot occur until the lime salts are added. 


\section{THE EFFECT OF GASTRIC JUICE ON CARBOHYDRATES}

On account of the fact that cane sugar undergoes inversion into equal molecules of glucose and fructose in the stomach, it has been sometimes thought that gastric juice contains a ferment invertase. It seems, however, that the inversion which takes place in the stomach can be completely accounted for by the action of hydrochloric acid present, and that there is no need to assume the presence of a special ferment.

In the same way inulin, the variety of starch which gives rise to the lævorotatory sugar fructose on hydrolysis, and is found in dahlia tubers and certain other reserve structures of plants, is converted by the acid of gastric juice into fructose. The inulin is therefore completely utilised in the alimentary canal of animals, although there is no definite ferment inulase provided for its hydrolysis.

\section{THE EFFECT OF GASTRIC JUICE ON FATS}

The chief action of this juice on fats is the solution of their connectivetissue framework and protoplasmic envelopes, so as to set the fat free in the stomach contents. After a fatty meal it is found moreover that a considerable proportion of the fat in the stomach has undergone hydrolysis and conversion into free fatty acid. In this hydrolysis two factors are involved, viz. (1) the action of the warm dilute hydrochloric acid; (2) the action of a special fat-splitting ferment or lipase, which is secreted by the walls of the stomach, and acts especially at the beginning of gastric digestion before the contents have attained a high degree of acidity. The action of this ferment is only marked if the fat be present in a finely divided form, e.g. as yolk of egg. The chief digestion of fat takes place in the next segment of the alimentary canal, namely, in the duodenum.

\section{THE SECRETION OF GASTRIC JUICE}

Pawlow has shown that if an animal provided with gastric and œesophageal fistulæ be given food, when hungry, it will eat with avidity, and since the food cannot reach the stomach and so satisfy its hunger, it will continue to eat for two or three hours. Five minutes after the beginning of this sham feeding, gastric juice begins to drop from the fistulous opening; and in this way large quantities of juice, free from any admixture with other substances, can be easily obtained. By this means we obtain a secretion of gastric juice, which is excited by the presence of food in the mouth. This method does not, however, enable us to determine whether the character of the juice will be altered in any way by the changes which the food undergoes in the stomach itself. In order to form an idea of the normal course of secretion of gastric juice, when food is taken into the stomach in the ordinary way, Pawlow has devised another procedure. A small diverticulum representing about onetenth of the whole stomach is made at the cardiac or pyloric end, in direct muscular and nervous continuity with the rest of the stomach, but shut off from the main part of the viscus by a diaphragm of mucous membrane. The method in which this operation is carried out will be evident by reference to 
the diagram (Fig. 336). In a dog treated in this way it is found that the amount of juice secreted by the small stomach always bears the same ratio to the amount secreted by the large stomach, while the digestive power of the juice obtained from the small stomach is equal to that obtained from the large. This is shown in the following Table:*

Secretion from Gastric Fistula after Sham Meal

\begin{tabular}{|c|c|c|c|c|}
\hline \multirow{2}{*}{ Hours } & \multicolumn{2}{|c|}{ Small stomach } & \multicolumn{2}{|c|}{ Large stomach } \\
\hline & Quantity & Strength $\uparrow$ & Quantity & Strength \\
\hline $\begin{array}{l}1 \\
2 \\
3\end{array}$ & $\begin{array}{l}7 \cdot 6 \text { c.c. } \\
4 \cdot 7 \text { c.c. } \\
1 \cdot 1 \text { c.c. }\end{array}$ & $\begin{array}{l}5 \cdot 88 \mathrm{~mm} \text {. } \\
5.75 \mathrm{~mm} \text {. } \\
5 \cdot 5 \mathrm{~mm} \text {. }\end{array}$ & $\begin{array}{l}68 \cdot 25 \text { c.c. } \\
41 \cdot 5 \text { c.c. } \\
14 \cdot 0 \text { c.c. }\end{array}$ & $\begin{array}{l}5 \cdot 5 \mathrm{~mm} \\
5 \cdot 5 \mathrm{~mm} \\
5 \cdot 38 \mathrm{~mm}\end{array}$ \\
\hline Total & $13 \cdot 4$ c.c. & - & $123 \cdot 75$ c.c. & - \\
\hline
\end{tabular}

B
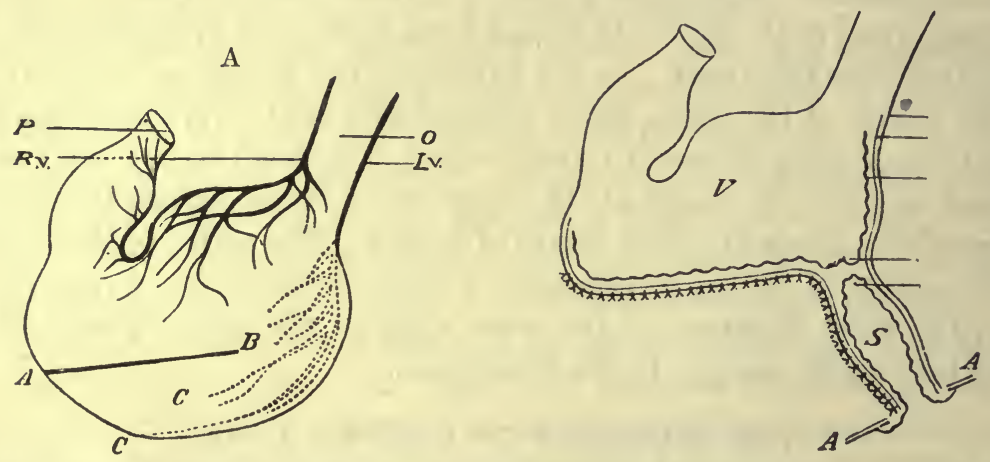

Fig. 336. Diagram to show Pawlow's method of making a cul-de-sac of the cardiac end of the stomach, with vascular and nerve supply intact.

In $\mathrm{A}$ the line of the incision into the stomach wall is shown. $\mathrm{B}$ represents the operation as completed. In A ; $O$, œsophagus ; $R . v, L . v$, right and left vagus nerves; $P$, pylorus; $C$, cardiac portion of stomach; $A, B$, line of incision. In B : $V$, main portion of stomach; $S$, cardiac cul-de-sac ; $A$, abdominal wall ; $e, e$, mucous membrane reflected to form diaphragm between the two cavities.

In this case a fistulous opening had been established into the large stomach, so that the juice could be obtained simultaneously from both sections of this organ. Secretion was excited by a sham meal, in which the food taken by the animal dropped out of an opening in the neck, and was not allowed to reach the stomach. It will be seen that the secretions in the two sections of the stomach run parallel to one another, while there is an almost exact equivalence between the strengths of the juices obtained from each

* Pawlow, "The Work of the Digestive Glands" (translated by W. H. Thompson, M.D.), p. 80.

$\dagger$ The strength of the juice was determined by measuring the number of millimetres of coagulated egg-white (in Mett's tubes) which were digested in eight hours. 
section. We may therefore regard the secretion obtained from the small stomach as a sample of that produced by the large, and from the changes in this small stomach judge of the effects occurring in the whole organ. By this method it is possible to study the effects of a normal meal in which the food is swallowed, or of a sham meal in which the food is merely masticated in the mouth, or of a meal in which the food is directly introduced into an opening into the large stomach.

The method which we must adopt for the collection of gastric juice shows that we have to do, in the first place, with a reflex nervous mechanism, since an active secretion is excited by the presence of food in the mouth and by its mastication. Moreover a secretion, which is at least as vigorous as that produced by a sham meal, can be evoked by merely arousing in the dog the idea of a meal. If the animal be hungry, it is sufficient to show it the food to produce a secretion. In the experiment from which the following Table is taken, the dog was continually excited by showing it meat during a period of an hour and a half. At the end of this time the animal, which had an œesophageal fistula, was given a sham meal. It will be observed that the psychical secretion obtained during the first period of the experiment was rather greater than the secretion produced by the introduction of food into the mouth.

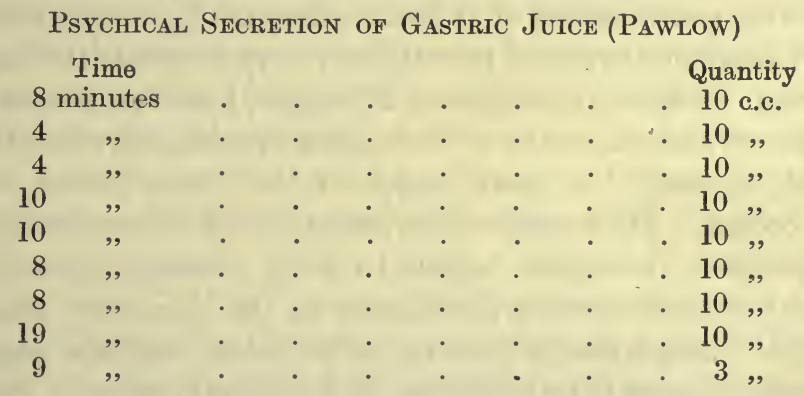

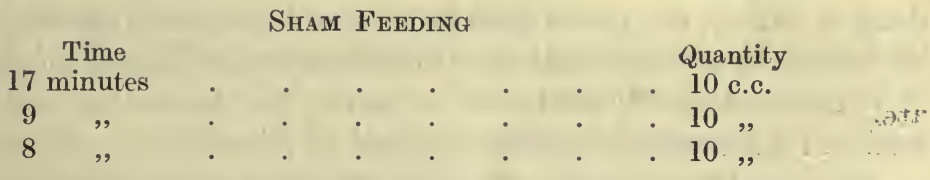

The afferent channels for this reflex may be therefore either the afferent nerves from the mouth, or, when the idea of food is involved, any of the nerves of special sense, such as sight, smell, or hearing, through which these ideas are called forth. The efferent channels can only be one of two nerves, viz. the vagus and the sympathetic, since these are the only two which are distributed to the stomach. That it is the former of these nerves which is involved is shown by the fact, recorded by Pawlow, that psychical secretion, as well as the results of a sham meal, is entirely abolished by division of both vagi. On this account division of both vagi may give rise to entire absence of gastric digestion, and death of the animal may ensue from inanition, or from poisoning by the products of decomposition of food in the stomach, even 
when care has been taken to avoid injury to the pulmonary and tracheal - branches of these nerves.

The converse experiment of exciting secretion by direct stimulation of the vagus presents greater difficulties. Stimulation of the vagus in the neck causes stoppage of the heart, and consequent anæmia of the mucous membrane of the stomach. Moreover, the stomach seems to be much more susceptible than the salivary glands to the action of poisons, such as anæsthetics. Its activity is also easily affected by inhibitory impulses arising in the central nervous system as the result of either painful impressions or emotional states of the animal. In order to avoid these disturbing factors Pawlow proceeded as follows: An animal with fistulæ of œsophagus and stomach had one vagus nerve divided. A thread was attached to the peripheral end of the cut vagus and allowed to hang out through the wound. Four days after the operation the vagus was drawn out of the wound by carefully pulling on the thread, so as not to hurt or frighten the animal in any way, and its peripheral end stimulated by means of induction shocks. No effect was produced on the heart, owing to the degeneration of the cardio-inhibitory fibres, which is well known to occur within this period after section. Five minutes after the commencement of the stimulation the first drop of gastric juice appeared from the gastric cannula, and a steady secretion of juice was obtained with continuation of the stimulation. This experiment furnishes the decisive and final evidence that the secretory nerves to the stomach run in the two vagi. There is one marked difference, however, between the action of these nerves and the action of the chorda tympani nerve on the submaxillary gland, namely, the great length of the latent period before gastric secretion occurs. The length of this latent period has not yet been satisfactorily explained. It cannot be due to delay occurring between the vagus fibres and the local nervous mechanism in the stomach. It may be that the chemical changes finally resulting in secretion require a longer period for their accomplishment than is the case in the salivary gland. Physiologically there is, indeed, no special need for a rapid secretion of gastric juice, whereas in the mouth it is essential that the introduction of food should be immediately followed by the production of saliva, for the tasting and testing of the food and for its subsequent mastication or rejection.

These experiments show conclusively that an important-probably the most important-part of the gastric secretion is determined by a nervous mechanism. This nervous secretion does not, however, account for the whole of the gastric juice obtained as the result of a meal. If an animal provided with two gastric fistulæ, one into a diverticulum and the other into the main stomach, has both its vagi divided, it is found that the introduction of meat into the large stomach is followed, after a period of twenty to forty-five minutes, by the appearance of a secretion of gastric juice from the small stomach. Moreover, when an animal is given a normal meal and is allowed to swallow the food after mastication, the total amount of gastric juice obtained is greater than that produced by the sham feeding alone and the flow is of longer duration. In fact, we may say that the gastric juice secreted 
in response to a normal meal consists of two parts, viz., (1) a large amount, the secretion of which begins within five minutes of the taking of the food and is determined by the reflex nervous mechanism described above; and (2) a smaller portion, the secretion of which is excited by the presence of the food in the stomach. This combined character of the gastric juice produced by a normal meal is shown in the following Table (Pawlow):

\section{Secretion of Gastric JuICe}

\begin{tabular}{|c|c|c|c|c|c|c|c|}
\hline \multirow{2}{*}{ Hours } & \multicolumn{2}{|c|}{$\begin{array}{l}\text { Normal meal. } \\
200 \text { grm. meat into } \\
\text { stomach }\end{array}$} & \multicolumn{2}{|c|}{$\begin{array}{l}150 \text { grm. meat direct } \\
\text { into stomach }\end{array}$} & \multicolumn{2}{|c|}{ Sham meal } & \multirow{2}{*}{$\begin{array}{c}\begin{array}{c}\text { Sum of two } \\
\text { last ex- } \\
\text { periments }\end{array} \\
\begin{array}{c}\text { Quantity } \\
\text { c.c. }\end{array}\end{array}$} \\
\hline & $\begin{array}{l}\text { Quantity } \\
\text { c.c. }\end{array}$ & $\begin{array}{l}\text { Strength } \\
\mathrm{mm} \text {. }\end{array}$ & $\begin{array}{l}\text { Quantity } \\
\text { c.c. }\end{array}$ & $\begin{array}{c}\text { Strength } \\
\mathrm{mm} \text {. }\end{array}$ & $\begin{array}{l}\text { Quantity } \\
\text { c.c. }\end{array}$ & $\begin{array}{l}\text { Strength } \\
\mathrm{mm} \text {. }\end{array}$ & \\
\hline 1 & $12 \cdot 4$ & $5 \cdot 43$ & $5 \cdot 0$ & $2 \cdot 5$ & $7 \cdot 7$ & $6 \cdot 4$ & $12 \cdot 7$ \\
\hline 2 & $13 \cdot 5$ & $3 \cdot 63$ & $7 \cdot 8$ & $2 \cdot 75$ & $4 \cdot 5$ & $5 \cdot 3$ & $12 \cdot 3$ \\
\hline 3 & $7 \cdot 5$ & $3 \cdot 5$ & $6 \cdot 4$ & $3 \cdot 75$ & $0 \cdot 6$ & $5 \cdot 75$ & $7 \cdot 0$ \\
\hline 4 & $4 \cdot 2$ & $3 \cdot 12$ & $5 \cdot 0$ & $3 \cdot 75$ & 0 & 0 & $5 \cdot 0$ \\
\hline
\end{tabular}

In the first column is given the result of a normal meal on the secretion from the gastric diverticulum. In the second column are given the amount and digestive power of the juice which is excited by the direct introduction of $150 \mathrm{grm}$. of meat into the large stomach of the animal, care being taken not to excite in any way the nervous reflex mechanism. In the third column are given the amount and digestive power of the juice which is evoked by a sham meal of $200 \mathrm{grm}$. of meat. In the fourth column is given the sum of the last two experiments. It will be seen that the total effect of the sham meal plus the direct introduction of meat into the stomach is almost identical with the secretion obtained when the food is taken in a normal way and allowed to pass through the œsophagus into the stomach.

The second phase of the gastric secretion cannot be ascribed to the intervention of the reflex vagal mechanism. Since it occurs after cutting off the stomach from its connections with the central nervous system, it must have its causation in the gastric walls themselves. That it cannot be due to mechanical stimulation is shown by the fact, previously mentioned, that it is impossible by local stimulation of the mucous membrane, by rubbing, or introduction of sand, or any other method, to evoke a secretion. Moreover it is not produced by all sorts of food. The introduction of white of egg, of starch, or of bread into the stomach causes no secretion. On the other hand, if bread be mixed with gastric juice and allowed to digest for some time, the introduction of the semi-digested mixture into the stomach evokes a secretion. We have already seen that meat produces a secretion; still more potent than meat, however, is a decoction of meat, or bouillon, or Liebig's extract of meat, or certain preparations of peptone. Pure albumoses and peptones have no effect, so that the exciting mechanism must be some 
chemical substances present in meat, and produced in various other foods under the action of the first gastric juice secreted in response to nervous stimuli. Popielski has shown that this secretion occurs after complete severance of the stomach from the central nervous system, as well as after destruction of the sympathetic nervous plexuses of the abdomen. Since the injection of bouillon directly into the circulation has no effect, this author concludes that the second phase of secretion is determined by the stimulation of the local nerve plexus, and that we have here, in short, a peripheral reflex action, the centres of which are situated in the walls of the stomach itself. There is, however, another possible explanation for this second phase of secretion. Although the peptogenic substances, those substances which evoke gastric secretion on introduction into the stomach, have no effect on the gastric glands whe injected directly into the blood stream, it is possible that they may have an influence on the cells which line the cavity of the stomach, and that they may produce, in these cells, some other substance which is absorbed into the blood, and acts as a specific excitant of the gastric glands. A process of this nature is known to occur in the next segment of the alimentary canal, viz. the duodenum, where it determines the secretion of the pancreatic juice and the bile.

Edkins has carried out a series of experiments to determine whether such a chemical mechanism may not also account for the secretion of gastric juice, which is excited by the introduction of substances into the stomach. Edkins' experiments were carried out in the following way: The animal, dog or cat, having been anæsthetised, the abdominal cavity was opened, and a ligature passed round the lower end of the csophagus so as to occlude the cardiac orifice and effectually crush the two vagus nerves. A glass tube was then introduced through an opening in the abdomen into the pyloric part of the stomach, and fixed in this position by a ligature tied tightly round the pylorus. The glass tube was connected by means of a rubber tube with a reservoir containing normal salt solution at the temperature of the body. By means of this reservoir, a certain amount of fluid was introduced into the stomach and kept there at a constant pressure ; the quantity of fluid introduced varied from 30 to 50 c.c. It has been shown by Edkins, as well as by von Mering, that no absorption of water or saline fluid occurs in the stomach. It is therefore possible to recover the whole of the fluid an hour after it has been introduced, by simply lowering the reservoir below the level of the animal's body. If secretion of gastric juice has occurred into the cavity of the stomach, the fluid will be increased in amount, and will contain hydrochloric acid as well as pepsin. In a series of control observations Edkins showed that the mere introduction of this fluid into the stomach caused no secretion of gastric juice, the fluid removed at the end of an hour having the same bulk and the same neutral reaction as the fluid which had been injected. Edkins then tried the influence of injecting substances into the blood stream. The injection of peptone, of acid, of broth, or of dextrin into the blood stream produced no secretion of gastric juice. If, however, in the course of the hour during which the fluid was allowed to remain in the 
stomach, a decoction made by boiling pyloric mucous membrane with acid, or with water, or with peptone was introduced in small quantities every ten minutes into the jugular vein, the fluid removed at the end of the hour was found to be distinctly acid in its reaction and to possess proteolytic properties. The injection of these substances had therefore caused the secretion of a certain amount of gastric juice containing both hydrochloric acid and pepsin. In order to produce this positive effect, it was necessary to employ pyloric mucous membrane, extracts made by infusing or boiling cardiac mucous membrane with any of these substances being without effect. Edkins concludes therefore that the secondary secretion of gastric juice is determined, not, as Pawlow and Popielski imagined, by a local stimulation of the reflex nervous apparatus in the gastric wall, but by a chemical mechanism. The first products of digestion act on the pyloric mucous membrane, and produce in this membrane a substance which is absorbed into the blood stream, and carried to all the glands of the stomach, where it acts as a specific excitant of their secretory activity. This substance may be called the gastric secretin or gastric hormone. It is noteworthy that it is produced in that portion of the stomach where the process of absorption is most pronounced.

The normal gastric secretion is therefore due to the co-operation of two factors. The first and most important is the nervous secretion, determined through the vagus nerves by stimulation of the mucous membrane of the mouth, or by the arousing of appetite in the higher parts of the brain. The second factor, which provides for the continued secretion of gastric juice long after the mental effects of a meal have disappeared, is chemical, and depends on the production in the pyloric mucous membrane of a specific substance or hormone, which acts as a chemical messenger to all parts of the stomach, being absorbed into the blood and thence exciting the activity of the various secreting cells in the gastric glands. It is still a moot point whether this gastric hormone is formed only in the pyloric mucous membrane, or whether it may not be also produced in the lower sections of the gut. Popielski has stated that the introduction of bouillon into the small intestine excites a secretion of gastric juice in animals, even after extirpation of the abdominal sympathetic plexuses and division of both vagi. On the other hand, introduction of the same substance into the large intestine has no influence on gastric secretion. Popielski ascribes this secretion again to a local reflex; but it is more probable that the mechanism in this case is the same as that involved in the secretion which is excited by the presence of semi-digested food in the stomach itself.

Pawlow has shown that the second phase of the gastric secretion is largely influenced by the character of the contents of the stomach. Thus the ingestion of large quantities of oil diminishes considerably the amount of gastric juice secreted, and Pawlow has suggested the administration of oil or oily foods as a possible remedy in cases where the production of gastric juice, and especially of hydrochloric acid, is in excess. It has long been imagined that the secretion of gastric juice was stimulated by the taking of alkalies. 
This idea has been shown by Pawlow to be erroneous. Whereas the formation of gastric juice is increased by the administration of acids, especially after a meal, it is largely diminished by the administration of alkalies such as sodium bicarbonate. In fact, sodium bicarbonate diminishes the activity of the digestive glands throughout the alimentary tract, and can be used as a means of diminishing the secretion of gastric juice as well as of pancreatic juice.

A further important question has been propounded by Pawlow, namely, whether there is any alteration in the constitution and amount of gastric juice with variations in the character of the food. So far as concerns the first phase of secretion, the psychical or 'appetite' juice, this observer has shown that, whatever the previous diet of the animal, the juice always has the same characters, the same digestive power, and the same percentage of hydrochloric acid. He finds, however, that in the case of the second, or what we may call ' chemical' secretion, i.e. that produced by local changes in the stomach, there is considerable variation in the nature of the juice. Whereas the secretion of juice is greatest in amount after a meal of meat, the digestive power of the juice is greatest after one of bread, and Pawlow regards these differences in the juice as determined by the variations in the stimulus applied to the gastric mucous membrane. It is doubtful, however, whether these results justify us in ascribing a number of specific sensibilities to the gastric mucous membrane. We have seen that the psychical juice depends merely on appetite, and therefore will be greater in amount the more welcome the food is to the animal. On the other hand, the juice secreted in the second phase must vary according to the quantity of gastric hormone produced in the pyloric mucous membrane, and therefore with the nature and amount of the substances produced in the preliminary digestion of the gastric contents by means of the psychic juice. The amount of juice may vary also with the salts contained in the food, according to their alkaline or acid character, and the percentage of pepsin in the juice may vary with the intensity of stimulus as well as with the quantity of fluid available for the formation of the gastric juice. These factors will co-operate in determining the characters of the whole juice secreted after any given meal, and it seems possible to explain the variations, observed on such different diets as meat and bread, without having recourse to the difficult assumption of a specific sensibility of the gastric mucous membrane to such inert substances as dextrin or egg albumin. 


\section{SECTION IV}

\section{THE MOVEMENTS OF THE STOMACH}

'THeSE can be best studied by Cannon's method-that is, by direct observation of the movements in a living unanæsthetised animal by means of the Röntgen rays. In order to make the shape of the stomach visible, the food-bread and milk-is mixed with a quantity of bismuth subnitrate or bismuth oxychloride (Hertz). The presence of this salt does not interfere with the processes of digestion, but renders the gastric contents opaque to the Röntgen rays. On examining by this means the stomach of a cat which has just taken a meal, the whole of the food is seen to be lying in the fundus. It is marked off by a strong constriction, the 'transverse band,' from the pyloric portion. In about twenty to thirty minutes faint waves of contraction begin a little to the cardiac side of the transverse band and travel slowly towards the pylorus. These waves succeed one another so that the pyloric part of the stomach may present a series of constrictions. Their effect is to force towards the pylorus the food which has been digested by gastric juice and detached from the surface of the mass in the fundus. The pylorus remaining closed, the food cannot escape, and therefore is squeezed back, forming an axial reflux stream towards the cardiac end. These contractions last throughout the whole period of gastric digestion, and become more marked as digestion proceeds. Their effect is to bring the whole of the food in close contact with every particle of pyloric mucous membrane and to cause a thorough mixture of food and gastric juice. At varying periods after a meal, according to the nature of the food taken, the arrival of one of these waves of contraction at the

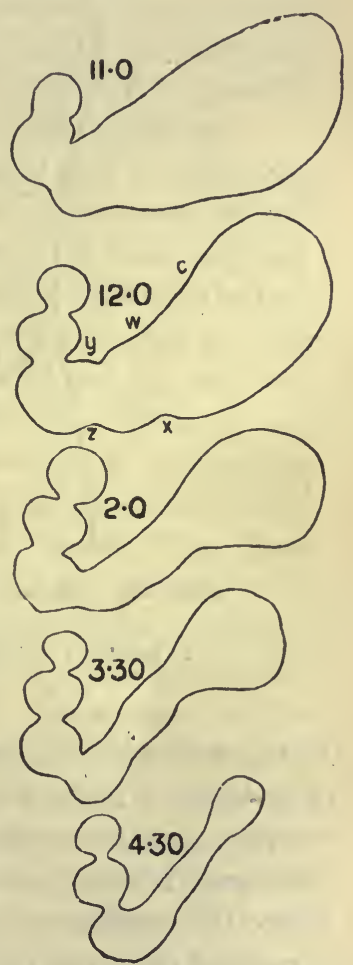

FIG. 337. Shadow sketches of the outlines of the stomach of a cat, immediately after a meal (11.0), at various intervals afterwards(12.0,2.0,3.30,4.30).

$c$, situation of cesophageal opening; $y z$, 'transverse band'; wx, junction of cardiac and pyloric por tions. (W. B. CANNoN.) pylorus causes a relaxation of the orifice, and a few cubic centimetres of gastric contents are squirted into the first part of the duodenum. 
While these movements of the pyloric mill are going on, the cardiac portion of the stomach is exercising a steady pressure on its contents in consequence of a tonic contraction of its muscular wall, so that each successive portion of the food mass which is loosened by the digestive action of the gastric juice is forced on into the pyloric mill. As digestion proceeds the opening of the pylorus becomes more frequent. The stomach empties itself more and more, until finally the whole of the viscus has the shape of a curved tube (Fig. 337).

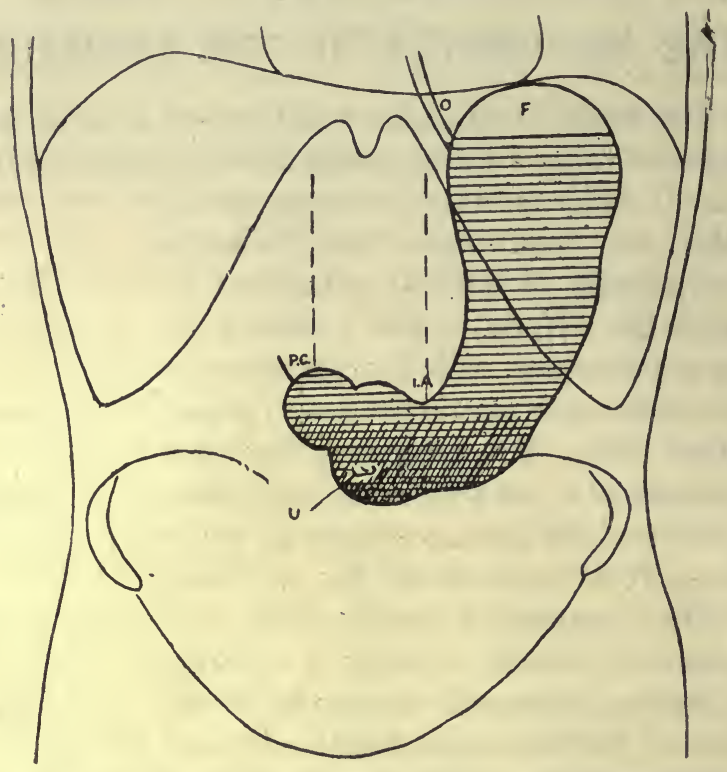

FIG. 338. Sketch of human stomach, in erect position, shortly after a bismuth meal. (HERTz.)

F, fundus ; U, umbilicus ; IA, incisura angularis ; PC, pyloric canal; o, œsophagus.

At the very end of digestion the pylorus may open to allow the passage even of undigested morsels of food.

1.: Very similar are the changes in the human stomach, as shown by Hertz. The term fundus is by him limited to that part of the stomach situated above the cardiac orifice (in the erect position). The body of the stomach is marked off, more or less, by the incisura angularis on the lesser curvature corresponding to what we have called the transverse band. The pyloric portion consists of the pyloric vestibule and the pyloric canal, the latter being a tubular portion about $3.0 \mathrm{~cm}$. in length, especially well marked in infants. When a small quantity of food has been swallowed (in the erect position) its weight is sufficient to overcome the resistance of the contracted gastric wall, and it rapidly passes to the pyloric part. Peristalsis begins almost at once, each constriction starting near the middle of the stomach, and deepening as it slowly progresses towards the pylorus (Fig. 338). About one inch from the pyloric canal it is so marked that part of the pyloric vestibule becomes almost completely separated from the rest of the stomach. 
The part thus cut off then diminishes in size in every direction, part of its contents being forced through the pyloric canal, while the remainder escapes back as an axial reflux stream into the stomach. The waves recur at regular intervals of fifteen to twenty seconds, and three or four are present simultaneously. They continue without cessation until the stomach is emptyfrom one to four hours after the meal according to its bulk and composition.

The foregoing descriptions apply especially to the events which succeed the taking of a considerable meal. If warm fluid alone, e.g. water, be swallowed, the opening of the pylorus occurs within a very short time after the fluid has reached the stomach. Thus if a large draught of water be taken to quench thirst it may arrive in the duodenum within a minute or two after being swallowed, and it is from the duodenum and small intestine that any absorption takes place. When a meal is undergoing digestion, there is a distinct relation between the amount of acid present in the gastric contents and the opening of the pylorus. One may indeed say that acidity of the gastric contents exercises a direct inhibitory stimulus on the pyloric sphincter.

These movements of the two portions of the stomach may be observed also on anæsthetised animals and even on a stomach which has been excised and placed in warm salt solution. They must therefore have their origin in the walls of the stomach itself. Although the co-ordination between the two parts of the stomach, between the tonic contractions of the fundus and the rhythmic contractions of the pyloric part, may be carried out by the local nervous system-Auerbach's plexus-situated between the layers of the muscular coat, it is probable that the advancing waves of contraction observed in the antrum are myogenic, i.e. directly originated in and determined by the muscle fibres themselves. Cannon has shown that these movements persist after complete division of Auerbach's plexus by 2 to 6 circular incisions carried through the entire muscular coat of the stomach ; and it is evident that they do not partake of the nature of a true peristalsis, since they are not preceded by a wave of relaxation. The opening of the pylorus, on the other hand, which occurs at increasingly frequent intervals at the end of a wave, must be ascribed to a nervous mechanism. Although the local mechanism probably plays the greater part in this act of relaxation, the normal emptying of the stomach is also largely dependent on the integrity of the connection of this viscus with the central nervous system. If both vagus nerves be divided in a dog below the point at which they give off their branches to the lungs and heart, a large amount of food may remain in the stomach in an undigested condition. The secretion of gastric juice is deficient, and the opening of the pylorus is not easily carried out. Such dogs therefore tend to die of sapræmia, being poisoned by the absorption of products of putrefaction from the gastric contents. . Pawlow has shown that animals can be kept alive for months after division of both vagi if a gastric fistula be made, the animals be carefully fed, and care be taken to wash out adherent non-digested portions of food from the stomach.

The opening of the pylorus depends not only on intragastric events but also on the condition of the duodenum. It has been shown by Serdjukow 
that the pylorus remains firmly closed so long as the contents of the duodenum are acid. If alkaline fluid be introduced into the stomach, this is rapidly passed into the duodenum. If, however, some acid be introduced at the same time into the duodenum by means of a duodenal fistula, the pylorus remains firmly closed, and no fluid passes into the duodenum until the acid

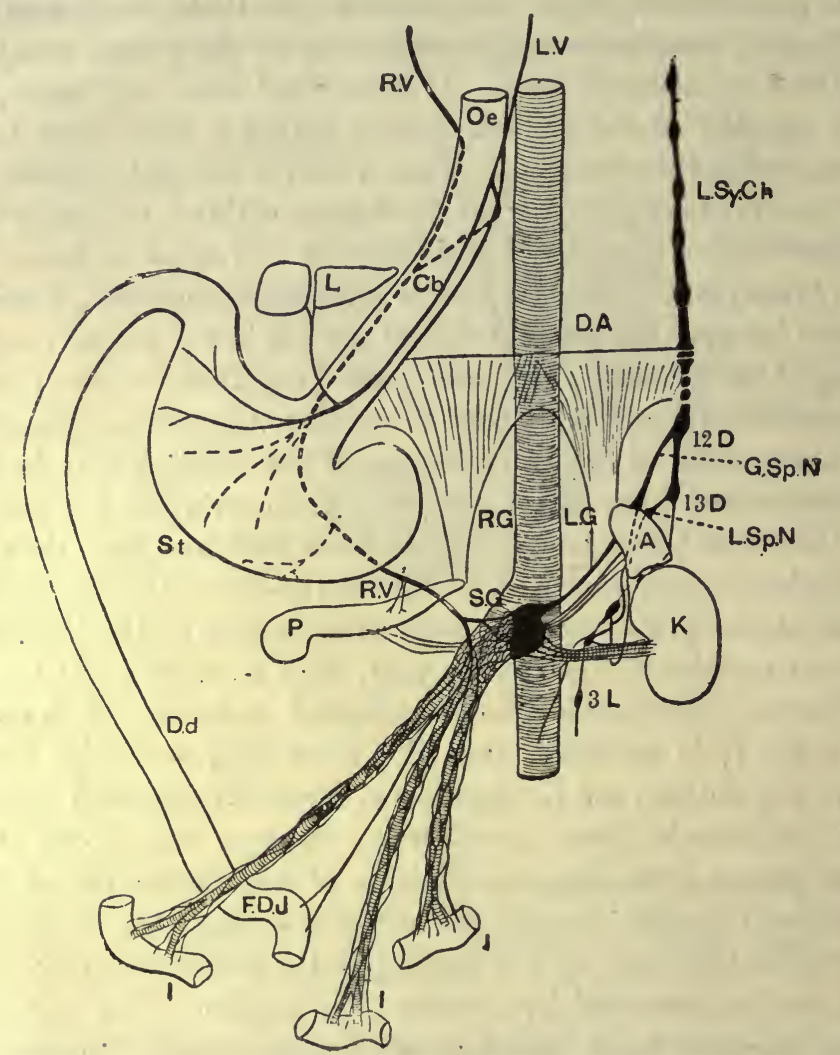

FIG. 339. Distribution of the vagus in the abdomen of the dog.

(M. H. NAYLOR.)

$\mathrm{RV}, \mathrm{LV}$, right and left vagi. The right vagus runs behind the œsophagus $(\mathrm{Oe})$ and stomach (St), and in those places is represented by a discontinuous line. $\mathrm{Cb}$, connecting branch between right and left vagi ; $\mathrm{P}$, pancreas; $\mathrm{Dd}$, duodenum; FDJ, flexura duodeno-jejunalis; I, I, I, intestine ; L, liver ; $\mathrm{K}$, kidney; A, suprarenal capsule ; RG, LG, right and left cura of diaphragm ; L.Sy.Ch, left sympathetic chain ; $12 \mathrm{D}, 13 \mathrm{D}$, twelfth and thirteenth dorsal ganglia; 3 L, third lumbar ganglion; G.Sp.N, L.Sp.N, great and small splanchnic nerves; S.G, left semilunar and superior mesenteric ganglia; D.A, dorsal aorta.

which was placed there has been neutralised by the secretion of pancreatic juice and succus entericus. We have probably in the walls of the alimentary canal a local nervous mechanism for the movements of the pyloric sphincter. This may be played upon by impulses starting either in the stomach or in the duodenum, probably by the contact of acid with the mucous membrane. Increasing acidity on the side of the stomach causes relaxation of the orifice, whereas acidity on the duodenal side causes contraction of the pyloric 
sphincter. The exact parts, however, played in this mechanism by the local system and by the central nervous system respectively have not yet been thoroughly made out, though there is no doubt that these movements may proceed independently of any connection with the central nervous system.

Stimulation of the peripheral end of the vagus nerves may exercise varying effects on the stomach wall as well as on its sphincters. In the normal animal stimulation of the peripheral end of the vagus as a rule causes strong contractions of the cesophagus as well as of the cardiac sphincter. After the administration of atropine, stimulation of the same nerve will occasion dilatation of the cardiac sphincter. On both cardiac and pyloric portions of the stomach the vagus exercises inhibitory as well as augmentor effects. So far as concerns the musculature of the fundus or body of the stomach, the most usual effect is an inhibition during stimulation of the vagus succeeded by an augmented tonus immediately the stimulus is removed. If the vagus be excited a number of times the tonus of the muscular wall augments with each stimulus. On the pyloric portion stimulation of the vagus also causes inhibition, followed by contraction. The inhibition may, however, be very short and in rare cases altogether absent, so that during the excitation this inhibition is followed by a series of large rhythmic contractions. The prevailing motor effect of the vagus therefore is in the fundus increased tonus, in the pyloric portion augmented peristaltic waves. On the pylorus itself we may obtain from vagal stimulation either increased or diminished contraction. The conditions under which each of these may be evoked have not yet been definitely ascertained. Whether the splanchnic nerve, i.e. the sympathetic system, has a direct influence on the movements of the stomach has been disputed. According to Page May any effect produced by stimulation of this nerve, generally consisting in diminished motor activity, is probably due to the simultaneous influence on the vascular supply to the organ; the blood-vessels being constricted, an artificial anæmia is produced which in itself is sufficient to account for diminished activity. Other observers regard the splanchnic as having an influence on the stomach similar to its action on the intestine, and regard it as the chief inhibitory nerve to this organ. It is possible that the extent to which the stomach is brought under the control of the sympathetic system may vary in different species of animals.

Carlson has shown that the 'pangs of hunger' are associated with and probably due to rhythmic contractions of the stomach wall, which come on about meal time especially if this be delayed. 


\section{IN'TESTINAL DIGESTION}

THE products of gastric digestion, after being worked up in the pyloric half of the stomach, are passed at intervals into the first part of the duodenum. Here they meet the secretions of three glands, namely, the pancreas, the liver, and the tubular glands of the intestine. In addition to these must be mentioned the secretion of Brunner's glands, which are situated at the very beginning of the duodenum. The glands of Brunner extend only over about half to one and a half inches in the carnivora, such as the dog or cat, but in the herbivora they may be found occupying the upper six inches of the intestine. The secretion of these various juices is practically simultaneous and is aroused by the very act of entry of the acid chyme into the duodenum. Although they co-operate in their action on the food-stuffs, it will be convenient to deal separately with each both as regards their action and the mechanism of their secretion.

\section{SECTION V \\ THE PANCREATIC JUICE}

PURE pancreatic juice can be obtained either from an animal with a permanent fistula or from one with a temporary fistula by the injection of secretin into the animal's veins. A flow of pancreatic juice may also be produced by the administration of pilocarpine. This drug acts, however, as a poison on many tissues of the body, not confining its action to the pancreas or even to the secreting glands. It is not to be wondered at therefore that the pancreatic juice obtained by its injection differs in quality from that obtained by the more natural method of injection of secretin. The average composition of pancreatic juice is shown in the Table on p. 703 .

It is a clear or slightly opalescent fluid, strongly alkaline from the presence of sodium carbonate, its alkalinity varying between $\frac{\mathrm{N}}{10}$ and $\frac{\mathrm{N}}{7} \mathrm{Na}_{2} \mathrm{CO}_{3}$. It is therefore about as alkaline as gastric juice is acid, and it will be found that equal quantities of gastric juice and pancreatic juice when added together practically neutralise one another. The proteins of the juice may be roughly divided into three groups, a small amount of nucleo-protein precipitated on acidification, a protein coagulating at $55^{\circ} \mathrm{C}$, and another at about $75^{\circ} \mathrm{C}$. The juice tends to become poorer in proteins and richer in alkali as secre. 


\begin{tabular}{|c|c|c|c|c|}
\hline & A & \multicolumn{2}{|c|}{ B } & C \\
\hline Alkalinity : & & (a) & (b) & \\
\hline $\begin{array}{c}\text { Number of c.c. } \frac{\mathrm{N}}{10} \mathrm{NaOH} \text { equal } \\
\text { to } 10 \text { c.c. juice }\end{array}$ & $12 \cdot 7$ & 12.4 & 9 & $5 \cdot 5$ \\
\hline I.e. in terms of $\mathrm{Na}$ in 100 c.c. & $0 \cdot 2921$ & 0.2852 & $0 \cdot 2587$ & $0 \cdot 1166$ \\
\hline Total solids in 100 c.c. & $\begin{array}{l}1 \cdot 6 \\
1 \cdot 56\end{array}$ & $2 \cdot 25$ & 1.5 & $6 \cdot 38$ \\
\hline Total proteins in 100 c.c. & 0.5 & - & - & $4 \cdot 8$ \\
\hline Ash in 100 c.c. & $\begin{array}{l}1 \cdot 00 \\
0 \cdot 92\end{array}$ & $1 \cdot 00$ & $1 \cdot 00$ & $1 \cdot 3$ \\
\hline Chlorides in 100 c.c. & $\left.\begin{array}{l}0 \cdot 2808 \\
0 \cdot 2966\end{array}\right\}$ & 一 & - & $0 \cdot 2695$ \\
\hline Total nitrogen & - & 一 & - & 0.735 \\
\hline
\end{tabular}

A. Secretin juice from three dogs. Sp. gr. 1014.

B. Secretin juice, specimen collected at beginning $(a)$, and at end $(b)$.

C. Pilocarpine juice.

tion proceeds. The concentrated juice obtained by injection of pilocarpin, which may contain as much as 6 per cent. total solids, is always considerably less alkaline than the more dilute juice got by injection of secretin. The most interesting and important constituents of the juice are its ferments or precursors of ferments. The juice on arrival in the intestine has; or develops, an effect on all three classes of food-stuffs, namely, proteins, fats, and carbohydrates.

\section{ACTION ON PROTEINS}

Although the digestive action of pancreatic juice on proteins was pointed out by Corvisart, little attention was paid to this action either by Claude Bernard or subsequent authorities until Kühne subjected the action of extracts of the gland to a thorough investigation. The neglect of this action by Claude Bernard must be ascribed to the fact that he worked with pancreatic juice. It has been shown more recently that pancreatic juice as secreted is free from proteolytic effects, and that for the development of this power it is necessary that some change should be brought about in the juice itself, namely, a conversion of trypsinogen into trypsin. This change under normal circumstances is brought about directly the juice enters the gut, by the action of a substance - enterokinase - contained in the succus entericus. The pancreatic juice thereby acquires a proteolytic activity superior to that of any other digestive juice, so that the proteins of the food undergo a very thorough disintegration. The different constituents of the protein molecule show a varying resistance to the action of trypsin. The greater part of the molecule is rapidly broken down into its proximate constituents, namely, amino-acids, and the same change is undergone by the proteoses and peptones resulting from the gastric digestion of proteins. Within a few minutes therefore after the chyme has reached the small intestine a certain amount of ${ }^{\prime}$ 
amino-acids will have been formed. Some of the groups present, however, a resistance to disintegration. After tryptic digestion for a few hours the mixture will be found still to contain a considerable quantity of peptone, which in consequence of its resistance to further alteration was designated by Kühne 'antipeptone.' The antipeptone of Kühne certainly included some of the diamino-acids, which at that time had not been isolated. There is always a part, however, which gives the biuret reaction and is only slightly broken down after the prolonged action of trypsin into the amino-acids. Even when the trypsin has acted for weeks and the biuret reaction has entirely disappeared, the mixture will be found to contain, in addition to the separate amino-acids, some members of the polypeptide class, composed of two or more molecules of amino-acid united together. One of these polypeptides has been isolated by Fisher and Abderhalden from the products of tryptic digestion of the protein of silk, and has been found to contain glycine, alanine, and proline. The stages therefore in tryptic digestion, e.g. on fibrin, may be set out as follows :

(1) After one hour's digestion-soluble coagulable protein, deuteroalbumose, peptone, amino-acids, with a small amount of alkali metaprotein produced by the action of the alkali of the juice.

(2) After digestion for one day-deutero-albumose, 'antipeptone,' amino-acids, polypeptides.

(3) After digestion for one month-amino-acids, polypeptides.

Among the amino-acids tyrosine is one of the first to be split off, and this substance, with leucine, was among the earliest known products of pancreatic digestion. The action of trypsin is thus seen to resemble very closely the action of boiling concentrated hydrochloric acid. Like the latter it attacks the protein molecule at the $-\mathrm{CO}-\mathrm{NH}$ - coupling, introducing water at this point and therefore breaking up the polypeptide groupings into simple amino-acids. Why it always leaves a certain remnant of the polypeptides unattacked is not at present explained. The investigation of its action on the polypeptides has shown that very minute differences in the grouping of the molecule may determine whether or not the molecule is attacked by trypsin. Apparently it will only attack such molecules as are present in the naturally occurring proteins. Thus under the action of trypsin the following polypeptides undergo hydrolytic dissociation : alanyl glycine, alanyl alanine, alanyl leucine A; while the closely similar polypeptides glycyl alanine, glycyl glycine, alanyl leucine B are left untouched.

\section{CONDITIONS OF TRYPTIC ACTIVITY}

Since the pancreatic juice is strongly alkaline it might be expected that trypsin would be most effective in an alkaline medium. It must be remembered, however, that the alkaline juice when secreted meets the correspondingly acid contents discharged from the stomach, and that the resulting mixture is practically neutral. This neutrality exists throughout the small intestine, the reaction of the contents of the gut being similar to that of a fluid containing alkali which has been saturated by the passage of carbonic 
acid, viz. alkaline to such indicators as methyl orange, and acid to such indicators as phenolphthalein. On investigating the action of trypsin outside the body, it is found that, at any rate as concerns its earlier stages, this ferment is more active in the presence of sodium carbonate. It is usual to make up an artificial digestive mixture by dissolving commercial trypsin in 0.2 to 0.3 per cent. sodium carbonate. The optimum amount of sodium carbonate depends on the strength of the solution in trypsin: the more trypsin present the higher is the optimum amount of sodium carbonate. It is stated that although an alkaline reaction is more advantageous for the earlier stages of tryptic activity, the later stages take place best in a neutral medium. This result is probably due to the fact that trypsin in alkaline medium is extremely unstable, so that when prolonged digestions are carried out the trypsin would be rapidly destroyed if the medium were strongly alkaline. The destructibility of trypsin, as well as its action, is largely affected by the presence of proteins or their digestion products in solution. Bayliss has adduced evidence to show that when trypsin acts upon protein it enters into some form of combination with the protein molecule. This combination protects the trypsin from the destructive action of alkali. The velocity of the reaction, which takes place under the influence of trypsin, gradually diminishes, owing probably to a combination of the trypsin with the products of digestion, e.g. with the peptones or amino-acids, and its consequent removal from the sphere of action. If by any means the aminoacids be removed the action of the trypsin is renewed. This destruction of ferment occurs in the intestine itself. If the intestinal contents be collected by means of a fistula at the lower end of the ileum, they show little or no proteolytic activity. The trypsin is therefore an extremely active ferment which carries out its function of protein hydrolysis at the upper part of the gut and is destroyed before reaching the lower end.

\section{THE ACTIVATION OF PANCREATIC JUICE}

It was observed by Kühne that extracts of the fresh pancreas did not develop their full activity for some considerable time, the development being aided by preliminary treatment with a weak acid. When a pancreatic fistula is made according to Pawlow's method, the juice obtained always presents some proteolytic activity. It was shown by Pawlow and Chepowalnikoff that the development of the activity of the juice was due to the action of a constituent of the succus entericus which they named enterokinase, and it has since been found that if care be taken to avoid contact of the juice with the mucous membrane surrounding the orifice of the duct, it is, when secreted, entirely inactive. The enterokinase acts like a ferment on a body, trypsinogen, present in the juice as secreted, converting this into trypsin. Pawlow therefore called this body the ' ferment of ferments.'

This view of the action of enterokinase has been challenged, especially by Delezenne, according to whom there is an actual combination between the enterokinase and the trypsinogen, trypsin itself being a mixture or combination of the two bodies. $\mathrm{He}$ compared the reaction to that of the hæmolysins, which, as is well known, involve in 
their action the co-operation of two bodies, the amboceptor and the complement. If this were correct there should always be a proportionality between the quantities of trypsinogen and enterokinase respectively which are necessary to form trypsin. It has been shown by Bayliss and Starling that this proportionality is not present. The smallest quantity of enterokinase is sufficient to activate any amount of trypsinogen if sufficient time be allowed. The effect of increasing or diminishing the amount of enterokinase is not to alter the total amount of trypsin finally produced, but merely the time taken for its production. This behaviour characterises a ferment, and we may therefore conclude that the view originally put forward by Pawlow is correct, namely, that trypsin is produced from trypsinogen under the action of a ferment enterokinase. If pancreatic juice be allowed to stand, even with the addition of toluol to prevent bacterial infection, it gradually acquires a certain degree of activity. If, however, sodium fluoride be used as an antiseptic the juice remains permanently inactive. The spontaneous activation of the juice may be hastened by neutralisation. The most potent means next to enterokinase is the addition of lime salts. If a few drops of 10 per cent. calcium chloride solution be added to fresh pancreatic juice, the calcium being in such a quantity as to suffice to combine with all the carbonate present in the juice, complete activation of the juice occurs within a couple of days, no further increase in its digestive powers being obtained on subsequent addition of enterokinase. It has been suggested that the action of calcium is in some way to assist in the production of an enterokinase from some precursor of this body already present in the juice. According to Mellanby, the calcium acts simply by neutralising the juice and thus allowing minute traces of enterokinase already present in the juice to exert their effect. It is not likely that this calcium activation plays any part in the normal processes of digestion, since for its completion it needs twelve to sixteen hours, whereas the enterokinase present in the succus entericus will effect the activation of the juice within a few minutes.

\section{THE ACTION OF PANCREATIC JUICE ON MILK}

On the addition of pancreatic juice to milk a clot is produced which speedily redissolves. If re-solution takes place too rapidly the production of a formed clot may be missed. In every case, however, on heating the milk a few minutes after the addition of the trypsin a clot is obtained. How far this action is to be ascribed to the proteolytic ferment trypsin, or how far it is due to the presence of a free rennet-like ferment in the juice, is not yet definitely settled. Since the rennet action is parallel to the proteolytic activity of the juice, it is probable that we must regard the clotting of milk as the first stage in its proteolysis.

\section{THE ACTION OF PANCREATIC JUICE ON CARBOHYDRATES}

The pancreatic juice, as well as fresh extracts of the pancreas itself, contains a strong amylolytic ferment, diastase, amylase, or amylopsin. If a few drops of pancreatic juice be added to a 1 per cent. solution of boiled starch, within a few seconds the solution clears, and in half a minute, on the addition of iodine, a red colour is obtained, showing the presence of erythrodextrin. At the end of a few minutes no colour is obtained with iodine, and the solution contains maltose. The stages in the hydrolysis of starch brought about with pancreatic juice are exactly similar to those effected by ptyalin. If the juice be neutralised, the process of hydrolysis goes on to the formation of dextrose or glucose. This further conversion is due to 
the presence in the juice of a second ferment-maltase-which converts the disaccharide maltose into the monosaccharide glucose. The juice in the gut is therefore able to effect the further digestion of the products of salivary digestion. On the other disaccharides pancreatic juice is without effect. It contains no invertase, nor does it, in spite of certain statements to the contrary, ever contain lactase. It has therefore no effect on either cane sugar or milk sugar.

\section{THE ACTION OF PANCREATIC JUICE ON FATS}

Fresh pancreatic juice contains a strong lipase or fat-splitting ferment, by means of which, in the presence of water, neutral fats, e.g. the triglycerides of palmitic, stearic, and oleic acids, are broken up into glycerin and the corresponding fatty acids. This ferment is active either in alkaline, neutral, or very slightly acid reaction. - If the reaction be alkaline the fatty acids produced by the lipolysis combine with the alkali present with the formation of soaps. The ferment may be obtained from extracts of the fresh gland, but is rapidly destroyed if active trypsin be present. It is also contained in some of the dried commercial preparations of trypsin. It is apparently insoluble in distilled water, and is therefore found in the residue after extracting these commercial preparations with water. It is easily soluble in glycerin. The velocity with which lipolysis occurs is much increased (four to five times) by the addition of bile. This adjuvant action of bile is not destroyed by boiling, and is due entirely to the bile salts. These act in two ways. In the first place, by their physical qualities they diminish the surface tension between water and oil, so enabling a closer contact to be effected between the watery solution contained in the juice and the oil which is presented to it. Moreover they may aid in the solution of the ferment itself. In the second place, bile salts have a solvent action on soaps as well as on fatty acids in slightly acid medium. Bile may be regarded therefore as a favourable excipient or medium for the interaction of the lipase and the neutral fats. The lipase of pancreatic juice will also hydrolyse the esters of the fatty acids, such as ethyl butyrate or monobutyrin. On the phosphorised fats or phosphatides, such as lecithin, its action is still a subject of doubt. According to certain authors extracts of the pancreas have the power of splitting off choline from lecithin. It is not known whether the same property is present in pancreatic juice itself, or whether any other dissociations are brought about in the complex molecule of lecithin under the action of this digestive fluid.

\section{THE SECRETION OF PANCREATIC JUICE}

In order to study the relation of the secretion of pancreatic juice to the other processes of digestion, observations must be carried out on an animal with a permanent pancreatic fistula.

Such a fistula was established by Claude Bernard by bringing the duct of the pancreas to the surface and inserting into it a lead or silver tube. The arrangement was, however, unsatisfactory since after a few days the tube dropped out and the natural course of the duct from pancreas to intestine was restored. In order to avoid the disadvantages 
of this proceeding Heidenhain and Pawlow independently devised another method to enable us to determine the causes of pancreatic secretion. The pancreas in most cases possesses two ducts, the upper one opening along with the bile duct, the lower one a short way down. The relative sizes of these two ducts vary in different animals, the lower one being larger in the dog, while in man and the cat the upper one is larger. In order to establish a pancreatic fistula in a dog, a small quadrilateral piece of the duodenal wall is exsected, having the papilla of the lower duct opening in the middle of its mucous surface. The integrity of the gut is restored by suturing in a single line of stitches the margins of the wound in the duodenum, and the exsected piece is brought to the surface and stitched in the middle of the abdominal wound. The greater part of the pancreatic secretion will escape by the fistula, and can be collected either by the insertion of a cannula into the duct or by attaching a glass funnel below its orifice. Great care has to be taken in the after treatment of such animals. The pancreatic juice, which flows over the papilla, acquires in so doing strong proteolytic powers, and tends therefore to dissolve and irritate the adjacent abdominal wall. This can be prevented by taking care to collect all the juice, and to allow none to flow away over the surface of the body. Another drawback is that the continual loss of pancreatic juice in many cases seriously affects the animal's health. This may be obviated to a certain extent by keeping the animal on a milk diet with the addition of sodium bicarbonate to replace the loss of this salt by the juice. With great care Pawlow has succeeded in keeping such animals in a perfectly healthy condition.

In the fasting condition there is, as a rule, no secretion of juice, though the escape of a few drops may be observed at long intervals. If a meal be administered to the animal, a flow of juice begins in one to one and a half minutes. From this time there is a steady, slow rise of the rate of secretion, which lasts for two to three hours, and then gradually diminishes. The greatest increase in flow is observed at the time when the first portions of digested food escape from the stomach into the duodenum. The secretion must therefore be determined in some way by the entry of this food into the duodenum. By experiments on dogs possessing a gastric as well as a pancreatic fistula, it has been shown that the introduction of acid, e.g. $0 \cdot 4$ per cent. $\mathrm{HCl}$, into the stomach evokes, as soon as it passes into the duodenum. a rapid flow of pancreatic juice. A similar, but smaller, effect is produced by the passage of oil from the stomach into the duodenum. The introduction of alkalies is without effect. Weak acids are also effective exciters of secretion if they be introduced directly into the duodenum itself or into a loop of small intestine. The effect gradually diminishes as the loop which is chosen comes nearer to the cæcum, and as a rule the injection of dilute acid into the lower foot or eighteen inches of ileum is without effect on the pancreas. The striking resemblance between the secretion thus evoked and that produced in the salivary glands by injection of acid into the mouth suggests that we have here to do with a reflex of the same kind as that which affects the salivary glands. In the search for the channels of this reflex Heidenhain showed that stimulation of the medulla oblongata occasionally produced a flow of pancreatic juice. He was unable, however, to obtain any secretion on stimulation of the vagus nerve. The pancreas receives fibres from the vagi as well as from the splanchnic nerves (sympathetic system). According to Pawlow the ill success of Heidenhain's experiments was due to the fact that in any operation a gland is played upon by reflex impulses 
partly of an inhibitory, partly of a secretory nature, in which the inhibitory predominate, and by the further fact that the pancreas is extremely susceptible to alterations in its blood-supply, so that any stimulation of the vagus which caused inhibition of the heart would ipso facto prevent the effect of simultaneous excitation of secretory fibres from making its appearance. Pawlow noticed that if in an animal with a permanent fistula the vagus on one side were cut and left for four days in order to allow the cardio-inhibitory fibres to degenerate, repeated stimulation of the peripheral end of the nerve evoked a flow of pancreatic juice. He obtained the same results by stimulating this nerve below the point at which it had given off its cardio-inhibitory fibres, in animals in which the reflex inhibitions from the operation itself were prevented by total section of the medulla. Under certain circumstances he obtained also secretion on stimulation of the splanchnic nerves, and therefore concluded that these two nerves-splanchnics and vagi-were the efferent channels in the reflex secretion set up by the introduction of the acid into the duodenum. It was shown later, however, independently, both by Popielski, a pupil of Pawlow, and by Wertheimer, that the injection of acid into a loop of small intestine was followed by secretion of juice even after section of both vagi and destruction of the sympathetic ganglia at the back of the abdominal cavity. On repeating these experiments Bayliss and Starling found that a secretion of juice was produced even when the acid was introduced into a loop of the small intestine entirely freed from any possible nervous connections with the rest of the body. It was evident therefore that the stimulus or message from the intestine to the pancreas which causes the secretion of the latter must be carried, not by the nervous system, but by the blood stream. Since the injection of acid into the portal vein was without effect on the pancreas, it was concluded that something must be produced in the epithelial cells of the gut under the influence of acid, and that this product of the epithelial cells was absorbed in the blood stream and was the active agent in exciting the pancreas. On pounding up some scrapings of the intestinal mucous membrane with dilute hydrochloric acid and filtering, and injecting the filtrate, a copious flow of pancreatic juice was produced. This chemical messenger or hormone from the intestine to the pancreas is called 'secretin,' or 'pancreatic secretin' to distinguish it from possible other members of the same class. It is produced in the mucous membrane from a precursor-pro-secretin. The latter has not been isolated, but that it is present in the mucous membrane is shown by the fact that secretin can be extracted by the action of acids from mucous membrane which has been killed by heat or by the prolonged action of alcohol.

Secretin itself is not a ferment. In order to prepare it the mucous membrane is ground up with sand, boiled with 0.4 per cent. hydrochloric acid, and then neutralised while boiling by the cautious addition of sodium hydrate. The coagulable proteins are in this way precipitated, and the filtered solution contains the secretin. It is not precipitated by the ordinary alkaline reagents, and diffuses slowly through animal membranes. Though stable in acid solutions, it is very rapidly destroyed in alkaline or neutral 
solutions, especially under the influence of bacteria. It is apparently oxidised with extreme ease. A similar, or more probably the same, body may be produced from intestinal mucous membrane by treating this with solutions of soap.

In this secreting mechanism we have a very striking example of a correlation between the activities of two different portions of the body effected by chemical means. The strongly acid chyme enters the first part of the duodenum. Immediately a certain amount of secretin is produced by the acid in the cells of the mucous membrane. The secretin is carried by the blood stream to the cells of the pancreas and excites there the secretion of strongly alkaline pancreatic juice. As soon as sufficient juice has been secreted to neutralise the acid chyme the formation of secretin, and therefore the further secretion of pancreatic juice, comes to an end. If the stomach still contains food the process is, however, renewed in virtue of the local reflex mechanism which we have just studied regulating the opening and closure of the pylorus. So long as the contents of the duodenum are acid the pylorus remains firmly closed. As soon as these are neutralised the pylorus relaxes and allows the entrance of a further portion of acid chyme. Thus the formation of secretin proceeds afresh, and the whole chain of processes goes on until the stomach is empty and all its contents have passed into the intestine.

In view of the efficacy of this chemical reflex mechanism, the question arises whether the results first obtained by Pawlow were really due in some way to the formation of secretin. Stimulation of the vagus may cause contraction of the stomach, opening or closing of the pylorus, and it seems possible that under its action there might have been an escape of acid gastric contents into the intestine, and therefore the formation of secretin, which would suffice to arouse the pancreatic secretion. Later experiments by this observer, in which the escape of any gastric contents was effectively prevented by ligature of the pylorus while the stomach itself contained an alkaline solution, have shown that even with these precautions a flow of juice may be obtained on stimulation of the vagus nerve. The flow, however, is very small in comparison with that obtained by injection of secretin, and one must conclude that, although the nervous system may play a small part in the excitation of the activity of this gland, the main factor involved is the chemical mechanism which has just been described.

The amount of pancreatic juice obtained after a meal varies with the nature of the latter. The Table on $p$. 711 represents the results obtained on an animal fed with 600 c.c. of milk, $250 \mathrm{grm}$. of bread, and $100 \mathrm{grm}$. of meat respectively.

The differences between these results seem, however, largely determined by the duration of gastric digestion, and therefore the amount of acid secreted in the stomach and passed on to the duodenum. It was suggested by Walther that apart from this quantitative adaptation there was a qualitative alteration in the constitution of the juice according to the nature of the food ingested, that, e.g., excess of protein causes an increase of the trypsin, 
while excess of carbohydrate would cause an increase in the amylase of the juice. Later researches have failed to confirm this view. Apparently when the pancreas is excited to secrete, it turns out its various ferments in constant proportion, depending on the amounts of these already present and stored up in the gland.

Secretion of Pancreatic Juice (Walther)

\begin{tabular}{|c|c|c|c|}
\hline Hours after meal & 600 c.c. milk & 250 grm. bread & 100 grm. meat \\
\hline 1 & 8.5 c.c. & 36.5 c.c. & 38.75 c.c. \\
2 & 7.6 c.c. & $50 \cdot 2$ c.c. & 44.6 c.c. \\
3 & 14.6 c.c. & 20.9 c.c. & $30 \cdot 4$ c.c. \\
4 & 11.2 c.c. & 14.1 c.c. & 16.9 c.c. \\
5 & 3.2 c.c. & 16.4 c.c. & 0.8 c.c. \\
6 & 1.0 c.c. & 12.7 c.c. & - \\
7 & - & 10.7 c.c. & - \\
\hline
\end{tabular}

\section{THE STRUCTURAL CHANGES IN THE PANCREAS WHICH ACCOMPANY SECRETION}

The ease with which secretin may be prepared and used to arouse the activity of the pancreas has rendered it possible to study more closely the changes which in this gland accompany activity. Kühne and Sheridan Lea succeeded in observing the gland of the rabbit in a living state under the
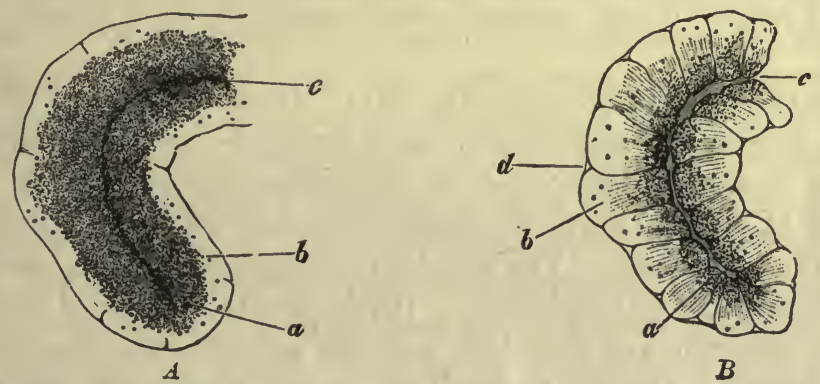

Frg. 340. A terminal lobule of the pancreas of the rabbit. (KÜHNE and SHERIDAN LEA.)

$\Delta$, in resting condition; $\mathbf{B}$, after active secretion.

microscope. They noted that activity, excited by pilocarpine, was associated with a discharge of granules, a clearing up of the cells, and a diminution in size and the appearance of a lumen to the gland alveoli (Fig. 340). A normal resting gland is of an opaque, yellowish-white colour, and of firm consistence. On section it is seen to consist of numerous secreting alveoli which open into narrow intercalary tubules, and these in their turn into wide collecting tubules. The lining epithelium of the intercalated tubules is often continued into the secreting part, where they lie internal to the secreting cells, as the so-called centro-acinar cells. The secreting cells themselves present 
two well-marked zones, a narrow peripheral zone in which the nucleus is embedded, which is strongly basophile, and a central part which is turned towards the lumen, occupying two-thirds or three-quarters of the cell, and is closely packed with highly refractive granules strongly acidophile and presumably containing or composed of the precursors of the various con-

A

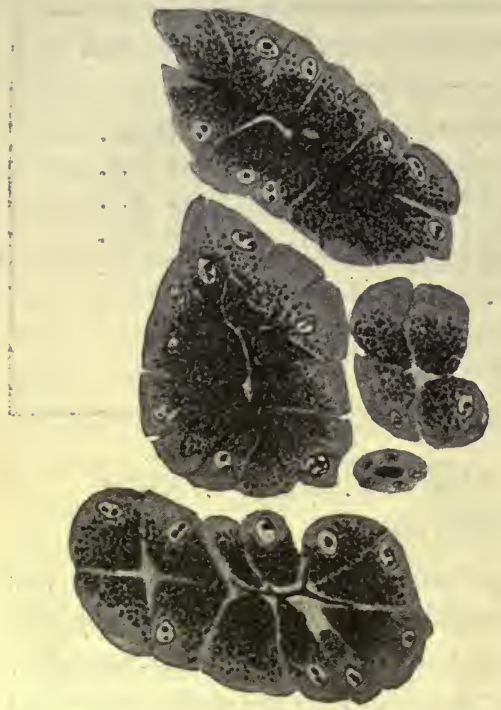

B

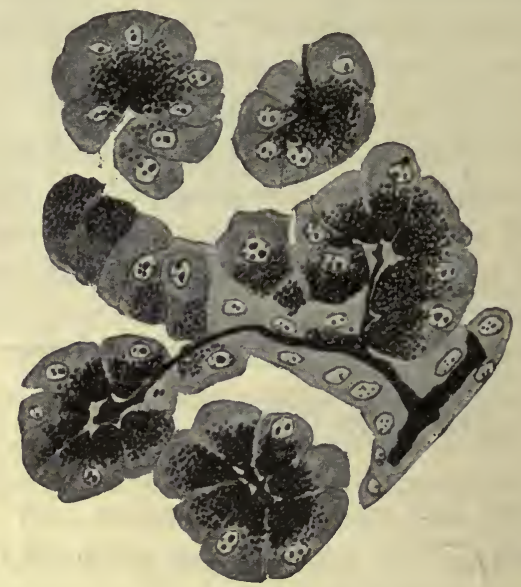

Fig. 341. Alveoli of dog's pancreas. (Babkin, Rubaschkin and Sawitsch.) A, resting; B, after moderate secretion with discharge of granules.

stituents of the pancreatic juice (Fig. 341). If the activity of the gland be aroused by injection of secretin and the injection be continued until the rate of secretion evoked by each injection diminishes considerably, i.e. the gland shows signs of fatigue, marked changes are observed both macroscopically and under the microscope. The gland is now pink and transparent in appearance, moist and soft in consistence. On section the lumen of each alveolus is enlarged, the cells are shrunken, and the granules are found to lie only along the border of the cell turned towards the lumen, the rest of the cell, which is much reduced in size, being made up of the basophile protoplasm. Similar effects are observed after long continued stimulation of the vagus (Fig. $341 \mathrm{~B})$. 


\section{SECTION VI}

\section{THE LIVER AND BILE}

THE liver, the largest gland in the body, is, like the other glands associated with the alimentary tract, formed in the embryo by an outgrowth of the hypoblast lining the alimentary canal. At first it resembles in structure other secreting glands, such as the pancreas, being composed of branch tubules which pour their secretion into a common duct. In the adult, however, the relation of the liver cells to the ducts is entirely subordinated to their relation to the blood-vessels of the liver, and it requires special histological methods to make out the relations between the liver cells and the bile-ducts. The liver, on section, is seen to be divided off into lobules composed of columns of polygonal cells, radiating from the centre like the spokes of a cart-wheel. The portal vein, which drains the blood from the alimentary canal, breaks up into branches which lie at the periphery of the lobules, forming the interlobular veins, and send off numberless capillaries which pass inwards between the columns of cells to join the intralobular vein lying at the centre of the lobule. From the intralobular the blood passes by the large sublobular vein into the hepatic veins and inferior vena cava. In an injected specimen it is easy to see that every liver cell is connected with at least one blood capillary, and the liver thus forms a blood gland, lying as it does at the gate of entrance of blood from the alimentary canal into the general circulation. The portal vein conveys only venous blood to the liver. In order to supply oxygen to the working liver cells, this organ receives a second supply of arterial blood by the hepatic artery derived from the coliac branch of the aorta. The branch of the hepatic artery runs with the branches of the portal vein in the connective tissue of Glisson's capsule surrounding the lobules, and breaks up into capillaries which are in free communication with the capillaries derived from the portal system and pour their blood finally into the hepatic vein.

As might be expected from its structure, the secretory functions of the liver represent but a small proportion of its activities in the body. The liver is, in fact, the greatest chemical factory of the body, receiving by the portal vein the products of digestion as they are absorbed from the alimentary canal. It converts these into other substances, breaking them down or building them up according to the needs of the body as a whole. Thus, when carbohydrates are being absorbed in quantity it converts the glucose contained in the portal blood into glycogen which it stores up, reconverting 
the latter into glucose and letting it loose into the circulation when this substance is required by the body tissues. In the complete absence of carbohydrate from the food the liver may, as we shall see later, actually convert the products of protein digestion into sugar. In the same way the liver plays an important part in the metabolism of proteins and of fats, so that its functions will have to be dealt with in the various chapters concerned with the fate of the different food-stuffs and different constituents of the animal body. In this chapter we are merely concerned with its action as a secreting gland. The fact that its secretion is in so many animals poured into the intestine by an orifice common to it and the pancreatic juice suggests that these two fluids cooperate in their actions on the ingested food-stuffs, and points to a direct use of the bile in the processes of digestion. In addition to this function, the bile must also be regarded as an excretion, representing as it does the channel by which the products of disintegration of hæmoglobin-the red colouring-matter of the blood-are got rid of from the organism. As an excretion the production of bile must be continuous, and related, not to the processes of digestion, but to the intensity of destruction of the red corpuscles. On the other hand, bile, as a digestive fluid, is needed in the gut only during the period that digestion is going on. The exigencies of the body therefore require a continuous excretion of bile by the liver, but a discontinuous entry of this fluid into the small intestine. This discontinuity in the entry of a continuous secretion into the intestine is secured, in the majority of animals, by the existence of the gall-bladder, a diverticulum from the bile-ducts, in which all bile, secreted during the intervals between the periods of digestive activity, is stored up. In the horse, where the gall-bladder is absent, its place is taken to some extent by the great size of the bile ducts. Moreover in such an animal the process of digestion is much more continuous in character than it is in carnivora. Since the bile accumulates in the gallbladder during the whole time that digestion is not going on, and is only poured into the gut during digestion, in a fasting animal the gall-bladder is distended, whereas in an animal some hours after a meal the gall-bladder is practically empty.

During the period that the bile remains in the gall-bladder it undergoes certain changes, as is shown by comparison of the composition of bile obtained from the gall-bladder with that obtained from a fistula of the bile-duct.

\section{Analyses of Bile (Human)}

From a biliary fistula (Yeo and Herroun) in 100 parts

Mucin and pigments

Sodium taurocholate

Sodium glycocholate

Cholesterin .

Lecithin

Fats

Inorganic salts

Water

$0 \cdot 148$

$0 \cdot 055$

$0 \cdot 165$

$0 \cdot 840$

$98 \cdot 7$
From the gall-bladder (Hoppe-Seyler) in 100 parts

Mucin

$1 \cdot 29$

$\{0 \cdot 038$
$0 \cdot 87$

$3 \cdot 03$

$1 \cdot 39$

0.35

$\begin{array}{lllllll}\text { Lecithin } & . & . & . & . & . & 0.53 \\ \text { Fats } & . & . & . & . & . & 0.73\end{array}$ 
During its stay in the bladder the bile is concentrated by the loss of water and by the addition to it of mucin or nucleo-albumen, derived from the cells lining the bladder. Of the other constituents of bile, the pigments must be regarded simply as waste products, and an index to the disintegration of hæmoglobin. Their mode of origin will be discussed in dealing with the history of the red blood corpuscles. They pass into the intestine and are there converted by the processes of bacterial reduction into stercobilin, which is excreted for the most part with the fæces, a small proportion being absorbed into the blood-vessels and turned out in a more or less altered condition as the pigments of the urine. From the point of view of digestion, the important constituents of bile are the bile salts, with the lecithin and cholesterin held in solution by these salts. The time-relations of the secretion, as well as of the flow of bile into the intestine in connection with the processes of digestion, can be learnt from animals in which the bile is conducted to the outside of the body by means of a permanent fistula.

For this purpose Pawlow has devised the following operation: In the dog the abdomen is opened, and the common bile-duct sought as it passes through the intestinal wall. The orifice of the duct, with a piece of the surrounding mucous membrane is cut out of the wall of the intestine, and the aperture thus made sutured. The excised portion of mucous membrane, with the opening of the duct, is then sewn on to the surface of the duodenum, and the loop of duodenum at this point is stitched into the abdominal wound. After healing, the natural orifice of the bile-duct is thus made to open on the surface of the abdomen.

In an animal treated in this way the flow of bile from the fistula is found to run parallel to the pancreatic secretion. Although smaller in amount, it rises and falls with the latter. Thus a meal of meat produces a large flow of bile, a meal of carbohydrates only a small flow. Moreover, beginning almost immediately after taking food, it attains its maximum with the pancreatic juice in the third hour and then rapidly declines.

In the production of this flow of bile two factors may be involved : (1) the emptying of the gall-bladder; (2) an increased secretion of the bile. In order to determine the relative importance to be ascribed to each factor, we must compare the results obtained on an animal possessing a Pawlow fistula with those obtained on an animal provided with a fistulous opening into the gall-bladder, the common bile-duct in the latter having been ligated to ensure that the total secretion of bile passed out by the fistula. In such animals we find, as we should expect, that the secretion of bile is a continuous process, but that, synchronously with the great outpouring of bile into the intestine during the third hour after a meal, there is an increased secretion of this fluid. The passage therefore of the semi-digested food from the stomach into the duodenum causes not only a slow contraction and emptying of the gall-bladder but also an increased secretion of bile by the liver. What is the mechanism involved in the production of these two effects? The muscular wall of the gall-bladder, as has been shown by Dale, is under the control of nerves derived both from the vagus and from the sympathetic, the former conveying motor and the latter inhibitory impulses. It is usual to suppose that the entry of acid chyme into the duodenum provokes reflexly 
the contraction of the gall-bladder, but the exact.paths and steps in this reflex act have not yet been fully determined. The increased secretion of bile, which is produced by the passage of the acid chyme through the pylorus, can be also evoked by the introduction of acid, such as 0.4 per cent. $\mathrm{HCl}$, into the duodenum, and occurs even after division of all connection between the liver and the central nervous system. Since the presence of bile is necessary for the full development of the activities of the pancreatic juice, and its secretion is initiated by the same sort of stimulus, i.e. acid applied to the mucous membrane of the gut, the question naturally arises whether the mechanism for the secretion of bile may not be identical with that for the secretion of pancreatic juice. In order to decide this point we must make a temporary biliary fistula by inserting a cannula into the hepatic duct. A solution of secretin is then prepared from an animal's intestine. In making this solution we must ke careful to avoid any contamination by bile salts, which may possibly be adherent to the mucous membrane of the gut and would in themselves, on injection, evoke an increased secretion of bile. It is therefore better to extract the pounded mucous membrane with boiling absolute alcohol, until this fluid, evaporated into a small bulk, shows no trace of bile salts. The dried and powdered gut is then boiled with dilute acid. On injecting the solution of secretin so obtained into the animal's veins, an increased flow of bile is at once produced. In one experiment, for instance, the injection into the veins of 5 c.c. of a solution of secretin, prepared in this way, increased the secretion of bile by the liver from twentyseven drops in fifteen minutes to fifty-four drops in fifteen minutes. The rate of secretion was therefore doubled. We may conclude that the mechanism, by which the increased secretion of bile is produced at the time when this fluid is required in the intestine, is identical with that for the secretion of pancreatic juice, and that in each case one and the same substance-secretin -is formed by the action of the acid on the cells of the mucous membrane, and, on absorption into the blood stream excites both the liver and pancreas to increased activity.

\section{THE DIGESTIVE FUNCTIONS OF THE BILE}

Bile contains a weak amylolytic ferment. Its uses in digestion are dependent, however, not on the presence of this ferment, but on the peculiar action of the bile salts on the fermentative powers of the pancreatic juice. It was shown long ago by Williams and Martin that the amylolytic power of pancreatic extracts is doubled by the addition of bile or of bile salts. Pawlow has stated that the same holds good of the proteolytic power of this juice. Most important, however, is the part played by the bile in the digestion and absorption of fats. The fat-splitting action of pancreatic juice is trebled by the addition of bile, whether boiled or unboiled. This quickening action of the bile probably depends, like its function in the absorption of fats, on the peculiar physical properties of the bile salts, with those of the lecithin and cholesterin which they hold in solution. Not only does such a solution diminish the surface tension between watery and oily fluids, so 
promoting the closer approach of the lipase of the pancreatic juice to the fats on which it is to act, but it has also the power of dissolving fatty acids and soaps, including even the insoluble calcium and magnesium soaps. It is probable that it aids also in holding in solution, and bringing in contact with the fat, the lipase of the pancreatic juice. It has been shown by Nicloux that the lipase contained in oily seeds, such as those of the castor plant, is insoluble in water, but soluble in fatty media. The dried ferment obtained from the pancreas in many cases yields no lipase to water, but gives a strongly lipolytic solution when extracted with glycerin. The digestive function of bile therefore lies in its power of serving as a vehicle for the suspension and solution of the interacting fats, fatty acids, and fatsplitting ferment. This vehicular function plays an important part in the absorption of fats. These pass through the striated basilar membrane bounding the intestinal side of the epithelium, not, as was formerly thought, in a fine state of suspension (an emulsion), but dissolved in the bile in the form of fatty acids or soaps and glycerin. On the arrival of these products of digestion in the epithelial cells, a process of resynthesis is set up. Droplets of neutral fat make their appearance in the cells, whence they are passed gradually into the central lacteal of the villus and so into the lymphatics of the mesentery and into the thoracic duct. The bile salts thus released from their function as carriers are absorbed by the blood circulating through the capillaries of the villi, and carried by the portal vein to the liver. On arrival they are once more taken up by the liver-cells and turned out into the bile. Owing to the fact of their ready excretion by the liver-cells, bile salts are the most reliable cholagoguies with which we are acquainted. By this circulation of bile between liver and intestine the synthetic work of the liver in the production of the bile salts is reduced to a minimum, and it has only to replace such of the bile salts as undergo destruction in the alimentary canal, under the influence of microorganisms, and are lost to the organism by passing out in the fæces as a gummy amorphous substance known as dyslysin. Further investigation is still wanted as to the exact method in which secretin acts on the liver-cells, and especially as to whether it actually excites in them the manufacture of fresh bile salts, or whether it simply hastens the excretion of such bile salts as have been formed by the spontaneous activity of the liver-cells or have arrived at them after absorption from the alimentary canal. Such questions can only be dẹcided by studying the action of secretin on animals possessing a permanent kiliary fistula.

The effect of various diets on the secretion of bile has been studied by Barbera. He finds that, whereas the secretion of bile is greatest on a meat diet, it is somewhat less on a diet of fat, and is insignificant on a purely carbohydrate diet. That is to say, the secretion of bile is greatest on those diets the digestion of which is attended by the passage of a large amount of acid chyme or of oil into the duodenum. Oil is almost as efficacious as acid in promoting the production of secretin in the living duodenum, the production in this case being probably determined by the formation of soap from the oil, and the direct action of the soap on the prosecretin in the epithelial cells of the gut. 


\section{SECTION VII}

\section{THE INTESTINAL JUICE}

For the development of one of its most important properties, namely, that of proteolysis, the pancreatic juice is dependent on the co-operation of the intestinal juice or succus entericus. Besides this activating power on the pancreatic juice, the intestinal juice has numerous other functions to discharge in the digestion of the food-stuffs. In spite of the great similarity which obtains between the microscopic structure of the wall of the gut at different levels from duodenum to ileocolic valve, functionally there are many differences between the upper, middle, and lower portions of the gut. Speaking generally, we may say that, whereas the processes of secretion are better marked in the upper portions of the gut, the processes of absorption predominate in the lower sections, i.e. in the ileum. Much of the divergence in the statements which have been made with regard to the factors determining secretion and absorption in the small intestine is due to the failure to appreciate this great difference between the activity of the mucous membrane at various levels.

The process of secretion in the small intestine may be studied by isolating loops by means of ligatures, and determining the amount of secretion formed in these loops in the course of a few hours' experiment on an anæsthetised animal. Better results, however, may be obtained by establishing permanent fistulæ. These fistulæ are of two kinds. Thiry's original method of establishing a fistula consisted in cutting out a loop of intestine, and restoring the continuity of the gut by suturing the two ends from which the loop had been severed. The upper end of the loop itself is closed and the lower end is sutured into the abdominal wound. For some purposes it is better to make a Thiry-Vella fistula. In this case the continuity of the gut is restored as in the simple Thiry fistula, but both ends of the excised loop are left open and brought into the abdominal wound. In such a fistula it is easy to introduce substances into the upper end and determine the flow of juice from the lower end, the constant emptying of the loop being provided for by the peristaltic contractions of its muscular coat.

In animals with intestinal fistulæ a number of different conditions have been found to give rise to a flow of succus entericus, and so far no qualitative difference has been recorded between the upper and lower ends of the gut. A condition which will cause a free flow of juice from a fistula high up in the intestine will generally cause a scanty flow from a fistula made from the ileum. In all cases it is found that a flow of juice is produced in consequence of a meal. If a dog with a fistula, which has been starved for twenty-four hours, be, given a meal of meat, a flow of juice may begin within the next 
ten minutes. The flow remains very slight for about two hours and then suddenly increases in amount during the third hour, corresponding thus very nearly to the flow of pancreatic juice excited by the same means. In this post-prandial secretion of juice it is not probable that the nervous system takes any very large share, though its intervention in the secretion has not been excluded by direct experiment.

There are certain facts which seem indeed to speak for an action of the central nervous system on the process of intestinal secretion, not in the direction of augmentation, but in the direction of inhibition of secretion. Thus it has been observed, on many occasions, that extirpation of the nerve plexuses of the abdomen or section of the splanchnic nerves causes a condition of diarrhœa which may last for two or three days. This condition might be determined either by an increased motor activity of the gut, or by removal of inhibitory impulses normally arriving at the intestinal glands, Such a view receives support from an experiment first performed by Moreau. The abdomen of a dog is opened under an anæsthetic, and three contiguous loops of small intestine are separated by means of ligatures from the rest of the gut. The middle loop is then denervated by destruction of all the nerve fibres lying on its blood-vessels, as they course through the mesentery, care being taken not to injure the blood-vessels themselves. The loops are then replaced in the abdomen and the animal left from four to sixteen hours. On killing the animal at the end of this time, it is often found that the middle loop, i.e. the denervated loop, is distended with fluid having all the properties of ordinary intestinal juice, whereas the other two loops.are empty. A series of comparative experiments by Mendel and by Falloise have shown that the secretion begins about four hours after the operation, increases for about twelve hours, and then rapidly declines, so that at the end of two days all three loops will be found empty. This has often been interpreted as due to the removal of inhibitory impulses passing from the central nervous system to the local secretory mechanism, and we have no direct evidence which can be adduced against such a view. It is possible, however, that the hyperæmia of the gut, which is produced by the process of denervation, may be sufficient to account for the increased formation of intestinal juice, since the hyperæmia will tend to pass off as the vessels recover a local tone.

It is not possible to explain the flow of intestinal juice which follows a meal by any assumption of nervous impulses transmitted through the local nerve plexuses of the gut, since these have been divided in the making of the fistula. It we exclude a nervous reflex action by the long paths, namely, through the spinal cord and the sympathetic or vagus nerves, the flow which attends the passage of food into the first part of the duodenum must be excited by the formation of some chemical messenger. As to the existence of such a chemical messenger or hormone for the intestinal secretion there can be no doubt, but the evidence as to its precise nature is at present conflicting. It is stated by Pawlow that the most effective stimulus to the flow of succus entericus is the presence of pancreatic juice in the loop of gut. No evidence has yet been brought forward that injection of pancreatic juice into the blood stream will cause any flow of intestinal juice. On the other hand, Delezenne and Frouin have shown that in animals provided with a permanent fistula involving the duodenum or upper part of the jejunum, intravenous injection of secretin always causes a secretion of intestinal juice. In the upper part of the gut therefore the simultaneous presence of the three juices necessary for complete duodenal digestion is 
ensured by one and the same mechanism, namely, by the simultaneous activity of the secretin, produced in the intestinal cells by the action of the acid chyme, on pancreas, liver, and intestinal glands. A further chemical mechanism for the arousing of intestinal secretion has been described by Frouin. According to this observer, the flow of juice can be excited by intravenous injection of intestinal juice itself. Since this juice is alkaline, and does not produce any effect on the pancreas, it must be free from pancreatic secretin. It would seem therefore that the flow of juice in the upper part of the gut, excited by the pancreatic secretin, causes also a production of a different secretin or hormone, which can be absorbed from the lumen of the gut, travel by the blood stream to other segments of the small intestine, and there excite a secretion in preparation for the on-coming food. Further experiments are, however, necessary on this point.

The glands of the small intestine can also be excited by direct mechanical stimulation of the mucous membrane. The easiest method of exciting a flow of intestinal juice from a permanent fistula is to introduce into the intestine a rubber tube. The presence of the solid object in the gut causes a secretion, and within a few minutes drops of juice can be obtained from the free end of the tube. The object of such a sensibility to mechanical stimuli is obvious; it is of the highest importance that the onward passage of any solid object, especially if it be indigestible, shall be aided by the further secretion of juice in the portions of gut which are immediately stimulated. This mechanical stimulation probably acts on the tubular glands of the intestine through the intermediation of the local nervous system, the plexus of Meissner. It is stated by Pawlow that a juice obtained by mechanical stimulation differs from that produced by the introduction of pancreatic juice into the loop in containing little or no enterokinase, so that the pancreatic juice excites the secretion of the substance which is necessary for its own activation.

\section{CHARACTERS OF INTESTINAL JUICE}

The intestinal juice obtained from a permanent fistula has a specific gravity of about 1010. It is generally turbid from the presence in it of migrated leucocytes and disintegrated epithelial cells. It contains about 1.5 per cent. total solids, of which 0.8 per cent. are inorganic and consist chiefly of sodium carbonate and sodium chloride. It is markedly alkaline in reaction, but less so than the pancreatic juice. The organic matter, besides a small amount of serum albumin and serum globulin, includes a number of ferments, all of which are adapted to complete the processes of digestion of the food-stuffs commenced in the stomach and duodenum. Of these ferments two are concerned in proteolysis. Enterokinase we have already studied in detail. Possessing no action itself on proteins, it is a necessary condition for the development of the full proteolytic powers of the pancreatic juice. In addition to this ferment another ferment has been described by Cohnheim under the name 'erepsin.' Erepsin or some similar ferment is present in the fresh pancreatic juice and in almost all tissues of the body. It 
is distinguished by the fact that, although it has no power of digesting coagulated protein or gelatin, and only slowly dissolves caseinogen and fibrin, it has a rapid hydrolytic effect on the first products of proteolysis, converting albumoses and peptones into amino- and diamino-acids-their ultimate cleavage products.

The other ferments of the intestinal juice are connected with the digestion of carbohydrates. In all mammals the intestinal juice is found to contain invertase, which transforms cane sugar into glucose and levulose nr fructose, and maltase, which converts maltose into glucose. In.young mammals, as well as in those in whom the milk diet is continued throughout life, the intestinal mucous membrane also contains lactase, i.e. a ferment converting milk sugar into galactose and glucose. Such a ferment can be extracted from the mucous membrane of all young animals, but may be very slight or even absent in the intestines of older animals, when it is no longer needed for the ordinary processes of nutrition. By means of these three ferments, coming as they do after the digestion of the starches by the amylase of the saliva and pancreatic juice, it is provided that all the carbohydrate food of the animal is transformed into a hexose, in which form alone carbohydrate can be taken up and assimilated by the cells of the body. The seat of origin of these various ferments has been the subject of special investigations by Falloise. Whereas secretin can be obtained from the whole thickness of the mucous membrane, and is probably therefore contained in the form of prosecretin in the epithelial cells covering the villi as well as in those lining the follicles of Lieberkuhn, a superficial scraping of the mucous membrane, which removes only the epithelial cells covering the villi with the adherent mucus and intestinal secretion, gives a much more active solution of enterokinaise than a deeper scraping. The most active solutions of enterokinase are, however, to be obtained from the fluid found in the cavity of the intestine after the injection of secretin. It seems therefore that enterokinase is not present as such in the epithelial cells, but is first produced in the process of secretion and formation of the intestinal juice. The other ferments, namely, erepsin, maltase, invertase, and lactase, probably pre-exist as such in the epithelial cells, especially in those lining the tubular glands of the gut, since pounded mucous membrane in water yields a solution of these ferments which is generally more powerful in its action than the succus entericus itself. So great is the difference, in fact, that many physiologists have suggested that the chief action of these ferments occurs, not in the lumen of the gut, but in the passage of the food-stuffs through the epithelial cells of the small intestine on their way to the blood-vessels. 


\section{SECTION VIII}

\section{FUNCTIONS OF THE LARGE INTESTINE}

GREAT differences are found in the structure of the large intestine of different animals, differences which depend, not on the zoological position of the animal, but entirely on the nature of its food. In the carnivora the large intestine is short and narrow and possesses little or no cæcum. In herbivora the large intestine is well developed with sacculated walls, and the cæcum, i.e. that part of the large gut distal to the opening of the ileum into the colon, is very large. Man occupies a somewhat intermediate position between these two classes. The differences between the total length of the alimentary canal in various animals are largely determined by the varying development of the large intestine. The relation of these differences to the diet is seen if we compare the length of the intestine with the length of the animal. Thus in the cat the intestine is three times the length of the animal, in the dog from four to six times, in man from seven to eight times, in the pig fourteen times, and in the sheep twenty-seven times. - The great development of the large intestine in vegetable feeders is due to the fact that in this class of food all the nutritious material is enclosed in cells surrounded by cellulose walls. In order that the food-stuffs- e.g. proteins, starch, \&c.may be dissolved by the digestive juices and absorbed by the wall of the gut, these cellulose walls must be disintegrated. In none of the higher vertebrates do we find any cellulose digesting ferment, cytase, produced in the alimentary canal. The cellulose has therefore to be dissolved either by the agency of bacteria or by means of cellulose-dissolving ferments which may be present in the vegetable cells themselves. Thus in ruminants the masses of grass and hay are first received into the paunch, where they are kept warm and moist with saliva. In the paunch opportunity is thus given for the development of huge numbers of micro-organisms which can dissolve cellulose. From time to time portions of the sodden mass are returned to the mouth, chewed and then swallowed again to be subjected to the action of the proper digestive juices. In the horse and rabbit the chief part of the digestion of the cellulose occurs in the cæcum. Even after abstinence from food for some time the cæcum is still found to contain food material. In the cæcum, under the action of bacteria, the cellulose is dissolved and the cells are opened up so as to allow their contents to escape. The products of digestion of cellulose include a number of organic acids, chiefly of the lower fatty acid series, as well as methane, carbon dioxide, and hydrogen. In the paunch the acids accumulate so that fermentation occurs in an acid medium, 
whereas in the cæcum the acids are neutralised by the secretion of alkalies and the reaction remains practically. neutral. The products of digestion of cellulose, as well as the contents of the vegetable cells set free by the solution of the cell walls, are gradually absorbed by the walls of the large gut. In carnivora the large intestine has very unimportant functions to discharge in digestion and absorption. The proteins of meat are practically entirely absorbed by the time that the food has arrived at the ileocolic valve, and the same applies to fat. In man the importance of the large intestine will vary with the nature of his food. Under the conditions of civilised life the food material is almost entirely absorbed by the time that it reaches the lower end of the ileum. If, however, a large quantity of vegetable food be taken, such as fruit or green vegetables, or cereals roughly prepared and coarsely ground, a considerable amount of material may reach the large intestine unabsorbed. A certain proportion of this may undergo absorption in the large intestine, while the rest will pass out with the fæces, increasing their bulk.

It is hardly possible to speak of a secretion by the mucous membrane of the large intestine. In herbivora alkaline carbonates are secreted to neutralise the acids produced in the bacterial fermentation of the food, but the processes of absorption and secretion keeping pace, there is no accumulation of the products of secretion in the intestine. A section of the mucous membrane shows a number of simple tubular glands. The greater number of the cells lining these glands are typical 'goblet' cells and contain plugs of mucin. The secretion of mucus not only aids the passage of the fæces along the gut, but probably impedes the propagation of the bacteria which are present in countless numbers in the fæces. This may account for the fact that although bacteria are so numerous in the fæces, it is difficult to cultivate any large numbers most of them being dead.

As an absorbing organ the large intestine of man is of little importance. From observations on fistulæ in man it has been calculated that about 500 c.c. of water pass the ileocolic valve in the twenty-four hours. Of these about 400 c.c. undergo absorption in the large intestine. The absorption of any of the food substances by this part of the gut is much slower than that which takes place on introduction by the mouth. Feeding by nutrient enemata is thus always very inadequate. In some cases after the introduction of large enemata into the large intestine a certain amount may escape backwards into the ileum and may there undergo absorption. The isolated large intestine of man is able to absorb only about six grammes of dextrose per hour and about 80 c.c. of water. If egg albumin or caseinogen solutions be introduced by the rectum no absorption can be detected after several hours. In observations extending over a considerable time some disappearance has been observed of proteins and emulsified fats, as well as of boiled starch. This was due, however, to the action of bacteria on these substances, and was probably of very little value for the nourishment of the individual. Feeding by nutrient enemata is thus merely a method of slow starvation. If it is employed it should be limited to administration of water, salines, or solutions of glucose. 
The chief value of the large intestine in carnivora and in civilised man would seem to be as an excretory organ, since it plays an important part in the excretion of lime, magnesium, iron, and phosphates. Lime salts are excreted partly with the fæces, partly in the urine. The path taken by the lime under different conditions varies with the character of the other constituents of the food. If phosphates are present in large quantities the greater part of the lime will be excreted by the large intestine and escape with the fæces as insoluble calcium phosphate. If acids be administered, such as hydrochloric acid, the amount of lime in the urine will increase, that in the fæces will diminish. Thus in herbivora normally only about 3 to 6 per cent. of the lime is excreted with the urine, whereas in carnivora with an acid urine the proportion leaving the body by this channel rises to 27 per cent. The excretion of magnesium is determined by very similar conditions. Its phosphates are somewhat more soluble than those of lime. In man about 50 per cent. of the magnesium leaving the body is contained in the urine, whereas the amount of lime in the fæces is ten to twenty times as much as that contained in the urine. It must be remembered that the whole of this difference is not due to excretion of lime into the gut, since a certain proportion of this substance may be precipitated as an insoluble phosphate or carbonate in the upper part of the small intestine and pass through the gut without undergoing absorption.

The absorption of iron takes place in the duodenum and upper part of the jejunum. Only 1 or 2 milligrammes appear in the urine, all the rest being excreted in the large gut and appearing in the fæces, chiefly as sulphide of iron.

Of the acid radicals phosphates may pass out either with the urine or with the fæces, the exact path taken being determined by the relative amount of calcium and alkaline metals present in the food. If there is an excess of calcium most of the phosphates will leave with the fæces.

The large intestine is the main channel of excretion for certain substances which cannot be regarded as normal constituents of the food, e.g. the heavy metals, such as bismuth and mercury. If bismuth be administered subcutaneously the fæces will be found to contain this substance, and the wall of the large intestine will be stained black from a deposit of sulphide of bismuth. This deposit stops short at the ileocolic valve. The excretion of mercury by the wall of the large intestine may account for the frequent presence of ulceration of this part of the gut in cases of poisoning by mercury. 


\section{SECTION IX}

\section{MOVEMENTS OF THE INTESTINES}

THE movements of the intestines can be investigated either by observation of the exposed gut, or by the shadow method introduced by Cannon, in which the nature of the movements is judged from the shadows of food containing bismuth which are thrown on a sensitive screen by means of the Röntgen rays. These movements have been the subject of experimental investigation for many years, but with varying results. The great discrepancy which obtained between the statements of earlier observers is due to the fact that they failed to exclude the many disturbing impulses which can play on any segment of the gut, either reflexly through the central nervous system, or from other parts of the alimentary canal itself through the local nervous system. In order to observe the normal movements of the gut, it is necessary to exclude the disturbing influences due to reflexes through the central nervous system either by extirpation of the whole of the nerve plexuses in the abdomen, or by division of the splanchnic nerves, or by destruction of the lower part of the spinal cord from about the middle dorsal region. If the abdomen of an animal which has been treated in this way be opened in a bath of warm normal salt solution, so as to exclude the disturbing influence of drying and cooling of the gut, the small intestine will be seen to present two kinds of movements. In the first place, all the coils of gut undergo swaying movements from side to side-the so-called pendular movements. Careful observation of any coil will show that these movements are attended with slight waves of contraction passing rapidly over the surface. If a rubber balloon, filled with air and connected with a tambour, be inserted into any part of the gut, it will reveal the existence of rhythmic contractions of the circular muscle repeated from twelve to thirteen times per minute. By means of a special piece of apparatus (the 'enterograph') it is possible without opening the gut to record the movements of either circular or longitudinal muscular coats ; and it is then found that both coats present rhythmic contractions at the same rate, the two coats at any point contracting synchronously. When the contractions are recorded by means of a balloon, the constriction which accompanies each contraction is seen to be most marked at the middle of the balloon, i.e. at the point of greatest tension, and the amplitude of the contractions is augmented by increasing the tension on the walls of the gut. These movements are unaffected by the direct application of drugs such as nicotine or cocaine, which we might 
expect to paralyse any local nervous structures in the wall of the gut. Bayliss and Starling concluded that these rhythmic contractions were myogenic, ${ }^{*}$ that they were propagated from muscle fibre to muscle fibre, and that they coursed down the gut at the rate of about $5 \mathrm{~cm}$. per second. Since, however, they may apparently arise at any portion of the gut which is subject to any special tension, it is not easy to be certain that a contraction recorded at any point is really propagated from a point two or three inches higher up. These contractions must cause a thorough mixing of the contents of the gut with the digestive fluids. On examining under the Röntgen rays the intestines of a cat which has taken a large meal of bread and milk mixed with bismuth some hours previously, a length of gut may be seen in which the food contents form a continuous column. Suddenly movements occur in this

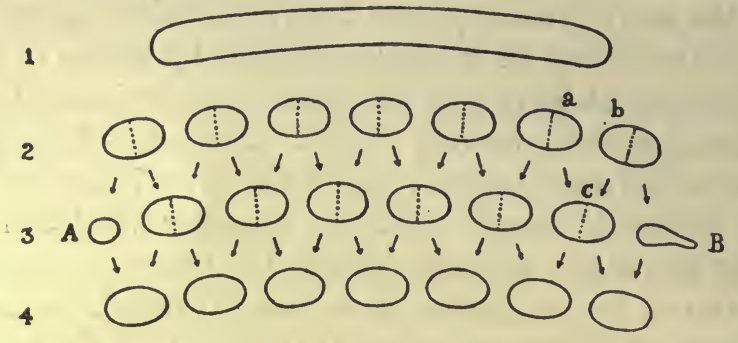

FI\%. 343. Diagram of the 'segmentation' (pendular) movements of the intestines as observed by the Röntgen rays, after administration of bismuth. (CANNON.)

1. A continuous column, intestinal movements being absent. 2. The column broken up into segments. 3. Five seconds later, each segment divided into two, the halves joining the corresponding halves of adjacent segments. 4. Condition (2) repeated five seconds later.

column, which is split into a number of equal segments. Within a few seconds each of these segments is halved, the corresponding halves of adjacent segments uniting. Again contractions recur in the original positions, dividing the newly formed segments of contents and re-forming the segments in the same position as they had at first (Fig. 343). If the contraction is a continuous propagated wave, it is evidently reinforced at regular intervals down the gut, so as to divide the column of food into a number of spherical or oval segments." The points of greatest tension immediately become the points which are midway between the spots where the first contractions were most pronounced. The second contractions therefore start at these points of greatest tension, and divide the first formed segments into two parts, which join with the corresponding halves of the neighbouring segments. In this way every particle of food is brought successively into intimate contact

* Magnus has shown that strips of the longitudinal coat, pulled off from the small intestine of the cat, may continue to beat regularly in oxygenated Ringer's solution. He stated that these contractions only occurred if portions of Auerbach's plexus were still adherent to the muscle fibres, and concluded therefore that the rhythmic, like the peristaltic, contractions, were neurogenic. Gunn and Underhill however have obtained well-marked rhythmic contractions from strips of muscle entirely free from any remains of the nerve plexus, thus confirming the view enunciated above. 
with the intestinal wall. These movements have not a translatory effect, and a column of food may remain at the same level in the gut for a considerable time.

The onward progress of the food is caused by. a true peristaltic contraction, i.e. one which involves contraction of the gut above the food mass and relaxation of the gut below. If a balloon be inserted in the lumen of the exposed gut, it will be found that pinching the gut above the balloon causes an immediate relaxation of the muscular wall in the neighbourhood of the balloon. This inhibitory influence of the local stimulus may extend as much as two feet down the intestine towards the ileocæcal valve. On the other hand, pinching the gut half an inch below the situation of the balloon causes a strong continued contraction to occur at the balloon itself ${ }^{7}$ (Fig. 344 ).

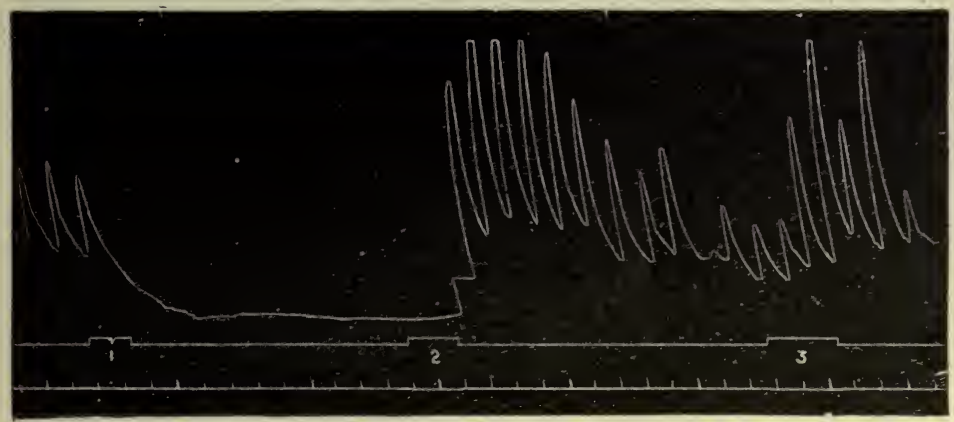

FIG. 344. Intestinal contractions (balloon method). In this dog all the abdominal ganglia had been excised, and both vagi cut. Showing propagated effects of mechanical stimulation, above and below the balloon.

(1) pinch above, (2) pinch below, (3) pinch below balloon.

Stimulation at any portion of the gut causes contraction above the point of stimulus and relaxation below the point of stimulus (the "law of the intestines '). The same effect is produced by introduction of a bolus of food, especially if it be large or have a direct irritating effect on the wall of the gut (Fig. 345). In this case the contraction above and the inhibition below cause an onward movement of the bolus, which travels slowly down the whole length of the gut until it passes through the ileocæcal opening into the large intestine. The peristaltic contraction involves the co-operation of a nervous system. Whereas in the œsophagus it is the central nervous system which is involved, the peristaltic contractions in the small intestine occur after severance of all connection with the brain and spinal cord. On the other hand, they are absolutely abolished by painting the intestine with nicotine or with cocaine. They must therefore be ascribed to the local nervous system contained in Auerbach's plexus, which we can regard as a lowly organised nervous system with practically one reaction, namely, that formulated above as the 'law of the intestines.' An antiperistalsis is never observed in the small intestine. Mall has shown that, if a short length of gut be cut out and reinserted in the opposite direction, a species of partial obstruction results, in consequence of the fact that the peristaltic waves, started above the point of operation, cannot travel down- 
wards over the reversed length of gut. The intestine above this point therefore becomes dilated. If however the reactions of the local nervous system be paralysed or inhibited, a reflux of intestinal contents is quite possible, since the contractions excited at any spot by local stimulation of the muscle have the effect of driving the food either upwards or downwards; the direction of movement of the food will be that of least resistance.

The movements of the small intestine are also subject to the central nervous system. Stimulation of the vagus has the effect of producing an initial inhibition of the whole small intestine, followed by increased irritability and increased contractions (Fig. 346). On the other hand, stimulation of the splanchnic nerves causes complete relaxation of both coats of the small gut (Fig. 347). It seems that the splanchnics normally exercise a tonic

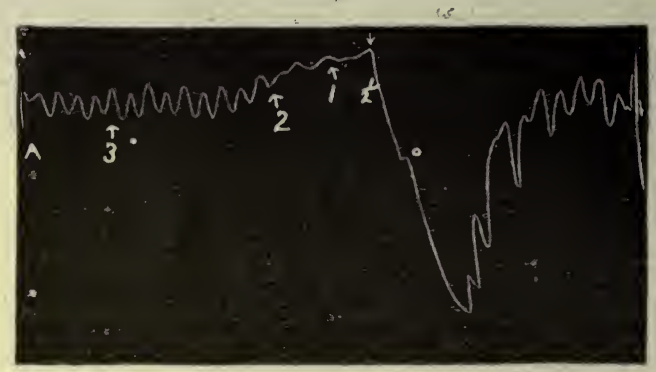

Fig. 345. Passage of bolus. Contractions of longitudinal coat (enterograph). The bolus (of soap and cotton-wool) was inserted into the intestine four inches above the recorded spot at $\mathbf{A}$. The figures below the tracing indicate the distance of the middle of the bolus from the recording levers. As the bolus arrived two inches above the levers there is cessation of the rhythmic contractions and inhibition of the tone of the muscle. This is followed, as the bolus is forced past, by a strong contraction in the rear of the bolus.

inhibitory influence on the intestinal movements, which can be increased by all manner of peripheral stimuli. On this account it is often impossible to obtain any movements in the exposed intestine so long as these remain in connection with the central nervous system through the splanchnic nerves. The relaxed condition of the gut which obtains in many abdominal affections is probably also reflex in origin, and is due to reflex inhibition through the splanchnic nerves.

As a result of the two sets of movements described above, the food is thoroughly mixed with the digestive juices, and the greater part of the products of digestion are brought into contact with the intestinal wall and absorbed. What is left-a proportion varying in different animals according to the nature of the food-is passed on by occasional peristaltic contractions through the lower end of the ileum into the colon, or large intestine. The lowest two centimetres of the ileum present a distinct thickening of the circular muscular coat, forming the ileocolic sphincter. This sphincter relaxes in front of a peristaltic wave and so allows the passage of food into the colon. On the other hand, it contracts as a rule against any regurgitation which might be caused by contractions in the colon. Although thus falling into 
line with the rest of the muscular coat, as concerns its reaction to stimuli arising in the gut above or below, it presents a marked contrast to the rest of the gut in its relation to the central nervous system. It is unaffected by stimulation of the vagus. Stimulation of the splanchnic, however, which

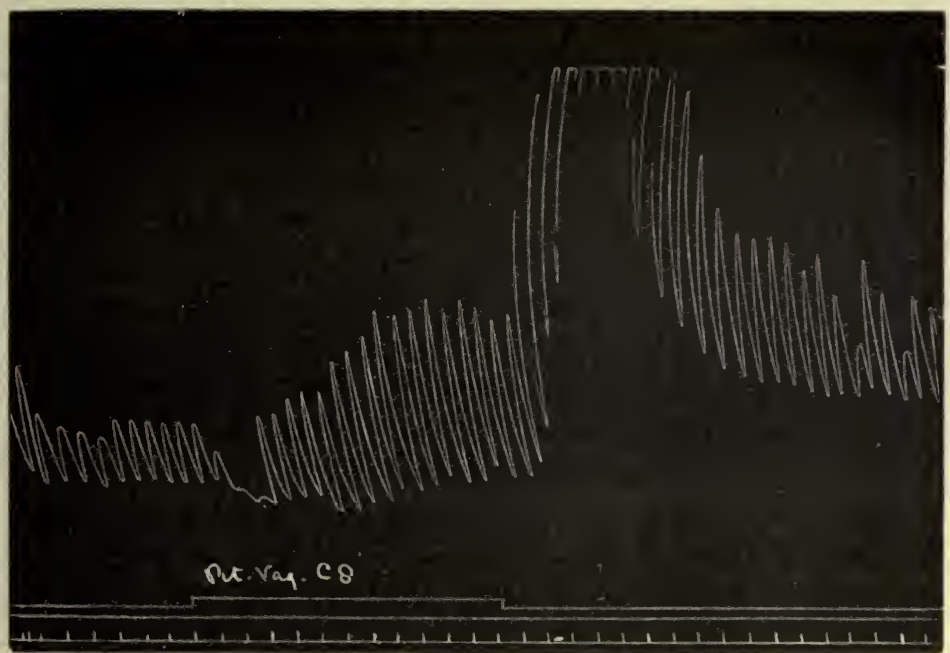

FIG. 346. Effect of stimulation of right vagus on intestinal contractions.

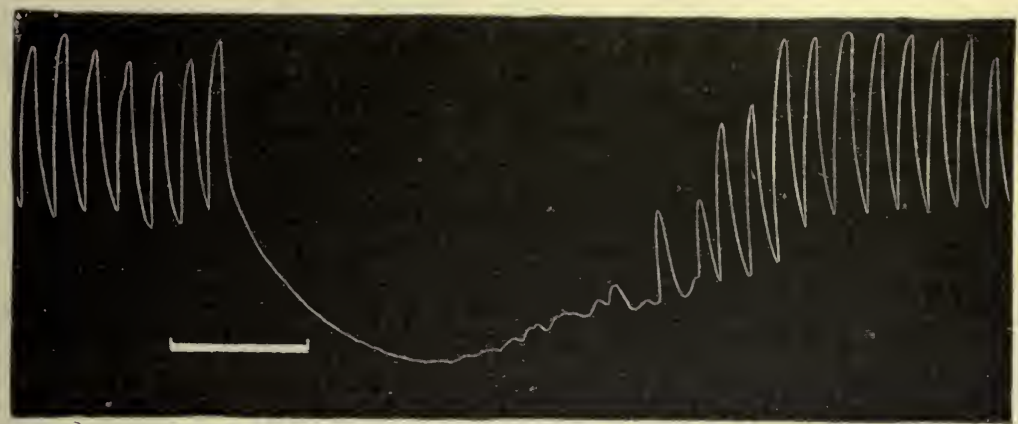

FIG. 347. Excitation of both splanchnic nerves. Balloon method. Intestine returned to abdomen.

causes complete relaxation of the lower part of the ileum with the rest of the small intestine, produces a strong contraction of the muscle-fibres forming the ileocolic sphincter (Elliott).

\section{MOVEMENTS OF THE LARGE INTESTINE}

By means of the occasional peristaltic contractions, accompanied by relaxation of the ileocolic sphincter, the contents of the small intestine are gradually transferred into the large. In man these contents are considerable in bulk, are semi-fluid, and, probably fill the ascending as well as the transverse colon. 
The large intestine is supplied with nerves from the central nervous system. These run partly in the sympathetic system along the colonic and inferior mesenteric nerves, partly in the pelvic visceral nerves or nervi erigentes, which come off from the sacral cord and pass direct to the pelvic viscera. In addition it possesses a local nervous system, presenting the same structure as that found in the small intestine. The movements of the large intestine differ considerably in various animals, as has been shown by Elliott, according to the nature of the food and the part played by this portion of the gut in the processes of absorption. In the dog the process of absorption is almost complete at the ileocolic valve, whereas in the herbivora a very large part of the processes of digestion and absorption occurs in the colon and cæcum. Man takes an intermediate position as regards his large intestine between these two groups of animals. Elliott and Barclay Smith divide the large intestine into four parts, according to their functions, viz. the cæcum, and the proximal, intermediate, and distal portions of the colon. Of these the dog possesses practically only the distal colon. We may take Elliott's account of the movements as they probably occur in man. They agree very closely with those observed by Cannon under normal circumstances in the cat by means of the Röntgen rays. The food as it passes from the ileum first fills up the proximal colon. The effect of this distension is to cause a contraction of the muscular wall at the junction between the ascending and transverse colon. This contraction travels slowly over the tube in a backward direction towards the cæcum, and is quickly succeeded by another, so that the colon may present at the same time several of these advancing waves. These waves are spoken of as anti-peristaltic; but as they do not involve also an advancing wave of inhibition, they must not be regarded as representing the exact antithesis of a peristaltic wave, as we have defined it. The effect of these waves is to force the food up into the cæcum, regurgitation into the ileum being prevented partly by the obliquity of the opening, partly by the tonic contraction of the ileocolic sphincter. As the whole of the contents cannot escape into the cæcum, a certain portion will slip back in the axis of the tube, so that these movements have the same effect as the similar contractions in the pyloric end of the stomach, causing a thorough churning up of the contents and its close contact with the intestinal wall. The movements are rendered still more effective by the sacculation of the walls of this part of the large intestine. The distension of the cæcum caused by this anti-peristalsis excites occasionally-a true co-ordinated peristaltic wave, which, starting in the cæcum, drives the food down the intestine into the transverse part. These waves die away before they reach the end of the colon, and the food is driven back again by waves of antiperistalsis. Occasionally more food escapes through the ileocolic sphincter from the ileum, so that the whole ascending and transverse colon may be filled with the mass undergoing a constant kneading and mixing process. The result of this process is the absorption of the greater part of the water of the intestinal contents, as well as of any nutrient material, and the drier part of the intestinal mass collects towards the splenic flexure, where it may be 
separated by transverse waves of constriction from the more fluid parts which are being driven to and fro in the proximal and intermediate portions. By means of occasional peristaltic waves these hard masses are driven.into the distal part of the colon. The distal colon must be regarded as a place for the storage of the fæces and as the organ of defæcation. In the transverse colon, in the descending and iliac-colon, the anti-peristaltic movements and consequent churning of the contents are probably slight. These therefore represent the intermediate colon, with propulsive peristalsis as its chief activity. The descending colon is never distended, and Elliott therefore

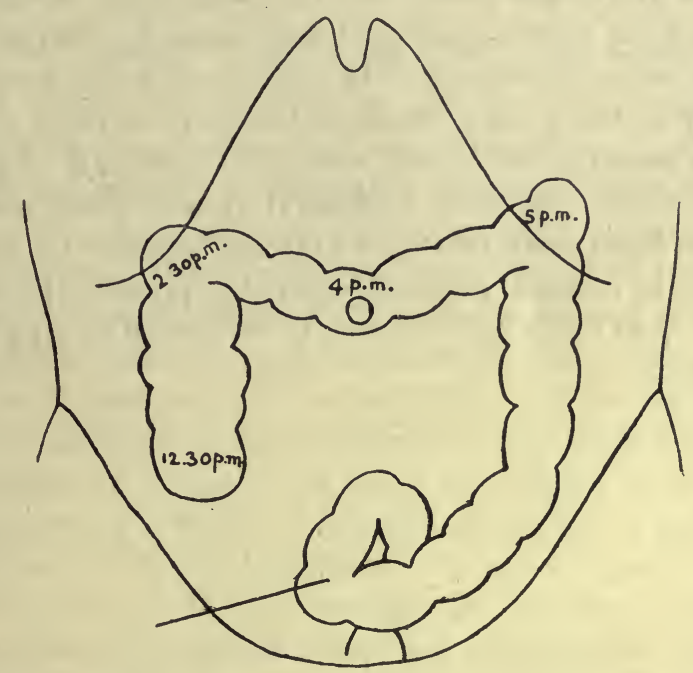

FrG. 348. Skiagram to show normal position of colon in man, and the position attained by its contents at different periods after a meal containing bismuth. The bismuth meal was taken at 8.A.M. The times of arrival at different levels are marked on the colon. (Hertz.)

regarded it as a transferring segment of exaggerated irritability. The storage of the waste matter takes place chiefly in the sigmoid flexure. This with the rectum represents the distal portion of the colon. The distinguishing feature of the distal colon is its complete subordination to the spinal centres. It probably remains inactive until an increasing distension excites reflexly through the pelvic visceral nerves a complete evacuation of this portion of the gut. Stimulation of these nerves in an animal, such as the cat, produces a rapid shortening of the distal part of the colon, due to contraction of the recto-coccygeus and longitudinal fibres of the gut, followed after some seconds by a contraction of the circular coat. This originates at the lower limit of the area of anti-peristalsis, i.e. probably at the upper end of the sigmoid flexure, and, spreading rapidly downwards, empties the whole of this segment of the gut. In-man the emptying of the rectum itself is largely assisted by the contractions of the voluntary muscles of the abdominal walls and pelvic floor.

The last section of the rectum is emptied at the close of the act by a 
forcible contraction of the levator ani and the other perinæal muscles, and this contraction also serves to restore the everted mucous membrane.

The carrying out of this reflex act is dependent on the integrity of a certain part of the lumbar spinal cord. If this ' centre' be destroyed, the tonic contraction of the sphincter muscles disappears. This centre may be either excited to increased action, or be inhibited by peripheral stimulation of various nerves, or by emotion, such as fear. Application of warmth to the region of the anus causes reflex relaxation of the sphincter; application of cold increases its tonic contraction.

In man, as Hertz has shown by the skiagraphic method, the pelvic colon becomes filled with fæces from below upwards, the rectum remaining empty till just before defæcation. In individuals whose bowels are opened regularly every morning after breakfast the entry of fæces into the rectum gives rise to a sensation of fulness and acts as the call to defæcation. If no response be made, the desire to defæcate passes away, since the rectum relaxes and the fæcal mass no longer exercises pressure on its wall. Hertz has shown that the minimal pressure required to produce the call to defæcate varies from 30 to $40 \mathrm{~mm}$. $\mathrm{Hg}$, according to the length of the gut which is the seat of distension. 


\section{SECTION $\mathrm{X}$}

\section{THE ABSORPTION OF THE FOOD-STUFFS}

\section{THE ABSORPTION OF WATER AND SALTS}

THE intake of water, and probably of salts, by the alimentary canal, in accordance with the requirements of the organism as a whole, seems to be regulated almost entirely by the central nervous system, the higher parts of this system, viz. those concerned with appetite, being particularly involved in the process. Thus in man any large loss of fluid to the body, as by sweating, diarrhœa, hæmorrhage, gives rise to an intense thirst that has its natural reaction in increased intake of water by the mouth. On the other hand, the property possessed by the alimentary canal of absorbing water and weak saline fluids contained in its interior is very little influenced by the state of depletion, or otherwise, of the water depôts of the body. It is practically impossible, however large the quantities of fluid ingested, to evoke the production of fluid motions, the greater part of the ingested fluid being absorbed on its way through the alimentary canal. Thus a man may keep himself in perfect health and maintain the water content of his body constant whether he take one litre or six litres of water daily. The whole process of regulation, apart from that determined by appetite, is carried out at the other end of the cycle, viz. by the kidneys. As concerns absorption of water there is no chenical solidarity between the alimentary surface and the rest of the body. Whenever water is presented to the surface it is absorbed and passes into the circulation.

The absorption of water in the stomach may be regarded as nil. Although from this viscus alcohol, and possibly peptone and sugar, may be absorbed to a slight extent, water or saline fluids introduced into it are passed through the pylorus either without change or increased by the secretion of fluid from the gastric glands. In no case is there a diminution of fluid in the stomach.

The chief absorption of water occurs in the small intestine. It is on this account that the salient features of cases of dilatation of the stomach with stenosis, absolute or relative, of the pyloric orifice can be nearly all referred to the starvation of the body in water, and can be often relieved by the administration of water either subcutaneously or by the rectum, i.e. by the channels through which absorption is still possible. The introduction of water into the stomach simply increases the dilatation, but does not relieve the intense thirst of the patient. Water that has been swallowed 
to quench thirst has first to be passed from the stomach into the small intestine before it can be absorbed and relieve the needs of the tissues. The intestinal contents at the ileocæcal valve contain relatively nearly as much water as they do at the upper part of the jejunum. Their absolute bulk is, however, much smaller, so that only a small proportion of the water that has been taken in by the mouth remains to be absorbed in the large gut-an amount probably much less than that which has been added to the contents of the small intestine in the form of secretion by the stomach, liver, pancreas, and intestinal tubules.

The main problem before us is therefore the mechanism of absorption of water and saline fluids by the villi of the small intestine. By means

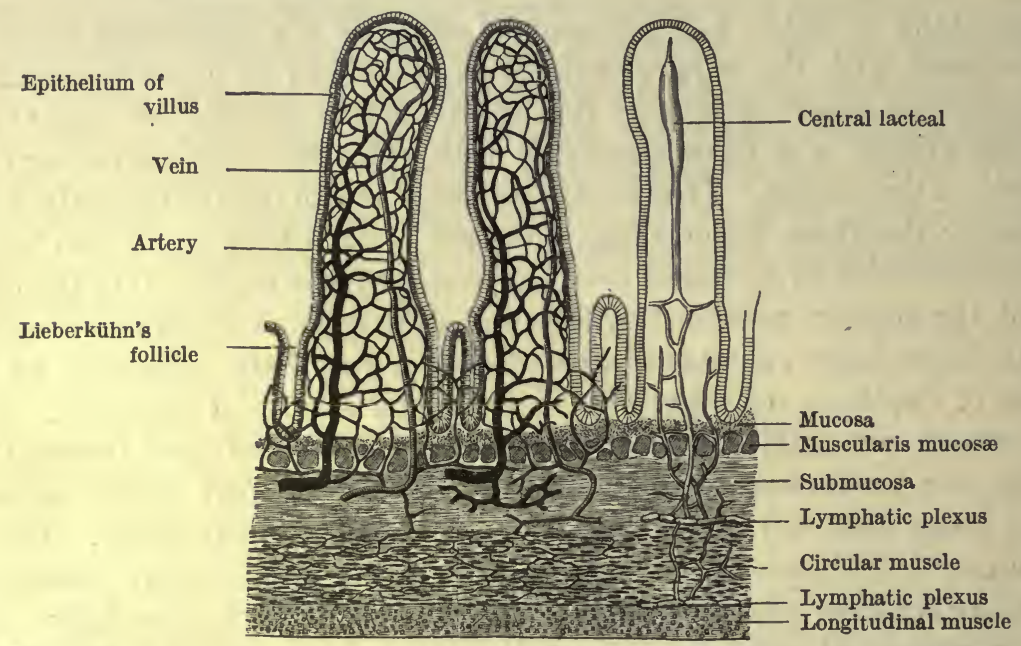

FIG. 349. Diagrammatic section through wall of small intestine to show vascular and lymphatic arrangements of mucous membrane. (After MALL.)

of these structures the absorbing surface of the intestine is largely increased. It has been calculated that each square millimetre of intestine represents an absorbing surface of 3 to $12 \mathrm{~mm}^{2}$ Each villus (Fig. 349) consists of a framework of reticular tissue containing many leucocytes in its meshes, separated from the lumen of the gut by a continuous layer of columnar epithelial cells. These cells rest on an incomplete basement membrane and present on the side turned towards the lumen of the gut a striated basilar border. The villus offers two channels by means of which material, which has passed through the epithelium, may be carried into the general circulation. In the centre of the villus is the central lacteal, a club-shaped vessel bounded by a complete layer of delicate endothelial cells. This leads into a plexus of lymphatics placed superficially to the muscularis mucosæ. From the superficial plexus communicating branches pass vertically to a corresponding plexus lying in the submucosa. The central lacteal and the superficial plexus are free from valves, which, however, are present in abundance in the deeper plexus, so that fluid can pass easily from the lacteal to the 
deeper plexus, but not in the reverse direction. From the muscularis mucosce unstriated muscle fibres pass up through the villus to be attached partly to the outer surface of the central lacteal, partly by expanded extremities to the basement membrane covering the surface of the villus. Contraction of these muscle fibres will tend to empty the central lacteal into the deep plexus of lymphatics and may also cause an expulsion of the contents of the spaces of the retiform tissue of the villus into the central lacteal. The alimentary canal represents one of the few localities where a formation of lymph is constantly proceeding, even in a condition of complete rest. On placing a cannula in the thoracic duct of a dog an outflow of lymph is obtained which may vary in different animals between 1 c.c. and 10 c.c. in the ten minutes. The greater part of this lymph is derived from the alimentary canal, so that any of the intestinal contents which have made their way into the spaces of the villus might be entrained in this lymph current and carried away with it into the thoracic duct and so into the general blood system.

The other possible channel of absorption is by the capillary bloodvessels of the villus. Each villus is supplied with blood from one or two arterioles which break up into a rich plexus of capillaries lying close under the basement membrane of the villus. The return blood is collected into one or two veins, which join the radicles of the portal vein in the submucosa and in the mesentery. In these capillaries the blood is circulating rapidly, so that a considerable amount of material may pass into them from the spaces of the villus within, say, one hour without altering appreciably the percentage composition of the blood. On the other hand, it must be remembered that the blood in these vessels is at a high pressure, probably not less than $30 \mathrm{~mm}$. Hg., so that any absorption into the blood stream must occur against this pressure. It is probable therefore that in explaining any absorption by the blood-vessels we shall have to place out of court any possibility of the passage occurring in consequence of hydrostatic differences of pressure, i.e. by a process of filtration.

When salt solutions are introduced into the small intestine they are rapidly absorbed without the production of any corresponding increase in the rate of lymph flow from the thoracic duct. On the other hand, the absorption of large amounts of fluid may cause an actual diminution of the solids of the plasma, so that we are justified in regarding the capillary network of blood-vessels at the surface of the villi as solely responsible for the absorption.

What are the forces which cause this transference of fluid and dissolved substances from one side to the other of the membrane composed of epithelial cells plus capillary endothelium? Like other cells, those of the intestinal epithelium are probably bounded on their free surface by a 'lipoid' membrane, i.e. one containing some complex of lecithin and cholesterin and permeable only by such substances as are soluble in lipoids. On the other hand, the cement substance between the cells may be of a different character and possibly permeable to water-soluble substances. The question has been 
propounded whether the greater part of the substances which enter the blood plasma from the gut pass between the cells or through the cells. Water could, of course, pass in either way. Most of the inorganic salts, such as sodium chloride, as well as the very important constituents of the food, the sugars, are insoluble in lipoids, and would have to pass between the cells. When the question is investigated by the use of dye-stuffs, soluble or insoluble in lipoids, it is found that the lipoid-soluble dye-stuffs, such as neutral red or toluidin blue, pass into the cells, whereas the dye-stuffs which are insoluble in such substances pass into the intercellular spaces. Too much stress, however, must not be laid on these experiments. All these dye-stuffs are abnormal so far as the body is concerned. We cannot imagine that at any time in the course of evolution of the properties of the intestinal epithelium the cells were ever presented with or had to discriminate between different dye-stuffs. The fact that absorption of these dye-stuffs is determined by the physical conditions of the cell membrane is no proof that the absorption of the normal food constituents is determined in the same way. In fact, it is quite legitimate to assume that the lipoid membrane or limiting layer round every cell has as its main office, not the regulation of the access of food-stuffs to the cell, but its protection from any of the food-stuffs which it does not require for its metabolism. If it were not for such a membrane the assimilation of a salt would be determined entirely by its concentration in the immediate surroundings of the cell, whereas we know that the assimilation of any living organism, whether uni- or multi-cellular, is regulated in the first place by the activity of the organism itself. According to this activity and the needs thereby induced the uptake of food material may be large or small whatever its concentration in the surrounding medium. It would indeed be strange that the whole absorbing surface of the intestine should be covered by a membrane, of which the greater part was useless for the absorption of the common food-stuffs, as would be the case if these could only penetrate the membrane by the narrow chinks between the cells. It seems more probable that the absorption of the different food-stuffs, and probably also of the normal salts of the body, is effected by the cells themselves, in accordance with their nutritional needs, and this view is strengthened when we come to examine into the absorption even of normal saline solutions. If 50 c.c. of normal sodium chloride solution be introduced into a loop of intestine, it is absorbed steadily, so that at the end of an hour not more than about 20 c.c. may be recoverable. The absolute amounts absorbed differ in various experiments, but are fairly uniform for repeated observations on one and the same animal. The absorption of such a solution could be ascribed to the osmotic pressure of the colloids in the blood plasma or lymph within the spaces of the villi. If, instead of using isotonic solutions, hypertonic solutions are employed, e.g. a 2 or 3 per cent. $\mathrm{NaCl}$ solution, absorption still takes place, but may be preceded by an interval in which there is an actual increase of the fluid contained in the gut. Here, again, we might ascribe the absorption to the physical factors present, were it not that absorption is found to commence before the fluid in the gut has attained isotonicity with 
the blood. In fact, employing a 1.5 per cent. salt solution, absorption may occur from the very beginning of the experiment. If such a solution is passed through the epithelial membrane into the blood plasma with a smaller tonicity, it is evident that work must be done in the process, work which can only be furnished by the cells of the epithelium. When sugar solutions are employed they behave in somewhat similar fashion to sodium chloride solutions, provided that the sugar is one of the absorbable hexoses, both sugar and water being rapidly absorbed. It is important to note that dextrose is absorbed from the gut almost as rapidly as sodium chloride, and quite as rapidly as sodium iodide, although its diffusibility is very considerably less than either of these salts. Moreover great differences are found between the rate at which different sugars are absorbed, differences which are not referable to the diffusibility of the sugars in question. Thus the monosaccharides glucose, fructose, galactose are absorbed with double the rapidity of solutions of cane sugar and maltose, and it seems that in the absence of hydrolytic splitting of the disaccharides absorption from the gut would be entirely abolished. Thus lactose disappears from the intestine much more slowly than either of the other two disaccharides, so that large doses may give rise to a laxative effect. In animals devoid of lactase, the lactose-splitting ferment, in their intestinal epithelium milk sugar is apparently not absorbed at all.

The most cogent argument perhaps in favour of an active intervention of the cells of the gut in the process of absorption is furnished by the study of the absorption of blood serum. It has been shown that if an animal's own serum be introduced into a loop of its intestine the serum undergoes absorption. This absorption affects the water and salts more than the protein, so that the percentage of the proteins in the fluid remaining in the intestine is increased. Finally, however, the whole of the serum is absorbed. In this case the fluid within the gut is identical with the fluid within the blood-vessels. There are no differences in concentration, quality of salts, or osmotic pressure of proteins. Nevertheless water passes through the cells of the gut from their inner to their outer sides, entraining with it the salts of the serum and a certain proportion of the indiffusible proteins. It is impossible to explain this result as due to the digestion of the proteins and their conversion into diffusible products, since the intestinal loops were washed free of any trypsin that they contained, and serum has itself a strong antitryptic action which would prevent its being attacked by even a strong solution of trypsin.

The active intervention of the cells in the absorption of salt solutions, as well as of serum, can be abolished by any means which diminishes or destroys their vitality, such as the addition of sodium fluoride to the fluid to be absorbed, or destruction of the epithelium by previous temporary veclusion of the blood-vessels supplying the loop of intestine.

We must conclude that, when a fluid is introduced into the intestine, an active transference of water from the lumen into the blood stream is effected by the intermediation of forces having their origin in the metabolism of the cells themselves. This work of absorption of the cells may be aided or 
hindered according to the physical conditions present. If these act against the cells, e.g. if the fluid be hypertonic, the absorption is effected more slowly, while with hypotonic solutions the physical conditions concur with the vital activity of the cells in bringing about a very rapid transference of fluid from the gut into the blood-vessels. Among these physical conditions we must reckon the nature of the salts present in the solution. If these can pass easily into and through the cells, e.g. ammonium salts, sodium chloride, absorption is carried out rapidly. If, on the other hand, the salts in the intestinal contents are but slightly diffusible or have very little power of penetrating into the cells, the absorption of water by the cells causes an increased concentration of the salts, and therefore an increased osmotic pressure, which offers a resistance to any further absorption; and the process comes to an end, when the absorptive power of the cells is exactly balanced by the increased osmotic pressure, or attraction for water, of the intestinal contents.

Cushny and Wallace, as the result of their experiments on the relative absorbability of salt solutions from the gut, divide the salts into four main classes as follows :

I

Sodium chloride,

bromide, iodide,

formate acetate, propionate, butyrate,

valerianate, caprate.
II

Ethyl sulphate, nitrate, lactate, salicylate, phthalate.
III

Sulphate, phosphate, ferrocyanide, caprylate, malonate, succi nate, malate, citrate, tartrate.
IV

Oxalate, fluoride.

Of these the first class contains those salts which are absorbed with great ease from the intestine. The second group of salts are absorbed with somewhat greater difficulty. The third group are absorbed so slowly, i.e. the salts retain the water in which they are dissolved so long, that they increase peristalsis and act as laxatives or purgatives. The members of the fourth class are not absorbed at all. It is evident that this classification is independent of the diffusibility of the salts. Sodium acetate has a much smaller dissociation value and a lower diffusibility than sodium chloride or iodide, and yet is absorbed at approximately the same rate as these two salts. There is, however, as Cushny pointed out, one physical or chemical character which apparently determines the non-absorbability (relative or absolute) of the members of the third and fourth classes. All these salts form insoluble compounds with calcium. This common character is not an explanation of the permeability of the cell wall, but is simply a general statement of one of the conditions which affect the power of the cells to take up salts from their solutions, this power being absent in the case of salts which furnish an insoluble calcium compound.

\section{THE ABSORPTION OF FATS}

Fats administered to an animal in excess of its diurnal requirements are stored up in the body in the form in which they are administered. Each cell of the body probably possesses in itself the mechanism for the utilisation of these neutral fats, and for effecting in them the various changes involved in the successive stages of their disintegration and oxidation through which they are finally converted to $\mathrm{CO}_{2}$ and water. The problem therefore of fat absorption is ultimately one of the simplest with which we have to deal, and involves merely the transference of the neutral fat of the food to the circulating fluids in such a form that it can be carried by them to the place 
where it is required for the metabolism of the body or where it may be stored up as a reserve substance.

The processes of digestion of fat result in the production of glycerin and fatty acids, if the reaction be neutral or slightly acid. If the reaction of the gut be alkaline the alkali will combine with the fatty acids to produce soaps. Analyses of the contents of the gut after a fatty meal show that the greater proportion of the fats are present as a mixture of fatty acids and soaps, the amount of these substances as compared with unchanged fat increasing as we descend the gut.

In studying the absorption of fats the investigator is able to take advantage of the fact that the micro-chemical detection of this substance is usually very easy. Globules of fats or fatty acids containing any proportion of the unsaturated fatty acids have the property of reducing osmic acid, and therefore of being stained black by this reagent. Practically all the fats which occur in the food or in the cells of the body contain oleic acid or the glyceride of this acid in association with palmitic or stearic acid, and therefore give the typical micro-chemical fat reactions. In many cases it is useful to employ the specific stains for fats, such as Sudan red or alkanna red. It is important to remember that the intensity of the fat reaction given by a cell is only an expression of the fat or fatty acid contained in a free state in the cell, and is no criterion of the total amount of fat which may be present. Thus a normal heart muscle in section gives only a diffuse light brown coloration with osmic acid. After poisoning by phosphorus or by diphtheria toxin, every muscle-cell may be found studded with minute black granules of fat. Chemical analysis shows, however, that the normal heart muscle contains as much fat as the degenerated muscle. Our microchemical methods will therefore throw no light on the amount of fat which is actually in combination with the cell protoplasm.

If an animal be examined a few hours after the administration of a meal rich in fats, the lymphatics of the intestine are seen to be distended with a milky fluid - chyle - and the same fluid is found filling the cisterna lymphatica magna and the thoracic duct. The lymph from the thoracic duct will also be milky, and chemical analysis shows that the opacity is due to the presence of minute granules of neutral fat. The fat in such chyle may amount to over 6 per cent., so that in a moderate-sized dog 12 grammes of fat may be carried in the course of an hour from the intestine to the blood by this means. This great access of fat to the blood during fat absorption introduces corresponding changes in the blood. The plasma itself becomes milky, and if the blood be allowed to clot, the serum expressed from the clot is also milky. On standing, a layer of fat globules like cream may rise to the surface of the serum. Fat is found in a free state in this finely divided condition in the blood plasma so long as it is being absorbed in the intestine. During starvation it disappears entirely, the serum becoming perfectly clear. Thus part, at any rate, of the fat which is absorbed from the gut is carried thence by the lymphatic channels in the form of neutral fat to the blood stream, by which it is distributed to the various tissues of the body, gradually 
leaving the blood stream in a manner which at present has not been determined. Not all the fat which is absorbed takes this path by way of the lymphatics and the thoracic duct. Ligature of the thoracic duct, if effective, certainly impedes the absorption of fat, but does not abolish it. If the thoracic duct lymph be collected during the absorption of a given quantity of fat from the intestine, not more than 60 per cent. of the fat which has disappeared from the gut can be recovered from the lymph. What happens to the remainder we do not know. Apparently it does not reach the blood in a finely divided condition. If the thoracic duct be ligatured the percentage of fat in the blood rapidly falls to a minimum which remains constant, even during starvation. If now fat be administered, although a considerable proportion of it may be absorbed, the percentage of fat in the

A

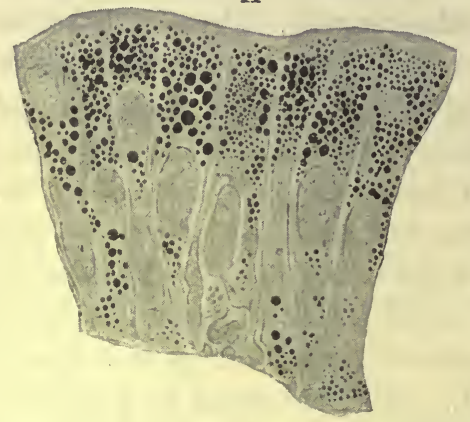

B

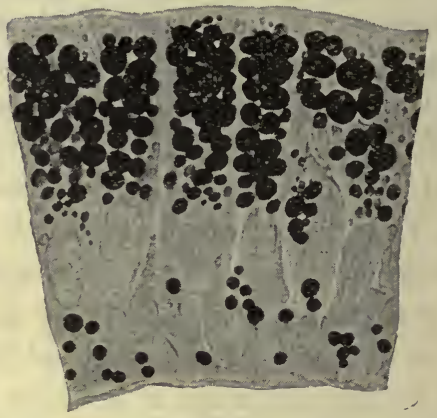

FIG. 350. Columnar epithelium from small intestine of frog stained with osmic acid to show fat-absorption.

A, five hours after a meal of olive oil ; B, three hours later. It should be noticed that the fat globules first formed grow in size in the course of digestion, pointing to a gradual deposition of fat on the globules from solution in the protoplasm. (SCHAFER.)

blood is not raised. If therefore the fat is absorbed directly into the blood it cannot be in the particulate condition, and it must be in such small quantities at a time that it is at once removed from the blood by the tissues through which this fluid flows. It is difficult to imagine that any large proportion of this lost fraction of the fat is absorbed into the blood stream in the form of soaps, since, as Munk has shown, soaps injected into the blood-stream act as poisons and give rise to a great fall of blood-pressure, incoagulability of the blood, and a condition of coma. We must therefore leave out of account for the present the mechanism of absorption of this lost fraction and endeavour to trace the course of the absorption of that part of the fat which makes its way into the lymphatics.

Microscopic examination of a section of the villus during fat absorption shows that the absorption occurs for the most part through the epithelial cells. These are found closely packed with fat granules (Fig. 350), which, small at the beginning of the process of absorption, rapidly enlarge till they occupy the greater part of the cell lying between the nucleus and the basilar striated border. Most observers are agreed that no fat globules are to be seen within the border itself. 
According to Altmann the fat granules found in the cells during absorption are themselves produced by a transformation of fuchsinophile granules which are present in the cell even during the fasting condition. At an early stage the small fat granules can be stained so as to show a distinct fuchsinophile envelope. Altmann interprets this appearance as showing that the epithelial cells take up the fat in a dissolved form, probably in a hydrolysed condition, and that a process of synthesis then occurs in the granules leading to the formation and accumulation of fat. When the process of absorption is proceeding actively the meshes of the villus contain a number of free fat granules, and the leucocytes in these meshes are generally found also full of these granules. Accorring
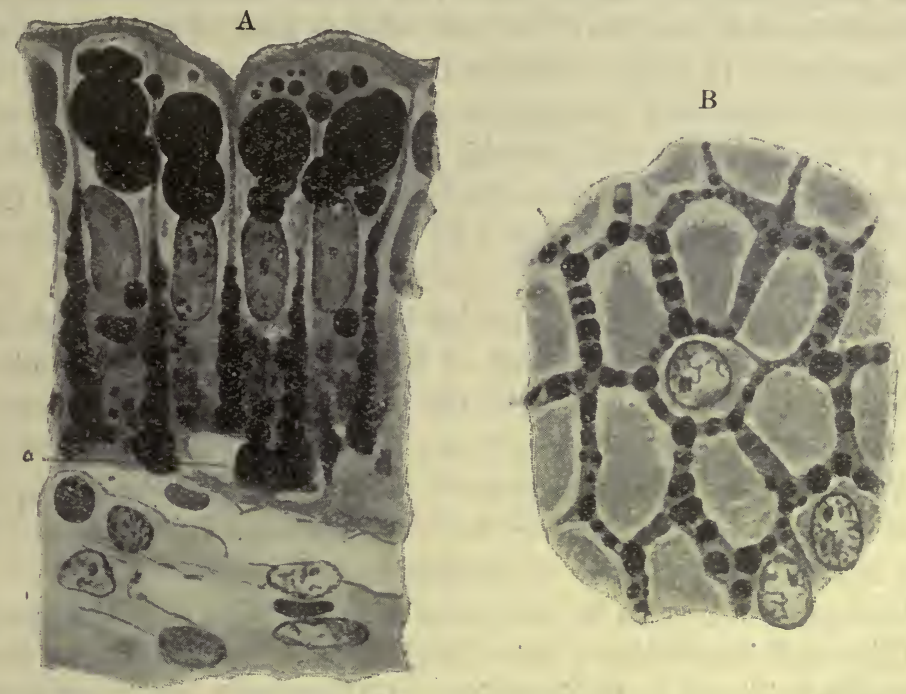

FIG. 351. A. Vertical section through intestinal epithelium of a rat during fat absorption. B. Horizontal section through deeper parts of the cells, showing excretion of fine fat globules into the intercellular clefts. (REUTER.)

to Zawarykin and Schäfer an important function in the transfer of the granules from epithelial cells to central lacteal was performed by the leucocytes. These were supposed to take up the fat granules extruded by the epithelial cells at the base of the villi, to wander into the central lacteal where they broke down, furnishing in this way the molecular basis of the chyle as well as its protein constituents. This view was strongly combated by Heidenhain, who pointed out that many of the granules staining darkly with osmic acid were not necessarily fat, and that the number of leucocytes within the villi were hardly sufficient to account for the amount of material observed. According to Reuter the epithelial cells take up fat in a dissolved condition through the striated border, and deposit it as granules of neutral fat in the inner portion of the protoplasm. From here the fat is passed on by the protoplasm by the side of the nucleus and extruded in the form of very fine granules in the deeper parts of the inter-epithelial clefts, which thus function as true excretory channels for the epithelial cells (Fig. 351).

It is probable that the muscular mechanism of absorption described many years ago by Brücke plays an important part in the absorption of fats, but it is difficult to furnish any experimental proof of the manner in which this mechanism works. Repeated contractions of the muscle fibres of the villus would tend to empty the spaces into the central lacteal, and this in its turn into the submucous plexus of lymphatics, so that the lymph in the spaces is constantly renewed and passes laden with absorbed fat particles into the valved lymphatics of the mesentery. 
It was long considered that the fats were taken up by the epithelial cells from the intestine as fine particles of neutral fat, the chief use of the pancreatic juice being to aid the formation of an emulsion of fat in the intestines. There seems to be little doubt that this was an error, and that the fats are absorbed, dissolved in the bile, either as soap or as fatty acid. The arguments for this view can be shortly summarised as follows ;

(1) Although the bile does not dissolve neutral fats, it has a strong solvent action on fatty acids, on soaps, and even on the insoluble calcium soaps. This solvent power is greatest in the case of oleic acid, of which bile can dissolve 19 per cent. It is very small in the case of pure stearic acid, but the solubility of the latter acid is largely increased if it be associated as usual with oleic acid. Moore has shown that this solvent action is chiefly conditioned by the bile salts, aided by the lecithin and cholesterin also present in the bile, a solution of lecithin and cholesterin in bile salts having a greater solvent power than the salts alone.

(2) The presence of bile in the intestine is essential for the normal absorption of fat. If the bile be cut off by occlusion of the bile ducts or by the establishment of a biliary fistula, the utilisation of fat sinks from about 98 per cent. to about 40 per cent., the unabsorbed fat appearing in the fæces. This large undigested residue of fat hinders also the absorption of the other food-stuffs by covering them with an insoluble layer, so that nutrition as a whole may suffer considerably.

(3) Absorption may also be interfered with by ligature of the pancreatic duct. This result is probably due to the absence of the fat-splitting ferments of the pancreatic juice from the intestine. If the fæces be analysed it is found that a very large proportion of the fat has been split into fatty acids in the course of its passage through the alimentary canal. This lipolysis has, however, been carried out by the agency of micro-organisms, i.e. in the lower segments of the gut where the greater part of the bile has been already reabsorbed into the portal circulation. If fat be given to animals deprived of their pancreas, in a finely divided form, such as cream or milk, a certain proportion of it is absorbed. Under these conditions a considerable degree of lipolysis may occur in the stomach itself, so that the fats would be already hydrolysed when they came in contact with the bile in the duodenum.

(4) It was shown by Schiff, by means of his amphibolic fistula, that the bile which is poured into the gut undergoes a circulation, being re-absorbed from the lower parts of the digestive tube, carried to the liver by the portal vein, and re-secreted in the bile. The same quantity of bile salts may therefore be used over and over again as a vehicle for the transfer of the fatty acid and soaps from the lumen of the gut into the epithelial cells.

(5) Substances which are physically almost identical with fats, e.g. petroleum or paraffin, are not absorbed even when introduced into the intestine in the finest possible emulsion. If neutral fat be melted with a soft paraffin and the resulting mixture made into a fine emulsion and administered it is found that the intestine rejects the paraffin, but takes up the neutral fat. This result can only be explained by assuming that the fat in the 
particles has been actually dissolved out by the digestive juices and has been absorbed in a state of solution.

We may sum up the processes involved in digestion and absorption of fat as follows. Neutral fat is hydrolysed into fatty acid and glycerin under the action of the gastric juice, the pancreatic juice, and the succus entericus, the effect of the gastric juice being, however, extremely limited unless the fat be presented to it in a finely divided condition. The lipolytic action of the pancreatic juice and succus entericus is largely aided and increased by the simultaneous presence of bile, which, in virtue of the bile salts lecithin and cholesterin it contains, enables the pancreatic juice to enter into close relation with the fat, and dissolves the products of the activity of the ferment, and so enables it to attack renewed portions of the neutral fat. As the result of this lipolysis there are formed glycerin, which is soluble in water, and fatty acids or soaps, according as the reaction of the medium is acid or alkaline. The alkaline soaps are soluble in water, the soaps of magnesium and calcium are soluble in bile, free fatty acids are soluble in bile acids. The fat is thus reduced to a condition in which it is soluble in the intestinal contents whatever their reaction. In this state of solution its constituents are taken up by the cells of the intestinal mucosa. Within the cells a process of synthesis takes place, the soaps being split and the fatty acids thus set free or absorbed, being combined with glycerin with the elimination of water to form neutral fat, which appears as fine granules in the cell protoplasm. By an active process of excretion these granules are extruded in a somewhat more finely divided form into the intercellular clefts and into the spaces of the villus, whence by the contractions of the musculature of the villus they are forced with the lymph transuding from the capillary bloodvessels into the central lacteal, and thence along the mesenteric lymphatics to the thoracic duct. This description would apply to about 60 per cent. of the fat which is absorbed. It is probable that all the fat which is absorbed is taken up in a dissolved condition, but whether the remaining 40 per cent. enters the blood stream, or is utilised and broken down in the tissues of the intestinal wall itself, we have no means of judging. Under normal circumstances the utilisation of fat is almost complete. By the time the intestinal contents have arrived at the lower end of the ileum 95 per cent. of the fat has been absorbed. Removal of the whole large intestine was found by Vaughan Harley not to affect fat absorption.

\section{THE ABSORPTION OF CARBOHYDRATES}

As a result of the action of the various digestive juices all the carbohydrate constituents of the normal diet of man are reduced to the state of monosaccharides. The absorption of these digestive products may take place at any part of the alimentary canal, the greatest part in the act of absorption being taken by the small intestine. By the time that the food has arrived at the ileocæcal valve practically the whole of the carbohydrate constituents of the food have been absorbed. All experimenters are agreed that the carbohydrates pass into the body by way of the vessels of the portal 
system. The lymph from the thoracic duct contains no more sugar than does the arterial blood taken at the same time, whereas several observers have obtained an increased percentage of sugar in the portal blood during the absorption of a big carbohydrate meal.

Of the carbohydrates of the food, some, like starch, dextrin, glycogen, are colloidal and indiffusible; others, such as the disaccharides cane sugar, milk sugar, and maltose, are soluble and diffusible, and the products of the action of digestive ferments on these two classes, namely, monosaccharides, mannose, fructose, glucose, galactose, are also soluble and diffusible. The problem as to the mechanism involved in the passage of these substances across the intestinal wall into the blood-vessels has been already dealt with in treating of the absorption of water and salts. The most striking fact is the relative impermeability of the intestinal wall to the disaccharides as compared with the monosaccharides. The intestinal wall is apparently only able to take up in any quantity such sugars as can be utilised by the cells of the organism. For this purpose the disaccharides are useless; cane sugar or lactose introduced into the blood-vessels or subcutaneously is excreted quantitatively in the urine, and, as might be expected, does not increase in any way the glycogen of the liver. When maltose is injected in the same manner a certain proportion of it is utilised owing to the fact that the blood and fluids of the body contain a ferment, maltase, capable of converting the disaccharide into the monosaccharide, glucose. The absorption of these disaccharides occurs therefore much more slowly from the intestine than does the absorption of monosaccharides, the process of absorption being always preceded by and waiting for the process of hydrolysis. Thus huge doses of cane sugar may be taken without causing the appearance of cane sugar in the blood or urine. It has been found that sugar does not appear in the urine until as much as 320 grm. of cane sugar have been ingested, whereas any quantity of glucose over $100 \mathrm{grm}$. may give rise to glycosuria. Lactose is absorbed still more slowly, and in animals whose intestine is free from the ferment lactase, is not absorbed; large doses of lactose in such animals therefore give rise to diarrhœa. The behaviour of the intestinal wall to the non-assimilable sugars of artificial origin has not yet been sufficiently investigated. It would be interesting to inquire whether the rate of absorption of the different sugars was in any way determined by their stereomeric configuration, whether, for instance, l-glucose would be absorbed as rapidly as the ordinary $d$-glucose.

\section{THE ABSORPTION OF PROTEINS}

In very few departments of physiology has there been so great a revolution in our ideas as in that relating to protein absorption, especially as to the form in which it is absorbed from the alimentary canal, and its fate after absorption. As to the channel by which it obtains entry into the circulation, practical agreement reigns that it is absorbed by the blood-vessels. Almost every physiologist who has occupied himself with the investigation of the lymph flow from the thoracic duct has been impressed by the fact that the 
variations in the amount of lymph to be obtained in this way bear no relation to the condition of the animal as regards the state of digestion. Nor do we find any appreciable increase in the amount of lymph flow or in the amount of proteins contained in this lymph during digestion. The small increase observed by Asher and Barbara would be sufficiently accounted for by the increased blood-supply to the intestines during digestion, and is insufficient to account for the absorption of any appreciable quantity of the protein which is being taken up from the alimentary canal. Moreover it was shown by Schmidt Mülheim that the absorption of proteins was not interfered with as the result of ligature of the thoracic duct, and that after this duct had been ligatured the ingestion of proteins was followed at the usual interval by the increased output of urea which is the invariable concomitant of protein absorption and assimilation. We must therefore conclude that the products of protein digestion are taken up by the epithelial cells and passed on by these into the blood-vessels.

During the absorption of a protein meal changes have been described by various observers in the structures of the villus. In nearly every case there is marked increase in the number of mitotic figures in the epithelium lining the follicles of Lieberkühn. According to Hofmeister there is during absorption an increase in the number of leucocytes in the villi, and this observer ascribed an important function to these eclls in the absorption of protein. Heidenhain showed that this increase of leucocytes was not constant in all animals, and bore no relation to the amount of absorption that was taking place, and was quite inadequate to account for the total absorption that was carried on. On the other hand, several observers have described changes in the epithelium as the result of protein digestion. According to Reuter the epithelial cells become swollen, their protoplasm stains less deeply, and at their basal ends the cells' limits disappear, the protoplasm being apparently distended with hyaline coagulable material (Fig. 352). Reuter regards this appearance as a direct expression of the taking up of proteins in a dissolved form and their conversion near the bases of the cells into coagulable proteins ; but further evidence on this subject is necessary before we can attach much importance to such an interpretation of the appearances observed.

Under the influence of the gastric juice the proteins of the food are resolved during their stay in the stomach into albumoses and peptones. In the small intestine the process of hydration is carried further, the trypsin of the pancreatic juice carrying the proteins through the stage of secondary albumoses and peptones, and converting them into a mixture of amino-acids and polypeptides. The same end-products result from the action of the erepsin of the intestinal wall on the albumoses and peptones produced by gastric digestion. The digestive juices finally reduce the proteins therefore to a mixture of amino-acids, with a certain remainder of polypeptides consisting of two or three of the amino-acids associated together, which do not undergo further disintegration under the action of the intestinal ferments. The final products give no biuret test. The first question we have to decide is to what extent the proteins are reduced to their ultimate hydration products before absorption. We have evidence that protein may be absorbed by the small intestine without having undergone any hydration whatsoever. The absorption of serum protein has been discussed already in dealing with the mechanism of absorption of salt solutions from the gut. In a series of 
experiments made by Friedländer the absorptions of various proteins were compared after their introduction into loops of the small intestine which had been washed free from ferment. During a period of three hours this author found that 21 per cent. of the proteins of egg white or of blood serum were absorbed. During the same period, of alkali-albumen which had been introduced into the loops, 69 per cent. was absorbed. On the other hand, when

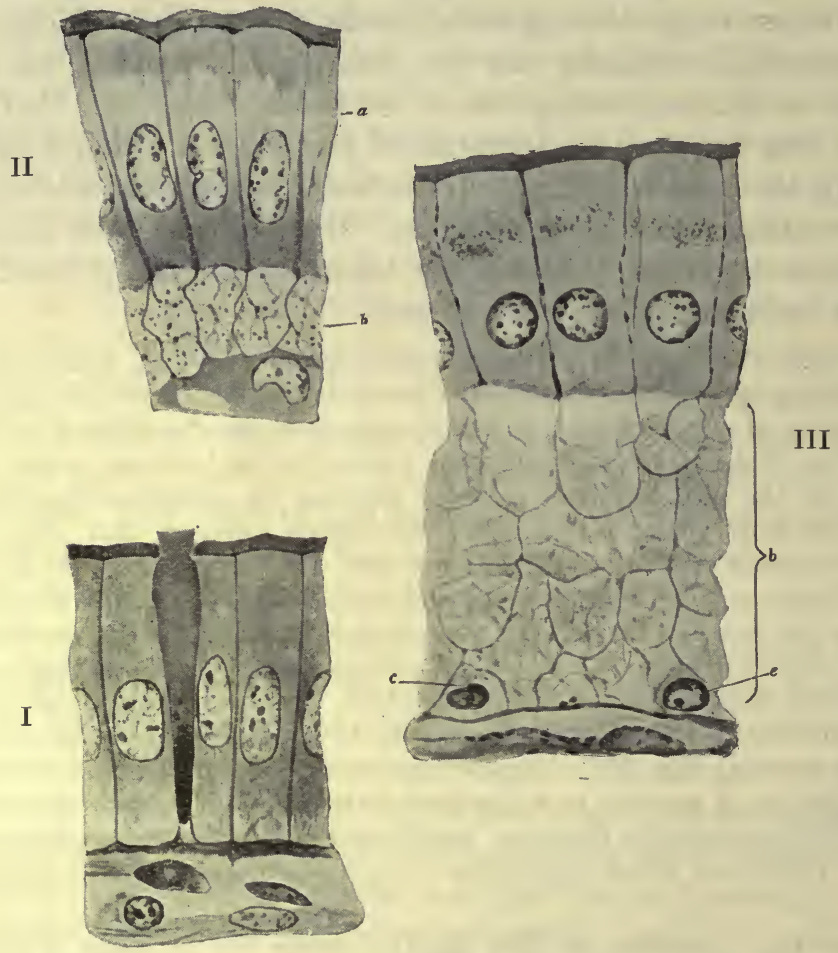

FIG. 352. Figures (from REUTER) showing changes in intestinal epithelium induced by absorption of protein.

I, epithelium of fasting rat; II, initial stage; III, later stage of protein absorption.

syntonin and casein were introduced into the intestine, no absorption whatever was observed. As to the condition in which such unchanged protein reaches the blood stream our knowledge is still imperfect. Foreign proteins, such as egg albumin, or the serum of other species introduced into the blood stream, may cause poisonous effects, and give rise to albuminuria, to lowering of blood pressure, or to alteration of the coagulability of the blood. If injected in small quantities they excite, as a reaction on the part of the organism, the production in the blood serum of a precipitin, and the presence of the precipitin may be looked upon therefore as a test by which we may decide whether these proteins have passed through the intestinal wall unchanged. In most cases it is found that, however abundant the amount of protein administered in the soluble form, none of it appears in the urine, 
nor is any precipitin formation aroused. Ascoli has, however, observed such events occasionally to follow the administration of large doses of egg white, and it has been shown that there is a difference in the behaviour of animals to the introduction of soluble protein into their alimentary. canal, according as they are new born or are more than a few days old. It seems that during the first few days of life the cellular lining of the alimentary canal is permeable to foreign proteins, whereas later on any protein which is taken up unchanged from the gut does not arrive in the same unchanged condition in the blood stream.

The absorption, however, of unchanged proteins can play but a small part in the assimilation of protein as a whole. Animals very rarely take coagulable proteins in a condition in which they will arrive at the small intestine in a state of solution unchanged. Even in the carnivora the living tissues taken into the stomach will undergo coagulation by the acid, and will then be dissolved by the gastric juice. In man practically all the proteins of the food are either insoluble or are rendered insoluble by the process of cooking. For absorption to take place it is therefore necessary that this insoluble or coagulated protein should be brought into solution, and this process is accomplished, together with hydration, by means of the ferments of the gastric and pancreatic juices. This process of solution has long been regarded as the chief object of the digestive ferments. Although both Kühne and Schmidt Mülheim were aware of the production of amino-acids, such as leucine and tyrosine, as the result of digestion, they regarded their production as evidence of a waste of material. Proteoses and peptones are soluble, diffusible, and rapidly absorbed from the alimentary canal, and there is no doubt that a large proportion of the products of protein digestion are taken up by the absorbing membrane in this form. For many years physiologists were occupied with the problem as to the fate of these peptones and proteoses after their entrance into the mucous membrane. They do not pass as such into the blood. The injection of small quantities of proteose and peptone into the blood gives rise to the excretion of these substances by the kidneys ; injection of larger quantities has pronounced poisonous effects, which were first studied by Schmidt Mülheim and Fano. If samples of blood be taken either from the portal vein or from the general circulation after a heavy protein meal, no trace either of proteose or of peptone is to be found in the blood. The observations of Hofmeister and others to the contrary depend on the fact that these observers employed a method for the separation of coagulable protein, as an antecedent to the testing for proteoses, which was in itself capable of producing small traces of these substances. Hofmeister showed that during the absorption of a protein meal the mucous membrane either of the stomach or of the intestine, if rapidly killed by plunging into boiling water directly it was taken from the animal, always contained a considerable amount of peptone, and similar observations were made by Neumeister. If, however, the mucous membrane was kept warm for half an hour after removal from the body, the peptone disappeared. Salvioli, under Ludwig's guidance, introduced peptone into a loop of gut 
which was kept alive by passing defibrinated blood through its vessels. At the end of some hours the loop was found to contain a certain amount of coagulable protein, but no trace of peptone, nor was any trace of the latter substance found in the blood which had been passed through the vessels. These observations were interpreted as pointing to a regeneration in the intestinal wall of coagulable protein from the proteose and peptone taken up from the gut, and opinions were divided whether the most important part of this regeneration was to be ascribed to the leucocytes of the villi (Hofmeister), or to the epithelial cells of the mucous membrane itself.

It is evident that such a conclusion was not justified by the experiments. All that these experiments showed was that the proteoses and peptones disappeared, i.e. were converted into something which did not give the biuret test. The discovery of the ferment erepsin by Cohnheim led this observer to repeat the experiments of Hofmeister and Neumeister with a view to testing the conclusions drawn by these physiologists. Cohnheim found that, although it was perfectly true that proteose and peptone disappeared when intestinal mucous membrane and peptone were placed together in the presence of either blood or of Ringer's fluid, this disappearance was due, not to a regeneration of coagulable protein, but to the fact that the erepsin of the mucous membrane carried the process of hydrolysis a step further, converting the proteoses and peptones into the ultimate crystalline products of protein hydrolysis. Similar observations were made by Kutscher and Seemann, who showed that at any time after a protein meal these end-products, especially leucine, tyrosine, lysine, and arginine, were to be found in the contents of the small intestine. A repetition of Salvioli's experiment by Cathcart and Leathes deprived this also of much of its significance. It was found that the artificial circulation, although sufficient to maintain the activity of the muscular wall of the intestine, as evidenced by the peristaltic movements, was insufficient to keep the mucous membrane alive. After one hour's experiment the loop contained a mass of epithelial cells mixed with the products of the action of erepsin on the introduced peptone solution. In no case was there any diminution in the amount of uncoagulable nitrogen, i.e. there was no formation of coagulable protein, while the processes of absorption had been brought by the desquamation entirely to a standstill.

These additional experiments caused a complete revolution in the attitude of physiologists towards the problem of protein absorption. All this evidence went to show that protein, however introduced, whether as coagulated protein or as albumose and peptone, underwent complete hydrolysis either in the gut or in the wall of the gut before entering the blood stream. If this were the case it should be possible to feed an animal on a diet in which the necessary protein had been replaced by the corresponding amount of ultimate products of protein hydrolysis, i.e. by a mixture which would give no biuret reaction. Such a possibility had previously been negatived on theoretical grounds by Kühne and by Bunge. It was thought by these observers either that the animal body lacked the power of synthesis of proteins from these crystalline products (hydration products), or that any complete 
hydration occurring in the intestine would involve such a loss of energy to the body as to be unteleological. Neither of these theoretical objections is justified in fact. We know from the researches of Fischer and others that although the different proteins in our food present a marvellous qualitative similitude, in that all of them yield on hydrolysis the same kinds of aminoacids, there are great differences in the relative amounts of these amino-acids contained in different proteins. Thus, in gelatin, glycine is contained in considerable quantities, but is absent in many of the other proteins. Caseinogen is distinguished by the large amount of leucine that it yields, while gliadin, the chief protein of wheat flour, contains very large amounts of glutamic acid. It is difficult to imagine how, for instance, muscle protein could be formed from wheat protein, a process continually occurring in the growing animal, unless we assumed that the protein molecule is first entirely taken to pieces, and that its constituent molecules are then selected by the growing cells of the body and built up in the order and proportions which are characteristic of muscle protein. Moreover, when we measure the amount of energy change involved in the hydrolysis of the proteins, we find it is relatively small. There is not a loss of 5 per cent. of the total energy available i.e. the heat of combustion of the products of pancreatic digestion would differ from that of the original protein submitted to digestion by less than 5 per cent. The energy of the protein as evolved in the body lies, not in the coupling of the amino-acids with one another, or indeed in the coupling of the nitrogen to the carbon, but, like that of the other food-stuffs, in the carbon itself, and is derived from the combustion of the carbon of the molecule under the influence of the oxidising processes of the body into carbon dioxide. The experimental decision of this question was first attempted by 0 . Loewi, who found that it was possible to keep a dog in a state of nitrogenous equilibrium on a diet containing fat, starch, and a pancreatic digest of protein which contained no substances which would give the biuret test. These results have been confirmed for carnivora by Henderson, by Lüthje, by Abderhalden and Rona, and by Henriques and Hansen. According to Abderhalden, it is possible to keep an animal alive when the nitrogen in his food is represented entirely by the end-products of pancreatic digestion. The same result cannot be attained by the administration of the products of acid hydrolysis of protein, but this may be due either to the racemisation of the amino-acids under the action of the strong acid, or to the fact that the acid splits up certain polypeptide groupings which are still contained in the trypsin digest, and which possibly cannot be synthetised by the cells of the body.

We are justified therefore in concluding that while a certain small proportion of the proteins of the food may be alosorbed unchanged, a much larger proportion is taken up as proteoses and peptones or as amino-acids. The proteoses and peptones are, however, rapidly changed in the mucous membrane itself into amino-acids, which we may regard as the form in which practically all the protein of the body is presented to the absorbing mechanisms of the alimentary canal for absorption and for passing on into the circulating fluids. 
THE FATE OF THE AMINO-ACIDS AFTER ABSORPTION BY THE INTESTINAL EPITHELIUM. During a condition of starvation the normal protein requirements of the body, or rather of the active tissues, are met at the expense of the less active tissues. The protein characteristic of any tissue can be taken down, removed to another part of the body, and built up into the protein characteristic of some other active tissue. It is difficult to conceive that such a transference and transformation could occur in any other way than by a more or less thorough disintegration of the protein molecule at one place and its synthesis at the other, and we know from the researches of Hedin and others that every tissue contains intracellular ferments which are capable of effecting the disintegration of the protein molecule, and are responsible for the autolytic degeneration of tissues after death. If therefore the normal interchange of protein between the tissues is accomplished, as we know it to be in plants, by the disintegration of the proteins into their constituent amino-acids and their subsequent reintegration, there is no a priori reason to believe that the blood carries the proteins from the alimentary canal to the tissues in any other form than that of amino-acids. The experimental proof of this conclusion was hardly possible before the invention of a reliable method for the detection of small quantities of amino-acids in the blood and tissues. This is rendered possible by van Slyke's method, in which after the separation of coagulable proteins by alcohol the amino-acids are determined by measuring the nitrogen evolved on addition of nitrous acid. Van Slyke has shown that the blood always contains a certain amount of amino-acids even during fasting. After a protein meal there is a considerable increase in the amount of amino-acids. Thus the blood of fasting animals contains from $3 \cdot 1$ to $5 \cdot 4$ milligrams aminoacid nitrogen per 100 c.c. Blood taken after food contains $8 \cdot 6$ to $10 \cdot 2$ milligrams amino-acid nitrogen per 100 c.c. of blood. The question of the fate of amino-acids thus absorbed from the intestine to the blood is decided by an estimation of the amino-acid content of the different tissues after the injection of amino-acids into the blood. Van Slyke has found that after the injection of amino-acids only a certain proportion is excreted with the urine, and that the rest of the amino-acids rapidly disappeared from the blood and are taken up by the tissues without undergoing any immediate chemical change, though in the case of certain tissues, such as the muscles, a definite saturation point exists which sets the limit to the amount of amino-acids that can be absorbed. On the other hand the capacity of the internal organs, and especially of the liver, for the absorption of amino-acid is much greater.

It is worthy of note, however, that the absorption of amino-acids by the tissues from the blood is never complete, i.e. the amino-acids of the blood must be in a state of equilibrium with those of the tissues, although the concentration in the latter may be much greater than in the former. If several hours be allowed to elapse after the injection of amino-acids before the analysis of the tissue is undertaken, it is found that the amino-acid nitrogen content of the liver may have returned to normal although the concentration 
in the muscles has suffered no appreciable fall. Since we have evidence that the circulation of amino-acids through the liver gives rise in this organ to the formation of urea, we must conclude that this organ is especially responsible for the breakdown of the products of protein digestion which are not directly required for replacing tissue waste. This breakdown must involve a process of deamination. We may therefore conclude that the amino-acids normally produced by a protein digestion are absorbed without further change into the blood-stream. They then circulate throughout the body, a certain proportion of them being built up in each tissue into the proteins characteristic of that tissue in order to replace the waste caused by wear and tear. The rest, probably the major part of the protein, is taken up by the liver, where it undergoes deamination, the nitrogen moiety being rapidly converted into urea and excreted by the kidneys, while the nonnitrogenous moiety is carried to the working tissues to which it serves as a ready and immediate source of energy.

The fact that not only the blood but also the tissues contain amino-acids, even after complete starvation for some days, shows that these substances are intermediate steps not only in the synthesis but in the breaking down of body proteins. Free amino-acids are thus the protein currency of the body, just as glucose is the carbohydrate currency. In the fasting body we must regard the processes of autolysis as the main source of the amino-acids found in the tissues, and it is by autolysis that the proteins of the resting tissues are made available in starvation for those whose continued working is essential for the maintenance of life. The fact that high protein feeding does not appreciably increase the amino-acid content of the tissues, shows that any storage of nitrogen in the organism must take place, not in the form of amino-acids, but as body protein.

It was formerly thought that the deamination of amino-acids occurred on a large scale in the wall of the alimentary canal, on the grounds that a larger amount of ammonia was present in the portal blood than in the arterial blood. It seems, probable, however, that the source of this excess of ammonia is to be found in intestinal bacterial changes, and that the major portion of the amino-acids is actually absorbed unchanged. The view of Abderhalden that the amino-acids are synthetised in the intestinal wall to serum proteins, and absorbed in that form into the blood-stream, need here only be mentioned, since it lacks experimental support.

\section{THE ACTUAL COURSE OF DIGESTION}

In a recent series of papers London describes the course of digestion of meals of various characters in dogs which had been provided with fistulæ in one of the following places: $(a)$ gastric fistula (into the fundus of the stomach); (b) pyloric fistula (on the duodenal side of the pylorus) ; (c) duodenal fistula (about one foot below the pylorus); (d) jejunal fistula (about the middle of the small intestine); (e) ileum fistula (just above the cæcum).

We may take as an example the course of digestion of a meal composed of $200 \mathrm{grm}$. of bread. This is eaten by the animal, mixed with the saliva and swallowed. On arriving at the stomach it gives rise to the secretion of gastric juice. In a series of special experiments London found that on the average $200 \mathrm{grm}$. of bread evoked the secretion of $20 \mathrm{grm}$. of saliva, about $10 \mathrm{grm}$. of mucus from the coats of the stomach, and about $315 \mathrm{grm}$. of gastric juice. The secretion of gastric juice is continuous during 
the whole time that the food remains in the stomach. In the animal with a pyloric fistula, one to two minutes after the meal had been taken, a few drops of alkaline fluid were extruded from the opening. From three to eight minutes after the conclusion of the meal small quantities of clear acid gastric juice were repeatedly extruded. The first admixture of the food with the outflow from the fistula occurred at eight to twelve minutes after the completion of the meal, and after this time the pylorus continued to open at regular intervals of ten to forty seconds, discharging each time a small amount of fluid composed of particles of undigested bread mixed with gastric juice. One and a half hours later the pylorus began to open less regularly and the fluid became of a more pasty consistence, devoid of lumps of undigested bread. In the fourth, fifth, and sixth hours after the meal the pylorus opened only once every one or two minutes, and towards the end of this period the fluid extruded was clear. The following Table. shows the percentage amount of food taken which had left the stomach at the end of each hour after the meal:

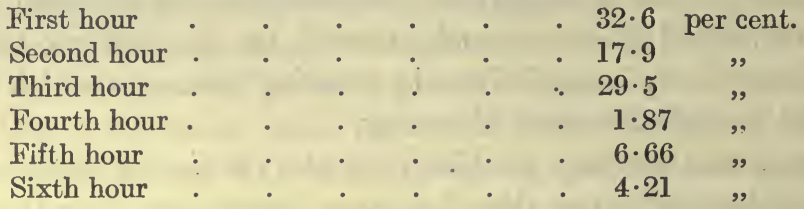

The large proportion of the ingested food leaving the stomach during the first two or three hours can hardly be regarded as normal. Since in these experiments there was a free outflow from the pylorus and the food was not allowed to enter the duodenum, the local reflex, evoked by the presence of acid in the duodenum, was absent. The gastric contents obtained in this way were analysed in order to find what changes had been wrought on the food by the gastric juice. It was found that 32 per cent. of the bread had been brought into solution. This solution had affected the proteins more than the carbohydrates. Thus 67 per cent. of the nitrogen had been brought into soluble form, consisting chiefly of proteoses and peptones. No amino-acids were formed. Only 25 per cent. of the starch of the bread had been rendered soluble, and of this, 21 per cent. was in the form of dextrine and 4 per cent. in the form of sugar. No absorption, however, either of the digested proteins or of the digested carbohydrates was ever found to take place in the stomach.

DUODENAL DIGESTION. The influence exerted by the pancreatic juice, bile, and succus entericus, poured out on the food in the duodenum, was studied by analysis of the intestinal contents leaving the intestine by a fistula, either at the lower end of the duodenum, or in the jejunum, or in the ileum. From the duodenal fistula the expulsion of food occurs at repeated intervals, but in a somewhat irregular fashion, its movements being determined partly by the contractions of the stomach and partly by those of the duodenal wall. Usually a large gush is followed by a series of small gushes: Although only a foot intervenes between the duodenal fistula and the pyloric fistula, a great difference is observed in the character of the intestinal contents obtained in the two cases. The outflow from the duodenum, being mixed with the pancreatic juice and the bile is yellow in colour and increased in amount. With a meal of $200 \mathrm{grm}$. there is secreted on the average $130 \mathrm{grm}$. of bile and $140 \mathrm{grm}$. of pancreatic juice. During its passage through the duodenum the carbohydrates of the food undergo considerable changes, so that even one foot below the pylorus we find that one-half to three-fifths of the carbohydrates have been converted into dextrine and sugar. A further digestion of the proteins also takes place amounting to about one-tenth of the whole protein taken with the food.

On deducting the amount of juices which have been added to the food it is found that even in this short length of intestine absorption has taken place of about one-sixth of the ingested food, about one-fourth of the carbohydrates having been absorbed and about one-eighth of the proteins.

In a dog with a fistula about the middle of its small intestine, the outflow began six to fifteen minutes after the meal, and lasted six or seven hours. The outflow was 


\section{THE ABSORPTION OF THE FOOD-STUFFS}

by small gushes repeated at intervals of five to ten seconds separated by intervals of one to five minutes, during which nothing appeared at the orifice of the cannula. The material obtained was quite different in character from that flowing from the duodenal fistula. The pasty character had disappeared, the material forming a frothy, orangeyellow, even jelly-like mass with practically no trace of undigested bread.

From a fistula in the ileum the outflow occurred at long intervals of three to fifteen minutes and was much scantier than that obtained from the jejunal fistula, consisting of a thick jelly-like, orange-coloured mass. Both proteins and carbohydrates were entirely digested, and in the case of the former the chief products of digestion consisted of amino-acids. Thus in one experiment, after four large meals of 500 grm. of meat each had been given, in order to obtain sufficient quantity for analysis, $175 \mathrm{grm}$. of soluble substances were obtained. From this were isolated tyrosine, leucine, alaninc, aspartic acid, lysine, and traces of arginine and histidine.

From a fistula in the cæcum there was no outflow until four or five hours after the meal had been taken. The material from the gut was then extruded in frecal like masses at long intervals of one half to one hour. This regular outflow lasted for about six hours. The reaction of the contents was strongly alkaline, with no food particles, and the material contained merely débris of cells, with small traces of sugar, dextrine and unaltered starch. The absorption of the food-stuffs is thus practically complete by the time that the food has reached the lower end of the small intestine.

The following Tablc gives the total amounts obtained in a series of experiments from the different fistulæ after administration of $200 \mathrm{grm}$. of bread, and also the percentage amount of food-stuffs which had been absorbed before the food had arrived at the level of the fistula in question :

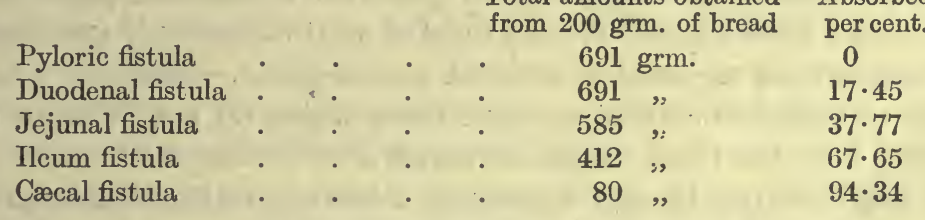

Absorbed per cent.

$37 \cdot 77$

$94 \cdot 34$ 


\section{SECTION $\mathrm{X}$ \\ THE FACES}

THE fæces are often regarded as representing the undigested or indigestible constituents of the food which have escaped solution and absorption in their passage through the alimentary canal. This view is hardly correct as applied to man or to the carnivora. In these the absorption of the constituents of a meal, whether consisting of fats, proteins, or carbohydrates, is practically complete by the time that the food has arrived at the lower end of the ileum. The fæces, in fact, are not derived from the food, but are produced in the alimentary canal itself. This is shown by the fact that on analysing the fæces no soluble carbohydrates or proteins, albumoses, peptones, or amino-acids are to be found. After a meal of meat microscopic examination of the fæces reveals no trace of striated muscle fibres. Moreover animals in a state of complete starvation form fæces which do not differ in their composition from the fæces which are found after feeding with meat, eggs, sugar, or cooked starch, though the amount is less in a state of inanition than under normal circumstances. In one experiment Hermann isolated a loop of gut, joining its ends together so that a continuous ring was formed. The continuity of the gut was then restored by suturing the two free ends. After some weeks the isolated loop was found to contain a semi-solid material similar to fæces in appearance, consistence, and chemical composition. It contained a large amount of phosphoric acid, lime, and iron.

So long as vegetables or coarsely ground cereals are excluded from the diet, the nature of the latter does not alter the chemical constitution or appearance of the fæces. Under these circumstances the fæces have the following composition :

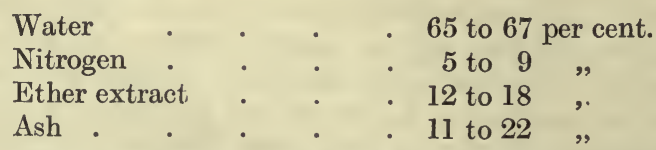

The ash consists chiefly of lime and phosphoric acid with some iron and magnesia. The ethereal extract contains fatty acids and a small amount of lecithin. Neutral fat is present in very small proportions. The fæces also contain small quantities of cholalic acid and its products of decomposition, dyslysin, and coprosterin, a body allied to cholesterin, and a certain amount of purine bases consisting of guanine, adenine, xanthine, and hypoxanthine. On the average the fæces contain about $0 \cdot 11 \mathrm{grm}$. of purine bases per diem, 
about seven times as much as is contained in the urine passed in the same time. The material basis of the fæces seems to be largely desquamated epithelial cells from the intestinal wall, and bacteria, of which countless numbers, chiefly dead, are present. It has been reckoned that as much as 50 per cent. of the fæces may consist of the dead bodies of bacteria.

Very different is the composition of fæces if the food contains a large amount of cellulose. Not only does the ingested cellulose pass unchanged into the fæces, but large quantities of other substances enclosed in the cellulose walls may also escape digestion and absorption. Moreover the increased bulk of the undigested residue stimulates peristalsis, and thus quickens the passage of the food through the gut to such an extent that the digestive ferments have not time to exert their full action on the digestible constituents of the food. The influence of the character of the food is well illustrated by a comparison of the amount and composition of the fæces on different kinds of bread (Rubner) :

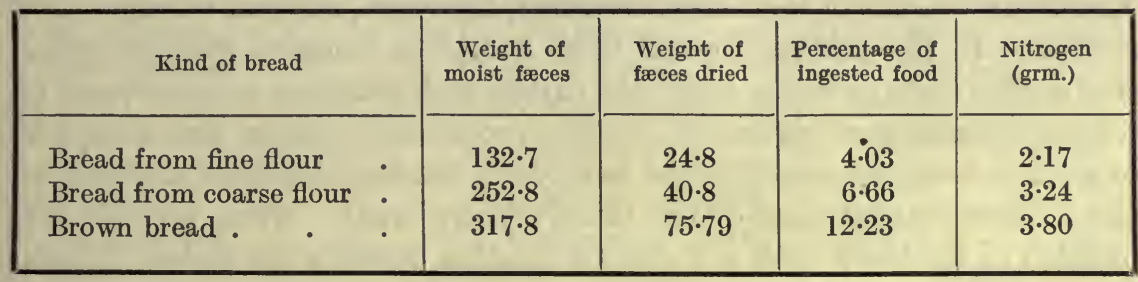

The following Table is also instructive. In this Table Rubner calculates the amount of fæces which a man would pass in twenty-four hours if he satisfied his energy requirements at the expense of one only of the different kinds of food enumerated. The numbers refer to the amount of organic material which would be excreted in the fæces :

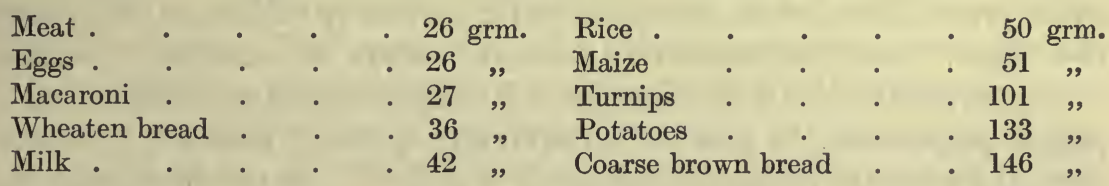

The indigestible cellulose in the food is not without value. It has been shown previously that the peristaltic contractions of the intestine are roused primarily by the mechanical stimulus of distension. If the food is capable of entire digestion and absorption the amount of fæces formed is limited to that produced by the intestinal wall itself. The small bulk exercises very little stimulating effect on the intestine, and the movements of the latter will therefore tend to be sluggish, especially in the absence of the mechanical stimulus determined by muscular exercise. The presence of a certain amount of cellulose in the diet may therefore be of considerable advantage by giving bulk to the fæces and ensuring the proper regular evacuation of the lower gut. It is probable that the constipation which is so common a disorder in civilised communities is due as much to the refinement in the preparation of the food as to the prevalence of sedentary occupations incident on the working of such a community. 


\section{CHAPTER XI}

\section{THE HISTORY OF THE FOOD-STUFFS}

\section{SECTION I \\ PROTEIN METABOLISM}

A PROTEIN consists of the elements carbon, hydrogen, oxygen, nitrogen, and sulphur. In the oxidation which these bodies, in common with the other food-stuffs, undergo in the body, the carbon and hydrogen are converted to carbon dioxide and water. A certain proportion escapes this complete oxidation, being excreted by the kidneys in combination with nitrogen as the essential constituents of the urine (chiefly urea). When proteins are oxidised in the body there is a definite relation between the carbon dioxide which is produced and the oxygen consumed. This respiratory quotient $\frac{\mathrm{CO}_{2}}{\mathrm{O}_{2}}$ in the case of proteins equals $0 \cdot 81$. Since the respiratory quotient on a pure consumption of fats is only 0.71 and on carbohydrates equals 1 , we may, by a study of the respiratory exchanges, arrive at some idea of the extent to which protein metabolism is responsible for the energy exchanges of the body as a whole. Such information by itself will always be somewhat uncertain, since it is possible, by a combination of fat and carbohydrate metabolism in proper proportions, to produce a respiratory quotient identical with that obtaining when the metabolism is purely of protein. On the other hand, the specific constituents of proteins, namely, nitrogen and sulphur, are excreted entirely by the urine (if we exclude the small traces which may leave the body in the sweat, or as scales of epidermis, hair, nails, \&c.). It has therefore been customary to take the nitrogen output in the urine as an index of the protein metabolism. This proceeding is only justified if we remember that we are dealing in the urine merely with the nitrogen and sulphur of the protein molecule, and that a large proportion of the carbon and hydrogen moiety of this molecule is being left unregarded.

Since we cannot follow all the stages in the changes undergone by proteins on their way through the body, it will be convenient to take the nitrogenous end-products of protein metabolism such as appear in the urine, and to determine, where possible, their precursors, and the conditions which determine their formation in the body. The chief nitrogenous constituents of urine are urea, ammonia, uric acid, creatinine, hippuric acid. There is a small residue 
of undetermined nitrogen which may include traces of purine bases, such as xanthine and hypoxanthine, traces of amino-acids, small amounts of pigment and of nucleo-protein from the wall of the bladder. The relative proportions in which these bodies occur are not invariable, but differ according to the nature of the protein foods taken and also according to the proportion which the protein metabolism bears towards the total energy requirements of the body. In the following Tables (Folin) are given the average composition of two specimens of urine from the same individual, one on a diet containing the ordinary proportion, and the other on a diet containing only a minimal amount of protein :

TABLES I AND II

Distribution of Nitrogen in Urine on Various Diets

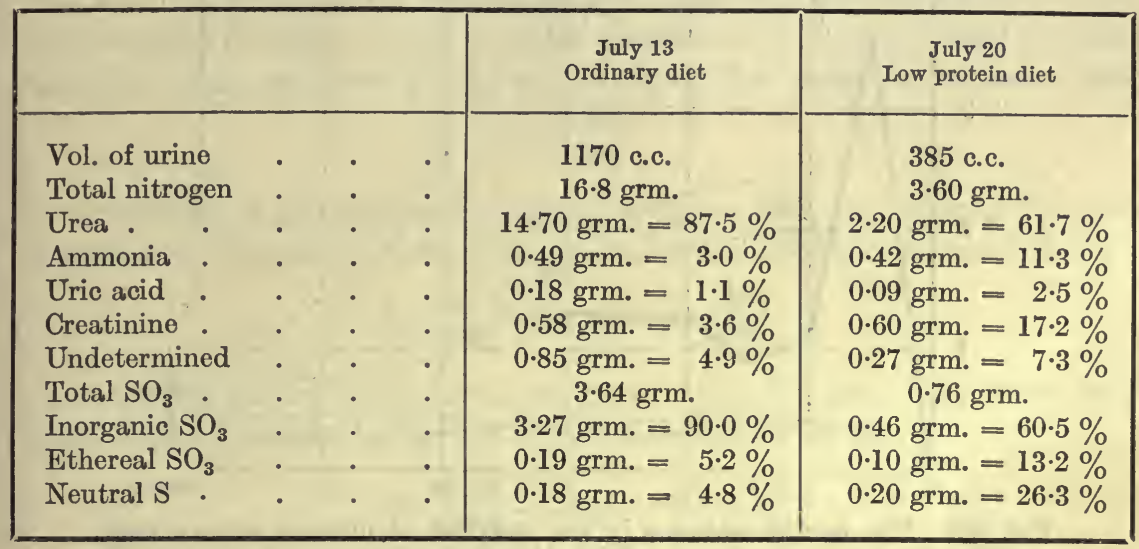

In dealing with the metabolism of the body as a whole we saw reason to believe that the proteins taken in with the food might be regarded as having a twofold destiny. One part, and under normal circumstances the greater part, is applied to the production of energy, in this respect discharging a function which might equally well be performed by the fats and carbohydrates of the food. In its second function protein cannot be replaced by any other food-stuff, since it alone contains the necessary elements as well as the groupings of these elements which are essential for the building up of the living tissues. We saw reason to believe that this tissue metabolism only accounted, however, for a small part of the nitrogen of the food. On this account it is possible to ensure health and a condition of nitrogenous equilibrium with amounts of protein in the diet of man which might vary between 40 and 200 grm. per diem. The more protein that is taken in with the food the greater is the relative amount which is applied to the energy needs of the body. If therefore we would attempt to find out what are the endproducts of the tissue metabolism we should confine the energy metabolism of proteins within the smallest possible limits by reducing the quota of protein in the diet to its minimum. Folin has shown that if we compare the composition of the urine obtained under these two conditions, namely, on a diet containing a normal quantity of protein and on a diet containing a minimal 
amount, we find evidence of a qualitative difference between the two kinds of metabolism. The difference is well brought out in the Tables just quoted. On a large diet the greater part of the nitrogen can be regarded as derived directly from the food, whereas on a small diet a relatively larger proportion of it must come from protein which has been previously built up into the tissues. Folin distinguishes these two sources of the nitrogen of the urine as exogenous, i.e. that from the food, and endogenous, i.e. derived from the tissues. Two facts stand out in comparing these two urinary

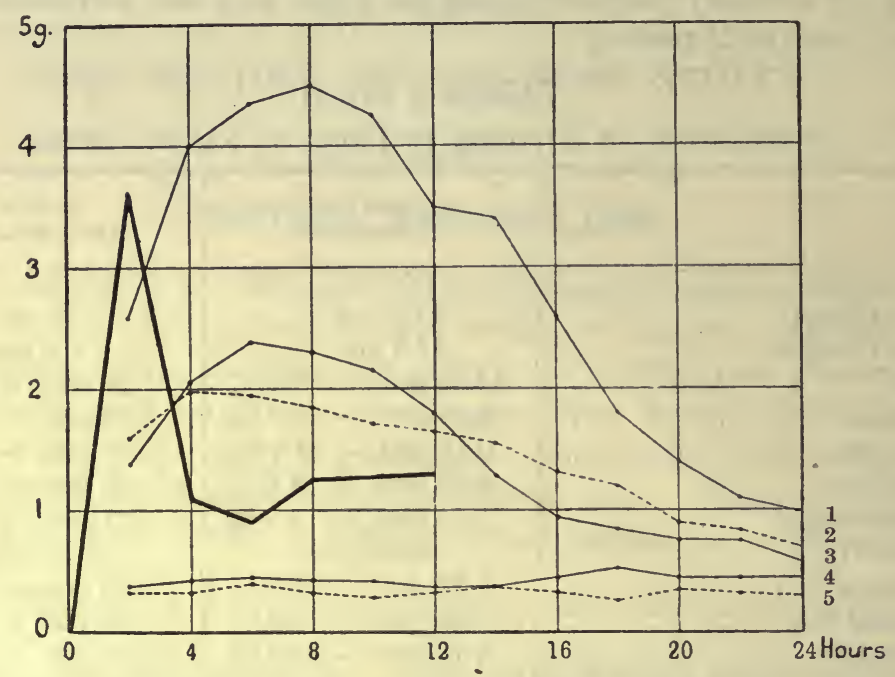

FIG. 353. The hourly variation in the excretion of nitrogen after a meal. The meal was given at 0 . The thick line represents the average absorption of the food from the alimentary canal. The thin-lined curves represent the N. excretion (1) after a meal of 1000 grm. meat ; (2) after 500 grm. meat and $150 \mathrm{grm}$. fat; (3) after a meal of $500 \mathrm{grm}$. meat ; (4) and (5) both represent the excretion in a fasting animal. (From TIGERSTEDT after FEDER.)

analyses. In the first place, on a normal protein diet the urea accounts for 87 per cent. of the total nitrogen of the urine. On an excessive protein diet this percentage may rise to 90 or 95 . On the low protein diet the percentage of nitrogen appearing as urea is reduced to 60 . On the other hand, practically identical amounts of creatinine are obtained under the two conditions, so that whereas on the full diet it amounts only to $3 \cdot 6$ per cent., on the low protein diet it forms as much as 17 per cent. of the total nitrogen output. We are therefore justified in regarding urea as to a large extent exogenous in origin, and as derived directly from the nitrogenous moiety of the protein molecule, which may not at any time have formed part of the living tissues of the body.

On giving a large protein meal to a dog the urea in the urine rapidly rises, and at the end of four or five hours 50 per cent. of the total nitrogen taken in with the food has appeared in the urine as urea (Fig. 353). If we take into account that the digestion of a meat meal in this animal may go on for eight hours, we are justified in the statement that by far the greater 
portion of the protein nitrogen taken with the food is excreted almost directly after absorption as urea in the urine. Urea is therefore to be regarded in the first place as an index to the amount of protein absorbed. We have seen that the end-products of protein digestion in the intestine are the amino-acids; and that these are the immediate precursors of the urea is shown by the fact that the administration of these bodies is followed very rapidly by the appearance of the whole of their nitrogen in the urine as urea. Many attempts have been made to explain the method by which urea may be derived from the amino-acids.

F. Hofmeister succeeded in preparing urea by oxidising amino-acids with potassium permanganate in the presence of ammonia and ammonium sulphate. He assumes that urea is formed by an oxidation synthesis. The first step would be the formation, by oxidation of protein or amino-acids, of the group $\mathrm{CONH}_{2}$, and this at the moment of formation would combine with the $\mathrm{NH}_{2}$ left over in the oxidation of the ammonia to form urea,

$$
\mathrm{CO}<\mathrm{NH}_{2}
$$

According to Drechsel and Nencki, the immediate precursor of the urea is probably ammonium carbamate, which loses a molecule of water, thus :

$$
\mathrm{CO}<\mathrm{NH}_{2} \mathrm{ONH}_{4}-\mathrm{H}_{2} \mathrm{O}=\mathrm{CO}<\frac{\mathrm{NH}_{2}}{\mathrm{NH}_{2}}
$$

Schröder, on the other hand, suggested that the urea was formed from ammonium carbonate by the loss of two molecules of water.

$$
\mathrm{CO}<\mathrm{ONH}_{4}-2 \mathrm{ONH}_{2} \mathrm{O}=\mathrm{CO}<\mathrm{NH}_{2}
$$

In all these views it is assumed that before urea can make its appearance in the urine there must be a complete destruction of the amino-acid. If we compare the structure of any amino-acid with that of urea we see that the proportion of carbon to nitrogen is very much greater in the former than in the latter, and that even after splitting off from two molecules of amino-acid the necessary elements to form urea, $\mathrm{CON}_{2} \mathrm{H}_{4}$, almost the whole of the molecule will be left in an unoxidised condition. A complete oxidation of the amino-acid would result in the production of ammonia, carbon dioxide, and water, so that if oxidation were the method adopted for the production of urea the immediate precursor of this substance would be a combination of ammonia and carbonic acid, either ammonium carbonate as suggested by Schröder, or carbamate as thought by Dreschel. We have distinct evidence that ammonia in one of these two forms is an important precursor of urea. If ammonium carbonate or carbamate be administered to man or to an animal the whole of it is turned out in the urine as urea. Although there is normally a small amount of ammonia in the urine, it is not increased by injections of ammonium carbonate. Schrödèr has shown that the liver, even after removal from the body, has the power of transforming ammonium carbonate into urea. Defibrinated blood mixed with ammonium carbonate was passed through a surviving liver. After a little time it was 
found that the ammonium carbonate had disappeared and that its place was taken by urea. If the liver is necessary for this conversion to take place and ammonia is a constant precursor of urea, we should expect to find that the abolition of the hepatic functions would cause the appearance in the urine of ammonium carbonate or carbamate in the place of urea. The cutting out of the liver is not, however, an easy matter in mammals. Ligature of the portal vein, which would be a necessary step in the extirpation of the liver, causes the blood to be dammed up behind the ligature in the portal area. The intestinal wall gets full of effused blood, the blood pressure falls steadily, and the animal dies within a few hours, being bled to death, so to speak, into its portal vessels. A way of obviating this difficulty was suggested by a Russian surgeon, Eck, and was successfully carried out by Pawlow. Before ligature of the portal vein, this vessel was joined to the vena cava and an artificial opening made connecting the lumen of the two vessels, so that, after the ligature, the blood could flow directly into the general circulation without passing through the liver. Some animals operated on in this way showed no abnormal symptoms whatsoever. There was a rapid formation of a collateral circulation so that the blood could get round the ligature to the liver. Under all circumstances a path to the liver was still open by the hepatic artery, but to arrive here the blood from the alimentary canal had first to pass through the general circulation. A certain number of animals were found to be particularly susceptible to the nature of their diet. On a diet largely consisting of carbohydrates they maintained good health. After a large meat meal, however, they became ill, and in many cases suffered from tremors and convulsions ending in coma. At the same time there was a definite increase of ammonia in the urine, chiefly in the form of ammonium carbamate. Analysis of the blood from a normal animal showed that during protein digestion there was a constant excess of ammonia in the blood of the portal vein above that in any other part of the vascular system. In the carotid blood the normal amount, according to Nencki, is about $2 \mathrm{mg}$. per 100 c.c., in the portal blood 4 to $6 \mathrm{mg}$.* During the morbid symptoms brought on by a protein meal in the animals in whom an Eck fistula had been produced, the ammonia in the carotid blood may rise to as much as $4 \mathrm{mg}$., i.e. to the amount normally found in portal blood. Pawlow and Nencki therefore ascribed the symptoms observed in these dogs after a heavy meat meal to a condition of ' ammoniæmia,' and regarded the liver as an organ which is normally concerned in protecting the rest of the body from ammonia produced in the alimentary tract by converting this substance into the innocuous neutral body, urea.

This view of the function of the liver is confirmed by Schröder's experiments on birds. In these animals the chief nitrogenous excretion is not urea, but ammonium urate, 60 per cent. of the nitrogen of the urine appearing in the form of uric acid. In birds there is naturally a communication between the portal system and the general venous system by means of the vein of

* According to Folin this excess of ammonia in the portal blood, when present, is due to bacterial fermentation processes occurring within the intestine. 
Jacobson, which connects the lower branches of the portal vein with, as a rule, the left renal vein (Fig. 354). On this account the liver can be cut out of the body or of the circulation without entailing the rapid death of the bird, which may live for three or four days, and pass urine after the operation. The urine is, however, fluid, and the uric acid, instead of accounting for 60 per cent. of the total nitrogen, now forms only 5 per cent. The place of the greater part of the uric acid has been taken by ammonium lactate, which therefore seems to be the chief immediate precursor of the uric acid in

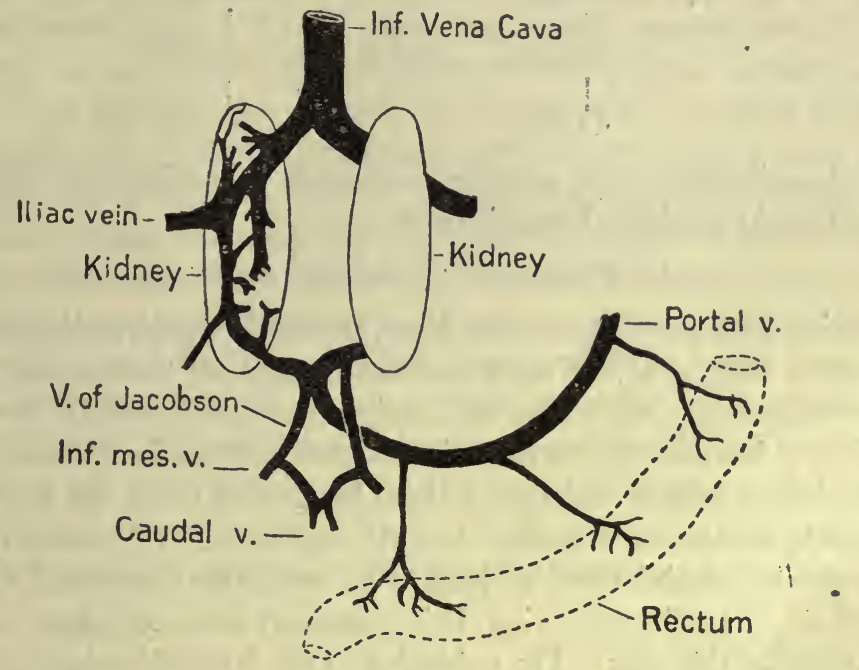

FIG. 354. Diagram to show the arrangement of the veins in the bird, with the communication of the renal and portal veins. (After Morat.)

the urine of birds. We shall have occasion to consider the method of transformation of ammonium lactate to uric acid more fully when dealing with the origin of the latter body.

Of late years evidence has been brought forward that the formation of ammonia from the amino-acids may involve no such profound changes of oxidative disintegration as were suggested in the theories of Hofmeister or Schröder. If amino-acids be treated with the pulp of various organs the amount of ammonia in the mixture is increased, an increase which is absent when no amino-acids are added. More ammonia, for instance, was found when leucine, glycine, tyrosine, or cystine was added to the pulp. On the other hand, phenylalanine gave rise to no production of ammonia. This conversion was ascribed by Lang to the presence of a deaminising ferment in the cells of these different tissues,* and Leathes and Folin have suggested that this process of deamination is the essential factor in the rapid conversion of the nitrogen of the ingested protein into urea. Viewed in this light,

* According to van Slyke, the liver plays the chief part in the breakdown of the amino-acids, though there is no reason to deny the possession of similar power to the other tissues (e.g. muscles) of the animal body. 
these results of Lang, and Folin, effect an entire revolution in our views of protein metabolism. Instead of regarding the urea which appears in the urine after protein ingestion as produced by the total disintegration of the protein molecule, we see now that it represents merely the throwing off of the nitrogenous part of this molecule. This deamination may be a purely hydrolytic change or it may be associated with oxidation or reduction. Deamination of alanine, for instance, by simple hydrolysis would result in the formation of lactic acid (an oxy-fatty acid).

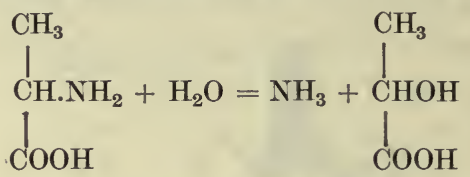

If the deamination were accompanied with oxidation the corresponding keto-fatty acid would be formed, thus

$$
\mathrm{CH}_{3} \cdot \mathrm{CHNH}_{2} \cdot \mathrm{COOH}+\mathrm{O}=\mathrm{NH}_{3}+\mathrm{CH}_{3} \mathrm{CO} \cdot \mathrm{COOH}
$$

If reduction took place at the same time, the result would be the production of a saturated fatty acid. Knoop has shown that all three cases may occur. The investigation of the stages in deamination, and indeed in the disintegration of fatty derivatives generally, is rendered difficult by the fact that all the intermediate products undergo further change and leave the body in a state of complete oxidation, as carbon dioxide and water. If, however, an aminoacid group be administered as part of an aromatic compound, i.e. forming a side-chain of the benzene ring, it is protected from complete oxidation by the stability of this ring. The oxidation of the fatty side-chain may proceed to a certain degree, so that intermediate products of metabolism may be excreted still attached to the benzene nucleus. In the $\alpha$-amino-acids the point where disintegration first occurs is the $a$-group. Deamination Knoop finds most usually associated with oxidation. The primary product

is therefore an $\alpha$-keto-acid. Further oxidation affects the $\mathrm{CO}$ group, so that carbon dioxide is eliminated and the next lower acid in the fatty acid series, is produced. Thus from alanine the body would produce pyruvic acid, $\mathrm{CH}_{3} \cdot \mathrm{CO} \cdot \mathrm{COOH}$, and this on further oxidation would form acetic acid, $\mathrm{CH}_{3} . \mathrm{COOH}$, and carbon dioxide. On the other hand, these keto-acids may undergo reduction to an oxy-acid, or even a step further, to a fatty acid, though the conditions which determine whether oxidation or reduction shall take place have not yet been fully studied.

Of very great importance is Knoop's discovery that deamination is a reversible process, so that, given a right molecular grouping, a fatty acid residue may react with ammonia to form an amino-acid. The proof of this fact was facilitated by the discovery that the next higher homologue of phenylalanine, namely, phenyl- $\alpha$-amino-butyric acid, when administered to an animal, was excreted in large quantities in the urine as an ether-soluble acetyl derivative, which was easily isolated in a state of purity. If then this 
amino-acid were formed in the body, one might expect to find it without difficulty in the urine. Knoop found that the administration of either phenyl- $\alpha$-keto-butyric acid or phenyl- $\alpha$-oxybutyric acid led to the excretion of the corresponding amino-acid in the urine. Since keto-acids occur as the ordinary products of the breakdown of amino-acids and also as the intermediate products of oxidation of oxy-acids, e.g. lactic acid, it is evident that the animal body can assimilate ammonia and form amino-acids, provided only that it is supplied with the proper non-nitrogenous acids. These latter need not be derived from proteins at all, but, like lactic acid, be a result of carbohydrate metabolism. Thus, if the fitting non-nitrogenous food be given (e.g. oxy-fatty acids, or carbohydrates, from which these bodies may be formed), part of the nitrogen set free by protein disintegration might be recombined with the formation of amino-fatty acids without giving rise to urea or appearing in any way in the nitrogen balance-sheet of the body. This possibility enjoins the necessity of caution in interpreting the results of metabolism experiments where the nitrogen excreted is taken to represent the total protein metabolism of the body. The fate of the nitrogen does not, however, matter much to the energy balance-sheet of the body, since so far as regards energy the residue of the protein molecule or the amino-acid molecule which is left behind after the process of deamination has taken place, has lost only from one-fifth to one-tenth of the total energy of the original molecule. This is shown in the following Table of the heat equivalents of some of the amino-acids and their corresponding fatty and oxy-acids :

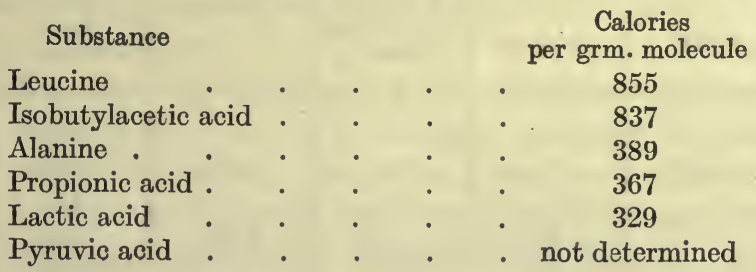

Even in the case of the smallest molecule the loss of energy attendant on simple deamination and conversion into the corresponding oxy-acid only amounts to about 20 per cent. We thus come to the conclusion that the urea output in the urine after a protein meal tells us nothing whatever about the fate of that part of the protein which contains 80 to 90 per cent. of the total energy of the protein food. So far as concerns the output of energy, the exogenous protein metabolism may be regarded as practically non-nitrogenous. The rise in the rate of excretion of urea after a protein meal was regarded both by Voit and Pflüger as a sign that the cells of the body preferred to use proteins for all their requirements if this substance were available. We see now that the big output of urea after a protein meal affords no basis for this view, but is rather a sign that the body has no need for all the nitrogen contained in its food and that this must be got rid of before the really valuable part, the energy-giving part of the protein molecule, is admitted into the metabolic cycle of the cells.

The important problem in the energy metabolism of protein is thus, not 
the origin of the urea, but the nature of the substances that are left after deamination and their subsequent fate in the body. Since they contain only the elements carbon, hydrogen, oxygen, one would expect to find that they could replace either fat or carbohydrate. So far as concerns the production of energy this is true. Moreover, as we shall see in dealing with the metabolism of carbohydrates, we have definite evidence that part of this nonnitrogenous moiety of the protein molecule may be converted into sugar or glycogen. Thus, of the amino-acids formed by the digestion of proteins, glycine, alanine, aspartic acid and glutamic acid can be converted quantitatively under appropriate circumstances into glucose. On the other hand, leucine, phenylalanine and tyrosine yield no glucose, even in the diabetic animal, but may in the liver undergo conversion into aceto-acetic acid, which is a stage in the oxidative disintegration of fats. In spite of this latter fact we have no evidence that fat may be formed from this part of the protein molecule ; at any rate, no fat which can be stored in the body and give rise to the production of adipose tissue. On the other hand, the proteins, more than either of the other two food-stuffs, cause a direct augmentation of the respiratory exchanges of the body. This is shown in the following Table by Rubner, in which isodynamic quantities of proteins, fats, and carbohydrates were administered during a period of starvation :

\begin{tabular}{|c|c|c|c|c|c|c|c|}
\hline \multirow{2}{*}{\multicolumn{2}{|c|}{ Day }} & \multicolumn{3}{|c|}{ Food } & \multirow[b]{2}{*}{ Calories } & \multicolumn{2}{|c|}{ Energy metabolism } \\
\hline & & N. grm. & Fat grm. & $\begin{array}{c}\text { Carbohydrate } \\
\text { grm. }\end{array}$ & & $\begin{array}{c}\text { Total } \\
\text { calories }\end{array}$ & $\begin{array}{c}\text { Per kilo } \\
\text { body weight } \\
\text { calories }\end{array}$ \\
\hline 2 & . & - & - & - & - & 969 & $40 \cdot 2$ \\
\hline 3 & . & $56 \cdot 8$ & - & - & 1543 & 1072 & $44 \cdot 8$ \\
\hline 4 & . & - & - & & - & 947 & 39.9 \\
\hline 5 & . & - & 167 & - & 1536 & 963 & $40 \cdot 9$ \\
\hline 6 & . & - & - & - & - & 922 & $39 \cdot 6$ \\
\hline 7 & . & - & - & 411 & 1446 & 982 & $42 \cdot 3$ \\
\hline 8 & . & - & - & - & - & 977 & $42 \cdot 1$ \\
\hline
\end{tabular}

It will be seen that the metabolism, i.e. the caloric output, of the body on administration of protein increased 11.9 per cent., whereas with fat the increase amounted only to 1.2 per cent., and with carbohydrate to 4.7 per cent. In another similar experiment the animal received $57 \cdot 4$ calories protein, 54.2 calories fat, and 57 calories carbohydrate respectively per kilo body weight. During hunger the total metabolism per kilo body weight amounted to 37.5 calories; with meat, to 46 calories; with fat, to $39 \cdot 4$ calories; with carbohydrates, to $39 \cdot 4$ calories. Compared with the metabolism during starvation the rise per cent. with protein was $24 \cdot 3$, and with fat and carbohydrates $5 \cdot 1$. This surplus output of energy resulting from the administration of protein cannot be ascribed to increased work thrown on the digestive organs. There is no evidence that this is greater in the case of proteins than it would be with carbohydrates or fats ; 
and even if the capacity of these organs be strained to their utmost by administration of large quantities of bones, the increase in the carbon dioxide output which results is not so great as that following a large protein meal. It seems therefore that the $\mathrm{CHO}$ moiety of the protein undergoes oxidation more rapidly than either dextrose or the ordinary fats of the diet, and that, as we concluded in an earlier chapter, the metabolism of these substances is really to a considerable extent dependent on the quantity presented to the organism rather than on the actual needs of the cells of the body. It is this rise in metabolism and respiratory exchanges after protein ingestion which justifies to a certain extent the idea that the proteins, more than any other food-stuff, have a stimulant action on metabolism. The reason why the $\mathrm{CHO}$ remainder of the protein molecule is so prone to oxidation and does not, like an excess of carbohydrates, undergo conversion into fats in the body, we shall have to consider in greater detail in dealing with the fate of this latter class of substances. We need, however, considerably more evidence, as to the extent to which deamination occurs and as to its conditions and end-products before we can hope to determine the cause for the rapid breakdown of these end-products in the body.

\section{ARE THE AMINO-ACIDS INTERCONVERTIBLE ?}

Although the animal organism is apparently capable of synthetising amino-acids from ammonia and the corresponding keto- or oxy-fatty acid, it is unable to convert one amino-acid into another. On this account many proteins are inadequate as food substances since they do not contain the necessary amino-acid groups. Life cannot be supported on such bodies as zein or gelatin, which are lacking in the tryptophane and tyrosine groups. The failure in these cases is not, as has been generally supposed, owing to an inability to assimilate, i.e. synthetise, nitrogen as ammonia, but to the fact that in the animal the apparatus is wanting for the manufacture of some of the oxy-fatty acids and other radicals which form the non-nitrogenous part of the amino-acids. This view receives confirmation from the fact that the simplest of the amino-fatty acids, namely, glycine, can be easily manufactured in the body, acetic acid being one of the latest stages in the oxidation of most carbohydrates and fats. It has been shown that alanine too can be easily manufactured by the body, by the amination of the three carbon acids or oxy-acids derived from the breakdown of glucose or glycogen.

\section{THE EXCRETION OF AMMONIA}

A large proportion of the urea appearing in the urine after a protein meal is exogenous and is derived by a rapid separation of ammonia from the proteins or their disintegration products almost immediately after their absorption. The greater part of the ammonia is converted in the liver into urea, which is excreted by the kidney. A certain small proportion of the nitrogen in the urine is generally turned out in the form of ammonia. This proportion is not increased by the administration of ammonium carbonate. If ammonium chloride be given to a starving rabbit it appears in the urine 
unchanged, and so increases the proportion of ammonia in this fluid. If, however, the ammonium chloride be administered at the same time as the animal is receiving its ordinary vegetable diet there is no increase in the ammonia in the urine, the whole of the ammonium chloride being converted into urea. The factor which determines the proportion of ammonia in the urine is the relative proportion of acids and bases which have to be eliminated from the body. The normal reaction of urine, though acid as regards certain indicators, can be regarded as neutral, since it contains no free hydrogen ions, the ' acidity' being due to the presence of such substances in solution as acid sodium phosphate. If the fixed alkalies in the food are sufficient to combine with the whole of the acids excreted from the body, then the ammonia will be completely converted into urea and eliminated as such. If, however, a dose of mineral acid be administered to an animal, this must be excreted in combination with a base. If the fixed alkalies available do not suffice for this purpose, the neutralisation of the acid is effected by coupling with ammonia. The ammonia of the urine is therefore an index to the amount of acids which are excreted. These acids may be introduced directly with the food, as when mineral acids are administered by the mouth, or may be the product of abnormal metabolic processes occurring in the body. Thus under certain circumstances, e.g. in complete carbohydrate starvation, there is a failure in the last stages of the oxidation of fats, and oxy-fatty acids, viz. oxybutyric acid and aceto-acetic acid, are produced in the body in large quantities, but cannot undergo further disintegration. The alkalescence (electrical neutrality) of the fluid media of the body is a necessary condition for the continuance of the life of the cells and especially of the normal processes of oxidation. It is therefore essential for the preservation of life that the acids thus formed and accumulating as a result of the impaired oxidative processes should be neutralised, carried to the kidneys, and excreted by them in combination with some base. When these acids are produced in large quantities the alkalies of the food and of the tissues do not suffice for their neutralisation. Ammonia, which is a constant intermediate stage in the production of urea, is then utilised for this purpose and the acids appear in the urine together with the corresponding amount of ammonia. The ammonia therefore of the urine gives valuable information, not as to the total nitrogenous exchanges of the body, but as to the formation of acids in abnormal quantities during the processes of metabolism.

There is one other method in which urea may be formed by a rapid alteration of the proteins taken in with the food. Nearly all the ordinary proteins contain arginine as an integral part of their molecule. This substance can be regarded as formed by a coupling of guanidine with aminovalerianic acid and as analogous to the most prominent extractive of muscle, namely, creatine, which is methyl guanidine acetic acid. On heating either of these substances with baryta water it undergoes hydrolysis and is decomposed with the formation of urea and, in the case of arginine, $\alpha$ - $\delta$-diaminovalerianic acid ; in the case of creatine, methyl amino-acetic acid or sarcosine. It has been shown by Dakin and Kossel that the same change may be 
effected under the agency of a ferment, arginase, which is contained in extracts of the intestinal wall or of the liver. We have every reason to believe therefore that a certain small proportion of the urea which appears in the urine after the ingestion of protein is due to this hydrolytic splitting of the arginine contained in the protein molecule. The other moiety of the arginine, namely, the diamino-valerianic acid, probably undergoes the same changes as the other amino-acids, such proportion of it as is not required for the building up of the tissues of the body being deaminised and giving rise to urea and some $\mathrm{CHO}$ group in the manner already discussed.

\section{THE ENDOGENOUS OR TISSUE METABOLISM OF PROTEINS}

On comparing the output of the various nitrogenous excreta given in Folin's Tables quoted above (p. 757), we see that on a low protein diet, when the exogenous or energy metabolism of this food-stuff is reduced to a minimum, the only substance which does not undergo simultaneous diminution is the creatinine. Whereas on an ordinary diet free from meat it only accounts for about 3 per cent. of the total nitrogen output, on the low diet it contains as much as 17 per cent. The conclusion at once suggests itself that creatinine, more than all the other constituents of the urine, must be regarded as an index of the tissue metabolism of protein. Let us see what facts can be adduced in favour of this view.

Creatinine has the formula:

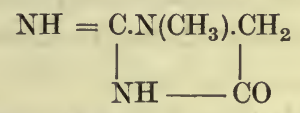

and may be regarded as derived by a process of dehydration from creatine (methyl guanidine acetic acid).<smiles>CC(N)(N)CCC(=O)O</smiles>

It may be formed from this latter substance by boiling for three hours with strong hydrochloric acid. Creatine has long been known as the most abundant nitrogenous extractive in the body. It exists in relatively large quantities in muscle, and in meat extracts, such as Liebig's, it occurs to the extent of 10 or 12 per cent. It has been calculated that the body of a man at any time contains about $90 \mathrm{grm}$. of this substance. On boiling creatine with baryta water it undergoes hydrolysis with the formation of urea and sarcosine or methyl glycine.<smiles>C[C@H](CC(=O)O)C(=O)NCCCCC(C)(N)C(=O)O</smiles>

Owing to the ease with which this formation of urea from creatine may be brought about outside the body, it was natural that this substance should be regarded as an important precursor of the urea in the urine. The view 
was held till recently, however, on the ground of experiments by Voit, that creatine administered in the food appeared in its entirety as creatinine in the urine, so that if creatine were liberated from the muscles in their normal processes of metabolism it would pass to the kidneys and be excreted as creatinine without undergoing further decomposition. On this account too the creatinine in the urine was regarded as derived almost exclusively from the creatine taken in with the food. The analyses given in Folin's Tables show that in one respect at any rate this view was incorrect. Creatinine is excreted in considerable quantities even when the man is on a creatine-free diet, or even when his food is almost free from protein. It has been found moreover by Folin that creatine administered by the mouth may disappear in the body. This is especially the case if the animal or man is on an insufficient protein diet, but there is no evidence of a corresponding increase in urea formation. If a larger amount be given creatine appears as such in the urine. In most cases a certain minute proportion escapes and causes an increase in the quantity of creatinine. Under abnormal circumstances, e.g. during illness, when the physiological activities of the body are lowered, a portion of the creatine may be found in the urine in an unchanged condition. If creatinine is to be regarded in any way as the index of tissue metabolism its amount ought to vary with the extent of this metabolism. Thus it should be increased when there is an exaggeration of the disintegrative processes in the tissues, and should be diminished when the nutritive changes in these tissues, especially in the muscles, are reduced to a minimum. The end-products of tissue metabolism therefore should be increased under the following conditions :

(1) Increased motor activity involving increased wear and tear of the muscular tissues.

(2) In fevers, especially in those where there is severe toxæmia and rapid wasting of the muscles of the body.

On the other hand, it should be diminished where the activity of the muscular tissue is reduced to a minimum, as under the influence of sleep or soporifics, or where the bulk of the muscular tissue is reduced as well as its activity, as in cases of widespread muscular atrophy and paralysis.

The excretion of creatinine has been investigated under these various conditions by Van Hoogenhuyze and Verploegh, and their results fully bear out the view expressed above as to the intimate relation of creatinine with the tissue metabolism of protein.

During protein starvation the uric acid output, though diminished, does not show a change which is at all proportional to that shown by the urea. This substance also might therefore represent an end-product of tissue metabolism. Since, however, uric acid is an outcome of the metabolism of a special group of bodies, the nucleins and purine bases, we shall have to devote a complete section to its consideration.

Although the urea is diminished in protein starvation, it still remains the most abundant nitrogenous constituent of the urine. We are therefore not justified in excluding this substance from the products of tissue metabolism. 
If any creatine undergoes complete oxidation in the body during protein starvation a certain proportion of the urea might be derived in this way. We shall see later that uric acid may possibly also undergo further oxidation with the formation of urea. Even during complete protein starvation some of the urea which is turned out may be the expression of a utilisation of protein through deamination for the energy needs of the body. The active cells are bathed everywhere with a tissue fluid in which proiteins form a preponderating constituent, and it is possible that, even in the times of greatest protein need, these cells utilise the proteins of their surrounding medium, though in a reduced degree, for the production of energy. In this case the active cell would initiate the utilisation by throwing off that part of the protein molecule, namely, $\mathrm{NH}_{2}$, which is useless to the cell as a source of energy, so that deamination would be carried out in the working tissues, and not, as in the rapid formation of urea after a heavy meal, in the liver.

\section{SULPHUR}

Sulphur occurs in the urine in three forms, namely, as ordinary inorganic sulphates, as ethereal sulphates (indoxyl- and skatoxyl-sulphates), and in an unoxidised condition often termed neutral sulphur. There is no doubt that part of the latter consists of cystine, part of sulphocyanates, and in some animais mercaptan compounds. The excretion of the inorganic sulphates rises pari passu with that of the urea, so that very soon after the throwing off of the $\mathrm{NH}_{2}$ group there must be also a removal and oxidation of the greater part of the sulphur contained in the cystine group of the protein molecule. So far as regards the metabolism of the body as a whole, the ethereal sulphates may be classed with the inorganic sulphates. They are excreted in varying quantity according to the extent of the decomposition processes which are occurring in the intestine. Under the influence of these processes the tryptophane, produced in the pancreatic digestion of proteins, is converted into indol and skatol. These two substances after absorption are deprived of their poisonous qualities by oxidation and conjugation with sulphuric acid to form the indoxyl- and skatoxyl-sulphates of the urine, both of which are innocuous. If the processes of putrefaction are increased, as in intestinal obstruction, the relative amount of sulphate appearing in the conjugated form is also increased. On administration of phenol a large proportion of the sulphate appears in the urine conjugated with phenol or with products of its oxidation. If the normal putrefactive processes which go on in the intestine are abolished by the administration of intestinal antiseptics such as naphthalene or calomel, the ethereal sulphates practically disappear from the urine. We cannot therefore regard the absence or diminution of the ethereal sulphates during protein starvation as throwing any light on the endogenous protein metabolism. On the other hand, the fact that the neutral sulphur undergoes no decrease suggests that this part of the sulphur output of the organism may be connected with tissue metabolism. "Further observations on the output of neutral sulphur during fever or wasting diseases are necessary before a definite conclusion can be arrived at on this point. 


\section{THE FATE OF THE AROMATIC AND OTHER CYCLIC GROUPS IN THE PROTEIN MOLECULE}

A typical protein such as can be utilised as a complete food-stuff contains, in addition to the amino-acids of the fatty series, a number of other nitrogenous derivatives of cyclic compounds, including benzene, indol, pyrrol, and iminazol. Substances such as gelatin, from which some of these groupings are absent, cannot, as we have seen, entirely replace protein in the food. So far we are acquainted with three compounds of the aromatic series among the products of disintegration of the protein molecule. These are tyrosine, phenylalanine, and tryptophane. Since these substances are also contained in the protein constituents of the tissues we may assume that, after they have been set free by the digestive hydrolysis of proteins, they are absorbed and built up again with the other amino-acids in appropriate groupings. Like these they are suisceptible of complete oxidation in the body, so that they can contribute to the supply of energy. Any one of these substances, administered with the food or subcutaneously, is entirely destroyed, with the production of urea, carbon dioxide, and water. In this respect they present a marked contrast to almost all other compounds of the aromatic series. In these we find that the benzene ring is extremely stable, so that, although changes may occur in its side-chains, the benzene ring itself appears intact in the urine, and is not broken up in the body. Thus benzoic acid, benzylalcohol, and phenyl propionic acid, when administered, are passed in the urine as hippuric acid (benzoyl glycine). Indol and skatol, which are closely allied to tryptophane, undergo oxidation in the body without further modification and appear in the urine as conjugated aromatic sulphates.

Some light is thrown on the conditions of breakdown of these aromatic bodies by the study of a rare disorder in metabolism, which may occur in certain families and is known as alcaptonuria. In this condition, which is congenital and lasts throughout life, the urine darkens considerably when made alkaline and exposed to the air. It has the power of reducing Fehling's solution, so that the presence of sugar might be suspected. On analysis the peculiarities of the urine are found to be due to the presence in it of a substance known as homogentisic acid. This is dioxyphenyl acetic acid.

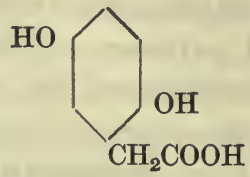

The amount of this substance in the urine bears a constant ratio to the nitrogen excreted. It does not disappear during starvation, and is much increased on a large protein diet. It must therefore be derived from the disintegration of proteins both exogenous and endogenous. If tyrosine or phenylalanine be administered to patients affected with this disorder, both substances are quantitatively converted into homogentisic acid. The ratio 
of this acid to the total nitrogen indicates that the whole of the tyrosine and phenylalanine of the protein molecule, whether set free in the alimentary canal or in the tissue metabolism, is converted into homogentisic acid. It is not possible to conceive of the direct conversion of

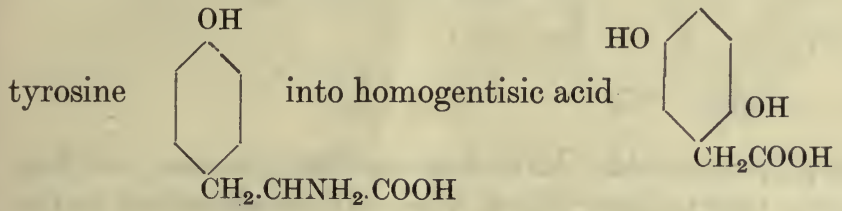

The tyrosine must first be reduced to phenylalanine

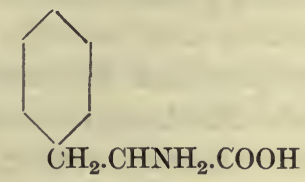

and then this substance must undergo oxidation into homogentisic acid. Since phenyl lactic acid and phenyl pyruvic acid, but not phenyl acetic acid, are also converted in alcaptonuric patients to homogentisic acid, it has been suggested that these two substances form stages in the conversion of phenylalanine into homogentisic acid. Thus

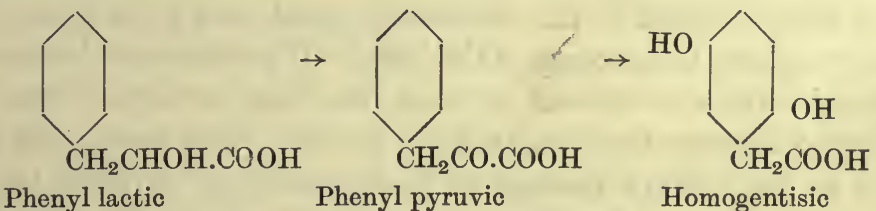

It is further thought that under normal circumstances the phenyl derivatives, tyrosine and phenylalanine, are oxidised to homogentisic acid as in the alcaptonuric patient. In the normal individual, however, the introduction of two hydroxyl groups into the benzene ring leads to some process, perhaps of a ferment character, which breaks up the ring. This ferment is absent in the alcaptonuric, so that the transformation of the phenyl derivatives stops short at the stage of homogentisic acid (Garrod). The eminently specific character of this process is shown by the fact that although these various substances undergo complete oxidation in the body, a slight modifcation in the chain of the processes renders the change impossible. Thus if the side group in phenyl lactic or phenyl pyruvic acid be converted to acetic acid before the introduction of the two $\mathrm{OH}$ groups into the phenyl ring, the phenyl acetic acid thus produced is incapable of undergoing further oxidation. Tyrosine in the intestine undergoes deamination to form oxyphenyl propionic acid and oxyphenyl acetic acid. These cannot be further oxidised, but appear in the urine as such or, after conversion into kresol or phenol, as sulphuric acid esters.

Somewhat similar conditions apply to the oxidation of tryptophane. 
This body is an indol derivative and consists of a benzene ring and a pyrrol ring having two of their carbon atoms in common. Its formula is

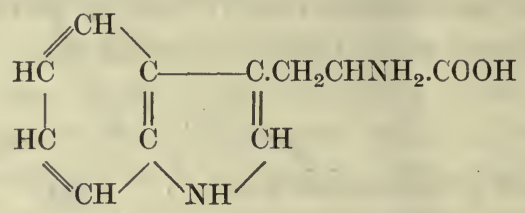

i.e. it is indol amino-propionic acid. It undergoes, like tyrosine, complete oxidation in the body. On the other hand, a very slight alteration in the molecule renders it incapable of this change. Thus the tryptophane set free by the tryptic digestion of proteins under the influence of the putrefactive bacteria of the intestine may undergo deamination and reduction with the production of indol propionic acid, and this by oxidation may be converted to indol acetic acid. The latter substance by decarboxylation may be converted into skatol, or by oxidation nearer the chain and further loss of carbon dioxide into indol. Of these products of bacterial change, indol acetic acid may be found in the urine, and indol and skatol are oxidised to the corresponding phenols and pass into the urine conjugated either with sulphuric acid or with glycuronic acid. Apart, however, from these putrefactive changes due to bacteria, no indol derivatives pass into the urine. The amount of the indol and skatol esters serves therefore merely as an index of bacterial decomposition in the alimentary canal, and gives us no clue to the total tryptophane metabolism of the body. If putrefaction be prevented by the administration of calomel or other intestinal antiseptic these esters may entirely disappear from the urine. On the other hand, the partial obstruction to the onward passage of food, caused by dividing the small intestine in two places a few inches apart and replacing the intervening length of intestine the wrong way round, causes the indican excretion to be increased twenty or thirty fold. Subcutaneous injection of tryptophane in rabbits does not increase the indoxyl and skatoxyl sulphates (urinary indican) in the urine, whereas a considerable increase is brought about by subcutaneous injection of indol.

The pyrrol ring which occurs in proteins as proline and oxyproline (i.e. pyrrolidine carboxylic acid and oxypyrrolidine carboxylic acid) appears to undergo complete disintegration in the body. The steps in this conversion are unknown, though it is possible that the ring may be unlinked so as to produce from the pyrrol ring amino-valerianic acid, which would then undergo the process of deamination with which we are already familiar. This ring is of interest since it appears to take an important part in the building up of the molecule of hæmatin, the essential prosthetic group of the hæmoglobin molecule.

Another ring grouping, iminazol, occurs in histidine, which is iminazol $a$-amino-propionic acid. This too undergoes complete oxidation in the body. It is important to bear in mind that this ring may be produced synthetically by very simple means, i.e. by the action of zinc oxide and 
ammonia on glucose, which results in a rich yield of methyl iminazol ( $v$. p. 117). The same grouping is found in creatinine, as is seen by comparing the formulæ :<smiles></smiles>

Creatinine<smiles>Cn1ccnc1</smiles>

Methyl-iminazol

and it is possible that this may furnish a clue to the mode of formation of creatinine in muscle. Creatine has generally been regarded as the primary product of muscular metabolism, but it is possible that the ring-grouping is the original one and that creatine is produced by hydrolysis occurring in this ring.

The iminazol group is at present chiefly interesting in that it contributes to the formation of the complex ring compounds known as the purines. Since the purine metabolism is closely connected with the question of the origin of uric acid we may consider these questions together. 


\section{SECTION II}

\section{NUCLEIN OR PURINE METABOLISM}

IN an undifferentiated cell the proteins, as such, form but a small part, the mass of the cell being composed of conjugated proteins. The nucleo-proteins are especially abundant constituents of nuclei, and therefore occur to a greater or less extent in all the ordinary animal foods, eggs and milk excepted. Just as the metabolism of proteins is the metabolism of the amino-acids, so the metabolism of the nucleo-proteins and nucleins is essentially comprised in the history of its main constituents, i.e. the purines.

The nucleo-proteins themselves are bodies of very varying composition. If any cellular tissue such as thymus or liver be extracted with water or salt solution, a fluid is obtained from which a precipitate can be thrown down by the addition of acid. This precipitate as a rule is soluble in excess of acid or in alkalies. If subjected to gastric digestion it undergoes solution, leaving behind a residue of nuclein which is rich in phosphorus. The amount of this residue varies with the strength of the artificial gastric juice employed, so that the method cannot be looked upon as in any way quantitative, and the question arises whether the original nucleo-protein is to be regarded as an association or a combination of nuclein with ordinary protein. The most convenient source for the preparation of nucleins is the heads of fish spermatozoa. All nucleins are associations or compounds of nucleic acids with proteins belonging to the class of protamines or histones. The nucleins of fish spermatozoa contain protamine as one of their constituents. On separating off the protamine, nucleic acids can be isolated. These acids have been named either according to their source or according to the purine base which is their most prominent constituent. Only from inosinic acid, the nucleic acid of muscle, has it been found possible to prepare crystalline derivatives, so that in all other cases it is difficult to decide whether we are dealing with chemical individuals or with mixtures.

On hydrolysing any of the nucleic acids by heating with strong mineral acid, they are broken down into a series of bodies belonging to the following four groups: (1) phosphoric acid, (2) purine bases, (3) pyrimidine bases, (4) a carbohydrate. The chief purine bases obtained from the hydrolysis of nucleic acid are guanine and adenine.

Hypoxanthine and xanthine are often obtained as products of decomposition of nucleic acid, but are generally formed by the deamination and oxidation of guanine and adenine. Fischer has shown that all these bodies 
are derivatives of a base purine. They contain a central chain of three carbon atoms to which is attached on each side a urea group, so that they may be regarded as diureides. Purine itself has the formula<smiles>c1ncc2[nH]cnc2n1</smiles>

The relation of the purine bases obtained from disintegration of nucleic acid to purine itself has been given on p. 103. From these formulæ we see that adenine and hypoxanthine are related to one another, adenine being 6-aminopurine, while hypoxanthine is 6-oxypurine. In the same way guanine and xanthine are related, guanine being 2-amino-6-oxypurine, while xanthine is 2-6-dioxypurine. The investigation of the relationships of these bases was of interest to physiologists since it brought to light the close relation which they have to uric acid, a substance which has been known as a constituent of urine and urinary calculi for a long time, having been discovered in 1776 by Scheele. Uric acid is 2-6-8-trioxypurine and has the formula<smiles>O=C1NC2NC(=O)C(=O)C(N1)N2</smiles>

Uric acid $=2 \cdot 6-8$-trioxypurine

In uric acid the two urea groups are attached to a central 3-carbon chain, and it is interesting to note that one of the first syntheses of uric acid, namely, that by Horbaczewski, was accomplished by melting together trichlorlactamide and urea in a sealed tube.

Belonging to the same group of purines are the two bases which form the essential constituents of tea, coffee, and cocoa. In tea and coffee is found caffeine, which is 1-3-7-trimethyl-2-6-dioxypurine, and in cocoa occurs the closely allied theobromine, which is 3-7-dimethyl-2-6-dioxypurine. Caffeine is thus methyltheobromine.
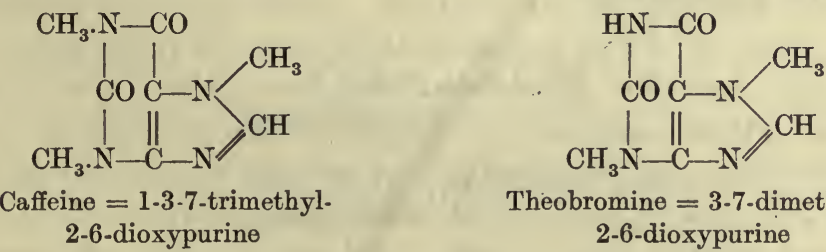

Theobromine $=3 \cdot 7$-dimethyl-

2-6-dioxypurine

The pyrimidine bases which are also obtained from the hydrolysis of nucleic acid are derived from a pyrimidine nucleus which is, so to speak, half a purine nucleus, consisting of a $C</ \mathrm{N}$ chain joined to a 3-carbon 
chain. Three pyrimidine bases have been isolated from the decomposition products of nuclein, namely, thymine, cytosine, and uracil.<smiles>O=c1cc[nH]c(=O)[nH]1</smiles>

Uracil 2-6-dioxypyrimidine<smiles>Cc1c[nH]c(=O)[nH]c1=O</smiles>

Thymine 5-methyluracil<smiles></smiles>

Cytosine 6-amino-

2-oxypyrimidine

Cytosine is easily converted by oxidation into uracil.

After separation of the purine and pyrimidine bases and phosphoric acid a substance is left over which gives the reactions of a carbohydrate.

This carbohydrate differs in different nucleic acids. In plant nucleic acid, as well as in guanylic acid from the pancreas and inosinic acid from muscle, the carbohydrate is a pentose, d-ribose. Most nucleic acids of animal origin yield lævulinic acid on hydrolysis and must therefore contain a hexose. The researches of Levene, Jones, and others have shown that the nucleic acids vary in complexity and consist of one or more so-called nucleotides linked together. The simplest nucleic acids are mononucleotides. Examples of these are inosinic and guanylic acid. Each consists of phosphoric acid and a purine or pyrimidine base linked together by carbohydrate. Upon hydrolysis with boiling mineral acid they are.decomposed into their three constituentis :

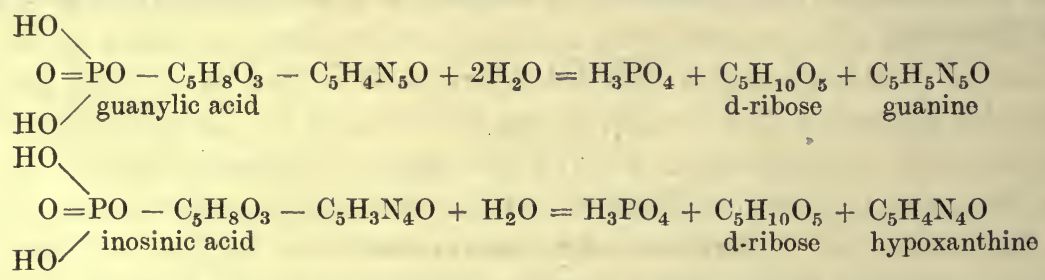

When submitted to neutral hydrolysis at $175^{\circ} \mathrm{C}$ under pressure, decomposition occurs in a different way, the phosphoric acid is split off and a glucoside-like body is left which is called a nucleoside. Thus guanylic acid yields guanosine, inosinic acid yields a body known as inosine or hypoxanthosine. Under the action of ferments, known as nucleases, which may occur in animal tissues, the mono-nucleotides may be split in one of two ways. The phosphonuclease removes the phosphoric acid, leaving a compound of purine and carbohydrate, while the purine nuclease sets free the purine base and leaves a hexose- or pentose-phosphoric acid. Of the more complex nucleic acids. which occur in cell nuclei, yeast nucleic acid has been the most carefully studied. According to Levene this nucleic acid is a tetranucleotide, having a structure represented by the following formula:

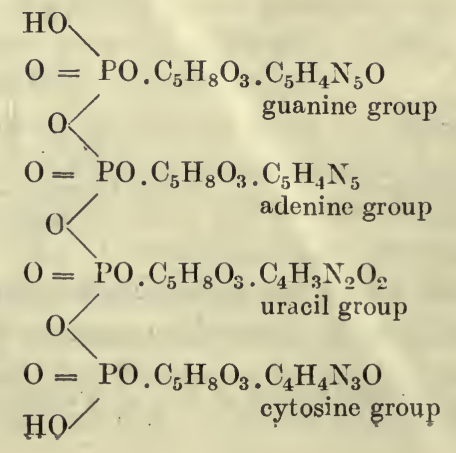


If this is subjected to neutral hydrolysis, it loses the whole of its phosphoric acid and sets free four nucleosides, viz. : guanosine, adenosine, uridine, and cytidine.

\section{FORMATION OF NUCLEINS IN THE BODY}

In the case of the proteins we saw reason to believe that in the higher animals, at any rate, there was no power of converting one amino-acid into another (with the exception of the lowest member of the series, namely, glycine), and that on this account the food had to contain representatives of every amino-acid (or perhaps of the corresponding oxy-fatty acid) necessary to the building up of the tissue proteins. The nucleins, on the other hand, can certainly be synthesised by the animal. This is shown by the fact that the hen's egg before incubation contains practically no nuclein or purine bases. During incubation tissues are formed, and there is a rapid increase in the number of nuclei, so that the chick just before it is hatched contains a considerable amount of nuclein from which purine bases can be extracted. This nuclein must have been formed by a synthesis from the phospho-proteins and phosphatides (phosphorised fats) which form so prominent a constituent of the egg-yolk, and in the same way the purines must have been formed by a process of synthesis. This synthesis may occur by a conjugation of two urea molecules with the 3 -carbon chain which is so prominent a feature in the proximate principles of the body (e.g. in lactic acid, alanine, and all the compound amino-acids of which alanine is a constituent). Methyliminazol, representing one-half of the purine ring, can be formed simply by allowing ammonia and glucose to stand in contact with zinc hydroxide. The power of synthesis of purines possessed by the body must complicate the question of their fate after ingestion, since it is evident that they can either be destroyed and excreted in some other form or that the products of their destruction may be built up into fresh purine or nuclein molecules. In the same way, in the growing child there is a rapid increase in the nuclein of the body, although the only food ingested is milk, which contains but an insignificant amount of nuclein.

\section{FATE OF NUCLEINS IN THE BODY}

Nucleins and nucleic acids are dissolved by the pancreatic juice, but no digestion of the nucleic acid occurs in the alimentary tract other than by the action of micro-organisms. We must assume therefore that the nucleic acid is taken up by the cells of the intestinal wall unchanged.

Ingestion of nucleic acid is followed by an increased excretion of uric acid in the urine, so that we regard this substance as the end-product of nuclein metabolism in the body. It is evident that the uric acid of the urine may be derived either from the nucleins of the food or from the nucleins of the tissues of the body, the uric acid in these two cases being spoken of as exogenous and endogenous respectively. By digestion of nucleic acids with animal tissues or extracts of animal tissues under varying conditions, it is possible to bring about all the changes involved in the conversion of the purine base contained in them into uric acid. In the 
intestinal wall, or after absorption into other tissues of the body, the nucleic acid is subjected to hydrolytic changes by the agency of ferments which may be classed as nucleases. These are, however, of different kinds, the phosphonuclease splitting off the phosphoric acid and leaving the nucleosides, while the purine nucleases, which are more effective in a slightly alkaline medium, split off the purines, leaving the phosphoric acid combined with the carbohydrate. The purines set free in this way undergo further changes. The hypoxanthin derived from inosinic acid is converted under the action of an oxidase first into xanthine and then into uric acid.

This was one of the earliest facts discovered in the metabolism of purines. Horbaczewski showed that if spleen pulp be digested with blood for some time, it is possible to extract a considerable amount of xanthine from the mixture. If, however, oxygen be bubbled through the fluid, the xanthine disappears, its place being taken by uric acid.

From the more complex nucleic acids the amino-purines, adenine, and guanine; are set free. These first undergo deamination under the action of special ferments, adenase and guanase, and are thus converted into hypoxanthine and xanthine respectively. These bodies then, under the action of oxidases, may be converted into uric acid.

All these changes occur in the living body, though not necessarily in the order just set out. Thus when the pyrimidine derivatives are administered to dogs, they pass out unchanged. If, however, free nucleic acid be administered to the animal, no trace of these derivatives can be found in the urine, so that they must have undergone complete oxidation. In the same way the dog's liver is able to deaminise completely the adenine group of nucleic acid, converting it into hypoxanthine, but is without effect on free adenine. It is evident, therefore, that the various ferments which have bəen described act partly on the whole nucleoside molecule, partly on the products of its decomposition, and that the results of the action of the body ferments are not the same in the two cases.

If we make this reservation, namely, that the constituent parts of the nucleic acid molecule may undergo changes while still bound to the other parts, we may represent diagrammatically the formation of uric acid from nucleic acid as follows:

Ferments

Nuclease

Nucleic acid

Phosphoric acid guanosine adenosine
deaminase
oxidase
$\begin{aligned} & \text { oxidase } \\ & \text { (uricase) }\end{aligned}$


purine nuclease

leaminase

pentose-phosphoric acid

(guanase and adenase)

oxidase

oxidase

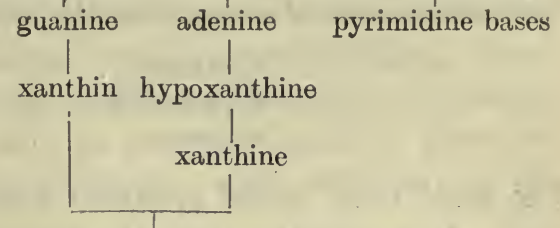

uric acid

The question arises whether the uric acid excreted by a man represents the whole of the nucleins which have been destroyed in the body. Although complete equivalence has been found between the amount of hypoxanthine ingested and the amount of uric acid excreted, the same equivalence has not been established in the case of nucleic acid, and the important question arises whether uric acid once formed is stable or whether it may undergo further changes before being excreted. In many animals, such as the dog, the amount of uric acid in the urine is only minute, the chief purine derivative in this fluid being allantoin. Allantoin is formed when uric acid is oxidised with potassium permanganate, the following changes taking place :

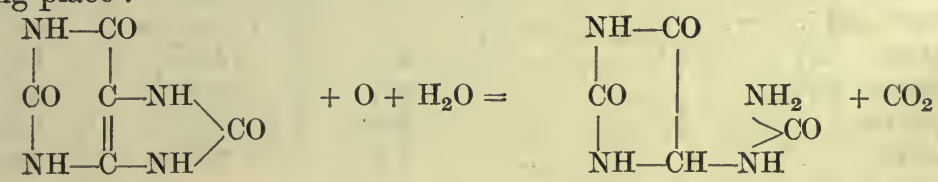

The same transformation can be effected by extracts made from the liver of the dog and probably of other animals. The ferment carrying out this change is known as uricase. No such ferment is found in human liver or any human organs, and, according to Jones and others, uric acid once formed in the human organism is not further oxidised. The small trace of allantoin which may occur in human urine is directly derived from the food. Modern research does not confirm the idea which was formerly held that a portion of the uric acid formed might undergo further oxidation in man with the production of urea.

On the other hand it is important to bear in mind the possibility that some of the uric acid which occurs in human urine may be formed by a process of synthesis. We have seen already that in the bird the greater part of the uric acid is formed not from purines at all but by a process of synthesis from lactic acid and ammonia, and though we have no evidence of a similar change occurring in the mammal, we are not able definitely to exclude its possibility.

\section{EXCRETION OF URIC ACID}

The complexity of these various processes in man renders it a difficult task to form a clear idea of the origin of the urinary uric acid and of the conditions which determine the variations in the amount excreted at 
different times. Under ordinary circumstances a nian excretes about half a gramme of uric acid per day. In addition the urine contains a certain small amount of purine bases, the ratio of these bases to the uric acid being generally about 1:6. From 10,000 litres of human urine Krüger and Salomon succeeded in isolating the following purine bases :

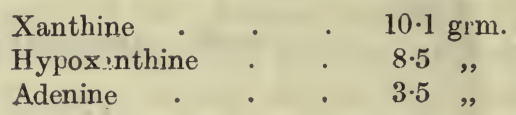

The same urine would probably have contained about $500 \mathrm{grm}$. of uric acid. As we should expect, the amount of uric acid in the urine varies with the diet. The following Tables from Bunge give the composition of the urine secreted (1) on a mixed diet, (2) on a diet mainly composed of meat, (3) on a diet mainly composed of bread:

\begin{tabular}{|c|c|c|c|c|c|}
\hline Twen & ty-four hours & & Mixed & Mcat & Bread \\
\hline Quantity c.c. & $\cdot \quad \cdot$ & . $\quad$. & 1500 & 1672 & 1920 \\
\hline Urea & . & . grm. & 33 & 67 & 20 \\
\hline Uric acid & . $\quad$. & . $\quad$, & .55 & $1 \cdot 3$ & .25 \\
\hline Ammonia & . & . & $\cdot 9$ & $2 \cdot 1$ & .9 \\
\hline Creatinine & - & . & .77 & $\cdot 9$ & $\cdot 4$ \\
\hline Hippuric acid & . & . $\quad . \quad$, & .4 & - & - \\
\hline Sulphates & . & ", & 2 & $4 \cdot 6$ & $1 \cdot 2$ \\
\hline Sodium chlorid & le & . $\quad$., & $16 \cdot 5$ & $7 \cdot 5$ & $8 \cdot 2$ \\
\hline Phosphates & . & . $\quad$. & $3 \cdot 16$ & $3 \cdot 4$ & $1 \cdot 6$ \\
\hline Potassium & . $\quad$. & . $\quad$. & $2 \cdot 5$ & $3 \cdot 3$ & $1 \cdot 3$ \\
\hline
\end{tabular}

Calcium, magnesium, iron, colouring-matter, gases, ferments.

An attempt has been made to arrive at the amount of uric acid produced endogenously, i.e. from the breakdown of the tissues, from a study of the quant ty of uric acid in the urine under varying conditions of food. During starvation, when the man is living on his own tissues, one might expect the uric acid to be increased in consequence of disintegration of the tissues. It has been suggested that the amount of the endogenous uric acid in the urine would be obtained by an analysis of the urine from patients taking a diet free from purine bases, but containing sufficient nitrogen to maintain nitrogenous equilibrium. It is impossible, however, to arrive at any constant figure for the endogenous uric acid. Even in the entire absence of purine derivatives from the diet the amount of uric acid increases with the total nitrogenous metabolism. This fact is well shown in the Tables by Folin (already quoted) of the composition of the urine on a low and a high protein diet respectively. Although in each case care was taken to exclude purine-containing bodies from the food, the output of uric acid on the high nitrogenous diet was double as much as on the low diet. All we can say is that uric acid is constantly 
being derived from the tissue disintegration, but that it varies under different conditions of nutrition as well as under different conditions of activity of the body.

There are two main conditions which give rise to a marked increase in the output of endogenous uric acid. These are (1) severe muscular activity, (2) febrile states accompanied by increased nitrogenous metabolism. Since both these conditions are associated with an increased breakdown of muscle substance we may regard the uric acid as derived especially from the hypoxanthine or its precursors, such as inosinic acid, contained in the muscle.

The foods which are especially effective in causing increase in the exogenous uric acid are those rich in nuclein, such as sweetbreads or liver,

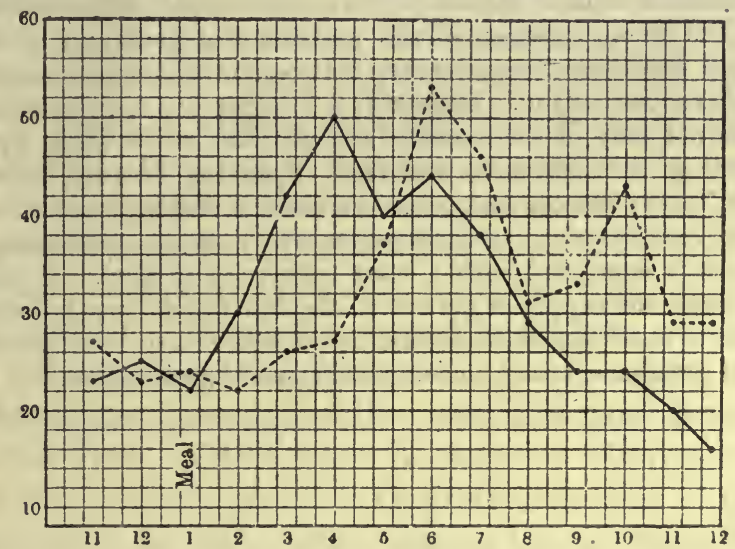

FIG. 355. Curves showing the hourly excretion of uric acid and urea after a single meal. (Hopkins.) The continuous line = uric acid output; the dotted line $=$ urea output.

and those rich in hypoxanthine or its precursors, such as meat or meat extract.

When these foods are taken, or when nucleic acid itself is administered, a condition of leucocytosis is generally produced, the number of leucocytes in the blood being increased as much as three times. It has been suggested that the uric acid is actually formed by a disintegration of the newly formed leucocytes and not by a direct conversion of the purines of the food. It is quite possible, as suggested by Schittenhelm, that the leucocytes play a part in the transference of the nucleins from the intestine to the circulation. But the absence of any absolute proportionality between the degree of leucocytosis and the amount of uric acid excreted points to the probability of a direct conversion of the purines of the food into uric acid.

\section{URIC ACID IN GOUT}

Gout is a condition in which deposits of urate of soda occur in the cartilages of the joints, the great toe joint being the seat of predilection for this disorder. The deposit is generally associated with an acute inflammation of the joint. In normal individuals the amount of uric acid in the blood is too small to be detected. Uric acid is readily excreted by the healthy kidneys. If the production of uric acid be largely increased by the administration in large quantities of food-stuffs rich in purines, it becomes 
possible to demonstrate the actual presence of uric acid in the blood. In gout there is constantly an increased amount of uric acid in the blood, probably in the form of sodium urate, even when the patient is on a purine-free diet, so that gout may be regarded, from one point of view at any rate, as a uricæmia of endogenous origin. On the other hand, the output of uric acid in the urine is not increased, and may in fact be somewhat smaller than normal. It might be thought that the presence of uric acid in the blood must therefore be due to diminished power of excretion of this substance by the kidneys. This view is difficult to reconcile with the fact that if uric acid be injected subcutaneously into gouty subjects it is stated to be excreted in the urine exactly in the same way and as rapidly as in normal persons. It has been suggested that gout consists essentially in a disturbance in the various fermentative mechanisms which are responsible for the changes undergone by the purines, so that there is an increased amount not only of uric acid itself but of various intermediate products in its formation from the purine bases of the food and of the tissues. The deposit of the uric acid in the joint cartilages characteristic of acute gout appears to be simply a crystallisation of urate of soda from a supersaturated solution of this substance in the blood. The whole question of the pathology of gout and of the disordered metabolism which may precede or intervene between actual acute attacks of the disease is in need of further investigation. Especially is it important to determine the influence on this condition not only of the nucleins and proteins of the food, but of the other constituents, such as carbohydrates and fats. Speaking broadly, gout is a disease of the well-to-do, of the person who, while pursuing a sedentary or no occupation, is not limited in his food-supply. It is almost unknown in the labouring class, where hard manual work is combined with a bare sufficiency of food. It seems therefore that it is not so much the supply of purines in the diet which must be controlled as the general conditions of nutrition which determine the fermentative changes in the purines, either of the food or tissues, under normal conditions of metabolism. 


\section{SECTION III \\ THE HISTORY OF FAT IN THE BODY}

FAT is found in the body in various situations. In a fat animal the largest amount occurs in the panniculus adiposus in the subcutaneous tissues. Large quantities are also found surrounding the abdominal organs and between the layers of the mesentery and great omentum. In this adipose tissue the fat is enclosed within and distends connective-tissue cells, the protoplasm of which is reduced to a thin pellicle round the fat globule. Fat is also found in the form of granules in more highly specialised cells, such as the secreting cells of the liver or the muscle-cells. The condition of these cells is often spoken of as fatty infiltration, or fatty degeneration, according to the circumstances which are responsible for bringing about the deposition of fat. We shall have to discuss later on how far we are justified in assuming any real distinction between these two processes. From the physiological standpoint the most important intracellular depôt of fat is in the liver. If this organ be deprived of glycogen and fat by starvation, a fatty meal gives rise to a great deposition of fat in its cells. There is apparently an antagonism between the processes which lead on the one hand to the deposition of glycogen and on the other to the deposition of fat. Thus an excessive carbohydrate diet, which induces great deposition of fat in the subcutaneous tissues, only causes the formation of glycogen in the liver. The glycogen must be got rid of before it is possible to cause the deposition of fat. On this account, the normal content in fat of the livers of different animals varies with their ordinary diet. Fishes, e.g. the cod, which take but little carbohydrate in their food, have generally a very large quantity of fat in their livers. Herbivorous animals, as a rule, have practically no fat in the liver.

Fat also occurs in certain secretions, e.g. the milk and the sebum, its function in the latter case being mainly protective.

Besides the visible deposit of fat found in adipose tissue and in other situations a large amount of fat is always present built up into the protoplasm of the cells in such a condition that its presence cannot be detected by histological means. The presence or absence of visible fatty globules affords very little clue to the total quantity of fat in the cells. Thus in one case the heart muscle, which had undergone extreme fatty degeneration and was loaded with fat globules, contained 19 per cent. of its dried weight of fat. A heart muscle taken from a normal animal at the same time, presenting no visible fat globules, contained 17 per cent. of fat. 


\section{COMPOSITION OF FAT}

The fats occur generally in the form of triglycerides of various fatty acids. In adipose tissue the acids are chiefly stearic, palmitic, and oleic, the consistency of the fat depending on the relative amount present of triolein, with its low melting-point. In certain animals the glycerides of more unsaturated fatty acids occur. Thus lard contains about 10 per cent. of fats belonging to the linoleic series. The fats of cows' milk, though consisting chiefly of the three above mentioned, include also the esters of butyric and caproic acids in fair amounts, and traces of the intermediate acids, caprylic, capric, lauric, and myristic acids.

The "fat" extracted from the tissues (e.g. heart muscle) includes a considerable amount of 'phosphatides' (lecithins, \&c.). It also contains a much larger proportion of unsaturated fatty acids, of the linoleic and even lower series, so that its 'iodine value' is generally found relatively high (120 as compared with 40 to 60 in adipose tissue).

\section{FUNCTIONS OF FAT}

First and foremost must be mentioned the significance of fat as a reserve food store. The power of the organism to store up reserve carbohydrate is strictly limited. The liver of man can probably not accommodate more than 150 grm. of glycogen, and assuming that the muscles of the body may contain an equal amount, $300 \mathrm{grm}$. represents the extreme limit of storage of carbohydrates in the body. On the other hand, in most animals there is practically no limit to the amount of fat which can be laid down, and over-feeding, whether with carbohydrates or fats, leads to the deposition of fat. This fat does not enter into the normal metabolism of the body, but is available for use whenever the needs of the body are increased above its income.

As to the part taken by fat, especially the hidden fat of the working cells, in the chemical processes which determine the life of the cell, our knowledge is still very scanty. Fats enter into the constitution of the complex bodies lecithin and myelin, which form important constituents of the limiting membrane of every living cell. As constituents of the membrane itself, fatty substances therefore have a protective action, and also regulate the passage of substances into the cell across the membranes.

The presence of lecithin as an integral constituent of all protoplasm, and of the first products of disintegration of protoplasm, suggests that this substance may play a part in the normal transformations which occur within the cell, and may represent, so to speak, the currency into which fat is transformed in order to participate in the vital processes, and that it is in this form that the energy of fat is utilised for the needs of the cell.

\section{ORIGIN OF FAT IN THE BODY}

Fat formation is the result of an excess of income over expenditure. As soon as the latter exceeds the former the fat store is drawn upon, so 
that adipose tissue is the one which presents the greatest loss during starvation. As much as 97 per cent. of the total fat of the body may disappear during this process. We have therefore to consider what part is played by each class of food-stuffs in the formation of fat. Can this substance be formed from all three classes of food-stuffs?

FORMATION FROM THE FAT OF THE FOOD. Experiment has shown that the composition of the fat of any animal is by no means constant and can be varied within wide limits by alterations in the nature of the fat presented in the food. This dependence of the composition of the fat on the fats of the food is shown strikingly in an experiment performed by Lebedeff. Two dogs, after a preliminary period of starvation, were fed, one on a diet containing a large amount of linseed oil, and the other on a diet containing much mutton suet. After some weeks, when the animals had put on a large amount of fat, they were killed, and it was found that whereas the fat of the dog which had been fed on mutton suet was solid at $50^{\circ} \mathrm{C}$., that of the $\operatorname{dog}$ fed on oil was still fluid at $0^{\circ} \mathrm{C}$. It has been shown moreover that by feeding animals with fatty acids not usually found in the body these are laid down in the adipose tissue. Thus colza oil contains a glyceride of erucic acid, and an animal, as Munk has shown, fed on colza oil lays on fat in which erucic acid is present. The same physiologist has observed that after the administration of various fatty acids to a man with a chylous fistula, the glycerides of the corresponding fatty acids made their appearance in the chyle, whether these fatty acids were those normal to man or consisted of substances, such as erucic acid, not generally found in human fat. One must conclude therefore that the fats taken with the food, if not immediately required for the energy needs of the body, are laid down without change in the adipose tissues, as well as in the cells of the body. The mechanisms involved in the translation of fat from the alimentary canal to the tissues are of the simplest possible description and only involve changes of hydrolysis and dehydrolysis. The fats are hydrolysed in the gut and are resynthetised to a certain extent in their passage into the epithelium. In the chyle and blood they probably wander chiefly as neutral fats, to be rehydrolysed for their passage into the cells of the body, which they may enter either in the form of soaps or possibly as fatty acids dissolved in some of the constituents of protoplasm.

FORMATION OF FAT FROM CARBOHYDRATES. It has long been the experience of farmers that animals might be fattened on a diet in which carbohydrates predominate. The chemical difficulty involved in the transformation of carbohydrates into fats has often led to a doubting attitude on the part of chemists towards this transformation. Voit put forward the view that when fats are formed in the body as a result of an excessive carbohydrate diet, they are formed, not directly by a transformation of carbohydrate, but from the proteins of the food, the rôle of the carbohydrates of the food being simply to protect the proteins from disintegration and oxidation, so that the whole of their carbon can be utilised for the formation of fat. 
Definite evidence has, however, been brought forward, especially by Lawes and Gilbert, for the transformation of carbohydrates into fats. In these experiments two young pigs, ten weeks old, of the same litter, with approximately equal weights, were taken. One was killed and the fat and total nitrogen in the body estimated. From the amount of nitrogen the maximum possible quantity of proteins present was calculated. The second was fed on barley for four months. The barley was measured and analysed, as well as the amount of undigested fat and protein that passed through the animal. At the end of the four months the second animal was killed and analysed. It was found that the animal contained 1.56 kilos more protein and 8.6 kilos more fat. It had taken up with the food $7 \cdot 49$ kilos more protein and 0.66 kilo fat. If we subtract the protein added to the body $(1 \cdot 56)$ from that taken up with the food $(7 \cdot 49)$ there is a remainder of 5.93 kilos which might possibly have given rise to fat. But 7.9 kilos of fat had been added in the body-a far larger amount than could possibly have arisen from the maximum amount of protein left over for the purpose. At least 5 kilos of fat in this experiment must have been derived from the direct conversion of the carbohydrates of the food. We must conclude that fat can be formed directly from carbohydrates; although how and where this conversion takes place is at present quite unknown. The fats formed on a carbohydrate diet are deposited chiefly in the subcutaneous tissue. For the reasons already given the liver is found free from fat under these conditions. In the fat formed from carbohydrate the two saturated acids, palmitic and stearic acid, predominate. On this account the fat has a firm consistency and a high melting-point. The fats of low melting-point, such as olein, are absorbed more readily from the intestine than those of high melting-point. Where the fat of the body is chiefly derived from the fat of the food it tends to be of the more fluid acids and contains a larger percentage of olein.

Although it is impossible to trace out all the steps in the process of conversion of sugar into fatty acid, we are acquainted with certain reactions which may throw some light on the nature of the changes involved. If we compare the formula of dextrose with that of the corresponding fatty acid, caproic acid,

$$
\text { }
$$

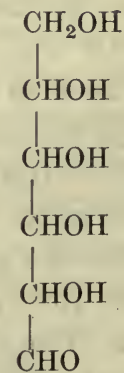

we see that the conversion involves a considerable loss of oxygen. In order to convert three molecules of glucose, $\mathrm{C}_{6} \mathrm{H}_{12} \mathrm{O}_{6}$, into one molecule 
of stearic acid, $\mathrm{C}_{18} \mathrm{H}_{36} \mathrm{O}_{2}$, it is necessary to split off 16 atoms of oxygen. That this setting free of oxygen actually occurs in the transformation of carbohydrate into fat is shown by the study of the respiratory exchanges of animals which are rapidly laying on a store of fat at the expense of a carbohydrate food. Thus the marmot, towards the end of summer, eats large quantities of carbohydrate food and very rapidly lays on a thick layer of subcutaneous fat to last it during the winter. If glucose were entirely oxidised in the body the amount of oxygen absorbed would be exactly equal to the amount of carbon dioxide evolved. Thus

$$
\mathrm{C}_{2} \mathrm{H}_{12} \mathrm{O}_{6}+6 \mathrm{O}_{2}=6 \mathrm{CO}_{2}+6 \mathrm{H}_{2} \mathrm{O} \text {. }
$$

In this case the respiratory quotient would be

$$
\frac{6 \mathrm{CO}_{2}}{6 \mathrm{O}_{2}}=1
$$

If, however, oxygen is being set free by the conversion of part of the carbohydrate into fat, this oxygen will be available for the oxidation of other portions of the carbohydrate. The animal will not need to take in so much oxygen from outside for the production of the same amount of carbon dioxide, and the carbon dioxide output of the animal will therefore be greater than its oxygen intake. Pembrey has shown that under these conditions the respiratory quotient may be as high as 1.5 . We cannot assume, however, that the process of conversion of glucose into fatty acids takes place by this simple process of deoxidation. The change is probably a more complex one, and occurs in separate stages. Glucose easily breaks up under the action of ferments into two molecules of lactic acid, and lactic acid can be equally easily converted into aldehyde and formic acid, thus :

$$
\begin{aligned}
& \mathrm{C}_{6} \mathrm{H}_{12} \mathrm{O}_{6}=2 \mathrm{C}_{3} \mathrm{H}_{6} \mathrm{O}_{3} \text { lactic acid, and } \\
& { }_{\mathrm{COOH}}^{\mathrm{CH}_{3}}={ }_{\mathrm{CHO}}^{\mathrm{CH}_{3}}+\left.\right|_{\mathrm{COOH}} ^{\mathrm{H}}
\end{aligned}
$$

Now aldehydes possess a marked tendency to combine with other molecules of other or the same substance, i.e. to undergo polymerisation. Thus from two molecules of aldehyde we get one molecule of aldol,

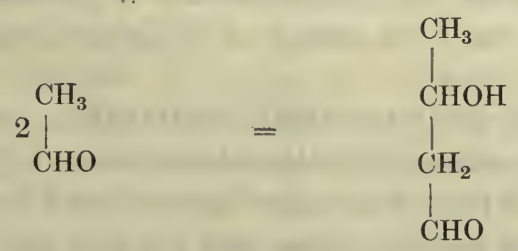

which by a simple transposition of oxygen would give butyric acid, or by oxidation would give $\beta$-oxybutyric acid, a substance which occurs during various abnormal conditions of metabolism.

The fats occurring in the body, e.g. in milk, include only the fatty acids 
with an even number of carbon atoms (v. p. 54). We may probably assume from this fact that the building up, as well as the breaking down, of fatty acids occurs by two carbon atoms at a time. Although heating aldehyde or aldol with potash or any other polymerising agent gives rise to a mixture of many substances, it is probable that under the catalytic agencies at the disposal of the living cell these synthetic changes are directed entirely in one direction, so that from butyric acid we shall have hexoic, caprylic, capric acid, and so on. The process would seem to take place more easily through pyruvic acid, as described on p. 121. Why the process comes to an end with the formation of the 16 and 18 carbon atoms it is difficult to see.* Possibly with the formation of acids whose melting-point is higher than that of the body temperature, a certain stability is imparted to them which prevents their further circulation and ready synthesis to the still higher acids.

With regard to the glycerine which is a necessary constituent of the neutral fats laid down in the body, there is no difficulty in accounting for its formation from the carbohydrates. By a simple splitting of glucose, we may obtain two molecules of glyceraldehyde,

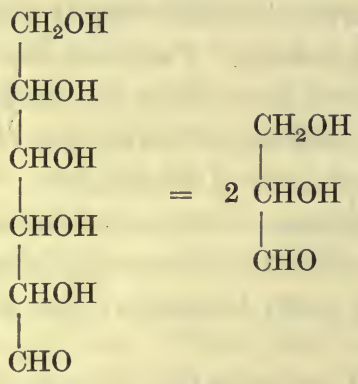

which by reduction is readily converted into the corresponding alcohol glycerine, $\mathrm{CH}_{2} \mathrm{OH}$. $\mathrm{CHOH}$. $\mathrm{CH}_{2} \mathrm{OH}$.

We may conclude then that fats are formed by the body with ease from carbohydrates, and that in all probability this change involves a building up of the fatty acid from the lower members by the successive addition of a group containing two atoms of carbon. The whole change, as Leathes has shown (v. p. 121), is an exothermic one. For the formation of one molecule of palmitic acid, four molecules of glucose would be required, and 12.5 per cent. of the total energy of the glucose would be set free as heat.

THE FORMATION OF FAT FROM PROTEINS. Among the decomposition products of proteins the amino-derivatives of the fatty acids take a prominent part. Of these some may be converted into carbohydrate in the body, while others such as leucine and tyrosine may give rise to acetoacetic acid. It seems therefore that these latter might in their turn be built up by the process we have just discussed into the higher members

* From the fats extracted from the kidney Dunham has isolated carnaubic acid, $\mathrm{C}_{24} \mathrm{H}_{49} \mathrm{O}_{2}$. 
of the series. For many years, as a result of the investigations of Voit, the proteins were indeed regarded as the chief, if not the sole, source of the fats of the body, and it needed the energetic assaults of Pflüger on this doctrine, in 1891, before it could be clearly examined by physiologists.

Let us see what are the grounds for assuming a formation of fat from protein. In the first place, there is a well-known experiment by Voit. A dog was fed with large quantities of lean meat for a considerable time. Voit found that the whole of the nitrogen of the intake was excreted, but that a certain percentage of carbon was retained in the body, and that the percentage of this carbon was greater than could be accounted for by the deposition of glycogen in the liver and muscles. He therefore assumed that it must have been laid down as fat. Pflüger showed that these conclusions were not justified by Voit's results, and were really based on the fact that too high a figure had been assumed for the carbon of the meat. Whereas Voit found that the animal had laid on $56 \mathrm{grm}$. of fat during one day of the experiment, a recalculation of the same results by Pflüger shows that the animal could not have put on more than $3.9 \mathrm{grm}$. of fat, an amount which might quite well be accounted for by the fat and glycogen present in the meat. Pflüger has shown moreover that an animal may be fed for weeks on the leanest meat that it is possible to procure, in any quantities, without putting on any fat at all, and, as we have seen, increasing the ration of protein increases simply the nitrogenous and general metabolism of the body.

Although therefore we must assume that the healthy body does not normally form fat from protein, there are certain pathological conditions which seem at first to tell in favour of such a conversion. Thus during certain diseases, such as diphtheria, pernicious anæmia, and as the result of poisoning by phosphorus, the majority of the organs of the body undergo acute fatty degeneration. The liver may be enlarged and all its cells are studded with fat granules which are apparently formed by a change in the protoplasm of the cells. This change was long interpreted as due to a direct conversion of protein into fat. More exact analyses have shown that during fatty degeneration the total fat in the body is not increased. Thus one observer took 124 pairs of frogs and poisoned one of each pair with phosphorus. The animals were then killed, and the whole of them analysed. The difference in the content of fat between the poisoned and unpoisoned animals fell within the limits of experimental error, so that there had been no increase in the fat of the body as the result of the poisoning. In some of these cases the liver is actually enlarged, but this deposition of fat in the cells is due to the immigration of the fat from other parts of the body and not to conversion of the protein of the cells. This is shown by the facts that the composition of the fat in the degenerated liver varies according to the composition of the fat in the rest of the body, and that, if abnormal fats are given with the food, such as erucic acid or iodine fats, these are found in the fat extracted from the liver. In fatty degeneration two processes are at work: one is the. immigration of fats 
from other parts of the body; the second, and probably the more important one, is a change in the relation of the fat to the protoplasm of the cell.

It was long stated that the fat of milk was not increased by feeding with fats, but only by feeding with proteins. More recent researches have given contrary results. The dependence of the composition of milk fat on the composition of the fat present in the body or administered in the food is shown by the fact that cows fed on oilcake may produce a butter which is useless for commercial purposes owing to its low melting-point. In one experiment, when a cow was fed on linseed oil, the iodine number of the milk fat rose from 30, its normal figure, to 70.4. After the introduction of iodine fat subcutaneously, iodine fats are found in the milk. In another experiment a bitch which had been fed with mutton suet and had deposited in its tissues a fat of high melting-point produced a milk the iodine number of which was the same as that of the mutton suet. In this case the fat of the milk had evidently been derived from the tissues, since during the lactation the animal was being fed on meat which was poor in fat. The same dependence of fatty secretion on diet has been found in geese, where the composition of the oil secretion of the feather glands has been altered by giving abnormal fats, such as sesame oil, with the food.

We must conclude that the protein of the food does not give rise to fat in the body. A nearer consideration of the composition of the proteins, taken in connection with our discussion as to the mechanism by means of which the fat is built up in the body, might help to account for this fact. The fatty acids formed by the disintegration of proteins are chiefly the lower acids of the series, such as acetic and propionic, which would undergo rapid oxidation in the body. Butyric acid has not yet been found among the products of disintegration of the proteins, and the 6-carbon acid, derived from leucine, is not the normal acid, but is a branched chain, viz. isobutyl-acetic acid.

\section{THE UTILISATION OF FATS IN THE BODY}

The constant presence of fat, and bodies allied to fat, in protoplasm, from whatever source obtained, suggests that these substances can enter directly into the chemical changes on which the life of the cell depends and that they play an essential part in vital phenomena. The direct utilisation of fat for the needs of the body is also indicated by the results of experiments on man and the lower animals. After a few days' starvation the body may be regarded as practically free from stored carbohydrate. The sole source of the energy which is evolved under these circumstances must be fats and proteins, and it is possible to determine by an estimation of the nitrogen output the exact fraction of the total energy evolved which is to be ascribed to protein metabolism. Thus in the case of Cetti, the professional faster, it was found that the nitrogenous metabolism per unit of body weight remained fairly constant between the fifth and tenth days of starvation, and corresponded to an average of $1 \mathrm{grm}$. of protein per kilo body weight daily. In order to convert this amount of protein into 
urea, carbonic acid, sulphuric acid, and water, nearly 2 grm. of oxygen would be required in the twenty-four hours, i.e. about 1 c.c. per minute. Cetti's total oxygen consumption was at the rate of 5 c.c. per kilo per minute, so that four-fifths of the oxygen absorbed was required for the oxidation of non-nitrogenous substances, and these, as we have seen, could only have been fats. In animals with a large store of fat the proportion of the energy obtained at the cost of the fats may be still greater. In dogs Rubner and Voit reckoned that only 10 to 16 per cent. of the total energy was derived from proteins, the rest, i.e. 84 to 90 per cent., being obtained from the oxidation of fats.

The oxidation of fats supplies energy not only for the production of heat but also for the performance of mechanical work, and it seems probable that the utilisation of the fat occurs in the muscular tissues themselves. Fat is found as a normal constituent of all muscle fibres, and the amount of this substance is greater in proportion to the activity of the muscles concerned. Thus the ever-active heart muscle, and the red muscles of the diaphragm, contain larger amounts of fat than the pale voluntary muscles which only have to undertake short periods of activity. In the human heart muscle 15 per cent. of the solids are soluble in ether, and more than one-half of the ether extract is composed of fat, and is sufficient to supply the energy of the contracting heart for six or seven hours' work.

The degree to which the muscles during contraction call upon each class of food-stuffs may be judged from the respiratory quotient. If the body has previously supplied the greater part of its needs at the expense of fats, it will continue to do so during muscular work. This is well shown in the following Table, in which the oxygen consumption and respiratory quotient are compared in a man resting and working on three different diets, one principally fat, one principally carbohydrate, and the other principally protein :

\begin{tabular}{|c|c|c|c|c|c|c|c|}
\hline \multirow{2}{*}{$\begin{array}{c}\text { Diet } \\
\text { principally }\end{array}$} & \multicolumn{2}{|c|}{ Resting } & \multicolumn{2}{|c|}{ Working } & \multirow{2}{*}{$\left|\begin{array}{c}\mathrm{m} . \mathrm{kg} \text {. of } \\
\text { work done }\end{array}\right|$} & \multicolumn{2}{|c|}{ Per m. kg. of work } \\
\hline & $\begin{array}{l}\text { c.c. oxy- } \\
\text { gen used } \\
\text { per min. }\end{array}$ & $\begin{array}{c}\text { Resp. } \\
\text { quo- } \\
\text { tient }\end{array}$ & $\begin{array}{c}\text { c.c. oxygen } \\
\text { used per } \\
\text { min. }\end{array}$ & $\begin{array}{c}\text { Resp. } \\
\text { quo- } \\
\text { tient }\end{array}$ & & $\begin{array}{l}\text { c.c. oxy- } \\
\text { gen used }\end{array}$ & Cal. \\
\hline Fat & 319 & 0.72 & 1029 & 0.72 & 354 & $2 \cdot 01$ & $9 \cdot 39$ \\
\hline Carbohydrate & 277 & 0.90 & 1029 & 0.90 & 346 & $2 \cdot 17$ & $10 \cdot 41$ \\
\hline Protein & 306 & $0 \cdot 80$ & 1127 & 0.80 & 345 & $2 \cdot 38$ & $11 \cdot 35$ \\
\hline
\end{tabular}

We may conclude then that the tissues of the body are able to obtain their energy by the direct utilisation of the fats which they contain. The changes in the fat molecules which are involved in the utilisation of their energy are still to be determined. The energy of fat is only available on its oxidation. The transformation of fats into fatty acids or glycerine, or the synthesis of fats from aldehydes or from carbohydrates, which we 
have discussed in the previous section, do not involve any large changes of energy. Weight for weight, butyric acid with its 4 carbon atoms has practically the same heat-value as stearic acid with its 18 carbon atoms, or stearine with its 57 carbon atoms. We have therefore to determine what changes the great fat molecule undergoes before it is brought into a condition in which it may undergo oxidation and set free the energy required for the purposes of the body. The general tendency of metabolic research of recent years is to show that the living cell is in a position to effect all changes which do not involve a large evolution or absorption of energy in either direction. In the plant cell, at any rate, the fatty acids may be converted into amino-acids, or the latter may be deaminised, as occurs in the liver, into fatty or oxyacids. Dextrose may pass into maltose and starch, or starch may be converted into maltose or dextrose. If therefore fats are constantly being made from carbohydrates, or from the lower molecules such as aldehyde, by a process of repeated addition of a group containing two carbon atoms, it is probable that the same change will go on in a reverse direction when fats are broken down previous to oxidation.

In the germination of oily seeds the utilisation of the fat is preceded by the splitting of the higher fatty acids into acids of lower molecular weight. Although we cannot trace out in the animal body the stages in the breakdown of a large fatty acid, such as stearic acid, we can, by a certain artifice much used in metabolic experimentation, bring forward evidence in favour of the view that the breakdown, like the building up of fats, occurs by two carbon atoms at a time. When, in the process of breaking down, a fat finally arrives at the four- or two-carbon stage, it is quickly oxidised and is therefore not traceable in the excretions or in the fluids of the body. This end stage may, however, be preserved from oxidation by hanging it, so to speak, on to an aromatic ring. If acetic acid or ethyl alcohol be administered in small quantities, it is entirely oxidised. If, however, these bodies be attached to a benzene ring and be administered as a phenacetic acid or phenylethyl alcohol, they are excreted in the oxidised form of phenaceturic acid, which is simply a combination of phenacetic acid with glycine. In the same way benzoic acid and benzyl alcohol are excreted in the form of hippuric acid, thus :

$$
\begin{aligned}
& \mathrm{C}_{6} \mathrm{H}_{5} \cdot \mathrm{COOH}+\mathrm{NH}_{2} \cdot \mathrm{CH}_{2} \cdot \mathrm{COOH}=\mathrm{C}_{6} \mathrm{H}_{5} \cdot \mathrm{CO} \cdot \mathrm{NH} \cdot \mathrm{CH}_{2} \cdot \mathrm{COOH}+\mathrm{H}_{2} \mathrm{O} \\
& \text { Benzoic acid Glycine Hippuric acid }
\end{aligned}
$$

Phenacetic acid, $\mathrm{C}_{6} \mathrm{H}_{5} \cdot \mathrm{CH}_{2} \mathrm{COOH}$, is excreted as $\mathrm{C}_{6} \mathrm{H}_{5} \cdot \mathrm{CH}_{2}$.$\mathrm{CO} . \mathrm{NH} . \mathrm{CH}_{2} \mathrm{COOH}$. In each case the fatty side-chain is protected from further oxidation by its attachment to the benzene ring and by the tacking on of the glycine molecule.

With phenylpropionic acid two carbon atoms of the side-chain are oxidised, and the remaining benzoic acid excreted as hippuric acid. Phenylbutyric acid undergoes a similar change; two carbon atoms are oxidised away, leaving phenylacetic acid, which is excreted as phenylaceturic acid. 
If phenyl valerianic acid be given, four carbon atoms are oxidised away and benzoic acid is left, and appears in the urine as hippuric acid. In each case the oxidation of the side-chain occurs by two carbon atoms at a time, and it seems probable that a similar change will occur in the ordinary fatty acid, the last stages, in the absence of any protective ring compound, being oxidised like the earlier groups and therefore not detectable in the excretions.

Evidence in the same direction is afforded by certain cases in which the oxidative power of the body for fats is inadequate, either by reason of morbid changes in the oxidative powers of the body, or as the result of what we may call an overstrain of the fat-oxidising powers. Such a condition is found in the acetonuria of acute acidosis, such as occurs in the end stages of diabetes. The oxybutyric and diacetic acids occurring in the urine in this condition were formerly thought to be derived from the carbohydrates of the food, or from sugar abnormally produced in the body. The condition of acidosis, however, is often brought on directly as the result of putting the patient on a strict anti-diabetic diet, i.e. one consisting chiefly or exclusively of fats and proteins, and may be produced in a healthy man by simple starvation, when the body has only at its disposal its stored-up fats and proteins. It occurs in a marked degree on the administration of a diet consisting almost entirely of fats. Thus in one experiment a healthy man took as his sole diet for five days a daily ration of $250 \mathrm{grm}$. of butter, $200 \mathrm{grm}$. of oil, and a little wine. The result was an intense acidosis, such as is only found in the severest cases of diabetes, diacetic acid, oxybutyric acid, and acetone being found in the urine in large quantities. On the last day of the experiment these acids caused so much of the nitrogen in the urine to appear as ammonia that of the $5 \cdot 8 \mathrm{grm}$. total nitrogen excreted only $2.7 \mathrm{grm}$. were in the form of urea, while as much as $2 \cdot 1 \mathrm{grm}$. were present as ammonia.

If, during a period of starvation in man, a day is interpolated on which $100 \mathrm{grm}$. of protein are taken, the amount of acetone excreted falls below that obtained on the other days when the individual is living chiefly at the cost of his own fat. These facts indicate that the chief source of the $\beta$-oxybutyric acid and the diacetic acid is the fats of the food or of the body. The condition of acidosis is more easily brought about by ingestion of butyric acid than of the higher acids, such as palmitic or stearic, suggesting that whatever fatty acid is given it is finally reduced to butyric acid before its oxidation, and that in the condition of acidosis it is merely the last stages of this oxidation which are at fault. We are thus justified in concluding that the oxidative breakdown of fats occurs always by an oxidation in the $\beta$ position.

We take, for instance, the 6 -carbon stage :

$$
\mathrm{CH}_{3} \cdot \mathrm{CH}_{2} \cdot \mathrm{CH}_{2} \cdot \mathrm{CH}_{2} \cdot \mathrm{CH}_{2} \cdot \mathrm{COOH}
$$

the first change which probably occurs is the oxidation :

$\mathrm{CH}_{3} \cdot \mathrm{CH}_{2} \cdot \mathrm{CH}_{2} \cdot \mathrm{CHOH} \cdot \mathrm{CH}_{2} \cdot \mathrm{COOH}$ 
A further change is the complete oxidation of the last two groups and the production of butyric acid:

$$
\mathrm{CH}_{3} \cdot \mathrm{CH}_{2} \cdot \mathrm{CH}_{2} \cdot \mathrm{COOH}
$$

This then undergoes again oxidation in the $\beta$ position, with the production of $\beta$-oxybutyric acid :

$$
\mathrm{CH}_{3} \cdot \mathrm{CHOH} \cdot \mathrm{CH}_{2} \cdot \mathrm{COOH}
$$

and then again is converted to diacetic acid,

$$
\mathrm{CH}_{3} \cdot \mathrm{CO} \cdot \mathrm{CH}_{2} \cdot \mathrm{COOH}
$$

In the normal individual this last stage undergoes complete oxidation, both oxybutyric acid and diacetic acid given to a healthy person being completely destroyed in the body. It is only under the abnormal conditions which we have mentioned above that these last stages fail of complete oxidation, and are excreted unchanged in the urine.

\section{THE QUESTION OF THE FORMATION OF SUGAR FROM FAT}

The ease with which the animal body performs the difficult chemical operation of transforming carbohydrate into fat suggests that under appropriate conditions it might effect the reverse change. Is there any evidence that in the animal body sugar may be derived from fat? Such a conversion is of normal occurrence during the germination of fatty seeds, starch sugar and cellulose being formed at the expense of the stored-up fats of the seeds. If such seeds be allowed to germinate over mercury in a confined volume of oxygen, they are found, like seeds containing chiefly carbohydrate reserves, to absorb oxygen and to give off carbon dioxide. Whereas, however, in the latter case the amount of carbon dioxide evolved is almost equal to the oxygen absorbed, in the case of the fatty seeds much less carbon dioxide is given out than would correspond to the volume of oxygen absorbed, so that the total volume of gas above the seeds diminishes:

The same change in the relation of oxygen intake to carbon dioxide output is found under certain conditions in animals. During hibernation, as Pembrey has shown, the marmot has a very low respiratory quotient, which may be not greater than 0.3 or 0.4 . This means that the animal takes in more oxygen than the carbon dioxide which it gives out, and this intake of oxygen can be so marked as to cause an appreciable increase in the weight of the animal, which under such circumstances is literally living on air. This retention of oxygen can only be explained by assuming that there is a conversion of substances containing a small amount of oxygen into substances containing a larger amount of oxygen going on in the body, such a conversion as that of fats into carbohydrates. Just as the high respiratory quotient obtained from a marmot during the period of putting on fat was shown to be associated with a conversion of carbohydrate into fat, so does the abnormally low quotient obtained during hibernation indicate the reverse change of fat into carbohydrate.

The same conversion has been alleged to take place in certain cases of 
diabetes. In many cases when the diabetic animal is living on a purely protein diet, a uniform ratio has been found to exist between the glucose or dextrose and the nitrogen. excreted.

\section{$\frac{\mathrm{D}}{\mathrm{N}}$ equals generally $2 \cdot 8$.}

In certain other cases a constant $\mathrm{D}: \mathrm{N}$ ratio of 3.65 has been found. The former represents a conversion of 45 per cent., the latter of 58 per cent., of protein into sugar. In a few cases, however, even during complete starvation, the ratio $\mathrm{D}: \mathrm{N}$ has been found to be much greater than that given above and to amount to as much as 10 or 12 . These animals are stated to be practically free from carbohydrates, so that the sugar excreted in the urine can only come from the breakdown of proteins or fats. It is impossible by any means whatever to break up a protein molecule so as to get from it ten times as much dextrose as corresponds to the nitrogen, and Pflüger concludes that in cases where such a high $\mathrm{D}: \mathrm{N}$ ratio exists the dextrose must have been derived by a conversion of the fats of the body. This conclusion is by no means generally accepted. If correct, it would bear out the general statement made above, namely, that in the living body practically all the chemical changes are reversible, and that the living cell can so regulate the conditions of the reaction that the reversible reaction becomes practically complete in either direction, the direction being determined by the needs of the body at the time.

Accepting this generalisation, the chemical mechanism by which fats are converted into carbohydrates must be the reverse of that by which carbohydrates are changed into fats. The 2-carbon group split off from the large fatty molecules are probably utilised for the building up of the sugar molecule. We know that such a synthesis can take place from such simple groups as formic, glycollic, or glyceric aldehyde. Though it is impossible to deny to any cell of the body the power of effecting the conversion of fats into carbohydrates, or carbohydrates into fats, the chief centre for such conversions is probably the chemical factory of the body, namely, the liver. It is significant that in the course of fatal diabetes, in which the fat disappears entirely from the body, and there is wasting of practically all the tissues, the liver is the only organ which retains its weight unchanged. During this disease there has been an enormous amount of work done in the conversion of proteins and possibly of fats into carbohydrates which could not be utilised by the body, and the large size of the liver at death suggests that the work of transformation has been performed by this organ. 


\section{SECTION IV}

\section{THE METABOLISM OF CARBOHYDRATES}

AlL the carbohydrates which are taken in with the food are ultimately transformed in the alimentary tract, or in its walls, into the three monosaccharides, glucose, fructose, and galactose. These three, together with mannose, are the only sugars which are directly fermentable and directly assimilable by higher animals. A consideration of their structural formulæ shows that they are fairly easily interconvertible, galactose presenting the greatest divergence from the general type. This conversion actually takes place in watery solution. If a solution of any one be left for some months, it will be found to contain all four at the end.

Since these monosaccharides, for the greater part glucose, must enter the blood in large quantities during the absorption of a heavy carbohydrate meal, one would expect to find a greater proportion in the blood during such a meal than during a period of starvation. The amount of reducing sugar in the blood, however, is practically constant, and varies between $0 \cdot 1$ and $0 \cdot 15$ per cent.

Searching for the origin of this constant proportion of reducing sugar, Claude Bernard found that the blood of the hepatic vein in a fasting animal contained more sugar than the blood taken at the same time from the portal vein. Although the reliability of this experimental result has been put in doubt by more recent investigators, it was important in that it attracted Bernard's attention to the liver. If the liver be taken from an animal which has been dead for some time, and extracted with water, the extract is found to contain a large quantity of reducing sugar (glucose). If, however, it be removed immediately the animal is dead, its vessels washed out with ice-cold saline fluid, and be then cut up and thrown into boiling. water, ground and extracted, the extract, after separation of the coagulable proteins, contains hardly a trace of sugar, and no more than is present in the blood. The fluid is, however, opalescent; and Bernard found that this opalescence was due to the presence of a substance at that time new to science, belonging to the class of polysaccharides. This substance he called glycogen, i.e. the sugar-former.

After a carbohydrate meal, glycogen may be present in very large amounts in the liver, up to 12 per cent. of the weight of the fresh liver. From its solution in water it can be thrown down by the addition of alcohol to 60 per cent When collected and dried, it forms a snow-white powder, 
tasteless and odourless, with a formula identical with that of starch, viz. $\mathrm{C}_{6} \mathrm{H}_{10} \mathrm{O}_{5}$. Like starch, it is hydrolysed by the action of acids and superheated water, or of amylolytic ferments, into dextrins, maltose, and finally glucose. It gives with iodine a mahogany-red colour, which disappears on boiling, but returns again on cooling.

It is not possible to extract the whole of the glycogen from a tissue by merely boiling it with water. Külz introduced on this account the method of dissolving the tissues in caustic alkali, then throwing down the protein with phosphotungstic acid, and in the filtrate precipitating the glycogen with alcohol. This method has been modified by Pflüger as follows : $100 \mathrm{grm}$. of the tissue (liver or muscle) are heated with 100 c.c. caustic potash containing 60 to 70 per cent. KHO for twenty-four hours in the water bath. The solution is then cooled, diluted with 200 c.c. of water, and treated with 800 c.c. alcohol of 96 per cent. The precipitate of glycogen is filtered off and washed several times with 66 per cent. alcohol. The precipitate of glycogen is now washed with a little water into a small beaker, neutralised carefully with acetic acid, and then introduced into a 100 c.c. flask. To the solution 5 c.c. of hydrochloric acid of $1.19 \mathrm{sp}$. gr. are added, and the mixture is made up to 85 c.c. The flask is then heated in the water bath for three hours. By this means the whole of the glycogen is converted into glucose, which can be estimated by Fehling's method or by Allihn's method. In practice it is more accurate to estimate the glycogen in the form of sugar than to weigh it directly. If large quantities of glycogen are expected in the tissue, the inversion of the glycogen must be carried out in a larger beaker, and only an aliquot portion taken for titration.

The large amount of sugar found in the liver which has been left in the body is due to the conversion of glycogen into glucose. This conversion has been variously ascribed to the activity of the surviving liver-cells, or to the action of an amylase ferment present in the liver-cells. That it is really a ferment action is proved by the fact that the liver may be dehydrated with alcohol, dried and powdered, and kept for months in this condition without any alteration occurring in the glycogen. If, however, the coagulated liver be mixed with water and allowed to remain at the temperature of the body for some hours, the glycogen is found to disappear and give place to glucose.

\section{FORMATION OF GLYCOGEN}

Glycogen is most readily formed from the carbohydrates of the food. In order to obtain a large amount from the liver, the animal is fed twelve to twenty-four hours previously on a meal which is rich in carbohydrates. Not all carbohydrates will give rise to the formation of glycogen. Only those which we have mentioned as directly assimilable, i.e. which will give rise in the alimentary tract to mannose, glucose, fructose, or galactose, will cause an increased formation of glycogen. The conversion involves a direct polymerisation of the glucose, produced either directly from the foods or by a molecular rearrangement taking place in one of the other three of these monosaccharides.

Glycogen can also be formed from the proteins of the food, or from the products of their disintegration, the amino-acids. By means which we shall consider shortly, it is possible to free the liver of animals entirely from glycogen : if such animals be fed on a diet of washed fibrin or of pure 
caseinogen, or even on the ultimate products of pancreatic digestion of proteins (containing therefore only amino-acids), and be killed shortly afterwards, the liver is found to contain glycogen. It does not seem to be possible for the liver to manufacture glycogen out of fats. At any rate, that is the interpretation which is generally placed on experiments on feeding with fats. In these experiments it is found that if fats be administered to an animal after the liver has been freed from glycogen, although the liver may store up fats it does not store up any glycogen.

If an animal be starved, the glycogen gradually disappears from the liver, although even at the end of ten or twelve days' complete deprivation of food small traces of glycogen may still be found in this organ. If starvation be combined with hard work; if, for instance, a dog be made to drag about a milk-cart on the second day of the starvation period, its liver becomes quite free from glycogen. The same disappearance of glycogen may be produced by any means which evoke an increased muscular activity, e.g. poisoning with strychnine. Of the various reserve materials which are available the carbohydrate is the first to be called upon to meet the increased needs of the tissues during functional activity, such as muscular work or increased heat production. Thus the glycogen rapidly disappears from the liver of a rabbit which has been immersed in a cold bath.

The glycogen of the liver represents a reserve material analogous to the reserve carbohydrates stored up as starch in different parts of plants. When the blood is loaded with carbohydrates, a considerable proportion is laid down as the inert polysaccharide glycogen. As soon as the supply of sugar to the blood is withdrawn, the tissues continue to use the sugar of the blood, which is made up at the expense of the glycogen in the liver. In every liver-cell therefore a twofold process is always going on, namely, a building up of glycogen by the activity of the liver-cells, and a breaking down of glycogen under the action of the ferment formed in the liver-cells. Which of these two processes preponderates depends, in the normal animal, on the percentage amount of sugar in the blood which is circulating through the organ.

On account of the importance of glycogen as a reserve material it is produced and stored up in almost all growing tissues, to be utilised in their subsequent development. Thus it is found in large quantities in the placenta during a certain period, in fœtal muscles, and in various other situations. It is found in yeast, in oysters, and in the muscles of the body generally. In fœtal muscles it may amount to as much as 40 per cent. of the total dried solids. The glycogen of the adult muscle is apparently utilised during muscular work, and diminishes in amount with activity of the muscle. In adult muscles it never reaches anything like the percentage which is found in the liver. The average amounts found by Schöndorf in the different tissues were as follows : 


\begin{tabular}{|c|c|c|c|c|}
\hline & - & 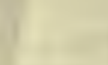 & $\begin{array}{l}\text { Maximum per cent. } \\
\text { of fresh tissue }\end{array}$ & $\begin{array}{l}\text { Minimum per cent. } \\
\text { of fresh tissue }\end{array}$ \\
\hline Liver & . & . & $18 \cdot 69$ & $7 \cdot 300$ \\
\hline Muscle . & . & . & $3 \cdot 72$ & 0.720 \\
\hline Heart . & . & . & $1 \cdot 32$ & $0 \cdot 104$ \\
\hline Bone . & . & . & 1.90 & $0 \cdot 197$ \\
\hline Intestines & . & . & $1 \cdot 84$ & 0.026 \\
\hline Skin & . & . & $1 \cdot 68$ & $0 \cdot 090$ \\
\hline Blood . & . & . $\quad$. & 0.0066 & 0.0016 \\
\hline
\end{tabular}

\section{THE UTILISATION OF SUGAR IN THE BODY}

Arterial blood is always found to contain between 0.12 and 0.15 per cent. of sugar in the form of glucose. The same amount is found whether the blood be taken from an animal after a heavy carbohydrate meal or from one in a condition of complete starvation. The constancy of the sugar content of the blood suggests that this substance is a necessary constituent of the circulating fluid, necessary, that is to say, for the nutrition of the tissues. That it is being used up in all the processes of the body is shown by the immediate alteration in the respiratory quotient which occurs when the food is changed from a mixed diet to one consisting mainly of carbohydrate. An important factor in the maintenance of a constant sugar content in the blood is the reconversion of the stored-up glycogen of the liver into sugar. The glycogen is not, however, the sole source of the sugar, since in complete starvation the sugar content of the blood remains constant even after the last traces of glycogen have disappeared from the liver. If the liver be cut out of the body or removed from the circulation, during the few hours that the animal survives there is a steady diminution in the bloodsugar, pointing to the liver being the chief, if not the sole, source of the blood sugar. In some animals, e.g. the carnivora, it would seem that the liver can continue to supply sugar to the blood on a diet which includes only proteins and fats, and we have already seen that in such animals glycogen itself can be stored up at the expense of protein. It is doubtful whether a perfectly normal existence is possible in man in the total absence of carbohydrates from the food, though there is no doubt that in the northern nations, e.g. the Eskimos, the amount of carbohydrate consumed is very small in comparison with the fats and proteins. During muscular exercise the increased output of energy is associated with a corresponding increase in the absorption of oxygen and in the output of carbon dioxide, pointing to a consumption of carbohydrate and fat in the contracting muscles. We might therefore assume that sugar is being normally released by the liver into the blood-stream so as to maintain the proportion of this substance in the blood at a certain level, and that the sugar is as constantly being taken up and oxidised in the muscles, where it serves as a source of energy. According to Chauveau and Kaufmann the venous blood flowing from a contracting muscle contains less sugar than the arterial blood flowing to the muscle. A 
similar consumption of glucose occurs in the isolated contracting mammalian heart when fed with Ringer's fluid containing a small trace of glucose. A heart, fed with blood and performing a normal amount of work, may use about $4 \mathrm{mg}$. sugar per gramme of heart muscle per hour. That the question of utilisation of sugar by the tissues is highly complex is shown by a study of the conditions under which sugar may appear in the urine. We learn thereby to appreciate to some extent the significance of carbohydrates both as sources of energy and as foods for the tissues, though we are still a long way from unravelling all the changes which the sugar must undergo in the cell before it appears once again in the oxidised products, carbon dioxide and water.

\section{GLYCOSURIA}

Normal urine always contains a small proportion of sugar, about 1 part per 1000 , i.e. about the same as the blood itself. For the detection of these small traces of sugar in the urine special methods are necessary. The term glycosuria is not employed unless sugar appears in quantities large enough to give a reaction with Fehling's solution or with the phenylhydrazine test. Such a condition may easily be brought about by the injection of sugar subcutaneously or intravenously. It is then found that any trace of the disaccharides, cane sugar or lactose, introduced in the circulation, is excreted in the urine. A rather larger quantity of maltose may be injected slowly without appearing in the urine, since the blood-serum contains a ferment maltase, which converts the maltose into glucose. Glucose, fructose, mannose, or galactose, if introduced slowly into the circulation, are stored up as glycogen in the liver. If, however, the percentage of sugar in the blood rises above 2 parts per 1000 , the sugar (generally glucose) appears in the urine. When this condition of hyperglycæmia (excess of sugar in the blood) is set up, the concentration of the sugar in the urine no longer corresponds to that in the blood. If the blood contains, e.g. 4 parts per 1000, the urine may contain from 2 to 7 per cent. of sugar. Up to a certain point, then, blood-sugar is kept back by the kidneys as a necessary food material for the tissues. Any excess above the normal apparently acts as a foreign substance and is excreted by the kidneys in a concentration much greater than that in which it exists in the blood-serum.

(1) ALIMENTARY GLYCOSURIA. A state of hyperglycæmia may be induced by the administration of abnormally large quantities of glucose by the alimentary canal. The amount has to exceed in a healthy individual $100 \mathrm{grm}$. in order that it shall appear in the urine. In certain individuals the power of assimilating glucose may be deficient so that an alimentary glycosuria may be caused by. any over-indulgence in carbohydrate food. In the healthy person it is hardly possible to produce glycosuria by the administration of starchy foods, since the liver can store up the excess of glucose as fast as it is produced from the starch by digestion and absorbed into the blood stream.

(2) DIABETIC PUNCTURE. It was shown by Claude Bernard that puncture of the floor of the fourth ventricle in rabbits is often followed 
immediately by an excessive secretion of urine and the appearance of sugar in this fluid. The glycosuria may last from twenty-four to thirty hours. If at the end of this time the animal be killed, the liver is found to be free from glycogen. A sample of blood taken during the height of glycosuria may contain from 3 to 4 parts of sugar per 1000. In order that the experiment may succeed it is important that the animal be previously well fed. If the puncture or 'piqûre' be carried out on an animal that has been starved or whose liver has been freed by any means from glycogen, no glycosuria is produced. It is evident that the effect of the puncture has been to cause a rapid conversion of the glycogen previously stored up in the liver into glucose. The glucose so formed escapes into the blood, raising the sugar content of this fluid above the normal, and the excess is immediately excreted by the kidneys together with an increased amount of water. A similar temporary hyperglycæmia and glycosuria may be brought about by fright, struggling or the administration of anæsthetics; but the effect is absent, if both splanchnic nerves have been previously divided above the suprarenals. It has been shown (Elliott, Cannon) that all these conditions are associated with an increased discharge of adrenalin from the medulla of the suprarenals into the circulation. Since the injection of adrenaline itself causes a condition of diabetes similar in all its limitations and aspects to 'puncture diabetes,' it is now generally believed that the two conditions are identical, and that the diabetic puncture acts through the splanchnic nerves on the suprarenals, setting free adrenalin, which passing to the liver causes a rapid 'mobilisation' of the stored-up glycogen, and a consequent hyperglycæmia and glycosuria, lasting as long as the glycogen store holds out.

$\mu$ (3) PHLORIDZIN DIABETES. Phloridzin is a glucoside extracted from the root cortex of the apple-tree. It may be decomposed into a sugar and phloretin. When phloridzin or phloretin is administered by the mouth or subcutaneously it gives rise to glycosuria, unaccompanied, at first at any rate, by any other symptom. The urine may contain from 5 to 15 per cent. of glucose. The glycosuria induced in this way differs from the forms already described in the fact that it is not due to hyperglycæmia. Analysis of the blood shows that the sugar is slightly diminished rather than increased. The excretion of glucose seems to be due to a specific effect of the drug upon the kidneys. If a cannula be placed in the two ureters so as to collect the urine from each kidney separately and a small dose of phloridzin be then injected by a hypodermic syringe into the left renal artery, the urine flowing from the left ureter will in two minutes be found to contain sugar, while the urine from the right kidney will not contain any sugar for another five or ten minutes. The effect therefore is rapidly to drain off sugar from the blood. In order to maintain the sugar content of the blood at its normal height the liver must rapidly manufacture fresh sugar to take the place of that lost by the kidneys. In the first instance the liver will utilise its stored-up glycogen for this purpose. If a dose of phloridzin be given to each of twe animals and one animal killed as soon as the excretion of sugar is coming to an end, the liver will be found free from glycogen. If now a second dose of phloridzin 
be given to the other, which may be regarded as glycogen-free, glycosuria is produced as before, and the excretion of sugar can be continued indefinitely by repeated administration of the drug. So long as sufficient food is given, including carbohydrates, the loss of sugar does not entail any increase in the destruction of the tissues; but if the drug be administered to starving animals the waste of sugar has to be made good at the expense of material other than carbohydrate. The source of the sugar excreted under these circumstances is the protein of the tissues. The nitrogen excreted in the urine rises in amount in proportion to the quantity of sugar excreted, and there is a constant ratio between the amount of nitrogen and the amount of sugar excreted in the urine. In different experiments this ratio $\mathrm{D}: \mathrm{N}$ varies from $2 \cdot 8: 1$ to $3 \cdot 6: 1$. If meat be administered to such starving animals with glycosuria, the $\mathrm{D}: \mathrm{N}$ ratio does not alter; the amount of nitrogen in the urine increases, but the sugar increases in the same proportion. The sugar production is therefore proportional to the protein metabolism and must be derived from protein. The source of the sugar is the amino-acids of which the protein is composed. It has been shown by Lusk, Embden and Dakin that the following amino-acids yield large amounts of glucose when administered to a phloridzinised animal: glycine, alanine, serine, cystine, aspartic acid, glutamic acid, ornithine, proline and arginine. We must assume that these amino-acids produced in digestion or by the autolysis of the tissues undergo deamination and that the sugar is formed by a process of synthesis from the oxyacids thereby produced. On the other hand leucine, tyrosine and phenylalanine give no increase in the output of sugar. It is however just these amino-acids which seem to follow the line of fat metabolism, since they are converted into aceto-acetic acid when perfused through a dog's liver-and the administration of fats to phloridzinised dogs is also without effect on the sugar excretion. The drain of sugar from the organism determined by the action of phloridzin on the kidneys thus necessitates a continued breakdown of the nitrogenous tissues of the body in the effort to maintain a normal supply of sugar to the tissues, and unless excessive feeding be employed the animal must waste. The great increase in the nitrogenous output resulting from the condition of phloridzin diabetes is shown in the following Table (Lusk) :

\begin{tabular}{|c|c|c|c|c|c|c|}
\hline \multirow{2}{*}{ 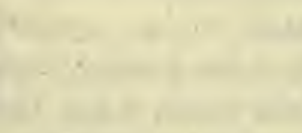 } & \multicolumn{3}{|c|}{ GOAT } & \multicolumn{3}{|c|}{ DOG } \\
\hline & D & $\mathrm{N}$ & $\mathrm{D}: \mathrm{N}$ & $\mathrm{D}$ & $\mathbf{N}$ & $\mathrm{D}: \mathrm{N}$ \\
\hline Fasting . & - & $3 \cdot 72$ & 一 & 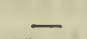 & $4 \cdot 04$ & - \\
\hline Fasting . & - & $3 \cdot 71$ & - & - & $4 \cdot 17$ & - \\
\hline Fasting and diabetic. & $20 \cdot 33$ & $4 \cdot 90$ & $4 \cdot 15$ & $63 \cdot 55$ & $12 \cdot 66$ & $5 \cdot 02 *$ \\
\hline Fasting and diabetic. & $26 \cdot 08$ & $8 \cdot 83$ & 2.95 & $65 \cdot 30$ & $18 \cdot 76$ & $3 \cdot 38$ \\
\hline Fasting and diabetic. & $23 \cdot 39$ & $8 \cdot 06$ & $2 \cdot 90$ & $65 \cdot 84$ & $18 \cdot 57$ & $3 \cdot 54$ \\
\hline Fasting and diabetic. & $19 \cdot 01$ & $6 \cdot 84$ & $2 \cdot 78$ & $64 \cdot 60$ & $17 \cdot 29$ & $3 \cdot 74$ \\
\hline
\end{tabular}

* The high D : N ratio on the first day is evidently due to the conversion of the glycogen still present in the body. 
The constant drain of sugar will in time involve a relative carbohydrate starvation of the tissues, which will make good their energy requirements as much as possible at the expense of protein and fat. The administration of meat will diminish the fat metabolism to a certain extent, but since it does not alter the $\mathrm{D}: \mathrm{N}$ ratio it would appear that the latter does not depend in any way on the quantity of fat undergoing oxidation. This is shown in the following respiration experiment (Mandel and Lusk) on a dog with phloridzin glycosuria, in which the metabolism during starvation and after ingestion of meat was determined :

\begin{tabular}{|l|c|c|c|c|}
\hline & $\mathrm{D}: \mathrm{N}$ & $\begin{array}{c}\text { Calories from } \\
\text { protein }\end{array}$ & $\begin{array}{c}\text { Calories from } \\
\text { fat }\end{array}$ & $\begin{array}{c}\text { Calories } \\
\text { total }\end{array}$ \\
\cline { 1 - 5 } & & & & \\
\hline Fasting & $3 \cdot 69$ & $80 \cdot 2$ & $274 \cdot 4$ & $354 \cdot 6$ \\
300 grm. meat & $3 \cdot 55$ & $161 \cdot 9$ & $261 \cdot 7$ & $423 \cdot 6$ \\
\hline
\end{tabular}

The enormous waste of energy involved in such a constant loss of sugar will be apparent if we consider that a $\mathrm{D}: \mathrm{N}$ ration of 3.65 means that 52.5 per cent. of the energy in the protein taken as food or set free from the tissues is lost to the organism in the form of glucose. According to Rubner 28.5 of the energy of meat protein is not utilised in the body, but is liberated simply as heat. This stimulating effect of protein on metabolism or on the processes of oxidation in the body is described by Rubner as the 'specific dynamic action' of proteins. If we accept this view and add this 28.5 per cent. lost as heat to the 52.5 per cent. lost as sugar there would remain a balance of only 19 per cent. actually available for the vital activities of the tissues. It is not to be wondered at that the nitrogenous metabolism may be increased three- to five-fold as a result of the artificial induction of the diabetic condition.

The carbohydrate starvation has other deleterious effects, since we have evidence that a certain amount of carbohydrate food is a necessary condition for both fat and protein metabolism. The necessity of carbohydrate for the assimilation of protein is brought out in an experiment by Cathcart. It has long been known that carbohydrate administration has a sparing effect on protein metabolism. If an animal or man be starved, the nitrogenous output sinks to a certain level and there remains practically stationary. If now pure carbohydrate food be administered sufficient to meet the energy requirements of the animal or man (about 35 calories per kilo), there is at once a rapid drop in the output of nitrogen and therefore in the protein waste of the tissues. Fat has a much slighter or no sparing effect on the nitrogenous metabolism. Indeed in certain experiments by Cathcart the administration of fat caused an actual rise in the nitrogenous output.

The importance of carbohydrates is borne out by the results of feeding animals with proteins which have been digested with pancreatic juice until the biuret reaction has disappeared. After Loewi had shown that it was possible to maintain nitrogenous equilibrium in dogs with such a digest. 
Lesser was unable to confirm his results; but it has been pointed out that the essential difference between the two observers was that Loewi gave an abundant supply of carbohydrates with the digest, while Lesser omitted carbohydrates altogether and administered fats and protein digest alone.

The evidence that the carbohydrates play a necessary part in the metabolic history of fats has already been mentioned ( $v$. p. 793). We have seen that in the absence of carbohydrates the last stages in the oxidation of fats make default so that the partially oxidised fatty acids, oxybutyric acid and aceto-acetic acid, accumulate in large quantities and are excreted as such or as acetone in the urine. Not only does this involve a loss of energy to the body, but these organic acids require other bases for their neutralisation. Up to a certain point they will be excreted in the urine in combination with the fixed alkalies. When these are no longer present in sufficient quantity they will be excreted in combination with ammonia, so that the ammonia of the urine is largely increased. If the condition of carbohydrate starvation be continued, this mechanism of neutralisation is insufficient and the phenomena of acidosis, dyspnœa and coma, ensue, resulting in the death of the animal.

Another effect of continued administration of phloridzin is fat infiltration of the liver. This is merely a result of the carbohydrate starvation. A similar condition of fat infiltration can be brought about by feeding with a pure protein plus fat diet. The liver seems to be able to act as a storehouse either of fat or of carbohydrate, so that there is an inverse ratio between the amount of glycogen and the amount of fat stored up in the liver at any given time. It has been shown that the fat in the liver under these circumstances is simply fat which has been transferred to this organ from the ordinary fat depôts, subcutaneous tissues, \&c., of the body.

(4) PANCREATIC DIABETES. Von Mering and Minkowski found that total excision of the pancreas gives rise to a severe and rapidly fatal diabetes, which presents many similarities to the severer cases of diabetes in man. Owing to the fact that the tissues of a diabetic are extremely prone to infection, it is often difficult after total excision of the pancreas, when diabetes has been set up, to procure healing of the wounds without suppuration. The operation is therefore usually carried out in two stages. In the first stage one small portion of the tail of the pancreas is transplanted under the skin of the abdomen, while the rest of the gland is excised. Such animals do not get diabetes and therefore recover quickly from the operation. When the wounds are quite healed the transplanted portion is removed through a simple skin incision. The second operation is followed in a few hours by the appearance of a large amount of sugar, 5 to 10 per cent. in the urine. The glycosuria persists, the animal rapidly wastes, and finally dies at the end of two to three weeks from diabetic coma. From the nature of the operation it is evident that the condition of diabetes observed under these circumstances has nothing to do with the presence or absence of the pancreatic secretion from the intestine, since this secretion is cut off at the first operation and diabetes does not make its appearance until the second small portion of the 
gland is removed. Moreover ligature of the ducts of the pancreas or obstruction of the ducts by the injection of melted paraffin does not give rise to diabetes. The excretion of sugar by the kidneys is due to an increase in the sugar content of the blood. The blood-sugar may amount to between 4 and 5 parts per 1000. This state of hyperglycæmia and the excretion of sugar in the urine persist even when the animal is completely starved or is fed on a pure protein or protein plus fat diet. Moreover, as in phloridzin glycosuria, we find a constant ratio between the sugar and the urinary nitrogen, the $\mathrm{D}: \mathrm{N}$ ratio being usually about $2 \cdot 8$. The administration of protein food to an animal previously starved increases the output of nitrogen, but increases at the same time the output of glucose. No similar increase in the glucose excretion is observed as a result of the administration of fat. We must conclude therefore that in the absence of carbohydrate from the diet the excess of sugar in the blood as well as that escaping by the urine is derived from the breakdown of the proteins of the tissues. On the other hand, the power of the animal to assimilate or utilise carbohydrate is diminished and sometimes entirely abolished, so that glucose administered to a starving animal with pancreatic diabetes may appear quantitatively in the urine. The amount taken by the alimentary canal is simply added to the amount which would have been excreted if no food had been given. In most cases, at any rate during the first week after total extirpation, there is apparently still some power of carbohydrate assimilation, since administration of glucose causes a transitory rise in the respiratory quotient (Moorhouse). Glycogen disappears entirely from the liver; but the muscles, especially the heart, may contain a normal or an increased amount of glycogen. There is a rapid wasting of all the tissues of the body, including the fats and proteins, and finally the animal is destroyed by the accumulation of the products of imperfect oxidation of the fatty acids.

It is still very difficult to say definitely why removal of the pancreas brings about this condition or what disturbance of metabolism is primarily responsible for it. Two views have been put forward. According to one, the primary disturbance is the diminished or absent power of utilisation of sugar by the tissues; according to the second, an increased production of sugar by the liver. There is no doubt that in the diabetic animal the power of utilising carbohydrates is deficient. This is shown by the low respiratory quotient and by the fact that administration of glucose to the animal causes an almost corresponding increase in the amount of glucose excreted in the urine. But the loss of power of utilisation is not absolute, at any rate in the first week of the disorder. Administration of glucose causes a slight and temporary rise in the respiratory quotient, and if $20 \mathrm{gms}$. of glucose be administered, it is often possible to recover only about 15 to $18 \mathrm{gms}$. from the urine. Moreover the increased amount of glycogen in the heart muscle of diabetic dogs points to a persistent power of assimilation of sugar by this organ. The heart from a diabetic animal, if fed with its own blood, can be shown to use up not only the sugar circulating in the blood but also its store of glycogen, and this utilisa- 
tion is especially marked if the heart be made to work excessively by raising the arterial resistance and administering adrenalin; but taken as a whole the power of utilising glucose is very inferior to that possessed by normal animals. One of the most striking features of the condition caused by total extirpation of the pancreas is the rapid diminution of the fat depôts of the body, attended by a marked condition of lipæmia and accumulation of fat in the liver. The blood is so full of fat globules that it has been compared in appearance to strawberries and cream. One of the first effects of extirpation of the pancreas is therefore a rapid fat mobilisation, and the respiratory quotient agrees with that obtained when the metabolic needs of the body are being mainly satisfied at the expense of the fat. The sugar of the urine, after the depletion of the glycogen store of the liver, is derived from the protein, and the protein tissues of the body therefore diminish as rapidly as the fat stores. On the theory of deficient utilisation, it is thought that these tissues suffer from carbohydrate starvation, even though they are bathed in a medium containing an increased amount of sugar, and that the liver in response to some call from the tissues turns first its glycogen and later on the proteins of the body into sugar to supply this lack-all to no purpose however, since the tissues are unable to avail themselves of the sugar or ferment.

According to the second view, the primary disorder affects only the liver. This organ is freed from some restraining influence on its power of manufacturing sugar from glycogen and from protein, so that the blood is flooded with sugar, which is therefore excreted in the urine. Any deficient utilisation of the sugar would be regarded as secondary to a poisoning of the tissues by this overloading of their nutrient fluids with sugar. It is certain that the sugar production in diabetes is excessive, as is shown by the rapid wasting of the protein tissues to give rise to the sugar, and that this over-production takes place in the liver is shown by the fact that extirpation of the liver in the diabetic animal causes a rapid disappearance of the sugar from the blood.

According to the Vienna school (Rudinger, Falta, and others), a close interaction exists between the thyroid, the suprarenals, the pancreas, and the liver, the thyroid to a slight extent, the pancreas still more, inhibiting the glycogenic functions of the liver, while the suprarenals through their excretion of adrenalin stimulate this function. Glycæmia and glycosuria caused by extirpation of the pancreas would therefore be ascribed to an unchecked activity of the suprarenals. An important difference however seems to exist between the two conditions. Adrenalin glycosuria comes to an end when the glycogen store of the liver is exhausted, whereas pancreatic diabetes continues until the death of the animal, long after all traces of glycogen have disappeared from the liver. We do not yet know how the pancreas affects sugar production or utilisation in the normal animal. It is generally assumed that it secretes into the blood stream a hormone which may, according to the view of the nature of diabetes which we adopt, pass to the tissues and enable them to utilise sugar, or pass to 
the liver and inhibit the sugar production in this organ. A very small portion of the pancreas is sufficient for this purpose, but we have been unable to imitate the action of the pancreas still in vascular connection with the body by injection or administration of extracts of this organ. Even connection of a healthy animal with a diabetic animal by means of its blood-vessels, so as to allow the healthy blood, presumably provided with the products of secretion of the pancreas, to circulate through the diabetic animal, does not abolish the condition of hyperglycæmia in the latter, though connection of the portal vein of the healthy animal with that

A

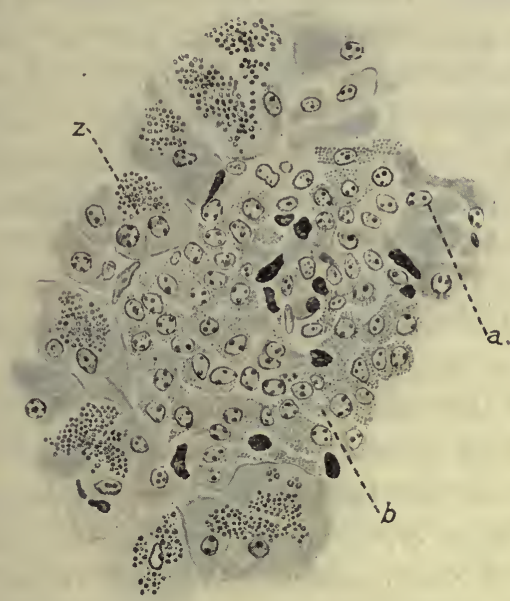

B

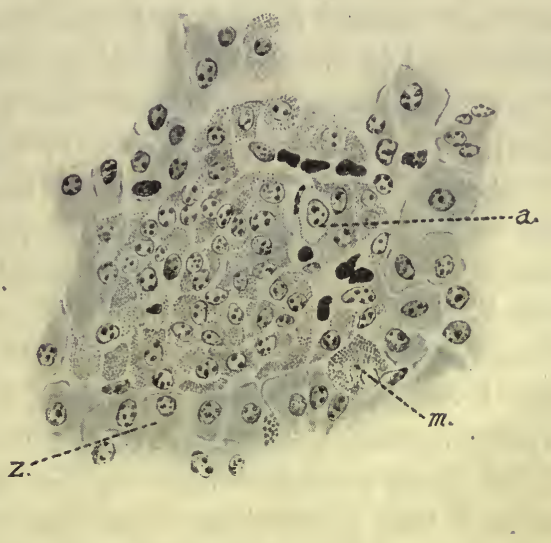

FIG. 356. (A) and (B) show an islet with the surrounding tissue in a resting gland (A) and after exhaustion with secretin (B). In (A) the secreting acini are charged with symogen granules. In (B) these have entirely disappeared. On the other hand no change is noticeable in the cells of the islet. In the latter the granular cells are the $b$ cells, and the clear hyaline cells are the $a$ cells, $(m)$ showing what are called Minkowski granules. The granulation of this cell is regarded by Bensley as due to postmortem changes.

of the diabetic animal has, according to Hédon, had the effect of stopping the condition of glycosuria. Further work is required on this point.

We thus see that the pancreas has a two-fold function, namely, the secretion of a digestive juice into the intestine and the exercise by some means or other of an influence on general metabolism, the absence of which is followed by the supervention of diabetes. Corresponding with this two-fold function, two kinds of structures are present in the gland, the secreting acini and the islets of Langerhans. These latter, though arising in connection with the ducts, are solid masses of cells and have no communication with the lumen of the ducts. According to Bensley and Lane the islet cells may be divided into two varieties which have been given the name of A and B cells, according as their granules are fixed respectively by alcoholic or watery solutions. It has been shown both by Bensley and by Homans that these cells undergo no alterations when the gland is excited to secrete by the injection of secretin. On the other hand, if four-fifths 
of the pancreas be removed, the remaining part may gradually become inadequate to prevent diabetes, and Homans has shown that when under these circumstances diabetes supervenes, the granules disappear from the B cells. Changes have also been found in the islets of Langerhans in fatal cases of diabetes in man. It seems therefore probable that what we may term, for lack of a better word, the antidiabetic functions of the pancreas, are associated with and dependent on the integrity of the islets of Langerhans.

(5) DIABETES IN MAN. In its severer forms the diabetes of man resembles very closely that produced in the dog by total extirpation of the pancreas. The output of urine is largely increased and the frequency of micturition is often the first symptom noticed. On examination the urine, though light in colour, is of a high specific gravity, 1030 to 1035 , and may contain from 5 to 10 per cent. of sugar. The appetite is largely increased, but in spite of the large amount of food taken the body wastes. The excessive quantity of fluid lost by the body gives rise to a constant thirst. The patient may die after some months or years in a condition of diabetic coma. Warning of the onset of this condition is given by the rise of ammonia in the urine and by the appearance of oxybutyric and diacetic acids. The breath may smell of acetone, and this substance may also be present in the urine. On the other hand, the diabetic state is attended by diminished resistance of the tissues to infection. A pimple may become a carbuncle; a slight sore on the foot may give rise to a rapidly spreading gangrene of the lower extremity; tubercular infection of the lungs spreads rapidly to the whole organ so as to stimulate pneumonia. The patient may thus die of some such intercurrent infection before the onset of coma. In a few cases the pancreas is found to be atrophied or diseased, but in the large majority no marked pathological change is to be observed in this organ. Yet the condition is essentially similar to that which occurs in pancreatic diabetes. The radical defect is the inability, relative or complete, of the organism to assimilate carbohydrate. We may find all grades between such cases and those in which there is still a considerable power of assimilation. In order to determine the grade of the disorder it is usual to give a test diet with a certain proportion of carbohydrate, e.g. 100 grm. of bread with meat, bacon, eggs, butter, green vegetables, cheese, lettuce, coffee and wine. If the urine remains free from sugar on this diet, the diabetes is mild in character. More bread may then be added to the diet from time to time until sugar appears in the urine and the limit of tolerance for carbohydrate has been reached. In many cases the sugar will disappear from the urine on the administration of a diet consisting entirely of proteins and fats. When this has been effected carbohydrates may be added in small proportions to the diet until the limit is found at which the assimilatory powers of the patient are reached. It seems that administration of any carbohydrate in excess of this limit is of disadvantage to the patient and hastens the progress of his disorder. When the power of assimilating carbohydrates is entirely abolished the prognosis is almost absolutely fatal. This point 
may be determined in two ways. In the first place, a patient with no power of carbohydrate assimilation will continue to excrete sugar in the urine on a pure protein fat diet, and the $\mathrm{D}: \mathrm{N}$ ratio will be $2 \cdot 8$ or higher. Information may also be obtained from a study of his respiratory quotient. The production of dextrose from protein involves the absorption of oxygen. Oxygen will therefore be taken in which will not reappear as carbon dioxide in the expired air. In severe cases of diabetes therefore the respiratory quotient will fall below that representing fat metabolism, i.e. below 0.7 . In most cases of diabetes, where there is still some power of assimilating carbohydrate and of storing up glycogen, the respiratory quotient will be found approximately normal. A very low respiratory quotient is a sign of the severity of the disorder.

This study of the conditions of carbohydrate metabolism shows how all three classes of food-stuffs co-operate in the maintenance of the chemical processes which lie at the root of the existence and the activities of living organisms. We see how fallacious were the ideas that the proteins alone were necessary for life and that protoplasm was simply living protein. Protoplasm, i.e. the material substrate of life, must be regarded as a complex in which proteins, fats, carbohydrates, nucleins, salts, and water all play a part and of which each is an essential constituent. In the higher animals proteins are necessary to furnish the proteins of the tissues, and the food must contain just those amino-acids which are requisite for the building up of the proteins characteristic of each separate tissue. Moreover certain groups of the protein molecule appear to be destined to serve as mothersubstances of hormones and other chemical compounds which play a dynamic rather than static part in the phenomena of life, and supply conditions of activity rather than material for the production of energy. The carbohydrates not only act as sources of energy, but are necessary to the building up of the proteins into the protoplasmic complex. Without them moreover this complex cannot properly utilise the fat contained in itself or supplied in its food. On the other hand, the carbohydrates by. themselves are not available as food, but require some connecting link, which may be protein or nitrogenous in character, to enable their association with the active part of the protoplasm and their utilisation by oxidation. At the same time there is a certain possibility of interconversion between these different substances; sugar may be formed from proteins, fats from carbohydrates. On the other hand, the formation of fats from proteins is apparently impossible in the cells of the higher animals, and the evidence for the formation of sugar from fat is limited to the study of the respiratory quotient in hibernating animals. With the exception of a few cases quoted by Pflüger and von Noorden, no support for such a conversion is obtained from the conditions observed in the glycosuria caused by the administration of phloridzin or by extirpation of the pancreas. 


\section{CHAPTER XII}

\section{THE BLOOD}

In the unicellular animals and in the lowest metazoa the cells are bathed by the medium in which the organisms live, and are therefore exposed to all the changes in the composition of this fluid which may be brought about by cosmic events. With the evolution of a body cavity filled with fluid the tissue-cells are set free from the necessity of adapting their metabolism to wide ranges of chemical composition, being bathed by an internal medium which is maintained practically constant in its characters for any given type. With increasing differentiation the fluid of the cœlom, which may be called blood, becomes enclosed in branching systems of tubes, and its circulation is provided for by the development of contractile chambers at some point or points of the tubes. In all the higher animals, the blood, the common medium and means of exchange for all parts of the body, circulates through a closed system of tubes, a constant flow being kept up by the action of the heart. It is separated from the tissue elements themselves by the walls of the blood-vessels. The free interchange of material between blood and tissues is facilitated by the tenuity of the vascular wall. The interstices of the tissues contain a fluid, the 'tissue fluid,' any excess of which is drained off by special channels known as lymphatics and carried back to the blood. Interchange between the -blood and the tissue-cells can be effected partly by diffusion, partly by a direct exudation or filtration of the fluid parts of the blood with certain of its constituents through the capillary walls. Since the function of the blood is to act as the common nutritive medium of all parts of the body, it has to convey food materials from the digestive organs and oxygen from the lungs to the tissues. From these it receives in exchange their waste products, namely, carbon dioxide and the results of nitrogenous metabolism, and carries them away to the excretory organs, such as the lungs and kidneys, by which they are eliminated. It is evident that the composition of the blood must vary from time to time and place to place according to the condition of activity and the function of the organ which it is traversing. The organs of the body are adjusted to respond to very minute changes in the composition of the circulating fluid, and add to or subtract from its constituents according as these are present in deficiency or excess. The changes are therefore kept within infinitesimal limits; in most cases they are within the limits of errors of analysis, 
and we may therefore treat the blood as a fluid of approximately constant composition and qualities.

Blood obtained from a mammal is an opaque fluid varying in tint according to the vessel from which it is derived, being scarlet when taken from an artery, purplish in colour when taken from a vein, the difference being determined by the degree of oxygenation of the blood. On shaking venous blood with air it takes up oxygen and acquires the scarlet colour characteristic of arterial blood. If examined in a thin layer under the microscope its opacity is seen to be due to the fact that it is not homogeneous, but consists of a number of corpuscles of different kinds suspended in a light yellow transparent fluid. In order to make out the characters of these corpuscles the blood should be diluted with some 'normal' fluid, such as 0.9 per cent. sodium chloride. It is then seen to contain two classes of corpuscles. Much the most numerous are the 'red corpuscles.' These differ in appearance according as the blood is derived from a mammal or from

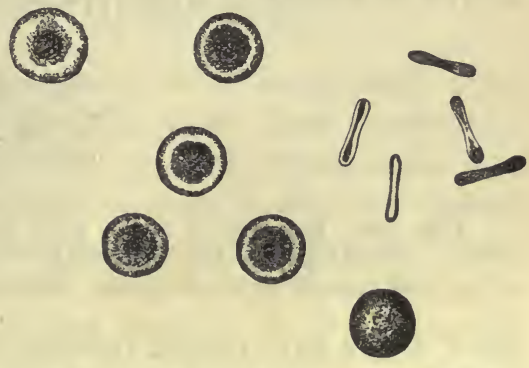

FIG. 357. Non-nucleated red blood-discs of human blood. On the right of the figure the corpuscles are seen on edge. (SWALE Vincent.)

one of the lower orders of vertebrates. In all the latter it is a nucleated cell. In the frog, for instance, it is an oval bi-convex disc containing an oval nucleus in the centre. In man and other mammals the red corpuscle is a bi-concave circular disc (Fig. 357), varying in size in different species. The average sizes of the corpuscles in man are given in the following Table :

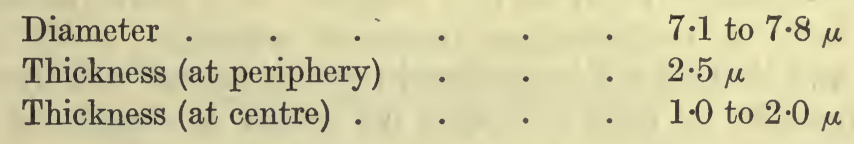

In the blood of man there is an average of five million red blood-discs in every cubic millimetre of blood.

The other kind of formed element, the white corpuscle, or leucocyte, is present in much smaller numbers than the red corpuscle, there being in human blood an average of one leucocyte to every 500 red corpuscles. These leucocytes are colourless cells, somewhat larger than the red blood-discs of man, presenting one or more nuclei and a granular or hyaline protoplasm. When examined on the warm stage they are seen to be amœboid, and many of them, like the amcba, have the power of ingesting granules of carmine, food, or dead bacteria with which they may come in contact.

In addition to these two classes, a third body is generally described under the name of 'blood-platelets' or hæmatoblasts. These are especially well seen when the blood has been received directly into an excess of osmic acid. It is still doubtful whether they are pre-existent in the circulating blood or are formed in the plasma by a process of precipitation. 
Unless special precautions are taken, the examination of blood obtained from a blood-vessel is interfered with by the process of clotting, which ensues shortly after the blood has left the blood-vessels. If blood be received into a beaker it is at first perfectly fluid, so that it can be poured from one vessel to another. After a space of time varying from three to eight minutes it begins to be viscous, and if poured out of the beaker leaves an adherent layer on the sides of the vessel. A minute later the whole mass of the blood becomes solid and the beaker can be inverted without spilling its contents. If a section be made of this blood-clot it is found to owe its solidity to a network of fine threads of a protein substance named

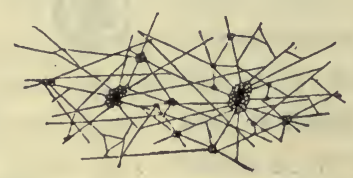

FIG. 358. Network of fibrin, after washing away the corpuscles from a film of blood that has been allowed to clot; many of the filaments radiate from little clumps of blood-platelets. (SCHÄFER.)

fibrin, which have formed throughout the plasma and enclose the corpuscles in their meshes (Fig. 358). On leaving the clot for some hours, drops of yellow fluid appear on its surface and run together. The whole clot contracts, and finally there is a reduced clot floating or suspended in a yellowish fluid known as serum. If after the blood has left the vessels it be whipped with a bunch of twigs, or stirred with a glass rod, the filaments of fibrin as they are formed are deposited on the twigs. After three or four minutes the twigs can be withdrawn and the spongy fibrin collected. The blood which is left consists only of the corpuscles, plus serum, and will not clot, since its fibrin has been removed. It is known as defibrinated blood. Since the corpuscles are apparently unchanged in the meshes of the clot and clotting can be produced in blood-plasma entirely separated from corpuscles, we must look upon the process of coagulation as determined in the main by changes in the blood-plasma. We can regard the blood therefore as a tissue consisting of a fluid matrix, which is extremely unstable and undergoes change when it leaves the vessels, and as having: embedded in its matrix, formed elements or cells of various kinds. 


\section{SECTION I}

\section{THE WHITE BLOOD-CORPUSCLES}

AмœBоID cells are a constant constituent of the cœlomic fluid in all classes of animals. Even in the lower metazoa, where there is not yet a body cavity, wandering mesoderm cells are present which apparently discharge functions analogous in all respects to those of the white bloodcorpuscles of mammals. On carefully examining a specimen of human blood, either fresh or in the form of a thin stained film, several varieties of these cells are seen to be present. In a fresh specimen we can distinguish the following varieties :

(a) A cell with a lobed nucleus and finely granular protoplasm;
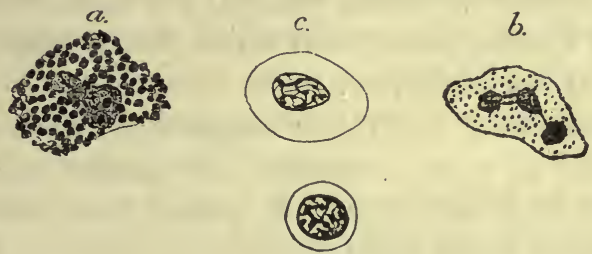

d

Fia. 359. Various forms of leucocytes.

$a$, eosinophile corpuscle ; $b$, ordinary polynuclear leucocyte (' neutrophile ') ; $c$, hyaline corpuscle; $d$, lymphocyte.

(b) A small cell consisting almost entirely of a nucleus surrounded by a thin layer of protoplasm;

(c) A cell with a single nucleus and clear hyaline protoplasm;

(d) A cell with a lobed or reniform nucleus, the cytoplasm being beset with large coarsely refracting granules.

These four types are known as the finely granular or polymorphonuclear cell, the lymphocyte, the hyaline corpuscle, and the coarsely granular corpuscle.

The differentiation of the various types of leucocytes is more easily made if recourse be had to staining with mixtures of aniline dyes. This method was introduced by Ehrlich, who classified leucocytes according to the staining characters of their granules, dividing the latter into :

(a) Those staining with acid dyes, such as eosin-acidophile or eosinophile granulation;

(b) Those staining with basic dyes-basophile; 
(c) Those staining only with a mixture of the acid and basic dyes and therefore spoken of as neutrophile.

An acid dye is generally a salt in which the colouring-matter plays the part of an acid radical. Thus eosin is the sodium salt of the coloured acid tetrabrom-fluorescein. Basic dyes possess basic colour radicals. An example of this class, methylene blue, is the chloride of the coloured base tetramethyldiphenthiazine. Neutral dyes, according to Ehrlich, are those in which a colour base is combined with a colour acid, such as the eosinate of methylene blue, or the picrate of rosaniline.

In preparations stained with mixtures of these dyes we may distinguish the following types :

(1) The polymorphonuclear cells. These preesnt a lobed nucleus, and their protoplasm contains abundant fine neutrophile granules. They form about 70 per cent. of the total leucocytes. If the specimen be overstained with eosin the granules may take on a red stain.

(1a) A few cells are sometimes seen with a horseshoe or hour-glass nucleus and presenting a few neutrophile granules. These are spoken of as transitional cells, and have been supposed to represent an intermediate stage between large mononuclear or hyaline cells and the polymorphonuclear leucocyte. They do not form more than 1 per cent. of the leucocytes.

(2) The lymphocytes are small cells with a round nucleus surrounded by a thin layer of hyaline protoplasm which is free from granules. These form 23 per cent. of the leucocytes.

(3) Large mononuclear or hyaline corpuscles. These cells are two or three times the size of a red corpuscle, and possess a large oval nucleus which stains feebly with basic dyes. In normal blood not more than 2 per cent. of the leucocytes are of this type.

(4) In every well-stained blood-film the eosinophile corpuscle is very evident, although not forming more than 3 per cent. of the white corpuscles. The nucleus is generally single, but is often crescent-shaped or reniform. The protoplasm is crammed with large discrete highly refractive granules which stain deeply with eosin and give micro-chemical reactions for iron as well as phosphorus. The granules, which in man, dog, and rabbit are spherical, are cuboidal in the horse, and in birds have the shape of short rods.

(5) A cell which is found with difficulty, but is apparently a normal constituent of human blood, is the basophile leucocyte. This, which is somewhat smaller than the polymorphonuclear cell, has a lobed or tri-lobed nucleus and presents a number of granules in its protoplasm which stain deeply with basic dyes. It is sometimes spoken of as a 'Mast' cell, the German term for the cell being used without translation. It does not form more than 0.5 per cent. of the total leucocytes of the blood. The granules are practically invisible in fresh specimens, in this respect presenting a contrast with the eosinophile granules. The leucocytes as a whole undergo variations in number according to the physiological state of the animal and are increased during digestion, specially of a protein 
meal. They vary from one in 300 to one in 600 red corpuscles, or, taken as a whole, from 18,000 to 9000 per cubic millimetre of blood.

\section{FORMATION OF THE LEUCOCYTES}

In classifying the white corpuscles of the blood it is essential to know whether the different varieties we have described represent phases in one and the same corpuscle or a number of different cells of separate origin. The question as to the specificity of each kind of leucocyte cannot be regarded as settled. According to some observers, Gulland and others, all the leucocytes are derived from one kind of cell, namely, the lymphocyte. Ehrlich and his school, on the other hand, regard each type as forming a tissue sui generis, originating in separate localities and from distinct kinds of cells. Since division of the leucocytes in the blood itself appears to be an occurrence of the utmost rarity, we must locate the original seat of formation of these cells in two tissues. Lymphocytes are derived from the adenoid tissue forming the lymphatic glands and the lymph nodules surrounding so many of the mucous cavities. These lymphatic nodules present towards their centre a clearer zone, consisting of cells rather larger than those of the periphery and known as the 'germ centre.' The nuclei in these cells present a well-marked reticular arrangement, and nuclear figures are often to be seen. By the division of these cells lymphocytes are formed, pushing towards the periphery of the nodule, where they make their way into the lymph-sinus and are carried slowly by the lymph into the blood. Some of these lymphocytes may possibly pass directly through the capillary wall into the blood-stream.

The other tissue concerned in the formation of leucocytes is the bonemarrow. This is the chief blood-forming tissue of the body, since it is responsible also for the production of all red blood-corpuscles which are formed during adult life. In the red marrow are seen a number of cells known as myelocytes. These contain a single rounded nucleus and a well-marked protoplasm which may be non-granular or may contain granules, generally eosinophile in character, but sometimes basophile. It is stated that all intermediate stages are to be found in the bone-marrow between these 'myelocytes' and the polymorphonuclear leucocyte as well as the eosinophile leucocyte. It is certain that in the disease leukæmia, which is associated with an increased number of leucocytes in the blood, there may be an increase either of eosinophile cells or of the neutrophile cells, and either condition is associated with changes in the red bone-marrow. We may therefore provisionally arrange the leucocytes of the blood according to their origin as follows :

(1) Small lymphocyte derived from lymphoid tissue.

(2) Large mononuclear or hyaline corpuscle : doubtful whether derived by a growth of (1) or from a myelocyte.

(3) Polymorphonuclear leucocyte formed in bone-marrow.

(4) Eosinophile cell derived from similar cells in the bone-marrow. This origin of the eosinophile corpuscle is rendered more probable by the 
fact that the shape of the granule, which differs from one species to another, is the same whether the cell be derived from the blood or the bone-marrow.

The intermediate or transitional cell may be derived either from the lymphocyte or from a myelocyte. In many cases of leukæmia the myelocyte passes into the blood in large numbers without undergoing the changes necessary to convert it into the typical blood-cell. We find then mononuclear cells which are either free from granules or contain eosinophile or basophile granules.

\section{FUNCTIONS OF THE LEUCOCYTES}

PHAGOCYTOSIS. We have seen that the leucocytes from whatever animal they be taken present two phenomena, viz. that of amoboid movement and that of ingesting foreign particles which may be presented to them. On account of this power of eating up foreign particles they are frequently spoken of as 'phagocytes,' in this respect resembling unicellular organisms and the undifferentiated cells of many kinds of tissue. All the phenomena connected with the process of inflammation in higher animals are directed to the assemblage of leucocytes at the spot which is the seat of injury or of infection, so that they may devour and remove either the injured tissue or the invading micro-organisms. This process plays therefore an important part in determining the immunity of any animal against infection; though in the higher animals it is assisted by a number of other mechanisms directed towards the same end, which we shall have to discuss in a subsequent chapter. The use of phagocytosis is not, however, confined to the protection of the organism against infection. Wherever any effete or dead tissue has to be cleared away, whether as the result of injury or in the course of metamorphosis of organs, the leucocytes play an important part. Thus in the great rearrangement of tissues which occurs in the larval state of insects, the removal of the muscle fibres which are no longer required is effected by the accumulation of phagocytes around them. The phagocytes may send processes into the muscle substance, which dissolve this tissue and then take it up. The absorption of the tail of the tadpole is effected in the same way by means of phagocytes. In mammals, including man, the moulding of the long bone which occurs in the process of growth is effected by continual and coincident processes of absorption and new formation of bone. The absorption is carried out by means of special phagocytes formed by the aggregation of a number of leucocytes, the well-known 'giant cells' or myeloplaxes which form so prominent a constituent of bone-marrow.

The blood-corpuscles represent the wandering phagocytes of the body. There are fixed phagocytes of which the myeloplaxes just mentioned may be regarded as a type. Other members of this class are the endothelial cells (Kupfer's 'Sternzellen') which line the capillaries of the liver. If a suspension of carmine or of micro-organisms be injected into the bloodstream these endothelial cells are found a little later to have taken up large numbers of the foreign bodies. Under normal circumstances these 
cells as well as some similar cells in the spleen take up effete red bloodcorpuscles and destroy them. During the process of degeneration of a peripheral nerve brought about by its separation from the ganglion-cells of which its fibres are the processes, a marked proliferation of the nervenuclei takes place. These become surrounded with protoplasm and act the part of phagocytes, loading themselves with the fat globules set free by the degeneration of the myelin sheath. To the same class of fixed phagocytes may possibly be ascribed certain of the plasma-cells of the connective tissues.

That the polymorphonuclear leucocytes are endowed with these phagocytic properties is universally acknowledged, but some doubt still exists as to how far the other types of leucocytes which we have described can function as phagocytes. It is probable that the lymphocytes, and certainly the large mononuclear or hyaline corpuscles, are endowed with these properties. The granular corpuscles, namely, eosinophile and basophile, are thought by some to function as unicellular glands and to react to infection, not by englobing the micro-organisms, but by discharging substances stored up in their granules which have a poisonous effect on the micro-organisms, and so prepare them for subsequent ingestion by the polymorphonuclear leucocytes.

The other functions which have been ascribed to leucocytes are unimportant as compared with their rôle as phagocytes, and are all of them questionable. Thus some authors ascribe to leucocytes an important part in the taking up of fat from the intestine and its carriage into the lymphatic system. In the coagulation of the blood the leucocytes have been supposed to act by the discharge of substances which may act as precursors of the fibrin ferment. In the invertebrata the wandering mesoderm cells not only remove the injured tissue but apparently give rise to new connective tissues. The same function was formerly assigned to the leucocytes of mammals by Ziegler, and Metchnikoff still believes that after the removal of any injured tissue the emigrated leucocytes undergo elongation to form so-called fibroblasts, by the further division of which are produced the white fibres of the connective tissues as well as the branched connective-tissue cells. Most authors at the present time have come to the conclusion that the work of the leucocytes is complete with the removal of dead or injured tissue, and that the process of regeneration is carried out by the plasma-cells of the connective tissue, which enlarge, undergo division, and form the fibroblasts of the developing tissue. These plasma-cells can change their position and act as phagocytes, eating up and digesting the polymorphonuclear leucocytes which have prepared the way for their regenerative activity. Their amœboid power is shown by the fact that if two sterile cover-glasses be introduced under the skin, new connective tissue is formed between the cover-glasses, and this method has been adopted by Ziegler for the study of the cellular changes involved in the new regeneration of this tissue. 


\section{SECTION II \\ THE RED BLOOD-CORPUSCLES}

THE red blood-corpuscles, or erythrocytes, in man and in mammals are nucleated bi-concave discs, about 7 to $8 \mu\left(\frac{1}{3200}\right.$ in.) in diameter and about one-third of this in thickness. The colour of a single corpuscle when viewed under the microscope is yellow, the red colour being only apparent when larger numbers are seen together. The red corpuscles form about 50 per cent. of the total mass of the blood, there being about 5,000,000 red corpuscles in every cubic millimetre of blood. They are soft, flexible, and elastic, so that they can readily squeeze through apertures and canals narrower than themselves without undergoing permanent distortion. Each red corpuscle eonsists of a framework or stroma, composed chiefly of protein material, containing in its meshes or in a state of loose chemical combination a red colouring-matter, hæmoglobin, to which is due the colour of the corpuscles and of the blood itself.

It is only in mammalia that the red corpuscles are of the character described. In the camel they are oval in shape, but otherwise resemble the corpuscles of other mammals. In all other classes of vertebrata the red corpuscles are oval, nucleated cells. The hæmoglobin is diffused through the protoplasm of the cell-body and does not extend to the nucleus. During the early part of fœtal life the corpuscles of mammals are also nucleated, but in the adult condition the erythrocytes, except under abnormal conditions, lose all traces of the nucleus before entering the blood stream. The small size and great number of the red corpuscles determine that a very large area of surface of red corpuscles is exposed to the plasma. The volume of each corpuscle has been estimated as $\cdot 0000000722 \mathrm{~mm}^{3}$, and its surface as $\cdot 000128 \mathrm{~mm}^{2}$, so that the total surface of red corpuscles in the blood of a man weighing about 70 kilos (assuming his total blood as $\frac{1}{13}$ of the body weight) would be about 3000 sq. metres, or 1500 times the surface of the body itself. This great extent of surface is of importance in facilitating the exchange of material, especially oxygen, between the corpuscle itself and the surrounding plasma.

On treating the blood with weak solutions of tannic or boracic acid a separation occurs between the hæmoglobin and the stroma, the former appearing as a small ball near the centre of a colourless disc or being extruded so as to lie just outside the stroma. Brücke, who first observed this appearance, gave the name of 'zooid' to the mass of hæmoglobin and of ' œecoid' to the stroma. 
OSMOTIC RELATIONSHIPS OF THE RED CORPUSCLE. If the bloodplasma be concentrated by evaporation or by the addition of neutral salts its osmotic pressure rises and water diffuses from the corpuscles into the plasma in order to equalise the osmotic pressure within and without the corpuscle. The latter therefore becomes wrinkled or crenated. On the other hand, dilution of the plasma diminishes its osmotic pressure below that of the corpuscle, and water therefore passes into the latter, which swell up and become spherical, and if the plasma be made sufficiently dilute the corpuscles burst with the liberation of the hæmoglobin they contain. The corpuscles of mammalian blood neither gain nor lose volume in a solution containing 0.9 per cent. sodium chloride. The osmotic pressure, as determined by the freezing-point, of such a solution is identical with that of the blood. For frogs' blood such a solution would be too concentrated and bring about crenation. The salt solution which is normal for frogs' blood only contains 0.65 per cent. sodium chloride.

Although the average molecular concentration of blood-plasma in mammals is equivalent to that of a 0.9 per cent. sodium chloride solution, it may vary even in one animal within fairly wide limits, as is shown by the following determinations of the freezing-point of blood-serum taken from animals under various circumstances :

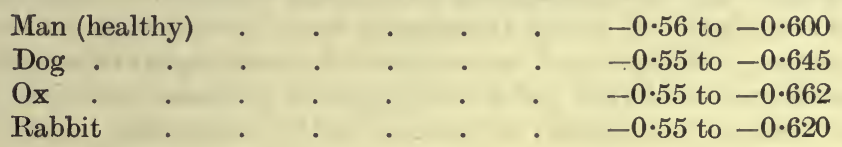

The behaviour of the red corpuscles when immersed in solutions of sodium chloride of different concentrations shows that its limiting membrane or most external layer is impermeable to sodium chloride. If this salt be added to defibrinated blood and the crenated corpuscles separated by the centrifuge, practically the whole of the added sodium chloride remains in the plasma or serum. The red corpuscle is impermeable to most neutral salts as well as to cane sugar and glucose. We may therefore make 'normal' solutions with sodium chloride, sodium sulphate, potassium nitrate, or cane sugar, taking care that each of the solutions shall be isotonic with a 0.9 per cent. solution of sodium chloride. On the other hand, a solution of urea behaves towards the corpuscles like distilled water. If some red corpuscles be added to a 1 per cent. solution of urea in normal salt solution, they neither shrink nor swell, and if the mixture be centrifuged and the corpuscles and supernatant fluid examined separately, the percentage of urea in the two cases will be found identical, though there would be a great preponderance of sodium chloride in the supernatant fluid. If a 1 or 2 per cent. solution of urea in water be added to defibrinated blood, the corpuscles will swell up and burst just as if distilled water had been added. There are a large number of substances to which the corpuscles are permeable, e.g. alcohol, chloroform, ether, \&c. In their permeability the corpuscles resemble most other vegetable and animal cells in permitting the passage of all those substances which are soluble in fats and the allied substances, cholesterin, lecithin, and protagon, which are invariable constituents of all living cells. According 
to Overton the external limiting pellicle of the red corpuscles, as in most living cells, is formed by a lecithin-cholesterin compound, whose solvent power determines the permeability of the cell by foreign substances. If therefore we wish to stain the living cell we must choose some dyestuff, such as methylene blue or neutral red, which is soluble in such lipoid bodies.

\section{CHEMISTRY OF THE RED BLOOD-CORPUSCLES}

The red corpuscles consist of two parts, hæmoglobin and stroma, probably in a state of loose chemical combination. By various means it is possible to destroy this combination and to dissolve out the hæmoglobin, leaving the colourless swollen-up stroma floating in the plasma. At the same time the blood becomes darker but more transparent, and is spoken of as 'laked' blood.

It has been thought by Schwann, Schäfer, and others that the red corpuscle consists of a solution of hæmoglobin contained within an envelope which contains lecitr in and cholesterin and forms the stroma. Though the hæmoglobin can be separated from the stroma by very simple means, it is difficult to believe that it is in watery solution. Thus the blood-corpuscles contain a greater percentage of solids than any soft tissue of the body. The blood-corpuscles contain 36.7 per cent. solids, as against muscular tissue with 25 per cent. or nervous with 22 per cent. solids. Of these solids, 95 per cent. consist of hæmoglobin, so that the solution would have to contain at least 30 per cent. hæmoglobin. No solution of hæmoglobin of this strength can be prepared. In many animals, such as the rat and guinea-pig, it is sufficient merely to 'lake' the blood, i.e. to bring the hæmoglobin into solution in the surrounding plasma or serum, in order to make the hæmoglobin crystallise out. Some form of combination is therefore necessary in the corpuscles if merely to keep the hæmoglobin from separating out in a crystalline form.

Blood may be ' laked' by any of the following means :

(a) Addition of a small amount of ether.

(b) Free dilution with water.

(c) Alternate freezing and thawing of the blood.

(d) Addition of bile-salts.

(e) The action of foreign blood-serum or of various hæmolysins whose nature we shall have to discuss later.

From such laked blood we may prepare either hæmoglobin or stroma.

In order to separate the stroma from the hæmoglobin, blood which has been defibrinated or prevented from clotting by the addition of a little sodium oxalate is centrifuged until all the formed elements form a solid cake at the bottom of the tube. The tube is then filled up with normal saline fluid and again centrifuged, and this process repeated twice in order to wash away adherent plasma or serum. After the final washing two volumes of distilled water saturated with ether are added to one volume of caked corpuscles. The corpuscles swell up and their hæmoglobin passes into solution into the surrounding fluid. The blood is laked. The fluid is once more centrifuged in order to throw down white blood corpuscles. A 1 per cent. solution of acid sodium sulphate is now added drop by drop until the solution acquires the opaque appearance presented by ordinary blood. The action of this salt, as of dilute acids, is to precipitate the swollen-up stromata, which reacquire the power of reflecting light from their surfaces and restore the opacity to the blood. On centrifuging, the stromata are thrown down, and can be collected and washed with distilled water several times on the centrifuge. 
The stroma protein only forms about 4 per cent. of the total solids of the corpuscle. It is insoluble in dilute acids, but easily soluble in dilute alkalies. On gastric digestion the greater part dissolves, leaving a residue which is rich in phosphorus, and has been called nuclein. Stroma protein is therefore spoken of as a nucleo-protein. Wooldridge, who devised the method given above for the preparation of pure stromata, regarded the protein as a compound of protein and lecithin. The substance certainly contains a large quantity of lecithin, the greater part of which is present in the precipitate obtained on gastric digestion. According to Wright the nuclein residue yields purine bases on hydrolysis, and is therefore rightly classed with the other nucleins from tissue-cells and contained in nuclei.

From the laked solution of corpuscles oxyhæmoglobin can be obtained in a crystalline form with varying readiness according to the animal from which the blood is derived. Thus in the case of the rat, the guinea-pig, the dog, and the horse it is sufficient merely to cool the laked blood, preferably in a freezing mixture to about $-10^{\circ} \mathrm{C}$. in order to obtain a large crop of hæmoglobin crystals. Crystallisation is facilitated by the addition of 25 per cent. of absolute alcohol to the mixture, though the use of alcohol certainly tends to interfere with the subsequent purification and solubility of the hæmoglobin. Oxyhæmoglobin can be recrystallised by dissolving it in weak alkali at $35^{\circ} \mathrm{C}$, , cooling the solution to $0^{\circ} \mathrm{C}$., and then adding cold alcohol to 25 per cent. and allowing the mixture to stand for some days at a temperature of $-5^{\circ}$ to $-10^{\circ} \mathrm{C}$. In the case of those bloods which yield oxyhæmoglobin crystals with greater difficulty, it is better to add to the laked blood an equal volume of a saturated solution of ammonium sulphate. The precipitate of globulins is filtered off and the filtrate allowed to stand in a cool place. Crystals of hæmoglobin then come down in quantity.

\section{PROPERTIES OF HAEMOGLOBIN}

The crystals thus obtained are as a rúle microscopic in size. Most animals yield an oxyhæmoglobin which crystallises in rhombic prisms or needles belonging to the rhombic system. In the guinea-pig the crystals are tetrahedral in form, while the oxyhæmoglobin of the squirrel crystallises normally in the form of six-sided plates. On recrystallisation, however, a squirrel's hæmoglobin can be obtained as a mixture of rhombic prisms with rhombic tetrahedra. The water of crystallisation of oxyhæmoglobin varies in different animals between 3 and 9 per cent. The solubility of the crystals differs according to the animal from which

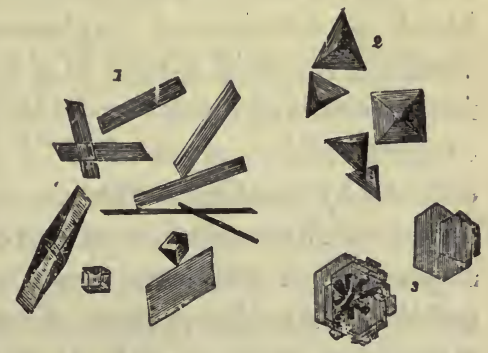

Fia.360. Crystals of oxyhæmoglobin. 1. From rat. 2. From guinea-pig. 3. From squirrel. they have been derived, and is in direct proportion to the difficulty with which the crystals are obtained. They are more soluble in highly diluted solutions of ammonia and the caustic alkalies and their carbonates than in water. A solution of hæmoglobin will not diffuse through parchment paper ; the elementary analysis of oxyhæmoglobin crystals gives somewhat varying 
results according to the animal employed. In the case of the oxyhæmoglobin of the dog Jaquet obtained the following figures:

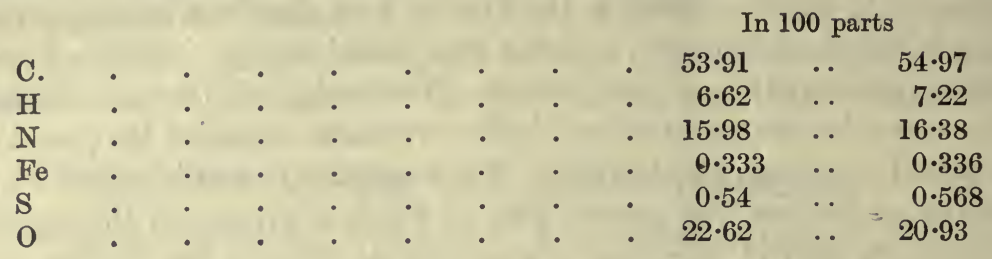

The chief differences between different animals appear to have relation to the sulphur. Hæmoglobin from the hen contains 0.857 per cent. sulphur. All specimens are alike in containing a constant proportion of iron, as is shown in the following Table :

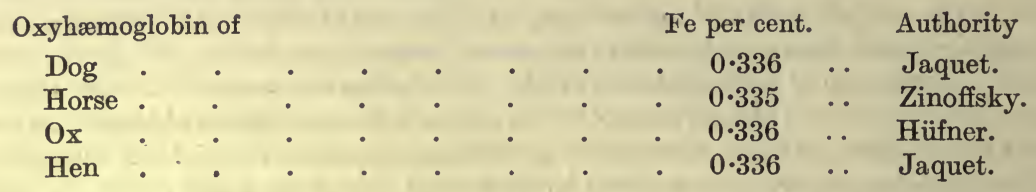

On the assumption that each molecule of oxyhæmoglobin contains one atom of iron, its molecular weight would be 16,660 , and this result is borne out by the volume of oxygen or carbonic oxide which can enter into combination with hæmoglobin. It has been suggested by Bunge that the enormous size of the hæmoglobin molecule finds a teleological explanation; if we consider that iron is eight times as heavy as water, a compound which would float easily along with the blood-current through the vessels could only be secured by the iron being taken up by so large an organic molecule. Oxyhæmoglobin is a compound in definite proportions of oxygen and hæmoglobin or reduced hæmoglobin. It can be easily dissociated and is split up by such simple means as exposure to a vacuum. If, for instance, some arterial blood or solution of oxyhæmoglobin be introduced into a Torricellian vacuum, the fluid is seen to give off bubbles of gas, and the colour changes from a brilliant scarlet to a dull bluish red. In this process each gramme of oxyhæmoglobin gives off 1.34 c.c. of oxygen. The same change can be effected by treating a solution of oxyhæmoglobin with reducing agents such as an alkaline solution of ferrous tartrate (Stokes's fluid) or ammonium sulphide; in the latter case reduction is aided by gently warming the solution. Another reagent of value for effecting the reduction of oxyhæmoglobin is a solution of hydrazine. The oxygen in oxyhæmoglobin can be replaced by equivalent quantities of other gases. Thus if carbon monoxide gas be led through a solution of oxyhæmoglobin, oxygen is given off and its place is taken by an equal volume of carbon monoxide with the formation of a more stable compound, carbon monoxide hæmoglobin. This body is only dissociated with extreme slowness and is unaffected by the addition of reducing agents. By using special precautions to prevent oxidation of the gas the carbon monoxide can be replaced in this compound by nitric oxide, 
NO. We have therefore a series of three compounds which can be arranged in order of stability, thus :

$$
\begin{aligned}
& \mathrm{NO} \text {-hæmoglobin. } \\
& \mathrm{CO} \text {-hæmoglobin. } \\
& \mathrm{O}_{2} \text {-hæmoglobin. }
\end{aligned}
$$

The poisonous properties of carbon monoxide are due to its power of turning out the oxygen from the oxyhæmoglobin, thus depriving the tissues of the oxygen which is normally carried to them by the red corpuscles.

Hæmoglobin and its derivatives give well-marked absorption spectra. Thus dilute solutions of oxyhrmoglobin placed in front of the slit of a spectroscope show two well-marked absorption bands between Fraunhofer's

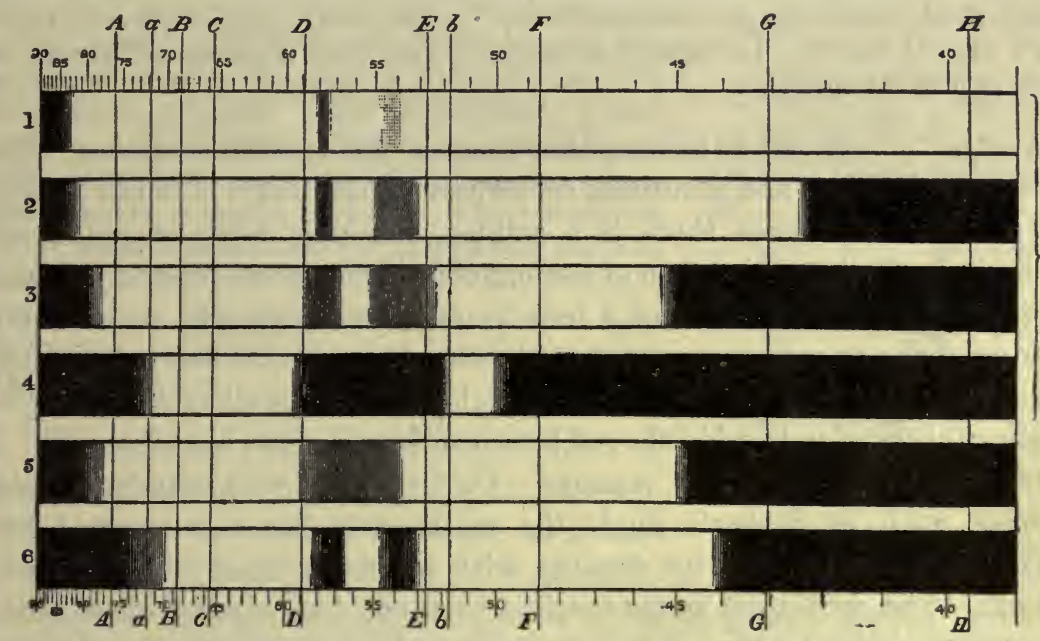

FIG. 361. The spectra of oxyhæmoglobin in different grades of concentration, of (reduced) hæmoglobin, and of carbon monoxide hæmoglobin. (After Preyer and Gamgee.)

1 to 4. Solution of oxyhæmoglobin containing (1) less than 01 per cent., (2) 09 per cent., (3) 37 per cent., (4) 8 per cent., (5) solution of (reduced) hæmoglobin containing about $\cdot 2$ per cent., (6) solution of carbon monoxide hæmoglobin. In each of the six cases the layer brought before the spectroscope was $1 \mathrm{~cm}$. in thickness. The letters $(A, a, \& c$.$) indicate Fraunhofer's lines and$ the figures wave-lengths expressed in $\frac{1}{100000}$ millemetre.

lines $\mathrm{D}$ and $\mathrm{E}$. The centre of the band nearest to $\mathrm{D}$ corresponds to $\lambda 579$, and is often spoken of as the band $\alpha$, while the second band, the one next to $\mathrm{E}$, which can be called the band $\beta$, is broader, has less sharply defined edges, and its centre corresponds approximately to $\lambda$ 544. On concentrating the solution or using thicker layers a point is reached at which the two bands fuse into one, and with a still stronger solution it will be found that the whole of the spectrum is absorbed with the exception of the red end.

The above figure shows the spectrum of oxyhæmoglobin in varying concentrations, a stratum one centimetre thick being examined. If a reducing agent be added to the solution of oxyhæmoglobin the two bands disappear and their place is taken by a more diffuse band lying midway between the two (Fig. 361, 5), its centre corresponding to $\lambda$ 555. This is the 
absorption spectrum of hæmoglobin or reduced hæmoglobin. The spectrum of carboxyhæmoglobin is very similar to that of oxyhæmoglobin, the bands, however, being shifted slightly towards the red end. This solution is of a brighter red than oxyhæmoglobin. Its tint is best observed on diluting the blood to a large extent, when oxyhæmoglobin acquires a yellowish tint, while the pink colour of CO-hæmoglobin is retained so long as any colour is visible. The fact that $\mathrm{CO}$-hæmoglobin is not altered by reducing agents can be shown by adding ammonium sulphide to CO-hæmoglobin and examining with the spectroscope, when no change is observed.

All these derivatives of hæmoglobin, besides their absorption bands in the visible spectrum, have characteristic absorption of light in the ultra-violet spectrum, as has been shown by Gamgee. In the case of oxyhæmoglobin this absorption causes a band (Soret's band) which occupies the greater part of the spectral region between Fraunhofer's lines $G$ and $H$. In reduced hæmoglobin this band is displaced towards the visible part of the spectrum.

Another compound of hæmoglobin with oxygen is methamoglobin. This substance, although not of normal occurrence in the body, is found in urine and in blood whenever there is a sudden breaking down of red bloodcorpuscles with the setting free of hæmoglobin in the blood-plasma. It may be prepared by the addition of a ferricyanide, permanganate, or nitrite, or other oxidising or reducing agents to the laked blood or to solutions of oxyhæmoglobin. It is a chocolate-brown substance, crystallisable, and gives a distinct absorption band in the red between Fraunhofer's lines $C$ and $D$. It is unaltered by exposure to a vacuum. On treatment with reducing agents, however, such as Stokes's fluid, the methæmoglobin is converted into hæmoglobin, from which by shaking with air oxyhæmoglobin can be reformed. The fact that methæmoglobin cannot be reduced by exposure to a vacuum indicates that it is a compound of oxygen with hæmoglobin in which the oxygen is in a different state of combination. According to Buckmaster methæmoglobin only contains half the oxygen contained by oxyhæmoglobin, so that the composition of the two bodies might be represented.

$$
\mathrm{Hb}<\mathrm{I}_{\mathrm{O}}^{\mathrm{O}} \text { (oxyhæmoglobin) and } \mathrm{Hb}=\mathrm{O} \text { (methæmoglobin). }
$$

The change from oxyhæmoglobin to methæmoglobin is not affected, however, by a simple shifting of the oxygen groups, but must be assumed to involve two distinct events. The whole of the oxygen in loose combination with hæmoglobin is given off, and the oxygen in the methæmoglobin molecule is derived from the oxidising agent added, so that ferricyanide of potash, for instance, is converted into ferro-cyanide.* Since the whole of the oxygen in the oxyhæmoglobin is given off on the addition of potassium ferricyanide, we may use this fact in order to determine the total amount of oxygen in combination in the blood ( $v$. p. 859).

* When the change is effected by reducing agents we must assume that the oxygen of the water or air is the source of that required for the oxidation of the reduced hæmoglobin to methæmoglobin. 
DERIVATIVES OF HÆMOGLOBIN. Hæmoglobin is a compound of an iron-containing coloured group (the prosthetic group) with a protein, which probably varies somewhat in different animals. The prosthetic group is identical in every case where it has been examined. A separation of the prosthetic group from the protein moiety can be effected with extreme ease, and occurs whenever the hæmoglobin is treated with weak acids, with alkalies, or is heated above $70^{\circ} \mathrm{C}$. . The protein group is known as globin.

In order to separate globin, oxyhæmoglobin crystals are dissolved in water and treated with small quantities of very dilute hydrochloric acid. A precipitate of pigment forms, which, if the hæmoglobin used be free from inorganic salt, rapidly dissolves in excess of the acid. Alcohcl and ether are then added in such relative quantities that the ether separates rapidly from the aqueous solution. The colouring-matter (hæmatin) dissolves in the ether, whilst the protein (globin) remains in solution in the water. The solutions are separated by a separating funnel and ammonia added carefully to the aqueous solution. This throws down a precipitate of the protein, which is soluble in acids and alkalies and coagulable on heating; the coagulum, however, is soluble in acids. It is precipitated. by ammonia in the presence of ammonium chloride. It contains as much as 16.89 per cent. nitrogen, and yields a considerable amount of the basic derivatives on hydrolysis. It is therefore classified with the histones.

Hæmoglobin yields about 94 per cent. of globin and about 4.5 per cent. of the chromogenic group, hæmatin. In order to obtain hoematin in a pure condition it is usual to

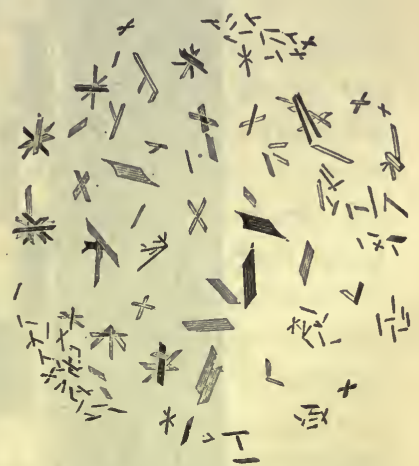

FIG. 362. Hæmin crystals. start with the crystalline derivative of hæmoglobin known as homin. When some dried blood is heated with a crystal of common salt and placed in acetic acid on a slide, a residue is obtained in which a number of reddish-brown needles are embedded known as Teichmann's crystals or hæmin crystals (Fig. 362). The preparation of these crystals is often used as a convenient test for the identification of blood.

In order to obtain them in large quantities the following method, devised by Chalfejew, is employed. One volume of defibrinated blood is added to four volumes of glacial acetic acid previously heated to $80^{\circ} \mathrm{C}$. As soon as the temperature has fallen to $60^{\circ} \mathrm{C}$. the liquid is again warmed, and then allowed to cool. Crystals are formed which are allowed to stand for twelve hours and are then separated and washed by decantation, first with distilled water and then with graduated strengths of alcohol. In order to purify these crystals the crude material is shaken for fifteen minutes with a mixture of chloroform and pyridine. The solution is filtered and then thrown into glacial acetic acid previously saturated with sodium chloride and heated to $105^{\circ} \mathrm{C}$. A few drops of concentrated hydrochloric acid are then added and the mixture allowed to stand for twenty-four hours. The crystals which separate out are filtered off, washed with dilute acetic acid, and then dried.

Hæmin crystals have been regarded as hydrochloride of hæmatin. Elementary analysis shows that they have the following formula (Willstätter) : $\mathrm{C}_{33} \mathrm{H}_{32} \mathrm{O}_{4} \mathrm{~N}_{4} \mathrm{Cl}$.Fe. By dissolving hæmin in alkalies and throwing 
the solution into an excess of acid a precipitate is obtained which is hæmatin. Hæmatin forms a powder of bluish-black colour and metallic lustre. It is insoluble in water, alcohol, or ether, but is slightly soluble in glacial acetic acid and in absolute alcohol. It is easily soluble in concentrated sulphuric acid, but undergoes decomposition, losing its atom of iron and being transformed into homatoporphyrin, which forms a deep purple solution. The formula of hæmatin has not yet been ascertained with certainty. It is probably

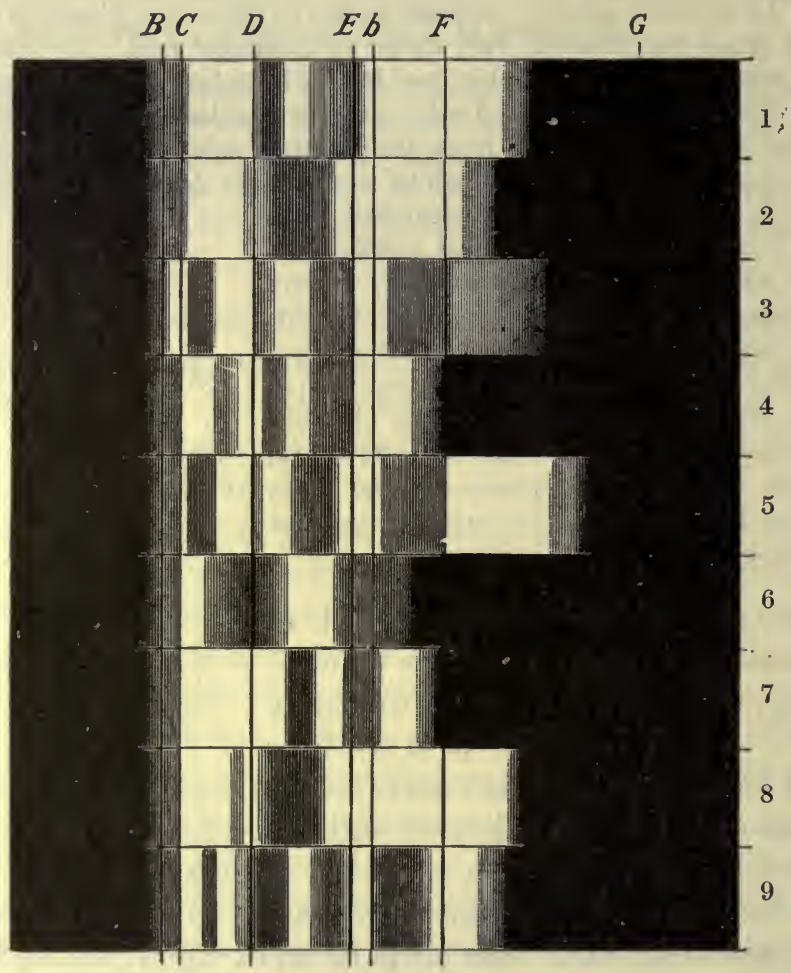

FIG. 363. Absorption spectra of hæmoglobin and its derivatives.

1. Oxyhæmoglobin. 2. Reduced hæmoglobin. 3. Methæmoglobin. 4. Alkaline methæmoglobin. 5. Acid hæmatin in ether. 6. Alkaline hæmatin in rectified spirit. 7. Reduced hæmatin. 8. Acid hæmatoporphyrin. 9. Alkaline hæmatoporphyrin. (From MACMUnN.)

$\mathrm{C}_{33} \mathrm{H}_{32} \mathrm{O}_{4} \mathrm{~N}_{4} \mathrm{Fe} . \mathrm{OH}$. Its compounds with acids and alkalies are spoken of as acid and alkaline hæmatin, and each gives a characteristic absorption spectrum (Fig. 363). The alkaline solutions exhibit one indistinct absorption band between $\mathrm{C}$ and $\mathrm{D}$, the acid solutions an absorption band also between $\mathrm{C}$ and $\mathrm{D}$ but nearer to $\mathrm{C}$, and resembling somewhat the band presented by methæmoglobin. According to Hoppe-Seyler and Gamgee perfectly pure solutions of hæmatin in alkalies are quite unaffected by reducing agents; in the presence of certain foreign matters, however, alkaline hæmatin, when treated with reducing agents, exhibits a spectrum known as that of reduced alkaline hæmatin, which is identical with that of hæmochromogen. The same 
change is further observed when alkaline hæmatin made by the action of alkalies on ordinary blood is treated with reducing agents such as ammonium sulphide. Since this substance hæmochromogen is responsible for the respiratory functions of the hæmoglobin, i.e. the power of its molecule to form unstable compounds with oxygen, its preparation merits fuller consideration.

Homochromogen is prepared by the action of caustic alkalies on hæmoglobin in the absence of oxygen. For this purpose a test-tube containing a solution of sodium or potassium hydrate is placed in a bottle with two necks containing a solution of hæmoglobin, care being taken not to spill any of the alkaline solution. Hydrogen is then passed through the larger bottle until the hæmoglobin is entirely reduced and all the air is replaced by hydrogen. The bottle is then inverted so as to mix its contents with the caustic alkali, when hæmochromogen is formed and can be recognised by its characteristic colour and spectrum. The hæmochromogen in solution has a cherry-red colour; and when sufficiently diluted shows two well-marked absorption bands identical with those given by reduced alkaline hæmatin (Fig. 363, 7). Of the two absorption bands which are situated between $\mathrm{D}$ and $\mathrm{E}$, that nearest to $\mathrm{D}$ has very sharply defined borders; the position of the two absorption bands may be given in terms of their wavelengths as follows : $\lambda 567$ to 547 and $\lambda 532$ to 518 . The band nearest D is given by hæmochromogen solutions diluted so that there is only one part of the pigment in 25,000 parts of water, so that the formation of reduced alkaline hæmatin is an even more delicate test for blood than the spectrum of oxyhæmoglobin itself. When CO-hæmoglobin is treated in the same way with alkali in the absence of oxygen, a body $\mathrm{CO}$-hæmochromogen is formed which contains exactly the same volume of $\mathrm{CO}$ in combination as the original CO-hæmoglobin. This fact, combined with the possibility of reducing ordinary alkaline hæmatin by the action of ammonium sulphide or Stokes's fluid, indicates that the group of atoms which in hæmoglobin is responsible for taking up oxygen or carbon monoxide gas passes unchanged into the hæmochromogen molecule. Hæmochromogen therefore represents an ironcontaining coloured radical which can combine with protein bodies to form hæmoglobin, and is responsible for the oxygen-combining powers of the latter. We may assume therefore that oxyhæmoglobin and CO-hæmoglobin contain oxyhæmochromogen and CO-hæmochromogen respectively.

Hcematoporphyrin. If hæmoglobin, hæmatin, or hæmin be mixed with concentrated sulphuric acid, it dissolves forming a purple-red solution. On pouring this solution into a large quantity of water, hæmatoporphyrin is thrown down in the form of a brown precipitate. In order to prepare hæmatoporphyrin, pure crystallised hæmin is added to a saturated solution of hydrobromic acid in glacial acetic acid. The whole is allowed to stand for three or four days and then thrown into distilled water. The resulting mixture is filtered and the hæmatoporphyrin thrown down by careful neutralisation of the hydrobromic acid with caustic soda. Hæmatoporphyrin is easily soluble in alkalies and somewhat less readily so in acids. 
forming alkaline and acid hæmatoporphyrin respectively. The formula of hæmatoporphyrin has been given by Nencki and Sieber as $\mathrm{C}_{16} \mathrm{H}_{18} \mathrm{~N}_{2} \mathrm{O}_{3}$, and is according to them isomeric with the chief bile-pigment, bilirubin. According to Willstätter its formula is $\mathrm{C}_{33} \mathrm{H}_{38} \mathrm{~N}_{4} \mathrm{O}_{6}$. An alcoholic solution of hæmatoporphyrin acidulated with hydrochloric acid shows two absorption bands: one, the fainter, between $\mathrm{C}$ and $\mathrm{D}$, and the other, broader and more defined, midway between D and E. Solutions of alkaline hæmatoporphyrin show four absorption bands: a weak band between $\mathrm{C}$ and $\mathrm{D}$, another between $\mathrm{D}$ and $\mathrm{E}$, a more strongly marked band nearer to $\mathrm{E}$, and a fourth band, darkest of all, between $b$ and F. It will be observed that in the formation of hæmatoporphyrin from hæmatin the iron of the latter has been split off by the action of the strong acid. Laidlaw has found that the splitting off of iron occurs much more readily in the absence of oxygen. If reduced hæmoglobin be taken, or defibrinated blood which has been allowed to stand until it is thoroughly reduced, it is sufficient to add 15 per cent. hydrochloric acid in order not only to convert the greater part of the hæmoglobin to hæmatin but to split off the iron of the latter and form hæmatoporphyrin.. Hæmotoporphyrin occurs in minute quantities in normal urine and in larger quantities in certain toxic conditions, especially in poisoning by sulphonal, when the urine may have a bright purple colour. It is important to remember that although urine is acid from the presence of acid sodium phosphate, urinary hæmatoporphyrin is always alkaline hæmatoporphyrin and gives the spectrum of this body.

CHEMICAL RELATIONSHIPS OF HÆMATIN. Hæmatin, or hæmochromogen, is widely diffused through the animal kingdom, occurring in the form of hæmoglobin in a large number of the invertebrata, as well as in all the vertebrata except, perhaps, Amphioxus. Since the respiratory function of hæmoglobin depends on the power of its iron-containing radical to combine with a molecule of oxygen, forming an easily dissociable compound, it becomes of interest to try whether by a study of its disintegration products we can throw any light on its chemical relationships and on the conditions of its formation in the living organism. When hæmatin is oxidised with sodium bichromate and acetic acid two new acids are formed, called the hæmatinic acids. One of these has the formula $\mathrm{C}_{8} \mathrm{H}_{9} \mathrm{O}_{4} \mathrm{~N}$, and the other $\mathrm{C}_{8} \mathrm{H}_{8} \mathrm{O}_{5}$. The first acid is converted into the second by the action of alkalies. The relationship of the two hæmatinic acids can be represented by the following formulæ :-
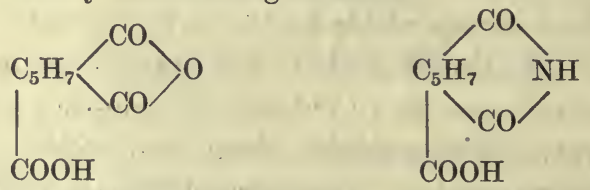

If, on the other hand, hæmin or hæmatoporphyrin be reduced by the action of hydriodic acid dissolved in acetic acid with the addition of phosphonium iodide, and the product be distilled with steam, the distillate contains a mixture of substituted pyrroles formerly known as hæmopyrroes. The mixture readily oxidises to a red substance on exposure to the air. If ammonia be added to the coloured solution the colour changes to yellow, which, on the addition of an ammoniacal solution of zinc chloride, changes to pink with a green fluorescences. These reactions are also given by urobilin, one of the urinary pigments and the chief pigment of the fæces, as well as by hydrobilirubin, a substance obtained by the action of tin and sulphuric acid on an alcoholic solution of hæmatin. 
The hæmopyrroles, according to Willstätter are three in number and have the following formula :-
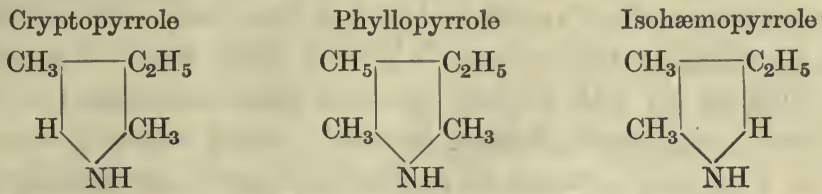

The same substances can be obtained from the chlorophyll of plants. On treatment with acid chlorophyll loses magnesium, and is converted into phacophytin. From this three $\mathrm{COOH}$ groups can be split off leaving a substance ætioporphyrin.

It is interesting that hæmatoporphyrin can be readily converted into the same substance. On treatment with pyridin and alcoholic potash it is converted into hæmoporphyrin, and this heated with soda lime gives ætioporphyrin $\left(\mathrm{C}_{31} \mathrm{H}_{36} \mathrm{~N}_{4}\right)$. Thus the same group forms the basis both of the substance which is responsible in the plant for the assimilation of carbon from carbon dioxide, and of the pigment which in the animal is the carrier of oxygen between the tissues and the surrounding medium. According to Willstätter, ætioporphyrin and hæmatoporphyrin are both built up of four substituted pyrrole rings.

Thus hæmatoporphyrin has the following structural formula :-

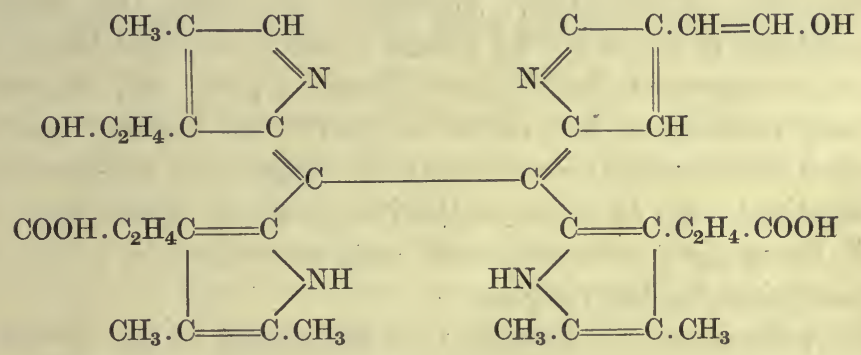

and the same worker suggests the following formula for hæmin :-

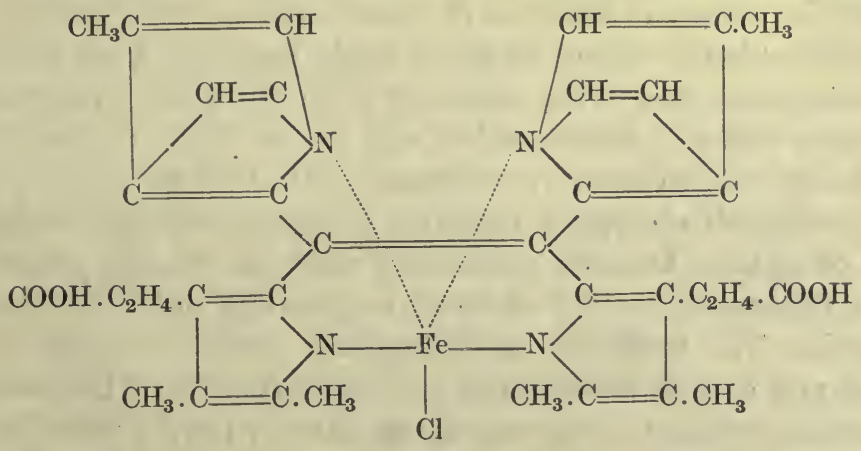

THE SYNTHESIS OF THE BLOOD-PIGMENTS. Chemists have not yet succeeded in the artificial formation of hæmatoporphyrin. Given hæmatoporphyrin, however, evidence has been brought forward both by Menzies and Laidlaw of the possibility of forming artificially both hæmatin and hæmoglobin, or some substance indistinguishable from the latter. 
Reduced hæmoglobin is a compound of hæmochromogen and a protein, globin. The splitting off of the prosthetic chromatogenic group-hæmochromogen - can be effected either by acid or alkali. When the latter is employed we obtain a red solution which is fairly stable, and can be converted by shaking up with air into ordinary alkaline hæmatin. With acids the decomposition is easily carried further. Even with 2 per cent. hydrochloric acid a certain amount of hæmatoporphyrin is formed, and if the strength of the acid be increased to 15 per cent. the whole of the iron is split off and the hæmochromogen is converted entirely into hæmatoporphyrin.

If oxyhæmoglobin be treated in the same way it yields acid or alkaline hæmatin directly, so that hæmatin must be regarded as an oxyhæmochromogen. The distinction drawn by Hoppe-Seyler between hæmochromogen and reduced alkaline hæmatin had its chief ground in the fact that pure hæmatin is not reduced to hæmochromogen by the action of such reducing agents as ammonium sulphide. The conversion can, however, be easily effected by using a strong reducing agent, such as hydrazine hydrate. Whether the hæmatin contains the whole of the oxygen of the oxyhæmoglobin is doubtful. According to Ham and Balean, when oxyhæmoglobin is converted by means of acids into acid hæmatin, exactly half of the oxygen of the oxyhæmoglobin is given off, so that hæmatin would only contain one-half of the oxygen of the oxyhæmoglobin. There is a marked difference between the stability of hæmatin and hæmochromogen. In the oxidised form of hæmatin the iron is firmly bound and can only be split off by using strong sulphuric acid, concentrated hydrochloric acid being insufficient for the purpose.

It has been shown by Laidlaw that the change in the reverse direction, i.e. the combination of iron with hæmatoporphyrin to form hæmochromogen, may be effected with equal ease. One gramme hæmatoporphyrin prepared by Nencki's method is dissolved in dilute ammonia and warmed in a flask on the water-bath. Some Stokes's fluid, prepared from about 2 grm. ferrous sulphate, and a few drops of a 50 per cent. hydrazine hydrate solution are added. At the end of one or two hours the solution is seen to be of a bright red colour when examined in thin layers, and on dilution shows the typical absorption spectrum of hæmochromogen, which changes to that of alkaline hæmatin on shaking with air. Strong potash is added, and the ammonia is boiled off in an evaporating dish with free exposure to the air. The hydrazine is decomposed, and a solution of hæmatin remains, and can be precipitated by acidification with hydrochloric acid. The pigment obtained in this way agrees in every respect with that prepared from oxyhæmoglobin. Analysis of the product gave 9.58 per cent. of iron, which agrees with Nencki's formula for hæmatin, $\mathrm{C}_{32} \mathrm{H}_{30} \mathrm{~N}_{4} \mathrm{O}_{3} \mathrm{Fe}$.

A pigment occurring in the wing feathers of certain birds, called turacin, was shown by Church to contain copper, and to yield, on treatment with strong sulphuric acid, a substance indistinguishable from hæmatoporphyrin. Laidlaw has succeeded in synthetising this pigment by treating ordinary hæmatoporphyrin obtained from blood 
with ammoniacal copper solution, showing that it is a compound corresponding to hæmatin, in which the place of iron is taken by copper.

It was stated some -years ago by Menzies that a solution of impure hæmochromogen, prepared by the action of ammonium sulphide on alkaline hæmatin obtained in the ordinary way from blood, on standing for some days was reconverted into reduced hæmoglobin. Ham and Balean have confirmed this observation, and have shown in addition that hæmochromogen, prepared by the action of ammonium sulphide on an alkaline solution of pure hæmin, though perfectly stable by itself, was rapidly reconverted into hæmoglobin if a solution of globin were added to the mixture. The same change took place if egg-white were used instead of globin. The hæmoglobin thus formed was changed into oxyhæmoglobin on shaking with air. Although in these experiments the oxyhæmoglobin was not separated in the crystalline form, its colour and spectral characters are so very distinctive that we are justified in concluding not only that it is possible to effect a recombination of the hæmochromogen and globin, but also that other proteins can take the place of globin in the hæmoglobin molecule.

\section{THE LIFE-HISTORY OF THE RED BLOOD-CORPUSCLES}

The growth of the embryo as well as of the young animal must be attended with a continual increase in the number of red blood-corpuscles present in the body. In the developing embryo the first formation of red corpuscles occurs in the vascular area. In the chick, about the twentieth hour of incubation, the area opaca, which surrounds the blastoderm, and will later become the area vasculosa, presents on examination under the low power a network of anastomising strands more opaque than the rest of the area. On section these strands are seen to be made up of cellular masses, the ordinary mesenchyma, with branched cells and amœboid corpuscles lying between. The cells in these cords are continually multiplying by indirect division. Those on the outer side of the cord become the endothelium of dilated blood-vessels, while those in the interior acquire a yellowish colour from the laying down of hæmoglobin in their cytoplasm. The cords become canalised, and, as soon as a connection is established with the vascular system of the embryo, the newly formed blood-corpuscles move slowly on into the general circulation. The red corpuscles in the bird are true erythrocytes, i.e. are nucleated cells. The leucocytes seem to arise by the immigration of wandering cells from the surrounding mesenchyma. Other places in the fotus where a similar growth of corpuscles proceeds throughout foetal life are the liver and the spleen, and later on the bone-marrow.

In the mammal the nucleated erythrocytes, though forming the majority of the red corpuscles in early fœtal life, become fewer and fewer in number as gestation advances, so that at birth practically the whole of the corpuscles are of the non-nucleated type. These, however, can be shown to be derived from nucleated red corpuscles by a process either of extrusion 
or of degeneration and solution of the nucleus (Fig. 364). The formation of red corpuscles does not cease with the end of fœtal life or even with the attainment of full stature by the animal. We have definite proof that a continual formation of red corpuscles can proceed and is proceeding throughout the whole of adult life. In an adult the total volume of blood and the total number of corpuscles remain approximately constant. By bleeding an animal we can diminish the total amount of corpuscles. The

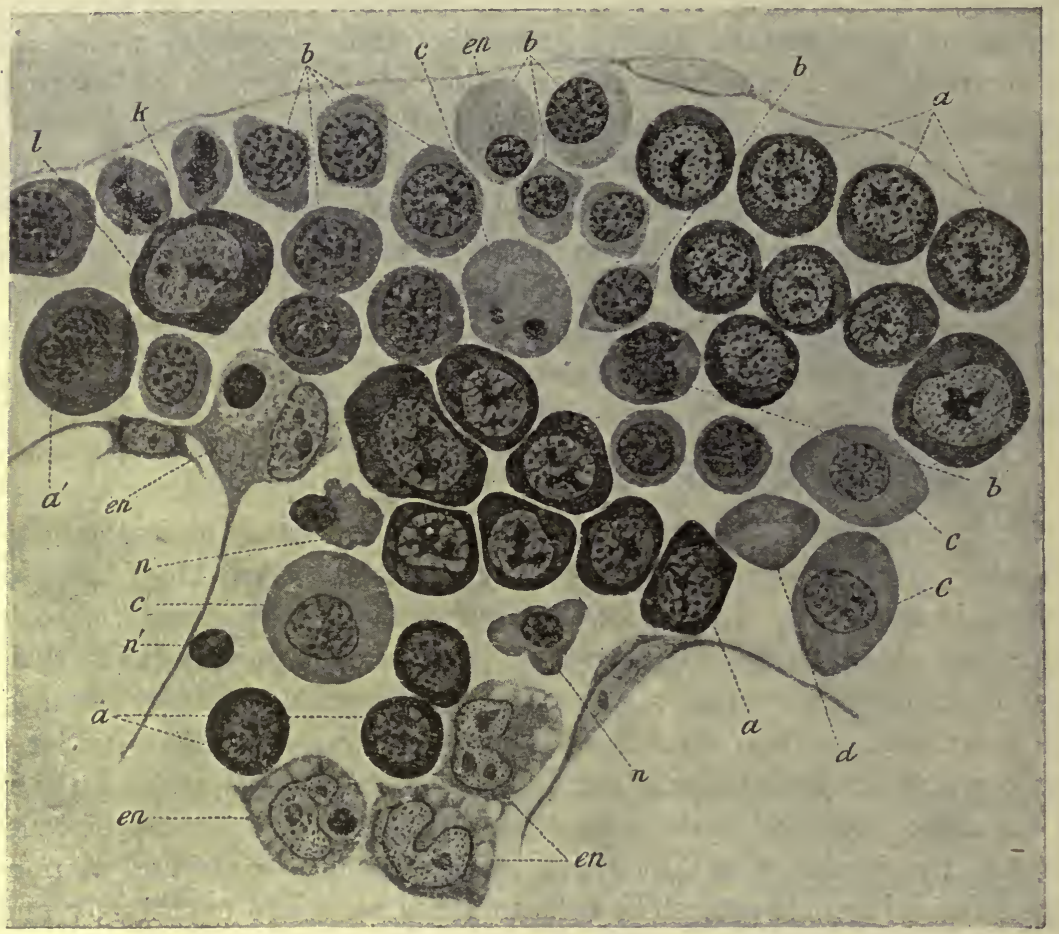

FIG. 364. Part of a blood-vessel from the yolk-sack of the rabbit embryo, showing the changes which occur in the formation of erythrocytes. (From ScHärer after MAXIMOW.)

$a$, megaloblasts ; $b$, normoblasts changing into erythroblasts ; $c$, erythroblasts, in which the nuclei are disappearing ; $d$, an erythrocyte fully formed, but not yet discshaped; $e n$, phagocytic endothelial cells; $l$, lymphocytes; $k$, a divided lymphocyte; $n$, erythroblasts, shrunken with atrophic nucleus.

first effect of such a bleeding is that the fluid parts of the blood are made up, so that the volume of the blood is restored to normal and the blood therefore becomes relatively poor in corpuscles. In a few weeks, however, the corpuscular content of the blood is found to be once more normal, showing that the loss of corpuscles has been followed by a compensatory regeneration. The fact that the pigments constantly leaving the body with the urine and fæces, namely, urochrome and urobilin or stercobilin, are derived by means of the liver from hæmoglobin, shows that a constant destruction of red corpuscles must be proceeding. Since the number of corpuscles remains unaltered, this loss of hæmoglobin must be made 
good by a continual regeneration of fresh hæmoglobin and new red corpuscles. The seat of the formation of red corpuscles in the higher vertebrates is the bone-marrow. Here we have a structure protected from pressure where the capillaries and veins are dilated and thin-walled, and allow a slow passage of blood and the entry of newly formed corpuscles through the imperfect walls into the blood-stream (Fig. 365). That the marrow is the tissue involved in the process is shown by the fact that it is the only tissue of the body which undergoes an alteration in appearance when blood formation is stimulated by such means as repeated bleeding or destruction of corpuscles by the injection of toxic agents. Under such

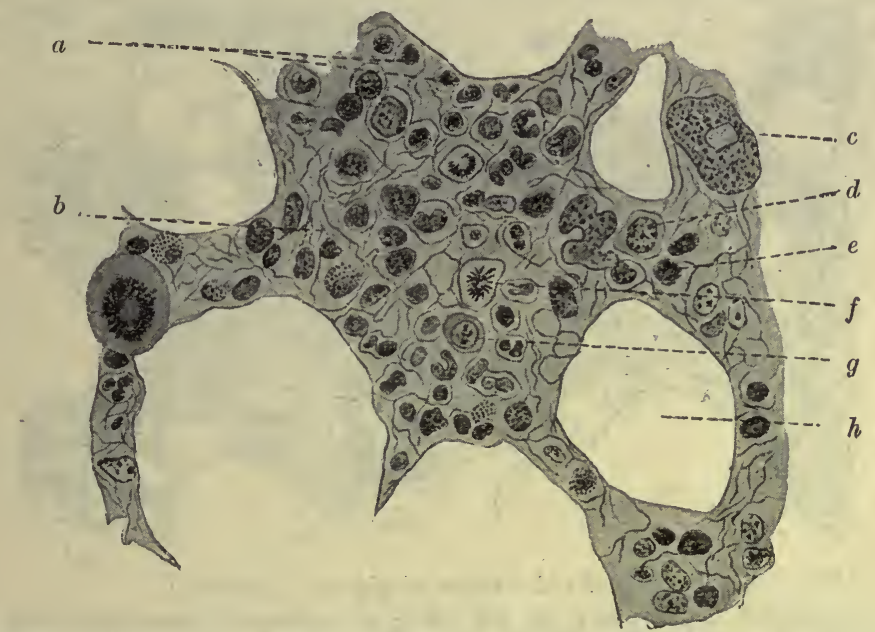

Fig. 365. Section of red marrow of mammal. (BöHM and DAvidofF.) $a$, e, erythroblasts ; $b$, recticulum ; $c$, myeloplax ; $d, g$, marrow cells ; $f$, a marrow cell dividing; $h$, a space which was occupied by fat.

conditions the red marrow, which in adult mammals is present only in the epiphyses, is found to have increased in extent and in many cases to occupy the greater part of the shaft of the bone, having taken the place of the yellow marrow. It is in the red marrow therefore that we must seek the precursors of the red blood-corpuscles. In the bird the erythroblasts, i.e. the precursors of the red blood-corpuscles, form a sort of inner lining to the dilated capillaries of the marrow (Fig. 366). Here we can see all grades between the colourless nucleated corpuscle which lies nearest the periphery and the fully formed red oval corpuscle containing hæmoglobin, lying next the lumen and ready to be carried away in the blood-stream. If blood formation has been stimulated by repeated bleeding, this bloodforming tissue is found to occupy the greater part of the lumen of the marrow capillaries. If, however, blood formation has been reduced to its lowest extent by a process of chronic starvation, the erythroblasts form a single layer of cells just inside the dilated capillaries and intermediate stages between the erythroblasts, and the fully formed erythrocytes are almost entirely wanting. In the frog this process of blood-corpuscle 
formation occurs only at one period of the year, namely, in the early summer, and it is only at this time that the bones are found to contain red marrow. In mammals the process is very similar. In the red marrow are a number of nucleated cells containing hæmoglobin, which are thought by Löwit to be themselves derived from colourless nucleated cells. In the confused medley of colourless cells which exists in the bone-marrow and are precursors of all the varied corpuscles found in the blood, it is difficult to be certain of the identity of the colourless erythroblasts and to distinguish them from the smaller colourless cells engaged in bone formation or in the production of leucocytes. The hæmoglobin-containing cells are often to

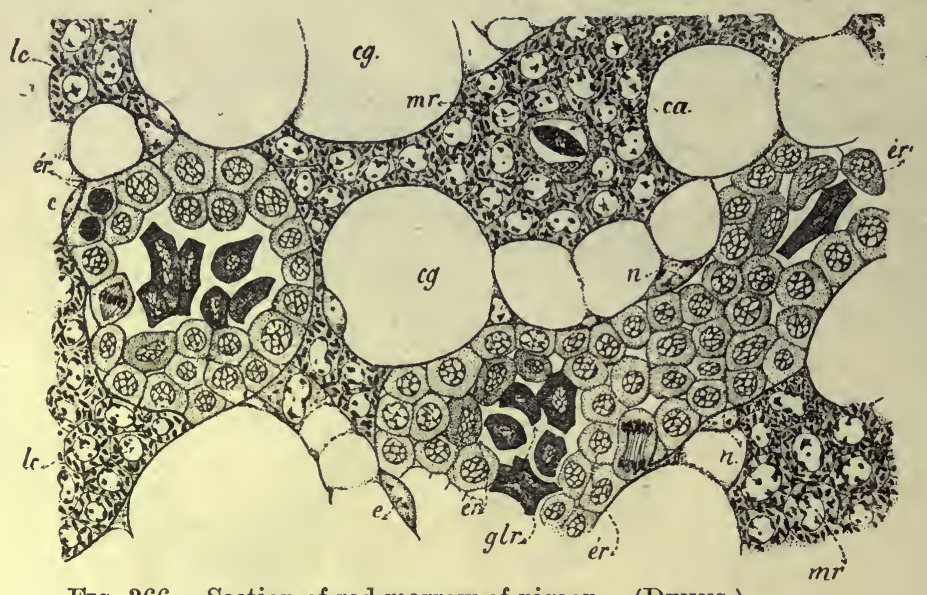

Fig. 366. Section of red marrow of pigeon. (Denys.)

$l c$, eosinophile leucocytes; $c g$, fat cells ; $e$, nucleus of endothelial cell of blood-vessel; $c a$, blood-capillary; $e r$, erythroblasts lying within vascular endothelium; $g l r$, fully formed red corpuscles.

be seen in process of division, and the nucleated daughter-cells appear to undergo a process of nucleolysis, the nucleus being extruded or dissolved. When blood formation is quickened as the result of previous destruction or loss, some of these immature nucleated blood-discs may make their way into the circulation and be found in the blood, where they are spoken of as normoblasts.

How long a corpuscle continues to exist in the circulating blood is not known. The experiments made to determine the length of time during which foreign corpuscles such as those of birds can be recognised after injection into the circulation of a mammal are evidently beside the mark, since these foreign cells will be destroyed by the serum and rapidly taken up by the phagocytes of the body. Sooner or later, however, every corpuscle undergoes disintegration, a process which is generally ushered in by the ingestion of the corpuscle by some phagocytic cells. Thus in the hæmolymph-glands and in the spleen we find large cells which have englobed red corpuscles and in which we can recognise pigment-granules obtained from their destruction. The chief place of disintegration of the hæmoglobin is certainly the liver, i.e. the organ where the hæmatin is converted into bile- 
pigment. Injection of hæmoglobin into the circulation causes increased secretion of bile-pigment. A section of normal liver immersed in potassium ferrocyanide and then in acid alcohol shows the presence of iron by the assumption of a blue colour. The amount of iron which can be demonstrated in the liver in this way is enormously increased by any condition which augments the rate of blood destruction. In the pathological condition known as pernicious anæmia, as well as after poisoning by the injection of pyrogallic acid or toluylene diamine, both of which agents cause a great destruction of red blood-corpuscles, the liver on treatment in this way assumes a deep blue colour. In some cases crystals of hæmoglobin have been seen within the nucleus of the liver-cell. In the destruction of the corpuscles the hæmoglobin is dissociated first into its protein and chromogenic moieties; the hæmochromogen then loses its iron and is converted into bile-pigment. The iron remains in the liver and is probably retained in the body and utilised for the formation of the fresh hæmoglobin necessary for the newly forming red blood-corpuscles in the bone-marrow. 


\section{SECTION III}

\section{THE BLOOD-PLATELETS}

The very existence of these, the third class of formed elements of the blood, is still a matter of dispute. If a drop of osmic acid be placed on the

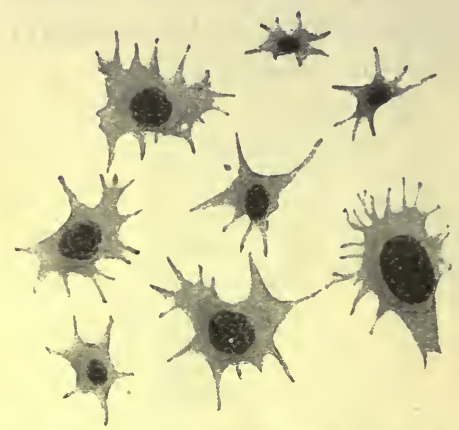

FIG. 367. Blood-platelets, highly magnified, showing the amœboid forms which they assume when examined under suitable conditions, and also exhibiting the chromatic particle which each platelet contains, and which has been regarded as a nucleus. (After КорSCH.)

finger, which is then prieked through the drop so that the shed blood may mix with the fixing fluid directly it leaves the vessels, a drop of the mixture when examined under high powers is seen to present a number of granular bodies from one-third to one-half the diameter of a red blood-corpuscle. Their number has been variously stated from 180,000 to 800,000 per cubic millimetre, so that they rank second in point of number among the morphological constituents of the blood. Their shape varies considerably. Some are bi-convex structures; others are flatter with numerous processes. They may be isolated or agglutinated into clumps. Their shape, size, and number vary according to the fluid with which the blood is mixed or the method adopted for their demonstration.

When blood is examined in Hayem's fluid* nearly all the blood platelets appear as bi-convex dises. The best method for the display of platelets is apparently that given by Deetjen. The drop of blood is received directly from the vessels on to a sheet of solid agar jelly which is made with $\mathbf{0 . 6}$ per cent. sodium chloride solution with the addition of sodium metaphosphate and bipotassium phosphate. When examined on this medium large numbers of platelets are seen, each of them provided with numerous processes (Fig. 367). Their central part is more strongly refracting than the periphery and stains with basic dyes, so that it has been regarded as a nucleus.

Similar platelets are observed when the blood is received into normal salt solution, and, as the mixture clots, the filaments of fibrin can be seen

* Hayem's fluid is made up as follows :

Distilled water

Sodium chloride

Sodium sulphate

Iodine in iodide of potassium 
often to radiate from a disintegrated blood-plate as from a centre. That the blood-platelets are concerned in the production of clots is shown by the fact that in the living vessels blood-platelets aggregate round any injured spot in the vessel wall, and later fuse together so as to form an adherent thrombus or clot which covers the seat of injury and helps to repair the damage and to prevent the escape of the contents of the bloodvessel. Blood-platelets have only been found in mammalian blood and are certainly absent from frogs' blood as well as from the blood of fishes and birds, nor can they be demonstrated in any of the serous fluids even in

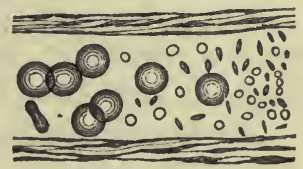

FIG. 368. Blood-corpuscles and blood-platelets, within a small vein.

(SCHÄFER after OSLER.)

mammals. Certain nucleated spindle-shaped cells have been described in frogs' blood as blood-platelets, but these are probably immature red blood-corpuscles and not homologous with the blood-platelets of mammals. (Blood-platelets themselves were regarded by Hayem as stages in the formation of red blood-corpuscles.) If the blood of an animal be defibrinated by bleeding, continually whipping the blood and returning it to the veins of the animal, it will be found for the next few days to be quite free from blood-platelets. There is no doubt therefore that it is possible by the most varied means to demonstrate the existence of blood-platelets in shed blood. According to the method adopted, so do the number and form of these platelets vary. Moreover they can be seen to be deposited from the circulating blood in the living animal on any injured portion of the vessel wall or on any foreign body introduced into the blood-current (Fig. 368). On the other hand, it is possible to obtain blood in an uncoagulated state from the vessels in which no trace of platelets is to be observed. As Buckmaster has shown, a film of blood examined in a platinum loop and kept carefully at the temperature of the body presents no platelets on microscopic examination; and the same absence of platelets is to be noted when blood is received into sterile bloodserum of the same species of animal and kept at the body temperature. On allowing these specimens of blood to cool, blood platelets make their appearance. Many specimens of non-coagulable plasma, such as peptone plasma or oxalate plasma, can be separated by means of the centrifuge from all formed elements. If the plasma be cooled to $0^{\circ} \mathrm{C}$. for twenty-four hours, a precipitate indistinguishable from blood-platelets is found to have been produced under the action of cold. We are therefore probably justified in concluding that the blood-platelets do not form a constituent of normal living blood, but are produced in the plasma either on contact with foreign bodies or lowering of its temperature from $37^{\circ} \mathrm{C}$. to $18^{\circ}$ or 
$20^{\circ} \mathrm{C}$. All the various fixing fluids which have been recommended for the display of blood-platelets may owe their virtues, not to the fact that they preserve, but to the fact that they produce platelets. These may therefore be regarded as precipitates produced in the plasma directly it undergoes alterations, their appearance being the first sign of changes in this fluid. They consist of some substance probably belonging to the class of nucleoproteins, and like these yield a precipitate on gastric digestion, and have a.specific affinity for basic dyes. Even after their first appearance they are unstable, tending to undergo further alteration and to give off substances to the surrounding plasma which play an important part in the formation of fibrin-ferment and in the coagulation of the blood. 


\section{SECTION IV}

\section{THE COAGULATION OF THE BLOOD}

In the process of coagulation, while the corpuscles remain to a large extent intact, the plasma becomes solid from the production in it of a network of fibrin, being converted into fibrin plus serum. The question of coagulation involves the consideration of the precursors of fibrin and of the conditions which determine the conversion of these precursors into fibrin. It is evidently impossible to arrive at any conclusions on these points during the few minutes which elapse between the time at which the blood leaves the vessels and the appearance of the clot. We must therefore find some means of retarding coagulation so that we may obtain the plasma free from corpuscles and be able to initiate coagulation in this cell-free fluid at will. Having succeeded in staying the process of coagulation, it is always possible to obtain a cell-free plasma either by allowing the blood to settle or, better still, by the employment of a centrifugal machine. Under the influence of centrifugal force the corpuscles are thrown rapidly down to the bottom of the tube and the clear supernatant plasma can be syphoned off.

\section{METHODS OF PREVENTING COAGULATION}

(1) So long as the blood is in contact with the uninjured vessel it remains fluid. If the jugular vein of a large animal such as the horse be tied in two places, the blood contained between the ligatures will remain fluid, sometimes for days. If the tube of vein be hung up, the corpuscles sink to the bottom and the plasma in the upper part of the tube can be poured from one vein to another without undergoing coagulation. On pouring it into a glass vessel or bringing it into contact with foreign substances, it undergoes coagulation.

(2) When an incision is made in the ordinary way into a blood-vessel of a bird the issuing blood clots very rapidly. The clotting is initiated by a substance contained in the tissues surrounding the vessels. If therefore the vessel be isolated and a perfectly clean glass cannula be inserted into it, care being taken not to bring the cannula in contact with any of the surrounding tissues, blood can be drawn off into a sterilised beaker perfectly free from dust and will remain unclotted for days. Such blood can be centrifuged and the cell-free plasma used for experiment. The same procedure does not apply to the mammal, where even the most scrupulous care to prevent contamination by the tissue juices will not prevent the blood from clotting on leaving the vessels.

(3) Clotting can be excited even in the living vein by introducing into the blood any solid substance which is wetted by the blood. If the contact of the blood with such substances be prevented by receiving it into vessels previously coated with oil or with paraffin and scrupulously free from dust, clotting may often be delayed for many hours.

(4) Cooled plasma. Horses' blood is received directly into a narrow vessel immersed 
in ice, so as to cool it rapidly to between $0^{\circ} \mathrm{C}$. and $1^{\circ} \mathrm{C}$. At this temperature it remains fluid for an indefinite time. The corpuscles sink, and the supernatant plasma can be decanted and filtered.

(5) Methods involving Mixture with Neutral Salts. (a) Magnesium sulphate. Blood from any animal is received into one-quarter its bulk of a 25 per cent. solution of magnesium sulphate.

(b) Sodium sulphate. Blood is mixed on leaving the vessels with an equal volume of half-saturated sodium sulphate solution. The plasma obtained in either of these ways is known as salt-plasma. Clotting is indefinitely delayed in either case, but it can be induced by suitable treatment of the separated plasma.

(6) Methods depending on Decalcification of the Blood. Oxalate plasma is obtained by receiving blood into a solution of sodium oxalate so that the total blood contains 1 per 1000 of the oxalate. Instead of oxalate we may use sodium fluoride, the proportion in this case being 3 parts of $\mathrm{NaF}$ per 1000 blood.

(7) Methods depending on the use of certain Substances of Animal Origin. (a) Peptone plasma is obtained by injecting rapidly into the veins of a dog or cat in a fasting condition a solution of commercial peptone in the proportion of $0 \cdot 3 \mathrm{grm}$. peptone per kilo of the animal. The effect of this injection is to cause a rapid fall of blood-pressure and hurried respiration, and the animal then passes into a state of coma which may last an hour or two. On drawing off the blood immediately after the fall of pressure has taken place it is found to be uncoagulable, and cell-free plasma can be obtained from it by the use of the centrifuge. A number of animal extracts act in a somewhat similar fashion, such as extract of crayfish, of mussels, \&c.

(b) Leech extract or hirudin plasma. Peptone is only efficacious in retarding clotting when it is injected into the animal's veins directly, and has no influence in this direction if it be mixed with the blood as it flows out of the vessels. It has long been familiar to physicians that the bites made by leeches continue to bleed for a considerable time, and it was shown by Haycraft that this is due to the presence of an anticoagulating substance in the buccal glands of the leech. This substance, which has the properties of an albumose, can be extracted by boiling from the anterior half of the leech. It will destroy the coagulability of the blood either when injected into the blood-stream or when blood is received into a solution of hirudin.

By any of these methods it is possible to obtain blood-plasma free from formed elements. The conditions which will bring about coagulation in such plasmata are strikingly diverse. Thus in cooled plasma a simple rise of temperature is often sufficient to bring about coagulation. If, however, the cooled plasma be filtered several times through two thicknesses of filter-paper, being kept at a temperature of about $1^{\circ} \mathrm{C}$. during the whole time, it loses this spontaneous coagulability on warming. It can still be made to clot by the addition of certain substances such as blood-serum or the washings of a blood-clot, and in some cases by the addition of tissue extracts.

Oxalate plasma clots on simple addition of lime salts. Sodium sulphate plasma clots on dilution. Magnesium sulphate plasma will not clot on dilution, but needs in addition the presence of some blood-serum or some substance derived from blood-serum. In both these cases tissue extracts have no influence. By a careful study of one or two forms of plasma we can arrive at a conception of the processes of coagulation which enables us to understand the behaviour of all these various types. We may take as our type oxalate plasma. Oxalate plasma, as procured by centrifuging a specimen of horse's blood containing $0 \cdot 1$ per cent. sodium oxalate, is a clear yellow fluid, perfectly free from formed elements, which evinces no tendency 
to clot. On adding a drop of calcium chloride to such plasma we see that it contains an excess of oxalate from the production of a precipitate of calcium oxalate. If calcium chloride be added drop by drop so as to be present in slight excess and the plasma be then kept warm, it will clot within a time varying from a few minutes to an hour. The clot thus formed is perfectly firm and the vessel can be inverted without any of its contents flowing out. Very often no retraction of the clot takes place, but if it be pressed with a glass rod or with the fingers a clear serum is squeezed out and a mass of pure fibrin remains. The place of lime can be taken by strontium, but barium and magnesium are powerless to initiate clotting. We must therefore conclude that the presence of a soluble calcium salt is one factor of those necessary for coagulation. This is borne out by the fact that a similar uncoagulable blood is produced by the action of sodium fluoride. Some difficulty, however, was felt when it was found that sodium citrate might be used instead of sodium oxalate or fluoride, since sodium citrate does not produce in the blood any precipitate of insoluble lime salts. Here therefore we have a blood containing lime in solution and yet uncoagulable. The difficulty has been cleared up by the work of Sabbatani and C. J. Martin. When sodium citrate is added to a lime salt a double salt of sodium calcium citrate is formed in which the calcium is in the anion and forms part of the acidic radical. The mere presence of dissolved calcium is not sufficient for clotting to take place. It is necessary that the calcium should be in the form of a salt and in an ionised condition, such as calcium chloride or calcium sulphate.

Though calcium is a necessary condition for the occurrence of coagulation, it cannot be regarded as a precursor of the protein fibrin. If the composition of the plasma before coagulation has been set up be compared with that of the serum which has separated from the clot, it is found that plasma contains a protein, fibrinogen, not represented in the serum, which must therefore be the precursor of fibrin. Fibrinogen belongs to the class of globulins. It can be separated from oxalate plasma by half-saturation with common salt. An equal volume of a saturated solution of sodium chloride is added to plasma so that the whole mixture contains 16 per cent. sodium chloride. The fluid gradually becomes turbid from the production of a precipitate which, at first granular, rapidly aggregates to form a stringy, slimy solid, and on stirring aggregates into masses which adhere to the glass rod used for stirring. This mass can be taken out of the fluid, washed by kneading with half-saturated sodium chloride solution, and then redissolved by the addition of distilled water. With the salt adhering to the precipitate a dilute saline fluid is formed in which the fibrinogen is soluble. The fibrinogen can be purified by repeating the precipitation and solution, though it tends to lose its solubility in the process, so that purification is always attended with some loss. The solution of fibrinogen thus obtained is perfectly clear and colourless. On warming, practically the whole of its protein is thrown down between $56^{\circ}$ and $60^{\circ} \mathrm{C}$. The same precipitate is produced on heating the original plasma, whereas serum obtained by the expression of the clot does not give any 
precipitate on heating until a temperature of $68^{\circ}$ to $70^{\circ} \mathrm{C}$. is reached. If a solution of fibrinogen, obtained by precipitating with sodium chloride and dissolving in distilled water, be treated with a drop or two of calcium chloride it rapidly clots with the formation of typical fibrin. We might conclude from this experiment that fibrin was a compound produced by the union of calcium salts with fibrinogen. Further experiments show, however, the untenability of this hypothesis. If the fibrinogen has been thoroughly purified by repeated precipitation and re-solution, calcium salts are found to have entirely lost their power of causing coagulation. Such a purified fibrinogen can still be made to clot by the addition either of serum or of the washings of a blood-clot, or of the watery extract of alcohol-coagulated serum. This power of serum to convert fibrinogen into fibrin is due to the presence in it of minute quantities of a substance which has been designated as ' fibrin ferment' or thrombin. It has been regarded as a ferment because it is active in minimal quantities and is stated not to be appreciably used up in the process of clotting. Thus if we add some serum to a fibrinogen solution we can cause clotting, and then on squeezing the clot obtain a serum which will bring about coagulation when added to a fresh portion of fibrinogen. Considerable doubt, however, has been thrown on the ferment nature of thrombin by the recent work of Rettger in Howell's laboratory. Rettger shows, in the first place, that the statement of the preceding sentence is only true if a large excess of thrombin solution be made use of in the first instance. If small quantities of thrombin solution be added to large quantities of plasma or of pure solutions of fibrinogen, the amount of fibrin obtained is proportional to the amount of thrombin added. This is shown in the following experiment :

5 drops of thrombin gave $0 \cdot 2046 \mathrm{grm}$. fibrin.

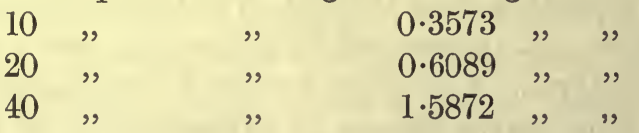

Moreover the action of thrombin on fibrinogen solutions is almost independent of temperature, occurring practically as rapidly at $17^{\circ} \mathrm{C}$. as at $40^{\circ} \mathrm{C}$. It has been found that there is only a slight increase in the rate of action of snake venom on fibrinogen solutions on warming from $20^{\circ}$ to $40^{\circ} \mathrm{C}$. Rettger therefore regards fibrin as formed by the union of thrombin and fibrinogen. This union is apparently very unstable. If fibrin be extracted with a 3 per cent. salt solution for some time, part of it goes into solution, and the solution is found to contain thrombin. In the same way the thrombin may be re-extracted from the fibrin if the latter be allowed to putrefy.

From all the different kinds of plasma which have been enumerated above the purified fibrinogen can be obtained by the use of sodium chloride, and in every case can be made to clot by the addition of serum or of a solution of thrombin. The last change in the act of clotting is therefore the change from fibrinogen to fibrin, and this event is brought about by the intervention of 
thrombin. It cannot be at this stage of the process that the calcium salts exercise their influence, since 'fibrin ferment' or thrombin will cause the coagulation of fibrinogen in the total absence of soluble calcium salts and even in the presence of a slight amount of ammonium oxalate. Moreover Hammarsten has shown that the calcium content of fibrin is no greater than that of the fibrinogen from which it is formed.

The fact that a solution of pure fibrinogen is made to clot by thrombin and by this alone renders such a solution an excellent reagent for the presence of the 'ferment.' By this means we can show that thrombin is absent in circulating blood. If blood be received direct from the vessels into absolute alcohol and the precipitate, after coagulation by alcohol, be extracted by water, the extract is found to contain no trace of ferment. The same statement applies to fresh oxalate plasma. If, however, oxalate plasma be made to clot by the addition of calcium salts, the serum squeezed from the clot is found to contain thrombin. In the process of coagulation therefore not only is there a conversion of fibrinogen to fibrin, but there is an actual formation of the agent which is responsible for this change, namely, thrombin or fibrin ferment. Our next step therefore must be to inquire into the precursors of the thrombin and the conditions of its formation.

If oxalate plasma be cooled to $0^{\circ} \mathrm{C}$. for two or three days a scanty granular precipitate is produced. This can be centrifuged off or separated by filtration through several thicknesses of paper. It is then found that the remaining plasma can no longer be made to coagulate by the addition of lime salts, although it still contains fibrinogen, which is converted into fibrin on the addition of thrombin. If the precipitate be collected and treated with calcium chloride and the mixture added to the oxalate plasma the latter clots. The same effect is produced if the precipitate plus calcium be added to a pure solution of fibrinogen. We must conclude that the precipitate, though itself not fibrin ferment, will give rise to fibrin ferment on treatment with lime salts. It was therefore designated by Hammarsten 'prothrombin,' and regarded as the precursor of thrombin.

Thus far practically all workers on the subject are agreed. Coagulation of the blood is due finally to the coagulation of thrombin and fibrinogen. The fibrinogen is present as such in the circulating plasma. Thrombin is not contained in the circulating blood, but is produced from some precursor or precursors after the blood has been shed. For the production of thrombin the presence of calcium salts is necessary. Further research on the nature of the precursor prothrombin has shown that the matter is not quite so simple as imagined by Hammarsten. In the following account we shall adhere chiefly to the account as given by Morawitz, reserving most of the criticisms and limitations to be made to this theory for the historical sketch. of the theories of clotting at the end of this section.

Oxalate plasma which has been separated from the precipitate of prothrombin can be made to coagulate by the addition of extracts of almost any animal tissues together with lime salts, and these therefore were supposed to contain prothrombin similar to that obtained by cooling oxalate plasma. 
These extracts even on mixture with calcium are, however, without effect on pure solutions of fibrogen, and moreover the precipitate produced by cold, if thoroughly washed before treatment with lime salts, loses its power of evoking coagulation in fibrinogen solutions. Prothrombin is therefore unable by itself, even on addition of lime salts, to produce fibrin ferment, but needs the co-operation of some other substance which is contained in oxalate plasma and which generally adheres in sufficient quantities to the precipitate produced by cooling. Three factors are therefore necessary for the production of fibrin ferment : first, lime salts ; secondly, a substance present in the precipitate of prothrombin as well as in most animal tissues ; and thirdly, a substance present in solution in oxalate plasma. These two latter substances have been designated by Morawitz thrombokinase and thrombogen. Thrombokinase is contained in tissues and also in the bloodplatelets. It can be obtained by extraction of the stroma of the red bloodcorpuscles or of the bodies of lymph-cells or leucocytes. Separation of the blood-platelets by cooling in the form of the disc-like precipitation abolishes the spontaneous coagulability of any form of plasma. The thrombogen is contained in solution in oxalate plasma. It is therefore concluded that when blood leaves the vessels there is a disintegration of the blood-platelets with the liberation of thrombokinase. This acts upon thrombogen in the presence of lime salts and produces thrombin. By the intermediation of the thrombin the fibrinogen also present in solution in the plasma is converted into fibrin. The changes occurring in shed blood and resulting in the production of a clot are therefore mainly concerned with the production of the fibrin ferment. This view of the essential characters of coagulation is borne out by observations on other forms of plasma, especially of plasma obtained from birds' blood. This when obtained with scrupulous cleanliness so as to avoid any contamination with dust or with the tissues remains permanently uncoagulable. In the plasma got by centrifuging the blood no blood-platelets are to be seen, and no precipitate is produced by exposure to a temperature of $0^{\circ} \mathrm{C}$. We may say therefore that bloodplatelets with their contained thrombokinase are absent from birds' blood, and with them the property of spontaneous coagulability. It is also free from fibrin ferment, but contains thrombogen as well as soluble lime salts. It is only necessary therefore to add thrombokinase in the shape of a watery extract of any tissue in order to cause the appearance of fibrin ferment and the conversion of the fibrinogen already present in the plasma into fibrin.

In every case the initiation of the act of clotting would seem to depend on the setting free of thrombokinase in the plasma. In mammalian blood, although thrombokinase can be derived from red or white corpuscles, we have no reason to believe that there is any appreciable disintegration of these formed elements when the blood leaves the vessels. In oxalate blood leucocytes can be seen alive and exercising amœboid movements two or three days after the blood has left the vessels, and although certain observers have assumed the presence of explosive corpuscles which break up directly the 
blood leaves the vessels, the presence of such corpuscles in the higher animals has not been demonstrated, though in some invertebrata they are certainly present. The sole source therefore of the thrombokinase is the very perishable formed elements of which we have spoken as the blood-platelets. The very existence of this element is doubtful in normal blood. What is certain is that any slight change in the plasma, whether due to contact with a foreign object or to cooling of the blood, causes the appearance of these elements. The first act therefore in coagulation is the appearance of the blood-platelet and its disintegration with the setting free of thrombokinase. The part played by the platelets in coagulation is of great importance in maintaining the integrity of the vascular system. If a fine needle be thrust through the wall of a venous capillary which is kept under observation by the microscope it will be seen that the blood, as it flows past the injured spot, deposits bloodplatelets on the side of the puncture. These aggregate to form a plug closely adherent to the wall of the vessel, which effectively prevents any escape of the contents of the capillary. The blood-platelets fuse so as to form a mass of fibrin, and later on by the growth of the adjacent endothelial cells the thrombus is organised, converted into connective tissue, and covered with a layer of endothelium continuous with the rest of the vessel. The same process occurs when any part of the lining membrane of a large vessel is injured. Thus destruction of a patch of endothelium in a vein leads to the deposition of blood-platelets over the patch and the formation of a 'thrombus' adherent to the wall. From this thrombus coagulation may spread through the rest of the contents of the vessel and produce thrombosis of the whole vein. Under healthy conditions the thrombus serves simply to cover the bare area in the wall of the vein and is grown over later by endothelium, so restoring the integrity of the vessel wall. If we believe in the pre-existence of blood-platelets in the circulating blood we must assume the first act in coagulation to be the disintegration of these elements and the setting free of thrombokinase. If we disbelieve in their pre-existence the first act in coagulation must be a change in the plasma itself (which perhaps can be regarded as a dropsical protoplasm), leading to the separation of an unstable substance, thrombokinase, in the form of a disc-like precipitate which rapidly undergoes further changes, reacting with the thrombogen remaining in solution in the plasma with the production of fibrin ferment.

Why does the blood not clot in the vessel? No theory of coagulation can be satisfactory which does not account at the same time for the preservation of the fluidity of the circulating blood. One factor at any rate in the prevention of intravascular clotting must be the nature of the surfaces with which the blood comes in contact. The blood, even of mammals, can be prevented for a time from clotting if it be kept carefully from contact with any foreign substance which is wetted by it, as, for -instance, when it is received into vessels free from dust and coated with a layer of oil or paraffin. On the other hand, free contact with such substances, as occurs when the blood is whipped, materially hastens the process of coagulation. One must 
therefore conclude that mere contact with a foreign body has a direct destructive action either on the plasma leading to the formation of bloodplatelets, or on the latter, leading to their disintegration and the discharge of thrombokinase. Birds' blood, for instance, can be made to clot without the addition of tissue juice if, by increasing the mechanical insult, as by violent whipping, filtration through a clay cell, or addition of water, we destroy the leucocytes and red blood-corpuscles, so leading to the liberation of their contained thrombokinase. Another factor is probably the presence of what we may call antithrombins in circulating blood. Although evidence of the existence of these bodies is still somewhat uncertain, the fact that blood-serum often has an inhibitory effect on the action of a solution of fibrin ferment points to the presence in the serum of some antibody to the ferment. One must assume too that processes of disintegration are continually occurring in the blood and involving the plasma, blood-platelets, and leucocytes, just as we know them to affect the red blood-corpuscles. In the healthy animal the liberation of thrombokinase which must take place under these circumstances has no influence in producing clotting. The organism therefore must possess means of neutralising the presence of small quantities either of kinase or of fibrin ferment. When small quantities of either of these substances are injected into the blood-stream no coagulation takes place, but the blood obtained after the injection clots with less readiness than before, a change which can only be ascribed to the production in the body of antikinase or of antithrombin. This production of anticoagulins must be continually taking place and must co-operate in the preservation of the fluid state of the blood while in the vessels.

INTRAVASCULAR CLOTTING. On account of the protective mechanisms with which the animal organism is endowed the production of clotting in the vessels of the living animal is not readily effected by the injection of thrombin. Solutions obtained by Schmidt's method from alcoholcoagulated serum are generally without effect, and we have to inject a very strong solution of thrombin or the strong fibrin ferment contained in the venom of certain snakes in order to bring about coagulation of the blood in the vessels. Intravascular clotting is more easily effected by the injection of thrombokinase. It was shown by Wooldridge that normal saline extracts of tissues rich in cells, such as the thymus, lymph-glands, or testis, causes invariably extensive thrombosis. If such extracts be acidified with acetic acid a precipitate is produced which is soluble in dilute alkalies, and on gastric digestion yields a precipitate rich in phosphorus. Alkaline solutions of the acid precipitate bring about intravascular clotting when injected into the blood-stream. According to Wooldridge the injected substance takes part in the formation of the clot, and he therefore gave it the name of ' tissue fibrinogen.' It is usual to regard these tissue fibrinogens as nucleo-proteins. They are certainly rich in lecithin, and the precipitate obtained from them"on gastric digestion may contain as much as 25 per cent. of this substance. They must be derived either from the cytoplasm or from the interstitial fluid of the tissues, and it is still doubtful whether we are justified in giving 
them the name of nucleo-proteins or whether we should not rather classify them with the phospho-proteins which play so great a part in the building up of the cytoplasmic part of the cell. We may explain the action of these tissue extracts as due to their containing thrombokinase.* Their injection would resemble therefore the liberation of thrombokinase which normally occurs when blood leaves the vessels. More difficult to understand is the result of injecting small amounts of these tissue extracts or large amounts in small doses. A minute quantity of tissue extract injected into the bloodstream produces, not intravascular clotting, but a delay of the coagulation time. Repeated injections of small doses may absolutely annul the coagulability of the blood, which can be collected by opening the blood-vessels and will remain unclotted for many days. The same double effect may be observed even with a larger dose. In rabbits and in dogs after a full meal the intravascular coagulation which occurs is complete, extending through the whole vascular system. If, however, the injection be made into a fasting dog the thrombosis produced is limited to the portal vein. There is a sudden fall of blood-pressure, from which the animal gradually recovers. If a vessel be opened during the period of low pressure the blood which flows out is totally uncoagulable, and if the animal be killed at this time a clot will be found filling up the whole portal vein. Wooldridge described these two effects of injection of tissue extracts, namely the coagulation and the loss of coagulability, as the positive and negative phases respectively. Since the negative phase has not been observed in any form of extra-vascular plasma we must ascribe it to a reaction on the part of the living cells, and probably, since it is so rapid in.its establishment, to the action of the cells lining the blood-vessels. The interest of these observations lies in the relation which they bear to the production of immunity. If a toxin such as that of diphtheria or tetanus be injected into an animal, an anti-toxin is produced in the course of two or three days. If now further doses of toxin be injected its first effect is to destroy the whole of the anti-toxin present in the circulating blood. In the course of a day or two the anti-toxin gradually returns, and at the end of three days is found in larger quantities in the blood than were present before the second injection. Every toxin has therefore a positive as well as a negative phase of action. Tissue extracts in their effect on coagulation have a similar positive and negative phase, but these phases are established within a few seconds instead of taking two or three days for their development. One cannot but believe that a renewed study of the conditions of intravascular clotting might shed important light on the chemical mechanism of production of immunity.

FATE OF THE FIBRIN FERMENT. The substances which interact for the production of thrombin in shed blood as well as thrombin itself are not entirely used up in the process of clotting. Blood serum, though free from fibrinogen, contains traces of thrombokinase (which can be precipitated by

* According to Howell, these tissue fibrinogens consist of phosphatides in association with protein. When separated from the protein they are thermostable, and their thromboplastic effect is due to their power of neutralising antithrombin. 
the addition of dilute acetic acid) and thrombogen, as well as a fairly strong solution of thrombin. Thrombin, however, rapidly disappears from serum, so that a blood-serum which has been kept for two or three days may be almost free from fibrin ferment. Such a serum can be reactivated by the addition of small traces either of acid or alkali. It has been suggested that the thrombin undergoes a modification into an inactive form which is called metathrombin. This substance has no relation to the precursors of fibrin ferment which we have already considered. It is unaltered by lime salts or by the addition of thrombokinase, but can be reconverted into thrombin by means of acids or alkalies. According to Rettger the disappearance of thrombin from serum is due to its combination with some of the proteins of the serum. This combination, like that of thrombin with fibrinogen to form fibrin, is unstable and can be broken up by the action of alkalies, acids, or even of putrefaction. Thrombin itself seems to be extremely stable and will even withstand the temperature of boiling water for a short time. If solutions containing thrombin be evaporated to dryness the dry residue can be heated to $135^{\circ} \mathrm{C}$. without destruction of the thrombin.

We are now in a position to see how far the theory of coagulation evolved from a study of two forms of plasma will serve to explain the behaviour of the many other kinds of plosma which have been the subject of investigation.

COOLED PLASMA contains the thrombokinase in the form of blood-platelets or a disc-like precipitate. This precipitate can be separated by centrifuging at a low temperature or by filtration. The remaining plasma contains only thrombogen, lime salts, and fibrinogen, and can be made to clot by the addition of tissue extracts or of fibrin ferment, but will not clot on warming.

In SODIUM SULPHATE PLASMA the interaction of the fibrin factors is merely impeded by the excess of salt. All are still present, and it is therefore sufficient merely to dilute the plasma in order to produce clotting.

MaginesiUm SUlPhate PLASMa behaves somewhat differently. If the blood be received directly into magnesium, sulphate solution and the mixture centrifuged while still warm, a clear magnesium sulphate plasma is obtained which will clot on simple dilution. If the blood be left for twenty-four hours before centrifuging, the plasma will not clot on dilution nor on addition of tissue extracts. It contains fibrinogen only and is therefore an excellent reagent for the presence of fibrin ferment. Magnesium sulphate not only hinders the interaction of the fibrin factors but actually slowly precipitates the thrombokinase, so that if time be allowed for this precipitation to be complete the remaining plasma contains only fibrinogen.

SoDIUM FLUORIDE PLASMA might be expected to act like oxalate plasma since sodium fluoride is a precipitant of lime salts. This salt has, however, the additional property of causing a certain amount of fixation of the formed elements of the blood as well as of the blood-platelets. If it be thoroughly centrifuged so that the plasma is obtained free from these constituents it will no longer clot with lime salts* nor even with lime salts plus tissue extracts, but will clot readily on addition of thrombin. Although it still contains a certain amount of thrombogen, this is entangled and carried down in the precipitate of calcium fluroide which is produced by the addition of lime salts, so that the thrombokinase has nothing on which to exercise its effect. Sodium fluoride plasma is therefore useful, like magnesium sulphate plasma, as a test for the presence of thrombin. If water be added to the sodium fluoride blood so as to destroy some of the formed elements and liberate their constituents into the plasma, it is possible to produce clotting by the simple addition of lime salts.

* According to Rettger this statement is incorrect. 
HiRudin PLASMa. The action of hirudin is that of an anti-thrombin. It apparently combines with and neutralises fibrin ferment. Hirudin plasma can therefore be made to clot by the addition of fibrin ferment in sufficiently large quantities to combine with all the hirudin present and leave an excess over in the fluid.

Peptone plasma presents many difficulties in the explanation of its behaviour. Peptone itself has apparently no influence on the coagulation of the blood. Blood received into peptone solution clots as rapidly as when received into salt solution. On the other hand, if blood be received into peptone blood obtained by the injection of large doses of peptone into the veins of another animal, the mixture does not clot, showing that peptone blood contains some substance which inhibits the processes of coagulation. This 'anticoagulin' must be produced by the organism itself as a result of the injection of peptone, and evidence has been brought forward by Delezenne and others that the seat of formation of the anticoagulin is in the liver. Whether the antisubstance partakes of the characters of an anti-thrombin or of an antikinase has not yet been definitely ascertained. Peptone plasma can be made to clot by the addition of tissue extracts even in small quantities. It still contains all the fibrin factors since it will clot on simple dilution or on the passage of a current of carbon dioxide. It needs, however, the addition of large quantities of fibrin ferment to bring about coagulation.

\section{THE TRANSUDATIONS}

The earlier work on the mechanism of coagulation was largely carried out on the fluids obtained from the pericardial or pleural cavities or on hydrocele fluid from the tunica vaginalis. These as a rule can be kept indefinitely without clotting, but will clot readily on addition of a few drops of blood or the washings of a blood-clot or fibrin ferment. They will not clot on the addition of tissue extracts containing thrombokinase. Though they contain leucocytes and even some red corpuscles, they are free from blood-platelets. Their behaviour is readily explained by the assumption that they contain fibrinogen, but are free from thrombokinase or thrombogen. In order to produce coagulation it is therefore necessary to add two fibrin factors, thrombokinase and thrombogen, as happens when we add blood, or to treat them with fully formed thrombin or fibrin ferment.

HISTORY OF THE COAGULATION QUESTION. It is not surprising that the coagulation of the blood, with the antecedent changes which lead to the appearance of thrombin and probably represent the successive stages in the disintegration of a fluid labile protoplasmic molecule, i.e. the change from life to death of the plasma, should have been the subject of a very large number of investigations, and that even at the present time the interpretation of the salient facts presents many difficulties. Some help may be given to the future clearing up of these difficulties by a study of the steps by which our present standpoint has been arrived at. The universal practice of bleeding as a therapeutic measure naturally afforded many opportunities to physicians for observing the processes of coagulation under diseased as well as healthy conditions. The general result was, however, in most cases, a crop of ill-founded and uncritical theories, and it is not till the time of Hewson (1772) that we meet with investigations of the question carried out on modern lines with reference to observation and experiment at each stage. We owe to Hewson the discovery that coagulation can be inhibited indefinitely by the addition of neutral salts, such as sodium sulphate, and it was by a study of such bloods that Hewson arrived at the conclusion that the formed elements of the blood take no part in the production of the clot. Johannes Müller in 1832 came to the same conclusion from a study of frog's blood. This he diluted with sugar solution and filtered through filter-paper. The large corpuscles were retained by the meshes of the filter-paper and the clear fluid which came through slowly underwent coagulation. 
The beginning of our modern ideas on the subject must be ascribed to Buchanan, though many of the facts discovered by this observer escaped general recognition and were later re-discovered by Alexander Schmidt. Buchanan worked chiefly on hydrocele fluid and showed that this could be made to yield fibrin by treatment with fresh blood or by adding it to the washings of a blood-clot. He compares the action of the latter to that of rennet on the protein of milk. His experiments showed that 'fibrin has not the least tendency to deposit itself spontaneously in the form of a coagulum, that, like albumin and casein, fibrin often coagulates under the influence of suitable reagents, and that the blood, like most other liquids of the body which appear to coagulate spontaneously, only do so in consequence of their containing at once fibrin and substances capable of reacting upon it and so occasioning coagulation.' He held therefore that the coagulation of the blood is due to the conversion of a soluble constituent of the liquor sanguinis into fibrin by an action exerted probably by the colourless corpuscles and comparable to the action which rennet exerts in effecting the coagulation of milk. Furthermore, the liquid which accumulates in certain serous sacs may be made to yield a coagulum of fibrin when subjected to the action of liquids or solids rich in the cellular elements with which the coagulent action appeared to be associated (Gamgee).

Denis in 1856 attempted to separate this precursor of fibrin. He received the blood into one-sixth of its volume of saturated sodium sulphate, allowed the corpuscles to settle, and filtered off the supernatant plasma. On saturating this with sodium chloride a precipitate was produced which Denis designated 'plasmine.' This precipitate, on solution in water, slowly underwent coagulation and was apparently split into two substances-a solid fibrin and a soluble protein. Clotting therefore, according to Denis, was dependent on the splitting of a single protein into two different proteins, one of which was insoluble. A few years later the subject was taken up by Alexander Schmidt, who devoted the remaining thirty years of his life to the investigation of the coagulation of the blood. Working first, as Buchanan had done, on fluids obtained from serous cavities, he noticed that these could be made to clot by the addition of serum, and he concluded that coagulation was due to the interaction or combination of two different proteins, one fibrinogen, contained in the serous fluid, and the other 'fibrinoplastin,' which was contained in the serum and could be precipitated by acidification or by dilution and passage of a stream of carbon dioxide. This fibrinoplastin was identical with what we should nowadays call paraglobulin, but had adherent to it fibrin ferment. Schmidt later on found that in many cases it was not sufficient to mix these two substances together, but that a third factor was necessary, which could be obtained either from serum or from blood-clot which had been coagulated by alcohol. This third factor he compared to a ferment, so that the theory put forward by Schmidt in 1872 was that coagulation depends on the interaction of two substances-fibrinogen and fibrinoplastin-under the influence of a third substance, fibrin ferment or thrombin. A few years later Hammarsten, of Upsala, in a very careful series of experiments, proved conclusively that the fibrinoplastin of Schmidt was not a necessary factor. Hammarsten discovered the method which we use at the present time for separation of fibrinogen, namely, precipitation by half-saturation with common salt, and showed that the fibrinogen obtained in this way and purified by repeated precipitation and resolution would yield a clot of fibrin on the addition of fibrin ferment prepared by Schmidt's process. According to Hammarsten, therefore, clotting was due to the conversion of the fibrinogen present in the circulating plasma into fibrin by the action of fibrin ferment, which was probably yielded by the disintegration of the white blood-corpuscles. Schmidt's later work was directed chiefly to determining the mode of origin of the fibrin ferment. Though his researches yielded a number of important facts, especially as to the part played by tissue-cells in furnishing the precursors of the ferment or in influencing the processes of clotting, they did not result in clarifying the views of physiologists generally on the subject of clotting. Perhaps their most useful effect was to demonstrate the complexity of the processes which occur in the blood after it leaves the vessels, and to show that in the maintenance of the fluid condition in the vessels as well as in the production of a clot outside the vessels, there must be an interaction between the opposing factors, some of which hinder and some of which favour 
the occurrence of coagulation. According to Schmidt the plasma is itself derived from the cells of the body, the fibrinogen being formed through the stages of paraglobulin and cytoglobulin. The thrombin is derived from a precursor prothrombin under the action of a zymoplastic substance also derived from the cells. In the presence of the proper concentration of salts the thrombin acts upon fibrinogen to produce fibrin. His views may be roughly expressed by the following schema given by Howell :

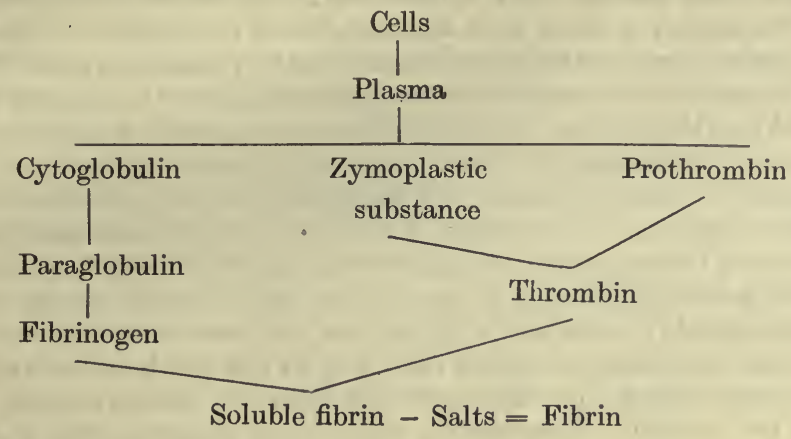

Some important light was thrown on the subject by the researches of Wooldridge. Working chiefly with peptone plasma, he showed in the first place that such plasma contained all the factors necessary for the production of fibrin, and therefore that the co-opration of leucocytes was not a necessary part of the process. Peptone plasma, separated entirely from leucocytes and red corpuscles, could be made to clot by dilution, by the passage of a stream of carbon dioxide or filtration through a clay cell. This powər of clotting without addition of any other substances depended on the presence in the plasma of a substance called by Wooldridge 'A-fibrinogen,' which was thrown down as a disc-like precipitate on cooling to $0^{\circ} \mathrm{C}$. On separating this precipitate, which he regarded as equivalent to the blood-platelets, by means of the centrifuge, the remaining plasma would only clot on the addition of extracts of tissues. Since neither the original plasma nor the plasma after separation of the A-fibrinogen would clot on the addition of fibrin ferment, Wooldridge thought that the fibrinogen of Hammarsten was absent from such plasma, which only contained two fibrinogens, A- and B-fibrinogen. Clotting therefore consisted essentially in an interaction between A- and B-fibrinogen, and was inaugurated by the appearance of A-fibrinogen as a disclike precipitate. In this interaction he showed that ferment was produced, and the weakest part of his theory was that it gave practically no office to the ferment produced during the first steps of the process imagined by him. The B-fibrinogen could bo thrown down by the action of dilute acid or of salt from the plasma after separation, of the A-fibrinogen. After precipitation and re-solution two or three times it would clot with fibrin ferment, and was coagulated at a temperature of $56^{\circ} \mathrm{C}$., and was therefore the typical fibrinogen of Hammarsten. According to Wooldridge, therafore, previous observers had been working, not with the fibrinogens of the plasma, but with a fibrinogen altered by repeated precipitation and re-solution. One fact discovered by him which at once attained universal recognition was the production of intravascular clotting by the injection of tissue extracts. These tissue extracts contained tissue fibrinogens which he compared with A-fibrinogen. According to him clotting could be inaugurated either by the action of A-fibrinogen on the B-fibrinogen, or by the action of tissue fibrinogen on the B-fibrinogen of the plasma. In every case fibrin ferment resulted and could therefore effect the conversion of any C-fibrinogen of Hammarsten which might be present in the fibrin. It will be seen that this theory of Wooldridge presents a striking similarity to that which is generally accepted at the present day. If we change the names of A-fibrinogen to thrombokinase, of B-fibrinogen to thrombogen, we see that the only difference between Wooldridge's theory and that of Morawitz is that the former ignored the importance of lime salts in the process and 
imagined that the interaction of thrombokinase and thrombogen resulted in the direct production of fibrin as well as ferment, instead of recognising that the interaction of the two substances was simply a first stage and that thrombin was formed in this process for the subsequent conversion of the fibrinogen into fibrin.

Attention, however, was largely diverted from Wooldridge's work by the discovery of the necessity of calcium salts for clotting. Green had already shown that the clotting of many forms of salt plasma could be hastened by the addition of calcium sulphate, whereas the coagulation of serous fluid was not affected by this salt. Green suggested that possibly a zymogen of the ferment was activated by the calcium salt. The absolute necessity of the presence of this salt was first demonstrated by Arthus and Pagés (1890) on oxalate and fluoride plasma. At first Arthus was inclined to regard the part played by calcium salts in the coagulation of the blood as analogous in all respects to that played in the coagulation of milk by rennet, and suggested that the conversion of fibrinogen into fibrin was actually the combination of fibrinogen with calcium salts, the combination being effected by the agency of the ferment. It was shown, however, by Pekelharing that the power of lime salts to produce clotting in oxalate plasma was annulled if the body precipitable by cold had been previously removed, and Hammarsten proved conclusively that the action of calcium salts was on this prothrombin and not on the fibrinogen, careful analyses of fibrinogen and fibrin respectively giving practically equal figures for calcium. Hammarsten pointed out moreover that fibrin ferment would convert fibrinogen into fibrin in the total absence of soluble calcium salts and even in the presence of a slight excess of oxalate.

Later experiments have had reference chiefly to the nature of the prothrombin precipitate and to the question of the origin of the fibrin ferment from the blood-plasma. Recent advances in the subject were much facilitated by the discovery by Delezenne that birds' blood could be prevented from clotting by the simple expedient of collecting it free from any contact with the tissues. A careful study of this blood and a comparison of its behaviour with that of other forms of uncoagulable plasma by Fuld and Spiro, and especially by Morawitz, have resulted in the further separation of the precursor of fibrin into two substances, thrombokinase and thrombogen. Further investigations by Nolf have dealt especially with the question of the interaction which is continually taking place between the vessel wall and the contained blood, and which may result, according to the circumstances, in the diminution or increase in the coagulability of the blood. According to Nolf the essential factors in the production of blood-clotting are three proteins, namely, fibrinogen, thrombogen, and thrombozym. The two former are produced in the liver, while the thrombozym is formed from the leucocytes. The clotting depends, not on a ferment action, but on a mutual interaction and precipitation of colloids with, as a result, either fibrin or thrombin. Thrombin differs from tibrin merely in containing less fibrinogen. For this reaction to take place the presence of calcium is necessary as well as certain thromboplastic substances which act as centres of precipitation. The fluid condition of the blood in the vessel depends on the presence of antithrombin formed in the liver. Nolf thus agrees with Wooldridge in regarding thrombin as a product of coagulation rather than a cause. Thrombin, according to him, is merely an unsaturated compound which is capable of taking up or uniting with more fibrinogen to form fibrin. Nolf would regard the formation of fibrin as an important preparatory step in the nutrition of the cells. He compares these actions occurring in the blood to the actions of digestive ferments on proteins. Just as casein is first precipitated and then digested by pepsin, so fibrinogen is first precipitated as fibrin by union with thrombozym and thrombogen. This fibrin is then hydrolysed and dissolved by the further action of the thrombozym, which he regards as essentially proteolytic in character. That the whole blood does not coagulate within the vessels he explains by assuming that the cells of the blood and tissues are covered normally with an ultramicroscopic layer of fibrin. This forms a neutral surface, like a paraffined vessel, which has no thromboplastic effect upon the plasma.

According to Mellanby the prothrombin in the plasma is constantly associated with the fibrinogen. It may be converted to thrombin either by the action of calcium and thrombokinase, or by the action of calcium and alkali, or possibly by the action of 
calcium alone. The latest work on the subject by Rettger has tended somewhat to the simplification of this extremely complex problem. In the first place, he regards the formation of fibrin as non-fermentative in character, thus agreeing with Nolf, fibrin being produced by the simple union of fibrinogen and thrombin, though a very small amount of thrombin may produce a much larger amount (about 200 times its weight) of fibrin. He finds no evidence of the pre-existence in the blood of a thrombin or pro-ferment, and is inclined to regard the action of so-called kinases, which can be extracted from animal tissues, as similar to that of such agents as dust, threads of linen, which can produce a similar coagulating effect in birds' blood. The thrombin he regards as derived from the formed elements at the moment of their rapid disintegration when placed under abnormal circumstances. For the formation of active thrombin a minimal amount of calcium salts must enter into the molecular complex. We thus return to the simpler expression of the processes of coagulation as given by Pekelharing and Hammarsten, the prothrombin which is formed from the platelets and leucocytes by secretion or process of disintegration being activated to thrombin by the calcium salts present, and the thrombin so formed combining quantitatively with the fibrinogen to form fibrin. The prothrombin is not readily destroyed. It may remain in calcium free serum for days and when activated form thrombin quickly. Thrombin, on the other hand, disappears very rapidly from active serum in consequence of combining with some of the proteins of the serum. This property of combining with the fibrinogen and disappearing from the serum is not shared by the prothrombin. 


\section{SECTION V}

\section{THE QUANTITY AND COMPOSITION OF THE BLOOD IN MAN}

\section{A. THE TOTAL QUANTITY OF BLOOD IN THE BODY}

THE amount of blood contained in the body can be estimated by Welcker's method. It is not sufficient simply to open one of the blood-vessels and allow the animal to bleed to death, because it is not possible in this way to obtain the whole of the blood present in the body, and the blood which is obtained gradually becomes more dilute in consequence of absorpticn from the tissue spaces as bleeding continues. A small sample of blood is therefore taken from a blood-vessel and diluted 100 times with distilled water to serve as a standard of comparison. The animal is then bled from a cannula placed in a large artery, while at the same time normal salt solution is led into a vein so as to maintain the vascular system as full as possible and allow of its being washed out by the action of the heart. When the heart ceases to beat, the blood-vessels are thoroughly washed out by a stream of normal salt solution from the aorta. The animal is then minced up thoroughly and extracted with distilled water, and filtered so as to dissolve out the hæmoglobin still adherent to the tissues and especially contained in the red marrow. These washings are mixed with the whole diluted blood and the strength of the mixture in hæmoglobin is compared with that of the standard solution. In this way it is possible to estimate the total hæmoglobin present in the body in terms of the sample and so find the total amount of circulating fluid. It has been found that the dog contains about 7.7 per cent. of his body weight as circulating blood, and although smaller figures were obtained on other animals, such as the rabbit, the number of one-thirteenth has been taken as applicable to man on the basis of two observations made long ago on executed criminals. Haldane has shown recently that this estimate is much too high, the average amount of blood in man being only about 4.9 per cent. of the body weight, i.e. about one-twentieth; in some cases, as in fat individuals, it may be as little as one-thirtieth. Since the determination of the total volume of the circulating blood plays an important part in the consideration of the pathology of certain diseases such as anæmia and heart disease, the ingenious method adopted by Haldane for this determination in the living animal may be here described. The method depends on the fact that carbon 
monoxide gas when inhaled combines with hæmoglobin, expelling the oxygen from the oxyhæmoglobin. If therefore we allow a man to breathe a certain volume of carbon monoxide until it is entirely absorbed and then find that one-fifth of the hæmoglobin in his blood is saturated with carbon monoxide, we know that the whole blood could take up five times the bulk of carbon monoxide which the man has inspired. We therefore in this way determine the total 'carbonic oxide capacity' of the blood, and since CO-hæmoglobin contains the same volume of carbon monoxide as oxyhæmoglobin does of oxygen, the same figure gives us the total 'oxygen capacity.' The total oxygen capacity enables us to determine the total

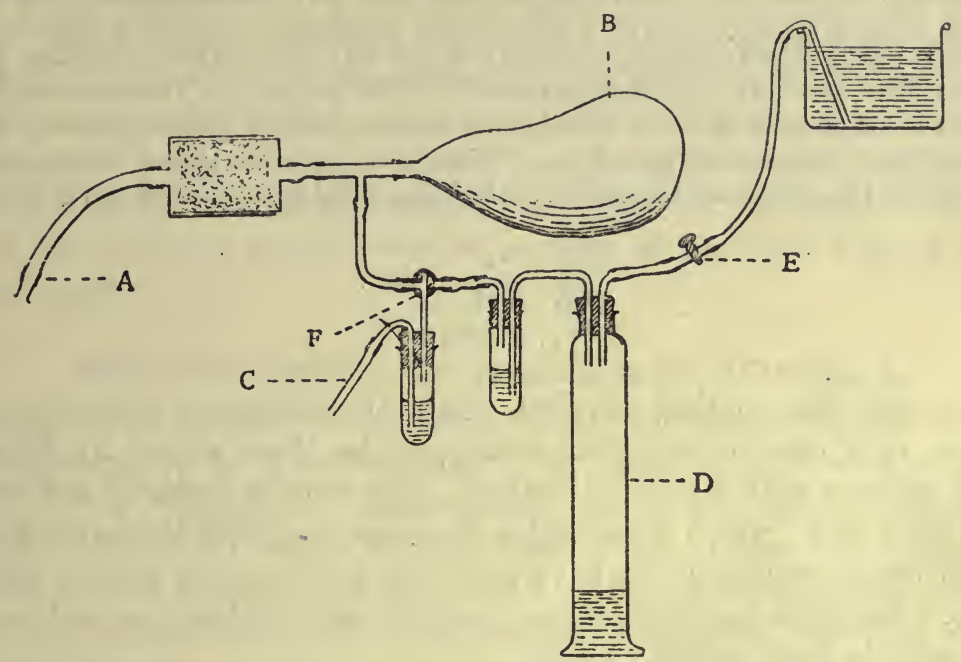

Fig. 369. Haldane's CO method for determining total blood volume in man.

amount of hæmoglobin in the body, and if we know the percentage amount of hæmoglobin in the blood it is easy to calculate the total volume of circulating fluid.

Before the carbonic oxide is administered the percentage oxygen capacity, i.e. the volume of oxygen capable of being taken up by the hæmoglobin of 100 c.c. of the blood, is determined as follows : The oxygen capacity of a sample of fresh ox blood is accurately determined by the ferricyanide method ( $v$. p. 859). The ox blood is then compared colorimetrically with blood obtained in the ordinary way by means of a hæmoglobinometer needle from the finger of the subject of the experiment, and the oxygen capacity of the latter blood calculated from the result of the comparison. The subject is now made to breathe through a mouthpiece A (Fig. 369) into a bladder B of about 2 litres capacity. The carbon dioxide produced during the experiment is absorbed by the soda lime vessel between the mouthpiece and the bladder. The oxygen as it is used up is replaced from an oxygen cylinder through the tube C. D is a graduated vessel containing pure carbonic oxide gas. While the subject is breathing in and out of the bag a given volume of carbon monoxide is admitted into the bag, being driven out from the tube $\mathbf{D}$ by allowing water to flow through the tap E. The requined volume of carbon monoxide is gradually driven in from the measuring cylinder at the rate of about 30 c.c. every two minutes. When the required quantity has been driven in and pushed forward by the oxygen an interval of two or three minutes is allowed to elapse. After this a 
drop of blood is taken for analysis. It contains a certain amount of CO-hæmoglobin. The relative saturation of the blood in carbon monoxide is determined by the colorimetric method. A number of narrow test-tubes of exactly equal diameter and each holding about 6 c.c. are taken, and 2 c.c. of water saturated with air measured off into each. Two cubic millimetres of the blood of the subject are measured off in the ordinary way by means of a hæmoglobinometer pipette into each of the six tubes, the solutions being well mixed. Four cubic millimetres of this blood are thoroughly saturated with coal gas and placed in another shorter tube, which is filled full and tightly corked. In this tube the hæmoglobin is completely saturated with carbon monoxide. After the subject has breathed the carbon monoxide, a sample of his blood is taken and diluted as before. The solution in this tube is, of course, pinker than those in the other tubes. A standard solution of carmine is now added from a narrow burette to one of the tubes of normal blood solution until its tint is the same as that of the blood taken after the inhalation. Addition of the carmine is then continued until the tint is equal to that of the blood solution which is entirely saturated with carbon monoxide. Supposing that 0.45 c.c. of carmine was required to produce equality of tint with that of the blood taken during the experiment and 2.5 c.c. to produce equality of tint with that of the saturated blood, then as 2.5 c.c. of carmine in 4.5 c.c. of liquid were required to produce saturation tint, and only 0.45 c.c. of carmine in 2.45 c.c. of liquid to produce the tint of the blood under examination, the percentage saturation of the latter could be calculated by the following sum :

$$
\begin{aligned}
& \frac{2 \cdot 5}{4 \cdot 5}: \frac{\cdot 45}{2 \cdot 45}:: 100: x \\
& \therefore x=33 \cdot 1
\end{aligned}
$$

Although this method requires careful execution in order to avoid fallacies, it is possible to attain results, as has been shown by Douglas, closely agreeing with Welcker's method. The error is probably not greater than 10 per cent., which is negligible in comparison with the large changes in total blood volume which have been found to occur in certain cases of disease. The total record of two such observations by Haldane and Lorrain Smith may be here quoted :

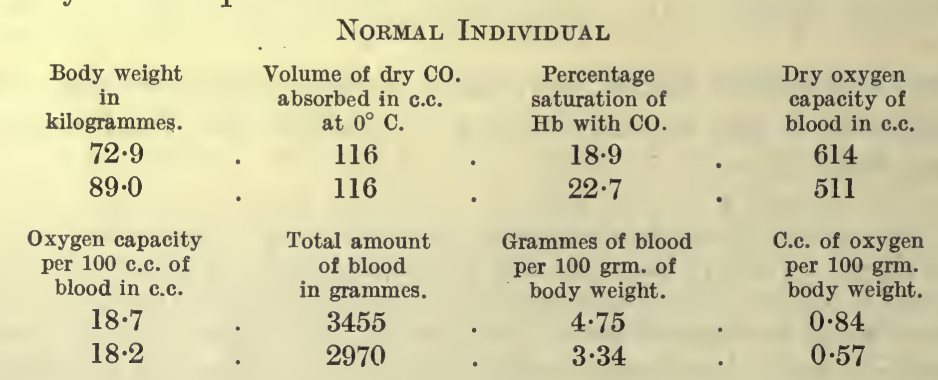

In applying this method in cases of disease it is important not to give too large a dose of carbonic oxide gas. In a normal individual 30 per cent. of the hæmoglobin may be combined with carbon monoxide before any oxygen hunger is felt, and it is possible to saturate half the hæmoglobin with this gas, though with considerable discomfort to the individual. In cases such as heart disease, where the patient is at the very margin of his resources, even 30 per cent. diminution of the oxygen capacity of the blood may have serious results, and the carbon monoxide inspired must be therefore kept at the lowest limit at which it is possible to carry out a reliable 
determination of the relative carbonic oxide saturation of the blood sample.

The total blood volume probably varies appreciably with alterations in the conditions of the animal, and may be found different on two succceding days. It is certainly influenced by the height of the blood pressure as well as by the oxygen tension in the air breathed, and therefore alters with the altitude. Some of these variations we shall have to consider more fully in a later section. Any lowering of blood pressure causes an absorption of fluid from the tissues into the blood, so that the latter becomes more dilute. The blood content during the last stages of bleeding may contain little more than 50 or 60 per cent. of the hæmoglobin which was present in the first samples of blood, pointing to a corresponding dilution of the blood during these few minutes by means of tissue lymph. By this means, i.e. the absorption of fluid from tissues, the volume of circulating blood after a limited hæmorrhage is rapidly brought up to normal, so that there is a circulation of a fluid impoverished in corpuscles. The latter are made up in the course of a few weeks as a result of increased activity in the bone-marrow.

\section{RELATIVE AMOUNT OF PLASMA AND CORPUSCLES}

The relative amount of corpuscles in a given sample of blood is most easily determined by Blix's method. The blood is mixed with a definite amount of 2.5 per cent. potassium bichromate, and the mixture is put into small graduated capillary tubes, which are then placed in a centrifuge revolving about 10,000 times per minute. The corpuscles rapidly accumulate in an almost solid mass at the bottom of the tube, and their volume can be directly read off. It is often possible by working quickly to receive blood into such graduated capillary tubes and to centrifuge it rapidly before it has had time to coagulate. The corpuscles are hurried down to the bottom of the tube within two or three minutes and their volume can be in this way directly determined. An indirect method for the same purpose was devised by Hoppe-Seyler. The total proteins of defibrinated blood are determined and compared with the total proteins of the washed corpuscles and of the serum. Thus in one experiment $100 \mathrm{grm}$. of defibrinated pigs' blood contained 18.90 grm. protein plus hæmoglobin. The blood-corpuscles of $100 \mathrm{grm}$. of the same blood contained $15.07 \mathrm{grm}$. proteins plus hæmoglobin; therefore the serum of the same $100 \mathrm{grm}$. of blood contained $18.90-15.07=3.83 \mathrm{grm}$. proteins. One hundred grammes of serum contain: $6.77 \mathrm{grm}$. protein. From these figures the amount of serum in the 100 grm. of defibrinated blood may be computed as follows :

$$
\begin{aligned}
& \frac{3 \cdot 83}{6 \cdot 77} \cdot 100=56 \cdot 6 \text { per cent. serum. } \\
& 100-56 \cdot 6=43 \cdot 4 \text { per cent. blood corpuscles. }
\end{aligned}
$$

The average volume of corpuscles in human blood can be taken as 50 per cent. of the total amount, different estimations having given figures varying 
from 48 to 54 per cent. In the horse the volume of corpuscles is 53 per cent., in the dog 36 per cent.

\section{THE ENUMERATION OF THE CORPUSCLES}

In order to enumerate the red corpuscles the blood is diluted with a known amount of an isotonic fluid and the number is counted in a measured volume of the mixture. The average number of red corpuscles is about $5,000,000$ per cubic millimetre in adult men and rather fewer, about $4,500,000$ in adult women. The enumeration of corpuscles is subject to considerable errors, probably not less than 10 per cent. Moreover different conditions of the circulation may cause variations in the relative distribution of plasma and corpuscles respectively in different parts of the circulation, so that the blood-count of a specimen from the capillaries of the finger or lobe of the ear may vary considerable from a similar count of the corpuscles in blood obtained directly from a minute vein or artery. More important therefore is the determination of the hæmoglobin. For this purpose a measured quantity of the blood, 2 to 5 c.mm., is obtained in a capillary pipette and mixed with a given volume of water. The red fluid thus obtained is compared with a standard. This latter in von Fleischl's instrument is a prism of coloured glass. In Oliver's instrument the standard consists of a series of tinted glasses, one of which represents the colour of a measured quantity of normal blood diluted with water and placed in a flat glass cell of a certain size, while the others represent percentages of hæmoglobin below and above the normal. The most accurate method is that due to Hoppe-Seyler and Haldane, namely, the conversion of the blood sample into CO-hæmoglobin and its comparison with a standard specimen of CO-hæmoglobin, which is stable in solution and can therefore be kept in a sealed glass vessel for any length of time.

\section{THE OXYGEN CAPACITY OF THE BLOOD}

Instead of determining the hæmoglobin we may measure directly the oxygen capacity of the blood, since the oxygen-binding power of this fluid is entirely dependent on the amount of hæmoglobin it contains. For this purpose we may make use of the fact discovered by Haldane, that the combined oxygen in oxyhæmoglobin is liberated rapidly and completely on addition of a solution of potassium ferri-cyanide to laked blood, and may thus be easily measured with the help of an apparatus similar to that used for determining urea in urine by the hypobromite method.

The following description of the method is given by Haldane :

"Twenty cubic centimetres of the oxalated or defibrinated blood, thoroughly saturated with air by swinging it round in a large flask, are measured out from a pipette into the bottle $\mathrm{A}$, which has a capacity of about 120 c.c. As it is important to avoid blowing expired air into the bottle the last drops of blood are expelled from the pipette by closing the top and warming the bulb with the hand." Thirty cubic centimetres are then added of a solution prepared by diluting ordinary strong ammonia solution (sp. gr. 0.88 ) with distilled water to $\frac{1}{500}$. The ammonia prevents carbonic acid from coming off, while the distilled water lakes the corpuscles. The blood and ammonia 


\section{QUANTITY AND COMPOSITION OF BLOOD}

solution are thoroughly mixed by shaking, and at the end of this operation the solution should appear perfectly transparent when tilted up against the sides of the bottle.* About 4 c.c. of a saturated solution of potassium ferricyanide are then poured into the small tube $\mathbf{B}$ (the length of which should slightly exceed the width of the bottle) and placed upright in A. The rubber stopper, which is provided, as shown, with a bent glass tube connected with the burette by stout rubber tubing of about $1 \mathrm{~mm}$. bore, is then firmly put in, and the bottle placed in the vessel of water c, the temperature of which should be as nearly as possible that of the room and of the blood and water in the bottle. If the stopper is not heavy enough to sink the bottle the latter should be weighted. Byopening to the outside the three-way tap (or T-tube and clip) on the burette, and raising the levelling tube, which is held by a spring clamp, the water in

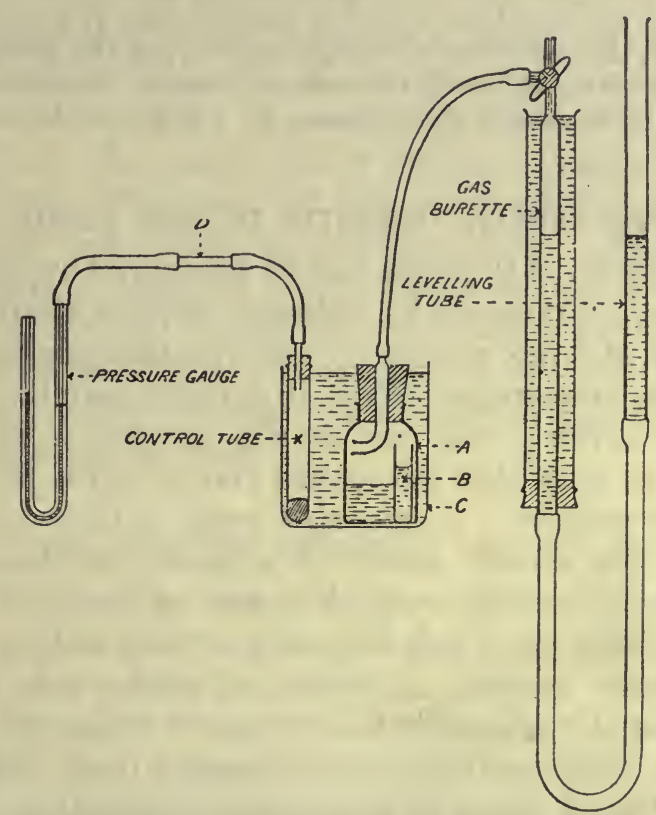

FIG. 370. Haldane's method for determining the oxygen capacity of the blocd.

the burette is brought to a level close to the top. The tap is then closed to the outside, and the reading of the burette (which is graduated to 05 c.c., and may be read to 01 c.c.) taken after careful levelling.

The water-gauge (which has a bore of about $1 \mathrm{~mm}$.) attached to the temperature and pressure-control tube is now accurately adjusted to a definite mark. This is easily accomplished by sliding the rubber tube backwards or forwards on the piece of glass tubing $\mathbf{D}$. The control tube is an ordinary test-tube containing some mercury to sink it, and connected with the gauge by stout rubber tubing of about $1 \mathrm{~mm}$. bore.

As soon as the reading of the burette is constant, which it will probably be within two or three minutes, the bottle is tilted so as to upset $\mathrm{R}$, and is shaken as long as gas is evolved. During this operation B should be repeatedly emptied, as otherwise the oxygen dissolved in its liquid might not be completely given off. When the evolution of oxygen has ceased the bottle is replaced in the water. If, as is probable, the pressuregauge indicates an alteration in the temperature of the water, cold water from the tap, or warmed water, is added till the original temperature has been re-established, and the

* If the solution were not-transparent this would indicate that the laking was incomplete, and more ammonia solution would need to be added. 
reading of the burette noted as soon as it is constant. The bottle is again shaken, \&c., until a constant result is obtained, for which about fifteen minutes from the beginning of the operations are required. The temperature of the water in the jacket of the burette, and the reading of the barometer, are now taken, and the gas evolved is reduced to its dry volume at $0^{\circ}$ and $760 \mathrm{~mm}$. To calculate the oxygen evolved from 100 c.c. of blood, allowance must be made for the fact that a 20 c.c. pipette does not deliver 20 c.c. of blood, but only about $19 \cdot 6$ c.c. The actual amount of shortage for a given pipette can easily be determined by weighing the pipette after water, and again after blood, has been delivered from it. A further slight correction is necessary on account of the fact that the air in the bottle at the end of the operation is richer in oxygen than at the beginning, so that, as oxygen is about twice as soluble as nitrogen, slightly more gas will be in solution. With a bottle of 120 c.c. capacity and 20 per cent. of oxygen in the blood, the air in the bottle at the end will evidently contain about 27 per cent. of oxygen, so that, assuming that the coefficients of absorption of oxygen and nitrogen in the 54 c.c. of liquid within the bottle are nearly the same as in water, the correction will amount at $15^{\circ} \mathrm{C}$. to .06 c.c. in the reading of the burette, or +0.30 per cent. in the result."

\section{THE SPECIFIC GRAVITY OF THE BLOOD}

The specific gravity of the blood may be determined by directly weighing a sample, or more conveniently by collecting blood in a capillary tube and discharging drops of it into a series of vessels containing glycerin and water mixed in varying proportions. When it is found that the drop of blood as it leaves the capillary vessel neither rises nor falls in the glycerin and water mixture, we know that the specific gravity of the blood is identical with that of the mixture. A graduated series of these mixtures is kept in bottles and their specific gravity is generally determined before the experiment. Hammerschlag's method consists in placing a drop of blood in a mixture of chloroform and benzene and then adding chloroform or benzene, as the case may be, until the drop neither rises nor falls. The specific gravity of the mixture is then taken. The specific gravity varies in man between 1057 and 1066, and in woman from 1054 to 1061. It is increased by loss of water, as after profuse perspiration, or by passive congestion of the part from which the sample is taken. It is also increased as a result of any operation upon a serous cavity in consequence of exudation of plasma in the inflamed or irritated part. It is diminished as the result of bleeding. The specific gravity of serum is 1028 to 1032, of corpuscles about 1090. It is interesting to note that the specific gravity of the blood is highest in the fœtus at full term, when it amounts to 1066 , contrasting with that of the mother at the same time, the specific gravity of whose blood is only 1050. The specific gravity rapidly falls to the latter figure after birth.

\section{THE REACTION OF THE BLOOD}

The -blood is alkaline to litmus. This fact can be demonstrated by allowing a drop to fall on a piece of glazed litmus paper and then wiping away the blood with a piece of linen moistened with distilled water or neutral saline solution. In order to estimate the alkalinity a small definite quantity of the blood is mixed with sulphate of soda solution containing a definite 
amount of tartaric acid. The acid mixture is then titrated against a decinormal solution of sodium hydrate until the mixture gives a blue stain when a drop of it is placed on glazed litmus paper. The alkalinity of normal blood as determined in this way amounts on the average to $0.2 \mathrm{grm}$. $\mathrm{NaHO}$ per 100 c.c. of blood. If the blood be laked the alkalinity rises to as much as $0.4 \mathrm{grm}$. NaHO per 100 c.c.

All these questions of reaction depend, however, on the indicator employed. A neutral salt might react alkaline to litmus if the acid radical of the salt were capable of being displaced by the coloured acid radical of the indicator with the production of a blue alkaline salt of litmus. Sodium bicarbonate may be acid or neutral to litmus and alkaline to methyl orange. The absolute alkalinity of any fluid may be expressed by the number of free $\mathrm{OH}$ ions which it contains, just as the acidity is a measure of the free $\mathrm{H}$ ions. The number of free $\mathrm{OH}$ ions in the blood can be determined by an electrical method, and is found to be very small, very little more in fact than that contained in distilled water. The alkalinity of the blood as ordinarily determined by the litmus method gives us, however, more important knowledge than this determination of its absolute alkalinity, since on its relative alkalinity to litmus depends to a large extent its power of combining with carbon dioxide and therefore acting as a carrier of this gas from the tissues to the lungs.

\section{THE OSMOTIC PRESSURE OF THE BLOOD}

Since the blood serves as a circulating medium by means of which the composition of the tissues juices forming the immediate environment of all the cells of the body is maintained constant, its osmotic pressure must be of considerable importance in regulating. the normal exchanges of the cells with their surrounding fluid. The osmotic pressure of the blood depends on its molecular concentration and can be determined by any of the methods mentioned earlier (p. 125). Of these the most convenient is the determination of the freezing-point. The depression of freezing-point, $\Delta$, of mammalian blood is about 0.56 and varies between 0.54 and 0.60 . The depression of the freezing-point observed in blood is equal to that of a 0.9 per cent. sodium chloride solution, which is therefore taken as isotonic with the blood. Since the corpuscles are in osmotic equilibrium with the plasma, their osmotic pressure must be equal to that of the plasma, and laking the blood does not alter its freezing-point or its osmotic pressure. The blood of the frog has a lower osmotic pressure, the normal saline fluid for the frog's tissues being equivalent to 0.65 per cent. sodium chloride solution.

\section{THE ELECTRICAL CONDUCTIVITY OF THE BLOOD}

In a solution it is only the dissociated ions which have the power of carrying electric discharges. The conductivity of a solution of pure urea or pure glucose would not differ appreciably from that of distilled water, 
since neither of these substances is ionised in solution. The conductivity of blood-serum is therefore determined almost entirely by its content in salts. Since this is approximately constant, the conductivity of serum varies within very narrow limits. The conductivity of defibrinated blood varies, however, within wide limits, since the outer limiting layer of the corpuscles is impermeable to many of the ions of the salts of the serum. The corpuscles present a resistance to the passage of the charged ions and therefore of the electric current through them, so that the larger the number of corpuscles contained in a given specimen of blood the lower will be the conductivity of the latter. Stewart has made use of this fact as a basis for a method of determining the relative volume of corpuscles and plasma.

\section{The Electrical Conductivity of the Entrie Blood as compared with that} of its Serum. (Stewart.)

The relative amount of serum can be given by the formula:

$$
\mathrm{p}=\frac{\lambda(b)}{\lambda(s)}(174-\lambda(b))
$$

where $p$ is the number of c.c. of serum in 100 c.c. of blood; $\lambda(b), \lambda(s)$, the conductivity respectively of the blood and serum (both measured at or reduced to $5^{\circ} \mathrm{C}$. and expressed in reciprocal $\mathrm{Ohms} \times 10^{8}$ ). A reciprocal $\mathrm{Ohm}$ is the conductivity of a mercury column 1.063 metres long and 1 square millimetre in section.

\section{THE GENERAL COMPOSITION OF THE BLOOD}

The general composition of the blood has been determined by Karl Schmidt in man, and by Abderhalden in the horse and bullock. The results are given in the Tables on pages 863 and 864 .

The important points to be drawn from these analyses may be summarised as follows. Blood contains from rather over one-third to one-half of its weight of corpuscles. It contains from 20 per cent. to 25 per cent. solids. Blood-plasma is resolved by clotting into serum and fibrin. The fibrin forms only 0.2 to 0.4 per cent. of the total weight of blood. The serum contains in 100 parts 8 to 9 parts of solids, of which 7 to 8 parts consist of proteins, while the salts make up about 1 part. The chief salt present in the serum is sodium chloride, which constitutes 60 per cent. of the ash. Next to this comes sodium carbonate, about 30 per cent., and besides these two we find traces of potassium, sodium, and calcium chlorides and phosphates. Traces of fats, cholesterin, lecithin, dextrose, urea, and other nitrogenous extractives are constantly found in the serum. The fats are much increased after a meal rich in them and may give the serum a milky appearance. The red corpuscles contain from 30 to 40 per cent. total solids. Of the solid constituents hæmoglobin forms nine-tenths ; the other tenth corresponds to the stroma consisting of stroma protein (nucleo-protein), lecithin, cholesterin, and salts. There is a striking contrast between the salts of the corpuscles and those in the serum, the former consisting chiefly of potassium phosphate, the latter of sodium chloride, which in some animals is entirely wanting in the corpuscles. 


\begin{tabular}{|c|c|}
\hline 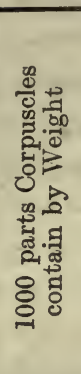 & 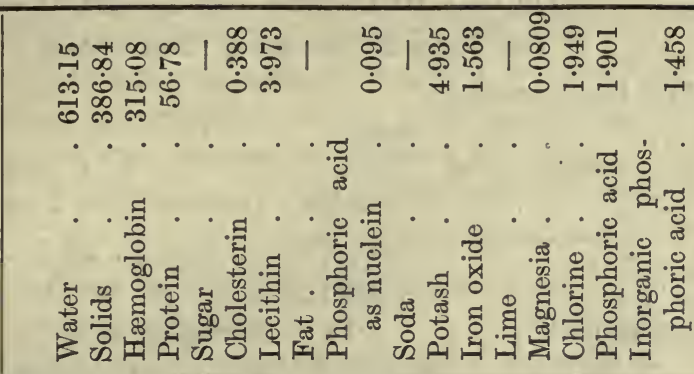 \\
\hline 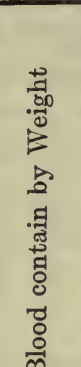 & 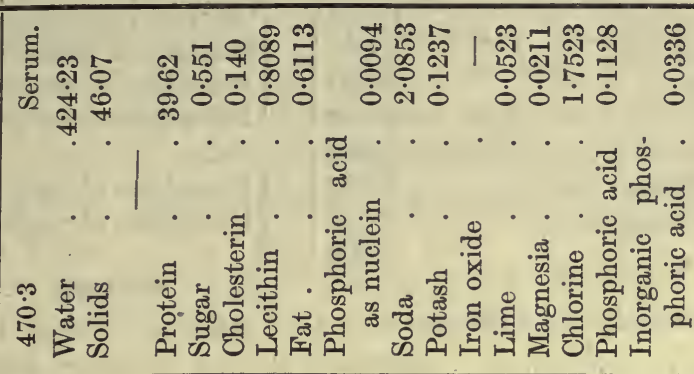 \\
\hline 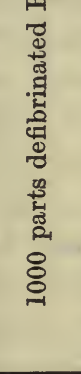 & 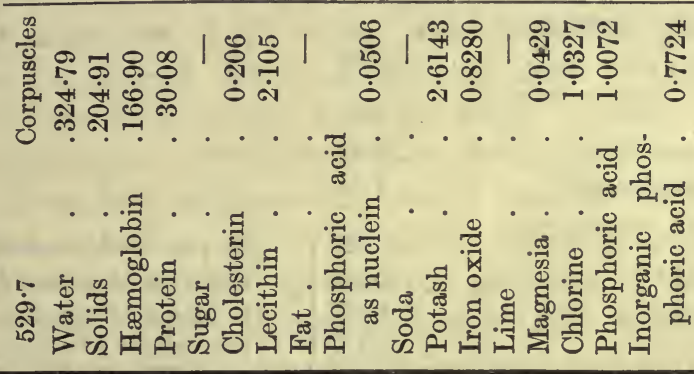 \\
\hline 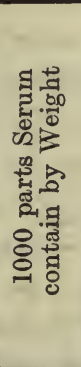 & 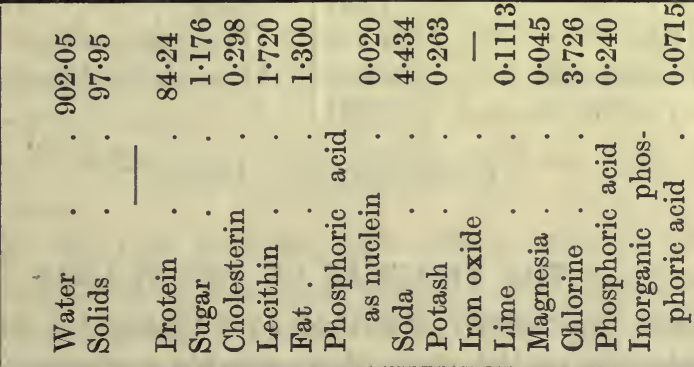 \\
\hline 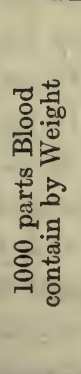 & 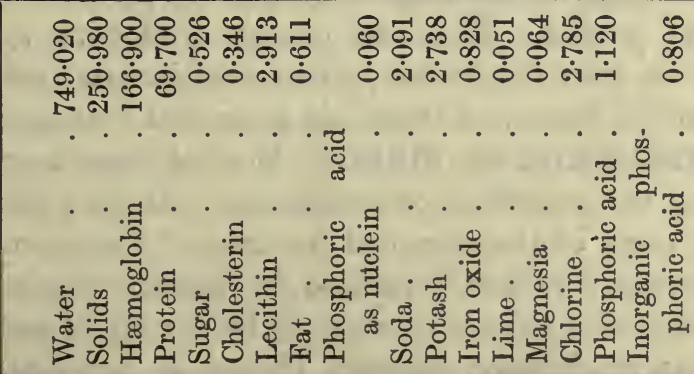 \\
\hline
\end{tabular}


Blood of a Man Twenty-five Years of age.

One thousand Grammes of Blood contain

513.02 Blood-corpuscles.

Water • . . . . $349 \cdot 69$

Substances not vapourising at $120^{\circ}$

Hæmatin

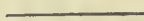

'Blood-casein,' \&c. . .

Inorganic constituents .

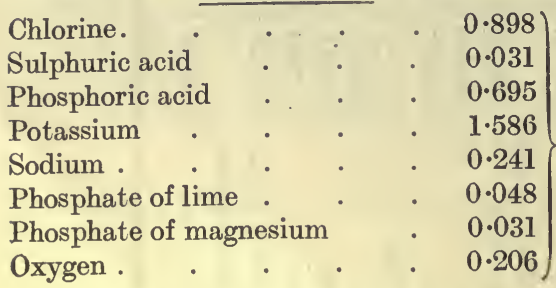

$163 \cdot 33$

$7 \cdot 70$ (including $0 \cdot 512$ iron)

$151 \cdot 89$

3.74 (excluding iron)

0.898 ) (Chloride of potassium . . 1.887

0.031 Sulphate of potassium . . 0.068

0.695 Phosphate of potassium . . $1 \cdot 202$

$1.586\}$ Phosphate of sodium . . 0.325

$0.241\}=\{$ Soda . . . . . 0.175

0.048 Phosphate of lime . . 0.048

0.031 Phosphate of magnesium . 0.031

486.98 Interstitial Fluid (Plasma).

Total .

$3 \cdot 736$

Water
Substances not vaporising at
$120^{\circ}$

Fibrin . . . .

'Albumen,' \&c. . .

Inorganic constituents .

Chlorine. . . .

Sulphuric acid . . $\quad$. 0.063

Phosphoric acid . . . 0.071

Potassium . . . . 0.153

Sodium . . . . . 1.661

Phosphate of lime . . . . 0.145

Phosphate of magnesium . $0 \cdot 106$

Oxygen . . . . 0.221

Specific gravity $=1 \cdot 0599$

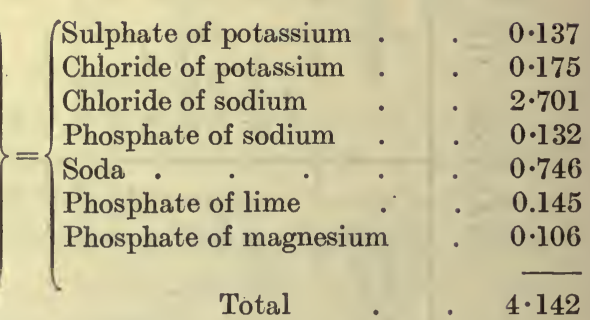

\section{THE PROTEINS OF THE PLASMA}

The plasma is generally described as containing a number of different proteins belonging to the class of coagulable proteins. No albumoses or peptones are present. Since the plasma in clotting gives rise to fibrin and serum we may divide its protein constituents into those which are the precursors of fibrin and those which are still contained in the serum.

THE PRECURSORS OF FIBRIN. Most of these have been dealt with in discussing the causation of coagulation. It only remains for us here to mention some of the chemical features of fibrinogen and its product fibrin. Fibrinogen is best separated by Hammarsten's method, namely, half-saturation with sodium chloride, or by the use of ammonium sulphate. Fibrinogen is precipitated between 13 and 28 per cent. saturation with 
ammonium sulphate, whereas no other globulins are precipitated until the saturation amounts to 29 per cent. of ammonium sulphate. Fibrinogen obtained in either of these ways can be purified by re-solution and reprecipitation, but loses its solubility in the process, so that every time it is precipitated some of the substance becomes insoluble. The insoluble fibrinogen resembles fibrin in many characters, but does not swell in the presence of dilute acids as fibrin does. Fibrinogen is soluble in dilute alkali, from which it may be precipitated by careful neutralisation. Fibrinogen in salt solution coagulates at $56^{\circ} \mathrm{C}$. A small amount, however, remains in solution and is not coagulated until $65^{\circ} \mathrm{C}$. is reached. Fibrinogen can be therefore described as a globulin occurring in the plasma and converted on coagulation into fibrin. The other precursors of fibrin, namely, those involved in the production of thrombin and called thrombokinase and thrombogen, seem to be phosphorus-containing proteins perhaps belonging to the class of nucleo-proteins. Their chief characteristics have already been dealt with.

FIBRIN. Fibrin is easily obtained by whipping blood as it flows from the vessels with a bundle of wires or twigs and then washing the stringy threads so obtained under a stream of water. As prepared in this way it always contains fragments of leucocytes, blood-platelets, and stromata, which have become entangled in its meshes. In order to prepare fibrin in a pure state it is necessary to get it by the action of fibrin ferment on a pure solution of fibrinogen. Fibrin is a white stringy substance insoluble in water and in dilute salt solutions. It slowly dissolves in 5 per cent. solutions of sodium chloride, sodium sulphate, potassium nitrate, \&c., but is converted in this process into soluble globulins. It is probable that its solution is effected by the agency of minute traces of proteolytic ferment present in the blood and adherent to the fibrin as it is precipitated. This probability is strengthened by the fact that a certain amount of albumoses is always found in the fluid along with the soluble globulins. In dilute acid, such as 0.2 per cent. hydrochloric acid, fibrin swells into a clear jelly which very slowly undergoes solution with the formation of acid albumen and proteoses.

THE PROTEINS OF THE SERUM. The serum proteins are generally grouped in two classes, namely, the serum albumens and the serum globulins. All the proteins are completely precipitated by saturation with ammonium sulphate. By half-saturation with this salt only the globulins are precipitated and can be separated from the serum albumens by filtration. The proportion of globulin to albumen as determined in this way is known as the 'protein quotient.' It varies in different animals, but in the same individual it is almost constant in the blood, serum, lymph, and serous transudations, though the total amounts of protein in these may be very different.

SERUM ALBUMEN. Serum albumen remains in the serum after halfsaturation with ammonium sulphate. It can be precipitated from this by complete saturation with ammonium sulphate or sodio-magnesium sulphate, 
or in the crystalline form by slight acidification, as in Hopkin's method described on p. 80. Serum albumen is soluble in distilled water. Its solutions therefore can be dialysed indefinitely without any precipitation taking place.

THE GLOBULINS. The globulins of serum, known as para-globulin or serum globulin, are obtained by half-saturation with ammonium sulphate. Their solutions in salt coagulate at about $75^{\circ} \mathrm{C}$. Since globulin is insoluble in distilled water it is precipitated on dialysing serum against distilled water. The precipitate obtained in this way is not, however, so great in extent as that obtained on half-saturation, and on this account the globulin fraction of the serum proteins has been divided into two fractions, namely, euglobulin, precipitable by dialysis, and pseudo-globulin, not precipitable by dialysis, but thrown down on half-saturation with ammonium sulphate.

A thorough study of serum globulin by Hardy has shown that this body forms adsorption combinations with acids, alkalies, or neutral salts. With acids and alkalies the globulin forms ' salts' which ionise in solution so that in an electric field the entire mass of protein moves. These salts cannot be precipitated by dialysis. In them the globulin acts much more strongly as an acid than as a base, so that a weak acid, such as acetic acid, has a much smaller dissolving power over globulin than has the equivalent amount of hydrochloric acid, and boracic acid has a very slight power indeed. The weak basic character of globulin causes its salt in weak acids to undergo hydrolysis with separation of globulin, so that in order to reach the same grade of solution with a weak acid as with a strong acid a great excess of the acid is necessary. Owing to the much stronger acid character of globulin it is found that weak ammonia dissolves it almost as well as strong alkalies. With neutral salts globulins form molecular compounds which are soluble, but are readily decomposed by water with liberation of the insoluble globulin. They are therefore only stable in the presence of a comparatively large excess of salt. The globulins differ from the albumens of the serum in containing constantly organic phosphorus as an integral part of their molecule. In all its solutions globulin is present in large molecular aggregates, so that it is impossible to filter a globulin solution through a porous clay cell.

\section{THE CONDITION OF THE PROTEINS IN THE BLOOD-SERUM}

Although it is easy by such simple means as the addition or removal of neutral salts to separate one or more different forms of protein from serum, we have strong evidence that these proteins do not exist side by side in the serum, but are combined to form what we may term serum protein, which acts as a whole and differs in its qualities from many of those of its constituent globulins or albumens. When a current is passed through blood-serum no movement of protein takes place (Hardy). Alkali globulin therefore cannot be present. Salt globulin might be assumed to be present since it does not ionise in solution, but serum is not precipitated by simple addition of acid, which would readily precipitate salt 
globulin in alkaline solution. Moreover serum can be readily filtered through a porous cell, and this method is adopted for obtaining it free from contamination by micro-organisms. Globulin in any of its solutions will not pass through a porous cell. If globulin be present as such in the serum it is therefore not ionised, but the agent which dissolves it must be something more than alkali or salt, since either alone or together they will not produce a solution which will pass through a porous cell. Serum has still the power of taking up globulin and will dissolve almost its own volume of precipitated globulin, though in oxalate serum there is not a trace of alkali globulin nor of any ionised protein. We are justified therefore in concluding that serum protein may be regarded as a complex unit. By simple means, such as dialysis, dilution, or addition of salt, this unit can be broken up with the separation of the various proteins which we have designated as serum albumen and serum globulin, \&c. The question naturally suggests itself whether in plasma we have not a similar combination of all its varied colloidal constituents to form one labile mass of fluid protoplasm. 


\section{CHAPTER XIII}

\section{THE PHYSIOLOGY OF THE CIRCULATION}

\section{SECTION I \\ GENERAL FEATURES OF THE CIRCULATION}

IN order that the nutrition of the tissues may be properly carried out, and that they may receive a continual supply of nourishment from the alimentary canal, and of oxygen from the lungs, and be able to free themselves of their waste products, the blood which flows through them must be continually renewed. For this purpose every part of the body is supplied with tubes-blood-vessels - of various sizes and structures.

In the tissues the blood is passing continuously through a thick meshwork of capillaries, hair-like vessels with walls consisting of a single layer of delicate endothelial cells which permit of a free interchange of material by diffusion between the blood within and the tissue fluid outside the vessel. The movement of the blood is maintained by a hollow muscular organ, the heart, placed in the chest, the blood being brought from the heart to the tissues by thick-walled tubes, the arteries, and being carried back from the tissues to the heart by a system of thin-walled vessels, the veins.

In all the vertebrates the vascular system is closed, i.e. communicates at no point with the tissue spaces or cœlomic cavity. It is found in its simplest form in fishes (Fig. 371, A), where the heart consists of one auricle and one ventricle. The blood is received from the great veins into the auricle. The walls both of auricle and ventricle contract rhythmically. By the contraction of the auricle the blood is forced into the ventricle, and this, when it contracts, sends the blood on into the bulbus arteriosus. From the bulbus the blood passes through the branchial arteries into the gills, where it takes up oxygen from the surrounding water, and then flows on into the aorta, by which it is distributed to the various organs of the body. From the capillaries of these organs the blood is collected by the veins and is carried once more back to the auricle. The fish heart is thus entirely on the venous side of the vascular system.

In amphibia, such as the frog, the heart consists of two auricles and one ventricle. The right auricle receives venous blood from the body by means of the venæ cavæ and forces it by its contraction into the ventricle. 
From the ventricle the blood passes into the aorta, whence it is carried partly by the pulmonary artery to the lungs, partly by arteries to the different organs of the body. The blood which has passed through the lungs and been arterialised flows through the pulmonary veins to the left auricle, whence it passes into the ventricle and mixes with the venous blood which is arriving from the right auricle. The pulmonary circulation is thus merely a branch of the general or systematic circulation. The bulbus aortæ in the frog is divided into two parts by means of a spiral

A

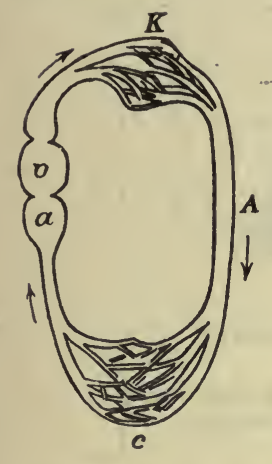

B

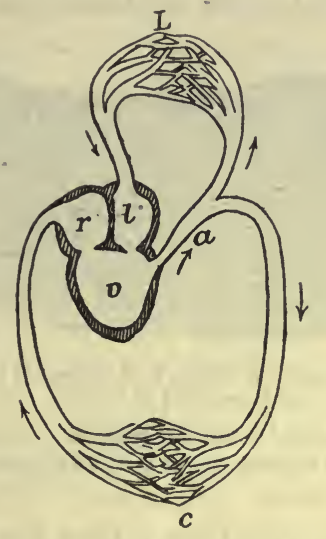

C

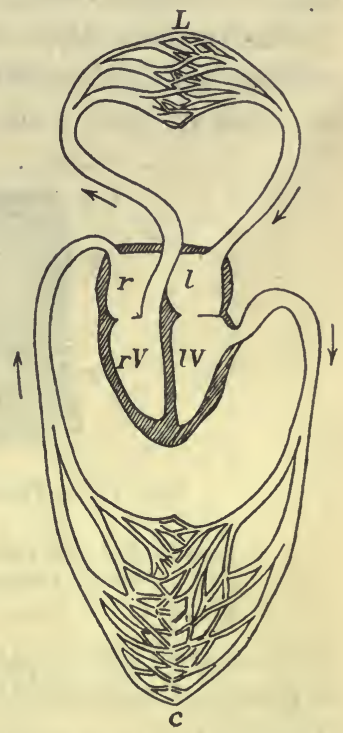

Fig. 371. Diagram of circulatory system in A, fish ; B, amphibian (frog); C, mammal. $v$, ventricle ; $a$, auricle ; $K$, gill capillaries ; $A$, aorta ; $c$, systemic capillaries ;

$L$, lung capillaries; $r, l$, right and left auricles; $r V, l V$, right and left ventrieles.

valve, by which a partial separation of the blood coming from the right and left auricles is effected, and the venous blood from the right auricle directed especially into the pulmonary artery.

In birds and mammals the heart has become entirely divided into two halves, right and left, which have no communication with one another except by way of the blogd-vessels and capillaries. The right auricle receives the venous blood from all parts of the body and sends it on to the right ventricle, whence it is forced into the lungs along the pulmonary artery. In the lungs it takes up oxygen and becomes arterial and is returned by the pulmonary veins to the left auricle and so to the left ventricle. The rhythmic contractions of the left ventricle then force the blood into the aorta, whence by the branching arteries it is carried to all parts of the body. The whole vascular system is distensible and elastic, so that its capacity will increase with the pressure of the blood contained in it. Since the driving force is furnished by the heart the pressure which. 
causes the flow of blood through the system must decline as we pass from the arterial to the venous side. The chief function of the large arteries is to serve as elastic conduits, whereas the small arteries or arterioles leading from the arteries to the capillaries have in addition the function of regulating the amount of blood flowing through the capillary area of the organs which they supply. The veins have the function of conducting blood at a low pressure from capillaries to heart and of storing up any excess of blood which is not immediately taken up by the heart. Corresponding to this difference in function we find variations in the structure of the blood-vessels according to their situation in the circuit.

The vessels which carry the blood from the heart to the tissues, the arteries, are thick-walled, and contain an abundance of muscular and elastic elements in their walls. The typical medium-sized artery is described as

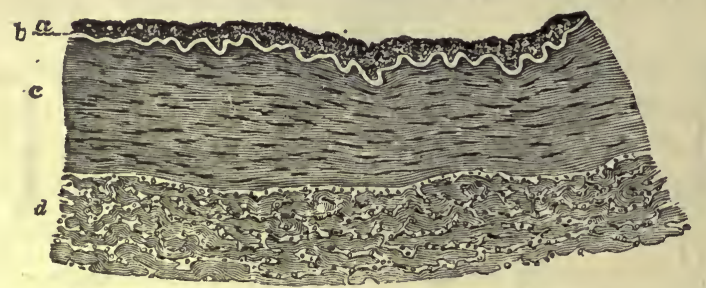

FIG. 372. Transverse section of part of the wall of the posterior tibial artery $(\times 75)$.

$u$, endothelial and sub-endothelial layers of intima ; $b$, lamina of elastic tissue ; $c$, media consisting of muscle fibres ; $d$, adventitia. (SCHÄFER.)

consisting of three coats (Fig. 372) : an intima lined by a continuous layer of flattened endothelial cells, which rest on a well-marked lamina of yellow elastic tissue; a media composed of unstriated muscular fibres arranged longitudinally and circularly; and an external coat or adventitia of fibrous tissue, with a number of longitudinal elastic fibres. Near the heart, in the great vessels such as the aorta and its larger branches, there is a preponderance of elastic tissue as compared to the muscular; and we find in the media alternate layers of muscle fibres and fenestrated elastic membranes. In the smallest arteries, on the other hand, the arterioles, the elastic element entirely disappears, so that the wall consists of muscle fibres, chiefly circular, lined by the endothelium. In the latter vessels a contraction of their walls may result in an entire obliteration of the lumen, so shutting off altogether the supply of blood to the capillaries beyond. In the veins the same three coats can be distinguished as in the typical artery, but the wall of the vessel is much thinner in proportion to the lumen. In the vein moreover there is a preponderance of the fibrous tissue elements, the muscular and elastic tissue being but little marked. On this account the vein collapses unless it is distended by some internal pressure. The histological difference between veins and arteries is of considerable importance for the understanding of the distribution of pressures in the vascular system, since the distensibility and reaction to pressure of these vessels 
are conditioned by their structure. In Fig. 373 is represented the extensibility, i.e. the increase in capacity of an artery and a vein under gradually increasing internal pressure. It will be seen that an artery which has a certain capacity at zero pressure gradually distends with increasing pressure. The increase in capacity is small at first, and becomes most rapid between 90 and $100 \mathrm{~mm}$. Hg. After this point every increment of pressure brings about a gradually diminishing increment of capacity. Thus a change of internal pressure causes the greatest change in capacity when the pressure in the artery corresponds, as we shall see, to the average arterial pressure in the normal animal. In the vein, on the other hand, the capacity, which is nothing at zero pressure, becomes considerable on raising the pressure to $1 \mathrm{~mm}$. Hg. A further rise of pressure to $10 \mathrm{~mm}$. $\mathrm{Hg}$ causes a consider-

Capacity in c.o.

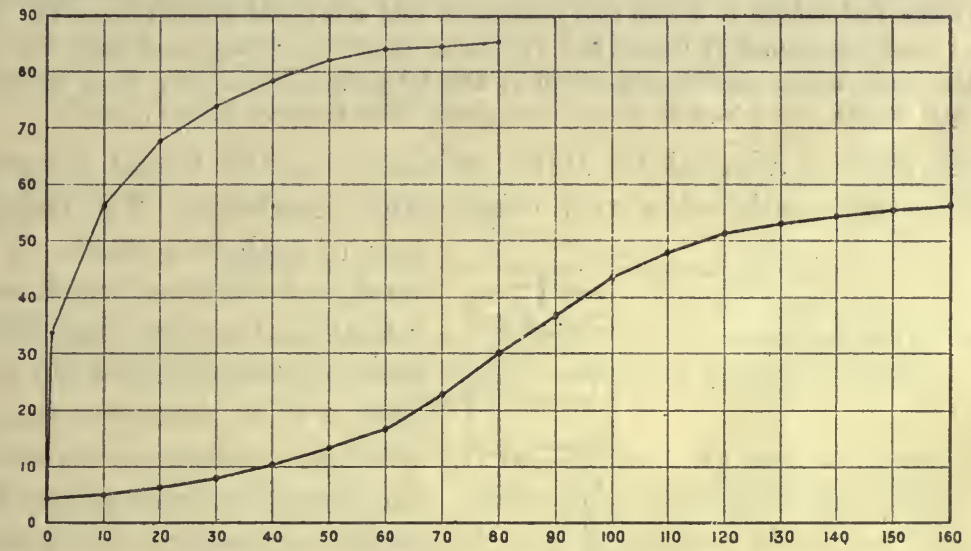

$\mathrm{mm} . \mathrm{Hg}$.

FIG. 373. Curves of distensibility of an artery (thick line) and of a vein (thin line). The figures at the left side of the diagram represent the capacity of a section of the vessel when distended under a certain pressure, expressed by the figures on the base line in mm. Hg. (Constructed from figures given by Rox.)

able increase in volume, but from this point the increments of volume with rising pressure rapidly diminish. Whereas the artery is most distensible at about $100 \mathrm{~mm}$. $\mathrm{Hg}$, the vein has its limits of optimum distensibility between 0 and $10 \mathrm{~mm}$. $\mathrm{Hg}$.

As the arteries branch, although each branch is smaller than the parent vessel, the total area of the two branches into which the vessel divides is greater. Thus there is a continual increase in the cross area of the bed of the blood-stream as we pass from the heart towards the periphery. This increase is especially marked at the junction between the capillaries and the arterioles at one side and the venules on the other, so that the total area of the bed in the region of the capillaries can be taken as about 800 times that of the area of the aorta where the blood leaves the heart.

On cutting through an artery, blood escapes from the central end, i.e. that nearest the heart, with great force and in a series of jerks, each of which corresponds to a contraction of the ventricles. This manner of 
escape shows that in the arteries the blood is at a high pressure, and that the flow from the heart to the periphery is a pulsatory one. The same lesson may be learnt by connecting a long tube with the central end of a divided artery. This experiment, which was first performed by the Rev. Stephen Hales, may be described in his own words :

"In December I caused a mare to be tied down alive on her back ; she was fourteen hands high, and about fourteen years of age, had a Fistula on her Withers, was neither very lean, nor yet lusty: Having laid open the left crural Artery about three inches from her belly, I inserted into it a brass Pipe, whose bore was one sixth of an inch in diameter; and to that, by means of another brass Pipe which was fitly adapted to it, I fixed a glass Tube, of nearly the same diameter, which was nine feet in length: Then untying the Ligature on the Artery, the blood rose in the Tube eight feet three inches perpendicular above the level of the left Ventricle of the heart: But it did not attain to its full height at once ; it rushed up about half way in an instant, and afterwards gradually at each Pulse twelve, eight, six, four, two, and sometimes one inch: When it was at its full height, it would rise and fall at and after each Pulse two, three, or four inches; and sometimes it would fall twelve or fourteen inches, and have there for a time the same Vibrations up and down at and after each Pulse, as it had, when it was at its full height ; to which it would rise again, after forty or fifty Pulses."

The method adopted by Hales of measuring the lateral pressure of blood in the vessels offers very considerable drawbacks. The manipula-

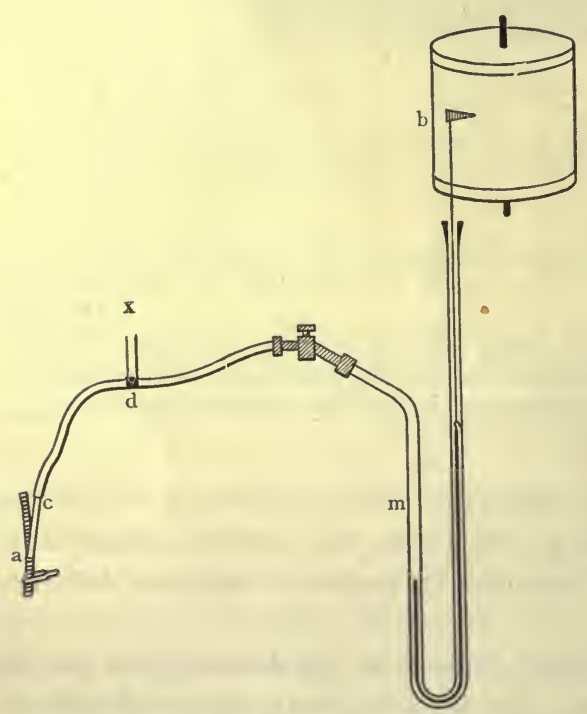

FiG. 374. Arrangement of an apparatus for taking blood-pressure tracing.

$a$, artery (carotid); $c$, cannula; $d$, threeway cock; $m$, mercurial manometer; $b$, drum covered with smoked paper; $x$, tube to pressure bottle.

\section{moving sheet of paper (Fig. 374).} a continuous movement to a sheet of glazed paper is known as a kymograph.) Instead of smoking the paper a pen may be fitted to the end of a rod and its excursions recorded in ink on a moving band of ward, and the blood which escapes into the tubes very soon clots and renders further observation impossible. It is therefore customary when we desire to gain an idea of the average pressure in any bloodvessel, especially in an artery, to use a mercurial manometer for this purpose. This instrument, which was first applied to physiological purposes by Ludwig, consists of a $U$-tube with two vertical limbs about eighteen inches in height, which is half-filled with clean mercury. On the surface of the mercury of one limb is a float of vulcanite from which a stiff fine rod of straw, glass, or steel rises, bearing on its upper end the writing-point. This point may be adjusted so as to write on the blackened glazed surface of a (The arrangement for imparting 
white paper. The other limb of the manometer is connected by a flexible inextensible tube with a small tube or cannula which is tied into the central end of an artery, a clip being previously placed on the artery so as to prevent the escape of blood during the insertion of the cannula. To the manometer is connected a three-way tap by means of which the manometer can be placed in communication with the artery alone, or with the artery and a pressure bottle. By means of the latter the whole system is filled with magnesium sulphate solution (25 per cent.) or a half-saturated solution of sodium sulphate, at a pressure of $150 \mathrm{~mm}$. Hg. The pressure bottle is then cut off so that the manometer remains in connection only with the cannula, the mercury in one limb being 150 millimetres above that in the other. The clip is then taken off the artery. The pressure in the cannula being greater than that in the artery, a small amount of the fluid used to fill the tubes runs into the circulation. The mercury in the

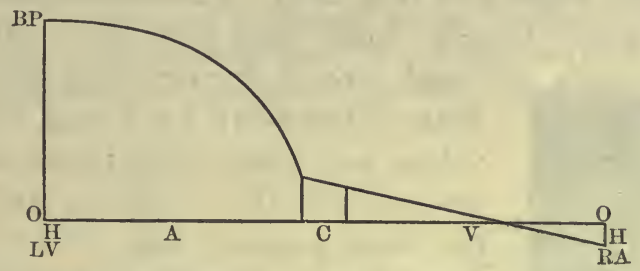

FIG. 375. Scheme of blood-pressure in-A, the arteries; $\mathrm{c}$, capillaries; and v, veins. 00 , line of no pressure; LV, left ventricle; $\mathrm{RA}$, right auricle; $\mathrm{BP}$, height of blood-pressure.

manometer drops to a height of 100 to $120 \mathrm{~mm}$. $\mathrm{Hg}$ and stays about that level, rising and falling slightly with each heart-beat (Fig. 376). The blood which enters the cannula at each heart-beat does not clot for a considerable time owing to its admixture with the saline fluid used for filling the cannula and connecting tubes.

If a vein be ligatured, it swells up on the distal side of the ligature. If the vein be cut across, blood escapes chiefly from the peripheral end, and instead of spurting out to a considerable distance with each heartbeat it flows steadily, but with very little force, so that light pressure by a bandage is sufficient to restrain the hæmorrhage. If a mercurial manometer be connected. with the vein the pressure in its interior is found to amount to only a few $\mathrm{mm}$. Hg.

By taking the pressure at different parts of the circulation we obtain a distribution which is represented roughly in the accompanying diagram (Fig. 375). The blood pressure, which is about 100 to $120 \mathrm{~mm} . \mathrm{Hg}$ in the large arteries near the heart, falls only slowly in these arteries, so that in the radial artery it is not very much below that in the aorta. Between the medium-sized arteries and capillaries there is a very extensive fall of pressure as the blood passes through the arterioles, so that in the capillaries the pressure on an average may be taken as 20 to $40 \mathrm{~mm} . \mathrm{Hg}$; from the capillaries to veins the blood-pressure falls steadily until in the big veirs near the heart it may be negative. 


\section{SECTION II}

\section{THE BLOOD-PRESSURE AT DIFFERENT PARTS OF THE VASCULAR CIRCUIT}

THE ARTERIAL BLOOD-PRESSURE. The arterial blood-pressure as recorded by a mercurial manometer exhibits a series of pulsations corresponding to each heart-beat (Fig. 376). These pulsations are due to the fact that the artery becomes fuller each time the ventricle forces more

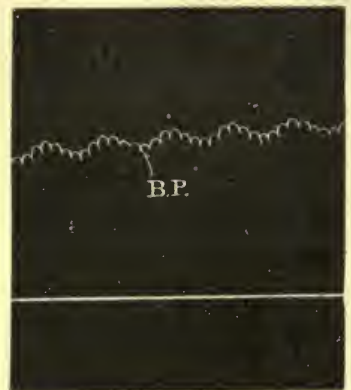

FIG. 376. Blood-pressure tracing taken with nercurial manometer (from carotid of rabbit).

A, abscissa or line of no pressure. blood into it during its systole. Between the beats of the heart, i.e. during diastole, the aortic valves are closed, and blood escapes from the arteries into the capillaries and veins, so that the blood-pressure falls. The mercurial manometer does not register these rapid changes of pressure in the artery with any accuracy. The A inertia of the mercury is such that it takes some time to be set into movement by the rise of pressure in the artery, and before it has attained its full height the pressure in the artery has already begun to fall. With a very wide-tubed manometer the oscillations may be almost imperceptible owing to the mass of mercury that has to be moved at each heart-beat. Such a manometer gives a true record of what is known as the 'mean arterial pressure.' In order to determine the true course of the pressure in the heart it is necessary to diminish to the utmost possible extent the inertia of the moving parts of the recording instrument, and to employ some manometer such as that of Hürthle or of Frank, in which the pressure is measured by recording the stretching of an elastic membrane. Such instruments will be described later in dealing with the changes of pressure in the ventricle during contraction.

In the living animal the variation in the arterial pressure at each heartbeat is much greater than would be anticipated from an inspection of the tracing given by the mercurial manometer. The highest pressure which occurs while blood is passing from the heart into the aorta is called the systolic arterial pressure; the pressure at the end of diastole, just before the heart begins to force a fresh quantity of blood into the aorta, is the diastolic pressure; and the range between these two extremes is known 
as the pulse pressure. Thus in the dog, with a mean pressure of about $120 \mathrm{~mm} . \mathrm{Hg}$ in the aorta, the systolic pressure may be as much as 160 , while the diastolic pressure is only $100 \mathrm{~mm}$. In this case the pulse pressure would be $60 \mathrm{~mm}$. $\mathrm{Hg}$. In man the systolic pressure, as measured in the brachial artery, is under normal conditions about $110 \mathrm{~mm}$., while the diastolic pressure is only 65 to $75 \mathrm{~mm}$., so that the pulse pressure is about $45 \mathrm{~mm}$. Hg. As we pass outwards towards the periphery the pulse pressure becomes less and less marked, until finally in the capillaries and veins there is no pulse-wave perceptible.

\section{THE DETERMINATION OF THE BLOOD-PRESSURE IN MAN}

It is important for clinical purposes to be able to determine even approximately the blood-pressure in the different parts of the vascular system in man, and various methods have bəen devised for this purpose. The determination of the systolic blood-pressure in the arteries is easily carried out by the use of Riva Rocci's sphygmomanometer. This apparatus (Fig. 377) consists of a leather or canvas band about $10 \mathrm{~cm}$. wide, which can be buckled closely round the upper arm. Inside this band is a rubber bag of the same shape, which communicates by a rubber tube with a mercurial manometer and by

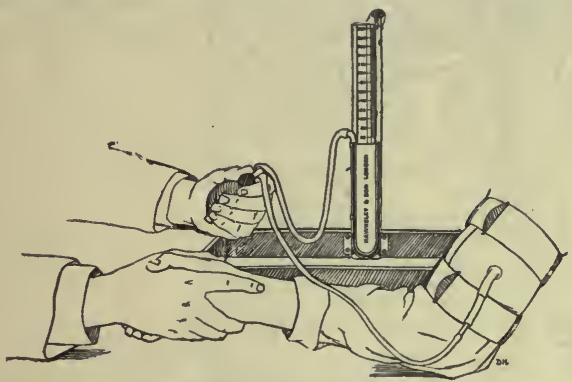

Fig. 377. Riva Rocci's sphygmomanometer.

(C. J. Martin's pattern. HAWKSLEY.)

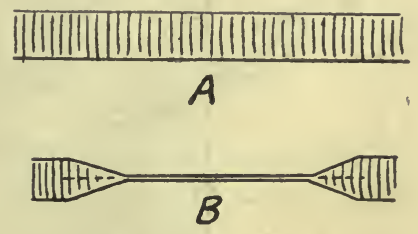

Fig. 378.

a three-way tap with a pressure bulb or bicycle pump, or with the external air. The band is buckled round the arm and the fingers of the observer are placed on the radial pulse. The bag is then distended with air so that it exercises a pressure on the arm, the pressure being indicated on the mercurial manometer. Air is forced in until the radial pulse disappears. By means of the three-way tap the air is then let slowly out of the bag until the radial pulse is just perceptible. The height of the mercurial manometer at this moment is equal to the systolic pressure in the main arterial trunk from which the brachial artery takes origin. The principle of this method will be made clear by reference to the diagram (Fig. 378). If we imagine $A$ as a segment of the brachial artery passing through the tissues which are surrounded by the rubber bag, we see that so long as the pressure in the interior of the artery is greater than that on the exterior exerted by the tissues, the artery will be patent and the pulse can pass through. If, however, the pressure in the tissues becomes greater than the maximum pressure inside the artery at any time of the heart-beat, the segment of artery will collapse (as in $\mathbf{B})$, thus stopping the transmission of blood and of the pulse-wave. If we exclude the elasticity of the tissues themselves we may take the pressure in the bag as representing the pressure in the tissue fluids surrounding the artery, so that. the pulse-obliterating pressure in the bag will correspond to the maximum or systolic pressure in the artery. By a slight modification of the apparatus it is possible to determine also the diastolic pressure. For this purpose the rubber bag is connected also with a manometer of small inertia, giving a true representation of the actual changes of pressure. It is evident 
that when the pressure in the bag and in the tissues surrounding the artery exactly corresponds to the diastolic pressure, the artery will be completely collapsed when the pressure arrives at its lowest point and will then dilate almost to the utmost with the systolic rise of pressure. If we are taking a record of the pressure changes in the bag in this way, the pulse-waves as recorded by the manometer will slowly increase in size as the pressure in the bag is gradually raised. At one point the waves rapidly increase and reach a maximum, marking the pressure at which the artery is just completcly

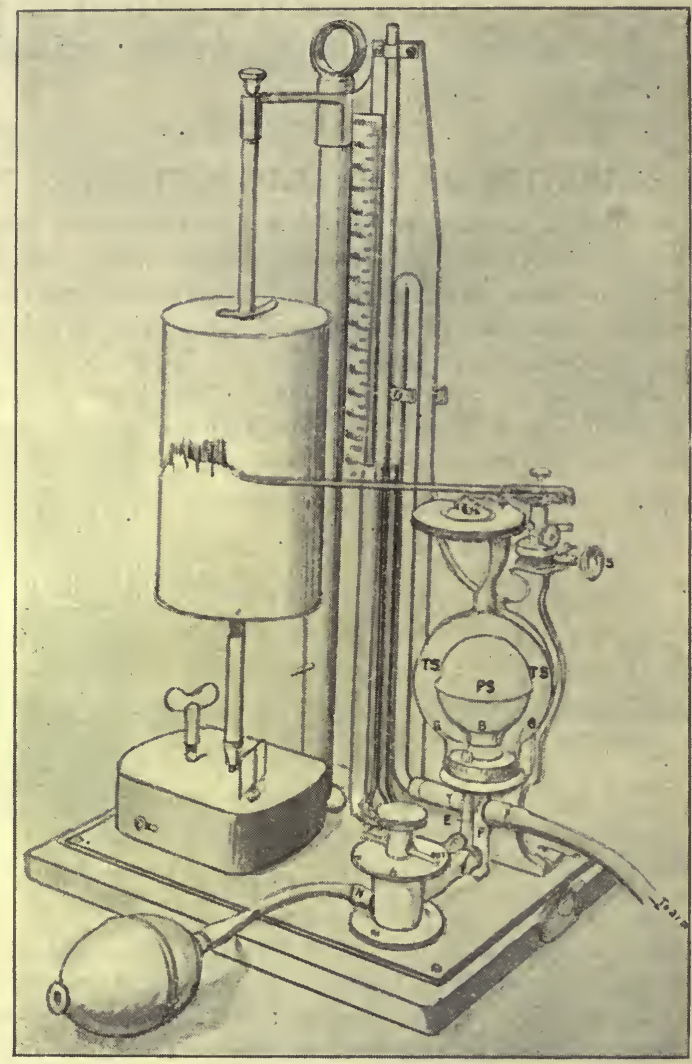

Fig. 379. Erlanger's apparatus for recording systolic and diastolic blood-pressures.

collapsed at the lowest point of each pulse-wave (the diastolic pressure). As the pressure is still further raised the excursions of the manometer tend to diminish in size, first slowly and then rapidly, and the point of rapid diminution corresponds to the systolic pressure. Above this point the manometer still shows small oscillations, due to the impact of the unoccluded stump of the artery on the upper border of the india-rubber bag.

Many different methods have been introduced for the purpose of recording the pressure oscillations in the bag. 'In Erlanger's apparatus the rubber bag is put into connection with a thick walled rubber ball PS contained in a glass chamber. The chamber (Fig. 379) communicates with a sensitive tambour and also by means of a capillary opening provided with a stop-cock with the external air. By this means the slow expansion of the ball PS is not recorded by the tambour, which only moves with the sudden oscillations of pressure due to each heart-beat. With this instrument it is easy to read on the accompanying mercurial manometer the point at which the oscilla- 
tions of pressure in the bag suddenly became maximal, and so to determine approximately the diastolic pressure in the artery.

VENOUS PRESSURE. To determine the venous pressure in man we may use some modification of von Recklinghausen's method. A circular, disc-shaped, incomplete rubber bag (Fig. 330) is made by cementing together at the circumference two rubber dises, each of which has a hole in the centre. This is placed over a peripheral vein and a glass plate laid on the top (Fig. 331). A tube leads from the interior of the annular rubber bag to a water manometer and to a bicycle pump or bellows for the injection of air. On blowing air into the bag the pressure in its interior rapidly increases. If the skin and glass plate have been previously smearcd with glycerine, the air does not escape but distends the bag, pressing it against the skin on the one hand and the glass plate on the other. Through the hole in the rubber bag it is easy to see the pressure at which the vein collapses-that is to say, the point at which the pressure in the bag is equal

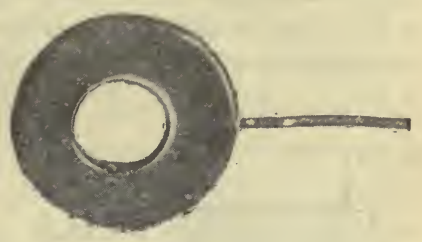
to the pressure within the vein. By a similar method, using a smaller bag, we may determine the pressure which is just sufficient to obliterate the capillaries in any given area of the skin, so causing a blanching of the skin lying under the bag.

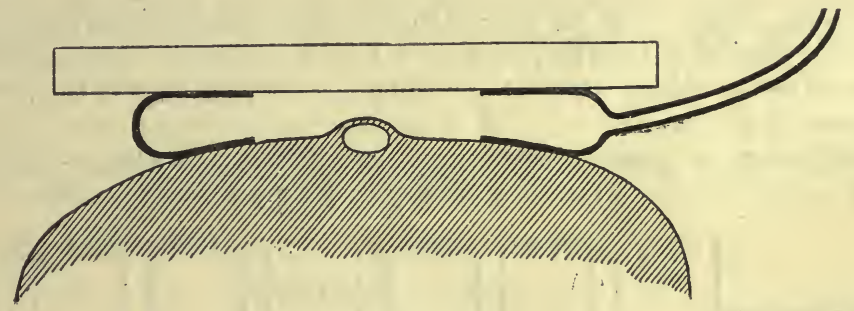

Fig. 381.

The following Table may serve to give an idea of the average height of the mean blood-pressure (not systolic) at different parts of the vascular system in man, in the horizontal position. The pressures are all subject to considerable variations according to the activity of the individual and the physiological activity of the various parts and organs of the body :

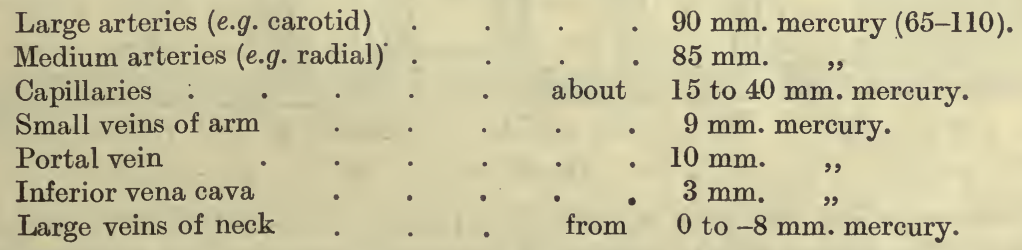

The cause of these peculiarities in the circulation in different parts of the vascular system will be rendered clearer by a study of a flow of fluid through a tube of uniform bore (Fig. 382). If the tube AG be connected with the reservoir $\mathrm{R}$, fluid will flow from $A$ to $G$ under the influence of the pressure difference between the fluid in the reservoir and that at $G$. The pressure on the fluid at each part of the tube can be measured by attaching at a series of points-e.g. at B, C, D, E, F-vertical tubes in which the fluid will rise to a height corresponding to the lateral pressure existing at these several points. When fluid is flowing from $\mathrm{A}$ to $\mathrm{G}$ it will be found that the heights of the fluid 
in the tubes show a continuous descent, so that the line joining the tops of the fluid in the various tubes is a straight one. The movement of the fluid from $\mathrm{B}$ to $\mathrm{C}$ can be regarded as due to the difference of the pressure between $\mathrm{B}$ and $\mathrm{C}$, i.e. $\mathrm{P}_{\mathbf{2}}-\mathrm{P}_{\mathbf{3}}$. It will be noticed in the diagram that the straight line joining the tops of the fluid does not strike the surface of the fluid in $\mathrm{R}$, but falls a little below it. Of the total pressure in $\mathrm{R}$, $\mathrm{H}$, the large portion $h^{\prime}$ is employed in overcoming the resistance of the tube AG, while a small portion $h$ represents the force necessary to give to the fluid as it leaves the rescrvoir

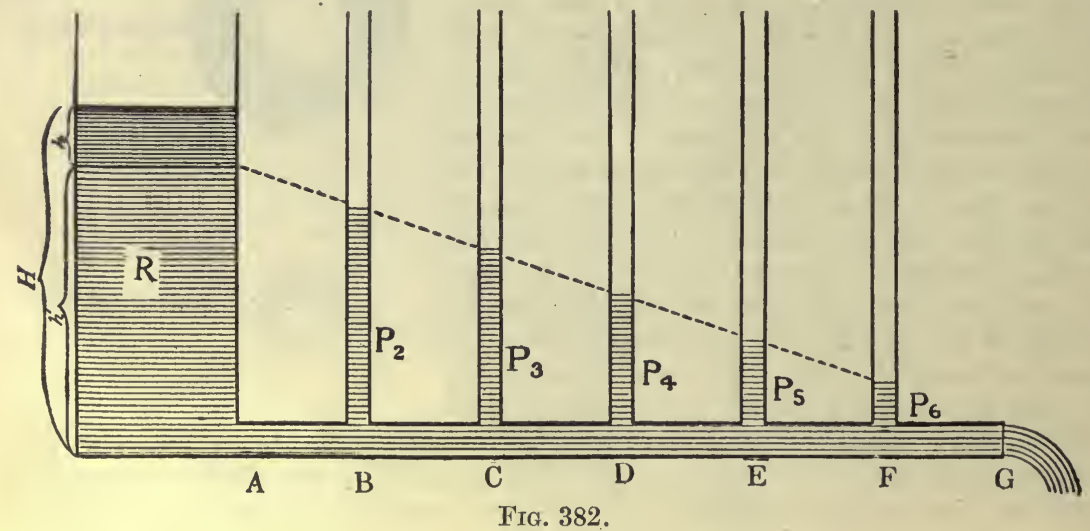

at $\mathrm{A}$ a certain velocity. If the flow of fluid be diminished by partially clamping the end at $\mathrm{G}$ the rate of fall of the pressures will be diminished. The same effect will be produced either by raising the level of $\mathrm{G}$ or by lowering the level of the reservoir and so the pressure at $\mathbf{A}$.

The difference of pressure between any two points, i.e. between $\mathrm{D}$ and $\mathrm{E}$, may be regarded as that pressure which is necessary to maintain a certain velocity of the fluid

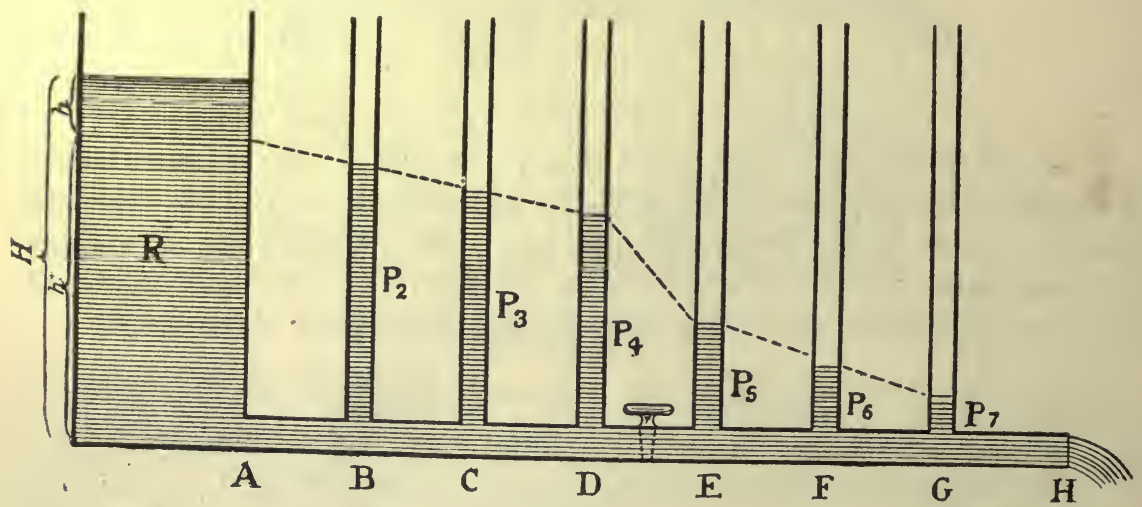

FIG. 383.

against the resistance offered by the friction of the fluid in contact with the walls of the tube. This friction, and therefore the resistance to the flow, can be altered by diminishing the diameter of the tube, when a larger difference of pressure will be necessary in order to maintain the same velocity of flow. This can be shown by introducing a resistance between $\mathrm{D}$ and $\mathrm{E}$ by partially clamping the tube at this point (Fig. 383). The continuity of the fall of pressures in the vertical tube is at once abolished. Between $\mathrm{A}$ and $\mathrm{D}$ there is a continuous fall, which is succeeded by a steep fall between $\mathrm{D}$ and $\mathrm{E}$, and this again by a gradual fall between $\mathrm{E}$ and $\mathrm{G}$. In any system of tubes therefore through which fluid is flowing the fall of pressure between any two points 
will be proportional to the velocity of the flow between these two points. The velocity, on the other hand, will vary directly as the difference of pressures, and inversely as the resistance between the two points, which may be expressed by the formula

$$
\mathrm{V} \propto \frac{\mathrm{P}}{\mathrm{R}}
$$

In the vascular system, while the circulation is maintained, the largest difference of pressure exists between the arteries on the one side and the small veins on the other, a great fall occurring between the arteries and the capillaries themselves. This distribution of pressure points to the chief resistance in the vascular system as being situated in the arterioles. The resistance presented by these vessels is due to the fact that they are maintained in a state of tonic contraction by the agency of the central nervous system. The total bed of the stream in the region of the arterioles, while greater than that of the arteries, is considerably less than that of the rich meshwork of capillaries, while the difference between the diameters of arterioles and capillaries is not very great. On this account the velocity of the blood in the arterioles is very much greater than that obtaining in the capillaries, and since friction and therefore the resistance varies as the square of the velocity, the resistance to the flow of blood through the arterioles must be much greater than that presented by the capillaries. The large part taken by the arterioles in determining the difference of pressure between the arteries and veins is shown by the fact that this difference can be diminished to one half by any means which causes a dilatation of the arterioles, as, for example, destruction of the vasomotor centre.

\section{THE CONVERSION OF AN INTERMITTENT INTO A CONSTANT FLOW}

Not only is the blood-pressure in the veins much lower than in the arteries, but the flow of blood has been converted on its passage through the peripheral resistance from a pulsatory into a continuous flow. This change is connected with the distensible elastic nature of the arterial walls.

Since this is a purely mechanical question it will be more easily understood by a simple illustration. The heart may be regarded as a pump, forcing a certain amount of blood (in man about 60 c.c.) into the circulation at each stroke. If a pump be connected with a rigid tube, every time that a certain amount is forced into the beginning of the tube an exactly equal quantity will be forced out at the other end. Increasing the peripheral resistance by partial closure of the end of the tube will not affect the intermittent character of the flow, but will merely serve to diminish the quantity thrown in, as well as the quantity which escapes at the other end of the tube, supposing that the work done by the pump is equal in both cases. If instead of a rigid tube we employ an elastic tube and the end be left open so that no resistance is offered to the outflow of the fluid, the effect will be the same as when we used the rigid tube; the outflow will correspond exactly to the inflow and will be just as intermittent. But now, if the end of the elastic tube be clamped so as to increase the 
resistance to the outflow, there will be a marked difference from the results obtained when the rigid tube was partially obstructed. Each stroke of the pump forces a certain amount of fluid into the tube. Owing to the peripheral resistance this cannot all escape at once, and so part of the force of the pump is spent in distending the walls of the tube, and part of the fluid that was forced in remains in the tube. The distended elastic tube tends to empty itself and forces out the fluid which over-distends it before the next stroke of the pump occurs. So now the outflow may be divided into two parts, one part which is forced out by the immediate effect of the stroke of the pump, and another part which is forced out by the elastic reaction of the tube between the strokes. If the strokes be rapidly repeated before the tube has time to empty itself thoroughly, it will get more and more distended. Greater distension means stronger elastic reaction, and therefore stronger outflow of the fluid between the beats. This distension goes on increasing till the fluid forced out between the strokes by the elastic reaction of the wall of the tube is exactly equal to that entering at each stroke, and the flow thus becomes continuous.

The same thing occurs in the living body. A man's heart at each beat or contraction forces about 60 c.c. of blood into the already distended aorta. The first effect of this is to distend the aorta still further. The elastic reaction of the walls drives on another portion of blood, which distends the next segment of the arterial wall, and so the wave of distension is transmitted with gradually decreasing force along the arteries. This wave of distension is what we feel on the radial artery, or any exposed artery, as the pulse. After each heart-beat the arteries tend to return to their original size, and drive the blood on through the arterioles (the peripheral resistance) into the capillaries and so into the veins. By the time the blood has reached the veins all trace of the heart-beat has disappeared and the pressure has fallen to a few millimetres of mercury.

\section{INFLUENCE OF THE CAPACITY OF THE VASCULAR SYSTEM ON THE CIRCULATION}

So far we have only considered the influence of changes of pressure and resistance in a system of tubes with a head of pressure at one end and a free outflow at the other. In the body, however, the vascular system is a closed circuit of elastic tubes presenting varying resistances to the flow of blood, and of varying distensibility at different parts of their course. In this closed system is inserted a pump, the heart, with the function of driving the blood through the system. Since all the blood-vessels are elastic and distensible the capacity of the system is not fixed, but must vary with the internal pressure to which the vessels are subjected. Moreover the position of the different parts of the circulation must have an influence on the capacity of the system, since the dependent vessels will be distended not only by the average pressure of the fluid throughout the system but also by the hydrostatic pressure due to the weight of the column of fluid pressing on them. The elasticity of the tubes is also a varying 
factor and can be considerably altered by the contraction of the muscular coats of the vessels, or by pressure on the vessels exerted by the surrounding muscular and elastic structures.

It will simplify the discussion of the main factors of the circulation in a closed system if, for the present, we neglect the variable factors and

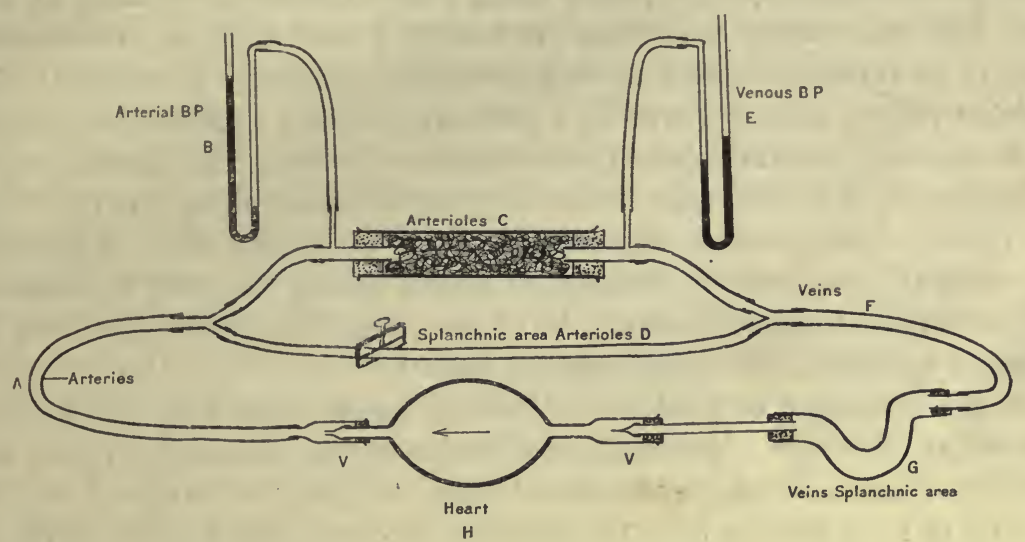

FIG. 384. Artificial schema to demonstrate the main features of the circulation.

The heart is an enema syringe with valves at $\mathrm{v}$ and $\mathrm{v}$. The artery is a thick-walled rubber tube. On the venous side is placed a length of wide thin-walled tubing, to represent the large thin-walled distensible veins. The arterioles and capillaries (peripheral resistance) are represented by wide glass tubes packed with sponges. By opening the clamp on the tube D ('splanchnic area arterioles') the peripheral resistance can be removed, and a free passage of fluid allowed from arterial to venous side.

see what would take place in such a system of elastic tubes all situated on one horizontal plane. Such a system is represented in the diagram (Fig. 385), and a working model of it in Fig. 384.

The heart $\mathrm{H}$ is interpolated at one part of the circuit, while the free outflow of the fluid from B to $\mathrm{D}$ is impeded by the presence of a peripheral resistance at c. Such a system would have a definite capacity at zero internal pressure, but a very much greater amount of fluid might be forced into it under a positive pressure. We will assume that the pressure throughout the system is equal to $10 \mathrm{~mm} . \mathrm{Hg}$, i.e. the elastic tubes are all slightly distended. If the heart $\mathrm{H}$ now begins to contract it will pump fluid from $\mathrm{E}$ into $\mathrm{A}$.

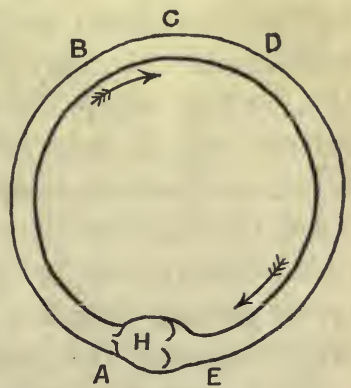

FIG. 385 . The pressure in $\mathrm{E}$ will fall from $10 \mathrm{~mm}$. to $0 \mathrm{~mm}$., while that in A will rise to a corresponding extent, the resistance at $\mathrm{c}$ preventing the free escape of fluid from $\mathrm{B}$ to $\mathrm{D}$ and so causing the heart to pile up the fluid which it has taken from $\mathrm{E}$ into $\mathrm{A}$.

If the texture of the tubes were uniform throughout the system it is evident that the rise of pressure in A would approximate very nearly to the fall of pressure in $\mathrm{E}$. In the vascular system the veins are, however, 
much more easily distended than the arteries. In Fig. 373 (p. 871) is shown the distensibility of corresponding sections of arteries and veins under gradually increasing internal pressures. An artery has a certain capacity even at zero pressure. As the pressure in its interior is increased the artery is distended, and its capacity rises first slowly and then more rapidly, the increment in capacity being greatest between 90 and $110 \mathrm{~mm}$. $\mathrm{Hg}$. The vein, on the other hand, is collapsed when there is no distending force in its interior, so that at zero pressure its capacity is nothing. The slightest rise of pressure, even of $1 \mathrm{~mm}$. $\mathrm{Hg}$ causes a considerable increase in its capacity, and the capacity rises rapidly with increasing pressure up to about $20 \mathrm{~mm}$. $\mathrm{Hg}$. Whereas the artery is most distensible at $100 \mathrm{~mm}$. $\mathrm{Hg}$., the vein is at its optimum distensibility at about $10 \mathrm{~mm}$. $\mathrm{Hg}$. If therefore the tubes at $\mathrm{E}$ are made of thin-walled rubber tubing they will be considerably distended under a pressure of $10 \mathrm{~mm}$. $\mathrm{Hg}$, which has practically no influence on the thicker-walled arterial tube A.

A small amount of fluid taken from $\mathrm{E}$ would cause very little fall of pressure on this side. A considerable force will be necessary to send this fluid into the more resistant arterial tube, so that on pumping a given amount of fluid from $\mathrm{E}$ to $\mathrm{A}$ the pressure in $\mathrm{E}$ may fall $5 \mathrm{~mm}$., while the pressure in $\mathrm{A}$ has to be raised from 50 to $100 \mathrm{~mm}$. $\mathrm{Hg}$ in order to distend the arteries to such an extent that they will accommodate the fluid taken from $\mathbf{E}$.

In such a system, when the heart is at rest, the pressure all over the system will be uniform, and in the example we have chosen the mean systemic pressure was $10 \mathrm{~mm}$. $\mathrm{Hg}$. When the heart contracts it takes up fluid from the venous side and piles it up on the arterial side until the pressure on the arterial side is sufficient to cause exactly the same amount of fluid to flow through the peripheral resistance into the veins as is taken by the heart from the veins at each beat. This rise of pressure in the arteries may be many times greater than the fall of pressure in the veins. If more fluid is injected into the system when the heart is at rest the whole system will be more distended and the mean systemic pressure will rise. When the heart contracts it will raise the pressure on the arterial side and lower that on the venous side as before, but it is evident that according to the force of the heart-beat the arterial pressure may be less than, equal to, or greater than the pressure attained before the introduction of fluid. Since, however, the mean systemic pressure is raised, the increased amount of fluid must be accommodated somewhere, so that if the arterial pressure is as great as before, the venous pressure must be greater. In the same way the withdrawal of a certain amount of fluid may lower the mean systemic pressure, say from 10 to $5 \mathrm{~mm}$. $\mathrm{Hg}$. It is still possible for the pump to maintain an arterial pressure equal to that produced when the mean systemic pressure was $10 \mathrm{~mm}$. $\mathrm{Hg}$, but to produce this effect the relative distribution of blood must be altered and the veins must be more empty than they were previously. The maintenance of a constant arterial pressure with varying amount of fluid in the system can therefore be 
accomplished either by alterations in the work of the heart or by alterations in the peripheral resistance, and therefore in the ease with which the blood is allowed to escape from the arterial to the venous side.

Alterations of the capacity of the system will have the inverse effect to alterations of its contents. Thus diminution in the volume of veins, such as might be caused in the living body by the contraction of their walls and which may be imitated in our model by pressure on the veins from without, will drive the fluid into other parts of the system and therefore raise the mean systemic pressure. This rise of pressure may be confined to the arteries by increased action of the heart, or it may be confined to the veins by diminished action of the heart or decreased constriction of the arterioles forming the peripheral resistance.

Similar change in capacity may be brought about if we bring in the effects of hydrostatic pressure. If in the model illustrated (Fig. 384) we allow the thin-walled vein to hang over the edge of the table the pressure of the column of fluid within it causes it to dilate and therefore to accommodate more fluid, and this increased capacity might be so great that the pressure in the section of the vein near the heart might sink to -nothing and the heart receive no blood when it started to contract. The whole arterial system might in this way be allowed to drain under the influence of gravity into the distensible dependent segment of the venous tube.

All the conditions in our artificial schema have their exact analogue in the living body. The determination of the mean systemic pressure in the living body is difficult to carry out with accuracy. If, for instance, we stop the heart, which we can do by stimulation of the vagus nerve, the arteries will gradually empty themselves through the peripheral resistance into the veins, and this process will tend to go on until the pressures are identical throughout the system. Before this equilibrium is arrived at, however, reaction takes place on the part of the animal, tending to restore the failing circulation. Thus the vessels contract strongly, so diminishing the capacity. Movements take place causing pressure on the veins of the abdomen and the suction of the blood into the big veins of the thorax. Moreover the vessels in an animal are not all on one plane, and if the animal is in a vertical position the hydrostatic pressure of the column of blood between the heart and the dependent parts of the body may distend the veins to such an extent that the whole of the blood is taken up in these veins and none returned to the heart. The fact that after stoppage of the heart the pressure is positive at all parts of the vascular system in the animal with open thorax shows that there is actually a mean systemic pressure, i.e. under normal circumstances, when the animal is in a horizontal position, all parts of the system are slightly distended. Direct measurement shows that this mean systemic pressure is about $10 \mathrm{~mm} . \mathrm{Hg}$. The smallness of this figure shows moreover that under the influence of gravity alone the pressure will be easily reduced to nothing at all in the upper parts of the body. In a man in the vertical position, in the absence of the nervous reactive mechanism which we shall consider later on, the 
whole of the blood would accumulate in the abdomen and lower parts of the body, and the circulation would come to a standstill. On the other hand, the pressure may be altered in any part of the vascular system in any of the following ways :

(1) Alteration of capacity of the total system either by contraction of walls of the vessels or by pressure on them from without.

(2) Alteration of the total volume of the circulating fluid.

Either of these two factors would affect in the first place the mean systemic pressure. The distribution of pressure, i.e. the relative pressure in the arteries and veins, will be determined by

(3) Alteration in the output of the heart.

(4) Alteration in the peripheral resistance and therefore in the ease with which the blood can escape from arterial to venous side.

In any change either in arterial or venous pressure at least two of these factors are involved. Every constriction of arterioles causes not only an increase in the peripheral resistance but also a diminished capacity of the whole system, so that the arterial pressure is raised at the same time as the mean systemic pressure. Nearly always such a change will involve as its immediate consequence some corresponding alteration in the heartbeat, so that at least three factors will co-operate in the production of the rise or fall of blood-pressure. We shall have occasion to deal with many examples of these complex conditions when we are discussing the reactions of the vascular system as a whole.

\section{THE DEPENDENCE OF ARTERIAL PRESSURE ON OUTPUT OF HEART}

The importance of the heart-beat in determining arterial pressure is connected with its output in a given time. The arterial pressure is due to the fact that the heart is taking up fluid from the venous side and pumping it into the arterial side. The pressure on the latter side must rise so long as the rate at which the fluid is put into the arterial system by the heart is greater than that by which it escapes through the peripheral resistance. Arterial pressure therefore is a resultant of the two effects :

(a) The amount of blood entering the arterial system from the heart;

(b) The amount of blood leaving the arterial system through the peripheral resistance.

It is evident that the pressure will be altered by altering either of the two factors-peripheral resistance or output of the heart. The cardiac output will depend on the amount of blood contained in the heart at the beginning of each contraction, on the strength with which the heart beats, and on the number of contractions which the heart gives in any given period of time. The filling of the heart at the beginning of each beat is in its turn dependent on the amount of blood which is available to fill the cavities and therefore on the pressure in the great veins. Increased frequency of heart-beat need not therefore necessarily increase the total 
output of the heart into the arterial system. If the heart is beating with optimum rate and force it will keep the venous system, 'at any rate that part nearest the heart, practically empty, and it is not possible for it to obtain more blood to put into the arterial side, however frequently it may beat. There will be an optimum frequency of the heart-beat which will depend on the state of filling of the great veins. The fuller these are the more rapidly the heart may beat and increase the total output. On the other hand, in a normal animal with the heart beating at its optimum rate and with effective contraction of its muscular walls, while slowing the heart-rate will diminish the total output and therefore the arterial pressure, increase in the frequency of the beat cannot raise the arterial pressure to any appreciable extent, though the heart may tend to wear itself out by beating at a greater rate than the optimum. 


\section{SECTION III}

\section{THE VELOCITY OF THE BLOOD AT DIFFERENT PARTS OF THE VASCULAR SYSTEM}

When fluid is flowing through a tube of uniform diameter, the amount passing between any two points is practically in proportion to the difference of pressure between these two points, and varies inversely as the resistance to be overcome. If the tube is of unequal bore, as represented in Fig. 386, since the amount of fluid passing $a$ during a given interval of time must be equal to the amount passing $b$-where the bed of the stream is wider-the velocity of the flow must be smaller at $b$ than at $a$. The same dependency

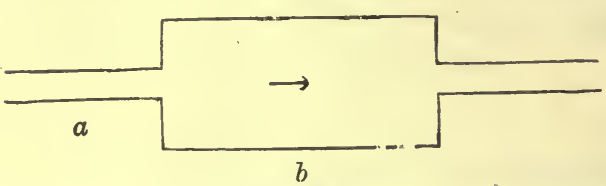

FIG. 386. of velocity on the total bed must apply in any closed system of tubes. Thus in a closed circuit (Fig. 385) with a steady flow from the arterial to the venous side, the amount of fluid leaving

the heart and passing A during a minute must be exactly equal to the total amount of fluid passing from arteries to veins through the peripheral resistance $\mathrm{B}$.

The total area at $\mathrm{C}$ is probably one thousand times that of the aorta at $\mathrm{A}$, and we should expect therefore a proportionate slowing of the blood-stream. As a matter of fact, while the velocity of the blood in the aorta of a large animal may be taken as about half a metre per second, the velocity of the blood in the capillaries is about half a millimetre per second. Moreover, since the total cross-section of the big veins near the heart under a normal distending pressure is about twice that of the first part of the aorta, the velocity of the blood in the great veins is only about half of that found in the aorta. In such a closed circuit increased output of the heart will increase the average velocity round the system, and the same effect may be produced by diminution of the peripheral resistance.

In the living body a great dilatation of the arterioles, causing a fall of the peripheral resistance, generally increases the total capacity of the system. The arterial relaxation therefore not only gives rise to an easier outflow from arteries to veins but also causes a diminished dilatation of the veins and therefore decreased filling of the heart during diastole. The heart output 
is therefore also lessened, so that a final result of a dilatation of the arterioles may be a diminished instead of an increased velocity throughout the system.

The foregoing discussion of the factors which determine the average velocity across a given cross-section of the whole vascular system must not be applied directly to the changes in the velocity following on local alterations in the resistance presented by some particular vascular area. In this case the local changes are insufficient to affect the general arterial blood-pressure, and the effect of diminution of peripheral resistance is to furnish a short cut for a small portion of the total output of the heart from the arterial to the venous side. Thus dilatation of the vessels of the submaxillary gland, while not altering the general blood-pressure as registered in the carotid artery, causes the blood-flow through the gland to be increased six to eight times, and the peripheral resistance in the gland may be so far diminished that the blood passes through the capillaries into the veins without losing the pulsatile force imparted to it by each heart-beat. The pressures therefore in arterioles, capillaries, and veins are all increased by this local vaso-dilatation. On the other hand, constriction of the arterioles of any given part will diminish the velocity of the blood through this part and also the pressure in its capillaries.

The larger the area affected by the change in the peripheral resistance, the more difficult it is to predict a priori what will be the result on the velocity of the blood and on the circulation as a whole, or in the parts specially affected. Thus section of one splanchnic nerve in the dog causes an increased flow of urine from the kidney on the same side, the paralysis of the vessels in this organ causing an increased flow of blood through it and an increased pressure in its capillaries. Section of the corresponding nerve of the rabbit may cause a diminution rather than an increase in the amount of urine secreted, owing to the fact that the total area supplied by the splanchnic nerve is much greater relatively in the rabbit than in the dog. Thus section of this nerve may cause such a widespread dilatation that the blood-pressure falls; and although the vessels in the kidney are relaxed, the arterial pressure is not sufficient to drive through these relaxed vessels as much blood as was previously driven through the normally contracted arterioles.

\section{METHODS OF MEASURING THE VELOCITY OF THE BLOOD}

The velocity in an artery is measured by placing some apparatus in the path of the blood without intercepting its flow; such an apparatus may be used to give the quick variations in the velocity which occur in the course of each heart-beat, or the average flow of blood through the cross-section of the artery in a given space of time. For the latter purpose Ludwig's Stromuhr, or current clock (Fig. 387), has been most used. This instrument consists of two bulbs of equal size, $a$ and $b$, communicating with one another above; their lower ends are clamped in the disc $c$, which is pierced by two openings serving to connect the lower orifices of the bulbs with the tubes $t, t$, cemented into the lower disc $a b$.

An artery such as the carotid, being clamped at its central end and divided, $a$ is inserted into its central end, and $b$ into its peripheral cut end. The tube $a$ is filled with 
oil and $b$ with salt solution or defibrinated blood. On clamping the artery, blood flows into $a$ and drives the contained oil over into $b$, the contents of $b$ being meanwhile forced into the peripheral end of the artery. When blood has com-

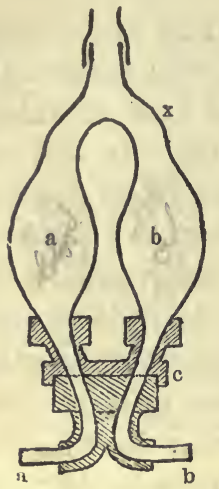

Fic. 387. Diagram of Ludwig's 'Stromuhr.' pletely filled the bulb $a$, the two bulbs are reversed, and the blood now entering the artery displaces the oil in $b$, and forces the blood which had entered $a$ on into the peripheral end of the artcry. Knowing the capacity of the bulbs and the number of times it has been necessary to turn them in the course, say, of one minute, we know also the amount of blood which has passed across the section of the artery under experiment.

In order to determine from this volume the velocity of the blood across the section, i.e. through the artery, the total volume passing in the minute must be divided by the crosssection. This will give the velocity per minute. Many modifications of this apparatus have been devised. A simple form of current measurer is shown in Fig. 388. The whole apparatus is constructed of glass. The tube $a$ is connected with the central end of a cut artery, and the tube $p$ with the peripheral end. The blood flows into $B$ and fills it. As soon as it is full and its level rises

over the level of the bend of the siphon tube $\mathrm{s}$, the blood is rapidly siphoned off into $\mathrm{c}$ whence it flows along $p$ into the peripheral part of the artery. The side tube $\mathbf{R}$ is connected with a mercury or membrane manometer. Every time that B is emptied into $\mathrm{C}$ a depression is produced on the manometer tracing, which thus records not only the average pressure but also the average velocity of the blood in the artery. Each instrument has to be calibrated in order to know how much blood passes from B to $\mathrm{C}$ each time that siphonage occurs.

None of these methods give any information of any rapid changes occurring in the velocity of the blood, e.g. during a single pulse-wave. For this purpose we must have recourse to some instrument such as Chauveau's hæmadromograph or Cybulski's photohæmatachometer. The hoemadromograph (Fig. 389) consists of a pendulum which is hung in a tube, through which the blood is allowed to flow, placed in the course of the artery. The deviation of this pendulum from the vertical will be in proportion to the velocity of the current, and if its upper end be connected, as in the diagram, with a tambour, the variations in velosity can be recorded on a blackencd surface by means of a lever. The photohoematachometer is based on an interesting application of Pitot's tubes. If a current

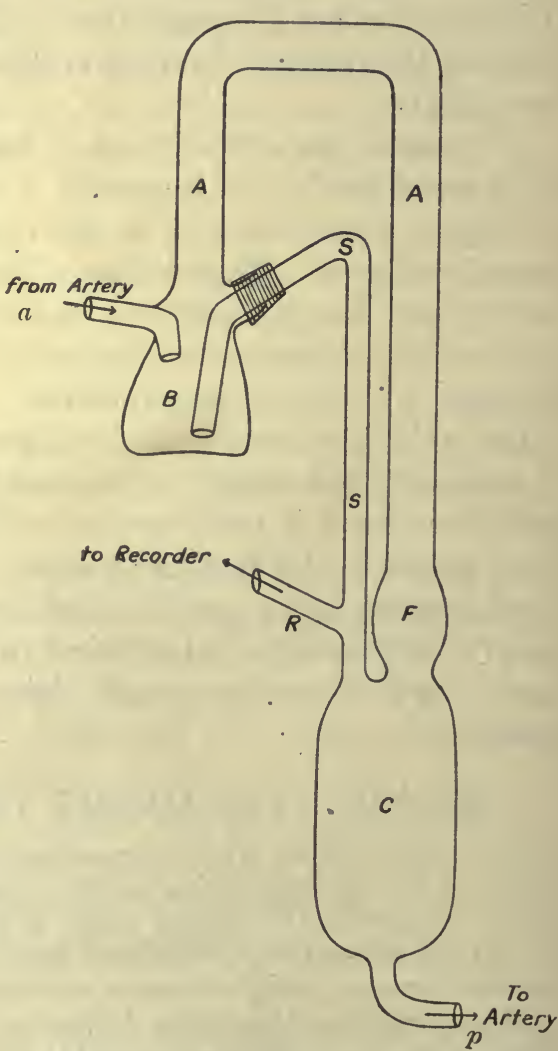

Fra. 385. A simple blood-current measurer. (IsHikawa and Starling.) of blood be directed along the tube $a b$ possessing two vertical side tubes $c$ and $d$ (Fig. 390), the pressure at $c$ will be greater than that at $d$, since at $c$ the 
momentum of the moving mass of blood is added to the lateral pressure of the fluid. A tube of this shape is connected with an artery, such as the carotid, and the tubes

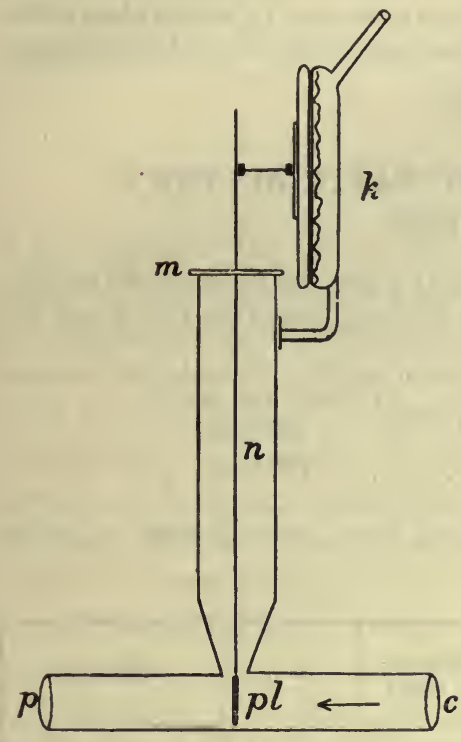

FIG. 389. Diagram showing the construction of Chauveau's hæmadromograph.

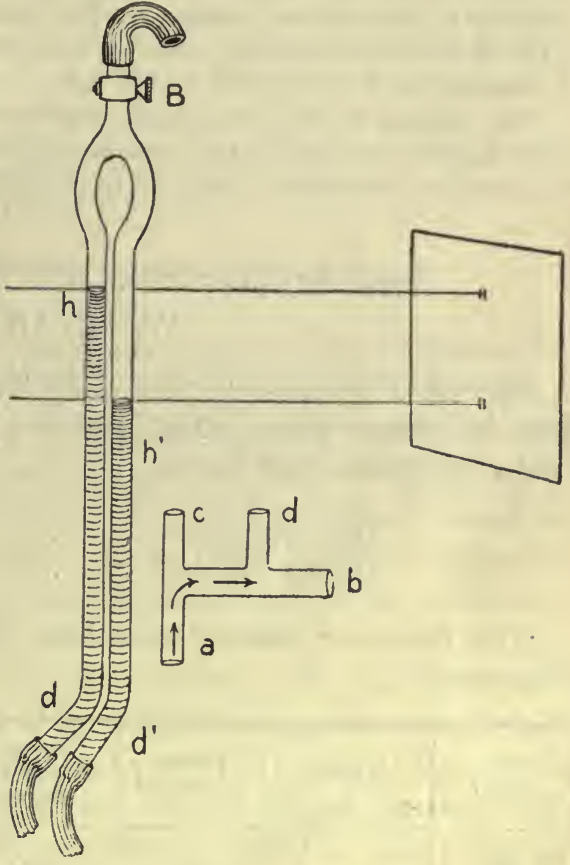

Fig. 390. Diagram to show principle of construction of Cybulski's photo-hæmatachometer.

$h$ and $h^{\prime}$ are attached at the points $c$ and $d$. These two tubes are united at their upper extremities. In this case so long as the blood flows from $a$ to $b$ the fluid in $h$ will rise higher than in $h^{\prime}$, and the difference in height of the fluid in the two tubes will be pro-

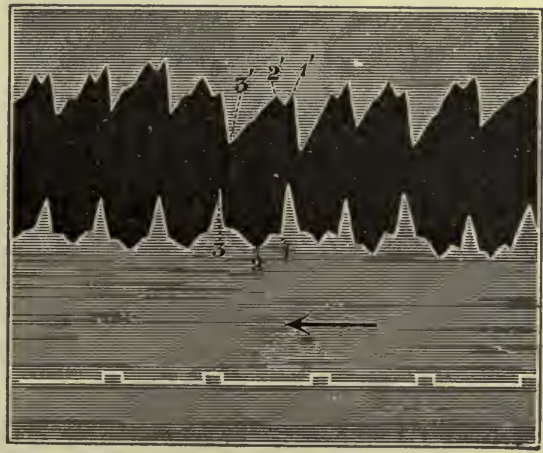

Fig. 391. Record of blood-velocity in the carotid artery of the rabbit. (СYвULSKI.)

portional to the velocity of the blood. A graphic record of this difference of pressure is obtained by allowing a narrow beam of light to throw an image of the menisci of the two columns of fluid through a slit on to a moving photographic plate. Such a record is given in Fig. 391. The width of the black space at any point is proportional to the 
velocity of the blood at the moment at which this part of the record was being taken. Of course this instrument has to be calibrated if we wish to determine the velocity of the blood in absolute measure. In Fig. 391 the velocity at the points 1 and $1^{\prime}$, corresponding to the cardiac systole, was $248 \mathrm{~mm}$. per second. At 2 and $2^{\prime}$, corresponding to the dicrotic elevation, the velocity was also $248 \mathrm{~mm}$. At 3 and $3^{\prime}$, towards the end of diastole, the velocity sank to $127 \mathrm{~mm}$.

The velocity of the blood in the capillaries can be measured by direct observation of the capillaries under the microscope, and noting the time it takes for a blood-corpuscle to move from -one edge of the field to the other.

\section{THE VELOCITY IN DIFFERENT PARTS OF THE VASCULAR SYSTEM}

During systole the velocity of the blood in any part of the arterial system must be greater than during diastole ; thus in the carotid of the horse the following figures were found :

Velocity per second

During systole . . . . . $520 \mathrm{~mm}$.

During diasto!e . . . . . $150 \mathrm{~mm}$.

The following figures have been obtained from experiments on dogs (Tigerstedt) :

\begin{tabular}{|c|c|c|c|c|c|c|}
\hline $\begin{array}{l}\text { Body } \\
\text { weight }\end{array}$ & Artery & $\begin{array}{l}\text { Volume } \\
\text { per second }\end{array}$ & $\begin{array}{l}\text { Linear } \\
\text { velocity per } \\
\text { second }\end{array}$ & $\begin{array}{l}\text { Diameter } \\
\text { of artery }\end{array}$ & B.P. & Remarks \\
\hline kg. & Crural. & $\begin{array}{c}\text { c.c. } \\
0^{*} 63\end{array}$ & $\begin{array}{l}\mathrm{mm} . \\
128\end{array}$ & $\begin{array}{l}\mathrm{mm} . \\
2 \cdot 5\end{array}$ & $\begin{array}{c}\text { mm.Hg. } \\
77\end{array}$ & $\begin{array}{l}\text { Nerves un- } \\
\text { injured }\end{array}$ \\
\hline $14 \cdot 6$ & Crural. & $1 \cdot 69$ & 275 & $2 \cdot 8$ & 88 & Nerves cut \\
\hline $14^{\bullet} 1$ & Carotid. & 1.95 & 241 & $3 \cdot 3$ & 93 & $\begin{array}{l}\text { Nerves un- } \\
\text { injured }\end{array}$ \\
\hline
\end{tabular}




\section{SECTION IV}

\section{THE MECHANISM OF THE HEART PUMP}

In the mammal the two sides of the heart are only in communication by means of the blood-vessels of the systemic and pulmonary area. Each side consists of an auricle into which the veins open, and a ventricle which receives the blood from the auricle and discharges it into the arterial trunkeither aorta or pulmonary artery. Since the auricles have to act merely as a receptacle for part of the blood which enters during the relaxation or diastole of the heart, their cavities are smaller than those of the ventricles, and their walls are thin, corresponding to the small amount of work thrown on them in propelling blood into the relaxed ventricle. The ventricles have the office of carrying on the main work of the circulation and of forcing blood through the peripheral resistance. Their walls are much thicker than those of the auricles. The right ventricle has a wall which is only about one-fourth the thickness of the left ventricle, in conformity with the much heavier work to be done by the latter. On cutting a section through the two ventricles in a contracted condition the thin wall of the right ventricle is seen to lie in the form of a crescent round the circular left ventricle. The capacity of both ventricles is approximately equal, and amounts in man to about 140 c.c. for each ventricle when the heart is completely relaxed.

The auricles are separated from the ventricles by a fibrotendinous ring. From this ring take origin most of the muscular fibres of the heart walls. The muscular fibres of the auricles run in both circular and longitudinal directions, the circular fibres being continued round both auricles, and special rings of circular fibres surrounding the openings of the great veins. From the fibrotendinous ring between the auricle and the left ventricle and from the sides of the aorta the muscular fibres forming the superficial layer of the ventricular wall pass obliquely downwards to the left towards the apex of the ventricle. Here they loop round into the interior of the ventricle and pass up near its inner surface to end either in the papillary muscles or in the auriculo-ventricular ring of fibrous tissue. Between these two layers we find a third median layer of muscular fibres which is in the form of a muscular cone. The fibres of this layer form complete loops round the left ventricle. The middle layer is connected by many strands of muscular fibres with both inner and outer layers.

Mall divides the muscular fibres of the mammalian heart into four groups, two superficial and two deep, as follows :

(1) The superficial bulbo-spiral fibres. These arise from the conus arteriosus, the 
left side of the aorta and the left side of the auriculo-ventricular ring, and take an oblique course to the apex, where they make a spiral turn (the vortex) and reach the interior of the left ventricle, ending for the most part in the intraventricular septum and the papillary muscles.

(2) The superficial sino-spiral fibres rise on the dorsal side of the heart from the right auriculo-ventricular ring and run obliquely on the anterior surface of the right ventricle

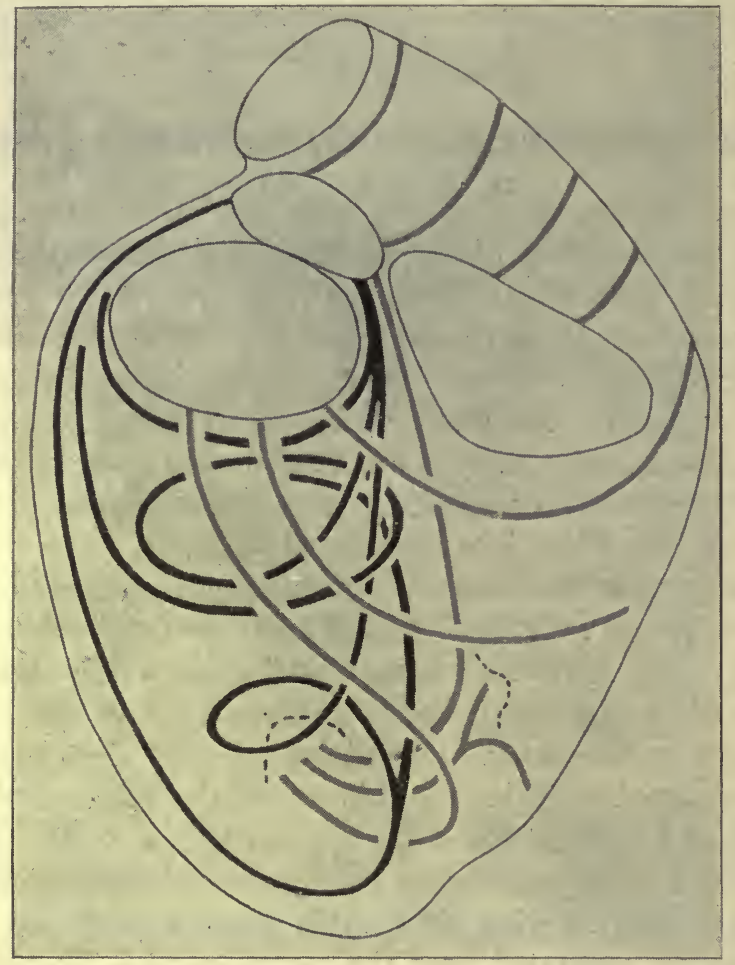

Fig. 392. View of the heart from behind, to show the course of the chief strands of muscle fibres. (MaLL.)

The black lines represent the bulbo-spiral fibres, the grey lines the sinospiral fibres.

to the apex, where they also turn inwards, forming the anterior horn of the 'vortex,' and end chiefly in the papillary muscles of the right ventricle.

(3) The deep bulbo-spiral fibres form a complete cylinder around the left ventricle, and are attached chiefly to the dorsal side of the aorta.

(4) The deep sino-spiral fibres are attached to the dorsal aspect of the left auriculoventricular ring, whence they enter the right ventricle and turn upwards towards the base. The uppermost of these fibres form circular rings round the conus arteriosus at the base of the pulmonary artery.

The fact that the muscular fibres are continuous over both auricles and over both ventricles respectively ensures the practically simultaneous contraction of each of these parts of the heart. Although on coarse dissection there seems to be absolute division between the muscular tissue of auricles and ventricles, it has been shown by Kent, His, and others that there is continuity of muscular tissue between the two parts of the heart by a special 
band of muscular fibres, 'the bundle of His,' which rises in the wall of the right auricle and passes beneath the foramen ovale and across the auriculoventricular junction into the inter-ventricular septum. The exact course of these fibres and their significance will be considered later.

The normal direction of the blood-flow through the heart is determined mainly by the valves which guard the auriculo-ventricular orifices and the openings of the aorta and pulmonary artery. The auriculo-ventricular

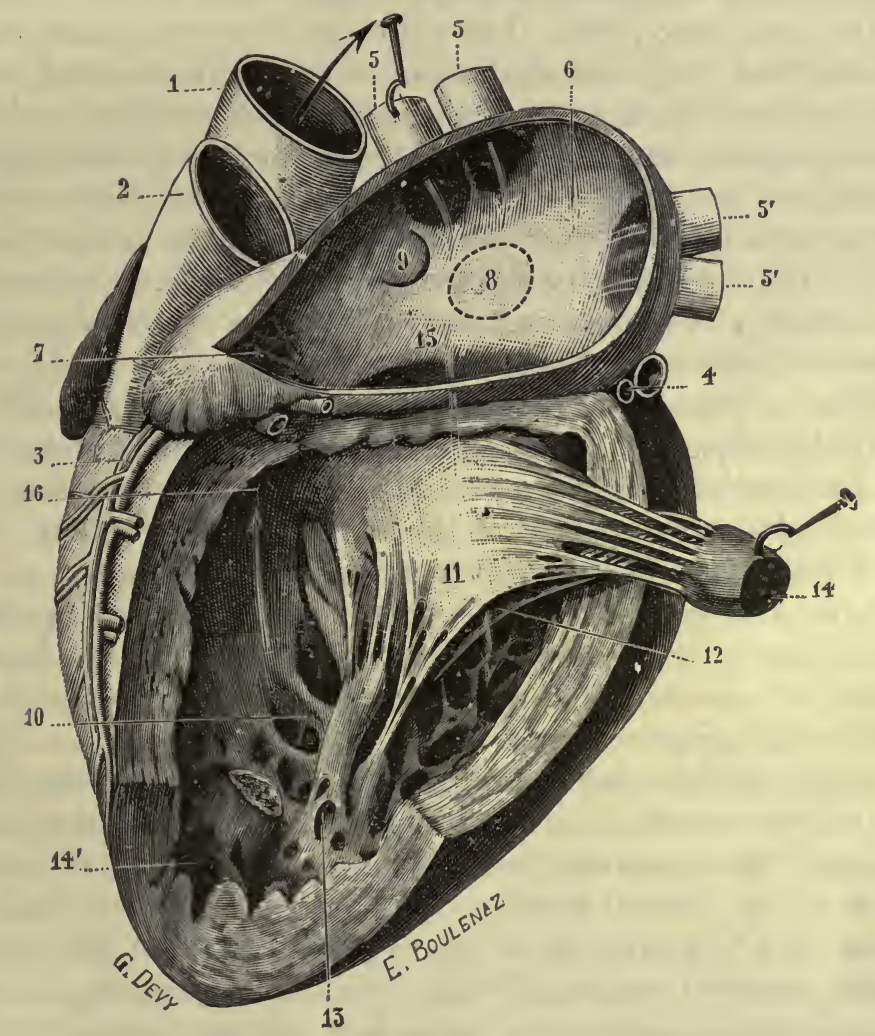

FIG. 393. Left auricle and ventricle, with outer side cut away to show chief points in anatomy of heart. (TESTUT.)

1 , aorta ; 2, pulmonary artery ; 3 , ant. coronary vessels ; $5,5^{\prime}$, pulmonary veins ; 6 , left auricle; 7 , auricular appendage ; 10 , cavity of left ventricle; 11,12 , mitral valves; 13, 14, papillary muscles; 16 , arrow pointing to aortic orifice.

valves are tubular membranes attached round the entire circumference of the auriculo-ventricular ring. They are composed of fibrous and elastic tissue, covered on each side with endocardium, and project downwards into the cavities of the ventricles. On each side the membrane is divided by deep incisions into large flaps, three in number on the right side (the tricuspid valves) and two in number on the left side (the mitral valves). The sail-like margins of these valves are connected by thin tendinous cords to the papillary muscles, which are nipple-shaped projections of the muscular walls of the ventricles. By this means the edges of the valves are kept close together 
and prevented from eversion under the strong pressure exerted by the contracting ventricle. By the downward pull of the papillary muscles on the valves during the contraction of the ventricles, closure is rendered more complete, the inner surface of the valves being apposed over a considerable area. The action of the valves is aided by the contraction of the fibres surrounding the base of the heart, so that the auriculo-ventricular orifice is much smaller during systole than during diastole.

From a purely mechanical standpoint the valves guarding the arterial orifices are much more perfect than those just described, which depend for their efficiency on the proper contraction of the ventricular wall and of the musculi papillares. Each orifice is provided with three valves, each of which is semilunar in shape and attached by its convex borders to the arterial wall, and presents in the middle of its free border a small fibro-cartilaginous nodule, the corpus Arantii, from which fine elastic fibres pass to all parts of the valve. The extreme margin of the valve, the lunula, on each side of the corpus Arantii is very thin, being formed of little more than the endocardium. Whenever the pressure in the arteries is greater than that in the ventricles, these valves are closed, and the thin margins come in contact with similar portions of the adjacent valves, so preventing the reflux of a single drop of blood. The borders of the valves under these circumstances come together in the form of a star composed of three lines at angles of $120^{\circ}$, the three corpora Arantii being pressed together at the centre of the star.

No valves are found at the orifices of the great veins into the auricles, a reflux of blood in this situation during contraction of the heart being limited by the contraction of the muscular rings round the veins, which always accompanies the auricular contraction.

The heart and the roots of the great vessels lie almost free in a special cavity, the wall of which is formed by a tough fibrous membrane, the pericardium. This is attached below to the central tendon of the diaphragm, and above to the arterial trunks. It is lined by a layer of endothelium continuous with a similar layer covering the surface of the heart. The two surfaces are kept continually moist by the pericardial fluid, so that the heart can move freely within the pericardium without friction. One of the chief functions of the pericardium appears to be to check an excessive dilatation of the heart during conditions attended by a great rise of venous pressure.

\section{THE SEQUENCE OF EVENTS IN THE CARDIAC CYCLE}

On opening the chest of an anæsthetised animal, while artificial respiration is maintained, the heart is seen contracting rhythmically within the pericardium. On incising this sac its restraining power on the dilatation of the heart is shown by the fact that during diastole the wall of the heart bulges through the opening, and the increased diastolic filling, consequent on the removal of this restraining influence, is at once apparent, if in any way the frequency of the contractions of the heart be diminished so as to prolong the diastolic period. 
Each beat of the heart begins by a simultaneous contraction of both auricles associated with a retraction of the auricular appendages, which become pale and bloodless. After a pause of not more than a tenth of a second the contraction of the auricles is followed by that of the ventricles, and blood is thrown out into the large arteries. The contraction of the auricles lasts about a tenth of a second, that of the ventricles about three-tenths of a second. The period of relaxation or diastole lasts about four-tenths of a second. During this cycle of changes the following events are taking place within the heart:

In the diastolic period the aortic valves are closed and the arterial system is open only towards the capillaries. In consequence of the high pressure established within the arteries by the previous heart-beats the blood flows steadily through the arterioles, capillaries, and veins into the right heart, and similarly the pressure in the pulmonary artery causes a partial emptying of this vessel with its branches through the pulmonary capillaries into the left heart. The flow into the heart is assisted by the elastic retraction of the lungs, which causes a negative pressure in the structures between them and the chest wall, so that the blood is sucked from the other parts of the body towards the thorax. During diastole there is a continuous flow of blood from veins into auricles and from auricles into ventricles, and as the walls of both these cavities are relaxed there is no impediment to the inflow of the blood until the dilating heart begins to stretch the pericardium.

Under normal circumstances the diastole comes to an end before the restraining influence of the pericardium can be effective. The contraction of the auricles drives their contents into the ventricles and so still further increases their distension, no resistance being offered by the widely dilated auriculo-ventricular orifices or by the flaccid wall of the ventricles. As the blood rushes from auricle into ventricle through the funnel-shaped opening of the membranous tube formed by the valves, eddies are set up in the ventricle tending to close the valves, so that they are held, as the resultant of the two opposing currents, in a condition midway between closure and opening. The onset of the ventricular contraction is extremely rapid. There is a quick rise of pressure in the ventricle, which presses together the flaps of the mitral or tricuspid valves, while the bases of these valves are approximated by the contraction of the circular fibres at the base of the ventricles. As the heart shortens in systole the papillary muscles also shorten, so that the valves are prevented from eversion into the auricles, while the blood is pressed, so to speak, between the cone of the ventricular wall and the cone formed by the tubular valves.

The outflow of blood from the ventricles does not, however, commence immediately. Whereas at the beginning of systole the pressure in the ventricle cavity is quite small (only 2 or $3 \mathrm{~mm}$. $\mathrm{Hg}$ ), there is a pressure in the aorta of 50 to $80 \mathrm{~mm}$. $\mathrm{Hg}$. Before the semilunar valves separating the lumen of the aorta from the ventricular cavity can be opened, the pressure in the left ventricle must rise to a point which is greater than that in the aorta, and 
similarly on the right side of the heart. As soon as this happens the valves open and the outflow of blood commences, and continues so long as the pressure in the ventricles is higher than that in the great arteries. Directly however, the ventricular pressure falls below the arterial pressure the valves must close and the output of blood come to an end.

In order to obtain an accurate idea of the exact duration of each of these events in the cardiac cycle it is necessary to study the changes occurring in the pressure within the auricles and ventricles during the various phases of the heart-beat.

\section{THE ENDOCARDIAC PRESSURE}

A manometer which shall register accurately the changes in the pressure within the heart must be capable of responding to very rapid changes. Thus in the left ventricle at the beginning of the systole there may be a rise of $130 \mathrm{~mm} . \mathrm{Hg}$ in $\cdot 06 \mathrm{sec}$, i.e. $2170 \mathrm{~mm}$. $\mathrm{Hg}$ per second. In a heart beating rapidly and forcibly under the action of adrenalin, the rise may be still more

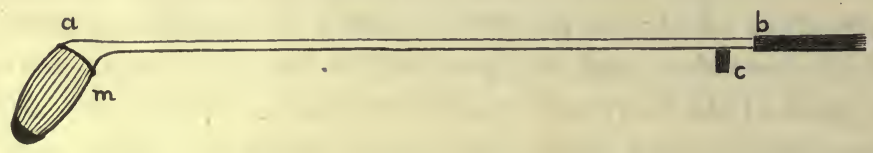

Fig. 394. Diagram of Marey's cardiac 'sound,' consisting of a long tube $a b$, terminating at one end in the ampulla $m$, which is covered with an elastic membrane. The side-piece $c$ serves to indicate the position of the ampulla after it has been introduced into the vessels.

rapid, e.g. $150 \mathrm{~mm}$. $\mathrm{Hg}$ in $.025 \mathrm{sec}$. A mercurial manometer with its great inertia would be quite unequal to registering such rapid changes of pressure and would moreover tend to enter into oscillations which would quite deform the curve. We require an instrument with very small weight of moving parts, so as to possess small inertia and be capable of registering a rapid rise of pressure without entering into oscillations of its own.

Several methods have been adopted for this purpose. In one (Chauveau and Marey) a cardiac 'sound ' (Fig. 394) is passed down the jugular vein into the right auricle or ventricle, or down the carotid artery into the left ventricle. The cardiac sound is a

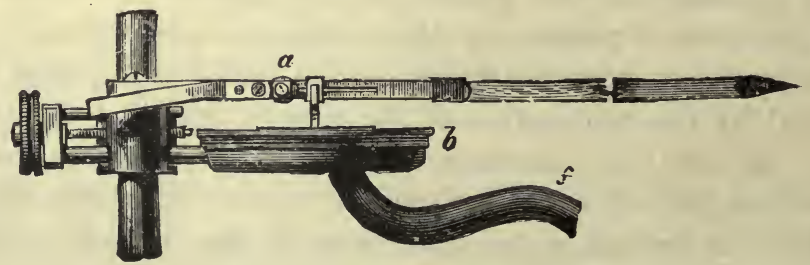

Fig. 395. Marey's tambour.

$a$, axis of lever; $b$, metal tray covered with rubber membrane, and communicating by tube $f$ with free end of cardiac sound.

stiff tube having an elastic bulb or ampulla at the end which is to be inserted into the heart. The bulb is supported by a steel frame, so that it is not completely compressible by external pressure. The free end of the tube is connected with a writing tambour (Fig. 395), a small round metal tray covered with a delicate elastic membrane. To the top of the membrane a lever is attached by which any change of pressure on the 
ampulla may be recorded on a moving smoked surface. The large size of these sounds makes it difficult to use them on any animal smaller than the ass or horse. In smaller animals, such as the dog, the question has been investigated by the use of a manometer such as that of Hürthle. In this instrument (Fig. 396) the changes of pressure are

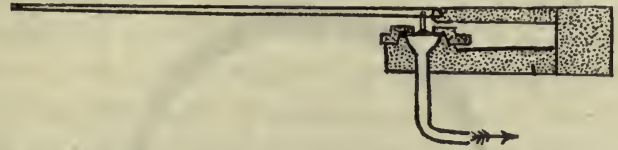

FIG. 396. Diagram to show construction of Hürthle's membrane manometer.

recorded by the oscillations of a thick rubber membrane which covers a very small tambour. The tambour is filled with magnesium sulphate solution, which is also used to fill the tube connecting with the heart. This tube can be inserted in the same way as Marey's cardiac sound.

Even Hürthle's instrument is inadequate to give a correct representation of the very rapid changes of pressure occurring in the contracting ventricle. A study of the theory of recording instruments by Otto Frank has enabled him to lay down certain fundamental requirements of such a recording instrument. In order that an instrument may reproduce correctly rapid changes of pressure, the mass moved must be as small as possible in order to reduce the momentum, and therefore the tendency to overthrow of the instrument, to the greatest possible extent. Moreover, the movement of fluid into and out of the instrument, which accompanies each change of pressure, must occur with the smallest possible friction. This is accomplished, as in Hürthle's instrument, by using a very small tambour, covered with a strong tightly stretched membrane connected by as short and wide a tube as is feasible, with the heart or blood-vessel where it is desired to register changes of pressure. A lever is entirely got rid of, the minute oscillations of the membrane being recorded by means of a beam of light which impinges

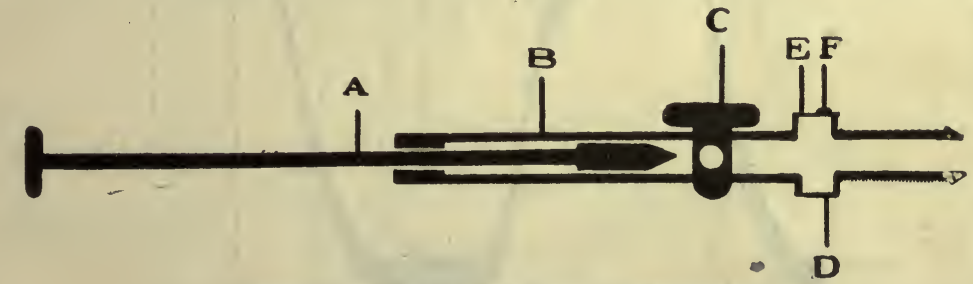

FIG. 397. Diagram of Piper's manometer.

on a mirror attached to the rubber membrane and reflected on to a moving photographic surface. In Fig. 397 is represented the construction of Piper's manometer, built on the principles laid down by Frank.

It consists of a tube armed with a stilette, $\mathbf{A}$, which fits it accurately. At $\mathbf{c}$ is a tap which, when opened, will permit the passage of the stilette, and can close the tube entirely when the stilette is withdrawn. About $2 \mathrm{cms}$. above the lower extremity of the tube is a small drum-like enlargement, closed on one side by a thick membrane, $\mathrm{E}$. On the edge of this membrane is fixed by means of shellac a minute mirror, $\mathrm{F}, 1 \mathrm{~mm}$. in diameter. With the stilette protruding, the manometer is thrust directly into the cavity of the heart, and fixed in position by a purse-string suture through the superficial part of the heart muscle, tied tightly round the end of the manometer. The stilette is then withdrawn and the tap turned off, but alterations in pressure in the cavity of the heart causes minute oscillations of the membrane, which can be recorded and magnified to any desired extent by means of a beam of light reflected from the mirror on to a moving photographic plate or paper. The advantage of this optical method of registration is that the magnification can be increased to any extent without altering the mass moved. The 'figure of merit' of this manometer, i.e. its own period of vibration, when filled with fluid, is about 250 per second with a thick memhrane, so that it can record with perfect accuracy all such rapid changes of pressure as may occur even in the left ventricle. 
On registering the endocardiac pressure by the optical method it is found that the curves vary in form according to the condition of the heart.

A
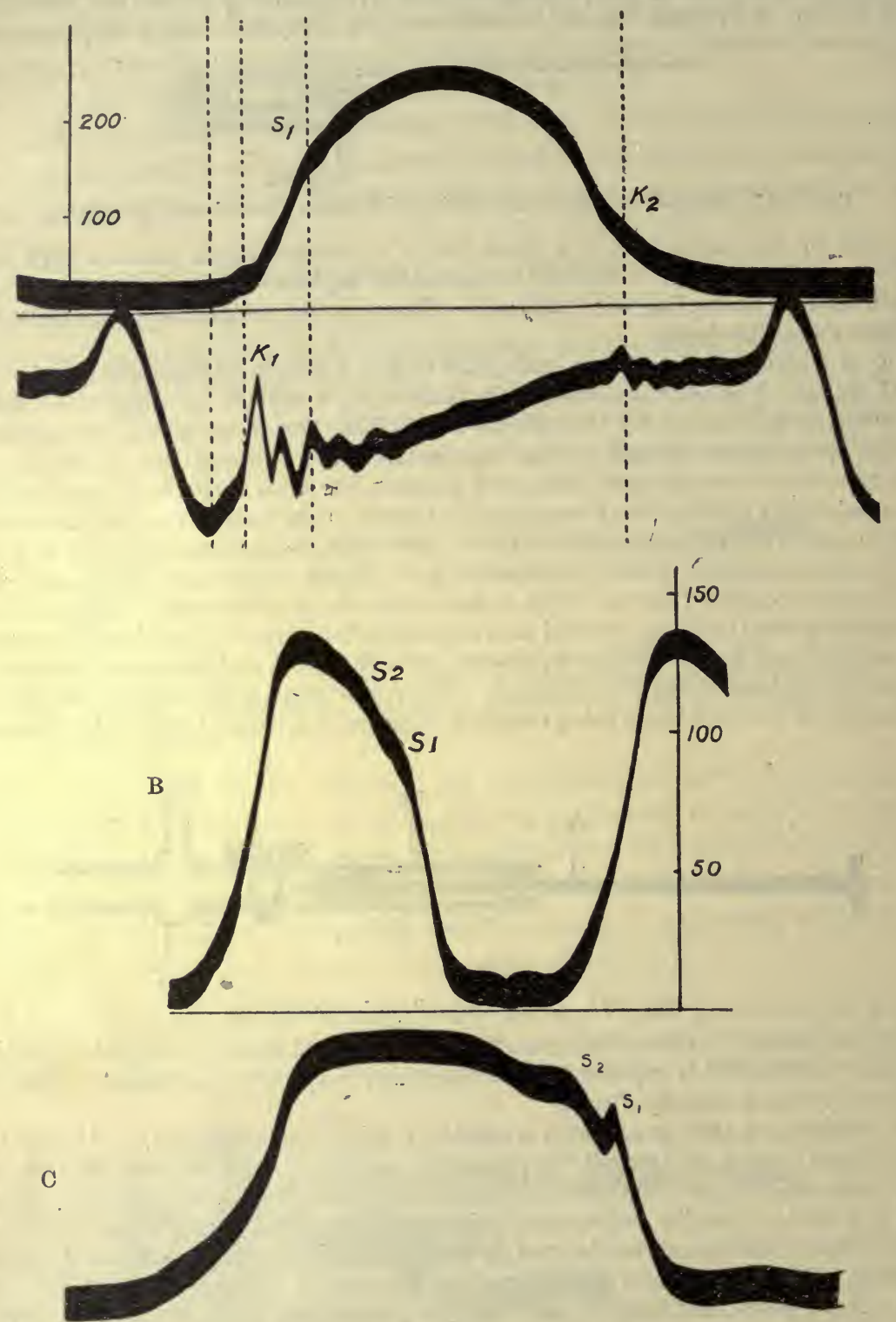

FIG. 398. Endocardiac pressure tracings, taken with Piper's manometer

A, Simultaneous tracings from left ventricle and left auricle. To be read from left to right. $\mathrm{B}$ and $\mathrm{C}$ taken from left ventricle, $\mathrm{C}$ at a faster rate of recording surface than $B$. To be read from right to left. $K_{1}=$ closure of $A . V$. valves; $S_{1}$ opening

of aortic valves: $S_{2}$, elastic oscillation or wave; $K_{2}$, opening of $A$. V: valves.

In order to interpret these curves we must utilise the knowledge obtained from a simultaneous record of the pressures in the auricle and ventricle, or in the ventricle and aorta. Figure 398 represents the different forms 
of curve obtained from the left ventricle. Very often, as in A, the curve is not unlike a single muscle twitch or the curve of contraction of the frog's heart muscle. Nearly always however it is possible to see on the upstroke one or two elevations, the most noticeable being the elevation marked $\mathrm{S}_{1}$. This can be shown to correspond to the opening of the aortic valves. This is still better marked in $\mathrm{B}$, where the heart was beating very forcibly and rapidly under the influence of adrenalin, and is also very evident in $\mathrm{C}$. In some cases, as in A, the rise of pressure occurs distinctly more slowly after $S_{1}$ than before. In $\mathrm{B}$ there is a further rapid rise of pressure after $\mathrm{S}_{1}$ before the curve begins to slope away, and at the change of velocity of rise, there is a second wave at the point marked $\mathrm{S}_{2}$. This is also marked in c. The slope of the curve after $\mathrm{S}_{1}$ or $\mathrm{S}_{2}$ varies considerably according to the

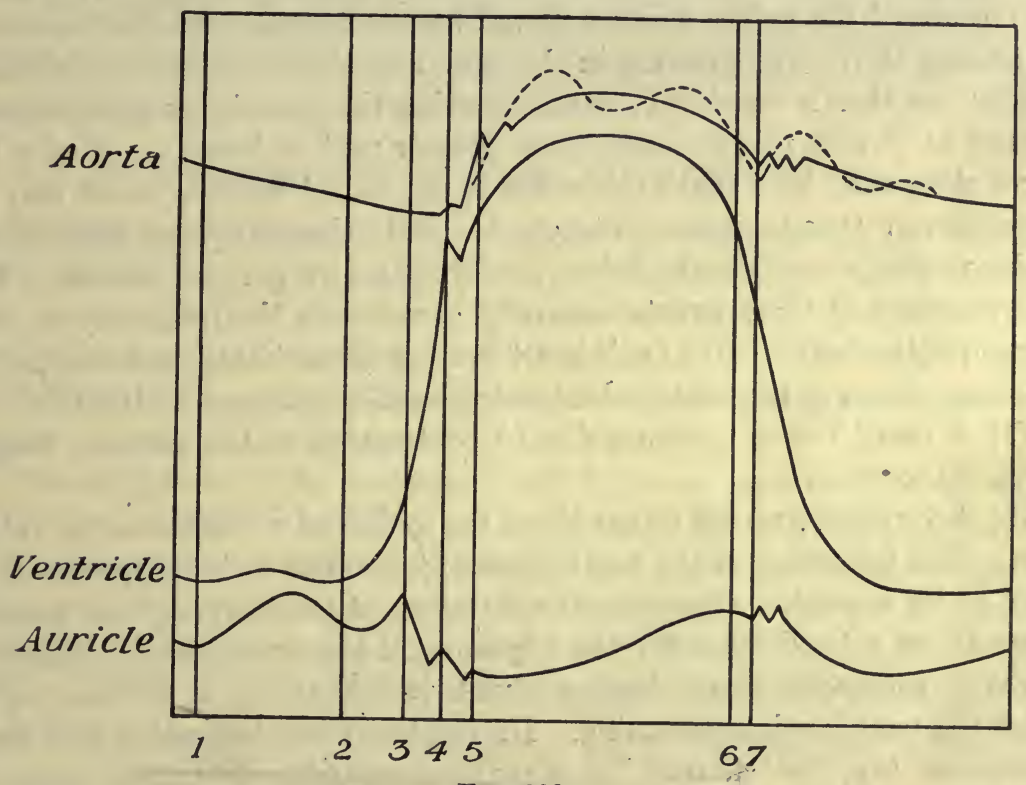

Fia. 399.

amount of blood the heart is sending out. In $\mathrm{c}$ the intraventricular pressure curve runs almost horizontal for a time and this part of the curve is known as the systolic plateau, but, as is evident from A, a plateau in the strict sense of the term is not always present. At the end of the 'plateau' the pressure rapidly falls, and the period where the lines thin out, i.e. the point at which the fall is occurring most rapidly, corresponds to the closure of the aortic valves.

The average course of the changes of pressure in the heart during each beat is shown diagrammatically in Fig. 399 (Piper). The cardiac cycle begins with the contraction of the auricle at 1 which may or may not give rise to a slight rise of pressure in the ventricles. As the auricular contraction dies away, the ventricular contraction begins at 2 . This causes a very rapid rise of pressure. Almost immediately after the beginning of the rise, or 
sometimes synchronously, the auriculo-ventricular valves close at the point marked 3. The pressure then rises rapidly in the ventricular cavity. Directly it exceeds the pressure in the aorta, the aortic valves open at the point marked 4 , and the aortic pressure thereafter rises with the ventricular pressure. During the whole duration of the ventricular contraction the aortic pressure remains somewhat below the ventricular pressure, showing that blood is flowing continuously from ventricle into aorta. The rise of pressure in the aorta may be at first rapid and then slow off gradually. With the change of velocity of the rise of pressure vibrations may be set up at 5 especially in the aorta and also, but to a less extent, in the ventricle. These vibrations are however often absent. At the end of the plateau the pressure in the ventricle falls rapidly as this organ begins to relax. Its pressure therefore falls below that of the aorta and the aortic valves close, the closure of the valves being followed by the so-called dicrotic elevation or incisure at 7. The pressure in the ventricles then continues to fall, first rapidly and then more slowly, until it reaches the line of zero pressure, and remains at or near this line during the greater part of diastole.. With a big inflow there may be a slight rise towards the end of diastole which may be accentuated by the auricular contraction. If the chest is opened the pressure in the ventricle never sinks below zero during any part of diastole. The time relations of these events naturally varies with the frequency of contraction of the heart. In a dog's heart beating about 100 times a minute the following phases in the ventricular tracing were determined by Hürthle.

(1) A small rise of pressure due to contraction of the auricles, lasting about $\cdot 05$ seconds.

(2) A very steep ascent rather above the middle of which the aortic valves open. The beginning of the rise is generally marked by a sharp secondary wave or by a sudden change in the direction of the curve. This point is about .02 to .04 seconds after the beginning of the ventricular contraction.

(3) A prolonged stage, lasting about two-tenths of a second during which the ventricle is contracting. During this time the tracing may show a flattened top, the 'plateau,' or a rounded summit. Near the beginning of this plateau, a second wave may make its appearance if the rate at which the pressure rises falls off suddenly. This second wave is therefore most marked with a considerable output, and a heart which beats forcibly and rapidly.

(4) A rapid fall due to the relaxation of the ventricular muscle. Near the upper part of this fall the aortic valves close, but it is generally difficult, without comparison with the simultaneous aortic pressure curve to make out the exact point of closure on the ventricular pressure curve.

(5) A period lasting about two-tenths of a second during which the ventricle remains relaxed and the pressure is approximately zero, in some cases rising slightly towards the end. The period of outflow of blood lasts from the moment at which the aortic valves open to the moment at which in the relaxing ventricle the pressure falls below that in the aorta and the aortic valves close. It therefore corresponds with the duration of that part 
of the curve which has been called the 'plateau.' The maximum pressure attained in the left ventricle naturally depends on the height of the aortic pressure and is always greater than this. Under normal conditions in the dog, with an average aortic pressure of $100 \mathrm{~mm}$. $\mathrm{Hg}$., the pressure in the ventricle may be 130 or $150 \mathrm{~mm}$. Hg. The difference between the average pressure in the aorta and the maximum pressure attained during contraction of the ventricle will naturally be greater the larger the amount of blood which is thrown out at each contraction. Thus in one case in a dog of 10 kilos, with an average aortic pressure of $100 \mathrm{~mm}$. $\mathrm{Hg}$., the maximum pressure in the left ventricle was $145 \mathrm{~mm}$. Hg. with an output of 2040 c.c. per minute, and $115 \mathrm{~mm}$. $\mathrm{Hg}$. with an output of 650 c.c. per minute. On the right side the maximum pressure is much less, corresponding to the low resistance of the pulmonary system of blood-vessels. Otherwise the general course of the curves is very similar on the two sides of the heart. The maximum pressure in the right ventricle may be taken as varying between 25 and $35 \mathrm{~mm}$. $\mathrm{Hg}$. under ordinary conditions.

\section{CHANGES OF PRESSURE IN THE AURICLES}

Owing to the absence of valves between the right auricle and the venæ cavæ, changes of pressure within this cavity are transmitted along the veins. The venous pulsation is especially marked in circumstances which give rise to high venous pressure, so that the veins, are not entirely emptied at any part of the cardiac cycle. The most superficial observation shows that the jugular vein pulsates twice for each heart-beat.

The exact form of the pressure tracing in the auricles varies considerably. according to the inflow of blood and the state of filling of their cavities. A typical tracing with a moderate inflow of blood is given in Fig. 398 A, p. 898, and in this figure the relations of the different elevations in the auricular tracing to the intraventricular events can be made out. A somewhat different curve is given in Fig. 400, but it will be noted that the essential features of the curves are identical. In every case the auricle curve presents the following features :

(1) The first positive wave (pre-systolic wave) corresponding to the auricular systole.

(2). The second positive wave $k$ (first systolic wave) occupying the beginning of the ventricular systole. This is caused by the sharp closure of the mitral valve.

(3) A third positive wave (second systolic wave) which may present secondary undulations. This rise of pressure is due to the gradual filling of the auricles while the auriculo-ventricular valves are still shut.

(4) A negative wave which corresponds to the ' post-systolic vacuum ' of Chauveau and Marey. At this point the ventricle is entirely relaxed and the auriculo-ventricular valves open, so allowing the blood to flow freely from the auricle into the ventricle. This negative wave is not always well marked, and is represented only by. a series of small undulations in Eig. 398 A. 
The pressure rises in the left auricle somewhat higher than in the right auricle. In the latter case the big veins act as a supplementary reservoir to the auricle, so that in no period of the cardiac cycle need the pressure in

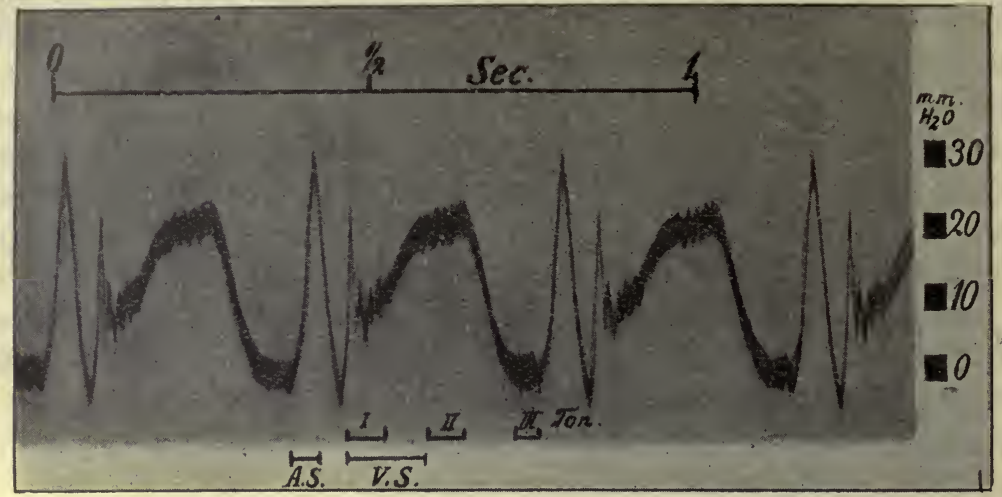

FIG. 400. Curve of pressures in left auricle of cat. (STRAUB.)

I, II, III Ton $=1$ st; 2 nd, and 3rd heart sounds.

the latter chamber rise to any extent. In both the auricular tracings given the heart sounds are apparent as small oscillations in the curve.

NEGATIVE PRESSURE. When the flow of blood through a tap is suddenly interrupted, the momentum of the fluid tends to make it continue its movement so that a diminution of pressure is produced in the rear of the moving column. In the tracings obtained by means of the older instruments, such as those of Hürthle, the lever at the end of the ventricular systole even descended below the base line, thus suggesting that for a short period of time there was actually a negative pressure in the ventricles. If the manometer be connected with the heart by a tube provided with a valve allowing the movement of fluid only in one direction, it becomes a maximum or minimum manometer according to the direction of the valve. This tube, however, increases the inertia of the whole system, so that the use of the minimum manometer tends to exaggerate the apparent negative pressure. According to some statements there may be a negative pressure of 40 to $60 \mathrm{~mm}$. $\mathrm{Hg}$. in the ventricle at some period during the cardiac cycle. Many explanations were put forward to account for this negative pressure, but they are all rendered unnecessary by the fact revealed by more perfect graphic methods that at no period in the cardiac cycle is there a negative pressure in the ventricles. Naturally under normal conditions the pressure in the chest outside the heart is negative owing to the elastic retraction of the lungs, and may vary from -3 to $-30 \mathrm{~mm}$. $\mathrm{Hg}$., according as it is measured during normal expiration or during forced inspiration. This negative pressure may be transmitted to the interior of the heart, but the pressure within the ventricle never falls below the pressure obtaining outside the ventricles.

\section{CHANGES IN FORM OF THE HEART}

As the heart walls are perfectly flaccid during diastole, the shape of this organ as a whole will depend upon the position in which the heart is lying and the direction of its support. Thus if the chest and the pericardium be opened and the animal be in the supine position, the heart during diastole will be flattened from before backwards as a result of the simple weight of its contents. In this position therefore systole will be accompanied by a 
shortening in the lateral and vertical directions and a lengthening in the sagittal direction. During systole, when the heart becomes tense and all its fibres are firmly contracted, the heart, whatever its previous condition, takes the form of a truncated cone. Under normal circumstances the heart in the unopened chest lies in the pericardium, which is attached above to the great vessels and below to the central tendon of the diaphragm. It is supported laterally by the lungs, which, however, owing to their elasticity, have very little influence on its shape during diastole.

When the heart is freed from the pericardium, the obliquity of its fibres causes the apex to move forwards and to the right during systole; this movement is normally prevented by the attachment of the pericardium to the central tendon of the diaphragm, so that the most movable part of the heart comes to be the base. If three needles be passed through the chest wall so that their points lie, one in the base, one about the centre of the ventricles, and one in the apex of the ventricles, each ventricular systole is found to be accompanied by a movement of the needle in the base of the heart downwards, a slighter movement in the same direction of the needle in the middle of the ventricles, and practically no movement at all of the needle which is thrust into the apex. During systole the base of the heart moves downwards towards the apex. "This movement is determined partly by the shortening of the fibres of which the ventricular wall is composed, partly by the lengthening of the great arteries as blood is forced into them under pressure from the ventricles.

The changes in the shape of the cavities of the heart during contraction have been studied in the stage of extreme contraction produced by heat rigour. In such hearts it is found that the cavities are never entirely obliterated, though the right ventricle is reduced to a narrow slit widening out slightly in the neighbourhood of the auriculo-ventricular orifices, while in the left ventricle a distinct cavity is left between the mitral valves and the free ends of the papillary muscles. During normal activity it is probable that the emptying of the cavities rarely proceeds to so great an extent.

\section{THE APEX BEAT}

The movement of the heart at each contraction is communicated to the chest walle over a limited area of which it may be felt and seen, except in fat individuals. The region where the pulsation of the chest wall is most marked lies in the fifth intercostal space, a little to the median side of the left nipple. The pulsation is spoken of as the 'apex beat,' and was formerly thought to be due to the twisting forward of the apex at each systole. The apex of the heart is really situated lower down, and, as we have already seen, so long as the pericardium is intact is relatively motionless. During diastole the ventricles form a flabby flattened cone lying against the chest wall and slightly deformed by the latter. In systole the ventricles contract forcibly on the contained fluid and become hard and rigid, assuming the form of a rounded cone. This sudden recovery of shape and hardening of the 
ventricular wall pushes out the part of the chest wall in immediate proximity to the ventricles and so gives rise to the 'apex beat.'

The cardiac impulse may be registered by means of a cardiograph. In nearly all forms of this instrument a button resting on the chest wall

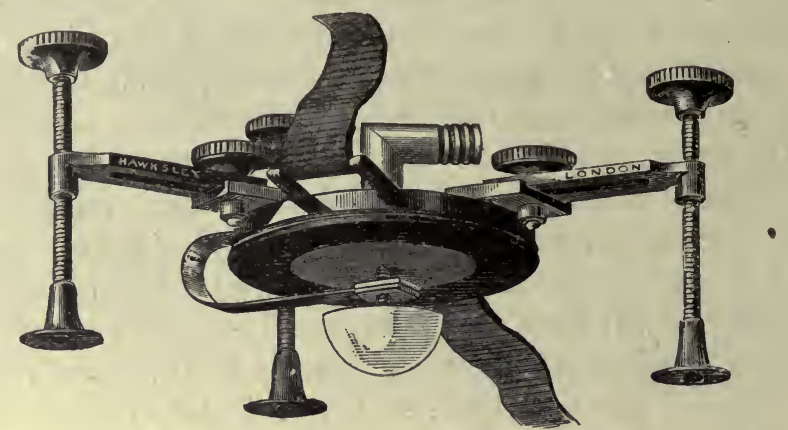

Fig. 401. A cardiograph. This is strapped round the chest, the central button is applied to the 'apex beat,' and its pressure on the chest wall regulated by means of the three screws at the sides. The tube at the upper part of the instrument serves to connect the drum of the cardiograph with a registering tambour such as that shown in Fig. 395.

transmits the movement of the latter to a tambour, which again is connected by a tube to a registering tambour. One such instrument is shown in Fig. 401.

The curves so obtained, which are known as cardiograms, may vary considerably in the same subject according to the pressure employed and the exact spot at which the tambour is applied. Their interpretation often presents difficulties owing to the fact that their form is conditioned by two factors, viz. (1) the actual size (antero-posterior diameter) of the ventricles,

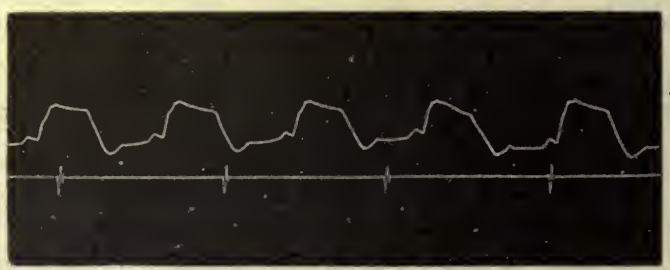

FIG. 402. Cardiogram. (HÜRTHLE.)

(2) the resistance to distortion (i.e. the tension) of the ventricular wall; this factor will increase in importance with increasing pressure of the cardiograph button on the chest wall. Fig. 402 represents a cardiographic tracing or cardiogram which may be spoken of as typical. In order to interpret this curve we must record at the same time either the intraventricular pressure in animals or the heart sounds in man. This cardiogram presents considerable similarities to the endocardiac pressure curve ; in both there is an ascending limb, a plateau, and a descending limb, and in many cases a small elevation occurs at the beginning of a curve during the contraction of the auricle. The exact point at which the auricular passes into the ventricular contraction 
varies from case to case and may be altered by altering the degree of pressure put on the recording button. In the first figure given the auricular systole finishes before the main rise of the lever occurs. In many cases, however. the elevation due to the auricular systole may take up the greater part of the ascending limb of the curve, as in Fig. 403.

In the experiment from which this figure was taken the heart sounds were recorded at the same time as the apex beat. It will be seen that the

1

2

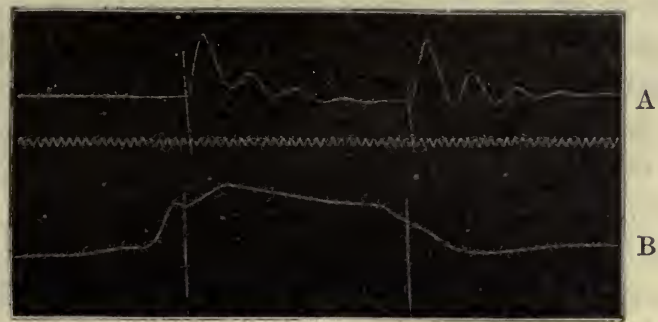

FIG. 403. Cardiogram (B) with simultaneous record of heart-sounds (A). (HÜRTHLE.)

1, position of first heart-sound; 2, position of second heart-sound.

first heart sound, corresponding to the ventricular systole, begins, not at the commencement of the rise of the cardiogram, but at the notch near the top of the ascent. The first part of the ascent is therefore caused by the increase in the volume of the ventricle, due to the sudden contraction of the auricles, the ventricular systole being marked by the notch near the top of the curve. Owing to the co-operation of the volume and pressure factors in the production of the cardiogram, the curve generally begins to decline with the diminution in volume which follows the sudden opening of the aortic valves. Here again, however, the effect will vary with the pressure of the button. If an actual deformation of the ventricular muscle can be effected, as in thin patients, the plateau of the curve may last during the whole of the cardiac cycle. Other forms of curves may be obtained which show considerable deviation from the endocardiac pressure tracing; these are spoken of as atypical, and are generally conditioned by a faulty position of the cardiograph, the button being applied to the chest wall in the immediate vicinity of the apex beat instead of to the apex beat itself.

\section{THE HEART SOUNDS}

If we apply our ear to the front of a person's chest (it is more convenient to use the stethoscope for the purpose) we hear two distinct sounds accompanying each beat of the heart, followed by a pause corresponding to the diastole. The sounds are compared to the syllables $l u b b, d u p$, the first sound being low-pitched and prolonged, the second sound high and sharp. Thus the heart sounds may be represented : lubb, dup (pause), lubb, dup (pause).

The causation of the second sound is very simple, and may be considered first. It is heard just over the second right costal cartilage, i.e. the place where the aorta lies nearest the surface. It comes at the end of the systole, 
as determined by the hardening of the apex of the heart, felt as the apex beat, and can be shown to be synchronous with the closure of the aortic valves. It is, in fact, caused by the sudden shutting and stretching of these valves that occur directly the heart ceases to contract and to force the blood into the aorta. If the valves be hooked back in an animal by means of a wire passed down a carotid artery, the second sound disappears and is replaced by a murmur caused by the blood rushing back into the ventricle at the end of the systole. The same disappearance of the normal second sound is observed in cases where the valves are prevented from closing by diseased conditions.

The pulmonary and aortic valves generally close simultaneously. In some cases, however, the aortic may close slightly before the pulmonary, giving rise to a 'reduplicated second sound.' The pulmonary element of this sound is best heard over the second left cartilage, or in the second left intercostal space.

The first sound has probably a twofold origin, viz. from the sudden closure of the auriculo-ventricular valves and from the contraction of the thick muscular wall of the ventricle.

If the veins going to the heart be clamped so that the heart can no longer be distended with blood nor the valves put on the stretch, the first sound is altered in character, but not abolished. The first sound may indeed be heard on listening with a stethoscope to the beat of an excised heart. It is said that two notes may be detected in the first sound-a high note of short duration due to closure of the valves, and a low-pitched note due to the muscular contraction. The muscular element of the first sound has the same pitch as the sound produced by contracting voluntary muscle, and therefore as the resonance-tone of the ear. This consideration prevents our arguing from the tone that a cardiac contraction is a tetanus. As we shall show later on, each ventricular contraction is analogous to a simple muscletwitch and not to a tetanus.

THE THIRD HEART SOUND. A number of observers have described a third heart sound as occurring in certain individuals during the diastole, a short time after the second sound. It is softer and of a lower pitch than the second sound, and is heard most distinctly over the apex beat. It is probably due to the vibrations set up in the fluid itself or in the auriculo-ventricular valves by the sudden inrush of blood from auricles to ventricles at the beginning of the diastole. The sound is shown objectively by the vibrations on the endocardiac pressure curve given in Fig 400 (Straub). It has also been registered electrically by Einthoven.

\section{CARDIAC MURMURS}

When a fluid escapes through a narrow orifice into a wider space vibrations are set up in the fluid and may be transmitted by any elastic medium to the ear, giving rise to the sensation of sound. Such a sound is produced when water is allowed to run from a tap into a vessel of water, or when air is blown out between the partially closed lips. The formation of such vibrations forms, indeed, the basis for the construction of many musical instruments. The same sort of vibration may be set up in the large vessels or in the heart, whenever the blood passes rapidly through a narrow orifice into a wider space. 
In the normal individual sounds produced in this way are so slight that they may be neglected; under abnormal conditions, as after diseases affecting the valvular orifices of the heart, this vibration may occur during every heart cycle and be heard with ease on applying the ear to the chest. These murmurs, or bruits as they are called, are of paramount importance in enabling the medical man to form a judgment as to the condition of the different valves of the heart. Thus injury to an aortic valve, so as to allow of leakage during diastole, involves the squirting of a small amount of fluid under high pressure from the aorta into the relaxed ventricle. On listening to the chest of a man with such a lesion this regurgitation during diastole is heard as a rushing sound occurring in the place of or continuing the second sound up to the beginning of the next first sound which denotes the beginning of systole.

In many cases the disease which occasioned the inadequacy of the valve is followed by processes of repair and cicatrisation in which the valves become puckered and contracted and perhaps adherent, so that the orifices can never become thoroughly patent or thoroughly closed. Under such circumstances vibrations will be set up in the current of blood as it escapes through the narrow orifice into the aorta during systole, and on listening to the chest over the second right costal cartilage a 'to and fro' bruit is heard composed of a systolic immediately followed by a diastolic murmur. In the same way incompetency of the mitral valve or dilatation of the mitral orifice, in consequence of weakness of the cardiac muscle, gives rise to a murmur which lasts during the whole of the ventricular contraction and is therefore systolic in character. Such a murmur is heard best over the apex beat, and is also transmitted backwards so that it can be heard on listening at the back of the patient. A narrowing of the mitral orifice in consequence of contraction of the valves will set up a resistance to the flow of blood from left auricle to left ventricle. The auricle becomes hypertrophied, its contraction prolonged, and the escape of blood through the contracted orifices gives rise to a murmur which is heard on listening over the apex beat as a presystolic bruit. This bruit is easily distinguished from a systolic murmur by noticing that it runs up to and ends with the apex beat, whereas a systolic murmur does not begin until the elevation of the apex commences.

Several physiologists have succeeded in recording heart sounds graphically. Hürthle's method consists in an application of the microphone. A special form of stethoscope is so arranged that by its means the vibrations corresponding to the heart sounds are transmitted to a contact between silver and carbon. Through this contact a strong current is passing. This also passes through an electro-magnet, which attracts an iron disc attached to the membrane of a Marey's tambour. Any vibration transmitted to the carbon-silver contact alters its resistance, and so the strength of the current passing through the electro-magnet. In this way the heart sounds can affect the pull exerted by the electro-magnet on the membrane of the tambour, and the change in the volume of the contained air is recorded by means of an ordinary registering tambour.

Similar results have been obtained by Einthoven, who has allowed the variations in the current passing through the microphone to be recorded directly by means of a very delicate capillary or string electrometer. 


\section{TIME-RELATIONS}

The time-relations of the various events of the cardiac cycle are indicated in the accompanying diagram (Fig. 404). In man the heart beats on the average about seventy-two times in the minute, so that each cardiac cyclei.e. systole plus diastole - can be regarded as occupying 0.8 sec. During five-tenths of a second the ventricles are relaxed; during the first four-tenths of this period, which corresponds to the diastole of the heart as a whole, blood is flowing in a steady stream from the veins through the auricles into the ventricles, so that the heart is gradually increasing in size. The systole of the

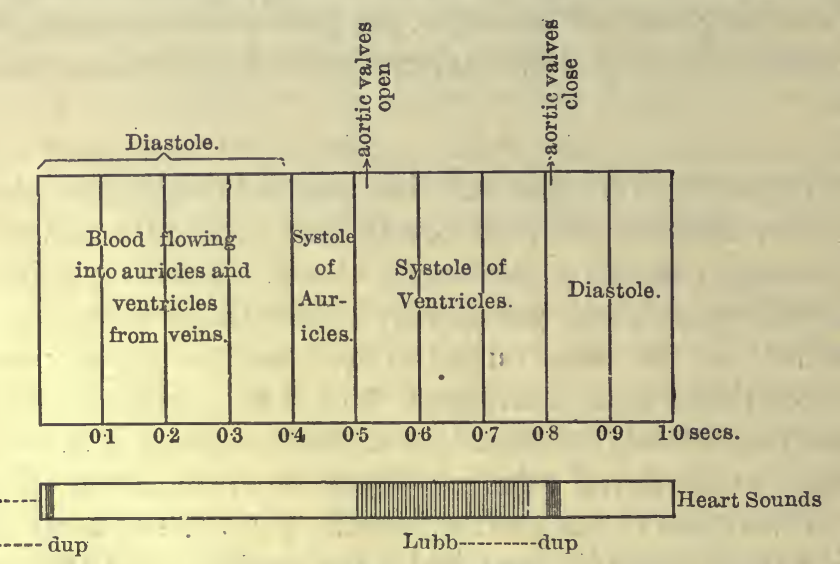

FrG. 404. Diagram of events constituting a cardiac cycle.

auricle then occurs and lasts about $0 \cdot 1 \mathrm{sec}$. This is followed by the ventricular systole, the immediate effect of which is to close the auriculo-ventricular valves on both sides of the heart. The whole ventricular contraction lasts 0.3 sec.; during the first period of this the ventricle is getting up pressure, the pressure rapidly rising until it equals the aortic pressure. This period, during which the ventricle is simply contracting isometrically on its contents without any flow of blood occurring, lasts between .02 and .04 sec., and is, of course, longer the higher the pressure in the aorta. Directly the intraventricular pressure rises above this point the aortic valves open and blood is driven into the aorta. The outflow of blood continues throughout the whole of the ventricular systole, and may be taken as lasting about $0.2 \mathrm{sec}$. The ventricle then suddenly relaxes, the period of relaxation occupying about $0.5 \mathrm{sec}$. ; the ' plateau' of the endocardiac pressure curve on the average lasts about $0.18 \mathrm{sec}$., and according to the condition of the heart and the peripheral resistance may present a gradual ascent or descent. Directly relaxation commences the ventricular pressure falls below the aortic pressure or the pulmonary pressure and the semilunar valves close, giving rise to the second sound. Systole is now at an end and the diastolic period of filling recommences. The first sound is synchronous with commencement of the ventricular contraction, and the same event is signalled by the occurrence of the apex beat. 
Although the pulse frequency may undergo considerable variations according to the condition of the individual, being higher during activity or under conditions of mental excitement, the greater part of the difference in duration of the cardiac cycle thereby induced falls upon the diastolic period. Thus to take wide limits the pulse-rate may vary between 32 and 124 beats in the minute, while under the same circumstances the period occupied by the systole only varies between 0.382 and $0 \cdot 190 \mathrm{sec}$ - - and these, of course, are extreme limits. Variation therefore in the time occupied by each cardiac cycle is determined mainly by variation in the time occupied by the diastole.

\section{FILLING OF THE HEART IN DIASTOLE}

Since the heart is perfectly flaccid during diastole it is unable to exert any suction force on the blood in the veins. Its filling during diastole depends on the existence of a positive pressure within the veins, or at any rate of a pressure greater than that in the auricles and ventricles; the greater the pressure within the large veins the more rapidly will the blood enter the heart during diastole and the larger the amount of blood in this viscus when it begins its contraction. In this process an important part is played by the mechanical conditions existing in the thoracic cavity. Owing to the elasticity of the lungs and the fact that they are constantly tending to contract, the pressure in the thorax is less than that of the external atmosphere by the amount which is required to distend the lungs to fill the cavity.

At the end of expiration this difference amounts to about $5 \mathrm{~mm}$. $\mathrm{Hg}$. rising to $9 \mathrm{~mm}$. $\mathrm{Hg}$. at the end of inspiration and to $30 \mathrm{~mm}$. $\mathrm{Hg}$. at the end of a forced inspiration. On the other hand, the veins outside the thorax are exposed to a pressure which is a little above that of the atmosphere. When the thorax is at rest the veins and auricles are therefore expanded and the flow of blood into them rendered more easy. The respiratory movements, by causing an alternating suction on the walls of the great veins, act like an accessory pump and cause an aspiration of blood into the veins of the thorax with each inspiration.

It is evident that if the pressure within the thorax be sufficiently raised so as to cause a positive pressure on the big veins and auricles, the return flow of blood to the heart must come to an end. Thus during extreme muscular efforts the glottis is fixed and a positive pressure is produced in the thorax. The deficient circulation and the deficient aeration of the blood thereby induced are shown by the engorgement of the superficial veins and the blueness of the surface. Weber showed that by a forcible expiration, with the glottis closed, the pulse might disappear at the wrist and the circulation be brought for a time to a standstill, so that even loss of consciousness might supervene.

Since the heart during its systole diminishes its own volume by the expulsion of blood from the thorax, it becomes smaller, and the space thus provided in the chest cavity is taken up by an expansion of the veins, auricles, and lungs. To this systolic 
diminution of intrathoracic pressure is due the 'cardiao-pneumatic' movements. These are recorded by attaching one nostril to a delicate tambour by means of a tube, while the other nostril and the mouth are kept closed. If a carotid pulse tracing be taken at the same time it will be found that there is a fall of the lever attached to the nasal cavity synchronous with the rise of the pressure in the arteries, due to the expulsion of blood from the heart.

The normal filling of the heart during diastole can be prevented by anything which hinders its expansion, such as the presence of fluid in the pericardial cavity. The same effect may be produced experimentally. If oil be allowed to flow into the pericardium under pressure, when the pressure of the oil rises to about $60 \mathrm{~mm}$. the pressure of the vena cava rises to a height just above that obtaining in the pericardial cavity. On increasing the pressure, a point is finally reached at which no more blood can be driven from the veins to the heart, so that the arterial blood-pressure falls to zero and death ensues.

In order to maintain the arterial pressure it is necessary that the amount of blood driven into the arterial system by the contraction of the left ventricle should be exactly equal to that leaving the arteries to pass into the capillaries during the period which elapses between each systole.

Over-filling of the heart is prevented to a certain extent by the resistance of its walls. The danger of over-filling is therefore most marked in the right ventricle. An important part is played moreover by the pericardium in this regard. Even when beating normally, the heart during diastole tends to protrude through a slit made in the pericardium, and Barnard has shown that the right auriculo-ventricular valve ceases to be entirely efficient when the pericardium has been freely opened, the closure of this valve being dependent on the support afforded to the heart by the pericardium.

\section{SYSTOLIC OUTPUT OF THE HEART}

The amount of blood which passes through the whole body and is available for the metabolic exchanges of all the tissues depends on the amount of blood which leaves the heart each minute. The height of the arterial pressure also depends on the relation between the amount of blood leaving the arterial system by the capillaries and that entering from the heart. The determination of the output of the left ventricle is therefore one of the most important problems in physiology. The output of the right ventricle must be equal to that from the left ventricle, otherwise the blood would accumulate on one or other side of the heart and bring the circulation to a standstill. It is therefore immaterial on which side of the heart the output be determined.

The methods which have been devised for determining the cardiac output fall into two classes. In the first class it is sought to determine the total volume of blood leaving the right or left ventricle in the course of a given time, say one minute. If this amount be divided by the number of heart-beats in the same time, the output of each ventricle per beat is at once obtained. A second method consists in the determination of the volume changes in the ventricles at each beat of the heart. During diastole 
the ventricles are receiving blood and increase in volume, during systole they expel blood and therefore diminish in volume. The change in volume at each beat gives therefore the combined output of right and left ventricles and must be divided by half in order to give the output of either ventricle separately.

METHODS OF DETERMINING OUTPUT. In a method devised by the author it is possible to determine the output of the left ventricle under all manner of conditions

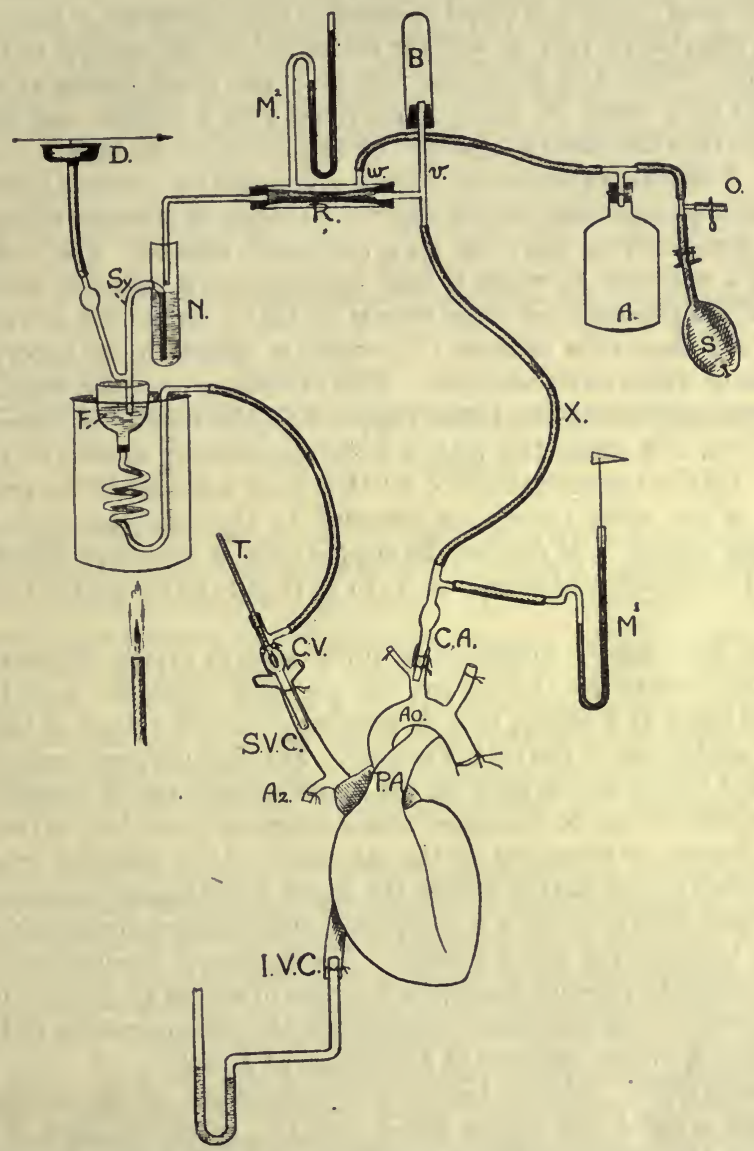

Fia. 405. Arrangement of apparatus forworking on the isolated mammalian heart. (KNowltoN and STARLING.)

and to vary at will the arterial resistance, the venous pressure, the filling of the heart, or the temperature of the blood-supply to the heart. The arrangement of the apparatus is shown in Fig. 405. Artificial respiration being maintained, the chest is opened under an anæsthetic. The arteries coming from the arch of the aorta-in the cat, the innominate and the left subclavian-are then ligatured, thus cutting off the whole blood-supply to the brain, so that the anæsthetic can be discontinued. Cannulæ are placed in the innominate artery and the superior vena cava. The cannulæ are filled beforehand with a solution of hirudin in normal salt solution so as to prevent clotting of the blood during the experiment. The descending aorta is closed by a ligature. The only path left for the blood is by the ascending aorta and the cannula $C A$ in the innominate artery. 
The arterial cannula communicates by a T-tube with a mercurial manometer $M^{\prime}$ to record the mean arterial pressure, and passes to another T-tube, $v$, one limb of which projects into a test-tube $B$. The air in this test-tube will be compressed with a rise of pressure and will serve as a driving force for the blood through the resistance. It thus takes the part of the resilient arterial wall. The other limb of the T-tube passes to the resistance $R$. This consists of a thin-walled rubber tube (e.g. a rubber fingerstall) which passes through a wide glass tube provided with two lateral tubulures $w, w$. One of these is connected with a mercurial manometer $M^{2}$ and the other with an air reservoir $A$, into which air can be pumped by the elastic bellows $S$. When air is injected into the outer tube the tube $R$ collapses, and will remain collapsed until the pressure of the blood within it is equal or superior to the pressure in the air surrounding it. It is thus possible to vary at will the resistance to the outflow of the blood from the arterial side. From the peripheral end of $R$ the blood passes at a low pressure and is collected in a vessel $N$, which is provided with a siphon, and can be made of such dimensions that the blood is siphoned off as soon as 10,20 , or 30 c.c. have collected in the vessel. A lateral branch on the siphon tube leads by a rubber tube to a tambour D. Every time that siphonage occurs there is a change of pressure within the tambour which can be registered by the lever on a blackened surface. The siphon discharges the blood into a reservoir $F$, which is kept immersed in a vessel of water maintained at any desired temperature by some source of heat. From the spiral below $F$, an india-rubber tube leads to a cannula $C V$, which is placed in the superior vena cava, all the branches of which have been tied. This cannula is provided with a thermometer to show the temperature of the blood supplied to the heart. A tube placed in the inferior vena cava and connected with a water manometer shows the pressure in the right auricle. On the recording surface we thus have a record of the arterial pressure, of the output of the whole system, as recorded by the tambour, and of the pressure within the right auricle. "If desired the simple current measurer described on p. 888 can be inserted in the arterial circuit at $X$, so as to give immediately the output of the left ventricle.

This method, although of considerable importance in giving information as to the conditions which determine the output of the left ventricle and the maximum capacity of the heart as a pump, tells us nothing as to the output of the left ventricle under normal conditions in the intact animal. For this purpose some indirect means must be adopted which can be used on the intact animal and if possible on man himself, so that the output can be measured under different conditions of rest and activity. Moreover, the output as-measured on the other side of the artificial arterial resistance represents the ventricular output minus the blood flow through the coronary arteries. It is possible, however, to insert a cannula into the coronary sinus, and so to measure the blood-flow through the heart muscle. The coronary circulation must be added to the flow through the arterial resistance in order to arrive at the correct total output of the left ventricle. The two chief methods for the determination of the ventricular output in the intact animal are those of Zuntz and of Krogh.

ZUNTZ'S METHOD. This is based on a comparison of the differences in gases contained in the arterial and venous blood and the actual amount of oxygen taken from the air in the lungs. Thus in one case he found that in a horse weighing 360 kilos 2733 c.c. of oxygen were taken up in he lungs per minute, while the arterial blood contained 10.33 per cent. more oxygen than the venous blood. Since therefore every 100 c.c. of blood that passed through the lungs had taken up $10 \cdot 33$ c.c. of oxygen, and 2733 c.c. had been taken up in the course of a minute, it is evident that

$$
\frac{100 \times 2733}{10 \cdot 33}=26,457 \text { c.c. }
$$

of blood must have passed through the lungs in the time. This therefore was the output of blood by the right ventricle in a minute and was equivalent to .00122 of the body. weight per second.

In a similar experiment on a dog the output per second of the right ventricle was found to be .00157 of the body-weight. In order to get the output at each beat it will 
be necessary to divide the output per minute by the number of heart-beats in the same time. From the results of determinations made in this way Zuntz concluded that the output of the right ventricle in man at each beat varies between 50 and 100 c.c. and may be taken on an average at 60 c.c.

KROGH'S METHOD. In Krogh's method an endeavour is made to determine the volume of blood flowing through the lungs in a given time by determining how much nitrous oxide is taken up from a mixture of nitrous oxide and air, with which the lungs are filled. Nitrous oxide is chosen because it can be breathed in considerable proportions without injury, and is itself very soluble in water or in the blood. The estimation is carried out in the following way. A small recording spirometer is filled with about $4 \frac{1}{2}$ litres of a gas mixture containing 10 to 25 per cent. $\mathrm{N}_{2} \mathrm{O}$ and 20 , to 25 per cent. oxygen. The subject, seated in a chair or on a bicycle ergometer, expires to the greatest possible extent, and then takes a deep inspiration from the spirometer. He holds his breath for five to fifteen seconds, breathes out sharply into the spirometer,

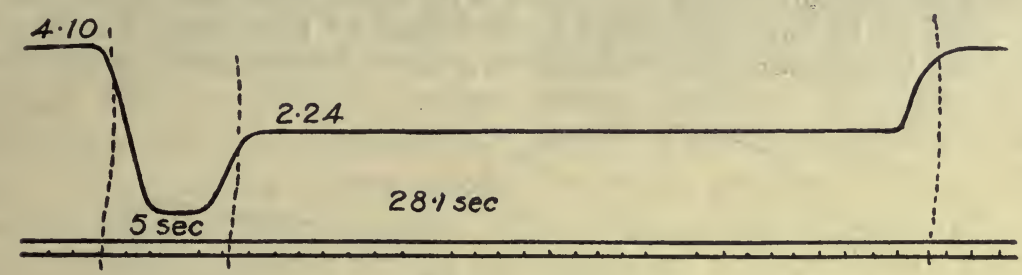

FiG. 406. (KROGH,)

expiring at least onelitre. At the end of this sharp expiration, a sample of his alveolar air is taken by connecting the tube from his face-piece with an evacuated glass bulb, as in Haldane's method of determining alveolar air. The breath is now held for a period, time varying between six and twenty-five seconds. He then makes a final sharp ample expiration into the spirometer, a sample of his alveolar air being taken at the end of this expiration. The excursions of the spirometer indicate exactly what volume of air he has breathed in and breathed out at each part of the experiment. These are recorded on a travelling surface, so that the duration of the experiment is represented by the horizontal distance between the lines showing the moments of sampling (Fig. 406).

By comparison of the composition of ordinary alveolar air with the alveolar air ob: tained after the first sharp expiration, the amount of residual alveolar air is determined, so that the total volume of gas contained in the lungs at each part of the experiment is also known. During the time when the breath is being held, nitrous oxide is being taken up in solution by the blood as it passes through the lungs, its solubility being such that 1 c.c. of blood, if exposed to an atmosphere of pure nitrous oxide will take up 0.43 c.c. of this gas. From the data obtained in this way, the amount of blood passing through the lungs during the period between the two expirations can be calculated. The following record of one experiment may serve as an example. The volume of air in the lungs at the beginning of the experiment was $3 \cdot 25$ litres and contained 12 per cent. nitrous oxide, so that the total quantity of nitrous oxide in the air of the lungs was 3250 c.c. $\times \frac{12}{100}=390$ c.c. At the end of the period the total volume of air in the lungs was three litres, containing only 10 per cent. nitrous oxide, so that the lungs now contained only 300 c.c. nitrous oxide, 90 c.c. nitrous oxide having been taken up by the blood. This 90 c.c. was taken up from an air in which the mean pressure of this gas was $\frac{12+10}{2}=11$ per cent. During the period of observation, from a gas 'containing at atmospheric pressure 11 per cent. of nitrous oxide, each c.c. of blood will take up $\frac{.43 \times 11}{100}=0.047$ c.c. In order to take up 90 c.c. therefore 1.9 litres of blood must have passed through the lungs during the time of the observation. The 
experiment lasted twenty-eight seconds. The amount of blood passing through the lungs per minute was therefore $4 \cdot 2$ litres. This figure represents the output from the right ventricle during one minute, and if the pulse rate is 70 per minute, the output per beat will be $\frac{4200 \text { c.c. }}{70}=60$ c.c. per beat. The figure arrived at in this way for the average output of each ventricle in man during rest, thus agrees with the figure obtained by Zuntz. The output of both ventricles is, of course, the same.

According to Krogh, the ventricular output per minute in man may vary from $2 \cdot 8$ litres to 21 litres of blood per minute. The latter is an extreme figure and was obtained in a powerful athelete doing hard work. In the case of Krogh himself, the maximum output was about 12 litres per minute. It is interesting to note that the same performance may be obtained from a dog's heart in the heart-lung preparation, allowing for the difference in size between the hearts of the dog and man respectively.

CARDIOMETRIC METHOD. Of the various methods which have been devised for recording plethysmographically the changes in the volume of the heart at each beat (as first carried out by Roy), the simplest is that devised by Henderson. The chest and pericardium being opened, a glass cardiometer, of the shape shown in Fig. 407, is slipped

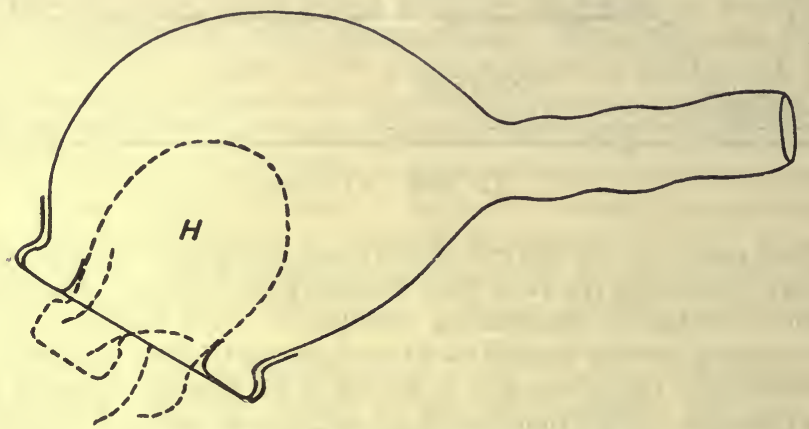

Fig. 407. Henderson's glass cardiometer.

over the heart. This cardiometer consists of a glass sphere with a wide opening. To the margin of the opening is tied a rubber diaphragm with a hole in it which accurately fits the heart as it lies in the auriculo-ventricular groove. The tube of the cardiometer is connected with some form of piston recorder or a tambour with a slack membrane. The disadvantage of this method is that the graphic record of rapid and ample changes in volume is one of the most difficult problems in experimental physiology, the inertia and friction of the moving piston tending to deform the shape of the curve obtained. Straub has therefore used a soap bubble as the volume measurer, photographing its edge and using the record as an index to the change in volume. It is possible, however, to obtain a pistol recorder moving sufficiently freely to give a fairly correct reproduction of the volume changes of the heart, provided that these do not occur with too great rapidity. It has been suggested by Piper to convert the volume changes into small pressure changes, and to record these latter by one of the methods described above.

The factors which determine the output of the left ventricle are best studied in the heart lung preparation. In this it can be shown that, provided the venous inflow remains constant, the output is also constant and is unaffected by considerable alterations of arterial resistance and of the rate of the heart. Thus with a moderate venous inflow the output remains constant whether we maintain the average arterial pressure at $60 \mathrm{~mm}$. $\mathrm{Hg}$. or at $160 \mathrm{~mm}$. Hg. It is also unaffected by altering the rate of the heart from 80 beats per minute up to 160 , or even 200 , beats per minute. On 
the other hand, the output is at once altered by alterations in the venous inflow and, as already stated, can be altered in a heart weighing 50 gms. from a few c.c. up to 3000 c.c. per minute. The only essential in this preparation is that the output from the left ventricle shall be sufficient to maintain a circulation through the coronary vessels and so keep the active muscle properly supplied with blood.

With increasing inflow of blood into the heart the large veins, auricles, and ventricles naturally become more filled during diastole, and during systole of the ventricles, when the auriculo-ventricular valves are closed, the blood rushing in from the venous system must accumulate in the big veins and auricles to a still greater extent. The venous pressure therefore rises with increased venous inflow. In so far then as venous pressure is an index of venous inflow, we may say that the output of the heart increases with the venous pressure so long as the heart is functionally capable of dealing with the blood it receives during diastole. But although the ventricular output is practically independent of the frequency of the heartbeat and a constant venous inflow, the venous pressure tends to fall as the heart-beat increases in rate. The optimum venous pressure is that which fills the ventricle during its diastole to the maximum extent to which it is able to respond. As the rate of the heart increases the inflow of blood can also be increased without causing over distension of the ventricles. The increase of heart rate therefore is an important factor in enabling this organ to deal with the maximum amount of blood. Although increase of rate does not alter the output with constant venous inflow, it does increase the maximum amount of inflowing blood which the heart is able to expel.

We thus see that alterations in the vigour of the circulation depend in the first instance on the venous circulation. The greater the volume of the blood that is brought up to the heart by the accessory factors of the circulation, the greater will be the output of this organ. The changes in rate and force of the heart which accompany its increased activity and increased output, e.g. during exercise, represent merely the means by which this organ is able to deal in the most advantageous manner with the increased inflow.

\section{THE WORK OF THE HEART}

The energy of the ventricular contraction is expended in two ways : first, in forcing a certain amount of blood into the already distended aorta against the resistance presented by the arterial blood-pressure, which itself is directly conditioned by the resistance in arterioles and capillaries; and secondly, in imparting a certain velocity to the mass of blood so thrown out. Thus the energy of the muscular contraction is converted partly into potential energy in the form of increased distension of the arterial wall and partly into the kinetic energy represented by the momentum of the moving column of blood. The work done at each beat may be calculated from the formula :

$$
\mathrm{W}=\mathrm{QR}+\frac{w \mathrm{~V}^{2}}{2 g}
$$


where $\mathrm{W}$ stands for work, $w$ for the weight, and $\mathrm{Q}$ for the quantity (volume in c.c.) of blood expelled at each contraction; $R$ is the average arterial resistance or pressure during the outflow of blood from the heart, and $\mathrm{V}$ is the velocity of the blood at the root of the aorta. In this equation $\mathrm{QR}$ is the work done in overcoming the resistance, ${ }^{*}$ and $\frac{w \mathrm{~V}^{2}}{2 g}$ is the energy expended in imparting a certain velocity to the blood.

If we take 60 c.c. as the average output of each ventricle, $100 \mathrm{~mm} . \mathrm{Hg}$. as the average pressure at the beginning of the aorta, and $500 \mathrm{~mm}$. per second as the velocity imparted to the blood thrown into the aorta, we can calculate the work done by the human heart at each beat.

$$
\mathrm{QR}=60 \times 0 \cdot 100 \mathrm{~m} \cdot \times 13 \cdot 6=81 \cdot 6 \text { grammetres, }
$$

or roughly 80 grammetres. On the other hand, the expression

$$
\frac{w \mathrm{~V}^{2}}{2 g}=\frac{60 \times(0.5)^{2}}{2 \times 9 \cdot 8}=0.7 \text { grammetres. }
$$

It is evident that this latter factor is negligible, and that for all practical purposes we may regard the work of the heart as proportional to the output multiplied by the average arterial blood-pressure. Taking the average pressure in the pulmonary artery at $20 \mathrm{~mm}$. Hg., the work of the right ventricle at each beat would amount to about 16 grammetres, a total for the two ventricles of about 100 grammetres per beat, which is equivalent to about 10,000 kilogrammetres in twenty-four hours for a man at rest.

This work is done by a contraction of the muscle fibres surrounding the cavities of the ventricles. It is important to remember that the strain or tension which is thrown on these fibres and which resists their contraction will simply be determined not by the blood-pressure which has to be overcome, but also by the size of the ventricle cavities. Since the pressure in a fluid acts in all directions, the tension caused by any given pressure on the walls of a hollow vessel will increase with the diameter of the vessel. Thus if we take a sphere with a radius of $10 \mathrm{~cm}$. filled with fluid at a pressure of $10 . \mathrm{cm} . \mathrm{Hg}$. there will be a pressure on each square centimetre of the inner surface of the sphere of $136 \mathrm{grm}$. The total distending force, i.e. the pressure on the whole of the inner wall of the sphere, will be equal to this pressure multiplied by the area, i.e. to $136 \times 4 \pi r^{2}=136 \times 4 \pi \times 100$. If by a contraction of the walls the radius be reduced to $5 \mathrm{~cm}$, the total pressure on the internal surface will be reduced to $136 \times 4 \pi \times 25$, i.e. will be one quarter of the previous amount. Moreover in the case of the heart, with increasing distension the wall becomes thinner and the number of muscle fibres in a given area fewer, so that the larger the heart the more strongly will each fibre have to contract in order to produce a given tension in the contained fluid. At the beginning of systole the distended heart must therefore contract more strongly than at the end of the systole, in order to raise the blood it contains to a pressure sufficient to overcome that in the aorta.

* This expression, QR, is only approximately correct. Supposing the pressure in the aorta at the beginning of systole is $50 \mathrm{~mm}$. Hg. and at the end of systole $150 \mathrm{~mm}$., the work could not be deduced accurately from the average pressure, but would need a simple application of the integral calculus for its determination. The expression employed above deviates from the real value only by about 10 per cent., and is therefore sufficiently accurate for our purpose. 
It is evident that an unrestricted diastolic filling of the heart is not of unqualified advantage to this organ. If during diastole the heart be too forcibly distended, as may easily occur after opening the pericardium, or in cases of enfeeblement of the heart's action by chloroform poisoning or otherwise, the muscle fibres of the heart may be quite unable to contract against the distending force represented by a pressure in the heart equal to that in the aorta. Under such conditions we may have sudden heart failure, which can only be relieved by diminishing the diastolic distension, as, e.g., by letting blood from the veins opening into the heart. 


\section{SECTION V}

\section{THE FLOW OF BLOOD THROUGH THE ARTERIES}

THE PULSE. Owing to the elasticity and distensibility of the arterial wall the rhythmic rise of pressure corresponding to each heart-beat causes an expansion, which can be felt by the finger placed on any exposed artery, such as the radial, and is spoken of as the pulse. Just as the blood-pressure diminishes from heart to periphery, so the amplitude of the pulse decreases as we go farther away from the heart.

If the arterial system were perfectly rigid the increased pressure due to the forcing of the blood into the arterial system at each ventricular systole would occur practically simultaneously at every point. The arteries are, however, elastic and distensible, so that the first effect of the flow of blood into the aorta is to distend the section of the aorta nearest to the heart. The elastic reaction of this forces a portion of the blood into the nearest section, so that the increased pressure is transmitted from segment to segment of the arteries in the form of a wave at the velocity of about seven metres per second.

It is important not to confuse the velocity of the pulse-wave with that of the blood-flow; the latter is never greater than 0.5 metre per second, and is very much less than this in the smaller arteries. Perhaps the difference between the two quantities may be made clearer by illustration : If the hindmost of a row of billiard-balls be struck sharply with a cue the foremost ball flies off and the others stop still; in this case the energy imparted to the first ball by the stroke has been transmitted from ball to ball, just as the effect of the ventricular contraction is transmitted from section to section of the arterial blood-stream. If the balls are struck so that the cue continues pressing on the hindmost after the stroke is delivered, the front ball flies off, while the others move slowly along in the direction of the stroke. In the arteries this continuous pressure is furnished by the elastic reaction of the arterial wall, and we see how the impact of the blood may travel quickly as a wave of increased pressure while the blood itself is moving slowly along, impelled by the reaction of the arterial wall.

If we imagine a rigid tube $\mathbf{A B}$ (Fig. 408) provided with a piston at the end $A$, and filled with an incompressible fluid, an inward movement of the piston at $\mathbf{A}$ will cause a simultaneous outflow of fluid at the end B. If the end $\mathrm{B}$ is closed the piston at A cannot be moved at all. Pressure applied to the piston will raise the pressure simultaneously at all points in the 
tube $\mathrm{AB}$. The increased pressure applied at $\mathrm{A}$ is therefore transmitted with practically no loss of time to all parts of the tube $\mathrm{AB}$. This immediate spread of the wave of pressure only applies to an incompressible fluid within a rigid tube. If the fluid were compressible, if it consisted, e.g. of air, a sudden movement inwards of the piston at $\mathbf{A}$ would not be felt immediately at B. The propagation of the wave of pressure from $\mathbf{A}$ to $\mathbf{B}$ would take a finite period of time, its velocity being identical with that of the velocity of propagation of a wave of sound in air, i.e. 1100 feet per second.

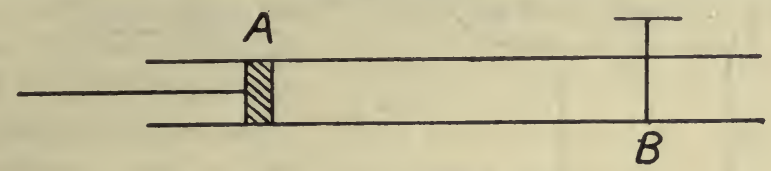

Fig. 408.

The same retarding effect will be produced if we have an incompressible fluid within a tube whose wall is distensible and elastic. If we imagine (Fig. 409) an elastic tube BC filled and distended with water and connected at $\mathrm{B}$ to a rigid tube, which is provided with a piston, the first effect of a rapid movement of fluid driven in by the piston will be a rise of pressure at the point immediately in front of the piston, viz. at $a$. The wall being distensible, and pressure being propagated along the fluid in every direction, the rise of pressure at $a$ will be spent partly on the particles of fluid in

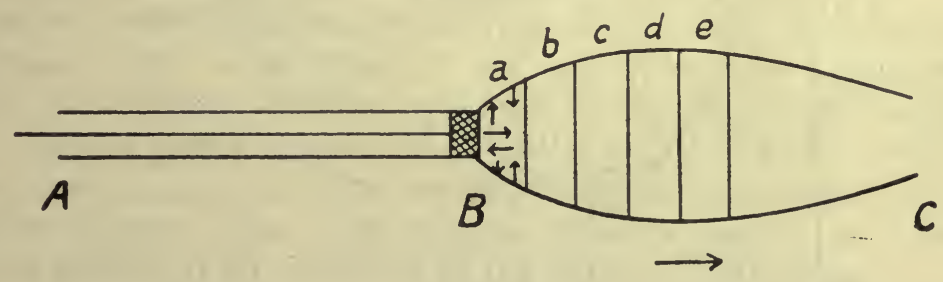

Fig. 409.

front of it, viz. at $b$, but also on the walls of the tube, so that this is stretched and the cross-section of the tube enlarged. The distended segment at $a$ will then exert a pressure on the contained fluid, driving this backwards and forwards. The fluid on its side towards the piston will tend to come to a stop, while that towards the distal end of the tube will be accelerated. The distended wall therefore returns to its original diameter, and the next segment at $b$ is stretched in its turn, so that a wave of increased pressure is propagated along the tube in the direction of the arrow.

The velocity with which this wave is propagated depends on the density of the fluid, i.e. its inertia, and on the resistance of the walls of the tube to distension, since this will determine the rapidity of its recovery. The velocity of propagation of the wave of increased pressure, or the wave of expansion of the artery, is expressed by the following formula :

$$
v=k \sqrt{\frac{\overline{g e a}}{\overline{\mathrm{D} d}}}
$$


where $v$ is the velocity per second,

$g$, the acceleration due to gravity,

$e$, the elastic coefficient of the wall,

$a$, the thickness of the wall,

$d$, the diameter of the tube,

$\mathrm{D}$, the density of the fluid,

$k$, a constant.

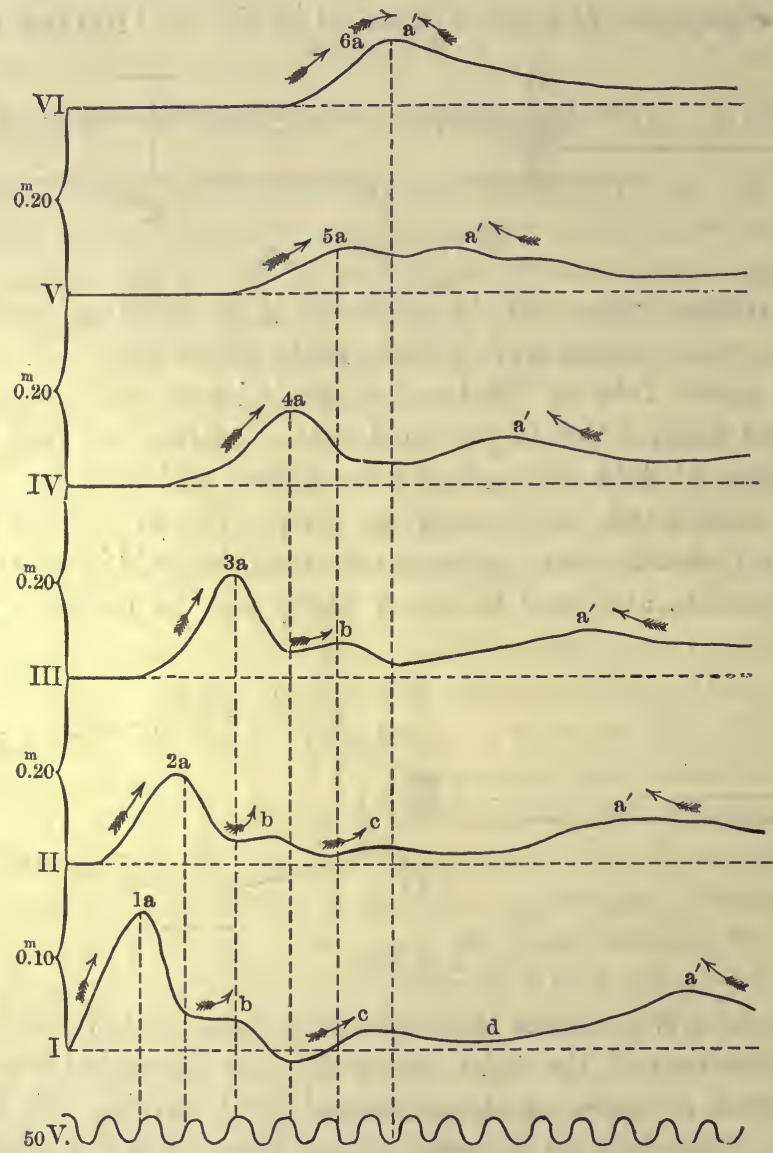

FIa. 410. Pulse-curves described by a series of sphygmographic levers placed at intervals of $20 \mathrm{~cm}$. from each other along an elastic tube, into which fluid is forced by the sudden stroke of a pump. The pulse-valve is travelling from left to right, as indicated by the arrows over the primary $(a)$ and secondary $(b, c)$ pulse-waves. The dotted vertical lines drawn from the summit of the several primary waves to the tuning-fork curve below, each complete vibration of which occupies $\frac{1}{50}$ sec., allow the time to be measured which is taken up by the wave in passing along $20 \mathrm{~cm}$. of the tubing. The waves $\left(a^{\prime}\right)$ are waves reflected from the closed distal end of the tubing; this is indicated by the direction of the arrows. It will be observed that in the more distant lever (vI) the reflected wave, having but a slight distance to travel, becomes fused with the primary wave, so that the rise of pressure in VI is actually greater than that in V. (From Foster, after MarEY.)

If the end $\mathrm{c}$ of the tube is closed, the wave of a positive pressure on arriving at $\mathrm{B}$ will be reflected back as a positive reflected wave. If a 
tracing be taken of the oscillations or variations of pressure in the tube, two waves at least are seen, one of which is the primary wave due to the movement of fluid caused by the piston; the other is the secondary wave reflected back from the periphery. The fact that the secondary wave is a reflected one is shown by the fact that the nearer to the peripheral resist. ance the pulse is recorded, the nearer is the secondary to the primary wave, as is seen in Fig. 410.

If the tube $\mathbf{B C}$ be widely opened a reflected wave is also observed, but this time of reversed sign, i.e. the wave is one of negative pressure. The production of this wave is dependent on the momentum of the moving column of fluid. If in the tube $a b$, with a tap at $c$ and a manometer $m$ (Fig. 411), the current of fluid be suddenly checked by turning the $\operatorname{tap} c$, the column in front of the tap, having a certain momentum, will tend to go on moving and therefore

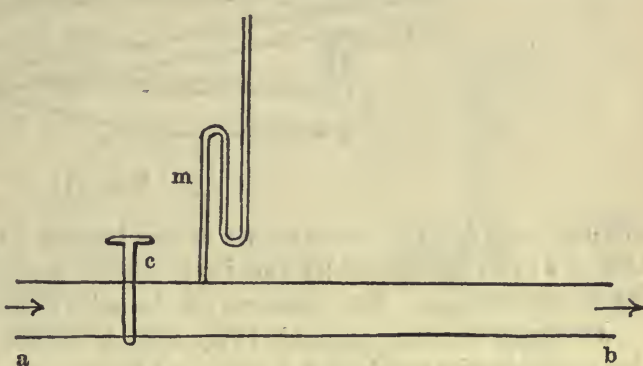

Fig. 411.

produce a suction or negative pressure behind it. When a wave of positive pressure arrives at the open end of a tube there is a sudden increase in the velocity of output, and the momentum of the mass of fluid which is thrown out causes a similar suction or negative pressure, which travels back the whole length of the tube. If the end of the tube is only partially closed, every primary positive wave will be transformed into a reflected one which is partly positive and partly negative. Since both these reflected waves travel through the tube with the same velocity and will mutually interfere, the result may be either a positive or a negative wave or nothing at all, according to the degree of constriction.

In a branching system of tubes, such as the arterial system, reflection of waves must take place at every dividing place. All the conditions for the origin of reflected waves and interference of such waves are present in the arterial system. It is impossible $a$ priori, however, to say whether any reflected wave will form a marked feature on the pulse-tracing. It is possible that the multitudinous reflections which must occur in every part of the arterial system may interfere with one another to such an extent that they mutually annul each other. The origin of any secondary wave in the pulse-tracing must therefore be determined by experiment.

To study the pulse more fully it is necessary to obtain a graphic record of the expansion of the arteries, or, what comes to the same thing, of the exact changes in pressure which produce this expansion. The curve obtained with the mercurial manometer shows elevations corresponding to the pulse; but the instrument is far too sluggish to record the finer: variations of pressure. For this purpose a manometer which has very, little inertia, such as Hürthle's or Piper's, must be used. The expansion 
of the artery is registered by means of a lever, which may be made to rest more or less heavily upon the artery, and the movements of which are recorded on a blackened surface. Such an instrument is called a sphygmograph. Of the many forms of sphymographs, Marey's or Dudgeon's is perhaps the most convenient for clinical purposes.

The principle of Marey's sphygmograph is shown in Fig. 412. The button $b$ is adjusted so as to press on the radial artery. Its movements are transmitted to a lever $m$. The screw on this works on a small cogged wheel at $o$, which is also the axis of the

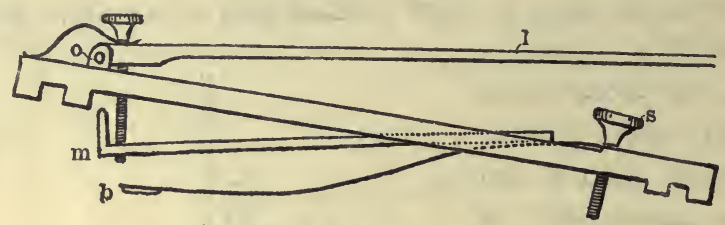

FIG. 412.

writing lever $l$. The movements of the button $b$ thus transmitted to a point near the axis of $l$ are reproduced by this lever highly magnified, and as such are recorded on a blackened surface. The pressure on the artery can be adjusted by means of a screw.

Dudgeon's sphygmograph (Fig. 413) is rather easier to use than Marey's, and is therefore largely employed for clinical purposes. It is provided with a dial by which the pressure on the artery can be graduated, and has a small clockwork arrangement for moving along the slip of smoked paper on which the records are taken. The

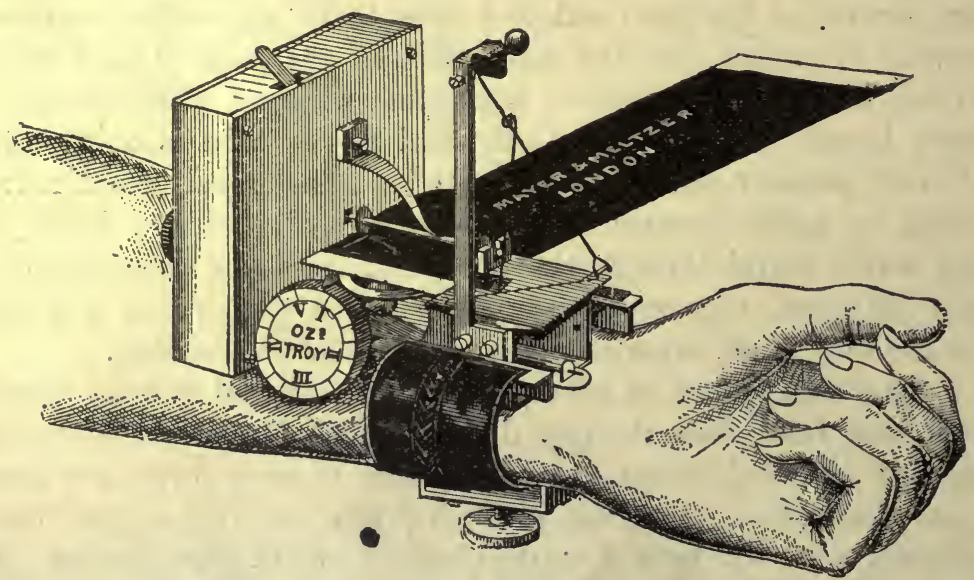

FIG. 413. Dudgeon's sphygmograph, showing its mode of application to the radial artery.

arrangement of the levers in this form of sphygmograph is shown in Fig. 414, where $\mathrm{F}$ is the (adjustable) spring bearing by its button $P$ on the artery. The up-and-down movements of $\mathrm{P}$ are transmitted to $\mathrm{s}$, being much magnified and converted into sideto-side movements. The point of s rests on the blackened surface represented in section at $\mathbf{A}$, and scratches on this, when moving, a magnified record of the expansion of the artery under the knob P.

In all these sphygmographs, even the most perfect, the moving parts have a considerable amount of inertia, so that the curve they give is always more or less deformed. This fact must be borne in mind when comparing the pulse-curves obtained by means of a sphygmograph with those given by the more perfect forms of manometer, such as Frank's or Piper's. 
Either form of sphygmograph is generally applied to the radial artery, since this is near the surface and is supported by bone, and the arm is well adapted for the application of the sphymograph. The pulse-curve obtained by means of a sphymograph varies according to the artery employed and the force with which the lever presses on the artery, but all the curves present the same general features.

The velocity of the pulse can be measured by taking simultaneous tracings from two arteries separated by some distance from one another, such as the femoral artery and the dorsalis pedis, or from the carotid and radial arteries. In a healthy individual the velocity varies between 7 and 10 metres per second. The more rigid the arteries the greater will be the velocity, so that the velocity of propagation gradually increases with advancing age, and is higher in the arteries of the lower extremities than in the more distensible arteries of the arm.

The length of the pulse-wave can be found by multiplying the velocity of transmission by the time occupied by the wave in passing any given point. The duration of the wave at any point corresponds to the time of a cardiac cycle, viz. 0.8 sec., so that if the velocity of transmission be taken as 7 metres per second, the length of the wave is about $5 \cdot 6$ metres.

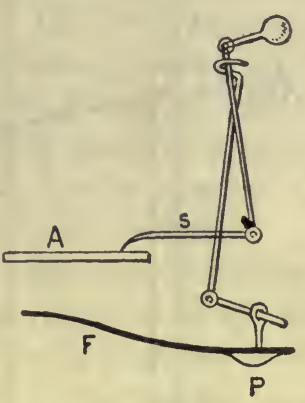

FiG. 414. Diagram of arrangement of recording lever in Dudgeon's sphygmograph.

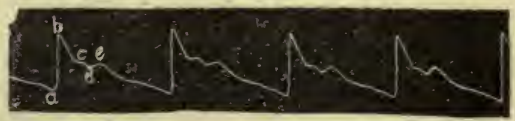

FIG. 415. Pulse-curve from radial artery. The pulse-wave thus reaches the periphery-long before it has been completed in the aorta. Fig. 415 represents a pulse-curve taken from the radial artery: The elevation due to the expansion of the artery is rapid and uninterrupted. We have already explained that this is due to the sudden pumping of blood into the first part of the aorta, whence the impulse is transmitted as a wave along the arteries. The curve descends gradually till the next beat occurs, since the elastic reaction of the arteries, which tends to keep up the pressure, acts more constantly and steadily than the heart-beat. On this descending part of the curve occur two or three secondary elevations : $b$ is the primary or 'percussion' wave, $c$ the predicrotic or 'tidal' wave, and $e$ the dicrotic wave. Elevations may occur on the curve after $e$ which are called post-dicrotic waves. It is better to class the elevations before the dicrotic notch $d$ as systolic elevations, and those afterwards, including the dicrotic elevation itself, as diastolic.

For the exact understanding of these elevations it is necessary to compare the pulse tracings taken from a small artery with the variations in pressure which occur at the same time in the aorta and in the left 
ventricle (Fig. 416). We are enabled in this way to dissociate the waves caused by the ventricular systole from those which have their origin in the arterial system. In Fig. 416 is given somewhat diagrammatically typical tracings of the intra-auricular, intraventricular, and aortic pressures during one heart-beat, and in dotted lines is represented approximately the sort of a curve which would be given by a sphymograph applied to the aorta, taking into account the greater inertia of the latter instrument. The auricular systole begins at the ordinate 1 . It gives a slight rise of

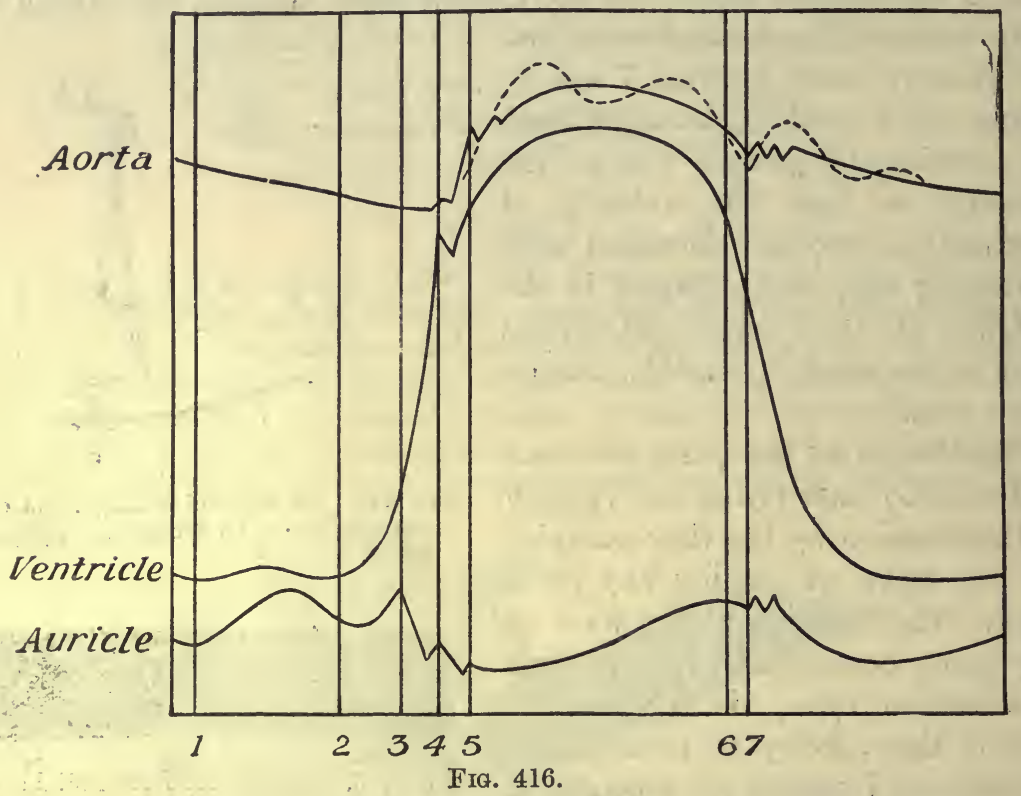

pressure in the ventricle, but as a rule is not transmitted to the aorta, though often some small traces of it can be seen. As the auricular contraction is dying away, the ventricular contraction begins at 2. The first effect of this rise of pressure is to close the auriculo-ventricular valves, as is shown by the elevation at 3 in the auricular curve, and the shock of the closure is occasionally transmitted to the aorta. The pressure in the ventricles then rapidly rises. At the point 4 it surpasses the pressure in the aorta and then rapidly rises above it. Since the aortic valves offer no resistance to the flow of blood from ventricles to aorta, they must open as soon as the intraventricular exceeds the aortic pressure, and this is shown by the rise of pressure in the aorta at 5. The shock of the inrush of blood may give rise to a distinct secondary wave at this point. The pressure then continues for a time to rise rapidly both in the ventricle and in the aorta, blood flowing from the heart into the arterial system. As the first rush of blood diminishes and as the blood begins to escape more rapidly under the influence of the rise of pressure from the peripheral end of the arterial system, the rise of pressure in the ventricle and aorta slows off and the junction between these two periods at 5, where the rise of 
pressure becomes suddenly slower, may be marked in the aortic curve by one or two secondary waves. It must be remembered however that all these secondary waves shown on the aorta at 4 and 5 may be absent, the one at 5 being the one which is most frequently seen. From 6 to 7 the ventricle is still contracting and forcing blood into the aorta. The curve of pressure is generally rounded. It may present a flat top, the plateau, or the top may be rounded with an inclination to fall or to rise (cf. Fig. 398). At 6 the ventricle relaxes, the intraventricular pressure falls rapidly, and at 7 falls below the aortic pressure. The aortic valves must now close since the pressure is greater on their aortic side. The pressure in the ventricle now continues to fall until it becomes zero. In the aorta however there is a sharp elevation immediately after 7 , i.e. immediately after the closure of the aortic valves. This is known as the dicrotic elevation, the previous depression being the dicrotic notch. It is at this point that the second sound of the heart is heard and is evidently due to the vibrations which are represented graphically in the record of intra-aortic pressure.

There are several factors at work tending to produce a secondary wave at this point. With the sudden cessation of the inflow of blood from the ventricles at the end of the ventricular contraction a negative wave must be produced at the beginning of the aorta, which, transmitted along the arterial system, will tend to produce a reflux of blood towards the heart. The movement so caused is reinforced by the elastic reaction of the arterial wall so that the returning blood is driven up against the aortic valves, closing them tightly and putting them on the stretch. Even in a rigid tube the sudden cessation of flow causes a negative wave, followed by a positive wave in the opposite direction in the aorta; this positive wave is increased by the elastic reaction of the stretched aortic valves. The blood is driven up against them by the wave of positive pressure and then rebounds, like a billiard ball from the elastic cushion, and gives rise to the dicrotic elevation. That the dicrotic elevation is for the most part a positive wave running in a centrifugal direction is shown by the fact that the distance between it and the primary wave does not alter appreciably from whatever part of the arterial system the tracing be taken. If it were a reflected wave the distance between it and the primary elevation of the pulse-curve ought to be less the nearer the periphery the pulse tracing is taken.

The predicrotic waves in the pulse tracing are evidently due to the instrumental exaggeration of the wave which may occasionally be seen even in a perfect pressure tracing at 5. The rapid rise of pressure in the aorta or in the more peripheral artery; which follows the opening of the aortic valves sets up a tendency to secondary oscillations at this point. The greater the inertia of the instrument, the greater is the exaggeration of these waves. As is shown by the dotted line in Fig. 416, the lever of the sphygmograph is jerked up, so that it practically leaves the artery, and then falls and rebounds again, so that the simple rounded top becomes resolved by instrumental error into a curve with two waves, which have 
been called the percussion wave and the predicrotic wave. In the same way the inertia of the instrument will tend to exaggerate the dicrotic elevation and possibly to give rise to slight postdicrotic waves.

The general form of the pulse-curve varies with changes in the heart; in the arteries, and in the peripheral resistance. Thus some curves may present secondary elevations on the ascending part, and are called anacrotic, while in others all secondary elevations occur on the descending part. The latter type is called catacrotic, and is the tracing usually obtained from a normal radial artery. By comparing these two types of curves with the corresponding intraventricular pressures, we find that in both cases blood is flowing into the aorta during the whole time from the beginning of the primary elevation to the notch just before the dicrotic elevation. This is shown by the fact that the intraventricular pressure is all this time slightly higher than the aortic pressure. So long as this is the case blood must flow from ventricle into aorta. (This fact proves that there is normally no part of the cardiac cycle during which the ventricle remains contracted and empty, the ventricle in all cases relaxing before it has completely emptied itself of blood.)

Now it is easy to see the conditions which determine whether the systolic plateau shall be ascending or descending, and therefore when the pulse shall be anacrotic or catacrotic. If, after the first sudden rise of pressure in the aorta, the blood can escape more rapidly through the peripheral resistance than it is thrown into the beginning of the aorta, the "systolic plateau' will sink, and a catacrotic pulse tracing is obtained. If, on the other hand, the peripheral resistance is high, or an extra large amount of blood be thrown into the aorta at each stroke of the heart (e.g. by prolongation of the diastole), the aortic pressure will rise so long as blood is flowing in, and we get an ascending systolic plateau and an anacrotic pulse. Thus we obtain an anacrotic pulse in old people with Bright's disease, in whom the peripheral resistance is very high, and also in animals when the heart is slowed by vagus action.

The production of the dicrotic elevation is favoured by any influence which increases the elastic resiliency of the arteries or causes the primary elevation of the pulse to be rapid and sharp. Thus it is much more pronounced in young people than in old people, whose arteries have become rigid. When the peripheral resistance is low through relaxation of the arterioles, and the heart is beating forcibly, as in many cases of fever and also to some extent after a good meal with alcohol, the dicrotic elevation becomes very marked. Under such circumstances it may be easily felt with the finger at the wrist, and in many cases the mistake has been committed of taking the dicrotic wave for a normal beat, and so doubling the rate of the pulse.

OTTO FRANK'S WORK ON THE PULSE. In the account given above of the peculiarities of the pulse-curve in different parts of the system I have adopted in the main the views of Marey and Hürthle, which have been generally accepted for a considerable time and have influenced most of the clinical work on this subject. According 
to these authors all the secondary waves on the pulse-curve are central in origin, and can therefore be traced with slight modification on the curves obtained from the aorta, the large and the small arteries. Although Marey investigated the propagation of reflected waves and showed their presence in the artificial schema of the circulation (vide p. 920), he considered that they could not contribute to the production of the waves on the pulse-curve, owing to the enormous number of points at which reflection might occur, so that the different reflected waves would tend mutually to annul each other's effects. Moreover the distance of the dicrotic notch from the primary elevation was found to be nearly the same at different parts of the system, pointing to a propagation of this wave from the centre to the circumference of the arterial system.

Frank points out that the effect of the propagation of the fairly simple wave started

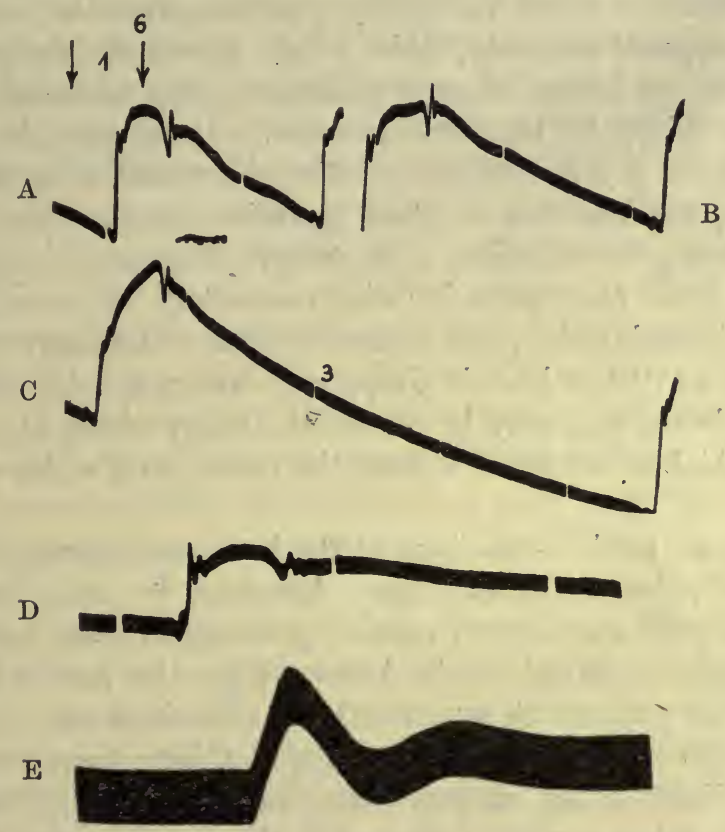

Fig. 417. Pulse-pressure curves taken by means of Frank's manometer (FraNK).

A, B, C, aortic pressure curves at different rates of the heart ; $D$ and $\mathrm{E}$, aortic pressure curve, $\mathrm{D}$, compared with simultaneous record of the pressure in the femoral artery E.

in the aorta in an endless system of elastic tubes would be to diminish the rapidity of onset of each vibration; and therefore to diminish the secondary vibrations on the curve. In a closed elastic system of tubes, such as the arterial system, there will be factors at work analogous in many respects to those responsible for the deformation of the curve given by an imperfect manometer. These will be of two kinds, namely, (1) oscillations of the column of fluid within the stretched arterial wall, (2) reflections of waves from different points in the periphery. These reflections we should expect to be evident in certain cases. Thus in the carotid there should be a reflected wave from the circle of Willis; in the descending aorta, from the bifurcation of this vessel into the two iliac arteries. As a result the pulse in the peripheral arteries, such as the radial or femoral, diverges considerably from the pulse in the aorta. In the figure (Fig. 417, D and $\mathrm{E}$ ) the primary rise of pressure in the femoral artery is even higher than the primary rise in the aorta, i.e. the primary wave must be augmented here by a reflected wave from the periphery. Frank acknowledges that the dicrotic depression in this curve is due to the propagation of the wave set up by the closure of the aortic valves ; 
but he would regard the more pronounced dicrotism of the pulse, of which examples have been given earlier, as due for the most part to the reflection of waves from the periphery.

From time immemorial the physician has sought by feeling the pulse to come to some idea as to the condition of the circulation. A number of different qualities have therefore been distinguished. According to the number of beats per minute the pulse is distinguished as frequent or rare. The size of the pulse has reference to the amplitude of excursions of each beat and the pulse is distinguished as large or small. The velocity of the pulse expresses the speed with which the excursion is accomplished. The quick pulse is one in which the artery presses against the finger suddenly and then disappears suddenly, while in the slow pulse the period during which pressure can be felt is more prolonged. The hardness of the pulse is determined chiefiy by the blood-pressure. If the pulse is compressible it is spoken of as soft; if it can only be obliterated with difficulty it is hard. Certain combinations of these qualities are also described. Thus a large and hard pulse is spoken of as strong, a weak pulse being both small and soft. A small hard pulse is called contracted. If the rhythm of the heart-beat is irregular the pulse is also irregular. An intermittent pulse is one in which one heart-beat is dropped occasionally, i.e. once in every four or eight beats, and may be due to the interposition of a ventricular contraction which is too weak to send the pulse along so far as the radial artery.

Judgments as to the conditions of the heart and circulation from the feeling of the pulse oscillations must, however, be made with extreme caution. The pulse-curve may, indeed, give approximate information as to the condition of things in the heart. Thus the period between the beginning of the primary elevation and the dicrotic notch corresponds to the outflow of blood from ventricle to aorta. A large pulse-curve does not necessarily indicate a big output, since the expansion of the artery is determined not only by events occurring in the aorta but also by the tonus of the artery under the finger and the resistance in the peripheral branches.

Perhaps the best-marked condition of the pulse is that known as the 'water-hammer' pulse, which is observed in cases where the aortic valves are injured or diseased so as to allow of regurgitation into the ventricle. The systolic rise of pressure in the arterial system is followed by an extremely rapid fall, so that towards the end of diastole the pressure in the arteries may' be insufficient to keep the arterial system filled. Under such conditions, if the arm be held above the head and the wrist of the patient be grasped, the pulse in the arteries of the wrist is felt as a smart blow coinciding with each beat of the heart.

\section{THE CIRCULATION THROUGH THE CAPILLARIES}

The capillary circulation is most easily studied by examining under the microscope the tongue of the frog or the web of the frog's foot. Under a.power of about 150 to 180 diameters a network of vessels is seen, 
consisting of small arteries, capillaries, and veins. The direction of flow in the arteries is opposite to that in the veins. In the capillaries the flow is from arteries to veins, though, on account of the reticular arrangement of these vessels, the direction of the stream through them is not quite constant and may occasionally be reversed. The flow of blood in the arteries is rapid, whereas in the veins it is generally possible to distinguish the individual blood-corpuscles. Through the capillaries the flow is very inconstant. If a group of capillaries be watched for some time the blood may at first hurry through a number of them with great rapidity; the flow then becomes slower and then may quicken up to a moderate pace again. These variations in the capillary flow are probably associated with spontaneous alterations in the condition of contraction of the small arteries supplying the group of capillaries. It is easy to observe that the arterial flow is pulsatile, the pulsation disappearing in the capillaries and veins. Another difference between the circulation in these three kinds of vessels is to be found in the condition of the peripheral zone. In the arteries the blood-stream is divided into two parts, the peripheral stream -about $.01 \mathrm{~mm}$. wide, consisting only of colourless plasma with occasionally a stray leucocyte-and an axial stream, in which all the red blood-corpuscles are being hurried along. In the veins there is a similar peripheral plasmatic zone, but here we find regularly scattered leucocytes which travel rather more slowly than the axial stream of red corpuscles. The formation of this axial zone is purely mechanical, and may be imitated in any fluid containing in suspension particles whose specific gravity is somewhat higher than that of the fluid. In the capillaries there is no separation of the two zones, since the lumen of these vessels as a rule allows only the passage of one or two corpuscles abreast, so that they are everywhere in contact with the wall. The corpuscles are evidently elastic structures, and may be seen to bend if they impinge on the dividing point of two capillaries before they are finally swept off by the stream into one or the other.

The capillary wall is composed of a single layer of elongated flattened cells which present little resistance to the passage through them by diffusion of dissolved substances, such as sugar, salts, oxygen, or carbon dioxide. In this way the tissue-cells obtain oxygen from the red blood-corpuscles and nutriment from the plasma, and give off to the circulating blood carbon dioxide and other effete substances as the products of their metabolism. There is evidence that in some situations the cells forming the capillary wall may be contractile. According to Stricker and others, the cell substance is arranged in strands running from the nuclei around the capillary. By the contraction of these strands the vessel may be narrowed to obliteration. These phenomena have been observed in the nictitating membrane of the frog, but it is doubtful how far they may be extended to the other capillary systems of the body. If the contractile power is at all universally present, it must play an important part in determining the amount of blood-flow through the capillaries of an organ, and will doubtless be largely affected 
by chemical substances produced as the result of the metabolism of the surrounding tissues.

The average length of a capillary is between 0.4 and $0.7 \mathrm{~mm}$. The velocity of blood-flow can be directly determined by observing under the microscope the time taken by any given corpuscle to travel a measured distance on the microscope stage. The mean velo-

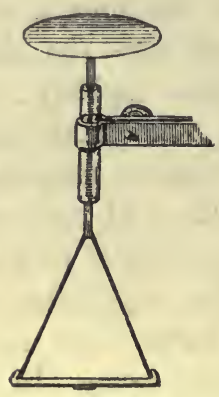

Fig. 418. Apparatus of von Kries for measuring capillary blood-pressure. city determined in this way varies from about 0.5 to $0.8 \mathrm{~mm}$. per second.

The blood-pressure in the capillaries may be measured approximately by applying pressure to the outer surface of the skin or mucous membrane and noticing the point at which blanching of the surface is produced.

In von Kries' method a small glass plate, from 2 to $5 \mathrm{sq} . \mathrm{mm}$. in area, is placed on the last joint of the finger. Attached to this glass plate is a small scale pan on which weights are placed until the pressure is just sufficient to blanch the underlying skin. In using this method the calculation of the capillary pressure is made as follows:

Supposing that the size of the glass plate is $4 \mathrm{sq} . \mathrm{mm}$. and $1 \mathrm{grm}$. in the scale pan is just sufficient to cause a change of colour in the skin, then

$$
\text { a weight of } 1 \text { grm. }=1 \text { c.c. } \mathrm{H}_{2} \mathrm{O}=1000 \text { c.mm. } \mathrm{H}_{2} \mathrm{O}
$$

is present on an area of $4 \mathrm{sq}$. $\mathrm{mm}$. The height of the column of water supported by 1 sq. mm. is therefore $\frac{1000}{4}=250 \mathrm{~mm} . \mathrm{H}_{2} \mathrm{O}$. The errors of this method are considerable, since the pressure thus determined is not the total capillary pressure, but this minus the pressure in the tissue spaces on the outer side of the capillary wall. The result will therefore vary not only with capillary pressure but also with the tension of the skin and the amount of fluid in the tissue spaces.

The pressure in the capillaries as found by this method necessarily varies with the position of the part under investigation, i.e. with the hydrostatic pressure of the column of blood between it and the heart. The following figures were found by von Kries:

Finger : $\quad \mathrm{Mm} . \mathrm{H}_{2} \mathrm{O}$

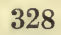

329

513

738
Distance of finger below head

$0 \mathrm{~mm}$.

$205 \mathrm{~mm}$.

$490 \mathrm{~mm}$.

$840 \mathrm{~mm}$.

Ear : $20 \mathrm{~mm}$. Hg.

Gums of Rabbits : $33 \mathrm{~mm}$. $\mathrm{Hg}$.

Frog's Web (Roy) : 100-150 mm. $\mathrm{H}_{2} \mathrm{O}$.

Capillary venous pressure of brain (Hill) :

(1) Animal in horizontal position : $10 \mathrm{~mm} . \mathrm{Hg}$.

(2) , , , feet-down position : zero or less.

(3) During strychnine convulsions : $50 \mathrm{~mm}$. Hg.

Owing to the fact that a varying and unknown resistance-that of the arterioles-lies between the capillaries and the arteries, the pressure in the capillaries must stand in much closer relationship to that in the veins than to that in the arteries. One cannot therefore argue that a fall of 
arterial pressure necessarily involves a fall of capillary pressure in all parts of the body. We can only judge of changes in the capillary pressure by taking simultaneously the pressures in both the afferent and efferent vessels. If these both rise or fall together we may be certain that the capillary pressure also rises or falls. Where the arterial and venous pressures move in opposite directions it is difficult to say what alterations, if any, will be produced in the capillary pressure.

The resistance to the flow of blood through the capillaries is determined by the internal friction, i.e. the viscosity of the blood; this varies in different animals between three and five times that of water. It has been calculated that the fall of pressure undergone by the blood in passing through any given capillary area is only about 20 to $60 \mathrm{~mm}$. of blood, and at the most is never more than $150 \mathrm{~mm}$. blood, i.e. about $10 \mathrm{~mm}$. $\mathrm{Hg}$. This bears out the conclusion to which we have already come, viz. that the chief seat of the resistance in the vascular system is the arterioles, and it is in this region that the chief fall of pressure occurs. 


\section{SECTION VI}

\section{THE FLOW OF BLOOD IN THE VEINS}

In the veins there is a constant decrement of pressure as we pass from the periphery towards the heart. This decrement of pressure is the consequence of the pumping action of the heart, so that the flow through the veins must be ascribed to the same force as that which determines the flow through the arteries, viz. the heart-beat. Owing to the fact that no appreciable resistance lies between the veins and the heart, the difference of pressure necessary to maintain a constant flow through these vessels is very small. Thus in the horizontal position the pressure in the femoral veins may be from 5 to $10 \mathrm{~mm}$. $\mathrm{Hg}$., and in the inferior vena cava from 1 to $5 \mathrm{~mm}$. The pressure in the great veins near the heart is generally negative owing to the aspiration of the thorax, and this negative pressure is naturally increased during inspiration. Opening the thorax therefore causes a rise of pressure in all the large veins. In the latter the pressure depends chiefly on the heart activity, being lowered by vigorous action of the heart pump and raised when this fails in any way. In the peripheral veins the pressure is more dependent on the flow through the corresponding arteries. If an artery of a limb be ligatured the pressure in the small veins of the limb sinks until it is reduced to the pressure in the nearest large trunk in which a flow of blood continues.

Each cardiac cycle causes variations in the pressure in the great veins next the heart in two ways :

(1) By the transmission along the veins of the alterations in the intraauricular pressure.

(2) By the diminution in the volume of the heart in consequence of the expulsion of its blood along the arteries with each heart-beat.

On this account the jugular veins show pulsations with each heart-beat which are somewhat complex in character and resemble closely those occurring in the auricle (vide p. 201). In Fig. 419 a tracing from the wall of the jugular vein is given. It will be seen that each heart-beat gives rise to three variations in pressure within the veins. These three undulations are evidently exactly analogous to those given in Fig. 400 as occurring in the auricular tracing. We should therefore regard $a$ as the auricular contraction, $c$ as the elevation due to the closure of the auriculoventricular valves, $v$ as the elevation due to the accumulation of blood in the auricles during the ventricular systole. The curve $c$ is often spoken 
of as the carotid elevation, and has been ascribed by Mackenzie to direct propagation to the jugular vein from the underlying carotid artery. $\mathrm{He}$ has come to this conclusion because he has not found it in tracings of the liver pulse in cases of incompetent tricuspid valves. There is no doubt, however, that the elevation can be seen on tracings from the inferior vena cava. The explanation of its absence from liver tracings is probably to be ascribed to the fact that the great mass of the liver substance is unable to transmit the very rapid oscillation of pressure due to the closure of the auriculo-ventricular valves. These venous pulsations are much more

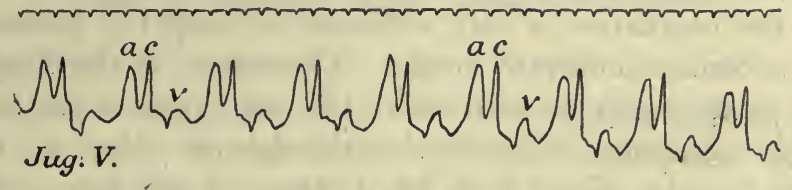

Rad. art.

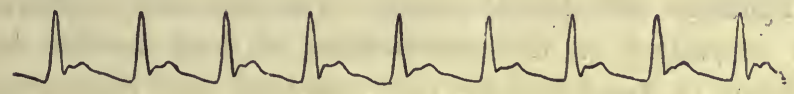

FiG. 419. Venous pulse-tracing from jugular vein compared with the arterial pulse-tracing from the radial artery.

marked in cases of heart disease, where there is partial failure of the heart pump and overfilling of the venous system, often combined with incompetency of the auriculo-ventricular valves.

Besides the favourable influences exercised on the circulation through the veins by the aspiration of the thorax and the momentary aspiration of the heart-beat itself, a considerable part is played in the venous circulation by the contraction of the muscles of the body as well as by the passive movements of different parts. The adjuvant effect of passive or active movement on the circulation through the veins is rendered possible by the existence in these vessels of valves, which are semilunar folds of the intima projecting into their lumen, and so arranged that they allow the passage of blood only towards the heart. Two such valves are as a rule situated opposite to each other. Every movement of a limb, active or passive, causes an external pressure on the veins, and therefore empties them towards the heart. Thus, in walking, each time the thigh is moved backwards the femoral vein becomes empty and collapses, and fills again as soon as the leg is brought forward to its former position or is flexed in front of the body. When muscular movements become general, as in walking or running, the active compression of the veins thus brought about plays an important part in hurrying the blood into the right heart, so that the output of this organ is increased and the arterial blood-pressure is raised.

Since the blood in the vessels is subject to the influence of gravity, we should expect to find that the pressure in the veins of the foot was equal to the pressure in the veins, say, of the hand at the level of the heart plus the pressure equivalent to the column of blood between these veins and 
the heart, i.e. about a metre of blood. On measuring the pressure by von Recklinghausen's or by Hill's method in these veins, this is not found to be the case. The pressure, indeed, in the veins of the foot is but little higher than that in the veins of the hand. Von Recklinghausen found that after subtracting the distance between the foot and the heart the pressure in the veins was negative by as much as $40 \mathrm{~cm}$. In the same way, as Hill has shown, the pressure in the capillaries of the foot is about the same as in the capillaries of the hand. When a man assumes the upright position the arteries of the leg and foot contract until, under the combined influence of the heart's contraction and gravity, the bloodsupply to the capillaries is only sufficient to keep the pressure in these vessels at a certain moderate height. The return of the blood from the dependent parts cannot be ascribed to the heart-beat at all, but is due to the extrinsic mechanism of circulation through the veins, i.e. the contractions of the muscles of the limb which press all the deep and superficial veins, and in virtue of the valves force the blood contained therein by Poupart's ligament into the abdomen. The fact that circulation through the legs is dependent on the contractions of their muscles explains why it is so difficult to stand still for any length of time without moving, and emphasises the need of moderate exercise for the maintenance of a normal circulation. 


\section{SECTION VII}

\section{THE PULMONARY CIRCULATION}

In the lungs there is an extensive system of wide capillaries presenting very little resistance to the flow of blood. The arterioles are wide and have only a slight amount of muscular fibre in their walls, so that a slight pressure suffices to drive the blood from the right to the left heart. The determination of the normal average pressure in the pulmonary artery presents considerable difficulties, but it probably does not exceed 15 to $20 \mathrm{~mm}$. Hg., i.e. about one-sixth of the mean aortic pressure.

The capillaries of the lungs may vary passively in size according to the condition under which they may be placed. Thus, whereas at the height of inspiration the blood contained in the lungs is about one-twelfth of the whole blood in the body, this amount is diminished during expiration to between one-fifteenth and one-eighteenth, and by forcible artificial inflation of the lungs may be lessened to one-sixtieth. These changes exercise a considerable effect on the systemic blood-pressure and are largely responsible for the respiratory variations observed in the systemic blood-pressure. On the other hand, the distensibility of the lung capillaries may play an important part in enabling the lungs to act, so to speak, as a reservoir for the left side of the heart. If, in consequence of raised arterial pressure or other factor, there is a temporary excess of output on the right side that cannot be dealt with at once by the left heart, the excess is taken up for a time in the lung eapillaries.

Vaso-motor fibres to the lung-vessels have been described as running in the anterior roots of the third, fourth, and fifth dorsal nerves. Their action is, however, of little importance, and their very existence is questioned by some observers. The fact that injection of adrenalin causes some vaso-constriction in the lungs, points to the presence of a vaso-motor sympathetic supply to those organs.

If we examine a tracing of the arterial blood-pressure, we notice that it presents certain periodic oscillations which accompany the movements of respiration. With each inspiration the blood-pressure rises; with each expiration it falls. The synchronism of the rise and fall with the respiratory movements is not exact, since the rise continues for a short time after the beginning of expiration before it begins to fall, and the fall continues right into the beginning of the next inspiration, so that the highest point of the curve occurs at the beginning of expiration and the lowest point at the 
beginning of inspiration. During the fall which accompanies expiration the heart-beats, as shown in the diagram (Fig. 420), become less frequent, and an obvious explanation of the fall of pressure would be to ascribe it to a reflex inhibition of the heart. On dividing both vagi, this difference in the pulse-rate during inspiration and expiration disappears, but the main features of the blood-pressure curve remain the same ; so that we must look for some mechanical explanation of the respiratory undulations.

We have already seen that under normal conditions the lungs are in a state of over-distension, and that in consequence of this condition they are constantly tending to collapse, and are therefore exerting a pull on the chest wall. As soon as we admit air

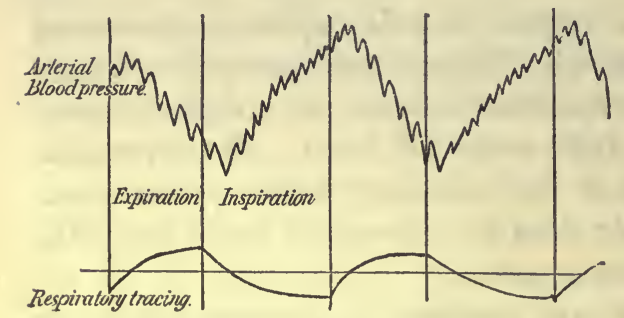

Fig. 420. Diagram of blood-pressure curve, showing effects of the respiratory movements on blood-pressure and pulse-rate. (The effects are purposely exaggerated.) into the pleural cavity by perforating the chest wall the lungs collapse. The force with which the lungs tend to collapse amounts to $6 \mathrm{~mm} . \mathrm{Hg}$. at the end of a quiet - expiration, so we say that in the pleural cavity there is normally a negative pressure of $6 \mathrm{~mm} . \mathrm{Hg}$. As the chest expands in inspiration it drags the lungs still more open. As these become more distended their pull on the chest wall becomes greater, and hence the negative pressure in the pleura may be increased during forcible inspiration to $30 \mathrm{~mm}$. Hg. It must be remembered that the heart and great veins and arteries are in the thorax separated from the pleural cavity only by a thin yielding membrane, so that they are practically exposed to any pressure, positive or negative, which may exist in the pleural cavity. Hence even at the end of expiration the heart and large vessels are subjected to a negative pressure of $6 \mathrm{~mm}$. Hg. Outside the thorax all the vessels are exposed to a positive pressure, conditioned in the neck by the elasticity of the tissues and in the abdomen by the contractions of the diaphragm and abdominal muscles.

Blood, like any other fluid, will always flow from a point of higher to a point of lower pressure. There must thus be a constant aspiration of blood from peripheral parts into the thorax. This aspiratory force will not influence arteries and veins alike. The arteries, having thick, comparatively non-distensible walls, will be very little affected by the negative pressure obtaining in the thoracic cavity, whereas the thin-walled distensible veins will be largely influenced by the same factor. The total result then of the negative pressure in the pleural cavities is to increase the flow of blood from the veins into the heart without affecting to any appreciable degree the outflow of blood from the heart into the arteries. The more pronounced the negative pressure in the thorax, the greater will be the amount of blood sucked into the heart from the veins. During inspiration therefore the heart will be better supplied with blood than during expiration, and this 
factor in itself will tend to raise the arterial blood-pressure. The inspiratory descent of the diaphragm will moreover tend to increase the inflow into the heart by raising the positive pressure in the abdomen, so that blood is pressed. out of the abdominal veins and sucked into the heart and the thoracic veins.

Another important factor is the influence of the respiratory movements on the circulation through the lungs. In trying to understand this influence it must be remembered that the pulmonary capillaries lie in a certain amount of elastic and connective tissue and are separated, on the one side by the alveolar epithelium from air at the ordinary atmospheric pressure, and on the other by the pleural endothelium from the pleural cavity, where the pressure varies from 6 to $30 \mathrm{~mm}$. $\mathrm{Hg}$. below the atmospheric pressure. We may therefore consider the pulmonary capillaries as lying between, and attached to, two concentric elastic bags. Under normal conditions, since these bags are always tending to collapse, the inner one must be pulling away from the outer one, and the outer one from the chest wall. Hence there must be a negative pressure in the tissues between these two bags-a negative pressure which in the expiratory condition will be something between 0 and $-6 \mathrm{~mm}$. $\mathrm{Hg}$., and in the inspiratory condition between 0 and $-30 \mathrm{~mm} . \mathrm{Hg}$. If we regard the average pressure within the pulmonary capillaries as constant, these capillaries must be more dilated in the inspiratory than in the expiratory condition. This dilatation of the pulmonary capillaries will have two effects. Their capacity will be increased and the resistance they present to the flow of blood will be diminished.

Let us now consider what effect these changes will have on the general arterial blood-pressure. We will assume that during expiration the pulmonary vessels have a capacity of 25 c.c. and that the beat of the right heart is forcing through them 10 c.c. of blood per second. So long as the chest remains in the expiratory condition 10 c.c. of blood will be flowing into the left heart and into the aorta, so that the systemic blood-pressure will remain constant. Now let us suppose that an inspiratory enlargement of the thorax takes place, the negative pressure in the pleura is increased, the two walls of the lungs are pulled farther away from one another, and there is a general enlargement of the pulmonary capillaries. We will assume that this enlargement increases the capacity of the pulmonary capillaries from 25 to 30 c.c. Owing to this increased capacity, the first 5 c.c. of blood which flows into the lungs after the beginning of inspiration will not flow out through the pulmonary vein, but will simply serve to bring the capillaries into the same state of distension as before. Hence at the beginning of inspiration the flow through the pulmonary vein will be diminished; there will be less blood discharged into the left heart, and therefore a fall in systemic pressure. As soon, however, as the increased capacity of the pulmonary vessels is made up, the dilating effect of the inspiratory movement of these vessels will aid the flow through the lungs, in consequence of the diminution of resistance, so that the same force of the right heart which drove 10 c.c. of blood per second through the former resistance during expiration will now 
drive more, say 12 c.c. of blood. There is thus more blood entering the left heart, and therefore a rise of systemic pressure during the last threequarters of the inspiratory movement. Expiration will have exactly the reverse effect. At the beginning of expiration there is a diminution of capacity in the pulmonary vessels from 30 to 25 c.c. Hence during the first second of expiration the outflow of blood from the pulmonary vein into the left heart will be 17 c.c. ( 12 c.c. +5 c.c.). After this, the increased resistance in the pulmonary capillaries in consequence of their constriction will come into play, and the flow of blood through them will fall once more from 12 c.c. to 10 c.c. Hence at the beginning of expiration the inflow of blood from the pulmonary vein into the left heart is greater than at any period. The arterial pressure will therefore rise to its greatest height at the beginning of expiration, and will fall during the last three-quarters of expiration, but will attain its minimum only at the beginning of the next inspiration.

In this way the effect of the respiratory movements on the systemic bloodpressure can be entirely explained by the influence they exert on the lungvessels or lesser circulation. On the other hand, Lewis regards the pericardial pressure, i.e. the direct influence of the thoracic movements on the heart, as playing a much more important part than changes in the pulmonary circulation in the production of the respiratory undulations in the bloodpressure. He shows moreover that in man the effect of respiration on arterial blood-pressure may vary according to the type of respiratory movement, a deep intercostal inspiration (not prolonged) causing a pure fall of pressure, while a deep diaphragmatic inspiration gives a pure rise of blood-pressure. In expiration the reverse effects hold. He concludes that it is not possible to make any general statement as to the nature of the blood-pressure response to a particular respiratory act. 


\section{SECTION VIII}

\section{THE CAUSATION OF THE HEART-BEAT}

IF the heart be cut out of the body of a cold-blooded animal, such as the frog or tortoise, it will continue to beat with the normal sequence of its different chambers for hours, or even days, provided that it be kept cool and moist. In the case of a warm-blooded animal the heart is similarly capable of continuing its rhythmic contractions for some little time after excision. The period of survival of the heart is less in warm-blooded than in coldblooded animals. The fact that in both cases the heart will continue to beat after removal from all its connections with the central nervous system, and when blood is no longer flowing through it, shows that the causation of the heart-beat is to be sought in the walls of the heart itself.-

The heart wall consists of a muscular tissue resembling in many respects voluntary muscle; like this, it presents longitudinal and transverse striations ; like this, it is capable of contracting in response to direct stimulation. Normally voluntary muscle only contracts in response to impulses from the central nervous system. When Remak described the existence of collections of ganglion-cells in the sinus venosus, it was natural that physiologists should ascribe to these collections of nerve-cells the same automatic rhythmic functions that had been found by Flourens and others to be associated with the grey matter of the medulla oblongata in connection with the maintenance of the respiratory movements.

\section{ANATOMY OF THE FROG'S HEART}

The hearts of the frog and of the tortoise have figured so largely in the researches on the causation of the heart-beat that it may be profitable to mention briefly the main points of their anatomy.

The frog's heart consists of the sinus venosus, which receives the anterior and posterior venæ cavæ, two auricles, one ventricle, and the bulbus arteriosus, which opens into the two aortæ. The venous blood from the body flows into the sinus venosus by the three venæ cavæ, and thence into the right auricle, while the left auricle receives the blood from the lungs. The ventricle thus receives mixed arterial and venous blood, the arterial blood being directed by the spiral valve of the bulbus aortæ so as to flow chiefly towards the head.

The muscular fibres of the heartareless highly developed thanthose of the mammalian heart. They are spindle-shaped, and only dimly cross-striated. The cross-striation becomes more distinctly marked as we proceed from sinus to ventricle, the sinus muscle fibre representing the most primitive condition. There is complete muscular continuity between all the cavities of the heart. The circular ring of muscle at the junction 
of sinus with auricles and of auricles with ventricles presents only slight traces of crossstriation (Gaskell).

The heart is well supplied with nerve fibres and ganglion-cells. The two vagi enter the sinus venosus and branch just under the pericardium. Here they become connected with a collection of nerve-cells, known as Remak's ganglion. From the sinus the two vagi, now called septal nerves, pass down in the interauricular septum, one in front and the other behind. Near the auriculo-ventricular groove they enter two collections of ganglion-cells, called Bidder's ganglia. From these ganglia non-medullated fibres are distributed to surrounding parts of the auricle and to the whole of the ventricle. In the upper third of the ventricle occur scattered ganglion-cells attached to the nerve fibres. These are quite absent in the lower half or two-thirds.

In the tortoise (Fig. 422) the two auricles are bound together by a flat band of tissue, which serves also to connect the sinus with the ventricle. The septum between
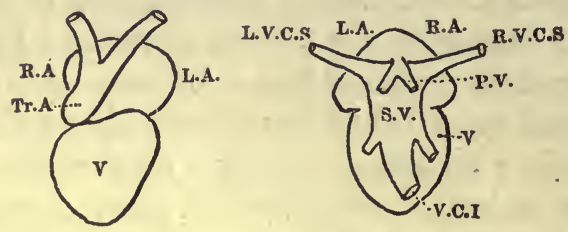

Fig. 421.

Diagram of frog's heart. Cyon.)

v, ventricle; R.A, L.A, right and left auricles (atrium); S.v, sinus venosus; P.v, pulmonary veins; L.v.c.s and R.v.c.s, left and right superior vena cava; V.C.I, vena cava inferior; Tr.A. truncus arteriosus.

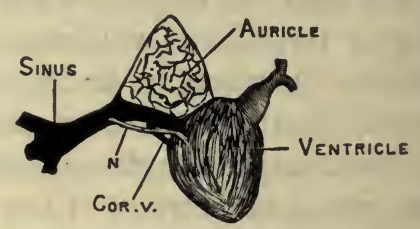

Fig. 422. Tortoise's heart (after GASkELL) as it appears when suspended for registering the auricular and ventricular contractions. $\mathrm{N}$, nerve-trunk with fibres connecting Remak's and Bidder's ganglia; COR. v, coronary vein.

the auricles arises from the central line of this junction wall. The two vagi nerves pass into a large accumulation of ganglion-cells in the sinus, and thence along the basal wall to the auriculo-ventricular groove, lying just under the pericardium. In the groove they pass into a collection of ganglion-cells, whence fibres are given off to both auricles and ventricle. As they leave the sinus, a branch is always given off by the right nerve to accompany the coronary vein, which conveys blood from the ventricular wall to the sinus. Thus the nerves of the tortoise's heart are altogether more accessible than those of the frog's heart: In other points the tortoise's heart is similar to the frog's heart, though considerably larger.

\section{THE AUTOMATIC CONTRACTION OF THE FROG'S HEART}

The frog's heart in the body, or when removed from the body intact, beats regularly, the contraction starting in the sinus, then travelling to auricles, ventricle, and bulbus. If, however, the heart be removed by cutting it across the sino-auricular junction, or if the auricles be functionally separated from the sinus by a ligature round this junction (Stannius's ligature), the auricles and ventricle stop in an uncontracted condition (diastole), while the sinus goes on beating regularly. After the lapse of a period varying from five minutes to half an hour the detached part of the heart begins to beat, at first slowly and then more rapidly, but never attaining the rate of the sinus. The auricles beat first, and then the ventricle. If now the ventricle be cut away by an incision in the auriculo-ventricular groove from the auricles the latter go on beating; while the former, after a few beats due to the excitation of the incision, stops still, and only after a considerable time may begin again to contract very slowly. 
On the other hand, a ventricle-apex preparation (that is to say, the lower two-thirds of the ventricle separated functionally from the rest of the heart) never beats again under normal circumstances. To single stimuli it responds with a single beat, not with a series of beats as the whole heart does. If the lower third of the ventricle be separated functionally in the living frog by crushing the ring of tissue between it and the upper third, it never gives a spontaneous beat again, although it is under the most normal eonditions possible in the circumstances. There is thus a descending scale of automatic power in the different parts of the frog's heart-from the sinus, where it is highest, to the lower parts of the ventricle, where it is apparently absent. From this fact it has been thought that the automaticity of the frog's heart is dependent on the ganglia present in it. The contraction was supposed to be started by impulses proceeding from the sinus ganglion. If this were cut off, Bidder's ganglia, or the scattered cells in the upper third of the ventricle, could, it was thought, take up its task of originating impulses. The muscle-cells under this hypothesis act as the servants of the ganglion-cells, just as the voluntary muscles wait on the commands of the cells in the spinal cord and brain.

The view that the ganglion-cell sends out rhythmic impulses had, however, to be discarded when it was discovered that the muscle forming the lower third of the ventricle either of the frog or the tortoise, though free from ganglion-cells, could be excited by various means to rhythmic contractions. Thus it could be set into rhythmic action when supplied with salt solution under pressure, through a perfusion cannula, or when excited by the passage ${ }^{*}$ of a constant current or of weak induction shocks. The fact that the heart muscle responded to continuous stimulation by a rhythmic discharge suggested that the function of the ganglion-cells was to furnish a constant stimulation to the muscle-cells and so maintain these in rhythmic activity.

The theory of the ganglionic origin of the cardiac rhythm was seriously affected by a series of researches carried out by Gaskell and by Engelmann. The arguments against the 'neurogenic' hypothesis may be summarised as follows :

(a) The cardiac muscle, free from any ganglion-cells whatsoever, can be excited by various means to rhythmic contraction. When, in the living frog, the apex of the ventricle is crushed off from the base so as to leave only material continuity between the two parts, the circulation of the blood is maintained by the contraction of all the parts of the heart except the apex, which never resumes its activity. If, however, the intraventricular pressure be raised by clamping the aorta the apex begins to beat at its own rhythm, which is independent of the rhythm of the rest of the heart. Moreover a strip can be cut from the apex of the tortoise's ventricle (Fig. 423), free from ganglion-cells, which on keeping in a moist chamber and moistening occasionally with normal salt solution enters into rhythmic contractions.

(b) In the frog it is possible to excise the inter-auricular septum with its ganglia, and a considerable portion of the ganglia in the sinus venosus and at the base of the ventricles, without interfering in any way with the cardiac 
rhythm. This experiment is still easier to carry out in the tortoise's heart where the nerves and ganglia run in the basal portion of the auricles. This can be excised, leaving the two auricular appendages in connection with the sinus venosus and with the ventricles. -

(c) The heart in the developing chick can be seen beating at a time when it is quite free from nerve-cells, which only extend into it at a later date.

(d) Remak's ganglia are situated at the point where the two vagus nerves enter the heart, and under the microscope can be seen to be connected with the fibres of these nerves. We have now, from the discovery of Langley and Dickinson, a means of judging of the action of ganglion-cells in the drug nicotine, which first stimulates and then paralyses nerve-cells themselves

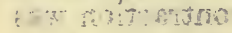

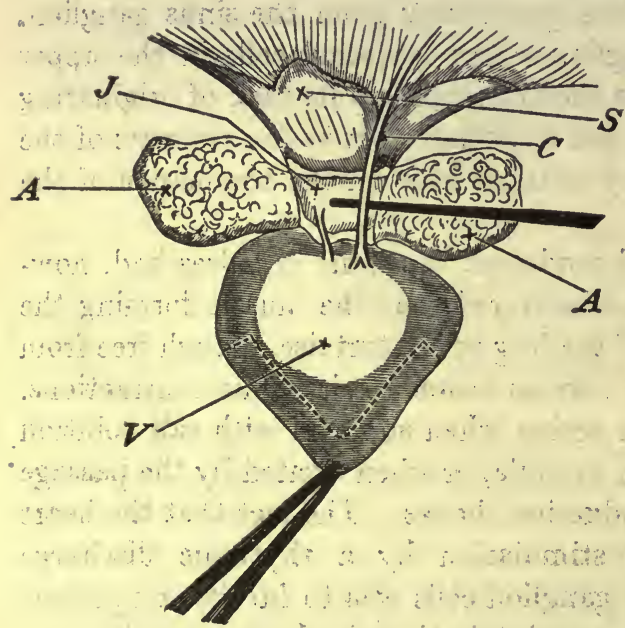

Fig. 423. Tortoise's heart from dorsal surfacc. (GASKELL.)

$\mathrm{S}$, sinus ; J, sino-auricular junction; A, auricles; C, coronary vein; V, ventricle. (The dotted line shows how a strip may be cut from the ventricle apex.) or the synapses between the cells and the nerve fibres in connection with them. Direct application of nicotine to the heart, after a primary period of slowing, leaves the heart-beat practically unaltered, the normal sequence of beat in the various cavities being unaffected. After the application of the drug, however, stimulation of the trunk of the vagus is without effect, though slowing or stoppage of the heart may still be produced by excitation of the post ganglionic nerve fibres of the vagus which arise from the cells of Remak's ganglia. These ganglia must therefore be regarded not as a motor centre for the heart, but merely: as a distributing-centre for the inhibitory fibres of the vagus. Since tetanisation of the heart with weak currents also causes local inhibition, it would seem that the finer nerve fibres ramifying throughout the muscular substance are, to a large extent at all events, inhibitory in their function. This is confirmed by the fact that atropine, which paralyses the inhibitory fibres of the vagus, also abolishes the direct inhibitory effect of tetanisation on the heart muscle.... Gaskell and Engelmann therefore came to the conclusion that the source of the cardiac rhythm was to be found, not in the ganglia scattered about its cavities, but in the muscular cells themselves.

. The normal sequence of events-i.e. the subordination of the ventricle to auricles and auricles to sinus, so that the beat always follows in the order, sinus, auricles, ventricle, bulbus - can be ascribed to the difference between the natural rhythms of these different cavities. It is possible to record the contractions of each of these parts of the heart separately, after having divided 
them, either functionally by crushing the intervening tissue, or by actual section. Under such conditions it is found that there is a descending scale of rhythm from sinus to bulbus, the contractions of the sinus being most frequent, those of the ventricle and bulbus the least frequent. Thus it is impossible for the ventricle to beat at its own rhythm, since before it is ready to beat again after performing one beat it receives an impulse from the auricles causing an excited beat. That the normal sequence of contractions is dependent simply on the natural rhythm of the sinus is shown by the fact that by exciting the ventricle by means of induction shocks repeated at a rhythm slightly greater than that of the sinus it is possible to excite a reversed rhythm, the order of the beat being now ventricle, auricles, sinus venosus.

The dependence of the ventricular rhythm on the beat of the sinus may be shown by a simple experiment. The ventricle is connected with a lever suspended by a spring so as to record its contractions on a drum. A platinum loop connected with a galvanic battery is put round the heart, either round the sinus or round the ventricle (Fig. 424). When a current is allowed to pass through the inner loop the corresponding part of the heart is warmed. When the ventriele alone is warmed the beats, become larger, but the rhythm is unaltered. On lowering the loop so as to warm the sinus, the

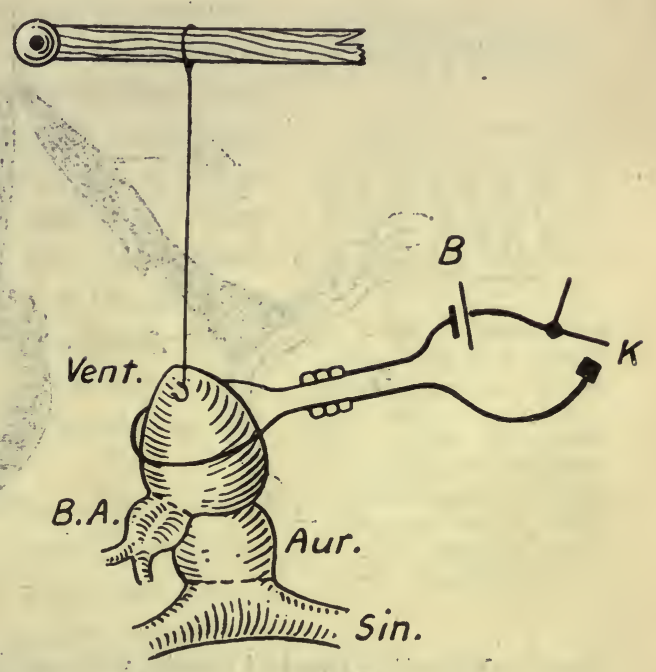

Fig. 424. rhythm of the whole heart is quickened, but the size of the ventricular beats is unaffected. The different rhythmic power of these parts of the heart is apparently connected with the histological characters of the muscle fibres at each part. The lowly differentiated sinus cell has wellmarked rhythmic power and a quick rhythm of beat, but is not a able to exert such force in its contraction. The more highly differentiated ventricle cell has only a slight rhythmic power, but beats forcibly and is a good servant of the sinus.

\section{THE PROPAGATION OF THE WAVE OF CONTRACTION}

The normal contraction started in the sinus venosus is propagated to the auricles, thence to the ventricle, and thence to the bulbus aortæ. Between the contractions of each of these cavities there is a slight pause, whereas the contraction spreads so rapidly over each cavity that all parts, say of the auricles or ventricle, appear to contract simultaneously. It is obvious that the excitatory wave might be propagated through the heart from one 
muscle-cell to another, or by means of nerve fibres, which would excite the muscular tissue of each cavity to contract.

The distinct pause which intervenes between the contractions of auricles and ventricle was long regarded as evidence for the nervous character of the contraction, and as showing the operation of a nerve-centre in the coordination of the contractions of different cavities. A contraction wave may, however, be started at any part of the heart and may travel from this to all other parts. Thus, although the normal direction of the contractions is from sinus to ventricle, it is possible, by stimulating the apex of the ventricle, to excite contractions in the reverse order, viz. from ventricle to sinus. Such a fact is at varianee with all our present knowledge of excitation

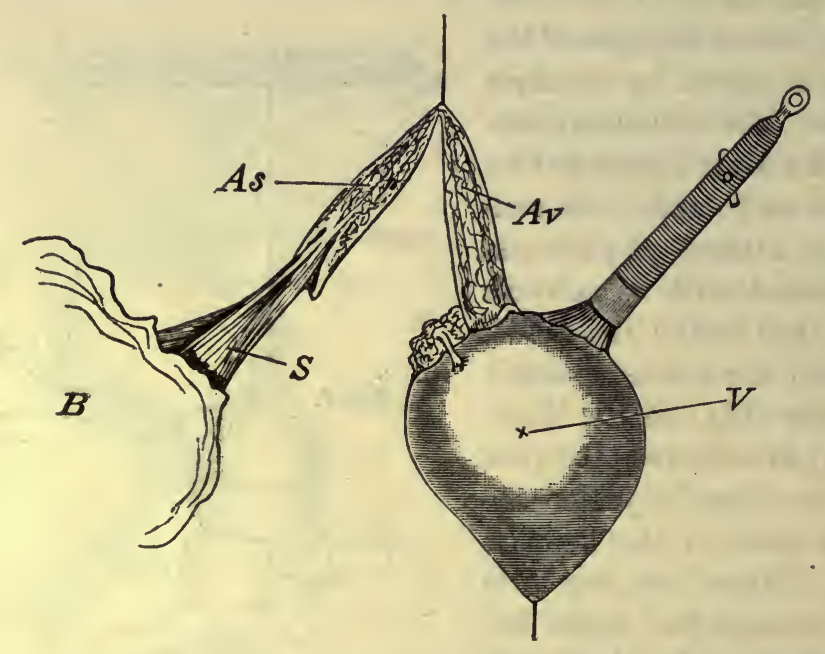

FIG. 425. Heart of tortoise with auricle slit up so as to cause a partial block. (GASKELL.)

of motor nerves. Excitation of the nerve going to the sartorius, or any part of the nerve, may excite contractions of all the fibres of which the muscle is composed. On the other hand, excitation of a part of the muscle which is free from nerve fibras causes a contraction which is limited to the muscle fibres directly excited and does not extend to the nerves. If motor nerves arose from the hypothetical motor ganglion of the heart and passed to the ventricular muscle, one would not expect that contraction of the ventricular muscle could excite these nerves and so cause the propagation of a wave of contraction in the reverse direction.

That the propagation cannot be due to any nerve-trunks running from sinus to ventricle is shown by various experiments of Engelmann and Gaskell. Thus, if the auricle is slit up by a series of interdigitating cuts, the contraction wave starting from the sinus travels along the auricular muscle around the end of each section, and finally, on arrival at the ventricle, causes a contraction of this cavity. In the heart of the tortoise the nervetrunks run, not in the interauricular septum, but in a band of tissue joining 
the sinus to the ventricles; this band can be exercised with all its contained nerves without interfering in any way with the normal sequence of contractions. Moreover the pause observed between the contractions of auricles and ventricles has been shown by Gaskell to be due to the retardation of the excitatory wave which occurs in its propagation through the muscular tissue in the auriculo-ventricular junction. A similar retardation of the wave can be produced at any point either in auricles or ventricle by diminishing the conducting muscular tissue to a sufficiently small extent. Thus, if the auricle of the tortoise be divided as in the diagram (Fig. 425), it will be noticed that the sinus first eontracts, then the auricular half $A s$; a distinct pause then occurs while the contractile process is passing over the 'bridge,' and finally $A v$ contracts, followed by the ventricle. The apparent pause between the contraction of the auricles and ventricle is due therefore to a partial 'block' at the auriculo-ventricular junction. If the block be increased in the experiment just quoted, as, for instance, by allowing the bridge of tissue to dry or by making it still narrower, it may be found that only one out of every two contractions passes across the bridge (Fig. 426), and the slightest increase in the resistance to the propagation of the wave may lead to the block becoming complete. On moistening the bridge again every contraction may be seen to pass.

By the methylene-blue method it is possible to demonstrate a close network of non-medullated fibres surrounding all the muscle-cells of the heart. It is

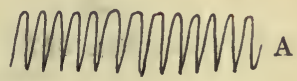
obvious that the experiment just quoted would not exclude the possibility of propagation occurring through such a nerve network. The properties of the network would have to differ from those of any of the nerve tissues with which we are acquainted; whereas we know that under certain circumstances impulses may be transmitted from fibre to fibre even in striated muscle, and such a mode of propagation is the most obvious explanation of the phenomena observed in the heart.

If the auricles be soaked for some time in distilled water they enter into a condition of what is known as water-rigor (Wasserstarre). In this condition they are incapable of contracting, but can still propagate the wave from sinus to ventricle. This experiment has been regarded as a demonstration of the part taken by nerve fibres in the propagation of the wave, but such an explanation is not necessary, since a similar condition of water-rigor in a voluntary muscle fibre has been shown to allow the passage of an excitatory wave through the affected part to the normal portion of the muscle, which then responds by a contraction.

A series of interesting researches by Carlson on the mechanism of the heart-beat in the king-crab Limulus have been thought to throw light on the vexed question of the automatism of the vertebrate heart. 
In Limulus the heart forms a segmented tube of ordinary striated muscular fibres. In large specimens the tube may be from 10 to $15 \mathrm{~cm}$. long and 2 to $2 \frac{1}{2} \mathrm{~cm}$. broad. Like the hearts of most other invertebrates and of all vertebrates, it has a local system of ganglion-cells, but so situated that they can be cut away entirely from the muscular portions of the organ. The arrangement of the cardiac nervous system in Limulus is shown in Fig. 427.

The ganglion-cells are collected chiefly in a dorsal nerve ganglicn cord which runs almost the whole length of the heart. From this cord non-medullated nerve fibres pass

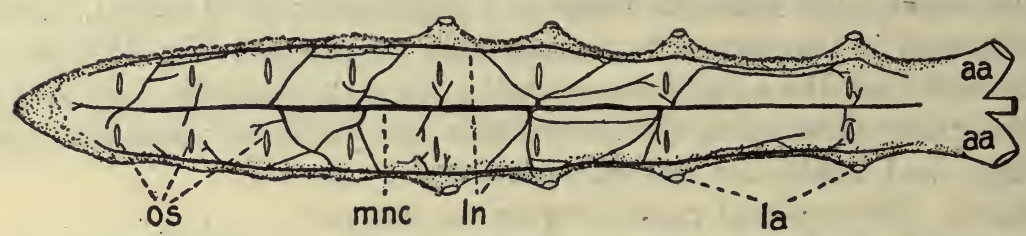

Fig. 427. Heart of Limulus from dorsal surface. (Carlson.) $m n c$, median nerve-cord; ln, lateral nerve-trunks.

directly into the substance of the heart, and also send branches to two lateral nervetrunks, by which fibres are distributed to all parts of the heart.

The heart normally contracts about forty times per minute. Each contraction affects all parts practically simultaneously, though in the dying heart the posterior portions apparently contract slightly before the anterior, and may continue to contract after the anterior end has come to a standstill.

Division of the muscular tissue leaving the nerve-strands intact does not alter in any way the synchronism of contraction of the two ends of the heart. Division of the nervous cord into two parts, the section being carried between the posterior third and anterior two-thirds, causes complete lack of co-ordination between the two ends; both ends of the heart continue to contract, but at different rhythms. Extirpation of the

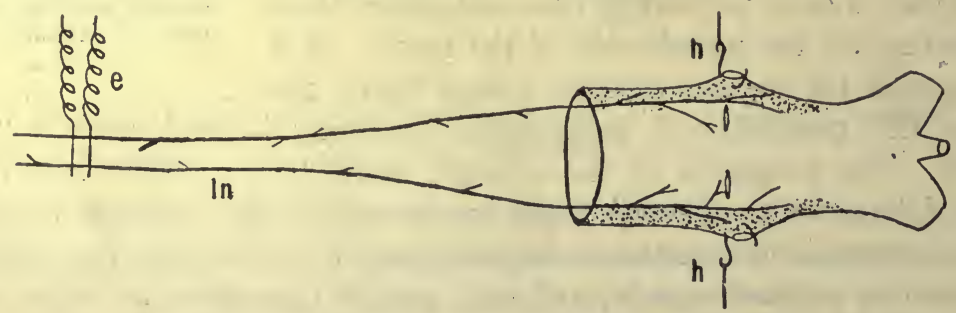

Fig. 428. 'Nerve-muscle preparation' of heart of Limulus consisting of the muscle of the two anterior segments, with the two lateral nerves. (CARLSON.)

nerve-cord abolishes spontaneous contractions. If the anterior half of the dorsal ganglionic cord be excised, all parts of the heart will continue to contract in unison. If now the lateral nerve-trunks be divided, the anterior half of the heart ceases to contract, showing that it was being excited by impulses arising in the posterior part of the ganglionic cord. It is possible therefore to make a nerve-muscle preparation of the anterior part of the heart, consisting of the muscle of the first two segments with a longer stretch of the lateral nerves (Fig. 428). Stimulation of the lateral nerves with a single shock causes a single beat of the anterior segments; tetanising shocks cause a continued contraction of the muscle preparation.

There seems to be no doubt that in this animal the beat of the heart is originated and co-ordinated by the action of the local ganglionic centres. Moreover Carlson has shown that the inhibitory nerve to the heart acts, not by direct influence on the muscle fibres, but by an inhibition of the automatic activity of the ganglionic cells, thus con- 
firming for this spccial case the general view of inhibition long ago put forward by Morat, but not now generally accepted.

The heart muscle does not show a refractory period, but on direct stimulation with repeated shocks there may be a summation of contractions, which may fuse to a complete tctanus. The question naturally arises how far the heart of Limulus is to be regarded as a spccial case, or how far we may transfer results gained from experience on this heart to those of other hearts in which a perfect separation between ganglicncells and muscle fibres is not so easily attainable. Carlson has sought to show the applicability of his results to the explanation of the cardiac mechanism in vertebrates by a scries of observations on other invertebrates' hearts; where the muscular and nervous tissues are not so easily dissociable. Such hearts present phenomena very analogous to those of the frog's heart. According to him the phenomenon of the refractory period, the 'all or none 'law of contraction, and the absence of tetanus in the heart of the frog is due, not to the peculiar functions of the muscle fibres, but to the fact that in all our experiments we are affecting muscular and nervous tissues simultaneously.

In the absence of more perfect knowledge of the properties of the nerve-nets which surround involuntary and cardiac muscle fibres a decision of the point is not yet possible. The muscle and nerve fibres of Limulus show, however, important differences from the cardiac muscle of the frog in their reaction to chemical stimuli. Acceptation of the neurogenic theory would necessitate the prediction of a type of nervous tissue endowed with propcrties for which we have no analogy in any of the nerve tissues which have been the subject of exact investigation, whereas the myogenic theory only ascribes to the musclc-cells of the heart properties which are the common attribute of all protoplasm or are displayed in a less marked degree by the ordinary skelctal muscle fibres. It would, at any rate, be premature to transfer unreservedly all the results obtained on the heart of the Limulus, the muscle fibres of which have the structure and behaviour of skeletal muscle fibres, to the explanation of the phenomena exhibited by the hearts of vertebrates.

\section{THE HEART-BEAT AS A WAVE OF CONTRACTION}

It the beat of the frog's ventricle, or a strip of mammalian ventricle, be recorded, the curve obtained resembles closely the twitch of a voluntary muscle produced in response to a single excitation. Whereas, however, a single contraction with the subsequent relaxation of voluntary muscle only lasts about one-tenth of a second, the contraction of the mammalian ventricular muscle lasts three-tenths of a second, of the frog's ventricle about half a second, and of the tortoise ventricle about two seconds.* In the heart, as in a voluntary muscle fibre, the contractile process originates at the stimulated point and travels thence to all other points.

The progress of the excitatory wave is well seen if a record be taken of the electrical changes resulting in the frog's heart from a single stimulation. If the two ends of a strip of ventricular muscle be connected with the two terminals of a capillary electrometer, stimulation at one end causes a diphasic variation, showing that the excitatory process starts at the stimulated end and travels to the other end of the heart. Thus if the acid of the electrometer be connected with the base of the ventricle and the mercury of the capillary be connected with the apex, stimulation at the base causes a wave passing from base to apex. Directly after the stimulation therefore the base

* The duration of the contraction depends on the temperature. The figures given are for the mammalian heart at $37^{\circ} \mathrm{C}$. and for the amphibian heart at about $15^{\circ} \mathrm{C}$. 
becomes negative and the column of mercury moves towards the acid; a moment later the contraction extends to the apex. All parts of the heart are now in a similar condition of excitation : there is no difference of potential between the two terminals and the mercury comes back quickly to the base line. Relaxation, like contraction, starts first at the base and proceeds thence to the apex. There is thus a small period during which the apex is still contracted while the base is relaxed and the apex is therefore negative to the base. This terminal negativity of the apex is shown on the capillary electrometer by the excursion of the column of mercury away from the point of the capillary (cp. Fig. 87, p. 230).

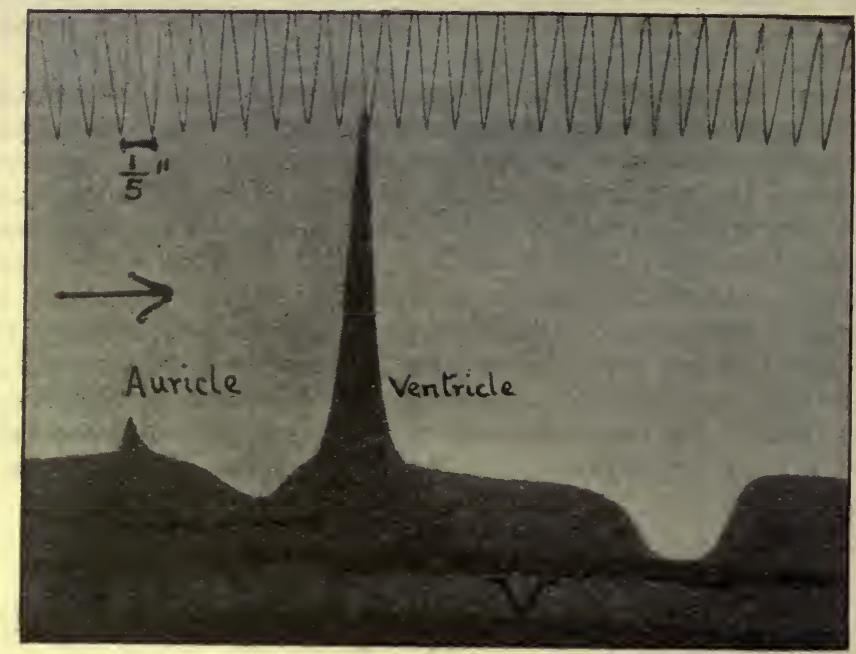

FIG. 429. Electrometer record of variation of spontaneously beating tortoise heart. (Gотсн.)

Analogous effects are obtained on leading off the spontaneously beating heart in the frog or tortoise (Fig. 429). The conditions are, however, rather more complex, and the most usual variation, as Gotch has shown, is triphasic. In its most primitive form the vertebrate heart is composed of a simple tube in which a contraction starts at the venous end and is propagated in a wave-like manner along the tube to the arterial end. In the higher vertebrates the heart at its first appearance has the same tubular form, but the simple tube very rapidly becomes modified, partly by twisting on itself, partly by the outgrowth of the dorsal or the ventral wall of the tube to form the cavities of the auricle and ventricle. Gotch suggests that the excitatory process follows the course of the original tube and that the typical form of the curve is due to the base becoming excited twice, first at the part in continuity with the auricle, and, secondly, when the wave sweeps up to the bulbus aortæ. But it is possible that in the cold-blooded, as in the mammalian, heart there may be a special conducting tissue which 
leads the excitatory process to many different parts of the ventricle almost simultaneously.

There is no doubt that the ventricular systole is comparable with a simple muscular twitch and cannot be regarded as the summation of several contractions. Since the excitatory process extends in the form of a wave not only to all parts of the same cavity but to all parts of the heart, it is evident that the musculature of the heart is to be compared, not with skeletal muscle composed of many fibres, but to a single muscle fibre in which all parts are in functional continuity.

\section{THE BEAT OF THE MAMMALIAN HEART}

The mammalian heart, like the heart of cold-blooded animals, will beat for some time after it has been cut out of the body, and a perfectly rhythmic activity may be maintained for hours by feeding the heart from the coronary arteries either with defibrinated blood or with oxygenated Ringer's solution, with or without the addition of glucose.

Ganglion-cells are found in the mammalian heart around the openings of the great veins, along the border of the interauricular septum, in the groove between auricles and ventricles, and in the basal parts of the ventricles.

The ventricles of mammals are endowed with a greater rhythmic power than the corresponding cavities in the frog and tortoise. It is possible to sever or crush all the nervous and muscular connections between auricles and ventricles without destroying their mechanical connection by means of fibrous tissue. Such a procedure does not, even for a moment, stop the contractions of the ventricles, which go on beating at a rhythm which is independent of and slower than that of the auricles. Porter has shown that a mere fragment of the ventricular wall, perfectly free from ganglion-cells, may maintain rhythmic contractions for some hours if fed by an artificial circulation through a branch of the coronary artery. We may therefore conclude that in the mammalian, as in the amphibian, heart the cause of the rhythm is to be sought in the properties of the muscle fibres themselves, and that every part of the heart-muscle possesses the power of rhythmic activity, the normal sequence of the beats being determined by the greater frequency of the natural rhythm of the venous end of the heart.

In the mammalian, as in the amphibian, heart the excitatory condition started at one point in the muscle spreads through the muscle in all directions, and the process of conduction of excitation seems to be independent of nerve fibres. The excitatory process may be conducted not only in the ordinary direction from auricles to ventricles, but also from ventricles to auricles. If the ventricles be excited at a rhythm of higher frequency than the natural beat which is starting at the venous end of the heart, we may obtain a reversed rhythm in which the order of the beats is ventricles-auricles. It is difficult to conceive of an arrangement of neurons which would propagate impulses impartially from auricles to ventricles or from ventricles to auricles. Such a condition would seem to be in contradiction to the law of forward direction which obtains throughout the nervous system. On the 
other hand the phenomena are easily explained on the assumption that the whole of the musculature of the heart acts in many respects as a single muscle fibre, along which an excitatory process may be propagated in any direction. But in the adult mammalian heart, on superficial dissection, the muscle fibres both of auricles and ventricles are seen to arise from a fibrocartilaginous ring surrounding the auriculo-ventricular junction, leaving apparently no muscular continuity between the two cavities. On this account it was thought for many years that the propagation of the contrac-

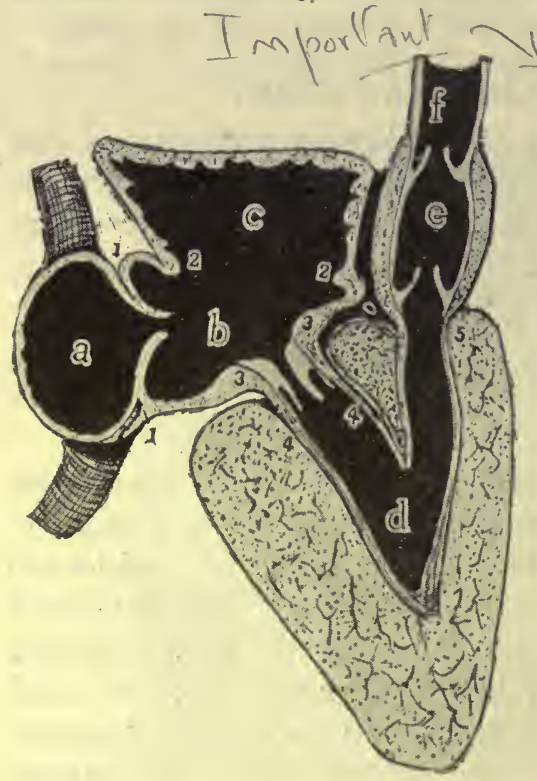

FIG. 430. A generalised type of vertebrate heart. (КеIтH.)

$a$, sinus venosus; $b$, auricular canal; $c$, auricle ; $d$, ventricle ; $e$, bulbus cordis; $f$, aorta; $1-1$, sino-auricular junction and venous valves; 2-2, canalo-auricular junction ; 3-3, annular part of auricle ; 4-4, invaginated part of auricle; 5 , bulbo-ventricular junction. tion from auricles to ventricles must occur by means of nerve fibres, and it was only with the discovery by His of a distinct band of modified muscle fibres, passing from the auricles to the ventricles, the ' auriculoventricular bundle, that an anatomical basis was furnished for the physiological behaviour of the heart. The heart is developed from a muscular tube in which at the beginning we must assume muscular continuity throughout. The primitive vertebrate heart is formed by a modification of this muscular tube. In this heart, as Keith has shown, we may distinguish five chambers, namely, the sinus venosus, the auricular canal, the auricle, the ventricle and the bulbus (Fig. 430). The musculature of these chambers is continuous throughout. In the adult heart, e.g. of man, the anatomical relations of the different cavities have become considerably modified in the course of development. The sinus venosus, i.e. the part where in the lower vertebrates the contraction wave takes its origin, is now represented merely by the termination of the superior vena cava and of the coronary sinus in the right auricle. These two veins are derived from the right and left ducts of Cuvier in the embryo. The sinus venosus is also represented by a small amount of tissue underlying the tonia terminalis of the right auricle, as well as by the remains of the Eustachian and venous valves. The auricular canal gives rise to the auricular septum and to the auricular ring surrounding the auricular-ventricular orifice, and in some hearts it is prolonged into the ventricle as the intraventricular or invaginated part of the auricular canal. In the adult heart two accumulations of more primitive tissue are found in the region corresponding to the sinus venosus of the embryo which are known 
as the sino-auricular node and the auriculo-ventricular node. The sinoauricular node (Fig. 431) lies in the groove between the superior vena cava and the right auricle. The auriculo-ventricular node lies at the base of the auricular septum on the right side, below and to the right of the opening of the coronary sinus. From this point a bundle of muscular fibres (the bundle of His or the auriculo-ventricular or A.V. bundle) runs along the top of the interventricular septum just below its membranous part and then

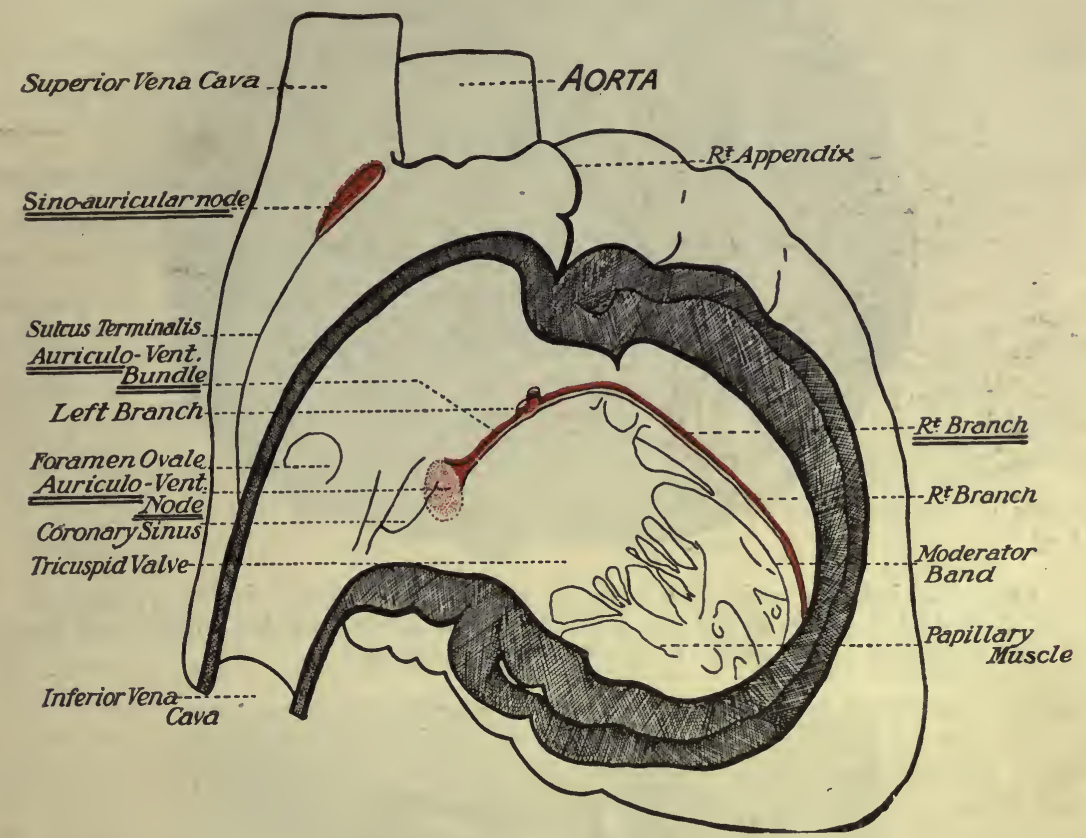

FrG. 431.

divides into the right and left septal divisions, which pass down in each ventricle on the interventricular septum into the papillary muscle arising from the septum. Each half of the bundle gives off several branches which break up more and more, finally forming a reticulated sheet of tissue over the greater part of the interior of the ventricles just below the endocardium. The fibres composing this tissue are more primitive in character than the rest of the cardiac musculature and have long been distinguished as the ' fibres of Purkinje.' In them the fibrillation is confined to the periphery of the muscle cell. They are distinguished by a high glycogen content. They may be regarded as a part of the muscular wall of the heart specially differentiated for the rapid conduction of the excitatory process to all parts of the ventricles (Figs. 432 and 433).

Numerous nerve fibres and ganglion-cells are found to accompany the muscle fibres of the auriculo-ventricular bundle. We have, however, no reasons for regarding the nerrous structures as concerned in the propagation of the excitatory wave. 
The auriculo-ventricular bundle forms the only continuous muscular tissue between the auricles and ventricles, and destruction of it causes complete abolition of the normal sequence of beat between auricles and ventricles.

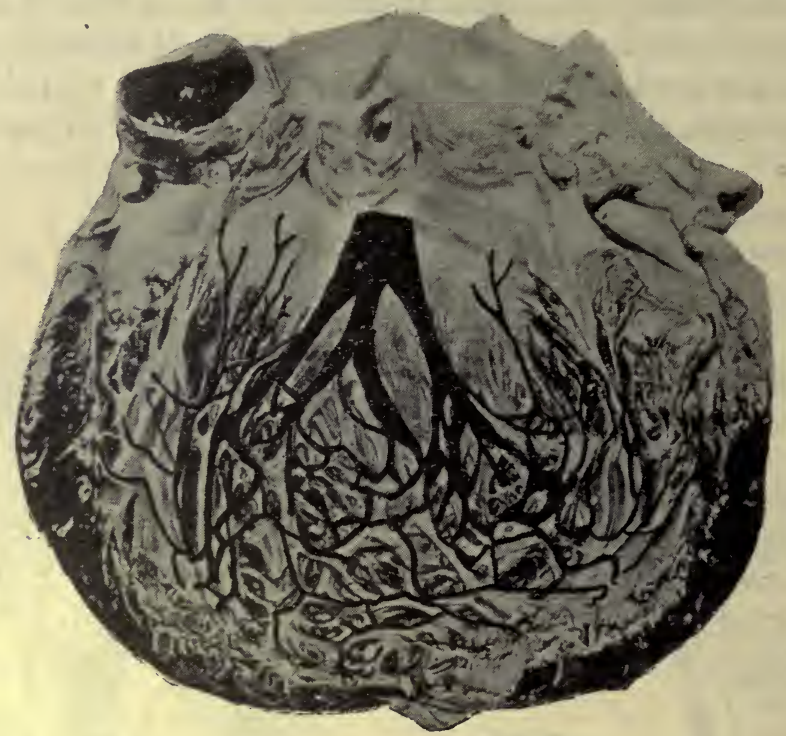

FIG. 432. Left ventricle laid open to display the interventricular septum on which the course of the left division of auriculo-ventricular bundle and its ramifications are shown in black. (After TAWARA.)

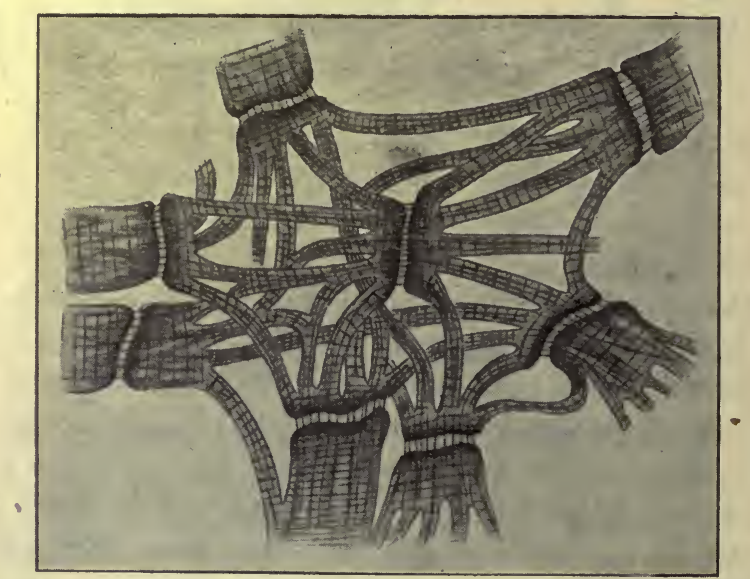

FIG. 433. Fibres of Purkinje, from the subendocardial network. (TAwara.)

By leading off different parts of the heart to the string galvanometer it is possible to determine the time relations of the excitatory process. It is then found that the mass of Purkinje tissue, known as the sino-auricular node, is the starting-point of the excitatory process concerned in each heartbeat. It is therefore spoken of as the 'pace-maker' of the heart. At each beat a contraction starts at the sino-auricular node, spreads a short way up 
the great veins, and along the auricular muscle in all directions. When it arrives at the auriculo-ventricular node, the impulse is carried on to the ventricles along the auriculo-ventricular bundle, spreading along the branches of this bundle to almost all parts of the ventricular muscle. Although normally the sino-auricular node initiates each heart-beat, this node can be put out of action by injury or cooling without stopping the rhythmic sequence of the heart-beat, the office of pace-maker being now taken up by the auriculo-ventricular node. A specialisation of function accompanies the differentiation in structure which we find in the auriculo-ventricular bundle and its branches. Lewis has shown that the conduction of the excitatory process along the auriculo-ventricular bundle of the Purkinje tissue occurs about ten times as fast as the conduction through the ordinary muscular tissue of the heart, the rates being about $5000 \mathrm{~mm}$. and $500 \mathrm{~mm}$. per second respectively. Although all parts of the ventricles receive the impulse to contraction almost simultaneously, the contraction wave, as judged by the electrical changes, is found to commence slightly earlier at two points, namely, on the anterior surface near the apex to the right of the groove separating right and left ventricles, and at the extreme apex of the heart where the endocardiac tissue comes very close to the surface. On the other hand, the conus arteriosus is the last part of the heart to begin contracting.

The limitation of the muscular continuity to a single narrow bundle which is endowed with greatly increased conducting powers, and ends in a network of tissue endowed with similar powers, is evidently designed (to use an old-fashioned but convenient word) to ensure that all parts of the ventricles contract practically simultaneously. If this were not the case the sudden contraction of the muscle fibres near the base of the ventricles would simply bulge out the still uncontracted portion near the apex, and there would be a risk of injury or even rupture of the uncontracted part of the ventricle under the stress of the pressure produced by the contracting part. On the other hand, if all parts of the heart were endowed with a similar rapid power of conduction, any part slightly more excitable or irritated than the rest, might serve as a centre for emitting excitatory waves which would interfere with those transmitted from the auricles, and the tendency to heart delirium would be enormously increased.

\section{THE HUMAN ELECTROCARDIOGRAM}

The passage of the excitatory wave over the different heart cavities is associated with corresponding electrical changes resulting in differences of potential. If we lead off any two parts of the heart's surface to a string galvanometer or capillary electrometer, we obtain movements which are caused partly by changes occurring in the muscle just underlying the electrode, partly by changes occurring at a distance and transmitted by the intervening muscle acting simply as a moist conductor. These two kinds of effect may be alluded to as direct and indirect. If we lead off, not from the heart itself, but from neighbouring tissues in contact with the heart, we shall still obtain the indirect effect of the electrical changes at each heartbeat, and these can be obtained, as Waller has shown, when the intact animal is led off to the electrometer or galvanometer by his limbs. In an animal such as the dog the two fore limbs may form one lead and the two 
hind limbs the other. In man, when the heart lies asymmetrically, it is usual to lead off the right arm, and left arm, the right arm and left leg, or the left arm and left leg, the hand or foot being immersed in salt water connected to the galvanometer by a zinc electrode contained in a porous pot full

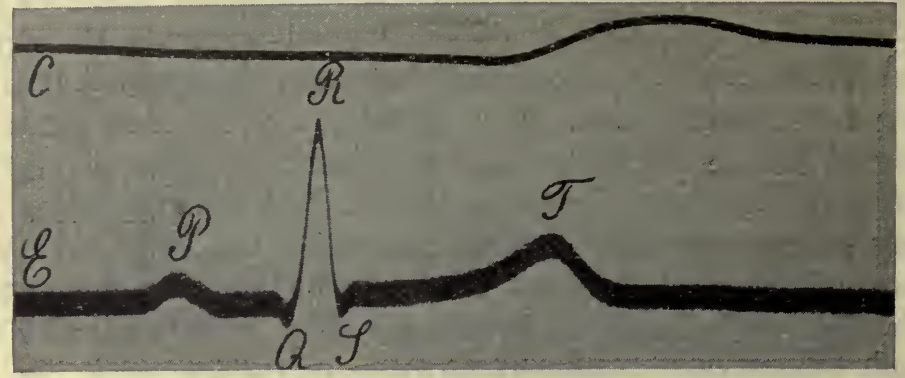

FIG.434. Electrocardiogram of man, obtained by leading off from the two hands to a string galvanometer.

C is the carotid pulse tracing. The different parts of the curve are designated by the letters $P, Q, R, S, T$, first applied to them by Einthoven.

of saturated zinc sulphate solution. By this means an electrocardiogram is obtained similar to that shown in Fig. 434.

In view of the mechanism of the propagation of the excitatory wave in the ventricle we should not expect the cardiogram obtained in this indirect fashion to be easy of interpretation, at any rate so far as regards the course of the wave through the ventricular muscle. Such an electro-

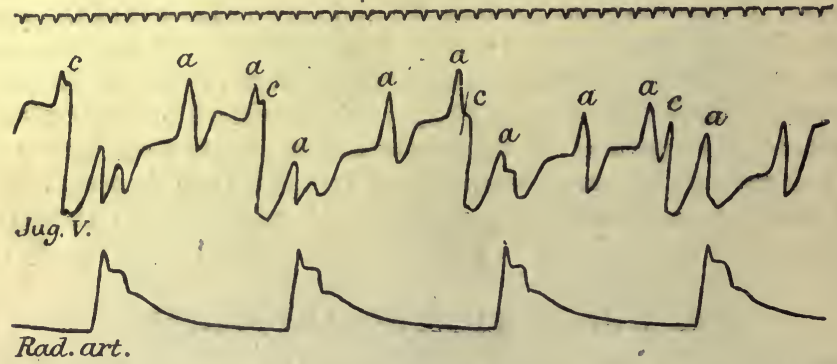

FIG. 435. Simultaneous tracings of the jugular venous pulse and the radial arterial pulse, from a case in which the A.V. bundle was destroyed by disease. The contractions of the auricles are marked by the $a$ waves on the venous pulse. They are more rapid than and quite independent of the ventricular contractions. (MACKENZIE.)

cardiogram however is of considerable use clinically, especially for the determination of the relation between the auricular and the ventricular contractions. The different points in a typical tracing, such as that contained in the figure, are designated by the letters $P, Q, R, S, T$, which were first applied to them by Einthoven and are retained because they do not involve any theoretical interpretation of the curves. Of these $\mathbf{P}$ is certainly due to the auricular contraction and $\mathbf{Q}$ marks the beginning of the ventricular contraction. The A.V. interval is given by the distance between $\mathbf{P}$ and $\mathbf{Q}$, 
the total duration of the excitatory condition in the ventricle by the distance between $\mathrm{Q}$ and $\mathrm{T}$.

The fibres of the auriculo-ventricular bundle may be destroyed by disease. In such cases we get a series of phenomena known under the name of Stokes-Adams's disease, the main characteristic of which is the slow contractions of the ventricle, accompanied by a rapid venous pulse at a rhythm entirely independent of the ventricular pulse. The automatic activities of auricle and ventricle are in fact dissociated (Fig. 435). At certain intervals, or at certain stages of the disease, the fibres of the bundle may present only a partial block, so that the ventricle responds once to every second contraction of the auricle. The existence of this disease is shown at once on the electrocardiogram by the dissociation of the normal relation between the auricular and ventricular variations. It may be also shown by a study of the venous pulse (Fig. 435).

\section{THE PHYSIOLOGICAL PROPERTIES OF THE CARDIAC MUSCLE}

\section{THE RESPONSE OF HEART-MUSCLE TO DIRECT EXCITATION}

When a skeletal muscle is directly stimulated with induction shocks of varying strength, within narrow limits the height of the contraction is proportional to the strength of the stimulus. If the frog's ventricle, rendered motionless by a Stannius ligature, be stimulated with a single induction shock, if it responds at all it will respond with a maximal contraction, no change in the extent of the contraction being obtainable, however the stimulus may be increased. There is thus no proportionality in the heart between strength of stimulus and height of contraction. The heart, if it contracts at all, always contracts to its utmost, the height of the contraction being dependent, not on the strength of stimulus, but on other conditions affecting the muscle at the time of its response.

Although much stress has been laid on this supposed difference between heart-muscle and voluntary muscle, a renewed investigation of the response of the latter to graded stimuli by Gotch and by Keith Lucas tends to show that the distinction is not so fundamental. According to these observers the fact that the response to a minimal stimulus in skeletal muscle is smaller than the response to a maximal stimulus is simply owing to the fact that in the former case only a small proportion of the muscle fibres is active and that increasing the strength of the stimulus merely increases the number of fibres thrown into contraction. According to this view therefore a maximal contraction of skeletal muscle would be one involving all the fibres. In the heart-muscle all the muscle fibres are functionally continuous, so that a stimulus, if it excites at all, must excite all the fibres, and every contraction must be analogous to the maximal contraction of a skeletal muscle. The existence of the ' all or none ' law in any contractile tissue would be therefore dependent on the existence of functional continuity between all the contractile elements of the tissue.

In the retractor penis of the dog it is possible to get graded contractions with graded strength of stimuli, and in this case it is easy to observe that with increasing strength of stimulus a greater extent of the muscle is thrown into the contractile state. Closely connected with this manner of response is the fact that in heart-muscle, under normal circumstances, it is not possible 
to get summation of contractions by putting in a stimulus, however strong, before the muscle has returned to rest. If, however, the propagation of the first contraction throughout the heart-muscle be retarded or prevented by a partial death of the tissue, or by stimulus of the vagus nerve, it is possible, as Frank has shown, to obtain an apparent summation of two stimuli, i.e. a curve in which the second contraction is superposed on and rises higher than the first. Such a result, on the explanation given above, would be due to a phenomenon of 'block' limiting the propagation of the first contractile wave, and yielding more to the second; though this is not the explanation given by the original observer.

\section{SUMMATION OF STIMULI}

If an isolated frog's ventricle which is not beating be stimulated with inadequate shocks, it may be found, on repeating these shocks at short intervals of time, that they become adequate and cause a contraction of the

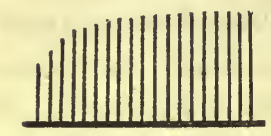

Fig. 436. Group of pulsations showing 'staircase' character.

ventricle. A stimulus therefore which is subminimal may nevertheless cause some change in the heartmuscle, so that the latter responds more readily to subsequent stimuli.

A similar improving effect of previous stimulation on the condition of the heart-muscle may be observed on the contractions themselves. Thus in a Stannius preparation, if the ventricle be excited with single induction shocks, once in every ten seconds, the first four or five contractions form an ascending series, each contraction being rather higher than the preceding one. This is often spoken of as the 'staircase phenomenon' (Fig. 436).

\section{THE REFRACTORY PERIOD}

At each contraction of the heart-muscle there is a sudden decomposition of contractile material which, so far at least as concerns the incidence of an external stimulus, is maximal, i.e. complete. Directly this has occurred a process of assimilation or re-formation of contractile material begins. This lasts throughout the diastolic period, and the store of contractile material is at its maximum just before the next contraction. A mechanical analogy is furnished by a bucket into which a stream of water is constantly flowing, and which tips up automatically and empties out its contents as soon as the water reaches a certain height. It is evident that the power of the heart-muscle to contract in response to a stimulus (its 'irritability ') must be at a minimum immediately after the automatic discharge or decomposition has taken place, and will continually increase from this point as the store of contractile material grows, until it arrives at such a height that the explosive discharge occurs spontaneously. Hence in each cardiac cycle there is a period, known as the refractory period, in which stimuli applied to the heart have no effect. This will be followed by a period in which a stimulus is followed by an extra contraction, but with a prolonged latent period. Just before the next spontaneous contraction the irritability is at its height, and the heart-muscle 
responds with a contraction to a minimal stimulus. These facts are well shown in Fig. 437.

When a tracing is being taken from part of the heart, e.g. the ventricle, which is beating rhythmically in consequence of a stimulus communicated to it from some other part, such as the sinus venosus, an extra contraction is

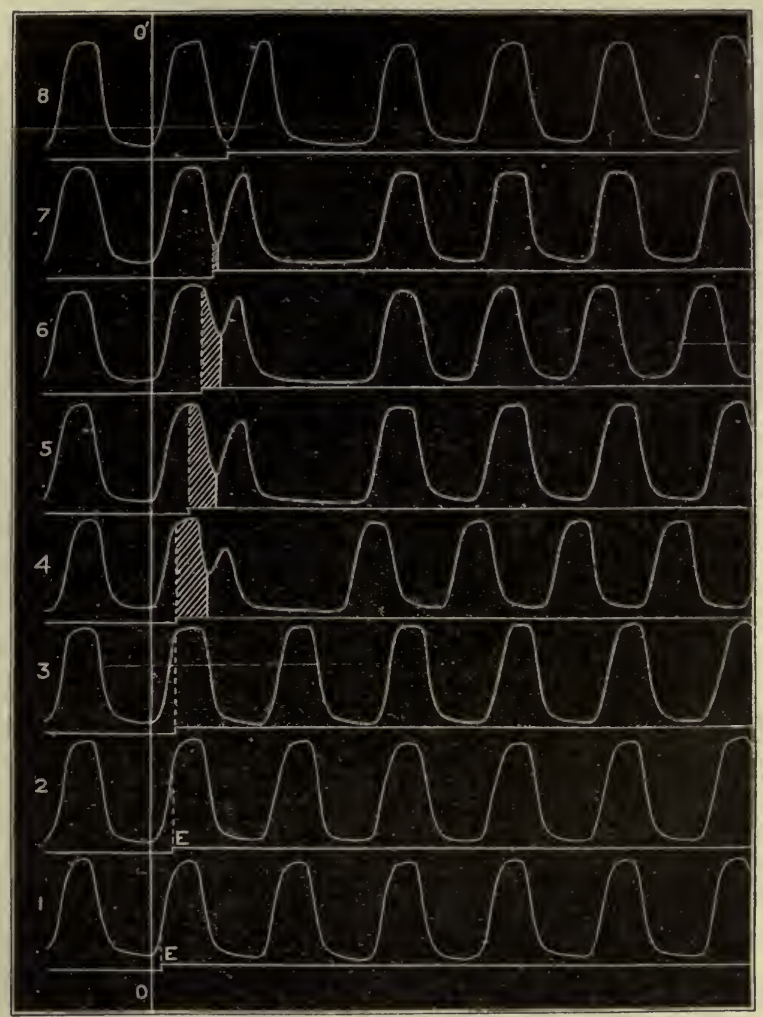

FIG. 437. Tracings of spontaneous contractions of frog's ventricle, to show refractory period. In each series the surface of the ventricle was stimulated by an induction shock at $\mathrm{E}$, as indicated by the tracing of the signal. In 1,2 and 3, this stimulus had absolutely no effect, since it fell during the refractory period. In 4, 5, 6, 7 the effect of the shock was to interpolate an extra contraction in the series, the latent period (shaded part) gradually diminishing from 4. to 7 (diastolic rise of irritability). In 8 the irritability of the preparation was already considerable, and the latent period inappreciable. The 'compensatory pause' after the extra beat is also well shown in $4,5,6,7,8$. (MAREY.)

followed by a 'compensatory pause,' and in certain cases the first contraction following the pause is considerably augmented. This is due to the fact that one of the impulses arriving from the sinus arrives at the ventricles during the refractory period ensuing on the application of the artificial stimulus ; hence it produces no effect and the ventricle has to wait.for the arrival of the next succeeding excitatory wave from the sinus before it gives its next beat. Hence the compensatory pause does not occur when we are testing the effects of artificial stimuli on the sinus venosus. 
On account of the refractory period which ensues on the commencement of the contractile process on heart muscle, it is impossible to throw the muscle into a tetanus, since all the stimuli which fall during systole are entirely ineffective. By using very strong stimuli it is possible to intercalate extra contractions before the heart has returned to the base line, i.e. before diastole is complete. So that in this way one may obtain almost a continuous contraction, presenting, however, waves on its summit, which differs from the tetanus of skeletal muscle in the fact that its height is no greater than the height of a single contraction.

Only when the functional continuity of the heart-muscle is impaired by the 'block' effect of vagal stimulation or the administration of muscarine - is it possible to obtain phenomena even superficially analogous to the summation of contractions in skeletal muscle.*

\section{FACTORS MODIFYING THE ACTIVITY OF CARDIAC MUSCLE}

\section{INFLUENCE OF TENSION AND DISTENSION}

When we examine the behaviour of a heart isolated from the central nervous system and from the rest of the body, às, for instance, in the heart-lung preparation (vide p. 911) we find that it has a marvellous power of adaptation, i.e. of regulating its activity according to the mechanical demands which are made upon it. Thus while we may maintain the venous inflow constant so that the heart is sending out a litre of blood per minute, it makes no difference to the output of the heart whether the average arterial pressure, and therefore the resistance to the outflow of blood, be maintained at 80 or $160 \mathrm{~mm}$. $\mathrm{Hg}$, although in the latter case the heart must do exactly twice as much work in order to maintain the outflow at the same level. In the same way we may maintain the arterial pressure constant and alter the venous inflow and we find that within very wide limits the heart is able to expel against the arterial resistance the whole of the blood which flows into it from the veins. In this way we can alter the output of a small heart of 50 gms. from 300 to 3000 c.c. per minute. As we should expect, this variation in the work done by the heart is associated with corresponding variations in the chemical changes which occur at each heart-beat. Evans has shown that the respiratory exchanges of the heart increase pari passu with the work it is said to do. Careful investigation of the volume and pressure changes of the heart under varying conditions of arterial resistance and venous filling enables us to throw some light on the mechanism of this power of adaptation.

Let us take first the changes in volume as recorded by the cardiometer. A heart is contracting 100 times per minute and forcing out at each beat 10 c.c. of blood into the aorta against an average pressure of $80 \mathrm{~mm}$. Hg., with systolic and diastolic pressures respectively of 100 and $60 \mathrm{~mm} . \mathrm{Hg}$. In order that the left ventricle may force 10 c.c. of blood against this resistance,

* According to Mines the effect of vagus excitation in enabling the prcduction of summation is due to the shortening of the refractory period which results from vagal stimulation. 
the pressure in its interior must rise at each heart-beat above the maximum systolic pressure in the aorta, e.g. to $110 \mathrm{~mm}$. Hg. The aortic valves will open as soon as the pressure rises above $60 \mathrm{~mm}$. Hg. The arterial resistance is now increased so as to raise the average pressure to $120 \mathrm{~mm}$. $\mathrm{Hg}$. The heart now may raise the pressure in its interior to $120 \mathrm{~mm}$. $\mathrm{Hg}$. This will be higher than the diastolic pressure in the aorta and a certain amount of blood will escape, but the outflow of blood will cease as soon as the pressure in the aorta is equal tc that in the ventricle. Diastole will then occur, the ventricle will relax before it has emptied out 10 c.c. of blood. Let us assume it has forced out 3 c.c. of blood-it will then contain an excess of 7 c.c. of blood at the end of diastole. Meanwhile the venous inflow is proceeding at the same rate as before so that at the end of diastole it has 7 c.c. more blood than it had at the end of the previous beat, i.e. its diastolic volume will be increased and the heart will be dilated. At the increased beat we find that the contraction of the ventricle is much more forcible. The maximum pressure now rises to $130 \mathrm{~mm}$. $\mathrm{Hg}$. and 8 c.c. of blood are sent out into the aorta. At the end of this beat the heart will be still fuller than before, containing an excess of 9 c.c. of blood. The third beat is still more forcible, the intra: ventricular pressure rising to a maximum of $140 \mathrm{~mm}$. Hg., and

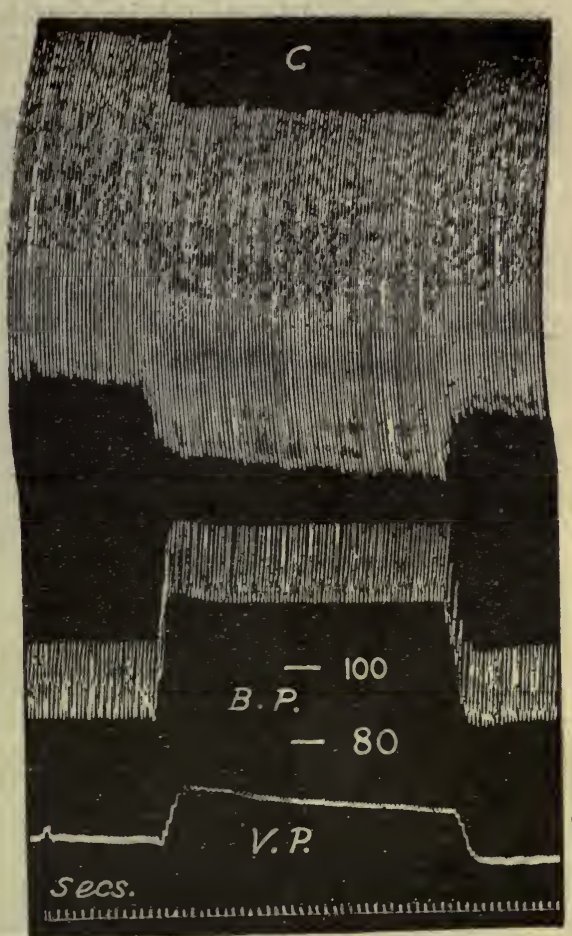

FiG. 438. Effect or increased arterial pres. sure on the volume changes of the heart, with a steady inflow of 154 c.c. blood per 10 seconds.

C. cardiometer curve. B.P. arterial bloodpressure. V.P. pressure in the inferior vena cava. The lines 100 and 80 show the height of the blood-pressure in $\mathrm{mm}$. Hg.

10 c.c. of blood are expelled. After this the heart goes on beating regularly expelling 10 c.c. of blood at each beat, i.e. the same amount as it receives from the veins, and the arterial pressure is maintained constant at an average of $120 \mathrm{~mm} . \mathrm{Hg}$. But the heart remains more dilated than it was previously since it contains an excess of 9 c.c. of blood. If now the arterial resistance be suddenly reduced to its previous amount, the first beat after the change may send out 17 c.c. of blood, the second beat 12 c.c. of blood and the third beat 10 c.c. as before. We see therefore that the energy set free at each contraction of the heart is increased by increasing the volume of the heart, but increased volume of the heart means increased length of the muscular 
fibres composing its wall, so we arrive at a statement similar to that made previously for voluntary muscles, namely, that the energy of contraction is a function of the length of the muscle fibres, i.e. to the extent of active surface involved. This reaction of the heart to increasing distension has long been known but was ascribed to the excitatory influence of tension on the muscle fibres. It is evident that in a resting heart increasing distension of its cavities will tend to stretch its muscle fibres and therefore to exert a tension

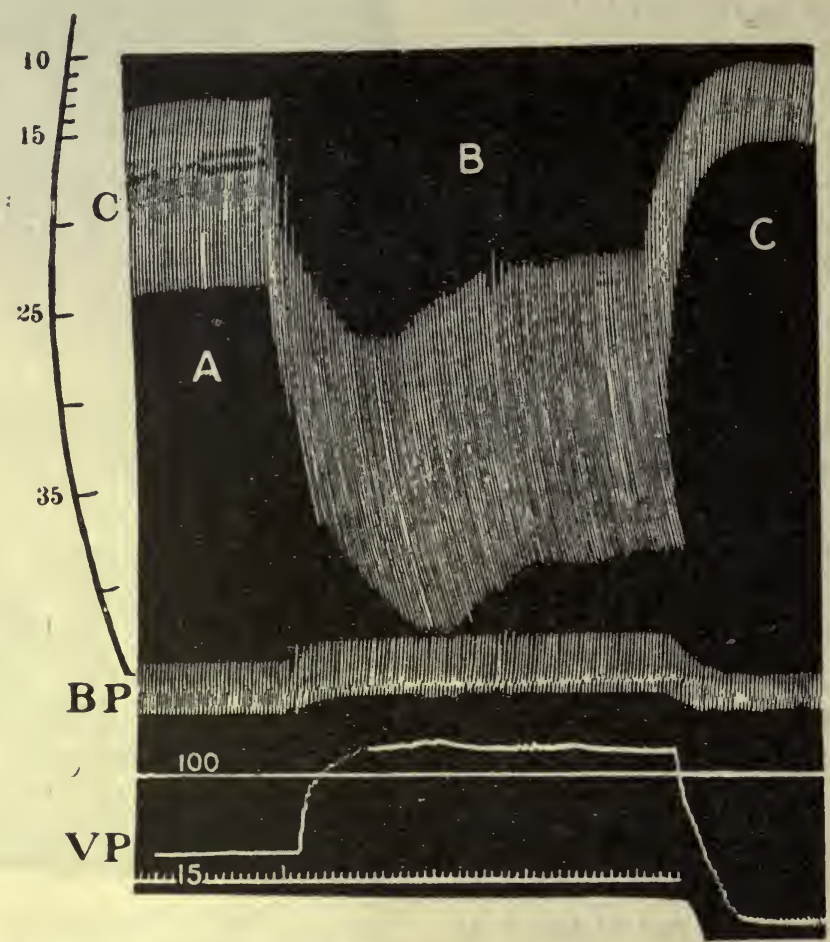

Fic.439. Effect of alterations in venous supply on volume of heart. Heart, 67 gms.

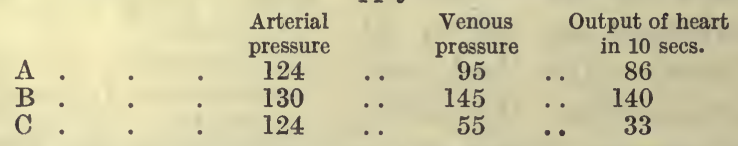

The curved line at the side represents the value of the cardiometer excursions in capacity of ventricles in c.c.

on them. By an accurate record of the pressure changes within the contracting ventricle under varying conditions it is possible however to exclude the tension on the fibres as the determining factor. In a heart beating regularly the inflow of blood is proceeding during diastole, during the relaxation of the ventricles, i.e. the muscles are giving before the inflowing blood. The latter is therefore able to distend the heart without exercising more than a minimal pressure on its walls and it is found that the pressure in the ventricles may be approximately zero at the end of diastole whether the heart is contracting against a resistance of $80 \mathrm{~mm}$. Hg. or a resistance of $120 \mathrm{~mm}$. 
$\mathrm{Hg}$., or whether it is receiving 5 c.c. or 10 c.c. during the period of diastole With a bigger outflow or a bigger resistance the energy of contraction is increased, although the tension on the heart wall at the beginning of the contraction is not altered. The only condition then which always changes pari passu with the energy of contraction is the distension of the heart cavities, i.e. the length of its muscle fibres, and we are therefore justified in regarding this last factor as the one which determines the energy of the response of the muscle to excitation. Naturally if the distension increased beyond a certain extent it would be associated with increased tension on the muscle fibres. But the changes of initial tension and excitatory response are not proportional. It is evident that the capacity of the heart for adapting itself to changes in mechanical demands made upon it will be limited by the inability of the heart to dilate further, as is probably the case in the intact animal, where its dilatation is limited by the pericardium, or by the mechanical disadvantage at which the further dilated heart acts. The more the heart attains a globular form, the greater the mechanical disadvantage of the muscle fibres in raising the pressure in the interior of the ventricles (vide p. 916) so that by continually increasing the demands on the heart we shall finally arrive at a stage at which this organ is unable to deal with the blood which is applied to it and rapidly fails to expel any of its contents.

The physiological condition of the heart is measured by the maximum pressure which it is able to produce in its cavities when it contracts, starting from a certain initial size or length of fibres. As the heart becomes fatigued this pressure falls so that the heart must dilate in order that each contraction shall produce the same maximum pressure as before. Fatigue of the heart is shown therefore, not by failure of it to do its work, but by the fact that it can only do its work when it is undergoing considerable dilatation. Dilatation is therefore a measure of fatigue. What is often spoken of as the tonus of the heart is really synonymous with physiological condition. A heart in good condition has a high tonus. It empties itself almost completely at each beat even when receiving a considerable quantity of blood during diastole. A heart with a low tonus is in the condition of a fatigued heart. It is widely dilated and when it has finished contracting still contains a large amount of residual blood.

This property of the cardiac muscle is responsible for the power of ' compensation ' possessed by a diseased heart. We may take as an example the destruction of one aortic valve, a lesion which can be produced experimentally in a dog. In this case, immediately after the lesion is established, no additional resistance is offered to the expulsion of the blood, and the ventricle will send the normal amount into the aorta. During the succeeding diastole the blood at a high pressure in the aorta will leak back into the ventricle through the damaged valve. The arterial pressure therefore falls rapidly, and the ventricle receives blood from two sides, i.e. by regurgitation through the aortic valves, and in the normal way from the auricles and veins. At the end of diastole the ventricle is therefore overfilled. Increased stretching of its fibres, however, has the effect of exciting an increased con- 
traction, and the heart at its next systole throws out not only the normal quantity of blood but also that which it has received back from the aorta. The arterial system thus receives at each beat the normal quantity of blood plus the amount which leaks back into the ventricle after each systole; so that the amount of blood remaining in the aorta and available for passage on to the capillaries is the same as in the normal animal. On this account, after a lesion of the aortic valves has been established, the average of the arterial pressure remains the same as before, although the oscillations of pressure with each heart-beat are increased in extent. The augmented output by the ventricles naturally involves increased work on the part of their muscular walls, which react in the same way as skeletal muscle does to increased work, i.e. by hypertrophy; the final effect therefore is a heart bigger than normal, with hypertrophied and thickened walls, but capable of maintaining an adequate circulation throughout all parts of the body; in other words, in the healthy animal complete compensation has taken place $=\ldots \ldots$

\section{THE INFLUENCE OF TEMPERATURE ON THE HEART RATE}

The frequency of the heart varies directly with the temperature. The higher the temperature the greater the frequency. At $40^{\circ} \mathrm{C}$. the contraction of the mammalian heart may be four times as frequent as at $25^{\circ} \mathrm{C}$.

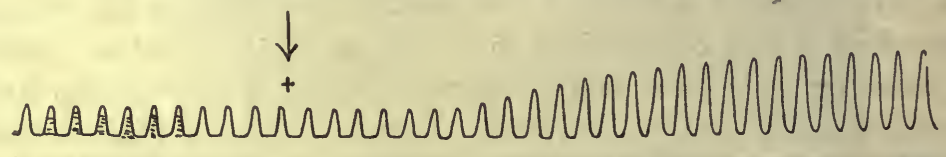

FIG. 440. Tracing of contractions of a frog's heart (by RINGER), showing effect of adding a trace of $\mathrm{CaCl}_{2}$ to the $\mathrm{NaCl}$ solution used previously for perfusion. The arrow marks the point at which the addition was made.

\section{INFLUENCE OF THE CHEMICAL COMPOSITION OF THE SURROUNDING MEDIUM ON THE HEART-MUSCLE}

The tissues of the heart, like all other cells of the body, require for the normal display of their functions a definite osmotic environment, i.e. a certain molecular concentration of the fluid with which they are bathed. This is equivalent to a 0.65 per cent. sodium chloride solution for the frog's heart, and to a 0.9 per cent. solution for the mammalian heart. As Ringer first showed, the nature of the neutral salt employed for making up the normal solution is all-important to the heart-muscle. Thus a strip of muscle from the apex of the tortoise's ventricle as a rule does not beat spontaneously. If, however, it be immersed in a 0.7 per cent. solution of sodium chloride it begins to beat rhythmically after a short latent period. The contractions soon reach a maximum and then gradually die away. Sodium chloride therefore acts as a stimulus to contraction, but is unable to maintain the beats for any considerable length of time. The strip of muscle ceases contracting in a condition of relaxation.: On now adding to the solution a trace of calcium chloride or calcium sulphate, the contractions 
begin again (Fig. 440). Now, however, the-relaxations after each contraction become more and more incomplete, until finally the heart-stops in a tonically contracted condition. If now a trace of potassium chloride or phosphate be added the contractions begin again and may last for many hours, although the solution contains nothing which can furnish energy to the contracting muscle. It has been suggested that the rhythmic contractions of the heart-muscle may be the result of the constant chemical stimulus of the inorganic salts present in the blood-plasma, sodium acting as a stimulus to contraction, while the calcium salts are necessary for the maintenance of the systolic tone, and the potassium salts for the occurrence of relaxation.

The exact significance of these different salts for the functions of cardiac and other forms of muscular tissue, though they have been the subject of many detailed investigations, must be still regarded as an open question.

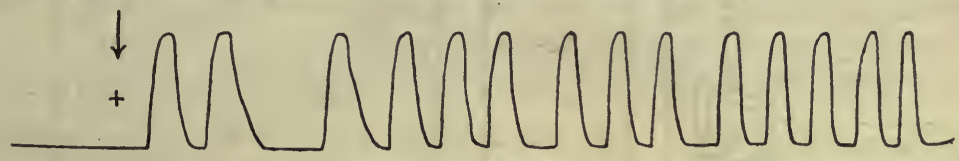

Fig. 441. A frog's heart poisoned by excess of calcium salts, recovers its spontaneous rhythm on adding a trace of KCl to the perfusion fluid. (RINGER.)

The fluids containing the three salts mentioned above in slightly varying proportions are commonly used to maintain the beat in an excised heart either of a cold- or of a warm-blooded animal. In the case of the latter it is necessary to keep the fluid saturated with oxygen. According to Locke the addition of glucose to the solutions enables the beats to go on for a longer period of time, and will in fact renew the rhythm of a heart which has ceased beating while being fed with pure saline solution.

The following represent the fluids most frequently used :

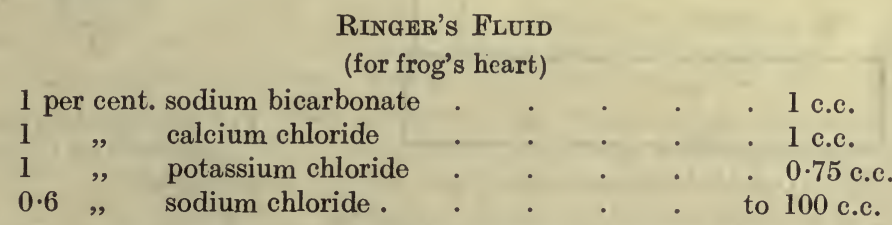

\section{LOCKE'S FLUID}

(for mammalian heart)

0.015 per cent. sodium bicarbonate,

$0.024 \quad, \quad$ calcium chloride,

$0.042 \quad "$ potassium chloride,

$0.92 \quad$ " sodium chloride,

$0 \cdot 1 \quad, \quad$ glucose,

in distilled water.

The influence of the chemical composition of the medium on the contraction of the heart may be investigated in the following ways :

One of the simplest methods is that employed by Gotch, represented in the diagram (Fig. 442). The apparatus consists of a small glass jar with inlet and outlet tubes. 
A disc of cork is fixed on to a brass rod so that it can be let down into the fluid. On the upper end of the brass rod is poised a light lever with a paper point. To fix the heart in the apparatus, the top of the ventricle is transfixed by a fine hook to which is attached a thread connected with the lever. The heart is fastened to the cork by a pin through the bulbus aortæ. The glass jar is filled with the fluid whose action it is desired to investigate. It is usual to start with Ringer's fluid in order to obtain a normal beat, and then to try in turn the various constituents of this fluid.

Another method of investigating the action of the heart of cold-blooded animals is by perfusing the heart cavities with the fluid under investigation. Two forms of perfusion are made use of. In the method first introduced by Williams a double

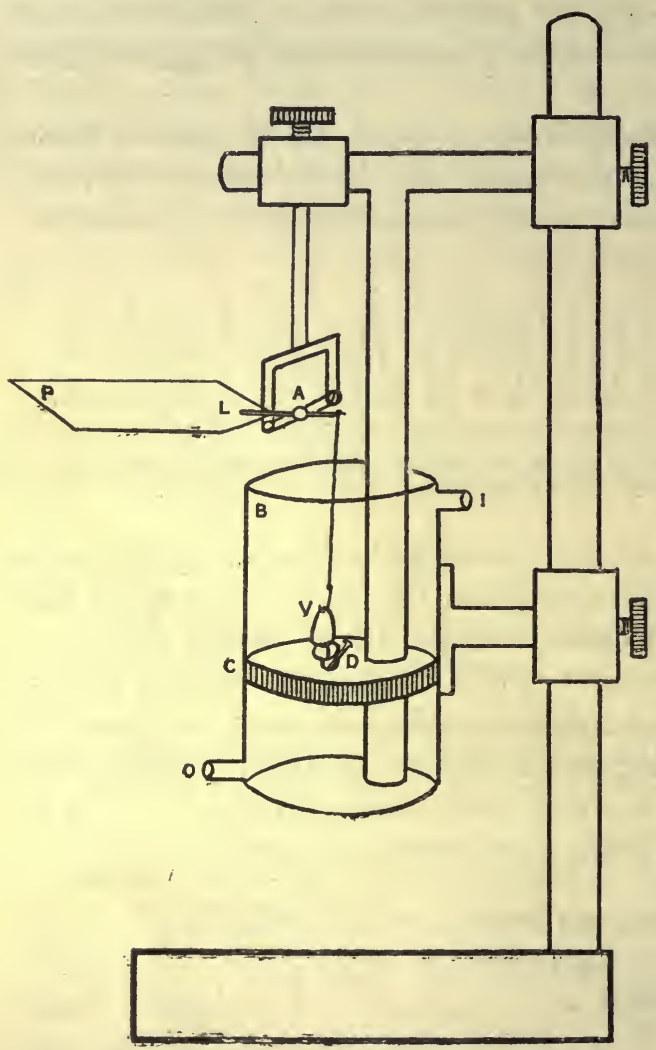

FIG. 442. Gotch's frog heart apparatus.

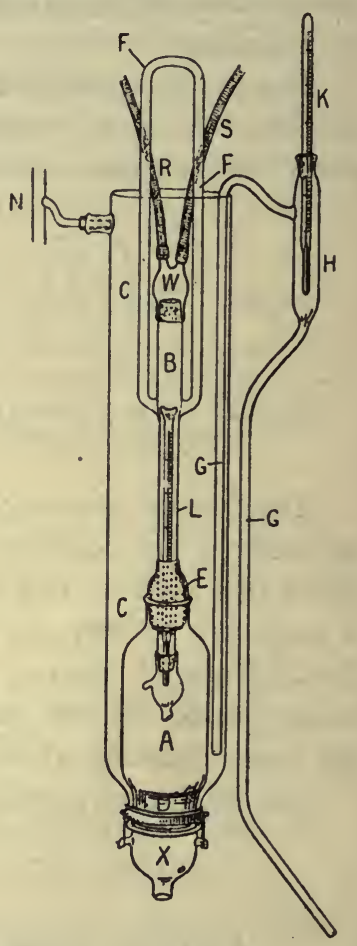

FIG. 443. Brodie's perfusion apparatus for the mammalian heart.

cannula is tied into the ventricle, the rest of the heart being cut away. The tubes leading to and away from the perfusion cannula are armed with valves so as to allow the fluid only to pass in one direction. The contractions of the ventricle may be recorded either by connecting the outgoing tube with a manometer, which may be a mercurial or a membrane manometer, or by connecting some form of recording apparatus with the vessel in which the heart is contained, so as to register changes in the volume of the ventricle. A large number of different forms of apparatus have been devised for these purposes.

In another method the fluid is allowed to flow through the whole heart, passing in by the sinus and out by the aorta. Here again the activity of the heart may be registered either by recording the pulsations in the arterial column of fluid or by connecting a tambour or piston recorder with the vessel in which the heart is contained.

The heart of warm-blooded animals can also be investigated by a somewhat similar 
method. It was shown by Porter that the mammalian heart could be kept alive by transfusing oxygenated blood serum through the coronary vessels, and Locke found that the same results could be obtained by using oxygenated Ringer's solution, modified so as to have the same tonicity as mammalian blood. A convenient apparatu s for this purpose has been devised by Brodie.

The apparatus (Fig. 443) consists of a chamber a to contain the heart, and of a tube $B$, through which the perfusion fluid is carried to the heart. Both are enclosed in a large outer jacket $\mathrm{c}$, through which is kept flowing a stream of water at body temperature. The chamber $\mathrm{A}$ is bell-shaped and is fitted into the jacketing tube $\mathrm{c}$ by a groundglass joint $\mathbf{D}$. Its upper orifice is closed by a piece of rubber tubing of such size that the perfusion tube $\mathbf{B}$ slips through it easily. By means of the glass handle $\mathbf{F}$, fused into the tube about half-way down, B can be drawn up or lowered into any desired position. To its lower end the heart cannula is attached by a ground joint. Its upper end is fitted, by a second ground joint, with a small bulb w, with two tubes, $\mathbf{R}$ and $\mathbf{s}$, fitted into it. These latter are connected by rubber tubing with aspirators containing the solutions to be perfused. The lower half of the tube B is nearly filled up with a thermometer $\mathrm{L}$, the bulb of which projects into the heart cannula T. The upper half is almost filled with a piece of glass tubing sealed at both ends, so that the perfusion fluid passes in a thin layer down the tube and thus offers a large surface for heating purposes. Also by filling the interior of the tube in this way its capacity is reduced to a very small amount.

The large outer tube $\mathbf{C}$ is kept supplied with warm water, entering through the tube $\mathrm{G}$ and overflowing through a side-tube at the top into a wide $T$-piece $\mathrm{N}$. By raising or lowering this $\mathrm{T}$-piece the level of the water in the jacket is adjusted. The watersupply comes from a cold-water tap, but on its passage to $\mathrm{G}$ passes through a metal spiral heated by a Bunsen burner. By varying the rate of flow and the position of the burner the temperature of the water can be regulated with considerable accuracy. The upper end of the supply-tube $G$ is provided with a thermometer so that the temperature of the inflowing water can be seen and regulated.

In using the apparatus the heart cannula is removed, and the tube $\mathrm{B}$ is then passed through $\mathrm{E}$ and pushed down until its lower end issues just below the level of the chamber A. The circulation of the warmed water through the jacket is then started and adjusted to the proper temperature. One of the rubber tubes, $\mathrm{s}$, is next attached to the reservoir containing the main perfusion fluid, and the tube B filled with fluid and left to warm while the heart is being prepared.

The heart having been excised and washed well in saline so as to remove as much blood as possible, the cannula is tied into the aorta. The cannula is now held under the perfusion tube, filled with the warm saline, and at once attached in its proper position and the perfusion started. A bent pin to which a long thread is tied is hooked into the apex of the heart, and the perfusion tube pulled up until the heart lies quite within the warm chamber. When thus drawn up the bulb w lies just below the surface of the water in the outer jacket. The tube is held firmly in position by a clamp which fixes one arm of the handle $\mathrm{F}$. The heart cannula is provided with a side opening $\mathrm{v}$, on to which a long piece of fine rubber tubing is passed. This renders possible the removal of any gas bubbles that may collect in the cannula, or the washing out of the cannula with a stream of fluid if necessary. The beats of the heart are recorded by means of a simple lever attached by the thread previously fixed to the heart.

\section{THE SIGNIFICANCE OF THE REACTION OF THE BLOOD FOR THE} HEART-BEAT. It was long ago shown by Gaskell that the reaction of the perfusing fluid has a marked influence on the frog's heart. When weak acids are transfused through this heart, there is a gradual diminution of tonus, the beats become smaller and finally disappear. A similar relaxation may be obtained as the result of the action of carbon dioxide. Weak alkalies on the other hand produce a gradual decrease of tonus, so that the 
heart is finally arrested in a contracted condition. There will therefore be some reaction intermediate between the weak acid and the weak alkaline fluids which will represent the optimum reaction for the beat of the frog's heart. Mines has shown that this optimum reaction differs for the different cavities of the heart, and also for the hearts from different animals, a shifting of the reaction of the transfusing fluid to the acid side always bringing about a diminished contraction and tonus, while the opposite effects are produced by an increase in the alkaline reaction. In the

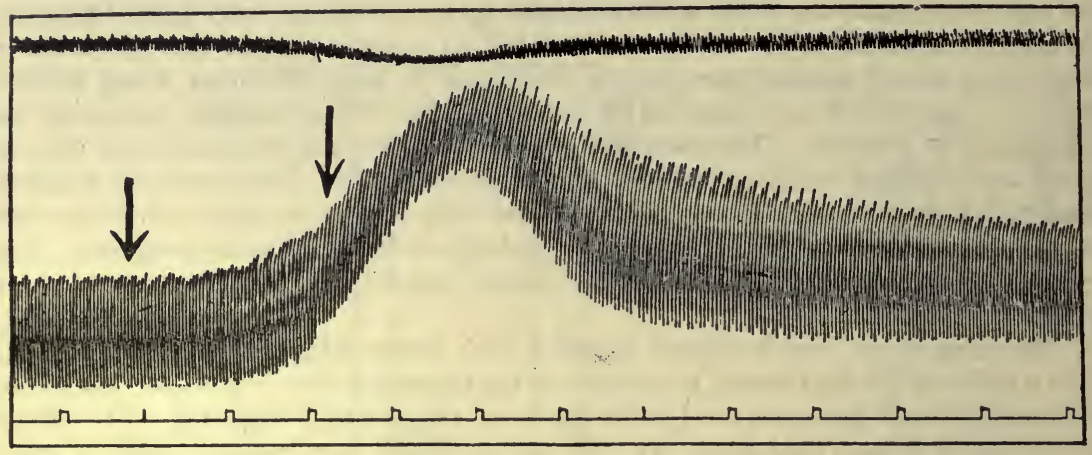

FIG. 444. Volume curve of ventricles (cat) (lower curve). The upper curve is the arterial pressure, maintained by an adjustable resistance at $130 \mathrm{~mm}$. $\mathrm{Hg}$. Between the arrows the air used for artificial respiration was replaced by a mixture containing 20 per cent. $\mathrm{CO}_{2}$ and 25 per cent. oxygen. Note the dilatation with impaired contraction, followed by increased amplitude of contractions.

mammal under normal conditions, the chief factor affecting the reaction of the blood is the tension of carbonic acid in this fluid, and an increase in the carbonic acid of the blood, when sufficiently pronounced, always brings about dilatation of the heart. The resistance of the hearts of different animals is however not of the same strength. Thus, a dog's heart is much more susceptible to the presence of a small excess of carbonic acid in the blood than is the cat's heart (cp. Fig. 444). There is probably an optimum tension of carbon dioxide in the blood, varying between 5 and 6 per cent. of an atmosphere, at which the physiological condition of the ventricular muscle is at an optimum, but the tension may be reduced considerably below this without causing any marked change in the action of the heart.

Yandell Henderson found that vigorous artificial ventilation of the lungs brought about a condition in which the heart's contraction was very forcible and the heart's cavities almost empty. He ascribed this condition to the hypertonicity of the heartmuscle produced by washing the carbonic acid out of the blood. These results were, however, probably due to the mechanical influence of the respiratory movements on the venous filling of the heart, and there seems no reason to believe that the condition of 'acapnia' (deficiency of carbon dioxide in the blood) had anything to do with the results observed. The improving effects of administration of carbon dioxide described in the first edition of this work have not been confirmed by more recent and accurate experiments. 


\section{THE NUTRITION OF THE HEART}

In the frng's heart the muscle fibres are supplied directly by the blood within the cavities, the spongy ventricular wall permitting the access of blood between the fibres. In the mammalian heart the muscular tissue is nourished through the coronary arteries, which break up into a meshwork of capillaries around all the fibres.

The flow of blood through the coronary circulation may be measured either in the whole animal or in the heart-lung preparation by introducing a cannula through the wall of the right auricle into the coronary sinus and collecting the blood from the latter outside the body. Another method is to feed a heart from the aorta through the coronary arteries with blood and collect the total outflow from the cut pulmonary artery. By a comparison of these two methods it is found in the dog that the blood flow through the coronary sinus forms about three-fifths of the total blood passing through the coronary arteries. It is therefore possible to measure the flow through the coronary sinus in the heart-lung preparation under varying conditions of pressure and output. The figures so obtained multiplied by $\frac{5}{3}$ will represent approximately the total flow through the coronary circulation.

Blood enters the coronary arteries from the aorta both during systole and diastole, though it is probable that the systole of the ventricles exercises a direct effect in increasing the resistance to the flow of blood through the heart and squeezes out the contained blood into the coronary veins. This may be one reason why the flow of blood through the coronary system is greater in a beating heart than in a heart which is quiescent. The most important factor in determining the flow through the coronary vessels is the arterial pressure. The marked effect of this factor is shown in the following Table :

$\begin{array}{cc}\text { Heart weight, } 107 \mathrm{gms} ., \text { total output per minute, } 1400 \mathrm{cc} . \\ \text { Arterial } & \begin{array}{c}\text { Coronary circulation } \\ \text { per minute }\end{array} \\ \text { pressure } & 50 \\ 60 & 90 \\ 100 & 124 \\ 128 & 208 \\ 166 & 500 \\ 190 & \end{array}$

We see from this Table that the heart muscle is supplied with blood in proportion to its needs, since its work and its respiratory exchanges increase continuously with the rise of arterial resistance. Indeed, under the severe test of contracting against an average pressure of $190 \mathrm{~mm} . \mathrm{Hg}$. over one-third of the whole blood leaving the heart was passing through its muscular walls, one gramme of muscular tissue being irrigated with 5 c.c. of blood per minute. Another important factor in determining the coronary flow is the effect of the metabolites produced by the contracting heart-muscle itself. This is well shown when the heart is asphyxiated. Thus in one experiment while the arterial pressure. was maintained constant, the total coronary flow was 56 c.c. per minute. Artificial respiration was then discontinued, and during the succeeding minutes the coronary circulation was $61,72,150,180$. The circulation then failed. Carbonic acid produces 
also an increase in the flow through the coronary arteries, but it is impossible with the highest attainable percentages of carbonic dioxide in the blood to produce such an increase in the coronary flow as is observed during asphyxia. The dilatation of the coronary vessels which occurs in the latter condition must therefore be ascribed to non-gaseous metabolites produced by the contracting muscle. Thus the heart contains in itself a mechanism for increasing the flow of blood through its tissues whenever this becomes inadequate and the muscle is suffering for lack of oxygen. Mechanical and physiological factors thus co-operate in providing the most important muscle in the body with oxygen sufficient for its needs.

If a coronary artery be ligatured, the heart very often beats for one or two minutes with unimpaired force, then a beat is dropped occasionally, and very shortly afterwards the heart stops altogether and the bloodpressure falls to zero. On inspection. of the heart immediately after the blood-pressure has fallen, its muscular wall is seen to be in a state of fibrillar contractions, or 'delirium cordis.' All the strands of muscle fibres are contracting more or less rhythmically, but the rhythms of no two parts coincide, so that the heart dilates and is incapable of carrying on the circulation. It is probably in this way that sudden deaths occur in cases where the coronary arteries are diseased or calcified. In such cases a man may drop down dead, having previously shown no symptoms of heart mischief.

Delirium cordis may be explained as the result of block, produced by interference with the nutrition of a large part of the heart-wall. The contractile wave arriving at this part, in some directions will not spread at all, in others will spread at a lower rate, so that different parts of the heart receive the impulse to contract at different times and a state of incoordination results. The same condition can be produced by freezing the apex of the ventricle, so causing a block, or by stimulating the surface of the ventricle at a rate which is greater than can be taken up by the ventricle as a whole, as, e.g., by tetanising currents. Such a condition in the higher animals, as the dog and man, is practically irrecoverable, although in the rabbit, and very rarely in the dog, it is sometimes possible to bring the heart back to a state of rhythmic contraction by kneading it rhythmically.

According to Mines delirium cordis is susceptible of a simpler explanation. This condition is easily brought on in the mammalian heart by stimulation of its surface by strong faradic currents. The effects of increased frequency of contraction on the heart muscle is to decrease the rate of propagation and to decrease the length of the wave of excitation. Ordinarily in the naturally beating heart the wave of excitation is solong and spreads so rapidly, that it excites the whole of the ventricle long before it has ceased in any one part. When, however, the muscle is stimulated more frequently the wave becomes slow and short, so that more than one wave can exist at one time in a single chamber. The main factor then in the production of delirium cordis after obstruction of the coronary artery would probably be a diminished rate of conduction through the affected part. 


\section{SECTION IX}

\section{THE NERVOUS REGULATION OF THE HEART}

In order that the activity of the heart may be adapted to the needs of the body as a whole, its automatic mechanism must be subject to the central nervous system, which must be able to affect the heart in either of two ways, viz. by increasing or diminishing its activity. This subjection to the integrative action of the central nervous system is also necessary for the sake of the organ itself ; otherwise the peripheral adaptation of the heartmuscle to changes in arterial resistance might result iu its exhaustion and permanent damage.

The regulation is effected through the intermediation of afferent and efferent nerve fibres connecting the heart with the central nervous system. The importance of these nerves is shown by the behaviour of animals in which they have been extirpated. Thus a dog in whom all the nerves of the heart had been divided survived the operation for eight months, the pulse reading during the time not having appreciably altered and the animal being in a fair condition of health. Although he regained his normal weight after the operation, he was found incapable of carrying out even a moderate amount of work, such as that represented by running, since the mechanism for increasing the action of the heart in response to the needs of the muscles had been lost.

\section{THE EFFERENT CARDIAC NERVES}

The heart in vertebrates is supplied with nerve fibres from two sources, from the medulla oblongata along the vagus nerve, and from the upper dorsal region of the spinal cord through the mediation of the sympathetic system.

The fibres which run through the sympathetic system take a somewhat different course in the animals on which the regulation of the heart's activity has been chiefly studied, viz. the frog and the mammal. In the frog (Fig. $445)$ the sympathetic fibres leave the spinal cord by the anterior root of the third spinal nerve; they then pass through the ramus communicans to the corresponding sympathetic ganglion, whence they run up through the second ganglion and the annulus of Vieussens to the first ganglion; they then pass into the cervical sympathetic strand to the ganglion trunci vagi; here they join the vague and pass down with the true vagus fibres to the heart. 
In the dog (Fig. 446) the sympathetic fibres leave the spinal cord by the anterior roots of the second and third dorsal nerves, run in the white rami communicantes to the stellate ganglion, and thence by the annulus of Vieussens to the inferior cervical ganglion. Cardiac branches convey the sympathetic fibres to the heart and are given off from the stellate ganglion, the inferior cervical ganglion, and from the trunk of the vagus.

By the nicotine method it is possible to trace out the cell connections of these fibres. As they leave the cord they are medullated nerve fibres,

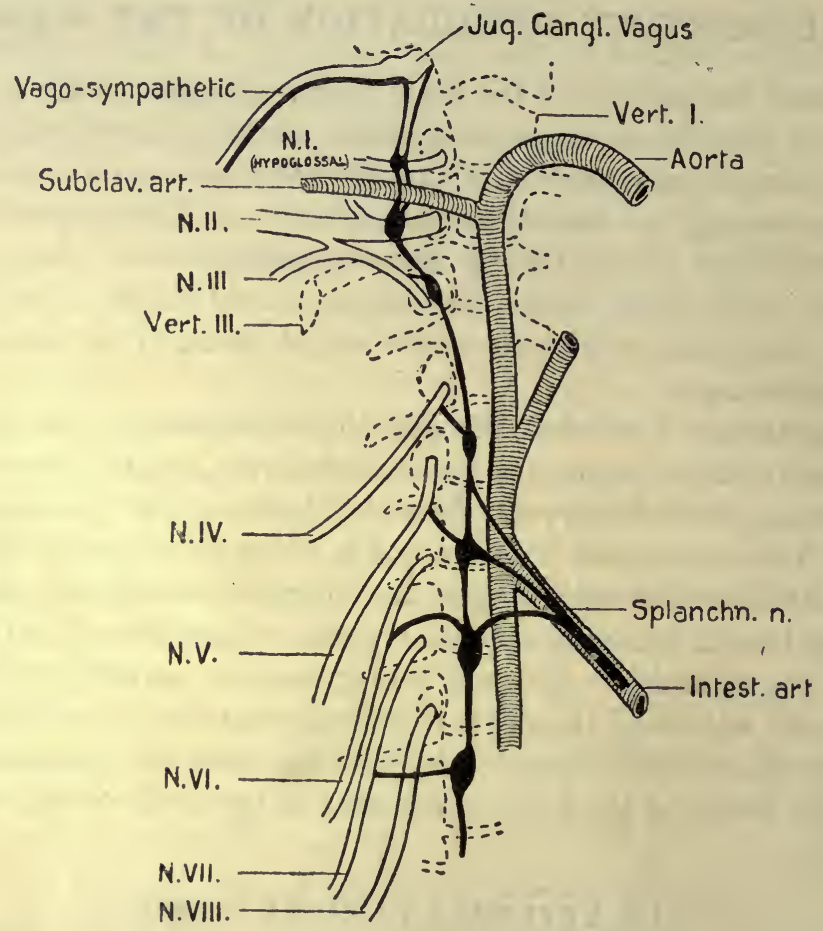

FiG. 445. Sympathetic chain of frog (right side) to show connection with vagus nerve. The sympathetic ganglia with their branches are black. Of the peripheral branches only the splanchnic nerve is represented. (Modified from EoKER.)

similar to the other fibres making up the visceral outflow throughout the dorsal region; the white fibres pass along the ramus communicans to the stellate ganglion, where they end, forming synapses with the cells of the ganglion. Here fresh relays of fibres, which are non-medullated, start and carry the impulses to the heart along the various cardiac nerves just mentioned. In the heart these fibres are distributed to the muscle fibres without the intervention of any other ganglion-cells. On the other hand, the fibres which leave the vagus to pass to the heart make connection with the cells of Remak's ganglion, and probably all the other intrinsic cardiac ganglia described above, whence non-medullated fibres carry their impulses to the heart-muscle. 


\section{ACTION OF THE VAGUS}

The action of the vagus fibres on the heart is almost identical in frog and mammal. If in the dog the peripheral end of the cut vagus be stimulated while the arterial blood pressure is being recorded by means of a mercurial manometer, the pulse is seen to become slower, or with a stronger stimulus to

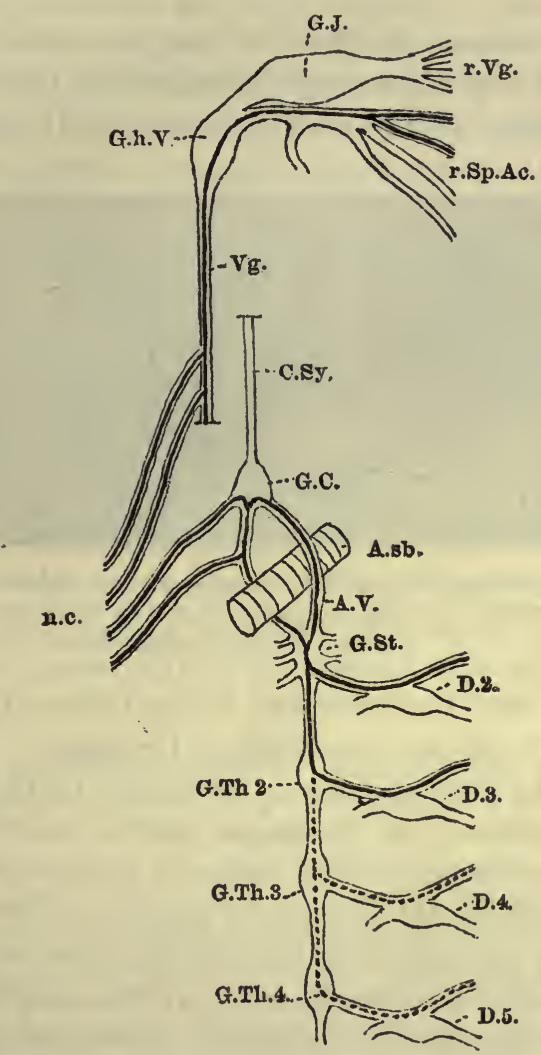

Fig. 446. Diagram of cardiac inhibitory and accelerator fibres in the dog. (From Foster.)

r. $\mathrm{Vg}$, roots of the vagus; r.Sp.Ac, roots of the spinal accessory; GJ, ganglion jugulare; G.h.V, ganglion trunci vagi ; Vg, trunk of vagus nerve ; C.Sy, cervical sympathetic; GC, inferior cervical ganglion; AV, annulus of Vieussens; A.sb, subclavian artery; nc, cardiac nerves; G.St, ganglion stellatum; D2, D3, D4, D5, second, third, fourth, and fifth dorsal spinal roots; G.Th, ganglia of the thoracic chain.

cease altogether, and the blood-pressure falls towards zero. On discontinuing the stimulus the heart begins to beat again and the pressure rises after a few beats to normal (Fig. 447).

If the stimulation of the vagus be prolonged, the blood-pressure, on discontinuance of the stimulus, may rise above normal owing to the asphyxia of the vaso-motor centres produced by the prolonged cessation of the circulation. Even during the application of the stimulus the heart often begins to beat again with a slow rhythm. In this case we speak of an 
'escape' of the heart from the vagus influence. This escape is generally confined to the ventricles and the heart-beats are found on opening the chest to be purely ventricular, the auricles and great veins remaining in a state of diastole. Vagus escape is favoured by distension of the heart cavities, and is often synchronous with the respiratory efforts, which supervene after a certain duration of inhibition as a result of the asphyxia of the respiratory centre.

When the arterial system is dilated, so that the mean systemic pressure, and consequently the venous pressure during cardiac inhibition, are low, or when the asphyxial gasps of the animal are prevented by anæsthesia or by a

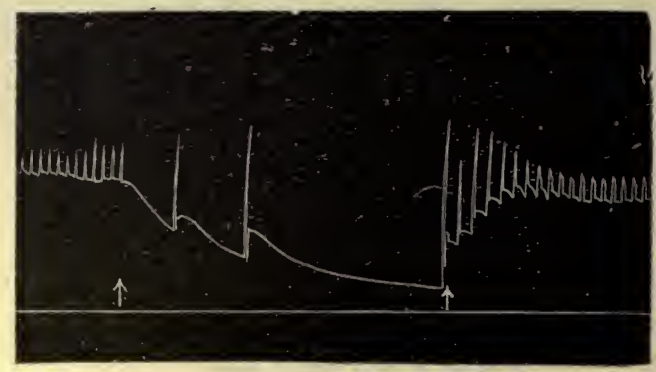

Fig. 447. Blood-pressure tracing from carotid of dog (taken with Hürthle's manometer), showing effect of excitation of vagus (between the arrows). $o$, abscissa line of no pressure.

section of the spinal cord, the heart may fail to recover from the inhibition produced even by a transitory stimulation of the vagus. In such cases it is necessary to knead the heart in order to restore its rhythmic action

To study the influence of the vagus on the auricles and ventricles respectively, it is necessary to work with the chest opened and to record separately the contractions of the different segments of the heart. It is then found that the vagus may affect the heart in one of several ways. Its most marked action is on that part of the heart where it enters, viz. the venous end. It may affect that part of the auricle corresponding to the primitive sinus venosus, where the rhythm of the whole heart is determined. In this case the sole effect of the vagus on the auricles and ventricles will consist in an alteration of rhythm. They may cease to beat altogether or they may give beats of normal strength but at a slower rhythm than before. Often indeed under these conditions the beats of the ventricles may be increased in size, since the strength and extent of their contractions are determined, not by the strength of the stimulus arriving from the auricles, but by the length of their fibres, and this will increase with any prolongation of the diastolic period, and consequent increased diastolic filling of the ventricle.

If the vagus acts on the auricles without affecting the sinus part of the auricles (sino-auricular node), the rhythm will be unaltered, but the response of the auricles to the impulses received by them will be diminished, and the amplitude of the excursions of the lever attached to them will therefore be 
considerably reduced. Indeed the auricular contractions may be reduced to such an extent that they cause no movement of the lever. It is only by observing their surface that one may perceive a slight contraction of their fibres. Under such circumstances the rhythm of the ventricles will be unchanged.

Generally the vagus absolutely stops the action of all parts of the auricles ; in such cases the ventricles also cease beating. Very often after a short pause the ventricles commence to beat at a slow rhythm, and it is then seen that they are contracting independently of the auricles and sinus. That the ventricle is really inaugurating the beat is shown by the fact that occasionally one may observe a reversed beat, i.e. a contraction of the auricle following instead of preceding each ventricular contraction. Whether the vagus has a direct action on the mammalian ventricle is still doubtful; its effect is at any rate very slight as compared with that on the venous end of the heart. The fact that stimulation of the vagus causes as a rule temporary cessation of the ventricular beat; while functional separation of the ventricles from the auricles causes no such temporary stoppage, would seem to indicate that this nerve has a direct, though slight, action on the ventricles.

Finally the vagus may affect the tissue which conducts the excitatory process from one cavity to another. Under vagus stimulation the auricles may beat at a greater rhythm than the ventricles, a block having been produced in the tissue passing from auricles to ventricles, viz. the auriculoventricular bundle.

Engelmann has described these effects of vagus excitation as negatively chronotropic (diminution of rhythm), negatively inotropic (diminished strength of contraction), and negatively dromotropic (diminished conductivity), and has distinguished a fourth action, viz. one on the irritability of the muscle to direct stimuli, which he calls negatively bathmotropic. He ascribes these four actions to four different sets of nerve fibres, but it is evident that they are due not so much to the difference in the nature of the impulse as to a difference in the place of incidence of the impulse.

Thus, if the vagus fibres which are distributed to the remains of the sinus are specially active, we shall get alterations of rhythm affecting the whole heart. If those which supply the A.V. bundle are excited, the most pronounced effect will be on the propagation of the excitatory process from auricles to ventricles.

Practically the same description will apply to the action of the vagus on the frog's heart. Since it is easy in this animal to register the contractions of the empty heart it is possible to show that the vagus has a direct inhibitory action on the ventricles, diminishing the strength of its contraction in response to the stimuli transmitted to it from the venous end. This action of the vagus on the ventricle is not, however, universal, and in the tortoise it is impossible to show any such action. In both these animals the auricles show the same effects as in the mammal, viz. an influence limited to the rhythm when only the sinus is affected, or a diminution of the strength of contraction when the sinus is unaffected and the chief action of the vagus is on the auricular muscle.

Ever since the discovery in 1845 by the brothers E. H. and E. F. Weber 
of the action of the vagus on the heart, much work has been expended with a view to determining the intimate nature of the inhibitory process. In the former neurogenic theory it was supposed that the vagus altered the activity, perhaps by a process of 'interference,' of the ganglion-cells responsible for the origination of the rhythm. Many facts, however, point to the inhibitory impulses as being continued to the heart-muscle itself. Thus tetanisation of any portion of the frog's ventricle, especially if it be filled with blood, causes an evident relaxation of the part between the electrodes. Application of nicotine to the heart prevents stimulation of the trunk of the vagus from having any influence on the heart, presumably from paralysis of the cells of Remak's ganglion, which lie at the termination of the vagus fibres, or of the synapses between the vagus fibres and the ganglion-cells. It is still possible to inhibit the heart by direct stimulation either of the fibres leaving this ganglion in the sino-auricular junction, or of the nerve-trunks which run in the inter-auricular septum. We must conclude therefore that the inhibition of the heart-muscle is peripheral and depends on the direct action of the nerve fibres on the muscle-cells themselves. These nerve fibres are paralysed by atropine, after administration of which no inhibitory effects can be produced by stimulation of nerve or muscle or any part of the heart. On the other hand, muscarine apparently stimulates the inhibitory nerve-endings, and when applied to the isolated auricle or ventricle causes weakening of the beat and finally complete inhibition, an effect which can be removed by its antagonist atropine.

Two views have been held as to the essential nature of the inhibitory process. According to that put forward by Claude Bernard, the natural tendency of any tissue during rest is towards anabolism. Activity involves disintegration or breaking down of the living material, and this disintegration must be succeeded by a process of building up or anabolism, which restores the tissue to its previous functional condition. On this view the state of inhibition would merely prolong the period of rest intervening between two periods of activity, so allowing a greater time for restitution to take place, with a corresponding improvement in the functional capacity of the tissue. According to Hering and Gaskell a state of anabolism can be induced in a tissue comparable to the state of sudden disintegration which is associated with activity. Excitation of the vagus nerve does not merely allow the normal process of building up, which goes on during rest, to take place, but actually hastens this process, just as the excitation of a motor nerve to a skeletal muscle induces an active breakdown of the contractile tissue, or the excitation of the augmentor nerve to the heart induces an increased rate of beat and therefore increased functional activity.

If stimulation of an inhibitory nerve induces the opposite chemical change to that occurring during activity, one would expect to find that, just as an active part of a tissue is negative to an inactive part, so a part of the tissue which is under the influence of an inhibitory stimulus should be electro-positive to any part which is not being so stimulated. According to Gaskell this condition is realised in the heart of the tortoise. The auricles 
are brought to a standstill by separating them from the sinus venosus. The apex of one auricle is then injured by heat, and the injured point and uninjured base are led off to a galvanometer. The usual demarcation current dependent on the difference of potential between the injured and uninjured portion is thus observed. If the vagus be now stimulated the auricles remain at rest, but the demarcation current is increased, i.e. a positive variation is produced, an electrical condition opposed in sign to that which would take place when the auricles contract. Doubt still exists, however, as to the exact interpretation to be put on this experiment.

It was mentioned above that potassium salts promote relaxation of the ventricle, so acting as antagonists to calcium salts. If potassium salts be present in a sufficient concentration in the circulating fluid, the heart is brought to a standstill in a condition. of diastole, as if the vagus mechanism were in action. On removal of the excess of $\mathrm{K}$ ions the heart at once starts beating again. Howell has shown that during stimulation of the vagus the amount of potassium in a diffusible form in the heart-muscle is increased. He has therefore suggested that the action of the vagus in stopping the heart is effected by the liberation of potassium salts. Potassium normally exists in a large percentage in the heart-muscle, but in a combined form, and Howell assumes that stimulation of the vagus effects a dissociation of this combined potassium, so that the liberated ions are able to exert their inhibitory influence on the heart.

\section{THE TONIC ACTION OF THE VAGUS}

If both vagi of a mammal be divided, the heart as a rule beats more frequently, showing that under normal circumstances tonic impulses are constantly descending the vagi and holding the heart's action in check. The extent of the quickening which is produced by section of the vagi varies in different animals and is apparently associated with the conditions of life of the animal and its powers of carrying out prolonged muscular exertions. Thus in the dog or horse the pulse, which is normally slow, may be doubled in frequency by section of the vagi. In the rabbit, which has a frequent pulse, and is only able to run for a short distance, division of both vagi causes very little alteration in the pulse-rate. It is stated that the tonic action of the vagi is much greater in the hare than in the rabbit.

This tonic action may be increased by various conditions of the blood, e.g. the presence of drugs, such as morphia.

\section{ACTION OF THE SYMPATHETIC CARDIAC NERVES}

Stimulation of the sympathetic cardiac nerves at any part of their course has an effect on the heart the exact reverse of that produced by stimulating the vagi. In most cases the pulse frequency is increased in consequence of the action of these nerves on that part of the heart from which the rhythm starts. The frequency which is attained by maximal stimulation of the accelerator nerves is independent of the previous rate of the heart-beat. The increase in rate involves a shortening of the time of the-cardiac cycle, which chiefly affects the diastolic period. The size of the auricular and ventricular contractions may be increased at the same time as their rate. In fact, like the vagus nerves, the sympathetic fibres of the heart can influence either 
rhythm or strength of contraction, or the conduction from auricle to ventricle, according to the part of the heart-muscle which is affected.

The augmentor effect on the strength of the ventricular beats is often very marked. The sympathetic fibres are much less easily tired than the vagus fibres, and have a longer latent period. Whereas the latent period

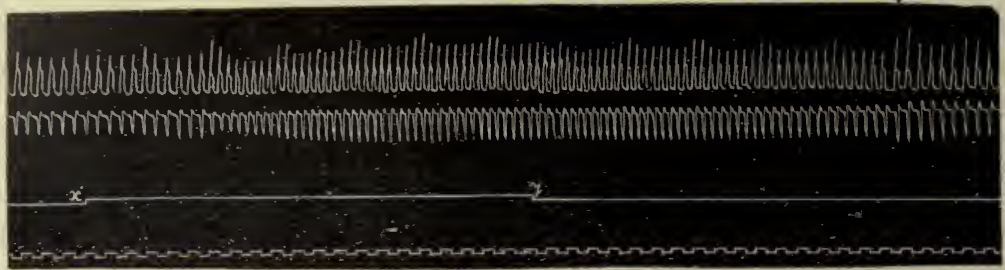

FIg. 448. Tracings of ventricular (upper curve) and auricular contractions (lower curve).

From $x$ to $y$ the accelerator nerves stimulated. Lowest line $=$ seconds.

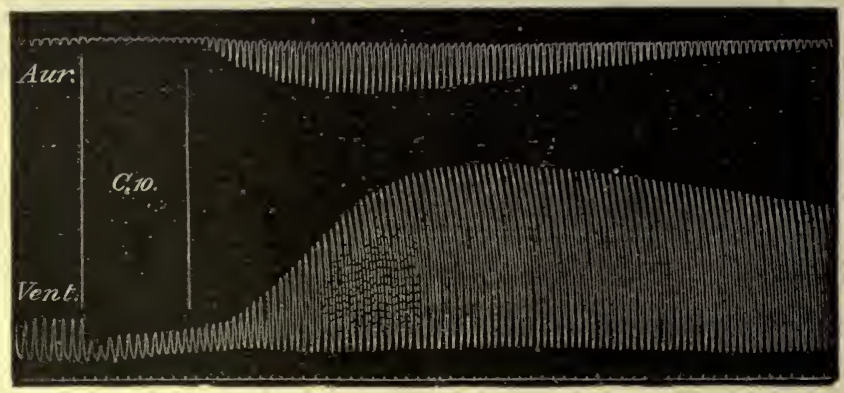

FIG. 449. Tracing to show effect of stimulation of the vago-sympathetic nerve on the frog's heart. The rhythm is unaltered, but the beats? of auricle and ventricle are much decreased in size. On ceasing the stimulation the beats become augmented. (GASKELL.)

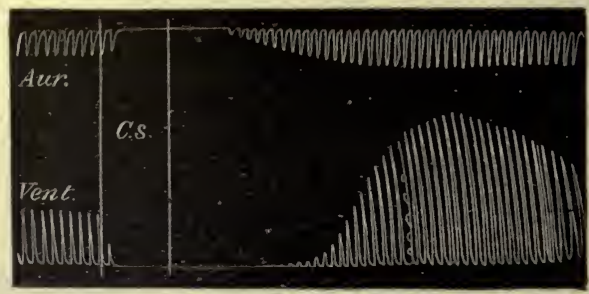

FIG. 450. A tracing similar to Fig 449. In this case, however, the stimulation caused complete stoppage (inhibition) of both auricular and ventricular beats. (GASKELL.)

of the vagus in the mammal is considerably less than one second, that of the accelerator nerves may amount to ten or even twenty seconds (Fig. 448). Hence if the vago-sympathetic of the frog be stimulated, the first effect is inhibition due to vagus action. The vagus nerve-endings then become fatigued, and the influence of the accelerator fibres makes itself apparent, and the heart commences to beat, and the beats become more rapid and forcible than before (Figs. 449, 450).

Like the vagus, the sympathetic nerve fibres appear to exercise a tonic 
influence on the heart, so that after extirpation of the stellate ganglion on each side, the pulse frequently becomes permanently slowed.

\section{THE ACTION OF ADRENALIN ON THE HEART}

The medullary part of the suprarenal glands forms and secretes into the blood-stream a substance, adrenaline, which has a marked action both on the heart and blood-vessels and plays therefore an important part in the regulation of the circulation. Whether this secretion is a constant one has not yet been fully ascertained, but there is no question that under certain specified

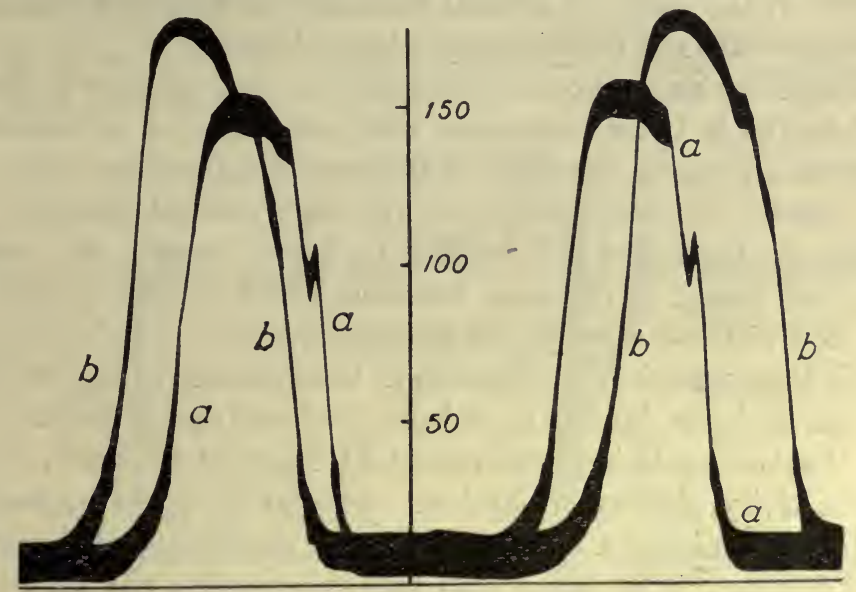

FIG. 451.

Intraventricular pressure tracings (left ventricle) from dog's heart (heart-lung preparation). The scale shows pressure in $\mathrm{mm}$. Hg.

$a$. Under influence of adrenaline.

$b$. Under simultaneous influence of adrenaline and $\mathrm{CO}_{2}$ (15 per cent.) (PATtersor). conditions there may be a marked influx of this substance into the bloodstream. The action of adrenaline on any part of the body is practically identical with that of excitation of the sympathetic nerve supply to the same part. Its isolated action on the heart is best studied on the perfused heart or in the heart-lung preparation. On adding $\frac{1}{10} \mathrm{mgm}$. of this substance to the 500 c.c. of blood circulating through the heart-lung preparation, a maximum effect is at once produced and this lasts for 15 to 20 minutes. The action is, like that of the sympathetic nerve, accelerator and augmentor. Through its influence on the sinus or the sino-auricular node the rhythm of the heart is markedly increased, in the dog to about 240 per minute. At the same time the energy of each contraction is increased. This is especially shown in a heart which is beginning to fail and is therefore undergoing a certain degree of dilatation. Directly the adrenaline reaches the heart the contractions become extremely energetic so that the heart rapidly diminishes in volume, the venous pressure falls, and the blood flowing into it at each diastole is thrown out with violence into the aorta. The more powerful beat enables the output of the heart at each beat to be maintained or even increased in spite of the shorter duration of the systole. With each beat the maximum 
pressure in the ventricle therefore rises to a marked extent (see Fig. 451). The strain on the ventricular wall of this sudden contraction which is necessary to empty the heart, during the period of systole, is often so great that small hæmorrhages are produced throughout the substance of the muscle. The stimulation effect of adrenaline is shown, moreover, by the considerable rise in the respiratory exchanges of the heart under the influence of this substance, the oxygen intake being increased two or three times above that which obtained before the administration of the adrenaline. The action of adrenalin therefore is in general to enable the heart to cope with a bigger strain either in the shape of arterial resistance or increased venous inflow than it could do without the stimulus of this substance.

The wonderful adaptation of the heart to its functions is illustrated, moreover, by the fact that adrenaline, which increases the metabolism of the heart to such an extent, exercises at the same time a dilator effect on the coronary vessels, so that apart from the high arterial pressure and the metabolites produced by the contracting heart muscle, the vessels are dilated by the action of the same hormone which evokes the need for an increased flow of blood through the working muscle.

There is thus a marked antagonism in the influence of the two common hormones on the heart, both of them being produced during general muscular activity. Carbonic acid in excess causes dilatation of the heart, diminished functional activity, slowing of rhythm. Adrenaline causes increased functional activity, diminution of cardiac volume, and increased rhythm. The action of adrenaline is so pronounced that it is possible to administer 20 or 30 per cent. carbonic acid to a heart-lung preparation without altering its output if adrenaline be administered at the same time. The heart is slowed by the carbonic acid, but the beat is maintained and contraction is effective in emptying the heart of its content.

\section{THE HEART REFLEXES}

The part of the nervous system chiefly concerned in the central coordination of the various afferent impulses which act on the heart is the medulla oblongata. It is in this situation that we find the nerve-cells giving origin to the efferent fibres of the vagus nerves, and also the collection of grey matter in which the afferent fibres of the vagus terminate. Direct stimulation of the vagus centre may cause slowing and stoppage of the heart. The tonic influence of the vagi can be abolished by destruction of this centre. In this region we also find the vaso-motor centre, so that the activity of one can affect that of the other. This cardiac centre may be played upon by impulses arriving at it through various afferent nerves or from the higher parts of the brain and giving rise to the changes of the pulse-rate associated with the emotional conditions, or it may be directly affected by the composition of the blood circulating through its capillaries.

The nerve-cells which give off the accelerator or augmentor fibres are situated in the intermedio-lateral tract of the spinal cord, near the point of origin of these fibres. We might therefore speak of an augmentor centre in 
this region; but it seems probable that the activity of these cells is subordinate to impulses arriving at them from the common meeting-place of visceral impulses, viz. the medulla.

The most important of the afferent nerves which affect reflexly the action of the heart are the nerves coming from the heart itself and the aorta. In the mammalian ventricle nerve fibres can be seen running over the surface of the ventricle which are entirely afferent, stimulation of their peripheral ends causing no effect on the heart-beat. Stimulation of their central ends may cause one of four conditions :

(a) Slowing of the heart.

(b) Rise of blood-pressure from constriction of the splanchnic area.

(c) Fall of blood-pressure by dilatation of the arterioles of the body.

(d) Reflex movements. The heart does not seem to be provided with the nerves of ordinary or tactile sensibility. There is no doubt, however, that

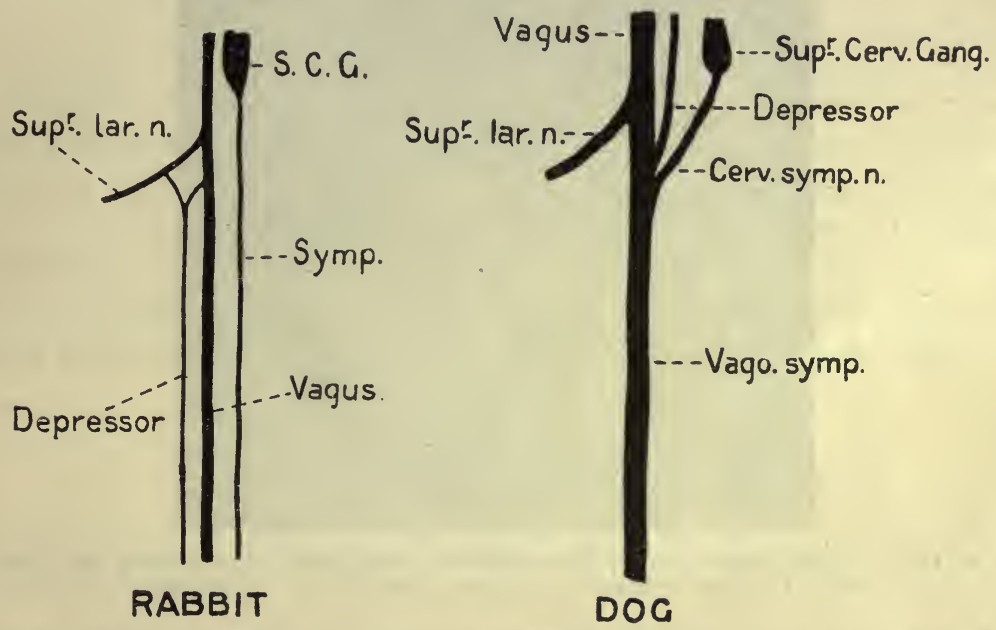

FIG. 452. Diagrams of the connections of the depressor nerve in the rabbit and dog, according to Cyon. It will be noticed that in the latter animal the depressor nerve runs in the vagus trunk together with the sympatbetic nerve, for the greater part of its course.

under abnormal circumstances impulses arising in the heart can give rise to sensations of pain which are referred not so much to the heart as to the surface of the body over the left side of the chest and left arm, in the region of the distribution of the cutaneous branches of the second and third dorsal roots.

An important afferent nerve coming from the heart, or rather from the beginning of the aorta, is the depressor nerve. In the rabbit this rises by two roots, one from the trunk and the other from the superior laryngeal branch of the vagus, and runs parallel with the vagus to the cardiac plexus (Fig. 452). It is purely afferent, stimulation of its peripheral end causing no effect. On stimulating its central end fall of blood-pressure (Fig. 453) and, reflex slowing of the heart are produced, the latter effect being abolished by section of both vagi. It has been shown by Bayliss that the depressor effect is due to universal dilatation of the blood-vessels of the body, the greater 
part, however, being played by the splanchnic area. This nerve is probably brought into action whenever the pressure in the aorta is so high as to constitute a serious check to the expulsive action of the heart. It is stated that under these conditions a current of action may be detected in the trunk of the depressor nerve, and that if both depressor nerves be cut when the aortic pressure is high the blood-pressure rises still higher. It presents a means by which the heart can be relieved of a load too great for its powers, and therefore dangerous to its future welfare. In many animals the depressor fibres are bound up with the trunk of the vagus and cannot be excited separately.

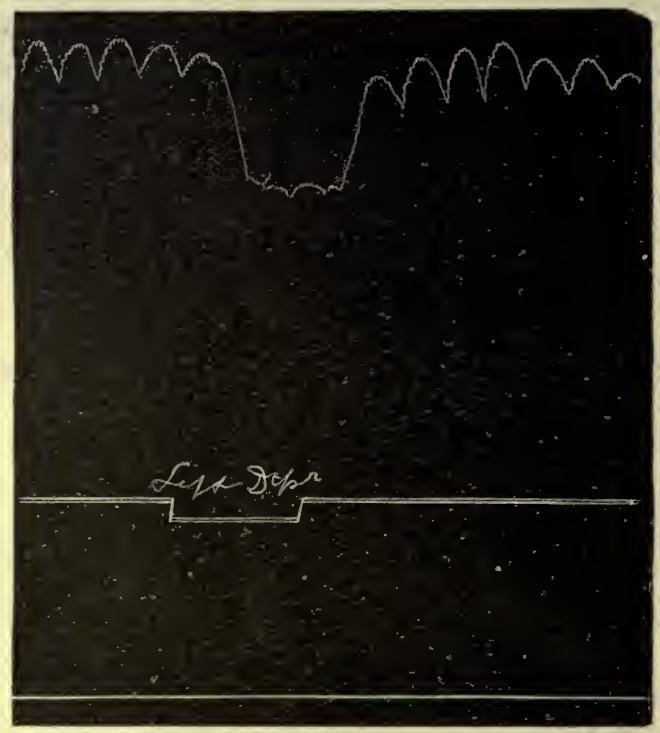

FIG. 453. Blood-pressure curve from rabbit showing effect of excitation of central end of depressor nerve (mercurial manometer). (BAYLISS.)

Stimulation of the central end of the vagus generally causes reflex slowing of the heart through the cardiac centre and the other vagus. In this reflex inhibition the chief fibres stimulated are those coming from the lungs (Brodie). Inflation of the lungs causes acceleration of the heart-whether due to diminution of the tonic action of the vagi, or to reflex excitation of the accelerator nerves, is not known. Most sensory nerves of the body when stimulated give either a slowing or a quickening of the heart. Stimulation of the fifth nerve, as in the nasal mucous membrane, always causes reflex inhibition.

The rate of the heart-beat in the normal animal is closely connected with the blood-pressure. Increase in blood-pressure due to a large vasoconstriction is associated as a rule (but not invariably) with a slowing of the heart-beat. In fact, 'Marey's law' states that the pulse-rate varies inversely as the blood-pressure. In this slowing of the heart the vagus nerves are of course active. Whether the blood-pressure acts directly on the cardiac centre in the medulla, or reflexly through afferent nerves distributed to the 
aorta and heart cavities, is not yet fully made out. The reflex slowing of the heart often fails to accompany rise of blood-pressure. Thus the rise in bloodpressure and the increased filling of the heart associated with muscular exercise are attended by an increased pulse-rate.

\section{THE PULSE-RATE IN MAN}

The normal pulse-rate in man is about 72 per minute. It is largely influenced by bodily movements. The pulse-rate varies considerably with age. The following Table represents the average pulse-rate in man at different ages :

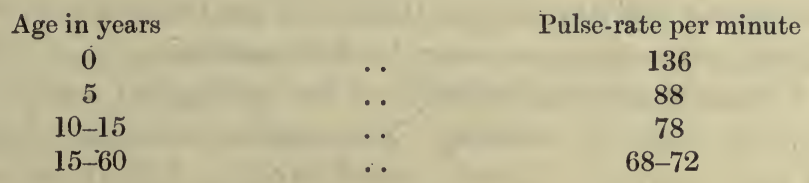

It must be remembered that marked differences in pulse-rate may be found in different individuals without having any pathological significance. Thus pulse-rates of 30 per minute and 120 per minute have been observed in men who were otherwise perfectly healthy. The pulse-rate is raised by warmth and diminished by cold applied to the surface of the skin. It is also increased somewhat by the taking of food. The act of swallowing causes a reflex quickening of the rate by inhibition of the tonic vagus action. 


\section{SECTION $\mathrm{X}$}

\section{THE NERVOUS CONTROL OF THE BLOOD-VESSELS}

DURING muscular activity the metabolism of the body as a whole, as judged by its gaseous interchanges, may be increased six- or eight-fold. This increase is due almost exclusively to the additional metabolic changes consequent on muscular activity. The muscles therefore during activity require a greater supply of blood in order to obtain from it the oxygen necessary for their contraction, and to get rid of the carbon dioxide, which is the end-result of their activity. In the same way every organ of the body requires an increased blood-supply during activity. Blood must be diverted from the inactive to the active tissues. All parts of the body must cooperate in subordination to the activity of that tissue whose function for the time being is of the greatest importance to the organism. This subordination of the part to the whole, i.e. of every part to the organ whose activity is specially evoked by the needs of the whole organism, is chiefly effected through the central nervous system, though local and chemical mechanisms also play some part in the process.

Our knowledge of the nervous control of the blood-vessels dates from the discovery by Claude Bernard that nerve fibres run in the cervical sympathetic to the blood-vessels of the head and neck, and maintain them in a state of tonic constriction. Bernard showed that if in the rabbit the cervical sympathetic on one side be divided, the vessels in the corresponding ear dilate. Vessels come into prominence which were previously invisible, and on account of the greater flow of blood thus produced, the ear on the side of the section becomes warmer than the normal ear. If the head end of the divided sympathetic nerve be stimulated, all the vessels of the ear contract, and the ear becomes colder than that of the other side. The fact that the dilatation of the vessels is produced by section of the cervical sympathetic and lasts for a considerable time after any irritant effect of the section must have passed off shows that the ear-vessels are continually under the influence of tonic constrictor impulses proceeding to them along the nerve fibres of the cervical sympathetic.

It can be easily shown that these impulses take their origin in the central nervous system. The paralysis of the ear-vessels, though lessening the resistance to the flow of blood there, affects too small a vascular area to have any marked influence on the total resistance of the circulation and therefore on the arterial blood-pressure. If the spinal cord be divided on a level 
with the origin of the first dorsal nerve, the blood-pressure sinks considerably. In the dog it may fall from $120 \mathrm{~mm}$. $\mathrm{Hg}$. to 40 or $50 \mathrm{~mm}$. Hg. The heart after the section beats more rapidly than before, so that the fall of pressure must be ascribed to a change affecting the blood-vessels and lowering the resistance to the flow of blood. Since a maximal effect on the blood-pressure is produced by section of the cord at this level, one may conclude that the tonic constrictor impulses to all the vessels of the body pass through this segment of the cord before leaving it to be distributed to the arterial walls. The source of these impulses may be made out by studying the effect of sections through different levels of the nervous system. Division of the cord at about the first or second lumbar nerve causes no effect on the bloodpressure. On making a section at the sixth dorsal root a considerable fall of pressure is produced, almost, but not quite, as great as that observed after section at the first dorsal segment; stimulation of the lower end of the cut cord causes almost universal vascular constriction and a large rise of bloodpressure. On the other hand, the fall of pressure is maximal when the section is carried through the first dorsal segment or through any part of the cervical cord. Section of the crura cerebri, or of the brain-stem at the upper border of the fourth ventricle, leaves the blood-pressure unaffected. Destruction of a small region of the medulla situated on each side of the middle line in the neighbourhood of the facial nucleus, i.e. in the forward prolongation of the lateral columns after they have given off their fibres to the decussating pyramids, causes an immediate and maximal lowering of the bloodpressure.

We must therefore conclude that all the vessels in the body are kept in a state of tonic contraction by impulses arising in this portion of the medulla oblongata, travelling down the cord as far as the dorsal region, and then passing out of the cord by the dorsal and upper lumbar nerves. This conclusion is confirmed by the fact that, whereas stimulation of the anterior roots of the cervical and lower lumbar and sacral nerves has no influence on the blood-pressure, a rise of arterial pressure can be obtained by stimulating any of the anterior roots from the first or second dorsal to the second or third lumbar. The same effect is produced by stimulation of the white rami communicantes from these roots to the sympathetic system, or by excitation of the sympathetic system itself.

The portion of the medulla concerned with the sending out of the tonic vaso-constrictor impulses is spoken of as the vaso-motor centre. In this region it is exposed to and played upon by afferent impulses from all portions of the body, from the higher centres of the brain and the cortex cerebri, and especially by afferent impulses travelling by the vagi from the viscera of the chest and abdomen. Whether in the absence of all afferent stimuli the centre would be active is doubtful; all we know is that the sum of the stimuli arriving at the centre produces a state of average continued activity, which is responsible for the maintenance of arterial tone and for the regulation of the arterial blood-pressure.

The centre may also be affected directly by changes in its blood-supply, 
or in the composition of the blood flowing through it. Thus anything which interferes with the gaseous exchanges of the centre, whether obstruction to respiration, absence of oxygen in the air breathed, or a failure of the bloodsupply, as by ligature of the cerebral arteries, calls forth an increased state of activity of the centre. This can be best studied by observing the changes in the blood pressure produced in a curarised animal by the cessation of artificial respiration.

These changes depend partly on the stimulation of the vaso-motor and vagus centres by the venous blood, and partly on the affection of the heart itself. We will first consider them with both vagi cut, in order to shut out the action of the vagus centre. The blood-pressure is registered by means of a mercurial manometer in connection with the carotid artery. On leaving off the artificial respiration, the blood-pressure may remain at the same height for some seconds, the only change noticed being the absence of the respiratory oscillations. Sooner or later the blood-pressure suddenly rises rapidly (Fig. 454, A), and in another ten seconds may reach a height twice as great as it was previously. The heart beats a little more forcibly in consequence of the increased cardiac tension, but its frequency is almost unaltered. The blood-pressure remains at this height for about a minute, and then gradually falls, the heart-beats becoming smaller and smaller, until the pressure has sunk to a point very little above the abscissa line (level of no pressure). This fall in pressure is due to the failure of the heart. The heart, badly supplied with oxygen, cannot overcome the high resistance presented by the contracted arterioles ; it gets overfilled, and gradually. loses the power of expelling any of its contents. If, when the blood-pressure has sunk to its lowest point, the heart be rapidly cut out of the body, it will begin to beat fairly forcibly, being relieved of the excessive internal tension. The vessels, however, remain constricted until the death of the animal. This is shown by two facts. If, while the pressure is sinking, artificial respiration be recommenced, the heart supplied with oxygen at once begins to beat more forcibly, and the blood-pressure may rise to an even greater height than immediately after the commencement of the asphyxia. Again, if the volume of the kidney be recorded by means of the oncometer, the rise of general blood-pressure produced by asphyxia is seen to be accompanied by a marked shrinking of the kidney, and this shrinking endures until the animal dies, showing that the fall of blood-pressure following the rise is due, not to a giving way of the arterial resistance, but to failure of the heart.

Similar results are obtained when the vessels to the brain are ligatured, or when the animal has to respire an indifferent gas free from oxygen, such as nitrogen (Fig. 454, B) or hydrogen. In the uncurarised animal the rise of blood-pressure is associated with increased respiratory movements and finally with convulsive spasms which may involve practically every muscle of the body.

We have spoken above of the phenomena of asphyxia as being due to the circulation of venous blood. There are, however, two factors which may 
be concerned and which may influence the medullary centres and the heart. When the renewal of the lung ventilation is stopped by ligature of the trachea or by cessation of the respiratory movements, the increasing venosity of the blood involves a diminished percentage of oxygen and an increased percentage of carbon dioxidc, and when asphyxia is excited by cessation of the circulation through the medullary centres, these centres may suffer at the same time from lack of oxygen and from the accumulation of carbon dioxide. The question arises whether one or both of these factors are concerned. It is easy to investigate the action of each separately. A pure oxygen lack may be brought about by allowing an animal to breathe some

A

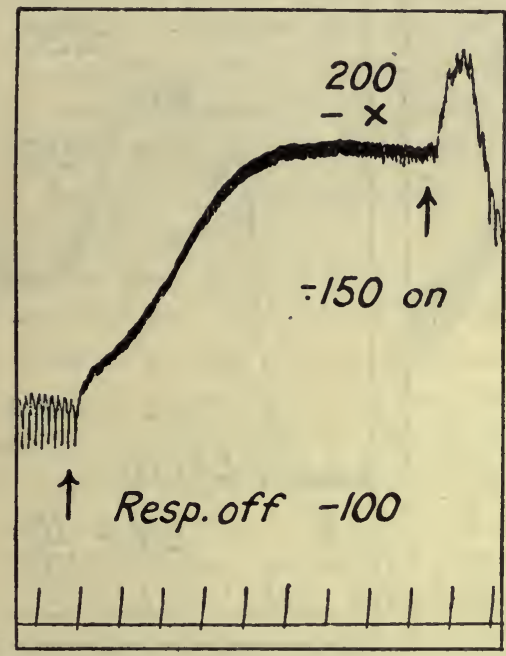

Fig. 454. Blood-pressure changes in a cat. A, after cessation of respiratory movements. B, as a result of artificial respiration with nitrogen. (MATHISON.)

inert gas, such as nitrogen or hydrogen, or in the curarised animal one of these gases may be administered by the pump used for artificial respiration. The effects of accumulation of carbon dioxide in the blood and tissues may be produced by the administration of gaseous mixtures containing excess of oxygen, i.e. 30 to 40 per cent., with varying percentages of carbon dioxide. In the first case, the tension of the carbon dioxide in the blood will be kept below normal ; in the second case, the tension of oxygen in the blood will be kept above normal. In order to obtain results uncomplicated by the influence of anæsthetics, the experiments may be carried out in animals which have been deprived of consciousness by destruction of the brain above the superior corpora quadrigemina. At different times physiologists have been inclined to ascribe the excitatory phenomenon of asphyxia either to absence of oxygen or to excess of carbon dioxide. Mathison has shown that both conditions may concur in the production of the rise of blood-pressure in asphyxia. In Figs. 454 and 455 the rise of arterial pressure produced by 
a short period of asphyxia is compared with that produced by oxygen lack, by a surplus of carbon dioxide, and by the injection of lactic acid into the circulation. There are certain minor details in these curves which are of interest. When the oxygen of the lungs is rapidly washed out with a neutral gas the asphyxial rise comes on about half a minute later than it would with pure asphyxia. In the latter case it seems that the first rise is due to the accumulation of carbon dioxide. The rise, however, under nitrogen when it occurs is extremely abrupt, and the subsequent fall of blood-pressure, i.e. the heart failure, is earlier in onset and more rapid than with ordinary asphyxia. When excess of carbon dioxide is administered, i.e. 5 to 10

A

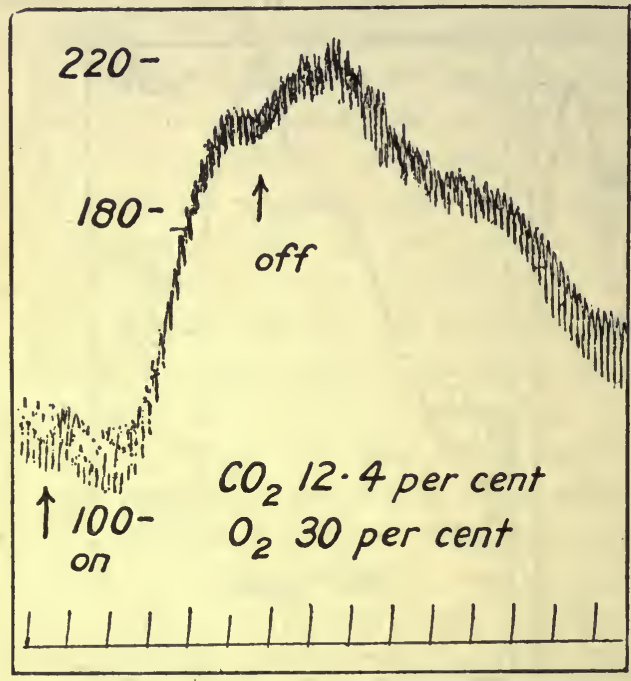

B

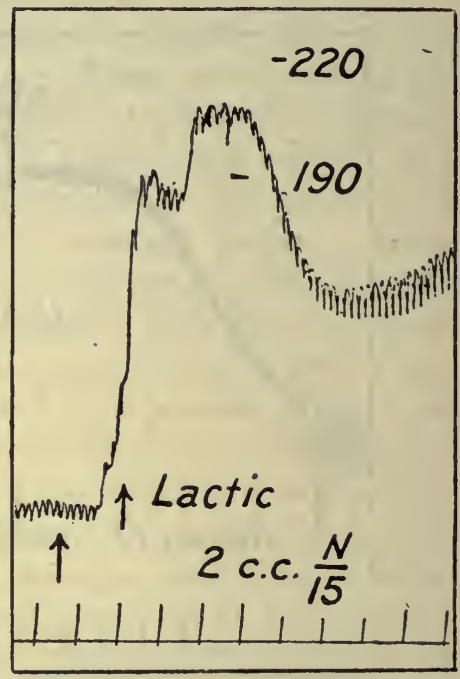

FIG. 455. Asphyxial blood-pressure changes in curarised cat. A, inhalation of $\mathrm{CO}_{2}$. B, injection of lactic acid. (Mathison.)

per cent., a marked rise of pressure occurs which, like that produced by oxygen lack, is almost entirely conditioned by stimulation of the vaso-motor centres and resulting constriction of the peripheral arterioles. If a loop of intestine be placed in a plethysmograph, it will be seen that the rise of pressure coincides with a shrinkage in volume of the intestine, pointing to a vascular constriction (Fig. 456). The rise of blood-pressure due to the vascular constriction may be maintained for a considerable period, e.g. ten to fifteen minutes, and we do not get the rapid fall of pressure due to failure of the heart that is observed in an ordinary asphyxia tracing. If partial oxygen lack or abnormally increased tension of carbon dioxide be continued for some time, a state of narcosis or paralysis finally ensues which affects not only the higher centres but also those of the medulla, so that death may ensue without convulsions or excessive rise of blood-pressure.

Is there any common factor in the two conditions of oxygen lack and carbon dioxide excess which may account for the similarity in their effects? It has been shown that whenever there is a deficiency of oxygen the metabo- 
lism of the tissues undergoes alteration, so that as a result of activity, e.g. in muscles, lactic acid is formed instead of carbon dioxide. Lactic acid can therefore be detected in the blood whenever violent exercise is taken sufficient to produce dyspnœea, or when the access of oxygen is diminished by poisoning with carbon monoxide, or by reducing the tension of this gas in the air breathed. Oxygen lack can be regarded therefore as synonymous with the production of lactic acid. Lactic acid introduced into the blood-stream, as is shown in the curve in Fig. 455, B, is equally efficacious with oxygen lack or with carbon dioxide excess in the production of a rise of bloodpressure indistinguishable from the asphyxial rise. It seems therefore that the common factor in asphyxia is the increased acidity or $\mathrm{H}^{\prime}$ ion concentration of the blood. We shall have occasion to return to this question in dealing with the regulation of the respiratory movements.

If in the dog, and to a less extent in other animals, the vagi be left intact, the blood-pressure tracing during asphyxia has quite another appearance. At the point of the tracing corresponding to the rapid rise in the previous experiment there is in this case only a slight rise of pressure, but the heart begins to beat very slowly. At each beat it necessarily sends out a greater volume of blood than when it is beating more frequently, and hence the oscillations on the blood-pressure curve caused by the heart-beats become very large. This slow beat is due to the action of the vagus

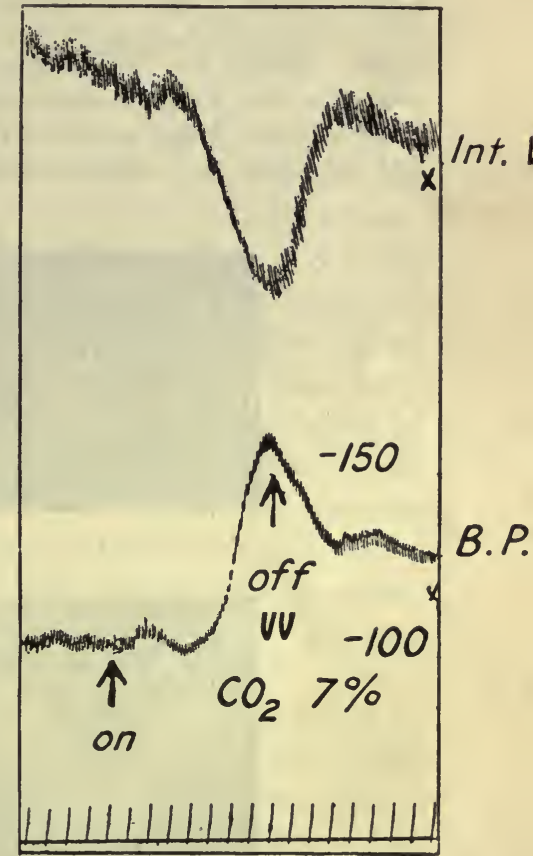

FIG. 456. Tracing of arterial bloodpressure and of intestinal volume, to show the influence of a moderate increase in the $\mathrm{CO}_{2}$ tension of the blood. (Mathison.) centre, and is at once abolished by section of the two vagi. The sparing of the heart by means of this vagus action enables it to last longer, and the final fatal fall of blood-pressure due to heart failure comes on rather later than when the vagi are divided. In the increased vagus action which occurs during asphyxia two factors are probably involved. The cardioinhibitory centre in the medulla probably partakes of the general excitation of the medullary centres due in the first place to carbon dioxide excess, in the second to oxygen lack. More important is the direct action of the rise of blood-pressure on the medullary centre. The rise of arterial pressure causes increased intracranial tension, and any increase of the latter excites the vagus centre and produces slowing of the pulse. The vagus slowing is therefore absent in asphyxia if the arterial blood be allowed to escape through a mercury valve so as to prevent any rise of pressure in the brain cavity. 
During the period of increased pressure waves are often observed on the bloodpressure curve. These are of two kinds. In the first place, in completely curarised animals we may observe oscillations of blood-pressure corresponding with the respiratory rhythm before the administration of curare, or if the vagi are cut, presenting a rhythm similar to that usual in animals with divided vagi. These waves are known as the Traube-Hering curves from the physiologists by whom they were first observed. They are certainly due to irradiation of impulses from the excited respiratory centre to the vaso-motor centre in the medulla. In fact, if the curarisation is not complete a slight twitch of the diaphragm, insufficient by itself to have any mechanical influence on the circulation, may be observed to accompany each rise on the blood-pressure curve. Besides the Traube-Hering curves others are occasionally seen which must arise in a slow rhythmic variation of the constrictor impulses sent out from the vasomotor centre. These waves are known as the Mayer curves, and are not to be confused with the waves on an nrdinary pressure curve due to respiration, being much slower in
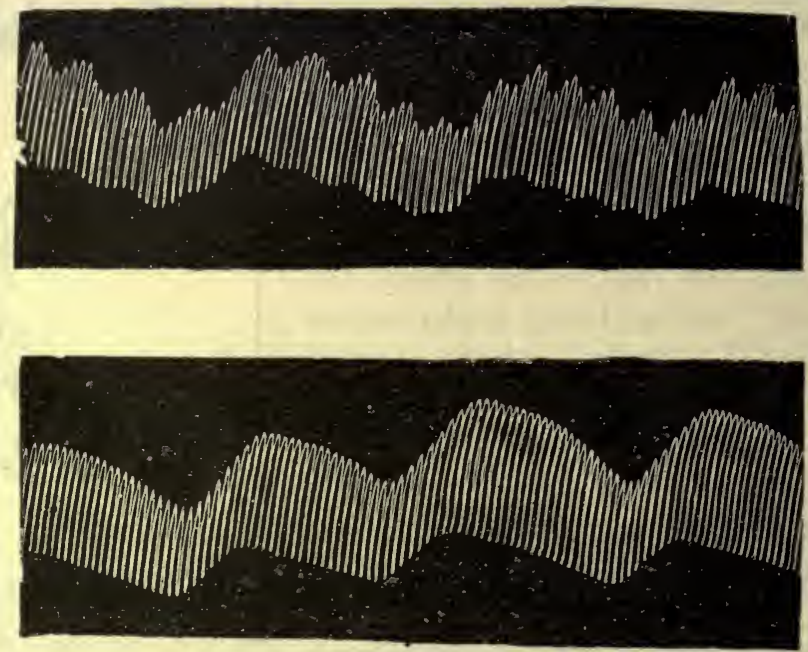

FIG. 457. Blood-pressure tracings showing S. Mayer curves. (C. J. MaRTIN.)

their rhythm than the latter. They are observed not only during asphyxia, but may occur in blood-pressure tracings from normal dogs, and are frequent in dogs poisoned with morphia. Fig. 457 represents tracings obtained from a dog under the influence of morphia and curare. The upper curve, taken while artificial respiration was being carried on, shows the three forms of curves-the oscillations due to the heart-beat next in size those due to the respiratory movements, which in their turn are superposed on the slow prolonged curves, i.e. the Mayer curves. The lower curve is taken immediately after cessation of the artificial respiration, and shows only the heart-beats and the Mayer curves. The presence of Mayer curves may generally be ascribed to a state of abnormal excitation of the vaso-motor centre. This excitation may arise in various ways. A very frequent cause is the one just described, viz. increased venosity of the blood supplied to the centre. Well-marked Mayer curves are often observed in cases of hæmorrhage. In spite of the loss of blood, the vaso-motor centres maintain a normal arterial blood-pressure by means of vascular constriction. As the bleeding continues, this means becomes inadequate, and at this point the 'efforts' of the centres take on a rhythmic character, giving well-marked Mayer curves, just as the arm of a man holding up a weight begins to shake before he is obliged to give way through fatigue. If the bleeding still continues, the pressure sinks steadily and the curves disappear. The curves may also be often observed during operations involving exposure of the cord, 
and may possibly be ascribed in this case to abnormal irritations ascending the posterior columns.

The vaso-motor centre may also be directly affected by drugs such as digitalis or strophanthus, both of which cause a rise in general blood-pressure from stimulation of the centre.

\section{SPINAL CENTRES}

The great fall of blood-pressure observed after section of the cord in the lower cervical region is not permanent. After one or two hours the pressure begins to rise, and if the animal be kept alive, may attain a height only a little inferior to that found in normal animals.

If the spinal cord of such an animal be destroyed, the blood-pressure sinks practically to zero and the circulation comes to an end, because the animal has been, so to speak, bled to death into its own dilated blood-vessels. In addition to the chief vaso-motor centre in the medulla there is a series of subsidiary centres in the spinal cord, centres which we may probably locate in the portions of grey matter situated in the lateral horns of the cord and giving origin to the fibres which go to make up the white rami communicantes. By means of these spinal centres a certain degree of adaptation is possible between the blood-supply of the various parts of the trunk. The important co-ordination between the state of the blood-vessels and the condition of the central pump, the heart, is, however, wanting, since the blood-vessels are now cut off from the cardiac centres and from the part of the central nervous system which receives the afferent impulses carried by the vagi.

The spinal centres, like the chief vaso-motor centre, are susceptible to changes in the composition of the blood supplied to them. If an animal be kept alive by means of artificial respiration for a little time after division of the cord just below the medulla, the blood-pressure slowly rises as the spinal centres begin to take on their automatic functions. If artificial respiration be now discontinued the asphyxia excites the centres of the cord. The motor discharge to the skeletal muscles reveals itself in a single prolonged spasm, since the respiratory centre is unable to take any part in directing the motor discharges. Simultaneously with the spasm of the skeletal muscles general constriction of the blood-vessels occurs which outlasts the muscular spasms and causes a considerable rise of blood-pressure (Fig. 458).

In this rise of pressure the main factor is lack of oxygen, and precisely similar curves are obtained whether the asphyxia be produced by cessation of artificial respiration or: by administration of nitrogen. The same effect may be produced by a very large excess of carbon dioxide, or by the injection of acids into the circulation. There is a striking difference between the sensibility of the spinal centres to these substances as compared with the medullary centres. Thus the medullary vaso-motor centre is readily excited by ventilation with 5 per cent. carbon dioxide, $\cdot$ whereas a rise of blood-pressure is only obtained from the spinal animal when mixtures containing 25 per cent. and upwards of carbon dioxide are employed. The excitation of the medullary centre comes on about thirty seconds after the 
administration of nitrogen has commenced in contrast to that of the spinal centres which does not occur until two minutes or more have elapsed. In the intact animal a maximal stimulation of the vaso-motor centre is produced in the cat by the injection of 2 c.c. $\mathrm{N} / 20$ lactic acid, whereas 5 c.c. of $\mathrm{N} / 2$ acid are required to excite spinal cord centres. Here therefore, as in

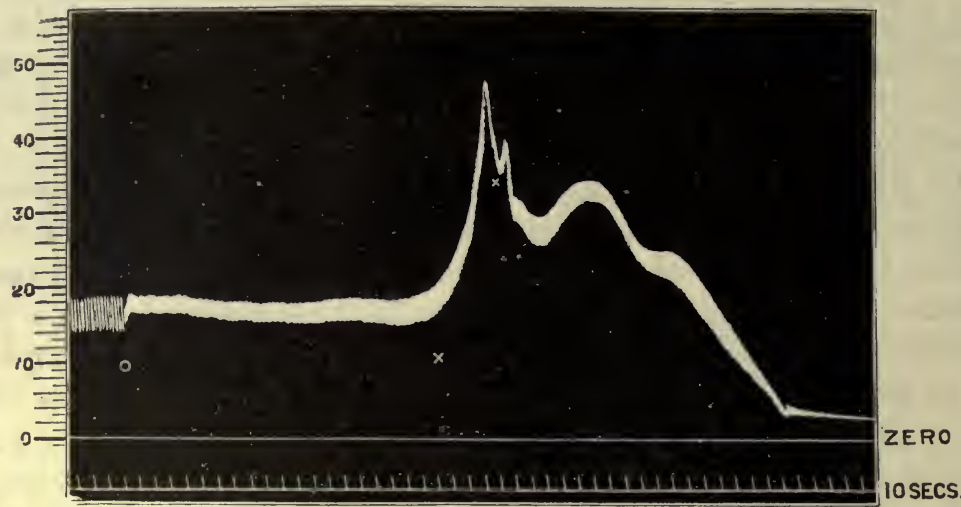

Fig. 458. Blood-pressure tracing taken by a mercurial manometer from carotid artery of a dog, three hours after section of the cord, just below the medulla oblongata. At o the artificial respiration was discontinued. A general spasm of the skeletal muscles occurred between $\mathbf{x}$ and $\mathbf{x}$. The muscles then relaxed, and were flaccid during the rest of the rise of blood-pressure.

the medulla, the common factor is probably increased $\mathrm{H}$ ion concentration, the excitation threshold for the medullary centres being only about onefifth that of the spinal centres.

The local spinal centres are connected with the medullary vaso-motor centre on each side by tracts of nerve fibres which descend in the lateral columns of the cord.

\section{THE PERIPHERAL TONE OF THE BLOOD-VESSELS}

Division of the sciatic nerve causes an immediate dilatation of the vessels of the lower limbs in consequence of their severance from the tonic activity of the vaso-motor centres. This dilatation passes off in a day or two and the vessels acquire a tone, i.e. remain in a state of average constriction which can be increased or diminished by local conditions. This recovery of tone has been ascribed by many physiologists to the existence of a third set of nerve-centres in the walls of the arteries. In the absence of any direct histological evidence of the existence of such centres it seems more rational to ascribe the tonus to the automatic activity of the muscular fibres themselves.

\section{THE COURSE AND DISTRIBUTION OF THE VASO-MOTOR NERVES}

Since the blood-vessels, like the heart, are the seat of an automatic activity, complete nervous control of these tubes can only be secured by the provision of two sets of nerves : one set-augmentor or motor-which will 
increase the state of constriction of the vessels; another set-inhibitor or dilator-which will diminish the tone of the arteriole muscle and cause vascular dilatation. Our knowledge of the existence of this second class of nerve fibres to the vessels we owe also to Claude Bernard, who observed that stimulation of the chorda tympani nerve not only evoked secretion from the submaxillary gland but also increased the blood-flow through its vessels five- or sixfold. Subsequent researches have revealed the fact that nearly all the vessels of the body receive vaso-constrictor fibres, and that many receive also vaso-dilator fibres. In order to determine the course and distribution of the vascular nerves it is necessary to have means at our disposal for investigating the condition of the blood-flow through different parts and organs of the body. Let us see what effects will ensue on the local circulation by constriction or dilatation of the arterioles with which it is supplied. If the arterioles $\mathbf{A}$ in the organ $\mathbf{B}$ dilate (Fig. 459), the first effect is a diminution of the resistance to the flow of blood into the capillaries beyond. Supposing that the arterial pressure in the trunk c remain constant, a local diminution of resistance in A will at once determine an increased flow of blood through the arterioles, and

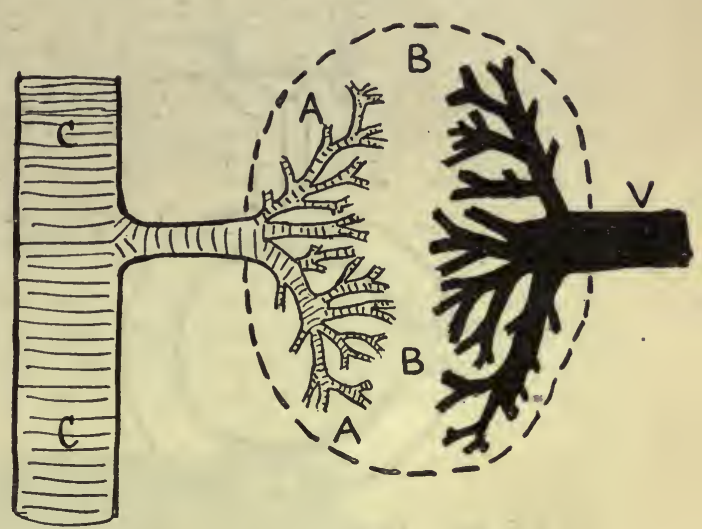

Frg. 459. the fall of pressure from $\mathrm{A}$ to the capillaries will be less than when the arteriole was constricted. If the organ is distensible and elastic, the increased pressure in the arterioles and capillaries will cause dilatation of these vessels, and a consequent dilatation of the whole organ. The same effect on intracapillary pressure, and therefore on the volume of the part, may be caused by obstruction to the flow of blood from the veins. Provided that there is no obstruction to the flow of blood through the vein, and that the general blood-pressure in $\mathrm{c}$ remains constant, dilatation of an organ may be taken as an expression of vasodilatation in the arteries with which it is supplied. The diminution of the resistance in A may also increase the velocity of the flow through the part, since the amount of blood flowing in a given period of time through any vessel varies directly as the difference of pressure, and inversely as the resistance in the vessel.

We can therefore use the following criteria for the occurrence of a vasodilatation in the arterial supply to any part or organ :

(1) If the surface of the part is translucent, the increased filling of the blood-vessels will cause redness or blushing.

(2) The increased size of the vessels will cause an increase in the volume of the organ concerned. 
(3) An increased velocity of blood-flow will, if the part be normally below the temperature obtaining in the central organs of the body, raise its temperature, and vaso-dilatation can thus be detected by the application of the hand or of a thermometer.

(4) Any of the methods mentioned in a previous chapter may be used to determine the velocity in the arteries going to the part, and an increased velocity may be interpreted as due to vaso-dilatation.

(5) The increased flow through the part may be detected by cutting the main efferent vein and measuring the total volume of blood which flows from it in a given time.

Of these methods the two most used are those based on determination either of the volume of the part, or of the venous outflow from the part.

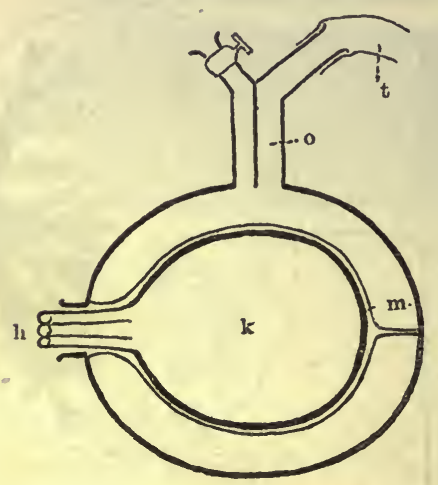

Fia. 460. Diagram of oncometer.

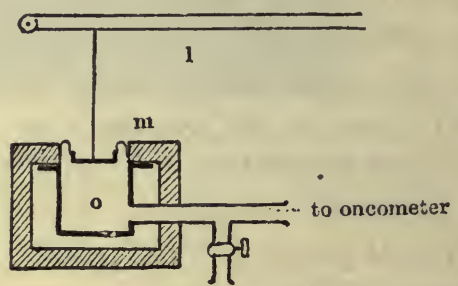

FIG. 461. Diagram of oncograph.

A fallacy may, however, arise, unless means be taken to ensure that the general arterial pressure remain constant during the experiment. A rise of general blood-pressure will cause an expansion of the vessels and of the part supplied, and also increased velocity of blood-flow through the part. In all cases therefore where it is desired to investigate the conditions of the local circulation, it is necessary to combine a determination of the general blood-pressure with some means of estimating changes in the local conditions. We may take as an instance an experiment on the blood-supply to the kidney.

For this purpose we may use a kidney plethysmograph or oncometer. The structure of Roy's oncometer is shown in Fig. 460. The oncometer is a metal capsule, the two halves of which are hinged together and come in contact at the whole of their circumference except at $h$, where a small depression is left in each half for the passage of the kidney vessels and ureter. A piece of peritoneal membrane is attached to the rim of each half of the oncometer, the space between this and the brass capsule being filled with warm oil. The kidney rests in the oncometer on this bed of warm oil, from which it is separated by a membrane. A tube leads from the cavity between the brass capsule and membrane to a registering apparatus, or oncograph (Fig. 461), which is a piston recorder containing oil. Any swelling of the kidney will drive oil out of the oncometer into the cylinder of the oncograph and so raise the piston, the excursions of which are recorded by a lever writing on a blackened surface. 
Schäfer's plethysmograph (Fig. 462), which can be adapted to almost any organ of the body, is made of vulcanite * previously moulded to the size of the organ whose volume is the object of investigation. In one side of the box a depression is left sufficient to accommodate easily the vessels, nerves, or ureter going to the organ. The oncometer is covered with a glass lid which is made air-tight by means of vaseline, the space

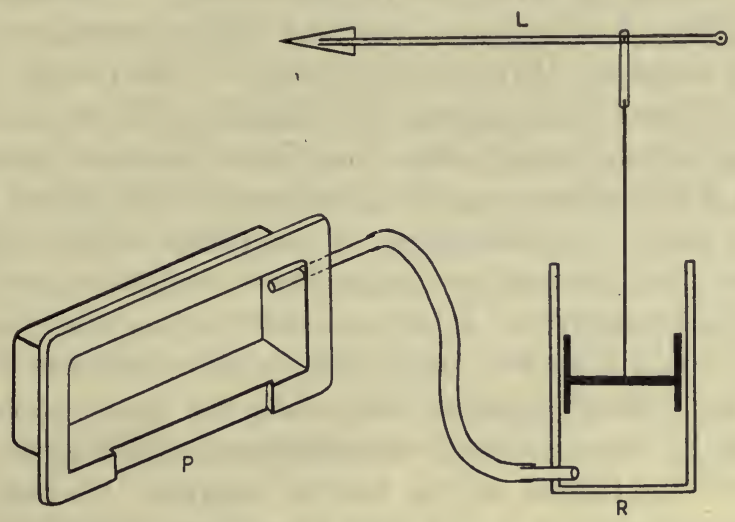

FIG. 462. Diagram of Schäfer's air plethysmograph.

between the lid and the vessels being also packed with cotton-wool and vaseline. A glass tube is fixed into one corner of the plethysmograph and leads to a piston recorder or tambour. Every variation in the volume of the organ causes a movement of air into or out of the oncometer and thus gives rise to a corresponding movement of the recording lever

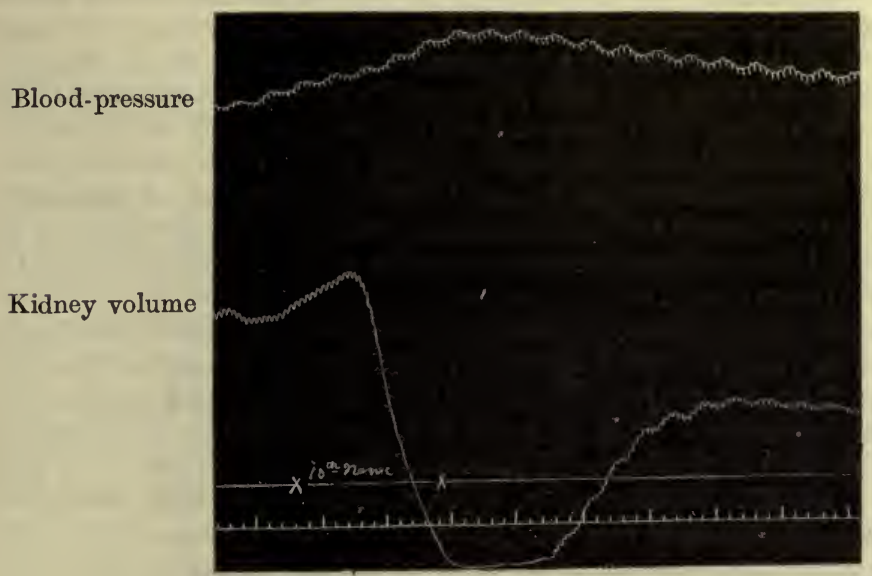

FIG. 463. Simultaneous tracings of carotid blood-pressure and volume of kidney. Between $x$ and $x$ the peripheral end of the divided tenth dorsal nerve was stimulated. Time-marking $=$ seconds. $($ BRADFORD.)

The kidney being placed in some such apparatus, a cannula is also placed in the carotid artery and connected with a mercurial manometer, so that two tracings are obtained at the same time on the moving blackened surface. In the figure given (Fig. 463), the upper curve represents the carotid blood-

* A very good material for this purpose is 'Stent's composition,' used by dentists for taking a mould of the jaw in fitting artificial teeth. 
pressure, while the lower is the tracing of the oncograph lever. At the beginning of the experiment the lower dorsal nerve-roots had been dissected out and prepared for stimulation. The peripheral end of the anterior root of the tenth dorsal nerve was excited by means of an interrupted current at the point marked with a cross on the tracing. This stimulation was followed by a rise of blood-pressure together with a diminution in the kidney volume. The increased blood-pressure would by itself tend to force more blood into the kidney and so increase its volume. The fact that the kidney volume diminished shows that there must have been active contraction of the arterioles of the kidney, emptying this organ of blood and so causing it to decrease in size. This contraction of the vessels would tend to cause a rise in general blood-pressure and must have taken some part at any rate in the rise actually observed. If the oncometer in this experiment had been used alone, it would have been impossible to have determined whether the shrinkage of the kidney might not have been due to a lowering of general blood-pressure, in consequence of vaso-dilatation occurring elsewhere, or in consequence of the failure of the heart's activity. On the other hand, without the oncometer it would only have been possible to determine that there was increased peripheral resistance somewhere or other in the body.

Instead of taking the volume of the kidney we might have determined the bloodflow through its vessels either directly by means of a cannula in the renal vein, or by the indirect method of Brodie. This method depends on the fact that under normal conditions the amount of blood leaving an organ is equal to that entering it during any short space of time. If the efferent vein be clamped for five or ten seconds, the blood entering the organ during this time cannot escape, and therefore accumulates in the organ and increases its volume. If the organ be in a plethysmograph the increase of volume during this period may be measured and is exactly equal to the volume of blood passing through the artery into the organ during the five or ten seconds of the closure. The vein must not be obstructed too long, otherwise the increasing distension of the organ will appreciably increase the resistance to the entry of blood, and so diminish the velocity of the blood in the artery.

The direct determination of the venous outflow is not well adapted to large organs on account of the very rapid loss of blood which occurs through the open vein. The method is, however, of great value in dealing with the circulation through small organs such as the submaxillary glands. In such a case it is usual to hinder or prevent the clotting of the blood by the preliminary injection of leech extract, and then, after placing a cannula in the efferent veins of the organ, to allow blood from the cannula to drop on to a mica disc attached to a Marey tambour. This tambour is connected by a tube with a registering tambour, every drop on the disc giving rise to a small elevation of the lever of the second tambour.

\section{COURSE OF THE VASO-CONSTRICTOR FIBRES}

In investigating the course of the vaso-constrictor fibres we have to determine :

(1) The origin of the fibres from the central nervous system;

(2) The course of the fibres on their way to their peripheral distribution in the blood-vessels ;

(3) Their connections with nerve-cells. 
The two first details can be found by stimulating various nerves and nerve-roots in different parts of their course and observing the effects produced on the local and general circulation. The importance of the third heading is due to the fact that the vascular nerves, like the visceral nerves generally, do not have their last cell-station in the spinal cord. The fibres carrying vaso-constrictor impulses, on leaving the cord, do not pass direct to the blood-vessels, but come to an end in a collection of ganglion-cells, which may belong to the main chain of the sympathetic, or be situated more peripherally and belong to the group of collateral or peripheral ganglia. These fibres, as they leave the central nervous system, are small medullated nerves. They end in the ganglion by arborising round ganglion-cells, whence a fresh relay of fibres starts and carries the impulses on to the muscle fibres of the blood-vessels. The post-ganglionic fibres differ from the preganglionic fibres in being non-medullated.

The discovery of the ganglia, with which any given set of nerve fibres is connected, is rendered easy by the fact that in many animals the sympathetic ganglion-cells are paralysed by nicotine (Langley). The nicotine may be painted on the ganglion or may be injected into the blood-stream. The first effect of the drug is a powerful stimulation of the ganglion-cells, so that, if the drug be injected, there is an enormous rise of blood-pressure owing to the universal vaso-constriction that is produced. The stimulation gives place to a condition of paralysis; the blood-pressure falls below normal, owing to the cutting off of the peripheral vascular nerves from the vasomotor centre. Stimulation of the pre-ganglionic fibre is now without effect, although the normal results follow stimulation of the post-ganglionic nonmedullated fibre.

By these methods it has been determined that all the vaso-constrictor nerves of the body leave the spinal cord by the anterior roots of the spinal nerves from the first dorsal to the third or fourth lumbar inclusive. From the roots they pass by the white rami communicantes to the ganglia of the sympathetic chain lying along the front of the vertebral column. Here they take different courses according to their destination.

The fibres to the head and neck leave by the first four thoracic nerves, pass into the sympathetic chain through the ganglion stellatum and ansa Vieussenii to the inferior cervical ganglion, and up the cervical sympathetic trunk to the superior cervical ganglion. Here they end, and the impulses are carried by a fresh relay of fibres, which start from cells in this ganglion and travel as non-medullated fibres on the walls of the carotid artery and its branches.

The constrictors to the fore limb in the dog leave the cord by the white rami of the fourth to the tenth thoracic nerves. The fibres run up the sympathetic chain to the stellate ganglion, where they all end in synapses round the cells of this ganglion. The impulses are carried on by nonmedullated fibres along the grey rami of the sympathetic to the cervical nerves which make up the brachial plexus, and run down in the branches of this plexus to be distributed to the vessels of the fore limb. 
The constrictor impulses to the hind limb in the dog arise from the nerve-roots between the eleventh dorsal and third lumbar roots. All the fibres end in connection with cells in the sixth and seventh lumbar and first and second sacral ganglia of the sympathetic chain, whence the impulses are carried by grey rami to the nerves making up the sacral plexus.

The most important vaso-motor nerve of the body is the splanchnic nerve. This nerve receives most of the fibres forming the white rami from the lower seven dorsal and upper two or three lumbar roots, the latter fibres often taking a separate course as the lesser splanchnics. The fibres can be seen to pass through the sympathetic chain of the thorax without interruption, and for the most part have their cell-station in the large ganglia, especially the semilunar ganglia, of the solar plexus, whence a thick meshwork of non-medullated fibres is distributed along all the vessels of the abdominal viscera. The area of the vessels innervated by this nerve is so large that section of this nerve on each side causes a large fall in the general blood-pressure. This fall is more marked in animals such as the rabbit and other herbivora, in which the alimentary canal is proportionately very much developed, and has consequently a very large blood-supply.

\section{VASO-DILATOR NERVES}

Since the arteries are in a constant condition of moderate contraction, a dilatation might be brought about by a relaxation of this tone by an inhibition of the normal constrictor impulses proceeding to the vessels from the vaso-motor centre. We find, however, in many parts of the body evidence of the existence of a nerve-supply to blood-vessels antagonistic in its function to the vaso-constrictors. Thus, if the chorda tympani nerve going to the submaxillary gland be cut, no change is evident in the bloodvessels of the gland. But if its peripheral end be stimulated there is instantly free secretion of saliva from the gland, and all the blood-vessels are largely dilated. In consequence of this dilatation the blood rushes through the capillaries so quickly that it has no time to lose much of its oxygen; the blood flowing from the vein is therefore bright arterial in colour, and is increased to six or eight times the previous amount. If atropine be injected into the animal, the action of the chorda tympani on the blood-vessels is unaffected, although the secretion on stimulation is abolished. The chorda tympani is therefore said to contain vaso-dilator fibres for the vessels of the submaxillary gland. Other examples of vaso-dilator (or dilatator) nerves are the small petrosal nerve to the parotid gland, the lingual nerve to the blood-vessels of the tongue, and the nervi erigentes or pelvic visceral nerves to those of the penis.

The course of these typical dilator nerves differs widely from that of the constrictors. Whereas the latter leave the central nervous system over a limited area of the cord, the vaso-dilators take their origin together with any of the cerebro-spinal nerves. Thus the chorda tympani fibres, and probably those contained in the petrosal nerve, arise from the nervus inter- 
medius between the seventh and eighth cranial nerves. The nervi erigentes leave the lower end of the cord by the anterior roots of the second and third sacral nerves. All of them, like the vaso-constrictors and probably all visceral nerve fibres, are interrupted by ganglion-cells before reaching to their destination. These cells, however, lie, not in the lateral chain of the sympathetic, with which the nerves have no connection at all, but peripherally, and are generally embedded in the organs to which the nerves are distributed. Thus the chorda tympani fibres to the submaxillary glands are interrupted by cells embedded in the gland itself. The nervi erigentes pass to ganglioncells in the hypogastric plexus lying on the neck of the bladder.

Whether any large numbers of the fibres making up the sympathetic system of nerves are vaso-dilator in function is still uncertain. In the dog dilatation of the vessels of the soft palate and gums can be produced by

A

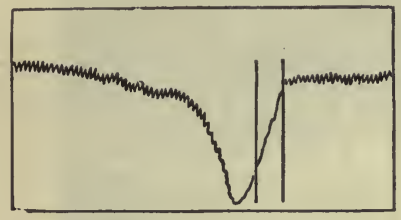

Nerve freshly divided. Constriction.
B

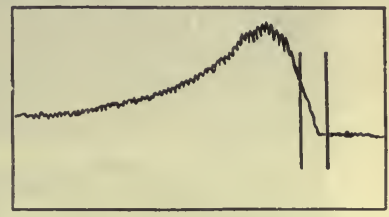

Nerve four days degenerated. Dilatation.

FIG. 464. Plethysmographic tracing of hind limbs, showing effect of stimulating the sciatic nerve on the volume of the limb, A, immediately after section of the nerve; $\mathrm{B}$, four days after section. The nerve was stimulated between the two vertical lines. Curves to be read from right to left. (BOWDITCH and WARREN.)

stimulation of the cervical sympathetic of the same side, or of the stellate ganglion or its rami communicantes. The effect has not yet been observed in any other animals. It is probable that the splanchnic nerves convey vasodilator fibres to the vessels of the abdomen, since stimulation of these nerves may cause a fall of blood-pressure, provided that the constrictor fibres, which predominate, have been paralysed by the previous administration of large doses of ergotoxin, derived from ergot.

The presence of vaso-dilator fibres in the nerves going to the limbs has been the subject of much debate. Since these nerves contain also constrictor fibres, the effect of the constriction overpowers any effects due to simultaneous stimulation of possible dilator fibres. Moreover the dilators apparently do not conduct any tonic influences to the blood-vessels, so that the only effect of section of a mixed nerve is that due to the removal of the tonic constrictor influences, and the vessels in the area of distribution of the nerves are dilated.

Various methods have been employed to show the presence of dilator fibres in such a mixed nerve-trunk. Of these the chief two are those depending on the unequal time taken for the two sets of fibres to degenerate and on the varying excitability of the two sets of fibres to different kinds of stimulation. Thus, if the sciatic nerve be cut, a primary dilatation of the vessels of the leg and foot is produced, which, however, passes off after two 
or three days. If now the peripheral end of the divided nerve is stimulated, dilatation of the vessels is produced (Fig. 464). Apparently the constrictor fibres degenerate before the dilator fibres, so that at a certain period after the nerve section only the latter respond to stimulation. On the other hand, it is often possible in the freshly cut nerve to obtain dilatation by stimulating its peripheral end with induction shocks repeated at slow intervals-

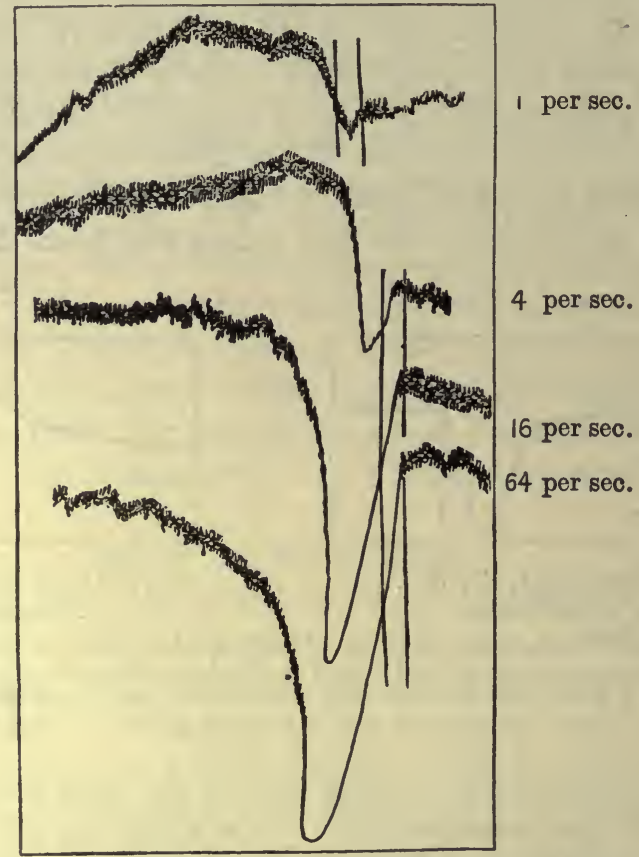

Fig. 465. Effect on the volume of the hind limbs of the cat stimulating of the sciatic nerve with induction shocks at different rates. It will be noticed that with one shock per second there is hardly any constriction, but considerable dilatation, whereas with 64 shocks per second the only effect produced is vaso-constriction.

Curves to be read from right to left. (BowDITCH and WARREN.)

one to four per second. The effects of different rates of stimulation on the limb-nerves of the cat are shown in Fig. 465.

When we endeavour to trace these limb dilator fibres back to the cord we find no trace of their passage through the sympathetic system. It was shown by Stricker and Morat that dilatation of the vessels of the hind limb can be produced by stimulating the posterior roots of the nerves going to the limb, i.e. far below the point of origin from the cord of the constrictor fibres to the same part of the body. Since it has been definitely shown by embryologists and histologists that in higher mammals all the fibres making up the posterior roots have their origin in the cells of the posterior root-ganglion, this observation was widely discredited, until it was confirmed by Bayliss for all manner of stimuli. Stimulation of the posterior roots, either before or after they have passed through the ganglia, causes dilatation of the vessels in the area of the supply of the roots, whatever be the nature of the stimulus employed, 
whether electrical, chemical, or mechanical (Fig. 466). This effect is not destroyed by previous section of the posterior roots on the proximal side of the ganglia, showing that the fibres by means of which the dilatation is produced have the same origin and course as the ordinary sensory nerves to the imbs. Since the vaso-dilator impulses pass along these nerves in a direction opposite to that taken by the normal sensory impulses, Bayliss has designated them as antidromic impulses. So far this phenomenon of a nerve-fibre functioning (not merely conducting) in both directions is almost without analogy in our knowledge of the other nerve-functions of the body. There is no doubt, however, that similar antidromic impulses are

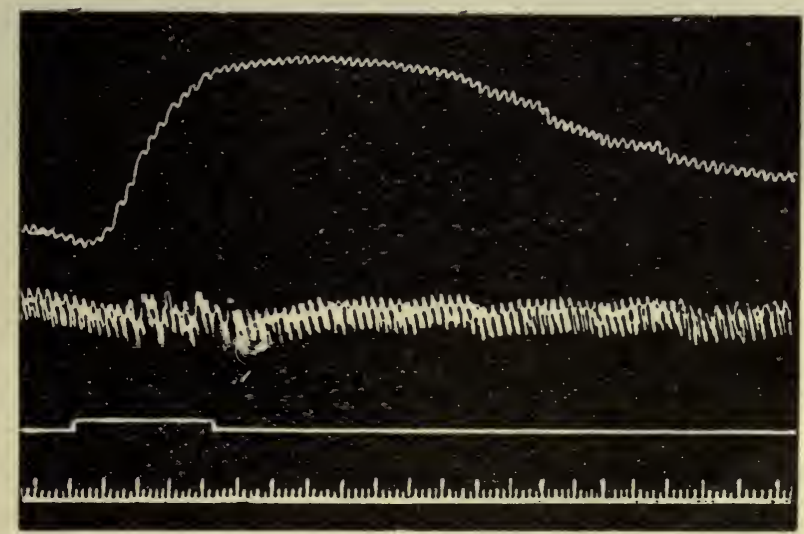

FIG. 466. Effect of excitation of peripheral end of the seventh lumbar posterior root in the dog. (BAYLISS.)

Uppermost curve, volume of left hind limb ; next below, arterial blood pressure ; the third line marks the period of stimulation; bottom line, timemarking in seconds.

involved in the production of the so-called trophic changes, such as localised erythema or the formation of vesicles (as in herpes zoster), which may occur in the course of distribution of a sensory nerve, and is always found to be associated with changes, inflammatory or otherwise, in the corresponding posterior root ganglia. Moreover evidence has been brought forward that these fibres may take part in ordinary vascular reflexes of the body, that in fact they are normally traversed by impulses in either direction.

Some observations by Hans Meyer and Bruce tend to indicate that in the antidromic vaso-dilatation, as well as in the reddening and inflammatory changes ensuing on local excitation, we are dealing with axon reflexes, perhaps the only remains of the local reflexes of a primitive peripheral subcutaneous nervous system. If croton oil or mustard oil be applied to the skin or to the conjunctiva, redness, swelling, and all the signs of a local inflammation are produced. The course of events is not altered by destruction of the central nervous system or by section of the sensory nerve-roots (posterior spinal root or trigeminus) on the central side of the ganglion. If, however, they be divided peripherally of the ganglion, and time be allowed for complete 
degeneration of the nerve fibres to their peripheral terminations, the application of croton or mustard oil, even to the delicate conjunctiva, is without effect. The same results may be produced if the peripheral terminations of the nerves be paralysed by the subcutaneous injection of local anæsthetics. We must assume that the axons of the peripheral sensory nerves branch, some branches going to the surface, others to the muscle-cells of the cutaneous arterioles, as indicated in the diagram (Fig. 467).

Gaskell has drawn an analogy between the nerves distributed to the blood-vessels and those going to the heart, which is indeed only a specialised part of the general blood-tubes of the body. These nerves, according to

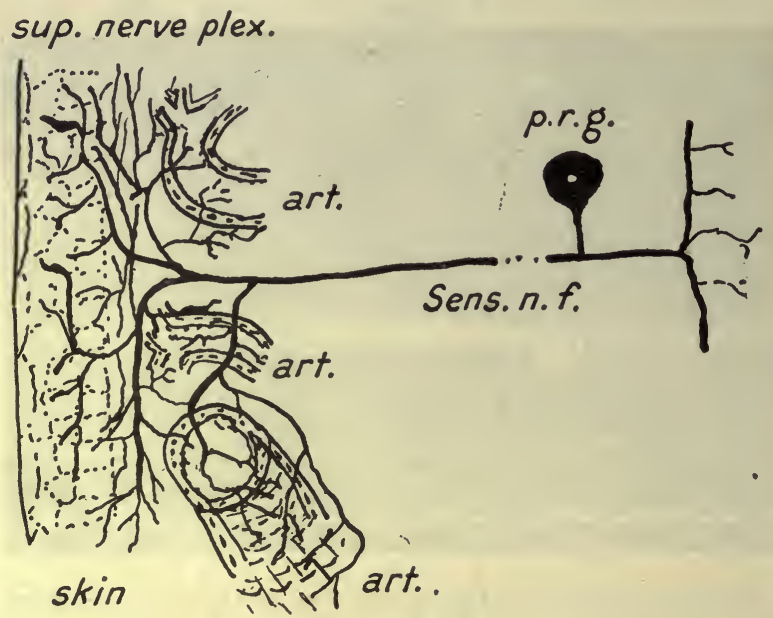

FIG. 467. Diagram to illustrate the production of vaso-dilatation in the area of distribution of a sensory nerve.

prg, posterior root ganglion; sens.nf, sensory nerve fibre, branching to supply dilator fibres to the skin arteries, and sensory fibres to the skin.

their action on the metabolic activity of the tissues supplied, are divided by Gaskell into anabolic and catabolic nerves. The anabolic nerves, as indicated by their name, cause a building up or regeneration of the contractile tissue. They therefore act as inhibitory nerves. This class would include the vagus and the vaso-dilator fibres. The catabolic nerves cause an increased activity of the contractile tissue, and active contraction is associated with and derives its energy from disintegration or catabolism of the muscular substance. An ordinary motor nerve to a muscle is therefore a catabolic nerve. This class would include the accelerator nerves to the heart, and the vaso-constrictors. The course of these two sets of nerves bears out this comparison, the path taken by the accelerator nerves being identical at first with that of the vaso-constrictor fibres to the head and neck.

\section{VASO-MOTOR REFLEXES}

The vaso-motor centre with its efferent tracts is constantly played upon by impulses arriving at it from the vascular system, including both heart and blood-vessels, from the viscera, from the muscles, and from the surface 
of the body. The reflex effects produced by stimulation of the various afferent nerves may be classified, according as they affect the general bloodpressure or the circulation through restricted areas of the body, as general and local.

The afferent impulses affecting the general blood-pressure are distinguished as pressor and depressor, and these names are sometimes applied

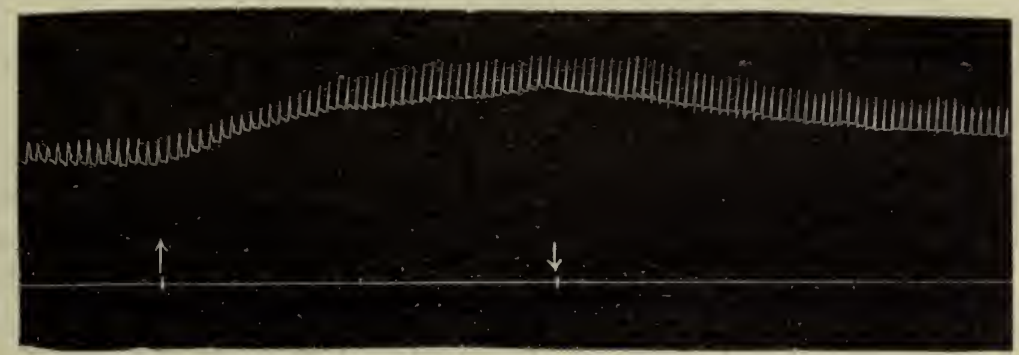

Fig. 468. Blood-pressure curve from carotid of dog. Between the arrows the central end of a sensory nerve was stimulated. (HÜRTHLE's manometer.)

to the nerves which carry the impulses. A pressor reflex is one which induces a rise of general blood-pressure by constriction of the blood-vessels, especially in the splanchnic area (Fig. 468). Effects of this kind are produced by stimulation of nearly all the sensory nerves of the skin. Practically all impulses which, if consciousness were present, would be attended with pain cause also a rise of general blood-pressure. A rise of pressure may be produced by the stimulation of such nerves as the fifth, the central end of the splanchnic nerves, or of the nerves distributed to the surface of the body. This rise occurs in all animals under morphia and curare. In the rabbit, when anæsthesia is induced by means of chloral or chloroform, stimulation of sensory nerves may cause a fall of bloodpressure.

The chief example of a depressor nerve we have already studied in

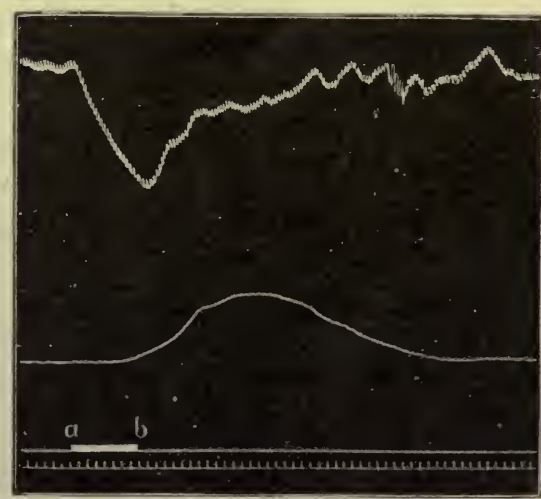

FIG. 469. Simultaneous tracing of arterial blood-pressure and splenic volume from a rabbit, showing the marked swelling of the spleen associated with fall of general blood-pressure on stimulation of the central end of the depressor nerve. The nerve was excited between $a$ and $b$. (BAYLISS.) dealing with the reflexes from the heart. The fall of pressure produced by stimulation of this nerve is effected chiefly by dilatation of the splanchnic area (Fig. 469), though, as Bayliss has shown, practically all the vessels of the body partake in the relaxation. The lowering of blood-pressure produced by stimulation of this nerve differs from that obtained on stimulating the sensory nerves of the rabbit under chloral, in that its effect lasts as long as 
the stimulation is continued, whereas in the latter case the effect shows signs of fatigue and disappears before the excitation is shut off.

So far as the general blood-pressure is concerned the most important impulses arriving at the centre are those from the vascular system, especially from the heart itself, and those from the higher parts of the brain. Whatever the condition of the heart the brain always demands a normal arterial pressure, since on this depends the supply of a proper quantum of blood to the master tissues of the body. A failing heart therefore evokes indirectly constriction of the blood-vessels, a fact which may lead to a vicious circle in cases where the heart is unable to perform its normal functions and to empty itself against the resistance of the blood-vessels. In this case the heart dilates more and more, until the slightest increase in the demands upon it, as by a slight muscular exertion, máy suffice to stop its action altogether.

Under normal circumstances every part of the body receives just so much blood as it needs for its metabolic requirements. Hence activity must be associated with an increased flow of blood through the part. Two mechanisms are involved in the production of this adaptation. In the first place, stimuli arising in any part of the body many affect the vascular system in two directions, causing reflexly dilatation of blood-vessels in the part which is the origin of the impulses and constriction of the blood-vessels in the rest of the body, so that a normal or raised blood-pressure is available for driving an increased supply of blood through the dilated vessels of the part. Thus, if both hind limbs of an animal be placed in a plethysmograph, it will be seen that stimulation of the anterior crural or peroneal nerve in the left leg causes dilatation of this leg and constriction of the leg of the other side. At rest the organs of the chest and abdomen contain more than half of the total quantity of blood in the body, so that very little change in the capacity of these organs suffices to furnish the extra supply of blood needed by any part during a state of increased activity.

\section{THE CHEMICAL REGULATION OF THE BLOOD-VESSELS}

Another factor which is possibly involved in the production of the increased blood-flow through active organs is a chemical stimulation of the vessels themselves, by means of substances (metabolites) produced as a result of the chemical changes accompanying activity. The great increase in the flow through the muscles which accompanies muscular exercise is probably brought about largely by this means. It has been shown that the passage of blood containing lactic acid or carbon dioxide (both results of muscular metabolism) causes a marked dilatation of the blood-vessels of a limb. The Table on the next page shows the influence of activity on the blood-fiow through various organs.

We thus see that carbon dioxide, which is the universal hormone set free in the circulation when the activity of the body as a whole is increased, has . a double effect on the blood-vessels-a central effect through the vasomotor centres, medulla and spinal cord, causing contraction of the bloodvessels, and a local peripheral effect causing dilatation of the blood-vessels. 
The general result therefore will be to cause dilatation of the blood-vessels of the part where the carbon dioxide is produced and where it is present !n greatest concentration, and vascular constriction elsewhere under the influence of the sensitive nervous centres.

Flow in Cubio Centimetres per Minute per 100 Grm. Tissue

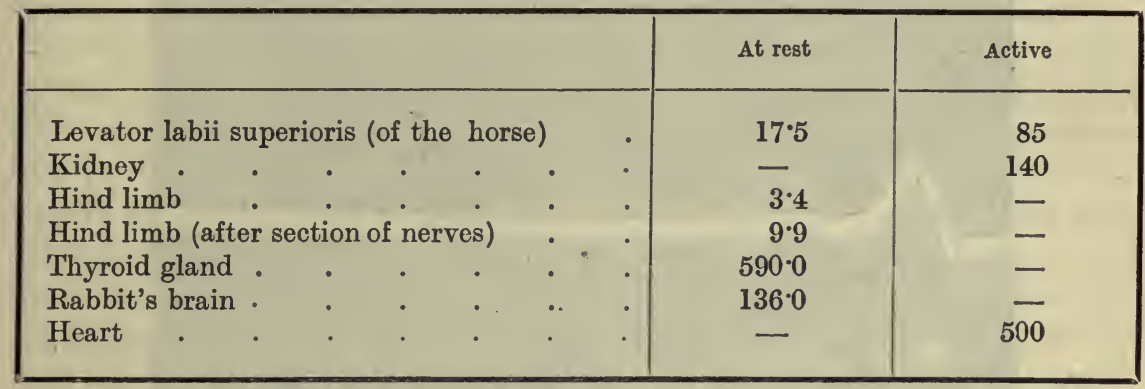

ACTION OF ADRENALINE. This substance, produced by the suprarenal glands, has also a marked influence on the calibre of the blood-vessels. If 1 c.c. of a 1 in 10,000 solution of this substance is injected into the jugular vein, there is at once a universal constriction of the arterioles with the exception of those of the brain. If the vagi are cut we obtain a simultaneous augmentor action of this drug on the heart and constrictor effect on the blood-vessels, so that the arterial pressure rises to an enormous extent, up to $300 \mathrm{~mm}$. Hg. or more. The same result occurs after section of the vasomotor nerves or after destruction of the brain and spinal cord, so that there is no doubt that adrenaline acts directly on the blood-vessel wall. The action of this drug as a whole is therefore largely to augment the energy of the circulation. The arterial pressure rises, and the blood will be therefore travelling at a much greater pace through any part of the body where the vessels are maintained in a dilated condition, e.g. in an active muscle, or where there are no vaso-motor nerves, as in the vessels of the brain. It is therefore not surprising that we have evidence of the secretion of adrenaline in increased quantities into the blood during any condition of stress. Whenever the splanchnic nerve is stimulated, there is an increased production of adrenaline. On this account the rise of pressure produced under these circumstances shows a stepped curve, the first rise being due to the direct action of the vaso-motor nerves of the blood-vessels, the second being brought about by the stimulation of the suprarenals and the discharge of adrenaline into the general circulation. Simultaneously with this second rise of blood-pressure we notice in the curve given in Fig. 473 a diminished volume of the heart due to more effective contraction of this organ.

This diminished volume of the heart is often associated with a marked quickening of the heart-rate, both effects being due to the action of adrenaline on the heart. During asphyxia the rise of arterial pressure is largely brought about through the intermediation of the splanchnic nerves and is therefore also associated with the discharge of adrenaline. It is on this account that 


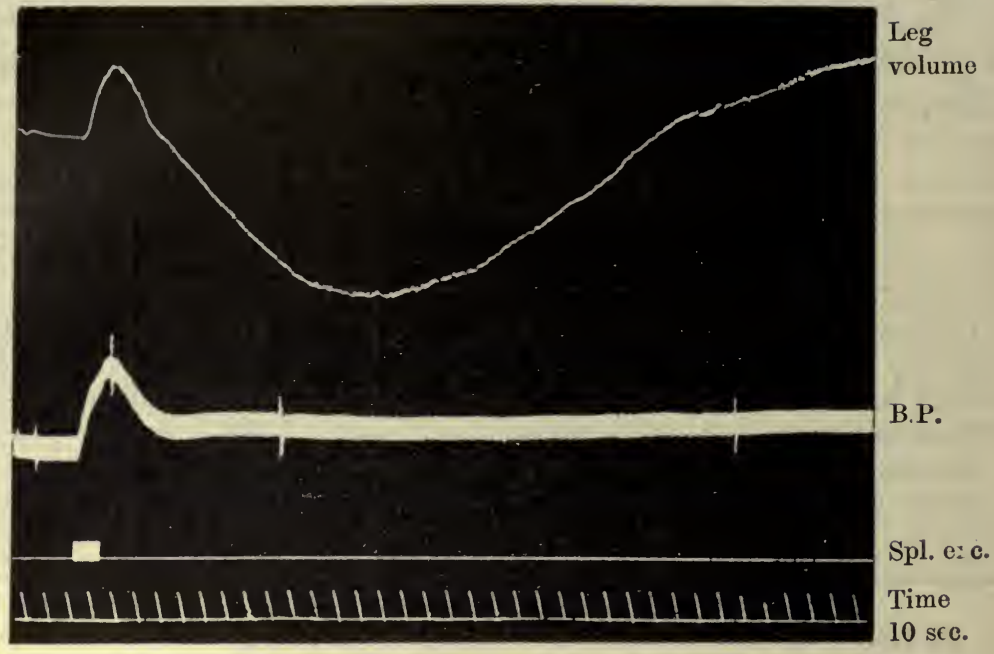

FIG. 470. Effect of excitation of splanchnic nerves on the blood-pressure and on the volume of the denervated hind limb of the cat. (BAYI.ISS.)

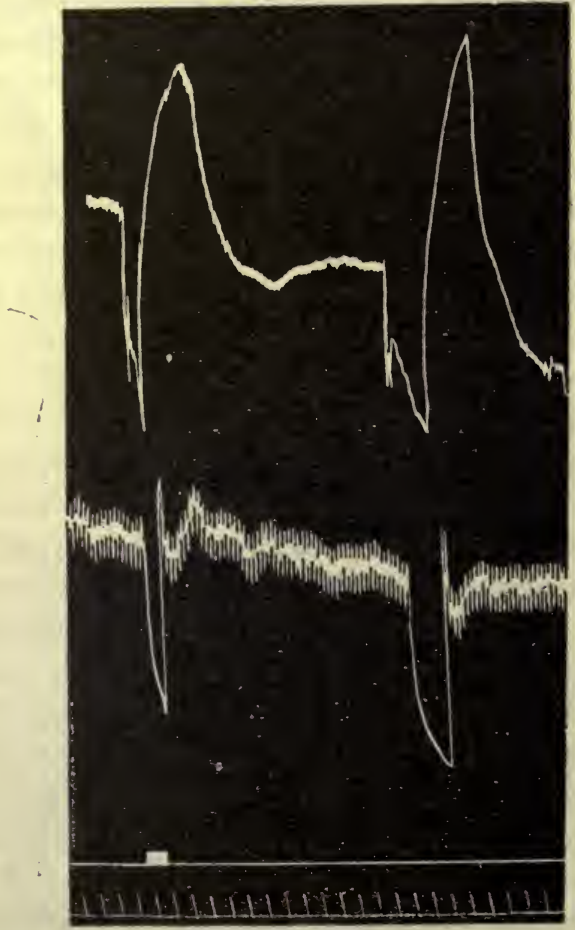

Hind limb

B.P.

Signal

Time 10 sec.

Fig. 471. Effect of temporary compression of the abdominal aorta on the volume of the denervated hind limb. Two compressions, the second not marked by the signal. Blood-pressure taken in the femoral artery of one hind limb, the other hind limb being in the plethysmograph. (BAYLISS.) 
in the whole animal, provided that sufficient oxygen is supplied, very large percentages of carbon dioxide may be inhaled without causing fatal dilatation of the heart, the effect of the adrenaline discharged into the blood-stream serving to counteract the injurious influence of carbon dioxide on the heart muscle. These two chemical influences, the local production of carbon dioxide and the discharge of adrenaline into the general circulation, must always be kept in mind in trying to account for the behaviour of the bloodvessels under the most various conditions. Thus in Fig. 470 is shown the effect of temporary stimulation of the splanchnic nerve on the blood-pressure and on the volume of the hind limb of the cat. It will be noticed that the volume of the hind limb increases passively with the rise of pressure and then diminishes much below its previous amount. This diminution is due to the discharge of adrenaline into the blood-stream as the result of stimulation of the splanchnic nerve and is absent if the suprarenals have been previously destroyed. The curve shown in Fig. 471, which with the foregoing one was taken by Bayliss, to indicate a local adaptation of the blood-vessels to their internal pressure, is probably brought about by the local production of carbon dioxide ( $v$. Anrep). Temporary occlusion of the abdominal aorta is here shown to cause first a diminution of the volume of the hind limb, followed by a marked increase. During the period of obstruction the circulation of the hind limb was interrupted and there was therefore accumulation of carbon dioxide in the tissues and around the blood-vessels. This caused a relaxation of the blood-vessel walls and a corresponding increased volume of the limb when the blood was allowed once more to flow by release of the aortic obstruction. 


\section{SECTION XI}

\section{THE EFFECT OF MUSCULAR EXERCISE ON THE CIRCULATION}

ANy muscular exercise, even moderate, produces rise of blood-pressure and acceleration of the pulse, associated with an increase of pulmonary ventilation-hyperpnœa. These effects can be readily shown by running up and down stairs for half a minute. The following Table by Pembrey and Todd shows the effect of such a form of exercise on the pulse-rate and systolic blood-pressure in two individuals, one trained and the other untrained :

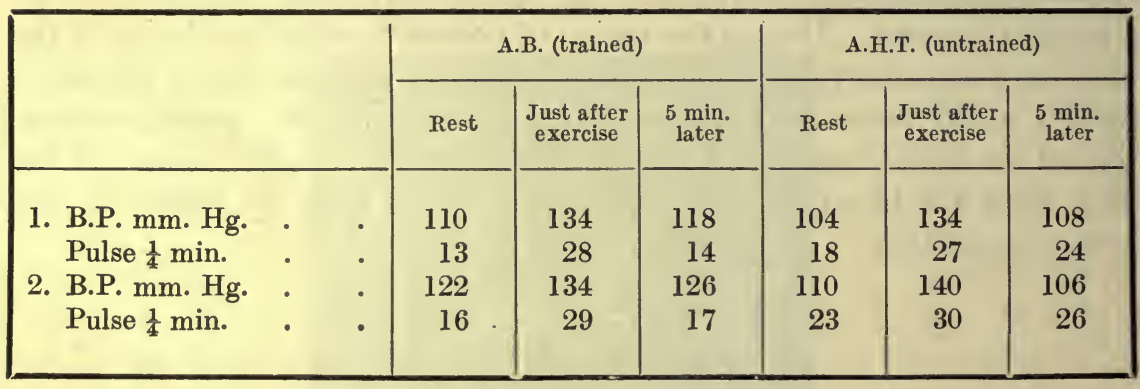

Several factors may concur in the production of these effects. Increased contractions of voluntary muscles will in the first place quicken the return of venous blood to the heart, and so will cause a greater diastolic distension of this organ and therefore a greater output of blood by the left ventricle. The increased respiratory movements will also aid the venous circulation and have a similar effect in increasing the systolic output. It must be remembered that the heart cannot put out more blood than it receives. Since during active exercise the output may be increased four to six times above the normal, it is evident that the venous circulation must be correspondingly increased, and this increase can only be ascribed to the pumping action of the contracting muscles and to the movements of respiration. All these factors will thus concur in producing a rise of pressure even when the heart and blood-vessels are cut off from the central nervous system. The latter also is concerned in the rise of pressure. The mere act of attention preparatory to muscular effort is in itself sufficient to raise the blood-pressure, and it seems probable that the increased activity of the motor centres actually spreads to the medullary centres which preside over the heart and blood- 
vessels, and that there is an active constriction of the vessels, especially of the splanchnic area, so that the greater part of the blood in circulation is available for the use of the actively contracting muscles. On this account hard exercise is not easily carried out after a meal, and, if forced, seriously interferes with digestion, by the diversion of the current of blood needed for the carrying out of this function.

It has been shown by Cannon that every state of excitement, and probably also the effort of concentration which precedes muscular effort, is attended with increased secretion of adrenaline into the blood. At the same time the contracting muscles are producing carbon dioxide in large quantities, and also if the supply of oxygen is not sufficient for their needs, lactic acid. Both these, substances co-operate in increasing the hydrogen ion concentration (the acidity) of the blood. Where they are present in greatest concentration they will produce local dilatation of the blood-vessels, i.e. in the contracting muscles. But as they are carried by the blood-stream to the medullary centres, they will cause a general vaso-constriction especially marked in the splanchnic area. On the heart, as already mentioned, the detrimental effect of increased acidity will be more than counterbalanced by the adrenaline which enters the circulation at the same time. But the nervous irritation of effort probably sets all these mechanisms in action at the same time. The quickening of the pulse, which is a normal concomitant of muscular effort, as well as the quickening of the respiration, and the rise of pressure, may be observed to begin before the actual muscular contractions, so that the brain, while sending impulses along the pyramidal tracts to the skeletal muscles, sends also impulses to the medullary centres which

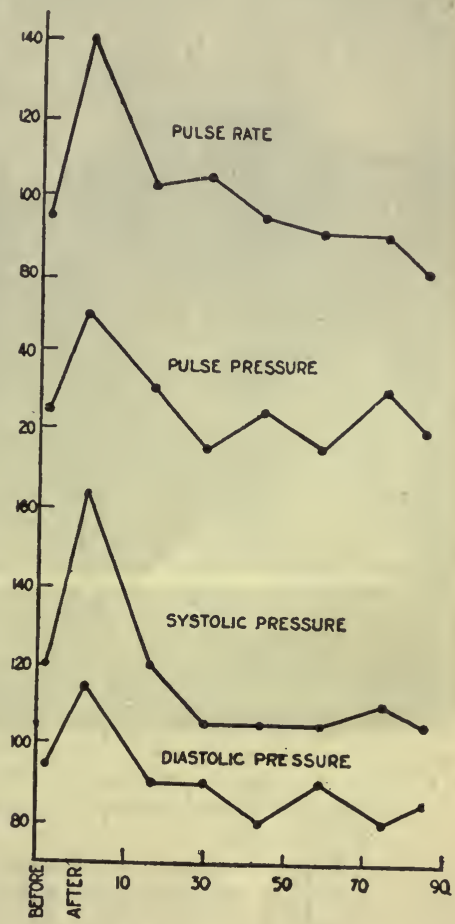

Fig. 472. Curves showing the influence of exercise on the circula. tion. The exercise was a six-mile run. Ordinates $=\mathrm{mm}$. Hg. pres. sure and rate per minute. (O. S. LOWSLEY.) quicken the respiration and the pulse and sends up the blood-pressure by constriction of the splanchnic area. Under these circumstances, therefore, there is an abrogation of the normal rule that a rise of blood-pressure is attended with a slowing of the pulse. Chemical mechanisms come in as a sort of second line in maintaining the conditions favourable for exercise, which are initiated by the direct action"of the central nervous system.

Slight acceleration of the heart is observed, after division of all its nervous connections, on tetanising the lower limbs. Mansfeld has shown that probably the chief, if not the only, factor in this case is the rise of temperature in the blood flowing to the heart. 
When exercise is discontinued the pulse-rate and blood pressure rapidly fall to normal, the return being quicker in the case of a trained individual, as is seen in the Table quoted above.

SECOND WIND. It is a familiar experience that in a running race of any duration the competitors after some time become less distressed than at the commencement of the race. The runner is now said to have got his

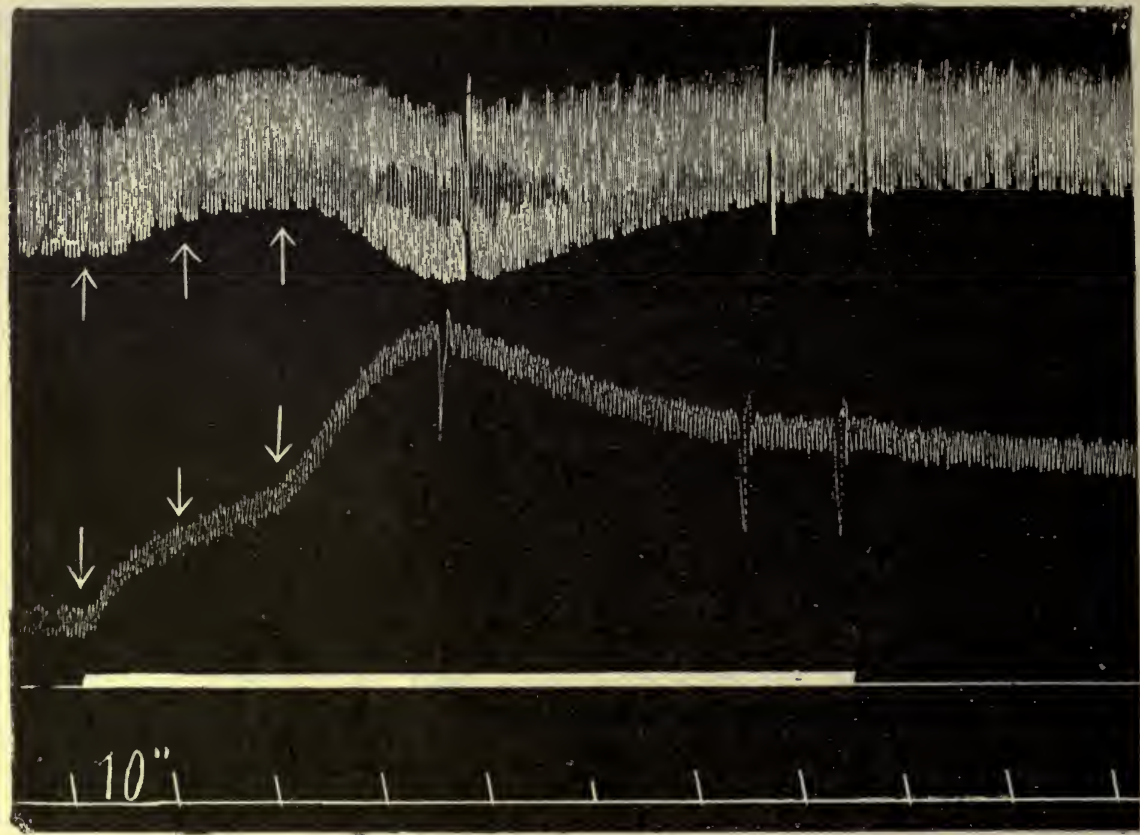

FIG. 473. Curve showing the effect of a sudden rise in the arterial resistance on the output and volume of the ventricles. Systole causes a downward movement of the lever.

H, heart volume ; BP, arterial blood-pressure ; $\mathrm{S}$, signal showing duration of stimulation of splanchnic nerve; T, time-marker, 10 secs.

'second wind,' and can continue running with comparative comfort. There are several factors which may account for this accommodation. In the first place, as a result of the production of metabolites in the contracting muscles, their vessels may be more dilated, so that the flow of blood through them is easier. More important is the change in the heart accompanying the onset of second wind. As Pembrey and Cook have shown, the onset of second wind is always attended with a diminution in the pulse-rate. At the same time there is an alteration in the respiratory quotient. During distress the respiratory quotient is high, i.e. more carbon dioxide is being given out than oxygen taken in. As the distress diminishes, the respiratory quotient also falls. The improved action of the heart may be partly due to the increased coronary circulation, partly to the entry of adrenaline into the circulation. 
SECTION XII

\section{THE INFLUENCE ON THE CIRCULATION OF VARIATIONS IN THE TOTAL QUANTITY OF BLOOD}

\section{PLETHORA AND HYDRÆMIC PLETHORA}

THE effects of increasing the total volume of circulating fluid may be studied by injecting several hundred cubic centimetres of defibrinated blood or normal saline fluid into a vein. In the latter case, since the blood is rendered

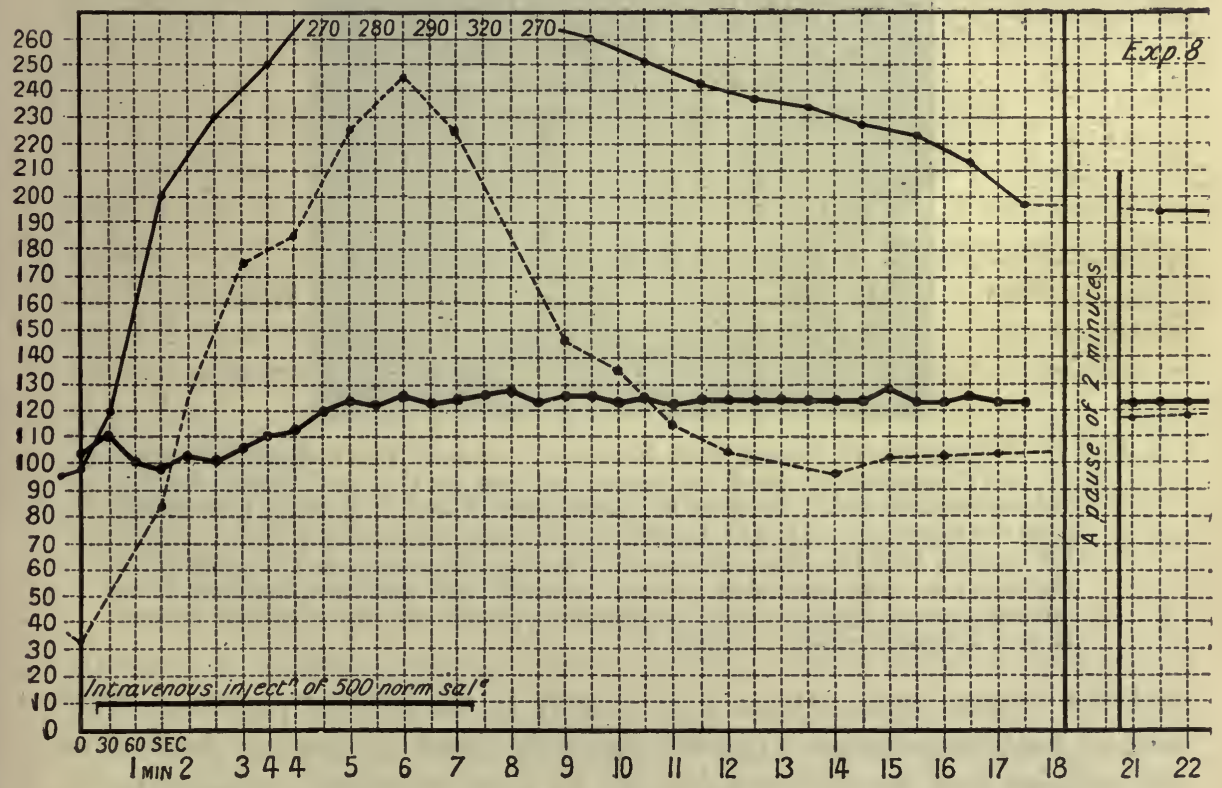

Fig. 474. Effects of hydræmic plethora on the pressures in the carotid artery (thick line), portal vein (thin line), and inferior vena cava (dotted line). (BAYLISS and STARLING.)

The arterial pressure is in $\mathrm{mm}$. $\mathrm{Hg}$. ; the venous pressures in $\mathrm{mm} . \mathrm{H}_{2} \mathrm{O}$.

more dilute, the condition is called hydræmic plethora (Fig. 474). On the arterial pressure the result of such an injection is not very marked. There is a slight initial increase in the pressure, but the increase is by no means proportional to the amount of fluid injected, showing that the fluid is not to any large extent contained in the arterial system. On examining the pressure in the veins, however, we find a very great relative rise of pressure, 
and on opening the abdomen it is seen that all the veins are distended and that the liver is swollen. The effect of increasing the volume of circulating fluid would be to increase the mean systemic pressure, and therefore one would expect to find a large increase both in arterial and venous systems. But the organism prevents the rise on the arterial side by relaxing the whole system of arterioles so that the distribution of pressures is altered,

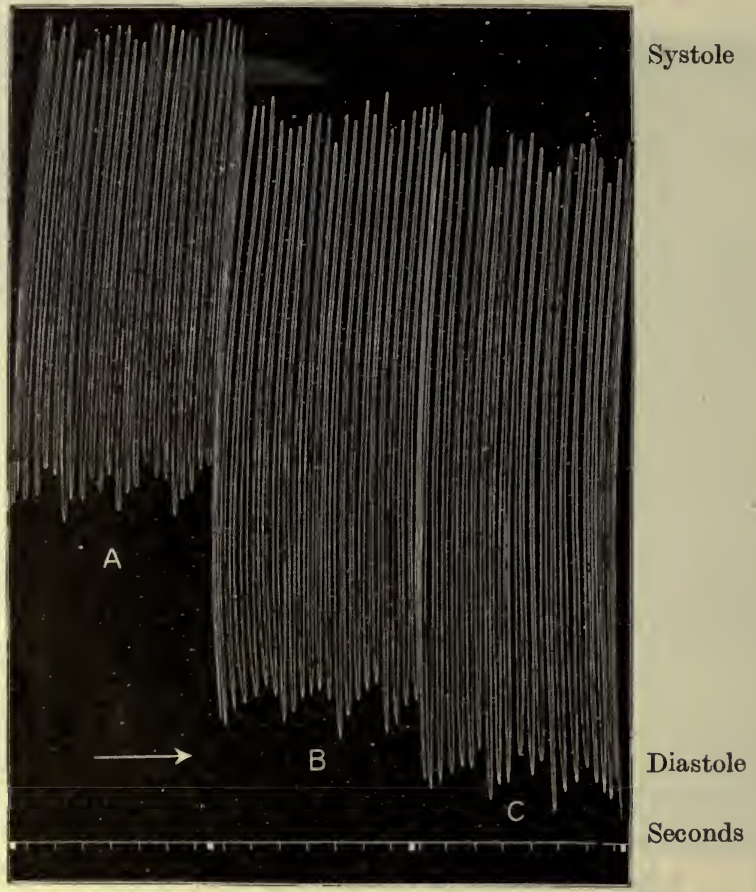

FIG. 475. Cardiometer tracing from dog's heart to show effect of increasing the volume of circulating blood (hydræme plethora) on the total output and the volume of the heart. Between the parts A and B 30 c.c. of warm normal salt solution were injected intravenously, and between $\mathrm{B}$ and $\mathrm{c} 20$ c.c. more. It will be noticed that both the systolic and the diastolic volume are increased, i.e. the heart is more distended during diastole, and does not contract to its normal size in systole. The contraction volume, and therefore the output, is very largely increased. (Roy.)

and the venous approximates more closely to the arterial pressure. This arterial dilatation augments the velocity of the blood: it has been found that the velocity may be accelerated to six or eight times the normal rate by injecting an amount of salt solution equivalent to 50 per cent. of the total blood.

The high venous pressure causes increased diastolic filling of the heart, and therefore augments the strength of the beat. The frequency is also generally increased if the vagi are intact. Thus the work of the heart is increased in three ways, viz. by

(1) Rise of arterial pressure.

(2) Greater frequency of beat.

(3) Increased output at each beat (Fig. 475). 
These series of changes result in the relief of the vascular system. The heightened pressure in the abdominal veins and capillaries causes a great leakage of fluid in the form of lymph from the capillaries of the intestines and liver, while the increased pressure and velocity of the blood in the glomeruli of the kidney induce a copious secretion of urine, so that within a couple of hours after the injection of salt solution the volume of the circulating fluid may have returned to normal.

This recovery is effected with geater difficulty if the plethora has been brought about by the injection of defibrinated blood, since this fluid cannot escape rapidly from the capillaries, nor can it be excreted unchanged by the kidneys. Hence it is easy to kill an animal by wearing out its heart, if too large quantities of defibrinated blood be injected. The ultimate fate of the injected blood is to be used as food by the tissues, and to be eliminated by the ordinary channels.

It must be remembered that the blood-serum of one animal is often poisonous for the corpuscles of another. Thus a few cubic centimetres of dog's serum injected into the peritoneal cavity of a rabbit will cause death. This poisonous action is also shown by mixing dog's serum with defibrinated rabbit's blood, in which case the red corpuscles of the latter are broken up, setting free hæmoglobin (hoemolysis).

\section{THE EFFECTS OF HÆMORRHAGE. ANÆMIA}

Any diminution of the total volume of the blood, as by bleeding, would tend to lower the pressure on both sides of the system. The vaso-motor centre, however, strives to maintain the normal arterial pressure, and so the circulation through the brain, unaltered. This object is attained by a general vascular constriction, which diminishes the total capacity of the system and alters the distribution of pressures throughout the system, so as to keep the blood as much as possible on the arterial side. Thus a slight loss of blood has no influence on the arterial blood-pressure, but causes a fall of pressure in the veins, blanching of the abdominal organs, and diminished flow of urine. The heart beats more frequently, and so aids in emptying the venous into the arterial system.

The deficiency of circulating fluid caused by bleeding is soon remedied by a transfer of fluid from the tissues to the blood. This transfer is independent of the flow of lymph from the thoracic duct into the blood, and is the direct consequence of the universal fall of capillary pressure which results from the bleeding. The abstraction of fluid from the tissues is responsible for the extreme thirst which is the result of hæmorrhage, and which directs the animal to take up by the alimentary canal the fluid which is wanting to the body. The transfer of fluid from tissues to blood is extremely rapid; even during the course of a bleeding it is found that the later samples of blood are more dilute than those obtained at the beginning. This mechanism suffices only to make up the supply of circulating fluid. After a 'bleeding, however, an animal" has lost proteins and blood-corpuscles, and these constituents of the blood are but slowly restored, the former directly from the food, the latter by an increased activity of the blood-forming cells in the red marrow. 


\section{CHAPTER XIV}

\section{LYMPH AND TISSUE FLUIDS}

Is no part of the body does the blood come in actual contact with the living cells of the tissue. In all parts the blood flows in capillaries with definite walls consisting of a single layer of cells, and is thus separated from the tissue-elements by these walls and by a varying thickness of tissue. In some organs, such as the liver and lung, every cell is in contact with the outer surface of some capillary; while in others, such as cartilage (which is quite avascular), a considerable thickness of tissue may separate any given cell from the nearest capillary. A middleman is thus needed between the blood and the tissues, and this middleman is the tissue-fluid or lymph which fills spaces between all the tissue-elements, so that any tissue can be regarded as a sponge soaked with lymph.

Throughout these spaces we find a close network of vessels lined, and separated from the tissue spaces, by a layer of extremely thin endothelial cells, and this plexus communicates with definite channels-lymphatics, by which any excess of fluid in the part is drained off. The lymphatics all run towards the chest, where those of the limbs join a large vessel (the receptaculum chyli), which receives the lymph from the alimentary canal, to form the thoracic duct. This runs up on the left side of the cesophagus, to open into the venous system at the junction of the left internal jugular with the subclavian vein. A small vessel on the right side drains the lymph from the right upper extremity and right side of the chest and neck.

The lymph may be looked upon as a part of the plasma which exudes through the capillary wall, bathes all the tissue-elements, passes between the endothelial cells into the peripheral lymphatic network, whence it is carried by lymphatic trunks into the thoracic duct, by which it is returned again to the blood.

It is easy to obtain lymph for examination by putting a cannula (a small tube of glass or metal) into the thoracic duct, and collecting the fluid that drops from it in a glass vessel.

We may also tap in a similar way one of the large lymphatic trunks of the limbs ; but in the latter case we have to use artificial means to induce a flow of lymph, since little or none can be obtained from a limb at rest, the only part of the body where there is normally a constant flow of lymph being the alimentary canal. And thus we cannot regard the flow of lymph from a part as any index of the chemical changes going on at that part. In a limb at rest 
food-stuffs are being taken up from the blood and being burnt up by the muscles with the production of $\mathrm{CO}_{2}$, although we may not be able to obtain a drop of lymph from a cannula in one of the lymphatics. The lymph is thus truly a middleman; as any substance, oxygen or food-stuff, is taken up by a tissue-cell from the lymph surrounding it, this latter recoups itself at once at the expense of the blood. Thus there would seem to be no need for lymphatics to drain the limb, were it not that under many conditions which we shall study directly, the exudation of lymph from the blood-vessels is so excessive that, if it were not carried off at once and restored to the blood, it would accumulate in the tissue spaces, give rise to dropsy, and by pressure on the cells and blood-vessels affect them injuriously.

\section{PROPERTIES OF LYMPH}

Lymph obtained from the thoracic duct of an animal varies in composition and appearance according to the condition of the animal, whether recently fed or fasting. From a fasting animal the lymph is a transparent liquid, generally slightly yellowish, and sometimes reddish from admixture of blood-corpuscles. When obtained from an animal shortly after a meal, it is milky from the presence of minute particles of fat that have been absorbed from the alimentary canal. In the latter case, if the intestines be exposed, the small lymphatics are to be seen as white lines running from the intestine to the attached part of the mesentery. It is owing to this fact that these lymphatics have received the special name lacteals, the lymph in them being called the chyle. The fatty particles form the molecular basis of the chyle.

On microscopic examination the transparent lymph of fasting animals presents colourless corpuscles similar to those of blood, or perhaps we ought to say identical, since the leucocytes of the blood are partly derived from the corpuscles that have entered with the lymph through the thoracic duct.

All the lymphatics pass at some point of their course through lymphatic glands, which we may look upon as factories of leucocytes, since these are much more numerous in the lymph after it has traversed the gland than before. Leucocytes are also formed in all the numerous localities where we find adenoid tissues, such as the tonsils, air passages, alimentary canal (Peyer's patches and solitary follicles), Malpighian bodies of the spleen, and thymus.

The lymph from the thoracic duct is alkaline, has a specific gravity of about 1015, and clots at a variable time after it has left the vessels, forming a colourless clot of fibrin, just like blood-plasma. It contains about 6 per cent. of solid matters, the proteins consisting of fibrinogen, paraglobulin, and serum albumen. The salts are similar to those of the liquor sanguinis, and are present in the same proportions.

\section{THE PRODUCTION OF LYMPH}

Many physiologists have thought that, in the transudation of the fluid which forms the lymph, there is an active intervention on the part of the 
endothelial cells forming the capillary wall, and that lymph is therefore to be regarded as a true secretion. A careful investigation of the known experimental facts has failed to show that the endothelial cells act otherwise than passively, as filtering membranes of variable permeability. The factors which are responsible for the transudation of lymph may be divided into two classes-mechanical and chemical, the former depending largely on the pressure of the blood in the vessels, and the latter chiefly on the metabolism of the cells outside the vessels.

According to the views here laid down, the formation of lymph may be compared to a process of filtration. If this be correct the amount of lymph formed in any given capillary area must be dependent on the difference of pressure between the blood in the vessels and the fluid in the extravascular tissue spaces. This latter pressure is normally extremely low, so that in attempting to test the truth of this view we must try the effects of altering the pressure inside the vessels, in the expectation of finding that the lymph production will rise and fall as the capillary pressure is increased or diminished. On attempting to carry out such experiments in different parts of the body, we have to recognise another factor besides the capillary pressure, viz. the permeability of the vessel-wall. Whereas the capillary walls in the limbs and connective tissues generally present a very considerable resistance to the filtration of lymph through them, and keep back the larger portion of the proteins of the blood-plasma, the intestinal capillaries are much more permeable, giving at moderate capillary pressures a continual flow of lymph and separating off only a small proportion of the proteins. It is in the liver, however, that we find the greatest permeability. Here a very small pressure suffices to produce a great transudation of lymph, containing practically the same amount of protein as the blood-plasma from which it is formed.

The ease with which fluid passes out from the capillaries of the liver is probably due to the fact that these vessels, unlike most other capillaries of the body, have not a complete endothelial lining. Thus it is impossible to display a continuous endothelial lining by means of silver nitrate. The cells surrounding the capillaries are large and branched, and possess marked phagocytic powers, so that after an injection of carmine granules or bacteria into the blood-stream these bodies are found in quantity within the cells. Owing to the incompleteness of this investment the liver-cells in many places abut on the lumen of the capillary. On injecting the blood-system of the liver the injection is found to run with ease into channels situated within the cells themselves, and it is reasonable to conclude that the blood-plasma takes the same course through these intracellular channels, by which it passes into the lymphatics which lie at the periphery of the lobules.

In experiments on the lymph production in the limbs alterations of capillary pressure have but slight effect. The lymph-flow from a limb lymphatic is practically unaltered by changes in its arterial supply, although a definite increase may be obtained by ligaturing all the veins of the limb so as to cause a very great rise of capillary pressure. The lymph-flow from the intestines can be measured by collecting the lymph from the thoracic duct. If the lymphatics which leave the liver in the portal fissure be 
previously ligatured, the whole of the thoracic duct lymph in an animal at rest is derived from the intestines. It will be found that lowering of the capillary pressure in these organs by obstructing the thoracic aorta stops the flow of lymph absolutely, whereas a rise of capillary pressure, such as that produced by ligature of the portal vein, causes a four- or fivefold increase of the lymph.

The effect of rise of capillary pressure on the lymph-flow is still more striking in the case of the liver. If the inferior vena cava be obstructed just above the opening of the hepatic veins, there is a great fall of arterial pressure, but, owing to the damming back of the blood, a rise of pressure in the liver capillaries to three or four times the normal height. This rise causes a large increase in the lymph-flow from the thoracic duct. The lymph may be increased eight to ten times in amount, and it contains more protein than before. If the portal lymphatics be previously ligatured, obstruction of the inferior vena cava has no effect on the lymph-flow, showing that the whole of this increase is derived from the one region of the body where the capillary pressure is increased, viz. the liver.

We must conclude that in those regions of the body where the capillaries are fairly permeable the most important factor in lymph production is the intracapillary pressure.

In the case of the limbs and connective tissues generally, the pressure factor is probably, under normal conditions, of less importance, so that the second condition, the chemical, comes here more into prominence. The capillary wall not only permits of filtration under certain pressures but also allows the passage of water and dissolved substances by diffusion and osmosis. These osmotic interchanges between blood and cell through the intermediation of the lymph are constantly going on in the normal life of the tissue, and are quite independent of the amount of lymph produced. Thus a gland-cell may use up oxygen, calcium, or sugar, and create a vacuum of these substances in the layer of lymph immediately surrounding the cell. There is at once a disturbance of the equilibrium, and a flow of these substances from blood to lymph is set up. In consequence of the wonderful arrangements in the tissues for ensuring the intimate contact of blood and lymph without intermingling, these changes can occur with great rapidity. We find, for instance, that if a very large amount ( $40 \mathrm{grm}$.) of dextrose be injected into the circulation, osmotic equilibrium between blood and lymph is established within half a minute of the termination of the injection. In this case the rise of osmotic pressure caused by the injection of the sugar attracts water from the tissue-fluid, and this in its turn from the tissue-cells, until the osmotic pressure inside and outside the vessels is the same. By this means the volume of the circulating blood is increased at the expense of the tissues. A process of this character may, however, work under normal circumstances in the reverse direction, and lead to a passage of fluid from blood to tissues and tissue spaces. Every active contraction of a muscle, for . instance, is attended by the breaking down of a few large molecules into a number of smaller ones, and this increase in the number of molecules causes 
a rise of osmotic pressure in the muscle fibre and surrounding lymph, and therefore a passage of fluid from blood to lymph. In the same way a cell of the submaxillary gland, when stimulated by means of its nerve, pours out a quantity of fluid into the gland-duct, and so into the mouth. This fluid comes in the first instance from the cell itself, but the cell recoups itself from the surrounding lymph, raising the concentration of this fluid, and the difference in concentration thus caused at once induces a passage of water from blood to lymph. Hence salivary secretion is associated with a large flow of fluid through the capillary walls of the gland. In this passage the endothelial cells of the capillaries play no part, the whole process being conditioned by changes in the extravascular gland-cell. We have only to paralyse the gland-cell by means of atropin in order to see that the active flushing of the gland which accompanies activity produces merely a minimal increase in the lymph-flow from the gland.

The influence of tissue activity in the production of lymph is still better shown in the case of a large gland, such as the liver. Stimulation of this organ by the injection of bile salts into the blood-stream causes a large increase in the lymph-flow from the organ, and therefore in the lymph-flow from the thoracic duct.

It is important to remember that the relative insusceptibility of the limb capillaries to pressure holds only for the absolutely normal capillary. Any factor which leads to impaired nutrition of the vascular wall, such as deficiency of supply of blood or oxygen, the presence of poisons in the blood or in the surrounding tissues, scalding or freezing, increases at the same time its permeability. Under such conditions the limb capillary reacts to changes of pressure like a liver capillary, the slightest increase of pressure causing an appreciable increase in the lymph production. This increased lymph production may be too great to be carried off by the lymphatic channels, so that the exuded fluid stays in the tissue spaces, distending them and causing the condition known as œdema or dropsy.

LYMPHAGOGUES. Among the substances which have a direct action on the vessel-wall are a number of bodies which were described by Heidenhain as lymphagogues of the first class. As their name implies, these bodies on injection into the blood-stream cause an increased flow of lymph from the thoracic duct. They may be extracted from the dried tissues of crayfish, mussels, or leeches by simple boiling with water. Commercial peptone has a similar effect. Heidenhain regarded these bodies as direct excitants of the secretory activities of the endothelial cells. They are, however, general poisons, having a special action on the vascular system, and their effect on lymph production is probably due simply to their deleterious action on the capillary wall. Although these bodies act chiefly on the liver capillaries, so that the main increase in the thoracic duct lymph is derived from the liver, they can be shown also to have some effect in the same direction on the intestinal and skin capillaries. In fact the injection or ingestion of these bodies often gives rise to a copious eruption of nettle-rash, i.e. swellings of 
the skin due to an increased exudation of lymph into the meshes of the cutis.

An increased lymph-flow from the thoracic duct may be produced also by the injection of large amounts (10 to $40 \mathrm{grm}$.) of innocuous crystalloids, such as dextrose, urea, or sodium chloride, into the circulation. In this case the lymph becomes much more dilute. The explanation of the action of these bodies is very simple. We have already seen that injection of large amounts of dextrose into the circulating blood raises the osmotic pressure of this fluid. The blood therefore imbibes water from the tissues and swells up, i.e. a condition of hydræmic plethora is brought about as surely as if several hundred cubic centimetres of normal salt solution were injected into the circulation. This increase in the total volume of the blood causes

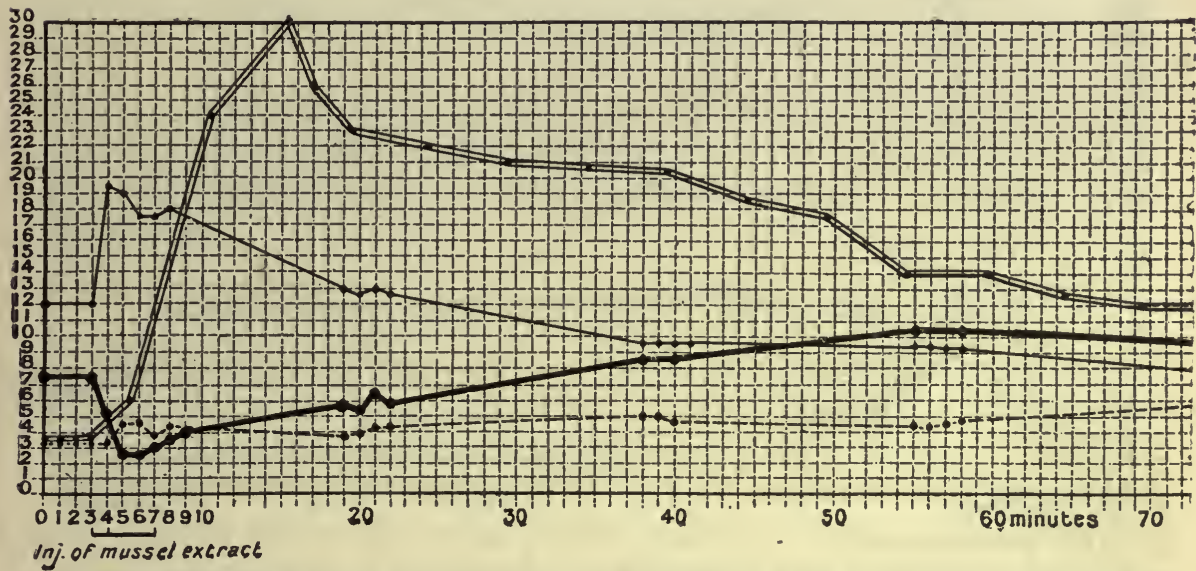

FIG. 476. Changes in lymph flow in portal, inferior cava, and arterial pressures, resulting from injection of a member of the first class of lymphagogues (extraet of mussels). (STARLING.)

a rise of pressure throughout the vascular system-arteries, capillaries, and veins - and the increased capillary pressure, combined with the watery condition of the blood, induces a great transudation of lymph, especially in the abdominal organs (Fig. 477). The lymph is more watery because the blood also is diluted. That the action of these bodies is purely mechanical is shown by the fact that, if the rise of capillary pressure be prevented by bleeding the animal immediately before the injection, the increase in the lymph-flow is also prevented (Fig. 477, B), although the concentration of the sugar or salt in the blood is still greater than in the experiments in which bleeding was not performed.

\section{MOVEMENT OF LYMPH}

In the frog the circulation of lymph is maintained by rhythmically contracting muscular sacs, which are placed in the course of the main lymphchannels, and pump the lymph into the veins. In the higher animals and in man the onward flow of lymph is effected partly by the pressure at which it is secreted from the capillaries into the interstices of the tissues, but also to 
a large extent by the contractions of the skeletal muscles. In the smaller lymph-radicles the pressure of lymph may attain 8 to $10 \mathrm{~mm}$. soda solution. In the thoracic duct, at the point where it opens into the great veins of the neck, the pressure is obviously the same as in these veins, that is to say,

A

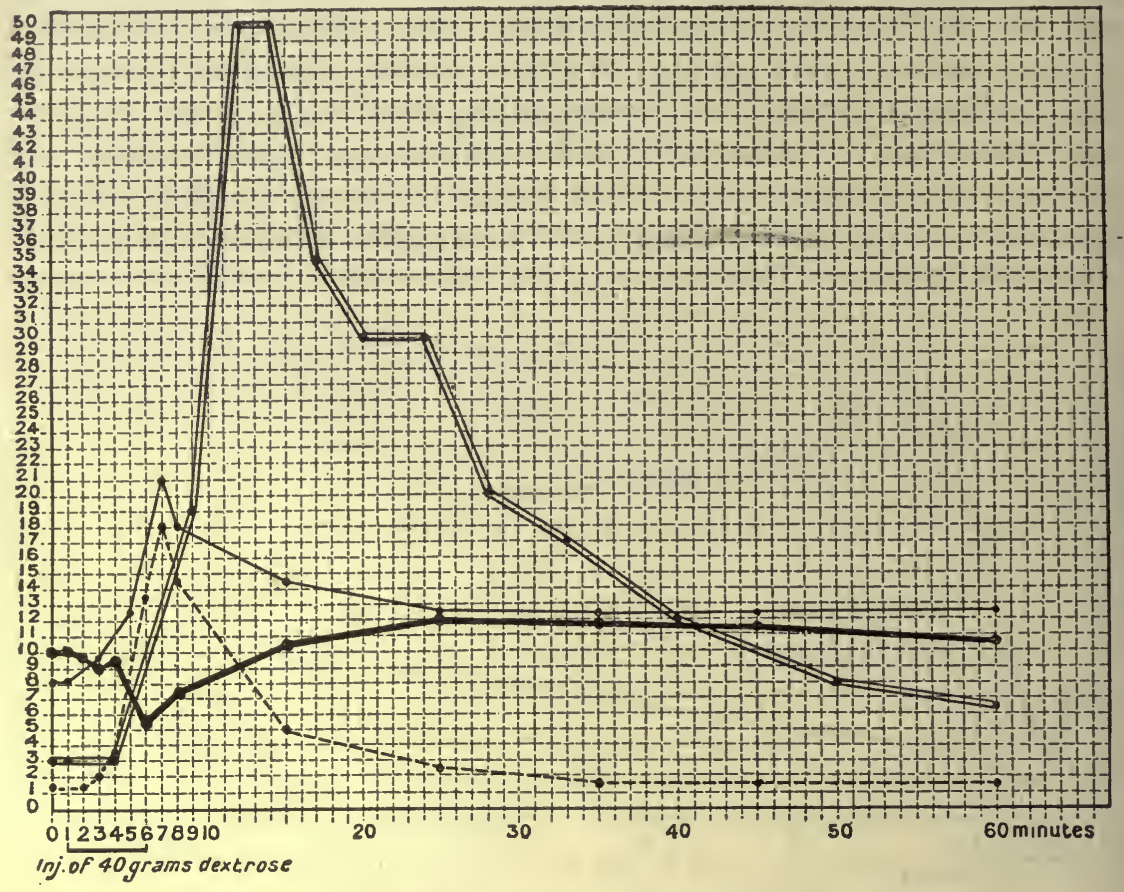

B

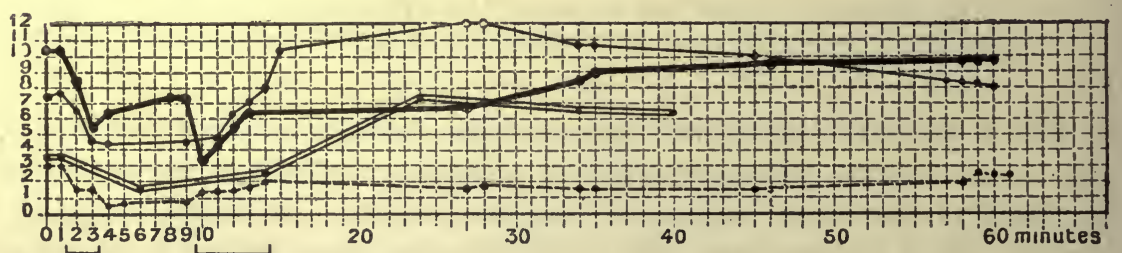

Bled to $240 \mathrm{ccm}$ ing.18 grams dextrose

FIG. 477. Effect on lymph flow and on arterial and venous pressures of injection of concentrated solution of glucose.

In $\mathrm{B}$ the animal was bled to 240 c.c. before the injection. The double line $=$ lymph flow in c.c. per ten minutes; thin line $=$ portal vein; thick line $=$ carotid artery; dotted line = inferior vena cava.

from -4 to $0 \mathrm{~mm}$. $\mathrm{Hg}$, the negative pressure being occasioned by the aspiration of the thorax. This difference of pressure is sufficient to cause a certain amount of flow. It must be remembered, however, that under normal circumstances no lymph at all flows from a resting limb. The only part of the body which gives a continuous stream of lymph during rest is the alimentary canal, the lymph in which is poured out into the lacteals, and thence makes its way through the thoracic duct. Movement, active or passive, of the limbs at once causes a flow of lymph from them. Since the lymphatics 
are all provided with valves (Fig. 478), the effect of external pressure on them is to cause the lymph to flow in one direction only, i.e. towards the thoracic duct and great veins. Hence we may look upon muscular exercise as the greatest factor in the circulation of lymph. The flow of lymph from the commencement of the thoracic duct in the abdominal cavity to the main part of it in the thoracic cavity is materially aided by the respiratory move-

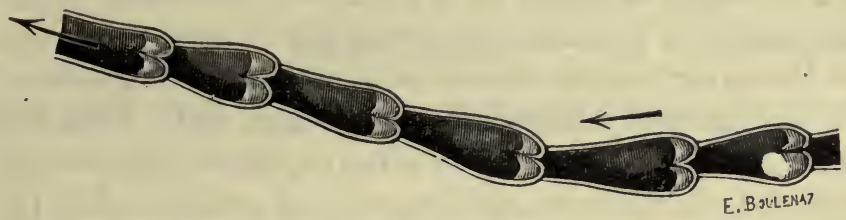

FIG. 478. A lymphatic vessel laid open to show arrangement of the valves. (TESTUT.)

ments ; since, with every inspiration, the lacteals and abdominal part of the duct are subjected to a positive pressure, and the intrathoracic part of the duct to a negative pressure, so that lymph is continually being sucked into the thorax.

\section{THE ABSORPTION OF LYMPH AND TISSUE FLUIDS}

On injecting a coloured solution or suspension into the connective tissues of any part of the body, and gently kneading the part, it is found that the fluid fills all the lymphatic channels running from the part; and we can in this way inject the lymphatics of the limb and trace their course on to the thoracic duct. The same path is taken by micro-organisms as they spread in the tissues, or by particles of carmine or Indian ink which have been introduced in tattooing. It is on account of these facts that the lymphatics are often spoken of as the ' absorbent system.'

This process of lymphatic absorption, except in the case of the pleural and peritoneal cavities is, however, a slow one, unless aided to a large extent by passive or active movements of the surrounding parts, and cannot therefore account for the rapid symptoms of poisoning which supervene within two or three minutes after the hypodermic injection of a solution of strychnine or other poison. That this absorption is not dependent on the lymphatics is shown by the fact that the symptoms occur almost as quickly when all the tissues of the limb have been severed with the exception of the main artery and vein. In the same way, after injecting methylene blue or indigo carmine into the pleural cavity or subcutaneous tissues, the dyestuff appears in the urine long before any trace of colour can be perceived in the lymph flowing from the thoracic duct. The absorption in these cases is by the blood-vessels, and consists in an interchange between blood and extravascular fluids, apparently dependent entirely upon processes of diffusion between these two fluids. So long as any difference in composition exists between the intra- and extravascular fluids, so long will diffusion-currents be set up, tending to equalise this difference. 
More difficulty is presented by the question of the mechanism of absorption by the blood-vessels of the normal tissue fluids - such an absorption as we have seen to occur after loss of blood by hæmorrhage. It seems probable that this absorption depends on the small proportion of protein contained in the tissue fluid as compared with the blood-plasma, and is due to the osmotic pressure of the protein. If blood-serum be placed in a bell-shaped vessel (the mouth of which is closed by a gelatinous membrane which does not permit the passage of protein), and suspended in normal salt solution, it is found that the serum absorbs the salt solution until the manometer attached to the bell-jar indicates a pressure of $25-30 \mathrm{~mm}$. $\mathrm{Hg}$. Thus we may conceive that there is normally a balance in the capillaries between the processes of exudation and of absorption, the former being conditioned by the capillary blood-pressure and the latter by the difference in protein content, and therefore of osmotic pressure between the blood-plasma and tissue-lymph. A rise of capillary pressure will upset this balance in favour of transudation and the blood will become more concentrated, whereas a fall of pressure will turn the scale in favour of absorption and the volume of blood will be increased at the expense of the tissue fluids.

\section{THE PART PLAYED BY THE LYMPH IN THE NUTRITION OF THE TISSUES}

The fact that the tissue-cells are separated by the lymph and the capillary wall from the blood shows that in all interchanges between the blood and tissues the lymph must act as the medium of communication. The lymphflow plays very little part in this process. The muscles of a resting limb are taking up nourishment as well as oxygen from the blood and giving off their waste products - carbonic acid and ammonia, though not a drop of lymph may flow from a cannula placed in a lymphatic trunk of the limb. In fact the interchange of material between tissue-cell and blood through the mediation of the lymph is carried out in the same way as are the gaseous interchanges, viz. by a process of diffusion. This explanation, however, holds good only for the diffusible constituents of the blood and will not account for the supply of the indiffusible protein molecules to the cell. Apparently the only way in which the tissues can obtain their supply of protein is from the small proportion of this substance which has filtered through the vesselwall into the lymph. The increased exudation of concentrated lymph to the tissues which occurs in inflammatory conditions or as the result of injury is therefore of advantage, since it furnishes an abundant supply of protein food to be used up in the regeneration of the damaged cells. 
CHAPTER XV

\section{THE DEFENCE OF THE ORGANISM AGAINST INFECTION}

\section{SECTION I \\ THE CELLULAR MECHANISMS OF DEFENCE}

ONE of the main distinctions, perhaps the most important, between the animal and vegetable kingdoms lies in the inability of animals to build up their tissues at the expense of inorganic salts, and especially to synthetise the various groups necessary for the formation of the protein molecule. They are thus rendered dependent on the assimilative powers of the vegetable kingdom, and have to supply their needs by using the members of this kingdom as food. The protozoa, for example, subsist largely on bacteria. To obtain a pure culture of any form of amœba it is necessary to cultivate this along with some form of bacteria. The power of the unicellular animals to digest bacteria meets with a response on the part of the latter, many of them developing, by way of self-defence, the habit of forming and excreting poisons which will deter the amœba from taking them up or injure it after it has ingested them. There is thus a continuous struggle among the various grades of unicellular organisms in which sometimes one, sometimes another type survives. An amœba placed in contact with most kinds of bacteria, living or dead, will rapidly englobe and digest them. There is, however, a small organism known as microsphera which is taken up by the amœba, but is not thereby destroyed. Retaining its vitality, it reproduces itself rapidly in the body of its host and finally leads to disintegration of the latter. In the same way the flagellate protozoa are often infected by a species of fungus known as chytridium, and die in consequence.

The liability of organisms to infection by others endeavouring to live a parasitic existence at their expense extends throughout the whole of the animal and vegetable kingdoms. In some cases the host and the parasite arrive at a compromise in which each benefits the other. This condition is known as symbiosis. We have examples of it in the union of fungi and algæ which occurs in lichens; in the association of nitrogenfixing bacteria with many plants, especially those belonging to the natural order Leguminosæ. In herbivorous animals the presence of specific bacteria in the paunch or cæcum causes the breakdown of the cellulose walls of the food and may indeed lead to a building up of protein from amino-acids or 
even from salts of ammonia. It is probable that in these cases the animal is decidedly benefited from the presence of these bacteria in its alimentary canal, so that here also we may speak of a symbiosis. In most cases invasion of a higher animal or plant by some lower organism is fraught with danger to the host, so that special mechanisms have to be provided for the protection of the tissues from infection. The most primitive means of defence, and one which is found throughout the whole animal kingdom, is exactly analogous to the process by which the amœba destroys and utilises any bacteria present in its environment. The prevention of infection is of course the function of the external layers of the organism, i.e. the epithelial covering, either of the skin or of the surface of the gut. Protection here may be

A

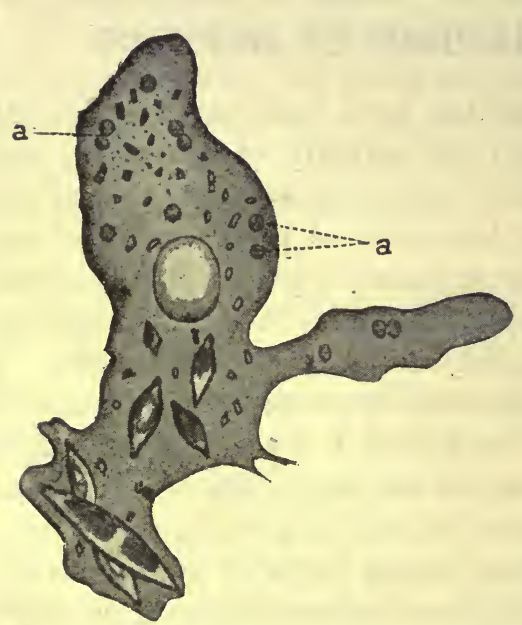

B

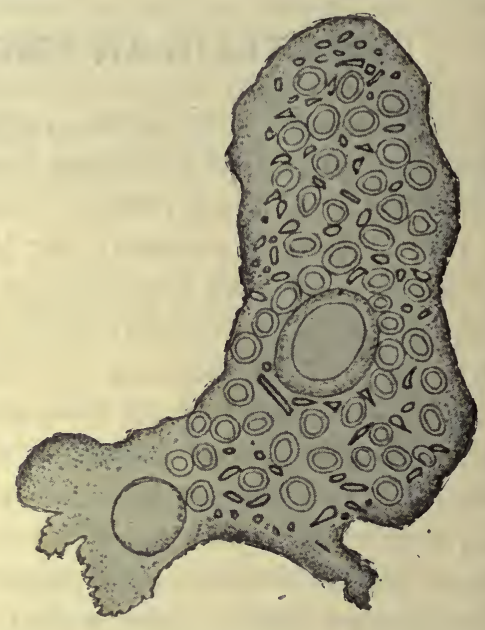

FIG. 479. A, amœba, infected by Microsphcera : $a$, early stage. B, a dying amœba, full of parasitic Microsphcerc. (METCHNIKOFF.)

of a physical or chemical character. The cells may secrete a horny or chitinous layer which presents a mechanical obstruction to the entry of bacteria. They may secrete mucin, which entangles and hinders the movements of invading micro-organisms, or they may secrete substances which actually destroy the life of such organisms. When, however, a microorganism has obtained entrance to the interior of the body, e.g. through a wound of the surface epithelium, the task of dealing with the invader becomes the office of a special type of cells belonging to the mesoblast. These cells are similar in character to the amœba. They have the power of extruding pseudopodia, of wandering from place to place, and of englobing and digesting particles of food or bacteria with which they come in contact. On account of these latter properties they have been called by Metchnikoff phagocytes, and the whole process by which foreign material or the animal's own dead tissues are got rid of is spoken of as phagocytosis. The process can be well studied, as has been shown by Metchnikoff, in the sponge or in the larva of the echinoderm. At one stage in the development of the latter the 
larva consists of a sack which is involuted at one extremity to form the alimentary cavity, while the mesoblast is represented by amœboid cells suspended in a semi-liquid substance filling the body cavity. If a particle of foreign substance be introduced into the body cavity the wandering mesoderm cells collect round the particle and fuse into plasmodial masses, thus forming a wall, as it were, around it. If bacteria be introduced, the phagocytes may be seen to adhere to and ingest the still living bacteria, which are then rapidly digested and destroyed. A similar process may be observed in the transparent crustacean known as the water-flea (Daphnia), and here it may be noted that the process of phagocytosis is not always successful in maintaining the health or life of the host. Thus if the spores

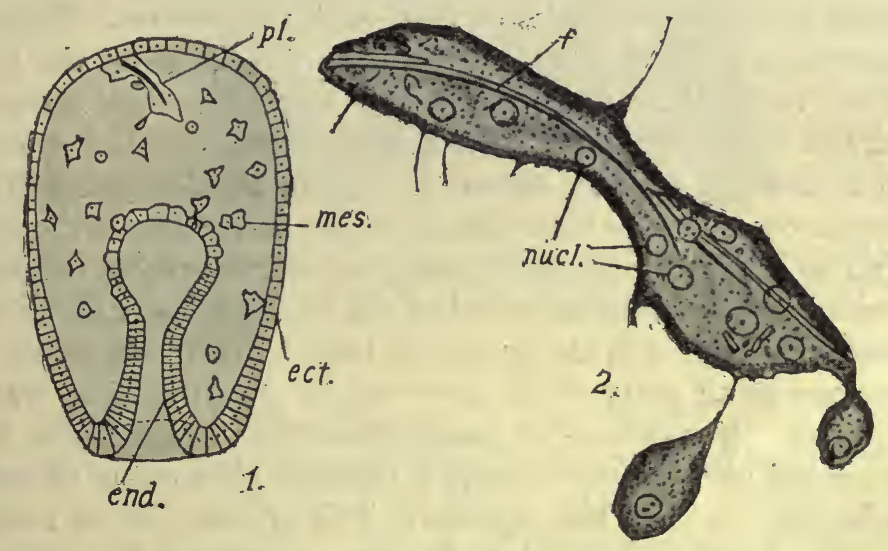

FIG. 480. 1, gastrula stage of starfish embryo, with a foreign substance, $p l$, in its body cavity; end, endoderm; ect, ectoderm; mes, wandering mesoblastic cells. 2 , the foreign body of 1 , surrounded by a plasmodium of phagocytes (highly magnified). (After METCHN 世KOFF.)

of a yeast-like organism, the Monospora, be introduced into the body cavity of Daphnia, the leucocytes may, if the spores be few in number, lay hold of the latter and digest them. If the spores be in excess the phagocytes may fail to ingest them or may indeed be destroyed as soon as they approach them. In this case the spores germinate, fill up the body cavity, and finally lead to the death of the host. The same process of phagocytosis may be studied in its simple form by injuring or infecting some tissue which is free from blood-vessels. Thus the tail fin of an embryonic axolotl may be cauterised with silver nitrate, or a small quantity of fluid containing carmine granules may be introduced by means of a hypodermic syringe. In either way a certain number of cells are destroyed and the dead tissue thereupon acts as a foreign body. As a result the wandering mesoderm cells or leucocytes move from the surrounding tissues towards the seat of the injury, and the day after the injury has been inflicted a collection of leucocytes can be seen, many of which contain particles of carmine or débris of the destroyed tissue which they have taken up. The cells finally wander away from the part, and the destruction is made good by the proliferation of the 
connective tissue-cells and of the epithelium immediately adjoining the injury. In the lowest types of metazoa it is impossible to speak of more than one type of wandering mesoderm cell. It is probable indeed that the same type of cell may at one time act as a scavenger and at another as the chief agent in the formation of connective tissues. Even in Daphnia, according to Hardy, only one form of leucocyte is present, whereas in the much more highly organised crayfish, belonging, however, to the same family, three different types of leucocyte may be distinguished. These leucocytes may be present free in the body cavity or they may form an element of the connective tissues. With the formation of a closed vascular system many of the wandering mesoderm cells became attached to this system, so that we may distinguish a group of blood leucocytes or phagocytes and a group of connective tissue or body-cavity leucocytes. Moreover by the formation of a blood vascular system all the tissues of the body are brought into material relationship with one another, so that distant parts may be drawn upon to supply the needs of any one part. It is evident that injury of a tissue in a higher animal containing blood-vessels will involve more complex consequences than a similar injury or infection of the avascular tissue of an invertebrate, and that the accumulation of cells for the defence of the organism against invading microbes will be much more effective if the blood-vessels participate in the process so that, by their means, the phagocytic resources of all parts of the body can be drawn upon to ward off a localised attack. The process of phagocytosis thus, in the higher animals, becomes merged into the more complex series of phenomena to which the term 'inflammation' has been applied. This process can be studied by observing the effects of slight injury to some transparent part of the body, e.g. the frog's tongue or mesentery, or the web of the frog's foot. For this purpose a small piece of the skin of the frog's web is snipped off with fine curved scissors, the section being sufficiently deep to remove the skin without causing hæmorrhage. The first effect noticed in the immediate neighbourhood of the injury is a dilatation of the vessels, especially of the venules, with acceleration of the blood-flow. In the course of an hour the capillaries also become dilated, and many capillary channels, previously invisible, are now occupied with blood. Through the dilated capillaries there is a rapid blood-stream, the corpuscles occupying the axis of the vessel, so that there is a periaxial layer of plasma. A little later this acceleration gives place to a slowing of the blood-stream, and simultaneously the leucocytes of the blood are seen to be adherent to the capillary wall. Apparently the latter becomes what we may call 'sticky,' the effect of the stickiness being to increase the resistance to the passage of the blood through the vessel and also to cause the adhesion of the leucocytes to the wall. As the current becomes still slower the distinction between axial and peripheral streams disappears. The corpuscles are closely packed together, the white cor. puscles being predominant at the margins of the capillary, where they form a lining to the vessel (Fig. 481). The next stage is the emigration of the leucocytes. These may be observed to thrust a process through the vessel- 
wall (according to Arnold this process of emigration always occurs through the stigmata, i.e. the points where the endothelial cells come in contactFig. 482). The prolongation enlarges on the outer side of the vessel, while the portion of the leucocyte within the vessel becomes smaller, so that finally

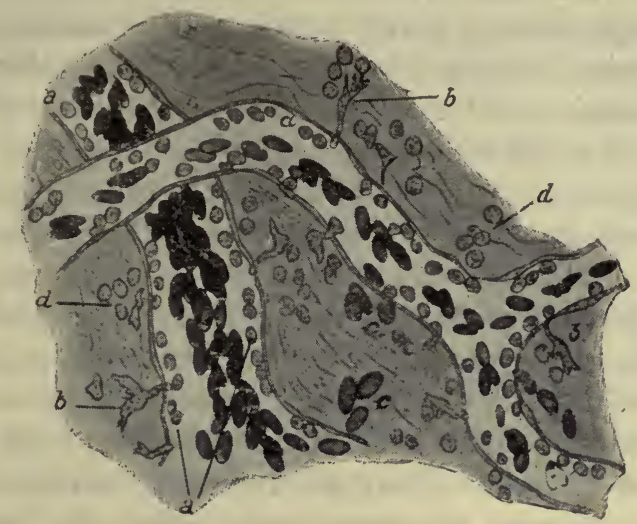

Fia. 481. Inflamed mesentery of frog, to show margination of leucocytes in the inflamed capillaries, $a$; migration of leucocytes, $b$; escape of red corpuscles, $c$; accumulation of leucocytes outside the capillaries, $d$. (From Adami after RIBBERT.)

the whole leucocyte passes through and lies in the lymph spaces outside the capillary. In the course of five or six hours all the capillaries and small veins in the neighbourhood of the injury may show a crowd of leucocytes along their outer surfaces. The use of this emigration seems to be to remove the tissue injured by the primary lesion. As soon as this is effected,

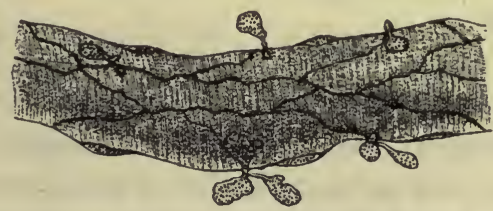

Fig. 482. Emigration of leucocytes through capillary wall. (ARNoLD.)

regeneration of the injured tissue occurs by a proliferation of the connectivetissue corpuscles and the epithelium, while the leucocytes move away and disappear. The essential phagocytic character of the inflammatory process may be shown if the primary lesion be attended with infection. Thus if a small quantity of the staphylococcus be injected into the subcutaneous tissue of the rabbit, the vessels surrounding the point of injection within four hours may be found densely filled with corpuscles. In ten hours' time the leucocytes are present in large numbers outside the vessels, while the injected cocci have spread for some distance along the lymphatic spaces and, while partly free, have been to a large extent ingested by the leucocytes. In 
twenty hours' time the connective-tissue fibrils at the point of injection are found to be widely separated by the aggregation of leucocytes. In fortyeight hours' time a well-defined abscess is produced. At the centre all traces of previous connective tissue have disappeared and its place has been taken by a dense mass of leucocytes, many in a state of degeneration, mingled with staphylococci, partly within, partly outside the cells. The margin of the abscess is formed by connective tissue infiltrated with living leucocytes. A certain number of cocci are to be seen free in the tissue outside this layer, but in the course of a day or two these free cocci disappear, and there is thus a continuous layer of phagocytes surrounding the abscess cavity and preventing any further invasion of the body as a whole from the seat of infection. The abscess subsequently discharges on to the exterior by a process of necrosis of the superjacent skin, and regeneration of tissue takes place in the same manner as in the more trivial injury. Inflammation in warm-blooded animals thus gives rise to dilatation of vessels and increased vascularity of a part, to alteration of a vessel-wall, and therefore to increased effusion of fluid. There are increased warmth and redness of the part from the vascular dilatation, swelling from the increased diffusion of lymph, and very often, as a result of the injury or the swelling and the consequent involvement of sensory nerves, pain. The four cardinal symptoms of inflammation, namely, rubor, calor, turgor, and dolor, which have been described for generations as typical of this condition, leave out of account altogether the phenomenon which Waller's and Cohnheim's observations, in the light of the comparative studies of Metchnikoff, have shown us to be the essential feature of the process, namely, phagocytosis, the accumulation of wandering mesoderm cells round the seat of injury with the objects of removing injured tissue, of destroying micro-organisms, of protecting the body from general infection, and of preparing the way for reintegration of tissue.

Prior to the work of Metchnikoff, the changes in the blood-vessels fettered the attention of physiologists, and the accumulation of leucocytes was regarded as secondary to these changes. Though the alteration of the capillary wall, by permitting the adhesion of the leucocytes, must no doubt favour their emigration and their passage from all parts of the body into the inflamed part, we know that the same accumulation of leucocytes occurs in the entire absence of a vascular system. The movement of the corpuscles towards dead or injured tissue must therefore have some other explanation. We have abundant evidence to show that the essential factor in this aggregation of leucocytes is their chemical sensibility, and that the phenomenon is simply one of chemiotaxis. A capillary glass tube containing a suspension of dead micrococci, or peptone, or broth extracted from dead tissue, if introduced into the anterior chamber of the eye or into the subcutaneous tissue, is found after a short time to be full of leucocytes. We must assume that the chemical products diffusing out of the ends of the capillary tube have acted like the malic acid discharged by the cells forming the female organ, the archegonium, of ferns. Just as the latter causes a movement of the anthero- 
zoids, the male cells, towards the ovule, so the chemical substances diffusing from the capillary tube have occasioned a positive chemiotaxis on the part of the leucocytes. It is worthy of note that the positive chemiotactic influence exerted by any given species of pathogenic bacterium is roughly inversely proportional to its virulence. A culture lacking in virulence may cause a very pronounced aggregation of leucocytes which speedily ingest and destroy the micro-organism, whereas if a culture of a more virulent variety of the same microbe be injected, there may be all the signs of inflammation, swelling, and large effusion of fluid, but the tissues may contain very few leucocytes. Under these circumstances the micro-organism rapidly proliferates and spreads from the seat of the lesion, giving rise finally to general infection.

So far we have spoken merely of leucocytes or phagocytes, and have not attempted to distinguish between the parts played by the various types of leucocyte which are found in the blood and connective tissues. In the higher animals there are, however, very many varieties of leucocytes belonging partly to the blood, partly to the connective tissues. The following Table, modified from Adami, enumerates the leucocytes which may be concerned with inflammation in a mammal or man :

Polymorphonuclear (polynuclear, finely granular oxyphile, neutrophile, or amphophile cell).

Eosinophile (coarsely granular oxyphile, macroxycyte).

Lymphocyte (? of two types).

Plasma-cell (? histogenous).

Endotheloid leucocyte (mononuclear leucocyte, hyaline cell (in part), 'epithelioid cell' (in part).

Connective tissue wandering cell (including clasmatocyte).
Originating in adult mammals from the bone marrow, and migrating from the blood into the inflammatory area.

Originating from lymphoid tissue and from vascular and other endothelia respectively; present in inflamed area either by migration from blood or as result of local proliferation.

Originating locally as result of tissue proliferation.

The part played by each of these forms is still to a large extent the subject of discussion. There is no doubt that in all active inflammations the polymorphonuclear leucocyte is the form which is attracted first and in largest numbers to the seat of injury. It is the characteristic cell from which pus is formed, and is actively phagocytic. It has nothing to do with the regeneration of the destroyed tissue. The eosinophile corpuscle is also present at an early stage around the inflammatory focus, but is never present in numbers at all comparable with those of the polymorphonuclear leucocyte. It is especially abundant in chronic inflammations of certain tissues, such as the skin. According to Kanthack and Hardy, these cells discharge their granules into the surrounding fluid, rendering this fluid toxic for bacteria. Although later observations have failed to confirm these views, no other satisfactory explanation has been given as to the part played by these cells. They are rarely seen to ingest bacteria and therefore cannot be spoken of as phagocytic. The lymphocyte predominates in certain chronic inflammations, 
especially in those caused by the tubercle bacillus. They do not ingest bacteria. The histogenous wandering cells appear in the inflammatory area at a later period than the polymorphonuclear and eosinophile cells. They are actively phagocytic and are motile. As a rule their phagocytic properties are exerted, not on bacteria, but on other cells and celldébris. After an acute inflammation their chief office is to clear away the remains of the polymorphonuclear leucocytes and dead tissues so as to prepare the way for subsequent regeneration. It is possible that these cells may take a part in the formation of new connective tissue. They are indistinguishable from the immature form of connective tissue-cells. It is therefore difficult to be certain whether the wandering and the fixed connective-tissue corpuscles are of identical or of different origin. Metchnikoff speaks of these cells as macrophages, to distinguish them from the polymorphonuclear type, which he terms microphages.

We thus see that several types of the wandering cells of mesoblastic origin which take part in inflammation do not exert active phagocytic properties and cannot therefore destroy bacteria or other invading organisms by the process of ingestion and digestion. Yet we have evidence that the part played by such cells in the defence of the organism is no less important than that of the actively phagocytic cells. In the alimentation of the more primitive invertebrata the cells lining the digestive cavity take up the particles of food directly, and the processes of digestion are carried out in vacuoles within the cells themselves. In the higher animals this process of intra-cellular digestion has almost disappeared, and the cells lining the alimentary tract have become differentiated into those which secrete digestive ferments and those which absorb the products of the action of the ferments on the food-stuffs. Digestion has thus become extra-cellular. It "seems that a similar modification has taken place to some extent in the means adopted by the organism for its defence from infection, and that the leucocytes destroy bacteria not only by the process of intracellular digestion but also by the excretion of substances into the surrounding body fluids which have a deleterious influence on bacteria. Thus normal blood-serum is found to have a strong destructive influence on most species of bacteria, whether pathogenic or not. Since this property is not shared to anything like the same extent by the blood-plasma, it may be ascribed to the breaking down of leucocytes in the process of clotting and the consequent liberation of bactericidal substances. Extracts made from any collection of leucocytes have a similar bactericidal effect, and it has been shown by Wright that the ingestion of bacteria by normal leucocytes goes on much more rapidly in the presence of blood-serum or if the bacteria have been previously subjected to the action of blood-serum. This adjuvant action of bloodserum on phagocytes is destroyed if the serum be heated to $55^{\circ} \mathrm{C}$., so that it must be due to the presence of some chemical substance in the serum which is unstable and destroyed by heat at a temperature far below the coagulation point of the serum proteins. Moreover, there are many species of pathogenic bacteria which cannot infect the animal as a whole. These 
nevertheless may multiply on the surface of the body or in an abscess cavity and lead to the death of the host, in consequence of the production by the bacteria of soluble toxins which are absorbed into the blood-stream. Examples of such micro-organisms are those which are associated with tetanus and diphtheria. The process of intracellular digestion is obviously inadequate to deal with such cases, and since we have the power of resisting and recovering from these diseases there must be other mechanisms at the disposal of the body for the neutralisation of these toxins. The protection of the body against destruction by bacterial toxins involves in fact a whole series of chemical mechanisms which we must regard as of equal importance and as co-operating with the phagocytic mechanism. 


\section{SECTION II}

\section{THE CHEMICAL MECHANISMS OF DEFENCE}

IMMUNITY. All infectious diseases are caused by the agency of microorganisms. The greater number of these, the bacteria, belong to the class of fungi or schizomycetes; a certain number must be classed with the yeasts, while others are protozoal in character. It is especially in the first class of diseases, namely, those due to bacteria, that the organism has developed chemical mechanisms of defence. In the protozoal diseases the micro-organisms occur for the greater part as intra-cellular parasites. One attack of the disease does not as a rule confer immunity, and the treatment has to be sought along the lines of medication by drugs rather than by the development of methods of protection normally displayed or developed by the animal which is the subject of the infection. The diseases due to bacteria include diphtheria, tetanus, tubercle, anthrax, pyæmia, and many others. In these diseases we have to deal with a number of phenomena more or less common to all. The infection in each case is due to the actual transference of the specific organism from one animal to another. After the microorganism has attained entrance into the system there is a period of incubation before the disease actually breaks out. When this occurs the specific microbe is to be found in large quantities either in the blood or in the tissues of the body. The disease is generally characterised by fever and often by local lesions, such as the intestinal ulcers of typhoid, or the glandular swellings of bubonic plague. The micro-organisms may develop in the animal until its death, or the disease may terminate in recovery and the total disappearance of the microbes from the body. After recovery it is found that the patient is protected from reinfection by the bacterium which was the cause of the disease, and this condition of immunity may last as long as the patient lives. The incidence of these bacterial diseases is not the same for all animals, so that in the case of many diseases we can speak of a natural immunity of certain animals for the diseases in question.

The pathogenic micro-organisms can, in a number of cases, be cultivated on artificial media outside the body. It is then found that they may be divided into two classes. One class, of which the diphtheria and tetanus bacilli are examples, secrete in the surrounding culture fluid substances which act as virulent poisons when injected into animals. Other bacteria do not form such extracellular toxins, but in their case it is found that if the bodies of the bacilli be broken up the injection of the contents of the 
bacteria is attended with poisonous effects. The bacteria may be thus classified according as they produce extracellular or intracellular toxins. We may deal first with the manner in which the body reacts to the toxins excreted by the first class. If a culture of diphtheria or tetanus bacilli be filtered, the clear filtrate free from bacilli is found to exercise as poisonous results as if the culture itself of the living bacilli had been employed. The toxins contained in these fluids are extremely potent. Thus five-millionths of a gramme of tetanus toxin is a fatal dose for a mouse, and 00023 grm. would kill a man. These weights apply to the mixture obtained by the evaporation of the solution of toxin, so that the pure toxin must be even more powerful than is represented in these figures. We have at present no means of preparing a toxin in a pure condition, nor do we know to what class of compounds it should be assigned. The toxin is an unstable body and is destroyed by heating to $65^{\circ} \mathrm{C}$. Similar toxins are widely distributed throughout the vegetable and animal kingdoms. Thus they form the active constituent of snake venom and of the poison of scorpions and spiders. They also occur in the seeds of castor oil and of jequirity, the toxins of which seem to be of protein character and are known as ricin and abrin. There is a great variability in the reaction of different animals to these toxins. Thus to the poison of tetanus the rabbit is weight for weight two thousand times and the hen twenty thousand times more resistant than the guinea-pig. As in the case of infection by bacteria themselves, a certain incubation time is necessary after the introduction of the toxin before its effects are displayed. There is a striking difference in this respect between the action of these complex bodies and the action of drugs, such as strychnine or morphine. Thus by increasing the dose of strychnine it is possible to kill an animal within half a minute. The period of survival after the injection of a dose of toxin cannot be reduced beyond a certain limit, however much toxin be injected. Thus a lethal dose of diphtheria toxin kills a guinea-pig in fifteen hours. If ninety thousand such doses be injected into a guinea-pig it is not possible to reduce the time of survival below twelve hours. Another characteristic of these toxins is the specificity of their action. One kind of toxin may act chiefly on the central nervous system, another on the peripheral nerves, another on the red blood-corpuscles. In this respect of course they resemble ordinary drugs. Associated with, however, and apparently a necessary condition of, this specific action is the actual combination which occurs between the toxin and the organ on which it exerts its effect. Thus tetanus toxin has a specific affinity for the central nervous system, and may be removed from a solution by shaking the latter up with an emulsion of brain. In spite of the excessively fatal character of these toxins it is possible to render an animal immune to their action. If a dose of diphtheria or tetanus toxin which is smaller than the fatal dose bc injected into an animal, the latter may show signs of injury from which it recovers. When recovery is complete it is found that three or four times the fatal dose may be injected without producing any evil effects, and this process of injection of toxin may be repeated in continually increasing doses 
until the animal is able to withstand a dose one hundred thousand times as large as that which would have been fatal to it in the first instance. When a condition of immunity has been produced in this way it is found that the blood-serum of the animal has the power of neutralising the toxin. Thus if the blood-serum from a horse which has been treated with large doses of diphtheria toxin be mixed with an equal quantity of the toxin itself, the mixture may be injected into susceptible animals without the production of any effect. It is possible in this way to get a serum 1 c.c. of which will neutralise many fatal doses of the toxin, and the antitoxic serum may be injected into a susceptible animal and used to confer an artificial immunity 'on the latter, or may be injected into a diseased animal and used thus as a curative agent. Antitoxin thus plays a great part in modern therapeutics, especially of diphtheria. In the case of tetanus the toxin has a specific affinity for the nervous system and apparently travels up the axis cylinders of the nerves to the central nervous system. By the time that it has arrived at the central nervous system, and the spasms typical of tetanus have broken out, the toxin is already so firmly bound to the reacting tissue that the injection of antitoxin into the blood-stream has little or no effect on the course of the disorder. The use of the tetanus antitoxin is therefore chiefly as a prophylactic agent.

The question of the manner in which the antitoxin is able to combine with and neutralise the toxin is one of considerable practical importance. In this process we have relations presenting marked analogies with the neutralisation of acids by bases. If we define a unit of toxin as that amount which possesses a certain power, i.e. which will kill a guinea-pig in so many days, or will cause the complete hæmolysis of 1 c.c. of blood in two and a half hours, we can find the amount of anti-body which is just sufficient to neutralise this effect, and this amount of anti-body can be regarded also as one unit. If instead of one unit of each we take 100 units, the neutralisation is effected in the same way. The process is found, however, to be more complex when we take 100 units of toxin or lysin and attempt to neutralise them by the fractional addition of antitoxin. In the case of a strong acid and strong alkali we know that if 100 c.c. of alkali are just sufficient to neutralise 100 c.c. of acid, the addition of 50 c.c. of alkali will leave half the acid unneutralised. If, however, we try the same experiment in the case of mixtures of toxin and antitoxin, it will be found that the addition of 50 units of antitoxin will neutralise much more than half of the toxin, and the same applies to other bodies of this class. Ehrlich has attempted to explain this result by assuming that in any toxin there is a mixture of substances, some having a strong affinity for the antitoxin, and others, which he calls toxones, possessing only a slight affinity. In the 50 units of toxin first added the toxins would satisfy all their combining powers, whereas the toxones would not begin to combine until they were present in large excess. Arrhenius and Madsen have drawn an analogy between the neutralisation of toxin by antitoxin and the neutralisation of a weak acid, such as boracic acid, by a weak base, such as ammnnia. They show that in this case the general 
course of events would be similar to that observed by Ehrlich. At no time would there be complete neutralisation, owing to the fact that hydrolysis constantly occurs, so that when equivalent quantities of each substance had been added, the fluid would still contain a certain amount of free base alongside of free acid, in addition to the salt produced by the combination of the two. It is impossible, however, to account for all the phenomena presented in the neutralisation of toxin by antitoxin in this simple manner. Thus seventeen parts of ammonia would neutralise exactly an equivalent quantity of boracic acid, whether these substances were dissolved in 10 c.c. or in 100 c.c. of water. If, however, it be found that 1 c.c. of antilysin exactly neutralises 1 c.c. of lysin, these two substances will no longer be in equilibrium when the whole is diluted up to 10 c.c. with water. If a neutral mixture of lysin and antilysin be taken and filtered under pressure through a gelatin filter, no lysin or antilysin passes through the filter, so that the residue on the filter becomes concentrated. On examining this residue it is found that it has a strong hæmolytic action, and the same is true of the substance which may be obtained by melting the gelatin out of the pores of the filter. It is evident that, even in a neutralised mixture, both free lysin and free antilysin, or free toxin and free antitoxin, are present, and it needs only the alteration of the physical condition of the mixture in order to display the action of one or other of these bodies. How then are we to regard this combination of toxin with antitoxin? Craw has pointed out that the combination is in all respects comparable to that which occurs between absorbing surfaces and many dyestuffs. If we place some filter paper in a solution of fuchsin or Congo red, the filter paper will take up the dye substance. The amount taken up by the paper will increase with increase in concentration of the solution. There will, however, be a tendency to the formation of false equilibrium points, as in the case of the reaction of toxin and antitoxin. Thus if two solutions of fuchsin be made and to each a sheet of filter be added, but in one case the paper be added at once, and in the other case in three parts at intervals of twelve hours, at the end of thirty-six hours the paper which has been added in parts will have removed more dyestuff from the solution than is the case where the whole amount of paper was added at once. In the same way, when treating a suspension of bacilli with an agglutinating serum, it is found that the successive addition of the bacillary suspension to the serum removes more agglutinin from the solution than when the addition is made at one time.

The interactions therefore between these bodies must be looked upon as special examples of the group of phenomena known as adsorption, such as the adsorption of iodine from solutions by charcoal, of iodine from water by starch, or of ammonia by charcoal. The exact adsorption which takes place must be a function of the chemical configuration of the substance forming the surface, since otherwise it would be impossible to account for the extremely specific character of the interaction between toxins and their corresponding antitoxins. The interaction must therefore be assigned to that special class, in which we have already placed the action of ferments, which is not 
entirely chemical nor entirely physical, but depends for its existence on a co-operation of both chemical and physical factors.

How are we to account for the production of the antitoxin as a result of the injection of toxins into the body, a production which is proportional to, but far transcending in amount, the toxin injected? In all the speculations on the mode of production and action of antitoxins an important part has been played by a conception put forward by Ehrlich in 1885 of the nature of the living protoplasmic molecule. According to this conception, which is spoken of as the 'side chain theory,' each unit of living matter consists of a centrally placed protein group with a number of side-chains attached to it, on the analogy of the hypothetical configuration of the benzene ring, to each corner of which may be attached an aliphatic chain. To explain the phenomena of nutrition and oxidation Ehrlich regarded some of these side-chains as corresponding to unoxidised food substances, while others of the side-chains had a strong affinity for oxygen and might be regarded, when fully saturated with this substance, as peroxide in character. Activity in such a unit would be associated with interaction between these two sets of side-chains. As a result the food chain would be converted to carbon dioxide and an affinity left unsaturated until it could take up another food-molecule. In the same way the oxygen side-chain, having lost the greater part of its oxygen, would have a strong affinity for this element and would re-saturate itself at the expense of the oxygen brought to it by the blood. Ehrlich regards the toxins as partaking essentially of the same character as the protoplasmic molecule, as being in fact protoplasmic fragments differing only from the protoplasm of the cell in the greater simplicity of arrangement of their side-chains. According to him the central group, or nucleus, of the toxin possesses two side-chains, one of which by its stereomeric configuration is peculiarly adapted to fit on to the organ or cell of the body which the toxin or active body attacks, and is known as the haptophore group, and another side-chain, the toxophore group, which is responsible, when the toxin is once anchored, for the destructive changes wrought by the toxin on the cell of the body. The antitoxins or antilysins are thus supposed to act in virtue of their adaptation to the haptophore group, so as to combine with the toxin or lysin and prevent these from exercising their injurious effects on the body. Ehrlich has shown that in many toxins the toxophore can undergo weakening or destruction without any alteration of the haptophore group; such modifications he designates as 'toxoids.' They have the same combining power for antitoxins as is possessed by the ordinary toxins, but are either without physiological effect, or their poisonous characters are only a fraction of that possessed by ordinary toxin.

The formation of antitoxins is accounted for (or rather described) on this hypothesis in the following manner. When a receptor side-chain of the cell is occupied by becoming attached to the haptophore group of the toxin, this side-chain is, so to speak, shut out from the normal activities of the cell. A defect is thus produced in the cell which the latter endeavours to adapt 
itself to by the production of other side-chains of the same character. It may be regarded as a general rule in living tissues that a reaction tends to be an over-reaction, so that the compensation by the cell should more than made good the defect produced by the attachment of the toxin. We thus get, not one, but a number of side-chains produced of the same character as that occupied by the toxin molecule, and therefore able also to act as receptors for the haptophore group of the toxin. These new receptor sidechains, being produced in excess, are supposed by Ehrlich to be thrown off
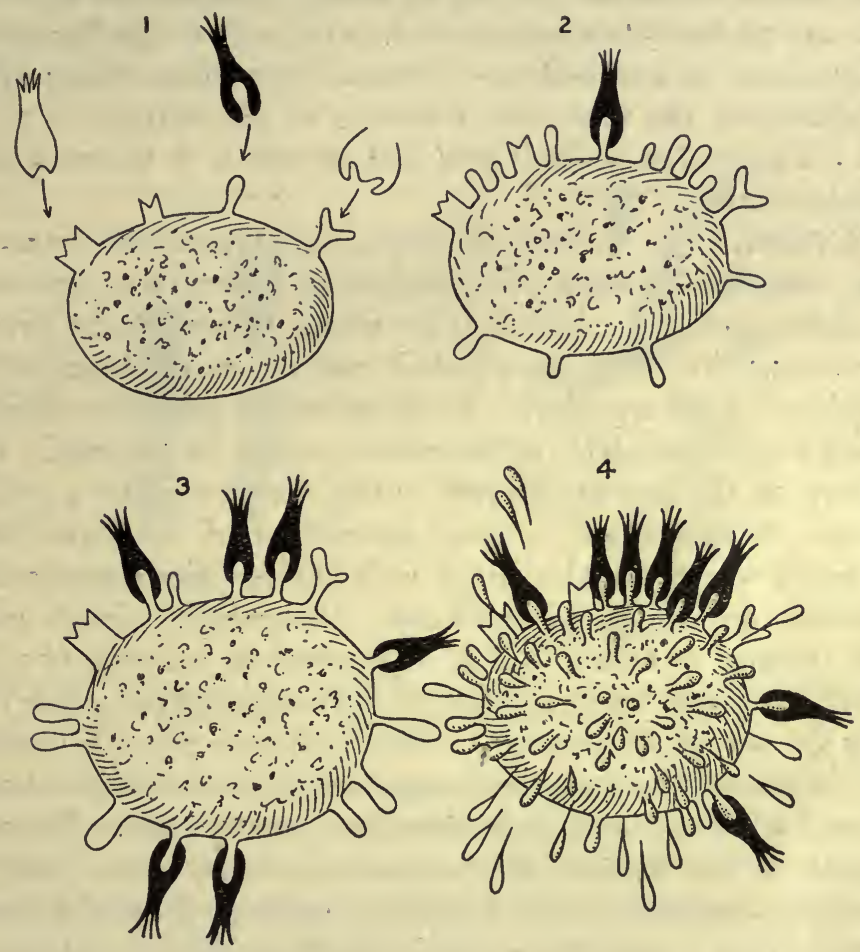

Fig. 483. Schematic representation of formation of antitoxin as side-chains of protoplasmic molecule. The black bodies are the toxin molecules which fit by their haptophore end on to the side-chains of the cell. (EHRLICH.)

from the cell and to circulate in the body-fluids (Fig. 483, 4). A number of protoplasmic fragments are thus set free which have a specific power of uniting with the toxin, and it is this excess of side-chains thrown off from the cell which represents the antitoxin molecules found circulating in the blood after the injection of toxins. It will be noted that this theory, though chemical in form, is really purely biological. It does not explain the phenomena by reference to the known laws of chemistry, but is a manner of viewing the biological phenomena which facilitates their description and discussion and enables us to classify the very complex phenomena of immunity in a more or less imperfect fashion.

The property of giving rise to anti-bodies on injection into an animal 
is not confined to toxins, a large number of substances, e.g. egg albumin, serum, proteins, ferments, albumoses, partaking of the same property. All such substances are classed together as antigens. Thus human serum injected into a rabbit produces in the rabbit's serum some body which will give a precipitate when mixed with human serum even in minute traces. This precipitin formation is specific, so that it may be used as a test for the origin of any unknown specimen of serum. In the same way rennet ferment when injected gives rise to the production of an anti-rennin which will neutralise the action of this ferment on milk. Antigens are all colloidal in character and probably.'optically active. Ordinary drugs do not give rise to the formation of anti-bodies, a necessary condition being apparently some similarity in the molecular structure of the antigen to the protoplasm of the animal on which it acts and to which it becomes linked by its haptophore group.

CYTOLYSINS. The bacteria of tetanus and diphtheria cannot exist in the body, infection by them being limited to a surface or abscess cavity. When a disease involves infection of the tissues themselves by living microorganisms, somewhat more complicated mechanisms are brought into play for the defence of the organism. We have already seen that normal bloodserum may exert a paralytic or destructive action on bacteria. Light has been thrown on the factors involved in this destruction by a study of the phenomenon of homolysis, i.e. the destruction of red blood-corpuscles. Normal goat's serum may be mixed with the red blood-corpuscles of the sheep without any injury to the latter. If, however, sheep's corpuscles, previously washed in normal saline, be injected at intervals of a few days into a goat, the goat's serum is found to have acquired the power of rapidly dissolving the red blood-corpuscles. This hæmolytic power is obvious, since it is only necessary to mix the serum and the washed blood-corpuscles together and allow the mixture to stand in a narrow tube. The corpuscles rapidly sink to the bottom, leaving the colourless serum above, unless hæmolysis has occurred, in which case the serum will be of a transparent red colour. If the hæmolytic serum be heated to $55^{\circ} \mathrm{C}$. it is found to have lost its power of dissolving sheep's corpuscles. This power is at once restored if to the heated serum be added any normal blood-serum, even of the sheep itself. It seems therefore that two substances are involved in the hæmolysis, namely, $(a)$ a substance present in most normal sera which is destroyed at a temperature of $60^{\circ} \mathrm{C}$. and has been called the complement, and $(b)$ a substance present in the serum only as a result of the previous injection of some species of red blood-corpuscle, which is resistant to the action of heat, and is called the amboceptor. The reason for these names will be at once apparent from the following experiment. Hæmolytic goat's serum is mixed with sheep's red blood-corpuscles and the whole mixture kept at $0^{\circ} \mathrm{C}$., at which temperature hæmolysis is indefinitely delayed. After some time the corpuscles are separated by means of the centrifuge. On testing the supernatant fluid it is found to have no action on sheep's corpuscles, though it still possesses the power of activating another specimen of serum which 
has been heated. The serum separated from the corpuscles has thus lost the amboceptor, but retained the complement. The amboceptor is found to have attached itself to the red blood-corpuscles. If these be washed and then added to normal sheep's serum, i.e. serum containing the complement, they are rapidly dissolved. When solution has taken place both complement and amboceptor are found to have disappeared. The function of the amboceptor thus seems to be to enable the complement already present in normal serum to act upon the red blood-corpuscles. We may regard the amboceptor therefore as having two haptophore groups, one of which anchors on to the red blood-corpuscle, while the other attaches itself to the complement (Fig. 484, 7). The amboceptor plus the complement thus comes to resemble
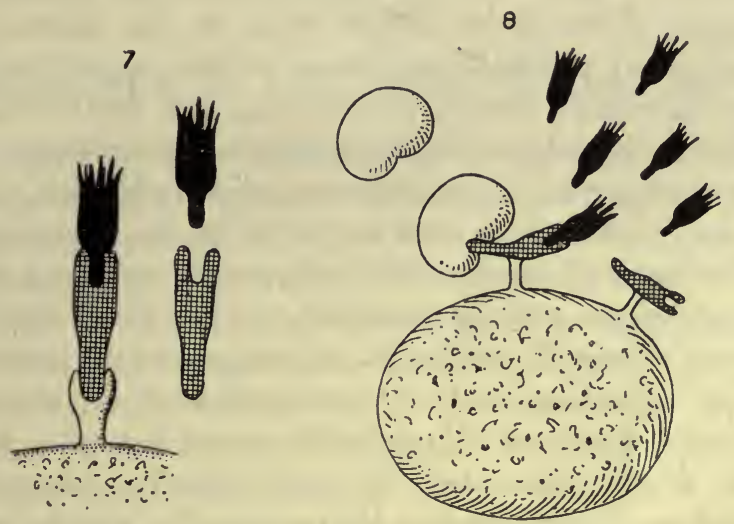

Fig. 484. Diagram to show the relation of amboceptor and complement to the animal cell (7) and to red corpuscles (8). (EHrLich.)

the toxin molecule, having a free haptophore group at one end and a toxophore group (the complement) at the other end. The reaction to the injection of the red blood-corpuscles consists in the formation of the amboceptor, which is essentially the anti-body of the red blood-corpuscle (Fig. 484, 8). Similar specific anti-bodies effecting the dissolution of cells or organisms may be produced by the injection of various species of bacterinm or of animal cells, such as leucocytes, spermatozoa, liver-cells, \&c., and there can be no doubt that bacteriolytic substances play a considerable part in acquired immunity.

OPSONINS. In some cases the antibodies produced by the injection of living or dead micro-organisms do not bring about actual destruction of the bacteria, but alter them in such a way as to make them more susceptible to the action of the phagocytes. If washed white blood-corpuscles be mixed with micrococci, such as those found in an ordinary boil, they are found to take up the micro-organisms in considerable numbers. The numbers taken up are much increased in the presence of serum derived from an individual who has received repeated minute injections of the dead micrococci in question. To the substances in the serum which thus prepares the micrococci for ingestion by the phagocytes Wright has given the name of opsonins. 
The opsonic index of the leucocytes of any individual in reference to a given species of microbe is determined by observing the number of the microbes taken up by the leucocytes after treatment with the serum of the individual and comparing it with the number taken up by the same leucocytes when the bacteria have been treated with the serum of an average individual.

We thus see that immunity, whether innate or acquired, is extremely complex in character and may depend on one or more of many factors. The immunity of an animal to any given infection may be determined by the absence of receptor groups in his body for the toxin excreted by the microbe responsible for the infection, or by the fact that the receptor groups are present but are confined to tissues on which the toxophore group can have no influence. Thus, e.g., an attachment of the tetanus toxin to a connective-tissue cell would be without effect on the health of the body. Again, immunity may be due to the efficacy of the phagocytes, either of the fluids or the connective tissues, in ingesting and destroying the microorganism, and this, as we have seen, may again be dependent on the presence or absence in the body-fluids of substances which, while not destroying the micro-organisms, render them more accessible to the action of the phagocytes. In those cases where the infecting organism secretes a specific toxin, the main line of defence and the main factor in the production of immunity is the formation of specific antitoxins to the poison in question. Finally there may be produced as a result of the excess of micro-organisms substances such as the amboceptors, which render the micro-organisms susceptible to destruction by the complements or cytases normally present in the circulating fluids and possibly themselves derived from the activity or destruction of the leucocytes and other phagocytes of the body.

In this short description we have only been able to touch upon the most salient features of the immunity problem. The question enters strictly into. physiology since, as we have seen, it involves adaptations on the part of the organism to change in itself or its environment. For the practical application of these facts, as well as the consideration of the minuter details and exceptions, we must refer the student to works especially dealing with the subjects of infectious diseases and immunity. 
CHAPTER XVI

\section{RESPIR A T I O N}

\section{SECTION I}

\section{THE MECHANICS OF THE RESPIRATORY MOVEMENTS}

IN unicellular animals the interchange of gases, i.e. the intake of oxygen and the output of carbon dioxide, is as a rule carried out by processes of diffusion occurring at the surface of the cell. With increased size of the organism the surface becomes insufficient for this purpose, and special organs make their appearance for presenting a large extent of surface to the surrounding medium. In the multicellular animals the actual process of tissue respiration is carried out between the internal medium, lymph, blood, \&c., and the individual cells, and the use of the special organ of respiration is to bring the circulating internal medium in intimate relation over a large area with the surrounding fluid, whether air or water. In insects we find a large branched system of tubes, the tracheæ, which contain air and are distributed to the finest tissues, renewal of the air in the tubes being provided for by special respiratory movements. In most water animals the respiratory organ is known as the gills, and presents a large surface well supplied with circulating blood over which a continual stream of the surrounding water is kept up. In all these animals therefore we can distinguish two processes, viz. (1) the interchange of gases between the tissue-cells and the surrounding lymph, 'internal respiration'; (2) the interchange of gases between the circulating fluid and the external medium, 'external respiration:' In all air-breathing vertebrates the organs of external respiration, the lungs, arise as paired diverticula of the anterior part of the alimentary canal. The renewal of the air in the air-sacs formed from these diverticula is effected by alternate increase and diminution in their size caused by the movements of respiration, while a rapid circulation of blood is carried out through a fine meshwork of capillaries just underneath the surface of the sacs. In man the organs of external respiration, the lungs, are built up in the following way :

The trachea or windpipe, a wide tube about $4 \frac{1}{2}$ inches long, divides below into two main branches-bronchi; and these subdivide again and again, becoming gradually smaller. The terminal ramifications or bronchioles open into rather wider parts-the infundibula, the walls of which are 
beset with a number of minute cavities, the alveoli. The larger tubes are kept patent by rings or plates of cartilage in their walls. The smaller tubes have no cartilage, their walls being composed of fibrous and elastic tissue and a coating of unstriated muscular fibres, which are able by their contraction to occlude the passage. The whole system of tubes is lined with a layer of epithelium-ciliated columnar in the trachea, bronchi, and bronchioles, and cubical over the parts of the infundibulum not occupied by air-cells. The alveoli are the special respiratory parts of the lung. Their walls are composed of connective tissue containing a large number of elastic fibres, and are covered internally by a single layer of extremely thin large flattened cells. The alveoli are closely packed together,

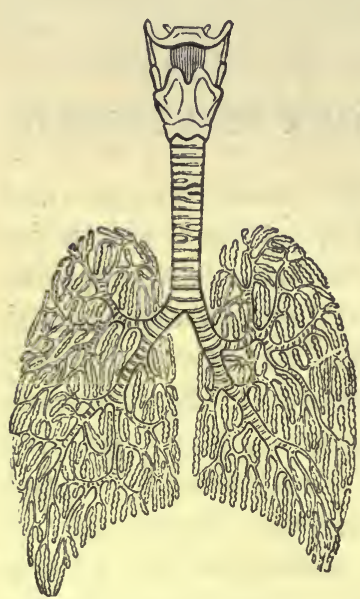

FIG. 485. Diagrammatic representation of the structure of the lungs. The trachea branches into two bronchi, which subdivide again and again before ending in the infundibula. (From YEo.) so that in a section of the lung an alveolus is seen to be in contact with others on all sides. Immediately below the squamous epithelium ramify blood-capillaries derived from the pulmonary artery. These form a close network, and the blood in them is in proximity to air on two sides, being separated from the air in the alveoli only by the thin endothelial cells of the capillary wall and the flattened cells lining the alveoli.

The lungs in their development grow out from the fore part of the alimentary canal into the front part of the body-cavity on each sidethe pleural cavity. The surrounding body-walls become strengthened by the formation of the ribs, so that the lungs are suspended in a bony cage-work, the thorax. Their outer surface is covered with a special membrane, the pleura, which is reflected on to the wall of the thorax from the roots of the lungs, and completely lines the cavity in which they lie. The surface of the pleura facing the pleural cavity is lined with a continuous layer of flattened endothelial cells, and is kept moist by the secretion of lymph into the cavity. Thus, being attached to the thorax only where the bronchi and great vessels enter, the lungs are able to glide easily over the inner surface of the thorax, with which under normal circumstances they are in intimate contact.

A constant renewal of the air in the lungs is secured by movements of the thorax, which constitute normal breathing. With inspiration the cavity of the thorax is enlarged, and the lungs swell up to fill the increased space. The capacity of the air-passages of the lungs being thus increased, air is sucked in through the trachea. The movement of inspiration is followed by that of expiration, which causes diminution of the capacity of the thorax and expulsion of air. At the end of expiration there is normally a slight pause. The number of respirations in the adult is about 17 or 18 a minute. 
This is, however, much influenced by various conditions of the body, and also by the age of the individual. Thus a new-born child breathes about 44 times a minute, a child of five about 26 times, a man of twenty-five about 16 , and of fifty about 18. The frequency is increased by any muscular effort, so that even standing up increases the number of respirations. These movements are much affected by psychical activity; they are to a certain extent under the control of the will, although they can occur in an animal deprived of its brain, and are normally carried out without any special act of volition. We can breathe fast or slow at pleasure, and can even cease breathing for a time. It is impossible, however, to prolong this respiratory standstill for more than a minute; the need of breathing becomes imperative, and against our will we are forced to breathe.

With every inspiration the cavity of the thorax is enlarged in all dimensions, from above downwards by the contraction of the diaphragm, and in its transverse diameters by the movements of the ribs.*

The diaphragm is a sheet separating the cavity of the chest from that of the abdomen. It consists of a central tendon which forms an arched double cupola, to the circumference of which are attached muscle-fibres. The diaphragmatic muscles present two main divisions, namely, (1) the spinal or crural part, the fibres of which arise from the upper three or four lumbar vertebræ and from

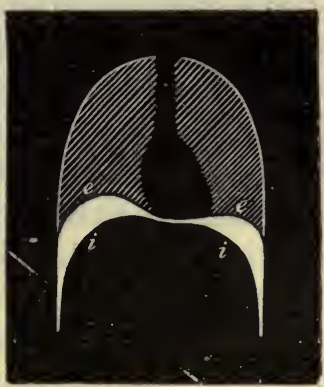

FIG. 486. Diagram showing movements of diaphragm in respiration.

$i$, inspiratory position ; $e$ e, expiratory position. (YEO.) the arcuate ligaments and are inserted into the posterior margin of the central tendon; and (2) the sterno-costal part, which arises by a series of digitations from the cartilages and adjoining bony parts of the lower six ribs and from the back of the ensiform process. These latter fibres pass backwards as they ascend. In the cavity of the larger dome on the right side lies the liver, while the smaller dome on the left side is occupied by the spleen and stomach. These viscera in the normal condition are pressed against the under-surface of the diaphragm by the elasticity of the abdominal walls. The central part of the diaphragm is thus pressed up into the chest, partly by the intra-abdominal pressure and partly by the elastic traction of the distended lungs. The upper surface of the central tendon is united to the pericardium. This part, during expiration, is the deepest part of the middle portion of the diaphragm. Towards the back of the pericardial attachment the central tendon is pierced for the passage of the inferior vena cava. In expiration the lateral muscular zone of the diaphragm lies in contact with the lower part of the thoracic wall. During inspiration the muscle fibres contract and draw the central tendon downwards, so that the

* The student is advised to consult the article by Keith on the 'Mechanism of Respiration in Man' for a fuller account of this subject (L. Hill's 'Further Advances in Physiology,' 1909). 
lower surface of the lungs descends. The enlargement of the lungs at the lower part of the thorax is aided by the abduction of the floating ribs, produced by the contraction of the quadratus lumborum and deep costal muscles. In this contraction the diaphragm presses on the contents of the abdomen, so that the abdomen swells up with each inspiratory movement. The middle of the central tendon, where the heart lies, moves less than the two domes, and the part where the vena cava passes through the tendon is practically stationary during normal respiration. In deep inspiration, however, both this part as well as the rest of the pericardial attachment is forcibly depressed towards the abdomen. In quiet breathing, when observed by the Röntgen rays, the mean descent of the right dome in inspiration has been found to be about $12.5 \mathrm{~mm}$., and of the left dome $12 \mathrm{~mm}$. We may say, roughly, that the average descent of the diaphragm during normal respiration is about half an inch. The viscera and the intra-abdominal pressure play an important part in determining the movement of the diaphragm, and especially in preserving the abduction of the lower ribs and so furnishing a fixed point for the muscular fibres of the diaphragm. If the contents of the abdomen are removed from a living animal the ribs are drawn inwards every time the diaphragm contracts. In children with weak chest walls and with respiratory obstruction we may often see a depression round the lower part of the chest corresponding to the lower border of the lungs. It corresponds to the line at which the diaphragm leaves the chest wall, so that the distending force of the abdominal pressure on the bony walls of the thorax abruptly gives place to the pull of the distended lung. The contraction of the diaphragm lasts four to eight times longer than a simple contraction or muscle-twitch. It may be regarded therefore as a short tetanus.

The enlargement in the other diameters is effected by an elevation of the ribs. Each pair of corresponding ribs, which are articulated behind with the spinal column and in front with the sternum, forms a ring directed obliquely from behind downwards and forwards. With each inspiratory movement the ribs are raised, the obliquity becomes less, and the horizontal distance between sternum and spinal column is therefore increased. Moreover the ribs from the first to the seventh increase in length from above downwards, so that when they are raised, the sixth rib, for instance, occupies the situation previously taken by the fifth, and the transverse diameters of the thorax at this height are increased. With each inspiration there is a rotation of the ribs. In the expiratory condition they are so situated that their outer surfaces are directed not only outwards but also downwards. As they are raised by the inspiratory movements, they rotate on an axis directed through the fore and hind ends of the rib, so that their outer surfaces are turned directly outwards. In this way a certain enlargement of the thoracic cavity is produced. As the thorax is raised there is always some stretching of the rib-cartilages.

In expiration the processes are reversed, and the cavity of the thorax is diminished in all three dimensions. 
The movements of the thorax are effected by means of muscles. Inspiration is performed by the following muscles :

The diaphragm, which is the most important, and almost suffices alone to carry out quiet respiration.

The external intercostal muscles, which shorten and so raise the ribs.

The serratus posticus superior.

It is probable that an important part is played even under normal circumstances in the respiratory movements by the extension of the spinal column. This movement, which is specially marked at the upper part of the thorax, causes an increase in all three diameters of this cavity. The levatores costarum, which are often included in inspiratory movements, are so inserted into the ribs as to be unable to influence their movements. They are concerned, not in respiration, but in lateral movements of the spine.

These muscles are the only ones normally engaged in carrying out inspiration. When, in consequence of muscular exertions or from any other cause, the inspiratory efforts become more forcible, a large number of accessory muscles are brought into play. These are :

The scaleni,

Sterno-mastoid,

Trapezius,

Pectoral muscles,

Rhomboids, and

The serratus anticus.

Normal expiration is chiefly effected passively. When the inspiratory muscles cease to contract, the lungs, which were stretched by the previous inspiration, contract by virtue of the elastic tissue they contain, and the thorax itself sinks by its own weight, and by the elastic reaction of the stretched costal cartilages.

It must be remembered, however, that in a position of rest the elasticity of the thorax is opposed to the elasticity of the lungs. Elasticity of the chest wall would therefore tend to produce inspiration. This factor would tend to make inspiration easier at its onset, but would also present an impediment to the carrying out of expiration, so that towards the end of this act there is need for the active co-operation of muscular contractions. It seems possible that more or less muscular activity of the expiratory muscles is alternated with that of the inspiratory muscles. In fact Sherrington's results on the co-ordination of muscular movements would tend to make us assume inhibition of the tone, e.g. of the abdominal muscles, during inspiration, and active augmentation of their tone during expiration. Where the tone of the muscles is entirely lost, e.g. in the condition of viscero-ptosis, it has been observed that the diaphragm is thrown out.of action, breathing being chiefly carried out by an elevation of the upper part of the thorax. Probably under normal circumstances the internal intercostal muscles also contract with each expiration. 
Although the action of the intercostal muscles has been a subject of debate, physiological experiments serve on the whole to confirm the view first put forward by Hamberger and based on a comsideration of the direction of the fibres. The external intercostals pass from one rib to the next below downwards and forwards. Hence if a pair of ribs be isolated from the rest of the chest wall, leaving the vertebral and costal attachments intact, contraction of these muscles will cause a rise of both ribs. This result will be evident from a consideration of Fig. 487, where $a b$ is a fibre of the external intercostal muscles, passing from the rib $v s$ to be attached to the rib $v^{\prime} s^{\prime}$ at $b$. When $a b$ contracts, the tension it exerts on its two attachments can be resolved into two components ac acting

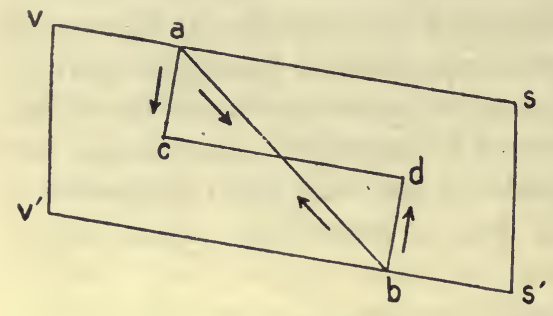

FIG. 487 .

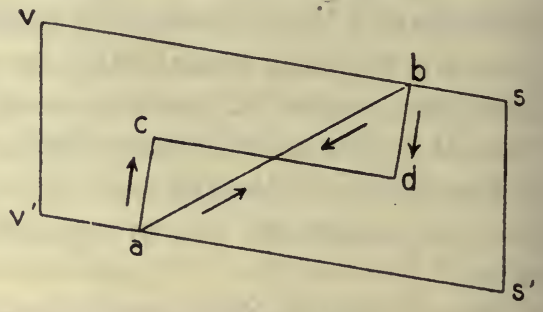

Fia. 488.

downwards and $b d$ acting upwards. $\quad b d$, however, acts at the end of the long lever $b v^{\prime}$, whereas $a c$ acts at the end of the short lever $a v$. Hence the raising effect will overcome the depressing effect, and both ribs will rise.

The fibres of the internal intercostals run in the opposite direction to the external muscles, and from a consideration of Fig. 488 it is evident that their effect will be to depress any pair of ribs, thus acting as expiratory muscles.

Owing to the fact that the costal cartilages make an angle with the bony ribs, the fibres of prolongation of the internal intercostals, musculi intercartilaginei, have the same relation to their attachments that the external intercostals have to the bony ribs. Their action therefore must be to raise the cartilages and flatten out the angle between the cartilaginous and bony ribs so that they must act with the external intercostals as inspiratory muscles.

In forced expiration a large number of muscles may take part-such as the serratus posticus inferior, and the muscles forming the wall of the abdomen, i.e. the rectus, obliquus, and transversus abdominis muscles.

As the lungs are distended with each inspiration their position changes in relation to the thoracic wall. All parts are not equally distensible in the normal position of the lungs. There are three areas which are in contact with the nearly stationary parts of the thoracic wall and cannot therefore be directly expanded. These are (1) the mediastinal surface in contact with the pericardium and structures of the mediastinum; $(2)$ the dorsal surface in contact with the spinal column and with the spinal segments of the ribs; (3) the apical surface lying in contact with the deep cervical fascia at the root of the neck. The roots of the lungs move with inspiration somewhat forwards and downwards. The front parts of the lungs move downwards and inwards, so that their inner borders in front approach one another. The extent and boundaries of the lungs can be easily ascertained in the living subject by means of percussion. On tapping the finger laid on the chest a sound is emitted which varies with the nature of the subjacent tissues. If this is lung-tissue filled with air, a clear-resonant tone is obtained; where it is solid tissue, such as the heart, or a lung consolidated with inflammatory 
products, or the liver, a dull sound is obtained. It is easy to show that the resonant area of the chest increases with each inspiration. The apices of the lungs extend about one inch above the clavicle anteriorly and behind reach as high as the seventh spinous process. During moderate expiration the lower margin of the lungs extends in front from the upper border of the sixth rib at its insertion to the sternum, and runs obliquely downwards to the level of the tenth rib at the back of the chest. During the deepest inspiration the lungs descend in front to the seventh intercostal space and behind to the eleventh rib, while during deepest possible expiration the lower margins of the lungs are elevated almost as much as they descend during inspiration. In the front of the chest a triangular space can be always marked out over the heart where the note obtained on percussion is dull. This space is bounded on the right by the left border of the sternum and extends out as far as the cardiac apex, being bounded above by the fourth costo-sternal articulation and below by the sixth costal cartilage.

BREATH SOUNDS. If the ear be applied to the chest wall, either directly or through the medium of a stethoscope, each inspiration is found to be accompanied by a fine rustling sound, the 'vesicular murmur.' It is thought to be caused by the sudden dilatation of the air-vesicles during inspiration or perhaps by the current of air passing from the narrow terminal bronchioles into the wider infundibula. It is important to remember that this sound is heard only during inspiration and over healthy lungs. On listening over the larger air-passages, i.e. the larynx, trachea, and bronchi, we hear a much louder sound which accompanies both expiration and inspiration and may be compared to a sharp whispered hah. This is known as the 'bronchial murmur.' It can be heard also at the back of the chest between the scapulæ at the level of the fourth dorsal vertebra, where the trachea bifurcates. In all other parts of the chest the healthy lung prevents the propagation of this sound to the chest wall. If, however, the lung is solid, as occurs in pneumonia, it conducts the sound easily from the large air-tubes to the chest wall. Bronchial breathing at any part of the chest other than that immediately over the air-tubes is therefore a distinctive sign of consolidation of the lung. Absence of breath sounds at any part of the chest implies either that air is not entering that part of the lung, or that the lung is separated from the chest wall by effused fluid.

INTRATHORACIC PRESSURE. Even at the end of expiration the lungs are in a stretched condition. This is shown by the fact that if in an animal or in the corpse an opening be made into the pleural cavity, air rushes into the opening and the lungs collapse, driving a certain amount of air out through the trachea. Since the lungs are always tending to collapse, it is evident that they must exert a pull on the thoracic wall. This pull of the lungs gives rise to a negative pressure in the pleural cavity. If we connect a mercurial manometer with the pleural cavity, we find that the pull of the lungs amounts in the corpse to $6 \mathrm{~mm}$. of mercury. If the lungs are fully distended, as after full inspiration, the elastic forces are more brought into play, and the negative 
pressure in the pleura may amount to $30 \mathrm{~mm}$. Since the lungs are always tending to collapse, respiration becomes impossible directly free openings are mades into the pleural cavities on both sides. With each inspiratory movement air rushes in through these openings, so that the thoracic movements can no longer exert any influence on the volume of the lungs. The negative pressure in the thorax is diminished by any factor decreasing the elasticity of the lung-tissue. Thus in an old man, where the elastic tissue is degenerated and the alveoli are enlarged, giving rise to the condition known as emphysema, the lungs may collapse only slightly or not at all on opening the chest. The lungs do not collapse on making an opening in the chest of a new-born mammal ; but this is owing to the fact that they completely fill the thorax in the expiratory position, and it is only later that, with the growth of the ribs, the thorax gets, so to speak, too large for the lungs, which are therefore stretched to fill it.

The force exerted by the inspiratory muscles is nearly all spent in overcoming the elastic resistance of the lungs and costal cartilages. A free access of air is provided for by contractions of certain accessory muscles of respiration. With each inspiration the glottis is widened by abduction of the vocal cords. When the glottis is observed by means of the laryngoscope, a rhythmical separation and approximation of the vocal cords are observed, synchronous respectively with inspiration and expiration (Fig. 255, p. 524). When inspiration is laboured, the alæ nasi are dilated by the action of the dilatator nasi. This movement of the nostril, which is constant in many animals, becomes very marked in children suffering from any respiratory trouble.

If a manometer be connected with one of the nostrils, so as to register the pressure in the air-cavities, it is found that there is a negative pressure of $-1 \mathrm{~mm}$. $\mathrm{Hg}$. with inspiration, and a positive pressure of 2 or $3 \mathrm{~mm}$. with expiration. With forced inspiration the negative pressure may amount to $-57 \mathrm{~mm}$. Hg., and with forced expiration there may be a positive pressure of $+87 \mathrm{~mm}$.

PULMONARY VENTILATION. Under no circumstances can we by forced expiration empty the lungs of air. At the end of the most forcible expiration, if the pleura were perforated, the lungs would collapse and drive more air through the trachea. When breathing quietly a man takes in and gives out at each breath about 500 c.c. of air, measured dry and at $0^{\circ} \mathrm{C}$. If measured moist and at the temperature of the body, viz. $37^{\circ} \mathrm{C}$, the volume would be about 600 c.c. This amount is known as the tidal air. By means of a forcible inspiratory effort it is possible to take in about 1500 c.c. more (complemental air). At the end of a normal expiration a forcible contraction of the expiratory muscles will drive out about 1500 c.c. more (supplemental air). These three amounts together constitute the 'vital capacity' of an individual. This total may be determined by means of the instrument known as the spirometer, which is merely a small gas-meter with a gauge by which the amount of air in it can be at once read off. The person to be tested fills his lungs as full as possible, and then expires to the utmost 
into the spirometer. The air left in the lungs after the most vigorous expiration is known as the residual air.

The residual air may be determined by letting a person expire to the utmost extent and then connecting with his mouth or nose a bag of known capacity filled with hydrogen. The subject of the experiment then inspires and expires into the bag two or three times, ending in the same state of forced expiration as he began. Any diminution of the total volume of gas in the bag will represent the gas lost during the experiment by diffusion into the blood-vessels. By analysis of the gaseous mixture in the bag, it is possible to determine the amount of air in the lungs at the beginning of the experiment. Supposing, for example, the bag held 4000 c.c. hydrogen, after two respirations the total volume is unaltered, but the gas is found to consist of 3000 c.c. hydrogen and 1000 c.c. oxygen, nitrogen, and $\mathrm{CO}_{2}$, i.e. pulmonary gases. Since the gas in the lungs must have the same composition and 1000 c.c. hydrogen have disappeared from the bag, it is evident that the lungs will contain 1000 c.c. hydrogen and $\frac{1000}{3}$, i.e. 330 c.c. pulmonary gases. Thus the total volume of gas left in the lungs at the end of the forced expiration was 1330 c.c., which is the residual volume for the individual.

The above example is purely imaginary. As a result of actual determinations carried out we may assume the residual air in the lungs as something between 600 and 1200 c.c.

Of the 500 c.c. of tidal air taken in at each inspiration, only a certain part reaches the alveoli, part being required to fill the air-tubes, trachea, bronchi, and bronchioles which lead to the air-cells. The volume of the air-tubes has been reckoned to amount to 140 c.c., so that of the 500 c.c. about 360 c.c. reach the alveoli. For the same reason the expired air represents the air from the alveoli (360 c.c.) diluted with 140 c.c. of air which has remained in the air-tubes and undergone very little change, other than the elevation of temperature and saturation with aqueous vapour. We have therefore to allow for this air contained in the so-called 'dead space' of the lungs when we seek to arrive at the composition of alveolar air from an analysis of expired air.

\section{THE BRONCHIAL MUSCULATURE}

Both the large and smaller air-tubes have a coating consisting of unstriated muscle-fibres which in the bronchioles is complete. Contraction of these fibres must have the following effects: (1) a constriction of the bronchi and bronchioles; (2) a diminution of the air space of the lungs and therefore of the volume of the lung; (3) an increased resistance to the passage of the air into and out of the alveoli. Changes in the condition of contraction of these muscle-fibres may be studied in two ways. In the first method artificial respiration is carried out, a constant volume of air being blown in and sucked out at each respiration. Any diminution in the calibre of the bronchioles must increase the resistance to the incoming current of air and so cause a rise of pressure in the tracheal tube. Einthoven in investigating this subject, has made use of an arrangement by means of which a mercurial manometer is connected with the trachea for a brief space of time during one part of the inspiratory phase. Any resistance to the current of air raises the pressure during the whole inspiration and 
therefore at the moment at which the manometer is put into connection with the tracheal tube, and a rise of the mercury in the manometer is therefore produced. By this method was obtained the tracing shown in Fig. 489. By the second method artificial respiration at a constant pressure is made use of. Any changes in the bronchioles will in this case affect the volume of air entering the lungs at each stroke of the pump, and can be measured by recording either the passive respiratory movements of the chest or the changes in volume of a lobe of the lung enclosed in a plethysmograph

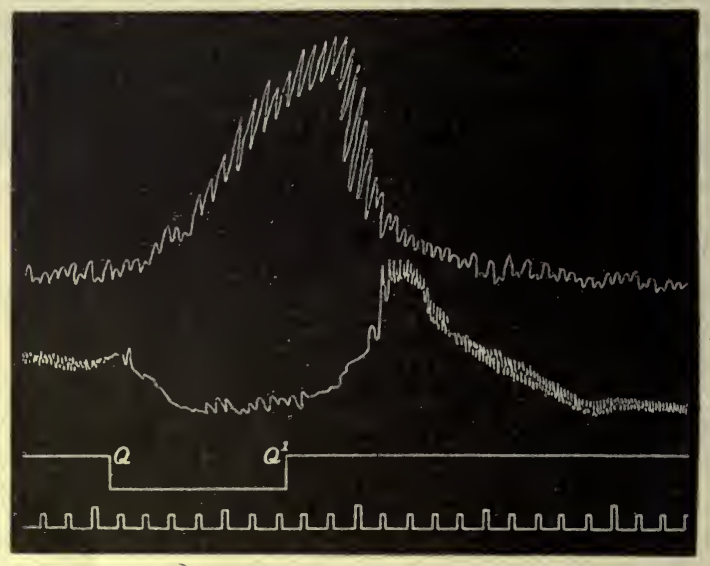

FIG. 489. Tracings of blood-pressure (middle curve) and of intratracheal pressure (upper curve) taken by Einthoven's differential manometer. Between $\mathbf{Q}$ and $\mathbf{Q}^{\prime}$ the peripheral end of one vagus was stimulated. Time marking $=$ seconds.

(Brodie and Dixon). By both these methods it has been shown that stimulation of the peripheral end of either vagus causes constriction of the bronchioles (vide Figs. 490 and 491). As a rule there is little tonic action of the vagi, section of both vagi leaving the respiratory pressure curve unaltered or lowering it slightly by 2 to $10 \mathrm{~mm} . \mathrm{H}_{2} \mathrm{O}$. It is very easy to bring about a vagus tonus by allowing the animal to inhale air containing 3 to 4 per cent. carbon dioxide. A peripheral tonus may also be produced by administration of muscarine or pilocarpine. In the latter case Brodie and Dixon have shown that stimulation of the vagus may cause relaxation of the bronchioles, so that this nerve appears to contain both motor and inhibitory fibres to the bronchioles.

THE EFFECTS OF BRONCHIIAL CONSTRICTION: ASTHMA. Under the influence of vagal stimulation or of carbon dioxide, the pressure necessary to drive the normal amount of air into the lungs may be raised in the dog from 125 to $300 \mathrm{~mm} . \mathrm{H}_{2} \mathrm{O}$. We should therefore expect that, in cases where bronchial constriction is present, there would be difficulty both in inspiration and expiration. There is, however, a difference in the mechanical conditions of the bronchi during the two phases of a respiratory movement. Normally the elastic structure of the lungs is drawing upon the bronchial wall, tending to maintain it patent, and so opposing the action of 
the bronchial muscle. During inspiration this expanding force is increased, so that in the presence of bronchial constriction the access of air

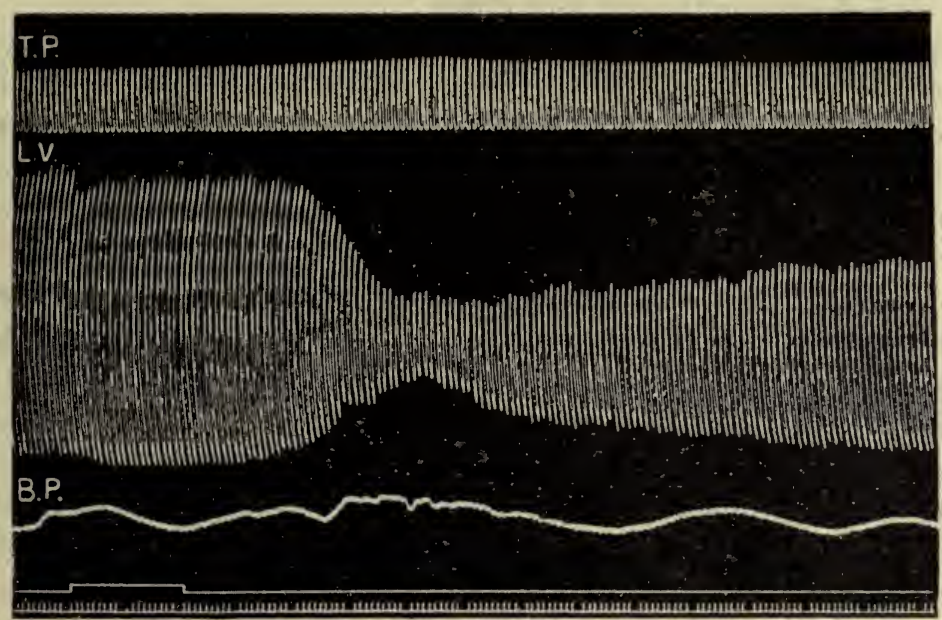

FIG. 490. Tracings of the volume changes of the lung, with constant variations of tracheal pressure. (BRodie and DixoN.) T.P. tracheal pressure. L.V. lung volume. B.P. blood-presure (Zero B.P. $17 \mathrm{~mm}$. below time marker). Showing constriction of bronchial musculature as a result of vagus excitation.

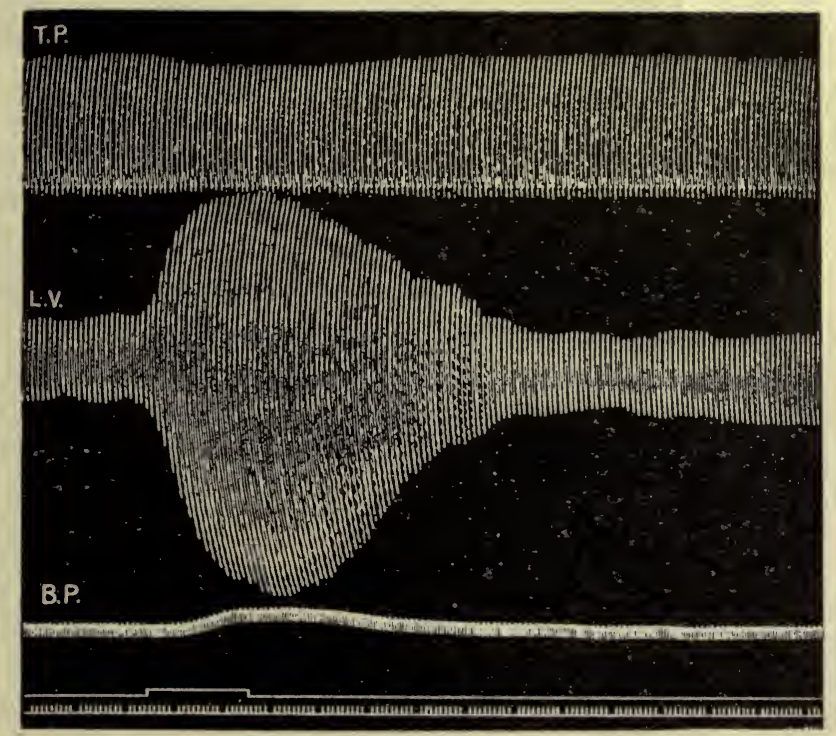

FIG 491. Tracing showing inhibitory effect of vagus on the bronchial tonus produced by $0.01 \mathrm{grm}$. pilocarpine.

is rendered the easier, the more powerful the contraction of the inspiratory muscles. In expiration all parts of the lung collapse, drawing with them the chest-wall ; the pull of the lung tissue on the bronchial wall is lessened, 
but is still present. If, however, the expiratory muscles contract vigorously, the intrapleural pressure becomes positive, and the pull of the lung-tissue on the bronchial walls is changed into a pressure tending to obliterate their lumen and so impede the out flow of air.

It is evident, therefore, that in the presence of a spasmodic contraction of the bronchial muscles the inspiration will be forcible and rapid, but all contractions of muscles must be avoided, so far as possible, during expiration, which must be left to the elastic reaction of the lungs, and becomes slow and prolonged. Moreover, it will be of advantage to keep the lung as nearly as possible in the inspiratory position, so as to reinforce the elastic forces which dilate the bronchioles and aid expiration. We thus get the typical breathing which occurs in man in cases of spasm of the bronchial muscles, known as asthma nervosum. This type of breathing is often described as being marked by expiratory dyspnza. This description is, however, erroneous. The muscles which in these cases are contracted to their uttermost, are the inspiratory muscles ; the expiratory muscles, such as the abdominal, will be found to be quite flaccid even during expiration. 


\section{SECTION II}

\section{THE CHEMISTRY OF RESPIRATION}

THE energy of the body is derived almost entirely from the oxidation of the carbon and hydrogen of the food-stuffs. An adult man during the twentyfour hours produces on the average 250 c.c. of carbon dioxide per kilo per hour. A man of 60 kilos will therefore excrete $250 \times 60 \times 24=360,000$ c.c. carbon dioxide in the course of twenty-four hours. During sleep the output of carbon dioxide is lowered with the diminution in all the metabolic processes of the body and amounts to only 160 c.c. per kilo per hour. If we assume that eight hours of the twenty-four are given to sleep, this will leave 295 c.c. per kilo per hour as the average excretion of carbon dioxide during the waking hours. Since the access of oxygen to the body and the removal of carbon dioxide is effected by the pulmonary ventilation, the expired air will differ from the inspired air in containing more carbon dioxide and less oxygen. The oxygen intake is not, however, absolutely proportional to the carbon dioxide output. This is owing to the fact that carbon is not the only element which leaves the body in an oxidised condition. Fats, for example, contain a number of unoxidised atoms of hydrogen, which in the metabolic processes of the body are fully oxidised, to be excreted as water. Oxygen will also leave the body in combination with carbon and nitrogen in the urine, so that a certain amount of oxygen which is taken in does not reappear as carbon dioxide in expired air. There is thus an absolute diminution in the volume of expired air as compared with that of inspired air. This diminution, due to loss of oxygen, is greater in carnivora, whose food consists mainly of proteins and fats, than in herbivora, which feed principally on carbohydrates, and depends on the respiratory quotient, i.e. the ratio $\frac{\mathrm{CO}_{2} \text { expired. }}{\mathrm{O}_{2} \text { inspired. }}$

In man the average respiratory quotient can be taken as $0 \cdot 85$. On this basis the amount of oxygen which will be taken in during the waking hours will be 347 c.c. per kilo per hour. Taking round figures, we may say that, when awake, a man takes in 350 c.c. oxygen and gives out 300 c.c. carbon dioxide per kilo per hour. From these figures we can calculate the normal composition of expired air when a man is breathing quietly. Under these conditions the tidal air amounts to 500 c.c. If he breathes seventeen times a minute the total pulmonary ventilation during the hour will be $500 \times 17$ $\times 60=510,000$ c.c. per hour. This will contain $300 \times 70$ c.c. $=21,000$ c.c. 
carbon dioxide. Hence the percentage of carbon dioxide in the expired air will be $4 \cdot 1$ per cent. In the same way we can reckon the percentage of oxygen in the expired air at 16.4 per cent. Exact experiments have shown that the volume of nitrogen is unchanged during respiration, this gas taking no part in the ordinary metabolic processes of the body. We may therefore compare the ordinary composition of inspired and expired air as follows :

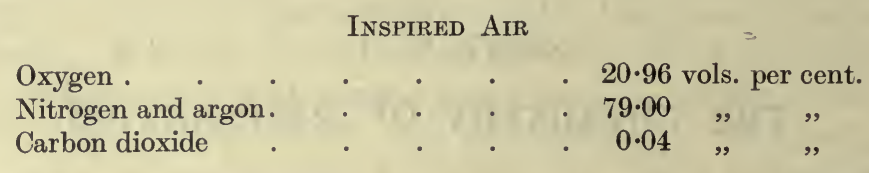

EXPIRED AIr

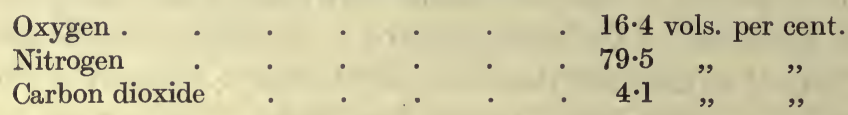

The increase in the figure for nitrogen refers of course only to the percentage amount, since the total volume of air breathed is decreased by the disappearance of a certain amount of oxygen without the production of a corresponding amount of carbon dioxide, so that the relative amount of nitrogen is slightly increased. These figures for the composition of inspired and expired air refer to dry air at a temperature of $0^{\circ} \mathrm{C}$. and a pressure of $760 \mathrm{~mm}$. Under normal circumstances inspired air contains a variable amount of aqueous vapour and has a variable temperature corresponding with the time of year. Expired air is fully saturated with aqueous vapour and has the temperature of the body, $37^{\circ} \mathrm{C}$. The aqueous vapour at this temperature is by no means negligible. Its tension amounts to $50 \mathrm{~mm}$. $\mathrm{Hg}$. Thus when a man is breathing dry air at a pressure of $760 \mathrm{~mm}$. $\mathrm{Hg}$., the pressure of the mixture of gases in the alveoli of his lungs will be only 760 - 50, i.e. $710 \mathrm{~mm}$. Hg.

Only a certain percentage of the 500 c.c. of tidal air reaches the alveoli, 100 to 140 c.c. being required to fill the trachea and bronchial tubes. Hence the alveolar air must contain more carbon dioxide and less oxygen than the tracheal air; and it is found that, if we take the air from the alveoli instead of that expired through the mouth or nose, the differences between it and the inspired air are much more pronounced.

A sample of alveolar air may be obtained for analysis in the following way (Haldane) : A piece of india-rubber tubing is taken of about 1 inch diameter and 4 feet long. Into one end (Fig. 492) is fitted a mouthpiece, the other being left open or connected with a spirometer. About 2 inches from the mouthpiece is fixed a gas sampling-bulb, which is provided with three-way taps at the upper and lower ends. Before an experiment the bulb is filled with mercury, if the lower end is open, or else it is completely exhausted. The subject of the experiment, after breathing normally a few times, at the end of a normal inspiration puts his mouth to the tube, expires quickly and deeply, and closes the mouth-piece with his tongue. The tap of the sampling-bulb is then turned, and the air last expelled from the lungs (which is therefore pure alveolar air) rushes into the bulb. The tap of the bulb is then turned off, and the gas may be removed for analysis. A similar sample is then taken, in whieh the subject expires deeply at the end of a normal 
expiration. This sample will, of course, contain more $\mathrm{CO}_{2}$ and less $\mathrm{O}_{2}$ than that obtained at the end of inspiration. The mean of the two samples is taken as the average composition of alveolar air.

The difference between the composition of expired air and alveolar air is determined by the dilution of the alveolar air with that contained in the dead space. Hence with shallow breathing there will be a large difference, but this will decrease with increased depth of respiration. Thus, if the alveolar air contained 6 per cent. $\mathrm{CO}_{2}$ and the dead space amounted to 150 c.c., the expired air would only contain 3 per cent. $\mathrm{CO}_{2}$ when the person was taking in only 300 c.c. at each respiration. If, however, he was breath-

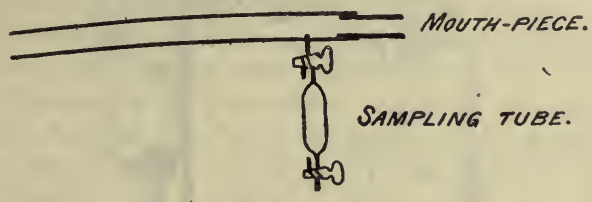

FIG. 492.

ing slowly and deeply so as to raise the tidal air to 1500 c.c., only one-tenth of this would be represented by the dead space, and the expired air would contain nine-tenths as much $\mathrm{CO}_{2}$ as the alveolar air, i.e. $5 \cdot 4$ per cent.

The changes in the composition of alveolar air with respiration are by no means so marked as those produced in the tidal air, since the latter forms only a small proportion of the total air in the lung alveoli. Thus at the end of a normal expiration the alveoli still contain 2500 c.c. of gases. In inspiration 360 c.c. atmospheric air is taken into this space and mixed with the 2500 c.c. already there. The 'ventilation coefficient' in quiet breathing is therefore only one-seventh, and the change in the oxygen and carbon dioxide content of the alveolar air produced by this access of 360 c.c. will amount to less than one-half per cent. This is illustrated by the following figures from Haldane, giving the alveolar content in carbon dioxide at the end of inspiration and at the end of expiration respectively.

Alveolar $\mathrm{CO}_{2}$ Tensions.

\begin{tabular}{|c|c|c|c|}
\hline Individual & $\begin{array}{c}\text { Alveolar } \mathrm{CO}_{2} \text { at end of } \\
\text { inspiration. - (Mean of } \\
\text { twelve observations) }\end{array}$ & $\begin{array}{c}\mathrm{CO}_{2} \text { at end of } \\
\text { expiration }\end{array}$ & Mean \\
\hline J. S. H. & $5 \cdot 54$ & $5 \cdot 70$ & $5 \cdot 62$ \\
J. G. P. & $6 \cdot 17$ & $6 \cdot 39$ & $6 \cdot 28$ \\
\hline
\end{tabular}

We can this speak of an average composition of alveolar air which in spite of the constant ventilation, differs from the external air in containing an excess of carbon dioxide and a relative lack of oxygen. Lavoisier, who was the first to study the chemical changes in respiration accurately, regarded the lungs as the seat of the formation of carbon dioxide and the consumption of oxygen. This view was generally accepted until it was shown by Magnus, in Heidenhain's laboratory, that the blood passing to the lungs contained more carbonic acid gas and less oxygen than that passing away 
from the lungs. The effects of this discovery were to transfer the chief seat of oxidation to the tissues of the body, and to show that the blood acts simply as a carrier of the oxygen from the lungs to the tissues, and of the carbon dioxide from the tissues to the lungs. We thus learnt to distinguish between external and internal respiratory processes. A consideration of the chemical mechanisms involved in the process of external respiration includes therefore an investigation of the manner in which gases are held by

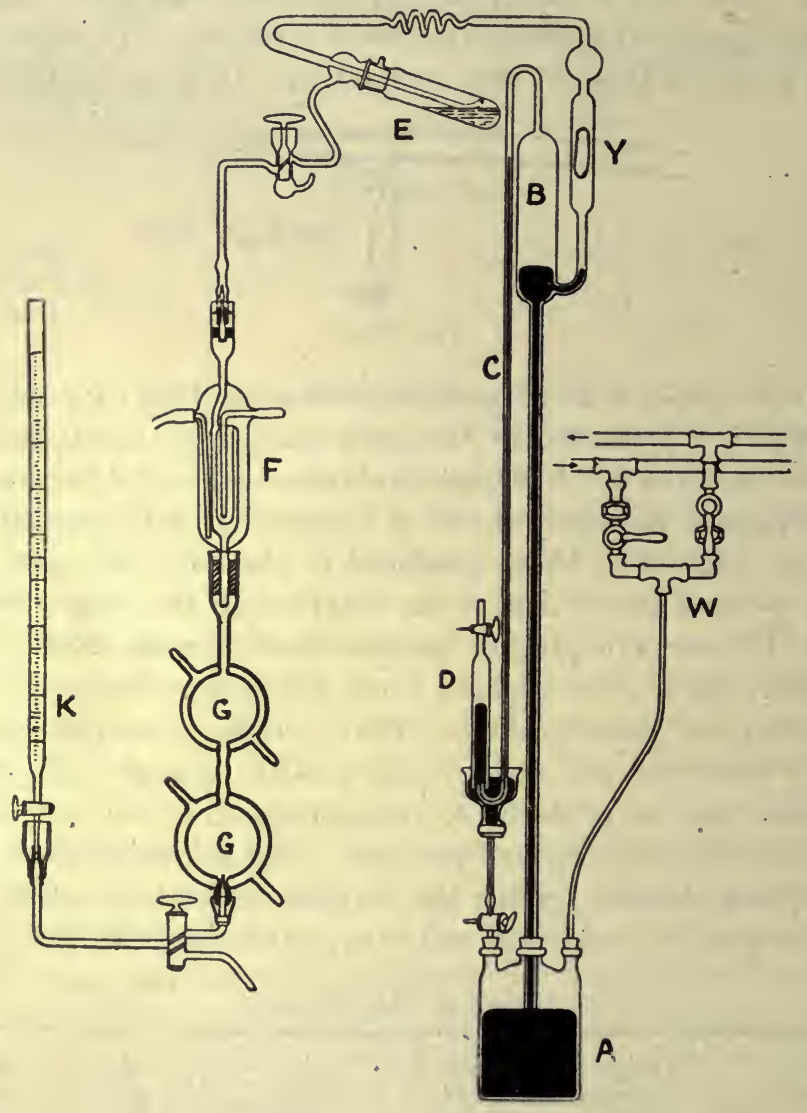

FIq. 493. Barcroft's modification of the Töpler pump.

the blood and of the factors which are responsible for the transfer of oxygen and carbon dioxide from blood to alveolar air, and from alveolar air to blood.

If blood be exposed to a Torricellian vacuum at the ordinary temperature, the whole of its contained gases is given off. For the purpose of extracting the blood gases a great variety of pumps have been devised. In every case a glass vessel is evacuated by means of the mercury pump, and is then put into connection with a reservoir containing blood which has been defibrinated, or has been prevented from clotting by the addition of oxalate or citrate. In all these pumps the main difficulty arises in the exclusion of atmospheric air, and it is therefore important to dispense so far as possible with taps. 
One of the best modifications of the Töpler mercury pump is that employed by Barcroft (Fig. 493), which differs little from the pump devised by Bohr.

The construction of the pump is shown in the diagram. The actual pump consists of the parts A, B, C, D. The bulb B is prolonged below by a wide tube dipping into the mercury in the Woulf bottle A. The upper part of the bottle is filled with water and connected by two taps at $w$ with the water-supply and with a sink. The water being turned on, mercury is forced up into $\mathrm{B}$; as it rises into $\mathbf{Y}$ it carries before it a glass valve which prevents its further passage, so that it can only escape by the tube $\mathrm{c}$, driving - before it all the air previously contained in B. The water-supply is now turned off, and the tap to the sink turned on. The mercury runs back. Air cannot enter by $\mathrm{c}$, since this tube is sealed by mercury. The valve $\mathrm{Y}$ therefore sinks and allows the air in the blood receivers G, G and the rest of the apparatus to escape into $B$. The process is repeated many times until a high vacuum is produced in the whole apparatus. A measured quantity of blood is now let into the lower bulb G. F is a condenser through which cold water is constantly flowing (to prevent all the blood boiling away), while warm water circulates round the bulbs $\mathrm{G}$, $\mathrm{G}$ to facilitate the giving off of the blood gases. The blood boils in the vacuum, and the gases escape into $\mathrm{B}$, and may be driven off and collected over mercury in a cylinder $\mathbf{D}$ by raising the mercury in $\mathbf{B}$. The process of exhaustion is repeated until no more bubbles rise into $\mathrm{D}$ on filling the bulb $\mathrm{B}$ with mercury. $\mathrm{E}$ is a sulphuric acid chamber for drying the gases as they pass from the blood to the bulb B.

In this way, from 100 c.c. of blood, about 60 c.c. of mixed gases may be obtained, consisting of oxygen, carbon dioxide, nitrogen, and argon. Argon is present only in insignificant quantities, about $\cdot 04$ volume per cent. The nitrogen also forms only between one and two volumes per cent. and is present in the same proportion in both arterial and venous blood. The amounts of oxygen and carbon dioxide in these two kinds of blood differ, however, within wide limits. The following Table represents the average composition of the gases obtained from an artery and a vein of the dog :

From 100 vols.

Of arterial blood.

Of venous blood

May be obtained
$\begin{gathered}\text { of oxygen } \\ 20 \text { vols. } \\ 8 \text { to carbon dioxide } 12 \text { vols. } 40 \text { vols. } 4 \\ \text { Measured at } 760 \mathrm{~mm} \text {. and } 0^{\circ} \mathrm{C} .\end{gathered}$

The principle introduced by Haldane (vide p. 859) for the determination of the oxygen combined in the form of oxyhæmoglobin may be successfully applied to small quantities of blood, such as 1 c.c. or even $0 \cdot 1$ c.c., and in the same sample of blood the carbon dioxide may also be determined. In this way it becomes practicable to make blood-gas analyses in a patient, or in experiments on small organs where it is desired to determine their gaseous metabolism by comparing the arterial with the venous blood. Barcroft's apparatus for dealing with 1 c.c. of blood is shown in Fig. $494 \mathrm{~A}$.

The apparatus consists of two bottles of identical size (about 30 c.c.) attached to a manometer, the tubing of which is $1 \mathrm{~mm}$. bore. The manometer is filled with clove oil of known specific gravity. To fill it take out the centre tube, put in clove oil at A, put in the centre tube with the glass tube B open and some pressure on the rubber tube $\mathbf{C}$. The oil should stand about half way up each tube; seal в in a flame. The constant of the apparatus must be determined, viz. the capacity of the bottles and with their connections.

It is determined by finding what rise of pressure in the apparatus is produced by the liberation of a known volume of oxygen from hydrogen peroxide, which is placed in the bottle, the liberation being effected by the addition of potassium. 
To determine the oxygen capacity of a sample of blood. Place 2 c.c. of ammonia solution (made by adding 4 c.c. of strong $\mathrm{NH}_{3}$ to a litre of water) in one of the bottles and add 1 c.c. of blood. Thoroughly lake the blood. Put vaseline on the large and small stoppers. Put 0.2 c.c. of a saturated solution of potassium ferricyanide in the small tube contained in the stopper of the bottle containing the blood (this is best done with a fine pipette which goes down these tubes). Put in the small stopper. Place the apparatus on the side of a large water bath (such as a pail) with both taps open. In about five minutes close the tap on the side of the blood and rotate the bottle on the stopper till the ferricyanide trickles into the laked blood. Shake thoroughly, replace in the bath, and repeat this several times till a constant difference of level is obtained. By means of the screw clamp

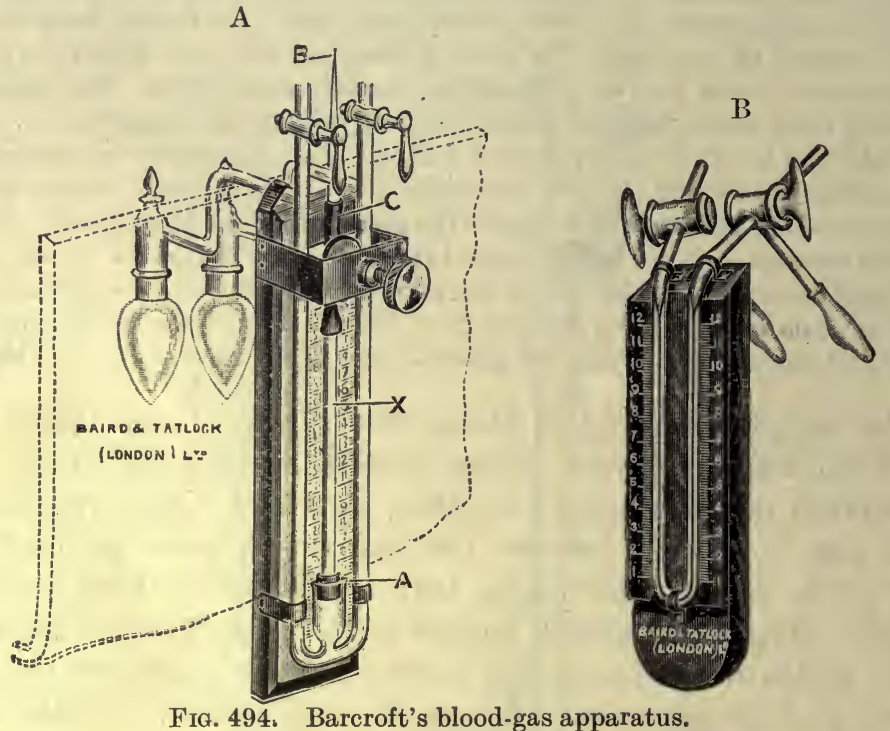

A, for 1 c.c.; $B$, for $0 \cdot 1$ c.c. blood.

bring the column of oil on the side of the blood to its original level, and then measure the difference of level between the two sides. 'Let this difference of level be $y \mathrm{~mm}$.; let $p$ be the height of the barometer in millimetres of clove oil, and $x$ the volume of oxygen given off in cubic millimetres ; then $x=y\left(\frac{V}{p}\right)$. Except in the most exact work $p$ may be taken as $10,000 \mathrm{~mm}$., in which case the expression $\frac{\mathrm{V}}{p}$ may be determined once for all and called $\mathrm{C}$, the constant of the apparatus : then $x=y \times \mathrm{C}$.

To determine the gaseous contents of a given blood. If we wish to determine the actual amount of oxygen as oxyhæmoglobin in the sample, the blood must be carefully introduced so as to lie below the ammonia and not to come in contact with the air. The stopper is then replaced in the bottle and immersed in the bath, with both taps open until it has attained a constant temperature. The tap is then closed and the height of the column of oil noted. The blood is then laked by rotating the apparatus, and after allowing five minutes for complete laking the ferricyanide is run in. The rest of the determination is carried out as above.

The carbon dioxide may be determined in the same sample of blood by running in tartaric acid in the same way as potassium ferricyanide was previously run in. It is necessary always to determine the oxygen before the carbon dioxide, since the mere acidification of the blood causes the evolution of a certain amount of oxygen. The results obtained for carbon dioxide are not so accurate as those for the oxygen, owing to the larger error introduced by the increased solubility of this gas in watery media.

The same apparatus may be used as a differential blood-gas manometer, where it is 
desired to compare the oxygen contents of two samples of blood, e.g. of arterial and venous blood. For this purpose 1 c.c. of the arterial blood is introduced into one bottle and 1 c.c. of the venous blood into the other bottle, in each case under $1 \frac{1}{2}$ c.c. of weak ammonia. The bottles are then placed on the apparatus and immersed in the water bath until no change occurs in the height of the column of oil. The two taps are then closed and the apparatus is vigorously shaken. The blood on each side is laked, and in contact with the air in the bottles becomes completely saturated with oxygen. No carbon dioxide is given off, since this combines with the weak ammonia. If the two bloods contain the same amount of oxyhæmoglobin no difference will be produced in the level of the oil in the two tubes. If, however, one be arterial and the other venous, the venous blood will absorb more oxygen from its bottle than the arterial blood from its side of the apparatus, so that the oil will rise in the tube on the side of the venous blood. From the amount of rise the difference in the amount of oxygen taken up by the blood on the two sides can be reckoned and this figure will express the relative saturation of the hæmoglobin in the two samples of blood.

For clinical purposes it is possible to work with $0 \cdot 1$ c.c. of blood. Fig. 494 B represents the form of apparatus devised by Barcroft for dealing with these minute quantities. The principle of the apparatus is the same as that of the larger type.

The condition of the gases in the blood can be judged by the amount of gas which the blood will take up when exposed to different pressures of the gas. If a gas is in simple solution the amount of it dissolved varies directly with the pressure. Thus, if water takes up a certain bulk of a gas at a given temperature and pressure, it will take up twice as much if the pressure of the gas be doubled. Since the volume of a gas varies inversely as the pressure, we may say that a fluid will dissolve the same volume of gas whatever the pressure. The absorption coefficient of a liquid for a gas is expressed by the number of cubic centimetres of gas which will be taken up at $0^{\circ} \mathrm{C}$. by 1 c.c. of the liquid when the gas is at a pressure of $760 \mathrm{~mm}$. $\mathrm{Hg}$. The absorption coefficient diminishes with rise of temperature. The following Table represents the absorption coefficients for oxygen, carbon dioxide, carbon monoxide, and nitrogen, in water at various temperatures between $0^{\circ}$ and $40^{\circ} \mathrm{C}$. :

\begin{tabular}{|c|c|c|c|c|}
\hline Temperature & Oxygen & Carbon dioxide & Carbon monoxide & Nitrogen \\
\cline { 2 - 3 } 0 & 0.0489 & 1.713 & 0.0354 & 0.0239 \\
10 & 0.0380 & 1.194 & 0.0282 & 0.0196 \\
20 & 0.0310 & 0.878 & 0.0232 & 0.0164 \\
30 & 0.0262 & 0.665 & 0.0200 & 0.0138 \\
40 & 0.0231 & 0.530 & 0.0178 & 0.0118 \\
\hline
\end{tabular}

From this Table we see that 100 c.c. of water at $0^{\circ} \mathrm{C}$. will absorb 4.89 c.c. oxygen at $760 \mathrm{~mm}$. $\mathrm{Hg}$., i.e. at one atmosphere. If the pressure be raised to two atmospheres the volume of gas absorbed will be the same, but if these gases be measured at the original pressure, i.e. at one atmosphere, the amount dissolved will be $9 \cdot 78$ volumes. If therefore we plot out the absorption of the gas on a curve of which the ordinates represent the amount of gas dissolved and the abscissa the different pressures of the gas, we shall find that the curve is a straight line. The relation between the amount 
absorbed is not altered by the presence of other gases at the same time. The pressure of the whole atmosphere is $760 \mathrm{~mm}$. Since the atmosphere consists roughly of four parts of nitrogen with one part of oxygen, the atmospheric pressure is due as to one-fifth to the oxygen and as to four-fifths to the nitrogen. If we shake up water at $0^{\circ} \mathrm{C}$. with the atmospheric air at the ordinary pressure, 100 c.c. of water will absorb 4.89 c.c. $\times \frac{1}{5}$ of oxygen, and of nitrogen 2.39 c.c. $\times \frac{4}{5}$. We may therefore extend our statement as to the solubility of gases in fluids and say that the amount of gas dissolved in a fluid is proportional to the partial pressure of the gas.

When water is shaken up with a gas until it will take up no more, i.e. until it is saturated for that pressure, a state of equilibrium exists between the gas dissolved in the fluid and the gas in contact with the fluid. In this state of equilibrium the number of molecules of the gas entering the fluid is exactly equal to the number of molecules of the gas leaving the fluid. If we remove the liquid after saturation, say, at one atmosphere, to a vessel where it is in contact with gas at a pressure of half an atmosphere, the liquid will give off gas until the amount left in solution is diminished to onehalf. The gas dissolved in a liquid thus has a pressure or tension which tends to make it escape from the liquid. The only way in which we can measure this tension is by finding what pressure of gas is in exact equilibrium with the liquid. Thus if we take some water containing carbon dioxide in solution, divide it into two parts, and shake up one part with a gaseous mixture containing 4 per cent. of carbon dioxide and the other part with a mixture containing 5 per cent. of carbon dioxide, and find that the solution loses gas to the former and takes up carbon dioxide from the latter, we may conclude that the tension of carbon dioxide in the original fluid was something between 4 and 5 per cent. of an atmosphere. It is by some such means that the tensions of gases in the blood are measured, the instruments for this purpose receiving the name of aerotonometers.

The solvent power of water for gases is diminished if the water contains other solid substances in solution. Blood-plasma or blood-corpuscles will therefore have a smaller solvent power for gases than has pure water. It has been shown by Bohr that the depression of solubility caused by the presence of proteins or salts in solution is the same for all gases. The absorption coefficient of blood-plasma for gases is reduced to 97.5 per cent. of pure water, and of blood to 92 per cent., that of the blood-corpuscles being as low as 81 per cent. We may thus reckon the absorption coefficient of bloodplasma, blood, and blood-corpuscles, for oxygen, nitrogen, and carbon dioxide.

From the following Table we see that 100 volumes of blood at $38^{\circ} \mathrm{C}$. might contain $2 \cdot 2$ c.c. of oxygen in solution if the blood had been exposed to oxygen at a pressure of one atmosphere. The blood in the lungs is, however, exposed to air which contains only about one-sixth of its volume of oxygen. so that the total amount of oxygen present in arterial blood in solution cannot be more than one-sixth of $2 \cdot 2$, i.e. about $0 \cdot 36$ c.c. per cent. Since arterial blood, or blood saturated with oxygen by shaking with air, will 
yield as much as twenty volumes per cent. of oxygen to a Torricellian vacuum, the oxygen cannot be in simple solution, but must be in some form of combination with some of the constituents of the blood. Of this oxygen practically the whole is contained in the red blood-corpuscles in combination with hæmoglobin, the plasma containing no more than could be accounted for by simple solution.

\begin{tabular}{|c|c|c|c|c|c|c|c|}
\hline & & \multicolumn{2}{|c|}{ Oxygen } & \multicolumn{2}{|c|}{ Nitrogen } & \multicolumn{2}{|c|}{ Carbon dioxide } \\
\hline & & $15^{\circ}$ & $38^{\circ}$ & $15^{\circ}$ & $38^{\circ}$ & $15^{\circ}$ & $38^{\circ}$ \\
\hline Blood-plasma & & 0.033 & $0 \cdot 023$ & 0.017 & 0.012 & 0.994 & 0.541 \\
\hline Blood & & 0.031 & 0.022 & $0 \cdot 016$ & 0.011 & 0.937 & 0.511 \\
\hline Blood-corpuscles & & 0.025 & 0.019 & 0.014 & 0.010 & 0.825 & 0.450 \\
\hline
\end{tabular}

One gramme of crystallised hæmoglobin can absorb 1.34 c.c. of oxygen. If a solution of oxyhæmoglobin be subjected in an air-pump to gradually diminishing pressure at the temperature of the body, very little oxygen is given off until the partial pressure of the oxygen is diminished to about $30 \mathrm{~mm}$. Hg. (Fig. 496). At this point a large evolution of gas begins, and continues at falling pressure until at $0 \mathrm{~mm}$. pressure all the oxyhæmoglobin is dissociated and converted into hæmoglobin. The same observation may be made in a reverse direction. If a solution of reduced hæmoglobin be exposed to gradually increasing pressures of oxygen, it will be found that the greatest absorption takes place between 0 and $30 \mathrm{~mm} . \mathrm{Hg}$. After this point the oxygen is more slowly absorbed up to the point of complete saturation.

Since there is no direct proportion between the partial pressure of the oxygen and the amount absorbed, it is evident that the oxygen combines with hæmoglobin to form an unstable chemical compound, and that this is not a mere question of solution. This is further proved by the fact that we can displace the oxygen $\left(\mathrm{O}_{2}\right)$ from the oxyhæmoglobin by equivalent amounts of $\mathrm{CO}$ or NO. Hæmoglobin is also supposed to form an unstable combination with carbon dioxide, since it takes up much more of this gas than the corresponding bulk of water or salt solution would do. Although carbon dioxide combines with hæmoglobin, it does not displace oxygen from the oxyhæmoglobin molecule. Thus we may have hæmoglobin saturated at the same time with oxygen and with carbon dioxide. The presence of carbon dioxide does, however, alter the case with which oxyhæmoglobin dissociates.

The relation between the partial pressure of oxygen and the amount of oxyhæmoglobin formed under varying conditions can be investigated in the following way (Barcroft) :

A large glass globe with a stop-cock at one or both ends (Fig. 495) is filled, with a gaseous mixture of known composition containing oxygen. Into it are introduced 2 or 3 c.c. of blood or of hæmoglobin solution. It is then tightly stoppered and immersed in a 
horizontal position in a pail of water kept at a constant temperature. In the pail it is suspended between its two ends, so that it can be slowly revolved by means of a piece of string passing round its neck. In this way the blood is continually spread in a thin layer over the sides of the vessel. At the end of a quarter to half an hour it will have attained equilibrium with the gaseous mixture. It is then turned into an erect position so that the fluid can run down into the neck closed by a stop-cock, whence 1 c.c. may be drawn off for analysis in a Barcroft apparatus. A further portion of the same blood may be shaken up with air so as to saturate it completely, and the saturation of the two samples may be compared in the differential gas apparatus.

Barcroft has shown that the dissociation curve of hæmoglobin is largely altered by slight variations in the fluid in which the hæmoglobin is dissolved. The most important of these conditions are (1) the saline content of the

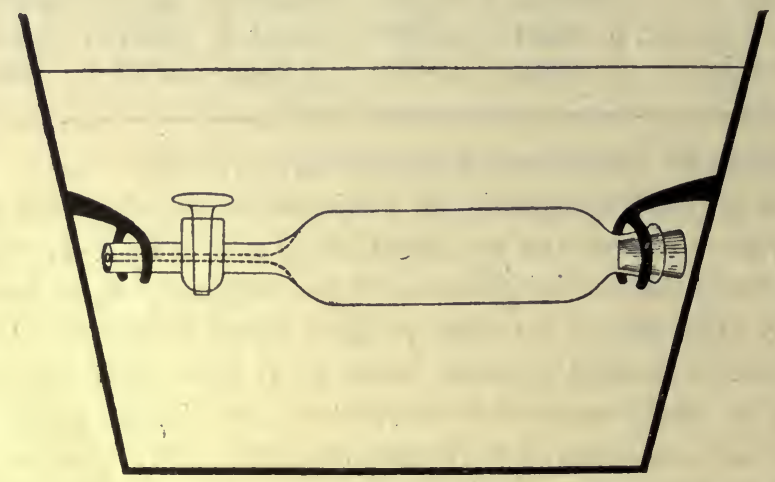

FIG. 495. Barcroft's apparatus for determining the curve of absorption of oxygen by hæmoglobin.

fluid, (2) the reaction of the fluid. Under this latter heading must be classed the amount of carbon dioxide present, since its action on the dissociation curve is similar to that produced by the presence of weak acids such as lactic acid. The influence of dissolved salts on the dissociation curve is shown in Fig. 496.

It is interesting to note that the differences between the dissociation curve of blood and of hæmoglobin solution, as well as between bloods of different animals, have been shown by Barcroft and Camis to be dependent on the different saline content of the solution in the various cases. Thus human hæmoglobin solution, with a concentration of salts similar to that of dogs' blood, gives the same dissociation curve as normal dogs' blood.

More important is the effect of reaction, since, as we shall see, it is the reaction of the blood, controlled especially by carbon dioxide tension, that determines the activity of the respiratory centres. In Fig. 497 is represented the influence of varying tensions of carbon dioxide, and in Fig. 498 the effect of slight additions of lactic acid on the dissociation curve. It will be seen that the more acid the blood, or the greater tension of carbon dioxide it contains, the more readily does it undergo dissociation. This is especially marked at the very high tension of $420 \mathrm{~mm}$. carbon dioxide. It plays an important part in the lower tensions. such as 40 and $80 \mathrm{~mm}$. Hg. 


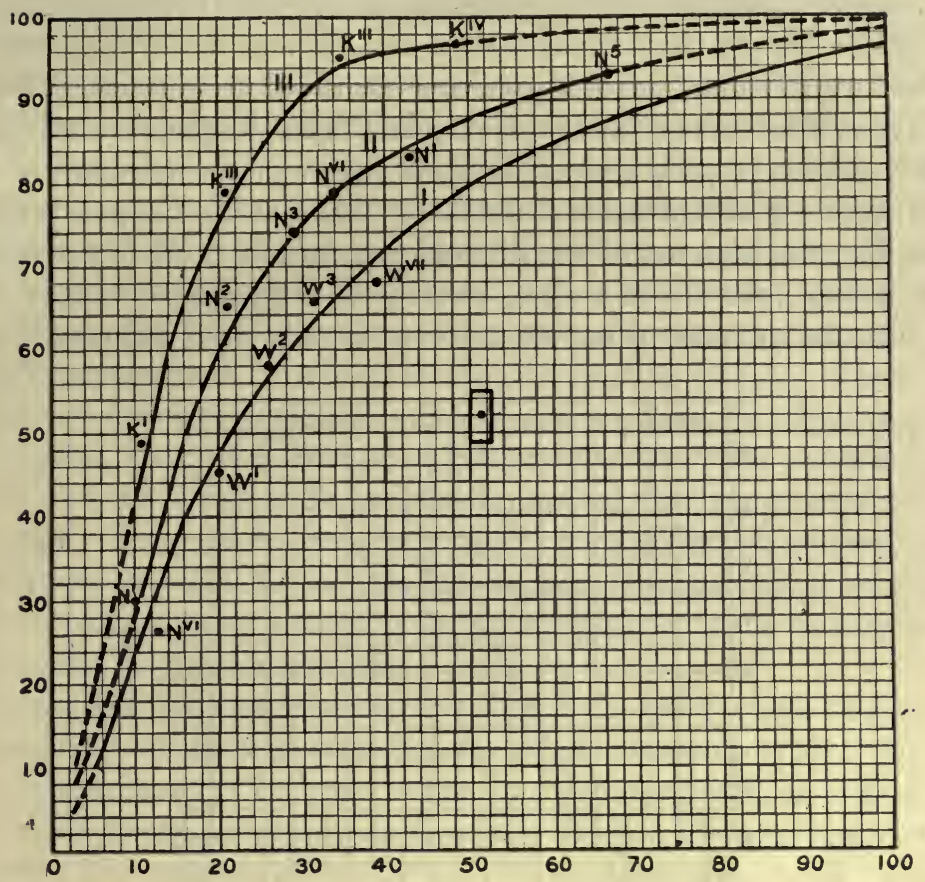

Fig. 496. Dissociation curve of hæmoglobin in various solvents. $\mathrm{I}$, in water; II, in 0.7 per cent. $\mathrm{NaCl}$; III, in 0.9 per cent. $\mathrm{KCl}$. (BARCROFT.)

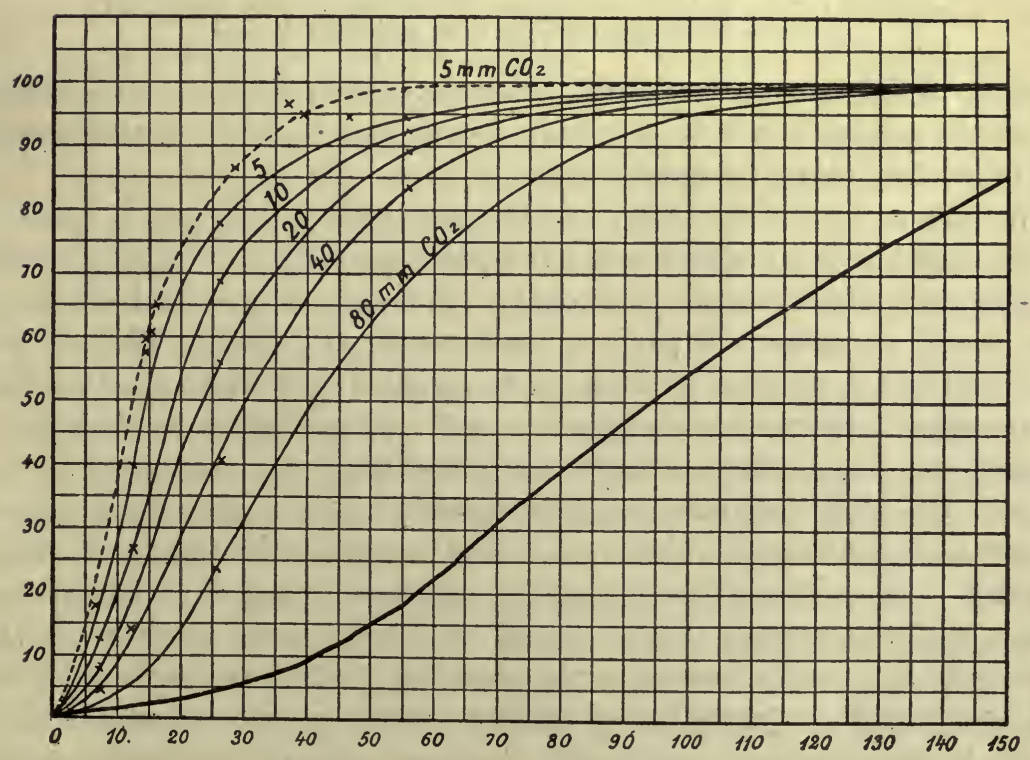

FrG. 497. Effect of varying tensions of $\mathrm{CO}_{2}$ on the dissociation curve of oxyhæmoglobin. The lowest curve is the dissociation at a $\mathrm{CO}_{2}$ tension of $420 \mathrm{~mm}$. $\mathrm{Hg}$. from observations by Barcroft. (BoHR.) 
carbon dioxide. It must be remembered that $40 \mathrm{~mm}$. carbon dioxide represents approximately the normal carbon dioxide tension in the blood. It is true that at $150 \mathrm{~mm}$. oxygen pressure the blood is practically saturated with oxygen whatever (within physiological limits) the pressure of the carbon dioxide. At lower pressures of oxygen, however, the pressure of carbon dioxide makes a considerable difference. Thus at an oxygen pressure of $20 \mathrm{~mm}$. $\mathrm{Hg}$. the amount of oxyhæmoglobin formed is 67.5 per cent. at a carbon dioxide pressure of $5 \mathrm{~mm}$., whereas at a pressure of carbon dioxide of $40 \mathrm{~mm}$. the amount of oxyhæmoglobin is only 29.5 per cent. In con-

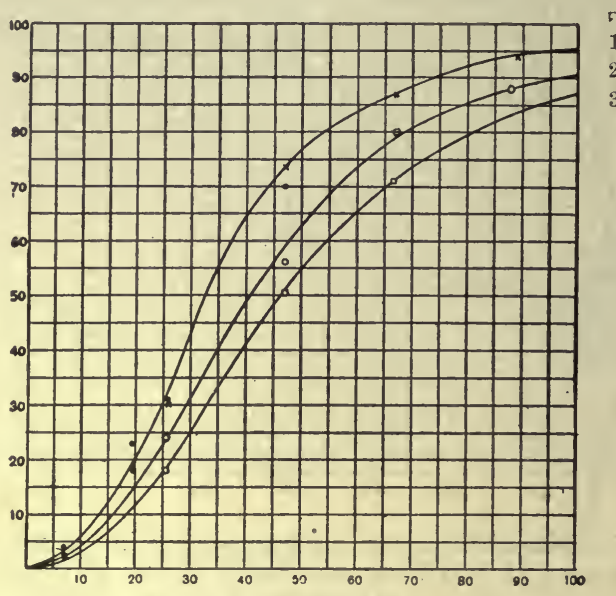

Fig. 498. Dissociation curve of sheep's blood.

1, normal blood; 2, blood containing 0.04 per cent. added lactic acid;

3 , blood containing 0.08 per cent. added lactic acid.

sequence of this fact, in the tissues, where the carbon dioxide tension is high, the oxyhæmoglobin will be dissociated with greater ease, so that oxygen will be set free where it is most wanted.

We are now in a position to understand how the oxygen is taken up by the blood as it circulates round the pulmonary alveoli. Arterial blood, such as that which fills the pulmonary veins and the systemic arteries, is very nearly (i.e. about 90 per cent.) saturated with oxygen, and will only take up about 2 volumes per cent. more on shaking it with air at the body temperature. Venous blood requires 8 to 10 volumes per cent. of oxygen to saturate it; but we have already mentioned that, at a tension of $30 \mathrm{~mm}$. oxygen, the blood becomes nearly saturated. The tension of oxygen in the alveoli is considerably above this. In the trachea the tension of oxygen is about $\frac{1}{6}$ of an atmosphere (since the air here contains 16 volumes per cent.), and the tension in the alveoli will be a little lower than this. If we take the oxygen tension in the alveoli at $\frac{1}{7}$ of an atmosphere, ${ }^{*}$ it will still be something over $100 \mathrm{~mm}$. Hence the venous blood brought to the alveoli by the pulmonary artery will, on there coming into intimate contact

* The oxygen tension in the alveoli has been reckoned at about $12 \cdot 6$ per cent. to 13.5 per cent. of an atmosphere. 
with the atmosphere, take up oxygen from it to saturation, or to a point not far removed from it.

The blood, thus laden with oxygen, travels to the left side of the heart, and from there is sent through the arteries to all parts of the body. It must be remembered that neither in the lungs nor in the tissues does the hæmoglobin come in actual contact with the source of the oxygen, nor with the cells which it is to supply. In both cases the interchange is effected through the intermediation of the plasma and, in the tissues, of the lymph as well. Since the tissue-elements are constantly using up oxygen, they absorb any oxygen that is present in the surrounding lymph. There is, in consequence, a descending scale of oxygen tensions from red blood-corpuscle through plasma, vessel wall, lymph, and tissue-element. The cell draws from the lymph, and the lymph from the plasma, so that the oxygen tension in the plasma sinks. This has the same effect as if we put the red corpuscles in a mercurial pump and lowered the pressure of gas. The immediate result is an evolution of oxygen, which is taken up by the plasma, to be in turn passed on to the lymph and the tissue-cell.

Under normal circumstances a blood-corpuscle never stays long enough in the proximity of the tissues to lose its whole store of oxygen. If, however, the further supply of oxygen to the blood be prevented, as in asphyxia, the last traces of oxygen disappear from the blood. The enormous avidity of the tissues for oxygen is shown by the following experiment (Ehrlich). If a saturated solution of methylene blue be injected into the circulation of a living animal and the animal be killed ten minutes later, it is found on first opening the body that most of the organs present their natural colour, although the blood is a dark blue colour. On exposure to the atmosphere all the organs acquire a vivid blue colour. The avidity of the tissues for oxygen has been so great that they have been able to decompose the methylene-blue molecule, with the formation of a colourless reduction-product, which on exposure to the air undergoes oxidation again and re-forms methylene blùe. If the tissues are able to effect the reduction of a comparatively stable body like methylene blue, it is easy to understand their power of reducing oxyhæmoglobin, which is so unstable that it is decomposed by simple physical means such as exposure to a vacuum.

It was long debated whether the chief processes of oxidation took place in the blood or in the tissues. Our experiences with muscle would alone serve to convince us that, in some tissues at any rate, processes of oxidation take place, and the methylene-blue experiment shows that these processes of oxidation are intense in all the chief organs of the body. It has been found moreover that it is possible to keep a frog alive after substituting normal saline solution for his blood, if he be placed in absolutely pure oxygen, and that in this case indeed the metabolism of the animal goes on as actively as before. As the frog has no blood, it is evident that its metabolic processes, consisting of the taking up of oxygen and the giving out of carbon dioxide, must have their seat in the tissues.

As a result of the oxidative changes in the tissues carbon dioxide is 
produced, and the tension of this gasin the tissues therefore rises. As Barcroft has pointed out, in cold-blooded animals the dissociation of oxyhæmoglobin with the setting free of oxygen must be largely conditioned by the rise of carbon dioxide tension in the tissues, since at the normal temperature of these animals the evolution of oxygen from hæmoglobin is extremely slow. The alteration in reaction of the blood caused by a rise in $\mathrm{CO}_{2}$ tension or by the presence of small amounts of lactic acid, markedly quickens the rate at which oxyhæmoglobin gives up its oxygen, as is shown in Fig. 499. The

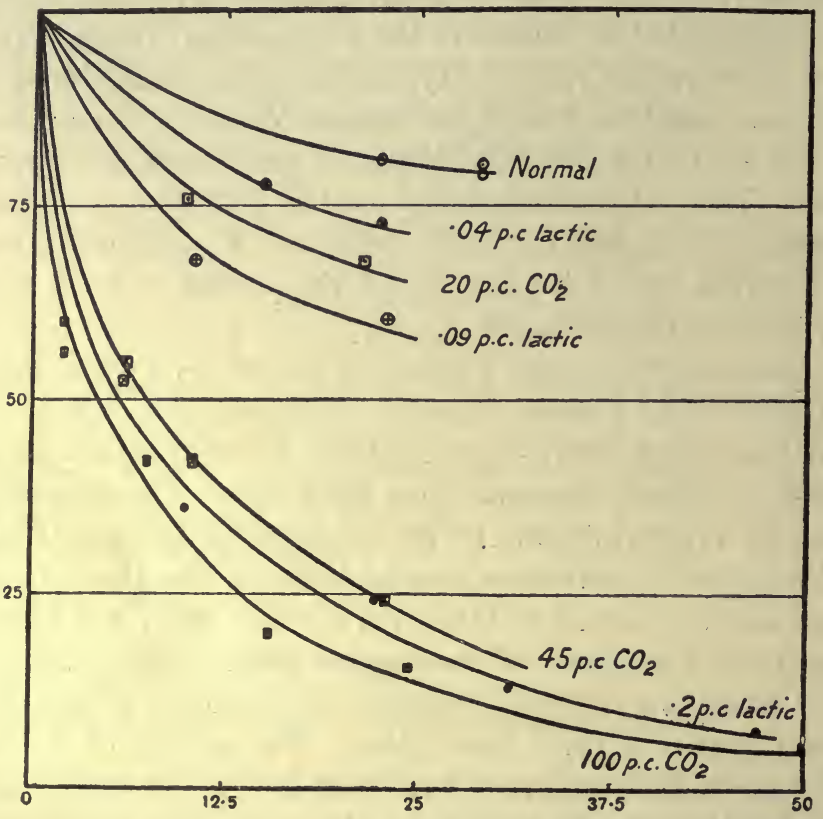

FIG. 499. Curves showing the rate at which arterial blood is reduced on bubbling through a gas free from oxygen, and the effect on the rate of the presence of $\mathrm{CO}_{2}$ and of lactic acid. Ordinates = percentage saturation of oxyhæmoglobin. Abscissæ = time in minutes. (MATHISON.)

carbon dioxide tension in the tissues may be approximately measured by taking the tension of this gas.in fluids such as the bile or urine. Here it may amount to 8 or 10 per cent. of an atmosphere, and since the carbon dioxide in venous blood is rarely above 6 per cent. of an atmosphere, there is a descending scale of tensions from tissue to blood, just as there is an ascending scale in the case of oxygen. This gas therefore passes from the tissues through the lymph into the blood by a simple process of diffusion.

The carbon dioxide carried by the blood is, like the oxygen, chiefly in a state of chemical combination. From dogs' venous blood we may obtain by means of the pump about 50 c.c. of carbon dioxide per 100 c.c. blood. Water at the temperature of the body, if shaken up with an atmosphere of carbon dioxide at a pressure of $760 \mathrm{~mm}$. Hg., would take up about 50 per cent. of the gas, and the plasma as a mere solvent would take up slightly less. The tension of carbon dioxide in the blood is, however, much less than 1 atmosphere. 
Shaken up with pure carbon dioxide at a pressure of 1 atmosphere, the blood would take up as much as 150 per cent. If we determine the tension of the carbon dioxide in the blood by one of the methods to be described later, we find that in venous blood this gas is at a pressure of only about 5 to 6 per cent. of an atmosphere (about $40 \mathrm{~mm}$. $\mathrm{Hg}$.). Taking the pressure of the carbon dioxide as $\frac{1}{20}$ of an atmosphere, and knowing that at a pressure of 1 atmosphere the blood might dissolve 50 volumes per cent., it is evident that at $\frac{1}{20}$ of an atmosphere the blood would only dissolve $\frac{50}{20}$ volumes per cent., i.e. about $2 \frac{1}{2}$ volumes. All the rest of the carbon dioxide in the blood must therefore be in combination (cp. Fig. 500).

The carbon dioxide is contained chiefly in the plasma, though a certain amount is also in combination in the corpuscles. Part of the carbon dioxide must be in combination with some constituent common to both plasma and corpuscles. When bloodplasma is calcined, the ash is found to be distinctly alkaline and to contain an amount of sodium greater than is necessary to combine with the other acid radicals, e.g. $\mathrm{Cl}, \mathrm{SO}_{4}$, and $\mathrm{PO}_{4}$, and this excess becomes greater if we consider that a great part of the $\mathrm{PO}_{4}$ and $\mathrm{SO}_{4}$ is derived from the oxidation of the sulphur and phos-

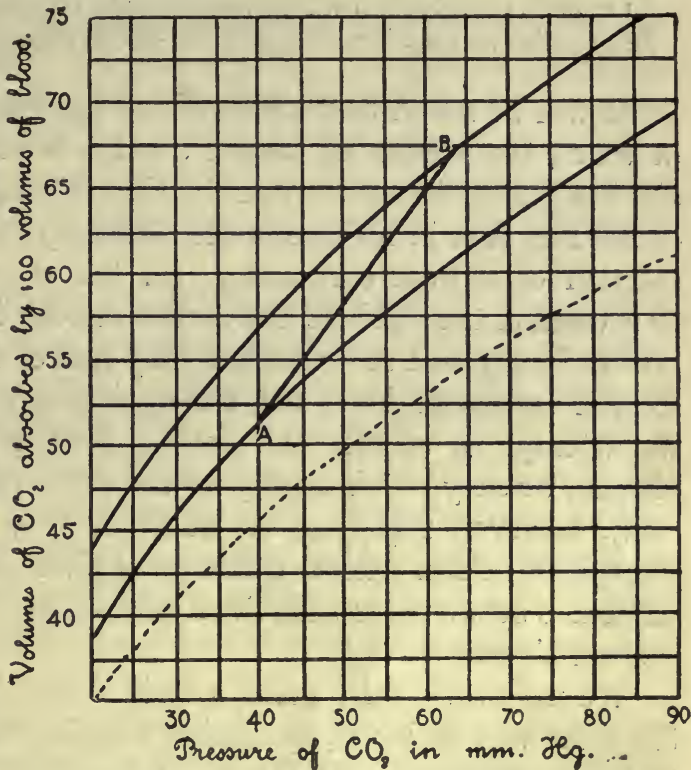

Fig. 500. Curve of $\mathrm{CO}_{2}$ tension in blood. (Christiansen, Douglas and Hatdane.)

This curve shows the influence of the saturation of the hæmoglobin with oxygen, on the amount of $\mathrm{CO}_{2}$ taken up by the blood at various pressures. Upper curve $=$ absorption of $\mathrm{CO}_{2}$ by human blood in presence of hydrogen and $\mathrm{CO}_{2}$. Middle curve $=$ absorption of $\mathrm{CO}_{2}$ by human blood in presence of air and $\mathrm{CO}_{2}$. Lower curve $=$ absorption of $\mathrm{CO}_{2}$ in blood of ox and dog in presence of air; the thick line $\mathrm{A}-\mathrm{B}$ represents the absorption of $\mathrm{CO}_{2}$ by human blood within the body (supposing the blood is completely deoxygenated in the tissues). "It is evident that an increase of 15 c.c. per cent. of $\mathrm{CO}_{2}$

- in the blood as it passes through the tissues would raise, the tension of this gas in the blood only $22 \mathrm{~mm} \mathrm{Hg}$ (from 40 to 62 ). Under normal conditions the rise of $\mathrm{CO}_{2}$ pressure in the blood on passing through the tissues is not more than 5-7 mm. Hg.

phorus present in organic combination in the plasma. We may therefore conclude that a considerable part of the carbon dioxide exists in the plasma as sodium carbonate or sodium bicarbonate. In the same way a certain proportion of the carbon dioxide which is held by the corpuscles is probably in combination, with sodium. Hæmoglobin also has the power of combining with carbon dioxide, and unstable compounds may be formed between carbon dioxide and the proteins of the plasma itself. According to Loewy 
one hundred cubic centimetres of arterial blood from the dog would yield normally about 40 c.c. of carbon dioxide. These 40 c.c. would be divided as follows :

In simple solution in the plasma and corpuscles .

As sodium bicarbonate $\left\{\begin{array}{l}(a) \text { in corpuscles . } \\ (b) \text { in plasma }\end{array}\right.$.

In organic combination with hæmoglobin in corpuscles

In organic combination with proteins of the plasma .

$1 \cdot 9$ c.c.

It will be noted that although we are dealing here with arterial blood, in which the tension of carbon dioxide is comparatively low, the carbon dioxide is stated to be in combination as bicarbonate. This is on account of the fact that in no case is the alkalinity of the blood equal to that of a solution of sodium carbonate. On the other hand, if we expose blood to a vacuum the whole of the carbon dioxide is given off. If sodium bicarbonate be exposed to a vacuum, only half of the carbon dioxide is evolved, sodium carbonate itself not undergoing any decomposition in vacuo. If we attempt to extract the carbon dioxide from blood-serum or bloodplasma, we may obtain nearly all the carbon dioxide present, the last 5 per cent., however, requiring the addition of a weak acid such as oxalic or phosphoric acid in order that it may be given off. How are we to explain the difference between the behaviour of blood and the behaviour of a solution of sodium bicarbonate?

We may artificially make a fluid which behaves to carbon dioxide in the same way as blood, by mixing together sodium carbonate and sodium hydrogen phosphate $\mathrm{Na}_{2} \mathrm{HPO}_{4}$. From such a mixture the whole of the carbon dioxide may be given off when exposed to a vacuum. On the other hand, a large amount of carbon dioxide will be taken up with a very small difference in tension of the gases. The behaviour of the mixture is due to an interaction which occurs between the acid radicals $\mathrm{PO}_{4}$ and $\mathrm{CO}_{3}$. When the mixture is exposed to a vacuum any sodium bicarbonate present will undergo dissociation, carbon dioxide being given off and the carbonate $\mathrm{Na}_{2} \mathrm{CO}_{3}$ formed. This then reacts with the sodium phosphate in the following way:

$$
2 \mathrm{NaH}_{2} \mathrm{PO}_{4}+\mathrm{Na}_{2} \mathrm{CO}_{3}=2 \mathrm{Na}_{2} \mathrm{HPO}_{4}+\mathrm{CO}_{2}+\mathrm{H}_{2} \mathrm{O} \text {. }
$$

In this way the whole of the sodium enters into combination with the $\mathrm{PO}_{4}$ and the carbon dioxide previously combined is given off. On exposing the mixture to an atmosphere containing carbon dioxide, the reverse change takes place, and we get once again sodium hydrogen phosphate and sodium carbonate, and finally sodium bicarbonate. It was formerly thought that in the blood-plasma phosphates were an important factor in the evolution of the carbon dioxide. Blood-plasma, however, contains the merest trace of phosphates, and the rôle of a weak acid competing with the carbon dioxide for the sodium is played chiefly by the proteins of the plasma. We can in fact, by adding proteins to a solution of sodium carbonate and exposing the mixture to a vacuum, obtain the evolution of practically all the carbon 
dioxide previously in combination with the sodium. In the corpuscles both hæmoglobin and proteins play the part of a weak acid. When plasma is exposed to a vacuum it is necessary, as we have seen, to add a little acid in order to obtain the last traces of carbon dioxide from the fluid. Instead of adding a weak acid, hæmoglobin or red blood-corpuscles may be employed. In the latter case it seems probable that the action on the carbonates of the plasma is due not so much to the hæmoglobin as to an interchange of acid radicals between the corpuscles and the plasma. If carbon dioxide be passed into defibrinated blood the alkalinity of the plasma increases while the chlorides diminish, and it is probably the reverse interchange of radicals between corpuscles and plasma which is responsible for the evolution of carbon dioxide on the addition of corpuscles to plasma in vacuo.

THE ALKALINITY OF BLOOD. Blood-plasma is generally described as slightly alkaline, and its alkalinity is measured in terms of deci- or centinormal acid. The term alkalinity is relative. Caustic alkali owes its alkalinity to the presence of $\mathrm{OH}$ ions. The neutrality of distilled water is due to the presence of practically equal amounts of $\mathrm{H}$ and $\mathrm{OH}$ ions in the fluid. We may measure the concentration of $\mathrm{H}$ or $\mathrm{OH}$ ions in a fluid electrically.* If this electrical method be applied to blood or blood-plasma it reveals either of these fluids as practically neutral, i.e. there is little or no greater concentration of $\mathrm{H}$ or $\mathrm{OH}$ ions in blood than in distilled water. The

* By the use of different indicators we may arrive at some conclusion as to the approximate concentration of hydrogen ions in any given liquid. In the following Table are set out, from a paper by Roaf, the colours of a number of different indicators, and the degree of acidity which is sufficient to change their colours :

\begin{tabular}{|c|c|c|c|c|}
\hline Indicator & Acid colour & $\begin{array}{l}\text { Transitional } \\
\text { colour }\end{array}$ & Alkaline colour & $\begin{array}{l}\text { Hydrogen ion concentra- } \\
\text { tion at which colour } \\
\text { change begins }\end{array}$ \\
\hline $\begin{array}{c}\text { Dimethylamido- } \\
\text { azobenzol }\end{array}$ & Red & Orange & Yellow & $1 \times 10^{-3}$ \\
\hline Congo red . & Blue & $\begin{array}{l}\text { Purple and } \\
\text { brown }\end{array}$ & Red & $1 \times 10^{-4}$ \\
\hline $\begin{array}{l}\text { Vesuvin brown. } \\
\text { Gallein . }\end{array}$ & $\begin{array}{c}\text { Brown } \\
\text { Colourless }\end{array}$ & Pink & $\begin{array}{l}\text { Yellow } \\
\text { Red }\end{array}$ & $\begin{array}{l}1 \times 10^{-4} \\
1 \times 10^{-4}\end{array}$ \\
\hline $\begin{array}{l}\text { Na alizarine } \\
\text { sulphonate }\end{array}$ & Yellow & Orange & Red & $1 \times 10^{-4}$ \\
\hline Lacmoid : & Red & Purple & $\begin{array}{l}\text { Blue } \\
\text { Red }\end{array}$ & $\begin{array}{c}1 \times 10^{-4}-1 \times 10^{-8} \\
1 \times 10^{-5}\end{array}$ \\
\hline $\begin{array}{l}\text { Rosolic acid } \\
\text { Litmus . }\end{array}$ & $\begin{array}{l}\text { Yellow } \\
\text { Red }\end{array}$ & $\begin{array}{l}\text { Orange } \\
\text { Purple }\end{array}$ & $\begin{array}{l}\text { Red } \\
\text { Blue }\end{array}$ & $\begin{array}{c}1 \times 10^{-5} \\
1 \times 10^{-5}-1 \times 10^{-8}\end{array}$ \\
\hline Neutral red & Red & Orange & Yellow & $1 \times 10^{-8}$ \\
\hline Alizarine & Yellow & Orange & Red & $1 \times 10^{-8}$ \\
\hline Phenolphthalein & Colourless & Pink & Red & $1 \times 10^{-9}$ \\
\hline
\end{tabular}

It should be remembered that in distilled water of the highest state of purity the concentration of $\mathrm{H}$ and $\mathrm{OH}$ ions respectively is about $1 \times 10^{-7}$. 
reaction of blood as normally taken depends as a matter of fact on the indicator which is used. Thus blood-plasma is acid to phenolphthalein, but alkaline to litmus. On the other hand, the carbon dioxide and proteins in combination with the sodium may be easily replaced by stronger acid radicals, and if the carbon dioxide be allowed to escape, very little change in the reaction, i.e. in the concentration of $\mathrm{H}$ or $\mathrm{OH}$ ions respectively, will be produced. We may thus speak of blood-plasma as actually neutral, though potentially alkaline in that it can neutralise a considerable amount of acid. This potential alkalinity is more important than the actual alkalinity, since on it depends the power possessed by the blood of carrying carbon dioxide from the tissues to the lungs. By adding a fixed acid to the blood we may use up this potential alkalinity and finally arrive at a point at which each addition of acid makes a proportionate increase in the actual acidity, i.e. in the concentration of $\mathrm{H}$ ions. From this time forward, however, the plasma has lost its power of binding carbon dioxide and can carry this gas only in simple solution. On exposure of such plasma to carbon dioxide the tension of the gas rapidly rises in the fluid, which becomes saturated when only a relatively small amount of gas has entered into the fluid. Any diminution of the so-called alkalinity of the blood or bloodplasma will seriously impair the function of the blood in respiration. An example of such a condition is the acidosis which occurs in diabetes, or in any other form of carbohydrate starvation.

\section{EXCHANGE OF GASES IN THE LUNGS}

A fluid gives off gas to or takes up gas from any other medium with which it is in contact, according to the relative pressures of the gas. The question arises whether the physical conditions in the lungs are such as to account for the absorption of oxygen and the evolution of carbon dioxide by the blood in its passage through these organs. In order to answer this question we must know the partial pressures or tensions of oxygen and of carbon dioxide in the alveolar air, in the venous blood coming to the lungs, and in the arterial blood leaving the lungs. In the alveoli the pressures are given by the analysis of alveolar air. The determination of the gaseous tensions in the blood presents, however, considerable difficulty. It is necessary to bring the blood in contact with gaseous mixtures containing various proportions of the gas whose tension in the blood it is desired to measure. By making various experiments a gaseous mixture will be found with which the blood is in equilibrium. If we know beforehand the amount of gas in this mixture, we know its tension and therefore the tension of the gas in the liquid.

Pflïger's aerotonometer (Fig. 501) consists of two glass tubes, $\mathrm{R}$ and $\mathrm{R}$, contained in a vessel filled with water at the temperature of the body. The upper ends of the tubes are connected by the tube $a$ with the artery or vein in which it is desired to estimate the tension of the blood-gases. If, for instance, we wish to determine the tension of $\mathrm{CO}_{2}$ in venous blood, where we expect the tension to amount to about 4 per cent. of an atmosphere, one tube $\mathrm{R}$ is filled with a gaseous mixture containing 3 per cent. $\mathrm{CO}_{2}$, and 
the other tube $\mathrm{R}$ with a mixture containing 5 per cent. $\mathrm{CO}_{2} . \quad a$ is now connected with the distal end of the jugular vein, or with the central end of the carotid artery, and blood is allowed to flow in a thin stream down the walls of the tubes $R$ and $R$, thus presenting a large surface to the contained gases. The blood collects in the lower narrower portions of the tubes, and runs out into the vessels $b, b$, whence after defibrination it is returned at intervals into the veins of the animal.

Bohr's aerotonometer was built on the plan of the Stromaiche devised by Ludwig, and could be inserted in the course either of an artery or of a vein. In using this instrument it is advisable to inject some substance like peptone or, better, hirudin, in order to prevent coagulation of the blood.

In all these instruments the main difficulty is in obtaining a sufficient surface of the blood exposed to the gaseous mixture. The interchange of gases is thus very slow, and it is difficult to be certain at any time that the blood and the gas with which it is in contact are really in equilibrium. "Krogh therefore adopted an ingenious device of limiting the volume of air to a small bubble, the superficial area of which is large in proportion to its bulk. This bubble, after it has been in a stream of blood for some minutes, is transferred to-a special

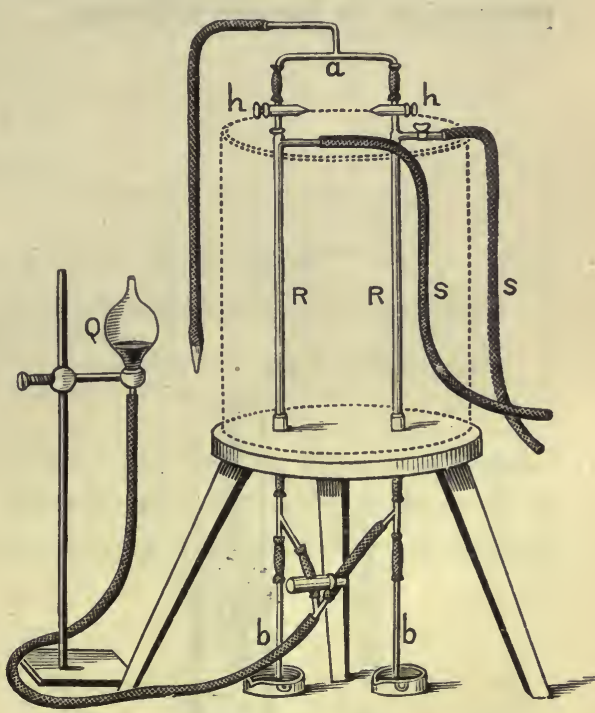

FiG. 501. Pflüger's aerotonometer. capillary tube in which its analysis can be carried out with a fair.degree of accuracy.

The performance of a tonometer may be expressed by the ratio of the surface of blood exposed to the volume of the air used. The 'srecific surface' of an aerotonometer

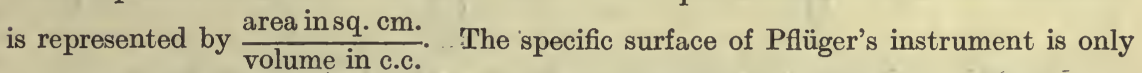
$3 \cdot 3$ and of Bohr's only $5 \cdot 2$. In Krogh's microtonometer the absolute volume of air employed is reduced to a bubble of about $2 \mathrm{~mm}$. in diameter, having a volume of .004 c.c. and a surface of $0.125 \mathrm{sq}$. cm., so that its specific surface is 30 . In such a bubble the equalisation of the tensions takes place with extreme rapidity and only a minute quantity of fluid is necessary. The microtonometer consists of the tonometer proper and the apparatus for the microanalysis of the gas bubble. In the latter the measurement of the gas bubble is carried out in a capillary tube, the absorption of carbon dioxide and of oxygen being carried out in the usual way with potash and with pyrogallic acid. The tonometer is represented in Fig. 502. It is kept in a small water-bath at the temperature of the blood to be examined. The tonometer is filled with saline solution and contains the gas bubble 2 , which can be drawn up by means of the screw 4 into the narrow graduated tube 3 , where its volume is measured. The blood from the artery or vein, in which we wish to examine the tension of the gases, passes by a cannula through the tube 1, and enters the tonometer as a fine jet. It forces its way up above the gas bubble, which is pressed a little down by the current, and kept oscillating with great rapidity. From the tonometer the blood flows back through the tube 7 and is collected in a vessel where it can be measured and afterwards drawn off and reinjected into the 
animal if necessary. Since the total pressure of the gases in the blood is nearly always negative, it is necessary to keep the pressure in the tonometer also negative.* This is accomplished by means of a mercury valve and can be regulated to any desired pressure.

During the course of a tonometric experiment the volume of the gas bubble is measured from time to time by drawing it up into the graduated tube, and the pressure is regulated until the volume of the bubble remains constant. After five minutes gaseous equilibrium will have been established between the gas bubble and the surrounding blood, and it is only necessary then to draw it up into the graduated tube and analyse it in order to determine the tension of the gases in the blood. Clotting of the blood is prevented by the injection of hirudin.

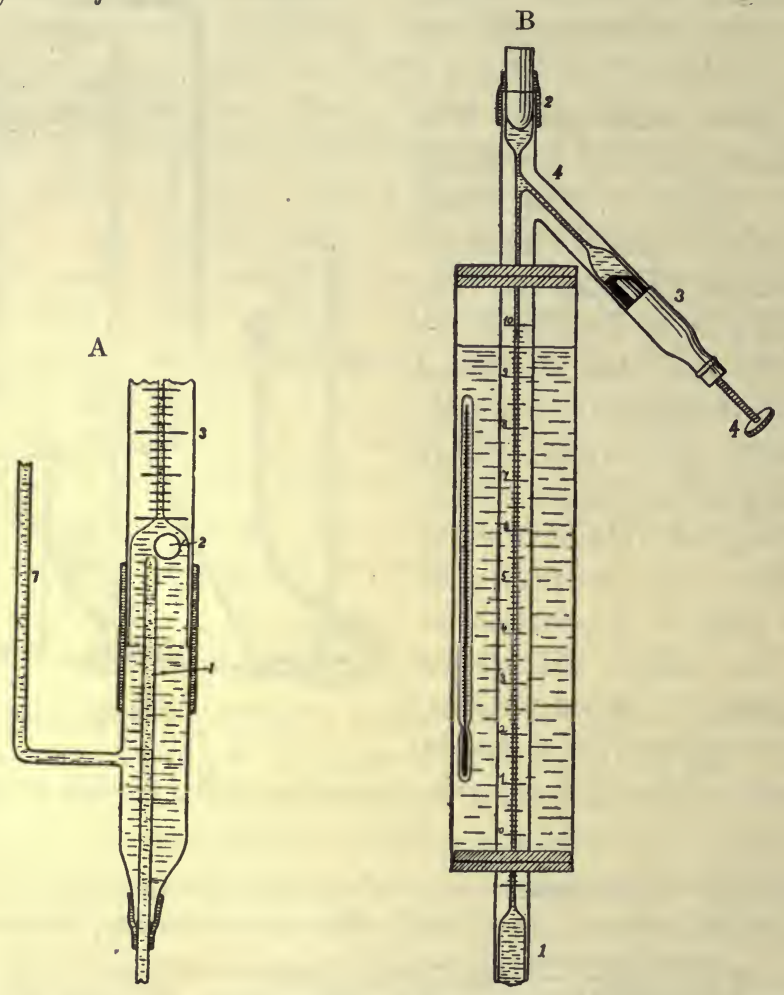

FIG. 502. A, Krogh's microtonometer. B, upper part of microtonometer showing capillary tube into which the bubble is returned for measurement and analysis.

In the experiments the tension of the air in the alveoli of the animal's lungs or in the bifurcation of the trachea was determined by taking samples of the air. The results of the experiments show that the tension of the gases in arterial blood follows closely the tension of the corresponding gases in the alveolar air. The tension of carbon dioxide in arterial blood is either identical with or very slightly above the tension of the gas in the alveolar air. The oxygen tension of the blood is always lower than the alveolar oxygen tension, and the difference is generally 1 to 2 - even 3 to 4 -per cent. of an atmosphere. The results of a series of determinations of the tensions of the gases in the blood and alveolar air respectively are given in Fig. 503. In Fig: $504 \mathrm{~A}$ and $\mathrm{B}$ (Krogh) the composition of the alveolar air was artificially

* Otherwise the whole bubble would gradually go into solution and disappear. 
altered by increasing the percentage of carbon dioxide and of oxygen respectively. It will be seen in each case that there was a corresponding alteration of the tension in the arterial blood, the tension of carbon dioxide

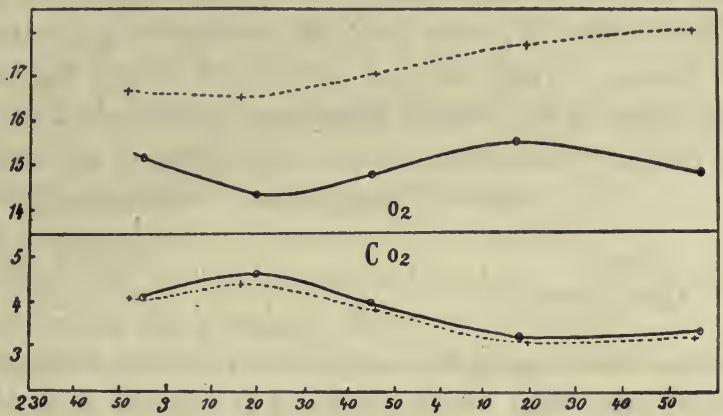

Fig. 503. Tensions of $\mathrm{O}_{2}$ and $\mathrm{CO}_{2}$ in alveoli compared with those in arterial blood of rabbit.

The dotted lines represent the tensions in the alveolar air, the uninterrupted lines the tensions of the gases in the arterial blood. (KROGH.)

being higher and that of oxygen lower in the blood than in the air throughout the experiment. We have no direct determinations of the tensions of the gases in the blood of man, though an approximate valuation of these tensions

A

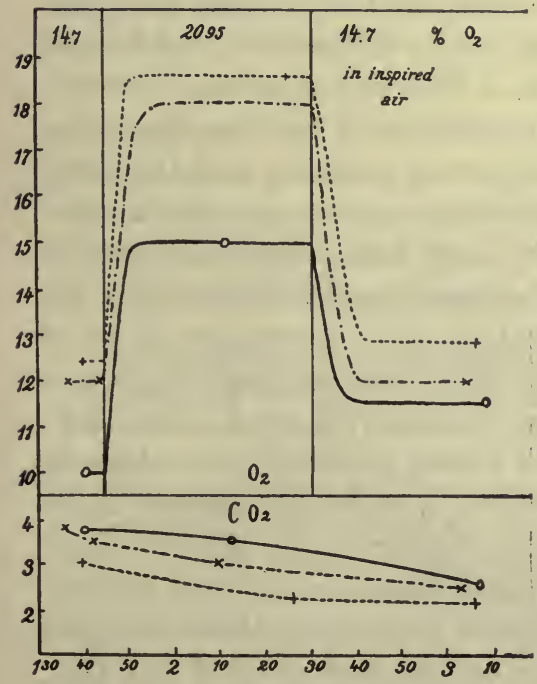

B

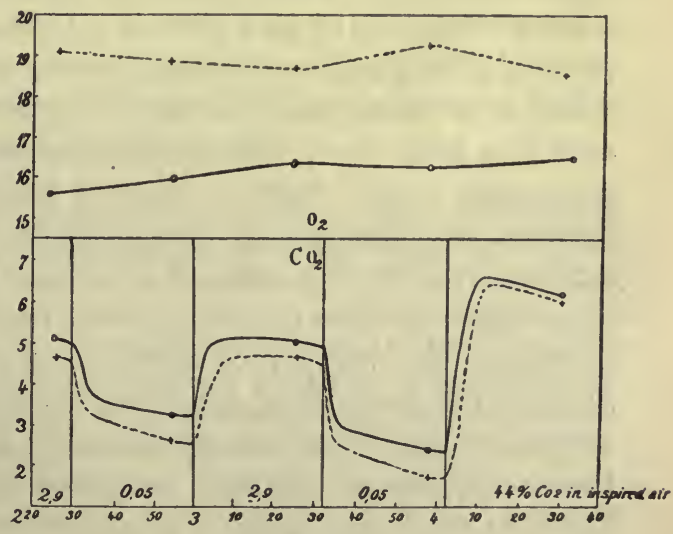

FIG. 504. Tensions of gases in alveolar air and in arterial blood.

A, during artificial increase of oxygen tension in alveoli ; $\mathbf{B}$, during artificial increase of $\mathrm{CO}_{2}$ tension in alveoli.

can be obtained by knowing the degree to which the arterial and venous blood respectively are saturated with oxygen or carbon dioxide. An indirect method may be employed to measure the gaseous tensions in the venous blood coming to the lungs. It is possible, as Loewy has shown, to block the 
right bronchus in man by introducing a catheter through the larynx and trachea, so that the renewal of air in the right half of the lung is entirely stopped for some time. A sample of air in the blocked lung can be taken at any time by means of the catheter. The interchange of gases between alveolar air and blood will go on until the tension of gases in the air is the same as that coming to the blocked portion of lung. By this means the tension of the oxygen in the venous blood was found to be $5 \cdot 3$ per cent. $=37$ $\mathrm{mm} . \mathrm{Hg}$., and that of the carbon dioxide 6 per cent. $=46 \mathrm{~mm}$. $\mathrm{Hg}$.

The tensions in the alveolar air of man may be taken as follows :

Oxygen . . . . . $107 \mathrm{~mm}$. Hg.

Carbon dioxide. . . . . 40 "

As the venous blood enters the lungs there is thus a difference of pressure of $107-37=70 \mathrm{~mm}$. $\mathrm{Hg}$., which will tend to cause a flow of oxygen from alveolar air to blood and a difference of $46-40=6 \mathrm{~mm}$. Hg., tending to cause a flow of carbon dioxide from blood to alveolar air. Is this difference sufficient to account for the amount of gas given off or taken up by the blood in its passage through the lungs? In a state of medium distension the 3000 c.c. of air contained by the lungs have been estimated to occupy seven hundred million alveoli, each of which has a diameter of $0.2 \mathrm{~mm}$., so that the total surface over which the blood is exposed to the alveolar air amounts to 90 square metres. This is a minimal figure, since no account is taken in the calculation of the augmentation of surface caused by the fact that the capillaries project into the lumen of the alveolus, and by Hüfner the total surface exposed is calculated at 140 square metres. The former figure, however, amounts to about 1000 square feet and is equivalent to the floor-space of a room 50 feet long by 20 feet wide. It is important to realise that the blood passing through the pulmonary artery suddenly spreads out into a layer which is not more than one blood-corpuscle thick, and is exposed to the air over this huge area, whence it is picked up again and collected into the pulmonary veins. Such a means of facilitating rapid interchange of gases between the blood and a given volume of air we cannot possibly imitate artificially. The thickness of the tissue separating this layer of air from the alveolar air is on the average $.004 \mathrm{~mm}$. Loewy and Zuntz have directly calculated the velocity of diffusion of carbon dioxide and nitrous oxide through the frog's lung and have calculated therefrom the rate at which oxygen would diffuse through a similar layer of tissue, taking into account the much greater solubility of carbon dioxide as compared ${ }^{-}$with oxygen. They calculate that under a constant difference of pressure of $35 \mathrm{~mm}$. $\mathrm{Hg}$., 6.7 c.c. of oxygen would pass in a minute through each square centimetre of the alveolar wall. Through the whole surface of the lung this would amount to an absorption of 6083 c.c. oxygen. The oxygen actually absorbed by a man-at rest amounts to about 300 c.c. per minute, so that the physical conditions allow an ample margin for any increase in the consumption of oxygen; in fact, a difference of pressure of a couple of millimetres would suffice to cause a passage of the 250 c.c. per minute which is required by the 
resting man. In the same way it is easy to account for the passage of carbon dioxide in the reverse direction. This gas diffuses through a wet membrane about twenty-five times as rapidly as oxygen, so that a difference of pressure between the blood and the alveolar air amounting to only $.03 \mathrm{~mm}$. $\mathrm{Hg}$. would suffice to cause a passage outwards of the 250 c.c. normally expired per minute.

It is evident that the only limitation for the absorption of oxygen is given by the power of the hæmoglobin to combine with the oxygen which passes through the alveolar wall into the blood-plasma.

If we look at the dissociation curve of the oxyhæmoglobin in mammalian blood given on p. 1061 we see that the amount of oxygen which can be taken up by hæmoglobin in the presence of the normal tension of carbon dioxide, i.e. $40 \mathrm{~mm}$. Hg., begins to diminish very rapidly when the pressure of the oxygen falls below $50 \mathrm{~mm}$. Hg. Thus at $40 \mathrm{~mm}$. oxygen pressure and a carbon dioxide tension of $40 \mathrm{~mm}$., oxyhæmoglobin is about 65 per cent. saturated, and at $30 \mathrm{~mm}$. it is only 50 per cent. saturated. Under normal circumstances the blood leaves the lungs over 90 per cent. saturated with oxygen. If the saturation falls to 60 per cent. we should expect to obtain evidence of failure of oxygen supply to the tissues. According to Loewy the oxygen tension in the alveoli can sink to between 30 and $35 \mathrm{~mm} . \mathrm{Hg}$. before any signs of oxygen lack make their appearance. These results were obtained by exposing a man in a state of complete rest to reduced pressure in an air-chamber. Under these conditions the slightest muscular exertion would at once tend to cause distress from deficient oxygen supply. The exact percentage of oxygen in the inspired air which would give an alveolar oxygen tension of 30 to $35 \mathrm{~mm}$. varies with the depth of respiration. Thus with shallow respiratory movements the pressure may sink to $35 \mathrm{~mm}$. $\mathrm{Hg}$, when the inspired air contains as much as 12 per cent. oxygen. If the movements be deeper the oxygen content of inspired air may be reduced to 9 or 10 per cent. before respiratory distress is observed.

The view that in the interchange of gases in the lungs the membrane between the blood and the alveolar air plays simply a passive part was till recently by no means unitersally aecepted. In Bohr's experiments on the tension of oxygen and carbon dioxide in the blood as determined with his aerotonometer, oxygen tensions were often found considerably higher in the blood than in the air of the alveoli, and in the same way the carbon dioxide tension of the blood leaving the lungs was found to be less than the carbon dioxide tension of the alveolar air. Krogh's experiments show conclusively, however, that these results are not reliable, and that the difference between the tensions in the alveoli and in the blood respectively is always such as to allow of the passage by diffusion of oxygen inwards and carbon dioxide outwards from the blood. Moreover, as Krogh points out, the structure of the pulmonary epithelium lends no support to the view that it acts as a secreting membrane. In mammals the cells are of two kinds, viz. small granular nucleated cells lying in the interstices of the capillaries, and larger, extremely thin structureless plates, without nuclei, covering the capillaries. In birds, where the gaseous exchange is of all animals the most rapid and efficient, the existence of a lung epithelium has never been demonstrated, and the capillaries appear to be almost completely free and to be surrounded with air on both sides.

Bohr's view as to the secretory function of the pulmonary epithelium was supported as concerns the intake of oxygen, by Haldane. This observer has devised a method of 
determining the oxygen tension of the blood in the lungs founded on the use of carbon monoxide. It has already been mentioned that carbon monoxide has the power of displacing oxygen from oxyhæmoglobin to form a much more stable compound, carboxyhæmoglobin. If blood be shaken up with a mixture of oxygen and carbon monoxide, the hæmoglobin distributes itself between the two gases. In order, however, to get an equal distribution, it is necessary to take a very small percentage of carbon monoxide, owing to its greater avidity for hæmoglobin. Thus, if hæmoglobin solution be shaken up with air containing 07 per cent. of $\mathrm{CO}$, the result is a mixture of equal parts of oxyand carboxyhæmoglobin. The affinity of $\mathrm{CO}$ for hæmoglobin would thus appear to be about $\frac{21}{\cdot 07}=300$ times the affinity of oxygen for hæmoglobin.

Carbon monoxide is not destroyed in the body, so that if a mixture containing a small proportion of $\mathrm{CO}$ be breathed, this gas will be taken up until a certain percentage of hæmoglobin is converted into $\mathrm{CO}$-hæmoglobin and the tension of $\mathrm{CO}$ in the tissues and fluids of the body is equal to that of the inspired air. The amount of hæmoglobin which is converted into carboxyhæmoglobin will serve as a measure of the relative tensions of $\mathrm{CO}$ and oxygen in the lungs. If the oxygen tension of arterial blood were the same as that of the alveolar air, we should expect that, with a given percentage of $\mathrm{CO}$ in the air breathed, the final saturation with $\mathrm{CO}$ of the blood within the body would be the same as the saturation of blood when shaken outside the body with air containing the same percentage of $\mathrm{CO}$ as in the air breathed. It was found by Haldane, however, that in all cases the percentage of $\mathrm{CO}$ hæmoglobin formed was much less in the body than outside the body. Thus in blood shaken up with air containing 20.9 per cent. oxygen and .045 per cent. $\mathrm{CO}$, the amount of carbon monoxide formed was 31 per cent. of the whole hæmoglobin. When the same mixture was inhaled for three or four hours by a man, the percentage of $\mathrm{CO}$ hæmoglobin in his blood rose only to 26 per cent., at which figure it remained stationary. This would correspond to an oxygen tension of about 25 per cent. of an atmosphere, whereas we have already seen that the oxygen tension in the alveoli cannot be greater than 15 per cent. He therefore concluded that the epithelial cells of the alveoli play an active part in the respiratory interchange, taking up the oxygen on one side at a tension of 15 per cent. and piling it up on the other until the pressure in the blood is much higher than that in the alveolar air. Theoretically there is no reason to deny the possibility of such powers to the pulmonary epithelium. We know that the secreting cells of the kidney take up urea from the blood which contains only about 02 per cent. of this substance, and excrete it into the renal tubule, into a medium containing about 2 per cent. ; and if the data given by Haldane are correct we must ascribe an analogous function to the pulmonary epithelium. These data, however, were obtained by a colorimetric method working with very minute quantities of blood, and lacked the support of control experiments. As a result of further experiments, Haldane has modified his position so far as to allow that under normal conditions the absorption of oxygen from the alveolar air takes place in accordance with the difference of pressure, i.e. by a process of diffusion. He is still of opinion that under abnormal conditions, when the oxygen tension in the alveolar air is very low, there is an active absorption and transference of oxygen to the blood on the part of the pulmonary epithelium. Why animals should evolve a function which can only be brought into play on climbing mountains seems difficult to understand, and it does not seem probable that a reinvestigation of the tensions of oxygen in the blood under such conditions by Krogh's method will lend any confirmation to Haldane's conclusions.

An analogy has been drawn between the processes of gas interchange in the lungs and that in the swim bladder of the fish. Bohr has shown that the gas obtained by puncturing the bladder often contains considerable excess of oxygen. If the bladder be punctured and the fish then left in the water, the gas rapidly reaccumulates, and it is found, on tapping a second time, that the percentage of oxygen is largely increased, and may amount to between 60 and 80 per cent. of the total gases. This reaccumulation of the gases does not take place if both vagi are cut, and is therefore ascribed to a direct secretory activity on the part of the epithelium lining the swim bladder under the influence $o$ the vagus nerves. Bohr, as the result of experiments by himself and some 


\section{THE CHEMISTRY OF RESPIRATION}

of his pupils, is inclined to endow the vagus nerves in the higher vertebrates, including mammals, with an analogous regulatory influence on the gaseous exchanges in the lungs. As regards the evolution of carbon dioxide the facts elucidated by Haldane himself would make one hesitate in ascribing any special secretory activity to the pulmonary epithelium. We find, namely, that the respiratory centre reacts immediately to the slightest increase in the tension of the carbon dioxide in the alveolar air. Since this behaviour of the respiratory centre is independent of any nervous connections between the lungs and the brain, it seems to indicate, as indeed Krogh has found, that the tension of the carbon dioxide in the blood follows closely the tension of the carbon dioxide in the alveolar air. If the carbon dioxide were secreted by the pulmonary epithelium we should expect the lungs to react to increased carbon dioxide in the alveoli by simply increasing their work so as to maintain the tension of carbon dioxide in the blood at a constant level. This at any rate is the way in which the kidney would behave under ana'ogous circumstances. Moreover there is no likeness between the thick typical secreting cells of the 'red gland,' which is the gas-secreting part of the swim bladder, and the thin structureless plates which separate the capillaries of the lungs from the alveolar air. 


\section{SECTION III}

\section{THE REGULATION OF THE RESPIRATORY MOVEMENTS}

EACH movement of inspiration involves the co-ordinated activity of a large number of muscles. Thus the diaphragm and the intercostal muscles must come into action at the same time, and the extent to which they contract will determine the depth of the inspiration. Similarly, they must cease to act simultaneously if the act of expiration is to take place. The rhythm and extent of the alternate contractions and relaxations of the respiratory muscles are determined, as we have seen, by the needs of the organism as a whole. These respiratory movements are regulated so that the total ventilation of the alveoli shall be sufficient to meet the gaseous exchanges of the body. Whether the organism consumes 250 or 1000 c.c. of oxygen per minute, the respiratory movements keep the composition of the gas in the alveoli at a practically constant level.

The muscles involved both in inspiration and expiration can only be thrown into activity by the intermediation of nerves. Each act of inspiration involves a discharge along a number of nerves, e.g. the facial to the muscles moving the alæ nasi, the vagus to the muscles of the larynx, the branches of the cervical and brachial nerves to the muscles of the neck, the phrenic nerves to the diaphragm, and the dorsal nerves to the intercostal muscles. The fibres making up these nerves are derived from nerve-cells of the anterior horn, situated at various levels in the medulla and spinal cord. In each act of inspiration or expiration the activities of all these groups of cells must be brought into relation among themselves, as well as with the needs of the organism for oxygen and for the elimination of carbon dioxide. It is conceivable that the co-ordination of the activities of the various motor nuclei might be attained by the provision of communicating nerve-paths joining the centres among themselves, and by a sensibility of all these centres to the gaseous contents of the blood as well as to the influence of afferent impressions from the periphery. A much more efficient co-ordination, however, would be effected by the subjection of these motor nuclei to the action of some specialised portion of the central nervous system which would act as a receiving centre for afferent impressions from the lungs and surface of the body, and would be endowed with a special sensibility to changes in the composition of the blood circulating through its vessels. Experiment shows that the latter method is employed in the organism for 
the regulation of the respiratory movements. If the spinal cord be cut across, below the seventh cervical nerve-roots, the action of the intercostal and abdominal muscles in respiration ceases permanently, although respiration is still continued by the rhythmic activity of the diaphragm and the other muscles supplied by nerves leaving the central nervous system above the point of section. Division of the cord at the first or second cervical nerve abolishes the action of the diaphragm, though the movements of the muscles supplied by the facial, vagus, and spinal accessory nerves continue. A section of the brain-stem through the mid-brain leaves the respiratory movements unaltered, and the same absence of effect as concerns these movements may often be obtained when a section is carried across the upper part of the medulla about the level of the strice acousticae. We must conclude from these experiments that the motor nuclei of the cord are subject to and normally thrown into activity by impulses originating in the medulla. oblongata and transmitted therefrom down the spinal cord.

Many experiments have been made with the idea of locating the position of the medullary respiratory centre more accurately. The first experiments on this point were made at the beginning of last century by Legallois, whose observations were confirmed and extended by Flourens. These observers described the respiratory centre as limited to a small area at the level of the apex of the calamus scriptorius, which they designated noud vital on account of the fact that destruction of this area was at once fatal by paralysis of respiration. Later experiments have shown that the centre is not quite so circumscribed. In the first place, it is bilateral, each centre presiding more especially over the muscles of the same side of the body, so that longitudinal section in the middle line does not destroy the respiratory movements. Other observers have located the centre in the situation of the solitary bundle ('respiratory bundle of Gierke '), which is made up of the descending branches of the vagus nerve after they have entered the medulla, while, according to Gad, the respiratory centre is diffused over a considerable area of the formatio reticularis on either side of the medulla. There is no doubt that this centre is in close connection with the central terminations of the vagus nerves.

From the centre on each side the efferent impulses to the motor nuclei of the respiratory muscles pass down in the deeper portions of the lateral columns of the cord. Hemisection of the cervical cord, e.g. on the right side, causes cessation of the contractions of the diaphragm on the same side. There must, however, be commissural fibres joining the motor nuclei on the two sides. If the right phrenic nerve be divided, after hemisection on the left side, the left half of the diaphragm at once commences to contract rhythmically with each respiraton (Porter). It is evident that the cessation of respiration after section of the cord is not due to a condition of shock of the lower spinal centres, since it is possible for impulses to pass down the cord and to cross over to the contra-lateral diaphragm nucleus immediately after hemisection of the cord on the side of the nucleus. 
THE QUESTION OF SPINAL RESPIRATORY CENTRES. Several physiologists e.g. Brown Séquard, Langendorff, and Wertheimer, have described respiratory centres in the spinal cord. There is no doubt that if the cord be cut across in the upper cervical region, and artificial respiration maintained for some time, cessation of the respiration may be followed by rhythmic contractions of the respiratory muscles. These are especially marked in young animals and if the activity of the cord has been heightened by the injection of small doses of strychnine. Careful observation of the movements shows, however, that they cannot be spoken of as respiratory, since although rhythmic, they are not co-ordinate. The diaphragm may contract either simultaneously or in alternation with the intercostals, and muscles which are essentially expiratory at the same time as those which we are wont to regard as inspiratory. These experiments show merely that the motor centres of the cord can enter into rhythmic activity under the influence of asphyxial conditions. The movements affect the muscles of the limbs as well as those essentially respiratory in function.

\section{THE AUTOMATICITY OF THE RESPIRATORY CENTRE}

We have now to inquire what it is that keeps the respiratory centre in activity. Is the rhythmic discharge of inspiratory impulses from the centre due to rhythmic or continuous stimulation of afferent nerves, or is the centre so constructed that under the normal conditions of its environment the metabolic activity of its constituent parts tends, like that of the heart-cells, to assume a rhythmic character? In other words, is the activity of the centre reflex or automatic? It has been found by Rosenthal that rhythmic respiratory movements are maintained even after complete section of the brainstem at the level of the superior corpora quadrigemina, section of the cord at the level of the seventh cervical nerve, and division of both vagi and of the posterior roots of all the cervical spinal nerves. It is true that if the sections of the brain-stem be placed as low as the strice acousticce, the respiratory movements are profoundly modified and give place to a series of inspiratory spasms. We might argue from this that the centre was capable of a very imperfect degree of automatic action, but needed the stimulus of afferent impulses from the vagi or from the higher parts of the brain to render these actions adequate for the respiratory needs of the organism.

In the above experiment the centre cannot be regarded as free from all afferent stimuli, since the mere closure of the demarcation current in the cut ends of the nerves would cause a certain amount of excitation, and the animal does not survive sufficiently long to allow this condition to pass off. Hering has shown that in the 'spinal cord frog' (i.e. one in which the brain has been destroyed) section of all the posterior roots absolutely destroys all mobility, the injection of strychnine being without effect. A typical spasm, however, can be at once produced by exposing and stimulating the stump of one of the cut posterior roots. We might suppose that the respiratory centre would be similarly devoid of automatism if absolutely free from afferent stimuli. It must be mentioned, however, that according to Sherrington it is possible to excite strychnine or asphyxial spasms in a dog or cat with isolated spinal cord, in which all the afferent roots below the transection have been divided six or seven hours previously. He therefore is of opinion that in the mammal the motor nervous mechanism can be set into activity 
apart from the incidence of afferent impressions. The respiratory centre tends to respond to all stimuli, continuous or rhythmic, by means of rhythmic discharges, and there can be no doubt that if we take the medulla in connection with the rest of the hind-and mid.brain we are justified in regarding its activity as automatic.

The automatic activity of the heart is intimately dependent on the saline constituents of the blood. It may be abolished or diminished by modifying these constituents, and can be maintained for a considerable length of time by perfusing the heart with solutions containing inorganic salts in the concentration in which they exist in the blood-plasma. When we speak of the automatic activity of the respiratory centre we imply in the same way that its activity is dependent on the normal composition of the blood circulating through its vessels. In this case, however, it is the gaseous contents of the blood which are of supreme importance. If the normal ventilation of the lungs be prevented, as by ligature of the trachea or opening both pleural cavities, the blood becomes more and more venous. As this venous blood circulates through the medulla the activity of the centre is continually increased, until finally the impulses discharged from the centre may set into activity practically every muscle of the body, producing asphyxial convulsions. On the other hand, the activity of the respiratory centre can be diminished or even abolished if, by an artificial ventilation of the alveoli, we maintain an over-arterialisation of the blood, so that the fluid passing to the brain contains more oxygen and less carbon dioxide than is the case under normal circumstances. What are the factors involved in this chemical regulation of respiration?

\section{THE CHEMICAL REGULATION OF THE RESPIRATORY MOVEMENTS}

If, the nervous centres being intact, the proper aeration of the respiratory centre be interfered with in any way, the respiratory movements increase in strength and frequency, and if the disturbing factor be not removed the animal dies, presenting a train of phenomena which are classified together under the term 'asphyxia.'

The phenomena of asphyxia may be dividea into three stages :

(1) In the first stage, that of hyperpnoea, the respiratory movements are increased in amplitude and in rhythm. This increase affects at first both inspiratory and expiratory muscles. Gradually the force of the expiratory movements becomes increased out of all proportion to the inspiratory, and the first stage merges into :

(2) The second, which consists of expiratory convulsions, in which almost every muscle of the body may be involved. Just at the end of the first stage consciousness is lost and almost immediately after the loss of consciousness we may observe a number of phenomena extending to almost all the functions of the body, some of which have been already studied. Thus the vaso-motor centre is excited, causing universal vascular constriction. 
There is often also secretion of saliva, inhibition or increase of intestinal movements, constriction of the pupil, and so on.

(3) At the end of the second minute after the stoppage of the aeration of the blood, the expiratory convulsions cease almost suddenly, and give way to slow deep inspirations. With each inspiratory spasm the animal stretches itself out and opens its mouth widely as if gasping for breath. The whole stage is one of exhaustion: the pupils dilate widely, and all reflexes are abolished. The pauses between the inspirations become longer and longer, until at the end of four or five minutes the animal takes its last breath.

If we increase the activity of the centre, and therefore its gaseous interchanges, by warming the blood in the carotid arteries, there may be a considerable quickening of respiration unaccompanied by any deepening, a condition which is spoken of as tachypnoea. On the other hand, we may slow the respiratory movements by placing a small piece of ice on the floor of the fourth ventricle.

In the production of the phenomena of asphyxia two factors must be at work. In the first place, there is an accumulation of carbon dioxide in the blood bathing the centre or an increased tension of this gas in the centres themselves, either as a result of deficient excretion or increased production. On the other hand, the centre is deprived of oxygen, either by failure of renewal of the oxygen supply, or by increased using up of this gas in the metabolism of the centre. The question arises, which of these two changes is responsible for the different physiological events which characterise asphyxia? At various times these phenomena have been ascribed either to the increased tension of carbon dioxide or to the diminished tension of oxygen in the centre. The view that the normal stimulus to the respiratory centre in asphyxia was the lack of sufficient oxygen and that the normal activity of this centre was determined by the tension of oxygen in the blood circulating through the brain was first put forward by Rosenthal. When sufficient oxygen was present, the centre, according to this observer, would cease to act, so that a condition of apnoea would be produced. According to Traube; on the other hand, the special respiratory stimulus was the excess of carbon dioxide in the blood, and this view was supported strongly by Miescher. The tendency of recent work, especially by Haldane and his pupils, has been to show that there is an element of truth in both views - that indeed the respiratory centre can be excited either by excess of carbon dioxide or by lack of oxygen, but that its sensitivity to carbon dioxide is by far the most important factor in the determination of the increased respiratory movements in asphyxia, and is the only chemical factor which can be regarded as playing any part in the regulation of the respiratory movements under normal conditions. This factor is well brought out if we investigate the effect on the respiratory movements of altering the tensions of the two gases in the air breathed. If by this means we succeed in altering the tension of the two gases in the alveolar air we may assume that the tensions of the gases in the arterial blood leaving the lungs are altered in the same ratio. The results of such experiments are very striking. Even 
a slight increase in the percentage of carbon dioxide in the air causes an increase first in the depth and later on in the rhythm of respiration (Fig. 505). This is shown in the following Table by Haldane, which represents the average depth and frequency of the respirations when the subject was breathing normal air and air charged with varying percentages of carbon dioxide.

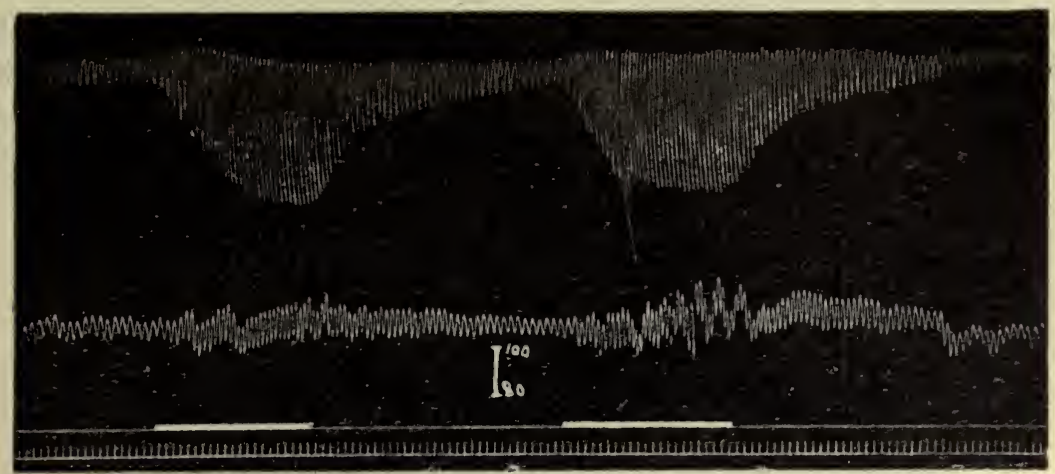

Fig. 505. Effect of $\mathrm{CO}_{2}$ on respiratory movements of rabbit. (Sсотт.)

Upper line, tracing of diaphragm slip (Head's method). Lower tracing, carotid pressure. During the first period indicated on the signal line the animal breathed $9 \cdot 6$ per cent. $\mathrm{CO}_{2}$ in air, and during the second period 10 per cent. $\mathrm{CO}_{2}$ with 33 per cent. oxygen. Time tracing $=2$ secs. Scale $=\mathrm{mm}$. Hg. blood-pressure.

A rise of carbon dioxide in the atmosphere to 2 per cent. increases the depth of respirations by 30 per cent., and the total alveolar ventilation by 50 per cent. A rise of carbon dioxide to 3 per cent. increases the total ventilation of the alveoli by 126 per cent. An amount of carbon dioxide equivalent to 6 per cent. increases the depth of each respiration by 272 per cent., and the total alveolar ventilation by 757 per cent.

\begin{tabular}{|c|c|c|c|c|}
\hline $\begin{array}{c}\text { Percentage } \mathrm{CO}_{2} \\
\text { in inspired air }\end{array}$ & $\begin{array}{c}\text { Average depth } \\
\text { of respirations }\end{array}$ & $\begin{array}{c}\text { Average } \\
\text { frequency of } \\
\text { respirations } \\
\text { per minute }\end{array}$ & $\begin{array}{c}\text { Ventilation of alveoli } \\
\text { with inspired air } \\
\text { (normal = 100) }\end{array}$ & $\begin{array}{c}\text { CO } \\
\text { in alvercentage }\end{array}$ \\
\hline $0 \cdot 04$ & 673 & 14 & 100 & $5 \cdot 6$ \\
$0 \cdot 79$ & 739 & 14 & $(6 \cdot 60$ litres per min.) & $5 \cdot 5$ \\
$2 \cdot 02$ & 864 & 15 & 116 & $5 \cdot 6$ \\
$3 \cdot 07$ & 1216 & 15 & 153 & $5 \cdot 5$ \\
$5 \cdot 14$ & 1771 & 19 & 226 & $6 \cdot 2$ \\
$6 \cdot 02$ & 2104 & 27 & 498 & $6 \cdot 6$ \\
\hline
\end{tabular}

If we examine the last column of figures in this Table, representing the percentage of $\mathrm{CO}_{2}$ in the alveolar air, it will be seen that, in spite of the very large variations in the air breathed, the alveolar content in $\mathrm{CO}_{2}$ remained practically constant until the $\mathrm{CO}_{2}$ in the atmosphere was increased to such an extent that the processes of compensation were no longer efficient. We must conclude therefore that the respiratory centre is so arranged as to 
react to the slightest increase of $\mathrm{CO}_{2}$ tension in the blood, any increase in this gas giving at once a compensatory increase in depth and frequency of respiration, so that the alveolar $\mathrm{CO}_{2}$ content may be maintained almost constant.

That it is the tension of $\mathrm{CO}_{2}$ in the alveolar air, and therefore in the blood bathing the centres, and not the percentage amount of this gas, which is the determining factor is shown by a comparison of the composition of the alveolar air under different atmospheric pressures. Thus, when the subject of the experiments, from which the above Table was derived, was placed in an air-chamber compressed to a pressure of $1261 \mathrm{~mm}$., the mean percentage

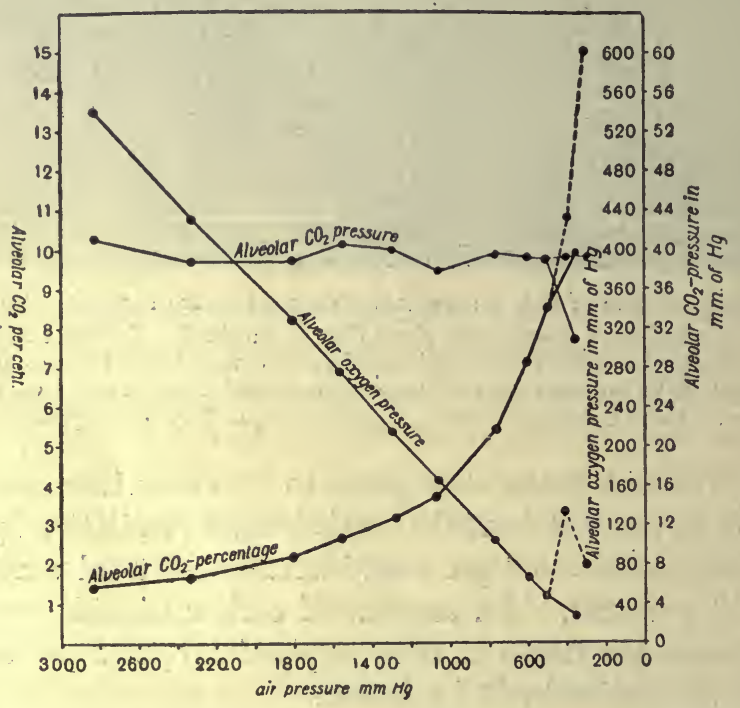

FIG. 506. Effects of alterations in the barometric pressure on the alveolar $\mathrm{CO}_{2}$ tension, the alveolar $\mathrm{CO}_{2}$ percentage, and in the alveolar $\mathrm{O}_{2}$ tension. Note that the excitant effects of $O$. lack are not seen until the pressure falls below $500 \mathrm{~mm}$. Hg. (BoycotT and HaLdaNe.)

of $\mathrm{CO}_{2}$ in the alveolar air was $3 \cdot 42$, corresponding, however, to a tension of $3.42 \times \frac{1261}{760}=5 \cdot 6$ per cent. of an atmosphere, a figure almost identical with those given in the last column of the Table above. At the top of Ben Nevis, where the barometric pressure was $646 \mathrm{~mm}$., the percentage of $\mathrm{CO}_{2}$ in the alveolar air was $6 \cdot 6$, corresponding to a tension of $6 \cdot 6 \times \frac{646}{760}=5 \cdot 2$ per cent. of an atmosphere, i.e. of $760 \mathrm{~mm}$. Thus the pressure of $\mathrm{CO}_{2}$ in alveolar air remains practically constant with widely varying limits of atmospheric pressure and with very different percentages of $\mathrm{CO}_{2}$ in the inspired air, showing that the reactions of the organism are directed so as to maintain, by alterations in the respiratory depth and rhythm, a constant tension of this gas in the alveoli and therefore in the arterial blood.

Very different are the phenomena observed on alteration of the partial 
pressure of oxygen (Fig. 506). Here, within wide limits, the partial pressure of oxygen in the alveolar air is determined by its pressure in the inspired air. Thus, if we take the same series of observations with a pressure of $646 \mathrm{~mm}$., the percentage of oxygen in the alveolar air was $13 \cdot 19$, corresponding to a tension of $13.19 \times \frac{646}{760}=10 \cdot 4$ per cent. At an atmospheric pressure of $755 \mathrm{~mm}$. the percentage of oxygen in the alveolar air was $13 \cdot 97$, corresponding to a tension of 13.06 per cent., which we may take as the normal figure at the

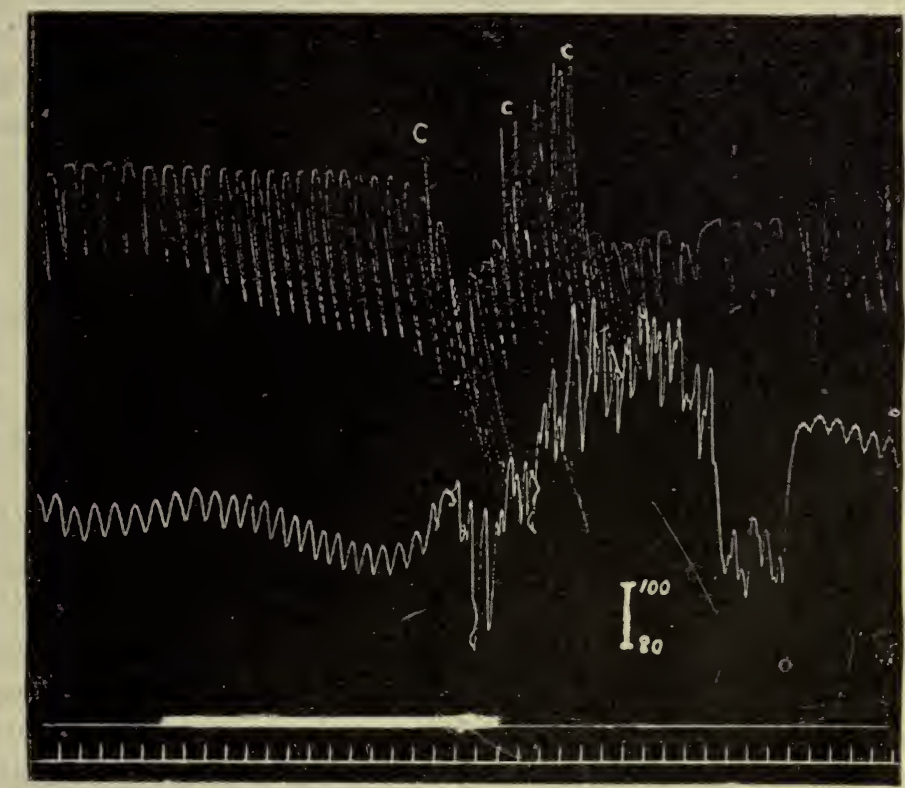

Fig. 507. Effects of oxygen lack. (Sсотт.)

During tracing, diaphragm slip; lower tracing, carotid blood-pressure. During time indicated by signal, 5 per cent. oxygen in nitrogen was inhaled. $\mathrm{c}=\mathrm{con}$ vulsion.

sea-level. In air compressed to a pressure of $1261 \mathrm{~mm}$. the percentage of oxygen was $16 \cdot 79$, corresponding to a tension of $16 \cdot 79 \times \frac{1261}{760}=26 \cdot 8$ per cent. of an atmosphere of $760 \mathrm{~mm}$.

Similar results are obtained by altering the percentage of oxygen in the air breathed. The oxygen tension or percentage in the inspired air can be lowered from its normal of 20.93 to 12 or 13 per cent. without altering in any way the depth or rhythm of respiration, and in fact without any change being noticed by the individual who is the subject of the experiment. A percentage of 13 per cent. of oxygen corresponds to an alveolar content in oxygen of 8 per cent., and with a further reduction of the oxygen content there is increased pulmonary ventilation (Fig. 507), but the diminution in oxygen may be pushed to such an extent that the patient becomes blue from the deficient aeration of his hæmoglobin, without any considerable distress 
being caused. In fact in many cases the subject of such an experiment may lose consciousness suddenly before he has been aware of any serious deficiency in his aeration.

The difference in the sensitiveness of the centre to increase of carbon dioxide and lack of oxygen respectively is well shown by an experiment of Haldane's, in which the same person breathed in and out of a bag, in the first place al'owing the carbon dioxide produced in respiration to accumulate, and in the second removing the carbon dioxide by means of soda lime, so that the sole effect of respiration was to produce a continual diminution in the percentage of oxygen. In the first case, when the carbon dioxide was allowed to accumu'ate it was found that extreme and intolerable hyperpnoa was produced when the gaseous content of the bag consisted of $5 \cdot 6$ per cent. carbon dioxide with $14 \cdot 8$ per cent. oxygen. When the carbon diox:de was absorbed it was possible to breathe in and out of the bag for a much longer period. No hyperpnœa was produced, and the experiment was stopped as soon as the subject was becoming blue in the face and experienced slight throbbing in the head. The pulse frequency had gone up from 80 to 108 . The bag was found to contain no carbonic acid and only $8 \cdot 7$ per cent. oxygen. In another similar experiment the oxygen had been reduced to 67 per cent. before it was necessary to stop the experiment.

We must conclude that the respiratory centre possesses a specific sensibility for carbon dioxide, which determines the normal depth and rhythm of the respiratory movements. Although the respiratory centre, in common with the rest of the central nervous system, is sensitive to and can be excited by lack of oxygen, this quality is rarely brought into play. Under all ordinary circumstances an increased need for oxygen is associated with an increased production of carbon dioxide in the oxidative processes of the body, and the augmentation of respiration, produced by the excitatory effect of a small excess of carbon dioxide tension in the blood, suffices to provide fully for the increased needs of the organism for oxygen. The reactions of the organism have not been evolved in order to adapt it to balloon ascents or experiments in respiratory chambers. As an example of a normal adaptation we may take the changes in respiration which occur in an animal as the result of muscular exercise. During their activity a large amount of carbon dioxide is produced in the muscles. The blood passing from the mu'scles to the heart will not be able to get rid of the excess of the carbon dioxide in passing through the lungs, and will reach the respiratory centre more highly charged with this gas, the tension of which will be raised. The respiratory centre is thus stimulated, and the increased pulmonary ventilation thereby produced lowers the alveolar carbon dioxide pressure, until a point is reached at which an equilibrium is maintained between the effect of the increased production of carbon dioxide in raising the arterial carbon dioxide tension and that of the increased respiratory activity in lowering it. Under these circumstances it is found that the increased consumption of oxygen in the contracting muscles is more than compensated, so that the oxygen tension in the alveoli and in the arterial blood is rather above than below normal.

In certain experiments Zuntz and Geppert found that during muscular exercise the respiratory movements were increased to such an extent as to bring the tension of carbon dioxide in the arterial blood below normal. 
In these experiments the muscular contractions were produced by tetanising, through the spinal cord, the lower limbs of an animal. Under these circumstances the activity of the muscle would be associated with a diminished blood-flow, so that the contractions would be carried out in the absence of a sufficient supply of oxygen. In the absence of sufficient oxygen muscular contractions result in the production, not of carbon dioxide, but of lactic acid, and it is highly probable that in the experiments in question there was a discharge of acid substances into the blood, diminishing the alkalinity of this fluid and therefore lowering its carrying power for carbon dioxide. As a matter of fact, one can produce dyspnœa by diminishing the alkalinity of the blood by the injection of acids, and attacks of dyspnœa are observed in the later stages of diabetes, when the alkalinity of the blood is decreased in consequence of the production of such bodies as oxybutyric acid. This dyspnœa has been ascribed to the fact that a diminished carrying power of the blood for carbon dioxide will raise the tension of this gas in the tissues where it is formed, so that a diminished alkalinity of the blood may cause a higher tension of carbon dioxide around the respiratory centre. It has been shown by Ryffel that even a short period of sufficiently violent muscular exercise, i.e. one giving rise to dyspnœa, causes a subsequent increase of lactic acid in the urine, and that the blood itself at the close of the period of exercise contains a demonstrable amount of this acid. Thus in one case the urine, passed thirty minutes after running one-third of a mile in two minutes, contained $454 \mathrm{mg}$. lactic acid as against a normal excretion of between 3 and $4 \mathrm{mg}$. of lactic acid per hour. In another experiment blood was obtained from the fore-arm before exercise, immediately after exercise, and three-quarters of an hour later. The exercise, which consisted of running rapidly, lasted two minutes forty-five seconds. The following Table represents the results obtained :

Blood before starting

Blood immediately after stopping

Blood 45 minutes later
Lactic acid per 100 c.c.

- $12 \cdot 5 \mathrm{mg}$.

- $70 \cdot 8$,

- $15 \cdot 9$,

The production of lactic acid during muscular exercise may thus be regarded as a second line of defence for the organism, tending to maintain the increased ventilation of the lungs even when the supply of oxygen is insufficient to oxidise completely the materials consumed in the production of the muscular energy. This acid mechanism is, however, only employed when the supply of oxygen lags behind the respiratory needs of the body (cp. Fig. 508). Ordinary exercise, even when considerable, e.g. a twentyfour hours' track walking race, does not cause, as Ryffel has shown, any appreciable increase in the elimination of lactic acid by the urine. Under normal circumstances the depth and rhythm of respiration depend on the carbon dioxide pressure in the respiratory centre, a rise of 0.2 per cent. of an atmosphere in the tension of this gas in the alveoli being sufficient to double the amount of alveolar ventilation during rest. 
The first phase in the phenomena of asphyxia is thus conditioned simply by the changes in the carbon dioxide tension. A little later the gradual exhaustion of oxygen in the blood round the centre begins to make itself felt. The respiratory centre shares with the rest of the central nervous system a sensitiveness to the absence of oxygen, deprivation of oxygen having first an excitatory and later a paralytic effect. In asphyxia the first centres to feel this effect are those of the cortex, and during the first stage there is mental excitation terminating rapidly in abolition of consciousness. During the second stage there is a discharge of energy, which spreads throughout the whole nervous system, beginning in the bulbar centres and causing a great rise of blood-pressure with slowing of the heart,

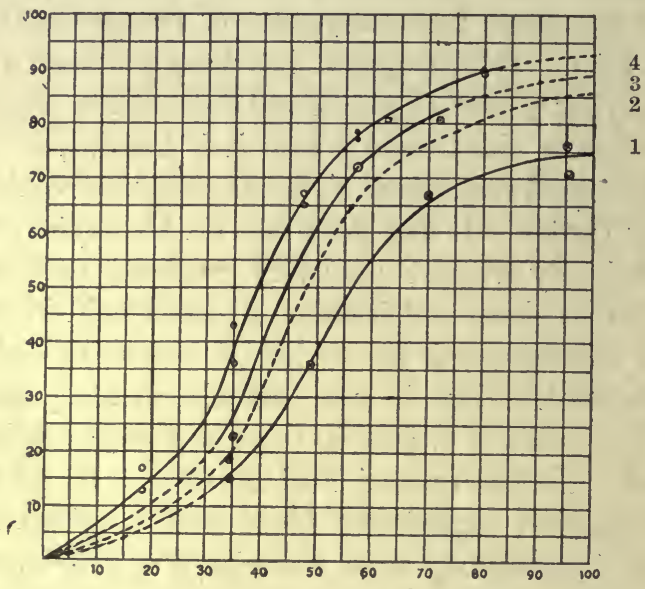

FIG. 508. Dissociation curve of oxyhæmoglobin in defibrinated cat's bleed. 1, cat I, after partial occlusion of trachea and fifteen minutes breathing of gas of increasing poverty in oxygen; 4, cat II, at beginning of experiment; 3 , cat II, after fifteen minutes gas respiration; 2 , after twenty-one minutes ditto.

and extending thence to all the spinal centres with the production of muscular spasms. At this stage too there is a discharge of impulses giving contraction of the pupil, and a discharge along the whole sympathetic system, producing the various phenomena of vaso-constriction, erection of hairs, sweating, salivation, which are generally brought about by stimulation of different parts of this system. The phenomena of the third stage are due to exhaustion of the nerve-centres, accompanied or preceded by exhaustion and dilatation of the heart, the circulation failing before the excitation of the lower centres has entirely come to an end. In this third stage it is impossible by the strongest stimuli to evoke any reflex.

Considerable discussion has taken place as to the exact nature of the stimulation brought about by want of oxygen. The blood of animals which have been killed by asphyxia is known to contain reducing substances, so that oxygen added to it disappears and cannot be recovered in a vacuum. Pflüger therefore suggested that it was these reducing substances themselves which were effective exciting agents. It was shown many years ago by Hoppe-Seyler and his pupils that in conditions of chronic oxygen 
starvation there was an excessive production of lactic acid in the body, and we have seen that the same is true for the isolated muscle and that to these substances has been ascribed (Zuntz and Geppert) the excitation of the respiratory centre which occurs in violent muscular exercise. Haldane has suggested that in the hyperpnœa and convulsions which occur as the result of breathing mixtures with very low percentages of oxygen the effective stimulus is also lactic acid. Experiments were carried out by Ryffel on individuals who had been subjected in a respiratory chamber to very low oxygen tensions, sufficient to cause cyanosis, so that their oxygen alveolar tension was only about 6 per cent. After an experiment, lasting four hours, there was a definite increase of lactic acid in the blood of the fore arm (up to $23.6 \mathrm{mg}$. lactic acid per 100 c.c.). After one lasting only fifteen minutes, in which the oxygen shortage became very marked, no increase could be detected. When we expose an animal such as a rabbit to low percentages of oxygen, the hyperpnœa so produced disappears almost immediately when a larger percentage of oxygen is supplied to the animal, whereas that produced by carbon dioxide excess dies away slowly on exposure to normal conditions. It would seem that when the exposure to low oxygen tensions is of short duration no lactic acid is produced in the blood. If therefore we ascribe the hyperpnoa to the production of lactic ac'd we must locate the production of this acid in the respiratory centre itself. There are no inherent improbabilities in such an assumption, but it is difficult at present to see how it can be put to the test of experiment.

In dealing with the question of the blood alkalinity we defined neutrality as a condition in which there were equivalent concentration of $\mathrm{H}$ and $\mathrm{OH}$ ions. In the blood the $\mathrm{H}$ ion concentration is about $0.3 \times 10^{-7} \mathrm{~N}$. The alkalinity is expressed by concentration $\mathrm{OH}$ ions

concentration $\mathrm{H}$ ions . The acids and bases of the blood-serum and of the tissue-fluids generally are in such proportions as to maintain the approximate neutrality of these fluids even after considerable additions of acid or alkali. This hydrochloric acid may be added to the extent of $\cdot 025 \mathrm{~N}$, or $\mathrm{NaOH}$ to the extent of $\cdot 005 \mathrm{~N}$, without causing any marked alteration in the reaction of the blood. Although, however, the change produced by the addition of acids or alkalies is so minute, it is appreciable by electrical methods, and it may still more readily be appreciated by and act as a stimulus for the cells of the body themselves. Thus we have not yet succeeded in determining electric:ally the change in hydrogen ion concentration caused by the change from arterial to venous blood. If, however, blood-serum be saturated with carbon dioxide at a full atmosphere, the concentration of the hydrogen ions rises to $1: 4 \times 10^{-7} \mathrm{~N}$, while after removing the greater part of the carbon dioxide from the same serum by the passage of a stream of air, the concentration of the hydrogen ions sinks to $008 \times 10^{-7} \mathrm{~N}$. As the respiratory centre responds to such minute changes of concentration as would be $\epsilon \mathrm{X}$. pressed by a difference of 0.2 per cent. of an atmosphere in the carbon dioxide tension of the circulating blood, it must possess a sensitivity greater than any of our physical means for measuring the concentration of hydrogen ions in a fluid. We may approach this delicacy of reaction by using a large molecule as our indicator. Thus, as we have seen, the dissociation curve of hæmoglobin is sensitive to the change in reaction caused by raising the tension of carbon dioxide in the hæmoglobin solution by $10 \mathrm{~mm} . \mathrm{Hg}$. (cp. Fig. 497).

The regulating factor in the blood is probably not carbon dioxide nor any special acid, but the concentration of hydrogen ions in this fluid or in the cells of the centre itself. Such a conclusion brings under one head all the several factors which we know to act upon the respiratory centre, namely, tension of carbon dioxide, presence of acids in the blood-especially lactic-and cons:derable diminution of oxygen supply to the cells. The respiratory centre would then not differ qualitatively from any other part of the central nervous system. Its special function would be determined simply by the evolution to a marked degree of a sensibility to hydrogen ions which is already possessed by the whole of the central nervous system and indeed by practically every tissue of the body.

We may conclude that mere lack of oxygen is not to be regarded in itself as an excitatory agent. Its influence will be rather to paralyse all 
activity. On the other hand, excitation is caused by the products of metabolism, which vary according as the oxygen supply is ample or insufficient for the needs of the cells. In the former case activity results in the production of carbon dioxide, in the latter of lactic acid, and perhaps other substances. Both these are acid substances and their production will therefore raise the concentration of the hydrogen ions in the cells where they are produced as well as in the blood. The nerve-centres are extremely sensitive to minute changes in the hydrion concentration either in themselves or in the fluids surrounding them, and are thrown into activity by excess of these ions and inhibited, or put to rest, by relative deficiency of the ions. In their relation to $\mathrm{H}$ and $\mathrm{OH}$ ions respectively the medullary centres have a sensibility five times as great as the spinal centres. The condition of apnœa, which is associated not only with cessation of respiratory movements but also with fall of blood-pressure, may be ascribed to relative increase in the $\mathrm{OH}$ ions or diminution in the $\mathrm{H}$ ions.

Since the animal has developed a mechanism by means of which changes in the reaction of the blood can be rapidly adjusted by varying the excretion of carbon dioxide, whilst the excretion of other acids is relatively slow, carbon dioxide may be regarded as the normal respiratory hormone, and so far we may agree with Henderson in regarding carbon dioxide as maintaining the activities of the various nerve-centres at their normal level. But it is the hydrion concentration which appears to be the essential factor, and the acid substances produced during oxygen lack are equally efficacious, but not so convenient. Thus their production is not a steady process like that of carbon dioxide, but, as Mathison points out, commences suddenly at a time when the executive side of the nerve-cell is feeling the effect of oxygen starvation, so that the cell may be too much disorganised to respond to stimulation. "The broad margin of safety protecting the organism against paralysis of its cells by oxygen starvation is assured by the sensitiveness of the medullary centres to hydrogen ion concentration and therefore to carbon dioxide in common with other acids."

On the other hand, it must be remembered that excessive production of hydrogen ions may finally result in a condition of paralysis, which in the nervous centres is expressed by narcosis. These effects can only be removed by a free supply of oxygen. The concentration at which these results occur varies, as we have seen, in different parts of the nervous system and also in different tissues. Thus on the heart a slight increase in $\mathrm{H}$ ion concentration causes diminished tone, which may lead to dilatation and failure of this organ. The same effect is produced on the unstriated muscle fibre of the bloodvessels. Since in the heart and blood-vessels the reverse effect is produced by increasing the $\mathrm{OH}$ ion concentration, it is evident that the line of 'physiological' neutrality at which neither stimulation nor paralysis is produced must vary in different tissues.

It is an interesting question whether the electrical excitation of nerves may not be due to a similar alteration in the hydrion concentration at the 
cathode which is the seat of stimulation. If this were so, all the activities of protoplasm might be regarded as determined by the relative concentration of the $\mathrm{H}$ and $\mathrm{OH}$ ions within the cells or in the medium surrounding the cells.

\section{THE REFLEX NERVOUS REGULATION OF RESPIRATION}

Although the specific sensibility of the respiratory centre to $\mathrm{CO}_{2}$ is the most important factor in determining the depth and rhythm of the respiratory movements, these movements and the condition of the respiratory centre

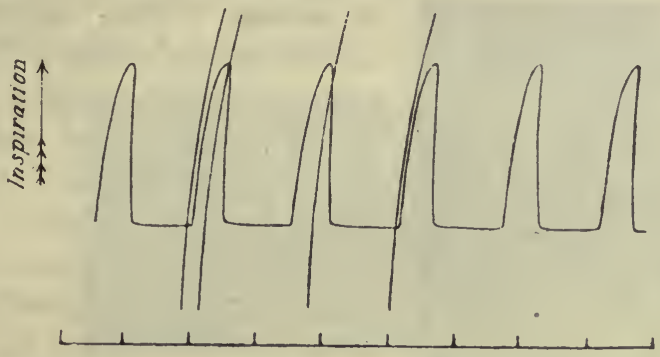

FIG. 509. Normal tracing of diaphragm slip (Head's method).

itself are modified in a large degree by impulses arriving at the centre along both vagi. Through other sensory nerves of the body the respiratory movements can be altered reflexly, but it is only through the vagi that a continuous stream of impulses passes to the centre under normal circumstances, so that every respiratory movement is modified by these impulses.

In studying the nervous mechanism of respiration, it is necessary to have some accurate method of recording the respiratory movements. They may be registered by means of a tambour applied to the chest, communicating with another tambour provided with a lever, which is arranged to write on a blackened surface ; or a side tube to a cannula in the trachea may be connected with the registering tambour. In the first case movements of the thorax are registered ; in the second changes of intra-pulmonary pressure. These methods are obviously useless when it is wished to study the effects of artificial distension or collapse of the lungs. In this instance we may use the ingenious method described by Head. In the rabbit a slip of the diaphragm on either side of the ensiform cartilage is so disposed that the end of it may be freed and attached by a thread to a lever without injury to its blood-or nerve-supply. It is found that this slip contracts synchronously with the rest of the diaphragm, so that it serves as a sample of the diaphragm, the contractions of which may be recorded uninfluenced by passive movements of the chest wall or artificial increase of intra-pulmonary pressure.

If, while the respiratory movements are being recorded in one of the afore-mentioned ways, both vagi be divided,* a marked change in the respiratory rhythm is at once seen. The first effect is an increased inspiratory tonus, but this rapidly disappears, and the respiratory move-

* The division of the vagi is best effected by putting them on a hooked copper wire, of which the upper end is inserted in a freezing-mixture. In this way complete functional division of the nerves is obtained without any excitation. If the nerves be cut, a certain amount of stimulation takes place in consequence of the closure of the demarcation current produced by the cross-section. 
ments become less frequent and are increased in amplitude. If now the central end of one of the vagi be stimulated with an interrupted current, the respiration may be quickened, or, as is more commonly the case, the inspiratory movements may be increased at the expense of the expiratory, so that finally a condition of inspiratory standstill is produced, and the slip of the diaphragm enters into prolonged contraction.

With a very weak stimulus it is sometimes possible to produce augmentation of the expiratory movements or rather inhibition of the inspiratory, and this is the invariable result of passage of

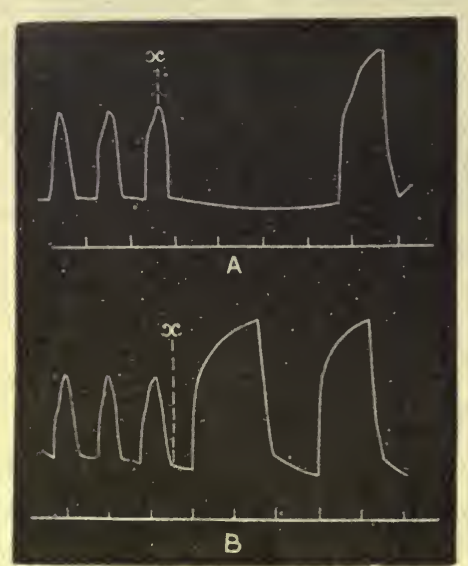

FIG. 510. Effects of distensioncollapse of lung. Both curves are described by a lever attached to a slip of the diaphragm of a rabbit. A contraction of the diaphragm (inspiration) raises the lever; during relaxation of the diaphragm the lever falls.

In $A$, the trachea is closed at $x$, the height of inspiration; a pause follows, during which the lever gradually sinks until an inspiration (a very powerful one) sets in.

In $\mathrm{B}$, the trachea is closed at the end of expiration, $x$; there follow powerful inspirations. (FosTER.) a constant current through the vagus in an ascending direction. This effect may be more strikingly brought about by stimulation of the central end of the superior laryngeal nerve, which produces first an inhibition of inspiration, so that the respiratory muscles come to a standstill in the position of expiration, and then a forcible contraction of the expiratory muscles. This illustration of the presence of expiratory fibres in the superior laryngeal nerve is not confined to laboratory experience, but is constantly occurring in everyday life. The superior laryngeal nerve supplies sensory fibres to the mucous membrane of the glottis, and we know that the slightest irritation of these fibres - the presence of a crumb or a particle of mucus - causes forcible expiratory spasms, with spasmodic closure of the glottis, which we term a cough.*

So we see that the vagus nerve contains two kinds of afferent fibres, or at any rate afferent fibres with two distinct functions. Stimulation of the one kind stops inspiration and produces expiration; stimulation of the other stops expiration and produces inspiration. Since section of both vagi causes slowing of respiration, impulses which exert some influence on the respiratory centre and quicken respiration must travel up the vagi from the lungs. The respiratory movements cause an alternate distension and contraction of the lungs, and it has long been thought that it is these changes in the volume of the lungs which start the accelerating impulses that travel

* It must not be imagined that the fibres of the superior laryngeal nerves are concerned in the reflex maintenance of the normal respiratory rhythm. They are cited here merely because the result of their stimulation resembles that which would be caused by stimulation of the analogous expiratory fibres which run in the trunk of the vagus from the lungs to the respiratory centre. 
up the vagus nerves. To test the truth of this hypothesis it is necessary to study the two phases of respiration separately; that is, to see first the result on the respiratory impulses of distension of the lungs, and, secondly, the result of a sudden collapse or a contraction caused by sucking air out of the lungs. The effects of distension or collapse of the lung may be shown by simply closing the trachea at the end of inspiration or of expiration. The results of such an experiment are shown in Fig. 510.

A still more marked effect is produced if the lungs, by means of a tube in the trachea, be artificially inflated or if air be sucked out of them. The inflation produces an instantaneous and complete relaxation of the diaphragm (Fig. 511) which by clamping the tracheal tube may be prolonged

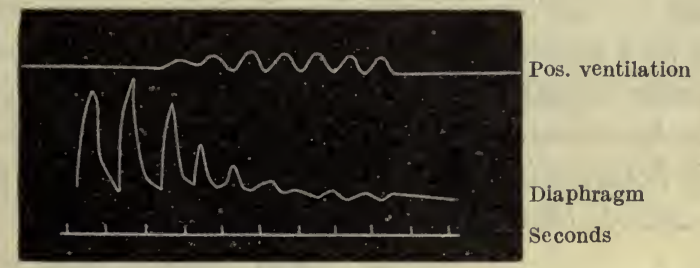

Fra. 511. Positive ventilation. (HeAd.)

Under the influence of positive ventilation, the inspiratory contractions of the diaphragm become less and less till they disappear completely.

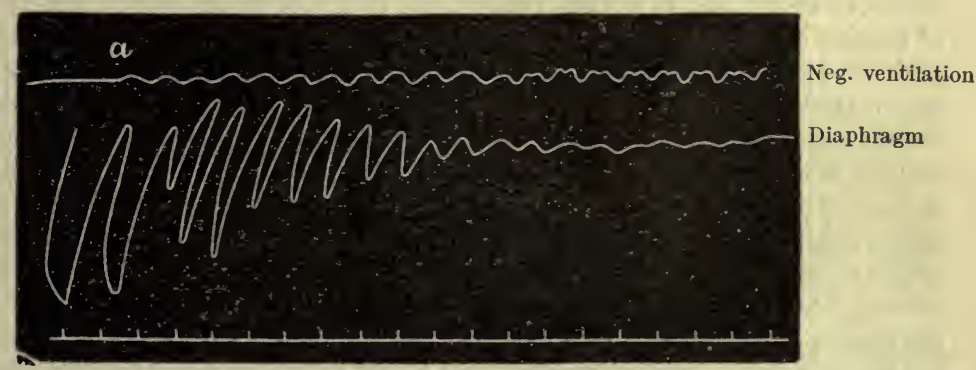

FIG. 512. Negative ventilation. (HEAD.)

At $a$ negative ventilation was commenced. The expiratory relaxation of the diaphragm is seen to become more and more incomplete, until it finally enters into continued contraction.

for several sconds, while sucking air out of the lungs causes a tonic contraction of the diaphragm (Fig. 512). Somewhat similar results may be obtained by repeatedly inflating or deflating the lungs (positive and negative ventilation). The effects here are complicated by the fact that one is dealing in both cases with alternating movements of the lungs, of expansion and contraction, both of which will have an influence on the respiratory centre. Moreover repeated forcible inflation of the lungs increases the ventilation of the pulmonary alveoli, thus lowering the normal carbon dioxide tension of the lungs. As a result of repeated ventilation we may obtain a condition of respiratory standstill. In this condition, however, as ẉe shall see later, the determining factor is rather chemical than mechanical.

These inhibitory and augmentor effects of changes in the volume of the lung must also result from the normal movements of these organs in 
respiration. Let us consider, for instance, what will happen if the influence of the two vagi could be suddenly thrown in after these nerves have been divided. (This experiment can, in fact, be realised more or less completely if the functional division of the vagi be effected by cooling or by ether narcosis). The animal would be breathing slowly and deeply. If at the beginning of an inspiration the vagi became functional the expansion of the lungs caused by the inspiratory movement would send inhibitory impulses up to the vagus centre, which would stop the movement of inspiration. The movement of expiration would then begin, and the collapse of the lungs thereby produced would itself send impulses up the vagi which would tend to excite an inspiratory movement. Both inspiration and expiration would therefore be shortened, and the successive movements would follow one another at a shorter interval than if the vagi were not functional. In this way, under normal circumstances, the rhythm of the respiratory centre must be determined reflexly through the agency of the vagi, while the chief factor in determining the total pulmonary ventilation is, as we have seen, the carbon dioxide tension of the blood.

In the foregoing account we have spoken of the expiratory and inspiratory effects of the vagus as if they were of equal importance. It seems probable, however, that the inhibitory or expiratory impulses started by the inspiratory movement, the only or the more active part of normal respiration, play a more prominent part in the regulation of respiration than do the inspiratory impulses ; and one observer (Gad) goes so far as to deny altogether the existence of two kinds of respiratory fibres in the vagus. According to Gad, the vagus, as regards the respiratory centre, is a purely inhibitory nerve. Hence the primary effect of dividing both vagi is an increased inspiratory tone. This view at first seems paradoxical, in that it explains the final slowing of respiration after section of the vagi as due to the cutting off of previous inhibitory mpulses. But inh1bition in all tissues has a twofold effect. Although the immediate effect is diminution of activity, yet the diminished disintegration necessarily associated with diminished activity means an increase of the anabolic at the expense of the catabolic processes of the tissues. In this way we explained the diminished excitability occurring in a nerve at the anode of a constant current, and it will be remembered that the secondary result of anelectroronus was increased irritability and consequent excitation at break of the constant current. The same sort of process must occur in the respiratory centre. A continued restraint of its rhythmic activity must lead to a heaping up of its irritable material, so that the final result is a state of hyperexcitability in which the centre, so to speak, boils over on the slightest provocation.

In this condition a cutting off of the inhibitory impulses must at first increase the activity of the centre, leading to the increased inspiratory tonus already described. But unchecked by any reign ing impulses, the centre enters upon a career of spendthrift activity. Each inspiratory contraction is maximal, but the centre, exhausted by the effort, has to wait a considerable time before it can accumulate sufficient energy for the next ; hence the final result of section of both vagi is deepening and slowing of respiration.

Although Gad has rendered great service in emphasising the importance of the inhibitory or expiratory impulses which ascend the vagi there is no doubt that he went too far in denying the existence of inspiratory fibres in the vagus. This is shown by the following experiment of Head. According to Gad's view, collapse of both lungs implies simply a removal of the normal inhibitory impulses ascending the vagi, and is therefore equivalent to division of these two nerves. If in the rabbit the left vagus be divided, a tube can be introduced into the left bronchus, and artificial respiration can be performed by alternate inflation and collapse of the left lung, without in any way affecting the respira- 
tory centre, all connections with the latter being destroyed ( $v$. Fig. 513). Meanwhile the animal carries out normal respiratory movements, which can be recorded by the diaphragm slip method. While the slip is contracting regularly, the right pleura is opened and the right lung allowed to collapse. The effect of this collapse, carried up by the right vagus to the centre, is an extreme contraction of the diaphragm, and since the onset of asphyxia is prevented by the artificial respiration carried out on the left lung, the tonic standstill of the diaphragm may last over a minute. In this case therefore:

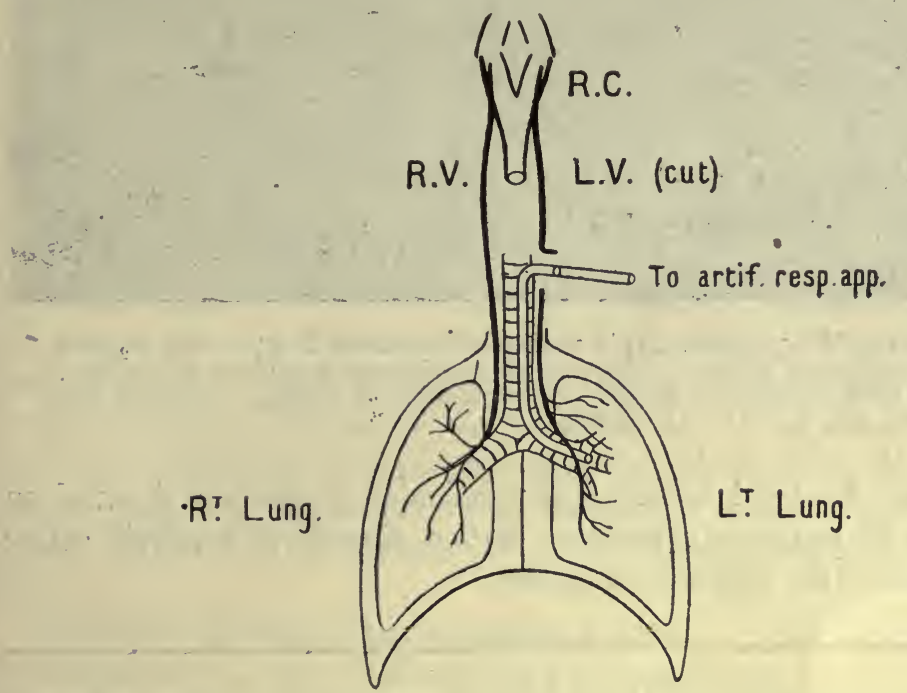

Fig. 513. Diagram to illustrate Head's experiment on the effect of collapse of the lung. R.C, respiratory centre; R.v, L.v, right and left vagi.

the effect of collapse of one lung is enormously greater than that produced by section of both vagi, showing that the effect is due, not to abolition of the ordinary tonic inhibitory stimuli, but to excitation of special inspiratory fibres in the vagus by the collapse of the lung.

By means of the string galvanometer it is possible to show definitely that a collapse 0 : 1 he lungs does set up a nervous impulse travelling up the vagus nerves. This impulse must be inspiratory in character, so that there is no reason to deny the existence of both kinds of fibres in these nerves. The effect of electrical stimulation, especially with an ascending constant current, is also strong evidence in the same direction.

After division of both vagi the total pulmonary ventilation does not as a rule undergo any marked changes, and in the absence of anæsthesia the aeration of the blood may be carried out almost, if not quite, as well as in the intact animal. The importance of the vagus action for the organism is shown, however, if we put an increased strain on the respiratory mechanism, as, for instance, by increasing the percentage of carbon dioxide in the air breathed. In the intact animal this procedure leads first to increased depth and later to increased frequency of respiration, the total ventilation being thereby augmented to such an extent as to keep the alveolar tension of carbon dioxide almost constant. If the same percentage of carbon dioxide be administered to an animal after section of both vagi the effect is deepening of respiration, but not quickening (Fig. 514). Each inspiratory movement, however, is already considerable so that the margin by which increase of 
pulmonary ventilation is possible, by increase of depth of respiration alone, is not so great as in a normal animal. Moreover, since no quickening of respiration takes place, the increased ventilation rapidly becomes inadequate for the maintenance of the normal alveolar carbon dioxide tension. In the

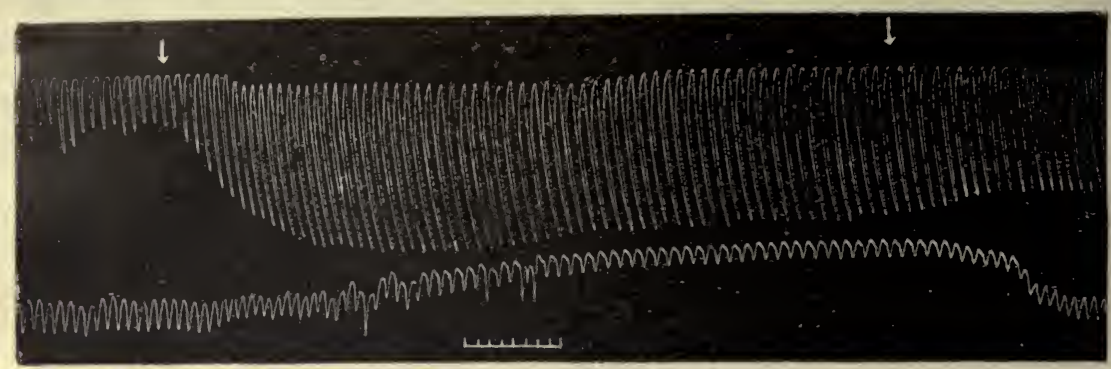

FIG. 514. Effect of 10.6 per cent. $\mathrm{CO}_{2}$ in a mixture containing 23.3 per cent. $\mathrm{O}_{2}$ on a rabbit with both vagi divided. The gas was administered between the arrows. Zero line of blood-pressure is $32 \mathrm{~mm}$. below bottom of tracing. Compare this figure with Fig. 505, p. 1081. (F. H. Scотт.)

following Table the total amounts of pulmonary ventilation obtamed on administration of mixtures containing carbon dioxide to a rabbit before and after section of the vagi are compared.

\begin{tabular}{|c|c|c|c|}
\hline \multicolumn{4}{|c|}{ RABBIT, 3 KILOS } \\
\hline & $\begin{array}{l}\text { Respirations } \\
\text { per minute }\end{array}$ & $\begin{array}{l}\text { Vol. of each } \\
\text { respiration }\end{array}$ & $\begin{array}{l}\text { Total ventilation } \\
\text { per minute }\end{array}$ \\
\hline $\begin{array}{cc}\text { Respiration with air } & . \\
, & 4 \cdot 2 \text { per cent. } \mathrm{CO}_{2} \\
, & 8 \cdot 6 \text { per cent. } \mathrm{CO}_{2} \\
, & \text { air }\end{array}$ & $\begin{array}{l}72 \\
96 \\
97 \\
72\end{array}$ & $\begin{array}{l}\text { c.c. } \\
19 \\
25 \\
29 \\
20\end{array}$ & $\begin{array}{l}1368 \\
2400 \\
2813 \\
1440\end{array}$ \\
\hline \multicolumn{4}{|c|}{ VAGI DIVIDed } \\
\hline $\begin{array}{c}\text { Respiration with air } \\
, \quad \\
, \quad 4.2 \text { per cent. } \mathrm{CO}_{2} \cdot \\
\quad 8.6 \text { per cent. } \mathrm{CO}_{2} .\end{array}$ & $\begin{array}{l}45 \\
45 \\
42\end{array}$ & $\begin{array}{l}29 \\
34 \\
38\end{array}$ & $\begin{array}{l}1305 \\
1530 \\
1596\end{array}$ \\
\hline
\end{tabular}

Whether we assume that the prevailing impulses travelling up the vagi are purely inhibitory or are both inhibitory and augmentor, the resultant effect, by reining in the activity of the centre, is to economise its energy and the energy of the respiratory muscles. The result of the vagal impulses will therefore be to increase the excitability of the respiratory centre and make it more susceptible to slight changes in the carbon dioxide tension of the blood, while maintaining a sufficient margin of energy to 
meet the increased needs thrown on the respiratory mechanism by augmented metabolism, such as occurs in violent muscular exercise.

The important part played by the vagi in the regulation of normal respiration is shown still more strikingly if the respiratory centre in the medulla be separated from the higher parts of the brain before the section of the vagi is carried out. Separation of the medulla from the higher parts of the brain, as by section just behind the corpora quadrigemina, has practically no influence on the respiratory rhythm. If now both vagi be divided the normal respiratory movements cease entirely, being replaced by a series of inspiratory spasms, each of which lasts several seconds and is followed by a pause of half to one minute's duration. These spasms are inadequate for the proper oxygenation of the blood. They become gradually less and less frequent, and in about half an hour the animal dies of asphyxia. We must conclude therefore that the medullary respiratory centre with the help of the vagi is able to carry out normal respiratory movements. If both vagi are cut, impulses arrive at the centre from the higher parts of the brain regulating its activity, and enabling it to carry out modified but sufficient respiratory movements. Removed from both these sources of afferent impulses, the centre discharges only a series of spasms which are totally inadequate for the renewal of the blood-gases, so that the animal dies.

We may summarise these results as follows :

Respiratory centre with vagi-normal respiration.

Respiratory centre with brain-modified respiration.

Respiratory centre alone-inadequate spasmodic contractions of respiratory muscles, and death of animal.

The nature of the supplemental action of the mid-brain on the medullary respiratory centre has not yet been made out. ' It is apparently not dependent on afferent impulses arriving at the brain, since section of no cranial nerve affects in any way the activity of the centres. Certain observers have described 'accessory respiraotry centres' in the mid-brain, in the region of the posterior corpora quadrigemina. Stimulation of this part causes increase in the rate of inspiratory movements and finally tonic spasm of the diaphragm. Expiratory effects have been produced by stimulation of the an:erior corpora quadrigemina, and it would seem that a section has to pass through or behind these bodies in order to produce the results, already described, of cutting off the higher centres from the medulla oblongata after division of the vagi. . Other localised spots in the brain from which effects on respiration have been obtained are the inner wall of the optic thalamus and the root of the olfactory tract. Further experiments are necessary before we can regard any of these centres as normally involved in the maintenance or regulation of the resp.ratory movements.

APNCEA. If artificial respiration be maintained so as to produce a somewhat greater ventilation than occurs by the normal respiratory move. ments of the animal, a standstill of respiration is brought about. This condition is called apnœa. The first explanation of this standstill was that it was due to over-oxygenation of the blood. The fact that it could be produced by artificial ventilation with inert gases, such as hydrogen and nitrogen, as well as the discovery of the inhibitory influence of distension 
of the lungs on the respiratory centre, led Head to ascribe it to the summation of a series of inhibitory stimuli. In these experiments, however, the fact was forgotten that forced ventilation of the lungs with air or any inert gases will reduce the carbon dioxide tension in the blood circulating round the pulmonary alveoli and therefore round the respiratory centre. A respiratory pause will therefore ensue and last until the increasing accumulation of carbon dioxide in the blood raises its tension to the normal height, at which the respiratory centre is 'set,' so to speak, to respond by a respiratory discharge. If the carbon dioxide content of inspired air be increased to about 4.5 per cent., it is impossible to produce an apnœic pause, however rapidly the respiratory movements be carried out. It would seem therefore that ordinary apnœea is entirely due to deficiency of carbon dioxide tension in the respiratory centre, and that although the vagus nerve is inhibitory of respiration, it is impossible to summate a series of vagus inhibitions by artificial respiration so as to produce a lasting cessation of respiratory movements. The chief use of the vagi in respiration seems to be for maintaining, by frequent inhibitions, the excitability of the respiratory centre at a maximum.

M'escher distinguished three types of apnœa, viz. :

A pnoea vera, due to the washing out of $\mathrm{CO}_{2}$ from the lungs, and the consequent reduction of the tension of this gas in the blood.

A pnoea vagi, a stoppage of respiration caused by stimulation of the inhibitory fibres of the vagi. This stoppage is limited, as we have seen, to the immediate duration of the stimulus (whether electric or produced by distension of the lungs).

Apnoea spuria. Stoppage of respiration by stimulation of other nervous or sensory surfaces. Thus when a duck plunges there is immediate stoppage of respiration, which may last four or five minutes if the animal remains so long under water. The same stoppage may be produced by pouring water on the beak.

\section{' CHEYNE-STOKES' BREATHING}

If a man desires to hold his breath for some time he takes first a series of deep breaths. The result is to diminish the carbon dioxide tension in the alveoli and therefore to take away the need and the desire to breathe until the carbon dioxide tension rises to normal as the result of the continued formation of carbon dioxide. By continuing forced respiratory movements for a minute or two the carbon dioxide tension both in the alveoli and in the blood may be brought down to a very considerable extent. As a result. there is a prolonged period of apncea. During this period of cessation of respirations, however, the oxygen is being used up, and the tension of this gas in the alveoli may fall to such an extent that the respiratory centre is excited by lack of oxygen before the carbon dioxide tension in the alveoli has risen to its normal value. As a result of the excitation by oxygen lack, a few breaths are taken and the carbon dioxide tension is once more lowered, and the stimulation due to the oxygen lack, disappears. There is therefore again a cessation of respiration. These periods of cessation alternate with periods of respiration so that we get a condition of periodic breathing 
which is spoken of as Cheyne-Stokes respiration. During the period of apnœea, resulting on forced breathing, the great diminution of oxygen tension in the alveoli is shown by the fact that the subject of the experiment becomes blue, and may indeed lose consciousness. There are at the same time rhythmic changes in the blood-pressure, which rises towards the

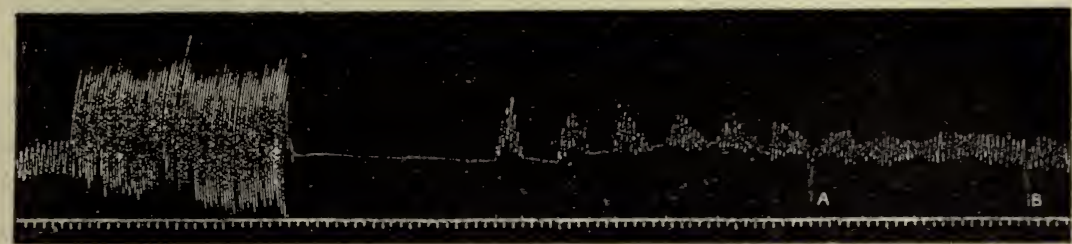

FIG. 515. Forced breathing of air for two minutes, followed by apncea for two minutes, and periodic ("Cheyne-Stokes ') breathing for about five minutes.

At $\mathrm{A}$, sample of alveolar air contained $\mathrm{O}_{2}, 11 \cdot 44$ per cent. $\mathrm{CO}_{2}, 5.58$ per cent. Second sample at $\mathrm{B}, \mathrm{O}_{2}, 13.55$ per cent.: $\mathrm{CO}_{2}, 5.57$ per cent. (DougLAS and HAIDANE.)

ends of the periods of the apnoea, falling during the periods of respiration. The first respiration after forced breathing is due to. oxygen lack. The period of apnœa may therefore be considerably prolonged, if the onset of oxygen lack be postponed, by increasing the tension of this gas in the alveoli at the commencement of the apnœic period. By forcibly breathing for a period of two minutes in an atmosphere of oxygen, men have succeeded in holding their breath for as long a period as eight minutes (Vernon).

'Cheyne-Stokes' breathing is almost invariably observed as one of the effects of exposure to high altitudes, and is then especially marked during sleep. It is often present when the activity of the respiratory centre is depressed, as in cases of uræmia or per-: nicious anæmia. Under these circumstances it may be temporarily removed by, administering either oxygen or carbon dioxide (in small percentage) to the patient. The oxygen improves the condition of the centre ; the carbon dioxide acts as an added stimulus and rouses its activity. 


\section{SECTION IV}

\section{THE EFFECTS ON RESPIRATION OF CHANGES IN THE AIR BREATHED}

$W_{E}$ have already seen that a moderate increase in the carbon dioxide percentage of the air breathed (e.g. up to 4 per cent.) causes a proportional increase in the ventilation of the lungs so as to maintain the tension of this gas in the alveoli at the normal level. The same effect is observed whether the mixture breathed contains 18 or 50 perr cent. of oxygen, showing that the slight diminution in oxygen content caused by mixing the air with carbon dioxide is in no way responsible for the effect. If the amount of carbon dioxide be increased to 12 or 15 per cent. it becomes almost impossible to continue the inhalation owing to the spasm of the glottis produced by the irritant effects of the carbon dioxide. If these high percentages be administered to an animal by a tracheal tube, violent dyspnœea is produced which gradually diminishes, and the animal passes into a condition of narcosis in which the respiratory movements become less and the oxygenation of the blood is ineffectively carried out even in the presence of excess of oxygen. The administration of larger percentages, such as 30 or 40 per cent., causes rapid death and failure of the circulation and respiration, often preceded by convulsions. Coincident with the increased respiration brought about by moderate percentages of carbon dioxide there is a. rise of bloodpressure, determined partly by vascular constriction, partly by an increased output from the heart. With high percentages of carbon dioxide the curve of blood-pressure obtained resembles that produced by lack of oxygen.

Oxygen itself exercises no excitatory effects on the respiratory movements. At the normal atmospheric pressure the tension of oxygen in the alveoli is about $107 \mathrm{~mm}$. $\mathrm{Hg}$, a pressure which, as we have seen, is amply sufficient to saturate the hæmoglobin passing through the vessels of the lungs. Since the depth and frequency of respiration are determined by the carbon dioxide tension in the alveoli no alteration in respiration will be produced by increasing the tension of oxygen in the air breathed above its normal amount. The respiratory movements in an atmosphere of pure oxygen will, in the normal individual, remain unchanged.

This statement is only true for the healthy individual. If from failure of the heart and circulation, or diminished oxygen tension, or severe loss of blood, the oxygenation of 
the blood is already insufficient, marked amelioration of the symptoms may be produced by inhalation of pure oxygen. Especially is this noticeable where there is failure of the heart. In these cases the heart, already affected, is unable to keep up an adequate circulation and to supply itself with sufficient oxygen. A vicious circle is thus established in which the heart tends to get steadily worse. By administration of oxygen an adequate supply of this gas to the heart-muscle is assured; the heart-beat therefore becomes more effective and the whole circulation is improved and therewith the provision of oxygen to the body at large.

If a warm-blooded animal be immersed in a chamber and submitted to pure oxygen at a pressure of four atmospheres, it dies as rapidly as if it were in an atmosphere of pure nitrogen. At this pressure the oxidative processes of the body as well as the intake of oxygen into the lungs are absolutely abolished. It is interesting to note that certain other oxidative phenomena, e.g. the spontaneous oxidation of phosphorus, also cease if the tension of the oxygen be sufficiently high. Exposure of an animal over a considerable period of time to a pressure of oxygen of two atmospheres may, as Haldane and Lorrain Smith have shown, set up severe inflammation of the lungs and thereby cause death indirectly.

CHANGES IN TENSION OF OXYGEN. If a man breathe a mixture of nitrogen and oxygen free from carbon dioxide, and the oxygen be gradually diminished, no feeling of ' want of breath' may be experienced. With percentages of oxygen as low as 12 per cent. there may be no change in the breathing, even though the deficient oxygenation of the blood may be shown by the blueness of the lips and face. If the oxygen be reduced still lower, a certain amount of hyperpnœa may occur, but in many cases the individual experimented on may not feel any ill effects until he suddenly becomes unconscious from lack of oxygen. If fresh oxygen be not supplied this unconsciousness may be followed by convulsive movements and death. If the administration of low percentages of oxygen, e.g. about 10 to 12 per cent. of an atmosphere, be continued for some time, the subject of the experiment may suffer considerable discomfort. One of the signs of oxygen lack is often severe headache, and this may be accompanied by vomiting or nausea and by a feeling of discomfort in the precordial region. Many experiments have been made both on animals and man by submitting them to a lowered atmospheric pressure in chambers specially built for the purpose. The limit to which the pressure may be reduced varies in different individuals, the variations being determined by the type of respiratory movement of the individual in question, since on the depth of respiration depends the relation between the tension of oxygen in the alveoli and that in the inspired air. The lowest limit at which life is possible corresponds to an oxygen tension in the alveoli of 27 to $30 \mathrm{~mm}$. $\mathrm{Hg}$.

MOUNTAIN SICKNESS. The phenomena just described as ensuing on exposure of an animal to low oxygen tensions in a respiratory chamber for some length of time are exactly similar to those which are regarded as characteristic of mountain sickness. The following Table shows the diminution in the atmospheric pressure at varying heights above the level of the sea : 


\begin{tabular}{|c|c|c|}
\hline $\begin{array}{c}\text { Height above sea level, } \\
\text { in metres }\end{array}$ & $\begin{array}{c}\text { Barometer } \\
\text { mm. Hg }\end{array}$ & $\begin{array}{c}\text { Per cent. of an } \\
\text { atmosphere }\end{array}$ \\
\hline 0 & 760 & 100 \\
1000 & 670 & 88 \\
2000 & 593 & 78 \\
3000 & 524 & 69 \\
4000 & 463 & 61 \\
5000 & 410 & 54 \\
6000 & 357 & 47 \\
7000 & 320 & 42 \\
\hline
\end{tabular}

At a height of 5000 metres the pressure of the air is reduced to little over half an atmosphere and the oxygen tension is therefore only about 11 per cent. of an atmosphere. It must be remembered that in most cases of mountain sickness, in addition to this absolute oxygen lack, there is increased consumption of oxygen, owing to the muscular exercise involved in climbing. Moreover a greater volume of the alveolar air must consist of carbon dioxide if the tension of this gas is to be kept constant (cp. Fig. 506, p. 1082). Since diminished oxygen tension, within fairly wide limits, does not excite any corresponding increase in the respiratory movements, there must, at these heights, be an actual diminution in the oxygen tension in the alveoli. This diminution in tension is shown by a series of observations carried out by Zuntz on himself and fellow workers at different localities. It may be noted that on Monte Rosa, where the oxygen tension in the alveoli was reduced to between 37 and $57 \mathrm{~mm} . \mathrm{Hg}$, as against the normal 101 to $105 \mathrm{~mm}$. Hg, all the members of the party were suffering from mountain sickness.

\begin{tabular}{|c|c|c|c|c|c|c|c|c|c|}
\hline \multirow{2}{*}{\multicolumn{2}{|c|}{ - }} & \multirow{2}{*}{$\begin{array}{c}\text { Height } \\
\text { above sea } \\
\text { level, } \\
\text { metres }\end{array}$} & \multirow{2}{*}{$\begin{array}{c}\mathrm{O}_{2} \text { tension } \\
\text { of air }\end{array}$} & \multicolumn{6}{|c|}{ Alveolar $\mathrm{O}_{\mathbf{2}}$ tension } \\
\hline & & & & A & $\mathbf{B}$ & c & $\mathbf{D}$ & $\mathbf{E}$ & $\mathbf{F}$ \\
\hline Berlin . & • & 54 & 157 & 105 & 101 & - & 105 & 103 & 104 \\
\hline Brienz . & . & 500 & 148 & $84 \cdot 5$ & 94 & 80 & 88 & 86 & 91 \\
\hline Brienzer Rotl & thorn & 2130 & 121 & 68 & 66 & 64 & 62 & 66 & 71 \\
\hline Col d'Olen & . & 2900 & 110 & 57 & - & - & 60 & 68 & 68 \\
\hline Monte Rosa & . & 4560 & 89 & - & 46 & 49 & 61 & 37 & 57 \\
\hline
\end{tabular}

As a result of the oxygen starvation there is inadequate supply of this gas to the heart, so that the circulation tends to fail, especially on making the slightest muscular movements. At the same time the oxygen starvation of the brain produces failure of judgment and inability to carry out or to co-ordinate muscular movements properly. The symptoms as a rule do not increase until death results, so that, although there is an oxygen starvation of the body, there must be some means by which the respiration is modified 
so as to obtain a sufficiency of this gas for the lowered requirements of the body. That the adaptation is effective is shown by the fact that most individuals, if they remain at a height, gradually recover from the mountain sickness and may finally be able to carry out muscular movements with almost as great precision and force as they could previously on the plains. The mechanism by which increased ventilation of the lungs is attained is that already mentioned in dealing with the effects of lack of oxygen, namely, the production of acid substances in the body. The respiratory centre is thus stimulated by these acid substances, especially lactic acid, as well as by the carbon dioxide tension of the blood, and the joint action of these two substances (which probably co-operate in raising the hydrion concentration of the blood) determines the marked increase in the lung ventilation. Since the carbon dioxide is no longer the sole factor responsible for the ventilation, the tension of this gas in the alveolar air is diminished.

ACAPNIA. This diminution of carbon dioxide tension in the blood and alveolar air has been regarded by Mosso as the essential factor in the causation of mountain sickness and has been designated acapnia. It may be absent, however, in the most marked cases of mountain sickness, where the respiratory centre has failed to respond to the additional acid stimulation, and may be present to a marked degree in individuals who are experiencing none of the ill effects of this disorder.

Another important means of rapid adaptation is by means of the circulation. This is noticeable even in the case of persons sitting quietly in a gas chamber who are subjected to gradually lower pressures. It is evident that a deficient passage of oxygen from the alveoli to the blood may, so far as the tissues and heart are concerned, be accommodated for by increasing the rapidity of the circulation, and this is effected by a quickening of the pulse-rate. The following Table shows the changes in the pulse-rate caused by exposure to varying pressures in a gas chamber :

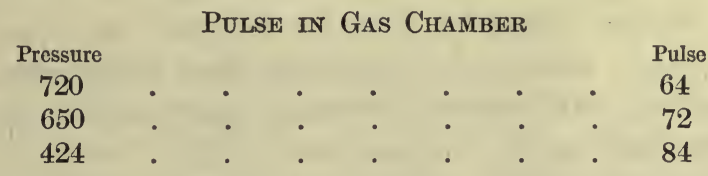

This quickening of the pulse is to be observed also in the trained mountain soldier, in individuals in whom there is no lowering of the alveolar carbon dioxide tension, so that apparently in such cases the whole adaptation to altered conditions is by means of the circulation. In cases where adaptation fails it is in the circulation that the failure is most marked, so that the symptoms of severe mountain sickness resemble closely those produced by rapid heart-failure. Dilated heart, cyanosis, muscular weakness, vomiting, mental torpor, inco-ordination, delirium, may all be observed in both cases. The disturbance of the central nervous system is shown by the almost invariable occurrence at great heights of Cheyne Stokes breathing.

If the animal is able to withstand the immediate effects of exposure to a rarefied atmosphere, a process of adaptation comes into play which finally 
fits him for discharging his functions normally even at the high altitude. From the lack of sensibility of the respiratory centre to small changes in oxygen tension, any diminution in oxygen tension must cause a corresponding diminution in the degree of saturation of the hæmoglobin of the blood. This change in oxygen saturation is at once felt by the blood-forming organs. As an immediate effect of change to a region of low atmospheric pressure there is a relative increase in the blood-corpuscles due to a concentration of the blood and a diminution of its plasma. Simultaneously, however, the blood-forming organs enter into a condition of increased activity, so that after a stay of four or five weeks' duration at a height both corpuscles and hæmoglobin are considerably increased in total amount. The following Table shows the average number of red corpuscles contained in one cubic millimetre of blood from the inhabitants of regions at varying altitudes :

\begin{tabular}{|c|c|c|c|}
\hline & & $\begin{array}{l}\text { Height above sea } \\
\text { level, } \\
\text { metres }\end{array}$ & Red corpuscles \\
\hline Christiania & & 0 & $4,970,000$ \\
\hline Zurich. & . . & 412 & $5,752,000$ \\
\hline Davos & . & 1560 & $6,551,000$ \\
\hline Arosa & . & 1800 & $7,000,000$ \\
\hline Cordilleras & . & 4392 & $8,000,000$ \\
\hline
\end{tabular}

There is of course a limit to the power of adaptation, a limit which varies in different individuals. Thus for some men it is impossible to stay any length of time in the high settlements in the Andes, while others, after two or three weeks' discomfort, become perfectly inured to their new conditions. It seems doubtful, however, whether any of the present race of men could become adapted to permanent residence at a height over 5000 metres, and though for a certain length of time by bringing into play the reserve mechanisms already described, they may raise themselves to a height considerably above 5000 metres, it seems questionable whether without artificial means, such as the inhalation of oxygen, it will be possible for any man to attain the highest points on the earth's surface, or at any rate to arrive there by his own unaided efforts. The highest summits in the Himalayas have a height approaching that attained by Tissandier with his two companions in his famous balloon ascent, namely, 8600 metres. In this ascent, although oxygen inhalation was used (somewhat ineffectively), two of the party succumbed.

The stimulating effect of oxygen lack on the blood-forming organs extends also to the muscular system, so that one of the effects of a residence in high altitudes is increased assimilation of nitrogen. For a time the nitrogen output is less than the nitrogen intake, and there is an actual building up of new tissue. The condition of the individual is similar to that of a growing animal, a fact which may explain the admirable results of a mountain holiday. We can hardly imagine that the power of the organism 
to react in this way was evolved through generations of mountain climbing. We are probably here making use of an adaptation which has been evolved for the purpose of retrieving loss of blood by hæmorrhage, such as must have been of continual occurrence in the struggle of individual against individual, which has resulted in the survival of the animals of to-day.

ALTERATIONS IN THE NITROGEN TENSION. The nitrogen of the atmosphere plays no part in the metabolism of the body, and must be regarded as a purely inert gas. It is a matter of indifference whether under normal atmospheric pressure we breathe an atmosphere of pure oxygen or one containing one-fifth part of this gas diluted with four-fifths of nitrogen. The very inertness of nitrogen may be of danger to the body under certain conditions. If a man or an animal be exposed, as in a diving-bell, to a pressure of three, four, or six atmospheres, the respiratory functions are unaffected, but the amount of nitrogen dissolved in the fluids of the body is increased in direct proportion to the pressure. If the pressure be now suddenly released, the nitrogen, which cannot be used up by the tissues, is given off from the body-fluids in the form of bubbles, just as carbonic acid gas rises in bubbles from soda-water when the pressure is removed by withdrawing the cork from the bottle. These bubbles occurring in all the capillaries obstruct the flow of blood, and therefore, if the evolution of gas is sufficiently large, the animal dies in convulsions. A similar evolution of gas may occur in the spinal cord, giving rise to destruction of the cord and paralysis (' divers' palsy'). In order to prevent this sudden evolution of gas it is necessary that the change from the high pressure to the ordinary atmospheric pressure should be carried out gradually, so as to give the blood-plasma, supersaturated with nitrogen, time to get rid of its excess of nitrogen without the formation of bubbles.

OTHER GASES. Hydrogen and methane are, like nitrogen, indifferent gases. They may be respired if mixed with 20 per cent. of oxygen, and either of the gases may be used instead of nitrogen to dilute the oxygen that we breathe, without harm or inconvenience.

Carbon monoxide is rapidly poisonous by its action on the red corpuscles. It combines with hæmoglobin, forming $\mathrm{CO}$-hæmoglobin, a compound which is much more stable than oxyhæmoglobin. The blood is therefore deprived of its oxygen carrier, and the animal dies of asphyxia. We have seen, however, that the displacement of oxygen by $\mathrm{CO}$ is not absolute, but only relative. Hence, although the avidity of $\mathrm{CO}$ for hæmoglobin is 140 times that of oxygen, we can convert the $\mathrm{CO}$ back into oxyhæmoglobin by increasing the mass influence of the oxygen. This may be done by giving the poisoned animal pure oxygen to breathe, or even oxygen under pressure. In pure oxygen at a pressure of two atmospheres an animal can breathe and live, even though the whole of its hæmoglobin is converted into $\mathrm{CO}$-hæmoglobin, the amount of oxygen which is simply dissolved by the blood-plasma being sufficient at this pressure for the respiratory needs of the animal (Haldane).

Other gases which have special poisonous properties are hydrocyanic 
acid, sulphuretted hydrogen, phosphuretted hydrogen $\left(\mathrm{PH}_{3}\right)$; arseniuretted hydrogen, \&c.

IRRESPIRABLE GASES are those which are so irritating that they produce spasm of the glottis. Such are ammonia, chlorine, sulphur dioxide, nitric oxide, and many others.

\section{VENTILATION}

A point of practical importance is the securing to each individual of sufficient fresh air, so that he may always have a plentiful supply of oxygen, and may be relieved of his waste products. It is found that a dwelling-room becomes unpleasant and stuffy when the percentage amount of $\mathrm{CO}_{2}$ has reached 0.1 per cent. This stuffiness is supposed to be due.to organic exhalations from the skin, lungs, and alimentary canal, some of which have a poisonous effect, giving rise to headache and sleepinesss. Since these cannot be measured, it is taken as a cardinal rule in ventilation that the amount of $\mathrm{CO}_{2}$ should never rise above $0 \cdot 1$ per cent.

Since in questions of ventilation we have generally to deal with trades in which the metric measure is not used, it may be convenient to give the data as to carbon dioxide production and the amount of air required in cubic feet.

An adult man gives off about $0 \cdot 6$ cubic foot of $\mathrm{CO}_{2}$ every hour. Hence in that time he raises the amount of $\mathrm{CO}_{2}$ in 1000 cubic feet of air from $\cdot 04$ per cent. (the normal amount in the atmosphere) to $0 \cdot 1$ per cent. He must therefore be supplied with 2000 cubic feet of air per hour in order to keep the amount of $\mathrm{CO}_{2}$ down to .07 per cent.

(Ordinary air contains $\cdot 04$ per cent. $\mathrm{CO}_{2}$, therefore 2000 cubic feet would contain 0.8 cubic foot $\mathrm{CO}_{2}$, which.with the 0.6 cubic foot.given off by the man would be $1 \cdot 4$, which is $\cdot 07$ per cent.)

In order that the air may be easily renewed without giving rise to excessive draught, a certain amount of cubic space must be allotted to each man. Each adult should have in a room 1000 cubic feet of space, and be supplied every hour with 2000 to 3000 cubic feet of air. 


\section{SECTION V}

\section{THE MECHANISMS OF OXIDATION IN THE TISSUES}

THE blood in its passage through the capillaries takes up carbon dioxide from the tissues, giving oxygen to the latter in exchange. This interchange is determined by the differences in tension of the gases on the two sides of the capillary wall. Whereas the tension of oxygen in the plasma varies from $100 \mathrm{~mm}$. $\mathrm{Hg}$ in arterial to $25 \mathrm{~mm}$. $\mathrm{Hg}$ in venous blood, the tension of oxygen in the tissues outside the vessels in most cases approaches 0 , as is shown by Ehrlich's methylene-blue experiment described on p. 1063. On the other hand, the tension of carbon dioxide in the tissues, as judged from the examination of fluids such as bile and urine, varies from 6 to 10 per cent. of an atmosphere. The continuous flow of oxygen into, and of carbon dioxide away from, the tissues points to the constant occurrence of oxidative changes in the tissue-cells. By the blood the tissues receive not only oxygen but also food-stuffs, namely, proteins or amino-acids, fats, and sugars, derived from the alimentary canal, or, in starvation, from other parts of the body. The activity of the tissues, whether motor, as in the case of muscle, or secretory, as in the case of glands, is derived from the energy set free in the partial or complete oxidation of these food-stuffs, which occurs within the active cells themselves. A study of the mechanism of oxidation in the body involves therefore a consideration of the processes which take place within the confines of each cell. The question is by no means an easy one. Although we speak of the 'burning' of food-stuffs, and compare the processes in the body to those which take place in combustion, e.g. in a candle-flame, the analogy is after all a very rough one. In the first place; the food-stuffs, even after absorption, belong to a class of substances which have been designated as dysoxidisable, since they present no tendency to combine with ordinary atmospheric oxygen. Thus sugars, proteins, or fats, if kept free from microbial infection, may be kept for years exposed to the air without undergoing any change. It is true that in certain cases, e.g. in alkaline solutions of sugar, we may obtain slow absorption of oxygen and oxidation of the sugar. The changes are, however, slight and limited in extent. All these food-stuffs are susceptible of combustion if raised to a sufficiently high temperature, but in the animal body the processes of oxidation have to go on at a temperature varying between $5^{\circ}$ and $40^{\circ} \mathrm{C}$., and in a solution which is almost neutral in reaction. It might be said that at the temperature of an ordinary flame the combustion of the food-stuffs is immediate and 
complete, whereas in the body the oxidation takes place by stages. Recent research has tended to remove this point of distinction by pointing out that even in an explosion of a mixture of methane and oxygen there is a series of intermediary products, and that the whole process, if analysed, is made up of stages in which hydrolysis and oxidation go on simultaneously, so that on this account it is difficult to cause a combination, even of hydrogen and oxygen, in the complete absence of any watery vapour. The oxidations in the body are strictly limited both in nature and extent. The mere fact that a substance is readily or even spontaneously oxidisable (autoxidisable) affords no guarantee that it will undergo oxidation in the animal body. Thus phosphorus or pyrogallol taken by the mouth can be recovered in an unoxidised form from the urine. Carbon monoxide is excreted unchanged. There must apparently be some definite relationship between the molecular structure of the food-stuff and that of the cells of the body. Thus ordinary proteins which undergo complete oxidation contain large quantities of leucine. This substance is lævorotatory and is designated $l$-leucine. If $l$-leucine be administered to rabbits it is completely oxidised. If its isomer $d$-leucine, resembling it in every particular so far as we can see except in its relation to polarised light, be administered to a rabbit, the greater part of the substance passes through the body unchanged. In the same way there are sixteen sugars of the formula $\mathrm{C}_{6} \mathrm{H}_{12} \mathrm{O}_{6}$. Of these only four, namely, glucose, fructose, galactose, and mannose, can be oxidised in the animal body. Other sugars differing in so slight a degree from these four as, e.g., $l$-glucose or $l$-fructose, cannot be utilised by the body. Not only must there be a distinct relation between the structure of the cell and the molecular structure of the food-stuff supplied, but there must be different mechanisms for the food-stuffs and their derivatives. Thus in certain cases of disease or of abnormal nutrition the body may lose absolutely the power of utilising, i.e. of oxidising, a whole class of food-stuffs. In severe diabetes, or after destruction of the pancreas, glucose behaves in the body as if it were one of the artificial unassimilable sugars. The normal oxidation of fats probably proceeds by stages in each of which two atoms of carbon undergo oxidation. The penultimate stage in the oxidation of any of the higher fatty acids is thus oxybutyric acid. In complete carbohydrate starvation, for some reason or other, the body loses its power of completing this last stage, so that the oxybutyric acid undergoes no further oxidation, and either accumulates in the body or is excreted combined with bases in the urine. In the normal individual tyrosine, whether administered separately or in combination in protein, is completely oxidised, the benzene ring being broken up. In certain rare cases of disordered metabolism the patient, who is otherwise apparently well, is unable to effect the total oxidation of tyrosine, which is therefore excreted as homogentisic acid, after undergoing only the first stage of its normal transformation in the body. These various mechanisms are adjusted in each case to the functional activity of the cell and are limited therefore, not by the supply of oxygen or of food-stuff to be oxidised, but by the necessities of the cell, i.e. the adaptations induced in it by its environ- 
mental changes. In discussing the mechanism of intracellular oxidation we have therefore to consider in the first place how the dysoxidisable foodstuffs are made to combine with the molecular oxygen diffusing into the cells from the blood in the capillaries, and in the second place the means by which these oxidative changes are strictly limited in accordance with the necessities of the cell, and finally the nature of the specific oxidative mechanisms for each kind of food-stuff and for the various stages in the oxidation of each food-stuff.

We are very far as yet from being able to give a definite answer to any one of these questions. Even in the first problem, namely, the oxidation of dysoxidisable substances, we have to confine ourselves almost exclusively to speculation on possibilities. Although these substances will not unite with the oxygen of the air, in which the combining activities of the oxygen are satisfied by the combination of two atoms to form one molecule, many of them readily undergo oxidation if subjected to the action of 'atomic" oxygen or 'active' oxygen, and it has been suggested that the problem of the oxidation of the body is really bound up with the question as to the mode of activation of the molecular oxygen derived from the oxyhæmoglobin. Thus Hoppe-Seyler suggested that the activation of oxygen might occur through the intermediation of reducing substances. He supposed that reducing substances might be formed under the influence of ferments by hydrolytic splitting of the food-stuffs. A reducing substance is one that has sufficient affinity for oxygen at the ordinary temperature to tear asunder the bonds which unite two atoms of oxygen to form one molecule, and to combine with one or both of the atoms so set free. If the combination is with only one atom, the other atom of the oxygen molecule is set free in an active form, and is therefore able to oxidise dysoxidisable substances which may be present. Thus, when a mixture of ammonia and pyrogallol is exposed to the atmosphere, the oxygen is rapidly absorbed, forming a dark brown solution, pyrogallol being therefore a reducing agent. But at the same time a certain amount of the ammonia (a dysoxidisable substance) undergoes oxidation with the formation of nitrite. In the slow spontaneous oxidation of phosphorus which occurs on exposing this substance to the atmosphere, ozone, $\mathrm{O}_{2} \mathrm{O}$, is always formed. As a type of the formation of reducing substances in hydrolytic fermentations may be adduced the butyric acid fermentation, in which sugar is converted into butyric acid, carbonic acid, and hydrogen :

$$
\mathrm{C}_{6} \mathrm{H}_{12} \mathrm{O}_{6}=\mathrm{C}_{4} \mathrm{H}_{8} \mathrm{O}_{2}+2 \mathrm{CO}_{2}+2 \mathrm{H}_{2} \text {. }
$$

The hydrogen produced in this process would act as a reducing agent. There is no doubt that reducing substances are formed under normal circumstances in the tissues, as is shown by the methylene-blue experiment, and it is possible that such reducing substances may aid in activating oxygen and in the induction of certain oxidative processes. The activation of oxygen would, however, not explain the specific character of the various oxidations, and the accurate gradation of these oxidations to the necessities of the cell. In many cases reducing substances may themselves act as 
carriers of oxygen, and their action be more or less specific. If, for instance, glucose be boiled with an ammoniacal solution of cupric hydrate, it undergoes oxidation, the cupric being reduced to cuprous hydrate. Cuprous hydrate in ammoniacal solution is a reducing substance; it absorbs oxygen from the air and is reconverted to cupric hydrate. A small amount of cupric hydrate therefore, in the presence of air, may act as a carrier of oxygen from the air to the sugar and may thus oxidise indefinitely large quantities of sugar. In the same way, if indigo in alkaline solution be boiled with sugar, it undergoes reduction with the formation of a colourless compound. On shaking the decolorised solution with air it absorbs oxygen with the reproduction of indigo, so that here again minute quantities of indigo blue may serve to oxidise large quantities of glucose. The mode of action of these oxygen carriers resembles closely that of the various ferments which effect the transference of water from the menstruum to the substrate (e.g. trypsin, invertase, \&c.). These hydrolytic ferments differ from ordinary hydrolytic agents, such as dilute acids, in the specific character of their action. Trypsin, for instance, will hydrolyse polypeptides of a type corresponding to those which make up the ordinary food-products, but is powerless to hydrolyse polypeptides composed of artificial amino-acids which are the optical isomers of those occurring in the body. It seems possible that we might explain the specific oxidations occurring in the cell by assuming the presence of a number of ferments, oxidases, which would act as oxygen carriers, but each of which would only be able to act on a certain type of food-stuff or on molecules of a given configuration.

Such oxidative ferments have been described as existing in many animal and vegetable extracts. Many species of fungus contain a ferment known as tyrosinase, from the fact that, when it is added to solutions of tyrosine in the presence of air, the tyrosine is oxidised with the formation of a brown pigment. The same ferment is able to effect the oxidation of other aromatic substances. The browning of a freshly cut potato or apple on exposure to the air is similarly ascribed to the oxidation of a chromogen by the oxygen of the air, through the intermediation of an oxidase present in the cells. If benzyl alcohol or salicyl aldehyde be added to a suspension of liver-cells in blood, and air be allowed to bubble through the mixture for some time, the alcohol or aldehyde is oxidised to the corresponding acid. In the same way xanthine $\left(\mathrm{C}_{5} \mathrm{H}_{4} \mathrm{~N}_{4} \mathrm{O}_{2}\right)$ added to a mixture of spleen pulp and defibrinated blood is converted into uric acid $\left(\mathrm{C}_{5} \mathrm{H}_{4} \mathrm{~N}_{4} \mathrm{O}_{3}\right)$.

Bach and Chodat have shown that in many cases the oxidase is not a single substance, but a mixture of an organic peroxide with a ferment, peroxidase, which has the property of splitting off atomic, i.e. active, oxygen from the peroxide. These peroxidases have the same effect on hydrogen peroxide. They must be distinguished from the ferment catalase, which is present in almost all animal and vegetable tissues, and which effects a rapid decomposition of hydrogen peroxide with the formation of molecular oxygen :

$$
2 \mathrm{H}_{2} \mathrm{O}_{2}=2 \mathrm{H}_{2} \mathrm{O}+\mathrm{O}_{2} \text {. }
$$


In the case of a peroxidase the equation would be represented :

$$
\mathrm{H}_{2} \mathrm{O}_{2}=\mathrm{H}_{2} \mathrm{O}+\mathrm{O}^{\prime} \text {. }
$$

Many reactions are known in chemistry in which the part of a peroxidase is played by an inorganic catalyst. Thus hydrogen peroxide effects a slow oxidation of many organic substances, but the oxidation is enormously hastened if to the mixture be added a trace of a ferrous salt (Fenton's reaction). The same part may be played by salts of manganese, and it is interesting to note that manganese forms an essential constituent of the peroxidase laccase, which is present in many plants and is responsible for the formation of the Japanese lacquer. It effects a specific oxidation of hydroquinone and pyrogallol. The oxidations carried out by the use of hydrogen peroxide, with or without a catalyst or peroxidase, present a close resemblance to the oxidations occurring in the animal body. Thus Dakin has shown that saturated fatty acids, even the higher members of the series, are gradually oxidised if warmed gently with hydrogen peroxide in the presence of ammonia, and the course of the reaction resembles in many respects that which, on other grounds, we have assumed to take place in the normal metabolism of the body.

We have no evidence that hydrogen peroxide is formed at any time in the body, though there is some reason to assume its formation in the process of carbon assimilation in the green leaf. If we adopt the views of Bach and Chodat we must assume that every animal cell contains organic peroxides as well as peroxidases, or else that it can under physiological conditions form these substances. Since there is also evidence of the presence of reducing substances in the cells, we may conveniently assume, with Ehrlich, that distinct side-chains of the protoplasmic molecule have specific affinities for oxygen. When all these affinities are saturated, these side-chains will act as peroxides, parting with their oxygen with extreme ease, whereas when the greater number are unsaturated, the resultant effect will be that of a reducing agent. The same protoplasmic molecule may therefore, according to its state of saturation with oxygen, act either as an oxidising or reducing agent, and can effect, probably through the intermediation of specifically adapted oxidases, the oxidation of the various food-stuffs stored up as the paraplasm of the cell. Since the oxidative processes are determined, not by the presence of oxygen, but by the functional activities of the tissue, we must assume that the peroxidases are not preformed in the cell, but exist as precursors, zymogens, from which they can be set free in accordance with the necessities of the cell.

It is probable that many of the food-stuffs or other proximate constituents are not directly accessible to oxidation, and that the first step in their utilisation is a process of cleavage or hydrolysis, which itself involves the presence of specific ferments. Thus, so far as we can tell, the amino-acids undergo deamination before oxidation. They can thus be stored up in the cell either free or in the form of protein and present no point of attack to oxygen until the process of hydrolysis and deamination has taken place. This course of events is certainly true for some of the members of the purine group. 


\section{CHAPTER XVII}

\section{RENAL EXCRETION}

\section{SECTION I}

\section{THE COMPOSITION AND CHARACTERS OF THE URINE}

THE main product of the oxidation of carbon, namely, carbon dioxide, is discharged by the lungs and to a slight extent by the skin. Water, taken as such with the food, but also derived to a slight extent from the oxidation of hydrogen, is got rid of by the lungs, skin, and kidneys. The salts of heavy metals when administered are excreted for the most part by the alimentary canal, e.g. iron, bismuth, mercury. A certain proportion of the pigmentary waste products of the body, derived from the breakdown of the blood-pigment, is also got rid of with the fæces. With these exceptions practically all the waste products resulting from metabolism are excreted in the urine by the kidneys. We have thus to seek in the composition of this fluid the last chapter in the metabolic history of a large number of the constituents of the body. Since, moreover, the kidneys may excrete almost any substance which circulates through their blood-vessels, many of the intermediate metabolites may be found in minute quantities in the urine and may be isolated by working up large quantities of this fluid. Under pathological conditions these metabolites may appear in the urine in larger amounts and serve then as an index to some interference with the later stages in the metabolism of fats, carbohydrates, or proteins.

The composition of the urine must therefore be a variable one, according to the activity of the body, the quantity and nature of the food taken, and the relative amount of water escaping by the kidneys, lungs, and skin respectively. But just as we can describe a normal diet for an adult man of average weight, so we can describe an average composition for the urine. The history of the urinary constituents has been given for the most part in the chapter dealing with the metabolism of the proximate constituents of the food. It will be useful, however, to enumerate in this chapter the various constituents of the urine and to summarise their properties, preparation, and normal significance.

The urine of man is a clear yellow fluid which froths when shaken. On standing, a cloud of mucus is deposited, consisting of a very small amount 
of nucleoprotein derived from the epithelial lining of the bladder and urinary passages. In concentrated urine a deposit occurs on cooling. This deposit dissolves when the urine is warmed, and consists of urates. Under certain circumstances urine is turbid as it is passed, but in this case the turbidity generally consists of earthy phosphates and is not cleared up by heating.

The colour of the urine varies with its concentration. After severe sweating the amount of water excreted by the kidneys is small, and the urine is therefore concentrated and of high colour. After copious draughts of liquid the urine may be very pale and dilute.

Ordinary urine has an aromatic odour, but this varies largely with the character of the food. Many food substances give characteristic odours, which may depend on alterations undergone by them in their passage through the body.

The specific gravity of the urine is proportional to its concentration. Normally it is 1016 to 1020 , though it may rise as high as 1040 or sink as low as 1002.

The molecular concentration of the urine is almost always greater than that of the blood. Its osmotic pressure may be measured by determining the depression of freezing-point. The $\Delta$ of urine normally varies between $0 \cdot 87$ and $2 \cdot 71$ ( $\Delta$ of blood $=0 \cdot 56$ ). After copious draughts of water the depression of freezing-point in the urine may be less than that of serum, and may be as small as $0 \cdot 25$.

The reaction of urine is generally described as acid. It is acid to litmus and to phenolphthalein. This is due to the fact that neutral constituents of the food give rise to acid end-products in metabolism. The sulphur of proteins is converted into sulphuric acid and the phosphorus of lecithin into phosphoric acid. There is thus a predominance of acid radicals over bases in ordinary urine. This statement, however, only applies to man and to carnivora. In the food of herbivora there is a predominance of alkaline bases. Vegetable acids, e.g. tartaric, malic, and citric acids, undergo oxidation to carbonic acid in the body, so that their bases leave the body as alkaline carbonates. The urine of such animals therefore contains an excess of alkaline carbonates, and is alkaline in reaction and froths on the addition of an acid. If a herbivorous animal be starved, so that it has to live on its own tissues, it becomes for the time, so to speak, carnivorous, and its urine becomes clear and acid. The urine of man can be made alkaline by the ingestion of large quantities of vegetables or fruits. Under such circumstances the urine as passed is generally turbid from the presence of precipitated earthy phosphates. In determining the reaction of urine it is usual to adhere to one indicator, e.g. phenolphthalein, and to give the acidity in terms of decinormal acid, naming the indicator used. The acidity (i.e. the concentration of $\mathrm{H}$ ions) can also be determined by the electrical method. In this way Hoeber found the acidity of human urine to vary between $4.7 \times 10^{-7}$ and $100 \times 10^{-?}$. On the average it was $49 \times 10^{-7}$ in the litre. 
THE AVERAGE COMPOSITION OF THE URINE. Several analyses of the day's urine under varying conditions of food have already been given (v. pp. 757,780 ). The following may be taken as a fair average for an adult man on ordinary mixed diet :

Total amount of urine $=1500$ c.c.

This contains about $60 \mathrm{grm}$. of solids, of which $25 \mathrm{grm}$. are inorganic and 35 grm. organic. These are distributed as follows :

Inorganic Constitueints

Sodium chloride .

Sulphuric acid

Phosphoric acid .

Potassium .

Ammonia

Magnesia

Lime .

Other substances .
Organic Constituents

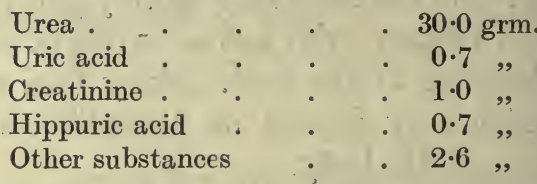

The quantity of urine will naturally vary with the water leaving the body by the kidneys, and therefore according to the habit of the individual with regard to the intake of fluids and with his occupation. Thus after copious sweating the total amount may fall to 400 c.c. in the course of the day. If large draughts of liquid be taken it may rise to 3000 c.c. or more. There are also diurnal variations in the amount secreted, depending probably largely on the circulation through the kidneys. The secretion is at a minimum during sleep, and especially between 2 and 4 o'clock in the morning. It is at its maximum during the first hours after rising, and increases generally after each meal. Muscular exercise may also give an initial increase owing to the greater vigour of the circulation associated with exercise. If the exercise is severe enough to cause sweating or is carried to fatigue, there may be a consequent diminution in the amount of urine secreted.

\section{THE INORGANIC CONSTITUENTS OF THE URINE}

(a) ACID RADICALS. The chlorides of the urine are derived almost entirely from the chlorides of the food. Though essential constituents of the body fluids, it does not seem that the chlorides enter into organic combination with the constituents of the cells. The output of chlorides, which normally varies from 6 to $10 \mathrm{grm}$. $\mathrm{Cl}$. in the course of the day, will therefore depend on the amount of chlorides taken in with the food. If these be withdrawn altogether, the chlorides may almost disappear from the urine, although the circulating blood contains practically the same amount of chlorides as in the normal individual, showing that the body retains the chlorides necessary for the proper carrying out of the vital processes as long as possible. Chlorides may also disappear from the urine temporarily under various pathological conditions. This is especially marked in cases of acute pneumonia. 
Sulphates. The salts of sulphuric acid do not form an important constituent of the food. The sulphates of the urine are derived almost entirely from the oxidation of the sulphur of the protein molecule. The output of sulphates is therefore, like that of urea, an index of protein metabolism. As the nitrogen of the urine goes up, so the sulphates will increase. On an average diet the ratio of urinary nitrogen to $\mathrm{SO}_{3}$ is about $5: 1$; though, owing to the varying content of different proteins in the sulphur, this ratio will naturally alter with the nature of the protein taken as food. The daily output of sulphuric acid varies between 1.5 and $3 \mathrm{grm} . \mathrm{SO}_{3}$. The greater part of the sulphate is present as sulphates of the alkaline metals. A certain proportion, about 10 per cent., is present in the form of conjugated or ethereal sulphates, chiefly indoxyl sulphate. A small proportion of the sulphur excreted in the urine is present in unoxidised form as so-called neutral sulphur. The neutral sulphur probably includes a number of different bodies, among which sulphocyanates and cystine are the best known.

Inorganic sulphates can be precipitated from the urine by the addition of hydrochloric acid and barium chloride. On filtering off this precipitate, the filtrate contains the ethereal sulphates. On boiling, the hydrochloric acid decomposes these substances, setting free sulphuric acid, which combines with the excess of barium present and is precipitated as barium sulphate. This second precipitate therefore, when weighed, gives the amount of ethereal sulphates present. To determine the neutral sulphur the fluid, after the separation of both kinds of sulphates, is treated with sodium carbonate to precipitate the barium, filtered, and the filtrate evaporated to dryness. The residue is then ignited with potassium nitrate, cooled and extracted with water. By this treatment all the neutral sulphur is converted into sulphates, which can be thrown down from the solution with barium chloride and weighed in the usual way.

Phosphates. The phosphates of the urine are derived partly from the phosphates of the food, partly from the oxidation of the organic phosphoruscontaining constituents of the food and of the tissues, e.g. nuclein, lecithin, \&c. If the food contains much calcium and magnesium, the amount of phosphates excreted by the urine diminishes, since these substances are excreted with the frces as calcium and magnesium phosphates. According to the diet therefore, phosphoric acid may be excreted either by the intestine or by the kidneys. The amount of phosphates, reckoned as $\mathrm{P}_{2} \mathrm{O}_{5}$, excreted in the course of the day may vary between 1 and $5 \mathrm{grm}$. In the urine the phosphates exist as a mixture of the mono- and di-sodium phosphates, the relative amounts of the two varying with the acidity of the urine. If the urine is neutral or alkaline there is very often a deposit of earthy phosphates. Whether this deposit is present or not depends on the varying solubility of the different calcium and magnesium phosphates. Thus the mono-magnesium phosphate $\mathrm{MgH}_{4}\left(\mathrm{PO}_{4}\right)_{2}$ and the mono-calcium phosphate $\mathrm{CaH}_{4}\left(\mathrm{PO}_{4}\right)_{2}$ are both fairly soluble in water, and their solubility is increased by the presence of neutral salts. With increased acidity of the urine the proportion of the two bases present in these forms is diminished. The di-magnesium and di-calcium phosphates are only slightly soluble in water, and the latter would, if present in the urine, be deposited. One may indeed, in slightly 
acid urine, find the di-calcium phosphate occasionally present as a crystalline deposit. On heating the urine the di-calcium phosphate breaks up into a mono-calcium phosphate and a tri-calcium phosphate, while the acidity of the urine is increased by the solution of the mono-calcium phosphate. Alkaline urine will always present a precipitate of tri-calcium phosphate $\mathrm{Ca}_{3}\left(\mathrm{PO}_{4}\right)_{2}$. When normal urine is allowed to stand, the urea is converted by the presence of micro-organisms into ammonium carbonate, and the urine becomes alkaline. Under such conditions we may often find a crystalline precipitate of ammonium magnesium phosphate, $\mathrm{NH}_{4} \mathrm{MgPO}_{4}$, the socalled 'triple phosphate.'

(b) THE BASES OF THE URINE. The bases include potash, soda, ammonia, magnesia, and lime.

The amount of potash excreted in twenty-four hours varies between 1.9 and $3 \cdot 2 \mathrm{grm}$, according to the nature of the food taken. With a large meat diet, which contains considerable quantities of potassium, the output of this base is increased. In fasting there is also an increase in the output of potash, owing to the utilisation of the tissues of the body which themselves are rich in potassium.

The amount of sodium excreted in the twenty-four hours varies on the average between 4 and 5 grm., but depends very largely on the quantity of sodium chloride taken with the diet. The alkaline earths, lime and magnesia, are invariably present in urine, but in much smaller quantities than the alkaline metals. The average amount of these two bases in the twenty-four hours varies in each case between $0 \cdot 1$ and $0.2 \mathrm{grm}$. Their output by the urine is no criterion of the amount taken in with the food or absorbed from the intestines, since both these bases may be re-excreted into the gut and appear as insoluble phosphates in the fæces.

Normal human urine always contains a small amount of ammonia, on an average between 0.6 and $0.8 \mathrm{grm}$. in the twenty-four hours. As we have already seen, in dealing with the origin of urea in the body, the quantity of ammonia in the urine is an index to the excess of acids over bases which have to be excreted by this fluid. Thus it is easily possible to increase the proportional amount of ammonia in the urine by the administration of mineral acids. An increase of the proportion of nitrogen excreted as ammonia, apart from the administration of acids with the food, is an important indication of the formation of abnormal acid substances in metabolism. Thus in diabetes, when the last stages of fat oxidation are in default, so that the oxy-fatty acids, $\beta$-oxybutyric and aceto-acetic acids, accumulate in the body, there is always a considerable rise in the ammonia of the urine.

It is usual to reckon iron among the bases which may be excreted by the urine. The amount of this substance in the urine is extremely small, as a rule less than $5 \mathrm{mg}$. in the day. It affords no clue to the iron metabolism of the body, since the main channel of excretion of this substance is the intestine. 


\section{ORGANIC CONSTITUENTS OF THE URINE}

Almost all these constituents contain nitrogen, which in man is distributed among the various urinary constituents as follows :

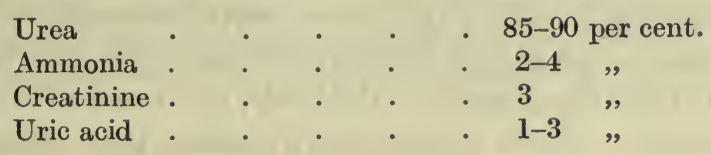

About 6 per cent. of the urinary nitrogen is in the form of other substances, such as hippuric acid, pigments, \&c.

UREA or CARBAMIDE, $\mathrm{CO} / \mathrm{NH}_{2}$ can be regarded as derived from carbonic acid, $\mathrm{CO} \angle \mathrm{OH}$ by the replacement of each $\mathrm{OH}$ group by an $\mathrm{NH}_{2}$ group. It is isomeric with ammonuim cyanate, $\mathrm{NH}_{4} \mathrm{CNO}$. If a solution of potassium cyanate and ammonium chloride be warmed together and evaporated, crystals of urea may be obtained in long colourless prisms (Fig. 516) without any water of crystallisation. It is soluble in water and alcohol, and insoluble in ether. Its solutions are neutral in reaction, but it forms crystalline salts with strong acids. Thus urea nitrate, which is produced by treating strong solutions of urea with concentrated nitric acid, forms microscopic rhombic plates which are extremely insoluble, so that their formation may be used as a test for urea (Fig. 517). With oxalic acid urea solutions yield an insoluble oxalate, also in typical crystals. Urea when heated melts at about $130^{\circ} \mathrm{C}$. On further heating, it undergoes decomposition, giving off ammonia and forming biuret, as follows :

$$
2\left(\mathrm{CO}<\frac{\mathrm{NH}_{2}}{\mathrm{NH}_{2}}\right)=\stackrel{\mathrm{CO}<}{\mathrm{CO}<} \underset{\mathrm{NH}_{2}}{\mathrm{NH}_{2}}+\mathrm{NH}_{3} .
$$

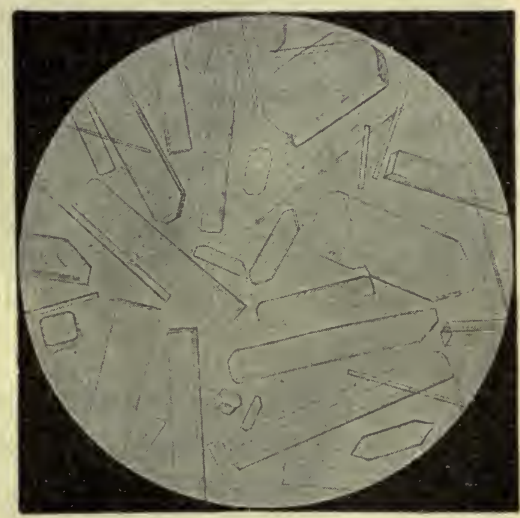

FiG. 516. Urea. (FUNKE.)

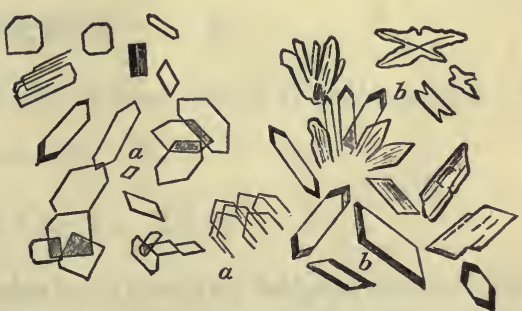

Fig. 517. Urea nitrate. Urea oxalate.

At the same time a certain amount of cyanic acid is formed, and this polymerises to cyanuric acid, $\mathrm{H}_{3} \mathrm{C}_{3} \mathrm{~N}_{3} \mathrm{O}_{3}$. On heating with alkalies or with strong acids urea undergoes hydrolysis, with the production of carbon dioxide and ammonia :

$$
\mathrm{CON}_{2} \mathrm{H}_{4}+\mathrm{H}_{2} \mathrm{O}=\mathrm{CO}_{2}+2 \mathrm{NH}_{3} \text {. }
$$

The same change is effected in urea by certain micro-organisms, e.g. the 
micrococcus ureæ, which is responsible for the ammoniacal change which occurs in urine when exposed to the air.

Urea, being the chief nitrogenous constituent of the urine, is the most important index to the protein metabolism of the body. As we have seen, urea may be regarded as partly exogenous, partly endogenous. The greater part of the 30 grm. excreted by a normal individual in the course of the day is derived directly from the proteins of the foods, as a result of the deamination of the amino-acids, which occurs shortly after their absorption. The ammonia thus formed is combined with carbonic acid and carried to the liver, where the ammonium carbonate undergoes dehydration with the production of urea. Its amount will therefore be directly proportional to the amount of protein taken as food. A smaller proportion is derived from the breakdown of the tissues of the body. This endogenous moiety may undergo considerable increase under any conditions which cause a rapid disintegration of the tissues. Thus there is a large rise in the urea output, even in a starving individeal, in febrile conditions.

In order to prepare urea from urine advantage may be taken of the insolubility of it s combination with nitric acid. Urine is concentrated to about one-sixth of its bulk. It is then cooled and treated with twice its volume of pure concentrated nitric acid, care being taken to keep the whole mixture cool. Urea nitrate is precipitated. The precipitate is collected, dried roughly by pressing between filter paper, and then rubbed up with fresh barium carbonate. Barium nitrate is formed and urea set free. The whole mass is dried and treated with hot absolute alcohol, in which the barium nitrate is insoluble. On filtering off the barium nitrate a pure solution of urea is obtained from which the urea will crystalline on allowing the alcohol to evaporate:

CREATININE. Creatinine is a normal constituent of urine, in which it occurs in quantities of 0.8 to $1.3 \mathrm{grm}$. in the twenty-four hours. It is easily produced from creatine by boiling the latter with strong hydrochloric acid, when a process of dehydration occurs. Creatine has the formula :

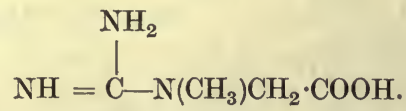

On dehydration it is converted into creatinine :<smiles>CC(=O)OCCC(C)C</smiles>

Creatinine may be obtained from urine in the following way. The urine is made alkaline with milk of lime and treated with calcium chloride and filtered. The filtrate is slightly acidified with acetic acid and evaporated on the water bath to a syrupy consistence. A little sodium acetate is added and the mixture extracted with alcohol. The filtered alcoholic extract is now treated with a concentrated neutral alcoholic solution of zinc chloride. A crystalline precipitate of creatinine zinc chloride is produced. After two days this precipitate is collected on a filter, wa shed with alcohol, dissolved in water, and then boiled for a quarter of an hour with lead hydrate. The mixture is then filtered and the filtrate evaporated to dryness. The dry residue is now extracted with cold alcohol and filtered. On allowing the alcohol to evaporate, crystals of creatinine separate out (Fig. 518). It gives the following tests : 
(1) WEYL'S TEST. On treating a solution of creatinine with a small quantity of very dilute sodium nitroprusside solution and then with weak caustic alkali, a rich ruby-red colour is produced which gradually changes to yellow. On now adding acetic acid and warming, the solution becomes green and then blue, and finally a precipitate of Prussian blue is formed.

(2) JAFFE'S TEST. On treating a solution of creatinine with a few drops of a watery solution of picric acid and dilute caustic potash, an intense red colour is produced. This reaction is made use of for the quantitative estimation of creatinine in the urine.

Like the other nitrogenous constituents, creatinine can be regarded as partly exogenous, partly endogenous in origin. The exogenous part is derived from the creatine contained in meat. When meat is excluded from

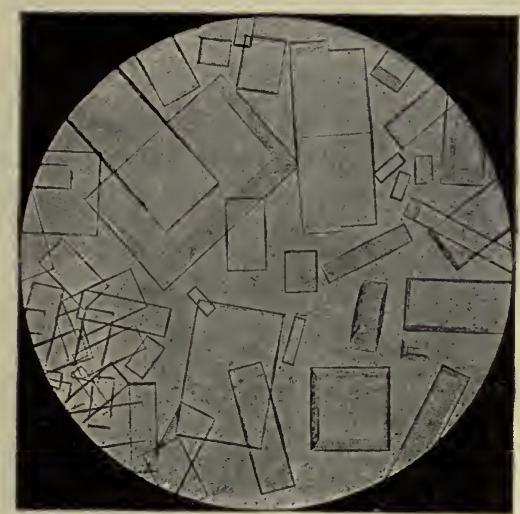

Fig. 518. Creatinine. (FuNKe).

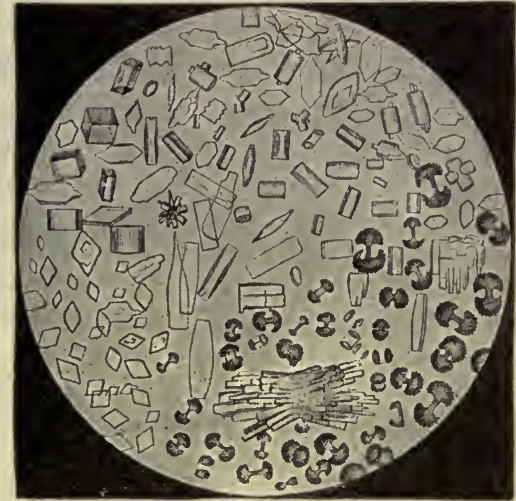

Frg. 519. Uric acid. (Funke.)

the diet the output of creatinine becomes remarkably constant and is little affected by the total amount of proteins taken, the amount excreted during starvation being practically identical with that excreted on a full protein diet. It is said to be increased during febrile conditions and as a result of violent muscular exercise.

URIC ACID. Uric acid is 2-6-8-trioxypurine.<smiles>O=CNC1=CNNC(=O)N1</smiles>

or<smiles>O=C(O)CNc1c2nnc(O)c1NC2O</smiles>

Uric acid forms small rhombic crystals. The crystalline form varies considerably in the presence of impurities. The different forms of uric acid crystal which may occur in the urine are shown in the accompanying figure (Fig. 519). It is extremely insoluble in pure water, one part of uric acid requiring 39,000 parts of water at $18^{\circ} \mathrm{C}$. for its solution. It is easily soluble in concentrated sulphuric acid and alkalies.

It may be prepared from human urine or from guano, which consists almost entirely of urates. In order to prepare it from guano, this is dissolved with the aid of heat in 
dilute sodium carbonate, filtered, and the filtrate treated with a few drops of concentrated hydrochloric acid and boiled. On allowing to cool, the uric acid crystallises out. From urine uric acid may be obtained by adding one-fiftieth of its volume of concentrated hydrochloric acid and allowing to stand for two days. The uric acid is thrown down in small dark-red or brown crystals. They can be collected on a filter, dissolved in alkali, decolorised by boiling with animal charcoal, and the pure acid thrown down as before by means of hydrochloric acid.

A more convenient method of preparation from human urine is based on the fact that ammonium urate is insoluble in concentrated solutions of ammonium chloride (Hopkins). The urine is saturated with crystals of ammonium chloride and a few drops of strong ammonia added. A gelatinous precipitate of ammonium urate is produced. This is collected on a filter, washed off with a minimum amount of hot water into a beaker, and a few drops of hydrochloric acid added. The mixture is boiled and then allowed to cool, when the pure acid crystallises out.

\section{TESTS FOR URIC ACID}

(1) MUREXIDE TEST. If a small quantity of uric acid be treated with a little strong nitric acid and the whole evaporated to dryness on the water-bath, an orangered residue is obtained, which on treatment with ammonia yields a fine purple colour. If a drop of sodium hydrate be now added the purple changes to blue. Instead of nitric acid, bromine water may be employed.

(2) SCHIFF'S TEST. If uric acid be dissolved in a little soda and a drop be placed on filter paper previously moistened with silver nitrate, a yellow or brown spot is produced.

(3) On boiling uric acid with Fehling's solution for some time, a yellowish precipitate of cuprous hydrate is produced.

(4) An alkaline solution of uric acid on treatment with a few drops of a solution of phosphomolyndic acid gives a dark blue precipitate with a metallic lustre, consisting of microscopic prismatic crystals.

(5) With sodium hypobromite uric acid is decomposed, giving off about half of its nitrogen as the free gas.

URATES. Of the four hydrogen atoms in uric acid two can be replaced by metallic radicals. Uric acid thus acts as a weak dibasic acid. It forms three orders of salts, namely, the neutral urates, the bi-urates, and the quadri-urates. The neutral urates, $\mathrm{M}_{2}^{\prime}{ }_{2} \overline{\mathrm{U}}$, are very unstable, and only exist in the presence of caustic alkalies. They are decomposed even by the carbonic acid of the atmosphere. The bi-urates, $\mathrm{MH} \overline{\mathrm{U}}$, are the most stable of the urates. They may be prepared by dissolving uric acid with the aid of heat in weak solutions of the alkaline carbonates, from which they separate, on cooling, in stellar crystals.

The qaudri-urates have the formula $\mathrm{H}_{2} \overline{\mathrm{U}}, \mathrm{MHU}$. They may be prepared by boiling uric acid with dilute solutions of potassium acetate. On cooling the mixture the quadri-urate separates as an amorphous precipitate or in crystalline spheres. The quadri-urates are extremely unstable, and in the presence of water are broken up into the bi-urates and free uric acid. It is probable that under normal conditions the greater part of the uric acid in the urine is present in the form of a quadri-urate (Roberts), and the socalled lateritious deposit, the brick-red amorphous precipitate of urates which occurs in concentrated urine on cooling, consists of these quadriurates. The exact condition of the urate, however, will depend on the 
reaction of the urine. A bi-urate, with acid sodium phosphate, is decomposed with the formation of uric acid in the following way:

$$
\mathrm{MH} \overline{\mathrm{U}}+\mathrm{MH}_{2} \mathrm{PO}_{4}=\mathrm{H}_{2} \overline{\mathrm{U}}+\mathrm{M}_{2} \mathrm{HPO}_{4} \text {. }
$$

Thus the quadri-urates present in the urine immediately after its secretion will tend to undergo spontaneous decomposition into uric acid and the bi-urate, and the latter itself may be decomposed with the formation of uric acid and alkaline phosphate. We see therefore that when the urine is acid, i.e. there is a predominance of acid phosphates, there will be a tendency to the precipitation of uric acid in the urinary passages. If, however, the disodium phosphate be in excess the uric acid may be kept in solution as the quadri-urate or even as the bi-urate.

The uric acid of the urine is derived almost entirely from the purine metabolism of the body. The uric acid may be endogenous or exogenous, i.e. may be derived from the breaking down of the nucleins of the cells or by a direct transformation of the nucleins contained in the food. The amount passed daily varies between 0.4 and 1 grm., according to the nature of the diet. It is not absent from the urine even during complete starvation. It is increased when foods are ingested rich in nucleins, such as liver or sweetbreads, or in any other precursors of uric acid, e.g. hypoxanthine, such as meat or meat extract. We have no evidence that the urinary uric acid in the mammal is formed by synthesis, though this is the manner in which the greater part of the uric acid excreted by birds and reptiles is formed.

Small traces of purine bases also occur in urine, namely, xanthine. hypoxanthine, and adenine. When tea and coffee are taken the methyl-purines may occur, namely, caffeine, theobromine, and their derivatives.

HIPPURIC ACID is a frequent, though not a constant, constituent of human urine. It is derived from benzoic acid or from an aromatic substance which on oxidation can give rise to benzoic acid. In the kidneys the benzoic acid is conjugated with glycine to form hippuric acid. The amount of hippuric acid excreted in the day may vary between 0.1 and $1 \mathrm{grm}$. After a diet rich in fruit or vegetables its amount may rise to $2 \mathrm{grm}$. It is present in considerable quantities in the urine of herbivora and may be most easily prepared from horses' urine. Hippuric acid has the formula :

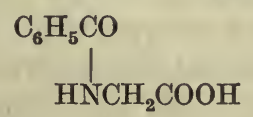

It can be obtained in milk-white crystals (Fig. 520), which are only slightly soluble in cold water, but easily soluble in alcohol, ether, and acetic acid. It is insoluble in petroleum, ether, and benzol. On heating, it is broken up into benzoic acid and glycine. On heating with concentrated nitric acid it forms nitro-benzol, which can be recognised by its characteristic smell of bitter almonds.

In order to extract it from the urine, the urine is made alkaline with sodium carbonate, filtered, and the filtrate evaporated to a syrupy consistence. This is then treated 
with alcohol, the alcohol evaporated, and the residue repeatedly extracted with acetic ether. The acetic ether is collected, evaporated to dryness, and the residue repeatedly extracted with petroleum ether to remove the benzoic acid and fat. What is left behind is hippuric acid, which can be purified by recrystallisation from alcohol or ether.

AMINO-ACIDS. According to Levene and van Slyke amino-acids are always present in the urine, and contribute about 1.5 per cent. of the total nitrogen.

OTHER AROMATIC SUBSTANCES. The chief of these is the so-called ' urinary indican,' or potassium-indoxyl-sulphate. This is derived from the indol produced in the intestines from the tryptophane contained in the proteins of the food, the change being effected by the influence of the microorganisms of putrefaction. The amount of the conjugated sulphates in

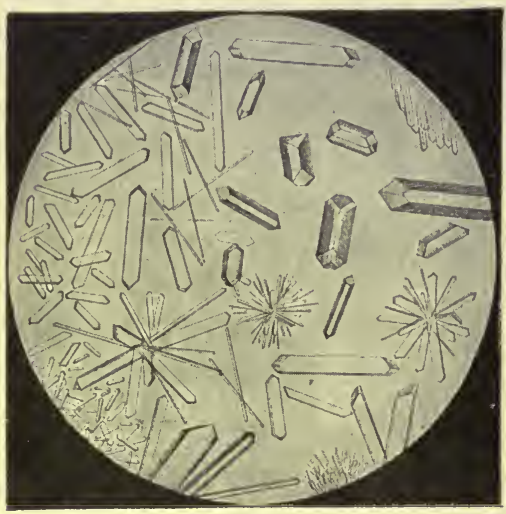

FIG. 520. Hippuric acid. (FUNKE.) the urine is thus an index of the extent of putrefaction in the intestines. In dogs, when the intestine has been disinfected by repeated doses of calomel, the conjugated sulphates entirely disappear from the urine. Urinary indican has the formula:

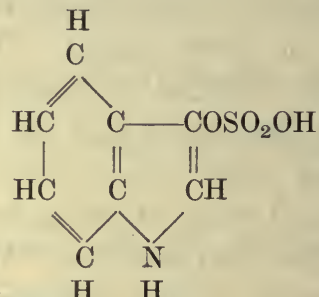

In addition to the tests for conjugated sulphates mentioned earlier, the indoxylsulphate can be detected by various methods dependent on the formation of indigo blue. The urine is treated with an equal volume of concentrated hydrochloric acid and several cubic centimetres of chloroform added. A solution of chloride of lime is now added drop by drop, shaking after the addition of each drop. A blue colour is produced which is extracted by the chloroform. It is important not to add too much chloride of lime, as otherwise the blue colour first produced will be destroyed by further oxidation.

THE URINARY PIGMENTS. Normal urine gives no definite absorption bands. It owes its colour to the presence of a yellow pigment, urochrome. In order to separate urochrome from urine, the urine is saturated with crystals of ammonium sulphate and filtered. The filtrate, which still contains nearly all the colour of the urine, is shaken up with alcohol, which withdraws the greater part of the colouring-matter. On concentrating the alcoholic solution and pouring it into an equal volume of ether, an amorphous brown precipitate falls, which is the urochrome. Urochrome, on treatment with aldehyde, yields a pigment closely similar to urobilin. On the other hand, urobilin, treated with potassium permanganate, is converted into a substance practically identical with urochrome. Urochrome must therefore be derived from the same source as urobilin. 
Urobilin is rarely present in normal urine, and then only in the form of a chromogen, from which it must be set free by acidification. In certain pathological conditions, especially in cirrhosis of the liver, urobilin may occur in the urine in considerable quantities.

In order to extract urobilin from such urine, the urates are first precipitated by saturation with ammonium chloride, and the filtrate is then saturated with ammonium sulphate and a drop of sulphuric acid added. On shaking the fluid up with a mixture of two parts ether and one part chloroform, the urobilin is taken up by the latter. The ether-chloroform solution is separated off and shaken up with caustic soda, when the urobilin passes entirely into the alkaline solution.

Urobilin in solution gives a single absorption band between the lines $b$ and $F$, i.e. at the junction of the green and blue of the spectrum. On treating with zinc chloride and ammonia its solutions show a well-marked green fluorescence. The urobilin of urine is identical with stercobilin, the colouring-matter of the fæces. It is formed from bile when the latter decomposes, and is probably produced in the intestines by the action of micro-organisms on bile pigment.

Other pigments which may occur in urine are uroerythrin and hæmatoporphyrin. Uroerythrin gives the pink colour to urate sediments. Its chemical nature is not known. It is distinguished by the fact that on addition of caustic soda the pink colour is changed to green. On suspending the red-coloured precipitate of urates in hot water and extracting with amyl alcohol, a pink solution is obtained which shows two absorption bands in the green part of the spectrum.

Hcomatoporphyrin is only present in very small amounts in normal urine, but under certain conditions, especially after poisoning with sulphonal, it may occur in such large quantities as to give the urine a deep purple colour. Under these circumstances it is found in the form of alkaline hæmatoporphyrin and gives the characteristic absorption bands of the latter.

Urorosein is a name that has been given to a pigment which is formed when the urine is treated with strong mineral acids. It is probably an indol derivative. It gives a single absorption band between the lines $\mathrm{D}$ and $\mathrm{E}$.

\section{ABNORMAL CONSTITUENTS OF THE URINE}

A very large number of substances occur in the urine in minute traces and may be detected when large quantities of this fluid are worked up at one time. Most of the socalled pathological constituents may be detected in this way in normal urine. It is only when they occur in easily detectable amounts that their presence becomes of any significance.

COAGULABLE PROTEIN. Under normal circumstances urine is free from any coagulable protein except the small traces of mucinous material, nucleoprotein, which gives the cloudiness to the urine. If the kidney-cells are damaged by disease, by interference with their blood-supply, or by circulating poisons, the glomerular epithelium permits the passage of a certain amount of the proteins of the plasma. Under these circumstances, if small pieces of the kidney be plunged into boiling water, the coagulated protein may be seen in Bowman's capsule. The presence of coagulable protein (generally spoken of as albumin) in the urine is significant of the pathological conditions of the kidney associated with Bright's disease. A small trace will generally be found in 
the urine which is passed shortly after taking muscular exercise. Under this condition the presence of albumin in the urine has no pathognomonic significance.

The proteins generally found are identical with those of the blood-plasma and con. sist of serum albumin and serum globulin. Their presence in the urine may be detected

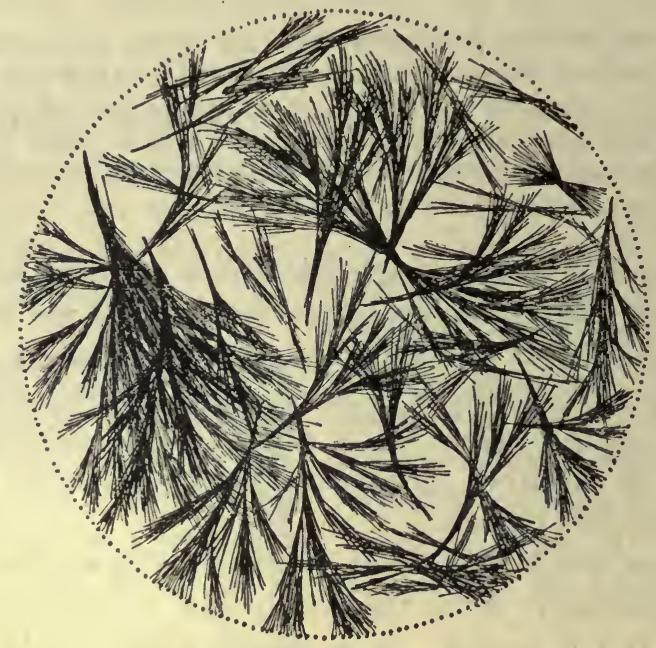

FIG. 521. Glucosazone.

by the precipitate produced on boiling. In carrying out this test a few cubic centimetres of saturated salt solution should be added and one or iwo drops of dilute acetic acid. A more delicate test is that known as Heller's. Some strong nitric acid is placed

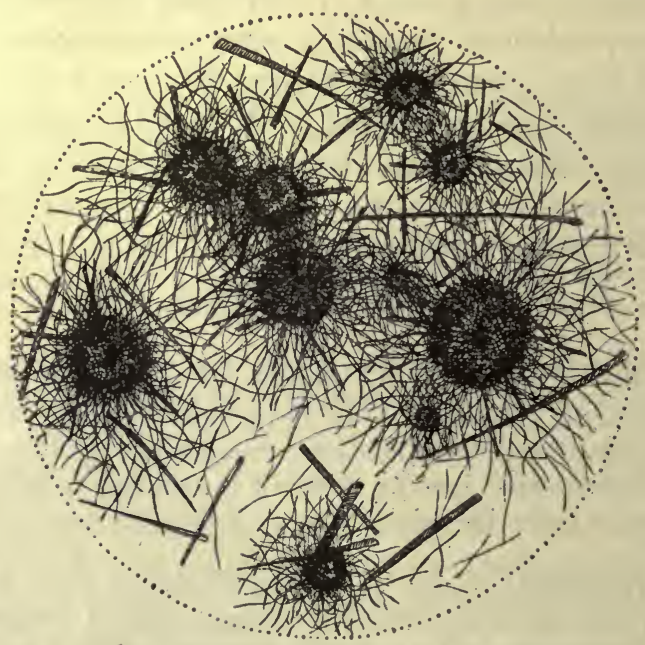

Fig. 522. Lactosazone. (Plimmer.)

in a test-tube and the urine is poured carefully down the side of the tube so as to form a layer on the surface of the nitric acid. If albumin be present a white ring is formed at the junction of the two liquids.

SUGAR. Normal urine contains about one part per thousand of glucose. In diabetes the power of assimilating carbohydrates is diminished or destroyed. The 
amount of sugar in the blood is increased, and sugar appears in large quantities in the urine. The sugar is practically always glucose or dextrose. Lactose may occur in the urine of nursing women even in conditions of health. Since both these sugars will reduce Fehling's solution it becomes important to be able to distinguish between them.

The following tests are used for the detection of abnormal amounts of sugar in the urine :

(1) FEHLING'S TEST. The urine is boiled with Fehling's solution (an alkaline solution of copper sulphate to which Rochelle salt has been added to keep the cupric hydrate in solution). Under the action of glucose or lactose the cupric hydrate is reduced to an insoluble cuprous hydrate, which forms a yellow or red precipitate.

(2) The phenylhydrazine test may be carried out as follows: 2 c.c. of 50 per cent. acetic acid, saturated with sodium acetate, and two drops of phenylhydrazine are added to 5 c.c. of urine. The mixture is evaporated down to 3 c.c., rapidly cooled, and again warmed in a water bath. It is then allowed to cool slowly. Crystals of the corresponding osazone separate out in the hot liquid in the case of glucosazone, on cooling in the case of lactosazone (Figs. 521, 522).

(3) The most convenient way of distinguishing between lactose and glucose is by adding a little yeast to the urine in an inverted test-tube. If glucose be the sugar present, it is fermented by the yeast with the production of carbon dioxide, which collects at the top of the test-tube.

In rare circumstances fructose or lævulose, or pentose may be found in the urine. The former would be detected by the fact that it rotates polarised light to the left, instead of the right, as is the case with glucose.

GLYCURONIC ACID. Small traces of this are present in normal urine. It occurs as a conjugated acid after the administration of various substances, e.g. camphor and chloral. If phenol, indol, or scatol be given to an animal which is receiving very little protein in its diet, these substances will leave the body conjugated, not with sulphuric acid, but with glycuronic acid. Glycuronic acid may be regarded as the first product of oxidation of glucose, having the formula :

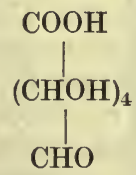

It reduces Fehling's solution and rotates the plane of polarised light to the left.

OXY-FATTY ACIDS AND ACETONE. These substances occur often associated with glucose in diabetes, especially towards the latter stages. They represent the penultimate stages in the oxidation of the fats. Their relation to one another is seen from their formulæ :

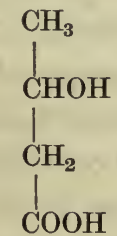

Oxybutyric acid

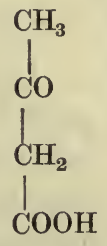

Aceto-acetic acid<smiles>CC(C)=O</smiles>

Acetone

They may also occur in any condition of carbohydrate starvation, relative or absolute. Thus they are found in the urine during absolute starvation as well as in individuals on a pure fat and protein diet. The two acids are generally found associated in the urine.

The presence of aceto-acetic acid may be detected as follows :

(1) To some urine add ferric chloride as long as a precipitate of ferric phosphate continues to form. Filter this off and to the filtrate add a few more drops of ferric chloride. If the acid be present a claret colour is produced. 
(2) On heating with dilute alkali, aceto-acetic acid is decomposed, with the production of acetone. This may be detected by its odour or by distilling off a small proportion of the fluid and testing the distillate in the following ways :

(a) On the addition of sodium hydrate and iodine and warming, iodoform is formed.

(b) Legal's test. A few drops of freshly prepared sodium nitroprusside solution is added and the mixture rendered alkaline with sodium hydrate. A deep red colour is formed. On acidifying with acetic acid this colour is changed to a reddish purple.

CYSTINE. This substance, which is a normal product of the hydrolysis of proteins, is found as a constant constituent to the amount of half a gramme a day in the urine of certain individuals. The condition of cystinuria represents, like alcaptonuria, an inborn error of metabolism. It is found in the child and persists throughout life. In such cases the cystine may give rise to urinary deposits or even to a urinary calculus.

HOMOGENTISIC ACID. This is an aromatic acid having the composition of dioxyphenyl acetic acid. Its formula is as follows :

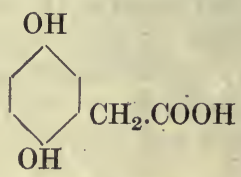

It occurs as a consitutent of the urine of certain individuals, who are said to be affected with alcaptonuria. The urine of these cases is remarkable for its resistance to putrefactive changes. It slowly darkens on exposure to the air, and on the addition of alkali and shaking with air it becomes rapidly brown or black. It reduces Fehling's solution, so that the presence of sugar may be suspected. Such urine contains homogentisic acid in a quantity of 3 to 6 grm. per day. The amount of the acid excreted varies with the protein food taken. It seems that in these cases the power of the organism to break up tyrosine and phenylalanine is entirely absent. If either of these substances be administered by the mouth it is converted almost quantitatively into homogentisic acid, which appears in the urine. Individuals with alcaptonuria continue to secrete homogentisic acid during starvation, so that the tyrosine and phenylalanine set free in the course of tissue disintegration undergo the same fate as when they are derived from the food. Alcaptonurics apparently suffer no ill effects as a result of their abnormal metabolism. The tyrosine and phenylalanine can be absorbed and play their part

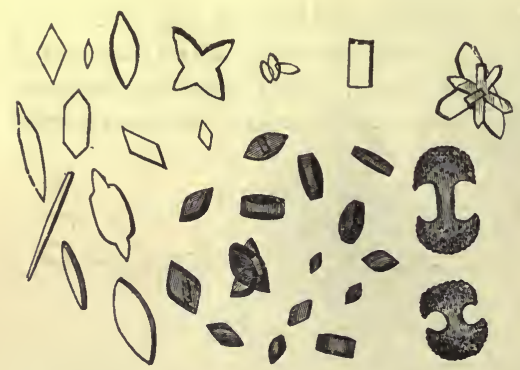

FIG. 523. Various forms of uric acid crystals. (FREY.) in building up the proteins of the tissues, but the process or ferment is wanting which is responsible for the further break-up of the first product of their oxidation, namely, homogentisic acid.

\section{URINARY DEPOSITS}

In addition to formed elements, such as blood-corpuscles, bacteria, or pus-cells, which may occur in abnormal urine, the following deposits may be found :

\section{(a) IN ACID URINE}

(1) Amorphous urates occur generally as a brick-red amorphous deposit thrown down as the urine cools. It is redissolved on warming the urine, and consists generally of the quadri-urates. The acid urate of sodium and of ammonium may occasionally occur in star-shaped clusters of needles or as spherules with small crystals adhering to them. 
(2) Uric acid. Whetstone, dumb-bell, or sheaf-like aggregations of crystals, generally deeply pigmented so as to resemble cayenne pepper (Fig. 523).

(3) Calcium oxalate (Fig. 524). Colourless, transparent, highly refrac-

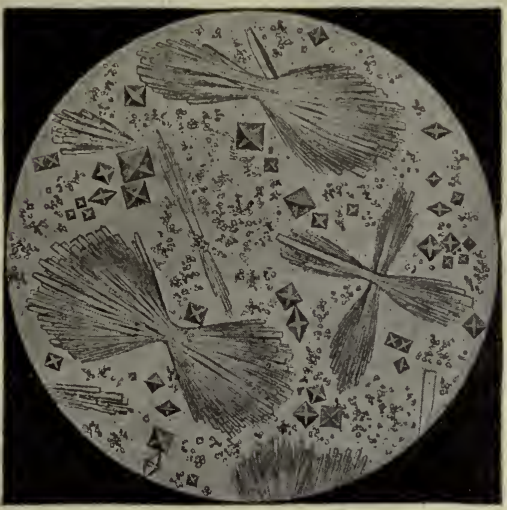

FIG. 524. Urinary deposit, containing uric acid, sodium urate, and calcium oxalate.

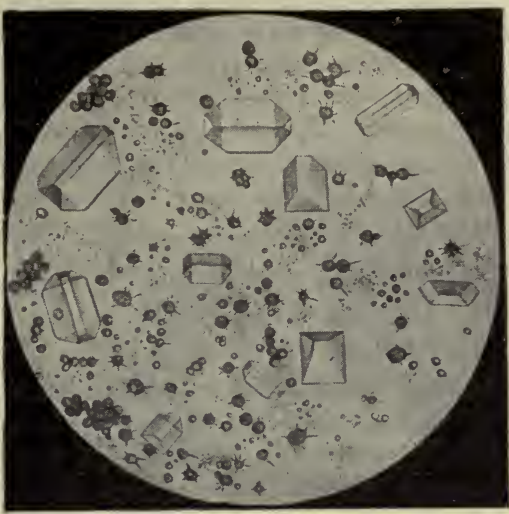

FIG. 525. Deposit of 'triple' phosphate and ammonium urate. (FUNkE.)

tive octahedral crystals (envelope-shaped). Insoluble in acetic acid, soluble in hydrochloric acid.

(4) Ammonium magnesium phosphates (in faintly acid urine). The crystals have been compared to knife-rests or coffin-lids (Fig. 525). They are soluble in acetic acid.

(5) Calcium hydrogen phosphate. $\mathrm{CaHPO}_{4}$. These are rare. They form large prismatic crystals often arranged in rosettes. Easily soluble in dilute acetic acid. On adding a solution of ammonium carbonate the crystals are eaten away and form an amorphous deposit.

(6) Tyrosine, fine needles in starshaped bundles, and cystine, in regular hexagonal plates, may occur under very rare circumstances.

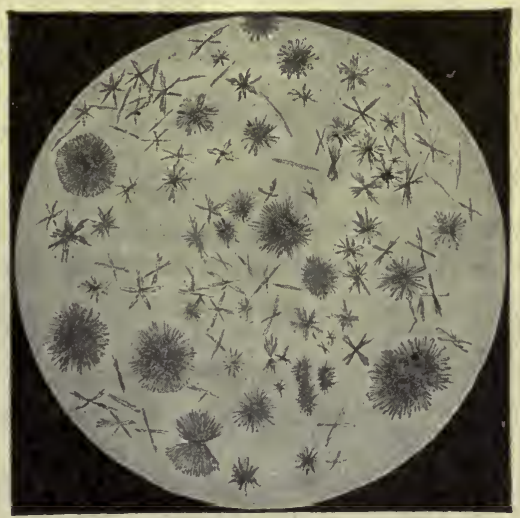

Frg. 526. Ammonium urate.

\section{(b) IN ALKALINE URINE}

(1) The commonest precipitate consists of earthy phosphates, amorphous, easily soluble in dilute acetic acid.

(2) Ammonium magnesium phosphate or triple phosphate is common in urine which has undergone ammoniacal fermentation.

(3) Acid ammonium urate (Fig. 526) may also occur in alkaline urine. $\mathrm{On}$ treatment with $\mathrm{HCl}$ it is dissolved and uric acid in crystals slowly separates out. 


\section{QUANTITATIVE ESTIMATION OF THE CHIEF URINARY CONSTITUENTS}

It may be useful here to summarise the most trustworthy methods which are employed for the estimation of the chief urinary constituents.*

The TOTAL 'ACIDITY' of the urine is measured by titrating it against decinormal alkali in the presence of an indicator, such as phenolphthalein. The indistinctness of the end-point is due to the presence of calcium salts and ammonium salts. Folin therefore recommends that the titration be carried out in the presence of potassium oxalate, which diminishes the error.

Method. To 25 c.c. urine add 15 to 20 grm. potassium oxalate and 1 to 2 drops of phenolphthalein. Shake thoroughly for one or two minutes, and whilst the solution is still cold from the effect of the oxalate, titrate with $\frac{n}{10} \mathrm{NaOH}$ until a permanent pink
remains.

TOTAL NITROGEN. In all metabolic experiments, the determination of the total nitrogen of the food, the urine, and the fæces is indispensable. In each case Kjeldahl's method is employed. This method depends on the fact that all the nitrogenous substances met with in the body, when heated for a considerable time with concentrated sulphuric acid, undergo oxidation, the nitrogen being finally converted into ammonia. On adding alkali to the mixture the ammonia is set free from its combination with the sulphuric acid and can be distilled off and received into a vessel containing a known amount of decinormal acid. By titrating this acid after the operation we can determine the quantity of ammonia which has been produced. To carry out this method 5 c.c. of urine are heated with 20 c.c. sulphuric acid and a small quantity of copper sulphate and potassium sulphate. The copper sulphate is to aid the oxidation of the organic substances, the potassium sulphate is to raise the boiling-point of the mixture. The boiling is continued for half an hour. The flask is then cooled and half filled with distilled water. A special form of distillation tube (Fig. 527) is now attached by a rubber cork which fits tightly, but just before this is done an excess of strong caustic soda sufficient to neutralise the concentrated sulphuric acid is run in under the acid. The other end of the distillation tube is at once arranged to dip under the surface of a measured quantity of standard acid (e.g. 10 c.c. $\frac{n}{10} \mathrm{H}_{2} \mathrm{SO}_{4}$ ), diluted with water, and contained in a 600 c.c. Erlenmeyer flask. The flask is then shaken and heated. In about a quarter of an hour the ammonia is completely distilled off, and its amount can be determined by titrating the acid in the flask with $\frac{n}{10} \mathrm{NaOH}$, methyl orange being used
as indicator.

UREA. The method usually adopted for estimating the urea is that devised by Hüfner. It depends on the fact that urea is decomposed by an alkaline hypobromite with the production of $\mathrm{CO}_{2}$ and nitrogen. In the presence of an excess of alkali the $\mathrm{CO}_{2}$ is absorbed and the nitrogen may be collected and measured, and serves as an index of the amount of urea present. The reaction which occurs is as follows :

$$
\underbrace{\mathrm{CO}\left(\mathrm{NH}_{2}\right)_{2}}_{\begin{array}{c}
60 \text { grm. } \\
1 \text { grm. }
\end{array}}+3 \mathrm{NaBrO}+2 \mathrm{NaOH}=3 \mathrm{NaBr}+\underbrace{\mathrm{N}_{2}}_{\begin{array}{c}
22 \cdot 4 \\
372 \text { litres }
\end{array}=28 \mathrm{grm} .}+\mathrm{Na}_{2} \mathrm{CO}_{3}+3 \mathrm{H}_{2} \mathrm{O}
$$

Actually, however, only $354 \cdot 33$ c.c. nitrogen are evolved by $1 \mathrm{grm}$. urea.

The disadvantage of this method is that other substances, such as ammonia, creatinine, and uric acid, give off a certain amount of their nitrogen with sodium hypobromite, so that the method is not strictly accurate, though enough so for most clinical purposes. In actually carrying out the method 5 c.c. of urine are treated with 25 c.c. of freshly

*Fuller details will be found in Plimmer's "Practical Physiological Chemistry," from which most of the methods here given are taken. 
prepared solution of sodium hypobromite, and the nitrogen evolved is collected in a graduated tube over water.

Folin's Method. In Kjeldahl's method all the nitrogenous constituents of the urine are converted into ammonia by boiling with strong sulphuric acid. This conversion occurs with extreme readiness in the case of urea, so that by using a weaker acid and carefully regulating the temperature the hydrolysis may be confined practically to the urea itself. This is the principle of Folin's method of estimating urea.

Five cubic centimetres of urine are measured into a 200 c.c. Erlenmeyer flask. Five cubic centimetres of concentrated hydrochloric acid, $20 \mathrm{grm}$. crystallised magnesium chloride, a piece of paraffin the size of a small hazel nut, and finally 2 or 3 drops of a 1 per cent. solution of alizarin red in water are added. A special safety tube is then inserted into the neck of the flask and the mixture boiled until each returning drop from the safety tube produces a very perceptible bump. The heat is then reduced somewhat, and the heating is continued for a full hour. The alizarin red is used in order to ensure that the contents of the flask do not become alkaline. At the end of an hour the contents of the flask are put into a litre flask with about 700 c.c. water and 20 c.c. of a 10 per cent. sodium hydrate, and the ammonia is then distilled off into a measured quantity of acid. The results obtained in this way will give us the total amount of urea together with any ammonia which was preformed in the urine. It is therefore necessary also to determine the amount of this preformed ammonia.

ESTIMATION OF AMMONIA. In Folin's method for the estimation of ammonia, this is set free by the addition of weak alkali (sodium carbonate) and is then removed from the urine at ordinary room temperature by passing a strong current of air through the liquid. The issuing

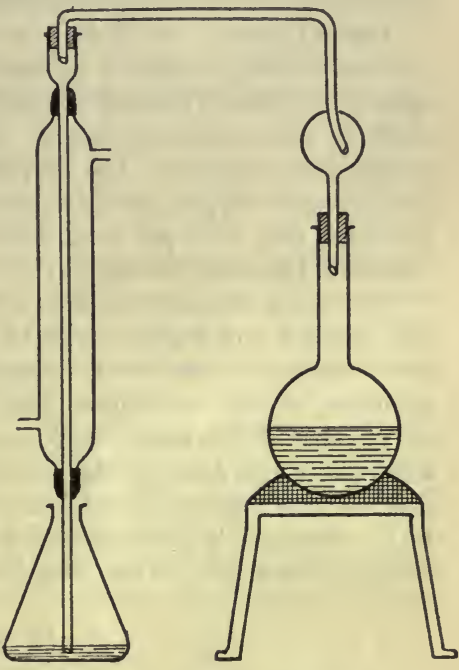

Fig. 527. current of air carrying the ammonia passes through a measured quantity of decinormal acid. If the air current be sufficiently strong, one and a half hours is sufficient to remove the whole of the ammonia from 25 c.c. of urine. The decinormal acid is then titrated and the amount of the ammonia reckoned. In carrying out the method 25 c.c. of urine is measured into a cylinder 30 to $40 \mathrm{~cm}$. high and about a gramme of sodium carbonate and some petroleum (to prevent foaming) are added. The upper end of the cylinder is then closed by a doubly perforated rubber stopper through which pass two glass tubes, only one of which is long enough to reach below the surface of the liquid. The shorter tube, about $10 \mathrm{~cm}$. in length, is connected with a calcium chloride tube filled with cotton, and this in turn is connected with a glass tube extending to the bottom of a wide-mouthed bottle, capacity about 500 c.c., which contains 20 c.c. decinormal acid in 200 c.c. of water.

A more convenient method for the estimation of ammonia is that originally proposed by Schiff and recently worked out by Malfatti. It depends on the fact that when a neutral solution of an ammonium salt is treated with formaldehyde, combination occurs with the formation of hexamethylene tetramine (urotropine) and the liberation of a corresponding amount of acid, which can be estimated by titrating with decinormal alkali. The reaction which occurs is as follows :

$$
\begin{aligned}
& 6 \mathrm{CH}_{2} \mathrm{O}+2\left(\mathrm{NH}_{4}\right)_{2} \mathrm{SO}_{4}=6 \mathrm{H}_{2} \mathrm{O}+\mathrm{N}_{4}\left(\mathrm{CH}_{2}\right)_{6}+2 \mathrm{H}_{2} \mathrm{SO}_{4} \text {. } \\
& \text { Formaldehyde Hexamethylene tetramine }
\end{aligned}
$$

In carrying out this method 25 c.c. of urine are measured by means of a pipette into a flask or beaker and diluted with five times its volume of water. Four or five drops of 
phenolphthalein are then added and decinormal sodium hydrate is run in until there is a slight permanent pink colour. The amount of alkaline solution necessary to produce this colour is a measure of the acidity of the urine. Ten cubic centimetres of formalin, diluted with three volumes of water and previously neutralised to phenolphthalein with decinormal alkali, are then added. The colour disappears owing to the setting free of the acid radicals previously combined with ammonia. Decinormal alkali is then run into the mixture until a permanent pink colour is again obtained. The number of cubic centimetres of the decinormal alkali required in this second case corresponds to the amount of decinormal ammonia previously present in the 25 c.c. of urine.

This method gives somewhat higher figures than the method of Folin just described, owing to the fact that the small traces of amino-acids, which may be present in the urine, react to formalin in a very similar way. The difference does not exceed 10 per cent., so that the method is amply delicate for clinical purposes.

CREATININE. In Folin's method for the determination of creatinine, which is now universally employed, advantage is taken of the colour reaction given by creatinine (and by no other normal urinary constituent) with picric acid in alkaline solution (Jaffré's reaction), the colour being compared with that of a standard potassium bichromate solution. The reagents employed are decinormal potassium bichromate containing 24.55 grm. per litre, saturated picric acid solution containing about 12 grm. per litre, and a 10 per cent. solution of sodium hydrate. For the comparison of the colours a Duboseq colorimeter is employed.

Ten cubic centimetres of urine are measured into a 500 c.c. flask ; 15 c.c. of picric acid and 5 c.c. of sodium hydrate are then added and the mixture allowed to stand for five minutes. Some of the potassium bichromate solution is placed into one of the cylinders of the colorimeter and its depth accurately adjusted to the $8 \mathrm{~mm}$. mark. At the end of five minutes the contents of the 500 c.c. flask are diluted up to 500 c.c. with water and some of the mixture placed into the other cylinder of the colorimeter, and the two colours are then compared. The calculation of the results is very simple. If, for example, it is found that it takes $9.5 \mathrm{~mm}$. of the unknown urine picrate solution to equal the $8 \mathrm{~mm}$. of the bichromate, then the 10 c.c. of urine contains

$$
10 \times \frac{8 \cdot 0}{9 \cdot 5}=8 \cdot 4 \mathrm{mg} \text {. creatinine. }
$$

ESTIMATION OF URIC ACID. The best method for this purpose is a slight modification by Folin of the method devised by Hopkins.

For this method the following reagents are required :

(1) A solution of ammonium sulphate, uranium acetate, and acetic acid, made up as follows : $500 \mathrm{grm}$. ammonium sulphate, 5 grm. uranium acetate, and 60 c.c. 10 per cent. acetic acid are dissolved in 650 c.c. water. The volume of this solution is almost exactly 1000 c.c.

(2) Ten per cent. ammonium sulphate solution.

(3) $\frac{n}{20}$ potassium permanganate solution made by dissolving $1.581 \mathrm{grm}$. pure potassium permanganate in one litre of water ; 1 c.c. $=\cdot 00375$ grm. uric acid.

Measure 200 c.c. urine with a pipette into a 500 c.c. flask and add 50 c.c of the ammonium sulphate and uranium acetate reagent. Mix the solutions and allow to stand for about half an hour so as to allow the precipitate to settle. This precipitate contains a mucoid substance (and phosphates) which, if not thus removed, renders the subsequent filtration and washing of the ammonium urate precipitate very slow. Filter off the supernatant liquid through a dry filter into a dry vessel, and measure out 125 c.c. (= 100 c.c. urine) of this with pipettes into a beaker. Add 5 c.c. concentrated ammonia, mix well, and allow to stand covered with paper for twelve to twenty-four hours.

Carefully decant the supernatant liquid upon a filter, wash the precipitate of ammonium urate on to the filter with 10 per cent. ammonium sulphate, and wash this once or twice with the same reagent to remove the chlorides as completely as possible.

Remove the filter from the funnel, open it, and with a fine stream of water wash 
the ammonium urate precipitate into a beaker. To the ammonium urate precipitate, suspended in about 100 c.c. water, add 15 c.c. strong sulphuric acid and titrate at once, without cooling, with the potassium permanganate solution. At first every small addition of the permanganate is decolorised before it diffuses through the liquid, but towards the end the decolorisation is slower and the permanganate should be added two drops at a time until a faint pink colour is seen throughout the whole solution. The amount of uric acid can then be calculated, 1 c.c of the permanganate solution being equivalent to $\cdot 00375$ grm. uric acid.

CHLORIDES. The chlorides of urine are estimated by Volhard's method. The principle of this method consists in precipitating the chlorides by excess of a standard solution of silver nitrate in the presence of nitric acid. The excess of silver is then estimated in an aliquot part of the filtrate with a solution of potassium or ammonium sulphocyanate which has been previously standardised against the silver solution, a ferric salt being used as indicator.

The following solutions are required : (1) Standard silver nitrate solution either $\frac{n}{10}$ or so that 1 c.c. corresponds to 01 grm.
$\mathrm{NaCl}$.

(2) Potassium sulphocyanate solution (8 grm. per litre).

(3) Pure $\mathrm{HNO}_{3}$ free from chlorides.

(4) A saturated solution of iron alum.

The potassium sulphocyanate solution must be standardised against the silver nitrate solution. This is carried out as follows: Place 10 c.c. $\mathrm{AgNO}_{3}$ solution with a pipette in a beaker, add 5 c.c. pure $\mathrm{HNO}_{3}, 5$ c.c. iron alum solution, and 80 c.c. water. Now run in the sulphocyanate solution from a burette until a permanent $\mathrm{r} \epsilon \mathrm{d}$ tinge is obtained. Note the amount required for the 10 c.c. $\mathrm{AgNO}_{3}$ solution.

The method of analysis is carried out as follows: Place 10 c.c. urine in a 100 c.c. measuring flask with a pipette. Then add about 4 c.c. pure nitric acid and 10 or 20 c.c. with a pipette of the standard silver nitrate solution. Now fill up to the mark with distilled water, mix thoroughly, and filter into a dry vessel through a dry paper. Take exactly 50 c.c. of the filtrate with a pipette and titrate with the sulphocyanate solution until a permanent red colour is obtained, iron alum having been added before the titration is commenced. Calculation of results :

$$
\begin{aligned}
& 50 \text { c.c. } \text { filtrate }=S \text { c.c. } \mathrm{KCNS} \\
& \therefore 100 \text { c.c. }, "=2 S \text { c.c. } "{ }^{\prime} \\
& \text { Now } x \text { c.c. } \mathrm{KCNS}=10 \text { c.c. } \mathrm{AgNO}_{3} \\
& \therefore 2 S \text { c.c. } \quad, \quad=\frac{10 \times 2 S}{x} \mathrm{AgNO}_{3}
\end{aligned}
$$

This is the excess not utilised to precipitate the chlorides

$$
\therefore\left(20-\frac{10 \times 2 S}{x}\right)=\text { amount of } \mathrm{AgNO}_{3} \text { solution used. }
$$

Hence $\mathrm{NaCl}$ in grammes per 10 c.c. in the volume passed in twenty-four hours.

ESTIMATION OF PHOSPHATES. The method depends upon the precipitation of all the phosphates by a standard solution of uranium acetate or uranium nitrate in the presence of sodium acetate and acetic acid as $\left(\mathrm{UrO}_{2}\right) \mathrm{HPO}_{4}$. The determination of the end-point, when soluble uranium salt is in solution, is shown by means of potassium. ferrocyanide, or by cochineal tincture, which becomes green.

The following reagents are required :

(1) Acid sodium acetate solution (100 grm. $\mathrm{Na} \overline{\mathrm{A}} \mathrm{c}, 30 \mathrm{grm} . \mathrm{HA} c, 1000$ c.c. $\mathrm{H}_{2} \mathrm{O}$ ).

(2) Cochineal tincture (5 grm. cochineal extracted for several days with 150 c.c. alcokol and 100 c.c. water and then filtered).

(3) Standard uranium solution (1 c.c. $=.005$ grm. $\mathrm{P}_{2} \mathrm{O}_{5}$ or $5 \mathrm{mg}$.).

This must be prepared by standardising against a standard phosphate solution. Generally sodium phosphate is employed; about $12 \mathrm{grm}$. are weighed out and dissolved in 1000 c.c. water ; 50 c.c. of this solution are evaporated to dryness, incinerated, and 


\section{PHYSIOLOGY}

weighed as pyrophosphate. From the weight of this the amount of $\mathrm{P}_{2} \mathrm{O}_{5}$ in 50 c.c. can be calculated and the remainder of the solution can be diluted, so that 50 c.c. contain $0.1 \mathrm{grm} . \mathrm{P}_{2} \mathrm{O}_{5}$. It is simpler to use acid potassium phosphate, $\mathrm{KH}_{2} \mathrm{PO}_{4}$, which can be weighed directly and dissolved in water, so that 50 c.c. contain 0.1 grm. $\mathrm{P}_{2} \mathrm{O}_{5}$. Fifty cubic centimetres of this solution are titrated with the uranium solution $(36 \mathrm{grm}$. in one litre) in the manner described below, and the uranium solution is then diluted so that 1 c.c. $=5 \mathrm{mg} . \mathrm{P}_{2} \mathrm{O}_{5}$.

The method of analysis is carried out as follows: Place 50 c.c. urine with a pipette in a 100 c.c. beaker, add 5 c.c. acid sodium acetate solution and a few drops of cochineal tincture. Heat the urine to boiling and run in slowly the standard uranium acetate solution from a burette as long as a precipitate is formed. Again heat to boiling and add the uranium solution drop by drop, until the red colour is changed to green. This end-point can also be tested by taking out a drop and placing it in contact with a drop of potassium ferro-cyanide solution or a little heap of finely powdered substance on a white piece of porcelain. A brown colour or precipitate is formed when excess of soluble uranium salt is present in the solution. (A few more drops may be required to reach this point than to turn the cochineal green.)

The principle of the estimation of sulphates has already been described. It is not advisable to attempt this volumetrically. 


\section{SECTION II \\ THE SECRETION OF URINE}

W Iтн the single exception of hippuric acid all the constituents of the urine are formed in parts of the body other than the kidneys. Extirpation of both kidneys leads to an accumulation of these specific urinary constituents in the blood and tissues. The work of the kidney is therefore confined to an excretion of preformed constituents. Considered from a broad standpoint, the function of this organ is the preservation of the normal composition of the circulating blood. Whenever the latter contains an abnormal constituent or any of its normal constituents are present in abnormal quantities, the kidney excretes the substance in question until the composition of the blood is restored. We have to determine the conditions which influence the quantity and quality of the urine secreted by the kidneys, and to ascribe to each element in these organs its proper share in the total work of the kidney.

In no other organ of the body are our views as to function so intimately dependent on our knowledge of structure as in the kidney. This organ is a branched tubular gland consisting in man of ten to fifteen nearly equal divisions, known as the Malpighian pyramids. In certain animals, such as the rabbit and rat, only one pyramid is present. It is divided into an outer portion or cortex, an inner portion, the medulla, and between these the 'boundary layer,' containing the larger branches of the renal bloodvessels (Fig. 528). From the outer boundary of the Malpighian pyramids of the medulla a

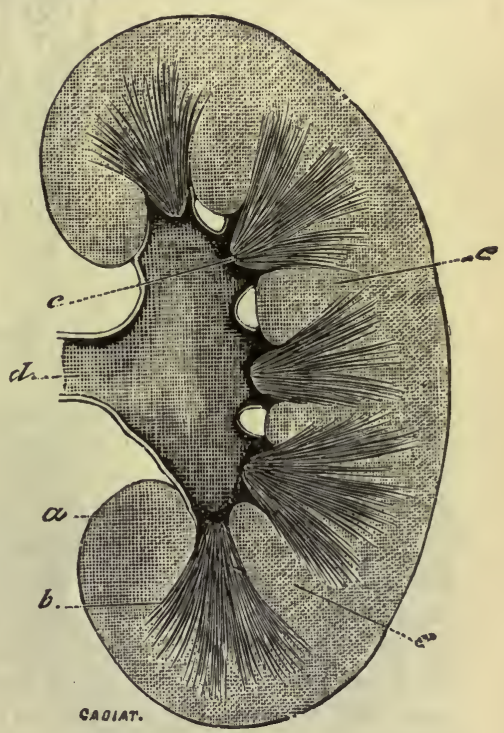

FIG. 528. Section of human kidney. (CADIAT.)

$a$, cortex ; $b$, medulla or Malpighian pyramids ; $c$, papilla ; $d$, ureter ; $e, e$, boundary zone.

number of processes, the medullary rays, pass out into the cortex towards the surface of the kidney. All parts of the kidney are made up of branched tubules embedded in scanty connective tissue and richly supplied with blood-vessels. Each tubule begins by a blind dilated extremity in the cortex, known as Bowman's capsule, which surrounds a bunch of capillary blood-vessels, the glomerulus, the two together forming the Malpighian body. From Bowman's capsule a short neck leads into a proximal convoluted tubule, and this into a $\mathrm{U}$-shaped portion which passes down in a medullary ray 
into the underlying portion of the medulla, and consists of straight descending and ascending limbs and the loop of Henle. The ascending limb passes into a distal convoluted tubule, and this by a 'junctional tubule' joins with a number of others to form a straight 'collecting tubule.' Several of these unite to form the papillary ducts, which open on the surface of the papilla in the expanded part of the renal duct or ureter (Fig. 529). The whole tubule consists of epithelium lying on a basement membrane ; the epithelium varies in structure in different parts of the tubule. The bunch of glomerular capillaries is covered with a very thin layer of endothelial cells, and a similar layer forms the lining of Bowman's capsule. The convoluted tubules contain cells which are roughly cubical or cylindrical in cross-section, but do not present very definite cell outlines. These cells, which are similar in the two sets of convoluted

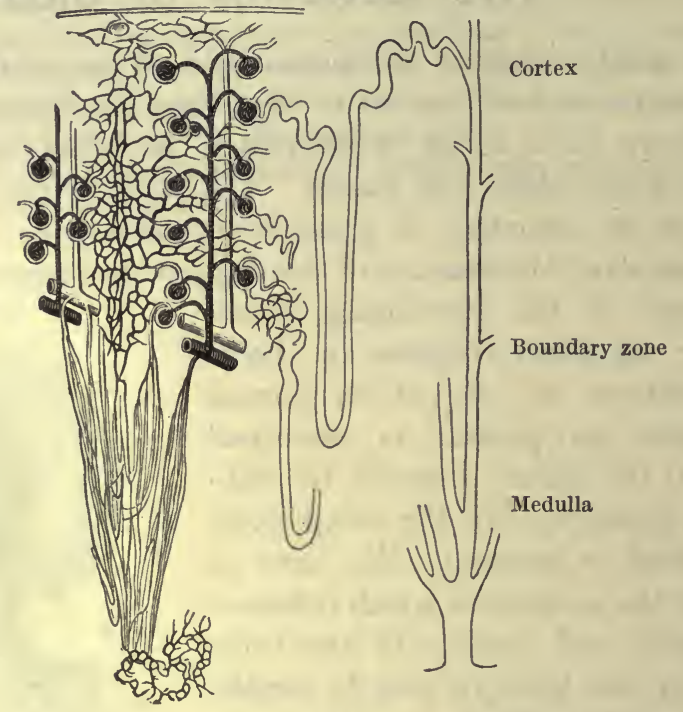

Fra. 529. Diagram showing course of urinary tubules, and the distribution of blood-vessels. (From YEo.)

tubules, have long been distinguished as 'rodded epithelium ' (Fig. 530) on account of the ease with which a radial disposition of rods or granules is demonstrated in their protoplasm. As ordinarily prepared, the free margin of these cells, where they abut on the lumen, is irregular. This appearance is due to the readiness with which the cells undergo alteration under the influence of different fixing reagents, especially of such as contain water. When properly fixed it is seen that the rodded structure as described by Heidenhain is due to rows of granules arranged vertically to the basement membrane. Moreover the free margin of the cells, instead of being irregular, consist of a well-marked striated border, formed of a number of very fine hairs closely set together and springing from a row of granules in the peripheral part of the cell (Fig. 531). The hairs, which make up the striated border (sometimes called the 'brush border '), have not been observed to present ciliary movement, and are probably comparable with the similar structures found clothing the free border of the epithelium of the intestinal villus. Such cells are characteristic features of the epithelium lining the urinary organs in all types of animals, and are well marked in the nephridia of worms. Besides these rows of granules other granules are found, especially towards the free margin of the cell and round about the nucleus. Some of the granules appear to be of a fatty, others of a protein character.

The descending limb of Henle's loop is narrow, and possesses flattened epithelial cells, while the ascending limb presents an epithelium similar to that of the convoluted 
tubules, but with less marked striation. The junctional and collecting tubules are lined with cubical or columnar cells with a clear protoplasm. The marked differences between the structure of these various parts point to a differentiation of function and division of labour among them in the preparation of the fully formed urine. This conclusion is borne out by a study of the blood-supply of the kidney. The large renal

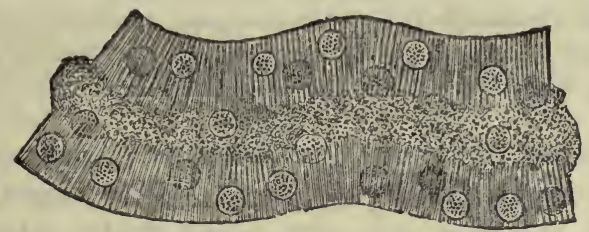

Fig. 530. A portion of convoluted tubule with ' rodded' epithelium.

(HEIDENHAIN.)

artery divides in the pelvis into four or five branches, which pass up to the boundary zone and there give off arteries in different directions; those which run towards the surface are the interlobular arteries. Each of these, which is an end-artery presenting no anastomoses with its fellows, gives off on all sides short wide branches, which pass to the glomeruli and constitute the vasa afferentia of these bodies. Each vas afferens has a thick muscular wall. The glomerulus itself consists of a number of anastomosing

A
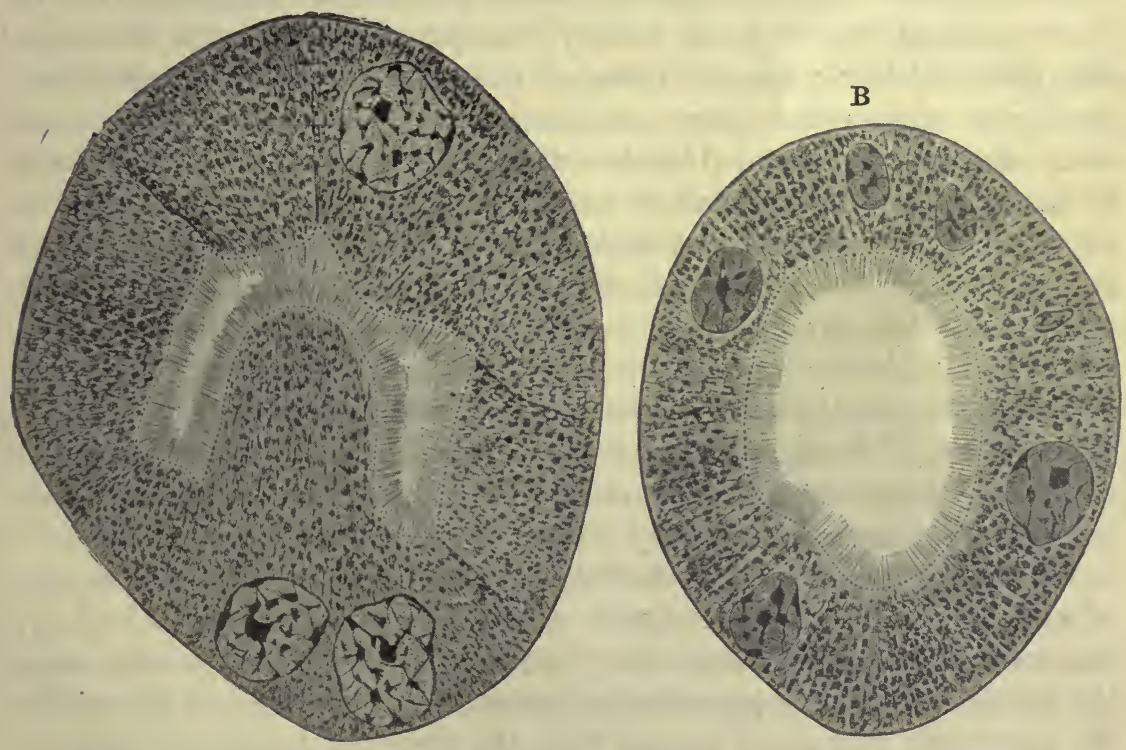

Fia. 531. Cross-sections of convoluted tubules from kidney of rat. (SAUER.) A, during slight secretion; B, during maximal secretion.

wide capillaries invested by an extremely thin wall, which is sometimes said to consist simply of a protoplasmic film devoid of nuclei. The glomerular capillaries are collected together to form an efferent vessel, the vas efferens, which is narrower than the vas afferens, but, like the latter, presents a well-marked muscular coat. The vas efferens breaks up again into a second set of capillaries, which ramify around the tubules of the cortex and communicate with a similar network round the tubules of the medulla. The medullary pyramids are also provided with blood by a plexus of capillaries taking their 
origin from little bunches of vessels, the vasa recta ( $v$. Fig. 529), which leave the concave side of the arterial arches of the boundary zone to run towards the papilla, and receive also a few vessels which spring from the vasa afferentia of the cortical vessels. From the capillaries of the tubules the blood is collected again into veins, which leave the kidney partly by the cortex and capsular vessels, partly by large venous trunks which join to form the renal vein at the hilum of the kidney. The kidney is richly supplied with nerves, which are chiefly distributed to the muscular walls of its blood-vessels. Some authors have described a fine nerve-plexus surrounding the tubules and sending branches between and into the cells of the convoluted tubules themselves.

The main points in the above description of the structure of the kidney were made out by Bowman in 1840, and suggested the theories of urinary secretion both of Bowman and of Ludwig (1844), theories which have furnished the basis of all our subsequent investigation of the subject. Both observers appreciated the great difference between the membrane covering the glomerular loop and the lining membrane of the tubule, and both drew attention to the difference in the circulation in these two portions of the kidney. The glomerular capillaries, supplied with blood through a short wide artery and drained by an efferent vessel smaller than the afferent, would represent a region of very high capillary blood-pressure, whereas the pressure in the capillaries surrounding the tubules must be low and similar to that in other capillary regions. Bowman therefore suggested that the urine consisted of two parts, namely, one part containing the water and salts produced by a process of filtration through the walls of the glomerular capillaries, and another part, containing the specific urinary constituents urea, uric acid, \&c., secreted by the cells probably of the convoluted tubules. To Ludwig, on the other hand, it seemed possible at first to account for the whole process of formation of urine without the assumption of any active intervention on the part of the cells of the tubules. He imagined that the whole of the urinary constituents passed from the blood to the urinary tubule in the glomerulus by a process of filtration. The glomerular transudate would represent therefore a very dilute urine containing the crystalloids of the blood in the same concentration as in the blood and with no more urea than the blood itself contained. The great difference in urea content between the blood and the fully formed urine he ascribed to a process of concentration taking place in the fluid in its passage through the tubules, in which water and certain of the salts were reabsorbed, a process of reabsorption conditioned by the difference in protein content between the urine within the tubules and the lymph under low pressure on the outside of the tubules. We know now that in its original form the theory of Ludwig is untenable. If a process of concentration takes place within the tubules it must involve the performance of work by the cells lining these tubules, and could not take place as a result of mere differences of colloid content between the two fluids. It was shown long ago by Hoppe-Seyler that, if urine be dialysed against serum, a passage of water takes place, not from urine to serum, but from serum to urine, i.e. the latter is much more concentrated than the former. The movement of water from one fluid to another through a colloid membrane depends on the relative osmotic pressures of the two fluids, and this 
in turn is determined by the molecular concentration of the two fluids. It is easy to estimate the molecular concentration of any sample of serum or urine. The method which is most convenient is to determine the depression of freezing-point in the two fluids. Whereas serum ordinarily freezes at $-0.56^{\circ} \mathrm{C}$. to $-0.59^{\circ} \mathrm{C}$., the freezing-point of urine is generally lower and may vary from this figure to as much as $-4 \cdot 5^{\circ} \mathrm{C}$. For the production therefore of urine from blood-plasma, a certain amount of work has to be done, and the seat of this work we can locate only in the cells of the kidney. We may determine the minimum work necessary to form a certain amount of urine of a given concentration by measuring the amount of heat that must be imparted to the blood-plasma in order to reduce it to the same concentration and volume, or we can calculate it if we know the freezing-points of the two fluids. A depression of freezing-point $\Delta=-1^{\circ} \mathrm{C}$. corresponds to an osmotic pressure of 122.7 metres of water. To concentrate 100 c.c. of a saline fluid such as urine so as to halve its bulk and double its depression of freezing-point, e.g. from $-1^{\circ} \mathrm{C}$. to $-2^{\circ} \mathrm{C}$., would therefore require the expenditure of work equivalent to that which would be required to compress 100 c.c. of a gas at a pressure of 122.7 metres of water to half its bulk.

The abandonment of Ludwig's view as to the mechanism of the concentration does not, however, place his theory out of court. The question will still have to be discussed whether the chief object of the tubules is the concentration of the fluid produced in the glomeruli, or whether they add to this fluid by a further secretory process, or whether they may not possibly possess both functions and in their various parts alter the fluid flowing through them either by addition or by withdrawal of water or dissolved constituents. The common point in the two theories is the sharp distinction which is drawn between the nature of the glomerular activity and the nature of the activity of the tubules. The questions which we have to decide by experiment are :

(1) The nature of the glomerular activity and the conditions which determine the amount of fluid formed by the glomeruli, and especially whether the energy required for the formation of the glomerular fluid is furnished by the heart through the blood-pressure within the capillaries or by the endothelium covering these capillaries.

(2) The function of the tubules, whether they secrete or absorb, and what part is played in these processes by the various segments of the tubules which differ so widely in their histological characters.

\section{THE SECRETION OF WATER AND SALTS. FUNCTIONS OF THE GLOMERULI}

It is generally assumed, as the best explanation of known facts with regard to the secretion of urine, that a watery exudation free from protein is formed in the glomeruli, and that this becomes concentrated on its way through the tubules either by the absorption of water and certain salts or by the secretion and addition of urea, uric acid, \&c., as well as such salts as 
acid phosphates. As to the nature of the glomerular functions two opinions have been held. According to the Ludwig school the process is one simply of filtration, in which, under the pressure of the blood in the glomerular capillaries, the water and crystalloid constituents of the plasma are filtered through the glomerular epithelium, leaving behind the protein constituents. According to Heidenhain the process cannot be regarded as one simply of filtration, but involves the secretory activity of the glomerular epithelium. If the glomerular urine is a filtrate it must resemble blood-plasma in practically all particulars except its protein content, since the blood pressure, which is the only force causing filtration, is too small to effect any appreciable separation of salts. On the other hand, a certain minimum difference of pressure between the two sides of the membrane must be present in order to separate the colloids from the other constituents of the plasma. We have seen in chapter iv (p. 143) that in order to produce a filtrate containing only water and salts from serum a minimum difference of pressure of $30 \mathrm{~mm}$. Hg. is necessary, corresponding to the osmotic pressure of the colloidal constituents of the blood-plasma or serum. Thus in order to produce a filtrate, free from protein, from the blood-plasma circulating through the glomerular capillaries, the pressure of the urine in the tubules and ureter must always be at least $30 \mathrm{~mm}$. lower than the pressure of the blood in the glomeruli. A direct determination of the latter figure is not possible. The anatomical arrangements are such as to bring this pressure up to a high point. Not only are the vasa afferentia very short, but the vasa efferentia are only twothirds of the diameter of the vasa afferentia. Moreover the sudden increase of bed which ensues as the blood passes from the vas afferens to the bundle of capillaries must itself cause a rise of pressure in the latter, due to the transformation of the kinetic energy of the moving fluid into the statical energy represented by pressure on the walls of the vessels.

This point can be rendered clearer by the following considerations. If fluid is flowing in a tube of continuous bore $a b$ (Fig. 532) there will be a continuous fall of pressure
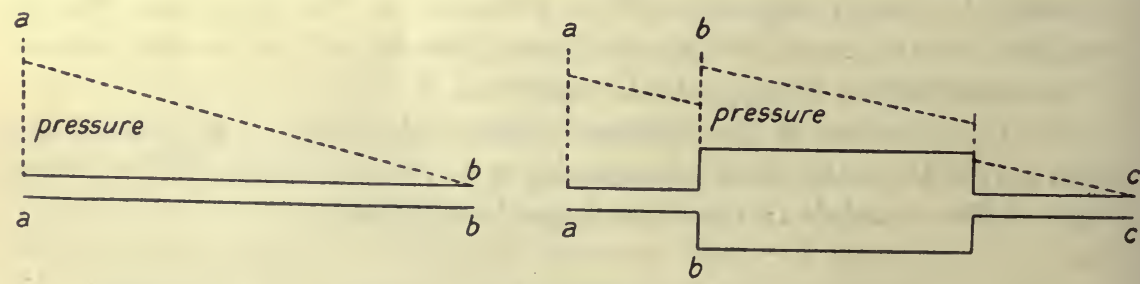

FIG. 532.

from $a$ to $b$. If, however, in the tube $a b c$ the segment $b$ be of much greater diameter than the segments $a$ and $c$, although while the fluid is at rest the pressures will be equal at all points of the system, as soon as the fluid moves from $a$ to $c$, although there is a fall of pressure between $a$ and $c$, a manometer attached to $b$ may show an actual greater pressure than a manometer inserted at $a$. Fluid is flowing from a place of lower to a place of higher pressure. The apparent paradox is due to the fact that the energy causing the fluid to move from $a$ to $b$ is of two kinds. It equals $\frac{1}{2} m v^{2}+\mathbf{P}$, i.e. represented by the kinetic energy of the moving mass of fluid as well as the difference of pressure 
between any two points of the tube. The total energy will diminish continuously from $a$ to $c$, and is used in overcoming the resistance of the system. We may say then that the sum of these two, namely, $\frac{1}{2} m v^{2}+\mathrm{P}$, is greater at $a$ than $b$, and is greater at $b$ than $c$; but as the fluid passes from the narrow tube $a$ into the wide tube $b$ there is a sudden fall of its velocity and a consequent diminution of the factor $\frac{1}{2} m v^{2}$. In order to provide for a continuous fall in the total energy of the fluid, namely, $\frac{1}{2} m v^{2}+\mathrm{P}$, the diminution in the factor $\frac{1}{2} m v^{2}$ must cause a corresponding increase in the factor $\mathrm{P}$, i.e. in the lateral pressure exercised by the fluid on the vessel wall. As the total diameter of the bed of the stream in the capillaries may be twenty times that of the bed in the vas afferens the velocity of the blood in these capillaries will be only one-twentieth of that in the artery and the kinetic energy of the blood only one four-hundredth. It is possible therefore that the pressure exercised by the blood on the walls of the capillaries may be even greater than that in the interlobular arteries, and this effect will be still further aided by the narrow diameter of the vas efferens. Although therefore the pressure in the ordinary capillaries of the body is probably not greater than 20 to $30 \mathrm{~mm}$. Hg., the glomerular capillaries might present a pressure little inferior to that in the main arteries of the body.

The pressure in the ureter is under normal circumstances approximately nil, whereas that in the glomerular capillaries is probably not more than $20 \mathrm{~mm}$. Hg. below that in the main arteries of the body, so that there is a difference of pressure on the two sides of the membrane more than sufficient to cause a constant filtration of a protein-free fluid from the blood-plasma coursing through these capillaries. On raising the pressure on the tubule side the filtration ought to come to an end when the pressure approaches a figure which is 30 to $40 \mathrm{~mm}$. below that in the glomerular capillaries. A number of observers have found that urinary secretion ceases when the blood pressure falls to between 40 and $50 \mathrm{~mm}$. $\mathrm{Hg}$. The urinary secretion can be stopped by raising the pressure in the tubules by means of ligature of the ureter. On applying the ligature the secretion continues for a time until the pressure in the ureter rises up to a certain point, when the secretion comes to an end. In one experiment the following pressures were obtained in a dog which was secreting urine copiously under the action of diuretin. Manometers were connected both with the carotid artery and with the ureters so that no outflow of urine was possible :

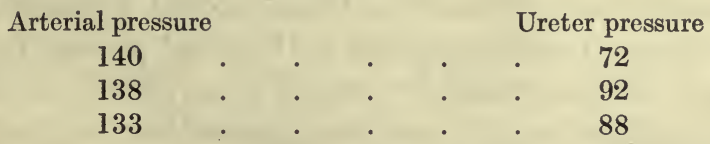

In this experiment therefore secretion came to an end with a difference of pressure between ureter and arteries of between 40 and $50 \mathrm{~mm}$. $\mathrm{Hg}$.

The absolute pressure attained within the ureter in any given experiment after ligature of these tubes will vary with several factors. In the first place, if the minimum secreting pressure is really conditioned by the colloid content of the blood-plasma, it will be less the smaller the proportion of colloids in the plasma. In some experiments (Magnus) a flow of urine was observed with a blood-pressure as low as $18 \mathrm{~mm}$. $\mathrm{Hg}$., but in this case the blood was extremely dilute as the result of the continuous injection into the blood-vessels of normal salt solution. Barcroft and Knowlton have shown that the diuresis brought about by injection of saline (Ringer's) solution is inhibited by mixing 
with the saline fluid colloids, such as gelatin and gum, which possess an osmotic pressure. Colloids such as starch, with no measurable osmotic pressure, have no such effect.

On the other hand, the ureters, or at any rate the urinary tubules, cannot be regarded as absolutely water-tight. Not only are the cells of these tubules capable of taking up fluid, but it is probable that at high pressures a certain amount of actual filtration takes place between these cells. This process of reabsorption will tend to diminish the actual pressure of the fluid in the ureters, so that the secretion of urine may apparently come to a standstill when there is still a difference of pressure between blood and urine considerably over $50 \mathrm{~mm}$. Hg. Under such circumstances the ureter pressure will be higher, and the difference of pressure between urine and blood less, the more rapid the formation of urine by the glomeruli. In a number of experiments by V. E. Henderson, it was found that the figure B.P. - U.P. tended to approximate $40 \mathrm{~mm}$. Hg. the more rapid the secretion of urine was. With a slow secretion the flow of urine apparently ceased when there was as much as $80 \mathrm{~mm}$. $\mathrm{Hg}$. difference of pressure on the two sides of the glomerular membrane.

We may conclude that, for the production of any urine by the kidney, a certain minimum difference of pressure is necessary between the blood in the glomeruli and the urine in the tubule, and that this difference becomes less the smaller the protein content of the blood. Since the only work required in the formation of a protein-free filtrate from the blood is that due to the osmotic pressure of the proteins themselves, and the observed difference of pressure during secretion is greater than this osmotic pressure, we are justified in concluding, provisionally at any rate, that the mechanical factors present at the upper end of the urinary tubule are sufficient to account for the production of a glomerular transudate free from protein, but containing the same proportion of water and salts as the blood-plasma circulating through the capillaries.

If the process occurring in the glomeruli is simply one of filtration, three conditions must be realised :

(1) The amount of filtrate, so long as the ureter pressure is constant, must depend on the pressure and rate of flow of the blood in the glomerular capillaries, and must fall or rise with the latter.

(2) The constitution of the fully formed urine as it appears in the ureters, after modification by addition or subtraction on the part of the tubular cells, must approximate more closely to the supposed glomerular transudate, containing the same proportion of salts as the blood-plasma, the more rapidly the formation of the glomerular transudate takes place : i.e. the quicker the flow of urine the more nearly must its composition, reaction, and osmotic pressure resemble those of the blood-serum.

(3) The total quantity of solids excreted in any given time must be increased with any increase in the urinary flow. For, whatever the activity of the tubules, the glomeruli must blindly turn out a certain proportion of solids with every cubic centimetre of fluid that they form.

We may deal first with the influence of alterations in the renal bloodsupply on the flow of urine. Ligature of the renal vein diminishes and soon stops the flow altogether. Since this procedure must cause a large rise of pressure in the capillaries of the kidney, this result was regarded by Heidenhain as disproving any possibility of the glomerular process being of the 
nature of a filtration. At any given time, however, the glomeruli contain but little blood. With total cessation of the renewal of this blood, their contents will rapidly become so concentrated that they will be little more than a mass of red corpuscles. No filtration of water and salts can take place unless there is a continual renewal of the fluid on the blood side of the filter.

On the other hand, alterations in the blood-supply to the kidney, determined by changes on the arterial side, have pronounced effects on the amount of urine formed. The pressure in the glomerular capillaries and the rate of flow through these capillaries can be increased in either of two ways :

(a) By increase of the driving force, i.e., the general blood-pressure;

(b) By a diminution of the resistance to the flow of blood through the kidneys, as by dilatation of the vessels of this organ.

The blood-flow through the kidney can be investigated either by recording the total volume of this organ or by determining the amount of blood which leaves it through the renal vein, according to the methods described in chapter xiii.

It is necessary at the same time to take a record of the arterial bloodpressure by means of a mercurial manometer. It is evident that an expansion of the kidney may be caused by a rise of general arterial pressure, or, the latter remaining constant, by a dilatation of the kidney-vessels; and, conversely, a fall of kidney volume may be due either to a fall of general blood-pressure or to a constriction of the renal blood-vessels. By taking these two records it is possible to tell whether a given increase of blood-flow through the organ is of local or of general causation, i.e. is active or passive. Thus the volume of the kidney gives us an indirect clue to the pressure in and the flow through the kidney-vessels. The flow through the vessels can be determined directly either by a cannula in the inferior vena cava, all veins other than the renal being clamped, or by Brodie's method already described (p. 994).

The results of the experiments carried out by these methods can be represented in the following tabular form :

\begin{tabular}{|c|c|c|c|c|}
\hline Procedure & $\begin{array}{l}\text { General blood- } \\
\text { pressure }\end{array}$ & Renal vessels & Kidney volume & Urinary flow \\
\hline $\begin{array}{l}\text { Division of spinal cord in } \\
\text { neck }\end{array}$ & $\begin{array}{l}\text { Falls to } \\
40 \mathrm{~mm} \text {. }\end{array}$ & Relaxed & Shrinks & Ceases \\
\hline Stimulation of cord & Rises & Constricted & Shrinks & Diminished \\
\hline $\begin{array}{l}\text { Stimulation of cord after } \\
\text { section of renal nerves }\end{array}$ & Rises & $\begin{array}{l}\text { Passively } \\
\text { dilated }\end{array}$ & Swalls & Increased \\
\hline Stimulation of renal nerves & Unaffected & Constricted & Shrinks & Diminished \\
\hline $\begin{array}{l}\text { Stimulation of splanchnic } \\
\text { nerve }\end{array}$ & Rises & Constricted & Shrinks & Diminished \\
\hline \multicolumn{5}{|l|}{$\begin{array}{l}\text { Division of one splanchnic } \\
\text { nerve : }\end{array}$} \\
\hline (a) In dog & Unaffected & Dilated & Swells·(?) & Increased \\
\hline (b) In rabbit & Falls & Relaxed & Shrinks (?) & Diminished \\
\hline Plethora & Rises & Dilated & Swells & Increased \\
\hline Hæmorrhage . & Falls & Constricted & Shrinks & Diminished \\
\hline
\end{tabular}


It will be seen that in every case where an increased blood-flow attended with a rise of blood-pressure in the glomerular capillaries, is brought about, the urinary flow is at the same time increased.

Another factor, altering the ease with which filtration of watery fluid and salts would take place through the glomerular capillaries, would be the composition of the blood-plasma. Any dilution of this plasma must render filtration more easy, while a concentration would make it more difficult. As a matter of fact hydræmia, and especially hydræmic plethora caused by injection of normal saline into the circulation, evoke an increased flow of urine. A smaller effect is produced by injection of defibrinated blood, and if the blood has been previously concentrated by depriving the animals of water, there may be little or no increase in flow, in consequence of the high osmotic pressure of the proteins of the plasma injected.

Experiments on the action of diuretics have a close bearing on the nature of the process occurring in the glomeruli. A large increase in the urinary flow can be brought about by the intravenous injection of saline diuretics such as sodium sulphate or potassium nitrate, or of neutral crystalloids such as urea or sugar. The question arises whether the mechanical changes thereby induced in the renal circulation are sufficient to account for the diuresis. Three factors might be concerned in promoting an increased glomerular transudation. These are :

(1) A rise of pressure in the glomerular capillaries.

(2) Acceleration of the blood-flow from the capillaries.

(3) Diminution of the amount of proteins in the blood-plasma.

When a concentrated solution of salt is injected into the circulation the osmotic pressure of the plasma is raised and water passes from the tissuecells into the blood-stream, in consequence of the osmotic differences between the blood and cells so induced. As a result the total volume of the circulating fluid is increased by the addition to it of water derived from the tissues, i.e. a condition of hydræmic plethora is set up, just as if a large bulk of normal saline fluid had been injected into the circulation. So long as this hydræmic plethora continues, so long is there a rise both in arterial and venous pressures and an increase in the velocity of the circulating blood. The kidney placed in an oncometer shows a great increase in volume. While the plethora lasts there are mechanical conditions at work in the kidneys, i.e. increased pressure, increased rate of flow, and diminished concentration of plasma-all of which would concur in producing an increased glomerular transudation. With certain salts, such as sodium chloride, the diuresis is coterminous with the hydræmic plethora, but with other members of this class, such as grape sugar, the diuresis outlasts the plethora, so that the continued increased secretion of urine leads to an actual concentration and diminution of the volume of the circulating blood, as is shown in Fig. 533. If the kidney be placed in an oncometer, it is found that the dilatation of the kidney outlasts the plethora, and comes to an end only with the cessation of the increased urinary flow. There must be local influences at work (perhaps the direct effect of the sugar on the blood-vessels) which lead to an 
active dilatation of the renal vessels, and a consequent rise of pressure and velocity of the blood in the glomeruli. That the vascular change is really responsible for the increased urinary flow is shown by the fact, determined by Cushny, that if the swelling of the kidney be prevented by means of an

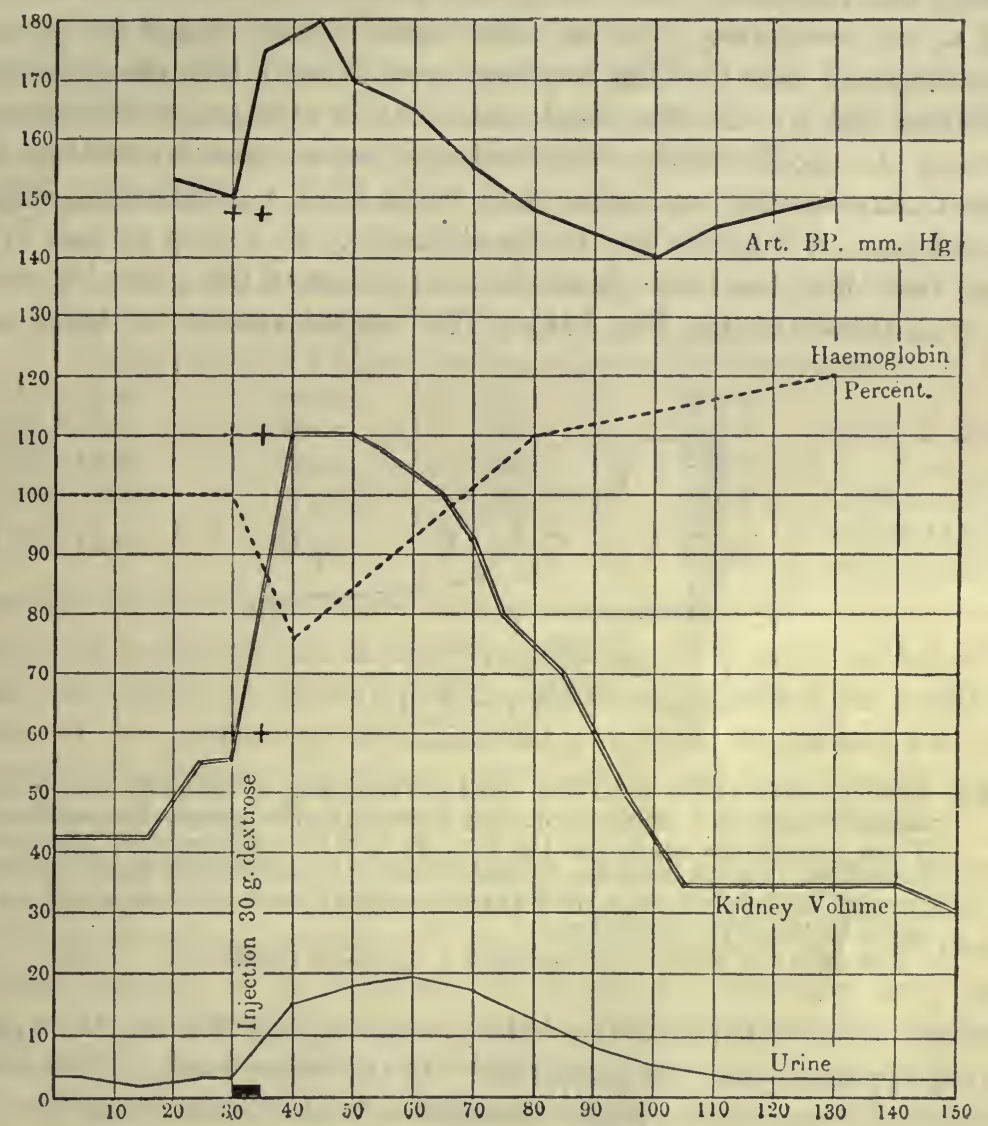

Fig. 533. A comparison of the effects of intravenous injection of $30 \mathrm{grm}$. glucose in concentrated solution on the arterial blood-pressure, the concentration of the blood, the kidney volume, and the urinary flow. Abscissa = time in minutes.

adjustable clamp on the renal artery, no diuresis is produced; so long as the kidney is kept at its normal size the flow of urine remains at the same rate as before.

With regard to the specific diuretics, such as caffeine, the question is not quite so clear. In most cases injection of caffeine in the rabbit brings about a dilatation of the kidney and a proportional increase in the secretion of urine. But cases have been recorded in which expansion of the kidney occurred without any increase in urinary flow, and, on the other hand, augmented urinary flow without any increase in the kidney-volume, or even in the rate of blood-flow through the kidney (as determined by Brodie's method). The general rule, however, is that a greater rate of blood-flow is 
obtained pari passu with the increased urinary flow ; and a consideration of certain peculiarities in the renal circulation must prevent us from laying too much stress on apparent exceptions to the rule. To the blood entering the kidneys by the renal arteries two ways are open. The blood may pass through the vasa afferentia, through the glomeruli and tubular capillaries, back to the renal vein. On the other hand, it may escape the glomeruli altogether, and pass through the vasa recta directly into the intertubular capillaries and so into the renal veins. It is a common experience, in injecting the blood-vessels of the kidneys post-mortem, to find the renal arteries, intertubular capillaries, and veins filled to distension with the injected mass, but hardly any in the glomeruli. One must assume in such a case that there has been spasmodic contraction of the muscular coats of the vasa afferentia (cp. Fig. 534). The normal amount of blood might

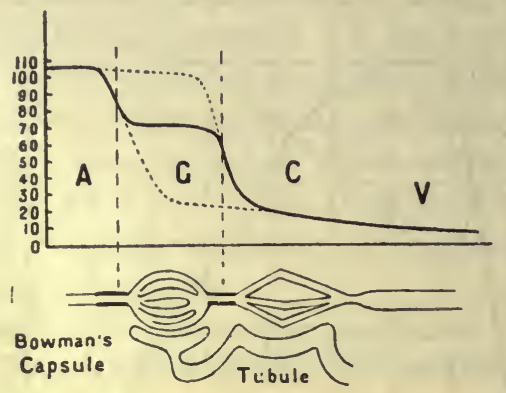

FiG. 534. Diagram (after MoraT) to illustrate the effect of active changes in the vasa afferentia and efferentia on the pressure in the glomerular capillaries. If the vas afferens constricts, the pressure will be represented by the lower dotted line. On the other hand, constriction of the vas efferens would raise the pressure in the glomerulus till it almost equalled that in the renal artery, as is shown by the upper dotted line.

A, arteries ; $\mathrm{G}$, glomerular capillaries ; c, tubular capillaries ; v, vein.

therefore circulate through the kidney without any flowing through the filtering apparatus, i.e. the glomeruli. On the other hand, a dilatation of the afferent vessels and a slight constriction of the efferent vessels would cause a considerable rise of pressure in the glomerular capillaries, and a consequent increased transudation, without necessarily altering to any marked extent the total circulation of blood through the whole organ. The changes in the afferent and efferent vessels and the glomeruli are, however, beyond our control or powers of observation, so that it is impossible to devise at the present time any crucial experiment which might decide the nature of the process occurring in the glomeruli.

THE COMPOSITION OF THE URINE. If the glomerular function is that of mere filtration, we should expect that the more rapidly the process occurs, the more nearly would the urine which is turned out into the ureters resemble the blood-plasma in composition, reaction, and osmotic pressure, since the glomerular filtrate hurried through the tubules would have very little time to undergo any changes resulting in its concentration. If, on the other hand, the diuresis, produced by salt or sugar solutions, is to be ascribed 
to a stimulation of the renal epithelium, the differences between bloodplasma and urine should be greatest at the height of the diuresis, when the concentration of the specific stimulant is also at its highest. The following experiment shows that the more rapid the secretion of urine, the more closely does its concentration, as indicated by its osmotic pressure and depression of freezing-point $(\Delta)$, approximate that of the blood-plasma.

A dog received $40 \mathrm{grm}$. of dextrose dissolved in $40 \mathrm{ccm}$. of water. The following Table represents the relative concentrations of urine and bloodserum at different stages in the diuresis thereby produced :

\begin{tabular}{|c|c|c|c|c|}
\hline Time & Urine & Rate of flow & $\Delta$ of urine & $\Delta$ of blood-serum \\
\hline $11.30-12$ & 10 c.c. & $3 \cdot 3$ & $2 \cdot 360$ & 0.625 (at 12.0 ) \\
\hline \multicolumn{5}{|c|}{ From 12.0 to 12.7 injected $40 \mathrm{grm}$. dextrose into jugular vein } \\
\hline $12.7-12.15$ & 35 c.c. & 45 & $1 \cdot 210$ & - \\
\hline $12.16-12.20$ & 20 c.c. & 50 & 0.975 & 0.700 (at 12.16) \\
\hline $12.20-12.30$ & 52 c.c. & 52 & 0.835 & - \\
\hline $12.30-12.40$ & 45 c.c. & 45 & 0.825 & 0.700 (at 12.30) \\
\hline $12.40-12.50$ & 22 c.c. & 22 & $0 \cdot 830$ & $0 \cdot 675$ (at 12.40 ) \\
\hline & & & & $0.010(\operatorname{arc} 12.00)$ \\
\hline
\end{tabular}

A still closer approximation of the concentration of the urine to that of the plasma was obtained by Galeotti in some experiments in which the modifying influence of the tubular epithelium on the glomerular transudate had been prevented by poisoning the animal with corrosive sublimate, which causes destruction of the epithelium, but is said to leave the glomeruli intact.

Since the glomerular transudate must have a concentration approximately identical with that of the blood-plasma, it would be impossible for a urine formed by mere filtration to have a concentration less than that of the blood-plasma. It is, however, of frequent occurrence that, after copious potations of tea or light beer, urine is passed with an osmotic pressure and a molecular concentration considerably below that of the blood. In one case Dreser obtained a urine with a freezing-point of $\Delta=0 \cdot 16^{\circ} \mathrm{C}$, and the same result has been obtained on one or two occasions when the diuresis has been produced by the administration of caffeine. If we assume that this hypotonic fluid is formed by the glomeruli, we must at once give up any idea of the process in these structures being essentially one of filtration. But it is possible that the epithelium of the tubules can secrete water as well as solid constituents. The fine adaptation of the kidney to slight changes in the composition of the blood is apparently.an endowment of the tubular epithelium, and in those cases where large quantities of hypotonic urine are passed there is not at any time any appreciable change either in the composition of the blood or in its total volume. Water is absorbed from the alimentary canal and is almost immediately excreted by the kidneys. When we attempt to produce the same effect by infusion of large quantities of water or hypotonic solutions into the blood-stream, we, get a flow of urine apparently determined entirely by the circulation through the kidney and 
having a concentration not inferior to that of the blood. The passage of hypotonic urine can be ascribed to a modification of the glomerular transudate as it passes through the tubules, a modification due partly to the absorption of salts from the fluid, partly, perhaps chiefly, to a secretion of water or extremely dilute salt solution by the cells of the tubules themselves.

Certain other observations accord with our hypothesis that in Bowman's capsule a fluid is transuded having the same molecular concentration as blood-plasma, and therefore considerably less concentrated than normal urine. Ribbert succeeded in extirpating the whole of the medullary portion of the kidney in the rabbit, leaving the cortex intact, and found in this case that during the survival of the animal the urine passed was much more dilute than normal. In cases where there is destruction of the tubular epithelium, while the glomeruli remain intact, either in consequence of disease or, as in Galeotti's experiments, as a result of poisons, we are accustomed to obtain a dilute copious urine ; and the continual passage of such urine is in man regarded as a sign of one form of renal disease.

The experimental facts which we have passed in review do not therefore negative the view that the glomerular epithelium plays the part of a passive filter in the formation of urine, and that the energy of the process by which ' urine' is produced in Bowman's capsule is entirely furnished by the heart in driving the blood at a high pressure through the glomerular capillaries.

\section{FUNCTIONS OF THE RENAL TUBULES}

Whatever the nature of the glomerular activity, it is evident that the multiform epithelium of the tubules may alter the glomerular transudate, either by the absorption or by the secretion of water or solid constituents. We may deal with the evidence for the occurrence of these two processes separately.

SECRETION BY THE URINARY TUBULES. Although it is impossible to collect the secretion of the glomeruli apart from that of the tubules, the arrangement of the blood-vessels in certain animals enables us to influence separately the circulation to these two parts of the kidney. The amphibian kidney receives a blood-supply from two sources. A number of renal arteries leaving the aorta enter the kidney and supply the whole of the glomeruli, the vasa efferentia from which pass, as in the mammalian kidney, into the intertubular capillaries. These are also supplied with blood of venous character by the renal portal vein. If all the renal arteries be divided or ligatured, the glomeruli, as was shown by Nussbaum, are entirely cut out of the circulation, though the tubules continue to receive venous blood through the renal portal vein. Nussbaum stated that ligature of all the renal arteries caused cessation of the urinary secretion, which could be reinduced by injection of urea. He concluded that urea with water was secreted by the tubules, whereas peptone, sugar, and hæmoglobin were turned out by the glomeruli. Beddard showed that these results of Nussbaum must have been due to the fact that he had not obstructed the whole of the renal arteries. One or two of these small vessels will suffice to supply blood to a considerable number of the glomeruli. After complete obstruc- 
tion of the arteries, no urinary flow could be induced even with subcutaneous injection of urea. But the cutting off of the arterial blood-supply from the tubules caused a rapid destruction of the tubular epithelium, so that the result of the experiment could not be taken as negativing the possibility of this epithelium having, when in a normal state of nutrition, some secretory power. He therefore carried out, with Bainbridge, another series of experiments of the same description, in which the frogs, after ligature of the renal arteries, were kept in an atmosphere of pure oxygen. Under these circumstances sufficient oxygen diffused into the blood of the renal portal

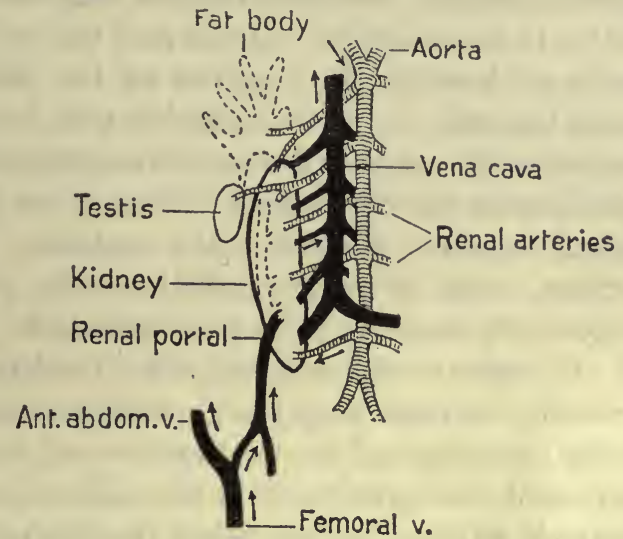

Fig. 535. The vascular supply to the kidney in the frog.

vein to maintain an adequate supply of this gas to the tubules. No desquamation of the epithelium resulted, and injection of urea produced a small flow of urine even when, by subsequent injection of the blood-vessels, it was proved that every glomerulus had been cut out of the circulation.

The view that a portion of the tubules is secretory in function is further supported by histological examination of these structures under various conditions of activity. In the cells of the convoluted tubules various kinds of granules and of vacuoles may be distinguished. Gurwitsch divides these vacuoles into three classes :

(1) Large granules staining densely with osmic acid, and probably rich in lecithin.

(2) Smaller very numerous granules consisting of some form of protein material.

(3) Large vacuoles lying close to the free margins of the cells, whose contents do not undergo coagulation with the ordinary fixing reagents, and therefore are free from protein, fat, or mucin. These vacuoles are especially marked in kidneys which are secreting at a great rate, in consequence of the injection of saline diuretics or of large quantities of normal salt solution. They may be regarded as excretory vacuoles, and consist of water or saline fluids which have been collected by the cells and are being passed on by them to the lumen of the tubules.

As a rule it is impossible to trace any definite constituent of the urine on its way through the cells of the tubules. But if a solution of uric acid in piperazin be injected intravenously into a rabbit, the kidneys, taken twenty to sixty minutes after the injection, present tubules full of uric acid concretions. In the medullary portion of the kidney this uric acid precipitate is confiner to the lumen of the tubules, but in the convoluted tubules granules 
of uric acid are to be found in the epithelial cells, especially towards their inner border. Since these cells are able to excrete uric acid when present in abnormal quantities in the blood, it is a reasonable assumption that they also undertake the secretion of this substance under normal conditions. Certain observers have in fact described the presence of urate granules in the cells of the convoluted tubules of the bird's kidney.

Although the larger number of the urinary constituents must escape detection on their way through the cells, we can throw some light on the excretory functions of the kidney by studying the mechanism by means of which it excretes certain dyestuffs, such as sulphindigotate of soda ('indigo carmine '). If the indigo be injected into the veins, it is excreted in a concentrated form, both by the liver and by the kidney, so that the urine assumes a dark blue colour. If the animal be killed when the excretion of the pigment is at its height, and the kidneys be rapidly fixed with absolute alcohol (which precipitates the dyestuff), all parts of the kidney present a blue colour, which is especially marked in the medulla. Under these circumstances the urine, which is being excreted by the glomeruli, rapidly carries down the dyestuff, wherever it may be turned out, into the tubules of the pyramids. In order to discover the exact locality of the cells involved in its excretion, we must stop the glomerular transudate by some means or other. This stoppage of the urinary flow can be effected in two ways, viz. by section of the spinal cord in the neck, so as to reduce the blood-pressure to about $40 \mathrm{~mm}$. Hg., i.e. below the minimum necessary for the production of urine, or by cauterising portions of the surface of the kidney by means of silver nitrate. If the indigo be injected into the veins, after section of the cord, and the animal be killed half an hour later, and the kidneys fixed with absolute alcohol, they are found to be of a bright blue colour, although no urine has been secreted. On cutting into the kidneys the colour is seen to be confined to the cortex, and on making microscopic sections granules of the pigment are found within the lumen and in the epithelial cells of the convoluted tubules. If the kidneys have been cauterised, the stain is confined to the convoluted tubules of the cortex only under those areas which have been cauterised, and where the glomerular functions have been abolished. It has been suggested that the appearances after the injection of indigo are due, not to the secretion, but to the absorption of water in the convoluted tubules. A certain amount of the dyestuff is thus rendered visible by becoming more concentrated, and is precipitated in a granular form, as soon as the salt concentration of the fluid reaches a certain height. The fact that these appearances are wanting after the injection of ordinary carmine, which stains the glomeruli as well as the tubules, combined with the histological facts mentioned in the last paragraph, render this a somewhat forced explanation; and we must take the results of the injection of indigo-carmine as telling rather in favour of a secretory than of an absorptive function on the part of the convoluted tubules.

The question as to the secretory activity of the kidney can be attacked from another side. The glomerular filtrate can contain only those crystal- 
loids of the blood which are diffusible and are not closely combined with its colloidal constituents. Loewi has shown that in this connection a contrast is to be drawn between the behaviour of substances, such as urea or sodium chloride, and certain other constituents of the blood such as phosphates or sugar. Any increase in the rate at which the glomerular secretion takes place must cause a corresponding increase in the total amount of the solid diffusible constituents of the blood-plasma which are turned out within a given time. Thus every diuresis increases the total output of chlorides and of urea. On the other hand, a diuresis caused, for example, by drinking large quantities of water does not increase the total output of phosphates in a given time, nor does it increase the very small amount of sugar which is normally excreted by the kidneys. If, however, sodium phosphate be previously injected into the blood, then any diuresis increases the output of this salt. The same thing holds for sugar. If an excess of free uncombined sugar be present in the blood, either in consequence of intravenous injection of this substance or as a result of previous extirpation of the pancreas, any form of diuresis will increase the rate at which it is turned out by the kidneys. Loewi concludes that phosphates, which must be present in minimal quantities in the glomerular transudate, are for the most part secreted by the activity of the cells of the convoluted tubules. Under abnormal conditions, e.g. as after administration of phloridzin, the cells of the kidneys can be excited to a similar activity with regard to sugar. After phloridzin injection the urine contains considerable quantities of sugar, but the rate at which the sugar is secreted is not affected in any way by raising the rate of urinary secretion, e.g. by the injection of such substances as sodium sulphate, which increases the rapidity of the glomerular process of transudation.

ABSORPTION BY THE RENAL TUBULES. The experiments of Ribbert, mentioned above, in which removal of the medullary portion of the kidney led to the formation of an increased quantity of a more watery urine, points to the possession by the tubules of a power of absorbing water. We have other evidence that this power of resorption is not confined to water, but may affect also the dissolved constituents of the glomerular transudate. It was pointed out by Meyer that, if two salts, such as sodium sulphate and sodium chloride, were present at the same time in the glomerular transudate, any process of resorption should affect chiefly the more diffusible salt, namely, sodium chloride. Such a differential resorption would account for the much greater diuretic power of sodium sulphate as compared with sodium chloride. In certain experiments Cushny produced a diuresis by the injection of equal parts of equivalent $\mathrm{NaCl}$ and $\mathrm{Na}_{2} \mathrm{SO}_{4}$ solutions into the veins of a rabbit. An increased flow of urine was produced which lasted two hours and a half. The chlorides of the urine rose with the diuresis and reached their maximum at the height of the urinary flow. They then sank, and in some experiments had practically disappeared from the urine towards the end of the observation. The concentration of the sulphates, however, continued to rise in the urine to the end of the experiment. Thus in the first of two identical experiments, when the rabbit was killed at the height of the 
diuresis, the serum contained 0.547 per cent. chlorine and $0 \cdot 259$ per cent. sulphate, while the urine contained 0.372 per cent. chlorine and 0.546 per cent. sulphate. In the second, in which the rabbit was killed when the rate of the urinary flow had considerably diminished, the serum contained 0.493 per cent. chlorine and $0 \cdot 191$ per cent. sulphate, while the urine contained .094 per cent. chlorine and 2.0 per cent. sulphate. These results are illustrated in Fig. 536.

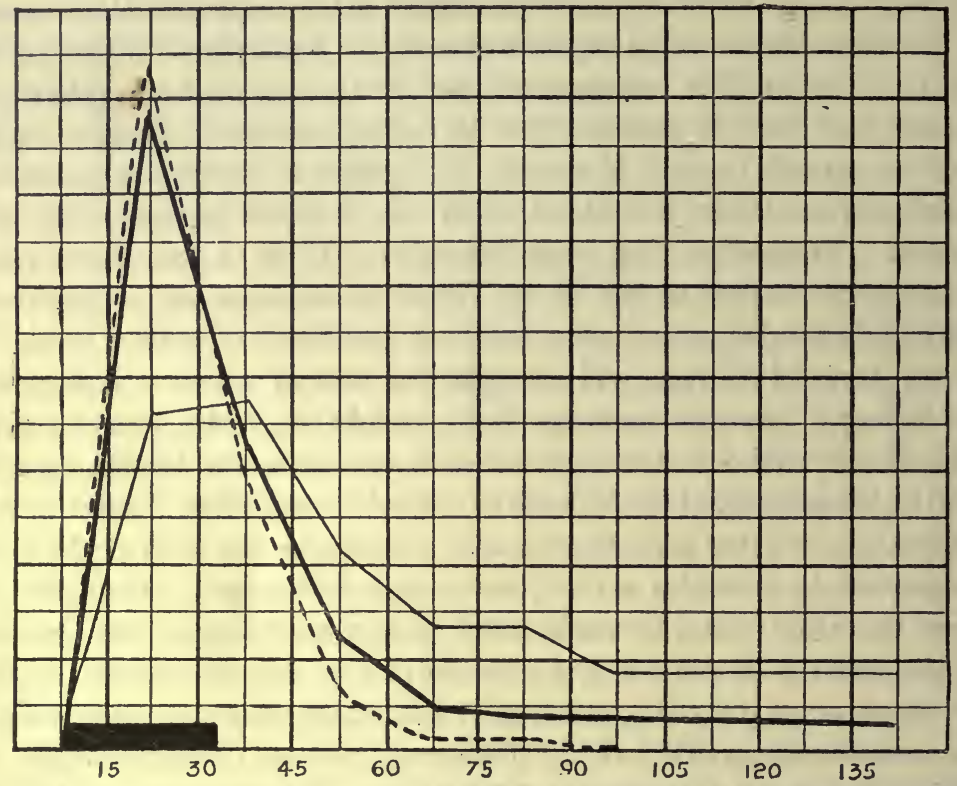

FIG. 536. Curves showing excretion of urine (thick line), of sulphate molecules ( $\frac{\mathrm{SO}_{4}}{96}$, thinline), and of $\mathrm{Cl}$ molecules $\left(\frac{\mathrm{Cl}}{35 \cdot 5}\right.$, dotted line), after injection of 50 c.c. of a solution containing $1 \cdot 775 \mathrm{grm}$. $\mathrm{Cl}$ and $4 \cdot 8 \mathrm{grm}$. $\mathrm{SO}_{4}$ per $100 \mathrm{c.c}$. The black line along the base marks the duration of the injection. (CusHry.)

The difference between the two salts can be made still more striking if the process of resorption be augmented by increasing the pressure within the tubules by partial obstruction of one ureter. Thus in one experiment, where diuresis was produced by the injection of 30 c.c. of a solution containing 5.85 per cent. $\mathrm{NaCl}+14 \cdot 2$ per cent. $\mathrm{Na}_{2} \mathrm{SO}_{4}$, the right ureter was partially clamped so as to make the right kidney secrete against a pressure of $31 \mathrm{~mm} . \mathrm{Hg}$. The following results were obtained :

\begin{tabular}{|c|c|c|c|c|}
\hline & & Urine c.c. & cl. g. & $\mathrm{SO}_{4} \mathrm{~g}$. \\
\hline 4.37 till 4.47 & $\left\{\begin{array}{l}\left\{\begin{array}{l}\text { Left kidney } \\
\text { Right kidney }\end{array}\right. \\
\text { Difference (absorption) }\end{array}\right.$ & $\begin{array}{r}24 \\
8 \\
16\end{array}$ & $\begin{array}{l}0.0809 \\
0.0142 \\
0.0677\end{array}$ & $\begin{array}{l}0 \cdot 1080 \\
0 \cdot 0667 \\
0 \cdot 0413\end{array}$ \\
\hline
\end{tabular}

We must conclude that the tubular epithelium possesses the power of modifying the glomerular transudate not only by the absorption of water 
but also by the absorption of dissolved constituents, and that the relative permeability of the cells to the constituents is at any rate one factor in determining the substances absorbed. It is not, however, the only factor. The function of the kidney is to preserve the normal constitution of the body fluids by turning out those substances which are abnormal or present in too great an amount. The behaviour of the tubule cells with regard to any given substance will therefore depend to a certain extent on the previous nutritive history of the body.

If, for instance, in consequence of the administration of sodium chloride in large quantities to the animal during the few days precect. ment, the body is overloaded with this salt, it becomes an abnormal constituent and the kidney secretes a urine far richer in sodium chloride than is the blood-plasma. Moreover, when diuresis is produced in such an animal by the injection of equivalent quantities of sodium chloride and sodium sulphate, there is no diminution of the $\mathrm{NaCl}$ in the urine towards the end of the diuresis, but its percentage rises steadily as the rate of urinary flow diminishes. On the other hand, a total deprivation of sodium chloride extending over several days, although not altering to any large extent the percentage amount of this salt in the blood-plasma, leads to a total disappearance of the salt from the urine, the whole of the sodium chloride present in the glomerular transudate being absorbed on its way through the urinary tubules.

It has been suggested that the effects of certain diuretics on the kidney, such as caffeine, diuretine, or theocine, may be largely conditioned not so much by their influence on the glomerular circulation as by a paralytic effect on the absorptive functions of the tubules. According to Loewi, on injection of caffeine or diuretine, the increase of total amount of urine is not accompanied by any diminution in the percentage amount of $\mathrm{NaCl}$. Perhaps, however, the strongest evidence in this direction is afforded by an experiment of Pototzky. A rabbit had been fed on a diet almost totally devoid of chlorides, and was therefore excreting a urine containing only .08 per cent. $\mathrm{NaCl}$. Under the influence of diuretine the urine was increased and the concentration of the $\mathrm{NaCl}$ rose to 0.64 per cent. The same increase in the percentage amount of sodium chloride in the urine has also been observed after the injection of theocine, which has therefore been specially recommended as a diuretic in cases of dropsy, where a diminution of the salt content of the body is a valuable means for the diminution of the dropsical fluid present in the tissue spaces.

\section{THE RENAL MECHANISM}

What conclusions can we draw from this mass of experimental data as to the functions of the kidney as a whole, and as to the part played by its various constituent elements in the secretion of urine?" The amazing adaptability of its functions to the needs of the organism has been abundantly illustrated in the facts with which we have dealt. Its ordinary activity is determined by the production, as a result of the normal processes of meta- 
bolism, of soluble non-volatile substances in every cell of the body. These substances, together with the excess of water taken in with the food above that lost by respiration and cutaneous transpiration, are turned out by the kidney as urine. The activity of this organ must therefore be determined in the first place by chemical stimuli. It must react to the slightest deviation from normal of the blood composition by excreting water or dissolved substances. This delicate sensibility is displayed in two directions :

(1) Under the influence of certain substances, such as urea, uric acid, or water, the cells of the convoluted tubules take up the substance, which is in excess, from the surrounding lymph and accumulate it in vacuoles, which are discharged on the inner surface of the cells into the lumen of the tubules.

(2) Besides this specific secretory activity of the cells of the convoluted tubules, the tubules as a whole are endowed with the power of absorbing both water and dissolved substances from the fluid in their lumen. Whether this absorptive power is limited to the cells of Henle's loop, as was first suggested by Ludwig, or occurs coincidently with secretion in the cells of the convoluted tubules, as might be imagined from the close analogy between the structure of these cells and that of the intestinal epithelium, we have not sufficient evidence to decide. We do know, however, that the quality of the absorption is strictly regulated according to the needs of the organism, so that the eonstituents which are precious are reabsorbed for service in the body, while those which are in excess or are of no value to the organism are allowed to pass out into the ureters. The process of resorption is indeed, as is shown by Cushny's experiments, largely dependent on the physical qualities of the substances undergoing absorption, and especially on the permeability of the renal cells to these substances. The physical conditions are, however, subordinated to the physiological, so that a salt so diffusible as potassium iodide is left in the fluid, while sodium chloride may be reabsorbed in large quantities.

The necessity for the endowment of the tubular epithelium with a resorptive as well as a secretory function is determined by the presence at the beginning of the tubule of a mechanism - the glomerulus, devoid of the fine selective power or chemical sensibility possessed by the cells of the convoluted tubules. The production of urine by the glomerulus is apparently regulated entirely by the pressure and velocity of the blood through its capillaries and by the colloid content of the blood-plasma. We may assume that in Bowman's capsule there is under normal conditions a constant production of a fluid free from protein, but having the same crystalloid concentration as the blood-plasma. With any rise of general blood-pressure the amount of this transudate is increased; . with any fall it is diminished. The small qualitative changes which are constantly occurring in the blood as the result of the taking of food or the activity of different organs, probably produce but little effect on the amount of glomerular fluid. Only indirectly, as the result of these events on the general blood-pressure, or possibly in consequence of the production of substances having a vaso-dilator effect on 
the renal vessels, will the amount of the urine turned out by the glomeruli be affected. These structures therefore have the twofold function of regulating the total amount of circulating fluid and of providing an indifferent fluid, which will, so to speak, flush the kidney tubules and carry down any constituents excreted in a concentrated form by the cells of these tubules. The constant production of a glomerular transudate might result, especially in terrestrial animals, in the loss to the organism of water, or, under certain nutritive conditions, of substances indispensable as normal constituents of the serum, such as sodium chloride, which could not be made good at the expense of the food. It is for this reason that an absorptive mechanism sensitive to and reflecting the nutritive condition of the whole body, especially as concerns water and salts, should be present in the tubules. As the result of the complementary processes of absorption and secretion in the tubules, the unchanging glomerular filtrate undergoes great modifications in its passage towards the ureter. It receives urea, uric acid, phosphates, and under certain conditions water, from the cells of the convoluted tubules. It gives up salts, especially sodium chloride, and generally water to the same or other cells of the tubules. So that finally, instead of a fluid isotonic with the blood and containing only about $0 \cdot 1$ per cent. urea, we have a fluid of deep yellow colour, with a molecular concentration four or six times greater than that of the blood, and containing between 2 and 3 per cent. urea. We have at the present time no means of judging the relative amounts of fluid furnished respectively by the glomeruli and the tubules to the fully formed urine. It is probable that, under ordinary circumstances, the processes of secretion and absorption of fluid go on pari passu in the urinary tubules just as they do in the mucous membrane of the small intestine. The demonstration of secretory powers in the cells of the convoluted tubules relieves us from the necessity of the assumption made by Ludwig as to the quantity of fluid normally turned out through the glomeruli. On the hypothesis that the sole function of the tubules was one of absorption, and that all the urinary constituents were derived from the glomerular transudate, thirty litres of fluid would have to be filtered through the glomeruli in order to excrete the $30 \mathrm{grm}$. urea which is the daily output of a man. Of these thirty litres, twenty-eight litres would have to be reabsorbed in the tubules. Since the amount of blood flowing through the two kidneys in a man probably varies between 1600 and 1800 litres in the twenty-four hours, there would be no difficulty in the production of such an amount as thirty litres, which would only represent a concentration in the blood in its passage through the glomeruli of under 2 per cent. The secretion and reabsorption of such large quantities of fluid seem, however, a clumsy way of arriving at a urine, whose composition should be adopted to the needs of the animal ; and as we have seen, the occurrence of an actual secretion of urea by the cells of the tubules removes the necessity for assum:ing any such wasteful proceeding. It is probable that the actual amount of the glomerular filtrate in the twenty-four hours may not exceed to any large extent the actual amount of urine formed by the whole kidney in this time. 


\section{SECTION III}

\section{THE PHYSIOLOGY OF MICTURITION}

THE urine as it is formed passes through the ureters to the bladder, where it gradually accumulates, and is voided at intervals.

The ureters are muscular tubes lined by transitional epithelium. The muscular coat is composed of three layers of unstriated fibres, a middle circular coat lying between external and internal longitudinal coats. If the ureter be exposed in the living animal, contraction waves are seen to pass along its muscular coat from the pelvis of the kidney to the bladder, driving the contained fluid in front of them. The frequency of the contractions is increased by warming the ureter, and to a certain extent by distension, so that the waves are more frequent when the secretion of urine is profuse. The ureters enter the bladder at or near its base, at the two posterior angles of the region known as the trigonum. Their entrance is oblique, so that a valvular orifice is formed, which effectively prevents reflux of urine from bladder to ureter. Rhythmic waves of contraction are observed also in the excised ureters, when these are kept warm in normal saline solution. By Engelmann they were regarded as myogenic, since they were present in the middle third of the ureter, which he imagined to be entirely free from ganglion-cells. As a matter of fact ganglion-cells are found throughout the ureter, though in larger numbers at its two ends. The ureters are supplied with nerve fibres from the splanchnic nerves by way of the renal plexus, and at their lower ends from the hypogastric nerves. Stimulation of the latter as a rule increases the rhythm of the contraction presented by the lower end of the ureter. The splanchnic nerves have been stated to produce either acceleration or inhibition of the contractions at the upper end; their action is, however, uncertain. It is by the rhythmic advancing waves of contraction of the ureter that the urine is continuously passed on to the bladder, so that the pelvis of the kidney is kept empty of fluid whatever the position of the animal.

The bladder is lined by transitional epithelium, closely adherent to the underlying muscular coat. It is usual to describe in the latter three layers of muscular fibres :

(1) An outer layer composed of bundles running longitudinally from the neck of the bladder to the fundus, sometimes distinguished by the name of the detrusor urince. At the neck of the bladder these bundles send some fibres to be attached to the pubes as the pubo-vesical muscles. On 
the dorsal surface some bundles in the male pass on to the prostate and the urethra, while in the female they end in the tough connective tissue in the urethro-vaginal septum.

(2) The middle layer, which is the thickest of the three, is composed of fibres arranged circularly and forming a continuous layer.

(3) The inner layer is thin and incomplete, and is composed of anastomosing bundles of fibres with meshes in between them which are covered by the folds of the mucous membrane. The bundles of fibres run freely from one layer to the other, and there is no doubt that the name of detrusor ought physiologically to be applied to the whole of the three coats, which act as one in diminishing the capacity of the bladder. At the base of the bladder the structure of the wall is modified over the triangular region lying between the orifices of the ureters and of the urethra (the trigonum) by the differentiation here of fibres which serve as a sphincter and prevent the escape of urine. Over the trigonum the mucous membrane of the bladder is smooth and closely adherent to the subjacent muscular fibres, which themselves are much more closely packed than the rest

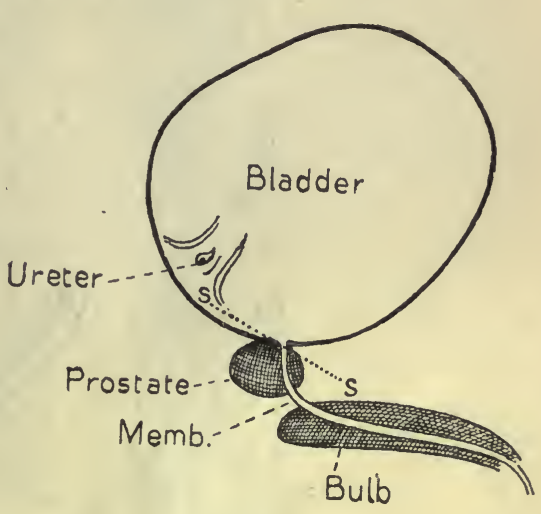

FIG. 537. of the bladder wall. From these muscular fibres the mostimportant sphincter, the sphincter trigoni, is formed. Bundles of muscle fibres pass from the trigonal muscle obliquely forwards and downwards (the individual being considered in the erect posture), and form a loop around the orifice of the bladder, lying on the ventral side of the bladder below and quite distinct from the thick coat of circular fibres belonging to the bladder itself (ss, Fig. 537).

This sphincter is the most important mechanism for the retention of urine. If a catheter be passed into the urethra no urine escapes until its orifice has actually entered the bladder. The wall of the urethra is surrounded by circular muscular fibres, which, by their tonic contraction, will also tend to prevent the escape of urine along the canal. This urethral muscle is strengthened by two sphincter muscles which are voluntary and composed of striated fibres. The chief one, which has been named by Kalischer the sphincter urogenitalis, but is better known as the compressor urethree, forms a flat ring around the second part of the urethra, extending in the male from the prostate to the bulb, where its function is taken up by the bulbo-cavernosus.

The bladder is therefore supplied with a powerful muscular wall, the contraction of which will cause its evacuation, and with sphincters of two kinds, one involuntary, the sphincter trigoni, at the upper neck of the bladder, and two voluntary, the sphincter urogenitalis and bulbo-cavernosus muscles, which can empty the lower parts of the urethra. 
The nerve-supply of the bladder (Fig. 539) is derived from two main sources, namely, from the upper four lumbar nerves through the sympathetic system, and from the second and third sacral nerves by means of

A

\section{Bladder}

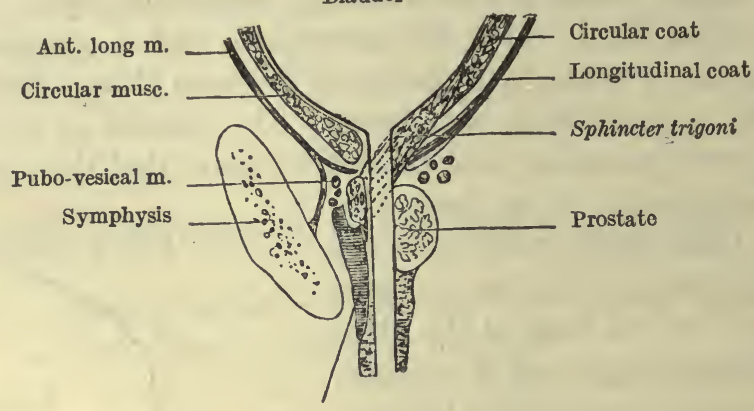

B

Bladder

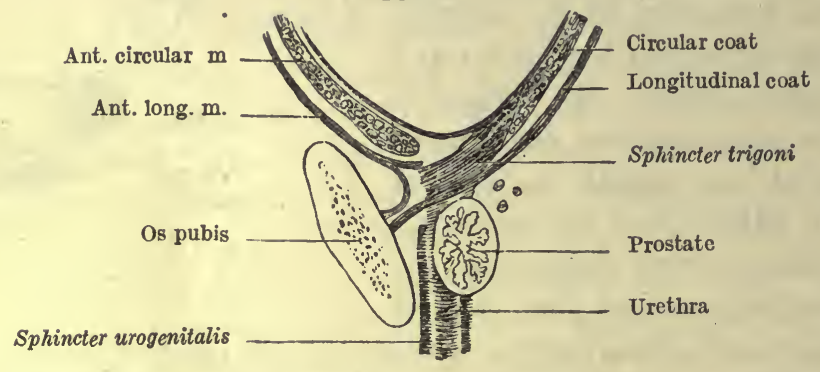

C

Bladder

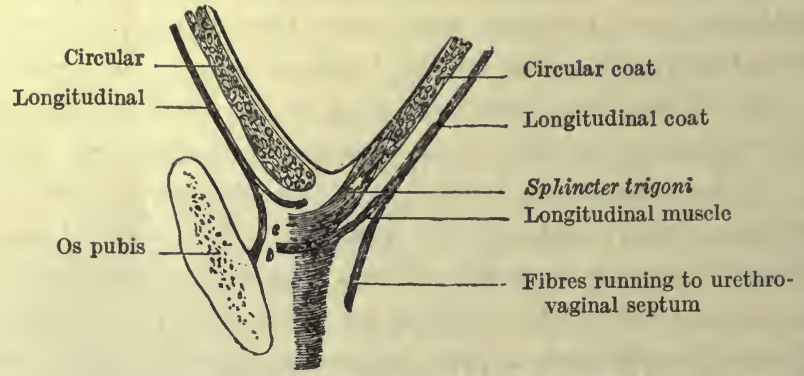

Fig. 538. Sagittal sections through ncck of bladder.

(METZNER after KaLISCHER.)

A, in middle line (male); B, slightly to left of middle line (malc); C, ditto (female).

the pelvic visceral nerves or nervi erigentes. The upper lumbar nerves send white rami communicantes to the lateral chain of the sympathetic, and thence to the collateral ganglia, which are grouped round the inferior mesenteric artery to form the inferior mesenteric ganglion. Most of the fibres end in this collection of ganglion-cells, and a new relay of axons passes by two main trunks, the hypogastric nerves, into the pelvis on each side of 
the rectum and ends in a plexus, the hypogastric plexus, at the base of the bladder. From this plexus fibres pass to the bladder wall. The pelvic visceral nerves are derived from the second and third sacral nerves. They make no connection with the sympathetic system, but pass directly to the

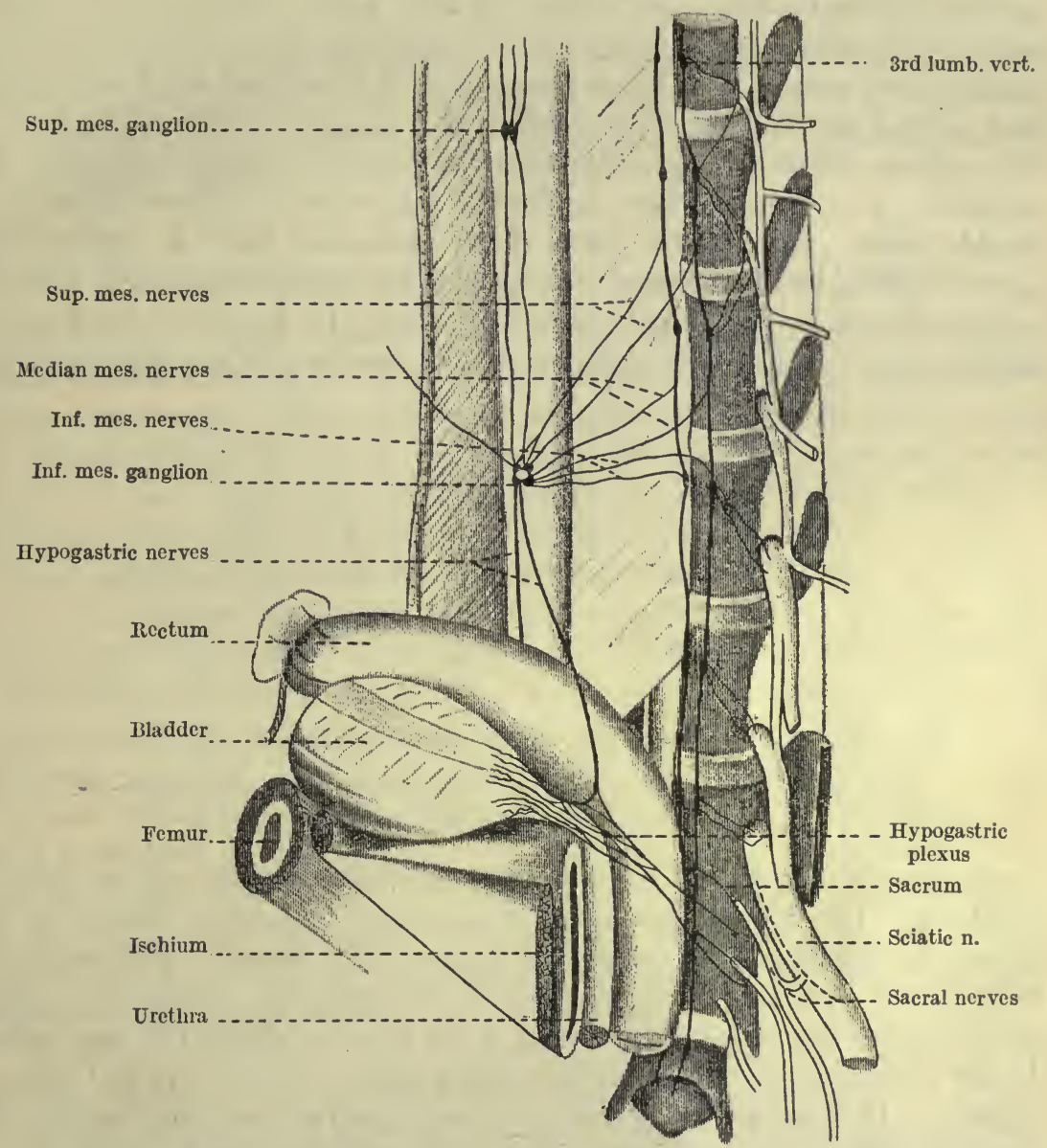

Fig. 539. Nerve supply to bladder of cat. (NAwrocki and SkabITSCHewSKy.)

hypogastric plexus and are carried with branches of this plexus to the neck of the bladder. The fibres do not run directly from the spinal cord to their ending in the bladder wall, but make connection with cells situated peripherally, partly in the hypogastric plexus, but chiefly in the walls of the bladder itself. Both sets of fibres supply also the rectum and the colon, and carry efferent impulses to the bladder. Afferent impulses from the bladder travel chiefly in the pelvic visceral nerves.

\section{THE FILLING OF THE BLADDER}

Under normal circumstances the sphincters at the neck of the bladder are in a state of tonic contraction, presenting a resistance to the emptying of 
this organ which will vary according to their degree of contraction. Thus it requires a considerably greater pressure in the bladder to overcome the resistance of the sphincters during life than after death of the animal. In some cases after death they may permit the passage of urine when the pressure of the bladder is only about $20 \mathrm{~mm}$. water, whereas in the normal animal the pressure has as a rule to be at least $160 \mathrm{~mm}$. of water before any escape takes place. The urine therefore as it is secreted must accumulate and distend the bladder. The bladder wall reacts to a distending force in the manner which is characteristic of all muscular tissue, especially unstriated. An extending force applied to an unstriated muscle fibre has a double effect. In the first place, if the stretching force is applied very slowly, a considerable increase in length of the muscle may occur with the application of a very small amount of force. If, however, the force be applied more rapidly, the sudden increase of tension acts as a direct excitant

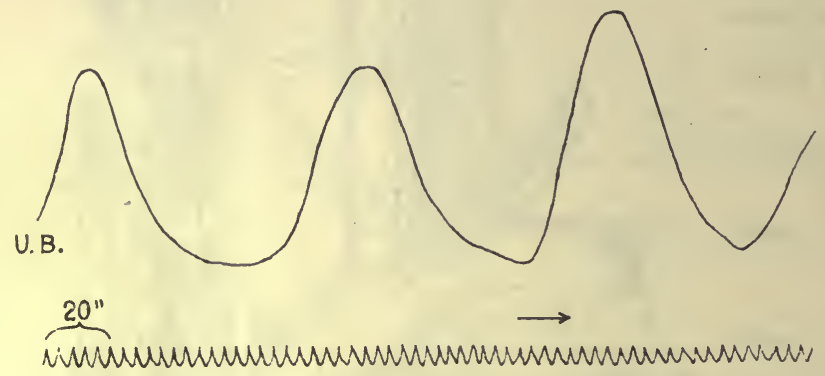

Fig. 540. Tracings of rhythmic contractions of urinary bladder. (SHERRINGTON.)

to the muscle, causing it to enter into contraction, which may be tonic or rhythmic. The effect of the entry of urine into the empty bladder on the tension in this organ will depend therefore on the rapidity with which the kidneys are secreting. Under normal circumstances micturition occurs in man when the intravesical pressure has risen to about $150 \mathrm{~mm}$. water. Under these conditions the bladder will contain between 230 and 250 c.c. of urine. If, however, the secretion of urine has occurred very rapidly, the same pressure may be attained with a much smaller bladder content, and if the bladder be artificially distended by the injection of fluid through a catheter, 50 c.c. of fluid may suffice to raise the pressure to this level. As the urine is slowly secreted, the bladder wall at first gives to the incoming fluid, so that a considerable amount can be stored without any marked rise of pressure. Later on the pressure begins to rise more rapidly, and finally attains a pressure of between 120 and $150 \mathrm{~mm}$. water. At this point the second effect of the stretching of the muscular wall makes its appearance. A manometer connected with the bladder shows a series of rhythmic contractions of the muscular wall (Fig. 540), each lasting about a minute, at first slight in extent, but becoming more marked as the distension of the bladder augments. In a bladder entirely cut off from its connection with the central nervous system these automatic rhythmic contractions gradually 
increase in force until one of them suffices to overcome the resistance presented by the tonically contracted sphincter. A partial emptying of the bladder therefore takes place, but the pressure falls below that necessary to overcome the resistance of the sphincter before the bladder has been quite emptied, so that there is always under these circumstances a certain amount of residual urine left in the bladder. This is the condition found in animals where the lower part of the spinal cord has been extirpated, or in man where the same part of the central nervous system has been destroyed as the result of accident or disease.

\section{THE MECHANISM OF EVACUATION OF THE BLADDER}

In the denervated bladder the factor finally causing partial evacuation is the gradual increase in the intravesical tension from the accumulation of fluid in this viscus. The same factor is prepotent in determining the onset of normal micturition in an animal with the nervous connections of its bladder intact. Apart from the control of the higher centres, micturition will take place each time that the tension in the bladder has reached a certain height, i.e. about $15 \mathrm{~cm}$. water, the amount of fluid in the bladder at the time depending on the one hand on the rate at which the fluid has entered this organ from the ureters, on the other hand on the irritability of the bladder wall itself and of the nervous centres concerned with its motor innervation. The effect of the gradual accumulation of fluid and rise of tension is twofold. In the first place, it acts on the bladder wall, causing rhythmic contractions of ever-increasing intensity ; in the second place, the mere stretching of the bladder originates impulses in the sensory nerveendings in its wall, which are reinforced at every rise of tension caused by the rhythmic contractions. These impulses travel up to the spinal centres, and are summated until they result in a sudden discharge of efferent impulses of two kinds, namely :

(1) Motor impulses to the whole musculature of the fundus of the bladder (the detrusor in its widest sense);

(2) An inhibition of the tonic contraction of the sphincter. This inhibition may be determined by inhibitory impulses travelling to the sphineter and causing its relaxation, or by the central inhibition of the impulses normally going to the sphincter and maintaining its tonic contraction. The resultant of these two processes, the contraction of the detrusor and the relaxation of the sphincter, is a complete emptying of the bladder, and the act is completed by the contraction of the involuntary and voluntary muscles surrounding the urethra and causing complete expulsion of the contents of this tube.

\section{THE INNERVATION OF THE BLADDER}

ACTION OF THE PELVIC VISCERAL NERVES. In all animals excitation of the peripheral end of one pelvic visceral nerve causes a strong 
contraction of the same side of the bladder, involving all its coats and sometimes extending to a slight extent to the contralateral half of the bladder. When both pelvic nerves are stimulated simultaneously contraction of both sides of the bladder causes a considerable rise of pressure in its interior (Fig. 541) which is always sufficient to overcome the resistance of the sphincter and to cause a complete emptying of the bladder. There is no doubt, therefore, that these nerves are the most important for the act of micturition. As to the action of these nerves, however, on the sphincter the results of different experimenters are somewhat at variance. In the cat there seems to be no doubt that inhibition of the sphincter may result from stimulation of the pelvic visceral nerves. On the other hand, Fagge, working on the dog, found that although micturition was excited by the stimulation of these nerves, the expulsion of urine did not occur until the

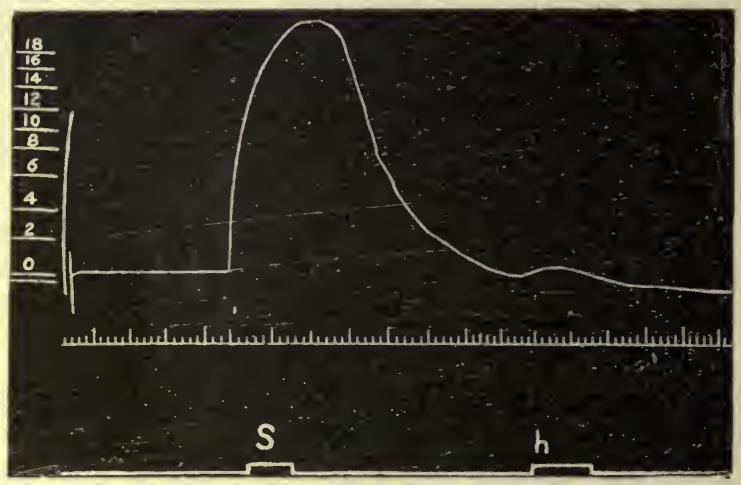

FIG. 541. Curve showing rise of pressure in the bladder caused by stimulation of $\mathrm{s}$, sacral nerves; $h$, hypogastric nerves. (FAGGE.)

The scale indicates centimetres of water.

intravesical tension had reached the point at which the resistance of the sphincter could be overcome without any alteration of its state of contraction, i.e. the point at which fluid injected into the bladder through the ureter began to escape from the urethra without stimulation of any nerves whatever.

Observations on man would support the view that an active relaxation of the sphincter trigoni is a necessary part of the act of micturition. Thus in experiments by Reyfisch a rigid catheter was introduced into the bladder, which was fully distended with fluid. On withdrawing the catheter until its opening lay just outside the bladder in the posterior urethra, the flow of urine stopped. The man, however, was able to micturate directly he was told to, and to stop again at will. It was impossible in this case for any of the urethral muscles to be concerned, since the rigid catheter impeded their action. The relaxation of the sphincter must therefore be brought about by impulses descending the pelvic visceral nerves, which we may regard as motor to the 'detrusor' and inhibitory to the sphincter of the bladder. 
Section of the nerve on one side causes no abnormality in micturition. After three weeks, stimulation of the intact nerve causes contraction of the whole bladder, owing to the outgrowth of preganglionic fibres from the sound trunk to the decentralised ganglia of the opposite side (Elliott). Section of both nerves paralyses micturition, but power of partial evacuation of the bladder may return in a few weeks. If now the hypogastrics be cut, or even the sacral cord extirpated, the bladder is not completely paralysed, but its evacuation becomes unconscious and incomplete.

ACTION OF THE HYPOGASTRIC NERVES. These nerves, which are derived from the sympathetic system, show marked differences in their action, according to the animal which is the subject of investigation. In the dog the hypogastric nerves cause a strong contraction of the muscle fibres at the base of the bladder, especially of the trigonum and of the sphincter trigoni. When these nerves are stimulated simultaneously with the pelvic visceral nerves a great rise of intravesical tension may be induced without any flow of urine taking place. In some cases prolonged stimulation of these nerves in the dog causes apparently an active relaxation of the sphincter of the bladder. On the other hand, in the rabbit and the cat these nerves cause an inhibition of the bladder wall. In other animals they may excite either contraction or relaxation (or both) of the detrusor. They always contain motor fibres to the sphincter of the bladder as well as to the constrictor fibres surrounding the urethra. Where this effect is tonic micturition must be associated with a central inhibition of their tonic activity. On the other hand, the retention of urine and the distension of the bladder may be aided by a reflex dilatation of the bladder wall and a reflex constriction of the sphincter in each case excited through these nerves. Normally, therefore, both sets of nerves are called into play. The hypogastrics play an especially active part during the accumulation of urine in the bladder, while the pelvic visceral nerves are necessary for the complete evacuation of the bladder which occurs at micturition.

\section{THE CENTRAL CONTROL OF THE BLADDER}

The nerve-centre which presides over the tonus and contraction of the bladder is situated in the lumbo-sacral spinal cord. If this centre and its connections be intact, micturition may be carried out normally even after section of the cord in the dorsal region. The centre can be excited reflexly by stimulation of almost any sensory nerve, such as the sciatic or the fifth nerve. In many cases where, in consequence of obstruction to the passage of impulses from the higher parts of the central nervous system, micturition is delayed, this act may be excited by the application of cold or hot sponges to the perineum, and it is well known that almost any irritation of the pelvic organs in children may give rise to reflex involuntary micturition.

In the adult the processes of retention and evacuation of urine are modified and controlled by voluntary effort. The normal action of the 
sphincter mechanism may be aided by the contraction of the perineal muscles which keep the urethra closed. The reflex process of evacuation may be set in motion by voluntary contraction of the abdominal muscles, by which the pressure in the bladder is increased and the normal sphincter action overcome. It is probable too that the individual has a certain degree of voluntary power over the unstriated muscles of the bladder, and that the contraction of the muscular wall may be directly augmented by impulses proceeding from the cortex to the upper part of the lumbar cord. This view is favoured by the fact that stimulation of the crus cerebri has been observed to cause contraction of the detrusor urinæ. In this experiment the abdomen was opened, so there could be no question of the contraction of the abdominal muscles. 


\section{CHAPTER XVIII}

\section{THE SKIN AND THE SKIN-GLANDS}

Is all classes of animals the skin performs two functions. In the first place, it serves to protect the more delicate underlying parts from injury and from penetration or invasion by foreign organisms. In the second place, it serves as a sense-organ, and is richly supplied with nerves, by means of which the activities of the body as a whole may be brought into relation with the changes going on in the environment and affecting the external surface of the body. In warm-blooded animals the skin plays an important part in the regulation of the body temperature, since the loss of heat to the body must occur almost entirely through its surface. In the present chapter we have to deal only with the first and third of these functions.

The development of the skin as an organ of protection shows wide modification in various classes of animals. Thus it may become the seat of formation of horny plates, as in the alligator; of poisonous glands, as in the toad; or of mucous glands, as in many varieties of fishes. In warmblooded animals the development of hair from the deeper layers of the epidermis serves to diminish the loss of heat. Since the hair-follicles are richly supplied with nerve fibres, the hairs act also as organs of sensation. In man, where the hairs are rudimentary, except in certain localities, practically only this tactile function is retained. The external layer of the skin in man consists of a tough horny layer formed by the keratinisation of the external layers of cells of the epidermis. The skin is composed of two parts, the epidermis and the cutis (Fig. 542). The epidermis is a stratified squamous epithelium. The deeper layers form the rete mucosum, being soft and protoplasmic, while the superficial layers forming the cuticle are hard and horny. The most superficial layer of the rete mucosum is formed of flattened cells filled with granules of a material staining deeply with hæmatoxylin and eosin, known as eleidin. This layer is called the stratum granulosum. Immediately superficial to this layer is another in which the cells are indistinct. The cells are clear in section and form what is known as the stratum lucidum. These two layers evidently form the intermediate stages in the transformation of the cells of the rete mucosum into the horny scales which make up the superficial cuticle. The cutis or corium is composed of dense connective tissue, which becomes more open in texture in its deeper part, where it merges into the subcutaneous connective tissue. The most superficial layer of the corium is prolonged into minute papillæ over which 
the epidermis is moulded. These papillæ contain for the most part capillary vessels; a few contain touch corpuscles, special organs of tactile sensation. The blood-vessels of the skin form a close capillary network immediately at the surface of the cutis, sending up loops into the papillæ. All parts of the skin, except the palms of the hands and the soles of the feet, are beset with hair-follicles. The hair-follicles are small pits which extend downwards into the deeper part of the corium, being down-growths of the rete mucosum. The hair grows from a small papilla of cells at the bottom of the follicle, the
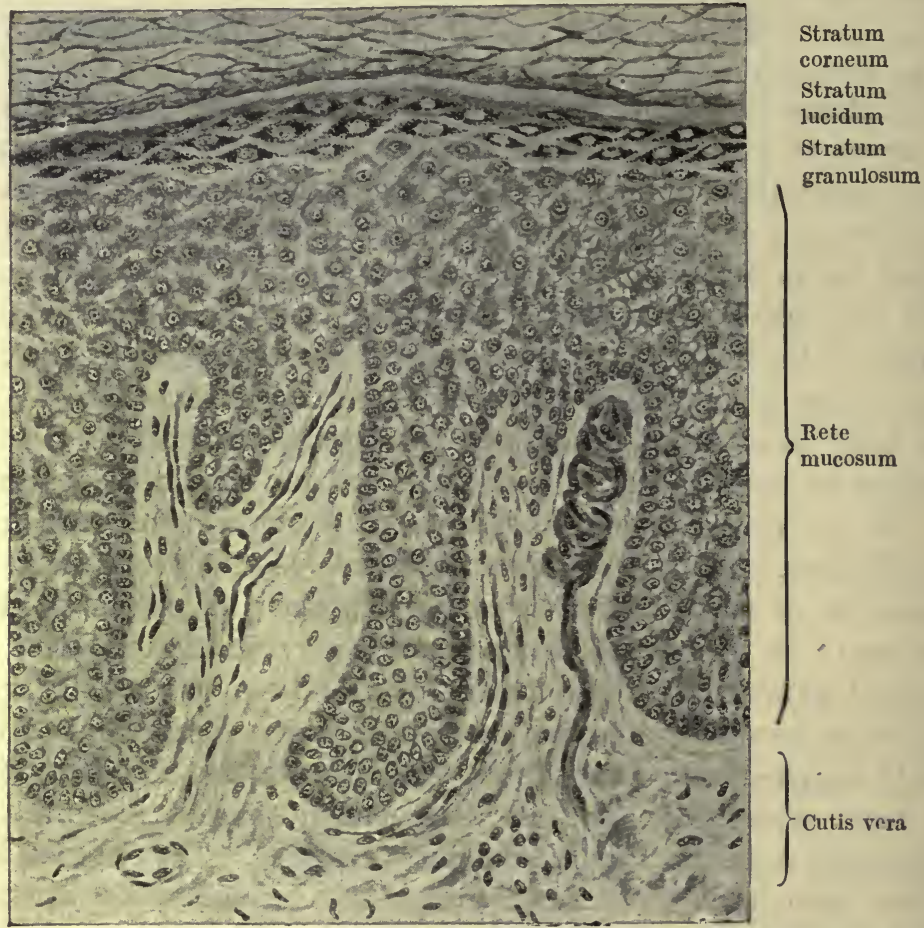

FIG. 542. Vertical section through the skin of the palmar side of the finger, showing two papillæ (one of which contains a tactile corpuscle) and the deeper layer of the epidermis. Magnified about 200 diameters. (ScHäFER).

part of the hair lying within the follicle being known as the hair-root. The hair itself consists of long tapering, horny cells, the nuclei of which are still visible, though the cell substance has been almost entirely converted into keratin.

In order to keep the cuticle supple and preserving it from the drying effects of the atmosphere, it is kept constantly impregnated with a fatty material known as sebum. This material is formed by the sebaceous glands, which are distributed all over the surface of the skin wherever hair-follicles are to be found, the mouths of the glands opening into the hair-follicles. A sebaceous gland is a pear-shaped body, consisting of a secreting part and a short neck, opening into the follicle. The gland proper is composed of a solid mass of cells. The outermost cells are flattened and generally show 
signs of proliferation. The cells lying internal to these are much larger, and their protoplasm is transformed into a network in the meshes of which are granules which may show the reaction of fat. Further inwards the protoplasmic network diminishes in amount, while the fatty granules increase in size, so that, in the lumen adjoining the duct, we find only a mass of cell débris and masses of fatty material. It has often been thought that the secretion of sebum depended simply on a fatty degeneration of the cells. The granules, however, when they first appear, stain with acid fuchsin rather than osmic acid, and one must regard the formation of sebum as an act of true secretion in which the secretory granules are gradually transformed into the special constituents of the sebum. For it must be noted that the sebum is not a true fat, nor does it correspond in composition with the fat found in other parts of the body. It is true that it contains fatty acids, but these are for the most part in combination, not with glycerin, but with higher alcohols, including cholesterol. A somewhat similar material may be extracted from wool, and is known as wool-fat or lanoline, as well as from the feather-glands of water birds, such as the goose and duck. It must be regarded rather as a wax than a fat. It presents many advantages over ordinary fat as a protective salve for the surface of the body. In the first place, it can take up a large amount, as much as 100 per cent., of.water. In the second place, it is not attacked by micro-organisms, so that it does not tend to become rancid or to furnish a nidus for the growth of these organisms on the surface of the body.

The secretion of sebum is a continuous process, though it is probably quickened in conditions of increased vascularity of the skin. The extrusion of the products of secretion is determined by the presence of unstriated muscle fibres, the arrector pili, which pass from the surface of the cutis obliquely over the outer surface of the sebaceous gland. When these muscle fibres contract the hair is erected and a certain amount of the sebum squeezed out on to the root of the hair and the surrounding skin. This contraction will occur whenever cold is suddenly applied to the skin. The contracted condition of all the muscles of the hair-follicles is shown by the ' goose-skin' produced under such circumstances. There is no evidence that the secretion of sebum is in any way under the control of the central nervous system.

THE SWEAT-GLANDS. Under normal circumstances in temperate climates the greater part of the water taken in with the food in the course of the day is excreted by the kidneys, a smaller proportion leaving by the lungs and by the surface of the skin. On an average we may say that about 700 c.c. are got rid of through the skin. The excretion of water by the skin is, however, mainly determined by the need for regulating the temperature of the body, so that the amount leaving in this way depends on the heat production of the body or on the external temperature, and is very little affected by alterations in the quantity of fluid drunk. A certain amount of water is constantly evaporated from the surface of the body as the so-called 'insensible perspiration.' If a man's body be enclosed in a vessel through 
which a current of air is passed, and the temperature of the air gradually raised, it will be noted that the amount of water given off rises slowly up to a certain degree and then rises rapidly. The sudden kink in the curve is due to the setting in of the activity of the sweat-glands, and we are therefore justified in regarding the insensible perspiration as being determined by evaporation of water from the surface of the cuticle itself apart altogether from the sweat-glands. The sweat-glands, which are distributed over the whole surface of the skin, are especially abundant on the palm of the hand and on the sole of the foot. They are composed of single unbranched coiled tubes, which lie in the subcutaneous tissue and send their ducts up through the cutis, to open on the surface by corkscrew-like channels which pierce the epidermis. The secreting part of the tube consists of a basement membrane lined by a double layer of cells; the innermost of these are cubical and represent the secreting cells proper. Between the secreting cells and the basement membrane is a layer of unstriated muscle fibres. The duct of the gland has an epithelium, consisting of two or three layers of cells with a well-marked internal cuticular lining, but there is no muscular layer.

The sweat formed by these glands is the most dilute of all animal fluids. As collected it generally contains epithelial scales and some admixture of sebum. After filtration it forms a clear colourless fluid of a specific gravity of about 1003. It contains over 99 per cent. of water. Among the solid constituents sodium chloride is the most prominent-it may contain from $0 \cdot 3$ to 0.5 per cent. of this salt. It is generally hypotonic as compared with the blood-plasma. It may also contain small traces of protein. This constituent is especially marked in the horse. It generally contains also a small quantity of urea, which may become a prominent constituent in cases of renal disease. The quantity of sweat excreted in the day is very variable. The secretion is under the control of the central nervous system and is almost entirely adapted to the regulation of the body temperature. The nervous mechanism can be set into activity either centrally or reflexly. The most usual factor is a rise of the body temperature. If a man sit in a warm room, e.g. of a Turkish bath, the secretion of sweat commences as soon as the temperature of the body has attained a height of $0.5^{\circ}$ to $1^{\circ} \mathrm{C}$. above normal. In the case of muscular exercise the temperature will generally be found to be raised if it be taken at the instant that sweating has commenced. The effect of rise of temperature may, however, be either local or central, so that one arm enclosed in a hot-air bath may sweat while the rest of the body is dry. Under ordinary circumstances the central stimulation by the warm blood is the predominant factor. This is shown by an experiment of Luchsinger. In the cat sweating is to be observed only on the hairless pads of the front and hind paws. If one sciatic nerve be cut and the animal be placed in a warm chamber, sweating will commence as the temperature of the animal rises in the three intact paws, while the paw with the nerve cut will be quite dry. Sweating moreover, as has been shown by Kahn, may be induced in the cat's paws by warming the blood passing through the 
carotid arteries on its way to the brain, at a time when the temperature of the blood circulating through the rest of the body, including the paws themselves, has undergone no alteration. Sweating may also be aroused by asphyxia, and this result is found even in the spinal cat, i.e. after separation of the spinal centres from the medulla. The secretion of sweat resulting from stimulation of the sweat-nerves, although generally associated with increased vascularity of the skin, is not in any way dependent thereon. Thus even in the amputated limb stimulation of the sciatic nerve may cause the appearance of drops of sweat on the pad of the foot. If the sciatic nerve be stimulated in the intact animal, the secretion of sweat which is produced is associated with constriction of the vessels of the skin, due to simultaneous stimulation of the vaso-constrictor nerves running in the sciatic nerve.

As Langley has shown, the sweat-nerves run entirely in the sympathetic system. Leaving the cord by the white rami communicantes from the second dorsal to the third or fourth lumbar nerves, they pass into the sympathetic chain. Here the first relay of fibres ends in connection with the cells of the sympathetic ganglia, and a fresh relay of fibres, which are nonmedullated, pass from the cells along the grey rami into the various spinal nerves, to be distributed to the whole surface of the skin. The secretion of sweat by the sweat-glands may be roused by the injection of pilocarpine even after division of the sweat-nerves, so that this drug must act peripherally on the glands. The action of pilocarpine, as well as the effects of artificial stimulation of the sweat-nerves, is abolished by the administration of atropine.

THE GASEOUS EXCHANGES OF THE SKIN. In any animal with a thin moist skin, such as the frog, the absorption of oxygen and the excretion of $\mathrm{CO}_{2}$ from the skin may be sufficient for the proper aeration of its blood, so that it may continue to live after the extirpation of its lungs. In man there is also a continuous output of $\mathrm{CO}_{2}$ through the skin, but the amount leaving the body in this way is negligible compared with that which is exhaled through the lungs. The loss of $\mathrm{CO}_{2}$ by the skin rises with increase of external temperature. Thus at a temperature of $29^{\circ}$ to $33^{\circ} \mathrm{C}$. the $\mathrm{CO}_{2}$ output by the skin is about $0.35 \mathrm{grm}$. per hour, i.e. about $8.4 \mathrm{grm}$. in the twenty-four hours. When the external temperature rises above $33^{\circ} \mathrm{C}$., the $\mathrm{CO}_{2}$ output increases, so that at $34^{\circ}$ it is doubled and at $38.5^{\circ}$ it may amount to as much as $1.2 \mathrm{grm}$. per hour (Schierbeck). It is just at this temperature of $33^{\circ} \mathrm{C}$. that a secretion of sweat begins to be noticeable, so it has been suggested that the increased $\mathrm{CO}_{2}$ output may be due directly to the increased work and metabolism of the sweat-glands during their activity.

ABSORPTION BY THE SKIN. In order to test the alleged influence of baths containing medicinal substances in solution, many experiments have been made to determine whether absorption is possible by the skin. It may be regarded as established that the uninjured skin is impermeable for watery solutions of salts or other substances. On the other hand, it is possible to produce a certain amount of absorption by the inunction of 
substances dissolved in fatty vehicles. Thus the administration of mercury is often carried out by the inunction of mercurial ointments, and the fact that mercurial salivation may be produced in these conditions shows that a certain amount of the mercury must have been absorbed. It is difficult to imagine that any appreciable amount of cod liver oil will be available for the nutrition of the infant when this substance is administered by rubbing it on the skin. On the other hand, the moist mucous surfaces, such as the conjunctiva or the mucous membrane of the respiratory passages, as well as raw surfaces of the skin, e.g. which have been deprived of their epidermal layer by the application of blisters, permit of the rapid passage of substances in watery or oily solution. 


\section{CHAPTER XIX \\ THE TEMPERATURE OF THE BODY AND ITS REGULATION}

IN dealing with the chemical changes in the body as a whole we have seen that the sum of the metabolic processes is associated with the evolution of heat. In man, under normal circumstances, while doing moderate work, the total energy requirements amount to about 3000 calories. The whole

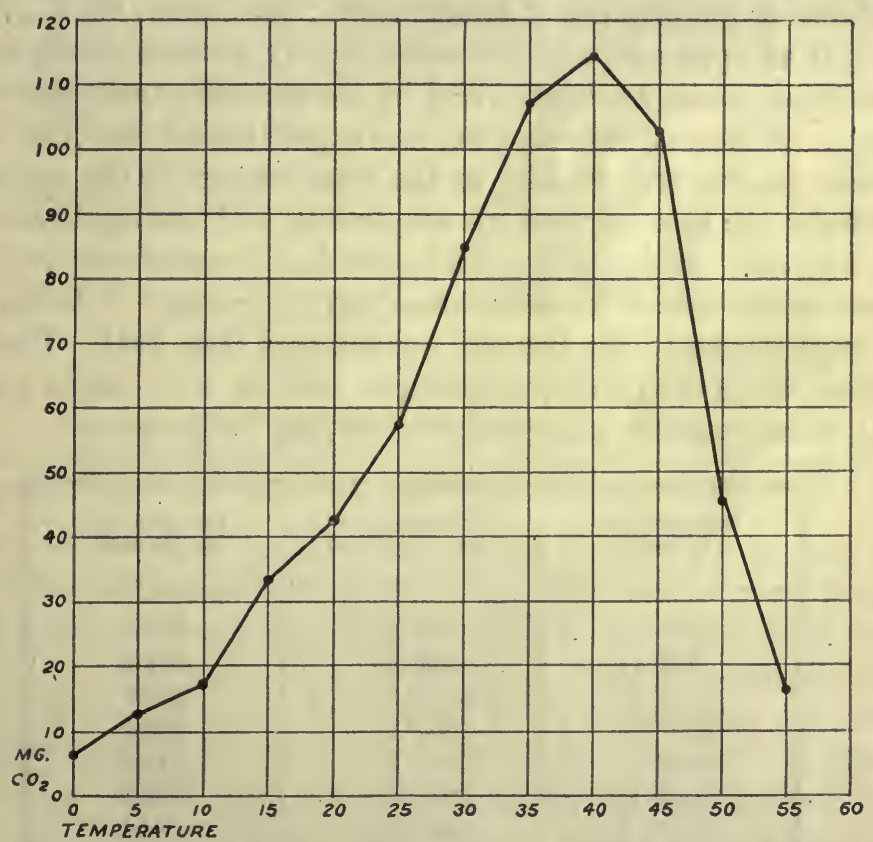

Fig. 543. Effect of temperature on the $\mathrm{CO}_{2}$ output of a lupin seedling.

Ordinates $=$ milligrammes $\mathrm{CO}_{2}$ per hour. Abscissæ $=$ temperature in degrees Centigrade.

of this is derived from the oxidation of the food, the combination of its carbon and hydrogen with oxygen to form $\mathrm{CO}_{2}$ and water, with the evolution of the corresponding amount of energy. Of this energy only a small proportion, on the average about one-twentieth, leaves the body as mechanical energy, the rest being evolved in the form of heat and being expended in the maintenance of the body temperature, or in the warming of the surrounding medium. 
The evolution of heat is not confined to the higher animals, but is common to all living beings. It is very evident, for instance, in the germination of peas or barley. The atmosphere of a bee-hive is often ten degrees above that of the surrounding atmosphere. Whenever we can excite increased activity in an organ, we are able to show, except in the single case of the nerve impulse, that such activity is associated with the evolution of heat. This heat is derived from the chemical changes which proceed in the living cells. Since all chemical processes are quickened by rise of temperature, we should expect to find that the heat produced in the metabolic processes of organisms would tend in itself to quicken these processes. In most chemical reactions a rise of about $10^{\circ} \mathrm{C}$. would increase the velocity of reaction from two and a half to three times; and the same rule is, within the limits of stability of living tissues, found to hold good for them also. The diagram (Fig. 543) shows the influence of temperature on the chemical changes in a lupin seedling as measured by the output of $\mathrm{CO}_{2}$ per hour per 100 grm. of plant. A marked increase in the rate of chemical decomposition is shown to follow a rise of temperature; but, about $40^{\circ} \mathrm{C}$., the rate of change is at an optimum, and thereafter rapidly declines, owing to the fact that the living tissues are being killed by the excessive temperature.

Hence in the animal organism we shall expect to find that the rate of the metabolism is also proportional to the temperature of the animal. This is universally the case whether we are dealing with warm-blooded or coldblooded animals. In ' cold-blooded' animals the temperature of the body, and therefore the rate of its metabolism and the amount of its heat production, is proportional to the external temperature (Fig. 544). The following Table gives the average $\mathrm{CO}_{2}$ output per hour of five lizards placed in a chamber which could be maintained at varying temperature :

\begin{tabular}{|c|c|c|}
\hline $\begin{array}{c}\text { Temperature } \\
\text { of bath }\end{array}$ & $\begin{array}{c}\text { Temperature of } \\
\text { lizards (average) }\end{array}$ & $\begin{array}{c}\mathrm{CO}_{2} \text { produced } \\
\text { in one hour }\end{array}$ \\
\cline { 2 - 3 } $5 \cdot 0^{\circ} \mathrm{C}$. & $5 \cdot 5^{\circ} \mathrm{C}$. & .0246 \\
$9 \cdot 0^{\circ} \mathrm{C}$. & $9 \cdot 2^{\circ} \mathrm{C}$. & .0790 \\
$15 \cdot 0^{\circ} \mathrm{C}$. & $15 \cdot 2^{\circ} \mathrm{C}$. & $\cdot 0981$ \\
$20 \cdot 5^{\circ} \mathrm{C}$. & $20 \cdot 4^{\circ} \mathrm{C}$. & $\cdot 1023$ \\
$25 \cdot 0^{\circ} \mathrm{C}$. & $24 \cdot 5^{\circ} \mathrm{C}$. & $\cdot 1193$ \\
$30 \cdot 0^{\circ} \mathrm{C}$. & $29 \cdot 3^{\circ} \mathrm{C}$. & $\cdot 1440$ \\
$35 \cdot 0^{\circ} \mathrm{C}$. & $34 \cdot 8^{\circ} \mathrm{C}$. & $\cdot 1814$ \\
$39 \cdot 0^{\circ} \mathrm{C}$. & $38 \cdot 5^{\circ} \mathrm{C}$. & .5454 \\
\hline
\end{tabular}

It might be thought that such a reaction in change of temperature would result in a vicious circle. Since the animal is continually producing heat and thus raising its temperature above that of its surroundings, one might expect to find that the higher the external temperature the greater would be the difference between this and the temperature of the animal, until finally the latter would rise to such a height that the animal would die of heat-stroke, its tissues being destroyed by the actual temperature attained. A certain 
protection is afforded to most cold-blooded terrestrial animals by the fact that their surface is moist, and that with a rise of external temperature the rate of evaporation on the surface increases, so that the increase in the rate of cooling by evaporation more than corresponds to the rate of increase in the heat production, which would tend to raise the body temperature. Most of these animals, however, escape from any extreme rise of external temperature by burrowing underground or taking to the water, while in plants a rise of external temperature assists transpiration to such an extent that the

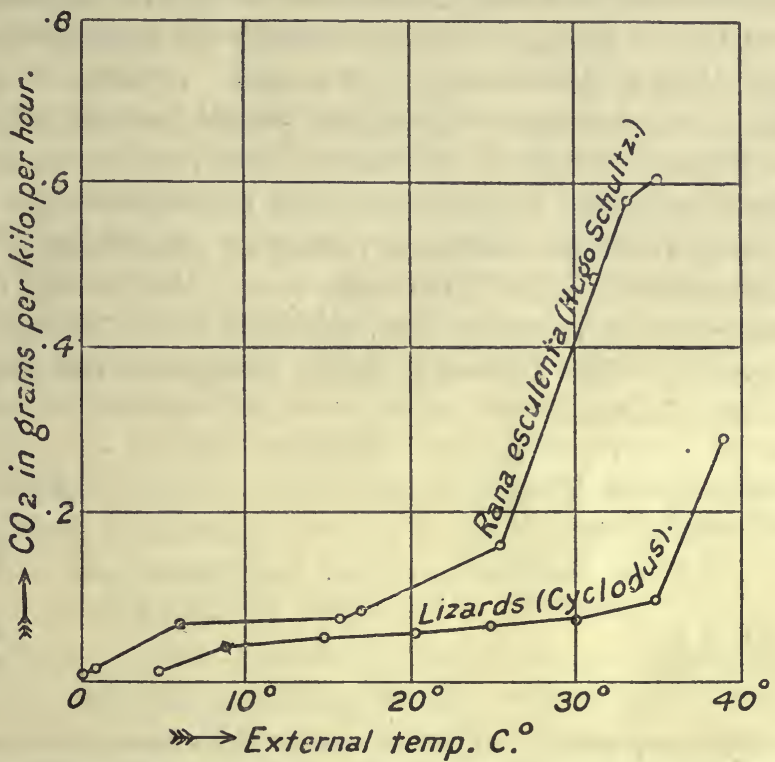

Fig. 544. Effect of alterations in the temperature of the surrounding medium on output of $\mathrm{CO}_{2}$ in cold-blooded (poikilothermic) animals. (C. J. MARTIN.)

temperature of the plant is generally several degrees below that of the surrounding atmosphere. The extreme variability in the metabolism of such animals implies a state of dependence of all the activities of the body on the environment, which would prevent the utilisation to the full of the available sources of energy. An animal whose metabolism was more or less independent of the surrounding temperature must have a great advantage over an animal liable to have his activities reduced and paralysed by a sudden spell of cold weather ; this greater independence of the environment which is characteristic of elevation of type, has been achieved by the warm-blooded animals including man. Such animals are often spoken of as homoiothermic, i.e. animals possessing a uniform temperature, in contradistinction to the cold-blooded animals, which are poikilothermic and possess a temperature varying with that of their surroundings.

Amongst the warm-blooded animals the body temperature may be very different according to the species. In birds it is generally from $39^{\circ}$ to $40^{\circ} \mathrm{C}$.; in mammals it varies from $35^{\circ}$ to $40^{\circ} \mathrm{C}$. The temperature of man varies within slight limits about $37^{\circ} \mathrm{C}$. $\left(98 \cdot 4^{\circ} \mathrm{F}\right.$.). 


\section{THE DIURNAL VARIATIONS IN THE BODY TEMPERATURE}

In any animal the seat of heat production, e.q. a contracting muscle, must be warmer than an inactive tissue, and this again than a tissue from which heat is being rapidly abstracted, such as the skin. Owing, however, to the rapidity of the circulation of the blood, the temperature of the internal organs can be regarded as approximately uniform. The temperature of man is usually taken in the mouth, rectum, or axilla. In the case of the mouth the temperature is liable to fluctuation with the rate of breathing, the mouth being cooled by the passage of the air through the nasal cavities. There is also probably loss of heat through the cheeks. In order to determine the temperature in this situation the mouth should be kept closed for a few minutes and then the bulb of the thermometer inserted under the tongue, and the lips kept closed on the stem of the thermometer for five minutes. Except in cases where the cutaneous vessels are much dilated, the temperature of a thermometer in the axilla takes a considerable time to rise to that of a thermometer in the mouth; it should never be left less than ten minutes in this situation. The following Table represents the temperature of different parts of the body :

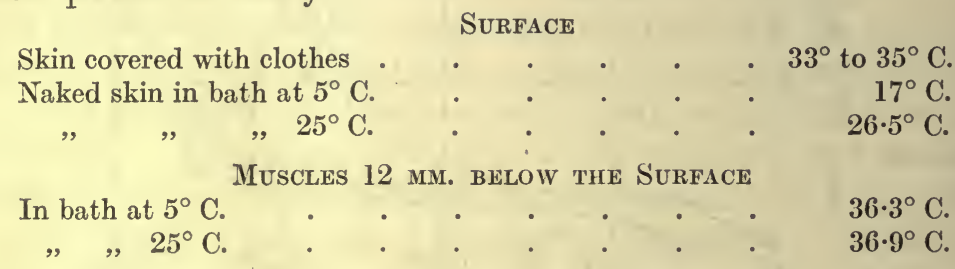

The body temperature of man shows variations of several tenths of a degree according to the time of day at which the temperature is taken. The highest temperature is obtained about six or seven in the evening, and the lowest at about four or five o'clock in the morning. With these diurnal changes in temperature are associated parallel oscillations in the rate of metabolism as shown by the elimination of carbon dioxide. They are probably determined by the changes in the movement and tension of the muscles occurring during the waking hours. If the habits of a man or animal be reversed, so that he sleeps in the daytime and performs his normal vocation by night, it is possible to reverse also the direction of the diurnal variations in temperature. The temperature may be also affected temporarily by various acts, such as the taking of food, or muscular work ; the influence of the latter factor is often very marked. In many individuals a hard game at tennis suffices to raise the temperature to $39^{\circ} \mathrm{C} .\left(102^{\circ} \mathrm{F}\right.$.), and even the healthiest individual will show some change in his temperature as the result of exercise. Pembrey found that the act of marching led to a considerable rise in temperature, a rise which is apparently responsible for the discomfort and fatigue observed under such circumstances. Such a change in the body temperature is merely temporary in its effects, and insignificant in comparison with the wonderful uniformity of temperature 
observed in men of all races and of all climes under most varying conditions of food and activity.

Just as there are linits to the power of the organism to regulate its temperature when there is an excessive formation of heat in the body, so there are limits to the regulation of temperature to severe alterations in the temperature of the external medium. Thus if the body is subjected to excessive external cold, or the loss of heat be increased by absence of clothes, by depriving an animal of its fur, or by immersion in a cold bath, the temperature of the body may sink continuously. This fall of temperature in the higher animals is very soon followed by paralysis of the highest nerve-centres and loss of consciousness. The centres in the medulla are later on affected, so that the respiration is slowed and the blood-pressure falls. If the temperature does not fall too low it is possible to revive the animal or man by checking the loss of heat or by supplying artificial warmth. Recovery has in fact been observed in men in whom, as a result of exposure, the body temperature had fallen to $24^{\circ} \mathrm{C}$. In the same way exposure to extreme heat, especially associated with muscular exercise and increased production of heat in the body, may cause a rise of the body temperature. A man, or animal, whose temperature is raised above the normal, is said to be in a state of pyrexia. A rise of $2^{\circ}$ or $3^{\circ} \mathrm{C}$. is associated with all the phenomena which characterise fever, i.e. quickening of pulse and respiration, malaise, headache, and loss of muscular power. If the temperature rise to a greater degree than this the patient may lose consciousness, and death ensues at a temperature of about $44^{\circ} \mathrm{C}$.

The very small variations presented by the body temperature in mammals, even under the influence of considerable variation in external temperature, or in the production of heat in the body, connotes an accurate adaptation between the production of heat in the body and the loss of heat from the body. The regulation of the temperature can be effected either by regulation of heat production, by alteration in the rate of loss of heat, or by a combination of both mechanisms. It will be convenient to deal with these two methods of regulation under separate headings.

\section{THE PRODUCTION OF HEAT IN THE BODY}

The reactions mainly responsible for heat production in the body are those associated with oxidation; the processes of disintegration, such as are effected by means of hydrolytic ferments, accounting for a very small part of the heat evolved.

In these processes of oxidation all the organs participate, the most important, especially in relation to the regulation of heat production, being the skeletal muscles. These represent more than half of the total weight of the soft tissues of the hody, and even during rest are the seat of oxidative processes and therefore of heat formation. Heat formation varies with the state of tone of the muscles, and is largely increased with every active contraction. The effect of muscular activity on the total output of energy of the body is well represented in the Table given on p. 639 . 
It is probable that, in relation to their size at any rate, the glands are still more effective as heat producers. The liver, and the blood flowing from the liver, have been stated to present a higher temperature than any other part of the body. On the other hand, the nervous system, although dependent on a constant supply of oxygen for its activities, does not appear to be the seat of extensive metabolic changes, nor does the heat produced in this system play any great part in maintaining the temperature of the body.

The skeletal muscles are controlled by the central nervous system; if separated from their centres in the cord they become flaccid and rapidly atrophy. The heat production in the muscles is therefore also dependent on their connection with the central nervous system. If this connection be severed either by curare or section of the cord, or if the reflex play of impulses on the muscles be abolished by anæsthetics, the animal will react like a coldblooded animal. The total metabolism of the body and the total production of heat sink to a minimum, and are diminished by application of cold, or increased by application of warmth, to the surface of the body. On the other hand, in the intact animal changes of temperature in the environment provoke, reflexly by their action on the muscles, changes in the opposite direction. Thus exposure to cold increases and to heat diminishes muscular metabolism and the heat production of the body.

The effects of variations in the external temperature on the metabolism of warm-blooded animals are well shown in the experiment, from which the following Tables are taken, on the $\mathrm{CO}_{2}$ output in the ornithorhynchus and in the rabbit (Martin) :

1. Orntthorhynchus. Whight. 693 Grm.; SURFACe, 876 sq. Centims.

\begin{tabular}{|c|c|c|c|c|}
\hline $\begin{array}{l}\text { Temperature } \\
\text { of } \\
\text { environment }\end{array}$ & $\begin{array}{c}\text { Temperature } \\
\text { of } \\
\text { animal }\end{array}$ & $\begin{array}{l}\text { Difference in } \\
\text { temperature, } \\
\text { animal and } \\
\text { environment }\end{array}$ & $\begin{array}{c}\mathrm{CO}_{2} \text { per hour, } \\
\text { in grammes }\end{array}$ & $\begin{array}{l}\mathrm{CO}_{2} \text { per hour } \\
\text { per } 1000 \\
\text { sq. centims., } \\
\text { in grammes }\end{array}$ \\
\hline 5 & $31 \cdot 8$ & $26 \cdot 8$ & $1 \cdot 090$ & $1 \cdot 244$ \\
\hline 10 & $32 \cdot 0$ & $22 \cdot 0$ & .722 & .825 \\
\hline 20 & $32 \cdot 6$ & $12 \cdot 6$ & .405 & .463 \\
\hline 32 & $33 \cdot 6$ & $1 \cdot 6$ & $\cdot 336$ & $\cdot 383$ \\
\hline 35 & $35 \cdot 3$ & $\cdot 3$ & $\cdot 377$ & .430 \\
\hline
\end{tabular}

2. RABBit. WEIGHT, 750 GRM.

\begin{tabular}{|c|c|c|c|c|}
\hline $\begin{array}{c}\text { Temperature } \\
\text { of } \\
\text { environment }\end{array}$ & $\begin{array}{c}\text { Temperature } \\
\text { of } \\
\text { animal }\end{array}$ & $\begin{array}{c}\text { Difference in } \\
\text { temperature, } \\
\text { animal and } \\
\text { environment }\end{array}$ & $\begin{array}{c}\text { CO }_{2} \text { per hour, } \\
\text { in grammes }\end{array}$ & $\begin{array}{c}\mathrm{CO}_{2} \text { per hour } \\
\text { per 1000 } \\
\text { sq. centims., } \\
\text { in grammes }\end{array}$ \\
\cline { 1 - 2 } 5 & 37.5 & 32.5 & 1.426 & 1.543 \\
10 & 38.0 & 28.0 & 1.038 & 1.124 \\
20 & 38.7 & 18.7 & .912 & .987 \\
35 & 40.5 & 5.5 & .766 & .829 \\
40 & 41.6 & 1.6 & .897 & .971 \\
\hline
\end{tabular}


In the former animal, where the regulation of the temperature of the body is effected almost entirely by changes in heat production, the effect of warming the environment of the animal on the $\mathrm{CO}_{2}$ output is extremely marked. It will be noticed that the $\mathrm{CO}_{2}$ per hour sinks continuously with rising temperature up to $32^{\circ} \mathrm{C}$. When the temperature of the chamber was raised to $35^{\circ}$ the temperature of the animal rose considerably, i.e. the regulatory mechanism was failing, so that the same effect was produced on metabolism as is observed in working with cold-blooded animals. The

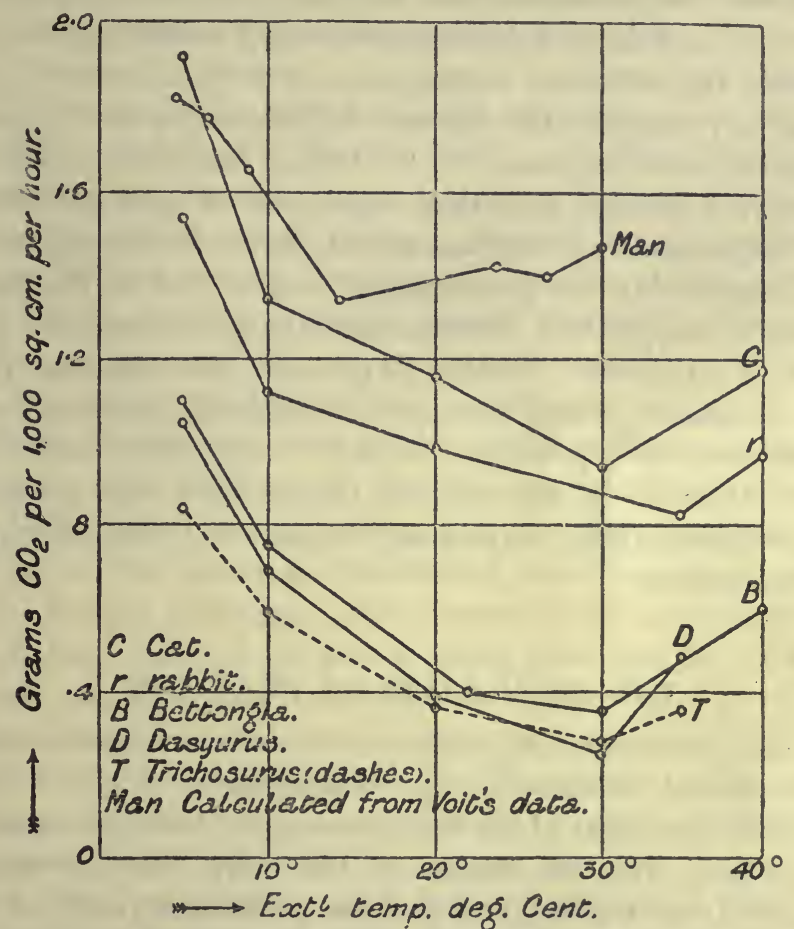

Fig. 545. Effect of variations in the external temperature on the $\mathrm{CO}_{2}$ output (per $1000 \mathrm{~cm}^{2}$ body surface) of warm-blooded animals. (C. J. MARTLN.)

same change, though less marked, is observed on exposing the rabbit to a gradual rising temperature. Here, however, the process of regulation is aided by alterations in the heat lost as well as in the heat production (Fig. 545). If the animals be observed, whilst subjected to changes of temperature, it will be evident to any one that the regulation is associated with changes in muscular activity. At $30^{\circ}$ to $35^{\circ} \mathrm{C}$. the animals will lie perfectly flaccid, breathing rapidly, or may go to sleep. On cooling they at once become more vigorous and perform active movements in their cage. The sams effects of changes in the external temperature are familiar in ourselves. The slackness and extreme disinclination to violent exercise observed in hot moist weather, contrasted with the stringing up of the tone of the muscles which follows exposure to cold, and which may be associated 
with voluntary exercise to keep ourselves warm, are indications of the important part played by the inuscles in determining the heat production of the body. As a rule the immersion of a man in a cold bath for a minute or two increases considerably his output of $\mathrm{CO}_{2}$. It is possible, however, to sit in a bath and by an act of the will keep all the muscles in a state of relaxation. Under these circumstances the temperature of the body rapidly falls, and with it the rate of metabolism, as judged by the output of carbon dioxide.

This process of adjustment of the body temperature by variations in the heat production, so long as it represents the only method, is extravagant of energy directly the difference in temperature between animal and environment attains any considerable degree. In the very perfect adjustment of the temperature which is present in the higher mammals, regulation of the heat loss plays a greater part than regulation of heat production. The economy of adjustment by heat loss is well shown if we compare in echidna and rabbit respectively the percentage alteration in $\mathrm{CO}_{2}$ production when the difference in temperature between animal and environment varies from $10^{\circ} \mathrm{C}$. to $20^{\circ} \mathrm{C}$. This is for echidna 72 per cent., for mammals 16 per cent. In echidna variations in heat loss can be practically neglected, so that the whole of the work of regulating the body temperature falls on the heat production. As soon as the external temperature falls below a certain degree the mechanism fails, the animal's temperature falls, and it passes into a state of hibernation.

\section{THE REGULATION OF HEAT LOSS}

In all temperate climates, and in fact in all climates except under certain exceptional conditions, the temperature of the warm-blooded animal is higher than that of his environment, so that there must be a constant loss of heat from the surface of the body. In the warm-blooded animals in the arctic regions, and in those which have adopted an aquatic existence, the thick layer of fat which underlies the skin protects the active portions of the body, the muscles and internal organs, from excessive loss of heat to the surrounding medium. In most terrestrial animals the loss of heat is also diminished by fur and feathers with which these animals are clothed, and in ourselves the same office is performed by clothes, which are capable of voluntary graduation to external conditions. The value of these different coverings depends on the fact that air is a very bad conductor of heat. In the case of a naked man the layer of air immediately in contact with the surface of the body is warmed, becomes lighter, and rises, its place being taken by fresh cold air. 'Loss of heat is increased by a draught, which quickens the rate at which the layers of air in contact with the surface are renewed. In the case of clothes the material encloses layers of air and hinders it from free circulation, and it is the air enclosed in the meshes of the garments and between the different layers of garments that plays the greater part in preventing loss of heat. The rapid cooling effect of wet 
clothes is partly due to the replacement of layers of air by water, which is a much better conducting fluid.

In addition to the loss of heat by convection, i.e. by warming the layers of air in contact with the body, heat is also lost by radiation, i.e. by an exchange of heat between the surface of the body and that of surrounding cooler objects. This loss is also prevented by clothing. Since the material of which clothes are made does not allow the passage of radiant heat, they absorb the heat leaving the body and become warm. This warm article of clothing may in its turn act as a centre for the loss of radiant heat, which may again be prevented by putting on another layer. It is a familiar experience that a multiplication of garments is more effective in retaining the heat of the body than merely increasing the thickness of the individual garments. The rate of loss of heat by radiation is diminished by a rise of the amount of watery vapour in the air, since this makes the air more opaque to the passage of radiant energy. Since the loss of heat depends on the difference of temperature between the surface of the body and the surrounding air, or objects, it will be largely affected by the temperature of the skin, and therefore by the amount of blood flowing through the skin. The blood-flow through the skin is under the control of the central nervous system, through the vaso-motor and vaso-dilator nerves, and it is by altering the size of these vessels that the central nervous system chiefly acts in regulating heat loss. In cold weather, or when the heat production in the body is low, the vessels are constricted, the skin is cold, and the heat loss is small. On the other hand, if the temperature of the surrounding air is high, or a large amount of heat is being produced in consequence of muscular exercise, the vessels are dilated and the skin is hot. In hot weather the dilatation in the cutaneous vessels is associated with muscular inactivity, so that there is a derivation from the muscles, where the blood is not required, to the skin, where a considerable circulation is necessary.

Loss of heat by radiation and convection can only happen when the temperature of the surrounding air is lower than that of the body. The body temperature can, however, be maintained at its normal height in an atmosphere with a temperature much higher than $37.5^{\circ} \mathrm{C}$., and this in spite of the fact that the production of heat in the body is still going on. In this case there is a profuse secretion of sweat on the surface of the body. In the evaporation of the sweat, especially if aided by a draught of air, a large amount of heat becomes latent and is abstracted from the body, which is therefore kept at a temperature below that of the surrounding atmosphere. If the secretion of swert is checked, by depriving an animal or man of water, or if its evaporation be impeded by placing him in an atmosphere already saturated with aqueous vapour, the temperature of the body runs up rapidly and death ensues from hyperpyrexia, or heat-stroke. Although a man can stand exposure to a temperature of $200^{\circ}$ or even $250^{\circ} \mathrm{F}$. for a considerable time, provided that the air is dry, a temperature of $89^{\circ} \mathrm{F}$. is rapidly fatal if the air be saturated with moisture. The same mechanism comes into play when the heat production in the body is very largely increased, as by 
violent exercise. Under these conditions a man may sweat profusely when the temperature of the surrounding atmosphere is at $0^{\circ} \mathrm{C}$.

The main regulation of heat loss thus takes place by the control of the nervous system over the cutaneous circulation and the sweat-glands. Besides these channels of heat loss, others may play an important part under certain conditions. Heat is lost to the body in warming the food and air which are taken in. It is also lost in respiration in the evaporation of water and the setting free of $\mathrm{CO}_{2}$ from watery solution into the expired air. The following estimate, by Tigerstedt, represents the proportion of losses in an adult man by these different ways :

\section{A. Warming the Food and AIr}

(1) $1500 \mathrm{~g}$. water drunk at $15^{\circ} \mathrm{C}$. and warmed to $37 \cdot 5^{\circ}$-raised therefore Cal. $22.5^{\circ}$. . . . . . . $=33.75$

(2) $1500 \mathrm{~g}$. food eaten at $25^{\circ} \mathrm{C}$. (mean) and warmed to $37 \cdot 5^{\circ}$-raised therefore 12.5 ; specific heat 0.8 . . . . . . $=15.00$

(3) 15,000 g. (=11,500 l.) air respired at $15^{\circ}$ C. and warmed to $37 \cdot 5^{\circ}-$ raised therefore $22.5^{\circ}$; specific heat 0.237 . . . . . . . $=79.95$

\section{B. Loss of Water and $\mathrm{CO}_{2}$ in the Breath}

$128 \cdot 70$

(4) It is assumed that the inspired air is half saturated with watery vapour at $15^{\circ} \mathrm{C}$. and that the expired air is fully saturated at $37 \cdot 5^{\circ} \mathrm{C}$. Approximately $450 \mathrm{~g}$. of water would be given off therefore in the form of vapour from the respiratory passages; the latent heat of the water vapour is $0.537 \mathrm{Cal}$.

(5) The absorption of heat in the liberation of $\mathrm{CO}_{2}$ from the lungs $(800 \mathrm{~g}$.); $0 \cdot 134$ Cal. per g.

$$
=241 \cdot 70
$$

From above

The sum of heat losses specified under these five headings amounts to 477.60 Cal. Estimating the total heat loss of an adult man at.2400 Cal., this sum represents only about 20 per cent. of the total. The remaining 80 per cent. (in round numbers) takes place through the skin.

If we estimate the total heat loss of an adult man at 2400 calories, we may say that about 5 per cent. of the total heat loss takes place by warming the food and air, about 15 per cent., by the evaporation of water and $\mathrm{CO}_{2}$ in respiration, and about 80 per cent. by radiation and convection and the evaporation of sweat from the skin. The proportion represented by the last factor will increase very largely in the presence of a high external temperature, or of an excessive heat production in consequence of violent muscular exercise.

\section{THE NERVOUS MECHANISM FOR HEAT REGULATION}

The accurate balance between heat production and heat loss which is responsible for the nearly constant temperature of man, indicates the active 
co-operation of the central nervous system in every step of the process. Whether this function of temperature regulation can be specially localised at any part of the central nervous system, so that it would be possible to speak of a heat-centre in the same way as we speak of a respiratory or vaso-motor centre, is doubtful. Many observers have found that injury to the corpus striatum causes a rise of temperature associated with increase both in heat production and in heat loss. On the other hand, injury to or pathological lesions of the pons Varolii often lead to an increased production of heat in the body, which is not compensated for sufficiently by heat loss, and so causes death by hyperpyrexia. It has been suggested that the thermogenic centre, i.e. that responsible for regulating heat production, is situated at a lower level in the nervous system than the thermotaxic system, i.e. that which presides over and determines the balance between heat production and heat loss. The observations of Meyer and Barbour that local cooling of the corpus striatum causes increased respiratory exchanges and heat production, while warming has the reverse effect, certainly point to a localisation of the thermotaxic centre in this part of the central nervous system. The centres for heat loss must be placed in the medulla, at any rate so far as concerns control of heat loss by alterations in the blood-supply to the skin or in the secretion of sweat. The facts at our disposal are, however, too meagre to warrant any definite localisation of the heat-regulating function in the central nervous system, or any such accurate analysis of the regulating function as has been just suggested.

In many warm-blooded animals the ability to maintain a constant temperature is not fully developed until some time after birth. Pembrey has shown that in the guinea-pig and chick, in which the nervous system is fully functional at birth, the heat-regulating mechanism is also completely adequate, whereas animals such as rats, pigeons, or the human child, which are born in a helpless condition, only acquire the power of regulating their own temperature some time after birth. As we should expect, the development of the power of regulating heat production runs pari passu with the acquisition of control by the nervous system over the muscles of the body. 


\section{CHAPTER XX}

\section{THE DUCTLESS GLANDS}

\section{INTERNAL SECRETIONS}

IN unicellular organisms, as in the rest of the living world, activity consists in adaptation to external conditions. The changes in the environment which determine the reactions of these organisms may occur at their surface or at some distance. Among the stimuli which, acting from a distance, evoke the reaction of unicellar organisms, probably the most important are those accompanied by chemical changes. The interrelation of microorganisms with one another is determined almost entirely by such chemical stimuli. Thus the antherozoids of ferns are attracted to the ovule in consequence of the production in the tissue surrounding the latter of substances such as malic acid. Among micro-organisms we find some which leave places rich in oxygen, while others move towards any spot in their environment where oxygen is most plentiful. These reactions of unicellular organisms to chemical stimuli are classed together under the term chemiotaxis. When the cells are united together to form cell colonies, or when, as in the metazoa, the multicellular aggregate is formed by the failure of the products of division of the ovum to separate one from another, the interrelations between the different cells forming the organism are still largely determined by chemical stimuli. In fact, in the lowest metazoa, such as the sponge, we know of no other means of correlating the reactions of different parts of the cell aggregate. If a foreign substance be introduced into the living tissue of a sponge it becomes speedily surrounded with a collection of wandering phagocytic cells, called from the surrounding parts by the diffusion of chemical substances from the seat of the lesion into the fluids of the body. The same chemical sensibility determines the aggregation of leucocytes around bacteria or dead tissue, which forms the essential feature of the process of inflammation in higher animals.

When the reaction of distant parts of the body to a change occurring in any one part depends on the diffusion of some substance from a stimulated part, the total reaction must require a considerable time for its full development. A much more effective method of correlation was acquired by the evolution of a nervous system, by means of which the consensus partium could be maintained by the rapid propagation of molecular changes along differentiated paths in the protoplasm. The development of this second mode of 
correlation of activities did not, however, do away with the necessity for the more primitive method. Even in the higher animals, where rapidity of reaction is not required, we find adaptations carried out in response to some change in distant parts of the body, the message having been chemical and not nervous in character (e.g. the secretin mechanism for pancreatic secretion).

When we speak of the chemical correlation of the activities of the different parts of the body, it is important not to confuse processes which have little or nothing in common. In one sense we may say that every cell in the body is chemically connected with and dependent on all the other cells in the body. This interdependence is a necessary consequence of the differentiation of function associated with increased complexity of the organism. Thus the food-stuffs are digested and absorbed by the cells lining the alimentary canal and are then transmitted, more or less changed by these cells, to all the other tissues of the body. The liver stores up glycogen and is ready to give of its store to any tissue in need of carbohydrate. All the tissues probably produce urea, which passes to the kidneys and there excites the act of excretion. All tissues produce carbon dioxide, which passes to the lungs to be excreted, but as it traverses the respiratory centre it arouses respiratory movements which are exactly proportioned to the tension of the carbon dioxide and therefore to the need of the whole body to eliminate this waste product. The liver receives ammonia from the alimentary canal and converts it into urea, thus shielding all the other tissues from the poisonous effects which would be produced by the entrance of the ammonia into the general circulation. Thus one organ may receive and modify any substance or food-stuff so as to prepare it for more ready assimilation by other tissues. It may shield these other tissues from the poisonous effects of certain waste products, either by converting these into harmless substances or by excreting them from the body. In all these cases the tissues are dealing with some substance which is utilised in bulk, or which, by its accumulation, could exert a toxic influence on other tissues. We are probably justified in treating apart a group of phenomena in which the substance transmitted from one part of the organism to another is significant almost entirely as an excitatory agent, and has little or no value as a source of energy. When the adaptation to a change of $\mathrm{A}$ consists in the activity of an organ $\mathrm{B}$, the activity of $\mathrm{B}$ can be evoked either by a nerve impulse passing from $A$ to the central nervous system and from this to $\mathrm{B}$, or by the production at $\mathrm{A}$, as a direct consequence of the stimulus, of a specific chemical substance, which passes into the circulating blood to $\mathrm{B}$, where in its turn it will excite the required state of action. Such chemical messengers are designated hormones, from ö $\rho \mu \alpha \omega$, 'I excite.' We have already met with several examples of such bodies. It may be interesting here to consider what must be their general character if they are to fulfil the part of chemical messengers.

(1) In the first place, they must not be antigens, i.e. their injection into the blood-stream must not evolve the production of an anti-body. If this were the case, the hormone, on entering the blood-stream, would meet 
its anti-body and would be unable to exert any effect on the appropriate reacting organ. Practically all the complex colloid bodies allied to the proteins, e.g. ferments, egg albumin, peptone, sera of different animals, when injected into the blood-stream, cause the production of the corresponding antibody. The hormones must be simpler in character than such substances and probably have a precise and comparatively simple chemical or molecular constitution.

(2) Since they must be carried by the blood-stream to the reacting organ they must in most cases be susceptible to easy passage through the walls of the blood-vessels if they are to excite a reaction within a fairly short space of time. This consideration would also tend to keep their molecular weight comparatively low.

(3) As a rule the chemical messenger must excite a state of activity in response to a change in some other part of the body. When the primary change passes away, the action of the hormone should also disappear. On this account it is necessary that the hormone should either be susceptible of easy destruction, by oxidation or otherwise, in the fluids of the body, or be readily excreted, so that its action may not be continued indefinitely.

In previous chapters we have already come across several examples of correlated activities of different tissues effected by chemical means. It is perhaps questionable whether we should regard carbon dioxide, or the lactic acid produced by a contracting muscle, as a hormone in the strict sense of the term, since both these products are produced in large quantities as the final product of oxidation or disintegration of the food-stuffs. Carbon dioxide is, however, rapidly eliminated from the body, and lactic acid is equally rapidly oxidised in the body, and there is no doubt that the activity of the respiratory centre is determined by the presence of this substance in the blood and is thereby perfectly co-ordinated with the activities of the whole of the rest of the organism. In the alimentary canal the secretion of pancreatic juice at the precise moment when it is required in the duodenum for the digestion of the food arriving there from the stomach is evoked by the production in the cells of the intestinal mucous membrane under the agency of the acid of the gastric juice (the specific stimulus) of a substance secretin. This substance, which is heat stable and diffusible, but is easily destroyed by oxidation, is absorbed into the blood and carried to the pancreas, where it acts as a specific stimulus for the secretory cells. The same substance excites also the secretion of bile by the liver-cells and the secretion of intestinal juice by the glands of the small intestine. These are perhaps the two best examples of chemical reflexes, i.e. adaptations effected by chemical means rather than by impulses passing along the nerve-channels. There are, however, many other examples of a chemical influence exerted by one organ on another, in which the interaction probably depends on the production of minute quantities of some substance acting in virtue of its excitatory qualities rather than of its value as a source of energy. For many of the organs of the body we know, in fact, no other function than the production of some substance the presence of which in the blood is a necessary 
condition for the carrying out of the normal functions either of growth or activity of many other parts of the body. In other cases an organ may have a twofold function. Thus the pancreas gives an external secretion which is used for the preparation of the food for absorption, and an internal secretion which, passing into the blood, exercises an important influence on the metabolism of the food-stuffs after absorption. Other instances of these chemical correlations may be cited. The secretion of gastric juice, which results from the presence of peptones or other substances in the stomach, has been ascribed by Edkins to the production in the pyloric mucous membrane of a gastric hormone, which travels by the blood to the glands of the fundus, where it excites secretion of gastric juice. According to Frouin the injection of boiled succus entericus, free from secretin, provokes the secretion of intestinal juice. In the reproductive system we have many examples of such chemical correlations. The pancreas, by its internal secretion, probably influences not only the oxidation of the carbohydrates but also the assimilation of the food-stuffs by all parts of the small intestine. All these examples are discussed in fuller detail in other parts of this book. There is one class of organs in which a chemical influence exerted on the rest of the body is the only function with which we are acquainted. These are included under the term ductless glands. As examples, we may cite the suprarenal bodies, the thyroid and parathyroids, the thymus and the pituitary body.

\section{THE SUPRARENAL BODIES}

The suprarenal capsules in mammals are two small masses lying on the upper or oral side of the kidneys. They consist of two parts, the cortex and the medulla. The cortex is composed of cells arranged in calumns or in a reticular fashion. The outermost layer of cells often presents an alveolar structure, the lumen, however, being but little marked. According to the arrangement of the cells the cortex is divided into three zones, the zona glomerulosa, zona fasciculata, and zona reticulata. The cells themselves are distinguished by the large amount of granules they contain, which give the ordinary reactions for fat, but consist probably of lecithin compounds. In some animals, e.g. the guinea-pig, the cells, especially towards the inner part of the gland, contain many yellow pigment granules. The medulla, much less extensive than the cortex, presents irregularly shaped cells, the outlines of which are but slightly marked. These cells contain granules which stain darkly with chromates and give a green colour with salts of iron. It is, hence, easy to delimit the area of the cortex in any section of a gland which has been hardened in a fluid containing chromates. The substance giving this reaction is known as chromophile or chromaffine substance. The suprarenals are richly supplied with blood, especially in the medullary part, the cells of which impinge directly on the endothelial lining of dilated capillaries. They also receive an abundant nerve-supply from the sympathetic system, the nerves forming a thick meshwork, especially in the medulla, and presenting at intervals ganglion-cells which may be isolated or combined to form small ganglia. 
A study of the development of the suprarenal glands shows that we have here to do with two distinct tissues, probably differing in the part they play in the animal economy. Whereas the cortex is derived from that portion of the mesoblast, the 'intermediate cell mass,' from which the mesonephros is also developed, the medulla is produced by an outgrowth from the sympathetic system and may be said indeed to consist of profoundly modified nerve-cells. In many fishes these two elements of the suprarenal gland remain separated throughout life, the cortex being represented by a series of paired interrenal bodies lying on the front of the spinal column, and the medulla by a number of collections of chromaffine cells lying in close juxtaposition to the spinal nerves. In some animals accessory suprarenals are not infrequent in which both cortex and medulla may be represented. In all animals we find masses of tissue, the so-called paraganglia, in close association with the sympathetic system, which present the chromaffine reaction typical of the medulla. Since a watery extract or decoction of these bodies has the same influence on injection into the blood-stream as an infusion of the medulla of the suprarenal body itself, we are probably justified in regarding these bodies as equivalent to accessory medullary portions of the suprarenal. They have the same-origin, the same staining reactions, and the same physiological effect as the latter.

The functions of the suprarenal bodies were a matter of pure hypothesis before Addison in 1855 drew attention to the coincidence of degenerative destruction of these bodies with a disease which has been known since that time as Addison's disease. The three cardinal symptoms of this disorder are (1) bronzing of the skin, (2) vomiting, (3) excessive muscular weakness and prostration. The disease is almost invariably fatal. Addison's observations have been amply confirmed since that time, but we are not yet in a position to account for the occurrence of all these symptoms as a result of interference with the cortex and medulla of the suprarenals. The experimental destruction or extirpation of these bodies has naturally been frequently carried out. The operation always leads to the death of the animal within twelve to twenty-four hours. Even when the destruction is carried out by degrees it has been impossible to reproduce the bronzing which is so characteristic of Addison's disease. The one symptom which is observed as a result of the experimental extirpation is the excessive prostration, which is attended with muscular weakness and a lowered blood pressure. In a few cases it has been found possible to keep rats alive after total extirpation of these organs, but this result is probably due to the frequent presence in these animals of accessory suprarenals.

Schäfer and Oliver in 1894 found that a watery extract or decoction of the suprarenal bodies, when injected into the circulation, caused a very great rise of blood-pressure, brought about chiefly by constriction of all the bloodvessels of the body. The active substance responsible for this rise was limited entirely to the medulla, infusions of the cortex being without influence on the blood-pressure. Later on Takamine succeeded in isolating the active substance, to which he gave the name of adrenaline, and since that time 
physiological chemists have succeeded not only in determining the constitution of adrenaline but also in preparing it synthetically. The constitution of adrenaline is shown by the following formula:

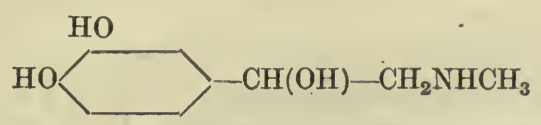

Since it possesses an asymmetric carbon atom, a substance of this formula may be either lævo- or dextrorotatory. Both forms, as well as the racemic modification; have been prepared synthetically. The substance which occurs in the suprarenal gland is the lævorotatory modification, and Cushny has shown that it is only this modification which is active, injection of the dextrorotatory compound having only one-twelfth the effect of the lævorotatory. Adrenaline is active in excessively minute doses, injection of one four-hundredth of a milligramme per kilo body weight sufficing to evoke a definite rise of blood-pressure. On injecting it into the circulation there is immediately a rise of blood-pressure which, if the vagi are intact, is only moderate in amount, but is accompanied by a marked slowing of the heart. This excitation of the vagus is, however, probably secondary to the rise of blood-pressure and is not due to direct action of the drug on the vagus centre. If the vagi be divided the injection of adrenaline evokes a huge rise of pressure which may amount to $300 \mathrm{~mm}$. Hg. It may indeed be so great that the animal dies from heart-failure or from pulmonary œdema. The rise of pressure is observed even after destruction of the central nervous system. The action is not limited to the blood-vessels. It has been shown by Langley and by Elliott that adrenaline injected into the circulation arouses every activity which can be normally excited by stimulation of the sympathetic system. A list of the actions of adrenaline is therefore identical with a list of the chief functions of the sympathetic nervous system. In the head it causes dilatation of the pupil, secretion of saliva, and erection of the hairs. On the heart it has a strong augmentor and accelerator influence, so that the heart beats more effectively as a rule even against the enormously increased resistance offered by the constricted arterioles. Whereas a rise of blood-pressure generally causes increased systolic volume of the heart, we may find after an injection of adrenaline and during the height of the rise of blood-pressure that the heart empties itself more effectively than it did before the injection. On the lung-vessels adrenaline has probably a slight constrictor influence. With regard to the vessels of the brain, we find the same divergence of opinion as in the case of excitation of possible vaso-motor nerves to this organ. Some observers, on perfusing the brain with defibrinated blood, have obtained constriction on adding adrenaline to the perfused blood, while others have been unable to obtain any positive results in this direction. In the abdomen intravenous injection of adrenaline causes complete relaxation of the musculature of the stomach, small and large intestines, but contraction of the ileocolic sphincter. On the bladider its effect varies according to the animal studied, 
but in every case is identical with that obtained by stimulting the hypogastric nerves. It has been shown by Dale that adrenaline may also excite vasodilator fibres or produce vaso-dilator effects when such effects are also obtained from stimulation of the sympathetic nerves. In order to evoke these results it is necessary to paralyse the vaso-constrictors by the injection of ergotoxin, one of the active principles of ergot. This drug, when injected, causes first active vaso-constriction and rise of blood-pressure, followed by paralysis of the vaso-constrictor mechanism. Excitation of the splanchnic nerves or injection of adrenaline will now bring about a fall of blood-pressure due to dilatation of the vessels in the splanchnic area.

The point of attack of the adrenaline appears to be in the muscular or glandular tissues themselves, since it may be obtained not only after destruction of the cord and sympathetic plexuses but even after time has been allowed for the peripheral (post-ganglionic) fibres to degenerate as a result of extirpation of the corresponding ganglia. Although the effect is not altered under these circumstances, and it may still produce either relaxation or contraction of muscles according to the original action of the sympathetic on these fibres, we are not justified in regarding it as acting on the contractile material of the cells themselves. Rather must we assume with Langley and Filliott that the action of adrenaline is on some substance mediating between the nerve and the responsive tissue. We may speak of this reactive material as the receptor substance (Langley), or we may locate it in the situation where the nerve joins the muscle or gland-cell and describe adrenaline as acting on the myoneural junction.

Each suprarenal receives a number of filaments from the splanchnic nerve on its own side. These pass to the medulla where they end apparently without the interposition of any ganglion cells on their course (Elliott), the cells of the medulla having themselves been developed by a modification of sympathetic ganglion cells. Stimulation of the peripheral end of the splanchnic nerve causes, as we have already seen, a discharge of adrenaline into the blood-stream. This discharge accounts for the secondary rise, often accompanied with quickening of the heart, observed on a bloodpressure tracing as the result of stimulating the splanchnic nerve. Through the splanchnic nerves a discharge of adrenaline can be excited by many general conditions, such as pressure on the brain, puncture of the fourth ventricle, administration of anæsthetics, mental conditions such as excitement or fright. Such a discharge is an important element in the reaction to environmental stress and enables the animal to react for the preservation of its life either by offence or flight. If one splanchnic nerve be cut before the administration of anæsthetics or the maintenance of a condition of irritation or fright, the suprarenal gland on the corresponding side will be found to contain two or three times as much adrenaline as the gland which has been left in connection with the central nervous system. It is interesting that no such condition of exhaustion can be produced by electrical stimulation of the peripheral end of the divided splanchnic. It has been suggested, therefore, that the splanchnic nerve contains two sets of fibres, anabolic 
and katabolic, that only the latter are stimulated by central irritation, whereas electrical stimulation, exciting both sets of fibres, causes an increased production of adrenaline in the gland, which exactly keeps pace with the increased output.

When adrenaline is injected into the blood-stream the effect is only temporary. It is not excreted in the urine, but rapidly disappears from the blood. Since it is easily oxidised and is extremely unstable in alkaline solution, we may conclude that after performing its excitatory function it is destroyed by oxidation in-the fluids. Adrenaline is thus a typical hormone, a body of comparatively low molecular weight, having a drug-like excitatory action on specific tissues of the body, easily diffusible, and rapidly destroyed after discharging its office.

Owing to the rapid destruction of adrenaline, relatively enormous doses have to be given by the mouth in order to produce any effect on the blood-pressure. There is, however, a whole series of substances, more or less allied to adrenaline in chemical constitution, which undergo less rapid destruction and can therefore be administered as drugs in the usual way. Dale and Barger have recently described three such sub. stances as occurring in infusions of putrid meat and as forming the most important of the active principles of ergot. The constitution of these substances is shown in the following formulæ :

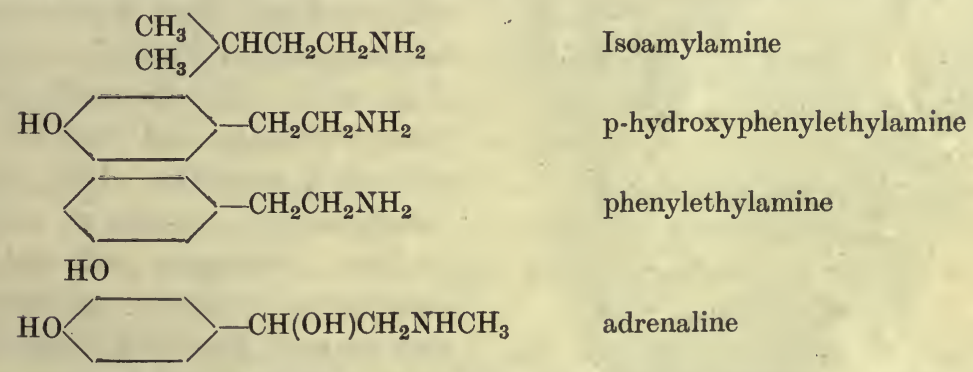

The formula of adrenaline is placed below in order to show the relation of these substances to the natural hormone. These bodies are produced from the amino-acids of proteins by a process of decarboxylation. Leucine would yield isoamylamine, tyrosine, hydroxyphenylethylamine, and phenylalanine would give phenylethylamine. Such substances may be formed in minute quantities during the normal processes of putrefaction which occur in the alimentary canal.

There seems little doubt that we must regard adrenaline as a true internal secretion, and therefore must ascribe to the medulla of the suprarenal capsules as well as to the other chromaffine tissue in the body, the function of maintaining the normal constriction of the arterioles and of facilitating in some way or other the functions of the sympathetic system generally. The absence of this secretion in cases of destruction by disease of the suprarenals would serve to account for the weakness, prostration, and lowered bloodpressure of Addison's disease. The two other symptoms of this disease, namely, bronzing and vomiting, still remain to be accounted for. It is possible that the latter may be due to some involvement by the morbid process of the numberless fibres of the solar plexus, which run in close proximity to the suprarenals. We have no knowledge whatsoever of the functions of the cortical portion of these organs. It is possible that future 
work may show some connection between the cortex and the destruction of pigment in the body. At present it is only by a process of exclusion that we may guess at a causal relationship between the destruction of the cortex and the bronzing which occurs in Addison's disease.

There seems little doubt that the rapidly fatal effects of extirpation of both suprarenals is to be ascribed rather to the removal of the cortex than of the medulla. The functions of the latter can be more or less effectively maintained by the other chromaffine tissues found at the back of the abdomen. In the few cases where animals have survived double extirpation small masses of accessory cortical substance have been found embedded in the kidney or elsewhere in the neighbourhood of the suprarenals.

\section{THE THYROID GLAND AND THE PARATHYROIDS}

The thyroid gland consists of two oval bodies lying on either side of the trachea, joined in many animals across the trachea by an isthmus. Surrounded by a capsule of connective tissue, it is made up of an aggregation of

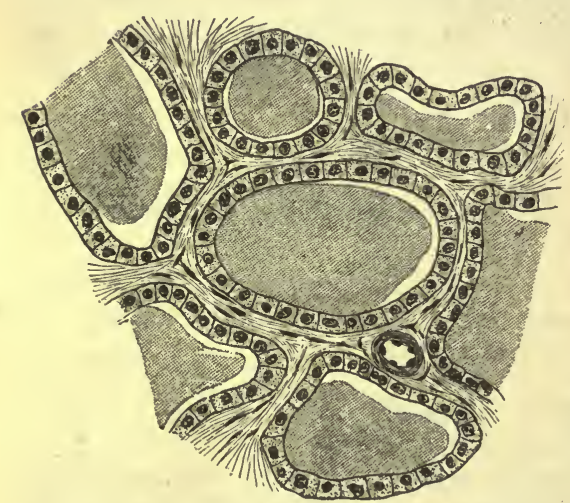

FIG. 546. Section of thyroid gland of dog. (Swale Vincent.) vesicles varying in size from 15 to $150 \mu$. The vesicles are lined by a single layer of cubical epithelial cells, and are filled with a translucent material known as colloid (Fig. 546). Of the cells, some present granules and resemble the cells of a secreting gland, while others contain masses of colloid, or have undergone colloidal degeneration. Between the vesicles may be seen, here and there, solid masses of cells which by some observers are regarded as destined to replace vesicles the epithelium of which has undergone complete degeneration. The colloid material can be traced between the cells into the lymphatics lying between the vesicles. Since the gland possesses no duct it is supposed that the cells furnish an internal secretion, which makes its way into the blood along the lymphatic efferents of the gland. The thyroid is richly supplied with blood by the superior, middle, and inferior thyroid arteries, and is surrounded with a plexus of veins lying immediately under the capsule. In development the thyroid is formed by an outgrowth from the fore-gut, but the connection with the gut disappears long before the end of fotal life. In rare cases part of the duct may persist, and, becoming gradually filled with fluid, give rise to a hyoid cyst which lies balow the tongue and may require excision by the surgeon.

As in the case of the other ductless glands, clinical observations have contributed materially to our knowledge of the functions of the thyroid. Although the gland had been extirpated in animals by Astley Cooper and 
by Schiff, the attention of physiologists and medical men was especially directed to the importance of this organ by the observations of surgeons, especially Kocher, on the untoward and even fatal effects following its complete removal in man in operations for extirpation of goitre. In this country attention had already been called to the connection of a disturbed condition of metabolism known as myxœdema with atrophy of the thyroid. A patient affected with myxœdema presents a gradually increasing blunting of his or her mental activities; speech is slow, cerebration delayed. With this nervous defect are associated changes in the connective tissues, the subcutaneous connective tissue becoming thickened, so that the face and hands appear swollen and puffy, looking at first sight as if œedema were present. The swelling is, however, due to newly formed connective tissue, and not to the presence of an excess of interstitial fluid in the tissues. The patient often presents a yellow, waxy appearance with a patch of colour on the cheeks. The hair falls out, the pulse is slowed, and the temperature tends to be subnormal owing to the diminution of the rate of metabolism in the body. The intake of food and the excretion of urea are diminished. If the atrophy of the thyroid occurs in early life during the period of growth, e.g. in young children, the growth of the skeleton practically ceases. The bones of the limbs may grow in thickness but not in length. There is early synostosis of the bones of the skull and complete cessation of development of mental powers. Children so affected may live for many years, but when twenty-five or thirty present still a childish appearance (Fig. 547, c). Stunted, pot-bellied, and ugly, they have the intelligence of a child of four or five. They often present fatty tumours above each clavicle, and similar subcutaneous tumours of fat or loose fibrous tissue are found in cases of myxœdema in the adult.

When the thyroid is extirpated in man the result is often the production of typical myxœdema. In some cases, especially in young individuals, the results are more severe, a condition of tetany being set up in which there are tonic spasms of the muscles of the body, especially of the extremities. When the thyroid gland is extirpated in animals the results more closely resemble these acute cases. In certain cases a chronic condition of malnutrition is set up, but a typical myxœdema with thickening of the subcutaneous tissues by new growth of connective tissue has only been described by Horsley in monkeys. The effects are more pronounced in carnivora than in herbivora. In the former a condition of tetany is produced accompanied with muscular tremors and clonic convulsions which come on at intervals and may be accompanied with severe dyspnoea leading to death within fourteen days. In herbivora, wasting, diminution of respiratory exchange, and disorders of nutrition are often the most prominent symptoms. These results were ascribed by Munk to interference with the recurrent laryngeal nerves during the operations, but the observations on man leave very little doubt that they are due entirely to the removal of the chemical influence of the thyroid gland. Many authorities were at first inclined to ascribe these results in man and animals to the circulation in the blood of 
toxic substances which would normally undergo destruction in the thyroid gland. This theory is put out of court by the results of administration of thyroid gland to patients with myxœdema or to animals deprived of their thyroids. Schiff first showed that the effects of extirpation of the thyroid might be prevented if, at the same time, the thyroid from another animal were transplanted into the subcutaneous tissue to take the place of the one removed. On removing the transplanted thyroid, the typical symptoms of thyroid destruction at once ensued. It was later found that similar good results could be obtained by subcutaneous injection of the expressed juice of the thyroid, and later that even this was not necessary, and that it

A

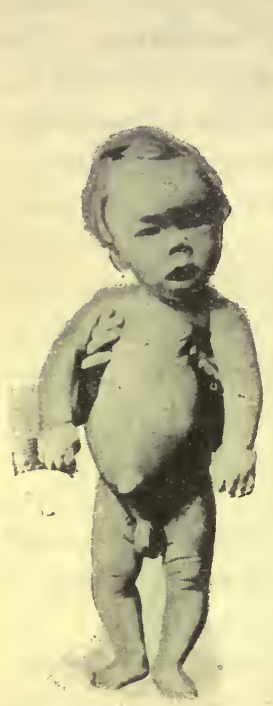

B

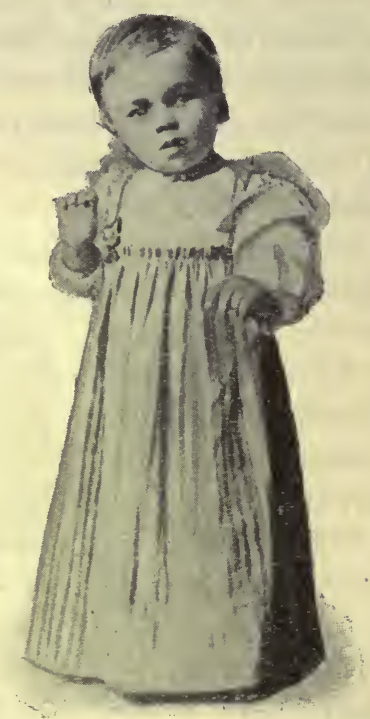

C

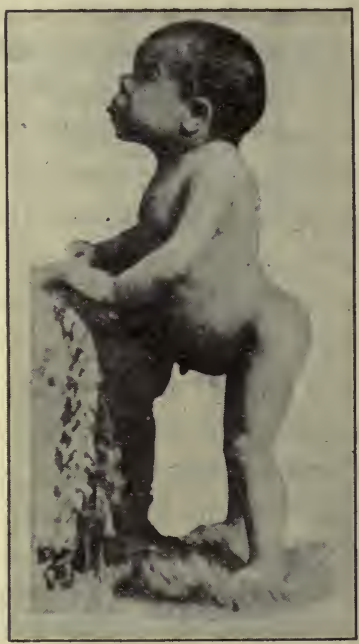

FIG. 547. A, a cretin, 23 months old. B, the same child, 34 months old, after administration of sheep's thyroids for 11 months. c, a cretin, untreated, 15 years old. (W. OsLER.)

was sufficient to administer the thyroid gland, either fresh, dried, or partially cooked, by the mouth. The administration of the thyroid gland in this way is indeed one of the therapeutic triumphs of the last twenty years. An ugly and idiotic cretin can be converted in this way into a child of ordinary intelligence with normal powers of growth (Fig. 547). Given to myx œdemic patients, the thyroid gland reduces the swelling of the subcutaneous tissues, causes a new growth of hair, and restores the patient to his or her former state of mental health. Nor is the thyroid gland without influence on the healthy individual. If given in large doses either to man or animals, it quickens the pulse, even causing violent palpitation, and increases the metabolic activities of the body, so that the appetite is increased, the nitrogenous output rises above the intake, and the subcutaneous fat is diminished or disappears. It is possible that a moderate degree of thyroid inadequacy is not infrequent and that the beneficial effects on general health, in removing excessive 
corpulence and in promoting the growth of hair, which are observed on administering the drug to people of middle life, may be due to the actual replacement of a function which is being insufficiently discharged. The symptoms caused by excessive doses of thyroid gland are strikingly similar to those occurring in the disease known as exophthalmic goitre, where there is a true hypertrophy of the gland associated with cardiac palpitation, proptosis (bulging of the eyes), wasting, and muscular weakness.

All these facts warrant us in including the thyroid body among the glands with an internal secretion, the presence of which in the blood-stream is a necessary condition for the normal growth and functions of almost all the tissues of the body. If this secretion is lacking we obtain the condition known as myxœdema in adults, as cretinism in young children. If it be present in excess the symptoms of exophthalmic goitre are produced. The exact character of the internal secretion cannot be regarded yet as definitely established. It seems possible that it is identical with a substance containing iodine in organic combination, which was isolated by Baumann from the thyroid gland and is known as iodothyrin. In certain experiments the results of administration of iodothyrin were found to be identical with those obtained by giving the whole gland. Doubt has been thrown on the specific nature of this body on account of the fact that iodine may be wanting in the thyroid gland in certain animals, though Reid Hunt has shown that the physiological effects of thyroid extract are proportional to the amount of iodine contained therein.

SIGNIFICANCE OF THE PARATHYROIDS. The parathyroids are small bodies, varying in number, situated on the border of the thyroid gland or actually embedded in its substance. In histological appearance they differ widely from the thyroid, and consist of solid masses or columns of epithelial cells surrounded with connective tissue and richly supplied with blood-vessels (Fig. 548). Considerable divergence of opinion still exists as to the significance of these bodies. In some animals, e.g. in the dog, where they are embedded in the gland. they will be necessarily removed in any operation for the extirpation of the thyroid. In others, such as the rabbit, where they lie outside the gland, it is easy to avoid them in the excision of the thyroid. To this varying distribution of the parathyroids have been ascribed the different results of extirpation of the thyroid in carnivora and herbivora respectively. Forsyth has shown that, in man, the situation of the parathyroids corresponds almost exactly with the places in which are found occasionally accessory thyroids ; and, according to Edmunds, after excision of the thyroid, the parathyroids undergo histological alteration and are converted into thyroid tissue, the cells taking on an alveolar arrangement and producing colloid material. According to this view the parathyroids would represent simply immature thyroid tissue. On the other hand, it has been suggested (Biedl) that the parathyroids have a function entirely distinct from that of the thyroid gland, removal of the thyroids producing simply a condition of cachexia and the changes associated with myxœedema, 
while removal of the parathyroids is responsible for the nervous disturbances and tetany observed after total extirpation of these organs. The matter cannot yet be regarded as definitely settled.

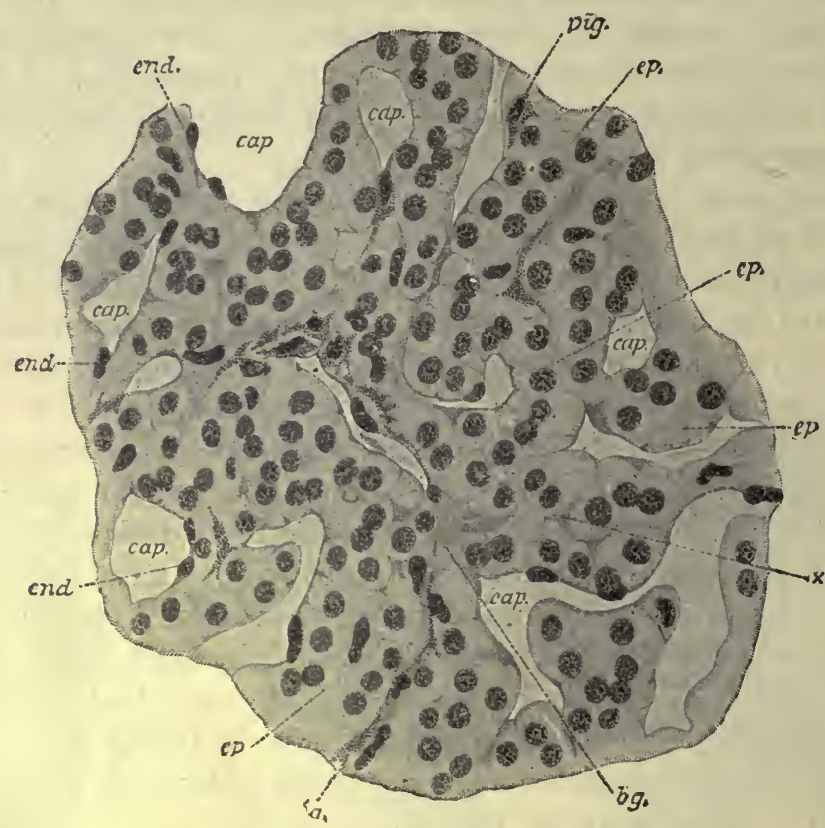

FIG. 548. Section of parathyroid. (KоHN.)

$e p$, secreting epithelium ; pig, cells containing pigment ; cap, sinus-like capillaries; end, endothelial cells.

\section{THE PITUITARY BODY}

The pituitary body consists of two parts which have separate modes of origin. An outgrowth from the buccal cavity in the embryo meets a hollow extension of the anterior cerebral vesicle. The buccal ectoderm gives rise to the anterior lobe and pars intermedia of the pituitary, while the neural epiblast becomes developed into the posterior lobe (Fig. 549). In some animals the posterior lobe remains hollow and retains its primitive connection with the third ventricle of the brain, but in man it becomes entirely solid. The anterior lobe in the adult consists of nests of epithelial cells (Fig. 550), many of which are filled with granules, and is richly supplied with large, thinwalled capillary blood-vessels. The anterior lobe is separated from the posterior lobe by a cleft which is the remains of the original hollow outgrowth from the buccal cavity. The epithelial tissue immediately surrounding this cleft differs somewhat from that constituting the anterior lobe. The cells, which present but few granules, are arranged in islets, separated by an intervening tissue continuous with the main mass of the posterior lobe. Many of the islets are hollow and enclose a colloid material. The colloid material has been traced by Herring into the interalveolar connective tissue and into the prolongation of the infundibulum which enters the posterior 
lobe. One must therefore conclude that the colloid material secreted by the cells of this part passes directly into the third ventricle. The amount of colloid material increases in animals which have undergone extirpation of the thyroid gland.

Our first clue to the importance of this organ in the normal processes of the body was furnished by the observations of Pierre Marie, who found that the morbid condition of 'acromegaly' is associated with tumours of the

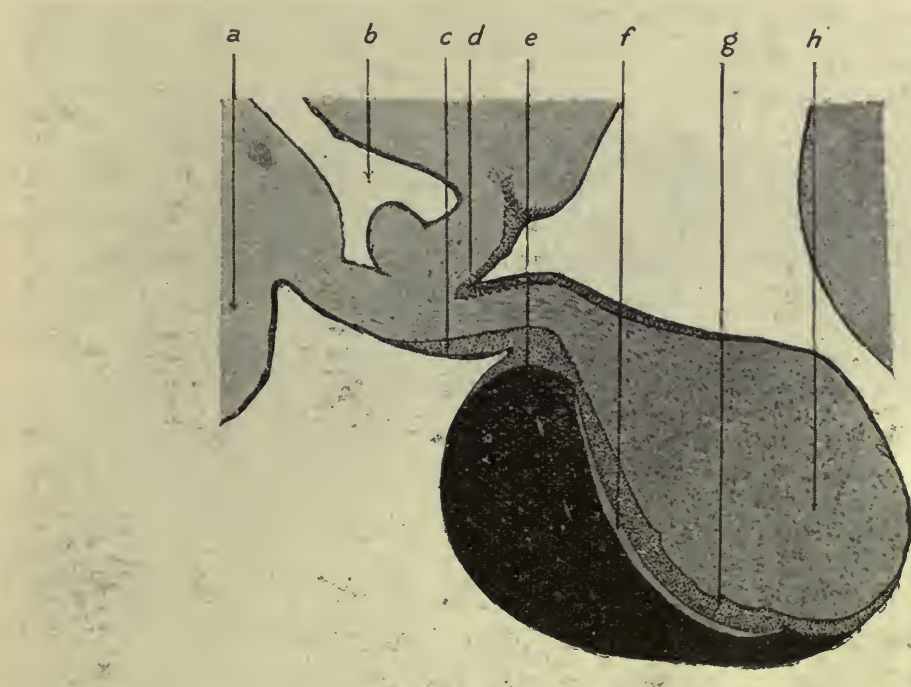

Fig. 549. Mesial sagittal section through the pituitary body of an adult monkey (semi-diagrammatic). (After Herring.)

$a$, optic chiasma ; $b$, third ventricle; $c$, tongue-like process of pare intermedia ; $d$, epithelial investment of posterior lobe; $\rho$, anterior lobe ; $f$, epithelial cleft; $g$, pars intermedia; $h$, posterior lobe.

pituitary gland. This disease consists in an increased growth of certain parts of the skeleton, especially the lower jaw and the extremities of the limbs. Headache is often present, and there may be polyuria and affection of the eyesight. When this disease occurs during the period of active growth there may be an increase in length both of the limb-bones and of the trunk, and most of the giants, which are shown from time to time, are examples of this pathological condition of 'gigantism.' It seems probable that this condition is due to an over-action of the gland. In cases of acromegaly the tumour is generally an adenoma, i.e. an enlargement of the ordinary gland tissue. Total extirpation of the pituitary body is generally followed by death within a few days, death which cannot be attributed to shock or any accidental features of the operation. One must regard the pituitary therefore as essential to the maintenance of life. It has not been possible by transplantation to replace a removed pituitary body, since the transplanted organ has hitherto always undergone degeneration. In a certain number of cases animals, especially if young, have survived extirpation of the 
pituitary body. In these the operation was followed by arrest of development- the animals remaining in an infantile condition, small with an excess of fat, and absence of sexual development.

The most definite evidence we have as to the mode of action of the different parts of the pituitary gland has been furnished by experiments on administration or injection of the dried gland or its extracts. The posterior lobe seems to be practically inactive, extracts made from this lobe having

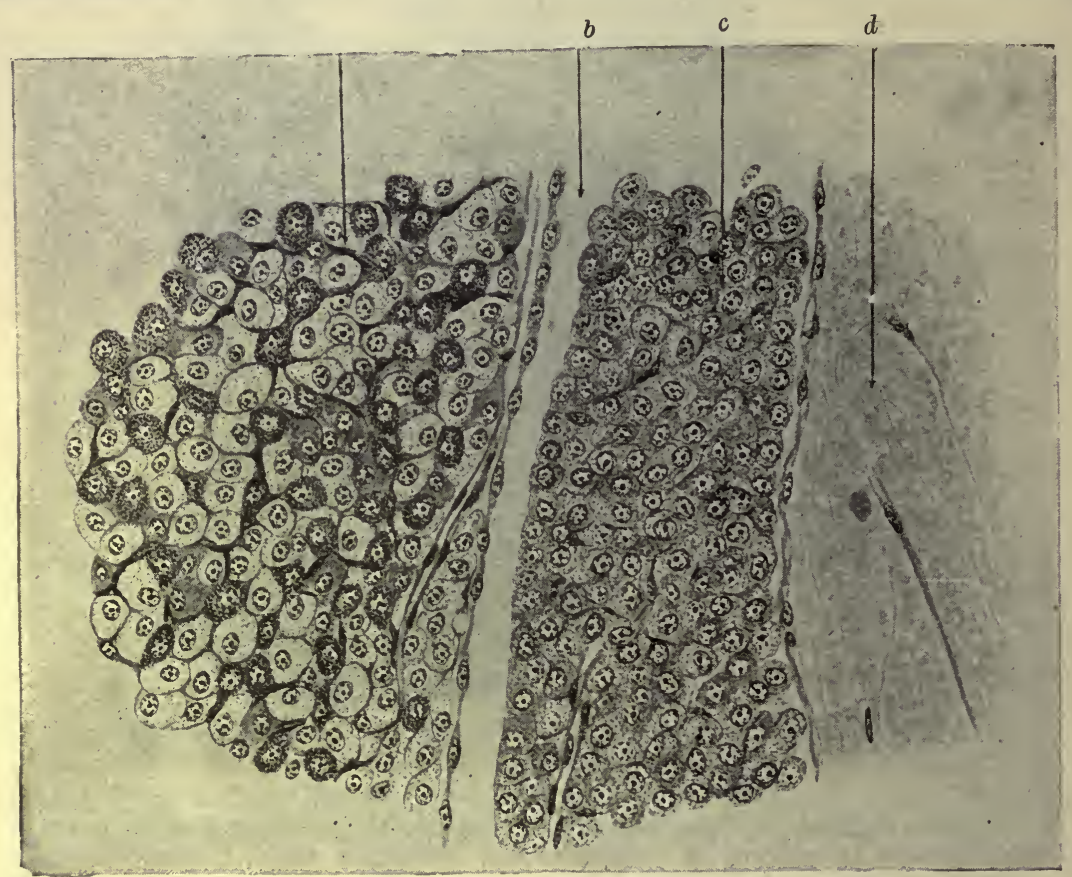

FIG. 550. Section of cat's pituitary body, passing through the cleft in the gland.

(P. T. Herring.)

$a$, pars anterior ; $b$, cleft ; $c$, pars intermedia ; $d$, pars nervosa (posterior lobe).

the same influence as extracts from nervous tissue generally. If, however, the intermediate epithelial substance is included in the posterior lobe, marked effects may be obtained from the intravenous injection. An extract of the posterior lobe (including pars intermedia) produces, as was shown by Schäfer, a rise of blood-pressure and diuresis. The latter result also follows administration of the posterior lobes by the mouth. Dale has shown that the active principle exercises a direct excitatory effect on all unstriated muscle, the effect being unaltered whether the nerve-supply to the muscle be present or not. Thus it produces contraction of the blood-vessels, of the intestinal muscle, and of the uterus, and will act upon muscular tissues, such as the arteries of the lungs or heart, which do not receive constrictor impulses from the sympathetic system. The active principle is much more stable than the other hormones we have already studied. It is not destroyed by boiling, and after injection into the blood-stream can be 
recovered from the urine. It is possible that the polyuria, which is not infrequently observed in association with head injuries or tumours of the brain, may be occasioned by an increased escape of this material into the general circulation.

Extracts from the anterior lobe have no definite effect when injected into the blood-stream. Schäfer found that the addition of the anterior lobe of the pituitary body to the food of young growing animals causes an increased rate of growth. In this experiment eight rats of a litter. were taken : four were fed with bread and milk to which the anterior lobes of pituitary bodies had been added, while the other four, which served as controls, received bread and milk with a corresponding quantity of testis or ovary. Later experiments have not however confirmed these results. According to Mackenzie extracts of the pituitary body have a marked excitatory effect on the secretion of milk by the mammary glands.

It is evident that much further work is necessary before we can regard the functions of the pituitary body as definitely ascertained. The evidence we have at present would seem to point to the following conclusions :

(a) The anterior lobe furnishes some substance to the circulation which promotes growth, especially of the bony and connective tissues of the body.

(b) The intermediate part surrounding the cleft between anterior and posterior lobes, in addition to the production of some substance which is a general excitant for unstriated muscle and produces diuresis, also furnishes a colloid secretion which passes directly into the ventricles of the brain and may be assumed to have some influence on the growth or functions of the central nervous system. Schäfer regards the principle giving rise to diuresis as distinct from that causing contraction of unstriated muscle, since diuresis may occur without corresponding rise of blood-pressure. The independence of the two phenomena, renal and vascular, cannot be regarded as proved.

(c) The posterior lobe consists mainly of neuroglia. We have no clue to its functions apart from the masses of intermediate cells which it may contain.

Very little can be said as to the other ductless glands. The thymus forms two large masses in the anterior mediastinum which in man grow up to the second year of life and then rapidly diminish so that only traces are to be found at puberty. It contains a large amount of lymphatic tissue and is therefore often associated with the lymphatic glands as the seat of formation of lymph-corpuscles. The epithelial remains of Hassell's corpuscles found in the medullary part of its globules have not had any function assigned to them. In certain cases of arrested development or of general weakness in young people the thymus has been found to be persistent. The effect of extracts made from the thymus do not differ from those of extracts made from any other cellular organ.

The pineal gland has, so far, not been proved to have any function in metabolism.* It is interesting as a vestigial remnant of a primitive dorsal eye. In certain lizards this organ still presents traces of its original

* Cases have been recorded in which tumours of the pineal body have been associated with obesity, premature sexual development and early maturity. 
structure, and is found to conform to the invertebrate type of eye. It is doubtful whether at any time in the history of vertebrates the pineal eye has been functional.

The carotid and coccygeal glands have often been grouped with the collections of chromaffine cells already described as associated with the sympathetic system. Their structure resembles more nearly that of the parathyroid bodies or the anterior lobe of the pituitary gland. They consist of a small collection of columns or masses of cells bound together by connective tissue with a rich supply of blood-capillaries. Nothing is known as to their function.

The lymph and hæmolymph glands, and the spleen, are often grouped with these ductless glands. The essential activity of these bodies, however, lies in the production, not of a diffusible chemical substance, but of formed elements, e.g. lymph-corpuscles, and they do not properly fall within the scope of this chapter. As a matter of convenience, we may deal shortly here with the functions of the spleen.

\section{THE SPLEEN}

This organ is similar in many respects to a lymphatic gland. It is formed of a framework of connective tissue and unstriated muscular fibres, in the interstices of which is contained the splenic pulp. This consists of a

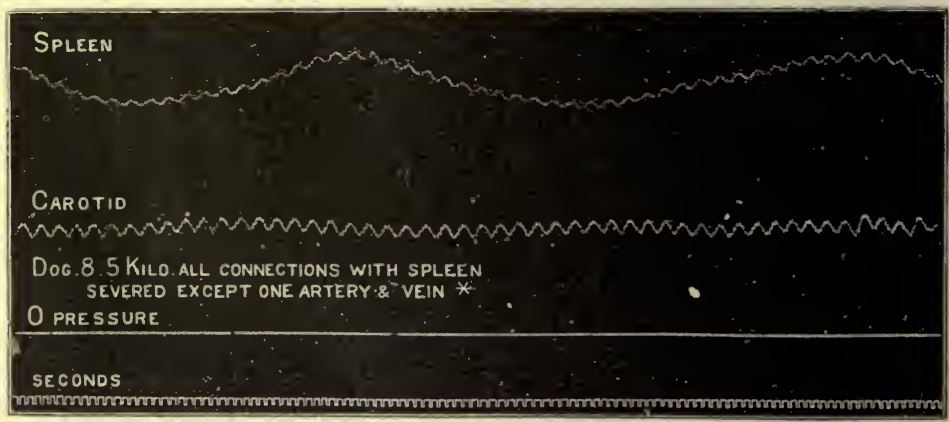

FIG. 551. Plethysmographic tracing of spleen (upper curve) from a dog, showing the spontaneous contractions of this organ (reduced from a tracing by SCHÄFER).

fine fibrillar network, on the fibrils of which lie endothelial cells. The meshes contain the cells of the splenic pulp, which are fairly large polygonal cells, and leucocytes. Just as in a lymphatic gland the cellular elements of the tissues are bathed by the lymph which flows through the gland, so in the spleen the walls of the capillaries become discontinuous, and the blood is poured out into the interstices of the tissue. The spleen is therefore the only tissue in the body where the blood comes in actual contact with the tissue-elements themselves. The blood from the splenic pulp is collected into large venous sinuses, which run along the trabeculæ to the hilum, where they unite to form the splenic vein. The arteries to the spleen are beset in their course along the trabeculæ with small nodules of lymphoid tissue, which are known as the Malpighian follicles. 
It is evident that the blood must meet with considerable resistance in passing through the close meshwork of the splenic pulp. In order to ensure a constant circulation through the gland, the muscular tissue of the capsule and trabeculæ has the property of rhythmic contraction. If the spleen be enclosed in a plethysmograph, or splenic oncometer, and its volume be recorded by connecting this with an oncograph, it will be seen to be subject to a series of large, slow variations, each contraction and expansion lasting about a minute, and recurring with great regularity (Fig. 551). Superposed on these large waves are smaller undulations due to the respiratory variations of the blood-pressure, and on these again the little excursions corresponding to each heart-beat. The contractile power of the spleen is under the control of the nervous system, and a rapid contraction may be induced by stimulation of the splanchnic nerves.

\section{FUNCTIONS OF THE SPLEEN}

The structure of this organ suggests that the splenic cells must exercise a constant influence on the blood which surrounds them, and that this influence is not purely of a chemical nature. In the liver and kidneys,

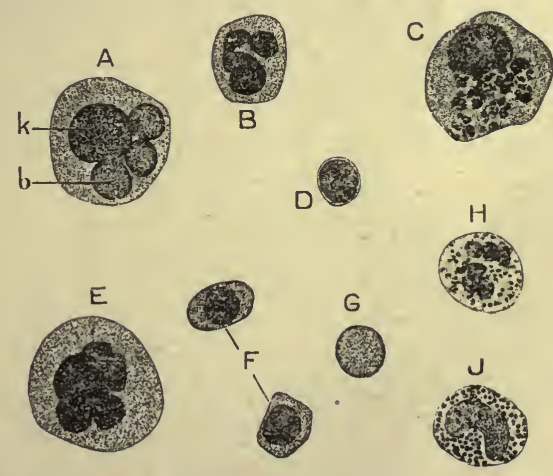

Fig. 552. Cells from a scraping of the spleen. (KöLLIKER.)

A, splenic pulp-cell containing red blood-corpuscles, $b$ ( $k=$ nueleus); $\mathbf{B}$, leueoeyte with polymorphous nueleus; $\mathrm{c}$, pulp-eell containing disintegrated red eorpuscles; D, lymphoeyte ; E, giant eell; F, nucleated red eorpuscles ; $G$, normal red corpusele ; $\mathrm{H}$, multinuclear leucocyte; J, eosinophile cell.

which exercise so powerful an effect on the composition of the blood passing through them, the proper cells of the organs are separated from the bloodstream by the capillary wall. Microscopic examination of the cells of the splenic pulp shows us that these are full of particles of brown pigment or fragments of red corpuscles (Fig. 552). In many cases of infectious disease, such as recurrent fever, the splenic cells are observed towards the end of the attack to be full of the organism-spirillum-which is the cause of the disease. In fact, these cells are so arranged that they can take up solid particles held in suspension in the blood-plasma. We must indeed look upon the spleen as the great blood-filter, purifying the blood in its passage by 
1196

PHYSIOLOGY

taking up particles of foreign matter and effete red corpuscles. The process of phagocytosis, which was described under the cellular mechanisms of defence (p. 1022), is in the spleen a normal occurrence.

A function has also been assigned to the spleen in the formation of red blood-corpuscles, but the evidence is not sufficient to determine whether such a process occurs normally.

Chemical analysis of the spleen reveals the presence of a large number of what are called extractives, such as succinic, formic, butyric, and lactic acids, inosit, leucine, xanthine, hypoxanthine, and uric acid. There is also a protein, combined with iron, as well as several pigments probably derived from the hemoglobin of the red corpuscles destroyed by the cells of the splenic pulp. The fact that, in cases where the spleen is pathologically enlarged as in leucocythæmia, the uric acid in the urine is largely increased points to a connection between the spleen and the formation of uric acid in the body. The numerous extractive which are formed probably owe their origin to the destructive changes effected on the effete constituents of the blood by the agency of the splenic pulp-cells. 
BOOK IV

REPRODUCTION 



\section{CHAPTER XXI \\ THE PHYSIOLOGY OF REPRODUCTION}

\section{SECTION I \\ THE ESSENTIAL FEATURES OF THE SEXUAL PROCESS}

THE two fundamental characteristics of protoplasm, which distinguish it above all others from unorganised matter, are growth and activity. Growth occurs at the expense of surrounding non-living material, while activity is in every case an adapted reaction to changes in the environment. The second characteristic would seem to involve a limitation of the first, and does, in fact, determine the conditions under which it may occur. In the process of growth of a minute spherical mass of protoplasm its bulk and mass increase as the cube, while the surface only increases as the square, of the radius. Thus the proportion of surface to mass diminishes with increased size of the protoplasmic unit, and since activity is a function of the surface the larger the unit the smaller must be its activity. It follows that there must be a limiting size to the living protoplasmic unit, and it is on this account that practically no unicellular animal or plant exceeds a fraction of a millimetre in diameter. If an organism is to attain any larger size, this can only be by a multiplication of units, each presenting the same relative amount of surface as a complete unicellular organism, though the surface may be exposed to an internal and not to an external medium. Another factor, limiting the size of the unicellular organism or of the unit of the multicellular organism, is the necessity for maintaining a certain proportion between the size of the nucleus and that of the cytoplasm composing the body of the cell. Observations on artificial division of cells have shown us that the functions of digestion, assimilation, and growth depend upon the presence of a nucleus. Hence, when for any reason it is advantageous that a cell should attain a large size, such a cell is almost always found to contain many nuclei. All the ' giant cells' found in the body of man under normal or pathological conditions are also multinuclear.

Thus the continuous display of the functions of assimilation and dissimilation, of growth and activity, is only possible so.long as cell division keeps pace with growth. In unicellular organismis, under favourable conditions, this growth and multiplication occur with prodigious rapidity. It has been computed that a paramœcium, freely supplied with food material, 
would, by growth and division, in the course of a year form a mass of protoplasm the size of the earth, assuming of course that no accidents or destructive agencies intervened to destroy the paramœcia which were being formed. This computation, which may seem a fanciful one, is useful as indicating the enormous number of individuals brought under the action of natural selection, which very few survive. In unicellular organisms, such as paramœcium or amœba, death cannot be regarded as a natural process. They may be eaten by higher organisms or serve as food to vegetable parasites, but so long as conditions are favourable and food-supply sufficient, they will continue to grow and reproduce themselves eternally. In the course of its existence each individual may be brought under many varieties of conditions ; some of these may be so harmful that the individual is destroyed and its race comes to an end. Other individuals, under circumstances of less severity, may undergo modifications in their molecular structure which will serve to neutralise the effect of the injurious invironment. Any such modification in structure, morphological or molecular, must be transmitted to the next generation, so that with slowly varying external conditions there is a possibility of a corresponding slow variation in type, which may finally attain a form altogether different from that with which it set out. A new species may in this way be formed by gradual alteration of environment. It is not therefore difficult to understand in the case of such organisms either the maintenance of type by heredity under constant conditions, or the change of type with gradually varying conditions.

Reproduction by continuous growth and division is not, however, the only means, even in the unicellular animals, by which new generations may be produced. If protozoa, such as paramœeia, be kept for a long time in nutrient solutions their rapidity of reproduction after a time falls off, while many die, and others become the easy prey to infectious diseases. Under these conditions a new phenomenon makes its appearance, viz. 'conjugation,' which is the analogue of the sexual reproduction of the higher animals. Infusoria contain two kinds of nuclei, a large and a small, known as the macro-nucleus and the micro-nucleus respectively. During conjugation the macro-nucleus breaks up and disappears in two cells, which become closely applied together, while in each the micro-nucleus divides twice to form four spindle-shaped bodies. Three of these degenerate (like the polar bodies of the ovum), while the fourth divides into two. This is followed by an exchange of micro-nuclei, one micro-nucleus from $\mathrm{A}$ passing into $\mathrm{B}$, while one micro-nucleus from в passes into $\mathrm{A}$. The two cells then separate, a single micro-nucleus being formed in each by the amalgamation of the two. This micro-nucleus then divides three times, so that eight nuclei are formed, while the cell itself divides into four, two nuclei passing into each of the daughter cells. Of these one enlarges to form the macro-nucleus, while the other remains as the micro-nucleus. After conjugation has occurred, the colony of infusoria takes on, so to speak, a new lease of life, and there is a rapid production of new generations by simple division of the cells, in which both macro-nucleus and micro-nucleus take part. Conjugation apparently only 
occurs in the presence of adverse conditions, and may be prevented almost indefinitely by maintaining the colonies in as favourable conditions as possible. In certain organisms, especially in Algæ, in which similar phenomena take place, each organism after conjugation may surround itself with a thickened wall and remain for a considerable length of time in a state of suspended animation. It is very difficult to understand the advantage of this interchange of nuclear material either to the individual or to the race.

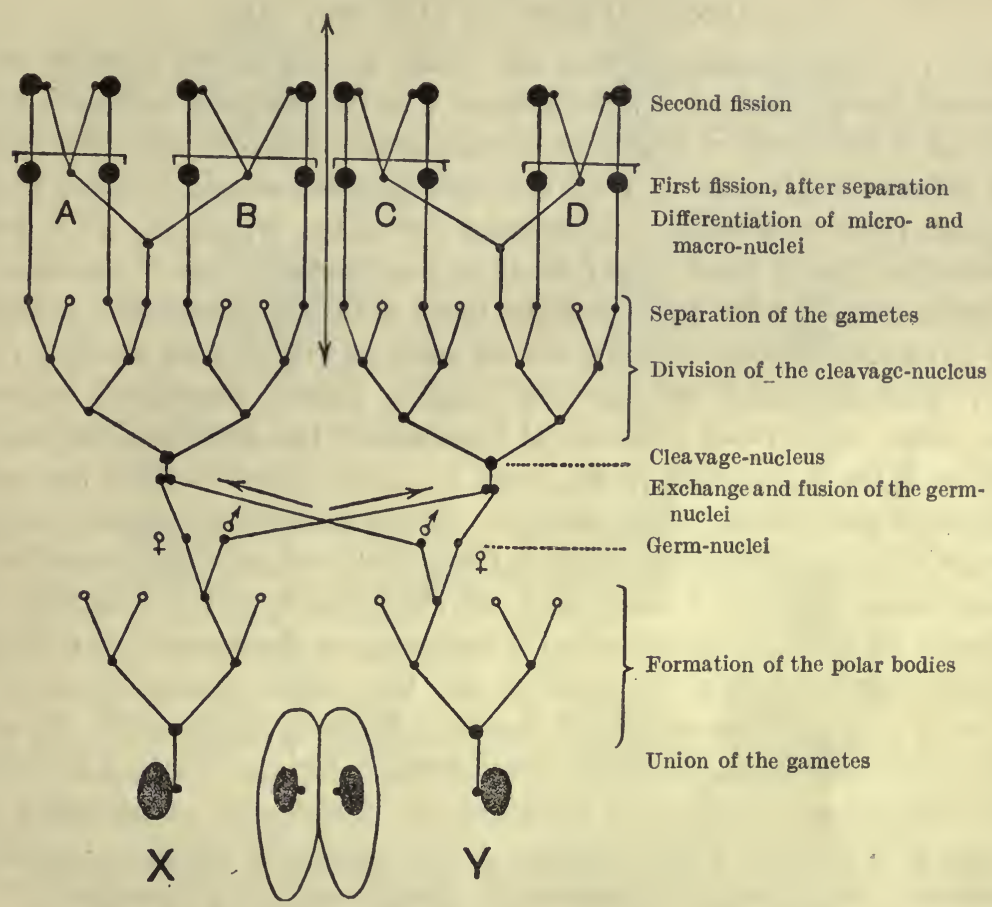

Fig. 553. Diagram showing the history of the micro-nuclei during the conjugation of paramœcium. (From WILSON after MAUPAS.)

$\mathrm{X}$ and $\mathrm{Y}$ represent the opposed macro- and micro-nuclei in the two gametes circles represent degenerating and black dots persisting nuclei.

It has been suggested that as soon as each individual concerned in the process receives the nuclear material from organisms which may have been exposed to slightly different circumstances, corresponding changes will be introduced into the tendencies to growth of the product of the union. Some of these tendencies may be more advantageous than before, while others may be the reverse. Increased possibility of variation is, however, introduced by this admixture of nuclear material, and this may be the advantage of the process to the race. It should be noted that the half of the nucleus lost by each conjugating organism is qualitatively different from that which it retains and probably from that which it receives. A gamete in which the nucleus can be represented by $a b$, and which by simple division will produce similar organisms with nucleus $a b$, conjugates with an organism 
of slightly different structure, and therefore with a nucleus which can be represented as $c d$. After conjugation, the $a b$ gamete will contain a nucleus represented by $a c$, while the $c d$ gamete will contain a nucleus represented by $b d$. $a c$ or $b d$ may be better or worse combinations than $a b$ or $c d$. If either of them is better, that organism will survive under the less favourable conditions, and the race will continue with a slight, and to us inappreciable, change of type.

\section{REPRODUCTION IN THE METAZOA}

The numberless cells forming the bodies of the higher animals are all produced by a series of divisions from a single cell, the fertilised ovum. This cell is the result of a process of conjugation between two cells derived from different individuals. With the multiplication of cells forming a single organism there is of course an increased size of the organism. It is doubtful whether this of itself would be of any advantage, were it not that the multiplication of cells goes hand in hand with differentiation, groups of cells being modified structurally and set aside for one or other function of the body. Differentiation of function implies higher functional capacity. As a motor organ or as a means of locomotion, the differentiated musclecells, with their attached parts, must be more effective than the undifferentiated protoplasm of the amœba. Specialisation of function involves changes of type in the cells resulting from the division of the primitive undifferentiated ovum. In most cases this change of type is permanent. An epithelial cell such as that forming the epidermis or the liver, when it divides, produces another cell of the same kind. One might almost speak of the evolution of a new species of cell but that it takes place within the short period of the development of the multicellular individual, instead of occupying a long space of time, and involving the destruction of countless individuals, as is the case when a change of type gradually occurs in unicellular organisms. Differentiation necessarily brings with it a limitation of the powers of reproduction. Any one of the descendants of a unicellular organism is in all respects equivalent to its ancestor, and can reproduce the same type of individual. The specialised liver- or muscle-cell can only produce a cell of the same type, one, that is to say, incapable of independent existence or of forming the divergent series of types necessary for the formation of an individual. Differentiation of function therefore involves the setting aside of certain cells, germ-cells, which retain their primitive character and are capable of indefinite division to form new generations each capable of developing into a complete individual. These germ-cells can often be recognised from the very earliest divisions of the fertilised ovum, which lead to the production of the mature individual. Thus, in Ascaris, the progenitor of the germ-cells differs from the somatic cells both by the greater size of its nucleus and in its mode of division (Fig. 554). In the cells destined to produce the somatic cells a portion of the chromatin is cast out into the cytoplasm, where it degenerates, so that only in the germ-cells is the sum total of the chromatin retained. Thus in the two-celled stage, in one cell 
all the chromatin is preserved, while in the other cell the thickened ends of the chromosomes are cast off into the cytoplasm and degenerate, only the thinner central portions being preserved. When these divide again, the same process is repeated in only one of the daughter cells derived from a germ-cell, and the process is repeated during five or six divisions, after which the chromatin elimination ceases, and the two primordial germ-cells
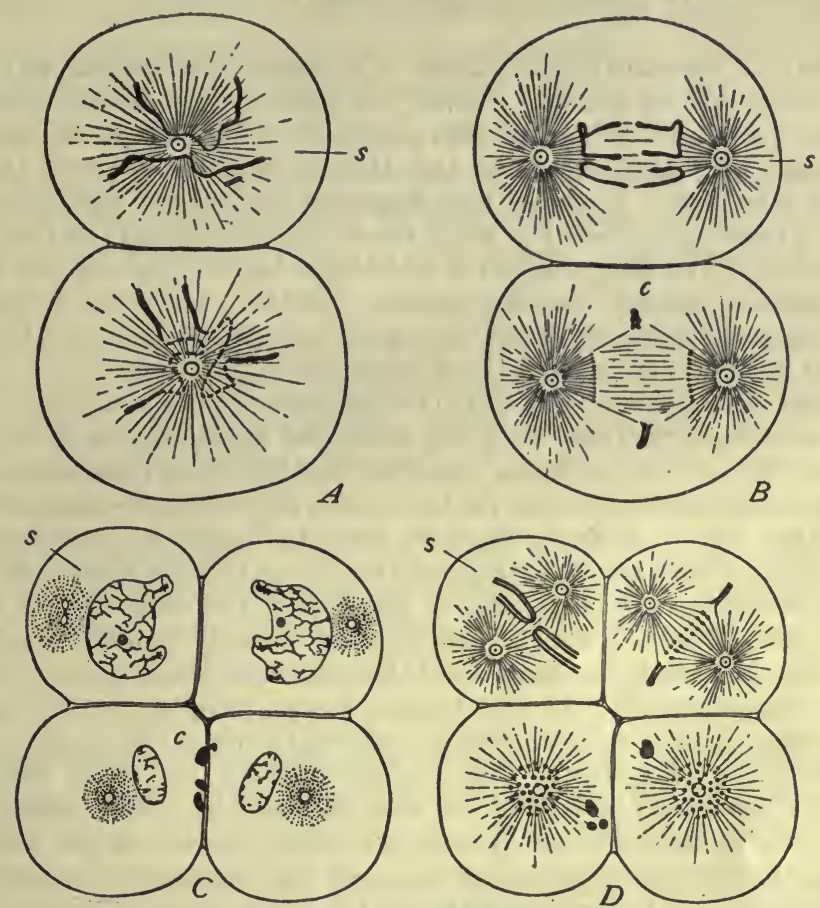

FIG. 554. Origin of the primordial germ-cells and casting out of chromatin in the somatic cells of $A$ scaris. (WILSON and BOVERI.)

A, two-cell stage dividing; $s$, stem-cell, from which arise the germ-cells. B, the same from the side, later in the second cleavage, showing the two types of mitosis and the casting out of ohromatin $(c)$ in the somatic cell. c, resulting four-cell stage; the eliminated chromatin at $c$. $D$, the third cleavage, repeating the foregoing process in the two upper cells.

thenceforward give rise only to other germ-cells in which the entire chromatin is preserved. Thus "the original nuclear constitution of the fertilised egg is transmitted, as if by a law of primogeniture, only to one daughter cell, and by this again to one, and so on, while in other daughter cells the chromatin in part degenerates, in part is transformed, so that all of the descendants of these side-branches receive small reduced nuclei" (Boveri, quoted by Wilson).

The immortality, which was the property of all the unicellular ancestors of the metazoa, has in the latter descended only to the germ-cells. All the other cells of the body, which form the nervous and muscular tissues, glands, skin, \&c., are mortal. They pass through a certain number of divisions ; but although this number is large, it is limited, and on the number 
of divisions which are possible depends the normal duration of life of the organism to which the cells belong. We may thus regard the egg-cell as dividing into two parts. From one part will be formed by differentiation all the complex somatic mechanisms of the adult animal; the other part will divide, but will remain in an undifferentiated form, until its descendants can conjugate with germ-cells from other individuals and form fertilised egg-cells, destined to undergo the same series of changes.

The metazoan individual thus consists of a mortal host holding within itself the immortal sexual cells or gonads. Gaskell has pointed out that the development of the fertilised ovum involves two parallel processes - on the one hand, the elaboration of the elements forming the host; on the other, of those derived from the free-living independent germ-cells. From the very beginning the somatic part of the organism, the host, is a reacting individual in which the nervous system acts as the integrator of all the activities of the body and as the middleman between the internal and external epithelial surfaces and the muscular system. The host may thus be regarded as a neuro-epithelial syncytium, every step in its evolution and differentiation being attended by increased control of all the units by a central nervous system.

The gonads were placed at first within the interstices of this syncytium, and escaped to form a new generation only after the death and disintegration of the host. But differentiation and division of labour affect also the free-living gonads. Some of these form a germ epithelium surrounding the body cavity, of which a few only of the elements pass out of the host as perfect germ-cells, while the others are subordinated to the metabolic needs of these germ-cells and are transformed into various elements, such as nurse-cells, wandering mesoderm cells or phagocytes, yolk-cells, and so on. Gaskell regards the greater part, if not the whole, of the connective-tissue framework of the body, as well as the wandering corpuscles of the blood and tissue-fluids, as derived from these primitive germ-cells. All these tissues, though useful to the host as well as to the finally successful germ-cells, present the common feature of an absolute independence of the central nervous system. Thus the evolution of the animal kingdom means essentially the evolution of the host, and must therefore be closely connected with the evolution of the central nervous system, the ruling element in the neuro-muscular syncytium. On these grounds Gaskell has used the comparative morphology of the central nervous system as a means of tracing the origin of the vertebrate from the invertebrate type, and has come to the conclusion that the immediate ancestor of the vertebrate must be sought in the invertebrate group presenting the most highly developed central nervous system, namely, the Arthropoda.

All the complex mechanisms which are concerned in maintaining the life of the individual have apparently been developed in order to give the potentially immortal germ-cells a better chance of survival in the struggle for existence. From the broad biological standpoint, as Foster points out, all the toil and turmoil of human existence may be regarded simply as the by-play of an ovum-bearing organism. From the same standpoint one must acknowledge that the mortality of the individual, resulting from the absence of an indefinite power of multiplication among the somatic cells, must be an advantage to the race. Throughout the living world the welfare of the individual is subordinated to that of the species. With each new generation there are possibilities of variation and of the production of individuals better or worse fitted for the maintenance of the race than those of the previous generation. Immortality of the individual would handicap the survival of the younger generations, and we should have the same retardation 
of progress in a race that we see in many civilised communities, where the power and the conduct of affairs are in the hands of the older members.

\section{THE FORMATION OF GERM-CELLS}

In multicellular organisms the cells which conjugate to form a new cell, capable of developing into an individual, are of two kinds. One, which has generally a certain amount of reserve material stored up in its cytoplasm, is the female element and is called the ovum. The other cell, which consists of little more than nuclear material, is the male element, and is called the spermatozoon. Both kinds of cells are derived from a mass of undifferentiated cells, the germ epithelium, which, as we have seen, can often be traced directly back to the first divisions of the fertilised egg. The use of the reserve material in the ovum is to serve as food for the developing individual. The ovum and spermatozoon cannot be regarded as corresponding to complete cells. Before their union or conjugation both male and female germ-cells undergo certain important changes which differentiate them from the ordinary somatic cells of the individual. The essential differences between a germ-cell and a somatic cell can be best seen by a study of the nuclear changes which precede their formation. In division the nuclei of all somatic cells, whether of plants or animals, undergo a series of changes which, in their broad outlines, are identical throughout both animal and vegetable kingdoms (Fig. 555). The nucleus of the resting cell in its vegetative condition is generally separated from the cytoplasm by a nuclear membrane, and contains irregular masses of a material staining deeply with basic dyes, and known as chromatin. In the cytoplasm of most animal cells may be seen a small particle known as the centrosome. When division is about to take place the clumps of chromatin arrange themselves into a filament which forms a continuous skein, the 'spireme stage.' This then breaks up into a number of segments, often $\mathbf{V}$-shaped, the chromatin filaments or chromosomes. Each of the filaments, in large nuclei, may often be seen to be composed of rows of granules. While this change has been occurring the nuclear membrane in most cases disappears, and the centrosome outside the nucleus divides into two parts, which travel to opposite ends of the nucleus. Round each centrosome the cytoplasm is modified and presents a radiate appearance, the aster, while joining the two centrosomes is a spindle of fine fibres, the achromatic spindle. The $\mathrm{V}$-shaped segments of chromatin arrange themselves in a circle at the equator of the spindle midway between the two centrosomes. Each of the loops then splits longitudinally, and each half travels towards one or other of the centrosomes, thus forming two daughter nuclei. The half-loops then join to form a skein, and may return to the form of a resting nucleus. These different phases in division are presented by all. somatic cells, and have received the following names :

(1) Prophase (the formation of the spireme and of the achromatic spindle, and the breaking up of the spireme into chromatin loops or chromosomes).

(2) Metaphase (the splitting of the chromosomes). 
(3) Anaphase (the travelling of each half-chromosome to the extremity of the spindle).

(4) Telophase (the retrogressive changes leading to the conversion of
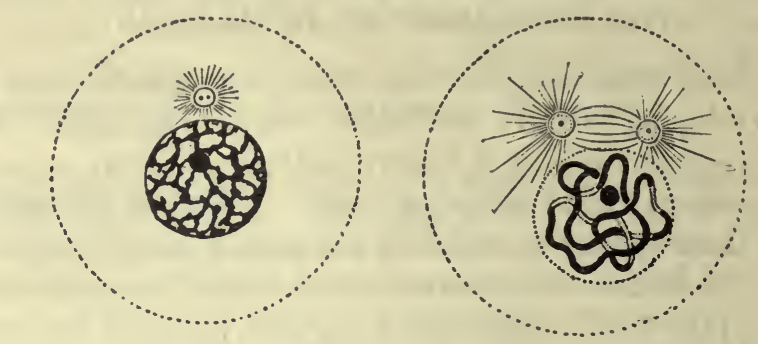

2
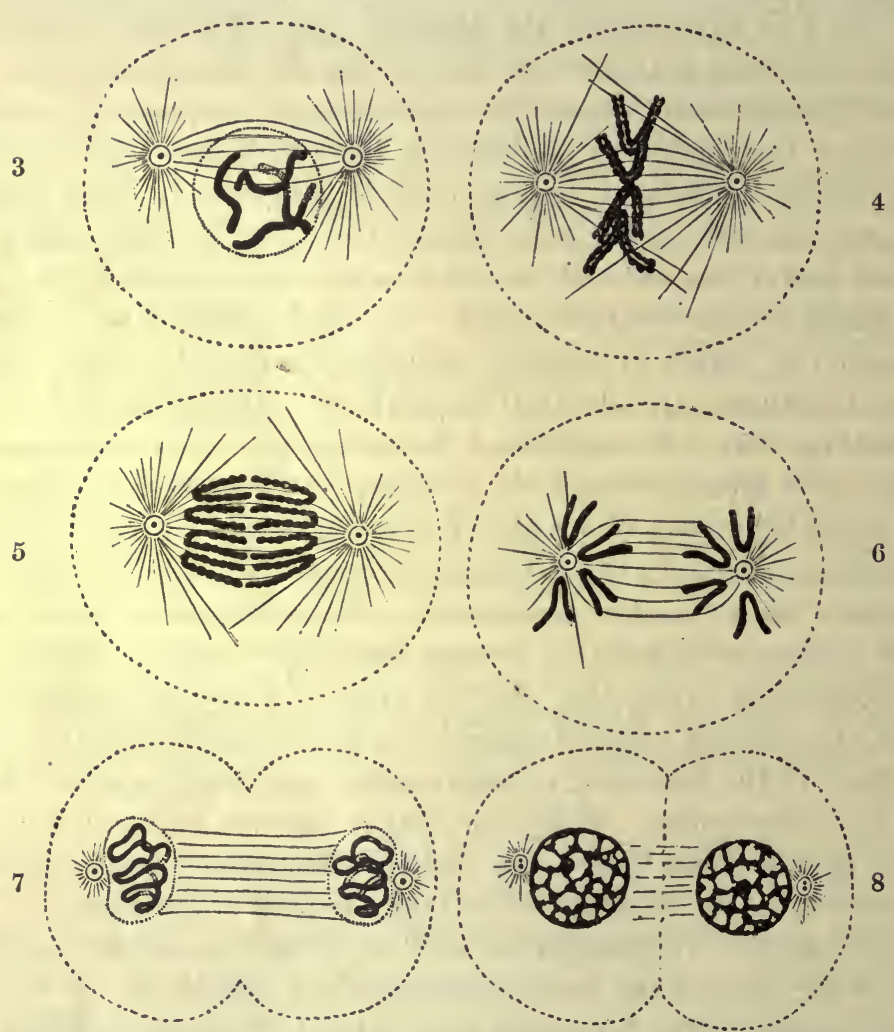

FIG, 555. Diagram showing the changes which occur in the centrosomes and nucleus of a cell in the process of mitotic division. (SCHÄFER.)

The nucleus is supposed to have four chromosomes.

the chromatin filaments into an ordinary resting nucleus, which are accompanied or preceded by a division of the cytoplasm across the equatorial part of the spindle).

When the spireme has broken up into separate chromatin loops, it is possible to count them, and it is found that the number present in any cell is constant for the species. Thus every human somatic cell has sixteen 
chromosomes in its nucleus. The same number is found in types so far apart as the ox, the guinea-pig, and the onion. In the mouse, the salamander, and the lily the number is twenty-four. Other types, such as the crustacean Artemia, are said to have as many as 168 chromosomes, while in Ascaris the cells only contain two or four chromosomes. All these changes, which are included under the term mitosis or karyokinesis, seem to be adapted to ensuring an equal qualitative as well as quantitative distribution of the nuclear chromatin among the daughter cells resulting from the division of any cell. As we have seen in an earlier chapter, the nucleus, by its interaction with the cytoplasm, determines the processes of assimilation
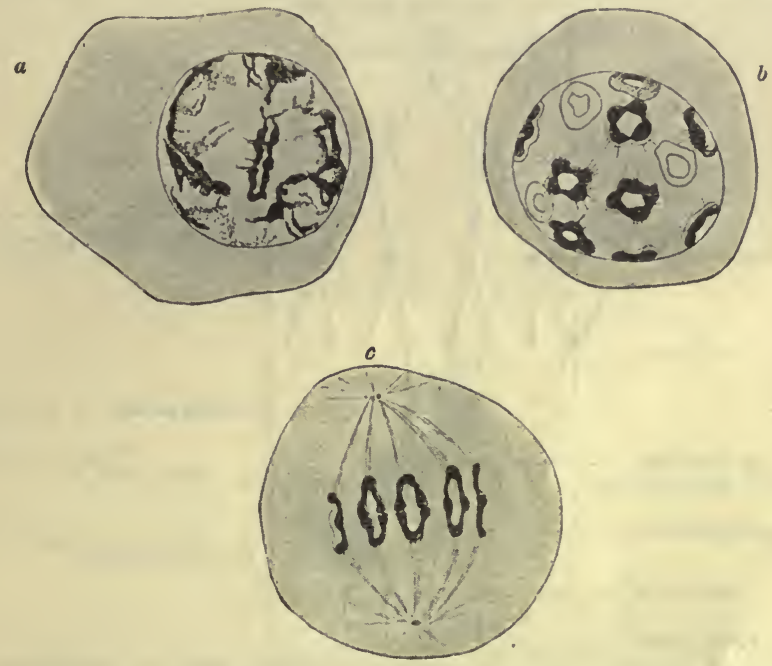

FiG. 556. Three stages of heterotype mitosis in spermatocyte of triton. (MoorE.) $a$, germinal condition of chromosomes; $b$, gemini arranged in quadrate loops or tetrads; $c$, separation of tetrads into the duplex chromosomes of the daughter nuclei.

and growth of the whole cell. For the preservation of type in cell division it is therefore essential that the nuclei of the daughter cells shall be identical in all respects with the nucleus of the mother cell.

Sexual reproduction involves the conjugation of two cells, with union of their nuclei. If each of these nuclei consisted of the normal number of chromosomes, the fertilised egg-cell would contain double the number characteristic of the species, and since these chromosomes would divide by splitting, the number of chromosomes in each cell would be doubled with each generation. This doubling is obviated by the fact that, in the formation of the germ-cells, the ovum and spermatozoon nuclei undergo a special type of division, which leads to the reduction of the chromosomes in the sexually mature cell to one-half of the number characteristic of the species. This mode of cell division is often called 'division by reduction,' or ' heterotype' mitosis, or 'meiosis' (Fig. 556). We may take as an example the development of spermatozoa. The mother cells of the spermatozoa, the 
spermatocytes, divide twice, giving rise to four daughter cells, the spermatids, each of which develops into a functional spermatozoon. In the nuclear changes preparatory to the first division, the spireme, when it breaks up, gives rise to only half the normal number of chromosomes. Thus if the somatic number of chromosomes were four we should find in the spermatocyte, after the breaking up of the spireme, only two chromosomes. These take up their position at the equator of the achromatic spindle and then divide, but the division is effected, not by splitting of the double chromosome, but by transverse division. Each chromosome breaks into half, one half going to each daughter cell. Since each of the reduced number of chromosomes can be regarded as made up of two normal chromosomes

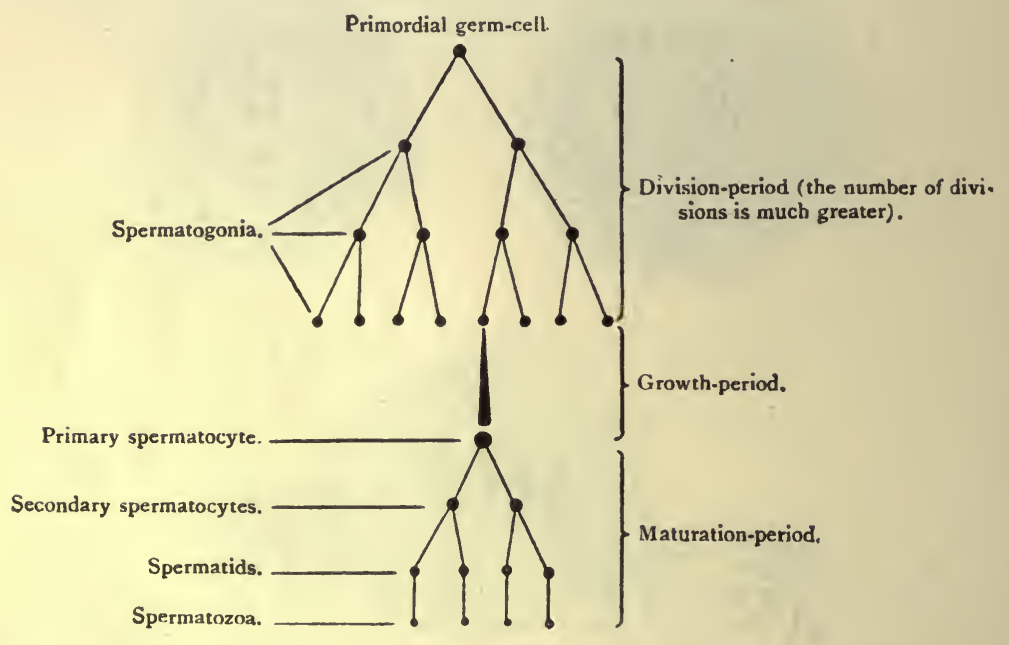

FIG. 557.

placed end to end or joined to form a ring, as in Fig. 556, $b$, the division in the middle provides for a qualitative difference between the two daughter cells. If we indicate the four normal chromosomes as $a, b, c, d$, in ordinary somatic division each daughter cell will also contain chromosomes which may be represented as $a 1, b 1, c 1, d 1$, and $a 2, b 2, c 2, d 2$. In the spermatocyte the two chromosomes may be represented as $a b$ and $c d$. When they divide one daughter cell receives $a$ and $c$, while the other daughter cell receives $b$ and $d$. The second division of these daughter cells takes place generally by splitting of the filaments, so that finally four spermatids are produced (Fig. 557), each containing two chromosomes, two of them containing $a$ and $c$, while the other two contain $b$ and $d$. In the ovum, during maturation, analogous changes take place. Two successive cell divisions occur as in the formation of spermatozoa, but the daughter cells are of very unequal size. In the first division, the heterotypical division, the chromosomes fuse in pairs and then divide as in the spermatocytes so that each of the daughter cells contains one half the somatic number of chromosomes. The larger of the two resulting cells, which retains most of the cytoplasm, 
is still called the ovum, while the smaller one is spoken of as the "first polar body.' The ovum now divides again and throws off a second polar body, the division being of the homotypical variety. The first polar body may also divide, so that from the original ovum three cells are produced, one of which retains the greater part of the cytoplasm, while the others are extruded, and degenerate (Fig. 558). The mature ovum has, however, only half the normal number of chromosomes, so that its nucleus is equivalent to the nucleus forming the head of the spermatozoon. The only difference therefore between the formation of ovum and spermatozoon is that in the former case three of the cells formed by the division of the primitive ovum are

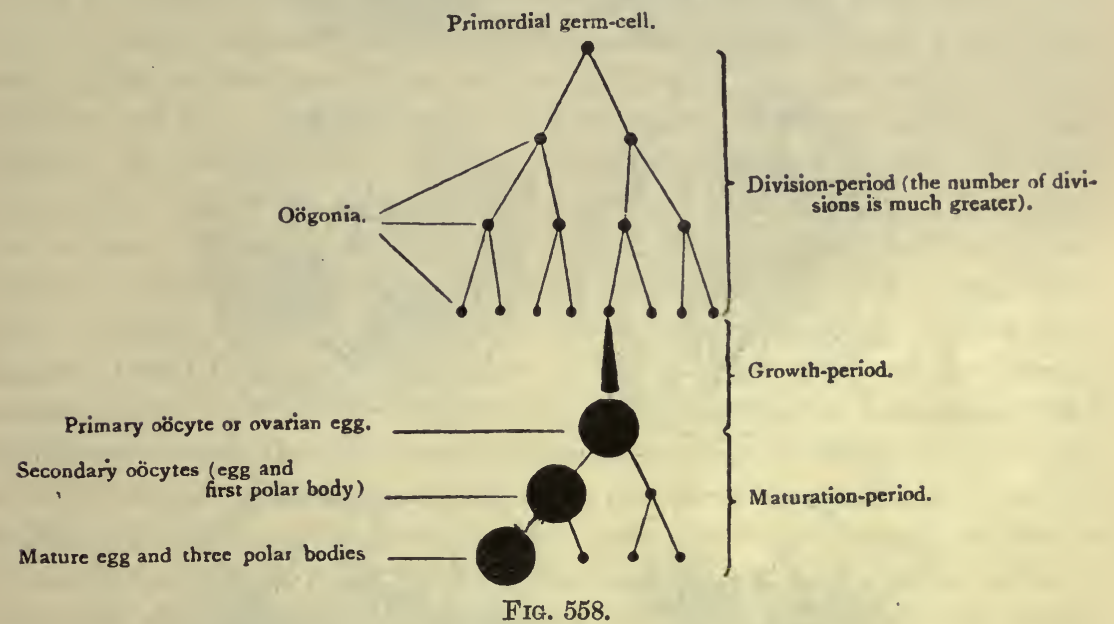

abortive, whereas in the spermatozoon all four daughter cells produced from the spermatocyte remain functional. The production of these two kinds of sexual cell is represented in Figs. 557 and 558.

Since the nuclei of the mature ovum and spermatozoon only contain half the normal number of chromosomes, they are generally spoken of as pronuclei.

\section{FERTILISATION}

The essential features of fertilisation, i.e. the union of the sexual cells, are best studied in some of the lower invertebrates, such as ascaris or echinoderms. In the latter fertilisation takes place in the sea-water, into which both ova and spermatozoa are extruded. The ovum of the echinoderm consists of a naked mass of protoplasm. Of the countless hordes of spermatozoa which may be in the neighbourhood of a given ovum only one as a rule enters. As soon as the spermatozoon has entered the ovum a tough membrane is rapidly formed round the latter, so preventing the entrance of any further spermatozoa. The head of the spermatozoon enters the egg, while the tail atrophies and disappears. The head of the spermatozoon enlarges and assumes the character of a nucleus, the dense mass of chromatin breaking up first into a thread and then into the characteristic number 
of chromosomes (Fig. 559). The egg now contains two nuclei or pro-nuclei, exactly similar in appearance, one derived from the male and the other belonging to the egg itself. The two nuclei approach one another and join.
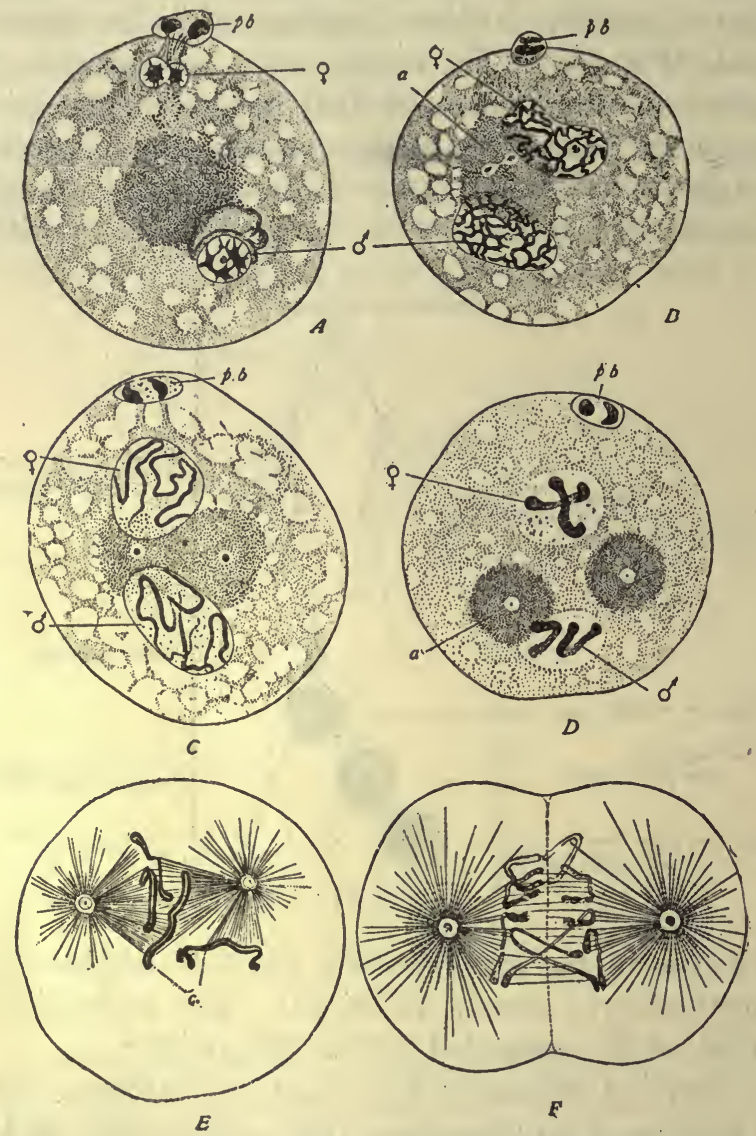

Fic. 559. Fertilisation and first division of ovum of Ascaris megalocephala. (Slightly modified from BoverI and WILSON.)

A, second polar globule just formed; the head of the spermatozoon is becoming changed into a reticular nucleus ( $\delta)$, which, however, shows distinctly two chromosomes; just above it, its archoplasm is shown: the egg-nucleus ( $q$ ) also shows two chromosomes. B, both pro-nuclei are now reticular and enlarged; a double centrosome $(a)$ is visible in the archoplasm which lies between them. c, the chromatin in each nucleus is now converted into two filamentous chromosomes; the centrosomes are separating from one another. $\mathrm{D}$, the chromosomes are more distinct and shortened; the nuclear membranes have disappeared; the attraction-spheres are distinct. E, mingling and splitting of the four chromosomes $(c)$; the achromatic spindle is fully formed. F, separation (towards the poles of the spindle) of the halves of the split chromosomes, and commencing division of the cytoplasm. Each of the daughter cells now has four chromosomes; two of these have been derived from the ovum nucleus, two from the spermatozoon nucleus.

In many cases there is an apparent fusion of the substance of the two nuclei. In others the chromatin filaments of male and female simply lie side by side, forming a complete nucleus with the somatic number of chromosomes. Fertilisation is rapidly followed by cell division. Each of the 
chromosomes splits longitudinally, half going to each of the daughter cells, and this process is repeated throughout the succeeding divisions which result in the formation of the new individual. Thus every cell of the body contains a nucleus of which exactly one half is paternal and the other maternal in origin. In ascaris it is often possible, in the first few divisions of the fertilised ovum, to distinguish in the daughter nuclei the chromatin filaments derived from the male from those derived from the female.

The strong impetus to cell division given by the process of fertilisation has naturally aroused much curiosity as to its intimate character. It might be thought that for cell division to take place a normal number of chromosomes is essential. As against this explanation may be adduced the fact that in many animals parthenogenesis occurs. The female pro-nucleus may, under certain conditions of environment or nutrition, start dividing and give rise to an embryo, each cell of which contains only half the normal number of chromosomes. In other cases of parthenogenesis only one polar body is extruded, or the second polar body joins again with the female pro-nucleus. In either case the ovum contains a nucleus, with a normal number of chromosomes, which divides and produces an individual resembling that resulting from the union of ovum and spermatozoon. It has been suggested that the impetus to division is given by the entry of the spermatozoon itself. In the series of divisions which precede the formation of the female pro-nucleus the centrosome of the ovum generally disappears, whereas, in the formation of the spermatozoon, the centrosome persists and forms the middle part of the spermatozoon. In many cases the centrosomes divide in the spermatozoon itself, so that this contains two centrosomes when it enters the egg. These two centrosomes then become the centres of attraction spheres. They diverge, and between them is formed an achromatic spindle, along the equator of which the chromatin filaments of male and female pro-nuclei arrange themselves. It is doubtful, however, how far the centrosome can be regarded as a permanent cell structure. In echinoderm eggs various modes of treatment will lead to the appearance of attraction spheres in the cytoplasm, and even to division of the non-fertilised egg. Loeb has suggested that the action of the spermatozoon is essentially chemical in character. By alteration of the medium in which the eggs are contained, Loeb has succeeded in imitating exactly the changes which normally need the entrance of a spermatozoon for their occurrence. On immersing the eggs in a weak solution of formic or lactic acid, a membrane is formed. The eggs are then taken from the acid, placed in concentrated sea-water for a short time, and then removed to ordinary sea-water. Division rapidly occurs with the production of a normal larva. He suggests that the spermatozoon brings with it ferments, or other chemical substances, which excite the egg-nucleus and cytoplasm in the same way as the chemical measures adopted for this artificial induction of segmentation. 


\section{SECTION II}

\section{DEVELOPMENT AND HEREDITY}

THERE is perhaps no phenomenon which is so impressive as the development from a minute speck of protoplasm, the fertilised egg, of an individual partaking of the minutest characteristics of both its parents. An egg-cell has much the same appearance whether it belong to an echinoderm, a fish, or a man. In the process of development, by a simple repetition of a series of cell divisions, this undifferentiated protoplasm is formed into the complex organs with the potentialities and habits which distinguish the type from which the protoplasm has been derived. We cannot wonder that the intimate nature of this process has been the subject of speculation from the very birth of science. Running through these speculations are two main ideas, which have been labelled the theories of 'evolution' and of 'epigenesis.' By the 'evolutionists ' the egg was believed to contain an embryo fully formed in miniature, as the bud contains the flower or the chrysalis the butterfly. Development was therefore only the unfolding of something already existing. If, however, this theory be pushed to its utmost and if the egg contain a complete embryo, this must itself contain eggs for the next generation, and so on ad infinitum, a conclusion which is of course absurd. According to the theory of epigenesis, the structure of the egg is wholly different from that of the adult, its development consisting in the continual formation one after the other of new parts previously non-existent as such. There is no doubt that this view is fundamentally correct. The difficulty with which we have to contend is the understanding of the orderly sequence and correlation of the cell divisions and differentiations which result in an adult individual of the same type as the parents. The fact that, under approximately identical conditions, one mammalian ovum will give rise to a mouse and the other to a man indicates that there must be some difference in structure, organisation, or composition of the primitive egg-cell in each case, and the theory of 'evolution' has reappeared in later days in a somewhat modified form, according to which the differentiation of the ovum is causally connected with a preformed differentiation in the nuclear structures, e.g. chromosomes, of the ovum itself. We have already seen that the germ-cells in some types are separated off from the rest of the embryo at a fairly early stage. If in the two-celled stage of the frog's egg one cell be destroyed by means of a hot wire. the other cell develops to form half an embryo, thus suggesting that each cell of the two-celled embryo could give 
rise only to the corresponding half of the body. This limitation of development, however, only occurs if the intact cell be left in connection with the cell that has been injured. If, in echinoderm larvæ, the cells be entirely separated, even as late as the eight-celled stage of division, each cell will give rise to a whole embryo, differing from a normal embryo only in respect of size. This difference suggests that the number of divisions that each cell can undergo is predetermined in the egg-cell itself, but shows also that the cells into which the egg divides are, at first at any rate, equipotential. We must assume therefore that the reason why one cell under ordinary circumstances only forms one half of the embryo is that its development is regulated and determined by the presence of the other cell in connection with it forming the other half of the embryo. That is to say, the development of the egg involves the reaction to environment of a protoplasm of certain properties and powers of reaction. The final product of development depends (1) on the nature of the protoplasm (including the nucleus) of which the egg is composed, and (2) on the environmental conditions to which the egg is subjected during the rapid growth and multiplication attending its development. We could therefore speak of a morphology of inheritance, but the morphology would be ultra-microscopic and have relation to the molecular structure of the protoplasm of which the egg was composed.

In the transmission of the potentialities of development from parent to fertilised egg we must regard the nucleus as the essential structure. In ordinary development the spermatozoon furnishes only a nucleus and centrosome, the ovum supplying the whole of the cytoplasm. There seems, however, no grounds for assigning any directive power to the latter structure. In echinoderm ova it is possible to get rid of the nucleus and then by the introduction of spermatozoa to have an individual entirely paternal in origin, which, on development, produces a larva of the paternal character. In division of the egg the only part of the cell which divides so that each daughter cell shall include an equal part of both parental germs is the nucleus. The constant number of the chromosomes in each species, and their accurate division on mitosis, suggest that the hereditary transmission of the potentialities of the cell is bound up with the chromosomes. It has been suggested that every character is located in a chromosome or part of a chromosome. If this be the case one might regard the differentiation into various tissues, which occurs in the process of development, as occasioned by an actual loss or degeneration of the constituent parts of one or more chromosomes. There is no doubt that many tissues do become thus differentiated at a fairly early period in development, having undergone in the process an absolute modification of their potentialities, which must be at any rate shared by the chromosomes of their constituent cells. The extent to which this limitation of powers of development occurs varies widely in different animals. In the higher animals, such as man, epithelium will reproduce epithelium, and liver-cells will reproduce liver-cells, while nerve-cells are absolutely incapable of multiplication On the other hand, in crustacea a whole limb may be torn off and be regenerated from the 
tissues of the stump. Destruction of the optic lens in the salamander is followed by its regeneration from the anterior part of the optic cup, a tissue which had no part in its primary formation. Worms will form a new head after decapitation. In these animals therefore the cells in many parts of the body possess the power of directed growth, if need arise, in case of injury, and are able to form tissues of many different kinds.

I have mentioned the small size of the larva formed from isolated cells of the segmenting egg as a proof that the number of cell.divisions of the somatic part of the developing animal is predetermined and limited. This conclusion must not be taken too absolutely. Many of the tissues, even of the highest animals, possess the power of almost unlimited regeneration by cell-multiplication as a response to injury. Under normal conditions the growth of such tissues is limited, not by absence of power to divide, but as a result of a mutual interaction between them and the surrounding cells. We might almost speak of a struggle for existence between the various tissues of the body, which in the healthy organism results in an equilibrium, or balance of multiplicative powers. If this balance is upset by any means, such as stimulation of certain cells by the presence of intra-cellular parasites, or their destruction by irritants or other abnormal conditions (e.g. exposure to X-rays), one tissue may enter into active growth at the expense of the surrounding tissues, and the result is a morbid growth such as cancer. It is possible that in the latter case a new factor comes into play. All tissues of the body, as we have seen, begin to die from the time that they are born. They have a certain span of life, a certain limitation to their generations, which results in the phenomenon of senescence, such as occurs in a culture of protozoa. In protozoa this phenomenon is the signal for rejuvenation by conjugation. It is possible that in cancer something of the same nature occurs. It is at any rate significant that in a rapidly growing cancer many of the dividing cells present the phenomenon of heterotype mitosis, a phenomenon which is otherwise found only in the sexual cells preparing for conjugation and for the production of a new individual. Given adequate conditions of nutrition, there seems to be no limit to the growth of cancer-cells. In mice a cancer may be transferred from one individual to another by inoculation, and this process may apparently go on indefinitely, so that finally a mass of cancer-cells may have been produced equal in volume to many thousands of mice, and persisting long after the mouse from which it was first taken would have died under natural conditions.

In sexual reproduction the new individual partakes of characteristics of both its parents. It therefore resembles neither of its parents in all details. The conjugation of the two parent cells from which it is derived has been preceded by a throwing out of half the chromosomes from each parent cell. It is therefore natural to ascribe the variations which occur among the members of one family to a qualitative difference in the chromosomes which have been eliminated in the formation of their respective egg-cells. Can we regard the chromosomes as representing separate qualities of the individual or must we assume that all qualities are represented to a greater or less 
extent in every chromosome? In the case of many qualities, especially those which distinguish the species as apart from the individual variation or family characteristic, we must probably accept the latter idea as correct. In this case the child can be regarded as representing an arithmetical mean of both its parents. In certain respects, however, a quality seems to be transmitted from parent to offpsring either completely or not at all. This is specially applicable to those characteristics which have been rapidly produced by artificial selection, characters which, if artificial selection be abandoned, rapidly disappear, with reversion to the type from which the special strain was ultimately produced. The way in which these characteristics are transmitted was first studied by Mendel and has been formulated as Mendel's law. Mendel's first experiments were carried out on peas. On crossing a tall plant with a dwarf plant seeds were obtained from which all the plants were tall. On recrossing the plants of this generation among one another a third generation was obtained in which 25 per cent. of the plants were dwarfs and 75 per cent. were tall. Crossing the dwarf plants among themselves led to the production of dwarf plants through successive generations. Of the tall plants 25 per cent. and all their descendants continued to produce tall plants when self-fertilised, whereas of the remaining 50 per cent. of the tall plants 25 per cent. produced dwarfs and the remaining 75 per cent. produced tall plants. On continuing the process of breeding, the dwarf plants when self-fertilised always produced dwarfs, whereas of the tall plants 25 per cent. produced tall plants, which bred true, while the remaining 50 per cent. produced the same percentage of tall and dwarf as in the preceding generations. Mendel explained these results by the assumption that a character could be dominant or recessive. If both characters were present together in one plant it would partake of the dominant type ; the fact that this plant possessed the recessive character would only be shown by the results of breeding. In the case of the peas the tall character was dominant over the dwarf. Thus when the tall and dwarf pea were crossed the first generation of plants would exhibit the dominant character and be tall. In the second generation, however, 25 per cent. of the individuals would be pure dominants $(\mathrm{D}+\mathrm{D}), 25$ per cent. would be pure recessive $(R+R)$, while 50 per cent. would be mixed $(D+R)$. The pure dominants bred together would always give rise to nothing but pure dominants, the recessive to recessive, while the mixed type would always, as before, give rise to 25 per cent. pure dominants, 50 per cent. mixed, and 25 per cent. pure recessives. These results may perhaps be made clearer by the following Table :

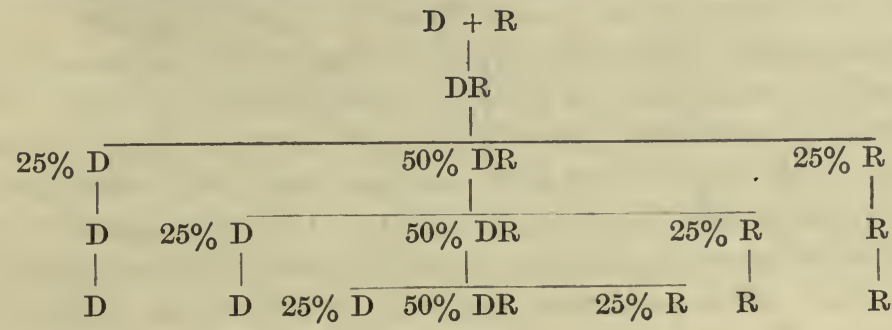


It has been suggested that a very large number, if not all, of the characters of an individual might be brought under this law. This might be done by indefinitely subdividing the characters, but the question would then become beyond the limits of analysis or experimental investigation. There is no doubt that many qualities are subject to Mendel's law, and that their study will be of considerable assistance in guiding the efforts of our breeders and horticulturists in the formation of new varieties desirable for their value to man. In respect of many qualities the Mendelian law seems to fail. Thus in man the progeny of a cross between a white and black race are more or less intermediate between the two and vary according to the amount of black and white blood introduced in succeeding generations. Definite black and white individuals are not produced, but merely individuals of various degrees of brownness. 


\section{SECTION III}

\section{REPRODUCTION IN MAN}

\section{THE DEVELOPMENT OF THE REPRODUCTIVE ORGANS}

THE most marked example of chemical correlation is found in the influence exerted by the genital glands upon the other parts of the reproductive apparatus and upon the body generally. Thus castration, i.e. removal of the testes or ovaries, if carried out before the time of puberty, prevents the development of the secondary sexual characters, which normally occurs at this epoch in both sexes. Puberty denotes the period at which ripe spermatozoa and ova are produced in the testis and ovary respectively. In the human species this period is marked or preceded in the male by increased growth of the skeleton, by growth of the larynx, leading to a lowering in pitch of the voice, by the growth of hair on the face and pubes, and by the development of sexual desire. In the female we find at puberty enlargement of the breasts, attended by some growth of the mammary glands and by a moulding of the whole form, making it more fit for the bearing of children. The chief sign of puberty in the female consists in the periodic changes in the uterus, which give rise to menstruation, i.e. a flow of blood and mucus from the genital organs, lasting three to five days and repeated every four weeks. Menstruation persists so long as the ovary is functional, and is producing ripe ova. The activity of the ovary comes to an end between the forty-fifth and fiftieth year (" the climacteric ' or " change of life '). With the cessation of its activity menstruation also stops, and the uterus undergoes a process of atrophy. These secondary sexual characters must be ascribed to the influence of chemical substances produced in the ovary and testis respectively. Castration after puberty, though not causing any change in the skeleton, which has already assumed its permanent form, brings about retrogressive changes in the other genital organs, analogous to those occurring in the female at the climacteric. In animals the phenomena of 'coming on heat' or 'rut' seem to be analogous with menstruation in the human female, and like this depend on the normal activity of the ovary. They are permanently abolished by extirpation of the ovaries, but may be reinduced by implantation in the peritoneum of an ovary from another animal of the same species. This fact shows that the changes in the uterus responsible for rut, as well as for menstruation, are independent of any nervous connections between the ovaries and the rest of the body, and must therefore be brought about by the circulation in the blood of specific 
chemical substances produced in the ovaries. According to some authors, the essential factors for the production of these genital hormones are the 'interstitial cells' found both in the testes and ovaries of various animals. These interstitial cells are not, however, universally present. It has been shown that, by means of the Röntgen rays, it is possible to destroy the germ-cells in either testes or ovaries, so rendering the animal sterile. The interstitial cells, when present, are not destroyed by these rays, yet the effects on the accessory genital organs are stated to be as marked as after complete extirpation of either ovaries or testes.

The chemical correlations between the ovaries and the other organs concerned in reproduction are perhaps best marked in the changes which attend pregnancy. In this case the fertilisation of the ovum by a spermatozoon is followed by a great development, first of the mucous membrane and later on of the muscular wall of the uterus. The mucous membrane thickens, apparently in order to form a bed for the developing fertilised ovum. With this growth of the uterus there is a corresponding growth of the other parts of the genital tract, e.g. the vagina. At the same time rapid changes take place in the mammary glands. These changes may be studied experimentally in the rabbit, in which animal gestation lasts only about twenty-nine days. In a virgin rabbit of a year old it is difficult with the naked eye to see any trace of the mammary gland in the tissue lying under the nipples. Each gland is limited to an area not more than $1 \mathrm{~cm}$. broad, and consists entirely of ducts lined with a single layer of flattened epithelial cells. With the occurrence of conception a marked change takes place. Four or five days after fertilisation, when it is still impossible with the naked eye to discover any embryos in the swollen uterine horns, on reflecting the skin from the abdomen each mammary gland appears as a circular pink area, about $3 \mathrm{c.m}$. in diameter. On section the gland consists of ducts which are in an active state of proliferation, their epithelial lining being two or three cells thick and presenting numerous mitotic figures. By the ninth day the whole abdomen is covered with a thin layer of glandular tissue ; by the twenty-fifth day this tissue is $\frac{1}{2} \mathrm{~cm}$. in thickness and consists for the greater part of secreting alveoli, lined with cells containing numerous fat-globules. At full term the alveoli contain ready-formed milk.

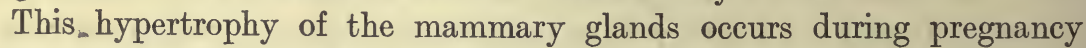
after complete division of all possible nervous paths between the glands of the ovaries or uterus. In the guinea-pig a mammary gland has been actually transplanted to another part of the body, thus severing all its normal nervous connections, and yet it enlarged as usual during a subsequent pregnancy. Ancel and Bouin have brought forward evidence that the corpus luteumthe tissue produced in the ovary as a result of the discharge of an ovumis intimately concerned with the growth of the mammary glands, and may indeed cause a certain degree of hypertrophy of these glands in the entire absence of any product of conception within the uterus.* The limited

* According to Ancel and Bouin, in the rabbit discharge of an ovum and formation of a corpus luteum only occur as a result of copulation. The same effect may be pro- 
growth of the glands, which occurs at puberty, can certainly not be ascribed to the presence of a fœtus in the uterus, and must be connected with the growth of ripe ova, or, as suggested by the two French authors, with the growth of the tissue of the corpus luteum, resulting from the discharge of ova.

There seem also to be obscure relationships between the activity of the sexual organs and that of certain so-called ductless glands. Thus castration at an early age leads to persistence of the thymus gland, whereas normally this gland atrophies just before the sexual organs commence their functional activity. The existence of a connection between the thyroid and the ovaries has been a popular belief for 2000 years. In many individuals the thyroid is perceptibly enlarged at each menstrual period. On the other hand, extirpation of the thyroid before puberty brings about, among the other signs of cretinism, failure of development of the ovaries, so that puberty is delayed partially or completely.

We must thus regard the germ-cells not only as representing the cells from which the individuals of the new generation may be developed, but also as concerned in the formation of chemical substances which, discharged into their hosts, affect many or all of the functions of the latter, with the object of finally subordinating the activities of the individual to the preservation and perpetuation of the species.

\section{THE MALE REPRODUCTIVE ORGANS}

In all the higher animals we may divide the reproductive organs into the essential organs, which form the germ-cells, the spermatozoa and ova respectively, and the accessory organs, which have as their office the facilitation of the access of the spermatozoa to the ova (fertilisation), and in the female the nutrition of the product of fertilisation during the early period of its development.

The essential sexual organ of the male is represented by the testis. This is made up of a collection of convoluted tubules, the seminal tubules, which are contained in a number of compartments separated by fibrous septa. The tubules present few or no branches, each one being about $500 \mathrm{~mm}$. long. The testis is formed in the first instance in the peritoneal cavity from the germinal epithelium, but early in life leaves the abdominal cavity by the abdominal ring to lie in a pouch of skin-the scrotum. Several tubules unite to form a straight tubule, which leads by a series of

duced by artificial rupture of a ripe follicle. Whenever this occurs there is a development of the mammary glands. If no impregnation has taken place (e.g. if the buck has been sterilised by ligature of the vas deferens), the glands develop for fourteen days and then begin to atrophy. This period corresponds to the period of active growth of the corpus luteum. The continued growth during the latter half of pregnancy these authors ascribe to the production of another hormone by a special glandular tissue ('myometrial gland') which makes its appearance about the fourteenth day in the wall of the uterus, at the site of implantation of the placenta, and lasts until the end of pregnancy. 
communicating spaces, the rete testis, into the vasa efferentia (Fig. 560). These unite to form the duct of the epididymis, which forms a mass lying at the back of the testis. The epididymis is composed of the convolutions of this single duct, which is about 20 feet long. From the lower end of the epididymis the vas deferens, a tube with thick muscular walls, leads by the abdominal ring to the base of the bladder, where it opens into the beginning of the urethra in its prostatic part. Just before it joins the urethra each vas

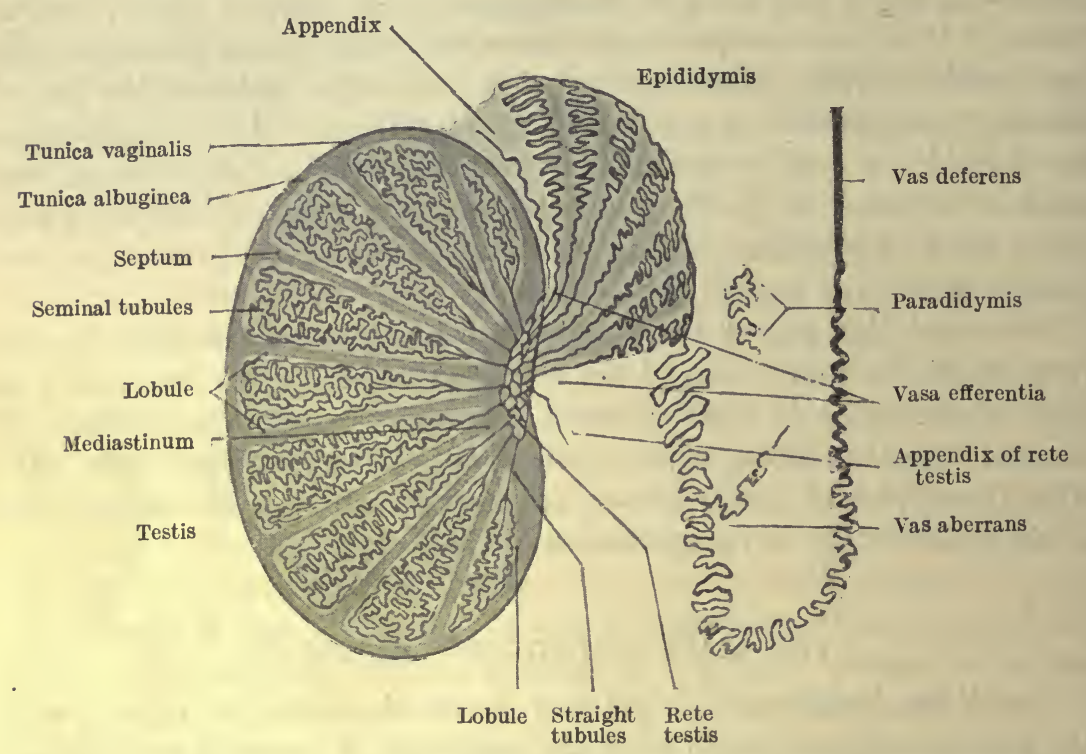

Frg. 560. Diagrammatic representation of the course of the seminal tubules in the testis and epididymis. (After NAGEL.)

deferens presents a diverticulum, the seminal vesicle, which lies along, and is attached to, the base of the bladder. The prostate itself, which surrounds the first part of the urethra, is composed of a matrix of unstriated muscular fibres, enclosing numerous branched racemo-tubular glands. From the point of entry of the vasa deferentia to its orifice, the urethra represents a common passage for the urine and for the sexual products-the semen. It passes therefore through tissues, forming the penis, which are especially adapted for the purpose of intromission, i.e. the introduction of the semen. containing the spermatozoal into the female. In the urethra we distinguish the prostatic, the membranous, and the penile portions. Into the beginning of the penile portion, the bulb of the urethra, open the ducts of the two glands of Cowper. In the penis itself the urethra is surrounded with erectile tissue, forming the corpus spongiosum, and lies between the two corpora cavernosa, which consist of the same kind of tissue. The erectile tissue is a spongy meshwork of elastic and unstriated muscle fibres, enclosing spaces in free communication with the efferent veins of the organ. The arterioles also open into these spaces, but under normal circumstances both the arterioles and the muscle-tissues of the framework are contracted, so 
that the blood trickles only slowly from the arterioles into the spaces, whence it escapes readily by means of the veins. If the muscle fibres be relaxed, so that blood can escape readily into and distend the spaces, the tissue swells and becomes harder, causing 'erection' of the organ.

In the immature testis, i.e. from birth up to puberty, the seminal tubules are filled with cells with large nuclei. Some of these are the spermatogonia, the mother cells of the future spermatozoa, while the others form the cells of Sertoli, whose function it is to act as nurse cells to the developing spermatozoa. The actual formation of spermatozoa begins at puberty, when the spermatogonia divide many times to form the spermatocytes, which in their turn undergo heterotype mitosis to form the spermatids, as already described. By a modification of the latter the fully formed spermatozoa are formed. These, when mature, pass by the tubules of the testis and of the epididymis into the vas deferens, whence they make their way into the seminal vesicles. Their movement is probably facilitated by the cells lining the tubule of the epididymis as well as by the secretion of the lining membrane of the seminal vesicles. It has been noted that the spermatozoa are practically motionless while in the seminiferous tubules of the testis, but become actively motile in the vas deferens, or when mixed with prostatic secretion. It is difficult to understand how the spermatozoa are conveyed through the resistance which must be offered by the huge length of the tubule of the epididymis, unless their onward motion is facilitated by the cilia-like structures attached to some of the cells lining this tubule. The formation of the spermatozoa is continuous, though the rate at which this occurs is variable and regulated by the sexual activity of the individual. In the fully formed semen the spermatozoa formed by the testis are mixed not only with the fluid secreted by the lining membrane of the epididymis and of the seminal vesicle but also with the mucous secretions of the prostatic glands and of Cowper's glands. Nevertheless it contains spermatozoa in enormous numbers, the semen emitted at a single act of coitus containing as many as $226,000,000$ spermatozoa. Though the vast majority of these are probably capable of fertilising an ovum, this act is carried out by only one-a fact characteristic of the prodigality of nature when dealing with the perpetuation of the type.

\section{THE FEMALE REPRODUCTIVE ORGANS}

The essential organ of reproduction in the female is the ovary, the seat of production of the ova. The accessory organs include the oviducts or Fallopian tubes, the uterus, in which the fertilised ovum is retained during the first nine months of its development, and the vagina, which is especially adapted for the reception of the male organ in the act of impregnation.

Among the accessory organs we may also reckon the mammary glands, which undergo a special development during pregnancy, and serve for the nourishment of the young individual during the first period of extrauterine life. 
ovUlation. At birth the ovary consists of a stroma of spindle-shaped cells, and is covered by a layer of cubical epithelium (the germ-epithelium) continuous with the endothelium lining the general peritoneal cavity. Embedded in the stroma, but especially numerous just underneath the epithelium, are a vast number of ' primordial follicles.' These are formed during fœtal life by down-growths of the germinal epithelium. Of the cells prolonged in this way from the germinal epithelium, some undergo enlargement to form the primordial ova, while the others are arranged as a single layer of flattened nucleated cells, the 'follicular epithelium,' as a sort of capsule to the ovum. Of the primordial follicles, about 70,000 are to be found in the ovary of the new-born child. During the first twelve to fourteen years of life they remain in a quiescent condition. With the onset of puberty one or more of the primordial follicles begin to develop. Indeed, this development may be regarded as the causative factor in the various phenomena which are characteristic of puberty in the female (v. p. 1217). The first stage in the growth of the follicle is a proliferation of the follicular epithelium, the cells of which become cubical and are arranged in several layers round the ovum. At one point in the mass of cells surrounding the ovum a cavity appears filled with fluid, the liquor folliculi. The epithelium thus becomes separated into two parts, i.e. the membrana granulosa, several layers thick, lining the whole follicle, and the discus proligerus, a mass of cells attached to one side of the follicle, in which is embedded the ovum (Fig. 561). Round the growing follicle the stroma assumes a concentric arrangement and forms a capsule, of which the internal layer consists chiefly of spindle-shaped cells richly supplied with blood-vessels, while the outer layer-the theca externa -is made up of a tough fibrous tissue. With the growth of the follicle the ovum also becomes larger and surrounds itself with a distinct membrane, known as the zona pellucida. This membrane presents a fine radial striation, which is supposed to indicate the existence of canals through which the ovum can obtain sustenance from the surrounding cells of the follicular epithelium. The nucleus also becomes larger, and forms the germinal vesicle containing one or two well-marked nucleoli-the germinal spot. The mature Graafian follicle projects from the surface of the ovary as a transparent vesicle about the size of a pea. (Its diameter is about $15 \mathrm{~mm}$.) In the process of growth the ovum has increased from a diameter of $25 \mu$ to $200 \mu$. Before the ovum can undergo fertilisation the double division of the nucleus, or germinal vesicle, has to take place, which leads to the formation and extrusion of the two polar bodies. This process probably occurs just before or just after the discharge of the ovum from the ovary.

With increasing size of the Graafian follicle the membrane covering it becomes progressively thinner. At certain periods, or under certain conditions, the membrane ruptures, and the ovum is discharged in the liquor folliculi, still surrounded by an adherent mass of the cells of the discus proligerus. In some animals this process of ovulation occurs at definite periods of the year. In others, such as the rabbit, the occurrence of ovulation depends upon coitus taking place during the period of sexual activity. 
We shall have later to discuss the relation of ovulation in the human female to the periodic changes occurring in the other parts of the reproductive apparatus.

After the discharge of the ovum the remaining portions of the follicle undergo a characteristic series of changes, which result in the production

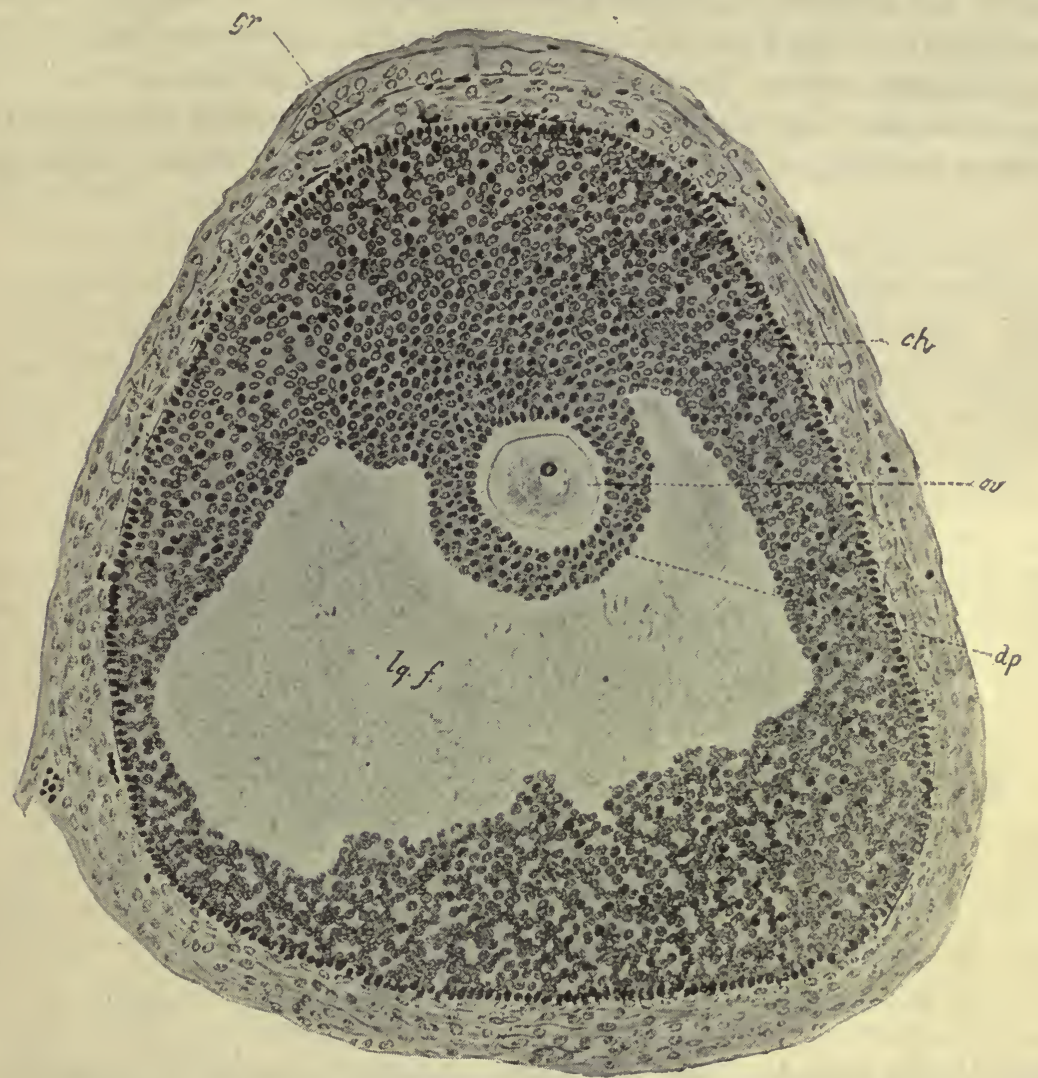

FiG. 561. Graafian follicle of mammalian ovary. (PRENANT and Bouin.) $o v$, ovum; $d p$, discus proligerus; $l q . f$, liquor folliculi ; $c h$, theca ; gr, membrana granulosa.

of the corpus luteum. Immediately after the rupture the follicle becomes filled with blood, apparently resulting from the sudden release of the pressure on the capillaries in the walls of the follicle. The cells of the membrana granulosa rapidly increase in size, a few of them undergoing mitotic division, so that a dense mass of cells is formed, nearly filling the original follicle. At the same time the cells of the internal theca proliferate, with the formation of connective tissue, which grows in among the cells filling the Graafian follicle. These cells finally attain a size four or five times that of the cells of the membrana granulosa in the mature follicle. Blood-vessels grow from the external theca towards the centre of the follicle. The cells within the follicle then undergo fatty degeneration and present a yellow colour due to 
a fatty pigment known as lutein. The corpus luteum, as the body so formed is called, attains its greatest size about a week after ovulation, and then gradually undergoes regressive changes. If, however, the ovum, which has been discharged, undergoes fertilisation, and pregnancy results, the corpus luteum continues to grow for a considerable time and attains its largest size at about the third month of pregnancy. It does not entirely disappear until after the end of pregnancy. The big corpus luteum found in pregnancy is often spoken of as the 'true' corpus luteum, and is distinguished from the corpus luteum spurium of menstruation or of ovulation without fertilisation. There is, however, no essential difference other than

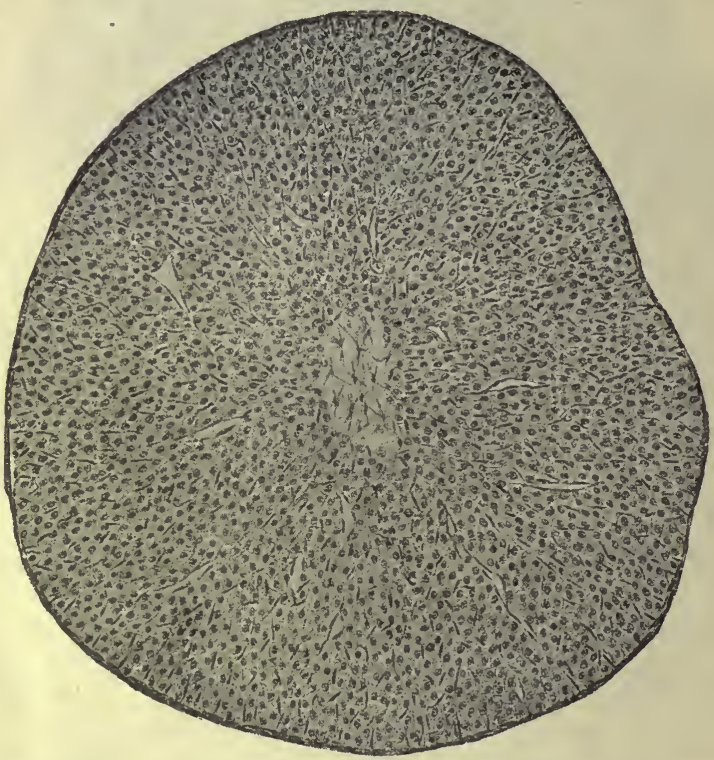

Fig. 562. Fully developed corpus luteum of the mouse. (Ковотта.

that of size between these two kinds of corpus luteum. It must not be imagined that all the 70,000 primordial follicles found in the ovary of a new-born child undergo this series of changes; it is probable that in the human female ovulation occurs, as a rule, once every four weeks during the thirty-five years of sexual life. A vast number of the Graafian follicles, after developing to a certain extent, undergo regressive changes, both during childhood and during adult life. The cellular elements degenerate, leucocytes wander into the follicle and attack the degenerating ovum, so that finally the follicle is replaced by connective tissue, without the formation of any corpus luteum.

MENSTRUATION. Puberty in the girl is marked by the onset of menstruation. Under this term is understood a flow of blood and mucus from the uterus, which recurs every four weeks and lasts each time from four to five days. Before the first menstrual period, other signs of puberty, i.e. of approaching sexual maturity, are usually observed. These include 
rapid growth, with changes in the skeleton, leading to the typically feminine type of pelvis, a development of the mammary glands, and the growth of hair on the pubes. At the same time there is increased development of the mental characteristics which are typical of the sex. The amount of blood lost at each menstrual period varies between 100 and 300 grm. During the 'period' there are often disturbances of other functions of the body, which are so common that to be 'unwell' is the recognised polite description of the menstrual period. Thus it is often attended with pains in the abdomen, a feeling of weight and fulness, disturbance of digestion, headache, and neuralgias of various distribution. At the same time there is a general disinclination for exertion.

Menstruation is due to periodic changes in the uterine mucous membrane. During the few days previous to the period the mucous membrane undergoes a rapid hypertrophy, increasing in thickness from $2 \mathrm{~mm}$. to $6 \mathrm{~mm}$. At the same time there is increased vascularity of the membrane in consequence of dilatation of its blood-vessels. At the commencement of the menstrual period there is an escape of the red blood-corpuscles, chiefly by diapedesis, but partly by actual rupture of the blood-capillaries into the spaces between the uterine glands. At this period sections of the uterine mucous membrane show numerous collections of red blood-corpuscles, lying immediately under the superficial epithelium. In some cases this stage is followed by an almost complete desquamation of the superficial epithelium. Generally the desquamation is only partial, but in either case the blood escapes into the cavity of the uterus, where it becomes mixed with the increased secretion from the uterine glands and is discharged into and from the vagina as the menstrual fluid. With the occurrence of the menstrual flow the mucous membrane begins to diminish in thickness. The vascularity decreases, and much of the blood in the deeper parts of the mucosa becomes reabsorbed. The desquamated epithelium is replaced by proliferation of the cells which remain intact, so that finally the mucosa is completely regenerated and brought back to its original condition. This period of regeneration lasts about fourteen days. During the next few days the condition of the membrane is stationary, but this period of rest lasts but a short time, since signs of the pre-menstrual swelling can be detected as early as three days before the onset of the next menstrual period.

\section{THE RELATION OF OVULATION TO MENSTRUATION}

There is no doubt that menstruation depends on the functional activity of the ovary. Its onset coincides with the first production of ripe ova in the ovary, and it ceases with the cessation of ovulation at the climacteric or menopause. In cases where the ovaries have been removed before puberty menstruation never occurs. Removal of both ovaries during adult life generally brings about a premature menopause. It seems probable that the ripening of the ova in the human ovary occurs at periods corresponding to those of menstruation. But there has been much division of opinion as to the exact relation between the two processes. Fairly definite clinical and 
post-mortem evidence has been brought forward for the theory that ovulation precedes the menstrual flow. On this theory the degeneration of the uterine mucous membrane, which occurs at each period, represents, so to speak, the undoing of a preparation for the reception of a fertilised ovum. The ovum has been discharged, the mucous membrane has been prepared for its reception, but fertilisation not having taken place, ovum and mucous membrane are cast out together in the menstrual flow. Unfortunately almost equally definite clinical evidence has been adduced for the view that ovulation occurs during or after the menstrual period. Light is thrown upon the question by the study of the phenomena of ' rut ' or ' heat' in the lower animals. In most mammals impregnation and conception can only occur at certain definite periods of the year. At these seasons the female presents a swelling of the mucous membrane of the external genitals, and often a flow of blood or mucus. As a rule it is only when in this condition that it will permit the approach of the male. Thus the bitch 'comes on heat' as a rule twice in the year ; the cat three or four times ; most carnivora only once a year. At these periods the uterus shows well-marked histological changes, which may be divided into the following periods :

(1) The period of rest. During this time, which extends over the greater part of the year, the mucous membrane is thin and pale. The period of heat being known as the cestrus, this first period is denoted by Heape the anostrum.

(2) The period of growth or congestion. This corresponds to the premenstrual thickening of the mucous membrane of the human female.

(3) Period of destruction, associated with hæmorrhages into the mucous membrane, desquamation of the superficial epithelial cells, and occasionally discharge of blood and mucus from the vagina. These two periods are grouped together as the pro-cestrum.

(4) Period of recuperation corresponding to the post-menstrual regeneration of the mucous membrane. It is during the first part of this period or at the very end of the last period that ovulation occurs in those animals where ovulation is independent of coitus. It is at this time, too, that the animal exhibits sexual desire and permits the approaches of the male. If fertilisation occurs, the mucous membrane undergoes rapid hypertrophy, much more marked than that occurring during the pro- œstrum. In the absence of impregnation the mucous membrane returns to the condition of rest, the stage of return being known as the metœstrum.

These results have been found by Heape and Marshall to apply to a large number of different mammals. We are therefore justified in concluding that menstruation is the physiological homologue of the pro-œstrum in the lower mammals, and that ovulation occurs, or at any rate that the ova attains maturity, after or at the very end of the menstrual flow. If we consider that the ovum may take some days to pass down the Fallopian tube to the uterus, and that the spermatozoa may retain their vitality for ten days or more in the Fallopian tubes or uterus, it is evident that in man impregnation may take place at any time between two menstrual periods. 
Sexual desire is thus not limited to certain seasons, as is the case with most of the lower animals.

\section{FERTILISATION}

The act of impregnation consists in the introduction of spermatozoa into the female genital tract, where they may come in contact with and fertilise the ovum, which is discharged from the ovary by bursting of a Graafian follicle. This is effected in the act of coitus or sexual congress by the insertion of the penis into the vagina of the female. Before this can occur erection of the male organ must take place. The mechanism of erection is twofold. The most important factor, as was shown by Eckhard and Lovén, is an active dilatation of the vessels of the penis, especially of the medium-sized and smaller arteries. If the penis be cut across while in the flaccid condition, venous blood merely trickles away from the cut surface, whereas, if erection be excited, the flow of blood from the cut surface is increased eight to ten times, and the blood becomes bright arterial in colour. It is thus possible to excite erection in an animal, in whom the second factor has been abolished by paralysing the muscles by means of curare. This second factor is the contraction of the ischio-cavernosus or erector penis muscle, certain fibres of which pass over the dorsal vein of the penis and compress this vessel when they contract. Since ligature of the veins coming from the penis does not produce erection, the contraction of this muscle must be regarded as simply aiding the effects of the arterial dilatation.

Before or at the beginning of coitus analogous changes occur in the female organs, leading to erection of the clitoris and of the erectile structures of the vulva. The glands of the vulva, especially the glands of Bartholini, secrete a mucous fluid, thus lubricating the passage into the vagina. The friction between the glans penis and the wall of the vagina causes a reflex aischarge of motor impulses in both male and female. In the former the muscular walls of the vasa deferentia and seminal vesicles enter into rhythmic contractions, thus forcing the spermatozoa they contain into the urethra. The spermatozoa, mixed with the secretions of the epididymis, the seminal vesicles, the prostatic glands, and the glands of Cowper, form the semen, which is pressed along the urethra by rhythmical contractions, from behind forwards, of the bulbo- and ischio-cavernosi muscles. It has been stated that movements take place coincidently in the uterus, so that its axis more nearly corresponds to that of the vagina. The movement of the semen along the uterus and Fallopian tubes is ascribed by certain observers to an antiperistaltic contraction of these organs. A more important factor is probably the movement of the spermatozoa themselves. As we have already seen, these are introduced into the female passage in countless numbers. They will be chemiotactically attracted by the alkaline mucus, secreted by and filling the cervix of the uterus. When they have entered this organ they will meet the downward stream of mucus impelled by the action of the cilia lining the uterus and Fallopian tubes. It seems 
probable that their reaction to this current is to swim * against it (positive rheotaxis), so that they reach the upper part of the Fallopian tubes or the surface of the ovary itself. Fertilisation of the ovum occurs in most cases in the Fallopian tube, and the fertilised ovum is then carried slowly down the tube into the uterus.

NERVOUS MECHANISM OF IMPREGNATION. Although, in both sexes, coitus is attended by a high degree of psychical excitement, yet it is essentially a spinal reflex, and can be carried out when all impulses from the higher centres are cut off by section of the cord in the dorsal region. The centre presiding over the act is situated in the lumbar spinal cord. The external generative organs, like the bladder, are supplied from two sets of nerve fibres-from the lumbar nerves through the sympathetic, and from the sacral nerves. The fibres from the lumbar nerves arise in the cat from the second, third, and fourth, or the third, fourth, and fifth lumbar nerveroots, and in the dog from the thirteenth thoracic, and the first to the fourth lumbar roots. They run in the white rami communicantes to the sympathetic chain, whence they may take two paths.

(a) The great majority of the fibres run down the sympathetic chain to the sacral ganglia, whence fibres are given off in the grey rami communicantes to the sacral nerves; their further course is by the pudic nerves, none running in the nervi erigentes.

(b) A few fibres go by the hypogastric nerves to the pelvic plexus.

Excitation of these fibres causes contraction of the arteries of the penis, nad of the unstriated muscles of the tunica dartos of the scrotum. In animals which possess a retractor penis muscle, excitation of the lumbar nerves causes strong contraction of the muscle.

The fibres from the sacral nerves can be divided into two classesvisceral and somatic. The visceral branches run in the pelvic nerves, or nervi erigentes. Stimulation of these fibres produces active dilatation of the arteries of the penis or vulva, and also inhibition of the unstriated muscle of the penis, the retractor muscle of the penis, when present, and of the vulva muscles. The somatic branches supply motor nerves to the ischio- and bulbo-cavernosi, as well as the constrictor urethræ. In the female they supply the analogous muscles, namely, the erector clitoridis (ischio-cavernosus) and the sphincter vaginæ (bulbo-cavernosus). Both these sets of fibres are therefore involved in the erection of the generative organs which accompanies coitus.

The internal organs, i.e. the uterus and vagina in the female, and vasa deferentia, seminal vesicles, and uterus masculinus in the male, differ from the external organs in receiving no efferent nerve fibres from the sacral nerves, as has been pointed out by Langley and Anderson. They are supplied with

* Spermatozoa move in a straight line, at the rate of 2-3 $\mathrm{mm}$. per minute. Thus they might traverse the distance of $16-20 \mathrm{~cm}$. between the os uteri and the trumpetshaped orifice of the Fallopian tubes in three-quarters of an hour. In animals spermatozoa have been found at the peritoneal end of the Fallopian tubes within an hour or two after coitus. 
fibres, which pass out through the anterior roots of the third, fourth, and fifth lumbar nerves (in the rabbit and cat), and run through the sympathetic to the inferior mesenteric ganglia, whence they proceed by the hypogastric nerves. On stimulating these fibres, two effects are produced on the uterus and vagina, namely, a contraction of the small arteries, leading to pallor of the organs, and a strong contraction of the muscular coats.* In the vagina the contraction can usually be seen to start from one end and spread to the other. The whole then remains for a time in a state of powerful tonic contraction, which affects both longitudinal as well as circular muscles. In the male stimulation of these nerves excites contraction of the whole musculature of the vasa deferentia and seminal vesicles, which may be strong enough to cause emission of semen from the penis. These effects on the uterus and seminal vesicles are not abolished by injection of atropine.

The course of the sensory fibres from the generative organs to the lumbo-sacral cord has not yet been fully made out, but it seems probable that it corresponds to the course taken by the efferent fibres.

An accessory genital muscle, the retractor penis, which is found in the dog, cat, horse, donkey, hedgehog (not in the rabbit or man), presents considerable physiological interest. It was first described by Eckhard as the Afterruthenband, and consists of a thin band of longitudinally arranged unstriated muscle (15 to $20 \mathrm{~cm}$. long in a spaniel weighing about 15 kilos), which is inserted at the attachment of the prepuce, and is continued backwards in a sheath of connective-tissue to the bulb, when it divides into two slips which pass on either side of the anus. A few striated fibres are found in the back part of this muscle, derived from the external sphincter of the anus and the bulbocavernosus muscles. This muscle is extremely sensitive to changes of temperature, and is at the same time very tenacious of life. Thus it may be cut out of the body and kept in serum or blood, in a cool place, for two days. At the end of this time it will, on warming, relax, and enter into spontaneous rhythmic contractions. At about $40^{\circ} \mathrm{C}$. the muscle is quite flaccid. On cooling slightly (to $35^{\circ}$ ) it will shorten, and at the same time may enter into slow rhythmic contractions. If cooled to $15^{\circ} \mathrm{C}$. the muscle will contract to about a quarter of its previous length. The same shortening may be produced on exciting the muscle with strong interrupted currents.

The muscle is innervated from two sources, the two nerves having antagonistic actions (cp. p. 247). The motor fibres to the muscle are derived from the lumbar sympathetic (i.e. the upper set of nerve-roots), and run to the muscle in the internal pudic nerve. The pelvic nerves, on the other hand, carry inhibitory impulses to the muscle, thus enabling the concomitant vascular dilatation to take effect in producing erection of the penis.

* Under some circumstances stimulation of the sympathetic nerves may cause relaxation of the uterus. 


\section{SECTION IV}

\section{PREGNANCY AND PARTURITION}

\section{PREGNANCY}

Fertilisation of the ovum takes place, as a rule, in the Fallopian tube. Directly one spermatozoon has penetrated into the ovum, a membrane is formed round the yolk, which prevents the entrance of any other spermatozoa. The fusion of the male and female pronuclei is followed immediately by division of the fertilised ovum, so that, by the time it arrives in the uterus (about eight days after fertilisation), it consists of a mass of cells known as the morula. At this time the ovum has a diameter of about $0.2 \mathrm{~mm}$. Pregnancy in the human being lasts about nine months, birth generally taking place 280 days, i.e. ten periods after the last menstrual period. During pregnancy menstruation is absent.

With the arrival of the fertilised ovum in the uterus, extensive changes begin in this and the neighbouring organs of generation. The virgin uterus is pear-shaped, and its cavity amounts to about $2-5$ c.c. Just before birth the volume of the uterus is about 5000-7000 c.c., and the walls of the organ are thickened in proportion. In the hypertrophy of the uterine wall all its elements are involved, but especially the muscle-cells. It is doubtful whether there is an actual new formation of muscle-fibres, but each fibre grows in length and thickness, becoming finally between seven and eleven times as long and three to five times as thick as in the unimpregnated uterus (Fig. 563). There is at the same time a great growth of the bloodvessels, which have to supply not only the growing wall of the uterus but also, by means of a special organ - the placenta - the nutritional needs of the developing fœtus.

CHANGES IN THE UTERINE MUCOUS MEMBRANE. At the moment of conception the uterine mucous membrane begins to undergo hypertrophy. Within fourteen days it has attained a thickness of $\frac{1}{2} \mathrm{~cm}$., and by the end of the second month, $\frac{3}{4} \mathrm{~cm}$. On section it shows a compact layer, lining the cavity of the uterus, and beneath this, abutting on the muscular tissue, is a spongy layer three times as thick as the compact layer. The superficial epithelium becomes flattened, loses its cilia, and degenerates. In the spongy layer the uterine glands proliferate, the stroma cells are enlarged, and the blood-capillaries are widely dilated. The stroma cells become converted into the large decidual cells. By the time the fertilised ovum arrives in the uterus the process of hypertrophy and lnosening of the layers of the 
mucous membrane has already made some progress. As it lies on the mucous membrane, the outermost cells of the developing ovum exercise a destructive influence on the adjacent cells of the mucous membrane, apparentiy through some sort of digestion, so that the ovum sinks in the membrane and reaches the subepithelial connective tissue. Round the margins of the depression, which the ovum has made for itself, the mucous membrane grows over the protruding part of the ovum (Fig. 564). When this has taken place, the different parts of the mucous membrane receive different names. Since (in man) they are all to be cast off with the fœtus at birth, each part is spoken of as the decidua, that lining the main body of the uterus being known as the decidua vera, that covering the protruding part of the egg as the decidua reflexa, while that to which the egg is immediately attached is the decidua serotina or basalis. It is from the latter that the placenta is formed. By the end of the second week the blood-vessels in this situation are considerably enlarged. This enlargement proceeds, affecting especially the capillaries and veins, until these form venous sinuses at the junction between the mucous membrane and the muscular coat. Changes take place at the same time in the embryo. When it sinks into the mucous membrane it has a diameter of $1 \mathrm{~mm}$. The blastoderm is fully formed with its three layers; the yolk-sac, the body cavity, and the amnion are present. The outermost layer of the epiblast becomes specially modified to serve for the nutrition of the embryo, and gives rise to the production of numerous villi, the chorionic villi, so that the whole ovum has a shaggy appearance. Since this tissue takes no part in the further development of the embryo, but serves simply for its nutrition, it is often spoken of as the trophoblast. With the formation of fœtal blood-vessels, these penetrate into the villi, together with mesoblast. The villi grow into the venous spaces, especially in

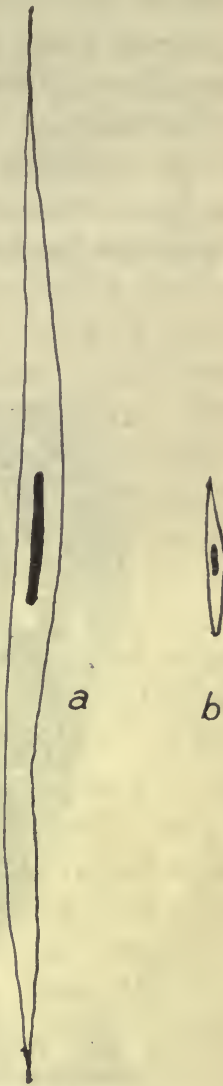

FIG. 563. Isolated muscle - cells from the uterus, showing the hypertrophy during pregnancy.

$a$, fibre from uterus in ninth month of pregnancy; $b$, fibre from a non-gravid uterus.

(After BUмm.) the basal part of the decidua, so that, at this period, the fœtal villi are immersed in maternal blood, the fotal blood-vessels being separated from the maternal blood by a double layer of epithelium, one layer of which is maternal and the other fotal in origin. Later these cells become reduced to a single layer.

NUTRITION OF THE EMBRYO. At the earliest period of its development the fertilised ovum is dependent for its nourishment on the remains of the cells of the discus proligerus adhering to it. or on the fluid of the Fallopian 
tube in which it is immersed. The first blood-vessels which are formed serve to take up nourishment from the yolk-sac. In man, however, this source of supply is insignificant, and from the second week onwards bloodvessels traversing the chorionic villi come into close relation with the maternal blood, from which, henceforth, the whole growth of the fœtus is to be maintained by a special development of these connections in the placenta.

In the fully formed fœetus blood passes from the fœtus to the placenta by the umbilical artery, and is returned by the umbilical veins. There is no communication between fœtal and maternal circulations. The placenta represents the fœtal organ for respiration, nutrition, and excretion. Thus

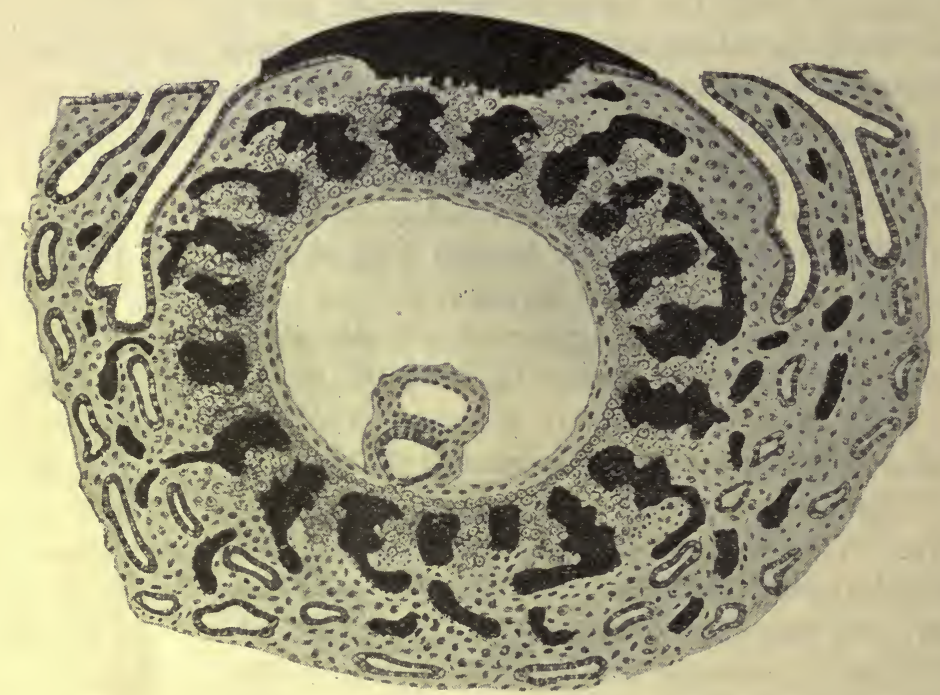

FIG. 564. Diagram to illustrate the imbedding of the ovum in the decidua, and the first formation of the fœtal villi in the form of a syncytial trophoblast (derived from the outer layer of the ovum) which is invading sinus-like blood-spaces in the decidua. (After T. H. BRYCE.)

the umbilical artery carries to the placenta a dark venous blood, which in this organ loses carbonic acid and takes up oxygen, so that the blood of the umbilical vein is arterial in colour. The oxygen requirements of the fœetus are, however, but small. It is protected from all loss of heat, movements are sluggish or for the most part absent, and the only oxidative processes are those required in the building up of the developing tissues. On the other hand, the fœetus has need of a rich supply of food-stuffs, which it must obtain through the placental circulation. It is imagined that the epithelium covering the villi serves as an organ for passing on the necessary food-stuffs from the maternal to the foetal blood in the form best adapted for the requirements of the foetus. We know, however, practically nothing as to the changes or mechanism involved in this transference. Although most of the organs of the fœtus are fully formed some time before birth, they are for the most part in a state of suspended activity. The nitrogenous excreta are 
turned out by the placenta, so that the fœtal secretion of urine is minimal or absent. The alimentary apparatus is for the most part ready. Thus pepsin can be extracted from the fœtal gastric mucous membrane. The pancreas contains trypsinogen and the intestinal mucous membrane prosecretin. Amylolytic ferments seem, however, to be absent both from the salivary glands and the pancreas. The liver stores up glycogen and secretes bile, which accumulates in the small intestine, forming the 'meco. nium.' This is generally voided by the child shortly after birth.

THE FCETAL CIRCULATION. In the fotus, from the middle of intrauterine life, we find certain arrangements of the circulation which are directed to providing the forepart of the body, especially the rapidly growing brain, with oxygenated blood, while the less important tissues of the limbs and trunk receive venous blood (Fig. 565). The arterial blood coming from the placenta along the umbilical vein can pass directly into the liver. The greater part of it, however, traverses the ductus venosus to enter the inferior vena cava, by which it is carried to the right auricle. Here it impinges on the Eustachian valve, and is directed thereby through the foramen ovale into the left auricle, whence it passes into the left ventricle to be driven into the aorta. As this arterial blood passes into the inferior cava, it is of course mixed with the venous blood, returning from the lower limbs and lower part of the trunk. By the aorta this mixture, containing chiefly arterial blood, is carried to the head and fore limbs. The venous blood from these parts is carried by the superior vena cava to the right auricle, and thence to the right ventricle, by which it is driven into the pulmonary artery. Only a small part of the blood, however, passes through the lungs, the greater part traversing the patent ductus arteriosus to be discharged into the aorta below the arch, whence it flows partly to the lower limbs and trunk, but chiefly to the placenta by the umbilical arteries. In the fœtus therefore the work of the circulation is largely carried out by the right ventricle. The greater thickness of the left ventricular walls, which is so characteristic of the adult, does not become evident until shortly before birth.

With the first breath taken by the new-born child all the mechanical conditions of the circulation are modified. The resistance to the blood-flow through the lungs being diminished, the blood passes from the pulmonary arteries through the lungs into the left auricle. The pressure in the left auricle is thus raised, while that in the right auricle falls, so that the foramen ovale is maintained closed. Even before birth proliferation of the lining membrane may be seen both in the ductus arteriosus and in the ductus venosus; and with the mechanical relief of the vessels afforded by respiration and the changed conditions of the fœtus, this proliferation goes on to complete obliteration of the vessels.

\section{PARTURITION}

As the uterus increases in size and becomes more distended, its irritability becomes greater, so that it is easily excited to contract. The stimulus may be supplied from adjacent abdominal organs, from the brain, as by emotions, 
or by direct excitation of the internal surface of the uterus, in consequence of movements of the fotus. In many cases no antecedent stimulus can be

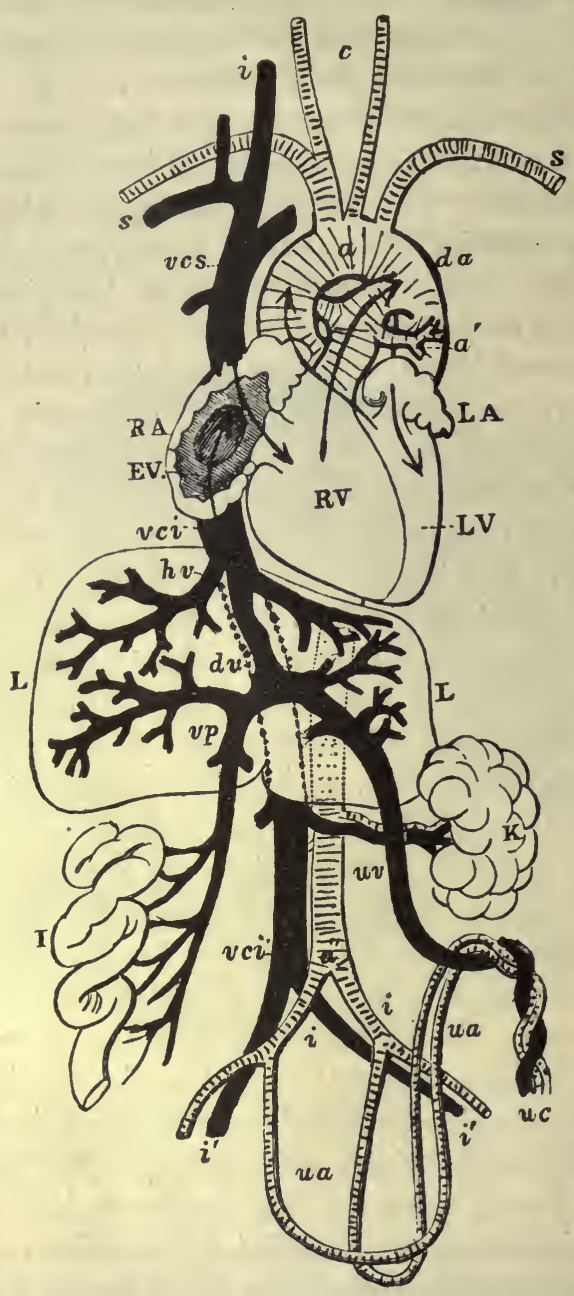

Fig. 565. Diagrammatic outline of the organs of circulation in the fotus of six months. (After ALLEN Thomson.)

RA, right auricle of the heart ; RV, right ventricle ; LA, left auricle ; EV, Eustachian valve ; $L V$, left ventricle ; $L$, liver; $\mathrm{k}$, left kidney ; $\mathrm{I}$, portion of small intestine; $a$, arch of the aorta ; $a^{\prime}$, its dorsal part ; $a^{\prime \prime}$, lower end ; vcs, superior vena cava ; $v c i$, inferior vena where it joins the right auricle; vci, its lower end; $s$, subclavian vessels; $j$, right jugular vein ; $c$, common carotid arteries ; four curved dotted arrow-lines are carried through the aortic and pulmonary opening and the auriculo-ventricular orifices; $d a$, opposite to the one passing through the pulmonary artery marlss the place of the ductus arteriosus ; a similar arrow-line is shown passing from the inferior vena cava through the fossa ovalis of the right auricle and the foramen ovale into the left auricle; $h v$, the hepatic veins ; vp, vena portæ ; $x$ to $v c i$, the ductus venosus ; $u v$, the umbilical vein; $u a$, umbilical arteries ; $u c$; umbilical cord cut short ; $i i^{\prime}$, iliac vessels.

discovered, and the automatic contraction of the uterus seems to be analogous to that which occurs in the distended bladder. These contractions 
ordinarily give rise to no sensations, and are only felt when they are augmented in consequence of reflex stimulation. During the greater part of pregnancy they have little or no effect on the contents of the uterus. During the last weeks or days of pregnancy, however, these contractions, which have now become more marked, have a distinct physiological effect. Not only do they, by pressing on the fœtus, cause it, in most instances, to assume a suitable position for its subsequent expulsion, but, affecting the whole body of the uterus, including the longitudinal muscular fibres surrounding its neck, they assist the general enlargement of the organ in dilating the internal os uteri, so that the upper part of the cervix is obliterated and drawn up into the body of the uterus some little time before labour has commenced.

With these changes in the uterus are associated changes in the round ligaments and in the vagina and vulva. The muscular fibres of the round ligaments become much hypertrophied and lengthened, and these structures can therefore aid appreciably the uterine contractions in the subsequent expulsion of the fœtus. The vaginal walls become thickened and of looser texture, so as to afford less resistance to distension during the passage of the fœtal head.

Considerable discussion has taken place as to the cause for the onset of the processes comprised under the heading of labour or parturition at a nearly constant period of two hundred and seventy-two days after conception: Most of the explanations which have been suggested, such as the great irritability of the uterus at the termination of pregnancy, the loosening of the fœtal membranes, the return of the menstrual congestion after ten months, thrombosis of the placental sinuses, simply replace one question by another. According to Spiegelberg the phenomena accompanying the birth of twins, which are often born at a considerable interval from each other, the onset of contractions of the uterus at the right time in normal as well as in extrauterine fœtation, the fact that the extra-uterine fœtus dies when it has become mature, all go to show that the reason why labour occurs at a definite time must be sought for in foetal rather than in uterine changes. This author suggests that some substances which had previously been used up by the fotus gradually accumulate in the maternal blood as the fœtus becomes mature, and provoke, by their direct action on the uterus or spinal cord, the uterine contractions which give rise to labour.

Actual parturition is in man generally divided into two stages. In the first stage the contractions are confined to the uterus, and chiefly act in dilating the os uteri. In this dilatation two factors are involved, namely, the active dilatation brought about by the contraction of the longitudinal muscular fibres which form the chief constituent of the lower part of the uterine wall; and in the second place, a passive dilatation by the pressure of the fœtal bag of membranes, which is filled with amniotic fluid, and forced down as a fluid wedge into the os by the contractions of the uterine fundus. The uterine contractions are essentially rhythmical, being feeble at first, and increasing gradually in intensity to a maximum which endures a certain 
time, and then gradually subsides. The frequency and duration of the contractions increase as labour advances.

As soon as the os is fully dilated and the fœtal head has entered the pelvis, the contractions change in character, being much more prolonged and frequent, and attended by more or less voluntary contractions of the abdominal muscles. This action of the abdominal muscles is associated with fixation of the diaphragm and closure of the glottis, so that pressure is brought to bear on the whole contents of the abdomen, including the uterus. No expelling force can be ascribed to the vagina, since it is too greatly stretched by the advancing fœtus. In this way the fœtus is gradually thrust through the pelvic canal, dilating the soft parts which impede its progress, and is finally expelled through the vulva, the head being born first. The membranes generally rupture towards the end of the first stage of parturition.

A third stage of labour is generally described. This consists in a renewal of uterine contractions about twenty to thirty minutes after the birth of the child, and results in the expulsion of the placenta and decidual membranes.

NERVOUS MECHANISM. We possess little experimental knowledge of the nervous mechanism of parturition. The most important observation on this point is the already quoted experiment by Goltz, in which this physiologist observed the normal performance of menstruation (heat), impregnation, and parturition in a bitch whose spinal cord had been completely divided in the dorsal region during the previous year. On the other hand, destruction of the lumbo-sacral cord completely abolishes the normal uterine contractions of parturition, so that this act must be regarded as essentially reflex, presided over by a controlling ' centre ' in the grey matter of the cord. The activity of the centre can be inhibited or augmented by impulses arriving at it from the peripheral parts of the body, as by the stimulation of sensory nerves, or from the brain, as under the influence of emotions. The nerve-paths from the centre to the uterus have been already described. 


\section{SECTION V}

\section{THE SECRETION AND PROPERTIES OF MILK}

\section{LACTATION}

DURING pregnancy the fœtus obtains the whole of its nourishment from the mother by means of the placenta. After birth the quality of the nutriment supplied to the young child depends on the activity of the cells of the mammary glands. Now, however, nutrition involves further activity on the part of the young animal, the alimentary canal being concerned in the digestion of the milk supplied by the mother, and the excretory organs, especially the kidneys, being made use of for getting rid of waste material. The preparation of the mammary glands for the subsequent nourishment of the new-born child begins in the first month of pregnancy, and is marked by swelling of the glands, rapid proliferation of the duct epithelium, and production of many new secreting alveoli. The developing of these glands in the rabbit has been already described, and there is no doubt that in the human species the process follows very much the same course, being, however, spread over nine months instead of four weeks, as is the case with the rabbit. During the latter half of pregnancy a watery fluid can generally be expressed from the nipple. In certain mammals this watery secretion gives place to a secretion of true milk at the end of gestation or during the process of parturition itself. In the woman the secretion does not begin as a rule until the second or third day after birth, though the formation of milk may be anticipated if a child has been put to the breasts during the latter part of pregnancy. Secretion begins on the second or third day, even if the child has been born dead and no attempt at suckling has taken place. For the maintenance of the secretion the process of suckling is absolutely necessary. If the woman does not nurse her child, the swelling of the breasts gradually passes off, the milk disappears, and the glands undergo a process of involution. Under normal conditions the secretion of milk lasts for six to nine months and may in rare cases extend over more than a year. The amount secreted increases at first with the growth and size of the child. The Table on p. 1238 represents the average amount of milk secreted during the thirty-seven weeks after birth. It will, of course, be greater with strong, big children, and smaller with weakly children.

COLOSTRUM. Before the secretion of true milk begins, the fluid which is obtained from the breast is known as colostrum. It may be expressed from the breasts immediately after birth and is ingested by the child during 
the first two days after birth. The colostrum is formed only in slight quantities. It is an opalescent fluid, often somewhat yellowish, containing fat globules, which, if the fluid be allowed to stand, form a yellowish layer on the top. Under the microscope, in addition to the fat globules, may be seen the so-called colostrum corpuscles, which consist of multinucleated cells loaded with particles of fat. They are probably leucocytes or phagocytes which have wandered into the alveoli and have taken up fat globules. Some of the corpuscles may be desquamated secretory cells. Colostrum is distinguished from true milk by containing little or no caseinogen. It contains about 3 per cent. of proteins, namely, lactalbumen and lactoglobulin, which coagulate on boiling. Lactose and salts are present in the same proportions as in ordinary milk. It is popularly supposed to have a laxative effect on the child.

Table Showing Amount of Milk Secreted by a Nursing Woman.

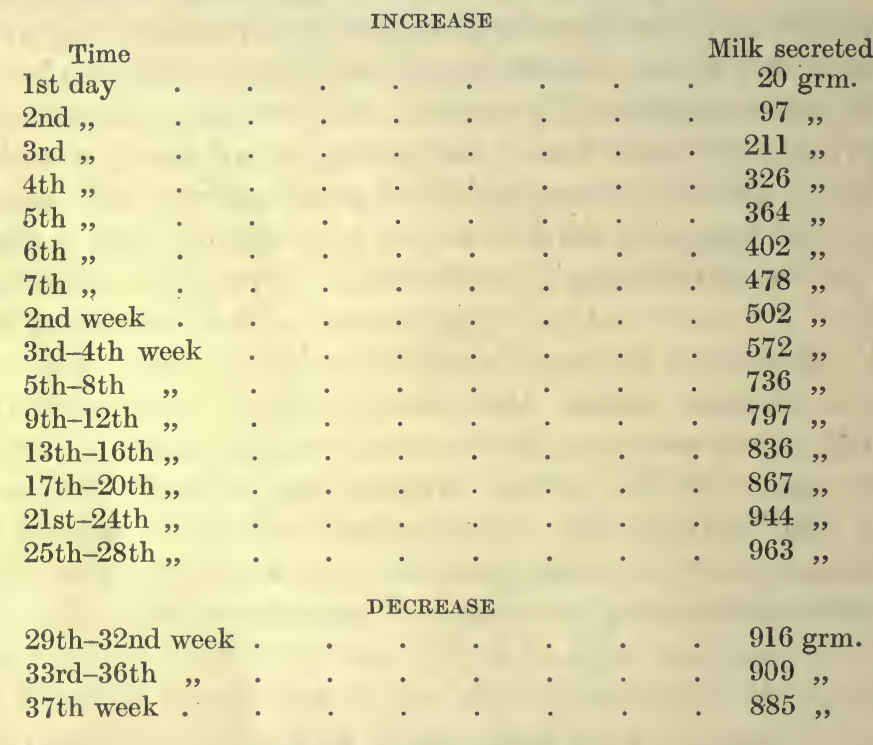

\section{PROPERTIES OF MILK}

Fully formed milk presents certain features which are common to all mammals. These have been chiefly studied in the case of cows' milk. We may therefore deal with the composition of cows' milk and point out later in what respects human milk differs therefrom. Milk forms an opaque white fluid with characteristic odour and sweetish taste. Its specific gravity varies between 1028 and 1034. Its reaction to litmus is neutral, to lacmoid it reacts alkaline, and to phenolphthalein, acid. One hundred cubic centimetres of fresh milk, when treated with lacmoid, requires 41 c.c. $n / 10$ acid for neutralisation. When treated with phenolphthalein the same amount requires $19.5 n / 10$ alkali for neutralisation. When exposed to the air milk rapidly undergoes changes in consequence of infection by micro-organisms. The most common of these changes is the formation of lactic acid by the 
bacillus lacticus. In some cases the milk may undergo a species of alcoholic fermentation, as in the formation of kephir, which is made by the fermentation of mares' milk.

The opaque appearance of milk is due chiefly to the presence of multitudes of fine fatty particles. On allowing the milk to stand the particles rise to the surface, forming "cream, and by mechanical agitation, especially if the milk is slightly sour, they may be caused to run together with the formation of butter. Much discussion has arisen as to the reason why the fat globules do not run together naturally. By many authors it has been imagined that they are clothed with a special protein membrane (haptogen membrane) originating from the protoplasm of the cell in which the fat globules were originally formed. It must be remembered that in any protein solution, such as that in which the globules are suspended, the protein tends to aggregate, with the formation of a pellicle, at the surface, so that an emulsion once produced in such a fluid will tend to be more or less permanent. There seems therefore no reason to assume the presence of a distinct membrane differing in composition from the proteins present in the surrounding fluid. The fats of milk consist for the greater part of the neutral glycerides, tripalmitin, tristearin, and triolein. In smaller quantities it contains the triglycerides of myristic acid, butyric acid (?), and capronic acid, with traces of caprylic, capric, and lauric acids.

The milk plasma, the fluid in which the fat globules are suspended, contains various proteins, a carbohydrate, lactose, and inorganic salts, with a small amount of lecithin and nitrogenous extractives.

THE PROTEINS OF MILK. The chief protein of milk is caseinogen, belonging to the class of phosphoproteins. Like other bodies of this class it presents distinct acid characteristics, being precipitated by acids and soluble in dilute alkalies. It may be prepared from separated milk by the addition of weak acids. A convenient method is to dilute one litre of milk with ten litres of distilled water and add to the mixture 10 c.c. of glacial acetic acid. The precipitate which is formed rapidly sinks to the bottom and may be washed two or three times by decantation. It may be purified by solution in dilute ammonia and precipitation by acetic acid two or three times. The precipitate finally obtained is extracted with alcohol and ether, and the dried caseinogen prepared in this way forms a snow-white powder which is practically insoluble in water and dilute salt solutions. It is easily dissolved on the addition of a little alkali, when it yields solutions which are acid to litmus. When rubbed up with chalk it dissolves, displacing the carbonic acid and forming a calcium caseinogenate. A solution of caseinogen in soda or potash is transparent and passes easily through a clay cell. The calcium caseinogenate forms only opalescent solutions. Apparently the compound is dissociated by water with the formation of caseinogen acid which is in a state of partial solution as swollen-up aggregates. It is impossible therefore to filter calcium caseinogenate through a clay cell. It is mainly in this form that caseinogen is contained in milk, hence the opalescent appearance of the milk-plasma. When calcium caseinogenate solution is 
boiled it forms a pellicle on the surface in the same way as milk does. On treating the caseinogen with rennet ferment it is converted into a modification known as paracasein, which in the presence of lime salts is thrown out as insoluble casein. To this process is due the clotting of whole milk by rennet, which is made use of in the preparation of cheese, the curd consisting of a network of casein enclosing fat globules in its meshes. On allowing the clot to stand it shrinks, pressing out a milk-serum.

From the milk-serum or whey may be obtained two other proteins, known as lactalbumen and lactoglobulin. These resemble very nearly the albumen and globulin of blood-serum. They are coagulated on heating. According to some authors a third protein is present in the whey, to which the name whey-protein has been given, and which is supposed to be split off from the caseinogen under the action of the rennet ferment.

Milk can be boiled without undergoing any coagulation. If it be allowed to stand and become sour by the formation of lactic acid, at a certain period boiling the milk causes its complete coagulation. Later on the acid produced is sufficient in itself to precipitate the caseinogen. Both these processes, namely, coagulation of half-sour milk by heating, and spontaneous clotting of milk by the production of acid, are made use of in different countries for the manufacture of cheese.

MILK SUGAR. The sugar of milk, or lactose, is most easily obtained from whey, which, after separation of the clot, is boiled to precipitate the remaining proteins. On filtering and evaporating slowly, the milk sugar crystallises out. Lactose is a disaccharide and has the formula $\mathrm{C}_{12} \mathrm{H}_{22} \mathrm{O}_{11}$. It is only known to occur in milk. It may be found in the urine of nursing women when the breasts are not kept empty, so that there is reabsorption of the lactose formed in the mammary glands. It is unaltered by ordinary yeast, so that the yeast test is the best means of distinguishing lactose from dextrose in the urine. It gives the ordinary tests for reducing sugar. The salts of milk include insoluble salts, soluble calcium salts, sodium and potassium, phosphates and chlorides.

Mere enumeration of the constituents of milk presents but little interest unless we realise how closely the composition of this fluid is adapted to the needs of the growing animal. In the first place, we find a proportionality between the total solids of the milk and the rate at which the young animal grows. It must be remembered that the milk taken by the animal serves only in part for the production of energy in its body, a great proportion of it being required for the building up of new tissue. In no respect is this correspondence seen better than in the comparative analyses of the ash of milk and of the young animal of the same species which were made by Bunge. The following Table shows the composition of the ash of a rabbit fourteen days old, of the milk which it was receiving from its mother, of the ash of rabbit's blood and blood-serum. Nothing could be more striking than the marvellous way in which the cells of the mammary gland have picked out from the salts of the circulating plasma exactly those salts which are needed for the growing animal and in the same proportion : 


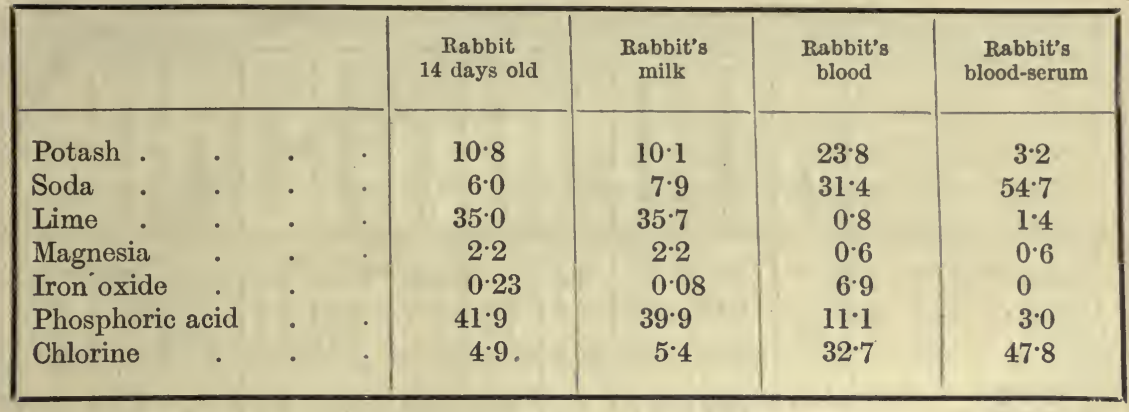

This close correspondence is only necessary where growth is very rapid, so that the greater part of the constituents of the milk have to be utilised in the building up of the animal tissues. As Bunge has shown, the slower the growth of the animal the greater the divergence between the composition of the milk and that of the new-born animal. We may compare, for instance, the rabbit, which doubles its weight in six days, with the dog, which doubles. its weight in ninety-six days, and the human infant, which takes one hundred and eighty days to double its weight at birth.

The last column of the following Table represents the composition of the ash of cow's milk, and shows how very inefficiently this milk can be regarded as replacing human milk, the natural food of the infant.

\begin{tabular}{|c|c|c|c|c|c|c|c|}
\hline & $\begin{array}{l}\text { Rabbit } 14 \\
\text { days old }\end{array}$ & $\underset{\text { milk }}{\text { Rabbit's }}$ & $\begin{array}{l}\text { Puppy few } \\
\text { hours old }\end{array}$ & $\begin{array}{c}\text { Bitch's } \\
\text { milk }\end{array}$ & $\begin{array}{c}\text { Infant } \\
\text { some } \\
\text { minutes } \\
\text { after birth }\end{array}$ & $\underset{\text { milk }}{\operatorname{Human}}$ & $\begin{array}{l}\text { Cow's } \\
\text { milks }\end{array}$ \\
\hline Potash. & $10 \cdot 8$ & $10 \cdot 1$ & $11 \cdot 4$ & $15 \cdot 0$ & $8 \cdot 9$ & $35 \cdot 2$ & $22 \cdot 1$ \\
\hline Soda & $6 \cdot 0$ & $7 \cdot 9$ & $10 \cdot 6$ & $8 \cdot 8$ & $10 \cdot 0$ & $10 \cdot 4$ & $13 \cdot 9$ \\
\hline Lime & $35 \cdot 0$ & $35 \cdot 7$ & $29 \cdot 5$ & $27 \cdot 2$ & $33 \cdot 5$ & $14 \cdot 8$ & $20 \cdot 0$ \\
\hline Magnesia. & $2 \cdot 2$ & $2 \cdot 2$ & $1 \cdot 8$ & $1 \cdot 5$ & $1 \cdot 3$ & $2 \cdot 9$ & $2 \cdot 6$ \\
\hline Iron oxide & $0 \cdot 23$ & 0.08 & $0 \cdot 72$ & $0 \cdot 12$ & $1 \cdot 0$ & $0 \cdot 18$ & 0.04 \\
\hline Phosphoric acid & $41 \cdot 9$ & 39.9 & $39 \cdot 4$ & $34 \cdot 2$ & $37 \cdot 7$ & $21 \cdot 3$ & $24 \cdot 8$ \\
\hline Chlorine. & $4 \cdot 9$ & $5 \cdot 4$ & $8 \cdot 4$ & $16 \cdot 9$ & $8 \cdot 8$ & $19 \cdot 7$ & $21 \cdot 3$ \\
\hline
\end{tabular}

The fitness of caseinogen for building up the tissues of the body is evident when we compare as in the Table on page 1242 the products of its hydrolysis with those of all the proteins in other food-stuffs. It will be seen that practically every amino-acid and allied substance employed in the building up of the various proteins is represented in caseinogen. The only exception is glycine, which can be easily formed from other amino-acids.

In another point we find an adaptation of the milk to the growth of the young animal, and that is in its lecithin content. Lecithin is probably employed to the largest extent in the building up of the central nervous system, where it forms the most important constituent of the medullary sheaths of the nerve fibres. There is a corresponding proportionality between the lecithin content of milk and the relative brain weight of the young 


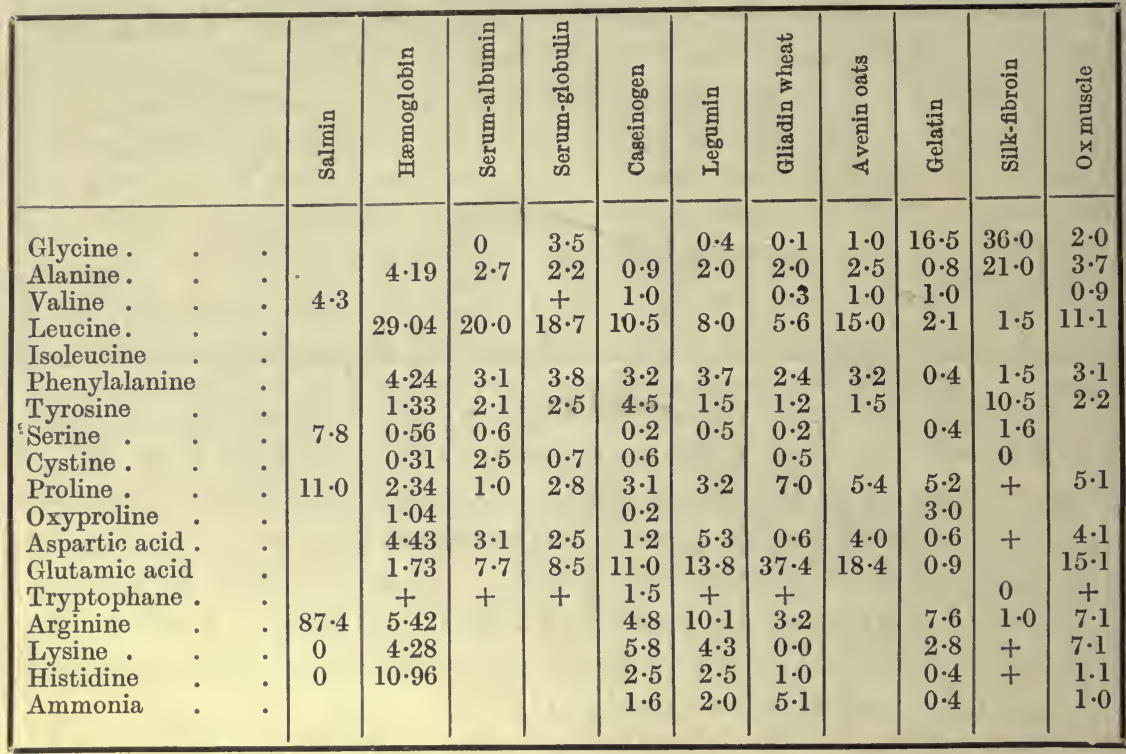

animal. Thus, in the calf the brain is only $\frac{1}{370}$ of the whole animal. In cow's milk lecithin is present in the proportion of 1.4 per cent. of the total protein. In the puppy the brain is $\frac{1}{30}$ of the whole body and the proportion of lecithin to protein in the milk is $2 \cdot 11$ per cent. In the infant, the brain forms $\frac{1}{7}$ of the body weight, while the lecithin is 3.05 per cent. of the protein of human milk.

\begin{tabular}{|c|c|c|c|}
\hline 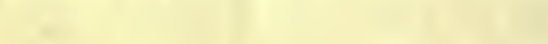 & Calf & Puppy & Infant \\
\hline $\begin{array}{l}\text { Relative brain weight . } \\
\text { Lecithin content of milk in percentage } \\
\text { of protein }\end{array}$ & $\begin{array}{r}1: 370 \\
1 \cdot 40\end{array}$ & $\begin{array}{l}1: 30 \\
2 \cdot 11\end{array}$ & $\begin{array}{l}1: 7 \\
3 \cdot 05\end{array}$ \\
\hline
\end{tabular}

We thus see that under normal conditions the young animal is supplied through its natural food with all the food-stuffs in the proportions which it requires for its normal nourishment and growth. It is impossible therefore satisfactorily to replace the natural milk of an animal by that of another species. In civilised communities it is becoming more and more the custom to endeavour to feed the child with cow's milk, more or less modified, in the vain endeavour to reproduce the properties of human milk. Among all classes this involves the administering of a milk differing in its qualities and in the relative proportions of its proteins, its fats, carbohydrates, and salts, from human milk. So-called ' humanised' milk is only a rough imitation of the natural mother's milk. Among the poorer classes this artificial feeding means the replacement of a natural sterile food, throwing very little work on the digestive organs of the child, by a foreign milk, very difficult 
to digest, and often teeming with micro-organisms. There is no doubt that of the children dying during the first year of life four-fifths are murdered by this unnatural method of feeding. In some cases it is necessary to adopt artificial feeding because the mother is abnormal, and there is an insufficient secretion of milk. It is therefore important to know what are the main differences in composition between human and cow's milk. In human milk the caseinogen is not only absolutely but also relatively less than in cow's milk, while the latter is relatively poorer in milk sugar. Human milk is poorer in salts, especially in lime, containing only one-sixth of the amount present in cow's milk. Human milk is also said to be poorer in citric acid. The main differences may be summarised as follows :

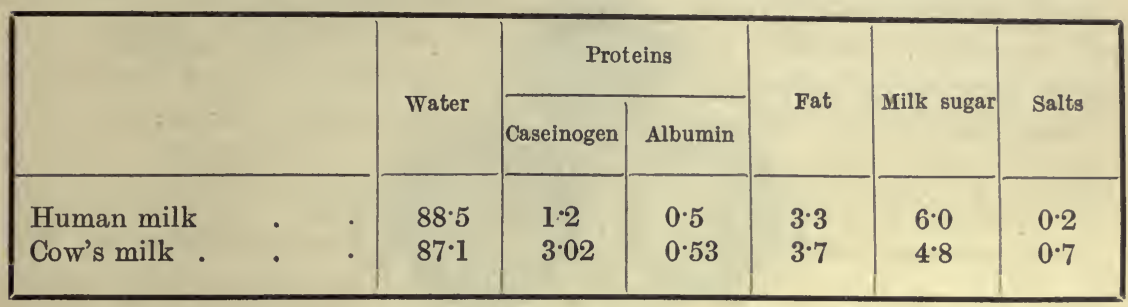

The caseinogen of human milk presents several points of difference from the caseinogen of cow's milk. It is less easly precipitated by acids. When coagulated by rennet it does not form a firm clot, but is thrown out in a flocculent form. It is thus much more susceptible to the action of gastric juice. Whereas the caseinogen of cow's milk generally gives a precipitate of 'pseudonuclein' on digestion with pepsin and hydrochloric acid, a smaller or no precipitate is formed with human caseinogen.

Another important advantage of human milk for the infant lies in the presence of antitoxins. It has been shown by Ehrlich that when a female animal has been immunised against any toxin and has produced in consequence antitoxins in its blood, these antitoxins will, if it has young, pass over into the milk. The same passage of anti-bodies into the milk has been proved in the case of various infective disorders. The ingestion of human milk will therefore not only nourish the infant, but will provide it with a certain measure of passive immunity against possible infection by diseases to which its species is liable.

THE SECRETION OF MILK. When fully formed, each mammary gland consists of fifteen to twenty lobes connected by connective tissue. Each lobe is made up of a mass of secreting alveoli which lead by narrow ducts into one large lactiferous duct. These lactiferous ducts, one from each lobe, open on the nipple, undergoing in the nipple itself an oval enlargement. Before secretion begins, the alveoli as well as ducts are lined with a cubical epithelium. When secretion commences a marked difference develops between the epithelium of the alveoli and that of the ducts. While.the latter retains its previous character, the cells of the secreting epithelium grow in length and project into the lumen of the gland. In the innermost part of the protoplasm numerous fat globules make their appearance. If sections be made of the 
gland during the various stages of its activity and stained by Altmann's method (acid fuchsin and picric acid), it will be seen that the commencement of activity is marked by the growth of the innermost part of the cells and the development in these of a number of granules (Fig. 566). These granules finally lengthen into shapes like spirilla, while others of them form fat and become metamorphosed into fat granules. The nuclei of the cells also divide, apparently in preparation for the replacement of some cells which undergo complete degeneration and are cast off into the secretion.

We know very little about the mechanism of milk secretion. It seems impossible at present to explain the very close adaptation between the
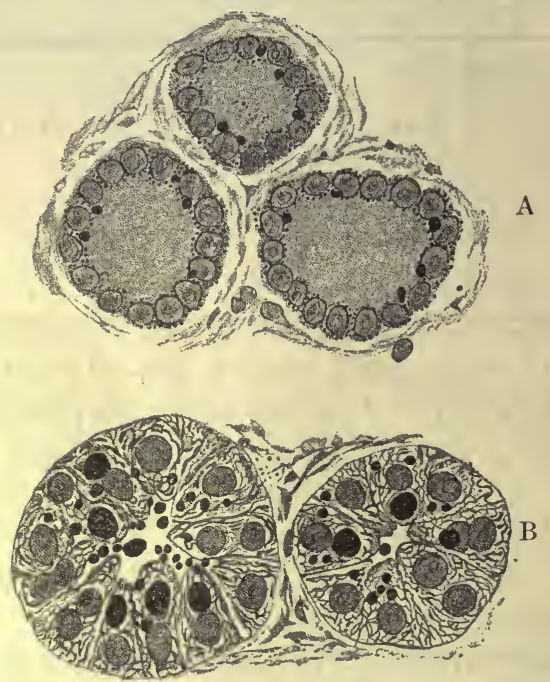

FIG. 566. Sections of mammary gland of guinea-pig (fat granules stained black with osmic acid).

A, during rest. B, during active secretion. It will be noticed that in this case the active formation of products of cell-metabolism (granules, \&c.) begins with the commencement of secretion, and does not occur almost exclusively during rest, as in the salivary glands. In the mammary gland, the active growth of protoplasm, the formation of granules from the protoplasm, and the discharge of these granules in the secretion appear to go on at one and the same time.

activity of the secretory cells and the needs of the infant or young animal. Two at least of the constituents of milk, caseinogen and lactose, are peculiar to this secretion. It has been assumed that the caseinogen is produced by some sort of alteration in the nucleo-proteins of the gland-cells, and that the lactose is derived in the same way from some sort of gluco-protein or gluconucleoprotein, but the evidence for either of these assumptions is very scanty. The growth of the mammary glands during pregnancy is largely determined by some form of chemical stimulation, the specific hormone being produced in the corpus luteum of the ovary and possibly also in the growing fœtus. It has been suggested by Hildebrandt that this stimulus is inhibitory in characterinhibitory, that is to say, of secretion-and therefore tending to the continuous growth of the gland-cells. With the removal of the fœetus at birth the 
source of the inhibitory stimulus is removed and the overgrown gland-cells enter into a condition of spontaneous activity. However this may be, there is no doubt that the secretion of the gland, once formed, is continued independently of the fœtus, or indeed of any of the pelvic organs. The onset of a new pregnancy brings the secretion to a close. Removal of the ovaries in a cow is sometimes employed as a means of prolonging the secretion of milk. The only condition which is necessary for secretion to continue during six to nine months after birth is the repeated emptying of the gland, i.e. the removal of the secreted milk. The process of suckling not only removes the milk already secreted but excites the secretion of more milk. The secretion is certainly subject to nervous influences, but physiologists have not succeeded in either producing secretion by stimulation of the nerves going to the glands, or in stopping secretion by section of these nerves. Moreover the food of the animal may be varied within very wide limits without altering the composition or amount of the milk secreted, provided only that the food is sufficient in amount. The only constituent of the milk for which we have direct evidence of alteration by changes in the food-supply of the mother is the fat. It is well known that the composition of butter may be affected according to the food supplied to the cow. A large supply of oilcake, for instance, may result in the production of a butter which is deficient in the higher fatty acids and is therefore oily at ordinary temperatures. Abnormal fats and fatty acids, such as iodised fats or erucic acid, when administered to an animal in lactation may appear among the fats of the milk. Not only can the secretion and composition of the milk be affected reflexly through the nervous system, as, e.g. under the influence of emotions, but the influence may be reciprocal. This is especially marked in the case of the pelvic organs. The act of suckling excites tonic contractions of the uterus. Putting the child to the breast shortly after birth is therefore an important means of causing contraction of the uterus and stopping any tendency to hæmorrhage from the venous sinuses opened by the separation of the placenta and fœtal membranes. The nursing of the child is therefore an important means of procuring a proper involution of the uterus after labour. Many uterine troubles among women may be ascribed to the previous neglect of this elementary duty. 



\section{NDEX}

A bSINThe, action of, 440

Absorption from connective tissues, 1019 in large intestine, 723

of amino-acids, 749

of carbohydrates, 743

of fats, 738

of food-stuffs, 731-753

of lymph, 1019

of proteins, 744

of water and salts, 731

significance of lipoids in, 735

Absorption-coefficient of gases, 1057

Acapnia, 1101

Accelerator nerves of heart, 975

Accessory olive, 367,382

Accommodation, 539-549 comparative physiology of, $547-549$ range of, 546

Acetamide, 49

Acetic acid, 48

Aceto-acetic acid, detection of, 1123 production from fats, 793

Acetone, 49 from amino-acids, 802 bodies in urine, 1123 in diabetes, 804

Achromatic spindle, 1205

Achroodextrin, 68, 662

Acid albumin, 98 amides, 49 hydrolysis of proteins, 76 number of fats, 56

Acidosis, 793 in diabetes, 804

Acromegaly, 1191

Acrose, 62 synthesis of, 153

Action-current, 226

Adaptation, 4 E.M.F. of, 233 sensory, 482 visual, 572

Addison's disease, 1185

Adenine, 103, 778

Adipose tissue, 53 action of gastric juice on, 688

Adrenaline, 51, 1182 action on blood-pressure, 1183 on blood-vessels, 1003 on heart, 977 on nerve terminations, 278 and sympathetic system, 1183 glycosuria, 801 instability of, 1185 isomers, action of, 1183

Adsorption, by colloids, 141
Adsorption, in ferment action, 168 law of, 148 of toxins, 1033

Adventitia of artery, 870

Aerotonometers, 1058, 1068 specific surface of, 1069

Afferent autonomic fibres, 476 impulses from muscles, 345 nerves, 255 path, 328 tracts, cerebellum, 402 cerebrum, 422

After-imagcs, 571 coloured, 576, 581

After-load, 207

Air, composition of, 1052 expired, 1052

Alanine, 48, 77, 81 deamination of, 154

Albuminates, 99

Albuminoids, 106

Albumins, characters, 97

Albuminuria, 1121

Albumoses, 100, 686

Alcaptonuria, 770, 1124

Alco-gels, 139

Alcohol, food-value of, 646

Alcohols, 46 polyatomic, 47

Aldehyde, 47 reactions of, 47 resin, 48

Aldol, 120, 785 condensation, 120

Aldoses, 60

Alexia, 456

Alkaline hæmatin, 98, 830

Allantoin, 779

'All or none' phenomenon, 205

Altitude, effect of, on red corpuseles, 1102 on respiration, 1082

Altmann's granules, 17

Aluminium, 44

Alveolar air, carbon dioxide in, 1081 composition of, 1053 sampling of, 1052

Amacrine cells, 560

Amboceptors, 1036

Amide nitrogen of proteins, 91

Amines, 49 action of, $1185^{\circ}$ formed in putrefaction, 155,1185

Amino-acids, 48, 77 absorption of, $745-749$ aromatic, 84 
Amino-acids, esters of, 80

fate after absorption, 750

food-value of, 645

heat equivalents of, 763

in digestion, 659

interconvertibility of, 765

separation of, 80

sulphur in, 86

synthesis of, 115, 154

Amino-isobutyl acetic acid, 82

a-amino-glutaric acid, 82

a-amino-succinic acid, 82

a-amino-thiopropionic acid, 86

Ammonia, estimation in urine, 1127

production from amino-acids, 761 in acidosis, 766

Ammonia-nitrogen of proteins, 92

Amœba, 14

Amœboid movement, 248

Amphoteric electrolytes, 80, 149

Amylase, 68

of pancreatic juice, 706

Amyloid substance, 106

Amyloplasts, 68

Amylopsin, 706

Anacrotic pulse, 926

Anæmia, 1011

Anaerobic organisms, 26

Analgesia, 495

Anal sphincter, 732

Anaphase of mitosis, 1206

Anarthria, 454

Anelectrotonus, 265

Anisotropous substance, 182

Ankle clonus, 335

Anodal contraction, 263

Anode, 187

Anœstrum, 1226

Anterior cerebellar tract, 354, 371 commissure, 426

Antidromic fibres, 323, 999

Antigens, 1036

Antilysins, 1033

Anti-peristalsis in large intestine, 730

Antithrombin, 846

Antitoxins, 150, 847, 1032

Antipeptone, 704

Apex-beat, 903

Aphasia, 454

Apnoea, 1080, 1095

spuria, 1096

vagi, 1096

vera, 1096

Aqueduct of Sylvius, 363, 373

Arabinose, 61

Arachidic acid, 54

Archipallium, 417

Arcuate fibres, 367

Arginase, 767

Arginine, 83, 84, 91

Aristotle's experiment, 493

Aromatic compounds, 49 substances, fate of, 770-773

' Arrest ' curves, 200, 208

Arterial pressure, 872,874

in $\operatorname{man}, 875$

and cardiac output, 884

Arterics, distensibility of, 871

flow in, 918-931

structure of, 870

Arterioles, structure of, 870
Arytenoid cartilage, 520

muscle, 521

Ascending tracts, 387

Ash, estimation in food, 614

Asparagine, 83

food-value of, 646

Aspartic acid, 82, 91

Aspergillus oryzæ, 168

Asphyxia, blood-pressure changes in, 984

factors in, 984-987

in decerebrate animal, 985

stages of, 1079

Assimilation, 425

Association areas, 452

cells of cortex, 427

centres, 433

fibres of cerebrum, 427

sensory, 451

Astasia, 404

Asthenia, 404

Asthma, 1048

Astigmatism, 538

Asymmetric carbon atoms, 51

Ataxy, 346, 598

Atonia, 404

Attraction sphere, 16

Atwater-Benedict calorimeter, 622

Auditory area, 447

fatigue, 518

localisation, 518

nerve, nucleus of, 379,380

ossicles, 513 mechanics of, 514

projection, 518

radiation, 423

sensations, 503

analysis of, 517

Audito-sensory area, 433

Auerbach's plexus, 469, 474

in intestine, functions of, 726,727

in stomach, 699

Auræ in epilepsy, 439, 446

Auricle, pressure-changes in, 901

Auriculo-ventricular bundle, 893, 952 node, 952

Autonomic fibres, afferent, 476

cranial, 472

of vagus, 473

sacral, 473

system, classification of, 470

Autonomic nervous system, 466-477

Autoxidisable substances, 1106

Average error method, 484

Avogadro's hypothesis, 126

Axis cylinder, 251

Axon, 251

reflexes, 474

in vaso-dilator fibres, 999

BACTERIA, action on amino-acids, 76 of putrefaction, 155

Bacterium nitromonas, 40

Bahnung, 305

in cortex, 441

Balanced reactions, 166

Balance-sheets of body, 613

Barcroft's blood-gas apparatus, 1055

Barger's method, 129

Barometric pressure, influence on alveolar $\mathrm{CO}_{2}, 1082$ 
Basal ganglia, definition of, 377 devolopment, 363

Basophile leucocytes, 813

Basilar membrane, functions of, 516

Bathmotropic effect of vagus excitation, 973

Bayliss on depressor reflexes, 979, 1001

Bayliss and Starling on intestinal move. ments, 726

on secretin, 709

Beats, 508

Bechterew's nucleus, 381, 402

Beckmann's apparatus for frcezing-point, 129,130

Behenic acid, 54

Bell and Magendie's law, 255, 323

Benedict's respiration apparatus, 617

Bensley on islots of Langerhans, 807

Benzene ring, 49 origin of, 118

Benzyl alanine, 155 pyrotartaric acid, 155

Bernard's experiment on vaso-motor nerves, 982

Betz cells, 428

Bichromato cell, 187

Bidder's ganglion, 940

Biedermann's fluid, 210

Bile, 713-717

acids, 715

composition of, 714

flow of, 715

functions of, 716

pigment, origin of, 83 t

salts, 714,716

circulation of, 717

effects of, on lipase action, 707

functions of, 717

secretion of, 715

Biliary fistula, 715

Bimolecular reaction, 163

Binocular vision, 597, 592

Biogen, 20

Biophor, 20

Biuret, 1115

base, 88

reaction, 93,100

Bladder, afferent impulses from, 1150

central control of, 1159

evacuation of, 1157

filling of, 1155

innorvation of, 1154,1157

musculature, 1152

rhythmic contractions of, 1156

sphincters of, 1153, 1159

Blind spot, 562

Blix apparatus, 197

on energy of muscular contraction, 201

Blood, 810-867

alkalinity of, 1067

amount in body, 854

carbon dioxide in, 1064, 1071

coagulation, $812,839-853$

effect of calcium on, 852

historical account, 849-853

negative phase, 847

positive phase, 817

corpuscles, relative amount, 857

electrical conductivity, 861

freezing-point of, 861
Blood, gases of, 1054

gas detcrinination, chemical methods, 1055

pumps, 1054

general composition of, 862

hremolysis, $23,127,820,834,1011,1036$

hydrogen ion concentration of, 1087

laking of, 820

life-history of red corpuscles, 831-835

methods of preventing coagulation, 839

osmotic pressure of, 861

oxygen capacity of, 855,858

pigments, 829

synthesis of, 825

plasma, coagulation of, 840

composition of, 864

protcins of, 846

relative amount, 857

platelets, 836-838

pressurc, alterations of, 884

apparatus, 872

in capillaries, 930

in $\operatorname{man}, 875$

in vascular systcm, 873, 874-885

reaction of, $860,1067,1087$

red corpuscles, $811,818-835$

chemistry of, 820

destruction of, 832

effect of altitude on number 1102

enumeration of, 858

life of, 834

osmotic properties of, 819

regeneration of, 833

stroma, 821

reducing substances in, 1087

serum, proteins of, 865

specific gravity of, 860

variations in amount of, 1009-1011

velocity of, $886-890$

vessels, nervous control of, 982-1005

volume, carbon monoxide method, 855 white corpuscles, 813-817

Body as a machine, 3

temperature, diurnal variations, 1170 regulation of, 1167-1177

Bone-marrow, structure of, 815,831

Boundary layer, 1131

Bowman's capsule, 1132 glands, 502

Boyle's law, 124

Brachium, superior, 377

Brain, 361-396

development of, 362

evolution of, 365,385

stem, ascending tracts, 387

association areas of, 452

cortical areas, 436

descending tracts, 389

evolution of, 361

functions of, 383-387, 392-396

long paths in, 383

structure of, 361-391

Break contraction, 192

excitation, 264

induction shock, 189

Broca's aphasia, 454 convolution, 435

Brodie's perfusion apparatus, 964

Bromine, 44

Bronchi, 1039 
Bronchial murmur, 1045

Bronchioles, 1039

innervation of, 1047

Brownian movement, 145, 146

Bruits, cardiac, 907

Bulbo-spinal animal, 393

Bulbo-spiral fibres of heart, 892

Bundle of His, 893, 952

Burch's capillary electrometer, 227

Burdon-Sanderson's electrodes, 224

Burdach's column, 324, 352, 354

Bütschli's emulsion theory, 19

Butyric acid, 48, 54

Cadaverine, 155

Caffeine, 103, 775

diuretic action of, 1141

Calcium salts, 43

Calcium salts, in milk coagulation, 688

effect on heart, 963,975

excretion of, 724

function in blood coagulation, 841

phosphate in urine, 1125

in starvation, 626

Calculi, biliary, 47

Callender recorder, 221

Calorific value of diet, 650

food-stuffs, 620

Calorimeter, 3

Atwater-Benedict's, 622

Canal of Schlemm, 543

functions of, 597

Cane-sugar, 67

inversion of, in stomach, 685,689

Cannon's shadow methods for movements of alimentary canal, 677, 697, 725

Capillary circulation, 928-931

electrometer, 174, 226

tracings, analysis of, 230

pressure, 930

wall, permeability of, 1014

Capric acid, 54

Caprylic acid, 54

Caproic acid, 54

Caput cornu posterioris, 316

Carbamide, see Urea

Carbamino-acids, 80

Carbohydrate metabolism, 796-809

radical in proteins, 94

in starvation, 628

Carbohydrates, action of gastric juice on, 689

influence of, on metabolism, 636

Carbohydrates, 59-70

absorption of, 743

imbibition by, 151

synthesis of, 37,110

Carbon assimilation, 109

importance of, 36

Carbon dioxide, assimilation of, 109

in atmosphere, 37

condition of, in blood, 1064

constancy of, in alveolar air, 1081

excretion of, 1051

tension in alveoli, 1071

tension in blood, 1065, 1071

tensions in tissues, 1064

Carbonic oxide hæmochromogen, 827

hæmoglobin, 823,824

Cardiac cycle, sequence of events, 894 time relations, 908
Cardiac impulse, 903

murmurs, 906

muscle, factors modifying activity of, 958-966

influence of tension on, 958

physiological properties of, 955958

nerves, 969

output, $910-915$

sound, 896

Cardinal points in schematic eye, 532

Cardiogram, 904

Cardiograph, 904

Cardio-inhibitory centre, 978

Cardiometer, 914

Cardio-pneumatic movements, 910

Carlson on heart of limulus, 946

Carotid gland, 1194

Casein, formation of, 688 hydrolysis of, 80

Caseinogen, 91, 100 action of gastric juice on, 688 preparation of, 1239

Catacrotic pulse, 926

Catalase, 1108

Catalysers, 159

Catalysis, 159

as a surface phenomenon, 161

by formation of intermediate products 161

of methyl acetate, 166

theories of, 160

velocity of, 162,163

Catechol, 50

Catelectrotonus, 265

Cathcart on carbohydrate metabolism, 803

Cathodal contraction, 263

Cathode, 187

Cell, 13-17

sap, 14

structure of, 16

wall, 14, 22

composition of, 22 electrical phenomena in, 173 permeability of, 22, 23, 127

Cells, chemical changes in, 153

galvanic, see Galvanic cells

growth of, 1199

histological differentiation of, 7,31

synthesis in, 168

vital phenomena of, 25

Cellulose, 70, 646

digestion of, 722

food-value of, 646

hydrolysis of, 70

Central nervous system, 288-477

Centres in medulla, 394

Centro-acinar cells, 711

Centrosome, 16, 19, 33

Cephalin, 57

Cetyl alcohol, 47, 56

Cerebellar ataxy, 405

gait, 405

path, 388

Cerebello-olivary fibres, 369

Cerebellum, ablation of, 404

afferent tracts, 402

corpus dentatum, 373

Cerebellum, efferent tracts, 402

functions of, 397

Golgi cell, 401 
Cerebellum, inferior peduncles, 369, 370, 402 middle peduncle, 402

nucleus emboliformis, 373,389

fastigii, 373,389

globosus, 373,389

Purkinje cclls, 400

roof nuclei, 373, 383, 389, 402

stimulation of, 403

structure of, 400

superior peduncles, $371,389,402,423$

vermis, 373,400

Cerebral axis, intermediatc grey matter, 382 cortex, associative functions, 451 excitability of, 435

function of layers, 431

lamination of, 427,430

motor areas in, 436

sensory areas in, 444

structure of, 416-427

thickness of, 431

hemispheres, 416-465 development of, 416

functions of, $434-460$

tracts in, 422

localisation, historical, 435

vesicles, 297

Cerebrum, afferent tracts of, 422

association fibres, 425

commissural fibres in, 422

development of, 362

fissures of, 417

lateral ventricles, 420

lobes of, 419

projection fibres, 423

structure of, 416-433

Cerumen, 512

'Characteristic' of excitability, 263, 271

Chemical changes in cells, 153

in living matter, 153-169

in muscle, 212-218

correlation of functions, 1178

stimulation of muscle, 186

Chemiotaxis, 6, 27, 498

in leucocytes, 1026

Cheyne-Stokes breathing, 1096

Chitin, 65

Chlorides, estimation of, in urine, 1129 resorption of, in kidney, 1149

Chlorine, 43

Chlorophyll, 6, 17, 37, 109, 829

Chloroplasts, 17, 33

Cholesterol, 47, 56

Choline, 57

Chondroitin, 106

Chondroitin-sulphuric acid, 106

Chondromucoid, 106

Chorda tympani, 472, 665 vaso-dilator fibres of, 996

Choroid coat, 542 plexus, 375

Chromaffine substance, 1181

Chromatic aberration, 537

Chromatin, 17, 31

filaments, 31

granules, 15

Chromatolysis, 320

Chromophile substance, 1181

Chromoproteins, 101

Chromosomes, 17, 31

number of, in somatic cells, 1206

Chronograph, 194, 195
Chronotropic effect of vagus excitation, 973

Chyle, 1013 fat in, 739

Ciliary ganglion, 552 movement, 248 muscle, 545 nerves, 552 processes, 542

Ciliated epithclium, 249

Cilio-spinal centre, 553

Cingulum, 417, 426

Circulating proteins, 642

Circulation, general features of, 868-873 in amphibia, 868

in fishes, 868

fœtal, 1233

influence of gravity on, 883

in lungs, 935-938

in mammals and birds, 869

physiology of, 868-917

effect of respiration on, 936

schema, 881 .

time, 912

Clarke's column, 318, 353, 354, 357, 388

Climacteric, 1217

Climate, influence of, on diet, 653

Clonus, 241

Closing tetanus, 273

Clostridium pasteurianum, 40

Coagulation of blood, 812, 839-853

of colloids, 72,150

of milk, 688

of proteins, 72,95

reversible, 72

Coccygeal ganglion, 466 gland, 1194

Cochlea, 515 development of, 603

Cochlear canal, 516 nerve, nucleus of, 380

Coefficient of partage, 24

Cœlom, 34

Coitus, 1227

Cold, actions of, on muscle, 208 sensations, conduction in cord, 359 spots, 487

Collagen, 106 action of gastric juice on, 687 food-value of, 645

Collateral sympathetic ganglia, 468

Collaterals in cord, 351,357

Collecting tubules of kidney, 1132

Colloidal metals, 140 particles, movements of, 145,146 size of, 144

solution, grades of, 151 solutions, phases in, 151

Colloids, 22, 72, 139-152 adsorption by, 141, 147 aggregation of, 147 charge of, 145, 147 coagulation of, 150,151 combination between, 149 electrical properties of, 145-149 heat coagulation of, 72,150 imbibition by, 151 of scrum, 150

Colloids, optical properties of, 144 osmotic pressure of, 142 precipitation of, 147 surface phenomena of, 141, 147 
Colon, movements of, 729-732

Colostrum, 1237

Colour-blindness, 578

Colour, contrast phenomena, 581 mixers, 576

saturation, 575

vision, 574-584

Edridge-Green's classification, 580

Hering's theory, 579

Young-Helmholtz' theorv, 577

Combination tones, 510

Comma tract, 353

Commissural cells of cord, 318

fibres of cerebrum, 426

Commissure of Gudden, 388

Commutator, see Reverser

Compasses test, 492

Complement, 1036

Complemental air, 1046

Complementary colours, 576

Compressor urethræ, 1153

Concentration cell, 171

Conchiolin, 108

Condenser, 191

discharges, use of, in excitation, 263

Conduction, irreciprocal, 275, 302

Cone cells, 559

function of, 574

Congo red, adsorption of, 148 colloidal properties of, 137, 143

Conjugate deviation, 438, 587 foci, 529

Conjugated proteins, 101

Conjunctival reflex, 594

Connective tissues, action of gastric juice on, 688

Consciousness, 9

Conservation of energy in body, 3,621 of mass, 3

Consonance, 509

Consonants, 525

Constant current, 186 flow in capillaries, 879

Constrictor fibres to limbs: 995

Contractile stress, 200, 207

tissues, 177-249

vacuole, 15, 33

Contraction, paradoxical, 283

remainder, 208

secondary, 233

voluntary, 239

wavc in muscle, 204

Contrast in sensation, 483

Conus arteriosus, 892

Convection, loss of heat by, 1175

Convoluted tubules, 1132

$$
\text { secretion by, } 1144
$$

structure of, 1133

Co-ordinated movements, mechanism of, 338-348

Copper, 44

ferrocyanide cell, 125

Cornea, 542

Corpora mammillaria, 374,375

Corpora quadrigemina, 363,373

Corpus Arantii, 894

callosum, 426

dentatum of cercbellum, 373

luteum, formation of, 1222

function of, 1218
Corpus luteum, spurium, 1224

subthalamicum, 377

striatum, 363, 421

trapezoides, $371,381,387$

Corresponding points, 587

Cortex, histological localisation in, 430 reciprocal innervation from, 437 thickness of, 430

Corti's organ, 517

Cortical areas, sensory, 444 motor, 436

epilepsy, 438

excitation, latent period of, 436,444

inhibition, 438, 441

motor functions, characters of, 449

Crampton's muscle, 547

Cranial autonomic fibres, 472

nerves, functions of, $410-415$ nuclei of, $378,410-415$

Crayfish, central nervous system, 293

Creatine, 84, 213, 766

Creatinine, 767

estimation of, 1128

in urine, 1116

tests for, 1117

Crescents of Gianuzzi, 664

Cretinism, 1187

Crico-arytenoid joint, 520

Cricoid cartilage, 520

Crico-thyroid muscle, 521

Crista acustica, 605

Crossed pyramidal tract, 352 reflexes, 304

Crura cerebri, 373 development of, 363

Crusta, 374

Crystallin, 98

Crystalline lens, 543

Crystallisation of egg albumin, 73

Crystalloids, 139 of serum albumin, 73

diffusibility of, 136

Crystals, mixed, 73

Cuorinc, 57

Curare, action of, 185, 259

Current, demarcation, 233 minimal gradient of, 272 of action, E.M.T. of, 233 of injury, 170, 225, 233 of rest, 225

Cushny on renal resorption, 1147

Cutaneous end-organs, 497 sensation, 486-497 sensibility, classification, 495

Cutis vera, 1161

Cyanuric acid, 1115

Cysteine, 86

Cystine, 86, 91, 1124

Cytase, 70, 646

Cytolysins, 1036

Cytoplasm, 14, 17

Cytosine, 104, 776

D : $\mathrm{N}$ RAтіo in diabctes, $795,803,805,809$

Daniell cell, 171, 186

Dark-adapted cye, 482, 572

D'Arsonval galvanometer, 228

Dead space (in lungs), 1047

Deamination, 76, 154, 762 energy changes in, 763 reversibility of, 762 
Decapitate animal, 329

Dccarboxylation, 155

Deccrebrate dog, 396

frog, 395

pigeon, 396

rigidity, $394,398,449$

Decidua, formation of, 1231

Decussation of fillet, 368 of pyramids, 352,366

Deep scnsibility, 345,495

Defects of eye, 535

Defæcation, 731

Defence, chemical, against infection, 10301038

Deglutition, 676-682

movements of larynx, 678

muscles of, 678

nervous mechanism of, 681

effect of, on respiration, 677,682

sounds, 677

study of, by Röntgen rays, 676,677

stages of, 677

time-relations, 679

Deiters' cells, 516

nucleus, $381,383,402$ connections, 404

Delirium cordis, 968

Dcmarcation current, 170, 225, 233 compensation of, 226 E.M.F. of, 226,233

Demilune cells, 664

Dendrites, 301

Dental consonants, 527

Depressor reflexes, 997, 1001

Descemet's membrane, 543

Descending tracts, 389

Detrusor urinæ, 1152

Deutero-albumose, 686

Development, 1212-1216

Dextrins, 68

Dextrose, 63

Diabetes, 800-809

in fasting animals, 802

in $\operatorname{man}, 807$

pancrcatic, 804

sugar consumption in, 805

Diabetic puncture, 800

Dialysis, 136

Diamino-acids, $83,91,97$ precipitation of, 93

Diamino-caproic acid, 155

Diamino-nitrogen of proteins, 93

Diamino-trioxydodecoic acid, $83,84,88$

a- $\delta$-diamino-valerianic acid, 79

Diaphragm, movements of, 1041 muscles of, 1041

Diastase, 68

Diastolic artcrial pressure, 874

Dichromatic vision, 578

Diencephalon, 363,392

Diet, normal, of man, 649-657

Dietaries, 649

Difference tones, 511

Differential blood-gas apparatus, 1056

Diffusion, 124, 131

coefficient, 131

of solutes, 131

Digestion, 658-755

course of, 751-753

in duodenum, 752

in intestine, $702-724$
Digestion, in stomach, 683-696

gastric, 685

pancreatic, 702

salivary, 661-675

Dihydroxybenzenes, 50

Dilatator pupillæ, 550

Dilemma, 459

Dimethylamine, 49

Dioptre, definition of, 530

Dipeptides, 89

Diphasic variation, 228

Direct cercbellar tract, 354 vision, 563

Disaccharides, 61,67

Discus proligerus, 1223

Dissimilation, 4, 25, 26

Dissociated colloids, 138

Dissonance, 508

Distearyl-lecithin, 57

Diuretics, action of, 1140

Divers' palsy, 1103

Dominant characters, 1215

Dorsal cerebellar tract, 354

Dromotropic effect of vagus excitation, 973

Dry cell, 187

Du Bois Reymond's key, 187

Ductless glands, 1178-1196

Ductus arteriosus, 1233

Dudgeon's sphygmograph, 922

Dulcite, 62

Dyne, definition, 262

Dysoxidisable substances, 1105

EAR, analysis of sounds by, 511 external, 512

internal, 515

physiology of, 511-519

Eck's fistula, 760

Edestin, 98 composition of, 91, 92

Edkins on gastric secretion, 694

Edridge-Green, classification of colour-vision 580

Efferent nerves, 255

path, 327

projection fibres, 423

tracts, cerebelluin, 402

Efficiency of machines, 234

Egg albumin, 97 composition of, 91 crystallisation of, 73

Ehrlich's side-chain theory, 1034 methylenc blue experiment, 1063

Eighth nerve, nucleus of, 380, 412

Einthoven galvanometer, 227

Elasticity of muscle, 203

Elastin, 108

action of gastric juice on, 688

Elastose, 100

Electrical capillarity, 174

changes in living tissues, 170-174

Elcctrical changes in muscle, 224

properties of colloids, 145-149

stimulation, nature of, 270

variations, effect of tcinperature on, $2: 0$

variation, time-relations of, 228

Electrocardiograms, 232, 953

Electrodes, non-polarisable, 224

Electrolytes, conductivity of, 129

Electrolytic dissociation, 170 
Electrolytic solution tension, 171

Electrotonic current, 280

Electrotonus, 264 influence of intrapolar length, 268

Electro-vagogram, 1093

Eleidin, 1161

Embryo, nutrition of, 1231

Emmetropic eye, 535

Einulsion, 56

theory of protoplasm, 18

Endocardiac pressure, 896-902

Endolymph, 515 negative phase, 902

End-plate delay, 276

End-plates, 183, 275

effect of nicotine on, 277

fatigue of, 260

End-products, effect of, on ferments, 166, 167

Enemata, nutrient, value of, 723

Energy balance-sheet of body, 620 exchange, 3,26

exchanges of, in body, 620-623

sources of, 25

total daily output of, 650

transformations of, 123

Engelmann's contractile strings, 234

Enterokinase, 703, 705

Enteroceptive nervous system, 479

Enterograph, 817

Entoptic phenomena, 536

Enzymes, see Ferments

Eosinophile leucocytes, 813

Ependyma, 362, 375

Epiblast, 33

Epicritic sensibility, 495

Epididymis, function of, 1220 structure of, 1220

Epilepsy, 438, 446

Epithelium, ciliated, 277-249

Equilibration, 399

dynamic, 606

functions of labyrinth in, 605 static, 608

Erectile tissue, 1221

Erection, 1221

Erepsin, 720

Erg, definition, 262

Ergastoplasm, 672

Ergotoxin, action of, 1184

Erlanger's sphygmomanometer, 876

Erythroblasts, 832

Erythrodextrin, 68, 662

Esbach's reaction, 95

Ester method for separation of amino-acids, 80

Esters, 46, 47, 53 mixed, 54 glyceryl, 54

Ethereal sulphates, excretion of, 769

Ethylamine, 49

Euglobulin, 866

Eustachian tube, 513 valve, 1233

Excitability, 26

Excitation, duration of current, 271 effect of intrapolar length, 268 minimal gradient, 272

Nernst's theory, 286 rate of change, 270

strength of current, 271
Excitation, time, 272 without contraction, 231

Excitatory process, nature of, 284 response, nature of, 271

Exophthalmic goitre, 1189

Extensibility of muscle, 203

Extensor reflex, 332

External auditory meatus, 512

Exteroceptive nervous system, 479

Eye, angle between axes in, 527 centring of, 537 constants of, in man, 533 dark-adapted, 482

filtration angle of, 597

movements, centres for, 407, 438 optical defects in, 535

Eyeball, dioptric mechanism of, 528530 electrical changes in, 566

nutrition of, 594-597

rotation of, 586

Eyeballs, movements of, 585-588

Eye-muscles, extrinsic, 585

intrinsic, 552 innervation of, 552

FACIAL nerve, nucleus of, $379,381,413$

Facilitation of reflexes, 305

Frees, composition of, 755 examination of, in metabolism experi. ments, 648

Faraday-Tyndall phenomenon, 144

Far point of vision, 546

Fasciculus retroflexus, 421 solitarius, 368,378

Fasting, metabolism in, 618, 624-630

Fatigue, muscular, 208

Fat, $45,53,55,784$

absorption of, 738

acid number of, 56

composition of, 784

depôts, 783

estimation in freces, 649

in food, 614

formation from food, 785

from carbohydrates, 785

from proteins, 788

functions of, 784

history of, in body, 783-795

in intestinal epithelium, 740

iodine number of, 56, 784

metabolism in starvation, 630

oxidation of, 792

saponification number of, 56

solubility of, 742

stains for, 739

synthesis of, 119,169

utilisation of, in body, 790

Fats, action of gastric juice on, 689

action of pancreatic juice on, 707

chemistry of, 53-58

influence of, on metabolism, 636

Fatty acids, 54 heat equivalents, 763 volatile, 56

Fatty degeneration, nature of, 739

Fechner's law, 485

Fehling's test, 64, 1123

Fenestra ovalis, 515

Fenton's reaction, 1109

Ferment action, adsorption in, 168 reversibility of, 167,169 
Ferment action, velocity of, 163, 164

Fermentation in stomach, 684

Fermentation test, 1123

Ferments, 157-169

action on optical isomers, 167

adenase, 778

amylase, 158

arginase, 158, 767

as catalysers, 159

catalase, 1108

characters, 157

classification, 158

deaminising, 154, 158

definition, 157

effect of end-products on, 166

cnterokinase, $15 \mathrm{~S}$

erepsin, 158

inverting, 158

isolation of, 158

laccase, 1109

lactase, 158

lactic, 158

lipase, 158, 169

maltase, 158, 168

nuclease, 776

optimum temperature for, 160

oxidases, 1108

pepsin, 158, 169, 685

peroxidase, 1108

properties of, 159

rennin, 688

specificity of, 160,167

as synthetic agents, 168

sucrase, 158

trypsin, 158

urease, 158

uricolytic, 779

zymase, 158

Fertilisation, 1209-1211, 1227

artificial, 1211

Fibre layers in cortex, 430

Fibrillæ of muscle, 179

Fibrin, 865 composition of, 92

ferment, 842

fate of, 847

Fibrinogen, 98, 841, 864

Fibroin, 107

Fick and Wislicenus's experiment, 638

Field of vision, 563

Fifth nerve, nucleus of, 380

Fillet, 368, 387, 423 decussation of, 368

lateral, 372,389

Final common path, 343

First focal plane, 531 point, 531

Fischer's method for separating amino-acids, 80

Flechsig's myelination method, 319

Flexion reflex, 332,340

Flicker phenomena, 571

Fluoride plasma, clotting of, 848

Fluorine, 44

Foci in eye, 533 of lenses, 529

Fœtal circulation, 1233

Folin's method for ammonia estimation, 1127

for urea estimation, 1127

for uric acid estimation, 1128
Fontana, spaces of, 543

Food, amount necessary, 649

analysis of, in metabolism experiments, 614

materials, utilisation of, 6

Food-stuffs, digestibility of, 649 changes in alimentary canal, 658-660

heat-values of, 620

history of, 756-809

proportions necessary, 6.52

significance of, 642-647

Foramen of Monro, 376

Foramen ovale, 514, 1233

rotundum, 515

Fore-brain, 362, 375

Formic acid, 48, 54

Fornix, 420

pillars of, 376

Fourth nerve, nucleus of, 379, 382, 411

Fovea centralis, 561

Frank's manometer, 897

Frank on pulse, 926

Fraunhofer's lines, 569

v. Frey, testing hairs, 490

Frontal lobe, 417

Frog, muscles of, 184

Frog's heart, anatomy of, 939

Fronto-pontine fibres, 424

Fructose, 61, 64

Fundamental tone, 507

Fungiform papilla, 499

GalaCtolipines, 57

Galactosamine, 105

Galactose, 64, 67

Gall-bladder, 713 innervation of, 715

Galvanic cells, bichromate, 187

concentration, 171, 172

Danicll, 171, 186

dry, 187

E.M.F. of, 173

Leclanché, 187

positive element, 186

positive pole, 186

source of energy, 171

Galvanic excitation, law of, 264

Galvanometer, D'Arsonval, 227 Einthoven's, 227

Ganglia, evolution of, 1293 sympathetic, 468

Ganglion habenulæ, 375 Gasserian, 380, 382

Gaseous diffusion, 134

exchange in lungs, 1068-1075 secretory theory, 1073

Gaseous metabolism of salivary glands, 674

Gases, tension of, in liquids, 1058

Gas laws, 123

Gastric digestion, 685

fistula, 683,690

hormone, 695

juice, 683 acid of, 684

action of, on carbohydrates, 689

action of, on fats, 689

action of, on proteins, 685

action on food-stuffs, 685

'appetite' secretion, 691, 696

composition of, 684 
Gastric juice, chemical excitants, 694 determination of hydrochloric acid in, 685

inverting power of, 685

rate of secretion of, 690

variations in, $690-693,696$

psychical secretion of, 691

secretion of, 690

sccretin, 695

Gastrocnemius of frog, 183

Gelatin, 107

composition of, 91, 92

food value of, 645

Gels, 139

Gemmules, 20

General physiology, 13-174

Geniculate bodies, $375,376,383,388$

Genital hormones, 1218

Geotaxis, 27

Germ cells, 1202

chromosomes in, 1208 division of, 1205

Germinal spot, 1222 vesicle, 1222

Gierke, respiratory bundle of, 1077

Glaucoma, 597

Gliadin, composition of, 91, 92

Gliadins, 98

Globin, 825 composition of, 91

Globulin, adsorption by, 149 salts of, 866

Globulins, 98

Glomerulus, filtration in, 1136 of kidney, 1131 pressure of blood in, 1137

Glossopharyngeal ncrve, nucleus of, 414

Glottis, 521, 524

Gluconic acid, 62

Glucosamine, $64,87,105$

Glucosazone, 63

Glucosazone crystals, 1122

Glucose, 61,63

in blood, 796

identification of, 63,1123

lactonic structure, 66,67

Glucosides, 65

Glutamic acid, 80, 82, 91

Glutelins, 98

Gluten peptone, 100

Glycerides, 53

Glycerophosphoric acid, 57

Glycerol, 47,53

Glyceryl aldehyde, 59, 122

Glyceryl esters, 53

Glycine, 78, 81, 91

ring formula of, 79

salts of, 79

ester, polymerisation of, 89

Glycogen, 69, 213, 796

preparation of, 797

formation of, 797-79s

Glycoproteins, 105

Glycosuria, 800-809, 1122

alimentary, 800

Glycuronic acid, 65, 1123

Alycyl-glycine, 88

Glyoxylic acid, 94

Goitre, 1187

Golgi cells, 318, 401

corpuscles, 497
Gorgi network, 301, 309

Golls' column, 324, 352

Gonads, 1204

Gotch's heart apparatus, 964

Gower's tract, 356, 371, 388

Graafian follicles, structure of, 1223

Gracilis experiment, 254 of frog, 184

Graham on colloids, 139

Grape-sugar, see Glucose

Graphic method, 194

Gravity, effect of, on circulation, 933

Green-blindness, 581

Grey matter of cortex, minute structurc of, 427 rami, 468

Growth, 4

of cells, 1199

food requirements in, 656

Guanine, 103, 105, 778

Guanylic acid, 105

Gudden's commissure, 388

Gunzberg's reagent, 684

Gustatory area, 449 sensations, 499

Guttural consonants, 527

Gymnema sylvestre, effect on taste, 500

HAIR FOLLICLES, 1162

Hairs, sensibility of, 490

Haldane-Pembrey respiration apparatus, 616

Hales' experiment, 872

Haploscope, 588

Haptogen, 1239

Haptophore group, 1034

Harmonic intervals, 509

Harmonics, 507

Hausmann's method for distribution of nitrogen, 91

Hayem's fluid, 836

Hæmatin, 97, 101, 826 chemical relationships, 828 synthesis of, 829

Hrmatinic acids, 828

Hæmatoporphyrin, 825, 827 combination with iron, 830 in urine, 1121

Hæmin, 825

structure of, 829

Hæmochromogen, 827, 830

Hæmocyanin, 44, 93

Hæmocytometer, 858

Hæmodromograph, 888

Hæmoglobin, 43

absorption spectrum, 823

composition of, 91,101

crystallisation of, 73

derivatives of, 825

dissociation curve, 1060

iron in, 822

molecular weight of, 75,144

oxygen capacity of, 822,1059

preparation of, 820

reduction of, 822

union with oxygen, 1059

Hæmoglobin-crystals, 821

Hæmoglobinometer, 855, 858

Hæmolysins, 1036

Hæmolysis, 23, 127, 820, 834, 1011, 1036

Hæmolytic scra, 1036

Hæmopyrrol, 829

Hæmorrhage, 1011 
Head on cutaneous scnsibility, 495

Head's diaphragm slip, 1089

Hearing, end-organ of, 514

Helmholtz's theory, 517

physiology of, 511

Rutherford's thcory, 518

telephone theory, 518

Waller's theory, 519

Heart, action of sympathetic nerves on, 975 action of vagus, 971

changes in form, 902

diastolic filling, 909

effect of calcium on, 963,975

muscarine on, 974

nicotine on, 974

potassium on, 963,975

electrical variations, 230

frog's, automatic contraction, 940

human, electrocardiogram of, 953

influence of temperature on rate of, 962

inhibition of, 972

mammalian, ganglion-cells in, 949

mechanism of, 891-917

nerve fibres in, 946

nutrition of, 966

primitive vertebrate, 950

rate in exercise, 1007

reflexes, 978

sounds, 905

graphic record of, 907

systolic output, $910-915$

theories of inhibition of, 974

work of, 915-917

Heart-beat, causation of, 939-968

contraction wave, 947-949

electrometer records of, 948

in mammals, 949-955

myogenic hypothesis, 942

neurogenic hypothesis, 941

propagation of contraction in, 943

significance of carbon dioxide for, 965

Heart-block, 955

Heart-fibrillation, 968

Heart-lung preparation, Starling's mcthod, 911

Heart-muscle, 'all or none' phenomenon, 955

excitation of, 955

influence of tension on, 958

of chemical substances on, 962

physiological properties of, 955-958

refractory period of, 956

summation of stimuli in, 956

Heart-musculature, 891

Heat-coagulation, 150

Heat-engines, efficiency of, 234

Heat-loss, regulation of, 1174

Heat, production in body, 1171

in muscle, 219

regulation, nervous mechanism of, 1176

rigor, 208, 214 in new-born, 1177

sensations, conduction in cord, 359

sexual, in animals, 1226

spots, 487

values of food-stuffs, 619

Heats of combustion, 156

Heller's test, 95, 1122

Helmholtz resonators, 508

side wire, 189

Helweg's bundle, 353
Hemianxsthesia, 446;

Hemianopia, 407, 447

Hemiplegia, 446

Henle's loop, 1132

Hensen's disc, 182

Hercdity, 1212-1216

basis of, 20

Hering's theory of colour-vision, 579

Herpes zoster, causation of, 999

Heteroalbumosc, 100

Heterocyclic amino-acids, 85

Heterotype mitosis, 1207

Hexachromic vision, 580

Hexone bases, 83

Hexoses, 61

classification of, 61

derivatives of, 64

reactions of, 62

Hind-brain, 362, 366-373

Hippocampal commissure, 427

Hippocampus, 419

Hippuric acid, 770, 1119

Hirudinised blood, 840,849

His' bundle, 893,952

Histidine, 86, 91, 104 fate of, 772

Histological differentiation, 7, 31 localisation in cortex, 431

Histones, 92, 97, 101

Hofmann's test, 85

Homans on islets of Langerhans, 807

Homogentisic acid, 51, 770, 1124

Homoiothermic animals, 1169

Hopkins' test for lactic acid, 215 for tryptophane, 94

Hopkins-Adamkiewicz reaction, 94

Hormones, genital, 1218

mammary, 1218

nature of, 1180

pancreatic, 709

Horopter, 588

Hüfner's method for urea estimation, 1126

Human stomach, form and movements, 698

Hürthle's manometer, 897

Hyaline corpuscles, 813

Hyaloid membrane, 543

Hyaloplasm, 18

Hydræmic plethora, 1009

effect on volume of

Hydrated proteins, 99 urine, 1140

Hydrazones, 62

Hydrocarbons, 45

unsaturated, 46

Hydrocele fluid, 849

Hydrochloric acid in gastric juico, 684,685

Hydrogels, 72, 140

Hydrogen, 39

Hydrogen ion concentration, determination of, 1067

Hydrolysis, 154 of cascin, 80 of proteins, $76,80,99$

Hydroquinone, 50

Hydrosols, 139 osmotic pressure $\cdot$ of, 142 properties of, 142,144

Hyperglycæmia, 805

Hypermetropia, 535

Hyperpnœa, 1079

Hypoblast, 33 
Hypogastric nerves, action on bladder, 1159 Hypogastric reflex, 475

Hypoglossal nucleus, 379,415

Hypoxanthine, 103, 778

IDENTICAL points in retina, 587

Idiopathic epilepsy, 439

Ileocolic sphincter, 729

Illusions of size, 591

Image, size of, 530

Imbibition by colloids, 151

pressure, 152 relation to constitution, 152

Iminazol, 104

ring, 86

synthesis of, 117

Iminazol-alanine, 86

Immunity, 1030-1038

Impregnation, nervous mechanism of, 1228

Incisura angularis, 698

Incus, 513

Indican, of urine, 1120

Indicators, 1067

Indigo as catalyser, 161

Indigo-carmine, excretion by kidney, 1146

Indirect vision, 563

Indol, fate of, 770

Induced currents, 188

Inductorium, 188, 189

Infection, cellular defence against, 1021-1029 chemical defence against, 1030-1038

Inferior cervical ganglion, 466 mesenteric ganglion, 466 oblique muscle, 585 peduncles of cerebellum, $368,369,370$

Inflammation, 816, 1024

Infundibula, 1039

Infundibulum in brain, 375

Inhibition, 26, 306, 339

Gaskell's theory of, 974

of heart, nature of, 974

in peripheral ganglia, 475

Injury, effect of, on nerve, 274

Injury-current, 170, 225

Inner cell-lamina of cortex, 428

fibre-lamina of cortex, 428

line of Baillarger, 430

Inorganic food-stuffs, 647

Inosinic acid, 774, 776

Inosit, 214

Inotropic effect of vagus excitation, 973

Instrumental inertia, 196, 927

Insular lobe, 417

Intercostal muscles, action of, 1044

Internal capsule, $364,376,421,423$

Internal ear, 515

respiration, 1039

restiform body, 370

secretions, 1178-1196

Intestine, absorption in, 733

law of, 727

movements of, 725-732

pendular movements of, 725

Intestinal fistula, 718

juice, 718

characters of, 720

secretion of, 719

Intima of artery, 870

Intra-auricular pressure, 901

Intra-molecular oxygen, 25

Intra-ocular fluid, 596
Intra-ocular pressure, 543, 595 pressure and blood pressure, 597

Intra-thoracic pressure, 1045

Intra-ventricular pressure curve, 898

Intra-vesical pressure, 1156

Intra-vitam staining, 23

Inulin, 69

Inversion, 67,160

Invertase, 67 of intestinal juice, 721

Invert sugar, 67

Iodine, 44 in thyroid gland, 1189 number of fats, 56

Iodothyrin, 1189

Ionisation, 128

Ions, 170 velocity of transport, 173

Iris, 542

as diaphragm, 537

functions of, 550

local stimulation of, 554 nerve-supply, 553

Irradiation, 339

Irreciprocal conduction, 275, 302

Iron, 42, 74 excretion of, 43 in large intestine, 724 in hæmoglobin, 74 in liver, 835

Island of Reil, 420, 423

Isoamylamine, 156

Isodynamic food-stuffs, 632

Isoleucine, 82

Isomaltose, 167,168

Isometric contraction, heat of, 221 lever, 197

Isosmotic solutions, 132

Isotonic lever, 197 solutions, 819

Isotropous substance, 182

JACKsONLAN epilepsy, 435, 439

Jaffé's test, 1117

Joints, sensibility of, 600

Jugular ganglion, 473

KaRyokinesis, see Mitosis

Karyosome, 16

Keratin, composition of, 91, 107

Keratins, 86, 107

'Kernleiter' model, 281, 284

Keto-acids, 49,154

Ketoses, 60

origin of, 762

Key, kick-over, 195

Keys, 187

Kidney, adaptability of, 1149

cells, structure of, 1145

functions of glomeruli, 1135-1142

nerves of, 1134

secretion in frog, 1145

of water and salts, 1135-1142

structure of, 1131-1134

tubules, functions of, 1144

absorption in, 1147

work of, 1135

Kinrsthetic areas, 442

Knce-clonus, 335

Knee-jerk, 333

abolition of, 334 
Knee-jerk, exaggerated, 451 latent period, 334

Knoop on deamination, 762

Krause's membrane, 179

Krogh's microtonometer, 1070

Kühne's gracilis experiment, 254

Kymograph, 872

LABLAL consonants, 527

Labour, 1235

Labyrinth, evolution of, 603 functions of, 398

Labyrinthine sensation, 603

Laccase, 1109

Lacrymal gland, 594

Lactalbumen, 1240

Lactase, action of, 158, 165 in intestinal juice, 721

Lactation, 1237-1245

Lacteals, 734, 1013

Lactic acid, 49

Hopkins' test, 215

in blood, 1085

in gastric juice, 684

in muscle, 215 in urine, 216,1085

Lactoglobulin, 1240

Lactosazone crystals, 1122

Lactose, 671240

Lævorotation, 52

Lævulinic acid, 104

Lagena, 603

Lamina cinerea, 375

Langerhans' islets in pancreas, 807

Lanoline, 56

Lardacein, 106

Large intestine, absorption in, 723 excretion in, 724

functions of, 722

movements of, 725-732

Laryngoscope, 523

Larynx, anatomy of, 521 movements of, in deglutition, 678

Latent period, 198, 207

Lateral columns of cord, 354

crico-arytenoid muscle, 522 fillet, 372,387

nucleus in medulla, 366 in pons, 370

sympathetic ganglia, 468

Lateritious deposit, 1118

Lauric acid, 54

Law of forward direction, 310 of the intestines, 727

Law of specific irritability, 480

Lecithin, 57

Leclanché cell, 187

Lemniscus, 368, 387

Lens, 543

elasticity of, 546 variation with age, 546

Lenses, formation of images by, 528

Lenticular ganglion, 552

Legumin, 98

Leguminous nodules, 41

Leucine, 82, 91

cones, 82

Leucines, oxidisability of, 1106

Leucocytes, chemiotaxis in, 1026 classification of, 813

origin of, 815
Lcucoplasts, 17

Icucosin, 98

Lever, momentum of, 197

Levulose, 64

Liebermann's rcaction, 94

Lifc, cvolution of, $5,6,33$ laws of, 7 without oxygen, 25

Ligamentum pectinatum iridis, 543

Light, nature of, 568 reflcx, 550

Lignin, 106

Lignoceric acid, 54

Limbic lobe, 417

Liminal intensity of stimulus, 482

Limulus heart, 946

Line of Gennari, 430

Lines of direction, 531

Linin, 17

Linoleic acid, 54

Linolinic acid, 54 series, 54

Lipase of pancrcatic juice, 707 in gastric juice, 689 reversibility of action of, 169

Lipines, 57

Lipoids, 23, 57

Liquor folliculi, 1223

Lissauer's tract, 324, 353, 357

Liver, bile formation in, 713

formation of urea in, 759 of uric acid in, 778

glycogen of, 797

hæmolysis in, 835

lymph production in, 1015

Living matter, chemical changes in, 153-169

Load, effect of a contraction, 203

Local reflexes, 474

sign of tactile sensation, 493

Locke's fluid, 963

Localisation of function in brain, 435

Locomotion, co-ordination of, 346

Locomotor ataxia, 346

Locus perforatus posticus, 375

Long ciliary nerves, 552 sight, 536

Longitudinal inferior fasciculus, 426 superior fasciculus, 426

Loudness of sound, 506

Ludwig's Stromuhr, 888 manometer, 872

Luminosity of spectral colours, 569,575

Lungs, circulation in, 935 distensibility of, 1044 exchange of gases in, 1068-1075

Lungs, vaso-motor fibres of, 935

Lutein, 1224

Luy's nucleus, 377

Lymph, absorption of, 1019 movements of, 1017 production, 1013

effect of capillary pressure on, 101 $\mathrm{j}$ in small intestine, 735 osmotic phenomena in, 1015

properties of, 1013

rǒle of, in nutrition, 1020

and tissuc fluids, 1012-1020

Lymphagogues, 1016

Lymphatic glands, 1013

tissuc, structure of, 815

Lymphatics, course of, 1012 
Lymphocyte, 813

Lysine, 83, 91

decarboxylation of, 155

Lysins, 1034

Macdonald's theory, 287

Macdougall's theory, muscular contraction, 235

Macro-nucleus of paramœeium, 1200

Macrophages, 1028

Macula acustica, 399, 605 lutea, 561

Magnesium, 43 excretion in large intcstine, 724

Major chord, 509

Make contraction, 192 excitation, 263 induction shock, 189

Mall on heart musculature, 891

Malleus, 513

Malpighian follicles of splcen, 1194 pyramids, 1131

Maltase, 167 of intestinal juice, 721 reversibility of action, 167

Malto-dextrin, 69

Maltose, 66, 67, 69

Mammary gland, structure of, 1243 hormones, 1218

Mannite, 62

Mannose, 61, 64

Manometer, Hürthle's, 897 Ludwig's, 872 Piper's, 897

Marchi's method, 320

Marey's law of blood-pressure, 980 sphygmograph, 922

Marginal lobe, 417

Marie's tract, 354

Mark-time reflex, 343

Marrow, 53

Martinotti cells, 428

Material basis of body, 36-122

Matter, exchanges of, in body, 613-657

Maximal stimulus, 193, 205

Mayer curves, 988

Mechanical changes in muscular contraction, 194-204

coagulation of colloids, 150

efficiency of muscle, 222

response of muscle, 205-211

Mechanism, 8

Meconium, 1233

Media of artery, 870

Medulla oblongata, 366-370 functions of, 392

Medullary sheath, 251

Medusa, nervous system of, 290

Meibomian glands, 594

Meiosis, 1207

Meissner's corpuscles, 497 plexus, $469,474,720$

Membrana granulosa, 1223 reticularis, 517 tectoria, 517 tympani, 512

Membranes, electrical phenomena at surface of, 173

permeability of, 22, 131

semi-permeable, 132

Membranous labyrinth, 515, 603
Mendel's law, 1215

Menstruation, 1224 relation of ovulation to, 1225

Mercurial manometer, 872

Mesencephalon, 363, 392, 394

Mesial fillet, 368,387

Mesotartaric acid, 52

Metabolism, effect of foods on, 631-636 during starvation, 624-630 experiments, analysis of excreta, 615 general, 613-647

influcnce of age on, 656 of carbohydrates on, 636 of fats on, 636 of muscular work on, 637 of protein on, 631 of temperature on, 1168,1172

methods, 614-623

of muscle, 216

of nucleo-proteins, 774-782

of proteins, 756-773

relation to body weight, 627 to surface of body, 628

Metakinesis, 9

Metal ' sols,' 140, 160

Metaphase of mitosis, 1205

Metaplasm, 16

Metaplasmic products, 18

Meta position, 50

Metaproteins, 98

Metcncephalon, 362, 392, 394

Methæmoglobin, 824

Methyl-acetate, catalysis of, 166

Methylamine, 49

Methyl glucosides, 66 glycine, 84 guanidin acetic acid, 84 purines, 103,775

Micellæ, 20

Micro-nucleus of paramœecium, 1201

Microphages, 1028

Microsome, 16

Microtonometer, 1070

Micturition, 1152-1160 nerves of, 1157 reflex, 1159

Mid-brain, 362,373 crusta, 374 functions of, 394 pes, 374 tegmentum, 374

Middle cell-lamina of cortex, 428

Milk, coagulation by pancreatic juice, $7 \mathrm{CE}$ fats of, 119, 1239 origin of, 1245

human, composition of, 657 properties of, 1238 proteins of, 1239 in relation to growth, 657 quantity secreted, 1238 salts of, 1241 secretion of, 1237-1245

Milk-sugar, 1240

Millon's reaction, $85,94,100$

Mineral salts, importance of, 647

Minimal difference method, 484 effective stimuli, 483

gradient, 272 stimulus, 193, 205, 482

Mitosis, 1207 heterotype, 1207 
Mitral cells, 421 valve, 893

Modality of sensation, 479

Molecular layer of cortex, 428

Molecules, energy in solution, 123 size of, 140,143

Molisch's test, 64,94

Molybdic acid as catalyser, 162, 163

Monakow's bundle, 353, 389

Mono-amino-dibasic acids, 82

Mono-amino-monobasic acids, 81

Mono-amino-nitrogen in proteins, 93

Monochromatic patches, 575 vision, 579

Monomolecular reaction, 163

Monophasic variation, 230

Monosaccharides, 61

Moore's test, 63

Morphotic proteins, 642

Moss fibres, 401

Motor aphasia, 454 arca, lamination, 431 cells of cord, 318 centres, ablation of, 440 end-plates, 183 sensibility, 446

'Motor points,' 269 somatic nuclei, 378

Mountain sickness, 1099

Movement, co-ordinated, 338 and sensation, 177-609 sensation 3 of, 598-602

Movements of alimentary canal, $676,697,725$ of deglutition, 676

of large intestinc, 729

of small intestine, 725

of stomach, 697

Mucin, action of gastric juice on, 688

Mucins, 105

Mucoids, 105

Mucous glands, 663

Müller's law of specific irritability, 255, 480

Multirotation, 65

Murexide test, 1118

Muscæ volitantes, 537

Muscarine, action on hcart, 974

Muscle, afferent nerves, 336

anistropic substance, 182

action of veratrin, 211

arrested contraction, 200

break contraction, 192

chemical changes in, 212-218

chemical stimulation of, 186

clotting of, 213

composition, 212

contractile stress, 200

contraction-remainder, 208

demarcation current, $170,225,233$

efficiency of, 222, 639

elasticity of, 203

electrical variation, effect of temperature, 230

excitation of, 185-193

excitation without contraction, 231

extensibility of, 203

heat-production in, 219-223

independent excitability, 185

injury-current, $170,225,233$

involuntary, 180, 243

of invertebrates, 248

isotropous substance, 182
Muscle, longitudinal striation, 180

make contraction, 192

mechanical response of, 205-211

metabolism of, 216

production of carbon-dioxide, 217

products of activity, 216-218

reciprocal innervation of, 355

red, 180

reflex tone, 398

respiratory quotient, 217

stimulation by constant current, 186 , 192

thickening of, 199

utilisation of encrgy, 221

voluntary, 177 structure of, 179

white, 181

Muscle-current, 170, 225, 228, 229

Muscle-curve, correction of, 197, 208

Muscle fibre, 179

Muscle-fibrillæ, 179 reversal of stripes, 182

Muscle-plasma, 212

Muscle-sound, 240

Muscle-spindle, 601

Muscle-twitch, 192, 194, 198

Muscle-wave, 204

Musical notes, vibration frequencies, 510

Muscular afferents, 600 contraction, ' all or none' phenomenon, 255

Engelmann's theory, 234

effect of drugs, 211

of fatiguc, 208

of load, 203

of salts, 210

of temperature, 207

of tension, 222

latent period of, 198

Macdougall's theory, 235

mechanical changes in, 194-204

nature of, 234-238

optical method, 198

osmotic theory, 235

point of stimulation, 194

Schäfer's theory, 182

strength of stimulus, 205

time-rclations of, 198

voluntary, 239

electrical changes in, 242

record of, 241

contractions, summation of, 206

energy, source of, 637

exercise, effect on circulation, 1006-1008

movements, co-ordination of, 345

relaxation, 198

sense, 600

psychology of, 601

sensibility, paths, 446

tone, 333,449

relation to labyrinthine sensations, 606

work, encrgy exchanges in, 639

effect on metabolism, 637

Muscularis mucosæ of small intestine, $73 \tilde{5}$

Musculi papillares, 894

Musculus vocalis, 522

Myelencephalon, 362, 392

Myelin sheath, 251-252

Myelination, 252

method, 319 
Myelocytes, 816

Mylohyoid of frog, 184

Myogen, 213

$$
\text { fibrin, } 213
$$

Myogenic movements of intestine, 726

Myohæmatin, 213

Myopia, 535

Myosin, 98, 213

Myosin fibrin, 213

Myosinogen, 212

Myristic acid, 54

Myxœdema, 1187

NASAL consonants, 527

Near point, 539,546

Nectocysts, 33

Negative after-image, 572

polarisation of nerve, 280

variation, 233

ventilation, 1091

Negativity, 229

Neopallium, 417 evolution of, 419

Nerve, core model, 281 double conduction, 250 electrotonic current, 280 electrotonus, 265

endoneurium, 252 excitability, 'characteristic' of, 263 , 271

effect of temperature on, 273

fatigue in, 259, 285

galvanic excitation, 262

human, stimulation, 268

law of forward direction in, 310

negative polarisation, 282

neurilemma, 252

nodes of Ranvier, 252

non-medullated, 252

polarisation in, 280

positive polarisation, 282

primitive sheath, 251

unipolar excitation, 268

Nerve-axon, 251

Nerve-block, 266

Nerve-cell, automaticity of, 314

functions of, 312

Golgi net, 301, 309

Nissl's granules, 300

pericellular network, 308

structure of, 300

Nerve-centres in medulla, 393

Nerve-eurrent, Macdonald's theory, 173

Nerve fibres, 250-287

excitation of, 262

size of, 252

Nerve fibres, structure, 250

Nerve-impulse, 253

conditions affecting, 258

effeet of temperature on, 258

electrical ehanges accompanying, 256

influenee of drugs on, 260

velocity of, 253

Nerve-injury, effect of, 274

Nerve-trunk, composition of, 327

Nerves, cranial, 410

nuclei of, $378,410-415$

grafting of, 255

Nervi erigentes, functions of, $473,996,1154$, 1158,1228
Nervous system, evolution of, 288-296 ganglia, 293 of medusa, 290

Neural canal, 297 of vertebrates, 297

Neurilemma, 251

Neurine, 57

Neuroblasts, 298

Neuro-fibrillar network, 428

Neuro-fibrils, 296

Neuroglia, 297 continuity of, 307

Neurokeratins, 107, 251

Neuro-muscular junction, 183, 275 spindles, 601

Neuron, definition of, 295, 301

Neurons, continuity of, 296 structure, 296

Neuropilem, 296

Neutrophile leucocytes, 813

Nicol's prism, 51

Nicotine, effect on end-plates, 277 method, 472 on heart, 974

Ninth nerve, nucleus of, 379,414

Nissl's granules, 301

Nitrates, 40

Nitrification of sewage, 40

Nitrifying organisms, 40

Nitrogen, 39 endogenous, in urine, 758

estimation of by Kjeldahl's method, 1126

excretion in starvation, 629,633

exogenous, in urine, 758

in food, 614 .

output, 756

requirements of body, 633

source of, 39

Nitrogenous constituents of urine, 757 equilibrium, $616,631,653$

Nociceptive stimuli, 341

Nodal point, 531, 533

Noud vital, 1077

Non-polarisable electrodes, 224

Normoblasts, 834

Nuclear sap, 17

Nucleic acid, 101

Nuclein, 97, 101 hydrolysis of, 774 metabolism of, 774-782

Nucleins, fate of, 777 formation in body, 777

Nueleolus, 16

Nucleoplasm, 17

Nucleoprotein, deeomposition of, 102

Nucleoprotein, digestion of, 104

Nucleus, 14, 27, 31

ambiguus, 379

of Bechterew, 381, 402

branched, 31

caudatus, 363

cuneatus, 367

emboliformis, 373

effect of removal, 28

fastigii, 373

function of, 27

globosus, 373

graeilis, 367

lentieularis, 363

nccessity for growth, 30 
Nuclcus of Luys, 377

of Rolando, 367

relation to cytoplasm, 27

Nutrition, mechanisms of, 613,809

Occipital lobe, 417

Occipito-frontal fasciculus, 426

Oculo-motor nuclcus, $379,407,410$

Oecoid, 818

Esophagus, inhibition of, in swallowing, 680

Estrus, 1226

Ohm's law of auditory analysis, 511

Oleie acid, 55,56

Oleyl-lecithin, 57

Olfactie, 504

Olfactometer, 503

Olfactory apparatus, 421

area, 448

bulb, 421

glomeruli, 421

lobe, 417,448

mucous membrane, 502

sensations, 498,502

tract, 421

tubercle, 421

Olivary body, 367,382

Olive, 368,382

Olivo-cerebellar fibres, 389

Olivo-spinal tract, $353,39 \mathrm{l}$

Oncometer, 992

Optic axis, 531

chiasma, 388, 406

dise, 561

nerve, decussation, 387,406 efferent fibres in, 560

radiation, 423

thalamus, $363,375,383,395,420$ nuclei of, 377

tracts, 387, 406, 447

Optical activity, 51

axis of lens, 528

centre of lens, 529

defects in eye, 535

isomers, aetion of ferments on, 167

Optimum temperature for ferments, 160

Opsonic index, 1038

Opsonins, 1037

Ophthalmometer, 541

Ophthalmoscope, 554-557

Optograms, 565

Ora serrata, 561

Orcin reaction for pentoses, 61,94

Organ of Corti, 517

Organic compounds in body, 45

Organic sensations, 598-609

Organic synthesis, mechanism of, 109

Organisation, 6

Ornithine, 79, 83

decarboxylation of, 156

Ortho position, 50

Osamines, 63

Osazones, 47, 62, 1123

Osmometer, 143

Osmotic pressure, 123, 133

Barger's method, 129

Beckmann's method, 129, 130

and boiling-point, 129

of colloids, 143

of electrolytes, 128

freezing-point method, 129

by hæmolysis, 127
Osmotic pressure, Hamburger's method, 127 measurement of, 125-130 by plasmolysis, 127 of proteins, 75,142 of scrum protcins, 142 and transport of water, 133 and vapour tension, 129

Osseous labyrinth, 515, 604

Osteoporosis, 626

Otie ganglion, 473

Otolith organ, 399, 605, 608

Otoliths, function of, 608

Outer cell lamina of cortex, 427

Outer fibre-lamina of cortex, 427

Outer line of Baillarger, 430

Overtoncs, 507

Overton's theory, 23

Ovulation, 1222

Ovum, 1205 maturation of, 1209

Oxalate crystals, 1125

Oxidases in tissues, 1108

Oxidation, 157, 1063, 1105-1109 by indigo, 161 mechanisms of, 1105-1109 seat of, in body, 1063

Oxyacids, 49 origin of, 761

Oxygen, 39 absorption of, in lungs, 1062 'active,' 1107 avidity of tissues for, 1063 capacity of blood, 1056 effect of ehanges in tension of, 1099 laek, in asphyxia, 985 production of lactic acid in, 1085 tension in alveoli, 1062, 1070 in blood, 1071 in blood by $\mathrm{CO}$ method, 1074

Oxyhæmoglobin, 821 absorption spectrum of, 823 crystals, 821 dissoeiation of, 1060 influence of acids on reduction of, 1064 p-oxyphenyl alanine, 84

Oxyproline, 85

Pacinian corpuscles, 497

Pain impulses, path of in cord, 359 sense, 494

Palmitic acid, 54

Pancreas, extirpation of, 804 internal secretion of, 806 struetural changes aecompanying secretion, 711

Panereatic diabetes, 804 fistula, 707

juice, 702 action on carbohydrates, 706 fats, 707 milk, 706 protcins, 703

activation of, 705 by calcium salts, 706 composition, 702 secretion of; 707 variations in, 710

secretion, effect of acids in duodenum, 708 regulation of, 710 secretin, 709

Pangene, 20 
Para position, 50

Paracasein, 1240

Paracentral lobc, 417

Paradox cold, 488

Paradoxical contraction, 283

Paraffins, 45

Paraglobulin, 98

Paramœecium, conjugation of, 1201

Paramucin, 105

Paramyosinogen, 98, 213

Paranuclein, 100, 688

Paraplasm, 16

Parathyroids, functions of, 1189

Parietal lobe, 417

Parotid gland, 663 innervation of, 666

Parthenogenesis, 1211

Partition coefficient, 24

Parturition, 1233-1236 nervous mechanism of, 1236

Passive movement, 600

Pawlow's gastric fistula, 690

Pelvic visceral nerve, 473 action on bladder, 1157

Pendular'movements of intestine, 725

Pendulum myograph, 195, 196

Pentachromic vision, 580

Pentamethylene diamine, 155

Pentosanes, 61, 106

Pentoses, 61, 105

Pentosuria, 61

Pepsin, action of, 685

Peptone, food value of, 645

Peptones, 100 fractionation of, 685

Peptonised blood, 840,849

Pericardium, use of, 894,910

Perilymph, 515

Perimeter, 563

Peripheral ganglia, inhibition in, 475 nerve nets; 469 reflexes, 474

Peripolar zone, 268

Peristalsis, 727

Permeability of membranes, 22, 125, 131

Peroxidases, 1108

Pes, 374

Pettenkofer's respiration apparatus, 618

Pfeffer's cell, 125

Pfiüger's law, 267

Phagocytes, 1022

Phagocytosis, 816, 1022

Phakoscope, 540

Phenylalanine, 85, 91

Phenylethylamine, 156

Phenylglucosazone, 63

Phenylhydrazine test, 123,162

Phenyllactosazone, 68

Phenylmaltosazone, 67

Phloridzin diabetes, 801

Phloroglucin reaction for pentoses, 61

Phonation, pressure, 523

Phosphates, estimation of, 1129 excretion in large intestine, 724 in urine, 1113

$$
\text { by kidney, } 1147
$$

Phosphatides, 57

Phospholipines, 57

Phosphoprotein, 100

Phosphoproteins, action of gastric juice on, 68

Phosphorus, 43, 57
Photochemical substances in retina, 567

Photo-hæmatachometer, 889

Phototaxis, 27

Phrenic ncrve, electrical variations in, 242

Phrenology, 434

Physiology, definition of, 1, 7 general, 13

Physiological heat-values, 621

Pick on fractionation of protcoses, 685

Picric acid, 51

Pilomotor nerves, 469

Pineal gland, 1193

Pinna, function of, 511

Piqûre diabetes, 800

Pituitary body, extirpation of, 1191 structure of, 1190

extract, effect of, 1192

Placenta, functions of, 1232

Plain muscle, 180, 243 chemical stimulation of, 246 contraction, time-relations of, 243 double innervation of, 247 influence of temperature on, 247 mechanical stimulation of, 246 stimulation of, 244

Plasina, muscle-, 212 blood, 811,857

Plasmahaut, 21

Plasmolysis, 22, 23, 127

Plasmosome, 16

Plasome, 20

Plasteins, 169

Plastids, 17, 33

Plethora, 1009

Plethysmograph for kidney, 993

Pleura, 1040 permeability of, 136

Pleural cavity, negative pressure in, 1045

Pneumogastric nerve, see Vagus

Pohl's reverser, 188

Poikilothermic animals, 1169

Polar bodies, 1209

Polar zone, 268

Polarimeter, 51

Polarisation, 51, 173, 224 by colloids, 145 in nerve, 280

Polarised light, 51

Polarising current, 265

Polymorphonuclear leucocytcs, 813

Polymorphous layer of cortex, 428

Polypeptides, 89, 90, 99, 704 action of trypsin on, 89 reactions of, 89 synthesis of, 88

Polysaccharides, 62, 68

Pons Varolii, 371

antero-lateral tract, 372

functions of, 394

pedal portion, 372

structure, 370

tegmentum, 372

Portal system in birds, 761

Position, sensations of, 603-609

Positive after-images, 571 polarisation of nerve, 282 ventilation, 1091

Posterior columns of cord, 354 crico-arytenoid muscle, 521

longitudinal bundle, $369,370,372,381$, 389,408 
Posterior root-ganglion, development, 298

Postganglionic fibres, 472

Postural reflexes, 449 tonus, 449

Potassium, 43

Potential energy of compounds, 156

Precipitins, 1036

Precuneus, 417

Preganglionic fibre, 471

Pregnancy, 1230-1236

Pre-pyramidal tract, 353

Presbyopia, 546

Pressor reflexes, 1001

Pressure impulses in cord, 359 sense, 489 slope in vascular system, 877

Primary coil, 189

Primitive sheath of nerve, 251

Primordial follicles, 1222 utricle, 14, 127

Principal focus of lens, 531 plane, 531 point, 531, 533

Projection fibres of cerebrum, 422

Projicient sense organs, 293, 384

Proline, 85, 91 fate of, 772

Pro-nuclei, 1209

Proøestrum, 1226

Prophase of mitosis, 1205

Propionic acid, 48, 54

Proprioceptive system, 397, 479, 598

Propriospinal fibres, 351

Pro-secretin, 709

Prostate, 1221

Prosthetic group, 101

Protamines, 92, 97, 101

Proteins, 45, 71

absorption of, 744

amide nitrogen in, 93

ammonia nitrogen in, 93

amount of nitrogen in, 616

biuret reaction, 93

carbohydrate radicle in, 94

classification of, 97-108

colour reactions of, 93

composition of, 71,90

conjugated, 101

constitution of, 90

copper compounds, 75

crystallisation of, 72

derivatives of, 98

diamino-nitrogen, 93

disintegration products of, 81

distribution of nitrogen in molecule, 91

effect of, on metabolism, 631, 653

halogen derivatives, 98

heat coagulation of, 72,95

hydrated, 98

hydrolysis, 76, 99

hydrolysis by cnzymes, 76

in urine, 1121

metabolism of, 756-773

effects of variation in diet, 633

endogenous, 767

Folin's theory, 644

in starvation, 630,633

Pfüger's theory, 642

Voit's theory, 642

minimum requirement, 654

molecule, structure of. 76,88
Proteins, synthesis of, 88

molecular weight of, 74

osmotic pressure of, 75

oxidation of, 764

physical characters, 72

precipitation by metallic salts, 94

putrefaction of, 76

salting out, 96

salts of, 72

scparation of, 95

specific dynamic effect of, 636

sulphur in, 74

synthesis of in body, 113-119

tests for, 93

Proteose, food value of, 645

Proteoses, 100

fractionation of, 687

hydrolytic products, 687

Prothrombin, 843

Protocerebrom, 294

Protopathic sensibility, 495

Protoplasm, 15

constituents of, 36

physical condition of, 20

salts of, 43

surface tension in, 21, 24

theories of structure, 17

ultra-microscopic structure, 20

Proximate constituents of the body, 45

Psalterium, 427

Pseudo-globulin, 866

Pseudo-ions, 149

Pseudomucin, 105

Pseudo-nuclein, 100, 688

Pseudopodia, 14

Pseudo-reflexes, 474

Psychical secretion of gastric juice, 691

Ptyalin, 661

Puberty, changes at, 1217

Pulmonary circulation, 935-938 ventilation, 1046

Pulse, 918-928

anacrotic wave, 926

catacrotic, 926

clinical features of, 928

dicrotic wave, $921,923,925$

in veins, 933

percussion wave, 923

pre-dicrotic wave, 923

primary wave, 923

Pulse-pressure, 875

Pulse-rate, in man, 981

Pulse-wavc, velocity of, $92: 3$ nature of, 918

Pulvinar, 376

Pupil, 543 contraction of, 550

dilatation of, 551

Purine bases, 102

in fæces, 754

in urine, 780

metabolism, 774-782

ring, 102, 775

Purkinje cells of cerebellum, 400 fibres of heart, 952

figures, 562

Putrescinc, 156

Pyloric canal, 698

sphincter, 699

vestibule, 698

Pylorus, nervous mechanism for opening, 699 
Pylorus, opening of, 697

Pyramidal cells of cortex, 427

decussation, 352,366 tracts, $352,366,423$

Pyrimidine, 103 bases, 775

Pyrogallol, 50

Pyrrol ring, 85 synthesis of, 118

a.pyrrolidin carboxylic acid, 85

Pyruvic acid, 49, 762 origin of, 762

QUADRI-URATES, 1118

Quellung, 151

RACEMIC compounds, 52

Rami communicantes, 468

Reaction, velocity of, 162,163 of blood, 987, 1087 of degeneration, 269 time, 457

Reactions, balanced, 166 reversible, 166,169

Recapitulation, Law of, 13

'Receptor' substance, 276 action of adrenaline on, 278, 1184

Recessive characters, 1215

Reciprocal innervation from cortex, 437 of antagonistic muscles,

Recti muscles, 585

Red-blindness, 578 corpuscles, see Blood marrow, 834 nucleus, 377,383

' Red reflex,' 555

Reduced eye, 533

Referred pain, 476

Reflex action, Bahnurg, 305

block in, 305

delay in, 303

facilitation of, 305

fatigue of, 304, 343

general characters of, 303

inhibition of, 306,339

in spinal animal, 329,332

localisation, 303

reinforcement, 341

successive spinal induction, 344

summation, 304

arc, 178, 295

movements, 177

scratch-, 332, 343

time, 304

tone, 398

Refractive indices in eye, 533

Refractory pcriod, 273

Regnault and Reiset's respiration apparatus, 617

Reissner's membrane, 516

Remak's ganglion, 940,942

Renal excretion, $1110-1160$ mechanism, 1149-1151

Rennin, 688

Pueproduction, 1199-1245

in $\operatorname{man}, 1217-1245$

in metazoa, 1202

in protozoa, 1200

Reproductive organs, development of, 1217 female, 1221
Reproductive organs, male, 1219

Residual air, 1047

Resonants, 527

Pesonators, 507

Respiration, 1039-1109

apparatus, Benedict's, 617

Haldane's, 616

Pettenkofer's, 618

Regnault and Reiset, 617

Zuntz and Geppert, 618

chemistry of, 1051-1075

effects of changes in air on, 1698-1104

effect on circulation, 937 of deglutition on, 681 of division of vagi, 1089

movements, co-ordination of, 1076

rate of, 1041

reflex regulation of, 1089-1097

'Respiration of swallowing,' 677

Respiratory centre, 1077

$$
\begin{aligned}
& \text { automaticity of, } 1078 \\
& \text { chemical excitants of, } \\
& 1079-1081,1087 \\
& \text { inhibitory action of vagus } \\
& \text { on, } 1092 \\
& \text { spinal, } 1078 \\
& \text { stimulation of, by acids, } \\
& 1087 \\
& \text { by oxygen lack, } \\
& 1083
\end{aligned}
$$

exchange, total, 616

movements, chemical regulation of, 1079-1089

Head's method, 1089

mechanics of, 1039-1050

regulation of, 1076-1097

muscles, 1043

quotient, 640, 1051

effect of foods on, 641

in diabetes, 809

in hibernation, 794

in muscular work, 217

sounds, 1045

Restiform body, 368, 402

Rete mucosum, 1161

Reticulin, 107

Retina, chemical changes in, 564

development of, 558

physical changes in, 564

structure of, 559

Retinal changes in vision, 558-567

image, path of rays, 534 induction, 582

Retractor lentis, 548 penis muscle, 247, 1229

Retrograde degeneration, 320

Reverser, Pohl's, 188

Reversibility of ferment action, 166,168

Revertose, 167, 168

Rheocord, 192

Rheonome, 270

Rheoscopic frog, 233

Rheotaxis, 1228

Rhinecephalon, 421

Rhodopsin, 559, 565

Rhombencephalon, 362

Ribs, movements of, 1042

Ricin, 1031

Rigor mortis, 208, 214

Rima glottidis, 521

Ringer's fluid, 963 
Ritter-Valli law, 274

Rods of Corti, 516

Rolandic fissure, 419

Roof nuclei of cerebellum, 383, 402

Rubro-spinal tract, 353, 389

Ruffiri's organs, 497

SacCharic acid, 62

Saccharose, 67

Saccule, 515

Saccus endolymphaticus, 604

Sacral autonomic fibres, 473

Salicylic acid, 50

Saliva, composition of, 661 energy involved in sccretion, 674 secretion of, 663

effect of nerves on, 666

Salivary secretion, energy changes in, 674

effect of metabolites, 675

histological changes in, 670

pressure, 668

theorics of, 668

Salivary digestion, 662

$$
\text { in stomach, } 663
$$

glands, innervation of, 665

changes accompanying secre. tion, 668-673

double nerve-supply, 673

electrical changes in, during secretion, 672

Salmin, composition of, 91

Salt hunger, 647 solutions, absorbability of, 738

Salts, absorption of, 753

Saponification, 46, 56 number, 56

Sarcolactic acid, 215

Sarcolemma, 179

Sarcomere, 179

Sarcoplasm, 179

Sarcosine, 84

Sarcostyles, 179, 181

Sarcous elements, 181

Sartorius of frog, 183

Scala media, 515

Scatol, fate of, 770

Schäfer's theory of contraction, 182, 236

Scheiner's experiment, 589

Schematic eye, 532

Schlemm, canal of, 543 functions, 596

Schwann's sheath, 251

Sclcroproteins, 106

Sclerotic coat of eye, 542

Scratch reflex, 332,343

Sebaceous glands, 1162

Sebum, 1162

Second wind, 1008

Secondary contraction, 233

Secretin, 709, 1179 action on intestinal secretion, 720

$$
\text { liver, } 716
$$

Secretion, energy changes in, 674

Semicircular canals, 399,515 destruction of, 606 functions, 605

Semilunar ganglion, 466 valves, structure of, 893

Semi-permeable cell, 125 membranes, 125, 132
Sensation, extent of stimulus, 482 localisation of, 481

modality of, 479

physiology of, 478-609

projection of, 481

quantitative study of, 482-485

relation to stimulus, 478-48.)

Sensations, organic, 598-609

Sensori-motor a reas, 444

Sensory adaptation, 482 aphasia, 454

arcas of cortex, 444

association, 451

paralysis, 346

path, 357

Septo-marginal bundle, 354

Serine, 81,91

Serous salivary glands, 663

Scrtoli, cells of, 1221

Scrum-albumin, 98, 865 composition of, 91 crystallisation of, 73

Serum-globulin, 98, 866

Scrum-proteins, osmotic pressure of, 143

Scventh nerve, nucleus of, $379,381,413$ visceral fibres, 472

Sexual organs, relation to ductless glands, 1219 process, essential features of, 1199-1211

Sham feeding, 690

Shock, 306 spinal, 330

Short ciliary nerves, 5.52 sight, 535

Sibilant consonants, 527

Side-chain theory, 1034

Side wire, Helmholtz, 189

Simultaneous contrast, 583

Sino-auricular node, 951

Sixth nerve, nucleus of, 379,411

Size, illusions of, 591 judgment of, 590

Skin, absorption by, 1165 functions of, 1161-1166 gaseous exchanges in, 1165 structure of, 1161

Small intestine, absorption from, 733 innervation of, 728 lymph production in, 735 movements in, 725-729

Smell, sense of, 501 sensations, 498, 502

Smooth muscle, see Plain musclc

Soaps, 46, 55 in digestion, 742

Sodium, 43

Solar plexus, 468

Solidity, judgment of, 593

Sols, 139

Solubility of gas, effect of pressure on, 1057

Solute, 131

Somatic nervous system, 466

Sorbite, 62

Sound, nature of, 505 pitch of, 506 timbre, 506

Spaces of Fontana, 543

Spastic gait, 335,450 paraplegia, 335,450

Specific dynamic action of proteins, 636 , 764,803 irritability, law of, 255 
Speech, 453, 520-527

intellectual basis of, 455

mechanism of, 525

Spermaceti, 56

Spermatids, 1208

Spermatocytes, 1208

Spermatozoa, development of, 1208

Spherical aberration, 537

Sphincter pupillæ, 550

trigoni, 1153

urogenitalis, 1153

Sphingomyeline, 57

Sphygmographs, 922

Spinal animal, 329, 393

Spinal cord, afferent path, 328

anterior cerebellar tract, 354 columns, 356

ascending tracts, 352,354

Burdack's column, 324

cells in 318 of columns, 318

central canal, 300

Clarke's column, 318

collaterals in, $324,351,357$

com $\sqrt{n a}$ tract, 353

commissural cells, 318

conduction in, 351-360

crossed pyramidal tract, 353

descending tracts, 352

development, 299

direct cerebellar tract, 354 pyramidal tract, 352

dorsal cerebellar tract, 354

effect of poisons, 347

efferent path, 327

endogenous fibres, 352

Golgi cells, 318

Golls' column, 324

Gower's tract, 356

Helweg's bundle, 353

hemisection, 359

lateral basis bundle, 371

lateral columns, 354

Lissauer's tract, 324, 352, 357

Marie's tract, 354

Monakow's bundle, 353

motor-cells, 318

olivo-spinal tract, 353

pain impulses, 359

path of impulses 357

postero-external column, 324

pre-pyramidal tract, 353

pyramidal tracts, 352

as reflex centre, 322

rubro-spinal tract, 353

scnsori-motor path, 357

septo-marginal bundlc, 354

structure, 315

thalamico-spinal tract, 353

tracts, methods of tracing, 319

trophic functions, 349

ventral cerebellar tract, 354

vestibulo-spinal tract, 353

Wallerian degeneration' 352

white matter, arrangement, 351

Spinal dog, 331 shock, 330

Spino-tectal tract, 356

Spino-thalamic tract, 356

Spiral ganglion, 517
Spiral lamina, 517

Splanchnic nerve, 996

Splecn, function of, 1195

rhythmic contractions of, 1195

structure of, 1194

Spongin, 108

Spongioblasts, 252, 298

Spongioplasm, 18

Staircase phenomenon, 245

in heart muscle, 956

Stannius' ligature, 940

Stapedius muscle, 514

Stapes, 513

Starches, 68

Starch, digestion by saliva, 662

hydrolysis of, 68,99

molecular weight, 68

soluble, 68

structurc of, 69

Starvation, carbohydrate metabolism in, 628

fat metabolism in, 630

loss in various organs, 625

metabolism in, 624-630

protein metabolism in, 630,633

Static ataxy, 346

Stearic acid, 54 ,

Stearyl-lecithin, 57

Stellate ganglion, 466

Stepping reflex, 332

Stercobilin, origin of, 715

Stereoisomerism, 52, 60, 78

Stereosomers, action of ferments on, 167

Stereoscope, 593

Stercoscopic vision, 593

Sternzellen, 816

Stimulation, electrical, nature of, 270 of human nerve, 268

Stimuli, summation of, 245

Stimulus, intensity of, 482

liminal, 482

locus of, 339

maximal, 193, 205

minimal, 193, 205

threshold, 483

Stokes-Adams disease, 955

Stomach, digestion in, 683-696

innervation, 700

movements of, 697

secretory nerves of, 691

sphincters, 701

Stratum granulosum, 1161

Stratum lucidum, 1161

Stria terminalis, 377

Striæ acousticæ, 379,380

Striated muscle, structure of, 179

of invertebrates, 248

String galvanomcter, 228

Stromuhr, Ludwig's, 888

Starling's, 888

Structural basis of body, 13

Strychnine, effect on cord, 347 spasm, electrical variation, 242

Sturin, composition of, 91

Sublingual gland, 663

Submaxillary ganglion, 473, 666 gland, 663

Substantia gelatinosa of Rolando, 316 nigra, $374,377,383$

Substrate, 164 effect of ferments on, 167 
Subthalamic region, 377

Successive contrast, 583 spinal induction, 344

Succus entericus, see Intestinal juice

Sugar in urine, 1122 formation of, from fat, 794 in plants, 111 from protein, 1122 utilisation of, in body, 799

Sugars, chemistry of, $60-68$ oxidisability of, in body, 1106

Sulphocyanate in saliva, 661

Sulphur, 42 excretion of, 769 test for proteins, 86,94

Summation, 245 of muscular contractions, 206 of sensation, 482 of stimuli, 245,272 tones, 510 in heart muscle, 956

Supplemental air, 1046

Suprarenal bodies, 1181-1186

Surface tension, 21, 24 in colloids, 141

Suspensory ligament, 543

Swallowing - see Deglutition

Sweat, amount and properties, 1163 glands, 1163

loss of heat by, 1176 secretion, 1164

Swim-bladder of fish, 1074 tension of oxygen in, 135

Sylvian aqueduct, 363,373 fissure, 419

Symbiosis, 1021

Sympathctic ganglia, 466 functions, 474 system, 969,975

Tachypnce, 1080

'Tactile localisation, 489 sensations, 489 sense area, 444 paths, 444

Talbot's law, 572

Tartaric acid, 52

Taste, 498

area, 448

buds, 499

nerves of, 500

sensation, classification, 499

'Tears, composition of, 594

Tecto-spinal tract, 391

T'enon, capsule of, 585

'T'ension, effect of on bladder, 1156 on heart, 958 on muscle, 201

Tensions of gases in liquids, 1059

'Tensor tympani muscle, 5141

Tenth nerve, nucleus of, 414

Testis, structure of, 1219

Tetanus, 207 toxin, action on cord, 348

Tetrachromic vision, 580

Tetrapeptides, 89

Tegmentum of pons, 372 of mid-brain, 374

T'eichmann's crystals, 825

Telencephalon, 363
Telophase of mitosis, 1206

Temperature, action of, on heart, 962

on muscle, 207

effect of, on metabolism, 1168, 1172 on electrical variations in muscle, 230

limits of, for life, 6

sense, 486 adaptation of, 488

Temporal lobe, 417

'Temporo-pontine fibres, 424

Tendon phenomena, 333

reflexes, 333

use of, 336

'Thalamencephalon, 392, 395

'Thalamico-spinal tract, 353

Thalamo-cortical tract, 422

'Thalamo-frontal fitres, 423

Thalamo-spinal tract, 391

Theobromine, 103, 775

Thermo-electric couple, 220

Thermogenic centres, 1177

'Thermopile, 219

Thermotaxic system, 1177

'Thigmotaxis, 27

Thiophene test, 215

Third nerve, functions of, 552 nucleus of, $379,382,408,410$ visceral fibres in, 472

Thiry-Vella fistula, 718

'Threshold stimulus, 483

Thrombin, 842

Thrombogen, 844

Thrombokinase, 844

Thymine, 104, 776

Thymus, structure and functions of, 1193

Thyroid gland, 1186 extirpation of, 1187 extract, effects of, 1188 structure of, 1186

Tidal air, 1046

Tissue-fibrinogen, 102, 846

Tissue-proteins, 642

Tone in muscle, 333 in heart, 958

Tonus, postural, 449

Tortoise heart. 040

Touch discrimination, 491 impulses in cord, 359 projection of, 493 sense, 489 spots, 489

Toxins, adsorption of, 1034 bacterial, 1030 mode of action, 1030

Toxoids, 1034

Toxones, 1032

Toxophore group, 1034

Traube-Hering curves, 988

Trapezium of pons, 371, 381, 387

'Trichromats, anomalous, 581

Trichromatic vision, 578

Tricuspid valve, 893

Trigeminal nerve, nucleus of, 380

Trigemino-thalamic tract, 387

Trimethylamine, 49 .

Tripeptides, 89

Triple phosphate, 1114, 1125

Tritoccrebrom, 294

Trommer's test, 62

Trophoblast, 1231 
Trypsin, 703

action on polypeptides, 704

on proteins, 703

velocity of action, 166, 705

Trypsinogen, 703

Tryptophane, 85, 91, 94 fate of, 772

Tuber cinereum, 363, 375

Tympanic membrane, 512 movements of, 513

Tympanum, 512

Tyrosinase, 1108

Tyrosine, 50, 84, 91

in urine, 1125

Uffelmann'\& reagent, 215

Ultra-microscope, 145

Umbilical cord, 1232

Uncinate fasciculus, 426

Uncus, 419

Unipolar excitation, 269

law of, 269

Unstriated muscle, 243

Uracil, 104, 776

propagation of wave in, 246

Urate deposit, 1124

Urates, 1118

Urea, 1115

estimation, 1126

Folin's method, 1127

hypobromite method, 1126

fermentation of, 1115

origin of, 759

output in starvation, 630

on protein diet, 758

preparation from urine, 1117

Ureter, contractions of, 1152

Uric acid, 103, 1117

crystals, 1125

daily amount, 1119

endogenous, 780

estimation, 1128

excretion, 779

in gout, 781

origin of, 777

preparation, 1117

production in birds, 760

structure of, 775

synthesis, 103

tests, 1118

Uricolytic ferment, 779

Urinary constituents, estimation of, 11261130

deposits, 1124

Urine, abnormal constituents in, 1121-1125

acetone in, 1123

ammonia in, 1115

average composition, 1112

bases of, 1114

chlorides of, 1112

composition of, 1110-1130, 1142

on various diets, 780

conditions of glomerular filtration, 1138

freezing-point, 1111

inorganic constituents of, 1112

lactose in, 1123

neutral sulphur in, 1113

organic constituents of, 1115

oxyacids in, 1123

phosphates of, 1113
Urine, pigments of, 1120

pressure of, in uretcr, 1137

reaction of, 1111

secretion of, 1131-1151

in glomerulus, 1135

influence of colloids, 1137

specific gravity of, 1111

kidney volume, 1139

sugar in, 1122

sulphates of, 1113

Uriniferous tubule, course of, 1131

tubule, functions of, 1134

Urobilin, 1121

Urochrome, 1120

Uroerythrin, 1121

Urorosein, 1121

Urotropine, 1127

Uterus, changes in, during pregnancy, 1230 nerve-supply, 1228

Utricle, 515 primordial, 14, 127

Uvea, 561

VACUOLE, contractile, 15

Vago-glossopharyngeal nucleus, 379, 414

Vagus, 473

action on auricles, 972

on heart, 971

on intestines, 729

on œsophagus, 682

on respiration, 1089

on stomach, 701

distribution in abdomen, 700

nucleus of, 414

proof of inspiratory fibres in, 1092

tonic action on heart, 975

Valerianic acid, 54

Valine, 81

Valve of Vieussens, 362, 371

Valves of heart, see Heart

Variation, monophasic, 230

Vasa afferentia, 1133

efferentia, 1133

recta, 1133

Vascular area of chick, 831

system, influence of capacity of, on circulation, 880

tone, effect of central nervous system on, 982

peripheral, 990

Vaso-constrictor fibres, course of, 994

Vaso-dilatation, criteria of, 991

Vaso-dilatation by metabolites, 1002

Vaso-dilator fibres in nerve-trunks, 997 nerves, 996

Vaso-motor centre, action of acids on, 989 location of, 983

variations in activity of, 983

centres in cord, 989

impulses, path of, in cord, 360

nerves, course of, 990

reflexes, 1000

Vegetable food, utilisation of, 649

Veins, blood-flow in, 932-934

capacity of, 883

distensibility of, 871

structure of, 870

valves in, 933

Velocity of reaction, 162, 163

in vascular system, 879

Venous pressure in man, 877 
Venous outflow, determination of, 994 pulse, 933

$$
\text { in heart block, } 955
$$

Ventilation, 1104

Ventral cerebcllar tract, 354

Ventricles, capacity of, 891 pressure in, 896

Veratrine, action of, on muscle, 211

Vesicular murmur, 1045

Vestibular nerve, Deiters' nucleus, 381, 402 functions of, 398

Vestibule, 515, 604 nucleus of, 380,413

Vestibulo-cerebellar fibres, 370,389

Vestibulo-spinal tract, 353,391

Vibrative consonants, 527

Vicq d'Azyr's bundle, 388, 421

Villus, changes in, during protein absorption, 745

Villuss, struoture of, 734

Viscera, innervation of, 473 sensibility of, 494

Visceral nervous system, $466-477$

Vision, 528-597

Visual adaptation, 572 angle, 534, 589

axis, 561

centre, 447

fatigue, 572

judgments, 589-593

localisation, 589

path, 387, 406, 447

purple, 559,565

reflexes, $406-409$

sensation, Weber's law, 570

sensations, 568-584

stimuli, time relations, 571

Visuo-psychic area, lamination, 431

Visuo-sensory area, lamination, 431

Vital capacity, 1046

Vitalism, 8

Vitamines, 652

Vitellins, 100

Vitreous humour, 532

Vocal cords, 521

Voice, 520-527

Volhard's method for chlorides, 1129
Voluntary contraction, 239

muscle, 177

clectrical changes in, 242

Vowel sounds, 525

IVALLERIAN degeneration, 35\% method, 320

Warm points, 486

Water, absorption of, 733 estimation in food, 614 necessity for, 6

and dissolved substances, passage across membranes, 131-138

rigor, 231, 945

Weber's law, 485, 491 in vision, 570

Wernicke's aphasia, 454

Weyl's test, 1117

White rami, 468 sensation of, 576

Word-blindness, 455

Work, relation to stimulus, 26

Xanthine, 103, 778

Xanthoproteic reaction, 93

Xylose, 61, 104

YeASTS, action on amines, 77 on carbohydrates, $64,65,67$

Yellow'spot, 561

Young-Helmholtz theory, 577

ZeIN, 98

food-value of, 646

Zincative, 229

Zollner's lines, 592

Zona fasciculata, 1181

glomerulosa, 1181

pellucida, 1222

reticulata, 1181

Zonula ciliaris, 543

Zonule of Zinn, 543

Zooid, 818

Zymins, 671

Zymogen granules, 671 




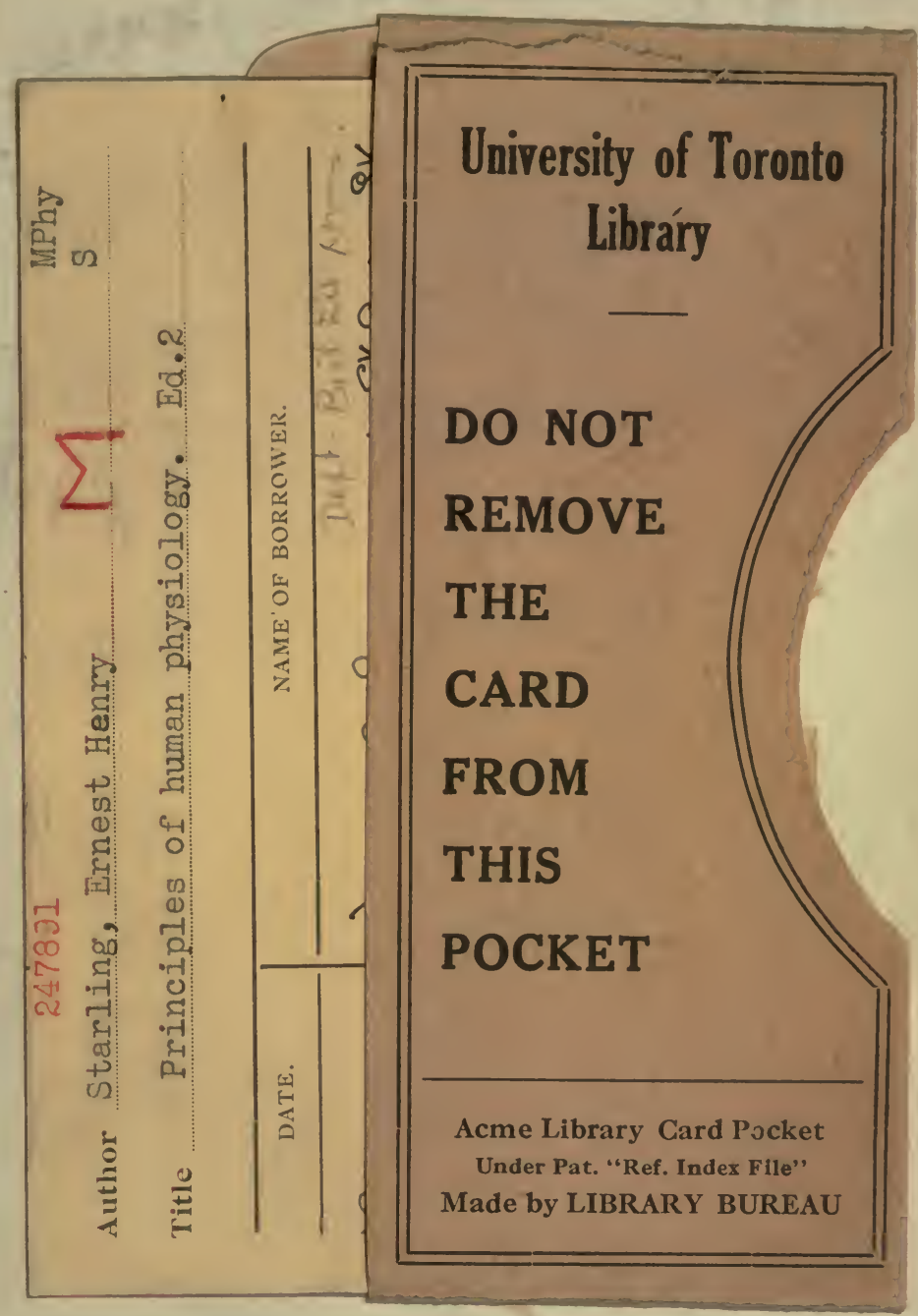


13.5 (1) H. (1) 13.

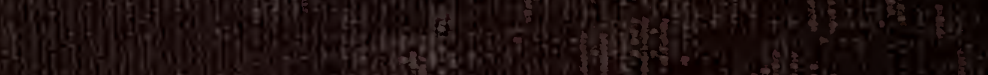
19.5 (17)

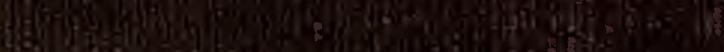
50 (3)

13:

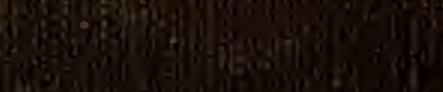

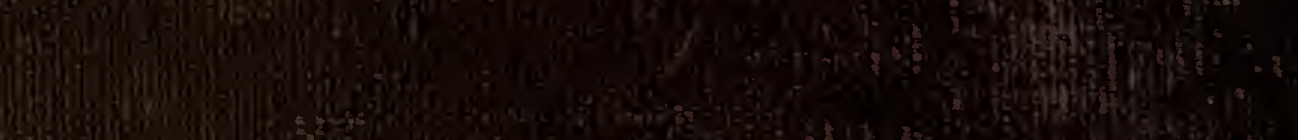
18:

S-

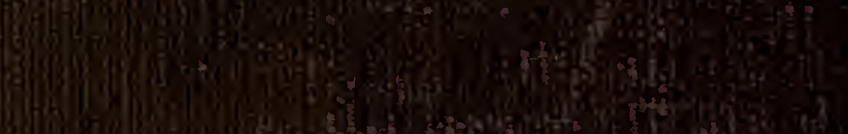

S1:

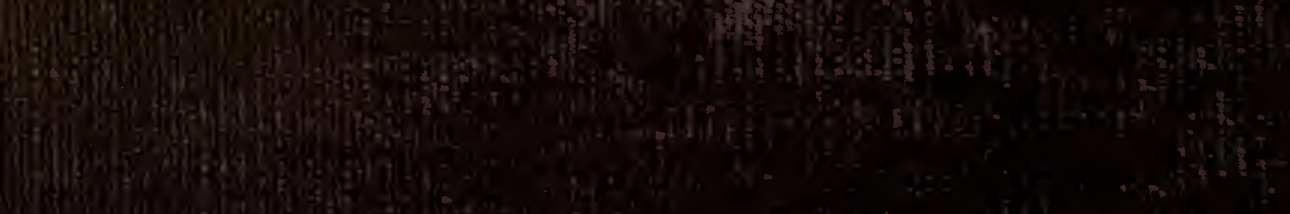

